
एक

EASE HANDLE WITH CARE

University of

onnecticut Libraries

कि

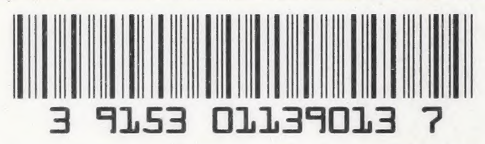



Digitized by the Internet Archive in 2013 


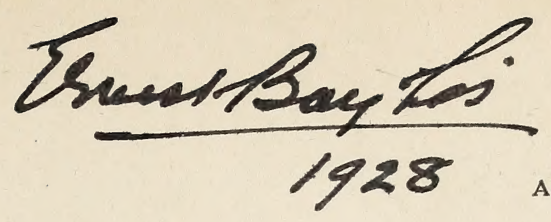

CORNELL UNIVERSITY

AGRICULTURAL EXPERIMENT STATION

\title{
A LIST OF THE INSECTS OF NEW YORK
}

\author{
WITH A LIST OF THE SPIDERS AND
}

CERTAIN OTHER ALLIED GROUPS

MORTIMER DEMAREST LEONARD

Editor-in-Chief

ITHACA, NEW YORK

PUBLISHED BY THE UNIVERSITY

Received for publication May 14, 1926

Date of issue, January, 1928 



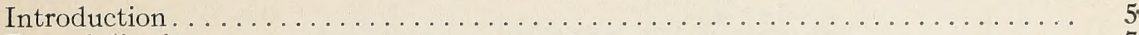

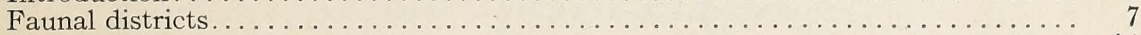

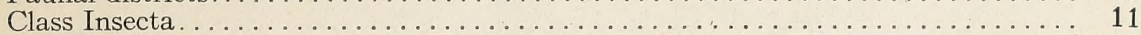

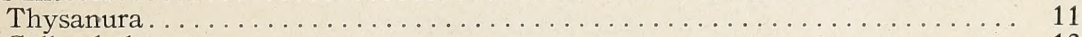

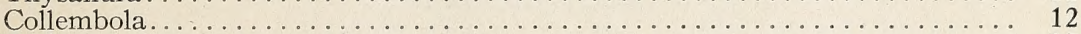

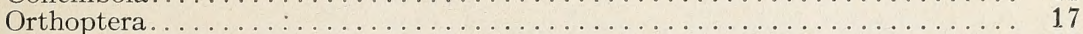

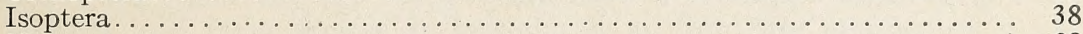

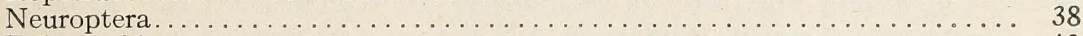

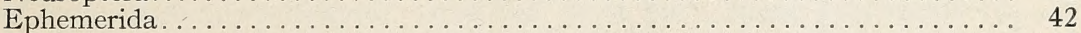

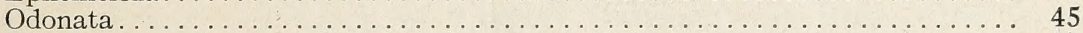

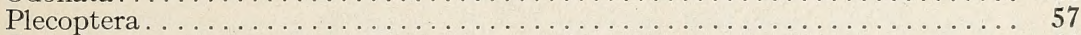

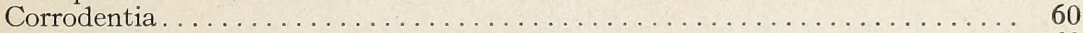

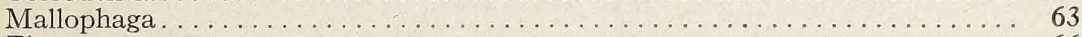

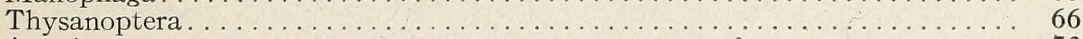

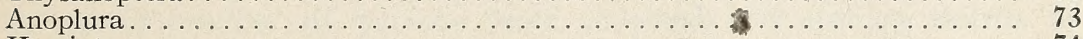

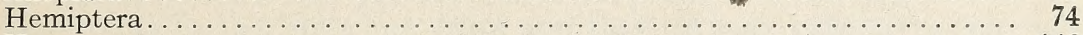

Homoptera. . . . . . . . . . . . . . . . . . . . . . . . . . . . . . 142

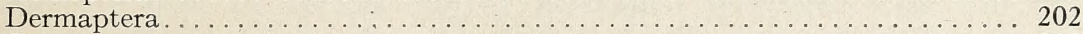

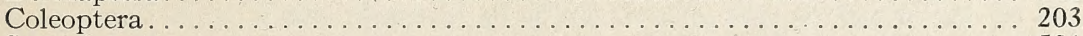

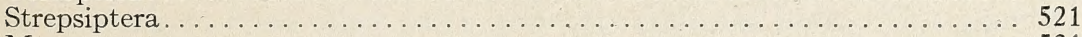

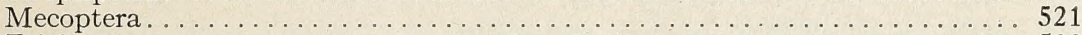

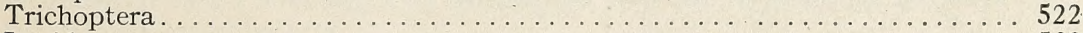

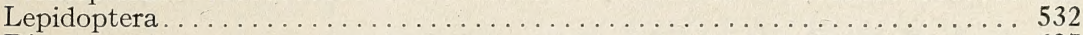

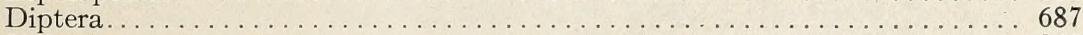

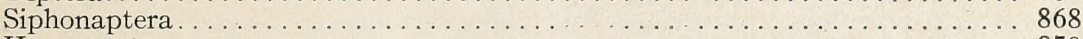

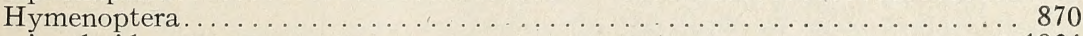

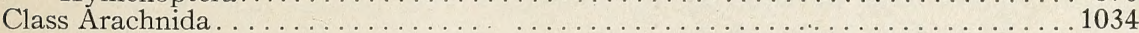

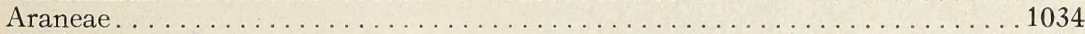

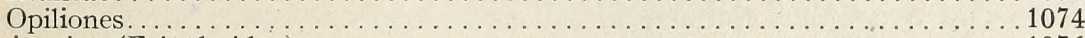

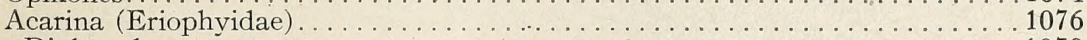

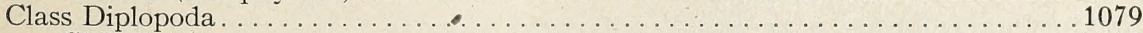

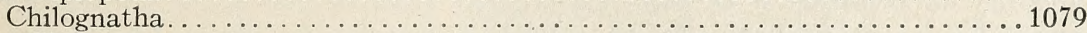

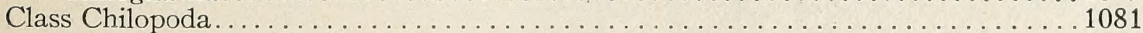

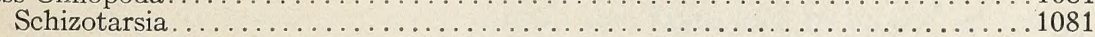

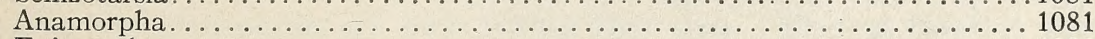

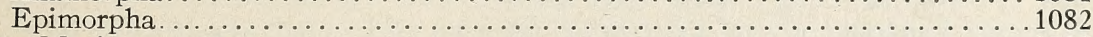

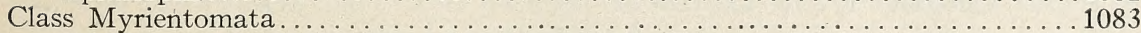

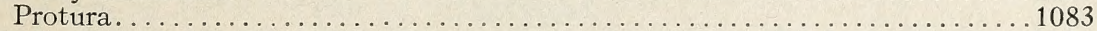

List of orders, and number of families, genera, and species recorded . . . . . . . . 1084

List of the more important collecting stations not in the United States Official

Postal Guide. . . . . . . . . . . . . . . . . . . . . . . . . . . . . . . . . . . 1085

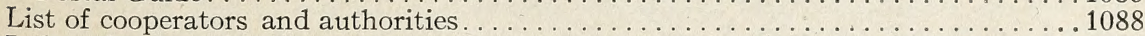

Index of orders, families, and genera $\ldots \ldots \ldots \ldots \ldots \ldots$

* The sequence of orders, and, unless otherwise indicated, of families, is that followed by Com. stock in $A n$ Introduction to Entomology. 



\title{
A LIST OF THE INSECTS OF NEW YORK
}

\author{
WITH A LIST OF THE SPIDERS AND CERTAIN OTHER ALLIEL) \\ GROUPS
}

\section{Mortimer Demarest Leonard ${ }^{1}$}

\section{INTRODUCTION}

New York State has for many years been a favorite collecting ground for insects, and from within its boundaries an immense number of specimens have been accumulated. For the most part, these specimens repose in the extensive collections of Cornell University at Ithaca, the New York State Museum at Albany, the American Museum of Natural History in New York City, the Brooklyn Museum, and Syracuse University. These institutions, however, by no means possess all of the material collected, for a number of persons have collected and studied insects at various points in the State for years and their private collections are rich in numbers both of species and of individual specimens. In addition there is much New York material in other States, due to exchange or removal by workers from other parts who have collected here. This material is included in the collections of museums and of specialists all over the United States and Canada.

Although New York material has either formed the basis, or been included in many pages, of monographic and revisional papers as well as of a number of local faunal lists, undoubtedly the bulk of it in collections is still to be mentioned in literature. Some of the best and most careful collecting in the State has been done by individuals who have published but little if anything.

Because of what had already been done, and as a stimulus to further coilecting and study, it seemed advisable to take inventory of all those species definitely known to occur within the State and to record as far as possible the distribution of each within this area. This in brief is the scope of the present list. The date of occurrence or the seasonal range is also given, as far as it can be determined.

This "List of the Insects of New York" is the outgrowth of a project initiated about twelve years ago by a committee of a number of the leading entomological specialists, including representatives of the various institutions and societies interested. Dr. J. Chester Bradley, of Cornell University, was appointed Editor-in-Chief of the Board of Editors, and a group of subeditors was selected composed of leading specialists in the major groups. The task was a considerable one, but these workers attacked it vigorousiy and thousands of records were transcribed to cards from the literature and from determined specimens.

\footnotetext{
1 Editor-in-Chief.
} 
Pressure of other work later prevented Dr. Bradley from continuing in charge, and the present editor was appointed in the spring of 1923, while he was Acting State Entomologist at Albany, New York. The Board of Editors was somewhat modified, for various reasons, and in the fall of 1924 the work was transferred to Cornell University, where it was completed under special appropriations by the New York State College of Agriculture and the Heckscher Research Fund. To these funds the New York Academy of Science added $\$ 150$ and the New York Entomological Society a like amount.

It is impossible here to express thanks individually to the large number of specialists and collectors, more than one hundred and fifty in all, whose work has made it possible to gather together the data from the great mass of published records and from specimens in public and private collections. We believe we have been successful in transcribing by far the greater part of these data, and no effort has been spared to include everything. Such acknowledgment is made in the text and in the list of cooperators beginning on page 1088. Special acknowledgment should be made, however, for assistance in many ways, to the authorities and officers of Cornell University, the American Museum of Natural History, the Brooklyn Museum, the New York Academy of Science, the Staten Island Institute of Arts and Sciences, the New York State Museum, Syracuse University, the New York Entomological Society, the Brooklyn Entomological Society, the United States Bureau of Entomology, and the United States National Museum.

The editor wishes to make particular acknowledgment to Professors O. A. Johannsen and C. R. Crosby for supervision of the many details in connection with seeing this publication through the press-a task to which the editor has been unable to attend personally. The editor wishes also to acknowledge with appreciation the painstaking effort of Miss Lela G. Gross, Assistant Editor of Publications at the New York State College of Agriculture. She has been responsible for the selection of the various kinds of type and for all other matters in connection with the mechanical arrangement of the List, and in immediate charge of all corrections and additions to the proof. Final typewritten copy was made by Mrs. H. M. Fridley. Her intelligent and continued interest in what proved to be far more than merely a clerical piece of work greatly facilitated its completion.

Because of the studies made by certain specialists and the availability of records in certain other groups besides insects, there have been included lists of the following: the millipedes and centipedes, the spiders and harvestmen, the eriophyid mites, and the ticks. In all, 16,124 species and varieties are included, of which 15,449 are insects. It is difficult to determine what percentage of the total number of species this figure represents. In many parts of the State but little collecting has as yet been done and many groups are still imperfectly known. It is not unlikely, therefore, 
that more intensive collecting and study should increase the present total by at least 25 per cent. It is hoped that the present list will increase both the number of collectors and the number of workers, and will stimulate further study along this line.

The name of a collector or its abbreviation is indicated by the use of italics, thus- $N d m$; that of the person responsible for a determination or as having supplied a record is placed in parentheses, thus- $(\mathrm{Ndm})$; italics in parentheses indicate that the same person is both collector and determiner, thus- $(N d m)$. Where the determiner is unknown the location of the material upon which the record is based is often given, and occasionally both determiner and location are indicated in parentheses; for example, (Vdyk CU), (VdykCU), or (Vdyk-CU), means determination by Van Dyke of specimens in the Cornell University collection.

The data in connection with each locality are separated by semicolons. Staten Island and Long Island localities come after a colon following SI and LI in bold-faced type.

Owing to a misunderstanding on the part of one of our editors, the species numbers which are used in the Hemiptera and the Coleoptera were omitted from the manuscript in some cases. It was thought that the slight inconvenience which might result from these omissions would not be sufficient to warrant the expense which would have been involved in making the corrections in the proof, and therefore the numbers are missing in these cases.

\section{FAUNAL DISTRICTS}

\section{W. T. M. Forbes}

For convenience in indicating localities, the State of New York may be divided into the districts shown on the accompanying map. They are distinguished partly by climate, but more by topography and geological substrata; and they are laid out with but little reference to life zones. The Adirondack, Appalachian, and Taconic districts, the Tug Hill plateau, and the small area of the New Jersey Highlands which extends into the State, represent the higher country, the intervening districts being the plains and the broader river bottoms. The Coastal Plain is represented by Staten Island and Long Island.

The St. Lawrence Plain is a broad open valley so far as New York is concerned, while on the Canadian side it is continuous with the broad St. Lawrence Plain of that region. Because of the northern latitude the climate represents the Transition zone, though the altitude is only a little above sea level. Relatively little collecting has been done here, the most important localities being perhaps Waddington and Ogdensburg near the northern boundary of the State, and Clayton near the mouth of Lake Ontario.

The Adirondack district comprises a great plateau of from 1500 to 1800 feet elevation, cut with valleys which are as a rule too narrow to shelter 
many southern forms, and rising in the eastern part into a group of mountains which reach an elevation of 5344 feet. The plateau shows in general the Canadian fauna, and is very largely still forest-covered, with many

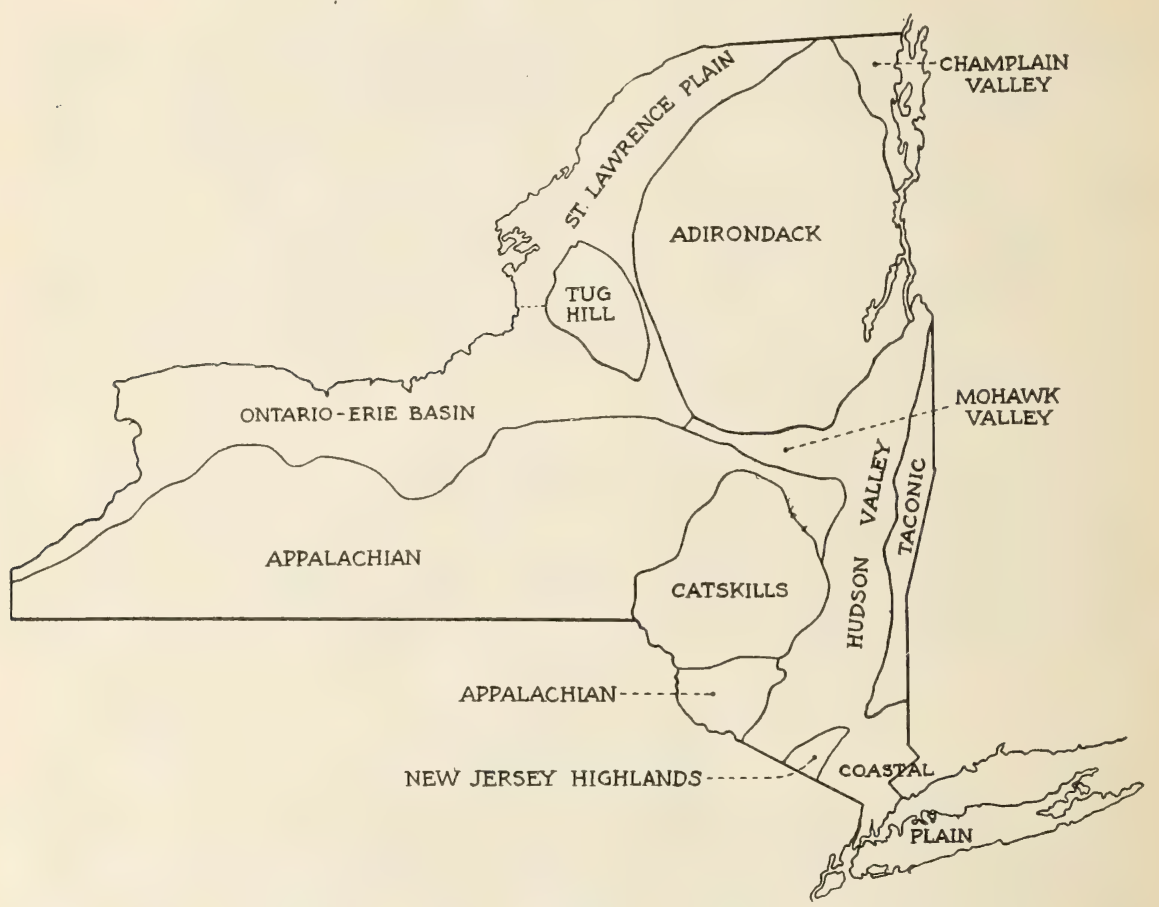

Figure 1. PRINCIPAL FAUNAL AREAS OF NEW YORK

lakes and cold bogs. The mountains higher than about 3500 feet rise into the Hudsonian, with conditions much like those of Labrador; while the tops of a few peaks (Marcy, McIntyre, and Whiteface) are alpine in character, largely as a result of the perpetual winds, which keep the forest down and allow the truly alpine plants and animals a foothold. The higher peaks of the northern group have been well explored entomologically, but the southern group, which should show cold Canadian conditions with some Hudsonian, will repay careful collecting. Collectors in many orders have worked in the plateau region, especially about Saranac and Cranberry Lakes and the Fulton Chain; but the most complete list 
in any large order is perhaps that due to Hill's many years of collecting of Lepidoptera at Fentons, on the west edge of the plateau.

Tug Hill is a plateau of about 1500 feet elevation, with a nearly level top and containing many lakes and swamps. Geologically it attaches not to the Adirondacks close by, but to the Appalachians, while in latitude it should be grouped with the Adirondacks. Entomologically it is unexplored.

The Ontario-Erie Basin is a broad plain bordering Lakes Ontario and Erie, and to the northward joining the St. Lawrence Plain. In spite of much snow and cold in winter, conditions are distinctly Upper Austral in the western part; while at North Fair Haven and eastward, Transition forms dominate. It is continuous with the Mississippi Valley on the west, but is reduced along Lake Erie to a strip two or three miles wide. On the north side of Lake Erie, southern Ontario shows the same mild climate and Austral forms. In most orders representatives of both faunal zones are present, especially for the region near Buffalo.

The Appalachian district, like the major part of the Adirondack district, is a plateau of from 1500 to 2000 feet elevation, but is cut into numerous blocks by deep through valleys. The characteristic insects represent the Transition zone, but many of the north-facing slopes show Canadian forms, and here and there in the valleys there are pockets with a few Austral species. This is the district for which general remarks as to dates and life cycles, particularly in the Lepidoptera, especially apply. The most thoroughly collected locality is Ithaca, lying in one of the deep through valleys. This fauna of the western part of the district is well indicated by collections made at Rock City, Cattaraugus County, at an elevation of 2500 feet. The interesting narrow strip lying between the Catskills and the Mohawk Valley was studied many years ago by the Albany group of workers. Sharon Springs represents this area, and shows typical Appalachian forms. The Appalachian area south of the Catskills has not been sufficiently studied.

The Catskills are a mass of mountains with peaks rising to an elevation of 4204 feet. Most of the collecting has been done at relatively low levels, and shows much the same mixture of Canadian and Transition forms as are found on the colder tops of the Appalachian plateau; but the peaks rise well toward the top of the Canadian zone, and a careful study would probably show Hudsonian forms. The most extensive list of insects in this district consists of the Lepidoptera of Big Indian Valley and Slide Mountain.

The Moharek Valley is a narrow valley separating the Adirondack and Appalachian districts. Most of the country is much broken, and the insects are not very different in character from those of the Appalachian district, but a few southern forms do appear. Such places as Trenton Falls and Wilmurt, in the southern foothills of the Adirondacks, are included in this area because their fauna is more southern in character. 
The Champlain Valley is a broad open valley similar in conditions and fauna to the St. Lawrence Plain. There is a good list of Lepidoptera from Plattsburg, showing a typical Transition fauna and containing many southern forms. The fauna of Peru is more northern in character, and represents the broad sandy area on the eastern side of the Adirondack district, with peculiar dune forms. The area as a whole has not been sufficiently studied.

The Hudson Valley is an open valley with a decidedly Austral character, especially south of Albany; northward it is narrow and broken, with a Transition fauna continuous with that of the Champlain Valley but showing a greater dominance of acid-soil forms in the Lepidoptera than any other part of the State in which the writer has collected. There are several good lists, especialiy those based on the material from the vicinity of Albany in the State Museum, and from a group of localities in the lower valley collected by Dyar and by the New York City entomologists. Florida, in Orange County, where S. W. Frost did some collecting, is in an extension of this district across to the Delaware River, and shows the same rather warm conditions.

The Taconic district consists of a sharp range of mountains extending along the eastern edge of the State, continuous with the Green Mountains of Vermont on the north, and with the Berkshire Hills on the east. To the southward they are separated from the Highlands of the Hudson only by the narrow gorge of the Hudson River. They have not been properly explored entomologically, the few short Taconic lists of insects being from the warmer deep valleys; but they show a true Canadian fauna with many acid-soil forms, like the Berkshires just across the state line.

The New Jersey Highlands and the Highlands of the Hudson represent the same wooded hilly area, continuous with the Taconics and Green Mountains on the north and the Blue Ridge on the south. The insects of this district have not been thoroughly collected, but the fauna of its extension into New Jersey shows a Transition character with woodland forms dominant because of the low altitudes, but with few of the cooler Transition forms.

New York City is not really a separate district, but it is particularly well known. The climate is practically Upper Austral, with some cooler areas in Westchester County. The country is hilly and originally was wooded, showing the forest type. The exact locality of many of the earlier records given as New York City and vicinity is very uncertain, a large region including Long Island, Staten Island, the lower Hudson River Valley, and part of New Jersey, being included.

The Coastal Plain comprises the whole of Long Island and Staten Island, with the mainland coast and the vicinity of New York City, just discussed. A great part of it is glacial moraine, more or less worked over by wind and water, and contains areas of sand and gravel and many square miles of 
salt marshes. The barrens have been fairly well surveyed, but the marsh needs more intensive study, and the relatively small areas of rich upland which have not yet been too much disturbed by man should receive special attention. It is certain that many forms now known only from Massachusetts and New Jersey, or even farther afield, will be discovered in this area. The climate is, as a whole, Upper Austral, but the easterly part of Long Island shows distinctly colder types and might best be assigned to the Transition zone. Unfortunately many of the early records are merely "L. I."

\section{CLASS INSECTA}

\section{ORDER THYSANURA}

Compiled by J. W. FoLsom

\section{FAMILY MACHILIDAE}

MACHILIS Latreille

M. variabilis Say. Southern end of Canandaigua L., Aug, Bish. Generally distributed: Mass, NY, Ind, Tenn, and NC.

M. (Trigonophthalmus) alternata Silv. LI: Cold Sp. Harb. (Silv).

\section{FAMILY LEPISMIDAE}

\section{SUBFAMILY LEPISMINAE}

\section{LEPISMA Linnaeus}

L. saccharina L. Macedon, Je, Hd; Ithaca, Ap, J1, Oct (MacG). Generally distributed: Europe; Mass, NY, Tex.

Ctenolepisma Escherich

C. quadriseriata Pck. Ithaca, Feb (MacG). Generally distributed: Mass, RI, Conn, NY, Tenn.

THERMOBIA Bergroth

T. domestica Pck. Ithaca, abundant in Roberts Hall for last 10 years at least; Ft. Plain, Aug-Sep, destroying paper (Felt); Albany (Felt); Peekskill?, eating laces and paper boxes (Lint); NYC, very abundant in house during the whole year of 1922 (Mutchler). Generally distributed: Mass, NY, I11.

\section{FAMILY CAMPODEIDAE}

\section{Campodea Westwood}

C. fragilis Mein. Ithaca, Je, in damp sandy earth, WSBarnard; Albany, Je, $Y g$, Bish; Voorheesville, Aug, Leon, is typical fragilis, not var. americana Pck. Generally distributed: Europe; eastern US.

C. fragilis var. americana Pck. Ithaca, Feb (MacG, Silv); LI: Cold Sp. Harb. (Silv). 


\section{ORDER COLLEMBOLA}

Compiled by J. W. Folsom

\section{FAMILY PODURIDAE}

\section{SUBFAMILY PODURINAE}

\section{PODURA (Linnaeus) Tullberg}

P. aquatica L. (gramilata MacG.). Ithaca, Oct-Nov, RubyBHughes. Common on surface of water of ponds and streams; adapted to a semi-aquatic existence; often appears in aquaria. Generally distributed: Europe; Siberia; Canada; Mass, NY, Ohio, Ind, Ill, Tenn, Wis, Minn, Wash.

\section{SUBFAMILY ACHORUTINAE}

\section{ACHORUTES Templeton}

A. packardi Fols. (nivicola Pck., 1873; Lint., 1885; MacG., 1891; Harv., 1893; Lint., 1896). Macedon, Ap-May, Hd ; Ithaca, Jan-Feb (Comstock\&MacG) ; Stockport, Ap; Monsey, Mar, HGlasgow. Generally distributed: Me, Mass, NY, Penn, Md, Ohio, I11; Canada.

A. packardi var. dentatus Fols. Potsdam, May, $Y g$; Ithaca, Ap (MacG); Ghent, Ap. Generally distributed: Me, Mass, NY, Ohio.

A. socialis Uzel (diversiceps Lint.). Otto, Comstock; Karner, Ap, Lint ; Voorheesville, Ap, on snow, Bish; Kingston, Mar, pools black with them, Leon. The "snow flea" was described by Fitch (1847) under the name Podura nivicola; Uzel gave, however, the first adequate description of the species. Generally distributed: common in most parts of Europe; Me, Mass, NY, Penn, Mich; Canada.

A. harveyi Fols. Ithaca, Ap (MacG). Generally distributed: Me, Mass, Conn, NY, Md; Canada.

A. macgillivrayi Fols. Ithaca, Ap-Nov (MacG). Generally distributed: NY, Ill.

A. manubrialis Tull. LI: Riverhead, Oct, frequently doing great damage to cauliflower plants, seedling cucumbers, and melons (Huck). Generally distributed:

Europe; S. America; NY.

A. armatus Nic. (boletivorus Pck., marmoratus Pck., texensis Pck., pratorum Pck.). Varna, Mar, Bks; Ithaca, Ap (MacG); NYC, J1 (Silv). Generally distributed: cosmopolitan. Europe; S. America; New Zealand; Sumatra; Canada; Cuba; Me, NH, Mass, Conn, NY, Penn, Ohio, I1l, Mo, Tex, Minn, Colo, Calif, Wash, Alaska.

A. (Schöttella) glasgowi Fols. Blauvelt, Mar, May, HGlasgorw; NYC, J1 (Silv).

\section{XENYLLA Tullberg}

X. humicola Tull. LI: Cold Sp. Harb., CBDavenport. Generally distributed: Europe; Canada; Mass, NY.

X. welchi Fols. LI: Bay Shore, Oct, in a greenhouse. Generally distributed: NY, IIl, Kans.

X. maritima Tull. Macedon, Ap, Hd. Generally distributed: Europe; NY. 


\section{SUBFAMILY NEANURINAE}

\section{PSEUDACHORUTES Tullberg}

P. dilatatus MacG. (Brachysius MacG.). Ithaca; types apparently lost. Generally distributed: NY.

\section{ANURIDA Laboulbène}

A. maritima Guér. LI: Sea Cliff, Bks; Cold Sp. Harb., CBDavenport. On seashore between tide marks; feeds on dead mollusks or crustaceans and is strictly maritime. Generally distributed: Europe; North America; Atlantic coast, Mass, NY, NJ, Fla.

\section{NEANURA MacGillivray}

N. muscorum Temp. (Anura gibbosa Pck., A. sextuberculata Harv.). Macedon, Ap, Hd; Ithaca, Mar-Ap (MacG) ; McLean, Je, Cy; Voorheesville, Aug, Leon, Bish. Generally distributed: Europe; Canada; Me, NH, Mass, Conn, NY, Penn, Ohio, Ill, Minn.

\section{SUBFAMILY ONYCHIURINAE}

\section{ONYCHIURUS Gervais}

O. armatus Tull. Ithaca, Feb (MacG). Generally distributed: Europe; Siberia ; Arctic region; E. Africa; Chile; Me, NY, Ill.

O. subtenuis Fols. Keuka L., Oct, $C y$; McLean Bogs, Ap, Je, $C y$; Voorheesville, Aug, Leon. Generally distributed: NY, Penn, I11.

O. fimetarius L. McLean Bogs, Ap, Cy; NYC, J1 (Silv). Generally distributed: cosmopolitan. Europe; Africa; Sumatra; Canada; Mass, NY, Penn, DC, Fla, Ohio, Calif.

\section{TULLBERGIA Lubbock}

T. sp. Central Nyack, Nov, 1918, BYSidoli (from WEBritton). Generally distributed: NY.

\section{FAMIIY ENTOMOBRYIDAE SUBFAMILY ISOTOMINAE}

\section{ANUROPHORUS Nicolet}

A. laricis Nic. NYC, J1, 1908 (Silv). Generally distributed: Europe; Siberia; NY, Minn.

\section{Folsomia Willem}

F. quadrioculata Tull. Mt. McIntyre, 4000-5000 ft., J1, Leon; NYC, J1 (Silv). Generally distributed: northern and middle Europe; Arctic regions, Canada; NY, I11, Minn.

F. fimetaria L. Ithaca, Mar, $N d m$, Nov; NYC, May. One of the commonest of the soil species. Often under stones, loose bark, etc. Common in flowerpots in dwellings and greenhouses. Often on well water. Generally distributed: Europe; Siberia; Arctic islands; Mass, NY, NJ, DC, Ohio, Ill, Ia, Kans, Minn, Calif, Alaska.

F. nivalis Pck. NYC, J1, 1908 (Silv). Generally distributed: Me, NY, Ia. 


\section{Isotoma Bourlet}

I. besselsi Pck. LI: Cold Sp. Harb., J1, CBDavenport. Strictly littoral; on seashore between tide marks; probably distributed by marine currents. Generally distributed: Finland; Norway; England; Scotland; Greenland; Spitzbergen; Nova Zembla, etc.; Tierra del Fuego; Mass, NY, Calif.

I. schäfferi Krausbauer. Voorheesville, Nov, Leon. Generally distributed: Europe; NY, Mass.

I. minuta Tull. Westport, May, FCSmith, "in hotbeds in large numbers"; Red Hook, Je (Felt); Nassau Co, Ap, Cy, "in greenhouse in masses." A soil species; minute, but sometimes occurring in masses in immense numbers. Generally distributed: Europe; Canada; Mass, Conn, NY, Penn, Ga, I11, Ia, Kans, Tex.

I. immersa Fols. LI: found in enormous numbers in a mushroom cellar (Lint). Generally distributed: NY.

I. albella Pck. Potsdam, May, $Y g$; Wells, J1, $Y g$; Geneva, J1, HGlasgoze; Albany, Feb, from a filter of city water (Lint).

I. walkeri Pck. (ieonina Pck.). Macedon, Ap, Hd; Ithaca (MacG). Common in colonies under recently loosened bark of trees or logs. Generally distributed: Mass, NY, Penn, Ohio, I1l, Ia, Minn ; Canada.

I. olivacea Tull. Albany, May, 7, 1924, $Y g$. Generally distributed: Northern Europe; Greenland; Canada; NY.

I. cinerea Nic. (MacGillivray's unica, dilatata, inclinata, and lateraria). Macedon, Ap-May, Hd; Ithaca (MacG) ; Albany, May, $Y g$. . Abundant in colonies under recently loosened bark of trees and logs. Generally distributed: Europe ; Siberia; Franz Josef Land; Canada; Me, Mass, NY, Ohio, Ill.

I. arborea L., Agren. (MacGillivray's synonymica and terminata). Potsdam, May, $Y g$; Macedon, Ap-May, Hd; Ithaca, Feb-Mar (MacG), Mar, Dobroscky (Fols). Generally distributed: Northern Europe; Me, Mass, NY, Ohio, Ill.

I. arborea var. nigra MacG. (nigra, brunnea, and speciosa). Ithaca, Mar, 1890 (MacG). Generally distributed: Me, Mass, NY, Ohio, I1l, Minn; Canada.

I. viridis Bourlet (Packard's glauca and plumbea). Potsdam, May, Yg; Macedon, Ap, Hd; Ithaca, Sep (MacG) ; McLean Bogs, Ap, Cy; Albany, Je, $Y g$; LI, $B k s$. Abundant in soil, under wood, stones, etc.; the largest species of its genus in NA and Europe, and easily seen with the naked eye. Generally distributed: Europe; Arctic islands; Canada; Me, NH, Mass, NY, DC, Ohio, Ill, Tenn, Ia, Tex, Minn, Colo, Calif, Wash, Alaska.

I. viridis var. riparia Nic. Mt. McIntyre, 4000-5000 ft., J1, Leon; Ithaca, Feb-Mar (MacG); LI, Dec, Bks. Generally distributed: Europe; Siberia; Canada; NY, NH, Va, Ia, Tex, Calif.

\section{ISOTOMURUS Börner}

I. palustris Müller (aequalis MacG.). Sodus Pt., Je, Hd; Ithaca, May-Je, Oct, RubyBHughes; Voorheesville, Aug, Leon; Pine Plains, Je, Yg; LI: Great Pond, Riverhead, May, Bish. A common species on the surface of pools and streams; varies greatly in coloration. Generally distributed: Europe; Greenland; Canada; Cuba; Me, Mass, NY, Md, Ga, Ohio, I1l, Ia, Wis, Tenn, Tex, Minn, Calif. 


\section{SUBFAMILY TOMOCERINAE}

\section{TOMOCERUS Nicolet}

T. flavescens Tull. var. separatus Fols. Mt. McIntyre, 4000-5000 ft., J1, Leon; Barneveld, Ap-Oct, GeoNWolcott; Penn Yan, May, Cy; Ithaca, Sep (MacG) ; McLean, J1, Cy ; Howard, J1, Cy; Ballston L., Aug, $W l f$; High Bridge Gorge, Schenectady, Aug, Wlf; Basin Bay, L. George, Aug, CarylHaskins; Karner, J1, Wlf; Voorheesville, Je, Bish, Wlf; Nassau L., Sep, Leon \& Wlf; Slide Mt., Je, $C y$; Highland, Aug, Wlf; West Park, Aug, Wlf; Ramapo Mts., Suffern, May, Cy; LI, Bks. Generally distributed: Me, NH, Mass, NY, Penn, DC, Ga, Tenn, Ohio, Ind, Ill, Ia, Mo, Mich; Nova Scotia; Ontario.

T. flavescens Tull. var. americanus Schött. Mt. McIntyre, 4000-5000 ft., J1, Leon; Adirondack Lodge, Je, Bish; Potsdam, May, $\mathrm{Yg}$; Barneveld, Ap-Oct, GeoNWolcott; Ithaca, J1, Sep (MacG) ; McLean Bogs, Ap, Je, Cy; Howard, J1, $C y$; Ballston L., Aug, $W l f$; Basin Bay, L. George, Aug, CarylHaskins; Nassau L., Sep, Leon \& Wlf; Tompkins Corners, J1, $W l f$; Karner, J1, Wlf; Voorheesville, Je, Wlf \& Bish, Aug, Nov, Leon; Slide Mt., 3000 ft., Je, Leon; White Plains, Sep, Leon; SI, Jan; LI: Great Pond, Riverhead, May, Bish. A soil species; not known to be of economic importance. Generally distributed: NH, Mass, NY, NJ, Va, Ohio, I11, Tenn, Mo, Mich, Minn, Miss, Tex, Colo, N. Mex, Calif, Ore, Wash, Alaska; Canada.

T. vulgaris Tull. Potsdam, May, $Y g$; Adirondack Lodge, Je, Bish; Wells, J1Aug, $Y g$; Ithaca, Ap, Sep (MacG); Freeville, Aug, Cy; Claverack, Mar, $C y$. Generally distributed: Europe; Canada; Me, NH, Mass, NY, Penn, Ill, Colo, Minn, Wash.

\section{SUBFAMILY ENTOMOBRYINAE}

\section{ENTOMOBRYA Rondani}

E. purpurascens Pck. Potsdam, May, $Y g$; Adirondack Lodge, Je, Bish; Wells, Je, Aug, $Y g$; Macedon, Ap, Hd; Ithaca, Mar, Dobroscky; Voorheesville, Aug, Leon; Nassau L., Sep, Wlf ; LI: Jamaica, Je, Wlf ; Great Pond, Riverhead, May, Bish. Generally distributed: Mass, NY, Ill; Canada.

E. griseo-olivata Pck. "NY", Mar 27, 1890 (MacG). Generally distributed : Mass, NY.

E. hexfasciata Harv: Adirondack Lodge, Je, Bish; Slide Mt., Je, Cy; Karner, J1, Wlf; Ballston L., Aug, Wlf; Voorheesville, Aug, Leon; Valley Cr. Swamp, near Voorheesville, Je, Bish \& Wlf; Nassau L., Sep, Wlf; LI: Riverhead, May, Bish; Mineola, J1, Wlf. Generally distributed: Me, Mass, NY; Canada.

E. ligata Fols. Ithaca, Aug (MacG) ; Karner, J1, Wlf; Voorheesville, Aug, Leon; LI: Mineola, J1, Wlf; Garden City, Je, Wlf. Generally distributed: NY.

E. multifasciata Tull. (decemfasciata Pck.). Potsdam, May, Yg; Ithaca, Mar-Ap (MacG) ; Voorheesville, Aug, Nov, Leon; LI: Garden City, Je, Wlf; Montauk, May, Cy. Generally distributed: Europe; Me, Mass, NY, Tenn, Tex, Calif, Wash.

E. assuta Fols. Macedon, Ap-May, Hd; Geneva, Je, HGlasgozo; Ithaca, Jan-Feb, Dobroscky. Generally distributed: Vt, NY, I11.

E. mineola Fols. LI: Mineola, J1 8, 1923, Wlf, type.

E. clitellaria Guthrie. Macedon, Ap 20, 1924, Hd. Generally distributed: NY, I1l, Minn. 
SIRA Lubbock

S. buski Lub. Nassau L., Sep 3, 1923, Wlf. Generally distributed: Europe ; Siberia ; Canada; Mass, NY.

\section{LEPIDOCYRTUS Bourlet}

L. albus Pck. Voorheesville, Aug 30, 1923, Lcon \& Bish. Generally distributed: Europe; Mass, NY.

L. cyaneus Tull. (metallicus Pck.). Wells, Je, Aug, Yg; Adirondack Lodge, Je, Bish; Mt. McIntyre, 4000-5000 ft., J1, Leon; Spy L., Je, Yg; Macedon, Ap, $H d$; Ithaca, May, Dec (MacG) ; Ballston L., Aug, Wlf; Voorheesville, Aug, Leon \& Bish, Nov, Leon; LI: Sea Cliff, J1, Bks. Generally distributed: Europe; Siberia; Africa; Me, Mass, NY, Ohio, Tenn, Alaska.

L. violentus Fols. Potsdam, May, $Y g$; Voorheesville, Nov, Leon. Generally distributed: Mass, NY, Ind, Ill.

\section{ORCHESELla Templeton}

O. cincta L. (flavopicta Pck.). Albany, JHEmcrton (Pck), also (Lint) ; LI: Sea Cliff, Bks. Generally distributed: Europe; Siberia; Mass, NY, Tenn.

O. ainsliei Fols. Voorheesville, Nov 9, 1923, Lcon. Generally distributed: NY, Tenn, I11, Ia.

\section{FAMILY SMINTHURIDAE SUBFAMILY SMINTHURIDINAE}

\section{PROSMINTHURUS Willem}

P. aquaticus Bourlet (amicus Fols.). Ithaca, spring and fall, RubyBHughes; Albany, Feb, CarylHaskins. Found on the surface of ponds and streams; structurally adapted for locomotion on the surface of the water. Generally distributed: Europe; Canada; Mass, NY, Ohio, I11.

\section{SMINTHURINUS Börner}

S. elegans Fitch, 1863 (quadrilineatus Tull., 1871). LI: Sea Cliff, Bks. Generally distributed: Europe; Mass, NY, W. Va, Ohio, Tenn.

\section{SUBFAMILY SMINTHURINAE}

\section{BOURLETIELLA Banks}

B. hortensis Fitch, 1863 (quadrisignatus Pck., pruinosus Tull., 1872). Macedon, Je, Hd; Oneonta (Felt); Albany (Fitch); LI: Sea Cliff, Bks. Abundant in gardens and fields, and occasionally of great economic importance locally; has caused considerable injury to young vegetable plants-cucumbers, onions, etc.-in Mass, Conn, Va. In Mass, May, 1922, it occurred in onion fields in astonishing numbers with serious injury, and fields had to be plowed up (Amherst, Mass, Fern). In Nova Scotia it wrought havoc, throughout the province, to turnips, mangels, etc., as these were emerging from the ground (WHBritton). Generally distributed: Europe; Japan; Canada; Me, Mass, Conn, NY, NJ, Va, Ohio, I11.

B. arvalis Fitch (luteus Lubb.). Rochester, J1, Hd; Macedon, Je, Hd; LI: Sea Cliff, Bks. Common in fields and gardens. Generally distributed: Europe; Canada; Mass, NY, Ohio, Mont. 
B. spinata MacG. Ithaca (MacG); Cinnamon L., J1, Wlf; LI: Sea Cliff, Bks. Adapted to live on the surface of water. Generally distributed: Mass, NY.

\section{SMINTHURUS Latreille ${ }^{2}$}

S. minutus MacG. Ithaca, RHPettit (MacG). Generally distributed: Mass, RI, NY.

S. quadrimaculatus Ryder. Ithaca, J1 20, 1889 (MacG). Generally distributed: NY, IIl.

S. clavatus Bks. LI: Sea Cliff $(B k s)$. Generally distributed: NY.

S. macgillivrayi Bks. LI: Roslyn, Bks. Generally distributed: NY.

S. nigripes Bks. LI: Sea Cliff, Bks. Generally distributed: NY.

S. sylvestris Bks. LI: Sea Cliff, Bks. Generally distributed: NY.

S. argenteornatus Bks. LI: Sea Cliff, Bks. Generally distributed: NY.

S. argenteornatus var. albescens Bks. LI: Sea Cliff (Bks). Generally distributed: NY.

S. dorsalis Bks. LI: Sea Cliff, Bks. Generally distributed: NY.

S. fraternus Bks. LI: Sea Cliff, Bks. Generally distributed: NY.

\section{SUBFAMILY DICYRTOMINAE}

\section{PTENOTHRIX Börner}

P. unicolor Harv. Ithaca, J1 (MacG) ; Howard, J1, Cy; Ballston L., Aug, Wlf; Voorheesville, Aug, Bish; Nassau L., Sep, Wlf; White Plains, Sep, Leon; LI: Sea Cliff, Bks; Great Pond, Riverhead, May, Bish. Common under damp logs. Generally distributed: Me, Mass, NY, Ohio, Ill, Minn.

P. purpurescens MacG. LI: Sea Cliff, Bks. Generally distributed: NY.

P. marmoratus Pck. Cinnamon L., J1, Wlf; Ithaca, J1 (MacG); McLean, Je, Cy; Howard, J1, Cy; LI: Sea Cliff, Bks.

\section{ORDER ORTHOPTERA}

\section{Compiled by William T. Davis}

The present list is no doubt fairly complete as far as the distribution of species on Staten Island and Long Island is concerned, but is quite the reverse for the remainder of the State, much of which appears never to have been visited by an entomologist with the object of collecting Orthoptera. The species of this order are most numerous in the southern part of the State, with the northernmost extension of several of them along the coast terminating on Staten Island or Long Island. Probably most of these will not be found in any other part of the State. Among crickets, for example, there are at least six species that are no doubt confined to these islands.

\footnotetext{
${ }^{2}$ Here used in the broad sense (Fols).
} 
A total of 136 species are recorded in this list, whereas Morse records 126 for New England, including 8 species of adventive cockroaches found but rarely. On the other hand, New Jersey, adjoining New York on the south, has an orthopterous fauna of about 150 species.

Taken together, Staten Island and Long Island have 112 species, and $2+$ species are recorded as not occurring on these islands. These species are usually of more northern range. A collector will discover that some of the species mature earlier on Staten Island than on the eastern part of Long Island.

The sequence of the families follows closely that of Morse's Mamual of the Orthoptera of New England (1920), which is nearly that of Westwood (1839). In Morse's book, or in the very useful Orthoptcra of Northeastern Amcrica, by Dr. W. S. Blatchley (1920), which covers a much wider area, the species here mentioned are all treated in detail, excepting three which have been recently described. Dr. Blatchley's book, moreover, contains a useful bibliography of the writings of Scudder, Rehn and Hebard, Caudell, and other authors who have mentioned the Orthoptera of the State of New York.

\section{FAMILY BLATTIDAE ${ }^{3}$ : COCKROACHES}

\section{Blattella Caudell}

B. germanica L. CROTON Bug. The adult of this common introduced household insect is most common in early summer, but in warm kitchens and among warm water pipes it may be found at almost any season. It is generally distributed in such situations throughout the State.

\section{PARCOBLATta Hebard ${ }^{4}$}

P. virginica Brunner (borealis Brunner). Ardsley-on-Hudson, Je, Winters; West Pt., Je, Sep 2, three females; Pine I., Je; Rochester, J1, Deveson; Coy Glen, Je 3, a male and its nymphal skin found under stone; Conesus L., Je 23; SI: adults, Je-Sep, females with their egg-capsules, Je-J1; LI: Wyandanch, J1; Coram, Aug; Selden, Aug; Yaphank, Je-Sep, J1 13, a female with oötheca; Wading R., Je-J1, Je 27, a female with oötheca, Je 27, 28, males on Pyrus bush, feeding on honeydew of aphids; Riverhead, J1-Aug; Gardiners I., Je, Aug. The females are much longer-lived than the males.

P. uhleriana Sauss. West Pt., Je; Ithaca, May (CU) ; SI: adults Je-Sep (females live the longer); Clove Val., J1 15, a female with oötheca; LI: Wyandanch, J1; Half Way Hollow Hills, J1 3, a female with oötheca; Coram, Aug ; Selden, Aug 31, six females; Yaphank, J1, Aug-Sep; Wading R., May-J1, Je 27, a

\footnotetext{
${ }^{3}$ In The Blattidae of North America, Hebard records Eurycotis caraibea (Bol.), Leucophaea maderae (Fab.), and Blaberus discoidalis Serv., as adventive species found within the limits of the State, and Professor Morse mentions still others as having been introduced into New England. None of these insects, however, are likely to become established.

${ }^{4}$ Species of this genus come at night to sugar bait spread on the trunks of trees and elsewhere.
} 
male on Pyrus bush, feeding at night on honeydew of aphids, J1 14, one male; Riverhead, J1; Orient, Je; Gardiners I., Je.

The nymphs of this and other Parcoblattae pass the winter under dead leaves and litter in the woods, and mature the following spring.

P. caudelli Hebard. ${ }^{5}$ LI: Selden, Aug 31, six females; Yaphank, J1-Sep, J1, females with oötheca, J1 8, six males; Wading R., Je 27 and 28, males on Pyrus bush feeding on honeydew of aphids.

P. pennsylvanica DeG. Bronx Pk., NYC; West Pt., Je; Hudson, Morse (Rehn \& Hebard) ; Ithaca, May-J1 (CU) ; Portageville, Je; Conesus Lake, Je ; Lewiston, J1, Wat; Rochester, Deveson; Thousand Isls., two males; SI: adults, under loose bark, etc., Je-Aug.

The males are especially active and are often hard to capture.

\section{BLATTA Linnaeus}

B. orientalis L. Generally distributed over the State. While in very warm houses this introduced species may be found at any time of year, in the average dwelling, which is cool in winter, it has decided seasons, and mature individuals are most common in the late spring and in summer. Hebard states that as its appearance is coincident with the arrival of the shad in the Delaware River, it is locally known as the "shac? roach." While the females cannot fly, they are excellent runners, and on SI are sometimes found under stones, etc., far from any dwellings. None, however, appear to survive the winter as far north as NY State, unless they are protected artificially by man.

\section{Periplaneta Burmeister}

P. americana L. As far north as NY this American species requires even more protection in order to survive the winter than does Blatta orientalis, and for that reason it is found in but few detached dwellings. In greenhouses, hotels, and any large buildings that are kept warm, it finds more congenial surroundings and multiplies accordingly. It is very common in the reptile house, Bronx $\mathrm{Pk}$, where it is trapped as living food for lizards, etc. As it is found on shipboard, it also gets into the sea, and so occasionally is washed ashore on SI and LF. Rochester, Deveson, is the most northern record for the State.

P. australasiae Fab. A nymph of this introduced species was found in the greenhouse, Prospect Pk., Brooklyn, Jan. 5, 1907, and no doubt it occurs in many such places. It is very common in parts of the Southern States. Ithaca, Aug 24, 1894, a female with its egg capsule (CU).

\section{Pycnocelus Scudder}

P. surinamensis L. This species is very common in parts of the Southern States and has been introduced in the reptile house, Bronx $\mathrm{Pk}$. It is reported also from several localities in New England. The many N. A. specimens thus far found have been females, with the exception of a single male collected in the reptile house, Jan 12, 1914.

\footnotetext{
5 The compiler has discovered that the females of caudelli, contrary to the description of the species, have short tegmina, and closely resemble the same sex in fulvescens Sauss. \& Zehnt.; so the published Long Island records of fulvescens must appear under caudelli.
} 


\section{PANCHLORA Burmeister}

P. cubensis Sauss. This beautiful green roach is adventive from tropical America and is usually introduced in bunches of bananas. It is an out-of-door roach and cannot establish itself as far north as NY State. It has been brought to SI in March, Aug, and Dec; all these four specimens were females. In the Brooklyn Museum there is a female taken in that city in a bunch of bananas, Jan, 1912. Other records are Albany, Mar (US), and Rochester, Ap, Deveson.

\section{CRYPTOCERCUS Scudder}

C. punctulatus Scud. This wingless shining black or brownish black roach, occurring in decayed moist logs in thick woods, is found from southwestern NY to northern Ga. It is found also along the Pacific coast. NY, one juvenile paratype (MCZ).

\section{Family Mantidae 6 : Praying Mantes, Soothsayers}

MANTIS Linnaeus

M. religiosa L. Introduced from the Old World. Rochester, Charlotte, Summerville, 1899, HFAtwood, reported in Ent. News, Dec, 1899, by M. V. Slingerland. Apparently now well established about Rochester and Ithaca. Will probably spread slowly.

\section{PARATENODERA Rehn}

P. sinensis Sauss. An Asiatic species, first introduced about 1896 at Meehan's Nursery, Germantown, Pa. It was brought to SI in 1902 and is now abundant in several places. It prefers dry hillsides with cat brier and other tangle. As the insect is beneficial, efforts have been made to establish it elsewhere near NYC.

\section{Family PHASMidAE: Walking Sticks \\ DIAPHEROMERA Gray}

D. femorata Say. Yonkers, Bno \& Farley; Ramapo, Oct; West Pt.; Ft. Montgomery, Aug, Schott; Monroe, Oct; Milton, Sep, Joutel; Minnewaska, Aug-Sep, Nic; Allaban, Catskills, Aug, Dow; Stuyvesant Falls (NYS); Albany, J1, Oct (NYS); Keeseville, Lg; Ithaca, Sep (CU) ; Watertown, Oct (NYS); Rochester, Deveson; Niagara Falls, the type locality (Say). This species is particularly fond of hazel, chestnut, linden, and some oaks. Not reported from SI, but occurs on LI: Orient, 1922, Lath; Shelter I. Elsewhere in the State it appears to be generally distributed, usually occurring in young trees and on bushes.

MANOMERA Rehn and Hebard

M. blatchleyi atlantica Ds. SI is the type locality of this race, or, more likely, distinct species. It is generally distributed over LI, mature individuals being found from J1 30 to Oct 1 . Often on Solidago rugosa as well as other goldenrods and associated plants growing in damp meadows.

The only other locality in the State thus far reported is Crotona Pk., Bronx, NYC, Oct 9, Wat.

No males are known; females collected from Conn to Va.

${ }^{6}$ Adventive species. In addition to Stagmomantis carolina (L.), which has occasionally been brought to N. Y. from the Southern States, Gonatista phyryganoides (grisea Fab.) and Phyllorates chlorophaed (Blanch.) are reported by Scudder in his Orthoptera of North America (1868), from N. Y. The type of chlorophaea came from Watertown, but, like grisea, is a southern species. A specimen of Thesprotia graminis (Scud.), collection US, is stated by Caudell (Proc. U. S. Nat. Mus., 1913) to be labeled "N. Y." It too is a southern species. 
FAMILY TETTIGONIIDAE: Katydids, and their kin

\section{SCUDDERIA Stål}

S. septentrionalis Serville. LI: Yaphank, J1 24, 1908 (two flew to light), J1, 1909; Riverhead, Aug, 1917; Greenport, Aug 3, 1913, at light; Wading R., J1, 1914.

S. pistillata Brunner. Callicoon, J1 26; Onteora Mt., Aug, Hoze; E Jewett, Aug, Ds; Saranac, $N d m$ (NYS); Keene Val., Aug-Sep, Not; Chateaugay L., Bowditch; Mountain View, Franklin Co, Sep, Not; Waddington, Aug, Woodr; Ithaca (Morse) ; Batavia, J1, Kngt. A northern form.

S. curvicauda curvicauda DeG. Yonkers, Aug, ERCasey; L. Mahopac, TDO'Connor (Rehn \& Hebard, Trans. Am. Ent. Soc., 1914) ; Waddington, Aug, Woodr; Batavia, Aug, Kngt. SI: Tottenville, J1 23, 1914, and J1 26, 1922. LI: Coram, Aug-Sep, common on sandy area; Smithtown, Aug; Wading R., Aug; Yaphank, J1-Aug; Riverhead, Aug; E. Quogue, Aug; Amagansett, Aug.

As this insect is found in northern New England, it probably has a wider distribution in the State than is here recorded.

S. texensis Sauss. \& Pict. Bronxville, Sep, Woodr; Ithaca, J1-Sep (CU) ; Geneva, Aug, Sherm (Hubbell). SI: Generally distributed; mature individuals J1-Oct; occasionally attracted to light. LI: Brooklyn, Sep, Zab; Coney I., Sep, Zab; Rockaway, Sep, Oct, Ds \& Ols; Fire I., Sep; Maspeth, Sep; Selden, Aug; Wading R., Aug; Patchogue, Sep; Riverhead, Aug; Amagansett, Sep; Montauk, Sep.

As this species has been recorded from the New England States, it is probably to be found more extensively in NY than is here recorded.

S. furcata Brunner. Vàn Cort. Pk., Aug; Mt. Vernon, Fit.ch; Bronxville, Aug; White Plains, Sep, Leon; W. Nyack, Aug; West Pt., Sep, Oct; Sullivan Co, Sep; Pine I., Sep, Brb; L. Minnewaska, Sep, Nic; L. Mahopac, TDO'Connor (Rehn \& Hebard); W. Hebron, Sep, Lg; Ithaca, J1-Aug, Oct (CU) ; Otto (CU) ; Elmira, Sep, common, Diven; Batavia, Aug-Sep, Kngt; Rochester, Deveson. SI: Common and generally distributed; mature individuals J1-Oct. LI: Brooklyn, Aug-Sep, Zab; Aqueduct, Sep; Maspeth, Ols; Flushing, Aug ; Oyster Bay, Aug; Selden, Aug; Coram, Aug-Sep; Yaphank, J1-Sep; Southold, Sep; Bay Shore, Aug-Sep, Ols; Patchogue, Sep; Amagansett, Sep; Promised Land, Sep; Gardiners I., Aug.

The black-striped variety fasciata Bt., is found in the northern part of the State, but not on SI.

\section{AMBLYCORYPHA Stål}

A. oblongifolia DeG. Bronx Pk., Mosholu, Sep, Mt. Vernon, Dunwoodie, Aug, ERCasey, L. Mahopac, Aug, TDO'Connor (Rehn \& Hebard, Trans. Am. Ent. Soc., 1914); W. Nyack, J1; Coeymans, EBSmith (NYS) ; Clifton Sps., Aug (CU). SI: Generally distributed; mature individuals Jl-Oct. Numerous examples of the pink variety have been found, particularly at W. New Brighton and in the Clove Valley. Brown individuals also have been collected. LI: Brooklyn, Aug-Sep; Aqueduct, Oct; Woodhaven, Aug; Maspeth, Aug-Sep; Flushing, Aug, Bell; Sands Pt., Sep, Burns; Wyandanch, Aug; Wading R., J1; Yaphank, J1, Sep; Riverhead, Aug; Southold, Sep; Amagansett, Sep; Montauk, Sep.

A. floridana carinata Rehn \& Hebard. LI: During a week spent on Gardiners I. in Aug, 1918, two males, three females, and a nymph were collected. The species is not uncommon in parts of the two forested regions of the island, namely the Great Forest and the Tobacco Lot Forest. 
Recorded also from Nantucket and Woods Hole, Mass, but more common southward.

A. rotundifolia Scud. Bronx, Sep, Ang; Ft. Montgomery, Aug, Schott; Marlboro, Sep (Bklyn Mus.-Rehn \& Hebard, Trans. Am. Ent. Soc., 1914) ; Ithaca, J1-Aug, Oct (CU) ; Rochester, Deveson. SI: Generally distributed, and found from late J1 until frost; latest date Oct 11, 1903; not so plentiful as oblongifolia. LI: Brooklyn, Aug, Zab; Pinelawn, Aug; Coram, Sep; Smithtown, Aug; Wading R., J1; Yaphank, J1-Aug; Calverton, Sep; Amagansett, Sep; Southold, Sep; Orient, Oct 30, Lath.

\section{MICROCENTRUM Scudder}

M. rhombifolium Sauss. Blauvelt, SBHusted. SI: Generally distributed. LI: Prospect Pk., Brooklyn, Sep; Maspeth, Sep; apparently the species is not found on the eastern part of the island.

Often found in wooded situations and on the wayside trees of well-shaded roads. It is more likely to be found in maple trees than is the true katydid, and lays its eggs on many species of plants. As the season advances, it, like Pterophylla camellifolia, often sings when the sun is shining brightly.

\section{PTEROPHYLla Kirby}

P. camellifolia Fab. Yonkers, EMWalker; West Pt., Sept-Oct, Robn; Milton, Sep, Joutel; Stuyvesant Falls, Coeymans (NYS); Dansville, Sep (CU); Niagara (EMWalker in Can. Ent. 36:330, 1904). SI: Once common, but now restricted, so far as known, to a small colony near the Moravian Cemetery, located in 1921 by Miss Miriam Campbell. An early record for the song is July 30 (1908), and the latest date is November 2 (1882). The insects stop singing when the thermometer falls to about $50^{\circ}$ (Journ. N. Y. Ent. Soc., Mar, 1922). LI: In Journ. N. Y. Ent. Soc., Sept, 1902, p. 174, is the statement by Zabriskie that this species was common some twenty-five years ago about his home in Brooklyn, but in recent years he had not seen nor heard a single one. Away from NYC it is generally distributed and often very commonJamaica; Center I., Oyster Bay; Selden, laying eggs in bark of locust, Sep 18; Bellport, Aug, Nic; Yaphank, Oct 1, common, five females laying eggs in the bark of pitch pines; Calverton, Sep, laying eggs in pine bark; Amagansett, Sep; Gardiners I., Aug; Wading R., Aug 6; Southold, Sep; Greenport, Aug 2, one singing; Orient, Nov 1, Lath.

\section{NeOCONOCEPHALUS Karney}

N. exiliscanorus Ds. SI: The type locality for this species is SI, where it was found on the edge of the salt meadows near New Dorp. It has also been found in many other parts of the island, including Clove Valley, J1-Sep. LI: New Utrecht; Brooklyn, Aug, many in cattail swamp; Woodhaven, Aug ; Maspeth, Sep 12, numerous, one male of the brown variety; Flushing, Aug.

N. lyristes Rehn \& Hebard. SI: Found in numerous localities along the edge of the salt meadows at Watchogue and Long Neck in Aug-Oct, but not inland; sometimes seen eating grass seeds. The brown form is darker-colored in this species than in any other native Neoconocephalus. LI: Woodhaven, Aug; Aqueduct, Oct; Bellport, Aug, Nic; Smith's Pt., Oct, Eng; Promised Land, Sep; Southold, Sep. 
N. ensiger Harr. White Plains, Sep, RFHussey; Ramapo, J1; Callicoon, J1; Oliverea, Sep; Oxford, SSHall (Rehn \& Hebard, Trans. Am. Ent. Soc., 1915); Ithaca, Aug-Sep (CU) ; Clifton Sps. (CU) ; Rochester, Sep, Deveson. SI: Generally distributed, J1-Oct. Brown individuals occasional. Sometimes sings in broad day when the sun is shining, but not so frequently as the other Neoconocephali. LI: Generally distributed; Brooklyn; Maspeth; Flushing; -Aqueduct; Central Pk.; Selden; Southold; Greenport; Orient; Yaphank; Amagansett; Montauk; Gardiners I., Aug-Sep.

N. robustus Scud. Hoffman I., NY Harbor, Sep 2, 1920, FMGaige (Hubbell). SI: Found in many localities, but especially on sandy ground near the border of the salt meadows and along the up-beach at New Dorp, Oakwood, etc., J1-Sep. Brown individuals are numerous. This species will sometimes sing from ten to fifteen minutes without a stop. LI: Brooklyn; Woodhaven; Aqueduct; Coney I.; Rockaway; Long Beach; Fire I.; Maspeth; Central Pk.; Selden; Coram; Wading R.; Southold; Greenport; Riverhead; Yaphank; Bellport; Càlverton; Amagansett; Promised Land; Montauk; Gardiners I., Aug-Sep.

N. retusus Scud. Manhattan I., Nov; Van Cort. Pk.; Bronx, Sep; Yonkers, Oct, HAAllard (Rehn \& Hebard, Trans. 'Am. Ent. Soc., 1915); White Plains, Sep, RFHussey. SI: Generally distributed, and sometimes attracted to light, Aug-Nov. Heard singing as late as Nov 9. Will occasionally sing for more than half an hour without intermission. LI: Woodhaven; Aqueduct; Rockaway Beach, Schott; Maspeth, Ols; Sands Pt., Sep, Burns; Orient, Schott; Yaphank; Montauk, Aug-Oct.

\section{ORCHELIMUM Serville}

O. vulgare Harr. Inwood, Manhattan I., Sep; Van Cort. Pk.; Bronx, Sep; White Plains, Sep, Leon; Ramapo, J1; Sullivan Co ; Wilmington, J1; Berkshire (Rehn \& Hebard, Trans. Am. Ent. Soc., 1915) ; Ithaca, Sep (CU) ; Elmira, Aug, Diven; Silver Creek, Sep, Leon; Geneva, Aug, HBSherman; Batavia, Sep, Kngt. SI: Generally distributed, J1-Oct (Oct 25 is the latest date). Females found laying eggs in pigweed (Chenopodium) Sep 28, and in an old fence rail Sep 15. Several of the long-winged form collected. Sings both by day and by night. LI: Generally distributed from Brooklyn to Montauk and Orient Pt., J1-Sep; Shelter I., Aug; Gardiners I., Aug.

O. gladiator Bruner. Ithaca, J1-Aug (CU); Batavia, J1-Sep, Kngt.

O. minor Bruner. LI: Yaphank, Oct 17, 1913, a female, Eng. A pine-tree species, more common among the pine trees of NJ and southward.

O. concinnum Scud. SI: Found in many places on the salt meadow and along its margin, J1-Sep. Song much like that of aulgare, only lower. LI: Brooklyn; Aqueduct; Rockaway ; Fire I.; Sands Pt., Sep, Bs; Wading R.; Orient, Lath; Wading R., Aug; Montauk, Aug-Sep. On Gardiners I., in Aug, 1918, it was found only in a single meadow bordering Bostwick Cr., where four males and three females were collected. On Aug 19, 1919, two females of this species were seen to dive into a pool of fresh water near the Peconic R., Riverhead, and one of them remained beneath the surface for one and a half minutes, the other not so long.

O. fidicinium Rehn \& Hebard. SI: On the salt meadow near Oakwood and Old Place, Aug-Sep. Lives in colonies. LI: Rockaway, Woodr; Wading R., Aug 29,1922 , a female captured by the wasp Tachytes mandibularis - wasp and grasshopper on the road, Bird \& Ds.

[O. nigripes Scud. has been found in southern Ontario and in Ohio, and therefore it may occur in the western part of New York State.] 


\section{CONOCEPHALUS Thumberg}

C. fasciatus DeG. Rye, Oct; W. Nyack, Aug; West Pt., Sep; Callicoon, J1; Keenc Val., Aug-Sep, Not; Saranac Inn, Ndm; Lowville; Ithaca, Aug-Oct (CU) ; Batavia, Aug-Sep, Kngt. In addition, Rehn and Hebard (1915) report it from Chateaugay L., Aug-Sep, Scudder; Clifton Sps. (CU); Cattaraugus, Sep $(\mathrm{CU})$. SI: Generally distributed and found both along the margin of the salt meadow and inland, J1-Oct. LI: Found from Brooklyn to Montauk and Orient on the salt meadow and inland, J1-Sep; Gardiners I., Aug, 1918.

C. brevipennis Scud. White Plains, Sep; W. Nyack, Aug-Sep; Ft. Montgomery, Sep, Schott; West Pt., Sep; Debruce, Sep; Onteora Mt., How; Oliverea, Sep; E. Jewett, Aug; Lowville; Ithaca, Aug-Oct (CU) ; Geneva, Aug, HBSherman; Batavia, Aug-Sep, Kngt; Chatham, Aug (Morse); Niverville, Aug (Morse). SI: Common both on the salt meadows and inland, Aug-Nov. Found as late as Nov 17 in 1888. Has a very faint little song, not to be heard unless one's ear is very close to the singer. As with other Orthoptera, this species eats and sings at the same time. LI: Found from Brooklyn to Montauk and Orient, J1-Sep. Some of the inland localities are Selden, Pinelawn, and Yaphank; Gardiners I., Aug.

[C. nemoralis Scud. "Eastern slope of the Palisades" (Bt). While taken on the NJ side of the state line, it probably extends into Rockland Co, NY.]

C. strictus Scud. SI: With the clearing of the wooded areas of the island and the consequent drying of the soil, the beard-grass, Andropogon, has become generally distributed and with it this grasshopper. It was first observed at Arrochar, Oct 1, 1904, by Woodr. Both long- and short-winged examples are found, but those having wings of intermediate length are rare. LI: Central Pk., Aug.

C. nigropleurum Bruner. Ithaca (CU \& Morse Coll. acc. to Rehn \& Hebard, Trans. Am. Ent. Soc., 1915); Junius, Aug; Rochester, Deveson; Batavia, Aug-Sep, Kngt.

C. attenuatus Scud. Ithaca, Oct 27, 1912, two males, three females (CU-Rehn \& Hebard, Trans. Am. Ent. Soc., 1915). One female from same locality, J1 28, 1906, in collection of Zool. Mus., Univ. of Mich.

C. nigropleuroides H. Fox. SI: On the salt meadows, Oakwood, Sep 14, 1914, Ds; Old Place, Sep 8, 1920, HHJohnson. LI: Wading R., Aug, in salt meadows, and at Long Pond.

C. spartinae H. Fox. SI: Abundant and generally distributed on the salt meadows, Old Place, Watchogue, Tottenville, J1-Sep. Long-winged form sometimes attracted to lights at a distance from the meadows. LI: Rockaway, Sep; Montauk, Aug; Bergen Beach, Dow; Sands Pt., Sep-Oct, Bs; Sea Cliff, Aug, Ols; Gardiners I., Aug 23, 1918, a female nymph.

C. saltans Scud. White Plains, Sep; LI: East Meadow, Central Pk., Sep 8, 1911. This insect may be either brownish or green in color, and there are occasional long-winged examples.

C. viridifrons Blatch. LI: Central Pk., Sep 8 (Ds). Also known from other localities outside the State.

\section{AtLanticus Scudder}

A. testaceus Scud. White Plains, J1, JMFarlcy; Croton-on-Hudson; West Pt., Je-Sep, Robn \& Ds. SI: On June 26, 1892, very common in a low wet field at Watchogue; found also in Oct. LI: Half Way Hollow Hills, J1; Yaphank, J1, Eng; Wading R., Je-J1, on J1 26, 1914, two males on bushes several feet from the ground and singing; Riverhead, Aug. 
Most of the older records for this species are under the name pachymerus Burm.; the true pachymerus, however, according to Rehn and Hebard, is a more southern species.

A. davisi Rehn and Hebard. The following is from Rehn and Hebard's revision of Atlanticus (Trans. Am. Ent. Soc., 1916): Ithaca, J1, Aug, OEPearce \& Comstock); Sullivan Co, Ds; E. Jewett, Aug, Ds; Catskills, Sep, CnJr; L. George, Aug, Zab. To the above may be added Mt. Whiteface, J1, 1914, numerous specimens nearly fully-grown. In parts of $\mathrm{Pa}$ and $\mathrm{Va}$, this is a common species.

A. americanus Sauss. Ft. Montgomery, Oct 2, Schott; West Pt., Je, nymphs; Peekskill, Sep. SI: Generally distributed in the wooded areas. Nymphs in $\mathrm{J} 1$ and adults in Aug-Oct. LI: Floral Pk., J1, HThurston; Half Way Hollow Hills; Wyandanch, Aug; Wading R., J1; Yaphank, Aug-Sep; Patchogue, Sep; E. Quogue, Aug.

Most of the older records for this species are under the name dorsalis Burm.; the true dorsalis, however, according to Rehn and Hebard, is a more southern form.

\section{Ceuthophilus Scudder}

C. nigricans Scud. E. Jewett, Aug; Mt. Whiteface, J1; Chateaugay L., Scud (Rehn and Hebard, Proc. Acad. Nat. Sci. Phila., 1916, p. 275-276) ; Onondaga Co, Ap 9, 1922 (Hubbel1) ; Rochester, J1, Deveson. LI: Yaphank, Sep.

Blatchley considers $C$. neglectus Scud., described from Ithaca, the same as nigricans, which has page priority.

C. maculatus Harr. In his paper on The North American Ceuthophili (Proc. Am. Acad. Arts and Sci., 1894), Scudder gives the following NY localities: Chateaugay L., F.C.Bowditch; Ithaca (CU) ; reported also from Howe Cave, Pck; Onteora Mt., J1 29, 1901, How (Caudell); Rochester, Aug-Sep, Deveson.

C. davisi Blatch. SI, J1-Aug. Davis states that he has taken specimens on 11umerous occasions in the forested parts of SI by baiting tin cans with molasses and fusel oil (Blatchley, Orthoptera of Northeastern America, p. 625). SI is the type locality, and specimens have been found in Clove Valley, in Reed's Valley, and near the Moravian Cemetery.

C. rehebi Blatch. Crotona Pk., Bronx, NYC, Sep 24, 1899, Wat; Nyack, Je; Ramapo, Oct. SI: Generally distributed in the forested area, on sandy ground, and collected in molasses traps, Je, J1, Sep. LI: Woodhaven, Aug; Selden, Aug 30-31, 1916, many on sandy roads, etc., and collected with a lantern at night; Coram, Aug-Sep; Yaphank, J1-Oct; Amagansett, Sep; Orient, Sep, Lath.

This is the species treated by Rehn and Hebard, 1916, as C. spinosus Scud. Blatchley, in his Orthoptera of Northeastern America (1920), identifies as the true spinosus specimens from Ga and Fla.

C. uhleri Scud. SI: Richmond, Aug, 1911, a female. A specimen sent to Scudder in 1894 was identified as uhleri.

Found in the "Middle States," according to Scudder, 1894.

C. latens Scud. Summit of Hook Mt., Nyack, Nov 26, 1916, Schott; Allaben, Catskills, Aug, Dow; Genesee Co, Je 24, 1916, nymph. "Ithaca and Enfield Falls, (Morse, CU)," see Scudder, 1894.

C. caecus Scud. SI: Richmond, Clove Val., Je, Aug, two males and one female.

C. lapidicola Burm. Saranac L., Sep; Oliverea, Sep, 1918, males and females at sugar bait; Taughannock, Oct. This species is reported from Conn (Walden), Mass (Morse), and Ontario as pallidipes (Walker), but it seems to be but poorly understood. $C$. pallidipes may be a northern race of $C$. lapidicola. 
C. gracilipes Hald. Manhattan I., near Inwood, Sep 9, 1905, and Oct 3, 1908, numerous; Bronxville, Aug; Rye, Oct 12, 1917, a female under bark of dead tree; Ardsley, Je, under bark, Winters; Croton, J1; Peekskill, Aug, Bs; Bear Mt, Aug, Schott; West Pt., J1; L. Mahopac, J1 20, 1903, two juvenile females, HFox; Oliverea, Sep, many at sugar bait; Caywood, Seneca Co, J1; Geneva, Aug; Ithaca, Comst (Scudd) ; Rock City, Je 6, 1915, a female at sugar bait, Je 10-11, two males; Rochester, Deveson. SI: Ft. Wadsworth, Sep 23, 1924, found about the foundation of an old house, nymphs, Ap, 1925. LI: Gardiners I., Je 14, 1911 (two males), Aug, 1918 (six males and three females).

C. terrestris Scud. Adirondacks, Saranac L., Sep; Ithaca, Aug 19, 1885 (Hebard) ; Batavia, Aug 31, 1913, two males, one female, Kngt.

[C. stygius Scudd. and some other species of Ceuthophilus that have been reported from adjoining or near-by States, doubtless occur in NY.]

\section{FAMILY GRYLLIDAE: CRICKETS}

GRYLLUS Linnaeus

G. assimilis Fab. SI: Immature individuals of the form pennsylvanicus Burm. live over winter under stones, etc., and mature in early May, when the males may be heard singing. The ovipositor in this form is short. The form luctuosus Serville appears about midsummer from eggs hatched in the spring, and survives until Nov. It has a long ovipositor. Long-winged examples of assimilis are often attracted by light. On Sep 26, 1908, a male and a female were seen eating a living Arphia sulphurea. They are more commonly vegetable feeters, perhaps from necessity.

LI: Form pennsylvanicus from Rockaway, Je; Half Way Hollow Hills, J1; Nissequogue, Je; Orient, Je, Lath; Yaphank, J1; Gardiners I., Je. Form luctuosus from Aqueduct, Hempstead, Fire I., Central Pk., Selden, Yaphank, Amagansett, Gardiners I., Cold Sp. Harb., Southold, Orient, Aug-Sep.

Away from the coast and at a greater altitude assimilis is smaller and darker-colored, and is represented by the form neglectus Scudd. Specimens have been examined as follows: West Pt.; Debruce; Callicoon; Oliverea; Keene Val., Not; Wilmington; Ithaca; W. Danby; Potter Swamp; Conesus L. ; Portageville; near Olean.

G. domesticus L. West Farms (Akhurst, in Psyche, vol - 9, p. 293, 1902). SI: This introduced species is usually not common, but is occasionally met with in houses, where it is sometimes regarded as a nuisance. Where there is sufficient heat it may be heard singing in winter, the song being much more rapid than that of assimilis. The long-winged form is sometimes found about lights. LI: Brooklyn, taken on a sugared tree, Aug 19, 1916; Flatbush, Nov 25, 1909 , found in a cellar, Zab; Yaphank, J1, 1909, in a house.

\section{Miogryllus Saussure}

M. verticallis Serv. SI, Je 17, a fully grown female.

\section{NEMobius Serville}

N. fasciatus DeG. (vittatus Harr., short-winged form). Manhattan I., Inwood, Oct; Van Cort. Pk. ; Rye, Oct; W. Nyack, Aug; West Pt., Sep; Suffern, Sep; Debruce, Sep; E. Jewett, Aug; Oliverea, Sep; Adirondacks, Aug; Ithaca, Aug, Oct; Clyde; Batavia, Sep, Kngt; Rochester, Aug ; Cattaraugus, Sep. SI: A common species of both upland and salt meadow. Mature individuals J1-Nov. The long-winged form is often attracted by electric lights. LI: 
Brooklyn; Aqueduct; Pinelawn; Rockaway; Fire I.; Cold Sp. Harb. ; Oyster Bay; Wading R.; Southold; Orient; Selden; Coram; Yaphank; Riverhead; Calverton; Springs; Montauk; Gardiners I., Aug-Oct.

[N. griseus E. M. Walker has been reported from Toronto (Ontario), several of the New England States, and Indiana. It should be found in New York State.]

N. maculatus Blatch. SI, Sep 19, a male and a female, Ds (Hebard); LI: Flatbush, Sep 30, Zab (Hebard).

N. cubensis Sauss. SI, Aug 22, a female, Ds (Hebard).

N. palustris Blatch. White Plains, Sep, Leon; LI: Yaphank, Aug 26, 1916, rather abundant in an old cranberry bog, on raised places among the moss; Southold, Sep 4, 1912, a male and a female.

N. carolinus Scudd. Bronxville, Aug; West Pt., Sep; Suffern, Oct; Ramapo, Oct; Catskills, Sep; Ithaca, Oct; Olcott, Aug; SI: generally distributed, J1-Nov; LI: Farmingdale, Oct; Pinelawn, Aug; Wading R., Sep; Springs, Sep.

\section{OECANTHus Serville}

O. niveus DeG. Snowy Tree Cricket. Ramapo, Oct; West Pt., Robn; Middie Hope (NYS) ; Elmira, Sep, Diven; Ithaca, J1-Aug (CU); Geneva ; Rochester; Alfred; Otto (CU) ; Ripley (NYS) ; "It is found all over the State of New York with the exception of forested regions in the northeastern part (B. B. Fulton, 1915). SI: Generally distributed, and may be heard singing, especially in gardens among the grapevines, raspberry bushes, and fruit trees, from $\mathrm{Jl}$ until frost. It sings all night and continues when the temperature is rather low. LI: Generally distributed: Brooklyn; Maspeth; Flushing; Central Pk.; Sea Cliff; Long Beach; Smithtown; Wading R.; Greenport; Southold; Coram; Yaphank; Riverhead; Amagansett; Montauk; on Gardiners I. it is probably the most plentiful species of the genus, occurring often in the clumps of bayberry bushes.

O. angustipennis Fitch. Bronxville, Sep (Woodr); White Plains, Sep, Leon; W. Nyack, Sep; Ithaca, Aug (CU); Rochester, Dereson; common in the lake region of the western part of the State (B. B. Fulton, 1915); SI: common Aug-Oct, but more of a woodland species than niveus; LI: Prospect Pk.; Brooklyn; Maspeth, Ols; Fire I.; Central Pk.; Cold Sp. Harb.; Bay Shore, Ols; Selden; Coram; Yaphank; Riverhead; Promised Land; Calverton; Amagansett; Greenport; Southold; Orient, Nov 1, 1914, in orchard, Lath; Gardiners I., Aug.

O. exclamationis Ds. Bronxville, Aug-Oct (Woodr) ; SI: (type locality) : Buck's Hollow, Sep; Long Neck, Aug-Sep; Watchogue, Aug; Eltingville, Sep; LI: Flushing; Kissena Pk., Aug; Central Pk., Aug-Sep; Cold Sp. Harb., Sep ; Selden, Aug; Yaphank, Sep-Oct; Springs, Aug; Smithtown, Aug; Deep Pond, Wading R., Aug; Shelter I., Aug; Southold, Sep; Gardiners I., Aug.

O. nigricornis Wlk. Van Cort. Pk., Sep; White Plains, Sep, Leon, Var. quadripunctatus Beut.: Rye, Oct; Karner, Aug; Onteora Mt., Aug (How); Ithaca, J1-Oct (CU) ; Rochester, Deveson; Otto (CU) ; Cattaraugus, Sep (CU). SI: Common on goldenrods, asters, and other low-growing plants along the edge of the salt meadows as well as inland, Aug-Oct; Huguenot, Oct 3, 1913, a male eating an aphid. Var. quadripunctatus Beut.: Watchogue; Long Neck; Tottenville. LI: Generally distributed, Aug-Oct: Queens; Maspeth; Flushing; Aqueduct; Rockaway; Long Beach; Fire I.; Pinelawn; Bay Shore, Ols; Coram, several on sugared trees; Yaphank; Patchogue; Southold; Orient, Lath. Var. quadripunctatus Beut.: Aqueduct; Rockaway; Long Beach; Fire 
I. ; Maspeth, Ols; Flushing; Queens; Central Pk.; Pinelawn; Selden; Yaphank; Amagansett; Montauk; Greenport; Southold; Shelter I. ; Gardiners I., Aug.

This species is said by B. B. Fulton (1915) to be widely distributed over $\mathrm{NY}$, and var. quadripunctatus to be found in most parts of the State with the exception of the northern forested area.

O. pini Beut. Karner, J1-Sep (Felt, NYS). SI: As the pines on which this species occurs have nearly all disappeared from the island, its presence is hardly expected. LI: Fairly common in places among pitch pines; Central Pk., Aug; Pinelawn, Aug; Coram, Aug-Sep; Yaphank, J1-Sep; Riverhead, Aug; Promised Land, Sep; Southold, near Great Pond, Sep.

O. latipennis Rly. SI: Once very common and still occasional on the low sandy ground near Old Place and Watchogue. Found on goldenrods, small white birches, etc., Aug-Oct. LI: Bay Shore, Oct, Ols; Calverton, Sep, 1910, two females.

NEOXABEA Kirby

N. bipunctata DeG. Van Cort. Pk., Aug 12; Ramapo Mts. (AM) ; Ft. Montgomery, Schott. SI, a few collected in Aug. LI: Flushing, Aug, Bell; Wyandanch, Sep, Schott; Yaphank, J1, Sep, Shoe; Promised Land, Sep, Ds; Southampton, Aug, Zab; Amagansett, Sep; Wading R., J1, nymph; Shelter I., Aug 3, nymph; Orient Pt., J1, nymphs; Gardiners I., Aug.

The range of this species is more to the southward, and it probably does not occur in the northern part of the State.

\section{Cycloptilum Scudder}

C. squamosum Scud. LI: E. Marion near Orient Bay, Aug 2, 1913, two wellgrown nymphs under $\log , D s \&$ Eng.

\section{AnAXIPHA Saussure}

A. exigua Say. SI: Formerly not uncommon in rank vegetation bordering the salt marshes, Aug, Sep. In Aug and Sep, 1901, it was found in considerable numbers in the woods near Egbertville, but of late years no specimens have been collected on the island. LI: Maspeth, Flushing, Ols; Sea Cliff, Oct (AM), and 19 specimens, Bks (CU).

\section{PHYLLOSCYRTUS Guérin}

P. pulchellus Uhi. SI: once fairly abundant at Old Place and Watchogue, J1Oct, but none have been collected recently.

In Proc. Ent. Soc. Phila., vol. 2, p. 544, Uhler records this species as "taken near the city of New York."

\section{HAPITHUS Uhler}

H. agitator Uhi. SI: Ward's Pt.; Tottenville, a female, Sep 21, 1902. This is the only record. It is a common species on Sandy Hook, NJ. LI: Fire I., opposite Bay Shore, Aug, 1917, Henry Thurston.

\section{GryLlotalpa Latreille}

G. hexadactyla Perty (borealis Harr.). West Pt., Sep, Robn; Kingston, Dr. C. $W$. Crispell (Rept. NY State Ent., 1888); Albany, Oct (Lint-NYS); Honeoye Falls, Aug 26, nymph, Deveson. SI: Found in all rural places, in moist ground, 
where it may be heard singing its krerr, krerr, krerr from late J1 until Oct. The long-winged form columbia Scud. is occasionally attracted to light. Rarely individuals are found running about on roads, etc., during the day. LI: Yaphank, Sep, four nymphs $5 \mathrm{~mm}$. long, which would probably pass the winter in an immature state; Calverton, Sep; Amagansett, Sep; Gardiners I., Je 13, 1911, nymph, Aug 7, 1918.

[G. gryllotalpa L. European Mole Cricket. This imported insect has been reported from Rutherford, NJ, as well as from Mass, so it may be looked for in NY.]

\section{TRIDACTYLUS Olivier}

T. apicialis Say. Ithaca, J1, Pearce (Scudd). SI: Old Place, Je, Sep, on damp ground. LI: Rockaway Beach, Je, Eng; Deep Pond, Wading R., May 31, mature individuals; Fort Pond, Montauk, Sep.

\section{FAmily ACRIDIDAE: Short-Horned Grasshoppers}

\section{TRYXALIS Fabricius}

T. brevicornis L. LI: Ravenswood Swamp, Long Island City, collecıed many years ago $(\mathrm{Bt})$.

PSEUdopomala Morse

P. brachyptera Scud. SI: Watchogue, Sep 17, 1911, Grossbeck; Old Place, on salt meadow, Sep 8 and 13, 1920, two females, one caught in the web of a spider (H. H. Johnson). LI: Central Pk., Aug 13, 1913 (Fulton); Bay Shore, J1, 1915, Ols; Orient, J1 1, 1917, a nymph, on sandy beach, Lath; Cedar Beach on Gardiners I., Aug 28, 1918, often in clumps of stunted bayberry bushes.

\section{ERITETTIX Bruner}

E. simplex Scud. LI: Top of High Hill, West Hills, Je 21, 1917, a considerable colony; Yaphank, May 28, 1911, J1 8, 1916, several in thick grass; Wading R., May 30, 1913, colony on hill near the great split rock in a one-time asparagus bed, neglected and overgrown; Bellport, Je 23, 1913, Nic.

\section{Chloealtis Harris}

C. conspersa Harr. Bronxville, J1-Sep. Woodr; Ft. Montgomery, Oct 20, Schott; Callicoon, J1; E. Jewett, Aug; Mt. Whiteface, J1; Ithaca, J1-Aug (CU) ; Rochester, J1, Deveson; Portageville, Je, three nymphs; Otto (CU). SI, from early J1 to the middle of Sep. On Sep 15, 1888, a female was found laying eggs in an old fence rail. The males stridulate while sitting on the ground. At one time not uncommon in Clove Valley, but of late years found principally on the low ground about Watchogue; Moravian Cemetery, J1; near Egbertville, J1 21, 1922, a male. II: Half Way Hollow Hills, J1; Wading R., Je-Aug, at both Deep Pond and Long Pond; Shelter I., Aug; Orient, J1, Lath; Yaphank, J1, Sep; Riverhead, Aug; Patchogue, J1; Calverton, Sep; Montauk, Sep; Gardiners I., J1-Aug.

\section{ORPhulella Giglio-Tos}

O. pelidna Burm. Van Cort. Pk., Bronx, Aug; White Plains, Sep; W. Nyack, Aug ; E. Jewett, Aug; Ithaca, J1-Aug (CU) ; Rochester, J1, Deveson. SI: Generally distributed, J1-Oct. One female at light, J1 16, 1912. Occurs in both dry and moist situations, and is often common along the edge of the salt meadows. Males generally brownish, many green females, also some females 
with elytra purplish. LI: A common species and found from Brooklyn to Orient and Montauk; also on Fire I. and Gardiners I. J1-Sep. Sometimes found in very dry situations.

O. olivacea Morse. SI: Arrochar, Oakwood, Old Place, Watchogue, J1-Sep. Often very common on the salt meadows. A long series does not contain any green males, which exist, however, though rare. The upper parts of the females may be of any shade between brown and green. LI: Ravenswood (Psyche, vol. 8, p. 318-Morse); Central Pk., Sep; Sands Pt., Sep, Bs; Orient, on salt meadow, Sep, Lath; Gardiners I., Aug 19, 1918, a male.

O. speciosa Scud. Van Cort. Pk., Aug; W. Nyack, Aug; Debruce, Sep; E. Jewett, Aug. SI: Generally distributed, but more of an upland species than either of the two last named, Aug-Sep. Many of the females are partly green in color. LI: Maspeth, J1, Ols; Central Pk., Sep; Southold, Sep; Amagansett, Aug; Montauk, Sep; Gardiners I., J1-Aug.

\section{DICHROMORPHA Morse}

D. viridis Scud. Bronxville, Aug-Oct (Woodr); Ramapo, Oct; W. Nyack, Sep; West Pt., Aug-Sep (Robn); Oliverea, Sep; Ithaca, J1-Aug (CU). SI: A common species; mature individuals from J1-Oct. Both sexes may be either green or brown. The female is usually short-winged, but one with long wings and tegmina was found on the sidewalk at St. George, Aug 16, 1908. LI, J1-late fall: Rockaway; Aqueduct; Maspeth; Flushing; Central Pk.; Wyandanch; Half Way Hollow Hills; Pinelawn; Coram; Selden; Yaphank; Patchogue; Riverhead; Center I.; Smithtown; Wading R.; Greenport. At the last-named place Mr. Latham found it Nov 1, 1914, in woods.

\section{Clinocephalus Morse}

C. elegans Morse. SI: Usually found on or near the salt meadows; Oakwood, Aug; Ward's Pt., Tottenville, Sep; Watchogue, Sep; Price's Meadow I., Sep. A long-winged female was found on the sidewalk at St. George, Aug 27, 1914, and a male on the turnpike near Silver L. Pk., Oct 13, 1917. LI: The type came from Ravenswood (Bt) (Psyche, vol. 7, p. 402, 1896, and Proc. Acad. Nat. Sci. Phila., Mar, 1915, p. 99).

\section{CHORTHIPPUS Fieber}

C. curtipennis Harr. Bronxville, Aug-Sep, Woodr; Ft. Montgomery, Oct 20, Schott; West Pt., Aug-Sep, Robn; Debruce, Sep; Oliverea and Slide Mt., Sep, both long- and short-winged males and females; Onteora Mt., Oct (How); Wilmington, J1; Ithaca, J1-Aug, Oct (CU); Otto (CU) ; Rochester, Sep (Deveson). SI: Generally distributed, and found in low meadows; often occurring on the borders of the salt meadows. Jl-Oct, inclusive. The males stridulate while sitting on the ground. LI: Aqueduct, Sep; Rockaway, J1, Schott; Maspeth, Aug, Ols; Flushing, Sep, Bell; Wading R., Aug-Sep; Riverhead, Aug, salt meadow.

\section{STEThophyMA Fischer}

S. lineatum Scud. Colden L., Aug; Essex Co, J1 (NYS); Ithaca, J1-Aug (WC Thro-CU). LI: Not uncommon on the border of the salt meadow near the upper part of Wading R., Aug-Sep, 1922.

S. gracile Scud. Saranac L., Sep; Adirondacks, Aug (NYS).

S. platypterum Scud. White Plains, Aug, 1923 (JMFarley, Jr). 


\section{ARPHiA Stål}

A. xanthoptera Burm. Bronxville, Aug-Oct, Woodr; W. Nyack, Aug; Ramapo Mts. SI: Generally distributed from St. George to Tottenville. A late-appearing species attaining full growth in Aug and continuing until Oct. Makes a crackling noise in flight. LI: Has been found all over the island in Aug-Sep. Maspeth, Ols; Central Pk., Aug 12, just matured; Cold Sp.; Smithtown; Selden; Coram; Yaphank; Southold; Orient, Lath; Amagansett; Springs; Montauk; Gardiners I.

A. sulphurea Fab. White Plains, Je-J1; Ft. Montgomery, May; West Pt., Je ; Croton, J1; Oliverea, Je; Elmira, Je, Diven; Ithaca, May-J1, also CU; Conesus L., Je; Rochester,.J1, Deveson. SI: Generally distributed. Arrives at maturity about the middle of May from over-wintering nymphs. A few survive until the first of Aug or a little later. LI: Generally distributed. Central Pk., J1; Massapequa, May; West Hills, Je; Half Way Hollow Hills, J1; Nissequoque, Je; Kings Pk., Je, Bell; Yaphank, Je-Jl, Sep 3 latest date; Riverhead, Aug; Wading R., May-Aug.

\section{Chortophaga Saussure}

C. viridifasciata DeG. Nyack, Je: Ft. Montgomery, May; West Pt., Je; Ramapo, Je; Pine I., Je; Albany (NYS) ; Axton (M\&H) ; Mt. Whiteface, Wilmington, Je; Baldwinsville, Je (CU) ; Spencer, May (NYS) ; Elmira, Je, Diven; Ithaca, May-J1 (CU) ; Rock City, Je; Portageville, Je; Bergen, GMGillette ; south shore of L. Ontario, Je. SI: Generally distributed, and overwinters in nymphal stages. Mature individuals from Ap-Nov; also nymphs on Nov 6 . Found laying eggs May 17; pair mated J1 23; recently matured Aug 27 and Sep 12. Crackles wings in flight. Males usually brown or brownish in color; females green, or brown and green. Occasionally a green male is found, and more frequently a brown female. Sometimes the pronotum is reddish and the general coloring very beautiful. LI: Generally distributed; adults from Mayfall, and nymphs at any season. Rockaway Beach, in wash-up; Maspeth; Baldwin; Pinelawn; Wyandanch; Half Way Hollow Hills; Nissequogue; Wading R.; Yaphank; Orient, Lath; Riverhead; Gardiners I.

\section{ENCOPTOLOPHUS Scudder}

E. sordidus Burm. Inwood, Manhattan I., Sep, Schott; Rockland L., Oct; West Pt., Robn; Debruce, Sep; Albany (NYS); Oliverea, Sep; Essex Co (NYS); Whitehall, Washington Co, Oct (Not); Elmira, Aug, Diven; Ithaca, AugSep (CU) ; Holcomb, Sep (Kngt); Batavia, Oct (Kngt). SI: Generally distributed. Matures in Aug and found as late as Nov 27 at Tottenville. Laying eggs in path, Ft. Wadsworth, Nov 6. Crackles its wings in flight. LI: Flatbush, Oct, Zab; Flushing, Oct, Bell; West Hills, Sep; Patchogue, Sep; Amagansett, Sep.

\section{CAMnula Stå1}

C. pellucida Scud. E. Jewett, Aug, common; Mt. Whiteface, J1, common; Keene Val., Aug-Sep, Not; Saranac Inn, Ndm; Jay, J1 (WEBritton in Jour. Econ. Ent., 1911, p. 545); McLean, Aug, Sib; Ithaca, Aug (CU).

\section{PARDALOPHORA Saussure}

P. apiculata Harr. Bronxville, May, Woodr; Ramapo, May; Nyack, Je; Crotonon-Hudson, May; Albany, May-Je (NYS) ; Elmira, May, Diven; W. Danby, May; Ithaca, May; L. Keuka, Je. SI: This beautiful insect, with hind wings 
partly red, used to be an ornament to the overgrown fields of the island, but it appears now to be extinct. The last one seen by the writer was a male at Dongan Hills, May, 1909. It passed the winter as a nymph and matured in the spring. A few survived until J1. On May 27, 1900, a female was found laying eggs. LI: Queens, May, Schott; Jamaica, May; Pinelawn, Je; West Hills, Ap 17, 1915, an early date; Half Way Hollow Hills, May; Wyandanch, May, Schott; Nissequogue, Je; Baldwin, May; Bellport, Je, Nic; Coram; Wading R., May-Je; Yaphank, Je.

\section{DISSOSTEIRA Scudder}

D. carolina L. This species is not only found over the entire State of NY, but occurs over the greater part of the U.S. Some of the more northern and western state records are: Debruce, Sep; Callicoon, J1; Oliverea, Sep; Keene Val., Aug-Sep, Not; Elmira, Je, Aug, Diven; Ithaca, J1; Batavia, J1-Aug, Kngt; Buffalo, J1. SI: Generally distributed. Mature individuals found from Je-Nov. The males are the first to mature; some of the records are Je 20 , 1885, Je 28, 1887, and Je 25, 1921. A male was found attracted by light J1 9, 1912, and two J1 16, 1912. They are often found dead on low bushes, etc., having been killed by the fungus Empusa. This is the "hovering grasshopper," the males sometimes poising in mid-air a few feet from the ground. Their hovering, however, is not sustained as in certain Diptera. LI: Generally distributed all over the island from Brooklyn to Orient, Montauk, and Gardiners I. Occurs in numbers even on the seashore, and in the Bulletin of the Brooklyn Ent. Soc., Ap, 1913, there is an account of "A Plague of Carolina Grasshoppers at Long Beach, L. I., in 1912." It is sometimes drowned in the ocean and washed ashore on Rockaway Beach.

\section{SPHARAGEMON Scudder}

S. bolli Scud. Van Cort. Pk., Aug; Callicoon, J1; Debruce, Sep; Ellenville; E. Jewett, Aug; Ithaca, J1-Aug (CU) ; McLean, Aug-Sep, Sib; Batavia, J1, Kngt. SI: This woodland species is found from Je-Oct and is generally distributed. It crackles its wings in flight. A male was found attracted to light, J1 16, 1912. LI: Aqueduct, J1; Central Pk., J1-Sep; Pinelawn, Aug-Sep; West Hills, Sep; Selden, Aug-Sep; Coram, Aug-Sep; Smithtown, Aug; Wading R., Aug; Southold, Sep; Yaphank, J1-Sep; Bay Shore, J1, Ols; Bellport, Aug (Nic) ; Patchogue, Sep; Riverhead, Aug; Calverton, Sep; Amagansett, Sep; Montauk, Aug.

S. saxatile Morse. Ramapo, Sep; W. Nyack, Aug; Ft. Montgomery, J1; West Pt., Sep. Found on rocky areas, sometimes of small extent.

S. collare wyomingianum Thomas. SI: At one time well distributed on the sandy areas of the island from Richmond Valley to Mariners Harbor, but now much more restricted. It is an insect of overgrown sandy fields and open sandy woodland. It has been found plentiful at Richmond Valley in Sep, and near Western Ave., Mariners Harbor, in Aug, also Oct 9, Oct 15, and Nov 6. Other localities are Long Neck, Sandy Br., Woodrow, Bogardus Corners, Kreischerville, and Tottenville.

Professor Morse states that typical examples of wyomingianum occur on SI. It has not been found on LI.

S. collare scudderi Morse. LI: Central Pk., J1-Sep, both to the north and to the south of the railroad track, and also on the undisturbed parts of Hempstead Plains; Montauk, on the grassy hills, also near Fresh Pond, Aug 26 and 27, 1917 (Eng, Ds); Gardiners I., very common on the grassy hills near the monument reading "Capt. Kidd's Treasure was buried in this hollow and 
recovered in 1699." The reddish brown form of both males and females is much less common at Central Pk. and on Gardiners I. than dark fuscous examples.

\section{SCIRTETICA Saussure}

S. marmorata Harr. SI: Tottenville, Sep; Rossville, Aug; Sand Ridges, Richmond Valley, Sep; Watchogue, Sep-Oct. On Sep 20, 1919, three males and one female were collected near the corner of Hughes Ave. and Lambert's Lane, but this beautiful species seems now nearly extinct on the island. Both sexes crackle their wings in flight. LI: Generally distributed on the sandy parts of the island. Central Pk., Aug; Cold Sp. Harb., in a sandy field, Sep; Smithtown, Aug; Selden, Aug-Sep; Wading R., Aug; Yaphank, J1, Sep; Patchogue, Sep; Riverhead, Aug; Calverton, Sep; Amagansett, Sep; Promised Land, Sep; Greenport, Aug; Shelter I., Aug; Orient, Aug, Nov, Lath.

\section{PSINIDIA Stål}

P. fenestralis Serville. SI: On sandy ground and on the up-beach of the seashore. Mature individuals from J1-Oct. Arrochar; South Beach; Oakwood; Crooke's Pt.; Great Kills; Richmond Valley; Tottenville; Kreischerville; Rossville ; Bogardus Corners; Price's I.; Long Neck; Watchogue. Irrespective of the sex, the central part of the hind wings may be red, yellow, or some intermediate color. LI: Generally distributed, and found wherever there are sandy areas. Jl-Oct. Aqueduct; Coney I. ; Rockaway ; Fire I. ; Patchogue; Yaphank; Riverhead; Calverton; Amagansett; Springs; Montauk; Bayville; Cold Sp.; Center I., in Oyster Bay; Selden; Coram; Wading R.; Southold; Greenport; Shelter I.; Orient; Gardiners I.

\section{TRIMEROTROPIS Stå1}

T. maritima Harr. SI: On the beach from the Fort at the Narrows to Tottenville. Mature individuals as early as J1 1; they continue into Oct. On J1 16, 1912 , a female was found under an electric light at Egbertville, about one and a half miles from the beach or any other sandy area. LI: Generally distributed along the sandy beach on the south shore, and occasionally on sandy areas farther inland. J1-Oct. Coney I.; Barren I.; Rockaway Beach; Fire I.; E. Quogue; Amagansett; Bayville; Sea Cliff (Bks); Wading R., both along the beach and on the sandy shore of Long Pond about two miles inland; Southold; Greenport ; Shelter I.; Orient, Nov 10, 1914, Lath; Gardiners I.; at Riverhead, Aug, 1917, individuals with pinkish hind tibiae were collected.

\section{Circotettix Scudder}

C. verruculatus Kby. L. Mohonk, Sep, Eng; Karner, J1 (NYS) ; Onteora Mt., Aug, How; E. Jewett, Aug; Keene Val., Aug, Not, Sep, Eng; Wilmington, J1; Mt. Whitef́ace, Aug (Kngt); Chateaugay L., Bowditch (Morse); Little Valley, Sep, Sherm.

\section{ScHISTOCERCA Stål}

S. americana Drury. SI: Our largest grasshopper. Not known to breed on the island; all individuals recorded supposed to be adventive from the South. Occasionally found in May, but more often in Sep and later. On Dec 23, 1894, a male was collected on Todt Hill, and on Dec 28, 1907, a female at Woodrow. The last record is Nov 27, 1923, of a male collected on Todt Hill, it having flown from the ground into a tree, as they often do when disturbed. LI: Aqueduct, Schott. 
S. alutacea Harr. and S. alutacea form rubiginosa Scudd. The striped form, W. Nyack, Aug; the brown form, White Plains, Sep. The latter is reported also from Sparkill, Rockland Co. (Baird). SI: The striped form, greenish in color with a lighter dorsal stripe, is found in moist meadows among rank vegetation: Old Place; Watchogue; Long Neck; Richmond; Arrochar; Richmond Valley. The all-brown or brownish form, rubiginosa, is confined more to dry areas: Richmond Valley; Kreischerville; Long Neck; Watchogue. Aug 5-Oct 20. LI: The striped form, Central Pk.; Wyandanch; Yaphank; Wading R.; Riverhead. The all-brown form, which is the more common, Rockaway; Aqueduct; Jamaica; Central Pk.; Wyandanch; Cold Sp.; Coram; Yaphank; Smithtown; Wading R.; Riverhead; Calverton; Amagansett; and Montauk. J1-Sep.

\section{HESPEROTETTIX Scudder}

H. brevipennis Thomas. LI: This beautiful pink and green species has usually been found among sweet fern, Comptonia, though it occurs also among other plants. On J1 27, 1909, a large colony was found at Yaphank in an almost circular clump of Comptonia about 36 feet in diameter. On Aug 31, 1912, it occurred in the same locality chiefly on goldenrod flowers, though also on sweet fern. Other localities are: Central Pk., Aug; Coram, Aug; Riverhead, Bald Hill, Aug; Bellmore, Calverton, Sep, Eng.

\section{DENDROTETTIX Riley}

D. quercus Pck. LI: on Sep 3, 1912, a single short-winged male was collected at Yaphank. This is the only record from NY of this sometimes very destructive grasshopper which is known on occasion to defoliate large forest trees.

\section{Podisma Latreille}

P. glacialis Scud. and form variegata Scud. Summit of Mt. Whiteface, J1, nymphs (Ds), adults, Aug 22, Kngt, and Sep, Lg; L. Placid, Sep, on hazel bush, Eng; summit of Mt. Marcy, Aug; Saranac Inn, "on bushes in openings in the woods", Ndm; Slide Mt., Sep. The considerable series from Slide Mt. approach variegata, but the antennae in the male are not longer than the hind femora. Variegata is regarded as a variety by Morse. In his Review of the Melanopli, Scudder reports glacialis from Mt. Marcy and Chateaugay L., and describes variegata from Ithaca and Enfield Falls.

\section{PAROXYA Scudder}

P. clavuliger Serville. Van Cort. Pk., Aug; Pine I., Sep, Brb. SI: Found in grassy swamps, and generally distributed; also along the edge of the salt meadows. Jl-Nov. LI: Maspeth, Sep; Flushing, Sep; Aqueduct, Sep; Canarsie, Sep, Ols; Riverhead, Aug.

\section{Melanoplus Stål}

M. viridipes eurycercus Hebard. Groton, Je 11, 1914, EAChapman, one male, three females (Ds) ; Mix Cr. Valley, Cattaraugus Co, Je 11, 1915 (Ds, Bdy), two males, one female (Ds) ; Rock City, Je 6, 1915 (Ds, Bdy), one female (Ds) ; Seneca Jct., Je 7, 1915 (Ds), two females (Ds); Ithaca, one female (Hebard); W. Danby, May 30, 1915 (Ds), one male (Ds); (Hebard in Trans. Am. Ent. Soc., vol. 46, p. 396, 1920). Slaterville, May 30, 1925, Cy\&Seeley. 
M. calloplus Hebard. West Pt., Je. 14, 1914, four males, seven females (Ds) (Hebard in Trans. Am. Ent. Soc., vol. 46, p. 401, 1920).

M. tribulus Morse. Ramapo Mts., Oct. SI: a wingless species of the woodlands found J1-Sep Clove Valley; Reeds Valley; Graniteville; near Lambert's Lane, Watchogue; Richmond.

M. mancus S. I. Smith. Kaaterskill High Peak, Catskills, $3800 \mathrm{ft}$. (Morse). A number have been collected in Sussex Co, northern NJ, so it should be sought between there and the Catskills (Ds).

[M. dazesoni Scud. Reported from Toronto, Ontario (W1k), Brunswick, Me (Pck), Manchester, NH (Morse), and Tobyhanna, Pa, so its discovery within NY is no doubt a matter of more thorough collecting (Ds).]

M. scudderi Uhler. Rockland L., Oct; Danby Hill, Aug, OEPearce (CU) ; Ithaca, Comst (Scudd). SI: More common of late years with the spreading of the beard grass consequent to the drier condition of the land. It matures late and is generally found from Aug-Oct. A male was collected in 1899 as late as Dec. 2. From Fort Hill, St. George, to Tottenville, in suitable localities. LI: Aqueduct, Oct; Pinelawn, Aug-Sep; West Hills, Sep; Selden, Aug ; Coram, Aug-Sep; Yaphank, Aug-Sep; Patchogue, Sep; Calverton, Sep; Riverhead, Aug.

M. fasciatus F. Walker. White Plains, Je, Farley; West Pt., Je 14 and 16, and Crow's Nest Mt., Je 5, an early date; Axton (M\&H) ; Mt. Whiteface, J1; Rock City, J1, Kngt; Chipmunk Swamp, Vandalia, Je. SI: Clove Valley, on dry hillside, J1 20, 1914, three males and two females, Aug 14, 1920, two males and one female; Watchogue, on sandy ground among huckleberry bushes, Oct 12, 1918, one male. LI: Common on some of the dry and sandy parts of the island. It matures early and has been found from Je 14-Sep. Central Pk.; Massapequa; Pinelawn; Wyandanch; Cold Sp.; Selden; Coram; Yaphank; Wading R.; Riverhead; Calverton.

M. mexicanus atlanis Rly. Callicoon, J1; Karner, J1 (NYS); E. Jewett, Aug; Mt. Marcy, J1, Beq; Keene Val., Aug, Not; Wilmington, J1; Mt. Whiteface, top, J1-Aug, Kngt ; Ithaca, Sep, Kngt; Batavia, J1, Kngt; Rock City, J1, Kngt ; Rochester, J1, Deveson. SI: Generally distributed, J1-Oct. LI: Aqueduct; Half Way Hollow Hills; Selden; Wading R.; Yaphank; Riverhead; Shelter I. ; Orient; Gardiners I. J1-Sep.

M. impudicus Scud. LI: In the pine-barren areas. Near Selden, Aug 30, 31, and Sep 1, 1916, three males and one female. Sandy ground north of Coram, Pennequid Barrens, Aug 17, 19, 1920, and Sep 17, 1920, eight males, two females. Deep Pond, Wading R., Aug 7, 1912; Long Pond, Wading R., Sep 3, 1919, and Je 28, 1922.

M. femur-rubrum DeG. Red-LEgGed Locust. Rye; W. Nyack; Debruce; Catskills; Adirondacks; Karner; Geneva; Ithaca; Batavia; Otto. SI: Generally distributed and common. Also on the salt meadows. Early Aug until frost. Has been found in numbers as late as Nov 27. LI: Probably the most common species on the island, and generally distributed. Found on Gardiners I.

M. borealis Fieb. Abundant in stagnant bog, Oswegatchie, Je, 1925, Hood (Caud). Hebard states that the northeastern specimens belong to the race junius Dodge.

M. confusus Scud. (minor Scud.). Van Cort. Pk., Aug; White Plains, Je, JMFarleyJr; Nyack, Je; Pine I., Je; Croton-on-Hudson, Je; West Pt., Je; Oliverea, Je; Allaben, Aug, Dow; E. Jewett, Aug; W. Hebron, Je, Lg; Mt. Whiteface, J1. SI: Clove Valley, Je 24, 27 ; Ocean Terrace, Je 24; Tottenville, Je 14, J1 23. LI: Wyandanch, J1; Half Way Hollow Hills, J1; Yaphank, Je-J1, Sep; Wading R., at both Long and Deep Ponds, Je-Aug. 
M. keeleri luridus Dodge. E. Jewett, Aug; Johnstown, Scp (NYS) ; Ithaca, Oct, EOPearce. SI: Richmond Valley, Sep 2; Watchogue, in a barren sandy field, Aug-Oct, but not found in recent years. On Sep 17, 1887, two males and two females were collected at this locality and a note was made that they were plentiful. LI: Generally distributed, Aug-Sep: Central Pk; Pinelawn; Wyandanch; Cold Sp.; Selden; Coram; Smithtown; Wading R.; Yaphank; Patchogue; Riverhead; Calverton; Amagansett; Promised Land; Montauk.

M. differentialis Thomas. On Aug 30, 1924, a male was found in the salt meadow among rank vegetation near Old Place on the north shore of SI. This was the first record for the State (Journ. N. Y. Ent. Soc., Dec, 1924). Now well established at Old Place.

M. bivittatus Say. Ft. Washington Pk., Manhattan I., Aug; Bronx Pk.; Hastings, J1, Joutel; Croton-on-Hudson, J1; West Pt., Aug, Robn; Debruce, Sep ; Callicoon, J1; Slide Mt., Sep; E. Jewett, Aug; Albany, J1 (NYS); Wilmington, J1 ; Cranberry L., J1 (H-Mus. Zoo. U. Mich); Ithaca, J1-Sep (CU) ; Rochester, J1-Aug, Deveson; Batavia, J1-Aug (Kngt). SI: Common in both dry and moist situations; most plentiful J1-Aug, but continuing into Oct. LI: Generally distributed, Je-Sep. Aqueduct; Rockaway ; Fire I.; Maspeth, Ols ; Flushing; Hempstead; Central Pk.; Pinelawn; Coram; Selden; Yaphank; Wading R.; Riverhead; Southold; Orient; Amagansett; Montauk; Gardiners I. An early date was a mature male, Deep Pond, Wading R., Je 26, 1913.

M. punctulatus Scud. Ellenville, Sep. Bt; Ithaca, Aug-Oct, Cy, OEPcarce (CU). SI: Rare in recent years. Found in Aug, Sep, Oct, usually on pines but sometimes on oaks. Sep 23, 1893, a male in grounds of the August R. Grote homestead, Egbertville, where many pines had been planted by the naturalist's father. Aug and Sep, 1899, a number near Richmond, mostly on oaks; Oct 8, 1911, a female on a white pine in the Moravian Cemetery; Eltingville, Sep 27, 1922, a male. LI: Cold Sp., Sep; Coram, Aug-Sep, some discovered feeding at night on pitch pines; Yaphank, Sep.

\section{Family ACRydiddae: The Grouse or Pygmy Locusts}

\section{Nomotettix Morse}

N. cristatus Scud. Mosholu; White Plains, J1; W. Nyack, Sep; Ramapo, May-Je; Suffern, May, Bell; Pearl R., May, Wat; Rye, Oct; Hastings, Joutel; Haverstraw, Oct, Nic; Ft. Montgomery, May, J1; West Pt., May 9, longwinged female, Je ; Callicoon, J1; Oliverea, Je, nymph; Ithaca, Aug-Sep (CU) ; Rock City, Je, J1 5, one female, long-winged form; Conesus L., Je. SI: Mature individuals in every month from Ap to Oct. Specimens with long pronotum and wings are rare. LI: Generally distributed and collected from Ap-Oct; nymphs found in J1-Sep. Forest Pk. (Hebard) ; Central Pk. ; Massapequa; Pinelawn; Wyandanch; Huntington, Schott; Cold Sp. Harb.; Coram; Kings Pk.; Wading R.; Baiting Hollow, Cy; Yaphank; E. Quogue; Riverhead; Calverton: Amagansett; Promised Land; Gardiners I.

\section{ACRYDIUM Geoffroy}

A. granulatum granulatum Kby. West Farms, Mar, Joutel; W. Nyack, Mar, Beq; Pine I., Sep, Brb; Saranac Inn, $N d m$; Saranac L., Sep; Mt. Whiteface, 22 individuals found on summit, J1 10-13, 1914; Keene Val., Aug, Not; McLean, May; Ithaca, Ap-May (CU); Tarbel, Je; Portage, May, Kngt; Batavia, May-Je, Sep, Kngt. SI: Rare; adults found from Mar-Oct. Buck's Hollow, border of pond, May 20, 1920 (Bs). LI: Brooklyn, Ap 17, 1915 (Shoe); North Beach, Ap 1, 1917, Schott. 
A. ornatum Say. Croton-on-Hudson, May; Slide Mt., Je, Shoe; Albany, J1 (NYS) ; Axton, Je (M\&H) ; Cascade L., Adirondacks, Aug; Keene Val., Aug, Not; Mt. Whiteface, J1, a long-winged male found on summit; Syracuse, May (H-Mus. Zoo. U. Mich); Montour Falls, Oct (Cy); Ithaca, Ap (Bks), Oct, Kngt; Rochester, Sep Deveson. SI, Ap-May, Aug. A male sent to Scudder was identified as his variety triangulatum. LI: Brooklyn, Eng; Rockaway, Aug, Woodr; Central Pk., Sep; Pinelawn, Je; Calverton, Sep.

A. acadicum Scud. (hancocki Morse). Axton, Je, M\&H (Morse). Blatchley regards hancocki as a variety of ornatum Say, but it is placed as distinct by Morse in his Orthoptera of New England. Hebard states that this species should be known under the name acadicum Scud.

A. arenosum angustum Hancock. Manhattan I., Beaver St., NYC, Ap 26, 1915; Van Cort. Pk., May; White Plains, May-Je, Bno; Nyack, Je; W. Nyack, Ap (Bs) ; Ramapo, May (Joutel) ; Pearl R., May, Wat ; Ft. Montgomery, J1; West Pt., Je 4, 1916, just matured; Debruce, Sep; Ithaca, Coy Glen, May-Je; Tarbel, Je; Egleston's Glen, L. Keuka, Je; Genesee Co, Je; Rock City, Je-J1 (Kngt, Ds). SI: Collected from Mar-Oct. Less common in recent years. LI: Rockaway Beach, Oct 5, 1912, and Oct 12, 1918, Schott; Wyandanch, May; Cold Sp., Eng; Orient, J1, Lath.

\section{NEOTETTIX Hancock}

N. femoratus Scud. SI, Je, Aug, two males. More common southward.

\section{PARATETTIX Bolivar}

P. cucullatus Burm. Manhattan I., Je; Croton-on-Hudson, Je-J1; Ft. Montgomery, May, J1 (Schott, Ds) ; West Pt., Je; Callicoon, J1; Ithaca, J1, Sep (CU) ; Tarbel, Je 1, 1915, 31 specimens on mud flat; Portage, Je, Kngt ; Batavia, J1, Kngt; Rochester, Sep, Deveson; Sylvan Beach, Oneida Co, Je, Not; Lowville, Je, Not.

\section{TETTIGIDEA Scudder}

T. lateralis parvipennis Harr. White Plains; Rye, Oct; Hastings, Je, Joutel; Nyack, Je; W. Nyack, Sep; Ramapo, May, Joutel; Croton-on-Hudson, MayJ1; Ft. Montgomery, May, Joutel; West Pt., May, Robn, Je; Callicoon, J1; Oliverea, Je, Sep; Saranac Inn, $N d m$; Wilmington, J1; Keene Val., Je, Sep, Not; Mt. Whiteface, top, J1 10 and 13, 1914; Ithaca, Aug, Sep (CU) ; McLean, May; Tarbel, Je 1, 1915, 19 examples, long- and short-winged individuals paired together; Potter Swamp, Yates Co, Je ; Rochester, J1, Deveson; Honeoye Falls, Je 26, 1916, 17 examples, 5 pairs; Batavia, Je-J1 (Kngt, Ds). SI: Generally distributed; collected from Ap-Oct, and known to hibernate in the mature state. Long-winged form found at St. George on sidewalk, Oct 6, 1916, and Oct 10, 1919. LI: Rockaway, Je, Oct, Eng, Schott; Pinelawn, Je, Sep; Wyandanch, May-Je, Sep, Dec, Ds, Schott; Melville, Oct, Schott; Sands Pt., Sep, Bs; Bellport, Aug, Nic; E. Patchogue, Sep; Yaphank, Aug 26, 1915, 19 individuals, some immature; Springs, Sep; Montauk, Aug 25, nymph; Orient, Oct 10, on salt meadow, Lath; Gardiners I., Aug. The form acuta Morse, with the anterior margin of the pronotum produced into an acutely pointed process, was described from "NY" (Journ. N.Y. Ent. Soc., Mar, 1895), and has been taken on SI, Ap (Ds), and LI, Cold Sp., Eng (Psyche, Ap, 1908).

T. armata Morse. SI: the following were part of the paratypes of $T$. davisi Morse, now considered a synonym of armata: female, Ap 24, 1897; male, Sep, 1900 ; male, Je 5 ; female, Je; in wet places. T. armata is more common southward. 


\section{ORDER ISOPTERA}

FAMILY TERMITIDAE

\section{RETiCUlitermes Holmgren}

R. flavipes Kollar. Ithaca; Binghamton, Je, destroying girders in a large office building (Chap); Karner (Felt); Albany, Ap (NYS); White Plains (Bno); Mt. Vernon, Ap (Felt); Bronx Pk.; Mosholu (Sf); NYC (Joutel); SI: Port Richmond; LI: Brooklyn; Bergen Beach; Long Island City; Cold Sp. Harb.; Floral Pk., Aug (Sirrine); Sea Cliff (Bks); Pinelawn, swarming, $\mathrm{Je}$ (Ds).

\section{ORDER NEUROPTERA \\ FAMILY SIALIDAE \\ SUBFAMILY SIALINAE}

SIALIS Latreille

S. americana Ramb. L. George (Ndm).

S. concava Bks. Ithaca (Bks).

S. infumata Newn. Saranac Inn (Ndm) ; Ogdensburg, Je (Felt); Sacandaga Pk., Je (Alex) ; Castile, May, Brad (Fbs) ; Ithaca (Ndm) ; Ft. Montgomery, May (Ds) ; Ramapo, May-Je (Ds) ; Croton-on-Hudson, May (Ds) ; SI: Richmond, May (Ds).

\section{SUBFAMILY CORYDALINAE}

\section{Chauliodes Latreille}

C. angusticollis Hagen. Highland L., Sullivan Co, Aug, Chapin (Ds) ; SI, J1-Aug (Ds).

C. concolor Ds. Ithaca, J1 (KCDavis).

C. fasciatus Wlk. (lunatus Hagen). West Pt., Je, Robn (Ds); Sterlington, Je (Ds).

C. pectinicornis L. Saranac Inn $(\mathrm{Ndm})$; Ithaca, Je (SmRC); Trenton Falls (Hagen); Schenectady, Je-J1 (Lint); Buskirk's Bridge (Lint); Tiashoke (Lint) ; Troy, J1 (Felt) ; Athens, J1 (Felt) ; Sullivan Co (Ds) ; West Pt., Je (Ds); SI: Tottenville and Watchogue, May-Aug (Ds) ; LI: Maspeth, Je, Olsen (Ds).

C. rastricornis Ramb. Saranac Inn, Je (Ndm) ; Baldwinsville, Je (Ndm) ; Fulton, $\mathrm{Je}(\mathrm{Ndm})$; McLean, Je (Ndm); SI, May-Je (Ds); LI, May (Ds).

C. serricornis Say. Saranac Inn, Je (Ndm); Ithaca, Jl (SmRC); McLean, May (Ndm) ; West Pt., Je (Ds) ; Ramapo, May (Ds) ; Ft. Montgomery, May (Ds) ; Bronx Pk., May (Ds); SI, May-J1 (Ds).

\section{CoRydalis Latreille}

C. cornuta L. Adirondacks (Felt); Ogdensburg, J1 (Felt); Oneonta (NYS); Rochester, J1 (Felt); Schuylerville, J1 (Felt) ; Ithaca; Peruville, Je (Felt) ; Schoharie, Je (Felt); Howe Cave, Je (Felt); Cobleskill, Aug (Felt); Shushan, J1 (Felt); Altamont, J1 (Felt); Albany, Je (Felt); Rensselaer, Je (Felt); Ghent, Aug (Felt); Grand Gorge, J1 (Felt) ; Kingston (Lint) ; Milton, J1 (Felt); Liberty (Ds); Cornwall (Lint); Pine I., Je (Ds); Ft. Montgomery (Ds). Rocky streams, generally (Ndm). 


\section{FAMILY MANTISPIDAE}

\section{MANTISPA Illiger}

M. brunnea Say. LI: Coram, on the Pennequid Barrens, Aug 19, 1920, Eng, and Yaphank, $W k s$ (Ds).

M. interrupta Say. LI: Long Pond, Wading R., Je 26, 1922, found by Schott on the coat of Wm T. Davis, who had been walking through scrub oaks (Ds).

\section{FAMILY SISYRIDAE}

SISYRA Burmeister

S. umbrata Ndm. Saranac Inn, Je-J1 (Ndm); Sacandaga Pk., Je-J1 (Alex).

S. vicaria Wlk. Sarrnac, Je (Bks); Ithaca, Je (Bks); Mosholu, Sep (Bks).

Climacia McLachlan

C. areolaris Hagen (dictyona Ndm). Saranac Inn, Jl-Aug (Ndm); Canada L., $\mathrm{J} 1$, and Sacandaga Pk., Je 22 (Alex).

\section{FAMILY SYMPHEROBIIDAE}

\section{PSECTRA Hagen}

P. diptera Burm. Gloversville, Aug 29 (Alex); Ithaca (Bks).

\section{SYMPHEROBIUS Banks}

S. amiculus Fitch. "NY," on peach trees, May-Oct (Fitch-Sm NJ List); Ithaca, Aug 1, Cy (SmRC); SI (Ds); LI: (Ds); Sea Cliff (Bks).

\section{FAMILY HEMEROBIIDAE}

\section{HEMEROBIUS Linnaeus}

H. castaneae Fitch. Mosholu, Mar.

H. conjunctus Fitch. "NY" (Fitch).

H. fidelis Bks. Gowanda, Je, EPV (Bks).

H. humuli L. (castanae Fitch, tutatrix Fitch). Wanakena, Aug, Drake (SmRC); Saranac Inn, Aug (CU); West Falls, Je (CU); Castile, May, Brad (Fbs); Ithaca; McLean, J1-Sep (SmRC); Pine Kill, Sullivan Co, May 22, Cy (SmRC) ; SI: Tottenville, etc., Je-Aug ; LI: Sea Cliff (Bks), May-J1 (Bks, SmRC) ; Ronkonkoma, Sep 9, Cy (SmRC).

H. hyalinatus Fitch. "NY," May-J1, on pine (Sm NJ List) ; L. Placid, Aug, EPV (NYS) ; Penn Yan, May 30, Cy (SmRC).

H. placidus Bks. L. Placid, Aug (Bks).

H. simulans Wlk. Ithaca (Bks).

H. stigmaterus Fitch. Old Forge, J1 (CU) ; Otto, Sep (CU); Castile, May, Brad (Fbs); Cinnamon L., J1 24, Cy (SmRC); Ithaca (Bks), in trap lantern, J1 24, Fbs (SmRC); McLean, Sep (SmRC); SI: New Brighton, May, Sep (Ds); LI: Sea Cliff'(Bks). 
BORIOMYIA Banks

B. disjuncta Bks. Keene Valley (Bks); Axton, $M a c G$ (Bks); Ithaca, MacG (Bks); Onteora Mt., J1, How (Bks).

B. longifrons Wlk. (alternatus Fitch). "NY," on pine and hemlock in Je (Fitch-Sm NJ List); Ithaca, Je (Bks).

\section{MICROMUS Rambur}

M. angulatus Steph. L. Placid, Aug, EPV (NYS); Cranberry L., J1, Drake (SmRC); Gloversville, Sep 9 (Alex).

M. jonas Ndm. Saranac Inn, J1 (Ndm); Woodworth's L., Aug 19 (Alex).

M. montanus Bks. L. Placid, Aug, EPV (NYS); Old Forge, J1 (CU); Mountain L., Fulton Co, J1 21 (Alex); Onteora Mt., How (Bks).

M. posticus Wlk. West Falls, J1 (CU) ; Ithaca, Jl, Sep-Oct (SmRC) ; Kingston, Aug, EPV (NYS).

M. subanticus Wlk. L. Keuka, Sep (CU).

\section{FAMILY BEROTHIDAE}

\section{LOMAMYIA Banks}

L. flavicornis W1k. SI: Clove Valley, J1, rare (Ds); LI: Central Pk., May, Aug (Ds); Coram, on the Pennequid Barrens, Aug 18, 1920 (Ds).

\section{FAMILY POLYSTOECHOTIDAE}

\section{Polystoechotes Burmeister}

P. punctatus Fab. Newport, Sep (Felt) ; Cranberry L., J1 (Ndm); Saranac Inn, common, J1 (Ndm) ; St. Lawrence Co, Aug (Ndm) ; Long L., Aug, abundant (Lint); Gloversville, J1 (Alex); N. Fair Haven, Aug (Ndm); Chautauqua (Ds); Ithaca, 1 specimen (SmRC); Troy, J1 (Felt); Stamford, Aug (Ds); SI, Ji-Aug (Ds); LI (Ds).

\section{FAMILY CHRYSOPIDAE ?}

\section{ALLOCHRYSA Banks}

A. virginica Fitch. SI, Aug (Bks-Ds).

\section{Chrysopa Leach}

C. chi Fitch (ypsilon Fitch). Newcomb, J1 (SmRC); Axton, Je (Bks) ; Cranberry L., J1 (SmRC) ; Old Forge (CU) ; Woodworth's L., Je 23, and Johnstown, Je 3 (Alex); Ithaca and McLean (SmRC); Black Br., Je (SmRC); Greene Co, J1 (SmRC) ; LI: Sea Cliff, May-Je (Bks).

C. emuncta Fitch. Onteora Mt., Aug, How (Bks).

C. harrisii Fitch. Newport, J1 (NYS); Pike, J1 (NYS) ; Albany, Oct (NYS); SI, Oct (Ds).

C. interrupta Fitch. Honeoye Falls, Sep $(\mathrm{SmRC})$; Ithaca, Aug, at trap lantern, Oct (SmRC).

${ }^{7}$ This list has been checked by Dr. Roger C. Smith, of the Department of Entomology, Kansas State Agricultural College. 
C. lineaticornis Fitch. Ithaca, on linden ( $\mathrm{SmRC})$.

C. nigricornis Burm. Sacandaga Pk., Je 27 (Alex) ; Buffalo (Bks); Ithaca, MayAug (Bks, SmRC); McLean, Je (Sib); SI, Aug-Sep (Bks-Ds); LI: Sea Cliff (Bks).

C. occulata Say (chlorophana Bks., albicornis Fitch). Generally distributed and common throughout the State. Wilmington, Aug (SmRC); Cranberry L., J1 (SmRC); Syracuse, J1 (SmRC); Old Forge and Baldwinsville; West Falls, J1; Ithaca, J1-Aug (Bks); McLean, J1-Sep (SmRC); Karner, Je (SmRC); Onteora Mt., How (Bks); SI, Je-Jl (Bks-Ds); LI: Sea Cliff, J1-Aug (Bks).

C. plorabunda Fitch. Not abundant in NY State (SmRC). Ithaca, J1 (CU).

C. quadripunctata Burm. Ithaca, Je, at trap lantern (SmRC); McLean, Aug (SmRC); SI, Sep (Bks-Ds); LI: Sea Cliff, Aug (Bks).

C. rufilabris Burm. Axton (Bks); Ithaca, Sep (Bks, SmRC); Trenton Falls (Wlk); SI, Sep (Bks-Ds); LI: Sea Cliff (Bks).

C. stenostigma Navas. LI: Yaphank, J1 25, Bno (Navas).

C. stichoptera Navas. LI: Yaphank, J1 10, Bno (Navas).

\section{MELEOMA Fitch}

M. signoretti Fitch. Old Forge, J1 (Ndm); Ithaca, Je (SmRC); Onteora Mt., Je, How (Bks); LI (Bks).

M. slossonae Bks. Old Forge, J1 (Ndm) ; Malone, Je ( $\mathrm{Ndm})$; Mountain L., Je 18 (Alex); Sacandaga Pk., Alex (SmRC).

\section{FAMILY MYRMELEONIDAE}

\section{MYRMELEON Linnaeus}

M. crudelis W1k. (rusticus Hagen). SI, J1 (Ds).

M. immaculatus DeG. Essex Co, J1 (NYS); Ithaca, Aug (Comstock); Albany; Coeymans, Je (Lint); SI, Je, Sep (Ds).

\section{DENDROLEON Brauer}

D. obsoletum Say. Ithaca, J1-Aug (Comstock, Ndm-CU); Taughannock, Aug $B d y$ (Ndm-CU) ; Coeymans, Sep (Hagen); Palenville, Aug (Lint); LI: Yaphank, Jl-Aug, and Gardiners I. (Ds).

\section{BRACHYNEMURUS Hagen}

B. abdominalis Say. Croton-on-Hudson, Je (Ds); SI: Arrochar, Je, Aug, and Watchogue, J1 (Ds) ; LI: Yaphank, J1, E. Quogue, Aug, Half Way Hollow Hills, J1, and Coney I., Sep (Ds).

\section{Cryptoleon Banks}

C. nebulosum Oliv. (conspersa Ramb). SI: Richmond Valley, May (Ds); LI: Wyandanch, J1; Bayshore, J1, Olsen, and Yaphank, J1 (Ds).

C. signata Hagen. SI (Bks-Ds). Mr. Davis states that this is the Dendroleon pumilis Burm. of the N. J. List, 1910 ed., which was based on a misidentification by Banks. 


\section{FAMILY ASCALAPHIDAE}

ULULODES Currie

U. quadripunctata Burm. SI (Ds).

COLOBOPTERUS Rambur

C. excisus Hagen. Ithaca, Ada E. Georgia (Ndm).

\section{FAMILY CONIOPTERYGIDAE \\ CONIOPTERYX Curtis}

C. hageni Bks. LI: Sea Cliff (Bks).

C. vicina Hagen. Hamburg (Bks); Woodworth's L. and Johnstown, Aug (Alex); Ithaca, Jl-Aug, common; LI: Sea Cliff (Bks).

MaLAComyza Wesmael

M. westwoodi Fitch. Gloversville, Je 13 (Alex).

Conwentzia Enderlein

C. hageni Bks. Speculator, J1, Yg (NYS).

\section{ORDER EPHEMERIDA ${ }^{8}$ \\ FAMILY EPHEMERIDAE SUBFAMILY BAETINAE}

BAETISCA Walsh

B. obesa Walsh. Northampton, May (Felt); Newport, May, $Y g$; Niagara R. (EPV); Fish House, May (Alex).

\section{Chirotenetes Eaton}

C. albomanicatus Ndm. Johnstown, Je-Jl (Alex); Fall Cr., Ithaca, J1 (Ndm, Morg); May-Aug, nymphs; Slaterville, J1 (Ndm).

\section{Ameletus Eaton}

A. ludens Ndm. Newport, May, $Y g$; Pleasant Br., Ithaca, nymphs abundant, Ap (Morg). An inhabitant of temporary streams, parthenogenetic, eggs aestivate five months. But one male known ( $\mathrm{Ndm})$.

\section{CHOROTERPES Eaton}

C. basalis Bks. Old Forge, Aug (Ndm); Woodworth's L., Aug (Alex); Wintergreen Pt.; Ithaca, J1 (Ndm); L. George, Aug (Ndm).

\footnotetext{
Edited by J. G. Needham.
} 


\section{Caenis Stephens}

C. diminuta Wlk. Thousand I. Pk. (M. R. Miller) ; Little Clear Pond, near Saranac Inn, $\mathrm{Jl}(\mathrm{Ndm})$;. Saranac Inn, J1 $(\mathrm{Ndm})$; Old Forge, J1-Aug $(\mathrm{Ndm})$; Fall Cr., Ithaca (Morg); McLean, Je-Aug, Sib (Ndm); L. George, Aug (Ndm).

C. hilaris Say. Thousand I. Pk. (M. R. Miller); Ithaca (Ndm, Morg), abundant.

C. lacustris Ndm. Oneida L., F. C. Baker (Ndm).

\section{SIPHLONURUS Eaton}

S. alternatus Say. Saranac Inn, Little Clear Pond, and Old Forge (Ndm); Bald Mt. Pond $(\mathrm{Ndm})$; First L., Je (Ndm) ; Sacandaga Pk., Je (Alex); Ithaca, nymphs common, Ap-Je (Morg); Slaterville, Je (Ndm); Trenton Falls (Wlk).

S. aridus Say. NY (Bks).

S. femoratus Say. "NY" (Bks).

S. mirus Eaton. Sacandaga Pk. and Fish House, May-Je (Alex).

S. typicus Eaton. Johnstown, Je (Alex).

\section{Siphloplecton Clemens}

S. flexus Clem. Shore of L. Ontario near N. Fair Haven, J1 19 (Ndm).

\section{LEPTOPHLEBIA Westwood}

L. betteni Ndm. Hamburg, J1 (Bet); Howard, J1 5, Cy (Ndm); Ithaca (Ndm) ; McLean, J1 (Emily R. Morrison), Aug, Sib.

L. mollis Eaton. Old Forge (Ndm); Fall Cr., Ithaca (Morg); L. George, Je $(\mathrm{Ndm})$.

L. praepedita Eaton. Fall Cr., Ithaca, May-Je (Morg).

\section{SiPHLONISCA Needham}

S. aerodromia Ndm. Sacandaga Pk., Alex (Ndm); Northampton, May (Felt); Johnstown, Alex $(\mathrm{Ndm})$.

\section{Callibaetis Eaton}

C. americana Bks. Van Cort. Pk., Je 2 (Ds) ; SI, Ap 30, May 10 (Ds) ; LI: Yaphank, J1 25 (Ds).

C. ferruginea Walsh. Saranac Inn, Aug (Ndm); Mountain L., Je (Alex); LI: Sea Cliff (Bks).

C. fluctuans Waish. Ithaca, Je (Ndm).

C. hageni Eaton. Old Forge, J1-Aug (Ndm).

C. undatus Pict. "NY" (Hagen).

\section{BAETIS Leach}

B. posticatus Say. Old Forge, J1-Aug ( Ndm).

B. propinquus Walsh. Ithaca, Murphy.

B. pygmaea Hagen. Saranac Inn $(\mathrm{Ndm})$; Sacandaga Pk., Je (Alex); Ithaca, J1 (Ndm, Morg). 


\section{BLASTURUS Eaton}

B. cupidus Say. Old Forge, Je, and Ithaca (Ndm, Morg); McLean, May-Je, Sib $(\mathrm{Ndm})$; Castile, Ap-May, Brad (Ndm); SI, Ap 16,27, and Je (Ds); LI: Wyandanch, Ap 21, Schott, and Yaphank, May 29 (Ds).

B. nebulosus Wlk. Fish House, May 28 (Alex).

\section{EPhemerella Walsh}

E. cornuta Morg. Ithaca (Morg).

E. deficiens Morg. Ithaca (Morg).

E. dorothea Ndm. Beaver Meadow Br., near Old Forge, extremely local (Ndm).

E. excrucians Walsh. Saranac Inn $(\mathrm{Ndm})$; Cayuga L., Je; L. George, Je (Ndm).

E. feminina Ndm. Ithaca (Ndm) ; McLean, J1-Aug, Sib (Ndm); Richfield Sps., May (Ndm). Apparently parthenogenetic.

E. lata Morg. Ithaca, Je (Morg).

E. rotunda Morg. Pleasant Br., Ithaca, Je (Morg).

E. serrata Morg. Ithaca (Morg).

E. simulans Wlk. Ithaca, Je (Morg).

E. tuberculata Morg. Fall Cr., Ithaca (Morg).

\section{TRICORYTHUS Eaton}

T. allectus Ndm. Old Forge, Ithaca, Je-J1 (Ndm).

\section{Cloeon Leach}

C. mendax Walsh. Old Forge (Ndm).

C. vicinum Hagen. Old Forge ( $\mathrm{Ndm})$.

HABROPHLEBIA Eaton

H. vibrans Ndm. Bald Mt. Pond, near Old Forge, J1 (Ndm).

\section{SUBFAMILY EPHEMERINAE}

\section{POLYMITARCYS Eaton}

P. albus Say. Fall Cr., Ithaca, Je (Morg); points on Susquehanna R. (Ndm); Corning, on Chemung R., Aug (Ndm).

\section{HEXAGENIA Walsh}

H. bilineata Say. Buffalo, J1, Bet (Ndm); Niagara Falls, J1, Watson, as limbata Guér. (Ds) ; Cayuga L. (Ndm) ; Seneca L. (Ndm) ; Schenectady, Je (Lint); L. George, in deep water, J1-Aug ( Ndm) ; Poughkeepsie, J1 (Felt).

H. recurvata Morg. Pine L., near Canada L., Herkimer Co. (Ndm); Michigan Hollow (Ndm); McLean (Ndm).

H. variabilis Eaton. Little Clear Cr., near Saranac Inn, Je-Aug (Ndm); Ithaca, May (Morg); ? Coxsackie, Je (Felt).

\section{EPHEMERA Linnaeus}

E. guttulata Pict. Sacandaga Pk., Alex (Ndm); Fall Cr., above McLean (Ndm); Ft. Montgomery, May 31, 1903 (Ds). 
E. simulans Wlk. Sacandaga Pk., Je 23 (Alex); N. Fair Haven (Ndm); Cayuga L., J1, Comstock (Ndm); Buffalo, Bet $(\mathrm{Ndm})$; Fall Cr., Ithaca, Je-J1 (Morg); L. George, late Je-J1 (Ndm).

E. varia Eaton (decora Hagen). Little Clear Cr. and Saranac Inn (Ndm); Moose R. $(\mathrm{Ndm})$; Old Forge $(\mathrm{Ndm})$; Gloversville, J1, Alex $(\mathrm{Ndm})$; Fall Cr., Ithaca (Ndm, Morg).

\section{Potamanthus Pictet}

P. diaphanus Ndm. Old Forge; Squaw I.; Buffalo, J1, Bet; Ithaca (Ndm).

P. inaequalis $\mathrm{Ndm}$. Schenectady, Je $(\mathrm{Ndm})$.

\section{SUBFAMILY HEPTAGENINAE}

ECDYONURUS Eaton

\section{(Ecdyurus Eaton)}

E. maculipennis Walsh. Mountain L., Fulton Co, J1 (Alex); Corning, Jl, Cy (Ndm) ; Ithaca, Je-J1 (Ndm, Morg); McLean, J1 29, Aug, Sib (Ndm).

\section{HEPTAGENIA Walsh}

? H. canadensis W1k. "NY," O. S. (Hagen).

H. interpunctata Say. Old Forge ( $\mathrm{Ndm})$; Trenton Falls, O. S. (Hagen); Ithaca, Ap (Morg) and J1 (Ndm); McLean, J1, Sib (Ndm); L. George, Aug (Ndm).

H. luridipennis Burm. Johnstown, Je, Alex (Bks); Westfield (Bks).

H. placita Bks. Sacandaga Pk., Je, Alex (Bks).

H. pleuralis Bks. Gloversville, May 15, Alex (Bks)-type; Ramapo, May 31, and SI, Je 7, as Iron (Ds).

H. pulchella Walsh. Saranac Inn (Ndm); Old Forge, Jl-Aug (Ndm); N. Fair Haven (Ndm); Fall Cr., Ithaca (Morg); L. George, Aug (Ndm).

H. tripunctata Bks. Westfield (Bks); Johnstown, Je 15 (Bks).

H. verticis Say. "NY" (Bks).

\section{IRON Eaton}

I. fragilis Morg. Coy Glen, Pleasant Br., and Fall Cr., near Ithaca (Morg).

\section{EPEORUS Eaton}

E. humeralis Morg. Ithaca; May-J1 (Morg); Slaterville, Theresa M. Robinson $(\mathrm{Ndm})$; Tarbel, near Lansing $(\mathrm{Ndm})$.

\section{ORDER ODONATA ${ }^{9}$ SUBORDER ZYGOPTERA \\ FAMILY AGRIONIDAE}

\section{CALOPTERYX Burmeister}

C. aequabilis Say. Old Forge ( $\mathrm{Ndm}$ ); Keeseville, Aug, Sheraton (Calv); Ithaca, Je (Kennedy); Bronx Pk., Je (Ds).

C. amatum Hagen. Axton, Je (M\&H); Keene Valley, J1 (Calv-NYS).

${ }^{9}$ Edited by J. G. Needham, with the collaboration chiefly of P. P. Calvert, E. M. Walker, and Wm, T. Davis. 
C. dimidiatum Burm. Axton, Je (M\&H) ; Rochester (Moore); NYC (Bt).

C. maculatum Beauv. Generally distributed. Axton, Je (M\&H); Keeseville, Je, Sheraton (Calv); Peru, Cy; Old Forge (Ndm); Saranac Inn, late Je $(\mathrm{Ndm})$; De Ruyter Res., Madison Co, Cy; Buffalo, EPV (Kellicott); Castile, Brad (Ndm); Ithaca (Bks); Schenectady, J1 (NYS); Trenton Falls, O.S. (Hagen) ; NYC, Je (Hagen); SI, Je-Aug (Ds); LI, general (Ds). Streams in deep woods.

\section{Hetaerina Hagen}

H. americana Fab. Keeseville, Aug, Sheraton (Calv); Ithaca, Salant (Bks); Albany Co (NYS) ; Slingerlands, Aug (Felt). Along sandy streams, AugSep; very local.

\section{LESTES Leach}

L. congener Hagen. L. St. Regis, Franklin Co, Sep, Moore (Calv); Castile, Brad $(\mathrm{Ndm})$; Ithaca (Ndm); Bronxville, Jl-Sep, Woodr (Ds); SI, Sep-Nov (Ds) ; LI: Flushing, Aug (Ds); Southold, Sep (Ds).

L. disjunctus Selys. Waddington, Aug (Woodr); Keene Valley (Not); L. St. Regis, Franklin Co, Sep, Moore (Calv); Keeseville, J1-Aug, Sheraton (Calv); Ithaca, Aug (Bks); Catskill L., Aug (Calv); Hunter, Mud, and Burnt Hill Ponds, and Beaver Br., Sullivan Co, J1 (Calv); LI: Wading R. and Long Pond, Aug (Ds); Calverton, Sep (Ds); Bridgehampton, Aug (Calv).

L. eurinus Say. Rochester (Moore); Dobbs Ferry, Willn (Calv); Riverdale, J1, Woodr (Ds); SI, Je-J1 (Ds); LI: Wading R., Je (Ds). Tree-shaded ponds.

L. forcipatus Ramb. Rochester (Moore); Ithaca, Je (Bks); Bronxville, Je-Aug, Woodr (Ds); SI, May-Aug (Ds) ; LI: Deep Pond, Wading R., Aug-Sep (Ds) ; Yaphank, Sep (Ds); Gardiners I., Aug (Ds).

L. inaequalis Walsh. SI, Je-J1 (Ds).

L. rectangularis Say. Dobbs Ferry, J1 (Calv); Rochester (Moore); Buffalo, Aug-Sep, EPV (Kellicott); Ithaca, J1-Aug (Bks); Schoharie (NYS); Saratoga L., Aug (Calv); Cairo and Stony Clove, Greene Co, Aug-Sep (Calv); Black L., Beaver Br., and Stump Pond, Sullivan Co, Aug (Calv); New Baltimore (NYS); Bronxville, J1 (Woodr); NYC, Calverley (Hagen); SI, Je-Aug (Ds) ; LI: Flushing and Gardiners I., Aug (Ds).

L. uncatus Kby. Mt. Marcy, $4500 \mathrm{ft}$. (Not); Keeseville, J1, Sheraton (Calv); Saranac Inn (Ndm); Batavia, Je (Ds); Buffalo, Je-Aug, EPV (Kellicott); Ithaca (Ndm); Bronxville, J1, Woodr (Ds).

L. unguiculatus Hagen. Keene Valley (Not); Saranac Inn, J1 (Ndm); Rochester (Moore); Ithaca (Ndm); NYC (Bt); Bronxville, Je-J1, Woodr (Ds) ; SI, Je-JI (Ds) ; LI: Yaphank, Sep (Ds) ; Long Pond, Wading R., Je-Aug (Ds) ; Orient, Sep, Lath (Ds); Gardiners I., J1 (Ds).

L. vigilax Hagen. Old Forge, Je-J1 ( Ndm); Green and Catskill Lakes, Aug (Calv); Folwood, Amber, Stevensville, and Black Lakes, Knapp and Stump Ponds, and Beaver Br., Sullivan Co, J1-Aug (Calv); LI: Yaphank, J1 (Ds); Wading R., Aug (Ds); Wyandanch, J1 (Ds); Cold Sp. Harb. (Ndm). 


\section{A RGIA Rambur}

A. apicalis Say. SI (Ds).

A. bipunctulata Hagen. "NY" (Bks).

A. moesta Hagen subsp. putrida Hagen. Axton, Je ( $\mathrm{M} \& \mathrm{H})$; Saranac Inn ( $\mathrm{Ndm}$ ) ; Keene Valley (Not); Rochester (Moore); Niagara, Slosson (Calv); Buffalo, J1-Aug, EPV (Kellicott); Ithaca, summer (Bks); Schoharie (NYS). Along creeks where rapid current is broken by projecting stones.

A. tibialis Ramb. Pine I., Je (Woodr).

A. translata Hagen. White L., Sullivan Co, Aug (Calv).

A. violacea Hagen. Waddington, Aug (Woodr) ; Axton (M\&H) ; Keeseville, J1Aug, Sheraton (Calv); Old Forge (Ndm); Saranac Inn, J1 (Ndm); Buffalo, Aug, EPV (Kellicott); Ithaca, J1 (Bks); L. George, Aug (Ndm) ; Green L. (Calv) ; Amber, White, and Black Lakes, Jenkins and Stump Ponds, and Beaver Br., Sullivan Co, J1-Aug (Calv); Dobbs Ferry, J1-Aug, Willn (Calv) ; Bronxville, J1, Woodr (Ds) ; SI, Je-Aug (Ds) ; LI: Yaphank, J1Sep (Ds) ; Smithtown, Aug (Ds); Wyandanch, J1 (Ds); Flushing, Aug (Ds). About most ponds.

\section{ENALlaGma Charpentier}

E. antennatum Say. Waddington, Aug (Woodr); Keene Valley (Not); Ithaca (Kellicott-CU).

E. aspersum Hagen. Dobbs Ferry, J1, Willn (Calv); Burnt Hill, Knapp, and Cranberry Ponds, Sullivan Co, J1 (Calv); NYC (Bt); SI, Je-Aug (Ds); LI: Yaphank, J1 (Ds); Bridgehampton, Aug (Calv).

E. calverti Morse (boreale Selys [Wlk.]). McLean Bogs (Bdy\&Hd).

E. carunculatum Morse. Waddington, Aug (Woodr); Keene Valley (Not) ; Piseco L., Aug (Calv); L. Pleasant, J1 (Calv); Squaw I., late Je, EPV (Kellicott) ; Niagara, Slosson (Calv); Saratoga L., Aug (Calv); L. George (Ndm) ; Bronxville, Je, Aug, Woodr (Ds).

E. civile Hagen. Rochester (Moore) ; Bronxville, Woodr (Ds) ; SI, Je, Aug-Sep (Ds); LI: Mecox Bay (Calv); Flushing, Yaphank, and Fire I. (Ds); Greenport, Aug (Ds); Amagansett, Sep (Eng); Rockaway, J1 (Woodr) ; Gardiners I., Aug (Ds).

E. cyathigerum Charp. (annexum Hagen). Keeseville, Je, Sheraton (Calv) ; Saranac Inn, J1 (Ndm) ; Ithaca, Aug (Bks); West Pt., Je (Ds).

E. divagans Selys. Rochester (Moore); Ithaca (Mary Lyon); LI (Bks).

E. doubledayi Selys. Ithaca (Mary Lyon); LI: Deep Pond, Wading R., Je, Aug (Ds); Bridgehampton, Aug (Calv).

E. durum Hagen. Stony Pt., Je (Ds); LI: Mecox Bay, Aug (Calv) ; Yaphank, Je, Sep (Ds).

E. ebrium Hagen. Waddington, Aug (Woodr); Keene Valley and Mt. Marcy, $4500 \mathrm{ft}$. (Not) ; Keeseville, Je-J1, Sheraton (Calv); Ithaca (Bks) ; L. George $(\mathrm{Ndm})$; Schoharie (NYS); Catskills (Calv-AES); ponds in Uíster, Sullivan, and Greene Counties, J1-Aug (Calv).

E. exsulans Hagen. Keene Valley (Not); Keeseville, Je, Aug, Sheraton (Calv) ; Saranac Inn (Ndm); Old Forge (Ndm); Black Rock Harb., Je, EPV (Kellicott); Rochester (Moore); Clarence, J1, EPV (Kellicott); Ithaca, J1Aug (Bks); Pine I., Je (Ds); Port Jervis (Calv); Saratoga L., Aug (Calv); Pine I., Je (Woodr); Bronxville, J1, Woodr (Ds); West Farms, Angus (Ds). 
E. geminatum Kellicott. Parish, Aug (Kellicott); Green L.. Aug (Calv); Amber L., Sullivan Co, Aug (Calv) ; SI, Aug-Sep (Ds), Je (Woodr) ; LI: Yaphank, Wyandanch, and Flushing, J1-Sep (Ds).

E. hageni Walsh. Axton, Je (M\&H) ; Saranac Inn (Ndm); Keene Valley (Not) ; Old Forge (Ndm); Squaw I., Je, EPV (Kellicott); L. Pleasant, J1 (Calv); Lake Bluff at Huron (Calv); Castile, Brad (Ndm); Ithaca (Bks-CU); Rensselaer, Je (Woodr); Knapp Pond, Sullivan Co, J1 (Calv).

E. hesperum Calv. (pollutum Hagen). Black L., Sullivan Co, Aug (Calv); LI: Yaphank, Sep (Ds).

E. minusculum Morse. LI: Long Pond, Wading R., Je 26, 1917, common (Ds).

E. pictum Morse. LI: Wyandanch, J1 (Ds).

E. piscinarium Willn. Mt. Marcy and Keene Valley (Not); Ithaca (Ndm).

E. recurvatum Ds. LI: Wyandanch, J1 (Ds).

E. signatum Hagen. Rochester (Moore); Williamsville, Sep, EPV (Kellicott); Ithaca (Ndm) ; Folwood, Amber, and Black Lakes, and Stump Pond, Sullivan Co, Aug (Calv); Green L., Aug (Calv); Bronxville, Aug (Woodr) ; SI, Je, Aug (Ds); LI: Yaphank, Sep (Ds) ; Flushing, Aug (Ds) ; Cold Sp. Harb., J1, Brb (Ds). Along sluggish creeks.

E. traviatum Selys. LI: (Bks); Wyandanch, Aug 21, 1917 (Ds).

\section{Nehalennia Selys}

N. carlotta Butler. Saranac Inn, Ndm (Hortense Butler).

N. gracilis Morse. Saranac Inn (Ndm); LI: Yaphank, J1 (Ds).

N. irene Hagen. Mt. Marcy, $4500 \mathrm{ft}$. (Not); Old Forge (Ndm) ; Saranac Inn, J1 (Ndm) ; Rochester (Moore); Ithaca, J1 (Bks); Nyack, Je (Ds); Bronxville, J1, Woodr (Ds); SI, Je (Ds); LI: Wyandanch and Yaphank, J1 (Ds) ; Forest Pk., Brooklyn, Je (Ds).

\section{AMPHIAGRION Selys}

A. saucium Burm. Rochester (Moore); Buffalo, J1-Aug, EPV (Kellicott); Ithaca (Bks); McLean (Sib); Trenton Falls, O.S. (Hagen); Karner (NYS) ; Schoharie, Je (Not); Big Pond, Delaware Co, J1 (Calv); SI, May-Je, Aug (Ds); LI: Half Way Hollow Hills, J1 (Ds); Yaphank, Je (Ds).

\section{COENAGRION Kirby}

C. resolutum Hagen. Cinnamon L., J1 ( $\mathrm{Ndm}$ ).

\section{CHROMAGRION Needham}

C. conditum Hagen. Saranac Inn (Ndm) ; Mt. Marcy (Not) ; Hamburg, Je, EPV Kellicott); Castile, Brad (Ndm); Ithaca (Bks); McLean, May (Ndm), Je (Sib) ; SI, J1 8, 1918 (Ds). Breeds in upland swales or in ditches of running water.

\section{ISCHNURA Charpentier}

I. posita Hagen. Rochester (Moore); Ithaca ( $\mathrm{Ndm}$ ); Central Pk. in NYC, Je (Woodr) ; SI, May-Sep (Ds) ; LI: Yaphank, J1 (Woodr).

I. ramburii Selys. NYC (Bt); SI, Sep-Oct (Ds); LI: Fire I., Sep (Ds).

I. verticalis Say. Generally distributed. Waddington, Aug (Woodr); Old Forge $(\mathrm{Ndm})$; Axton (M\&H); Mt. Marcy and Keene Valley (Not); Keeseville, 
Je, Aug, Sheraton (Calv); Mooers, Sep (Not); Saranac Inn, J1 (Ndm); Squaw I. and along Niagara R., May-Sep, EPV (Kellicott); Niagara, Slosson (Calv); Castile, Brad (Ndm); Ithaca, Jl-Aug (Bks); Saratoga L., Aug (Calv) ; many ponds in Delaware, Ulster, and Sullivan Counties, J1 (Calv); Dobbs Ferry, Je, Aug, Willn (Calv) ; Bronxville, J1 (Woodr); Central Pk. in NYC, J1 (Woodr); SI: May-Sep (Ds); Annadale, Je (Troop) ; LI: Mecox Bay and Bridgehampton, Aug (Calv).

\section{ANOMALAGRION Selys}

A. hastatum Say. Keeseville, J1, Sheraton (Calv); Ithaca (Ndm); NYC (Bt); Bronxville, J1, Woodr (Ds); SI, Jl-Nov (Ds); LI: Gardiners I., Jl-Aug (Ds).

\section{SUBORDER ANISOPTERA \\ FAMILY AESCHNIDAE}

\section{TACHOPTERYX Selys}

T. thoreyi Hagen. Portage, Je (Ds); Coy Glen, Ithaca, Je (DtAS); McLean (Kennedy); West Pt., Je (Ds) ; Ft. Montgomery, Jl, Schott (Ds) ; Ramapo, Je, Watson (Ds).

\section{CORDULEGASTER Leach}

C. diastatops Selys. Ithaca and McLean, May-J1 (Ndm, DtAS).

C. erroneus Hagen. Keene Valley (NYS); Ithaca, Je (DtAS).

C. maculatus Selys. Keene Valley (Comstock); Axton, Je (M\&H) ; Saranac Inn, J1 (Ndm) ; Ramapo, May (Ds) ; Bronxville, Je, Woodr (De') ; Nyack, Je (Ds) ; SI, May-Je (Ds) ; LI: Yaphank, May, Eng (Ds).

C. obliquus Say. Pine I., Je (Ds); Bronxville, May-Je (Woodr); Bronx Pk., Je (Ds) ; Van Cort. Pk., Je, Watson (Ds).

\section{GoMphoides Selys \\ (Progomphus Selys)}

G. obscura Ramb. LI: Wading R. and Deep Pond, Je-Aug (Ds).

\section{HAGENIUS Selys}

H. brevistylus Selys. Old Forge $(\mathrm{Ndm})$; Saranac Inn, Je (Ndm) ; Parish, Aug (Kellicott); Rochester (Moore); Ithaca (Bks-CU); L. George (Sib); LI, Eng (Ds).

\section{OPHIOGOMPHUS Selys}

O. aspersus Morse. Saranac Inn, Je-J1 (Ndm); Van Cort. Pk., Bronx, Je (Ds).

O. carolus Ndm. Castile, Brad (Ndm); Ithaca, May, Yg, Martin (Ndm); W. Danby, Je, abundant (Kennedy); Schoharie, Je (Not).

O. johannus Ndm. Wilmurt ( $\mathrm{Ndm}-\mathrm{CU})$.

O. mainensis Pck. Keene Valley, $1000 \mathrm{ft}$. (NYS).

O. rupinsulensis Walsh. Ithaca (Bks), Je, Martin ( $\mathrm{Ndm})$; Schoharie (SelysNYS); Callicoon, J1 (Ds). 


\section{LANTHUS Needham}

L. albistylus Selys. Bear Swamp, Ramapo Mts., close to state line, Je, C.E. Sleight (Ds).

L. parvulus Selys. Indian Pass, Adirondacks, J1, Watson (Ds); Ithaca (Bks, $\mathrm{Ndm}$ ) ; E. Jewett, Aug (Ds) ; Portage, Je (Ds) ; Ramapo, May (Ds).

\section{Gomphus Leach}

G. abbreviatus Hagen. Fall Cr., Ithaca, Je (Ndm); Greenwood L., Je, Schott (Ds).

G. adelphus Selys. Bethlehem, Je (Selys); Kenwood, Je (NYS); Coeymans, Sep (Lint).

G. amnicola Walsh. Bethlehem, J1 (Willn).

G. borealis $\mathrm{Ndm}$. Saranac Inn $(\mathrm{Ndm})$.

G. brevis Selys. Axton, Je (M\&H); Saranac Inn, Je-J1 (Ndm); Schoharie, Lint (Hagen).

G. cornutus Tough. McLean Res., Sib (Ndm).

G. descriptus Bks. Saranac Inn (Ndm); Ithaca, May (Bks), May-Je (Ndm); W. Danby, May (Ds); McLean, May, Hd (Ndm).

G. exilis Selys. Axton, Je (M\&H) ; Keeseville, Je, Sheraton (Calv) ; Saranac Inn, Je-Jl (Ndm); Rochester (Moore); Ithaca, Je-J1. (Bks); L. George, Je $(\mathrm{Ndm})$; Cinnamon L., J1 12 (Ndm); Coeymans, Sep (Felt) ; West Pt., Je (Ds) ; Pine I., Je (Woodr) ; Bronxville, Je (Woodr); SI, May-J1 (Ds) ; LI: Wading R., Je (Ds).

G. fraternus Say. Niagara R., Je, EPV, Schaum (Kellicott, Hagen).

G. furcifer Hagen. Ithaca ( $\mathrm{Ndm}-\mathrm{CU}$ ).

G. lividus Selys. Ithaca, May (Ndm); McLean, May (Ds); Pine I., Je (Ds); Bronxville, May-Je, Woodr (Ds).

G. notatus Ramb. Rochester (Moore).

G. plagiatus Selys. Bethlehem, Sep (Calv); L. George, Aug (Ndm); LI, Olsen (Ds).

G. quadricolor Walsh. Ithaca (Ndm); Schoharie, Je (Not).

G. scudderi Selys. Saranac Inn, Je-Aug ( Ndm); Ithaca, upper Cascadilla Cr., J1 (Kennedy).

G. spicatus Hagen. Axton, Je (M\&H) ; Old Forge, Je-J1 (Ndm) ; Saranac Inn, Je-Jl (Ndm); Clarence, J1, EPV (Kellicott); Black Rock Harb., Je, EPV (Kellicott) ; Ithaca (Mary Lyon) ; Cinnamon L., J1 12 (Ndm) ; Slide Mt., Je (Woodr). Taken in a tamarack swamp ( $\mathrm{Ndm})$.

G. spiniceps Walsh. Ithaca ( Ndm); Bethlehem, Sep, Willn (Lint-MCZ); Albany (Willn-MCZ).

G. vastus Walsh. "NY" (Hagen).

G. ventricosus Walsh. Old Forge $(\mathrm{Ndm})$; Pine I., Je (Ds).

G. villosipes Selys. Grand I., Fischer (Kellicott); Ithaca, May (Ndm, Bks); Pine I., Je (Woodr); SI: Ft. Wadsworth, Je 18 (Ds).

\section{DROMOGOMPHUS Selys}

D. spinosus Selys. Old Forge ( $\mathrm{Ndm}$ ); Baldwinsville, Pettit (Bks); Castile, Brad $(\mathrm{Ndm})$; Canandaigua L., Je (Herr); Ithaca (?) (Bks-CU) ; Schoharie, Je (Not); Karner, Je (Hagen) ; White L., Sullivan Co, Aug (Calv) ; Pine I., Je (Ds); Van Cort. Pk., J1, Watson (Ds). 
BOYERIA McLachlan

B. grafiana Willn. Old Forge, Aug, Willn (Ndm); E. Jewett, Aug (Ds); Ft. Montgomery, Sep (Schott).

B. vinosa Say. Waddington, J1 (Woodr); Old Forge, Aug (Ndm); Keeseville, J1, Sheraton (Calv); Saranac Inn, Je $(\mathrm{Ndm})$; Piseco L., Aug (Calv); Parish, Aug (Kellicott); Colden, Aug, EPV (Kellicott); Ithaca, J1 (Bks); Schoharie (Calv); Elk L., Aug (Calv); SI, Jl-Aug (Ds); LI: Yaphank, J1, Sep (Ds) ; Smithtown, Aug (Ds).

\section{Basiaeschna Selys}

B. janata Say. Keeseville, Je, Sheraton (Calv); Saranac Inn, Je-J1 (Ndm); Conesus L., Je (Ds) ; Etna, May (DtAS); Ithaca, bred (Ndm); L. George, Je (Ndm); West Pt., Je (Ds); Pine I., Je (Ds); Ramapo, Je (Ds) ; Hillburn, May, Schott (Ds) ; Brewster, J1 12 (Ndm); Bronxville, Je, Woodr (Ds) ; SI, May (Ds) ; LI: Yaphank, May-Je (Ds).

\section{Gomphaeschna Selys}

G. furcillata Say. Rochester (Moore); Ringwood Hollow near Ellis, Je (Mrs $\mathrm{Ndm}$ ); May, abundant (Kennedy); Freeville, McLean, and Ithaca, Je (DtAS); West Pt., Je (Ds); Nyack, Je (Ds); Ramapo, Je (Ds); LI: Yaphank, May-Je (Ds).

\section{ANAX Leach}

A. junius Dru. Generally distributed and common. Keene Valley (Comstock); Old Forge, Je (Ndm) ; Saranac Inn (Ndm) ; Olcott, Je-Sep (DtAS); Buffalo, Je-J1, EPV (Kellicott); Caledonia Cr., Dec, Lint (Hagen); Ithaca, (Bks); McLean, Je-Sep (DtAS); Schoharie (NYS); Coeymans, Aug (Felt); Cinnamon L., J1 $12(\mathrm{Ndm})$; many lakes and ponds in Ulster, Sullivan, and Greene Counties, J1-Aug (Calv) ; Bronxville, Je-J1 (Woodr); SI, Ap-Oct (Ds); LI (Weeks).

A. longipes Hagen. Not common. SI: not uncommon near Moravian Cemetery (Ds); Clove Valley, Je, Aug (Ds) ; LI: Wyandanch, Je (Ds); Long Pond, Wading R., Je (Ds).

\section{Aeschna Fabricius}

A. canadensis W1k. L. St. Regis, Franklin Co, Sep (Wlk); Ithaca, Aug (Wlk); SI, Je (Ds).

A. clepsydra Say. Waddington, Aug (Woodr); L. St. Regis, Franklin Co, Sep, Moore (Calv); Saranac Inn, latter part of summer (Ndm); Ithaca, Aug (Bks); Schoharie (NYS); Albany, Je (Felt, 31st Rept., 1916, p. 98); Catskills, Aug, Aaron (Calv-AES); Greenwood L., J1 (Ds). LI: Deep Pond, Wading R., J1 (Ds).

A. constricta Say. Hotel Champlain, Sep, Moore (Calv); Saranac Inn, Je-Aug (Ndm); Olcott, Aug-Sep (DtAS); Buffalo, J1, EPV (Kellicott); Ithaca, J1-Sep (Bks, DtAS); N. Spencer, Aug (DtAS); Watkins Glen, Slosson (Calv); Schoharie (NYS); Catskills, Aug, Aaron (Calv); Dobbs Ferry, Aug-Sep, Willn (Calv); Bronxville, J1, Woodr (Ds); NYC (Bt); SI, Je, Sep-Oct (Ds) ; LI: Flatbush, Sep (Ds).

A. eremita Scud. Loch Bonnie, Adirondacks, Sep; Saranac Inn, J1 (W1k).

A. interrupta Wlk. Franklin Co, Sep (W1k); Catskills, Aug.

A. tuberculata Wlk. Castile, Brad (Ndm). 
A. umbrosa W1k. L. St. Regis, Franklin Co, Sep (W1k) ; Saranac Inn, Aug (Woodr); Oneida; Aug (Wlk); Clyde, Aug (Wlk); Naples, Aug 14 (Bish) ; Ringwood, J1 (DtAS) ; Albany, Oct (Felt); Catskills, Aug (W1k) ; Cairo, Aug (Wlk) ; Ellenville, Aug (Wlk); West Pt., Sep (W1k); Dobbs Ferry, Aug (Wlk) ; Bronxville, J1-Oct, Woodr (Ds) ; NYC, Sep (Woodr) ; SI, Aug-Oct (Ds) ; LI: Yaphank, Oct (Ds); Pinelawn, Sep (Ds); Rockaway, Sep (Woodr).

A. verticalis Hagen. N. Spencer, Aug (DtAS); Caledonia Cr. (Hagen); Schoharie (NYS); Mooers, Sep (Not); NYC (Bt); SI: Je-Oct (Ds); Clove Valley, Oct (Ds) ; LI: Brooklyn, J1-Sep, Eng (Ds) ; Cold Sp. Harb., Aug, Brb (Ds), Sep (Wlk).

\section{NASIAESCHNA Selys}

N. pentacantha Ramb. Baldwinsville, Je, Pettit (Bks).

\section{EPIAEschna Hagen}

E. heros Fab. Lewis Co (NYS-Hill); Buffalo, Je-Jl, EPV (Kellicott) ; Rochester (Moore); Batavia, Je (Ds) ; Ithaca, Je (Bks-CU) ; Albany, Je (NYS); Ft. Edward, Je, King (Lint); Stuyvesant Falls (NYS); West Pt., Je (Ds) ; Dobbs Ferry, Aug, Willn (Calv); commonly seen in NYC (Woodr); SI, May, Aug (Ds) ; LI: Rockaway Beach, Aug 17, 1916, many drowned (Ds) ; Orient, Aug, Lath (Ds).

\section{FAMILY LIBELLULIDAE \\ DIDYMOPS Rambur}

D. transversa Say. Keeseville, Te, Sheraton (Calv); Saranac Inn, Je (Ndm) ; Old Forge, Je (Ndm) ; Conesus L., Je (Ds); Ithaca, Je-J1 (Bks); L. George, $\mathrm{Je}(\mathrm{Ndm})$; Ramapo, Je (Ds).

\section{MACROMIA Rambur}

M. illinoiensis Walsh. Saranac Inn ( $\mathrm{Ndm}$ ); Oswego Co, Je (Willn-US) ; Grand I., Fischer (Kellicott); Buffalo, J1 (EPV); Taughannock, Aug (DtÁS); L. George, Je (Ndm); Albany, J1 (Ds) ; Pine I., Je (Ds); Ft. Montgomery; May (Ds) ; Ramapo, Je (Ds).

\section{EPICORDULIA Selys}

E. princeps Hagen. Waddington, Aug (Woodr); Saranac Inn (Ndm); Black Rock Harb., Je-J1, EPV (Kellicott); Rochester (Moore) ; Ithaca, Je (BksCU) ; McLean, J1 (DtAS) ; Albany, J1 (Lint) ; Kenwood (NYS) ; Greenwood L., Je-J1, Watson (Ds) ; Benmar L., near Brewster (Ndm); Pine I, Je (Ds); NYC, Je, Woodr (Ds).

\section{NEURocordulia Selys}

N. obsoleta Say. L. George, Je, Aug (Ndm); Benmar L., near Brewster (Ndm) ; White L., Sullivan Co (Calv).

\section{HELOCORDULIA Needham}

H. uhleri Selys. Saranac Inn (Ndm) ; N. Fair Haven, J1 (Ndm) ; Greenwood L., May, Schott (Ds). 


\section{TETRAGONEURIA Hagen}

T. canis McL. Saranac Inn, Je (Ndm).

T. cynosura Say. Old Forge $(\mathrm{Ndm})$; Saranac Inn (Ndm); Black Rock Harb., Je, EPV (Kellicott); Rochester (Moore); Olcott, May, Aug (DtAS); Ithaca (Bks); Canandaigua (Herr) ; L. George (Calv-AES); West Pt., Je (Ds) ; Pocantico Hills, Je, Woodr (Ds) ; Benmar L., near Brewster (Ndm) ; Pine I., Je (Woodr) ; Tuxedo, May-Je (Woodr); NYC, Je, Woodr (Ds); SI, Je-Ji, Ds (Calv).

T. cynosura var. simulans Mutt. Axton (M\&H); Saranac Inn, Je (Ndm); Baldwinsville, Pettit (Bks); Black Rock Harb., Je, EPV (Kellicott); Ithaca (?) (Bks-CU) ; L. Mohonk and Pine I., Je (Woodr); Ramapo, May-Je (Ds); Benmar L., near Brewster (Ndm).

T. spinigera Selys. Saranac Inn, Je (Ndm); Oswego, May (Mutt); Buffalo, May, EPV (Kellicott); Castile, Brad (Ndm); Ithaca, Je (Ndm); Albany, Sep (NYS).

\section{DOROCORDULIA Needham}

D. lepida Hagen. Albany (Selys); Burnt Hill and Mud Ponds, and Amber L., Sullivan Co, J1-Aug (Calv) ; SI: Eltingviile, Je (Ds) ; LI: Wading R., Je (Ds).

D. libera Selys. N. Elba, J1 (Ds) ; Saranac Inn, J1 (Ndm) ; Catskills (Calv-AES) ; Cinnamon L., J1 12 (Ndm) ; West Pt., Je (Ds); Bronxville, May, Woodr (Ds) ; NYC, May, Woodr (Ds) ; SI: Ft. Wadsworth, Je 18 (Ds) ; LI: Deep Pond, Wading R., Je (Ds); Wyandanch, Je (Ds).

\section{WILLIAMSONIA Davis}

W. lintneri Hagen. Karner, May, Lint (Hagen).

\section{CORDUlia Leach}

C. shurtleffi Scud. Old Forge (Ndm); Saranac Inn, J1 (Ndm) ; Cinnamon L., J1 12 (Ndm); Catskills, Je (Eng); Slide Mt., Je (Ds); Tunis L., Ulster Co, Je (Calv); Oliverea, Je (Ds).

\section{SOMATOCHLORA Selys}

S. albicincta Burm. Mt. Marcy, J1, Not (Ds); L. Tear, J1, Watson (Ds).

S. elongata Scud. Ithaca, Je (Bks-CU), reared Ap, Kennedy (W1k).

S. linearis Hagen. Grand I., Fischer (Kellicott); Oswego Co, Aug (Kellicott); Varna and Ithaca (Ndm); SI: Green Ridge, Jl-Aug (Ds).

S. minor Calv. Harrietstown, J1, R. P. Currie (W1k).

S. semicircularis Selys. Wilmington, Adirondacks, J1, Ds (Willn).

S. tenebrosa Say. Oswego Co, Aug (Kellicott); N. Fair Haven, J1, Kennedy (Wlk); Clarence, J1, EPV (Kellicott); E. Jewett, Aug (Ds); Mt. Joy, Ramapo Mts., Aug (Ds) ; West Pt., Sep (Wlk); LI: Hauppauge and Wyandanch, Aug (Ds).

S. walshii Scud. Keene Valley, Aug (Calv); Chilson L. (Ds); Castile, Aug 23, 1924, Brad (Ndm); Ellis Hollow, Ithaca, Aug, Kennedy (Wlk).

S. williamsoni E. M. Wlk. Bone Pond, Saranac Inn, J1 (Ndm); Fair Haven, J1, Kennedy (E. M. Wlk). 


\section{LADONA Needham}

L. deplanata Ramb. LI: Amityrille, Je, Bather (Ds); Wyandanch, Ap (Ds) ; Wading R., Je (Ds).

L. exusta Say. L. George (Calv-AES) ; Croton-on-Hudson, J1; West Pt., Je (Ds) ; Ramapo, Je (Ds); SI, Je (Ds).

L. julia Uhler. Axton, Je (M\&H) ; Saranac Inn, Je (Ndm) ; Cinnamon L., J1 12 $(\mathrm{Ndm})$.

\section{LIBELLULA Linnaeus}

L. auripennis Burm. NYC (Bt); SI, May-J1 (Ds); LI: Deep Pond, Wading R., Je-Aug (Ds); Coney I. (Ds); Greenport, Aug (Ds); Shelter I., Aug (Ds).

L. axillena Westw. SI: Buck's Hollow, May (Ds).

L. cyanea Fab. Riverdale, J1, Woodr (Ds) ; NYC (Bt) ; Bronxville, Je-J1, Woodr (Ds); SI, Je-Aug (Ds); LI: Flushing, Aug (Ds); Yaphank, J1 (Ds) ; Wading R., Je (Ds); Half Way Hollow Hills, Jl (Ds).

L. flavida Ramb. NYC (Bt); LI: Long Pond, Wading R., Je (Ds) ; Riverhead, J1 (Ds).

L. incesta Hagen. Croton-on-Hudson, Je (Ds); Amber L. and Stump Pond, Sullivan Co, Aug (Calv) ; Ramapo, Je (Ds); LI: Yaphank, J1 (Ds) ; Wading R. and Long Pond, Aug (Ds); Riverhead, Aug (Ds).

L. luctuosa Burm. (basalis Say). Old Forge and Saranac Inn (Ndm); Olcott, Je-Aug (DtAS) ; Niagara R. and Tonawanda Cr., Je-J1, EPV (Kellicott) ; Danby, Je-Aug (DtAS); Ithaca, Aug (Ndm); Kenwood (NYS); Dobbs Ferry, J1, Willn (Calv); Bronxville, J1, Woodr (Ds) ; Van Cort. Pk., J1 (Ds) ; SI, Je-Aug (Ds); LI: Flushing, Aug (Ds); Wading R., Je (Ds).

L. pulchella Dry. Ponds everywhere $(\mathrm{Ndm})$. Saranac Inn and Old Forge (Ndm); Keeseville, J1-Aug, Sheraton (Calv); Axton, Je (M\&H); Keene Valley (Comstock); Olcott, J1-Aug (DtAS); Buffalo, Je-Aug, EPV (Kellicott); Portage, Je (Ds) ; Ithaca (Ndm); McLean, J1-Aug (DtAS) ; many ponds and lakes in Delaware, Ulster, Sullivan, and Greene Counties, J1-Aug (Calv); Schoharie (NYS); Dobbs Ferry, Je-J1, Willn (Calv); Bronxville, J1, and NYC, Je (Woodr); SI, May-Sep (Ds); LI: Coney I., Aug (Weeks) ; Wyandanch, J1 (Ds); Wading R., Je (Ds); Bridgehampton, Aug (Calv).

L. quadrimaculata L. Saranac Inn ( Ndm); Wilmington, J1 (Ds); Portage, Je (Ds) ; Olcott, J1-Aug (DtAS) ; Buffalo, EPV (Kellicott); Ithaca (Bks-CU), J1-Aug (DtAS); Schoharie (NYS); Karner, May (NYS); Ellenville $(\mathrm{Ndm})$; Bronxville, Je-J1, Woodr (Ds); SI, May-Je (Ds).

L. semifasciata Burm. Rochester (Moore); Buffalo, Je-Jl, EPV (Kellicott) ; Dobbs Ferry, J1, Willn (Calv) ; Bronxville, May-J1, Woodr (Ds) ; SI, Ap-Sep (Ds) ; LI: Yaphank, J1 (Ds); Wading R., Je (Ds) ; Aqueduct, J1 (Ds); Rockaway, Je (Woodr).

L. vibrans Fab. SI, Je-Aug (Ds).

\section{Plathemis Hagen}

P. lydia Dry. (trimaculata DeG.). Generally distributed. Keene Valley (Comstock); Old Forge (Ndm); Fairmount, Je (Felt); Olcott, Je-J1 (DtAS) ; Buffalo, EPV (Kellicott); Portageville, Je (Ds); Ithaca (Bks); Brookton (Theresa M. Robinson); Schoharie (NYS); Albany, Je (Felt, Lint-NYS); Stuyvesant Falls (NYS); Beaver Br., Sullivan Co, and Cairo, Aug (Calv) ; Dobbs Ferry, Je, Willn (Calv) ; NYC (Bt) ; Bronxville, Je, Van Cort. Pk., Woodr (Ds) ; SI, May-Sep (Ds) ; LI: Wyandanch and Yaphank, J1 (Ds); Flushing, Aug (Ds); Bridgehampton, Aug 18 (Calv). 


\section{PERIThemis Hagen}

P. tenera Say. Tarrytown, J1, Woodr (Ds) ; Benmar L., near Brewster (Ndm) ; Van Cort. Pk., J1, Woodr (Ds) ; Bronxville, J1-Aug, Woodr (Ds); NYC (Bt) ; SI, Je-J1 (Ds) ; LI: Wading R. and Long Pond, Aug (Ds) ; Shelter I., Aug (Ds) ; Flushing, Aug (Ds); Prospect Pk. L., Brooklyn, Aug (Ds).

\section{NANNOTHEMIS Brauer}

N. bella Uhl. Mt. Marcy, 5000 ft., Not (Ds) ; NYC (Bt) ; SI: Four Corners, Je-J1 (Ds) ; LI: Yaphank and Wyandanch, J1 (Ds).

\section{ERYTHRODIPLAX Brauer}

E. berenice Dry. Thousand Isls., Slosson (Calv); NYC (Bt) ; Riverdale, J1, Woodr (Ds) ; SI, May-Aug (Ds) ; LI: Sheepshead Bay, J1 (Calv) ; Aqueduct, J1 (Woodr); Rockaway Beach, J1-Aug (Ds); Flushing and Cold Sp. Harb., J1-Aug, Brb (Ds) ; Orient, J1, Lath (Ds), and Shelter I., Aug (Ds); Gardiners I., Aug (Ds).

\section{ERYTHEMIS Hagen}

E. simplicicollis Say. Saranac Inn (Ndm); Tonawanda R., Aug, EPV (Kellicott); Rochester (Moore); Olcott, Je-J1 (DtAS); Ithaca (Ndm); NYC (Bt); SI, May, Jl-Sep (Ds) ; LI: Wading R., Aug (Ds); Yaphank, J1 (Ds); Riverhead, Aug (Ds); Flushing, Aug (Ds); Bridgehampton, Aug (Calv).

\section{SYMPETRUM Newman}

S. corruptum Hagen. Rochester (Moore); Buffalo, EPV (Kellicott); SI: shore at Ellingville, May (Ds); New Dorp, Aug, and Dongan Hills, Sep (Ds).

S. costiferum Hagen. Saranac Inn, Aug (Ndm); Amherst, Sep, EPV (Kellicott); LI: Wyandanch, Aug, Long Pond, Wading R., J1-Aug, Riverhead, Aug (all Ds).

S. decisum Hagen. (See footnote under S. rubicundulum.)

S. obtrusum Hagen. Waddington, Aug (Woodr); Mt. Marcy, Je (NYS) ; Keene Valley, Jl-Aug (Calv); Saranac Inn (Ndm); Piseco and Elk Lakes, Aug (Calv); Hastings, Aug-Sep (Kellicott); Clarence, J1, EPV (Kellicott); Elma, Sep, EPV (Kellicott); Karner (NYS); SI, Je-J1 (Ds).

S. rubicundulum Say.10 Keeseville, J1, Sheraton (Calv); Saranac Inn (Ndm); Keene Valley (Comstock) ; L. St. Regis, Franklin Co, Sep, Moore (Calv); Olcott and Howland I., Je-J1 (DtAS); Buffalo, Je-Sep, EPV (Kellicott); Ithaca (Bks); Danby and McLean, Je-J1 (DtAS); ponds in Sullivan Co, J1-Aug; Cairo, Aug (Calv); Dobbs Ferry, J1, Willn (Calv); Bronxville, J1 (Woodr); NYC (Bt); SI, J1-Sep (Ds); LI: Coney I., Aug (Weeks); Gardiners I., Aug (Ds).

S. semicinctum Say. Waddington, Aug (Woodr); Keene Valley (Not) ; Olcott, Aug (DtAS); Castile, Brad (Ndm); Ithaca, Aug (Ndm); L. George, Aug (Ndm); Cairo, Aug (Calv) ; Riverdale, Jl, Woodr (Ds); Croton, Jl (Ds); Bronxville, Aug, Woodr (Ds); Van Cort. Pk., Aug (Ds); SI, J1-Sep (Ds); LI: Long Pund, Wading R., and Riverhead, Aug (Ds).

S. vicinum Hagen. Keeseville, J1-Aug, Sheraton (Calv); Saranac Inn (Ndm); Piseco L., Aug (Calv); L. St. Regis, Franklin Co, Sep, Moore (Calv); Pt. Abino, EPV (Kellicott) ; Olcott, Sep (DtAS) ; Ithaca, J1-Aug (Ndm);

\footnotetext{
10 "Some of theze records doubtless belong, to $S$. decisum which has been constantly confused with S. rubicundulum." -E. M. WALKER.
} 
Saratoga L., Aug (Calv) ; Schoharie (Hagen) ; Catskills, Aug (Calv-AES) ; Catskill L. and Cairo, Aug (Calv) ; Alder L., Ulster Co, Je; ponds in Sullivan Co; West Pt., Oct (Ds); Dobbs Ferry, Nov, Willn (Calv); NYC (Bt) ; Bowling Green, NYC, Oct (Ds) ; SI, Sep-Nov (Ds); LI: Yaphank, Oct (Ds). Low wet meadows and along small streams.

\section{PACHYDIPLAX Brauer}

P. longipennis Burm. Black Rock Harb., Je, EPV (Kellicott); Rochester (Moore) ; Olcott, J1 (DtAS) ; Ithaca (Bks) ; Dobbs Ferry, J1, Willn (Calv) ; Benmar L., near Brewster (Ndm) ; Riverdale, J1, Woodr (Ds) ; Bronxville, Je (Woodr) ; NYC (Bt) ; SI, May-Sep (Ds); LI: Forest Pk., Brooklyn, Je (Ds) ; Flushing, Aug (Ds).

\section{LEUCORHINIA Brittinger}

L. frigida Hagen. Adirondacks, near Redfield Mt., J1 (Beq) ; L. Tear, J1, Watson (Ds) ; Old Forge ( $\mathrm{Ndm})$; Knapp, Hunter, Mud, and Cranberry Ponds, Sullivan Co, Aug (Calv); SI: Tottenville, May (Ds); LI: Deep and Long Ponds, Wading R., Je (Ds).

L. glacialis Hagen. Old Forge (Ndm); Saranac Inn, late Je-early Aug (Ndm).

L. hudsonica Selys. Mt. Marcy, $4500 \mathrm{ft}$. (Not); Old Forge (Ndm).

L. intacta Hagen. Waddington, Aug (Woodr); Keeseville, J1, Sheraton (Calv); Rochester (Moore) ; Olcott, Je-J1 (DtAS) ; Niagara R. and Black Rock Harb., Je-early J1, EPV (Kellicott); Portage, Je (Ds); Ithaca (Ndm) ; McLean and Freeville (Ndm) ; Danby (DtAS) ; Karner, J1 (Hagen) ; Croton-on-Hudson, May, Ds (Calv); Cinnamon L., J1 (Theresa M. Robinson) ; Folwood L., Sullivan Co, Je (Calv); Benmar L., near Brewster (Ndm) ; Bronxville, Je, Woodr (Ds) ; NYC, May-Je, Woodr (Ds) ; SI, May-Aug (Ds) ; LI: Rockaway and Wading R., Je (Ds).

\section{Celithemis Hagen .}

C. elisa Hagen. Axton, Je (M\&FI) ; Ithaca, Morgan (Bks); ponds in Sullivan Co, J1-Aug (Calv); West Pt., Je (Ds) ; Tarrytown, J1, Woodr (Ds) ; Pocantico Hills, Je (Woodr) ; NYC (Bt) ; Bronxville, Je, Woodr (Ds); SI, Je-Aug (Ds); LI: Yaphank, (Ds); Wading R., May-Je, Aug (Ds); Flushing, Aug (Ds); Montauk, Aug (Ds).

C. eponina Drury. Lake Bluff, Wayne Co (NYS) ; Tarrytown, J1 (Woodr) ; NYC (Bt) ; SI, May-Aug (Ds); LI: Flushing and Cold Sp. Harb., Aug (Ds).

C. monomelaena Willn. LI: Wading R., Je-Aug, and Riverhead, Aug (Ds).

C. martha Willn. (ornata Ramb.). LI: Yaphank, J1-Aug (Ds); Wyandanch, Aug (Ds) ; Wading R. and Long Pond, Aug (Ds); Riverhead, Aug (Ds). A species of the pine-barren regions of LI (Ds).

PANTALA Hagen

P. flavescens Fab. Rochester (Moore); Bronxville, J1 (Woodr); SI, J1-Sep (Ds).

TRAMEA Hagen

T. carolina L. Schoharie (NYS) ; NYC (Bt); Bronxville, May, Woodr (Ds) ; SI, Ap-Oct (Ds) ; LI: Yaphank, J1 (Ds); Wading R., Aug (Ds) ; Bridgehampton, Aug (Calv).

T. lacerata Hagen. Rochester (Moore); Buffalo, Aug, EPV (Kellicott) ; Freeville, May (Bks); West Pt., Je (Ds); SI, May-Oct (Ds). 


\section{ORDER PLECOPTERA}

Compiled by J. G. Needham and P. W. Claassen

\section{FAMILY PTERONARCIDAE}

\section{PTERONARCYS Newman}

P. dorsata Say. "NY" (LucyW.Smith).

P. nobilis Hagen. General throughout NY, and ranging from Canada to Ga $(N d m)$.

P. proteus Newm. Trenton Falls; Ithaca.

P. biloba Newm. Trenton Falls; Ithaca.

P. comstocki Smith. Trenton Falls; Wilmurt; Tarbel, Ds.

\section{FAMILY PERLIDAE}

\section{PERLODES Banks}

P. lineata Smith. Old Forge, Aug $(N d m)$.

\section{ISOGENUS Newman}

I. frontalis Newm. Niagara Falls, Je.

\section{PERLA Geoffroy}

P. postica Wlk. Fall Cr., Ithaca, May (Ndm); Ft. Montgomery, May.

P. hastata Bks. Axton, Je (M\&H) ; Old Forge, Aug $(N d m)$; Woodworth's L., Je, Alex; Easton, May, Leon; Ringwood Hollow, near Ellis, May, $W u$.

P. bilobata Ndm. \& Claas. Old Forge, J1 $(N d m)$.

P. capitata Pict. West Branch of Ausable R., Essex Co, J1 (Claas); Ogdensburg, J1, Bet; Gloversville, Je, Alex; N. Fair Haven; Clyde, J1; West Falls, J1; Ithaca, May-J1; McLean, Je; Malloryville, Je; Binghamton, J1; Nassau, Je.

P. ramosa $\mathrm{Ndm}$. \& Claas. Ithaca (CU).

P. immarginata Say. Keene Val., Aug; Wilmington, Aug; Mt. Whiteface, Aug; Ogdensburg, J1; Hamilton Co; Enfield; Gloversville, Je, Alex; Ithaca, MarSep; Coy Glen, Ithaca, J1; Ludlowville, Aug.

P. media Wlk. Ogdensburg, J1; Crane Pond, Je; Wilmurt, Je; Mayfield, Je, Alex; Oswego, Je; Fulton, Je; Niagara Falls, J1; Buffalo, J1; Ithaca, May.

\section{ALLOPERLA Banks}

A. marginata Bks. Colden, J1 (Bks); Ithaca, Je, Sib.

A. Lateralis Bks. Gloversville, Je, and Woodworth's L., Je, Alex; Mix Cr. Val., Cattaraugus Co, Je; Ithaca, May-Je; De Ruyter, Je; Danby, Je; Ramapo, Je.

A. mediana Bks. Newport, May; Cranberry L., J1 (Drk); Gloversville, Je, Aler ; Clinton, May, Pozvell; Enfield Falls and Ithaca, May; Niagara Falls, Je.

A. imbecilla Say. Alcohol Br., Adirondack Lodge, J1; Sacandaga Pk., Je; Newfield, Je.

A. nanina Bks. West Branch, Ausable R., J1; Elizabethtown, J1; Adirondack Lodge, J1; Ithaca, May-J1; Oxford, J1. 
CHLOROPERLA Newman

C. cydippe Newm. Sport I., Je, Alex; Hamburg; J1; Ithaca, Je; Newfield.

\section{NEOPERLA Needham}

N. clymene Newn. Charlotte, Je; West Falls, J1; Martinsburg, J1 (Sib) ; Ithaca, Je-Sep; Ancram, Je; Ft. Montgomery, Jl.

\section{Clioperla Needham and Claassen}

C. similis Hagen. Moore's Br., near Ithaca, May, Sib; Ringwood Hollow, near Ellis, Ap (Claas).

C. annecta Ndm. \& Claas. Black Br., Je, $C y$.

\section{ISOPERLA Banks}

I. signata Bks. Elizabethtown, J1 (Claas); Clinton, May, Powell; Woodworth's L., Je, Alex; Buffalo, J1; Ithaca, May-Je; Ringwood, May (Dt); Greene Co, Je.

I. bilineata Say. A common eastern species, found everywhere in small streams. Ithaca, Je-Aug.

I. montana Bks. Ogdensburg, J1; Gloversville, Je, Alex; Lakeside, Je; Ithaca, May-Je (E1lis); Coy Glen, Je; Slaterville, Je; Enfield, May; Moore's Br., near Ithaca, May, Sib; Taughannock Falls, May (Dt); Newfield, Je.

\section{Perlesta Banks}

P. placida Hagen. Youngstown, J1, Alex; Ithaca, May-Aug.

ATOPERLA Banks

A. ephyre Newm. Canandaigua L., Naples, J1, Leon; Ithaca, Je-J1.

PERLinella Banks

P. drymo Newm. Deer R., May; Sport I., Sacandaga R., Je, Alex; Ithaca, May-J1.

\section{PELTOPERLA Needham}

P. cornelia Ndm. and Smith. Ramapo, May (Ds).

P. arcuata Ndm. Ithaca, Je (Pettit-CU).

\section{ACRONEURIA Pictet}

A. abnormis Newn. Keene Val., Je; Oswegatchie; North Cr., J1; Sacandaga Pk., Je, Alex; Ithaca, Je-Aug; Greene Co, Je.

A. arenosa Pict. Ithaca, J1; LI.

A. trijuncta Wlk. Juanita I., L. George.

A. arida Hagen. Ithaca, Je.

A. lycorias Newn. Artist's Br., Chapel Pond, Essex Co, Je (Claas); Gloversville, Je, Alex; Castile; Colden, Je (EPV) ; Otto, Je; McLean Bogs, Je; Ithaca, May-J1; The Hermitage, Cayuga L. (Bet) ; Trenton Falls, type locality; Ft. Montgomery, May; Ramapo, May. 


\section{FAMILY NEMOURIDAE}

\section{NEMOURA Latreille}

N. divergens Claas. Clinton, Mar (Powell-CU); Ithaca, Ap.

N. sinuata Wu. Alcohol Br., Adirondack Lodge, J1; Newcomb, J1; Cranberry L., Je, Drk; Old Forge, J1-Aug; Gloversville, Je; Ithaca, Je-Aug (CU) ; Etna; McLean; Greene Co.

N. venosa Bks. Alcohol Br., Adirondack Lodge, J1 (Claas) ; Newport, May ; Cranberry L., Je, Drk; Old Forge, Je $(N d m)$; Woodworth's L., Je-Aug, Alex; Colden, Je (Bks); Ringwood, Je; Enfield Glen, May; McLean, Je; W. Danby, Je (MacG).

N. trispinosa Claas. Mud Cr., Tompkins Co, Je (CU); McLean, Je.

N. vallicularia Wu. Ilion, Mar; Clinton, Ap ; Ithaca, Ap-May (CU) ; Nassau, Mar; Mosholu, Mar.

N. serrata Claas. Cranberry L., Drk (CU).

N. punctipennis Claas. Axton, Je $(\mathrm{M} \& \mathrm{H})$; Old Forge, Je.

\section{LEUCTRA Stephens}

L. decepta Claas. Old Forge, Je; Ringwood, Je (CU).

L. duplicata Claas. Adirondack Lodge, Je; Axton, Je (M\&H); Woodworth's L., Je; Labrador L., Cy; Chipmunk Swamp, Vandalia, Je; Ringwood, May.

L. hamula Claas. Mt. Marcy, Aug; Old Forge, J1 (CU); Sacandaga Pk., Alex; Woodworth's L., Je.

L. sibleyi Claas. Moore's Br., Ithaca, May (Sib); Taughannock, May.

L. tenuis Pict. Wilmington; Saranac Inn, J1; Woodworth's L., May, Alex; West Falls.

L. triloba Claas. McLean, Sep-Oct (Jensen-CU); Ithaca, Sep.

L. truncata Claas. Old Forge, Aug (CU).

\section{TAEnIOPTERyX Pictet}

T. maura Pict. Gloversville, Mar, Alex.

T. nivalis Fitch. Adams Center, Mar (Claas); Honeoye Falls, Mar, Cy; Pike, Mar; Ithaca, Feb-Mar; Binghamton, Ap; Albany, Mar-Ap.

T. fasciata Burm. Hamburg, Ap (EPV) ; Ithaca, Mar-Ap; Karner, Ap; E. Greenburgh, Ap.

\section{FAMILY CAPNIIDAE}

\section{CAPNIA Pictet}

C. vernalis Newport. Mountain L., Ap (Claas); Gilman L., Ap (Claas); Clinton, Mar, Powell; Coy Glen, Mar; Highland, Mar.

\section{CApnella Claassen}

C. granulata Claas. Adams Center, Mar (Claas); Johnstown, Mar (CU); Fulton Co, Alex.

C. recta Claas. Ithaca, Mar (CU); Interlaken, Nov.

C. incisura Claas. Ithaca, Mar (CU).

C. vivipara Claas. Bluff Pt., Ithaca, Mar.

C. pygmaea Burm. Northville, Ap; Pike, Ap-May; Ithaca, Feb-Ap; McLean, May; Mosholu, Mar; SI, Feb. 


\section{ORDER CORRODENTIA}

Compiled by P. J. Chapman and A. M. Nadler

\section{FAMILY PSOCIDAE}

\section{SUBFAMILY PSOCINAE}

\section{Cerastipsocus Kolbe}

C. trifasciatus Prov. "NY" (Bks).

C. venosus Burm. Westport, Aug (Felt); Newport, Aug (Felt); Johnstown, Aug $(B k s)$; Grand Gorge, Aug (Felt); Ithaca, Aug (Chap-AM); Middleburg (Chap-NYS); Berne, Aug (Felt); Ellenville, J1 (Nad); LI: Quogue, Aug $(Z a b-\mathrm{AM})$.

\section{Psocus Latreille}

P. amabilis Walsh. Geneseo, Scp; Olcott, Sep; Barcelona, Sep; Silver Cr., Sep; Richburg, Sep; Clarksburg, Sep; Stow, Sep; Ceres, Sep; Rock City, Sep; Painted Post, Sep; Ithaca, Sep; Elmira, Oct; (all Chap); LI: Sea Cliff, Sep (Chap). In dead or dying leaves.

P. atratus Aaron. Sacandaga Pk., Aug, Alex (Bks); Painted Post, Sep (Chap). On tree trunks.

P. leidyi Aaron. Woodworth's L., Fulton Co, Aug, Alex (Bks); L. Placid, Aug, EPV (NYS); Fairhaven, Sep; Clarksburg, Sep; Wellsville, Sep; Richburg, Sep; Stow, Sep; Ceres, Sep; Geneseo, Sep; Hornell, Sep; Painted Post, Sep; Montour Falls, Sep-Oct; Ithaca, Sep-Oct; Elmira, Oct; McLean, Aug; Hunter, Aug (all Chap) ; Ellenville, J1 (Nad) ; Bronx, J1, Grossb (Chap); LI: Sea Cliff, Sep (Chap). On tree trunks.

P. moestus Hag. Stow, Sep; Wellsville, Sep; Clarksburg, Sep; Hornell, Sep; Painted Post, Sep; Hall, J1; Ithaca, Sep (all Chap); LI: Sea Cliff, Sep $(N a d)$; Riverhead, Oct (Chap).

P. novaescotiae Wlk. L. Placid, Aug, EPV (NYS); Painted Post, Sep (Chap); Ithaca, Sep (Chap).

P. pollutus Walsh. Sacandaga Pk., Gloversville, Aug, Alex (Bks).

P. purus Walsh. Fairhaven, Sep (Chap); Ithaca, Aug, Bks (Chap) ; Cairo, Aug $(C h a p)$. Under loose stones and on tree trunks.

P. quietus Hag. Phoenicia, Aug, EPV (NYS).

P. semistriatus Walsh. LI: Sea Cliff, Aug (Bks).

P. stigmosalis Bks. Gloversville, Sep, Alex (Bks); LI: Sea Cliff, Sep (Nad).

P. striatus W1k. Ithaca, Aug-Sep (US) ; Van Cort. Pk., Sep; LI: Sea Cliff, Sep (Nad).

P. variabilis Aaron. Johnstown, Aug (Bks); Larchmont, J1 (Nad).

\section{AMPHIGERONTIA Kolbe}

A. confraterna Bks. (Psocus). Sacandaga Pk., Je (Bks); West Barre, Sep; Little Valley, Sep; Wellsville, Sep; Rock City, Sep; Ceres, Sep; Stow, Sep; Richburg, Sep; Painted Post, Sep; (all Chap); Phoenicia, Aug, EPV (NYS); Larchmont, J1 (Nad).

\section{TRICHADENOTECNUM Enderlein}

T. slossonae Bks. (Psocus). Ithaca, Sep (Bks); Ellenville, J1 ( Nad). 


\section{FAMILY CAECILIIDAE \\ SUBFAMILY CAECILIINAE}

\section{Caecilius Curtis}

C. aurantiacus Hag. Speculator, J1, L. Placid, Aug, EPV (NYS); Woodworth's L., Aug, Alex (Bks); Olcott, Aug, Cinnamon L., J1, Montour Falls, Sep, Howard, Jl, Louck's Pond, Steuben Co (Chap) ; Hammondsport, J1, Freeville, Oct, Cy (Chap) ; Penn Yan, J1-Aug, Enfield Glen, Aug, Ithaca, Jl-Aug, Danby, Oct, Woodwardia Swamp, Aug, Catskill, Hunter, Aug (Chap); Larchmont, Je-J1 (Nad); Ellenville, J1 $(N a d)$; LI: Sea Cliff, Sep (Nad, Chap).

C. confluens Walsh. Fair Haven, Sep, Ithaca, Sep, Woodwardia Swamp, Tompkins Co, Aug (Chap); Hunter, Aug (Chap); LI: Sea Cliff, Sep, Nad (Chap).

C. posticus Bks. LI: Sea Cliff, Aug (Bks).

C. umbrosus Bks. Hillside, Alex (Bks); LI: Sea Cliff (Bks).

LACHESILLA Westwood

(Pterodela Kolbe)

L. pedicularia L. Gloversville, Aug-Sep (Bks); Ithaca, Aug (AM).

L. rufa Walsh. Larchmont, Je (Nad).

\section{SUBFAMILY POLYPSOCINAE}

\section{PoLyPSOCUS Hagen}

P. corruptus Hag. Johnstown, Aug (Bks) ; Hamburg, J1, EPV (NYS) ; Letchworth Pk., J1, Cy (Chap); Wells, J1, Yg (Chap); Montour Falls, Sep (Chap) ; Ithaca, Enfield Glen, Aug (Chap); Albany (Chap-NYS) ; Poughkeepsie, Je, $Y g$ (Chap); LI: Sea Cliff, Sep (Nad).

\section{SUBFAMILY PERIPSOCINAE}

\section{PERIPSOCUs Hagen}

P. madidus Hag. Speculator, Aug, EPV (NYS); Saranac Inn, J1 (Ndm); L. Placid (EPV); Wells, J1, Yg (NYS); Avalanche L., Essex Co, Cy\&्Gish (Chap); Mt. McIntyre, Cy\& Bish (Chap); Woodworth's L., Aug (Bks); Fair Haven, Sep (Chap); Cinnamon L., J1, Penn Yan, Aug, Montour Falls, Sep, Ithaca, J1-Sep, Danby, Oct, McLean, J1-Sep, Howard, J1, Enfield Glen, Aug, Hunter, Aug (Chap); High Falls, Je, Ellenville, J1 (Nad) ; LI: Sea Cliff, Sep (Nad).

P. permadidus Walsh. L. Placid, Speculator, Aug, EPV (NYS); Ellenville, J1 (Nad); LI: Sea Cliff, Sep (Nad).

\section{FAMILY MYOPSOCIDAE SUBFAMILY MYOPSOCINAE}

LICHENOMIMA Enderlein

L. lugens Hag. Ithaca, Woodwardia Swamp, Tompkins Co, Aug (Chap).

L. sparsa Hag. Ithaca, Aug (Chap); Hunter, Aug (Chap) ; Poughkeepsie, Aug (Chap-NYS). 


\section{FAMILY MESOPSOCIDAE}

\section{SUBFAMILY MESOPSOCINAE}

Mesopsocus Kolbe

M. unipunctatus Mull. Sacandaga Pk., Je, Alex (Bks); Newport, Je (ChapNYS); Cinnamon L., J1 (Chap); McLean, Je-J1, Sib (Chap); Karner (Chap-NYS) ; Howard, J1 (Chap); Larchmont, Je (Nad).

\section{ELIPSOCUS Hagen}

E. pumilis Hag. "NY” (Hag).

\section{PHILOTARSUS Kolbe}

P. maculosus Aaron (Elipsocus). Montour Falls, Sep (Chap).

\section{FAMILY LEPIDOPSOCIDAE}

\section{ECHMEPTERYX Aaron}

E. hageni Pck. L. Placid, Aug, EPV (NYS); Fair Haven (Chap) ; Johnstown, Aug, Alex (Bks); Montour Falls, Sep (Chap); Ithaca, J1-Aug, Hunter, Aug (Chap); Ellenville, J1 (Nad); LI: Riverhead, Oct (Chap).

\section{FAMILY PSYLLIPSOCIDAE}

\section{PSYLLIPSOCUS Selys}

P. ramburi Selys. NYC, Jan, Je (Felt); Manhattan, J1 (Nad); LI: Brooklyn, Je-J1 $(N a d)$.

NyMPHOPSOCUS Enderlein

(Ocellataria Weber)

N. gravinympha Web. LI: Brooklyn, throughout the year (Nad).

\section{FAMILY PSOQUILLIDAE \\ DORYPTERYX Aaron}

D. pallida Aaron. Ithaca, Aug-Oct, in old papers and books (Chap).

\section{FAMILY TROGIIDAE SUBFAMILY TROGIINAE \\ TROGIUM Illiger}

T. pulsatorium L. The so-called "deathwatch." Hunter, Aug (Chap); LI: Sea Cliff, Sep, Nad (Chap). 


\title{
FAMILY LIPOSCELIDAE
}

\section{LIPOSCELIS Motschulsky}

(Troctes Burmeister)

L. divinatorius Mull. Fayetteville, Mar (Chap); Ithaca, May, in nests of English sparrow (Chap); Otsego (Lint); Albany, Oct (Felt); Wakefield, Oct (Felt); NYC (Felt).

\section{ORDER MALLOPHAGA ${ }^{11}$}

\author{
Compiled by F. H. WILSON
}

\section{SUBORDER AMBLYCERA \\ FAMILY MENOPONIDAE}

\section{MENOPON Nitzsch}

M. aegialitidis Durr. Hebron, Sep, on killdeer (Oxyechus vociferus vociferus L.). M. leucoxanthum Nitz. Ithaca, Oct, on black duck (Anas rubripes Brewst.).

M. stramineum Nitz. (biseriatum Piaget). LARGe Body Louse. General throughout the State, on hen (Herr); also Ithaca, Ap, on captive ring-necked pheasant (Phasianus torquatus Gmel.) (Herr) and domestic turkey.

M. pallidum Nitz. Common Hen Louse. General throughout the State, on hen (Herr), ring-necked pheasant, and guinea fowl.

\section{MYRSIDEA Waterston}

M. subaequalis Lyonet. Ithaca and Hebron, on crow (Corvus brachyrhynchos brachyrhynchos Brehm) and red-shouldered hawk (Buteo lineatus lineatus Gmel.).

\section{Colpocephalum Nitzsch}

C. laticeps Kell. L. Cossayuna, Sep, on great blue heron (Ardea herodias herodias L.).

C. pustulosum Piaget. Ithaca, Sep, on pectoral sandpiper (Pisobia maculata Vieill.).

\section{TRINOTON Nitzsch}

T. querquedulae L. (luridum Nitz.). Ithaca, on redhead (Marila americana Eyt.), pintail (Dafila acuta L.), and wood duck (Aix sponsa L.); Hebron, Sep, on green-winged teal (Nettion carolinensis Gmel.).

\section{PSEUdOMENOPON Mjöberg}

P. pacificum Kell. Ithaca, on coot (Fulica americana Gmel.) and pied-billed grebe (Podilymbus podiceps L.).

\footnotetext{
${ }^{11}$ The order of arrangement of families and genera here is that adopted by L. Harrison in his article entitled "The Genera and Species of Mallophaga," in Parasitology, vol. 9, p. 1-156, 1916. The species are arranged alphabetically in each genus. The list is based upon careful collecting by Mr. Wilson (chiefly at Hebron and at Ithaca), upon material already in the Cornell collection, and upon such published records as seemed trustworthy.
} 


\section{FAMILY RICINIDAE}

\section{Ricinius DeGeer}

R. leptosomus Carriker. Ithaca, on kingbird (Tyranmus tyrammus L.).

R. lineatus Osb. Recorded from Ithaca by Osb., on ruby-throated humming bird (Archilochus colubris L.).

Trochiloecetes Paine and Mann

T. prominens Kell. \& Chap. Ithaca, on ruby-throated humming bird.

\section{SUBORDER ISCHNOCERA \\ FAMILY TRICHODECTIDAE}

\section{TRICHODECTES Nitzsch}

T. bovis L. (scalaris Nitz.). Biting Cattle Louse. Common throughout the State, on domestic cattle.

T. equi L. Biting Horse Louse. Probably common throughout the State, on horse, but definitely recorded only from Ithaca.

T. ovis L. (sphaerocephalus Olfers). N. Blenheim and Liberty, on sheep (Lint. Rept. 1889).

T. subrostratus Nitz. Probably common throughout the State, on cat, but definitely recorded only from Ithaca.

\section{FAMILY PHILOPTERIDAE SUBFAMILY GONIODINAE}

GONIODES Nitzsch

G. dissimilis Nitz. Malone, Ap, on ring-necked pheasant (Phasianus torquatus Gmel.).

G. meleagridis L. (stylifer Nitz.). Ithaca, common on .domestic turkey (Herr).

GonIocotes Burmeister

G. bidentatus Scop. (compar Nitz.). Ithaca, very common on domestic pigeon (Herr).

G. gigas Tasch. Ithaca, on hen and guinea fowl, but not common (Herr).

G. hologaster Nitz. Lesser CHICKen Louse. Ithaca, fairly common on hen, and probably widely distributed throughout the State.

\section{SUBFAMILY PHILOPTERINAE}

\section{PHILOPTERUS Nitzsch}

P. agelaii Osb. Ithaca, on red-winged blackbird (Agelains phoenicens phoeniceus L.).

P. ceblebrachys Nitz. Avon, Jan, on snowy owl (Nyctea nyctea L.), GAFrench. P. corvi Osb. Ithaca and Hebron, on crow (Corvus brachyrhynchos brachyrhynchos Brehm).

P. cursor Nitz. Ithaca, on barred owl (Strix varia varia Barton). 
P. dentatus Scopoli (icterodes Nitz.). Ithaca, on domestic duck (Anas domestica), canvasback (Marila vallisneria Wils.), redhead (M. americana Eyt.), greater scaup duck (M. marila L.), old squaw (Harelda hyemalis L.), king eider (Somateria spectabilis L.), Canada goose (Branta canadensis canadensis L.), mallard (Anas platyrhynchos L.), black duck (Anas rubripes Brewst.), and pintail (Dafila acuta L.) ; Hebron, on green-winged teal (Nettion carolinensis Gmel.); NY, on hooded merganser (Lophodytes cucullatus L.).

P. jungens Kell. Ithaca, on northern flicker (Colaptes auratus luteus Bangs).

P. major Waterston. Lansing and Ithaca, on Wilson's snipe (Gallinago delicata Ord.).

P. pertusus Nitz. Ithaca, on coot (Fulica americana Gmel.).

P. quiscali Osb. Ithaca, on bronzed grackle (Quiscalus quiscula aeneus Ridgw.).

\section{DegeERIELla Neumann}

D. boephila Kell. Hebron, on killdeer (Oxyechus vociferus vociferus L.).

D. cedrorum Piaget. Hebron, on cedar waxwing (Bombycilla cedrorum Vieill.).

D. fusca Nitz. Hebron, on red-shouldered hawk (Buteo lineatus lineatus Gmel.).

D. interrupta Piaget. L. Cossayuna, on solitary sandpiper (Helodromas solitarius solitarius Wils.).

D. illustris Kell. Ithaca, on red-winged blackbird (Agelaius phoeniceus phoeniceus L.), bobolink (Dolichonyx oryzivorus L.), and bronzed grackle (Quiscalus quiscula aeneus Ridgw.).

D. nebulosa Burm. Ithaca, on starling (Sturnus vulgaris L.) and robin (Planesticus migratorius migratorins L.).

D. ornatissima Giebel. Ithaca, on red-winged blackbird.

D. vulgata Kell. Hebron, on English sparrow (Passer domesticus domesticus L.).

RALLICOLA Johnston and Harrison

R. advena Kell. Ithaca, on coot (Fulica americana Gmel.) and bufflehead (Charitonetta albeola L.).

R. bisetosa Piaget var. californica Kell. Ithaca and Hebron, on Virginia rail (Rallus virginianus L.) (Kell \& Chap).

\section{SUBFAMILY ACIDOPROCTINAE}

\section{ACIDOPROCTUS Piaget}

A. kelloggi Carr. Ithaca, on canvasback (Marila vallisneria Wils.).

\section{ORNITHOBIUS Denny}

O. goniopleurus Denny. Ithaca, on Canada goose (Branta canadensis canadensis L.).

\section{SUBFAMILY ESTHIOPTERINAE}

ESTHIOPTERUM Harrison

E. anseris L. (jejunum Nitz.). Ithaca, on domestic goose (Anser domesticus) (Herr).

E. columbae L. (baculum Nitz.). Rochester, French; Ithaca (Herr). On domestic pigeon. 
E. botauri Osb. Hebron, on American bittern (Botaurus lentiginosus Montag.).

E. comstocki Kell. \& Chap. Ithaca and Hebron, on Virginia rail (Rallus virginianus L.) (Kell\&Chap).

E. crassicorne Scop. (squalidum Nitz.). Ithaca, on king eider (Somateria spectabilis L.), mallard (Anas platyrhynchos L.), black duck (Anas rubripes Brewst.), pintail (Dafila acuta L.), and redhead (Marila americana Eyt.); W. Hebron, on green-winged teal (Nettion carolincnsis Gmel.).

E. infuscatum Osb. Ithaca, on American woodcock (Philohela minor Gmel.).

E. luridum Nitz. Ithaca, on coot (Fulica americana Gmel.).

E. thoracicum Piaget. Ithaca, on bufflehead (Charitonetta albeola L.).

\section{LIPEURUS Nitzsch ${ }^{12}$}

L. caponis L. (variabilis Nitz.). VARIABLE HEN LOUSE. Generally distributed throughout the State, on hen; also taken at Newfield on ring-necked pheasant (Phasianus torquatus Gmel.).

L. gallipavonis Geoff. (polytrapesius Nitz.). Slender Turkey Louse. Ithaca, common on domestic turkey (Herr).

L. heterographus Nitz. Chicken HeAd-Louse. Recorded definitely only from Ithaca, but common throughout the State, on hen (Herr).

\section{ORDER THYSANOPTERA}

\section{Compiled by J. Douglas Hood and Glenn W. Herrick}

While the present list of New York thrips is nearly twice as long as that of Hinds for all of North America (Proc. U. S. Nat. Mus., vol. 26, p. 79-242, 1902), it certainly represents not more than half of the species which could be taken in a few months of really careful collecting. New York material of the group would be welcome indeed, and collectors are urged to send specimens (preferably in 80-per-cent alcohol) to the compilers of this list, for identification.

The first attempt to compile a list of New York Thysanoptera was made by Jacobus C. Faure, now Professor of Entomology in the Transvaal University College, Pretoria, Union of South Africa, while he was an undergraduate student at Cornell University. Mr. Faure collected about thirty species, most of which had not previously been taken in the State.

\section{SUBORDER TEREBRANTIA \\ Superfamily AEOLOTHRIPOIDEA \\ FAMILY AEOLOTHRIPIDAE}

\section{AEOLOTHRIPS Haliday}

A. albicinctus Hal. Canastota, J1-Aug, on corn leaves, Faure (Hd); Macedon, Je, common on grass $(H d)$; Elmira, J1, on onion, Faure (Hd) ; Chester, Aug, on onion, Faure (Hd); Ithaca, May, on Pinus, Babiy (Hd), and in sweepings from grass $(H d)$.

${ }^{12}$ The genus Lipeurus is placed by Harrison in the subfamily Goniodinae. 
A. bicolor Hinds. Ithaca, on red clover, Wehrle (Herr); Chester, on onion, Faure ( $\mathrm{Hd}$ ).

A. nasturtii Jones. Ithaca, May, in sweeping grass $(H d)$.

A. fasciatus L. Cranberry L., Je, Drk (WatsonJR) ; Sodus Pt., Aug, in dandelion flowers $(H d)$; Macedon, Je-Oct, abundant on onion, feeding on Thrips tabaci $(H d)$; Ithaca, May, in sweeping $(H d)$; Geneva, $\mathrm{Jl}$, on cherry foliage, GlasgowH (Hd) ; Canastota, Aug, on clover, Faure (Hd) ; Elmira, J1, on onion, Faure (Hd); Pine I., Aug, on onion, Faure (Hd) ; Chester, Aug, on onion, Faure (Hd); Pulaski, Je, on grass, Babiy (Herr).

A. melaleucus Hal. (annectans Hd.). Macedon, Je, on Tilia $(H d)$; Monsey, J1, on foliage of pear, GlasgowH (Hd).

\section{Superfamily THRIPOIDEA}

\section{FAMILY THRIPIDAE}

\section{HETEROTHRIPS Hood}

H. limbatus Hd. Macedon, Je, in flowers of wild cherry $(H d)$.

H. arisaemae Hd. Macedon and McLean, May, in flowers of Jack-in-the-pulpit $(H d)$.

H. azaleae Hd. Suffern, May, in flowers of Azalea, KalmbachER (Hd).

\section{HELIOTHRIPS Haliday}

$\mathrm{H}$. femoralis Reut. Ithaca, in greenhouses, Ap- $\mathrm{Jl}$, on a large variety of plants, div. ( $\mathrm{Hd}$ and $\mathrm{Herr}$ ).

H. haemorrhoidalis (Bouché). The Greenhouse Thrips. Watkins, J1, presumably in greenhouse (Felt); Ithaca, in greenhouses, Jan, on fern, Faure (Hd), May, on Croton, Griswold (Hd).

\section{PARTHENOTHRIPS Uzel}

P. dracaenae (Heeger). Ithaca, in greenhouses, Mar, Faure (Hd), Ap, on calla lily, Joh (Hd), May, on palm, Griswold (Hd), Nov, Dec, on banana and Anthurium (Herr); Ogdensburg, on palm and rubber plant in greenhouse, LarerenceFW (Hd).

\section{SERICOTHRIPS Haliday}

S. cingulatus Hinds. Ithaca, May, in stomach of very young trout, $P c k(\mathrm{Hd})$. A grass species.

ECHINOTHRIPS Moulton

E. subflavus Hd. Little Valley, Sep, under bark of beech sapling, Chap (Hd).

\section{LIMOTHRIPS Haliday}

L. cerealium Hal. Sheldrake, Aug, on oats (Herr) ; LI: Riverhead, J1, in flight, abundant, RussellHM (Hd).

L. denticornis Hal. Macedon, in winter hibernating under bark of willow and in fallen leaves, in summer abundant on wheat and grasses $(H d)$; Ithaca, on wheat, RiesD (Hd), in fallen leaves, Mar-Ap, BarnesTC (Hd), in robin's nest, Mar, Dobroscky (Hd), on grass, Je (Hd); McLean, on grass, Babiy (Herr). 


\section{Chirothrips Haliday}

C. manicatus Hal. Rochester, $\mathrm{Jl}$, in heads of Dactylis glomerata $(H d)$; Canastota, $\mathrm{J} 1$, in milkweed flower, Faure $(\mathrm{Hd})$; Macedon, Je, on wheat $(H d)$; Elmira, $\mathrm{J1}$, on tobacco, Faure (Hd) ; Ithaca, May, in stomach of very young trout, Pck (Herr), Je, on grass $(H d)$; Slaterville, May, on grass $(H d)$; McLean, May, on grass $(H d)$.

C. obesus Hinds. Canastota, J1, on grass, Faure (Hd).

\section{APTINOTHRIPS Haliday}

A. rufus (Gmelin). Canastota, Nov, on grass, Faure (Hd) ; Macedon, May-Je, on wheat and grass $(H d)$; LI: Riverhead, Je, on grass, RussellHM (Hd).

A. rufus forma stylifera Trybom. McLean, May, common on grass, Leon $\mathcal{F} H d$ (Hd).

\section{ANAPHOTHRIPS Uzel}

A. obscurus (Müller). The Grass Thrips. Albion, J1 (Felt); Macedon, Oct, on corn $(H d)$; Canastota, J1, Nov, on grass, Faure (Hd); Taughannock, J1, on heads of timothy (Herr) ; Ithaca, Je, on grass $(H d)$; LI: Riverhead, Je, on grass, RussellHM (Hd); Cold Sp. Harb., Je, And (Hd).

\section{SCOLOTHRIPS Hinds}

S. 6-maculatus (Perg.). Chester, Aug, on elm leaves, predacious on mites, Faure ( $\mathrm{Hd})$.

\section{FRANKLINIELLA Karny}

F. fusca (Hinds) (Scirtothrips owreyi Watson). THE ToBAcco Thrips. Wells, May, on buttercup, $\mathrm{Yg}$ (Hd) ; Elmira, J1, on tobacco, Faure (Hd); Florida, Aug, on onion, Faure ( $\mathrm{Hd}$ ); Chester, Aug, on grass, Faure (Hd).

F. tenuicornis (Uzel) (nervosa Uzel, maidis Beach). Canastota, J1, on corn, Faure (Hd) ; Macedon, May-Oct, common on wheat and corn $(H d)$; Elmira, $\mathrm{J} 1$, on tobacco, Faure (Hd).

F. tritici (Fitch). "NY" (Fitch) ; Speculator, May, on strawberry, Yg (Hd) ; Williamson, Je, on Ranunculus bulbosus, Faure (Hd) ; Macedon, May-Oct, on flowers and foliage of various plants $(H d)$; Ithaca, Aug, on red clover, Wehrle (Herr); Taughannock, Jl, on Hypericum (Herr); McLean, May, in catkin of willow $(H d)$; LI: Riverhead, Aug, on various plants, RussellHM ( $\mathrm{Hd}$ ).

F. stylosa Hd. Suffern, May, on Azalea and dogwood, KalmbachER (Hd).

TAENIOTHRIPS Amyot and Serville

T. salicis (Reut.). Slaterville, May, on Salix $(H d)$.

T. inconsequens (Uzel) (Euthrips pyri Daniel). THE PEAR Thrips. Macedon, May-Je, on wild and cultivated cherry $(H d)$; Batavia, May, on pear, Faure (Hd) ; E. Bethany, May, on apple foliage, Herr (Hd) ; Geneva, Ap, GlasgowH, and May, ParrottPJ, on apple (Hd); Ithaca, May, on pear (Herr) ; Ravena and Athens (Felt); Tivoli, May, on pear, Rupert (Herr); Ulster Co, Ap, MillsWD ( $\mathrm{Hd})$. 


\section{CTENothrips Franklin}

C. bridwelli Frank1. Rose, May, on leaves of Cypripedium hirsutum, WeedAC (Hd) ; Macedon, May, on leaves of Podophyllum peltatum (Hd) ; Ithaca, Je, on Podophyllum peltatum, Herr ( $\mathrm{Hd}), \mathrm{Jl}$ and Sep, on skunk cabbage (Herr); Taughannock, J1, on Trillium (Herr); Ludlowville, May, on skunk cabbage $(H d)$; McLean, May, on May apple and skunk cabbage, Leon $\& H d(\mathrm{Hd})$.

THRIPS Linnaeus

T. impar Hd. Macedon, Aug, on Impatiens $(H d)$; McLean, Sep, on flowers and leaves of Impatiens $(H d)$.

T. nigropilosus Uzel. Canastota, Aug, on castor-oil plant in greenhouse, Faure (Hd) ; Macedon, Ap, on leaves of mullein $(H d)$; Rochester, Mar, on lemon verbena in greenhouse $(H d)$; Berkshire, Je, on lettuce, EasonNH (Hd). Apparently introduced from Europe.

T. physapus L. Speculator, May, $Y g(\mathrm{Hd})$; Sodus Pt., Aug $(H d)$; Rochester, May-Aug $(H d)$; Wallace, Ap $(H d)$; Ithaca, Je, Aug, Oct, Faure $(H d)$. A common dandelion-flower species.

T. tabaci Lindeman. The Onion Thrips. Newport, J1 (Felt); Canastota, Nov, on grass near onion fields, Faure (Hd) ; Marion, Je, on onion, Faure (Hd); Macedon, Oct, on onion $(H d)$; Geneseo, Je (Felt); Ithaca, Oct, on daisy, Faure (Hd); LI: Je, infesting cauliflower seedbeds, Huck (Hd).

T. monotropæ Hd. Sodus Pt., Aug 10, in flowers of Monotropo uniflora ( $H d)$.

T. veratri Herr. Ithaca, on Veratrum viride (Herr).

T. flavus Schrank. Ithaca, J1, in flowers of Angelica atropurpurea, JacksonLO ( $\mathrm{Hd}$ ).

T. fuscipennis Hal. Ithaca, J1, in flowers of Angelica atropurpurea, JacksonLO ( $\mathrm{Hd}$ ).

T. calcaratus Uzel. McLean, May 19, in grass, etc., Leon \&Hd (Hd).

BALIOTHRIPS Uzel

B. dispar (Hal.). Chester, Ap, on grass, Faure (Hd).

\section{FAMILY MEROTHRIPIDAE}

MEROTHRIPS Hood

M. morgani Hd. Macedon, Oct, under bark of fallen elm tree $(H d)$.

\section{SUBORDER TUBULIFERA \\ Superfamily PHLAEOTHRIPOIDEA \\ FAMILy PHLAEOTHRIPIDAE}

HAPLOTHRIPS Amyot and Serville

H. graminis Hd. Barneveld, on grasses, WolcottGN (Hd).

H. faurei Hd. Ithaca, Oct, on ivy foliage, Faure (Hd), and on Chenopodium album, Chap ( $\mathrm{Hd}$ ), May, on willow foliage $(H d)$; Florida, Aug, on willow foliage, Faure (Hd) ; W. Danby, May, on willow $(H d)$; Cinnamon L., Mav, on pine $(H d)$. 
H. leucanthemi (Schrank) (niger Osb., statices auct. nec Hal.). Speculator, Je, in flowers of red clover, daisy, and yarrow, $Y g$ ( $\mathrm{Hd}-\mathrm{NYS}$ ) ; Northampton, $\mathrm{Je}$, on yarrow, $\mathrm{Yg}$ ( $\left.\mathrm{Hd}-\mathrm{NYS}^{\prime}\right)$; Wells, May, on buttercup and blackberry, Yg (Hd-NYS) ; Canastota, Jl-Aug, on daisy, Faure (Hd) ; Rochester, Je-J1, on Chrysanthemum leucanthemum, Melilotus officinalis, and Trifolium repens, MaynardEA (Hd), on wild carrot (reared), and in flowers of Achillea millefolium $(\mathrm{Hd})$; Macedon, Je, on Chrysanthemum leucanthemum and Trifolium spp. $(H d)$; Ithaca, Je, in flowers of daisy and rhubarb, Faure (Hd), J1, on yarrow (Herr), Aug, on red clover, Wehrle (Herr) ; Taughannock, Jl, on ox-eye daisy (Herr); LI: Riverhead, Je, on daisy, RussellHM (Hd).

H. verbasci (Osb.). Speculator, Je, on mullein, $\mathrm{Yg}$ (Hd) ; Rochester, Nov, on mullein $(H d)$; Macedon, throughout the year, on mullein $(H d)$; Wallace, May, on mullein ( $H d$ ) ; Ithaca, May, on mullein (Herr), and in stomach of very young trout, Pck (Herr); McLean, May, on mullein, Leon $\& H d(\mathrm{Hd})$; LI: Riverhead, Je-J1, common on mullein, one specimen from black-eyed daisy, RussellHM (Hd).

\section{Hindsiana Karny}

H. dodgei Hd. Macedon, May 4, under rotting elder bark ( $H d)$.

H. pullata Hd. Macedon, Jan 23, under bark on apple tree $(H d)$.

\section{ZYGOTHRIPS Uzel}

Z. americanus Hd. Macedon, Jan-Ap, Aug, under bark of maple, hickory, apple, and ash trees $(H d)$; Slaterville, May, under maple bark $(H d)$.

\section{LEPTOTHRIPS Hood}

L. mali (Fitch) (adirondacks Watson, in part). "NY" (Fitch) ; Cranberry L., J1, on yellow birch, Drk, "type, male," of $L$. adirondacks Watson ( $\mathrm{Hd}$ ); Macedon, Je, on leaves of grape and basswood $(H d)$; E. Bethany, May, on apple, Herr ( $\mathrm{Hd}$ ) ; Geneva, on grape clusters (Hd); Ithaca, Oct, on grape foliage, Faure (Hd); Suffern, May, on Azalea and dogwood, KalmbachER (Hd).

\section{LIOTHRIPS Uzel}

L. caryae (Fitch). "NY", in galis of Phylloxera on hickory (Fitch).

L. citricornis (Hd.) (Leptothrips adirondacks Watson, in part). Cranberry L., Je, on Viburnum alnifolium, Drk, paratype of L. adirondacks Watson ( $\mathrm{Hd})$; Macedon, Je, on grape ( $H d)$; Suffern, May, on grape, JacksonLO (Hd).

L. vaneeckei Pries. LI: Brooklyı, Oct, on bulbs of Lilium canadense, Stout (Hd).

\section{RHYNCHOTHRIPS Hood}

R. russelli Hd. Keene Val., Je, on Virginia creeper, Mrs. W. S. Stewart (Hd).

R. pruni Hd. Macedon, throughout the year $(H d)$; Ithaca, Mar $(H d)$; Slaterville, May $(H d)$; McLean, May, Leon $\hat{\sigma} H d(\mathrm{Hd})$. Common under bark of wild cherry.

\section{NEOTHRIPS Hood}

N. corticis Hd. Macedon, Feb, Ap, Aug, under bark of hickory, maple, apple, and buckeye $(H d)$. 


\section{HOPLOTHRIPS Amyot and Serville}

H. beachi (Hinds). Macedon, Aug, under bark of dead maple saplings ( $H d$ ) ; Rochester, Je, reared from pupae taken under dead willow bark, TreherneRC $(H d)$; Mumford, Ap, under bark on dead willow branch $(H d)$; Ludlowville, May, under willow bark (Hd); Elmira, Oct, under bark of beech, Chap (Hd) ; Clarksburg, Sep, under bark of beech, Chap (Hd) ; Barcelona, Sep, under bark of beech, Chap ( $\mathrm{Hd})$.

H. corticis (DeG.). Cinnamon L., May, under bark of birch, Brad\&Hd (Hd) ; LI: Bergen Beach, Jan, under bark of oak, Dow (Hd).

H. karnyi karnyi (Hd). Macedon, Ap, under bark $(H d)$; Slaterville, May, under bark of apple $(H d)$.

H. karnyi major Hd. (Trichothrips drakei Watson, at least in part). Macedon, throughout the year, under bark of various trees, usually associated with fungus $(H d)$; Lakeville, May, under willow bark, MaynardEA ( $\mathrm{Hd})$; Syracuse, Oct, under bark of black locust, OsbornE, paratype of Trichothrips drakei Watson (Hd) ; Ithaca, Mar, in shelf fungus (Herr), Nov, under bark of tree, Babiy (Herr), Feb-Je, on Polyporus ( $H d)$, Je, in rotten stump, $C y$ (Hd) ; Taughannock, Aug, under bark of beech stick (Herr) ; McLean, May, under bark on fungus-covered stump, Leon $\mathcal{F} H d$ (Hd) ; Cinnamon L., May, under bark of birch, Brad\&Hd (Hd); LI: Sea Cliff, Sep, oak branches, Chap ( $\mathrm{Hd})$.

\section{TRICHOTHRIPS Uzel}

T. americanus $\mathrm{Hd}$. Ludlowville, May, under willow bark $(H d)$.

T. angusticeps Hd. Macedon, Ap, Aug, under bark of fungus-covered maple and hickory stumps $(H d)$; Rochester, Nov, on fungus under bark of beech stump $(H d)$.

T. fumiceps Hd. Potter, Ap, under rotting willow bark $(H d)$.

T. flavicauda Morgan. Macedon, Oct, under elm bark $(H d)$; W. Danby, Ap, under poplar bark $(H d)$.

\section{LISSOTHRIPS Hood}

L. muscorum Hd. Ithaca, May, in moss, BarnesTC (Hd).

\section{BOLOTHRIPS Priessner}

B. bicolor (Heeger). Canastota, Mar, on grass, Faure (Hd) ; Macedon, Mar, under fallen leaves $(H d)$; Ithaca, Mar-May, J1, on grasses and in fallen leaves and other débris, Babiy (Herr), BarnesTC ( $\mathrm{Hd})$.

\section{CRYPTOTHRIPS Uzel}

C. rectangularis Hd. (Gastrothrips salicis Watson). Cranberry L., J1, on willow, Drk, holotype of $G$. salicis Watson (Hd) ; Macedon, May-Je, under willow bark $(H d)$; Kenwood, J1, on peach bark, Faure (Hd) ; Ithaca, May, under willow bark $(H d)$.

C. (?) breviventris Hd. Macedon, Oct 26, in moss on fallen tree in lowland (muck) woods $(H d)$.

\section{PhLAEOTHRIPS Haliday}

P. chapmani Hd. Cinnamon L., Je, under bark of poplar, Chap (Hd) ; Elmira, $\mathrm{J} 1$, on tobacco foliage, Faure (Hd).

P. vittatus $\mathrm{Hd}$. Parkers, Lewis Co, Sep 2, Chap (Hd). 


\section{HOPLANDROTHRIPS Hood}

H. xanthopus Hd. Macedon, May; reared from nymph taken under maple bark $(H d)$.

H. proximus Hd. Macedon, May 18-31, Ithaca, May 27, under dead bark of Salix $(H d)$.

H. gynandrus Hd. Macedon, May 18 and 29, under dead bark of Salix sp. infested with Cryptorhynchus lapathi. $(H d)$.

\section{PHLOEOBIOTHRIPS Hood}

P. tumiceps Hd. Macedon, May, Oct-Nov, under bark of fallen branches and saplings $(H d)$.

\section{ACANTHOTHRIPS Uzel}

A. nodicornis (Reut.). Cranberry L., J1, in top of fallen maple tree, Drk (WatsonJR); Macedon, May, under willow bark $(H d)$.

\section{NEUROTHRIPS Hood}

N. magnafemoralis (Hinds). Cranberry L., J1, in top of fallen maple tree, Drk (WatsonJR) ; Macedon, Feb-Ap, under bark of maple and hickory $(H d)$; Canastota, Mar, under bark, Faure (Hd) ; Chester, on onion, Faure (Hd) ; Slaterville, May, one dead female under sycamore bark $(H d)$.

\section{POECILOTHRIPS Uzel}

P. ornatus (Hd.). Sodus Pt., Sep, one female taken in flight $(H d)$; Rochester, $\mathrm{J} 1$, one female taken in flight $(H d)$; Macedon, Ap, several nymphs under bark on hickory stump $(H d)$.

\section{GLYPTOTHRIPS Hood}

G. flavescens Hd. Chester, Ap, in grass, Faure (Hd).

\section{MEgALOTHRIPS Uzel}

M. spinosus Hd. (Idolothrips fuscus Watson). Syracuse, Oct, in burrows of cerambycid-beetle larva in twig of basswood, Drk, "type" and paratype of Idolothrips fuscus Watson (Hd); Macedon, Mar-Je, under bark of maple, in burrows of Cryptorhynchus lapathi in willow, and in stem galls on goldenrod $(H d)$; Ithaca, Mar, in hole in dead twig, FletcherPB (Hd); Nyack, in nest of mining bee, $Z a b$ (Hd) ; Rock City, Sep, under maple bark, Chap (Hd) ; LI: Sea Cliff, Sep, on oak branches, Chap (Hd).

\section{ELAPHROTHRIPS Buffa}

E. tuberculatus (Hd). LI: Long Pond, Sep 19, in dry oak leaves on broken limb, Chap (Hd); Wading River, Sep 19, Chap\&BBoyce (Hd). 


\section{ORDER ANOPLURA}

Compiled by G. F. Ferris

The published records of the occurrence of species of sucking lice in New York are apparently very few. However, the distribution of these insects is determined rather by the range of their hosts than by any other factor, and consequently we may be reasonably sure that those species whose normal hosts occur within the State will be found there also. The compiler has therefore included in this list the names of those species which may reasonably be expected to occur. Such species are indicated by brackets and are in italics.

The general classification of the Anoplura is in a rather unsatisfactory state and will probably require some modification. Two families are recognized for the New York species.

\section{FAMILy PEDICULIDAE \\ PEDICULus Linnaeus}

P. humanus (L.). Head And Body Louse of Man. Generally distributed throughout the State. Usually two species are recognized, capitis and corporis, but it has been shown that at best these are nothing more than races which should probably not be recognized.

\section{PhTHIRIUS Leach}

P. pubis (L.). Pubic Louse. Not common, but known from various places in the State where sanitary conditions are bad.

\section{FAMILY HAEMATOPINIDAE}

\section{HAEMATOPINUS Leach}

[H. asini (L.). HorSE LouSE. Occurs on horses in the State, but definite locality records are not available.]

H. eurysternus (Nitz.). Short-Nosed Ox Louse. Throughout the State, on domestic cattle. Watertown (Bishopp); Otto, Mar (Comst); Oswego (Comst); Ithaca.

H. suis (L.). Hor Louse. Throughout the State, on domestic pigs. Otto, Je (CU); Ithaca (Laura Florence).

\section{LINOGNATHUS Enderlein}

[L. pedalis (Osb.). Doubtless to be found in the State on domestic sheep.]

[L. stenopsis (Burm.). Doubtless to be found in the State on domestic goats anc perhaps on sheep.]

L. setosus (von Olfers) (piliferus (Burm.)). Dog Louse. LI: Brooklyn, on unnamed host, doubtless dog, GHFrank (Bishopp).

L. vituli (L.). Long-Nosed Ox Louse. Common throughout the State, on domestic cattle. Herkimer; Watertown and Oriskany Falls (Bishopp). 


\section{SOLENOPOTES Enderlein}

S. capillatus End. On domestic cattle. Herkimer; Churchville; Ithaca (Bishopp).

\section{POLyPLAX Enderlein}

$[P$. auricularis Kellogg \& Ferris. In all probability to be found on the white-footed mice of the genus Peromyscus.]

P. spinulosa (Burm.). LI: Brooklyn, host not given, but doubtless domestic rat, GHFrank (Bishopp). To be expected on the meadow mice of the genus Microtus.

\section{HOPLOPLEURA Enderlein}

H. acanthopus (Burm.). "NY", on Pitymys pinetorum (Ferris, from skins in U.S.). Certainly present also on meadow mice of the genus Microtus.

H. erratica (Osb.). Sing Sing, on Tamias striatus (Ferris, from skins in U. S.).

$[H$. hesperomydis (Osb.). Certainly to be found in the State on white-footed mice of the genus Peromyscus.]

[H. sciuricola Ferris. To be expected in the State on any of the tree squirrels.] [H. trispinosa Kellogg \& Ferris. To be expected in the State on flying squirrels.]

\section{NeOHAEMATOPINUS Mjöberg}

[N. sciurinus Mjöberg. To be expected in the State on any of the tree squirrels.] [N. sciuropteri (Osb.). To be expected in the State on flying squirrels.]

ENDERLEINELLUS Fahrenholz

[E. longiceps Kellogg \& Ferris. To be expected in the State on Sciurus carolinensis.]

[E. marmotae Ferris. To be expected in the State on woodchucks.]

[E. nitsschi (Burm.). To be expected in the State on Sciurus hudsonicus.]

[E. tamiasis Fahrenholz. To be expected in the State on Tamias striatus.]

\section{MICROPHTHIRUS Ferris}

[M. uncinatus (Ferris). To be expected in the State on flying squirrels.]

\section{EuHAEMATOPINUS Osborn}

E. abnormis Osb. Rochester, from mole (Ferris, from skins in U.S.), and from an unnamed host, certainly a mole, GHFrank (Bishopp).

\section{HAEMODIPSUS Enderlein}

[H. ventricosus (Denny). Certainly present in the State on both domestic and wild rabbits.]

\section{ORDER HEMIPTERA}

The list of Hemiptera has been prepared under the general editorship of H. G. Barber, of the New York Entomological Society, who has compiled the lists in all families not otherwise credited. J. R. de la TorreBueno, of the Brooklyn Entomological Society, has compiled the lists in 
the families of aquatic and semi-aquatic Hemiptera. The lists of Miridae and Isometopidae are by Dr. H. H. Knight, and those of Piesmidae and Tingitidae are by Dr. Carl J. Drake. Mr. Barber has determined much of the material in local public and private collections, except in the groups handled by Mr. Bueno, and has collected extensively on Long Island. Many records in all groups have been supplied by Dr. Knight, chiefly from Batavia, Ithaca, and Rock City; by Mr. Bueno from White Plains; and by William T. Davis, chiefly from Staten Island and Long Island but also from other parts of the State where he has collected. Ernest Shoemaker and F. M. Schott have supplied many records, and others also have furnished many, as credited. The editors are indebted to E. P. Van Duzee for an enormous amount of work in the determination of much material from all parts of the State, and for placing at their disposal all of the records which he had available.

Hemiptera have been actively collected by some of the leading specialists for many years in many places in the State, and the list is undoubtedly fairly representative. There is need, however, as will be readily seen, of much more collecting in certain groups and in certain regions.

The sequence of families and genera in the present list follows as closely as possible that in Van Duzee's Catalog of the Hemiptera. This is practically the reverse of the order used in Comstock's Introduction to Entomology. The order of arrangement of species, in general, follows Van Duzee's catalog, and for convenience the catalog numbers are attached. The synonymy has been changed only where later studies have seemed to warrant doing so. Species not preceded by a number have been described since Van Duzee's catalog was published.

\section{FAMILY SCUTELLERIDAE}

\section{SUBFAMILy TETYRINAE Stål}

\section{TETYRA Fabricius}

3 T. bipunctata H-S. LI: Central Pk., Woodr, Ds; Huntington, Schott; Bellport, Nic; Huntington, Schott; Wading R., Ols, Ds; Bay Shore, Ols; Yaphank, Bno, Eng; Massapequa, Ds; Wyandanch, Sf; Pinelawn, SWheat; Montauk, Ds; Promised Land, Eng, Ds; (all Brb). Ap-Sep. Adults more common on pitch pine in spring and fall; nymphs in midsummer, and often found on young pine cones (Ds).

\section{Acantholoma Stål}

8 A. denticulata Stål. West Pt., Ap-May, in lake drift, Robn (Bno, Brb).

\section{Stethaulax Bergroth}

11 S. marmoratus Say. LI: Flushing, Ols; Kissena Pk.; Rockaway Beach, Ds, Eng; (all Ols). May-Oct. Usually beaten from cedar (Brb). 
Homaemus Dallas

14 H. aeneifrons Say. Long L., Ds, Bno (Brb); L. Placid, Ds, Eng (EPV); Saranac L., Ds (Brb) ; Cranberry L. (Drk) ; Keene Val., Not (Brb); Cascade L., Indian L. (Brb); Ramapo Mts. (Brb-AM).

\section{SUBFAMILY ODONTOTARSINAE Stål}

EURYGASTER Laporte

19 E. alternatus Say. Found all over the State (Brb). Cranberry L. (Drk); L. Placid, Eng; Keene Val., Moss Pond, Not (Brb); Indian L., Cascade L. $(B r b)$; Niagara Falls, Buffalo (EPV); Ithaca, $D s$ $(K n g t)$; Mud Cr., Tompkins Co $(E P V-C U)$; L. George (Brb-AM) ; E. Jewett, Debruce, Ds (Brb-AM); W. Hebron, Lg (Brb); Phoenicia $(E P V)$; West Pt., Robn (Brb); White Plains (Bno); SI (Ds); LI: Bellport, Nic (Sf-BM); Amagansett, Sf (Brb).

\section{FAMILY CYDNIDAE SUBFAMILY THYREOCORINAE VanDuzee}

Galgupha Amyot and Serville

(Thyreocoris Schrank)

27 G. unicolor Pal. de Beauv. (atra Amyot \& Serv.). Occurs all over the State (Brb). Potsdam, J1, Houghton (EPV) ; Cranberry L., J1 (Drk); Newport, May, $Y g$ (EPV); Buffalo, Sep (EPV); Boston, Aug, Hamburg, May, E. Aurora, Sep, Colden, Aug, Chautauqua, May, Gowanda, Aug (EPV); Cattaraugus Co, J1, Ds (Brb) ; Nassau, Jl, Yg (EPV); Debruce, Dow (Bno) ; W. Hebron, Aug, Lg (EPV); Pine I., May-Je (Brb, Ols); White Plains, Armonk, May-Sep (Bno); Scarsdale, May-Aug (Bno); Bronxville, Je, Woodr (Brb); Van Cort. Pk., Je, Woodr (Brb); SI, Je-Sep, Ds, Bno (Brb); LI: Rockaway Beach, May, Ds (O1s); Long Beach, Je, Eng (Sf); Wading R., May, Maspeth, Je-Aug, Yaphank, May (Ols); Pinelawn, Sep, Montauk, Sep, Ds (Brb); Flatbush, May-Sep, Mosholu, Sep (Brb-AM). Probably some of these records should refer to the next species (Brb).

- G. aterrima Mall. Buffalo, Hamburg, J1 (EPV).

28 G. nitiduloides Wolff. Keene Val., Aug, Not, Mt. Whiteface, J1, Ds (Brb) ; Buffalo, Colden, Aug (EPV); SI, Je-Sep (Ds); LI: Rockaway Beach, May, div. (Ols); Long Beach, May (Ds).

31 G. nigra Dall. LI: Cold Sp. Harb., May, Aug, Yaphank, Sep (Brb).

CoRimelaena White

(Thyreocoris Schrank)

34 C. lateralis Fab. Axton, Je (M\&H) ; Niagara Falls, Je (EPV); Debruce, Dow (Bno) ; LI: Long Beach, May, Eng (Sf-BM).

- C. marginella Dall. (nanella McAtee, vide McAtee). Grandview, near Nyack, Je (Brb).

37 C. pulicaria Germ. Generally distributed throughout the State (Brb). Cascade L., Indian L., Aug (Brb); Mt. Whiteface, Lg (Brb); Cranberry L., J1 (Drk) ; Potsdam, J1, Houghton (EPV) ; Rochester, Wendt 
(Ols) ; Buffalo, Aug (EPV, Brb) ; Boston, Aug (EPV) ; Aurora, J1 (Brb-AM) ; Karner, J1 (EPV) ; Pine I., Je (Brb) ; White Plains, MayDec 23, hibernating on latter date (Bno); W Nyack, May (Ols); SI, J1-Aug (Ds) ; LI: Brooklyn, Wyandanch (Ds) ; Maspeth, sifting (Ols) ; Yaphank (Ds, O1s) ; Kissena Pk. (Ols); Rockaway Beach, div. (Bno) ; Bay Shore (Ols) ; Flatbush (Brb-AM) ; Long Beach, Promised Land, Eng (Bno); Cold Sp. Harb., EPButler (Huss); Fire I. (Ds) ; Feb-Sep.

\section{SUBFAMILY CYDNINAE Dallas}

\section{Cyrtomenus Perty}

44 C. mirabilis Perty. SI, Aug, Ds (Ashm).

\section{Aethus Dallas}

\section{(Cydnus)}

53 A. obliquus Uhl. LI: Aqueduct, Sep, Ds (EPV).

\section{Pangaeus Stål}

P. bilineatus Say. Oliverea, J1, Shoe (O1s); West Pt., Je (Sf-BM) ; Yonkers, Je (Brb); NYC, May, Van Cort. Pk., Je (Brb); Bronxville, Oct, Woodr (Brb); Inwood, May (Schott); SI, Sep, Ds (Brb); LI: Brooklyn, Eng (BM); Flatbush, Zab (Ols, Brb-AM); Kissena Pk. (Ols) ; North Beach, Schott. (O1s); Ap-Oct.

\section{Geotomus Mulsant and Rey}

62 G. pennsylvanicus Signoret. Mosholu, May (Brb).

\section{Amnestus Dallas}

67 A. spinifrons Say. Occurs all over the State (Brb). Indian L., Aug (Brb) ; Hamburg, Je, Aug (EPV); White Plains, May (Bno); Bronxville, J1-Aug, Woodr (Brb) ; SI, May-Je, Ds (EPV); LI: Yaphank, Aqueduct (Brb) ; Calvary Cemetery, ENielson (Ols) ; Flushing, Ols (Brb) ; Rockaway Beach (Ds); Huntington, Hempstead. Plains (Schott); Yaphank, Long Beach, Eng (Bno); Far Rockaway, Woodr (Brb); May-Oct.

68 A. pusillus Uhl. Keene Val., May, Not (Brb); Indian L. Aug (Brb); Rochester, Wendt (O1s); Buffalo, Ap-May, Hamburg, Je (EPV); Batavia, May, Kngt (Bno); Gowanda, May (EPV); Ithaca, May (EPV-CU); Albany, May (EPV-NYS); W. Hebron, Lg (Brb); Yonkers, Ap, Ang (Ols) ; Scarsdale, May, Eng (Bno); LI: Rockaway Beach, Ols (Brb) ; Far Rockaway, Woodr (Brb); Maspeth (Ols); Long Beach, Eng (Bno); May.

69 A. pallidus Zimm. Mosholu, May (Brb); Pine I., Je (Brb).

\section{SEHIRUS Amyot and Serville}

71 S. cinctus Pal. de Beauv. Occurs all over the State (Brb). Long L., Ds, Cascade L., Aug (Brb); Wilmington, J1 (Ds) ; N. Elba, J1 (Brb-AM); 
Saranac L., Sep, Lg (Ds) ; Cranberry L., Je-Sep, H (Huss, Drk) ; L. Placid, Aug-Sep (EPV) ; Chazy L., Je (NYS); Buffalo, Hamburg, $\mathrm{Jl}$, and Ithaca, J1 (EPV) ; L. George, J1 (Brb-AM) ; Oliverea, Je (Ds); Orange Co, Ap, JCFaure (Kngt); Tarrytown, J1' (Bno); SI, Aug (Ds); LI: Wading R. (Brb, Ds); Montauk, Schott (Ols); Je-Aug.

\section{FAMILY PENTATOMIDAE}

\section{SUBFAMILY GRAPHOSOMATINAE Jakowleff}

\section{Podops Palisot de Beauvois}

74 P. (Amaurochrous) cinctipes Say. Buffalo, May-Sep (EPV-NYS, CU) ; Ithaca, J1 (EPV-CU); White Plains, Ap-Sep (Bno); SI, Feb-Oct (Brb, Ds) ; LI: Rockaway Beach, Eng, Ds (Ols, Brb-AM) ; Far Rockaway, Woodr (Brb) ; Canarsie (Ols) ; Long Beach, Jamaica, Flatbush, Eng (BM) ; Bellport, Nic (BM); New Lots, Martin (BM); E. New York (Ds); Ap-Oct. Common on cattails (Ds), in salt meadows, and on Juncus gerardi and doubtless other rushes (Parsh).

75 P. (Amaurochrous) parvulus VanD. White Plains (Bno); "NY" (EPV).

\section{SUBFAMILy PENTATOMINAE Stål}

\section{ScIocoRIs Fallén}

79 S. microphthalmus Flor. Indian L., Aug (Brb); Cranberry L., J1-Aug (Drk). Sifted from moss.

\section{BROCHYMENA Amyot and Serville}

81 B. arborea Say. Lansing, Je-Sep (EPV-NYS) ; Honeoye Falls, Je (Ds) ; Buffalo, Ap-Sep (EPV) ; Hamburg, Je-Sep (EPV-NYS); Gowanda, Aug (EPV); Ithaca, May-Aug (Vdyk-CU); Nassau, Je-Sep (EPVNYS); White Plains, Aug (Bno); Ramapo, Oct (Ds); Pine I., Sep (Brb); West Pt., J1, Sep (Brb, Robn); SI, Aug-Nov (Ds); LI: everywhere (Brb); Huntington, Wyandanch, Schott (Ols); Queens, Ols (Brb); Yaphank, Eng (Ds-BM); Long Beach, Calverton, Eng (BM) ; Amagansett, Sf, Bellport, Nic (BM); Baldwin, nymphs (Ds) ; Rockaway Beach, Southold, Massapequa, Wading R., Farmingdale (Ds) ; Ap-Oct.

85 B. quadripustulata Fab. Mt. Whiteface, Je (Ds); Essex Fells, Aug, Nic (BM) ; Elma, Sep (EPV) ; Hamburg, Sep (EPV); Spring Br., Je, $M C V$ (EPV); Batavia, May-Sep, Geneva, Aug (Kngt); Ithaca, FebNov (EPV, Kngt-CU); Clinton Hgts., Ap (EPV); W. Hebron, Je (Lg); Pittstown, Aug (EPV-NYS); Nyack, Sep (Brb-AM); Pine I., Je, Sf (BM) ; Cruger, J1 (Brb-AM); White Plains, Mar-Sep (Bno) ; West Pt., Je (Ds) ; Ft. Montgomery, May (Brb) ; NYC (Brb) ; SI, Feb, Mar, J1-Sep (Ds); LI: Queens Village, Aug, Ols (Brb); Rockaway Beach (Ds) ; Port Washington, Sep (Ols) ; Kings Pk., Je, EBell (O1s) ; Coney I., Zab (O1s) ; Massapequa, Central Pk., Montauk (Ds) ; Parkerville, $B t$ (O1s).

89 B. carolinensis Westw. (annulatus Fab.). Albany Ap, $\mathrm{Yg}$ (EPV) ; Clinton Hgts., Ap (EPV); southern NY (EPV). 


\section{Peribalus Mulsant and Rey}

94 P. limbolarius Stål. Cranberry L., J1 (Drk); Onondaga Co, May, H (Huss); Buffalo, May-Sep (EPV); Hamburg, Aug-Sep (EPV); Batavia, May-Sep (Kngt); Ithaca, Ap-Aug (EPV-CU); Norton's Landing, May, SmHH (EPV); L. George, Aug (Brb-AM) ; Albany, Oct (EPV-NYS); Kingston, Aug (EPV); Mosholu, Aug (Bno); White Plains, J1-Sep (Bno, Ols); Ramapo, May, Aug (Ds); SI, May-Je, Oct (Ds) ; LI: generally distributed on Solidago and shepherd's-purse (Ols); Yaphank, Sep (Bno); Cold Sp. Harb., EPButler (Brb, Huss); Aqueduct (Ds, Brb) ; Kings Bridge (Brb); Rockaway Beach, Shoe (Ols, Ds); Queens Village, Schott, Hempstead, Canarsie (Ols) ; Pinelawn, Massapequa, Half Way Hollow Hills, Orient (Ds) ; May-Oct.

\section{TRICHOPEPLA Stål}

98 T. semivittata Say. Essex Fells, J1, Nic (BM); Buffalo, Je-Nov (EPV) ; Hamburg, J1, Colden, Aug (EPV); Salamanca, J1 (EPV); Ithaca, J1-Aug, Sep (EPV-CU) ; Binghamton, J1 (Brb-AM) ; Saugerties, J1 (Brb); Pine I., Je (Brb); Ramapo Mts. (Brb-AM); White Plains, Valhalla, May, Rye Beach, Aug (Bno) ; Yonkers (Brb-AM) ; Mosholu, J1-Oct, Bno (EPV) ; W. Nyack, Sep (Ols) ; Ft. Montgomery, J1, Bno (EPV) ; Bronxville, May-Sep, Woodr (Brb) ; Van Cort. Pk., Je-Sep (Brb) ; SI, Je-Sep, Bno (Ds, EPV); LI: Yaphank, Bno, Eng, Ds (Bno); Cold Sp. Harb., J1-Aug, EPButler (Huss, Brb) ; Maspeth (Ols); Wading R. (Brb, Ols); Bay Shore, Hempstead (Ols) ; Huntington, Schott (Ols) ; Flatbush, Zab (Brb) ; Pinelawn, Schott (O1s) ; Half Way Hollow Hills (Ds); Amagansett, Sf (BM) ; Jamaica, Flushing, Long Beach, Eng (Brb); May-Sep. Throughout LI on Daucus Carota (Ols).

\section{Rhytidolomia Stål}

101 R. saucia Say. Rye Beach, J1 (Bno); SI: salt meadow, May, Sep-Nov (Brb, Ds) ; LI: Rockaway Beach, div. (Ols, Brb-BM); Springs (Ds); Aqueduct (Ds, Brb, Ols) ; Bellport, Nic (BM) ; Coney I., Schott (Ols) ; Flatbush, Amagansett, Eng (BM) ; Bergen I., Cypress Hills (Brb-AM) ; Long Beach, Montauk, Schott (Ds) ; Sea Cliff, Bks; Orient Pt. (EPVNYS) ; May-Nov. Confined mostly to salt marshes; often taken on Spartina patens on LI (Parsh).

102 R. senilis Say. Rye Beach, Aug (Bno) ; NYC, Oct (Brb) ; SI, Jan, Ap-May, Sep, Dec (Brb, Ds) ; LI: Sands Pt., Bs (Ds); Rockaway Beach (Ds, Brb, Ols) ; Orient, Lath (Ds) ; Aqueduct, Ds (Ols) ; Central Pk., Sf, Eng (Ols-BM); Flatbush, Eng, Promised Land, Eng (BM); Coney I., Zab, Cypress Hills (Brb-AM) ; Hempstead (Ols) ; Brooklyn, Schott (Ols); Melville, Schott; Pinelawn, Eng (Ols); Amityville, Ols (Brb); Yaphank, near beach in rushes and grasses (Bno); Ap-Jan.

\section{ChLOROCHROA Stål}

106 C. persimilis Horv. (uhleri Stål, vide VanD.). Keene Val., Sep, Not (Brb); Cranberry L., Aug (Drk) ; Lewiston, J1, Wat (Brb) ; New Russia, Je, EABlunt (Felt); Rochester, Aug, Wendt, HESummers (Ols, EPV) ; Niagara Falls, Je, Lockport, Aug, Elma, Aug, Hamburg, May (EPV); Pike, Ap (EPV-NYS); Batavia, Je-Sep (Kngt); 
Buffalo, May-Oct (EPV); Brockport (Lint); Otto, Sep, Gowanda, Ithaca, May-Sep (EPV-CU) ; N. Chatham, May (Felt); Saratoga (Horv-NYS) ; Callicoon, J1 (Ds); W. Hebron, Aug, Lg (Ds) ; Helderbergs, J1, Wat (Brb) ; Ramapo Mts. (Sf-BM); Pine I., Je (Brb); LI: Bayville, Bks (Ols); Amagansett, Eng (Ols-BM); Promised Land, Ds (Ols); Wading R. (Ols); Central Pk. (Ds); Calverton, Eng (Sf-BM); May-Sep.

\section{MoRmideA Amyot and Serville}

111 M. lugens Fab. Keene Val., Aug, Not (Brb) ; Cranberry L., Je-Sep, H (Drk, Huss); Mt. Whiteface, Je (Ds); Hastings, J1 (Ds); Buffalo, Hamburg, Je (EPV); Lancaster (EPV); Batavia, Je-Aug (Kngt); Ithaca, J1-Aug, Comst, Atkinson (EPV-CU); Motts Corners, Aug, Comst (EPV-CU) ; L. George, Aug, Zab (Brb-AM); Poughkeepsie, Je (EPV-NYS) ; Debruce, Sep (Ds) ; Ulster Co, Sep; Kingston, Aug (EPV) ; Ramapo, May, Bno (EPV); Scarsdale, Aug (Bno) ; Pine I., Je (Brb); White Plains, Mar-Aug, Dec, under stones, Carmel, Aug 1 (Bno); Larchmont, Aug (Brb); Crugers, J1, Nyack, Aug (BrbAM) ; Mt. Vernon, Je (Woodr) ; Bronxville, J1 (Woodr) ; SI, Je-Aug (Ds, Ols) ; LI: (Ols) ; Forest Pk., Bno (EPV) ; Cold Sp. Harb., EPButler (Huss, Brb) ; Flushing (Ols) ; Yaphank (Ols, Ds) ; Hempstead (Ols); North Beach (Ols); Flatbush, Zab (Brb-AM); Southport, Wading R., Montauk (Ds); S. Huntington, Aug (Bno); Ap-Nov.

\section{SOLUBEA Bergroth}

117 S. pugnax Fab. Rye Beach, Aug (Bno); LI: Long Beach, Eng (BM); Rockaway Beach; Cold Sp. Harb. (Brb) ; Rockaway Pk. (Ols) ; MayOct.

\section{Euschistus Dallas}

121 E. euschistoides Voll. Generally distributed throughout the State. Wilmington, Saranac L., J1 (Ds); Keene Val. (Not); Cranberry L. (Drk); Axton, Je $(\mathrm{M} \& H)$; Indian L., Cascade L., Aug (Brb); Buffalo, Hamburg, Gowanda (EPV); Batavia, Jl-Sep (Kngt); Lewiston, Wat (Brb); Portageville, Aug (Kngt); Ithaca; Oliverea, Nov (Ds) ; Debruce, Dow (Bno); L. George, W. Hebron, Sep (Lg); Karner (NYS) ; Albany, May (EPV); Rye, Sep (Ds); White Plains, Carmel, Armonk (Bno); Scarsdale (Bno); Pine I., Sep (Brb); Ramapo, Suffern, Sep (Brb) ; Grand View, Nyack, Je (Brb) ; Bronxville, JeSep (Woodr) ; Mosholu, May-Aug (Bno) ; Bronx Pk., May (Brb) ; LI: Cold Sp. Harb.; Wading R.; Wyandanch; Montauk; Long Beach; Rockaway; Yaphank; Amagansett; Half Way Hollow Hills; May-Sep.

124 E. politus Uhl. Karner, Sep (NYS); West Pt., May-Je; SI, Je (Ds) ; LI: Flatbush (Ols); Rockaway Beach, May (Ds); Pinelawn, Je (Ds); Smithtown, Je (Ds) ; Yaphank (div.); Amagansett, Sep (Ds) ; Wading R. (div.); Half Way Hollow Hills (Ds); Wyandanch, Schott (Ols).

125 E. tristigmus Say. Generally distributed throughout the State. Thousand Isls. (Ds) ; Keene Val., Je (Not, Eng) ; Axton, Je (M\&H) ; Manlius, SmHH (EPV); Cascade L., Indian L., J1-Aug (Brb); L. Placid, Aug (EPV) ; Mt. Whiteface, J1 (Ds) ; Cranberry L. (Drk, etc.) ; Saranac L., Sep (Ds) ; Rochester, Wendt (Ols); Albion, Aug (NYS) ; Batavia, May-Sep (Kngt); Buffalo, Lancaster, Hamburg, Gowanda, 
J1-Aug (NYS) ; Rock City, Je (Kngt, Ds) ; Portageville, May (Kngt) ; Geneva, Aug (Huss); Ithaca, May-Sep (CU); McLean, Jl, Mud Cr., $\mathrm{Je}$, Ellis to Slaterville, Slaterville to Caroline, Je (EPV-CU); Callicoon, Debruce, E. Jewett, Oliverea, Je-Sep (Ds) ; L. George, J1 (AM) ; W. Hebron, Aug-Sep (Lg); Albany (NYS); Poughkeepsie (NYS); West Pt., Ft. Montgomery, May-J1 (Ds); White Plains, Sep (Huss); Armonk, Je, Scarsdale, Aug (Bno); Grand View, Nyack, Je (Brb) ; Pine I., Je (Ds) ; Mosholu, Jl, Bno (EPV); Van Cort. Pk., Sep (Ols) ; SI, May-Oct (Ds); LI: Cold Sp. Harb. (Brb, Huss): Flushing, Bay Shore, Rockaway Beach, Hempstead (Ols); Queens, Schott (Ols); Flatbush (AM); Yaphank, Eng (BM); May-Sep.

E. variolarius Pal. de Beauv. Common throughout the State, feeding on numerous kinds of plants (Brb); Cascade L., Aug (Brb); Pike (NYS) ; Fulton, Je (CU) ; Cranberry L., J1-Aug (Drk); Rochester, Wendt (Ols) ; Batavia, Je-Sep (Kngt); Portageville, Aug (Kngt); Buffalo, Otto, Hamburg, Colden, Gowanda (EPV-CU); E. Aurora, J1 (Brb-AM) ; Rock City, Je (Ds); Ithaca, Caroline to Harford (CU); L. George, Aug (Brb-AM); W. Hebron, Aug-Sep, Lg (EPV); Karner, Round L. (NYS) ; Albany, Coeymans (NYS); Debruce, Dow (Bno); Oliverea, Callicoon, J1, Sep (Ds) ; West Pt., May-Oct (Ds); Hastings, J1, Joutel (Ds) ; Scarsdale, White Plains, Aug (Bno, Huss); Ramapo, J1 (Ds); Van Cort. Pk., Mosholu, Je-Sep, Bno (EPV); Kings Bridge, Sep (Brb); SI, Mar-Oct, also found hibernating in old nest of red-eyed vireo, Feb. 24 (Ds); LI: Brooklyn (Ds, Ols); Aqueduct, Oct (Brb) ; Cold Sp. Harb. (Huss, Brb); Forest Pk. (Bno, EPV); Rockaway Beach (Ds, Ols); Wading R. (Ds, Ols); Yaphank (Ds); Wyandanch (Ols) ; Orient (AM) ; May-Sep.

127 E. ictericus L. Batavia, Je-Aug (Kngt); Buffalo, Cheektowaga, Je-Sep (EPV) ; White Plains, Aug (Bno); Pine I., Sep (Brb); Inwood, Oct (Ds) ; Bronx Pk. (Brb-AM); SI, Je-Sep (Ds); LI: Aqueduct, Flushing R., Promised Land; Wading R. (Ds); Maspeth, Rockaway Beach (O1s); Je-Oct.

\section{Coenus Dallas}

139 C. delius Say. Generally distributed throughout the State, collected on mullein (Brb); Mt. Whiteface, Wilmington, J1 (Ds); Cascade L., Indian L., Aug (Brb) ; Cranberry L., J1 (Drk); Onondaga Co, May, $H$ (Huss) ; Rochester, Wendt (Ols); Batavia, Je, Sep (Kngt); Oakfield, Je (Not) ; Buffalo, Hamburg, Ap-Sep (EPV); Portageville, Je (Ds); Ithaca, Mar-Jl; Karner, Sep (NYS); W. Hebron, Sep, Lg (Ds) ; E. Jewett, Aug (Ds) ; Ft. Montgomery, J1 (Brb) ; Saugerties, Aug (Brb); White Plains, Ap, Sep (Ols, Huss); W. Nyack, Sep (Ols) ; Pine I., Je (Ds) ; Bronxville, Je (Woodr); Mosholu, Sep, Ds, Eng (Ols) ; SI, May-Sep (Ds) ; LI: Montauk, Deep Pond at Wading R., Half Way Hollow Hills (Ds) ; Flatbush, Rockaway Beach (AM); Amagansett, Greenport, Central Pk. (BM); S. Huntington, Aug (Bno).

\section{HyMENARCys Amyot and Serville}

140 H. aequalis Say. NYC (EPV); LI: Long Beach, May, Eng (BM).

143 H. nervosa Say. Albany, May (EPV-NYS); SI, Je, Aug, Bno (EPV); LI: Yaphank, Ds (Ols, Brb-AM), Flatbush, Rockaway Beach, Schott (Brb, Ols-AM); Wading R. (Ols) ; Cold Sp. Harb. (Brb); May-Oct. 


\section{NeOtTIGLOSSA Kirby}

145 N. undata Say. Throughout the State (Brb). Cascade L., Indian L., Aug (Brb); Axton, Je (M\&H) ; Cranberry L., Je-Aug (Drk, Huss); L. Placid, Phoenicia, Aug (EPV); Essex, Je (NYS); Rochester, Jl, Wendt (Ols); Batavia, Je-Sep (Kngt); Oakfield, Je, Not (Brb); Portageville, May (Kngt); Buffalo, Gowanda, Hamburg, Lancaster, Colden, Je-Sep (EPV) ; Ithaca, Je-J1 (CU) ; LI: Yaphank, Oct (Ds).

\section{COSMOPEPLA Stål}

149 C. bimaculata Thom. Throughout the State, common on mints and other plants (Brb). Potsdam (EPV); Keene Val., Je, Not (Brb); Cranberry L., $H$ (Huss) ; Cascade L., Aug (Brb); Wilmington, J1 (Ds); Axton, Je (M\&H) ; Rochester, Wendt (Ols); Batavia, Portageville, May-Sep (Kngt); Buffalo (EPV); Sonyea, J1, Lint (NYS); Rock City, Je (Ds) ; Ithaca, May-Aug (CU) ; Binghamton, J1 (Brb) ; Oliverea, Je-Sep (Ols, Ds) ; Pine Hill (AM) ; Callicoon, E. Jewett, Jl-Aug (Ds) ; L. George, J1 (AM) ; W. Hebron, Je-Sep, Lg (CU); Albany, Je, Nassau, Aug (NYS); Hudson, Je, Eng (BM); Loomis (Huss); White Plains, May-Sep (Bno); Armonk, Je (Bno), as lintneriana Kirk.; Suffern, Aug, Sf (BM) ; Mosholu, Aug, Bno (EPV); LI: Flatbush, Huntington (Ols) ; Forest Pk., Je (EPV, Ols) ; Cold Sp. Harb., J1 (Huss, Brb) ; Winfield, Maspeth (Ols); Wading R., Je Schott (Ols) ; Je-Sep.

\section{MENECLES Stål}

155 M. insertus Say. Lewiston, May (EPV); Ithaca, Mar, Dec (Kngt); West Pt., Je, Sf (BM); White Plains, Mar, Feb 22, hibernating, Farley (Bno); SI: Richmond, Nov, St. George, Aug (Ds); LI: Rockaway Beach, May, Oct, Schott, Ds (Ols); Wading R., Je, Schott (Ols); Flatbush, May, Zab (Brb) ; Long Beach, Ap-Je, L. Ronkonkoma, May, Sichott (Ols) ; Ap-Je.

\section{ThyanTA Stål}

158 T. custator Fab. Spread throughout the State (Brb). Cranberry L. (Drk); Buffalo, Ap (EPV) ; Mosholu, Je-Sep, Bno (EPV); SI, J1 (Ds); LI: Huntington, May, Schott, Yaphank (Ols); Rockaway Beach (Ols); Calverton, Eng (BM); Amagansett, Sf (BM); Montauk, Riverhead, Wading R. (Ds); Flatbush, Eng (Ols); May-Sep.

160 T. calceata Say. Ft. Montgomery, J1, Ds (Brb); LI: Flatbush, Nov, Schott (Bno); Cold Sp. Harb., Aug (Brb).

\section{MuRgantia Stål}

172 M. histrionica Hahn. Harleguin Cabbage Bug. Oswego (How); Elmira, Beckwith (Felt); LI: Jamaica (How); Orient Pt., Lath (Felt). Occasional (Brb). Sometimes injurious to cabbage on LI. 


\section{ACROSTERNUM Fieber}

\section{(Nezara Amyot and Serville, in part)}

179 A. pennsylvanicum DeG. SI: Feb, Watchogue, Sep (Ds) ; LI: Huntington, Schott, Eng (Ols); Yaphank (Ols, Brb) ; Rockaway Beach, Wading R. (Brb); Calverton (Ds); Baldwin, Hein (Ols); May-Sep. Swept from young oaks, not common (Brb).

181 A. hilare Say. A common species in the southern part of the State, throughout LI and SI (Brb). Buffalo, J1-Aug, Oct (EPV); Batavia, J1-Sep (Kngt); Albany, Sep (EPV-NYS); L. George, Sep, Zab (Brb-AM); West Pt., Je (Brb) ; Ft. Montgomery, J1, Ds (EPV); White Plains, Ap-Sep (Bno); Scarsdale, Sep (Bno); W. Nyack, Sep (O1s); Grand View, Je (Brb); Rye, Oct (Ds); Katonah, J1, Zab (Brb-AM); SI, Ap-Óct (Ds); LI: Rockaway, Oct, Schott (Bno); Cold Sp. Harb., $\mathrm{Jl}$ (Brb); Oyster Bay (Bno); Cypress Hills, Flatbush, Zab (BrbAM) ; Little Neck, Schott (O1s); Wading R., Schott (O1s); Calverton, Eng (BM); Yaphank, Eng (Ols); Wyandanch, Schott (O1s); Hempstead (Ols); Promised Land, Amagansett (Ds); Orient, Lath (Ds) ; May-Oct.

\section{BANASA Stål}

182 B. dimidiata Say. Throughout the State (Brb). Essex Fells, Aug, Nic $(\mathrm{Sf}-\mathrm{BM}) ; \mathrm{Mt}$. Whiteface, J1 (Ds) ; Old Forge, Je (EPV-NYS); Newstead, Je (EPV); Cranberry L. (Drk); Axton, Je (M\&H); Rochester, Je, Wendt (Ols) ; Albion, Aug, Niagara Falls, Je (EPV); Buffalo, Aug-Sep (EPV); Batavia, Je-J1 (Kngt); Portageville, Je (Kngt); Portage, Je (Ds); Gowanda (EPV); Cattaraugus, Je (Kngt); Rock City, Je (Ds); Oliverea, Je, Aug-Sep, Shoe (Ds); L. George, Aug-Sep, Zab (Brb-AM); Sullivan Co, Lg (Brb); West Pt., Je, on shadbush (Ds); White Plains, Sep (Brb); Marlborough, J1 (Sf-BM) ; SI, Je-Aug (Ds); LI: Brooklyn, Dow, Wyandanch (Ols) ; Quogue, Zab, North Beach, Palmer (Brb) ; Rosedale, Gardiners I. (Schott); Rockaway Beach (Ols, Ds) ; Calverton (Ds); Orient, Lath (Ds); Coram (Ds); Amagansett, Eng (BM); May-Sep.

184 B. calva Say. Buffalo, Aug-Oct (EPV); Hamburg, Aug-Oct, Gowanda, Aug (EPV); Wyoming Co, Je (Kngt); Ithaca, Je-J1 (EPV-CU); Orange Co, J1 (Brb) ; Scarsdale (Bno) ; Ft. Montgomery, May (Brb); W. Nyack, Aug (Bno); SI, Oct (Ds); LI: Harbor Hill, Sea Cliff, Bks (Ols); North Beach, Plm (Brb); Amagansett, Eng (Ols); Mar-Sep.

186 B. sordida Uhl. LI: Huntington, Feb, Schott (O1s). An unusual record (Brb).

188 B. euchlora Stål. LI: Long Beach, Mar, Schott (O1s). Occurs on cedar in the South; an unusual record (Brb).

\section{DENDROCORIS Bergroth}

194 D. humeralis Uh1. Karner, May (EPV-NYS); Ulster Co, Sep, Nic (Ds), found on oaks; West Pt., Je (Ds); Ft. Montgomery, May-JI, Ds (Brb, EPV) ; LI: Yaphank; Huntington, Wyandanch, Schott (O1s) ; Wading R., Ds, Ols (Brb); Kings Pk., Shelter I. (Brb); Amagansett, Eng (Ds, Sf-BM); Pinelawn, Eng (Ds, Sf-BM); Bay Shore (Ols); Southold, Rockaway Beach, Central Pk., Riverhead, Massapequa (Ds); Bayville Beach (Bks); Deer Pk., Aug (Bno); May-Sep. 


\section{SUBFAMILY ACANTHOSOMATINAE}

\section{MEADORUs Mulsant and Rey}

203 M. lateralis Say. Cascade L., Indian L., Aug (Brb) ; L. Tear, J1, Keene Val., Aug, Not (Brb) ; Mt. Whiteface, Jl (Ds) ; Cranberry L., Je-Aug, H (Huss, Drk); L. Placid, Aug (EPV); Axton, Je (M\&H); Hamburg, Boston, Aug (EPV); Ithaca, J1 (Kngt) ; Oliverea, Je (Ds) ; White Plains, Oct, sifting (Huss); Ft. Montgomery, May (Brb); SI, Sep (Ds); LI: Rosedale and Fire I. Beach (Schott); Rockaway Beach (Ds, Ols); Yaphank (Ds); Wyandanch, Nov, Schott (Bno); May-Aug. Occurs particularly on yellow birch and beech (Brb).

\section{Elasmostethus Fieber}

204 E. cruciatus Say. Axton, Je $(\mathrm{M} \& \mathrm{H})$; Plattsburg, Wilmington, Mt. Whiteface, J1 (Ds) ; Keene Val., Je, Not (Brb); Cascade L., Aug (Brb) ; Cranberry L., Aug (Drk); Brant L., Aug, Doll (Sf-BM); Ithaca, Je-J1, Oct, div. (EPV-CU) ; McLean, Je-J1 (Kngt) ; L. George, Aug, Zab (Brb) ; LI: Yaphank (Brb); Rockaway Beach, Bno (Ols); Je-Sep. Occurs commonly on alder (Brb).

206 E. atricornis VanD. Cranberry L., Aug (Drk); Boston, Aug (EPV); Ithaca, J1-Aug (EPV-CU).

\section{SUBFAMILY ASOPINAE}

\section{STIRETRUS Laporte}

207 S. anchorago Fab. Rochester, J1, Wendt (O1s) ; Ithaca, Je, Aug (EPVCU) ; NYC, Wat (Brb): LI: Rockaway, Je, Eng (Ols). These belong to var. fimbriatus Say. Predacious, as are all of the members $(\mathrm{Brb})$.

\section{PERILlus Stål}

215 P. splendidus Uh1. SI: St. George, J1 5, 1917, in house at light, Ds (Brb).

216 P. bioculatus Fab. Buffalo, Nov, Elma, Aug, $M C V$ (EPV) ; Batavia, AugSep (Kngt) ; LI: Yaphank (Bno).

217 P. circumcinctus Stål. St. Lawrence (EPV) ; Cranberry L., J1-Aug (Drk) ; Ilion (EPV-NYS); Buffalo, Je-Aug (EPV); Hamburg, Lancaster, Elma, Je (EPV); Batavia, J1 (Kngt) ; Ithaca, J1 (EPV-CU) ; Callicoon, J1 (Ds); White Plains, J1 (Bno); SI, Je (Ds); LI: Rockaway Beach, Eng (Ds); Roslyn (Bks); Montauk (Schott); Long Beach, Eng (Sf-BM); Central Pk., Doll (Sf-BM); Yaphank, usually in company with bright blue larvae of a chrysomelid beetle, often numerous (Bno) ; May.

218 P. exaptus Say. New Russia (EPV-NYS); Mt. Whiteface, Aug (Ds) ; Cranberry L., Aug (Drk) ; Mosholu, Je, Bno (EPV) ; Van Cort. Pk., May (Brb); SI, J1 (Ds) ; LI: Montauk, Little Neck (Schott) ; Long Beach, Eng (Sf-BM) ; Rockaway Beach, Ds, Sf (Ols); Ap-Jl.

\section{Mineus Stål}

221 M. strigipes H-S. Essex Fells, Aug, Nic (Sf-BM) ; Cranberry L., J1-Aug (Drk); White Plains, Ap, J1, Sep (Bno); Mosholu, Bno (EPV); Van Cort. Pk., J1 (Brb); SI, Feb, Je-J1, sifting (Brb, Ds); LI: 
Huntington, Schott (O1s) ; Wading R. (Ds) ; Massapequa, Shoe (Ds) ; Cold Sp. Harb. (Brb); Flatbush, Zab (Brb-AM); Maspeth (Ols); Rockaway Beach (Brb); Long Beach, Eng (Sf-BM); Wyandanch, Mar, Schott (Bno); Mar, May-Oct.

\section{Apateticus Dallas}

224 A. cynicus Say. Buffalo, Je, Sep, Gowanda, Colden, Lancaster, Aug (EPV); predacious, particularly on caterpillars; Newport, Aug (EPV-NYS); Manlius, Aug, SmHH (EPV-CU); Batavia, Aug (Kngt); Jamestown (Lint); Ithaca, J1-Sep (EPV-CU); New Baltimore, J1, Zab (Brb) ; Callicoon, J1, E. Jewett, Aug (Ds); Allaben, Aug, Pears (Ols) ; Debruce, Dore (Bno); West Pt., J1, Sf (Brb); White Plains, Aug (Bno); Sloatsburg (Brb); SI, J1-Aug (Ds); LI: Yaphank, J1, Ds, Schott (Ols) ; Fire I. (Schott); Amagansett (Sf) ; North Beach, Schott (Ols) ; Orient, Montauk, Wading R., Central Pk. (Ds); Long Beach, Shoe (Ds); J1-Sep.

225 A. bracteatus Fitch. L. Placid, Sep, Eng (Sf-BM); Batavia, J1, Sep (Kngt) ; Hamburg, Arg (EPV); Jamestown (Lint) ; E. Jewett, Aug (Ds); SI, Sep (Ds) ; LI: Rockaway Beach (Brb, Ols); Glendale, Doll (Sf-BM); Fire I. (Ds); Flatbush, Zab (Brb); J1-Oct.

\section{Podisus Herrich-Schaeffer}

227 P. maculiventris Say. Common all over the State (Brb). Mt. Whiteface, Lg (Brb) ; Axton, Je, $M \mathcal{E} H$ (EPV) ; Cranberry L. (Drk) ; Cascade L., Aug (Brb-AM); Onondaga Co, May, H (Huss); Fayetteville (EPV-CU) ; Rochester, J1, Wendt (Ols) ; Buffalo, May-Sep (EPV) ; Gowanda, Aug, Hamburg, Jl (EPV) ; Batavia, Je-Sep, Portageville, Je (Kngt) ; Ithaca, May-Oct, div. (EPV-CU); Albany, May (EPVNYS); Karner, Oct (EPV-NYS) ; L. George, Sep, Zab (Brb-AM) ; Coeymans, EBS mith (Lint); W. Hebron, Aug-Sep, Lg (EPV, Ds) ; Callicoon, J1, Oliverea, Sep (Ds) ; Saugerties, J1 (Brb); Pine I., Sep (Brb); Scarsdale, Aug (Bno); Kings Bridge, Sep (Brb); White Plains, Feb, Sep (Bno, Huss) ; Mosholu, Je-Oct (Bno-AM) ; Ft. Montgomery, J1, Ramapo, May (Ds) ; SI, Ap-Nov (Ds) ; LI: Long Beach, Amagansett, Eng (Sf-BM) ; Flatbush, Zab (Ols, Brb-AM) ; Cold Sp. Harb. (Brb) ; Aqueduct (Brb, Ds) ; Shelter I., Plm (Brb); Rockaway Beach; Yaphank, Wading R., Montauk (Ds); Maspeth (Ols, Ds) ; Orient, Ds, Hempstead, Wyandanch (Ols); Huntington, Schott (Ols) ; Canarsie (Ols) ; Central Pk. (Ds) ; May-Oct.

$227 \frac{1}{2}$ P. fretus O1s. LI: Wyandanch, Schott (O1s) ; Rockaway Beach, Nic (O1s) ; Promised Land, Sep (Ds) ; Ap-Sep.

228 P. serieventris Uhl. All over the State (Brb). Axton, $M \& H$ (EPV); Mt. Whiteface, J1 (Ds); Cranberry L., Je-Sep (Drk); L. Placid, Aug (EPV) ; Rochester, J1, Wendt (Ols); Helderbergs, J1, Wat (Brb); Slide Mt., J1 (Ds); White Plains, Dec, sifting (Bno); Bronx Pk., Mar, Wat (Brb) ; LI: Huguenot (Brb-AM) ; Farmingdale, Patchogue (Ds) ; Bay Shore (Ols) ; Long Beach, Eng (Sf-BM); Yaphank, Bno, Eng (Sf-BM) ; Sea Cliff (Bks); Amagansett, Eng (Ols) ; Rockaway Beach, Bno, Dickerson (Bno, Brb); Oyster Bay, Aug (Bno); May-Oct.

229 P. modestus Dall. All over the State (Brb). Axton, Je (M\&H) ; Ilion, Newport, Johnstown, Elm L., Aug-Sep (EPV-NYS); Cranberry L., 
Je-Sep (Drk) ; Cascade L., Aug (Brb); Mt. Whiteface, J1 (Ds) ; Conesus, Aug (Ds) ; Buffalo, May-Sep, Gowanda (EPV); Batavia, Aug, Portageville, May (Kngt) ; Branchport, Je, Snow (Lint) ; Ithaca, Je-Sep (Kngt, EPV-CU) ; Mud Cr., Tompkins Co, Je (EPV-CU) ; Four Mile, J1 (Kngt); Phoenicia, Aug (EPV); Slide Mt., J1 (Ds); Oliverea, Je, Shoe, Ulster Co, Sep, Nic (Ds); Stanford, Aug (EPV); West Pt., J1, Sep (Brb, Ds) ; Kiamesha; Bangall, Sep, Eng (Sf-BM); Scarsdale, Aug-Sep (Bno) ; Ramapo, Sep (Ds); Ft. Montgomery, Sep (Schott); LI: W. Nyack, Aug (Bno) ; Fire I. (Schott); Amagansett, $D s$, Eng (Sf-BM) ; Selden (Ds); Yaphank, Ds (Brb) ; Flatbush, Zab (Brb, Ols); Brooklyn, Dow (Ols); Wading R., Rockaway Beach (Brb) ; Long Beach, Schott (O1s) ; Ap-Sep.

230 P. placidus Uhl. Spread all over the State (Brb). Cranberry L., Je-Aug (Drk) ; Rochester, Jl, Wendt (Ols) ; Batavia, Je-Aug (Kngt) ; Rock City, J1 (Kngt) ; Four Mile, Je (Kngt) ; Karner, May (EPV-NYS) ; West Pt., Je (Ds) ; Pine I., Je (Brb); SI, Je-Sep (Ds); LI: Fire I., Aug (Ds, Schott); Wyandanch (div.); Rockaway Beach, Eng (Ds, Sf-BM) ; Amagansett, Eng (Sf, Ds-BM) ; Yaphank, div. (Brb, BnoAM) ; Wading R., Ds (Brb, Ols) ; Pinelawn, Ds (Brb, Ols) ; Flushing, Zab, Shelter I., Plm (Brb) ; Riverhead, Coram, Central Pk., Half Way Hollow Hills, Massapequa (Ds); Oakdale, Schott (O1s); Aqueduct, Ds, Wyandanch, Schott (O1s).

\section{FAMILY COREIDAE}

\section{SUBFAMILY MEROCORINAE}

\section{MEROCORIS Perty}

236 M. distinctus Dall. Hamburg, Mar, J1, Oct, Boston, Aug (EPV); Ithaca, (Morr) ; L. George, Aug, Zab (Brb-AM) ; Karner, Sep (EPV-NYS); Ulster Co, Sep, Nic (Ds) ; Saugerties, J1 (Brb) ; Sullivan Co, GFrank (Ols) ; Pine I., Sep (Brb); Nyack, Zab (Brb-AM); White Plains, Sep-Oct, Ds (Bno); Mosholu, J1, Bno (Heid); Ft. Montgomery, J1 (Bno, Ds) ; Van Cort. Pk., Sep (AM); SI, Je-Sep (Ds) ; LI: Yaphank, Amagansett, Wading R., Montauk, Half Way Hollow Hills, Pinelawn (Ds); May-Sep. Considered as a var. of typhaeus Fab. by EPV and as a subspecies of the same by McAtee.

\section{SUBFAMILY COREINAE}

\section{ACANTHOCEPHALA Laporte}

242 A. terminalis Dall. West Pt., Je (Ds) ; Scarsdale, Sep (Bno) ; Ft. Montgomery, May-Je (Brb, Schott) ; Crugers, Je (Brb-AM) ; Ramapo, May (Ds) ; White Plains, J1 (Bno) ; Mosholu, Sep, Bno (Heid) ; Van Cort. Pk., J1 (Ds) ; NYC (Brb); SI, May-Sep (Ds) ; LI: Wyandanch, Schott (O1s, Bno) ; Hempstead (O1s); Wading R., Ds (Brb); Je-Sep.

\section{LEPTOGLOSSUS Guérin}

244 L. phyllopus L. LI, Gibbs (Uh1). This is a very unusual record, possibly an error (Brb).

246 L. corculus Say. SI: St. George, Oct (Ds) ; LI: Hempstead (O1s) ; Rockaway Beach, div. (Ds, Ols); May-Sep. 
248 I. oppositus Say. SI, Oct (Ds) ; LI: Cold Sp. Harb., from mulberry (Brb); J1.

249 L. fulvicornis Westw, Buffalo, Oct (EPV); White Plains, J1 (Bno) ; LI: Rockaway Beach, Oct, Schott (Brb). Usually on magnolia.

\section{ARCHIMERUS Burmeister}

271 A. alternatus Say. West Pt., Je-J1 (Brb, Ds) ; Nyack, Je-J1 (Ds, Brb) ; Ft. Montgomery, May (Brb); White Plains, J1-Sep (Bno); Mosholu, May-J1, Bno (Heid); Van Cort. Pk., Sep (Brb-AM); SI, Ap, Jl, Sep (Ds) ; LI: Forest Hills, Sep-Oct (Parsh, O1s) ; Brooklyn, May, Nic (Ols); Cypress Hills, May, Zab (Brb-AM); Rockaway Beach, Je, Schott (Bno).

\section{Euthochtha Mayr}

276 E. galeator Fab. Hastings, J1, Joutel (Brb); Karner, Je (EPV-NYS) ; Pine I., Je, Ds (Brb); Big I., Sep (Brb-AM); White Plains, J1-Aug (Bno); Ft. Montgomery, Oct (Schott); Mosholu, Sep, Bno (Heid); SI, Je-Sep (Ds); LI: Aqueduct (Ds); Forest Hills (Ols) ; J1-Sep.

Chariesterus Laporte

283 C. antennator Fab. Van Cort. Pk., J1 (Brb-AM) ; SI, Je (Ds) ; LI: Rockaway Beach (Schott); Brooklyn, Eng (Sf-BM); Jan, Oct. Not common, found on dogbane (Brb).

AnASA Amyot and Serville

301 A. tristis DeG. Souash Bug. All over the State, injurious to squash, melons, and other cucurbits (Brb). Honeoye Falls, Je (Ds) ; Buffalo, Je (EPV); Colden, Mar (EPV); Karner, Oct (EPV-NYS); Alfred, Aug, HCCoon (Lint-NYS); Coeymans, SmEB (Lint-NYS); White Plains, Je-Sep (Bno); Yonkers, Sep (Huss); White L., Sep (Brb-AM); West Pt., Oct (Ds) ; SI, Aug-Oct (Ds) ; LI: Flatbush, J1-Sep, Zab (Brb-AM) ; Maspeth, Bay Shore, Aqueduct, Yaphank (Ols) ; Queens Village (Ols); Lynbrook, Nic (Ols); Je-Oct.

304 A. repetita Heid. Inwood, Oct (Ds) ; NYC, Oct, Schott, Wat (O1s, Brb) ; LI: Maspeth, Aug-Oct (Ols). Wild cucumber.

307 A. armigera Say. LI: Cold Sp. Harb. (Brb); Rockaway Beach (div.) ; J1, Oct.

\section{SUBFAMILY CORISCINAE}

\section{(Alydinae)}

\section{PROTENOR Haglund}

312 P. belfragei Haglund. Saranac L., Sep (Ds); L. Placid, Aug-Sep, Eng (Sf, EPV-BM, NYS) ; Wilmington, Aug (EPV) ; Cranberry L., J1-Sep (Dke); Buffalo, Nov (EPV); Lancaster, Aug (EPV-CU); McLean, Sep (Ruckes); White Plains, May-Oct (Bno); SI, Aug (Ds); LI: Aqueduct, Brooklyn (Ds); Jamaica, Aug, Eng (Sf-BM); Aug.

\section{Megalotomus Fieber}

320 M. 5-spinosus Say. Keene Val., Sep, Not (Brb) ; Chilson L., J1, Wat (Brb) ; Hastings, J1, Eng (Sf, Ds-BM) ; Rochester, Aug (EPV); Niagara 
Falls, Aug, Kilman (EPV); Salamanca, J1 (EPV); Ithaca, J1-Aug (EPV-CU) ; Binghamton, J1 (Brb-AM); Karner, Aug (EPV-NYS) ; Callicoon, J1 (Ds) ; Huguenot, Aug, Zab (AM) ; Ramapo, Je, Sep, Bt (Ds, Brb-AM) ; Ft. Montgomery, Sep-J1 (Schott, Ds); Bryn Mawr Pk., J1 (Brb-AM) ; White Plains, J1-Sep (Bno); Mosholu, Je-Aug, Bno (Heid); SI, J1-Sep, Ols (Ds); LI: Flatbush, Zab (Brb); Queens (Schott); Selden (Ds); Yaphank, Eng (Ds, Sf); Calverton (Ds); Amagansett, Smithtown (Ds); Forest Hills (Ols); Wyandanch, Oakdale, Schott (Ols, Bno); J1-Oct.

\section{CoRIscus Schrank}

\section{(Alydus Fabricius)}

323 C. eurinus Say. Distributed throughout the State (Brb). Essex Co, Je (EPV-NYS) ; N. Elba; Oct, Lg (Ds) ; Wilmington, J1 (Ds) ; Cascade L., Aug (Brb) ; Cranberry L., J1 (Dke); Hastings, J1, Joutel (Ds); Buffalo, Je-J1 (EPV); Hamburg, Sep, Conesus L., Aug (EPV); Callicoon, J1 (Ds) ; Catskills, J1, Eng (Sf-BM) ; Grand View, Nyack, Je (Ds); White Plains, J1-Sep (Bno); Mosholu, Mar-Oct, Bno (Heid) ; Scarsdale, J1-Sep (Bno); Ft. Montgomery, J1 (Ds); Bangall, Sep, Eng (Sf-BM) ; Kings Bridge, Sep (Brb) ; Bryn Mawr, Sep (Brb-AM) ; Van Cort. Pk., Sep (Ds) ; SI, Je-Sep (Ds) ; LI: Pinelawn, Bellport (Ds) ; Yaphank (Ols, Ds) ; Rockaway Beach, Kissena Pk., Flushing, Hempstead (Ols); Long Beach (Brb-AM); Wading R. (Schott); Je-Sep.

324 C. conspersus Montandon. Cranberry L. (Dke) ; Long L. (Ds) ; Cascade L., Indian L., Aug (Brb); Keene Val., Not (Brb); Buffalo, Hamburg, J1-Sep (EPV); Ithaca, J1, Shull (Huss); L. George, Aug, Zab (Brb); White Plains, Je--Sep (Bno). Mostly northern part of the State.

327 C. pilosulus H-S. Wilmington, J1 (Ds) ; Ithaca, J1, Shull (Huss) ; Karner, Aug, Kingston, Aug (EPV) ; White Plains, J1-Oct (Bno) ; Grand View, Nyack, Je (Brb); Ft. Montgomery, J1 (Ds); Mosholu, Je-J1, Bno (Heid); Scarsdale, J1-Sep (Bno); Kings Bridge, Sep (Brb); SI, Je-Sep (Ds) ; LI: Brooklyn, Aqueduct, Half Way Hollow Hills, Pinelawn, Yaphank (Ds); Amagansett, Eng (Ds); Bellport, Nic (Ds) ; Central Pk., Eng (Sf-BM) ; Rockaway Beach, Eng (Sf-BM) ; Flushing, Wyandanch, Wading R., Maspeth, Hempstead, Bay Shore, Pinelawn (Ols) ; Forest Pk., Bno (Heid) ; Oakdale, Schott (Bno) ; Je-Sep.

\section{TOLLIUS Stål}

328 T. curtulus Stål. Kings Bridge, Sep (Brb). Rare.

\section{STACHYOCNEMUS Stål}

330 S. apicalis Dall. Uncommon (Brb). White Plains, Sep (Bno) ; LI: Aqueduct (div.); Woodhaven (Brb-AM); Ap, Sep.

\section{Ceraleptus Costa}

332 C. americanus Stål. Rare (Brb). LI: Rockaway Beach, Oct, Schott (O1s). 


\section{SUBFAMILY CORIZINAE}

\section{HARMOSTES Burmeister}

336 H. reflexulus Say. Gowanda, Aug, Ithaca, J1-Aug (EPV); Carmel, Aug (Brb-AM) ; Poughkeepsie, Sep (EPV-NYS) ; Ulster Co, Aug, Nic (Ols); Pine I., Je (Brb); Saugerties, Aug (Brb); Ramapo, May, Bno (Ds) ; Ft. Montgomery, J1, Bno (Ds); White Plains, J1-Oct (Bno) ; Scarsdale, Aug (Bno); Mosholu, Aug-Sep (Bno); SI, J1-Oct (Ds); LI: Yaphank (Brb-AM) ; Orient (Brb-AM) ; Aqueduct (Ds, Brb) ; Massapequa, Manorville (Ds) ; Cold Sp. Harb. (Ds, Brb) ; Wading R. (Brb, Ds) ; Pinelawn, Calverton, Eng (Sf-BM); Bay Shore (Ols); Huntington, Schott (Ols); Little Neck, Feb, Wyandanch, Oct, Schott (Bno); May-Oct.

\section{CORIzUs Fallén}

345 C. crassicornis L. Keene Val, Aug, Not (Brb) ; Cranberry L., J1-Aug (Dke); L. Placid, Sep (EPV); Axton, Je (M\&H) ; Wilmington, J1, Saranac L., Sep (Ds) ; Cascade L., Indian L., Aug (Brb) ; Hamburg, May, J1, Boston, Elma, Aug, Lancaster, Gowanda, Aug (EPV); Batavia, Aug (Kngt) ; Wyoming Co, Sep (Kngt) ; Rock City, Je (Ds) ; Otto, J1 (Hambleton-CU) ; Ithaca, J1-Aug, Freeville, Aug (HambletonCU) ; Phoenicia, Aug (EPV-NYS); Debruce, Sep (Ds).

348 C. lateralis Say. All over the State (Bks). Cranberry L., J1 (Dke); Buffalo, Je, Gowanda, Aug, Hamburg, May-Je (EPV); Batavia, Je (Kngt); Ithaca, J1 (Hambleton-CU) ; Coy Glen, Je (Ds) ; Albany, Je (NYS) ; Mosholu, J1-Aug, Bno (Heid); White Plains, J1-Sep (Bno) ; Scarsdale, Aug (Bno); Kings Bridge, Sep (Brb); Van Cort. Pk., Sep (Bno) ; SI, Aug-Oct (Ds) ; LI: Winfield (Ols); Little Neck (Schott); Orient (Brb-AM) ; Flatbush, Eng (Sf-BM); Long Beach, Pinelawn, Eng (Sf-BM); Maspeth, Yaphank, Hempstead, Canarsie, Bay Shore (O1s) ; Aqueduct, Central Pk., Massapequa (Ds) ; May-Nov.

349 C. bohemanii Sign. Axton, Je (M\&H) ; L. Placid, Sep (EPV) ; Keene Val., Je, Not (Brb) ; Cascade L., Aug (Brb) ; Hamburg, Colden, J1, Buffalo, May-Oct, Gowanda, Aug (EPV); Genesee Co, Wyoming Co, Je (Ds); Ithaca, Je-Sep (Hambleton-CU) ; Ellis, Slaterville, Mud Cr., Tompkins $\mathrm{Co}$, Je (Hambleton-CU); L. George; Aug, Zab (Brb-AM); Phoenicia, Aug (EPV-NYS); Oliverea, J1, Shoe. (Ols) ; Saugerties, Aug, West Pt., J1 (Brb) ; White Plains, Aug-Sep (Bno).

355 C. hirtus Bno. LI: Yaphank, Sep, Eng, Fire I., J1 (Bno). Found hibernating under Hudsonia (Brb).

\section{FAMILY ARADIDAE ${ }^{13}$}

\section{ARADUS Fabricius}

360 A. aequalis Say. Niagara Falls, May (EPV); Ithaca, Ap, Alex (Parsh); McLean, May (Parsh); W. Hebron, Je, Lg (Heid).

379 A. crenatus Say. Keene Val., Sep, Eng (Parsh); Ithaca, May, West Pt., Je, Ds, Catskills (Parsh).

361 A. quadrilineatus Say. Cranberry L., J1 (Drk); Cobble Hill, Aug (EPV) ; Mt. Whiteface, J1, Ds (Parsh); Fulton Co, Alex (Morr); Niagara

${ }^{13}$ The arrangement of species in this family is that of Parshley's Essay. 
Falls, Ap (EPV) ; Buffalo, Oct, EPV (Parsh) ; Colden, May, Hamburg, Je, EPV (Parsh) ; Lancaster, EPV (Bergroth); Ithaca, Ap, Plunkett (Parsh) ; L. George, J1, Zab (Parsh); Schenectady, Aug (Parsh); West Pt., Je, Ds (Parsh) ; Southfields, May, Schott (Parsh); Sloatsburg, Nov (Parsh); Ft. Montgomery, May (Parsh); White Plains, Ap, Bno (Parsh); SI, Ap-May, Ds (Parsh); LI: Rockaway Beach, Brb (Parsh); Wyandanch (Schott); L. Ronkonkoma (Schott); Yaphank, Nic (Parsh); Promised Land, Eng (Bno); Ap-Dec.

365 A. ornatus Say. Cranberry L., J1 (Drk).

362 A. robustus Uhl. Occurs throughout the State (Brb). Cranberry L., J1 (Drk); Wilmington, J1 (Ds) ; top of Mt. Whiteface, J1, Ds (Parsh); Orleans Co, Je, Ds (Parsh); Gowanda, Aug, Colden, May, EPV (Parsh); E. Concord, May, EPV (Bergroth); Ithaca, Ap, Plunkett (Parsh) ; Batavia, May (Kngt) ; Debruce, J1, Ds (Parsh) ; Sloatsburg, Nov, Schott (Ols); White Plains, Je, Dec, Bno, Ols (Parsh, Huss), from sifting; Ft. Montgomery, Je, Ds (Heid) ; LI: Rockaway Beach (Ds) ; Flatbush, Zab (Brb) ; Wyandanch, Cold Sp. Harb., Sf, Schott (Parsh); Mar-Je.

364 A. duzeei Bergr. Rochester, Wendt (Bergr); Ithaca (Bergr).

392 A. proboscideus W1k. (hubbardi Heid.). Cranberry L., J1, Drk, Saranac L., Sep (Parsh).

A. basalis Parsh. Saranac L., Sep, Ds (Parsh).

371 A. similis Say. Top of Mt. Whiteface, J1 (Ds) ; Cranberry L., Drk (Parsh); Lake Bluff, Je (EPV); Lancaster, EPV (Bergr); Ithaca (Parsh); Chauncey, Mar, Not (Parsh); White Plains, Mar-Ap, Bno (Parsh); Van Cort. Pk., Ang (Parsh); SI: Ap, Ds (Parsh); LI: Yaphank, May, Ols (Parsh); Rockaway Beach, May (Brb); Huntington, Ap, Schott (O1s); Ap-May. Under spruce and hemlock bark (Drk), and in shelf fungus on birch.

375 A. acutus Say. LI: Huntington, Wyandanch, Schott (Bno).

377 A. shermani Heid. LI: Yaphank, Je, Eng (Bno).

A. approximatus Parsh. LI: Wyandanch, Je, Schott (Parsh).

384 A. uniformis Heid. West Pt., Je, Sf (Parsh) ; LI: Flatbush, Zab (Parsh); Rockaway, Brb, Ds, Fire I., Bno, Wyandanch, Sf (Parsh) ; Huntington, Ronkonkoma (Schott) ; May-J1.

382 A. tuberculifer Kby. Cranberry L., J1, Drk (Parsh); Ithaca, Je (Uh1) ; LI: Rockaway Beach, Je, Ds (Parsh).

383 A. funestus Bergr. Buffalo, Feb, hibernating (EPV).

386 A. lugubris Fall. Cranberry L., J1 (Drk); Rochester Jct., Je, Leon (Parsh) ; Buffalo, Je, Hamburg, Aug, Colden, J1, EPV (Bergr) ; Ithaca, May (Parsh); Nyack, Zab (Parsh); White Plains, J1 (Bno); LI: Bay Shore, Ols, Flatbush, Schott (Parsh); Je, Sep.

387 A. abbas Bergr. All over the State (Brb) ; Axton, Je (M\&H) ; Cranberry L., Je-J1 (Drk); Saranac L. (Parsh); Colden, J1, EPV (Bergr); Hamburg, Je (EPV); Buffalo, May, MCV (EPV); Ithaca, J1, Plunkett (Parsh); Huguenot, J1 (Brb-AM); Saugerties, J1, Brb (Parsh) ; White Plains, Aug, Bno (Parsh); LI: Bay Shore, Ols (Parsh); Fire I., Bno, Wading R., Schott, Maspeth, Ols (Parsh); Rockaway Beach, Eng (Bno); Ap-Sep.

- A. uniannulatus Parsh. LI: Pinelawn, Je, Ds (Parsh). 
389 A. falleni Stål. White Plains; Feb (Bno), sifting; SI (Ds); LI: Rockaway Beach, Yaphank, Bno (Parsh).

391 A. cinnamomeus Panz. SI, Je (Ds) ; LI: Bay Shore, Ols, Wyandanch, Schott, Ds, Yaphank, Ols, Bno, Central Pk., Pinelawn, Ols (Parsh); Cold Sp. Harb., Aug (Bno); Amagansett (Ds); Huntington, Wyandanch, Wading R., L. Ronkonkoma, Schott (Bno); Ap-Sep. Frequents pine (Brb).

395 A. niger Stål. Ausable R., Aug (EPV) ; Cranberry L., J1 (Drk) ; Buffalo, Sep (EPV); Hamburg, Sep, Ithaca, Ap, EPV (Parsh); L. George, Aug, $Z a b$ (Parsh). Bark of dead pines (Brb).

\section{NeURoctenus Mayr}

408 N. simplex Uhl. Ithaca, May (Morr); White Plains, Aug (Bno); NYC, Ap (Brb) ; SI, Ap-May (Ds) ; LI: Hempstead Plains (Eng) ; Massapequa, Shoe, Yaphank (Ds); Wyandanch, Schott (Bno); May-Sep.

\section{ANEURUS Curtis}

414 A. inconstans Uhl. Cascade L., Aug (Brb); Keene Val., Je, Not (Brb) ; Cranberry L., Je-J1 (Drk); Niagara Falls, Ap (EPV); Buffalo, Feb-Ap, Hamburg, May, Sep, Colden, May, Gowanda, Aug (EPV); E. Jewett, Aug (Ds); White Plains (Bno); Rye L., Sep (Bno); Grand View, Nyack, Je (Brb); Tuckahoe, Nov, Schott (Parsh); LI: Rosedale, Schott (Parsh); Rockaway Beach (Ds); Yaphank (Brb) ; May-Nov.

415 A. simplex Uhl. Cranberry L., J1 (Drk); Van Cort. Pk., Mar (Brb) ; LI: Queens Village, Wyandanch, Schott (Parsh); Central Pk., Eng, Jamaica, Huntington, Schott (Parsh); Northport, Schott (Ols); Cypress Hills, Zab (Brb-AM) ; Feb-Aug. Common under bark (Brb).

417 A. fiskei Heid. West Pt., Aug, Robn (BM); White Plains, Jan-Ap, SepNov (Bno); Rye L., Sep (Bno); SI, Je (Ds); LI: Wyandanch, Schott (Ols); Yaphank (Brb); Queens, Sf (Bno); Cold Sp. Harb., Butler (Huss); May-Sep.

\section{FAMILY NEIDIDAE or BERYTIDAE SUbFAmily NEIDINAE Van Duzee}

NEIDES Latreille

419 N. muticus Say. Cascade L., Indian L., Aug (Brb) ; Keene Val., Sep, Not (Brb) ; L. Placid, Sep, Eng (Bno); Cranberry L., J1-Aug (Drk); Colden, J1, Lancaster, Aug (EPV-CU); Batavia, Je-Aug (Kngt); Portageville, May-Aug (Kngt) ; Rock City, Je (Ds) ; Ithaca, J1, Oct, Bks (EPV, Kngt-CU) ; McLean, Ap (Morr) ; Taughannock Falls, J1, Summers (EPV-CU): Phoenicia, Aug (EPV-NYS); L. George, J1-Sep, Zab (AM) ; West Pt., Je (Ds) ; Pine I., Je-Oct (Brb) ; Nyack, Je (Brb, Ds) ; Ft. Montgomery, Sep, Schott (Ols); White Plains. Jl-Sep (Bno); SI, Aug (Ds); LI: Cold Sp. Harb., Aug (Brb). 


\section{SUBFAMILY METACANTHINAE}

\section{JALYSUS Stål}

420 J. multispinus Ashm. LI: Bayville (Brb-AM).

421 J. spinosus Say. Lancaster, Aug (EPV); Ithaca, Aug (CU); Debruce, Sep (Ds); West Pt., Je-J1 (Brb, Ds) ; Ramapo, May (Ds); Nyack, May (Schott); White Plains, Ap-Sep, Scarsdale, Aug-Sep, Rye L., Sep (Bno) ; SI, Sep (Ds) ; LI: Yaphank (Bno, Brb) ; Cold Sp. Harb. (Brb) ; Bay Shore, Maspeth, Queens Village, Wyandanch (Ols) ; Flatbush, Zab (Brb-AM) ; Melville, Wading R., Schott. (Bno); May-Oct.

\section{FAMILY LYGAEIDAE}

\section{SUBFAMILY LYGAEINAE}

\section{ONCOPELTUS Stål}

432 O. fasciatus Dall. NYC, Geisell (O1s); SI, Je-Oct (Ds); LI: North Beach, Schott (Ols); Coram (Ds); Cold Sp. Harb. (Brb); Glendale, Kch, Bellport, Nic, Floral Pk. (Sf-BM); Aug-Sep.

LyGAEUS Fabricius

434 L. turcicus Fab. Lancaster, Palmer,Jr (EPV); LI: Flatbush, Dec, Eng (Bno).

437 L. kalmii Stål var. angustomarginatus Parsh. Throughout the State, on milkweed (Brb). Cranberry L., J1-Aug (Drk); Axton, Je (M\&H); top of Mt. Whiteface, J1, Wilmington, J1 (Ds); Hastings, J1, Joutel (Ds) ; Lancaster, Aug (EPV-CU) ; Buffalo, Je (EPV-CU); Hamburg, Ap (EPV) ; Batavia, Je-Aug (Kngt) ; Aurora, J1 (Brb-AM) ; Ithaca, Aug, Oct (EPV, Morr, Huss) ; Callicoon, J1 (Ds) ; Fallsburg (Huss); Saugerties, J1 (Brb) ; Pine I., Je (Ds); White Plains, J1 (Bno); Rye Pond, Je (Brb-AM) ; SI, Je-Aug (Ds) ; LI: Flatbush, Zab (BrbAM); Maspeth, Bay Shore (Ols); Rockaway Beach, Shoe (Ols) ; Aqueduct, Ds (Brb) ; Massapequa, Ds (Brb) ; Calverton (Ds) ; Orient, Lath (Ds); Wading R., Cold Sp. Harb. (Brb); Je-Oct.

452 L. bicrucis Say. SI: Watchogue, May (Ds) ; LI: Rockaway Beach, May-Je, Eng (Ds, Sf-BM), May, Nic, Shoe; Half Way Hollow Hills, J1 (Ds).

$460 \frac{1}{2}$ L. tripunctatus Dall. LI: Yaphank, J1, Bno (Brb).

\section{ORTHOLOMUS Stål}

O. scolopax Say (longiceps Stål). Throughout the State, on grasses (Brb). Indian L., Cascade L., Aug (Brb); Keene Val., Aug, Not (Brb); Cranberry L., J1-Aug (Drk); Buffalo, J1, Sep, Hamburg, J1-Sep (EPV); Aurora, J1 (Brb-AM) ; L. George, Aug, Zab (Brb-AM); Karner, Aug, J1, Wat, Yg (Brb-NYS); Kingston, Aug (EPV-NYS); Saugerties, Aug, Pine I., Sep (Brb); White Plains, Je-Sep (Bno); SI, Sep (Ds); LI: Winfield, Maspeth, Bay Shore, Yaphank (Ols); Brooklyn (Ds) ; Orient, Schott, Aqueduct (Brb); J1-Oct. 


\section{Nysius Dallas}

466 N. californicus Stål. Cascade L., Aug (Brb).

468 N. thymi Wolff. Cascade L., Aug (Brb); Keene Val., Aug, Not (Brb); L. Placid, Sep (EPV, Ds); Buffalo, May-Aug (EPV); L. George, Aug, $Z a b$ (Brb-AM); E. Jewett, Aug (Ds); LI: Wyandanch, Je, Schott (Bno).

470 N. ericae Schill. False Chinch Bug. All over the State, on grasses, etc. (Brb). Axton, Je (M\&H); Speculator, Aug (NYS); L. Placid, Aug-Sep (EPV); Cranberry L., J1-Aug (Drk); Hamburg, Aug (EPV); White Plains, Mar-Oct (Bno); LI: Sea Cliff, Bks (EPV); Cold Sp. Harb., Butler (Brb, Huss); Orient (Brb-AM); Yaphank, Eng (Ols, Ds, Bno); Huntington, Schott (Brb, Ds); Wyandanch, Schott (Brb, Ols); Bay Shore, Maspeth, Hempstead (Ols); Aqueduct, Ds (Brb); Ap-Oct.

\section{BELONOCHILUS Uhler}

473 B. numenius Say. Buffalo, May, Colden, Aug (EPV).

\section{SUBFAMILY CYMINAE}

\section{ISCHNORHYNCHUS Fieber - see hithat}

476 477

483

C. luridus Stål. Cranberry L., J1-Dec (Drk); Cascade, Aug (Brb) ; Buffalo, J1 (EPV); Pine I., Je (Brb); White Plains, May-Aug (Bno); Stony Pt., Je (Ds); LI: Babylon, Aug (Bno); Yaphank, May, Ols (Brb) ; Aqueduct, Fire I. (Ds) ; May-Oct.

C. robustus Brb. Carmel (type locality), Mt. Kisco, J1, Bno (Brb); LI: Cold Sp. Harb., J1.

484 C. angustatus Stål. Throughout the State (Brb). Cascade L., Aug (Brb) ; Cranberry L., Je-Aug (Drk); Adirondacks (EPV); L. Placid, Sep (EPV); Newport, May, $Y g$ (NYS); Lancaster, Aug (EPV-CU); Buffalo, May-Oct (EPV) ; Batavia, Je-J1, Portageville, May, Aug, Cattaraugus Co, Je (Kngt) ; Karner, May, Yg (NYS); Saugerties, Aug, Pine I., Je (Brb); Tuxedo, May (Brb); White Plains, May-Je, Scarsdale, May, Rye Beach, J1 (Bno) ; SI, Mar, Aug (Ds) ; LI: Cold Sp. Harb. (Brb); Flushing (Ols, Ds); Massapequa (Ds); Yaphank (Ols, Ds) ; Wading R. (Ds); Maspeth, Forest Hills (Ols); May-Aug

14 Members of this genus occur on grasses and sedges. 
485 C. discors Horv. Throughout the State (Brb). Axton, Je (M\&H); L. Placid, Sep, Adirondacks, Aug-Sep (EPV); Long L., Cold R. (Horv) ; Cranberry L., Je-Sep (Drk, Brb) ; Cascade L., Aug (Brb) ; Keene Val., Je, Not (Brb) ; Newport, May, Yg (NYS) ; Buffalo, J1 (EPV) ; Hamburg, May, J1, Colden, J1, Gowanda, Aug (EPV); Batavia, J1 (Kngt); L. George, Aug, Zab (Brb); White Plains, MayAug (Bno); Mosholu, Nov, sifting (Brb); LI: Yaphank, Je (Brb) ; Bay Shore, J1, Wyandanch, Aug (Ols) ; Je-Aug.

487 C. breviceps Stål. LI: Cold Sp. Harb., Aug (Bno).

\section{SUBFAMILY BLISSINAE}

\section{ISCHNODEMUS Fieber}

489 I. falicus Say. Oneida L., Drk (Brb) ; Buffalo, Je (EPV) ; Cayuga L., Oct, Kngt (Bno) ; Pine I., Je (Brb); White Plains, May-Je, Oct (Bno); Kensico, May (Bno); SI, Oct-Nov, sifting on edge of salt meadow (Brb); LI: Brooklyn, Rockaway Beach (Ols); New Lots (Brb-AM); Flatbush, Mar, Schott (Bno); May.

\section{BLIssus Burmeister}

496 B. leucopterus Say. Chinch Bug. Throughout the State, on wild grasses (Brb). Cascade L., Indian L., Aug (Brb); Wallface Mt., Je, Not (Brb); Hammond (Lint); Morristown (Lint); Oakfield, Je, Not (Brb) ; Buffalo, Ap, J1-Aug, Hamburg, May-Je (EPV); Pike, Mar (NYS) ; Batavia, May-Je, Portageville, May (Kngt); Lancaster (EPV) ; Gowanda, Aug (EPV); Norton's Landing, SmHH (CU) ; Freeville, Aug (CU) ; Peruville (Felt); Watervliet (Lint) ; Debruce, Sep (Ds) ; Carmel, Aug (Brb-AM); White Plains, May-Sep (Bno); NYC, Oct (Huss) ; LI: Maspeth (Ols) ; Flushing, Dow (Ols) ; Winfield (Ols); Rockaway Beach, May-Sep, Ds (Ols); Long Beach, Huntington (Schott, Bno); Flatbush, Zab (Brb-AM); Cold Sp. Harb. (Brb); Brighton Beach (Brb); Wading R., Ds (Brb); Ap-Nov.

- B. leucopterus var. arenarius Brb. SI, Sep, Ds (Brb) ; LI: Coney I., Zab, Rockaway Beach (Brb); Wading R. (Ds); May-Sep.

\section{SUBFAMILY GEOCORINAE}

\section{GEOCoRIs Fallén}

499 G. bullatus Say. Speculator, J1, $Y g$ (NYS) ; Cranberry L., J1 (Drk) ; Cascade L., Indian L., Aug (Brb); Buffalo, Je, Sep (EPV); E. Aurora, Aug (EPV); Batavia, Sep (Kngt); West Pt., J1 (Brb) ; White Plains, May-Sep (Bno); LI: Little Neck, Schott (Brb); Aqueduct, Ds (Brb); North Beach, Flushing (O1s); Massapequa, Wading R. (Ds); Amagansett (Ds); May-Nov.

499c G. bullatus var. discopterus Ståi. White Plains, May, Aug-Sep (Bno); SI (Ds); LI: Amagansett, Sep. Ds (Brb).

502 G. uliginosus Say. Cascade L., Aug (Brb) ; Cranberry L., J1 (Drk) ; L. Placid, Aug (EPV-NYS); Colden, J1, Buffalo, Je, Ithaca, Sep (EPV-CU); White Plains, Sep (Bno); LI: Cold Sp. Harb., Aug, Aqueduct, Oct (Brb) ; Flatbush, Sep, Zab (Brb-AM) ; Huntington, Schott (Brb); Maspeth, Sea Cliff, Rockaway (Ols); Yaphank (Ols); Feb-Dec. 
G. uliginosus var. lateralis Fieb. Cascade L., Indian L., Aug (Brb) ; Cranberry L., Aug (Drk).

G. uliginosus var. speculator Montd. Boston, Hamburg, Aug (EPV).

G. uliginosus var. limbatus Stål. Buffalo, Sep, Hamburg, J1-Aug, Lawtons, Colden, Aug (EPV).

\section{HYPOGEOCORIS Montandon}

(Isthmocoris McAtee)

507 H. piceus Say. Carmel, Aug, White Plains, Ap-Oct (Bno); Tuxedo, May (Brb); Rye Beach, W. Nyack Aug (Bno); LI: Flushing, Aug, Canarsie, Ap (Ols) ; Wyandanch, Schott (Brb, Bno); Huntington, Aug (Bno); Cypress Hills, Zab (Brb-AM); Yaphank, Eng (Bno); Ap-Dec.

\section{SUBFAMILY PACHYGRONTHINAE}

\section{PhLEgyas Stål}

513 P. abbreviatus Uhl. Throughout the State (Brb). Debruce, Aug, Mutchler (Brb-AM) ; Cascade L., Indian L., Aug (Brb) ; Keene Val., J1, Not (Brb) ; Wilmington, $\mathrm{Jl}$ (Ds) ; Axton, Je (M\&H) ; Buffalo, Je-Sep (CU); Hamburg, May-Je, Lancaster, Aug, Gowanda, Aug (EPV); Batavia, Je, Cattaraugus Co., Je (Kngt); Ithaca, Je-Sep (CU) ; L. George, Aug, Zab (Brb-AM); W. Hebron, Aug, Lg (Ds); E. Jewett, Aug (Ds); Poughkeepsie, Je (NYS) ; Saugerties, Aug, Pine I., Je (Brb); White Plains, J1 (Bno); Tuxedo, May (Brb); Ramapo, May (Ds); Marlborough, J1, Sf (BM); SI, Je (Ds); LI: Bellport, Nic (Sf-BM); Cold Sp. Harb. (Brb) ; Bay Shore, Yaphank (Ols) ; Pinelawn, Wading R. (Ds); May-Aug.

\section{Oedancala Amyoto and Serville}

515 O. dorsalis Say. Throughout the State (Brb). Cranberry L., J1 (Drk); Buffalo, May-Aug, Lancaster, J1, Hamburg, May, Conesus L., Aug (EPV); Karner, Je (NYS) ; Saugerties, Aug, Brb, Ft. Montgomery, J1, Schott (Brb); White Plains, Mar, Je-J1, Dec, sifting, Armonk; Je (Bno); Charlotte, Je (Brb-AM); LI: Fishers I. (Brb-AM); Wyandanch (Ols); Bay Shore (Ols); Aqueduct, Shoe (Ols); Yaphank (Ols, Ds); Wading R. (Brb); Cold Sp. Harb., Butler (Huss).

\section{SUBFAMILY OXYCARENINAE}

\section{CROPHIUS Stål}

519 C. disconotus Say. Cranberry L., Aug (Drk); Indian L., Aug (Brb); Colden, Aug (EPV); Tompkins Co, Feb, sifting (Ruckes); Phoenicia, Aug (EPV); Pine I., Sep (Brb); White Plains, Sep-Oct (BrbBno); Nyack, J1, Zab (Brb); LI: Huntington, Schott (Brb); Wyandanch, Schott (Brb, Bno); Yaphank, Eng (Bno); Fire I. (Ds); Feb-Nov. 


\title{
SUBFAMILY RHYPAROCHROMINAE
}

\author{
(My'odochinae)
}

Myodocha Latreille

526 M. setripes Oliv. Cranberry L., Jl (Drk); Buffalo, May-Je, Albany, May (EPV-NYS) ; Ft. Montgomery, JI (Ds); White Plains, Dec, sifting (Bno); Nyack, J1, Zab (Brb-AM); Scarsdale, Aug (Bno); Van Cort. Pk. (Sf-BM) ; SI, May-Nov (Ds) ; LI: Astoria, Zab, Cold Sp. Harb. (Brb) ; Flushing, Forest Hill (Ols) ; Rockaway Beach, Dow, Schott (Brb, Ols); Queens, Flatbush, Wyandanch, Schott (Bno); May-Sep.

\section{HeRAEUS Stål}

528 H. plebejus Stål. Lancaster, Sep, Elma, Je, Hamburg, Ap, Aug (EPV) ; Batavia, Aug, Portageville, Aug (Kngt); White Plains, Ap-Oct (Bno); SI, Feb, May-Je, Dec (Ds), taken sifting in Feb (Brb) ; LI: Cold Sp. Harb. (Brb); Wyandanch (div.); Flatbush, Zab (Brb-AM); Aqueduct, Shoe (Ds); Flushing, North Beach (Ds); Mar-Oct.

\section{SPHAEROBIUS Uhler}

531 S. insignis Uhl. Indian L., Aug (Brb).

\section{LIGYROCORIS Stå1}

537 L. sylvestris L. (contractus Say). Cascade L., Indian L., Aug (Brb) ; Cranberry L. (Drk); Keene Val., Aug, Not (Brb); Phoenicia, J1 (EPV-NYS); top of Mt. Whiteface, J1, Saranac L., Sep (Ds); Long L. (Ds) ; L. Placid, Aug (EPV); Speculator, Aug, Yg (NYS) ; Buffalo, Je-Sep (EPV); Salamanca, J1 (EPV); Ithaca, Aug (EPVCU) ; L. George, J1-Aug, Zab (Brb-AM); E. Jewett, Aug (Ds) ; Oliverea, Sep, Beq (Ols); White Plains, Je-Aug (Bno); Stamford, J1 (EPV); LI: Rockaway, Wyandanch, Oct, Schott (Bno).

538 L. diffusus Uhl. Throughout the State (Brb). Cranberry L., Je-Aug (Drk) ; Cascade L., Aug (Brb) ; Keene Val., Aug, Not (Brb) ; L. Tear, J1, Not (Brb); L. Placid, Aug-Sep (EPV); Niagara Falls, J1, Lancaster, Aug, Buffalo, Je, Elma, Je, Hamburg, Aug-Sep, Conesus L., Aug, Salamanca, J1-Sep (EPV) ; Batavia, J1-Sep (Kngt) ; Oakfield, Je, Not (Brb) ; Canisteo, Je, Not (Brb) ; L. George, J1-Aug, $Z a b$ (Brb-AM); West Pt., J1 (Brb); Carmel, Aug (Brb-AM); Yorktown Hgts., Sep (Ols); SI, Je-Sep (Ds); LI: Cold Sp. Harb., Butler (Huss); Amagansett, Sf (Bno); Bellport, Nic (Sf-BM); Maspeth, Schott (Ols, Brb); Flushing (Ols, Ds); Huntington, Schott (O1s) ; Bay Shore, Rockaway Beach (Ols) ; Aqueduct, Central Pk., Brooklyn, Yaphank, Montauk, Southold, Orient (Ds); Winfield (Ols) ; Ap-Sep.

- L. depictus Brb. Ithaca, Aug (Brb); White Plains, Je, Bno (Brb).

\section{PERIGenes Distant}

543 P. constrictus Say. Cranberry L., Aug (Drk); Indian L., Aug (Brb) ; Lewiston, J1, Wat (Brb) ; Phoenicia, Aug (EPV-NYS); Buffalo, Sep, Hamburg, Aug-Sep, Albion, Aug, Clarence, Sep (EPV) ; Batavia, J1-Sep (Kngt); White Plains, Je-Aug (Bno); Van Cort. Pk., Sep (Bno); SI, Je (Ds) ; LI: Southold (Ds); Flatbush, Rockaway, Schott (Bno); Rockaway, Winfield (Ols); Je-Oct. 


\section{ZERIDONEUS Barber}

545 Z. costalis VanD. Cascade L., Aug (Brb) ; Indian L., Aug (Brb) ; Batavia, Aug (Kngt) ; L. George, Aug, Zab (Brb); Ft. Montgomery, J1 (Ds); White Plains, J1-Oct (Bno); LI: Huntington, Schott (Ols).

\section{ORThAEA Dallas}

554 O. basalis Dall. Indian L., Aug (Brb) ; L. Placid, Aug, Buffalo, May-Aug, Lancaster, Aug (EPV); Hamburg, Aug (EPV); Scarsdale, Sep (Bno); White Plains, Je-Oct (Bno); Pine I., Sep (Brb); Mosholu, J1, Sf (Brb); NYC, Ap (Brb) ; SI, May (Ols); LI: Cold Sp. Harb. (Brb) ; Mineola, Wyandanch, Melville, Rockaway, Huntington, Schott, Flushing, Jamaica (Ols); Mar-Sep.

\section{PTOCHIOMERA Say}

557 P. nodosa Say. LI: Aqueduct, Cold Sp. Harb. (Brb) ; J1-Oct.

570 P. ferruginea Stål (Carpilis). Indian L., Aug (Brb); LI: Yaphank, Sep (Bno).

558 P. clavigera Uhl. NY (Uhl); LI: Aqueduct, Oct (Brb).

KOLENETRUS Barber

K. plenus Distant (Rhyparochromus). Indian L., Aug (Brb).

CNEmodus Herrich-Schaeffer

566 C. mavortius Say. Lancaster, Oct (EPV); NYC (Brb) ; SI, May (Ds) ; LI: Bellport, Nic (Sf-BM); Yaphank, Wading R. (Ds); Rockaway Beach, Brookhaven, Schott (Ols, Bno); Je-Oct.

\section{Pseudocnemodus Barber}

569 P. canadensis Prov. Phoenicia, Aug, Niagara Falls, Aug (EPV); LI: Flatbush, Zab (Brb-AM) ; Fire I. (Bno); Yaphank, Eng, Wyandanch, Nov, S. Huntington (Bno); J1-Sep.

\section{OZOPHORA Uhler}

571 O. picturata Uhl. White Plains, Nov, sifting (Bno) ; LI: Cold Sp. Harb., (Brb) ; Yaphank, Eng (Brb, Bno) ; Bellport, Nic (Sf-BM); Wyandanch, Rockaway, Schott (div.) ; Springfield (Ols) ; Ap-Aug.

\section{ANTILLOCORIS Kirkaldy}

578 A. pilosulus Stål. Buffalo, Ap, Gowanda, May, White Plains, Ap (EPV) ; Greenwood L., May, Not (Brb) ; SI, Nov (Ds); LI: Wading R., Schott, North Beach (Ols) ; Yaphank, Eng (Bno); Mar-Sep. In some cases probably confused with next species (Brb).

579 A. pallidus Uhl. Should occur all over the State; usually taken in sifting (Brb). Indian L., Aug (Brb); Cranberry L., Aug (Drk); Pine I., Je (Brb) ; Scarsdale, May (Bno); Tuxedo, May (Brb); Mosholu, Nov (Brb); White Plains, Armonk, Ap-Sep (Bno); SI, Feb (Ds); LI: Cold Sp. Harb. (Brb) ; Flushing, Aqueduct (Ols); Wyandanch, 4 sifting (Ds); Ap-Aug. 


\section{PLinthisus Fieber}

584 P. compactus Uhl. (americanus VanD.). Indian L., Aug (Brb).

\section{PERITRECHUS Fieber}

585 P. fraternus Uhl. Buffalo, Dec, hibernating, $M C V$ (EPV); SI, Je, Sep, Nov (Ds); LI: Cold Sp. Harb. (Brb); Maspeth, Rockaway Beach (Ols); Long Beach, Schott (Ols, Bno); Promised Land, Greenport (Ds) ; Jan-Aug.

586 P. paludemaris Brb. SI, May (Brb); LI: Rosedale, Flatbush, Schott (Brb); Cypress Hills (Brb-AM); Bellport, Nic (Sf); Rockaway Beach (Bno); Feb-May. A salt-marsh species (Brb).

\section{STYGNOCORIS Douglas and Scott}

588 S. rusticus Fall. Indian L., Aug (Brb) ; Cranberry L., Aug (Drk).

S. pedestris Fall. Cranberry L., Aug (Drk); Indian L., Aug (Brb); Elka Pk., Catskills, Aug, Drk (Brb).

\section{TRAPEZONOTUS Fieber}

589 T. arenarius L. Indian L., Aug (Brb) ; top of Mt. Whiteface, J1, Ds (Brb).

\section{SPHRAgISTICUS Stål}

592 S. nebulosus Fall. Buffalo, Sep (EPV); Batavia, J1-Sep (Kngt); LI: Brooklyn (Ds); Mineola, Schott (Bno); Maspeth (Ols); Wyandanch, Schott (Brb); J1-Aug.

\section{MALEZONOTUS Barber}

- M. fuscosus Brb. LI: Fire I. Beach, Bno, Huntington, Schott (Brb); J1.

\section{APHANUS Laporte}

594 A. umbrosus Distant. Top of Mt. Whiteface, J1, Ds (Brb) ; Colden (Horv).

\section{EMBLETHIS Fieber}

597 E. vicarius Horv. White Plains, Sep, Nov (Bno) ; LI: Aqueduct (Brb) ; Woodhaven, Springfield, Schott (Brb); Oct-Nov.

\section{DRYMUS Fieber}

598 D. unus Say. Cascade L., Indian L., Aug (Brb); N. Evans, Oct (EPV); S. Buffalo, Fischer (EPV); Lancaster, Sep (EPV); Gowanda, Aug (EPV); Ithaca, Oct (Kngt); W. Hebron, Lg (Brb); Ft. Montgomery, Sep, Schott (Brb); White Plains, Mar, Oct, Dec, sifting (Bno, Huss); Mosholu, Nov, sifting (Brb); LI: Sea Cliff, Bks (Brb) ; Yaphank (Brb, Bno) ; Flushing (Ols); Flatbush, Zab (Brb); Bellport, Nic (Sf-BM); Little Neck, Nov, Schott (Bno).

599 D. crassus VanD. White Plains, Dec, sifting (Bno); W. Hebron, Sep, Lg, Inwood, NYC, Sep (Ds). 


\section{EREMOCORIS Fieber}

600 E. ferus Say. Cranberry L., J1, Sep (Drk) ; Cascade L., Indian L., Aug (Brb); Wallface Mt., J1, Keene Val., Je, Not (Brb) ; top of Mt. Whiteface, JI (Ds) ; Colden, J1 (EPV) ; E. Aurora, Wild (EPV) ; Jamestown, Aug (EPV); Canisteo, Je, Not (Brb); W. Hebron, Sep, Lg (Ds); Ft. Montgomery, Aug, Schott (Brb); Suffern (Brb) ; White Plains, Ap, Sep-Oct, Dec, Carmel, Aug (Bno) ; SI, Ap, J1 (Ds) ; LI: Maspeth, Rockaway Beach (Ols); Brooklyn, Shoe (Ols); Long Beach, Shoe (Ds) ; Wyandanch, Wading R. (Ds); Flatbush, Cypress Hills, Zab (Brb-AM) ; Huntington, Schott (Bno).

\section{XESTOCORIS Van Duzee}

604 X. nitens VanD. Indian L., Aug (Brb); Colden, Aug (EPV); Buffalo, Mar, Fischer, Hamburg, Ap (EPV); Ithaca, Bks (Brb); Tuxedo, May, beneath clumps of dead grass (Brb). Gerhard has collected this species in burrows of field mice near Chicago (Huss).

\section{CRYPHULA Stål}

608 C. parallelogramma Stål. White Plains, Aug, Oct, Bno (Brb) ; SI, Feb, sifting (Ds, Brb) ; LI: Flushing, Forest Pk. (Ols) ; Little Neck, Rockaway Beach, Dec, Schott (Bno) ; Yaphank (Ds) ; Mar-May.

\section{Scolopostethus Fieber}

610 S. thomsoni Reuter. Indian L., Aug (Brb); Buffalo, May-Aug, Hamburg, Je, Chautauqua, May, Gowanda, Aug, Salamanca, J1 (EPV); White Plains, Je, Bno (EPV) ; LI: Yaphank (Brb) ; Wyandanch, Sf (Bno) ; May. This and the next two species are usually taken sifting dead leaves (Brb).

611 S. atlanticus Horv. N. Elba, Lg (Brb) ; Lancaster, Aug (EPV) ; E. Jewett, Ap (Ds); White Plains, May-J1 (Bno); Orangeburg, Ap, Not (Brb); SI, Aug, Ds (Brb) ; LI: Flushing, Aqueduct (Ols); Wyandanch, Ds (Brb) ; Mar-May.

612 S. diffidens Horv. Indian L., Aug, Mosholu, May (Brb) ; SI, Feb, sifting (Brb) ; LI: Wyandanch, Schott (Ols, Bno); Jamaica, Nic (Bno); Feb.

\section{FAMILY PYRRHOCORIDAE}

\section{EURYOPHTHALMUS Laporte}

617 E. succinctus L. Hague (Brb-AM); LI: Baldwin, Christian Hook (Ds); Rockaway Beach (Schott); May-Oct.

\section{FAMILY PIESMIDAE}

Compiled by Carl J. Drake

Piesma Le Peletier and Serville

636 P. cinerea (Say). Cranberry L., J1 (Drk); Syracuse, J1-Sep (Drk); Buffalo and vic., Je-Aug (EPV); Ithaca; Pine I., Je (Bno); White Plains, Mar-Oct (Bno); NYC (Bno); LI: Cold Sp. Harb., Aug (Brb). Common on its preferred food plant, lamb's-quarters (Chenopodium album L. ) ; hibernating individuals found under bark of sycamore and birch, and among fallen leaves. 


\title{
FAMILY TINGITIDAE Costa ${ }^{15}$
}

\author{
Compiled by Carl J. Drake
}

AcAlypta Westwood

$637 \frac{1}{4}$ A. lillianis Bno. Adirondacks, Indian L., Aug (Brb) ; Corinth; Pine I., Je (Brb); White Plains, May-J1 (Bno); LI: Sea Cliff. Food plant, moss; collected by sifting moss and sphagnum.

\section{CORYTHuCha Stål}

638 C. ciliata Say. Canastota, Mar, Faure; Syracuse, May-Je, Sep, Dec (Drk) ; Buffalo (EPV) ; Lancaster, Aug, Colden, J1 (EPV), Hamburg, May, Aug (EPV) ; Enfield Falls, J1, MacG; Ithaca, Je-J1, Sep, Oct, Summers (Morr); Poughkeepsie, Jl, Yg (NYS); White Plains, Feb-Oct (Ols\&Bno); LI: North Beach, Sep (Plm); Flushing, Sep (Plunk); Wyandanch L., Aug (Ols). Very abundant on sycamore or buttonwood (Platanus occidentalis L.) ; hibernates beneath bark of sycamore and among fallen leaves on the ground.

639 C. arcuata (Say). Fulton Co, Alex; Syracuse, Sep-Oct (Drk) ; Buffalo and vic., J1, Sep (EPV) ; Lime L., Aug (EPV); Machias (EPV); Ithaca, J1, Dec; Poughkeepsie, May; Scarsdale, Aug (Bno) ; White Plains, May, Oct (Bno); LI: Cold Sp. Harb., J1 (Brb). Breeds on various species of oaks, and occasionally on chestnut, very rarely on maple and apple. Hibernates beneath leaves and in other secluded places on the ground.

C. arcuata var. mali Gib. Common, and perhaps more abundant than typical form; same distribution and food plants.

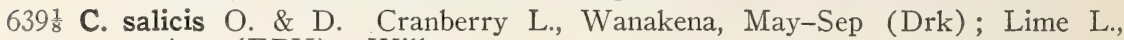
Aug (EPV). Willow.

- C. ulmi O. \& D. Nassau, Oct.

640 C. pergandei Heid. Wanakena, Cranberry L., May-Sep (Drk); Nassau, Oct; Syracuse, May-Je, Sep (Drk); Jamestown, J1; Ithaca, Je-J1, Summers; Karner, May, Oct; Voorheesville, all stages very abundant on hoary alder, Leon (Drk) ; Saugerties, J1 (Brb); White Plains, May-Je (Bno) ; Van Cort. Pk., Aug (EPV); SI, Feb, Je (Brb \& Ds) ; LI: Wading R., May (Brb); Bay Shore (Ols); Flushing, Sep (NYS). Common on alder (Alnus incana (L.) Moench and Alnus rugosa (Du Roi) Spreng.), and rarely on plum (Prunus americana Marsh.) ; sometimes taken at light; adults found on ground during winter by sifting fallen leaves.

$641 \frac{1}{4}$ C. pruni O. \& D. Colden, J1 (EPV); Salamanca, Aug (EPV). Wild black cherry (Prunus serotina Ehrh.).

645 C. marmorata (Uhl.) (decens VanD., in part). Syracuse, Aug-Sep (Drk) ; Rochester, J1; Lancaster, Sep (EPV); Buffalo, J1-Sep (EPV) ; Ithaca, J1-Aug, Summers; Albany, Je-Sep (Fitch, Felt); Karner, Je; Coeymans, Je-Aug; White Plains (as gossypii Bno.); West Pt., J1 (Brb) ; LI: Cold Sp. Harb., Aug (Brb) ; Bay Shore, Hempstead, J1 (O1s); Yaphank, J1, Flushing, Je (Ols). Occurs in large numbers on aster, goldenrod, Ambrosia trifida, and other Compositae.

${ }^{15}$ In addition to the species listed herein, Corythucha pallida O. \& D., C. coryli O. \& D., C. celtis O. \& D., C. bulbosa O. \& D., Gargaphia solani Heid., Gelchossa oblonga (Say), Teleonima nigrima Champ., Atheas insignis Heid., Leptoypha costata Parsh., Physatocheila major O. \& D., and Dictyonota tricornis var. americana Parsh., probably occur in New York State. 
649 C. juglandis (Fitch). Speculator, Je, $Y g$ (NYS); Delaware L., May, Cy; Mountain L., Ap 26, Cy; Hague, Aug, Leon (NYS); Wolcott, May (Leon); Syracuse, Sep-Oct (Drk); Niagara Falls, Aug, Lawtons, Sep; Colden, J1, Gowanda, Aug (EPV); Ithaca, Je-Oct; Albany, Je-Áug, Leon; Phoenicia, Aug (EPV); Northfield, Ap 23 (NYS); Millerton, May (Leon); Amity, Je (NYS); Thomson, May (Leon); Pine I., Dec (Brb); White Plains (Bno\&Ols); LI: Yaphank, Sep (Bno\&Eng). On walnut and basswood.

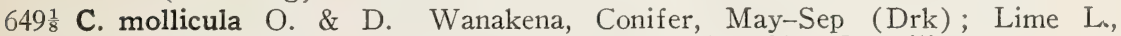
Jamestown, Aug (EPV); Karner, Aug (NYS). On willow.

- C. pallipes Parsh. (C. betulae Drk., C. cyrta Parsh., C. juglandis VanD. not Fitch; juglandis and pallipes were confused by Fitch in his original description, also by various authors since then). Chapel Pond, Je, Leon; Delaware Lodge, May, Cy; Big Moose, Aug (NYS); North Elba, Je, Leon; L. Placid, Aug (EPV); Cranberry L., May-Oct (Drk); Hamburg, Je (EPV). White birch, beech, ironwood, and occasionally mountain ash and hard and soft maple; adults hibernate among fallen leaves.

- C. heidemanni Drk. Syracuse, Sep-Oct (Drk); Ithaca, Aug, Summers; White Plains, Sep (Bno). Elm (Ulmus americana and U. fulva).

- C. bellula Gib. Wanakena, Barber Pt., Cranberry L., Je-Aug (Drk) ; Ithaca, May-Sep '(Wellhouse); Buffalo, Sep (EPV). Crataegus neofluvialis, C. pruinosa, C. punctata, etc., Almus incana, Ribes oxyacanthoides; adults found in crevices of bark and among fallen leaves during winter months.

- C. cydoniae Fitch (6391/4 C. crataegi O. \& D., and spp. of various other authors). Syracuse, Sep-Oct (Drk); Ithaca, Je-J1, Summers; Albany, Je-Oct; Nassau, May (NYS); LI: Yaphank (Bno). Hawthorn (Crataegus spp.), buttonbush (Cephalanthus occidentalis), Juneberry (Amelanchier); hibernates on ground among leaves.

- C. elegans Drk. Keene Val., Aug, Not; Adirondack Lodge, Je, Leon; Cranberry L., "The Plains," Conifer, May-Oct (Drk). Willow, poplar (Populus tremuloides Michx. and $P$. grandidentata Michx.); adults found among fallen leaves on ground during winter.

$649 \frac{1}{2}$ C. aesculi O. \& D. Rochester, Aug; Salamanca, Sep (EPV). Horsechestnut (Aesculus hippocastamum L.); also feeds on buckeye ( $A$. glabra Willd.).

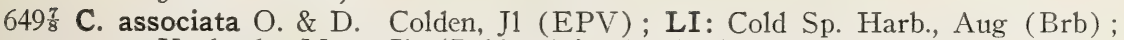
Yaphank, May, J1 (Brb); Riverhead, Ap 11 (NYS). Wild black cherry (Prunus serotina Ehrh.).

- C. cyrta Parsh. Big Moose, Aug (NYS); L. Placid, Aug (EPV); Chapel Pond, Je, Leon (NYS) ; N. Elba, J1, Leon (NYS); Delaware L., May, $C y$ (NYS); Voorheesville, J1, Leon (NYS).

\section{Galeatus Curtis}

651 G. peckhami (Ashm.). Cattaraugus Co (Kngt); Barber Pt., Cranberry L., J1-Sep (O\&D). Aster (Aster macrophyllus L.) and boneset (Eupatorium sp.). Locally distributed, but occurs in large numbers on knolls in semi-shady, dry situations.

\section{Stephanitis Stål}

652 S. pyrioides Scott. LI: Lynbrook (LCGriffith). Common laurel (Kalmia latifola) and Rhododendron sp. 


\section{GARGAPHIA Stål}

654 G. tiliae Walsh. Keene Val. (Not); Syracuse, Sep-Oct (Drk) ; Lancaster, Sep, Hamburg, J1, Gowanda, Aug, Buffalo and vic., J1 (EPV) ; Ithaca, J1, Summers (NYS) ; Trenton Falls, Je (NYS); White Plains, May (Bno).

657 G. angulata Heid. Karner, May (NYS); Albany, Aug; Montgomery, Aug (Heid). Ceanothus americanus and Solanum sp.

- G. amorphae Walsh. "NY" (EPV, 1917, as var. of G. tiliae).

\section{Gelchossa Kirkaldy}

- G. heidemanni O. \& D. LI: Wading R., May, Yaphank, Ap (Brb) ; Orient, May (NYS). Baptisia tinctoria.

\section{Physatocheila Fieber}

665 P. plexa Say. White Plains, Je (Bno); LI: Yaphank, Sep (Bno).

- P. plexa var. variegata Parsh. "NY" (EPV, 1917, as a distinct species, 6651/2) ; Northampton, Je, $Y g$ (NYS) ; Hamburg, Je, Gowanda, Aug (EPV); Salamanca, J1 (EPV); Suffern, May. Food plant unknown, but specimens have been collected on hickory.

$665 \frac{1}{4}$ P. brevirostris O. \& D. "NY" (EPV, 1917). Food plant unknown.

\section{CORYTHAICA Stål}

6581 C. bellula Bno. White Plains, Je-J1 (Bno\&O1s).

\section{LEPTOYPHA Stål}

666 L. mutica (Say). Keene Val., Je (Not); Hamburg, J1, Gowanda, Aug (EPV); Millerton, May, Cy (NYS). Fringe tree (Chionanthus virginica L.) and ash (Fraximus spp.). Sometimes, rarely, taken at light.

\section{LEPTOBYRSA Stål}

- L. rhododendri Horvath. Rhododendron LAcE Bug. Rochester (Felt); Ithaca, Je (Sling); West Pt., J1 (Brb) ; Port Jervis, Ap (Sling); Westchester Co, Sep (Bno); White Plains, Je-Sep (Bno); NYC (Bno); LI: Sea Cliff (Brb); Brooklyn, J1 (Griffith\&Sanford). Rhododendron and laurel (Kalmia latifola).

\section{MelanoRhopala Stål}

667 M. clavata (Stål). West Pt., J1 (Brb); Cold Sp. Harb., J1-Aug (Brb, Parsh); White Plains, Je-Aug (Bno). Swept from rank weeds and grasses; food plant unknown.

\section{Hesperotingis Parshley}

- H. antennata Parsh. SI: Huntington, J1 (Schott); LI: Fire I. (Bno): Food plant unknown. 
Tingitidae-EnicocePhalidae-P'hymatidae-Reduviddae 103

Alveotingis Osborn and Drake

$670 \frac{1}{2}$ A. grossocerata O. \& D. White Plains, Aug (Bno); LI: Smith's Pt. (Bno). Food plant unknown; Bueno captured a specimen on a thistle.

\section{FAMILY ENICOCEPHALIDAE}

\section{SYSTELLODERUS Blanchard}

684 S. biceps Say. Potter Swamp, Je, Fbs (Joh) ; Ithaca, J1-Aug (Joh) ; Interlaken, J1, Cy (Joh, MacG-CU).

\section{FAMILY PHYMATIDAE}

\section{Phymata Latreille}

685 P. vicina Handl. Pine I., Je (Brb); Ramapo, May (Ds); White Plains, Aug, Farley (Bno); Van Cort. Pk., J1 (Brb-AM); Bronxville, May (Woodr).

689 P. erosa L. Batavia, J1-Sep (Kngt); Ithaca, Barnard (CU) ; Coeymans, $S m E B$ (Lint); W. Hebron,. Aug (Bno); Ft. Montgomery, Aug (Schott) ; Scarsdale, Sep, W. Nyack, Aug (Bno) ; SI, J1-Oct (Ds) ; LI: Brooklyn, Pinelawn, Yaphank, Wading R., Aqueduct, Flushing, Greenport, Riverhead (Ds); North Beach, Schott (Bno); Mar-Sep. Subspecies are not indicated in this series (Brb).

689a P. erosa subsp. fasciata Gray. Keene Val., Aug, Not, L. George, Aug, Zab, Nyack, Aug, Zab (Brb-AM) ; LI: Flatbush, Zab (Brb-AM); Cold Sp. Harb. (Brb) ; Oyster Bay (Bno) ; J1-Aug.

689b P. erosa subsp. wolffii Stål. Keene Val., Aug, Not (Brb) ; Buffalo, J1-Oct, Hamburg, Aug (EPV); Ithaca, J1-Aug (EPV-CU); Albany, Coleman (Lint-NYS); Pine I., Sep (Brb); LI: Cold Sp. Harb., Aug (Brb).

\section{FAMILY REDUVIIDAE SUbFAMILy PLOIARIINAE 16}

(Emesinae)

\section{EMPICORIS Wolff}

\section{(Ploiariola Reuter)}

697 E. errabundus Say ( $P$. tuberculata Bks.). White Plains, Aug, Bno (McAtee \&Mall) ; LI: Cold Sp. Harb., Meiner, Sea Cliff, Bks (McAtee\&Mall) ; J1-Aug.

\section{EmesAya McAtee and Malloch \\ (Emesa auct. not Laporte)}

709 E. brevipennis Say (longipes DeG.). Aurora, J1 (Brb-AM); Ithaca, Aug (CU) ; Coeymans, SmEB (Lint) ; Nyack, Sep (Brb-AM) ; W. Nyack, J1 (Ols); White Plains, Aug-Sep (Bno); Scarsdale, Sep (Bno) ; LI: Cold Sp. Harb. (Brb); Yaphank, Wading R. (Ds); Pinelawn, Eng (Sf-BM) ; Central Pk., Schott (Bno); Aug-Oct.

${ }^{16}$ The arrangement of genera and species in this subfamily, and the nomenclature, are those of McAtee and Malloch's "Revision" in Proc. U. S. Nat. Mus., vol. 67, p. 1-153, 1925. 
Metapterus Costa

(Barce Stål)

714 M. uhleri Bks. Ithaca, J1-Aug, White Plains, Oct, Bno (McAtee\&Mall-CU) ; Tuxedo, May (Brb); LI: Sea Cliff, Bks, Central Pk., Eng (McAtee\&Mall-CU) ; Huntington (Schott) ; Ap.

- M. neglectus McAtee \& Mall. White Plains, Mar-Ap, Aug, Bno (McAtee\&Mall); SI, Mar, Bno (McAtee\&Mall).

713 M. annulipes Stål. Indian L., Aug, Brb (McAtee\&Mall); Ithaca, Alex (EPV) ; White Plains, Mar, Oct, Bno (McAtee\&Mall) ; SI (Ds) ; LI: Cold Sp. Harb., Parsh, Cypress Hills, Martin (McAtee\&Mall); Sea Cliff (Bks); Huntington (Schott); May-Aug.

715 M. fraternus Say. White Plains, Aug-Nov, Bno (McAtee\&Mall); LI: Cold Sp. Harb., Brb (McAtee\&Mall) ; Flatbush (Schott) ; Aqueduct (Brb) ; J1-Nov.

\section{SUBFAMILY SAICINAE}

\section{ONCEROTRACHELUS Stål}

717 O. acuminatus Say. White Plains, Sep (Bno, Brb) ; Orangeburg, Ap, Not (Brb).

\section{SUBFAMILY STENOPODINAE}

\section{PyGOLAMPIS Germar}

723 P. sericea Stål. Ithaca, Aug (E.PV-CU) ; LI: Wyandanch, Schott, Cedarhurst, Schott (Brb) ; Rockaway Beach, Ds (Brb).

724 P. pectoralis Say. LI: Yaphank (Brb) ; Huntington, Wyandanch, Little Neck, Schott (Ols, Brb) ; Rockaway Beach (div.); Long Beach, Eng (Sf-BM).

\section{STENOPODA Laporte}

727 S. cinerea Lap. (culciformis Fab.). White Plains, J1 (Bno); Bronx Pk., Wat (Brb) ; SI, Je-J1 (Ds) ; LI: Rockaway Beach (Brb) ; Huntington, Wading R., Melville, Wyandanch, Bay Shore, Schott (Ols, Bno); Coram, Yaphank (Ds); Wading R., Fire I. (Schott); Je-Nov.

\section{SUBFAMILY REDUVIINAE}

REDuvius Fabricius

733 R. personatus L. The Kissing Bug. Mt. Whiteface, J1 (Ds) ; Cranberry L., J1 (Drk, Brb) ; Olcott, Aug (Kngt); Buffalo, Je (EPV) Lewiston, Jl, Wat (Brb); Batavia, Je-JI (Kngt); Ithaca, Je-J1 (MacG-CU); Albany, J1, Lint (EPV); W. Hebron, Aug, Lg (Ds); Catskills, J1, Shoe (Ds); Nyack, Zab (Brb-AM); Katonah, Je (Brb-AM); White Plains, J1 (Bno); SI, May-J1 (Ds); LI: Bayside (Ds); Flatbush, Mar, Je (Schott).

\section{SUBFAMILY PIRATINAE}

\section{Melanolestes Stål}

745 M. picipes H-S. Ithaca, Je (EPV-CU) ; Nyack, Zab (Brb-AM) ; West Pt., Ap, Robn (Ds) ; White Plains, Ap, Oct (Bno) ; Van Cort. Pk., May, Wat (Brb); SI, Mar-Ap, Sep, Nov (Ds); LI: Cold Sp. Harb. (Brb); Cypress Hills, Zab (Brb); Pinelawn, Eng (Bno); Rockaway Beach (Ols, Schott); Huntington, Wyandanch (Schott); Ap-Sep. 
746 M. picipes var. abdominalis H-S. White Plains, Ap-May, Aug-Sep (Bno); Nyack, $Z a b$ (Brb-AM) ; Ramapo, Sep (Ds); SI, Mar-Ap, Nov, Dec (Ds); LI: Cold Sp. Harb. (Brb); Wyandanch, Schott, Queens (Ols); Rockaway Beach (Ds, Schott); Melville (Schott); May-Oct.

\section{SUBFAMILY ECTRICHODIINAE}

\section{RHIGINIA Stål}

751 R. cruciata Say. LI: Rockaway Beach, May, Oct, Ds, Schott (Brb).

\section{SUBFAMILY APIOMERINAE}

\section{APIOMERUS Laporte}

764 A. crassipes Fab. LI: Half Way Hollow Hills, Wading R. (Ds) ; Yaphank, Eng (Ds, Sf); Rockaway Beach (Brb); May-J1.

\section{SUBFAMILY HARPACTORINAE}

\section{ZELUS Fabricius}

771 Z. exsanguis Stål. Ithaca, May-Aug (EPV-CU) ; Buffalo, May-Je, Hamburg, Je-J1, Boston, Aug, Colden, J1 (EPV); Portage, May, Geneva (EPV); Genesee Co, Je (Ds) ; Batavia, May-J1 (Kngt) ; Portageville, May, Aug (Kngt); Potter Swamp, Je (Ds); Ithaca, May-Aug (Ds, EPV-CU) ; E. Jewett, Aug (Ds) ; West Pt., Je (Ds) ; Ft. Montgomery, May (Brb); White Plains, Mar, Dec, sifting (Bno); Scarsdale, May (Bno); SI, Je-J1 (Ds) ; LI: Yaphank; Wading R., Ols (Brb) ; Huntington, Yaphank (Schott); Bay Shore (Ols) ; Massapequa, Wyandanch (Ds) ; Southold, Amagansett, Orient, Shelter I. (Ds); Je-Sep.

774 Z. socius Uhl. Ithaca, Je-J1 (EPV); Highland Falls, Je, Schott (Ols) ; White Plains, J1 (Bno).

775 Z. audax Bks. Gowanda, Aug, Ithaca, J1 (EPV); L. George, Aug, Zab (Brb-AM); Tuxedo, May, Nad (Brb); LI: Sea Cliff (Bks).

\section{PSELLIOPUS Bergroth}

779 P. cinctus Fab. Nyack, May, Schott, Pine I., Sep (Brb) ; SI, Sep (Ds); LI: Wyandanch, J1, Pinelawn, Calverton, Massapequa, Sep, Yaphank, May (Ds); Central Pk., Aug (Ols); Hempstead, Ap (Eng-BM).

\section{Fitchia Stål}

789 F. aptera Stål. Hillburn, Dec (Schott); White Plains, Ap, Je (Bno) ; SI, Nov, sifting in salt meadow, (Brb); LI: Coney I., Nov (Schott); Long Beach, Mar, May-Je, Eng (Sf-BM); Yaphank, J1 (Ols, Ds); Flushing, J1, Wading R., May, Rockaway Beach, May (Ols).

\section{RocConota Stål}

791 R. annulicornis Stål. Van Cort. Pk., Wat (Brb) ; LI: Yaphank, Sep, Enc (Bno). 


\section{ARILUS Burmeister}

796 A. cristatus L. The Wheel Bug. Buffalo, Sep, Pohlman (EPV); SI, SepOct (Ds) ; LI: Rockaway Beach, Eng (Sf-BM); Wyandanch (Schott, Ds) ; Huntington (Schott).

\section{Acholla Stål}

797 A. multispinosa DeG. Clifton Sps., Aug (EPV-CU); Buffalo, J1-Oct, Lancaster, J1, Ithaca, Jl-Oct (EPV); Batavia, Ang-Sep (Kngt) ; Malcolm, May, Little (Lint-NYS) ; White Plains, Ap (Bno) ; Nyack, $Z a b$ (Brb-AM); SI, Aug (Ds); LI: Flatbush, Zab (Brb-AM); Wyandanch, Yaphank (Ds); Huntington, Schott (Ols); Flushing (O1s); Hempstead (Brb); Aug-Sep.

\section{SinEA Amyot and Serville}

800 S. diadema Fab. Cranberry L. (Drk); Keene Val., Aug, Not (Brb) ; L. Placid, Aug-Sep, Eng (EPV, Bno); Saranac L., Sep, Lg (Ds); Cascade L., Aug (Brb) ; Buffalo, J1-Sep (EPV-CU); Colden, Aug, Hamburg, Lancaster, Aug (EPV); Otto, Comst (Śummers-CU); Ithaca, J1-Aug (EPV-CU) ; L. George, Aug, Zab (Brb-AM); W' Hebron, Aug, Lg (Ds) ; E. Jewett, Aug, Ft. Montgomery, J1 (Ds) ; White Plains, Aug-Oct (Bno); Kings Bridge, Sep (Brb); SI, Jl-Aug (Ds) ; LI: Maspeth (Schott, Ols); Yaphank, Eng (Sf-BM); Cold Sp. Harb., Butler (Huss); Pinelawn, Bay Shore (Ols); Wading R. (Ols, Ds); Aqueduct, Half Way Hollow Hills, Flushing, Southold, Amagansett, Shelter I. (Ds); Wyandanch, Schott (Bno); May-Nov.

807 S. spinipes H-S. - Ithaca, Aug (EPV) ; LI: Maspeth (Schott) ; Shelter I., Plm (Brb); Yaphank (Brb, Ds); Southold, Amagansett (Ds); Aug-Sep.

\section{FAMILY HEBRIDAE 17}

\section{(Naeogeidae)}

\section{Compiled by J. R. DE LA TORRE-Bueno}

\section{HEBRUS Curtis}

810 H. burmeisteri L. \& S. Lancaster, J1 12, 1899 (EPV) ; White Plains, Ap-J1 $(B n o)$; SI, May (Ols) ; LI: Yaphank, Sep, Bno\&Eng (Bno).

\section{MERRAGATA White}

$813 \frac{1}{4}$ M. foveata Drk. White Plains, May 12, Aug 12, 16, 30 (Bno) ; SI, May 7, 16 (Bno); LI: Cold Sp. Harb., J1 29, in enormous numbers in and among duckweed (Bno\&Parsh); Yaphank, J1 2, Sep 25 (Bno).

$813 \frac{1}{2}$ M. brunnea Drk. White Plains, May-Aug (Bno).

${ }_{17}$ According to the footnote on page 272 in Van Duzee's catalog, this family may more properly follow the Veliidae. 


\section{FAMILy MESOVELIIDAE}

Compiled by J. R. DE LA TORRE-Bueno

Mesovelia Mulsant and Rey

814 M. bisignata Uhl. Cranberry L. (O\&D) ; Ithaca, J1-Aug (CU) ; Squaw I., Niagara R., Sep (EPV); Buffalo, Sep (EPV); Carmel, Aug (Bno); White Plains, common and abundant from late spring to fall (Bno); Kensico L., May (Bno) ; Van Cort. Pk., Aug-Sep (Bno) ; SI, Aug (EPV, Bno); LI: Flushing, J1 (Ols); Yaphank, J1-Sep (Bno, Ols).

\section{FAMILY NABIDAE}

Compiled by J. R. DE LA TORRe-Bueno

\section{SUBFAMILY PROSTEMIMINAE}

\section{PAGASA Stål}

816 P. fusca Stein. Cascade L., Aug (Brb) ; Cranberry L. (Drk) ; Hamburg, Sep (EPV) ; Lancaster, Jl, EPV (Harris); Stamford, Aug (EPV); White Plains, J1-Oct (Bno, Ols); NYC, Nov (Brb); LI: Cold Sp. Harb. (Brb); Rockaway Beach (Ols); Flatbush, Zab (Brb-AM).

NABIS Latreille

\section{(Reduviolus Kirby)}

818 N. subcoleoptratus Kby. Spread all over the State (Brb). Cascade, Aug (Brb) ; Cranberry (Drk) ; Axton, Je, Wilmington, J1 (Ds) ; N. Elba, Oct (Ds); Rochester, J1, Wendt (O1s) ; Colden, J1 (EPV-NYS); Buffalo, J1-Sep (EPV); Clarence, Sep (Harris); Batavia, Je-Aug (Kngt) ; Gowanda, Aug, Otto, J1 (EPV) ; Ithaca, J1-Aug (EPV-CU); E. Jewett, Aug (Ds); W. Hebron, Aug-Sep, $M \mathcal{F} H, L g$ (Ds, EPV$\mathrm{CU})$; Greene Co, Aug (Harris) ; Saugerties, Aug (Brb); Allaben, Aug, Pears (Ois); Callicoon, J1 (Ds); Debruce, Aug (Brb-AM); Nyack, $Z a b$ (Brb-AM) ; Ramapo, J1 (Ds) ; Pine I., Je (Sf-BM); White Plains, May-Aug (Bno); SI, Sep (Ds); LI, Eng (Sf-BM).

820 N. sordidus Reut. L. Placid, Sep, Eng (Bno); Oneida L., Aug, Drk (Harris); West Pt., J1, Mosholu, Nov, Suffern, Ap, Grand View, Nyack, Je (Brb); White Plains, Oct, sifting (Huss); SI, Aug, Ds (Heid) ; LI: Cold Sp. Harb. (Brb) ; Rockaway Beach, Schott (Brb) ; Hempstead (Eng); Central Pk., Eng (Bno).

823 N. annulatus Reut. N. Elba, Oct (Ds); Lancaster, Aug, Buffalo, Aug, EPV (Harris); Batavia, J1-Aug, Kngt, Ithaca, Aug (Harris); Saugerties, J1 (Brb) ; SI, Aug (Ds) ; LI: Cold Sp. Harb. (Brb); Amagansett, Sf (Bno); Queens, Sf (Bno); Flatbush, Aug-Sep, Zab (Brb-AM); Aug-Sep.

825 N. propinquus Reut. Cranberry L., J1, OsbornH (Harr); L. Placid, Sep, Wilmington Notch, Sep (EPV); Grand I., Sep (EPV); Buffalo, Ji (EPV).

826 N. limbatus Dahlb. Cranberry L., J1-Sep, OsbornH (Harr, Drk); Cascade L., Aug (Brb); L. Placid, Aug (EPV). 
$829^{\prime}$ N. ferus L. Throughout the State (Brb). L. Placid, Aug (EPV); Cranberry L., J1-Sep (Drk) ; Cascade L., Aug (Brb) ; Buffalo, May-Oct, Hamburg, May-J1 (EPV); Batavia, Je-Sep, Kngt (Harr) ; Colden, J1, Lancaster, May, Aug, Oct (EPV); Otto, J1 (MacG-CU); Ithaca, Mar, May, Oct, Coy Glen, May (Morr) ; McLean, Ap, Kngt (Morr) ; W. Hebron, Aug, Lg (Ds) ; Saugerties, Aug, Pine I., Sep (Brb) ; Ft. Montgomery, J1 (Ds) ; Crugers, Oct (Brb-AM) ; SI, Je-Nov (Ds); LI: Long Beach, Central Pk., Eng (Sf-BM); Yaphank, Eng (Ds, Sf-BM) ; Brooklyn, Mineola, Schott (Kngt, Bno) ; Cold Sp. Harb., Butler (Huss, Brb); Flatbush, Zab (Brb); Aqueduct (Brb, Ds); Ap-Oct.

830 N. roseipennis Reut. All over the State (Brb). Cranberry L., J1-Sep (Drk); Cascade L., Aug (Brb); L. Placid, Sep (EPV); Buffalo, May-Sep, Hamburg, Ap-J1, Gowanda, Aug (EPV) ; Lancaster, May-Je, Aug, Portage, May, EPV (Harr); Grand I., Sep, Concord, May (Harr); Ithaca, Je-Aug (Summers-CU); Saugerties, Aug (Brb); White Plains, Dec, sifting (Bno) ; Armonk, Je (Bno); Kings Bridge, Sep (Brb) ; SI, Je-J1, Ds (Heid) ; LI: Huntington, Nov, Schott (Brb); Flatbush, J1-Oct, Zab (Brb-AM); Cold Sp. Harb., J1-Aug (Huss, Brb, etc.) ; J1-Oct.

831 N. rufusculus Reut. Throughout the State (Brb). Cascade L., Aug (Brb); Cranberry L., Je-Sep (Drk); L. Placid, Aug-Sep (EPV); Madison Barracks, Grand I., Sep (Kngt) ; Rochester, Aug (Summers) ; Colden, J1-Aug, Buffalo, May-Sep, Hamburg, J1, Gowanda, Aug, Salamanca, J1 (EPV) ; Portage, May, Lancaster, Sep (Harr) ; Lime L., Aug (Brb) ; Pine I., Oct (Brb); Tuxedo, May (Brb); White Plains, Dec, sifting (Bno) ; LI: Flatbush, Zab (AM); Rosedale, Schott (Brb-AM).

832 N. inscriptus Kby. Axton, Je, $M \mathcal{E} H$ (EPV-CU) ; Buffalo, Aug, Hamburg, May, Lancaster, Aug (EPV).

\section{MetATROPIPHORUS Reuter}

834 M. belfragii Reut. Saugerties, J1 (Brb); LI: Yaphank, J1 (Ds). Rare.

Carthasis Champion

835 C. decoratus Uhl. White Plains, May (Bno); LI: Massapequa, Ap, Ols (Bno). Rare.

\section{FAMILy CIMICIDAE}

\section{Cimex Linnaeus}

836 C. lectularius L. The Bedbug. Generally distributed throughout the State (Brb).

837 C. pilosellus Horv. Lewis Co (Horv-US). Host, Vesperugo noctivagus.

\section{Oeciacus Stål}

838 O. vicarius Horv. LI: Cold Sp. Harb., J1-Aug, Butler (Huss), masses of eggs, hundreds of nymphs in first and second stages, and one or two adults, in a chimney swift's nest (Bno). 


\section{FAMILY ANTHOCORIDAE SUBFAMILY LYCTOCORINAE \\ LYCTOCORIS Hahn}

840 L. campestris Fab. Lancaster (EPV); Batavia, Aug, Kngt (Bno) ; Ithaca, Sep (Morr); LI: Wyandanch, Je, Schott (Bno).

843 L. stalii Reut. Hamburg, Aug (EPV); Lancaster, Jan, Palmer (EPV); Ithaca, J1 (EPV).

\section{LASIOCHILUS Reuter}

845 L. fusculus Reut. Hamburg, Oct (EPV).

\section{XYLOCORIS Dufour}

846 X. galactinus Fieb. L. Placid, Sep (EPV).

847 X. sordidus Reut. White Plains, Aug-Sep, Hartsdale, May (Bno); SI, May, Eng (Bno); LI: Wyandanch, Montauk, Ds (Ols); Jamaica, Nic (Bno-BM).

847 a X. sordidus var. binotatus Reut. Salamanca, J1 (EPV).

851 X. cursitans Fall. Buffalo, Oct, Hamburg, Je-Oct, Colden, May, Gowanda, Aug (EPV); SI, Ap (Ds).

\section{Asthenidea Reuter}

852 A. temnostethoides Reut. Cranberry L., Aug-Sep (Drk).

\section{SUBFAMILY ANTHOCORINAE}

\section{ANTHOCORIS Fallén}

860 A. borealis Dall. Cranberry L., J1 (Drk); Fulton Co, Alex (EPV); Lancaster, Stamford, Aug (EPV); Ft. Montgomery, J1, Schott (Brb); Armonk, W. Nyack, Aug (Bno).

861 A. musculus Say. Keene Val., Sep, Eng (Bno) ; Axton, Je, M\&H (EPVCU) ; L. Placid, Aug, Buffalo, Lime L., Aug (EPV); Otto, J1 (Uh1).

\section{TETRAPHLEPS Fieber}

T. americana Parsh. Top of Mt. Whiteface, J1, Ds (Brb).

T. osborni Drk. Cranberry L., J1-Sep (Drk).

T. uniformis Parsh. Cranberry L. (Drk).

\section{MACROTRACHELIELLA Champion}

M. nigra Parsh. SI, Aug, Ds (Brb).

\section{ORIUS Wolff}

(Triphleps Fieber)

$864^{\prime}$ O. insidiosus Say. Cascade L., Aug (Brb); Cranberry L. (Drk); Keene Val., Aug, Not (Brb) ; Axton, Je (M\&H) ; Mt. Whiteface, Aug, Buffalo, Je-Sep, Honeoye Falls, Rochester Jct., Lancaster, J1 (EPV); 
Batavia, Je-Sep (Kngt) ; Colden, J1 (EPV) ; Jamestown, Aug, Ithaca, Je (EPV); White Plains, May-Sep, Scarsdale, Aug, Rye, Aug (Bno); SI, Oct-Nov, Ds (Heid) ; LI: Sea Cliff, Je, Atkinson (EPV-CU) ; Little Neck, Wyandanch, Schott (Brb) ; Cold Sp. Harb. (Brb); Half

Way Hollow Hills (Ds); Amagansett (Sf-BM); Flatbush, Eng (Bno).

865 O. insidiosus var. tristicolor White. Aurora, J1 (Brb-AM); Ithaca, J1-Aug (EPV-CU).

\section{SUBFAMILY DUFOURIELLINAE}

DUFOURIELLUS Kirkaldy

871 D. ater Duf. Hamburg, Oct, Buffalo, J1 (EPV).

\section{FAMILY MIRIDAE}

Compiled by HARRY H. KNIGHT

\section{SUBFAMILY PHYLINAE}

\section{CAMPylomma Reuter}

1267 C. verbasci (Meyer). Batavia, Je-Aug (Kngt) ; Hilton, Je (Kngt) ; Buffalo, $\mathrm{Je}(E P V)$; E. Aurora, Aug $(E P V)$; Hamburg, Je-J1 $(E P V)$; Hamburg, Je-J1 (EPV); Nassau, Je (EPV); White Plains, J1, Bno (Kngt); LI: Huntington, J1, Schott (Kngt). Verbascum spp., potato, and occasionally apple; is sometimes attracted to colonies of aphids, where it feeds on honeydew.

\section{ChLAMYdatus Curtis}

1260 C. associatus (Uh1). Batavia, Je-Sep (Kngt); Buffalo, Je-Sep (EPV); Honeoye Falls, Je (Kngt); McLean, J1 (Kngt); White Plains, Sep, Bno (Kngt) ; Mosholu, Bno (Heid). Ragweed (Ambrosia spp.).

1259 C. suavis (Reut.). SI, J1, Ds (Kngt).

1258 C. pulicarius (Fall.). L. Placid, Aug (EPV); Cranberry L., Je-J1, Wanakena, J1-Aug, Drk (Kngt); Four Mile, Ji (Kngt).

\section{MICROPHYLELLUS Reuter}

M. elongatus Kngt. Batavia, Je-J1 (Kngt), type locality. Acer saccharum.

M. longirostris Kngt. Ithaca, J1 (Kngt), type locality; White Plains, J1, Bno (Kngt). Hazelnut (Corylus americana).

M. maculipennis Kngt. Batavia, Je $(K n g t)$; Conesus L., Je $(K n g t)$. Quercus alba.

1225 M. modestus Reut. Batavia, Je-J1 (Kngt) ; Hamburg, J1 (EPV) ; Gowanda, Je (EPV); Wyoming, Je $(K n g t)$; Conesus L., J1 (Kngt) ; Ithaca, Je (Kngt) ; Freeville, J1 (Kngt-CU) ; McLean, J1 (Kngt); Grand View, Je, Brb (Kngt). Elm (Ulmus) and Quercus alba; observed to be predacious on eggs of the elm leaf-beetle (Galerucella luteola) at Ithaca, Je, 1911.

M. nigricornis Kngt. Batavia, Je $(K n g t)$; Four Mile, J1 (Kngt) ; Ithaca, J1 (Kngt), type locality. Aster macrophyllus.

M. tsugae Kngt. Ithaca, J1 (Kngt); McLean Bogs, J1 (Kngt), type locality; Taughannock, J1 (Kngt). Hemlock (Tsuga canadensis). 


\section{Plagiognathus Fieber}

1237 P. albatus VanD. Batavia, J1 (Kngt); Hamburg, J1 (EPV), type locality ; Gowanda, Aug $(E P V)$; Salamanca, J1 $(E P V)$; Ithaca, Je (Kngt); McLean, J1 (Kngt) ; White Plains, J1, Bno (Kngt); LI: Gardiners I., J1, Schott (Kngt). Sycamore (Platanus occidentalis).

P. albatus var. vittiscutis Kngt. Ithaca, Je (Kngt), type locality. Butternut (Juglans cinerea).

P. albonotatus Kngt. Batavia, J1-Aug (Kngt); McLean, J1 (Kngt), type locality. Meadowsweet (Spiraea salicifolia).

P. albonotatus compar Kngt. Batavia, J1-Aug (Kngt), type locality; McLean, J1 (Kngt).

P. alboradialis Kngt. Mt. Whiteface, Aug (Kngt) ; Cranberry L., Wanakena, J1-Aug, Drk (Kngt); Batavia, Je (Kngt), type locality; Four Mile, J1 $(K n g t)$; Ithaca, J1 (Kngt).

1240 P. annulatus Uhl. Batavia, J1-Aug (Kngt); Four Mile, J1 (Kngt); Ithaca, $\mathrm{J1}$ (Kngt); LI: Kissena Pk., Je, Ols (Kngt); North Beach, J1, Ols (Kngt).

P. annulatus var. cuneatus Kngt. Batavia, J1 (Kngt); Ithaca, J1 (Kngt), type locality. Aster sp.

P. annulatus var. nigrofemoratus Kngt. Ithaca, J1 (Kngt), type locality.

1234 P. blatchleyi Reut. Batavia, Aug (Kngt); Hamburg, Je-J1 (EPV); Elma, $\mathrm{J1}(E P V)$; Gowanda, Aug (EPV); Ithaca, J1 (Kngt).

P. blatchleyi var. nubilus Kngt. Ithaca, J1 (Kngt), type locality.

P. brevirostris Kngt. Ithaca, J1 (Kngt), type locality.

P. caryae Kngt. Batavia, Je $(K n g t)$; Portageville, Je (Kngt) ; Ithaca, Je-J1 (Kngt), type locality. Hickory (Carya spp.).

P. chrysanthemi Wolff. Cranberry L., Wanakena, J1, Drk (Kngt); Ithaca, Je-J1 (Kngt) ; McLean, J1 (Kngt-CU). Chrysanthemum leucanthemum.

P. cornicola Kngt. Batavia, Je-J1 (Kngt), type locality; Ithaca, Je $(K n g t)$; White Plains, Je, Bno (Kngt). Cornus spp.

P. davisi Kngt. Pine I., Je, Ds (Kngt), type locality.

1230 P. delicatus Uhl. Batavia, Je (Kngt). Honey locust (Gleditsia triacanthos).

P. dispar Kngt. Batavia, Je-J1 (Kngt), type locality; Conesus L., J1 (Kngt); Hartsdale, J1, Bno (Kngt). Hickory (Carya sp.).

P. flavicornis Kngt. Batavia, Je-J1 (Kngt), type locality.

P. flavoscutellatus Kngt. Ithaca, Je, Buys (Kngt); Batavia, Aug (Kngt), type locality. Salix longifolia.

P. ilicis Kngt. Ringwood, J1 (Kngt), type locality. Ilex verticillata.

P. intrusus Kngt. Cranberry L., Je, Drk (Kngt); McLean, J1 (Kngt).

P. laricicola Kngt. Cranberry L., J1, Drk (Kngt); Ithaca, Je-J1 (Kngt), type locality; White Plains, J1, Bno (Kngt). Larix laricina.

P. nigronitens Kngt. Batavia, J1-Aug (Kngt), type locality; Wyoming Co, J1 (Kngt) ; Ithaca, J1 (Kngt); White Plains, Je-J1, Bno (Kngt).

1239 P. obscurus Uhl. Mt. Whiteface, Ang (Kngt) ; Cranberry L., Jl-Aug, Drk (Kngt); Cascade L., Aug, Brb (Kngt); Batavia, J1-Aug (Kngt); Ithaca, J1 (Kngt); McLean, J1 (Kngt). Occurs on herbaceous weeds.

P. obscurus var. albocuneatus Kngt. Batavia, J1-Aug (Kngt), type locality ; Portageville, Je (Kngt); Four Mile, J1 (Kngt); Ithaca, J1 (Kngt); McLean, J1, Bno (Kngt); White Plains, J1, Bno (Kngt). LI: Babylon, J1, Parsh (Kngt); Cold Sp. Harb., J1, Parsh, Aug, Bno (Kngt). 
1238 P. obscurus var. fraternus Uhl. Mt. Whiteface, Aug (Kngt) ; Cranberry L., Wanakena, J1-Aug, Drk (Kngt); Cascade L., Aug, Brb (Kngt); Batavia, Je-Sep (Kngt); Portageville, Aug (Kngt); Ithaca, J1-Aug (Kngt); McLean, J1 (Kngt); White Plains, Je, Bno (Kngt); LI: Cold Sp. Harb., J1, Parsh (Kngt).

1241 P. politus Uhl. Cranberry L., J1, Wanakena, Aug, Drk (Kngt) ; Batavia, Je-Oct (Kngt) ; Hamburg, J1 (EPV) ; Elma, Aug (EPV); Gowanda, Aug $(E P V)$; Four Mile, Jl (Kngt) ; Ithaca, Je-Oct (Kngt) ; McLean, J1 (Kngt); White Plains, J1, Bno (Kngt); SI, J1, Ds (Kngt); LI: Cold Sp. Harb., Aug, Brb (Kngt); Mamaroneck, Sep, Bno (Kngt). Ragweed (Ambrosia spp.).

P. politus var. flaveolus Kngt. Batavia, Aug-Oct (Kngt), type locality ; Ft. Montgomery, J1, Schott (Kngt); White Plains, Sep, Bno (Kngt) ; SI: Arrochar, Sep, Ds (Kngt); LI: Cold Sp. Harb., Aug, Brb (Kngt); Forest Hills, Sep, Ols (Kngt); Orient, Sep, Schott (Kngt). Ragweed (Ambrosia spp.).

P. politus var. pallidicornis Kngt. Mt. Whiteface, Aug (Kngt) ; Cranberry L., Wanakena, J1-Aug, Drk (Kngt); Batavia, Je-Aug (Kngt); Ithaca, Je-J1 (Kngt), type locality; McLean, J1 (Kngt); Hartsdale, Je, Bno (Kngt).

P. punctatipes Kngt. Batavia, J1 (Kngt); Ithaca, Je (Kngt), type locality. Collected on flowers of an ornamental Spiraea.

P. repetitus Kngt. Mt. Whiteface, Aug (Kngt), type locality; McLean Bogs, J1, Buys (Kngt); Conifer, J1, Drk (Kngt); Wanakena, J1-Aug, Drk (Kngt).

P. repletus Kngt. Batavia, Je (Kngt), type locality.

P. repletus var. apicatus Kngt. Conesus L., J1 (Kngt), type locality.

P. suffuscipennis Kngt. Rochester Jct., Je, Leon (Kngt), type locality ; White Plains, Je, Bno (Kngt). Spruce (Picea spp.).

\section{Psallus Fieber}

P. alnicenatus Kngt. Cranberry L., J1, Drk (Kngt); Conifer, J1, Osb (Kngt); Ithaca, J1 (Kngt); McLean Bogs, J1 (Kngt), type locality. Alder (Alnus incanus).

P. alnicola Douglas \& Scott. Ithaca, J1 (Kngt); McLean Bogs, J1 (Kngt). Alder (Alnus sp.).

1243 P. ancorifer Fieb. White Plains, J1, Bno (Kngt) ; LI: Cold Sp. Harb., J1, $B r b$ (Kngt).

P. drakei Kngt. Cranberry L., Je, Drk (Kngt), type locality, Je-J1, Htly (Kngt). Willow (Salix sp.).

P. morrisoni Kngt. Cranberry L., Aug, Drk (Kngt). LI: Cold Sp. Harb., Aug, Bno (Kngt). Collected on Myrica sp.

P. parshleyi Kngt. Cranberry L., J1, Htly (Kngt).

P. piceicola Kngt. Ithaca, J1, Bno (Kngt); White Plains, Je-J1, Bno (Kngt), type locality. LI: Cold Sp. Harb., J1, Parsh (Kngt). Spruce (Picea sp.).

P. strobicola Kngt. Cranberry L., J1, Drk (Kngt) ; Portageville, Je (Kngt) : Ithaca, Je-J1 (Kngt); Taughannock, J1 (Kngt); White Plains, J1, Bno (Kngt). Pinus strobus. 


\section{LEPIDOPSALLUS Knight}

L. minusculus Kngt. White Plains, J1, Bno (Kngt), type locality.

1088 L. rubidus Uhl. Batavia, J1-Aug $(K n g t)$; Honeoye Falls, Aug (Kngt); Ithaca, J1 (Kngt). Salix sp.

L. rubidus var. atricolor Kngt. Cranberry L., J1-Aug, Drk (Kngt), Aug, Htly (Kngt); Wanakena, Aug, Drk (Kngt); Batavia, J1-Aug (Kngt), type locality. Salix sp.

\section{CRIOCORIS Fieber}

1223 C. saliens (Reut.). Cranberry L., J1, Drk (Kngt); Batavia, Aug (Kngt); Buffalo, Je (EPV).

\section{MicRosynamMA Fieber}

M. bohemanni (Fall.). Honeoye Falls, Je, Leon (Kngt); Rochester Jct., Je, Leon (Kngt). Salix spp.

\section{RHINOCAPSUS Uhler}

1228 R. vanduzeii Uhl. Mt. Whiteface, Aug (Kngt); Ausable R., Aug (EPV); Cranberry L., J1-Aug, div. (Kngt); Wanakena, J1, Drk (Kngt); Fulton Co, Alex (EPV) ; Batavia, Je-Aug (Kngt); Buffalo, Lancaster, Colden, Je-J1 (EPV) ; Hamburg, J1 (EPV); Gowanda, Aug (EPV); Portageville, Je (Kngt). Raspberry (Rubus spp.).

\section{ATRACTOTOMUS Fieber}

A. magnicornis (Fall.). Ithaca, J1 (Kngt); White Plains, Je-J1, Bno (Kngt); Hartsdale, J1, Bno (Kngt). Pinus sylvestris and Picea.

A. magnicornis var. buenoi Kngt. White Plains, Je, Bno (Kngt), type locality. Hemlock (Tsuga canadensis).

\section{REUTEROSCOPUS Kirkaldy}

1213 R. ornatus (Reut.). Ithaca, J1 (Kngt); Buffalo, J1-Aug (EPV); White Plains, J1-Oct, Bno (Kngt); Mosholu (Heid) ; Yorktown Hgts., Sep, Ols (Kngt); LI: Cold Sp. Harb., J1, Bno (Kngt) ; Pinelawn, Sep, Ds (Kngt); Woodhaven, J1 (Kngt-Brooklyn Museum). Ragweed (Ambrosia spp.).

MACROTYLUS Fieber

1200 M. sexguttatus (Prov.). Cranberry L., J1-Aug, Drk (Kngt) ; Batavia, Je-J1 $(K n g t)$; Four Mile, J1 (Kngt). Aster undulatus, A. acuminatus.

\section{Lopus Hahn}

1207 L. decolor (Fall.). Cranberry L., Aug, Drk (Kngt); Elka Pk., Wanakena, Aug, Drk (Kngt); Fulton Co, Alex (EPV); Rochester Jct., J1, Leon (Kngt) ; Ithaca, J1, Bno (Kngt) ; McLean, J1 (Kngt); White Plains, J1, Bno (Kngt); Mosholu (Heid). LI: Huntington, J1, Schott (Kngt); Orient, J1, Ds (Kngt). Juncus dudleyi at McLean. 
ORECTODERUS Uhler

1101 O. obliquus Uhl. LI: Bay Shore, J1, Ols; Half Way Hollow Hills, J1, Ds (Kngt).

O. obliquus var. ferrugineous Kngt. LI: Bay Shore, J1, Ols (Kngt), type locality.

\section{TELEORHINUS Uhler}

T. tephrosicola Kngt. LI: Yaphank, J1, Ds (Kngt), type locality.

\section{SUBFAMILY DICYPHINAE}

\section{Dicyphus Uhler}

1108 D. agilis (Uhl.). L. Placid, Aug (EPV); Cranberry L., J1-Aug, Htly (Kngt); Wanakena, Jl-Aug, Drk (Kngt); Fulton Co, Alex (EPV); Batavia, Je-Aug (Kngt); Hamburg, Je-J1 (EPV); Lancaster, J1-Aug $(E P V)$; Gowanda, Aug $(E P V)$; Portageville, Je $(K n g t)$; Four Mile, J1 (Kngt) ; Conesus L., Je (Kngt); Ithaca, Je-J1 (Kngt) ; McLean, J1 (Kngt); White Plains, J1, Bno (Kngt) ; Hartsdale, J1, Bno (Kngt); Grand View, Je, Brb (Kngt). Rubus odoratus, and possibly other species.

D. discrepans Kngt. Cranberry L., Jl, Drk (Kngt), type locality (vestitus, Drk '22), Je, Htly (Kngt).

1109 D. famelicus (Uhl.). Axton, Je, MacG (EPV) ; Cranberry L., J1-Aug, Drk (Kngt); Batavia, Je-Aug (Kngt); Buffalo, May-Aug (EPV); Labrador L., May, Buys (Kngt) ; Colden, J1 (EPV) ; Hamburg, Je-Aug (EPV); Gowanda, Aug (EPV); Chautauqua, Je $(E P V)$; Otto, Jl, Comst (Kngt); Cattaraugus Co, Je (Kngt); Portageville, Je-Aug (Kngt); Honeoye Falls, Je (Kngt); Ft. Montgomery, Sep, Schott (Kngt); Bear Mt., Sep, Schott (Kngt); White Plains, Sep, Bno (Kngt); Grand View, Je, Brb (Kngt). Rubus odoratus.

\section{MACROLOPHUS Fieber}

1116 M. separatus (Uh1.). Cranberry L., Wanakena, J1, Drk (Kngt); Fulton Co, Alex (EPV); Niagara Falls, Aug (EPV); Hamburg, J1 (EPV).

\section{Hyaliodes Reuter}

1106 H. vitripennis (Say). Cranberry L., Aug, Htly (Kngt); Wanakena (Drk '22); Saranac L., Aug (EPV); Batavia, J1-Aug (Kngt); Niagara Falls, Aug (EPV); Buffalo, Sep (EPV); Colden, Jl (EPV); Elma, Sep (EPV); Boston, Aug (EPV); Gowanda, Aug (EPV); Salamanca, J1 (EPV); Ithaca, Jl, Kngt (EPV); White Plains, J1-Aug, Bno (Kngt) ; Mosholu (Heid); W. Nyack, Aug, Bno (Kngt) ; Bronxville, J1 (Wood) ; SI (Heid) ; LI: Yaphank, Jl, Ds (Kngt); Wading R., Aug, Ds (Kngt). Occurs on several plants, but is frequent on Vitis; predacious on plant lice.

1106a H. vitripennis var. discoidalis Reut. Batavia, J1-Aug (Kngt); Buffalo, Aug (EPV); Johnstown, Aug, Alex (EPV); Hamburg, Aug (EPV). LI: High Hill, Aug (Bno); W. Nyack, Je, Ols (Kngt). 


\section{SUBFAMILY BRYOCORINAE}

MONALOCORIS Dahlbom

1082 M. filicis (L.). L. Placid, Aug (EPV); Cranberry L., J1-Aug, Drk, Htly (Kngt); Wanakena, Aug, Drk (Kngt); Fulton Co, Alex (EPV); Mt. Whiteface, Aug (Kngt) ; Niagara Falls, Sep, $M C V$ (EPV); Buffalo, J1-Sep (EPV); Hamburg, J1 (EPV); Lancaster, Aug (EPV); McLean, J1 (Kngt) ; White Plains, J1, Bno (Kngt); Ft. Montgomery, Sep, Schott (Kngt). SI: (Heid) ; Tottenville, Aug, Ds (Kngt); LI: Cold Sp. Harb., J1, Brb (Kngt), Aug, Bno (Kngt); Yaphank, J1-Sep, Ds (Kngt).

PyCNODERES Guérin

1079 P. dilatatus Reut. W. Nyack, Aug, Ols (Kngt).

SixeONOTUS Reuter

1074 S. insignis Reut. NY (Reut); Fulton Co, Alex (EPV); Hamburg, Je $(E P V)$; Gowanda, Aug (EPV).

SYSINAS Distant

1083 S. linearis Distant. LI: Sea Cliff (Bks, Kngt).

\section{SUBFAMILY CYLAPINAE}

Cylapus Say

1090 C. tenuicornis Say. Niagara Falls, J1 (EPV); Gowanda, Aug (EPV); Ithaca, J1, Plunk (Kngt). SI: Richmond, Aug, Ds (Kngt); LI: Queens, J1, Ds (Kngt); Sea Cliff, Aug (Bks).

\section{Fulvius Stål}

1091 F. brunneus (Prov.). Catskill, Brb (EPV); Ithaca, Mar (Kngt-CU); Saugerties, Aug, Ds (Kngt); LI: Wyandanch, Aug, Schott (Kngt).

\section{SUBFAMILY CLIVINEMINAE}

\section{LARGIDEA Van Duzee}

1089:3 L. davisi Kngt. Bear Mt., Sep, Schott (Kngt). LI: Promised Land, Sep, Ds (Kngt); Yaphank, Sep, Ds (Kngt). Occurs on Pinus.

\section{SUBFAMILY DERAEOCORINAE -}

\section{EUSTICTUS Reuter}

1054 E. grossus (Uhl.). SI, Jl, Ds (Kngt); LI: Yaphank, Sep, Ds (Kngt).

E. necopinus Kngt. White Plains, Jl, Bno (Kngt), type locality. Occurs on aspen.

1050 E. venatorius VanD. Hamburg, Aug (EPV), type locality. LI: Central Pk., J1-Aug, Ds (Kngt). 


\section{EURYCHILOPTERELIA Reuter}

1056 E. luridula Reut. Holley, J1, Harel (Kngt); Batavia, J1 (Kngt); Buffalo, J1, EPV (Reut), type locality; White Plains, Je-J1, Bno (Kngt). Occurs on apple trees.

\section{DERAEOCORIS Kirschbaum}

\section{(Subgenus CAMPTOBROCHIS)}

1063 D. histrio Reut. Ithaca, Aug (Kngt-CU). LI: Cold Sp. Harb., Aug, Parsh (Kngt). Breeds on Polygonum.

1058 D. nebulosus Uhl. Axton, Je, MacG (EPV); L. Placid (EPV) ; Cranberry L., J1, Osb (Kngt) ; Fulton Co, Alex (EPV); Batavia, Je-Sep (Kngt); Niagara Falls, Je, EPV (Kngt); Buffalo, J1-Aug (EPV); Salamanca, J1 (EPV) ; Ithaca, Feb, under bark of log, Ap, under sycamore bark, J1, Oct (Kngt) ; McLean, J1 (Kngt); West Pt., Aug, Brb (Kngt); Schenectady, Aug, Heid (Kngt); L. Mahopac, Aug, Bno (Kngt); Oliverea, Sep, Ds (Kngt); White Plains, Je-J1, Bno (Kngt); Mosholu (Heid), Nov, by sifting, Brb (Kngt). SI, J1-Aug, Ols (Kngt), J1, Ds (Kngt). LI: Brooklyn, Oct, Ds (Kngt); Maspeth, Aug, Ols (Kngt) ; Rockaway Beach, May, Ds (Kngt), Aug, Ols (Kngt); Cold Sp. Harb., J1, Brb (Kngt); Port Washington, Sep, Ols (Kngt); Yaphank, Sep, Ds (Kngt). Occurs on white oaks; predacious on Phylloxera rileyi Rly.

D. nubilus Kngt. Wanakena, Aug, Drk (Kngt); Batavia, J1 (Kngt) ; Portageville, Je (Kngt); Taughannock, J1 (Kngt); Ithaca, Feb, under bark of log, May-J1 (Kngt), type locality; White Plains, Ap-Aug, Bno (Kngt); Saugerties, J1, Brb (Kngt). Breeds on Pinus resinosa.

D. poecilus McAtee. Ithaca, J1 (Kngt); McLean, J1, Sep, Sib (Kngt). Occurs on Almus rugosa; probably predacious.

\section{(Subgenus DERAEOCORIS)}

D. albigulus Kngt. Ithaca, Je (Kngt), type locality; Bear Mt., Je-J1, Schott (Kngt); Hartsdale, Jl, Bno (Kngt). Breeds on Pinus sylvestris.

D. alnicola Kngt. Batavia, Je-J1 (Kngt) ; Portageville, Je (Kngt) ; McLean, J1 (Kngt), type locality; White Plains, Je-J1, Bno (Kngt). Breeds on Alnus; probably predacious.

D. aphidiphagus Kngt. Cranberry L., Je, Drk (Kngt); Batavia, Je-J1 (Kngt) ; Lancaster, Jl, EPV (Kngt) ; Portageville, J1 (Kngt) ; Conesus L., J1 (Kngt) ; Ithaca, Je-J1 (Kngt), type locality; McLean, J1 (Kngt); White Plains, J1, Bno (Kngt); Hartsdale, J1, Bno (Kngt). Occurs on Ulmus and is predacious on Eriosoma americanum Rly.

D. betulae Kngt. Cranberry L., Je-J1, Drk (Kngt), J1-Aug, Htly (Kngt); Long L., Ds (Kngt); Mt. Whiteface, J1, Ds (Kngt); Four Mile, J1 (Kngt); Ithaca, Je-J1 (Kngt), type locality; Ringwood, Je-J1 (Kngt); McLean, J1 (Kngt); White Plains, Je-J1, Bno (Kngt); Ft. Montgomery, Je, Schott (Kngt); Hartsdale, J1, Bno (Kngt); SI, Je-J1, Ds (Kngt). LI: Cold Sp. Harb., J1, Parsh (Kngt). Breeds on beech (Betula lutea); is predacious on aphids.

D. borealis (VanD.). Cranberry L., J1, Drk (Kngt); Wanakena, J1, Drk (Kngt) ; Plattsburg, J1, Ds (Kngt) ; Batavia, Je-J1 (Kngt) ; Portageville, Je (Kngt); Ithaca, J1 (Kngt); McLean, J1 (Kngt); White Plains, Je, Bno (Kngt); Oliverea, J1, Ds, Shoe (Kngt) ; LI: Rockaway Beach, Je, Ols (Kngt). 
D. davisi Kngt. SI, Je, Ds (Kngt), type locality.

D. fasciolus Kngt. Cranberry L., Jl-Aug, Drk, Htly (Kngt); Wanakena, Aug, Drk (Kngt); Batavia, Je-Aug (Kngt); Ithaca (Kngt), type locality, Je-J1, Wellhouse (Kngt); White Plains, J1, Bno (Kngt). Occurs on Crataegus, apple, and occasionally other plants; is predacious on aphids.

D. fasciolus var. castus Kngt. Cranberry L., Aug, Htly (Kngt); Batavia, Je-Aug (Kngt); Ithaca (Kngt); SI, Je-Jl, Ds (Kngt). Predacious on Phyllaphis fagi L.

1070 D. grandis (Uhl.). Batavia, J1 (Kngt); Conesus L., J1 (Kngt); Ithaca, Je (Kngt); White Plains, Je-J1, Bno (Kngt); W. Hebron, Aug, Bno (Kngt) ; LI: Rockaway Beach, J1, collected in wash-up, Ols (Kngt). Breeds on hickory (Carya spp.).

D. laricicola Kngt. Conifer, J1, Osb (Kngt); Wanakena, Aug, Drk (Kngt) ; Batavia, Je-J1 (Kngt); Ithaca, Je-J1 (Kngt), type locality. Breeds on larch (Larix laricina).

D. nitenatus Kngt. Ithaca, J1 (Kngt) ; Lancaster, J1, EPV (Kngt); White Plains, J1-Aug, Bno (Kngt); SI, Jl-Aug, Ds (Kngt); LI: Bay Shore, J1, Ols (Kngt) ; Maspeth, J1-Aug, Ols (Kngt); Kings Pk., J1, Bell (Kngt); Roslyn, Aug, Ols (Kngt); Aqueduct, J1, Ds (Kngt). Predacious on Eriosoma lanigerum.

D. pinicola Kngt. Cranberry L., J1, Drk (Kngt) ; Conifer, J1, Osb (Kngt); Wanakena, Aug, Drk (Kngt); Ithaca, Je-J1 (Kngt), type locality; Ringwood, Je-J1 (Kngt); White Plains, May, Bno (Kngt); LI: Wyandanch, J1, Ds (Kngt). Occurs on Pinus strobus; predacious on Chermes pinicorticis Fh.

D. quercicola Kngt. Batavia, J1 (Kngt) ; Four Mile, J1 (Kngt) ; Portageville, Je $(K n g t)$; Conesus L., J1 (Kngt), type locality; Ithaca, Je-J1 (Kngt); White Plains, J1, Bno (Kngt); Pine I., Je, Ds (Kngt); Hartsdale, J1, Bno (Kngt); SI: Roseville, J1, Ds (Kngt); Richmond, Je, Ds (Kngt); LI: Bay Shore, J1, Ols (Kngt); Massapequa, J1, Ds (Kngt); Yaphank, J1, Ols (Kngt). Breeds on Quercus alba.

D. quercicola var. pallens Kngt. Batavia, J1-Aug (Kngt), type locality ; Buffalo, J1, EPV (Kngt). Breeds on Qucrcus macrocarpa.

1067 D. sayi (Reut.). Ithaca, Je (Kngt). Occurs on Quercus.

1071 D. ruber (L.). White Plains, Je, Bno (Kngt); SI, J1, Ds (Kngt); LI: Brooklyn, Jl, Ds (Kngt); Maspeth, J1, Ols (Kngt). Predacious on aphids.

D. ruber var. bicolor Kngt. LI: Brooklyn, J1, Ds (Kngt).

$1071 \mathrm{~b}$ D. ruber var. segusinus (Muller). White Plains, Je, Ols, Je, Bno (Kngt). LI: Maspeth, Je-J1, Ols (Kngt).

\section{SUBFAMILY ORTHOTYLINAE}

\section{PARTHENICUS Reuter}

1214 P. juniperi (Heid.). Taughannock, Jl (Kngt); W. Nyack, Sep, at light, Ols (Kngt); Mosholu, J1, Bno (Heid), type locality; LI: Rockaway Beach, Sep, W AHoffman (Kngt) ; Cold Sp. Harb., J1, Parsh (Kngt); Coram, Jl, Eng (Kngt). Juniperus virginiana.

1199 P. vaccini (VanD.). LI: Sea Cliff, Bks (Kngt). Vaccinium. 
Halticus Hahn

1122 H. citri (Ashm.). Fulton Co, Alex (EPV); Buffalo, Sep (EPV); Boston, Aug $(E P V)$; Hamburg, J1-Aug $(E P V)$; Gowanda, Aug ( $E P V)$; Cattaraugus Co, Aug (Kngt) ; Ithaca, Jl (Kngt-CU); White Plains, Je-Sep, Bno (Kngt) ; Yorktown Hgts., Sep, Ols (Kngt). White clover, beans, Plantago lanceolata; recorded also on many other plants.

$1120 \mathrm{H}$. intermedius Uhl. Batavia, J1-Aug (Kngt); Elma, Je (EPV); Cattaraugus Co, Aug. (Kngt); Wyoming Co, Aug (Kngt) ; McLean Bogs, Ithaca, J1, Buys (Kngt); Ringwood, J1 (Kngt); White Plains, $\mathrm{J} 1$, Bno (Kngt). Clematis virginiana.

\section{STRONGYLOCORIS Blanchard}

1123 S. stygica (Say). Cranberry L., J1, Drk (Kngt) ; Batavia, Je-Aug (Kngt) ; Ithaca, J1 (Kngt). Solidago spp.

\section{ORTHOCEPHALUS Fieber}

1189 O. mutabilis (Fall.). Ithaca, Je-J1 (Kngt) ; Ringwood, J1 (Kngt) ; McLean, J1 (Kngt); Owego, J1, JLFrank (Kngt). Chrysanthemum leucanthemum.

\section{LABOPS Burmeister}

L. hirtus Kngt. Axton, Je, MacG (Kngt) ; Cranberry L., J1, Drk (Kngt) ; Conifer, J1, Drk (Kngt) ; Wanakena, Aug, Drk (Kngt); Wilmington, J1, Ds (Kngt) ; Clinton Co, Je (Kngt); Peru, Je, Ds (Kngt); Fulton Co, Alex (Kngt).

\section{HADRONEMA Uhler}

1153 H. militaris Uhl. LI: Yaphanak, May, Ols, Je, Ds (Kngt). Baptisia tinctoria.

\section{ILNACORA Reuter}

1185 I. malina (Uh1.). Cranberry L., J1, Drk (Kngt); Wanakena, J1, Drk (Kngt); Adirondacks, Aug (EPV); Fulton Co, Alex (EPV) ; Hamburg, J1 (EPV); Lancaster, J1 (EPV); Four Mile, J1 (Kngt); Ithaca, Je-J1 (Kngt); Pine I., Je, Brb (Kngt); White Plains, Je, Bno (Kngt); Oliverea, Je, Ds (Kngt). Solidago rugosa, growing in shaded damp places at Four Mile.

1184 I. stalii Reut. "NY", Belfrage (Reut '76) ; Buffalo, J1-Aug (EPV) ; Lancaster, J1 (EPV); Ithaca, J1 (Kngt) ; LI: Brooklyn, Aug, Ds (Kngt). Occurs on cocklebur and other herbaceous weeds.

\section{LOPIDEA Uhler}

1143 L. caesar (Reut.). Ithaca, J1 (Kngt); Enfield Glen, Aug, Buys (Kngt); West Point, Sep, Ft. Montgomery, J1, Ds (Kngt).

1147 L. confluens (Say). Batavia, J1 (Kngt); Enfield Glen, Aug, Buys (Kngt) ; Colden, J1, EPV (Kngt); Salamanca, Aug, EPV (Kngt); White Plains, J1, Bno (Kngt) ; LI: Cold Sp. Harb., J1-Aug, Parsh (Kngt). Polymnia uvedalia, and probably $P$. canadensis.

1152 L. cuneata VanD. Lancaster, Sep (EPV), type locality. Populus balsamifera and $P$. deltoides. 
10893 L. davisi Kngt. LI: Half Way Hollow Hills, Ds (Kngt). Both wild and cultivated Phlox.

L. minor Kngt. Ithaca, Je, Buys (Kngt).

L. heidemanni Kngt. Batavia, Je (Kngt), type locality; Lancaster, Je, EPV (Kngt); Four Mile, J1 (Kngt) ; Portageville, Je (Kngt); Conesus L., J1 (Kngt) ; Honeoye Falls, Je (Kngt) ; Rochester Jct., May, Leon (Kngt) ; Ithaca, Je (Kngt-CU); West Pt., Je, Ds (Kngt); Pine I., Je, Ds (Kngt); White Plains, Je, Bno (Kngt); Grand View, Je, Brb (Kngt); Oliverea, Je, Ds, Shoe (Kngt); W. Nyack, Je, Ols (Kngt); LI: Farmingdale, J1, Morrow (Kngt); Yaphank, J1, Ds (Kngt). Ulmus, and Achillea millefolium.

$1145 a$ L. marginalis Reut. Elka Pk., Aug, Blkm (Kngt); Ithaca, J1 (Kngt) ; Enfield Glen, Aug, Buys (Kngt); Ft. Montgomery, J1, Ds (Kngt); White Plains, J1-Aug, Bno (Kngt) ; Saugerties, J1, Brb (Kngt); LI: Cold Sp. Harb., Jl, Parsh (Kngt).

1146 L. media (Say). L. Placid, Aug (EPV); Cranberry L., J1-Aug, Drk, Htly (Kngt) ; Conifer, Jl, Osb (Kngt); Wanakena, Aug, Drk (Kngt); Cascade L., Aug, Brb (Kngt) ; Wilmington, Jl, Ds (Kngt); Batavia, $\mathrm{J} 1(K n g t)$; Niagara Falls, Aug $(E P V)$; Buffalo, J1 (EPV); Colden, J1 $(E P V)$; Hamburg, J1 (EPV); Elma, Aug ( $E P V)$; Lancaster, J1 $(E P V)$; Gowanda, Aug $(E P V)$; Salamanca, J1 (EPV); Otto, J1, Comst (Kngt); Four Mile, J1 (Kngt); McLean, J1, Buys (Kngt); Ithaca, Aug (Kngt-CU) ; McLean, J1 (Kngt) ; Pine I., Je, Ds (Kngt); White Plains, Je-J1, Bno (Kngt); Mosholu (Heid); SI, J1, Ds; LI: Bellport (Kngt-Brooklyn Museum); Bay Shore, Jl, Ols (Kngt); Cold Sp. Harb., Jl-Aug, div. (Kngt). Solidago rugosa, and probably other herbaceous plants.

L. reuteri Kngt. L. George, J1, AKFisher (Kngt) ; Ramapo, J1, Ds (Kngt); Bear Mt., Aug, Schott (Kngt). Witch-hazel (Hamamelis virginiana).

1149 L. robiniae (Uhl.). Batavia, Jl-Aug (Kngt); Niagara Falls, Aug (EPV); Gowanda, Aug $(E P V)$; Wyoming Co, Sep (Kngt); Honeoye Falls, Aug (Kngt); Ithaca, J1 (Kngt); L. Mahopac, Aug (Bno); White Plains, J1-Aug, Bno (Kngt) ; Yonkers, J1, Bell (Kngt) ; LI: Gardiner's I., Aug, Ds (Kngt) ; Flushing, J1, Ds (Kngt); Kings Pk., J1, Bell (Kngt); High Hill, Aug (Bno); Farmingdale, J1, Morrow (Kngt); Cold Sp. Harb., J1-Aug, Bno, Brb (Kngt); Wading R., Jl, Ds (Kngt); Yaphank, May (Kngt-Brooklyn Museum). Black locust (Robinia pseudacacia).

L. salicis Kngt. Batavia, Je (Kngt); Portageville, Je (Kngt); Honeoye Falls, Je (Kngt), type locality; Rochester Jct., Je, Leon. (Kngt); Ithaca, Je (Kngt); Owego, J1, JLFrank (Kngt). Salix nigra.

L. staphyleae Kngt. Batavia, J1 (Kngt), type locality; Lancaster, EPV (Kngt). Staphylea trifolia.

\section{MECOMMA Fieber}

1188 M. gilvipes (Stål). Cranberry L., J1, Drk (Kngt); Wanakena, J1-Aug, Drk (Kngt); Ausable R., Aug (EPV); Fulton Co, Alex (EPV).

\section{CyRTORHINUS Fieber}

C. vagus Kngt. LI: Rockaway Beach, Sep, W AHoffman (Kngt), type locality; Sea Cliff, Aug-Sep, Bks (Kngt); Orient, Sep, Schott (Kngt). Occurs on sedges. 


\section{ORTHOTYLUS Fieber}

O. alni Kngt. Cranberry L., J1, Drk (Kngt); Mt. Marcy, 5000 ft., J1, Ds (Kngt) ; McLean Bogs, J1 (Kngt), type locality; Lancaster, Aug, EPV (Kngt). Alnus rugosa.

O. basicornis Kngt. Cranberry L., J1, Drk (Kngt) (viridis, Drk '22) ; Lancaster, Aug, EPV (Kngt) ; Batavia, Aug (Kngt), type locality. Salix longifolia.

$1178 \frac{1}{4}$ O. candidatus VanD. Cranberry L., Aug, Htly (Kngt). Populus tremuloides.

$1175 \frac{1}{2}$ O. catulus VanD. Wanakena, J1, Osb, Aug, Drk (Kngt); Niagara Falls, Je $(E P V)$; Hamburg, Je (EPV). Gnaphalium uliginosum.

$1179 \frac{3}{8}$ O. cruciatus VanD. White Plains, Je, Bno (Kngt); LI: Bay Shore, J1, Ols (Kngt). Found on Betula populifolia by Bueno.

1176 O. dorsalis (Prov.). Cranberry L., J1-Aug, div. (Kngt); Wanakena, J1, Osb (Kngt); Batavia, J1-Aug (Kngt); Honeoye Falls, Je (Kngt); McLean, J1 (Kngt); Ithaca, Je (Kngt); Ringwood, J1 (Kngt); White Plains, Je, Ols, Bno (Kngt).

1172 O. flavosparsus (Sahlb.). Batavia, J1-Sep (Kngt); Buffalo, Je (EPV); Hamburg, J1 (EPV) ; Tonawanda, Aug (EPV); Ithaca, J1 (Kngt); White Plains, Je-Aug, Bno (Kngt). Chenopodium album.

$1179 \frac{3}{8}$ O. knighti VanD. Batavia, J1, Kngt (EPV), type locality (actually nearer to Attica). Populus candicans.

$1175^{\frac{7}{8}}$ O. modestus VanD. Batavia, Jl, Kngt (EPV); Buffalo, Aug (EPV); Salamanca, J1 (EPV) ; Honeoye Falls, J1, Leon (EPV), type locality; Ithaca, J1 (Kngt) ; L. Mahopac, Aug, Bno (Kngt); White Plains, Je, Bno (Kngt); LI: Cold Sp. Harb., Parsh (Kngt). Salix fragilis and S. nigra.

O. modestus var. immaculatus Kngt. Batavia, J1 (Kngt), type locality; Honeoye Falls, Aug (Kngt); White Plains, Bno (Kngt). LI: Cold Sp. Harb., J1, Parsh (Kngt). Breeds on Salix.

$1178 \frac{1}{2}$ O. necopinus VanD. Cranberry L., J1, Htly (Kngt); Mt. Whiteface, J1, Ds (Kngt); Buffalo, Je (EPV); Ringwood, J1 (Kngt). Betula lutea.

O. neglectus Kngt. Cranberry L., J1, Drk, Htly (Kngt); Clayville, Je, Buys (Kngt) ; Batavia, Je (Kngt); Honeoye Falls, Je (Kngt); McLean, J1 (Kngt), type locality. Salix nigra.

$11799_{4}^{3}$ O. ornatus VanD. Honeoye Falls, Je-J1, Leon (EPV, Kngt), type locality; White Plains, Je, Bno (Kngt). Salix fragilis.

O. serus VanD. Batavia, Je-J1 (Kngt); Colden, J1 (EPV); Hamburg, J1 $(E P V)$; Gowanda, Aug (EPV); Salamanca, J1 (EPV); Four Mile, $\mathrm{J1}$ (Kngt); Ithaca, Je-J1 (Kngt); McLean, J1 (Kngt). Crataegus, and cultivated apple.

1178 O. submarginatus (Say). Hamburg, J1 (EPV); Ithaca, J1 (Kngt); LI: Cold Sp. Harb., Jl-Aug, div. (Kngt). Robinia pseudacacia.

1174 O. translucens Tuck. Batavia, J1 (Kngt); Buffalo, Je (EPV); Elma $(E P V)$. Salix longifolia.

$1175 \frac{1}{8}$ O. viridis VanD. Batavia, J1-Aug, Kngt (EPV), type locality; LI: Cold Sp. Harb., J1-Aug, Bno (Kngt). 


\section{DIAPHNIDIA Uhler}

1168 D. capitata VanD. Cranberry L., J1-Aug, Drk (Kngt) ; Wanakena, J1-Aug, Drk (Kngt) ; Johnstown, Aug, Alex (EPV), type locality; Batavia, J1 (Kngt) ; Ithaca, J1 (Kngt) ; LI: Cold Sp. Harb., J1, Bno (Kngt).

Witch-hazel (Hamamelis virginiana); hazelnut (Corylus), acc. Drk '22.

1166 D. pellucida Uhl. L. Placid, Aug (EPV); Cranberry L., J1-Aug, Drk, Htly (Kngt); Wanakena, J1-Aug, Drk (Kngt); Fulton Co, Alex (EPV); Mt. Whiteface, Aug (Kngt); Batavia, Je-Aug (Kngt); Hamburg, J1 (EPV) ; Gowanda, Aug (EPV) ; Conesus L., J1 (Kngt) ; Ithaca, J1 (Kngt); McLean, J1 (Kngt); White Plains, Je-J1, Bno (Kngt). Apple, Ostrya virginiana, and probably other plants.

1167 D. provancheri (Burque). Cranberry L., J1-Aug, Drk, Htly (Kngt); Batavia, Aug (Kngt); Hamburg, Sep (EPV); Boston, J1 (EPV); Gowanda, Aug (EPV); White Plains, Ji, Bno (Kngt). White oak (Quercus alba); beech and yellow birch (Drk, 1922).

\section{REUTERIA Puton}

1169 R. irrorata (Say). Cranberry L., Aug, Drk (Kngt); Batavia, J1-Aug $(K n g t)$; Gowanda, Aug (EPV) ; Salamanca, J1 (EPV) ; Saugerties, J1, Brb (Kngt); White Plains, J1, Bno (Kngt); Central Pk., NYC, J1, Ds (Kngt); LI: Cold Sp. Harb., J1, Parsh (Kngt). Tilia, and Quercus alba.

NOCT,UOCORIS Knight

11783 N. fumidus (VanD.). Batavia, J1-Aug (Kngt). Nocturnal in habits.

\section{HETEROTOMA Latreille}

$1180 \frac{1}{2}$ H. merioptera (Scop.). Honeoye Falls, Aug, Leon (Kngt). Probably imported on nursery stock.

\section{HETEROCORDYLUS Fieber}

1127 H. malinus Reut. Ithaca, Brockport, Syracuse, LaFayette, Batavia, Waterloo, West Palmyra, Albany (Cy) ; Batavia, Je-J1 (Kngt) ; Colden, J1, EPV (Reuter), type locality; Hamburg, Je (EPV); Portageville, Je (Kngt); Wyoming Co., Je (Kngt) ; Ithaca, Je (Kngt) ; Ringwood, Je-J1 (Kngt); McLean, J1 (Kngt); White Plains, Je, Bno (Kngt).

Native on Crataegus; breeds on wild apple; frequent on apple trees.

\section{Ceratocapsus Reuter}

C. digitulus Kngt. Cranberry L., Aug, Htly (Kngt) ; Batavia, J1 (Kngt) ; Cattaraugus Co, Aug $(K n g t)$; LI: Wyandanch, Aug, Ols (Kngt); Yaphank, J1, Ds (Kngt). Breeds on Salix.

C. drakei Kngt. Wanakena, Aug, Drk (Kngt), type locality.

1142 C. fasciatus (Uhl.). Batavia, Aug (Kngt); Niagara Falls, Aug (EPV); Hamburg, Aug $(E P V)$; Boston, Aug (EPV); Gowanda, Aug (EPV); Salamanca, Aug (EPV); Ithaca, J1 (Kngt); SI, J1, Ds (Kngt). Breeds on hickory (Carya).

C. fuscinus Kngt. Batavia, Aug (Kngt); Honeoye Falls, Aug (Kngt) ; LI: Cold Sp. Harb., Aug, Bno (Kngt). Occurs on Salix nigra. 
C. incisus Kngt. Batavia, J1-Aug (Kngt); Cattaraugus Co, Aug $(K n g t)$; Ithaca, J1 (Kngt) ; McLean, J1 (Kngt) ; LI: Cold Sp. Harb., Aug, Bno (Kngt). Occurs on Salix.

1137 C. lutescens Reut. LI: Bellport, Sep, Eng (Kngt).

C. Iuteus Kngt. White Plains, J1, Bno (Kngt), type locality.

1225 C. modestus Uh1. Cranberry L., Aug, Htly (Kngt); Wanakena, Aug, Drk (Kngt) ; Batavia, J1-Aug (Kngt); Portageville, Aug (Kngt) ; Ithaca, J1 (Kngt) ; Ft. Montgomery, Aug, Schott (Kngt); White Plains, J1, Bno (Kngt) ; Central Pk., NYC, Jl, Ds (Kngt) ; LI: Greenport, Aug, Eng (Kngt); Cold Sp. Harb., Aug, Bno (Kngt) ; Bear Mt., Aug, Schott (Kngt). Occurs on basswood (Tilia) and grapevine (Vitis sp.).

C. nigellus Kngt. Batavia, J1-Aug (Kngt), type locality; Conesus L., J1 (Kngt) ; Ithaca, J1 (Kngt) ; White Plains, Aug, Bno (Kngt). Breeds on hickory (Carya).

C. pilosus Kngt. Ithaca, Je $(K n g t)$; LI: Bay Shore, Je, Ols (Kngt). Occurs on Ostrya virginiana.

1139 C. pumilus (Uhl.). Cranberry L., Aug, Drk, Htly (Kngt) ; Madison Barracks, Aug (Kngt) ; Batavia, J1-Aug (Kngt); Hamburg, J1 (EPV) ; Gowanda, Aug (EPV) ; Salamanca, J1 (EPV); Honeoye Falls, Aug (Kngt) ; McLean, J1 (Kngt); White Plains, J1, Bno (Kngt); W. Nyack, Aug, Bno (Kngt) ; LI: Cold Sp. Harb., Aug, Bno (Kngt). Occurs on $V$ itis spp.

C. sericeus Kngt. Mosholu, Aug, Ds (Kngt); LI: Pinelawn, Aug, Schott (Kngt).

C. vicinus Kngt. SI, J1, Ds (Kngt), type locality; LI: Wyandanch, Aug, Ols (Kngt); Pinelawn, Aug, Ds (Kngt); Yaphank, Aug, Ds (Kngt).

\section{Alepidia Reuter}

1133 A. gracilis (Uh1.). Taughannock, J1 (Kngt); White Plains, J1, Bno (Kngt) ; LI: Bellport, Eng (Kngt); Bay Shore, J1, Ols (Kngt); High Hill, Aug $(B n o)$. Breeds on Pinus resinosa.

\section{PILOPHORUS Westwood}

1131 P. amoenus Uhl. LI: Bay Shore, Jl, Ols (Kngt); Bellport, Eng (Kngt) ; Yaphank, J1, Ds (Kngt). Breeds on Pinus virginiana.

P. brunneus Poppius. Cranberry L., J1, Drk (Kngt); Wanakena, Aug, Drk (Kngt); Batavia, J1-Aug (Kngt); Ithaca, J1 (Kngt) ; McLean, J1 $(K n g t)$; White Plains, J1-Aug, Bno (Kngt). Occurs on Salix.

1128 P. clavatus (L.). Cranberry L., J1-Aug, Drk, Htly (Kngt) ; Batavia, J1-Aug $(K n g t)$; White Plains, J1, Bno (Kngt); Oliverea, Je, Ds (Kngt). Occurs most frequently on Quercus.

P. strobicola Kngt. (crassipes Kngt., Hemip. Conn., p. 542, 1923). L. Placid, Aug, EPV (Kngt) ; Cranberry L., J1-Aug, Drk (Kngt) ; Mt. Whiteface, Aug (Kngt); Madison Barracks, Aug (Kngt); Ithaca, Je-J1 (Kngt) ; Ringwood, J1 (Kngt). Breeds on Pinus strobus.

P. juniperi Kngt. White Plains, J1, Bno (Kngt), type locality. Breeds on Juniperus virginiana.

P. laetus VanD. LI: Bay Shore, J1, Ols (Kngt). Breeds on Pinus virginiana. 
P. perplexus Douglas \& Scott. Batavia, J1-Aug (Kngt); Geneva, J1, Fulton (walshii, Fulton '13); White Plains, J1, Bno (Kngt). Occurs on goldenrod (Solidago), and has been taken on cherry, apple, and red cedar; predacious on aphids (Fulton, 1913, Ann. Ent. Soc. Am.).

P. piceicola Kngt. White Plains, Je, Bno (Kngt) ; Hartsdale, J1, Bno (Kngt), type locality; LI: Cold Sp. Harb., J1, Bno (Kngt). Breeds on Picea excelsa.

P. uhleri Kngt. Cranberry L., Aug, Drk (Kngt); Batavia, J1 (Kngt) ; Ithaca, Je-J1 (Kngt), type locality; White Plains, J1 (Kngt). Breeds on Pinus sylvestris.

P. vanduzeei Kngt. Taughannock, J1 (Kngt), type locality; Ithaca, J1 (Kngt) ; LI: Amagansett, Aug, Ols (Kngt); Bay Shore, J1, Ols (Kngt); Wyandanch, Sep, Ols (Kngt); Riverhead, Aug, Ds (Kngt). Breeds on Pinus resinosa.

\section{SERICOPHANES Reuter}

S. heidemanni Poppius. Fulton Co, Alex (EPV); Kingston, Aug (EPV); Buffalo, Je (EPV) ; Batavia, Je-Aug (Kngt); McLean, J1 (Kngt-CU). Nocturnal; males frequently attracted to lights.

\section{PSEUdoxenetus Reuter}

1159 P. regalis (Uhl.). LI: Yaphank, Je, Sf (Kngt). Occurs on live oak (Quercus virginiana).

1160 P. scutellatus (Uhl.). Syracuse, Je, Basinger (Kngt); Batavia, Je-J1 $(K n g t)$; Buffalo, Je (EPV); Gowanda, Aug (EPV); Portageville, Je $(K n g t)$; Conesus L., Je (Kngt) ; Ithaca, Je (Kngt); Grand View, Je, Brb (Kngt); Bronxville, Je (Wood); LI: Nissequogue, Je, Ds (Kngt) ; Coram, Bno (Kngt) ; Yaphank, Je, Ds (Kngt) ; Pine I., Je, Sf (Kngt). Quercus alba, Q. Muhlenbergii, and occasionally Fraxinus.

\section{SUBFAMILY MIRINAE}

\section{PithanUs Fieber}

P. maerkelii H-S. Honeoye Falls, Je (Kngt); White Plains, Kngt\&Bno (Kngt) ; LI: Maspeth, Je, Ols (Kngt). Grasses.

\section{MIMOCEPS Uhler}

M. insignis Uh1. Cranberry L., J1, Drk (Kngt); Wanakena, Aug, Drk (Kngt) ; Buffalo, Je-Sep, Cheektowaga, Je, Hamburg, J1, Conesus L., Aug, Springbrook, Je (EPV); Armonk, Je, Bno (Kngt); White Plains, Je, Bno (Kngt). Sedges.

\section{COLLARIA Provancher}

C. meilleurii Prov. Cranberry L., J1-Aug, Wanakena, Aug, Drk (Kngt); L. Placid, Aug-Sep (EPV); Conifer, J1, Osb (Kngt); Sodus Point, Aug, Buys (Kngt) ; Batavia, J1-Aug (Kngt); Buffalo, Je-Aug (EPV) ; Hamburg, J1 (EPV) ; Lancaster, J1-Aug, Colden, J1, EPV (Kngt); Otto, J1, Comst (Uh1) ; Ithaca, Ringwood, J1 (Kngt) ; Fulton Co, Alcx (EPV); E. Jewett, Aug, Ds (Kngt); Mosholu (Heid). Calamagrostis canadensis and other grasses in similar situations. 
C. oculata Reut. Cranberry L., J1, Drk (Kngt); Fulton Co, Alex (EPV); Hamburg, J1-Sep, Colden, J1 (EPV); White Plains, Je-Oct, Bno (Kngt); Mosholu (Heid) ; SI, Aug, Ds (Kngt); LI: Forest Hills, Sep, Ols (Kngt); Gardiners I., Je, Ds (Kngt). Grasses in dry sandy meadows.

\section{MIRIS Fabricius}

M. dolabratus L. Wilmington, J1, Ds (Kngt) ; Axton, Je, MacG (EPV) ; L. Placid, Aug (EPV); Wanakena, Je-J1, Drk (Kngt); Sodus Point, Je, Buys (Kngt); Batavia, Je (Kngt); Victor, Je, EPV (Kngt-CU); Buffalo, May-Aug (EPV); Hamburg, Je (EPV); Wyoming Co, Je (Kngt); Otto, Jl, Comst (Uhl) ; Ithaca, Je-Jl (Kngt-CU) ; Four Mile, McLean, Jl (Kngt); Albany, Je (Kngt); Bronxville, Je (Wood) ; LI: Gardiners I., Je, Ds (Kngt). Blue grass (Poa pratensis), timothy, and frequently other grasses.

M. ferrugatus Fall. Ithaca, Je-J1, Ringwood, McLean, J1 (Kngt).

\section{TERATOCORIS Fieber}

T. discolor Uhl. Buffalo, Aug-Sep, EPV (Kngt); Lancaster, Aug-Sep, Hamburg, Sep (EPV); Ithaca, Je (Kngt). Sedges; attracted to light.

T. paludum J. Sahlb. Cranberry L., Jl, Drk (Kngt) ; Buffalo, Aug, EPV (Kngt) ; Conifer, Jl, Osb (Kngt); Schuyler L., Aug, Buys (Kngt). Carex vesicaria; attracted to light.

\section{TRIGONOTYLUS Fieber}

T. uhleri Reut. Mamaroneck, Oct, Bno (Kngt); SI, Je, Sep, Ds (Kngt); LI: Sea Cliff, Aug, Ols (Kngt) ; Rockaway Beach, Sep, Ds, Cold Sp. Harb., Aug, Bno (Kngt). Salt meadows.

T. ruficornis Geoff. Axton, Je, MacG (EPV); Mt. Whiteface, Aug (Kngt); L. Placid, Aug (EPV); Cranberry L., Jl, Drk (Kngt); Fulton Co, Alex (EPV); Honeoye Falls, Je (Kngt); Batavia, Je-Aug (Kngt); Buffalo, Je-J1 (EPV, Kngt) ; Hamburg, Aug (EPV) ; Colden, J1 (EPV, Kngt) ; Lancaster, Je, EPV (Kngt); Wyoming Co, Je (Kngt); Ithaca, Je, McLean, Jl (Kngt) ; White Plains, J1, at light (Bno); LI: Maspeth, Je, Ols (Kngt). Oats and closely related wild grasses.

\section{MESOMIRIS Reuter}

M. curtulus Reut. SI, Aug, Ds (Kngt); LI: Bellport, Aug, Eng (Kngt); Sea Cliff, Aug, Ols (Kngt).

\section{STENODEMA Laporte}

S. trispinosum Reut. Cranberry L., Je-Aug, Drk (Kngt); Fulton Co, Alex (EPV); Buffalo, May-Aug (EPV); Portageville, May-Je, EPV (Kngt); Lime L., Aug, EPV (Kngt); Ithaca, Oct (Kngt); Mamaroneck, Oct, Bno (Kngt); Schuyler L., Aug, Buys (Kngt); White Plains, J1-Oct, Bno (Kngt); LI: Yaphank, May, Ds (Kngt); Wyandanch, Aug, Ols (Kngt); May-Sep, Ds (Kngt). Grasses in moist meadows; adults hibernate.

S. vicinum Prov. Axton, Je, MacG (EPV); Mt. Whiteface, Aug (Kngt); Cranberry L., J1-Aug, Drk (Kngt); Schuyler L., Aug, Buys (Kngt); L. Placid, J1-Aug (EPV); Fulton Co, Alex (EPV); Batavia, Ap-Sep (Kngt); Conesus L., J1 (Kngt); Buffalo, Aug (EPV); Hamburg, J1-Aug (EPV); Lancaster, Je-Sep, EPV (Kngt); Colden, J1, EPV (Kngt); Portageville, May (Kngt); Four Mile, Jl (Kngt); Ithaca, Jl (Kngt); McLean, Jl (Kngt); Enfield Glen, Aug, Buys (Kngt); Ft. Montgomery, Sep, Schott (Kngt): White Plains, Oct (Kngt); LI: High Hill, Aug (Bno). 


\section{SUBFAMILY CAPSINAE}

\section{OpISTHEURIA Reuter}

O. clandestina VanD. Ft. Montgomery, J1, Ds (Kngt).

O. clandestina var. ventralis Kngt. Ithaca, J1 (Kngt).

\section{Platytylellus Reuter}

P. borealis Kngt. Cranberry L., J1-Aug, Drk (Kngt); Wanakena, Aug, Drk (Kngt) ; Colden, J1, EPV (Kngt); Conifer, J1, Osb (Kngt); McLean, J1, $M a c G$ (Kngt).

P. circumcinctus Say. Wanakena, Aug, Drk (Kngt).

P. fraternus Kngt. Ft. Montgomery, Je, Schott (Kngt).

P. fraternus var. rubromarginatus Kngt. Ft. Montgomery, Je, Schott (Kngt).

P. insignis Say. Cranberry L., Aug, Drk (Kngt); Wanakena, Aug, Drk (Kngt); LI: Cold Sp. Harb., J1, Parsh (Kngt).

P. insitivus Say. Cranberry L., J1, Drk (Kngt); Buffalo, J1, EPV (Kngt); Portageville, Je (Kngt); Pine I., Je (Wood); LI: Bellport, Eng (Kngt); Rockaway, Je, Ds (Kngt).

P. nigricollis Reut. Cranberry L., Wanakena, Aug, Drk (Kngt); Syracuse, Aug, Basinger (Kngt); Springbrook, Je (EPV); Ringwood, Aug, Buys (Kngt); Ithaca, J1 (Kngt); McLean, J1, Jessiz Knight (Kngt); Elka Pk., Aug, Drk (Kngt) ; Catskills, Aug, Ds (Kngt) ; West Pt., J1, Eng (Kngt); LI: J1, Ds (Kngt); Bellport, J1, Eng (Kngt); Kings Pk., J1, Bell (Kngt).

P. nigroscutellatus Kngt. Batavia, Aug (Kngt); Enfield Glen, Aug, Buys (Kngt); Lancaster, J1, EPV (Kngt); Ithaca, J1-Aug (Kngt-CU), type locality.

P. rubrovittatus Stål. Cranberry L., Wanakena, Aug, Drk (Kngt); LI: Bellport, Eng (Kngt).

\section{TROPIDOSTEPTES Uhler}

T. cardinalis Uhl. Honeoye Falls, Je (Kngt); Batavia, Je (Kngt); Hamburg, Elma, Lancaster, Je (EPV) ; Conesus L., Je (Kngt); Four Mile, J1, Ithaca, Je (Kngt) ; Ellis, Je, MacG (Kngt) ; Pine I., Je, Ds (Kngt); Ft. Montgomery, Je, Schott (Kngt). Fraxinus americana.

\section{NEOBORUS Distant}

N. amoenus Reut. Cranberry L., J1, Drk (Kngt); Batavia, Je-Aug (Kngt); Conesus L., J1-Aug (Kngt, EPV); Buffalo, Boston, Hamburg, Gowanda, Aug (EPV); Salamanca, J1 (EPV); Wyoming Co, Sep (Kngt); Ithaca, J1 (Kngt); L. Mahopac, Aug, Bno (Kngt); Pine I., Je (Brooklyn Museum); White Plains, Sep, Bno (Kngt); LI: High Hill, Aug (Bno). Fraxinus americana, $F$. pennsylvanica; two generations.

N. amoenus var. scutellaris Reut. White Plains, Je, Bno (Kngt).

N. amoenus var. signatus Reut. Mt. Whiteface, Aug (Kngt); Batavia, Aug (Kngt).

N. canadensis VanD. Batavia, Aug (Kngt); Geneva, Je (EPV); Ithaca, Je (Kngt). Fraxinus americana.

N. geminus Say. Batavia, Je (Kngt); Colden, Je (EPV); Ithaca, Je, Four Mile, J1 (Kngt). Fraximus americana.

N. glaber Kngt. Batavia, Je (Kngt), type locality; Ithaca, Je (Kngt). Fraximus americana. 
N. palmeri Reut. Honeoye Falls, Je, Batavia, Je-Aug (Kngt) ; Hamburg, Aug (EPV); Ithaca, Je (Kngt). Fraximus americana, and probably other ash trees.

N. pubescens Kngt. Cranberry L., Je-Aug, Drk (Kngt) ; Fulton Co, Alex (Kngt) ; Batavia, J1 (Kngt) ; Elma, Je, Hamburg, J1-Aug, Salamanca, J1, Gowanda, Aug (EPV) ; Ithaca, J1 (Kngt), type locality ; Bear Mt., Aug, Schott (Kngt); White Plains, Je-J1, Bno, Parsh (Kngt). Fraximus americana, but found developing only on young plants in shaded situations.

N. rufusculus Kngt. Batavia, Je, Portageville, Je, Wyoming Co, Je (Kngt); Ithaca, May-Je (Kngt), type locality. Fraximus americana.

\section{XENOBORUS Reuter}

X. commissuralis Reut. Fulton Co, Alex (EPV); Batavia, J1-Aug (Kngt); Salamanca, J1 (EPV); Ithaca, J1 (Kngt). Fraximus nigra.

X. neglectus Kngt. Batavia, Je (Kngt); Tompkins Co, Je, MacG (Kngt), type locality.

X. pettiti Reut. Honeoye Falls, Je (Kngt) ; Batavia, Je (Kngt) ; Colden, Hamburg, Geneva, Je (EPV); Conesus L., Je (Kngt); Portageville, Je (Kngt); Wyoming Co, Je (Kngt); Ithaca, Je (Kngt) ; White Plains, Je, Bno, Parsh (Kngt).

X. plagifer Reut. Fulton Co, Alex (EPV); Batavia, Aug (Kngt). Fraximus nigra.

\section{LYGIDEA Reuter}

L. mendax Reut. Apple Redbur. LaFayette, Syracuse (Cy) ; W. Palmyra, Brockport (Cy) ; Batavia, Je-J1 (Kngt); Colden, J1, EPV (Reut) ; Hamburg, J1, $E P V$ (Reut), type locality; Portageville, Wyoming, Je (Kngt); Gowanda, J1 (Reut); Waterloo, Ithaca (Cy); McLean, J1, Ringwood, Je (Kngt); Albany (Cy); White Plains, Je, Bno (Kngt); LI: Yaphank, J1, Ds (Kngt). Crataegus, Pyrus coronaria, cultivated apple, and to some extent quince.

L. obscura Reut. Cranberry L., Wanakena, J1-Aug, Drk (Kngt) ; L. Placid, Aug, EPV (Kngt), type locality; Honeoye Falls, Je, Batavia, J1 (Kngt); Tompkins Co, Je, $M a c G$ (Kngt); White Plains, J1, Bno (Kngt). Salix nigra.

L. viburni Kngt. Cranberry L., J1, Osb (as rubecula Uhl. det. Drk) ; Batavia, Je-J1 (Kngt), type locality; Wyoming Co, J1 (Kngt). Nannyberry (Viburnum lentago); breeds especially on young growth.

\section{СоссоварнеS Uhler}

C. sanguinareus Uhl. Mt. Whiteface, J1, Ds (Kngt) ; Cranberry L., J1-Aug, Drk, Htly (Kngt); Fulton Co, Alex (EPV); Batavia, Je-J1 (Kngt) ; Honeoye Falls, Je (Kngt); Conesus L., Je (Kngt); Buffalo, J1-Aug, Hamburg, Je-J1, Colden, J1, Boston, J1, Salamanca, J1 (EPV) ; Ithaca, J1 (Kngt) ; Ringwood, J1 (Kngt) ; Four Mile, J1 (Kngt); Oliverea, Je-J1, Shoe, Ds (Kngt); Central Val., J1 (Bno); Ft. Montgomery, J1, Schott (Kngt).

\section{Capsus Fabricius}

C. ater L. Wilmington, J1, Ds (Kngt) ; Cranberry L., Wanakena, J1, Drk (Kngt) ; Fulton Co, Alex (EPV); Niagara Falls, Je (EPV) ; Batavia, Je (Kngt) ; Buffalo, May-Aug (EPV); Hamburg, J1 (EPV); Portageville, Je (Kngt) ; Otto, J1, Comst (CU) ; Ithaca, Je (Kngt-CU); McLean Bogs, May, Buys (Kngt); Four Mile, J1 (Kngt); White Plains, Je, Bno (Kngt); SI, Je, Ds (Kngt); LI: Wading R., May-J1, Ds (Kngt). Poa compressa, Agropyron repens, and probably other grasses. 
C. ater var. tyrannus Fab. Niagara Falls, Buffalo, Je (EPV); Batavia, Je (Kngt).

C. ater var. semiflavus L. Cranberry L., J1-Aug, Drk (Kngt); Niagara Falls, Je (EPV); Batavia, Je (Kngt); Colden, Je (EPV); Four Mile, J1, Ithaca, Je (Kngt).

\section{Platylygus Van Duzee}

P. luridus Reut. Cranberry L., J1-Aug, Drk (Kngt); L. Placid, Aug, EPV (Reut), type locality; Ringwood, J1 (Kngt). Pinus strobus.

\section{LyGUS Hahn}

L. approximatus Stål. Mt. Whiteface, Aug (Kngt); Cascade L., Aug, Brb (Kngt) ; Cranberry L., Wanakena, J1-Aug, Drk (Kngt). Hemlock (Tsuga canadensis); frequently attracted to flowers of Solidago macrophylla.

L. campestris L. Syracuse, J1, Basinger (Kngt); Batavia, May-J1 (Kngt); Hamburg, Je, Colden, J1 (EPV); Ithaca, McLean, J1 (Kngt); West Pt., J1, Brb (Kngt); Schuyler L., Aug, Buys (Kngt); Mosholu (Heid). Conium maculatum, also on other plants of the family Umbelliferae.

L. fasciatus Reut. LI: Bayville, Bks (Kngt), taken on Baccharis halimifolia.

L. pabulinus L. Mt. Whiteface, Aug (Kngt); Cascade L., Aug, Brb (Kngt); L. Placid, Aug (EPV); Wanakena, J1, Drk (Kngt); Fulton Co, Alex (EPV); Johnstown, Ds (Kngt); Batavia, Jl-Aug (Kngt); Boston, Aug, EPV (Kngt) ; Buffalo, J1-Aug, E. Aurora, Sep, Hamburg, J1-Aug, Salamanca, J1, Gowanda, Aug (EPV); Portageville, Je (Kngt); Wyoming, Je (Kngt); Howland I., Cayuga Co, under bark, Nov, Cy (Kngt); Ithaca, J1 (Kngt); Four Mile, J1 (Kngt); McLean, J1, Bno, Sib (Kngt); Oliverea, Je, Sep, Ds (Kngt) ; SI (Heid) ; LI: Cold Sp. Harb., J1, Bno, Flushing, Aug, Ds (Kngt). Breeds on Impatiens biflora.

L. plagiatus Uhl. Cranberry L., Je-Aug, Wanakena, J1-Aug, Drk (Kngt); Batavia (Kngt) ; Buffalo, Je-Sep (EPV); Ithaca, Jl (Kngt); White Plains, AugOct, Bno (Kngt); LI: Maspeth, Mar, Ols, Forest Hills, Oct, Ols (Kngt). Breeds on great ragweed (Ambrosia trifida).

L. pratensis var. oblineatus Say. Common throughout the State. This species is a pest on nursery stock, ornamental plants, and cultivated crops.

L. pratensis var. strigulatus Wlk. Cranberry L., J1, Drk (Kngt); McLean, Aug (Kngt-CU).

L. rubicundus Fall. Honeoye Falls, Je (Kngt); Batavia, J1 (Kngt); Buffalo, J1, Lancaster, May-J1, Colden, Aug (EPV); Ithaca, Ap-J1 (Kngt); White Plains, Ap, J1, Bno (Kngt). Breeds on Salix amygdaloides, also occurs on other willows; hibernates.

L. vanduzeei Kngt. Mt. Whiteface, Aug (Kngt); Wilmington, J1, Ds (Kngt); Cranberry L., Je-J1, Htly (Kngt); Wanakena, J1 (Kngt) ; Clayville, Je, Buys (Kngt); Fulton Co, Alex (EPV); Batavia, J1 (Kngt); Portageville, Je (Kngt), type locality; Hamburg, Je, EPV (Kngt); Wyoming, Je (Kngt); Gowanda, Aug, EPV (Kngt); Four Mile, J1 (Kngt); Labrador L., May, Buys (Kngt); McLean, J1, Sib (Kngt); L. George, J1, Fisher (Kngt); Catskills, Sep, Oliverea, Je, Sep, Ds (Kngt).

L. vanduzeei var. rubroclarus Kngt. Cranberry L., Je-Aug, Drk (Kngt) ; Oliverea, Je, Ds (Kngt).

L. (Neolygus) alni Kngt. Wanakena, J1, Ols, Aug, Drk (Kngt) ; Cranberry L., J1-Aug, Drk (Kngt); Ithaca, Ringwood, J1 (Kngt); McLean, J1 (Kngt), type locality. Breeds on Alnus incana. 
L. (Neolygus) atritylus Kngt. Cranberry L., J1-Aug, Drk, Htly (Kngt). Salix.

L. (Neolygus) belfragii Reut. Cranberry L., Wanakena, J1-Aug, Drk (Kngt); Syracuse, J1, Basinger (Kngt) ; Conesus L., Je, Batavia, J1-Aug (Kngt); Colden, Je, Hamburg, Aug (EPV); Portageville, Je (Kngt); Wyoming, Je (Kngt) ; Gowanda, Aug, Salamanca, J1 (EPV) ; McLean, J1, Ithaca, Je-J1, Ringwood, J1 (Kngt); Four Mile, J1 (Kngt); White Plains, Aug, Bno (Kngt). Breeds on Acer spicatum and Viburmum acerifolium.

L. (Neolygus) canadensis Kngt. Wanakena, J1-Aug, Drk (Kngt). Breeds on hazelnut (Corylus rostrata), according to Drk.

L. (Neolygus) canadensis var. binotatus Kngt. W. Nyack, Je, Ols (Kngt).

L. (Neolygus) caryae Kngt. Honeoye Falls, Je, Leon (Kngt) ; Batavia, Je (Kngt), type locality; Portageville, Conesus L., Je (Kngt); Hamburg, Je, EPV (Kngt); Ithaca, Je-J1, McLean, J1 (Kngt). On hickory (Carya).

L. (Neolygus) caryae var. subfuscus Kngt. Batavia, Je-J1 (Kngt), type locality; Byron, Portageville, Je (Kngt); Conesus L., Wyoming, Je (Kngt); Spring Brook, Je, EPV (Kngt); Ithaca, Four Mile, Je-J1 (Kngt). Carya.

L. (Neolygus) communis Kngt. The Pear Plant-Bug. Cranberry L., Wanakena, J1-Aug, Drk (Kngt); Batavia, Je-J1 (Kngt), type locality; Geneva (as invitus Say), ParrottEHodgkiss; Honeoye Falls, Je (Kngt), Hamburg, J1, Spring Brook, Je, EPV (Kngt) ; Conesus L., Je (Kngt); Wyoming, Portageville, Je (Kngt); Salamanca, J1, EPV (Kngt); McLean, J1, Bno, Ithaca, Je-J1 (Kngt) ; Four Mile, J1 (Kngt) ; Oliverea, Je, Ds (Kngt) ; Bear Mt., Je, Schott (Kngt). Breeds on Cormus stolonifera, C. paniculata, and cultivated pear.

L. (Neolygus) confusus Kngt. Cranberry L., J1-Aug, Drk, Htly, "white birch" (Kngt).

L. (Neolygus) fagi Kngt. Cranberry L., Je-Aug, Drk, Htly (Kngt) ; Honeoye Falls, Je (Kngt); Batavia, J1 (Kngt) ; Conesus L., Je (Kngt) ; Wyoming, Je (Kngt); McLean, Ringwood, Ithaca, type locality, J1 (Kngt); Enfield Glen, Aug, Buys (Kngt); Four Mile, J1 (Kngt); Hartsdale, J1, White Plains, Je-J1, Bno (Kngt). Breeds on Fagus grandifolia and perhaps Betula lutea.

L. (Neolygus) geneseensis Kngt. Batavia, Je (Kngt); Conesus L., J1 (Kngt), type locality; Portageville, Je (Kngt); Ithaca, Je-J1 (Kngt); Hartsdale, White Plains, Je, Bno (Kngt); LI: Wading R., Je, Yaphank, J1, Ds (Kngt). Breeds on Quercus alba.

L. (Neolygus) hirticulus VanD. Ausable R., L. Placid, Aug (EPV) ; Cranberry L., J1-Aug, Drk, Htly (Kngt); Wanakena, Aug, Drk (Kngt); Batavia, Je-J1 (Kngt) ; Boston, J1-Aug, Hamburg, J1, Lancaster, Aug (EPV) ; Conesus L., Je, Portageville, Aug, Wyoming, Je (Kngt); Salamanca, J1 (EPV); Four Mile, Ithaca, McLean, J1 (Kngt); Stanford, Aug (Kngt); Ashland, Aug, Frost, Oliverea, Je, Ds (Kngt); Grand View, Je, Brb (Kngt) ; LI: Cold Sp. Harb., J1, Bno (Kngt). Breeds sparingly on several plants; reared from chestnut, beech, and woodbine (Kngt); reared from yellow birch (Drk).

L. (Neolygus) inconspicuus Kngt. Honeoye Falls, Je (Kngt) ; Batavia, Je-J1 (Kngt), type locality; Conesus L., Je (Kngt); Ithaca, Je, Ringwood, J1 (Kngt); White Plains, J1, Bno (Kngt). Vitis rotundifolia.

L. (Neolygus) invitus Say. Honeoye Falls, Batavia, Je (Kngt) ; Conesus L., Je (Kngt); Buffalo, Niagara Falls, Colden, Je (EPV) ; Hamburg, Je-J1 (EPV) ; Portageville, Wyoming Co, Je (Kngt); Salamanca, J1, Gowanda, Aug, EPV (Kngt); Ithaca, Je-J! (Kngt); White Plains, Je-J1, Bno (Kngt). Ulmus americana. 
L. (Neolygus) johnsoni Kngt. Batavia, J1 (Kngt) ; Conesus L., (Kngt); McLean, J1, Ithaca, Je, Ringwood, Je (Kngt) ; Oliverea, Je-J1, Ds, Shoe (Kngt) ; Bear Mt., Je, Schott, White Plains, Je, Parsh (Kngt). Carpinus caroliniana.

L. (Neolygus) laureae Kngt. Four Mile, J1 (Kngt), type locality; Bear Mt., Je, Schott (Kngt); West Pt., Je, Ds (Kngt). Mountain laurel (Kalmia latifolia).

L. (Neolygus) neglectus Kngt. LI: Cold Sp. Harb., J1, Bno (Kngt). Carpinus caroliniana.

L. (Neolygus) omnivagus Kngt. Conesus L., Je, Batavia, Je-Aug (Kngt); Portageville, Je (Kngt); Ithaca, Je-J1 (Kngt), type locality; Four Mile, J1 (Kngt); Bear Mt., J1, Schott; Ft. Montgomery, Je, Schott (Kngt); White Plains, Je, Bno, Hartsdale, Ji (Kngt); LI: Bay Shore, J1, Ols (Kngt); Wading R., Je, Ds, Cold Sp. Harb., J1-Aug, Parsh (Kngt). Quercus alba, $Q$. rubra, $Q$. coccinea, and probably other oaks; reared from Cornus, Castanea, and Viburnum.

L. (Neolygus) ostryae Kngt. Cranberry L., Aug, Drk (Kngt); Honeoye Falls, Conesus L., Je (Kngt); Batavia, Je (Kngt), type locality; Portageville, JeAug (Kngt); Gowanda, Aug, EPV (Kngt); Ithaca, Je-J1, Four Mile, J1 (Kngt); McLean, J1, Kngt, Sep, Sib (Kngt); Stamford, Aug, EPV (Kngt). Ostrya virginiana.

L. (Neolygus) parrotti Kngt. Geneva, Je, Parrott (Kngt), type locality. Viburnum sterilis and $V$. opulus.

L. (Neolygus) parshleyi Kngt. Cranberry L., Jl, Drk (Kngt).

L. (Neolygus) quercalbae Kngt. Batavia, J1 (Kngt); Conesus L., Portageville, Je (Kngt) ; Ithaca, Je-J1 (Kngt), type locality; Four Mile, J1 (Kngt) ; White Plains, Je, Bno (Kngt); SI, Je, Ds (Kngt). Quercus alba.

L. (Neolygus) semivittatus Kngt. Conesus L., Portageville, Je (Kngt); Ithaca, Je (Kngt), type locality; LI: Pinelawn, Je, Ols (Kngt). Quercus alba.

L. (Neolygus) tiliae Kngt. Batavia, Je-J1 (Kngt); Conesus L., J1, Byron, Je, Portageville, Je (Kngt), type locality; Hamburg, Spring Brook, Je-J1, EPV (Kngt); Wyoming, Je (Kngt); Honeoye Falls, Je (Kngt); Elma, Aug (EPV); Four Mile, J1, McLean, J1, Ithaca, Je (Kngt). Tilia americana.

L. (Neolygus) univittatus Kngt. Portageville, Je (Kngt), type locality; Ithaca, Je-Jl, Kngt\&W ellhouse (Kngt); Ringwood, Je (Kngt). Crataegus.

L. (Neolygus) viburni Kngt. Sodus Pt., Aug, Buys (Kngt); Batavia, Je-J1 (Kngt), type locality; Portageville, Je, Aug (Kngt); Wyoming Co, J1 (Kngt); Ithaca, Je (Kngt). Viburnum lentago.

L. (Neolygus) vitticollis Reut. Honeoye Falls, Je (Kngt); Batavia, Je (Kngt); Colden, J1, E. Aurora, Je, Gowanda, Aug (EPV); Four Mile, J1 (Kngt); McLean, Je-J1, MacG (Kngt); Ithaca, J1, Ringwood, Je (Kngt) ; White Plains, Je, Bno (Kngt); LI: Bay Shore, J1, Ols, Orient, J1, Ds (Kngt). Acer saccharum and A. rubrum.

\section{DICHROOSCYTUS Fieber}

D. elegans Uhl. Batavia, Je (Kngt); Buffalo, Je (EPV); Portageville, Je (Kngt); Taughannock, J1 (Kngt); White Plains, Bno (Kngt); Mosholu (Heid). Red cedar (Juniperus virginiana) and white cedar (Thuja occidentalis).

D. rufipennis Fall. Ithaca, Je-J1 (Kngt). Pinus sylvestris. 
D. suspectus Reut. Wilmington, J1, Ds (Kngt); Ithaca, Je (Kngt). Pimus resinosa.

D. viridicans Kngt. Taughannock, J1 (Kngt); Saugerties, J1, Brb (Kngt); White Plains, J1, Bno (Kngt), type locality; LI: Lloyds Neck, J1, Ds (Kngt) ; Cold Sp. Harb., J1, Parsh, Bno (Kngt). Juniperus virginiana.

\section{POLYMERUS Hahn}

P. basalis Reut. Batavia, Je-Sep (Kngt) ; Buffalo, Je-Sep (EPV) ; Wyoming Co, Je (Kngt); Ithaca, J1-Aug (Kngt-CU); McLean, J1, Sib (Kngt); Ithaca, Four Mile, J1 (Kngt) ; Enfield Glen, Aug, Buys (Kngt) ; White Plains, Je, Bno (Kngt); Mosholu (Heid) ; Central Pk., NYC, Sep, Ds (Kngt) ; LI, Sep, Ds (Kngt). Dog fennel (Anthemis).

P. fulvipes Kngt. Four Mile, J1 (Kngt), type locality; Grand View, Je, Brb (Kngt) ; LI: Bay Shore, Ji, Ols, Pinelawn, Je, Ds (Kngt).

P. opacus Kngt. Wanakena, J1-Aug, Drk (Kngt); McLean Bogs, J1 (Kngt), type locality. Aster umbellaius.

P. punctipes Kngt. Cranberry L., J1, Drk (Kngt); Four Mile, J1 (Kngt), type locality. Lysimachia quadrifolia.

P. unifasciatus var. lateralis Hahn. Wilmington, Aug (EPV); Wanakena, Cranberry L., J1, Drk (Kngt). Galium boreale.

P. brevirostris Kngt. Lancaster, J1, EPV (Kngt).

P. venaticus Uhl. Cranberry L., Je, Wanakena, Jl-Aug, Drk (Kngt) ; Niagara Falls, Aug, Buffalo, Je, Elma, Je, Hamburg, J1, Lancaster, Je (EPV); Batavia, Je-Aug (Kngt); Wyoming, Je (Kngt) ; Ithaca, Je-J1, Four Mile, J1 (Kngt) ; White Plains, Je-J1, Bno (Kngt); SI, Je, Ds (Kngt); LI: Maspeth, Je, Ols (Kngt). Solidago altissima.

\section{PoEcilocapsus Reuter}

P. lineatus Fab. Cranberry L., J1, Wanakena, J1, Drk (Kngt); L. Placid, Aug (EPV); Fairmount (Sling); Walworth, Yeomans (Felt); Wayne Co, J1 (Kngt) ; Batavia, Je-J1 (Kngt, Lint); Buffalo, Je (EPV, Sling); Portageville, Je (Kngt); Hamburg, Je-J1 (EPV); S. Byron (Lint); Geneva (Sling); Otto, J1, Comst (Uhl) ; Peruville, Utica, Salem, Albany (Sling); Ithaca, Je-J1 (Sling, Kngt-CU) ; Enfield Glen, Aug, Buys (Kngt); Clayville, Je, Buys (Kngt) ; Four Mile, J1 (Kngt) ; Oliverea, Je, Ds (Kngt) ; Hartsdale, Je, White Plains, Je, Bno (Kngt); Mosholu (Heid); SI, Je, Ds (Kngt); LI: Wyandanch, Ji, Ds (Kngt). A large number of herbaceous plants, but especially Rumex; occasionally a pest on currant bushes.

\section{HORCIAS Distant}

H. dislocatus Say. Mt. Whiteface, J1, Ds (Kngt) ; Cranberry L., J1, Drk (Kngt) ; Conesus L., Je (Kngt); Portageville, Je (Kngt); McLean, J1, Ithaca, Je, Four Mile, J1 (Kngt); Catskills, Aug, Ds (Kngt); Valhalla, May, Bno (Kngt) ; LI, J1, Ds (Kngt). Smilacina racemosa, Geranium maculatum, Scrophularia leporella, and occasionally Caulophyllum thalictroides.

H. dislocatus var. affinis Reut. Cranberry L., Wanakena, Aug, Drk (Kngt) ; Honeoye Falls, Je (Kngt); Batavia, Je-J1 (Kngt); Conesus L., Je (Kngt); Buffalo, Colden, Je, Hamburg, Jl (EPV); Portageville, Je (Kngt); Four Mile, J1, McLean, J1 (Kngt); Ithaca, Je-J1 (Kngt-CU) ; Sodus Pt., Je, Buys (Kngt). 
H. dislocatus var. coccineus Emmons. Niagara Falls, Je (EPV); Batavia, Je-J1 (Kngt); Ithaca, Je (Kngt).

H. dislocatus var. flavidus Kngt. Batavia, J1, Portageville, Je (Kngt); Four Mile, J1 (Kngt), type locality.

H. dislocatus var. goniphorus Say. Fulton Co, Alex (EPV) ; Niagara Falls, Colden, Hamburg, Je, Buffalo, Jl (EPV); Sodus Pt., Je, Buys (Kngt); Batavia, Je-J1 (Kngt); Portageville, Wyoming, Je (Kngt); Four Mile, J1 (Kngt); Ithaca, Je, Ruckes (Fbs, Kngt-CU); Oliverea, Je, Ds (Kngt); White Plains, J1, Bno (Kngt); Mosholu (Heid); SI: Arrochar, Je, Ds (Kngt).

H. dislocatus var. limbatellus W1k. Wanakena, Aug, Drk (Kngt); Batavia, Je-J1 (Kngt); Portageville, Wyoming, Je (Kngt); Four Mile, McLean, J1 (Kngt).

H. dislocatus var. marginalis Reut. Gloversville, Je, Alex (EPV); Batavia, J1 (Kngt); Hamburg, J1, Buffalo, Je-J1 (EPV); Portageville, Je (Kngt); Ithaca, Je (Kngt); Four Mile, Ringwood, J1 (Kngt); Oliverea, Je, Ds (Kngt).

H. dislocatus var. nigriclavus Kngt. Batavia, Je (Kngt) ; Portageville, Je (Kngt); Four Mile, J1 (Kngt), type locality; Ithaca, Je (Kngt); Ringwood, Jí (Kngt); Bear Mt., J1, Schott (Kngt).

H. dislocatus var. nigritus Reut. Portageville, Wyoming, Je (Kngt) ; Sodus Pt., Je, Buys (Kngt); Batavia, Je-J1 (Kngt); Four Mile, J1 (Kngt); Ithaca, McLean, J1 (Kngt); Catskills, Oliverea, Je, Ds (Kngt); Pine I., Je, Ds (Kngt); Mosholu (Heid).

H. dislocatus var. pallipes VanD. Niagara Falls, Hamburg, Je (EPV); Batavia, Je-J1, Portageville, Je (Kngt) ; Four Mile, J1 (Kngt).

H. dislocatus var. scutatus Kngt. Four Mile, J1 (Kngt), type locality.

\section{ADELPHOCORIS Reuter}

A. rapidus Say. Adirondacks (EPV) ; Mt. Whiteface, Aug (Kngt) ; Cranberry L., J1, Wanakena, Aug, Drk (Kngt); Fulton Co, Alex (EPV); Buffalo, Je (EPV) ; Sodus Pt., Aug, Buys (Kngt) ; Batavia, Je-Sep (Kngt); Wyoming, Sep, Portageville, Je (Kngt); Otto, Four Mile, J1 (Kngt) ; Enfield Glen, Aug, Buys (Kngt); Ithaca, J1-Aug, Comst (Uh1); White Plains, Je-J1, Bno (Kngt); LI: Gardiners I., Je, Ds (Kngt); Schuyler L., Aug, Buys (Kngt); Rockaway, Je (Wood); Yaphank, J1, Ds (Kngt).

\section{CALOCORIS Fieber}

C. norvegicus Gmelin. White Plains, Je, Sep, Bno (Kngt); Mosholu (Heid); LI: Maspeth, Je, Ols (Kngt). Grasses; also abundant on daisy heads (Bno).

\section{Stenotus Jakovlev}

S. binotatus Fab. Wanakena, Cranberry L., J1-Aug, Drk (Kngt) ; Batavia, Je-J1, Portageville, Je (Kngt); Four Mile, J1 (Kngt); Ithaca, Je (Kngt); White Plains, Je, Bno (Kngt); Mosholu (Heid); LI, J1, Ds (Kngt). Breeds on Dactylus glomerata.

\section{PARACALOCORIS Distant}

P. scrupeus Say var. typ. Niagara Falls, J1, Lockport, Je, Hamburg, J1 (EPV); Batavia, Je-Jl, Portageville, Je (Kngt); Ringwood, J1 (Kngt); Pine I., (Kngt-Brooklyn Museum); West Pt. (Kngt-Brooklyn Museum); LI: Bellport (Brooklyn Museum). Vitis spp. 
P. scrupeus var. bidens McAtee. Batavia, Je (Kngt) ; Ithaca, Je-J1, Ringwood, Je (Kngt) ; Bear Mt., J1, Schott (Kngt); Pine I., Je, Ds (Kngt).

P. scrupeus var. cunealis McAtee. Rochester Jct., Je, Lcon (Kngt); Batavia, Je-J1 (Kngt), type locality; Ithaca, Je (Kngt).

P. scrupeus var. lucidus McAtee. Rochester Jct., Je, Leon (Kngt); Batavia, J1 (Kngt), type locality.

P. scrupeus var. nubilus McAtee. Portageville, Je (Kngt), type locality.

P. scrupeus var. par McAtee. Rochester Jct., Je, Leon (Kngt); Batavia, Je (Kngt) ; Portageville, Je (Kngt); Ithaca, Je (Kngt), type locality.

P. scrupeus var. rubidus McAtee. Batavia, Je (Kngt), type locality; Stony I., Je (Kngt).

P. scrupeus var. sordidus McAtee. Batavia, Je (Kngt), type locality.

P. scrupeus var. triops McAtee. Pine I., Je, Ds (Kngt), type locality; SI, Je, Ds (Kngt).

P. scrupeus var. varius McAtee. Rochester Jct., Je, Leon (Kngt) ; Honeoye Falls, Je (Kngt) ; Batavia, Je (Kngt), type locality.

P. hawleyi Kngt. Waterville, J1-Sep, Hazcl (Kngt), type locality; Hamburg, J1-Aug (EPV). Cultivated hop.

P. hawleyi var. ancora Kngt. Syracuse, J1, Basinger, Waterville, J1-Aug, Hawl (Kngt).

P. hawleyi var. fissus McAtee. Waterville, J1-Aug, Hazol (Kngt); SI, Ds (Kngt).

P. hawleyi var. pallidulus McAtee. Rochester Jct., J1, Leon (Kngt) ; Batavia, Je-J1 (Kngt), type locality. Apple.

P. colon Say. SI, Je, Ds (Kngt).

P. colon var. castus McAtee. Haverstraw, J1 (McAtee); SI, Aug, Ds (Kngt).

P. colon var. colonus McAtee. Lancaster, Aug, EPV (Kngt) ; Haverstraw, J1 (McAtee); LI: Wyandanch, Aug, Ols (Kngt) ; Cold Sp. Harb., Aug, Parsh, Riskel (Kngt).

P. multisignatus Reut. LI: Wading R., Je, Ds (Kngt).

\section{GARGANUS Stål}

G. fusiformis Say. Cranberry L., Aug, Drk (Kngt); Batavia, J1 (Kngt) ; Buffalo, J1-Aug, Boston, Aug, Colden, Hamburg, JI (EPV); Gowanda, Aug (EPV); Ithaca, J1 (Kngt); White Plains, Je-J1, Bno (Kngt); LI: Wading R., Jl, Ds (Kngt). Tussilago farfara.

\section{NEUROCOLPUS Reuter}

N. nubilus Say. L. Placid, Aug (EPV); Cranberry L., J1, Drk (Kngt) ; Fulton Co, Alex (EPV); Batavia, Je-Aug (Kngt); Buffalo, Jl-Aug, Colden, J1, Lancaster, Aug (EPV); Portageville, Aug (Kngt); Gowanda, Aug (EPV); Ithaca, Je-Aug (Ruckes, Kngt-CU); Enfield Glen, Aug, Buys (Kngt); Catskills, Aug, Ds (Kngt) ; Ft. Montgomery, J1, Schott (Kngt); Palisades, Mosholu (Heid); NYC, Je (Wood); SI: Eltingville, Jl, Ds (Kngt) ; LI: Bay Shore, J1, Maspeth, J1, Ols (Kngt); Hempstead, Sep, Ols (Kngt); Montauk, Aug, Ds (Kngt). Cephalanthus occidentalis, Rhus sp., and probably other plants. 


\section{PHYTOCORIS Fallén}

P. angustulus Reut. Ithaca, Taughannock, J1 (Kngt); McLean, Sep, Sib (Kngt); Hartsdale, Jl, Bno (Kngt); White Plains, Je, Bno (Kngt). Tsuga canadensis.

P. antennalis Reut. SI, Sep, Ds (Kngt); LI: Central Pk., Sep, Ds, Cold Sp. Harb., J1, Parsh (Kngt); Wyandanch, Aug, Bay Shore, Jl, Ols (Kngt).

P. borealis Kngt. Jamestown, Aug, EPV (Kngt).

P. brevifurcatus Kngt. Batavia, J1-Aug (Kngt), type locality.

P. buenoi Kngt. White Plains, J1, Bno (Kngt), type locality; LI: Cold Sp. Harb., J1, Parsh (Kngt).

P. caryae Kngt. Batavia, J1 (Kngt), type locality. Carya.

P. confluens Reut. Batavia, Aug (Kngt); Forest Hill, Sep, Ols (Kngt); White Plains, J1, Bno (Kngt); SI: Arrochar, J1, Ds (Kngt) ; LI: Flushing, Sep, Ols (Kngt); Port Washington, Sep, Ols (Kngt); Wading R., Je, Ds (Kngt). Hickory (Carya).

P. conspurcatus Kngt. Batavia, Aug (Kngt), type locality; Ithaca, Sep (Kngt); White Plains, J1, Bno (Kngt); LI: Wyandanch, Aug, Ols (Kngt). Bark of Tilia and Acer.

P. corticevivens Kngt. Waterville, J1, Harol (Kngt) ; Batavia, Je-J1 (Kngt), type locality; Hartsdale, J1, Bno (Kngt); SI, J1, Ds (Kngt). Bark of Acer saccharum.

P. cortitectus Kngt. Cranberry L., Jl, Drk (Kngt); Batavia, Jl (Kngt), type locality.

P. davisi Kngt. LI: Central Pk., J1, Ds (Kngt).

P. depictus Kngt. Batavia, Aug (Kngt), type locality. Quercus macrocarpa.

P. diversus Kngt. Madison Barracks, Aug (Kngt), type locality; Mt. Whiteface, Aug (Kngt); Ithaca, J1, Sep (Kngt); LI: Cold Sp. Harb., Aug, Parsh (Kngt). Pinus strobus.

P. erectus VanD. Wanakena, Aug, Drk (Kngt); Cranberry L., J1-Aug, Drk, Htly (Kngt); Cattaraugus Co, Aug (Kngt); Salamanca, J1 (EPV), type locality; McLean, Aug, Sib (Kngt).

P. eximius Reut. Cranberry L., Aug, Drk, Htly (Kngt) ; Batavia, Jl-Aug (Kngt); Portageville, Aug (Kngt); Ithaca, McLean, J1 (Kngt); LI: Cold Sp. Harb., J1-Aug, Parsh, Bno (Kngt); Yaphank, J1-Aug, Smithtown, Aug, Ds (Kngt); Wyandanch, Aug, Ols (Kngt).

P. fulvus Kngt. Cranberry L., Wanakena, Aug, Drk (Kngt); Ithaca, J1 (Kngt), type locality.

P. fumatus Reut. Ft. Montgomery, Je, Schott (Kngt) ; LI: Bay Shore, J1, Ols (Kngt); Wading R., Je, Yaphank, Jl, Ds (Kngt).

P. infuscatus Reut. Niagara Falls, Boston, Aug (EPV); Batavia, J1 (Kngt); Conesus L., J1 (Kngt); Gowanda, Aug (EPV); Ithaca, J1 (Kngt); White Plains, J1, Bno (Kngt). Carya.

P. lacunosus Kngt. Cranberry L., Aug, Drk (Kngt) ; Batavia, J1 (Kngt) ; McLean, J1 (Kngt), type locality.

P. lasiomerus Reut. Cranberry L., Aug, Drk, Htly (Kngt); Long L., Horv (Reut); Wanakena, Aug, Drk (Kngt); Fulton Co, Alex (Kngt) ; Cold R., Horv (Reut); Honeoye Falls, Aug (Kngt); Batavia, Jl-Aug (Kngt).

P. minutulus Reut. LI: Cold Sp. Harb., Aug, Parsh (Kngt); Maspeth, Sep, Ols (Kngt).

P. neglectus Kngt. Batavia, Je-Sep (Kngt), type locality; Wyoming Co, Je (Kngt) ; Four Mile, J1 (Kngt); Ithaca, Je-J1 (Kngt); Greene Co, J1 (Kngt); 
Ft. Montgomery, Sep, Schott (Kngt); White Plains, Aug, Bno (Kngt) ; LI: Amagansett, Je, Ds (Kngt). Apple trees.

P. obtectus Kngt. Honeoye Falls, Je (Kngt), type locality.

P. olseni Kngt. LI: Bay Shore, Yaphank, Jl, Ols (Kngt); Wyandanch, Aug, Ols (Kngt); Cold Sp. Harb., J1, Parsh (Kngt).

P. onustus VanD. Mt. Whiteface, Aug (Kngt); Batavia, J1 (Kngt); Portageville, Aug (Kngt); Boston, Aug (EPV); Salamanca, J1 (EPV), type locality; Four Mile, J1 (Kngt); Ithaca, J1 (Kngt). Bark of hickory trees.

P. pallidicornis Reut. Mt. Whiteface, Aug (Kngt); Fulton Co, Sep, Alex (EPV); Cranberry L., Wanakena, Aug, Drk (Kngt).

P. pinicola Kngt. Batavia, J1 (Kngt), type locality; Taughannock, J1 (Kngt) ; LI: Bay Shore, Jl, Ols (Kngt); Promised Land, Sep, Ds (Kngt) ; Cold Sp. Harb., Aug, Parsh (Kngt) ; Wyandanch, J1, Ds (Kngt) ; Amagansett, Sep, Ds, Coram, Ds (Kngt). Pinus sylvestris, $P$. resinosa.

P. puella Reut. "NY" (Reut); Ithaca, Sep (Kngt); White Plains, Je, Bno (Kngt) ; LI: Yaphank, J1, Ds, Ols (Kngt); Bay Shore, Aug-Sep, Ols (Kngt) ; Cold Sp. Harb., J1, Parsh (Kngt); Wyandanch, Aug, Ols (Kngt).

P. quercicola Kngt. Batavia, Aug (Kngt), type locality; LI: Fire I., Aug, Ds (Kngt). Quercus macrocarpa.

P. rubropictus Kngt. Wanakena, Aug, Drk (Kngt), type locality.

P. salicis Kngt. Cranberry L., J1-Aug, Drk, Htly (Kngt); Wanakena, J1, Drk (Kngt) ; Honeoye Falls, Aug (Kngt); Batavia, J1-Aug (Kngt), type locality ; Ithaca, Je-J1, McLean, J1 (Kngt); Ringwood, J1 (Kngt) ; W. Nyack, Aug, Ols (Kngt); LI: Cold Sp. Harb., Aug, Bno (Kngt); Orient, J1, Ds (Kngt). Salix.

P. spicatus Kngt. Batavia, J1-Aug (Kngt), type locality; Four Mile, J1 (Kngt).

P. sulcatus Kngt. Madison Barracks, Aug (Kngt); Batavia, Aug (Kngt), type locality; Ithaca, J1 (Kngt); SI, J1, Ds (Kngt).

P. tibialis Reut. Elma, Aug, Hamburg, J1-Sep, Lancaster, Aug, Colden, Aug, Buffalo, J1-Aug (EPV); Batavia, J1-Aug (Kngt); Portageville, Aug (Kngt); Salamanca, J1 (EPV); Ithaca, J1, Bno (Kngt-CU); McLean, J1-Aug, Sib (Kngt); Saugerties, J1-Aug, Brb (Kngt); White Plains, J1-Sep, $B$ no (Kngt) ; LI: Riverhead, Aug, Ds (Kngt); Flushing, Aug, Ds (Kngt); Cold Sp. Harb., J1, Butler, Bno, Parsh (Kngt).

P. tuberculatus Kngt. Four Mile, J1 (Kngt), type locality; Pine I., Je, Ds (Kngt).

P. uniformis Kngt. LI: Wyandanch, Aug, Ols (Kngt), type locality; Bear Mt., Aug, Schott (Kngt); Yaphank, Aug, Ds, Cold Sp. Harb., Aug, Bno (Kngt).

P. venustus Kngt. Syracuse, Sep, Drk (Kngt).

P. vittatus Reut. L. Placid, Aug (EPV), type locality; Lewis, Aug (Kngt). Ribes cynosbati.

\section{PARAXENETUS Reuter}

P. guttulatus Uhl. W. Nyack, Aug, Bno (Kngt); LI: Cold Sp. Harb., Aug, Brb (Kngt); Aqueduct, J1, Ds (Kngt).

BARBERIELla Poppius

B. apicalis Kngt. SI, Jl, Ds (Kngt), type locality; LI: Central Pk., Aug, Ds (Kngt). 
ISOMETOPIDAE-CRYptostemATIDAE-HydrometridAe-Gerridae 135

\author{
FAMILY ISOMETOPIDAE Fieber \\ Compiled by Harry $\mathrm{H}$. Knight \\ CORTICORIS McAtee and Malloch
}

C. pulchellus (Heid.). LI: Sea Cliff (Heid).

\title{
HEIDEMANNIA Uhler
}

H. cixiiformis Uhler. Batavia, J1, Kngt. During July of 1913 and 1914, twentyseven female specimens were found in scraping rough bark from the trunks of apple trees; the bugs appeared to be associated with psocids under the bark.

\section{FAMILY CRYPTOSTEMATIDAE}

(Dipsocoridae, Schizopteridae)

\section{CERATocombus Signoret}

C. (Xylonannus Reut.) vagans McAtee \& Mall. Keene Val., Je, Not (McAtee \& Mall). This species occurs in damp, rotten wood (Brb).

\section{FAMILY HYDROMETRIDAE}

\section{Compiled by J. R. DE LA TORRE-Bueno}

\section{HYDROMETRA Lamarck}

1281 H. martini Kirk. Ithaca, May-Aug (Bno, MacG-CU); McLean, J1 14 (Bno); White Plains, Mar-Aug, in great abundance (Bno); Van Cort. Pk., Ap, J1 (Bno) ; SI, May, Bno \& Ds (Bno), Ap 11 in plowed field, Ds (Bno); LI: Cold Sp. Harb., J1 (Bno).

\section{FAMILY GERRIDAE}

Compiled by J. R. DE' LA Torre-Bueno

\section{SUBFAMILY GERRINAE}

\section{GERRIS Fabricius}

1283 G. remigis Say. Mt. Whiteface, Aug (EPV); Axton, Je (M\&H); Old Forge (Bno); Cranberry L. (O\&D); Caledonia Cr., Dec (Lint); Colden, Gowanda, Jl-Aug (EPV); Ithaca, J1-Aug (CU); Fly Cr., Aug $(B n o)$; White Plains, Scarsdale, Port Chester, Feb-late Nov (Bno); Mosholu, Van Cort. Pk., Central Pk., NYC, Ap-Je (Bno); SI, Feb-Mar (Bno), J1 (Ols) ; LI: Flushing, Mar, Hempstead, J1, $(O l s)$; Cold Sp. Harb., J1, in brackish stream (Bno); Yaphank, Sep, Eng (Bno).

1285 G. conformis Uhl. Ithaca, Aug (CU) ; Croton-on-Hudson, Aug (Ols); White Plains, Je-J1 (Brio); Mamaroneck, May (Bno); LI: Cold Sp. Harb., J1, in brackish stream $(B n o)$.

1288 G. marginatus Say. Old Forge, Je-J1 (CU) ; Cranberry L. (O\&D) ; Fulton, Je, Mary Hill (CU) ; Otto (CU) ; Buffalo, Colden, Je-Sep (EPV); Ithaca, May-Sep (CU) ; Carmel, Aug (Bno); White Plains, Mar and throughout the year, adults in spring and fall (Bno) ; Van Cort. Pk., Central Pk., NYC, Mar-Oct (Bno); SI, Mar-Sep (Bno, Ols); LI: Maspeth, Flushing, Newtown Hgts., Hempstead, Lynbrook, Mar-Nov $(\mathrm{Ols})$; Cold Sp. Harb., J1, in brackish stream (Bno); Yaphank, Sep, Eng (Bno). 


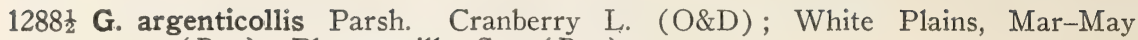
$(B n o)$; Pleasantville, Sep $(B n o)$.

1289 G. buenoi Kirk. Cranberry L. (O\&D); Ithaca, Aug (CU); McLean, J1, Kngt (Bno); White Plains, Mar-Nov, adults in spring and fall, nymphs in early summer $(B n o)$; Van Cort. Pk., Oct $(B n o)$; LI: Queens, Newtown Hgts., Yaphank, Mar-J1 (Ols); Cold Sp. Harb., J1 (Bno).

1290 G. canaliculatus Say. Putnam Co, Sep (Bno); White Plains, May-Nov $(B n o)$; Van Cort; Pk., Central Pk., NYC, Mar-Oct (Bno) ; SI, May $(B n o)$; LI: Rockaway Beach, Je (Ols); Cold Sp. Harb., J1 (Bno).

G. insperatus D, \& H. LI: Cold Sp. Harb., Brb (Drk\&Hottes).

\section{LIMNOPORUS Stål}

1291 L. rufoscutellatus Latr. Old Forge, Aug (CU) ; Buffalo, Hamburg, J1 $(E P V)$; Ithaca, J1-Sep (Bno); Enfield, Sep (CU) ; Putnam Co, Sep (Bno); White Plains, May-J1 and off and on throughout the year $(B n o)$; Van Cort. Pk., Je (Bno); LI: Jamaica, Feb, Dow (Bno).

\section{SUBFAMILY HALOBATINAE}

Metrobates Uhler

1295 M. hesperius Uhl. Franklin Co (EPV); Fulton, Alex (Bno) ; Cranberry L. (O\&D) ; Ithaca, J1-Aug (CU) ; L. Mahopac, both adults and nymphs in enormous numbers, Sep 1 (Bno).

\section{TREPOBATES Uhler}

1296 T. pictus H.-S. Cranberry L. (O\&D) ; Hamburg, J1 (EPV); Ithaca, JeSep (CU); L. Mahopac, Sep (Bno); Carmel, Aug (Bno); White Plains, Oct, adults generally found in late summer and early fall (Bno);

LI: Cold Sp. Harb., J1 (Bno); Yaphank, Sep, Eng (Bno).

\section{Rheumatobates Bergroth}

1297 R. rileyi Bergr. Cranberry L. (O\&D) ; Ithaca, J1-Aug (CU); White Plains, Aug-Oct (Bno) ; LI: Prospect Pk., Brooklyn, Aug, Ds (Ols); High Hill, Aug $(B n o)$; Cold Sp. Harb., J1, in brackish stream (Bno); Flatbush, Zab (Heid); Yaphank, Sep (Bno, Ols).

\section{FAMILY VELIIDAE}

\section{Compiled by J. R. DE LA TORRe-Bueno}

\section{Microvelia Westwood}

1303 M. borealis Bno. Cranberry L. (O\&D) ; Carmel, Aug (Bno); Tarrytown, Je (Bno); White Plains, throughout the year (Bno); SI, May (Bno), Aug (Ols) ; LI: Cold Sp. Harb., J1 (Bno).

1304 M. albonotata Champ. Fly Cr., Cooperstown, Aug (Bno); Valhalla, Oct, Armonk, Je (Bno); White Plains, May, winged form, Sep-Oct (Bno); LI: Cold Sp. Harb., J1 (Bno).

$1306 \frac{1}{2}$ M. fontinalis Bno. White Plains, Mar, May, Sep, Dec, but most abundant in spring in woodlands (Bno).

M. buenoi Drk. Cranberry L. (O\&D) ; White Plains, May-Sep, Feb 22, from among leaves at bottom of a pond, in great abundance on Mar 11, Nov 4, sluggish (Bno). 
1307 M. americana Uh1. Cranberry L. (O\&D) ; Adirondacks (EPV) ; Colden, Pipe Cr., Hamburg, E. Aurora, May-Aug (EPV); Fly Cr., Otsego Co, Aug (Bno); Tarrytown, Je, White Plains, the commonest species, Aug, Scarsdale, Sep, Port Chester, Aug, Armonk, Je (Bno); Bronx, Central Pk., NYC, Mar, Sep (Bno); SI, Mar (Bno); LI: Flushing, North Beach I., Yaphank, Mar-Oct (Bno, Ols).

M. heinei Drk. White Plains, May-Oct (Bno).

\section{Rhagovelia Mayr}

1309 R. obesa Uhl. Ausable R., Aug (EPV); L. Placid, Aug (EPV) ; Cranberry L. (O\&D) ; Niagara Falls, Colden, Hamburg, J1-Aug (EPV); Ithaca, Jl-Aug (CU) ; Fly Cr., Otsego Co, Aug (Bno) ; Tarrytown, White Plains, Armonk, in favorable situations from early spring to late fall (Bno) ; SI, Aug (Ols) ; LI: Yaphank, Sep (Bno).

\section{FAMily SALDIDAE}

Compiled by J. R. DE LA TORRE-Bueno

\section{PENTACORA Reuter}

1316 P. signoretii Guér. Dutchess Co, Je, Whiting (Bno); Rye, Jl, nymphs and adults (Bno); LI: Long Beach, Aug, Schott (Bno, Ols); Freeport, Dow (Ols); Gilgo Beach, Whiting (Bno).

1317 P. hirta Uhl. Rye, J1-Aug, Mamaroneck, Oct (Bno) ; LI: Gilgo Beach, J1 (Bno); Rockaway Beach, Sep, Cold Sp. Harb., J1 (Bno).

1318 P. ligata Say. Axton, Je (M\&H) ; Cranberry L., on stones (O\&D) ; Thousand Isls., Sep (CU) ; Batavia, Sep, Portageville, Je (Kngt); shores of L. Erie, Niagara R., J1-Sep $\left(E P V^{\prime}\right)$; Buffalo, Oct (EPV); Ithaca, Je-Sep (CU): Dutchess Co, Whiting, Je (Bno); Ft. Montgomery, Je, Schott (Bno); White Plains, J1, Sep (Bno).

1334 P. sphacelata Uhl. (Saldula). Rye, J1-Aug (Bno); LI: Gilgo Beach, Jl, Whiting (Bno); Babylon, Aug (Bno); Rockaway Beach, Sea Cliff, Bay Shore, Je-J1 (Ols).

\section{SALDA Linnaeus}

S. littoralis L. Pipe Cr., J1 (EPV).

S. coriacea Uhl. (anthracina Uh1., teste Bno.). Wallface Mt., J1, Not (Bno) ; Cranberry L. (O\&D); Oakfield, Je, Not (Bno); Batavia, Je, Kngt (Bno); Hamburg, J1 (EPV); Ithaca, J1 (CU) ; Ft. Hunter, May, Not (Bno); White Plains, J1 (Bno).

\section{Saldula Van Duzee}

1323 S. major Prov. Wallface Mt., Meadow Pond, Essex Co, Not (Bno) ; Cranberry L. (O\&D) ; Fulton Co, Alex (Bno) ; Axton, Je (M\&H); Buffalo, Hamburg, Je-Aug (EPV); Ithaca, J1 (CU); White Plains, J1Sep (Bno); Mt. Kisco, Je (Bno); LI: Je (Bno); Rockaway Beach, Je, Shoe (Ols); Bay Shore, Je (Ols).

1324 S. confluenta Say. Fulton Co, Alex (Bno); Cranberry L. (O\&D) ; Lancaster, Je, MCV (EPV); White Plains, Je-Sep (Bno); LI: Yaphank, $\mathrm{J} 1$, Ols (Bno). 
1325 S. orbiculata Uhl. Cranberry L. (O\&D); Buffalo (EPV); White Plains, Je-J1 (Bno); Mosholu, Je-Oct (Bno); LI: Amityville, J1 (Bno).

1328 S. interstitialis Say (pallipes auctt. Am.). Axton, Je (M\&H); L. Placid, Aug $(E P V)$; Cranberry L. (O\&D) ; Batavia, Aug, Kngt (Bno) ; Buffalo, Hamburg, Colden, May-Oct (EPV) ; Canisteo, Je, Not (Bno); Ithaca, Jl-Sep (CU) ; Carmel, Je-Aug (Bno); White Plains, Aug-Oct (Bno); Valhalla, Aug (Bno) ; LI: Maspeth, Bay Shore, J1-Nov (Ols) ; Cold Sp. Harb., J1 (Brio); Rockaway Beach, Ols (Bno); Gilgo Beach, J1 (Bno) ; Yaphank, J1 (Bno).

1329 S. separata Uhl. Wallface Mt, L. Tear, Indian Pass, Essex Co, J1, Not (Bno) ; Cranberry L., J1 (O\&D) ; Oakfield, Je, Not (Bno).

1330 S. reperta Uhl. Wallface Mt., J1, Not (Bno) ; Cranberry L. (O\&D) ; Buffalo, Colden, J1-Sep (EPV); White Plains, May (Bno); Scarsdale, Sep (Bno); Mosholu, Mar-J1 (Bno); SI, J1 (Bno).

1331 S. xanthochila Fieb. Elma, Je (EPV).

1335 S. opacula Zett. Cranberry L., J1 $(D r k)$; White Plains, by sifting, Mar-Sep (Bno); LI: North Beach, Jan 14, Schott (Bno).

1341 S. saltatoria L. White Plains, Mar 22-23, active, Dec 2, sifted from drifted leaves about tussocks in swamp $(B n o)$; Valhalla, May $(B n o)$; LI: Huntington, Feb 10, Schott (Bno).

\section{MICRACANTHIA Reuter}

1342 M. humilis Say. Wallface Mt., J1, Not (Bno); Cranberry L. (O\&D); Rochester, Je (CU); Buffalo, Hamburg, Gowanda, May-Aug (EPV); Canisteo, Je, Not (Bno); Nichols, May, Not (Bno); White Plains, Valhalla, Mt. Kisco, and Tarrytown, throughout spring and summer $(B n o)$; W. Nyack, Je (Ols); Mosholu, J1 (Bno); LI: Sea Cliff (CU) ; Rockaway Beach, Bay Shore, May-Je (Ols) ; Cold Sp. Harb., J1 (Bno); Amityville, Gilgo Beach, J1 (Bno); Yaphank, J1 (Bno), Je (Ols).

\section{LAMPRACANTHIA Reuter}

1345 L. crassicornis Uhl. Ithaca (Hgfd).

\section{CHARTOScIRTA Stål}

C. (Chartolampra) cursitans Bno. L. Tear, J1 27, Not (Bno), type locality.

\section{FAMILY NOTONECTIDAE}

Compiled by J. R. DE LA TORRE-Bueno

\section{SUBFAMILY NOTONECTINAE}

Notonecta Linnaeus

1349 N. irrorata Uhl. Keene Val. (Bno); Buffalo, Colden, J1 (EPV); Ithaca, Aug (CU); White Plains, Sep-Nov (Bno); Bronx Pk., Van Cort. Pk., Ap-Sep (Bno) ; NYC (Brb) ; SI, Aug-Oct (Bno); LI: Forest Pk., Nov (Bno); Queens, Flushing, Maspeth, Mar-Nov (Ols) ; Cold Sp. Harb., J1 (Bno).

1350 N. uhleri Kirk. Putnam Co, Sep (Bno); White Plains, Sep-Nov (Bno); Van Cort. Pk., Ap (Bno); LI: Flushing, Aug-Oct, Schott (Ols). 
1351 N. variabilis Fieb. Cranberry L., J1-Aug (O\&D) ; Keene Val., May, Not (Bno) ; Lowville, Je, Not (Bno); Ithaca, Aug (Bno) ; Tivoli, May, Not (Bno); White Plains, Ap-Sep, Nov (Bno); Valhalla, May (Bno); Van Cort. Pk., Mar-Nov (Bno) ; Central Pk., NYC, Ap (Bno); SI: May (Bno); Concord, Mar, Ang (Ols) ; LI: Flushing (Ols), Yaphank, J1-Sep (Ols, Bno).

1352 N. undulata Say. Mt. Marcy, J1, Not (Bno); Keene Val., May, Not (Bno); L. Placid (EPV) ; Cranberry L., J1 (O\&D) ; Buffalo and vic., J1-Aug $(E P V)$; Ithaca, Je-Nov (CU); Danby, Spencer, Aug, Comst (CU) ; Fly Cr., Otsego Co, Aug (Bno); Albany (NYS); Tivoli, May, Not (Bno); Putnam Co (Bno); White Plains, Feb-Dec (Bno); Bronx Pk., Central Pk., NYC, Bno, Mar-Sep (Bno); SI: Oct-Nov (Bno) ; Concord, Mar, Ang (Ols); LI: Forest Pk., Nov (Bno); Flushing, Maspeth, Sep-Nov (Bno); Cold Sp. Harb., J1 (Bno); Yaphank (Bno \& Eng).

1352c N. undulata var. maculata Fieb. Central Pk., NYC, Mar (Bno); SI, in autumn, Bno\&Ds (Bno).

1352e N. undulata var. latona Kirk. Gowanda, Aug (EPV); SI, Oct-Nov (Bno). 1354 N. raleighi Bno. White Plains, Mar 21, at thawed edges of a pond (Bno). 1357 N. insulata Kby. Mt. Marcy, J1, Not (Bno); Cranberry L., J1 (O\&D) ; Buffalo, Gowanda, J1-Aug (EPV); Albany (Bno); White Plains, Ap-Nov (Bno), Mar (Ols); NYC, Brb (Bno); SI, Sep, Ds (Bno); LI: Maspeth, Mar-Nov (Ols).

\section{BUENOA Kirkaldy}

1362 B. margaritacea Bno. Adirondacks (EPV); Cranberry L. (O\&D); White Plains, Feb-Dec (Bno); Pleasantville, Sep 9 (Bno); LI: Flushing, Huntington, May-Aug (Ols).

1363 B. platycnemis Fieb. "NY" (VanD. Cat.).

1364 B. elegans Fieb. White Plains, Aug-Sep $(B n o)$; Pleasantville, Sep-Nov $(B n o)$; Van Cort. Pk. (Bno); SI, Sep (Bno); LI: Cold Sp. Harb., J1 (Bno); Yaphank, Sep, Eng (Bno).

\section{SUBFAMILY PLEINAE}

\section{PLEA Leach}

1365 P. striola Fieb. Rochester, Moore (EPV); Ithaca, May-J1 (CU); White Plains, Oct, etc. (Bno) ; Hartsdale, Aug (Bno) ; Van Cort. Pk., ApSep (Bno); SI, May-Oct, Ds (Bno); LI: Cold Sp. Harb., J1 (Bno); Maspeth (Ols); Yaphank, J1 (Ols), Sep, Eng (Bno).

\section{FAMILY NAUCORIDAE}

Compiled by J. R. DE LA TORRE-Bueno

\section{PELOCORIS Stål}

1366 P. femoratus Pal. de Beauv. Baldwinsville, Sep, Pettit (CU); Carmel, Aug $(B n o)$; White Plains, May-Nov (Bno); Van Cort. Pk., Mar-J1 $(B n o)$; SI, May-Oct (Bno), Je (Ols) ; LI: Maspeth, Je, Rockaway Beach, Nov (Ols); Yaphank, J1 (Ols); Sep, Eng (Bno). 


\title{
FAMILY NEPIDAE
}

\author{
Compiled by J. R. DE LA TORRE-Bueno
}

NEPA Linnaeus

1379 N. apiculata Uh1. Fulton Co, Alex (Bno); Lancaster (EPV); Ithaca, Aug (CU) ; White Plains, Aug 16, nymph (Bno); Valhalla, Oct 2 (Bno); Mt. Vernon, Eng (Bno); Yonkers, Ang (Ols) ; Van Cort. Pk., Ang (Ols) ; SI, Ds (Bno) ; LI: Flushing, FWinters (Ols); Cold Sp. Harb., $\mathrm{J} 1$ (Bno).

RANATRA Fabricius

1381 R. kirkaldyi Bno. (fusca Am. auctt. not Pal. de Beauv.). E. Aurora, Oct (EPV) ; Ithaca, Jl-Aug (CU); L. Mahopac (Bno), type locality; Central Pk., NYC (Bno).

1383 R. americana Montd. Cranberry L., J1 (O\&D) ; Squaw I., Niagara Falls, Sep (EPV); Lancaster (EPV); Ithaca, May-Aug (CU); White Plains, from early spring to late fall (Bno); Van Cort. Pk., Central Pk., NYC, Mar (Bno); LI: Forest Pk., Nov 7 (Bno).

1385 R. protensa Montd. Ithaca, Nov (CU); Central Pk., NYC (Bno).

\section{Family BELOSTOMATIDAE}

\section{Compiled by J. R. DE LA TORre-Bueno}

\section{Benacus Stål}

1387 B. griseus Say. Cranberry L. and vic. (O\&D); Buffalo, Hamburg, Ap-Sep $(E P V)$; Ithaca, May-J1 (CU) ; White Plains, May-Sep (Bno); Tarrytown, May (Lint-NYS); NYC, J1 (Bno, Ols); SI: New Brighton, Mar (Ols); LI: Maspeth, Oct (Ols).

\section{LETHOCERUS Mayr}

1388 L. americanus Leidy. Adirondacks (Felt) ; Lockport, J1 (Lint-NYS) ; Buffalo, Hamburg, Ap (EPV); Caledonia Cr. (Lint); Johnstown, Ap (NYS) ; Ithaca, May-J1 (CU) ; Albany, Ap-Sep (NYS); E. Chatham, Je (Lint-NYS) ; Kingston (Lint-NYS); Stuyvesant Falls (NYS); White Plains, May-Oct (Bno).

1389 L. obscurus Duf. Hamburg, Mar (EPV); White Plains, Ap-Oct, on Nov 4, out of water on vegetation and torpid $(B n o)$; Mt. Vernon, Je $(B n o) ;$ SI: New Brighton, Mar (Ols).

\section{Belostoma Latreille}

1393 B. flumineum Say. Cranberry L. and vic. (O\&D); Buffalo, Sep (EPVNYS); Black Rock Harb., Sep (EPV); Mooers, Sep, Not (O1s-AM) ; Ithaca, Aug-Sep (CU) ; L. Mahopac, Mar (Bno); White Plains, J1Nov (Bno); Yonkers (Ols); Van Cort. Pk., Bronx Pk., Mar-Sep (Bno); SI, Mar (Ols), Aug-Oct (Bno); LI: Flushing, Rockaway Beach, Maspeth, Mar-Nov (Ols); Forest Pk., Yaphank, Jl-Nov (Bno).

1394 B. lutarium Stål. Yaphank, J1, Nov (Bno).

1401 B. testaceum Leidy. "NY" (VanD. Cat.). 


\section{FAMILY GELASTOCORIDAE}

Compiled by J. R. DE LA TORRE-Bueno

Gelastocoris Kirkaldy

1407 G. oculatus Fab. SI, Ds (Bno).

\section{FAMILY OCHTERIDAE}

Compiled by J. R. DE LA TORre-Bueno

\section{OCHTERUS Latreille}

1412 O. americanus Uhl. White Plains, May-Sep (Bno); Mosholu, May (Bno); SI, Ds (Bno); LI: Jamaica, J1, Huntington, J1, Schott (Ols).

1413 O. banksi Brb. White Plains, Je-J1 (Bno).

\section{FAMILY CORIXIDAE}

Compiled by J. R. DE LA TORRE-Bueno

\section{CALLICORIXA White}

1416 C. praeusta Fieb. Cranberry L., Bean Pond, Beaver Meadow (O\&D).

\section{ARCTOCORIXA Wallgren}

1422 A. alternata Say. White Plains, Mar-Je (Bno) ; LI: Cold Sp. Harb., J1 (Bno) ; Flushing, Sep-Oct, Maspeth, Dec (Ols).

1424 A. calva Say. Ithaca (Plunk); LI: Flushing, Mar, Ols (Plunk).

A. compressa Abb. Cranberry L. (O\&D).

$1427 \frac{1}{2}$ A. dubia Abb. Ithaca, Claas ( Hgfd).

1434 A. harrisii Uhl. White Plains, Valhalla, Mar, Sep, Nov (Bno) ; LI: Cola Sp. Harb., J1 (Bno).

1437 A. interrupta Say. Sodus Bay, Aug, ACWeed (Hgfd); Ithaca, J1-Aug (Hgfd); White Plains, Mar, Plunk (Ols); SI, Mar (Ols), J1 (Bno); LI: Flushing, Aug, Flunk (Hgfd); Maspeth, Oct, Plunk (O1s).

1438 A. kennicottii Uhl. White Plains, Sep-Oct (Bno); Van Cort. Pk., May, J1 (Bno).

1440 A. laevigata Uhl. "NY", three specimens (Hgfd).

1442 A. lineata Forst. "NY" (VanD. Cat.).

$1443 \frac{1}{2}$ A. modesta Abb. Ithaca, J1 (Bno); White Plains, Nepperhan, Je (Bno).

1444 A. nitida Fieb. White Plains, Mar, Ols (Plunk); LI: Maspeth, Mar, Ols (Plunk).

$1444 \frac{1}{4}$ A. ornata Abb. Ithaca, Claas (Hgfd).

$1444 \frac{1}{2}$ A. parshleyi Abb. Ithaca, paratypes.

1445 A. pectenata Abb. LI: Fire I. Beach, J1 19, in wash-up (Bno).

A. scabra Abb: Bean Pond, Cranberry L. (O\&D).

A. lobata Hgfd. LI ( $\mathrm{Hgfd}$ ).

A. obliqua Hgfd. Ithaca, J1-Aug ( $\mathrm{Hgfd}$ ).

A. vulgaris Hgfd. Ithaca, Mar-Nov, Alex, Plunk (Hgfd); White Plains, Bno (Hgfd), 


\section{PALMACORIXA Abbott}

1462 P. buenoi Abb. Cranberry L. (O\&D); Ithaca, J1 (Bno) ; Carmel, Aug (Bno); White Plains, Mt. Kisco, Valhalla, May-Sep (Bno); Van Cort. Pk., Je (Bno) ; Central Pk., NYC, Ap (Bno); SI, Aug (Bno); LI: Cold Sp. Harb., Parsh (Bno).

\section{CORIXA Geoffroy}

1465 C. macrocerops Kirk. LI: Cold Sp. Harb., Parsh (Hgfd).

1467 C. verticalis Fieb. LI: Maspeth, Dec 28, 1907, Ols (Plunk).

\section{ORDER HOMOPTERA}

The list in the order Homoptera has not had, properly speaking, a general editor, although Mr. E. P. Van Duzee was originally designated as in charge of this order at the time when the preparation of the state list as a whole was first contemplated. Pressure of other duties, and the transferral of Mr. Van Duzee's residence to California, have prevented him from continuing to take the general responsibility. While the Editorin-Chief has assembled the data for many of the groups, Mr. Van Duzee has done an immense amount of work in making determinations, furnishing records, and correcting synonymy, with the exception of the specialized families Coccidae and Aphiidae, which have been checked over by Messrs. Harold Morrison, and A. C. Baker and P. W. Mason, respectively. Mr. Van Duzee has been kind enough to look over the proof also.

The following families of this order are especially well represented in the State, due to the collecting and study by the specialists mentioned in connection with them: Cicadidae, Wm. T. Davis; Membracidae, W. D. Funkhouser, L. B. Woodruff, C. E. Olsen, and J. L. Buys; Cicadellidae, Herbert Osborn, D. M. DeLong, C. E. Olsen, and J. L. Buys; and for all three of these groups, of course, E. P. Van Duzee.

\section{FAMILY CICADIDAE 18}

\section{Compiled by William T. Davis}

\section{TIBICEN Latreille}

1473 T. linnei (Smith \& Grossbeck). Clyde; Aug, Bish; Albion, Ontario, Aug (NYS); Hamburg, Aug (EPV); Buffalo, Aug (EPV); Geneva, Canandaigua, Sep, Fulton; Gowanda, Aug (EPV); Ithaca, Aug (Univ. of Minn.), Aug, Sep, (CU), Aug (Ia. State Coll.), Aug (Morse); Coeymans, Aug-Sep (NYS); Saugerties, Aug, Brb; West

\footnotetext{
18 There are but nine species of cicadas in New York State, whereas more inland areas, as Kansas and Colorado, have about twenty-five species each, and on the Pacific coast they are even more numerous. In Tibicen auletes, however, the State has one of the largest of North American cicadas, and in $T$. chloromera one of the most noisy and energetic. With the exception of the seventeen-year cicada, practically nothing is known of the life cycle of any of the species,
} 
Pt., Sep, Robn; Yonkers; White Plains, Aug 29; Rye, Sep, several specimens, Junius Bird; W. Nyack, Aug; Central Pk., 1906; Inwood, Sep, Ds; Van Cort. Pk., Aug, Ds; City I., Lesisski; SI: generally distributed, but less plentiful where the soil is dry, late J1-Sep; LI: found in places from Brooklyn along the north shore to Orient; occurs on Gardiners I. and at Montauk, but is probably absent or unusual in the pine-barren regions of the island.

1479 T. canicularis (Harr.). Crown Pt., Aug, Not; Keene Val., J1-Aug (NYS), thirty-nine females, six males, Aug 18-Sep 29, six collected in Aug, thirty-nine in Sep, Not, Aug 11-26, Diven, Sep, Eng; Jay, J1 (WEBritton); Upper Jay, Aug, Not; Mt. Whiteface, Aug, FHBenjamin; Ausable Chasm, Sep, Not; Hope, Aug (NYS); Newport, Sep (NYS) ; Lowville, J1-Aug, RBHough; Clinton, three females, PBPowell (Ds); Utica, J1 (NYS); Syracuse, J1, AJBasinger, AugSep, Drk, Sep 11 (Ia. State Coll.); Baldwinsville, Sep (Mich. Agr. Coll.); Belle Isle, J1 (NYS); Oneida, Aug (US); Arcadia, Aug (Mich. Agr. Coll.); Clyde, Aug, Bish; Lake Bluff, Sep, Bish; Honeoye Falls, two males, one female, Aug, Kngt; Batavia, J1-Aug, Kngt; Hamburg, Aug (EPV); Buffalo, Aug (EPV); Geneva, two males, one female, Aug, VHLowe, Aug-Sep, BBFulton; Portage, J1, JLHoward; Wyoming Co, Sep, Kngt; Jamestown, J1, (NYS); Rock City, Sep, GIWheeler; Ithaca, eight specimens, J1 9, Sep 4 (CU), Aug (APMorse), J1, HPMorrison, Aug (Miss. Agr. Coll.), J1 (Univ. of Mich.), Aug, Eng; Elmira, Aug, Diven; Preble, Aug (Ia. State Coll.) ; Saratoga Sps., Angus (AM); Albany, J1, Aug; Karner, J1, Aug, Sep (NYS); Nassau, Je (NYS); Big Ind. Val., Aug, Pears; Allaben, Aug, Dow, Aug, AHHelme; E. Jewett, Aug, Ds; Elk Bushkill Val., Sep; Debruce, Sep, Ds; Callicoon, J1, Ds; L. Mahopac, Aug, TDO'Connor; West Pt., Sep, Robn; Valley Cottage; Peekskill, Aug, Sherm; Yonkers; SI: when there were many groves of pines on the island, this was not an uncommon species, but it has become the rarest of the genus; on Sep 18, 1917, one was heard singing from a pine tree in the Moravian Cemetery; LI: there are no records for the western part of the island, but from Central $\mathrm{Pk}$. eastward it is a common species, especially in the pine areas; it is less plentiful along the morainal hills of the north shore, but has been found as far east as Orient and Promised Land and also on Shelter I.

1477 T. Iyricen (DeG.). Ilion (Titus); Sodus, Foster (AM); Niagara Falls, Aug 18, 1909 (EPV); West Pt., Sep, Robn; White Plains, Aug, Ols, $J B K n a p p$; Pleasantville, Kessler; Tarrytown, J1, Wood; W. Nyack, Aug; Bronxville, J1, Wood, Aug ; Bronx Pk., Aug, Ds; Van Cort. Pk., Aug; Central Pk., NYC (AM); SI: generally distributed over the island, and in 1922 heard singing as early as J1 8; is more of a treeinhabiting species than chloromera; LI: found all over the island, including Orient Pt., Montauk, Shelter I., and Gardiners I. In late Aug, 1917, this species occurred in great numbers in places in the woods between Fresh Pond and Montauk; it was so abundant that the singing was a continuous performance.

1477 a T. lyricen var. engelhardti (Ds.). Syracuse, J1, AJBasinger (Ia. State Coll.) ; Ithaca, Je (CU) ; West Pt., Sep 14, three males, Robn; LI: Montauk, Sep, $D s$, Aug. In the South this variety occurs in the mountains and is rare in the lowlands.

1475 T. chloromera (W1k.) (Cicada sayi Smith \& Grossbeck). Gowanda, Aug, EPV; Albany, J1 (NYS); Aug, JSBailey (AM); Castleton, Aug (NYS) ; Phoenicia, Aug, EPV; Saugerties, Aug, fifteen specimens, 
Brb; Ft. Montgomery, Sep, Schott; Mt. Vernon, Aug, GBeyer; White Plains, Aug-Sep, nine males, two females, JBKnapp; Pleasantville, Sep, Kessler; Rye, Sep, Bird, Aug, Ds; Tarrytown, J1, Wood; W. Nyack, Aug, OlsEDDs; Central Pk., Bronx Pk., Van Cort. Pk.; Throg's Neck, Aug, thirty-seven males, eleven females, ASkinner; SI: distributed all over the island and often found on low vegetation; $\mathrm{J} 18$ (in 1913) is the earliest date that one has been heard singing on the island; on Oct 11, 1919, a very warm day, two were heard at St. George; LI: common on the westerly part of the island, but not recorded east of Oyster Bay and Hempstead; appears to prefer rather damp, heavy soil; occurs in Prospect Pk., Brooklyn.

1484 T. auletes (Germ.). Van Cort. Pk., Aug, Ds; Manhattan I., Sep, Shoe; SI: rather partial to sandy ground; Arrochar, Old Place, Watchogue, Long Neck near Travisville, Richmond Val., J1-Sep. On Sep 10, 1916, there were a considerable number of these insects in the trees to the north of the Finger Board Road near the corner of Sand Lane, Arrochar. They were so numerous that their songs were continuous. LI: Pinelawn, Patchogue, Farmingville, Selden, Coram, Yaphank, Wading R., Riverhead, Montauk, J1-early Oct. No doubt generally distributed over the island, especially in the areas of loose soil. Often sings in the late afternoon and into the evening.

\section{CicAdA Linnaeus}

1501 C. hieroglyphica Say. LI: A few seen among the pines near Bald Hill, Riverhead, Aug, 1917, and six males captured in the same locality, J1 18, 1918. The species no doubt occurs elsewhere on LI.

\section{MAGICICADA Davis}

1510 M. septendecim (L.) and form cassinii (Fisher). Periodical Cicada, or Seventeen-Year Cicada. Occurs in certain years very plentifully on SI, LI, in the Hudson R. Val., and in the central and western parts of the State. Occurs more sparingly elsewhere in the State, and appears to be absent from the higher regions. Only a few of the broods recorded as occurring in the State are really of prime importance, the others feebly representing some outlying swarms, or being perhaps belated individuals or else those that have appeared prematurely.

The small form cassinii appears with the larger septendecim and is usually considered as a variety of it. Its song is totally different from that of the large septendecim, being a series of chirps. It occurs in colonies in the area occupied by its larger relative, and is not so numerous or so widely spread.

On SI: brood 2 (1911-1928) appears in countless numbers; brood 6 (1915-1932) is the next largest, while broods $1,3,4,5,10,13,14,15$, and 17 have also been recorded, but not in large numbers.

On LI: brood 10 (1919-1936) and brood 14 (1923-1940) are the largest. Broods 1, 2, 4, 5, 8, and 9 have also been observed. Brood 1 (1910-1927) has a considerable swarm in the Half Way Hollow Hills, and brood 5 (1914-1931) in several localities from Wyandanch to Wading $\mathrm{R}$.

Elsewhere in the State, brood 2 (1911-1928) is the great swarm in the Hudson R. Val., extending as far north as Washington and Saratoga Counties. Brood 3 (1912-1929) has been reported from Geneseo (NYS 
Ent., 1912), brood 4 (1913-1930) from West Pt., brood 5 (1914-1931) from West Pt., brood 6 (1915-1932) from West Pt. (Robn), also from Dutchess, Greene, and Schenectady Counties (Rept. State Ent., 1915, and Bul. 71 of U. S. Dept. Agr., 1907); brood 7 (1916-1933) occurs in the central and western parts of the State, and has been reported from the following counties: Madison, Monroe, Onondaga, Ontario, Livingston, Yates, Cayuga, and Tompkins. Wyoming Co is given, as well as the above, in Bul. 71 of the U. S. Dept. Agr., 1907. For brood 8 (1917-1934) there is a doubtful record for Chautauqua Co. Brood 10 (1919-1936) is recorded from Monroe, Ontario, Niagara, and Columbia Counties. Brood 15 (1924-1941) is recorded from Saratoga and Dutchess Counties, in Bul. 71 of the U. S. Dept. Agr., 1907.

\section{OKanAgana Distant}

1518 O. rimosa (Say) (O. noveboracensis Emmons). Wilmington, J1, Ds; Ithaca; 1523

Windsor, Je, two females, Not; West Pt., pupa skin, Robn, and heard singing on several occasions (Ds) ; top of Bear Mt., Interstate Pk., Je, Schott; LI: Rockaway Beach, in drift, Je, Eng, Shoe.

The following records are supposed to belong to this species, though possibly they may belong to the next; Egleston's Glen, near Keuka L., Je, singing (Ds); near Potter Swamp, Je, a number singing; Letchworth Pk., Je 13, 1915, pupa skin, also a number singing. This species is distributed across the northern part of North America from Me to B.C.

1518 O. canadensis (Prov.). Valcour I., L. Champlain, Aug (PLaurent); Niagara Falls (AM) ; Buffalo, Je (EPV); Canandaigua L., J1; Ithaca, J1 (NYS), Je (Iowa State Coll.) ; Enfield Falls, Aug; Indian Ladder, J1, Schoharie, Je, Not.

\section{FAMILy CERCOPIDAE 19 SUBFAMILY CERCOPINAE}

MONECOPHORA Amyot and Serville

1545 M. bicincta Say. Ramapo, J1 (Ds) ; Mosholu, Bronx Pk., J1, Joutel (Ds); Wyandanch, Aug (Ds, Ols); Yaphank, Aug (Ds).

1545a M. bicincta var. ignipecta Fitch. Mosholu, J1 (Ball).

\section{SUBFAMILY APHROPHORINAE}

\section{APHROPHORA Germar}

1546 A. quadrinotata Say. Cranberry L., J1, Wanakena, Aug, on a variety of plants (Osb) ; White L., Zab (AM) ; L. Placid, Aug (EPV); Batavia, Aug, Kngt (Morr); Buffalo (EPV); Lime L., Salamanca, Gowanda, J1-Aug (EPV); Ithaca, J1-Aug (CU); Oliverea, Je, Shoe (Ols); L. George, Aug, Zab (Ols-AM) ; SI, Je-Oct (Ds) ; LI: Flatbush, J1-Áug, Zab (Ols-AM) ; Flushing, North Beach, Maspeth, Hempstead, J1 (Ols) ; Yaphank, Jl (Ds, Ols); Massapequa, Fire Place, Wading R., Southold, Orient, J1-Sep (Ds).

10 L. A. Stearns, of the New Jersey Agricultural Experiment Station, has been so kind as to check the manuscript for the family Cercopidae. 
1548 A. parallela Say. Pine Spittle Bug. Cranberry L., J1-Aug, on many conifers, breeding on spruce and pine (Osb); Batavia, J1, Kngt (Morr); Lancaster, Colden, Hamburg, Elma, Gowanda, Salamanca, Je-Aug (EPV); Ithaca (div.); Karner (Felt); E. Jewett, Aug (Ds); SI, Je-J1 (Ds); LI: common and generally distributed on pines, J1-Oct (Ds) ; Bay Shore, Yaphank, Wyandanch (Ols).

1551 A. saratogensis Fitch. Hamburg, Gowanda, Salamanca, Aug (EPV) ; Ithaca (WJBaerg) ; E. Jewett, Aug (Ds); Karner, J1 (Felt); SI, Je-Jl (Ds) ; LI: Bay Shore, Yaphank, Wyandanch, J1 (O1s). On pine.

1553 A. signoreti Fitch. Keene Val., J1, Not (Ols) ; Wilmington, J1 (Ds); Ithaca (WJBaerg); Ramapo, J1 (Ds); SI, Je-Aug (Ds); LI: Bay Shore, Yaphank, Wyandanch, J1-Aug (Ols).

\section{LEPYRONIA Amyot and Serville}

1555 L. quadrangularis Say. Keene Val., Aug, 1895 (Ball-NYS) ; L. Placid, Aug-Sep (EPV); Axton, Je (M\&H); Saranac L., Sep (Ds) ; Rochester, J1, Wendt (Ols) ; Portage, May, Kngt (Morr); Batavia, May, Kngt (Morr) ; Buffalo, Hamburg, Gowanda, Jl-Aug (EPV); Ithaca (CU) ; L. George, Aug, Zab (AM); W. Hebron, Sep, Lg (Ds); Karner (Felt); Yorktown Hgts., Sep (Ols) ; Bronx Pk., Aug (Ols); SI, J1-Oct (Ds) ; LI: Sea Cliff (EPV); Sands Pt., Oct, Bs, Flushing, Aug (Ds) ; Flushing, Hempstead, Wyandanch, Fishers I., J1-Sep (Ols).

\section{Philaronia Ball}

1559 P. bilineata Say. Cranberry L., J1; Wanakena, Aug.

\section{Philaenus Stål}

1560 P. leucopthalmus L. (spumarius L.). Cranberry L., Wanakena, nearly all varieties occur, J1-Aug (Osb); Ithaca; Salem, J1 ( Ball).

1560a P. leucopthalmus var. falleni VanD. Indian L., near Sabael, Aug, Brb (O1s), as form biguttatus Fab.

$1560 \mathrm{~b}$ P. leucopthalmus var. ulstulatus Fall. Salem, J1 (Ball).

1560d P. leucopthalmus var. leucocephalus L. L. Placid, Oct (Ds) ; Wilmington, J1 (Ds); Genesee Co, Je (Ds); Salem, J1 (Ball).

1560 h P. leucopthalmus var. pallidus Zett. Salem, J1 (Ball).

1561 P. lineatus L. Potsdam, J1, Houghton (O1s) ; L. Placid, Sep (EPV) ; Saranac L., Sep (Ds); Cranberry L., Wanakena, J1-Aug (Osb) ; Batavia, J1Aug, Kngt (Lawson); Hamburg, Lancaster, Elma, Salamanca, Je-Sep (EPV) ; Ithaca, J1 (div.) ; Oliverea, J1, Shoe (Ols) ; Debruce, Aug-Sep; L. George, Aug, Zab (Ols-AM) ; Salem, J1 (Ball) ; LI: Fishers I., Zab (Ols-AM).

\section{Clastoptera Germar}

1562 C. obtusa Say. L. Placid, Aug (EPV) ; Cranberry L., Wanakena, J1-Aug (Osb) ; Albion (EPV) ; Portage, Aug, Kngt (Morr) ; Batavia, Kngt (Morr) ; Buffalo, Niagara Falls, Gowanda, Lancaster, Colden, Hamburg, Lawtons (EPV); Otto (Uhl-CU); Ithaca (CU); Salem, Je (Ball); W. Albany (Lint); Scarsdale (Lint); LI: Sea Cliff (EPV-CU); Flushing, Bay Shore, Winfield, Maspeth, Yaphank, Wyandanch, J1-Sep (Ols). 
1562a C. obtusa var. achatina Germ. Colden, Boston, Gowanda, Salamanca (EPV); LI: Hempstead, Flushing, Maspeth, J1-Sep (Ols).

1562b C. obtusa var. testacea Fitch. "NY" (Fitch) ; Ithaca (WJBaerg) ; LI: Bay Shore, Wyandanch, J1-Aug (Ols).

1566 C. proteus Fitch. Ausable R. (EPV); Keene Val., Je (Ball-NYS); Cranberry L., Wanakena, J1, Aug (Osb); Portage, Aug, Kngt (Morr); Batavia, Kngt (Morr); Buffalo (EPV); Hamburg, Aug (EPV); Ithaca (Kngt, Baerg); Ft. Montgomery, J1 (Ds).

1566- C. proteus var. flava Ball. Keene Val., J1 (Ball-NYS).

1566b C. proteus var. vittata Ball. Hamburg, Aug (EPV); SI, J1 (Ds); LI: Flushing, Bay Shore, J1 (Ols).

1566 c C. proteus var. pini Fitch (var. nigra Ball). Hamburg, Elma, Je-J1 (EPV); Ithaca (WJBaerg); Matteawan, Je (Lint); LI: Bay Shore, Yaphank, $\mathrm{J} 1(\mathrm{O} 1 \mathrm{~s})$.

1566- C. proteus saint-cyri Prov. Ithaca, Baerg (Ball).

\section{FAMILY MEMBRACIDAE 20}

\section{SUBFAMILY SMILIINAE}

\section{Ceresa Amyot and Serville}

1570 C. diceros Say. Keene Val., Elizabethtown, Long L. (NYS) ; Saranac L., Kngt (Funk); Wells, Yg (Funk); Severance, CSBanks (NYS); Cranberry L. (Osb); Wilmington (NYS); Wanakena (Osb); Potsdam, Aug (EPV-CU) ; L. Placid, Aug (EPV); Rochester, Wendt (Ols); Hamburg, Boston, Colden, Elma, J1-Aug, Buffalo (EPV); Naples, Bish (Wood); Pike (NYS); Ithaca (EPV, Funk); Binghamton, Cortland (Funk); Karner (NYS); Normansville, Leon (Wood); Danby Hill, Comst, Aug, 1885 (CU); Watervliet Reservoir, Leon (Wood); Phoenicia (EPV); Callicoon, Ds (Wood); Poughkeepsie (NYS); West Pt., Ds (Wood); Millerton, Dickerson (Wood-AM); White Plains, J1, Bno (Ols, Wood); Allaben, Aug, Pears (Ols); Bronxville (Wood); SI, Ds (Wood); LI: Ds (Wood); Flushing, Aug (O1s) ; Maspeth, Aug (Ols); Hempstead, Sep, Rockaway Beach, Aug (Ols). Locust, elder, oak, sycamore, sweet clover, blackberry, butternut, goldenrod, wild cherry, grape, joe-pye weed.

1571 C. albescens VanD. Saranac L. (Wood); Wilmington (Funk); Niagara Falls (EPV); Hamburg, Elma, Jl-Aug (EPV); NYC, Ds (Wood).

1572 C. bubalus Fab. Buffalo Tree Hopper. Waddington (Wood); Cranberry L. (Osb) ; Barber Pt., Wanakena (Osb); Wilmington (Funk) ; Long L., Keene Val., Clinton Hgts., Hope (NYS); Albion, Aug (EPV); Clyde, Bish (Wood); Rochester (Funk); Churchville (Funk); Niagara Falls (EPV); Buffalo, Hamburg, Lancaster, Gowanda, J1-Sep (EPV); Pike (NYS); Geneva, Hodg (Funk); Ithaca (Funk); Cortland (Funk) ; L. George, Leon (Wood) ; Albany, Karner (NYS); Nassau L., Leon. (Wood); Salem (NYS); Normansville, Leon (Wood); Watervliet Reservoir, Leon (Wood); Callicoon, Ds (Wood); Sullivan Co, GFranck (Ols); White Plains (Ols); High Bridge, Kirkaldy;

20 The list in this family has been chiefly compiled from records, published and unpublished, furnished by E. D. Ball, W. D. Funkhouser, L. B. Woodruff, and C. E. Olsen. The first three specialists have looked over the manuscript and made changes and corrections in synonymy which the editor has attempted to harmonize as far as he has been able. 
NYC, Ang (O1s); Bronxville (Wood); SI, Ds (Wood) ; LI: Far Rockaway (Wood); Woodlawn, Dickerson (Wood-AM); Maspeth, Flushing (Ols). Sycamore, aster, poplar, potato, butternut, hazelnut, pear, sumac, oak, locust, elm, willow, elder, sweet clover, hickory, pignut, apple, white oak, joe-pye weed, grape, sweet pepperbush.

1574 C. brevitylus VanD. LI: Brooklyn (Funk).

1576 C. taurina Fitch. Wilmington (Funk) ; Keene Val. (NYS) ; Batavia, J1-Aug, Kngt (Funk) ; Buffalo, Colden, Hamburg, Boston, Lime L., Gowanda, and Salamanca, Je-Aug (EPV); Geneva, Hodg (Funk); Ithaca (Funk); Ballston L., Leon (Wood); Watervliet Reservoir, Leon (Wood); Oliverea, Ds (Wood); NYC, Mtch (Funk); Bronxville (Wood) ; SI, Ds (Wood, Ols) ; LI: Maspeth, Winfield, Flushing, Forest Hills (Ols); Brooklyn (Funk). Raspberry, hickory, potato, blackberry, dahlia, hazelnut, locust, witch-hazel, blue grass, white and red oak, beech, pear, apple, sweet clover, bittersweet, morning glory, quince.

1577 C. constans Wlk. Wilmington (Funk) ; Buffalo, Hamburg, Boston, Aug-Sep (EPV); Ithaca (Funk); Bronxville (Wood); SI, Ds (Wood); LI: Ds (Wood) ; Yaphank (Wood) ; Wyandanch (O1s).

1578 C. palmeri VanD. Olcott, Dt (Funk); Rochester (Funk); Batavia, Kngt (Funk); Buffalo (EPV); Salamanca, J1 (EPV); Ithaca (EPV); Coy Glen (Funk) ; W. Hebron, Ds (Wood) ; Hebron (EPV) ; Bronxville (Wood) ; Riverdale (Wood) ; Bronx, Ds (Wood) ; NYC (Mtch) ; SI (Wood); LI: Wyandanch, Flushing (Ols). Hickory, wild cherry, red oak, scarlet oak, swamp white oak, grass.

1579 C. borealis Fairm. Mt. Whiteface (Funk); Saranac L. (Funk); Rochester (Funk); Hamburg, E. Aurora, Elma, Buffalo, Boston, Lancaster, Gowanda, Salamanca, J1-Sep (EPV); Batavia, Jl-Aug, Kngt (Funk); Geneva, Hodg (Funk); Otto, Sep. (EPV-CU); Danby, Aug (EPV-CU) ; Ithaca, Cortland (Funk) ; Scarsdale (Funk); Nassau L., Leon (Wood) ; Catskills, Ds (Wood) ; W. Nyack (Ols) ; Bronxville (Wood) ; Millerton, Dickerson (Wood-AM) ; Riverdale (Wood) ; NYC (Mtch); SI, Ds (Wood); LI: Ds (Wood); Flushing, Bay Shore, Maspeth, Port Washington, Queens, Wyandanch, Hempstead, Forest Hills (Ols); Lott Wood, Zab, Flatbush, Zab, Schott (O1s); Woodlawn, Dickerson (Wood-AM). Wild grape, locust, elder, red, white, and swamp white oak, willow, hickory, pignut, raspberry, sycamore, apple, pear, beech, grass.

1580 C. basalis W1k. (turbida Goding). Speculator, Wells, $\mathrm{Yg}$ (Funk); Wilmington (Funk); Keene Val. (NYS); Saranac L., Ds (Ols); North Cr. (NYS) ; Mt. Whiteface (Funk); Wanakena (Osb) ; New Russia, Bdy (Funk) ; Cranberry L. (Osb) ; Albion, Aug (EPV) ; L. Placid, Aug (EPV); Waddington (Wood); Buffalo, Hamburg, Boston, Gowanda, J1-Sep (EPV) ; Phoenicia (EPV); Kingston (EPV); Catskills, Ds (Wood) ; LI: Jamaica (EPV-NYS). Sweet clover, rose, clematis.

1582 C. brevis Wlk. Gowanda, Aug (EPV).

1583 C. brevicornis Fitch. Buffalo and vic., J1-Oct (EPV); LI: Ds (Wood) ; Yaphank, Wyandanch, Ds (Wood); Bay Shore (Óls).

\section{STICTOCEPHALA Stål}

1587 S. inermis Fab. Keene Val. (NYS); Saranac L., Rochester (Funk); Batavia, J1, Kngt (Funk) ; Niagara Falls, Buffalo, Hamburg, Lancaster, Colden, Jl-Sep (EPV); Clyde, Bish (Wood); Geneva, Hodg (Funk); 
Ithaca (EPV); Karner (Wood-NYS); White Plains (Funk, Ols); W. Nyack (Ols) ; Fit. Montgomery, Ds (Wood) ; Riverdale (Wood); LI: Hempstead (Ols). Sweet clover, apple, timothy, red clover.

$\checkmark 1598$ S. lutea Wlk. Axton, Je, $M \mathcal{E} H$ (EPV-CU); Rochester, Wendt (O1s) ; Baldwinsville, Buffalo, Hamburg, Portage Falls, Fulton, Gowanda, Mud Cr., Ithaca, Caroline to Harford, Slaterville to Caroline, McLean, May-Sep (EPV-CU) ; Clyde, Bish (Wood); Genesee Co (Wood) ; Westfield, Dickerson (Wood-AM); Binghamton (Funk); Ilion (NYS); Albany (Wood) ; Poughkeepsie, Yg (Wood) ; Thacher Pk., Leon (Wood); Nassau (Wood); E. Jewett, Ds (Wood) ; Oliverea, Shoe (Ols) ; Tivoli (Wood) ; West Pt., Ds (Wood) ; Ft. Montgomery, Ds (Wood); Bronxville (Wood); White Plains (Ols); SI, Ds (Wood) ; LI: Yaphank, Bay Shore, Wyandanch, Pine Lawn, Maspeth (O1s) ; Brooklyn (Funk); E. Hampton and West Hills (Funk). Oak, daisy.

1600 ?S. nervosa Buckt. "NY" (Buckton).

S. sp. Woodruff states that specimens belong here which are identified as substriata W1k. in collection. LI: Central Pk. (Wood); Bay Shore, Ols (Wood).

\section{ACUTALIS Fairmaire}

1602 A. tartarea Say. Ithaca (EPV-CU); Wampus (Wood); White Plains, Bno (Wood); SI (Ols); LI: Maspeth, Ols (Wood); Winfield, Maspeth, Flushing (O1s). Locust.

A. tartarea var. semicrema Say. Batavia, Kngt (Funk); Albany (NYS); Yonkers, Ols (Wood); White Plains (Funk); Van Cort. Pk., Bronxville, NYC (Wood) ; SI (Ols); LI: Winfield, Ols (Funk); Maspeth, Flushing (Ols); Aqueduct (O1s); Brooklyn (Funk). Boneset, goldenrod.

\section{MicRutalis Fowler}

1604 M. dorsalis Fitch. Johnstown (NYS); Buffalo, Gowanda, Aug (EPV); Ithaca (EPV); Calders (Ball); E. Jewett, Ds (Wood). Clematis.

1605 M. calva Say. Ithaca (Funk); Scarsdale (Wood); Poughkeepsie, Yg (NYS); Bronxville (Wood); NYC (Mtch); LI: Flushing (Ols, Funk); Sea Cliff (EPV-CU). Alfalfa, locust, boneset.

\section{CARYNOTA Fitch}

1609 C. mera Say. Batavia, Kngt (Funk) ; Buffalo, Boston, Hamburg, Gowanda, Salamanca, J1-Aug (EPV); Ithaca, J1-Aug (EPV); Coy Glen, Dt (Funk); Albany (Yg); White Plains (Ols); NYC (Mtch); Bronxville (Wood); SI (Ols); LI: Yaphank (Wood); Flatbush, Dow (Mtch). Oak, hickory, butternut.

1611 C. stupida Wlk. Cranberry L., Wanakena (Osb).

1613 C. marmorata Say. Near Ausable R., Aug (EPV); Cranberry L. (Osb) ; Wells, Blue Mt., Yg (NYS); L. Placid (EPV); L. Pleasant (NYS) ; Bronxville, Wampus (Wood); LI: Aqueduct (Wood). Alder.

1614 C. porphyrea Fairm. Michigan Hollow, Ithaca (Funk); Saugerties, J1 (Funk). White oak. 
TheliA Amyot and Serville

1615 T. bimaculata Fab. Potsdam (EPV); Rochester (Funk); Buffalo, Gowanda (EPV); Wyoming Co, Kngt (Funk); Ithaca, Cortland, Binghamton (Funk); Albany, Yg (NYS); W. Hebron, Ds (Wood); White Plains (Funk); Riverdale (Wood); NYC (Mtch); SI, Ds (Wood, Mtch); LI: Cold Sp. Harb., Sep (Mtch, Funk); Flushing, Port Washington, Hempstead (O1s). Black locust.

1616 T. uhleri Stål. Buffalo and vic. (EPV); Ithaca (Funk). Plum.

\section{Glossonotus Butler}

1617 G. acuminatus Fab. Buffalo, Colden, Lancaster (EPV); Ridgeway (Ball); Ithaca (Funk); Karner, J1 (Felt); Bronxville (Wood); NYC (Mtch); LI: Yaphank, Ds (Wood); North Beach (Ols). Black, white, and red oak.

1618 G. turriculatus Emns. Batavia, Kngt (Funk); Karner, J1 (Wood); SI, Ds (EPV); LI: Bay Shore (Ols); Wyandanch, Central Pk. (Ols); Huntington (Wood); Yaphank, Ds. (Wood). Scrub oak.

1619

1620

G. univittatus Harr. (godingi VanD.). Sacandaga Pk., Alex (Funk); Gloversville, J1 (NYS); Rochester, Wendt (Ols), Moore (EPV); Batavia, Kngt (Funk); Buffalo, Gowanda, Je (EPV); Ithaca (Funk); Karner, J1 (Felt); Pine I., Ft. Montgomery, Ds (Wood) ; Riverdale (Wood) ; Bronxville (Wood) ; NYC (Mtch); LI: Bay Shore, Central Pk. (Ols) ; Rockaway Beach, Schott (O1s); Yaphank (Wood).

1621 G. crataegi Fitch. Kendall, J1 (NYS) ; Batavia, J1, Kngt (Funk) ; Hamburg, Lancaster, J1 (EPV); Albion (Funk); Kingston (Wood-AM) ; SI, Ds (Wood). Hawthorn, crab apple, quince.

G. nimbatulus Ball. Karner (Ball-NYS); LI: Massapequa, Ds (Ball). Scrub oak.

\section{HELIRIA Stål}

1622 H. cristata Fairm. "NY", probably Salem (Fitch-NYS); LI: Wading R., Ds (Wood).

1623 H. scalaris Fairm. Lancaster, Colden, J1 (EPV); Ithaca (Funk); Pine I., Sf (Ball); Bronx (Wood).

1624 H. projecta Butler. Ithaca, Je-J1 (EPV).

- H. cornutula Ball. Knoll, DeL, Pine I., Sf, Bronx; Oct, Mtch, LI, Ols (all Ball).

H. fitchi Ball. "NY" (Fitch-US).

\section{TELAMONA Fitch}

1629 T. pyramidata Uhl. Wilmington, Saranac L. (Wood) ; Gloversville (WoodNYS); Ithaca (EPV); Albany (Ball-NYS). Chestnut, oak, willow.

1628 T. pyramidata var. declivata VanD. Cranberry L. (Osb); Rochester, Wendt (Ols); Honeoye Falls, Kngt (Funk); Spring Brook, Je $(E P V)$; Ithaca (CU). This synonymy is Ball's.

T. westcotti Goding (obsoleta Ball). Keene Val. (Wood-NYS); Ithaca, J1 (EPV); LI: Central Pk., Ds (Wood). Oaks.

T. dubiosa VanD. Ithaca (EPV-CU); Rye, $D s$, Bronxville (Wood). White oak. 
1635 T. reclivata Fitch. Wanakena (O1s); Saranac L., Ds (Wood); Cobleskill (EPV-CU); Mt. Whiteface, Ds (Wood); Batavia, Kngt (Funk); Niagara Falls, Buffalo, Hamburg, Colden, Hamburg, Ashford, Gowanda, J1-Aug (EPV); Ithaca, Aug (EPV); Big Ind. Val., Ds (Wood); L. George (Wood-AM) ; Pine I., Ds, Bronxville (Wood) ; SI (Wood) ; LI: Central Pk., Cold Sp. Harb., Ds (Wood). Dr. Ball writes that almost all references to reclivata, especially those referring to basswood, belong to tilliae Ball. White oak, swamp white oak, red oak.

1630 1637

1644 T. monticola Fab. (querci Fitch). Mt. Whiteface, Ds (Wood); Rochester, 1645

T. decorata Ball (barbata VanD.). Cranberry L., Osb (Funk); Medina, LHWeld (Funk); Batavia, Kngt (Funk) ; Boston, Aug (EPV); Ithaca, Je (Funk, EPV); Scotia, Yg (Wood-NYS); L. Mohonk (Wood); Pine I., Ds (Wood); Central Pk., NYC, J1, Sf (Ball-Brooklyn Museum). Chestnut oak, white oak, linden, basswood. Dr. Ball states that most of these references are from males of tilliae Ball. Wendt (Ols); Olcott, Dt, Batavia, Aug, Kngt (Funk); Buffalo, Lanicaster, J1 (EPV); Medina (Ball); Portage, Kngt (Funk); Gowanda, Aug (EPV); Conesus L., Ds (Wood); Rock City, Kngt (Funk); western NY. (EPV); Ithaca, J1-Aug (Funk-CU); L. Mohonk (Wood); Pine I., Ds (Wood); Nyack (Wood-AM); Ft. Montgomery, May (Ball); Bronxville (Wood); SI, Ds (Wood); LI: Half Way Hollow Hills, Ds (Wood); Wyandanch, Schott (Ols); Flatbush (Wood); Yaphank, Ds (Wood). White, chestnut, and red oak.

1646 T. ampelopsidis Harr. Potsdam, Houghton (O1s); Ogdensburg (NYS); Wilmington (Funk); Oswego (NYS); Rochester (Funk); LeRoy, Cy (Funk); Buffalo, J1-Aug (EPV); Ithaca, Je-Oct (CU); Interlaken, Cortland (Funk); Binghamton (Funk); Trumansburg (Funk); Albany (NYS); Menands (NYS); Ft. Montgomery, Schott (Ols); NYC, Ds (Wood); SI, Ds (Wood); LI: Yaphank, Ds (Wood); Brooklyn (Wood); Maspeth (Ols). Virginia creeper.

1647 T. tristis Fitch. Batavia, Kngt (Funk); E. Aurora, Sep (EPV); Ashford, Gowanda, J1-Aug (EPV); Ithaca (Funk); SI (Wood-AM). Witchhazel, basswood, oak, hazelnut.

T. tristis var. coryli Fitch. Wanakena (Osb); Batavia, Aug, Kngt (Funk); Lancaster, Otto, Gowanda, Jl-Aug (EPV); Ithaca (Funk); Slide Mt., Ds (Wood); Orange Co, WTBather (Ols); SI (Wood). Oak, hazelnut.

1648 T. concava Fitch. Lewiston, Jl (EPV); Ithaca, GFAtkinson (EPV); Salem (Ball-NYS, US).

T. ornata Emm. Bronxville (Wood). Quercus alba and Q. velutina. Woodruff considers this distinct from concava.

1649 T. gemma Ball. Adirondacks, Brb (Ball); Lancaster, EPV (Ball); Ithaca (Funk); Catskills, Ols, Drk (Ball).

1651 T. unicolor Fitch. Rochestcr, Wendt (O1s) ; Batavia, J1-Aug, Kngt (EPV); Hamburg, Colden, E. Aurora, Spring Brook, Je-Sep (EPV); Portage, Kngt (Funk); Ithaca, Albany (Funk); Pine I., Ds (Funk); W. Nyack (Ols, Wood); Bronxville (Wood); LI: Rockaway Beach, Schott (Ols). Hickory, butternut, walnut, basswood.

1651a T. unicolor var. irrorata Fitch. "NY" (EPV).

1652 T. extrema Ball. Batavia, Kngt (Funk); Karner (Wood-NYS); LI: Winfield (Ols); Orient Pt., Coram, Ds (Wood). Scrub oak. 
1653 T. collina Wlk. Hamburg, J1 (EPV); Ithaca (EPV, Funk); Pine I., Bronxville (Wood). Sycamore, bur oak.

1654 T. maculata VanD. Ithaca, Aug (Funk); Pine I., Ds (Funk, Wood); Bronxville (Wood) ; SI, Ds (Wood) ; LI: Wading R., Schott (Wood); Yaphank (Funk). Swamp white oak, white oak.

T. tremulata Ball. Cranberry L., $O \& D$, as barbata (Ball); Salem, J1 (Ball). T. woodruffi Ball. Pine I., J1 (Ball-BM).

\section{ARCHASIA Stål}

1661 A. galeata Fab. Buffalo, J1 (EPV); Karner (NYS) ; LI: Wyandanch, J1, Ds (Ball); Bay Shore, Ols (Wood); Pinelawn, Wading R., Central Pk., Ds (Wood). Oaks.

1662 A. belfragei Stål. Medina, LHW cld (Funk); Buffalo, Hamburg, Gowanda, Je-J1 (EPV); Ithaca (EPV, Funk); Pine I., Ds (Wood); White Plains (Funk); Bronxville (Wood) ; SI, Ds (Wood) ; LI: Wyandanch, Ds (Funk); Coram, Eng (Wood); Massapequa, Yaphank, Ds (Wood). Oaks.

\section{Smilia Germar}

1664 S. camelus Fab. Rochester, Wendt (O1s); Batavia, Kngt (Funk); Buffalo (EPV); Ithaca (CU); Glenmont, HDHouse (Wood-NYS); Nassau (NYS); Catskills (Mtch); Ramapo Mts. (Mtch); Bronx Pk., Wat (Ols) ; LI, Je, Eng (Mtch, Ols). Locust, oak.

$1664 a$ S. camelus var. guttata Fitch. "NY" (Fitch); New Foundland, Je, Wat (Mtch).

1664d S. camelus var. silvestri Mtch. Otis Summit, Catskills, Je, Silvester (Mtch).

\section{Cyrtolobus Goding}

- C. funkhouseri Wood. Ft. Montgomery, Schott (Wood); SI, Ds (EPV) as celsus belongs here (Wood); LI: Central Pk., Shoe (Wood); Yaphank, Massapequa, Wading R., Ds (Wood); Bay Shore, Ols (Wood).

1668 C. ovatus VanD. Ithaca (Funk); SI, Ds (EPV); LI: Bay Shore, Ols (Wood); E. Hampton (Funk); Yaphank, Ds (Wood).

1669 C. fuliginosus Emns. Rochester, $M \mathcal{E} W$ (Wood, EPV); Indian Falls, Ds (Wood); Ithaca (Funk); Salem, J1 (Ball); Bronxville (Wood); SI, Ds (Wood); LI (Wood). All large oaks.

1671 C. arcuatus Emns. Ithaca, Kngt (Funk); SI, Ds (Funk); LI: Yaphank (Wood).

1672 C. fenestratus Fitch. Rochester, Wendt (Wood); Batavia, Ds (Wood) ; Catskills (Wood); Bronxville (Wood); LI: Yaphank, Ds (Wood). Large oaks.

1673 C. tuberosus Fairm. Rochester, Wendt (Wood); Ithaca (EPV); Danby (Funk) ; Spencer (Funk); Pine I. (Wood) ; LI: E. Hampton (Funk); Yaphank, Ds (Wood); Coram, Eng (Wood); Kings Pk., Bell (Ols).

1676 C. discoidalis Emns. Genesee Co, Ds (Wood); Boston, Aug (EPV); Ithaca (EPV, Funk); LI: Farmingdale, Morrow (Funk).

C. acuminatus Wood. Pine I., Ds (Wood).

C. auroreus Wood. Pine I., Ds (Wood) ; Bronxville (Wood) ; LI: Yaphank, Ds (Wood). White oak.

1677 C. cinctus VanD. Ithaca, Je (EPV), type; Bronxville (Wood). White oak. 
1678 C. vau Say (sculptus Fairm.). Albion, Conesus L., Aug (EPV) ; Rochester (Funk); Buffalo, Hamburg, Lancaster, Boston, Salamanca, Je-Aug (EPV); Olcott, Dt (Funk); Red House, Not (Wood); Ithaca (EPV, Funk); Caroline to Harford, Je (EPV-CU); Trumansburg (Funk); Cortland (Funk); Karner, Albany (Wood-NYS); Tivoli (WoodNYS); Pine I., Ds (Funk); Bronxville (Wood); West Pt., Ds (Wood); Ft. Montgomery, Schott (Ols); NYC (Mtch); LI: E. Hampton (Funk); Central Pk. (Ols); Bay Shore, Ols (Funk); Farmingdale, Morrow (Funk). White, red, chestnut, and scarlet oak.

C. pulchellus Wood. Pine I., Ds (Wood); W. Nyack, Ols (Wood).

C. puritanus Wood. LI: Yaphank, Ols, Half Way Hollow Hills, Ds, Central Pk., Shoe, Wyandanch, Ols (Wood).

1681 C. maculifrontis Emns. Rochester, Wendt (O1s) ; Portage, Kngt (Funk) ; Batavia, Kngt (Funk); Ithaca (Funk); Pine I., Ds (Wood) ; Bronxville (Wood); LI: West Hills, Wading R., Central Pk., Wyandanch, Ds (Wood).

1682 C. pallidifrontis Emns. Mt. Kisco (Wood-NYS); Rochester, Wendt (Wood) ; Red House, Not (Wood); Poughkeepsie (Wood-NYS) ; Pine I. (Wood) ; Nyack, Ds (Wood); W. Nyack (Ols) ; Bronxville (Wood) ; LI: Rockaway Beach (Ols) ; E. Hampton, Ds, Central Pk., Ols, Kings Pk. (Wood); Yaphank (Wood).

1683

C. gramatanus Wood. Pine I., Ds (Wood) ; Bronxville (Wood) ; SI (Wood). C. intermedius Emns. Mt. Whiteface, $D s \cdot$ (Wood); Wells, $Y g$ (Wood); Rochester, Moore (EPV); Buffalo, Hamburg, Gowanda (EPV); Batavia, Ds (Wood); Ithaca (Funk); Salem, J1 (Ball); Bronxville (Wood) ; SI (Wood); LI: Yaphank, Ds, Bay Shore, Ols (Wood).

1684 C. inermis Emns. Colden, Hamburg, Salamanca, J1-Aug (EPV) ; Ithaca (Funk); LI: Farmingdale, Morrow (Funk); Wading R., Ds (Wood); Central Pk. (Ols).

1685 C. griseus VanD. Genesee Co, Batavia, Ds (Wood).

1686 C. cinereus Emns. Batavia, Kngt (Funk); Buffalo, Je (EPV); Ithaca (EPV, Funk) ; LI: E. Hampton (Funk). Oak.

C. rufulus Wood. SI, Ds (Wood); LI: Central Pk., Ds, Wyandanch, Schott (Wood).

C. parvulus Wood. Karner (Wood-NYS), paratypes; LI: Massapequa, Ds, Bay Shore, Ols, Central Pk., Shoe (Wood). Quercus prinoides.

1687 C. fuscipennis VanD. Batavia, Kngt (Funk); Buffalo, Je (EPV); Red House, Not (Wood); Ithaca (EPV, Funk); Albany, Lansingburg (Wood-NYS) ; West Pt., Ds, Pine I. (Wood); Ft. Montgomery, Ds (Wood); Bronxville (Wood); SI, Ds (EPV, Wood); LI: Deep Pond, Ds, Bay Shore, Rockaway, Maspeth, Ols (Wood). Oak.

1688 C. maculatus Buckt. "NY" (Buckt).

C. dixianus Wood. Rochester Jct., Leon (Funk).

\section{(Subgenus Aтyma Stål)}

1690 C. castaneae Fitch. Gloversville, Ap (NYS); Olcott, Dt (Funk) ; Buffalo, Boston, Jamestown, Gowanda, Salamanca, Jl-Aug (EPV); Springwater, Not (Wood); Red House, Not (Wood); Rock City, Kngt (Funk); Freeville, J1 (EPV-CU); Ithaca (EPV, Funk); Karner, 
Poughkeepsie (NYS); Slide Mt., Ds (Wood); Ft. Montgomery (Wood); Slide Mt., Ds (Wood); Westchester Co (Wood); SI, Ds (Wood); LI: Maspeth, Ols (Wood). Scrub oak, chestnut, white oak.

C. castaneae var. viridis Emns. Olcott, $D t$, Ithaca (Funk). Woodruff regards viridis as a color form of castaneae.

$1690 \mathrm{a}$ C. castaneae var. lineatus Buckt. Hamburg, Gowanda, J1-Aug (EPV).

1691 C. helena Wood. L. Keuka, Cy (Funk); Bronxville, Je (Wood); LI: Farmingdale, Morrow (Funk). Quercus bicolor.

1692 C. querci Fitch. Hastings, Ds (Wood); Rochester Jct., Leon (Funk) ; Niagara Falls, Aug (EPV); Boston, Gowanda, Salamanca, J1-Aug (EPV); Portage, Kngt (Funk); Attica (Funk); Red House, Not (Wood); Trumansburg. Ithaca, Cortland (Funk); Binghamton (Funk) ; Karner, Albany (NYS); Salem, J1 (Ball); Poughkeepsie. (WoodNYS); West Pt., Ds (Funk) ; Pine I. (Wood) ; Southfields (Wood) ; Bronxville (Wood); SI, Ds (Wood); LI: Yaphank (Wood) ; Central Pk., Ds, Bay Shore, Kissena Pk., Hempstead, Ols (Wood) ; Wyandanch, Schott, Coram, Eng, Wading R. (Wood). Scrub, white, and chestnut oak.

1693 C. inornata Say. Batavia, Kngt, Ithaca, Plunk (Funk); Gowanđa, Aug (EPV-CU); Poughkeepsie (NYS); LI: Brooklyn (Funk). White oak, hickory.

\section{Xantholobus VanDuzee}

1695 X. muticus Fab. Rochester, Wendt (Wood) ; Rochester Jct., Leon (Funk) ; Batavia, Kngt (Funk); Buffalo, Je (EPV); Conesus L., Ds (Wood); Portage, Kngt (Funk); Ithaca (EPV, Funk) ; Nassau (Wood-NYS) ; Bronxville, Pine I. (Wood); SI, Ds (EPV); LI: Maspeth, Ols (Wood). Oak.

1696 X. lateralis VanD. Rochester, Wendt (Wood); Ithaca (EPV-CU).

1698 X. nitidus VanD. SI, Ds (EPV); LI: Bay Shore, Ols (Wood).

\section{OPHIDERMA Fairmaire}

1700 O. salamandra Fairm. Mt. Whiteface, Ds (Wood); Rochester, Wendt (Wood) ; Olcott, Dt (Funk); Buffalo, Je (EPV); Ithaca (CU) ; Albany (NYS); Slide Mt., Ds (Wood); Bronxville (Wood); LI: Kings Pk. (Wood); Rockaway, Ols (Wood). Oak.

1701 O. pubescens Emns. (flaviguttula Goding). Albion, Aug (EPV) ; Rochester, Wendt (Wood); Olcott, Dt (Funk); Fulton, Je (EPV-CU); Ithaca (CU) ; Caroline to Harford, Je (EPV-CU) ; Slide Mt., Ds (Wood) ; Bronxville (Wood); SI, Ds (Wood); LI: Farmingdale, Morrow (Funk); Wading R. (Wuod); Bay Shore, Ols, Wyandanch, Schott (Wood). White oak.

1703 O. flavicephala Goding. Ithaca, Je-J1 (EPV, Funk); SI, Ds (Wood) ; LI: Wyandanch, Schott, Farmingdale, Morrow (Funk); Bay Shore (Ols, Wood).

1704 O. flava Goding. Mt. McIntyre, summit, Bronson EChrisp (Wood-NYS) ; Mt. Whiteface, Ds (Wood); Woodworth, Alex (NYS); Ithaca (EPV, Funk); Albany (NYS); Bronxville (Wood); SI, Ds (EPV.CU).

O. definita Wood. Mt. Whiteface, Ds (Wood); Hamburg, Aug (EPV), paratype; Ithaca (Funk); Bronxville, Wood (Funk); SI (Wood) ; LI: Yaphank (Wood). Red oak. 
O. evelyna Wood. Rochester, Wendt (Wood); Ithaca (Funk); Bronxville (Wood); SI (Wood); LI: Wading R., Ds (Wood). Quercus velutina.

O. grisea Wood. Rochester Jct., Leon, Olcott, Dt (Funk). Quercus coccinea, Q. rubra.

O. fraterna Gibson \& Wells. "NY" (Gibson\&Wells). This is the male of flava Goding (Wood).

\section{VANDUZEA Goding}

1709 V. arcuata Say. Rochester (Funk); Buffalo (EPV); Ithaca (EPV, Funk) ; Cortland, Binghamton (Funk); White Plains (Funk); Bronxville (Wood); SI (Wood) ; LI: Cold Sp. Harb., Sep (Mtch); Jamaica, Ols, Farmingdale (Funk); Yaphank (Wood); Mt. Vernon, Je; Wading R., Ds (Wood); Port Washington, Maspeth, North Beach, Flushing, Hempstead, Central Pk. (Ols).

\section{ENTYLIA Germar}

1716 E. carinata Forst. (bactriana Germ.). Mt. Whiteface, Wilmington, Buffalo (Funk) ; Newport (Yg-NYS) ; Lake Bluff, L. Pleasant (NYS) ; E. Aurora, Hamburg, Colden, Lancaster, Lawtons, Clarence, Gowanda, Otto, Millville, Dryden L., Ithaca (EPV); Trumansburg, Binghamton (Funk); Trenton Falls, Dbl (Wlk); Albany, Nassau, Poughkeepsie, Stockport, Phoenicia, West Hills, Hoversville (NYS); Yonkers (O1s, Wood); NYC (Mtch); Bronx, Mueller (Funk); Bronx Pk., Ols, Van Cort. Pk. (Wood) ; SI, Ols (Mtch, Funk); LI: Queens (Mtch); Maspeth (O1s); Central Pk. (O1s). Thistle, joe-pye weed, sunflower.

1716a E. carinata var. torva Fitch. Lancaster, Lawtons, E. Aurora, Gowanda, JeOct (EPV).

1716b E. carinata var. reducta W1k. Gowanda, Aug (EPV); Ithaca (Funk).

1717 E. concisa Wlk. Ithaca (Funk); LI: Yaphank, Ols (Funk).

1718 E. sinuata Fab. "NY" (Fitch).

\section{Publilia Stål}

1719 P. concava Say. Severance, Bks, and Beede's, Keene Val. (NYS); Ilion (NYS); Norwood, Not (Wood-AM); Rochester (Wendt); Medina, LHWeld (Funk); Sodus Pt., Not (Wood-AM); Batavia, Kngt (Funk) ; Oakfield, Nol (Wood) ; Buffalo (EPV); Westfield (WoodNYS) ; Otto, Comst, Cattaraugus (EPV-CU); Freeville, Dt (Funk); Ithaca (EPV, Funk); Slaterville to Caroline, (EPV-CU); Trenton Falls (NYS); Thacher Pk., Bish (Wood) ; Ravena, Nassau (NYS) ; New Baltimore, $Y g$ (NYS); White Plains (Funk, Ols); W. Nyack, Ols (Wood); LI: Brooklyn (Funk). Goldenrod, skunk cabbage.

1719a P. concava var. nigridorsum Goding. Batavia, Kngt (Funk) ; Buffalo, Lancaster, Hamburg, E. Concord, Gowanda, May-Aug (EPV); Freeville, Dt (Funk) ; Ithaca (EPV, Funk); Dryden L., Je (EPV-CU).

\section{SUBFAMILY MEMBRACINAE}

\section{CAMPYLEnCHIA Stål}

1734 C. latipes Say (curvata Stål). Keene Val. (NYS); L. Placid, EPV (NYS); Speculator, $Y g$ (Funk); Potsdam, Houghton (Ols); Mt. Whiteface, Saranac L. (Funk); L. Champlain, Bish (Funk); near Ausable R. 
(EPV) ; Cranberry L. (Osb) ; Rochester (Funk) ; Olcott, Dt (Funk) ; Medina, LHWeld, Rochester Jct., Leon (Funk) ; Batavia, Kngt, Albion (Funk) ; Thousand Isls.; Buffalo, Hamburg, Gowanda, J1-Aug (EPV) ; Otto (EPV) ; Ithaca, Cortland, Trumansburg, Binghamton (Funk); Trenton Falls (Wlk-Brit.Mus.); Washingtonville, Poughkeepsie, Slingerlands, North Cr., Conquest, Clinton Hgts., Rensselaer, Albany, Nassau (NYS) ; Pine I. (Wood) ; W. Nyack, Ols (Wood) ; Oliverea (Wood) ; Bronxville, Riverdale (Wood); White Plains (Osb) ; NYC (Mtch) ; Bronx, Mueller (Funk) ; LI: Maspeth, Ols, Flushing, Plunk, Brooklyn, Woodhaven (Funk); E. New York (Funk); Bay Shore, Ols (Wood) ; Port Washington, Hempstead, Wyandanch (Ols) ; Cold Sp. Harb. (Osb). Aster, daisy, joe-pye weed, alfalfa, prickly lettuce, wild carrot, Helenium tenuifolium.

\title{
EnCHENopa Amyot and Serville
}

1735 E. binotata Say. Speculator, $Y g$ (NYS) ; Rochester, Moore (EPV, Funk); Buffalo, Hamburg, Gowanda, Jl-Aug (EPV); Otto, Comst (EPVCU) ; Ithaca, J1-Aug (Funk, EPV-CU) ; Utica, Sep (Lint) ; Chevioton-Hudson (Lint) ; Schenectady, Glens Falls, Karner, and New Paltz (NYS); Watervliet Reservoir, Leon (NYS); Scarsdale (Funk); White Plains (Funk); W. Nyack (Ols, Wood); Bronxville (Wood); Central Pk., NYC (Mtch); LI: Maspeth, Ols (Funk); Flushing, Port Washington, Hempstead, Central Pk. (Ols) ; Kings Pk., Bell (Wood); Jamaica, Wlf (NYS). Locust, wild grape, bittersweet, hickory, sycamore, butternut, dogwood, daisy, joe-pye weed.

\section{SUBFAMILY CENTROTINAE}

\section{MICROCENTRUS Stål}

1747 M. caryae Fitch. Cranberry L. (Osb); Batavia, Kngt (Funk); Buffalo, Elma, Hamburg, Gowanda, Aug-Sep (EPV) ; Coy Glen, Dt, Ithaca (Funk); SI (Mtch, Ols); LI: Cold Sp. Harb., Parsh (Funk); Pinelawn, Wyandanch (Ols); Queens (Mtch). Hickory, oak.

1748 M. perdita A. \& S. Bronxville (Wood); LI: Yaphank (Wood) ; Flushing (Ols); Huntington, Schott (Ols).

\section{FAMTLY CICADELLIDAE 21}

\author{
(Jassidae)
}

\section{SUBFAMILY BYTHOSCOPINAE}

\section{Agallia Curtis}

1762 A. novella Say. Axton, Je (M\&H) ; Cranberry L. (Osb); L. Placid, Aug (EPV); Batavia, May-Aug Kngt (O1s); Buffalo and vic., May-J1 (EPV) ; Rock City, Je, Kngt (Ols) ; Ithaca, McLean, May-J1 (Buys) ; Oliverea, May, Shoe (O1s); Phoenicia (Osb) ; LI: Bay Shore, Yaphank, Jl (Ols).

21 The list in the family Cicadellidae has been compiled from records, both published and unpublished, chiefly of E. P. Van Duzee, C. E. Olsen, E. D. Ball, J. L. Buys, D. M. DeLong, and Herbert Osborn. Apparently there is still some disagreement concerning the exact status of certain of the species, but the editor has attempted to harmonize the views of these specialists to the best of his ability. 
1763 A. oculata VanD. Niagara Falls, Hamburg, Salamanca, Jl-Aug (EPV); Cranberry L. (Osb).

1764 A. quadripunctata Prov. Axton, Je (M\&H) ; L. Placid, Aug-Sep (EPV); Cranberry L. (Osb) ; Rochester, Jl, Wendt (Ols) ; Batavia, Je-J1, Kngt (Ols) ; Buffalo and vic., May-Aug (EPV); Grand I., Clarence, Colden, Hamburg, Lawtons, May-Aug (EPV); Lancaster, EPV (Lint); Portage, May, Kngt (Ols) ; Rock City, J1, Kngt (O1s) ; Ithaca, Ap-Je (CU) ; McLean, Ringwood, May-Je (Buys); Labrador L., May (Buys); Oliverea, Jl, Shoe (Ols) ; White Plains, Je (Ols); W. Nyack, May (Ols) ; LI: Forest Pk., Jl, Bno (Osb) ; Jamaica, Cold Sp. Harb. (Osb); Maspeth, North Beach, Flushing, Yaphank, Wading R., May-Je (Ols).

1765 A. constricta VanD. White Plains, May (Ols); SI, May, Ols (EPV), Aug (EPV) ; LI: Maspeth, Forest Hills, Bay Shore, Hempstead, Winfield, May-Sep (Ols); Cold Sp. Harb. (Osb); Sea Cliff (EPV-CU).

A. lingulata Ols. W. Nyack, May 30, 1921 (O1s).

1766 A. deleta VanD. (immaculata Lathrop). White Plains, May 2, 1915 (Ols).

1767 A. sanguinolenta Prov. L. Placid, Aug (EPV); Cranberry L. (Osb); Batavia, May-J1, Kngt (Ols); Buffalo and vic., including Lancaster, Hamburg, N. Evans, Je-Oct (EPV); Phoenicia (EPV); Itkaca, May, Morr (Osb), J1-Aug (Buys); Karner (Osb-NYS); Eagle Bridge, Nassau (Osb); White Plains, Yorktown Hgts., Je, Sep (Ols); Mosholu, Oct, Bno (Osb) ; SI (EPV); LI: Jamaica, Cold Sp. Harb. (Osb) ; Maspeth, Flushing, Central Pk., Bay Shore, Bayville, Hempstead, Flatbush, Forest Hills, Yaphank, May-Nov (Ols).

\section{IDIOCERUS Lewis}

1776 I. amabilis Ball. Cranberry L. (Osb) ; Batavia, Aug, Kngt (O1s) ; Salamanca, J1 (EPV); LI: Bay Shore, Yaphank, J1 (Ols).

1777 I. nervatus VanD. Albion, Aug (EPV); Lancaster, Hamburg, J1-Aug (EPV); Albany (Osb-NYS); NYC, Southwick (EPV); SI (Osb).

1778 I. pallidus Fitch. L. Placid, Aug (EPV); Cranberry L. (Osb); Sodus Pt., Aug (Buys); Albion, Aug (EPV); Batavia, J1-Aug, Kngt (Osb, Ols); Buffalo and vic., including Hamburg, J1-Aug (EPV); Chatauqua Co, Sep $(D e L)$; Salamanca, J1 (EPV); Portage, Aug, Kngt (Ols); Ithaca, Aug (Buys); Oliverea, Aug, Ang (AM); Salem (Osb); White Plains, Je (Ols); W Nyack, J1 (Ols) ; SI, Sep, Ds (Ols) ; LI: Yaphank, Aug, Ds. Willow and poplar.

1779 I. suturalis Fitch. L. Placid, Aug (EPV); Cranberry L. (Osb) ; Keene Val., Aug, Not (O1s) ; Batavia, J1-Aug, Kngt (Ols, Osb); Buffalo and vic., including Hamburg, Gowanda, Elma, Colden, Je-Sep $(E P V)$; Chautauqua Co, J1 (DeL); McLean, J1 (Buys) ; Karner (Osb-NYS); Salem (Osb) ; Phoenicia (EPV). Willow.

1779a I. suturalis var. lunaris Ball. L. Placid, Aug (EPV); Batavia, J1-Aug, Kngt (Ols, Osb) ; Gowanda, Hamburg, Je-Aug (EPV); Chautauqua Co, J1 (DeL) ; Salamanca, J1 (EPV); McLean, J1 (Buys); Salem (Osb).

1779c I. suturalis var. vagus Ball. Hamburg, Salamanca, J1-Aug (EPV).

I. subnitens S. \& D. Cranberry L. (Osb).

1780 I. duzeei Prov. Lancaster, J1 (EPV). 
1781 I. alternatus Fitch. L. Placid, Aug (EPV); Cranberry L. (Osb) ; Buffalo and vic., including Hamburg, May-Aug (EPV); Chautauqua Co, J1 $(D e L)$; Ithaca, Ap-J1 (Buys); McLean, Ap, Morr (Osb); Salem $(O s b)$. Willow.

I. formosus Ball. Cranberry L. (Osb).

1782 I. verticis Say. Salem (Osb).

I. cognatus Fieb. Chautauqua Co, J1 (DeL) ; LI: Winfield, Maspeth, Port Washington, Je-Sep (Ols). Introduced from Europe.

1793 I. scurra Germ. Buffalo, Oct (EPV); Ithaca (Leon\&Cy); Bronx Pk.; Aug, Matausch (EPV); LI: Brooklyn, Aug, Schott (Ols); Maspeth, Flushing, Aug-Sep (Ols). Poplar.

1795 I. lachrymalis Fitch. L. Placid, Aug (EPV); Cranberry L. (Osb) ; Keene Val., Not (Ols) ; Batavia, Je-Sep, Kngt (Ols, Osb); Portage, Je, Kngt (Ols) ; Hamburg, Lancaster, Elma, Gowanda, Je-Aug (EPV); Chautauqua Co, J1 (DeL); Ithaca, Aug, McLean, J1, Taughannock Falls, J1 (Buys); Atwater, J1 (Buys); Salem (Osb); Phoenicia $(\mathrm{EPV})$; LI: Bay Shore, J1 (Ols). Poplar.

1797 I. snowi G. \& B. Lancaster, Aug (EPV).

1800 I. crataegi VanD. Niagara Falls, Aug (EPV); Buffalo and vic., including Lancaster, Hamburg, J1-Sep (EPV) ; Chautauqua, May, Salamanca, J1 (EPV) ; Conesus L., Aug (EPV); Ithaca, Aug (Buys). Crataegus.

1801 I. provancheri VanD. Black Apple-Leaf Hopper. L. Placid, Aug (EPV) ; Cranberry L. (Osb); Severance (Osb-NYS); Oswego (Leon); Newark (Leon); Wolcott (Mat); Williamson (Cy); Spencerport (Cy) ; Honeoye Falls, Rochester Jct. (Leon) ; Holley (Cy) ; Batavia, Je-J1, Kngt (Osb, Ols) ; Buffalo and vic., including Lancaster (EPV); Penn Yan (Cy); Ithaca (Leon); McLean, J1 (Buys); Ulster Co, J1 (Ols); Kinderhook, Stuyvesant Falls (Cy); LI: Babylon (Leon); Fishers I., Aug, Zab (AM). Apple, quince, pear.

1802 I. fitchi VanD. Batavia, Aug, Kngt (O1s); Buffalo, Hamburg, Salamanca, J1-Aug (EPV); White Plains, Je (Ols); W. Nyack, Je (Ols); Mosholu, Bno (Osb); LI: Hempstead, Sep. (Ols).

\section{MACROPSIS Lewis}

\section{(Pediopsis Burmeister)}

1806 M. reversalis O. \& B. Colden, EPV (O\&B).

1807 M. gleditschiae O. \& B. Chautauqua Co, Je ( $\left.D_{e} L\right)$; White Plains, Je $(O l s)$; LI: Yaphank, J1 (Ols).

1808 M. virescens Gmel. var. graminea Fab. Hamburg, Lancaster, J1-Aug (EPV); Salem, Aug (Osb). Willow.

1809 M. viridis Fitch. L. Placid, Aug (EPV); Cranberry L. (Osb) ; Batavia, Je-J1, Kngt (Ols) ; Buffalo, Hamburg, Colden, Elma, Gowanda, Je-Aug $(E P V)$; Chautauqua Co, Je $(D e L)$; Salamanca, J1 (EPV); Ithaca, Aug (CU), Je (Buys); Karner (Osb-NYS); Salem, Aug (Osb); LI: Flushing, $\mathrm{Jl}(\mathrm{Ols})$. Willow.

1811 M. suturalis O. \& B. Colden, EPV (O\&B); Hamburg, J1 (EPV); Chautauqua Co, Je (DeL); Ringwood, Je (Buys).

1812 M. crocea O. \& B. Hamburg, J1 (EPV). 
1815 M. basalis VanD. Cobble Hill, Adirondacks, Aug (EPV); Cranberry L. (Osb) ; Batavia, Je-J1, Kngt (Ols) ; Colden, Elma, Hamburg, Lancaster, Je-J1 (EPV); Salamanca, Aug (EPV). Aspen.

1817 M. canadensis VanD. L. Placid, Aug (EPV); Cranberry L. (Osb) ; Batavia, J1-Aug, Kngt (Ols); Portage, Je, Kngt (O1s) ; Lancaster, Je $(E P V-C U)$. Willow.

1818 M. bifasciata VanD. Axton, Je (M\&H); Karner (Osb-NYS); Salem $(O s b)$.

1819 M. nigricans VanD. Hamburg, J1-Aug (EPV); Colden, J1 (EPV); Gowanda, Je (EPV). Poplar.

1821 M. trimaculata Fitch. Axton, Je (M\&H) ; Sodus Pt., Sep (Buys) ; Batavia, Je-Aug, Kngt (Ols, Laws); Portage, Je, Kngt (Ols); Buffalo, Boston, Lancaster, Hamburg, Gowanda, Colden, Je-Aug (EPV); McLean (Buys); Highland, Felt (Osb). Wild plum.

1822 M. sordida VanD. Cranberry L., Htly (Osb \& DeL); White Plains, Je (Ols).

\section{ONCOPSIS Burmeister}

1824 O. variabilis Fitch. Cranberry L. (Osb) ; Plattsburg, J1, Ds (O1s); L. Tear, J1, Not (Ols) ; Upper Ausable L., J1, Beq (Ols) ; Fourth L., Je (Ols) ; Buffalo, Otto, Lancaster, Hamburg, May-J1 (EPV); Chautauqua Co (DeL); Gloversville (EPV); West Pt., Je, Ds (Ols) ; Bronxville, Je, Wood (Ols); LI: Bay Shore, Yaphank, Wyandanch, J1 (Ols). Birch.

1825 O. sobrius W1k. Cranberry L. (Osb); Genesee Co, Je, Ds (Ols) ; Niagara Falls, Colden, Lancaster, Salamanca, May-J1 (EPV); Chautauqua Co, Je (DeL); SI, Je, Ds (Ols) ; LI: Wyandanch, Schott; Bay Shore, Yaphank, Je-J1 (Ols). Birch.

1826 O. cognatus VanD. Plattsburg, J1, Ds (O1s) ; Cobble Hill, Adirondacks, Aug (EPV); Cranberry L. (Osb) ; Hamburg, Colden, Gowanda, Lancaster, May-Aug (EPV); Wyoming, Je, Ds (Ols); Ithaca, Ringwood, Je (Buys); Albany (Osb); Phoenicia (Ols).

1827 ?O. truncatus Baker. Batavia, Je-J1, Kngt (O1s).

1828 O. fitchi VanD. Cranberry L. (Osb); Wilmington, Aug (WHBrittain); Buffalo, Hamburg, Colden, Boston, May-Aug (EPV); Wyoming Co, Je, Ds (Ols).

1829 O. pruni Prov. Cranberry L. (Osb) ; Mt. Marcy, J1, Not (Ols) ; Axton, Je $(\mathrm{M} \& \mathrm{H})$; Upper Ausable L., J1, Beq (Ols); Batavia, Je, Ds (Ols) ; Buffalo (EPV); Rock City, J1 (Kngt); Ellis, Ithaca, Je (Buys); Albany (Osb) ; Oliverea, J1, Shoe (Ols) ; Bronxville, J1, Wood (Ols) ; LI: Hempstead, Central Pk., Bay Shore, Maspeth, Yaphank, Je-Oct (Ols).

1830 O. minor Fitch. Upper Ausable L., J1, Beq (O1s) ; Upper Ausable R., Aug $(E P V)$; Cranberry L. (Osb) ; Buffalo and vic., Je-Aug (EPV); Oliverea, J1, Shoe (O1s) ; W. Nyack, May-Je (O1s) ; SI: Richmond, Je, Ds (Ols); LI: Wading R., May (Ols).

1831 O. nigrinasi Fitch. Cranberry L. (Osb); Batavia, Je-Aug, Kngt (Ols); Portage, Je, Kngt (Ols) ; Buffalo, Colden, Hamburg, Lancaster, Gowanda, Je-Aug (EPV); Salamanca, J1, Rock City, Aug (EPV); LI: Flatbush, Je, Zab (AM). Hornbeam. 
1832 O. fagi Fitch. Mt. Marcy, J1, Not (O1s); Batavia, Je, Kngt (Ols) ; Cattaraugus, Je, Kngt (Ols); Ellis, Ithaca, Je (Buys); Oliverea, J1, Shoe (Ols); Ft. Montgomery, Je, Schott (Ols).

1833 O. distinctus VanD. Buffalo, Lancaster, Hamburg, J1-Sep (EPV) ; Chautauqua Co, J1 (DeL) ; Ft. Montgomery, J1, Schott (Ols) ; Pine I., Je, Ds (Ols). Poplar.

\section{SUBFAMILY CICADELLINAE}

\section{ONCOMETOPIA Stål}

1846 O. undata Fab. Oswego (Osb-NYS).

1847 O. lateralis Fab. Axton, Je (M\&H); Adirondacks, Brb (Ols) ; Cranberry L. (Osb); L. Placid, Aug (EPV); McLean, Aug (Buys).

1847a O. lateralis var. limbata Say. White Plains, Je 29, 1918 (Ols).

Aulacizes Amyot and Serville

1851 A. irrorata Fab. LI: Cold Sp. Harb. (Osb) ; Queens, Wyandanch, Je, Dec 25, under a stone, Schott (Ols); Pinelawn, Kings Pk., Je, Bell (Ols); Roslyn, Aug, Ballou (Ols).

\section{Cicadella Latreille}

1855 C. gothica Sign. Axton, Je (M\&H) ; L. Placid, Aug-Sep (EPV); Keene Val. (Osb) ; Cranberry L., Htly (Osb \& DeL) ; Rochester, J1, Wendt (Ols) ; Batavia, May-Aug, Kngt (Ols) ; Colden, Lancaster, Gowanda, May-Sep (EPV) ; Rock City, Je, Kngt (O1s) ; Portage, May, Kngt (EPV-Ols) ; Ithaca, Je (Buys) ; Oliverea, Je, Beq (Ols) ; Phoenicia, EPV (Osb) ; Karner (Osb); Salem, Aug (Osb) ; Clinton Hgts., Nassau (Osb) ; Kingston, EPV (Osb) ; W. Nyack, May-Oct (Ols) ; LI: Hempstead, Melville, Bay Shore, Wyandanch, Yaphank, May-Oct (Ols) ; Oyster Bay (Osb).

\section{Kolla Distant}

1859 K. bifida Say. Keene Val. (Osb-NYS), Aug, Not (O1s) ; Batavia, Jl, Kngt (Ols) ; Buffalo, Hamburg, Lancaster, Gowanda, J1-Aug (EPV); Ithaca, J1-Aug (Buys-CU); Oliverea, Aug, Zab (AM) ; Phoenicia, EPV (Osb) ; Ballston L., Aug, Leon (NYS) ; L. George, Aug, $Z a b$ (AM) ; Salem (Osb) ; Saugerties, J1, Brb (Ols) ; W. Nyack, J1 $(O l s)$; Bronxville, J1, Wood (Ols); NYC, Sep, Bno (Osb) ; LI : Jamaica, Cold Sp. Harb. (Osb) ; Flatbush, Oct, Zab (Ols).

\section{HELOCHARA Fitch}

1863 H. communis Fitch. L. Placid, Aug (EPV) ; Saranac Inn, J1, Ndm (Osb) ; Cranberry L. (Osb) ; Sodus Pt., Aug (Buys); Rochester, J1, Wendt (O1s) ; Batavia, Aug, Kngt (Osb, Ols); Buffalo, Hamburg, Ap-Sep (EPV) ; Portage, May, Kngt (Ols) ; Ithaca, May-Je, Kngt (Ols, Buys) ; McLean, Ap, Morr (Osb), May, Jl (Buys) ; Ringwood, J1 (Buys) ; Phoenicia (Osb) ; Salem, Ang (Osb) ; Karner, Albany, Nassau (OsbNYS) ; Eagle Bridge (Osb); Kingston (Osb-NYS); White Plains, May (Ols); W. Nyack, Je (Ols) ; SI (Osb-NYS) ; LI: Flushing, Maspeth, Bay Shore, Huntington, Forest Hills, Wyandanch, Je-Sep (Ols). Juncus. 


\section{GRAPHOCEPHALA Van Duzee}

\section{(Diedrocephala Woodworth)}

1864 G. coccinea Forst. Ausable R., L. Placid, Aug-Sep (EPV); Saranac Inn, Keene Val., Wilmington (Osb) ; Cranberry L., Htly (Osb \& DeL); Sodus Pt., Aug (Buys); Rochester, J1, Wendt (Ols) ; Batavia, Je-J1, Kngt (Osb); Buffalo, Hamburg, Gowanda, J1-Aug (EPV); Portage, Aug, Kngt (Osb); Ithaca, Je-J1 (Buys); Salem (Osb); Albany, Nassau, Eagle Bridge (Osb) ; Poughkeepsie (Osb) ; W. Nyack, Je-Sep (Ols) ; Mosholu (Osb) ; SI, J1 (Osb, Ols) ; LI: Forest Pk., Oyster Bay (Osb) ; Flatbush, Je-J1 (Buys) ; Bay Shore, Forest Hills, Huntington, Schott, Flushing, Central Pk., Sea Cliff, Kings Pk., Bell, Cold Sp. Harb., Brb, Rockaway Beach, Queens, Yaphank, Je-Oct (Ols).

$1864 a$ G. coccinea var. teliformis W1k. L. Placid, Sep (EPV); Boston, Gowanda, Aug (EPV); Trenton Falls (W1k-Brit. Mus.); LI: Bay Shore, J1, Ols (EPV).

\section{DRAECUlaCePhala Ball}

1873 D. angulifera Wlk. Batavia, Aug, Kngt (Ols); Ithaca, Je-Sep (Ols, Buys) ; McLean Bogs, May, Ji (Buys); Nyack, Aug, Zab (AM) ; LI: Flushing, Winfield, Maspeth, Je-Sep (Ols).

1874 D. mollipes Say. Axton, Je (M\&H) ; L. Placid, Aug (EPV) ; Cramberry L. (Osb) ; Sodus Pt., Aug (Buys) ; Batavia, Je-Aug, Kngt (Ols, Osb); Buffalo, Hamburg, Colden, May-Sep (EPV) ; Portage, Je, Kngt (Ols) ; Rock City, Je, Kngt (Ols); Ithaca and vic., Je-Oct, div. (CU) ; McLean Bogs, May, J1 (Buys) ; Ringwood, J1 (Buys); Phoenicia (EPV) ; Trenton Falls (W1k); Salem (Osb); Karner, Albany, Nassau, Eagle Bridge (NYS); Poughkeepsie (NYS); White Plains, May (Ols); NYC, Bno (Osb) ; SI, May (Osb, Ols); LI: Sea Cliff (EPV-CU) ; Jamaica, Cold Sp. Harb. (Osb); Maspeth, Flushing, Bay Shore, Rockaway Beach, Little Neck, Huntington, Schott, North Beach, Yaphank, Ap-J1 (Ols).

1875 D. minor Wlk. Buffalo and vic. (EPV) (as D. mollipes var. producta W1k.).

1877 D. manitobiana Ball. Batavia, Aug, Kngt (O1s).

1879 D. noveboracensis Fitch. L. Placid, Aug (EPV); Keene Val. (Osb) ; Cranberry L. (Osb); Piseco L. (Osb); Albion, Aug (EPV); Honeoye Falls, Leon (Buys); Batavia, J1, Kngt (O1s); Squaw I., Niagara R. $(E P V)$; Buffalo, Elma, Colden, Hamburg, Je-Aug (EPV); Otto, J1 (Uhl-CU) ; Portage, Aug, Kngt (Osb) ; Ithaca, Je-Sep (CU) ; McLean, J1, Buys (Ols); Salem (Osb); Nassau (Osb); Carmel, Aug, Buys (Ols) ; LI: Cold Sp. Harb., J1, Brb (Osb, Ols); Wyandanch, Aug (O1s).

PAGARONIA Ball

1883 P. tripunctata Fitch. Gowanda, Aug (EPV); Ithaca (CU); Phoenicia, Aug $(E P V)$; Salem, Aug (Osb); Albany (Osb); Saugerties, J1, Brb (Ols); White Plains, Je (Ols); W. Nyack, Oct (Ols); Mosholu (Osb); SI (Osb) ; LI: Jamaica, Cold Sp. Harb. (Osb); Sea Cliff (EPV-CU) ; North Beach, Glen Head, Wyandanch, Je-Sep (Ols).

Evacanthus LePeletier and Serville

1884 E. acuminatus Fab. Cranberry L. (Osb) ; Mt. Whiteface, Cobble Hill, Aug $(E P V)$; Batavia, Je, Kngt (Ols); Niagara Falls, Aug (EPV); Lancaster, Gowanda, J1-Aug (EPV); Salamanca, J1 (EPV); Chautauqua Co, Ji (DeL) ; Oliverea, J1, Shoe (Ols); Slide Mt., Debruce, Aug-Sep (AM). 


\section{SUBFAMILY GYPONINAE \\ PENTHIMia Germar}

1894 P. americana Fitch. Rochester, J1, Wendt (O1s) ; Batavia, Je, Kngt (Osb) ; Buffalo and vic., May-J1 (EPV); Portage, May, Kngt (Osb); Ft. Montgomery, J1, Schott (Ols); W. Nyack, May (Ols) ; LI: Wyandanch, Schott, Pinelawn, Yaphank, Wading R., May-J1 (Ols).

\section{GyPonA Germar}

1896 G. rugosa Spångb. Grand I., Sep (EPV); Niagara Falls, Aug (EPV) ; Buffalo, Aug (EPV); W. Nyack, Oct (Ols); LI: Flushing, Oyster Bay, Bay Shore, Hempstead, Huntington, Wyandanch, Aug-Sep (Ols). Oak.

1897 G. octolineata Say. Cranberry L. (Osb) ; Keene Val., Aug, Not (Osb, Ols) ; Hope (Osb) ; Sodus Pt., J1 (Buys) ; Batavia, J1-Sep, Kngt (Osb, Ols); Niagara Falls, Oct (EPV) ; Portage, Aug, Kngt (O1s) ; Buffalo, Lancaster, Hamburg, Aug-Oct (EPV) ; Ithaca, Aug, Sep (O1s, Buys) ; Karner (Osb); Salem (Osb); W. Nyack, Je-Sep (Ols) ; Dunwoodie, Oct, Ang (O1s) ; NYC, Sep (Osb, Ols) ; LI: Jamaica (Osb) ; Flushing, Maspeth, Bay Shore, Hempstead, Pinelawn, Port Washington, Glen Head, Wyandanch, Yaphank, Pennequid Barrens, div. (O1s).

1913 G. octolineata var. geminata Osb. (teste Ball). Sodus Pt., Aug (Buys) ; Hamburg, Sep (EPV) ; Ithaca, J1-Aug (Buys); LI: Oyster Bay $(\mathrm{Osb})$, types.

1898 G. octolineata var. cana Burm. Elma, Hamburg, Gowanda, Salamanca, Je-Aug $(E P V)$.

1899 G. octolineata var. quebecensis Prov. Isham House, Adirondacks, Aug $(E P V)$; Buffalo, Hamburg, J1 (EPV).

1904 G. octolineata var. striata Burm. Buffalo, Lime L., Hamburg, Gowanda, Salamanca, J1-Aug (EPV); LI: Sea Cliff (EPV-CU).

1906 G. melanota Spångb. (bipunctulata Woodw.). Gowanda, Aug (EPV) :

1910 Ithaca, Aug-Sep (EPV, Buys); Mosholu (Bno); SI (Bno); LI: Maspeth, Aug (O1s).

1907 G. verticalis Stål (bimaculata Spångb. teste Ball). White Plains, Pelham Pk., NYC, Sep, Ang (O1s). Both records are with the query that they may refer to melanota Spångb.

1923 G. scarlatina Fitch. Buffalo and vic., Jl-Aug (EPV) ; Ft. Montgomery, Je, Schott (O1s) ; LI: Maspeth, Aug, Schott (O1s).

1920 G. scarlatina var. puncticollis Spångb. (albosignata Uh1.). Niagara Falls,

1922 Hamburg, Ang (EPV); Batavia, Aug, Kngt (Ols); NYC, Sep, Ang (O1s).

1917 G. scarlatina var. pectoralis Spångb. Hamburg, J1, Gowanda, Aug (EPV) ; Ithaca, J1 (Buys); Bronx Pk., Aug (Ols).

1925 G. sanguinolenta Spångb. LI: Bay Shore, J1 (Ols).

\section{XEROPHLOEA Germar}

1930 X. viridis Fab. Rock City, J1, Kngt (Ols) ; Kingston (EPV) ; Mosholu, J1 (Osb) ; LI: Sea Cliff (EPV-CU).

1931 X. major Bak. White Plains, May $(O l s)$; W. Nyack, Ap-Je (Ols) ; Mosholu (Osb) ; LI: Flushing, Bay Shore, J1 (Ols) ; Cold Sp. Harb. (Osb) ; Wading R., Je, Schott (Ols). 
SUBFAMILY JASSINAE

STROGGYLOCEPHALUS Flor

1932 S. agrestis Fall. E. Concord, May (EPV); Ithaca, May (Buys).

\section{Acucephalus Germar}

1933 A. nervosus Schrank. Cranberry L. (Osb) ; Johnstown, Aug, Alex (EPV); Batavia, J1, Kngt (Ols) ; Ithaca, Aug, Ringwood, J1 (Buys).

1935 A. flavostrigatus (Donov.). Indian L., near Sabael, Aug, Brb (O1s); Eagle Bridge, Aug $(O s b)$; Phoenicia, Aug, EPV (Osb).

1936 A. albifrons (L). Cranberry L. (Osb); Wells, J1, Yg (Ball-NYS); Rochester, Moore (EPV); Conesus L., Buffalo, Lancaster, Colden, Hamburg, Portage, Gowanda, J1-Aug (EPV); Ithaca, J1-Aug (Buys, Baker, EPV-CU); Phoenicia, EPV (Osb); Salem $(O s b)$; White Plains, Je (Ols) ; NYC (Southwick); LI: Cold Sp. Harb., Brb (Osb) (Bul. 97, NYSMus); Queens, Aug, Maspeth, Je, Aug, Flushing, J1, Sep, Winfield, Sep, Bay Shore, J1, Rockaway Beach, Aug (Ols) ; Kings Pk., Bell (Ols); North Beach, J1, Schott (Ols); Sea Cliff (EPV-CU).

1937 A. placidus Prov. W. Nyack, Ap (Ols).

\section{Xestocephalus Van Duzee}

1940 X. pulicarius VanD. Cranberry L. (Osb) ; L. Placid, Sep, Hamburg, Sep, Buffalo, Je-Sep, Elma, Je, Stamford, Aug $(E P V)$; Lancaster, Colden, Aug (EPV-CU); Chautauqua Co, J1-Aug (DeL); Ithaca, J1-Aug (Buys, Baker-CU); Phoenicia, Aug (Osb); NYC, Aug, Southwick (EPV-CU); LI: Sea Cliff (EPV-CU); Jamaica, Aug (Osb).

1941 X. superbus (Prov.) (fulvocapitatus VanD.). Hamburg, Aug (EPV); Lancaster, Aug (EPV-CU); W. Nyack, Sep (Ols).

1944 X. nigrifrons Osb. W. Nyack, Je-Sep (O1s) ; Cranberry L. (Osb).

1945 X. brunneus Osb. Hamburg, J1 (EPV); Batavia, May, Kngt (Dickerson).

\section{Nionia Ball}

1946 N. palmeri (VanD.). Stony Pt., Je, Ds (O1s); White Plains, Je (Ols); W. Nyack, Je (Ols) ; Yonkers, Oct, Ang (Ols) ; LI: Forest Pk., Bno $(\mathrm{Osb})$; Sea Cliff (EPV).

\section{Hecalus Stål}

1952 H. lineatus (Uh1.). "NY" (VanD. Cat.).

\section{SPANGBERGIELLA Signoret}

1953 S. vulnerata Uh1. "NY" (Osb, Baker-US).

\section{PARABOLOCRATUS Fieber}

1955 P. viridis Uhl. Cranberry L. (Osb) ; Rochester, Wendt (Ols) ; Niagara Falls, Je, Hamburg, May-Je, Buffalo, Je (EPV); Batavia, Je, Kngt (Laws); Chautauqua Co, Je (DeL); Ithaca, Je (Buys); Mud Cr., Tompkins $\mathrm{Co}, \mathrm{Je}$ (Buys); White Plains (Ols); LI: Jamaica, EPV (Bul. 97, NYSMus); Maspeth, Ap-Sep (Ols) ; Flushing, J1, Rockaway Beach (Ols).

1956 P. major Osb. Cranberry L. (Osb) ; Wyoming, Je, Cattaraugus Co, J1, Kngt $(\mathrm{Ols})$. 
MESAMIA Ball

1975 M. vitellina Fitch. Cranberry L. (Osb) ; Cobble Hill, Niagara Falls, Lancaster, Elma, Buffalo, Gowanda, Salamanca, Je-Aug (EPV).

\section{SCAPHOIDEUS Uhler}

1980

S. sanctus Say. LI: Cold Sp. Harb., Brb (Osb).

1982

S. cruciatus Osb. LI: Cold Sp. Harb., Brb (Osb).

1983 S. auronitens Prov. Cranberry L. (Osb); Albion (Osb); Hamburg, Niagara Falls, Lancaster, Elma, Buffalo, Stamford, Gowanda, Je-Sep $(E P V)$; Chautauqua Co, J1-Aug (DeL); Ithaca, Aug (Buys) ; Albany (Osb); Phoenicia (EPV); W. Nyack, Sep (Ols); SI: Jl (Ols); Clove Val., J1 (Osb-AM); LI: Cold Sp. Harb., Brb (Osb) ; Maspeth, Aug, Port Washington, Sep, Ols (EPV); Hempstead, Sep, Kissena Pk., Oct, Forest Hills, Sep (Ols) ; Sea Cliff, Aug (Ols, EPV-CU) ; Flatbush, Sep (Osb-AM); Jamaica, Aug (EPV, Osb).

1984 S. jucundus Uhl. Lancaster, Aug (EPV); Karner, Aug (Osb-NYS) ; LI: Hempstead, J1, Maspeth, Je (Ols).

1985 S. unicolor Osb. SI (Osb).

1987 S. consors Uh1. Boston, Gowanda, Aug (EPV); Oliverea, Sep, Dickerson (AM).

1988 S. scalaris VanD. Cranberry L. (Osb); Ithaca, Aug (Buys); Phoenicia $(E P V) ;$ W. Nyack, Aug (Ols).

1989 S. lobatus VanD. Cranberry L. (Osb); Lancaster, Sep, WJPalmerJr (EPV-CU) ; Hamburg, Oct, $M C V$ (EPV, Osb); Boston, E. Aurora, Aug-Sep (EPV); Gowanda, Aug (EPV, Osb); Chautauqua Co, Aug (DeL) ; Ithaca, Aug (Buys, Osb); Poughkeepsie (Osb) ; Mosholu (Osb) ; Bryn Mawr Pk., Sep (AM); NYC, Southwick (EPV); LI: Jamaica, Cold Sp. Harb. (Osb); Kissena Pk., Flushing, Maspeth, Aug $(O l s)$; Waterworks, Flatbush, Oct, Lott Wood, Flatbush, Aug-Sep, Zab (Osb-AM).

1990 S. ochraceus Osb. Cranberry L., Aug (O\&D) ; Batavia, J1, Kngt (Ols) ; Rochester, Oct, Colden, J1 (EPV); Buffalo, Aug (EPV, Osb); Gowanda, EPV (Osb); W. Nyack, Je (Ols); LI: North Beach, J1 (O1s).

1991 S. productus Osb. Cranberry L. (Osb) ; Mt. Whiteface, Aug (DeL) ; Hamburg, E. Aurora, Gowanda, Aug (EPV); LI: Cold Sp. Harb., J1, Brb (Osb) ; Sea Cliff (EPV-CU).

1992 S. carinatus Osb. Hamburg, J1, EPV (Osb) ; Gowanda, Aug, Ithaca, J1Aug (EPV); LI: Cold Sp. Harb., Brb (Osb); Bay Shore, J1 (Ols).

1994 S. intricatus Uhl. "NY", Aug, EPV (Osb); LI: Maspeth, Aug (Ols); Wyandanch, Sep, Schott (O1s).

1995 S. luteolus VanD. Hamburg, J1 (EPV); near NYC, J1-Aug, Southwick (EPV) ; SI, J1 (Ols).

1996 S. immistus Say. Keene Val., Aug, Not (Ols); Cranberry L. (Osb) ; L. Placid, Aug (EPV); Sodus Pt., Aug (Buys); Lancaster, J1, EPV (Lint); Batavia, J1, Kngt (Osb, Ols); Niagara Falls, Hamburg, Buffalo, E. Aurora, Gowanda, Salamanca, J1-Aug (EPV); Chautauqua $\mathrm{Co}, \mathrm{Jl}(\mathrm{DeL})$; Ithaca, J1 (Buys); Karner (Osb); Phoenicia (EPV); Kingston (EPV); Eagle Bridge, Salem (Osb); W. Nyack, Aug, Yorktown Hgts., Sep (Ols); LI: Cold Sp. Harb., Jamaica (Osb); 
Bay Shore, J1, Ols (EPV); Hempstead, Aug-Sep, Wyandanch, Aug, Maspeth, Aug, Flushing, J1, Sep (Ols); Flatbush, Jl, Zab, Kissena Pk., Aug (Osb-AM).

1996a S. immistus var. minor Osb. Batavia, Aug, Kngt (O1s); Lancaster, Hamburg, Aug (EPV); LI: Báy Shore, Aug, Ols (EPV); Wyandanch, Aug (Ols).

1996b S. immistus var. major Osb. Boston, Gowanda, Aug (EPV).

1998 S. obtusus Osb. Lancaster, Hamburg, Aug-Sep (EPV); LI: Bay Shore, $\mathrm{J} 1$, Flushing, Je (Ols).

2000 S. opalinus Osb. Gowanda, Aug (EPV) ; LI: Cold Sp. Harb., on red cedar $(\mathrm{Osb})$.

\section{Platymetopius Burmeister}

2008 P. hyalinus Osb. Yonkers, J1, Nyack, Aug (Ols).

2014 P. acutus Say. Cranberry L. (Osb); L. Placid, Aug (EPV); Sodus Pt., Aug (Buys); Batavia, Je-Aug, Kngt (Laws-EPV); Wyoming Co, Je, Kngt (Ols); Hamburg, Colden, Lancaster, Buffalo, Gowanda, Salamanca, Je-Aug ( $E P V)$; Chautauqua Co, Jl-Aug $(D e L)$; Ithaca, Je, McLean, J1 (Buys); Karner, Felt (Osb); Phoenicia, EPV (Osb); Oliverea, J1, Shoe (O1s); Salem, Eagle Bridge $(O s b)$; Kingston, EPV (Osb); W. Nyack, Je, Oct (Ols); SI, Aug (Ols); LI: Cold Sp. Harb., Jamaica (Osb); Maspeth, Je, Sep-Oct, Bay Shore, J1, Sep, Rockaway Beach, J1, Canarsie, Sep, Hempstead, J1, Sep, Flushing, J1, Sea Cliff, Aug (Ols); Huntington, Sep, Schott (Ols).

2014a P. acutus var. dubius VanD. Niagara Falls, Hamburg, Elma, Salamanca, Jl-Sep (EPV) ; Ft. Montgomery, Sep, Schott (Ols) ; LI: Bay Shore, $\mathrm{J} 1(\mathrm{Ols})$.

P. acutus var. cinnamoneus Osb. Cranberry L. (Osb).

2017 P. cuprescens Osb. Cranberry L. (Osb) ; Indian L., near Sabael, Aug, Brb (Ols); Niagara Falls (EPV); Chautauqua Co, Aug (DeL); Salamanca, J1 (EPV); McLean, J1 (Buys); Phoenicia, EPV (Osb); Ft. Montgomery, Sep, Schott (Ols); LI: Sea Cliff (EPV-CU).

2020 P. angustatus Osb. LI: Cold Sp. Harb., Aug, on pine (Osb) ; Oyster Bay, Aug (Osb); Bay Shore, Sep (Ols).

2021 P. fulvus Osb. Colden, Gowanda, Salamanca, Phoenicia, Jl-Aug (EPV); LI: Cold Sp. Harb., J1-Aug (EPV); Oyster Bay (Osb).

2023 P. frontalis VanD. Niagara Falls, Buffalo, Hamburg, Je-Sep (EPV); Chautauqua Co, Je-J1 (div.-CU) ; Ithaca, Je (Buys); Karner, Nassau (Osb); Poughkeepsie, Phoenicia (Osb); Kingston (EPV); Salem (Osb); White Plains, Je, W. Nyack, Oct (Ols); Mosholu, NYC (Osb) ; SI (EPV); LI: Jamaica, Cold Sp. Harb., Nassau Co (Osb); Maspeth, Flushing, Hempstead, Newtown Hgts., Huntington, Winfield, Bay Shore, Forest Hill, Yaphank, Sea Cliff, Bayville, Je-Sep $(\mathrm{Ols})$.

2029 P. magdalensis Prov. (obscurus Osb.). Salamanca, J1 (EPV); Yorktown Hgts., Sep, W. Nyack, Oct (Ols) ; LI: Cold Sp. Harb., Aug, Pinelawn, Je, Ols (Osb); Pinelawn, Wyandanch, Ols (Osb); Bay Shore, Maspeth, Forest Hill, J1-Sep (Ols).

\section{Deltocephalus Burmeister}

D. delector S. \& DeL. Cranberry L. (Osb).

D. areolatus Ball. Vic. of NYC (Osb).

D. sandersi Osb. LI: Sea Cliff (EPV-CU). 
2042 D. pictus Osb. SI (Osb).

2051 D. configuratus Uhl. Axton, Je, $M \& H$ (EPV-CU) ; Cranberry L. (Osb) ; L. Placid, Aug (EPV-CU); Lancaster, Hamburg, Buffalo, Colden, Je-Aug $(E P V)$; Chautauqua Co, Aug $(D e L)$; Cattaraugus Co, Je, Kngt; Ithaca, Je, McLean Bogs, May (Buys).

2052 D. ocellaris Fallén. Cranberry L. (Osb) ; Mt. Marcy, J1, Not (DeL).

2053 D. sayi Fitch. Axton, L. Placid, Je-Aug (EPV-CU); Cranberry L. (Osb) ; Albion, Lancaster, Buffalo, Hamburg, Gowanda, May-Aug (EPV); Batavia, May-J1, Kngt (Ols); Chautauqua Co (DeL) ; Rock City, J1, Kngt (Ols) ; Ithaca, Je-Aug (Buys, Morr); McLean, May (Buys); Karner, Phoenicia, Kingston, Poughkeepsie; Eagle Bridge, Salem (Osb) ; White Plains, Je, W. Nyack, May-Je (Ols); SI (Osb) ; LI: Cold Sp. Harb., Jamaica, Maspeth, Flushing, Winfield, Newtown Hgts., Port Washington, Bay Shore, Yaphank, North Beach, Forest Hills, Bayville, May-Sep (Ols).

2055 D. weedi VanD. Chautauqua Co, J1 (DeL).

$2055^{\frac{1}{2}}$ D. interruptus DeL. Chautauqua Co $(D e L)$; W. Nyack, Je, Ols (DeL).

2057 D. obtectus O. \& B. Hamburg, Aug (EPV) ; Eagle Bridge (Osb) ; Chautauqua Co, Je $(D e L)$; Salem (Osb).

- D. misellus Ball. Cranberry L. (Osb).

2059 D. compactus O. \& B. L. Placid (EPV); Chautauqua Co, Je (DeL); Phoenicia (Osb) ; SI (Osb) ; LI: Cold Sp. Harb. (Osb).

2062 D. apicatus Osb. Cranberry L. (Osb) ; Mt. Whiteface, Hamburg, Gowanda, Aug-Sep (EPV) ; Chautauqua Co (DeL) ; Salem (Osb) ; W. Nyack, Je, Ols; LI: Forest Hills, Sep, Ols (DeL).

2063 D. inimicus Say. Cranberry L. (Osb) ; L. Placid (EPV) ; Sodus Pt., AugSep (Buys) ; Batavia, Je-Aug, Kngt (DeL) ; Buffalo, Hamburg, Lancaster, Je-Aug $(E P V)$; Portage, Aug, Kngt (Ols) ; Chautauqua Co, Je-Sep (DeL) ; Ithaca, Je-Aug, McLean Bogs, J1 (Buys); Karner, Kingston, Phoenicia; Nassau, Salem (Osb) ; White Plains, W. Nyack, Je, Ols (DeL); SI (Osb); LI: Cold Sp. Harb., Jamaica (Osb); Maspeth, Winfield, Flushing, Bay Shore, Sea Cliff, Je-Sep, Ols (DeL).

2065 D. flavicosta Stål. Batavia, J1, Kngt (DeL); Lawtons (EPV); Eagle Bridge (Osb) ; Chautauqua Co, J1 (DeL) ; Eagle Bridge, Nassau, Salem (Osb); Yorktown Hgts., Sep, Ols (DeL) ; LI: Nassau Co, Cold Sp. Harb., Jamaica (Osb).

2071 D. debilis Uhl. L. Placid, Buffalo, Hamburg, Lancaster, Je-Aug (EPV).

2072 D. abdominalis Fab. Cranberry L. (Osb) ; Batavia, Je, Kngt (Ols); W. Nyack, J1, Sep, Ols (DeL) ; LI: Cold Sp. Harb. (Osb) ; Maspeth, Je (Ols).

2073 D. pascuellus Fall. Cranberry L. (Osb) ; L. Placid, Sep (EPV) ; Batavia, Rock City, J1, Kngt (Ols) ; Ithaca, Ringwood, Trenton Falls, Je-J1 (Buys); Phoenicia, Kingston, Aug $(E P V)$; Salem, Eagle Bridge (Osb) ; LI: Cold Sp. Harb. (Osb).

2075 D. melsheimerii Fitch. Axton, Je, $M \& H$, Cranberry L. (Osb) ; L. Placid, Buffalo, May-Aug (EPV-CU) ; Hamburg, Lawtons, Gowanda, AugOct $(E P V)$; Chautauqua Co, Aug 9 (DeL); Ithaca, Je (Buys); Eagle Bridge, Salem (Osb); W. Nyack, Je, Ols (DeL).

2082 D. littoralis Ball. LI: Sea Cliff (EPV-CU).

2083 D. sylvestris O. \& B. Ausable R., Cobble Hill, Hamburg, Aug-Sep (EPV); Cranberry L. (Osb); Batavia, Je, Kngt (DeL) ; Chautauqua Co, J1Aug (DeL); W. Nyack, Je, Ols (DeL); LI: Maspeth, Je, Ols (DeL). 
2089 D. simplex VanD. (simplarius O. \& B.). LI: Astoria, J1, Southrerick, Sea Cliff (EPV).

D. nominatus S. \& DeL. (oculatus O. \& B.). Cranberry L. (Osb).

D. flavo-virens G. \& B. Cranberry L. (Osb).

2090 D. osborni VanD. Lancaster, Gowanda, Aug-Sep (EPV); Chautauqua Co, J1-Aug (DeL).

2097 D. nigrifrons Forbes. Lancaster, Hamburg, Phoenicia, Je-Sep (EPV).

D. acus S. \& DeL. Cranberry L. (Osb) ; Chautauqua Co, Jl-Aug (DeL); LI: Bay Shore, J1, Ols (S\&DeL).

2079 D. striatus L. (affinis G. \& B.). Batavia, Je, Kngt (S\&DeL) ; Niagara Falls, Buffalo, Hamburg, Salamanca, Je-Aug (EPV); Chautauqua Co, JeAug (DeL); Rock City, Jl, Kngt (Ols); White Plains, Je, Ols (S\&DeL); LI: Yaphank, Maspeth, May, Ols (DeL); Sea Cliff (EPV-CU).

D. nigriventer S. \& DeL. Cranberry L. (Osb).

\section{ACONURA Lethierry}

2117 A. acuticauda Bak. Axton, Je, $M \mathcal{E} H$ (EPV); Cobble Hill, Aug (EPV).

\section{DRIOTURA Osborn and Ball}

2126 D. gammaroides VanD. Hamburg, Mosholu (Osb); White Plains, W. Nyack, J1 (Ols) ; LI: Bay Shore, J1 (Ols) ; Cold Sp. Harb., Jamaica (Osb).

2126b D. gammaroides var. fulva Ball. LI: Hempstead, J1 (O1s).

\section{EuSCELIS Brullé}

2131 E. obscurinervis Stål (exitiosus Uh1.). "NY" (VanD. Cat.).

2132 E. striolus Fall. Cranberry L. (Osb); Batavia, J1, Kngt (Ols) ; Buffalo, Hamburg, Lancaster, Gowanda (EPV); Chautauqua Co, Aug (DeL); Phoenicia (EPV); Karner, Aug (Osb).

E. stactogalus Fieb. (Eutettix osborni Ball). NYC, Sep (Ols, Ball); SI: Westerleigh, J1, Bs (Ols); LI: Maspeth, Je (Ols).

2133 E. parallelus VanD. McLean, J1 (Buys).

2134 E. extrusus VanD. Cranberry L. (Osb) ; summit of Mt. Whiteface, Aug (EPV) ; Axton, Je, $M \mathcal{E} H$ (EPV-CU) ; Rock City, Jl, Kngt (Laws); Oliverea, W. Nyack, May, J1 (Ols) ; Mosholu, Sep (AM) ; SI, May (O1s) ; LI: Yaphank, Wading R., Bay Shore, Wyandanch, May-J1 (Ols).

2136 E. relativus G. \& B. Buffalo, Lancaster, J1 (EPV).

$2136^{3}$ E. deceptus S. \& DeL. Cranberry L. (Osb); Chautauqua Co, J1 (DeL). 2138 E. uhleri Ball. Speculator, J1, Yg (Ball-NYS); Cranberry L. (Osb); L. Placid, Hamburg, Buffalo, Salamanca, Gowanda, May-Aug (EPV); Batavia, Aug, Kngt (O1s) ; Ithaca, J1 (Buys) ; Oliverea, J1, Shoe (Ols) ; White Plains, Je (Ols); Mosholu (Bno); LI: Maspeth, Flushing, Bay Shore, Huntington, Je-Aug (Ols). 
2139 E. anthracinus VanD Adirondacks, How (EPV); Cranberry L. (Osb); Axton, $M a c G$ (EPV); Hamburg, J1 (EPV).

2142 E. arctostaphyli Ball. Cranberry L. (Osb) ; Indian L., near Sabael, Aug, $\mathrm{Brb}$ (Ols).

E. humidus Osb. Cranberry L. (Osb).

2144 E. striatulus Fall. Hamburg, J1 (EPV); Chautauqua Co, Je-J1 (DeL); LI: Bay Shore, J1, Hempstead, Aug (Ols); Cold Sp. Harb. (Osb).

2145 E. vaccinii VanD. Cranberry L. (Osb) ; Mt. Whiteface, Cobble Hill, Hamburg, Salamanca, J1-Aug (EPV); Karner, Poughkeepsie (Osb-NYS); White Plains, Je (O1s); LI: Bay Shore, Yaphank, Hempstead, May-J1 (Ols).

2146 E. instabilis VanD. Cranberry L. (Osb) ; Hamburg, Colden, Je-J1 (EPV); McLean, J1-Aug (Buys).

2147 E. angustatus Osb. "NY" (VanD. Cat.) ; Cranberry L. (Osb).

2148 E. elongatus Osb. Cranberry L. (Osb); Chautauqua Co, J1 (DeL).

2156 E. curtisii Fitch. Cranberry L. (Osb) ; L. Placid, Buffalo, Hamburg, Lancaster, Lawtons, Gowanda, Je-Aug (EPV) ; Sodus Pt., Aug (Buys); Batavia, Aug, Kngt (Laws); Portage, Aug, Kngt (Laws); Wyoming, Kngt (Ols); Otto, Jl (Uh1-CU) ; Chautauqua Co, Je (DeL); Ithaca, Je, Ringwood, J1 (Buys); Nassau, Eagle Bridge, Salem (Osb); Karner; Clinton Hgts.; Phoenicia; Kingston; Warwick; W. Nyack, May, White Plains, Je (Ols); NYC; SI, Aug, Ols (EPV); LI: Maspeth, Huntington, Je (Ols); Cold Sp. Harb., Jamaica (Osb).

E. comma VanD. Cranberry L. (Osb).

\section{EutetTix Van Duzee}

2160 E. luridus VanD. "NY" (Ball) ; Karner (Osb-NYS) ; LI: Bergen I., May, Zab (AM).

2161 E. marmoratus VanD. LI: Melville, Sep, Schott (Osb); Yaphank, Central Pk., Ap-May, Ols (Osb).

2162 E. southwicki VanD. Hamburg, Boston, Salamanca, J1-Aug (EPV); Gowanda, Karner, Aug (Osb); Ft. Montgomery, Sep, Schott, W. Nyack, Je (Ols) ; NYC (Ball) ; LI: Bay Shore, Jl (Ols); Wyandanch, Huntington, Aug-Sep, Schott (O1s).

2163 E. subaeneus VanD. LI (Ball-Osb).

2164 E. querci G. \& B. LI: Hempstead, J1 (Ols).

2167 E. pictus VanD. LI: Bay Shore, Hempstead, J1, Ols (EPV).

2168 E. slossoni VanD. LI: Bay Shore, J1, Ols (EPV).

2170 E. johnsoni VanD. Gowanda, Aug (EPV); White Plains, W. Nyack, Je, Sep (O1s); Mosholu, Mount Castle, Bryn Mawr Pk., Je, Sep (AM);

SI, Aug (EPV, Osb) ; LI: Jamaica $(O s b)$; Flatbush, Je-J1 (Buys); Flushing, North Beach, Forest Hills, Winfield, Maspeth, Hempstead, Bay Shore, J1-Oct (O1s).

2179 E. seminudus Say. Batavia, Je-Aug, Kngt (Ols); Hamburg, Buffalo, Lancaster, Je-Aug (EPV); Wyoming, Je, Kngt (O1s) ; Chautauqua Co, J1 (DeL) ; Ithaca, Je (Buys); Albany (Osb); E. Albany, Oct (Lint-NYS); Nassau, Eagle Bridge, Salem, NYC (Osb); W. Nyack, Je-J1 (Ols); SI (Osb) ; LI: Cold Sp. Harb. (Osb) ; Huntington, Schott (Ols). 
2180 E. cinctus O. \& B. LI: Glen Head, Sep, Bade (O1s); Wyandanch, Aug, Ols (Osb); Huntington, Sep, Schott (Ols).

2181 E. chenopodii Osb. (strobi Fitch). Buffalo (EPV); eastern NY, EPV (Ball); LI: Maspeth, Aug, Ols (EPV); Yaphank, May, Ols (Osb). This synonymy is that of DeLong.

2182 E. scitulus Ball. Cranberry L., Htly (Osb\&DeL).

\section{Phlepsius Fieber}

2195 P. majestus O. \& B. Mosholu (Osb); LI: Glen Head, Sep, Bade (Ols). 2197 P. excultus Uhl. "NY" (Uhl).

2201 P. decorus O. \& B. Cranberry L. (Osb) ; Hamburg, Niagara Falls, Elma, Gowanda, Salamanca, J1-Aug ( $E P V)$; Chautauqua Co, Aug $(D e L)$.

2212 P. uhleri VanD. LI: Yaphank, May, Ols (Osb).

P. strobi Fitch (Eutettix strobi Fitch, 2181). "NY" on pine (Fitch); NYC, J1, Southwick (EPV).

2215 P. maculosus Osb. (maculatus Osb.). Bronxville, J1, Wood (Ols). The synonymy is Olsen's.

2223 P. incisus VanD. Indian L., near Sabael, Aug, Brb (Ols); Ausable R., Saranac L., Lancaster, Buffalo, Boston, Gowanda, Salamanca, J1-Sep $(E P V)$; Batavia, Aug, Kngt (Osb); Chautauqua Co, Aug (DeL); Ithaca, Aug (Buys).

P. maculellus Osb. Cranberry L. (Osb).

2226 P. fuscipennis Vand. Montgomery, Sep, Schott (Ols) ; Albany, Bno (Osb-

2227 P. turpiculus Ball. Buffalo, Sep (EPV); LI: Bay Shore, J1, Ols (EPV).

2228 P. irroratus Say. Cranberry L. (Osb); Batavia, Je-J1, Portage, Aug, Kngt (Ols) ; Niagara Falls, Elma, Hamburg, Lawtons, Buffalo, Lancaster, Gowanda, Salamanca, Je-Sep (EPV); Ithaca, Je (Buys); Nassau, Salem (Osb); Nyack, Zab (AM); W. Nyack, Je, Oct, White Plains, Je (Ols) ; NYC, Bno (Osb); SI (Osb) ; LI: Cold Sp. Harb., Jamaica (Osb) ; Flatbush, Oct, Zab (AM); Wyandanch, Oct, Schott (Ols) ; Newtown Hgts., North Beach, Winfield, Maspeth, Forest Hills, Yaphank, Bay Shore, Port Washington, Je-Oct (Ols).

2230 P. collitus Ball. Gowanda, Salamanca, J1-Aug (EP $\dot{V})$; Chautauqua Co, J1-Aug ( $D e L$ ); White Plains, Je, W. Nyack, Oct (Ols); Bronxville, Je, Wood (Ols) ; Mosholu, Sep (AM) ; SI, Sep (AM) ; LI: Flushing, Bay Shore, Forest Hills, Sea Cliff, J1-Aug (Ols).

2234 P. lobatus Osb. LI: Sea Cliff, Aug, Maspeth, Sep (Ols).

$2235 \frac{1}{2}$ P. tennessa DeL. LI: Flushing, Aug (Ols) ; Pennequid Barrens, Coram, J1, Eng (Ols).

2236 P. apertus VanD. Ausable R., Aug (EPV); Cranberry L. (Osb); Keene Val., Felt (Osb); L. George, Aug, Zab (AM).

2237 P. fulvidorsum Fitch. Cranberry L. (Osb); L. Placid, Elma, Lancaster, Boston, Gowanda, Salamanca, J1-Sep (EPV); Chautauqua Co, Je-Aug $(D e L)$; L. Waccabuc, J1, Zab (AM); L. George, Aug, Zab (Ols-AM); NYC, Je, Southreick (EPV); LI: Wyandanch, Aug (Ols).

2241 P. slossoni Ball. LI: Bay Shore, J1 (Ols).

2244 P. nebulosus VanD. LI: Cold Sp. Harb. (Osb). 
2246 P. solidaginis Wlk. (humidus VanD.). Cranberry L. (Osb) ; Batavia, Aug, Kngt (O1s); Buffalo, Aug (EPV-CU); W. Seneca (EPV); Chautauqua $\mathrm{Co}$, Je $(D e L)$; Ithaca, Aug (Buys); Phoenicia; W. Nyack, J1 (Ols); NYC, Southreick (EPV-CU) ; LI: Maspeth, Bay Shore, J1-Aug, Ols (EPV).

2247 P. ramosus Baker. Gowanda, Aug (EPV).

\section{THAMNOTETTIX Zetterstedt}

2251 T. kennicotti Uhl. Cranberry L. (Osb) ; Cobble Hill, Oct (EPV); Batavia, J1, Kngt (Osb, O1s); Buffalo, Hamburg, Colden, Elma, Gowanda, Salamanca, Je-Sep (EPV); Ithaca, Aug, Baker (CU) ; LI: Sea Cliff (EPV-CU) ; Winfield, Bay Shore, Aug-Sep, Ols (EPV) ; Huntington, Sep, Schott (Ols); Hempstead, J1 (Ols).

2252 T. subcupraeus Prov. Oliverea, Sep, Dickerson (AM).

2253 T. brittoni Osb. Boston, Aug (EPV); Cattaraugus Co, J1, Kngt (Ols) ; Oliverea, 2300 ft., Sep, Dickerson (AM) ; LI: Yaphank, Bay Shore, Aug, Ols (Ball).

2263 T. morsei Osb. McLean, Aug, Sib (Buys); Ft. Montgomery, Bear Mt., Sep, Schott (Ols).

2265 T. clitellarius Say. Rochester, J1, Wendt. (O1s) ; L. Placid, Buffalo, Hamburg, Niagara Falls, Colden, Salamanca, Gowanda, May-Aug (EPV); Batavia, May-J1, Kngt (Ols) ; Chautauqua Co, Je-Oct (DeL) ; Ithaca, Je-J1, Ringwood, Je (Buys) ; Albany, Highland, Clinton Hgts., Phoenicia, Eagle Bridge, Salem, Mosholu (Osb); White Plains, Sep $(\mathrm{Ols})$; Yorktown Hgts., Sep (Ols); LI: Forest Pk. (Osb); Flatbush, J1 (Buys) ; Cold Sp. Harb. (Osb); Maspeth, Flushing, North Beach, Bay Shore, Forest Hills, Je-Sep (O1s).

T. cockerelli Ball. Cranberry L. (Osb).

2266 T. eburatus VanD. Johnstown, Aug, Alex (EPV); Indian L., near Sabael, Aug, Brb (Ols) ; Cranberry L. (Osb) ; Boston, Salamanca, J1 (EPV) ; Bear Mt., Sep, Schott (Ols).

2269 T. collaris Ball. Hamburg (Osb) ; Chautauqua Co, Aug (DeL) ; Ithaca, Hine (Osb) ; NYC, Park (Ball).

2275 T. belli Uhl. Cranberry L. (Osb) ; "NY" (Osb-NYS).

2275a T. belli var. gilletti VanD. Speculator, Colden, Je-J1 $(E P V)$.

T. belli var. brunneus Osb. Cranberry L. (Osb).

2278 T. aurantiacus Prov. Speculator, Aug (EPV).

T. waldanus Ball. Cranberry L. (Osb).

2292 T. chlamydatus Prov. L. Placid, Aug, EPV (Osb); Johnstown, Je, Alex (EPV).

2305 T. melanogaster Prov. Cranberry L. (Osb) ; Batavia, J1, Kngt (O1s) ; Hamburg, Buffalo, Lancaster, Elma, Gowanda, Je-Sep (EPV) ; Chautauqua Co, Aug (DeL); Ithaca, Aug (Buys); McLean, J1 (Buys); White Plains, Je, Ols (Ball); LI: Sea Cliff (EPV-CU).

2307 T. decipiens Prov. Cranberry L. (Osb) ; L. Placid, Ausable R., Aug-Sep $(E P V)$; Mountain View, Sep, Not (Dickerson-AM); McLean, Sep, Sib (Buys).

2312 T. fitchii VanD. Buffalo, Lancaster, Colden, Lawtons, Albion, Gowanda, Hamburg, Aug-Sep $(E P V)$; Chautauqua Co, Sep $(D e L)$; Phoenicia 
(Osb) ; White Plains, Je, Ols (DeL) ; SI; LI: Cold Sp. Harb. (Osb) ; Maspeth, Sea Cliff, Winfield, Je-Sep, Ols (Ball); Flushing, Bay Shore, Aug-Oct (Ols).

T. pallidulus Osb. Cranberry L. (Osb).

T. smithi VanD. Cranberry L. (Osb).

T. ciliatus Osb. Cranberry L. (Osb).

2314 T. nigrifrons Forbes (perpunctata VanD.). Batavia, J1, Kngt (Ball) ; Hamburg, Buffalo, Lancaster, Aug-Sep (EPV); Chautauqua Co, J1-Sep (DeL); NYC; LI: Sea Cliff (EPV-CU); Newtown Hgts., Ols (EPV); Maspeth, J1, Oct, Ols (DeL); Bay Shore, J1, Ols (Ball).

2318 T. inornatus VanD. Ausable R., Lancaster, Buffalo, J1-Sep (EPV) ; Indian L., near Sabael, Aug, Brb (Ols) ; Batavia, Sep, Kngt (O1s) ; Chautauqua Co, Aug (DeL); Ithaca, McLean, J1 (Buys); Salem (Osb); LI: Sea Cliff, Aug, Ols (Ball).

2319 T. placidus Osb. Ausable R., L. Placid, Aug (EPV); Cranberry L. (Osb).

2320 T. cypraceus Osb. Cranberry L. (Osb); Fulton Co, Sep, Alex (EPV); Hamburg, Buffalo, J1-Oct (EPV).

\section{Chlorotettix Van Duzee}

2324 C. unicolor Fitch. Lewiston; Cranberry L. (Osb) ; Keene Val., Saranac Inn (Osb); Rochester, Aug, Baker (CU); Sodus Pt., Aug (Buys); L. Placid, Hamburg, Buffalo, Niagara Falls, Lancaster, Gowanda, Je-Sep $(E P V)$; Batavia, J1-Aug, Kngt (Osb, Ols); Chautauqua Co, Aug $(D e L)$; Ithaca, Je-Aug, McLean, J1 (Buys); White Plains, J1 (Ols); Phoenicia, Eagle Bridge, Salem, Mosholu; SI; LI: Cold Sp. Harb., Oyster Bay, Jamaica (Osb); Hempstead, Winfield, Flushing, J1, Ols (EPV) ; Bay Shore (Ols) ; Central Pk., Je, Shoe (Ols); Kings Pk., J1, Bell, Coram, J1, Eng (O1s).

2327 C. tergatus Fitch. L. Placid, Buffalo, Hamburg, Lancaster, J1-Aug (EPV); Batavia, J1-Aug, Portage, Aug, Kngt (O1s) ; Chautauqua Co, J1 (DeL) ; Ithaca, J1 (Buys); W. Nyack, Aug-Sep (Ols) ; Karner, Eagle Bridge, Salem, Nassau, Mosholu, NYC (Osb); SI; LI: Cold Sp. Harb., Jamaica (Osb); Hempstead, J1 (Ols); Maspeth, Aug (Ols).

2329 C. viridius VanD. L. Placid (EPV-CU) ; W. Nyack, Sep (Ols) ; NYC, J1, Southwick (EPV); SI (EPV); LI: Cold Sp. Harb., Jamaica (Osb); Bay Shore, Canarsie, J1, Sep, Ols (EPV); Floral Pk., Sep, Bell (Ols) ; Glen Head, Sep, Bade (Ols); Sea Cliff, Aug (Ols).

2331 C. galbanatus VanD. Chautauqua Co, Aug (DeL) ; Nassau, Salem (Osb) ; W. Nyack, Sep, White Plains, Je (Ols) ; SI: Ft. Wadsworth, J1, Bs (Ols) ; LI: Jamaica, Sea Cliff (EPV); Maspeth, Ols (EPV); Bay Shore, Je, Sep (Ols); Pennequid Barrens, Coram, J1, Eng (Ols); Hempstead, Wyandanch, Aug-Sep (Ols).

2332 C. balli Osb. Boston, Hamburg, Gowanda, Salamanca, J1-Sep (EPV) ; Chautauqua Co, Aug (DeL); LI: Jamaica, Aug (Osb).

2336 C. Iusorius O. \& B. Cranberry L. (Osb) ; Ausable R., L. Placid Club, Niagara Falls, Hamburg (EPV); Chautauqua Co, Aug (DeI); Phoenicia (EPV); Poughkeepsie (Osb). 


\section{JASSUS Fabricius}

2340 J. clitorius Say. Albion, Niagara Falls, Buffalo, Salamanca, Gowanda, J1-Sep $(E P V)$; Batavia, Aug, Kngt (Ols); Hamburg (Osb); Chautauqua Co, Aug (DeL); Ithaca, Aug (Buys); L. George, Aug (AM); Karner, Salem (Osb) ; Phoenicia (EPV); Mosholu (Bno); SI; LI: Cold Sp. Harb., Jamaica (Osb) ; Hempstead, North Beach, Flushing, Bay Shore, Maspeth, Wyandanch (Ols); Flatbush, Aug-Sep (AM).

\section{PARACOELIDEA Baker}

2353 P. tuberculata Baker. LI: Oyster Bay (Osb).

\section{Cicadula Zetterstedt}

2356 C. punctifrons Fall. Lancaster, J1, EPV (Baker); Gowanda, Aug (EPV); Ithaca, J1 (Baker-CU); Callicoon, Jl, Ds (Ols).

2356a C. punctifrons var. repleta Fieb. (americana VanD.). Batavia, Aug, Kngt (Ball) ; Keene Val. (Osb-NYS) ; Lancaster, Buffalo, Hamburg, Colden, Gowanda, J1-Aug (EPV); Ithaca, J1 (Buys).

2358 C. variata Fall. Cranberry L. (Osb) ; L. Placid, Lancaster, Lime L., Hamburg, Elma, Phoenicia, Je-Sep (EPV); Batavia, J1, Kngt (Osb); Chautauqua Co, J1 (DeL) ; Ithaca, Je (Buys); Nassau (Osb); Oliverea, Sep (AM); W. Nyack, Je, Ols (DeL); LI: Maspeth, Ols (Ball); Flushing, Sep, Winters, Bay Shore, J1-Sep, Ols (Ball); Newtown Hgts., Je, Ols (EPV).

2359 C. lepida VanD. Sodus Pt., Aug, Buys; Ithaca, Aug (Baker-CU) ; NYC, Je, Southrick (EPV).

2362 C. sexnotata Fall. Cranberry L. (Osb); Saranac Inn, J1, Ndm (Osb); Axton, L. Placid, Hamburg, Buffalo, Je-Aug (EPV); Big Moose, Keene Val., Karner, Nassau, Eagle Bridge, Salem (Osb) ; Sodus Pt., Aug (Buys); Batavia, J1-Aug, Kngt (Ball); McLean Bogs, May, Ithaca, Je (Buys); Phoenicia, Kingston (EPV); Oliverea, Sep, Dickerson (AM) ; LI: Cold Sp. Harb., Jamaica (Osb) ; Maspeth, Bay Shore, May-J1, Ols (EPV); North Beach, Flushing, Je, Oct, Ols (Ball); Forest Hills, Yaphank, Je, Ols (DeL); Orient, Sep, Schott $(\mathrm{Ols})$.

2364 C. divisa Uhl. Saranac Inn, J1, Ndm (Osb).

2368 C. slossoni VanD. Cranberry L. (Osb); L. Placid, Clarence, Hamburg, Lancaster, Gowanda, J1-Sep $(E P V)$; Chautauqua Co, Aug $(D e L)$; High Bridge, NYC, Je, Southwick (EPV-CU) ; LI: Forest Hills, Flushing, Je, Ols (DeL).

2369 C. potoria Ball. "NY" (VanD. Cat.).

- C. pallida Osb. Cranberry L. (Osb).

\section{BALCLUTHA Kirkaldy}

2370 B. punctata Thunb. Axton, Je, $M \& H$ (EPV-CU); Cranberry L. (Osb); L. Placid, Buffalo, Hamburg, Colden, Gowanda, Stamford, May-Aug $(E P V)$; Sodus Pt., May, Aúg (Buys); Labrador L., May (Buys); Batavia, Ap, Je, Kngt (DeL); Portage, May-Aug, Kngt (Laws); Ithaca, May-Aug, McLean, May (Buys); Albany, Poughkeepsie (Osb); 
Phoenicia, Kingston (EPV); Oliverea, Aug, Dickerson (AM) ; Slide Mt., Sep, Dickerson (AM) ; Ft. Montgomery, Sep, Schott (Ols); White Plains, W. Nyack, Ap-May, Ols (DeL); LI: Yaphank, May, Ols (DeL) ; Jamaica, Aug (Osb).

2371 B. osborni VanD. Summit of Mt. Whiteface, Aug, EPV (Osb) ; W. Nyack, Je, Ols (DeL) ; Colden, Lancaster, Hamburg, Gowanda, J1-Aug (EPV);

LI: Sea Cliff (EPV-CU).

2372 B. confusa G. \& B. Hamburg, Aug (EPV); Ithaca, J1-Aug (Baker-CU).

2373 B. impicta VanD. Cranberry L. (Osb) ; Hamburg, Lancaster, Gowanda, Stamford, J1-Aug (EPV); Portage, Aug, Kngt (DeL); Ithaca, J1 (Baker-CU); LI: Maspeth, May, North Beach, Je, Ols (EPV).

2373a B. impicta var. flava Baker. Ithaca, J1-Aug (Baker-CU).

\section{EUGNATHODUS Baker}

2377 E. abdominalis VanD. LI: Sea Cliff (EPV-CU).

\section{SUBFAMILY TYPHLOCYBINAE 22}

\section{ALEBRA Fieber}

2380 A. albostriella Fall. Cranberry L. (Osb); Batavia, Aug, Kngt (DeL); Hamburg, Kingston, J1-Aug (EPV); Ithaca, Ap-May (Buys); LI: Maspeth, Bay Shore, Yaphank, May-J1, Ols (DeL).

2380a A. albostriella var. fulveola H.-S. (aurea Walsh). Lancaster, Hamburg, Elma, Gowanda, Salamanca, Je-Aug (EPV); Phoenicia, Kingston; LI: Jamaica (EPV).

2382 A. fumida Gill. Cranberry L., Htly (Osb\&DeL) ; Buffalo, Hamburg, Boston, Elma, Je-Aug (EPV); Ithaca, J1 (Gill-CU).

\section{DIKRANEURA Hardy}

2384 D. cruentata Gill. Ithaca, Ap-May, Aug (Buys, Gill-CU); Cranberry L. (Osb).

2386 D. mali Prov. (communis Gill.).' Summit of Mt. Whiteface, Lancaster, Hamburg, Buffalo, Conesus L., May-Aug (EPV) ; Batavia, Aug, Kngt (DeL) ; Ithaca, J1 (Gill-CU); White Plains, May-Je, Ols (DeL); Phoenicia $(E P V)$; LI: Maspeth, Flushing, Yaphank, May-Oct, Ols (DeL); Sea Cliff (EPV-CU).

2393 D. fieberi Lw. Cranberry L. (Osb) ; Sodus Pt., Aug (Buys) ; Batavia, Aug, Kngt (DeL) ; Hamburg, Lancaster, Lawtons, Buffalo, Je-Sep (EPV); Ithaca (Gill, Buys); McLean (Buys); White Plains, Je, Ols (DeL); LI: Forest Hills, Je, Ols (DeL); Maspeth, Ols (EPV, DeL) ; Flatbush, Nov, Schott (O1s).

\section{EMPOASCA Walsh}

2394 E. trifasciata Gill. Lancaster, Gowanda, Aug (EPV).

2395 E. smaragdula Fall. Cranberry L. (Osb); Sodus Pt., Aug, Buys (McAtee) ; Buffalo, Hamburg, Je-Sep (EPV); McLean, J1, Buys (McAtee); Salem (Osb); Oliverea, Aug, Diven (AM); White Plains, Je, Ols (DeL).

${ }_{22}$ Considered as a tribe-Typhlocybini-in Van Duzee's catalog, but by several recent workers considered as a subfamily and therefore so used here. 
2399 E. maligna Walsh (unicolor Gill.). Hamburg, Salamanca, J1-Aug (EPV); LI: Sea Cliff (EPV-CU).

2398 E. obtusa Walsh. Batavia, Je-Sep, Kngt, Morr (Osb) ; Hamburg, J1-Sep $(E P V)$; Ithaca, Morr (Osb); Phoenicia, Kingston (EPV); White Plains, Je, Ols (DeL) ; LI: Maspeth, Ols (EPV); Yaphank, North Beach, Hempstead, Je-Sep, Ols (DeL).

- E. aureoviridis Uhl. Cranberry L. (Osb).

2400 E. unica Prov. (splendida Gill.). Gowanda, Aug (EPV); Ithaca, Je, Buys (McAtee).

2401 E. atrolabes Gill. Cranberry L. (Osb) ; L. Placid, Gowanda, Salamanca, J1-Aug $(E P V)$.

2406 E. coccinea Fitch. Cranberry L. (Osb); "NY" (Fitch, W1k-Brit. Mus.).

2418 E. alboneura Gill. "NY" (EPV); LI: Jamaica, EPV (NYS).

2421 E. fabae Harr. (mali Le B., viridescens Walsh, consobrina Walsh, malefica Walsh). Apple Leaf Hopper. Cranberry L. (Osb) ; Batavia, Jl-Aug, Kngt (DeL) ; Fulton, Buffalo, Phoenicia, Hamburg, Lancaster, Geneva, Je-Aug (EPV) ; Waterville, Aug, Harel (EPV); Ithaca; SI (EPV); LI: Sea Cliff (EPV-CU); Bay Shore,. Forest Hills, J1-Oct, Ols (DeL).

2422 E. flavescens Fab. Near Ausable R., Hamburg, Je-Oct (EPV); Sodus Pt., Aug, Buys (McAtee); Batavia, Oct, Kngt (DeL); Ithaca, Je-Jl, Buys (McAtee); McLean, J1, Buys (McAtee); LI: Sea Cliff (EPV-CU) ; Maspeth, Yaphank, Aug-Sep, Ols (DeL).

2424 E. birdii Godg. Cranberry L. (Osb) ; Batavia, Aug, Kngt (DeL) ; Hamburg, May $(E P V)$; Ithaca, Aug (Gill-CU); Albany (Osb-NYS); LI: Lynbrook, Ap, Nic, Queens Village, Mar, Schott (DeL).

\section{TYPHLOCYBA Germar}

(Eupteryx Curtis)

2426 T. collina Flor. Fredonia, Nov, $C y$ (EPV).

T. melissae Curt. Batavia, Aug, Kngt (DeL); LI: Maspeth, Newtown Hgts., May-Je (Ols).

2427 T. vanduzeei Gill. Cranberry L. (Osb) ; Isham House, Adirondacks, Aug (EPV) ; Hamburg, J1-Aug, Alex (EPV, Gill).

2429a T. flavoscuta var. nigra Osb. Cranberry L., (Osb) ; LI: Jamaica, Oct 20, 1904 (Osb). The synonymy is McAtee's.

2429 T. flavoscuta Gill. Cranberry L. (Osb) ; L. Placid, Hamburg, Colden, Gowanda, Je-Aug (EPV); Sodus Pt., Aug, McLean, J1, Ithaca, Je (Buys) ; Phoenicia (Osb) ; LI: Sea Cliff (EPV-CU) ; Wyandanch, Oct, Schott (DeL); Hempstead, Sep, Ols (DeL).

\section{EMPOA Fitch}

\section{(Typhlocyba Germar)}

2430 E. querci Fitch. Cranberry L. (Osb); Mt. Whiteface, Stamford, Lancaster, Gowanda, Phoenicia, Kingston, J1-Aug (EPV) ; Ithaca, Oct (OsbMorr) ; Slide Mt., Sep, Diven; Bronxville, Wood (Ols); LI: Sea Cliff $(\mathrm{EPV}-\mathrm{CU})$. 
2430a E. querci var. gillettei VanD. (bifasciata G. \& B.). Essex Co, J1, Not (DeL) ; Cranberry L. (Osb) ; L. Placid, Hamburg, Lancaster, Gowanda, Phoenicia, Kingston, Aug (EPV); Batavia, Je-Aug, Kngt (DeL); LI: Maspeth, Je, Ols (DeL).

2433 E. ulmi L. Albany, Oct (Gill).

2435 E. tenerrima H.-S. Cranberry L. (Osb) ; Ausable R., Aug (EPV).

2436 E. lethierryi Edws. Cranberry L. (Osb); Hamburg, J1-Aug (EPV).

2437 E. rosae L. Rose Leaf Hopper. Cranberry L. (Osb) ; L. Placid, Buffalo, Hamburg, Je-Oct (EPV) ; Batavia, Jl-Sep, Kngt (DeL, Osb) ; Ithaca, Oct, Morr (Osb); Ghent, Oct (Felt); Albany, EPV (NYS); LI: Sea Cliff (EPV-CU); Maspeth, Oct, Ols (DeL).

\section{Hymetta McAtee}

2440 H. trifasciata Say. Lancaster, Hamburg, Gowanda, May-Sep (EPV); Batavia, Aug, Kngt (DeL) ; Ft. Montgomery, Sep, Schott (Ols).

\section{ERYTHRONEURA Fitch}

2441 E. tricincta Fitch. Rochester, J1, Wendt (DeL) ; Batavia, Aug, Kngt (DeL) ; Lancaster, Jan (div.) ; Ithaca, Aug (Gill-CU).

2443 E. hartii Gill. Bronxville, Aug, Wood (Ols).

2445 E. comes Say. Grape Leaf Hopper. Cranberry L. (Osb) ; Batavia, Aug, Kngt (Osb); Buffalo, Hamburg, Ap-May (EPV); Albany, Oct (NYS) ; LI: Sea Cliff (EPV-CU) ; North Beach, Maspeth, Flushing, Mar-Ap, Ols (EPV) ; Forest Pk., Yaphank, May, Ols (DeL) ; Lynbrook, Ap 27, Nic (DeL).

2445a E. comes var. vitifex Fitch. Ithaca, Ap, Aug, Buys (McAtee) ; Pawling, ALMarshall (McAtee).

2445b E. comes var. ziczac Walsh. Lancaster, Lawtons, Gowanda, Stamford, J1-Oct $(E P V)$.

2445c E. comes var. vitis Harr. Batavia, Aug, Kngt (DeL); Lancaster, May (EPV) ; Rochester, J1, Wendt (Ols); Ithaca, May, Aug, Buys (McAtee, Gill-CU) ; LI: Aqueduct, May, Ols (DeL) ; North Beach, Yaphank, Mar, May, Ols.

E. comes var. corona McAtee. Hamburg, Aug (EPV).

2445 d E. comes var. basilaris Say. "NY" (Osb, McAtee) ; Gowanda, Aug (EPV) ; Lancaster, Aug (EPV); Ithaca, May, Buys (McAtee).

$2445 \mathrm{f}$ E. comes var. rubra Gill. Batavia, Aug, Kngt (DeL) ; LI: Brooklyn, Forest Pk., Mar, Oct, Ols (DeL).

2445 g E. comes var. maculata Gill. LI: Forest Pk., Maspeth, Mar, Ols (DeL).

- E. comes var. gemina McAtee. Ithaca, Aug, Buys (McAtee).

2445 E. octo-notata Walsh. Ithaca, J1, MacG (Gill-CU); LI: Sea Cliff (EPV-CU).

2446 E. illinoiensis Gill. Hamburg, EPV (Osb).

2447 E. obliqua Say. Cranberry L. (Osb) ; L. Placid, Hamburg, Colden, MaySep $(E P V)$; Buffalo, May-Sep $(E P V)$; Ithaca, Ap, Buys (McAtee, Gill-CU); LI: Sea Cliff (EPV).

2447a E. obliqua var. dorsalis Gill. Colden, Stamford, May-Aug (EPV) ; LI: Sea Cliff (EPV-CU). 
2447c E. obliqua var. fumida Gill. Gowanda, Stamford, J1-Aug (EPV); LI: Sea Cliff (EPV-CU).

E. obliqua var. noevus Gill. Cranberry L. (Osb).

2448 E. vulnerata Fitch. Lancaster, Aug (EPV); Ithaca, Aug (Gill-CU); Albany, Oct, EPV (NYS); LI: Sea Cliff (EPV-CU).

2448 a E. vulnerata var. nigra Gill. Lancaster, Aug $(E P V)$.

2450 E. crevecoeuri Gill. "NY" (McAtee, EPV-CU).

\section{Family FUlgoridae \\ Compiled by Z. P. Metcalf \\ SUBFAMILY DICTYOPHARINAE \\ DICTYOPHARA Germar}

2462 D. microrhina Wlk. SI, Aug (EPV, Ds, Zab-AM); LI: Aqueduct, Aug (Ds); Maspeth, Sep (Ols); New Lots, Zab (AM).

2463 D. lingula VanD. SI, Aug-Sep (Ds); LI: Maspeth, Sep (O1s).

\section{Scolops Schaum}

2466 S. sulcipes Say. Wells (Yg); Cranberry L. (Osb) ; White L., Zab (AM); L. Placid, Aug (EPV); Lancaster (EPV); Nassau, Salem, Albany, Karner (NYS); Kingston (EPV); Phoenicia (EPV); New Baltimore (Yg); W. Nyack, Sep; Mosholu, West Farms, Kissena L. (AM); SI, Jl (Ols); LI: Kissena Pk., Maspeth, Winfield, Port Washington, J1-Sep (Ols) ; Aqueduct, Je, Shoe; Sea Cliff, Aug, Forest Hills, Sep (Ols).

2469 S. desiccatus Uhl. SI (Ds); also other localities (O1s).

2470 S. perdix Uhl. LI: Bay Shore, Maspeth, Winfield, Aug-Sep (Ols).

2477 S. angustatus Uhl. Ithaca (CU) ; Mosholu (Sm); Van Cort. Pk., Aug; SI (EPV); LI: Cold Sp. Harb., Jamaica (EPV); Winfield, Wyandanch, Rockaway, Maspeth, J1-Aug (Ols) ; Orient, Flatbush, J1, Aug, Zab (AM).

\section{Phylloscelis Germar}

2484 P. atra Germ. Cranberry Toad Bug. Carmel, Aug (AM); White Plains (Ols) ; LI: Sea Cliff (CU); Calverton, Riverhead (Sirrine \& Fulton). 2484a P. atra var. albovenosa Melich. White Plains (Ols); Cold Sp. Harb., Sep (Ds).

2485 P. pallescens Germ. LI (Sirrine \& Fulton).

\section{SUBFAMILY ACHILINAE}

\section{EPIPTERA Metcalf}

2498 E. colorata VanD. Gowanda (EPV).

2499 E. opaca Say. Phoenicia (EPV); near L. Erie (Say).

2502 E. slossoni VanD. Cranberry L. (Osb).

2503 E. septentrionalis Prov. LI: Bay Shore, Sep (Ols); Calverton, Sep (Ds),

2504 E. floridae W1k. LI: Bay Shore, Aug (Ols); Wyandanch, J1, Schott.

2505 E. variegata VanD. LI: Bay Shore, Sep (O1s).

- E. brittoni Metc. West Pt., Sep. 


\section{CATONIA Uhler}

2507 C. nava Say. Gowanda, Hamburg, Aug.

2508 C. cinctifrons Fitch. LI: Sea Cliff (EPV); Melville, Wyandanch, Sep, Oct, Schott; Forest Hills, Sep (Ols).

2510 C. picta VanD. LI: Huntington, Sep, Schott

2511 C. grisea VanD. Niagara Falls, Hamburg, Gowanda, Phoenicia (EPV); L. George, Aug-Sep, Zab (AM).

2512 C. pumila VanD. LI: Jamaica (EPV).

2514 C. impunctata Fitch. Buffalo, Colden, Phoenicia (EPV); L. George, Sep; Ramapo, J1 (Ds) ; LI: Bergen I., Orient, J1, Aug, Zab (AM); Wyandanch, Aug (Ols).

2515 C. dimidiata VanD. Phoenicia (EPV); Ft. Montgomery, Sep, Schott.

\section{SUBFAMILY CIXIINAE}

\section{OlIARUS Stål}

2528 O. quinquelineatus Say. Buffalo, E. Aurora (EPV); Rock City, Ithaca, J1 (Ds) ; Karner (EPV) ; SI, Je-J1 (Ols) ; LI: Pinelawn, May-J1 (Ds); Bay Shore, Yaphank, Je-J1 (Ols); Ft. Montgomery, J1, Sep, Huntington, Schott.

2529 O. placitus VanD. LI: Flatbush, Zab (AM).

2531 O. franciscanus Stål. Niagara Falls, near Buffalo, Boston, Gowanda (EPV); Crugers, J1 (AM) ; White Plains, W. Nyack, Je (Ols); LI: Yaphank, Bay Shore, Flushing, J1 (O1s).

2540 O. humilis Say. Buffalo, Hamburg (EPV); Goshen, Carmel, Aug, Sep (AM).

2541 O. cinnamomeus Prov. "NY" (VanD. Cat.).

\section{Cixius Latreille}

2545 C. misellus VanD. Speculator, Wells (Yg); Cranberry L. (Osb) ; Buffalo, L. Placid, Salamanca, Gowanda, Hamburg, Chautauqua, Colden, Lewiston (EPV); Elizabethtown, Trenton Falls (NYS).

2546 C. basalis VanD. L. Placid, Hamburg, Gowanda, Salamanca (EPV); Phoenicia (NYS) ; L. George, Zab (AM); NYC (AM); LI: Flatbush, J1, Zab (AM).

2547 C. coloepium Fitch. Mt. Marcy, J1 (O1s) ; Cranberry L. (Osb) ; Speculator, Blue Mt.; L. Placid, Lime L., Lewiston, Salamanca (EPV); Oliverea, J1 (Ois); SI, J1 (Ols).

C. apicalis Metc. Ithaca, Je.

\section{Oecleus Stål}

2554 O. borealis VanD. Hamburg (EPV) ; LI: Yaphank, Port Washington, May, J1, Sep (Ols).

\section{MyNDUS Stå1}

2565 M. pictifrons Stål. Buffalo, Hamburg, Aug.

2566 M. sordidipennis Stål. Near Buffalo, Hamburg, Je, J1, Colden,. Je. LI: Lott Wood, Flatbush, J1, Zab (AM). 
SUBFAMILY ISSINAE

Fitchiella Van Duzee

2580 F. robertsoni Fitch. "NY" (VanD. Cat.).

\section{BRUCHOMORPHA Newman}

2583 B. oculata Newm. Ithaca (CU) ; Cranberry L., J1, Aug (Osb) ; Speculator, Wells, $Y g$; Buffalo, Boston, Colden, Hamburg, Gowanda, Salamanca, Phoenicia (EPV); Salem (NYS); W. Nyack, Je (Ols).

2585 B. dorsata Fitch. "NY."

2588 B. tristis Stål. Niagara Falls (EPV).

\section{APHELONEMA Uhler}

2591 A. histrionica Stål. Cranberry L., Aug (Osb) ; Hamburg, L. Placid, Cobble Hill, Lancaster, Buffalo (EPV).

\section{ThIONIA Stål}

2634 T. bullata Say. Niagara Falls, Gowanda, Hamburg (EPV) ; LI: Maspeth, Jl, Sep, Port Washington, Sep (O1s); Flatbush, Aug, Zab (AM); Aqueduct, J1 (Ds).

SUbFAmily ACANALONIINAE Amyot and Serville

Acanalonia Spinola

2639 A. bivittata Say. Ithaca (Ols, CU) ; Schenectady (Ols) ; Albany, Karner (Yg) ; Phoenicia (EPV); Salem (NYS); Poughkeepsie (CU) ; West Pt., Sep, Robn (Ds) ; Ft. Montgomery, J1 (Ds) ; W. Nyack, Sep (Ols); Mosholu; Bryn Mawr Pk. (AM); SI (EPV); LI: Bay Shore, Maspeth, Winfield, Sea Cliff, Wyandanch, Port Washington, Pelham Parkway, Yonkers, Bayville, J1-Oct (Ols) ; Promised Land, Wading R., Southold, Amagansett, Pinelawn, Yaphank, Brooklyn, Flushing, Aug-Oct (Ds).

\section{Subfamily FLATINAE Spinola \\ ORMENIS Stål}

2649 O. pruinosa Say. Mealy Lantern Fly. Rochester, Wendt; Buffalo, Gowanda, Lancaster (EPV); Ithaca (Ols); Albany ; Nassau, Troy (NYS) ; Normanskill, Leon; Poughkeepsie; W. Nyack, Aug (Ols); Bronxville; NYC (Ols); West Farms (AM) ; Kingston; Bronx, Aug (Ols) ; LI: Maspeth, Jl, Aug (Ols) ; Yaphank, Wading R., Ft. Montgomery, Fire Place, J1-Oct (Ds); Wyandanch, Port Washington, J1Aug (Ols).

2656 O. septentrionalis Spin. Mt. Sinai (Ds); Albany; Poughkeepsie; White Plains, Sep, Dunwoodie, Yonkers, Sep (Ols) ; West Farms, Aug (AM) ; MacLean Ave., NYC, Aug (Ols); SI, Jl-Oct (Ds); LI: Sea Cliff (EPV) ; Jamaica (NYS) ; Syosset (NYS) ; Maspeth, J1, Sep, Flush.ing, Sep (Wlk); Wading R., Orient, Aug-Oct (Ds); Yaphank. 


\section{SUBFAMILy DERBINAE Spinola}

PATARA Westwood

2671 P. vanduzei Ball. Cranberry L., Htly (Osb\&DeL). Gowanda (EPV).

\section{Cedusa Fowler}

2672 C. vulgaris Fitch. L. Placid, Buffalo, Boston, Hamburg, Salamanca, Ithaca (EPV); Batavia, Kngt; Otto; Karner, Albany (NYS) ; L. George, Aug, Zab (AM) ; Oliverea, J1, Shoe; LI: Jamaica (EPV) ; Bay Shore, Yaphank, J1-Sep (O1s).

2673 C. obscura Ball. Elma, Hamburg, E. Aurora (EPV) ; W. Nyack, Sep (Ols) ;

LI: Bay Shore, Wyandanch, J1-Aug (O1s).

C. incisa Metc. Cranberry L., Htly (Osb\&DeL).

\section{Cenchrea Westwood}

2678 C. uhleri Ball. LI: Hempstead, Sep (OIs).

\section{Amalopota Van Duzee}

2681 A. uhleri VanD. Lancaster, Niagara Falls (EPV); Hamburg, Gowanda, Aug (EPV).

2682 A. fitchi VanD. Colden, Buffalo (EPV).

\section{AnOtia Kirby}

2683 A. bonnetii Kby. Buffalo (EPV); Karner (NYS).

2684 A. westwoodi Fitch. "NY" (Fitch).

2685 A. burnetii Fitch. Albion (EPV).

2687 A. sayi Ball. Albion (EPV).

\section{OTIOCERUS Kirby}

2689 O. degeerii Kby. New Russia, Bdy; Buffalo, Lancaster, Stamford, Salamanca, Gowanda (EPV); Phoenicia (EPV); SI, Aug (Ds); LI: Amagansett, Fire Place, Yaphank, Aug-Sep (Ds).

2690 O. stollii Kby. Buffalo Plains (EPV).

2691 O. abbotii Kby. Rye, Sep (Ds).

2693 O. coquebertii Kby. Old Forge (Yg) ; Cranberry L., J1, Aug (Osb) ; Colden, Hamburg, Gowanda, Stamford, Salamanca, Buffalo, Poughkeepsie (EPV); S. Wales, $M C V$; Otto (Uhl) ; Ithaca (NYS); Phoenicia (NYS).

2695 O. signoretii Fitch. Lancaster, Hamburg, Conesus L. (EPV).

2696 O. wolfii Kby. Lancaster, Gowanda, Hamburg, Albion, Aug, Sep (EPV) ; SI, Aug (Ds).

2697 O. amyotii Fitch. LI: Port Washington, Bay Shore, Sep (O1s) ; Yaphank, J1, Wood.

2698 O. kirbyii Fitch. "NY".

2699 O. schellenbergii Kby. Gowanda (EPV). 


\section{SUBFAMILY DELPHACINAE 22a}

\section{STENOCRANUS Fiebér}

2701 S. dorsalis Fitch. Cranberry L., J1 (Osb) ; Buffalo, L. Placid, Colden, Hamburg, Gowanda, Aug, Sep (EPV); Phoenicia (EPV); Johnstown, Albany, Karner, Newport (NYS) ; W. Nyack, Sep (Ols); Ft. Montgomery, Schott.

2703 S. felti VanD. Cranberry L., Aug (Osb); Speculator (Felt).

2704 S. palaetus VanD. "NY" (EPV).

S. vittatus Stål. NYC (EPV).

\section{KELISIA Fieber}

2706 K. axialis VanD. Cranberry L., Aug (Osb) ; Lancaster, Gowanda, Aug (EPV) ; LI: Maspeth, Sep (Ols).

\section{Megamelanus Ball}

2712 M. elongatus Ball. LI: Winfield, J1 (Ols).

2713 M. spartini Osb. SI; LI: Oyster Bay, Cold Sp. Harb. (Osb) ; Sea Cliff (EPV).

\section{PROKELISIA Osborn}

2715 P. setigera Osb. LI: Sea Cliff (EPV).

2716 P. marginata VanD. SI (WMG); LI: Rockaway Beach, Sep (Ols) ; Cold Sps., Sea Cliff (WMG).

\section{Megamelus Fieber}

2717 M. notulus Germ. Colden, L. Placid, Hamburg, Lancaster, Concord, Buffalo, May-J1 (EPV).

2718 M. piceus VanD. Potsdam, Je, J1 (EPV); Lancaster, Clarence, Grand I. (EPV) ; Colden, Je, J1 (EPV); LI: Yaphank, Je-J1 (EPV).

M. inflatus Metc. NY.

\section{Pissonotus Van Duzee}

2723 P. marginatus VanD. East Lake (Yg); Lancaster, Hamburg, Phoenicia (EPV); NYC; LI: Sea Cliff (EPV); Ravenswood, Long Island City, Southwick.

2724 P. basalis VanD. Johnstown, Alex; Lancaster (EPV); White Plains; W. Nyack, Je (O1s).

2726 P. ater VanD. Speculator (Yg) ; Cranberry L., Aug (Ols) ; Grand I., Buffalo, Hamburg, Elma, Je... (EPV).

2727 P. divaricatus Spnr. Middletown, Spooner.

2731 P. delicatus VanD. Niagara Falls, Aug; LI: Sea Cliff (CU).

2732 P. guttatus Spnr. Ithaca (CU).

2733 P. dorsalis VanD. Cranberry L., Aug (Osb) ; L. Placid, Lancaster, Colden, Boston, Phoenicia, Jl (EPV); Ithaca (CU).

22a F. Muir, of the Hawaiian Sugar Planters' Experiment Station, has assisted in the preparation of the list in the subfamily Delphacinae. 
2735 P. brunneus VanD. Clarence (CU) ; Grand I. (EPV); White Plains (O1s); NYC, Southrick; SI (NYS) ; LI: Sea Cliff (CU) ; Jamaica (NYS); Maspeth, Je (Ols).

2736 P. piceus Spnr. Middletown, Spooner.

2737 P. aphidioides VanD. Elm L. (Yg) ; Salamanca, Colden, Niagara Falls, Hamburg, Boston, Buffalo, J1-Aug (EPV); Ithaca (CU) ; Washingtonville $(\mathrm{Yg})$; LI: Sea Cliff (CU).

\section{Phyllodinus Van Duzee}

2739 P. nervatus VanD. Newport (Yg); Buffalo, Lancaster, Hamburg, Colden, Je, J1 (EPV); Caroline to Harford (CU); White Plains, Je (Ols).

2743 P. flabellatus Ball. Ithaca; White Plains (O1s); SI, Aug (EPV); LI: Maspeth, Je, Aug (Ols).

\section{LACCOCERA Van Duzee}

2745 L. vittipennis VanD. Axton, Cobble Hill, L. Placid (EPV); Speculator, Corinth (Yg); Cranberry L., Aug (Osb).

\section{STOBAERA Stål}

2750 S. tricarinata Say. Lancaster, Hamburg, Lawtons, J1 (EPV); N. Evans, Oct (MCV); LI: Flushing, Winfield, Maspeth, Sep-Oct (Ols); Orient, Wyandanch, Sep, Oct, Schott.

2753 S. pallida Osb. LI: Oyster Bay (Osb).

\section{LIBURNIELLA Crawford}

2758 L. ornata Stål. Cranberry L., J1-Aug (Osb) ; Hamburg, J1 (WMG); Lawtons, Buffalo, Oct (EPV); White Plains, W. Nyack, Je (Ols); SI, Oct (Ds) ; LI: Sea Cliff (EPV); Maspeth, Je (Ols).

\section{DELPHACODES Fieber}

(Liburnia Stål)

2762 D. laminalis VanD. Mt. Whiteface; L. Placid, Phoenicia (EPV).

2763 D. lateralis VanD. Lancaster, Colden, Niagara Falls, Hamburg, E. Aurora, Buffalo, Gowanda, Je-Oct (EPV).

2764 D. kilmani VanD. Colden, Elma, Buffalo, Hamburg, May-Je (EPV).

2766 D. pellucida Fab. Cranberry L., Aug (Osb) ; Saranac Inn, Ndm; Ithaca, Mt. Whiteface, Lancaster, Niagara Falls, Buffalo, Hamburg, May, Aug (EPV).

2768 D. puella VanD. Mt. Whiteface, Lancaster, L. Placid, Buffalo, Gowanda, Lawtons, Aug-Oct (EPV); LI: Sea Cliff (EPV); Wyandanch (Ols).

2769 D. basivitta VanD. White Plains (O1s).

2771 D. osborni VanD. Lancaster, Phoenicia, Aug (EPV).

2773 D. campestris VanD. Cranberry L., Je, Aug (Osb); Lancaster, Portage, Ithaca, Isham House, Kingston, L. Placid, Niagara Falls, Hamburg, Gowanda, Buffalo, Je-Aug (EPV); Karner (NYS); White Plains, May (Ols); LI: Maspeth, Yaphank, May (O1s). 
2774 D. furcata Prov. About Buffalo (EPV).

2776 D. detecta VanD. NYC (EPV); LI: Sea Cliff (EPV).

2779 D. foveata VanD. Portage, Lancaster, Clarence, Hamburg, Je (EPV).

2780 D. lutulenta VanD. Axton, MacG; Speculator ( $\mathrm{Yg}$ ); Cranberry L., Aug (Osb) ; Buffalo, Ithaca, L. Placid, Portage Falls, Hamburg, Colden, Niagara Falls, Lawtons, Gowanda, Aug (EPV); W. Nyack, White Plains, May-Je (Ols) ; LI: Sea Cliff (EPV) ; Bay Shore, Maspeth, May-J1 (Ols).

2781

2785

2790

2792

2793

D. obscurella Bohm. Concord, Colden, Portage Falls (EPV).

D. incerta VanD. Buffalo (EPV).

D. lineatipes VanD. Lancaster, Buffalo, Hamburg, Jl, Oct (EPV).

D. nigridorsum Crawf. White Plains, May (Ols).

D. dolera Spnr. Ithaca (EPV).

Pentagramma Van Duzee

2807 P. vittatifrons Uhl. Axton, Je, MacG; Oswego (CU).

\section{FAMILy CHERMIDAE}

\section{(Psyllidae)}

\section{SUBFAMILY LIVIINAE}

LIVIA Latreille

2810 L. maculipennis Fitch. Speculator, L. Placid, Aug (EPV) ; Phoenicia, Aug: EPV (Patch); Oneida, Aug (NYS); Hamburg, Lawtons, Aug (EPV) ; Belden Hill, May, Cy (Yg) ; Karner, May (Felt) ; Nassau, Ap (Felt); Stony Pt., Ap (NYS); NYC (Felt) ; LI: Orient, J1 (NYS).

2812 L. vernalis Fitch. Speculator, Aug, $Y g$ (EPV) ; Newport, J1, $Y g$; L. Placid, Aug, EPV (NYS) ; Hamburg, Gowanda, Je-Sep (EPV); Stamford, Aug (EPV); Karner, Ap (NYS); Nassau, Ap (NYS) ; New Baltimore, $\mathrm{Jl}, \mathrm{Yg}$.

\section{APHALARA Foerster}

2815 A. calthae L. Hamburg, Aug (EPV); Hudson Falls, J1, on Polygonum (Patch-NYS).

2818 A. angustipennis Crawf. Speculator, J1, $Y g$, Karner, Je (Felt).

2822 A. veaziei Patch. Speculator, Aug, $Y g$; L. Placid, Aug, EPV (NYS); Cranberry L., Je-J1 (Osb) ; Colden, Je (EPV); Freeville, J1, Caroline, Je (Patch); Karner, Je (NYS); Phoenicia, Aug, EPV (NYS) ; SI, Aug, EPV (NYS).

2826 A. fascipennis Patch. Mud Cr., Tompkins Co, Je (Patch).

\section{SUBFAMILY PAUROPSYLLINAE}

HETEROPSYLLA Crawford

H. quassiae Crawf. LI, Ap, on Quassia sp. (Crawford). 
Calophya Loew

2844 C. nigripennis Rly. Karner, Je, on white birch (Patch); N. Chatham, Je (Felt).

\section{SUBFAMILY TRIOZINAE}

TrIOzA Foerster

2857 T. diospyri (Ashm.). Buffalo, May 25, 1900 (EPV).

2860 T. quadripunctata Crawf. Canastota, Mar 12, hibernating on grass stems, Faure (Crawford).

2867 T. tripunctata Fitch. Axton, Je $(\mathrm{M} \& \mathrm{H})$; Penn Yan, Sep, Cy (Felt) ; Karner, Ap (Patch); LI: Sea Cliff (CU) ; Jamaica, Oct, Sirrine (Crawford).

2869 T. maura Foerst. Cranberry L., Htly (Osb).

$2869 \frac{1}{4}$ T. forcipula Patch. Ithaca, May (Patch).

2870 T. nigrilla Crawf. Cranberry L., Aug (Osb).

\section{SUBFAMILY PSYLLIINAE}

\section{PACHYPSYLLA Riley}

2886 P. venusta O. S. Peekskill (Bt); vic. NYC (Bt).

2887 P. celtidis-mamma Rly. Ithaca, Sep (CU) ; Athens, Aug (Felt); vic. NYC (Bt).

2889 P. celtidis-vesiculum Rly. Ithaca (Crawford); Stony Pt., Ap (NYS); vic. NYC (Bt).

2890 P. celtidis-gemma Rly. Albion, May (CU) ; Rochester, Ap, John Dunbar (Felt); Ithaca, Cy (EPV); Kingston, Mar (CU) ; White Plains, May, $\mathrm{J} 1$ (Felt); vic. NYC (Bt).

P. coagulata Uhl. MS. Ithaca, Oct, Dudley (Uhl-CU).

\section{PSYLLOPSIS Loew}

2913 P. fraxinicola Foerst. Rochester, Je, on ash (NYS) ; Richfield Sps., Aug, on walnut (Crawford).

\section{PSYLLIA Kirkaldy}

2922 P. pyricola Foerst. Pear-Tree Psylla. Bluff Pt.; W. Brighton, May; Lincoln, Ap ; Lewiston, Act; Mt. Morris; S. Byron; Ithaca, Je, Aug; Glenmont; Kinderhook; Menands; Clinton Hgts., Mar; Ghent; Medway; Athens, Je; Catskills; Ulster Co, Aug; Milton, Je; New Baltimore, May; SI: Annadale.

2924

P. quadrilineata Fitch. "NY" (Fitch).

2930 P. striata Patch. Elizabethtown, Aug (Patch) ; Cranberry Cr., J1, abundant on yellow birch (Osb); L. Placid, Aug, EPV (Patch); Northville, Je (NYS) ; Mud Cr., McLean, Ithaca, Je-Aug (Patch); Karner, Aug (Patch).

2931 P. carpinicola Crawf. Barber Pt., Cranberry L., Jl, on yellow birch, white pine, and fire cherry (Osb) ; Karner, Je, on white birch (Felt).

2932 P. cephalica Crawf. Elizabethtown, Aug, on ironwood, $Y g$; L. Placid, Aug (NYS) ; Mud Cr., and several other localities, Tompkins Co (Patch); Karner, Je (NYS). 
2933 P. annulata Fitch. Karner, Je, on white birch (NYS).

2936 P. floccosa Patch. Cranberry L., Barber Pt., Childrold\&Ranger (Osb).

2938 P. trimaculata Crawf. L. Placid, Aug, CSSheldon (Crawford); Cranberry Cr., abundant on Prunus pennsylvanica; Elma, Gowanda, Salamanca $(E P V)$.

$2940 \frac{1}{2}$ P. buxi L. NYC, abundant on box hedge (Bks).

2942 P. galeaformis Patch. Speculator, Aug, Yg; Blue Mt., J1 (NYS) ; L. Placid, Aug, EPV (NYS); North Cr., Aug (NYS); Freeville, J1 (Patch); Karner, Mar (Patch); Stamford, Aug (EPV).

P. quadrisignata Wlk. "NY" (Wlk).

\section{FAMILy APHIIDAE 23}

\section{SUBFAMILY APHIINAE}

Tribe LACHNINI

ANOECIA Koch

A. querci (Fitch). Ithaca, very common on leaves of red oak (Morr).

\section{Eulachnus Del Guercio}

E. agilis (Kalt.). Albany, NYC, J1, on pine (Gill-NYS).

\section{DilachNuS Baker}

D. abietis (Fitch). L. Placid, Sep, CHPeck (NYS).

D. laricifex (Fitch). Ithaca, Je, on larch (Morr).

D. strobi (Fitch). Ithaca, on white pine (Morr, with query); LI: Wading R., May, on pine (Ols).

LONGISTIGMA Wilson

L. caryae (Harr.). S. Byron, Je, JFRose (NYS).

\section{LACHNUS Burmeister}

L. curvipes Patch. Old Forge, on balsam, Aug (Felt).

L. viminalis (Fonsc.) (dentatus Le B.). Poughkeepsie, May; Ossining, May (NYS); LI: Flushing, Sep, on willow (NYS).

\section{Tribe CALLIPTERINI}

\section{PHYLLAPHIS Koch}

P. fagi (L.). A woolly species which appears to be very common on the leaves of copper beech in various parts of the State; probably widely distributed: Albany, Tivoli, Rochester, Brewster, Tarrytown.

${ }^{23}$ The present list is based upon one prepared by Dr. E. P. Felt several years ago, compiled from literature and from specimens and records in the New York State Museum, which has been further added to by the editor. A. C. Baker and P. W. Mason, of the United States Bureau of Entomology, have kindly rearranged the synonymy in the present list and have gone over the manuscript for possible errors. Dr. Edith M. Patch also has looked over the manuscript and made several changes and suggestions. 
CALAPHis Walsh

C. betulaecolens (Fitch). Buffalo, Je; Rochester, Je (Gill); E. Greenbush, Aug; Karner, Leon (Patch), on gray birch; Newark, Je ; Cranberry L., on yellow birch (Osb) ; reported also from Newport, Geneva, Rochester, and Poughkeepsie, Je (Gill).

?C. betulae (Buckt.). Albany, J1; Geneva, Je (Gill).

C. castaneae (Fitch). Albany, Ithaca, very common (Morr).

\section{THERIOAPHIS Walker}

T. bellus (Walsh). Rochester, Je, on Quercus rubra (Gill); Ithaca (Morr); White Plains, Nov (Ols); LI: Flushing, Oct, on Quercus rubra (Ols).

T. tiliae (L.). Geneva, Je (Gill); Ithaca, common (Morr).

T. ononidis (Kalt.). Albany, Geneva, Je-J1 (Gill).

\section{EUCERAPHIS Walker}

E. betulae (Koch). Geneva, Je (Gill); Ithaca, May (Patch); White Plains, Nov, on Betula lutea (O1s).

E. lineata Baker. Karner, on gray birch, Leon (Patch).

\section{Monellia Oestlund}

M. caryella' (Fitch). Ithaca, very common on hickory (Morr).

M. costalis (Fitch). Watervliet, Aug, abundant on pignut, Leon (Patch).

\section{MyZOCALLIS Passerini}

M. alnifoliae (Fitch). "NY" (Fitch).

M. asclepiadis (Mon.). Geneva, J1 (Gill); Sheridan (Hayhurst).

M. discolor (Mon.). Ithaca, Je, very common and injurious on white oak (Morr); LI: Maspeth, Oct, Quercus primus.

M. fumipenellus (Fitch) (caryfoliae Davis). "NY" (Fitch).

M. ulmifolii (Mon.). Common and frequently destructive on the American elm: Albany, Broome, Chautauqua, Fulton, Monroe, Montgomery, Oneida, Ontario, St. Lawrence, and Warren Counties, probably generally distributed throughout the State.

M. punctatellus (Fitch). "NY" (Fitch).

M. walshi (Mon.). Geneva, Je, on Quercus rubra (Gill).

\section{DREPANAPHis Del Guercio}

D. acerifoliae (Thos.). A common, though rarely abundant, species on soft-maple leaves, probably widely distributed throughout the State since it has been recorded or reported from Albany, Dutchess, Herkimer, Rensselaer, Saratoga, Tompkins, and Westchester Counties. LI: Maspeth, Je, Oct, on Acer saccharinum (Ols).

\section{DREPANOSIPHUM KOCh}

D. platanoides (Schrank). Ithaca, Je (Morr); LI: Orient, J1, Lath (Felt). Sycamore, maple. 


\section{ChaitophoRUs Koch}

C. populifoliae Oest. Ithaca, on Carolina poplar (Morr).

C. viminalis Mon. Geneva, J1 (Gill).

\section{PERIPHyLLUS Van der Hoeven}

P. lyropictus (Kess.). It is presumably this species which has been extensively recorded in economic literature in recent years as Chaitophorus aceris $\mathrm{L}$. Ogdensburg (JJDavis); Albany, Greene, and Ontario Counties. Probably this is the species which has been very destructive to Norway maples in many widely separated localities in the State.

P. negundinis (Thos.). Box-Elder ApHID. Newport; Ithaca (Morr); Albany, J1 (Gill) ; Nassau. Box elder.

\section{NEOTHOMASIA Baker}

N. populicola (Thos.). Ithaca (Morr) ; Karner, J1, abundant on the common aspen ; LI: Maspeth, Nov, on Populus sp. (Ols).

\section{Clavigerus Szépligeti}

C. smithiae (Mon.). LI: Wading R., May, on Salix fragilis (Ols); Winfield, Oct, on willow (Ols).

C. populifoliae (Fitch). "NY" (Fitch).

C. salicis (L.), Buffalo, Sep; Karner, Je (NYS).

C. saliceti (Kalt.). Ithaca, very common on willow (Morr).

\section{Tribe APHIINI}

\section{ANURAPHIS Del Guercio}

A. cardui (L.) (pruni Koch). Sheridan, Hayhurst; Geneva, Je (Gill) ; Ithaca, Oct (Morr) ; Schoharie, Je ; Rensselaer.

A. crataegifoliae (Fitch). Sheridan, Hayhurst.

A. maidi-radicis (Fbs.). Aibany, on aster (NYS).

A. persicae-niger (Smith). Portland (Sling).

A. roseus Baker (sorbi Am. auct.). Rosy Apple Aphis. Very injurious in apple orchards, and probably generally distributed throughout the State.

A. tulipae Boyer (gladioli Felt). Berlin, on gladioli (Felt).

A. viburnicola (Gill.). LI: Maspeth, Je, on Viburnum sp. (O1s).

\section{APHIS Linnaeus}

A. asclepiadis Fitch. Sheridan, Hayhurst; Geneva (Gill).

A. carduella Walsh. Ithaca, Oct, on Cirsium lanceolatum (Morr).

A. cerasifoliae Fitch. Sheridan, Hayhurst; Geneva, Je (Gill) ; Ithaca (Morr) ; Albany, Je. Probably widely distributed on chokecherry.

A. cephalanthi Thos. LI: Wading R., May, on Cephalanthus occidentalis (O1s).

A. coreopsidis (Thos.). Sheridan, Hayhurst; LI: Maspeth, Aug, on Bidens frondosa (Ols). 
A. forbesi Weed. LI: Maspeth, Je, on Fragaria virginiana (Ols).

A. gossypii Glover. Melon Aphis. Albany, Aug, SHPeck; Fredonia, Hayhurst: Menands, Aug (Felt); Ithaca; NYC, J1 (Gill); LI: Maspeth, Oct, on Hibiscus syriacus (Ols). Common and widely distributed in the State.

A. helianthi Mon. Ithaca, on sunflower (Morr); Blissville, Oct, Nov, on Helianthus rigidus. Probably widely distributed.

A. maidis Fitch. LI: Orient Pt., Nov, on corn, Lath (NYS).

A. lutescens Mon. LI: Winfield, Je, on milkweed (O1s).

A. pomi DeG. Apple Aphid. LI: Wading R. (Ols). A widely distributed, very common, and occasionally rather destructive, orchard pest.

A. pseudoavenae Patch. Ithaca, Mar, in greenhouse on wheat, Griswold (Patch).

A. rumicis L. Bean Aphid. Albany; Port Kent, May; Geneva, Je (Gill) ; Elmira, Sep, on buckwheat (Cy); Ithaca (Morr); LI: Maspeth, Je, on Arctium lappa, Oct, on Chenopodium sp. (Ols).

A. sanborni Patch. LI: Maspeth, May, on Sambucus canadensis.

A. spiraecola Patch. Albany; Geneva, Je-Jl (Gill).

A. salicicola Thos. Albany; Geneva, on willow (Gill).

A. illinoisensis Shimer. Grape-Vine Aphid. Troy, J1 (Leon); Middletown, Aug (Chap). LI: Maspeth, Je, J1 (Ols). Sometimes injurious to grape.

\section{BREVICORYNE Das}

B. brassicae (L.). Cabbage Aphis. Widely distributed throughout the State.

Cavariella Del Guercio

C. pastinaceae (L.) (Siphocoryne). Ithaca, very common on wild parsnip (Morr) ; LI: Maspeth, Nov (O1s), on Lonicera japonica.

C. capreae Fab. LI: Maspeth, J1, on cultivated parsnip (Ols).

\section{Hysteroneura Davis}

H. setariae (Thos.). LI: Maspeth, Nov (Ols), on Digitaria sanguinalis.

\section{HYALOPTERUS Koch}

H. atriplicis (L.). Fredonia, Jamestown, Lilly Dale (Hayhurst), on Chenopodium.

H. arundinis (Fab). Mealy Plum Aphid. Fredonia, on plum (Pergande); probably this species in Montezuma Marshes, HDHouse (Felt), on Phragmites; Ithaca, Je, $C y$ (Patch, as pruni Fab.).

H. trirhoda Wlk. Ithaca, Jl, on cultivated columbine, Griszold (Patch).

\section{LIOSOMAPHIS Walker}

L. rhois (Mon.) (Rhopalosiphum, Amphorophora). Ithaca, Je, on the terminal shoots and leaves of sumac (Morr).

\section{RHOPALOSIPHUM Koch}

R. rubiphila (Patch). Ashokan Reservoir, Je, abundant on wild raspberry plants affected with mosaic, Leon (Patch). 
R. pseudobrassicae (Davis). Turnip ApHID. Geneva; Ithaca; LI. Probably widely distributed throughout the State, since it is readily confused with the common cabbage aphid, Brevicoryne brassicae (L.).

R. prunifoliae (Fitch) (avenae Fab. and Am. auctt.). Apple Grain Aphid. Reported from many localities, and undoubtedly present wherever wild or cultivated apple occurs in the State.

R. angelicae (DelG). LI: Maspeth, Aug (O1s), very abundant on Artemisia absinthium.

R. rufomaculata (Wils.). Ithaca, Dec, in greenhouse on Chrysanthemum indicum hybrid, Griswold (Patch).

\section{AMPHOROPHORA Buckton}

A. solani (Thos.). Port Jervis, Je (Felt); sometimes prevalent and injurious on potatoes (Felt). Dr. Patch writes: "A. solani Thomas of most American authors is only Myzus persicae. The type slide is not a Solanum species at all -probably accidental. The description of colony applies to another Solanum aphid."

Francoa Del Guercio

F. rosarum (Kalt.) (Myzus rosarum auct.). Ithaca, Oct, in greenhouse on rose, Griswold (Patch).

\section{ILLINOIA Wilson}

I. liriodendri (Mon.). Sometimes common on tulip leaves, at least in the vicinity of NYC (Felt).

I. pisi (Kalt.) (Macrosiphum). PEA APHID. Probably widely distributed throughout the State. Definitely recorded from: Albany; Rensselaer; LI: Floral Pk.; Wading R. Pea and red clover, often very injurious.

I. solanifolii (Ashm.) (Macrosiphum). Ротато Aрнір. Widely distributed throughout the State.

I. lilii (Mon.). LI: Orient, Aug, Lath (Patch), on Lilium canadense.

\section{Macrosiphoniella Del Guercio}

M. sanborni (Gill.). Ithaca, Feb, on chrysanthemum (Patch).

\section{TRITOGENAPHIS Oestlund}

T. chrysanthemi Oest. Sheridan, Hayhurst (Felt).

T. rudbeckiae (Fitch). Probably widely distributed throughout the State. Bemis Hgts.; Albany, Je; Nassau; LI: Maspeth, Je-Nov, on Silene noctiflora, cultivated aster, goldenrod, Lactuca, Xanthium canadense, Rudbeckia laciniata, cultivated lettuce (Ols); Rockaway Beach, J1, on goldenrod; Wading R., May, on Antennaria neodioica.

T. taraxici Kalt. Ithaca, Je, Joh (Patch); LI: Maspeth, Je, on dandelion (Ols).

T. solidaginis Fab. Probably widely distributed throughout the State. Albany, J1, on aster (Gill).

\section{MACROSIPHüM Passerini}

M. cornelli Patch. Ithaca, throughout the year, in greenhouse on Pelargonium quercifolium hybrid, $P$. odoratissimum, $P$. domesticum, $P$. graveolens, $P$. peltatum, and $P$. radula, Griswold (Patch).

M. rosae (L.). LI: Maspeth, on rambler rose (Ols). 
M. granarium Kby. (not avenae). English Grain ApHid. Doubtless widely distributed throughout the State. Canaseraga; Chatham; Hudson; Kinderhook; Niverville; Stone Ridge; LI: Glen Cove.

M. dirhodum W1k. Ithaca, Mar, on greenhouse roses (Morr).

M. pseudodirhodum Patch. Ithaca, Ap, May, Nov, in greenhouse on roses, Griszold (Patch).

\section{Myzus Passerini}

M. cerasi (Fab.). BlACK CHERRY APHID. Generally distributed throughout the State, definite records extending from Saranac Inn to Wading R., LI.

M. circumflexus (Buckt.). Ithaca, on liliaceous greenhouse plants in Mar and May (Morr).

M. persicae (Sulz.). Green PeAch Aphid. Widely distributed throughout the State on greenhouse plants and on a variety of outdoor fruits and vegetables.

M. pseudosolani Theo. Ithaca, Jl, on potato, Nov, in greenhouse on Vertena hybrida, Griswold (Patch).

\section{CRyptomyzus Oestlund}

C. ribis (L.). CURRAnt Apirid. Widely distributed throughout the State and destructive to currants; recorded from St. Lawrence Co southward to Westchester Co and across the State.

C. galeopsidis (Pass.). LI: Maspeth, Je-Aug, on Polygonum (O1s).

Capitophorus Van der Goot

C. fragaefolii Ck11. LI: Maspeth, Je (O1s), on Fragaria virginiana.

C. hippophoes Koch (Rhopalosiphum). LI: Maspeth, Nov, on Polygonum pennsylvanicum.

\section{PHORODON Passerini}

P. humuli (Schrank). Hop ApHid. Probably widely distributed over the State, since it has been reported on hop in Cattaraugus, Dutchess, Madison, Montgomery, Otsego, and Schoharie Counties.

\section{IDIOPTERUS Davis}

I. nephrolepidis Davis. Ithaca, Feb, Mar, Dec, in greenhouse on Adiantum, Nephrolepis exaltata var. bostoniensis, Cyrtomium, and Pteris, Griswold (Patch).

\section{SUBFAMILY MINDARINAE}

MINDARUS Koch

M. abietinus (Koch). Star L., St. Lawrence Co, Je; L. Clear, Paul Smiths, Franklin Co, Elizabethtown, Je; Watertown, J1; Mt. Kisco, Je, on balsam and Scotch pine (NYS); Warwick, J1.

\section{SUBFAMILY ERIOSOMATINAE \\ Tribe ERIOSOMATINI}

Colopha Monell

C. ulmicola (Fitch). Known to occur in Onondaga, Genesee, Erie, Ontario, Washington, Albany, Rensselaer, and Westchester Counties, and probably moderately abundant and generally distributed throughout the State (Felt). 
ERIOSOMA Leach

E. lanigerum (Hausm.). Woolly Apple Aphid, or Elm Rosette Aphid. Widely distributed throughout the State from St. Lawrence and Essex Counties southward to Maspeth, LI. Apple, Crataegus, and elm, often injurious.

E. rileyi (Thos.). Probably generally distributed throughout the State, but recorded only from Watertown and Northville (Felt). Elm.

E. americanum Rly. Elm LeAf-Curl Aphid. Probably generally distributed throughout the State, availabie records including from St. Lawrence and Essex Counties southward to Westchester Co and Maspeth, LI (Felt). Probably blamable for the early summer curling of American elm leaves (Felt).

E. ulmi (L.). The distribution of this species within the State is probably coincident with that of $E$. americamum Rly., but $E$. ulmi is probably less common (Baker \& Mason); common on certain English elms (Patch).

\section{GoBAISHIA Matsumura}

G. ulmifuscus (Walsh). Reported from Warren, Ontario, Fulton, Greene, and Westchester Counties, but is presumably widely distributed in the State though rarely abundant (Felt). Produces a good-sized, somewhat irregular, gall on leaves of slippery elm (Felt).

\section{TETRANEURA Hartig}

T. graminis Mon. Albany, Sep (Felt), on heads of rice cut-grass, Leersia (Homalocenchrus) oryzoides; LI: Maspeth, on elm, Winfield, Je (Ols).

T. ulmisacculi Patch. Elm Sack Gall. Kenwood, May, HDHouse (Felt), gall on Ulmus montana; LI: Roslyn, Maspeth, Je (Ols).

\section{Tribe PEMPhigini}

\section{MoRdwilkoja Del Guercio}

M. vagabundus Walsh. Reported from Monroe, Washington, Saratoga, and Rensselaer Counties, and is probably rather generally and commonly present in sections of the State where poplars grow (Felt). Produces a very irregular, frequently massed, deformation of poplar leaves (Felt).

\section{Pemphigus Hartig}

P. popularius Fitch. Saranac Inil, J1, gall on poplar.

P. populicaulis Fitch. Ithaca, Albany, Je (Morr); LI: Mineola, J1 (Morr).

P. populiglobuli Fitch. New Rochelle, Je, TJWade, gall on Lombardy poplar.

P. populimonilis Rly. Alder Cr., Aug, gall on balm of Gilead.

P. populitransversus Rly. Rochester, Je (Gill); Cambridge, Jl; Albany, Je. Galls on cottonwood.

P. lactucae Fitch ('Tychea breviiornis Hart, Rhizobius lactucae). A common lettuce root-louse, and probably rather generally distributed (Felt); Fredonia (Pergande). 


\section{Tribe MELAPHINI}

\section{Melaphis Walsh}

M. rhois Fitch (Pemphigus). Little Falls, Sep (Felt); Clinton (Lint) ; Canandaigua, Sep (Felt); Schenectady, Sep (Lint). Produces a red-tinted pouch gall on sumac leaves. Has been observed in the lower Hudson Valley, and is probably generally distributed though comparatively rare (Felt).

\section{Tribe PROCIPHILINI}

\section{NEOPROCIPHILUS Patch}

N. aceris (Mon.) (Pemphigus). Union, Aug, rather injurious on maple, $C y$ (Patch).

N. attenuatus O. \& S. Karner, Oct, $C y$ (Patch), on Smilax herbacea.

\section{PROCIPHILUS Koch}

P. imbricator (Fitch). BEEch Blight. Probably widely distributed and somewhat common to abundant. Newport, Oct; Ithaca (Morr); Richfield Sps., Sep (Felt) ; Barneveld, Aug; Indian Ladder, Albany Co; Scarsdale, Aug.

P. corrugatans Sirrine. Ithaca, May (Patch), on Amelanchier.

P. tessellata (Fitch) (Pcmphigus tessellata and P. acerifolii Rly.). Alder Blight. Somewhat generally distributed, but especially abundant in the Adirondacks (Felt) ; Essex, Monroe, Tompkins, Orange, and Suffolk Counties.

\section{Tribe FORDINI}

FORDA Heyden

F. occidentalis Hart. LI: Maspeth, Je, on Polygonum roots (Ols).

\section{GeorcA Hart}

G. radicicola Essig (Triphidaphis). Warsaw, Aug, on bean roots, $C y$ (Patch).

\section{SUBFAMILY HORMAPHIINAE}

\section{HORMAPHIS Osten Sacken}

H. hamamelidis Fitch. A common and probably widely distributed species, producing a characteristic conical gall on the leaves of witch-hazel (Felt). Newport; Oneonta; Nassau; LI: Wading R., May (Ols).

H. papyraceae Oest. Cranberry L., galls on willow birch (Osb). This may be a synonym (Baker \& Mason).

\section{HAMAMELISTES Shimer}

H. spinosus Shim. Probably widely distributed, though not usually abundant excepting possibly on birch, its alternate food plant. Albany, J1 (Gill); Hudson (Felt) ; Canandaigua, Aug; Tarrytown, Je. Produces a peculiar spiny budgall on witch-hazel. 
CeRATAPHis Lichtenstein

C. lantaniae (Bdv). Ithaca, Dec-Mar, in greenhouse on Sobralia macrantha, Griswold (Patch).

\section{FAMILY PHYLLOXERIDAE SUBFAMILY ADELGINAE}

\section{ADELGES Linnaeus}

A. abietis L. Spruce-Gall Aphid. Moderately abundant to injurious from the Adirondacks to Westchester Co and presumably LI, probably occurring in most places where Norway spruce is growing.

A. cooleyi Gill. Watertown, J1 (NYS); White Plains, Aug, on Colorado blue spruce; LI: Westbury, J1 (Felt).

A. floccus Patch. Star L., St. Lawrence Co, Aug; St. Huberts, Essex Co, Dec (Patch).

A. piceae Ratz. Rochester, May, on Nordmann's fir, probably a recent introduction (Felt).

A. pinicorticis Fitch. PINE-BARK APHID. A moderately common species recorded definitely only from Albany, Chenango, Fulton, Saratoga, Dutchess, Ulster, Putnam, Rockland, Westchester, and Suffolk Counties, but widely distributed and occasionally destructive even to large pine trees.

A. pinifoliae Fitch (abieticolens Thos.). Pine-LeAF Aphid. Forms a cone-like gall with the needles modified to thin scales on black spruce, the adults maturing in mid-June and the migrants ovipositing on white pine (Patch). St. Huberts, J1 (Felt); Utica, J1 (NYS); Woodstock (Felt); Berlin, Jan.

A. strobilobius Kalt. (laricifoliae Fitch). This species has been referred to the European genus Gnaphalodes. The woolly aphid is sometimes very abundant on larch. Reported only from Ontario, Tompkins, Oneida, Dutchess, and Rockland Counties, but presumably somewhat generally distributed and occasionally rather injurious.

\section{SUBFAMILY PHYLLOXERINAE}

\section{PHYLLOXERA Boyer}

P. caryaecaulis Fitch. HICKORY-GALL APHid. Widely distributed throughout the State, probably occurring wherever hickory grows and often injurious to it.

P. caryae-fallax Walsh. Rochester, Je (Gill).

P. caryaefoliae Fitch. Salem (Fitch); Ithaca (Morr), on Hicoria glabra.

P. caryaeglobuli Walsh. LI: Cold Sp. Harb., Je, Lutz (NYS).

P. caryae-venae Fitch. Rochester, Je (Gill-NYS).

P. depressa Shim. Howe Cave, Schoharie Co, Je (NYS).

P. foveola Perg. Canandaigua, Je (NYS).

P. intermedia Perg. Rochester, Geneva, Je (Gill).

P. vitifoliae Fitch (vastatrix Rly.). Grape Phylloxera. Moderately common and widely distributed. Recorded from Chautauqua, Onondaga, Ontario, Chenango, Rensselaer, and Ulster Counties. Probably occurs in most sections of the State where grapes grow. 


\section{FAMILY ALEYRODIDAE 24}

\section{ALEUROCHITON Tullgren}

A. forbesii Ashm. Ithaca, Nov (CU), on silver maple.

\section{ALEYRODES Latreille}

A. asarumis Shim. (acteae Britton). Taughannock Falls, J1 (CU), on Asarum canadense; Fort Plain (Felt); probably widely distributed.

\section{TRIALEURODES Cockerell}

T. coryli Britton. Nassau, J1 (Felt), on hazel.

T. morrilli Britton. Tarrytown, Aug (Britton), on Impatiens fulva.

T. packardi Morrill. Sparkill (Sling); SI: Richmond (Sling).

T. vaporariorum Westw. GREenhouse Whitefly. Generally distributed in greenhouses throughout the State, infesting a variety of plants.

\section{TETRALEURODES Cockerell}

T. mori Quaint. Mt. Kisco, J1 (Felt), abundant on Kalmia latifolia; New Rochelle, Sep, on Norway maple (Felt).

\section{FAMILY COCCIDAE 25}

\section{Compiled by E. P. FeLt and Harold Morrison}

\section{SUBFAMILY MONOPHLEBINAE}

\section{ICERYA Signoret}

I. purchasi Mask. Cottony Cushion Scale, or Fluted Scale. Rochester, Ithaca (USBE); Albany (NYS); New Rochelle (USBE); LI: Lynbrook, LCGriffith (NYS). On Acacia and grapefruit. A greenhouse species in this latitude, and well known in the citrus-growing sections of the country. It has been recorded from a large variety of plants and has an extended distribution in the warmer sections of the world.

\section{SUBFAMILY MARGARODINAE}

\section{Matsucoccus Cockerell}

M. matsumurae (Kuw.). This species has caused considerable damage to the pines on the De Forest Estate on LI (Herbert, Proc. Ent. Soc. Wash., vol. 23, p. 19).

\footnotetext{
24 The list in this family was originally prepared by Dr. E. P. Felt, and the manuscript has been looked over by Dr. W. E. Britton.

${ }^{25}$ To the list of the Coccidae compiled by Dr. E. P. Felt several years ago, has been added, through Harold Morrison, a summary of the numerous New York species, host, and distribution records contained in the coccid note files of the Bureau of Entomology of the United States Department of Agriculture. About fifty new species and many additional locality records have been obtained from this source. The majority of these are based on determinations by Mr. Morrison. No special attempt has been made to separate the statements made by each, nor is either of these authorities to be held responsible for errors in the list. All dates are omitted, since many specimens are collected long after their death.
} 


\section{SUBFAMILY ORTHEZIINAE}

OrThezia Bosc d'Antic

O. insignis Dougl. Ithaca (Comst); NYC (Pettit\&King). On Coleus. This cosmopolitan species occurs in nearly all greenhouses in the State on a very large variety of plants, mostly herbaceous.

O. solidaginis Sanders. Ithaca. On Solidago, red clover, mint, and Prunella vulgaris.

\section{SUBFAMILY DACTYLOPIINAE}

\section{AsteroleCaniUm Targioni-Tozzetti}

A. bambusae Bdv. NYC (USBE). On Bambusa in greenhouse.

A. pustulans (Ckll.). Chatham (NYS). A subtropical species occurring on a variety of plants.

A. variolosum (Ratz.). Rochester, Geneva, Cortland, Newburgh, Yonkers (NYS) ; Tarrytown (USBE); NYC (NYS); LI: Flushing (NYS); Westbury, Brooklyn (USBE). This species is limited to oak, and is an introduction from Europe.

\section{Cerococcus Comstock}

C. parrotti (Hanter). LI: Brooklyn (USBE). On horse-chestnut.

\section{KERMES Boitard}

K. galliformis Rly. Ithaca (CU) ; Karner, Middletown (NYS) ; LI: Brooklyn (NYS). On black oak. This name has been indifferently applied to species of Kermes, and the records here given may therefore include more than one species.

K. kingii Ckll. Middletown (NYS), On red oak.

K. pettiti Ehrh. Ithaca, Pettit (Ehrh); Middletown, on oak, Thro (EEBogue).

K. pubescens Bogue. Scarsdale (USBE). On oak.

K. trinotatus Bogue. Albany (NYS). Apparently widely distributed in the United States. On Quercus nigra.

\section{GosSYPARIA Signoret}

G. spuria (Mod.) (ulmi Geoff.). Eurcpenn Elm Scale. Many localities show this species to be widely distributed throughout the State from LI and NYC along the Hudson R., northward to Round L., and westward to Newfane. Its occurrence at Binghamton and Ogdensburg also indicates a wide distribution, which may be restricted only by the lack of its food plant.

\section{ERIococcus Targioni-Tozzetti}

E. azaleae Comst. Canton (NYS) ; Geneva (Comst-CU) ; Ithaca (Comst-CU) ; LI: Brooklyn (NYS). On wild azalea, Azalea mendula, Rhododendron, huckleberry, Crataegus coccinea.

E. borealis var. Ckll. \& Robn. "NY" (Ckll-USBE). On Gaylussacia.

E. coccineus Ckll. NYC (USBE). On various greenhouse Cactaceae. 


\section{GYMNOcoccus Douglas}

G. agavium (Doug1.). NYC (USBE). On Agave spp.

\section{DACTYLOPIUS Costa}

D. indicus (Green). NYC (USBE). On Opuntia sp. in greenhouse.

\section{Phenacoccus Cockerell}

P. acericola (King). Faise Maple Scale. Numerous records covering a series of years indicate the common occurrence of this insect in the Hudson Val. northward to Troy. Sometimes very abundant on hard maple, and erroneously referred in earlier years in this country to Pseudococcus aceris Geoff. Also reported from Johnstown, Oneonta, and Batavia, indicating a wide distribution and suggesting that the area of greatest injury is in the southern part of the Hudson Val. The U. S. Bur. Ent. has many records throughout the State. On maple, hornbeam, lime, and horse-chestnut.

P. dearnessi King. Albany (USBE). On Crataegus.

\section{TRIONYMUS Berg}

T. americanus (Ck11.). Barneveld (USBE).

\section{PSEUDOcoccus Westwood}

P. adonidum (L.) (frequently referred to as longispinus). A common mealy-bug recorded from Ithaca, Albany, and one LI locality and Brooklyn, in greenhouse (USBE). Like citri, it is probably widely distributed throughout the State on a considerable variety of subtropical and tropical plants.

P. citri (Risso). Citrus Mealy-Bug. This common mealy-bug of the greenhouse is represented from Ithaca, Albany, LI (Maspeth, Brooklyn, Flushing), on many plants (USBE). This must be only a very small fraction of localities in the State where this insect occurs, since it is presumably world-wide in distribution and occurs on a very large variety of tropical and semitropical plants.

P. comstocki (Kuw.). Palisades, NYC, SI, and LI (Brooklyn, Mineola, Bayside) (all USBE). On a variety of plants.

P. gahani Green. NYC (USBE). On ampelopsis.

P. ledi Ckll. Oneida, HDHouse (NYS) ; Sand L., Rensselaer Co (Ckll-CU). On Ledum groenlandicum.

P. maritimus (Ehrh.). NYC (USBE). On a variety of plants.

P. nipae (Mask.). Coconut Mealy-Bug. New Rochelle, NYC (USBE); LI: Locust Val. (USBE). On a variety of plants in the greenhouse.

P. trifolii Fbs. Clover-Root Mealy-Bug. Ithaca (CU). On roots of red clover.

RIPERSIELla Tinsley

R. maritima (Ckll.). LI: Sea Cliff (USBE). On roots of Spartina. 


\section{SUBFAMILY COCCINAE}

Pulvinaria Targioni-Tozzetti

P. acericola Walsh \& Rly. Ithaca, Pettit\&Lochhead (CU); Albany (USBE) ; Tarrytown (NYS); LI: Maspeth (USBE). Occurs on the leaves of soft maple and on Cornus; is relatively scarce, and probably widely distributed throughout the State.

P. amygdali Ckll. Cottony Peach Scale. Williamson, Morton, Lockport, 1925, several infested peach orchards in each. Here first definitely recorded from the State (Morr-CU).

P. psidii (Mask.). Green Shield Scale. NYC (USBE). On several greenhouse plants.

P. vitis (L.). Cottony Maple Scale. Recorded by numerous observers from many localities in the State. Particularly abundant on LI and in the Hudson Val. localities northward to Hudson Falls. Reported in addition from: Adams; Rome; Phoenix; Rochester; Batavia; Buffalo; Salamanca; Ithaca; Auburn; Schenectady. Thrives on soft maple, is frequently reported on grape and under the name $P$. inmumerabilis Rathv., and has been recorded from a large number of other food plants.

\section{PROTOPULVINARIA Cockerell}

P. pyriformis (Ck11.). Pyriform Scale. NYC (USBE). On Antidesma in greenhouse.

\section{PSEUdophilipPia Cockerell}

P. quaintancii Ckll. Port Jervis (NYS). On pitch pine.

\section{ERIOPELTIS Signoret}

E. festucae Fonsc. Gravesville (USBE). On grass.

E. lichtensteinii Sign. Stark (NYS). On grass.

\section{Ceroplastes Gray}

C. grandis Hemp. Lancaster, EPV (CU). On Azalea in greenhouses. A Brazilian species previously recorded on Zanthoxylum, Baccharis, Ilex, and Psidium.

\section{Eucalymatus Cockerell}

E. tessellatus (Sign.). NYC (USBE). On many greenhouse plants.

\section{Coccus Linnaeus}

C. acuminatus (Sign.). NYC (USBE). On Jasminum in greenhouse.

C. elongatus (Sign.). NYC (USBE). On many greenhouse plants.

C. hesperidum L. Soft Scale. A common soft scale on greenhouse and house plants. Ogdensburg, Oswego (NYS) ; Sinclairville (USBE); Rogers Rock, L. George (USBE) ; Albany, Schenectady (NYS) ; Richfield Sps. (USBE) ; Chatham Center (NYS) ; Lebanon Sps. (USBE) ; Kingston (NYS) ; Milton, Monticello (USBE) ; Warwick (NYS) ; New Rochelle (USBE) ; Irvington 
(NYS) ; White Plains (USBE); Kenilworth (USBE); Nyack (USBE) ; NYC (NYS); SI: Rosebank (USBE); LI: Roslyn (USBE). This species is cosmopolitan and infests a considerable variety of plants.

C. pseudohesperidum (Ck11.). Ithaca, Garrison (USBE). On orchid.

C. viridis (Green). NYC (USBE). On citrus in greenhouse.

\section{Neolecanium Parrott}

N. cornuparvum Thro. Magnolta Scale. E. Aurora, Aurora, Jamestown (USBE); Trumansburg (Thro-CU); Elmira, Mt. Vernon (USBE).

\section{TOUMEYELLA Cockerell}

T. corrugatum neglectum Pett. \& McDaniel. W. Danby (USBE). On pine.

T. liriodendri (Gmel.). Tulip-Tree Lecanium. Recorded from many localities in the State, principally on LI and in the Hudson Val. Probably distributed coincident with its food plant. On tulip tree and magnolia.

T. pini (King). Rochester (NYS); Ithaca (CU); Karner (NYS). On. Pimus divaricata. and $P$. rigida.

\section{LECANIUM Burmeister}

L. caryae Fitch. Otto (CU); Albany (NYS); NYC (Fitch). On black cherry and hickory.

L. corni Bouché, and its synonyms, varieties, etc. European Fruit Lecanium. Throughout the State on many hosts (USBE).

L. fletcheri Ck11. ${ }^{26}$ Valcour, Canandaigua (NYS); has been taken also by Pettit, presumably at Ithaca; Yonkers (USBE); LI (USBE). On cedar and arbor vitae.

L. nigrofasciatum Perg. The Terrapin Scale. This is a common species occurring on a variety of trees. It has been recorded from widely separated localities in the State, such as Port Jervis, various places in the Hudson Val., Elmira, Unadilla Forks, and Stanley. Particularly abundant on plum, apple, linden, birch, olive, and Vaccinium.

L. persicae (Fab.). ${ }^{27}$ NYC; LI: Brooklyn (USBE). On kudzu vine and Berberis.

L. quercifex Fitch. ${ }^{28}$ New Russia, on chestnut (NYS) ; Otto, on elm (CU); Ithaca, on Rubus and elm (CU); Albany, on black currant (NYS); LI: Wading R., on oak (O1s).

\section{SAissetia Deplanches}

S. hemisphaerica (Targ.). Hemispherical Scale. Gouverneur, Rome, Rochester, Buffalo, Jamestown, NYC, SI (Prince Bay), LI (Locust Val., Flushing, Bayside) (all USBE) ; LI: Maspeth, on fern in greenhouse (Ols).

S. nigra.(Nietn.). NYC (USBE). On a variety of greenhouse plants.

S. oleae (Bern.). Black Scale. Rochester, NYC, LI (Brooklyn) (USBE); Maspeth, on lemon (NYS). A subtropical or tropical species occurring on a variety of greenhouse plants and on citrus fruits. It is cosmopolitan in distribution and is recorded from a very large variety of food plants.

\footnotetext{
${ }^{26}$ Pettit and McDaniel have recently (Michigan Tech. Bul. 48, Mar., 1920, p. 12-14) regarded this as corni Bouché.

${ }^{27}$ All the early records of the occurrence of this species in New York State apparently relate to $L$. corni Bouché.-Morr.

${ }_{28}$ There is a question as to the correctness of records on hosts other than oak (Morr).
} 


\section{PHYSOKERMES Targioni-Tozzetti}

P. piceae (Schr.). Spruce Bud-Scale. Port Henry (NYS); Ithaca (common), Yonkers (USBE); Blauvelt (NYS); Mt. Vernon, on spruce (NYS). A European species which has been recorded, in addition, on pine.

\section{SUBFAMILY DIASPINAE}

\section{ChIONASPIS Signoret}

C. americana Jn. White Elm Scale. Newark (NYS); Geneva (USBE); Schenectady (NYS) ; Albany (NYS) ; Cohoes (USBE) ; Tarrytown (NYS) ; NYC (USBE) ; LI: Brooklyn, Westbury (NYS) ; Maspeth (Ols). Appears to be confined to elm and is probably widely distributed, though the infestation is usually inconspicuous and generally overlooked.

C. corni Cool. Rochester (USBE) ; Geneva (NYS) ; Youngstown, Ithaca, Albany (USBE) ; LI: Westbury (NYS). Apparently occurs only on Cornus.

C. euonymi Comst. Evonymus Scale. Hudson (NYS); Lexington, Fishkill (Felt) ; Irvington, Mt. Kisco, Tarrytown, Felt, Blauvelt, Nyack (NYS); NYC, Mamaroneck (USBE) ; SI: Tottenville (Ds, Ols) ; LI: Roslyn, Maspeth (Ols) ; Great Neck (NYS) ; Flatbush, Brooklyn, Babylon, Westbury, Baldwin, Stony Br. (USBE). On Evonymus, Celastrus, privet, and lilac.

C. furfura (Fitch). Scurfy Scale. Is represented by specimens in the NYS and $\mathrm{CU}$ collections, and has been received from many contributors in different localities from the following counties: Madison, Wayne, Monroe, Niagara, Erie, Chautauqua, Ontario, Tompkins, Cayuga, Albany, Rensselaer, Greene, Columbia, Ulster, Dutchess, Orange, Westchester, New York, Richmond, Nassau, Suffolk (Felt); and from many points in the State (USBE). These records indicate a general distribution in the southern and more temperate sections of the State. The insect does not appear to have attracted notice in the Adirondacks. It is recorded from a large variety of food plants, has a wide distribution in Canada and the United States, and has become established in England.

C. lintneri Comst. Rochester, on Cornus (NYS); Buffalo (Felt) ; Ithaca (USBE); Albany, on Alnus (Comst-CU). Occurs, in addition to the above food plants, on willow, Viburnum, benzoin, Amelanchier, Betula, etc.

C. longiloba Cool. Ithaca (EEBogue). Recorded from Texas on cottonwood.

C. pinifoliae (Fitch). PINE-LEAF Scale. Apparently widely distributed in the State. Has been reported by a number of observers from the following counties: Clinton, Monroe, Ontario, Tompkins, Schenectady, Saratoga, Albany, Columbia, Dutchess, Orange, Westchester, Kings, Queens, Nassau, Suffolk (Felt); and from many points in the State (USBE). It occurs in particular on the Scotch, Austrian, and white pines, especially in parks, and also on spruce.

C. pinifoliae heterophyllae Cool. LI: Glen Cove, Southold (USBE). On Pinus densiflora and $P$. rigida.

C. salicis-nigrae (Walsh). "NY" (USBE). On poplar.

C. spartinae Comst. LI: Orient Pt., Lath (NYS). On Spartina alterniflora, S. glabra, and S. stricta.

C. sylvatica Sanders. NYC (USBE). On Benzoin (?).

HowARDIA Berlese and Leonardi

H. biclavis (Comst.). Ithaca, NYC (USBE). On a number of greenhouse plants. 


\section{Diaspis Costa}

D. boisduvalii Sign. Gouverneur, on orchid (NYS) ; Rochester (USBE) ; Albany (NYS) ; Garrison (USBE) ; Millbrook, on cycad (Morr) ; Ithaca (USBE) ; NYC (USBE); LI: Brooklyn (USBE). Probably somewhat widely distributed within the State on greenhouse plants, such as palms, cattleya, etc.

D. bromeliae (Kern.). Pineapple Scale. NYC (USBE). On Bromelia.

D. carueli Targ. Rochester, on Juniperus virginiana and Pinus aristata (John Dunbar); Ithaca (CU); Ossining, on juniper (NYS) ; NYC (NYS); LI: Garden City, on arbor vitae (CU); Lynbrook, on Juniperus chinensis (Cy) ; several localities (USBE). A moderately common and widely distributed species.

D. echinocacti (Bouché) var. cacti Comst. Rochester (USBE); Ithaca, on cactus (Comst-CU) ; NYC, on cactus (Joutel-NYS).

D. zamiae Morg. Altamont, on Cycas revoluta (NYS); Albany, on Cycas (USBE) ; Ithaca; NYC, on Encephalartos villosus (USBE).

\section{Aulacaspis Cockerell}

A. pentagona (Targ.). WEST INDIAN PEACH Scale. Chatham, on flowering cherry (NYS); Kingston, on imported Japanese flowering cherry (NYS); New Rochelle, on Prunus pseudo-cerasus (NYS) ; NYC, on cherry (NYS) ; LI: Brooklyn, on peach and cherry (USBE). A southern form, and probably unable to breed in the open except possibly in the southern part of the State.

A. rosae (Bouché). Rose Scale. Pompey, on rose (NYS) ; Brighton, on blackberry (NYS) ; Stanley, on blackberry and rose (NYS) ; Ithaca; Cobleskill, on rose (NYS); Ballston Spa, on rose (NYS); Albany, on raspberry (NYS) ; Castleton, on rose (NYS); Troy, on rose (NYS) ; Hudson, on blackberry (NYS); Kingston, on rose (NYS); Fishkill, on rose (NYS) ; Cornwall, on blackberry and raspberry (NYS); NYC, on blackberry (NYS); SI: New Brighton, on blackberry (NYS); LI: Brooklyn, on rose (NYS); Maspeth (Ols); many records from State (USBE).

\section{HEMICHIONASPIS Cockerell}

H. aspidistrae (Sign.). Fern Scale. Geneva (USBE); Jamestown (USBE); Ithaca, on grass in a greenhouse (CU); Albany, on grass in a greenhouse (NYS) ; New Rochelle and Hyde Park (USBE) ; LI: Brooklyn, Locust Val., Jamaica (USBE). On fern and other greenhouse plants.

\section{Pinnaspis Cockerell}

P. buxi (Bouché). Irvington-on-Hudson, NYC (USBE). On Spathyllum, Cordyline, and Phoenicophorium, in greenhouse.

\section{LEUCASPIS Targioni-Tozzetti}

L. cockerelli (de Charmoy). NYC (USBE). On Sabal, Trachycarpus.

\section{FIORINIA Targioni-Tozzetti}

F. fioriniae (Targ.). New Rochelle (USBE). On Kentia belmoreana.

F. japonica Kuw. Albany, on Japanese hemlock (USBE) ; LI: Queens, on Tsuga and Japanese hemlock (USBE).

F. theae Green. "NY" (USBE). On Camellia japonica. 


\section{EPIDIASPIS Cockerell}

E. piricola (DelG.). Albany, Garrison, Hunt's Pt., LI (USBE). On pear.

\section{Aspidiotus Bouché}

A. abietis (Schr.). Hemlock Scale. Ithaca, on Pimus rigida and Abies canadensis (Comst-CU); Karner, on pine (NYS); NYC, on hemlock (NYS).

A. aesculi Jn. Buffalo, on horse-chestnut (USBE).

A. ancylus Putn. Putnam's Scale. Recorded from many localities in the following counties: Oswego, Wayne, Monroe, Orleans, Genesee, Erie, Chautauqua, Ontario, Seneca, Tompkins, Cayuga, Albany; Greene, Columbia, Ulster, Rockland, Queens. Presumably widely and generally distributed throughout the State, except possibly in the Adirondack section. Has been recorded in NY from the following host plants: apple, beech, purple-leaved chestnut, Cornus, Florida currant, elm, hemlock, Ilex, linden, maple, mountain ash, nectarine, olive, Osage orange, pear, white birch, willow.

A. camelliae Sign. (rapax Comst.). Geneva, on bay tree (CU); NYC, on bay and other plants (USBE); LI: Roslyn, Brooklyn (USBE).

A. comstocki Jn. "NY" (Fern. Cat.). On maple.

A. cyanophylli Sign. NYC (USBE). On palm.

A. forbesi Jn. Cherry Scale. Syracuse (USBE) ; E. Aurora, on apple (NYS) ; Geneva, on pear (NYS) ; Manchester, on apple (NYS); Crescent Station, on apple (NYS); Albany (NYS); Highland, on peach (NYS) ; Cornwall, on Japanese plum (NYS); Blauvelt, NYC (USBE). Recorded from a considerable variety of plants, and presumably widely and somewhat generally distributed throughout the State.

A. hederae (Vall.). Oleander Scale. Gloversville (USBE) ; Lockport (NYS) ; Buffalo, Alden, Geneva (USBE) ; Ithaca, on English ivy (NYS); Watervliet, on ivy (NYS); Albany, on Cyperus (NYS) ; Idlewild (USBE) ; Mt. Kisco, on Cattleya; Kenilworth (USBE) ; NYC (USBE) ; LI: Edgemore (USBE) ; Maspeth (Ols). Subtropical; presumably generally distributed throughout the State in greenhouses, and recorded as occurring on a great variety of plants.

A. juglans-regiae Comst. WALNuT ScAlE. Brighton, on European mountain ash (NYS) ; Lockport, Fredonia, on willow (USBE); Ithaca, on willow (USBE) ; NYC, on Tilia; LI: Brooklyn (USBE). Recorded also from the State by others, but without definite record as to locality or food plants.

A. lataniae Sign. Lancaster, on palm, EPV (CU) ; Albany (NYS); NYC, on several plants (USBE).

A. osborni Newell \& Ckll. Mt. Vernon, on white oak (NYS). A very inconspicuous species, evidently with a greater range than is indicated by the foregoing record.

A. ostreaeformis Curt. European Fruit Scale. Recorded from many localities in the following counties: Oswego, Monroe, Orleans, Niagara, Erie, Chautauqua, Ontario, Columbia, Rockland (Felt); records from many places and hosts in the State (USBE). Probably generally distributed throughout the State. Has been recorded in NY from the following food plants: apple, cherry, currant, elm, pear, plum, poplar, willow.

A. perniciosus Comst. The San José Scale. An introduced species which has become established in nearly every county in the State, with the possible exception of Clinton Co, where it has not yet been found. Lives on a great 
variety of trees and shrubs. Has been recorded in NY as occurring on the following: apple, birch, cherry, Crataegus, currant, elm, hornbeam, Japanese quince, pear, peach, plum, lilac, Osage orange, rose, spiraea, willow. Will probably become generally distributed in most regions except the Adirondacks.

A. townsendi Ckll. LI: Westbury (USBE). On tulip tree.

A. ulmi Jn. Buffalo, on catalpa (NYS); Albany (NYS); NYC (USBE).

A. uvae Comst. Grape Scale. Ithaca (Ck11); LI: Greenbush (Felt-NYS). On grape.

\section{CRYTOPHYLLASPIS Cockerell}

C. liquidambaris Kot. NYC (USBE). On sweet gum (Liquidambar styraciflua).

\section{PSEUdAONIDIA Cockerell}

P. paeoniae (Ckll.). NYC (USBE). On Camellia.

\section{Chrysomphalus Ashmead}

C. aonidum (L.). Gloversville, Lyndonville, Menands (NYS); Ithaca; New Rochelle (USBE); Yonkers (NYS); NYC (USBE); LI: Locust Val. (USBE). Recorded from several of the above localities on rubber plant; well known as a greenhouse pest on a considerable variety of plants.

C. aurantii (Mask.). California Red Scale. “NY” (Fern. Cat.).

C. dictyospermi (Morg.). Morgan's Scale. Rochester (USBE) ; Albany, on sago pine and palms (NYS); Millbrook, on cycad and palms (Morr); New Rochelle and NYC (USBE). A greenhouse species occurring on a variety of hothouse plants.

C. odontoglóssi (Ck11.). NYC (USBE). On Albizsia lebbek.

C. perseae (Comst.). NYC (USBE). On Anthurium and Spathyllum in greenhouse.

C. rossi (Mask.). NYC (USBE). On Macrozamia moorci in greenhouse.

C. smilacis (Comst). NYC, on smilax, Southwick (NYS); LI: Maspeth, on smilax (O1s-NYS).

C. tenebricosus (Comst.). Buffalo (USBE). On soft maple.

\section{Pseudischnaspis Hempel}

P. bowreyi (Ck11.). NYC (USBE). On Agave and Livistona in greenhouse.

\section{Aonidia Targioni-Tozzetti}

A. lauri (Bouché). Albany (USBE); NYC, on bay tree (NYS) ; LI: Roslyn (USBE). Probably brought in on its European host.

\section{GyMNASPIS Newstead}

G. achmeae Newst. NYC (USBE). On Achmea, Ananas, Hohenhergia, Bromelia, and Billbergia, in greenhouse. 


\title{
LEPIDOSAPHES Shimer
}

L. beckii (Newm.). Puryle Scale. Rochester, Geneva (USBE) ; Albany (NYS) ; NYC, Inwood (USBE) ; LI: Maspeth, on orange and lemon (Ols, Felt). On Citrus and Elaeagnus in the greenhouse or market.

L. gloverii (Pck.). NYC, on orange and lemon (NYS). Undoubtedly brought in on its food plant.

L. lasianthi (Green). NYC' (Sasscer-USBE). On Camellia japonica.

L. newsteadi (Sulc.). Rye (Sasscer-USBE). On Sciadopitys.

L. philococcus (Ck11.). NYC (USBE). On Opuntia.

L. ulmi (L.). Oyster-Shell Scale. Recorded from the following counties: St. Lawrence, Herkimer, Lewis, Oswego, Fulton, Monroe, Orleans, Genesee, Erie, Ontario, Cayuga, Otsego, Schoharie, Saratoga, Washington, Albany, Rensselaer, Greene, Dutchess, Putnam, Sullivan, Orange, Westchester, Richmond, Queens, Nassau, Suffolk (Felt); records from many localities and hosts in the State (USBE). Is presumably generally distributed throughout the State. Occurs in NY on the following food plants: apple, ash, balm of Gilead, white birch, butternut, Cornus, Crataegus, grape, horse-chestnut, lilac, maple, Pennsylvania maple, silver magnolia, pear, privet, syringa, willow. Has been recorded in literature from a considerable variety of other plants.

\section{ISCHNASPIS Douglass}

I. longirostris (Sign). Thread Scale. Albion, on Chinese fan palm, JBAchilles (NYS) ; Fishkill, Pawling, Mamaroneck, NYC (USBE). SI: New Brighton (USBE). On various plants in greenhouse.

\section{PARLATORIA Targioni-Tozzetti}

P. pergandii Comst. Chaff Scale. Rochester; Geneva; NYC.

P. pergandii crotonis Ckll. Rochester (USBE). On Codiaeum variegatum.

P. proteus Curt. Geneva (CU) ; Ossining (NYS) ; NYC, on tangerine (NYS) ; LI: Maspeth, on orange (Ols-NYS).

P. theae Ckll. Rochester, on Japanese maple (NYS) ; Schenectady, on Japanese maple (NYS).

\section{ORDER DERMAPTERA ${ }^{29}$}

\author{
Compiled by William T. Davis
}

\section{FAMILY FORFICULIDAE}

\section{SUBFAMILY LABIDURINAE}

\section{ANISOLABIS Fieber}

A. maritima Géné. Larchmont, Nov, TDO'Connor (Hebard); Palisades of the Hudson, along the shore, May $(D s)$; along the East River under rubbish, very common (Joutel); SI: collected and still to be found at numerous localities along the easterly shore of the island from St. George to Totten-

20 Adventive species. - The following adventive species have been reported in New York State: Labidura riparia (Pallas), SI, male, in head of imported lettuce, Jan, 1916; Prolabia pulchella (Serv.), original type from Niagara; Doru lineare (Esch.), SI, male, in spinach, Feb 4, 1920. Euborellia annulipes (Lucas) has been found in Mass. 
ville; in 1880 it was a common species under stones and difift on the shore at St. George Point where the present ferry slips are; LI: Rockaway Beach, Sep; North Beach, Je, Ols; Center I., Oyster Bay, May; Southold, Sep; Greenport, Aug; Orient, J1, eggs and young (Ds), Sep, Lath; Gardiners I., May, nymph, $C y$.

\section{SUBFAMILY LABIINAE}

LABIA Leach

L. minor L. Ithaca, May-Aug, under a light (CU) ; Tuxedo, May, TDO'Connor (Hebard); Mosholu, Van Cort. Pk., Sf; Bronx, Aug, in numbers at light, Wat; Riverdale, Sep, Woodr (Ds); Manhattan I., Central Pk., Je, Sep, Joutel; SI, a male taken in May, a female in Aug, and a second male in Je; it sometimes comes to light and is also taken on plants; LI, represented in the collection of the Brooklyn Institute of Arts and Sciences.

\section{SUBFAMILY FORFICULINAE}

\section{DORU Burr}

D. aculeatum Scud. "NY (Coll. Uhler)" is cited by Scudder (Proc. Boston Soc. Nat. Hist., vol. 18 (1875-76), p. 263) and by Rehn and Hebard (Journ. N. Y. Ent. Soc., Je, 1914) ; SI, one female found on the edge of the meadows near Dongan Hills, Sep; LI: Hewlett, Nov, in rotten birch log, Schott.

\section{Forficula Linnaeus}

F. auricularia L. European EArwig. E. Aurora, Aug, numerous specimens of both sexes captured out-of-doors by JBAchilles. This introduced earwig has become well established at Newport, RI, where Morse states the occupied area is ten square miles.

\section{ORDER COLEOPTERA}

General Editor, Charles W. Leng

Staten Island Institute of Arts and Sciences

The list of Coleoptera is based on published lists and numerous manuscript communications. It is fairly complete in some families, especially for the southeastern part of the State where records have been obtained from many collectors in New York City and vicinity. In the Rhynchophora and in the Staphylinidae and other families of small beetles, however, many names may be added by further study and collecting. On the other hand, a few of the names included may eventually prove to be synonyms. The distribution of some species within the State is imperfectly shown because records for them are lacking from the central and western counties. Using as a guide those species for which the records are comparatively complete, it is evident that there are on Staten Island, on Long Island, and in the lower Hudson Valley, a considerable number of southern species; and in the higher parts of the Catskill and Adirondack Mountains, a number of northern species. Otherwise there is no clear evidence in the records assembled of any strong faunal boundary within 
the State. The sequence of families, genera, and species is that of Leng's Catalog, except in a few cases in which genera and species have been changed about for reasons explained in the text. The Leng catalog numbers precede each species, for convenience in referring to that work.

\section{FAMILY CICINDELIDAE}

\section{TETRACHA Hope}

38 T. virginica L. LI: Central Pk., Sep (Ds).

\section{Cicindela Linnaeus}

39b C. formosa Say var. generosa Dej. Keene Val., Je (Not); Elizabethtown, Aug (Harr); Horicon, J1 17, 1925 (Frost) ; Luzerne, J1 (HarrED); Rochester (M\&W) ; Ballston, Je (Harr) ; Schenectady (Dt) ; Albany (Lint); Karner, Je (NYS); Hoosick, Je (Harr); Yonkers, Sep (Harr); White Plains (Sq); Bloody Pond, Sep (Harr); Guymard, Je (HarrED) ; Mosholu, J1 (Bno); Van Cort. Pk., Oct (Bno); SI: Watchogue, Mar-Oct (L\&D); LI: Springfield Gardens, Sep (HarrED) ; Cold Sp. Harb., Sep (Ds) ; Aqueduct, Sep (Ds) ; Amagansett, Sep (Ds); Yaphank, Je (Ds); Wading R., May-Aug (Ds); Hauppauge, Je (Ds); Quogue, Sep (HarrED).

42 C. purpurea Oliv. Keene Val., J1, Sep (Not); Jay, Aug (Harr); Lewis Co, J1 (NYS-Hill); New Russia, Aug (CU); Potsdam, Ap (HarrED) ; Newport, Ap (NYS); Axton, Je (M\&H) ; Onondaga Co, Mar $18(\mathrm{H})$; Rochester (M\&W); Avon, Feb; May (Cls); Olcott (Dt) ; Buffalo (Z\&R,HarrED) ; Pike, Je (NYS); Castile, Je, Brad (Flet); Rock City, Je (Ds) ; Potters, Je (Ds) ; Ithaca, May-Je, Sep (Morr) ; Stamford, J1 (HarrED); Saratoga Sps., Je (HarrED); Haines Falls, Jl (HarrED); Debruce, Je, Sep (Ds,HarrED); East Branch, Oct (Ang); West Pt. (Robn); White Plains (Sq); NYC $(\mathrm{Lg}, \mathrm{Bt})$; SI, Mar-Sep, not common in recent years (L\&D); LI: Bethpage, May (HarrED) ; Islip, Sep (HarrED) ; E. Hampton, Sep (HarrED) ; Amagansett, Sep (Ds) ; Calverton, Sep (Ds) ; Yaphank, May-Oct (Ds) ; Wyandanch, May (Ds) ; Southold, Sep (Ds); Wading R., May-Je (Ds) ; Gardiners I., Je (Ds).

44 C. splendida Hentz. "NY" (Lec), also from Schaupp Coll. (HarrED). It is possible that these records refer to limbalis ( $\mathrm{Lg}$ ).

45 C. limbalis Klug. Buffalo (Z\&R); Westfield, May (Not); Rock City, Je (Ds); West Pt., Robn (Ds); Peekskill, J1 (Harr); Bear Swamp, Ramapo, Ap-Sep (Ds); Southfields, Oct (Ds).

48 C. ancocisconensis Harr. Buffalo (HarrED); Ebenezer, Je (Rein); Cazenovia Cr., May-Aug (Rein); Debruce, Je, Sep (HarrED, Ds).

49 C. duodecimguttata Dej. Raquette L., J1 (Harr); Wilmington, J1-Aug (Ds, Dt); Elizabethtown, Aug (HarrED); Jay Mt., Aug (HarrED); Cranberry L., Je-Aug (H) ; Keene Val., May-Aug (Not); Luzerne, Je (HarrED) ; Clearwater, J1 (HarrED) ; Buffalo (Z\&R); Ebenezer, Je (HarrED); Salmon Cr. (M\&W) ; Tarbel, Je (Ds) ; Ithaca, Aug (Dt); Stamford, Aug-Sep (Harr); North Branch, Aug (Schaupp); Pawling, Aug-Sep (HarrED); L. George, Aug (HarrED); SI, MayOct (L\&D) ; LI: Far Rockaway, Sep (HarrED) ; Coram, May (Ds); Yaphank, Sep (Ds). 
50 C. repanda Dej. Abundant in partly damp localities throughout the State (Lg). Keene Val. (Lint) ; Potsdam (Houghton) ; Axton, Je (M\&H); Ausable Pt., Sep (Not); Raquette L., J1 (HarrED); Wilmington, J1 (Ds) ; Elizabethtown, Aug (HarrED) ; Long L , Piseco, L. Pleasant, J1-Aug (NYS) ; Cranberry L., Je (H) ; Mountain View, Sep (Not); Clearwater, J1 (HarrED); Rochester (M\&W) ; Rochester Jct., JI (CU); Buffalo (Z\&R); Ebenezer, Je (HarrED); Westfield, May (Not); Castile, Brad (Flet); Geneva, Middlesex, Aug (Cls); Ithaca, J1 (CU) ; Karner, Ap-Sep (NYS); Guymard, Je (HarrED); L. George, J1 (HarrED) ; Hoosick, Je (Harr); Saratoga, Je (HarrED); Debruce (Dow); Callicoon, J1 (HarrED,Ds); Quaker Hill, Sep (HarrED); West Pt. (Robn); Newburgh (Robn); Peekskill, Hastings, Yonkers, Dobbs Ferry, May-Jl (HarrED); White Plains (Sq); Van Cort. Pk., Oct (Bno); SI, Mar-Oct, often rather numerous on moist areas (L\&D); LI: inhabits the loose sand beyond the beach (Lg) ; Flatbush, Coney I., Rockaway, Lawrence, Ruffle Bar, Long Beach, Oak I., Patchogue, Edgemere, Bay Shore, Quogue, Easthampton, Nissequogue, May-Sep (HarrED); Amagansett, Wading R. (Ds); Fire I., Sep (Ds) ; Gardiners I., Je (Ds); Orient, Lath (NYS).

51 C. hirticollis Say. SI, on seashore from early spring until late fall (L\&D); LI: Coney I., Aug (Ds); Rockaway, Long Beach, Je-Aug (Harr); Oak I., Fire I. (Ds): Patchogue, Sep (HarrED); Promised Land, Wading R. (Ds).

51b C. hirticollis var. rhodensis Calder. LI: Gardiners I. (Ds) ; Far Rockaway (HarrED).

53 C. tranquebarica Hbst. (vulgaris Say). Wilmington, J1 (Ds, Dt) ; Elizabethtown, Aug (HarrED); Axton, Je (M\&H); Raquette L., J1 (Harr); Jay Mts., Aug (HarrED); Potsdam (Houghton); Keene Val., Aug (Not); Mountain View, Sep (Not); Cranberry L., Je-J1 $(\mathrm{H})$; Rochester (M\&W) ; Buffalo (Z\&R) ; Avon, May (Ols); Rock City, Je (Ds); Ithaca, Mar (Morr); Debruce, Je, Aug-Sep (HarrED); Cooks Falls and East Branch, Oct (Ang); Newburgh (Robn) ; Yonkers, J1 (HarrED) ; White Plains (Sq) ; SI, Mar-Sep, found only on a sand dune at Old Place in recent years (L\&D); LI: West Hills; Wyandanch (Sf); Yaphank; Southold; Calverton, Springs, Gardiners I., Montauk, Mar-Sep (Ds); W. Hampton, E. Hampton, Quogue, Amagansett, Islip, Sep (Harr) ; Orient, Oct, Lath (Ds).

53c C. tranquebarica var. horiconensis Lg. Cranberry L., J1 (H) ; L. George (Lg); Pembroke (CU).

59 C. longilabris Say. Jay, J1-Aug (HarrED, Britton); Mt. Whiteface, J1 (Ds) ; Keene Val., J1-Aug (Not); Axton (M\&H) ; Long L. (Ds).

68a C. scutellaris Say var. lecontei Hald. Elma, May (NYS).

68c C. scutellaris var. rugifrons Dej. Confined to the pine-barren regions of LI ( $\mathrm{Lg})$. Immaculate forms have been found at Aqueduct, Quogue, Southampton, and Amagansett in Sep (HarrED). The Aqueduct locality published by Joutel (Journ. N. Y. Ent. Soc., vol. 10, p. 249) is now destroyed $(\mathrm{Lg})$. A form intergrading this with var. modesta very rarely found at Aqueduct and Southampton (HarrED). LI: Jamaica (Schaupp) ; Cold Sp. Harb., Aqueduct, Valley Stream, Springs, Calverton, Manorville, Yaphank, Wading R., May, Aug, Sep (Ds); Amagansett, E. Hampton, Southampton, Quogue, Islip, Springfield, Sep (HarrED); Shinnecock Hills, Sep, $L g(B t)$. 
68 C. scutellaris var. modesta Dej. Confined to pine barrens on LI and SI. Immaculate specimens mimicking nigrior formerly found at: Aqueduct (Lg) ; SI, Ap, Sep-Oct (L\&D); LI: Cold Sp. Harb., May, Sep (Sf, Ds); Aqueduct, Aug-Sep (HarrED) ; Valley Stream, Aug (HarrED) ; Springfield, Sep (HarrED).

69 C. sexguttata Fab. Wilmington, J1 (Ds) ; Keene Val., May, Aug (Not); Newport, May (NYS); Elizabethtown, Aug (HarrED) ; Potsdam (Houghton) ; Cranberry L., Je-JI (H) ; Lewis Co, Je (Hill-NYS); Luzerne, Je (HarrED); Rochester (M\&W) ; Syracuse, Ap, May (H) ; Buffalo (Z\&R); Avon, Geneva (Cls); Castile, Brad (Flet); Pike, May (NYS) ; Westfield, Je (NYS) ; Rock City, Je (Ds) ; Elmira, May (Diven); Ithaca, May-Je (Morr); McLean, Mar (Morr), May (Ds); Tarbel, Je (Ds); Saratoga, Je (HarrED); De Bruce (NYS); Schoharie (Not); Albany (Lint); Delmar, May (NYS) ; Nassau, May (NYS) ; Hoosick, Je (HarrED) ; West Pt. (Robn) ; Peekskill, Je; Hastings, Cornwall, May-J1 (HarrED); Haines Falls, J1 (HarrED); Guymard, Je (HarrED); White Plains (Sq); West Farms, May (Harr); SI, Mar-Aug (L\&D); LI: Flushing, Wyandanch, Smithtown, Wading R., Yaphank, Gardiners I., Mar-Sep (Ds) ; Nissequogue, Je (HarrED); Farmingdale, J1 (CU). Inhabits climax forests, openings, paths, etc., sometimes in gardens ( $\mathrm{Lg}$ ). Rare, without spots, at Hoosick, Je; with two spots at Peekskill and Hastings, May; often with eight spots (HarrED).

69c C. sexguttata var. harrisi Lg. Elizabethtown, Aug ( Lg) ; Luzerne, J1 (HarrED); L. George, Aug (Lg); Debruce, Je, Sep (Dow,Ds); Quaker Hill, Aug (HarrED); Cornwall, Jl (HarrED); Cooks Falls, Aug (Ang); E. Jewett, Aug (Ds); Stamford, Catskills, Aug (HarrED). An altitudinous variety of sexguttata ( $\mathrm{Lg})$.

70 C. patruela Dej. Conesus L., Je (Ds) ; Karner, May, Sep (NYS) ; West Pt. (Robn); Peekskill (HarrED). Confined to mountains; local ( Lg).

70a C. patruela var. consentanea Dej. Confined to pine-barren regions of LI and known from few localities (Lg). LI: Port Jefferson (HarrED); Wyandanch, Mar-Ap, Schott (HarrED); Riverhead, Aug (Ds); W. Hampton, Sep (Wood); Quogue, Sep (HarrED).

74 C. punctulata Oliv. Upper Jay, Aug (Not); Jay Mts., Aug (HarrED); Crown Pt., Aug (Not); Elizabethtown, Aug (HarrED); Luzerne, J1 (HarrED) ; Keene Val., J1 (Not) ; Geneva (Cls); Rochester (M\&W) ; Ithaca, Sep (CU) ; Saratoga, Aug (Dt); L. George, Aug-Sep (HarrED); Albany, Aug (Felt) ; Debruce, Sep (HarrED); Quaker Hill, Aug-Sep (HarrED) ; Haines Falls, J1 (HarrED); Kinderhook, J1 (HarrED); Yonkers, J1 (HarrED); Montrose, J1 (HarrED); West Pt. (Robn); White Plains (Sq); Mosholu, J1 (Bno); SI, Je-Sep, the most common species (L\&D); LI: Far Rockaway, E. Hampton, Yaphank, Sep (HarrED) ; Farmingdale, J1 (CU) ; Southold, Montauk, Sep, Gardiners I., Aug (Ds). Widely distributed; flying to light and occasionally seen even in city streets and garden paths ( Lg).

79 C. abdominalis Fab. LI: Riverhead, near Bald Hill; also in sandy road near Peconic R., Aug (Ds).

84 C. marginipennis Dej. Callicoon, J1 (HarrED, Lg, Ds); confined to pebbly flats of 'Delaware R. ( $\mathrm{Lg})$.

93 C. unipunctata Fab. Ft. Green, Je (Schaupp); Bronx Pk., Je (Lg, Bt) ; LI: Brooklyn (Schaupp). The records for this species are few and ancient, and do not clearly indicate it as an inhabitant of this State. 
101 C. dorsalis Say. Albany, 1867 (NYS); LI: Coney I., Rockaway Beach, J1-Sep (Sf) ; Fire I., Oak I., Long Beach, Edgemere, Arverne, J1-Aug (HarrED). Confined to sea beach of LI, Montauk, etc.; formerly found also on SI, but probably exterminated about 1895 by commercial use of beaches (Ds). C. signata Dej. is a nearly immaculate form of dorsalis, found at Fire I., Rockaway, Coney I. (HarrED).

104 C. marginata Fab. Confined to muddy beaches of SI and LI ( $\mathrm{Lg}$ ) ; NYC, Ang (H) ; SI, Jl-Aug (L\&D); LI: Coney I. (Lg); Sheepshead, Aug (NYS); Northport, Canarsie, Jl-Sep (Schaupp); Baywater, Far Rockaway, Ruffle Bar, Edgemere, J1-Aug (HarrED); Wading R., J1 (Ds) ; Gardiners I., Aug (Ds) ; Montauk, J1 (Ang).

108b C. cuprascens Lec. var. puritana Horn. "NY" (Schaupp). The only record is ancient and indefinite. This species lives on river banks in sandy beaches and occurs in midsummer ( $\mathrm{Lg}$ ).

110 C. lepida Dej. Buffalo (Z\&R); LI: Coney I., Je--J1 ( $\mathrm{Lg}, \mathrm{Bt})$; Rockaway Beach (Lg); Long Beach, Arverne, J1 (HarrED). Confined in this State to limited areas of white sand near the beach $(\mathrm{Lg})$.

\section{FAMily CARABIDAE}

\section{Compiled by Howard Notman \\ SUBFAMILY CARABINAE}

\section{SCAPHINOTUS Dejean}

125 S. elevatus Fab. Bronxville, Aug (Ds) ; NYC and vic. (Not); Van Cort. Pk., Aug (Woodr) ; SI, formerly common (L\&D) ; LI: Jamaica Woods, May (Schaupp); Yaphank, May-Oct, Shoe (Eng); Brooklyn, Central Pk., May-Oct, Shoe ; Syosset, Nassau Co, Nov, Eng (Not).

128 S. viduus Dej. Axton, Je (M\&H); Whiteface Mt., $4200 \mathrm{ft}$., Oct ( $\mathrm{Lg})$; Keene Val., 1000 ft., Aug (Not); Crown Pt., Aug (Not); Keeseville, Sep (Lg); Hope (Castle); Mt. Hopkins, 2500 ft., Je (Not); Batavia, Je, Kngt (Lg); Buffalo and vic. (Z\&R); Cattaraugus Co, Je, Ds; Tompkins Co, several localities (Dt, Vdyk) ; Ithaca, Mar, Kngt ; Hunter, Je; Catskills, $V d y k$ (Pears, Roeschke); North Branch (Schaupp); Yonkers (L\&B); Mosholu (L\&B); W. Dunwoodie; Manhattan I., Joutel; NYC, Oct (Lg); SI, rare (L\&D); LI: Inwood, J1, Sep, Skinner, Oct, Joutel.

\section{NomaRETUS LeConte}

134 N. bilobus Say. Whiteface Mt., J1, Ds; Wallface Mt., 3000 ft., J1. Nic (Not); Buffalo vic., Rein; Catskills, J1 (Beyer).

\section{SPHAERODERUS Dejean}

160 S. canadensis Chd. Mt. Whiteface (Lg); Mt. Marcy, 3500 ft., J1 (Not); Wilmington, 1500 ft., Aug (Dt); Keene Val., 1500 ft., Je (Not); Sodus Pt., Je (Not); Monroe Co, Moore (Wendt); Rock City, Je, Dt (Vdyk); Ithaca, Ap (Vdyk), Oct 9, Walton (Vdyk); Coy Glen, J1, Dt (Vdyk) ; Caroline, Ap, Dt (Vdyk); Hebron (Lg); Slide Mt., 2500 ft., J1, Shoe; North Branch (Schaupp); Debruce, J1, Shoe (Lg); Minnewaska, Aug (Nic). 
162 S. lecontei Dej. Upper Jay, Sep (Lg); Keene Val., Je, Aug, Oct (Not); Chateaugay, Sep $(\mathrm{Lg})$; Johnstown, Oct $(\mathrm{Vdyk})$; Syracuse, Ap $(\mathrm{H})$; Monroe Co $(M \& W)$; Karner, Nov (Vdyk); Olcott, Ap (Dt); Buffalo vic. (Z\&R) ; Erie Co, May (Dnd); Rock City, Je; Red House, Je (Not); Ithaca, Ap (Kngt); McLean, May, Ds; Albany, Lint; Whitehall, Oct (Not); W. Hebron, Oct (Lg); Catskill (Lg); Ulster Co, Sep (Lg); Peekskill, Sherman; West Pt., Robn; Greenwood L.; Ramapo, Ap, Ds; Mosholu; White Plains, Ap (Sq); Bronxville, Mar-Ap (Woodr); NYC, Sep (Lg); Inwood, Manhattan I., Oct, Sep, Ds; Manhattan I., Sep, Joutel; Van Cort. Pk., Mar-Ap (Not, Woodr); SI: Mar-May, Nov, once not uncommon, now rare (L\&D); Sandy Brook, Ap; LI: Yaphank, Jl-Aug, Wading R., May, Ds; Brooklyn, Ap-Oct, Shoe.

162a S. lecontei var. niagarensis Lap. "NY", Lec. Probably only a synonym of lecontei (Nic).

164 S. nitidicollis Chev. var. brevoorti Lec. Mt Whiteface, Sep ( Lg); Wallface Mt., Je (Quirsfeld \& Nic); Green Mt., 3000 ft., J1 (Not); Mt. Marcy, 5000 ft., J1 (Not).

\section{Carabus Linnaeus}

170 C. sylvosus Say. Keene Val., Sep (Not); Cascade Lakes, Aug, Brb; Gloversville, May; Monroe Co, May, $M \mathcal{E} W$; Buffalo (Dnd); Carmel, Aug ;.LI: near Ridgewood, Greenville, Je (Schaupp); Yaphank (Eng); Bellport, Wading R. (Nic). Apparently occurs wherever pines are found (Nic).

171 C. serratus Say. Keene Val., May-J1, Mooers, Sep (Not); Gloversville, May (Sf); Monroe Co, $M \& W$; Buffalo vic. (Z\&R); Rock City, Je (Vdyk); Ithaca, Aug (Vdyk); Butternut Cr., May (Dt); Larch Meadows, Tompkins Co, Je (Dt) ; Catskill, J1, Catskills, Aug (Sf); West Pt., Robn; Morrisania ; Peekskill (Vdyk) ; Ramapo Mts.; Lowerre, Ap (Woodr); NYC vic., Ap (Woodr); SI, Mar-Je (L\&D); LI: Bronx Pk., Brooklyn, Ap-Aug, Shoe.

172 C. limbatus Say. Gloversville, Aug (Lint) ; Olcott, Je (Dt) ; Monroe Co (M\&W) ; Buffalo (Z\&R); Westfield, May (Not); Ithaca, Ap, Aug, Larch Meadows, May, Six Mile Cr., Sep (Dt); Windsor, Je (Not); Karner, Ap, Kenwood, May (Lint); Washington Co, Je, Lg; W. Hebron, Je (Lg); Greenwood L.; Poughkeepsie, Je (Lint); West Pt.; Pine I. (Ang); Peekskill (Vdyk); White Plains, Mar-Aug, Brozenell; Carmel, Aug; Mosholu; NYC, Ap-May, Sep, Shoe; Van Cort. Pk., Mar-Ap (Woodr); SI, Mar-Aug (L\&D) ; Clifton, Ap; Clove Val., J1-Aug, Ds (Not); LI: Greenville, Jamaica Woods, Bay Ridge, May-Sep (Schaupp); Yaphank, Sep, Oct, Ds (Not); Brooklyn, Ap-May, Sep, Shoe; Parkville; Selden, Aug, Ds; Central Pk., Ap (Not); Half Way Hollow Hills, Jl, Wading R., May, Ds (Not). Common everywhere under stones and boards, and in bait bottles (Nic).

173 C. vinctus Web. Buffalo (Z\&R); Cedar Hill, Ap; Karner, J1, Hill; West Pt., Robn; Nyack, Je, Brownell; Westchester Co, May, Ap (Woodr); Van Cort. Pk., Mar-Ap (Woodr); NYC, Inwood, Ds; SI, Ap-Je, Sep (L\&D, Not) ; LI: Pennybridge, E. New York, Bay Ridge, May-Sep (Schaupp). Found with preceding species but not so abundant, and, although fairly generally distributed, more local (Nic). 
175 C. nemoralis Mull. Monroe Co, May (M\&W); White Plains, Mar-Nov (NYS) ; Bronx (Ang) ; Van Cort. Pk.; May, Ang; SI, May, Ds; LI: Winfield, J1 (Ols); Brooklyn, Ap-May, Schott, Shoe.

\section{Calosoma Weber}

176 C. externum Say. Newburgh, Martin; NYC; Central Pk., Ds (Martin); SI, Je-J1, Ds ; LI: Ridgewood, Greenville, J1-Sep (Schaupp).

180 C. scrutator Fab. Buffalo (Z\&R) ; Monroe Co, $M \mathcal{E} W$; Ithaca, J1 (Vdyk); Albany, Lint; Poughkeepsie, Je (Sf); West Pt. (Robn); Nyack, Je, Brownell; White Plains (Sq); NYC, May-J1, Ds (Woodr); SI, Je-Aug, Nabenhauer (L\&D) ; LI: Orient, May, Jl, Lath (Not-NYS); Rockaway Beach, Je-Jl, Shoe (Vdyk); Coney I. (Schaupp); Greenbush, Jordan (Lint); Yaphank, Je, Ds; Long Beach, Babylon, Bradford; Brooklyn, Je-Jl, Shoe; seashore (Not). Often common in wash-up on seashore, Je-Sep (Nic).

181 C. wilcoxi Lec. Buffalo (Z\&R); West Pt. (Robn); White Plains (Sq); NYC, May-Je (Schaupp\&Woodr); LI: Bellport, Wading R., May, Nic \&Ds (Nic) ; Rockaway Beach, May-Je, Shoe (Woodr, Not); Wading R., Je (Not); Orient Lath (NYS); Long Beach, Brownell (Lg).

182 C. sycophanta L. Albany (Lint); White Plains (Frost, Sq); LI: Flatbush (Schott, Sf).

183 C. frigidum Kby. Newport, May, Saranac Inn, Je (Felt); Wallface Mt., $3100 \mathrm{ft} ., \mathrm{Jl}$ (Nic); shore of L. Ontario, near Pulaski, Je, Chapman; Olcott, J1 (Dt); Monroe Co, $M \& W$; Buffalo (Z\&R); Pike (Felt); Batavia, Ap, Je, Kngt; E. Aurora (Dnd) ; Chipmunk Swamp, Vandalia, Je, Ds (Not); Rock City, Je, Ds (Not); Castile, (Brad, Flet); Tarbel, Je, Ds; Elmira, May (Diven); Nichols, May (Not); Ithaca, Je (Dt) ; Albany, Je (Felt); Garrison, Cattell; West Pt., Je (Robn); Bronxville, Je (Woodr); White Plains, Je (Sq) ; LI: Bellport (Nic); Wading R., May, Ds (Nic); Nissequogue, Je, Ds.

184 C. sayi Dej. Vic, NYC tollgate, May (Schaupp); SI, Ds; LI: Babylon, Je (Bradford).

204 C. calidum Fab. Peru, Je, Cy\& Fibs (Vdyk); Axton, Je, M\&H; St. Lawrence Co (Houghton); Wilmington, J1, Ds; Keene Val., May-Je (Not) ; Lowville, Hough; Monroe Co, M\&W; Olcott, May, J1 (Dt) ; Batavia, Je, Jl, Aug, Kngt; E. Aurora, Boston (Dnd); Buffalo (Z\&R); Ithaca, Ap-Je, Hamilton (Vdyk, Dt); Butternut Cr., May (Dt); McLean Bogs, Je (Dt); Tarbel, Je, Ds; Groton, Je, Chapman; Elmira, May (Diven); Windsor, May (Not) ; E. Jewett, Aug, Ds; Orange Co, Ap, Faure; Albany, Lint ; West Pt. (Robn); Nyack, Je, Brownell; White Plains, Ap (Sq); NYC, May-Oct (div.); SI: Ap-Je, Ds; Castleton Grounds, St. George, Sep, Ds; LI:-Brooklyn (Vdyk) ; Orient, May-Je, Lath; Hauppauge, Je, Ds; Bergen Beach, May; Brooklyn, Apr-J1, Shoe. Common in fields (Nic).

\section{ELAPHRUS Fabricius}

222 E. clairvillei Kby. Wallface Mt., J1, common (Not\&Nic) ; Ausable L., J1 (Not); Mt. Redfield, $3000 \mathrm{ft}$., J1 (Not) ; top of Whiteface Mt., J1, Ds; Cape Vincent, Sep (Not); Oakfield, Je (Not); Buffalo, Rein; Ithaca, May (Dt); McLean Bogs, J1 (Dt). 
230 E. riparius Linn. Tompkins Co: Inlet Val., J1 (Dt) ; Beebe L., J1 (Vdyk); Ithaca, Je (Vdyk); N. Lansing, Je ( ${ }^{\top}$ dyk).

225 E. olivaceus Lec. L. Tear, $4200 \mathrm{ft}$., Jl, Moss Pond, $4200 \mathrm{ft}$., Mt. Redfield, Wallface Mt., 3000 ft., J1 (Not); McLean Bogs, May, Diven (Vdyk., $\mathrm{Dt})$; Catskills, Ulke ( $\mathrm{Lec})$.

226 E. cicatricosus Lec. Buffalo, Je; Red House, Je (Not) ; Larch Meadows, Aug, Hamilton (Vdyk); Ringwood, J1, Dt (Vdyk); Windsor, May (Not); Orangeburg, Ap-May (Not\&Nic); LI: Steinway, Julich (L\&B); Brooklyn, May-J1, div.

228 E. fuliginosus Say. Lower Ausable L., Keene Val. (Not); Tarbel, Je, Ds; Catskill; LI: Brooklyn, Ap, May, Shoe (Sf); Jamaica Bay Marshes, Ap (Woodr) ; Canarsie, Ap-May (Not).

233 E. ruscarius Say. Common everywhere along mud flats, Ap-Je (Nic) ; Ausable L., J1, Moss Pond, 4200 ft., J1, Crown Pt., Jay, Aug, Mountain View, Sep (Not); Newport, May; St. Lawrence, Houghton; Waterville, Je (Not); Syracuse, May (H); Monroe Co, $M \mathcal{E} W$; Sodus Pt., Je (Not); Batavia, May, Je, Kngt; Buffalo (Z\&R); Erie Co (Dnd) ; Pike, Je (NYS) ; Westfield, May; Mayville, May (Not) ; Chautauqua, Thompson; Inlet Val., J1, Dt (Vdyk); Cascadilla Cr., May (Vdyk); Ithaca, May-Je (Vdyk); Tarbel, Je, Ds; Windsor, Je (Not); Schoharie, Je (Not); Greenville (Schaupp); Wingdale (Vdyk); West Pt., Robn; Ft. Hunter, May-Je (Not); Westchester, Ap (Woodr); White Plains (Sq); Bronxville, May (Woodr); Van Cort. Pk., Je (Woodr); NYC (Martin); West Farms, Mar, Joutel; LI: E. New York, Brooklyn, Ap-Je, May, Ds, Shoe; Ft. Hamilton, Ap (Woodr).

\section{BLETHISA Bonvouloir}

237 B. quadricollis Hald. Buffalo (Z\&R); McLean Bogs, J1 (Dt, Vdyk); Catskill, Sep.

238 B. multipunctata L. St. Lawrence Co, Houghton.

\section{LORICERA Latreille}

241 L. caerulescens L. (pilicornis Fab.). Ausable L., J1-Aug, Crown Pt., Aug, Mountain View, Sep (Not); Rochester (M\&W); Batavia, Je, Lg; E. Aurora (Dnd) ; McLean, Ap (Flet).

\section{Notiophilus Dumeril}

246 N. aeneus Herbst. Keene Val., Je-J1, Mooers, Sep (Not); Tarbel, Je, Ds ; McLean, Ap (Flet); West Pt. (Robn); NYC, Je-Sep (Schaupp); SI, Ap, J1, Lg; LI: Rockaway Beach, Shoe (Vdyk) ; Wading R., May, Ds; Parkville; Aqueduct, May-Je, Shoe; Far Rockaway, Je (Woodr).

247 N. semistriatus Say. Mt. Whiteface, $2000 \mathrm{ft}$., Aug, Bdy; St. Lawrence Co, Houghton; Peekskill (Vdyk); Nyack, Je, Brownell; NYC (Schaupp) ; SI, Je, Lg (Fall, Say) ; LI: Wading R., May, Yaphank, Sep, Half Way Hollow Hills, J1, Ds.

248 N. aquaticus Linn. Keene Val., Ap (Not); Mt. Marcy, $5300 \mathrm{ft}$., J1, Oct (Not\&Lg), 4500 ft., J1, Lg (Fall\&Not); Mt. Redfield, 4500 ft., Moss Pond, $4200 \mathrm{ft} ., \mathrm{Ji}$ (Not); Mt. View, Sep (Not). 
251 N. novemstriatus Lec. Batavia, Je, Lg; West Pt. (Robn); Peekskill (Vdyk) ; Bronxville, Mar-Ap (Woodr); SI, Ap, Je, Nov (Lg\&Shoe); LI: West Hills, Ap, Ds; Aqueduct, Mar, Shoe; Yaphank, May (Not); Farmingdale, Mar (Not).

N. parvus Csy. Catskills, SmHH (Csy). NY, type locality (Lg).

252 N. nemoralis Fall. Wallface Mt., 3000-4000 ft., J1 (Not).

NEBRIA Latreille

276 N. sahlbergi Fisch. Summit of Mt. Marcy, J1 (Not) ; Monroe Co (M\&W) ; Pike (Say).

285 N. suturalis Lec. Buffalo ( $Z \& R)$.

292 N. pallipes Say. Upper Ausable L., J1-Aug, Ausable Pt., Sep, Mooers, Sep (Not); Wilmington, Plattsburg, Jl, Ds (Not) ; Mt. Marcy, Dec; Mountain View, 1500 ft., Sep, Chaumont, Sep (Not) ; Chateaugay L., 2000 ft., Aug-Sep; Waterville, Je (Not) ; Monroe Co (M\&W) ; Forest Lawn, Je, Zab (Not); Buffalo (Z\&R); Conesus, Aug; Pike, Je ; Montour Falls, Je (H) ; Chautauqua, Thompson; Westfield, May (Not) ; Ithaca, Jl, Aug-Sep, Hamilton (Vdyk\&Dt); Buttermilk Cr., J1 (Dt) ; Inlet Val., J1 (Dt\&Vdyk); Ft. Hunter, Je (Not); Schoharie, Je (Not) ; Albany, Lint; W. Hebron, Je (Woodr); Washington Co, Je, Lg; West Pt., Je, Aug, Robn\&Ds (Not); Peekskill; White Plains, Sep, $S q(\mathrm{Sf})$; Bronx R. (L\&B); Bronx Pk:, Jl, Shoe; SI, Je-Sep, Nov, Ds; LI: Forest Pk., Je (Not). Common everywhere near water all the year (Nic).

\section{PASIMACHUS Bonvouloir}

302 P. sublaevis Beauv. SI, Bischoff; LI: Coney I., Je (Schaupp); Rockaway Beach, Je-J1, Shoe (L\&B); Fire I., Sep, Ds; Long Beach, J1 (Lg); Rockaway.

302a P. sublaevis var. substriatus Hald. LI (Hald\&Lec); Coney I. (Schaupp).

308 P. depressus Fab. SI (Bischoff, Stortz \& Not); LI: Yaphank, Wks (Sf) ; Coram, Yaphank, Aug (Nic).

\section{SCARITES Fabricius}

316 S. subterraneus Fab. Common everywhere, even in cities, almost all the year around (Nic); Buffalo ( $Z \& R)$; Boston (Dnd); Albany, Oct, Lint (Say); W. Hebron, Je (Lg) ; West Pt., Born; Peekskill (Vdyk); Bronx, Ap; Dunwoodie; White Plains, J1 (Sq); NYC vic. (Schaupp); NYC, Aug, Oct (Woodr) ; Central Pk., Nov, Ds; SI, spring-Dec, Lg (Vdyk); LI: Nissequogue, Je, Wading R., Aug, Ds; Astoria ; Brooklyn, Ap; Orient Pt, May, Lath (Say).

\section{DYSCHIRIUS Bonvouloir}

323 D. aeneus Dej. (integer Lec.). Croton, Je, Brownell ( $\mathrm{Lg})$.

324 D. nigripes Lec. Mooers, Sep, Ausable Pt., Sep, Chapel Pond, Je, Upper Jay, Aug, Crown Pt., Aug, Lowville, Je, Waterville, Je (Not); Syracuse, May (H) ; Sodus Pt., Je (Not) ; Monroe Co (M\&W) ; Oakfield, Je (Not); McLean Bogs, Aug, Dt (Vdyk); Ithaca, J1, Howland I., 
Nov (Vdyk); Washington Co; Peekskill (Vdyk); SI, J1, Ds; LI. Common in spring. Generally distributed (Nic).

330 D. longulus Lec. Keene Val., May-Aug, Wallface Mt., J1, Mt. Skylight, 4900 ft., J1, Mt. Colden, 4500 ft., J1, Mt. Marcy, 5300 ft., J1 (Not); Batavia, Je, $L g$.

331 D. globulosus Say. Common everywhere in spring (Nic); Whiteface Mt., Jl, Mt. Marcy, Oct, Lg; Keene Val., Aug (Not); St. Lawrence Co, Houghton; Sodus Pt., May (Not); Monroe Co (M\&W); Olcott, Oct (Dt); Buffalo (Z\&R); Ithaca, Jan, May, Jl-Aug, div. (Vdyk) ; Canisteo, Je (Not); McLean Bogs, Aug, Dt (Vdyk); Nichols, May (Not); Windsor, Je (Not); W. Hebron, Je (Lg); West Pt., Robn; Ft. Montgomery, May (Not); Peekskill (Vdyk); Bronxville, Mar (Woodr); NYC vic., Je, Ds (Schaupp); LI: Rockaway Beach, May-Je, Ds, Shoe (Not); Far Rockaway, Aug (Woodr); Farmingdale, Mar, Canarsie, May (Not); Wyandanch, May, Ds.

333 D. terminatus Lec. Monroe Co (M\&W); Hudson Falls, Aug (Vdyk); Peekskill (Vdyk).

334 D. haemorrhoidalis Dej. Sacandaga, Aug (H) ; Sylvan Beach, Je (Not); Pike, Aug $(\mathrm{H})$; Peekskill, Sherm.

336 D. affinis Fall. Peekskill, Sherm.

339 D. sphaericollis Say. Crown Pt., Aug (Not); St. Lawrence Co, Houghton; Sylvan Beach, Je, Waterville, Je (Not); Monroe Co (M\&W); Buffalo $(Z \& R)$; E. Aurora (Dnd) ; Westfield, May, Canisteo, Je (Not); Ithaca, May, J1, div. (Vdyk); Inlet Val., Dt (Vdyk); Ft. Hunter, May-Je, Schoharie, Je (Not); Washington Co (Vdyk); Wingdale (Vdyk); NYC, May-Sep (Schaupp); SI, Je, Lg; LI: Coney I. (Schaupp); Rockaway (L\&B); Long Beach (L\&B).

340 D. edentulus Putz. Ithaca, J1, Hamilton (Vdyk).

- D. aureolus Not. Schoharie, Je (Not).

342 D. erythrocerus Lec. Canisteo, Je (Not); W. Hebron, Je ( Lg); NYC, May-Sep (Schaupp); SI, J1, Ds; LI: Coney I., Rockaway, and Long Beach (L\&B); Rockaway Beach, May, Ds (Not).

345 D. sellatus Lec. LI: Rockaway Beach, J1, Shoe, Ds (Vdyk); Coney I., Je-Sep, Julich (Schaupp); Long Beach (L\&B).

346 D. paliipennis Say. Sylvan Beach, Je (Not); LI: Coney I., Schaupp, Long Beach (L\&B).

349

D. filiformis Lec. LI: Coney I., Je-Sep, Julich (Lec\&Lg); Rockaway Beach, May-Je, div. (Not).

352 D. pumilus Dej. LI: Coney I., Rockaway Beach (L\&B).

355 D. hispidus Lec. Rochester (M\&W).

356 D. setosus Lec. Batavia, Je, Lg; Oakfield, Je (Not); Red House, Je, Nichols, May (Not); LI: Brooklyn, May, Ds (Not).

\section{Clivina Latreille}

358 C. dentipes Dej. LI: Orient Pt., Je; Rockaway.

359 C. impressifrons Lec. Sacandaga R., Aug; Rochester (M\&W) ; Olcott, Je-J1 (Dt) ; Batavia, Aug, Kngt; E. Aurora (Dnd); Buffalo (Z\&R); Croton, Je, Brozenell; Windsor, Je (Not); Durlandville, Ap, SW Frost (Vdyk) ; LI: Rockaway (Vdyk); Babylon; Rockaway Beach, May, Je, Shoe (Woodr); Crient, Lath (NYS). 
366 C. rufa Lec. Ausable Pt., Sep (Not); Monroe Co (M\&W) ; SI, May, Ds.

369 C. americana Dej. Ausable Pt., Sep, Crown Pt., Aug (Not); Hessian L. (Vdyk) ; Lowville, Je, Waterville, Je, Oakfield, Je, Westfield, May (Not); Olcott, May, Ji (Dt) ; Ithaca, May, Sep, Dt (Vdyk); McLean Bogs; J1-Aug, Hamilton, Dt (Vdyk) ; Ft. Hunter, May (Not); W. Hebron, Je (Lg); Tivoli, May, Ft. Montgomery, May (Not); West Pt. (Robn); Peekskill; Bronx Pk. (Not); NYC vic. (Schaupp); SI, May, Lg; LI: Lg; Brooklyn, Mar, Je, Shoe.

372 C. striatopunctata Dej. Fishkill, May, Brownell (Lg); Orangeburg, May (Nic); SI, Ap, Lg; LI: Brooklyn, Ap-May, Shoe; Rosedale, Je 3 (Nic).

375 C. bipustulata Fab. Monroe Co (M\&W); NYC, Bt; SI, May-J1, L\&D (L\&B) ; LI: Rockaway Beach, Ap-Je, Shoe (Woodr).

370 ?C. morula Lec. LI (Lec, Horn).

\section{Schizogenius Putzeys}

382 S. planulatus Lec. E. Aurora (Dnd); Canisteo, Je (Not); Taughannock Gorge, May, Aug, Dt (Vdyk) ; LI: Coney I. (Schaupp).

383 S. lineolatus Say. Mooers, Sep, Jay, Aug (Not); Newport, May, Yg; Lowville, Je (Not) ; Monroe Co (M\&W) ; Sodus Pt., Je (Not); Buffalo (Z\&R) ; Portage, $Y g$ (Lint) ; Westfield, May (Not) ; Canadice, Je, Canisteo, Je (Not); Inlet Val., May, J1, Taughannock Gorge, May, Aug, Hamilton, Dt (Vdyk); Ithaca, Je, Aug, Hamilton (Vdyk); Norton's, Cayuga L., May, SmHH (Vdyk); Nichols, May, Windsor, Je, Ft. Hunter, May-Je, Schoharie, Je (Not); Albany, Lint; W. Hebron, Je (Lg); Esopus (Vdyk); West Pt. (Robn); Stony Pt., Ramapo, Je, Ds.

385 S. ferrugineus Putz. Monroe Co (M\&W); Buffalo (Z\&R); SI, May (Bischoff) ; LI: Rock Beach (Vdyk).

386 S. amphibius Hald. Newport, May, $Y g$; Lowville, Je (Not) ; Keene Hgts., J1 (Not) ; Sodus Pt., Je (Not) ; E. Aurora (Dnd) ; Westfield, May (Not); Canadice, Je (Not); Canisteo, Je (Not); Ithaca, May, J1, Hamilton (Vdyk); Taughannock Gorge, May, Dt (Vdyk); Inlet Val., May, J1, Dt, SmHH (Vdyk); Norton's, May, $S m H H$ (Vdyk); Croton, May, Je, Browell; Nichols, May (Not); Windsor, Je (Not); Ft. Hunter, Je (Not) ; Albany, Lint; Esopus (Vdyk) ; West Pt. (Robn) ; Stony Pt., Je, Ds; Ft. Montgomery, May (Not); Peekskill, Sherm (Vdyk).

\section{ARDISTOMIS Putzeys}

395 A. viridis Say. Buffalo (Z\&R); Esopus (Vdyk); Peekskill, Je, Sherm (Vdyk); LI: Rockaway, Je (Schaupp).

\section{SUBFAMILY HARPALINAE}

PANAGAEUS Latreille

398 P. crucigerus Say. LI: Cypress Hill Cemetery, May-Je, under stone (Schaupp); Amityville, May (NYS); Rockaway Beach, Brooklyn, May, Shoe, at beach, in wash-up and under stone (Sf). May-Je, under stones (Schaupp); Rockaway Beach, Je, Ds. 


\section{Nomius Laporte}

404 N. pygmaeus Dej. Keene Val., Je (Not); Saranac, Aug (NYS); St. Lawrence Co, Houghton; Ithaca, Je, Sep, at light (Vdyk).

\section{BEMBIDION Latreille}

731 B. fraternum Lec. Callicoon ( $\mathrm{Lg})$; LI: Newtown, Je ( Lg).

408 B. inaequale Say. Adirondack (Hayw); Keene Val., Je (Not); Crown Pt., Aug (Not); Mountain View, Sep (Not); Sodus Pt., May, Je (Not); Sylvan Beach, Je (Not); Waterville, Je (Not); Monroe Co (M\&W); Buffalo (Hayw) ; Lancaster, J1 (Lg); E. Aurora (Dnd); Westfield, May (Not); Canisteo, Je (Not); Ithaca, Ji (Dt) ; Windsor, Je (Not); Ft. Hunter, Je (Not); Schoharie, Je (Not); Orangeburg, Ap (Not); Bronx Pk., Ap; West Farms.

411 B. littorale Oliv. Saranac Inn, Je (NYS); Buffalo (Z\&R).

412 B. lacustre Lec. Ausable L., Je, J1 (Not); Keene Val., Je (Not); Indian Pass, J1 (Not) ; "NY" (ANSP).

416 B. carinula Chd. Adirondacks (Hayw); Crown Pt., Aug (Not); Ausable L., J1 (Not) ; Hamilton Co; Monroe Co, rare (M\&W); Buffalo and vic. $(Z \& R)$; Caldwell, L. George, Jl, Zab (Not).

419 B. punctatostriatum Say. Inlet of Upper Ausable L., J1, on sand bank, (Not); Lower Ausable L., Je (Not); Keene Val., Je (Not); Indian Pass, J1 (Not); Newport, Sep (NYS) ; Sylvan Beach, Je. (Not) ; Sodus Pt., May, Je (Not); Monroe Co (M\&W) ; Buffalo (Z\&R); E. Aurora (Dnd); Canisteo, Je (Not); Ithaca, May, J1 (Morr, Dt) ; Nichols, May (Not); Windsor, Je (Not); Albany, Je (NYS); Ft. Hunter, May, Je (Not).

426 B. confusum Hayw. (coxendix Say). Sylvan Beach, Je, very abundant on beach, L. Oneida (Not); Pike, Aug (NYS).

B. americanum Dej. Sodus Pt., Je (Not); Monroe Co, rare (M\&W); Red House, Je (Not); Whitehall, Oct (Not); Van Cort. Pk., Bradford; Bronx Pk.; SI: May-Je, Aug (Nic) ; Clove Val., Sep, on mud flat, flying when approached, Ds; LI: Jamaica (Nic) ; Far Rockaway, Je (Woodr) ; Flushing, Je, Bell (Not); Calverton, Sep, Ds.

438 B. nitidum Kby. Keene Val., Je-Jl (Not); summit of Mt. Whiteface, Jl, Ds (Not); N. Elba, Oct (Lg); Sodus Pt., Je (Not); Monroe Co $(M \& W) ; B u f f a l o(Z \& R)$.

440 B. laevigatum Say. Buffalo (Dnd); Olcott, Sep (Dt).

- B. basicorne Not. Keene Hgts., Je-J1 (Not); Springwater, Je (Not); Westfield, May (Not); in "the Gulf," Chautauqua Co; Windsor, May (Not); Rensselaerville, May (Not).

476 B. rusticum Csy. Catskilis, SmHH (Cy).

486 B. simplex Hayw. Ausable L., Keene Val., Je-J1 (Not) ; John's Br., Aug (Not) ; Crystal Br., Ausable L., Je-Aug (Not) ; Indian Pass, J1 (Not) ; Keene Hgts., Je-J1 (Not); E. Marcy Br., 3200 ft., J1 (Not); Feldspar Br., Mt. Marcy, 4000 ft., J1 (Not) ; Cliff Mt., 3500 ft., Jl (Not) ; Lowville, Je (Not); Monroe Co (M\&W) ; Springwater, Je (Not); Westfield, May (Not); Canisteo, Je (Not); Ithaca, Aug, Dt (Vdyk); Windsor, May, Je (Not); Ft. Hunter, Je (Not); Hebron, Je ( $\mathrm{Lg})$; Catskills, Nic (Not); LI: Jamaica, Nic (Not). 
B. occultator Not. Mt. Redfield, J1 (Not).

B. notmani Csy. NY, type locality.

B. essexense Csy. NY, type locality.

B. keeneanum Csy. NY, type locality.

B. planiusculum Mann. Keene Val., J1 (Not); Indian Pass, J1 (Not); Keene Hgts., J1 (Not) ; Keene, J1 (Not) ; Waterville, Je (Not); Westfield, May (Not); Windsor, Je (Not) ; Canisteo, Je (Not); Buttermilk Glen, Ithaca, J1 (Lg); Windsor, Je (Not); Schoharie, Je (Not).

489 B. complanulum Mann. Ausable L., J1 (Not); Indian Pass, J1 (Not); Keene Val., 1500 ft., J1-Aug (Not); Keene Hgts., J1 (Not); Waterville, Je (Not); Westfield, May (Not); Windsor, Je (Not).

512 B. nigrum Say. Indian Pass, J1 (Not); Ausable Forks, Aug (Not) ; Upper Jay, Aug (Not) ; Jay, Aug (Not) ; Ausable L., J1 (Not) ; Keene Hgts., 1500 ft., Jl (Not); Keene, J1 (Not); Mooers, Sep (Not) ; Keene Val., Je (Not); Lowville, Je (Not); Saranac L., Sep, Shoe, under pebbles on beach (Sf); Waterville, Je (Not); Sodus Pt., Je (Not); Monroe Co $(M \& W)$; Erie CG (Dnd); Canadice, Je (Not); Westfield, May (Not); Waccabuc, J1 (Not); Canisteo, Je (Not); Tarbel, Je, Ds (Not); Nichols, May (Not); Windsor, Je (Not) ; Ft. Hunter, May-Je (Not); Bolton, Aug ( $\mathrm{Lg})$; W. Hebron, Je ( $\mathrm{Lg})$; Whitehall, Oct (Not); Catskills, Nic (Not); Ulster Co, Sep, Nic (Lg) ; Ft. Montgomery, May (Not); Bronxville, May (Woodr); NYC and vic.; SI, J1, Sep; LI: Jamaica, Nic (Not).

521 B. concolor Kby. Buffalo, vic. (Z\&R); Ausable Pt., abundant, Whitehall, Crown Pt., Aug-Oct (Not). Abundant on beach, L. Champlain.

522 B. consessar Csy. Bluff Pt., L. Champlain (Csy).

533 B. planum Hald. Keene Hgts., 1500 ft., Crown Pt., Keene, Upper Jay, Jay, Mooers, Cape Vincent, Lowville, Waterville, Sodus Pt., Je-Sep (Not); Monroe Co (M\&W); Buffalo (L\&B) ; E. Aurora (Dnd) ; Canadice, Westfield, Canisteo, May, Je (Not) ; Ithaca, May, Dt (Morr, Vdyk); Taughannock, Nichols, Windsor, Ft. Hunter, Whitehall, Catskills, MayOct (Not); NYC (Hald).

536 B. fugax Lec. Monroe Co, rare (M\&W); Buffalo (Z\&R); Canisteo, Nichols, May, Je (Not); Taughannock Gorge, J1 ( $\mathrm{Lg})$; Ft. Hunter, May-J1, Not (Lg) ; Esopus, Aug, Sherm,Jr. (Lg); Suffern; Ramapo, Je; West Pt. (Robn).

540 B. transversale Dej. Newport, Albany, May (Say); Inwood, May, Oct.

560 B. nitens Lec. Mt. Marcy, $5000 \mathrm{ft}$., Wallface, $3500 \mathrm{ft}$., Keene Val., $1000 \mathrm{ft}$., summit of Mt. Dix, Mt. Marcy, 5300 ft., Mt. Haystack, $4900 \mathrm{ft}$., Mt. Colden, $4500 \mathrm{ft}$., Mt. Whiteface, top, Ap-Oct (Not).

567 B. militare Csy. LI: Willetts Pt. (Csy).

572 B. picipes Kby. Port Kent, in small brook near L. Champlain, Sep (Not); Newport, Je, $Y g$ (NYS) ; St. Lawrence Co, Houghton; Chaumont, Lowville, Utica, Waterville, Je-Sep (Not); Monroe Co (M\&W); Olcott, Ap (Dt) ; Buffalo (Z\&R); Montour Falls, Ap (H) ; Pike, Jl, $Y g$ (NYS); Canadice, Springwater, Westfield, Canisteo, Windsor, Schoharie, Whitehall, May-Oct (Not); Ithaca, Spencer, Dt (Vdyk); Poughkeepsie, Je, Yg (NYS) ; Peekskill, Sherm (L\&B); Tivoli, May (Not); Newport, Je, Yg (NYS) ; LI: Coney I., Rockaway, Je-J1, Lg (Schaupp, L\&B). 
573 B. scopulinum Kby. John's Br., Crystal Br., Upper Ausable L., Ausable Lakes, 2000 ft., Ausable Chasm, Wallface Mt., 3000 ft., Keene Hgts., Cliff Mt., 3000 ft., Shanty Br., shore of L. Ontario, near Pulaski, Lowville, on Roaring Br., Je-Sep (Not); Johnstown, Gloversville, Ap (NYS) ; Sodus Pt., W'estfield, May, Je (Not); Olcott, J1, Sep (Dt).

577 B. postremum Say. Monroe Co (M\&W); Springville, Marilla (Dnd); Pike (NYS); Canisteo, Windsor, Schoharie, Je (Not).

582 B. canadense Hayw. On beach, L. Erie, Sodus Pt., Westfield, Ft. Hunter, Ft. Montgomery; very rare, May-Je (Not).

588 B. tetracolum Say. Ausable Chasm, Ausable Lakes, Keene Hgts., Mt. Redfield, 3000 ft., J1-Sep (Not) ; St. Lawrence Co, Houghton; Gloversville, May, Aug (NYS) ; Monroe Co, Sodus Pt., May-Je (Not) ; Olcott, Mar, J1, Sep (Dt) ; Puffalo (Z\&R) ; Westfield, Canisteo, Nichols, MayJe (Not); Ithaca, May, Dt (Morr, Vdyk); Westchester, J1 (Lg) ; Yonkers; Bronxville, Mar-May (Woodr) ; West Farms; NYC (Dej); SI, Feb-Nov (Lg); LI: Canarsie, Ap (Not); Brooklyn, Mar-J1, Shoe (Sf); Flatbush, May (Not); Manitou Beach, Je, Zab (Not); Jamaica, Rockaway Eeach, May (Nic); Ft. Hamilton, Ap (Woodr).

588b B. tetracolum var. nactum Csy. Lower Hudson Val. (Csy).

591 B. honestum Say. Elizabethtown, Je (NYS) ; Ausable Forks, Upper Jay, Jay, Keene, Mooers, Wilmington, Stony Pt., Canisteo, Nichols, Whitehall, Ft. Hunter, Waterville, Windsor, Tarbel, Ft. Montgomery, MayAug (Not); Ithaca, Ap, J1, Dt (Vdyk); W. Hebron, Je-J1 (Lg) ; Taughannock Gorge, Je-J1 (Lg); Howasacra, Je (NYS).

592 B. chalceum Dej. Ausable Pt., Crown Pt., Upper Jay, Jay, Ausable Lakes, Keene Hgts., Keene, Mooers, Lowville, Waterville, Westfield, Nichols, Schoharie, Ft. Hunter, Whitehall, Ft. Montgomery, May-Sep (Not); Monroe Co, rare (M\&W); Buffalo (Z\&R); Ithaca, Mar, Aug, Dt (Vdyk); West Pt. (Robn).

600 B. grandiceps Hayw. Lowville, Cape Vincent, L. Ontario, Westfield, Canisteo, Nichols, Windsor, Ft. Hunter, Whitehall, May-Oct (Not); Ithaca, Aug, Dt (Vdyk).

B. rolandi Fall. NY, type locality $(\mathrm{Lg})$.

604 B. semistriatum Hald. Waterville, Windsor, Ft. Montgomery, May (Not); McLean Bogs, May (Dt); Ulster Co, Nic (Not); LI: Jamaica, Nic (Not).

612 B. nigripes Kby. Ausable Lakes, $2000 \mathrm{ft}$., J1 (Not).

648 B. posticum Hald. Wilmington, Mt. Wallface, Lowville, Ausable Pt., Ausable Lakes, Ausable Forks, Chaumont, Mountain View, Norwood, Cape Vincent, Crown Pt., Ausable Chasm, Keene Val., Mt. Marcy, Waterville, Mt. Skylight, Upper Jay, Jay, Keene Hgts., Keene, Mt. Redfield, Mooers, Sodus Pt., Mayville, Westfield, Red House, Oakfield, Canadice, Canisteo, Windsor, Nichols, Schoharie, Whitehall, Pine I., Tivoli, May-Oct (Not); Ithaca, J1, Dt (Vdyk); Callicoon, J1 ( Lg); LI: Rosedale, Canarsie, Ap-May (Not) ; Coney I., Rockaway, Je, J1 (Schaupp).

651 B. patruele Dej. Crown Pt., Ausable Forks, Upper Jay, Jay, Waterville, Lowville, Sylvan Beach, N. Brookfield, Sodus Pt., Mayville, Red House, Westfield, Canisteo, Nichols, Windsor, Schoharie, Ft. Hunter, Orangeburg, May-Aug (Not); Buffalo (Z\&R); Batavia, Ds; E. Aurora (Dnd); White Plains, Sq (Frost). 
655 B. intermedium Kby. Oneida L., Aug (Drk); O1cott, May-J1 (Dt) ; Buffalo (L\&B); E. Aurora (Dnd); Ithaca, J1, McLean, Aug, Dt (Vdyk) ; LI: Coney I., Rockaway, Je-J1 (Schaupp).

657 B. marcidum Csy. Lower Hudson Val. (Csy).

659 B. versutum Lec. Wallface Mt., Indian Pass, 2200 ft., Waterville, Nichols, Ft. Hunter, May-J1 (Not).

660 B. variegatum Say. Syracuse, Ap-May (H) ; Monroe Co (M\&W); Red House, on Allegheny R., Nichols, Windsor, Orangeburg, May-Je

(Not); Ithaca, Jl, Dt (Vdyk); West Pt. (Robn) ; SI, Mar-Sep (L\&D).

679 B. arcuatum Lec. Indian Pass, 2200 ft., Wallface Mt., Waterville, J1 (Not); Monroe Co (M\&W).

680 B. graciliforme Hayw. Axton, Je, $M \& H$; Adirondacks, Houghton; Ausable Pt., Chaumont, Lowville, Waterville, Sodus Pt., Oakfield, Canadice. Whitehall, Orangeburg (Not); E. Aurora, Springville (Dnd); Olcott, Ap-Je (Dt); Ithaca, Feb (Lg, Dt); Tivoli; Mosholu (Not); SI, Je-Sep (Not); LI: Ap, Hamilton (Woodr).

B. lengi Not. NY, type locality ( $\mathrm{Lg})$.

686 B. cordatum Lec. Monroe Co, rare (M\&W); SI, Nic (Not).

694 B. contractum Say. Westchester, Mar (Lg); Canarsie, May (Not); West Farms; Van Cort. Pk., Nic; SI: Oakwood, in salt meadow, Sep, Ds; Watchogue (Lg) ; LI: Rockaway Beach, May-Sep, Shoe (Sf); Rockaway, Babylon, Je, Bradford; Bellport, Je-Aug, Nic ; Jamaica, May, Nic; Far Rockaway, Ap-May (Woodr) ; Nissequogue R., Ap (Not); Center I., May, Ds (Not).

B. proximum Not. NY, type locality.

695 B. constrictum Lec. SI: Oakwood, Mar, Nic; LI: Coney I., Rockaway, Je-J1 (Schaupp) ; Rockaway Beach, May, Shoe, Nic (Sf); Bellport, J1 (Nic); Wading R., May, Nic; Far Rockaway, May, Jamaica Bay, Ap (Woodr).

705 B. affine Say. Canadice, Oakfield, Westfield, Red House, Tivoli, Canisteo, May-Je (Not); Oneida L., Aug (Drk); Catskills, Ulster Co, Nic; Orange Co, Ap, Faure (Morr); Bronxville (Woodr); Mosholu; Van Cort. Pk.; SI: Ds (Not): seashore and south shore; LI: Brooklyn, May, Oct, Shoe (Sf); Jamaica, Nic; Rockaway, May (Woodr); Rosedale, Wyandanch, Ap-Oct (Not).

713 B. decipiens Dej. Wallface Mt.; Indian Pass; Ausable Pt.; Mountain View; Norwood; Cape Vincent; Keene Val.; Ausable Forks; Mt. Marcy, 5000 ft.; Moss Pond; Upper Jay; Keene; Chapel Pond; Mt. Redfield; Mt. Colden; Mt. Skylight; Mooers; Windsor; Schoharie; Lowville; Waterville; Sylvan Beach; Sodus Pt.; Oakfield; Westfield; Mayville; Nichols; Ft. Hunter; Whitehall; Tivoli; Orangeburg; May-Sep; (all Not).

714 B. rotundiceps Csy. LI, Shoe (Csy).

723 B. versicolor Lec. L. Tear, Ausable Pt., Mountain View, Crown Pt., Norwood, Cape Vincent, Keene Val., Ausable Forks, Mt. Marcy, $5000 \mathrm{ft}$., Moss Pond, Keene Hgts., Upper Jay, Jay, Chapel Pond, Ausable Lakes, Mt. Redfield, Mt. Colden, Mt. Skylight, Cliff Mt., Mooers, Mt. Whiteface, Lowville, Waterville, Sodus Pt., Oakfield, Red House, Westfield, Windsor, Tarbel, Ft. Hunter, Stony Pt., Whitehall, Rensselaerville, 
May-Sep (Not) ; Oneida L., May-Aug (H) ; western NY, common on mud flats (Dnd); Olcott, Ap-J1, Sep, Ithaca, May, Aug, McLean Bogs, May, Aug (Dt); Durlandville, May, FrostSW (Dt); White Plains, J1, $S q$ (Frost); SI, south shore, May-Sep (Not).

725 B. muscicola Hayw. Cape Vincent, Waterville, abundant in cedar swamp, Je-Sep (Not); Johnstown, Gloversville, Mar-Dec (NYS) ; Montour Falls, Je (H); Olcott, Ap, J1 (Dt); Ithaca, Nov, Dt (Vdyk).

- B. semicinctum Not. Mooers, Orangeburg, Ap-Sep (Not).

\section{-}

730

B. quadratulum Not. Mt. Redfield, $4200 \mathrm{ft}$., J1 (Not). (B. proximum is probably synonymous.)

B. oberthuri Hayw. Crown Pt., Mountain View, Upper Jay, Windsor, Schoharie, Sylvan Beach, Red House, Canisteo, Nichols, Ft. Hunter, Whitehall, May-Oct (Not); Marilla (Dnd).

737

B. quadrimaculatum L. Castile, Brad (Flet); generally distributed and common throughout the State.

744 B. pedicellatum Lec. West Pt. (Robn); Bronx Pk., Ap, Nic (Not); Bronxville, Ap (Woodr); SI: Bulls Head, Ap (Woodr).

747 B. mutatum G. \& H. Mt. Whiteface, Aug, Dt (Vdyk) ; Keene Val., Moss Pond, Chapel Pond, Mt. Colden, Mt. Marcy, 5300 ft., May-J1 (Not); St. Lawrence Co, Houghton.

754 B. sulcatum Lec. Keene Val., Westfield, May-J1 (Not); Monroe Co $(\mathrm{M} \& W)$; McLean Bogs, May (Dt).

759 B. frontale Lec. Ausable Chasm, Lowville, Waterville, Utica, Oakfield, - Westfield, Red House, Nichols, Whitehall, Sodus Pt., Tivoli, Orangeburg, Clove Val., May-Oct (Not); Batavia, Je (Lg); Monroe Co (M\&W) ; Olcott, May, J1 (Dt); Buffalo (Z\&R); Ithaca, Nov, Dt (Vdyk); Albany, Ap; West Pt., Je, Ds (Not); Mosholu; Van Cort. Pk., May, Oct, Nic; LI: Brooklyn, May, Shoe, (Sf) ; Greenport, Aug, Ds (Not).

772 B. cautum Lec. Summit of Mt. Skylight, 4900 ft., J1 (Not) ; Genesee Co, Je ( Lg).

776 B. anguliferum Lec. Keene Val., Cape Vincent, Mt. Skylight, Waterville, very abundant under leaves in cedar swamp, McLean, May-Sep (Not); Westport, Aug; West Pt. (Robn); LI: Flatbush, J1, Zab (Not).

B. kuprianovi Mann. Buffalo $(Z \& R)$. The type locality is doubtfully $N$. Âm. ( $\mathrm{Lg})$.

\section{AMERIzUS Chaudoir}

778 A. oblongulus Mann. Mt. Whiteface, Aug, Dt (Vdyk); Mt. Hopkins, Mt. Skylight, Mt. Wallface, Mt. Marcy, Norwood, very unusual, taken under stones, shore Raquette R., Mt. Redfield, Mt. Whiteface, May-Sep (Not); Westfield (Not); N. Elba, Oct (Lg); Monroe Co, rare (M\&W) ; NYC, Palm (L\&B).

\section{Pericompsus LeConte}

792 P. ephippiatus Say: Monroe Co, not common (M\&W) ; Nichols, abundant on Susquehanna R., May (Not); near Hudson R., Sherm (L\&R); Hudson Falls, Sherm,Jr (Lg); SI (L\&D). 


\section{TACHYURA Motschulsky}

805 T. incurva Say. Keene Val., Norwood, Ausable Forks, Upper Jay, Keene, Ausable Lakes, Mt. Redfield, Mt. Colden, Mt. Skylight, Cliff Mt., Mooers, Sodus Pt., Westfield, Egleston's Glen, L. Keuka, Waterville, Windsor, Schoharie, Tivoli, Ft. Hunter, W. Hebron, Van Cort. Pk., May-Sep (Not); Newport, Gloversville, Ap-Nov (NYS); Olcott, Feb-Oct (Dt); Buffalo (Z\&R); Batavia, Je; E. Aurora (Dnd); Taughannock (Lg, Vdyk); Ithaca, May-J1 (div.); McLean Bogs, J1-Aug, Caroline, Ap, Inlet Val., May-Jl, Coy Glen, May, Dt (Vdyk); Catskills, Nic; West Pt. (Robn) ; SI: seashore, May-Sep, Clove Val., Mar (Not) ; LI: Flatbush, Ap (Not); Jamaica, Brooklyn, Nic (Not); Amagansett, Sep, Ds (Not); E. New York, May (Not); Wyandanch, Ap, Ds (Not).

811 T. granaria Dej. Keene Val., Westfield, May-Aug, Keene Hgts., Moss Pond, Mt. Colden, Mt. Skylight, Mt. Marcy, Je-Aug (Not); Monroe Co (M\&W) ; Catskili, Ap, Ang (Not) ; Catskills, Nic (Not); Westchester, Feb (Lg); Bronx, NYC; Bronxville, Mar-Ap (Woodr); SI, Mar (Lg); LI: Brooklyn, Ap-May, Shoe; Jamaica, Nic (Not).

813 T. laetifica Csy. NYC, Julich (Csy).

819 T. xanthopus Dej. Esopus, Peekskill, Ap (Vdyk) ; Tivoli, Van Cort. Pk., Mar (Not); SI, Jl (Lg).

820 T. levipes Csy. LI: Bellport, Je, Nic (Not).

825 T. capax Lec. West Pt. (Robn).

829 T. tripunctata Say. Waterville, Sodus Pt., Westfield, Red House, Ft. Hunter, Schoharie, Whitehall, Windsor, Tivoli, Ft. Montgomery, MayOct (Not); Taughannock Gorge, May-Jl, Dt; Ithaca, May (Vdyk); Tarrytown, May, Bradford; SI, Nov (Lg).

830 T. vivax Lec. Ausable Forks, Jay, Waterville, Westfield, Nichols, Schoharie, Whitehall, Tivoli, May-Oct (Not); Monroe Co (M\&W); Buffalo (Z\&R); E. Aurora (Dnd); Ithaca, Je, Hamilton (Vdyk); Taughannock Gorge, May-Aug, Dt, Hamilton (Vdyk); Inlet Val., May, J1, McLean Bogs, Aug, Dt (Vdyk); Coy Glen, May (Morr).

832 T. serva Csy. Bluff Pt. (Csy).

834 T. ferrugineus Dej. Peekskill, Mar (Vdyk); Greenwood L., May (Not); Riverdale, Je (Woodr); SI, Ap, Shoe.

835 T. frontalis Hayw. Canisteo, Je (Not); Peekskill, Je (Vdyk) ; LI: Jamaica $(\mathrm{Lg})$; Rockaway Beach, May, Nic (Lg).

\section{TACHYS Stephens}

841 T. cuneatus Csy. NYC (Csy).

849 T. proximus Say. Monroe Co $(M \& W)$; Buffalo (Z\&R) ; Olcott, Mar (Dt); Oakfield, Westfield, May-Je (Not); Ithaca, May-Je, McLean Bogs, Jl-Aug (Vdyk); Sandy Hill, Aug (Vdyk); Peekskill, May, Dt (Vdyk); SI: seashore, Je, Ds (Not).

T. rectangulus Not. Sodus Pt., Je, Tivoli, May, Je (Not).

851 T. scitulus Lec. Manlius, May (Vdyk); Monroe Co (M\&W); Buffalo (Z\&R) ; Westfield, Canisteo, Windsor, Ft. Hunter, Schoharie, May-Je (Not); Inlet Val., J1, Sep, Dt; Peekskill, Dt (Vdyk); Catskill, Ap, Ang (Not); West Pt. (Robn); Bronx Pk.; SI, May-Je (Lg); LI: Brooklyn, Ap, May, Shoe (Sf).

854 T. temporalis Csy. NYC (Csy). 
856 T. obliquus Csy. LI: Willetts Pt. (Csy).

857 T. coruscus Lec. Waterville, Sylvan Beach, Sodus Pt., Windsor, Schoharie, May-Je (Not); Peekskill, May (Vdyk); SI, Ap, Shoe (Sf); LI: Canarsie, Rockaway Beach, E. New York, May (Not).

T. reflexicollis Mots. Vic. NYC (Motschulsky).

883 T. laevus Say. Manlius, Ap, Comst (Vdyk); Newport, Ap (NYS); Ausable Chasm, Westfield, Ft. Hunter, Windsor, Schoharie, Whitehall, Je-Oct (Not) ; Monroe Co (M\&W) ; Olcott, Ap (Dt) ; Buffalo (Z\&R) ; McLean Bogs, Aug, Dt (Vdyk) ; Ithaca, Ap (div.) ; Six Mile Cr., Ap (Morr) ; Peekskill (Vdyk) ; Van Cort. Pk., Nic (Not); SI: Shoe (Sf); Arrochar, Je-Oct (Not); LI: Brooklyn, Bronx Pk., Ap, Shoe (Sf).

\section{TACHYTA Kirby}

890 T. nana Gyll. Cranberry L., J1 (Hatch); Cortland Co (Dnd).

892 T. inornata Say. Axton, M\&HH; St. Lawrence Co, Houghton; N. Elba; Keene Val., Wallface, Mt. Skylight, Red House, Westfield, W. Hebron, Sloatsburg, Catskills, Big Ind. Val., Van Cort. Pk., Feb-J1 (Not); Monroe Co $(\mathrm{M} \& W)$; Olcott, Ap (Dt) ; Syracuse, Ap (H); Buffalo (Z\&R) ; Pike, Ap (NYS) ; McLean, Ap (Morr) ; Ithaca, Aug, Hamilton (Vdyk); Fall Cr., Renwick, Freeville, Ap-Je, Dt (Vdyk); Shokan (Lg) ; Karner, Mar (NYS) ; Peekskill (Vdyk) ; SI: Clove Val., Feb-J1 (Not) ; LI: Flatbush, Sep-Oct, Zab (Not) ; Jamaica, Bellport, J1-Aug, Nic (Not); E. Hampton, J1 (Woodr); Brooklyn, May, Shoe (Sf).

895 T. debilicollis Csy. Ausable Pt.; Mountain View; Keene Val.; Red House; Westfield; Sloatsburg; LI: Yaphank; Ap-Sep; (all Not).

\section{TACHYMENIS Motschulsky}

898 T. flavicauda Say. Keene Val., Ausable Pt., Sodus Pt., Oakfield, Je-Sep (Not); Olcott, Aug, Oct (Dt) ; Windom, Oct, Knapp (Vdyk) ; Westfield; Red House, Schoharie, Ft. Hunter, Je (Not) ; Syracuse, Ap-May $(\mathrm{H})$; Monroe Co (M\&W) ; Buffalo (Z\&R) ; Ithaca, Ap, Aug, $M \mathcal{E} H$ (Vdyk); McLean, Ap (Morr); Renwick, Mar-Ap, Dt (Vdyk); Croton L., Ap (Not); Peekskill (Vdyk); Nassau, May (NYS); Mosholu, Nov, Ang (Not); Tarrytown; Van Cort. Pk., Oct, Nic (Not); SI: Clove Val., May (Not); Arrochar, Ap (Woodr); LI: Brooklyn, Ap-May, Shoe; Flatbush, Ap-Oct, Zab; Jamaica, Ap-May, Bellport, Aug, Nic; Central Pk., Ap (Woodr) ; Yaphank, May (Not); Hauppauge, Ap (Not); Baldwin, Amagansett, Sep, Ds (Not); Orient, Lath (NYS). Common under bark of dead trees (L\&D).

\section{PATROBUS Dejean}

901 P. longicornis Dej. Keene Val., Crown Pt., Jay, Mooers, Long L., Norwood, Waterville, Conesus, Conesus L., Westfield, Schoharie, May-Sep (Not) ; St. Lawrence Co, Houghton; Newport, May, Holiday (NYS); Monroe Co (M\&W); Olcott, May, Jl, Oct (Dt); Buffalo (Z\&R); western NY, common in damp places (Dnd) ; Pike (NYS) ; Batavia, Je, $D s$; Ringwood, Inlet Val., Je, J1, Dt (Vdyk) ; Ithaca, Ap-Sep, Dt, Hamilton \& Yuasa (Vdyk); Windom, Oct, Knapp (Vdyk); Hebron (Lg) ; Catskills, Nic (Not); White Plains, Aug-Sep, Sq (SfNYS); Van Cort. Pk., Nic (Not); Bronx (Woodr); Bronx Pk. (Not); NYC, 
May-Sep (Schaupp) ; SI; LI: Brooklyn, May-Je, Shoe; Jamaica, May, Nic (Not); Forest Lawn, J1, Zab (Not); Bellport, J1, Aug, Nic; E. New York, Je (Not).

902 P. septentrionis Dej. var. tenuis Lec. Mt. Marcy; Mt. Redfield; Moss Pond; J1; (all Not).

\section{Platidius Chaudoir}

908 P. rugicollis Rand. Keene Val., Westfield, May-Aug (Not); Catskills, ex coll. Nic; Hemlock Mit., Je, Woodr (Not); Callicoon (Schaupp); NYC (Rand).

- P. longipalpus Not. Keene Hgts., J1 (Not), type.

\section{TRECHUS Clairville}

909 T. borealis Schaef. Whiteface Mt., J1-Sep, Bdy (Lg\&Vdyk); Cliff Mt., Mt. Redfield, Mt. Marcy, Jl-Oct (Not); Chapel Pond, Je, Bish (Dt); Olcott, Mar (Dt) ; Howland I. (Vdyk); Ulster Co, J1, Sep, Nic; LI: North Beach (Sf).

910 T. chalybeus Dej. Mt. Whiteface, Mt. Marcy, Sep-Oct (Lg); Rochester $(\mathrm{M} \& \mathrm{~W})$; LI: Bellport, Nic (Sf).

T. hydropicus Horn. Catskill, Oct (Not).

\section{MYAS Dejean}

929 M. coracinus Say. Manlius (Vdyk); Rock City, Je, trapped in molasses jar (Not); Ithaca, May (Dt) ; Coy Glen, Jl, one under stone and another under log in deep woods, $D t$ (Vdyk); North Branch (Schaupp); SI, Je-Sep (L\&B); Silver L., Sep (Sf) ; Montauk, J1 (Ang); Huntington, Je, Schott (Sf).

930 M. foveatus Lec. "NY" (Lec); Catskills, Aug, HarrED (Sf); LI: Wading R., Eng (Sf).

\section{CyLINDRocharis Casey}

933 C. rostrata Newn. Old Forge, Aug (Vdyk); Westfield (Not); Rock City, Caroline, Ringwood, Coy Glen, Je-J1, Dt (Vdyk); Ithaca, Ap-Sep, div. (Vdyk).

935 C. piceata Csy. Saranac L., Keene Val., Upper Ausable L., Wilmington, Sodus Pt., Westfield, Rock City, Je-Sep (Not); Johnstown, Ap, Pike, Sep (NYS) ; Hebron; North Branch (Schaupp); LI: Jamaica, Nic (Not).

C. grandiceps Lec. "NY" (Lec).

\section{Pterostichus Bonvouloir}

P. rejectus Lec. Keene Val., Red House, Tivoli, Rensselaerville, May-Je (Not).

1006 P. adoxus Say. Keene Val., Norwood, Saranac L., Long L., Mt. Marcy, Wilmington, Lowville, White L., Sodus Pt., Westfield, Rock City, Rensselaerville, Hebron, Catskills, Catskill, Ramapo, Je-Sep (Not); Westport, Sep, Nic; St. Lawrence Co, Houghton; Rochester (M\&W); Saranac Inn (NYS); Boston, Erie Co (Dnd); Buffalo (Z\&R); 
Ithaca, Je-Sep, div (Vdyk); Ringwood, Coy Glen, Windom, McLean Bogs, Cortland Co, McLean Bogs, Junius, Aug, Norton's, May-Aug, Dt (Vdyk) ; Peekskill (Vdyk); West Pt. (Robn) ; SI: North Branch (Schaupp) ; Clove Val., J1-Aug, Ds (Not) ; LI: Flatbush, Zab (Not) ; Bellport, J1-Aug, Nic; Yaphank, Je, Sep, Ds (Not).

\section{Gastrellarius Casey}

1024 G. honestus Say. Keene Val., Upper Ausable L., Saranac L., N. Elba, Wilmington, Lowville, White L., Parkers, Waterville, Sodus Pt., Westfield, Canisteo, Windsor, Je-Oct (Not) ; Olcott, Mar, Je, Aug, Oct (Dt) ; Taughannock Gorge, Aug, Hamilton (Vdyk); Hebron; St. Lawrence Co, Houghton ; Rochester (M\&W) ; Buffalo (Z\&R) ; E. Aurora, Allegheny R. (Dnd) ; Pike, Nov (NYS) ; Portageville, Je (Vdyk) ; Ithaca, McLean Bogs, Coy Glen, Caroline, Ap-Nov, Dt (Vdyk); Catskills, Nic; Big Ind. Val., Aug, Pears (Not); Haines Falls (Vdyk); North Branch (Schaupp) ; Slide Mt., Je-J1, Shoe; Rensselaer, Houghton (NYS) ; LI: Jamaica, Nic (Not).

ANAFERonia Casey

1030 A. substriata Lec. "NY", Julich, Say (div.).

\section{Cyclotrachelus Chaudoir}

1045 C. unicolor Say. "NY" (Schaupp, L\&B).

\section{Eumolops Casey}

1056 E. furtiva Lec. Westfield, May (Not).

1058 E. sodalis Lec. Boston, Hamburg, Marilla (Dnd); Windom, Oct, Knapp (Vdyk).

\section{EVARTHRUS LeConte}

1067 E. sigillatus Say. Albany (Say).

\section{Monoferonia Casey}

1077 M. osculans Csy. (diligendus Lec. nec Chd.). Keene Val., Upper Ausable, Parkers, Saranac L., Ausable Lakes, Canisteo, May-Sep (Not) ; Manlius, May, SmHH (Vdyk); Rochester (M\&W); Ithaca, McLean Bogs, Ringwood, Caroline, Ap-J1, Dt (Vdyk); Catskills, Sep, Nic; Big Ind. Val., J1, Pears (Not); West Pt. (Robn).

\section{Euferonia Casey}

1085 E. relicta Newn. Mt. Marcy (NYS); Ausable, Westfield, May-Je (Not) : Ithaca, Coy Glen, Ringwood, Ap-Sep, Dt (Vdyk) ; Ramapo Mts. (AM).

1088 E. vapida Csy. Adirondack Mts., Kemp (Csy).

1089 E. stygica Say. Crown Pt., Ausable Chasm, Waterville, Ausable Forks, Jay, Batavia, Rock City, Windsor, Whitehall, Sodus Pt., May-Oct (Not); Rochester (M\&W) ; Olcott, Feb, Je, Oct (Dt); western NY. 
common in damp places (Dnd); Pike, Ap (Say); Buffalo (Z\&R); Olean, Sep (Vdyk); Westfield, May (Not); Castile, Brad (Flet); Ithaca, Ap-Sep, div. (Vdyk) ; Honeoye Falls, May (Vdyk); McLean Bogs, Caroline, Ringwood, Ap-J1 (Vdyk); Albany, May (Say); E. Jewett, Ds (Not); Peekskill (Vdyk); West Pt. (Robn); Mosholu, Bronx, May; Forest Lawn, Huguenot, J1, Zab (Not); Bronxville, Bronx, NYC, Ap-J1 (Woodr) ; Van Cort. Pk., Mar-Oct, Nic (Not) ; SI (L\&D) ; LI: Babylon, Je, J1, Bradford; L. George, Aug, Zab; Jamaica, Nic (Not).

1093 E. coracina Newm. Essex Co, Dt (Vdyk); St. Lawrence Co, Houghton; Keene Val., Upper Ausable, Ausable Chasm, Ausable Forks, Mountain View, Mt. Marcy, N. Elba, Whiteface Mt., Port Kent, Parkers, Batavia, Rock City, Red House, Westfield, Forest Lawn, Tivoli, May-Sep (Not) ; Syracuse, Ap (H) ; Manlius, May, $\mathrm{SmHH}$ (Vdyk); Rochester (M\&W) ; Olcott, May-Oct (Dt); Niagara (Lec); Buffalo, Ithaca, Ap-Sep, div. (Vdyk); Taughannock Gorge, Caroline, Coy Glen, Smith Hill, Ringwood, Junius, Ap-Aug, Dt (Vdyk); McLean, Ds (Not) ; Long Eddy, Cy (Vdyk); Haines Falls (Vdyk); Catskills, Nic; Big Ind. Val., Aug, Pears; Poughkeepsie (NYS); E. Jewett; West Pt. (Robn) ; LI: Yaphank, Je (Not); E. Patchogue, Wyandanch, J1-Sep, Ds (Not).

1095 E. adjuncta Lec. "NY" (Lec).

1097 E. lachrymosa Newm. Newport, May (NYS) ; Syracuse, Ap (H) ; Manlius, Aug, SmHH (Vdyk); Olcott, Ap, Sep-Oct (Dt); Pike, May (Vdyk, NYS) ; E. Aurora (Dnd); Westfield, May (Not) ; Canisteo, Je (Not); Ithaca, Ap-Sep, Hamilton, Dt (Vdyk); McLean Bogs, Coy Glen, Caroline, Ap-J1, Dt (Vdyk) ; Callicoon, J1; West Pt. (Robn).

\section{LYPEROPHERUS Motschulsky}

1098 L. punctatissimus Rand. Mt. Whiteface, 4000 ft., J1-Aug, div. (Vdyk) ; Mt. Marcy, $4500 \mathrm{ft}$., Wallface Mt., $3000 \mathrm{ft}$., Upper Ausable L., $2000 \mathrm{ft}$, J1-Sep (Not).

\section{Refonia Casey}

1100 R. moesta Say. Pike, Mar, Yg (NYS); Rock City, Je (Not); Ramapo, Suffern, Ap-Oct; NYC, Je-Sep (Schaupp); SI (L\&D).

1101 ?R. superciliosa Say. Ithaca, Ap (Morr).

\section{ABAcidus LeConte}

1103 A. sculptus Lec. "NY" (Wickham).

\section{Gastrosticta Casey}

1110 G. obscura Say. "NY" (Lec).

\section{CRYobIUS Chaudoir}

1137 C. hudsonicus Lec. Summit of Mt. Marcy, Whiteface Mt., J1, Oct (Not).

1141 C. arcticola Chaud. Whiteface Mt., Sep (div.); Mt. Marcy, Mt. Hopkins, Mt. Skylight, Moss Pond, Green Mt., J1-Oct (Not).

1145 C. mandibularis Kby. Mt. Marcy, Mt. Whiteface, Sep-Oct (Vdyk). 


\section{PoEcilus Bonvouloir}

1161 P. chalcites Say. Lower Ausable L., Ausable Lakes, Mt. Marcy, Sodus Pt., Westfield, Pulaski, May-Jl (Not); Rochester (M\&W); Honeoye Falls, Je (Vdyk) ; Montour Falls, Ap (H) ; E. Aurora (Dnd); Buffalo (Z\&R) ; Ithaca, Ap-Sep, Hamilton (Vdyk); W. Hebron; Orange Co, Ap, Faure (Morr) ; Peekskill (Vdyk); West Pt. (Robn) ; NYC, Mar-J1 (Woodr); Van Cort. Pk.; SI, May-Sep, Ds; LI: Farmingdale, J1, Morrow (Vdyk); Rockaway Beach, May-J1, Shoe, Nic (Not, Woodr) ; Babylon, Bradford; Flatbush, J1, Coney I., Oct, Zab; Jamaica, Feb, Nic (Not).

1162 P. lucublandus Say. Castile, May, Brad (Flet); generally distributed and common throughout the State.

1163 P. bicolor Lec. "NY" (Lec).

1164 P. convexicollis Say. St. Lawrence Co, Houghton; Batavia, Je, Ds.

\section{LOPHOGLOSSUS LeConte}

1165 L. gravis Lec. SI: Watchogue, edge of salt meadow (L\&D).

1167 L. tartaricus Say. SI (L\&D).

1168 L. strenuus Lec. Buffalo (Z\&R); LI: Ridgewood, May (Schaupp); Brooklyn (Fuchs); Astoria, Sep (Bt); Cypress Hills, May; Zab.

1169 L. scrutator Lec. Ausable Pt., Crown Pt., Cape Vincent, Oakfield, Aug-Sep (Not); Batavia, Je, Ds; Buffalo (Z\&R); Ithaca, Aug, Dt (Vdyk); LI: Jamaica, Mar, Queens, Nic.

\section{OMaseus Ziegler}

1174 O. caudicalis Say. Peru (Vdyk); Axton, Je, MEH ; Newport, May (NYS) ; Keene Val., Ausable Pt., Lowville, Norwood, Crown Pt., Mooers, Port Kent, Ausable Chasm, Red House, Westfield, Canisteo, Nichols, Whitehall, Inwood, May-Sep (Not); Olcott, Feb, Sep (Dt); Pike, Oct (NYS) ; Batavia, Je, Ds; E. Aurora (Dnd) ; Ithaca, Ap-Sep, Diven (Vdyk); McLean Bogs, Aug, Yuasa (Vdyk); West Pt. (Robn); NYC, Ap (Vdyk).

1173 O. ebeninus Dej. Rochester (M\&W); Ithaca, Ap-May (Morr).

1175 O. luctuosus Dej. Axton, Je (M\&W) ; Plattsburg, Ausable Chasm, Upper Ausable, Unadilla, Norwood, Cape Vincent, Batavia, Canadice, Oakfield, Sodus Pt., Potter Swamp, W. Hebron, Whitehall, Schoharie, E. Jewett, Tivoli, Forest Lawn, Catskills, Windsor, Je-Sep (Not); Syracuse, Montour Falls, Ap (H) ; Rochester (M\&W) ; Olcott, Mar, May, J1, Oct (Dt); Ithaca, South Hill, Turkey Hill, Taughannock Gorge, Renwick, McLean Bogs, Jl-Nov, Dt (Vdyk) ; Van Cort. Pk., Bronx, Mar, Ap (Woodr) ; LI: Rockaway Beach (Vdyk); Canarsie, Ap, May (Not); Brooklyn, Ap-Oct, Ds, Shoe (Not); Rockaway Beach, May, Ds, Nic (Not); Greenport, Aug, Ds; Rosedale, Ap (Not) ; Jamaica, Ap, Bellport, Ap-Je, Nic (Not); Jamaica Bay, Ap (Woodr).

1176 O. corvinus Dej. Generally distributed and common throughout the State, except at elevations above $500 \mathrm{ft}$. in northern $\mathrm{NY}$, where it is absent.

O. brevibasis Csy. NY, type locality. 


\section{Dysidius Chaudoir}

1177 D. purpuratus Lec. Elmira, May, on ground in field (Diven).

1178 D. mutus Say. Castile, Brad (Flet); generally distributed and common throughout the State.

\section{PSEUdARGUTOR Casey}

1181 P. erythropus Dej. Axton, Je, $M \mathcal{F} H$; St. Lawrence Co, Houghton; Keene Val., Whiteface Mt., N. Elba, Oakfield, Batavia, Windsor, Whitehall, Je-Oct (Not) ; Syracuse, Ap (H) ; Olcott, Ap, Sep (Dt) ; Buffalo (Z\&R) ; Chaffee (Dnd) ; E. Aurora (Dnd) ; Ringwood, McLean Bogs, Taughannock, South Hill, May-Nov, Dt (Vdyk) ; Albany, May, SmHH (Vdyk) ; West Pt. (Robn); Dunwoodie, Ap (Woodr); SI (L\&D) ; LI: Rockaway Beach, Ap-May (div.) ; Cypress Hills, May, Zab (Not) ; Coney I., May, Nic; Brooklyn, Jamaica, Mar-Ap, Nic; Jamaica Bay, Cedarhurst, Ap (Woodr); Yaphank, Je (Not); E. New York, Je (Not); Rosedale, Ap (Not).

\section{Micromaseus Casey}

1182 M. patruelis Dej. St. Lawrence Co, Honghton; Upper Ausable, Cape Vincent, Sodus Pt., Batavia, Oakfield, Whitehall, Tivoli, Orangeburg, Je-Sep (Not) ; Syracuse, Ap (H) ; Rochester (M\&W) ; Olcott, Feb-J1 (Dt) ; Buffalo (Dnd) ; Pike, Ap (NYS) ; Howland I., Nov (Vdyk); Ithaca, Mar-Aug, Hamilton (Vdyk) ; Ringwood, McLean Bogs, Renwick, Mar-Nov, Dt (Vdyk) ; McLean, Ap (Morg); Peekskill (Vdyk); Ramapo, Je (Not); Bronxville, Je (Not); Van Cort. Pk., Nic (Not); SI, Feb-Nov; LI: Rockaway Beach, Ap-May, Diven (Not) ; Pinelawn, Mar, Ds (Not) ; Rosedale, Ap (Not) ; Jamaica, Brooklyn, Jamaica Bay, Mt. Hamilton, Ap (Woodr); Canarsie, Ap-May (Not); Brooklyn, May, Ds (Not).

1183 M. femoralis Kby. Rochester (M\&W) ; Pike, Aug (NYS) ; Norton's, Oct, $S m H H$ (Vdyk); Taughannock Gorge, May, Dt (Vdyk).

1186 M. corrusculus Lec. Rochester (M\&W) ; LI: Wyandanch, Oct, E. New York, May, Rosedale, Ap (Not).

\section{BOTHRIOPTERUS Chaudoir}

1188 B. pennsylvanicus Lec. Keene Val., Mountain View, Waterville, Ausable Chasm, Parkers, Forest Lawn, Whitehall, Je-Oct (Not) ; Olcott, Ap, Oct (Dt) ; Grand I., Niagara R. (Dnd); Batavia, Je; Coy Glen, Turkey Hill, South Hill, Ithaca, McLean Bogs, Caroline, Ap-Nov, Dt (Vdyk) ; LI: Babylon, Je, Bradford; Manitou Beach, Je, Zab (Not).

1190

1192

1194

B. oblongonotatus Say. Hebron (Not).

B. adstrictus Esch. St Lawrence Co, Houghton; Buffalo (Z\&R).

B. luczoti Dej. Wilmington, Aug, Dt (Vdyk); Mt. Whiteface, $2000 \mathrm{ft}$., Aug-Sep (div.) ; St. Lawrence Co, Houghton; Ilion (NYS); Buffalo (Z\&R) ; Keene Val., Upper Ausable L., Mountain View, N. Elba, Saranac L., Mt. Marcy, Long L., May-Oct (Not).

\section{LOXANDRUS LeConte}

1214 L. erraticus Dej. SI, Feb, Lg, a single specimen. 8 


\section{CuRtonotus Stephens}

1251 C. brunnipennis Dej. NY, Edw (AM).

1258 C. elongatus Lec. NY, Edw (AM).

1259 C. pennsylvanicus Hayw. Port Kent, Cape Vincent, Sep (Not) ; Olcott, Sep (Dt); Ithaca, Sep, Lg; West Pt. (Robn); Van Cort. Pk. (Woodr) ; SI: Mar, Ds; Tottenville, Sep, Ds (Not) ; LI: Rockaway Beach, Sep, Ds, Nic (Not); Brooklyn, Je-Aug, Shoe.

\section{STEREOCERUS Kirby}

1260 S. haematopus Dej. "NY" (AM).

\section{LEIRONOTUS Ganglbauer}

1261 L. arenarius Lec. Keene Val., Lower Ausable L., Indian Pass, Wallface Mt., L. Tear, Westfield, Je-J1 (Not); Buffalo (Z\&R).

\section{LEIOCNEMIS Zimmermann}

1262 L. avida Say. Axton, Je, M\&H; Lower Ausable L., Sodus Pt., Nichols, May-Aug (Not); Rochester (M\&W); Olcott, Ap (Dt) ; E. Aurora (Dnd); Buffalo (Z\&R) ; Olean, Sep (VdykCU); Ithaca, Mar (Vdyk); W. Hebron, Je, Lg; Mosholu (Not-AM) ; Bronx, Ap-Nov (Woodr); NYC, Ds (Not); SI, Je, Ds (Not); LI: Rockaway Beach (AM); Ravenswood (AM) ; Jamaica, May (Not); Bellport, Aug (Not).

\section{BRADYTUS Stephens}

1265 B. exaratus Dej. Rochester (M\&W) ; Red House, Ithaca, Mar-Je (Not); Mosholu (AM) ; Dunwoodie, Ap, Bronxville, Je (Woodr); Van Cort. Pk., Oct-Nov (Not); SI, Sep, Lg; LI: Brooklyn, Je-Sep, Shoe ; Ravenswood (AM) ; Rockaway (AM) ; Jamaica, May (Not) ; Port Washington, Oct (Not); Rockaway Beach, Aug-Sep (Woodr); Half Way Hollow Hills, Selden, J1-Aug, Ds (Not); Orient, J1, Lath (Not).

1267 B. apricarius Payk. Adirondack Mts. (Rehn\&Hebard); Axton, Je (M\&H); Crown Pt., N. Elba, Keene Val., Batavia, E. Jewett (Not); Olcott, Je-J1 (Dt); Ithaca, Je-J1, Sep, Dt, Hebron, W. Hebron, Je ( Lg); SI, May-Aug (Not); LI: Brooklyn, Oct (Not-AM); Woodside, Ds; Orient, J1-Sep, Lath (Not).

1271 B. schwarzi Hayw. Buffalo, Boston (Dnd); LI: Babylon, J1 (AM).

1272 B. latior Kby. Keene Val., Mountain View, Norwood, Je-Sep (Not); Olcott, Oct (Dt) ; Batavia, Je, $L g$; E. Aurora (Dnd) ; Castile, Apr, Brad (Flet); Ithaca, Je (Dt); Albany, Aug (NYS); Catskills (Not).

\section{PERCosia Zimmermann}

1281 P. obesa Say. Keene Val., Mountain View, Norwood, Ausable Lakes, Long L., Waterville, Schoharie, Whitehall, Debruce, Je-Sep (Not); Rochester (M\&W) ; Olcott, May (Dt); Buffalo (Z\&R); E. Aurora (Dnd); Genesee Co, Je, Ds; Batavia; Orleans Co, Je (Ds); Sullivan Co (Ds); Hebron (Lg); NYC, May-Nov (Schaupp); SI (Not); LI: Rockaway Beach, Je (NYS).

1284 P. ventricosa Csy. LI (Csy). 


\section{CELIA Zimmermann}

1285 C. patruelis Dej. Mt. Whiteface (Not); St. Lawrence (Houghton); Erie Co (Dnd); NYC (Schaupp) ; SI: Watchogue, Ap-J1, Ds (Not); LI: Jamaica, Rockaway Beach, May, Nic (Not).

1289 C. erratica Sturm. Keene Val.; Lower Ausable; Whiteface Mt.; Wilmington; Mt. Marcy; Ap-Sep; (all Not).

1302 C. laxicollis Csy. Keene Val., Je (Not); Johnstown, Ap (NYS) ; Van Cort. Pk., Nic (Not); SI, Nov (Lg); LI: Sheepshead Bay (Csy); Rockaway Beach (Csy, Lg-AM) ; Babylon, Je (AM) ; Jamaica, Wading R., May, Nic (Not); Orient, Aug, Ds (Not).

1308 C. mimica Csy. Ausable Pt., Sep (Not).

1309 C. remotestriata Dej. - Buffalo, scarce (Dnd).

1318 C. sodalis Csy. SI (Csy).

1319 C. chalcea Dej. Ausable Pt., Sep (Not); Axton, Je (AM); Peekskill, Dec, Lg (AM) ; Westchester, Ap, Lg; Bronxville, Mar (Woodr) ; NYC, May-Nov (Schaupp); SI, Feb-Sep, Lg (Not) ; LI: Rockaway (AM) ; Jamaica, Bellport, Farmingdale (Not); Rockaway Beach, Baldwin, May-Je, Ds (Not).

1324 C. schotti Csy. LI: Jamaica, Schott (Csy).

1325 C. sphaerops Csy. LI: Wyandanch (Csy).

1334 C. brunnea Gyll. Mt. Whiteface (Not, Lg).

1348 C. nupta Csy. L. Champlain (Csy).

1350 C. subaenea Lec. Keene Val., J1 (Not); SI, Sep (Lg); LI: Rockaway Beach, May (Woodr).

1354 C. lyncea Csy. LI: Sheepshead Bay (Csy).

1358 C. liquida Csy. LI (Csy).

1360 C. shoemakeri Csy. LI, Shoe (Csy).

1361 C. pallida Csy. LI: Willetts Pt. (Csy).

1362 C. politissima Csy. LI (Csy).

1367 C. nigripennis Csy. LI: Willetts Pt. (Csy).

1372 C. musculus Say. N. Elba (Not) ; Rochester (M\&W) ; Olcott, Je, Aug (Dt) ; Buffalo (Z\&R); Pike (NYS) ; L. George, Sep, Zab (AM); SI, Sep (Lg) ; LI: Coney I., May-Sep, Zab (SchauppAM) ; Rockaway Beach, May-Sep (div.) ; Jamaica, Nic; Wading R., Jamaica, MaySep (Not).

1375 C. paulula Csy. LI: Wading R. (Csy).

1378 C. rubrica Hald. Keene Val., Aug-Sep (Not) ; N. Elba (Not) ; Rochester $(\mathrm{M} \& W)$; L. George, Aug, Zab (Not); Nyack, Van Cort. Pk., Sep, $Z a b$ (Not); SI (Lg) ; LI: Rockaway Beach, May (div.) ; Brooklyn, Je, Shoe; Long Beach (AM); Babylon, Je (AM); Flatbush, Zab (Not) ; Bellport, Aug, Nic (Not) ; Maspeth, Sep, Baldwin, May, Yaphank, May, Ds (Not).

AMARA Bonvouloir

1384 A. arcuata Csy. LI: Willetts Pt. (Csy).

1385 A. impuncticollis Say. Castile, Brad (Flet); generally distributed and common throughout the State. 
1389 A. fallax Lec. Keene Val., N. Elba, Mt. Whiteface, Mt. Marcy, Sodus Pt., Genesee Co, Westfield, Bronx Pk., Ap-J1 (Not); Rochester Jct., Honeoye Falls, Je, Leon (Vdyk); Rochester (M\&W); Buffalo (Z\&R); SI, Ap-J1 (Not); LI: Brooklyn, Rockaway Beach, May-Sep, Shoe; Tarrytown (AM); Jamaica, Bellport, Forest Pk., Aug, Nic (Not).

1398 A. humilis Csy. Keene Val., Canisteo, Nichols, May-Je (Not) ; LI: Rockaway Beach, May, Ds, Nic (Not); Jamaica, Forest Pk., Nic (Not); Wading R., May (Not).

1400 A. cupreolata Putz. Keene Val., Mt. Haystack, Mt. Marcy, Wilmington, Ap-Aug (Not) ; Syracuse, Ap-May (H) ; E. Aurora (Dnd) ; Castile, May, Brad (Flet); Ithaca, Ap-J1 (VdykCU); White Plains, May (Sf) ; Bronx, Ap, Van Cort. Pk., May (Woodr); SI: Clove Val., May, Aug, Lg; LI: Brooklyn (Vdyk); Rockaway Beach, May, Nic (Not); Flatbush, May, Zab (AM).

1402 A. marquettensis Csy. Ithaca, Ap, Kngt (Not).

1403 A. aeneopolita Csy. Mt. Marcy, Keene Val., Mt. Whiteface, Windsor, May-J1 (Not).

1415 A. polita Lec. Mt. Reserve, Keene Val., Ausable Forks, Pulaski, Sodus Pt., Je-Aug (Not) ; Rochester (M\&W); Olcott, Mar-Oct (Dt); western NY, common (Dnd) ; Ithaca, Ap (Dt); West Pt. (Robn) ; Bronxville, NYC, Van Cort. Pk., Ap-May (Woodr).

1423 A. crassispina Lec. Turkey Hill, Ithaca, Nov, Dt (Vdyk); SI, Mar (Woodr).

1424 A. parviceps Hayw. West Pt. (Robn).

1429 A. basillaris Say. Bronx, Bronxville, Ap (Woodr); LI: Brooklyn, Rockaway Beach, Sep, Shoe.

A. edax Not. Windsor (Not).

A. teres Not. Westfield, May (Not).

\section{TRIAENA LeConte}

1430 T. pallipes Kby. Keene Val., W at; Windsor, Je-J1 (Not); St. Lawrence Co, Houghton; northern NY (Lec); West Pt. (Robn).

T. lawrenceana Csy. NY, type locality.

T. parallela Csy. NY, type locality.

1433 T. angustata Say. Generally distributed and common throughout the State.

\section{REMBUS Latreille}

1441 R. major Lec. Buffalo (Z\&R); Ithaca, J1 (Vdyk).

1442 R. laticollis Lec. Norwood, Sodus Pt., Westfield, May-Sep (Not) ; Syracuse (Lint) ; Rochester (M\&W) ; Buffalo ( $Z \& R)$; Batavia, Je ( Lg) ; E. Aurora (Dnd) ; Montour Falls, Ap (H) ; McLean, J1-Aug, Ithaca, Aug, Dt (Vdyk); SI (Lg) ; LI: Rockaway Beach, Brooklyn, Ap, Je, Shoe.

1442c R. laticollis var. assimilis Lec. "NY" (Lint).

1443 R. impressicollis Dej. Waterville, Je; Sodus Pt., May, Je (Not) ; Genesee Co, Je, Ds; Batavia, Je (Lg); Taughannock, Je, and Ithaca, Aug (Vdyk); Whitehall, Oct (Not); SI, May (L\&D). 
1444 R. obtusa Lec. Keene Val., Sep (Not); Syracuse, May (H); Sturgeon Pt., on L. Erie (Dnd); Ithaca, Aug (Vdyk).

1446 R. alternans Csy. Cape Vincent, Waterville, Sodus Pt., May-Sep (Not); Genesee Co, May (Lg); McLean Bogs, Ithaca, J1 (Vdyk).

R. brevicollis Csy. Ogdensburg (Csy).

R. foveata Csy. NY, type locality.

\section{Dicaelus Bonelli}

1450 D. dilatatus Say. Syracuse, May, Oct (H) ; Rochester (M\&W) ; Buffalo (Z\&R) ; Ithaca, Ap-Sep, div. (Vdyk); Fall Cr., Cascadilla Cr., Larch Meadows, Ap-May (Dt) ; Karner, May (NYS) ; Greenwood L., May (AM) ; Schoharie, Ft. Montgomery, May-Je (Not) ; Catskills, Je, Nic (NotNYS) ; Big Ind. Val., Aug (Not); Peekskill (VdykCU) ; North Branch (Schaupp); West Pt. (Robn).

1454 D. purpuratus Bon. "NY" (AM); SI, Aug, L\&D (Not).

1463 D. crenatus Lec. Buffalo (Z\&R).

1464 D. ovalis Lec. Buffalo (Z\&R); McLean Bogs, Nov (Vdyk).

1465 D. elongatus Bon. Newport, Je (NYS); Erie Co, frequent in woods under cover (Dnd) ; Buffalo (Z\&R) ; Ithaca, May-Sep, div. (Vdyk); McLean Bogs, J1, div. (Vdyk) ; Cascadilla Cr., Ap-Mar (Dt) ; Freeville, Ringwood, Caroline, Ap-J1 (Vdyk); Schoharie, Whitehall, Je-Oct (Not); Peekskill (Vdyk); Catskills, Nov, Nic (Not, Lg); Hebron (Lg) ; Van Cort. Pk., Ap (Woodr) ; Ramapo, Rockland, Sep-Oct (Ds) ; SI: Clove Val., Mar-Aug (Lg) ; LI: Bellport, Jamaica, Je-Aug, Nic (Not); Bergen Beach, May, Je (Not AM); Woodhaven, Je, Zab (AM) ; Flatbush, May, Zab (AM) ; Brooklyn, May-Je, Shoe; Orient Pk., May, Lath (NYS) ; Yaphank, Je (Not); Oyster Bay, Aug (Ds) ; Selden, Half Way Hollow Hills, Orient, Ds (Not).

1466 D. ambiguus Laf. Streetroad, Je (NYS).

1467 D. teter Bon. Upper Jay, Aug (Not) ; Syracuse, Montour Falls, Ap (H) ; Rochester (M\&W) ; Olcott, Je, Aug (Dt) ; E. Aurora (Dnd) ; Buffalo (Z\&R); Westfield, May (Not); Rock City, Je, Ds (Not); Ithaca, May-Aug, Dt (Vdyk); Caroline, Ap, Dt (Vdyk); Schoharie, Je (Not); Catskills, Nic (Not,Lg); Peekskill, Ap. (Vdyk CU) ; Ramapo, Ap, Ds.

1468 D. politus Dej. Keene Val., Je (Not); Syracuse, Montour Falls, Ap (H); Rochester (M\&W) ; E. Aurora, Allegany Pk. (Dnd); Westfield, May (Not); Ithaca, div. (Vdyk); Coy Glen, Larch Meadows, May (Dt); Ringwood, J1, Taughannock, May, Dt (Vdyk); W. Hebron ( Lg); Catskills, Sep, Nic (Not); West Pt. (Robn); Inwood, NYC, Ap (Woodr) ; SI, Ap; LI: Queens, Ap, Wyandanch, May, Nic (Not); Half Way Hollow Hills, May, Ds (Not).

\section{BADISTER Clairville}

B. gilvipes Csy. LI (Csy).

1470 B. notatus Hald. St. Lawrence Co, Houghton; Syracuse, Ap-May (H) ; Ithaca, Mar-Oct (Vdyk CU); Turkey Hill, Nov (Vdyk); Six Mile Cr., Ap (Not) ; Schoharie, Je (Not); Van Cort. Pk., Mar (Woodr) ; SI, May, Je (div.) ; LI: Brooklyn, Ap, May, Shoe; Forest Pk., Ap, Nic (Not). 
1471 B. pulchellus Lec. Keene Val., Cape Vincent, Wilmington, Je-Sep (Not); Syracuse, Ap (H) ; Rochester (M\&W) ; Batavia, Jl-Aug, Kngt (Not); E. Aurora (Dnd); Buffalo (Z\&R); Ithaca, Je, Snn (Vdyk); McLean Bogs, Aug, Hamilton (Vdyk); West Pt. (Robn); Suffern (AM) : Bronx, Dec, Nic (Not); SI, May (Lg).

1472 B. bipustulatus Fab. St. Lawrence Co (Houghton).

1476 B. ferrugineus Dej. Buffalo (Z\&R). Known from Alaska and Calif.

1478 B. flavipes Lec. "NY" (Wickham).

1479 B. micans Lec. Ausable Pt., Cape Vincent, Waterville, Je-Sep (Not); Olcott, Oct (Dt); Ithaca, Ap, J1, SmHH (Vdyk); Peekskill, Ji (Vdyk CU); NYC, Bronx, Ap-Je (Woodr); Van Cort. Pk., Mar (Woodr); LI: Jamaica Bay, Ap (Woodr).

1480 B. laticeps Blatch. Taughannock, May, Dt (Vdyk).

1481 B. reflexus Lec. Howland I. (VdykCU) ; McLean Bogs, Aug, Dt (Vdyk).

\section{Calathus Bonelli}

1482 C. gregarius Dej. Castile, Ap, Brad (Flet); generally distributed and common throughout the State.

1483 C. ingratus Dej. Wilmington, Aug, Dt (Vdyk) ; Mt. Whiteface, J1-Sep, div. (Vdyk, Not); Parkers, Je (Not); Green Mt., J1 (Not).

1484 C. opaculus Lec. Syracuse, Ap (H,VdykCU); Forest Lawn, J1 (Not) ; McLean Bogs, Nov, Dt (Vdyk); Ithaca, Aug (Vdyk); Bronx, Ap (Woodr) ; SI, Mar (Lg) ; LI: Yaphank, Aug, Nic (Not); Shinnecock Hills, Ds.

\section{PRISTODACTYLA Dejean}

1488 P. advena Lec. Mt. Whiteface, trail, Aug, Dt (Vdyk, Not) ; Mt. Marcy. Mt. Redfield, Moss Pond, Mt. Redfield, Ji (Not).

1489 P. impunctata Say. Axton, Je, $M \mathcal{E} H$; Wilmington, Aug, $D s$ (Vdyk) ; Keene Val., Ausable Forks, Mooers, W. Hebron, Parkers, Sodus Pt., Catskills, Ulster Co, Debruce, Je-Aug (Not); Olcott, Sep (Dt) ; L. Champlain (Sf); Hamilton Co, Ds; Crugers, Je (Not AM); Ithaca, Sep (VdykCU); McLean, Ap (Flet); Albany (NYS AM); North Branch (Schaupp); Peekskill (VdykCU); SI: Clove Val., Aug, Ds (Not) ; LI: Long Beach (Vdyk) ; Bellport, J1, Nic (Not); Rockaway Beach, Wyandanch, Yaphank, Je-Oct, Ds (Not).

\section{Platynus Bonelli}

1507 P. hypolithos Say. Gloversville, Je (NYS); Oneida Co, Sep, Shoe: Rochester (M\&W) ; Olcott, Je-J1, Sep-Oct (Dt); E. Aurora and Allegany Pk., common (Dnd); Batavia, Je, Kngt (Ds,Lg); Buffalo ( Lg,Z\&R); western NY (Lint); Chautauqua (VdykCU); Manlius, Aug, SmHH (Vdyk); Red House; Unadilla, Westfield, May (Not); Olean, Sep (VdykCU); Castile, Brad (Flet); Ithaca, Aug, Larch Meadows, J1 (Hamilton); McLean Bogs, J1-Aug, Dt (Vdyk).

1508 P. angustatus Dej. John's Br., and Upper Ausable L., Aug-Sep, Nic (Not); Wilmington, Aug, Dt (Vdyk); Fourth L., Je (NYS); Haines Falls (VdykCU); Pike (VdykCU); western NY (Lint); Buffalo (Z\&R) ; Olean, Sep (Vdyk) ; Chautauqua (Ds) ; Rock City, Jl, Kngt 
(Not); Canisteo, Windsor (Not); Ithaca, May, Sep, Hamilton, Dt (Vdyk); McLean Bogs, Ringwood, Coy Glen, Je-J1 (Dt); Catskilis (Lint); North Branch (Schaupp); L. George, Aug, Zab (AM); Peekskill (Vdyk CU) ; Oliverea, Je-J1, Shoe; West Pt. (Robn) ; Ulster Co, Aug-Sep, Nic (Not); SI (L\&D); LI: Jamaica, Nic (Not).

P. aesopus Csy. Adirondack Mts. (Csy).

1511 P. stygicus Lec. Keene Val.; Ausable Lakes; Mountain View; Mt. Marcy Whiteface Mt.; J1-Oct; (all Not).

1513 P. decens Say. Mt. Marcy, Whiteface Mt., N. Elba, Keene Val., Upper Ausable L., Mountain View, Waterville, Cattaraugus Co, Rock City, Windsor, Catskills, W. Hebron, Orangeburg, Whitehall, Ap-Oct (Not); Rochester (M\&W) ; Olcott, Ap (Dt); Pike, Oct (NYS); Batavia, Sep, Kngt (Not) ; Renwick, Ap, Ithaca, Sep (Vdyk) ; Schenectady, Dec (Dt); Haines Falls (Vdyk CU) ; Bronx Pk. (AM) ; Palisades-on-Hudson, Ap, Van Cort. Pk. (Woodr, Not AM) ; Bronxville, Ap (Woodr) ; SI, Ap-Oct (Not) ; LI: Flatbush, Ap, Nov, Zab (Not); Parkville, (Not AM) ; Jamaica, Mar, Queens, Nov, Nic (Not); Hauppauge, Ap, Nic (Not); Wyandanch, J1, Long Pond, Wading R., Je, Ds (Not).

1514 P. sinuatus Dej. Generally distributed and common throughout the State.

1516 P. opaculus Lec. Keene Val., Je (Not) ; NYC (Schaupp) ; Van Cort. Pk., May (Not AM) ; LI: Rockaway, May (Woodr).

1517 P. tenuicollis Lec. Manlius, May, SmHH (Vdyk); NYC (Lg).

1518 P. cincticollis Say. Keene Hgts., John's Br., Mooers, Lowville, Port Kent, Ausable Pt., Sodus Pt., Westfield, Schoharie, Ft. Montgomery, MaySep (Not); Rochester (M\&W) ; Pike, Je (NYS) ; Inlet Val., Ithaca, May (Not); Renwick, Ap, Dt (Vdyk); Canisteo, Je (Not) ; NYC (Not, Lg); SI, Shoe, Ds (AM) ; LI: Brooklyn, Rockaway Beach, Ap-Oct, Shoe (Not): Forest Pk., Nic (Not); Jamaica, Mar, Nic (Not); Far Rockaway, May (Woodr); Wading R., May, Ds (Not).

1519 P. reflexus Lec. Upper Ausable L., Mountain View, Cape Vincent, Keene Hgts., Mooers, Waterville, Ausable Pt., Lowville, Tivoli, Westfield, Catskills, Windsor, May-Sep (Not); Syracuse, Ap-May (H); Rochester (M\&W) ; Montour Falls (H); Buffalo (Dnd) ; Ithaca, Renwick, May-Nov, Dt (Vdyk); Taughannock, Ap, Snn (Vdyk) ; McLean Bogs, Je, Dt (Vdyk); Egleston's Glen, Je, Peekskill, May (Vdyk CU) ; Albany, May (AM); L. George, Aug, Zab (Not); West Pt. (Robn) ; SI, Ap (Not, Lg) ; LI: Newtown Hgts., May (Not AM) ; Rockaway, Aug, Flatbush, Je, Oct, Zab (Not) ; Jamaica, Nic (Not); Far Rockaway, May (Woodr); Rockaway Beach, May-Je, Wading R., Aug, Ds (Not).

1520 P. parmarginatus Ham. Pike, Mar (NYS); LI: Orient, Lath (NYS).

1521 P. brunneomarginatus Mann. Buffalo (Z\&R). A well-known Calif. sp. $(\mathrm{Lg})$.

1522 P. extensicollis Say. Generally distributed and common throughout the State.

$1522 \mathrm{~b}$ P. extensicollis var. viridis Lec. Buffalo (Z\&R).

P. gaudens Csy. (Anchomenus). L. Champlain (Csy).

1523 P. decorus Say. Crown Pt., Oakfield, Nichols, May-Aug (Not); L. Onondaga (Lint) ; Syracuse, Ap-May, Oct ( $\mathrm{H})$; Rochester (M\&W) ; Olcott, Ap, J1 (Dt) ; Six Mile Cr., Ap (Not); McLean Bogs, Jl-Aug, 
Hamilton, Dt (Vdyk) ; Inlet Val., Sep, Dt (Vdyk); Ithaca, J1 (Vdyk CU) ; Peekskill (Vdyk CU) ; West Pt. (Robn); White Plains, Aug (Sq) ; NYC, May (Woodr) ; SI: May (Woodr, Ds) ; New Brighton, Aug (Ds) ; LI: Rockaway Beach (Vdyk CU) ; Brooklyn, Ap, Jl, Shoe (AM); Cypress Hills, Aug (AM); Queens, Ap, Bellport, J1, Nic (Not); E. New York, Je (Ds).

1529

P. decorus ab. syracusensis Hatch (Anchomenus). Syracuse, Ap, Oct $(\mathrm{H})$.

P. anchomenoides Rand. Keene Val., Ausable Lakes, Waterville, Red House, Nichols, Canisteo, Windsor, Schoharie, May-Je (Not); Rochester (M\&W); Niagara, Buffalo (Dnd); Ithaca, Je, Hamilton (Vdyk) ; Inlet Val., J1, Renwick, Ap, Dt (Vdyk CU); NYC (Rand ANSP).

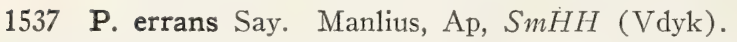

1537a P. errans Say var. subcordatus Lec. Rochester (M\&W).

1538 P. moerens Dej. St. Lawrence Co, Houghton; Oneida L., Aug (Drk) ; LI (Dej ANSP).

1539 P. tenuis Lec. N. Elba (Not,Lg); Ithaca, Aug (Vdyk CU); White Plains, May (Sq); SI (Not,Lg); LI: Rockaway Beach, Brooklyn, Ap-Nov, Shoe.

P. militaris Csy. West Pt., Robn (Csy).

1540 P. collaris Say. Buffalo (Z\&R).

1541 P. mutatus G. \& H. (atratus Lec.). St. Lawrence Co, Houghton; Keene Val., Upper Ausable L., Windsor, Chauncey, Sloatsburg, N. Elba, Tarbel, Mar-Je (Not) ; Syracuse, Ap (H) ; Rochester (M\&W) ; Rock City, Je (Vdyk CU) ; McLean Bogs, Inlet Val., Ringwood, J1-Oct, Dt (Vdyk) ; Ithaca, J1-Aug (Vdyk CU) ; Catskills, Nic (Not); Bronxville, Mar (Woodr) ; SI, May (Lg); LI: Brooklyn, May-Sep, Shoe; Jamaica, Bellport, Wading R., Mar-Aug, Nic (Not); Jamaica Bay, Ap, Ft. Hamilton, Ap (Woodr).

1542 P. melanarius Dej. Generally distributed and common throughout the State.

1543 P. propinquus G. \& H. Cape Vincent, Crown Pt., Mt. Marcy, Waterville, Batavia, May-Sep (Not); Howland I., Cayuga Co (Dt).

1546 P. affinis Kby. Peru, J1, M\&E (Vdyk); St. Lawrence Co, Houghton; Keene Val., Indian L., Norwood, Keene Hgts., Ausable Lakes, Mt. Skylight, Lowville, Ausable Pt., Batavia, Sodus Pt., Canadice, Westfield, Nichols, Tivoli, Whitehall, May-Oct (Not); Rochester (M\&W); Olcott, J1 (Dt); Buffalo (Z\&R) ; E. Aurora (Dnd) ; Montour Falls (H); McLean Bogs, Ringwood, Inlet Val., Ithaca, Keuka Pk., MarOct, Dt (Vdyk) ; Nyack, Zab (Not AM) ; Bronxville, Mar-Ap, Bronx, Ap, Van Cort. Pk., Ap (Woodr) ; NYC, Schaupp (Lg) ; LI: Babylon, Je $(\mathrm{AM})$.

1547 P. carbo Lec. Pine I., Sep (Not AM); LI: Newtown Hgts., Ap (Not AM).

1548 P. corvus Lec. Mt. Redfield, J1 (Not); Van Cort. Pk., Mar (AM) ; NYC (Schaupp) ; LI: Canarsie, Ap (Not) ; Jamaica (Lg); E. New York, May, Nic (Lg); Brooklyn, Ap-Sep, Shoe (AM).

1551 P. metallescens Lec. Mt. Marcy, J1, Keene Val., May (Not) ; St. Lawrence Co, Houghton.

P. fidele Csy. Keene Val., Moss Pond, Parkers, May-J1 (Not).

1552 P. deceptivus Lec. Mt. Marcy, Mt. Redfield, J1 (Not). 
1553 P. cupripennis Say. Castile, Ap, May, Brad (Flet); generally distributed and common throughout the State.

1555 P. excavatus Dej. Lowville, Keene Val., Waterville, Canadice, Oakfield, Sodus Pt., Canisteo, Sylvan Beach, Red House, Nichols, Tarbel, Ft. Hunter, Orangeburg, Van Cort. Pk., Ap-Je (Not); Rochester $(\mathrm{M} \& W)$; Olcott, May, J1 (Dt); Ithaca, J1-Aug, Hamilton (Vdyk); Butternut. Cr., Je, Dt (Vdyk); McLean Bogs, May, J1 (Dt) ; Hudson Falls, Aug (Vdyk CU); West Pt. (Robn); Suffern (AM); NYC (Schaupp); SI (L\&D); LI: Rockaway Beach, Brooklyn, May-Je, Shoe.

1556 P. ferreus Hald. Ithaca, Je, Dt (Vdyk CU) ; Tivoli, May (Not); Orangeburg, Ap (Not); Nyack, Zab (Not); Bronxville, Ap (Woodr) ; Peekskill, Ap (Vdyk CU); West Pt. (Robn); Mosholu, Sep, Bronx Pk., Van Cort. Pk. (AM) ; SI: Mar-Je, Nic (Not) ; Clove Val., Mar (Lg); LI: Brooklyn, May, Nov, Shoe; Flatbush, Ap (Not AM) ; Jamaica, May, Nic (Not) ; Forest Pk., May (Vdyk CU).

1557 P. basalis Lec. "NY" (Lint,Csy).

1558 P. nutans Say. Waterville, Je (Not); St. Lawrence Co, Houghton; Olcott, Ap, Je (Dt) ; Buffalo (Z\&R) ; E. Aurora, Cortland Co (Dnd); Westfield, May (Not); Ithaca, Je, Ringwood, J1, Turkey Hill, Je-Nov, Dt (Vdyk); West Pt. (Robn); NYC, Bronx, Bronxville, Mar-J1 (Woodr); SI, May (Lg) ; LI: Rockaway Beach, May, Nic (Not); Brooklyn, Ap, Far Rockaway, Ap-May (Woodr).

1561 ?P. maculicollis Dej. Buffalo (Z\&R). Known from Kans. and Calif. ( $\mathrm{Lg}$ ). 1562 ?P. variolatus Lec. Buffalo (Z\&R). Known from southern Calif. $(\mathrm{Lg})$.

1566 P. octopunctatus Fab. Axton, Je $(\mathrm{M} \& H)$; Mt. Skylight, Wallface Mt., Keene Val., Sylvan Beach, Nichols, May-J1 (Not); Newport, Je, Aug (NYS) ; St. Lawrence Co, Houghton; Rochester (M\&W); Olcott, Feb (Dt) ; Buffalo (Z\&R) ; Pike, Je (NYS) ; E. Aurora (Dnd); Ithaca, Sep (Dt); McLean, J1, Dt (V dyk); Albany, Je, Nassau, Aug (NYS); White Plains (Sq); Nyack, Zab (Not); High Bridge (AM); Van Cort. Pk. (AM) ; Bronx, Ap (Woodr); Peekskill, Sep, NYC (Lg); SI (Lg) ; LI: Brooklyn, May, Shoe (AM); Jamaica, Ap, Nic (Not); Babylon, J1 (AM) ; Flatbush, Ap, Zab (AM) ; Bergen Beach, Ap-May (Not AM) ; Forest Lawn, J1, Zab (Not); Rockaway Beach (div.).

1567 P. placidus Say. Peru, J1, M\&E (Vdyk); Ausable Pt., Port Kent, Sep (Not); Wilmington, Je (Ds) ; Syracuse, Ap-May (Vdyk,H) ; Rochester Jct., Je, Leon (Vdyk); Sodus Pt., May (Not) ; western NY, common (Dnd); Olcott, Mar, J1, Oct (Dt); Batavia, May-J1, Kngt (Not); Castile, Brad (Flet); Geneva, Nov (Vdyk CU); Cattaraugus Co, J1 (Ds); Ithaca, Coy Glen, Renwick, Taughannock Gorge, Ap-Sep, Dt (Vdyk); Whitehall, Oct (Not); Peekskill (Vdyk CU); West Pt. (Robn); Bronxville, NYC, Je-J1 (Woodr); Bronx, SI, Mar-Je (Woodr) ; LI: Jamaica, E. New York, Port Washington, Mar-Sep, Nic (Not) ; Far Rockaway, Lawrence, Ft. Hamilton, Ap-Je (Woodr); Orient, Lath (NotNYS) ; Flushing, Ap, Kngt (Not).

1573 P. bogemanni Gyll. Keene Val., Mt. Whiteface, Lowville, Je-Aug (Not); St. Lawrence Co, Houghton; Saranac Inn, Je (Woodr); Gloversville, Ap (NYS); Rochester (M\&W) ; Buffalo (Z\&R); Ithaca, May-Aug (div.); Karner, Mar, Bish (Dt); Pine I., Je, Ds (Not); West Pt. (Robn) ; NYC, Van Cort. Pk., Je, Nic (Not); SI: May (div. NYS); Tottenville, Je, Ds (Not); LI: Babylon, Je-J1 (AM); Flatbush (Not AM) ; Bellport, Jamaica, May-Je (Not); Rockaway Beach, Wyandanch, Half Way Hollow Hills, Je-J1, Ds (Not). 
1574 P. quadripunctatus Dej. Keene Val., Wallface Mt., J1, Oct (Not); Mt. Marcy, May (Not,Lg) ; Mt. Whiteface, Sep (div.); N. Elba, Oct (Lg); St. Lawrence Co, Houghton; Buffalo (Z\&R); Green Mt., J1 (Not).

1577 P. aeruginosus Dej. Ausable Pt., Sep, Westfield, May (Not); Catskills, J1, Pears (Nic); Orangeburg, Ap (Not); West Pt. (Robn); Mosholu, Bronx Pk. (AM); Bronxville, Je (Woodr); NYC (div.); SI, Nic (Not); LI: Brooklyn, May-Je, Shoe; Bellport, Rockaway Beach, May-Ji, Nic (Not); E. New York, May, Flushing, Aug, Ds (Not).

1579 P. crenistriatus Lec. Cape Vincent, Sep (Not) ; Rochester (M\&W) ; Buffalo (Lec-ANSP); Pike, Aug (NYS); Batavia, J1, Kngt (Not); Ithaca, Jl, Dt (Vdyk) ; Junius, Aug, Dt (Vdyk) ; Catskills, Nic (Not); Peekskill (Vdyk CU); Westchester Co, Sep ( $\mathrm{Lg})$; Bronxville, Ap (Woodr); SI: Nov-Dec (Lg); Tottenville, Sep, Ds (Not) ; LI: Rockaway Beach, Je-J1 (div.); Long Beach (AM); Sheepshead Bay, Aug, Long Beach, Forest Lawn, J1 (Not); Bergen I., Forest Lawn, Jl, Zab (Not).

1580 P. rubripes Lec. St. Lawrence Co, Houghton; Ithaca, Ap (Vdyk CU); Inlet Val., May, Oct, Dt (Vdyk); Unadilla, Sep, Nic (Not AM); Bronxville, Mar (Woodr); NYC (Schaupp,Lg); SI, Feb-Dec (Lg); LI: Far Rockaway, J1-Sep (Woodr).

1581 P. punctiformis Say. Erie Co (Dnd); Rochester (M\&W); Ithaca, J1, Hamilton (Vdyk); Hebron (Lg); Peekskill (Vdyk CU); Nyack, Zab (Not); Tarrytown (AM) ; Van Cort. Pk., Bradford (AM); NYC, Sep (Woodr); SI, Feb-Dec (Lg); LI: Jamaica, May, Nic '(Not); Jamaica Bay, Ap (Woodr) ; E. New York, May (Not,Ds); Brooklyn, May, Ds (Not).

P. politissima Csy. subsp. statenensis Csy. SI, type locality.

1582 P. sordens Kby. L. Tear, Keene Val., Keene Hgts., Mt. Whiteface, and Wilmington, May-J1 (Not); Rock City, Je (Vdyk CU).

1584 P. ruficornis Lec. Mountain View, Cape Vincent, Keene Val., N. Elba, Waterville, Sodus Pt., Batavia, Canadice, May-Oct (Not) ; Wilmington and base of Mt. Whiteface, Aug, Dt (Vdyk); St. Lawrence Co, Houghton; Rochester (M\&W) ; Olcott, J1 (Dt) ; Buffalo (Z\&R); Howland I., Nov (Vdyk CU); McLean, Ap, Kngt (Not); Renwick, Inlet Val., Taughannock Gorge, Ithaca, Ringwood, J1-Nov, Dt (Vdyk); Unadilla, Sep, Nic (Not); Karner, Mar, Bish (Dt); Catskills, Nic (Not); West Pt (Robn); NYC (Schaupp); Bronx, Ap (Woodr); LI: Brooklyn, Ap-May, Nov, Shoe; Richmond Hill (AM); Flatbush, Ap (Not AM) ; E. New York, Wading R., May, Nic (Not); Flushing, Mar, Rosedale, Ap (Not); Orient, Lath (NYS).

1587 P. elongatulus Hald. Keene Hgts., Keene Val., Upper Ausable L., Ausable Forks, N. Elba, Mt. Hopkins, Batavia, Catskills, Big Ind. Val., Parkers, May-Oct (Not); St. Lawrence, Houghton; Pike, Ap (NYS); Ithaca, Renwick, Ap-Nov, Dt (Vdyk); Buttermilk Cr., Mar (Vdyk CU) ; Peekskill (Vdyk CU); NYC (Schaupp); SI (L\&D); LI: Brooklyn, May, Shne; Flatbush, Sep, Zab (Not); Jamaica Bay, Ap (Woodr); Wyandanch, J1, Pinelawn, Mar, Ds (Not).

1585 P. retractus Lec. Adirondacks, Houghton; Sodus Pt., May (Not).

1586 P. gemellus Lec. Ithaca, Mar-Aug, Hamilton, Dt (Vdyk CU).

1587 P. picipennis Kby. Syracuse, Ap, Je (H) ; Cape Vincent, Tivoli, Canadice, Westfield, Canisteo, Windsor, Orangeburg, Ap-Sep (Not); Glovers- 
ville, Mar (NYS) ; Rochester (M\&W) ; Buffalo (Z\&R) ; E. Aurora (Dnd) ; Batavia, Je ( Lg) ; Ithaca, Ap (Not) ; Bronx Pk., West Farms (AM) ; Van Cort. Pk., Tarrytown, Bradford (AM) ; NYC (Not,Lg) ; SI, Je (Woodr.Lg); LI: Brooklyn, May, Nov, Shoe; Far Rockaway, May, Ft. Hamilton, Ap (Woodr); Canarsie, Ap, E. New York, Je (Not); Flushing, Mar (Not).

1588 P. lutulentus Lec. St. Lawrence Co, Houghton; Sodus Pt., May (Not); Batavia, J1, Kngt (Not); Buffalo (Z\&R); Westfield, May (Not); Renwick, Ithaca, Inlet Val., Mar-Oct, Dt (Vdyk); Peekskill, J1 (VdykCU); LI: Brooklyn, Ap-May, Shoe (NotAM); Babylon, Je (AM) ; Bellport, Je (Not) ; Jamaica Bay, Ft. Hamilton, Ap (Woodr) ; Canarsie, E. New York, Ap-May (Not).

1589 P. nigriceps Lec. "NY", Rein (L\&B).

1591 P. quadrimaculatus Horn. Northwestern NY, Hamilton (L\&B).

1592 P. obscurus Hbst. Adirondacks, Houghton; L. Onondaga (Lec) ; Syracuse, Ap, Oct (H) ; Canastota, March (Not); Genesee Co, Je (Ds) ; Batavia, Je (Lg,Ds); Oakfield, Je (Not); Buffalo (Z\&R, Lec); E. Aurora (Dnd) ; Pike (AM) ; Red House, Je (Not) ; Howland I., Nov (Vdyk) ; Ithaca, Mar-Ap, Nov, Hamilton, Dt (Vdyk); Renwick, Mar-Ap, Nov, Dt (Vdyk); W. Hebron (Woodr,Lg); Whitehall, Ort (Not); West Farms (AM); SI (Lg); LI: Orient, Lath (NYS).

\section{OLISThOPUS Dejean}

1595 O. parmatus Say. Keene Val., Whiteface Mt., Sodus Pt., May-J1 (Not); Rochester (M\&W); Olcott, Sep (Dt); E. Aurora, Allegany Pk. (Dnd) ; Spencer, Oct, Dt (Vdyk); Howland I. (VdykCU); NYC (Lg) ; Bronxville, Nov (Woodr) ; LI: Rockaway Beach, Mar-May, Shoe, Nic (Not); Far Rockaway, May (Woodr).

1596 O. micans Lec. Buffalo (Z\&R); West Pt., Je, Ds (Not); NYC (LecANSP) ; LI: Rockaway Beach, Je, Shoe.

\section{Atranus LeConte}

1609 A. pubescens Dej. Rochester (M\&W) ; Buffalo (Z\&R); Westfield, May (Not) ; Howland I., Je, Dt (Vdyk); Taughannock Gorge, May, Aug, Hamilton (Vdyk); Ithaca, J1-Sep, Dt (Vdyk); Canisteo, Je (Not); Peekskill (VdykCU); West Pt (Robn); LI: Rockaway Beach, Ap, Shoe; Brooklyn, May, Shoe.

\section{LEPTOTRACHELUS Latreille}

1610 L. dorsalis Fab. NYC (LgAM); SI (Lg); LI: (Schaupp); Brooklyn, Shoe (Ds).

\section{CASNONIA Latreille}

1612 C. pennsylvanica L. Rochester (M\&W); Olcott, Ap (Dt); Buffalo (Z\&R) ; Pike, Je (NYS); Ithaca, Je-Sep (VdykCU); Albany, Je, Nassau and Poughkeepsie, May (NYS); Catskills, Nic (Not); Callicoon, J1, Ds (Not); Florida, Je, FrostSW (Vdyk); Peekskill, Ap (VdykCU,Lg) ; West Pt. (Robn); White Plains (Sq); SI: May-Sep (div.) ; Tottenville, May, Ds (Not); LI: Rockaway Beach, May-Je (div.) ; Brooklyn, Ap-Je, Shoe (AM); Flatbush, Aug-Oct, Zab (AM) ; 
Prospect Pk., Feb, Queens (AM) ; Jamaica, May, Nic (Not); Half Way Hollow Hills, Jl, Ds (Not); E. New York, Je, Long Beach, J1 (Ds).

\section{Galerita Fabricius}

1616 G. janus Fab. Newport, May (NYS); Pulaski, Je (Not) ; Syracuse, Ap (VdykCU) ; Rochester (M\&W); Olcott, Ap (Dt); Buffalo (Z\&R); western NY (Dnd); Rock City, Windsor, Je (Not); Ithaca, Caroline, Ringwood, Ap-Sep, Dt (Vdyk) ; Six Mile Cr., Ap (Not); Spencer, Nov (VdykCU,NYS) ; Karner, May, Hill (NYS) ; West Pt. (Robn); Carmel, West Farms (AM) ; White Plains, Mar (Sq) ; NYC, Bronxville, Van Cort. Pk., Aug-Sep (Woodr); SI: Nic (Not, Ds) ; Clove Val., Ap (Not,Lg); LI: Rockaway Beach, May-Je, Shoe; Parkville (AM) ; Jamaica, Bellport, Je-Aug, Nic (Not); Yaphank, J1 (Woodr,Not,Ds); Cold Sp., May (Not,Ds); Orient, May, Lath (Not).

1620 G. bicolor Drury. Ausable Pt., Sep (Not); Sodus Pt., Je (Not) ; western NY (Dnd); Ithaca, Ap-Sep, Dt (Vdyk); Whitehall, Oct (Not); Windsor, May (Not); NYC (Lg); SI: May-Sep (Ds) ; Clove Val., J1 (Not,Ds); LI: Bellport, Jl-Aug, Nic (Not); Farmingdale, Mar, Wading R., May (Not); Yaphank, Oct, Ds (Not).

\section{TETRAGONODERUS Dejean}

1636 T. fasciatus Hald. Buffalo (Z\&R) ; Sylvan Beach, Je (Not) ; LI: Aqueduct, Mar, Shoe; Jamaica, May, Rockaway Beach, Sep, Nic (Not).

\section{LEBIA Latreille}

1641 L. grandis Hentz. Newport, Je, Lewis Co, Aug (NYS); Keene Val., (Not) ; Syracuse, Ap (H) ; Rochester (M\&W); Olcott, Je, Sep-Oct (Dt) ; Buffalo (Z\&R) ; E. Aurora (Dnd) ; Ithaca, Mar-J1, Dt (Vdyk) ; Peekskill (VdykCU) ; Port Jervis, Oct (Lint); West Pt. (Robn); White Plains, Mar (Sq); Nyack, Je (Not,Lg); Van Cort. Pk., Je (NotAM); Inwood, Feb, Westchester Co, Ap, Bronxville, Sep (Woodr) ; NYC (Ds,Lg); SI: (Ds,Lg) ; Watchogue, Aug (Ds) ; LI: Brooklyn, May-Je, Sep-Nov, Shoe (AM); Parkville, Long Beach (AM); Jamaica (NYS); Flatbush, Ap-Oct, Zab (AM); Bergen Beach, Ap-May, Oct (AM) ; Queens, Port Washington, Bellport, Mar-Nov, Nic (Not) ; Far Rockaway, May, E. Hampton, J1 (Woodr) ; Rockaway Beach, Aqueduct, Fiushing, Je-Aug, Maspeth, Orient, MayAug, Ds (Not).

- L. enormis Csy. NYC (Csy).

1642 L. atriventris Say. Cranberry L., Sep (Drk) ; Wilmington, Pulaski, Waterville, Westfield, Windsor, Tivoli, May-J1 (Not); Rochester (M\&W) ; White L., Aug, Zab (AM) ; Olcott, Mar, Je (Dt) ; Buffalo (Z\&R) ; E. Aurora (Dnd) ; Castile, Brad (Flet) ; Ithaca, Mar-Sep, Dt (Vdyk) ; Albany, Poughkeepsie, Je (NYS); W. Hebron (Lg); Catskill (Lg); Esopus (Vdyk,Dt); West Pt. (Robn); Florida, J1, Frost $W$ (VdykNYS) ; White Plains, May (Sq) ; Westchester Co, Ap, Bronxville, Aug-Sep (Woodr) ; West Farms (AM) ; Goshen, Sep (NotAM); Van Cort. Pk., Dec-Feb, Nic (Not); NYC, Je-Aug (Woodr); SI (div.); II: Rockaway Beach (div.); Brooklyn, May, Oct, Shoe; Manitou Beach, Coney I., Flatbush, Je-Sep, Zab (AM); Bergen Beach, Jamaica, Mar-May, Nic (Not); Yaphank, Je (Not). 
1643 L. tricolor Say. Keene Val., May-Je (Not); Wilmington, J1, Ds (Not) ; Axton, Je (M\&H) ; St. Lawrence Co, Houghton; Canisteo, Je (Not) ; Olcott, Ap (Dt) ; Buffalo (VdykCU) ; Ulster Co, Shoe; Van Cort. Pk., Ap (Woodr); West Pt. (Robn); SI - Ds) ; LI: Rockaway Beach, Brooklyn, May, Sep, Shoe.

1646 L. atriceps Lec. Mosholu, Aug (NYS).

1649 L. pulchella Dej. Peekskill, Ap, Oct (div.) ; Morrisania, Julich (Schaupp); NYC (Woodr, LgAM); SI, Ap (Lg); Silver L., Ap (Ds); LI: Yaphank, May-Sep, Shoe; Bellport, Rockaway, May, Sep, Nic (Not) ; Amagansett, Sep (Ds).

1654 L. marginicollis Dej. LI: Flatbush (Ent. News, 1909).

1655 L. viridis Say. Generally distributed and common throughout the State.

$1655 \mathrm{~b}$ L. viridis Say var. moesta Lec. Buffalo (NYS,AM).

1656 L. cyanea Dej. "NY" (NYS).

1658 L. pumila Dej. Adirondacks, Houghton (NYS) ; Essex Co, Aug (NYS) ; Saranac L., Sep, Shoe; White L., Sep, Zab (AM); Ausable Forks, Mt. Marcy, Keene Val., Mt. Whiteface, Sodus Pt., Port Kent, Bronx Pk., Crugers, Carmel, Je-Sep (Not); Rochester (M\&W) ; Buffalo $(Z \& R)$; Pike (NYS) ; E. Aurora (Dnd) ; Rock City, Je (Not) ; L. George, Aug, Zab (AM) ; Washington Co (Lg,Ds) ; West Pt. (Robn) ; Greenville; Van Cort. Pk. (NotAM); SI (Lg); LI: Yaphank, Wyandanch, Bellport, May (Not).

1659 L. pleuritica Lec. Buffalo (Z\&R); West Pt. (Robri); White Plains, Mar (Sq) ; Westchester Co, May (AM); Van Cort. Pk., Dec, Nic (Not) ; SI, Ap (L\&D) ; LI: Flatbush; Brooklyn, Ap-Sep, Shoe; Jamaica, Queens, Central Pk., Aug-Nov, Nic (Not) ; Massapequa, Rockaway Beach, May-Je (Woodr); Nassau Co, May-Je, Orient, Je (Not,Ds).

1664 L. viridipennis Dej. Rochester (M\&W); Buffalo (Z\&R); Peekskill (div.); LI: Jamaica woods, Mar, Oct-Nov (Schaupp); Aqueduct, Aug-Sep, Shoe; Bergen Beach, Je (NotAM) ; Jamaica, Nic (Not); Fire I., Sep, Ds (Not).

1666 L. lobulata Lec. West Pt., Je, Ds (Not); Peekskill, Je (Lg).

1667 L. ornata Say. Keene Val., Aug-Sep (Not) ; Newport, Je (NYS) ; Wi1mington, J1, Ds (Not) ; Mt. Whiteface, Sep (Lg) ; White L., Aug-Sep, $Z a b$ (AM) ; Buffalo (Z\&R); Pike, Je (NYS); western NY, scarce, but generally distributed (Dnd) ; Rock City, Je (Not, Ds) ; Castile, May, Brad (Flet); Ithaca, May-Sep, Dt (Vdyk); L. George, Aug, $Z a b$ (AM); Seneca and Howland I., Je-Nov (Vdyk); Karner, Je (NYS) ; Windsor, May, and Debruce, Sep (Not); Catskills, Nic (Not) ; New Baltimore, Zab (AM) ; Ramapo Mts., Suffern (AM) ; Greenville (Schaupp); Van Cort. Pk., Nic (Not); Bronxville, Sep (Woodr) ; NYC (Lg) ; SI: Sep (AM) ; Watchogue (Ds) ; Richmond, Je (Woodr) ; LI: Riverhead, Sep (VdykCU) ; Yaphank, Rockaway, Je, Sep, Shoe (AM) ; Flatbush, Sep, Fishers I., Aug, Zab (AM) ; Jamaica, Nic (Not); Bellport, Aug, Nic (Not); Orient, Lath (NYS); Amagansett, Montauk, Sep (Ds).

L. reperta Csy. "NY" (Csy).

1669 L. collaris Dej. "NY" (Lec).

1671 L. analis Dej. Batavia, Je (Ds) ; Poughkeepsie, May (NYS) ; Callicoon, J1 (Ds); Florida, J1, FrostSW (Vdyk); White Plains (Frost,Sq); NYC ( Lg) ; Van Cort. Pk., Nic (Not); West Farms (AM); LI: Jamaica (Schaupp); Westbury, Aug (Champlain); Bellport, Aug, Nic (Not); Flushing, Mar (Not). 
1672 L. fuscata Dej. Generally distributed and common throughout the State.

L. esurialis Csy. Catskills, Pears (Sf).

1675 L. scapularis Dej. Manlius, Aug, SmHH (VdykNYS) ; Oneida L., Aug (Drk); Sylvan Beach, Je (Not); Oswego, J1; Rochester (M\&W); Buffalo (Z\&R) ; Ithaca, Aug (VdykCU) ; Tivoli, May (Not) ; NYC, Ap ( $\mathrm{LgAM}$ ) ; SI, Je, Aug ( $\mathrm{Lg})$; LI: Jamaica woods, Mar, Oct-Nov (Schaupp); Jamaica, Nic (Not); Rockaway Beach, May-Je, Shoe; Long Beach (AM); Forest Hills, Jan (Woodr).

1677 L. furcata Lec. Keene Val., Sep, Ds (Not) ; Long L., Aug, Gloversville, Je (NYS) ; Rochester (M\&W) ; Catskills, Nic (Not); LI: Quogue, Zab (Not); Bellport, Aug, Nic (Not); Selden, Aug, Wading R., Je, Rockaway Beach, May, Ds (Not); Yaphank, Jl, Ds.

1681 L. vittata Fab. "NY" (L\&B); Peekskill (VdykCU).

$1681 b$ L. vittata var. conjungens Lec. "NY" (Lec).

1682 L. pectita Horn. Shokan (Lg); West Farms (AM); Van Cort. Pk., Nic (Not); SI (div.); LI: Yaphank, May, Sep, Shoe; Richmond Hill, Jamaica, Bellport, J1-Aug, Nic (Not); Rockaway Beach, May, Ds (Not).

1686 L. bivittata Fab. Peekskill (VdykCU); LI: Rockaway Beach, Yaphank, May-Je, Oct, Shoe; Jamaica, Nic (Not).

\section{COPTODERA Dejean}

1688 C. aerata Dej. SI, Je ( Lg).

\section{DROMIUS Bonelli}

1691 D. piceus Dej. Keene Val., May-Aug (Not); Olcott, Je (Dt) ; Buffalo (Z\&R,Lg) ; E. Aurora (Dnd); Penn Yan, Mar, Cy (Vdyk); Ithaca, Fall Cr., Feb, Nov (Vdyk) ; White Plains, Mar, Ols (Not) ; Mosholu, Je (AM) ; Van Cort. Pk., Nov (Woodr); NYC (Lg); SI (Lg); LI: Wyandanch, Cold Sp., Yaphank, Pine Lawn, Promised Land, May-Sep, Ds (Not); Rockaway Beach, Sep (Woodr); Huntington, Dec (Not).

1692 D. atriceps Lec. LI: Rockaway Beach (Dietz).

\section{APRISTUS Chaudoir}

1693 A. cordicollis Lec. Newport, May (NYS) ; Lowville, Sodus Pt., Canadice, Westfield, Nichols, Windsor, Tivoli, Ft. Hunter, Catskills, May-Je (Not) ; Rochester; Pike, May, Oct (NYS) ; Norton's, May (VdykCU) ; Taughannock Gorge, Jl-Aug, Ithaca, May (Vdyk,Lg) ; SI, May, Shoe (Lg); LI: Rockaway Beach (VdykCU).

1694 A. subsulcatus Dej. Sodus Pt., Canadice, Mayville, Canisteo, Westfield, Nichols, May-Je (Not); Rochester (M\&W); Peekskill (VdykCU); West Pt. (Robn) ; SI: Je-Aug (L\&D) ; south shore, Aug (Lg); LI: Peconic Bay, Aug, Nic (Not); Southold, Sep, Shelter I., Aug, Ds (Not).

\section{BLECHRUS Motschulsky}

1696 B. glabratus Duft. St. Lawrence Co, Houghton; Mt. Whiteface, Aug (VdykCU) ; Olcott, Je, Sep (Dt) ; Canastota, Mar (Not) ; Oakfield, Je (Not); Ithaca, Ap (VdykCU); Catskills, Nic (Not); West Pt. (Robn) ; LI: Rockaway Beach, May, Shoe (Not,Ds).

1698 B. pusio Lec. LI: Jannaica, Nic (Not). 


\section{METABLETUS Schmidt-Goebel}

1699 M. americanus Dej. Keene Val., Keene Hgts., Mt. Marcy, Upper Ausable L., Mt. Haystack, Mt. Whiteface, Waterville, May-Sep (Not) ; Rochester $(\mathrm{M} \& \mathrm{~W})$; N. Elba, Oct ( Lg) ; St. Lawrence Co, Houghton; E. Concord (Dnd) ; Olcott, Ap, Je, Sep-Oct (Dt) ; Buffalo (Z\&R) ; Ithaca, Je-Aug, Hamilton, Fall Cr. Val., Nov, Dt (Vdyk); Karner, Mar (NYS); Catskills, Nic (Not); Peekskill (VdykCU); West Pt. (Robn); W. Hebron, NYC (Lg); SI (L\&D); LI: Bellport, Je, Aug, Nic (Not); Yaphank, Je, Ds (Not); Wyandanch, Oct (Not).

\section{Axinopalpus LeConte}

1700 A. biplagiatus Dej. St. Lawrence Co, Houghton; Keene Val., May, Lowville, Je (Not); Olcott, Aug (Dt); Peekskill (VdykCU); NYC (Schaupp) ; LI: Central Pk., Je, Shoe.

\section{Calleida Dejean}

1707 C. viridipennis Say. Buffalo (Z\&R, Say).

1710 C. punctata Lec. Norwood, Sep (Not); St. Lawrence Co, Houghton; Rochester (M\&W) ; Olcott, May (Dt) ; Buffalo (Z\&R) ; Ft. Erie, Sep, Julich (LgAM) ; Hamburg, Aug (NYS) ; Cattaraugus, Sep (Vdyk,Dt); Gowanda (Dnd); Castile, Brad (Flet); Canisteo, Je (Not); Tivoli, May (Not); White Plains (Frost,Sq); Bronx Pk. (NYS); NYC, Julich (Lg); SI (Lg); LI: Shoe; Brooklyn, Sep, Shoe.

1712 C. purpurea Say. LI: Jamaica woods, Je (Schaupp); Yaphank, May, Sep, Shoe; Babylon, Je (AM) ; Hempstead Bay, Aug (Not); Bellport, Ap, Nic (Not); Central Pk., May (div.); Fire I., Sep, Promised Land, Je, Riverhead, Aug, Ds (Not); Pinelawn, Sep (Ds); Hicksville (Champlain).

\section{Philophuga Motschulsky}

P. obscura Csy. NY, type locality.

\section{Plochionus Dejean}

1723 P. timidus Hald. Buffalo (Z\&R); NYC (Schaupp); SI, Je, Pears (Nic,Lg); LI: Jamaica, Pears (Nic); Brooklyn, Ji, Shoe (AM).

\section{PINACOEERA Schaum}

1725 P. limbata Dej. Rochester (M\&W) ; White L., J1, Zab (AM) ; Buffalo (Z\&R) ; Coy Glen, Aug, Inlet Val., Sep, Dt (Vdyk); Ithaca, Aug (VdykCU) ; Peekskill (VdykCU); West Pt. (Robn) ; Ramapo, Ap, Ds; Carmel, Aug (AM); NYC (Lg); SI, May-Je (div.) ; LI: Coney I., Je (Schaupp); Orient, May (NYS); Brooklyn, Rockaway Beach, May-Sep, Shoe; Babylon, Long Beach, Flatbush, May-Je (AM); Jamaica, Ap, Bellport, J1, Belle Terre, Je, Nic (Not) ; Far Rockaway, Yaphank, May-Aug (Woodr); Wading R., May, Ds (Not).

1726 P. platicollis Say. Rochester (M\&W) ; Ithaca, Mar-Ap, Dt (VdykCU); W. Hebron, Je (Lg); West Pt., Je, Ds (Not); Peekskill (VdykCU); Bronxville, Ap (Woodr); NYC (Lg); Arlington, Ap (Woodr; ; 
SI, Oct (L\&D) ; LI: Rockaway Beach, Je (VdykCU) ; Jamaica, Mar, Bellport, J1, Nic (Not); Yaphank, May (Not); West Hills, Ap, Ds (Not).

1726a P. platicollis var. fuscata Dej. "NY" (L\&B); Bronx Pk., SI, Ap, Shoe.

\section{CyMindis Latreille}

1738 C. cribricollis Dej. Mt. Whiteface, Shoe, Dt (Vdyk); N. Elba (Not); Minerva, Aug (Lg); Saranac L., J1, Sep, Shoe; Buffalo (Z\&R) ; Ithaca (VdykCU) ; Hebron ( $\mathrm{Lg}$ ).

1746 C. pilosa Say. Mountain View, Sep (Not); Westport, Sep, Nic (Not); Minerva, Aug ( $\mathrm{Lg}$ ); Gloversville, Sep (NYS); Manlius, SmHH (VdykCU); Buffalo (Z\&R); Ithaca, Jl-Aug, Dec, Hamilton (VdykCU) ; Albany, J1 (NYS) ; Catskills, Nic (Not); W. Hebron (Lg); Debruce, Sep (Not); West Farms (AM); Bronxville, Mar (Woodr); NYC (Lg); SI, May-Je (L\&D) ; LI: Rockaway Beach, Je, Shoe (Sf); Bellport, Aug-Sep, Nic (Not).

1747 C. borealis Lec. Elmira (NYS); NYC (Lg); LI: Rockaway Beach, Je, Shoe (Sf).

1748 C. neglecta Hald. Syracuse, Ap (H) ; Rochester (M\&W) ; Ithaca, Sep, Dt (Vdyk) ; Catskills, Nic (Not); Manlius, Feb (VdykCU); Shokan (Lg) ; NYC, SI ( Lg).

1749 C. cribrata Lec. St. Lawrence Co, Houghton.

1751 C. venator Dej. "NY" (Lec).

1752 C. americana Dej. Manlius, Nov, SmHH (Vdyk); Rochester (M\&W) ; Olcott, Ap (Dt) ; Buffalo (Z\&R) ; E. Aurora (Dnd) ; Caroline, McLean, Inlet Val., Ithaca, Ap-Sep, Dt (Vdyk); Schenectady, Dec (Dt) ; Catskills, Nic, Big Ind. Val., Aug (Not); West Pt (Robn); Mosholu (AM) ; Van Cort. Pk., Mar, Bronxville, Ap (Woodr); SI: (Lg) ; Clove Val., Ap (Lg) ; LI: Brooklyn, Ap, Oct, Shoe; Flatbush, Ap (AM).

\section{APENES LeConte}

1753 A. lucidula Dej. NYC, J1 (Woodr); SI, Je, Ds (Not); LI: Coney I., May-Je (Schaupp); Rockaway Beach, May, Shoe '(SfAM); Long Beach (NotAM); Fire I. ( Lg).

1756 A. sinuata Say. Peekskill (VdykCU); LI: Coney I., May-Je (Schaupp); Rockaway Beach, May-Je, Shoe (Sf AM); Long Beach (AM); Jamaica, Mar, Nic (Not); Far Rockaway, Sep (Woodr).

\section{HeLluomoRPHA Laporte}

i. \pm 5 H. nigripennis Dej. LI: Rockaway Beach, May, Shoe (Sf); Bellport, Je, Nic (Not).

1766 H. ferruginea Lec. Greenville, Je, Sep (Schaupp).

1769 H. praeusta Dej. "NY" (NYS).

1770 H. bicolor Harris. "NY" (Lec); West Pt. (Robn) ; Nyack, May (Lg) ; Mosholu (L\&B); Van Cort. Pk., Nic (Not); LI: Massapequa, ApMay, Shoe; Yaphank, Sep (Ds). 


\section{BRACHINUS Weber}

1774 B. americanus Lec. Rochester (M\&W) ; Pike, Je (NYS); Nassau, Oct (NYS).

1775 B. janthinipennis Dej. L. Onondaga (Lint); L. George, Nic (Not); Inlet Val., J1, Butternut Cr., May, Dt (Vdyk); Schoharie, Je (Not).

1776 B. viridipennis Dej. Unadilla, Sep, Nic (Not); Buffalo (Z\&R); Albany, Poughkeepsie, May (NYS) ; NYC (Schaupp) ; Croton R., May (Ds) ; SI, May-Je (div.).

1776a B. viridipennis var. perplexus Dej. Olcott, Ap (Dt); McLean Bogs, Caroline, Ithaca, Ap-Sep, Dt (Vdyk); LI: Orient, Lath (NYS).

1777 B. pulchellus Blatch. Olcott, Ap-May, Oct (Dt) ; LI: Jamaica, Ap, Nic (Not); Flushing, Mar (Not); Orient, Lath (NYS).

1778 B. minutus Harris. L. Onondaga (Lec); Chautauqua (Lg); Schoharie, Je (Not); W. Hebron (Lg AM); Catskills, J1 (AM) ; NYC (Schaupp) ; LI: Wading R., Aug, Ds (Not).

1779 B. medius Harris. L. Onondaga (Lec); Rochester (M\&W) ; Buffalo (Lg AM) ; Ithaca, Aug, McLean Bogs, J1-Aug, Dt (Vdyk); W. Hebron (Lg); West Pt. (Robn); SI (Lg); LI (Lg).

1780 B. quadripennis Dej. NYC, Schaupp (L\&B); Westfield, May (Not).

1782 B. conformis Dej. Buffalo (Z\&R, Dej ANSP); Pike, May (NYS); White Plains, Aug (Sq).

1783 B. cyanipennis Say. Olcott, Ap, J1 (Dt) ; Batavia, Je (L\&D) ; Genesee Co, Je (Ds); Buffalo (Z\&R); McLean Bogs, Ringwood, Ithaca, Caroline, Ap-Aug, Dt (Vdyk); Schoharie, Je (Not); NYC (Schaupp).

1784 B. gracilis Blatch. Sodus Pt., Je (Not); Westfield, May (Not).

1786 B. alternans Dej. Buffalo (Z\&R); West Pt. (Robn) ; NYC (Schaupp); LI: Rockaway Beach (Not AM).

1788 B. ballistarius Lec. Olcott, Ap, Sep (Dt); E. Aurora, common (Dnd) ; Ithaca, McLean Bogs, Taughannock Gorge, May-Sep, Dt (Vdyk); Windsor, May (Not); Catskills, Nic (Not); LI: Jamaica, Nic (Not).

1789 B. fumans Fab. Lowville, Je (Not) ; Olcott, J1 (Dt) ; E. Aurora, common (Dnd) ; Buffalo (Z\&R); Pike, May (NYS); Batavia, Westfield, Windsor, Schoharie, May-Je (Not); Danby, McLean Bogs, Caroline, Taughannock Gorge, Ithaca, May-Sep, Dt (Vdyk); Karner, J1 (NYS) ; Croton R., May (Ds); W. Hebron (Lg); NYC, May, J1 (Woodr); SI, Nic (Not) ; LI: Brooklyn, May, Shoe; Flatbush, May, Zab (AM); Jamaica, Bellport, Nic (Not); Far Rockaway, May (Woodr).

1789a B. fumans var. similis Lec. Buffalo (Z\&R).

1790 B. cordicollis Dej. Mooers, Sep, Schoharie, Je (Not); Rochester (M\&W); Buffalo (Z\&R); Six Mile Cr., Ap (Morr); Ithaca, McLean, Taughannock Gorge, May-Sep, Dt (Vdyk); Kenwood, May (NYS); West Pt. (Robn) ; LI: Jamaica, Ap (AM) ; E. New York, May, Ds (Not); Rockaway Beach, May, Ds, Flushing, Mar (Not).

B. ovipennis Lec. Buffalo ( $Z \& R)$.

B. rejectus Lec. SI: Clove Val., May, Ds (Not).

MiscoderA Eschscholtz

1801 M. arctica Payk. Keene Val., Je-J1 (Not). 


\section{Chlaenius Bonelli}

1806 C. tomentosus Say. Axton, Je (M\&H) ; Severance, Je, Gloversville, Sep (NYS) ; Wilmington, Aug (Dt) ; Keene Val. (Not) ; Pulaski, Je, Ds (Not); Olcott, Je, Aug (Dt); Buffalo (Z\&R); Ithaca, Je (Dt); W. Hebron, Je (Lg); Unadilla, Sep, Nic (Not); West Pt. (Robn); Mosholu (AM) ; NYC, Bronx, Ap (Woodr) ; SI (div.) ; LI: Rockaway Beach, Brooklyn, May-Je, Shoe; Long Beach (AM) ; Jamaica, Bellport, J1, Nic (Not); Amagansett, Sep (Not, Ds); Orient, May (Not, Ds).

1808 C. purpuricollis Rand. LI: Far Rockaway, Sep (Woodr).

1810 C. niger Rand. Rochester (M\&W) ; Ithaca, Sep (Dt) ; Van Cort. Pk., Bradford (AM) ; Manhattan, Je (AM) ; LI: Brooklyn, Ap (Woodr): Canarsie, Ap (Not); E. New York, May-Je (Not, Ds) ; Flushing, Mar (Not).

1814 C. impunctifrons Say. Essex Co, Aug (NYS); Crown Pt., Waterville, Westfield, Mayville, Canadice, Nichols, Orangeburg, Tivoli, Ap-Aug (Not) ; Olcott, Ap, J1 (Dt) ; E. Aurora, Buffalo (Dnd) ; Genesee Co, Je (Ds); Batavia, Je, Chautauqua (Lg); Bronx Pk., West Farms (AM) ; Van Cort. Pk., Ap (Not, Woodr) ; Bronx, Mar (Woodr) ; SI, Je (Ds) ; LI: Flatbush, Ap (Not); Brooklyn, May, Nic (Not).

1817 C. pennsylvanicus Say. Generally distributed and common throughout the State, Ap-Sep.

1819 C. brevilabris Lec. Lowville, Westfield, Nichols, Canisteo, Whitehall, MayOct (Not); Syracuse, Ap (H) ; Batavia, Je, Kngt (Morr); Ithaca, Ap-May (Morr); Unadilla, Sep, Nic (Not); LI (Lec).

1821 C. tricolor Dej. Generally distributed and common throughout the State, Ap-Oct.

1822 C. nemoralis Say. Rochester (M\&W); western NY, frequent (Dnd) ; Batavia, Je (Lg); Genesee Co, Je, Ds (Not); Buffalo (Z\&R) ; Westfield, May (Not); Ithaca, Ap-Aug (div.); McLean, Caroline, Ap, Aug (Dt); Catskills, Nic (Not); West Pt. (Robn); West Farms, Mosholu (AM) ; NYC, Bronx, Mar-Aug (Woodr); SI, Nic (Not, Lg); LI: Brooklyn, May-Aug, Shoe; Long Beach (AM) ; Flatbush, May-Aug, Zab (AM) ; Jamaica, Nic (Not); Hauppauge, Ap (Not); Rockaway, Central Pk., Ap-May, Ds (Not); Calverton, Sep, Yaphank, J1, Ds (Not).

1827 ?C. vafer Lec. Buffalo (Z\&R). Described from Tex. (Lg).

1830 C. solitarius Say. Buffalo (Z\&R).

1831 C. leucoscelis Chev. Ausable Pt., Crown Pt., Sodus Pt., Westfield, Canisteo, Nichols, Whitehall, Ft. Hunter, May-Oct (Not); Rochester (M\&W); Lakes Erie and Ontario; Olcott, Ap, J1 (Dt); Buffalo (Lg) ; Ithaca, May-Je (div.) ; Taughannock Gorge, May, Aug (Dt) ; North Branch, Aug (Schaupp); Croton R. (L\&B); Suffern, Sf (Lg AM); LI: Forest Lawn, Aug (Not AM).

1831a C. leucoscelis var. cordicollis Kby. Buffalo (Z\&R).

1934 C. prasinus Dej. Rochester (M\&W) ; Buffalo (Z\&R); E. Aurora (Dnd) ; Westfield, May (Not).

1838 C. aestivus Say. Buffalo (Z\&R); Ithaca, Sep (Dt); Albany, Poughkeepsie, May (NYS) ; Tivoli, May (Not); Ft. Montgomery, May (Not) ; Peekskill (Lg); Palisades-on-Hudson, Ap (Not AM); West Pt., Je, Ds (Not); Nyack, Zab (AM); White Plains, Ap (Sq); Van 
Cort. Pk. (div.); Bronx, Mar-Ap (Woodr); Mosholu, May (Ds);

NYC, Sep (Vdyk); Yonkers, Ap,-Bronxville, Ap-May (Woodr); SI: May-Sep (div.) ; Clove Val., J1, Ds (Not); LI: Jamaica, Nic (Not); Wading R., Aug, Ds (Not).

1841 C. diffinis Chd. Van Cort. Pk., Nic (Not); LI: Rockaway Beach, Ap (AM) ; Jamaica, Brooklyn, E. New York, Ap, Nic (Not).

1842 C. laticollis Say. Buffalo (Z\&R); Ithaca, Oct, Flet (Dt); Van Cort. Pk. (Not AM) ; SI, Ap, Ds; LI: Rockaway Beach, Brooklyn, Ap-May, Shoe (AM); Bergen Beach, Ap (Not AM) ; Jamaica (div.) ; E. New York, Rockaway, May, Ds (Not).

1846 C. sericeus Forst. Generally distributed and common throughout the State, Mar-Oct.

1847 C. fuscicornis Dej. Buffalo ( $Z \& R)$.

1848 C. erythropus Germ. Buffalo (Z\&R); LI: Jamaica, Nic (Not).

\section{ANOMOGLOSSUS Chaudoir}

1854 A. delectans Csy. Central New York (Csy).

1856 A. emarginatus Say. Wallface Mt., J1, Nic (Not) ; Keene Val., J1 (Not); Rochester (M\&W) ; Olcott, Je (Dt) ; E. Aurora (Dnd) ; Castile, Brad (Flet) ; Pike, May (NYS) ; McLean, May, Ds (Not) ; Ithaca, Freeville, Taughannock Gorge, May-Sep (Dt); Peekskill (Lg) ; Mosholu, West Farms, Dunwoodie, Van Cort. Pk. (AM) ; NYC, Van Cort. Pk., Ap (Woodr) ; SI, Ap (L\&D) ; LI: Jamaica, May-Oct (Schaupp); Rockaway Beach, Je, Shoe; Parkville, Long Beach (AM) ; Rockaway, Aug, Zab (AM); Half Way Hollow Hills, J1, Yaphank, Je, Ds (Not).

1858 A. pusillus Say. Buffalo (Z\&R) ; Mosholu (AM); Bronx, Ap, NYC, Aug (Woodr) ; SI, Mar-Oct (Lg) ; LI: May-Sep (Schaupp); Rockaway Beach, Je, Shoe (AM) ; Coney I., Lg.

A. coeruleicollis Chd. Van Cort. Pk. (AM) ; SI, Nic (Not) ; LI: Babylon, J1 (AM); Jamaica, Nic (Not).

\section{BRACHYLOBUS Chaudoir}

1860 B. lithophilus Say. St. Lawrence Co, Houghton; Mooers, Waterville, Canisteo, Westfield, Nichols, Tivoli, Schoharie, May-Sep (Not); Rochester (M\&W) ; Buffalo (Z\&R); Olcott, May (Dt); McLean, Ithaca, Aug-Sep (Dt); W. Hebron, Je (Lg); Van Cort. Pk., J1 (Not AM) ; Suffern, West Farms (AM) ; SI (L\&D) ; LI: Brooklyn (Not) ; Jamaica, Ap-May, Ft. Hamilton, Ap (Woodr); Canarsie, Ap (Not); E. New York, May, Ds (Not).

\section{LACHNOCREPIS LeConte}

1862 L. parallelus Say. Rochester (M\&W) ; Sodus Pt., May-Je (Not) ; Buffalo (Z\&R) ; Erie Co, May-Aug (Dnd); Ithaca, Mar-Ap, Dt (Vdyk); Tivoli, May (Not); White Plains, Mar (Sq); LI: Ridgewood, Je (Schaupp); Brooklyn, Ap-May, div. (Not); Jamaica Bay, Ap (Woodr); Canarsie, May (Not); E. New York, May-Je (Not).

ANATRICHIS LeConte

1863 A. minuta Dej. LI: Huntington (Schott). 


\section{OODES Bonvouloir}

1867 O. amaroides Dej. Rochester (M\&W) ; Ulster Co, May, Ji, Shoc; White Plains, May-Aug (Sq); SI (Lg) ; LI: Ridgewood (Schaupp); Brooklyn, May, J1, Shoe; Babylon (AM); Wading R., Aug, Ds (Not).

1868 O. americanus Dei. Rochester (M\&W); Buffalo (Z\&R); McLean Bogs, Jl, Dt (Vdyk) ; Tivoli, May (Not); Catskill (Lg); Pine I., Je, Ds (Not); Van Cort. Pk., Ap (Woodr); NYC (Lg); LI: Brooklyn, May (div.).

1869 O. fluvialis Lec. Buffalo (Z\&R); Catskill (Not, Lg); NYC (Lec).

1870 O. cupreus Chd. Buffalo (Z\&R).

\section{Geopinus Leconte}

1878 G. incrassatus Dej. Gloversville, May (NYS); Rochester (M\&W) ; Buffalo (Z\&R) ; Karner, May (NYS); Greenville (Schaupp); Catskill (Not, Lg) ; SI, Je (Lg); LI: Coney I., Rockaway, May-Sep (Schaupp); Rockaway Beach, Je. (AM); Jamaica, May, Nic (Not).

\section{CRATACANTHUS Dejean}

1886 C. dubius Beauv. Buffalo (Z\&R); NYC (Lg); LI: Coney I., Je-J1 (Schaupp); Jamaica, Nic (Not).

HARPALUS Latreille

1896 H. caliginosus Fab. Keene Val. and Port Kent (Not); Glens Falls, Je, Nassau, Sep, Albany, Je (NYS); Junius, Aug (Dt); Ilion, Pike, Clinton Hgts., Aug (NYS); White L., Aug, Zab (AM); Monroe Co $(\mathrm{M} \& W)$; Olcott, Je-J1 (Dt) ; Buffalo (Z\&R); E. Aurora, common about dry fields (Dnd); Batavia, Je (Lg); Castile, Brad (Flet); Elmira, Je (Diven) ; Ithaca, May-J1 (Dt); Nichols, May (Not); W. Hebron, Je ( $\mathrm{Lg}$ ); Columbia Co, Sep; Whitehall, Oct (Not); West Pt. (Robn); Nyack, Zab (AM); Van Cort. Pk., J1 (NotAM) ; NYC, Je-J1 (Woodr); SI, Je-Sep (Lg,Ds); LI: many localities and collectors.

1897 H. erraticus Say. Keene Val., J1 (Not); Essex Co, J1 (NYS); St. Lawrence Co, Houghton; Mountain View, Norwood, Ausable Forks, Keene, Ausable Pt., Mooers, Aug-Sep (Not); Rochester (M\&W); Olcott, May-J1, Oct (Dt) ; Buffalo (Z\&R); Schenectady (Dt) ; L. George, Aug, Zab (AM); New Baltimore, Jl, Shoe (AM); Whitehall, Oct (Not); SI, Aug (L\&D); LI: Aqueduct, Sep, Shoe; Woodhaven (AM) ; Fishers I., Aug, Zab (AM); Bellport, Nic (Not); Selden, Ds (Not).

1898 H. caudalis Csy. "NY" (C.sy).

1903 H. viridiaeneus Beauv. Castile, Brad (Flet); generally distributed and common throughout the State, Je-Sep.

1907 H. vagans Lec. Mooers, Lowville, Canisteo, L. Waccabuc, Nichols, MayAug (Not); Glens Falls, J1 (NYS) ; Monroe Co (M\&W) ; Inlet Val., McLean Bogs, Freeville, Ithaca, Renwick, May-Sep (Dt).

1909 H. haldemani Csy. LI (Csy). 
1910 H. erythropus Dej. Keene Val., J1 (Not); Buffalo (Z\&R); Westport, Newport, Pike, Albany, Poughkeepsie, Je-Aug (NYS); Montour Falls, Ap (H) ; E. Aurora, Aug-Sep (Dnd); Big I., Sep (Not) ; Olcott, Sep (Dt); Ithaca, Ringwood, J1-Sep (Dt) ; Carmel, Aug (AM) ; NYC, Aug-Sep (Woodr); Bronxville, Aug (Woodr); SI, Aug (L\&D) ; LI: Aqueduct, Sep; Brooklyn, Aug, Shoe; Bergen Beach (NotAM):; Jamaica, Oct, Bellport, Je-Oct, Nic (Not).

1913 H. effetus Csy. LI: Willetts Pt. (Csy).

1915. H. compar Lec. Saranac L., Sep (AM); Wilmington, J1, Ds (Not); Syracuse, Ap (H) ; western NY, common (Dnd) ; Olcott, Je-J1 (Dt) ; Monroe Co (M\&W) ; Buffalo (Z\&R); Oakfield, Je (Not) ; Canisteo, Je (Not) ; Ithaca, Ap, J1, Sep (Ds) ; Inlet Val., Butternut Cr., MaySep (Dt); Whitehall, Oct, Parkers, Je (Not); Windham, Oct (Dt) ; Ft. Hunter, Je (Not) ; NYC (Schaupp, Lg); LI: Astoria (AM); Forest Pk., Je (NotAM); Brooklyn, Oct (Not); Bellport, J1-Aug, Jamaica, Nic (Not) ; Selden, Aug, Ds (Not).

1920 H. longicollis Lec. Keene Val., J1 (Not); Newport, J1 (NYS); Thousand Isls. (Ds); Genesee Co, Ap, Kngt (Not,Ds); Batavia, May, Kngt (Not) ; western NY (Dnd) ; Elmira, Je (Diven) ; Ithaca, J1, Hamilton (Vdyk) ; L. Waccabuc, J1 (NotAM) ; Whitehall, Oct (Not) ; SI, J1Aug (L\&D) ; LI: Flushing, Ap (Not).

1921 H. dolosus Csy. Plattsburg (Csy).

1922 H. faunus Say. Ausable Chasm, Sep (Not); Monroe Co (M\&W) ; Buffalo (Z\&R) ; Ithaca, Aug (Dt,Lg); Albany, J1 (NYS); Whitehall, Oct (Not); Van Cort. Pk., Nic (Not) ; SI, Ap, Sep (div.) ; LI: Jamaica, Bellport, Aug-Sep, Nic (Not).

1925 H. pennsylvanicus Dej. Generally distributed and common throughout the State, Mar-Sep.

1926 H. convivus Lec. Wilmington, J1, West Pt., Je, Ds (Not) ; LI: Rockaway Beach, Je, Ds (Not).

1933 H. viduus Lec. Rock City, Je (Not) ; Ithaca, McLean Bogs, May, Aug, Hamilton (Vdyk); Windsor, Je (Not); Ramapo, Ap (Ds).

1934 H. laticeps Lec. Keene Val., May (Not); Axton, Je (M\&H-AM); E. Aurora (Dnd); Ithaca, May, Butternut Cr., J1 (Dt); Caroline, Ap (Dt).

1936 H. rufimanus Lec. Keene Val., Je-Aug (Not) ; Elmira, Ap (Diven) ; Ithaca, Ap, Alex (Not); Catskills, Nic (Not).

1944 H. pleuriticus Kby. Generally distributed and common throughout the State, Ap-Sep.

1956 H. herbivagus Say. Generally distributed and common throughout the State, Ap-Sep.

1957 H. fallax Lec. Batavia, Je, Ds ( Lg) ; Castile, Brad (Flet) ; Ithaca, Sep, Caroline, Ap (Dt) ; Karner, May, Albany, Je (NYS); Catskills, Nic (Not); Peekskill (Ds) ; LI: Rockaway Beach, May (Woodr).

1959 H. placidus Csy. LI: Willetts Pt. (Csy).

1968 H. opacipennis Hald. Keene Val., May-J1 (Not) ; Mt. Skylight, J1 (Not); Sodus Pt., May (Not) ; Ithaca, Ap, Alex (Vdyk); Debruce, Sep (Ds) ; NYC (Lg).

1971 H. nitidulus Chd. LI: Coney I. (Schaupp).

1978 H. fulvilabris Marn. Mt. Hopkins, Je, Mt. Marcy, Jl, Sodus Pt., May (Not). 
1981 H. lewisi Lec. Keene Val. (Not).

1982 H. aesopus Csy. Plattsburg (Csy).

2000 H. gravis Lec. Buffalo (Z\&R); LI: Orient Pt., May (NYS).

2006 H. obesulus Lec. Sodus Pt., May (Not).

2007 H. spadiceus Dej. Newport, J1, Ilion, May, Pike, Je, Albany, Je (NYS); Monroe Co (M\&W); Buffalo (Z\&R) ; Chautauqua (Lg); Ithaca, May, Sep (Dt); W. Hebron (Lg); Catskill (Not, Lg); NYC (Not, Lg); SI, Ap (Lg) ; LI: Brooklyn, Ap, Sep-Oct, Shoe; Jamaica, Nic, Yaphank, J1, Ds (Not).

2009 H. vulpeculus Say. Monroe Co (M\&W) ; Chautauqua (Lg); Rock City, Je, Ds (Not); Ithaca, Je-Sep (Vdyk, Dt) ; Windsor, May (Not); W. Hebron (Lg); Catskills, Nic (Not); West Pt. (Robn); Van Cort. Pk., Nic (Not); Bronxville, Ap (Woodr); SI, Mar (L\&D); LI: Jamaica, E. New York (Schaupp) ; Brooklyn, Aug, Oct, Shoe; Queens, Nov, Nic (Not).

2010 H. dichrous Dej. Monroe Co (M\&W) ; Olcott, Je, Oct (Dt) ; Ithaca, J1 (Dt); W. Hebron (Ds); New Baltimore, J1, Shoe (AM); Van Cort. Pk., Sep (NotAM) ; SI, Je-Sep (Ds) ; LI: Jamaica (Not, Schaupp); Rockaway (AM).

H. immixtus Csy. NY, type locality ( $\mathrm{Lg})$.

H. admissus Csy. NY, type locality $(\mathrm{Lg})$.

H. amiculus Csy. NY, type locality ( $\mathrm{Lg})$.

\section{SELENOPHORUS Dejean}

2013 S. opalinus Lec. Monroe Cc (M\&W) ; Olcott, May-Je (Dt) ; Ithaca, Je-J1, McLean, J1 (Dt) ; Coy Glen, Je, Ds (Not); L. George, Aug, Zab (Not); Shokan ( $\mathrm{Lg})$; Huguenot, Aug, Zab (Not); Orange Co, Ap (Morrison); Van Cort. Pk. (AM); NYC, Je-Jl, Bronxville, Ap (Woodr) ; SI, Ap, Je-J1 (L\&D) ; LI: Long Beach (AM) ; Jamaica, Je (Schaupp); Yaphank, Aug (NotAM); Wading R., May (AM); Far Rockaway, Je (Woodr); Rockaway Beach, Je, Ds (Not).

2015 S. depressulus Csy. Monroe Co (M\&W) ; Buffalo (Z\&R); Ithaca, J1.

2017 S. gagatinus Dej. E. Aurora (Dnd); Ithaca, Ap, J1 (Dt); Catskills, Nic (Not); LI: Coney I. (Schaupp); Rockaway Beach, Je-Jl, Shoe (AM); Wyandanch, J1, Ds (Not).

2024 S. fossulatus Dej. LI: Rockaway Beach, May, Shoe.

2026 S. ellipticus Dej. NYC (AM); Bronxville, Ap, Van Cort. Pk., Je (Woodr); SI, Sep (Lg); LI: Brooklyn, Rockaway Beach, May-Je, Shoe (DsAM); Jamaica, Nic (Not).

2027 S. currens Csy. Catskills (Csy).

2039 S. pedicularius Dej. Monroe (M\&W); SI (div.); LI: Long Beach (AM) ; Jamaica, Bellport, Je-Sep, Nic (Not).

\section{GYNANDROPUS Dejean}

2051 G. hylacis Say. Monroe Co (M\&W) ; Olcott, May (Dt); E. Aurora, rare (Dnd); Pike, Mar, Albany and Karner, Ap (NYS); Ithaca, J1-Aug (Vdyk); Peekskill (Lg); West Pt. (Robn) ; Huguenot, Aug, Zab (Not) ; NYC, Je (Woodr) ; Van Cort. Pk., Sep (NotAM) ; SI, Feb (div.) ; LI: Woodhaven, May (Vdyk); Rockaway Beach, Ap-J1, Shoe (Not); Jamaica, Ap, Nic (Not); Far Rockaway, Je (Woodr). 


\section{DISCODERUS LeConte}

2064 D. parallelus Hald. Catskills, Nic (Not); LI: Babylon, J1 (AM).

\section{TRIPLECTRUS LeConte}

2070 T. carbonarius Say. Newport, Je, Gloversville, Ap, Albany, May (NYS) ; Monroe Co (M\&W); Westfield, May (NotNYS); NYC, Aug (Woodr) ; SI, Ap, Lg, Ds (Not) ; LI: Flatbush, Ap (NotAM); Prospect Pk., Feb (Not); Rockaway Beach, Mar, Nic (Not); Brooklyn (Lec).

2071 T. rusticus Dej. Castile, Brad (Flet); generally distributed and common throughout the State, Ap-Sep.

2072 T. crassus Lec. "NY" (Lec).

T. kempi Csy. NY, type locality ( $\mathrm{Lg}$ ).

\section{Anisodactylus Dejean}

2087 A. harrisii Lec. St. Lawrence Co, Houghton; Norwood, Sep . (Not) ; Newport, Pike, Albany, May (NYS) ; Olcott, Ap, Je, McLean, J1, Ithaca, Ap-J1 (Dt); Johnstown, Genesee Co, Windsor, Centre I., Chauncey, Canisteo, Whitehall, Orangeburg, Catskill, Ft. George, Ft. Hunter, Sloatsburg, NYC, Ap-Sep (Not); Syracuse, Ap (H); West Pt. (Robn); Tarrytown, May (AM); Bronx, Bronxville, Ap (Woodr) ; SI, Ap-Je (Ds) ; LI, fairly common, Ap-Je (div.).

2088 A. nigèrimus Dej. Norwood, Johnstown, Westfield, Greenwood L., Windsor, Nichols, Whitehall, Sloatsburg, Dunwoodie, Ap-Oct (Not); Monroe (M\&W) ; E. Aurora, Hamburg (Dnd) ; Ithaca, Mar-J1 (Dt, Vdyk) ; Catskills, Nic,Lg (Not) ; Van Cort. Pk., Mar (NotAM) ; SI, Mar-May (Lg) ; LI: Bergen Beach, May (NotAM) ; Cypress Hills, May, Zab (Not); Rockaway Beach, Ap, Bellport, J1-Aug, Nic (Not).

2089 A. melanopus Hald. Westfield, May, Greenwood L., May, Chauncey, Mar (Not); West Pt. (Robn); White Plains, Mar, Ols (Not); Van Cort. Pk., Mar (WoodrAM) ; LI: Bergen Beach, May, Cypress Hills, Je-J1, Nov (NotAM) ; Rosedale, Ap (Not).

2090 A. nigrita Dej. St. Lawrence Co, Houghton; Waterville, Je (Not) ; Buffalo (Z\&R) ; Monroe Co (M\&W); Ithaca, May, Nov (Dt) ; Nichols, May (Not); West Pt. (Robn); NYC; Bronx, Mar, Nic (Not); SI, Ap (div.) ; LI: Brooklyn, May, Oct, Shoe (AM) ; Cypress Hills, Ji (Not) ; Jamaica, Ap-May, Nic (Not); E. New York, May (Not); Calverton, Sep (Not).

2091 A. interpunctatus Kby. Axton, Je (M\&H) ; Batavia, May, Kngt (Not) ; Olcott, Je (Dt); Niagara Falls (Lec); Montour Falls, Ap (H) ; western NY, common (Dnd) ; Potter Swamp, Je (Not); McLean Bogs, J1, div. (Vdyk) ; Whitehall, Oct (Not); White Plains, Sep, Sq (Sf) ; LI: Jamaica, Mar, Nic (Not) ; Brooklyn, May, Ds (Not) ; E. New York, May, Rockaway Beach, Je, Ds (Not); Flushing, Mar (Not).

2092 A. agricola Say. Olcott, Aug-Sep (Dt) ; Pike (NYS) ; Bronx, May, Nic (Not).

2094 A. semipunctatus Lec. Western NY, common (Dnd).

2097 A. sericeus Harr. LI: Orient, Lath (NYS). 
2107 A. coenus Say. Monroe Co (M\&W) ; NYC (div.); SI, Je ( $\operatorname{LgAM}$ ); LI: Rockaway Beach (AM); Flatbush, Ap-Je, Zab (Not); Jamaica, Nic (Not); Brooklyn (Lec).

2108 A. laetus Dej. NYC (L\&W).

2109 A. similis Lec. Buffalo (Z\&R). Described from Oregon ( $\mathrm{Lg})$.

\section{XESTONOTUS LeConte}

2110 X. lugubris Dej. St. Lawrence Co, Houghton; Keene Val., Ap-Je (Not); Olcott, Je (Dt) ; E. Aurora, Mar (Dnd) ; Niagara Falls (Lec) ; Westfield, May (Not); Cortland Co (Dnd); Ithaca, Oct, McLean, Aug, Nov (Dt); Catskills, Nic (Not) ; Catskill (Not,Lg) ; Croton L., Ap (Not); White Plains, Ap-May (Sq); Bronx Pk., Ap (Woodr); SI, May (Lg); LI: Parkville (AM); Bergen Beach, Ap (NotAM); Jamaica, Ap, Nic (Not); Flushing, Mar (Not).

\section{AMPHASIA Newman}

2111 A. interstitialis Say. Monroe Co (M\&W); Syracuse, Montour Falls, May-Je (H) ; Olcott, Oct (Dt); Buffalo (Z\&R); Genesee Co, Je (Lg); Batavia, Je (Lg); Castile, Brad (Flet); Ithaca, Ap, Sep (Dt); Trenton Falls (Not); Rensselaer, Albany, Poughkeepsie, Ap, Oct (NYS) ; Concord (NotAM); Schoharie, Whitehall, Tivoli, L. Mohonk, Je-Sep (Not); West Pt. (Robn); Ramapo, Je (Lg); Van Cort. Pk., May (div.) ; Bronx, Mar-Ap (Woodr); SI, Feb-Nov (L\&D); LI: Queens, Nov, Jamaica, Mar, Nic (Not); Far Rockaway, May (Woodr); Pinelawn, Je (Ds).

\section{Pseudamphasia Casey}

2112 P. sericea Harr. Olcott, May (Dt); Monroe Co (M\&W) ; Buffalo (Z\&R); Ithaca, Ap-J1 (Dt) ; Albany, J1, Hill (NYS); Ft. Hunter, Je (Not); West Pt. (Robn); Nyack, Zab (NotAM); NYC (Schaupp); SI, May-Je (L\&D); LI, rather common.

\section{Anadaptus Casey}

2126 A. discoideus Dej. Ausable Lakes, J1-Aug, Keene, Mountain View, Lowville, Pulaski, Canisteo, Westfield, Windsor, Schoharie, Nichols, Ft. Hunter, May-Oct (Not); Monroe Co (M\&W); Buffalo (Z\&R); E. Aurora, Gowanda (Dnd) ; Newport, Albany, Je (NYS) ; Ithaca, Je-J1 (Dt); West Pt. (Robn); W. Hebron, Je (Lg); NYC, Je (Woodr); SI, May (Lg).

2127 A. baltimorensis Say. Castile, Brad (Flet); generally distributed and common throughout the State, Ap-Sep.

\section{ANISOTARSUS Chaudoir}

2132 A. sayi Blatch. (piceus Lec.). Junius, Aug (Dt); Ithaca, Aug (Lg); West Pt. (Robn); SI, Ds (Not); LI: Fishers I., Aug, Zab (Not); Calverton, Yaphank, Wading R., J1, Sep, Ds (Not). 
2139 A. terminatus Say. Monroe Co (M\&W) ; Buffalo (Z\&R) ; Cattaraugus Co, J1 (Not); Ithaca, J1-Aug (Dt); White Plains, Sq (Sf); NYC, J1-Sep (div.) ; Bronx Pk., Yonkers (AM); SI, Jl-Aug (L\&D) ; LI: Cold Sp. Harb., J1 (NotAM) ; Flatbush, Aug, Zab (Not); Bellport, J1-Aug, Nic (Not); Rockaway Beach, May, Far Rockaway, J1 (Woodr).

2143 A. nitidipennis Lec. E. Aurora, Boston (Dnd); Ithaca, May (Dt); Mosholu, May (Not,Ds); LI: Wading R., May, Nic (Not).

\section{SPONGOPUS LeConte}

2144 S. verticalis Lec. Monroe Co (M\&W) ; Batavia, May, Kngt (Not) ; Westfield, May (Not); Ft. Hunter, Je (Not); NYC (Schaupp); Bronx, Van Cort. Pk., Mar-Ap (Woodr); LI: Lawrence, Rockaway, Ap (Woodr).

TRICHOCELLUS Ganglbauer

2146 T. cognatus Gyll. West Pt., Robn (Csy); NYC (Schaupp).

\section{Glycerius Casey}

2151 G. nitidus Dej. "NY" (DejANSP).

\section{EPiscopellus Casey}

2156 E. autumnalis Say. Buffalo (Z\&R); Ithaca, Je-J1, Sep (Dt,Lg) ; Cortland Co (Dnd); West Pt (Robn); SI: Tottenville, May, Ds (Not); LI: New Jamaica, E. New York (Schaupp); Babylon, J1 (AM); Rockaway Beach, Queens, Nic (Not); Canarsie, Ap (Not).

\section{TACHyCELLUS Morawitz}

2158 T. nigrinus Dej. Keene Val., Keene Hgts., Ausable Lakes, Sodus Pt., Mayville, May-J1 (Not); Whiteface Mt., Sep (Lg); Renwick, Ap, Caroline, Ap, Dt (Vdyk); West Pt. (Robn).

\section{TRILIARTHRUS Casey}

2159 T. badiipennis Hald. Whiteface Mt., Upper Ausable L., Keene Val., Portage, May-Aug (Not); N. Elba, Oct (Lg); Monroe Co (M\&W); Buffalo (Z\&R) ; Caroline, Ap, Ithaca, Mar-May, Dt (Vdyk); Catskills (Lg); West Pt (Robn); Ramapo Mts., May (AM); NYC (Schaupp); Bronx (Woodr) ; Van Cort. Pk., May (Not) ; SI, May (Lg); LI: Far Rockaway, May (Woodr); Rockaway Beach, May, Long Beach, J1, Ds (Not); Jamaica (Lg); Rosedale, Ap (Not).

2161 T. properus Csy. West Pt. (Csy).

2162 T. kirbyi Horn. Waterville, Je, Sodus Pt., Je, Batavia, May, Canisteo, Je, McLean, Ap (Not) ; Ithaca, May-Je, Sep, Dt (Vdyk); LI: Rosedale, Ap (Not).

2163 T. atrimedius Say. Monroe Co (M\&W); Buffalo (Z\&R); Canisteo, Nichols, Orangeburg, Tivoli, Ap-Je (Not); NYC (Schaupp). 


\section{Catharellus Casey}

2167 C. cordicollis Lec. Keene Val., May-J1 (Not); L. Tear, J1 (Not); Manitou Beach, Je, Zab (Not).

\section{Stenocellus Casey}

2169 S. antennalis Csy. Catskills (Csy).

2170 S. insulsus Csy. NYC (Csy).

2171 S. rupestris Say. Generally distributed and common throughout the State, Feb-Oct.

2172 S. occultus Csy. Batavia, J1-Aug, Kngt, Canisteo, Je (Not) ; L. Champlain, Catskills (Csy).

2173 S. debilipes Say. Lowville, Nichols, Sodus Pt., Schoharie, Windsor, Ft. Hunter, May-Je (Not); LI: Nissequogue, Canarsie, Wading R., May-Je (Not); Syracuse, Montour Falls, Ap-May (H).

S. cinctus Say. "NY" (Csy).

2175 S. congener Lec. West Pt., Robn (Csy).

2195 S. tantillus Dej. Keene Val., L. Tear, Cape Vincent, Port Kent, Sodus Pt., Oakfield, Nichols, Ft. Hunter, Windsor, May-Sep (Not); Olcott, Feb, J1 (Dt) ; E. Aurora (Dnd) ; Ithaca, Mar, J1, McLean, J1 (Dt) ; SI, Dec-Mar (AM,Lg); LI: Rockaway Beach, Ap-May, Shoe; Bellport, J1, Nic (Not).

2198 S. neglectus Lec. Buffalo (Z\&R) ; Ithaca, Je-J1, Hamilton (Vdyk) ; West Pt., Robn (Csy).

S. nigriceps Lec. Ithaca, May, Hamilton (Vdyk).

\section{AmERINUS Casey}

2203 A. linearis Lec. Monroe Co (M\&W).

\section{Philodes LeConte}

2205 P. testaceus Lec. Olcott, Mar (Dt) ; Canisteo, Yates Co, Windsor, Nichols, May-Je (Not); Taughannock Gorge, Coy Glen, May, Dt (Vdyk).

\section{Goniolophus Casey}

2209 G. rectangulus Chd. Nichols, May (Not).

\section{Acupalpus Latreille}

2211 A. hydropicus Lec. St. Lawrence Co, Houghton; Monroe Co (M\&W); West Pt. (Robn); Bronx Pk., Mar-Ap, Shoe; Van Cort. Pk., Ap (Woodr) ; SI, Feb-Ap (div.) ; LI: Brooklyn, Mar-Ap, Shoe; Jamaica, Ap, Nic (Not).

2213 A. carus Lec. Keene Val., Ausable Pt., Mt. Marcy, Waterville, Sodus Pt., Batavia, Oakfield, Nichols, Tivoli, May-Sep (Not) ; Gloversville, Mar, Johnstown, Ap (NYS) ; Monroe Co (M\&W) ; Olcott, Mar-Ap (Dt) ; Buffalo (Z\&R); Ithaca, May, McLean Bogs, J1 (Dt); West Pt. (Robn); Bronx, Ap (Woodr); SI, Feb-Sep (div.); LI: Brooklyn, May, Jamaica, Ap, Nic (Not).

2215 A. trivialis Csy. L. Champlain (Csy). 


\section{STENOLOPHUS Dejean}

2216 S. carbonarius Dej. SI (Lg); LI: Hauppauge, Ap, Nic (Not).

2218 S. ochropezus Say. Rather generally distributed throughout the State, Ap-Nov.

2220 S. fuliginosus Dej. Keene Val., Upper Ausable L., Wallface Mt., L. Tear, Canadice, Sodus Pt., Canisteo, Genesee Co, Tivoli, Orangeburg, Ap-Sep (Not); St. Lawrence Co, Houghton; Monroe Co (M\&W); Olcott. Feb-Ap (Dt); E. Aurora, scarce (Dnd) ; Pike, Ap (NYS) ; Renwick, Ap, Nov, Dt (Vdyk); Ithaca, McLean, J1-Aug, Dt (Vdyk); West Pt. (Robn) ; Van Cort. Pk. (AM) ; SI: Feb, Ap, Oct (L\&D,Woodr); Watchogue, Ap, Ds (Not); LI: Jamaica, Rockaway Beach, May, Nic (Not).

2232 S. dissimilis Dej. Buffalo (Z\&R).

2234 S. plebejus Dej. Pike, Albany, Poughkeepsie, Ap-May (NYS); Ithaca. Mar, Je, Dt (Vdyk); Ramapo, Ap (Not,Ds) ; Van Cort. Pk. (NotAM); SI, Feb, Je (Woodr,Not); LI: Jamaica, Nic (Not); Wyandanch, Oct (Not).

2235 S. fuscatus Dej. L. Tear, J1, Windsor, Je, Van Cort. Pk. (Not).

2236 S. humidus Ham. "NY" (Lg Cat.).

2238 S. conjunctus Say. Generally distributed throughout the State, Ap-Sep.

2243 S. scitulus Csy. Keene Val., May-Je, Westfield, May (Not).

\section{TACHISTODES Casey}

2245 T. indistinctus Dej. Olcott, Ap, Je (Dt); E. Aurora, May (Dnd) ; NYC (Schaupp); SI (Lg).

2246 T. testaceus Dej. SI, Je ( Lg); LI: Rockaway Beach, Je, Shoe, May, Nic (Not).

2247 T. pauperculus Dej. Keene Val., Mt. Skylight, Cliff Mt., Canadice, Lowville, Oakfield, Nichols, Tivoli, May-J1 (Not); St. Lawrence Co, Houghton; Buffalo (Z\&R) ; Batavia ( $\mathrm{Lg})$; Ithaca, J1 (Lg) ; Charlotte, Je, Zab (Not); White Plains, May (Sq); Suffern (AM); SI (Lg); LI: Jamaica, Ýaphank, Nic (Not).

2248 T. humilis Dej. "NY" (Not,Lg); Batavia, Jl, Kngt (Not).

T. obscurus Csy. NY, type locality ( $\mathrm{Lg})$.

T. convergens Csy. NY, type locality $(\mathrm{Lg})$.

2249 T. partiarius Say. Syracuse, May (H) ; Monroe Co (M\&W) ; Buffalo $(Z \& R)$; Olcott, Ap, Je (Dt) ; Taughannock Gorge, J1 (Lg); Albany, Nassau, May (NYS); West Pt. (Robn); West Farms (AM) ; SI, Ap, Je (L\&D) ; LI: Jamaica, Rockaway Beach, Bellport, May-Je, Nic (Not); Far Rockaway, May (Woodr); Coney I. (Lg).

\section{AGONODERUS Dejean}

2251 A. lineola Fab. Rochester (M\&W); Buffalo (Z\&R); Peekskill, May (Lg) ; West Pt. (Robn) ; Mosholu, Je (NotAM) ; High Bridge (AM); NYC (Schaupp, Woodr) ; SI (Lg); LI: Jamaica, Bellport, Je-J1, Nic (Not); E. Hampton, J1 (Woodr).

2254 A. infuscatus Dej. "NY" (Lec); E. Aurora, J1 (Dnd). 
2256 A. pallipes Fab. St. Lawrence Co, Houghton; Axton, Je, Monroe Co (M\&W) ; western NY, common (Dnd); Olcott, Feb-J1 (Dt) ; Castile, Brad (Flet); Six Mile Cr., Ap (Morr); McLean, Aug, Dt (Vdyk) ; Ithaca, Je-J1 (Vdyk, Dt); Taughannock Gorge, Sep (Vdyk) ; Renwick, Ap, Dt (Vdyk); Newport, Nassau, Albany, Ap-Je (NYS); Windsor, Je (Not); New Baltimore, Nyack, Zab (AM); Orange Co, Ap (Morr); Durlandville, Je, FrostSW (Dt); West Pt. (Robn); Stony Pt., Je (AM); Mosholu, Je (Not); Bronxville, Mar-Ap (Woodr); Bronx Pk., Ap-May, Sep, Shoe (AM); SI, Feb-J1 (Woodr,Lg); LI: Brooklyn, Ap-May, Sep, Shoe (AM) ; Flatbush. May-Sep, Zab (AM); Orient, Lath (NYS); Forest Pk., Bergen Beach, May-Je (NotAM); Bellport, Jamaica, J1, Sep, Nic (Not).

2261 A. comma Fab. Generally distributed throughout the State, May-Oct.

2263 A. gracilitarsis Csy. "NY" (Csy).

\section{FAMILY OMOPHRONIDAE}

\section{OMOPHRON Latreille}

2282 O. labiatum Fab. SI, May, Shoe; LI: Coney I., Lg; Rockaway Beach, Sep, Shoe. Confined to the extreme southern part of the State. Lives in sandy soil near water; dislodged by day by pouring water over the soil; active on the surface at night.

2284 O. americanum Dej. The most common species. Keene, J1, Not; Lowville, Je (Not); Manlius (VdykCU) ; Sylvan Beach, Je, Sodus Pt., Je (Not) ; Monroe Co (M\&W) ; Buffalo (Z\&R) ; E. Aurora, Je (Dnd) ; Pike, J1 (NYS,AM) ; Oakfield, Je, Westfield, May, Canisteo. Je. Nichols. May (Not) ; Inlet Val., May, Jl (Dt,Wendelkin); Taughannock Falls. J1 (Dt, Wendelkin) ; Ithaca, Ap-May, div. (Vdyk,MacG-CU,NYS) ; Schoharie, J1, Ft. Hunter, May-Je (Not) ; Albany (Lint) ; Washington Co, Je, Lg; L. Waccabuc, Aug (NotAM) ; Peekskill, May, Sherm (VdykCU) ; Nyack, Je, White Plains, May, Brownell (Lg): SI: May-Je, Aug, Lg (Ds-NYS, AM) ; Bulls Head, Sep (Woodr) ; LI: Brooklyn, May, Shoe; Canarsie, Ap-May (Not); Coney I., Rockaway, May-Oct (Schaupp). On sandy banks of brooks and pools.

2287 O. tessellatum Say. Sylvan Beach, Je (Not) ; Buffalo (Z\&R) ; E. Aurora, Je (Dnd) ; Nichols, May (Not); Inlet Val., J1 (Dt, Wendelkin); Schoharie, Je (Not); SI, Lg (Ds) ; LI: Edgemere, May (Woodr); Coney I., Rockaway, May-Oct (Schaupp). Not common. Ecology same as in labiatum.

\section{FAMILY HALIPLIDAE}

\section{HALIPLUS Latreille}

2299 H. fasciatus Aubé. Warren Co (Sherm); Syracuse (H) ; Olcott (Dt) ; Buffalo (Rein) ; E. Aurora, Dnd (Mat); Washington Co, Lg (Sherm); Hessian L., Bear Mt. (Sherm); Peekskill (Sherm); West Pt. (Robn); White Plains (Sq); Esopus, Sherm (Mat); Tomkins Cove (Rbts); NYC (NotAM); SI (L\&D); LI: Cypress Hills, Zab (NotAM). Ap-Sep.

2300 H. connexus Mat. Warren Co (Sherm); Fish Cr., Oneida Co (Dt) ; Buffalo, Dnd (Mat); Ithaca, Taughannock Gorge, Ringwood (Mat); Washington Co, Je (Lg, Sherm); Esopus (Sherm). Je-Aug. 
2301 H. triopsis Say. Sodus Pt., May (Not) ; Buffalo (Rein) ; E. Aurora, Dnd (Mat); Ithaca, Ringwood (Mat, Dt); Washington Co (Lg, Sherm); W. Hebron, Je (Ds); Wingdale (Sherm); Peekskill (Sherm) ; Esopus (Sherm); West Pt. (Robn); White Plains (Sq); Hessian L., Bear Mt (Sherm); NYC (L\&B); SI (NotAM). May-Oct.

2303 H. leopardus Rbts. Olcott (Dt); Washington Co (Sherm); Catskills (Nic); Esopus (Sherm); Peekskill (Sherm); Hessian L., Bear Mt. (Sherm); White Plains (Sq); NYC (Rbts).

2305 H. cribrarius Lec. Warren Co (Sherm); Syracuse, in cedar and sphagnum bog $(\mathrm{H})$; E. Aurora, Dnd (Mat); Ithaca, Ringwood, McLean (Mat, Dt); Catskills (Sherm).

2306 H. subguttatus Rbts. Recorded from Tyngsboro, Mass., by Roberts, but undoubtedly occurs in NY collections mixed with the preceding species (Mat).

2317 H. borealis Lec. Mooers, Oct (Not); SI, Oct 9 (L\&D).

2318 H. ruficollis DeG. Probably common all over North America (Mat); Syracuse, in cedar bog, as immaculicollis, $\operatorname{Harr}(\mathrm{H})$; Sodus Pt., May-Je (Not); Olcott (Dt) ; Buffalo, E. Aurora, E. Concord, Dnd (Mat); Oakfield, Je (Not); Ithaca (Mat); McLean (Dt); Oneida Co (Dt); Esopus (Sherm); Peekskill, Chatham (Sherm); West Pt. (Robn); White Plains (Sq); Bronx Pk. (Lg); SI (L\&D); LI: Astoria; Flatbush (AM); Cypress Hills (AM); Fishers I. (AM). May-Oct.

2319 H. blanchardi Rbts: Syracuse, in sphagnum bog (H) ; SI (Robts, L\&D). May-Je.

2322 H. longulus Lec. Pike (NYS); Buffalo (Rein); Grand I., E. Concord, Dnd (Mat); Batavia (Kngt); Ithaca (Mat); Ringwood (Dt); Karner (NYS); Washington Co (Sherm); Peekskill (Sherm); West Pt. (Robn); SI (AM). May-Oct.

\section{Peltodytes Regimbart}

2324 P. tortulosus Robts. Buffalo, J1 20, 1918, Dnd (Mat).

2328 P. muticus Lec. Sodus Pt., Je (Not) ; E. Aurora, E. Concord, Dnd (Mat); Murray Br., Oneida Co (Dt); Syracuse, in cedar bog (H); Colden (Mat); Ithaca (Mat); Esopus (Rbts); West Pt. (Robn); White Plains (Sq); NYC and vic (AM); SI, Sep, Sherm (L\&D); LI: Flushing (Mat). May-Oct.

2331 P. shermani Robts. White Plains (Sq); NYC and vic (Robts); SI, May-Sep, types (L\&D).

2332 P. pedunculatus Blatch. Ithaca (Mat).

2335 P. lengi Rbts. Hessian L., Bear Mt (Sherm); SI, Sep, type (L\&D).

2336 P. duodecimpunctatus Say. Oneida Co (Dt); Sodus Pt., May-Je (Not); Olcott (Dt); Buffalo (Rein) ; E. Aurora, Dnd (Mat); Castile, Brad (Flet); Ithaca (Mat); W. Hebron (AM); Poughkeepsie (Mat); Esopus (AM); West Pt. (Robn); Tomkins Cove, Zab (AM); Yonkers (Lg); Bronx Pk. (Lg) ; SI, AD-May, Sep, Sherm (L\&D); LI: Flushing (Mat); Flatbush, Cypress Hills, Fishers I., Zab (AM). Ap-Sep.

2337 P. edentulus Lec. Keene Val. (Dt); Pike (Mat); White L., Onondaga Co $(\mathrm{H})$; Sodus Pt., May-Je (Not); Buffalo, Dnd (Mat); Colden (Mat); Ithaca, McLean (Mat); Washington Co ( $\mathrm{Lg})$; West Pt. (Robn); White Plains (Sq); SI (L\&D). May-Nov. 


\section{FAMILY DYTISCIDAE 30 \\ SUBFAMILY NOTERINAE}

\section{CANTHYDRUS Sharp}

2340 C. puncticollis Cr. L. Waccabuc (AM); NYC (L\&B); SI, May (L\&D).

2342 C. gibbulus Aubé. Westchester Co (ShermCU).

\section{HYDROCANTHUS Say}

2346 H. iricolor Say. Sodus Pt., Je (Not); Buffalo (AM); Peekskill; Whitc Plains, May 30-Oct 25 (Sq); Esopus, Sep (AM); Van Cort. Pk. (Sherm) ; NYC (AM) ; SI, Ap-Je, Aug-Sep (L\&D); LI: Richmond Hill (Sherm); Fishers I., Aug (AM).

\section{SUBFAMILY LACCOPHILINAE}

\section{LACCOPHILUS Leach}

2351 L. maculosus Germ. Keene Val., J1, Saranac Inn, J1 (NYS) ; Newport (NYS); Adirondack Lodge, J1, Bish (NYS); Mooers, Sep (Not); Cranberry E., Je 18 (H); Syracuse, Ap-May $(\mathrm{H})$; Sodus Pt., Je (Not) ; Buffalo (Z\&R) ; Pike (NYS) ; Castile, Brad (Flet) ; Batavia, Je (Kngt) ; Caledonia Cr., Livingston Co, Dec (Lec); Westfield, May (Not) ; Ithaca, J1-Sep (Sherm-CU) ; McLean Bogs, Dt (NYS) ; Windsor, Je (Not); Schoharie (Not); Albany, Ap, Karner, Nassau, May (NYS) ; Peekskill, Aug (ShermCU) ; Poughkeepsie, Ap (NYS); W. Hebron, Je ( $\mathrm{Lg}$ ); Binghamton, Ap (AM) ; West Pt (Robn) ; Nyack, Oct (AM) ; Esopus (AM); White Plains, Mar 10-Oct 12 (Sq) ; Tomkins Cove (Lg); Bronx Pk., Aug-Sep (AM); SI, Mar-Je (L\&D); LI: Flatbush, Ap-May, Cypress Hills, May, Fishers I., Aug (AM); Calverton, Sep (Ds).

2353 L. proximus Say. E. Aurora, Sep, in ponds, scarce (Dnd) ; Ithaca, Je-J1 (Dt) ; Peekskill (Sherm); SI, Jan-Ap, Sep, Nov (L\&D).

2354 L. inconspicuus Fall. Ringwood, May, Senning (Flet).

2359 L. fasciatus Aubé. Peekskill (Sherm); NYC (AM); SI, May, J1 (L\&D).

2363 L. undatus Aubé. Buffalo (AM); Peekskill (Sherm); Esopus, Sep (Sherm); West Pt. (Robn); NYC (AM); SI, Ap-Je, Aug-Oct (L\&D).

\section{SUBFAMILY HYDROPORINAE}

HydRovatus Motschulsky

2367 H. cuspidatus Kunze. Sodus Pt., Je (Not) ; E. Aurora, Aug, in a marshy pool (Dnd) ; Esopus (Sherm); West Pt. (Robn); Peekskill (Sherm); SI, Ap-May, Aug-Sep (L\&D).

\section{DESMOPACHRIA Babington}

2374 D. convexa Aubé. Mooers, Sep (Not) ; Gloversville, May (NYS) ; Syracuse, Ap-May (H) ; Sodus Pt., Je (Not); Buffalo (Z\&R) ; Concord, Erie Co, Aug (Dnd); Castile, Brad (Flet); Ithaca, Aug 12, McLean, May 21 (ShermCU); Ringwood, Je-J1 (Dt); Peekskill (Sherm); NYC (L\&B); SI, Ap-May (L\&D); LI: Jamaica, May (AM).

${ }^{30}$ H. C. Fall and John D. Sherman, Jr., have gone over the manuscript for this list and made many corrections. Mr. Sherman has supplied all of his records from his extensive collecting and study of this family in New York. 


\section{BIDEssus Sharp}

2385 B. flavicollis Lec. Chaumont, Sep (Not); SI: Sep 8 (L\&D) ; 1000 specimens of this species were taken in one single haul of the net from the shallow water, close to the shore of the L\&D locality, Sep 8, 1900 (Sherm).

2388 B. pulicarius Aubé. Ithaca, Oct (Kngt); Hessian L., Aug, Esopus (AM); Bear Mt. L., Peekskill (Sherm) ; SI, May (L\&D).

2390 B. affinis Say. Newport, May (NYS) ; Cranberry L., Je 23 (H) ; Mooers, Sep (Not) ; Syracuse, Ap-May (H) ; Sodus Pt., Je (Not); Olcott, J1 (Dt) ; Buffalo (Z\&R) ; E. Aurora, J1-Aug, common (Dnd) ; Caledonia Cr., Dec (Lec); McLean, Aug (Dt); Ithaca, Ap, Oct (ShermCU); Cortland Co, J1-Aug, common (Dnd); Windsor, Je (Not); Oneida Co, Aug, Clms (Dt); W. Hebron, Je (Lg); West Pt. (Robn); Bear Mt. L. (Sherm); Esopus, NYC (AM); SI, J1, Sep (L\&D); LI: Rockaway Beach (AM).

2394 B. lacustris Say. Buffalo (Z\&R); Batavia, J1 (Kngt); Chautauqua, Thompson (Sherm); NYC (L\&B); SI, Je, Aug (L\&D).

2395 B. fuscatus Cr. Sodus Pt., Je (Not) ; E. Aurora, Sep, Nov; Oneida Co, J1 25, Clms (Dt); Ringwood, Je (Dt); Peekskill (ShermCU); SI, May, Jl-Aug (L\&D). Occurs always in woodland pools (Sherm).

2398 B. granarius Aubé. Esopus (Sherm); Peekskill, J1 21 (ShermCU); SI, May, J1-Aug, Nov (L\&D).

2399 B. suburbanus Fall. SI, J1 (L\&D); type locality in woodland pools (Sherm).

Celina Aúbé

2400 C. angustata Aubé. NYC (AM); SI, May, J1 (L\&D).

\section{CoElambus Thomson 31}

2407 C. acaroides Lec. E. Aurora, Sep, scarce (Dnd).

2403 C. punctatus Say (inaequalis Fab.). Adirondacks (Fall); Mooers, Sep (Not); Syracuse, May (H) ; Sodus Pt., Je (Not); Olcott, Je (Dt); Buffalo (Z\&R); Westfield, May (Not); Ithaca, May 8 (ShermCU); McLean, May 21 (ShermCU); West Pt. (Robn) ; Peekskill (Sherm); White Plains, Ap 27-Oct 25 (Sq); near NYC (L\&B); SI, Ap-May, Aug-Oct (L\&D).

2425 C. laccophilinus Lec. Mooers, Sep (Not) ; Ringwood, Je-J1 (Dt) ; Peekskill, in woodland pools (Sherm); SI, J1-Aug (L\&D).

2426 C. sylvanus Fall. Peekskill, type locality, in woodland pools (Sherm).

2408 C. turbidus Lec. Mooers, Sep (Not); Ringwood, Je (Dt); near NYC (L\&B); SI, Sep (L\&D).

2409 C. dispar Lec. (dissimilis G. \& H.). Westfield, May (Not); McLean, Aug (Dt) ; Washington Co (AM) ; Schoharie (Not) ; Esopus, Sherm (Fall); West Farms (AM).

2420 C. nubilus Lec. Common and widely distributed from New England to western Texas (Fall). Buffalo (Z\&R); E. Aurora, Aug-Sep, frequent in pools and brooks (Dnd); Ithaca (ShermCU); West Pt. (Robn); near NYC (L\&B); SI, Jan, Oct (L\&D).

${ }^{31}$ The arrangement of species in Coelambus is that of Fall in his paper on The North American Species of Coelambus, published in 1919. 
2424 C. impressopunctatus Schall. Syracuse (H); Buffalo, Aug (Z\&R, Dnd) ; E. Aurora, Aug (Dnd) ; Sodus Pt., May-Je, and Westfield, May (Not); Ithaca, Sep (Dt); NYC (AM); SI, Mar-Ap, J1 (L\&D).

\section{HYDROPORUS Clairville ${ }^{32}$}

2444 H. mellitus Lec. Peekskill (AM); Esopus, in running brooks, Sherm (Fall) ; SI, Aug (L\&D).

2440 H. pulcher Lec. E. Aurora, J1, in brooks, scarce (Dnd); Chautauqua (Lg); Esopus, Aug, in running brooks (Sherm); White Plains, Oct (Sq); SI, J1-Aug (L\&D).

2437 H. wickhami Zaitz. (concinnus Lec.). E Aurora, J1-Aug, common in brooks (Dnd); Chautauqua (Fall); Sodus Pt., Je, Westfield, May (Not); Ithaca, Sep (Fall,Dt); near NYC (L\&B).

2441 H. oppositus Say (integer Shp.). Livingston Co, Dec (Lec); Ithaca, Oct

244815 (ShermCU); Catskills (Fall); Esopus, Sep, Sherm (AM); Washington Co, Aug (AM); SI, Sep (AM).

2473 H. vitiosus Lec. Mooers, Sep, Not (Fall); Ithaca, J1, Sep (Fall,Dt) ; Washington Co., Sherm (Fall); SI, Je-J1 (L\&D).

2442 H. shermani Fall. SI, type locality (L\&D).

2474 H. blanchardi Sherm. (vitiosus Shp.). Peekskill, in running brooks, Shcrm (Fall); SI (Fall).

2471 H. striatopunctatus Melsh. Ithaca, Oct 15 (ShermCU); West Pt. (Robn); Esopus, in running brooks, Sherm (Fall); NYC, Aug (AM); SI, May, J1-Aug (L\&D).

2460 H. sulcipennis Fall. Peekskill, in running brooks, Sherm (Fall).

2458 H. spurius Lec. Buffalo (Z\&R); Oneida Co, J1 25, Clms (Dt); West Pt. (Robn) ; Esopus, Sep, in running brooks (Sherm); LI: Yaphank (Ds).

2459 H. dilatatus Fall. Esopus, in running brooks, Sherm (Fall).

2449 H. lobatus Sharp. SI, May, J1-Aug (L\&D).

2447 H. undulatus Say. Generally distributed (Sherm). Newport, May (NYS) ; Syracuse, Ap-May (Wallis); Oneida Co, Je, Clms (Dt) ; Castile, Brad (Flet); Ithaca, J1-Aug, Sherm (Fall, Dt); McLean Bogs (Fall, Dt) ; Esopus, Sherm (Fall); West Pt. (Robn); Peekskill (Fall); White Plains, May 3-Oct 13 (Sq); Van Cort. Pk., Bronx Pk., Aug (AM) ; SI, Feb, Ap, Je-Oct (L\&D) ; LI: Cypress Hills, Je, Fishers I., Aug (AM); Calverton, Sep (Ds).

2454 H. consimilis Lec. Westport, Aug (NYS); Mooers, Sep (Not); Warren Co, Sherm (Fall); Sodus Pt., Not (Fall); Buffalo (Z\&R), J1, in marsh, scarce (Dnd) ; Ithaca, Oct (Kngt).

2457 H. carolinus Fall (proximus Aubé). Cranberry L., Je-J1 ( $\mathrm{H})$; Washington 2464 Co, Sherm (Fall); Warren Co, Sherm (Fall); Westchester Co (ShermCU).

2452 H. clypealis Shp. Ithaca, Oct 9-10 (ShermCU); McLean Bogs, Aug (Fall, Dt) ; Washington Co (Sherm) ; Esopus, Sep (AM) ; Peekskill. Sherm (Fall); SI, J1-Sep (L\&D).

2463 H. mixtus Lec. ?Avon, Jan (Cls); Ithaca, Oct, McLean, May 21 (ShermCU) ; NYC (L\&B); SI, Aug (L\&D). Fall writes, "I have not recognized this form from New York, but these records may be all right."

${ }^{32}$ The arrangement of species in Hydroporus is that of Fall in his paper on A Revision of the North American Species of Hydroporus and Agaporus, published in 1923. 
2466 H. sericeus Lec. Buffalo (Z\&R). Possibly a misidentification (Fall).

2467 H. solitarius Shp. Ithaca, Oct 5 (ShermCU); Esopus (Sherm); Wingdale (Sherm) ; LI: Wading R., May, Nic (Lg).

2461 H. hybridus Aubé. "NY" (AubéANSP). Fall writes, "I doubt this somewhat."

H. deflatus Fall. SI, Sherm (Fall).

H. paugus Fall. Warren Co; Peekskill; Esopus; Wingdale; SI. All Sherm (Fall).

2523 H. oblitus Aubé. Peekskill (L\&B); SI (L\&D).

2521 H. stagnalis G. \& H. Cranberry L., Je (H); Peekskill, J1 (ShermCU) ; NYC (L\&B); SI, J1 (L\&D).

H. filiolus Fall. McLean Bogs, Aug (Fall, Dt) ; Peekskill, Sherm (Fall).

H. aequus Fall. Peekskill, Kaaterskill, Sherm (Fall).

H. rufiplanulus Fall. Rock City, types, Ds (Fall); Peekskill, Sherm (Fall).

2510 H. dichrous Melsh. Syracuse, May (H) ; Buffalo (Z\&R) ; Ithaca, J1, Sep (Fall, Dt); Esopus, Sep (AM); NYC (L\&B); SI, May, J1-Aug (L\&D).

2524 H. brevicornis Fall. Peekskill, Sherm (Fall); SI, Sherm (Fall).

2505 H. americanus Aubé. Newport, Pike, Je (Yg); Ithaca, Aug, Karner, J1 (NYS) ; Peekskill (L\&B); NYC (L\&B); SI, May-J1, Sep (L\&D).

2506 H. dentellus Fall. Ithaca, Je-J1 (Fall, Dt).

2516 H. ruficeps Shp. SI, J1-Aug (L\&D).

2514 H. niger Say (modestus Aubé). Cranberry L., Je 23 (H) ; Mooers, Sep (Not) ; Syracuse, Ap-May (H) ; Sodus Pt., Je (Not) ; Buffalo (Z\&R); Ithaca, J1, Sep (Fall, Dt); McLean, Oct 21 (ShermCU) ; Windsor, Je (Not); Washington Co, W. Hebron, Peekskill, Sep (AM); White Plains, Mar 10-Oct 20 (Sq) ; Kaaterskill, Sherm (Fall); SI, Jan, Ap-May, J1-Oct (L\&D) ; LI: Cypress Hills (AM).

2495 H. despectus Shp. Ithaca, Je-J1 (Fall, Dt); SI (AM).

2493 H. tenebrosus Lec. Mooers, Sep (Not); Syracuse, May (H).

2490 H. melanocephalus Gyll. (morio Shp.). Mooers, Sodus Pt., May (Not).

2508 H. signatus Mann. not Shp. (inornatus Shp.). Syracuse, Ap-May (Wallis) ; Buffalo (Z\&R) ; Ithaca, Je, McLean Bogs, Aug (Fall, Dt); West Pt. (Robn); Peekskill (L\&B); Wingdale (Fall); SI, Feb, May-J1, Sep (L\&D) ; LI (Fall).

2509 H. fuscipennis Kies. Syracuse, Ap (Wallis). (Fall gives the author of this species as Schaum.)

H. striola Gyll. Cranberry L., Je (H) ; Syracuse, Ap-May (H) ; Ithaca, J1 (Fall, Dt), as vitulus Er.

2502 H. striola var. subtonsus Lec. Peekskill (AM); SI (Sherm).

2501 H. tristis Payk. Widely distributed (Sherm). Pike, Ap (NYS); Peekskill (Sherm); NYC (L\&B); SI, J1 (L\&D).

2430 H. (Deronectes) griseostriatus DeG. var. catascopium Say. Pike, Je

2429 (NYS) ; Buffalo (Z\&R); McLean, Oct 21, Ithaca (ShermCU); W. Hebron, Je (Lg) ; Peekskill, Sherm (L\&B); NYC (AM); SI, May, Oct (L\&D).

2428 H. (Deronectes) depressus Fab. (rotundatus Lec.). "NY" (Fab) ; Buffalo (Z\&R); W. Hebron, Je, in running water (Sherm, Lg). 
2483 H. (Oreodytes) septentrionalis Gyll. Westfield, May (Not); Catskills, in running water, Sherm (Fall).

\section{AgAPORUS Zimmerman}

2531 A. difformis Lec. (Hydroporus). Buffalo (Z\&R); Peekskill, May-Sep (Sherm); White Plains, Ap (Sq); SI, Ap-Je (Sherm). Occurs in woodland pools, among rotted leaves, and is very sluggish in its movements (Sherm).

2532 A. conoideus Lec. Ringwood, J1, Senning (Flet).

\section{SUBFAMILY COLYMBETINAE}

\section{AGABUS Leach ${ }^{33}$}

2548 A. semivittatus Lec. E. Aurora, J1-Aug, in brooks (Dnd); Westfield, May (Not); Ithaca, Ap-May, J1 (ShermCU); Schoharie (Not).

2546 A. stagninus Say. "NY" (AM); Clarence, Erie Co, Aug, one specimen (Dnd); Batavia, Je (Kngt).

2543 A. obtusatus Say. Newport (NYS); Chautauqua, Thompson (Sherm); Westfield, May (Not); Peekskill, J1, in springs (ShermCU); White Plains, Ap 13-Sep 13 (Sq); near NYC (L\&B); Spuyten Duyvil (Sherm); SI (L\&D).

2544 A. planatus Shp. SI, Rbts (Fall).

2539 A. seriatus Say. Newport, May (NYS); Manlius, Mar (ShermCU); E. Aurora, May-J1, in brooks, scarce (Dnd); Buffalo (Z\&R); Ithaca, Aug 5 (ShermCU); Gloversville, Ap (NYS); Peekskill, Sherm (L\&B); SI, Ap-Aug (L\&D).

2550 A. punctatus Melsh. NYC (AM); SI, Ap-Je, Aug (L\&D); LI, L\&D (Sherm).

2551 A. punctulatus Aubé (aeneolus Cr.). Pike, May 20 (Fall, Frost); Buffalo (Z\&R) ; McLean, J1 (Dt) ; Cortland Co, May 21 (ShermCU); Washington $\mathrm{Co}$, Je (AM) ; W. Hebron (Sherm).

2553 A. semipunctatus Kby. (stridulator Shp.). Buffalo (Z\&R); Cortland Co, $\mathrm{J1}$, in pasture pools (Dnd); Peekskill, in woodland pools (Sherm); NYC (AM); SI (Sherm).

2556 A. taeniolatus Harr. Buffalo (Z\&R); NYC (AM); SI (L\&D).

2557 A. disintegratus $\mathrm{Cr}$. E. Aurora, Sep, one in large pond (Dnd); Ithaca, Ap 21 (ShermCU); McLean Bogs, Aug (Dt); West Pt. (Robn); Peekskill (AM) ; NYC, Oct (AM); SI, May, J1, Dec (L\&D) ; LI: Forest Pk., Shoe (Sherm).

2562 A. ambiguus Say (reticulatus Aubé, fimbriatus Lec.). Cranberry L., Je $(\mathrm{H})$; Mooers, Sep (Not) ; Syracuse, Ap-May (H) ; Buffalo (Z\&R); E. Aurora, Sep, in ponds, scarce (Dnd); Ithaca, Oct 6 (ShermCU); McLean, Aug, Ringwood, Je-J1 (Dt); Warren, Sep (ShermCU); Peekskill (Sherm); White Plains, Ap (Sq); SI, Ap, J1 (L\&D).

2572 A. erythropterus Say. Newport (NYS) ; Cranberry L., Je (H) ; Ithaca (ShermCU); West Pt. (Robn); Peekskill (Sherm); White Plains, Oct $25(\mathrm{Sq})$; NYC (L\&B); SI (L\&D).

\footnotetext{
${ }^{33}$ The arrangement of species in Agabus follows Fall's paper on A Review of the North American Species of Agabus, published in 1922, and differs considerably from that in the Leng catalog.
} 
2564 A. discolor Harr. Warren Co, Sherm (Fall) ; Cranberry L., Je (H) ; Buffalo, J1 10, MCV (Fall); Washington Co, Sherm (Fall); W. Hebron $(\mathrm{Lg})$.

2563 A. confinis Gyll. "NY" (Gyll); Washington Co, Aug (ShermCU).

2575 A. anthracinus Mann. Cranberry L., Je (H); White Plains, Sep (Sq).

2579 A. nigroaeneus Er. Ringwood, Je (Dt).

2580 A. gagates Aubé. Newport, Je (Yg); Cranberry L., Je-Jl (H) ; Ilion (NYS); Pike (NYS); E. Aurora, J1, one in pond (Dnd); Chautauqua, Thompson (Sherm); Ithaca, J1 30 (ShermCU) ; McLean, Aug, Ringwood, J1 (Dt); Esopus, Sep (AM) ; Poughkeepsie, May (NYS); West Pt. (Robn); Peekskill, in spring holes (Sherm); White Plains, Ap 20-Oct $4(\mathrm{Sq})$; NYC (L\&B); SI, Je-Aug (L\&D).

\section{ILyBIOSOMA Crotch}

2587 I. bifarius Kby. Buffalo (Z\&R); Waterville, Je (Not); Karner, Ap (NYS); LI: Forest Pk., May (Sherm).

\section{ILYBIUS Erichson}

2590 I. pleuriticus Lec. Cranberry L., Je $(H)$.

2593 I. quadrimaculatus Aubé. Peekskill (L\&B). This determination should be verified (Fall).

2594 I. ignarus Lec. Peekskill, J1 26, in a woodland pool (Sherm); SI (Sherm).

2595 I. angustior Gyll. Cranberry L., Je $(H)$.

2598 I. biguttulus Germ. Keene Val., J1 (NYS); Saranac Inn, J1 (NYS); Newport, Je (Yg) ; Cranberry L., Je-Aug (H) ; Mooers, Sep (Not); Buffalo (Z\&R) ; Pike (NYS); E. Aurora, Aug-Sep, frequent in ponds (Dnd); Oneida Co, J1 11, Clms (Dt); Ithaca, Je-Aug (ShermCU); Ringwood, J1 7 (Vdyk); Windsor, Je (Not); W. Hebron, Je (Lg); Albany, Je (Yg) ; Wingdale (Sherm); Kaaterskill (Sherm); Peekskill (Sherm); White Plains, May 11-Nov 11 (Sq); near NYC (L\&B); SI, Feb, May-Oct (L\&D).

2600 I. confusus Aubé. Sodus Pt., Je (Not); Ithaca, May 4 (ShermCU); Valley Falls, May (NYS); Esopus, Sep (Sherm); Kaaterskill (Sherm) ; Bear Mt. L., J1 (Sherm); White Plains, Je 6-J1 11 (Sq); NYC (AM); SI, J1 (L\&D).

2601 I. oblitus Shp. Cranberry L. (H) ; Buffalo, Je, Esopus, Sep (AM); White Plains, Sep-Oct (Sq).

\section{Agabetes Crotch}

2602 A. acuductus Harr. Ausable Pt., Sep 23 (Not); Warren Co (Sherm); Buffalo (Z\&R) ; Ringwood, Je (Dt); West Pt. (Robn); Peekskill, May-Je (ShermCU); White Plains, Mar 30-May 3 (Sq); SI, May-J1, Sep (L\&D); LI: Forest Pk. (Sherm). Usually in woodland pools (Sherm).

\section{Matus Aubé}

2603 M. bicarinatus Say. Warren Co, Aug (Sherm); Nassau, Ap (NYS); West Pt. (Robn) ; Peekskill, May, J1, Sep (ShermCU); White Plains, Mar $12-\mathrm{Nov} 11(\mathrm{Sq})$; near NYC (L\&B); SI, May (AM); LI: 
Orient, J1 (NYS). Usually in woodland pools, and has been taken in various localities in very moist and damp mossy places in the woods where there is usually very little water, this species being a crawler rather than a swimmer (Sherm).

\section{Copelatus Erichson}

2605 C. glyphicus Say. Syracuse, Ap (H) ; Cazenovia Cr., E. Aurora, Sep (Dnd); Waterville, Je (Not); Westfield, May (Not); Ithaca, J1 24 (ShermCU) ; McLean, J1 (Dt) ; Karner, Mar (NYS); Esopus, Sep (Sherm); West Pt. (Robn) ; Peekskill (Sherm) ; SI, Ap-May, Aug (L\&D) ; LI: E. New York, Je (Ds). Under stones, logs, etc., on muddy shores of river at Peekskill and Esopus, between tide lines (Sherm).

\section{Coptotomus Say}

2610 C. interrogatus Fab. Keene Val., J1 (NYS); Cranberry L., May 15 (H); Syracuse, Ap (H) ; Olcott, J1 (Dt); Batavia, J1 (Kngt) ; Buffalo $(Z \& R)$; E. Aurora, J1, Sep, common in ponds (Dnd); Castile, Brad (Flet); Avon, Jan (Cls) ; Canandaigua, J1 (NYS); Ithaca, Ap, Oct (ShermCU) ; Karner, J1 (NYS); W. Hebron, Je (Lg) ; Esopus, Sep (AM) ; West Pt. (Robn) ; Peekskill (Sherm); Bear Mt. (Sherm); White Plains, Mar 12-Nov 11 (Sq); near NYC (L\&B); SI, Ap, Je-Oct (L\&D) ; LI: Forest Pk. (Sherm).

\section{SCUTOPTERUS Crotch ${ }^{34}$}

2612 S. angustatus Lec. Adirondacks, Not (Sherm).

\section{RHANTUS Lacordaire}

2616 R. binotatus Harr. Keene Val., J1 (NYS); Newport, May (Yg) ; Cranberry L., Je (H) ; Sodus Pt., Je (Not); E. Aurora in Cazenovia Cr., one, Sep (Dnd); Buffalo (Z\&R); Westfield, May (Not); Ithaca, AugSep, Ringwood, Je (Dt) ; Albany, May, Karner, J1 (Yg) ; Delmar, May (NYS); Esopus, Sep, Sherm (AM); White Plains, Mar 4-Oct 2 (Sq); NYC (AM) ; SI, Ap (L\&D).

2619 R. calidus Fab. Occurs in N. and S. Am. from Me. (Peak I.) to Brazil (Sherm). Peekskill, Aug (Sherm); near NYC (L\&B); SI, May (L\&D) ; LI: Rockaway Beach (AM).

2620 R. sinuatus Lec. Peekskill (Sherm); SI (Sherm); LI (Sherm).

2623 R. bistriatus Bergst. E. Concord, Aug, one in a weedy pond (Dnd) ; Ithaca, Sep (Dt); Wingdale (Sherm); White Plains, Sep (Sq).

2624 R. tostus Lec. Pike, Ap (NYS); Ithaca, J1 12 (ShermCU); Karner, Ap (NYS) ; LI: Forest Pk. (Sherm).

\section{Colymbetes Clairville}

2632 C. sculptilis Harr. Ogdensburg, Je (NYS); Gloversville, May (NYS); Oswego, Je, Bks (NYS) ; Syracuse, Ap (H); Pike (NYS); Buffalo $(Z \& R)$, Je (Dnd) ; E. Aurora, Je-Sep, common in ponds (Dnd) ; Avon,

${ }^{34}$ The species of this genus live in sphagnum pools in the deep forests of the North (L. Superior, White Mts., Adirondacks, and Hudson Bay), and fly considerable distances but are very sluggish in the water (Sherm). 
Nov-Dec, Geneva, Mar (Cls); Ithaca, Ap-May, J1-Aug, Oct (ShermCU); Albany, Karner, Je (NYS) ; Hermitage, J1 (Mat); White Plains, Mar 30-Oct 11 (Sq) ; NYC (AM) ; SI, Ap-Je (L\&D); LI (Sherm).

\section{SUBFAMILY DYTISCINAE}

\section{Dytiscus Linnaeus}

2636 D. fasciventris Say. Oswego, Oct, Bks (NYS); Buffalo (Z\&R); E. Aurora, Sep, in ponds, scarce (Dnd) ; Avon, Dec (Cls) ; Batavia, J1 (Kngt); Canandaigua L., J1 11 (ShermCU); Canandaigua (NYS); Ithaca, Je-Aug (ShermCU); Utica, Nov (LintNYS); Washington Co (Sherm) ; Albany, Oct (LintNYS) ; Chatham, Nov (NYS) ; West Pt. (Robn); Peekskill (Sherm); White Plains, Oct (Sq); NYC (L\&B); SI, Ap, Je, Nov (L\&D) ; LI: Orient Pt. (NYS); Astoria (AM).

2637 D. hybridus Aubé. Keene Val., Nov (Not); Oswego, Oct, Bks (NYS); Olcott, J1 (Dt); Buffalo (Z\&R); Pike (Sherm); Niagara R., May (Dnd) ; Canandaigua, J1 (NYS) ; Orchard Pk., J1 (NYS) ; Ithaca, J1-Aug, Oct (ShermCU); McLean, Oct 21 (ShermCU); West Pt. (Robn); White Plains, Mar 19-Sep 6 (Sq); NYC (L\&B) ; SI, J1, Sep-Oct (L\&D).

2638 D. verticalis Say. Keene Val., Saranac Inn, J1 (NYS); Gloversville, Sep (NYS) ; E. Aurora, Buffalo, Aug, frequent (Dnd) ; Pike (NYS) ; Canandaigua L., J1 (NYS) ; Ithaca, J1-Aug, Oct (ShermCU) ; McLean Bogs, Aug (Vdyk); Albany, Aug, Nassau, J1 (NYS) ; Esopus, Peekskill (Sherm); West Pt. (Robn); White Plains, Oct (ShermCU); Van Cort. Pk., Aug (ShermCU); NYC (L\&B); SI, Mar-May, II (L\&D) ; LI: Forest Pk. (Sherm).

2641 D. marginalis L. Avon, Dec (Cls); Canandaigua, J1, Pike, Ap (NYS) ; Ithaca, Sep (Dt); Pittstown, Je (ShermNYS).

2645 D. dauricus Gebl. Ithaca, Aug 28 (ShermCU).

2646 D. harrisii Kby. Adirondacks, Sep (Eng) ; Wells, Aug (NYS) ; Olcott, J1 (Dt) ; Buffalo (Z\&R) ; E. Aurora, J1, in ponds, scarce (Dnd) ; Aurora, Ap, Bish (NYS) ; Johnsburg, Sep (NYS) ; Caledonia Cr., Dec (Lec) ; Geneva, J1 (LintNYS); Ithaca, J1, Oct (ShermCU); McLean, Oct 21 (ShermCU); Hadley, Feb (NYS); Utica, May, Chatham, Nov (NYS); East Branch, Aug, Ds (Ang); Saratoga Sps., Oct (LintNYS) ; LI: Forest Pk. (Eng).

\section{SUBFAMILY HYDATICINAE}

\section{HydATICUS Leach}

2647 H. stagnalis Fab. Buffalo (Z\&R); Ithaca, Mar, Aug (Dt) ; New Salem, Ap, Bish (NYS); Karner, J1 (NYS); Peekskill, Sep (Sherm); White Plains, Ap (Sq); near NYC (L\&B); SI, Ap-May (L\&D).

2648 H. laevipennis Thom. Syracuse, May (H).

2649 H. piceus Lec. Ithaca, Sep (Dt) ; West Pt. (Robn); Peekskill (Sherm) : White Plains, Ap 22-May 20 (Sq); Van Cort. Pk., Oct (Lg); SI. Ap, Je, Aug, Oct (L\&D).

2650 H. bimarginatus Say. Near NYC (L\&B); SI, Je (L\&D). 


\section{AcIlius Leach}

2651 A. fraternus Harr. (scmisulcatus Aubé, acc. Sherman). Mt. Marcy, Keene 2652

Val., Saranac Inn, J1 (NYS); Wilmington, Aug (Dt); Cranberry L. (H) ; Pike, Ap (NYS) ; Syracuse, May (H) ; Sodus Pt., Je (Not); Batavia, Je (Kngt); E. Aurora, Buffalo, Je, Sep, common in ponds (Dnd) ; Canandaigua L., J1 (NYS); Livingston Co, Dec (Lec); Geneva, Avon, Dec (Cls) ; Westfield, May (Not); Ithaca, Sep (Dt), Oct (Sherm); McLean, Aug (Dt); Karner, May (NYS) ; Esopus, Sep (AM); West Pt. (Robn); Peekskill (Sherm); White Plains, J1-Nov (Sq); SI, Ap-May, Oct-Nov (L\&D).

2653 A. mediatus Say. Ithaca, Aug 28 (ShermCU); Peekskill, Sep (Sherm); SI, Ap-Aug (L\&D).

\section{THERMONECTES Crotch}

2654 T. ornaticollis Aubé. Ithaca, J1 18, Stedman (ShermCU); NYC (L\&B).

2655 T. basilaris Harr. Buffalo (Z\&R); Canandaigua L., J1 (NYS); Ithaca, Sep (Dt) ; Karner, May (NYS) ; Peekskill (Sherm); White Plains, Oct (Sq); NYC (AM) ; Van Cort. Pk. (SI Mus); SI, Ap-May, J1-Oct (L\&D); LI: Brooklyn, May (Ds).

\section{GRAPHODERES Thomson}

2659 G. liberus Say. Syracuse, May (H) ; Buffalo (Z\&R); Ithaca (ShermCU); McLean, Aug (Dt); Oneida, Je 29, Clms (Dt); Peekskill (Sherm); White Plains, Mar 12-Oct 15 (Sq); NYC (L\&B); SI, Ap-May, Aug (L\&D) ; LI: Forest Pk. (Sherm).

2660 G. cinereus L. Ithaca (Dt) ; McLean, Aug (Dt) ; West Pt. (Robn) ; NYC (L\&B) ; SI, Ap, Je, Aug (L\&D) ; LI: Astoria (AM).

\section{SUBFAMILY CYBISTERINAE}

\section{Cybister Curtis}

2667 C. fimbriolatus Say. Oswego Co, Je, Bks (NYS); Ithaca, Aug-Sep (ShermCU) ; West Pt. (Robn); White Plains, Sep 2 (Sq) ; Van Cort. Pk. (SI Mus); NYC (L\&B); SI, May, Aug (L\&D); LI: Orient Pt. (NYS).

\section{FAMILY GYRINIDAE 35}

\section{Dineutes MacLeay}

2671 D. vittatus Germ. Ithaca (AM); Poughkeepsie (NYS); White Plains, Je-Oct (Ang, Sq) ; Putnam Co, May (Lg); Nyack, Oct (AM); Van Cort. Pk., May (Lg); SI, Ap-Oct (L\&D); LI: Yaphank (Ang).

2674 D. discolor Aubé. Mooers, Sep (Not); Oneida Co, Clms (Dt); Avon, Geneva (Cls); Ithaca (Dt); L. George, Aug (AM); Windsor, Je (Not); Schoharie, May-Je (Dt); Albany, Poughkeepsie (NYS); White Plains, Je (Sq); NYC and vic. (AM); SI (L\&D); LI: Cypress Hills, Babylon, May-Je (AM).

${ }^{35} \mathrm{H}$. C. Fall has kindly read the manuscript for this list, and has made some changes in synonymy and added some records. 
2679 D. nigrior Robts. Ithaca, Sep (Dt); White Plains, May-Oct (Sq) ; NYC and vic. (AM) ; SI (L\&D) ; LI: Astoria (AM) ; Deep Pond, Wading R., J1, Ds (Fall).

2680 D. americanus Say (assimilis Kby.). Oneida Co, May-Je, Clms (Dt); Buffalo (Z\&R) ; Sodus Pt., Je (Not); E. Aurora, Je, Sep, common (Dnd) ; Pike (NYS); Castile, Brad (Flet); Westfield, May (Not); Ithaca, Sep (Dt); W. Hebron, Rockland L. (Lg); West Pt. (Robn); White Plains, May-Je (Sq); Nyack, West Farms (AM); NYC and vic. (AM) ; SI, Ap-Oct (L\&D) ; LI: Flatbush, Coney I., Cypress Hills, May-Sep (AM); Orient, Lath (NYS).

2681 D. horni Rbts. Axton (MacG); Saranac L. (AM) ; Sodus Pt., Je (Not); Spencer, Aug (Dt); Ithaca (AM); Peekskill (Pollard); White Plains, May-Aug (Sq); Van Cort. Pk. (AM); SI, Ap-Oct (L\&D).

2682 D. emarginatus Say. Poughkeepsie (NYS); Putnam Co, May (Lg); Peekskill (Pollard); SI, May-Oct (L\&D).

\section{GYRINUS Geoffroy ${ }^{35 a}$}

2684 G. minutus Fab. (rockinghamensis Lec.). Pike (NYS); Catskills, Pears (AM).

2685 G. confinis Lec. Chaumont R.; Waddington, Ausable Pt. (Fall); Mooers, Sep, Sodus Pt., Je (Not); Buffalo (Z\&R).

2686 G. fraternus Coup. Sodus Pt., May-Je, Westfield, May (Not); Carmel, Aug, Sq (Fal1); E. Aurora, May, Sep, scarce (Dnd); West Pt. (Robn); White Plains (Fall, Sq); SI, Ap-May (L\&D); LI: Amagansett, Sep, Riverhead (Fall).

2687 G. aeneolus Lec. Rochester (Regimbart); Buffalo (AM); Ithaca, Je (Frost, Vdyk) ; Esopus; Pine I., Windsor (Fall) ; West Pt. (Robn); NYC and vic. (NotAM); SI (Lg).

2688 G. limbatus Say. Not identified by Fall; records are under his latilimbus $(\mathrm{Lg})$. Hamilton Co, Sodus Pt., Je, Mooers, Sep, Windsor, Je (Not); Ulster L. (Ds); White Plains (Fall, Sq) ; LI: Rockaway Beach, Je (Ds).

G. latilimbus Fall. Ithaca (Fall); White Plains, Oct, $S q$ (Fall); Ramapo Mts. in AM as borealis (Fall); NYC and vic. (NotAM); SI, Oct (Woodr). If limbatus Say is the same, it occurs also in western NY.

2689 G. dichrous Lec. (fratellus Not.). Mooers, May, Windsor, Je (Not); Chaumont R.; Pine I., Je ( $\mathrm{Lg})$; White Plains (Fall, Sq); NYC $(\mathrm{L} \& \mathrm{~B})$.

G. fraterculus Not. (dichrous Fall). Mooers, Sep (Not).

2690 G. elevatus Lec. SI (L\&D). Fall gives no localities for this species except Harris Coll. It is possible that specimens heretofore called elevatus are woodruffi Fall.

2691 G. ventralis Kby. Newport, Pike (NYS); Ithaca (Fall); Chaumont R. (Fall); Peekskill (NotAM); White Plains (Fall, Sq); Van Cort. Pk. (AM); NYC and vic. (NotAM); SI, Mar-Aug (L\&D); LI: Riverhead (Fal1).

2692 G. aquiris Lec. Ausable Pt.; Crown Pt.; Chaumont R.; Westfield, May (Not); Ithaca; Tivoli; White Plains, Sep, $S q$ (Fall); NYC and vic. (AM); SI (Fall).

35a The sequence of species in Gyrinus is that of Leng's catalog, which differs from the arrangement in Fall's paper in Trans. Am. Ent. Soc. 47:269-306, 1922. 
2695 G. maculiventris Lec. Mooers, Sep (Not); Buffalo (Z\&R); Westfield, May (Not); Ithaca (Fall); McLean, May (Fall).

- G. falli Not. Mooers, Sep (Not).

2696 G. affinis Aubé. Mt. Marcy, Keene Val. (Fall) ; Sodus Pt., May-Je, Westfield, May (Not); Ithaca; West Pt. (Robn); SI (L\&D).

2699 G. pernitidus Lec. Not rare from Me. to N.J. (Fall). West Pt. (Robn); Ramapo Mts. (AM); SI (AM) ; LI: Amagansett, Sep (Ds).

2700 G. analis Say. Sodus Pt., May-Je (Not); Buffalo (Z\&R) ; E. Aurora, Je, one specimen (Dnd); Ithaca (Fall); Putnam Co ( $\mathrm{Lg}$ ); West Pt. (Robn); Ramapo Mts. (AM); SI, May-Aug (L\&D).

2705 G. marinus Gyll. L. George, Aug (AM); White L., Jl, Zab (AM); Nyack, Oct, $Z a b$ (AM); SI (Robts Coll.) ; LI: Flatbush, Ap-May, $Z a b$ (AM); Fishers I., Aug (AM).

2707 G. borealis Aubé. Axton (MacG); Oneida Co, May, Aug, Clms (Dt); Ithaca (Fall); West Pt. (Robn); White Plains, Oct, $S q$ (Fall); SI (L\&D).

2707a G. borealis var. lugens Lec. (falli Not.). The commonest Gyrinus (Fall). Keene Val., Mt. Marcy, JI, Not (Fall) ; Crown Pt.; Hamilton Co (Ds); Ithaca, McLean, May; Westchester Co; White Plains (Fall,Sq); SI (L\&D) ; LI: Riverhead.

- [G. picipes Aubé, 2704. Confined to the Pacific district according to Fall. Has been often cited from NY, the specimens possibly being lugens Lec.] G. frosti Fall. Ithaca (Fall); SI (AM) as gibber Lec, goes here (Fall); also specimens from White L. (AM), L. George, Nyack, SI, and LI (Fishers I.), all as opacus Sahlb., go here according to Fall.

G. woodruffi Fall. Willow Br.; SI, Je 14, Woodr, type (L\&D).

G. bifarius Fall. Waterville, Je, Ithaca (Fall).

G. pugionis Fall. Chaumont R. (Fall).

\section{FAMILY HYDROPHILIDAE SUBFAMILY HYDRAENINAE}

\section{OCHTHEBIUS Leach}

2718 O. tuberculatus Lec. Cayuga L. Basin (Rich); SI, J1 (L\&D).

2726 O. holmbergi Mann. Cayuga L. Basin (Rich).

\section{HydRAENA Kugelann}

2729 H. pennsylvanica Kies. Mooers, Sep (Not); Oneida Co, J1 14, Clms (Dt); Olcott, J1 (Dt); Cayuga L. Basin (Rich); West Pt. (Robn); SI (L\&D).

H. angulicollis Not. Westfield, May (Not).

\section{SUBFAMILY LIMNEBIINAE}

Limnebius Leach

2737 L. discolor Csy. Westfield, May (Not); Cayuga L. Basin (Rich). 


\section{SUBFAMILY HELOPHORINAE}

HELOPHORUS Fabricius

2739 H. tuberculatus Gyll. Buffalo (Z\&R); SI, J1 19 (L\&D).

2740 H. inquinatus Mann. Mooers, Sep, Sodus Pt., Je., Schoharie (Not).

2743 H. lacustris Lec. Syracuse, Ap, flying $(H)$; Buffalo $(Z \& R)$; E. Aurora, J1-Sep, frequent in brooks (Dnd); Cayuga L. Basin (Rich); NYC (L\&B).

2744 H. obscurus Lec. SI, Je (L\&D). According to Winters this should replace lacustris.

2745 H. nitidulus Lec. E. Aurora, Williamsville, Clarence, J1-Aug, common (Dnd) ; Cortland Co, J1-Aug, common (Dnd).

2746 H. linearis Lec. Cranberry L., Je 23 ( $H)$; Syracuse, May, in cedar and sphagnum bog (H); E. Aurora, J1, scarce (Dnd); Cayuga L. Basin (Rich); Ithaca, May-Sep (Dt).

2748 H. granularis L. Cayuga L. Basin (Rich).

2749 H. obsoletesulcatus Mots. "NY."

2750 H. lineatus Say. Newport, Je (NYS) ; Cranberry L., Je 23 (H) ; Mooers, Sep (Not) ; Syracuse, Ap-May, in pond (H); Buffalo (Z\&R); E. Aurora, Aug, scarce (Dnd); Schoharie (Not); Ithaca, Jl-Aug (Dt) ; Albany, Ap (NYS) ; Karner, May (Yg); West Pt. (Robn); NYC (L\&B) ; SI, Jan, May-Je (L\&D).

H. viridicollis Steph. Cayuga L. Basin (Rich).

\section{SUBFAMILY HYDROCHINAE}

\section{HYDROCHUS Leach}

2754 H. scabratus Muls. Cayuga L. Basin (Rich); West Farms (AM) ; NYC (L\&B) ; SI, May, Aug (L\&D) ; LI (Muls).

$2758 \mathrm{H}$. inaequalis Lec. Cranberry L., Je 23 ( $\mathrm{H})$; West Pt. (Robn) ; NYC (L\&B) ; SI, Ap-May, Sep (L\&D) ; LI: Cold Sp. Harb., Aug 6 (H).

2759 H. excavatus Lec. Mooers, Sep, Sodus Pt., Je (Not); E. Aurora, Sep, frequent in brooks (Dnd); Cayuga L. Basin (Rich).

2760

2761

H. laticollis Not. Mooers, Sep (Not).

H. rufipes Lec. LI (Winters).

H. subcupreus Rand. Cranberry L., Je 23 (H) ; Buffalo (Z\&R); Pike, J1 (NYS); Caledonia Cr., Dec (Lec); Westfield, May (Not); Albany, May, Kinderhook, Je (NYS); NYC (L\&B); SI, Ap (L\&D); Rockaway Beach (AM); Cypress Hills, May, Zab (AM).

2762 H. granulatus Blatch. LI (Winters).

2764 H. squamifer Lec. Mooers, Sep (Not); Buffalo (Z\&R) ; Pike, J1 (AM) ; Cayuga L. Basin (Rich); McLean, Aug (Dt); LI (Lec).

2767 H. simplex Lec. Buffalo (Z\&R); Washington Co (AM); SI (AM).

\section{SUBFAMILY HYDROPHILINAE}

\section{BEROSUS Leach}

B. aculeatus Lec. Mooers, Sep (Not); SI, Je 30 (L\&D).

B. pantherinus Lec. West Pt. (Robn); NYC (L\&B). 
2777 B. peregrinus Hbst. Cranberry L., Je 18 (H) ; Buffalo (Z\&R); Ithaca, $\mathrm{Jl}$, McLean, Aug (Dt); Cayuga L. Basin (Rich); Washington Co (AM) ; West Pt. (Robn) ; NYC (L\&B) ; SI, Ap-May (L\&D) ; LI: Fishers I., Aug (AM).

2781 B. exiguus Say. LI (Winters).

2782 B. pallescens Lec. "NY" (Lec).

2783 B. infuscatus Lec. West Pt. (Robn); "NY" (AM).

2784 B. striatus Say. Essex Co, Aug (NYS); Cranberry L., Je (H); Syracuse, Ap-May, in cedar and sphagnum bog $(\mathrm{H})$; Mooers, Sep, Sodus Pt., Je (Not); Buffalo (Z\&R); E. Aurora, J1, Sep, frequent (Dnd); Westfield, May (Not) ; Cayuga L. Basin (Rich); Ithaca, Aug (NYS); Ringwood, McLean, J1-Sep (Dt); West Pt. (Robn); White Plains, Ap 9-Sep $2(\mathrm{Sq})$; NYC (L\&B); SI, Ap-Aug (L\&D).

\section{HydRous Dahl}

2789 H. triangularis Say. Buffalo, Aug, at light (Dnd, Z\&R); Pike, J1 (NYS) ; Avon, Geneva, Sep (Cls) ; Cayuga L. Basin (Rich); Ithaca, J1-Sep (Rich); Albany, J1 (NYS); New Baltimore, Zab (AM); West Pt. (Robn); White Plains, Aug 30 (Sq) ; Port Jervis (Lint); NYC (AM); SI, May, Aug-Oct (L\&D) ; LI: Cypress Hills, Je (AM) ; Huntington, Ap (AM) ; Rockaway, Aug, Zab (AM) ; Babylon, J1 (AM); Flatbush, Ap (AM).

\section{Dibolocelus Regimbart}

2792 D. ovalis Ziegl. West Pt. (Robn); SI (L\&D).

\section{HydROPHILUS DeGeer}

2795 H. obtusatus Say. Mooers, Sep (Not) ; Cranberry L., Je 18 (H) ; Syracuse, Ap-May, in ponds and bogs $(\mathrm{H})$; Olcott, J1 (Dt); Buffalo (Z\&R) ; Pike, Ap (NYS); Castile, Brad (Flet); E. Aurora, J1-Oct, common in pools (Dnd); Avon, Mar (Cls) ; Cayuga L. Basin (Rich); Ithaca, Aug (Vdyk); Ringwood, Je (Dt, Rich); Hermitage, J1 (Mat); West Pt. (Robn); West Farms, Van Cort. Pk., May (AM); NYC (L\&B) ; SI, Mar-Aug, Dec (L\&D) ; LI: Babylon, Je (AM); Flatbush, May (AM); Cypress Hills, May, Zab (AM); Orient, Lath (NYS).

\section{TROPISTERNUS Solier}

2797 T. striolatus Lec. "NY" (Sharp).

- T. blatchleyi Orych. (sublaevis Blatch. not Lec.). Syracuse, May, in sphagnum bog $(\mathrm{H})$.

2805 T. glaber Hbst. Mooers, Sep (Not); Pike, J1 (NYS); Olcott, J1 (Dt, Richmond) ; Buffalo (Z\&R); E. Aurora, Jl 9, common (Dnd); Syracuse, May, in sphagnum bog $(\mathrm{H})$; Cattaraugus Co, J1 (Ds); Avon, Dec-Jan, Geneva, Sep (Cls) ; French's Mill, Je (NYS); Canandaigua L., J1 (NYS) ; Westfield, May (Not) ; Cayuga L. Basin (Rich) ; Ithaca, J1 (Vdyk); Windsor, Je (Not); Albany, Ap (NYS) ; West Pt. (Robn); White Plains, Mar 30-Nov 2 (Sq); Nyack, Oct, Zab (AM) ; NYC (L\&B) ; SI, Je (L\&D) ; LI: Babylon, Je (AM) ; Flatbush, Ap, Oct, Zab (AM); Cypress Hills, Sep (AM); Calverton, Sep (Ds) ; Fishers I., Aug (AM); Orient, Lath (NYS). 
2806 T. mixtus Lec. Essex, J1 (NYS) ; Cranberry L., Je-J1 (H) ; Mooers, Sep, Sodus Pt., Je (Not); Olcott, Ap (Dt); Buffalo, Je-Sep, common (Dnd) ; E. Aurora (Dnd) ; Oneida Co, Sep, Clms (Dt); Westfield, May (Not); Cayuga L. Basin (Rich); Ithaca, J1 12 (Vdyk); West Pt. (Robn); White Plains, May 20-Oct 13 (Sq).

2807 T. lateralis Fab. Syracuse, Oct, in pond (H) ; Buffalo (Z\&R) ; E. Aurora, Aug-Sep, common (Dnd); Buffalo (Z\&R); Batavia, J1 (Kngt); Cayuga L. Basin (Rich); Ithaca, Aug (NYS); West Pt. (Robn); Tomkins Cove, Aug (AM); White Plains, Mar 21-Oct 13 (Sq) ; NYC (L\&B); Van Cort. Pk., Aug, West Farms (AM); SI, Ap-Aug (L\&D) ; LI: Flatbush, Ap, Babylon, Je-J1, Cypress Hills, May, Fishers I., Aug (AM).

T. quadristriatus Horn. LI (Winters).

\section{HYDROBIUS Leach}

2808 H. fuscipes L. Newport, Je (NYS) ; Mooers, Sep (Not) ; Olcott, Ap, Je $(\mathrm{Dt})$; Pike, Ap (NYS) ; Oneida Co, Clms (Dt) ; Syracuse, Ap-May, in pond and in sphagnum bog $(\mathrm{H})$; Buffalo $(\mathrm{Z} \& \mathrm{R})$; E. Aurora, Aug, rare (Dnd) ; Westfield, May (Not); Cayuga L. Basin (Rich); Ringwood, Je (Dt); Hermitage, J1 (Mat); Albany, Oct (Yg); Karner, May (NYS); White Plains, J1 26 (Sq); SI, May (L\&D).

2809 H. tumidus Lec. "NY" (Lec).

2810 H. globosus Say. Adirondacks, Sep (Eng) ; Olcott, Mar (Dt) ; Livingston Co, Dec (Lec); Buffalo (Z\&R); Oneida Co, Je-Aug, Clms (Dt); Cayuga L. Basin (Rich); Ithaca, Ap (MatCU) ; McLean, Aug (Dt); Michigan Hollow, May (MatCU); West Pt., Sep (AM); White Plains, Ap, Sep (Sq) ; Van Cort. Pk., Sep (AM) ; SI, Feb, May-Je (L\&D).

2813 H. tesselatus Zimm. Hunt's Pt., NYC (L\&B).

\section{AnACAEna Thomas}

2814 A. infuscata Mots. Cranberry L., Je 23 (H) ; Mooers, Sep (Not) ; Syracuse, Ap-May, in ponds and bogs $(\mathrm{H})$; Sodus Pt., Je (Not); Olcott, Je-Oct (Dt) ; Erie Co, Je, Aug, common in brooks (Dnd) ; Oneida Co, J1-Sep, Clms (Dt); Westfield, May (Not); Cayuga L. Basin (Dt, Rich); Ringwood, McLean, Je-Oct (Dt, Rich).

\section{PARACYMUS Thomas}

2817 P. suturalis Lec. Peekskill (L\&B); West Pt. (Robn).

2818 P. despectus Lec. E. Aurora, Aug-Sep, rare (Dnd); Livingston Co, Dec (Lec).

2819 P. subcupreus Say. Cranberry L., Je 23 (H) ; Syracuse, Ap-May, in ponds and bogs $(\mathrm{H})$; Mooers, Sep, Sodus Pt., Je (Not); Olcott, Je-Oct (Dt); Oneida Co, Je, Aug, Clms (Dt); Cayuga L. Basin (Rich); McLean, Ringwood, Je-Oct (Dt, Rich); West Pt. (Robn); SI, Feb-Ap (L\&D); LI (AM).

2825 P. digestus Lec. Cranberry L., Je (H); Pike (NYS); Niagara (Lec); Westfield, May (Not); West Pt. (Robn). 


\section{CRenitis Bedel}

2827 C. monticola Horn. Catskills, Nic (Winters).

\section{HeLochaRes Mulsant}

2828 H. maculicollis Muls. Buffalo (Z\&R).

\section{ENOCHRUS Thomson}

2834 E. nebulosus Say. Syracuse, May, in cedar bog (H) ; Mooers, Sep, Sodus Pt., Je (Not) ; Olcott, J1 (Dt, Rich); Buffalo (Z\&R); E. Aurora, Aug, scarce (Dnd); Livingston Co, Dec (Lec); Cayuga L. Basin (Rich) ; Ithaca, Aug, Normanskill, May (NYS); NYC (L\&B); SI, May (L\&D) ; LI: Rockaway Beach (AM).

2835 E. ochraceus Melsh. Cranberry L., Je-J1 (H); Mooers, Sep (Not); Syracuse, May, in cedar and sphagnum bogs $(\mathrm{H})$; Sodus Pt., Je (Not); Olcott, J1-Sep (Dt); Pike, Aug (NYS) ; E. Aurora, Je-Sep, very common (Dnd); Buffalo (Z\&R); Oneida Co, Aug 7, Clms (Dt); Cayuga L. Basin (Dt, Rich); Westfield, May (Not); Ithaca, Aug 9 (Vdyk) ; Ringwood, McLean, J1-Sep (Dt, Rich); Albany, Aug (NYS); West Pt. (Robn); White Plains, May 3-Sep 2 (Sq) ; SI, Ap-May, Sep (L\&D) ; LI: Rockaway Beach, Babylon, J1 (AM).

2836 E. perplexus Lec. Newport, Je ( $\mathrm{Yg})$; Syracuse, May, in cedar bog (H); Buffalo (Z\&R) ; E. Aurora, Sep, scarce (Dnd) ; Westfield, May (Not); Cayuga L. Basin (Rich); McLean, J1-Aug (Dt, Rich); Cortland Co, J1, scarce (Dnd); Windsor, Je (Not); West Pt. (Robn); NYC (L\&B) ; SI, Mar-Je, Sep, Dec (L\&D) ; LI: Babylon, Cypress Hills, Je, Flatbush, May (AM).

2837 E. cinctus Say. Pike, Aug (NYS) ; Buffalo (Z\&R); E. Aurora, May, Sep, scarce (Dnd); Cayuga L. Basin (Rich); Ithaca, J1-Aug (Vdyk); McLean, Ringwood, Je, Aug (Dt, Rich); Windsor, Je (Not); West Pt. (Robn); White Plains, May 3-Aug 16 (Sq); NYC (L\&B); SI, May (L\&D) ; LI: Cypress Hills, May-Je, Babylon (AM).

2838 E. consors Lec. Cayuga L. Basin (Rich); Ithaca, McLean, Je-J1 (Dt, Rich). 2841 E. hamiltoni Horn. Cranberry L. (H) ; Mooers, Sep (Not); Syracuse, May, in cedar bog $(\mathrm{H})$; E. Aurora, Je, Aug, common (Dnd) ; Sodus Pt., Je (Not); Oneida Co, Aug, Clms (Dt) ; Cayuga L. Basin (Rich); Ithaca, Aug 9 (Vdyk); West Pt. (Robn) ; NYC (L\&B); SI, Ap-Je, Aug (L\&D) ; LI: Rockaway Beach, J1, Babylon (AM).

2843 E. reflexipennis Zimm. Livingston Co, Dec (Lec); NYC (L\&B); SI. Ap-May (L\&D); LI: Babylon (AM).

\section{Cymbiodyta Bedel}

2849 C. rotunda Say. West Pt. (Robn).

2850 C. frmbriata Melsh. Mooers, Sep (Not); Olcott, Sep (Dt, Rich); Buffalo (Z\&R); Pike, Je (AM); E. Aurora, May-Aug, common (Dnd); Westfield, May (Not); Cayuga L. Basin (Rich); Windsor, Je (Not); White L., Jl, Zab (AM) ; West Pt. (Robn); NYC (L\&B) ; SI, Mar, May, Aug (L\&D) ; LI: Wyandanch, May (Ds).

C. minima Not. Mooers, Sep (Not). 
2851 C. blanchardi Horn. Olcott, J1-Sep (Dt, Rich) ; E. Aurora, J1, Sep, common (Dnd); Westfield, May (Not); Oliverea, Sep, Ds (Not); Woodland, Oct (Ds) ; Windsor, Je (Not) ; Cayuga L. Basin (Rich); Watkins Glen (L\&B); Van Cort. Pk. (AM) ; LI: Babylon (AM).

2852 C. lacustris Lec. Axton, Je $(M \& H)$; Buffalo ( $Z \& R)$; E. Aurora, Aug, common (Dnd); Cayuga L. Basin (Rich); Cortland Co, J1, common (Dnd) ; Schoharie (Not) ; NYC (L\&B); SI, Mar (L\&D).

C. vindicata Fall. SI, type locality.

\section{HELOCOMBUS Horn}

2853 H. bifidus Lec. Cranberry L., J1 (H) ; Mooers, Sep (Not); Buffalo $(Z \& R)$; E. Aurora, Jl-Sep, common (Dnd); Cayuga L. Basin (Rich); Ringwood, Je-J1 (Dt, Rich); Cortland Co, J1-Sep, common (Dnd); West Pt. (Robn); White Plains, Ap 21-May 18 (Sq); NYC (L\&B); SI, May, J1 (L\&D).

\section{LACCOBIUS Erichson}

2854 L. agilis Rand. Newport, Je ( $\mathrm{Yg}$ ) ; Syracuse, Ap, on clay bank (H) ; Mooers, Sep, Sodus Pt, Je (Not); Buffalo (Z\&R) ; E. Aurora, Aug, common (Dnd) ; Livingston Co, Dec (Lec) ; Westfield, May (Not); Cayuga L. Basin (Rich); Cortland Co, J1, common (Dnd); Oneida Co, May, J1, Clms (Dt); Windsor, Je (Not); Schoharie (Not); Suffern (AM) ; Albany, Je (Yg); NYC (L\&B); SI (L\&D).

\section{Chaetarthria Stephens}

2859 C. atra Lec. Westfield, May (Not); Cayuga L. Basin (Rich).

\section{SUBFAMILY SPHAERIDIINAE}

\section{PHAENONOTUM Sharp}

2864 P. estriatum Say. NYC (L\&B).

\section{SPHAERIDIUM Fabricius}

2867. S. scarabaeoides L. Axton, Je (M\&H) ; Potsdam, Je-Aug (Houghton); Syracuse, May, on cattle dung $(\mathrm{H})$; E. Aurora, Aug, common in manure (Dnd); Pike, May (NYS); Castile, May, Brad (Flet); Cayuga L. Basin (Rich); Ithaca, May (Houghton); Windsor, Je (Not); Catskills, Jl (Pears); West Pt. (Robn); West Farms (Sf); SI, May-Je (L\&D) ; LI: E. New York, early May (Sf, Ds) ; Rockaway Beach, Wyandanch.(Ds).

2868 S. bipustulatum Fab. E. Aurora, Aug-Sep, common in manure (Dnd); Cayuga L. Basin (Rich); West Pt. (Robn); LI: Brooklyn, May, Shoe (AM) ; E. New York, May (Sf).

\section{CERCYON Leach}

2869 C. littoralis Gyll. SI, Je 5 (L\&D) ; LI: Rockaway (L\&B); Long Beach (L\&B) ; Stony Pt., Sep (Winters) ; Coney I. (Horn).

2873 C. unipunctatus L. N. Fair Haven (Rich); McLean, Aug (Dt, Rich); Windsor, Je, Schoharie (Not); Ramapo, Je (Ds). 
2875 C. ocellatus Say. Sodus Pt., Je, Schoharie (Not); Cayuga L. Basin (Rich); NYC (L\&B).

2876 C. praetextatus Say. Buffalo (Z\&R); Aug, scarce (Dnd); Cayuga L. Basin (Rich); SI, Ap-Je (L\&D).

2878 ?C. fulvipennis Mann. Buffalo (Z\&R). Determination doubtful; this is a West Coast species ( $\mathrm{Lg}$ ).

2879 C. lateralis Marsh. Syracuse, May, on cattle dung (H)?; SI, May 25, Ds (L\&D).

2880 C. indistinctus Horn. Cranberry L., Je 30 (H) ; Newport, Je, Gloversville, Sep (NYS).

2883 C. haemorrhoidalis Fab. Potsdam (Houghton); Cranberry L., J1 (H); Syracuse, May, on cattle dung $(\mathrm{H})$; Buffalo $(\mathrm{Z} \& \mathrm{R})$; E. Aurora, Cortland Co, J1-Aug, common (Dnd); Cayuga L. Basin (Rich); West Pt. (Robn); SI, May (L\&D).

2884 C. melanocephalus L. Syracuse, May, on cattle dung $(\mathrm{H})$; E. Aurora, Aug, common (Dnd).

2885 C. pygmaeus Illig. Cranberry L., Je 25 (H) ; Cayuga L. Basin (Rich); SI, Je 7 (L\&D).

2886 C. nigriceps Marsh. Ithaca, Sep (Dt, Rich); NYC (L\&B).

2887 C. convexiusculus Steph. West Pt. (Robn) as lugubris.

2892 C. navicularis Zimm. Cayuga L. Basin (Rich); Peekskill (L\&B).

2893 C. analis Payk. Newport, Je (Yg) ; Cranberry L., Je-J1 (H) ; West Pt. (Robn); SI, Ap, Je, Dec (L\&D).

C. incrematus Not. Windsor, Je (Not).

C. basillaris Not. Windsor, Je (Not).

\section{OOSTERNUM Sharp}

$2894 \frac{1}{2}$ O. pubescens Lec. NY (Winters).

\section{CRYPTOPLEURUM Mulsant}

2899 C. minutum Fab. Cranberry L., Je-J1 (H); Newport, May (NYS); Syracuse, Ap, a skeleton (H); Buffalo (Z\&R); Cayuga L. Basin (Rich); Windsor, Je (Not); McLean, Aug (Dt, Rich); West Pt. (Robn); LI (AM).

\section{FAMILY BRATHINIDAE}

\section{BRATHINUS LeConte}

2903 B. nitidus Lec. Mt. Whiteface, $2000-4000$ ft., Aug (CU); Slide Mt. Je, Oliverea, Sep (Ds); Peekskill, May, Sherm (AM, CU).

2904 B. varicornis Lec. Utica (Lec).

\section{FAMILY SILPHIDAE}

\section{NECROPHORUS Fabricius}

2911 N. americanus Oliv. Gloversville (NYS); West Pt. (Robn); Bronx (AM) ; NYC; SI (L\&D); LI: E. New York, Schott; Yaphank, Sep (BM); Brooklyn, Shoe. May-Aug. 
2912 N. sayi Lap. Wallface Mt.; Cranberry L.; L. Pleasant, Aug; Rochester; Ithaca (Dt) ; Batavia, Je-J1 (Kngt) ; Ft. Montgomery; NYC; SI (L\&D) ; LI: Wyandanch; Babylon (AM) ; Montauk, beach wash-up, J1 (Ang); Bellport; Brooklyn (Shoe). Ap-Oct. On carrion.

2913 N. orbicollis Say. Wilmington (Ds) ; Cranberry L.; Rochester; Sodus Pt., Je (Not); Olcott (Dt); Buffalo (Z\&R); Castile, J1, Brad (Flet); Batavia, Je (Kngt); E. Aurora, Je (Dnd); Pike; Crosby (CU); Ithaca, McLean Bogs (Dt); L. George, Zab (AM); West Pt. (Robn); Ft. Montgomery; NYC; SI (L\&D); LI: Bergen I., Zab (AM); Cold Sp. Harb.; Bellport; Yaphank, J1 (AM); Brooklyn, May (BM) ; Riverhead (Ds). May-Aug. On carrion.

2914 N. marginatus Fab. Rochester; Avon, J1 (Cls) ; Ithaca (Dt) ; Windsor, $\mathrm{Je}$ (Not); Kinderhook (CU); White Plains; Westchester Co; West Pt. (Robn) ; Ft. Montgomery, Schott ; NYC; SI (L\&D) ; LI: Aqueduct; Jamaica; Flushing; Long Beach; Flatbush; Bellport; Rockaway Beach; Gardiners I.; Brooklyn, Yaphank (BM).

2918 N. pustulatus Hersch. Cranberry L.; Rochester; Olcott; Buffalo; Ithaca; L. Pleasant (AM) ; NYC; SI (L\&D) ; LI: Jamaica; Babylon; Riverhead (Ds); Yaphank (Ds); Bellport; Promised Land (BM); Amagansett; Good Ground; Gardiners I.; Orient, Lath (NYS). May-Sep.

2919 N. vespilloides Hbst. Thousand Isls.; Wallface Mt.; Wilmington; Rochester; Castile, Je, Sep, Brad (Flet); Cooks Falls; L. Pleasant (BM); Slide Mt., Shoe; Oliverea; LI: E. New York, J1-Sep.

2920 N. tomentosus Web. Keene Val. (BM); Cranberry L.; Wilmington (Shoe); Olcott; Honeoye Falls (CU); Buffalo (Z\&R); Pike; Castile, Brad (Flet); Batavia, Aug (Kngt); Ithaca (Dt); Albany (Lint); West Pt. (Robn) ; Ft. Montgomery; Yonkers; Peekskill; Nepperhan; Nyack; Mosholu; White Plains (Sq); SI, Pollard (L\&D) ; LI: Cold Sp. Harb.; Bellport; Wyandanch; Huntington; Flatbush (BM). Je-Oct. On carrion.

\section{SILPHA Linnaeus}

2922 S. surinamensis Fab. Wilmington (Ds) ; Long L. (Ds) ; Cranberry L.; Buffalo (Z\&R); Batavia, J1 (Kngt); Montour Falls; Ithaca (Dt); Oliverea (Ds) ; West Pt. (Robn) ; SI (L\&D) ; LI: Coney I., Bergen I., Flatbush (AM) ; Cold Sp. Harb.; Yaphank (BM); Brooklyn, Dow; Orient, Lath (NYS).

2923 S. lapponica Hbst. Axton (M\&H) ; Wilmington (Ds, Shoe) ; Whiteface Mt. (Shoe); Cranberry L.; Syracuse (BM); Rochester Jct. (CU); Sodus Pt., Je (Not) ; Buffalo (Z\&R) ; E. Aurora, May-Je (Dnd); Pike (AM); Montour Falls; Ithaca (Dt); LI (BM). May-Aug. On carrion.

2926 S. inaequalis Fab. Lowville (Ds) ; Avon (Cls) ; Sodus Pt., Je (Not) ; E. Aurora, May-J1 (Dnd); Batavia, Je (Kngt); Castile, Brad (Flet); Westfield, May (Not); Ithaca (Dt); Windsor, Je (Not); Halcottsville, New Baltimore (AM); Tuxedo (Ds); West Pt. (Robn); White Plains (Sq) ; NYC; SI, at willow sap (L\&D) ; LI: Cold Sp. Harb.; Yaphank (BM) ; Babylon (AM) ; Jamaica, Forest Pk. (BM); Brooklyn (Shoe); Rockaway Beach, Center I. (Ds) ; Queens, Dow; Orient, Lath (NYS). Mar-Sep. 
2927 S. noveboracensis Forst. Wilmington (Ds) ; Cranberry L.; Wanakena, Aug (Drk) ; Chateaugay L., 2000 ft. (AM) ; Syracuse; Olcott (Dt); Buffalo (Z\&R); Avon (Cls); Castile, Brad (Flet); Batavia, Ji (Kngt) ; E. Aurora, May (Dnd); Montour Falls; Canandaigua (CU) ; McLean Bogs; Windsor, Je (Not); West Pt. (Robn); New Baltimore, Mosholu (AM) ; NYC; SI (L\&D) ; LI: Cold Sp. Harb.; Flushing; Queens, Pinelawn, Dow; Massapequa (Ds); Orient, Lath (NYS). Mar-Sep. On carrion.

2928 S. americana L. Wilmington, Conesus L. (Ds) ; E. Aurora, May-J1 (Dnd); Cranberry L.; Buffalo (Z\&R); Batavia, Je, on dead corn (Kngt); Ithaca; McLean Bogs (Dt); Albany (Lint); Cropseyville (NYS); Peekskill (Sherm); Avon (Cls); Hudson (BM); Sullivan Co (Ds); White Plains (Sq); West Farms (AM); SI (L\&D); LI: Cold Sp. Harb.; Brooklyn (Shoe); Babylon; Montauk (Ds, Ang); Orient, Lath (NYS).

2930 S. opaca L. Buffalo (Z\&R). Probably an error, since the species occurs in Siberia, Hudson Bay territory, and Calif. (Lg).

NECROPHILUS Latreille

2932 N. pettiti Horn. Cooks Falls, Sep, Sue Davis (H) ; Allegany Pk., J1 (Dnd).

\section{Choleva Latreille}

2947 C. luridipennis Mann. Keene Val., J1 (Not).

2950 C. simplex Say. Olcott (Dt); Ithaca (Dt); Ludlow, Sep (Thompson); Keene Val., May, Je (Not); White Plains (Ang); SI, Shoe (L\&D). Ap-Je.

2951 C. basillaris Say. Keene Val., May, J1 (Not) ; Newport, on store cheese (Yg); L. Pleasant (AM); Ludlow (Thompson); Mosholu (BM); SI (L\&D). Feb-Je.

2952 C. clavicornis Lec. Keene Val., J1, Sep (Not) ; Cranberry L., on decaying fungi; West Pt. (Robn); Mosholu (AM); Spuyten Duyvil, May (Ang); LI: Bellport (Nic).

2955 C. terminans Lec. Axton $(\mathrm{M} \& \mathrm{H})$; Cranberry L.; L. Placid (BM); L. Pleasant (AM); Keene Val., May (Not); Olcott (Dt); Ithaca (Dt); Etna; Catskill, Ap (Lg); SI, on three occasions in owl pellets (L\&D). Mar-Oct. On carrion.

\section{PRIONOCHAETA Horn}

2957 P. opaca Say. Keene Val., May (Not); Cranberry L., on carrion; L. Pleasant (AM); Olcott (Dt); Batavia, Je (Kngt); Gowanda, May (Dnd) ; Ithaca, Ringwood (Dt); Cortland Co, Je (Dnd); McLean, May, Je (Flet); Bangall (BM); Peekskill (Sherm); West Pt. (Robn); White Plains; Mosholu (BM); SI, Shoe (L\&D); LI: Brooklyn (Shoe); Yaphank (BM). Ap-Sep.

\section{Ptomaphagus Illiger}

2958 P. consobrinus Lec. Olcott (Dt) ; Ithaca (CU) ; West Pt. (Robn) ; Peekskill (Sherm); Mosholu (BM).

2964 P. pusio Lec. Buffalo (Z\&R); Ithaca (Dt); West Pt. (Robn). 
2965 P. parasitus Lec. Syracuse, under a stone, perhaps associated with ants; Olcott (Dt); Buffalo (Z\&R) ; West Pt. (Robn) ; Peekskill (Sherm); Bronx Pk., Sf (AM); Bronxville, in nest of Formica sericea, Wheel; SI (L\&D); LI: Jamaica (Shoe); Pinelawn (BM).

2967 P. brachyderus Lec. "NY" (Horn); Katonah, Sherm (CU); Mosholu ( Lg).

CoLon Herbst

2970

c. bidentatum Sahlb. "NY", Clke (Horn).

2973

C. dentatum Lec. Keene Val., J1 (Not).

2976

C. putum Horn. Keene Val., J1 (Not).

2977

C. magnicolle Mann. Keene Val., May (Not).

2978

C. pusillum Horn. Peekskill, Sep, Sherm (Sz).

2982

C. asperatum Horn. Windsor, Je (Not).

\section{SUBFAMILY ANISOTOMINAE}

HYDNOBIUS Schmidt

H. laticeps Not. Schoharie (Not).

2991 H. substriatus Lec. Keene Val., J1 (Not).

2992 H. latidens Lec. Keene Val., Je-J1 (Not).

\section{Anisostoma Illiger}

2996 A. alternata Melsh. West Pt. (Robn); SI, Oct (L\&D).

2998 A. valida Horn. Danby, Je, AndersonEG (Dt).

3000 A. assimilis Lec. Axton, Je (M\&H) ; Keene Val., Je, Aug (Not); McLean, Je (Flet).

3001 A. punctatostriata Kby. Keene Val., May, J1 (Not); Windsor, Je (Not); E. Jewett, Aug (Lg).

3003 A. collaris Lec. Keene Val., J1 (Not).

3009 A. obsoleta Melsh. Keene Val., J1 (Not).

\section{Colenis Erichson}

3014 C. impunctata Lec. Keene Val., Aug, Oct (Not) ; E. Aurora, Dnd (Dt); West Pt. (Robn); Mosholu (BM); SI (L\&D). May-Je.

\section{CyRTUSA Erichson}

3015 C. picipennis Lec. Northern NY, Ulke (Lec); Keene Val., J1 (Not).

3018 C. egena Lec. Keene Val., J1 (Not).

\section{Cainosterum Notman}

C. imbricatum Not. Westfield, May (Not). 


\section{LEIODES Latreille}

3021 L. globosa Lec. Keene Val., Je (Not); Peru (CU) ; Cranberry L. (H) ; Olcott (Dt). Je-Sep.

3022 L. polita Lec. SI, J1 (L\&D).

3023 L. discolor Melsh. Axton (M\&H) ; Buffalo (Z\&R) ; E. Aurora, J1 (Dnd) ; Ithaca (Dt); Harrison (AM) ; SI, May-Je (L\&D). Je.

3025 L. obsoleta Horn. Keene Val., J1 (Not); Sodus Pt., Je (Not) ; Olcot: $(\mathrm{Dt})$; Cortland $\mathrm{Co}$, Je (Dnd); Windsor, Je (Not); SI (L\&D). Je-Sep.

3026 L. basalis Lec. Keene Val., J1 (Not) ; E. Aurora, J1 (Dnd) ; Ithaca (Dt) ; SI, on slime mold (L\&D); LI: Bellport, Babylon (BM). Je-Aug.

3026a L. basalis var. dichroa Lec. Buffalo ( $Z \& R)$; Ithaca (Dt). Je.

3027 L. geminata Horn. Keene Val., J1 (Not) ; Cranberry L., Je, under bark $(\mathrm{Dt}, \mathrm{H})$; McLean, Je (Flet).

\section{Agathidium Illiger}

3029 A. oniscoides Beauv. Cranberry L., from Acer saccharum and Pimus strobus; Olcott (Dt) ; Buffalo (Z\&R) ; Harrison, Mosholu (AM) ; Katonah, Peekskill (Ang); SI, common under bark of dead trees, rolled into a ball (L\&D); LI (BM). May-Sep.

3030 A. exiguum Melsh. Axton (M\&H); Keene Val., Je-J1 (Not); Olcott, Ithaca (Dt) ; Peekskill (Sherm); West Pt. (Robn) ; Mosholu (AM); SI (L\&D); LI (BM). May-Je.

3034 A. revolvens Lec. Keene Val., May-Je, Westfield, May (Not).

3043 A. pulchrum Lec. Keene Val., J1 (Not).

3044 A. difforme Lec. Cranberry L., J1 6; Keene Val., J1 (Not).

\section{AgLyPtinus Cockerell}

3045 A. laevis Lec. Olcott, Sep 10, Oct (Dt).

\section{FAMILy CLAMBIDAE}

\section{Clambus Fischer}

3047

3048

C. gibbulus Lec. Keene Val., May (Not).

C. puberulus Lec. Keene Val., May (Not).

\section{FAMILY SCYDMAENIDAE SUBFAMILY SCYDMAENINAE}

\section{EUCONNUS Thomson}

3058 E. ventralis Csy. "NY" (Csy).

3059 E. clavipes Say. Peekskill, Sherm (AM) ; vic. NYC (AM) ; LI: Ap 16May 2, Shoe (Nic); Jamaica, May 15 '(Pears) ; Coney I., Schott (Nic).

3060 E. consobrinus Lec. "NY" (Lec); Ithaca, Mar (Lg).

3061 E. semiruber Csy. Sylvan Beach, Je (Not). 
3063 E. bicolor Lec. Buffalo (Z\&R) ; Peekskill (Sherm); LI: Ap 9-19, Shoe (Nic) ; Jamaica, Sf, Dow, Pears (Nic).

3064 E. cavipennis Csy. NY, SmHH (Csy).

3065 E. occultus Csy. Westfield, May (Not).

3067 E. nigrescens Csy. "NY" (Csy).

3068 E. affinis Csy. LI: Wyandanch, Ap 3, under pine bark (Nic).

3069 E. gratus Csy. Keene Val., J1, Whitehall, Oct (Not).

3073 E. salinator Lec. SI, Mar, on salt meadows (L\&D); LI: common in salt marshes; in early spring found along edge of snow; usually taken under boards and stones during May-Je (Nic); Brooklyn, Dow (Ds); Flatbush (Ds) ; Bellport, Je, Jamaica (BM).

3074 E. haedillus Csy. Keene Val., Je, J1 (Not).

3075 E. fatuus Lec. Keene Val., Je (Not); Schoharie (Not).

\section{PycNophus Casey}

3080 P. rasus Lec. Buffalo (Z\&R); Mosholu (BM); SI, Mar-Ap (L\&D); LI: Farmingdale, Ap, Eng. (Nic).

\section{CONNOPHRON Casey}

3085 C. longipenne Csy. LI: Yaphank (Not).

3087 C. formale Csy. LI: Yaphank (Not).

3090 C. flavitarse Lec. "NY" (Lec) ; LI: Rosedale, Ap, Wyandanch (Not).

3099 C. fossiger Lec. Buffalo (Z\&R); LI: Jamaica, Pears, Dow (Nic) ; Huntington, Je 18, Schott (Nic); Flatbush, Ap 15, Schott (Nic); Wyandanch, Ap 30, Dow, Shoe (Nic) ; Brooklyn (Ds); Yaphank, May, Newtown, Ap (BM).

3101 C. brevicorne Say. LI: Wyandanch, Ap 3, Oct 30, not rare in sphagnum bog (Shoe, Nic).

3106 C. clavicorne Csy. Peekskill (Sherm); Westchester Co, Dow (Ds); Mosholu, Sep (BM).

3107 C. abducens Csy. Tivoli, May (Not).

3109 C. laetulum Csy. Hudson R. Val., SmHH (Csy).

3115 C. frontale Csy. Mosholu, Sep (BM); SI, Nov (L\&D); LI: Jamaica, May 15, Pears (Nic).

3130 C. castaneum Csy. Keene Val., Je, J1 (Not).

3136 C. parcum Csy. Croton L., Ap (Not).

3139 C. biceps Csy. LI: Wyandanch, Ap 3, under pine bark (Nic).

3142 C. trinifer Csy. "NY" (Csy); LI: Jamaica, Pears (Nic).

3145 C. fulvum Lec. "NY" (C'sy) ; Westfield, May (Not).

3150 C. capillosulum Lec. Mosholu, Sep (BM) ; LI: Huntington, Je 18, Schott (Nic); Yaphank, Ap (BM).

3153 C. lynceum Csy. "NY", SmHH (Csy).

3155 C. politum Say. LI: Wyandanch, Oct (Not).

3165 C. pumilum Csy. Hudson R. Val. (Csy). 


\section{SCYDMAENUS Latreille}

3168 S. perforatus Schaum. Johnstown, Ap (NYS) ; Ithaca, Nov, Bish (NYS) ; Windsor, Je (Not); Westchester Co, May, Dow (Ds) ; Van Cort. Pk. (BM) ; SI, common, sifting leaves, Ap, Woodr, Nic (L\&D); LI: Jamaica, May 11, Pears (Nic).

3171 S. cribrarius Lec. Keene Val., J1 (Not).

3172 S. turbatus Csy. Keene Val., Parkers in Lewis Co, Je (Not).

3183 S. corpusculum Csy. Ramapo (Sf).

3184 S. caducus Csy. Westfield, May (Not).

\section{OpREsus Casey}

3188 O. othonus Csy. Bronx Pk., Je (Sf).

\section{Cephennium Müller and Kunze}

3194 C. corporosum Lec. Near NYC (Csy); Bronx Pk., Mar, Sf (AM) ; Van Cort. Pk. (Nic).

\section{Chevrolatia Duval}

3208 C. amoena Lec. Vic. NYC (Lec).

\section{ACHOLERops Casey}

3222 A. zimmermanni Schaum. Sodus Pt., Je (Not); LI: Yaphank, Sep (BM).

\section{FAMILY ORTHOPERIDAE}

(Corylophidae)

\section{BATHONA Casey}

3229 B. sphaericula Csy. Mooers, Sep (Not); LI: Yaphank, Je (Not).

\section{CORYLOPHODES Matthews}

3230 C. marginicollis Lec. Peekskill (LgAM); SI, Mar (L\&D) ; LI: Yaphank, Je (Not).

\section{Gronevus Casey}

3234 G. sticticus Csy. Mooers, Sep (Not).

3236 G. truncatus Lec. NY, under stones (Csy).

\section{Rypobius LeConte}

3239 R. marinus Lec. SI, Mar 18, Ap 30, in salt meadow (L\&D) ; LI: Rockaway Beach (LgAM) ; Coney I. (Lec).

\section{ORTHOPERUS Stephens}

3244 O. glaber Lec. Buffalo (Z\&R); Canastota, Mar 13, J, C. Faure (Kngt); SI, Je (L\&D).

3245 O. suturalis Lec. Newport (NYS). 
6476a O. scutellaris Lec. var. piceus Csy. NY, type locality. Axton, Je (M\&H), as scutellaris, belongs here $(\mathrm{Lg})$. The reference in $\mathrm{Lg}$ Cat. to Sphaeriidae is erroneous.

\section{SERICODERUS Stephens}

3252 S. lateralis Gyll. (flavidus Lec.). Buffalo (Z\&R); Keene Val., J1 (Not).

3253 S. obscurus Lec. Buffalo (Z\&R); Keene Val., May (Not).

SACIUM LeConte

3258 S. lugubre Lec. Keene Val., Sep (Not).

\section{Molamba Casey}

3265 M. biguttata Lec. "NY" (Csy); West Pt. (Robn).

3266 M. lunata Lec. Newport (NYS); Keene Val., Sep (Not) ; Cranberry L., Je-J1 (H) ; Buffalo (Z\&R) ; SI, ex Rbts Coll. (L\&D) ; LI: Central Pk., Ap (Eng).

3268 M. lepida Lec. Vic. NYC (AM).

3269 M. ornata Csy. Pike, Ap (NYS) ; Niverville, May, Leon (NYS).

3271 M. fasciata Say. Buffalo (Z\&R); vic. NYC (AM); SI, Je (Chitt).

\section{FAMILY STAPHYLINIDAE 36 SUBFAMILY MICROPEPLINAE}

MICROPEPLUS Latreille

3290 M. tesserulus Curtis. Keene Val., Je (Not).

\section{SUBFAMILY OXYTELINAE \\ Tribe PIESTINI \\ SIAGONIUM Kirby}

3298 S. americanum Melsh. Newport, 1898 (NYS).

3299 S. punctatum Lec. Cranberry L., J1-Aug (H); Fourth L., Je (NYS).

TRIGA Fauvel

3305 T. picipennis Lec. Keene Val., J1, Westfield (Not) ; Delmar, May (NYS); Peekskill (AM); Normanskill, Ap (NYS); West Pt. (Robn); SI, Ap-May, under bark of beech log (L\&D); LI: Queens, Ap, Shoe (Sf).

\section{ELEUSis Castelnau}

3307 E. pallida Lec. "NY" (Horn); Windsor, Je (Not); Nichols, May (Not).

\section{LISPINUS Erichson}

\section{L. prolixus Lec. "NY" (Lec).}

${ }^{36}$ The list in this family has been gone over critically by Howard Notman and H. C. Fall, and Mr. Notman has made many additions from his extensive collecting and study of this group in the State. 
THORACOPHORUS Motschulsky

(Glyptoma Erichson)

3315 T. costalis Er. Newport, May (NYS); Sodus Pt., Je (Not); Buffalo (Z\&R) ; Westfield (Not); Albany, Nov 3 (NYS); Peekskill (AM); SI, Ap 26, under bark (L\&D) ; LI: Yaphank, May (Not).

\section{PSEUdOPSIS Newman}

3319 P. sulcata Newm. Keene Val., Je (Not) ; Gloversville, Sep (NYS) ; Westfield (Not); L. Pleasant, Love (Sf).

\section{OlisthaERUS Heer}

3321 O. nitidus Lec. (substriatus Gyll.). Keene Val., Je (Not); Herkimer Co, Yg (AM).

\section{Proteinus Latreille}

3322 P. atomarius Er. Westfield (Not).

\section{Megarthrus Stephens}

3330 M. excisus Lec. Cranberry L., J1 3, in decaying fungi $(H)$; Gloversville, Sep (NYS).

3331 M. sinuaticollis Boisd. Keene Val., Je-J1 (Not).

3332 M. americanus Sachse. Peekskill (AM).

\section{Tribe OMALIINI}

\section{TRIGONODEMUS LeConte}

3335 T. striatus Lec. Buffalo ( $Z \& R)$.

\section{AnthoвiUm Stephens}

3339 A. convexum Fauv. Axton, Je (M\&H) ; Keene Val., Je (Not) ; Cranberry L., Je (H) ; Newport, May (NYS); Castile, May, Brad (Flet); Katonah (Fall).

3344 A. horni Fauv. Cranberry L., Je-Aug, extremely abundant on flowers of Acer, Spiraea, Rubus, etc. $(\mathrm{H})$; Red House, Je (Not) ; Karner, May (NYS).

3347 A. pothos Mann. (dimidiatum Melsh.). Mt. Marcy, J1 (Not); Fourth L., Je (NYS); Buffalo (Z\&R).

3351 A. orientale Bnhr. Mt. Marcy, Jl, Keene Val., Je, Red House, Je (Not).

\section{PyCNOGLYPTA Thomson}

3355 P. lurida Gyll. Keene Val., Je (Not); Cranberry L., Je 28, in sweeping $(\mathrm{H})$.

PHYLLODREPA Thomson

3359 P. punctiventris Fauv. LI: Orient, Ap (NYS).

3363 P. florale Payk. West Pt. (Robn). 


\section{OMALIUM Gravenhorst}

3379 O. humerosum Fauv. Newport, 1898 (NYS); Albany, Je (NYS).

3386 O. repandum Er. Peekskill (Fall, Sherm); LI: Orient, Nov (NYS).

3387 O. rivulare Payk. Sodus Pt., Je, Westfield (Not).

\section{PhLOEONOMUS Heer}

3393 P. lapponicus Zett. Mt. Marcy, J1 (Not).

3394 P. pusillus Grav. Trenton Falls (Hamilton).

\section{OLOPHRUM Erichson}

3412 O. obtectum Er. Newport (NYS); Keene Val., Sep (Not); Syracuse, May (H) ; Pike (NYS) ; Batavia, J1 (Kngt) ; Maratanza L., Ulster Co, May (NYS); Peekskill (AM); SI, in salt meadows (LgAM).

3413 O. rotundicolle Sahlb. Buffalo (Z\&R); SI, Mar-Ap, at border of salt meadow (L\&D).

\section{ARPEDIUM Erichson}

3420 A. schwarzi Fauv. Newport, May (NYS); Washingtonville, Oct (NYS). 3423 A. brachypterum Grav. Moss Pond, Mt. Marcy, J1 (Not).

\section{Acidota Mannerheim}

3424 A. crenata Fab. Cranberry L., Je 28, sweeping in woods (H) ; LI: Rosedale, Ap (Not).

3425 A. subcarinata Er. Tivoli, May (Not).

\section{Paralesteva Casey}

3431 P. pallipes Lec. Mt. Marcy, Oct 1 (LgAM); Sodus Pt, Je (Not); Windsor, Je (Not).

\section{MicRoedus LeConte}

3446 M. austinianus Lec. Keene Val., J1 (Not).

\section{GEODROMICUS Redtenbacher}

3461 G. brunneus Say. Buffalo (Say); Howe Cave, May (NYS); Windsor, Je (Not); Peekskill (Fall, Sherm); SI, May (L\&D).

3462 G. strictus Fauv. Sodus Pt., Je, Westfield (Not); McLean, Aug 12 (Flet); SI $(L \& D)$.

3464 G. plagiatus Fab. "NY" (Hamilton); Sodus Pt., May-Je (Not).

G. plagiatus var. nigritus Müll. Westfield (Not).

BOKEAPHILUS Sahlberg

3474 B. americanus Not. LI: Rosedale (Not).

RENARDIA Motschulsky

$3474 \frac{1}{2}$ R. jubilosa Mots. "NY" (Lg Cat.). 


\section{Tribe OXYTELINI \\ SYNTOMIUM Curtis}

3475 S. confragosum Mak1. Keene Val., Mt. Skylight, J1 (Not).

\section{ANCYROPHORUS Kraatz}

3483 A. planus Lec. Mt. Marcy, J1 (Not); Washington Co (AM).

\section{TROGOPHLOEUS Mannerheim}

3487 T. corvinus Csy. Westfield, May (Not).

3496 T. bilineatus Steph. (riparius Lac.). "NY" (Fauvel in Hamilton).

3498 T. quadripunctatus Say. Syracuse, May $(\mathrm{H})$; Sodus Pt., Windsor, Je (Not) ; LI: Brooklyn (Csy).

3500 T. morio Er. Syracuse, May (H); Westfield, May (Not); Schoharie (Not); Windsor, Je (Not).

3501 T. rivularis Mots. Sodus Pt., Windsor, Je (Not).

T. lacustris Not. Cranberry L., Je $23(\mathrm{H})$. Type.

3514 T. difficilis Csy. Sodus Pt., Windsor, Je (Not).

3518 T. temporalis Csy. "NY" ( $\mathrm{Sm})$.

3520 T. nanulus Csy. Cranberry L., Je 23, sweeping in marshy area $(H)$.

3525 T. subtilis Er. Sodus Pt., Windsor, Je (Not).

3531 T. pudicus Csy. Windsor, Je (Not); SI, Je (L\&D).

3532 T. robustulus Csy. "NY" (Csy).

3537 T. simplarius Lec. LI: Coney I., in salt marsh (Lec).

3540 T. convexulus Lec. "NY" (Lec).

— T. volans Not. Windsor, Je (Not).

T. vespertinus Not. Windsor, Je (Not).

\section{APOCEllus Erichson}

3556 A. sphaericollis Say. Crown Pt., Aug (Not); Mooers, Sep (Not); SI, on sidewalk (L\&D).

\section{Oxytelus Gravenhorst}

3571 O. sculptus Grav. Newport (NYS); Fourth L., Je (NYS); Buffalo (Z\&R) ; Peekskill, Ap-May, Sherm (AM); Mosholu (Sf).

3572 O. insignitus Grav. Newport, May-Je (NYS) ; Pike, Sep (NYS); Albany, May; Clinton Hgts., Ap (NYS); Ulster Co, Sep, Nic (Sf); West Pt. (Robn); West Farms, NYC, Oct, Zab (AM); SI (L\&D); LI: Flatbush, Oct-Nov, Zab (AM); Jamaica, Pears (Sf); Orient, Ap (NYS).

3574 O. breviceps Csy. Newport, May (NYS); Catskills (Csy).

3576 O. laqueatus Marsh. Windsor, Je (Not).

3579 O. invenustus Csy. Ulster Co, Sep, Nic (Sf).

3580 O. pennsylvanicus Er. Newport, May (NYS); Fourth L., Je (NYS). 
3582 O. rugosus Fab. Newport, May (NYS) ; Westfield, May (Not) ; Albany, Ap (NYS) ; Peekskill, Ap, under small stones in gardens (Sherm); Mosholu (Sf).

3587 O. nitidulus Grav. "NY" (Hamilton); Windsor, Je (Not).

3589 O. suspectus Csy. Albany, May (NYS); Bronx Pk. (Sf).

3592 O. nanus Er. NY ( Lg Cat.).

3593 O. exiguus Er. Newport, 1898 (NYS); Peekskill, Mar, Sherm (AM).

3595 O. tetracarinatus Block. Windsor, Je (Not).

\section{Platystethus Mannerheim}

3596 P. americanus Er. Keene Val., Je (Not) ; Cranberry L., Je 25, in decaying fungi $(\mathrm{H})$; Buffalo (Z\&R,Horn,Er); Windsor, Je (Not); SI (L\&D).

BLEdius Mannerheim

3599 B. pallipennis Say (mandibularis Er.). "NY" (Lec); LI: Rockaway Beach, Dietz (Sf).

3601 B. brevidens Lec. "NY" (Lec).

3615 B. politus Er. LI: Coney I., J1 (Lec).

3622 B. semiferrugineus Lec. Sodus Pt., Je (Not); Buffalo (Z\&R); Albany, Ap (NYS).

3625 B. fumatus Lec. Buffalo (Rein); Peekskill, Mar, Sherm (AM).

3636 B. analis Lec. Peekskill, Sherm (AM).

3637 B. nitidicollis Lec. "NY" (Lec).

3643 B. opacus Block. "NY" (Fauv).

3651 B. sinuatus Lec. Westfield, May (Not).

3656 B. stabilis Csy. Schoharie (Not).

3657 B. confusus Lec. Syracuse, Ap $(H)$.

3668 B. tau Lec. Sodus Pt., Je (Not); Hudson Falls, Sherm (Fall).

3676 B. emarginatus Say. Windsor, Je, Schoharie (Not); Nassau, J1 (NYS); Hudson Falls, Aug, Sherm (AM).

3677 B. honestus Csy. Schoharie (Not); Catskills (Csy).

3679 B. basalis Lec. LI: Coney I., in salt marsh (Lec); Rockaway Beach (AM).

3685 B. opaculus Lec. LI: Coney I. (Lec).

3686 B. cordatus Say. Northampton, Karner, Je (NYS) ; Hudson Falls, Aug, Sherm (AM) ; SI: Sandy Hill, $L g$ (Sf).

B. fracticornis Payk. Windsor, Je (Not).

B. verticalis Not. Westfield, May (Not).

\section{ThINobius Kiesenwetter}

3690 T. flavicornis Lec. Oneida L., Aug (Drk); LI: Coney I., in salt marsh (Lec).

3691 T. grossulus Csy. Near NYC (Csy).

3694 T. fimbriatus Lec. Westfield, May (Not).

— T. amphibius Not. Westfield, May (Not). 
T. tardus Not. Westfield, May (Not).

T. grandicollis Not. Westfield, May (Not).

T. caseyi Not. Westfield, May (Not).

T. apicicornis Not. Westfield, May (Not).

$3702 \mathrm{~T}$. delicatulus $\mathrm{Kr}$. Westfield, May (Not).

\section{SUBFAMILY STENINAE}

\section{STENUS Latreille}

3711 S. bipunctatus Er. (comma Lec.). Newport, J1 (NYS) ; Syracuse, May (H) ; Buffalo (Z\&R) ; Sodus Pt. (Kngt); Waterville, Je, Westfield, May (Not); Windsor, Je. (Not); Ithaca, Oct (Kngt); Schoharie (Not); Ft. Hunter, Je (Not); West Pt. (Robn).

S. fulvoguttatus Not. Windsor, Je (Not).

3712 S. colon Say. Keene Hgts., J1 (Not); Buffalo (Z\&R); Westfield, May (Not).

3714 S. semicolon Lec. Syracuse, Ap (H) ; Pike, Ap (NYS); Westfield, May (Not).

3718 ?S. laccophilus Csy. Syracuse, May (H).

3719 S. juno Fab. Keene Val., Keene Hgts., J1 (Not) ; Newport (NYS) ; Gloversville, Mar (NYS); Waterville, J1, Sodus Pt., May (Not); Buffalo (Z\&R) ; Westfield, May, Windsor, Je (Not) ; Utica, Je, Tivoli, May (Not); SI, Feb, Woodr, sifting (L\&D).

3722 S. austini Csy. Mt. Marcy, J1 (Not).

3727 S. femoratus Say. Sodus Pt., Je (Not).

3728 S. similiatus Blatch. Parkers, Je, Tivoli, May (Not).

3730 S. strangulatus Csy. "NY" (Ent. Amer. 1, 74, see Csy, Stenini, 1884, 39).

3736 S. erythropus Melsh. Rensselaer, May, Kinderhook, Je (NYS) ; SI, Feb, sifting, Woodr (L\&D).

3737 S. rugifer Csy. Keene Hgts., J1, Ausable Forks, Crown Pt., Aug, Lowville, Je, Sodus Pt., Je, Tivoli, May, Sylvan Beach, Je (Not).

S. edax Not. Windsor, Je (Not). May not be distinct from rugifer Csy. (Not).

3753 S. placidus Csy. Johnstown, May (NYS).

3757 S. militaris Csy. Westfield, May (Not).

3758 S. pluto Csy. "NY", Je, Sherm (AM); Ausable Lakes, J1 (Not).

3769 ?S. nanus Steph. "NY" (AM).

3776 S. obstrusus Csy. "NY" (Csy).

3780 S. colonus Er. Cranberry L., Je 23, sweeping (H) ; Ashland, Aug (Frost) ; West Pt. (Robn).

3782 S. humilis Er. Cranberry L., J1 5, in dying birch (H).

- S. perexilis Not. Windsor, Sodus Pt., Je (Not).

3787 S. stygicus Say. Mooers, Sep (Not) ; Newport, Johnstown, Aug (NYS) ; Windsor, Je (Not); Ramapo, Je (Ds); SI, Je (L\&D).

3788 S. egenus Er. Common in Essex, Franklin, St. Lawrence, Lewis, and Oneida Counties, Je-Sep (Not); Cranberry L., Je, sweeping ( $\mathrm{H}$ ) ; Sodus Pt., Je (Not).

3789 S. dolosus Csy. Ausable Lakes, J1, Ausable Forks, Aug, Crown Pt., Aug, Sodus Pt., Je, Sylvan Beach, Je (Not). 
3791 S. sectilifer Csy. (milleporus Csy.). "NY" (Csy).

3792 S. vinnulus Csy. Cranberry L., Je, sweeping (H) ; Moss Pond, Ausable Lakes, J1, Sodus Pt., May (Not).

3795 S. argus Grav. Keene Val., Mt. Marcy, Sylvan Beach, Je-J1 (Not) ; LI: Yaphank, Mar (Not).

3796 S. pudicus Csy. "NY", Je, Sherm (AM).

3797 ?S. minor Csy. Syracuse, Ap (H).

3799 S. parallelus Csy. Newport, 1898 (NYS).

3802 S. juvencus Csy. Keene Val., Je (Not).

3809 S. gratiosus Csy. Waterville, Je (Not).

3811 S. curtus Csy. Waterville, Je (Not).

3832 S. flavicornis Er. Newport, May (NYS); Keene Val., Keene Hgts., Ausable Lakes, Jay, Waterville, Sylvan Beach, Lowville, Sodus Pt., Windsor, Je-Aug (Not); Chapel Pond, Je, Leon (NYS); Syracuse, Ap-May $(\mathrm{H})$; Bridgeport, Ap-May (H) ; Buffalo ( $\mathrm{Z} \& \mathrm{R})$; Schoharie (Not); Albany, Karner, Voorheesville, New Baltimore, Poughkeepsie, May-Je (NYS); Whitehall, Oct, Ft. Hunter, Tivoli, May (Not); West Pt (Robn); SI, Feb, in sifting, Woodr (L\&D).

3833 S. annularis Er. Keene Va1., Je, Mt. Marcy, J1, Sodus Pt., May (Not); Normanskill, May (NYS); SI, May (L\&D).

3837 S. tarsalis Ljungh. Keene Val., Mt. Marcy, Moss Pond, Ausable Pt., Ft. Hunter, Whitehall, Je-Oct (Not); Cranberry L. (H) ; Washington Co, Aug, Sherm (AM); Rensselaer, May (NYS); Peekskill, Mar (AM); Hudson Falls, Sherm (AM).

3838 S. canadensis Csy. Newport (NYS) ; Keene Val., Sylvan Beach, Windsor, Whitehall, Ft. Hunter, Je-Oct (Not).

S. reconditus Csy. Ashland, Aug (Fall). Fall believes it a good species.

3841 S. punctatus Er. Ausable Lakes, Crown Pt., Mt. Marcy, Keene Val., Waterville, Sodus Pt., Windsor, Tivoli, Ft. Hunter, May-Aug (Not); Newport, Je (NYS) ; Elm L., Aug (NYS) ; Washington Co, Aug, Sherm (AM) ; Kinderhook, Je, Poughkeepsie, May (NYS); Peekskill (AM) ; Ramapo, May (Ds) ; Ashland, Aug (Frost); SI, Oct (L\&D).

3842 S. artus Csy. "NY" (Csy).

3844 S. callosus Er. Syracuse, May $(\mathrm{H})$.

3846 S. arculus Er. Crown Pt., Keene, Whitehall, Tivoli, May-Aug (Not); Washington Co, Peekskill, Hudson Falls (AM); Kenwood, J1 (NYS); SI, Oct (L\&D).

3848 S. hubbardi Csy. Mt. Marcy, Keene Val., Moss Pond, Ausable Lakes, Mountain View, Ft. Hunter, Tivoli, May-Sep (Not).

S. caenicolus Not. Mooers, Keene, Keene Hgts., Ausable Forks, Lowville, Sylvan Beach, Waterville, Sodus Pt., Windsor, Rensselaerville, Tivoli, May-Je (Not).

3853 S. croceatus Csy. Sodus Pt., Je (Not). SI, Feb, in sifting (L\&D).

\section{Dianous Samouelle}

3857 D. coerulescens Gyll. Buffalo (Robts); Suffern, May, Schott (Not); NYC and vic. (Frost); SI, Ap-Nov; clings to boards and stones below falls (L\&D).

3858 D. nitidulus Lec. Ausable Lakes, J1 (Not); East Bay, Wayne Co, Sep, Bish (NYS). 


\section{SUBFAMILY EUAESTHETINAE}

\section{STICTOCRANIUS LeConte}

3859 S. puncticeps Lec. SI, Ap (Sf).

\section{EUAESTHETUS Gravenhorst}

3869 E. robustulus Csy. LI: North Beach, Feb, Schott (Sf); New Lots, Cypress Hills, Ap, Shoe (Sf).

3872 E. americanus Er. Sodus Pt., Windsor, Je (Not); Peekskill (AM); Bronx Pk. (Sf) ; SI, Oct (L\&D).

\section{SUBFAMILY PAEDERINAE \\ PINOPHILUS Erichson}

3874 P. latipes Grav. "NY" (GravANSP).

\section{PALAMinus Erichson}

3891 P. luteus Csy. LI (Csy).

3892 P. testaceus Er. SI, May-Je (L\&D).

3894 P. hudsonicus Csy. LI (Csy).

3900 P. larvalis Lec. LI, under moss (Lec).

\section{Gastrolobium Casey}

(Cryptobium)

3903 G. carolinum Er. "NY" (AM).

3908 G. bicolor Grav. Mooers, Sep (Not) ; Newport, May (NYS) ; Gloversville, Je (NYS) ; Pike (NYS) ; Buffalo (Z\&R) ; Sodus Pt., Je, Westfield, May (Not); Ithaca, May-Oct (DtNYS); Windsor, Je (Not); Schoharie (Not); Clinton Hgts., Ap (NYS); West Pt. (Robn); SI, May, J1 (L\&D).

3909 G. melanocephalum Er. "NY" (Csy).

3910 G. badium Grav. SI, Ap, Je (L\&D).

3914 G. parallelum Csy. LI (Csy).

\section{Hesperobium Casey}

3926 H. pallipes Grav. Gloversville, Mar-Sep (NYS); Syracuse, Ap, Oct $(\mathrm{H})$; Buffalo (Z\&R); Westfield, May (Not); McLean, Ap 17, Babiy (Flet) ; Ithaca, Ap-Sep (Dt) ; Auburn (H) ; Poughkeepsie, Je (NYS); West Pt. (Robn); Mosholu, May (Ds); SI, Mar-Ap, Sep, Nov (L\&D) ; LI: Orient, Mar 17 (Ds).

3926a H. pallipes var. capito Csy. Ithaca, May-Oct (Dt).

3927 H. flavicorne Lec. "NY" (Csy).

3928 H. cinctum Say. "NY" (Csy); Syracuse, Ap (H) ; SI, Feb, in sifting (L\&D).

3932 H. sellatum Lec. Olcott, Ap (Dt). 
3933 H. cribratum Lec. Newport, May (NYS); Gloversville, Mar (NYS); Syracuse, Ap $(\mathrm{H})$; Buffalo (Z\&R) ; Ithaca, Mar-Nov (Dt) ; Windsor, Je (Not); Albany, May (NYS); Rensselaer, May (NYS); Poughkeepsie, Je (NYS); West Pt. (Robn); Peekskill, Mar (AM).

\section{PAEDERUS Fabricius}

3950 P. obliteratus Lec. "NY" along ocean beach (Csy); LI: Ap, Shoe (Sf); Brooklyn, Nic (Sf).

3952 P. littorarius Grav. Mooers, Sep (Not) ; Axton, Je (M\&H) ; Newport (NYS) ; Cranberry L., Je-Jl, under damp stones (H) ; Oneida L., Aug (Drk); Syracuse, Ap (H) ; Sodus Pt., Je (Not); Olcott, Howland I., Ds (Dt); Buffalo (Z\&R); Pike, Mar (NYS); Castile, Brad (Flet); Ithaca, common in early spring (Dt); McLean, Ap, Babiy, Je, Cy (Flet) ; Windsor, Je (Not); Karner, Je (NYS) ; Cedar Hills, Ap (NYS); Nassau, Mar (NYS); Ramapo, May (Ds); West Pt. (Robn) ; SI, Mar, May-Je, Nov (L\&D) ; LI, Ap, Shoe (Sf).

3954 P. canonicus Csy. LI (Csy).

\section{Cryptobiella Casey}

3959 C. pusilla Lec. LI: from the seashore (Lec).

\section{LATHROBIUM Gravenhorst}

3964 L. amplipennis Csy. (deceptivum Csy.). Westfield, May (Not) ; Ithaca, Ap, Jl (Dt); Hudson Val. (Csy); White Plains, Mar, Bno (Sf).

3966 L. armatum Say (procerum (Csy.). Gloversville, Mar (NYS) ; Syracuse, Ap (H) ; Ithaca, May (Dt) ; McLean, J1 (Dt) ; Windsor, Je (Not); Delmar, Mar (NYS).

3969 L. nigrolucens Csy. Westchester Co, Dow (Sf); LI (Csy).

3981 L. simile Lec. Oneida L., Aug (Drk) ; Pike, Ap (NYS) ; Ithaca, Nov (Dt); Clinton Hgts., Oct (NYS); Peekskiil, Mar, J1 (AM) ; Hudson Falls, Sherm (AM); near NYC (Csy); LI, May, Shoe (Sf).

3983 L. neglectum Csy. Ringwood, May (Dt).

3990 L. bicoloratum Bnhr. Newport, Je (NYS).

3991 L. puncticolle Kby. "NYS" (Horn, Kby).

L. lintneri Not. Westfield, May (Not).

\section{LITOLATHRA Casey}

3992 L. rhodeana Csy. Near NYC (Csy).

3995 L. cruralis Csy. Ithaca, Mar (Dt).

3999 L. subgracilis Csy. "NY" (Csy).

4001 L. suspecta Csy. Near NYC (Csy).

\section{LATHROBIOMA Casey}

4008 L. othioides Lec. Windsor, Je (Not).

4009 L. tenuis Lec. "NY" (Lec); Windsor, Je (Not); Peekskill, Mar, as Lathrobium (AM). 
4010 L. nigrolinea Csy. Cranberry L., Je 23, under bark of dead $\log (\mathrm{H})$.

4012 L. scolopacea Csy. Cranberry L., Je 23, under bark of dead log (H).

4013 L. inops Csy. "NY" (Sf).

\section{LATHROLEPTA Casey}

4014 L. debilis Lec. Near NYC (Csy).

\section{TETARTOPEUS Czwalina}

4016 T. punctulatus Lec. Westfield, May (Not); Gloversville, Mar (NYS); Sodus Pt., Je (Not); Buffalo (Rein); West Pt. (Robn); SI, Sep 30, in sifting (L\&D); LI: Brooklyn, Mar, Shoe (Sf).

4022 T. angularis Lec. Buffalo (Z\&R); Albany, May (NYS) ; Clinton Hgts., Oct (NYS); SI, Jan 19, Ap, in sifting (L\&D).

4024a T. tetricus Csy. var. agitans Csy. Near NYC (Csy).

\section{Eulathrobium Casey}

4031 E. grande Lec. Sodus Pt., Je (Not); Ringwood, McLean, J1 (Dt); Peekskill (AM); LI: Cypress Hills, Ap, Shoe (Sf).

\section{LATHROTAXIS Casey}

4045 L. longiuscula Grav. Newport, May (NYS); Gloversville, Ap (NYS); Olcott, Ap-May (Dt); Windsor, Oct (Dt) ; Westfield, May (Not); Ithaca, Ap-May (Dt) ; Poughkeepsie, May (NYS) ; Bronx Pk. (Sf); LI: Rockaway Beach, May, Shoe (Sf).

\section{LATHRobiella Casey}

4068 L. ventralis Lec. Newport, May (NYS); Nassau, Je, Normanskill, May (NYS).

4074 L. collaris Er. Axton, Je (M\&H); Newport, May (NYS); Sodus Pt., May (Not) ; Syracuse, Ap (H) ; Olcott, Ap, Ithaca, May (Dt) ; Westfield, May (Not) ; Windsor, Je (Not) ; Albany, May, Clinton Hgts., Ap (NYS) ; West Pt. (Robn); Westchester Co, Dow (Sf); NYC, May (NYS); SI, May (L\&D); LI, Ap, Shoe (Sf).

4081 L. fragilis Csy. Windsor, Je (Not); near NYC (Csy).

4082 L. rubida Csy. Westfield, May (Not).

4087 L. depressula Csy. Catskills (Csy).

\section{MicRolathra Casey}

4088 M. pallidula Lec. Fourth L., Je, Gloversville, Ap (NYS) ; Karner, Oct, Normanskill, May (NYS); SI (Csy); LI: Brownsville, Ap, Dow (Sf).

\section{ADEROCHARIS Sharp}

4093 A. corticinus Grav. Ithaca, Sep (Dt); Schoharie (Not); SI, Je (L\&D) 


\section{LITHOCHARIS Lacordaire}

4094 L. ochracea Grav. Schoharie (Not); Peekskill, Je (AM).

\section{TRACHYSECTUS Casey}

4105 T. confluentus Say. Axton, Je (M\&H with query); Newport, May, Gloversville, Ap (NYS); Syracuse, Ap (H) ; Cranberry L., Je, under bark of dead $\log (\mathrm{H})$; Pike, Ap (NYS) ; Sodus Pt., Je, Westfield, May (Not); Karner, Nassau, May, Normanskill, Ap (NYS); West Pt. (Robn); Westchester Co (Ds) ; SI, Ap-May (L\&D) ; LI: Queens, Flatbush, Rockaway, Mar (Ds).

\section{TETRAMEDON Casey}

4151 T. rufipenne Csy. Hudson Val. (Csy).

\section{HYPOMEDON Rey}

4173 H. debilicornis Woll. NYC, Jan 30 (NYS).

\section{Pseudomedon Rey}

4174 P. thoracicum Csy. Peekskill, Je, as obsoletus Nordm. (AM); SI, Jan (L\&D).

4174a P. thoracicum var. ruficolle Csy. "NY" (Csy).

\section{SCOPAEUS Erichson}

4207 S. crassulus Csy. Sodus Pt., Je (Not).

4210 S. longipennis Fall. Sodus Pt., Je (Not).

4215 S. hudsonicus Csy. Hudson Val. (Csy).

- S. quadriceps Not. Schoharie (Not); Windsor, Je (Not).

4216 S. exiguus Er. Hudson Val. (Csy).

4218 S. degener Csy. Hudson Val. (Csy).

4221 S. delicatus Csy. "NY" (Csy).

\section{Scopaeoma Casey}

S. pallida Not. Westfield, May (Not).

\section{Scopaeopsis Casey}

4234 S. ventralis Csy. LI (Csy).

\section{STILICUS Latreille}

4240 S. latiusculus Csy. Sodus Pt., Je (Not); LI: Flatbush, Mar, Rockaway Beach, Dore (S).

4241 S. rudis Lec. Westchester Co, May, Dow (Sf); Aqueduct, Mar, Shoe (Sf); Huntington, Je, Schott (Sf). 
4243 S. angularis Lec. Peekskill, May (AM); LI: Bellport, J1, Nic (Sf).

4247 S. biarmatus Lec. LI: Forest Pk, Ap, Pears (Sf).

4247a S. biarmatus var. abbreviellus Csy. Near NYC (Csy).

4248 S. dentatus Say. Westchester Co (Ds); Peekskill (AM); SI, Ap (L\&D); LI: Brooklyn, Flatbush, Feb (Ds).

\section{Megastilicus Casey}

4251 M. formicarius Csy. West Pt., in ants' nests $(\mathrm{Lg})$.

\section{Pachystilicus Casey}

4250 P. hanhami Wick. Syracuse, Ap (H).

\section{STAMNODERUS Sharp}

4257 S. monstrosus Lec. SI, Jan 19, Feb, in sifting (L\&D).

\section{Astenus Stephens}

\section{(Sunius Erichson)}

4260 A. prolixus Er. Peekskill, Mar (AM); Westchester Co, May, Dow (Sf); SI, Mar, Sep (L\&D).

4262 A. americanus Csy. "NY to Va" (Csy) ; LI: Jamaica, May, Pears (Sf).

4263 A. linearis Er. Buffalo (Z\&R).

4265 A. binotatus Say. SI, Jan 19, in sifting (L\&D).

4266 A. cinctus Say. Albany, Ap (NYS).

4270 A. brevipennis Aust. Mooers, Sep (Not); SI, May (L\&D).

4272 A. discopunctatus Say. Mooers, Sep (Not); Newport, May-Je (NYS); Syracuse, Montour Falls, Ap (H); Westfield, May (Not); Windsor, Je (Not) ; Ithaca, Mar, McLean, J1 (Dt) ; Catskills, Pears (Sf); Delmar, May (NYS as Paederus); Normanskill, Mar (NYS); SI, Pears (Sf).

4278 A. longiusculus Mann. Buffalo (Z\&R); Peekskill (AM); SI, Mar-Ap, Nov (L\&D).

\section{SUBFAMILY STAPHYLININAE}

\section{GAUROPTERUS Thomson}

4284 G. fulgidus Fab. Peekskill, late Ap-early May (Sherm); West Farms (AM) ; LI: Rockaway, May, Shoe (Sf).

\section{Nudobius Thomson}

4285 N. cephalus Say. Mt. Seward, 4500 ft., Je (M\&H) ; Mt. Whiteface, Aug (Dt) ; Newport, Wells, May (NYS) ; Cranberry L., Je-J1 (H); Syracuse, in hickory (B\&S); Buffalo (Z\&R); Castile, May, Brad (Flet) ; Ithaca, Mar-Oct (Dt); McLean, Sep 20 (Flet); Unadilla, Nic (Sf); West Pt. (Robn); SI, Ap, Sep (L\&D).

4293 N. luridipennis Csy. LI: Wyandanch, Ap, Schott (Sf). 


\section{GYROHYPNUS Mannerheim}

4296 G. obsidianus Melsh. Sodus Pt., Je (Not); Oswego, Sep, Bks (NYS); Pike (NYS) ; Ithaca, Sep (Dt); Pinelawn, Dore (Sf); Wyandanch, Oct, Schott (Sf).

4302 G. emmesus Grav. Newport, May (NYS); Buffalo (Z\&R) ; Karner, Ap (NYS) ; Peekskill, Sherm (AM) ; LI: Little Neck, Ap, Schott (Sf).

4304 G. fusciceps Lec. "NY" (Csy) ; Syracuse, Ap (H) ; Sodus Pt., May, Westfield, May, Windsor, Je (Not).

- G. pallipennis Not. Westfield, May (Not).

4307 G. melanops Csy. Windsor, Je (Not).

4308 G. hamatus Say (obscurus Er). Mooers, Sep (Not); Gloversville, Ap (NYS) ; Olcott, Ap (Dt); Buffalo (Z\&R) ; Batavia, Sep (Kngt); McLean, Ap 17, Babiy (Flet); Peekskill (AM); West Pt. (Robn); SI, Sep 15 (L\&D) ; LI: Jamaica, May, Dow (Sf) ; Orient, Lath, Ap (NYS).

- G. davisi Not. SI, on gravestones, Aug 31, Sep 28, type (L\&D).

4312 G. sanguinipennis Lec. LI: Brooklyn, May, Dow (Sf).

4313 G. gilvipennis Csy. "NY" (Csy); Westchester Co, Mar, Dow (Sf).

4314 G. pusillus Sachse. Sodus Pt., Je, Westfield, May (Not).

4319 G. gularis Lec. Lansingburg, Oct (NYS); West Pt. (Robn).

\section{LEPTACINUS Erichson}

4324 L. cephalicus Lec. Hudson Val. (Csy).

\section{LEPTACINODES Casey}

4325 L. flaviceps Lec. Peekskill, Sherm, as batychrus Gyll. (AM); LI (Csy).

\section{Stictolinus Casey}

4331 S. scolopaceus Csy. "NY" (Csy).

\section{Nematolinus Casey}

4334 N. longicollis Lec. Ithaca, Ap-May (Dt).

\section{LEPTOLINUS Kraatz}

4347 L. rubripennis Lec. Newport (NYS); Montour Falls, Ap (H).

\section{BAPTOLINUS Kraatz}

4351 B. macrocephalus Nord. Moss Pond, Wallface Mt., Keene Val., J1 (Not).

4352 B. americanus Csy. (pilicornis Payk.). Cranberry L., J1 6-10, under bark of dead stump (H); Sodus Pt., Je (Not); Schoharie (Not); Ulster $\mathrm{Co}, \mathrm{Sep}, \mathrm{Nic}$ (Sf).

4355 B. fraternus Csy. (longiceps Fauv.). "NY" (Fauv). 


\section{Diochus Erichson}

4356 D. schaumi Kr. Peekskill, Sherm (AM) ; SI, on salt meadow, Nov 6 (L\&D) ; LI: Yaphank, Je (Not).

4358 D. thoracicus Csy. LI (Csy).

\section{NEOBISNIUS Ganglbauer}

4361 N. sobrinus Er. Newport, May, Sodus Pt., May-Je, Westfield, May, Windsor, Je (Not); McLean, Aug (Dt); Schoharie (Not); W. Hebron, Je ( $\operatorname{LgBM})$.

4362 N. agnatus Er. LI: Forest Pk., Pears (Sf).

4364 N. paederoides Lec. Newport (NYS) ; Sodus Pt., May-Je, Westfield, May, Windsor, Je (Not) ; Ithaca, May-Nov, McLean, Aug (Dt); Schoharie (Not); Poughkeepsie, Je (NYS); Peekskill, Sherm (AM); LI (Lec, Sf).

$4366 \mathbf{N}_{\mathrm{e}}$ jocosus Horn. Sodus Pt., Je (Not); Bronx Pk. (Sf).

4367 N. jucundus Horn. LI (AM).

4369 N. terminalis Lec. Mooers, Sep (Not); Newport (NYS); Westfield, May, Windsor, Je (Not); Schoharie (Not); Ithaca, Ap, J1-Aug (Dt).

N. fulvicornis Not. Sodus Pt., Je (Not):

\section{Actoвius Fauvel}

4372 A. cinerascens Grav. Mooers, Sep (Not); Sodus Pt., Je, Westfield, May (Not) ; Ithaca, J1 (Dt) ; Peekskill, May (Fall, Sherm); SI, Feb, in sifting, Woodr (L\&D); LI: Jamaica, Dow (Sf).

4373 A. nanus Horn. Sodus Pt., Je (Not); LI: Brooklyn, Mar, Shoe (Sf).

4374 A. inutilis Horn. SI, Feb, in sifting, Woodr (L\&D).

4375 ?A. fraterculus Horn. SI, Sep 18, in sand about grass roots (L\&D).

4379 A. patella Horn. "NY" (Horn).

4380 A. loxatus Horn. SI, $L g$ (Sf).

4383 A. parcus Horn. Cranberry L., J1 3, in decaying fungi (H); SI, Oct 27 (L\&D) ; LI: Cold Sp. (Sf).

\section{Philonthus Curtis}

4384 P. politus L. (aeneus Rossi). Axton, Je (M\&H); Newport, May-Je $(\mathrm{Yg})$; Johnstown, Sep (NYS) ; Cranberry L., Je-J1, in decaying fungi (H) ; Gloversville, May (Yg); Oswego, Aug, $-B k s$ (NYS) ; Olcott, May-Je (Dt) ; Buffalo (Z\&R) ; Interlaken, Ap (NYS) ; Ithaca, ApSep, McLean, Ap, Aug (Dt) ; Albany, Aug (Yg) ; Clinton Hgts., Ap (NYS) ; West Pt. (Robn) ; SI, Je, Aug (L\&D) ; LI: Eastern Parkway, Martin (Sf); Flatbush, Coney I., Oct-Nov, Zab (AM) ; Center I., May, Amagansett, Aug, Eng (Sf); Orient, Je (NYS).

P. tetragonocephalus Not. Cranberry L., Je-Jl, in decaying fungi $(\mathrm{H})$.

4385 P. chalceus Steph. LI: Bellport, Nic (Sf); Wyandanch, Ap, Schott (Sf).

4387 P. sericinus Horn. Cranberry L., J1 3 (Not, H) ; Bronx Pk., Ap, Shoe (Sf) ; LI: Flatbush, May, Dow (Sf).

P. cruentatus Gmel. Cranberry L., J1 $3-4$, in decaying fungi and on carrion $(\mathrm{H})$; Syracuse, Ap-May (H) ; Westfield, May (Not). 
4392 P. umbratilis Grav. Ithaca, Nov (Dt) ; Albany, May (NYS) ; LI: Rockaway Beach, May, Shoe (Sf) ; Wyandanch, Ap, Je, Schott (Sf); Orient, Ap, Lath (NYS).

4393 P. laetulus Say. SI, Je (L\&D).

4395 P. asper Horn. Fourth L., J1 (NYS); West Pt. (Robn); SI (L\&D); LI: Little Neck, Dec, Schott (Sf).

4396 P. fuscipennis Mann. Olcott, Ap (Dt).

4403 P. hepaticus Er. LI: Flatbush, Feb, Schott (Sf); Flushing, Dow (Sf).

4412 P. umbrinus Grav. Gloversville, Sep (NYS); West Pt. (Robn); LI: Wyandanch, Aug, Schott (Ds).

4414 P. palliatus Grav. Mooers, Sep (Not); McLean, May, Ithaca, Je (Dt).

4418 P. quadricollis Horn. Mooers, Sep (Not); Newport (NYS); Olcott, Je (Dt); Sodus Pt., Je (Not); Pike, May (NYS); McLean, May, J1 (Dt); Windsor, Je (Not).

4420 P. debilis Grav. Olcott, Ap (Dt); Ithaca, Sep (Dt); Peekskill, Sherm $(\mathrm{AM})$; SI, Dec, under weeds in garden (L\&D).

4421 P. varians Payk. Syracuse, in hickory (B\&S) ; Olcott, May (Dt); SI, May, Woodr (L\&D) ; LI: Bellport, Nic (Sf) ; New Lots, May, Shoe (Sf) ; Flatbush, J1, Schott (Sf).

4422 P. cervicalis Csy. "NY" (Csy).

4423 P. longicornis Steph. Potsdam (Houghton) ; Ilion, May (NYS) ; Gloversville, Ap, Oswego, Sep (NYS); Westfield, May (Not); Albany, May (NYS); West Farms, Van Cort. Pk. (AM); SI (L\&D).

4424 P. discoideus Grav. Peekskill (AM); West Pt. (Robn).

4427 P. alumnus Er. "NY" (Horn); Mt. Whiteface, J1, Shoe (Sf).

4427 a P. alumnus var. rufulus Horn. "NY" (Horn).

4430 P. fusiformis Melsh. Westfield, May (Not); Ithaca, Mar (Dt) ; Schoharie (Not); Peekskill, Ap (Fall, Sherm); Van Cort. Pk., Bronx Pk. (AM); LI: Rockaway Beach, May, Shoe (Sf).

4431 P. fulvipes Fab. Mooers, Sep (Not).

4433 P. schwarzi Horn. Gloversville, Ap (NYS); Sodus Pt., Je (Not) ; Olcott, Ap (Dt) ; Ithaca, Ap (Dt); West Pt. (Robn) ; LI: North Beach, Jan, Schott (Sf).

4441 P. micans Grav. Newport, May-Je (NYS) ; Buffalo (Z\&R) ; Ithaca, Mar, Sep-Nov (Dt); McLean, Aug, Ringwood, May (Dt).

4443 P. lomatus Er. Mooers, Sep (Not) ; Newport, May (NYS) ; Olcott, FebAp, J1-Sep (Dt); Westfield, May, Windsor, Je (Not); Gloversville, Mar (NYS) ; Glens Falls, Je (NYS) ; Pike (NYS); Ithaca, Mar-Ap, Nov, Ringwood, May (Dt); Albany, Ap, Rensselaer, May (NYS); West Pt., Ap, Robn (Sf); Peekskill (AM); West Pt., Van Cort. Pk., West Farms (AM) ; SI (L\&D).

4444 ?P. cunctans Horn. Gloversville, Ap; Westfield, May (Not).

4445 P. aequalis Horn. "NY”, Mar 28, Sherm (AM); West Pt., May, Robn (Sf).

4446 P. brunneus Grav. Newport, May (NYS); Westfield, May (Not) ; Oswego, Aug, Bks (NYS) ; McLean, Ap 17, Babiy (Flet); West Pt. (Robn); Mosholu (AM); SI (L\&D); LI: Jamaica, Je, Schott (Sf) ; Flatbush, Feb, Dow (Sf). 
4447 P. cyanipennis Fab. Fourth L., Je (NYS) ; Cranberry L., under bark of dead $\log (\mathrm{H})$; Ilion, May (NYS) ; Sodus Pt., Je (Not) ; Olcott, JeSep (Dt); Buffalo (Z\&R); Pike, J1 (NYS); Ithaca, Ringwood, Je-Sep (Dt); McLean, J1 17 (Dt); L. George, Aug, Zab (AM); Catskills, Pears (Sf); Poughkeepsie, (NYS); White L., J1, Zab (AM); West Pt. (Robn); Mosholu, J1 (Sf); SI, Je-Sep (L\&D); LI: Flatbush, Nov (AM).

4448 P. blandus Grav. Newport, May, Elizabethtown, Aug (NYS) ; Fulton Chain, Je (NYS); Buffalo (Z\&R); Westfield, May, Windsor, Je (Not); Poughkeepsie, J1 (NYS); Peekskill, Harrison (AM); SI, Sep (L\&D); LI: E. New York, May, Martin (Sf).

4450 P. sordidus Grav. Fourth L., Je (NYS); Southfields, May, Shoe (Sf); E. New York, May, Martin (Sf).

4452 P. cephalotes Grav. Newport, May (NYS); Olcott, May (Dt); LI: Wyandanch, Ap, Schott (Sf).

4455 P. ventralis Grav. Buffalo (Z\&R).

4458 P. fallaciosus Horn. Sodus Pt., Je, Westfield, May (Not).

4470 P. nigritulus Grav. (aterrimus Grav.). Buffalo (Z\&R); Westfield, May (Not).

4473 P. microphthalmus Horn. Newport (NYS); Gloversville, Sep (NYS) ; Oswego, Bks (NYS); Syracuse, in hickory (B\&S); Olcott, Ap 10 (Dt); Sodus Pt., Westfield, May (Not); Peekskill, Ramapo Mts. (AM) ; LI: Brooklyn, May, Dow (Sf).

4483 P. validus Csy. "NY" (Csy).

4488 P. viridanus Horn. Mooers, Sep (Not); Schoharie (Not); West Farms (AM) ; LI, Johnson (Sf).

4495 P. vulgatus Csy. "NY" (Csy).

4497 P. aurulentus Horn. Syracuse, Ap ( $H)$; Sodus Pt., Je (Not) ; Olcott, J1 (Dt); Ithaca, Je, Sep, Ringwood, May (Dt); McLean, May, Aug (Dt).

P. strigicollis Not. Westfield, May (Not).

P. opacipennis Not. Mooers, Sep (Not).

Cafius Stephens

4506 C. bistriatus Er. SI, Je (L\&D).

4509 C. sericeus Holme. LI: possibly Coney I. (Horn); Rockaway Beach, Dow (Sf).

\section{Hesperus Fauvel}

4512 H. apicalis Say. Albany, Ap (NYS) ; West Pt. (Robn) ; LI: Yaphank, Oct, Eng (Sf).

4513 H. baltimorensis Grav. West Pt. (Robn) ; Mosholu, May (Ds) ; SI, ApMay, Sep (L\&D); LI: Flatbush, Parkville, Ap-May, Zab (AM).

\section{BELONUCHUS Nordmann}

4514 B. formosus Grav. Peekskill, Mar, Sherm (AM); West Pt. (Robn); Bronx Pk. (AM); SI, Je (L\&D). 


\section{StAPHYLINUS Linnaeus}

4526 S. badipes Lec. (carbonatus Lec.). Syracuse, May (H) ; Sodus Pt., MayJe (Not); Olcott, Ap-Sep (Dt); Buffalo (Horn); Castile, May, Brad (Flet) ; Batavia, Je (Kngt) ; Windsor, Je (Not); Cayuga L., Mar, Bish (NYS); Ithaca, Ap (Dt); McLean, Ap 17, Babiy (Flet); Schoharie (Not); Suffern, Ap (Sf).

4527 S. pleuralis Lec. Buffalo (Z\&R). Occurrence in NY doubtful (Fall).

4532 S. vulpinus Nordm. Syracuse, Ap (H) ; Buffalo (Z\&R) ; Westfield, J1 (NYS) ; Ithaca, Ap (Dt), Sep (NYS); New Salem (NYS); Albany, Medusa, Ap (NYS); West Pt. (Robn); SI, May, Oct (L\&D) ; LI, Ap, Eng (Sf).

4533 S. maculosus Grav. Syracuse, May (H) ; Olcott, May-Je (Dt) ; Buffalo (Z\&R) ; Ilion, May (NYS) ; Windsor, Je (Not) ; Ithaca, Ap-Sep, Bish (NYS); McLean, Je (Flet); Albany, Ap-May, Karner, May, Clinton Hgts., Ap (NYS); Schodack Landing, Poughkeepsie, Je (NYS) ; West Pt. (Robn) ; Mosholu, West Farms (AM) ; SI, ApSep (L\&D); LI: Flatbush (AM).

4534 S. mysticus Er. Newport, May (NYS); Gloversville, May (NYS) ; Syracuse, Ap (H) ; Sodus Pt., Je (Not) ; Ithaca, Ap-J1 (Dt) ; Poughkeepsie, May (NYS); West Pt. (Robn); Mosholu, West Farms (AM); SI, Ap-Je (L\&D); LI: Brooklyn, May, Eng (Sf).

4537

S. tomentosus Grav. Peekskill, Tarrytown, West Farms (AM); SI, (L\&D).

4543 S. fossator Grav. Mt. Whiteface, Aug (Dt); Oswego, May, Bks (NYS) ; Poughkeepsie, Aug (NYS); West Pt. (Robn); Peekskill, J1, Sherm (AM) ; Mosholu, J1 (Sf); SI, Aug (L\&D); LI, Eng (Sf).

4543a S. fossator var. immaculatus Horn. "NY" (DeKay).

4545 S. cinnamopterus Grav. Mooers, Sep (Not); Syracuse, Ap-May (H) ; Olcott, May-Oct (Dt) ; Buffalo (Z\&R) ; Batavia, Aug (Kngt); Westfield, J1 (NYS) ; Castile, Brad (Flet) ; Ithaca, Ap-Sep (Dt) ; McLean, J1 (Dt); Albany, Je, Coeymans (NYS) ; Clinton Hgts., Ap (NYS); Catskills, Sep (Ds); West Pt. (Robn); Inwood, J1, Sep (Ds); SI, Je, J1, Sep (L\&D); LI, Je, Eng (Sf).

4546 S. violaceus Grav. Newport (NYS); Olcott, Je-Aug (Dt); Buffalo (Z\&R); Batavia, J1-Aug (Kngt); Pike (NYS); Westfield, May (Not); Ithaca, May, McLean, J1 (Dt) ; Nassau, May (NYS); West Pt. (Robn) ; SI (L\&D); LI: Yaphank, May, Eng (Sf).

4548 S. viridanus Horn. "NY" (Horn).

4549 S. exulans Er. Westfield, May (Not); West Pt., Je, Robn (Sf).

4550 S. praelongus Mann. Tarrytown (AM); LI: Rockaway Beach (Sf); Orient Pt., Je, Lath (NYS).

\section{OCYPUS Kraatz}

4551 O. ater Grav. Olcott, J1-Sep (Dt); Buffalo (Z\&R) ; Ithaca, J1 (NYS) ; Nassau, Aug (NYS) ; Hudson Falls, Sherm (AM); SI, Aug (L\&D) ; LI: Rosedale, Oct, Rockaway Beach, Je, Amagansett, Eng. (Sf).

\section{ONTHOLESTES Ganglbauer}

4552 O. cingulatus Grav. Keene Val., Je; Axton, Je (M\&H) ; Newport, Je (NYS) ; Crane Pond, Je, Bks (NYS); Cranberry L., J1, in decaying fungi and on carrion $(\mathrm{H})$; Olcott, May-Je (Dt); Buffalo (Z\&R); 
Pike (NYS) ; Windsor, Je (Not) ; Ithaca, May-Sep (Dt); McLean, Aug-Sep (Flet); Poughkeepsie, Aug (NYS); L. George, Aug, Zab (AM) ; Peekskill (AM); West Pt. (Robn); West Farms, Angus (AM) ; SI, May-Oct (L\&D) ; LI: Flatbush, Aug, Zab (AM).

4553 O. capitatus Bland. Newport, May (NYS); Johnstown, Sep (NYS) ; Buffalo (Z\&R); West Farms, Angus (AM).

\section{TYMPANOPHORUS Nordmann}

4554 T. puncticollis Er. LI: Rockaway, Je, Dietz (Sf).

\section{Creophilus Mannerheim}

4555b C. maxillosus L. var. villosus Grav. Newport, May (NYS) ; Cranberry L., Je-J1, on carrion (H) ; Olcott, May (Dt) ; Buffalo (Z\&R) ; Montour Falls, Ap, carrion (H) ; Windsor, Je (Not) ; Ithaca, Ap-Sep, Bish (NYS) ; Crane Pond, Je, Bks (NYS) ; Interlaken, Ap (NYS); Nassau, Je (NYS) ; Stamford, Oct, Bish (NYS) ; West Pt. (Robn); SI, Ap-Sep (L\&D) ; LI: Prospect Pk., Flatbush, Coney I., May-Nov, $Z a b$ (AM); Orient, Lath (NYS).

\section{ACYLOPHORUS Nordmann}

4564 A. flavicollis Sachse. Ringwood, May (Dt).

4565 A. pronus Er. Mooers, Sep (Not); Fourth L., Je (NYS) ; Johnstown (NYS) ; Sodus Pt., May (Not); Buffalo (Z\&R); Windsor, Je (Not); McLean, May, Aug (Dt) ; Peekskill, J1 (AM) ; Hudson Falls, Sherm (AM) ; Esopus, Aug, Sherm (AM).

4566 A. caseyi Lg. "NY" (Csy).

4567 A. densus Lec. LI: Flushing, Mar, Schott (Sf).

\section{HETEROTHOPS Stephens}

4569 H. fumigatus Lec. Keene Val., Aug-Sep (Not); Catskills, Pears (Sf).

$4569 \mathrm{a} H$. fumigatus var. fusculus Lec. Westfield, May (Not).

4571 H. pusio Lec. "NY" (Lec).

\section{Quedius Stephens}

4574 Q. ferox Lec. SI (L\&D); LI, Schaupp (AM).

4575 Q. (Quedionuchus) laevigatus Gyll. Cranberry L., Je-J1, under bark of dead logs $(\mathrm{H})$; Sodus Pt., Je, Westfield, May (Not); Ithaca, Ap, J1 (Dt) ; West Pt., Ap, Robn (Sf).

4578 Q. vernix Lec. Ithaca, May, Oct, Ringwood, J1, McLean, J1-Aug (Dt) ; Clinton Hgts., Ap (NYS) ; Mosholu (Sf); SI, Je (L\&D).

4579 Q. molochinus Grav. Ithaca, Mar-Ap, Oct (Dt); Slide Mt., J1, Pears (Sf); Peekskill, Aug (AM); West Pt. (Robn); SI (L\&D).

4586 Q. capucinus Grav. Buffalo (Z\&R).

4589 Q. bruneipennis Mann. Ithaca, Ap (Dt); Southfields, Oct, Schott (Sf). 
4595 Q. peregrinus Grav. Ithaca, Aug-Oct (Dt); West Pt. (Robn) ; Peekskill, Mar, Sherm (AM).

4599 Q. erythrogaster Mann. "NY" ( Lg Cat.).

4600 Q. mesomelinus Marsh. SI, Je (L\&D) ; LI: Flatbush, Aug, Zab (AM), May, Schott (Sf), as fulgidus Fab.

4601 Q. iracundus Say (fulgidus Horn). "NY" (Horn); Ithaca, Je (Dt).

4621 Q. spelaeus Horn. "NY" (Horn). Questioned by Fall.

\section{SUBFAMILY OXYPORINAE}

OXYPORUS Fabricius

4628 O. femoralis Grav. Newport, J1 (NYS); Olcott, Je-Aug (Dt) ; Ithaca, J1-Sep (Dt); White L., Nov, Zab (AM); West Pt., Je (Sf) ; Peekskill, Sherm (AM); West Pt. (Robn); SI, Je-Óct (L\&D); LI: Queens, Sep 26 (Frost); Flatbush, Zab (AM); Yaphank, Oct, Eng (Sf).

4631 O. major Grav. Cranberry L., Je (H); Peekskill, J1 (AM); West Pt. (Robn) ; SI, Sep (L\&D); LI: Queens, Sep 19 (Frost), Sep, Schott (Sf) ; Richmond Hill (Sf).

4632 O. rufipennis Lec. Johnstown, Sep (NYS); Pike (NYS); Debruce, Aug (Ds) ; West Pt., Je, Robn (Ds).

4633 O. stygicus Say. Keene Val., Je (Not) ; Ft. Montgomery, Je, Schott (Sf) ; West Pt. (Robn).

4634 O. vittatus Grav. White L., Nov (AM) ; Albany, Karner, Sep (NYS); West Pt. (Robn); SI (L\&D); LI: Flatbush, Nov, Zab (AM).

4635 O. fasciatus Horn. LI: Brooklyn, Je (Ds); Wyandanch (Sf).

4637 O. lateralis Grav. Keene Val., Je (Not) ; Newport, May (NYS) ; Olcott, Oct (Dt); Pike, May (NYS); McLean, May (Flet); West Pt. (Robn) ; Mosholu (Sf) ; SI, Oct (L\&D); LI, Eng (Sf).

4637a O. lateralis var. brevis Mielsh. "NY" (Melsh).

4638 O. occipitalis Fauv. Newport (NYS); Albany, Sep (NYS).

4639 O. lepidus Lec. "NY" (Lec).

4640 O. quinquemaculatus Lec. West Pt., Je, Robn (Ds, Sf).

\section{SUBFAMILY TACHYPORINAE}

\section{TACHINUS Gravenhorst}

4652 T. memnonius Grav. Gloversville, Sep (NYS) ; Buffalo vic. (Z\&R) ; Pike, Nov 11 (Frost); Ithaca, Sep (Dt); Albany, Oct (NYS); Peekskill (AM); SI, Nov' (L\&D).

4657 T. repandus Horn. Winḋsor, Je (Not); McLean, J1 (Dt); Trenton Falls (Horn); Peekskill, May (AM); West Pt. (Robn); SI (AM).

4660 T. addendus Horn. Newport, May (NYS).

4661 T. parallelus Horn. Mt. Skylight, J1 (Not).

4663 T. flavipennis Dej. Newpert, May (NYS) ; Ilion (NYS) ; Cranberry L., Je-J1 (H) ; Ithaca, Ringwood, May-Je (Dt); McLean, Je (Flet); Peekskill, Sep (AM) ; Suffern, May, Schott (Sf) ; SI, Mar-Ap, J1, in horse manure (L\&D).

4664 T. luridus Er. Newport, Je (NYS); Fourth L., Je (NYS); Buffalo (ErANSP); Westfield, May (Not); Karner, Ap (NYS); McLean, Je (Flet). 
4665 T. fimbriatus Grav. Newport, Je, Johnstown, Sep (NYS) ; Buffalo (Z\&R) ; Pike, Je (NYS); Ithaca, Je-Sep (Dt); McLean, Sep 12 (Flet); White L., J1, Zab (AM) ; Poughkeepsie, Je (NYS) ; Peekskill, J1, Sep (AM) ; West Pt. (Robn); West Farms (AM); SI, Je-Sep (L\&D); LI: Flatbush, Zab (AM); Orient, Lath (NYS).

4666 T. picipes Er. Wilmington, Aug (Dt) ; Johnstown, Sep (NYS) ; Woodworth's L., J1 (NYS) ; Cranberry L., Je 23, in decaying fungus; Buffalo (Z\&R) ; L. George, Aug, Zab (AM); White L., J1, Nov, Zab (AM).

4668 T. limbatus Melsh. Mooers, Sep (Not); Newport, May-Je, Wells, J1 (NYS) ; Wilmington, Aug (Dt); Gloversville, Sep (NYS) ; Johnstown, Ap (NYS); Buffalo (Z\&R); Ithaca, J1, Sep (Dt) ; Catskills, Aug (Ds) ; Hudson, J1, Eng (Sf); Mosholu (Sf); West Pt. (Robn); SI, May-Je (L\&D) ; LI, Sep, Shoe (Sf).

4669 T. fumipennis Say. SI, May (L\&D).

4670 T. pallipes Grav. (frigidus Er.). Wilmington, Aug (Dt) ; Cranberry L., $\mathrm{Je}-\mathrm{J1}$, in decaying fungus $(\mathrm{H})$; Buffalo ( $\mathrm{Z} \& \mathrm{R})$; White L., J1, Zab (AM) ; Ithaca, Ap, Ringwood, May (Dt); Peekskill (AM); West Pt. (Robn) ; LI, Je, Shoe, Eng (Sf).

4673 T. basalis Er. (circumcinctus Mäkl.). Gloversville, Sep (NYS) ; Ringwood, May (Dt).

4674 T. nitiduloides Horn. "NY" (Horn) ; Mosholu, Je (Sf). Questioned by Fall.

TACHYPORUS Gravenhorst

4678 T. maculipennis Lec. 'Trenton (Horn); Peekskill, Sherm (AM).

4679 T. elegans Horn. Ithaca, Oct-Nov, Ringwood, May (Dt); SI (L\&D); LI, Ap, Aug, Shoe (Sf).

4681 T. jocosus Say. Mt. Whiteface, Aug (Dt); Axton, Je (M\&H) ; Sodus Pt., May (Not); Olcott, Ap (Dt); Buffalo (Z\&R); McLean, Nov (Dt) ; Windsor, Je (Not) ; Peekskill, May, J1 (AM); SI, Jan (L\&D); LI: Flushing, Mar (Ds).

4682 T. chrysomelinus L. Axton, Je (M\&H) ; Cranberry L., J1 (Drk) ; Gloversville, Nov (NYS); Olcott, Ap (Dt); Buffalo (Z\&R); Castile, May, Brad (Flet); McLean, May (Dt); Windsor, Je (Not); Washington Co, Aug (AM).

4682a T. chrysomelinus var. acaudus Say. Cranberry L., Je (H) ; Syracuse, Montour Falls, Ap-May, under stones, herbage $(\mathrm{H})$.

4686 T. nitidulus Fab. (brunneus Fab.). Syracuse, Montour Falls, Ap-Je (H) ; Sodus Pt., May (Not); Olcott, Ap (Dt); Westfield, May, Windsor, Je (Not); Peekskill, Mar-Ap (AM); SI, Mar, Nov, on salt meadow (L\&D).

\section{LEUCOPARYPHUS Kraatz}

4687 L. silphoides L. Gloversville, Oct (NYS); Oswego, Aug, Bks (NYS); Buffalo (Z\&R).

\section{ERCHOMUs Motschulsky}

4690 E. ventriculus Say. Axton, Je (M\&H); Elizabethtown, Aug, Newport, Je (NYS) ; Cranberry L., Je, under bark of dead logs (H) ; Herkimer Co, Yg (AM) ; Syracuse, under bark (H); Buffalo (Z\&R) ; Batavia, Sep (Kngt); Castile, Brad (Flet); Olcott, Ithaca, very common under 
bark of logs in damp woods (Dt); Westfield, May (Not); McLean, Sep 20 (Flet) ; Albany, Oct, Delmar, May, Cedar Hill, Ap, Nassau, Ap, Normanskill, Ap (NYS) ; SI, Ap-May, under bark (L\&D) ; LI: Aqueduct, May, Shoe (Sf).

\section{Conosoma Kraatz}

4695 C. knoxi Lec. Newport, May (NYS) ; Cranberry L., J1 4, under piece of bark on ground $(\mathrm{H})$; SI (L\&D).

4696 C. littoreus L. Potsdam, May (NYS) ; Newport, May (NYS) ; Cranberry L., Je 6, in decaying fungi $(\mathrm{H})$; Peekskill, May-Je (AM).

4697 C. opicus Say. Newport, May (NYS); Windsor, Je (Not); West Pt. (Robn) ; SI, Ap, Je, Woodr (L\&D).

4698 C. imbricatus Csy. Newport, May (NYS); Ithaca, May, Bish (NYS) ; Albany, Nov, Greenville, Ap (NYS); Peekskill, Sherm; Van Cort. Pk., West Farms, as pubescens Payk. (AM) ; SI, Oct, Dec, under weeds in garden and on fungus (L\&D) ; LI: Mar, Eng (Sf) ; Riverhead, Ap (NYS).

4700 C. velocipes Csy. Near NYC (Csy).

4705 C. basalis Er. Syracuse $(H)$; Ithaca, Ap (Dt) ; West Pt. (Robn) ; SI, Ap, Je, Sep (L\&D) ; LI: Central Pk., May, Eng (Sf).

4706 C. occulatus Csy. Westfield, May (Not).

4707 C. crassus Grav. Newport, May (NYS) ; Cranberry L., Je 25, in decaying fungi (H) ; Johnstown, Sep (NYS); Buffalo (Z\&R); Pike (NYS); Ithaca, Sep (Dt) ; McLean, J1 (Dt); Schoharie (Not); Kinderhook, Je (NYS) ; SI, May-Sep (L\&D); LI: Rosedale, Oct, Eng (Sf); Riverhead, Ap (NYS).

4713 C. scriptus Horn. Newport (NYS); Johnstown, Sep (NYS).

\section{Bolitobius Stephens}

4716 B. niger Grav. Newport (NYS).

4718 B. axillaris Grav. Johnstown, Sep (NYS).

4719 B. dimidiatus Er. Fourth L., Je (NYS) ; Ft. Montgomery, J1 (Ds) ; SI, Sep (L\&D) ; LI: Wyandanch, Aug, Schott (Sf).

4720 B. nepigonensis Bnhr. Cranberry L., J1 2-7, in dying beech ( $\mathrm{H})$.

4721 B. intrusus Horn. Sodus Pt., Je (Not); Ithaca, May (NYS) ; Ramapc (Sf).

4722 B. cingulatus Mann. Newport, May (NYS); Tuckahoe, Nov, Schott (Sf); SI, Ap, Shoe (Sf); LI: Bellport, Aug, Nic (Sf).

4723 B. cincticollis Say. Axton, Je (M\&H) ; Newport, May (NYS) ; Fourth L., Je (NYS) ; Cranberry L., Je (H) ; Buffalo (Z\&R) ; Windsor, Je (Not) ; Karner, May (NYS); West Pt. (Robn) ; SI (L\&D).

4724 B. anticus Horn. Newport (NYS); Gloversville, Sep (NYS); Mosholu (Sf) ; SI, Aug, Nov (L\&D).

4727 B. trinotatus Er. Cranberry L., Je 23, in decaying fungus (H) ; Newport, May (NYS) ; Johnstown, Sep (NYS) ; Albany, Sep and S. Westerlo, May (NYS); L. George, Aug, Zab (AM); Peekskill, J1, Sherm (AM) ; West Pt. (Robn); Mosholu (Sf) ; SI (L\&D) ; LI: Flatbush, Nov 8, Zab (AM). 
4729 B. obsoletus Say. Poughkeepsie, Aug (NYS); Peekskill, Sep (AM).

4730 B. cinctus Grav. Newport, May (NYS); Buffalo (Z\&R); Peekskill, J1 (AM) ; West Pt. (Robn) ; LI: Yaphank, Oct, Eng (Sf).

4732 B. quaesitor Horn. Newport (NYS); Windsor, Je (Not).

\section{BRYOPORUS Kraatz}

4737 B. rufescens Lec. Elizabethtown, Aug, Big Moose, J1, Newport, May, Ilion, May (NYS); Olcott, Ap (Dt); Westfield, May, Windsor, Je (Not); Karner, Je (NYS); Peekskill, May, Sherm (AM); West Pt. (Robn); Mosholu, Je (Sf).

\section{MYCETOPORUS Mannerheim}

4738 M. humidus Say. LI: Rockaway Beach, Je, Schott (Sf) ; Forest Pk., Ap, Pears (Sf).

4739 M. horni Lg. Keene Val., J1 (Not).

4740 M. consors Lec. Ringwood, May (Dt).

4742 M. americanus Er. Cranberry L., Je $(H)$; Syracuse, May $(H)$; LI: Little Neck, Ap, Schott (Sf).

4742a M. americanus var. lucidulus Lec. Keene Val., Je, Tivoli, May (Not).

4743a M. flavicollis Lec. var. pictus Horn. McLean, May 16 (Flet).

4744 M. splendidus Grav. Syracuse, May $(H)$.

\section{SUBFAMILY HABROCERINAE}

HABROCERUS Erichson

4752 H. schwarzi Horn. "NY" (Horn); Van Cort. Pk., Oct, Nic (Sf); LI: North Beach, Jan, Schott (Sf).

\section{SUBFAMILY TRICHOPHYINAE}

TRICHOPHYA Mannerheim

4755 T. pilicornis Gyll. Westfield, May (Not); Red House, Je (Not); near NYC (Csy).

\section{SUBFAMILy ALEOCHARINAE \\ DEINOPSIS Matthews}

4757 D. americana Kr. NY (ANSP); Ithaca, May (Dt, Fenyés).

\section{GyMNUSA Gravenhorst}

4760 G. brevicollis Payk. Ithaca, May (Dt, Fenyes); McLean, May (Flet).

\section{MyLLAENA Erichson}

4764 M. dubia Grav. Peekskill, Sherm (AM).

4765 M. intermedia Er. Westfield, May (Not). 
4766 M. minuta Grav. SI, Je (L\&D).

4770 M. vulpina Bnhr. Mooers, Sep (Not); Westfield, May, Windsor, Je (Not); Schoharie (Not).

4772 M. audax Csy. Near NYC (Csy).

4773 M. abdita Csy. Westfield, May (Not).

4776 M. procidua Csy. "NY” (Csy).

\section{LEPTOBAMONA Casey}

4797 L. pertenuis Csy. Keene, J1, Jay, Aug, Canisteo, Je (Not).

\section{GYRONYCHA Casey}

4798 G. valens Csy. Ithaca, Aug (Dt, Fenyes).

4801 G. fusciceps Csy. Catskills (Csy).

4803 G. longicornis Csy. Westfield, May (Not); Ithaca (Csy).

\section{Somatium Wollaston}

4814 S. nugator Csy. Peekskill, Dec (Sherm).

Oligota Mannerheim

4825 O. pusillima Grav. NYC (Julich).

4826 O. parallela Csy. LI: Willetts Pt. (Csy).

4827 O. linearis Csy. "NY" (Csy).

\section{Placusa Erichson}

4842 P. tachyporoides Walt1. Cranberry L., J1 5 (H) ; Olcott, Aug, Dt (Fenyes).

4844 P. despecta Er. Albany, May (NYS).

4845 P. tacomae Csy. Olcott, Dec, Dt (Fenyes).

\section{Silusa Erichson}

4857 S. valens Csy. Catskills (Csy).

\section{Thecturota Casey}

4875 T. demissa Csy. Catskills (Csy).

THECTURA Thomson

4880 T. cuspidata Er. Westfield, May (Not).

4881 T. americana Csy. "NY" (Csy).

\section{LEPTUSA Kraatz}

L L. laticollis Not. Westfield, May (Not).

4892 L. opaca Csy. Olcott, Aug, Dt (Fenyes).

4893 L. seminitens Csy. "NY" (Csy).

4899 L. pavida Csy. "NY" (Csy). 
BOLITOCHARA Mannerheim

4928 B. marginella Csy. Mooers, Sep (Not); Cranberry L., Je (H) ; Olcott, Sep, $D t$ (Fenyes).

4929 B. picta Csy. W. Hebron (Lg); near NYC (Csy) ; SI, Je (L\&D).

4930 B. blanchardi Csy. Olcott, Aug-Sep, Dt (Fenyes); Windsor, Je (Not).

- ?B. trimaculata Er. Olcott, Aug, Dt (Fenyes). The tristigma of Casey, which may or may not be the trimaculata of Er. (Fall).

Homalota Mannerheim

4937 H. plana Gyll. Cranberry L., J1 (H) ; Westfield, May (Not).

4942 H. humilis Csy. "NY" (Csy).

\section{Eumicrota Casey}

4947 E. corruscula Er. Olcott, Je, Dt (Fenyes); Catskills (Csy); SI, Sep (L\&D).

4950 E. socia Er. Tivoli, May (Not).

4952 E. pallidula Csy. Catskills (Csy).

Gyrophaena Mannerheim

4956 G. affinis Sahlb. Newport, May (NYS); Olcott, Aug, Dt (Fenyes).

4959 G. vitrina Csy. Catskills (Csy).

4960 G. attonsa Csy. Catskills (Csy).

4961 G. flavicornis Melsh. Cranberry L., Je (H); Windsor, Je (Not) ; Catskills (Csy).

4963 G. gaudens Csy. Red House, Je (Not).

4966 G. lobata Csy. Mooers, Sep (Not); Albany, Sep (NYS); Catskills (Csy); SI (L\&D).

4967 G. involuta Csy. Catskills (Csy).

4968 G. antennalis Csy. Catskills (Csy).

4970 G. fuscicollis Csy. Catskills (Csy).

4971 G. modesta Csy. Catskills (Csy).

4972 G. gilvicollis Csy. "NY" (Csy).

4977 G. sculptipennis Csy. Catskills (Csy).

4978 G. subpunctata Csy. Catskills (Csy).

4980 G. laetula Csy. Catskills (Csy).

4982 G. fustifer Csy. Peekskill (Csy).

4983 G. centralis Csy. Catskills (Csy).

4987 G. subnitens Csy. Keene, Keene Val., J1, Tivoli, May (Not).

4988 G. compacta Csy. Tivoli, May (Not).

4995 G. fasciata Say (vimula Er.). Mooers, Sep (Not); Newport, May (NYS); Olcott, Aug, Dt (Fenyes); Parkville, J1 (AM); SI (L\&D).

4996 G. ocularis Csy. Catskills (Csy).

4999 G. dissimilis Er. SI, Je (L\&D). 


\section{XENODUSA Wasmann}

5008 X. cava Lec. Johnstown, Oct (NYS); Ithaca, J1, Dt (Fenyes).

\section{MYRMEDONIA Erichson}

5018 M. caliginosa Csy. Catskills (Csy).

5022 M. obliqua Csy. "NY" (Csy).

5026 M. lauta Csy. "NY" (Csy).

\section{HOPLANDRIA Kraatz}

5037 H. lateralis Melsh. Westfield, May, Windsor, Je (Not); SI, Oct (L\&D).

5038 H. pulchra Kr. W. Hebron ( Lg).

5039 H. ochracea $\mathrm{Kr}$. "NY" ( $\mathrm{Lg})$.

\section{TRICHIUSA Casey}

5058 T. transversa Csy. Westfield, May (Not).

5070 T. robustula Csy. Cranberry L., Je $28(\mathrm{H})$.

\section{Tinotus Sharp}

5083 T. caviceps Csy. SI (Fenyes).

5085 T. pallidus Csy. Olcott, Je-J1 (Dt); Ithaca, Je (Dt).

5089 T. imbricatus Csy. Catskills (Csy).

T. lateralis Not. Westfield, May (Not).

\section{Philotermes Kraatz}

5098 P. pilosus Kr. LI: Flatbush, May, from nest of Termes flavipes, Zab (AM).

Atheta Thomson

5099 A. divisa Märk. Cranberry L., Je-J1 (H); Olcott, J1, Dt (Fenyes).

5102 A. coriaria Kr. Olcott, Aug, Dt (Fenyes).

5104 A. ventricosa Bnhr. N. Elba (AM) ; Cranberry L., Je (H); Ithaca, Aug, Good (Fenyes).

5106 A. nigritula Grav. Windsor, Je (Not); LI: Brooklyn, J1 (Bnhr).

5112 A. virginica Bnhr. Cranberry L., Je (H) ; Ithaca, Je, $D t$ (Fenyes).

5120 A. frosti Bnhr. Olcott, Sep, Dt (Fenyes).

5122 A. obsoleticollis Bnhr. Olcott, Sep, Dt (Fenyes).

5132 A. klagesi Bnhr. Olcott, Sep-Oct, $D t$ (Fenyes); SI (L\&D).

5138 A. vaticina Csy. LI: Willetts Pt. (Csy).

5139 A. iterans Csy. Near NYC (Csy).

5140 A. enitescens Csy. Ithaca (Csy).

5149 A. rusticula Csy. Catskills (Csy).

5151 A. catula Csy. Catskills (Csy).

5153 A. candidula Csy. Catskills (Csy). 
5154 A. diffisa Csy. Catskills (Csy).

5161 A. gnoma Csy. Catskills (Csy).

5162 A. elota Csy. Catskills (Csy).

5163 A. insidiosa Csy. Catskills (Csy).

5164 A. ducens Csy. Catskills (Csy).

5165 A. tradita Csy. Catskills (Csy).

5169 A. auguralis Csy. Catskills Csy).

5171 A. dama Csy. Catskills (Csy).

5173 A. tractabilis Csy. Catskills (Csy).

5174 A. ordinata Csy. Catskills (Csy).

5181 A. strigulosa Csy. Catskills (Csy).

5187 A. discipula Csy. Catskills (Csy).

5209 A. nupera Csy. LI: Willetts Pt. (Csy).

5211 A. citata Csy. Catskills (Csy).

5212 A. nympha Csy. Catskills (Csy).

5213 A. discreta Csy. Catskills (Csy); W. Hebron ( $\mathrm{Lg}$ ).

5219 A. spadix Csy. Near NYC (Csy).

5230 A. irvingi Csy. Catskills (Csy).

5230a A. irvingi var. affluens Csy. LI (Csy).

$5230 \mathrm{~b} \mathrm{~A}$. irvingi var. clarescans Csy. Catskills (Csy).

5235 A. sororella Csy. Catskills (Csy).

5236 A. officiosa Csy. Catskills (Csy).

5237 A. tuta Csy. Ithaca (Csy).

5239 A. spuriella Csy. Catskills (Csy).

5248 A. alutacea Csy. Ithaca, $\operatorname{Sm}$ (Csy).

5250 A. dentata Bnhr. Cranberry L., Je 30 (H) ; Westfield, May, Windsor, Je (Not); Schoharie (Not).

5300 ?A. festinans Er. Olcott, Feb, Dt (Fenyes).

5302 ?A. polita Melsh. Olcott, J1, Dt (Fenyes).

5307 A. pennsylvanica Bnhr. Ithaca, Ap, Aug, Good (Fenyes); Catskills (Csy).

5308 A. unigena Csy. Catskills (Csy).

5314 A. palustris Kies. Mooers, Sep (Not) ; Cranberry L., Je 27-29 (H) ; Buffalo (Hamilton); Westfield, May, Windsor, Je (Not); Olcott, Mar, Dt (Fenyes) ; Westfield, May, Windsor, Je (Not); Brookton. Ap, Dt (Fenyes); Schoharie (Not).

5316 A. repanda Csy. Ithaca (Csy); Catskills (Csy).

5318 A. frugalis Csy. Catskills (Csy).

5319 A. leviceps Csy, Catskills (Csy).

A. inconspicua Er. "NY", Sherm (AM). A European species.

A. luridipennis Mann. West Farms, Oct (AM).

\section{TRAumoecia Rey}

5338 T. obsequens Csy. Catskills (Csy).

5354 T. ithacana Csy. Ithaca (Csy).

5357 T. asperiola Csy. "NY" (Csy). 
5366 T. fecunda Csy. Catskills (Csy).

5368 T. tincta Csy. "NY" (Csy).

5370 T. ingratula Csy. Windsor, May-Je (Not).

5371 T. recessa Csy. Catskills (Csy).

AMPhibitherion Notman

A. demissum Not. Westfield, May (Not).

DINARAEA Thomson

5384 D. subdepressa Bernh. Westfield, May (Not).

D. angustula Gyll. Buffalo (Hamilton).

\section{Metaxya Rey}

5401 M. dichroa Grav. Mooers, Sep (Not); SI, Feb 22, in sifting, Woodr (L\&D).

5413 M. impotens Csy. Ithaca (Csy).

5413a M. impotens var. albanica Csy. Catskills (Csy).

5425 M. complana Mann. SI: Watchogue, Ap 22 (L\&D).

M. vilis Er. Westfield, May (Not).

M. magniceps Sahl. Windsor, Je (Not).

M. obscuricornis Not. Mooers, Oct, Dec (Not).

\section{HYDROSMECTA Thomson}

5432 H. caduca Csy. Westfield, May (Not); Catskills (Csy).

5439 H. rarula Csy. Ithaca (Csy).

5447 H. scenica Csy. Near NYC (Csy).

H. torrida Not. Westfield, May (Not).

H. tincta Not. Westfield, May (Not).

\section{Aloconota Thomson}

5453 A. sulcifrons Steph. Buffalo (Hamilton).

5454 A. cambrica Woll. Westfield, May, Windsor, Je (Not).

5459 A. brunneipes Csy. Ithaca, Sm (Csy).

5461 A. ventralis Csy. Ithaca, $\operatorname{Sm}$ (Csy).

\section{AMISCHA Thomson}

5473 A. analis Grav. N. Elba, Oct (Lg); Cranberry L., Je 23 (H); Westfield, May (Not); Ithaca, Aug, Good (Fenyes); Mosholu (AM).

5478 A. normalis Csy. Near NYC (Csy).

\section{DATOMICRA Rey}

5507 D. decolorata Csy. Catskills (Csy).

5508 D. inopia Csy. Catskills (Csy).

5511 D. diffidens Csy. Catskills (Csy). 
5512 D. vacans Csy. Catskills (Csy).

5517 D. hebescens Csy. Catskills (Csy).

5522 D. filiformis Csy. Catskills (Csy) ; LI: Bellport, J1 (Nic).

5523 D. particula Csy. Catskills (Csy).

5526 D. atomica Csy. Catskills (Csy).

\section{SyNAPTINA Casey}

5531 S. consonens Csy. Near NYC (Csy).

DimetRota Rey

5560 D. novella Csy. LI: Willetts Pt. (Csy).

SABLETA Casey

5570 S. flaveola Melsh. SI, Aug (L\&D).

5572 S. ornator Csy. Catskills (Csy).

Pseudota Casey

5588 P. puricula Csy. Catskills (Csy).

5589 P. fascinans Csy. Catskills (Csy).

\section{ARISOTA Casey}

5598 A. tetricula Csy. Ithaca (Csy); Catskills (Csy).

\section{PANCOTA Casey}

5604 P. collaris Csy. Catskills, Sm (Csy).

5605 P. redundans Csy. Catskills (Csy).

5610 P. cupiens Csy. Catskills (Csy).

5611 P. laetabilis Csy. Catskills (Csy).

5614 P. luteola Er. N. Elba, Oct ( Lg).

5618 P. euphonia Csy. Catskills (Csy).

5620 P. panda Csy. Windsor, Je (Not).

5623 P. reclusa Csy. Catskills (Csy).

\section{ACROTONA Thomson}

5627 ?A. fungi Grav. Olcott, Mar 26, Ap 19, J1 23, Dt (Fenyes).

5630 A. clientula Er. Westfield, May (Not).

5631 A. modesta Melsh. Cranberry L., Je 30 (H); Ithaca, May-Je, Sep, Dt (Fenyes); SI, Mar (L\&D).

5636 A. fusiformis Csy. NYC, Sm (Csy). 
COLPODOTA Rey

5650 C. puritana Csy. Mooers, Sep (Not).

5653 C. acutella Csy.. Catskills (Csy).

5654 C. egregiella Csy. Ithaca, Catskills (Csy).

5659 C. assecla' Csy. Catskills (Csy).

— C. parva Sahlb. Westfield, May, Windsor, Je (Not).

\section{COPROTHASSA Thomson}

5678 C. sordida Marsh. Cranberry L., Je-J1 (H) ; Olcott, Je, Sep, Dt (Fenyes); Buffalo, as luridipennis, fide Fenyes ( $\mathrm{Z} \& \mathrm{R})$; Montour Falls, Je $(\mathrm{H})$; Ithaca, Je, Sep, Dt (Fenyes); Windsor, Je (Not); West Farms (AM).

5679 C. smithi Csy. Catskills (Csy).

\section{STRIGota Casey}

5681 S. oppidana Csy. Near NYC (Csy).

\section{GNYPETA Thomson}

5702 G. nigrella Lec. (brunnescens Csy.). Olcott, J1, Dt (Fenyes); Ithaca, $\mathrm{J} 1, D t$ (Fenyes); near NYC (Csy).

5703 G. baltifera Lec. Olcott, Je, $D t$ (Fenyes); LI: Coney I. (Lec).

5708 G. atrolucens Csy. Catskills (Csy, Fenyes).

\section{TACHYUSA Erichson}

5722 T. smithi Csy. Catskills, Sm (Csy).

5725 T. americana Csy. Olcott, J1, Dt (Fenyes); Westfield, May (Not); Ithaca, Catskills (Csy).

5726 T. silvatica Csy. Ithaca (Csy).

5730 T. cavicollis Lec. Ithaca, J1, Dt (Fenyes); Windsor, Je (Not).

5736 T. pruinosa Csy. Catskills, Sm (Csy).

\section{MERONERA Sharp}

5743 M. venustula Er. Mooers, Sep (Not); Buffalo (Z\&R); Olcott, Feb, Ap, $D t$ (Fenyes); Ithaca, Je, Dt (Fenyes); Cortland, J1, Bks (NYS); West Pt. (Robn) ; SI, Feb 22, in sifting, Woodr (L\&D).

5745 M. obliqua Csy. Catskills, Sm (Csy).

BORBOROPORA Kraatz

5747 B. quadriceps Lec. "NY", Ap (Lec, Csy).

\section{CARDIOLA Rey}

5750 C. obscura Grav. NY (from Jour. NY Ent. Soc., $20: 25$ ). 


\section{Aleodorus Say}

5751 A. bilobatus Say. "NY" (Lg); LI: Coney I. (Lec).

5753 ?A. partitus Lec. Buffalo ( $Z \& R)$.

\section{FALAGRIA Mannerheim}

5755 F. dissecta Er. (ithacana Csy.). Cranberry L., Je-J1 (H) ; Mooers, Sep (Not); Gloversville, Mar (NYS) ; Syracuse, Ap, skeleton (H) ; Buffalo (Z\&R); Windsor, Je (Not); Ithaca, Catskills (Csy); Peekskill, Mar (AM); Mosholu (AM); SI, Ap (L\&D).

\section{IJORINOTA Casey}

5757 L. cingulata Lec. Newport, May (NYS) ; Olcott, Aug, Oct, Dt (Fenyes); Westfield, May (Not); Ithaca, Nov, Dt (Fenyes).

\section{Anaulascaspis Ganglbauer}

5762 A. perexilis Csy. (hudsonica Csy.). Catskills (Csy).

\section{Aleochara Gravenhorst}

5763 A. curtula Goeze. SI (L\&D) as brachyptera Fourc.; LI (AM).

5764 A. lustrica Say. Ithaca, Sep-Oct, $D t$ (Fenyes); McLean Bogs, Aug, Dt (Fenyes); LI (Csy).

5764a A. lustrica var. sternalis Csy. Ithaca, Catskills (Csy).

5766 A. lata Grav. Newport, Je (NYS) ; Ilion, May (NYS) ; Buffalo (Z\&R) ; Pike, Ap (NYS); Montour Falls, Ap, on carrion (H) ; Westfield, May (Not); Ithaca, Mar, Nov, Dt (Fenyes) ; West Pt. (Robn) ; West Farms (LgAM) ; SI, Ap, in dead log (L\&D) ; LI: Astoria (AM) ; Orient, Lath (NYS).

5767 A. rubripes Blatch. Newport, May (NYS); Olcott, Mar, Dt (Fenyes).

5771 A. medialis Csy. Catskills (Csy).

5774 A. pleuralis Csy. Newport (NYS); McLean, Je, Aug, Dt (Fenyes) ; near NYC (Csy).

5775 A. americana Csy. SI (L\&D).

5780 A. puberula Klug. Peekskill, May, Sherm (Fęnyes).

5784 A. rubripennis Csy. Westfield, May (Not).

\section{BARYODMA Thomson}

5797 B. defecta Csy. Catskills (Csy).

5799 B. sculptiventris Csy. Cranberry L., J1 2 (H) ; Olcott, Je, Dt (Fenyes); Montour Falls, Je (H) ; Westfield, May (Not); Ithaca, May-Je, Sep, Dt (Fenyes); Catskills (Csy).

5806 B. bimaculata Grav. Newport, May (NYS) ; Potsdam (Houghton) ; Cranberry L., Je $25(\mathrm{H})$; Oswego, J1, Bks (NYS); Buffalo (Z\&R); Windsor, Je (Not); SI, May-Je (L\&D).

5820 B. nitida Grav. Shokan (Thompson); SI, Aug (L\&D). 


\section{EMPLENOTA Casey}

5833 E. maritima Csy. NY to Va (Csy).

\section{Homoeusa Kraatz}

5858 H. crinitula Csy. NY (Jour. NY Ent. Soc., 8:54).

\section{Crataraea Thomson}

5866 C. suturalis Mann. Keene Val., Je (Not).

\section{IsCHNOGLOSSA Kraatz}

5872 I. abscissa Csy. Westfield, May (Not).

\section{THIASOPHILA Kraatz}

5876 T. laticollis Csy. Olcott, May, $D t$ (Fenyes).

T. parvula Not. Westfield, May (Not).

\section{STICHOGLOSSA Fairmaire}

5882 S. corticina Er. Olcott, May-Oct, Dt (Fenyes).

DASYGLOSSA Erichson

5884 D. fenyesi Bnhr. Mt. Marcy, Oct (Lg).

\section{Hylota Casey}

5885 H. ochracea Csy. Near NYC (Csy).

\section{OXYPODA Mannerheim}

5889 O. sagulata Er. "NY" (Er).

5896 O. convergens Csy. Catskills (Csy).

5900 O. hudsonica Csy. Near NYC (Csy).

5905 O. stygica Csy. Near NYC (Csy).

5910 ?O. perexilis Csy. "NY" (Csy).

5921 O. rubescans Csy. Catskills (Csy).

5937 O. canora Csy. Catskills (Csy).

O. schaefferi Not. Windsor, Je (Not).

\section{Callicerus Gravenhorst}

C. puberulus Csy. "NY" (Csy). 


\section{ChILOPORA Kraatz}

5992 C. americana Csy. Peekskill (Csy).

\section{PACHycerota Casey}

6017 P. duryi Csy. Ithaca, Je, $D t$ (Fenyes).

\section{PHLOEOPORA Erichson}

6020 P. corticalis Grav. (ferruginea Csy.). Olcott, May, Dt (Fenyes).

\section{FAMILY PSELAPHIDAE 37}

\section{TRIMIOMELBA Casey}

6054 T. dubia Lec. Peekskill, Ap (Sherm); Ramapo (Sf).

\section{Actium Casey}

6065 A. foveicolle Lec. LI: Yaphank, May (Not).

6066 A. angustum Csy. Ramapo (Sf).

6071 A. globifer Lec. Ramapo (Sf); NY, type locality; LI: Baldwin, May 10, Ds (Nic).

\section{Pseudactium Casey}

6073 P. mellinum Csy. Keene Val., Lowville, Je (Not).

\section{Melba Casey}

6078 M. fossiger Csy. LI: Wyandanch, Oct 30 (Nic).

\section{TRIMIOPLECTUS Brendel}

6090 T. obsoletus Bndl. Brooklyn, Je 16, Franck (Nic).

\section{EUPLECTUS Leach}

6093 E. confluens Lec. Peekskill, Je (Sherm).

6095 E. elongatus Bndl. Keene Val., May, Je, J1 (Not).

6100 E. rotundicollis Bndl. Keene Val., Je (Not).

6109 E. hudsonicus Csy. NY (Csy).

\section{BIBLOPORUS Thomson}

6126 B. bicanalis Csy. NY (Csy).

\section{BIBLOPLECTUS Reitter}

6127 B. ruficeps Lec. LI: Wyandanch, Ap 3, in sphagnum bog (Nic).

${ }^{37}$ Alan S. Nicolay has gone over the manuscript for this family, made some changes, and supplied many records. 


\section{RAMECIA Casey}

6131 R. crinita Bndl. Buffalo (Z\&R), as Euplectus crinitus; Westfield, Je (Not).

\section{RHEXIDiUs Casey}

6155 R. canaliculatus Lec. Ramapo, May (AM); Peekskill, Sep, Sherm (Lg); LI: Wyandanch, Oct 30 (Nic).

\section{Rhexius LeConte}

6158 R. insculptus Lec. Westfield, May (Not); Windsor, Je (Not).

\section{BATRISODES Reitter}

6174 B. monstrosus Lec. Buffalo ( $Z \& R)$.

6174a B. monstrosus var. ferox Lec. SI (L\&D) ; LI: Jamaica, Ap 12, in ant's nest under stone (Nic).

6188 B. schaumi Aubé. Greenwood L., May (Not).

6189 B. riparius Say. Peekskill, Mar, Sherm ( Lg).

6190 B. uncicornis Csy. NYC (Csy).

6191 B. scabriceps Lec. LI: Flushing, Dow (Nic); Amagansett, Sep 21, from nest of Formica exsectoides, Ds (Nic).

6194 B. lineaticollis Aubé. LI: Jamaica (Nic).

6200 B. globosus Lec. Westfield, May (Not); Peekskill, Sherm (Lg); Buffalo (Z\&R) ; Ramapo Mts., May 5 (AM) ; Suffern, May 26 (AM); SI, Ap, with ants under a $\log$ and also under bark (L\&D); LI: common throughout the island, under bark, stones, etc. (Nic).

6201 B. spretus Lec. Windsor, Je (Not).

6202 B. foveicornis Csy. LI: Flushing, Amagansett, Ds (Nic).

6204 B. punctifrons Csy. Keene Val., May, Je, J1 (Not); LI: Hauppauge, Ap (Not).

6211 B. denticollis Csy. NY (Csy).

6212 B. triangulifer Bndl. LI (type locality): Wyandanch, Ap 3, from sphagnum bog (Nic).

6213 ?B. nigricans Lec. SI, Mar, Nov, on salt meadow (L\&D).

6216 B. striatus Lec. Rochester (M\&W) ; SI, Mar, Ap, Nov, several times on salt meadow (L\&D); LI: Wyandanch, Nov 7 (Nic), Ap 3, Shoe (Nic); Queens (Dow).

\section{Nisaxis Casey}

6225 N. tomentosa Aubé. LI: Jamaica, May, Pears (Nic). Reichenbachia minuta Bndl. is identical with this species, fide Casey in Ann. N. Y. Acad. Sci., vol. 9, p. 593.

\section{BRACHYGLUTA Thomson}

6230 B. abdominalis Aubé. Peekskill, May 30, Sherm (AM); SI, Feb-May (L\&D) ; LI: common in salt marshes at Flatbush, Brooklyn, Rockaway Beach, etc., Ap-May (Nic). 
6232 B. intermedia Bndl. "NY" (Bndl).

6233 B. labyrinthea Csy. "NY" (Csy).

6235 B. illinoisensis Bnd1. "NY" (Bndl).

6237 B. intricata Csy. "NY" (Csy).

6238 B. terebrata Csy. (perforata Bnd1.). Madison Co (?), May 20 (H) ; LI: (Csy); Jamaica, May, Pears (Nic).

6247 B. luniger Lec. Peekskill, May, Sherm (Lg); SI, May, on seashore (L\&D) ; LI, May .30, Shoe (Nic).

6248 B. cavicornis Bndl. Peekskill (Ds); SI, May, on salt meadow (L\&D), Mar 17 (Frost).

B. elegans Bndl. LI: Jamaica (Frost).

\section{REICHENBACHIA Leach}

6251 R. gemmifer Lec. Keene Val., May (Not).

6257 R. divergens Lec. Peekskill, Mar 26 (AM), Ap (Sherm) ; LI: Jamaica; Ap 27, Pears (Nic); Queens (Dow); Wyandanch, Nov, in sphagnum, Sf, Ds, Shoe (Nic); Yaphank, Ap (Brb).

- R. minuta Bndl. NY (Bndl).

6260 R. congener Bndl. Peekskill, Mar (Sherm); SI (L\&D); LI: (Bndl); Jamaica, May, Pears (Nic).

6261 R. facilis Csy. LI (Csy); Queens, Dow (Nic); Jamaica, Ap 27, Pears (Nic); Wyandanch, Ap-Nov, in sphagnum bog, Sf, Ds, Shoe (Nic).

6262

R. scabra Bndl. LI: (Bndl); Wyandanch, Ap 3, rare, in sphagnum, Shoe (Nic).

6264 R. rubicunda Aubé. Westchester Co, May, Dow (Nic); SI, Feb 22 (L\&D).

6265 R. fusciventris Csy. Westchester Co, May, Dowe, as gracilis (Nic); LI: Brooklyn, May 8, in salt marsh, North Beach, Dec, Feb (Schott). Most of the rubicunda and gracilis records belong to this species (Nic).

R. insolita Csy. Suffern (AM).

6269 R. gracilis Csy. Keene Val., Je, J1 (Not).

6276 R. puncticollis Lec. LI: E. New York, May, Wyandanch, Ap, May (Ds, Sf), in sphagnum bog, Nov 7 (Nic); Queens, Dow.

6277 R. borealis Csy. Sylvan Beach, Je, Tivoli, May (Not); LI: Canarsie, Rosedale, Ap (Not).

6278 R. corporalis Csy. Keene Val., Je (Not).

6298 R. polita Bndl. "NY" (Bndl).

6300 R. spatulifer Csy. Mt. Marcy, J1 (Not).

6301 R. propinqua Lec. Keene Val,, May, Je, J1 (Not).

\section{RyBaXIS Saulcy}

6309 R. valida Bndl. NY (Lg Cat.).

6310 R. conjuncta Lec. Ramapo (Sf) ; LI: Yaphank, Ap, Brb (Lg).

6311 R. clavata Bndl. West Pt. (Robn); Suffern (Sf) ; LI, usually common: Huntington, Je (Schott); Wyandanch, Ap-May, Sf (Nic).

6312 R. mystica Csy. Keene Val., May, Je, J1, Lowville, Je (Not) ; LI: Rosedale Ap (Not). 


\section{DECARTHRON Brendel}

6314 D. formiceti Lec. LI: Brooklyn, Dow, probably in marshes on outskirts of city (Nic).

6317 D. abnorme Lec. West Pt. (Robn) ; Catskill (AM) ; Suffern (AM) ; LI: Brooklyn, Mar 31 (Shoe); Jamaica, May, Pears (Nic).

6319 D. brendeli Csy. LI: Yaphank, Mar (Brb).

6320 D. longulum Lec. Peekskill (Sherm); SI (L\&D).

6321 D. exsectum Bndl. NY (Bndl).

6322 D. strenuum Bndl. SI, May (L\&D).

- D. exiguum Not. Windsor, Je (Not).

\section{ByTHINOPSIS Raffray}

6330 B. tychoides Bndl. Near NYC (Bnd1); LI: Jamaica, Wyandanch, Mar, Ap, Oct, Nic (Sf), Ap 3, several specimens from sphagnum bog, Shoe, Nic (Nic).

\section{Tychus Leach}

6333 T. minor Lec. Ramapo (Sf); LI: Wyandanch, Ap (Sf), Nov 7 (Nic). 6338 T. spiculifer Csy. LI: Rosedale, Ap (Not).

Pselaphus Herbst

6350 P. bellax Csy. Mosholu, Sep (Sf); Suffern (Smith, N. J. List).

6351 P. erichsoni Lec. NY (Lec); SI, Feb (L\&D).

6353 P. fustifer Csy. SI (L\&D); LI: Brooklyn May 8, Bellport Je 8 under board in salt marsh (Nic).

\section{Pilopius Casey}

6359 P. lacustris Csy. Windsor, Je (Not); SI, Feb 22, Woodr (L\&D).

6362 P. piceus Lec. Buffalo (Lec, Z\&R); West Pt. (Robn); Suffern (AM); LI: Jamaica, May 8, Pears, Mar, Schott, Wading R., May 30, in sweeping at border of pond, Nic, Huntington, May, Schott, Wyandanch, May-Je, Ds (Nic).

6364 P. granicollis Csy. Madison Co, Mar 20, with query (H); LI: Willetts Pt. (Csy).

6367 P. consobrinus Lec. Buffalo (Z\&R); Westchester Co, May, Dorw (Nic).

\section{Cedius LeConte}

6375 C. ziegleri Lec. West Pt., Sep (Robn); LI: Brooklyn, Dow (Nic); north of Amagansett, Sep 21, in nest of Formica exsectoides, Ds (Nic).

6377 C. spinosus Lec. Cranberry L., Je 18 (Hatch); LI: Massapequa, Ap 26, Eng (Nic).

\section{TyRus Aubé}

6382 T. humeralis Aubé. Newport (NYS) ; Westfield, May (Not) ; Gloversville, Mar (NYS); Schoharie (Not); LI: Central Pk., Ap, Eng, Bellport, Aug 13, under pine bark, Nic, Massapequa, Ap, Shoe, Wyandanch, Ap, Shoe (Nic). 


\section{TMESIPHORUS LeConte}

6379 T. costalis Lec. Bellport, Je 28, sparingly under boards along border of oak woods (Nic).

\section{FAMILY CLAVIGERIDAE}

ADRANES LeConte

6388 A. coecus Lec. SI, Mar 29, with ants, Je 9, under bark of stump (L\&D); LI, very rare (Nic); Jamaica, Je 10, in ant's nest under bark, Pears (Nic); Farmingdale, Ap, Eng (Nic); Wyandanch, Nov 7, one specimen found in sifting leaves around pond (Nic).

6389 A. lecontei Bndl. SI (L\&D).

\section{FAMILY PTILIIDAE 38 \\ (Trichoptergidae) \\ SUBFAMILY PTILIINAE}

NANOSELla Motschulsky

[6396 N. fungi Lec. Recorded from Ohio.]

[6397 N. atrocephala Dury. Recorded from Ohio.]

\section{Actidium Matthews}

6399 A. crotchianum Matth. Westfield, May (Not).

6401 A. granulosum Csy. Westfield, May (Not).

\section{PTILIUM Erichson}

[6410 P. hornianum Matth. Recorded from N. J.]

[6411 P. fungicola Csy. Recorded from Pa.]

[6412 P. truncatum Csy. Recorded from Pa.]

[6415 P. ulkei Matth. Recorded from N. J. and D. C.]

\section{Ptenidium Erichson}

6417 P. pusillum Gyll. Cranberry L., Je 29-J1 3, in box of decaying fungi and in sweeping $(\mathrm{H})$.

6421 P. foveicolle Lec. Cranberry L., Je 29, in sweeping (H); Keene Val., May (Not); Windsor, Je (Not).

6422 P. glabratum Mots. "NY" (Mots).

P. simplicicolle Not. Westfield, May (Not).

\section{Priliola Haldeman}

6424 P. collani Mann. Keene Val., Je (Not).

38 Definite records for this family are so few that the editors have included those species known from adjoining States, as a guide to the users of the list. These are italicized and olaced within brackets. 
PTERyX Matthews

5426 P. brunnea Lec. Keene Val., J1 (Not).

6428 P. duvali Matth. Schoharie (Not).

\section{ACRATRICHIS Motschulsky}

(Trichopteryx Kirby)

6432 ?A. moerens Matth. Voorheesville, Je, Bish (NYS); Little Pond, Orange Co, May, Bish (N.YS).

6433 A. aspera Hald. Windsor, Je (Not).

[6435 A. longipennis Csy. Recorded from $\mathrm{Pa}$.]

6436 A. vicina Matth. Westfield, May (Not).

6438 A. sericans Heer. (ambigua Matth.). Cranberry L., Je 25-J1 3, in box of decaying fungi, identity queried $(\mathrm{H})$; Schoharie (Not). Also recorded from Can.

6455 A. discolor Hald. Schoharie (Not); Windsor, Je (Not).

[6456 A. fungina Csy. Recorded from $\mathrm{Pa}$.]

6457 A. dohrni Matth. NY (Lg Cat.) ; E. Aurora (Dnd).

6458 A. glabricollis Matth. NY (Lg Cat.).

6459 A. haldemani Lec. Newport, May (NYS) ; (?) Cranberry L., Je 30-J1 2, in box of decaying fungi, and in sweeping, identity queried $(\mathrm{H})$; Johnstown, Sep (NYS); Schoharie (Not); Delmar, Mar (NYS); Albany, Ap (NYS); SI, Ap, in sifting leaves (L\&D). Also recorded from N. J. and Conn.

6465 A. nanula Mots. "NY" (Lg).

\section{SMICRUS Matthews}

6468 S. filicornis Fairm. Windsor, Je (Not).

Ptinella Motschulsky

6470 P. quercus Lec. Keene Val., J1 (Not).

\section{NEPHANES Thomson}

[6472 N. laeviusculus Matth. Recorded from N. J.]

6473 N. flaviventris Mots. "NY" (Mots).

\section{LIMULODES Matthews}

6475 L. paradoxus Matth. "NY" (Lg Cat.). Also recorded from N. J.

\section{FAMILY SCAPHIDIIDAE}

\section{SCAPHIDIUM Olivier}

6482 S. quadriguttatum Say. Newport (NYS); Cranberry L., J1 24 (H) ; Onondaga, Sep (NYS); Buffalo (Z\&R); Mosholu, May (Ds); SI, Je 7, Woodr (L\&D); LI: Orient, May (Ds). 
6482b S. quadriguttatum var. quadripustulatum Say. "NY” (Lec).

6482c S. quadriguttatum var. obliteratum Lec. West Pt. (Robn).

6482d S. quadriguttatum var. piceum Melsh. Johnstown, Aug (NYS) ; Buffalo (Rein) ; Ithaca, Je 23, Hamilton (Vdyk) ; Schoharie (Not) ; L. George, J1 (NotAM) ; Albany, May (NYS); West Pt. (Robn); SI, Jan, Ap-Je, Nov (L\&D) ; LI: Orient Pt., Je, Lath (NYS).

\section{ScAPHISOMA Leach}

6489 S. convexum Say. Newport (NYS); Glover̨sville, Oct (NYS); SI (L\&D).

6502 S. rubens Csy. Windsor, Je (Not); Catskills (Csy); LI (Csy).

6504 S. suturalis Lec. Westfield, May (Not).

6506 S. terminatum Melsh. "NY" (Csy).

\section{BAEOCERA Erichson}

6513 B. apicalis Lec. Buffalo (Z\&R).

6514 B. punctipennis Blatch. SI, J1 23 (L\&D).

6515 B. concolor Fab. Geneva, Aug (Cls); West Pt. (Robn); SI, Je 5, seashore (L\&D).

6516 B. congener Csy. LI (Csy).

6517 B. deflexa Csy. Cranberry L., J1 7 (H).

\section{Toxidium LeConte}

6528 T. gammaroides Lec. SI, Je, Sep (L\&D).

\section{FAMily HISTERIDAE SUBFAMILY HOLOLEPTINAE}

Hololepta Paykull

6530 H. aequalis Say (fossularis Say). Buffalo (Z\&R); Ithaca, Sep (Dt) ; White Plains, J1 (Sq); SI, Je-J1 (L\&D); LI: Orient Pt., May, Lath (NYS).

6531 H. lucida Lec. West Pt. (Robn); LI (Lec).

\section{SUBFAMILY HISTRINAE}

HISTER Linnaeus

6546 H. arcuatus Say. NYC (NYS) ; SI, Je, on seashore (L\&D) ; LI: Coney I. (Schaupp); Rockaway Beach, Wading R., Je (Ds); Long Beach ( Lg).

6554 H. biplagiatus Lec. Jamesport, Mar (NYS) ; LI: Rockaway (Lg).

6554a H. biplagiatus var. instratus Lec. Ithaca, Aug (Dt). Described from Kans and Ariz.

6569 H. harrisii Kby. Newport, Aug, Johnstown, May (NYS) ; Ithaca, May (CU) ; West Pt. (Robn).

6570 H. stygicus Lec. Newport, Je (NYS); Karner, Ap (NYS); SI, May (Sf, L\&D). 
6571 H. interruptus Beauv. Keene Val., Je (NYS) ; Cranberry L., J1, on carrion (H); Fulton Co, May (HCU); Newport, Je (NYS); Rochester Jct., Je, Leon (HCU) ; Buffalo (Z\&R); E. Aurora, Je (Dnd) ; Castile, Brad (Flet); Cattaraugus Co, J1 (Ds); Ithaca, May, J1 (H) ; Interlaken, Ap (NYS); Albany, May (NYS); Nassau, May (Yg); West Pt. (Robn) ; Ft. Montgomery, May, attacking dead caterpillar (Ds) ; SI, May (L\&D).

6572 H. obtusatus Harr. Wilmington, Aug (Dt); O1cott, May-Je (Dt) ; Ithaca, McLean, May-Aug (Dt); West Pt. (Robn); SI, Feb, Je (L\&D) ; LI (Lg).

6573 H. immunis Er. Cranberry L., J1 (H) ; Newport, May (NYS) ; Rochester Jct., Je, Leon (HCU); Penn Yan, Je, Cy (HCU); Renwick Swamp, Mar, Bish (NYS); Ithaca, Mar-Je (HCU); Karner, Je (Yg); Ashland (Frost); West Pt. (Robn); SI, May (L\&D); LI: Orient Pt., Je, Lath (Yg).

6574 H. memnonius Say. Buffálo (Z\&R); E. Aurora, Je (Dnd); Schoharie (Not), as merdarius Hoffm.; Nassau, Je (NYS); West Pt. (Robn); LI: Orient, Lath (NYS).

$6586 \mathrm{H}$. foedatus Lec. Cranberry L., Je-Jl, on decaying fungi and on carrion (H) ; Newport, Je (Yg) ; Fourth L., Je (NYS); Johnstown, Sep (Yg) ; Buffalo (Z\&R) ; E. Aurora, J1-Aug (Dnd) ; Otto, J1 (HCU) ; Rock City, Je (HCU); Ithaca, May-Sep (H, Dt); Ashland (Frost); Peekskill (Ds).

6588 H. hudsonicus Csy. NY (Csy), type; Ringwood, May (Dt).

6590 H. unicus Csy. Catskills (Csy); SI, in toadstools (Sf, L\&D).

6591 H. marginicollis Lec. Olcott, Mar, Oct (Dt); Rock City, Je (HCU) ; Ithaca, May (HCU); West Pt. (Robn).

6592 H. cognatus Lec. Newport, May (Yg); Johnstown, Sep, Alex (Yg) ; Ilion, Fourth L., Je (NYS) ; Buffalo (Z\&R); Albany, May (NYS) ; Karner, Je (Yg) ; West Pt. (Robn).

6596 H. abbreviatus Fab. Cranberry L., Je-J1, carrion (H) ; Pike, J1 (NYS) ; Potsdam (Houghton); Newport, May (NYS); Oswego, Aug, Bks (NYS) ; Oswego Falls, Jl (Bks) ; Syracuse, May, on cattle dung (H) ; Rochester Jct., Je, Leon (HCU); Olcott, Je (Dt); Buffalo (Z\&R); Ithaca, Je-Aug (HCU); McLean, Aug (Dt); Windsor, Je (Not); Albany, Poughkeepsie, May (NYS); West Pt. (Robn); SI, Je-Aug (L\&D); LI: Orient Pt., Je, Lath (Yg).

6601 H. furtivus Lec. Wilmington, Aug (Dt); Buffalo (Z\&R); Ithaca, Aug 7 (HCU) ; West Pt. (Robn); SI, Aug (L\&D).

6602 H. incertus Mars. Ringwood, May (Dt).

6606 H. depurator Say. Cranberry L., J1 (H) ; Gloversville, Johnstown, Sep, Alex (Yg); Buffalo (Z\&R); E. Aurora, Sep (Dnd); Ithaca, Aug (HCU) ; West Pt. (Robn); Ludlow (Thompson); SI (L\&D).

6613 H. defectus Lec. "NY" (Lec).

6623 H. bimaculatus L. Johnstown, Nov, Alex (Yg); Ithaca, Mar (HCU) ; Peekskill, May (HCU) ; West Pt. (Robn) ; Ludlow, Ap (Thompson); LI: Coney I. (Schaupp). Under cattle or horse dung.

6624 H. sedecimstriatus Say. Syracuse, May, cattle dung (H); Buffalo (Z\&R); Pike (Yg); Ithaca, Ap, J1, Nov (H) ; Ringwood, May (Dt); Peekskill (Ds); West Pt. (Robn); LI: Orient, Lath (NYS). 
6627 H. americanus Payk. Mooers, Sep (Not) ; Newport, Je (Yg); Old Forge, J1 (NYS) ; Rochester Jct., Je, Leon (HCU) ; Buffalo (Z\&R); Pike, May (Yg); Ithaca, Ap-May (HCU) ; Delmar, Karner, Rensselaer, May (NYS); West Pt. (Robn); SI, Ap-May, Aug (L\&D).

6627a H. americanus var. perplexus Lec. Newport, Je (NYS); Buffalo (Z\&R) ; Ithaca, Ap ( $\mathrm{Lg}$ ); West Pt. (Robn); SI (L\&D).

$6627 \mathrm{~b} \mathrm{H}$. americanus var. exaratus Lec. West Pt. (Robn).

6630 H. biabbreviatus Csy. NY, type locality ( Lg).

\section{TERETRIOSOMA Horn}

6637 T. americanum Lec. Cranberry L., May-Jl, from Pinus strobus, Betula lutea, and Picea rubens (H) ; Newport, May-Je, Fourth L., Je ( $\mathrm{Yg})$; Rochester (M\&W) ; Buffalo (Rein); Albany, May (Yg); Ludlow, West Farms (Lg); SI (Lg).

\section{TERETRIUS Erichson}

6646 T. latebricola Lew. Central NY, Wick (Lew).

\section{PLATySOMA Leach}

6651 P. carolinum Payk. Olcott, Je-Sep (Dt) ; Buffalo (Z\&R); E. Aurora, Grand I., May (Dnd); Ithaca, May-Je (H, Dt); West Pt. (Robn); SI (L\&D).

6652 P. aurelianum Horn. Olcott, Aug, Ithaca, Je (Dt) ; LI: Rockaway, May $(\mathrm{Lg})$.

6653 P. depressum Lec. Axton, Je (M\&H) ; Cranberry L., Je-Jl, under bark, herbage $(\mathrm{H})$; Peru, Je (HCU) ; Newport, May (NYS); Syracuse, Ap-May, under stones and bark (H); Olcott, May-Je (Dt); Buffalo $(Z \& R)$; E. Aurora, May, Je, Sep (Dnd); Westfield, May (Not); Castile, Brad (Flet); Pike, May (NYS) ; Ithaca, May-Aug, Spring L., J1 (HCU) ; Cortland Co, Je (Dnd) ; Windsor, Je (Not); Wileyville, Ap-Sep (Dt); Karner, in galleries of Tomicus calligraphus (Felt); Manor, Oct (NYS); Clinton Hgts., Je (NYS) ; Shandaken, Je, Cy (NYS) ; Poughkeepsie, Je (Yg) ; Ft. Montgomery, May (Ds); West Pt. (Robn); SI, Mar-Oct, under bark of sycamore and beech (L\&D) ; LI: Central Pk., on dead pines (Eng) ; Roslyn, J1, Orient, Lath (NYS).

6656 P. aequum Lec. Vic. NYC (AM).

6657 P. basale Lec. Cranberry L., Je-J1 (H).

6658 P. parallelum Say. Cranberry L., Je, from Pinus strobus (H) ; E. Aurora, May (Dnd); Albany, Nov (Yg); Delmar, May (NYS); West Pt. (Robn) ; SI, Mar (L\&D); LI: Central Pk., Ap, on dead pines (Eng).

6659 P. coarctatum Lec. Cranberry L., Je-Jl, from Pinus strobus, Picea rubens, and Abies balsamea (H); Olcott, Aug (Dt); Buffalo (Z\&R); Ithaca, J1, Nov (Dt); West Pt. (Robn); SI, Mar (L\&D).

\section{Cylistix Marseul}

6661 C. cylindrica Payk. Round L., J1 (NYS); Manor, Oct (NYS); Ft. Montgomery, J1 (Lg). 
6662 C. attenuata Lec. Buffalo (Z\&R).

6663 C. gracilis Lec. Newport (Yg); Cranberry L., J1, from Pinus strobus and Picea rubens $(\mathrm{H})$.

C. parvula Csy. NY, type locality.

\section{Phelister Marseul}

6666 P. subrotundus Say. Cranberry L., Je 30, on herbage (H) ; Syracuse, May, under stones and flying $(\mathrm{H})$; on dead hickory $(\mathrm{Blkm})$; Honeoye Falls, Mar, Leon (HCU) ; Olcott, Je-Sep (Dt); Buffalo (Z\&R); Westfield, May (Not); Ithaca, May, Aug (HCU); West. Pt. (Robn) ; Shokan (Lg) ; SI (L\&D) ; LI: Coney I. (Lg).

6666a P. subrotundus var. frosti Carn. NY, type locality.

6673 P. vernus Say. West Pt. (Robn).

6674 P. saunieri Mars. West Pt. (Robn); SI (L\&D); LI: Flatbush, May, Zab (AM).

6678 P. hospes Lew. Ulster Co (Lew).

6679 P. conquisitus Lew. "NY" (Lew).

\section{Psiloscelis Marseul}

6684 P. harrisi Lec. Johnstown, May (NYS); West Pt. (Robn); Peekskill, Ap (Sherm); Katonah, under the name planipes (Sherm).

6685 P. incurva Csy. "NY" (Csy).

\section{EPIERUS Erichson}

6696 E. pulicarius Er. Rochester (M\&W).

Xestipyge Marseul

6705 X. geminatum Lec. Olcott, May (Dt); SI (Sf, L\&D).

Carcinops Marseul

6714 C. quatuordecimstriata Steph. Rochester (M\&W); Albany, May (Yg).

\section{PAROMALUS Erichson}

6723 P. aequalis Say. Syracuse, Ap, under bark (H); Rochester, (M\&W); Buffalo (Z\&R); Ithaca, Ap, Sep (H,Dt) ; West Pt., Ap, Robn (Ds); SI, Ap (L\&D).

ISOLOMALUS Lewis

6724 I. bistriatus Er. Cranberry L., J1, from Pinus strobus (H); Ithaca, Mar-Ap (Dt); West Pt. (Robn) ; vic. NYC (AM,Palm Coli.).

DENDROPHILUS Leach

6732 D. punctulatus Say. Lansingburg, Oct (Yg); Albany, May (Yg); West Pt. (Robn). 


\section{Caerosternus LeConte}

6734 C. americanus Lec. Fourth L., Je (NYS).

\section{HETAERIUS Erichson}

6745 H. brunneipennis Rand. Ithaca, May (Dt); Catskills, Ap (Ang) ; Bronxville (Wheeler); SI, from ant's nest, Ap (L\&D); LI: Half Way Hollow Hills, J1 (Ds).

\section{ONTHOPHILUS Leach}

6755 O. alternatus Say. Peekskill, Aug (Lg); Rockland Co, Sep (Lg); SI (L\&D).

\section{Plegaderus Erichson}

6756 P. sayi Mars. Cranberry L., J1 (H).

6765 P. transversus Say. Newport, May ( $\mathrm{Yg})$; Cranberry L., J1 (H) ; Ithaca, Ap (Dt) ; Albany, Ap (NYS); Karner, in galleries of Tomicus calligraphus (Felt); SI, Ap, Shoe (L\&D); LI: Central Park, Ap, on dead pine (Eng); Manorville, Oct, on dead pine; Pinelawn, May. (Ds).

\section{BaCanius LeConte}

6772 B. punctiformis Lec. Newport, with query (Yg); Buffalo (Z\&R); Lancaster, $M C V$ (Frost); Ithaca, Ap (Dt).

6773 B. tantillus Lec. Westfield, May (Not).

6775 B. misellus Lec. "NY” (Lec).

\section{ACRITUS LeConte}

6781 A. politus Lec. Olcott, Ap (Dt) ; Buffalo (Rein); Westfield, May, Windsor, Je (Not); SI, Oct, in ant hill (L\&D).

6790 A. exiguus Er. Syracuse, May, under bark (H); Rochester (M\&W); Ithaca, Nov 14 (HCU) ; SI, Feb-Oct, under bark of beech (L\&D).

6797 A. strigosus Lec. Olcott, Sep (Dt); Buffalo (Z\&R); Ithaca, Ap (Dt). 6802 A. sternalis Csy. NY, type locality (Lg).

\section{SAPRINUS Erichson}

6819 S. posthumus Mars. Rochester (M\&W) ; Olcott, May-Je (Dt) ; Ithaca, Sep (Dt); West Pt. (Robn).

6820 S. obsoletus Csy. NY, type locality $(\mathrm{Lg})$.

6827 S. lugens Er. Cranberry L., J1, on carrion (H) ; Rochester (M\&W).

6828 S. imperfectus Melsh. Newport, Je (NYS).

6829 S. pennsylvanicus Payk. Rochester (M\&W); SI, Je-J1, often seen on seashore (L\&D); LI: Shinnecock Hills (Ds); Orient, Lath (NYS).

6831 S. oregonensis Lec. var. distinguendus Mars. Axton, Je (M\&H) ; Cranberry L., Jl, on carrion $(\mathrm{H})$; West Pt. (Robn). 
5836 S. assimilis Payk. Cranberry L., Jl, on carrion (H); Newport, Oct (Yg) ; Johnstown, Je (NYS) ; Gloversville, Sep; Buffalo (Z\&R); Pike, J1; Ithaca, May-Sep (H, Dt); McLean, Aug (Dt); Peekskill (Ds); West Pt. (Robn); SI, May-Aug (L\&D); LI: Oyster Bay, Mar (NYS) ; Orient, Je, Lath (NYS).

6838 S. conformis Lec. Rochester (M\&W); Buffalo (Z\&R); Ithaca, May, Sep (H,Dt).

6875 S. sphaeroides Lec. SI, J1, Woodr (L\&D).

6885 S. fraternus Say. Rochester (M\&W); Buffalo (Z\&R); NYC, May (NYS) ; SI, Je-J1, often on seashore (L\&D) ; LI: E. New York, Rockaway, Je (Ds).

6896 S. patruelis Lec. Rochester (M\&W); Newport (Yg); Pike (Yg); SI, Je-J1 (L\&D).

6901 S. dimidiatipennis Lec. Buffalo (Z\&R); vic. NYC (AM-Palm); SI, Je-Aug (L\&D).

\section{GNathoncus Duval}

6908 G. communis Mars. Newport (Yg); Olcott, May (Dt); Albany, type (Dt) ; LI: Orient Pt., May, Lath (Yg).

6909 B. deletus Lec. Peekskill, Sherm (Csy).

\section{FAMILY LYCIDAE}

\section{Calopteron Guérin-Ménéville}

6925 C. terminale Say. Keene Val., Aug (NYS); L. George, Zab (AM); Karner, Aug (NYS); West Pt. (Robn); White Plains, Aug (Sq); Van Cort. Pk., Aug, Pears (Nic); SI, Jl-Aug (L\&D); LI, Aug, Eng (Sf).

6926 C. reticulatum Fab. Adirondacks (Felt); Newport (NYS); Buffalo (Z\&R) ; Naples (NYS); Canandaigua L., Aug, Bish, Canandaigua, J1 (NYS); Penn Yan (FletCU); Ithaca, Je-Aug (CU) ; Hague, Aug, Leon (NYS); Karner, Aug (NYS); Albany, J1, Lansingburg, Poughkeepsie (NYS) ; West Pt. (Robn); White Plains, Aug (Sq); Van Cort. Pk., Aug (Nic) ; SI, Je, Aug (L\&D) ; LI: Bellport, Je (Nic).

\section{Caeniella Cockerell}

6929 C. dimidiata Fab. Axton, Je (M\&H) ; Newport, Yg (NYS) ; Cranberry L., J1 (Drk) ; Gloversville, J1 (NYS) ; Caroline, May, Ithaca, J1-Aug (FletCU); Catskills, Je, Pears (Nic); SI, Je, Aug (L\&D).

\section{Celetes Newman}

6931 C. basalis Lec. Axton, Je $(\mathrm{M} \& \mathrm{H})$; Big Moose, J1, Newport, Je, Yg (NYS); Chapel Pond, Je, Bish (NYS); Buffalo (Z\&R); Ithaca, Je-J1 (FletCU) ; Catskills, Je (Nic); West Pt. (Robn); SI, Je (L\&D) ; LI: Bellport (Nic), J1, Eng (Sf).

\section{LOPHEROS LeConte}

6933 L. fraternus Rand. Newport, $Y g$ (NYS); Buffalo (Z\&R). 


\section{EROS Newman}

6935 E. thoracicus Rand. Saranac Inn, J1 (FletCU); Keene Val., J1 (NYS); SI, Joutel (L\&D).

6939 E. aurora Hbst. Mt Whiteface, on trail, J1 (Ds) ; L. Clear, Yg (NYS); Newport (NYS) ; Wilmington, Je (NYS); Gloversville, May (NYS); Boston, under bark, scarce, Ap (Dnd); Westfield, May (Not); Rock City, Je (FletCU); Castile, Brad (Flet); Ithaca, Ap-Je (FletCU); McLean, May (Vdyk,Dt); Slide Mt., Je, Leon (NYS); Catskills, Je Pears (Nic); West Pt. (Robn) ; SI: Great Kills, Ap (L\&D); LI: Jamaica (Nic).

6940 E. sculptilis Say. Old Forge, J1 (FletCU); Woodworth's L., Dug Mt., Newport, J1-Aug (NYS); Hamburg, Je, MCV (Frost); Buffalo $(Z \& R)$.

6941 E. humeralis Fab. Peru, Je (FletCU); Newport, Je (NYS); Buffalo $(Z \& R)$; Albany, Je (NYS).

6942 E. trilineatus Melsh. Newport (NYS) ; Naples, J1, Bish (NYS) ; Catskills, Je, Pears (Nic); Callicoon (AM); SI, J1 (L\&D); LI: Je, Eng (Sf); Flatbush, Zab (AM).

6943 E. crenatus Germ. Newport, Je (NYS); Dug Mt., Aug, Yg (NYS); Buffalo (Z\&R); Broadalbin, Je, Yg (NYS); Big Ind. Val., Pears (Sf, Nic).

\section{Plateros Bourgeois}

6944 P. timidus Lec. Ithaca, J1, Dt (Vdyk,Dt); SI, Je (L\&D).

6945 P. modestus Say. Newport, Dug Mt., Elm L., Augur Flats, J1-Aug (NYS) ; Buffalo (Z\&R); Coy Glen, Ithaca, Aug (Vdyk,Dt); McLean, J1 (FletCU); Nyack, Je (Ds); SI, Je-J1 (L\&D).

6946 P. canaliculatus Say. Newport, Je (NYS); Buffalo (Z\&R); Ithaca, J1 (Vdyk,Dt); Ithaca, J1 (Vdyk,Dt); Karner, J1 (NYS); SI, J1 (L\&D); LI: Flatbush, J1, Yaphank, Je, Eng (Sf); Bellport, Je, Pears (Nic).

6949 P. lictor Newn. (mollis Lec.). Keene Val., Newport, J1 (NYS); E. Aurora, J1, rare (Dnd); Buffalo (Z\&R); White Plains, Je-J1 (Sq); LI: Bellport, Je (Nic).

6950 P. floralis Melsh. Cattaraugus Co, J1 (Ds); McLean, J1 (FletCU); West Pt. (Robn); SI (L\&D).

\section{CALOchromus Guérin-Ménéville}

6958 C. perfacetus Say. Plattsburg, J1 (Ds); Clinton Co, Je, Mix\&Everett (FletCU); White Plains, Je-J1 (Sq); LI: Bellport, Je (Nic) ; Farmingdale, Je, Morrow (FletCU); Yaphank, Wading R., J1 (Sf).

\section{FAMILY LAMPYRIDAE}

\section{Calyptocephalus Gray}

6967 C. bifaria Say. Newport, Je (NYS).

\section{LuCIDOTA Laporte}

6971 L. atra Fab. Keene Val., L. Placid, Wells, Je (NYS); Potsdam, May, Ilion (NYS) ; Newport, Je (NYS) ; Cranberry L., Je-J1 (H) ; Long L. (AM) ; Chapel Pond, Je, Bish (NYS); Niagara Falls, May, larva; 
Buffalo (Z\&R) ; Pike, Je (NYS); Ithaca and vic., including Ringwood, J1-Aug (Vdyk); Scotia, Je, Schenectady, J1. (NYS) ; Karner, Je, Kenwood, Jl; Schodack Landing, Je; L. Minnewaska (Ds) ; West Pt. (Robn); Poughkeepsie, Je, Mosholu, Aug (NYS) ; Ramapo, Je (Ds) ; Nyack (AM) ; SI, Je (L\&D) ; Jamaica, Je (NYS) ; Brooklyn (Vdyk,Dt); Flatbush (AM).

6971a L. atra var. tarda Lec. Castile, Brad (Flet); "NY" (Horn Coll.).

6972 L. punctata Lec. Newport, Je, Long L., Aug, Ilion, May (NYS) ; Ithaca, J1 8 (Vdyk,Dt); Windsor, Je (Not); Indian Fields, J1 (NYS).

6975 L. corrusca L. Mooers. Sep (Not); Elizabethtown, Keene Val., Ausable Forks, Aug (NYS); Adirondacks (Felt); Mt. Whiteface, 1500 ft., Aug 25 (Dt) ; Cranberry L., Je-J1 (H); White L., L. George, Zab (AM) ; E. Aurora, Je, on beech trunks (Dnd); Pike, Ap (NYS); Castile, May, Brad (Flet) ; Montour Falls, Je (H) ; Ithaca, McLean, Ap 8-Aug 25 (Dt); Windsor, Je (Not); Trenton Falls, Je (NYS); Karner, Voorheesville, May (NYS) ; Ballston Spa, Je, Clinton Hgts., Mar (NYS) ; Debruce (Ds); West Pt. (Robn); Poughkeepsie, Jl, Gilman, Ap, Mt. Kisco, Oct, Slide Mt., Je (NYS); SI, Mar-Oct (L\&D); LI: Orient, Lath (NYS).

$6975 a$ L. corrusca var. autumnalis Melsh. Buffalo (Z\&R); Nyack (Ds); SI, Ap-J1 (L\&D) ; LI: Bellport, Aug 30 (Nic).

6975 b L. corrusca var. lacustris Lec. Cranberry L., Je, Sep (Drk) ; Buffalo (Z\&R).

6976 L. luteicollis Lec. "NY" (Horn Coll.). Described from Fla.

6977 L. fenestralis Melsh. Axton, Je (M\&H) ; White L. (AM) ; Erie and Allegany Counties, J1 (Dnd); Ithaca, Je-Aug (Vdyk); LI: Flatbush (AM).

6978 L. nigricans Say. Johnstown, J1 (NYS); Ithaca, J1-Aug (Vdyk); West Pt. (Robn).

6979 L. decipiens Harr. Keene Val., J1 (NYS) ; Cranberry L., Aug (Drk) ; White L., Zab (AM) ; Ithaca, Jl (Vdyk); Stony Pt., Je (Ds); Newcastle and Van Cort. Pk. (AM); SI, Je-J1 (L\&D).

\section{PyRACTOMENA LeConte}

6984 P. angulata Say. Keene Val., J1 (NYS) ; Cranberry L., Je 20 (H) ; Buffalo (Z\&R) ; Pike, May (NYS) ; Castile, Je, Brad (Flet) ; Ilion, May, Canajoharie, Je (NYS) ; Allegany Pk., J1 (Dnd) ; Cattaraugus Co, J1 (Ds) ; Ithaca, J1 (Vdyk); Windsor, Je (Not); Karner, J1 Nassau, May (NYS); Catskills, Aug, Pears (Nic) ; Poughkeepsie, Je (NYS); West Pt. (Robn); Nyack, Je (Ds).

6985 P. borealis Rand. Keene Val., Jl (NYS); Axton, Je (M\&H) ; Buffalo (Z\&R) ; Catskills, J1, Pears (Nic).

6987 P. lucifer Melsh. Newport, Pike (NYS); Sullivan Co (Ds).

\section{Photinus LeConte}

6988 P. consanguinius Lec. Newport, Je (NYS); Ithaca, Ringwood, Je-Aug (Dt) ; Poughkeepsie, Je (NYS) ; Nyack, Je (Ds); SI, Je-Jl (L\&D) ; LI: Bellport, Je (Nic); Flatbush, Forest Pk. (AM).

6990 P. ardens Lec. Newport, Je (NYS); Buffalo (Z\&R); Ithaca, Ringwood, J1 (Dt). 
6996 P. pyralis L. Oswego, J1, Bks (NYS) ; Ithaca, J1 (Vdyk) ; SI (L\&D) ; LI: Parkville, Flatbush (AM).

6997 P. marginellus Lec. Rochester, J1, Geneva, Je (Cls) ; E. Aurora, J1 (Dnd) ; Ithaca, J1 (Dt); Van Cort. Pk. (AM); LI: Flatbush (AM).

6999 P. scintillans Say. Newport, J1 (NYS); W. Winfield, Je, Leon (NYS) ; Buffalo (Z\&R) ; Avon, Je (NYS) ; Cattaraugus Co (Ds) ; Albany, Karner, Je-J1, Watervliet Reservoir, Aug, Leon, Rensselaer, Middleburg, J1 (NYS) ; Normansville, Poughkeepsie, J1 (NYS); West Pt. (Robn) ; Stony Pt. (Ds) ; SI, Je-Aug, the common "firefly" of lawn and shrubbery (L\&D).

\section{PHOTURIS LeConte}

7013 P. pennsylvanica DeG. Keene Val., J1, Leon (NYS) ; Newport, Cranberry Cr., Je (NYS) ; Conesus L., Aug (Cls) ; Erie Co, Jl, scarce (Dnd) ; Buffalo (Z\&R) ; Middlesex, Aug (Cls) ; Ithaca, J1 (Vdyk) ; Windsor, Je (Not); Albany, Nassau, Thompson, Poughkeepsie, Je (NYS); West Pt. (Robn) ; Mosholu (AM) ; Cornwall, Oct (Lint) ; SI, Je-J1, makes the brighter greenish light among the higher trees (L\&D); LI: Orient, Ap, Lath (NYS); Shinnecock Hills (Ds); Flatbush (AM).

\section{FAMILY PHENGODIDAE}

\section{Phengodes Illiger}

7023 P. plumosa Oliv. Ithaca, Je, Babiy (Kngt); West Pt. (Robn) ; SI, Je, male (L\&D) (H. S. Barber believes this may be distinct from the plumosa of the South) ; LI: Flatbush, Zab (AM) ; Yaphank, May, Eng (Sf).

\section{TYTTHONYX LeConte}

7034 T. erythrocephala Fab. Boston, Je, rare (Dnd) ; LI: Yaphank, Jl, Eng (Sf).

\section{OMETHES LeConte}

7037 O. marginatus Lec. SI, Je (L\&D).

\section{FAMILY CANTHARIDAE}

\section{Chauliognathus Hentz}

7051 C. pennsylvanicus DeG. (americanus Forst.). Soldier Bug. Keene Val., Aug (NYS) ; Hope, Aug, Bks (NYS); Newport, Aug (NYS) ; Cranberry L., J1 (H) ; Elka Pk., Aug (Drk) ; Buffalo (Z\&R) ; Batavia, J1-Sep (Kngt) ; Pike, Aug (NYS) ; Castile, Brad (Flet); Ithaca, McLean, J1-Sep (Vdyk); L. George (AM); Karner, Aug (NYS); Poughkeepsie, Je (NYS) ; Debruce (Ds); West Pt. (Robn) ; Pine I. (AM) ; Mosholu, Je (NYS); White Plains, Aug-Sep (Sq); SI, Aug-Oct (L\&D); LI: Mineola, J1, Wolf (NYS).

7052 C. marginatus Fab. Schodack Landing, Je (NYS) ; Poughkeepsie, May-Je (NYS) ; Red Hook, Je, Leon (NYS) ; West Pt. (Robn); SI, Je-J1 (L\&D) ; LI: Brooklyn (Vdyk, Dt); Bergen Beach (Ds); Flatbush (AM). 


\section{PODABRUS Westwood}

7055 P. tricostatus Say. Keene Val., J1 (NYS) ; Buffalo (Z\&R) ; Pike (NYS); Montour Falls, Je $(\mathrm{H})$; Ithaca, J1 6 (Vdyk); Trenton Falls, Je (NYS); Schenectady, Albany, Poughkeepsie, Je (NYS); Red Hook, Je, Leon (NYS); West Pt. (Robn) ; SI, Je-Jl (L\&D) ; LI: Bellport, Mar 10 (Nic); Jamaica, Je, Wolf (NYS).

7056 P. rugulosus Lec. E. Aurora, May-Je (Dnd); Buffalo (Z\&R); Schuyler Co, Je, with query $(\mathrm{H})$; Ithaca, J1 5 (Vdyk); Windsor, Je (Not); West Pt. (Robn) ; L. Waccabuc, Nyack, Rye, Lutz (AM) ; SI, Je-J1 (L\&D) ; LI: Flatbush (AM).

7057 P. frater Lec. Big Moose, Je (NYS); Buffalo (Z\&R); Ithaca, Je, Coy Glen, Aug (Vdyk).

7058 P. basillaris Say. Buffalo (Z\&R); E. Aurora, Je-J1 (Dnd); Ithaca, Je (Vdyk); Windsor, Je (Not); Poughkeepsie, Je (Yg); Tivoli, Je (NYS); Dunwoodie (AM); West Pt. (Robn); Ramapo, Je (Ds); SI, May-Je (L\&D).

7058a P. basillaris var. discoideus Lec. "NY" (Lec).

7061 P. diadema Fab. Axton, Je (M\&H) ; Cranberry L., Je 14-27 (H) ; Howland I., Je (Vdyk); Buffalo (Z\&R); Erie Co, Je-Jl, scarce (Dnd); Ithaca, Ringwood, Inlet Val., Je-J1 (Vdyk); Ft. Montgomery, May (Ds); West Pt. (Robn) ; White Plains, Je-J1 (Sq); SI, Je (L\&D).

7062 P. modestus Say. Axton, Je (M\&H) ; Newport, Je-J1 (NYS) ; Ilion, May (NYS) ; Cranberry L.,, Je $30(\mathrm{H})$; White L. (AM) ; Buffalo (Z\&R); Erie Co, Je-J1, scarce (Dnd).; Pike, Je (NYS) ; Castile, Brad (Flet); Ithaca, May-J1 (Vdyk); Montour Falls, Je (H) ; McLean, Inlet Val., Je-Jl (Vdyk) ; Windsor, Je (Not) ; Trenton Falls, Albany, Je (NYS); Riders Mills, May, Bish (NYS); Catskills, Pears (Nic); White Plains, Je-J1 (Sq); West Pt. (Robn); vic. NYC, Palm (AM); SI, May-J1 (L\&D).

7062a P. modestus var. flavicollis Lec. Cranberry L., J1 (H); Valley Falls, Je (NYS) ; Nassau, May, Poughkeepsie, Je (NYS); West Pt. (Robn).

7066 P. tomentosus Say. Albany, Je-Aug, Nassau, Je (NYS); West Pt. (Robn).

7068 P. brunnicollis Fab. Ithaca, J1 (Dt) ; Inlet Val., Aug 12 (Vdyk) ; Poughkeepsie, Je (NYS).

7071 P. limbellus Lec. Poughkeepsie, Je (NYS).

7072 P. punctatus Lec. Westfield, May (Not); Windsor, Je (Not) ; Catskills, Pears (Nic).

7074 P. puncticollis Kby. Monadnock, Je (NYS).

7078 P. piniphilus Esch. L. Tear, J1 (NYS).

7080 P. puberulus Lec. Cranberry L., Je $9(\mathrm{H})$.

7082 P. simplex Couper. Karner, Je (NYS).

7083 P. laevicollis Kby. Buffalo (Z\&R).

7086 P. pattoni Lec. Newport, Je (NYS); Westfield, May (Not) ; Freeville, Je 5 (Vdyk). Described from Calif. 


\section{CANTHARIS Linnaeus}

(Telephorus Schaeffer)

7091 C. dentiger Lec. Elizabethtown, Je, Newport, May (NYS) ; Long L. (Ds) ; Thompson, May, Leon (NYS); Pike, Je (NYS); Castile, Brad (Flet); Valley Falls, May (NYS); Ithaca, May-Je (Vdyk); Enfield Glen, May (NYS); Windsor, Je (Not) ; Schenectady, Je, Albany, May, Karner, Je, Nassau, May, Kinderhook, Je, Shandaken, Je, Poughkeepsie, May (NYS); West Pt. (Robn); Bronx Pk., Suffern (AM); NYC (AM).

7092 C. excavatus Lec. Poughkeepsie, Je, Ionia (NYS); West Pt. (Robn) ; SI (L\&D).

7093 c. vilis Lec. "NY" (Lec).

7095 C. walshi Lec. Ithaca, J1 (Vdyk).

7096 C. fraxini Say. Axton, Je (M\&H); Mt. McIntyre, J1, Leon (NYS) ; Speculator, Aug, Northville, L. Clear, Je (NYS); Wanakena, Aug (Drk); Buffalo (Z\&R); Castile, May, Brad (Flet); Windsor, Je (Not); Catskills, J1, Pears (Nic).

7097 C. carolinus Fab. Axton, Je (M\&H); Keene Val., J1, Newport, May (NYS) ; Cranberry L., Je-J1 (H) ; Long L. (Ds); Buffalo (Z\&R); Erie Co, Je, common (Dnd) ; Pike, Je (NYS); Westfield, Je (NYS); Schuylerville, Je (NYS) ; Montour Falls, Je (H); Ithaca, Ringwood, McLean, Je-J1 (Vdyk); Windsor, Je (Not); Albany, Rensselaerville, May, Nassau, May, Schodack Landing, Je, Kinderhook, Je, Poughkeepsie, Je (NYS); West Pt. (Robn); Nyack, Ramapo (Ds); Van Cort. Pk. (AM); SI (L\&D).

C. neglectus Fall. Albany, Je (NYS).

7098 C. lineola Fab. E. Aurora, May-Je, scarce (Dnd) ; Karner, Je (NYS) ; SI, Je (L\&D); LI: Bellport, Aug (Nic); Orient, Lath (NYS).

7100 C. nigritulus Lec. Chazy L., Rainbow L., Je (NYS) ; Cranberry L., Je 9J1 $10(\mathrm{H})$; Port Byron, Je (Vdyk); Howland I., Je (Vdyk); Ithaca, Freeville, Je, Ringwood, J1 7 (Vdyk); Windsor, Je (Not); Suffern (AM) ; SI, Je (L\&D).

7101 C. rectus Melsh. (pusillus Lec.). Newport, Je (NYS); Buffalo (Z\&R); Ithaca, Ringwood, Inlet Val., Je-J1, Coy Glen, Aug 2' (Vdyk) ; Albany, Je, Poughkeepsie, May (NYS); Catskills, Je 25 (Nic); Nyack, Je (Ds) ; SI, Je (L\&D); LI: Bellport (Nic).

7105 C. flavipes Lec. Buffalo (Z\&R); Castile, Brad (Flet); Windsor, Je (Not).

7106 C. scitulus Say (imbecilis Lec.). Keene Val., Aug (NYS) ; Wells, J1, Newport. Je (NYS) ; White L., Zab (AM) ; Buffalo (Z\&R) ; Aurora, $\mathrm{Zab}$ (AM) : Ithaca, Ringwood, Butternut Cr., Jl (Vdyk); Coy Glen, Aug 2 (Vdyk); Karner, J1 (NYS); Catskills, Pears (Nic); Ft. Montgomery (Ds); West Pt. (Robn); Van Cort. Pk. (AM); SI, Je-Aug (L\&D) ; LI: Flatbush (AM).

7107 C. pusillus Lec. SI, J1 (L\&D).

7110 C. longulus Lec. Niagara (Lec).

7113 C. rotundicollis Say. Axton, Je ( $\mathrm{M} \& \mathrm{H})$; Essex Co, Aug, Elizabethtown, J1, Mt. McIntyre, J1, Newport, Je, Wells, J1, Saranac Inn, J1 (NYS); Cranberry L., Je-J1 (H) ; Chapel Pond, Je, Leon (NYS); Buffalo (Z\&R) ; Cincinnatus, JI (Flet, Dnd) ; Ithaca, J1 (Vdyk); Windsor, Je (Not); Corinth, Albany, Karner, Je (NYS); West Pt. (Robn) ; SI, May-J1 (L\&D). 
7119 C. tuberculatus Lec. (impressus Lec.). Cranberry L., Je $11(\mathrm{H})$; E. Aurora, Protection, May-Je, frequent (Dnd); Ithaca, Je (Vdyk); Albany, Karner, Nassau, Poughkeepsie, May (NYS); N. Chatham, Je (NYS) ; West Pt. (Robn) ; Ft. Montgomery, Ramapo (Ds); SI, May-Je (L\&D) ; LI: Babylon (AM).

7119a C. tuberculatus var. armiger Couper. Buffalo (Z\&R).

7121 C. bilineatus Say. Axton, Je (M\&H) ; Newport, May, Wells, Je (NYS) ; Cranberry L., Je $16(\mathrm{H})$; Herkimer Co, Feb, larvae (as bilineatus?) (Lint) ; Syracuse, May (H) ; Buffalo (Z\&R) ; E. Aurora, May-Je, common, in beating (Dnd) ; Westfield, May (Not) ; Castile, May, Brad (Flet); Windsor, Je (Not) ; Ithaca, May-Je (Dt); Corinth, Albany, Poughkeepsie, May (NYS); Germantown, Je, Leon (NYS); West Pt. (Robn) ; SI, Ap-May (L\&D) ; LI: Orient, Lath (NYS).

7127 C. marginellus Lec. Cranberry L., Je $(H)$. Described from Ga. and Fla.

\section{Polemius LeConte}

7137 P. laticornis Say. Rensselaer, J1 (NYS); SI, Je (L\&D).

7139 P. limbatus Lec. SI, Je (L\&D).

\section{SILIS Latreille}

7152 S. percomis Say. Newport, Ilion, May (NYS); Cranberry L., Je (H) ; Buffalo (Z\&R); Catskills, Pears (Nic); West Pt. (Robn) ; LI: Orient Pt., May, Lath (NYS).

7157 S. spathulata Lec. "NY" (Lg Cat.).

7161 S. bidentatus Say. Newpcrt, Ilion, May (NYS); Wells, J1, Hope, Salem, Phoenicia, Aug (NYS); West Pt. (Robn); SI, May-Je (L\&D).

\section{TRYPHERUS LeConte}

7169 T. latipennis Germ. Northampton, Je, Oswego, J1 (NYS); Ithaca, Aug 4 (Vdyk) ; West Pt. (Robn) ; SI, Je-J1 (L\&D) ; LI: Jamaica, Pears (Nic).

\section{MaLThinus Latreille}

7172 M. occipitalis Lec. West Pt. (Robn).

\section{Malthodes Kiesenwetter}

7178 M. concavus Lec. "NY" (Lec); Ft. Montgomery (Ds).

7179 M. fragilis Lec. (transversus Lec.). Cranberry L., Je-J1 (H); Niagara (Lec); vic. NYC, Palm (AM).

7180 M. exilis Melsh. Windsor, Je (Not); "NY" (Melsh); SI: Buck's Hollow, J1 12 (L\&D).

7188 M. fuliginosus Lec. Windsor, Je (Not).

7193 M. parvulus Lec. "NY" (Lec); Cranberry L., Htly (H). 


\section{FAMILY MELYRIDAE \\ SUBFAMILY MALACHIINAE}

COLlops Erichson

7196 C. tricolor Say. Catskills, Van Cort. Pk., Je-Jl, Pears (Nic) ; Ft. Montgomery, Il (Ds); West Pt. (Robn); LI, Je, Eng (Sf).

7207 C. nigriceps Say. "NY" (Lec), as cximius Er.; Suffern (AM); SI, JeSep (L\&D); LI: Orient Pt., Aug (NYS); Rockaway, Yaphank, Je, Eng (Sf); Islip, Johnson (Sf); Babylon (AM); Wading R., Jl-Aug (Ds).

7215 C. vittatus Say. Axton, Je (M\&H) ; Potsdam (NYS) ; Saranac Inn, Newport, J1 (NYS) ; Cranberry L. (H) ; SI, Je (L\&D) ; LI: Bellport, Je (Nic) ; Orient Pt., Sep, Lath (NYS); Shinnecock Hilis, J1 (Ds).

7227 C. quadrimaculatus Fab. Newport (NYS) ; Essex Co, J1 (NYS) ; Gloversville, J1 (NYS) ; Syracuse, J1 (NYS); Olcott, Jl-Sep (Dt); Buffalo (Z\&R); Batavia, J1-Aug (Kngt); Aurora (AM) ; Geneva, Avon, J1Aug (Cls) ; Westfield, J1 (NYS) ; Ithaca, J1 2, Yuasa (Vdyk) ; Ringwood, J1 (NYS) ; Schenectady, J1, Scotia, Je (NYS) ; North Creek, Aug, Nassau, J1 (NYS) ; West Pt. (Robn); Ft. Montgomery, J1 (Ds); White Plains, Je, Sq (Frost); SI, J1 (L\&D); LI: E. New York, Je, Martin (Sf); Rockaway Beach, Je, Eng (Sf) ; Yaphank, May-Sep, Eng (Sf); Long Beach, Orient (AM) ; Central Pk., Wading R., Half Way Hollow Hills (Ds).

\section{Malachius Fabricius}

7238 M. aeneus L. Potsdam, May, Speculator, Je (NYS) ; Canton, L. Pleasant, Je (Babiy); L. Clear, Je, Ogdensburg, J1 (NYS); Northville, Je (NYS) ; Cranberry L., Je 18, on blossoms of trefoil and flying about camp $(\mathrm{H})$; Syracuse, Je; Castile, May, Brad (Flet); Batavia, Je (Kngt); Geneva, Avon, Ap-May (Cls); Schuylerville, Je (NYS); Ithaca; Ringwood, J1 3, Yuasa (Vdyk); Windsor, Je (Not); Waterford, May, Albany, Je, Nassau, May (NYS).

\section{Anthocomus Erichson}

7276 A. erichsoni Lec. Buffalo (Z\&R); SI: Old Place, Je (L\&D).

7277 A. flavilabris Say. NYC and vic. (Ds).

7279 A. apicalis Say. West Pt., J1, Eng (Sf).

\section{Pseudobaeus Horn}

7281 P. pusillus Say. Schoharie (Not).

7280 P. bicolor Lec. LI: Yaphank, May (Ds).

7282 P. oblitus Lec. Buffalo (Z\&R); Windsor, Je (Not); Schoharie (Not); Peekskill, among lichens on rocks, late May (Sherm); West Pt. (Robn); Nyack, Je (Ds) ; SI (L\&D) ; LI: Bellport, in sweeping, Aug (Nic); Yaphank, Je (Ds).

\section{Attalus Erichson}

7290 A. nigrellus Lec. Mt. Seward, 4500 ft., Je 22 (M\&H).

7294 A. terminalis Er. Newport, Je (NYS) ; Ithaca, Aug (Dt) ; Fort Montgomery (Ds) ; West Pt. (Robn); NYC, Ap (NYS); LI: Yaphank, J1, Eng (Sf). 
7300 A. granularis Er. Ithaca, Aug (Dt).

7303 A. morulus Lec. "NY" (Lec).

7304 A. pallifrons Mots. Schoharie (Not); SI: Watchogue, Je 28 (L\&D).

7312 A. rufiventris Horn. Plattsburg (Ds); Buffalo (Z\&R); Stony Pt. (Ds); LI: Wading R. (Ds).

7314 A. otiosus Say. West Pt. (Robn) ; LI: Yaphank, Je, Eng (Sf).

7322 A. scincetus Say. Poughkeepsie, May (NYS); Peekskill, in flowers of Viburnum prunifolium (Sherm); Ramapo (Ds); West Pt. (Robn); vic. NYC (AM); SI (L\&D); LI: Greenport, May (NYS); Yaphank, Je, Eng (Sf); Orient, Lath (NYS).

\section{SUBFAMILY MELYRINAE}

Dolichosoma Stephens

7503 D. foveicollis Kby. Buffalo (Z\&R).

\section{FAMILY CLERIDAE 39 SUBFAMILY CLERINAE}

MONOPHYLLA Spinola

7516 M. terminata Say. Rochester Jct., Je, Leon (WolcCU); West Pt. (Robn); Bronx Pk. (Sf); SI, May (Chitt), larvae predatory on borers in various trees and shrubs.

\section{CYMATODERA Gray}

7544 C. bicolor Say. Keene Val., Je (Not); Olcott, Je-J1 (Wolc, Dt) ; Buffalo (Z\&R, Wolc); Kinderhook, Aug, Peekskill (WolcCU); West Pt. (Robn) ; Mosholu (Sf) ; LI: Cold Sp. Harb., Je, Eng (Sf).

7545 C. inornata Say. Olcott, Je-J1 (Wolc, Dt) ; Ilion, May (NYS) ; Buffalo (Z\&R, Wolc) ; Ramapo (Sf) ; Greenwood L. (Sf) ; West Pt. (Robn); SI, larvae predatory on secondary borers in dead trees of several kinds (L\&D) ; LI (Linell).

7566 C. undulata Say. "NY" (Sm, Wolc); West Pt. (Robn), as balteata, under which name a species is recorded also in the N. J. List.

\section{PRIOCERA Kirby}

7577 P. castanea Newn. Buffalo (Z\&R).

\section{THANASIMUS Latreille}

7584 T. trifasciatus Say. Keene Val., Je (Not); Buffalo ( $Z \& R)$, as nigripes; "NY" (Ashton Coll., Wolc-Purdue Univ.).

7585 T. dubius Fab. Mt. Seward, Je (M\&H) ; Keene Val., Je-J1 (Not) ; Black Br., Clinton $\mathrm{Co}$, Je (WolcCU); Buffalo (Z\&R); Ithaca, Je (WolcCU); Peekskill, Sherm (Wolc); West Pt. (Robn); LI: Jamaica, Aug, Pears (Nic); Wyandanch, Ap, Schott (Ds).

39 A. B. Wolcott, besides supplying many records, has gone over the list for this family and is responsible for several changes in synonymy and spelling of names, which differ from those in Leng's catalog. 
7587 T. undulatus Say. Black Br., Je (WolcCU); Buffalo (Z\&R); SI, larvae in several kinds of trees infested by cerambycid borers (L\&D).

7587 a T. undulatus var, nubilis Klug. Buffalo (Z\&R).

\section{PLACOPTERUS Wolcott}

7589 P. thoracicus Oliv. Olcott, Je-J1 (Wolc, Dt); Buffalo (Z\&R), also C.F.Baker (Wolc); E. Aurora, Je (Dnd); Montour Falls, Je (H); Schoharie (Not); Ithaca, May (WolcCU); Ft. Montgomery, Je, Schott (Ds); West Pt., Je (Sf) ; Mosholu (AM) ; SI, Je (L\&D); LI: Huntington, Wading R., Je (Ds); Yaphank, May-Je, Eng (Sf).

\section{ENOCLERUS Gahan}

7594 E. nigrifrons Say. E. Aurora (Dnd); Ithaca, Je (Wolc, Dt); West Pt., Je (Ds) ; SI, Je-J1 (L\&D) ; LI: Bayshore, J1 (O1s); Huntington, Je, Wyandanch, Mar, Oakdale, Aug, Schott (Ds, Sf); Farmingdale, Je, Morrow (WolcCU); Pinelawn, Yaphank, Je, Eng (Sf).

7596 E. nigripes Say. ${ }^{40}$ Buffalo, Sherm (Wolc); Ithaca, May-J1 (Wolc, Dt, CU) ; West Pt., Je (Sf) ; LI: Central Pk., Yaphank, Ap, Je, Eng (Sf).

7596b E. nigripes var. rufiventris Spin. Black Br., Je (WolcCU); E. Aurora (Dnd) ; Ithaca, May, J1 (Wolc, Dt, CU).

7597 E. rosmarus Say. Buffalo (Z\&R); Ft. Montgomery, Je, Schott (Ds); West Pt. (Robn) ; Stony Pt., Mar (Ds) ; White Plains (Sq) ; Bronx Pk. (Sf); SI, Je, Aug (L\&D); LI: Maspeth, Je (Ols); Flatbush (AM); Massapequa, J1, Wyandanch, Je (Ds); Flushing, Je, Eng (Sf).

7605 E. ichneumoneus Fab. Cranberry L., J1 (Drk) ; NYC (Wolc); LI: Bellport, Wading R. (Nic).

7610 E. quadrisignatus Say. LI: Central Pk., Ap, Yaphank, Sep, Eng (Sf).

7612 E. lunatus Klug. LI: Rockaway Beach (Shoe).

7614 E. analis Lec. Pike, Je, Albany, Je, Poughkeepsie, May (NYS).

7616 E. laetus var. coccineus Schklg. "NY" (Fitch), but both Wolcott and Leng regard the record as doubtful since the species is more western.

E. liljebladi Wolc. LI: Bellport, Nic (Sf).

\section{TRICHODES Herbst}

7629 T. nutalli Kby. Wilmington, J1 (Ds); Honeoye Falls, Je, Leon (WolcCU); New Russia, Aug, Bdy (Wolc); Potsdam (Wolc-Ill. Lab. Nat. Hist.); Otto, J1 (CU); Buffalo (Z\&R); E. Aurora (Dnd) ; Batavia, J1-Aug (Kngt); Ithaca, J1-Aug (Wolc, Dt, CU); W. Hebron, Aug (Ds); $\mathrm{SI}, \mathrm{J} 1$, in fresh meadows (L\&D).

${ }^{40}$ E. quadriguttatus Oliv., 7595. Newport, Je (NYS); Cranberry L., Je-Jl, on pine and spruce (H) ; Buffalo (Frison): Karner, J1 (NYS): West Pt., Te (Sf): SI, Mar 29, on cedar (L\&D); LI: Central Pk., Ap (Eng); Bellport, Je (Nic); Wyandanch, Mar, Oakdale, Aug, Schott (Ds). A. B. Wolcott writes: "This is not a North American species and was not intended to be included in Leng's catalog. The records should be transferred to E. nigripes Say. See Trans. Am. Ent. Soc., vol. 48, pp. 70-71, 1922." 
7630 T. apivorus Germ. (mutalli Say). Ithaca (Chitt); Saratoga (Lec); Albany, Karner, Je (NYS).

7630a T. apivorus var. borealis Wolc. \& Chap. "NY", as var. intermptus (Wolc) ; Binghamton, Aug, Franck (Sf); Ithaca, Áug (Wolc, Dt).

\section{HYDNOCERA Newman}

7637 H. unifasciata Say. LI: Loyd's Neck, J1, Brb (Sf).

7642 H. humeralis Say. Buffalo (Z\&R); West Pt., Ft.' Montgomery, Je, Schott (Ds); Peekskill, Sherm (Wolc); SI, May (L\&D); LI: Rockaway Beach, Je-Jl (Chitt), on Myrica cerifera; Smithtown, Je, Bayshore, Je, Maspeth, Huntington, May, Massapequa, J1, Yaphank, Orient, J1 (Ds) ; Yaphank, Amagansett, Wyandanch, May-Je, Eng (Sf).

7642a H. humeralis var. difficilis Lec. Axton, Je (M\&H); Peru, Je, Aug (WolcCU) ; Buffalo (Z\&R), as cyanescens Lec.; Ithaca, Je, Aug (Wolc, Dt, CU) ; Peekskill, Sherm (Wolc); West Pt. (Robn) ; Ft. Montgomery, Je-J1, Schott (Ds) ; LI: Huntington, Sep, Wyandanch, J1, Schott (Ds) ; Yaphank, Pinelawn, Je, Eng (Sf).

7648 H. lecontei Wolc. Bear Mt., J1, as subaenea Spin., Nic (Sf).

7649 H. maritima Wolc. LI: Amagansett, Aug (Sf).

- H. commixta Chapin. "NY" Chapin (Wolc). Type.

7675 H. verticalis Say. Mt. Whiteface, 2000-4000 ft., Aug 22-24 (WolcCU); Plattsburg, J1 (Ds) ; Olcott, Je-J1 (Wolc, Dt) ; Buffalo (Z\&R, Wolc) ; Castile, Brad (Flet); Ithaca, Feb, Babiy, Je, Wolc (CU) ; Schoharie (Not); Ft. Montgomery, Je-Jl, Schott (Ds) ; Peekskill, Sherm (Wolc) ; Nyack, Je (Ds); NYC (Wolc) ; Bronx Pk., Van Cort. Pk. (Sf); SI, Je-J1, predatory on small cerambycid larvae in grape and Celastrus (L\&D) ; LI: Parkville, Sherm (Wolc) ; Bellport, Je (Nic) ; Pinelawn, Yaphank (Sf); Riverhead, Sep (WolcCU).

7677 H. pallipennis Say. White L. (AM); Buffalo (Z\&R, Wolc); Ithaca, Aug (Wolc, Dt) ; Hudson Falls, J1, Sherm (Wolc) ; L. George (AM) ; West Pt. (Robn); Ft. Montgomery, Ramapo, J1 (Ds); Bronx Pk., Van Cort. Pk. (Sf) ; SI, J1-Aug (L\&D) ; LI: Greenport, Bellmore, Yaphank (Sf); Flatbush, Quogue (AM).

\section{ISOHYDNOCERA Chapin}

7688 I. curtipennis Newn. Cranberry L., Je 29 (H) ; Buffalo (Z\&R) ; Montour Falls, Je (H) ; Ithaca, J1 (Wolc, Dt, CU) ; Windsor, Je (Not); Peekskill (Fall) ; Ramapo (Sf) ; LI: Rockaway Beach, Je, Eng (Sf). Also as longicollis Zieg1. from Batavia, J1 (Kngt); Schoharie (Not); West Pt. (Robn).

7689 I. tabida Lec. Rochester Jct., Je, Leon (WolcCU); Spring Lake, J1 (WolcCU) ; Ithaca, May-Je (Wolc, Dt, CU) ; Windsor, Je, (Not); Greenwood L. (Sf); SI, Je, predatory on mordellid larvae in stems of annual plants (L\&D); LI: Rockaway, Je (Sf).

\section{ZENODOSUS Wolcott}

7694 Z. sanguineus Say. St. Lawrence Co, J1 (Drk); Keene Val., May, Aug (Not) ; Cranberry L., Je-J1, also in burrows of bark beetles $(\mathrm{Blkm}, \mathrm{H})$; Şyracuse, Ap, under bark (H); Geneva, Feb-Je (Cls); 
Buffalo (Ashton Coll., also Bolter Coll., Univ. I11.) (Z\&R), as Enoclerus; E. Aurora (Dnd) ; Batavia, Sep (Kngt) ; Bronx Pk. (Sf) ; LI: Flushing (Ds).

\section{SUBFAMILY CORYNETINAE}

Phyllobaenus Spinola

7696 P. dislocatus Say. Keene Val., Aug (Not) ; Cranberry L., J1, in burrows of bark beetles (H) ; Syracuse, Je (Drk); Olcott, Je-J1 (Wolc,Dt) ; Buffalo (SayANSP) ; Ithaca, Ap-May (WolcCU); West Pt. (Sf) ; Ft. Montgomery, Je, Sichott (Ds) ; Peekskill (AM) ; Mosholu (Sf) ; Bronx Pk. (Ds); SI, Je, larvae predatory on small borers in deciduous trees (L\&D) ; LI: Wyandanch, Je, Schott (Ds); Bellport, Aug (Nic).

\section{NEICHNEA Wolcott and Chapin}

7698 N. laticornis Say. Buffalo (Z\&R); Greenwood L. (Dietz, Sf); West Pt. (Robn) ; LI: Bellport, Aug (Nic).

\section{Chariessa Perty}

7708 C. pilosa Forst. Honeoye Falls, Je, Leon (WolcCU) ; Castile, Brad (Flet) ; Syracuse (B\&S); Buffalo (Z\&R); Ithaca, Je 30 (WolcCU) ; West Pt., Je (Ds) ; Peekskill, J1, Sherm (Wolc) ; SI: Clove Val., Je 27, from hickory (L\&D) ; LI: Bellport, Aug (Nic).

7708 a C. pilosa var. marginata Say. Buffalo (Z\&R), as onusta; Peekskill, Je, Sherm (Wolc); West Pt., Je (Ds); LI: Wyandanch, Je, Schott (Ds).

\section{CoRINTHISCUS Fairmaire and Germain}

7711 C. leucophaeum Klug. Rochester (Moore); Buffalo (Z\&R), as Cregya vetusta; Bronx Pk. (Sf).

\section{Cregya LeConte}

7716 C. oculatus Say. Buffalo (Z\&R); Peekskill, Sherm (Wolc); Mosholu (Sf) ; NYC (Wolc, Wickham) ; SI, Mar, Je, J1, on cedar and deciduous trees; larvae predatory on borers (L\&D); LI: Montauk Beach, Wyandanch, Half Way Hollow Hills, Je (Ds).

\section{ORTHOPLEURA Spinola}

7719 O. damicornis Fab. Buffalo (Z\&R); Peekskill, Sherm (Wolc); LI: Jamaica, Pears (Nic).

\section{NECROBIA Olivier}

7727 N. rufipes DeG. Perry, Dec (WolcCU) ; Buffalo (Z\&R) ; Ithaca, Aug-Sep (WolcCU) ; SI, J1-Sep, often found on beach, on dead animals (L\&D) ; LI: Rockaway Beach, May, Schott (Ds) ; Brooklyn, Long Beach (Sf); Coney I. (AM). 
7728 N. ruficollis Fab. Buffalo (Z\&R) ; Oswego, Sep, Sheldon (Wolc) ; Ithaca, Aug (WolcCU); Peekskill, Sherm (Wolc); NYC (AM); SI, Aug-Sep, habitat same as for rufipes (L\&D); LI: Rockaway Beach, Sep, Schott (Ds) ; Coney I. (AM).

7729 N. violacea L. Axton, Je (M\&H) ; Syracuse, Ap (H) ; Olcott, May (Wolc,Dt) ; Potsdam (Wolc-Ill. Lab. Nat. Hist.) ; E. Aurora (Dnd) ; Montour Falls, Ap (H) ; Ithaca, Ap, May, Aug (Wolc, Dt, CU) ; West Pt. (Robn); SI, Aug (L\&D); LI: Brooklyn, Yaphank, May, Eng (Sf).

\section{FAMILY LYMEXYLIDAE}

\section{HyLecoetus Latreille}

7739 H. lugubris Say (americanus Harr.). Keene Val., May-Je (Not) ; Newport, May-Je (NYS) ; Trenton Falls, May 20, larva boring in elm (Hagen); Albany, May (NYS); vic. NYC (AM).

\section{Melittomma Murray}

7740 M. sericeum Harr. NY", larva in oak, Horn (Fitch); LI: Rockaway Beach, Je, Long Beach, J1 (Sf).

\section{FAMILY CUPESIDAE}

\section{Cupes Fabricius}

7743 C. capitatus Fab. Newport (NYS); Buffalo (Z\&R) ; Albany, J1 (NYS) ; vic. NYC (AM); North Branch (Schaupp).

7744 C. concolor Westw. Newport (NYS) ; Syracuse, in dying hickory (B\&S) ; Buffalo (Z\&R); Ithaca, Jl-Aug (Vdyk); North Branch (Schaupp); LI: Westbury, J1 (bred by Champlain-H) ; Greenport, Aug, at light (Ds).

$7744 a$ C. concolor var. oculatus Csy. "NY" (Csy).

\section{FAMILY CEPHALOIDAE}

\section{Cephaloon Newman}

7747 C. lepturides Newn. (lepturcides Hald.). Mt. Seward, Axton, Je (M\&H) ; Newport (Yg); Chapel Pond, Keene, Je, Leon, Bish (Yg); Peru (FletCU) ; Olcott, Je-J1 (Dt); E. Aurora, Je (Flet,Dnd) ; Buffalo (Z\&R) ; Rock City, Je (FletCU); Ithaca, May-JI (Dt); Windsor, Je (Not); Trenton Falls (Newn); Schoharie (Not) ; Ft. Edward, Je (FletCU) ; Ramapo, Nyack, Je (Joutel) ; SI: Green Ridge, Je, common in flowers of the tulip tree (Liriodendron) (L\&D).

\section{TyPITIUM Casey}

7748 T. ungulare Lec. Whiteface Mt., J1 (Lg); Essex Co, J1 (FletCU); E. Aurora, Je (Flet,Dnd). 


\section{FAMIIY OEDEMERIDAE SUPFAMILY OEDEMERINAE}

NACERDA Stephens

7763 N. melanura L. Oswego, J1 (NYS); Lockport, Mar (FletCU); Buffalo, Aug (NYS); Castile, Brad (Flet); Ilion, May (NYS); Ithaca, J1 (Dt); Albany (Lint); West Pt. (Robn); NYC (FletCU); SI, Je-J1 (L\&D); LI: Bergen Beach, Greenport (Ds) ; Flatbush, Long Beach (AM) ; Rockaway Beach, Je (Sf); Barren I. (AM).

\section{Ditylus Fischer}

7765 D. coeruleus Rand. Buffalo (Z\&R).

\section{Copidita LeConte}

7769 C. thoracica Fab. Greenwood L. (Sf); West Pt. (Robn) ; LI: Wyandanch, a variety $(\mathrm{Sf})$.

\section{Alloxacis Horn}

7776 A. dorsalis Melsh. West Pt. (Robn); vic. NYC (AM); SI, Jl-Aug, once rather abundant, under logs (L\&D); LI: Fire I., Rockaway Beach, Aug (Sf).

\section{ASCLERA Stephens}

7799 A. puncticollis Say. Mt. Seward, 4500 ft., Je (M\&W); Whiteface Mt., J1 (Sf) ; Buffalo (Z\&R); Trenton Falls (Newn); SI, May 8, 1881 (L\&D).

7800 A. ruficollis Say. Cranberry L., Je (H) ; Buffalo (Z\&R) ; Ithaca, Ap-May, McLean Bogs, May (FletCU); Taughannock, Gorge, Ap 21, Dt (Snn); Trenton Falls (Newn); Albany, Nassau, E. Schodack, Ap-May (NYS); Ramapo, West Pt. (Robn); NYS (AM); SI, Ap-May (L\&D) ; LI: Yaphank, May (Sf).

\section{FAMILY MORDELLIDAE 41}

\section{TOMOXIA Costa}

7804 T. bidentata Say. Peru, Je (CU) ; Rochester, Honeoye Falls, Leon (CU) ; Castile, Brad (Flet) ; Batavia, Je (Kngt); Ithaca, J1 (CU) ; Albany, Je (NYS); West Pt. (Robn); Nyack, Ds (Nic); Palisades (Nic); Van Cort. Pk. (Nic) ; SI, J1 31, on dead oak log (L\&D) ; LI: Maspeth, Je, Shoe (Nic). On decaying hickory (Felt).

7805 T. lineella Lec. Peru, Je 21 (CU); Rochester Jct., J1 7, Leon (CU); Buffalo (Lec); Ithaca, J1 (CU); Poughkeepsie, Je (NYS); Catskills (Nic); SI, Je (L\&D). On decaying hickory (Felt).

7806 T. inclusa Lec. Rochester Jct., J1, Leon (CU); Buffalo (Z\&R); Catskills, $\mathrm{Jl}$ (Nic).

\footnotetext{
${ }^{41}$ Emil Liljeblad has seen the manuscript of the list for this family, and has made some changes
} in synonymy as well as determined all of the material from New York in the Cornell collection. 


\section{MORDELLA Linnaeus}

7808 M. quadripunctata Say. Cranberry L., J1 10 (H).

7809 M. borealis Lec. Big Moose, J1 (NYS) ; Newport (NYS) ; Cranberry L., Aug (H) ; Albany, Nassau (NYS) ; Catskills, Pears (Nic). On dying spruce and in decaying maple stumps (Felt).

7810 M. melaena Germ. Chazy L., Saranac Inn, L. Placid, Severance, Hope, Wells, L. Pleasant, Gloversville, Northampton, Pike, Je-Aug (NYS); Castile, Brad (Flet); Cranberry L., J1 (H); Buffalo (Z\&R); Wales, J1 (Dnd) ; Canandaigua, J1 (NYS); Ithaca, J1-Aug (CU); McLean, J1 (Dt) ; Saratoga Sps., J1 (NYS) ; Karner, J1 (NYS) ; Catskills (Nic); Pine I. (Ds); Van Cort. Pk., very local and not common (Nic); SI, on wild rose (L\&D).

7811 M. atrata Melsh. (Liljeblad writes that scutellaris Fab. is a South American species.) Peru, Je (CU) ; Black Br., Je.(CU) ; Axton, Je (M\&H) ; Newport, Severance, Hope, Je-Aug (NYS) ; Saranac, Aug (CU) ; Long L. (Ds) ; Cranberry L., Wanakena (H) ; Erie Co, Je (Dnd) ; Ithaca, Enfield, J1 (CU); McLean Bogs, J1 (Dt); Kenwood, J1 (NYS) ; Karner, J1 (NYS) ; Poughkeepsie, Je-J1 (NYS) ; West Pt. (Ds) ; SI, Je, Sep (L\&D) ; LI: Orient, Lath (NYS).

7811a M. atrata var. lecontei Csiki. Ithaca, Je, $D t$ (CU); Middletown, Je (CU). 7814 M. octopunctata Fab. Rochester Jct., Aug, Lcon (CU) ; E. Aurora, J1 (Dnd); Geneva, Je (Cls) ; Ithaca, J1 (CU) ; Albany, Je (NYS); West Pt., Ft. Montgomery (Ds) ; SI, Je-J1, bred from hickory (L\&D) ; LI: Mineola, Je, Wolf (NYS); Farmingdale, Je (Lilj). Larvae in oak stumps (Felt).

7817 M. marginata Melsh. Keene Val., L. Placid, Saranac Inn, Wells, Hope, Je-Aug (NYS) ; Wilmington (Dt); Wanakena, Aug (Drk) ; Cranberry L., J1 (H) ; Rochester Jct., Je, Leon (CU) ; Olcott (CU) ; Buffalo (Z\&R); E. Aurora, Je-J1 (Dnd); Pike, Sep, Knull (Lilj) ; Castile, Brad (Flet); Otto, J1 (CU) ; Crosby, Je (CU); Montour Falls, Je (H) ; Ithaca, J1-Aug (CU) ; McLean (Dt); Danby, J1 (Dt); Spring Lake, J1 (CU) ; Schoharie (Not) ; L. George (AM) ; Catskills (Sm) ; Poughkeepsie, Je-J1 (NYS) ; Rhinebeck, J1, Cy (CU); West Pt. (Robn) ; SI, Je-Jl, on hickory (L\&D) ; LI: Jamaica, Je (NYS) ; Flatbush, Long Beach (AM); Farmingdale, Je (CU).

M. marginata var. lineata Melsh. Cranberry L., J1 10 (H).

7818 M. lunulata Hellm. Crosby Landing, L. Keuka, Je, Cy (CU) ; Albany, Je (NYS).

7819 M. serval Say. Newport, Je (NYS) ; Cranberry L., J1 (Drk) ; Buffalo (SayANSP); Ithaca, J1 15 (CU) ; Albany, Je (NYS); Catskills, May (Nic); SI, Aug (L\&D); LI (Sm).

7824 M. triloba Say. Ithaca, Taughannock Gorge, J1 (CU); Spring Lake, J1 (CU).

7825 M. undulata Melsh. Buffalo (Z\&R).

7827 M. discoidea Melsh. LI ( Sm).

\section{MoRdellistena Costa}

7834 M. confusa Blatch. Ringwood, May 27, McLean Bogs, May 30 (Dt).

7836 M. arida Lec. Spring Lake, JI (CU); LI: Bellport, Jl-Aug, in beating dead pines, Shoe (Nic).

7837 M. lutea Melsh. Buffalo (Z\&R). 
7838 M. trifasciata Say (lepidula Lec., according to Lilj). Buffalo (Z\&R); Danby, J1 (Dt); Ithaca, J1 (CU); Catskills (Nic); Van Cort. Pk. (Nic) ; SI, Je-J1 (L\&D) ; LI: Bellport, J1 (Nic).

7841 M. limbalis Melsh. Ithaca, J.W.Green (Lilj); SI (L\&D).

7842 M. elegantula Sm. LI ( Sm).

7844 M. dimidiata Hellm. Olcott, J1 4 (Dt).

7845 M. biplagiata Hellm. E. Aurora, May (Dnd); Castile, Je, Brad (Flet).

7846 M. intermixta Hellm. Adirondacks ( $\mathrm{Sm})$.

7848 M. vapida Lec. "Vic. NYC" (Sm) ; SI (Nic).

7850 M. bipustulata Hellm. Olcott, J1 (Dt).

7851 M. atriceps Sm. Newport (NYS); "NY" (Sm).

7853 M. picipennis Sm. NY ( Sm).

7854 M. pallipes Sm. Ithaca (Sm).

7855 M. fulvicollis Melsh. Buffalo (Z\&R).

7856 M. ornata Melsh. SI, Je (L\&D).

7858 M. scapularis Say (biplagiata Hellm., according to Lilj). Peru (CU); Keene Val., J1, Newport, Je (NYS) ; Cranberry L., Je (H) ; Olcott, Je-Jl (Dt); Buffalo (Z\&R) ; Geneva, Je (Cls) ; Port Byron, Je (Dt); Ithaca, May-Je (CU) ; Schoharie (Not); Catskills (Nic); Poughkeepsie, May-Je (NYS) ; Middletown (CU) ; Ramapo, Je, Ds (Nic) ; Peekskill, Je (CU); West Pt. (Robn); Highlands of the Hudson (Nic); Van Cort. Pk. (Nic); SI, May-Je (L\&D).

7859 M. comata Lec. Newport, Je (NYS); Hope, Aug, Bks (NYS) ; Ilion, May (NYS) ; Olcott, JI (Dt) ; E. Aurora, Je, Protection, Jl (Dnd); L. George (AM); Nassau, May (NYS); Kinderhook, Je (NYS); SI, Je-Sep (L\&D); LI: Bellport, Je-J1 (Nic).

7859a M. comata var. cervicalis Lec. Axton, Je (M\&H) ; Cranberry L., J1 (H) ; Buffalo (Z\&R) ; Ithaca, May-J1, Ringwood, Je (CU); Freeville, J1 (CU) ; Rhinebeck, J1, Cy (Lilj); Middletown, J1, Spooner (CU).

7860 M. aspersa Melsh. Axton, Je (M\&H) ; Newport, J1 (NYS); Cranberry L., J1 (H) ; Olcott, Je-J1 (Dt) ; Ithaca, May-Aug, McLean, J1 (CU); Windsor, Je (Not); Caroline to Harford, Je (CU) ; Cortland, J1 (NYS); Schoharie (Not); Nassau, Karner, Menands, J1 (NYS) ; Rhinebeck, J1, Cy (Lilj); Mosholu, Je (NYS); LI: Forest Pk., Je (NYS).

7861 M. indistincta Sm. "Adirondacks" (Sm) ; Fall Cr., Ithaca, Aug 2 (Dt).

7862 M. tosta Lec. E. Aurora, J1 (Flet,Dnd); Ithaca, J1 (CU).

7869 M. picilabris Hellm. LI: Bellport, Je 24, evidently one of the few maritime species (Nic).

7870 M. infima Lec. Mt. Whiteface, 2000-4000 ft., Aug 22-24 (CU) ; Ithaca, Aug 3, Ringwood, Je 26 (CU).

7871 M. andreae Lec. Poughkeepsie, Je (NYS).

7872 M. grammica Lec. Wells, J1 (NYS); SI, in blossom of wild parsnip, Ds (Nic, L\&D) ; LI: Jamaica, Floral Pk., Je (Nic).

7872a M. grammica var. varians Lec. Newport, Je (NYS); Ithaca, J1 (NYS) ; Karner, J1 (NYS); Ramapo Mts. (AM); SI, on flowers of wild parsnip, Ds (Nic, L\&D).

7874 M. ancilla Lec. "NY" (Lec).

7877 M. impatiens Lec. NY ( $\mathrm{Sm})$. 
7879 M. nigricans Melsh. Newport, Je (NYS); Ithaca, Je 28-J1 19 (CU); Albany, Je (NYS); Poughkeepsie, Je (NYS); SI, on seashore, Je (L\&D).

7881 M. ruficeps Lec. "Around NYC” (Sm); SI, Aug (Nic, L\&D).

7883 M. splendens Sm. SI, Aug, on Spartina (Nic,L\&D).

7888 M. pustulata Melsh. Axton, Je (M\&H) ; Cranberry L., Je 9 (H); E. Aurora, May 1 (Dnd); Montour Falls, Je (H); Ithaca, Je-J1 (CU); Windsor, Je (Not); Scotia, Je (NYS); Rensselaer (NYS); West Pt. (Ds) ; SI, Je (L\&D).

7889 M. convicta Lec. Olcott, Je 5 (Dt) ; Ithaca, Je, J1 2 (CU) ; Windsor, Je (Not); West Pt. (Robn).

7891 M. fuscipennis Melsh. "Around NYC" (Sm).

7893 M. morula Lec. Ithaca, Je-J1 (CU); Windsor, Je (Not); West Pt., Je, Ds (Nic); SI, Je 26 (Nic, L\&D).

7894 M. ambusta Lec. Olcott, J1 (Dt); Buffalo (Rein); Ithaca, J1 (CU) ; Poughkeepsie (NYS); "near NYC" (Sm); SI, J1 (L\&D).

7899 M. unicolor Lec. Cranberry L., J1 (H) ; West Pt. (Robn).

7901 M. marginalis Say. "Common throughout the State" (Nic); Boston, Je (Dnd); Rock City, Je 9 (Lilj); Allegany Pk., Aug (Dnd); Ithaca, Ellis, Ap-Je (CU); Woodwardia Bog, Tompkins Co (CU); McLean (CU) ; SI, Je (L\&D) ; LI: Farmingdale, Je (CU).

7903 M. schauppi Sm. "NY" (Sm).

7904 M. vera Lilj. "NY", one specimen in Univ. of Ill. (Lilj).

7905 M. aequalis Sm. Ithaca, Je 12 (CU).

7910 M. pubescens Fab. Common everywhere (Nic); Mt. Merino, Columbia $\mathrm{Co}, \mathrm{Jl}$ (CU) ; Poughkeepsie, Je (NYS); West Pt. (Robn); Ft. Montgomery (Ds); Peekskill, Ap 9 (Lilj); vic. NYC (Sm); SI, Je (L\&D); LI: Yaphank, Orient (Ds).

7912 M. liturata Melsh. Buffalo (Z\&R); SI, J1 (Nic, L\&D); LI: Aqueduct, Jamaica, Shoe (Nic).

7913 M. fuscata Melsh. Around NYC (Sm).

7915 M. pityptera Lec. Catskills, Aug 3, a very rare insect hitherto recorded only from $\mathrm{Pa}$. and Tex. (Nic).

7917 M. cinereofasciata Sm. Ithaca ( Sm).

7925 M. attenuata Say. "NY" (Sm).

7926 M. discolor Melsh. SI, J1 23, Shoe (Nic, L\&D).

\section{ANASPIS Geoffroy}

7938 A. nigrina Csiki. Ithaca, Je-J1 (CU); Schoharie (Not).

7942 A. flavipennis Hald. Mt. Whiteface, summit (Ds); Axton, Je (M\&H); Newport, Cranberry Cr., May-Je (NYS) ; Cranberry L., Je-J1 (H); Long L. (Ds) ; Buffalo (Z\&R); Batavia, J1 (Kngt); Ithaca, May-Je (CU) ; Caroline to Harford, Je (CU) ; McLean, May (CU) ; Ringwood, J1 24 (CU) ; Spencer L., Je (CU) ; Albany, May (NYS); Catskills (Nic); West Pt. (Ds); Peekskill, on flowers of Viburnum prunifolium (Sherm); SI, May, on Veratrum (L\&D). On willow catkins, also on shadbush when in bloom (Felt).

7943 A. rufa Say. Cranberry L., Je 14-20, extremely abundant on blossoms of Spiraea, Rubus, Acer, Sambucus, etc. (H) ; Olcott, Je-J1 (Dt) ; Buffalo (Z\&R) ; Protection, J1 (Flet,Dnd) ; Portageville, Je; Montour 
Falls, Je (H) ; Ithaca, Ringwood, May-Aug (CU); Enfield, J1 (Lilj) ; Windsor, Je (Not); Kenwood, Je (NYS); Rensselaer, Je (NYS); Poughkeepsie, Je (NYS); West Pt. (Robn); SI, Je (L\&D).

\section{FAMILY RHIPIPHORIDAE}

PELECOTOMA Fischer

7946 P. flavipes Melsh. NY (ANSPHorn).

\section{MACROSIAGON Hentz}

7949 M. flavipenne Lec. West Pt. (Robn) ; SI, J1 (L\&D) ; LI: Aqueduct, J1, Shoe (Sf).

7950 M. dimidiatum Fab. Newport, Lg (NYS); Peekskill (Sherm); SI, J1-Aug (L\&D).

7953 M. pectinatum Fab. West Pt. (Robn); SI, Aug (Sf); LI: Rockaway Beach (Shoe).

7954 M. cruentatum Germ. Peekskill (Sherm).

7956 M. limbatum Fab. West Pt. (Robn); Peekskill (CU); Mosholu, Aug (NYS); Nyack (AM); White Plains, J1 (Fall, Sq); SI, J1-Aug (L\&D) ; LI: Southold, Orient Pt., Aug-Sep (NYS).

\section{RHIPIPHORUS Bosc d'Antic}

7958 R. luteipennis Lec. Karner, Aug 13, Yg (NYS); Mosholu, Aug (Sf); SI, Aug (L\&D) ; LI: Montauk, Aug, Eng (Sf).

7967 R. fasciatus Say. Speculator, J1 (NYS); Ithaca, Jl-Aug (CU); Albany, Nassau, Jl-Aug (NYS); New Baltimore (AM); LI: Riverhead, Huck (Flet).

7968 R. schwarzi Lec. Buffalo (Z\&R).

7969 R. walshi Lec. "NY" (Lec).

7970 R. stylopides Newn. Greenwood L. (Sf).

\section{FAMily MELOIDAE SUBFAMily MELOINAE \\ PoMpHOPOEA LeConte}

7990 P. sayi Lec. Newport, Je (NYS) ; Cranberry L., Je-J1 (H) ; Herkimer, Je (Cy); Wilmurt (CU); Frankfort (NYS); Gloversville, May (NYS); Boonville, Je, Utica, Je (Cy); Syracuse, Je (Cy); West Rush; Fairport, Je (Cy) ; Pittsford, Je (Cy); Batavia, Je (Cy); Lime, Je (Cy); Pavilion, Je (Cy) ; Williamson; Avon, Genesee, May (Cls) ; Mt. Morris, Je; Caledonia, Je; Tuscarora, Je; Livonia Center, Je; Portage (CU); Sonyea, J1 (Cy); Scottsville, Je; Perry, Je (CU) ; Castile, Je, Brad (Flet) ; Hammondsport; Weedsport, Je ; Branchport, Je (Cy) ; Lakemont, Je (Cy); Valois, May (Cy) ; Schuylerville (Felt); Lowman, Je (Cy); Wellsburg, J1 (Cy); Owego, Je (Cy) ; Binghamton, Je (Cy) ; Nunda, Je (Cy); Nineveh, Je; Berkshire, Je (Cy); Elmira, Je (Cy); Waverly, Je (Cy); Ithaca, Je; McLean Bogs, Je; Newfield, Je (Cy); Interlaken, Je (Cy) ; Cortland, Je (Cy); Moravia, Je; Cazenovia, Je (Cy); Windsor, Je (Not); Canajoharie, Je (Fitch) ; Fonda, May-Je (Felt); Schoharie, Je (Not, Lint); Schenectady, Je (Cy); Quaker Street, Je (Felt); Corinth, Je (NYS); Hartford, May-Je (Felt); Cambridge (Felt); 
Clarksville (Felt); Slingerlands, May-Je (NYS); Castleton, May-Je (NYS) ; Voorheesville, J1 (NYS) ; Elsmere, May (NYS) ; Philmont, Je (Felt); Poughkeepsie, Je (NYS); West Pt. (Robn); Pine I. (AM) ; Ft. Edward, Je (Cy) ; western SI, May (L\&D) ; I.I: Mineola, $\mathrm{Je}(\mathrm{Cy})$.

7993 P. aenea Say. Frankfort (Lint); E. Auburn (Dnd) ; Ithaca, May, Bish (NYS); New Windsor (AM); West Pt. (Robn); Van Cort. Pk. (AM).

Epicauta Redtenbảcher

8018 E. vittata Fab. Buffalo (Z\&R) ; Cayuga Co (Lint) ; Valatie, Aug ; Bethlehem, Je (Lint); West Pt. (Robn); SI, J1-Aug (L\&D); LI: Flatbush (AM).

8024 E. cinerea Forst. Buffalo (Z\&R); Ithaca, Sep (Dt) ; West Pt. (Robn) ; SI, J1-Aug (L\&D).

8024a E. cinerea var. marginata Fab. Gowanda, Aug (Cy); Castile, Brad (Flet) ; Ithaca, J1, Sep (Dt) ; Albany, Aug (NYS) ; Karner, J1, Kenwood, J1, Coeymans, Sep (NYS) ; Normanskill, Aug, Leon (NYS); White Plains, J1-Sep (Cy); Tarrytown, Aug (Cy); LI: Brooklyn (Dt) ; Flatbush (AM) ; Hempstead, J1 (Cy) ; Roslyn (CU) ; Floral Pk., J1 (NYS).

8033 E. pennsylvanica DeG. Wilmington (Lint); Severance, Aug, Bish (NYS) ; Hope, Keene Val., Aug (NYS); L. Chazy, Je (NYS) ; Buffalo (Z\&R); Avon, Aug (Cls); E. Aurora (Dnd) ; Cobleskill, Aug (Felt); Charleston Four Corners, Aug (Felt); Otto, Je (CU); Castile, Brad (Flet); Oswego, Aug (Cy); Worcester, Aug (Cy) ; Schenevus, Aug (Cy) ; Ithaca, Aug-Sep (CU) ; Watkins, Aug (NYS) ; Danby, Aug (CU); Candor, Aug (Cy); L. George (AM) ; Pawling, Sep (Cy) ; Albany, Sep (NYS); West Pt. (Robn) ; White Plains, Aug-Sep (Fall, Sq) ; Baldwin (AM); SI, Aug-Sep, on goldenrod (L\&D) ; LI: Floral Pk., Aug (Leon) ; Mineola, Aug (Cy) ; Flatbush (AM); Orient, Lath (NYS).

\section{MACROBASIS LeConte}

8042 M. unicolor Kby. Plattsburg, J1, Northville, Je, Ausable Forks, Je (NYS) ; Cranberry L., Je (H); Bulls Head (Lint); Buffalo (Z\&R); E. Aurora (Dnd); Ithaca, J1 (Dt) ; Schoharie (Not) ; Canton, J1 (Leon) ; Elmira, J1 (CU) ; Waverly, J1 (Leon) ; Factoryville, Je (NYS) ; Saratoga Sps., J1 (NYS) ; Altamont, J1 (CU); Schenectady, J1 (CU) ; Gouverneur, Je (Leon) ; Albany, J1, Nassau, Karner, Je (NYS); Clinton Corners, J1 (CU) ; Chatham, J1 (NYS) ; Florida (Cy); SI, Je-J1 (L\&D) ; LI: Orient, J1, Lath (NYS); Flatbush (AM).

$8042 \frac{1}{2}$ M. debilis Lec. "NY" (Lec. in Proc. Acad. Nat. Sci. Phila. 6: 344, 1854).

LyTTA Fabricius

8066 L. nuttalli Say. SI: Eltingville, May 21, 1922, Clarence Busteed (L\&D).

Meloe Linnaeus

8142 M. impressus Kby. Syracuse, Ap, under stones (H); Westfield, May (Not).

8142a M. impressus var. niger Kby. "NY" (ANSP). 
8147 M. angusticollis Say. Keene Val., Aug (NYS); Cranberry L., J1-Aug (H) ; Newport, Je (NYS) ; Ausable Forks, Aug (Lint) ; Axton, Je $(\mathrm{M} \& \mathrm{H})$; Ilion, Sep (NYS) ; Hannibal, Sep (Cy); Syracuse, Sep (Leon) ; Buffalo (Z\&R) ; E. Aurora (Dnd) ; Avon, Aug (Cls) ; Pike, Je (NYS) ; Castile, Brad (Flet); Perry (Lint); Ithaca, Sep (Dt) ; L. George (AM) ; Karner, E. Schodack, Ap (NYS) ; E. Greenbush, Ap (NYS) ; Ft. Plain, Sep (NYS); White L. (AM); West Pt. (Robn); White Plains, Ap (Sq); SI (L\&D); LI: Babylon (AM) ; Orient, Lath (NYS).

8148 M. americanus Leach. Cranberry L., Sep (Drk); Ithaca, Ap-May (Dt); SI, May (L\&D).

8149 M. moerens Lec. "NY" (Lec); Lewis, Mar (NYS).

ZoNITIS Fabricius

8158 Z. bilineata Say. SI, Aug, on wild sunflower (L\&D).

NEMOGNATHA Illiger

8182 N. nemorensis Hentz. "NY" (Lec).

\section{TRICRANIA LeConte}

8194 T. sanguinipennis Say. SI, found dead on sandy path at Watchogue, Ap 22, 1917 (L\&D).

\section{FAMILY PYTHIDAE}

Рутно Latreille

8203 P. niger Kby. Buffalo (Z\&R); Ithaca, Nov (Flet); vic. NYC (AM).

8204 P. planus Hbst. Cranberry L., Je, common under bark of pine (H) ; Buffalo (Z\&R); Pike, Je-J1 (NYS); Ithaca, Mar-Je (DtCU), Nov (Flet); Albany, May, Lansingburg (NYS); SI, Nov 1 (L\&D).

\section{SALPINGUS Gyllenhal}

8211 S. virescens Lec. Mt. Whiteface, Je (Ds); Ithaca, Aug 15 (Flet); Coy Glen, Ithaca, Aug 2, Dt (Vdyk); LI: Woodside, Je (Sf).

\section{RHINosimus Latreille}

8215 R. viridiaeneus Rand. Newport, Ap 28 (NYS) ; Buffalo (Z\&R) ; McLean, Ap 17, Babiy (Flet); Ringwood, May (Vdyk); Catskills (Sf); West Pt. (Robn).

\section{FAMILY PYROCHROIDAE}

\section{IsChalia Pascoe}

8218 I. costata Lec. Wallface Mt., J1 8, Quirs (Frost); E Jewett, Ap 18 (Ds). 


\section{NEOPYROCHROA Blair}

8220 N. flabellata Fab. Newport (NYS); Buffalo (Z\&R); Geneva, J1 (Cls); Ithaca, J1 (FletCU); West Pt. (Robn); SI, Je-Aug (L\&D); LI: Half Way Hollow Hills (Ds).

8221 N. femoralis Lec. Buffalo (Z\&R); SI, May-Je (L\&D); LI: Gardiners I. (Ds).

\section{Schizotus Newman}

8224 S. cervicalis Newn. Axton, Je $(\mathrm{M} \& \mathrm{H})$; Newport, May, L. Clear, Je (NYS) ; Cranberry L., Je (H); Buffalo (Z\&R); Castile, May, Brad (Flet); Trenton Falls (Newn); Nassau, May (NYS).

\section{DENDROIDES Latreille}

8225 D. bicolor Newn. Axton, Je (M\&H) ; Newport, Je-J1 (NYS) ; Old Forge, J1 (FletCU) ; Cranberry L., Je-J1 (H) ; Olcott, Je, E. Aurora, J1 (Dnd); Buffalo (ANSP); Pike (NYS); Batavia, Je-J1 (Kngt); Genesee Co (Ds) ; Cattaraugus Co, Je (Ds) ; Ithaca, Je-J1 (DtCU); Broadalbin, Je, Menands, Aug, Rensselaer, J1, Clinton Hgts., J1 (NYS); Trenton Falls (Newn); SI, Je, Aug (L\&D); LI: Aqueduct (Ds).

8226 D. concolor Newn. Peru, Cy, Fbs (FletCU); Axton, Je (M\&H) ; Newport, Keene Val., Je (NYS); Saranac Inn, J1 (NYS); Cranberry L., Je-J1 (H) ; Mt. McIntyre, J1, Leon (NYS); Olcott, Je (Dnd); Conesus L. (Ds) ; Buffalo (ANSP) ; Geneva, Jl (Cls) ; Allegany Pk., J1 (Dnd); Rock City (Ds); Castile, Brad (Flet); Ringwood, Je, Ithaca, Jl, at trap lantern (FletCU) ; Cortland Co, Je (Dnd); Trenton Falls (Newn); West Pt. (Robn); LI: North Beach (Olsen); Flushing (Ds).

8228 D. ephemeroides Mann. Olcott, Je-J1 (Dt); Ithaca, J1 12 (Dt).

\section{FAMILY PEDILIDAE}

\section{PEDILUS Fischer}

8243 P. canaliculatus Lec. Newport, Je (NYS); Erie Co, May-Je (Dnd); Castile, May, Brad (Flet).

8244 P. elegans Hentz. Ilion, May (NYS); Batavia, Je (Kngt); Ithaca, Je, Horn (Vdyk) ; Nassau, N. Chatham, Wappingers Falls, Je (NYS); Rensselaer, May (NYS); Sunnyside, May (NYS).

8245 P. terminalis Say. Buffalo (Z\&R); Ithaca, May-Je (FletCU); Trenton Falls (Newn); Albany, Je (NYS); West Pt. (Robn).

8247 P. fulvipes Newn. Buffalo (Z\&R); Pike (NYS); Marilla, Je (Dnd); Trenton Falls (Newn).

8248 P. newmani Lec. Axton, Je (M\&H) ; Newport, May (NYS); Buffalo (Z\&R); Batavia, Je (Ds); Marilla, Je (Dnd); Ithaca, May-Je (FletCU); McLean Bogs, Je (FletCU); Trenton Falls (Newn); Albany, Nassau, Je (NYS) ; Rensselaer, May (NYS).

8249 P. cyanipennis Bland. Catskills, Je (Ds).

8250 P. labiatus Say. Rochester Jct., Je, Leon (Flet); Buffalo (Z\&R) ; Geneva, Je (Cls); Montour Falls, Je (H) ; Ithaca, J1 (Dt); McLean Bogs, Je (FletCU) ; Albany, Je, Rensselaer, May (NYS) ; West Pt. (Robn), as pulcher. 
8251 P. lugubris Say. Newport, May (NYS) ; Northampton, Je (NYS) ; Ilion, May (NYS) ; Rochester Jct., Je, Leon (FletCU); E. Aurora, May (Dnd); Pike (NYS); Castile, Brad (Flet); Batavia, Je (Kngt); Windsor, Je (Not); Spencer, Port Byron, Je (Dt); Schoharie (Not); Corinth, May (NYS).

8252 P. collaris Say. Newport (NYS); Buffalo (Z\&R); Port Byron (Dt); Ithaca, Je, Ham (Vdyk); Spencer, Je (Dt); Trenton Falls (Newn); Nassau, May (NYS); West Pt. (Robn).

\section{STEREOPALPUS LaFerte-Senectère}

8264 S. mellyi Laf. Buffalo (Z\&R).

8265 S. vestitus Say. Buffalo (Z\&R).

\section{MACRATRIA Newman}

8278 M. confusa Lec. Ithaca, Je (FletCU); Schoharie (Not).

8279 M. murina Fab. Buffalo (Z\&R); SI, J1 (L\&D).

8280 M. ovicollis Csy. "NY" (Csy).

\section{FAMILY ANTHICIDAE}

\section{Notoxus Geoffroy}

8289 N. bifasciatus Lec. Buffalo (Z\&R); Lockport, J1, Ithaca, Je (NYS); Karner, Je (NYS).

8302 N. anchora Hentz. Axton, Je $(M \& H)$; Ausable Forks, May, Wells, J1 (NYS) ; Saranac Inn, J1 (Ndm) ; Cranberry L., Je-J1 (H) ; Northampton, Je (NYS) ; Buffalo (Z\&R) ; Batavia, Je (Kngt); McLean, Aug (Flet); Letchworth Pk., J1 (NYS); Schoharie (Not); Corinth, Je, Albany, J1, Karner, Je (NYS).

8304 N. monodon Fab. Albany, Ap, FTHartmann, Cedar Hill, Je, E. Schodack, Mar, Normanskill, Ap (NYS); West Pt. (Robn); Ramapo (Ds); Suffern (AM) ; SI, Mar, May, Nov (L\&D); LI: Central Pk., Ap (Eng).

MECYNOTARSUS LaFerte-Senectère

8325. M. elegans Lec. Buffalo ( $Z \& R)$; Ramapo (Ds). Possibly the same as flavicans Csy. $(\mathrm{Lg})$.

TOMODERUS LaFerte-Senectère

8334 T. constrictus Say. Phoenicia, Aug (NYS) ; SI, Feb, May (L\&D).

AMBLYDERUS LaFerte-Senectère

8340 A. granularis Lec. Buffalo (Z\&R).

8342 A. pallens Lec. SI, Aug-Sep, about plant roots at seashore (L\&D).

ANThicus Paykull

8347 A. formicarius Laf. Mooers, Sep (Not); Lake Bluff, Sep, Bish (NYS); Buffalo (Z\&R); Pike, Mar (NYS); SI, May, Sep, Nov (L\&D); LI: Orient, Ap, Lath (NYS). 
8348 A. cinctus Say. Chautauqua, Shokan (Lg); West Pt. (Robn); SI, Nov 6, Joutel (L\&D) ; LI: Orient, Lath (NYS).

8350 A. blandus Csy. SI, May 18 (L\&D); LI: Willetts Pt. (Csy).

8353 A. obscurus Laf. Buffalo (Z\&R); Schoharie (Not) ; LI: Coney I., J1 (Lec).

8359 A. sturmi Laf. Cranberry L., Je 28, in box of fungi $(H)$.

8372 A. rejectus Lec. "NY" (Lec).

8387 A. floralis L. Newport, Aug, Herkimer, Sep (NYS) ; Buffalo (Z\&R); Batavia, J1-Sep (Kngt); West Pt. (Robn); SI, J1 (L\&D).

8397 A. ephippium Laf. Buffalo (Z\&R) ; Batavia, J1 (Kngt); Westchester Co, Palm (AM) ; SI, Je 4 (L\&D); LI: Orient Pt., May, Lath (NYS).

8402 A. scabriceps Lec. Montour Falls, Je (H) ; SI, Aug, under log on seashore, and in sand (L\&D).

8403 A. compositus Csy. LI: Willetts Pt. (Csy).

8417 A. cervinus Laf. Cranberry L., Je (H) ; Buffalo (Z\&R) ; E. Aurora, Je, Dec (Dnd); Batavia, J1 (Kngt) ; Westfield, May (Not) ; SI, Aug, on seashore, Je 4 (L\&D); LI: Orient Pt., May, Lath (NYS); Coney I., Ap (NYS).

8448 A. melancholicus Laf. SI, Je 5, on seashore (L\&D).

8454 A. pubescens Laf. Johnstown, Sep, Albany, J1 (NYS); SI, Nov 6, on salt meadow (L\&D).

8461 A. festinans Csy. Mooers, Sep (Not); Windsor, Je (Not).

\section{FAMILY EUGLENIDAE}

ELonus Casey

8475 E. basalis Lec. Buffalo (Z\&R); Ludlow, Thompson ( $\mathrm{Lg})$; LI: Cold Sp. Harb., J1, Brb (Sf).

\section{ZoNANTES Casey}

8480 Z. signatus Hald. NY (Hald).

8481 Z. subfasciatus Lec. Mosholu (Sf).

8484 Z. fasciatus Melsh. NY (Hald); Newport, May (NYS); Buffalo (Z\&R); Ludlow, Thompson $(\mathrm{Lg})$.

\section{VANONUS Casey}

8492 V. piceus Lec. NY (Lec); Cranberry L., Je 23-Aug 4, in sweeping in marshy area, also flying about camp $(\mathrm{H})$; Washington $\mathrm{Co}(\mathrm{Lg})$.

8494 V. vigilans Csy. Hudson Val. (Csy).

8495 V. fusciceps Csy. "Near NYC" (Csy).

\section{TANILOTES Casey}

8501 T. densus Csy. McLean, Aug (Flet). 


\section{FAMILY RHIPICERIDAE}

\section{SANDALUS Knoch}

8544 S. petrophya Knoch. Near NYC; SI, on beach; Aug 27, on sidewalk (L\&D).

8546 S. niger Knoch. Ithaca, Sep (Dt), late Aug, P. Miller, Fbs (FletCU); West Pt. (Robn).

\section{FAMILY ELATERIDAE 42}

\section{SUBFAMILY PYROPHORINAE}

\section{Adelocera Latreille}

8553 A. aurorata Say. Buffalo (Z\&R) ; Olcott, Je (Dt) ; Lake Bluff, J1 (NYS) ; Catskills, Je (Nic).

8554 A. impressicollis Say. Rochester (M\&W).

8557 A. brevicornis Lec. Axton, Sep (M\&H) ; Ausable Chasm, Sep, Eng (Sf) ; Batavia, J1 (Kngt); Catskills, Je-Jl, Aug (Nic, Ds).

8558 A. obtecta Say. Cranberry L., J1 (H) ; Rochester (M\&W) ; Buffalo (Say-ANSP) ; Castile, Brad (Flet) ; E. Aurora, Nov (Dnd) ; Windsor, Je (Not); Ithaca, Je (Vdyk); Catskills, Je (Nic) ; Big Ind. Val., J1, Pears (Sf); West Pt. (Robn); Pine I., Je (Sf); Mosholu, Wat $(\mathrm{Lg})$.

8560 A. marmorata Fab. Paradox, J1, Essex Co, Aug, Ilion, Je (NYS) ; Gloversville, Je (NYS) ; Rochester (M\&W) ; Buffalo (Z\&R) ; E. Aurora, Je (Dnd) ; Albany; J1 (NYS).

8561 A. discoidea Web. Olcott, Je (Dt) ; Rochester (M\&W) ; Buffalo (Z\&R) ; Ithaca, J1, Ap (Vdyk); Taughannock Falls, Ap (Dt,Vdyk); Albany, Ap (NYS) ; Peekskill (Sherm); West Pt. (Robn); SI, Mar-Sep (L\&D) ; LI: Aqueduct, May, Wyandanch, Feb (Quirs); Parkville, Dietz (Sf); Central Pk., Aug, Eng (Sf).

8562 A. avita Say. Cranberry L., J1 (H) ; Protection, Je (Dnd).

8563 A. modesta Boisd. Big Ind. Val., May, Pears (Sf).

\section{LACON Castelnau}

8564 L. rectangularis Say. Buffalo (Z\&R).

\section{Alaus Eschscholtz}

8571 A. oculatus L. Eyed Click Beetle. Adirondacks (Felt); Newport, May (NYS) ; Herkimer, J1 (Cy); Rochester (M\&W); Leroy, Je (Cy); Honeoye Falls, Je (Ds); Buffalo (Z\&R); Olcott, Oct (Dt) ; Castile, J1, Brad. (Flet); E. Aurora, Je-J1 (Dnd); Geneva, Ap, Je, Dec (Cls) ; Lorraine, Je (Cy) ; Ithaca, Je, Sep (CU) ; McLean Bogs, May 30 (CU) ; Albany, Je, Schenectady, Aug, Castleton, Dec (NYS); Poughkeepsie, J1 (Cy) ; Ramapo, May (Ds) ; Middletown, Je (Cy) ; West Pt. (Robn) ; Croton L., Je (Cy) ; SI, May-J1 (L\&D) ; LI: Maspeth, Je (Ds) ; Flatbush, Babylon (AM); Jamaica, May (Quirs).

42 The list for this family has been checked by J. A. Hyslop, Alan S. Nicolay, and E. D. Quirsfeld. Mr. Nicolay and Mr. Quirsfeld have supplied many records. 
8572 A. myops Fab. Gloversville, May-Je (NYS); Buffalo (Z\&R) ; SI (L\&D); LI: Central Pk., Ap (Eng) ; Coney I., Rockaway (Schmelter); Wyandanch, Wading R., May (Quirs); Pinelawn, Ap, Je, Yaphank, May, Eng (Sf).

\section{HEMIRHIPUS Latreille}

8576 H. fascicularis Fab. NY (Horn ANSP); NYC $(S f)$.

MONOCREPIDIUS Eschscholtz

8596 M. lividus DeG. SI, J1 (L\&D) ; LI: Manor, Sep, Eng (Sf).

8601 M. vespertinus Fab. SI (L\&D).

8607 M. auritus Hbst. Newport, Je (NYS) ; Olcott, Ap, Je (Dt) ; Buffalo (Z\&R); Pike, Je (NYS); Ringwood, J1, McLean Bogs, May (Dt); Windsor, Je (Not); Albany, Je (NYS); West Pt. (Robn); Van Cort. Pk. (AM); SI, Mar (L\&D); LI: Rockaway Beach, May-Je (Ds, Sf); Astoria, Flatbush (AM) ; Wading R., May, Little Neck, Ap (Quirs).

8609 M. bellus Say. Ithaca, May-Je (Dt); West Pt. (Robn); Van Cort. Pk. (AM) ; SI, Mar-May, Oct (L\&D).

\section{AEOLuS Eschscholtz}

8613 A. amabilis Lec. Vic. NYC (AM) ; SI, Mar, Je (L\&D) ; LI: Rockaway, May-Je, Eng (Sf).

8614 A. dorsalis Say. Olcott, Feb-Oct (Dt); Buffalo (Z\&R); Castile, Aug, Brad (Flet) ; Ithaca (US); West Pt. (Robn), as elegans; vic. NYC (AM) ; SI, May (L\&D); LI: Rockaway Beach, May-Je, Eng (Sf); Flatbush, Ap, Eng (Sf).

\section{Pityobius LeConte}

8617 P. anguinus Lec. Buffalo (Z\&R); Ithaca, Je (Dt); Haines Falls, Franck (Sf) ; Catskills, Aug (Nic) ; LI: Bellport, Aug (Nic) ; Bayshore, J1, Olsen (Ds); Yaphank (Sf); Wading R., J1, Eng (Sf).

\section{LIMONIUS Eschscholtz}

8619 L. auripilis Say. Nassau, Poughkeepsie, May (NYS); Peekskill, Je, on Rumex (Sherm).

8624 L. aurifer Lec. Newport, Je (NYS) ; Cranberry L., Htly (H) ; Crown Pt., Je (NYS); Buffalo (Z\&R);Westfield, May (Not); Catskills (Nic); North Branch (Schaupp); West Pt. (Robn).

8625 L. propexus Cand. Olcott, J1 (Dt) ; Buffalo (Z\&R); SI, May-J1 (L\&D).

?L. griseus Beauv. Buffalo (ANSP) ; E. Aurora, Allegany Pk (Dnd); Windsor, Je (Not); West Pt. (Robn); Ithaca, Ap, J1-Aug (Dt); Ramapo, Nyack, Je (Ds); Van Cort. Pk. (AM) ; LI: Parkville (AM); Flatbush, Astoria (AM); Yaphank, Pinelawn, Je, Eng (Sf).

8627 L. confusus Lec. E. Aurora, Je (Dnd) ; Macedon, May, on quince blossoms (Horn); Ithaca, May-Je (Dt); Taughannock Falls, May (Dt); Ringwood, J1 (Vdyk); vic. NYC (AM); SI, Je (L\&D); LI: Flatbush (US). 
8629 L. plebejus Say. West Pt. (Robn) ; SI, May (L\&D) ; LI: Flatbush (AM).

8631 L. aeger Lec. Cranberry L., Je-J1 (H) ; Schoharie (Not) ; Ramapo, May-Je (Ds); Catskills (Nic); Slide Mt., Jl, Shoe (Sf); West Pt. (Robn); vic. NYC (AM).

8632 L. pectoralis Lec. Mt. Seward, $4500 \mathrm{ft}$, Je (M\&H). Described from Calif.

8633 L. quercinus Say. Ithaca, Aug, Taughannock Falls, Aug (Dt) ; Ft. Montgomery, Nyack, Ramapo, Je (Ds); West Pt. (AM); Mosholu (Sf) ; Van Cort. Pk., May (Nic) ; SI, May-Je (L\&D) ; LI: Flatbush (AM) ; Yaphank, May, J1, Eng (Sf).

8635 L. basillaris Say. West Pt., Ft. Montgomery, Je (Ds) ; Bear Mt., J1 (Quirs) ; Ramapo, Je (Ds) ; Bronx Pk. (Sf); SI, May-Je (L\&D); LI: Bellport, Je (Nic); Yaphank, May, Pinelawn, Je, Eng (Sf).

\section{Nothodes LeConte}

8639 N. dubitans Lec. E. Aurora, Je (Dnd); Castile, Brad (Flet); Ithaca, May-Je (Dt); Trenton (US).

\section{Pheletes Kiesenwetter}

8640 P. stigma Hbst. Castile, Brad (Flet); Ithaca, Ap-Je (Dt) ; Catskills, Ap (Flet); West Pt. (Robn) ; LI: Wading R., May, Schott (Sf).

8649 P. anceps Lec. Western NY (Lec); Windsor, Je (Not).

8650 P. ectypus Say. Pike (NYS) ; Castile, Brad (Flet) ; Danby, Je, Ithaca, May-Je (Dt); Callicoon (AM).

8653 P. refinitus Ziegl. Ramapo, May (Ds); West Pt., May, Robn (Sf) ; SI, May (L\&D) ; LI: Flatbush (AM).

8654 P. nimbatus Say. West Pt. (Robn) ; SI, May (L\&D) ; LI: Flatbush, May, $Z a b$ (Sf); Pinelawn, Je, Eng (Sf).

\section{LEPTOSCHEMA Horn}

8657 L. bicolor Lec. "NY" (Lec).

8658 L. discalceatum Say. Buffalo (Z\&R) ; Ft. Montgomery, Aug, Schott (Sf).

8659 L. oberndorferi O. Schw. Described from NY.

\section{AтHOUs Eschscholtz}

8662 A. brightwelli Kby. Buffalo (Z\&R); western NY, generally distributed (Dnd) ; Ringwood, J1 (Vdyk) ; Olcott, J1 (Dt); Catskills, Aug (Nic) ; Bear Mt., J1 (Quirs) ; LI: Bellport, Aug (Nic).

8663 A. acanthus Say. Newport, Je (NYS) ; Cranberry L., Je-J1 (H) ; West Pt. (Robn); LI: Bellport, J1 (Nic); Yaphank, Jl, Eng (Sf).

8663b A. acanthus var. maculicollis Lec. Buffalo (Z\&R); SI (L\&D).

8667 A. cucullatus Say. Cranberry L., J1 (H) ; Olcott, Aug (Dt) ; Buffalo (Z\&R) ; Castile, Brad (Flet); Ithaca, Ji (Vdyk); Catskills, J1 (Nic); Callicoon (AM) ; West Pt. (Robn) ; SI, Je-J1 (L\&D) ; LI: Jamaica, Bellport, J1 (Nic).

8668 A. scapularis Say. Cranberry L., Je (H) ; Olcott, Je (Dt) ; Buffalo (Z\&R) : E. Aurora, Jl (Dnd) ; Ithaca, Je (Dt) ; Catskills, Aug (Nic); West Pt. (Robn). 
8676 A. rufifrons Rand. Axton, Je $(\mathrm{M} \& \mathrm{H})$; Cranberry L., Je $(\mathrm{H})$; Wanakena, Aug (Drk); Buffalo (Z\&R); Enfield Glen, J1 (Dt); Catskills, J1 (Nic); LI, Pears (Sf).

\section{LEPTUROIDES Herbst}

8698 L. productus Rand. Cranberry L., J1 (H) ; Catskills (Nic); Oliverea, J1 (Quirs).

8699 L. denticornis Kby. Axton, Je (M\&H) ; Wallface Mt., Je (Quirs) ; Cranberry L., Je-J1 (H) ; Hamilton Co (Ds); Castile, Je, Brad (Flet); McLean, May (Dt); Catskills, Pears (Sf); Slide Mt., J1 (Quirs).

\section{LUDIUS Eschscholtz}

8705 L. virens Schr. Mt. Whiteface (HyslopUS).

8706 L. vernalis Hentz. Adirondacks (Felt); Buffalo (Rein); Westfield, May (Not); Catskills, Je (Nic); Ithaca, May (Flet).

8707 L. resplendens Esch. Whiteface Mt., Shoe (Sf) ; Cranberry L., J1 (H) ; Catskills (Nic); Oliverea, J1 (Quirs); LI: Central Pk., Wading R., Wyandanch, May, as splendens (Ds).

8708 L. sjaelandicus Müller. Buffalo ( $Z \& R$ ), as tesselatus; E. Aurora, Je (Dnd); Ithaca, May (Dt); Windsor, Je (Not); Saratoga, as cuprascens (Lec); West Pt. (Robn); LI: Bellport, Je (Nic).

8715 L. pyrrhos Hbst. Buffalo (Z\&R) ; Dunkirk (HyslopUS); E. Aurora, Aug (Dnd) ; Ringwood, J1 (Dt) ; Lava, J1, Eng (Sf) ; Ft. Montgomery, J1 (Ds); SI, Je-Aug (L\&D); LI: Coney I. (US); Jamaica, Pears (Sf); Jl-Aug (Nic).

8721 L. cylindriformis Hbst. Cranberry L., J1 (H); Syracuse, Ap-May (Quirs,H) ; Olcott, May (Dt) ; Buffalo (Z\&R) ; E. Aurora, Ap (Dnd); Onondaga Co, May (Quirs); Ithaca, Ap-Je (Hyslop,Dt) ; Cortland Co, Je (Dnd); West Pt. (Robn) ; Tarrytown, Ap (Dt) ; Ramapo, Mar (Ds) ; Mosholu (Sf) ; SI, Ap-May (L\&D) ; LI: Central Pk., Ap, on dead pines, Eng (Sf); Flatbush (AM); Wyandanch (Quirs); Pinelawn, Je, Eng (Sf).

8722 L. copei Horn. Catskills, Je, Nic (Sf); Pine I., May, Schott (Sf).

8727 L. fulvipes Bland. Ithaca, May (Vdyk); Big Ind. Val., Oct, Pears (Sf); Catskills, Pears (Nic); West Pt. (Robn); vic. NYC (AM).

L. exilis Not. Schoharie (Not).

8729 L. appressus Rand. Mt. Seward, Je (M\&H) ; L. Clear, Saranac Inn, Je (NYS).

8731 L. vulneratus Lec. Crusoe L., Wayne Co, Bish (Dt).

8734 L. elongaticollis Ham. Catskills, J1, Shoe (Sf).

8738 L. tarsalis Melsh. Wallface Mt., J1 (Quirs); Buffalo (Z\&R); Ithaca, Ap, Je (Dt) ; Catskills, Ap (Nic) ; West Pt., Je (Sf) ; Ft. Lee (US); NYC, Mar (Quirs); SI, Ap (L\&D) ; LI: Jamaica, Ap (Nic) ; Wading R. (Quirs); Long Beach, May, Eng (Sf).

8739 L. spinosus Lec. Mt. Seward, 4500 ft., Je (M\&H) ; Cranberry L., Je-J1 $(\mathrm{H})$.

8741 L. insidiosus Lec. Wallface Mt., J1 (Quirs); Slide Mt. (US) ; Catskills, Pears (Sf). 
8745 L. falsificus Lec. Catskills, Pears (Nic).

8748 L. sulcicollis Say. Cranberry L., J1 (H) ; Buffalo (Z\&R) ; E. Aurora, May (Dnd); Geneva, Mar (Cls) ; Ithaca, Ap-May (Dt) ; Trenton (US); Catskills, Aug, Pears (Nic) ; West Pt. (Robn) ; LI: Central Pk., Ap, Eng ( $\mathrm{Sf})$.

8761 L. aethiops Hbst. West Pt., J1, Eng (Sf) ; Bronx Pk. (Sf) ; SI, May-J1 (L\&D) ; LI: Yaphank, Je (Ds).

8762 L. hamatus Say. Cranberry L., Htly (H) ; Olcott, May-Je (Dt) ; Buffalo $(Z \& R)$; Ithaca, May (Dt); West Pt., Je (Ds); Catskills, Je, Pears (Nic); Oliverea, Je (Quirs); Highland Falls (Quirs); Bronx Pk. (Sf).

8763 L. propola Lec. Buffalo (Z\&R); Ithaca, May, Jl-Aug, Eng (Dt).

8766 L. triundulatus Rand. Wallface Mt., J1 (Quirs); Cranberry L., J1 (H); Wanakena, Aug (Drk); Catskills (Nic); LI: Rockaway, May, Schott (Sf).

8769 L. medianus Germ. Buffalo (Z\&R); West Pt. (Robn).

8771 L. fallax Say. Slide Mt., J1, Shoe (Sf); West Pt., J1, Eng (Sf).

8772 L. mirabilis Fall. Keeseville (US). Described from Calif.

8776 L. splendens Ziegl. West Pt., Robn (Sf); LI: Wyandanch, Wading R., May (Quirs); Rockaway Beach, May, Eng (Sf).

8777 L. aereipennis Kby. Jay, J1 (Britton); New Baltimore, May (NYS); Buffalo (Z\&R); Ithaca, Ap-Je, Caroline, Ap (Dt); West Pt. (Robn).

8778 L. nigricornis Panz. Cranberry L., Je (H); Wallface Mt., Shoe (Sf).

8779 L. aratus Lec. West Pt. (Robn).

8780 L. inflatus Say. Buffalo, (Z\&R); Erie Co, May-Aug (Dnd); Westfield, May (Not) ; Ithaca, Je-J1 (Dt); Schoharie (Not); West Pt., Je-JI, Eng (Ds, Sf) ; Nyack, Je (Ds) ; Mosholu (Sf); Bronx Pk., Je (Ds); SI, Je (L\&D) ; LI: Jamaica, Pears (Nic) ; Yaphank, May, Pinelawn, Je, Eng (Sf).

8791 L. rotundicollis Say. Buffalo (Z\&R); Ithaca, Nov (Dt).

8793 L. cruciatus L. Catskills, May, Pears (Nic); Big Ind. Val., Je, Pears (Sf).

8796 L. hieroglyphicus Say. Whiteface (AldrichUS) ; Cranberry L., Je (H) ; Olcott, May-J1 (Dt) ; Buffalo (Z\&R) ; E. Aurora, Ap-Aug, generally distributed (Ang) ; Geneva, Je (Cls) ; Elmira, Je, Diven (Dt); Ithaca, May-J1 (Vdyk); Windsor, Je (Not); Schoharie (Not); Eddyville, J1 (Hyslop, Dt) ; West Pt., Je (Ds) ; Pine I., Je (Ds) ; SI, Je (L\&D) ; LI: Bellport, J1 (Nic); Orient Pt., Je (Ds); Rockaway Beach, Je, Eng (Sf); Long Beach, Yaphank, Je, Eng (Sf).

8802 L. costalis Payk. Wallface Mt., J1 (Quirs); Slide Mt., Je (Quirs).

8803 L. estriatus Lec. Wallface Mt., J1 (Quirs); Whiteface Mt., Je, Shoe (Sf).

\section{HEMICREPIDIUS Germar}

8813 H. decoloratus Say. Buffalo (Rein) ; Erie, Allegany, and Cortland Counties, J1 (Dnd) ; Ithaca, J1-Aug, Ringwood, J1, Taughannock Falls, J1 (Dt); Danby, J1 (Dt) ; Schoharie (Not); Catskills (US) ; West Pt. (Robn); Tarrytown, Je (Dt); SI, Je (L\&D). 
8814 H. memnonius Hbst. Cranberry L., J1 (H) ; Buffalo (Z\&R) ; Castile, Brad (Flet); E. Aurora, May-J1 (Dnd); L. Minnewaska, J1 (Ds) ; West Pt. (Robn); SI, J1-Aug (L\&D); LI: Flatbush, Yaphank, J1, Eng (Sf); Orient, Lath (NYS).

8815 H. brevicollis Cand. SI, J1 (L\&D).

8817 H. bilobatus Say. Buffalo (Z\&R) ; Ithaca, Aug, $V d y k$ (Dt) ; Windsor, Je (Not); Callicoon (AM); LI: Flushing, Aug, Dow (Sf).

\section{CRYPTOHYPNUS Eschscholtz}

8818 C. hyperboreus Gyll. Westfield, May (Not); Ithaca, Je-J1 (Dt) ; Oliverea (US).

8821 C. exiguus Rand. Newport, May (NYS) ; Axton, Je (M\&H) ; Rochester $(\mathrm{M} \& W)$; Buffalo (Z\&R); Westfield, May (Not); Ithaca, Ap-J1 (Vdyk); Freeville, Je (Vdyk); SI, May, on oak (L\&D).

8826 C. abbreviatus Say. Wallface Mt., J1 (Quirs); Westfield, May (Not) ; Ithaca, Ap (Dt) ; Fireeville, Je (Dt); Cortland (Dnd) ; Schoharie (Not); West Pt. (Robn) ; LI: Long Beach, May, Eng (Sf).

8828b C. nocturnus Esch. var. bicolor Esch. Whiteface Mt., 4000 ft., Aug 24, (Hyslop, Dt).

8831 C. lecontei Lg. Herkimer Co, Dietz (Sf); Pike (NYS); Ithaca, Ap, Je-J1, Taughannock Falls, Ap (Dt) ; Catskills, J1 (Nic); Oliverea, Je, Shoe (Sf).

\section{HyPNOIDUS Stephens}

8832 H. striatulus Lec. Ithaca, Je-JI (Vdyk); Windsor, Je (Not).

8834 H. choris Say. Windsor, Je (Not); LI: Wading R., May (Quirs).

8837 H. melsheimeri Horn. Newport, Je (NYS) ; Westfield, May, Windsor, Je (Not); Ithaca, May (Dt); Taughannock Falls, May (Vdyk).

8841 H. tumescens Lec. Catskills, J1 (Nic); Stony Pt., Je (Ds).

8844 H. aestivus Horn. Altamont, Sep (NYS).

8848 H. obliquatulus Melsh. Windsor, Je (Not); N. Evans, May 14, MCV (Frost); Nassau, May (NYS); Catskills, J1 (Nic) ; Peekskill, Je (Ds) ; SI, usually on moist sandy banks (L\&D).

8849 H. pectoralis Say. Newport, Je (NYS) ; central NY (US) ; Catskills, J1 (Nic); Oliverea, J1 (Quirs); West Pt. (Robn).

H. felti Not. Westfield, May (Not).

\section{Oedostethus LeConte}

8850 O. femoralis Lec. Buffalo (Z\&R); Irving, Je (Dnd); Schoharie (Not).

\section{Melanactes LeConte}

8853 M. piceus DeG. E. Aurora, Aug (Dnd) ; West Pt., Je (Ds) ; Bear Mt., Je (Quirs); SI, May-J1 (L\&D).

8855 M. morio Fab. West Pt. (Robn); SI, J1 (L\&D).

8856 M. puncticollis Lec. LI: Jamaica (Nic). 
Oestodes LeConte

8859 O. tenuicollis Rand. Essex Co, J1 (Quirs); Cranberry L., J1-Aug ( $\mathrm{H}$ ) ; Buffalo (Z\&R); Irving, Je (Dnd); Ithaca, J1 (Vdyk); Schoharie (Not); Catskills (HyslopUS).

\section{SUBFAMILY ELATERINAE}

\section{Parallelostethus O. Schwarz}

8861 P. attenuatus Say. West Pt. (Robn); Bronx (Sf).

\section{CRIGMUS LeConte}

8866 C. abruptus Say. Speculator, Aug, Lake Bluff, J1 (NYS) ; Buffalo (Z\&R, US); Batavia, J1 (Kngt); Castile, Aug, Brad (Flet); Poughkeepsie, Jl (NYS) ; West Pt. (Robn) ; LI: Bellport, Aug (Nic) ; Yaphank, Jl, Eng (Sf).

\section{OXYGONUS LeConte}

8875 O. obesus Say. Buffalo (Z\&R) ; E. Aurora, May-J1 (Dnd) ; Ithaca, ApMay (Vdyk); McLean Bogs, May (Dt); Schoharie (Not).

8876 O. montanus Sf. Slide Mt., Catskills, J1, Shoe (Ds, Sf).

\section{DOLOPIUS Eschscholtz}

8878 D. lateralis Esch. Axton, Je (M\&H) ; Cranberry L., Je, on Viburnum, maple, beech, willow, and goldenrod $(\mathrm{H})$; Wanakena, Aug (Drk); Olcott, Ap-J1 (Dt) ; Buffalo (Z\&R) ; E. Aurora, May-Aug (Dnd) ; Westfield, May (Not); Ithaca, May-J1 (Dt); McLean Bogs, May (Dt) ; Ringwood, J1 (Dt) ; Windsor, Je (Not) ; Ft. Montgomery, May (Ds); West Pt. (Robn); SI, May-J1 (L\&D); LI: Woodside, May (Sf); Rockaway Beach, May, Eng (Sf).

\section{SERICUS Eschscholtz}

8881 S. viridanus Say. West Pt. (Robn) ; Bronx Pk., Dictz (Sf) ; SI (L\&D); LI: Yaphank, May (Ds).

8882 S. incongruus Lec. Cranberry L., Je 18 (H); Redford, Martin (Sf); Windsor, Je (Not).

8883 S. silaceus Say. Buffalo (Z\&R); E. Aurora, Je, Boston, May, Allegany Pk., Aug (Dnd) ; Windsor, Je (Not) ; Ithaca, Je-J1 (Dt); West Pt., Je (Ds); SI (L\&D) ; LI: Pinelawn, Yaphank, May-Je, Eng (Ds); Babylon (AM).

8884 S. honestus Rand. Catskills, J1, Shoe, Pears (Nic, Sf); Oliverea (Shoe, US).

\section{AGRIOTES Eschscholtz}

8885 A. mancus Say. Buffalo (Z\&R); Erie Co, Je-J1 (Dnd) ; Cooperstown, Je (Cy); Locke, May (Felt); Castile, July, Brad (Flet); Bath, Aug (Hyslop-CU Econ) ; Ringwood, J1, Sep (Dt); Ithaca, Je-J1 (Leon); Windsor, Je (Not); West Pt. (Robn) ; Florida, May (CU) ; Pine I., J1 (Ds) ; NYC (US) ; SI, Mar-Je (L\&D) ; LI: Huntington, Ap, Rockaway, Je, Glendale, Ap (Quirs); Flatbush, Ap, Jamaica, May, Eng (Sf). 
8886 A. stabilis Lec. Axton, Je (M\&H); Cranberry L., Je-J1 (H) ; Olcott, Je-J1 (Dt) ; Buffalo (Z\&R) ; Avon, Je (Cls); Catskills (Nic).

8887 A. insanus Cand. West Pt., Ramapo, May-Je (Ds); SI, May-Je (L\&D) ; LI: Wading R., May 30 (Nic).

8888 A. fucosus Lec. Axton, Je (M\&H) ; Cranberry L., Je-J1 (H) ; Olcott, Je (Dt); Buffalo ( $\mathrm{Z} \& \mathrm{R})$; Allegany $\mathrm{Pk}$. and Cortland $\mathrm{Co}, \mathrm{Je}$ (Dnd); Catskills, Pears (Nic); Ithaca, May (Dt).

8888a A. fucosus var. collaris Lec. "NY", Dietz (Sf).

8893 A. pubescens Melsh. Cranberry L., Je, on maple blossoms (H) ; Buffalo $(Z \& R)$; E. Aurora, Je (Dnd); Windsor, Je (Not); Ithaca, Je-J1 (Dt); Catskills, Pears (Nic) ; West Pt. (Robn); SI, May (L\&D).

8894 A. limosus Lec. Whiteface Mt., J1, Shoe (Ds, Sf) ; Mt. Seward, 4500 ft., Axton, Je (M\&H) ; Cranberry L., Je-J1 (H); Wallface Mt., J1 (Quirs); West Pt. (Robn).

8897 A. oblongicollis Melsh. Castile, Brad (Flet) ; E. Aurora, Je (Dnd) ; Bear Mt., J1, Hewlett, Je (Quirs) ; Ft. Montgomery, Nyack, Ramapo, MayJe (Ds) ; West Pt. (Robn) ; SI, May-Je (L\&D) ; LI: Bellport, Je (Nic) ; Yaphank, Je, Eng (Sf).

8900 A. avulsus Lec. "NY", Dietz (Sf).

\section{BETARMON Kiesenwetter}

8904 B. bigeminatus Rand. Bear Mt., J1 (Quirs); Ft. Montgomery, Je, Schott, West Pt., Je, Eng (Sf).

\section{GLyPhONYX Candeze}

8906 G. recticollis Say. Schoharie (Not); SI, Je (L\&D).

8909 G. testaceus Melsh. SI, J1 (L\&D).

8910 G. inquinatus Say. Montour Falls, Je (H).

\section{ELATER Linnaeus ${ }^{43}$}

8929 E. cordatus Horn. Cranberry L., J1 (Drk).

8932 E. pullus Germ. Axton, Je (M\&H) ; Cranberry L., Je, on willow (H) ; Whiteface Mt., Sep (AM); Buffalo (Z\&R); Catskills, Pears (Sf).

8934 E. nigricollis Hbst. Cranberry L., J1 20 (H) ; Olcott, May, Sep (Dt) ; Buffalo (Z\&R) ; E. Aurora, Mar, Je (Dnd) ; Geneva, Ap, Oct-Nov (Cls); Ithaca, Ap, Je, Nov (Dt); McLean Bogs, May (Dt) ; Cooks Falls, Oct, Yonkers, Ap-Nov (Quirs); West Pt. (Robn); SI, Feb-Je (L\&D) ; LI: Little Neck, Nov, Queens, Nov, Wyandanch, Ap (Quirs) ; Pinelawn, Central Pk., Ap, Eng (Sf); Hewlett, Ap-May (Quirs).

8935 E. linteus Say. Syracuse, Ap, under bark (H) ; Buffalo (Z\&R) ; E. Aurora, May, Sep-Oct (Dnd) ; Montour Falls, Ap 23 (H) ; Geneva, Sep-Oct (Cls) ; Ithaca, Je (Dt) ; Catskills, Je (Nic) ; Yonkers, Nov, Valhalla, Ap (Quirs) ; SI, Ap-Je (L\&D) ; LI: Bellport, Je (Nic); Wyandanch, Ap, Little Neck, Ap-Nov (Quirs); Cold Sp. Harb., Ap, Eng (Sf).

\footnotetext{
${ }^{43} \mathrm{H}$. S. Barber points out that the Elater of authors is not the Elater of Linnaeus, wherefore Ampedus Dejean must be used.
} 
8936 E. sellatus Dej. E. Aurora, May (Dnd) ; Castile, Je, Brad (Flet) ; Ithaca, Je (Vdyk, Dt); Freeville, Je (Vdyk, Dt); vic. NYC (AM); SI, Feb 2, under bark (L\&D).

8937 E. vitiosus Lec. Buffalo (Z\&R) ; Ithaca, Je (Dt); Windsor, Je (Not) ; Trenton (US) ; Yonkers, Feb (Quirs).

8938 E. laesus Lec. Buffalo (Z\&R); E. Aurora, May-Je (Dnd) ; Ithaca, Je (Dt); Catskills, Je, Pears (Nic).

8939 E. sayi Lec. Buffalo (Lec-ANSP) ; NYC, May (Quirs) ; Bronx Pk. (Sf) ; SI, May (L\&D).

8940 E. verticinus Beauv. Buffalo (Z\&R) ; West Pt. (Robn) ; Mosholu (Sf) ; vic. NYC (AM) ; SI (L\&D) ; LI: Wyandanch, Ap (Quirs); Yaphank, May-Je, Eng (Sf).

8941 E. semicinctus Rand. Olcott, Je-J1 (Dt) ; Buffalo (Z\&R) ; E. Aurora, May-Je, Grand I., Je (Dnd); Ithaca, Ap-May, Sep (Dt) ; Taughannock Falls, Ap (Dt); McLean (US); Trenton (US) ; Catskills, Je, Pears (Nic); West Pt. (Robn); Ft. Montgomery, May (Ds); Cooks Falls, Sep, Yonkers, Feb-Mar (Quirs).

8942 E. militaris Harr. Ithaca, Sep 16 (Dt); West Pt. (Robn); SI (L\&D).

8943 E. rubricus Say. Axton, Je $(\mathrm{M} \& H)$; Cranberry L., Je-J1 (H) ; Buffalo (Z\&R) ; E. Aurora, Allegany Pk., Je-Aug (Dnd) ; Geneva, Sep-Oct (Cls); Ithaca, Ap-May (Dt) ; McLean Bogs, May (Dt); Windsor, Je (Not); Otsego Co (US); Trenton (US); Pine I., Je (Ds); SI, May-J1 (L\&D); LI: Woodside, Je (Sf) ; Yaphank, May, Eng (Sf).

8948 E. collaris Say. Buffalo (Z\&R); West Pt., Je (Ds) ; SI, May-J1 (Nic, L\&D) ; LI: Bellport, J1 (Nic) ; Central Pk., May (Ds) ; Yaphank, May (Ds); Wyandanch, J1 (Quirs); Hempstead Plains, May, Eng (Sf).

8950 E. sanguinipennis Say. Cranberry L., Je-Jl (H) ; E. Aurora, May (Dnd) ; Castile, Brad (Flet); Geneva, Mar, Oct (Cls); SI, Ap-May (L\&D) ; LI: Jamaica, J1, Pears (Nic); Woodside, Ap (Sf).

8951 E. xanthomus Germ. SI (US) ; LI: Bellport, Je (Nic) ; Central Pk., May (Ds); Melville (US); Wyandanch, Ap (Quirs).

8953 E. apicatus Say. Saranac Inn, Je (NYS); Whiteface Mt. (US) ; Wallface Mt., J1 (Quirs); Johnstown, Oct (NYS); Buffalo (Z\&R).

- E. longipennis Not. Cranberry L., Htly, Montour Falls, Je, with query (H) ; Bear Mt., J1 (Quirs).

8955 E. obliquus Say. Buffalo (Z\&R); E. Aurora, Jan, E. Elma, Je (Dnd) ; Madison Co, May 20, Quirs (H) ; Windsor, Je (Not); Callicoon (AM) ; West Pt. (Robn); SI (L\&D) ; LI: Jamaica, Pears (Nic).

8955 a E. obliquus var. areolatus Say. Castile, Brad (Flet); Ithaca, J1 (Vdyk); Schoharie (Not); SI, Sep (L\&D).

8956 E. pusio Germ. LI (US).

8959 E. manipularis Cand. Olcott, Sep (Dt) ; E. Aurora, May, Allegany Pk., J1 (Dnd); Ithaca, Mar-Ap (Dt) ; LI: Bellport, J1 (Nic).

8960 E. pedalis Germ. Axton, Je (M\&H); Buffalo (Z\&R); Ithaca, May-Je (Dt) ; Pine I., Je (Ds); SI, Je (L\&D); LI: Jamaica, J1 (Nic).

8964 E. mixtus Hbst. Axton, Je (M\&H) ; Wallface Mt. (Quirs) ; Cranberry L., Je-J1 (H) ; Buffalo (Z\&R) ; Ithaca, J1 (Dt); Ringwood, J1 (Dt) ; McLean Bogs, J1, as fusculus (Hyslop, Dt); West Pt. (Robn); SI, May (L\&D). 
8965 E. impolitus Melsh. Ithaca, Je-Jl (Vdyk, Dt) ; Ringwood, J1 (Vdyk, Dt) ; Catskills, Je, Pears (Nic) ; LI: Jamaica (Nic).

8966 E. socer Lec. Mt. Seward, Je (M\&H) ; Schoharie (Not) ; Ft. Montgomery, J1, Schott (Sf).

8968 E. luctuosus Lec. Cranberry L., Je-J1 (H); Buffalo (Z\&R) ; McLean Bogs, May (Dt).

8969 E. nigricans Germ. Wallface Mt., J1 (Quirs) ; Mt. Seward, Je (M\&H) ; Cranberry L., Je-J1 (H) ; Olcott, Sep (Dt) ; E. Aurora, Je (Dnd) ; Windsor, Je (Not); West Pt. (Robn); Ithaca, May-Je (Dt); Ft. Montgomery, Je, Schott (Sf); SI: May-Je (L\&D); Silver L., J1 (Sf) ; LI: Flatbush (AM).

E. longipennis Not. Westfield, May (Not).

\section{ECTAMONOGONUS Buysson}

8971 E. melsheimeri Lg. Cranberry L., J1 17 (H) ; E. Aurora, May, Boston, Je (Dnd) ; Trenton (US) ; SI, Dec (L\&D) ; LI: Aqueduct, May, Forest Pk., Ap, Schott (Sf); Port Jefferson, Aug, Murphy (Sf); Orient, Lath (NYS).

\section{MEGAPENTHES Kiesenwetter}

8980 M. limbalis Hbst. SI, J1-Aug (L\&D) ; LI: Bellport, J1 (Nic) ; Aqueduct (Ds) ; Flatbush (AM); Babylon, Johnson (Sf).

8983 M. rufilabris Germ. Mosholu ( $S f)$.

8988 M. stigmosus Lec. Cranberry L., Aug (Drk).

\section{Melanotus Eschscholtz}

9015 M. castanipes Payk. Cranberry L., Je-J1 (H) ; Buffalo (Z\&R) ; E. Aurora, May-Je (Dnd) ; Debruce, J1 (Quirs); West Pt. (Robn) ; SI, Je (Quirs) ; LI: Bellport, J1 (Nic) ; Sayville, J1 (Quirs).

9016 M. corticinus Say. E. Aurora, Je (Dnd) ; LI: Jamaica, Pears (Nic).

9018 M. macer Lec. "NY" (Lec).

9019 M. decumanus Er. Protection, Je (Dnd) ; NYC, Ap (Quirs) ; SI, J1 2, at electric light (L\&D).

9020 M. canadensis Cand. E. Aurora, May-Sep, common under bark (Dnd).

9022 M. clandestinus Er. LI: Orient, Lath (NYS).

9025 M. ignobilis Melsh. Ft. Schuyler, Je (Quirs); LI: Bellport, Aug (Nic).

9026 M. depressus Melsh. E. Aurora, May-J1 (Dnd).

9029 M. trapezoideus Lec. Bear Mt., Je (Quirs) ; LI: Wading R., May 20 (Nic).

9030 M. taenicollis Lec. SI, Je (L\&D); LI: Flatbush (AM).

9031 M. leonardi Lec. West Pt. (Robn); Bear Swamp, Ramapo Mts., J1 (Ds) ; Bear Mt., J1 (Quirs).

9033 M. glandicolor Melsh. Catskills, J1, Pears (Nic).

9034 M. divarcarinus Blatch. Schoharie (Not).

9035 M. communis Gyll. Buffalo (Z\&R); Erie Co, May-Sep (Dnd) ; Montour Falls, Je (H); Orange Co (Felt); NYC, Mar (Quirs); West Pt. (Robn); SI, Ap-Je, Nov (L\&D); LI: Orient Pt., Je, Lath (Ds) ; Hewlett, Je (Quirs). 
9036 M. fissilis Say. Cranberry L., Je-J1 (H) ; Syracuse, May, under bark (H) ; Buffalo (Z\&R) ; E. Aurora, Je-J1 (Dnd) ; Ithaca, J1 (Vdyk); Ringwood, J1 (Vdyk); West Pt. (Robn); NYC, Ap (Quirs); SI, May-Je, Sep (L\&D).

9040 M. parumpunctatus Melsh. Catskills, Je, Pears (Nic); West Pt. (Robn); Bear Mt., J1 (Quirs); SI, Je-J1 (L\&D).

9048 M. pertinax Say. Buffalo (Z\&R); SI, J1 (L\&D); LI: Bellport, Je (Nic).

9049 M. dubius Lec. "NY" (Lec).

9051 M. americanus Hbst. Pike (NYS); West Pt. (Robn); Bear Mt., J1 (Quirs) ; SI, Je-J1 (L\&D).

9059 M. sagittarius Lec. E. Aurora, May (Dnd); LI: Bellport, Je-Aug (Nic).

9060 M. difficilis Blatch. Westfield, May (Not); Windsor, Je (Not).

9061 M. pilosus Blatch. E. Aurora, Cortland Co, May (Dnd).

9066 M. similis Kby. "NY" (Fitch).

M. hyslopi VanD. Greenwood L., J1 (AM).

\section{SUBFAMILY CARDIOPHORINAE}

\section{CARDIOPHORUS Eschscholtz}

9071 C. cardisce Say. Rochester (M\&W) ; Buffalo (Z\&R) ; SI, Je (L\&D) ; LI: Coney I. (F. Blanchard); Woodhaven, May (Vdyk,Dt); Babylon (AM) ; Rockaway, Je (Quirs) ; Amagansett, May, Eng (Sf) ; Rockaway Beach, Je, Sep, Olsen, Schott, Eng (Sf).

9082 C. convexus Say. Olcott, J1 (Dt); Karner, Je-J1 (NYS); West Pt. (Robn); SI, May-Je (L\&D); LI: Wading R., May, Schott, Rockaway Beach, May, Eng (Sf).

9087 C. gagates Er. Axton, Je (M\&H); Rochester (M\&W) ; Boston, May (Dnd) ; Ithaca, J1 (Vdyk, Dt); Karner, Je (NYS); Mosholu (AM);

Fishkill (US); West Pt. (Robn); SI, May-Je (L\&D) ; LI: Wading

R., May, very common (Nic); Hewlett, Je (Quirs); Pinelawn, Je, Yaphank, May, Eng (Sf).

9092 C. convexulus Lec. Newport, May (NYS); Rochester (M\&W); West Pt. (Robn) ; vic: NYC (AM) ; LI: Wading R., May (Nic).

9098 C. robustus Lec. Karner, Ap-May (NYS) ; LI: Jamaica, May, Pears (Nic).

\section{HoRIstonotus Candeze}

9104 H. vulneratus Horn. Buffalo (Z\&R); LI: Wading R., May 30 (Nic). Hyslop queries these records, since this is an Arizona species.

9114 H. curiatus Say. West Pt. (Robn); Bronx Pk. (Sf).

\section{FAMILY MELASIDAE}

\section{SUBFAMILY EUCNEMINAE}

\section{Melasis Olivier}

9125 M. pectinicornis Melsh. Mosholu (Sf). Oak (Felt). 
ISORHIPIS Lacordaire

(Tharops Castelnau)

9127 I. ruficornis Say. Pike, Je (NYS) ; Newport, Je (NYS) ; Cranberry L., J1 (Hatch); Rochester (M\&W); Olcott, Je (Dnd); Buffalo (Say ANSP) ; E. Aurora, Je (Dnd); Ithaca, Je-J1 (Dt); Greenwood L. (Sf) ; West Pt., Je (Sf) ; Catskills, Je-J1 (Pears); SI, Je-J1, often on beech (L\&D); LI: J1, Eng (Sf); Montauk Pt., Aug, Eng (Not).

\section{STETHON LeConte}

9130 S. pectorosus Lec. Rochester (M\&W); Batavia, J1 (Kngt).

\section{DELTOMETOPUS Bonvouloir}

9133 D. amoenicornis Say. Newport, Je (NYS) ; Cranberry L., J1 (H) ; Wanakena, Aug (Drk); Rochester (M\&W); Buffalo (Z\&R); Olcott, Je (Dt) ; E. Aurora, Ji (Dnd) ; Canadaigua (Bonv) ; Ithaca, J1-Aug (Vdyk); McLean, Aug (Vdyk); Catskills, Je-Ji (Nic); Van Cort. Pk., Je-J1 (Nic); Mosholu (Sf); SI, on dead beech (L\&D); LI: Yaphank, Je (Sf).

9134 D. rufipes Melsh. Olcott, Je (Dt); NYC (Sf).

\section{DROMAEOLUS Kiesenwetter}

9136 D. sejunctus Bonv. Canandaigua (Bonv); LI (Horn).

9141 D. striatus Lec. "NY" (Horn).

9142 D. harringtoni Horn. "NY" (Horn).

9143 D. cylindricollis Say. Buffalo (Z\&R); Mosholu (Sf).

9144 D. marseuli Bonv. Canandaigua (Bonv).

\section{FORNAX Castelnau}

9147 F. calceatus Say. Cranberry L., J1 (H).

9150 F. orchesides Newn. Saranac Inn, J1 (NYS) ; Cranberry L., Je-J1 (H) ; Wanakena, Aug (Drk); Trenton Falls (Newn); Rochester (M\&W); Buffalo (Z\&R); LI: Bellport, Aug (Nic).

\section{MICRORHAGUS Eschscholtz}

9154 M. pectinatus Lec. "NY" (Horn).

9155 M. audax Horn. NYC (Sf).

9156 M. imperfectus Lec. (subsimuatus Lec.). Johnstown (Yg NYS) ; Rochester $(\mathrm{M} \& W)$; E. Aurora, Aug (Dnd); Bellport, J1 (Nic); Mosholu, Greenwood L. (Sf).

9157 M. triangularis Say. Bronx Pk (Dt); Mosholu (Sf); SI (L\&D).

9159 M. bonvouloiri Horn. Rochester (M\&W); Buffalo (Z\&R); NYC and vic. (Sf).

ENTomophthalmus Bonvouloir

9161 E. rufiolus Lec. NYC and vic. (Sf). 
Nematodes Latreille

9167 N. penetrans Lec. LI: Gardiners I., J1, Eng (Sf).

\section{HyPocoelus Eschscholtz}

9172 H. frontosus Say. Adirondacks, Martin (Sf) ; NYC and vic. (Sf) ; SI, J1 (L\&D).

9173 H. terminalis Lec. "NY" (Lec ANSP).

SCHIZOPHILUS Bonvouloir

9174 S. subrufus Rand. NYC and vic. (Sf).

\section{EPIPHANIS Eschscholtz}

9175 E. cornutus Esch. "NY" (Lec); Cascade (Springville, Erie Co), J1, MVD (Frost).

\section{PHLEGon Castelnau}

9176 P. heterocerus Say. LI: Bellport, Aug, Nic (Sf).

\section{SUBFAMILY PEROTHOPINAE \\ PEROTHOPS Erichson}

9180 P. mucida Gyll. LI: Queens, Je, Eng (Sf).

\section{FAMILY THROSCIDAE \\ DRAPETES Dejean}

9182 D. geminatus Say. Buffalo (Z\&R); Catskills, Pears (Nic) ; Ramapo, May (Ds) ; Van Cort. Pk. (Pears, Nic).

9185 D. quadripustulatus Bonv. Buffalo (Z\&R).

\section{Aulonothroscus Horn}

9191 A. punctatus Bonv. Newport (NYS) ; Albany, Je (NYS) ; Catskills, J1 Pears (Nic).

9195 A. constrictor Say. Newport, Speculator, Je (NYS); Axton, Je (M\&H); Cranberry L., Je-J1 (H) ; Buffalo ( $Z \& R$ ); Ithaca, Je (CU); Windsor, Je (Not); Schoharie (Not); Catskills, J1, Pears (Nic).

9198 A. distans Blanch. Je-J1, Cranberry L. (H).

9200 A. convergens Horn. "NY" (Lg Cat.).

\section{Throscus Latreille}

9204 T. chevrolati Bonv. Axton, Je (M\&H) ; Cranberry L., Je (H) ; Syracuse, May (H) ; Olcott (Dt); Westfield, May (Not); Buffalo (Z\&R); Ithaca, Feb-Aug (Dt); Nassau, May (NYS); SI, Ap, in sifting (L\&D) ; LI: Rockaway Beach, May (Nic). 


\section{FAMILY BUPRESTIDAE 44}

\section{ACMAEODERA Eschscholtz}

9286 A. tubulus Fab. Common throughout the State (Nic). Rochester (M\&W); L. Keuka, May (Vdyk-CU); Coy Glen, May, Ithaca, May-Je (Vdyk-CU) ; W. Danby, May (Vdyk-CU) ; Nassau, May (Yg); W. Hebron, Je (Lg); Peekskill, May (Sherm); West Pt., Je (Ds); Ramapo, May (Lg) ; Suffern (AM); SI, May (L\&D); LI: Half Way Hollow Hills, J1 (Ds); Yaphank, May-J1 (Ds, Sf). Larva in hickory, white oak, and redbud (Fisher).

\section{Chalcophora Solier}

9316 C. virginiensis Dry. Locally common wherever pines occur (Nic). Peru, Je (Vdyk-CU) ; New Russia, Je (NYS) ; top of Mt. Whiteface, J1 (Ds); Cranberry L., J1 (Mund); Syracuse (B1km); Rochester $(M \& W)$; Buffale (Z\&R); Yates Co (AM); Ithaca, Je-Oct (Vdyk-CU) ; Karner, Je-Aug (Yg) ; Slingerlands, Oct (NYS) ; New Baltimore (AM); SI, May (L\&D). Larva in both hard and soft pines (Fisher).

9317 C. lacustris Lec. Ausable Chasm, Je (Vdyk-CU); Hague, Oct (AM). Nicolay regards this as a synonym of virginiensis.

9320 C. fortis Lec. Local and rare (Nic). Keene Val., Je (Ds) ; Long L. (Lg) ; Axton, Je (M\&H) ; Newport, J1 (NYS) ; Valcour I., J1, Black Br. (Lg); Fulton (Vdyk-CU) ; Rochester (M\&W); Buffalo (Z\&R); Conesus, Aug (Ds); Ithaca, May-J1 (Vdyk-CU); Pittstown, Oct (NYS) ; Croton-on-Hudson, Je-J1 (Ds) ; West Pt., Je (Ds, Robn). Larva in white pine (Fisher).

9321 C. liberta Germ. Locally common (Nic). New Russia, Je (NYS) ; Brant L., Doll (Sf); Syracuse (Blkm) ; Buffalo (Z\&R) ; Enfield Glen, Je (Dt); Karner, Je, Schuylerville, Je (NYS); Albany, May (NYS).

\section{Chalcophorella Kerremans}

9323 C. campestris Say. Somewhat rare in NY (Nic). Buffalo (Lg-AM); West Pt. (Sf); vic. NYC (AM). Larva in beech, willow, buttonwood, and sugar maple (Fisher); in tulip tree (Felt).

\section{TRACHYKELE Marseul}

9332 T. lecontei Gory. LI: Brooklyn, one specimen from introduced lumber $(\mathrm{Lg})$. Larva in cypress in the South (Fisher).

\section{DICERCA Eschscholtz}

9333 D. divaricata Say. Widely distributed and common. Mt. Whiteface, J1 (Ds) ; Mt. Seward, $4500 \mathrm{ft}$., Je (M\&H) ; Axton, Je (M\&H) ; Cranberry Cr., Je, $\mathrm{Cr}$ (Vdyk); Cranberry L., Je-Aug (Mund); Keene Val., J1, Newport, May, Big Moose, J1, Fultonville, Je (NYS); Redford, Peru, Black Br., Je (Vdyk); Ilion, Jl (NYS); L. Clear, J1 (Yg);

\footnotetext{
${ }^{44}$ W. S. Fisher and Alan S. Nicolay have gone over the list for this family and have made many suggestions and changes in synonymy. Mr. Fisher particularly has supplied the New York State food-plants for most of the species, where these were known.
} 
Manlius, May (Vdyk) ; Syracuse, Je-J1 (Blkm); Holley, Je (Vdyk) ; Honeoye Falls, Je, Leon (Vdyk); Rochester (M\&W) ; Olcott, Je-Aug (Dt) ; Conesus L., Je (Ds) ; E. Aurora (Dnd) ; Batavia, Kngt (Morr) ; Buffalo (Z\&R) ; Portageville (Ds) ; Westfield, Je (NYS) ; Rock City, Je (Vdyk); Castile, Brad (Flet); Ithaca, May-J1 (Vdyk, etc.); McLean Bogs, May-Aug (Vdyk) ; Elmira (Diven) ; W. Hebron (Lg) ; Schuylerville, Je (Yg); Albany, J1, Karner, Je, Nassau, Aug, Glenmont, Je (NYS) ; New Baltimore, Zab (AM) ; E. Jewett, Aug, West Pt., May-Je (Ds) ; Croton-on-Hudson, J1 (Ds) ; Debruce (Shoe) ; Peekskill (Vdyk); Nyack, Zab (AM); Ramapo Mts. (AM); Mosholu (Ds) ; Grahamville, J1 (NYS) ; Bronx Pk. (AM) ; SI, Mar-Sep (L\&D) ; LI, J1, Eng (Sf). Larva in black birch, ironwood, linden, white ash, sugar maple, redbud, American elm, and black ash (Fisher); in apple, pear, cherry, and plum (Felt).

9334 D. caudata Lec. Adirondacks (Csy) ; E. Aurora, Je (Dnd) ; Ithaca, May, Oct (Vdyk) ; Catskills (Nic); Bangall, Sep, Eng (Sf); Ludlow (Lg); White Plains, Oct (Lg); Pine I., Je (Ds); NYC, J1 (Lg); SI: Richmond (L\&D) ; LI: Bay Shore, JI, Ols (Frost); Flushing, Je (AM). Larva in black birch (Fisher).

9335 D. prolongata Lec. Plattsburg, Jl (Ds); Mt. Whiteface, J1, Shoc (Sf) ; Wilmington, J1-Aug (Ds); E. Aurora, Je (Dnd); Ithaca, Je-Aug (Vdyk, Sf); Windsor, Je (Not). Larva in aspen and willow (Fisher).

9337 D. punctulata Schön. Taken commonly in beating pine, May-Sep (Nic) ; Buffalo (Z\&R) ; L. Keuka, May, Cr (Vdyk); Ithaca, Ap-Aug (Vdyk) ; Karner, Mar, Je (NYS); Peekskill, Feb (Sherm); West Pt., Je' (Ds, Not); SI: Richmond, Ap-J1 (L\&D); LI: Central Pk., Aug (Ds) ; Rockaway (AM) ; Astoria (Lg); Bellmore, Sep, Eng (Sf); Massapequa, May (Shoe); Pinelawn, Bald Hill, Aug (Ds); Bellport, Je-Aug (Nic) ; Yaphank, J1-Sep (div.); Amagansett, Sep (Ds) ; Deep Pond, Wading R., J1-Aug, Eng (Ds). Larva in pitch pine (Fisher).

9340 D. pugionata Germ. A rather rare species (Nic). Rochester (M\&W); Ithaca, Je-Sep (Vdyk); Catskills, J1 (Nic); Albany, May, Lint (NYS) ; West Pt., May (Ds) ; Minnewaska, Aug (Nic) ; Ft. Montgomery, Sep, Schott (Frost); Phoenicia, Aug, MCV (NYS) ; Bangall, Sep, Eng (Sf) ; Greenwood L., May (Sf); LI: Wyandanch, Oct, Schott (Frost). Larva in witch-hazel, alder, and Spiraea (Fisher); in oak and maple (Felt).

9341 D. obscura Fab. Buffalo (Z\&R, Csy) ; E. Aurora (Dnd) ; Geneseo, Je (NYS); Ithaca (Vdyk); West Farms, Aug (AM); Bronx Pk., May-J1 (Lg); SI: Richmond, Long Neck, Feb-Sep, on hickory and often on persimmons (L\&D). Larva in persimmon (Fisher).

9342 D. lurida Fab. The most abundant species in the State (Nic). Newport, J1 (NYS) ; Syracuse, hundreds bred from dead hickory (Blkm); Rochester (M\&W); Honeoye Falls (Vdyk); Hamburg, Aug (NYS); Sonyea, Je (Vdyk); Ithaca, Ap-Oct, McLean Bogs, May (Vdyk); Elmira, J1 (Diven); Hague, L. George, Aug (Diven); Schoharie (Not); W. Hebron, Je (Lg); Poughkeepsie, May (NYS); West Pt., Je, Sep (Ds), J1, Eng (Sf); Pine I., Je (Ds) ; Nyack (AM); Shokan, J1' (Lg); Croton-on-Hudson (Ds); West Farms, Aug (AM); Van Cort. Pk., May (Woodr); SI: Richmond, Feb-Sep, often on hickory (L\&D) ; LI: Aqueduct, J1, Half Way Hollow Hills, J1 (Ds) ; Rockaway Beach, May (Shoe); Flatbush, Zab (AM); Parkville (AM); Central Pk., Ap (Eng) ; Rosyln, J1 (NYS) ; Promised Land, Yaphank, Je, Eng (Sf); Amagansett, Sep, Eng (Sf). Larva in hickory, blue beech, and alder (Fisher). 
9344 D. spreta Gory. Buffalo (Z\&R, Knull). Nicolay believes this refers to tuberculata.

9345 D. tenebrosa Kby. L. Placid, Je, Speculator, Aug (NYS) ; Keene Val., Aug (Diven); Axton, Oct (M\&H) ; Big Moose, Saranac Inn, Je-J1, Yg (Not); Potsdam (Knull) ; Cranberry L., Je-J1 (Mund); Black Br., Je (Vdyk); E. Aurora, Je (Dnd); Pike (NYS). Larva in white pine, spruce, and arbor vitae (Felt).

- D. distinguenda Cast. "NY" (Frost). This species is distinct from tenebrosa Kby., according to Frost.

9346 D. chrysea Melsh. Mt. Whiteface, J1 8 (Ds); L. George (Csy). This probably refers to tenebrosa, according to Nicolay.

9348 D. dumolini Cast. Ithaca, May 17 (Vdyk). Nicolay says, "unknown to me, may refer to tuberculata:"

9349 D. tuberculata Cast. Long L., Aug (Lint); Murray Bay, Essex Co, Aug (NYS); Keene Val., Aug, Big Moose, Jl (NYS); Syracuse, Ap $(B 1 \mathrm{~km})$; Rochester (M\&W); Buffalo (Lec, Z\&R); Elmira, Je (Diven); Ithaca, May (Vdyk); Albany, Je (NYS) ; Peekskill (Vdyk); NYC (Csy) ; Bronx Pk., Van Cort. Pk. (Sf) ; LI: Brooklyn (Lec); Jamaica (Nic). Larva in hemlock (Fisher).

9349a D. tuberculata var. scobina Chev. Buffalo ( $\mathrm{Lg})$; West Farms (AM) ; SI: Richmond, Ap-Je (L\&D). Larva in sour gum (Fisher); oak and hickory (Felt). Nicolay says this is the same as tuberculata Cast.

9350 D. mutica Lec. LI: Brooklyn, a single specimen reported many years ago by LeConte.

\section{POECILONOTA Eschscholtz}

9351 P. cyanipes Say. Mt. Whiteface, J1 (Shoe); Wilmington, J1-Aug, Dt (Vdyk, Ds) ; Keene Val., Aug (NYS) ; Brant L., Doll (Sf) ; Buffalo (Lg-US) ; Hemlock L., Aug (Vdyk); Ithaca, Aug, Eng (Sf). Larva in aspen (Fisher).

9351a P. cyanipes var. erecta Gory. Buffalo ( $\mathrm{Lg})$. Same as cyanipes, according to Nicolay.

9353 P. thureura Say. Buffalo (Z\&R); Bear Mt., J1 4, Schott (Nic). Larva in willow (Fisher).

\section{Ciny RA Castelnau and Gory}

9355 C. gracilipes Melsh. Iona I., Je, Schott (Frost) ; Bronxville, Je (Woodr) ; vic. NYC (AM) ; SI: Richmond, Great Kills, Old Place, on Quercus bicolor (L\&D); LI: Rosedale, J1, Schott (Nic); Half Way Hollow Hills, J1 (Ds). Larva in white oak, swamp oak, ironwood, and ash (Fisher).

\section{BUPRESTIS Linnaeus}

9360 B. sulcicollis Lec. Ithaca, May 26 (Vdyk). Larva in pine (Fisher).

9363 B. striata Fab. Schroon L., Je, Paradox, J1 (NYS); Gloversville, Ap, Alex (NYS); Syracuse, Je (Blkm); E. Aurora, J1 (Dnd) ; Rock City, Je (Vdyk); Ithaca, Ap-Je (Vdyk); W. Danby, Je (Vdyk); Albany, Je (NYS); Catskills, Pears (Sf); Minnewaska, Jl (Nic); Valhalla, Ap, Schott (Frost) ; Bear Mt., J1 (Quirs) ; Greenwood L., Je (Nic) ; LI: Bay Shore, Ols (Nic). Larva in hemlock and in both soft and hard pines (Fisher). The record by Fitch of aurulenta L. from NY on spruce, May-Je, probably belongs here. 
9363a B. striata var. impedita Say. Cranberry L., Je, rare (Mund) ; Greenwood L., Je (Nic); West Farms (AM). Larva in pine, found with the preceding species (Fisher).

9365 B. decora Fab. SI: Tompkinsville, May, in introduced lumber (L\&D). Larva probably lives in pine in the South (Fisher).

9366 B. salisburyensis Hbst. Black Br. (Lg); Bear Mt., Jl, Schott,Quirs (Nic). Larva in pine (Fisher).

B. salisburyensis var. Ft. Montgomery, J1, Schott.

9367 B. lineata Fab. Syracuse (Blkm); Karner, J1 ( Lg); LI: Flatbush, Mar (AM); Pinelawn, Aug (Sf), Champlain (US); Bellport, J1-Aug (Nic); Yaphank, J1-Sep, Bay Shore, J1 (Shoe); Long Pond, Wading R., J1 (Ds). Larva in pitch pine and scrub pine (Pinus virginiana) (Fisher).

9368 B. maculipennis Gory. Adirondacks (Csy) ; Black Br., Je (Lg); Wanakena, Aug (Drk); Ithaca, Je-J1 (Vdyk); Greenwood L., J1 (Nic); LI: Massapequa, Je (Shoe); Bellport, J1-Aug, on pine (Nic). Larva in pitch pine and hemlock (Fisher).

9370 B. maculativentris Say. Peru, Je (V dyk); Westport (Uhl-US); Mt. Whiteface, summit, J1 (Ds); Wallface Mt., J1 (Nic); Murray Bay (AM) ; Keene Val., Aug (Diven); Wilmington, Aug (Dt); L. Placid, J1 (NYS) ; Jay, J1 (Britton); L. Pleasant, J1, Long L., Aug, Old Forge, J1 (NYS) ; Saranac L., Sep (Lg); Severance, J1-Aug (AM) ; Wells, Newport, J1 (NYS); Cranberry L., Je-Aug (Mund); Gloversville, Aug (NYS); Syracuse (Blkm); Buffalo (Lg); Catskills $(\mathrm{Lg})$; LI (Sf). Larva in pine (Fisher).

9372 B. nuttalli Kby. Cranberry L., J1 (Drk).

9372a B. nuttalli Kby. var. consularis Gory. "NY", Dt (Sf) ; Cranberry L., Aug (Mund). Larva in pitch pine (Fisher).

9374 B. rufipes Oliv. "NY" (Not-AM). Not rare farther south in the U. S. (Nic). Larva in chestnut, American elm, beech, sour gum, hickory, white oak, tulip tree, and sugar maple (Fisher).

9377 B. fasciata Fab. Wilmington, J1 (Shoe); Keene Val., J1-Aug (NYS) ; Saranac Inn, Aug (Woodr); Severance, J1, Paradox L., May, Aug (NYS) ; Brant L., Doll (Sf); Wells, Jl (NYS) ; Cranberry L., Jl-Aug (Knull); Wanakena, Nov (Drk); Syracuse (Blkm) ; Rochester (M\&W); Buffalo (Z\&R); Pike, J1 (NYS); Ithaca, J1-Aug (Vdyk) ; Binghamton, Sep (Lg); L. George, Aug (AM) ; Gouverneur, J1 (Lint) ; L. Minnewaska, J1 (Ds); Nyack (AM) ; NYC (Knull). Larva in pine (Fisher); in maple and poplar (Felt).

\section{XENORHIPIS LeConte}

9379 X. brendeli Lec. Probably the rarest of our eastern buprestids (Nic). LI: Brooklyn (Horn). Larva in hickory (Fisher); in oak (Felt).

\section{Melanophila Eschscholtz}

9384 M. aeneola Melsh. Common throughout the State, J1-Aug, taken beating pine (Nic). Black Br., Je ( Lg) ; West Pt., Je (Not) ; Ft. Montgomery, J1, Schott (Frost); LI: Wyandanch, Yaphank, J1 (Ds) ; Bay Shore, Ji (Sf); Loyd's Neck, Jl, Brb (Sf). Larva in scrub pine (Fisher). 
9387 M. fulvoguttata Harr. Very common in the northern woods on cut pine logs in Je-J1 (Nic). L. Placid, Je, Potsdam, J1, Newport, Je (NYS); Mt. Marcy, 5344 ft., Aug (Diven); Mt. Whiteface, J1 (Shoe); Big Moose, J1 (Felt) ; Cranberry L., Je-Sep (Mund) ; Black Br. (Vdyk); Crittenden, from hemlock and spruce (B\&S); Buffalo ( $\mathrm{Lg})$; Boston, May (Dnd) ; Ithaca, Je-Jl, Dt (Vdyk); Slide Mt., Jl (Shoe); Big Ind. Val., J1, Pears (Sf) ; Poughkeepsie, J1 (NYS); West Pt., Je (Ds) ; Ft. Montgomery, Je, Schott (Frost); Scarsdale, Ap (NYS); Ramapo (Lg); NYC, Ap, Eng (Sf) ; LI: Rockaway Beach, Je, Eng (Sf). Larva in white pine, red spruce, hemlock, and American larch (Fisher).

M. drummondi Kby. var. abies Champ. \& Knull. Mt. Whiteface, J1 10, Shoe; Wallface Mt., J1 15, N. Elba, J1 (Nic). Rather rare and local (Nic).

9391 M. acuminata DeG. Old Forge, Je, Murray Bay, J1, Newport (NYS); Rochester (M\&W); Buffalo (Z\&R); Ithaca, Sep, Cy (Vdyk); Newburgh, May (Sf); Albany, Sep (NYS); Nyack (AM); SI lL\&D). Larva in pine and fir (Fisher).

\section{AnTHAXIA Eschscholtz}

9392 A. viridifrons Gory. Syracuse, Je (Drk); Rochester (M\&W) ; Buffalo (Z\&R, Lg) ; Batavia, Je (Kngt) ; Conesus L., Je (Ds) ; Portageville, Je (Not); Castile, Brad (Flet); Ithaca, May-J1 (Vdyk); West Pt., Pine I., Je (Not); Bronxville, Je (Woodr); NYC (Lg); SI, Je (Lg) ; LI: Massapequa, Yaphank, J1 (Woodr). Larva in American elm and hickory (Fisher).

9394 A. aeneogaster Cast. Apparently rare in the East, but common on the western coast (Nic). Newport, Gloversville, Aug (NYS); Rochester $(M \& W)$. Although this western species has been recorded from the East, there is some doubt of its occurrence in New York, species in this genus being easily misidentified (Fisher).

9395 A. viridicornis Say. A rare species (Nic). Peru, Je (Vdyk); Rochester $(\mathrm{M} \& W)$; Ithaca, May (Vdyk); Schoharie (Not). Larva in willow, hickory, and elm (Fisher).

9396 A. quercata Fab. Common everywhere in Je on oak leaves (Nic). Rochester $(M \& W)$; Buffalo ( $Z \& R)$; Crittenden, bred from larch (B\&S); Ithaca, May, J1-Aug (Chitt, Vdyk); Windsor, Je (Not); Schoharie (Not) ; Karner, J1 (NYS) ; Mosholu (AM) ; SI: Richmond, Je, on oak leaves (L\&D) ; LI: Massepequa, J1 (Ds, Shoe); Wyandanch, J1, Deep Pond, Wading R., Je (Ds) ; Yaphank, Je-Jl, Eng (Sf, Ds) ; Bayshore, $\mathrm{J} 1$, Ols (Frost). Larva in redbud, Crataegus coccinea, white pine, American larch, and chestnut (Fisher).

\section{AGRILAXIA Kerremans}

9399 A. flavimana Gory. Bronxville, J1 (Woodr). Larva in white oak (Fisher).

\section{Chrysobothris Eschscholtz}

9405 C. sexsignata Say. Locally common throughout the State (Nic). Mt. Whiteface, J1 (Shoe) ; Cranberry L., J1 (Mund) ; Syracuse, bred from hickory (B\&S); Rochester (M\&W); Rochester Jct., J1 (Vdyk); 
Crittenden, Je-J1, from larch (B\&S); Buffalo ( $\mathrm{Lg}, \mathrm{Z} \& \mathrm{R})$; Ithaca, Je-Aug (CU) ; Cropseyville, Je (NYS) ; Big Ind. Val., J1, Pears (Sf, Nic); West Pt., Je (Ds); Bronxville, J1-Aug (Woodr); SI: Richmond, J1 (Ds) ; LI: Aqueduct, J1 (Shoe); Flatbush, J1 (AM). Larva in hemlock, black and white oak, red maple, white swamp oak, walnut, butternut, and American larch (Fisher); in beech and birch (Fisher).

9407 C. scitula Gory. Some, at least, of the following localities may not refer to scitula, since there are no NY specimens in the USNM (Fisher). Rochester (M\&W) ; Hastings, Je (Lg); Buffalo (Z\&R); Ithaca, JeJ1 (CU) ; Karner, May (NYS) ; Bronxville, Je (Woodr); Van Cort. Pk. (Lg); SI, May-Je, on dogwood (L\&D); LI: Brooklyn, Je-JI (Shoe); Rockaway (AM); Jamaica (Nic); Montauk Beach, J1 2 (Frost). Nicolay says scitula should be cut out and the records credited to lecontei Lg. Larva in white swamp oak and alder (Fisher); in white birch (Felt).

9408 C. lecontei Lg. Batavia, Je (Kngt); E. Aurora, J1 (Dnd) ; Ithaca, Je (NYS) ; Karner, May, Nassau, Je (NYS). Larva in alder, white swamp oak, and sumac (Fisher).

9436 C. blanchardi Horn. Rochester (M\&W) ; Crittenden (B\&S); West Pt., Je (Not, Robn-AM) ; Greenwood L., Jl (Nic); NYC (Lg). Larva in pitch pine and white pine (Fisher).

9448 C. pusilla Cast. Common everywhere, May-Aug (Nic). Big Moose, J1 (NYS) ; Cranberry L., Je-Aug, not common (Mund); Black Br. (Lg) ; Syracuse, J1 (Drk) ; Ithaca, Je-J1 (Vdyk); West Pt., Je (Ds) ; Ft. Montgomery, Je, Schott (Frost); SI: Richmond, Je (L\&D); LI: Pinelawn, Wading R., Yaphank, Je-Jl (Ds,Sf). Larva in pitch pine and hemlock (Fisher).

9458 C. trinervia Kby. Mt. Whiteface trail, J1 10, Shoe (Frost) ; Cranberry L., Je-J1, bred from spruce, rather common (Mund); LI (Linell-US). Larva in pine (Fisher).

9459 C. verdigripennis Frost. Minerva, J1 20, 1925, on balsam fir (Frost) ; Wallface Mt., J1 (Nic); Ft. Montgomery, J1, Schott (Sf); Bear Mt., J1, Schott (Nic).

9461 C. dentipes Germ. Common throughout the State on pine in Je (Nic). Mt. Whiteface, J1 (Shoe); Newport (NYS) ; Black Br., Je, Cy (Vdyk); Cranberry L., from larch (Mund); Broadalbin, Je (NYS); Crane Pond, J1, Bks (NYS) ; Syracuse, J1 (Drk) ; Buffalo (Lg) ; Crittenden, from larch (B\&S); Ithaca, May, J1 (Vdyk); Karner, Je-J1 (Felt); NYC (Lg) ; LI: Bellport, Je (Nic). Larva in white pine and American larch (Fisher).

9464 C. floricola Gory. Common wherever pine grows, May-Aug (Nic). Black Br., Je (Lg) ; Buffalo (Z\&R); E. Aurora, Je (Dnd); Karner, Je-J1 (Lg); Ft. Montgomery, J1, Schott (Sf); LI: Yaphank, Wading R., May, J1, on pine (Ds); Central Pk., May (Woodr); Pennequid Barrens, Aug, Eng (Sf). Larva in pitch pine (Fisher).

9466 C. femorata Oliv. Flat-Headed Apple-Tree Borer. Generally distributed and often injurious. Big Moose, J1; Newport, J1 (NYS); Peru; Black Br.; Waddington; Cranberry L., Wanakena, J1-Aug (Drk); Syracuse (Blkm) ; Ilion, J1 (NYS) ; Rochester, Rochester Jct.; Buffalo; Batavia, J1 (Kngt); Ashville, J1 (Cr); Castile, June, Brad (Flet); Egleston's Glen; Ithaca; Freeville; Elmira; Windsor, Jie (Not) ; L. George; Albany; W. Hebron; New Baltimore; Pine I.; West Pt.; Dunderberg Mt.; Bronx Pk.; Bronxville; Riverdale; SI: 
Richmond; Watchogue; Clove Val.; LI: Astoria; Aqueduct; Rockaway Beach; Hempstead; Yaphank; Half Way Hollow Hills; various collectors, dates ranging from May to Sep. Larva attacks nearly all hardwood trees and has been recorded also from coniferous trees; probably more than one species is confused under this name (Fisher).

9467 C. lesueuri Cast. Cranberry L., Nov (Drk) ; Honeoye Falls, Je (Ds) ; West Pt., Je (Robn); Peekskill (Lg); Ft. Montgomery, May 30 (Lg) ; NYC (Woodr); SI: Richmond (L\&D) ; LI: Central Pk., Je (Not); Yaphank, J1 (Not). Nicolay regards this the same as femorata.

9468 C. scabripennis Cast. Common in the northern part of the State in pine logs (Nic). Keene Val., Aug (NYS); Mt. Whiteface, summit, J1, Shoe (Ds); Mt. Marcy, summit, Je-J1 (L\&D); Mt. McIntyre, Ji (Bish-NYS) ; Wilmington, Je, Wells, Big Moose, Pike, J1 (NYS) ; Vandalia, Je (Vdyk); Ithaca, May-Sep; LI: Jamaica, May, Pears (Nic). Larva in white pine and hemlock (Fisher); in spruce (Felt).

9479 C. harrisi Hentz. "NY" (Fitch, Lg). Larva in scrub pine and white pine (Fisher).

\section{ACTENODES Lacordaire}

9481 A. acornis Say. NYC ( Lg); SI: Richmond, Je (L\&D) ; LI: Rockaway Beach, Je (Shoe); Long Beach (AM); Yaphank, Je, Eng (Sf), J1 (Ds); Bellport, Wading R., Je (Nic); Orient Pt., J1 (Ds). Beetles often found on beach in wash-up (Nic). Larva in red maple, beech, black birch, hickory, and black oak (Fisher).

\section{EUPRISTOCERUS Deyrolle}

9485 E. cogitans Web. Ithaca (Chitt); W. Albany, Karner, Je (NYS) ; West Pt., Je (Ds) ; Van Cort. Pk., Je (Nic, Sf); NYC (Lg); SI (Nic), forms galls on alder stems (Knull, L\&D); LI: Aqueduct, J1 (Shoe). Larva makes galls on alder, both Almus rugosa and A. incana.

\section{AgRILUS Stephens}

9491 A. bilineatus Web. Two-Lined Chestnut Borer. Common everywhere (Nic). Syracuse, J1 (Blkm) ; Rochester (M\&W) ; Rochester Jct., Je (CU) ; Buffalo (Z\&R) ; Protection, E. Aurora (Dnd) ; L. Keuka, May (CU) ; Ithaca, May-J1, Taughannock Falls, J1 (CU) ; Albany, Je, Nassau (NYS) ; West Pt., Je, Nyack, Je (Ds); Mosholu (AM); Bronxville, Je (Woodr) ; SI, Je-J1, on oak (L\&D); LI: Old Westbury, Garden City (Felt); Flatbush, Je (AM); Aqueduct, Brooklyn, Je-J1 (Shoe); Rockaway Beach, May, Eng (Sf); Yaphank, May-Je (Ds, Sf) ; Half Way Hollow Hills, Deep Pond, Wading R., Je-Ji (Ds). Larva very injurious to oak, chestnut, and beech (Fisher).

9498 A. acutipennis Mann. Windsor, Je (Not) ; LI: Huntington, May, Schott, a variety (Frost) ; Central Pk., May (Shoe); Flatbush, J1 (AM) ; Yaphank, May (Lg), Je, Eng (Sf); Bellport, Je (Nic).

9503 A. horni Kerr. "NY" (Horn).

9504 A. anxius Gory. Bronze Birch Borer. Keene Val., Aug (NYS) ; Mt. Whiteface, Wilmington, J1 (Ds) ; Axton, Je (M\&H) ; Elk L., $2000 \mathrm{ft}$., Aug (Lint) ; Jay, J1 (Britton); Wells, J1 (NYS) ; New Russia, Aug (CU) ; Cranberry L., Je-J1 (Mund) ; Broadalbin, Je (NYS) ; Syracuse $($ Blkm) ; Rochester (Felt); Buffalo (Lg-US); E. Aurora (Dnd) ; 
Geneva (Felt) ; Elka Pk., Aug (Drk); Elmira (Felt) ; Ithaca, May-Je (CU) ; Karner (Felt); Hudson Falls, Je (NYS); Troy (Felt); E. Jewett, Aug (Ds); Feekskill (Lg); Ft. Montgomery, Je, Schott (Frost); NYC (Lg); SI: Richmond, Je (L\&D); LI: Bellport, Je 30 (Nic) ; Bayshore, J1, Ols (Frost), as torpidus Lec. Larva in white birch, aspen, willow, and various species of birch and poplar, often very injurious to white birch (Fisher).

9508 A. lacustris Lec. Schoharie (Not).

9512 A. pensus Horn. Niagara Falls, J1, $M C V$ (Frost).

9513 A. ruficollis Fab. RED-NECKed CANE-Borer. Generally distributed and common. Cranberry L., Je (Mund) ; Speculator, Aug (NYS) ; Newport, Je, Wells, J1, Gloversville, May (NYS); White L. (AM) ; Luzerne, May (Cr); Honeoye Falls, Je (Ds) ; Syracuse, Je (Blkm); Rochester (M\&W) ; Rochester Jct., Je (Vdyk); Olcott, J1 (Dt) ; Buffalo (Z\&R) ; Erie Co, Je-Aug (Dnd) ; Batavia, Je-Jl (Kngt); Rock City, J1 (CU) ; Castile, Brad (Flet); Painted Post, May (Cr) ; Ithaca, Je-Aug, Taughannock Falls, Aug (CU) ; Schoharie, J1 (Lint); Albany, Delmar, Karner, Je (NYS); Nassau, Je (NYS); Athens (Lint) ; Poughkeepsie, May-Je (NYS); West Pt., Je (Ds); White Plains, May (Frost, Sq) ; Mosholu, Van Cort. Pk. (AM) ; NYC (Lg) ; SI: Richmond, Watchogue, Je (L\&D) ; LI: Brooklyn, Je (Shoe) ; Flatbush, Je, Astoria (AM); Jamaica, Je (NYS) ; Pinelawn, Aug, Wyandanch, J1, Massapequa, J1, Half Way Hollow Hills, J1 (Ds); Farmingdale, Je (CU) ; Yaphank, Je, Eng, J1 (Ds). Larva injurious to blackberry, raspberry, and dewberry (Fisher).

9514 A. lateralis Say. Rochester (M\&W); Buffalo, Je ( Lg); NYC (Lg); LI: Massapequa, J1 (Shoe); Half Way Hollow Hills, J1 (Ds); Yaphank, Je, Eng (Ds, Sf) ; Bayshore, J1, Ols (Frost); Bellport, J1 (Nic). Larva in bayberry and sweet fern (Fisher).

9515 A. masculinus Horn. Cranberry L., Je (Mund) ; Rochester (M\&W) ; Buffalo, Horn (Lg); Rock City, Je (Ds); Ithaca, Je (Ds) ; Schoharie (Not); Pine I., May (Frost); SI: Richmond (L\&D). Larva in box elder and red maple (Fisher).

9516 A. pusillus Say. Rochester (M\&W) ; vic. NYC (AM).

9517 A. defectus Lec. Buffalo, Je ( Lg, Frost); NYC (Lg); LI: Pinelawn (Shoe); Massapequa, May (Shoe); Wading R., Je (Nic). Larva in white oak (Fisher).

9518 A. crinicornis Horn. Ft. Montgomery, Je 17, Schott (Frost).

9520 A. egenus Gory. Common throughout the State on oak in Je (Nic). Axton, Je (M\&H) ; Wanakena, Aug (Drk) ; Rochester (M\&W) ; Olcott, JeJ1 (Dt) ; Batavia, Je (Kngt) ; Ithaca, May (Chitt); Schoharie (Not) ; West Pt., Je (Ds), J1, Eng (Sf) ; Nyack, Mosholu, Je (AM) ; Bronxville, Je (Woodr); NYC (Lg); SI: Richmond, Je (L\&D); LI: Brooklyn, May-Je (Shoe); Maspeth, May, Ols (Frost); Wyandanch, Je, Yaphank, J1, Half Way Hollow Hills, J1, Deep Pond, Wading R., Je, Aug (Not, Ds). Larva in locust (Fisher); in Virginia creeper (Kirk); in hickory (Chitt).

9521 A. puncticeps Lec. Buffalo (Z\&R). Larva in butternut (Fisher). This is possibly an error according to Nicolay, but Fisher thinks the species should occur in the State.

9522 A. cephalicus Lec. Ft. Montgomery, J1 11, Schott (Frost). 
9523 A. arcuatus Say. Rochester (M\&W) ; Olcott, Je (Dt); Buffalo (Lg) ; Elka Pk., Aug (Drk); E. Aurora, Aug (Dnd) ; Ithaca, J1 (Vdyk) ; Schoharie (Not) ; West Pt., Je (Ds) ; Greenwood L., J1 (Nic) ; White Plains, J1 (Frost, Sq) ; Mosholu (AM); Bronxville, Je (Woodr) ; NYC (Lg); SI, Je (L\&D); LI: Central Pk.; Wyandanch; Brookhaven, Sep, Schott (Frost) ; Half Way Hollow Hills; Wading R., Je-J1 (Ds). Larva in beech, hickory, and black oak (Fisher).

9523c A. arcuatus var. coryli Horn. Van Cort. Pk., Je (Woodr); LI: Wyandanch, Aug 21, Ols (Nic). Larva forms galls on hazel, both Corylus americana and $C$. rostrata (Fisher).

9529 A. imbellis $\mathrm{Cr}$. A rare species (Nic). Van Cort. Pk., Je (Ds); SI: Richmond, Je (L\&D); LI: Queens, J1 (Frost); Flatbush, May-Je (AM) ; Central Pk., J1 (Ds) ; Farmingdale, Je-J1 (CU) ; Wyandanch, J1 (Ds) ; Yaphank, Je-J1 (Sf-AM).

9531 A. fallax Say. A rare species (Nic). Newport (NYS); Batavia, J1 (Vdyk); Buffalo (Z\&R); NYC (Lg); SI: Richmond, J1, Woodr (L\&D) ; LI: Pinelawn, Je (Lg); Half Way Hollow Hills, J1 (Ds). Larva in honey locust and hackberry (Fisher).

9536 A. subcinctus Gory. White Plains, May 30, Sq (Frost); SI: Richmond, Je (L\&D). Probably breeds in poison ivy, as adults are always taken on this plant (Fisher).

9537 A. putillus Say. Olcott, J1-Aug (Dt) ; Cortland Co, Je (Dnd) ; Hoosick, $\mathrm{J} 1$ 15, 1925, in sweeping (Frost).

9539 A. otiosus Say. Very common throughout the State, May-Je (Nic). Syracuse, Je-J1 (Blkm); Rochester (Z\&R); Rochester Jct., Je (CU); Olcott, Je-J1 (Dt); Batavia, Je (Ds); Buffalo (M\&W, Lg); E. Aurora, Je-Aug (Dnd); Ithaca, May-Aug (CU); Schoharie (Not); Albany, May-Je, Karner, Je, N. Chatham, Je, Nassau, Je, Clinton Hgts., Je, St. Ile, Je (NYS) ; Ft. Edward, Je (Vdyk) ; Tivoli, May (NYS); Ft. Montgomery, J1 (Ds) ; West Pt., Jl (Sf); Pine I., Je (Ds); Poughkeepsie, W. Nyack, Je (NYS) ; Nyack, Je (Ds); Ramapo, May (Lg); Bronxville, Je (Woodr); vic. NYC (AM); SI: Richmond, Watchogue, May-J1 (L\&D) ; LI: Cedarhurst, J1 (Woodr); Yaphank, Je-J1 (Ds) ; Wading R., May (Ds), Je (Nic) ; Peconic, May (NYS). Larva recorded from hickory, persimmon, dogwood, redbud, black walnut, butternut, and maple, but some of these host plants may apply to other species due to erroneous identification (Fisher).

A. frosti Knull. White Plains, Je (Frost, Sq).

9540 A. sinuatus Oliv. Sinuate Pear Borer. An introduced species. Confined to lower Hudson R. Val. as follows: Poughkeepsie; New Paltz; Marlboro; Newburgh; Middletown; Monsey; Port Chester; White Plains; Fishkill; Cornwall; Blauvelt; Yonkers; Ossining; Croton Falls; New Rochelle (Frost); Mt. Kisco; NYC. These records are from adults bred from pear, to which the insect is injurious, and also from quince, hawthorn, and Cotoneaster. Beetles appear in June (H. Glasgow).

9542 A. politus Say. Common, Je-J1 (Nic). Mt. Whiteface, 1000-4000 ft., Aug (CU) ; Chazy L., Je (NYS) ; Plattsburg, J1 (Shoe, Ds) ; Wilmington, J1 (Ds) ; Cranberry L., J1 (Drk); Newport, Je (NYS) ; Rochester (M\&W); Rochester Jct., Je (CU); Buffalo 'Z\&R); Allegany Pk., 
Aug (Dnd) ; Cortland Co, J1 (Dnd) ; Windsor, Je (Not) ; Schoharie (Not); Elka Pk., Jl (Blkm); E. Jewett, Aug (Ds); Callicoon (AM) ; West Pt., Je (Ds); Ft. Montgomery, Je, Schott (Frost); West Pt., Je (Ds); Bronxville, Je (Woodr); Van Cort. Pk., Je (Woodr). Larva in striped maple and willow (Fisher); Felt records the species as making galls on twigs of white thorn (Crataegus sp.).

9547 A. obsoletoguttatus Gory. Occurs everywhere (Nic); Peru, Je (CU) ; Hague, J1, Leon (NYS) ; Honeoye Falls, Je (Ds) ; Rochester Jct., Je (CU) ; Buffalo, Je (Z\&R, Lg) ; Olcott, Je-J1 (Dt) ; E. Aurora, Je (Dnd); Ithaca (Chitt); Windsor, Je (Not); Nassau, Je (NYS) ; West Pt., Je, Ft. Montgomery, J1, Nyack, Je (Ds); NYC (Lg) ; SI: Richmond, Je (L\&D); LI: Pinelawn, Je, Half Way Hollow Hills, J1, Yaphank, May-J1 (Ds). Larva in beech, blue beech, ironwood, red oak, hickory, and birch (Fisher).

9548 A. vittaticollis Rand. Ausable Chasm, Je (Vdyk); Portage, Je (Kngt); Ithaca, J1, Dt (Vdyk); Windsor, Je (Not); West Pt., Je (Ds, Robn). Larva in shadbush (Fisher); in apple, pear, wild thorn, and wild crab apple (Brooks).

9551 A. granulatus Say. SI, Je (L\&D). Larva in Lombardy poplar and cottonwood (Fisher).

9553 A. lecontei Saunders. LI (Horn). Larva in hackberry (Fisher).

\section{RHAEBoscelis Chevrolat}

9561 R. tenuis Lec. SI (Sf).

\section{PACHYSCHELUS Solier 45}

9564 P. purpureus Say. Rochester (M\&W) ; E. Aurora, J1 (Dnd); Windsor, Je (Not); Poughkeepsie, May (NYS); Ramapo, May (Ds); Mosholu (Sf) ; NYC (Soltau-US) ; SI: Richmond, May-J1 (L\&D) ; LI: Flatbush, Sep (AM); Newtown, Aug, Doll.(Sf). Larva mines in leaves of bush clover (Lespedeza sp.).

9567 P. laevigatus Say (ovatus Say). Rochester (M\&W); Ithaca, Aug, Dt (Vdyk); Albany, J1 (NYS); Garrisons, Je (Woodr); Mosholu, Je (Lg) ; Bronxville, Je (Woodr)'; Van Cort. Pk., J1 (AM)'; NYC (Lg); SI: Richmond, Je-Aug (Ds); LI: Brooklyn, Aug (Shoe); Flatbush. J1 (AM); Yaphank, Je-Aug (Ds, Sf). Larva mines leaves of Desmodium canadense, D. pendula, Meibomia canadensis, Lespedeza capitata, and L. bicolor (Fisher).

\section{BRACHYS Solier ${ }^{46}$}

9572 B. aerosus Melsh. Common everywhere on oak (Nic). Mt. Whiteface, summit, J1 (Ds); Clayville, Je, Leon (NYS); Rochester (M\&W); Rochester Jct., Je (Vdyk); Olcott, Je-J1 (Dt); Buffalo (Lg) ; E. Aurora, Je-J1 (Dnd); Portageville, Je (Vdyk); Castile, May, Brad

\footnotetext{
45 According to Fisher, the adults of all species of this genus are found feeding on the foliage of a great variety of plants.

46 According to Fisher, the adults of this genus are found feeding on the foliage of a great variety of plants.
} 
(Flet) ; Westfield, May (Not) ; Rock City, J1 (Vdyk) ; Ithaca, JeAug (CU); McLean Bogs, May (CU) ; Dryden. L., Je, Slaterville to Caroline, Je (CU); Montour Falls, Je $(\mathrm{H})$; Windsor, Je (Not) ; Schoharie (Not); Nassau, May-Je, Albany, Je, Poughkeepsie, May (NYS) ; West Pt., Je (Ds, Robn) ; Staatsburg, Je (Woodr) ; Stony Pt., Je (AM) ; Ramapo, Je (Ds) ; Bronxville, May-Je (Woodr) ; Ft. Montgomery, May (Ds); Pine I., Je (Ds); Wappingers Falls, Je, Leon (NYS) ; West Lawn, J1, Zab (AM) ; SI: Richmond, Je-Aug (L\&D) ; LI: Brooklyn, Je (Shoe) ; Massapequa, J1 (Ds) ; Farmingdale (CU) ; E. Hampton, Je (CU) ; Central Pk., May (Shoe) ; Pinelawn, Je, Half Way Hollow Hills, J1, Nissequogue, Je, Smithtown, Je, Wading R., May, Yaphank, J1 (Ds).

9572 B. aerosus var. rufescens N. \& W. Olcott, Je (Dt) ; Buffalo (Nic, Weiss) ; Catskills (Nic); LI: Jamaica (Fisher). Rarer than the typical species (Nic).

9573 B. aeruginosus Gory. Rochester (M\&W); Allegany Pk., Aug (Dnd) ; Windsor, Je (Not); West Pt., Pine I., Je (Ds) ; SI: Richmond, Je' (L\&D); LI: Massepequa, May (Shoe); Central Pk., May (Shoe, Woodr, Sf); Pinelawn, Smithtown, Je (Ds); Yaphank, Je-J1 (Ds, $\mathrm{Sf})$; E. Hampton, Je (CU) ; Bellport, Je-J1 (Nic).

9574 B. ovatus Web. Common on oak everywhere (Nic). Rochester (M\&W) ; Olcott, J1 (Dt) ; Buffalo (Z\&R) ; Batavia, Je-J1 (Kngt) ; Ithaca, Je-Ji (CU) ; Karner, May (NYS); Pine I., Je (Ds); Bronxville, Je (Woodr); Bronx Pk., May (AM); SI: Richmond, May-Je (L\&D); LI: Flatbush, May-Je (AM) ; Rockaway Beach, Je, Eng (Sf) ; Massapequa, Central Pk., May-Je (Ds); Farmingdale, Je (CU) ; Pinelawn, Wyandanch, Central Pk., Bayshore, Smithtown, Selden, Wading R., Yaphank, May-Aug (Ds) ; Babylon (AM) ; E. Hampton, Je, Riverhead, J1 (CU). As B. tessellatus Fab. are the following: SI: Richmond (L\&D); LI: Farmingdale, Je (Vdyk); Pinelawn, Central Pk., May-Je (Shoe); Riverhead, J1 (Vdyk). Larva mines leaves of oak (Fisher).

9574 B. ovatus var. bellporti N. \& W. Windsor, Je (Not) ; LI: Pinelawn, Je (Lg); Yaphank, Wyandanch, J1 (Sf); Wading R., Bellport, Je, type locality (Nic). Not nearly so common as the typical species (Nic).

\section{TAPHROCERUS Solier}

9578 T. gracilis Say. Common throughout the State (Nic). Newport (NYS); Gloversville, Je (NYS) ; Rochester (M\&W) ; Buffalo (Z\&R) ; Ithaca, May-Oct (CU) ; Windsor, Je (Not); Poughkeepsie, Je (NYS); White Plains, Je (Frost, Sq) ; Suffern, May, Love (Sf-AM) ; Ramapo Mts. (AM) ; Bronxville, May (Woodr) ; SI: Richmond, J1 (L\&D) ; LI: Rockaway Beach, May (Shoe); Yaphank, Sep (Ds). Larval host plant unknown, but adult habits indicate coarse grasses (Fisher).

\section{Mastogenius Solier}

9584 M. subcyaneus Lec. Local and rare (Nic). Rochester (M\&W) ; White Plains, J1 (Sq); Bronxville, J1 (Woodr); Bronx Pk. (Sf); NYC (Lg); SI: Richmond (L\&D): LI: Aqueduct, J1 (Shoe); Jamaica (Frost). Larva in oak (Fisher). 


\section{FAMILY PSEPHENIDAE}

\section{PSE'PHENUS Haldeman}

9586 P. herricki DeKay (lecontei Lec.). Probably generally distributed throughout the State. Pike (NYS); Trenton Falls (Horn); Batavia, J1 (Kngt); Buffalo (Lec); Niagara Falls (Horn); Conesus L., J1 (Kngt) ; Oneida Co, Jl, Clms (Kngt) ; Oswego, Je-Sep (West); Ithaca and vic.; Schoharie (Not); Windsor, Je (Not); SI, J1 (L\&D).

\section{FAMILY DRYOPIDAE}

\section{(Parnidae)}

\section{HELICHUS Erichson}

9603 H. lithophilus Germ. Mooers, Sep (Not); Rochester (M\&W); Oneida Co, Aug (Dt,Clms); Buffalo (Z\&R); Ithaca, Oct (Kngt), Nov (West-CU) ; Spencer L., Oct, Lloyd (Kngt); McLean, Oct (Kngt); Schoharie (Not); West Pt. (Robn); Greenwood L. (AM) ; vic. NYC (Ds).

9604 H. fastigiatus Say. Mooers, Sep (Not) ; Rochester (M\&W) ; Oneida Co, Aug (Dt, Clms); Buffalo (Z\&R); Ithaca, J1 (Kngt); Ludlow (Thompson); West Pt. (Robn); Troy (Lec) ; SI (L\&D) ; LI: Babylon (AM).

9605 H. striatus Lec. Mooers, Sep (Not); Oneida Co, May-Sep (Dt, Clms) ; Batavia, Jl (Kngt); Buffalo (Z\&R); Ithaca, Sep, Kngt (Dt) ; Freeville, May (Kngt).

\section{FAMILY HELMIDAE}

\section{Stenelmis Dufour}

9607 S. linearis Zimm. Mooers, Sep (Not); Schoharie (Not).

9608 S. sinuata Lec. Schoharie (Not).

9609 S. crenata Say. Mooers, Sep (Not); Saranac Inn, Aug (NYS); Oneida Co, May-Sep (Dt, Clms) ; Rochester (M\&W); Olcott (Dt); Buffalo $(Z \& R)$; Ithaca, Je, Sep (Kngt, Dt) ; Freeville, May (Kngt) ; Schoharie (Not) ; Albany, Je (NYS); SI, May, J1 (L\&D).

9610 S. bicarinata Lec. Mooers, Sep (Not); Ithaca, J1 (West-CU, Kngt, Dt) ; McLean (Dt).

9611 S. quadrimaculata Horn. Buffalo (Z\&R) ; Ithaca, J1 (West), Sep (Kngt) ; Schoharie (Not).

9612 S. vittipennis Zimm. Batavia, J1 (Kngt); Oneida Co, Aug (Dt, Clms); Buffalo (Z\&R) ; Ithaca, Ap, Jl, Oct (Kngt); SI, Oct, Ds (Kngt).

S. n. sp. Esopus, Aug, Ds? (Kngt).

S. n. sp. Batavia, Sep, 1915 (Kngt).

\section{HELMIS Latreille}

9615 H. vittata Melsh. Mooers, Sep (Not) ; Batavia, Spencer L., Ithaca, Esopus, Sep-Oct (Kngt).

9617 H. bivittata Lec. Mooers, Sep (Not) ; Oneida Co, Aug-Sep (Dt, Clms); Rochester (M\&W); Buffalo (Z\&R); Spencer L., Oct (Kngt); Ithaca, Sep-Oct (Kngt); Washington Co, Aug (West). 
9618 H. quadrinotata Say. Mooers, Sep (Not) ; Oneida Co, Jl-Sep (Dt, Clms) ; Rochester. (M\&W); Buffalo (Z\&R) ; Ithaca, May, Sep (Kngt, Dt);

Michigan Hollow, Je, Lloyd (Kngt); Washington Co, Aug (West); NYC (AM); SI, Aug, Oct (L\&D).

9622 H. pusilla Lec. In rapids of Niagara R., Je (Lec); Batavia, Sep (Kngt); SI, J1 (L\&D).

\section{LIMNIUS Erichson}

9633 L. elegans Lec. Ithaca, Sep (Kngt).

9634 L. fastidius Lec. Johnstown, Aug (NYS).

9635 L. ovalis Lec. Johnstown, Mar (NYS) ; Oneida Co, May-Sep (Dt, Clms); Ithaca, Je-Sep (Kngt, Dt) ; Freeville, Je (Dt-NYS), Je, Lloyd, May (Kngt).

\section{HeTERELMis Sharp}

9637 H. nitidulus Lec. "NY" $(\mathrm{Lg})$.

9638 H. latiusculus Lec. Schoharie (Not).

\section{MACRONYCHUS Müller}

9640 M. glabratus Say. Mooers, Sep (Not); Saranac Inn, Aug, Ndm (NYS); Oneida Co, Je-Aug (Dt, Clms); Rochester (M\&W); Olcott (Dt); Buffalo (Z\&R); Castile, Brad (Flet); Ithaca, Aug (West), Oct (Kngt); McLean, May-Aug (West); Peekskill (Yg); SI, May (L\&D) ; LI: Yaphank, Sep (Ds).

\section{ANCYRONYX Erichson}

9642 A. variegatus Germ. Ithaca, Oct (Kngt); SI, Sep 30, Shoe (L\&D) ; LI: Babylon (Csy).

\section{FAMILY HETEROCERIDAE}

\section{HETEROCERUS Fabricius}

9645 H. ventralis Melsh. Westfield, May, Windsor, Je (Not).

9646 H. undatus Melsh. Ithaca, J1 24, Yuasa (Vdyk); West Pt. (Robn) ; SI, May-Je, on salt meadow Ap 1 (L\&D) ; LI: Long Beach, J1 17 (Frost); Rockaway (AM).

9646a H. undatus var. cuniculus Kies. Buffalo (Z\&R).

9646c H. undatus var. mollinus Kies. Buffalo (Z\&R); LI: Coney I., eggs (Sanborn).

9648 H. brunneus Melsh. Newport (NYS) ; Syracuse, May (H) ; Ithaca, May, Bish (NYS).

9649 H. schwarzi Horn. Westfield, May (Not).

9650 H. collaris Kies. Syracuse, May (H); Westfield, May (Not).

9651 H. tristis Mann. Schoharie (Not); Westfield, May (Not); Hudson Falls, Aug (Sherm-CU) ; vic. NYC (AM); LI: Yaphank, Sep (Ds).

9653 H. auromicans Kies. SI (Horn); LI: Rockaway (AM). 


\section{FAMILY GEORYSSIDAE}

\section{GeoRyssus Latreille}

9654 G. pusillus Lec. Schoharie, three specimens (Not).

\section{FAMILY DASCILLIDAE}

\section{SUBFAMILY DASCILLINAE}

EURYPOGON Motschulsky

9659 E. niger Melsh. Rochester (M\&W) ; Buffalo (Z\&R); Peekskill (FrostCU); West Pt. (Robn); Ft. Montgomery, May (Ds).

\section{AnchytaRsus Guérin-Ménéville}

9663 A. bicolor Melsh. Buffalo (Z\&R); LI: Yaphank, J1 (Sf).

\section{SUBFAMILY EUBRIINAE}

DicRanopselaphus Guérin-Ménéville

9673 D. variegatus Horn. Van Cort. Pk., Je (Sf).

ECTOPRIA LeConte

9674 E. nervosa Melsh. Newport ( $\mathrm{Yg}$ ); Mud Cr., Dryden L., Je (NYS)'; Schoharie (Not); Ramapo (Sf); Batavia, Je (Ds).

9674a E. nervosa var. tarsalis Lec. Buffalo ( $Z \& R$ ).

9674b E. nervosa var. thoracica Zieg. Rochester (M\&W).

\section{SUBFAMILY EUCINETINAE}

\section{EUCINETUS Germar}

9678 E. oviformis Lec. Ithaca, May (Wolc,Dt).

9682 E. terminalis Lec. Buffalo (Z\&R); Ithaca, May-Je (Wolc,Dt); SI, Sep 9 (L\&D); LI: Jamaica, May, Pears (Sf).

\section{FAMILy HELOdidAE}

\section{(Cyphonidae)}

\section{SUBFAMILY HELODINAE}

\section{HELODES Latreille}

9,687 H. pulchella Guér. Buffalo (Z\&R); West Pt. (Robn); SI (L\&D); LI: Yaphank (Eng,Sf).

9688 H. fuscipennis Guér. (thoracicus Melsh.). Rochester (M\&W); Buffalo (Z\&R); West Pt. (Robn) ; SI (L\&D).

9689 H. thoracica Guér. Olcott, J1 (Wolc, Dt); Geneva, Je (Cls). 


\section{MICROCARA Thomson}

9690 M. explanata Lec. Rochester (M\&W); Rochester Jct., Je, Leon (Flet-CU); Buffalo, Je 27, $M C V$ (Frost).

\section{Cyphon Paykull}

9691 C. robustus Lec. LI: Bellport, Je, Nic (Sf).

9692 C. ruficollis Say. Newport, May-Je (Yg); Rochester (M\&W) ; Rochester Jct., Je, Leon (CU); Mud Cr., Je (NYS); Pike, Je (Yg) ; Olcott, Je (Wolc,Dt) ; Buffalo (Z\&R) ; Schloer Cr., Oneida Co, Je (Dt) ; Westfield, May (Not); Ithaca, May-Je (Wolc,Dt); Montour Falls, Je (H) ; Nassau, May 25-26 (NYS); Catskills, Pears (Sf); West Pt. (Robn); LI: Yaphank, Je, Eng (Sf).

9693 C. brevicollis Lec. Rochester (M\&W).

9696 C. obscurus Guér. Elizabethtown, Je 8 ( $\mathrm{Yg}$ ) ; Axton, Je (M\&H) ; Black Br., Je (CU) ; Cranberry L., Je (H) ; Loon L., Je (CU); Wells, J1 12 (Yg); Newport, May-Je (Yg) ; Castile, Brad (Flet); Pike, Je; Rochester (M\&W) ; Olcott, Je (Wolc,Dt) ; Buffalo (Z\&R) ; E. Aurora, May (Flet,Dnd) ; Ithaca, May-J1 (Wolc,Dt); Montour Falls, Je (H); Mix Cr. Val., Cattaraugus Co, Je (CU); McLean Bogs, May (CU); Woodwardia Bog, Tompkins Co (CU) ; Slaterville, Je (CU) ; Dryden L., Je (NYS); Schoharie (Not); Nassau, May 25-26 (NYS); Dug Mt., Aug 8 (Yg) ; Karner, Je (NYS) ; SI, Je (L\&D) ; LI: Riverhead, Ap 11 (NYS).

9697 C. collaris Guér. Chazy L., Je 28 (NYS) ; Cranberry L., Je (H) ; Wanakena, Aug (Drk) ; Cold Br., Oneida Co, Je (Dt) ; Rochester (M\&W) ; Buffalo (Z\&R); Newport, May-Je (Yg); Corinth, Je 22 (Yg); Ft. Edward, Je (CU) ; Karner, Je 6 (NYS); Poughkeepsie, May 18, 28 (Yg) ; West Pt. (Robn); W. Nyack, Je 14 (NYS); SI, May-Je (L\&D) ; LI: Farmingdale, Je, Morrow (CU).

9698 C. variabilis Thunb. Cranberry L., Je, J1 (Dury, Drk, H) ; Oneida L., Aug (Drk); Rochester (M\&W) ; Syracuse, flying, Ap-May (H) ; Olcott, Ap-Sep (Wolc, Dt); Buffalo (Z\&R); Castile, Brad (Flet); Hilton (CU) ; Geneva, May (CU) ; Newport, May-Je (Yg) ; Pike, Je 1-Sep 28 (N YS) ; E. Aurora, May, J1 (Flet,Dnd) ; Clinton Hgts., Oct 8 (NYS); McLean, Ap (Kngt) ; Karner, Mar, Sep 18 (Bish); N. Chatham, Je 6 (NYS); Ithaca and vic., May-Aug (Wolc,Dt,CU); McLean, May (CU) ; Schoharie (Not); West Pt. (Robn); SI, May-Nov (L\&D) ; LI: Wyandanch (Sf); Rockaway Beach, May, Eng (Sf); Montauk, Nov (NYS).

9699 C. padi L. Cranberry L., Je (H) ; Lake Bluff, Sep 9, Bish (Hoffman); Rochester (M\&W); Ithaca, May-Je (Wolc,Dt); Slaterville, Je (NYS) ; Peekskill (CU) ; "LI", May (Sf).

C. n. sp. McLean, J1 (Sf-CU,BM) ; LI: Cold Sp. Harb., Brb (Sf).

9702 C. coarctatus Payk. Ithaca, Je (Wolc,Dt).

\section{PRIONOCYPHON Redtenbacher}

9703 P. discoideus Say. Peru, Je (Good-CU); Rochester (M\&W) ; Newport $(\mathrm{Yg})$; Buffalo (Z\&R); E. Aurora, J1 (Flet,Dnd); Ithaca, Aug (Vdyk,Wolc,Dt); Karner, Je 4 (Yg); Mosholu, Je (Sf). 
9704 P. limbatus Lec. Cranberry L., J1 (H) ; Mud Cr., Je (NYS) ; Ithaca, May-J1 (Wolc,Dt); Ringwood, Ap 16-Je 25 (Good); Catskills, Je Pears (Sf); SI, Je, Sep (L\&D).

\section{SCIRTES Illiger}

9708 S. tibialis Guér. Ogdensburg, J1 (NYS); Rochester (M\&W) ; Linden, J1 (CU) ; Pike (NYS); Ithaca, Je-J1 (CU) ; Albany, Je (NYS); Warwick, J1 (NYS); Kinderhook, J1 (NYS); Ilion, May.(Yg); SI, J1 9 (L\&D).

9709 S. orbiculatus Fab. Rochester (M\&W) ; Wadsworth L., Je 13 (NYS); Sacandaga R., Je 27 (NYS); Catskills, Pears (Sf); Mosholu (Sf); vic. NYC (AM); SI, Je (L\&D).

\section{SUBFAMILY PTILODACTYLINAE}

\section{PTILODACTYLA Illiger}

9716 P. serricollis Say. Newport, Je 1 ( $Y g$ ); Rochester (M\&W) ; Buffalo (Z\&R); Ithaca, Je (Wolc,Dt); SI, Je (L\&D); LI: Brooklyn, Aug (Eng); Yaphank (Sf).

\section{FAMILY DERMESTIDAE SUBFAMILY BYTURINAE}

\section{BYTURUS Latreille}

9718 B. unicolor Say. American Raspberry Beetle or Fruit-worm. Axton, Je $(\mathrm{M} \& \mathrm{H})$; Wilmington, Wells, J1 (NYS) ; Cranberry L., Je-J1, on foliage of Rubus (H); Wanakena, Aug (Dnd); Gloversville, Mar (NYS) ; Syracuse, May (H); Wayne Co, J1, locally abundant ( $\mathrm{Cy}$ ); Buffalo (Z\&R) ; E. Aurora, May (Flet,Dnd); Westfield, May (Not); Chautauqua $\mathrm{Co}$, injurious (Cy); Rock City (Fbs-CU); Ithaca (Fbs-CU) ; Albany, Je, Nassau, May (NYS); Ulster Co, especially at Milton and Marlboro, also Kingston, injurious to raspberries; Debruce, J1, Eng (Sf); Rhinebeck, Cy (Fbs-CU) ; West Pt. (Robn); Newburgh, injurious; Mosholu (Sf) ; SI, May-JI (L\&D) ; LI: Orient Pt., Je (NYS).

\section{SUBFAMILY THYLODRIINAE}

THYLODRIAS Motschulsky

9723 T. contractus Mots. The well-known Ignotus aenigmaticus Slosson. NYC (Ds); SI (L\&D) ; LI: Brooklyn (BM).

\section{SUBFAMILY DERMESTINAE}

\section{DERMESTES Linnaeus}

9725 D. caninus Germ. Gloversville, May (NYS) ; Buffalo, Je (Dnd) ; Karner, Je (NYS); Nyack (AM); Ithaca (Fbs-CU); West Pt. (Robn); Peekskill, in vacated bird's nest (Sherm); vic. NYC (AM); SI, May-Je, on dead animals (L\&D); LI: Bergen Beach, Dec 1, in rotten $\log$ (Dow); Orient Pt., J1, Lath (NYS); Wading R., Amagansett, Greenport, Center I., May-Aug (Sf). 
9725c D. caninus var. nubipennis Csy. Buffalo (Z\&R), as mubilus; E. Jewett (Ds) ; Ft. Montgomery (Ds) ; LI: Wading R., Central Pk., Orient (Ds).

9730 D. talpinus Mann. Chafee, May, on dead hawk (Dnd).

9731 D. carnivorus Fab. "NY" (Lec), as mucoreus.

9732 D. vulpinus Fab. Buffalo (Z\&R, Lec); Pike, May (NYS); Gowanda, May (Dnd); Ithaca (Fbs-CU); Albany, Jan-May (NYS); West Pt. (Robn); SI, Aug (L\&D) ; LI: Rockaway (AM) ; Brooklyn (Sf).

9734 D. frischi Kug. Gloversville, May (NYS) ; SI, J1-Sep, often under dead fish, etc., on seashore (L\&D) ; LI: Sea Cliff (Fbs-CU); Center I., Rockaway Beach, Promised Land, Orient (Ds); Amagansett, Aug (Sf).

9735 D. nidum Arrow. Buffalo (Z\&R), as elongatus.

9737 D. lardarius L. LARDER BEeTLE. Throughout the State, injurious to stored meats, skins, etc., and a well-known pest in insect collections. Axton, Je $(M \& H)$; Little Falls; Erie Co (Dnd) ; Watertown (Lint-NYS); Geneva (Cls); Buffalo (Z\&R); Castile, Brad (Flet); Ithaca (Fbs-CU); Windsor, Je (Not) ; Utica; Cortland Co; Chenango Co; Delaware Co; Dutchess Co; SI, all seasons, mostly on stored provisions; LI, several localities (Sf).

9739 D. pulcher Lec. Buffalo (Z\&R); Ithaca (Fbs-CU); Poughkeepsie, J1 (NYS).

\section{AtTAgenus Latreille}

97,41 A. pellio L. "NY" ( $\mathrm{Lg}$ Cat.) ; Buffalo (Z\&R).

9742 A. piceus Oliv. Black Carpet Beetle. Elizabethtown, Je (NYS) ; Gloversville, Sep (NYS); Oswego Falls, Aug, Bks (NYS); Louisville (Lint); Buffalo (Z\&R); E. Aurora, May (Dnd); Ithaca (CU); Cincinnatus, J1 (Flet,Dnd) ; Albany, Ap (NYS); Schenectady (Lint); Coeymans (Lint); Poughkeepsie (Lint); West Pt. (Robn); NYC (Ds) ; Roseville (Ds) ; SI, in houses at all seasons, adults often common in spring (L\&D); LI: Brooklyn, Flushing, Eng (Sf) ; Flatbush, Zab (AM).

9745 A. deficiens Csy. Windsor, Je (Not).

9749 A. elongatulus Csy. West Pt. (Robn); vic. NYC (AM).

\section{TROGODERMA Latreille}

9770 T. ornata Say. Newport, Je (NYS); Honeoye Falls, Leon (Fbs-CU); Buffalo (Z\&R); Albany, Sep (NYS); vic. NYC (AM); West Pt. (Robn); SI, May-J1 (L\&D).

9772 T. procera Csy. LI (Csy).

9774 T. tarsalis Melsh. Albany, May (NYS); West Pt. (Robn); Nyack, Zab (AM) ; SI, Je, in stored nuts, J1 (L\&D); LI: Flatbush, Zab (AM).

9785 T. versicolor Creutz. Ithaca (Fbs-CU), as inclusa Lec.; Albany, Ap-May, Aug (NYS); West Pt. (Robn); vic. NYC (AM).

\section{CRYPTORHOphalum Guérin-Ménéville}

9816 C. ruficorne Lec. SI, May 31, on strawberry blossoms at Watchogue (L\&D).

9824 C. triste Lec. Peekskill, May (Sherm), on dandelion; West Pt. (Robn).

9825 C. picicorne Lec. SI, Sep 21 (L\&D); LI: Flatbush, Manor, Yaphank, May-Sep, Eng (Sf). 


\section{ANTHRENUS Fabricius}

9829 A. scrophulariae L. Carpet Beetle or Buffalo Moth. Denmark, May (NYS); Gloversville; Oswego, Je (NYS) ; Syracuse; Rochester; Buffalo; Castile, Brad (Flet) ; E. Aurora, May-Je (Dnd); Lakewood; Hornell; Montour Falls, May-Je (H); Spencer L. (Fbs-CU) ; Caroline to Harford (Fbs-CU) ; Ithaca; Schoharie; Utica; Albany; Coeymans; Nyack (AM) ; Poughkeepsie; Suffern (AM) ; West Pt. (Robn) ; SI, out of doors on flowers, often on Pyrus in May; LI: Flatbush (AM); Yaphank, Sep (Eng).

9829a A. scrophulariae var. flavipes Lec. "NY" (Lec).

9830 A. thoracicus Melsh. Buffalo (Z\&R).

9832 A. lepidus Lec. Buffalo ( $Z \& R$ ). Described from Calif., and possibly misdetermined or introduced.

9835 A. verbasci L. Cranberry L., Je-J1, on blossoms of Sambucus and yarrow; Syracuse, Montour Falls, in house and on flowers $(\mathrm{H})$; Buffalo $(Z \& R)$; E. Aurora, Je (Dnd); Ithaca, Mar-May (div.); Albany (Lint); West Pt. (Robn); Suffern (AM); SI, Mar-May (L\&D); LI: Flatbush (AM).

9837 A. museorum L. Schoharie (Not); White L., Je, Zab, L. George, Zab (AM) ; Albany (Felt); SI, J1 (L\&D); LI: Flatbush, J1 (Eng).

9838 A. castaneae Melsh. Speculator, Je, Aug, Wells, J1 (NYS); Cranberry L., Oct (Drk); Ithaca (CU); Schoharie (Not); Albany, Je (NYS); Poughkeepsie, Je (NYS).

9839 A. caseyi Dalla Torre (rotundulus Csy.). "NY" (Csy).

\section{ORPHILUS Erichson}

9843 O. subnitidus Lec. Buffalo (Z\&R). Described from Calif., also from Col., possibly misdetermination for ater.

9844 O. ater Er. Buffalo (Z\&R); Ithaca (Fbs-CU).

9846 O. niger Rossi. West Pt. (Robn); SI, May (L\&D). According to Casey, this and ater are identical.

\section{FAMILY BYRRHIDAE}

\section{SUBFAMILY NOSODENDRINAE}

\section{NOSODENDRON Latreille}

9847 N. unicolor Say. LI: Long Beach, Je, Schott (Sf); Flatbush, Ap, Eng (Sf).

\section{SUBFAMILY BYRRHINAE}

\section{Cytilus Erichson}

9864 C. alternatus Say. "Northern NY" (Lec, in Proc. Acad. Nat. Sci. Phila., vol. 7, p. 115, 1856, as varius of Europe); Axton, Je (M\&H); Newport, May (NYS) ; Potsdam (NYS) ; Saranac Inn, May (NYS) ; Rochester (M\&W) ; Buffalo (Z\&R); Pike (NYS); Castile, Ap, Brad (Flet); Batavia, Je (Kngt); E. Aurora, May-Je (Dnd); Westfield, May Windsor, Je (Not); Cinnamon L. (NYS); Rock City (Ds); Tarbel, Je (Ds) ; Ringwood, Jl, Leon (NYS); Ithaca Ap (Bish-NYS) ; Al- 
bany, Karner, Nassau, May-Je (NYS); Rensselaer, May (NYS); West Pt., Ap, Robn (Ds); SI, Je, on ground among grass roots (L\&D) ; LI: Long Beach, May, Eng (Sf).

\section{BYRRHUS Linnaeus}

9866 B. pettiti Horn. Cranberry L., J1 21, Blkm (H); Buffalo (Z\&R).

9867 B. cyclophorus Kby. Catskills, Pears (Sf); Buffalo (Z\&R).

9869 B. americanus Lec. Keene Val., J1 (NYS) ; Newport (NYS) ; Woodworth's L., J1 (NYS) ; Wanakena, Aug (Drk) ; Rochester (M\&W) ; "Niagara"

(Lec) ; E. Aurora, J1-Aug (Dnd) ; Aurora, Ap (Bish-NYS); Ithaca, J1 (Vdyk) ; Canaan, Je (NYS); Big Ind. Val., Je, Pcars (Sf); West Pt. (Robn); vic. NYC (AM).

9873 B. geminatus Lec. Buffalo (Lec).

9889 B. kirbyi Lec. Albany, Je (NYS) ; Buffalo (Z\&R).

\section{PORCINOLUS Mulsant}

9893 P. undatus Melsh. "NY" (J. Hamilton); Buffalo (Z\&R), as murimus.

\section{SYNCALYPTA Stephens}

9895 S. spinosa Rossi. Rochester, Moore (Sf); Batavia, Je (Lg); McLean, May (Flet); Genesee Co, Je, Ds (Sf); Windsor, Je (Not).

\section{SUBFAMILY LIMNICHINAE}

\section{Limnichites Casey}

9905 L. punctatus Lec. McLean, May, Je (Flet); Mooers, Sep (Not) ; Schoharie (Not).

\section{Eulimaichus Casey}

9913 E. obscurus Lec. NY (Lec).

\section{FAMILY RHYSODIDAE}

\section{RHYSODES Dalman}

9944 R. americanus Lap. Buffalo, as exaratus (Z\&R); McLean Bogs, Nov (Vdyk).

\section{Clinidium Kirby}

9946 C. sculptile Newn. North Branch (Schaupp).

\section{FAMILY OSTOMIDAE \\ (Trogositidae) \\ CoRTICotomus Sharp}

9954 C. parallelus Melsh. West Pt. (Robn); Mosholu (Sf); SI (L\&D); LI: Jamaica (Pears); Rockaway Beach, May (Eng).

9957 C. cylindricus Lec. Buffalo (Z\&R). 
AIRORA Reitter

9966 A. cylindrica Serv. Buffalo (Z\&R); E. Aurora (Dnd); Ithaca, Jan 2 (Dt-CU).

\section{TEMNOCHILA Westwood}

9976 T. virescens Fab. Karner (Felt); SI (L\&D) ; LI: Bellport, Wading R. (Nic); Rockaway Beach, Je, 'Yaphank, Sep (Ds).

\section{TENEBRoIDES Piller and Mitterpacher}

9977 T. mauritanicus L. The Cadelle. Throughout the State, infesting stored grains, cereals, etc.

9984 T. bimaculatus Melsh. Buffalo (Z\&R); Bronx Pk. (Sf); SI, Je (L\&D).

9989 T. collaris Sturm. SI (L\&D).

9990 T. americanus Kby. Syracuse, in dying hickory (B\&S) ; Buffalo (Zimm) ; Schuylerville, Oct (NYS); SI (L\&D).

9991 T. laticollis Horn. Buffalo (Zimm); West Pt. (Robn); LI, Ap (Eng); Half Way Hollow Hills, J1 (Ds).

9994 T. corticalis Melsh. Rochester (M\&W); Buffalo (Z\&R); Syracuse, in dying hickory (B\&S), and Ap, under bark (H); Peekskill (Sherm); SI, Ap-J1, Nov (L\&D) ; LI: Yaphank, J1 (Eng) ; Bellmore, E. Hampton, Sep (Eng); Half Way Hollow Hills, J1 (Ds); Orient, Lath (NYS).

\section{Calitys Thomson}

9998 C. scabra Thunb. Rochester (Moore); Buffalo (Z\&R).

\section{OsToma Laicharting}

10003 O. ferruginea L. Keene Val., Sep (Eng) ; Cranberry L., Je-J1, under bark of dead tree $(\mathrm{H})$.

10005 O. pippingskoeldi Mann. Cranberry L., J1 (H), but Schaeffer queries this determination since the species occurs in Alaska and Calif.

10007 O. quadrilineata Melsh. Buffalo (Z\&R) ; E. Aurora, Chafee, May (Dnd) ; Mosholu (Sf); Catskills (Pears).

\section{ThyMALUS Latreille}

10010 T. marginicollis Chev. Cranberry L., J1 7, under bark (H); Rochester (M\&W) ; Buffalo (Z\&R) ; Oakdale, Je (AM) ; Cortland Co, Je (Dnd) ; West Pt. (Robn) ; Bronx Pk. (Sf) ; SI, May-Je (L\&D) ; LI: Wyandanch (Sf, Eng). Often found on the fungus Polyporus betulinus on white birch.

\section{FAMILY NITIDULIDAE SUBFAMILY CATERETINAE \\ BRACHYPTEROLUS Grouvella}

B. pulicarius L. (Heterostomus). Cranberry L., Je, in sweeping (H); Syracuse, May, in numbers on dandelion flowers $(H)$; present in Niagara, Saratoga, and Schoharie Counties, and in parts of Albany 
Co, also injuring strawberries in southern Columbia Co (Felt, Ins. Pest Survey, Bul. 2, p. 89) ; Dutchess Co, generally distributed in strawberry beds (Hervey); feeding on apples in Greene Co, May, Mills (Flet). Possibly these records all refer to mordelloides (Leng).

B. mordelloides Not. Keene Val., Je (Not).

\section{CAteretes Herbst}

10012 C. pennatus Murr. Buffalo (Z\&R); Ithaca, Ap-May (CU) ; Normanskill, May, on willow blossoms (NYS); Ft. Montgomery, May, Schott (Sf).

\section{Cercometes Reitter}

10016 C. abdominalis Er. Wells, Newport, May (NYS) ; Rochester (M\&W); Buffalo (M\&W) ; Allegany Pk., J1 (Dnd); Corinth, May (NYS); Durlandville, Je (Dt) ; Karner, J1 (NYS); West Pt. (Robn) ; Mosholu (Sf); SI, May-Je, on elder blossoms (L\&D).

\section{BRACHYPTERUS Kugelann}

10017 B. urticae Fab. Newport, May (NYS); Rochester (M\&W); Buffalo (Z\&R); Ithaca and Ellis to Slaterville, May-Aug (CU); Schoharie (Not); Burnt Hills (NYS); West Pt. (Robn); SI (L\&D); LI: Cold Sp. Harb., J1 (Sf).

Amartus LeConte

10022 A. rufipes Lec. Buffalo (Z\&R); LI: Astoria (AM).

\section{SUBFAMILy MELIGETHINAE}

MELIGethes Stephens

10025 M. mutatus Harold. Westfield, May (Not); Catskills (Sf); West Pt. (Robn), as brassicae.

\section{SUBFAMILY CARPOPHILINAE}

\section{Colopterus Erichson}

10029 C. maculatus Er. Catskills, Pears (Sf); Bronx (Joutel).

10030 C. semitectus Say. Newport, Wells, May (NYS); Olcott, Je (Dt) ; Rock City, Je (CU) ; Poughkeepsie, Je (NYS); SI, Ap 24 (L\&D); LI: Woodside, May, Aug (Sf).

10031 C. unicolor Say. Buffalo (Z\&R).

10032 C. truncatus Rand. Big Moose, Aug (NYS) ; Cranberry L., Je-J1, on birch (H) ; Rochester (M\&W) ; Olcott, May-Je (Dt) ; Boston, Ap (Dnd) ; Rock City, Je (CU) ; Karner, Aug (NYS) ; West Pt. (Robn); SI, Mar, on cedar, Ap-May (NYS) ; LI: Central Pk., Eng (Sf). 


\section{CONOTELUS Erichson}

10035 C. obscurus Er. Wells, Pike, Jl (NYS); Rochester (M\&W) ; Buffalo (Z\&R) ; E. Aurora, J1 (Dnd) ; Portage, J1 (NYS) ; Ithaca, May 16, Good (H) ; Scotia, J1 (NYS); West Pt. (Robn); Mosholu, Bronx Pk. (Sf); SI (L\&D).

\section{Carpophilus Stephens}

10040 C. floralis Er. SI, Je 14, on flower of prickly pear (L\&D).

10042 C. hemipterus L. Rochester (M\&W); Olcott, Je-Jl (Dt); E. Aurora, Mar (Dnd) ; Albany, Sep (NYS); NYC, in Turkish figs (Ds); SI, from dried figs (L\&D); LI: Lawrence, J1 (NYS).

10043 C. dimidiatus Fab. Newport, May (NYS); Poughkeepsie, J1 (NYS).

10052 C. niger Say. Elizabethtown, Aug, Newport, Je (NYS) ; Cranberry L., Je-J1 (H) ; Rochester (M\&W) ; Olcott, Je (Dt) ; Buffalo (Z\&R) ; Rock City, Je (CU); Ithaca, Ap (NYS); Poughkeepsie (NYS); West Pt. (Robn); SI, May-JI (L\&D); LI: Cold Sp. Harb., Ap, Schott (Sf).

10056 C. marginatus Er. Poughkeepsie, Je (NYS) ; LI: Jamaica (Nic).

10057 C. corticinus Er. Olcott, May (Dt); Ringwood, May (Dt) ; SI, May, Aug (L\&D) ; LI: Jamaica, Pears (Sf); Bellmore, May, Eng (Sf).

10058 C. brachypterus Say. Chazy L., L. Clear, Je (NYS); Newport, Pike, May (NYS) ; Buffalo (Z\&R) ; Westfield, May (Not); Ithaca, May (CU); Albany (NYS); West Pt. (Robn); Mosholu (Sf); SI, Ap-May (L\&D); LI: Central Pk., Ap, Eng (Sf); Yaphank, May, Eng (Sf).

10060 C. antiquus Melsh. SI, Je (L\&D).

\section{SUBFAMILY NITIDULINAE}

\section{Nitidula Fabricius}

10065 N. bipunctata L. Axton, Je (M\&H) ; Newport, Denmark, Pike, May (NYS) ; Rochester (M\&W) ; Buffalo (Z\&R); Batavia, Je (Kngt); Nassau, May (NYS); West Pt. (Robn); SI, Je 23, in peaches not grown on SI (L\&D).

10067 N. rufipes L. Axton, Je (M\&H) ; Newport, May-Je (NYS) ; Cranberry L., Jl., on carrion (H) ; Rochester (M\&W) ; Olcott, Sep (Dt) ; Buffalo $(Z \& R)$; E. Aurora, Je (Dnd); Batavia, Je (Kngt); Montour Falls, Ap, carrion (H); Westfield, May (Not); Ithaca, Ap-May (CU); Karner, Poughkeepsie, Je (NYS); Peekskill (CU); West Pt. (Robn); SI, Ap-May, Sep (L\&D) ; LI: Riverhead, Ap (NYS) ; Orient, May (NYS).

10068 N. ziczac Say. Rochester (M\&W) ; Olcott, Je, Sep (Dt) ; LI: Amagansett, Aug, Eng (Sf).

N. carnaria Schall. "NY" (Sf) (not in Lg Cat.) ; West Pt. (Robn).

\section{Omosita Erichson}

10069 O. colon L. Axton, Je $(\mathrm{M} \& \mathrm{H})$; Cranberry L., Je-J1, in carrion and in decaying fungi $(H)$; Newport, Ilion, Oswego (NYS) ; Syracuse, ApMay (H); Rochester (M\&W); Buffalo (Z\&R); Erie Co (Dnd); 
Batavia, Je (Kngt); Pike (NYS) ; Westfield, May (Not) ; Ithaca, Ap-Sep (CU) ; McLean Bogs, Aug (Dt); Schoharie (Not); Clinton Hgts., Ap (NYS) ; Albany, Karner, Ap, Poughkeepsie, Je (NYS) ; West Pt. (Robn); Bangall, White Plains, Eng (Sf) ; NYC (NYS) ; SI, Mar-Sep, often on dead animals (L\&D); LI: Jamaica, Rockaway Beach, North Beach, Long Beach, Forest Pk., Yaphank, Greenport, Mar-Aug (Sf); Orient, Mar, on rotten cabbage (Ds).

\section{EPUREA Erichson}

10073 E. helvola Er. Gloversville, May (Alex-NYS); Rochester (M\&W) ; Olcott, J1 (Dt) ; Buffalo (Z\&R) ; Eden, Erie Co, Aug (Dnd) ; Ithaca (CU); Greenwood L., Je (Sf) ; SI, Aug-Sep (L\&D); LI: Rockaway Beach, May (Sf); Yaphank, Oct, Eng (Sf).

10074 E. rufa Say. Buffalo (Z\&R) ; West Pt. (Robn) ; SI, Mar, May, Dec, Ap 4 , in sifting leaves in garden (L\&D); LI: Jamaica, Rockaway Beach (Sf).

10077 E. erichsoni Reit. Cranberry L., Je, on maple blossoms (H) ; Olcott, Je (Dt) ; Rock City, Je (CU) ; Ithaca, May-J1 (Dt) ; West Pt., May, Robn (Sf) ; Little Pond, Orange Co, May, Bish (NYS) ; LI: Central $\mathrm{Pk}$, Ap (Eng).

10078 E. rufida Melsh. Buffalo (Z\&R); SI, Feb, Je (L\&D).

10080 E. corticina Er. Wells (NYS); Rochester (M\&W); Buffalo (Z\&R); West Pt. (Robn).

10083 E. avara Rand. Axton, Je (M\&H) ; Cranberry L., Je, on Betula lutea (H) ; Olcott, Je (Dt); Boston, Ap (Dnd).

10087 E. truncatella Mann. Cranberry L., Je-Jl, on maple and pine ( $\mathrm{H})$; Axton, Fourth L., Je, Albany, Ap (NYS); West Pt., Mar, Robn (S'f) ; LI: Queens, Ap (Sf).

10089 E. ovata Horn. Cranberry L., J1-Aug (H) ; Olcott, Je-J1, Dec-Jan (Dt) ; Mosholu, Sep (Sf).

10091 E. peltoides Horn. Olcott, Aug-Sep (Dt); Windsor, Je (Not); West Pt. (Robn).

10092 E. depressa I1l. Cayuga L., Je (CU); Ithaca, May (Dt).

10093 E. labilis Er. Axton, Je (M\&H) ; Cranberry L., Je-J1 (H) ; Rochester $(\mathrm{M} \& W)$; Olcott, Je-J1 (Dt); Westfield, May (Not); Windsor, Je (Not) ; SI, May (L\&D).

E. ornatula Not. NY, type locality.

\section{STELIDOTA Erichson}

10098 S. geminata Say. Denmark, Sep (NYS); Rochester (M\&W) ; Olcott, JeOct (Dt); Buffalo (Z\&R) ; Ithaca, Aug-Sep (Dt); Menands, Sep, Middleburg, May (NYS); West Pt. (Robn); Nyack, Je (Ds); SI, Aug, on decaying pears (L\&D) ; LI: North Beach, Rockaway Beach, Long Beach, Jamaica, May, Shoe, Schott, Pears (Sf).

10099 S. octomaculata Say. Johnstown (Alex-NYS); Wolcott, May, $C y$ (NYS); Ithaca, Sep (CU); Karner, Mar, Bish (Dt); West Pt. (Robn); SI, Je, trapped in molasses jar (L\&D). 


\section{PROMETOPIA Erichson}

10101 P. sexmaculata Say. Olcott, Je (Dt); Buffalo (Z\&R) ; Ithaca, Je (Dt) ; West Pt. (Robn) ; Nyack, Je, Ramapo, May (Ds); SI, Ap-Oct, under bark of dead beech (L\&D) ; LI: Jamaica, Woodside, Rockaway Beach, May-Je, Eng (Sf); Long Beach (AM).

\section{LOBIOPA Erichson}

10105 L. undulata Say. Rochester (M\&W) ; Olcott, Aug-Sep, Ithaca, J1 (Dt) ; E. Aurora, May (Dnd) ; Windsor, Je (Not); West Pt. (Robn); SI, Je-J1 (L\&D) ; LI: Flatbush, Jamaica, Aug (Sf) ; Greenport, Eng (Sf).

\section{SORONIA Erichson}

10106 S. guttulata Lec. Albany, Ap, $Y g$ (NYS).

Phenolia Erichson

10109 P. grossa Fab. Newport, Je (NYS) ; Cranberry L., J1 (H) ; Dexter, J1 (NYS) ; Olcott, Je, Aug (Dt) ; Buffalo (Z\&R); Ithaca, J1-Sep (CU) ; McLean, May' (CU) ; Rensselaer, Aug (NYS); West Pt. (Robn); White Plains, Mar, Eng (Sf) ; SI, Ap-Je (L\&D); LI: Central Pk., Ap, Eng (Sf).

\section{Amphotis Erichson}

10110 A. ulkei Lec. Ithaca, Ap (Dt); LI: Barton Hollow, in sifting, Aug (Flet-CU).

AMPHICROSSUS Erichson

10113 A. ciliatus Oliv. Ithaca, Je, Sep (CU); White Plains, Mar, Eng (Sf) ; SI, May (L\&D); LI: Brooklyn, May (Dt); Jamaica, Pears, Eng (Sf).

\section{CyChRAMUS Kugelann}

10115 C. adustus Er. Newport, $Y g$ (NYS) ; Ithaca, Je, Sep (CU) ; Windsor, Je (Not) ; West Pt., Robn (Sf) ; LI: Yaphank, Ap (Sf).

\section{POCADIUS Erichson}

10117 P. helvolus Er. West Pt. (Robn); Mosholu (Sf); SI (L\&D).

\section{Cyllodes Erichson}

10121 C. biplagiatus Lec. Buffalo (Z\&R) ; Ithaca, Je (Dt) ; Windsor, Je (Not); West Pt., Robn (Sf). Occurs on a fungus on poplar.

\section{OXYCNEMUS Erichson}

10122 O. histrina Lec. E. Aurora, Nov (Dnd); West Pt. (Robn); SI, Aug (L\&D) ; LI: Valley Stream, Yaphank, J1 (Sf) ; Melville, Oct, Schott, Riverhead, Aug, on Phallus impudicus (Ds).

10123 O. nigripennis Lec. LI: Yaphank, J1 (Sf). 


\section{PALLODES Erichson}

10125 P. silaceus Er. Mosholu, Bronx Pk. (AM); SI, Je-Nov, often in fungi (L\&D) ; LI: Cold Sp. Harb., Amagansett, Orient, Aug, Oct (Sf).

\section{SUBFAMILY CRYPTARCHINAE}

\section{CRYPTARCHA Shuckard}

10129 C. ampla Er. Newport, Je, Plattsburg, Sep (NYS) ; Syracuse, May (H) ; Rochester (M\&W) ; Olcott, Ap-Sep (Dt) ; Buffalo (Z\&R) ; E. Aurora, May (Dnd) ; Batavia, Je-Sep (Kngt); Westfield, J1 (NYS) ; Windsor, Je (Not); Ithaca, May-Aug (NYS); West Pt. (Robn); SI, Ap-J1, on sap (L\&D); LI: Brooklyn, May' (Dt); Jamaica, Flatbush, May (Sf) ; Yaphank, Eng (Sf).

10131 C. strigata Fab. Buffalo (Z\&R); Peekskill, Sep (Sherm); West Pt. (Robn) ; LI: Yaphank, May, Eng (Sf).

10132 C. concinna Melsh. E. Aurora, May (Dnd); Boston, Ap, Dnd (Dt); Karner, Aug (NYS) ; SI, May-Je (L\&D); LI: Jamaica, Pears, Yaphank, May, Eng (SE).

\section{GLISCHROCHILUS Reitter}

(Ips Fabricius)

10136 G. obtusus Say. SI, Aug 25, under bark of dying red maple (L\&D).

10137 G. fasciatus Oliv. Cranberry L., Je-Jl, at sap on birch stump (H) ; Axton, Je (M\&H) ; Big Moose, J1, Newport, Wells, May-J1 (NYS) ; Gloversville, Je (NYS) ; Syracuse, May-Je (H) ; Olcott, Ap-Oct (Dt) ; Buffalo (Z\&R) ; Erie Co, May-Sep (Dnd) ; Geneva, Avon, Ap, Je (Cls) ; Batavia, Je-Aug (Kngt); Castile, Sep, Brad (Flet); Binghamton, Ap (NYS) ; Ithaca, Ap-Sep (Dt) ; Interlaken, Ap (NYS) ; Montour. Falls, May-Je (H); Windsor, Je (Not); Schenectady, Dec (Dt); Albany, Menands, Poughkeepsie, J1 (NYS); West Pt. (Robn); SI, Ap-Aug (L\&D) ; LI: Flatbush, Lawrence (NYS); Rockaway Beach, Central Pk., Greenport, Ap-Aug, Eng (Sf).

10138 G. sanguinolentus Oliv. Newport, May, Saranac Inn, J1 (NYS) ; Cranberry L., Je-J1, at birch sap and from Pinus strobus $(\mathrm{H})$; Fourth L., Je (NYS); Olcott, Sep (Dt); Geneva, Ap (Cls); Buffalo (Z\&R) ; E. Aurora, May-Je, Allegany Pk., Aug (Dnd); Batavia, J1-Aug (Kngt) ; Schuyler, May (OS-NYS) ; Ithaca, May-J1 (Dt); Catskills, Pears (Sf); West Pt. (Robn); SI, Ap-Sep (L\&D) ; LI: Jamaica, Central Pk., Ap, Eng (Sf).

10139 G. confluentus Say. Buffalo (Z\&R) ; Boston, Ap (Dnd) ; West Pt., Mar, Robn (Ds) ; LI: Jamaica (Schott); Queens, Mar-Ap, Eng (Sf).

10140 G. vittatus Say. Newport, Ap (NYS).

\section{FAMILY RHIZOPHAGIDAE}

\section{RHIzophagus Herbst}

10144 R. bipunctatus Say. Cranberry L., J1 2 (H); West Pt. (Robn) ; SI (L\&D).

10147a R. parallelicollis Gyll. var. robustus Sf. LI: Woodside, Ap (Sf). 
10149 R. sculpturatus Mann. Big Moose, J1 (NYS) ; Cranberry L., May-J1 $(\mathrm{H})$; Rochester (M\&W) ; Buffalo (Z\&R); SI, Ap 24, Woodr (L\&D).

10150 R. dimidiatus. Mann. Newport (Yg-NYS); Cranberry L., Je-J1 (H, Dury).

10151 R. approximatus Lec. Buffalo (Lec).

10152 R. remotus Lec. Cranberry L., J1 (H), J1 (Dury.); West Pt., Ap, Robn

(Sf) ; LI: Queens, Hempstead, Bellmore, Ap-May (Sf).

10155 R. brunneus Horn. Fulton Chain, Je (NYS).

10157 R. cylindricus Lec. LI: Central Pk., Ap, Eng (Sf); Wyandanch (Sf).

\section{FAMILY MONOTOMIDAE}

\section{SUBFAMILY MONOTOMINAE}

\section{MONOTOMA Herbst}

10158 M. producta Lec. Vic. NYC (AM) ; SI, Je, along shore or under bark of old logs; LI: Flatbush, Ap (Eng); Rockaway Beach, Je (Eng).

10159 M. picipes Hbst. Wells, Je (NYS); Syracuse, May, on herbage (H) ; Rochester (M\&W); West Pt. (Robn); vic. NYC (AM); SI (L\&D).

10163 M. fulvipes Melsh. SI, May (L\&D).

10165 M. americana Aubé. Cranberry L., Je 28 (H), in decaying fungi.

10167 M. parallela Lec. NY (Lec).

10171 M. longicollis Gyll. Cranberry L., Je 25 (H), in decaying fungi.

\section{EUROPS Wollaston}

10179 E. pallipennis Lec. Mosholu (Sf); vic. NYC (AM).

\section{BACTRIDIUM LeConte}

10183 B. ephippigerum Guér. Newport (NYS); Westfield, May (Not) ; SI, Ap (L\&D) ; LI: Hempstead Plains, May (Eng).

10184 B. erythropterum Melsh. Buffalo (Z\&R).

10185 B. convexulum Csy. (? striolatum Reit.). Newport (NYS); Olcott, May (Dt) ; Buffalo (Z\&R), as striatum; West Pt. (Robn); SI, May, under bark of beech (L\&D).

10186 B. hudsoni Csy. NY (Csy).

\section{Pycnotomina Casey}

10190 P. cavicolle Horn. Newport (NYS); Ringwood, Je (Dt); West Pt. (Robn) ; Bronx Pk. (Sf) ; SI, Aug (Shoe); LI: Jamaica, Pears (Sf).

\section{FAMily CUCUJIDAE}

\section{ORYZAEPHILUS Ganglbauer}

10194 O. surinamensis L. Saw-Toothed Grain Beetle. Newfane (Dt-CU); Castile, Brad (Flet); Ludlowville, Nov (CU) ; Ithaca, Sep (Dt); West Pt. (Robn) ; SI, in dried fruits at all seasons (L\&D) ; LI: Flushing, Queens, Eng (Sf). Probably rather generally distributed in the State, infesting grains and dried fruits. 


\section{SILVANUS Latreille}

10198 S. bidentatus Fab. Newport (NYS) ; Johnstown, Oct (NYS) ; Cranberry L., Je $23(\mathrm{H})$; Olcott, May-Oct (Dt) ; West Pt. (Robn); SI, Ap, Je, Aug (L\&D) ; LI: Central Pk., Ap, Eng (Sf).

10199 S. planatus Germ. Cranberry L., Je 23 (H) ; Olcott, Ap, Jl (Dt) ; Buffalo (Z\&R) ; Ithaca, Sep (Dt); Windsor, Je (Not); Van Cort. Pk., Nic (Sf) ; SI, May, under bark (L\&D).

10200 S. imbellis Lec. Cranberry L., Je-J1 (H) ; Olcott, May, Oct (Dt) ; Buffalo, Peabody (Fris) ; E. Aurora, May. (Dnd); SI, Feb 22, in sifting, Woodr (L\&D), J1, Eng (Sf) ; LI: Huntington, Sep, Sichott (Sf).

10201 S. gemellatus Duv. (quadricollis Lec.). E. Aurora, Je (Dnd) ; Glens Falls, Oct (Felt); Westfield, May (Not); Schenectady, Je (NYS).

CATHaRtus Reiche

10203 C. cassiae Reiche. "NY" (J. Hamilton).

10204 C. advena Waltl. Newport, Sep (NYS) ; Sanborn, Nov (CU, Lg) ; Olcott, Sep-Nov (Dt); Buffalo (Z\&R) ; E. Aurora, Nov (Flet, Dnd) ; New Baltimore, Sep (NYS); SI, in dried pears from Calif. (L\&D).

10207 C. longulus Blatch. Mosholu (Sf).

\section{NAUSIBIUS Redtenbacher}

10209 N. clavicornis Kug. Mosholu (Sf); Van Cort. Pk., Nic (Sf).

\section{Catogenus Westwood}

10211 C. rufus Fab. Gloversville, Aug, Alex (NYS) ; Buffalo (Z\&R) ; E. Aurora, May, Nov (Dnd) ; Ithaca, Mar, Oct (CU) ; Albany, May (NYS);

West Pt. (Robn); Peekskill (Sherm); SI, Je, on seashore (L\&D);

LI: Hempstead, May, Pinelawn, Ap, Eng (Sf); Yaphank, May, Sep, Wading R., J1 (Ds).

10211d C. rufus var. parvus Csy. Ithaca, Ap-May (Dt).

\section{Cucujus Fabricius}

10221 C. clavipes Fab. Newport, Ap-Je (NYS); Oswego L., Nov. (NYS) ; Olcott, May (Dt); Buffalo (Z\&R) ; E. Aurora, Mar, Sep (Dnd) ; Avon (Cls) ; Pike, Ap-Je (NYS); Batavia, Sep (Kngt); Geneva, Mar-May, Dec (Cls); Westfield, May (Not); Ithaca, Mar-May (CU) ; Waterford, Jan (NYS) ; Albany, Nov, Shandakin, Je (NYS); West Pt. (Robn); White Plains (Fall, Sq) ; Bronx Pk. (Sf) ; SI, Jan-May, under bark (L\&D); LI: Flushing (Ds, Sf, Eng).

\section{LAEMOPHLOEUS Laporte}

10225 L. biguttatus Say. L. Placid, Aug (Vdyk) ; Newport, May (NYS) ; Cranberry L. (H), J1 (Dury); Olcott, May, Aug (Dt); Buffalo (Z\&R) ; Batavia, J1-Sep (Kngt); Pike, May (NYS); West Pt. (Robn); SI, Ap-May (L\&D) ; LI: Central Pk., Ap; Hempstead, May, Yaphank, Sep (Eng).

10226 L. fasciatus Melsh. Newport, Mar-Ap (NYS); Cranberry L. (H) ; Olcott, May (Dt) ; Buffalo (Z\&R) ; Batavia, Aug-Sep (Kngt) ; Pike, Mar-Ap (NYS); Westfield, May (Not); SI, Ap-May (L\&D). 
10236 L. convexulus Lec. Mt. Seward, $4500 \mathrm{ft}$., Je (M\&H); Glens Falls, Oct (NYS) ; Olcott, May-Je (Dt) ; Buffalo (Z\&R); Windsor, Je (Not) ; West Pt. (Robn); SI, May 6, under bark (L\&D); LI: Central Pk., Ap-May, Hempstead, May, Eng (Sf).

10238 L. sphaerops Csy. Olcott, Ithaca, May (Dt).

10239 L. adustus Lec. Newport, Ap (NYS) ; Johnstown, Ap, Alex (NYS) ; Cranberry L., Je 30, in sweeping (H); Wanakena, Aug (Drk) ; Syracuse, May, on herbage (H); Olcott, Ap (Dt) ; Ithaca, May (Dt) ; Windsor, Je, Schoharie (Not); Nassau, May (NYS); West Pt. (Robn); Mosholu (Sf) ; SI, Je, under willow bark (L\&D); LI: Central Pk., Ap (Eng); Orient, Lath (NYS).

10242 L. modestus Say. Buffalo (Z\&R); SI, May (L\&D).

10243 L. apertus Csy. Syracuse, Ap-May, under bark (H); Westfield, May (Not); Mosholu (Sf); LI: Wyandanch, Nov, Schott (Sf).

10245 L. sobrinus Csy. Westfield, May (Not).

10246 L. liquidus Csy. Newport, May (NYS); Castorland; Olcott, May-Je (Dt); Ithaca, Ap-Je (CU); Ilion, May (NYS); Buffalo (Z\&R); SI, Ap-Je (L\&D) ; LI: Wyandanch, Feb, Schott (Sf).

10247 L. nitens Lec. LI: Wyandanch, Oct-Nov, Schott (Sf).

10248 L. punctatus Lec. West Pt. (Robn).

10254 L. angustulus Lec. Peekskill, Sherm (Sf).

10258 L. ferrugineus Steph. Buffalo (Z\&R).

\section{DENDROPHAGUS Schönherr}

10272 D. cygnaei Mann. Green L., Jan (Dt); Buffalo (Mann-ANSP).

\section{BRontes Fabricius}

10273 B. dubius Fab. Keene Val., Je (Not) ; Newport, May (NYS) ; Cranberry L., Je $23(\mathrm{H})$; Syracuse, May, under bark (H) ; Batavia, Aug-Sep (Kngt) ; Westfield, May (Not) ; Castile, Brad (Flet) ; E. Aurora, May-Je, Oct (Dnd) ; Buffalo (Z\&R) ; Ithaca, Mar-Ap (CU) ; Albany, Nassau, May, Nov (NYS) ; Rensselaer, J1 (NYS); West Pt. (Robn); NYC (Martin); LI: Central Pk., Ap, Yaphank, Je, Eng (Sf).

10274 B. debilis Lec. Olcott, May-Je (Dt) ; E. Aurora, May, Sep (Dnd) ; Ithaca, Mar, Sep (Dt) ; Mosholu (Sf) ; Van Cort. Pk. (AM) ; SI, May-Je, Sep, under bark (L\&D); LI: Rockaway, Je, Yaphank, May (Ds); Flatbush (AM).

\section{TELEPHANUS Erichson}

10276 T. velox Hald. Syracuse, Ap-Je, under stones (H) ; Olcott, Ap-Sep (Dt) ; Lockport, Sep (NYS) ; Buffalo (Z\&R); Castile, Brad (Flet); Oneida L., Aug (Drk); Ithaca, Mar-Aug (CU); Taughannock Falls, Aug $(\mathrm{Vdyk})$; Montour Falls. Ap-Je, under stones $(\mathrm{H})$; Albany, Rensselaer, Kenwood, May-Je, Nov (NYS); Peekskill, Mar (CU); West Pt. (Robn); White Plains, in sifting in autumn (Fall, Sq); Mosholı (Sf); SI, Mar-Oct (L\&D) ; LI: Rockaway Beach, Ap, Eng (Sf); Flatbush (AM).

PSAMMOECUS Latreille

10278 P. desjardinsi Guér. NYC (Sf). 


\section{FAMILY EROTYLIDAE}

\section{SUBFAMILY LANGURIINAE}

\section{LANGURIA Latreille}

10279 L. bicolor Fab. Buffalo (Z\&R).

10282 L. mozardi Latr. Clover-Stem Borer. Syracuse, May-Je (H) ; Buffalo (Z\&R) ; Pike (NYS); Rock City, Je (CU); Ithaca, Caroline, MayJ1, Sep (Dt-CU) ; Montour Falls, May-Je (H); Windsor, Je (Not) ; Karner, Je (NYS); Mosholu (Sf); New Baltimore, Albany (AM); Bangall, Sep (Sf); Poughkeepsie, Je (NYS) ; West Pt. (Robn); White Plains, May (Fall, Sq); SI, May-Je, often common on dandelion flowers (L\&D) ; LI: Farmingdale, Je (CU); Pinelawn, Ap, Eng (Sf) ; Yaphank, May, Eng (Sf).

10284 L. taedata Lec. Near NYC (Lec); SI, J1 (L\&D) ; LI: Rockaway Beach, Je, Eng (Sf).

10286 L. angustata Beauv. Buffalo (Z\&R); Greenwood L., Dt (Sf); SI, Jan 19 , under stones (L\&D).

10286a L. angustata var. uhleri Horn. "NY" (Horn).

10287 L trifasciata Say. SI (L\&D).

\section{ACROPTEROXYS Gorham}

10292 A. gracilis Nevin. Rochester Jct., Je, Lcon (CU) ; Buffalo (Z\&R); E. Aurora, May, J1 (Dnd); Batavia, Je (Kngt); Windsor, Je (Not) ; Ithaca, Je-J1, Sep (Dt-CU); White Plains, Je-J1 (Sq); Van Cort. Pk., Je (Sf); SI, Je-J1 (L\&D).

10292a A. gracilis var. inornata Rand. Newport, Je (NYS); Buffalo (Z\&R).

10293 A. lecontei Cr. Karner, J1 (NYS); Rensselaer, May (NYS) ; Clayville, Je, Leon (NYS).

\section{SUBFAMILY EROTYLINAE}

ISCHYRUS Lacordaire

10301 I. quadripunctatus Oliv. Buffalo (Z\&R); Pike, May (NYS); Batavia, Aug (Kngt).

\section{TrITOMA Fabricius}

10308 T. sanguinipennis Say. Newport (NYS) ; Johnstown, Sep (NYS) ; Olcott, Je, Aug (Dt); Buffalo (Z\&R) ; E. Aurora, Je (Dnd) ; Pine I., Je (Ds); SI (L\&D); LI: Orient, Nov (Ds).

10309 T. pulcher Say. Newport (NYS); Cranberry L., Je 11-J1 6, in fungi $(\mathrm{H})$; West Pt. (Robn) ; Olcott, Oct (Dt) ; Buffalo (Z\&R) ; E. Aurora, Aug (Dnd); North Branch (Schaupp); SI, Aug, many in a fungus, Sep (L\&D).

10309a T. pulcher var. dimidiatus Lac. Mosholu (Sf).

10310 T. dissimulator Cr. Wilmington, Il (Ds).

10314 T. angulata Say. Pike, Ap-May (NYS); Albany, May, J1 (NYS); Mosholu (AM) ; SI, J1, in a fungus. (L\&D) ; LI: Flatbush, Parkville $(A M)$. 
10317 T. biguttata Say. Poughkeepsie, Aug (NYS); West Pt. (Robn); SI, Aug (L\&D); LI, J1, Eng (Sf).

10319 T. humeralis Fab. Newport, Je (NYS); Buffalo (Z\&R); Pike (NYS); Ithaca, Je, Aug (Dt); Corinth, Albany, Wemple, Ji (NYS); SI (L\&D) ; LI: Yaphank, Je (Sf).

10323 T. unicolor Say. Johnstown, Sep (NYS); Buffalo (Z\&R); Mosholu (Sf).

\section{TRIPLAX Herbst}

10328 T. flavicollis Lac. Buffalo (Z\&R); E. Aurora, Aug (Dnd) ; Oneida L., Aug (Drk); Windsor, Je (Not); West Pt. (Ds); White Plains, J1 (Fall, Sq) ; Mosholu (Sf); SI (L\&D).

10332 T. macra Lec. Newport (NYS); Buffalo (Z\&R); West Pt. (Robn).

10334 T. thoracica Say. Cranberry L., Je 23-Aug (H) ; Olcott, Sep (Dt); Buffalo (Z\&R) ; E. Aurora, Je-Oct (Dnd) ; Batavia, Sep (Kngt); Ithaca, Je (Dt); Windsor, Je (Not); Albany, J1 (NYS); West Pt. (Robn); White Plains, J1 (Fall, Sq) ; SI, Je-J1 (L\&D).

\section{DACNE Latreille}

10342 D. quadrimaculata Say. Newport (NYS); Johnstown, Aug (NYS); Olcott, May (Dt); E. Aurora, Aug (Dnd); Buffalo (Z\&R); Batavia, Jl-Aug, Kngt (AM); Albany, J1, Ballston Spa, Je (NYS).

\section{HYPODACNE LeConte}

10346 H. punctata Lec. Buffalo (Z\&R).

\section{MEGALODACNE Crotch}

10347 M. fasciata Fab. White L. (AM) ; Olcott, Sep (Dt); Buffalo (Z\&R); E. Aurora, Je-J1 (Dnd) ; Batavia, Sep (Kngt) ; Ithaca, Jan, Ap (DtCU) ; Hudson Falls, Sep (NYS) ; Pine I., Je (Ds) ; West Pt. (Robn); SI (L\&D) ; LI: Queens, Mar, Schott (Frost); Newtown, Je, Eng (Sf); Orient (Ds). Larvae occur in the fungus Polyporus lucidus, according to Weiss.

10348 M. heros Say. Newport, Je (NYS) ; Buffalo (Z\&R); E. Aurora, May-Je (Dnd) ; Rock City (Ds); White L. (AM) ; Ithaca, May-Aug (Dt); Trenton Falls, Je (NYS); Albany, Je, Bish (NYS) ; Rensselaer, Aug (NYS) ; Rockland Co, Aug, Martin (Sf).

\section{FAMILY DERODONTIDAE}

\section{LARICOBIUS Rosenhauer}

10350 L. erichsoni Rosenh. Ithaca, May (Blaisdell, Dt); SI, Dec (L\&D).

\section{DERODONTUS LeConte}

10353 D. maculatus Melsh. E. Aurora, Je (Dnd) : Pike, Oct 11 (Frost); Johnstown, Alex (Yg-NY); Rochester (M\&W); Buffalo (Z\&R); Valhalla, Nov, Schott (Sf); SI, Nov, Shoe (L\&D). 


\section{FAMILY CRYPTOPHAGIDAE}

\section{SUBFAMILY CRYPTOPHAGINAE}

Diplocoelus Guérin-Ménéville

10356 D. brunneus Lec. SI, on dry fungus (L\&D).

10357 D. rudis Lec. LI: Orient, Feb (NYS).

\section{Telmatophilus Heer}

10360 T. americanus Lec. Batavia, J1 (Kngt); SI, Je (L\&D).

\section{LOBERUS LeConte}

10361 L. impressus Lec. Buffalo (Rein).

\section{TOMARUS Erichson}

10365 T. pulchellus Lec. Keene Val., J1 (NYS) ; Oneida L., Aug (Drk) ; Buffalo $(\mathrm{Z} \& \mathrm{R})$; Montour Falls, Je $(\mathrm{H})$; Westfield, May, Windsor, Je (Not); Karner, J1, Nassau, May, Albany, Aug (NYS); Schoharie (Not) ; Rockland L., Aug (NYS); SI, Mar, in mouse nest, Ap, SepNov (L\&D); LI: Central Pk., Ap (Eng); Orient, Lath (NYS).

\section{ANTHEROPHAGUS Latreille}

10370 A. ochraceus Melsh. Axton, Je (M\&H), as Atomaria; E. Aurora, J1 (Flet, Dnd); Peekskill (Ds).

\section{Crosimus Casey}

10376 C. obesulus Csy. "NY" (Csy); Johnstown, Aug (NYS).

10377 C. hirtus Csy. Westfield, May (Not).

\section{CRYPTOPHAGUS Herbst}

10384 C. acutangulus Gyll. Newport (NYS) ; E. Aurora, Je (Dnd) ; Batavia, J1 (Kngt); Albany, Ap (NYS); West Pt. (Robn).

10385 C. cellaris Scop. Buffalo (Z\&R); Albany, May, Hartman (NYS); LI: Orient, Feb (NYS).

10393 C. cicatricosus Csy. "NY" (Csy).

10398 C. croceus Zimm. Newport (NYS); Buffalo (Z\&R); Menands, Feb (NYS); SI, J1 (L\&D); LI: Orient Pt., May (NYS).

\section{HENOTICUS Thomson}

10418 H. serratus Gyll. Cranberry L., Je, on maple blossoms (H) ; Sacandaga Pk., Aug (NYS); West Pt. (Robn). 


\section{SUBFAMILY ATOMARIINAE}

CaEnoscelis Thomson

10427 C. macilenta Csy. "NY" (Csy); Cranberry L., Je (H).

10428 C. basalis Csy. "NY" (Csy).

\section{ATOMARIA Stephens}

A. lineola Not. Schoharie (Not) as Agathengis; Windsor, Je (Not).

10436 ?A. pumilio Csy. Cranberry L., Htly (H).

A. parviceps Not. Westfield, May (Not).

\section{ANCHICERA Thomson}

10463 A. ephippiata Zimm. Axton, Je (M\&H) ; Newport, Je (NYS); Cranberry L., Je (H) ; Buffalo (Z\&R) ; Batavia, J1 (Kngt); Castile, Brad (Flet); Montour Falls, May-Je $(\mathrm{H})$; Bridgeport, May-Je $(\mathrm{H})$; Schoharie (Not); Windsor, Je (Not); Delmar, Mar (NYS); Clinton Hgts., Ap (NYS) ; West Pt. (Robn); SI, Ap, in sifting, Nic (L\&D); LI: Orient, Lath (NYS).

10467 A. distincta Csy. SI, Ap, in sifting, Nic (L\&D).

10471 A. ochracea Csy. Windsor, Je (Not).

10471b A. ochracea var. pennsylvanica Csy. Schoharie (Not); Westfield, May, Windsor, Je (Not).

10473 A. gonodera Csy. Cranberry L., Je $(\mathrm{H})$.

10479 A. oblongula Csy. Mooers, Sep (Not); Schoharie (Not); Westfield, May, Windsor, Je (Not).

10481 A. pusilla Payk. Newport, May (NYS); Schoharie (Not); Windsor, Je (Not).

10483 A. ovalis Csy. Newport, May (NYS) ; Cranberry L., Je 25(H) ; Batavia, Jl-Sep (Kngt); Schoharie (Not); West Pt. (Robn).

A. ebenina Csy. NY, type locality.

A. nebulosa Csy. NY, type locality.

\section{EPHISTEMUS Stephens}

10488 E. apicalis Lec. Newport (NYS); Ithaca, Je (NYS).

\section{FAMILY MYCETOPHAGIDAE}

\section{Mycetophagus Hellwig}

10490 M. punctatus Say. Newport, May (NYS); Ilion (NYS); Buffalo (Say); Pike, J1 (NYS) ; E. Aurora, Aug (Dnd) ; Batavia, J1 (Kngt) ; Albany, J1 (NYS); West Pt. (Ds); SI, Aug (L\&D).

10491 M. flexuosus Say. Newport, May (NYS) ; Cranberry L., Je, in fungus Collybia platyphylla (H); Buffalo (Say); Pike, May, Aug (NYS); Johnstown, Sep (NYS); E. Aurora, Aug (Dnd); Batavia, J1-Aug (Kngt); Ballston Spa, Je (NYS) ; SI, Ap, Je (L\&D); West Pt., Ft. Montgomery, Je (Ds). 
10493 M. pictus Csy. Windsor, Je (Not).

10501 M. pluripunctatus Lec. Syracuse, Ap, in bracket fungus $(H)$; Newport (NYS); Buffalo (Z\&R); SI, J1 4, in fungus on maple (L\&D).

10503 M. bipustulatus Melsh. Newport (NYS); Nassau, Je (NYS) ; NYC, May (Ds) ; LI: Huntington, Dec, under log (Schott).

10507 M. tenuifasciatus Horn. LI: Rockaway Beach, May (Shoe); Yaphank, Sep (Sf).

10508 M. obsoletus Melsh. LI: Rockaway Beach, May (Shoe).

\section{TYPHAEA Curtis}

10509 T. fumata L. Newport (NYS); Canton, Sep (NYS); Batavia, J1-Sep (Kngt); Castile, Brad (Flet); Buffalo (Z\&R); Valley Falls, May (NYS); Cooperstown, Aug (NYS); West Pt. (Robn); SI (L\&D); LI: Brooklyn (Sf).

\section{LITARGUS Erichson}

10511 L. tetraspilotus Lec. Buffalo (Z\&R); SI, Aug (L\&D); LI: Pinelawn, Aug (Sf); Bellport, Aug (Nic); Riverhead, Ap, Cy (NYS).

10512 L. sexpunctatus Say. Olcott, Aug (Dt); Buffalo (Z\&R); West Pt. (Robn); SI, Ap (L\&D); LI: Yaphank, J1 (Sf); Central Pk., Ap (Eng).

10516 L. pallens Csy. "NY" (Csy).

10514 L. nebulosus Lec. SI, Ap 27 (L\&D); LI: Flatbush, Sep (Schott).

10517 L. didesmus Say. Albany, Ap (NYS); SI, May-Je, Thompson (L\&D); LI: Bellport, J1-Aug (Nic); Yaphank, Ap (Eng).

\section{FAMILY COLYDIIDAE 47}

\section{SynCHITA Hellwig}

10526 S. laticollis Lec. NY (Lec).

10527 S. variegata Lec. Mosholu (Sf). Described from southern Calif., but Schaeffer states that this single specimen, as well as some from Canada, agrees with the description of variegata but not with that of parvula.

10529 S. parvula Guér. Ithaca, Je (Chitt).

10530 S. fuliginosa Melsh. Buffalo (Z\&R); West Pt. (Robn); Bronx Pk. (Sf); SI, Je, in old fungus (L\&D); LI: Rockaway Beach, Je, Eng (Sf); Long Beach, Je, Eng (Sf).

\section{EUCICONES Sharp}

10533 E. marginalis Melsh. NYC (Julich).

\section{Bitoma Herbst}

10539 B. pinicola Schffr. LI: Central Pk., Sep, Rockaway Beach, May (Eng); Wyandanch, Je, Schott (Sf).

10541 B. quadriguttata Say. Pike, Newport (NYS); Buffalo (Z\&R); SI (L\&D) ; LI: Yaphank, Nov, Eng (Sf).

\footnotetext{
${ }^{47}$ Charles Schaeffer has gone over the list for this family, and has supplied many records and made certain changes in synonymy.
} 
Coxelus Latreille

10551 C. guttulatus Lec. Buffalo (Z\&R); LI: Central Pk., Ap, Eng (Sf).

\section{LASCONOTUS Erichson}

10558 L. borealis Horn. Cranberry L., Je-J1 (H).

\section{Aulonium Erichson}

10576 A. parallelopipedum Say. SI, under elm bark (L\&D).

\section{Colydium Fabricius}

10580 C. lineola Say. Cranberry L. (H); Buffalo (Z\&R); Bronx Pk. (Sf); SI, $\mathrm{Je}(\mathrm{L} \& \mathrm{D})$.

\section{Xuthia Pascoe}

$\mathrm{X}$. brevipes Shp. LI: Astoria, Aug, introduced in mahogany logs, Sz (FeltJour. N. Y. Ent. Soc. 21:275, 1913).

\section{BOTHRIDERES Erichson}

10592 B. geminatus Say. Syracuse, Je (Drk); Buffalo (Z\&R); Genesee Co., Ap (NYS); Mosholu, May, in hickory (Ds).

\section{CERYLON Latreille}

10598 C. castaneum Say. Fourth L., Je (NYS) ; Newport (NYS) ; Cranberry L., Je-J1 $(H)$; Syracuse, May, under bark $(H)$; Westfield, May, Windsor, Je (Not); Buffalo (Z\&R); Schoharie (Not) ; Albany, Nassau, Kinderhook (NYS); West Pt. (Robn) ; SI, Ap, Nov (Shoe, Woodr), May, Oct, under bark (L\&D) ; LI: Queens, Sep (Schott); Flatbush, Ap, Eng (Sf).

10601 C. sticticum Csy. SI, J1 (L\&D); Catskills, Pears (Sf).

\section{Philothermus Aubé}

10604 P. glabriculus Lec. Newport (NYS) ; Montour Falls, under bark, Ap (H) ; Westfield, May, Windsor, Je (Not); Delmar, Karner, Ap (NYS); West Pt. (Robn); SI, Ap-May (L\&D) ; LI: Pinelawn, Central Pk., Ap, Eng (Sf); Southold, Ap (NYS) ; Orient, Lath (NYS).

\section{FAMILY LATHRIDIIDAE 48 \\ Holoparamecus Curtis}

10614 H. kunzei Aubé. NY (Fall).

${ }^{48} \mathrm{H}$. C. Fall has read the manuscript for this family, and has suggested one or two changes in synomyny and added some records. 


\section{LATHRIDIUS Herbst}

10627 L. breviclavus Fall. LI (Fall).

10631 L. liratus Lec. Newport, May-Je (NYS); Cranberry L., Je (H) ; Olcott, Mar-J1 (Dt); Schoharie (Not); Johnstown, Sep (NYS); McLean, Je (Flet); Ithaca, Mar-J1 (Dt) ; Windsor, Je (Not); West Pt. (Robn) ; SI, J1 (L\&D).

\section{Coninomus Thomson}

10633 C. constrictus Gyll. Cranberry L., Je 25, in decaying fungi $(H)$.

10635 C. nodifer Westw. Olcott, Je (Dt).

\section{ENICMUS Thomson}

10639 E. hirtus Gyll. NY (Fall).

10642 E. minutus L. Newport, May-Je (NYS); Speculator, Je (NYS); Buffalo $(Z \& R)$; Rochester (M\&W).

10646 E. aterrimus Mots. "NY" (Fall); Peekskill, Je (Sherm), as opaculus.

10656 E. tenuicornis L. Otsego (Fall); Olcott, Je (Dt).

CARTODERE Thomson

10659 C. ruficollis Marsh. Albany, Ap, J1 (NYS); Nassau, Ap (NYS); Blauvelt, Aug (NYS).

10662 C. argus Reit. Schoharie (Not).

10664 C. filum Aubé. NY (Fall); Ithaca, Sep (Dt).

10665 C. elegans Aubé. NYC, Oct, Woodr (Not).

\section{Adistemia Fall}

10666 A. watsoni Woll. NYC, infesting boxes of moldy beetles in collection of L. B. Woodruff, Oct 5, 1920 (Fal1).

CORTICARIA Marsham

10669 C. pubescens Gyll. NY (Fall); Newport, May-Je (NYS).

10679 C. serricollis Lec. Peekskill, Jan, Sherm (Sz).

10685 C. serrata Payk. Buffalo (Z\&R); Windsor, Je (Not).

10689 C. dentigera Lec. Cranberry L., in fungi $(H)$.

10693 C. elongata Gyll. Rochester (M\&W); Schoharie (Not); SI, Oct 9, in sifting (L\&D).

10694 C. ferruginea Marsh. "NY" (Lec), as obtusa; Cranberry L., Je 29 (H); Buffalo (Z\&R); West Pt. (Robn).

\section{Melanophthalma Motschulsky}

10696 M. picta Lec. Mooers, Sep (Not); Schoharie (Not); Westfield, May, Windsor, Je (Not); Olcott, Feb, Ap (Dt); West Pt. (Robn).

10699 M. villosa Zimm. Mooers, Sep (Not) ; Cranberry L., Je-J1 (H) ; Westfield, May, Windsor, Je (Not). 
10701 M. distinguenda Com. Mooers, Sep (Not); Axton, Je (M\&H) ; Cranberry L., Je (H) ; Newport, May-Je (NYS); Syracuse, May-Oct $(\mathrm{H})$; Rochester (M\&W) ; Montour Falls, May-Oct $(\mathrm{H})$; Westfield, May (Not); Schoharie, Windsor (Not); Bridgeport, May-Oct (H); Ithaca, Ringwood, McLean, Feb-Nov (Dt); LI: Orient, Ap (NYS).

10703 M. pumila Lec. Cranberry L., Je-J1 (H) ; Buffalo (Z\&R) ; Schoharie (Not); West Pt. (Robn); SI, Joutel (L\&D); LI: Central Pk., Ap (Eng); Rockaway Beach (AM, Lg); Coney I. (Lg).

10705 M. gibbosa Hbst. Cranberry L., Je-J1 (H); Syracuse (H) ; Bridgeport (H).

10711 M. longipennis Lec. Montour Falls, Je (H) ; Olcott, Je (Dt) ; Westfield, May, Windsor, Je (Not).

10712 M. americana Mann. Johnstown, Sep (NYS) ; Olcott, Feb, Je-J1 (Dt) ; Westfield, May (Not); Ithaca, Feb, Je-J1 (Dt); Catskills (Lg); SI, Je (L\&D) ; LI: Coney I. ( Lg).

10713 M. cavicollis Mann. Cranberry L., Je-J1 (H) ; Syracuse, May, queried $(\mathrm{H})$; Olcott, Feb-Oct (Dt); Buffalo (Z\&R) ; Ithaca, Saratoga, Feb-Oct (Dt) ; Albany, Ap (NYS); Windsor, Je (Not); West Pt. (Robn); SI, Feb 22 (Woodr), Sep (L\&D).

\section{FAMILY MYCETAEIDAE}

Mycetaea Stephens

10716 M. hirta Marsh. Buffalo (Z\&R); SI, Mar, May, in fungus on logs and stumps (L\&D) ; LI: Wyandanch, J1, Schott (Sf).

\section{RHANIS LeConte}

10717 R. unicolor Zieg1. Newport (NYS); Olcott, Sep (Dt); Buffalo (Z\&R) ; SI, Ap, Je, J1, Nov (L\&D); LI: Yaphank, Je, Eng (Sf).

\section{PHYMAPHORA Newman}

10718 P. pulchella Newn. Newport, May (NYS) ; Cranberry L., J1 6 (H) ; Olcott Sep (Dt); Trenton Falls (Newn); Buffalo (Z\&R); Johnstown, Oct (NYS); Ithaca, Ap 30 (CU); North Branch (Schaupp); Bronx Pk. (Sf).

\section{FAMILY ENDOMYCHIDAE SUBFAMILY ENDOIMYCHINAE}

LYCOPERDINA Latreille

10720 L. ferruginea Lec. Keene Hgts., Je (Not); Olcott, Oct (Dt); Buffalo (Z\&R) ; Pike, Sep (NYS) ; E. Aurora, Cortland Co, Je-Sep (Dnd); Ithaca, Ap 14 (CU); White L., Zab (AM); W. Hebron (Lg); West Pt. (Robn) ; Mosholu, Nov (Ang); SI, Je, Dec (L\&D) ; LI: Central Pk., May, Eng (Sf); Yaphank, Je, Eng (Sf); Flatbush, Zab (AM). In puffball fungus. 


\section{APHORISTA Gorham}

10726 A. vittata Fab. Newport (NYS); North Cr., Je (NYS); Buffalo (Z\&R) ; Erie Co, May, Sep (Dnd) ; Batavia, Ap (Kngt) ; Castile, Brad (Flet); Ithaca, May 3 (CU); Meredith, May, Cy (NYS); W. Hebron (Lg); Middlesex, Aug 13 (Cls); SI (L\&D); LI: Wyandanch, Ap (Ds); Central Pk., Ap, Pinelawn, Yaphank, May, Eng (Sf) ; Orient Pt., Ap, Sep (NYS).

\section{Mycetina Mulsant}

10727 M. perpulchra Newn. Newport, Je (NYS); Cranberry L., Je-J1 (H) ; Gloversville; Ap (NYS); Olcott, Sep (Dt); Wolcott, May, Cy (NYS) ; Buffalo (Z\&R) ; E. Aurora, Je (Dnd) ; Alpine, Nov (NYS); Ithaca, Je (Dt); Trenton Falls (Newn); North Branch (Schaupp); Voorheesville, May (NYS); West Pt. (Robn).

10730 M. testacea Zieg1. Newport (NYS); Ithaca, Mar (Dt); W. Hebron (Ds Coll).

\section{Stenotarsus Perty}

10739 S. hispidus Hbst. LI: Huntington, Aug 22, Schott (Frost).

ANAMORPHUS LeConte

A. sp. LI: Yaphank, Je (Sf).

RHYмBUs Gerstaecker

10752 R. pilosus Csy. Bluff Pt., L. Champlain (Csy).

\section{ENDOMYCHUS Panzer}

10753 E. biguttatus Say. Elizabethtown, Pike, Newport (NYS); Long L. (Ds Coll) ; Cranberry L., J1 20 (H) ; Buffalo (Z\&R) ; Batavia, Je-Sep (Kngt); Geneva, Mar (Cls) ; Cattaraugus Co (Ds Coll); Schuyler, Mar (NYS); Ithaca, J1 30, Yuasa (Vdyk); L. George, Aug, Zab (AM) ; Phoenicia, Aug, MCV (NYS) ; West Pt. (Robn) ; Ramapo Mts. (AM) ; White Plains (Fall, Sq); SI, Jan-Oct, under beech bark (L\&D) ; LI: Riverdale, Oct, Eng (Sf).

\section{FAMILY PHALACRIDAE}

\section{PHALACRUS Paykull}

10772 P. pumilio Lec. Axton, Je (M\&H) ; Syracuse, May (H) ; SI, May-Je, on seashore (L\&D).

10773 P. politus Melsh. Axton, Je (M\&H); Syracuse, May (H); Buffalo (Z\&R); L. George, Aug (Not-AM) ; Albany, J1 (NYS) ; White L., Sep (Not-AM) ; SI, Je (L\&D) ; LI: Rockaway Beach, Je (Ds).

\section{OLIBRUS Erichson}

10776 O. vittatus Lec. NY (Csy).

10777 O. lecontei Csy. Buffalo (Z\&R).

10779 O. abstinens Csy. LI (Csy). 
10780 O. semistriatus Lec. Juanita I., L. George, J1, Bish (NYS); SI, Aug (L\&D).

10782 O. frustratus Csy. NY ( Lg).

10783 O. bullatus Csy. (sternalis Csy.). LI ( $\mathrm{Lg})$.

10784 O. impotens Csy. NY ( Lg Cat.).

10785 O. neglectus Csy. NY (Csy).

10786 O. pallipes Say. NY (Csy); SI (L\&D).

\section{ACYLOMUS Sharp}

10813 A. ergoti Csy. Axton, Je (M\&H) ; Cranberry L., Je (H) ; Syracuse, Montour Falls, Bridgeport, May-Je (H); SI, May (L\&D).

\section{STILbUS Seidlitz}

10828 S. pallidus Csy. "NY" (Lg).

10829 S. apicalis Melsh. Newport, May (Yg) ; Cranberry L., Je-J1 (H) ; Oswego, J1, Bks (NYS) ; Syracuse, Ap, Je (H) ; Buffalo (Z\&R); Oswego, J1 (Yg); Batavia, Je-Aug (Kngt); Montour Falls, May-Je (H); Westfield, May (Not); West Pt. (Robn); Gloversville, Ap (NYS); White Plains, Aug (Frost, Sq); SI, May, Aug (L\&D); LI: Orient, May (NYS).

10831 S. probatus Csy. "NY" ( $\mathrm{Lg})$.

10834 S. ludibundus Csy. "NY" (Csy).

10836 S. finitimus Csy. NY (Csy).

10841 S. obtusus Lec. Mooers, Sep (Not); Schoharie (Not). Described from southern Calif.

10844 S. nitidus Melsh. Gloversville, Sep (NYS); Syracuse, Ap, Je, under stones $(\mathrm{H})$; Buffalo (Z\&R); Pike, Ap (NYS) ; Montour Falls, Ap, Je (H); SI, Ap (L\&D) ; LI (Csy).

10849 S. thoracicus Csy. "NY" (Csy).

10851 S. quadrisetosus Csy. NY ( Lg).

S. atomarius L. Schoharie (Not); Windsor, Je (Not).

\section{FAMILy COCCINELLIDAE SUBFAMILY COCCINELLINAE}

HyPERASPIS Chevrolat

10874 H. laterailis Muls. Buffalo (Z\&R).

10876 H. bigeminata Rand. Cranberry L., Htly (H); Mt. Seward, Je (M\&H) ; Mt. Whiteface, J1, Shoe (Sf); Mt. Marcy, J1, Wat (Lg); Buffalo (Z\&R).

10879 H. signata Oliv. Mt. Marcy, J1 (NYS) ; Syracuse, May (H) ; Olcott, May-J1 (Dt) ; E. Aurora, May-J1 (Dnd); Westfield, May (Not); Ithaca, May-Aug (CU) ; Albany, Sep (NYS); Karner (NYS); West Pt. (Robn); Ludlow, Thompson (Lg); SI, May-Aug (L\&D); LI: Rockaway Beach, Eng (Sf); Yaphank, May, Pollard (Lg).

H. binotata Say. Buffalo (Lg); West Pt. (Robn) ; Ludlow (Lg); Mosholu $(\mathrm{Lg})$; LI: Yaphank, May, Pollard $(\mathrm{Lg})$. 
10880 H. proba Say. Buffalo (Z\&R); Ithaca, J1-Aug (CU); Montour Falls, Je (H) ; West Pt. (Robn); SI, Aug-Sep (L\&D); LI: Yaphank, Je, Amagansett, Aug, Eng (Sf); Orient, Lath (NYS).

10908 H. fimbriolata Melsh. Minerva, J1 20, 1925, on Solidago (Frost); White Plains, Sep (Sq); SI, two at Ward's Pt., Je 5 (L\&D) ; LI: Rockaway Beach, Long Beach, Yaphank, May, J1, Eng (Sf).

10914 H. serena Csy. Mt. Whiteface, Sep (Lg); Mosholu, Je, Aug (Lg).

10921 H. disconotata Muls. Cascade L., Aug ( Lg).

10922 H. troglodytes Muls. SI, J1 (L\&D).

10923 H. lugubris Rand. Ithaca, May-Aug (CU).

10930 H. undulata Say. Wilmington, J1, Cascade L., Aug ( Lg) ; Newport, May (NYS); Gloversville, Johnstown, Ap-May (NYS); Oswego, May (NYS) ; Syracuse, Ap-May, under stones (H) ; Olcott, Je (Dt) ; Buffalo (Z\&R); Ithaca, Ap-J1 (CU); West Pt., J1, Eng (Sf); Crotonon-Hudson, Je, Ang (Lg) ; LI: Rockaway Beach, Long Beach, Yaphank, Ap-J1, Eng (Sf); Orient Pt.

\section{BRACHYACANTHA Chevrolat}

10967 B. dentipes Fab. NY (NYS).

10972 B. ursina Fab. Newport, Je (NYS) ; New Russia, Aug (Brad-CU) ; Cranberry L., J1 (Drk) ; Otto, J1 (CU) ; Pike, Je (NYS) ; Erie Co, May-J1 (Dnd); Westfield, May (Not); Ithaca, Je-Aug (CU); Schoharie (Not); Albany, Je (NYS); Nassau, J1 (NYS) ; Poughkeepsie, Je (NYS) ; Bangall, Sep (Eng); Peekskill (Sherm); White Plains (Sq); SI, May-J1, common (L\&D) ; LI: Flushing, Bayshore, Yaphank (Sf).

10972a B. ursina var. congruens Csy. Ithaca (Pack).

10974 B. felina Fab. var. decempustulata Melsh. Ogdensburg, J1 (NYS) ; Newport, Je (NYS) ; Castile, Brad (Flet); E. Aurora, Cortland Co, Je-J1 (Dnd) ; Olcott, Ithaca (Dt) ; Schoharie (Not) ; Karner, Je (NYS); Ellenville, Sep-Nov, Nic (Frost); West Pt. (Robn); White Plains (Sq) ; SI, Je-J1 (L\&D); LI: Yaphank, J1 (Eng).

10977 B. indubitabilis Cr. Ft. Montgomery, J1 (Ds) ; LI: Massapequa, J1, Shoe (Sf).

10979 B. quadripunctata Melsh. Pike, Je (NYS); Ithaca, Aug (CU); Poughkeepsie, Je (NYS); Mosholu (Sf); LI: Yaphank, Oct, Eng (Sf).

10979b B. quadripunctata var. flavifrons Muls. W. Hebron, J1 (Lg).

\section{MICROWEISEA Cockerell}

10988 M. marginata Lec. Ithaca, Ap (Pettit,Horn).

10989 M. misella Lec. Cranberry L., Je-J1 (H) ; Ithaca, May, Aug (Dt) ; Albany, Sep (Felt); Germantown (NYS); West Pt. (Robn); Orange Co (Felt); Mosholu (Sf); SI, J1-Aug (L\&D).

\section{STETHORUS Weise}

10998 S. punctum Lec. Cranberry L., Je (H); Olcott (Dt); West Pt. (Robn); SI, Aug-Sep, many specimens (L\&D). 


\section{SCymus Kugelann}

11011a S. creperus Muls. var. fraternus Lec. Ithaca (Pack); Montour Falls, Je $(\mathrm{H})$, with query; Ithaca, Aug (Dt); Schoharie (Not).

11012 S. brullei Muls. NY (Horn).

11013 S. haemorrhous Lec. Buffalo (Z\&R); Ludlow, Thompson (Lg); SI (L\&D).

11016 S. chromopyga Csy. SI, May-Aug (L\&D).

11018 S. cervicalis Melsh. West Pt. (Robn); SI, Sep (L\&D).

11024 S. caudalis Lec. Ithaca (Pack).

11028 S. collaris Melsh. Cranberry L., J1, on Sambucus blossoms (H); Syracuse, May-Je (H) ; Buffalo (Z\&R); Montour Falls, May-Je (H); Ithaca (Pack); SI, May-Aug (L\&D); LI: Yaphank, May, Pollard (Lg).

11034 S. indutus Csy. Ithaca, Je (Dt).

11035 S. puncticollis Lec. Cranberry L., Je-J1, on Spiraea and Prunus (H) ; Montour Falls, Je, queried $(\mathrm{H})$; Schoharie (Not); West Pt. (Robn).

11036 S. agricola Csy. Ithaca, May (Dt).

11055 S. tenebrosus Muls. Axton, Je (M\&H) ; Cranberry L., Htly $(\mathrm{H})$; Ithaca, May-Sep (Dt); Schoharie (Not); West Pt. (Robn); SI, Je-Aug (L\&D).

11060 S. lacustris Lec. Buffalo (Z\&R).

11077 S. punctata Melsh. Castile, Brad (Flet); Ithaca, May (Dt); SI (Lg).

11079 S. nanus Lec. Cranberry L., Je, on willow $(\mathrm{H})$; West Pt. (Robn).

11082 S. americanus Muls. Cranberry L., Je $(\mathrm{H})$; Syracuse $(\mathrm{H})$; Olcott, J1 (Dt) ; Buffalo (Z\&R); Ithaca, J1 (Dt); West Pt. (Robn); Ludlow, Thompson (Lg); SI, May-Aug (L\&D).

11093 S. flavifrons Melsh. NY (Lec).

11093a S. flavifrons var. bioculatus Muls. Buffalo (Z\&R); SI (L\&D).

11094 S. ornatus Lec. McLean, May, June (Flet).

11115 S. terminatus Say. Buffalo (Z\&R); Bronxville, J1 (Woodr).

\section{Delphastus Casey}

11143 D. pusillus Lec. Cranberry L., Htly $(\mathrm{H})$; Montour Falls, Je (H) ; Ithaca, Je (Pack); Woodhull L., Aug, Love (Lg); Van Cort. Pk., Aug (Sf).

Coccidula Kugelann

11147 C. lepida Lec. Ashland, Aug 4 (Frost); LI: Yaphank, J1, Eng (Sf).

PSYLLOBORA Chevrolat

11150 P. viginti-maculata Say. Mooers, Sep (Not); Wilmington, Aug (Dt); Newport, Je (NYS); Keene Val., Sep, Eng (Sf); Cranberry L., Je $(\mathrm{H})$; Syracuse, Ap-May, under log $(\mathrm{H})$; Olcott, Mar-Sep (Dt); Buffalo (Z\&R); Batavia, Je-Aug (Kngt); Westfield, May (Not); Ithaca, May-Oct (CU); Ilion, May (NYS); Schoharie (Not); Nassau, May (NYS); West Pt. (Robn); White Plains (Sq); SI, J1-Oct (L\&D) : LI: Jamaica, Aug, Rockaway Beach, Je, Eng (Sf); Orient, Lath (NYS). 


\section{AnIsosticta Chevrolat}

11154 A. bitriangularis Say. Saranac, Sep (Ds, Sf); Wanakena, Aug (Drk); Cranberry L., Je (H); E. Aurora, Ap (Dnd); Montour Falls, Je (H) ; Batavia, Je-Aug (Kngt); Ithaca, May-Je (Dt, Pack) ; McLean Bogs, May, Aug (CU); W. Hebron, Je (Lg); West Pt.' (Robn); White Plains, May ( $\mathrm{Lg})$; SI, in swampy places (L\&D).

\section{NAEmia Mulsant}

11155 N. seriata Melsh. SI, May, Aug-Sep, often on Solidago sempervirens (L\&D); LI: Aqueduct, Forest Pk., Rockaway Beach, Je-Sep (Sf); Orient, Lath (NYS).

\section{Ceratomegilla Crotch}

11158 C. fuscilabris Muls. (Megilla maculata auct. not DeG.). Syracuse, Ap-Je (H); Oswego Falls, Aug (NYS); Olcott (Dt); Buffalo (Z\&R) ; Pike, Mar (NYS); Lockport, May (Cy); Batavia, Je-Aug (Kngt); Castile, J1, Brad (Flet); Montour Falls, Ap-Je (H); Elmira, Aug (Cy); Ithaca, Mar-Sep (CU); Windsor, Je (Not); Albany, Karner, Aug (NYS); Nassau, Ap (Sherm); West Pt. (Robn); White Plains (Sq) ; Pine I., Je, Pollard (Lg) ; SI, Je-Oct (L\&D); LI: Hempstead Plains, Central Pk., Rockaway Beach, Ap-Je (Sf); Orient, Lath (NYS). Often hibernates in numbers under stones $(\mathrm{Lg})$.

\section{Hippodamia Dejean}

11162 H. tredecimpunctata L. Essex Co, J1 (NYS); Cranberry L., J1-Aug (Drk); Olcott, May (Dt); Buffalo (Z\&R) ; Erie Co, J1, Nov (Dnd); Batavia, J1-Sep (Kngt); Westfield, May (Not); Lyons Falls, Aug (Mat) ; Ithaca, Je-Oct (CU) ; West Pt. (Robn); Croton-on-Hudson (Ang); White Plains (Sq); SI, J1-Aug (L\&D); LI: Rockaway Beach, Ap, Montauk Pt., Eng (Sf).

11163 H. parenthesis Say. Axton, Je (M\&H); Wilmington, Aug (Dt) ; Wanakena, Aug (Drk); Cranberry L., J1 (H); Newport, Je (NYS); Oswego, J1 (NYS); Syracuse, Ap-Oct 6 (H); Olcott, Mar-Aug (Dt); Buffalo (Z\&R); Castile, Brad (Flet) ; E. Aurora, May-Aug (Dnd) ; Pike, J1 (NYS); Westfield, May (Not); Ithaca, Ap-Sep, Dec (CU) ; McLean Bogs, J1 (Dt) ; Albany, May (NYS) ; West Pt. (Robn) ; Peekskill (Sherm); Clinton Cr., J1 (NYS); White Plains (Sq); Croton-on-Hudson (Ang); SI, May-Aug (L\&D); LI: Rockaway Beach, Yaphank, Je-J1, Eng (Sf); Orient, Lath (NYS).

11171 H. glacialis Fab. Buffalo (Z\&R); Elka Pk., Aug (Drk); Westfield, May (Not); Ithaca (Pack); Nassau, Ap (NYS); Poughkeepsie, Je (NYS); Peekskill (Sherm); West Pt. (Robn); White Plains (Sq) SI, Je-Sep (L\&D) ; LI: Rockaway Beach, Oct (L\&D) ; Jamaica, Pine lawn, Eng (Sf); Orient Pt., Je (NYS).

11172 H. quindecim-maculata Muls. Batavia, J1-Aug (Kngt); Portage, Aug (Kngt); McLean, May (CU).

11173 H. convergens Guér. Batavia, J1 (Kngt); Port Kent (Lint); Pike (NYS); Buffalo (NYS); Woodlawn Beach, L. Erie, J1 (Dnd); Windom, Oct (Dt); Castile, Brad (Flet); Ithaca, May-Sep (CU); 
Coeymans (Lint); Peekskill (Sherm); West Pt. (Robn); White Plains (Sq); SI, Je-Nov, common (L\&D); LI: Rockaway, May, Pollard (Lg); Orient, Lath (NYS).

11175 H. quinquesignata Kby. Adirondacks (Lg); Cranberry L., J1 (H) ; L. Placid, J1 (NYS); Syracuse, Montour Falls, May-Je (H).

\section{CocCinella Linnaeus}

11181 C. perplexa Muls. Axton, Je (M\&H) ; Wilmington, Aug (Dt) ; Newport, May (NYS) ; Cranberry L., Je-Jl (H) ; Syracuse, Ap-Je (H) ; Buffalo (Z\&R) ; Batavia, J1-Aug, as trifasciata L. (Kngt) ; Erie Co, MayAug (Dnd); Montour Falls, Ap-Je (H); Ithaca, McLean, May (Dt) ; Schoharie, as trifasciata L. (Not); Karner, May (NYS); West Pt. (Robn); White Plains, Pollard (Lg); SI, May-Aug, often plentiful on white birches infested with plant lice (L\&D); LI: Central Pk., Ap, Eng (Sf) ; Rockaway, May, Pollard (Lg); Orient, Lath (NYS).

11183 C. tricuspis Kby. Chateaugay L., Aug-Sep, Cascade L., Aug (Lg) ; Cranberry L., J1 (H).

11184 C. novemnotata Hbst. Wilmington (Dt); Denmark, May (NYS) ; Cranberry L., Je-J1 (H); Gloversville, Aug (NYS); Syracuse, Ap-Je $(\mathrm{H})$; Rochester, Aug (Cy) ; Olcott, Windom (Dt); Buffalo (Z\&R); E. Aurora (Dnd); Pike, Ap (NYS); Batavia, Je-Aug (Kngt); Castile, J1, Brad (Flet); Montour Falls, Ap-Je (H); Westfield, May (Not); Ithaca, May-Sep (NYS, CU) ; McLean, May (CU) ; Dansville, Sep (CU); Schoharie (Not); Washington Co (Ashton) ; Albany, Nassau, May, J1 (NYS) ; Karner, Sep (NYS) ; Coeymans (Lint) ; Peekskill (Sherm); West Pt. (Robn); White Plains (Sq) ; SI, MarAug, common (L\&D); LI: Jamaica, May, Flatbush, Dec, Eng. (Sf) ; Rockaway, May, Poliard (Lg); Orient, Lath (NYS).

11185 C. transversoguttata Fald. Mt. Marcy, J1 (NYS); Mooers, Sep (Not); Saranac (Lg); Wilmington, Aug (Dt); N. Elba, J1, Wat (Lg) ; Olcott (Dt); Buffalo, Aug (NYS); Erie Co, May-Aug (Dnd) ; Westfield, May (Not); Ithaca (Pack); Highland, Aug (Cy); West Pt. (Robn) ; SI, J1 (L\&D) ; LI: Orient, Lath (NYS).

$11185 a$ C. transversoguttata var. quinquenotata Kby. Essex Co, J1 (NYS) ; Washington Co (AshtonTB); Thousand Isls., Sep (CU) ; Castile, May, Brad (Flet); Ithaca, May (CU).

11187 C. monticola Muls. Mt. Whiteface, J1 ( $\mathrm{Lg}$ ) ; Wanakena, Cranberry L., Aug (Drk).

\section{Cycloneda Crotch}

11189 C. sanguinea L. Newport, Je (NYS) ; Cranberry L., J1 (H) ; Syracuse, May, Oct $6(\mathrm{H})$; Olcott, Aug (Dt) ; Buffalo (Z\&R) ; E. Aurora, JeSep (Dnd); Pike, Je (NYS); Batavia, Je-Aug (Kngt); Castile, Sep Brad (Flet); Ithaca, J1-Sep (CU); Coeymans (NYS); West Pt. (Robn); White Plains (Sq); LI: Yaphank, May, Pollard (Lg); Orient Pt., May (NYS).

11189a C. sanguinea var. immaculata Fab. LI: Brooklyn, J1 (Nic).

11190 C. munda Say. Ithaca (Pack); SI, Je-Oct (L\&D); LI: Pinelawn, Ap, Yaphank, Sep, Eng (Sf). 
Olla Casey

11192 O. abdominalis Say. "NY" (Lec). This record is doubtful (Lg).

AdALIA Mulsant

11193 A. bipunctata L. Cranberry L., Je-J1 (H) ; Green L., Jan (Dt) ; Pittsford, May (Leon); Olcott, Je-J1 (Dt) ; Buffalo (Z\&R); E. Aurora (Dnd) ; Batavia, Ji (Kngt) ; Westfield, May (Not) ; Forestville, Dec (Cy); Castile, Brad (Flet); Fayetteville, Oct (NYS); Ithaca, MarSep (NYS, CU) ; Windsor, Je (Not) ; Wampsville, J1 (Cy); Sherburne, Je (Cy); Albany, Nassau (Felt); Poughkeepsie (Sherm) ; Bangall (Sf); West Pt. (Robn); White Plains (Sq) ; Mt. Vernon (Flet) ; Croton-on-Hudson, May (Ang); NYC (Felt); SI, Ap-Oct, often hibernates in dwellings (L\&D); LI: Westbury; Forest Pk., Je (Lg); Orient, Lath (NYS).

11194 A. frigida Schn. Saranac ( $\mathrm{Lg})$; Axton, Je (M\&H); L. Placid, Aug (NYS) ; Brant L., Doll (Sf); Buffalo (Z\&R); E. Aurora, J1 (Dnd).

11194a A. frigida var. disjuncta Rand. Saranac (Lg).

11194b A. frigida var. humeralis Say. LI, Sep, Pears (Sf).

\section{Cleis Mulsant}

11196 C. picta Rand. Mt. Marcy, J1 (NYS); Mt. Whiteface, J1 (Lg); Mt, Seward, $4500 \mathrm{ft}$., Je (M\&H) ; Speculator, J1 (NYS) ; Cranberry L., Je-Jl (H) ; Buffalo (Z\&R) ; Ithaca, Je (CU) ; West Pt. (Robn); SI, Ap, Je (L\&D) ; LI: Rockaway Beach, Long Beach, Wyandanch, Yaphank, May-Nov, Eng (Sf).

11197 C. hudsonica Csy. Mt. Whiteface, J1, Shoe (Sf).

\section{Anisocalvia Crotch}

11200 A. duodecim-maculata Gebl. Mt. Whiteface, J1, Shoe (Sf) ; Cranberry L., Htly (H).

11201a A. quatuordecimguttata L. var. similis Rand. Wilmington, J1 (Lg) ; Cranberry L., Htly (H).

11201b A. quatuordecimguttata var. cardisce Rand. "Adirondacks" (Csy); Mt. Whiteface, J1 ( Lg).

A. quatuordecimguttata var. exoleta Weise. Mt. Whiteface, J1, Shoe (Sf).

A. quatuordecimguttata var. bicordifera Csy. NY, type locality.

A. quatuordecimguttata var. uniformis Csy. NY, type locality.

11201d A. quatuordecimguttata var. obliqua Rand. Mt. Whiteface, J1, Shoe (Sf).

A. quatuordecimguttata var. scutulata Weise. Mt. Whiteface, Jl, Shoe (Sf) ; Big Ind. Val., J1, Pears (Sf).

\section{Anatis Mulsant}

11202 A. quindecimpunctata Oliv. Plattsburg (NYS); Cranberry L., Je (H) ; Wilmington, Aug (Dt) ; Olcott, Je, Aug (Dt); Buffalo (Z\&R); Pike (NYS) ; Batavia, Je-J1 (Kngt) ; Castile, Brad (Flet) ; Ithaca, Ap-J1 (NYS, CU) ; Albany, Karner (NYS) ; Poughkeepsie (NYS) ; West Pt., J1 (Eng); White Plains (Sq); Port Jervis (Lint); Pine I., Je, 
Pollard (Lg); SI, May-Oct (L\&D) ; LI: Mattituck (CU) ; Flatbush, Amagansett, Eng (Sf); Rockaway, May, Pollard (Lg); Orient, Lath (NYS).

11202a A. quindecimpunctata var. mali Say. Webster, J1 (CU); SI, on shore at Ward's Pt., Je 5 (L\&D).

\section{NEOMysia Casey}

11205 N. pullata Say. Mt. Whiteface, J1, Shoe ( Lg); Buffalo (Z\&R) ; Ithaca, Ap-Aug (CU); W. Hebron (Lg); West Pt. (Robn); SI, Mar, Je-Ji (L\&D); LI: Far Rockaway, Central Pk., Promised Land (Sf).

11209 N. randalli Csy. Ithaca (Lg-CU).

\section{Axion Mulsant}

11212 A. tripustulatum DeG. SI, Je, Sep (L\&D) ; LI: Coney I. (Lg) ; Yaphank, May (Sf); Rockaway, Je, Pollard (Lg).

\section{CHILocorus Leach}

11217 C. bivulnerus Muls. Mt. Whiteface, J1 (Lg) ; Cranberry L., Je-J1 (H) ; Olcott, May-Je (Dt); Buffalo (Z\&R); Batavia, Aug (Kngt); Castile, May, Brad (Flet); Ithaca, Mar-Sep (CU); Bolton, Thompson (Lg); Albany, Karner, Nassau (NYS) ; Kingston, J1 (Sf); Poughkeepsie (NYS); West Pt. (Robn); SI, Ap-J1 (L\&D); LI: Aqueduct, Babylon, Aug (Sf); Orient, Lath (NYS).

\section{Exосномus Redtenbacher}

11220 E. marginipennis Lec. "Ind. Jct." (NYS); SI, Je-J1, Sep, usually on oak (L\&D) ; LI: Pinelawn, Yaphank (Sf).

11226 E. davisi Lg. LI: Yaphank (Sf); Wyandanch, May (Ds).

\section{SUBFAMILY EPILACHNINAE}

EPILACHNA Chevrolat

11229 E. borealis Fab. Ithaca, J1-Aug (CU); Peekskill, Sep (CU) ; West Pt. (Robn); White Plains, Sep 9 (Sq); SI, Ap-Aug (L\&D) ; LI: Glen Cove, Dosoris, Bellport (Lint); Sea Cliff (CU); Orient, Lath (NYS).

\section{FAMILY ALLECULIDAE (Cistelidae) SUBFAMILY ALLECULINAE \\ LOBOPODA Solier}

11236 L. punctulata Melsh. Callicoon (AM); Bronx Pk. (Sf) ; LI: E. Patchogue, Yaphank, May-Sep, Central Pk. (Ds).

11239 L. atra Say. Newport, Je (Yg); Greenwood L. (Sf); LI: Jamaica, Je (NYS); Wading R., JI (Ds); Yaphank, Je (Sf). 


\section{HYMENORUS Mulsant}

11246 H. niger Melsh. Newport, Je (NYS); Cranberry L., Wanakena, Aug (Drk); Olcott, Aug (Blaisdell); Buffalo (Z\&R); Castile, Brad (Flet); E. Aurora, J1, Allegany Pk., Aug (Flet,Dnd); Cincinnatus, J1 (Flet,Dnd); West Pt. (Robn); SI (L\&D); LI: Jamaica (Sf).

11250 H. melsheimeri Csy. Minnewaska, Aug 25, Nic (Frost).

11251 H. pilosus Melsh. Catskills (Sf); West Pt., Je (Sf); Bronx Pk. (Sf); SI, Je-Aug (L\&D); LI: Central Pk., Wading R., Yaphank, Jl-Sep (Ds).

11252 H. difficilis Csy. "NY" (Csy).

11253 H. perforatus Csy. NY (AM).

11255 H. obscurus Say. Buffalo (Z\&R); Mosholu (Sf); SI, Je (L\&D).

11267 H. discretus Csy. Mosholu (Sf).

11287 H. punctatissimus Lec. Buffalo ( $Z \& R)$. Described from Ariz. and southern Calif.

\section{Pseudocistela Crotch}

11299 P. brevis Say. Mt. Whiteface, J1 (Ds); Buffalo (Z\&R); Conesus L., Je (Ds) ; Ramapo, Je (Ds); West Pt., Je (Ds).

11301 P. pinguis Lec. Ramapo (Sf).

11303 P. marginata Ziegl. West Pt., May, Je, Robn (Sf).

IsOMIRA Mulsant

11306 I. tenebrosa Csy. Buffalo (Csy); Portage, Je (Ds); NYC (Csy).

11307 I. sericea Say. Newport, Je (Yg); L. Placid; Mt. Whiteface, J1 (Ds); E. Aurora, J1, Allegany Pk., Aug (Flet,Dnd); Buffalo (Z\&R); Pike (NYS); Ithaca, Jl-Aug (Dt); Taughannock Falls, Aug 11 (Vdyk); Albany, J1 (NYS); White Plains, J1 (Fall,Sq); West Pt. (Robn); Mosholu (Sf); SI, Je-J1 (L\&D); LI: Yaphank, J1 (Sf); Jamaica, Mineola, J1, A. Wolf (NYS); Flatbush (AM).

11311 I. quadristriata Couper. Axton, Je (M\&H) ; Speculator, Je (NYS); Hope, Aug, Bks (NYS); L. Placid, Je (NYS); Long L., Aug (NYS); Newport, Je (NYS) ; Cranberry L., Je-J1 (H); Severance, Aug, Bks (NYS); Gloversville, J1 (NYS); Northampton, Je (NYS); Sodus, J1 (NYS) ; Olcott, Je-Jl (NYS) ; Buffalo (Z\&R) ; Batavia, Je (Kngt); Pike, J1 (NYS) : Rock City (Ds); Montour Falls, Je (H); Ithaca, Je-J1 (Dt); Windsor, Je (Not); Schoharie (Not); Albany, J1 (NYS) ; Corinth, Je (NYS) ; Karner, J1 (NYS) ; West Pt., Ft. Montgomery, Stony Pt. (Ds); Mosholu, Je (NYS); SI, J1 (L\&D); LI: Flatbush (AM).

11314 I. oblongula Csy. Olcott, Je-J1 (NYS); Buffalo (Csy).

Chromatia LeConte

11319 C. amoena Say. Buffalo (Z\&R).

\section{CAPNOCHROA LeConte}

11320 C. fuliginosa Melsh. Newport, Je (Yg); Buffalo (Z\&R); North Branch, (Schaupp); Greenwood L. (Sf); West Pt., Je (Ds) ; Peekskill (Ds); SI, Je (L\&D); LI: Yaphank, Je (Sf); Half Way Hollow Hills, Ji (Ds). 
MycetochaRA Berthold

11321 M. haldemani Lec. Buffalo (Horn); West Pt. (Robn).

11322 M. fraterna Say. Cranberry L., J1 2, from dying spruce $(\mathrm{H})$; Buffalo $(Z \& R)$; Ithaca, Je (Dt); West Pt. (Robn); SI, Je (Ds); LI: Brooklyn, Je (Sf).

11324 M. megalops Csy. Northampton, Je (NYS); Batavia, J1 (Kngt); West Pt. (Robn).

11326 M. tenuis Lec. Newport, Je (Yg); Buffalo (Z\&R); Schenectady, J1 (NYS) ; Albany, Feb (NYS).

11327 M. foveata Lec. Buffalo.

11329 M. nigerrima Csy. "NY" (Csy); Cranberry L., J1 12 (H). Under dead bark of witch-hazel (Felt).

11330 M. lecontei Borchm. Rochester (Moore).

11331 M. rufipes Lec. Rochester (Moore); Ithaca, J1 (Dt).

11332 M. marginata Lec. "NY" (Lec).

11333 M. binotata Say. Newport (NYS) ; Cranberry L., J1 (Drk); Geneva, J1 (Cls); Buffaio (Z\&R); Ithaca, Je (Dt); Schoharie (Not); West Pt. (Robn) ; SI, Je (L\&D). Under poplar bark (Felt).

11336 M. bicolor Coup. Mt. Whiteface, J1 (Sf).

11337 M. analis Lec. West Pt. (Robn); Nyack, Je (Ds); Bronx Pk. (Sf) ; SI, $\mathrm{Je} 4$, on seashore (L\&D).

11338 M. lugubris Lec. Ithaca, J1 (Dt).

\section{SUBFAMILY OMOPHLINAE}

\section{ANDRIMUS Casey}

11353 A. convergens Csy. "NY" (Csy).

\section{ANDROCHIRUS LeConte}

11355 A. erythropus Kby. Cranberry L., J1 21 (H) ; Ithaca, J1 (Dt); North Branch (Schaupp); West Pt. (Ds); Mosholu, Je (Sf); SI (L\&D); LI: Half Way Hollow Hills (Ds).

\section{FAMILY TENEBRIONIDAE \\ Compiled by F. C. Fletcher \\ SUBFAMILY TENTYRIINAE}

BOTHROTES Casey

11551 B. arundinis Lec. Buffalo (Z\&R); West Pt. (Robn); SI, Sep (L\&D).

\section{Schoenicus LeConte}

11591 S. puberulus Lec. E. Aurora, Allegany Pk., Je (Dnd); LI: Coram, Selden, Riverhead, Amagansett, Deep Pond, Wading R., Aug-Sep (Ds); Promised Land, Sep, Eng (Sf). 


\section{Phellopsis LeConte}

11671 P. obcordata Kby. Axton, Je, 1901 (M\&H) ; Keene Val., Aug, Diven (Flet); White L., Zab (AM); Newport, Je (NYS); Cranberry L., J1 (Drk), Je (H); Buffalo (Z\&R); Ithaca, May (Dt); E. Jewett, Ap (Ds); North Branch (Schaupp).

\section{SUBFAMILY BLAPTINAE}

BLAPS Fabricius

11922 B. mucronata Latr. NYC, $D t$ (Sf).

11923 B. lethifera Marsh. NYC, Dt (Sf).

\section{SUBFAMILY PEDININAE \\ OPATRINUS Latreille}

12187 O. aciculatus Lec. SI, May-Nov (L\&D).

12188 O. minimus Beauv. Bangall, Sep, Eng (Sf); West Pt. (Robn); SI, Sep (L\&D); LI, Eng (Sf).

\section{Blapstinus Latreille}

12207 B. metallicus Fab. Cranberry L., J1 (H) ; Rochester (M\&W); Buffalo (Z\&R) ; Olcott, Ap-May, Aug (Dt) ; Ithaca, May (Flet); West Pt. (Robn); SI, May-Je (L\&D) ; LI: Greenport, Aug, Promised Land, Je, Eng (Sf) ; Babylon, Jl (AM).

12208 B. interruptus Say. Rochester (M\&W); Olcott, J1 (Dt); Buffalo (Z\&R); Ithaca, J1 (Dt) ; Peekskill, Ap, Schaupp (Blais); SI, Je (L\&D) ; LI: Babylon, Je (AM).

12227 B. moestus Melsh. Rochester (M\&W); West Pt. (Robn); Peekskill, Ap, Sherm (Flet); SI, May, Sep (L\&D); LI: Shinnecock Hills, Orient (Ds) ; Kockaway Beach, Ap, Eng, Queens, Schott (Sf).

\section{SUBFAMILY OPATRINAE}

AMmodonus Mulsant and Rey

12275 A. fossor Lec. Rensselaer (Lec); SI, May, Sep (L\&D); Tottenville, Je, Ds (Sf).

\section{EPHALUS LeConte}

12279 E. latimanus Lec. LI: Wading R., on shore, Ols (Ds) ; Amagansett, Aug, Promised Land, Eng (Sf); Rockaway Beach, Dt (Sf).

\section{SUBFAMILY PHALERIINAE}

PhALERIA Latreille

12286 P. testacea Say. SI, May-Aug (L\&D); LI: Rockaway Beach, Je, Long Beach, May (Ds); Babylon, Coney I. (AM). 


\section{SUBFAMILY BOLITOPHAGINAE}

\section{BolitotheRUS Candeze}

12295 B. cornutus Panz. Axton, Je (M\&H) ; Keene Val., Diven (Flet) ; Wilmington, Aug (Ds); Cranberry L., Je-J1 (H) ; Newport, May (NYS); White L., Zab, J1, abundant (AM) ; Rochester (M\&W) ; Olcott, Je-J1, Sep (Dt); Buffalo (Z\&R); Pike, May (NYS); Batavia, Aug-Sep (Kngt) ; Ithaca, Ap-Je, Sep (Dt, Flet); McLean, May (Blais-CU); Michigan Hollow Swamp, May (Flet); North Branch (Schaupp); West Pt. (Robn) ; SI (L\&D); LI: Rockaway Beach, Orient (Ds).

\section{BOLITOPHAGUS Illiger}

12296 B. corticola Say. Cranberry L., J1 (Drk); Buffalo (Z\&R); Allegany Pk., Je (Dnd); Ithaca, Feb (Blais-CU); North Branch (Schaupp).

\section{Eleates Casey}

12297 E. depressus Rand. Cranberry L., J1 (H) ; Rochester (M\&W) ; North Branch (Schaupp).

\section{SUBFAMILY RHIPIDANDRINAE}

\section{RHIPIDANDRUS LeConte}

12301 R. flabellicornis Sturm. Mosholu (Sf).

\section{SUBFAMILY DIAPERINAE}

\section{DIAPERIS Geoffroy}

12305 D. maculata Oliv. Cranberry L., Ji (H) ; Newport, Je (NYS) ; Rochester $(\mathrm{M} \& W)$; Batavia, Je-Ji (Kngt) ; Buffalo (Z\&R); E. Aurora, Je-Aug (Dnd); Pike, May (NYS) ; Ithaca, May, Jl, Sep (Flet, Dt); Karner, Je (NYS); West Pt. (Robn); Mosholu (AM); NYC (AM); SI, Ap-Sep (L\&D) ; LI: Yaphank, May, Eng, Wyandanch (Sf); Flatbush, Oakdale (AM).

\section{Hoplocephala Castelnau and Brullé}

12308

12309

H. viridipennis Fab. Buffalo ( $Z \& R$ ) ; SI, Ap-May (L\&D).

H. bicornis Fab. Cranberry L., J1 $(\mathrm{H})$; Syracuse (B\&S); Rochester $(\mathrm{M} \& W)$; Olcott, Mar, May, Oct, Dec (Dt); Batavia, Sep (Kngt) : Buffalo (Z\&R) ; Castile, Brad (Flet); Pike, May (NYS); Onondaga Co $(\mathrm{H})$; Westfield, May (Not); Geneva, Nov (Blais-CU); Ithaca, May (Flet); Taughannock Falls, Oct (Blais-CU); Albany, Ap (NYS); West Pt. (Robn) ; SI, Feb-May (L\&D); LI: Orient (Ds); Flatbush (AM).

SCAPHIDEMA Redtenbacher

12311 S. aeneolum Lec. Catskills, Pears, Oliverea, J1, Shoe (Sf); West Pt. (Robn). 
Platydema Castelnau and Brullé

12314 P. excavatum Say. Rochester (M\&W); E. Aurora, Oct-Nov (Dnd); Buffalo (Z\&R): Batavia, Aug (Kngt); Ithaca, Sep (Dt); Six Mile Cr., Ithaca, May (Flet); West Pt. (Robn); SI, Ap-May, J1 (L\&D) ; LI: Hempstead Plains, Yaphank, May (Cf) ; Forest Pk., Mar, Martin (Sf).

12314a P. excavatum var. parvulum Csy. LI, type locality.

12318 P. ruficorne Sturm. Rochester (M\&W) ; Buffalo (Z\&R); E. Aurora, Aug (Dnd); Pike, May (NYS); Ithaca, Ap-May (Flet); West Pt. (Robn); SI, Ap-Je, Sep (L\&D).

12321 P. ellipticum Fab. Rochester (M\&W) ; SI, Ap, J1 (L\&D) ; LI: Aqueduct, Flushing, Dec (Ds).

12323 P. americanum Cast. \& Brll. Peru, Je (Blais-CU) ; Cranberry L., J1 (H) ; Rochester (M\&W); Buffalo (Z\&R); Protection, Je, Dnd (Flet); Ithaca, May, Sep, Nov (Flet, Dt); Selkirk, Ap (NYS); SI, Je (L\&D).

12327 P. picilabrum Melsh. West Pt., May (Robn).

12328 P. subcostatum Cast. \& Brll. Newport, Je (NYS); Rochester (M\&W) ; Olcott, Sep-Oct (Dt) ; Buffalo (Z\&R) ; E. Aurora, Aug, Dnd (Flet); Ithaca, Sep-Oct (Dt); Albany, Aug (NYS); West Pt. (Robn); SI, May, J1 (L\&D) ; LI: Rockaway Beach, Yaphank, May, Eng (Sf); Flatbush (AM).

\section{Alphitophagus Stephens}

12332 A. bifasciatus Say. Howard, Dec (Blais-CU).

\section{PENTAPHYLLUS Latreille}

12333 P. pallidus Lec. Rochester (M\&W) ; Buffalo (Z\&R); Westfield, May (Not); Catskills, May (NYS).

\section{SUBFAMILY ULOMINAE}

GNATHOCERUS Thunberg

12336 G. cornutus Fab. SI (L\&D).

\section{TRIBOLIUM MacLeay}

12343 T. ferrugineum Fab. Buffalo (Z\&R); Albany, Oct (Fab) ; SI, Ap, SepOct (L\&D).

12345 T. confusum Jacq. Duv. Rochester, Jan (Flet-CU); Castile, Brad (Flet); Ithaca, May, Sep, Nov (Flet); West Pt. (Robn); SI (L\&D).

\section{DIOEDUS LeConte}

12346 D. punctatus Lec. Buffalo (Z\&R); Ithaca, Mar (Blais); Ramapo (AM).

PALORUS Mulsant

12348 P. depressus Fab. LI: Brooklyn (Chitt); Astoria, Aug, Felt (NYS).

12349 P. ratzeburgi Wissm. NYC (Chitt); LI: Howard, Dec (Blais-CU). 
ULOMA Castelnau

12353 U. impressa Melsh. Newport, May (NYS); Oswego, May (NYS); Rochester (M\&W) ; Olcott, May (Dt) ; E. Aurora, May (Dnd); Cattaraugus Co, Jl (Ds) ; Ithaca, Ap-Je (Blais, Dt); McLean Bogs, J1 (Blais, Dt); L. George, White L., Zab (NYS); SI, Mar-Sep (L\&D) ; LI: Jamaica, Pinelawn, Ap, Yaphank, May, Eng (Sf); Forest Pk., Mar, Martin, Shetland I., Jl, Eng (Sf).

12354 U. imberbis Lec. "NY to Kans." (Lec); West Pt. (Robn).

12357 U. punctulata Lec. Rochester (M\&W) ; Ithaca, J1 (Dt) ; LI: Yaphank, May (Sf).

\section{Alphitobius Stephens}

12360 A. diaperinus Panz. NYC (Blais).

12361 A. piceus Oliv. "NY" (Oliv).

\section{Eutochia LeConte}

12362 E. picea Melsh. Peekskill, Sherm (Blais-CU); Mosholu (Sf); SI, Ap (L\&D).

\section{HyPOPHLOEUS Fabricius}

12372 H. parallelus Melsh. Cranberry L., Jl (H); Olcott, Dec (Dt); Buffalo (Z\&R) ; SI, Mar (L\&D) ; LI: Central Pk., Ap, Eng (Sf) ; Rockaway (AM).

12376 H. tenuis Lec. Cranberry L., Je-J1 (H).

12377 H. thoracicus Melsh. Buffalo (Z\&R); SI, May (L\&D).

\section{SUBFAMILY TENEBRIONINAE}

\section{Scotobates Horn}

12386 S. calcaratus Fab. Essex Co, Aug (NYS); Peru, Je (Blais-CU); New Russia, Aug, Bdy (Blais); Malone, Oct (Blais-CU); White L., Zab $(A M)$; Syracuse (B\&S); North Fairhaven, Aug, Babiy (Flet); Honeoye Falls, Je (Ds); Rochester (M\&W); Erie Co, Jl-Sep, Allegany Pk., Aug (Dnd); Buffalo (Z\&R); Canandaigua L., J1 (NYS); Ellicottville, Aug (Ds) ; Ithaca, J1-Sep (Dt, Vdyk); McLean Bogs, Aug, Yuasa (Vdyk); Saratoga Sps., Aug (Dt); Albany (NYS); Oliverea (Ds) ; West Pt. (Robn) ; SI, Je-Aug (L\&D) ; LI: Cold Sp. Harb., Coram, Half Way Hollow Hills, J1 (Ds); Yaphank, J1, Sep, Eng (Sf); Flatbush, Zab (AM).

\section{MERINUS LeConte}

12389 M. laevis Oliv. Buffalo (Z\&R) ; Egleston's Glen, Keuka L., Je (Ds); Ithaca, Aug (Blais-CU); LI: Smithtown, Deep Pond, Wading R. (Ds) ; Yaphank, May, J1, Sep, Eng (Sf, Ds). 


\section{XyLopinus LeConte}

12390 X. saperdioides Oliv. Peru, J1, Mix \&Everett (Blais) ; Cranberry L., J1 $(\mathrm{H})$; Syracuse (B\&S); Rochester (M\&W) ; Batavia, J1 (Kngt); Buffalo (Z\&R); Pike, Je (NYS); Ithaca, May, Aug (Flet, Blais); Rensselaer, J1 (NYS) ; Pine I., Je (Ds) ; West Pt. (Robn) ; LI: Yaphank, J1, Eng (Sf, Ds).

12390a X. saperdioides var. rufipes Say. Buffalo (Z\&R); West Pt. (Robn); LI: Yaphank, J1, Eng (Sf).

12391 X. aenescens Lec. Ithaca, Aug, Oct, Reynolds (Blais); Bronx Pk. (Sf); SI, J1 (L\&D); LI: Yaphank, J1 (Ds).

\section{HAPLANDRUS LeConte}

12392 H. fulvipes Hbst. Rochester, Je, Leon (Flet, M\&W) ; Olcott, J1, Sep (Dt); Buffalo (Z\&R); Ithaca, Je, Aug (Blais-CU); Peekskill, Schaupp (Blais); West Pt., J1, Eng (Sf); Bronx Pk., Jí (Ds); Sİ, Je, Aug (L\&D) ; LI: Babylon, Flatbush (AM).

12393 H. ater Lec. LI: Far Rockaway, Mar, Dow (Sf).

\section{IPHTHIMUS Truqui}

12405 I. opacus Lec. Saranac Inn, J1, Newport, May (NYS); Mt. Whiteface, 2000-4000 ft., Aug (Dt); Wanakena, Aug (Drk) ; Cranberry L., JeJ1 (H) ; Rochester (M\&W) ; Pike, May (NYS); Ithaca, Je (Flet); Windsor, Je (Not); Big Ind. Val., Sep, Eng (Ds); Karner, J1 (NYS).

\section{Alobates Motschulsky}

12407 A. pennsylvanica DeG. Chateaugay L., Sep (AM) ; Cranberry L., Je-J1 (H); Wells, Je, Nov, Babiy (Flet); Syracuse, Aug, Oct (Drk); Rochester (M\&W) ; Buffalo (Z\&R); Batavia, Je (Kngt); Erie Co, Je-Dec (Dnd) ; Westfield, May (Not) ; Castile, Brad (Flet); Summit, Crouse (Lint); Penn Yan, Cy (Blais); Coy Glen, May (Blais-CU); Ithaca, May, J1 (Dt) ; McLean, May (Blais-CU) ; Windsor, Je (Not); Saratoga Sps., Aug (Dt); Poughkeepsie, Je (NYS); West Pt. (Robn); SI, Feb, J1 (L\&D) ; LI: Pinelawn, Central Pk., Yaphank, Wyandanch, Amagansett, Half Way Hollow Hills, Mar-Je (Ds); Brooklyn, Oct, Eng (Sf); Flatbush (AM).

12408 A. barbata Knoch. Ithaca, Oct (Blais-CU) ; LI: Yaphank, May, Pinelawn, Ap, Eng (Sf).

\section{UPIS Fabricius}

12411 U. ceramboides L. Axton, Je $(\mathrm{M} \& \mathrm{H})$; Peru, J1, Mix\& Everett (Blais) ; Wilmington, Aug (Ds) ; Cranberry L., Je-J1 (Drk, H) ; Mt. Whiteface, 4000-4872 ft., Aug (Dt); Saranac Inn, Je (NYS); Rochester $(\mathrm{M} \& W)$; Buffalo (Z\&R); Pike (NYS); Fulton, Ap, Cy (Blais); Karner, Mar, J1 (NYS); LI: Orient, May, Lath (Ds).

\section{IDIOBATES Casey}

12412 I. castaneus Knoch. Rochester (M\&W) ; Buffalo (Z\&R) ; Ithaca, Je (Dt) ; LI: Yaphank, May, Sep, Eng (Sf). 


\section{TENEBRIO Linnaeus}

12413 T. obscurus Fab. Severance, Aug, Newport, May (NYS); Rochester $(M \& W)$; Buffalo (Z\&R) ; Victory, J1 (NYS) ; Ithaca, Je (BlaisCU) ; Albany, Je, Ilion, May (NYS); Nyack (AM); SI, May-Je, Aug (L\&D) ; LI: Yaphank, J1 (Ds) ; Brooklyn, J1 (Sf) ; Flatbush (AM).

12414 T. molitor L. Essex Co, J1, Newport, Je (NYS); Peru, J1, Mix \& Everett (Blais); Keene Val., Diven (Flet); Rochester, J1, Leon (Blais); Buffalo (Z\&R); Otto (Blais-CU); Castile, J1, Brad (Flet); Ithaca, J1Aug, $D t$ (Blais, Flet) ; Newark Val. (Lint) ; Albany, Wadham (Lint); Coeymans, SmEB (Lint); Mt. Vernon, May (NYS); SI, Je-J1 (L\&D) ; LI: Orient, Lath (NYS) ; Flatbush, Je, Eng (Sf) ; Brooklyn, Oct, Rockaway Beach, May, Eng (Sf).

\section{NeAtus LeConte}

12415 N. picipes Hbst. (Tenebrio). Essex Co., Aug, Newport (NYS) ; Cranberry L., Je (H) ; Keene Val., Diven (Flet-CU); Peru, Je (Blais-CU); Oswego, May (NYS) ; Syracuse (B\&S); Rochester (M\&W) ; Olcott, May-Aug (Dt) ; Buffalo (Z\&R) ; Castile, Brad (Flet) ; E. Aurora, Je, Dnd (Flet); Canandaigua, Aug (Blais-CU); Ithaca, Ap-Sep (Dt); Enfield Falls, Ap (Blais); Albany, Ilion, Karner, Mar (NYS) ; Catskills, Aug (NYS) ; West Pt. (Robn); SI, Ap, J1 (L\&D) ; LI: Yaphank, Deep Pond, Long Pond at Wading R., Pennequid Barrens, Coram (Ds).

\section{BoRos Herbst}

12423 B. unicolor Say. Buffalo (Z\&R); Ithaca, Mar (Dt) ; Ap 13 (Flet) ; Peekskill, Sherm (Flet-CU); SI, Ap, Nov (L\&D);LI: Melville, Feb, Schott (Frost) ; Central Pk., Ap, Eng, Parkville (Sf).

\section{SUBFAMILY HETEROTARSINAE}

ANAEDUS Blanchard.

12425 A. brunneus Ziegl. E. Aurora, May (Dnd); West Pt. (Robn); SI, Ap, Nov (L\&D), Sherm (Flet-CU); LI: Selden, Aug (Ds); Yaphank, Sep, Eng, Forest Pk., Mar, Martin (Sf).

\section{Prateus LeConte}

12431 P. fusculus Lec. "NY" (Lec).

\section{PaRatenetus Spinola}

12432 P. punctatus Spin. Keene Val., Sep, Eng (Sf); Buffalo (Z\&R); E. Aurora, J1, Dnd (Flet); Ithaca, Je-Aug (Dt); Ft. Edward, Je (NYS); Kenwood, Je (NYS); West Pt. (Robn); SI, Je (L\&D) ; LI: Yaphank, Sep, Eng (Sf).

12433 P. fuscus Lec. Peekskill, Ap, Sherm (Blais-CU). 


\section{SUBFAMILY HELOPINAE}

\section{TARPELA Bates}

12446 T. micans Fab. Rochester (M\&W); Buffalo (Z\&R); Erie Co, May-J1 (Dnd) ; Ithaca, May (Flet-CU); West Pt. (Robn) ; Peekskill, Schaupp, Sherm; Sterlington, May, Cy (Flet-CU) ; SI, Ap-J1 (L\&D); LI: Lynbrook, Je, Cy (Flet-CU) ; Orient, Lath (NYS) ; Wading R., Massapequa, Yaphank, Wyandanch, Central Pk. (Ds); Flatbush (AM).

12448 T. venusta Say. SI, Aug (L\&D).

\section{HELOPS Fabricius}

12455 H. gracilis Bland. LI: Yaphank, Je-Jl, Rockaway Beach, May, Eng (Sf). 12469 H. aereus Germ. Peekskill (Schaupp); West Pt., Je (Ds) ; Bronxville, Je (NYS) ; SI, May-Je (L\&D) ; LI: Central Pk., Massapequa, Half Way Hollow Hills, May-J1 (Ds) ; Flatbush (AM); E. New York, Pinelawn, May, Eng (Sf) ; Central Pk., Ap, Eng (Sf).

\section{SUBFAMILY MERACANTHINAE}

\section{MeRACANTHA Kirby}

12485 M. contracta Beauv. Rochester (M\&W) ; Olcott, Je-J1 (Dnd, Dt); Buffalo $(Z \& R)$; Lake Bluff, Ap, Je (CU); Aurora, Aug, Foster (Flet-CU); SI, J1 (L\&D) ; LI: Parkville (AM) ; J1, Eng (Sf).

\section{SUBFAMILY STRONGYLIINAE}

\section{STRONGYLIUM Kirby}

12489 S. terminatum Say. Ithaca, J1 (Flet).

12488 S. tenuicolle Say. Rochester, Jl, Leon (Blais-CU); Olcott, Je (Dt); E. Aurora, Je (Dnd); Ithaca, Jl, Babiy (Flet); West Pt. (Robn) ; SI, Je-J1 (L\&D); LI: Je, Eng (Sf); Parkville (AM).

\section{FAMILY LAGRIIDAE}

\section{ARTHROMACRA Kirby}

12497 A. aenea Say. Speculator, Je, Wells, Je, Old Forge, L. Placid, Je (NYS); Saranac Inn, Je (NYS); Axton, Je (M\&H) ; Cranberry L., Je-Jí $(\mathrm{H})$; Olcott, Je-J1 (Dt); Buffalo (Z\&R); Pike (NYS); Westfield, Je (NYS); Montour Falls, Je (H) ; Ithaca, Ringwood, McLean Bogs, Je-J1 (Vdyk); Windsor, Je (Not); Albany, Corinth, Lansingburg, North Chatham, Poughkeepsie, Je (NYS); West Pt. (Robn); Mosholu, Je (Sf); Huguenot, White L. (AM); West Pt., J1 (Sf); White Plains, Je (Sq); vic. NYC (AM); SI, May-Je (L\&D).

\section{STAtiRa Serville}

12502 S. gagatina Melsh. Mosholu (Sf); SI, Je-J1 (L\&D); LI: Flatbush, Long Beach (AM). 


\section{FAMILY MELANDRYIDAE}

\section{TETRATOMA Fabricius}

12519 T. truncorum Lec. Johnstown, Oct (NYS); Buffalo (ANSP).

\section{AbSt RULia Casey}

12521 A. tessellata Melsh. Buffalo (Z\&R); West Pt. (Robn); Mosholu (Sf); SI, Je (L\&D).

\section{Pisenus Casey}

12525 P. humeralis Kby. Syracuse, Ap, bracket fungus $(\mathrm{H})$; Olcott, Mar, Aug (Dt); Westfield, May (Not); Howland I., Nov (CU); Shokan, Thompson (Lg); West Pt. (Robn); LI: Bellport, Aug, Nic (Woodr).

\section{Penthe Newman}

12527 P. obliquata Fab. Cranberry L., Je-J1 (H) ; Buffalo (Z\&R) ; Olcott, May, Aug-Sep (Dt) ; Castile, Brad (Flet); Ithaca, May, Sep (Dt-CU) ; Schenectady, May, Aug-Sep (Dt) ; Peekskill, J1 (Sherm); West Pt. (Robn) ; Bronx Pk., Sep (NYS) ; Bronxville, Van Cort. Pk., Mar-J1 (Woodr); SI, Jan-Nov (L\&D); LI: Flatbush, Ap, Oct (Sf, Woodr-AM); Jamaica, Bellport, Nic (Woodr); Parkville (Woodr-AM) ; Flushing, Dec, Bell (Ds); Melville, Sep, Yaphank, May-Je (Ds).

12528 P. pimelia Fab. Mayfield, May (NYS) ; Cranberry L., Je 23 (H) ; Syracuse, Ap-May, under bark (H) ; Olcott, Je, Sep (Dt); Buffalo (Z\&R); Batavia, J1 (Kngt); Ithaca, Je-Sep (Dt); W. Hebron, Je (Lg); Ulster Co, Nic (Woodr); Van Cort. Pk. (Woodr); LI: Flatbush (Woodr-AM). Under beech bark (Felt).

\section{SYNCHROA Newman}

12529 S. punctata Newn. Ilion, May (NYS); Olcolt, May-Aug (Dt); Buffalo (Z\&R) ; Castile, Brad (Flet); Cincinnatus, Ap (Flet,Dnd); Ithaca, Aug (CU); Trenton Falls (Newn); Albany, May (NYS); Catskills (Woodr); West Pt. (Robn); West Farms (Woodr-AM); SI, MayAug (L\&D) ; LI: Rockaway Beach, Je (Sf); Bellport, Jamaica, J1, Nic (Woodr); Yaphank, J1 (Woodr). Larvae and pupae under maple bark in $\mathrm{Je}(\mathrm{Yg})$.

\section{EUSTROPHINUS Seidlitz}

12531 E. bicolor Fab. Johnstown (NYS); Olcott, Aug-Dec (Dt); Buffalo (Z\&R) ; Pike (NYS); Batavia, J1 (Kngt); E. Aurora, May, Oct (Flet,Dnd) ; Ithaca, Aug-Dec (Dt); Van Cort. Pk., Ap (Woodr); SI, Ap-Sep (L\&D); LI: Queens, May (Eng); Jamaica, May (Lg); Rockaway, J1 (Woodr). Under bark of linden (Felt); on the fungi Pleurotus sapidus and Polyporus squamosus in N.J. (Weiss).

12534 E. confinis Lec. West Pt. (Robn). 


\section{EUSTROPHUS Illiger}

12535 E. tomentosus Say. Johnstown, Gloversville, May, Aug (NYS) ; Newport (NYS); Syracuse, May, under bark (H); Olcott, Je, Aug (Dt); E. Aurora, Je (Flet,Dnd); Batavia, Je-J1 (Kngt); Buffalo (Z\&R); SI, May (L\&D).

\section{SYNSTROPHUS Seidlitz}

12536 S. repandus Horn. Johnstown, Aug (NYS); Olcott, Je-Sep (Dt); E. Aurora, Aug (Flet,Dnd); Pike, Aug 15 (Frost); West Pt. (Robn).

\section{HOLOSTROPHUS Horn}

12537 H. bifasciatus Say. Newport (NYS); Buffalo (Z\&R); West Pt. (Robn); Peekskill, Mar, Jl, Joutel (Woodr); White Plains, Mar (Sf); SI: Clove Val., May, Bischoff (L\&D); LI: Wyandanch (Sf); Jamaica (Woodr); Forest Pk., Yaphank (Lg); Bellport, Je, Nic (Woodr).

\section{Hallomenus Panzer}

12540 H. scapularis Melsh. Buffalo (Z\&R); Ithaca, Aug (Sf).

12541 H. punctulatus Lec. Ludlow, Thompson (Lg); SI, J1 (L\&D).

12542 H. serricornis Lec. LI: Montauk Beach, J1 (Sf).

\section{ORCHESIA Latreille}

12546 O. castanea Melsh. Wilmington, J1-Sep (Dt) ; Old Forge, J1 (CU) ; O1cott, J1-Sep (Dt); Buffalo (Z\&R); Pike, Sep (NYS); Ithaca, Sep (CU) ; Bolton, Aug, Thompson (Lg) ; Nassau, Sep (NYS) ; Callicoon, Mosholu (Woodr-AM); West Pt. (Robn); Shokan, Thompson (Lg): Bronxville, Je (Woodr); SI, Je-J1, Nov (L\&D) ; LI: Flatbush, Jamaica (Woodr); Yaphank, Sep (Sf). On birch fungus, also linden (Felt); on Trametes suaveolens on willow (Weiss); on hard fungus on beech $(\mathrm{Lg})$.

12547 O. gracilis Melsh. Cranberry L., Je 23 (H); Buffalo (Z\&R); LI: Orient Pt., Je (NYS). On birch fungus (Felt).

MICROSCAPHA LeConte

12548 M. clavicornis Lec. Mosholu (Sf); Bronxville, J1 (Woodr).

\section{Prothalpia LeConte}

12550 P. undata Lec. Cranberry L., Je, $H$ (Dt) ; Buffalo (Z\&R) ; Big Ind. Val., J1, Pears (Woodr); West Pt. (Robn); Ramapo (Sf).

\section{MELANDRYA Fabricius}

12552 M. striata Say. Peru, Je (CU) ; Mt. Whiteface, J1 (Ds) ; Axton (M\&H) ; Olcott, May-J1 (Dt) ; Pike, May (NYS) ; Batavia, Je (Kngt); Rock City, Je (CU) ; Ithaca, May (CU); Honeoye Falls, Je (Ds); McLean Bogs, May (CU) ; Windsor, Je (Not); Karner (NYS); Oliverea, Je (Ds); Peekskill (Sherm); West Pt., Ft. Montgomery, May-Je (Ds); Tarrytown, J1 (Woodr); Bronxville, May (Woodr); SI, May-Je (L\&D) ; LI: Queens, Promised Land, Je (Sf). 


\section{EMMESA Newman}

12553 E. labiata Say. Mt. Whiteface, J1 (Woodr); Ithaca, Je (Vdyk); Wordsworth (?), J1 (NYS) ; Oliverea, J1 (Woodr) ; West Pt. (Robn) ; SI, May-Je (L\&D); LI: Valley Stream, May 16, Schott (Frost); Forest Pk., Je (Ds); Wading R., May, Nic (Woodr).

12554 E. connectens Newn. Mt. Whiteface (Woodr-AM), Je (Ds) ; Cranberry L., Je-J1 (H) ; Buffalo (Z\&R) ; Big Ind. Val., Sep, Pears (Woodr); Debruce, Slide Mt., J1 (Sf) ; LI: Jamaica, J1, Nic (Woodr).

\section{Amblyctis LeConte}

12556 A. praeses Lec. Near Buffalo, type locality (Z\&R); Cascade (Springville, Erie Co), J1 12, $M C V$ (Frost); Ithaca, J1 (Sf).

\section{XyLita Paykull}

12557 X. laevigata Hellen. Mt. Whiteface, J1, Quirs (Woodr); Mt. Seward, Je $(\mathrm{M} \& \mathrm{H})$; Cranberry L., J1 (Dt).

\section{ZILORA Mulsant}

12560 Z. canadensis Hausen. Mt. Whiteface, J1, Shoe ( Lg); Slide Mt., Je ( Lg).

\section{RUSHIA Forel}

12561 R. longula Lec. Buffalo (Z\&R) ; E. Aurora, J1, Dnd (Dt) ; LI: Wyandanch, J1 (Sf).

\section{SPILOTUS LeConte}

12564 S. quadripustulatus Melsh. Olcott, Je (Dt); Buffalo (Z\&R); Rockland Co, Je, Joutel (Lg); Mosholu (Sf); SI, Je (L\&D).

\section{SCOTOCHROA LeConte}

12565 S. atra Lec. Mt. Whiteface, J1 (Sf); Buffalo (ANSP).

\section{ENCHODES LeConte}

12567 E. sericea Hald. Cranberry L., J1 19 (H) ; Buffalo (Hald) ; Ithaca, Je-J1 (Dt). On maple (Felt).

\section{SERROPALPUS Hellenius}

12568 S. barbatus Schall. Mt. Whiteface, J1 (Ds); Cranberry L., Je-Aug (H); Ithaca, Je (CU) ; Slide Mt., J1 (Sf); West Pt. (Robn). Enters wounds on living trees, common on blazed balsam, fir, and spruce (Felt).

\section{Mystaxús Kugelann}

12569 M. simulator Newn. Herkimer Co (Lg); Buffalo (Z\&R); Pike (NYS); W. Hebron, Je ( $\mathrm{Lg})$; Trenton Falls (Newn); Big Ind. Val., May, Pears (Woodr); West Pt. (Robn); Peekskill (Sherm); Katonah $(A M)$; SI (AM); LI: Jamaica (Woodr). In rotting beech and maple stumps (Felt). 


\section{DircaEA Fabricius}

12570 D. quadrimaculata Say. Big Moose, J1 (NYS) ; Cranberry L., Je-J1 (H); Peru, Je (CU) ; Buffalo (Z\&R) ; Batavia, J1-Sep (Kngt) ; Ithaca, J1 (CU) ; Catskills (Woodr); Oliverea, Je (Ds); Florida, Aug, FrostSW (CU); West Pt. (Robn); SI, Je-Aug; reared from blue beech, Je (L\&D); LI: Pinelawn, Aug (Ds); Yaphank, J1 (Ds,Sf). Under dead maple bark (Felt).

\section{Phloeotrya Stephens}

12571 P. vaudoueri Muls. West Pt. (Robn); Nyack, Je (Joutel); Cos Cob (Rbts); Bronxville, J1 (Woodr); LI: Bellport, Je (Nic).

\section{HyPulus Paykull}

12573 H. concolor Lec. West Pt. (Robn); Bronxville, J1 (Woodr).

\section{MICROTONUS LeConte}

12576 M. sericans Lec. Buffalo (ANSP); Ithaca, J1 (CU); SI, Je (L\&D); LI: Farmingdale, J1, MorrowND (CU).

\section{SyMPHORA LeConte}

12577 S. flavicollis Hald. Newport, Je (NYS) ; Cranberry L., Je 11 (H) ; Olcott, Je, Aug (Dt) ; Montour Falls, Je (H) ; Peekskill, Je (Sherm) ; Catskills (Woodr); Mosholu (Sf); SI, Je (L\&D); LI: Jamaica (Woodr).

12578 S. rugosa Hald. Buffalo (Z\&R); Catskills (Sf,Woodr); SI (L\&D).

\section{Anisoxya Mulsant}

12579 A. glaucula Lec. "NY" (Lg) ; Bronxville, J1 (Woodr) ; LI: Aqueduct, J1, Shoe $(\mathrm{Lg})$.

\section{Allopoda LeConte}

12580 A. lutea Hald. "NY" (Hald); Bronx Pk. (Dt); LI: Rockaway Beach (Woodr-AM).

SCRAPTIA Latreille

12583 S. sericea Melsh. Buffalo (Z\&R); Peekskill (Sherm); Mosholu (Sf).

\section{CANifa LeConte}

12585 C. plagiata Melsh. "NY" (Lg); LI: Wading R., May, Nic (Woodr).

12586 C. pusilla Hald. "NY" (Lg); Olcott, Je-J1 (Dt); Ithaca, Je-J1 (Dt); Bronxville, J1 (Woodr); LI (Woodr).

12587 C. pallipennis Lec. Ludlow, Thompson ( Lg); SI, Je (L\&D).

12588 C. pallipes Melsh. Cranberry L., Je-J1 (H); Ithaca, May-Je (CU); Windsor, Je (Not); Schoharie (Not); Middletown, J1 (CU); Ramapo, May (Ds); Ludlow, Thompson (Lg); Peekskill, Thompson (Lg); Catskills (Woodr) ; Mosholu (Sf) ; SI, J1 7 (L\&D) ; LI: Bellport, Aug, Nic (Woodr). 


\section{OSPHYA Illiger}

12589 O. varians Lec. Olcott, Je (Dt); Buffalo (Z\&R).

\section{MycteRUS Clairville}

12594 M. scaber Hald. Gloversville, Aug (NYS) ; Ithaca, J1-Aug (Dt) ; Karner, Je (NYS); Washington Co (Lg); Cairo, J1 (Lg).

12595 ?M. concolor Lec. Newport, Je (NYS); Oliverea, Je, three specimens doubtfully placed (Ds).

\section{FAMILY PTINIDAE \\ Gibium Scopoli}

12600 G. psylliodes Czemp. NYC, in Produce Exchange, Ds (Joutel).

\section{Mezium Curtis}

12601 M. americanum Lap. NYC (Fall); "NYC", Aug, Dec (Sf).

\section{TRIGONOGENIUS Solier}

12604 T. globulum Sol. NYC (Ds).

\section{Ptinus Linnaeus}

12610 P. villiger Reit. "NY" (Fall); LI: Jamaica (Sf).

12612 P. brunneus Dufts. Axton, Je (M\&H) ; Honeoye Falls, F. Mather (Rly); SI (L\&D).

12613 P. fur L. White-Marked Spider-Beetle. Buffalo (Z\&R); Waterville, May, I. M. Hawley (Kngt); SI, Ap (L\&D); LI: Rockaway, May, Yaphank (Sf); Orient, Lath (NYS).

12625 P. bimaculatus Melsh. "NY" (Fall).

12629 P. quadrimaculatus Melsh. Middleville, Jan, in green calfskins (Lint).

\section{FAMily ANOBIIDAE 49 SUBFAMIIY ANOBIINAE}

EUCRADA LeConte

12639 E. humeralis Melsh. Newport (NYS); Cranberry L., J1 (Drk) ; Wells, J1 (NYS) ; Buffalo (Z\&R); Batavia, Je (Kngt); McLean, May (CU); Greenwood L., Je (Sf); West Pt. (Robn); Ft. Montgomery, May (Ds); Peekskill, Je (CU); Bronx, Je (Lg); SI, May, on dead oak (L\&D) ; LI: Westbury (Champlain). On beech.

\section{Xestobium Motschulsky}

12641 X. rufovillosum DeG. Cranberry L., J1 (H); NYC (Palm). On black spruce (Felt).

49 The manuscript for this family has been checked by H. C. Fall. 


\section{ERNOBIUS Thomson}

12649 E. mollis L. Windsor, Je (Not); Karner, May (NYS); Albany, Je (NYS); Nassau, Je (NYS); NYC vic. (Lg). On hard pine (Felt). [12664 E. filicornis Lec. Coast region from Mass. to Va. (Fall).]

12665 E. granulatus Lec. West Pt. (Robn). On pine (Felt).

[12667 E. luteipennis Lec. Coast region from Mass. to Va. (Fall). On pine (Felt).]

12668 E. tenuicornis Lec. Mass. to Pa.; Karner, May (NYS). On pine (Felt).

\section{OLIGOMERUS Redtenbacher}

12684 O. sericans Melsh. NY (Fall); SI, Aug 6 (L\&D).

12686 O. obtusus Lec. Buffalo (Fall); Albany (Fall); Catskills, Je, Pears (Nic); SI (Fall); LI (Fall).

12688 O. alternans Lec. LI: Mastic, Je, as alternatus (Nic).

\section{SITODREPA Thomson}

12689 S. panicea L. Drug-Store Beetle. Castile, Brad (Flet); Geneva, J1 (NYS); Buffalo (Z\&R); Albany, May, Je (NYS); Valley Falls, May (NYS) ; Chatham, Je, Branion (NYS); Van Cort. Pk., Sep (Joutel); NYC, in red pepper (Lutz); SI (L\&D); LI: Brooklyn (Sf). A few years ago this species became so numerous in a storage warehouse in NYC that thousands were swept from the pavement in front of the store $(\mathrm{Lg})$.

\section{TRICHODESMA LeConte}

12695 T. gibbosa Say. Syracuse (B\&S); Buffalo (Z\&R); Pine I., Je, on hickory (Ds) ; Mosholu (Sf) ; Van Cort. Pk., Pears (Nic); SI (L\&D). On elm (Felt).

\section{HADROBREGMUS Thomson}

12705 H. carinatus Say. Newport, Je (NYS); Cranberry L., Je 23 (H); Spencerport, Dec 5 (Vdyk); Buffalo (Z\&R); Avon, Je 19 (Cls); Ithaca (Palm); Schoharie (Not); Albany, Ji (NYS); Nassau, Ji (NYS); Oliverea, J1 (Sf) ; West Pt. (Robn) ; Ludlow, Je (Thompson); SI (L\&D); LI: Bellport, Je (Nic). On hickory (Felt).

12706 H. linearis Lec. Buffalo (Z\&R); Peekskill (Sherm); West Pt. (Robn).

12707 H. pusillus Fall. Cranberry L., J1, maple (H); Ithaca (Fall).

12709 H. umbrosus Fall. "NY" (Fall).

\section{Coelostethus LeConte}

12712 C. notatus Say. Buffalo (Z\&R); Ithaca ( $\mathrm{Lg})$; West Pt. (Robn); LI: Yaphank, Je (Sf). On oak'(Felt).

\section{TRYPOPITYS Redtenbacher}

12717 T. sericeus Say. Newport, Je (NYS); Buffalo (Z\&R); Cattaraugus Co, J1 (Ds); Ithaca, not rare at light (Fbs); Albany, Aug (NYS); Nassau, J1 (NYS) ; Ludlow (Thompson); NYC and vic. (Palm); Bronx Pk. (Sf) ; SI (L\&D) ; LI: Greenport, Aug (Sf). 
XYLETINUS Latreille

12734 X. peltatus Harr. Nassau, J1 (NYS); Catskills, Je, Pears (Nic); Bronx (Joutel).

12735 X. harrisi Fall. NY (Fall); Van Cort. Pk., Aug (Sf).

$12736 \mathrm{X}$. fucatus Lec. Buffalo (Rein).

12738 X. lugubris Lec. West Pt. (Robn); NYC vic. (Blanchard).

\section{LASIODERMA Stephens}

12742 L. serricorne Fab. NY (Fall); Castile, Brad (Flet); Elmira, on tobacco (CU Econ); Albany, Jan (NYS) ; Middletown, on tobacco (CU Econ); NYC, on cigars (Cy); SI, in stored paprika (L\&D); LI, Feb (Sf).

12743 L. falli Pic. Peekskill, Sherm (Fall).

\section{Petalium LeConte}

12752 P. seriatum Fall. SI (Fall).

12754 P. bistriatum Say. LI: Aqueduct, J1 (Sf).

\section{Eupactus LeConte}

12761 E. nitidus Lec. Catskills, Je (Sf); Peekskill, Sherm (Lg).

Catorama Guérin-Ménéville

12786

12802

12804

C. dichroum Fall. Greenwood L., Je (Sf).

C. vexatum Fall. NY (Fall).

C. grave Lec. Buffalo (Z\&R); NYC (Rbts); SI, Je 26 (L\&D); LI (Fall).

\section{ProtheCA LeConte}

12837 P. hispida Lec. NY (Lec).

12838 P. puberula Lec. Keene Valley, Je (Not); Buffalo (Z\&R).

\section{DORCATOMA Herbst}

12839 D. dresdensis Hbst. Cranberry L., Je-J1 (H) ; Valley Falls, May (NYS).

12841 D. setulosum Lec. Newport, Je (NYS); Ithaca, Je (NYS). In woody fungus on birch (Felt).

\section{Eutylistus Fall}

12843 E. intermedius Lec. Valley Falls, May (NYS).

12845 E. tristriatus Lec. "NY"? (Chitt, Fall).

\section{CAENOCARA Thomson}

12852 C. scymnoides Lec. Nassau, May (NYS); Poughkeepsie, Je (NYS).

12855 C. oculata Say. Cranberry L., Je 9, from Betula (H) ; Newport (NYS); Buffalo (Z\&R) ; Ithaca (Lg); West Pt. (Robn); SI, May, Aug, Nov (L\&D). 


\section{PTILINUS Geoffroy}

12864 P. ruficornis Say. Saranac Inn, injuring birch and maple floors (Felt); Newport, Je (NYS); Cranberry L., Je-J1, common in seaweed and dead beech $(\mathrm{H})$; Northampton, Je (NYS) ; Clay, in timbers, J1 (CU); Buffalo (Z\&R); Westfield, Je (NYS); West Pt. (Robn); NYC (Palm). Beech, maple, cherry, and ironwood (Felt).

\section{FAMILY BOSTRICHIDAE}

\section{EndeCATomus Mellie}

12870 E. reticulatus Hbst. West Pt. (Robn); SI, in Polyporus gilvus (L\&D).

12871 E. rugosus Rand. Newport, Je (NYS) ; Johnstown, Aug (NYS); Buffalo $(Z \& R)$; Karner, Mar (NYS) ; vic. NYC (AM); LI: Flatbush, Nov (Sf).

\section{Scobicia Lesne}

12873 S. bidentata Horn. West Pt. (Robn); SI, Je (L\&D); LI: Jamaica, Je Pears (Nic); Long Island City, Aug, $S z$ (NYS); Flatbush, Pinelawn, Je (Sf).

\section{XYLOBIOPS Casey}

12877 X. basillare Say. Syracuse, in hickory (Rhoades); Albany, Je (NYS); West Pt., Je, beating scrub pine (Robn); Bronx Pk. (Sf) ; LI: Rockaway Beach, Je (Sf); Long Beach, May (Sf). Makes short curved galleries in the branches of deciduous trees (Felt).

\section{AMPHICERUS LeConte}

12888 A. bicaudatus Say. Apple Twig-Borer. Buffalo (Z\&R); LI: Long Beach, Je (Sf).

\section{LICHENOPHANES Lesne}

12900 L. truncaticollis Lec. Syracuse, in hickory (Rhoades); Buffalo (Z\&R).

\section{BOSTRICHUS Geoffroy}

12902 B. bicornis Web. Syracuse, in hickory (Rhoades); Buffalo (Z\&R); Peekskill (CU); Mosholu (Sf) ; SI, Je 26 (L\&D); LI: Rockaway Beach, Je (Ds); Yaphank, Je (Sf). On oak, elm, and apple (Felt).

12903 B. angustus Csy. "NY" (Sf).

\section{STEPHANOPACHYS Waterhouse}

12908 S. substriatus Payk. Albany, Je (NYS).

12913 S. rugosus Oliv. Axton, Je (M\&H) ; Buffalo (Z\&R); West Pt. (Robn) ; SI, May (L\&D); LI: Cold Sp. Harb. (Sf).

12915 S. hispidulus Csy. LI: Long Beach, May (Sf).

12916 S. densus Lec. NY (Lec); LI: Wyandanch, May (Sf).

12918 S. cribratus Lec. LI: Central Pk., Ap, about cut pine (Eng) ; Orient Pt., May, Lath (NYS); Coney I., May, Long Beach, Je (Sf):

12919 S. punctatus Say. Sterling L., May (Sf) ; West Pt. (Robn); Peekskill, vic. NYC (AM); LI: Wyandanch (Sf); Central Pk., Je (Sf). 
RHIZOPERTHA Stephens

12921 R. dominica Fab. Karner, Je (NYS).

\section{FAMILY LYCTIDAE}

Lyctus Fabricius

(Trogoxylon LeConte)

12932 L. punctatus Lec. "NY", J1 (Lec).

12934 L. parallelopipedus Melsh. Buffalo, Oct, in black walnut lumber (Felt); LI (Hopk).

12938 L. opaculus Lec. Westfield, Je (NYS) ; Pine I. and West Pt., Je (Ds); Mosholu (Sf); SI, Je (Hopk) ; LI: Jamaica, May (NYS) ; Rockaway Beach, Je (Sf).

12940 L. planicollis Lec. Hudson, May, destroying shovel handles (Cy) ; SI, boring in maple in Mus. SI Inst. Arts and Sci., adult emerged Ap (L\&D).

12943 L. linearis Goeze. Hudson, May, Grahamville, Je (NYS) ; Lowville, in stored wood (Ds); West Pt. (Robn).

\section{Minthea Pascoe}

12945 M. stichothrix Reit. "NY" (Kraus-Horn Coll.).

\section{FAMILY SPHINDIDAE \\ SPHindus Chevrolat}

12949 S. americanus Lec. Buffalo (Z\&R); Ithaca, Aug 25 (Fbs-CU); SI, Je (L\&D).

\section{FAMILY CISIDAE SUbFamily CISINAE}

\section{Cis Latreille}

12960 C. falli Blatch. SI, Oct 8, under bark of dead tree (L\&D).

12966 C. julichi Dury. NYC (Dury), type locality.

12972 C. fuscipes Mell. Newport, Je (NYS); Cranberry L., Je, in Polyporus lucidus (H); Syracuse, in hickory (B\&S); Buffalo (Z\&R); Westfield, May (Not); Glen (Lint), as fulvipes (Mell) ; Albany, Ap (NYS); West Pt. (Robn); SI, Sep (L\&D) ; LI: Jamaica (Pears).

12974 C. pistoria Csy. Cranberry L., Je 25, in decaying fungi (H).

12979 C. creberrima Mell. Buffalo (Z\&R); Syracuse, from dead hickory (B\&S); West Pt. (Robn); Mosholu (Sf).

12981 ?C. horridula Csy. Albany, Ap (NYS).

12988 C. curtula Csy. "NY" (Csy), type locality; West Pt., Ap (Robn).

\section{ORTHOcIS Casey}

12992 O. punctata Mell. Schoharie (Not); Bronx Pk. (Sf); SI, Mar, from dead grapevine (L\&D). 


\section{Xestocis Casey}

12997 X. levetti Csy. Newport, J1 (NYS) ; Cranberry L., Je-J1, in fungu.s (H) ; Syracuse, May, in bracket fungus ( $\mathrm{H})$; Castile, Brad (Flet); Westfield, May (Not); Mosholu (Sf); vic. NYC (AM); SI, May, from fungus (L\&D).

12998 X. davisi Dury. SI, type locality (L\&D).

\section{BRACHYCIS Casey}

13009 B. brevicollis Csy. Ithaca (Csy), type locality. In the fungus Pleurotus ostreatus in N. J. (Weiss).

\section{Plesiocis Casey}

13010 P. cribrum Csy. Albany, Aug (Frost). Described from southern Calif.

\section{SulCaCis Dury}

[13011 S. lengi Dury. Found in Bennington Co, Vt., and Mercer Co, N. J., and sure to occur in NY.]

\section{EnNEARTHRon Mellie}

13018 E. thoracicorne Ziegl. Ithaca, Nov (NYS); Nassau, May, Schaghticoke, Aug (NYS); West Pt. (Robn); Mosholu (Sf); vic. NYC (AM); SI, Je, Aug, from Polyporus versicolor (L\&D); LI: Little Neck, Mar (Schott); Orient, Mar (Ds).

\section{Ceracis Mellie}

13030 C. sallei Mell. Valley Falls, Ap (NYS); West Pt. (Robn); Mosholu (Sf) ; SI, Aug (L\&D). According to Weiss, this species breeds in the fungus Fomes applanatus.

\section{SUBFAMILY OROPHIINAE}

OCTOTEMNUS Mellie

13037 O. laevis Csy. Syracuse (B\&S); Catskills, Je, Pears (Nic); Bronx Pk. (Sf); "NY", type locality.

\section{FAMILY SCARABEIDAE SUBFAMILY COPRINAE}

\section{CANTHON Hoffmansegg}

13047 C. vigilans Lec. LI ( Jn).

13048 C. laevis Drury. Buffalo (Z\&R); Albany, Je (Lint); West Pt. (Robn); Bronx Pk. (Sf) ; SI, formerly common, Aug 19, 1903 (Woodr), also 1910 and 1914 (L\&D); LI: Flatbush, Sep (Eng).

13052 C. viridis Beauv. West Pt., Je (Sf); SI, Aug 7 (L\&D) ; LI: Yaphank, J1 7 (Ds). 


\section{ChoERIDIUM Geoffroy}

13057 C. histeroides Web. Buffalo (Z\&R); SI, Je, on dead fish (L\&D); LI: Yaphank, J1 (Ds).

\section{PINOTUS Erichson}

13059 P. carolinus L. West Pt. (Robn); SI, Je (L\&D); LI: Bellport, Aug (Nic); Riverhead (Ds).

\section{COPRIS Geoffroy}

13062 C. minutus Drury. Buffalo (Z\&R); West Pt. (Robn); SI, Ap, Sep (L\&D) ; LI: Amagansett, Sep (Eng) ; Wyandanch, May (Sf) ; Orient, Lath (NYS).

13065 C. tullius Oliv. (anaglypticus Say). Adirondacks (Felt); Hope, Aug (NYS) ; Gloversville, May, Oswego, Je (NYS) ; Pike, Sep (NYS); E. Aurora, May, Sep, common in burrows beneath manure, also at light (Dnd) ; Buffalo (Z\&R) ; Ithaca, Je-Aug (Dt); West Pt. (Robn); SI, May-Sep (L\&D) ; LI: Orient Pt., Je (Ds) ; Brooklyn, May (Eng).

\section{Phanaeus MacLeay}

13072 P. carnifex L. West Pt. (Robn); Oscawana Hgts., Putnam Co, May (Flet); Bronx Pk. (Sf); SI, Je-Sep (L\&D); LI: Bellport (Nic); Montauk, Sep (Ds, Ang).

ONTHOPHAGUS Latreille

13080 O. hecate Panz. Axton, Je (M\&H) ; Cranberry L., J1 24 (H) ; Potsdam (Houghton) ; Buffalo (Z\&R) ; E. Aurora, Aug-Sep, scarce (Dnd) ; West Pt, May (Not); Ithaca, May-Aug (CU); Ringwood, McLean, May-J1; Windsor, Je (Not); Debruce, Sep (Ds); West Pt., Je (Robn); White L. (AM) ; SI, May-Aug (L\&D) ; LI: Wyandanch, May, Yaphank (Sf); Orient, Je (Ds).

13082 O. orpheus Panz. West Pt., Je, Robn (Ds).

13084 O. janus Panz. Buffalo (Z\&R); Ithaca, Jl-Aug (Vdyk-CU) : West Pt., Je (Robn) ; White Plains (Sq); SI, J1-Aug (L\&D) ; LI: Yaphank, J1 (Eng) ; Wading R. (Sf) ; Flatbush (AM) ; Orient, Lath (NYS).

13084a O. janus var. striatulus Beauv. SI, Jl-Sep (L\&D) ; LI: Wyandanch (Sf) ; Flatbush, Zab (AM).

13086 O. pennsylvanicus Harold: E. Aurora, Boston, Je, Aug, scarce (Dnd) ; Windsor, Je (Not); West Pt. (AM) ; SI, Aug (L\&D) ; LI: Wyandanch, May (Sf); Flatbush, Zab (AM).

13087 O. cribricollis Horn. LI: Yaphank (Sf).

13088 O. nuchicornis L. Cranberry L., Je-J1 (H) ; LI: E. New York, May (Shoe) ; Rockaway Beach, May (Ds); New Lots, May (Shoe).

13090 O. tuberculifrons Harold. LI: Wyandanch (Sf); Greenport, Aug (Eng). O. ovatus L. "NY" (Fitch); Buffalo (ANSP). A common European species which may have been introduced.

Aegialia Latreille

13102 A. conferta Horn. Rochester (Moore). 


\section{SUBFAMILY APHODIINAE}

\section{APHODIUS Illiger}

13107 A. fossor L. Axton, Je (M\&H) ; Cranberry L., Je 30 (H) ; Syracuse (H) ; Olcott, May-Sep (Dt); Castile, May, Brad (Flet); Ithaca, May-J1 (Vdyk, Dt) ; McLean, May-Sep (Dt); Windsor, Je (Not) ; E. Jewett, Aug (Ds); Peekskill, May-Je (Sherm); West Pt. (Robn) ; Katonah (Sherm, Dt, CU) ; West Pt., J1 (Eng) ; SI, Je (L\&D) ; LI : Rockaway Beach, May (Ds); Long Beach, Je (Eng); Wyandanch, May (Sf).

13110 A. erraticus L. Wilmington, J1 (Shoe); Cranberry L., Je (H) ; Syracuse, May $(\mathrm{H})$.

13111 A. haemorrhoidalis L. Cranberry L., J1 3 (H) ; LI: Wading R., Je (Sf, Schott); Huntington, Je (Schott).

13119 A. fimetarius L. Keene Val. (Lint); L. Pleasant, J1 (Lint) ; Potsdam (Houghton) ; Cranberry L., Jl (Drk); Syracuse, Ap-May (H) ; Buffalo (Z\&R) ; Batavia, J1-Sep (Kngt); Westfield, May (Not) ; Castile, Brad (Flet); Geneva, May (Lint); Ithaca, Ap-Oct, McLean Bogs, Aug (CU); Windsor, Je (Not); Bangall, Sep (Eng); West Pt. (Robn); Nyack (AM) ; White Plains, Ap (Sq); SI, Ap-J1 (L\&D) ; LI: Jamaica, Flatbush (AM); Orient, Lath (NYS).

13123 A. putridus Hbst. Syracuse, May, on cattle dung (H) ; E. Aurora, J1, Sep, scarce (Dnd).

13127 A. ruricola Melsh. Axton, Je (M\&H) ; Potsdam (Houghton); Olcott, Je, Aug (Dt); Westfield, May (Not); Cortland Co, J1, rare (Dnd) ; Ithaca, Je-Aug (Dt-CU) ; McLean (Dt); Schoharie (Not); West Pt. (Robn); SI (L\&D); LI, J1 (Eng).

13131 A. granarius L. Honeoye Falls, Je (Ds) ; Rochester Jct., Je, Leon (DtCU) ; Westfield, May (Not); Batavia, Je (Ds) ; Erie Co, May-J1, common (Dnd); Castile, May, Brad (Flet); Ithaca, Taughannock Falls, Mar-Je (Dt-CU); Schoharie (Not); West Pt. (AM); Nyack (AM) ; Ramapo, Je (Ds) ; NYC, Feb (Dt-CU) ; SI, Mar-Aug (L\&D) ; LI: Flatbush (AM) ; Hempstead Plains, Ap (Eng) ; Central Pk., Yaphank, Ap (Eng); Orient, Lath (NYS).

13132 A. vittatus Say. Batavia, J1 (Kngt) ; E. Aurora, May, J1, common (Dnd) ; Ithaca, May, J1 (Dt-CU); SI (L\&D); LI: Long Beach, May (Eng).

13147 A. lutulentus Hald. "NY" (Hald-ANSP).

13159 A. rubeolus Beauv. New City, J1, Carn (Frost); SI, Je (L\&D).

13162 A. stercorosus Melsh. Syracuse, May, on cattle dung (H) ; E. Aurora, J1-Sep, frequent, Sep (Dnd); Peekskill, J1, at light (Sherm); West Pt. (Robn); Ramapo, Je (Ds); SI, Je (L\&D).

13170 A. consentaneus Lec. Cortland Co, J1, one (Dnd).

13173 A. phaleroides Horn. LI: Coney I. (Horn) ; Bellport, J1 (Nic) ; Rockaway (AM).

13180 A. bicolor Say. Olcott, Oct (Dt); Buffalo (Z\&R) ; E. Aurora, Aug-Sep, common (Dnd); Castile, May, Brad (Flet).

13182 A. serval Say. "NY" (Fitch).

13184 A. distinctus Müll. Potsdam (Houghton); Syracuse, May, on horse dung $(\mathrm{H})$; Olcott, Mar-Ap, Oct (Dt); Buffalo (Z\&R) ; E. Aurora, Oct, common (Dnd) ; Castile, May, Brad (Flet); Ithaca, Ap-May (DtCU) ; Spencer, Mar-Ap, Oct (Dt); West Pt. (AM); SI, Ap, Oct, 
found once in a dead blacksnake (L\&D); LI: Rockaway Beach, Ap (Eng); Yaphank, May (Eng); Forest Pk., Mar (Martin); Orient, Lath (NYS).

13186 A. leopardus Horn. E. Aurora, Sep (Dnd) ; Ithaca, Ap (Dt-CU) ; West Pt., Sep (Robn).

13189 A. terminalis Say. SI (Sf).

13192 A. rufipes L. E. Aurora, under manure, J1 (Dnd); Ithaca, J1, Yuasa (Vdyk).

13193 A. depressus Kug. "NY" (Horn); Cranberry L., Je 17 (H).

13198 A. rubripennis Horn. Wilmington, Aug (Dt); Rock City, Je (Dt-CU); Slide Mt. (Shoe).

13201 A. femoralis Say. Syracuse, Oct (Drk); Buffalo (Z\&R); Westfield, May (Not); West Pt. (Robn).

13202 A. prodromus Brahm. Axton, Je (M\&H) ; Potsdam (Houghton) ; Syracuse, Ap 9, very abundant $(\mathrm{H})$; Westfield, May (Not); Ithaca, ApMay (Dt-CU).

13204 A. oblongus Say. Ithaca, Ap 23, 1882 (Dt-CU).

\section{Oxyomus Castelnau}

13210 O. silvestris Scop. (porcatus Fab.). Peekskill, Ap (Sherm); West Pt. (Robn); White Plains, Mar (Sq); vic. NYC (AM); SI, Ap-May, Dec (L\&D) ; LI: Flatbush, Je-J1 (Nic).

\section{Ataenius Harold}

13216 A. abditus Hald. Westfield, May (Not).

13220 A. imbricatus Melsh. Bronx Pk. (Sf); SI (L\&D).

13226 A. gracilis Melsh. Buffalo (Z\&R); E. Aurora, Oct, rare (Dnd); LI: Jamaica, May, Pears (Nic).

13231 A. strigatus Say. Windsor, Je (Not); "NY" (Fitch), as Aphodius. Probably belongs here (Fall).

13232 A. stercorator Fab. Buffalo (Z\&R); Westfield, May (Not); vic. NYC (AM).

13233 A. cognatus Lec. Potsdam (Houghton); Olcott, Ap-Sep (Dt) ; Syracuse, May (H) ; Ithaca, Ap-Sep (Dt); Peekskill, May (Fall); West Pt. (Robn); SI (L\&D); LI: Flatbush (Sf).

\section{Dialytes Harold}

13237 D. truncatus Melsh. LI, Aug (Schott).

13239 D. striatulus Say. E. Aurora, Sep, one beneath dung (Dnd) ; Ithaca, Oct (Dt-CU) ; Windsor, Je (Not).

PSAMмовIUS Heer

13240 P. cruentus Harold. "NY"? (Horn-ANSP).

13242 P. aegialioides Hald. "NY" (Hald-ANSP).

Rhyssemus Mulsant

13251 R. scaber Hald. Peekskill, May (Sherm); LI: Rockaway Beach (Schott). 


\section{PLEUROPHORUS Mulsant}

13255 P. caesus Creutz. "NY" (Fall); Olcott, Je, Oct-Nov (Dt).

13256 P. parvulus Chev. LI: Jamaica (Ds), as namus DeG.

\section{SUBFAMILy GEOTRUPINAE}

\section{Odontaeus Klug}

13285 O. corniger Melsh. Buffalo (Z\&R); Catskills, Je (Nic); Ft. Montgomery, J1 (Schott); SI (L\&D) ; LI: Bellport, Je (Nic); Yaphank, J1 (Ds); Amagansett, Oct (Eng).

13286 O. filicornis Say. Cranberry L., Je $(\mathrm{H})$; Indian Falls, Jl, always at light, scarce (Dnd); Windsor, Je (Not); LI (Jn).

13287 O. obesus Lec. Jay, J1, Britton (Frost) ; Ithaca, May (Kngt). Fall questions these records, since this is a California species.

\section{Bolbocerosoma Schaeffer}

13288 B. farctum Fab. SI, May 6, J1 14 (L\&D); LI: Forest Pk., Aug (Eng).

13288a B. farctum var. tumefactum Beauv. "NY" (Sf).

\section{EUCANTHUS Westwood}

13289 E. lazarus Fab. Peekskill, J1 (Sherm); West Pt. (Robn) ; SI (L\&D); LI: Rockaway, Je (Eng); Far Rockaway (Jn).

\section{Geotrupes Latreille}

13290 G. balyi Jek. Cranberry L., J1 (H) ; Ithaca, Sep (CU) ; Ringwood, MaySep (Dt); Catskills, Aug, Pears (Nic); Yankee L., Wirtsboro, Sullivan Co, Sep (Ds); Carmel, Aug (Sf); Brant L., Aug (Doll); West Pt. (Robn) ; LI: Yaphank, J1, Oct (Ds); Melville, Oct (Schott).

13291 G. horni Blanch. West Pt. (Robn); SI (L\&D) ; LI: Deep Pond, Wading R., Aug (Ds); Wyandanch, Aug (Schott); Yaphank, Oct (Eng).

13293 G. blackburni Fab. Buffalo (Z\&R); Sullivan Co, Sep (Ds) ; Bronx Pk., Ap (Shoe); SI, Aug, Oct (L\&D); LI: Brooklyn (Jn).

13294 G. egeriei Germ. Adirondacks (Feit) : Buffalo (Z\&R); SI, May, Aug-Sep (L\&D) ; LI: Flatbush, Aug, Yaphank, Oct (Eng).

13298 G. semiopacus Jek. Buffalc (Z\&R) ; E. Aurora, Aug-Sep (Dnd); Ithaca, Sep (Dt) ; Debruce, Sep (Ds); West Pt (Robn).

13299 G. splendidus Fab. Buffalo (Z\&R); Ithaca, Aug (Dt); Windsor, Je (Not); Peekskill, in fungus (Sherm); Catskills, Aug (Ds); Pine I., Je (Ds); West Pt., Je (Ds, Sf); SI, May-Oct (L\&D) ; LI: Amagansett, Sep, Yaphank, Oct (Ds) ; Wyandanch, J1 (Schott).

13267 Ochodaeus biarmatus Lec. (Subfamily Ochodaeinae.) Buffalo (Z\&R). This is probably a misdetermination, as the range is from Kansas to Arizona (Fall). 


\section{SUBFAMILY GLAPHYRINAE}

AMPHICOMA Latreille

13313 A. lupina Lec. SI, Sep (Sf, L\&D) ; LI: Rockaway (Dt).

13315 A. vulpina Hentz. Lava, J1 (Eng); Van Cort. Pk., Pears (Nic).

\section{SUBFAMILY ACANTHOCERINAE}

Cleotus Germar

13320 C. aphodioides Ill. Buffalo (Z\&R). Leng queries this.

\section{SUBFAMILY TROGINAE}

TROX Fabricius

13328 T. scabrosus Beaut "NY" (Lec); SI, on seashore (L\&D).

13329 T. monachus Hbst. Peekskill, Ap (Sherm).

13330 T. asper Lec. LI: Calverton, Sep (Eng).

13331 T. suberosus Fab. SI, Je, seashore (L\&D) ; LI: Bellport, Je (Nic) ; Rockaway, J1, Orient, J1, Sep (Ds) ; Promised Land, Yaphank, Je (Eng).

13333 T. tuberculatus DeG. LI: Montauk Pt. (Eng).

13336 T. erinaceus Lec. Buffalo ( $Z \& R$ ); SI, Feb, Ap, May, in owl pellets, and Sep, under dead rabbit (L\&D); LI: Bellport, Aug (Nic);'Yaphank, May, Oct (Sf, Eng); Flatbush, May (Eng).

13337 T. capillaris Say. Cranberry L., J1 (Drk); Buffalo (Z\&R); Gowanda, May (Dnd); West Pt. (Robn); SI (L\&D).

13338 T. unistriatus Beauv. Cranberry L., Je-J1 (H) ; Buffalo (Z\&R) ; Ithaca, J1 (CU); New Paltz (Lint); Peekskill, Ap (Sherm); SI, Je, Aug (L\&D) ; LI: Bellport, Aug (Nic) ; Bedford Pk., Flatbush, J1 (Eng).

13339 T. sordidus Lec. West Pt. (Robn); SI, Ap (L\&D); LI: Bellport, Je (Nic).

13340 T. insularis Chev. (foreicollis Harold). West Pt. (Robn); SI, Aug (L\&D); LI: Yaphank, J1, Flatbush, May (Eng).

13341 T. terrestris Say. Peekskill, Ap (Sherm); West Pt. (Robn); SI, J1 (L\&D) ; LI: Aqueduct, Sep (Ds) ; Flatbush, May (Sf).

13342 T. aequalis Say. Cortland Co, Je, under bird carcass (Dnd); West Pt. (Robn) ; SI (L\&D); LI: Yaphank (Eng).

13344 T. scaber L. Buffalo (Z\&R); E. Aurora, Je (Dnd) ; Catskills, May, Pears (Nic) ; West Pt. (Robn); New City, Mar (Carn, Frost) ; SI, Ap-May, in owl pellets (Ds) and sometimes at light (L\&D); LI: Flatbush, May (Eng).

13346 T. laticollis Lec. "NY" (Lec).

\section{SUBFAMILY MELOLONTHINAE}

\section{SERICA MacLeay}

13354 S. vespertina Gyll. Buffalo (Z\&R); Gowanda, Allegany Pk. (Dnd); Ithaca, May-Je (Daw); Windsor, Je (Not); West Pt. (Robn); SI, Ap-J1 (L\&D) ; LI: Orient, Je, Lath (Ds); Flushing, Mar (Ds). 
S. cucullata Daw. Cranberry L., Je-J1 (H); Saranac L., Sep (Daw); Peru, Je (Daw); Ithaca, May-Je, Aug, McLean, May (Daw); Sullivan Co, Shaler (Daw).

13355 S. georgiana Lg. Buffalo (Daw); Ithaca, May (Daw); Hudson, Ramapo (Daw); Bronx Pk., Bt, NYC (Daw); SI, Bt (Daw); LI: Brooklyn (Daw).

13356 S. evidens Blatch. SI, May, Aug (L\&D).

- S. lecontei Daw. Peru, Wilton, May, Ithaca, Ap-Jl, SI, paratype (Daw).

13357 S. intermixta Blatch. Peru, Aug, Mix \& Everett (Daw); Ithaca, (Ap-J1 (Daw).

13361 S. iricolor Say. Ithaca, May, West Pt. (Robn) ; Peekskill, Ft. Montgomery, May, Bronx Pk., Bt (Daw) ; SI, Je (L\&D) ; LI: Central Pk., Ap (Eng); Wading R., May, Nissequogue, Je (Ds); Wyandanch, May (Sf); Yaphank, Jl, Pinelawn, Je (Eng).

S. elusa Daw. Cranberry L., Je-J1, Ithaca, Ap-May, Taughannock Falls, Ap (Daw).

S. mystacea Daw. Hudson, Dbl, Bronx Pk., Ap (Daw).

13363 S. tristis Lec. Peru, May-Je, Ithaca, Je (Daw); LI: Orient, Lath (NYS).

13364 S. sericea Ill. Wilmington, J1 (Daw) ; Buffalo (Z\&R); E. Aurora, Mar, hibernating, Niagara Co, J1 (Dnd) ; Castile, May, Brad (Flet) ; Ithaca, Ap-J1 (Daw) ; Nyack (Daw); West Pt. (Robn); Mosholu, May (Ds); Bronx Pk., Bt (Daw); SI, Ap-J1 (Daw); often at light (L\&D); LI: Shinnecock Hills (Ds); Orient, Je, Lath (Ds).

S. parallela Csy. A distinct species (Daw). New Rochelle (Fisher); Bronx Pk., Bt (Daw); SI and LI (Daw).

13372 S. trociformis Burm. Wilmington, Je (Daw); Buffalo (Z\&R); Ithaca, May (Eng) ; Karner, May, J1 (Daw) ; West Pt., Je (Ds) ; Ft. Montgomery, May (Ds); Bronx Pk., Bt (Daw); SI, May-Je (Daw); LI: Nissequogue, Je (Ds); Pinelawn, Je, Yaphank, May, J1 (Eng).

S. sponsa Daw. Ghent, Je, Kenwood, May, Albany, Ap (NYS).

\section{AutosericA Brenske}

A. castanea Arrow. Yonkers, Mt. Vernon, New Rochelle (Arrow).

\section{Alobus LeConte}

13375 A. fulvus Lec. "NY" (Lec).

\section{Diplotaxis Kirby}

13376 D. sordida Say. West Pt. (Robn); SI, Je. (L\&D) ; LI: Yaphank, Sep (Ds); Wyandanch (Sf).

13405 D. atlantis Fall. Castile, Brad (Flet); Windsor, Je (Not); SI, Ap-Je (Fall, L\&D) ; LI: Bellport, Je (Nic) ; Far Rockaway, Shinnecock Hills (Ds) ; Yaphank, May-Je (Eng).

13409 D. tristis Kby. Horicon, J1 17, 1925 (Frost); Syracuse, Ap, under stone $(\mathrm{H})$; Peekskill; West Pt. (AM) ; LI: Babylon, Bradford (AM) ; Long Beach, Jamaica (AM). 
13410 D. liberta Germ. E. Aurora, May, in numbers about roots of Populus grandidentata (Dnd); Ithaca, Mar-Aug (Dt); Peekskill (Fall); West Pt. (Robn) ; vic. NYC (AM) ; LI: Hauppauge (Felt); Cold Sp. Harb. (Sherm).

13446 D. frondicola Say. "NY" (Lec).

13455 D. harperi Blanch. "NY" (Fall).

PHYLLOPHAGA Harris

\section{(Lachnosterna Hope)}

13486 P. glaberrima Blanch. LI: Coney I. (Sm).

13487 P. ephilida Say. "NY". (Lec).

13496 P. gracilis Burm. West Pt. (Robn) ; Peekskill (Ds) ; SI, Je-Aug (L\&D) ; LI: Yaphank, Riverhead, Aug-Sep (Ds); Forest Pk., J1 (Martin).

13497 P. futilis Lec. (gibbosa Burm.). Buffalo (Z\&R); Ithaca, J1 (CU) ; SI, Je (L\&D) ; LI: Coney I. (Lec) ; Rockaway Beach, May (Nic); Elmhurst (Ds) ; Brooklyn Bot. Garden, May (Sf); Yaphank, Je (Eng).

13499 P. congrua Lec. "NY" (Lec).

13503 P. crassissima Blanch. "NY" (Sm, Felt).

13506 P. inversa Horn. "NY" (Dt); West Pt. (Robn).

13508 P. micans Knoch. Bronxville (Felt); SI, May-Je (L\&D) ; LI: Brooklyn (Dt) ; Woodside, May (Sf).

13511 P. fusca Froelich. Peru, Fbs, Cy (CU) ; Essex Co, Je (NYS) ; Warrensburg (Lint) ; Newport, Dec, larva (Lint); Syracuse, Ap-May (H) ; Glenfield, May 27 (JJDs) ; Buffalo (Z\&R) ; Perry, Je, Hawley (CU); Pike (JJDs); Albany, Coeymans, Lint (Felt); Nassau, Je, Fielt (JJDs) ; West Pt., Je, Ft. Montgomery, May (Ds) ; Bronxville, Felt (JJDs) ; SI, Ap-J1 (L\&D) ; LI: Hollis, Je (JJDs) ; Orient Pt., Je, Lath, Wingfield, Ap, Sedgwick (JJDs); Yaphank, May-Je (Eng).

13515 P. fervida Fab. (arcuata Sm.). "NY" (Sm, Fitch, et al.) ; Bronxville (Felt) ; SI (L\&D); LI: Elmhurst (JJDs).

13516 P. anxia Lec. (dubia Sm.). Axton, Je (M\&H); Peru, Je, $C y$ (Dt); Cranberry L., Je-J1 (H) ; Glenfield, Lewis Co, (JJDs); Newport, Je (NYS) ; Marion, Je, Leon (Dt) ; Syracuse, Ap-May (H) ; Pike, May (JJDs) ; Castile, Ap, Brad (Flet); Perry, Je, Hawley (Dt); Buffalo (Glasgow, Z\&R) ; E. Aurora, Je, scarce (Dnd) ; Ithaca, Mar-J1 (Dt-CU); Windsor, Je (Not); LI: Orient Pt., Lath (JJDs).

13517 P. drakei Kby. (grandis Sm.). Buffalo (Glasgow); Ithaca, Je (Sling); Albany, May (Felt-NYS); SI, Je (L\&D).

13519 P. barda Horn. Ramapo, J1 (Ds); Wading R., May (Nic) ; Huntington, Ap (Schott).

13520 P. marginalis Lec. "NY" (Lec, Sm); Ithaca, Je-J1 (JJDs) ; West Pt. (Robn); Ramapo, Je (Sf).

13522 P. fraterna Harr. Buffalo (Z\&R), as cognata; Albany, May, Cy, Fbs (CU) ; Wēst Pt. (Robn) ; Mosholu, May (Ds) ; Bronxville, Je (Felt); SI, Ap-Je (L\&D) ; LI: Yaphank, J1 (Eng).

13523 P. forsteri Burm. (lugubris Lec.). "NY” (Lec,Sm); SI, Ap 22, Woodr (L\&D). 
13528 P. knochi Gyll. "Around NYS" (Sm); SI (L\&D); LI: Wading R., Coram, Riverhead, Aug (Ds); Yaphank, May (Eng).

13530 P. rugosa Melsh. E. Aurora, May-Je, scarce (Dnd); LI: Wading R., May-Je (Nic).

13531 P. rugosioides Linell. LI (Linell-US).

13532 P. hirsuta Knoch. West Pt (Robn); Mosholu, May (Ds); SI, May-J1 (L\&D) ; LI: Yaphank, Je (Eng).

13535 P. balia Say. Buffalo (Z\&R); Ithaca, Ap-J1 (Dt).

13536 P. villifrons Lec. Buffalo (Z\&R); Ramapo, May (Ds).

13537 P. nitida Lec. Albany, J1 (NYS).

13538 P. hirticula Knoch (hirsuta Say). Syracuse, Ap-May (H); E. Aurora, Ap-May, frequent under cover (Dnd); Ithaca, Ap-Aug (Dt, Sling, at trap lantern); Albany, Je (Felt); West Pt., Robn (JJDs); Grant Pt., May (NYS); Bronxville (Felt); SI, May (L\&D); LI: Brooklyn (Dt); Hollis (JJDs); Elmhurst (JJDs) ; Orient Pt., Robn (Ds).

13540 P. ilicis Knoch. Ithaca, May-J1 (Sling, Dt); West Pt. (Robn); SI (L\&D); LI: Brooklyn (Sm); Wading R., May (Nic); Flatbush, May (Eng).

13541 P. subtonsa Lec. "NY" (Lec); Yaphank, Je-J1 (Eng).

13544 P. crenulata Froel. Albany, Aug (NYS); West Pt. (Robn); Bronxville (Felt) ; SI, Ap-Aug (L\&D); LI: Far Rockaway (Ds) ; Yaphank, J1, Greenport, Aug (Eng); Wading R., Aug (Sf).

13556 P. quercus Knoch. Ithaca, Je (Sling); Nyack (Frost).

13561 P. crinita Burm. Marlboro, May 16 (Dt-CU). Described from Tex. and Mex.

13563 P. tristis Fab. Syracuse, Ap, under stones (H); Cambridge (Lint); Deposit (Lint); Marlboro, May (Sz); West Pt. (Robn); SI, May (L\&D) ; LI: Brooklyn, Yg (Dt); Elmhurst (JJDs); Orient, May, Lath (Ds); Yaphank, May (Eng).

\section{POLYPHYLLA Harris}

13624 P. variolosa Hentz. Peru, J1 (CU) ; Buffalo (Z\&R) ; Karner, Schenectady, J1-Aug, Yg (Felt) ; SI, Je-Jl, at light (L\&D); LI: Bellport, Coney I., Je (Nic); Rockaway, Je (Ds); Riverhead, J1 (Ds).

\section{DICHELONYX Harris}

13649 D. elongata Fab. Axton, Je $(M \& H)$; Cranberry L., Je $(H)$; Charlotte (AM) ; Olcott (Dt); Buffalo (Z\&R) ; Erie Co, common; May (Dnd); Batavia, Je (Kngt); Castile, May, Brad (Flet); Conesus L., Je (Ds); $\mathrm{S}$. Salem, Je (Lint); McLean, Ringwood, Ithaca, May-Je (Dt); Windsor, Je (Not); West Pt. (AM); Ramapo (Sf); SI, Je (L\&D); LI: Amagansett, May, Yaphank, Je (Eng); Babylon, Flatbush (AM).

13650 D. canadensis Horn. "NY"? (Horn).

1365.1 D. subvittata Lec. Cranberry L., Je 13 (H) ; West Pt. (Robn) ; Ft. Montgomery, May (Ds); SI, May-Je (L\&D).

13652 D. diluta Fall. Windsor, Je (Not); Schoharie (Not); Ramapo, Je 12, identity queried (Ds); LI (Jn). 
13653 D. fuscula Lec. Vic. NYC (AM); LI: Val. Stream, J1, Deep Pond, Wading R., Yaphank, May (Ds); Bellport, Je (Nic); Wyandanch (Sf).

13654 D. testacea Kby. Perry, Je, Hawley (Vdyk-CU); Windsor, Je (Not).

13655 D. backi Kby. Recorded from "NY" by Fitch, but Fall and Leng query the determination.

13676 D. albicollis Burm. Cranberry L., Je-J1 (H) ; Buffalo (Z\&R); Westfield, Je (NYS); Ithaca, Je (Vdyk); Karner, Je (NYS); West Pt. (Robn); Nyack, Je (Ds) ; Brant L., Aug (Doll); SI, Je, on pine (L\&D) ; LI: Yaphank (Sf).

\section{MACRODACTYLUS Latreille}

13685 M. subspinosus Fab. Rose Bug. Common and general in sandy areas. Cranberry L., Htly (H) ; Oswego (Lint) ; Rochester (Felt) ; Batavia, Je (Kngt); Ithaca; Utica (Fitch); Stillwater (Fitch); Albany, Schodack, Loudonville, and Peekskill (Lint); West Pt. (Robn); Grahamville; NYC (Fitch); SI, Je-JI (L\&D); LI, general.

\section{HOPLIA Illiger}

13694 H. trifasciata Say. Cranberry L., J1 (H) ; Olcott, May-Je (Dt) ; Greece, May (Felt); Genesee Co, Je (Ds); Buffalo " (Z\&R) ; Windsor, Je (Not); SI, Ap, sometimes common on hickory buds (L\&D); LI: Orient, Lath (NYS).

13694a H. trifasciata var. tristis Melsh. "NY" (Lec); SI (AM).

$13695 \mathrm{H}$. trivialis Harold. "NY" (Lec); SI, May (L\&D).

13697 H. limbata Lec. (modesta Hald.). Schoharie (Not); SI, Je (L\&D); LI (Schaupp); Long Beach (AM); Rockaway (Dietz, Fall); Hempstead Plains, Ap (Eng).

13698 H. equina Lec. SI, J1 (L\&D).

\section{SUBFAMiLy RUTELINAE}

Anomala Samouelle

13709 A. binotata Gyll. SI, May 4 and 28 (L\&D) ; LI: Rockaway Beach, Je (Ds) ; Far Rockaway (Jn).

13730 A. undulata Melsh. LI: Flatbush, Barren I. (AM).

13732 A. innuba Fab. LI: Bellport, Je (Nic); Yaphank, Je (Eng).

A. orientalis Waterh. New Rochelle; Mt. Vernon; White Plains; LI: Westbury; Jericho, Boyce.

\section{PACHySTETHUS Blanchard}

13739 P. marginata Fab. LI (Eng).

13741 P. lucicola Fab. Buffalo (Z\&R) ; Karner, Je-J1 (Felt) ; Croton-on-Hudson (Ds) ; West Pt. (Robn) ; White Plains, Je-J1 (Sq) ; SI, Je-J1 (L\&D) ; LI: Orient, May, J1 (Ds); Woodhaven (Dt); Greenport, Aug (Eng); Rockaway Beach, Je (Eng). The Anomala maculicollis recorded from N. Y. by Fitch possibly belongs here ( $\mathrm{Lg})$.

13742 P. oblivia Horn. SI, Je (L\&D); LI: Bellport, J1 (Nic); Yaphank, J1 (Eng). 
STRIGODERMA Burmeister

13747 S. arboricola Fab. Ithaca, Anderson (Dt) ; SI, Je-J1 (L\&D) ; LI: Rockaway Beach, Je (Ds); Woodhaven, Benjamin (Dt); Long Beach (AM) ; Orient, Lath (NYS).

\section{STRIGODERMELLA Casey}

13750 S. pygmaea Fab. SI, J1 (L\&D); LI: Gardiners I., J1 (Eng).

Popillia Serville

13754 P. japonica Newn. Japanese Beetle. East bank of Hudson R. northw. to Ossining; Port Chester; LI: western third of island.

\section{PeLidnota MacLeay}

13755 P. punctata L. Adirondacks (Fitch); Olcott, May-Aug (Dt); Honeoye Falls (CU) ; Buffalo (Z\&R); Batavia, Aug-Sep (Kngt); E. Aurora, J1-Sep, common (Dnd) ; Ithaca, Saratoga, May-Aug (Dt); Branchport (Lint) ; Kenwood, Je (NYS) ; West Pt. (Robn); White Plains, J1-Sep (Sq); SI, Je-Aug (L\&D) ; LI: Promised Land, Sep (Ds); Flatbush, Queens, Babylon (AM); Orient, Lath (NYS).

13755b P. punctata var. brevis Csy. LI (Csy).

13757d P. lutea Oliv. var. hudsonica Csy. "NY" (Csy).

13758 P. tarsalis Csy. "NY" (Csy).

\section{CotalPa Burmeister}

13769 C. lanigera L. Buffalo (Z\&R); West Pt. (Robn); Croton-on-Hudson, May (Ds); NYC (Martin) ; SI, May-J1 (L\&D); LI: Southampton, Je (CU); Wyandanch, Half Way Hollow Hills, May-Jl, Wading R., Orient (Ds); Promised Land, Je (Eng).

13769 b C. lanigera var. vernicata Csy. "NY". (Csy).

Polymoechus LeConte

13787 P. brevipes Lec. "NY" (Lec).

\section{SUBFAMILY DYNASTINAE \\ OCHROSIDIA Casey}

13801 O. immaculata Oliv. SI, Je-Aug (L\&D); LI (Eng).

13803 O. villosa Burm. SI (Csy).

\section{Dyscinetus Harold}

13829 D. trachypygus Burm. Nyack, Catskills (AM); SI, Ap-J1 (L\&D); LI: Babylon (AM); Orient, Lath (NYS). 


\section{LIGYRODES Casey}

13834 L. relictus Say. Rochester (Lint); Buffalo (Z\&R); Ithaca (CU); West Pt. (Robn) ; SI, May-Sep, Oct, one on sidewalk (L\&D); LI: Flatbush, Barren I. (AM); Brooklyn, Oct (Eng).

\section{LIGYRUS Burmeister}

13838 L. longulus Csy. LI (Csy).

13843 L. gibbosus DeG. SI, May-Aug (L\&D); LI: Brooklyn (Yg, Dt); Rockaway Beach, Ap (Eng).

\section{Aphonus LeConte}

13874 A. politus Csy. "NY" (Csy).

13878 A. castaneus Melsh. "NY" (Lg Cat., as obesus Burm.). SI, J1 (L\&D); LI: Flatbush, J1 (Eng).

13879 A. cubiformis Csy. LI (Csy).

\section{Strategus Hope}

13896 S. antaeus Fab. LI: Bellport, Aug (Nic); Selden, Aug (Ds); Deep Pond, Wading R., Aug, Sf (Ds); Riverhead, Aug. This species should be called septentrionis if the differences noted by Casey are specific $(\mathrm{Lg})$.

XYLORYCTES Hope

13902 X. satyrus Fab. Buffalo (Z\&R); Ithaca, Jl-Aug (Dt, Flet); Schoharie (Not) ; West Pt. (Robn) ; SI, Je-Aug (L\&D) ; LI: Babylon, injurious to young ash trees (Glover); Bellport, J1-Aug (Nic); Montauk, Sag Harbor (Dimmock); Glen Cove, Far Rockaway, Amagansett, Yaphank, J1 (Ds) ; Orient, Lath (NYS).

\section{DyNASTES Kirby}

13908 D. tityus L. Kingston, J1, at light (Fernow). A solitary record for this southern species $(\mathrm{Lg})$.

\section{SUBFAMILY CETONIINAE \\ Cotinis Burmeister \\ (Allorrhina Burmeister)}

13931 C. nitida L. Green June Beetle. Irvington, J1 (NYS); NYC (AM); SI, Je-J1, swarming about beds of Canna (L\&D); LI: Glen Cove (NYS); Central Pk. (AM).

\section{EUPHORIA Burmeister}

13937 E. fulgida Fab. Little Falls (Lint); Trenton Falls (Dbl) ; Gloversville, Je (NYS); Buffalo (Z\&R); E. Aurora, Je, at sap, scarce (Dnd); Ithaca, Je (CU); Windsor, Je (Not); Albany, Je (NYS); Catskills, Pears (Nic); West Pt. (Robn); Middletown, Je-J1 (CU); L. Waccabuc (AM); SI (L\&D) ; LI: Brooklyn (Ds); Yaphank, J1 (Eng). 
13938 E. herbacea Oliv. SI, May-Aug, not uncommon some years ago at Silver L. (L\&D) ; LI: Shelter I. (Sf).

13940 E. inda L. Brown Frijt Chafer. Adirondacks (Felt); Warrensburg; Syracuse, May (H) ; Rochester, Sep (CU) ; Honeoye Falls; Olcott, Ap-Sep (Dt) ; Buffalo (Z\&R) ; Batavia, Aug-Sep (Kngt) ; E. Aurora, May, Aug, scarce (Dnd); Brocton (Cy); Ithaca, May-Sep (CU); Schenectady; Greenfield Center; Albany, Ap, Sep (NYS) ; Stuyvesant Falls (NYS); Poughkeepsie; Millbrook; West Pt. (Robn); White Plains (Lint); Yonkers; Mt. Kisco, larvae in manure pile (Sling); Bronx Pk. (AM); SI, Mar-Sep, sometimes attracted to sap on birch stumps (L\&D) ; LI: Wading R., May, Amagansett, Sep, Southold, Sep, Orient (Ds); Babylon, Quogue, Flatbush (AM).

\section{STEPHANUCha Burmeister}

13964 S. areata Fab. SI, Sep (L\&D); LI: Jamaica, Pears (Nic); Aqueduct (Ds).

\section{Cremastocheilus Knoch}

13972 C. harrisi Kby. Peekskill, Sep (CU) ; Tarrytown (AM) ; LI: Wading R., May 30 (Nic); Promised Land, Je, Cold Sp. Harb., Ap (Eng); Far Rockaway (Jn).

13973 C. canaliculatus Kby. Mooers, Sep (Not); Saranac, Newport; Westfield, May (Not); Phoenicia (Vdyk); Gloversville, Hunter, May-Je (NYS); Windsor, Je (Not) ; Schoharie (Not); Peekskill, Sep (CU); West Pt. (Robn); Sterling L., May (Kneff).

13974 C. castaneae Knoch. Catskills, Pears (Nic); Greenville, J1 30 (Joutel); Greenwood L., May (AM); West Pt. (Robn); Ft. Montgomery, May (Schott); Dutchess Co, Ap (Eng); Peekskill (Fall); Bronxville, Ap (Joutel).

13980 C. variolosus Kby. West Pt. (Robn); LI: Wyandanch, Ap (Sf).

\section{OSMODERMA Serville}

14010 O. scabra Beauv. Adirondacks (Felt); Wilmington, J1-Aug (Dt) ; Cranberry L., Je-J1 $(\mathrm{H})$; Buffalo (Z\&R) ; E. Aurora, Je, Aug, scarce (Dnd); Batavia, J1 (Kngt); Ithaca, J1-Aug (Vdyk); McLean, Aug, Yuasa (Vdyk); Saratoga, N. Spencer (Dt); Albany, Je-J1 (NYS); West Pt. (NYS); Catskills, J1 (Shoe); SI, Je-Aug (L\&D).

14010a O. scabra var. rugosa Kby. Ithaca, J1 (Dt).

14012 O. eremicola Knoch. Essex Co, J1-Aug (NYS); Buffalo (Z\&R); E. Aurora, Aug, scarce (Dnd); Batavia, Aug (Kngt); Castile, Aug, Brad (Flet) ; Lake Bluff (NYS) ; Ithaca, J1-Aug (Vdyk); West Pt. (Robn); Huguenot, Mosholu (AM); SI, Jl-Aug (L\&D); LI: Yaphank (NYS).

\section{Gnorimella Casey}

(Gnorimus auct.)

14013 G. maculosa Knoch. Buffalo (Z\&R) ; Allegany Pk., J1, one (Dnd) ; West Pt. (Robn) ; Van Cort. Pk., Pears (Nic); SI, May-Je (L\&D); LI: Coram, May (Bell). 


\section{TRICHIOTINUS Casey}

14016 T. piger Fab. Cranberry L., J1 (Drk); E. Aurora, Buffalo, Je-J1, common on flowers (Dnd); Batavia, Je (Kngt); Castile, Brad (Flet) ; Ithaca, J1 (Dt); Schoharie (Not); West Pt. (Robn) ; Stony Pt. (AM) ; SI, Je-J1 (L\&D) ; LI: Yaphank, Je (Eng) ; Orient, Lath (NYS).

14017 T. texanus Horn. SI, Eng (Joutel); LI (Eng). Fall queries these records, since the species is listed from Tex. and Fla.

14022 T. assimilis Kby. Catskills, J1 (Eng).

14023 T. viridans Kby. "NY" (Fitch). Leng suggests that this possibly refers to assimilis.

14025 T. affinis Gory. Mt. Marcy; Saranac Inn, Old Forge, J1 (NYS) ; Long L. (Ds) ; Hewitt, Je (Ds); Axton, Je (M\&H) ; Cranberry L., Je, Aug (H) ; Olcott, Je-Aug (Dt); Buffalo (Z\&R); Syracuse, Je 27, in hickory (B\&S); Ithaca, J1 22, Yuasa (Vdyk); Ringwood, Je-Aug (Dt); Windsor, Je (Not); Schoharie (Not); L. George (AM); Albany, J1 (NYS) ; West Pt., Stony Pt., Je, Ft. Montgomery, Callicoon, J1 (Ds); SI, Je-Aug (L\&D) ; LI: Yaphank, Long Pond, Wading R., J1 (Ds).

14026 T. parvulus Csy. "NY" (Csy).

14027 T. bibens Fab. Buffalo (Z\&R); Ramapo, May (Ds); Greenwood I. (Dietz).

\section{VALGUS Scriba}

14030 V. squamiger Beauv. West Pt. (Robn); SI, Ap 9, in decayed log; also found May-Je (L\&D) ; LI: Central Pk., Ap (Eng) ; Pinelawn, Yaphank, Ap, Central Pk., Sep (Eng); Farmingdale (Sf).

14031 V. canaliculatus Fab. Peekskill, May (Sherm); Rensselaer, May (NYS).

\section{FAMILY LUCANIDAE}

\section{Compiled by JoHn W. AngelL}

\section{SUBFAMILY LUCANINAE}

\section{Pseudolucanus Hope}

14034 P. capreolus L. (dama Fab.). Formerly common throughout the State; now becoming rarer. L George, Je, Shoe; Medina, J1 (CU) ; Tonawanda, J1 (CU) ; Olcott, May-Aug (Dt) ; Buffalo (Z\&R) ; Elmira, Je (CU) ; Ballston, J1 (NYS) ; Coeymans (NYS) ; Albany, Je-J1 (NYS) ; Nassau, Je, Felt; Athens, J1 (Ang); Catskills, J1 (NYS) ; Nyack, Zab; Fishkill; West Pt., Robn; Croton, Je (Ds); NYC: West Farms (AM) ; Clason Pt., J1; Washington Hgts., J1 (Ang); Bronx Pk. ; Van Cort. Pk., Nic; SI: May-Aug, L\&D; Princess Bay, Je (Ang); Clove Valley, Je-Aug, Lg; LI: Brooklyn, Aug, Shoe; Jamaica, Nic; Hollis: Rockaway Beach, Je, Shoe; Shelter I., Aug, Ds; Babylon; Orient Pt., J1, Lath; Flatbush, Aug (Áng).

The larva lives in the wood of deciduous trees such as maple, oak, and apple. Adults are often taken by beating the foliage of oak, or by "sugaring" at light or on the bark or at the base of the trees mentioned. The species exhibits considerable variation in size and color. Specimens vary from deep chestnut to pale reddish brown. 
14036 P. placidus Say. Generally considered a western species, but said to be fairly common in northern localities such as Buffalo. Dr. Melville $\mathrm{H}$. Hatch records it at Ann Arbor, Mich., at light and in beach drift. Buffalo (Ang); Olcott, May-Je (Dt).

The larva lives in oak, according to Carl Selinger.

\section{SUBFAMILY DORCINAE}

\section{DoRcus MacLeay}

14039 D. parallelus Say. Formerly a common species throughout the State, but now becoming rarer. Mt. Marcy, Not; Herkimer, J1 (Geo. Franck Coll.); Rochester (Ward Coll.); Hodgeville; Buffalo, Ashm, Z\&R; Batavia, Je (Kngt); Otto, J1 (CU) ; Aurora, Je, Zab; Ithaca, MayAug (CU) ; Schoharie, Not; Schenectady, J1; Catskills, Je; North Branch, Schaupp; West Pt., Robn; Cooks Falls, Oct (Ang); White Plains; Dunwoodie, Je (Ang); Nepperhan, Ap (Ang); NYC, Allerton Ave., Ap; Clason Pt., May-J1 (Ang); Washington Sq. (Ang); West Farms (Ang-AM); Bronx; Van Cort. Pk., May, Diven; SI: May, L\&D; Bulls Head, May (Ang); LI: Rockaway Beach, Je, Diven; Corona, Je; Babylon, Je; Flatbush, Oct, Zab; Wading R., Aug, Schott; Jamaica, Pears; Cold Sp. Harb., Brb; Brooklyn, J1, Shoe.

The larva lives in the wood of deciduous trees, such as oak, maple, and sycamore, boring in the hard part of the wood. Adults are often taken in or under charred logs or stumps or at light.

14039a D. parallelus var. carnochani Ang. Evidently a local race or variety. Three specimens, all male, were taken by F. G. Carnochan while blasting stumps on a farm at New City. Differs from typical form in slightly heavier body, smoother elytra, and blunter mandibles.

\section{PlatyceRUS Geoffroy}

14041 P. quercus Weber. Fairly common throughout the State. Newport, $\mathrm{Yg}$; Syracuse, Ap, H (Ang); Olcott, Je-Sep (Dt); Batavia, Je (Kngt); Westfield, May (Not); Buffalo, Z\&R; Ithaca, Sep (CU); Taughannock Falls, May (CU) ; McLean, Je-Sep (Dt); Windsor, Je (Not); W. Hebron ( Lg Coll.); Catskills, May, Pears; Big Ind. Valley, Je, Pears; Southfields, May, Schott; West Pt., Robn; Suffern, May, Schott; Hillburn, Nyack, May, Schott; Ramapo Mts., May (AM); Hartsdale (AM); SI, Je, Joutel, L\&D; LI: Jamaica, Ap; Queens, Schott.

The larva lives in oak, and, rarely, in hickory. Adults are often found resting on flowers, such as wild blackberry. Also occurs in spring flights of many individuals. Adult male insects show two forms, a black and a dark green.

14045 P. depressus Lec. Axton, Je, $M \& H$; Crown Pt., J1 (NYS); Ticonderoga, May, Bks; Northampton, $Y g$; Mt. Marcy, Not; Buffalo, Z\&R; Pike, Je (NYS) ; Catskills, May, Sep, Pears; Pine I., Je-J1, FrostSW, Sf; NYC, Je.

Differs considerably from the typical form, being more elongate, with heavier mandibles and striations of the elytra, and may prove to be a new species; color varies from deep bronze to black. 


\section{SUBFAMILY AESALINAE}

\section{Ceruchus MacLeay}

14058 C. piceus Weber. Common throughout the State. Mt. Marcy, Not; Cranberry L., Je, $H$; Newport, $Y g$; Peru, Je (CU); Gloversville, Sep (NYS) ; Batavia, Je-J1, Ds (Kngt); Buffalo, Ashm, Z\&R; Ithaca, Mar-Ap, Sep, Bish, Dt. (CU) ; Freeville, Windsor, Je (Not); W. Hebron, Je, Lg; Coeymans, Ap (NYS) ; Slide Mt., Catskills, Je, Sep, Ds, Shoe; Cooks Falls (Ang); North Branch, Schaupp; Katonah, Lg; Mosholu (AM) ; West Pt., Robn; Yonkers, Oct (Ang) ; Nepara Pk., Yonkers, Mar, Diven; NYC, Pelham Parkway; Van Cort. Pk., Nov (Ang); Rattlesnake Cr., Ap (Ang); West Farms (AM); SI, J1 (L\&D); LI: Queens, Nov, Schott; Bellport, J1, Nic; Yaphank, J1, Ds; Orient, Sep, Lath; Montauk, J1 (Ang).

The larva lives in the decayed wood of white birch, elm, oak, hemlock, maple, and butternut. Specimens recorded from northern or mountainous parts of the State often exhibit considerable rugosity of elytra, particularly noticeable in the females, and attain a greater size.

\section{NiCAGUS LeConte}

14062 N. obscurus Lec. Locally common. Usually considered a seashore species, although occasionally found inland. Wm. T. Davis records this species as found on the plant vegetation of the up-beach at Wading R., LI. Keene Val., May (Not); Mt. Marcy, Not; Buffalo, $Z \mathcal{E} R$; Westfield, May (Not); LI: Wading R., May-Je, Diven; Montauk, Je, Schott.

\section{FAMILY PASSALIDAE}

\section{PASSALUS Fabricius}

14064 P. cornutus Fab. Common all over the State in the decaying, and generally moist, wood of deciduous trees (Ang). Representative localities are: Adirondacks (Felt); Batavia; Olcott, May, Aug, Nov (Dt); West Pt. (Robn); SI (L\&D).

\section{FAMILY CERAIMBYCIDAE 50}

\section{SUbFAMILy PRIONINAE}

PARANDRA Latreille

14067 P. brunnea Fab. Parandra Borer. Adirondacks (Felt); Syracuse, Onondaga Hill (Blkm); Rochester (M\&W); Buffalo (Z\&R); E. Aurora, Wild (Dnd); Olcott, J1-Aug, Oct (Dt); Castile, Brad (Flet); Ithaca, J1-Sep (Dt), Spring L. Beach, Ithaca, J1, under bark of Pinus rigida (Chitt); West Pt. (Robn) ; SI, Aug-Sep (L\&D) ; LI: Brooklyn, Aug (Ds), in hollow trunks of Ailanthus (Linell); Greenport, Aug (Ds); Prospect, Aug, Central Pk., Je (BM). Larva in nearly all kinds of coniferous and hardwood trees (Fisher).

50 W. S. Fisher has gone over this list, and has supplied many New York food-plant notes. Dr. M. W. Blackman also has supplied records. 


\section{DEROBRACHUS Serville}

\section{(Orthosoma Serville)}

14081 D. brunneus Forst. Lesser Prionus. Adirondacks (Felt); Cranberry L., J1 (Mund); Syracuse, Sodus Bay (Blkm); Rochester (M\&W): Buffalo (Z\&R); Avon, J1, Cls; Cattaraugus and Allegany Counties (Dnd); Ithaca, Aug (Dt-CU); McLean, N. Spencer, Jl-Aug (CU); W. Hebron (Lg); West Pt. (Robn); SI, J1-Aug (L\&D) ; LI: Riverhead, Aug (Ds); Greenport, Aug (Ds); Orient, Je, Lath (Ds). Twenty more records $(\mathrm{Lg})$. Larva in decaying logs and stumps of nearly all hardwood trees (Fisher).

\section{PRIONUS Fabricius}

14084 P. laticollis Drury (kempi and oblongus Csy.). BroAd-NECKed Prionus. Adirondacks (Felt); Syracuse (Blkm); Buffalo (Z\&R); Geneva, Cls; Ithaca, J1-Aug (Dt-CU) ; L. George, J1 (Zab) ; Schenectady (Dt-CU) ; Oak Hill, J1-Aug, in roots of oak (Clarkson); West Pt. (Robn): White Plains, J1 (Frost, Sq) ; Peekskill (Blkm); Bronx (Ds); SI, J1-Aug (L\&D); LI: Half Way Hollow Hills, Wading R., Yaphank, J1 (Ds) ; Orient, Latk (NYS) ; Oyster Bay, 'Selden, Aug (Ds); Croton, Je-J1 (Ds). Twenty-five more records $(\mathrm{Lg})$. Larva works within the roots of various hardwoods, especially oak, poplar, and chestnut (Fisher).

P. nigrescans Csy. Adirondacks, L. Champlain (Csy). ${ }^{51}$

14085 P. pocularis Dalm. Saratoga, Aug (Dt-CU); Bronxville, J1 (Woodr); LI: Yaphank, Aug, at light (Ds); Bay Shore, Aug (Olsen). Larva in dead pine logs (Fisher).

1408? P. imbricornis L. LI: Bay Shore, Aug, Ols (BM). Larva in the roots of oak and chestnut (Fisher).

\section{TRAGosoma Serville}

14097a T. depsarium L. var. harrisi Lec. Adirondacks (Felt) ; Cranberry L., J1 (Mund) ; Long L. (Ds) ; Wanakena, Aug (Drk) ; Lewiston, J1 (Lg Coll.) ; Buffalo (Z\&R); Ithaca, J1 (CU); North Branch (Schaupp); Bronx (BM); LI: Yaphank, Aug (AM); North Beach, Aug (BM). Larva in sapwood and decaying wood of coniferous trees.

\section{Distenia Serville}

14098 D. undata Fab. West Pt. (Robn); SI (L\&D); LI: Westbury (Champlain). Chestnut, hickory, and hornbeam (Felt). Larva in hickory, elm, and redbud (Fisher).

\section{SMODICUM Haldeman}

14099 S. cucujiforme Say. SI (L\&D). Oak, beech, hackberry, and willow (Felt). Larva in the heartwood of very old, dry, and seasoned hardwoods, including oak, hickory, beech, elm, etc. (Fisher).

51 The species of Prionus described by Casey in 1912 were treated as synonyms in the Leng catalog. Casey in 1924 reiterated his opinion that they were valid species, and added nigrescans to the category. It is here retained, as it has not been studied since its description. 


\section{ASEMUM Eschscholtz}

14107 A. moestum Hald. Axton (Houghton); Cranberry L., May-J1 (Mund); Mt. Whiteface, Jl (Ds), Sep (Lg); Mt. Seward, 4500 ft., Je (Houghton); Syracuse (Blkm); Crittenden $(B 1 \mathrm{~km})$; Buffalo $(Z \& R)$; Allegany Pk. (Dnd); Ithaca, J1 (Dt-CU); Nassau, Troy, breeds in pine (Felt); West Pt. (Robn); Bronx (AM); SI, Je (L\&D); LI (Nic, Ds). Larva in spruce, fir, and larch (Fisher).

14109 A. ebenum Csy. Cranberry L., Je (Mund); Mt. Whiteface trail, J1 (Ds), as atrum Esch., an allied western species. Larva in spruce in the West (Fisher).

\section{TETROPIUM Kirby}

14124 T. cinnamopterum Kby. Adirondacks, Je-J1 (NYS); Axton (Houghton); Wanakena, Aug (Drk) ; Cranberry L., Je-Jl, bred from spruce (Mund) ; Syracuse (Blkm). Larva in pine, spruce, and fir (Fisher).

14128 T. velutinum Lec. Mt. Whiteface trail, J1 (Ds); Cranberry L., Je (Mund). Larva in pine (Fisher).

\section{CRIocephalus Mulsant}

14132 C. agrestis Kby. Adirondacks (Hill) ; Cranberry L., J1 (Mund) ; Oswego (NYS); Buffalo (Z\&R); Ithaca, Je-J1 (Dt-CU); Albany, Aug (NYS); SI, Je (L\&D); LI: Bay Shore, Aug (BM). Infests roots of pine and spruce (Felt).

14139 C. obsoletus Rand. Buffalo (Z\&R); E. Aurora (Dnd) ; LI, Je-Aug (Nic, Shoe). Larva in pine (Fisher).

C. champlainianus Csy. L. George (Csy).

\section{OEME Newman}

14146 O. rigida Say. Bronxville, Je (Woodr); SI, J1, bred from cedar (L\&D); LI: Je-Ji, in wash-up (Shoe); Rockaway Beach, Je (BM). Larva in juniper or red cedar (Felt).

\section{DRYobIUS LeConte}

14153 D. sexfasciatus Say. "NY". Larvae mine inner bark of dead elm, exceedingly rare (Fitch). Larva in basswood (Fisher).

TESSAROPA Haldeman

(Dysphaga LeConte)

14168 T. tenuipes Hald. SI, Je, from terminal twig of ash (L\&D). On oak, walnut, and redbud (Felt). Larva in twigs of oak, hickory, and beech; pupates in late summer or fall, and adults remain in twigs over winter, emerging very early in spring (Fisher).

TyLONOTUS Haldeman

14177 T. bimaculatus Hald. Northern NY (Pack); Gloversville, Aug (Yg); Rochester (M\&W); Buffalo (Z\&R); E. Aurora (Dnd); West Pt. (Robn). Larva in ash, hickory, black walnut, tulip, and birch (Felt). 
StRomatium Serville

14178 S. fulvum Villers. Cosmopolitan.

14179 S. pubescens Hald. Buffalo (Z\&R); LI (Ds).

\section{CHION Newman}

14181 C. cinctus Drury. Syracuse (Blkm); Buffalo (Z\&R); Penn Yan (Dt); Ithaca (Philip Miller); Albany (NYS); Hudson, May, larva on hickory, oak, and plum (NYS) ; West Pt. (Robn); NYC, Mar-May, from firewood (Woodr); SI (L\&D); LI, Je (BM). In cut timber, especially hickory (Felt). Larva in walnut, oak, hickory, and chestnut (Fisher).

\section{EBURIA Serville}

14190 E. quadrigeminata Say. Syracuse, bred from hickory (B\&S); West Pt. (Robn); LI (Lg). On honey locust, hickory, ash, beech, elm, and oak (Felt).

\section{RoMALEUM White}

14196 R. atomarium Drury. Syracuse (Blkm); Charlotte, Sep (NYS); LI: Bellport, J1-Aug (Nic); Wading R., J1 (Ds). Larva in stumps and logs of recently dead oak (Felt) and chestnut (Fisher).

14199 R. rufulum Hald. Syracuse (Blkm); Buffalo (Z\&R); Ithaca, Sep (CU) ; W. Hebron (Lg); New Paltz, Aug (Ds); West Pt. (Robn); Bronxville, J1 (Woodr); SI, J1-Aug (L\&D); LI: Brooklyn, J1 (Shoe); Yaphank, J1 (Ds). Larva in oak (Fisher).

\section{HYPERMALLUS Lacordaire}

14207 H. incertus Newn. Syracuse, bred from hickory (B\&S); Bronxville, J1 (Woodr) ; LI: Yaphank, Sep (Ds) ; Rockaway, Aug (Woodr) ; Bellport, Je (Nic). Larva in mulberry (Fisher).

14210 H. abruptus Csy. West Pt., Je (Not-AM); LI (Not-AM).

$14211 \mathrm{H}$. villosus Fab. The OAK Pruner. Syracuse (Blkm); Rochester (M\&W) ; Olcott, J1 (Dt) ; Buffalo (Z\&R) ; E. Aurora (Dnd); Ithaca, J1 (Dt-CU); L. George (Felt); Lawrence (Blkm); Nyack, Je (Ds); West Pt. (Robn) ; SI, May-Aug (L\&D) ; LI: Wading R., Je (Ds); Wyandanch, Yaphank, J1 (Ds) ; Flatbush, Ap, Yaphank, May, Amagansett, May (BM). Twenty-five other records $(\mathrm{Lg})$. Not very injurious (Felt). Larva in a great variety of hardwoods (Fisher).

14212 H. militaris Csy. "NY" (Csy).

14214 H. medialis Csy. Huguenot, Aug, bred from Quercus rubra (Not-AM).

14217 H. parallelus Newn. Lewiston, J1 ( $\mathrm{Lg})$; Buffalo $(\mathrm{Z} \& \mathrm{R})$; Ithaca, May (Dt); Albany (NYS); West Pt., Je (Ds); Newburgh, Blauvelt (NYS) ; Thousand Isls., May-J1 (Ds) ; LI: Wading R., May (Ds); Bellport, Sea Cliff, Bayport (NYS). Larva in a variety of hardwoods (Fisher). 


\section{ELAPHIDION Serville}

14219 E. irroratum L. "NY" (Lec, Hald).

14220 E. mucronatum Say. L. Minnewaska, J1 (Ds); Buffalo (Z\&R); West Pt. (Robn); SI, Je, J1, Aug (L\&D) ; LI: Half Way Hollow Hills, J1 (Ds) ; Brooklyn, Jl-Aug (Shoe); Flatbush, Je (BM). Larva in chestnut, redbud, maple, oak, poplar, mulberry, and many other hardwoods (Fisher).

\section{ANEFLOMORPhA Casey}

14227 A. subpubescens Lec. LI: Rockaway Beach, Central Pk., May (Ds) ; Wyandanch, J1, Yaphank, May, Jl, Deep Pond, Wading R., Je (Ds). Larva in chestnut and oak (Fisher).

\section{Anoplium Haldeman}

14240 A. cinerascens Lec. Buffalo (Z\&R); West Pt. (Robn); Bronxville, J1 (Woodr); SI (L\&D). (A. pumilum record probably belongs here.)

\section{PSEUdibidion Casey}

14263 P. unicolor Rand. Adirondacks, J1 ( Lg); Syracuse, bred from hickory (B\&S) ; Rochester (M\&W) ; Buffalo (Z\&R) ; Avon, Je, Cls; Ithaca, Je (Dt-CU) ; Clinton Hgts., Je (NYS); West Pt. (Robn); Mt. Kisco, Aug (NYS); SI, Je 29 (L\&D) ; LI: Brooklyn (Shoe); Central Pk., Yaphank, J1 (Ds); Bellport, Aug, beating scrub oak (Nic); Thousand Isls. (Ds). Larva in oak, beech, walnut, and hickory (Fisher).

\section{HETERACHTHES Newman}

14268 H. ebenus Newn. SI, Je (L\&D) ; LI: Rockaway, May, Wyandanch, Je (Ds) ; Babylon, Je-J1, Long Beach (AM) ; Bellport, J1 (Nic) ; Hicksville, Aug (Champlain). Larva in pine (Fisher).

14271 H. quadrimaculatus Fab. Syracuse, bred from hickory (B\&S); Buffalo (Z\&R); West Pt. (Robn); Bronx (BM); Bronxville, Je (Woodr); Riverdale, J1 (Woodr); West Farms (AM); NYC (Ds); SI, Je-J1, hatched from hickory (L\&D); LI, J1 (Shoe). Larva in hickory and tulip poplar (Fisher).

\section{GRACILIA Serville}

14282 G. manca Lec. Type of manca from NY. NYC ( Lg Coll.). Possibly identical with minuta.

14283 G. minuta Fab. Syracuse (Blkm); Buffalo (Z\&R); NYC (AM).

\section{OBRIUM Curtis}

\section{(Phyton Newman)}

14286 O. maculatum Oliv. (pallidum Say). Buffalo (Z\&R); Mosholu (BM); SI, J1, on oak (L\&D); LI: J1 (Ds); Aqueduct, J1 (Ds). Larva in oak, hackberry, hickory, mulberry, and chestnut (Fisher).

14291 O. rufulum Gahan (rubrum Lg.). Buffalo (Z\&R); White Plains, J1 (Frost, $\mathrm{Sq}$ ) ; Bronx (BM); SI, J1, on privet (L\&D) ; LI: Flushing, Aug (Ds). Larva in ash (Felt). 


\section{RHAGIUM Fabricius}

14298 R. lineatum Oliv. Ribbed Pine Borer. Mt. Whiteface, J1 (Ds) ; Cranberry L., Je, Sep (Mund); Syracuse (Blkm); Buffalo (Z\&R); Geneva, Je-J1, Cls; Ithaca, Oct (Ds); Taughannock Falls, Ithaca, Ap (Dt-CU); Schenectady, Dec (Dt); Albany (Felt); Elka Pk (Blkm); West Pt. (Robn) ; SI, Ap-Nov, under bark of white pine (L\&D); LI (Eng); Wyandanch, Ap, pitch pine (Ds) ; Yaphank, May (Ds); Central Pk., Ap (BM). Larva in pine (Fisher).

\section{Hapalosalia Casey}

14304 H. aurata Horn. Genesee Co, Je (Lg) ; Portageville, J1 (Ds); Rock City, Je (Ds) ; Ithaca, Je-J1 (Dt); Ringwood, Je-J1 (Dt-CU); Windsor, Je (Not); Catskills, J1 (Blk).

14308 H. vibex Newn. Mt. Whiteface, J1 (Shoe); Protection (Dnd); Newport (NYS) ; Buffalo (Z\&R) ; Castile, Brad (Flet) ; Potter Swamp, Yates Co, Je (Ds); Ithaca, Coy Glen, Ringwood, Danby, Je-J1 (Dt-CU); Kenwood, Albany (NYS); Trenton Falls (Newn); Schoharie (Not); Catskills, J1 (Blk); West Pt. (Robn).

14310 H. sphaericollis Say. Mt. Whiteface, J1 (Ds) ; Rochester (M\&W) ; Buffalo (Z\&R); Olcott, Je (Dt); Portageville, Je (Ds); Rock City (Ds); Catskills, J1 (Blk); West Pt. (Ds).

14310a H. sphaericollis var. ? ruficollis Say. Olcott, May-J1 (Dt); Ringwood, Je (Dt); Catskills; W. Hebron; West Pt. (Robn); Ramapo, May; Ft. Montgomery.

\section{Centrodera LeConte}

14311 C. decolorata Harris. Barneveld, Oct (Yg); Sodus Bay (B1km); Niagara (Lec) ; Buffalo (Z\&R); Ellington, Je ( $\mathrm{Lg})$; Seneca Jct., Rock City, Je (Ds); Ithaca, Je (Lg), J1 (Dt-CU) ; Catskills (Pears); Binghamton (BM). On butternut and beech (Felt). Larva in oak, chestnit, and tulip poplar (Fisher).

14313 C. picta Hald. Rochester (M\&W) ; Buffalo (Z\&R); Portageville, Je (Ds); Rock City (Ds) ; Montour Falls (H) ; Taughannock Falls, Ringwood, May (Dt); West Pt. (Robn) ; Ramapo (AM) ; SI, Je, at light (Grossbeck); LI (Ds). On hickory (Felt, Bish).

STENOCORUS Fabricius $\mathbf{5 2}$

(Toxotus LeConte)

14320 S. cinnamopterus Rand. "NY" (Lg Cat.).

14321 S. schaumi Lec. Newport (NYS); Batavia, J1 (Kngt); Buffalo (Rein); Danby, Cy (Flet) ; Cortland Co (Dnd) ; Catskills, Ji, Pears, very rare on dead beech (Nic); Shokan ( $\mathrm{Lg})$. On beech (Felt).

14321a S. schaumi var. croceus Lg. Wilmington, Aug (Ds).

14323 S. trivittatus Say. Mt. Whiteface, J1 (Ds) ; Cranberry L., J1 (Mund); Minerva, J1 20, 1925, one specimen on milkweed flowers (Frost) ; Rochester (M\&W) ; Ithaca (Philip Miller); Binghamton (BM); West Pt., Je (Ds); Croton, Je (Ds); Ft. Montgomery, May 30 (AM).

52 The larval habits of this genus are unknown (Fisher). 
14324 S. vittiger Rand. West Pt. (Robn); Croton, Je (Ds); Ramapo (Sm).

14343 S. cylindricollis Say. Trenton Falls (Newn); Nyack (Zab); Van Cort. Pk., Pears.

\section{PACHYTA Zetterstedt}

14348 P. rugipennis Newn. Homadaga L., Essex Co, May, 1924, very rare (Frost); Cranberry L., three specimens on bark of dead spruce, Je (Htly).

\section{Evodinus LeConte}

14349 E. monticola Rand. Axton (Houghton); Cranberry L., Je (Mund) ; Big Moose (NYS) ; Boston, Protection (Dnd) ; Buffalo (Rein) ; Portageville, Je (Ds); Ithaca, Je (Dt-CU); W. Danby, May, Tarbel, Je (Ds); Windsor, Je (Not); Schoharie (Not); Catskills, Je-J1 (Ds, Blk). Larva in hemlock (Fisher).

\section{ACMAEOPS LeConte}

14395 A. directa Newn. Adirondacks, Aug (Martin); Keene Val., Aug (BM); Rochester (M\&W) ; Buffalo (Z\&R); Holland, Allegany Pk. (Dnd); Ithaca, J1 (Dt-CU); Albany (NYS); New Baltimore (AM) ; Catskills, Aug (Ds) ; Elka Pk. (Blkm); Poughkeepsie, Je (NYS) ; Peekskill (AM); West Pt. (Robn); Nyack (Ds); Ramapo, Je (Ds).

14396 A. pratensis Laich. Axton (Houghton); Mt. Whiteface, Wilmington, J1 (Ds) ; Cranberry L., Je-J1 (Mund); Catskills (Pears).

14402 A. cavicollis Csy. Cranberry L., Je-J1, bred from spruce and balsam (Mund).

14403 A. proteus Kby. Adirondacks (Shoe-NYS); Cranberry L., Je (Drk); Ithaca, Je (Dt-CU) ; LI: Yaphank, Je, Rockaway Beach, May (BM).

14405 A. discoidea Hald. LI: Wading R., May (Ds); Rockaway, Je (Shoe).

14406 A. thoracicus Hald. Mt. Whiteface, J1 (Shoe); Windsor, Je (Not); Big Ind. Val., Je (Shoe); West Pt. (Robn); Ramapo (Sm).

14407 A. bivittatus Say. Lewis Co, J1 (NYS); Rochester (NYS); Pike, Je (NYS); West Pt. (Robn).

14408 A. nigripennis Lec. West Pt. (Robn).

14409 A. fusciceps Lec. Cattaraugus Co (Lec), type.

\section{GaUROTES LeConte}

14411 G. cyanipennis Say. Mt. Whiteface, J1 (Ds); Cranberry L., Je, Aug (Mund) ; Geneva, Middlesex, Je, Cls; Avon, May, Cls; Olcott, Je (Dt) ; Buffalo, Je (Ds, Z\&R) ; Ithaca, Je-J1 (Dt-CU); Groton, Je (Ds); Schoharie (Not); West Pt. (Robn) ; Croton, Je (Ds) ; Ramapo (Ds) ; SI, Je-J1, on chestnut (L\&D) ; LI (BM). Thirty-four more records (Lg). In butternut, oak, and birch (Felt). Larva in dogwood, sour gum, oak, wild cherry, walnut, and mulberry (Fisher).

14414 G. abdominalis Bland. Mt. Whiteface trail, J1 (Ds); Rochester (M\&W); Allegany Pk. (Dnd); Catskills (BM); West Pt., in laurel blossoms, Je (Robn). 


\section{Anthophilax LeConte}

14415 A. attenuatus Hald. Mt. Whiteface, J1 (Ds) ; Keene Hgts., Aug-Sep (Lg) ; Cranberry L., Je-J1, on male flowers of red pine (Mund) ; Newport, on dead beech (Yg); Lewis Co, J1 (NYS); Holland (Frost) ; Catskills, May (Pears); Big Ind. Val., J1 (Shoe). Larva in beech, birch, maple, and poplar (Fisher).

14416 A. malachiticus Hald. Cranberry L. (Blkm); Rochester (M\&W) ; Rock City, Je (Ds); Ithaca, Ringwood, Je (Dt-CU) ; Slide Mt., J1 (Lg) ; Catskills, May 30 (Knull Coll.) ; Big Ind. Val., J1 (Shoe) ; Ft. Montgomery (Brb).

14417 A. viridis Lec. Mt. Whiteface, J1 (Shoe); Slide Mt., Je (Ds) ; Cranberry L. (Mund); Big Ind. Val., J1 (Shoe). Larva in beech, birch, and maple (Fisher).

14417a A. viridis var. viridipennis Csy. Northern NY (Csy).

Judolia Mulsant

14424 J. sexmaculata L. Long L. (Ds) ; Mt. Whiteface, J1 (Ds) ; Cranberry L., Je-J1 (Mund).

14426 J. cordifera Oliv. Cranberry L., J1-Aug (Mund); Rochester (M\&W) ; Canandaigua Center (NYS); Allegany Pk. (Dnd); Ithaca, Coy Glen, Ringwood, Je-J1 (Dt-CU) ; Albany (NYS) ; Catskills, J1-Aug (Blk, Ds); Staatsburg, Je (Woodr); Callicoon, J1 (Ds) ; West Pt. (Robn); Stony Pt., Je (Ds) ; SI, Je-J1, on flowers of New Jersey tea (L\&D); LI, Je-Ji (Ds).

\section{BRACHYLFPTURA Casey}

14438 B. rubrica Say. Cranberry L., J1-Aug (Mund); Saranac (Lg); Syracuse, bred from hickory (B\&S) ; Geneva, Jl, Cls; Buffalo (Z\&R); Ithaca, Je-J1 (Dt-CU); Albany (Yg); Catskills, J1-Aug (Blk,Ds); North Branch (Schaupp); West Pt. (Robn); Mosholu (AM); SI, Je-J1, on flowers of New Jersey tea (L\&D) ; LI: Pinelawn, Aug, Wyandanch, Je (Ds). Twenty-five more records ( $\mathrm{Lg})$. Larva in a great variety of dead hardwoods and conifers (Fisher).

14441 B. vagans Oliv. Adirondacks; Old Forge (NYS); L. Pleasant (NYS) ; Cranberry L., J1-Aug (Mund) ; Buffalo (Z\&R); Canandaigua (NYS); Ithaca, Dec (Chitt); Buttermilk Cr., J1 (Dt-CU) ; Karner (NYS); Catskills, J1-Aug (Blk, Ds) ; Calicoon, J1 (Ds); West Pt. (Robn); SI, bred from branch of pitch pine (L\&D). In oak (Felt). Larva in decaying pine (Fisher).

14445 B. circumdata Oliv. Cranberry L., Je (Mund) ; Ithaca, Je (Dt) ; Catskills, J1 (Blk):; West Pt. (Robn) ; LI: Wyandanch, Yaphank, Je (Ds).

14447 B. canadensis Fab. Wilmington, Aug (Ds, Dt); Keene Val., Aug (BM) ; Long L. (Ds) ; Tahawus, Aug (Lg); Cranberry L., J1-Aug, bred from balsam and spruce (Mund); Saranac L. (Lg); Mt. Whiteface, 4000 ft., Aug (Dt); Syracuse (Blkm); E. Aurora (Dnd); Avon, J1, Cls ; Buffalo (Z\&R); Albany, J1 (NYS) ; Catskills, Aug (Blk,Ds) ; Sullivan Co ( $\mathrm{Lg})$; North Branch (Schaupp). In spruce and hemlock (Felt). Larva in hemlock and pine (Fisher).

14447a B. canadensis var. cinnamoptera Hald. Cranberry L., J1-Aug (Mund) ; Big Ind. Val., Aug (BM); Catskills (Blkm). 
14448 B. cribripennis Lec. Buffalo ( $Z \& R$ ).

14450 B. sanguinea Lec. Cranberry L., J1-Aug (Mund); Mt. Whiteface, J1 (Shoe); Catskills, J1 (AM); West Pt. (Robn).

14452 B. lacustris Csy. Cranberry L., J1-Aug, bred from spruce (Mund).

\section{PaRallelina Casey}

14455 P. chalybea Hald. Buffalo (Z\&R); Ithaca, Je (Kngt).

14456 P. exigua Newn. (sancia Lec.). Newport, Je (NYS); Syracuse (Blkm); Buffalo (Z\&R); Ithaca, Ringwood, Je (Dt); Kenwood, Albany, Je (NYS); Schoharie (Not); Poughkeepsie, Je (NYS); Peekskill, May (Lg); West Pt. (Robn); Ft. Montgomery, May (Lg).

14457 P. nana Newn. Catskills, J1 (Blk); Trenton Falls (Newn).

14457a P. nana var. haematites Newn. Newport, Je (NYS); Rochester (M\&W) ; Boston (Dnd); Buffalo (Z\&R); Pike, Je (NYS); Portageville, Je (Ds) ; Ithaca, May-Je (Dt, Kngt); Trenton Falls (Newn); Catskills, Je (Pears); Mosholu, Je (BM); Elka Pk. (Blkm); West Pt. (Robn) ; Ft. Montgomery, May (AM) ; Bronx, May (AM); SI, May, on flowers (L\&D); LI: Yaphank, Je (Ds).

14459 P. subargentata Kby. Plattsburg, Wilmington, J1 (Shoe); Rochester (M\&W) ; Buffalo (Z\&R) ; Allegany Pk. (Dnd); Catskills (Pears).

14459c P. subargentata var. ruficollis Prov. Castile, Je, Brad (Flet); Allegany Pk., Boston (Dnd); West Pt. (Robn).

14461 P. similis Kby. "Western NY," frequent (Dnd).

\section{Charisalia Casey}

14463 C. americana Hald. Albany, J1 (NYS).

14464 C. capitata Newn. Axton (Houghton) ; Cranberry L., Je (Mund) ; Rochester (M\&W) ; Genesee Co, Je (Lg); Olcott, Je (Dt); Buffalo (Z\&R); Ithaca, Je (Dt-CU) ; Trenton Falls (Newn); West Pt. (Robn); NYC and vic. (AM).

\section{STRANGALEPTA Casey}

14465 S. lineola Say. Western NY, common (Dnd) ; Buffalo (Z\&R); Portageville, Je (Ds) ; Rock City, Je (Ds); Ithaca, May-J1 (Dt); McLean, Ringwood, J1 (Dt) ; Windsor, Je (Not) ; Schoharie (Not); Catskills, J1 (Blk); West Pt. (Robn) ; Ft. Montgomery, May, Ramapo, Je (Ds); SI, May-Je, on flowers (L\&D) ; LI: Yaphank, Je (Ds). Fifty-three more records (L). Larva in dead birch (Fisher).

14466 S. pubera Say. N. Elba, J1 (Ds); Mt. Whiteface, J1 (Ds) ; Cranberry L., Je (Mund); western NY, frequent (Dnd); Portageville, Je (Ds); Castile, Brad (Flet); Cattaraugus, Je (Ds) ; Ithaca, Je-J1, Ringwood, Je, McLean, J1 (Dt); Schoharie (Not); Catskills, J1 (Blk); West Pt. (Robn); SI, May-Je, on flowers (L\&D). Twenty-six more records $(\mathrm{Lg})$.

14469 S. vittata Oliv. Long L. (Ds); N. Elba, J1 (Ds) ; Cranberry L., Je-J1, bred from spruce and larch (Mund); Syracuse, Crittenden (Blkm); Olcott, Je-J1 (Dt); Genesee Co, Je; Buffalo (Z\&R); Portageville, $\mathrm{Je}$; Conesus L., Je; Geneva, Je, Cls; Ithaca, Je-Jl, McLean, J1 (Dt) ; Catskills, J1-Aug (Blk); West Pt. (Robn); White Plains, Je (Frost, 
Sq) ; Pine I., Je; Ft. Montgomery, J1, Ramapo, Je; SI, May-Je, on flowers (L\&D); LI: Wyandanch, Half Way Hollow Hills, Yaphank, Je-J1 (Ds). Sixty-seven more records ( $\mathrm{Lg})$. Larva in cedar, pine, fir, and chestnut (Fisher).

\section{STRANGALIA Serville}

14482 S. subhamata Rand. Adirondacks, J1 (NYS); Long L. (D's); Cranberry L., J1, on flowers (Mund); Minerva, J1 20, 1925, on Spiraea flowers (Frost); Rochester (M\&W); Buffalo (Z\&R); Java, E. Aurora (Dnd); Pike (NYS); Ithaca, Je-J1 (Dt); Catskills, Aug (Ds); Sullivan Co (Schaupp). In hemlock, tamarack, and beech (Felt). Larva in dead pine and hemlock (Fisher).

14483 S. elegans Hald. Cranberry L., J1-Aug, on flowers (Mund); Saratoga (Lec).

14484 S. cruentata Hald. Hastings, May-Je (Joutel); Ithaca, Je (Dt); West Pt. (Robn).

14487 S. plebeja Rand. Essex Co, J1 (Yg); Minerva, J1 20, 1925, on Spiraea flowers (Frost); Cranherry L., Je-J1, Sep, on flowers (Mund); Long L. (Ds); Catskills, J1 (Blk).

\section{Xestoleptura Casey}

14493 X. octonotata Say. Rochester (M\&W) ; Olcott, Je (Dt) ; Buffalo (Z\&R); Ithaca, Je (Dt-CU); Poughkeepsie, Je (Yg); West Pt. (Robn); Mosholu, Je (Ds); Ramapo, Je (Ds); SI, Je, on flowers (L\&D).

\section{STROPHIONA Casey}

14508 S. nitens Forst. (zebra Felt). Syracuse (Blkm); Olcott, Je (Dt) ; Buffalo (Z\&R); Conesus L., Je (Ds); Geneva, J1, Cls; Ithaca, Je (Dt); Catskills, Aug (Ds) ; West Pt. (Robn) ; Ft. Montgomery, J1, Nyack, Ramapo, Je (Ds); SI, May-Je, on flowers (L\&D); LI: Rockaway Beach, Je, Half Way Hollow Hills, J1, Yaphank, Je (Ds). Thirtyeight more records $(\mathrm{Lg})$. Larva in chestnut and oak (Fisher).

\section{LEPTURA Linné}

14514 L. emarginata L. Syracuse (Blkm); Buffalo (Z\&R); Ithaca, Aug (E. A. Richmond, Philip Miller); Rensselaer, Je (NYS); West Pt. (Robn); Bronx, J1 (AM); SI (L\&D). Larva in red oak (Joutel), elm (Felt), birch, beech, oak, sour gum, and tulip poplar (Fisher).

14516 L. nigrella Say. Cranberry L., Je-J1 (Mund); Oswego, L Placid, Je (NYS) ; Geneva, Je-JI, Cls.

14520 L. chrysocoma Kby. Adirondacks (Shoe, Not, Yg); Mt. Whiteface, Wilmington, N. Elba, J1 (Ds) ; Axton (Houghton) ; Cranberry L., Je$\mathrm{J} 1$, on flowers (Mund). Larva in pine (Fisher).

14525 L. proxima Say. Adirondacks, Je-J1 (Ds) ; Cranberry L., Je-Aug, on flowers (Mund); Olcott, Je (Dt); Buffalo (Z\&R) ; E. Aurora (Dnd); Elka Pk., Aug (Drk); Conesus L., Je-J1 (Ds) ; Ithaca, J1 (Dt) ; Catskills, J1-Aug (Blk, Ds); West Pt. (Robn); SI, on flowers (L\&D). Twenty-nine more records ( $\mathrm{Lg})$. Larva in chestnut, maple, hickory, 
and linden (Fisher). (Many specimens from Cranberry $L$. and the Catskills would fall under L. minnesotana Csy. if this be considered valid-Blkm.)

- L. kempiana Csy. L. George (Csy).

14530 L. biforis Newn. Cranberry L., Je-J1 (Mund); Speculator, J1 (NYS); Syracuse, Onondaga Hill (Blkm); Sodus Bay, J1 (NYS); Rochester $(\mathrm{M} \& W)$; Buffalo (Newn); Ithaca, J1-Aug (Dt-CU) ; Helderbergs, J1 (NYS); Trenton Falls (Newn); Catskills (Lec, Pears); Elka Pk. (Blkm). Larva in chestnut (Fisher).

14531 L. laurentica Csy. "Northern NY" (Csy).

14532 L. mutabilis Newn. Axtcn (Houghton); Cranberry L., Je, bred from yellow and white birch (Mund); Syracuse, bred from alder (B1km); E. Aurora, Allegany Pk. (Dnd) ; Castile, Brad (Flet); Portageville, Ji (Ds); Ithaca, Je-J1 (Dt); Windsor, Je (Not); Schoharie (Not); Catskills, J1 (Blk); West Pt. (Robn); Nyack, Je (Ds) ; SI, Je, on flowers, bred from blue beech and dogwood (L\&D). Larva in oak, maple, chestnut, and birch (Fisher).

14532 a L. mutabilis var. luridipennis Hald. Cranberry L., Je-J1, cut from hemlock (Mund); Allegany Pk., scarce (Dnd); Elka Pk. (Blkm). Twentynine more records $(\mathrm{Lg})$.

14533 L. aspera Lec. Catskills (Knull). Larva in dead birch (Fisher).

14534 L. pedalis Lec. Cranberry L., Je-Jl, on flowers (Mund); Mt. Whiteface, J1 (Ds); Newport, Old Forge, Je (NYS); Elka Pk. (Blkm).

\section{BELLAMiRA LeConte}

14537 B. scalaris Say. Cranberry L., J1-Aug, cut from birch (Mund) ; Mt. Whiteface, J1 (Ds) ; Mt. Marcy, J1 (Ds); Buffalo (Z\&R); E. Aurora (Dnd); Ithaca, Aug (CU); Catskills, J1 (Blkm); Saratoga (Lec); West Pt., bred from poplar (Robn); Sullivan Co (Schaupp). Larva in many varieties of coniferous and hardwood trees (Fisher).

\section{TyPOCERUS LeConte}

14540 T. zebra Oliv. NY (Lg, Lec). On white oak (Felt).

14543 T. velutina Oliv. Cranberry L., J1-Aug, on flowers (Mund) ; Syracuse, bred from hickory (B\&S); Olcott, Je-J1 (Dt); Buffalo (Z\&R); Elka $\mathrm{Pk}$., Aug (Drk); Geneva, J1, Cls; Ithaca, J1-Aug (Dt); Karner, on scrub oak (Felt); Caliicoon (Ds); Catskills, J1-Aug (Blk); West Pt. (Robn) ; SI, Je-Aug, on roses (L\&D); LI: Massapequa, Half Way Hollow Hills, Wading R., Yaphank, J1-Aug (Ds). Larva in many varieties of coniferous and hardwood trees (Fisher).

14544 T. acuticauda Csy. Cranberry L., J1-Aug, with preceding on flowers (Mund); Elka Pk. (Blkm).

14548 T. lugubris Say. Olcott, Je (Dt); Buffalo (Z\&R) ; Greene Co, J1 (Lg) ; Ulster Co, Je (Lg); Bronx (AM); SI, Je-Ji (L\&D).

\section{OpHISTOMIS Thomson}

14553 O. famelica Newn. Staatsburg, Je (Woodr); West Pt. (Robn) ; Bronxville, Je (Woodr); SI, Je-J1, on flowers (L\&D); LI: Wyandanch, Half Way Hollow Hills, J1 (Ds); Yaphank, Je (BM). Larva in decaying oak and yellow birch (Fisher). 
14554 O. acuminata Oliv. Poughkeepsie, Je (NYS); Staatsburg, Je (Woodr) ; West Pt. (Robn); Ft. Montgomery, May 30 (Lg); Bronxville, Je (Woodr) ; Bronx (AM); SI, Je, on flowers (L\&D); LI: Yaphank, Je-J1 (Ds). Bred from dead Viburnum stems (Fisher).

14556 O. luteicornis Fab. Rochester (M\&W) ; Coy Glen, Ithaca, J1-Aug (DtCU) ; Catskills, J1-Aug (Blk); West Pt. (Robn); Ft. Montgomery, J1 (Ds) ; Callicoon (Ds) ; Stony Pt., Je (Ds) ; SI, Je-Aug, on flowers (L\&D) ; LI: Wyandanch, Half Way Hollow Hills, Yaphank, Je-J1 (Ds). Thirty-five more records ( $\mathrm{Lg})$. Larva in decaying elm, beech, grape, and Viburmum dentatum (Fisher).

14560 O. bicolor Swed. LI: Yaphank, J1 (Ds). Larva in oak and maple (Fisher).

\section{ENCYCLOPS Newman}

14573 E. coerulea Say. Slide Mt., J1 (Knull); Egleston's Glen, L. Keuka, Je (Ds) ; Olcott, Je (Dt) ; Kenwood, Je (Yg) ; Buffalo (Z\&R) ; Portageville, Je (Ds) ; Ithaca, Je (Kngt) ; Trenton Falls (Newn); Schoharie (Not); Big Ind. Val., Je--Jl (Shoe); West Pt. (Robn) ; SI, May 30, on flowers (L\&D). Larva in walnut, sour gum, chestnut, maple, oak, and tulip poplar (Fisher).

\section{DESMOCERUS Serville}

14574 D. palliatus Forst. The Cloaked Knotty-Horn. Adirondacks (NYS); Syracuse (Blkm); Rochester (M\&W); E. Aurora (Dnd); Buffalo (Z\&R) ; Branchport, Je-J1 (NYS); Castile, Je, Brad (Flet) ; Elka Pk., Aug (Drk); Geneva, Je, Cls; Ithaca, Je-Sep, Cayuga Co (DtCU) ; Karner, Albany, Je-J1 (NYS); Big Ind. Val., Je-J1 (Shoe) ; New Baltimore (AM); Elka Pk. (Blkm); West Pt. (Robn, Ds); Pine I., Je (Ds) ; Nyack (AM); White Plains, May-Je (Frost, Sq); Bronxville, Je (Woodr) ; Van Cort. Pk. (AM) ; SI, J1 (L\&D) ; LI: Elmhurst, Aug (Ds). Larva in roots of elder (Fisher).

Necydalis Linné

14580 N. mellitus Sav Buffalo (Z\&R); West Farms (AM). Larva in chestnut and oak (Fisher).

\section{MOLORCHUS Fabricius}

14584 M. bimaculatus Say. Syracuse, bred from hickory (B\&S); Rochester (M\&W) ; Olcott, Je (Dt) ; Buffalo (Z\&R) ; Boston (Dnd) ; Pike, Je (NYS); Castile, Brad (Flet); Kenwood, Je (NYS); Aurora, Je (AM); Schuyler Co, Je (H); Ithaca, Je-J1 (Dt-CU); W. Danby, May (Ds) ; Poughkeepsie, Je (NYS) ; Peekskill, May (Sherm) ; West Pt., Je (Robn, Ds) ; Ramapo, Je (AM) ; Bronxville, May (Woodr) ; SI, May, bred from dogwood (L\&D) ; LI: Yaphank, May (Ds). Larva in redbud, dogwood, chestnut, oak, grape, walnut, maple, and sweet gum (Fisher).

\section{Callimoxys Kraatz}

14586 C. fuscipennis Lec. (sanguinicollis Oliv.). Rochester (M\&W); Buffalo (Z\&R) ; Aurora (AM) ; Ithaca, Je-J1 (Kngt, Dt-CU); Harford, Je (BM); Schoharie (Not); Catskills, Je-J1 (Shoe). 


\section{Gonocallus LeConte}

14606 G. collaris Kby. "NY" (Lec); Portage (Frost). Larva in pine and spruce (Fisher).

\section{PHYSOCNEMUM Haldeman}

14608 P. brevilineum Say. Clinton, Oswego, J1 (NYS); Olcott, Je (Dt) ; Buffalo (Z\&R, Dnd); Geneva, J1, Cls; Ithaca, J1 (Dt-CU) ; Albany, J1 (NYS) ; West Pt. (Robn, Ds); West Farms (AM) ; LI: Yaphank, Je, in scarlet oak, Wading R., Je (Ds). Larva in elm (Fisher).

\section{Ropalopus Mulsant}

14612 R. sanguinicollis Horn. Mt. Whiteface (Ds); Saranac, Je (NYS) ; Syracuse (Blkm); Elka Pk., May (Drk); Big Ind. Val., Je (Ds); Catskills, Pears (Shoe).

\section{HYLOTRUPES Serville}

14613 H. bajulus L. Buffalo (Z\&R); Yonkers, Je (NYS); West Farms (AM); Bronx (BM); SI (L\&D); LI: Rockaway Beach, J1 (Ds); Moriches, damaging flooring of house (Zab). Larva in dry, seasoned wood of conifers, doing considerable injury to finished woods (Fisher).

\section{Anocomis Casey}

14614 A. lignea Fab. Syracuse, from arbor vitae (Blkm); Buffalo (Z\&R); West Pt. (Robn); SI, Mar, common on recently felled cedar, also Ap (L\&D) ; LI: Rockaway Beach, May (BM); Orient, Lath (NYS). Larva in red cedar (Fisher).

\section{MERIUM Kirby}

14619 M. proteus Kby. "NY" (Lec). Larva in spruce (Fisher).

\section{Callidium Fabricius}

14620 C. antennatum Newn. Axton (Houghton) ; Cranberry L., Je-J1 (Mund); Old Forge, May-Je (NYS); St. Lawrence Co, Je (Drk); Rochester $(M \& W) ; B u f f a l o(Z \& R)$; Karner, May-Je (NYS); Albany, May-Je (NYS) ; Elka Pk. (Blkm) ; West Pt. (Robn) ; SI, Ap, Je, larva lives in pine (L\&D); LI: Rockaway Beach, May (Ds); Central Pk., common on dead pine, Ap (Eng); Long Beach, Wyandanch, May (BM). Bred in large numbers from a young dead pine at Karner (Felt). Larva in pine and spruce (Fisher).

14621 C. schotti Sf. LI: Huntington, Sep (Schott); Long Beach, May (BM). (C. janthinum of Buffalo list perhaps belongs here; also Mt. Whiteface, $\mathrm{Jl}$, record by Mr. Davis, here or with the next.)

14622 C. violaceum L. Lowville, J1 (Ds); Keene Val., Je (BM); Mt. Whiteface, J1 (Shoe); Saranac (Woodr); Syracuse (Blkm); LI: Brooklyn (BM).

14625 C. frigidum Csy. Pine I., May (BM); NYC, from cedar introduced for rustic work, May (Frost); LI: Yaphank, May (Ds). 


\section{Phymatodes Mulsant}

14630 P. amoenus Say. Syracuse (Blkm); Rochester (M\&W); Buffalo (Z\&R); E. Aurora (Dnd) ; Albany, May-Je (NYS); West Pt. (Ds); Mosholu (AM) ; SI, May-Je, larva in dead grapevines (L\&D); LI: Rockaway, Je (Shoe); Wading R., May (Ds); Yaphank, Long Beach, Montauk, May (BM).

14630a P. amoenus var. hudsonicus Csy. Type locality, NY ( Lg).

14631 P. lengi Joutel. Egleston's Glen, L. Keuka (Ds); Coy Glen, Ithaca, Je (Ds); SI, Je (L\&D); LI (BM).

14634 P. testaceus L. Buffalo (Ham); Ithaca, Je (Dt-CU); W. Hebron, Je (Lg); Ft. Montgomery, May 30 (Ds); West Farms (AM); SI, Je, larvae under bark of oak logs (L\&D); LI: Babylon (AM).

14634 P. testaceus var, variabilis L. Buffalo (Z\&R); West Pt. (Robn); Ft. Montgomery, May, Nyack, Je (Ds); SI, with testaceus (L\&D). Twenty-eight more records ( $\mathrm{Lg})$. Larva mines in the inner bark of dead and dying oak (Felt).

14635 P. aereus Newn. Syracuse, bred from hickory (B\&S); Rochester (M\&W); Olcott, Je (Dt) ; Buffalo (Z\&R) ; Coy Glen, Ithaca, Je-J1 (Dt-CU); W. Hebron (Lg Coll.); Peekskill (Lg Coll.); West Pt. (Ds); Ramapo (Lg Coll.); LI: Rockaway (Sherm); Yaphank, Je (BM). Larva in chestnut and oak (Fisher).

14640 P. dimidiatus Kby. Adirondacks, May, Aug; Axton (Houghton); Mt. Whiteface, Jl (Ds); Cranberry L., Je-Jl (Mund); Syracuse, Crittenden, bred from larch (Blkm); Buffalo (Z\&R); Catskills, Aug (Ds); Van Cort. Pk. (AM); SI, larva bores in oak (L\&D); LI: Rockaway Beach, Je (BM). Emerging from spruce siding (Felt). Larva in spruce, hemlock, and larch (Fisher).

14646 P. varius Fab. Olcott, Je (Dt); Buffalo (Rein); W. Danby, May (Ds); Peekskill, Je (Rein); West Pt., Je (Robn, Ds); Ft. Montgomery, May (Ds); Nyack, Je (Rein, Ds); SI, larva bores in oak (L\&D); LI: Wading R., May (Ds); Amagansett, May, Flatbush, Je (BM). Fitch also records variety albofasciatus Bland. Oak and hickory (Felt).

14650 P. ater Lec. Buffalo (Horn).

14655 P. lividus Rossi. SI (Joutel); LI, introduced ( $\mathrm{Lg}$ ).

\section{Cyllene Newman}

14670 C. caryae Gahan (picta Horn). Rochester (M\&W); Buffalo (Z\&R); Ithaca, Je-J1 (Dt-CU); L. George (AM) ; Albany, Je, Aug (NYS); West Pt. (Robn) ; Katonah, Nyack (AM) ; NYC, J1 (Woodr); SI, May, larva in hickory (L\&D); LI (AM). Larva in hickory (Fisher).

14671 C. robiniae Forst. (pictus Drury). Locust BoRer. Oswego (NYS) ; Syracuse (Blkm) ; Rochester (M\&W) ; Buffalo (Z\&R); E. Aurora (Dnd); Castile, Brad (Flet); Geneva, Avon, Cls; Ithaca, Sep (CU); Utica (Fitch); Karner, Albany (NYS); Stuyvesant (NYS); Poughkeepsie, Sep (NYS); West Pt. (Robn); White Plains, Aug-Sep (FrostSW, Sq) ; Westchester Co (Woodr); SI, Aug, Oct, larva in locust (L\&D); LI: Brooklyn (Blkm); Patchogue, Yaphank, Sea Cliff, Sep (Ds); Orient, Nov, Lath (Ds). Thirteen more records (Lg). Larva in locust, often injurious. 


\section{ARHOPALUS Serville}

14672 A. fulminans Fab. Syracuse (Blkm); Buffalo (Z\&R); Portage, J1 (NYS) ; Geneva, Je, Cls; Ithaca, Je-Aug (Dt-CU); W. Hebron, Je (Lg); West Pt. (Robn); Westchester, J1 (NYS) ; Nyack, Je (Lg); SI, Je-J1, larva in chestnut (L\&D); LI: Je (Ds); Flatbush; Rockaway Beach, Je (BM). Larva mines inner bark and sapwood of chestnut and oak (Felt).

\section{Glycobius LeConte}

\section{(Plagionotus LeConte not Mulsant)}

14673 G. speciosus Say. Sugar-Maple Borfr. Mt. Whiteface, J1 (Ds) ; Delano (NYS); Glens Falls (NYS); Fulton (Felt) ; Syracuse (NYS, Blkm); Rochester (M\&W) ; Batavia (NYS); Buffalo (Z\&R); E. Aurora (Dnd); Castile, Brad (Flet); Williamsville (NYS) ; Freeville, J1 (Ds) ; Elmira, Je (Diven) ; Canajoharie (NYS) ; Albany, J1 (NYS) ; Ballston, Je, Stuyvesant Falls (NYS); Rensselaer, Je (Woodr) ; Schoharie (NYS) ; Adams (NYS) ; Mt. Riga (NYS); White Plains, J1 (Frost, Sq). Breeds in sugar maple, often injurious.

\section{Calloides LeConte}

14674 C. nobilis Harris. Gloversville (NYS); Syracuse (B1km); Buffalo (Z\&R); Geneva, Je-Jl, Lodi, J1, Cls; Ithaca, Je (CU); Albany (NYS); West Pt. (Robn); Greenwood L., Nyack (AM); LI: Rockaway Beach, Je (Ds); Wyandanch, J1 (Ds); Forest Pk., Je (BM). Larva in oak (Fisher).

\section{XYLOTRECHUS Chevrolat}

14679 X. colonus Fab. Rustic Borer. Cranberry L., Je-J1, yellow birch (Mund) ; St. Lawrence Co, J1 (Dnd); Syracuse (Blkm); Buffalo (Z\&R); E. Aurora (Dnd) ; Castile, Je, Brad (Flet) ; Avon, Geneva, Je, Aug, Cls ; Cattaraugus Co, Je (Ds); Ithaca, J1-Aug (Dt); W. Hebron (Ds) ; Elka Pk. (Blkm); West Pt. (Robn); White Plains, J1 (FrostSW, $\mathrm{Sq}$ ) ; SI, Je, Aug, larva in many hardwood trees (L\&D). Forty-four more records $(\mathrm{Lg})$. Larva in nearly all hardwoods, and has been recorded from hemlock (Fisher).

14680 X. sagittatus Germ. Buffalo (Z\&R) ; Ithaca (Dt-CU) ; Rensselaer (NYS); Catskills, May-Je (Nic) ; West Pt., Je (Ds) ; Nyack, Aug-Sep (AM) ; LI: Wading R., Jl (Ds); Flatbush, Aug-Sep (AM) ; Yaphank, Sep (BM). Larva in pine, spruce, and fir (Fisher).

14683 X. quadrimaculatus Hald. Cranberry L., on yellow birch logs (Htly); Buffalo (Z\&R) ; E. Aurora (Dnd) ; Big Ind. Val., J1 (Pears) ; SI, J1 (L\&D). Larva in birch, beech, and alder (Fisher).

14684 X. aceris Fisher. SI, J1 (L\&I)); LI (Sf). Larva in red, silver, and sugar maple (Fisher).

14691c X. undulatus Say var. fuscus Kby. Keene Val., J1 (BM) ; Cranberry L., May-J1, bred from larch and spruce (Mund); Big Moose, J1-Aug (Felt); Lewis Co, J1 (NYS); Buffalo (Z\&R); Big Ind. Val., Je (BM) ; Elka Pk. (Blkm); West Pt. (Robn). Larva in fir and hemlock (Fisher). 
14691e X. undulatus Say var. interruptus Cast. Cranberry L., J1 (Drk); Ithaca, J1 (Dt-CU); Catskills, J1 (AM).

$14696 \mathrm{X}$. convergens Lec. Buffale (Rein). Larva in dead thorn, Crataegus sp. (Fisher).

\section{NEOCLYTUS Thomson}

14701 N. longipes Drury (angulatus Fab., rhombifer Oliv.). Has been recorded from NY. Record probably based on West Indian individuals hatching from imported timber.

14703 N. scutellaris Oliv. NY (Lec) ; LI: Long Beach, J1 (Frost) ; Coney I., Je (BM). On hickory, elm, and grape (Felt). Larva in white oak (Fisher).

14704 N. mucronatus Fab. (luscus Fab.). Buffalo (Z\&R). Larva in hickory (Felt).

14707 N. caprea Say. Scottsville, injurious to black ash (Felt) ; West Pt. (Robn); West Farms (AM). Larva in ash and oak (Fisher).

14708 N. kirbyi Auriv. (longipes Kby.). Wanakena, Crittenden, bred from larch (B\&S). Larva in oak (Fisher).

14713 N. muricatulus Kby. Essex Co, J1 (NYS) ; Cranberry L., bred from larch, Je-J1 (Mund) ; Syracuse, Crittenden, bred from larch (B1km); Buffalo $(Z \& R)$. Larva in spruce, larch, and pine (Fisher).

14717 N. acuminatus Fab. (erythrocephalus Fab.). Cranberry L., Je (Mund); Syracuse, from hickory (Blkm); Honeoye Falls (Ds); Buffalo $(Z \& R)$; E. Aurora (Dnd) ; Castile, Je, Brad (Flet); Schuyler Co, Je (H) ; SI, Je-J1, common on various hardwoods (L\&D) ; LI, Je-J1, Sep (Ds). Forty-nine more records (Lg). Larva in a great variety of hardwoods (Fisher).

\section{EURYSCELIS Chevrolat}

14726 E. suturalis Oliv. Brooklyn, undoubtedly from imported wood, being a native of the West Indies $(\mathrm{Lg})$.

\section{Antновоscus Chevrolat}

\section{(Clytanthus Thomson)}

14728 A. ruricola Oliv. Adirondacks, Je-Aug (Blk); Cranberry L., Je, Aug (Mund); Wanakena, Aug (Drk); Syracuse, J1-Aug (Blk); western NY (Dnd); Olcott, Je-JI (Dt); Buffalo (Z\&R); Castile, Brad (Flet); Conesus L., Je-Aug (Ds); Port Byron, Cayuga Co, Je (Dt); Geneva, Je, Cls; Ithaca, Je-J1 (Dt-CU) ; Catskills, Je-Aug (Ds) ; West Pt. (Robn); LI: Wading R., May (Ds). Thirty-seven more records (Lg). Larva in maple, hickory, birch, and beech (Fisher).

\section{Clytoleptus Casey}

14730 C. albofasciatus Lap. "NY" (AM); Cranberry L., J1 (Mund); LI $(\mathrm{BM})$. Larva in dead grape (Fisher). 


\section{Clytus Laicharteg}

14734 C. marginicollis Cast. "NY" ( $\mathrm{Lg})$; Cranberry L., one specimen, J1. This is a southern species and may have emerged from southern lumber shipped in $(\mathrm{B} 1 \mathrm{~km})$. Breeds in pine (Fisher).

\section{MicRoclytus LeConte}

14739 M. gazellula Hald. Boston (Dnd). Occurs in Canada, Ohio, and Pa. (Journ. NY. Ent. Soc. 26:8 (Lg). Larva in oak and hickory (Fisher).

14740 M. gibbulus Lec. (insinuans Csy.). "NY" (Hald); Adirondacks (Not). On oak (Felt).

\section{CYRTOPHORUS LeConte}

14741 C. verrucosus Oliv. Onondaga Co, May (H) ; Buffalo (Z\&R); E. Aurora (Dnd) ; Rock City, Je (Ds); Castile, Brad (Flet); Middlesex, Je, Cls; Ithaca, May-J1 (Dt-CU); Windsor, Je (Not); Schoharie (Not); Big Ind. Val., May (BM); West Pt. (Robn); SI, Je (L\&D); LI: Yaphank, Central Pk., May (Ds); Amagansett, May (BM). Twentytwo more records $(\mathrm{Lg})$. Bred from various hardwood limbs at Syracuse $(B \& S)$. Larva in dogwood, maple, birch, spicebush, persimmon, pear, cherry, blue beech, and redbud (Fisher).

\section{EUDERCES LeConte}

14745 E. picipes Fab. Adirondacks, May, J1 (Ds); Syracuse, Je (Drk) ; Rochester (M\&W) ; Olcott, Je-J1 (Dt) ; Buffalo (Rein); Portageville, Je (Ds) ; Avon, Je, Waterloo, Je, Geneva, Je-J1, Cls; Conesus L., Je (Ds) ; Allegany Pk. (Dnd); Ithaca, Je-J1 (Dt-CU); Schoharie (H); W. Hebron, Je (Lg) ; Pine I., Je (Ds); West Pt. (Robn) ; Ft. Montgomery, May, J1 (Ds) ; Bronx Pk., Je (Ds) ; SI, Je, common on flowers $(\mathrm{L} \& D)$. Fifty more records $(\mathrm{Lg})$. Bred from hickory, chestnut, beech, and linden, at Syracuse (B\&S). Larva in a great variety of hardwoods-chestnut, hickory, oak, redbud, hackberry, walnut, locust, sour gum, pear, and dogwood (Fisher).

14746 E. pini Oliv. NYC, Mar (Woodr).

\section{TILLOCLYTUS Bates}

14750 T. geminatus Hald. West Pt. (Robn); SI, May-Je (L\&D); LI: Bellport, Je (Nic); Brooklyn, May 27 (Shoe); Amagansett, May (BM). On white oak (Felt). Larva in oak, hickory, grape, dogwood, and sour gum (Fisher).

\section{STENOSPHENUS Haldeman}

14757 S. notatus Oliv. Syracuse, from hickory (B\&S); Poughkeepsie, May (Yg); West Pt., Je (Ds); West Farms, Nyack (AM) ; LI: Half Way Hollow Hills, Jl, Rockaway, May (Ds); Yaphank, May (BM). Breeds in dead limbs of hickory (Felt).

\section{Atimia Haldeman}

14772 A. confusa Say. Syracuse (Blkm); West Pt. (Robn). In cedar and pine (Felt). Two broods of adults each year in the southern States, one in the spring and the other in the early fall (Fisher). 


\section{ELyTROLEPTUS Duges}

14777 E. floridanus Lec. LI: Yaphank, Central Pk., on Qucrcus nana (Ds) ; Pinelawn, Huntington, Bellport, Wading R. (Schott, Nic, Shoe, Ds).

\section{TRAGIDION Serville}

14792 T. coquus L. LI: Yaphank, Sep, Riverhead, Aug (Ds); Coram (Eng); Rockaway, Sep (Shoe, Schmelter); Coney I. (Schmelter); Jamaica (Nic). Larva in oak (Felt).

\section{PURPURICENUS Germar}

14795 P. humeralis Fab. Mt. Whiteface, J1 (Ds); Syracuse (Blkm); Geneva, $\mathrm{Jl}, \mathrm{Cls}$; Croton, J1 (Ds) ; Albany (NYS) ; West Pt. (Robn) ; SI, Je (L\&D) ; LI, Je (Shoe, Woodr); Rockaway, Je (Ds). Larva in oak, chestnut, and birch (Fisher).

14796 P. axillaris Hald. Syracuse, bred from hickory (B\&S); vic. NYC (AM). Larva in oak and chestnut (Fisher).

\section{BATYLEOMA Casey}

14857 B. suturale Say. Karner, Je, on pine (Felt); Mosholu, J1 (AM) ; Garrison, J1 (AM) ; Peekskill (Sherm) ; West Pt., Jl (BM) ; Ft. Montgomery, Yonkers, J1 (AM); SI, J1-Aug (L\&D); LI, J1 (Shoe). Larva in chestnut, oak, and hickory (Fisher).

\section{CyRTINUS LeConte}

14893 C. pygmaeus Hald. Buffalo (Z\&R); Westchester Co (AM); SI, Je (L\&D) ; LI, J1 (Shoe, Ds) ; Yaphank, J1 (Ds) ; Greenport, Aug (Ds) ; Bellport, J1 (Nic). In oak, hickory, box elder, and locust (Felt). Larva in maple, locust, dogwood, hickory, oak, and tulip poplar (Fisher).

\section{PSENOCERUS LeConte}

14894 P. supernotatus Say. Plattsburg, J1 (Ds) ; Cranberry L., J1 (Drk) ; Rochester (M\&W); Olcott, May-Jl (Dt); Buffalo (Z\&R); E. Aurora (Dnd) ; Batavia, Je (Ds) ; Castile, Je, Brad (Flet); Ithaca, Je-J1 (Dt-CU) ; Windsor, Je (Not); West Pt. (Robn) ; SI, May-J1 (L\&D); LI, May-Je, Shoe (Ds). Thirty more records (Lg). On dogwood (Felt). Injurious to stems of currant $(\mathrm{Lg})$. Larva in elm, tulip poplar, willow, Virginia creeper, and currant (Fisher).

\section{Monochamus Serville}

14895 M. titillator Fab. Cranberry L., Je (Mund); Syracuse (Blkm); Buffalo (Z\&R) ; West Pt. (Robn) ; SI, Je-Aug, on pine (L\&D) ; LI: Rockaway Beach, Je, Yaphank, J1 (Ds). Twenty-two more records ( $\mathrm{Lg}$ ). Larva in pine (Fisher).

14901 M. notatus Drury (confusor Kby.). The Sawyer. Cranberry L., Jl-Aug, under spruce bark (Mund); Syracuse (Blkm); Buffalo (Z\&R); Ithaca, Je-J1 (CU) ; L. George (BM); Elka Pk (Blkm); West Pt. (Robn); Westchester (Ds); SI, Aug, on pine (L\&D). Thirty more records 
(Lg). Injurious to pine, spruce, and balsam (Felt). Larva in pine (Fisher).

14904 M. scutellatus Say. White Spotten Sawyer. Axton (Houghton); Mt. Whiteface, Jl (Ds) ; Long L., Wilmington, Aug (Ds) ; Cranberry L., Je-Jl, common in balsam (Mund); Syracuse (Blkm); Buffalo (Z\&R); Elka Pk. (Drk); Ithaca, Je-J1 (Dt); Greene Co (Drk) ; Catskills, Je-Sep, in spruce and larch (B\&S); West Pt., bred from white pine (Robn); SI, Je, on pine (L\&D). Thirty more records (Lg). Larva in pine and spruce (Fisher).

14905 M. marmorator Kby. Wilmington, Aug (Dt) ; St. Lawrence Co (Drk) ; Mt. Whiteface, Aug (Dt); Cranberry L., Je, from dead balsam (Mund); Elka Pk. (Blkm). Larva in balsam fir (Fisher).

\section{DoRCHASCHEMA LeConte}

14909 D. alternatum Say. SI, Je-Aug, on osage orange and mulberry (L\&D). Larva in mulberry (Fisher).

14910 D. nigrum Say. Syracuse, Je-J1, bores in hickory limbs (B\&S) ; Rochester $(M \& W)$; Buffalo (Z\&R); Poughkeepsie, Je (NYS); 'West Pt. (Robn); Pine I., Je, on hickory (Ds); Nyack, Je (Ds); LI, Jl (BM). Larva in hickory (Fisher).

\section{HeTOEMIS Haldeman}

14911 H. cinerea Oliv. Syracuse, Je-Jl, from hickory (B\&S) ; Poughkeepsie, J1 (NYS) ; Peekskill (Sherm); Pine I., on hickory (Ds) ; SI, J1 (L\&D). Bred from osage orange, mulberry, and hickory (Hamilton). On walnut (Felt). In linden and hackberry (Fisher).

\section{Cacoplia LeConte}

14912 C. pullata Hald. Rochester (M\&W); Buffalo (Z\&R). On oak (Felt).

\section{HAMMODERUS Thomson}

14915 H. tessellatus Hald. SI, J1, on oak shoots (Linell). Larva irı oak, chestnut, and shadbush (Fisher).

\section{Goes LeConte}

14917 G. tigrinus DeG. Syracuse (Blkm); Buffalo (Z\&R); Ithaca, J1 (Fisher-US); West Pt. (Robn); Bronx, J1 (AM); SI, J1, on oak (L\&D) ; LI: (Shoe) ; Jamaica, J1-Aug (Pears). Larva in hickory, oak, and walnut (Felt).

14918 G. pulcher Hald. Rochester (M\&W) ; Buffalo (Z\&R); Menands, J1 (NYS) ; West Pt. (Robn) ; Nyack (AM); West Farms (AM); SI, J1-Aug, on hickory (L\&D) ; LI: Je (Ds) ; Flatbush, Je (BM). Larva in hickory (Fisher).

14919 G. pulverulentus Hald. Syracuse (Blkm); Rochester (M\&W) ; Buffalo (Z\&R) ; Ithaca, J1, on black oak (CU); Saratoga (Lec); Albany, Je (NYS); West Pt. (Robn) ; Ramapo, J1 (Ds) ; SI, J1 (L\&D) ; LI: Brooklyn, J1, on scarlet oak (Shoe); Wyandanch, Jl (Schott). In oak, ironwood, hornbeam, beech, and wild cherry (Wickham). Larva in elm, sycamore, blue beech, and ironwood (Fisher). 
14921 G. laurenticus Csy. "Northern NY" (Csy).

14922 G. debilis Lec. Rochester (M\&W); Buffalo (Z\&R); Albany, May (Yg); North Castle (AM); LI: Bellport, J1 (Nic); Brooklyn, Je-Jl, on oak trees (Shoe). Larva in oak (Fisher).

\section{MicRogoes Casey}

14923 M. oculatus Lec. Mt. Whiteface, J1 (Ds) ; Cranberry L., Je-J1, bred from maple (Mund); Rochester (M\&W); Buffalo (Z\&R); Ithaca, Je-J1 (Dt); L. Henderson, Aug (Lg); Catskills (Pears); Big Ind. Val., $\mathrm{Jl}$ (Shoe). Larva in dogwood, chestnut, and beech (Fisher).

\section{PSAPHAROCHRUS Thomson 53}

14931 P. quadrigibbus Say. Rochester (M\&W); Katonah, Aug (AM); LI: $(\mathrm{Lg})$; New City, J1 (Frost). Larva in many varieties of hardwoods (Fisher).

\section{AEGOMORPHUS Haldeman ${ }^{54}$}

14932 A. decipiens Hald. Syracuse, Je, bred from hickory (B\&S); Rochester (M\&W) ; Lake Bluff, J1 (NYS) ; Buffalo (Z\&R) ; Cattaraugus Co, J1 (Ds) ; Karner, May (NYS); West Pt. (Robn); SI, Je, reared from hickory and maple (L\&D) ; LI: Je-Jl (Shoe); Yaphank, J1-Aug (Ds). Larva in many varieties of hardwoods (Fisher).

\section{LEPTOSTYLUS LeConte}

14943 L. aculifer Say. Buffalo (Z\&R); West Pt. (Robn); SI (L\&D); LI: Jamaica (Pears). Larva in apple, elm, walnut, hickory, dogwood, tulip poplar, Rhus, and redbud (Fisher).

14954a L. collaris Hald. var. interruptus Hald. "NY" (Guex) ; West Pt. (Robn); Bronxville, $\mathrm{Jl}$ (Woodr). Larva in chestnut (Fisher).

14957 L. biustus Lec. SI (L\&D); LI: Aug-Sep (Ds); Brooklyn, J1 (Shoe); Jamaica, J1 (Pears); Shelter I., Aug, Orient, Sep (Ds); Greenport, Aug (BM). Larva in hackberry, mulberry, and Rhus (Fisher).

\section{AstYLOPSIS Casey}

14960 A. macula Say. Mt. Whiteface, J1 (Ds) ; Cranberry L., J1-Aug, bred from maple (Mund); Syracuse (Blkm); Rochester (M\&W); Olcott, J1-Aug (Dt); Buffalo (Z\&R); E. Aurora (Dnd); Ithaca, Aug (Dt); W. Hebron, Je (Lg) ; Big Ind. Val., Aug (BM); West Pt. (Robn); Pine I., Je (Ds) ; Ft. Montgomery, May (Ds); SI, Je-Ji (L\&D); LI: Half Way Hollow Hills, Yaphank, J1 (Ds). Larva in apple, elm, hickory, dogwood, chestnut, etc. (Fisher).

14961 A. guttata Say (sexguttata Say, commixta Hald.). Cranberry L., J1 (Mund); Syracuse, bred from pine (Blkm); Rochester (M\&W); Crittenden (Blkm); Olcott, Aug (Dt); Buffalo (Z\&R); West Pt. (Robn); White Plains, Je (Frost,Sq) ; SI, Je-Jl (L\&D) ; LI: Wading R., J1 (Ds); Oakdale, Aug (BM). Bred from larch (Blk). Larva in pine and spruce (Fisher).

63 Formerly included in Acanthoderes.

54 Formerly included in Acanthoderes. 


\section{Astylidius Casey}

14963 A. parvus Lec. "NY" ( $\mathrm{Lg})$. Larva in bittersweet, mulberry, poison ivy, and box elder (Fisher).

\section{LEIOpUS Serville}

14968 L. variegatus Hald. Wilmington, J1 (Shoe); Syracuse, J1 (Drk); Rochester (M\&W) ; Buffalo (Z\&R) ; Pike, J1 (NYS) ; Albany, J1 (NYS) ; West Pt. (Robn); Van Cort. Pk. (AM) ; SI, Je, Aug (L\&D) ; LI: Yaphank, J1 (Ds). Larva in many varieties of hardwoods (Fisher).

14971 L. crassulus Lec. W. Hebron, Je ( Lg) ; Callicoon, J1, on willow (Schaupp); Catskills, Je (Pears); SI, J1 (L\&D); LI: Yaphank, J1 (Ds).

14975 L. punctatus Hald. Karner, Je (NYS); SI (L\&D); LI: Je-J1 (Ds) ; Half Way Hollow Hills, Yaphank, Riverhead (Ds). Larva in Virginia creeper, dogwood, and plum (Fisher).

14976 L. fascicularis Harris. Ithaca, Sep (Dt); W. Hebron, Je (Lg); West Pt. (Robn); Bronxville, Je (Woodr); SI (L\&D); LI: Brooklyn, J1 (Shoe).

14979 L. vicinus Hald. "NY" (Fitch).

14983 L. obscurellus Csy. Northern NY (Csy).

14984 L. cinereus Lec. Syracuse (Blkm); Rochester (M\&W); Buffalo (Z\&R) ; LI, Je-J1, Nic (Schaupp).

14985 L. alpha Say. Syracuse, bred from hickory (B\&S) ; Rochester (M\&W) ; Olcott, Je, Aug (Dt); Buffalo (Z\&R); E. Aurora (Dnd) ; Ithaca, Ji (Dt); West Pt., Je (Ds); Pine I., Je (Ds); SI, Je-Aug, hatched from sumac (L\&D); LI, J1-Aug, widely distributed (Ds). Twentyseven more records $(\mathrm{Lg})$. Larva in many varieties of hardwoods (Fisher).

\section{LEPTURGES Bates}

14994a L. confluens Hald. var. angulatus Lec. Buffalo (Z\&R) ; Big Ind. Val., Je-J1 (Shoe); Mosholu (BM).

14995 L. symmetricus Hald. Cranberry L., J1 (Mund); Newport, Je (Yg); Rochester (M\&W) ; Buffalo (Z\&R) ; Pine I., Je (Ds); Van Cort. Pk. (Pears) ; SI, Je (L\&D). Larva in walnut, hickory, chestnut, hackberry, mulberry, linden, and box elder (Fisher).

14998 L. signatus Lec. Rochester (M\&W); Olcott, Je, Aug (Dt); Buffalo $(Z \& R)$; E. Aurora (Dnd) ; Ithaca, Aug (Dt) ; Coy Glen, Aug (Dt) ; W. Hebron, Je (Lg); West Pt. (Robn); Big Ind. Val., Je-J1 (Shoe); Bronxville, Je-J1 (Woodr); SI, Je-Aug (L\&D); LI, Je (BM). Larva in maple, beech, mulberry, poison ivy, box elder, and hickory (Fisher).

14999 L. tenebrosus Csy. Northern NY (Csy).

15001 L. querci Fitch. Syracuse, bred from hickory, oak, butternut, and redbud (B\&S) ; E. Aurora (Dnd) ; Olcott, J1-Aug (Dt); Buffalo (Z\&R) ; Avon, Je, Cls; Ithaca, Aug (Dt); W. Hebron, Je (Lg); West Pt. (Robn); White Plains, Je-J1 (Frost,Sq) ; Pine I., Je (Ds) ; Nyack, Je (Ds) ; SI, Je, J1, Aug (L\&D) ; LI: Aqueduct, Yaphank, J1, Greenport, Aug (Ds). Larva in chestnut, walnut, apple, bittersweet, oak, elm, butternut, ironwood, poison ivy, and linden (Fisher).

15003 L. facetus Say. Rochester (M\&W); Buffalo (Z\&R); West Pt. (Robn); Mosholu (BM); Van Cort. Pk. (AM); SI (L\&D). Larva in Crataegus Crus-galli (Fisher). 


\section{HYPERPLATYS Haldeman}

15005 H. maculata Hald. Plattsburg, J1 (Ds) ; Mt. Whiteface, J1 (Ds) ; Cranberry L. (Mund); Syracuse (Blkm); Rochester (M\&W); Olcott, J1Aug (Dt); Erie Co (Dnd); Ithaca, Je (Dt-CU); Albany (Lint) ; Karner, Je (Yg) ; near Athens, Je (Yg); Bronxville, J1 (Woodr); Bronx (BM); LI: Brooklyn, Je-J1 (Shoe); Greenport, J1 (BM). Bred from dead twigs of oak and other trees (Felt). Larva in Rhus, locust, dogwood, walnut, box elder, and sumach (Fisher).

15011 H. nigrella Hald. Mt. Whiteface, Slide Mt., J1 (Shoe); Bronxville, May (Woodr); SI (L\&D).

15013 H. variolata Csy. NY, type locality (Lg Cat.).

15015 H. binocularis Csy. NY, type locality (Lg Cat.).

15016 H. aspersa Say. Syracuse (B\&S) ; Olcott, J1-Aug (Dt) ; Erie Co (Dnd) ; Buffalo (Z\&R) ; Castile, Brad (Flet); Wyoming Co, Je (Ds) ; Avon, Je, Geneva, J1, Cls; Ithaca, Aug (Dt); West Pt. (Robn) ; Ft. Montgomery, J1 (Ds); West Plains (Fall, Sq); SI, Je-J1, Sep, hatched from hickory, chestnut, poplar, apple; currant also given as food plant (L\&D) ; LI: Shelter I., Aug (Ds) ; Aqueduct, Half Way Hollow Hills, J1, Wading R., Je, Yaphank, Sep (Ds). Thirty more records (Lg). Larva in chestnut, currant, willow, butternut, dogwood, and wild cherry (Fisher).

\section{Ceratographis Gahan}

15020 C. biguttatus Lec. "NY" (Lec); Ithaca, Je 12, 1915 (Dt).

\section{GRAPHISURUS Kirby}

15021 G. fasciata DeG. Cranberry L., Je, larva in yellow birch, J1, from dead spruce, cedar, beech (Mund); Syracuse, bred from hickory (B\&S); Olcott, May-Aug (Dt) ; Buffalo (Z\&R); Castile, Brad (Flet); Ithaca, Je-J1 (Dt) ; Mosholu, May (Ds) ; West Pt. (Robn) ; NYC, Sep (Brb) ; SI, Je-Sep, hickory, oak, chestnut, and maple (L\&D) ; LI: Aqueduct, J1 (Ds). Twenty-five more records ( $\mathrm{Lg})$. An oak and pine borer (Felt). Larva in a variety of hardwoods (Fisher).

15021a G. fasciata var. reducta Csy. NY, type locality.

15022 G. hebes Csy. NY, type locality (Lg Cat.); Syracuse (B1km). Larva in hickory (Fisher).

15023 G. triangulifera Hald. Whiteface Mt., Je (Shoe) ; LI: Aqueduct, J1 (Shoe). Bores in hackberry (Felt, quoting Packard). Larva in hackberry (Fisher).

\section{Acanthocinus Stephens}

15024 A. obsoletus Oliv. Buffalo (Z\&R); Karner (NYS); West Pt. (Robn) ; Nyack (Zab) ; LI: Bellport, J1 (Nic); Rockaway, J1 (Shoe); Ronkonkoma, Aug (US).

15026 A. pusillus Kby. Severance, J1 (NYS) ; Cranberry L., Je-J1, bred from spruce and hemlock (Mund); Buffalo (Z\&R); Albany, Jan (NYS).

15034 A. nodosus Fab. Geneva, Je-Aug, Cls; LI: Bay Shore, Aug 25, 1910, at light (Ols). Larva in pine (Fisher). 


\section{Dectes LeConte}

15037 D. spinosus Say. Rochester (M\&W) ; Buffalo (Z\&R) ; Kenwood, Je (Yg); Pine I., Je (Ds); Mosholu (BM); SI, breeds in stems of ragweed and Xanthium? (L\&D) ; LI: Amagansett, Aug (BM). Larva in living stems of ragweed, Eupatorium, and Xanthium (Fisher).

15041 D. brevis Csy. LI, type locality (Lg Cat.).

\section{Hoplosia Mulsant}

15047 H. nubila Lec. Mt. Whiteface, J1 (Ds); Syracuse, bred from basswood (Blkm); Buffalo (Z\&R) ; E. Aurora (Dnd) ; Big Ind. Val., Je (Shoe); West Pt. (Robn); West Farms (AM). On basswood twigs (Felt). Larva in linden (Fisher).

\section{POGONOCHERUS Latreille}

15049 P. penicillatus Lec. Mt. Whiteface, J1 (Ds) ; Cranberry L., Je (Mund); Syracuse, bred from spruce twigs (Blkm). On sumac (Felt). Larva in spruce (Fisher).

15053 P. parvulus Lec. Buffalo (Z\&R).

15054 P. salicicola Csy. Ithaca, Je (Dt). Larva in willow (Fisher).

15056 P. mixtus Hald. Mt. Whiteface, Jl (Ds); Saranac, Feb, Aug (NYS); Cranberry L., J1 (Drk); Syracuse, Crittenden, bred from white pine and larch (B\&S); Rochester (M\&W); Buffalo (Z\&R); Ithaca, J1 (CU) ; Karner, J1 (NYS) ; W. Hebron, Je (Lg) ; West Pt. (Robn) ; SI, Je (L\&D); LI: Massapequa, J1 (Shoe); Rockaway, Je, Aug (Woodr); Yaphank, Long Beach (BM). On hard pine and willow (Felt). Larva in spruce and pine (Fisher).

\section{ECYRUS LeConte}

15067 E. dasycerus Say. Rochester (M\&W); Buffalo (Z\&R); Ithaca, J1-Aug (Dt) ; L. George (Zab) ; West Pt. (Robn) ; Ft. Montgomery, J1 (Ds); SI, Je, on hickory, tulip, and locust (L\&D) ; LI: Aqueduct, Central Pk., Half Way Hollow Hills, Yaphank, J1 (Ds). Larva in locust, hickory, and oak (Fisher).

\section{Eupogonius LeConte}

15069 E. tomentosus Hald. Wilmington, Je ( $\mathrm{Lg}$ ); Rochester (M\&W) ; Buffalo (Z\&R) ; Westfield, Je (NYS) ; Mosholu (BM) ; LI: Riverhead, Aug (Ds); Bellport, J1 (Nic); Flatbush, J1 (Zab); Rockaway Beach, Je $(\mathrm{BM})$. Larva in pine and spruce (Fisher).

15070 E. vestitus Say. Syracuse, J1 (Drk) ; Rochester (M\&W) ; Buffalo (Z\&R); E. Aurora, one only, rare (Dnd) ; Mosholu (AM) ; Pine I., Je (Ds) ; SI, J1, dogwood, hickory, walnut, chestnut (L\&D); LI: Jamaica (Pears); Aqueduct, J1 (Shoe); Greenport, Aug (BM). Larva in walnut, dogwood, mulberry, ash, redbud, maple, honey locust, poison ivy, elm, linden, and oak (Fisher).

15072 E. subarmatus Lec. Rochester (M\&W) ; Buffalo (Z\&R); E. Aurora, rare (Dnd); Catskills (Pears); Nyack, on linden ( $\mathrm{Lg})$. Larva in linden (Fisher). 


\section{LYPSIMENA LeConte}

15075 L. fuscata Lec. "NY" (Lec).

\section{ONCIDERES Serville}

15088 O. cingulatus Say. Buffalo ( $Z \& R$ ); Ithaca, Aug (Dt); Coy Glen, Fall Cr., Aug (Dt) ; West Pt. (Robn); White Plains, Aug (Frost,Sq); Bronxville, Aug (Woodr); SI, in autumn, on hickory (L\&D); LI: Yaphank, Aug, Oct (Ds) ; Prospect Pk., Aug (Yg) ; Westbury, Sep (Champlain); Flushing, Cold Sp. Harb. (BM). Larva in hickory, elm, poplar, and various hardwoods (Fisher).

\section{Hippopsis Serville}

15098 H. lemniscata Fab. Rochester (M\&W) ; SI: Richmond, J1 15, 1919 (Bs); LI: Jamaica (Pears) ; Floral Pk., Jl (Not). Larva in stems of ragweed (Fisher).

\section{SAPERDA Fabricius}

15109 S. obliqua Say. Cranberrry L., J1 (Mund) ; Elka Pk., Aug (Drk); Syracuse (Blkm); Olcott, J1 (Dt); Buffalo (Z\&R); West Pt. (Robn); Bronx Pk. (Joutel); SI, Jl, makes galls on alder near roots (L\&D); LI: Newtown (Ds); Queens, May-Je (Eng); Flushing, Jamaica, Je (BM).

15110 S. mutica Say. "Western NY" (T.ec); Buffalo (Z\&R). Larva in willow (Fisher).

15112 S. candida Fab. Round-heAded Apple Borer (L\&D). Mt. Whiteface, J1 (Ds) ; Syracuse (H) ; Rochester (M\&W); Buffalo (Z\&R); Branchport (Lint); McLean Bogs, Aug (Yuasa); Washington Co (Fitch); W. Hebron, Je ( Lg); Oliverea, Je (Ds); Elka Pk. (Blkm); West Pt. (Robn) ; Pine I., Je (Ds) ; Ft. Montgomery, J1 (Ds) ; SI, Je-Aug (L\&D) ; LI: Brooklyn, Je (Ds). Twelve more records (Lg). Larva in apple, often injurious; also in pear, thorn, and most pomaceous trees and shrubs.

15113 S. calcarata Say. Poplar Borer. Keene Val., Aug (BM); Syracuse (Blkm) ; Mt. Whiteface, J1 (Ds); Rochester (M\&W); Olcott, J1 (Dt) ; Buffalo (Z\&R) ; E. Aurora (Dnd); Cattaraugus Co, J1 (Ds); Ithaca, Sep (Reed) ; Albany (Joutel); West Pt. (Robn) ; LI: Brooklyn, Aug, on Populus alba (Ds, Joutel). Eighteen more records ( $\mathrm{Lg}$ ). Larva in poplar (Fisher).

15113a S. calcarata var. adspersa Lec. Keene Val., Sep (BM); Mt. Whiteface, J1 (Ds): Brant L. (Doll); Albany (Joutel).

15114 S. tridentata Oliv. Elm Borer. Waddington, Aug (Woodr); Gloversville (Lint); Olcott, May (Dt) ; Rochester ('M\&W); Buffalo (Z\&R); Castile, Brad (Flet); E. Aurora, Cortland Co (Dnd): Geneva. Je-JI, Cls; Ithaca. Je-J1 (Dt) ; Albany (Lint): Hudson (BM); West Pt. (Robn) ; Mott Haven (Joutel); SI, May-Je, on elm (L\&D) ; LI, Je (BM). Larva in elm (Fisher).

15115 S. cretata Newn. Spotted Apple-Tree Borer. Ithaca (Philip Miller); Elmira, Je (Diven). Larva in apple, sometimes injurious. 
15116 S. discoidea Fab. Hickory Saperda. Syracuse, bred from hickory (B\&S); Genesee, Ap (NYS) ; Buffalo (Z\&R) ; West Pt. (Robn) ; Pine I., Je (Ds) ; Bronx Pk. (Joutel) ; SI, J1-Sep, on hickory and walnut (L\&D) ; LI: Aqueduct, J1 (Ds). Larva in hickory and walnut (Fisher).

15117 S. vestita Say. LINDEN Borer. Adirondacks (Felt) ; Newport, Je (NYS); Syracuse (Blkm); Rochester (M\&W); Olcott, Je-(Dt); Buffalo (Z\&R) ; E. Airora, Protection (Dnd) ; Hamburg, Aug (NYS) ; Avon, Geneva, Je, Cls; Ithaca, J1, Sep (Dt-CU) ; W. Hebron, Je (Lg) ; Greene Co (Zab) ; Sullivan Co, Je (Ds) ; West Pt. (Robn) ; Nyack, Je (Ds); LI: Flushing, Je (BM). Larva in linden (Fisher).

15118 S. imitans Felt \& Joutel. Cranberry L., II (Drk); Syracuse, bred from hickory (B\&S). Larva in hickory, willow, linden, and cherry (Fisher).

15119 S. lateralis Fab. Buffalo (Z\&R) ; Conesus L., Je (Ds) ; Schoharie (Not) ; Albany, Je (NYS); W. Hebron, Je (Lg); Poughkeepsie, Je (NYS); West Pt. (Robn); Pine I., Je (Ds); Ft. Montgomery, May (Ds); Nyack, Je (Ds); Bronx Pk. (Joutel) ; SI, Je, on hickory (L\&D) ; LI Wyandanch, J1, Yaphank, Je-J1 (Ds); Brooklyn (Joutel). Larva in hickory, elm, maple, linden, ash, and oak (Fisher).

15119a S. lateralis var. connecta Felt \& Joutel. "NY" (Joutel).

15120 S. fayi Bland. Rochester (M\&W); Buffalo (Z\&R); Schenectady, Je (NYS); Bronx (BM). Breeds in small limbs of wild thorn (Joutel).

15121 S. puncticollis Say. Syracuse (Blkm); Rochester (M\&W); Buffalo $(Z \& R)$; Geneva, Cls; SI, Je (L\&D). Larva in Virginia creeper (Fisher).

15122a S. populnea L. var. moesta Lec. Wilmington, J1 (Shoe) ; Gloversville, May (NYS) ; Rochester (M\&W) ; Buffalo (Z\&R) ; Karner, May (NYS); Feeds on balsam poplar (Joutel).

15123 S. concolor Lec. Syracuse (Blkm); Allegany Pk., Cortland Co (Dnd); Windsor, Je (Not); White Plains, Je (Frost, Sq). Larva makes galls on poplar and willow (Fisher).

15123a S. concolor var. unicolor Felt \& Joutel. Mt. Whiteface, J1 (Ds) ; Rochester (M\&W); Genesee (NYS); Buffalo (Z\&R); Albany (NYS); West Pt. (Robn) ; Van Cort. Pk. (Bt) ; SI, on dwarf willow (L\&D); LI: Yaphank, May, Wyandanch, Jl (Ds) ; Flatbush, Hempstead Plains, May (BM).

$15123 b$ S. mecasoides Csy. NY, type locality (Lg Cat.) ; SI (Soltau-US by Fisher). S. shoemakeri Ds. Slide Mt., J1; LI: Wyandanch, J1, Yaphank, May (Ds).

\section{OBEREA Mulsant}

15134 O. schaumi Lec. Wilmington, J1 (Shoe) ; Mt. Whiteface, J1 (Ds) ; Albany, May (NYS) ; Karner, Je (NYS). Larva in poplar (Fisher).

15137 O. pallida Csy. Cranberry L., Sep (Stage).

15138 O. tripunctata Swed. Olcott, Je-J1 (Dt); Ithaca, Je, Aug (Dt) ; McLean, J1 (Dt) ; Elka Pk., J1, on flower (Blk) ; Callicoon, J1 (Lg); West Pt., Je (Robn); Pine I., Je (Lg); Ramapo, May, Nyack, Je (Lg); White Plains, Je-Jl (Frost, Sq); SI, Je-J1 (L\&D) ; LI: Flushing, Je (BM). Thirty-six more records $(\mathrm{Lg})$. The varieties myops Hald. and man- 
darina Fab. are not differentiated in the records $(\mathrm{Lg})$. Larva in plum, apple, quince, peach, elm, and dogwood (Fisher).

15139 O. praelonga Csy. "Northern NY" (Csy).

15144 O. filum Csy. Windsor, Je (Not).

15145 O. basalis Lec. Buffalo (Z\&R); Schuyler Co, Je (H); Schoharie (Not); West Pt. (Robn) ; SI, J1 (Ds) ; LI: Half Way Hollow Hills, J1 (Ds); Bellport, Je-J1 (Nic); Richmond Hill (Logan). Twelve more records

$(\mathrm{Lg})$. This is perhaps only a variety of bimaculata Oliv.

15146 ?O. iowensis Csy. Nyack, Je (Ds) ; SI, Je-J1 (Ds).

15147 ?O. insignis Csy. LI: Massapequa, J1 (Ds).

15148 O. bimaculata Oliv. var. tripunctata Fab. Raspberry-Cane Borer. Cranberry L., J1 (Mund) ; Syracuse, J1, on flowers (Blk); Buffalo (Z\&R); E. Aurora (Dnd); Ithaca, Aug (Dt-CU); Windsor, Je (Not); West Pt. (Robn); White Plains, May-J1 (Frost,Sq); SI, Je-J1 (L\&D). Twenty-two more records $(\mathrm{Lg})$.

O. bimaculata var. affinis Harris. Redford, Je (Shoe); Batavia, J1 (Ds); McLean Bogs (CU); Taughannock Falls, Aug (CU); Debruce, J1 (Shoe).

15152 O. ocellata Hald. Eye-spotted Apple-twig Borer. Wilmington, Aug (Ds); Rochester (M\&W) ; Karner (Yg); Peekskill, Je (Sherm); West Pt. (Robn) ; Orange Co, Je (Zab) ; Bronx (Woodr) ; Van Cort. Pk., Aug $(\mathrm{BM})$; LI: Smithtown, Aug (Ds); Jamaica (Pears); Rockaway, Je (Shoe). The var. discoidea Horn should also be found ( $\mathrm{Lg}$ ). Larva sometimes injurious to new growth or twigs of apple; also in Rhus (Fisher).

15153 O. ruficollis Fab. Rochester (M\&W) ; Buffalo (Z\&R); White Plains, J1 (Frost, Sq) ; Riverdale, J1 (Woodr); SI, Je (L\&D); LI: Brooklyn, J1 (Shoe); Flushing, Je (BM). Larva in sassafras and spicebush (Fisher).

15154 O. gracilis Fab. SI (L\&D); LI: Yaphank, Je, Riverhead, Aug (BM).

\section{TETRAOPES Serville}

15162 T. canteriator Drap. West Pt. (Robn); White Plains, J1 (Frost,Sq); SI, Je-J1 (L\&D) ; LI: Wading R., Orient (Ds); Selden, Half Way Hollow Hills, J1-Aug (Ds). Twenty-seven more records ( $\mathrm{Lg}$ ). Larva in roots of milkweed (Fisher).

15170 T. tetraophthalmus Forst. Plattsburg, J1 (Ds) ; Wilmington (Ds) ; Cranberry L., J1 (Mund) ; Olcott, Je (Dt); Buffalo (Z\&R); E. Aurora (Dnd); Castile, Aug, Brad (Flet); Geneva, Avon, Je, Cls; Ithaca, J1; Freeville (Ds); West Pt. (Robn); White Plains, J1 (Frost, Sq); SI, Je-J1 (L\&D) ; LI: Woodhaven, Je (Dt) ; Orient, Wading R., J1 (Ds). Fifty-six more records ( $\mathrm{Lg})$. Larva in roots of milkweed (Weiss).

\section{AMPHIONYCHA LeConte}

15186 A. flammata Newn. E. Aurora, beaten from sprouts about basswood stump, Je (Dnd); Olcott, J1 (Dt); West Pt. (Robn); Nyack, Je (Ds). 


\section{FAMILY CHRYSOMELIDAE 55 SUBFAMILY DONACIINAE}

\section{Compiled by Chas. Schaeffer}

\section{DONACIA Fabricius}

15199 D. militaris Lac. (floridae Lg.). Carmel, White Plains, Aug, Sq (Sf). On flowers and leaves of white water lily, Castalia odorata.

15202 D. piscatrix Lac. Saranac Inn, Lake Bluff, Big Moose (NYS) ; Horicon, Brant L., Doll; L. George, J1, Fisher; Ballston L., Je, Leon; Pine I., Je, Ds; White Plains, May-J1, Bno, Sq (Sf); SI, Je, L\&D; LI: (Eng); Ronkonkoma, Je, Schott. On flowers and leaves of yellow water lily, Nymphaea advena.

15199 D. palmata Oliv. Keene Val., J1, Not; Potsdam (NYS) ; Long L., Ds ; Adirondack Lodge, Je, Leon; Horicon, Doll; L. George, J1, Fisher (US Biol. Surv.) ; Athens, J1, Schott; Carmel, Aug, Sq; Rockland L., J1, Schott; White Plains, May-Aug, Bno, Sq (Sf); SI, Ds. On flowers and leaves of yellow water lily.

D. texana Cr. var. minor Sf. Rockland L., J1, Schott; White Plains, J1, Sq, Aug, Bno; SI (Lg). On yellow water lily.

15201 D. rufescens Lac. L. George, Aug, Sherm; Peekskill, Sherm; SI: Silver L. (L\&D) ; LI: Wyandanch, J1-Sep, Schott (Sf). Generally on flowers and leaves of white water lily; occasionally on Brasenia.

D. edentata Sf. Crum L., Je, Ds; LI: Montauk Beach, J1, Schott. On flowers and leaves of white water lily.

D. parvidens Sf. LI: Yaphank, May (Sf); Wyandanch, May, Schott, Dec, sifting, Not (Sf) ; Wading R., Je, Shoe. On flowers and leaves of white water lily.

15198a D. cincticornis Newm. Our most common species; occurs almost everywhere in the State. Ausable L., Aug, Not; Saranac L., Sep, Shoe; Long L., Ds ; Horicon, Doll; Ballston L., Áug, Leon; Carmel, Aug, * Sq; SI: Je, LED ; Silver L.; LI: Cold Sp. Harb., Brb; Bellport, J1, Nic; Yaphank, Je-J1, Eng; Wading R., Je, Shoe, Schott; Wyandanch, J1-Sep; Greenport, May, Schott. On flowers and leaves of white water lily, and on yellow water lily when the two occur together; also on Brasenia.

D. cincticornis var. tenuis Sf. Tupper. L., Aug, Dyar (Sf-US); Spencer L., Aug, Dt; Peekskill, Sherm; LI: Wyandanch, Wading R., Schott. On white water lily and Brasenia.

D. cincticornis var. rufipennis Lac. "NY" (Dietz Coll.) ; SI: New Pond, Britton's Upper Pond, $L \mathcal{E} D$. Adults and larvae found on Potamogeton by Boving and Craighead.

15198 D. proxima Kby. Ausable L., J1, Not; Horicon, Doll; Pike (NYS) ; Spencer L., Aug-Sep, Dt; Franklinton, Je, Leon; Ballston L., Aug, Leon; Orange L., Sep; White Plains, Aug, $S q$ (Sf). On flowers and leaves of the yellow water lily.

15199 D. coerulea Oliv. White Plains, May-Aug, $S q, B n o$; Van Cort. Pk., May, Nic; LI: Baldwin, May, Eng. On leaves of the yellow water lily.

\footnotetext{
${ }^{55}$ Chas. Schaeffer of the Brooklyn Museum, well-known authority on the Chrysomelidae, has gone over the manuscript for this family, is responsible for some changes in synonymy, and has supplied many records and passed on the validity of as many as was practicable.
} 
15194 D. hirticollis Kby. Rochester, Moore; Buffalo; Catskills; LI: Yaphank, Je, Eng.

15196 D. pubescens Lec. "NY" (Lec). This species is recorded by Blatchley as occurring on pickerel weed.

15203 D. quadricollis Say (D. cupraea Kby., D. curticollis Knab). Plattsburg;

15204 Rochester, Wendt; Buffalo; Ithaca, Aug, Marshall; Spencer L., May, Aug, $D t(\mathrm{CU})$. Reported by Fred Knab on bulrushes.

15206 D. rugosa Lec. Hamilton Co (CU) ; Lake Bluff, Sodus, J1 (NYS) ; Esopus, Aug, Sherm; Peekskill, Sherm; W. Nyack, Aug, Beq; SI, Jl, on pickerel weed, $L \& D$. On Sagittaria (Lg) and Pontederia cordata (Wenzel).

15216 D. biimpressa Melsh. (torosa Lec., distincta Lg. not Lec.). Wallface Mt., J1, Not, Nic; Ithaca, McLean, May (CU) ; Peekskill, May, Sherm; White Plains, May-Je, Pollard, Sq (Sf); Van Cort. Pk., Nic; SI, Je, LED; LI: Long Beach, Rockaway Beach, Eng; Yaphank, May. On Carex stricta (Frost), and on various grasses, bur reeds (Sparganiaceae), and Sagittaria (Wenzel).

D. biimpressa var. limonia Sf. Indian Pass, J1, Quirs; Concord, May; Ithaca, May; White Plains, Je, $S q$; Van Cort. Pk.

15203 D. subtilis Kunze. Oakfield, Je, Not; Ithaca, Je, New Berlin (CU); Voorheesville, J1, Watervliet Reservoir, Aug, Leon; Easton, Thomson, May, Leon; W. Nyack, Aug, Beq; Pine I., Je, Pollard, Ds; White Plains, Je, $S q$; SI, Je-Jl, on Sparganium, LED ; LI: Yaphank, May, Eng; Kissena Pk., Mar, Ols. On Sparganium (Ds, Wenzel).

15203 D. subtilis var. fulgens Lec. Old Forge, Je (NYS); Saranac L., Shoe; Black R., Lowville, Not; Ithaca, Dt (CU); White Plains, $S q$; LI: Yaphank, Eng.

D. tuberculifrons Sf. Ithaca, May-Jl, Kngt (CU). On yellow pond lily (Wenzel, Wolc), bulrushes (Liebeck), and Sparganium (Ds).

D. liebecki Sf. LI: Wyandanch, Je, Ds, Schott; Wading R., Je, Shoe. On flowers and leaves of white water lily, also on yellow water lily and Orontium aquaticum.

15205 D. megacornis Blatch. (megalocera Weise). "NY" (AM); Long L., Ds; Kingston, J1 (NYS); Rockaway, Shoe. On flowers and leaves of white and yellow water lily (Wenzel).

15209 D. aequalis Say. Black R., Lewis Co, Je, Not; Oakfield, Je, Not; Voorheesville, Je, Leon; SI, J1, swept from subaquatic plants, $L \& D$. On Sparganiaceae and Sagittaria (Wenzel).

15218 D. rufa Say (tuberculata Lac.). Cranberry L., J1 (Drk); Voorheesville,

15210 Jl, Leon; White Plains, Jl, Sq; SI: Clove Val., Jl-Aug, on Sagittaria, $L \mathcal{E} D$.

15208 D. harrisi Lec. McLean, J1 (CU); White Plains, J1 (Sq); LI: near Brooklyn, J1, Shoe. On Carex (Frost).

15218 D. sulcicollis Lac. (rufa Lg. not Say). Newport, May (Yg); Keene Val., May-Jl, Mt. Skylight, J1, Not ; Portage, May, Kngt; Rock City, Je, $D s$, Ithaca, May, Dt, McLean, May (CU) ; Tarbel, Je, Ds; Freeville, Je, Dt ; Catskills, Pears; Nassau, J1 (NYS); Thomson, May, Leon; Voorheesville, May, Leon; Mud Hollow Pond, Je, Leon; Peekskill, May, Sherm; White Plains, Je-J1, Sq ; Suffern, Ramapo, Greenwood L.; LI: 
Queens, Dow (Sf, Ds). Found on Caltha palustris and Ranunculus acris (Ds), on Carex stricta (Frost), and on leaves of skunk cabbage (Blatchley).

15217 D. flavipes Kby. Saranac L., Sep, Shoe; Sodus Pt., Je, Not; Rochester, $M \mathcal{E} W$; Batavia, Je, Kngt; Oakfield, Je, Not; Ithaca, May-Je, $D t$; Tivoli, May, Not; White Plains, Je (Sq); Greenwood L.; Bronx Pk., Ds; SI, Je, L\&D. Occurs on Peltandra (Ds, Liebeck).

15217 D. flavipes var. shoemakeri Sf. Iona I., Rockland Co, May, Schott; Bronx Pk., Je, $D s$.

D. nitida Germ. Freeville, Je, Knab, Dt.

D. notmani Sf. Wallface Mt., J1, Not, Nic, Quirs.

15216 D. emarginata Kby. Elizabethtown, Je (NYS); Keene Val., May, Not; L. Clear, Je (NYS) ; Black R., Lowville, Je, Not; Cranberry L., Je (H); Ithaca (NYS); Ballston L., Aug, Leon; Catskills, Pears ; Karner, Nassau, May (NYS) ; Mosholu, Ap; Bronxville, May, Shoe; LI: Brownsville, May, Woodr.

D. emarginata var. frosti Sf. Sullivan Co, Ds; Peekskill, Sherm; Ramapo; LI: Queens, Dow (Sf, Ds).

15215 D. metallica Ahr. (femoralis Kby.). Elizabethtown, $Y g$ (NYS) ; Newport, Je, Yg (NYS); Keene Val., Je, Not; Gloversville, J1, Alex (NYS); Deer R., May (NYS); Portage, May, Kngt; Mud Cr., Tompkins Co, Je, Ds; West Pt., May, Robn; Mud Hollow Pond, Je, Leon. On Sparganiaceae and Sagittaria (Wenzel), on Carex stricta (Frost), and on Ranunculus acris (Ds).

15214 D. germari Mann. (scrricauda Sf.). McLean Bogs, May, Dt; LI: Rockaway Beach, May, Shoe.

15213 D. fulvipes Lac. (cuprea Lec. not Kby.). Keene Val., J1 (NYS) ; Catskills, Pears; Suffern; LI: Wyandanch, Ap, sifting, Shoe; Orient, Oct, Schott. Swept from grasses and weeds growing in a low, moist meadow near a small creek.

15213 D. pusilla Say. Elizabethtown, Je (NYS) ; Black Br., Lowville, Je (Not) ; Remsen, Je (NYS); Pike, Je (NYS); Ithaca, May (CU). On rushes (H), and on Carex stricta (Frost).

\section{HAEMONIA Latreille}

15193 H. nigricornis Kby. Onondaga Co, May (H) ; Pike, Je, Yg (NYS) ; LI: Yaphank, Je, Eng; Wading R., May, Schott; Wyandanch, May.

\section{SUBFAMILY ORSODACNINAE}

\section{ORSODACNE Latreille}

15219 O. atra Ahr. Speculator, Je (Yg); Mt. Whiteface, J1 (Shoe); Ithaca, May-Je (Dt); Slide Mt., Je (Ds, Shoe) ; West Pt. (Robn); SI, Ap (L\&D) ; LI: West Hills, Ap (Ds); Central Pk., Aug (Eng); Wyandanch, May (Sf) ; Massapequa, Ap (Shoe); Huntington, Ap (Schott). Abundant on willow catkins, Ap 3-19 (Robn). 
15219a O. atra var. childreni Kby. Mt. Whiteface, J1 (Shoe); Adirondack Lodge, Leon, Newport ( $\mathrm{Yg})$.

15219b O. atra var. hepatica Say. Newport, Je (Yg); Slide Mt., Je-J1 (Shoe); LI: Central Pk., Ap-May (Eng, Sf); Wyandanch, May' (Sf).

15219d O. atra var. tibialis Kby. Mt. Whiteface (Shoe); Newport, May (Yg); Oliverea (Shoe); LI: Central Pk., May (Sf).

15219e O. atra var. trivittata Lac. Mt. Whiteface, J1 (Shoe); Newport, May $(\mathrm{Yg})$; Oliverea, J1 (Shoe); LI: Massapequa, Ap (Shoe).

$15219 \mathrm{f}$ O. atra var. vittata Say. Boston, Je (Flet, Dnd); Schuyler Co, Je (H); LI: Central Pk., Ap-May (Eng, Sf); Wyandanch, May (Sf).

\section{SYNETA Lacordaire}

15223 S. ferruginea Germ. Newport, May-Je, Speculator, Je (Yg); Mt. McIntyre, Je (Leon); Denmark, May (Yg); L. Placid, Sep (Shoe); Castile, Brad (Flet); Wyoming, Je (Ds); Wolcott, May (Cy) ; Westfield, May (Not); Spencer, Je (Ds) ; Ithaca, May-Je (Dt); McLean, May (Ds); Windsor, May, Je (Not); Oliverea, Je (Ds); Slide Mt., J1 (Shoe); West Pt. (Robn); Nyack, May 1 (Schott); Nassau, May (Yg); Ramapo (Sf); SI (L\&D); LI: Massapequa, May (Shoe); Wyandanch, Ap (Sf).

\section{ZEUGOPHORA Kunze}

15227 Z. kirbyi Baly. Western NY ( $\mathrm{Lg}$ Cat.).

15228 Z. reineckei Grt. Buffalo (Rein).

15230 Z. consanguinea Cr. L. Placid, Sep (Shoe); Mosholu (Sf); SI, J1 (L\&D) ; LI: Aqueduct, J1 (Shoe).

15233 Z. varians Cr. Newport (Yg); L. Placid, Aug, EPV (Yg); Chazy L., Je (Yg) ; Horicon, Brant L., Aug (Doll) ; SI, May (L\&D) ; LI: Brooklyn, Aug (Shoe); E. New York, Ap (Martin).

\section{SUBFAMILY CRIOCERINAE}

\section{CRIOCERIS Geoffroy}

15234 C. asparagi L. Common Asparagus Beetle. Rochester (Lint); Brighton, May, Robbins (Lint) ; E. Aurora, on asparagus (Dnd); Castile, Brad (Flet); Geneva, Je, Goff (Lint, Cls) ; Avon, May (Cls); Ithaca, AugSep (Dt) ; Albany, J1 (NYS) ; Tuxedo, May (Ds) ; West Pt. (Robn); White Plains, May-Sep (Sq) ; SI, May-Je, Aug (Ds) ; LI: Forest Pk., J1 (Martin); Flatbush, Aug (Eng); Queens, J1 (Sf); Brooklyn, Aug (Shoe) ; Huntington, Aug (Schott); Orient, Lath (NYS); Wading R., May (Schott); Selden, Aug (Ds); Rockaway Beach, May, Amagansett, Sep (Ds).

15235 C. duodecimpunctata L. Penfield (How) ; Lockport, Darrison (Felt); N. Hector, Je, Sm (Felt); Avon, J1 (Cls) ; Pike (Felt); Brighton, May, Robbins (Lint) ; Buffalo (Felt) ; E. Aurora (Dnd); Fredonia, Almond (Barden); Westfield (Felt); Ithaca, J1-Sep (Dt); Coeymans, Albany, Sm (Felt); Manor, Oct (Felt); West Pt. (Robn); White 
Plains, May-Sep (Sq) ; SI, Je, Sep (Ds) ; LI: Flatbush, Aug (Eng); Rockaway Beach, May (Eng, Shoe); Jamaica (Sf); Brooklyn, Ji (Shoe); Wading R. (Ds); Huntington, Je (Schott); Orient, Lath (NYS) ; Selden, Sep (Ds).

\section{LEMA Fabricius}

15240 L. palustris Blatch. Horicon, Brant L., Aug (Doll) ; Catskills, Je (Pears); Ramapo Mts. (Sf); SI, Je, on thistle (L\&D), as brunnicollis Lec.

15253 L. trilineata Oliv. Three-Lined Potato Beetle. Newport, Je (Yg) ; Ilion, May (Yg) ; Clyde, J1 (Lint) ; Olcott, J1 (Dt) ; Buffalo (Lint) ; Geneseo, J1 (Ds); Orleans Co, Je (Ds); E. Aurora, May (Dnd) ; Avon, J1 (Cls) ; Westfield, Je (NYS) ; Castile, Brad (Flet) ; Schuyler Co, Je (H) ; Ithaca, Je-Jl, Y uasa (Vdyk); Schoharie, Je (Not) ; Karner, J1-Aug (NYS) ; Coeymans, Sm (Lint) ; Nassau, J1 (NYS) ; West Pt. (Robn); Nyack, Je (Ds) ; Rockland Co, May (Ds); Rye (Sq); SI, J1 (L\&D) ; LI: Rockaway Beach, May (Shoe, Eng); Brooklyn, Aug (Shoe); Yaphank, Flatbush, Jamaica, J1 (Eng). Abundant on nightshade (Robn).

15255 L. nigrovittata Guér. Brooklyn Bot. Garden, Eng (Sf). Described from southern Calif. and Ariz., doubtless introduced.

\section{SUBFAMILY CLYTRINAE}

\section{Antipus DeGeer}

15262 A. laticlavia Forst. Hamburg, Je (Eng); Ithaca, Je-J1 (Dt) ; Windsor, May, Je (Not); Schoharie, Je (Not); Poughkeepsie, Je (Yg) ; Pine I., Sep, West Pt., Je (Ds); Ft. Montgomery, Ji (Schott, Ds); Mosholu (Sf); Bronx Pk. (Sf); SI, Je (L\&D); LI: Massapequa, Je (Shoe); Wading R., Je, Yaphank, J1 (Ds).

\section{COSCINOPTERA Lacordaire}

15267 C. dominicana Fab. Ithaca, Je (Ds) ; Schoharie, Je (Not) ; Hudson, Aug (Eng); Nassau, Je (Yg); West Pt. (Robn); LI: Pinelawn, Je, Amagansett, May (Eng); Woodhaven, May (Martin); Yaphank. Wading R., May (Ds). Larva lives with ants.

\section{BABIA Lacordaire}

15282 B. quadriguttata Oliv. Ithaca, Aug (Dt) ; Schoharie, Je (Not) ; Karner, Je, Kenwood, J1, N. Chatham, May, Poughkeepsie, Je (Yg); West Pt. (Robn); Ft. Montgomery, May (Ds); Greenwood L., Je (Love); West Pt., Je (Ds); Pine I., Je (Ds) ; Ramapo, Je (Ds) ; Nyack, Je (Ds) ; LI: Central Pk., Je (Shoe); Wading R., Yaphank, Je (Ds). Abundant on hickory (Robn).

\section{SAXINIS Lacordaire}

15292 S. omogera Lac. West Pt., Je (Sf) ; Ft. Montgomery, May (Ds) ; LI: Yaphank, Je-Jl (Eng, Ds); Central Pk., Je (Shoe); Wyandanch, J1 (Ds). 


\section{SUBFAMILY CHLAMYDINAE}

\section{ChLamys Knoch 55a}

15297 C. gibbosa Fab. Pike (Yg); E. Aurora, Je (Dnd); Windsor, May, Je (Not); Corinth, Springville, Je (Yg); Poughkeepsie, May (Yg); Ulster Co, Sep (Shoe); West Pt. (Robn); White Plains, May (Sq); Mosholu (Sf); SI, Ap-Je (L\&D); LI: (Eng); Wyandanch, Ap (Schott); Calverton, Sep (Ds). On blackberry, the larva living within a case.

15299 C. cribripennis Lec. LI: Yaphank, May (Eng), J1 (Sf) ; Pinelawn, Je; Promised Land, Sep; Central Pk., May (Shoe); Amagansett, Sep (Ds).

\section{EXEMA Lacordaire}

15306 E. conspersa Mann. Westfield, May (Not); Ithaca, J1 (Dt).

15306 a E. conspersa var. dispar Lac. Honeoye Falls, Je (Ds) ; Windsor, May, Je (Not) ; Greenwood L. ; West Pt. (Robn) ; Nyack, Je (Ds) ; Ramapo, Je (Ds) ; Suffern (Sf) ; SI: Tottenville, Oct, Clove Val., May (Ds); LI: Manorville, Sep (Eng); Wading R., May-Je (Ds).

\section{SUBFAMILY CRYPTOCEPHALINAE}

\section{PACHYBRACHYS Redtenbacher}

15312 P. pubescens Oliv. Ithaca, May (Ds); West Pt., Je (Ds) ; Westchester Co, J1 (Ds) ; SI, Je (L\&D) ; LI: Central Pk., Je, Massapequa, J1 (Shoe); Wading R., May (Ds, Schott); Huntington, J1 (Schott); Yaphank, Je (Ds).

15357 P. pectoralis Melsh. Greenwood L., J1 (Sf); SI, J1 (Lg, Fall); LI: Huntington, Sep (Schott).

15358 P. sobrinus Hald. E. Aurora, Je (Dnd); SI (Lg, Fall); LI: Rockaway Beach, Massapequa, Je (Shoe).

15369 P. femoratus Oliv. Slide Mt., J1 (Shoe); SI (Lg); LI: Coney I. (FallUS); Massapequa, J1, Yaphank, Aug (Shoe).

15369 a P. femoratus var. aquilonis Fall. "NY" (Fall-US).

15371 P. subfasciatus Lec. Slide Mt., J1 (Shoe) ; Peekskill, May (Sherm) ; West Pt., May (Robn), Je (Ds); SI, Soltan (Fall-US); LI: Pinelawn, Je (Eng); Central Pk., May (Ds, Shoe) ; Massapequa, Je (Ds) ; Yaphank, May (Shoe); Wyandanch, J1, Wading R., May (Ds, Schott).

15382 P. confederatus Fall. "NY" (Fall).

15393 P. peccans Suffr. Buffalo (Fall,Lg) ; LI: Cold Sp. Harb. (Brb).

15407 P. atomarius Melsh. Protection, J1 (Dnd) ; W. Hebron, Je (Fall,Lg); West Pt. (Robn) ; Mt. Vernon (Sherm); Van Cort. Pk., Je (Sf); SI (Lg).

15409 P. obfuscatus Fall. "NY" (Fall).

15412b P. cephalicus Fall var. parvus Fall. LI ( Lg).

15413 P. roboris Fall. Buffalo (Fall); Ft. Montgomery, Je (Schott).

15415 P. relictus Fall. "NY" (Lg); LI: Cold Sp. Harb. (Brb). 
15432 P. tridens Melsh. Western NY (Dnd); Oliverea, Je (Shoe); Debruce, J1 (Shoe); West Pt. (Robn); Bronx Pk. (Sf); SI, Je (Lg, Ds); LI: (US); Brooklyn, May (Shoe). On poison ivy (L\&D).

15433 P. obsoletus Suffr. Buffalo (Am. Ent. Soc. Coll., Fall) ; Bluff Pt. (Csy, Fall); SI, J1, Shoe (Sf); LI: Cold Sp. Harb., J1 (Sherm, Fall); Brooklyn, JI (Shoe); Wyandanch, J1 (Schott).

15440 P. bivittatus Say. Keene Val., J1 (Yg); Windsor, May, Je (Not); Ithaca, Je, Aug (Dt).

15451 P. othonus Say. Protection, J1 (Dnd) ; Ithaca, Je (Dt); Elmira (EPV, Fall); W. Hebron, Je ( $\mathrm{Lg})$; Nassau, Jl (Yg); Peekskill (Sherm in Lg Coll., Fall); West Pt. (Sf); SI, J1 (L\&D); LI: Pinelawn, Je (Eng, Ds); Yaphank, May-Je (Ds, Eng), Sep (Shoe); Cold Sp. Harb. (Brb); Wyandanch, J1 (Schott); Wading R., May (Ds).

15455 P. carbonarius Hald. Peekskill, Sherm (Fall); Van Cort. Pk., J1, Zab (Fall); SI, Je (L\&D); LI: Yaphank, Je (Eng).

15458 P. m-nigrum Melsh. Keene Val., J1 (Yg) ; Newport, Woodworth's L., J1 ( $\mathrm{Yg}$ ); Washington Co, Lg; White L., Jl, Zab (US); SI, Lg; LI: Cold Sp. Harb. (Brb) ; Massapequa, J1 (Ds, Shoe) ; Riverhead, J1 (Ds).

15459 P. trinotatus Melsh. Potsdam, J1 (Liebeck) ; Colden, EPV (US) ; Protection, J1 (Dnd) ; Peekskill, Sherm (Lg Coll.) ; LI: Yaphank, J1, Amagansett, Aug (Eng); Cold Sp. Harb. (Brb); Central Pk., Je (Shoe); Wyandanch, Sep (Schott).

15462 P. luridus Fab. Ithaca, Je (Ds); W. Hebron, Je, Aug (Ds, Lg); West Pt. (Robn); Ft. Montgomery, Je (Schott); SI, Je (L\&D); LI: Pinelawn, Je (Eng); Yaphank, May, J1 (Ds, Eng); Massapequa, J1, Brooklyn, J1 (Shoe); Wyandanch, Je (Schott).

15462a P. luridus var. nigrinus Blatch. LI: Brooklyn, Je, Massapequa, J1 (Shoe).

15470 P. hepaticus Melsh. "NY" ( Lg).

\section{MoNACHULUS Leng}

15472 M. ater Fab. Karner, N. Chatham, Je (NYS); Southfields, Aug (Schott); Westchester Co (Ds); West Pt. (Robn); SI, south shore, J1 (Shoe, Ds) ; LI: Cold Sp. Harb. (Brb).

15473 M. saponatus Fab. Allegany Pk., J1 (Dnd) ; Karner, J1, Hope, Aug, Nassau, J1, Woodworth's L., J1 (NYS); SI, J1 (Shoe), Je (L\&D) ; LI: Brooklyn, J1, Aqueduct, Aug (Shoe).

15474 M. thoracicus Cr. West Pt. (Robn). Determination queried by Sf, since the species is known only from Fla.

\section{Cryptocephalus Geoffroy}

15479 C. notatus Fab. Keene Val., J1 (Yg); Horicon, Brant. L., Aug (Doll); Corinth, Je ( $\mathrm{Yg})$; Karner, Je (Yg); Poughkeepsie, May ( $\mathrm{Yg})$; White Plains, Aug (Sq); Nyack, Je (Ds) ; LI: Yaphank, May-Je (Eng, Ds); Hempstead Plains, May (Eng); Central Pk., May (Ds, Shoe); Huntington, May (Schott); Wyandanch, J1, Wading R., May (Ds).

15479a C. notatus var. quadrimaculatus Say. Western NY (Dnd) ; Olcott, May-J1, Ithaca, May-Aug (Dt); Wilmington, J1 (Shoe); Catskills, J1 (Shoe); West Pt. (Robn); White Plains, Je-J1 (Sq) ; LI: Woodhaven (Dt) ; Orient, Lath (NYS). 
15480 C. quadruplex Newn. Newport (Yg); Western NY, Je (Dnd); Rock City, Je (Ds) ; Ithaca, J1 (Eng) ; W. Danby, May (Ds) ; Windsor, May, Je (Not); Corinth, Je (Yg); Karner, Je (NYS); West Pt. (Robn) ; SI, Je-J1 (Shoe, Ds) ; LI: Central Pk., May (Shoe).

15480a C. quadruplex var. quadriguttulus Suffr. West Pt., Je (Ds); SI, Je (Ds); LI: Pinelawn (Eng); Huntington, Je (Schott); Wyandanch, Jl, Wading R., May, south of Smithtown, Je (Ds).

15483 C. guttulatus Oliv. "NY" (Sf); West Pt. (Robn); LI: Central Pk., Je (Shoe).

15495 C. venustus Fab. Keene Val., Aug ( $\mathrm{Yg}$ ); Cortland, J1, Bks (Yg); Albany, Nassau, Kinderhook, J1 (Yg); West Pt. (Robn); White Plains, J1 (Sq) ; SI, J1-Aug (Ds) ; LI: Cold Sp. Harb. (Brb) ; Brooklyn, J1 (Shoe); Wyandanch, May (Schott) ; Central Pk., Sep, Wading R., J1 (Ds).

15495a C. venustus var. cinctipennis Rand. "NY" (Sf); Woodworth's L., J1 (Yg) ; LI: Fire I., J1 (Ds).

15495b C. venustus var. hamatus Melsh. SI, Aug (Ds); LI: Babylon (Jn); Cold Sp. Harb. (Brb); Brooklyn, Aqueduct, Ji (Shoe).

15495d C. venustus var. simplex Hald. SI, Aug (Ds) ; LI: Yaphank, J1-Aug (Eng, Shoe); Cold Sp. Harb. (Brb) ; Central Pk., Je (Shoe); Half Way Hollow Hills, Ji (Ds).

15500 C. calidus Suffr. White Plains, Je (Schott); SI, J1 (L\&D) ; LI: Wading R., J1 (Sf), Je (Ds) ; Cold Sp. Harb., J1 (Brb) ; Yaphank, Je (Eng).

15501 C. gibbicollis Hald. LI: Massapequa, Je, Yaphank, Aug (Shoe).

15503 C. trivittatus Oliv. Mosholu, Je (Sf); LI: Wyandanch, J1 (Schott); Bayshore, J1 (Ols).

15505 C. incertus Oliv. LI: Yaphank, Aug (Eng, Shoe); Amagansett, Aug (Sf), Sep (Ds).

15507 C. mutabilis Melsh. Newport (Yg); Pike (Yg); Castile, Sep, Brad (Flet); White Plains, Aug (Sq) ; Ft. Montgomery, J1 (Schott); SI, J1-Aug (Shoe); LI: Wading R., Jl, Ds (Sf); Central Pk., Je, Yaphank, Aug (Shoe); Huntington, Aug (Schott); Riverhead, Aug (Ds); Pinelawn, Sep (Ds).

15515 C. schreibersi Suffr. West Pt. (Robn); Bear Mt., Sep (Schott); LI: Wyandanch, Aug (Schott, Sf); Central Pk., Aug (Ds).

15516 C. tinctus Lec. Ft. Montgomery, Sep (Schott); SI, Sep (L\&D); LI: Yaphank, Sep-Oct (Eng, Shoe).

15518 C. striatulus Lec. "NY" (Sf); SI, Je-J1 (L\&D).

\section{DIACHUS LeConte}

15521 D. auratus Fab. Speculator, Aug, Newport, Je ( $\mathrm{Yg})$; Horicon, Brant L., Aug (Doll) ; Mooers, Sep (Not); Schuyler Co, Je (H) ; Oliverea, Je (Ds, Shoe); Catskills (Pears); West Pt. (Robn); Mosholu (Sf); SI, Je-J1 (L\&D); LI: Wyandanch (Sf); Rockaway Beach, Je (Ds); Brooklyn, Je (Shoe); Central Pk., Aưg (Ds).

15526 D. aeruginosus Lec. Redford, Je (Martin). Described from Colo.

15527 D. catarius Suffr. Newport, Je, Wells, J1 (Yg); Mt. Whiteface, J1 (Shoe); Corinth, Je (Yg); Oliverea, Je (Shoe); Catskills (Pears).

15528 D. pallidicornis Suffr. "NY" (Sf); Olcott, Je-J1 (Dt). 


\section{BASSAREUS Haldeman}

15530 B. brunnipes Oliv, var. clathratus Melsh. SI, J1 (L\&D) ; LI: Yaphank, J1, Wading R., Je, Selden, Aug (Ds). On alder and Clethra.

15532 B. formosus Melsh. Keene Val., Je, Karner, J1 (Yg); Van Cort. Pk. (Nic) ; LI: Pinelawn, Je (Eng); Babylon (Jn); Massapequa, Je-J1 (Shoe); Wyandanch, J1, Oakdale, Aug (Schott); Riverhead, J1 (Ds).

15532 B. formosus var. sulphuripennis Melsh. Protection, JI (Dnd); LI: Yaphank, J1 (Eng); Wyandanch, Je (Schott).

15534 B. mammifer Newn. Newport, J1 (Yg); Horicon, Brant L., Aug (Doll); Erie Co, Je-J1 (Dnd) ; Ithaca, Je (NYS) ; West Pt. (Robn) ; SI, Je (L\&D) ; LI: Wyandanch, Je (Schott).

15534 b B. mammifer var. luteipennis Melsh. "NY" (Dt).

15534c B. mammifer var. pretiosus Melsh. SI, J1 (L\&D); LI: Cold Sp. Harb. (Brb); Yaphank, Aug (Shoe); Jamaica, J1 (Schott).

15534d B. mammifer var. sellatus Suffr. Castile, Sep, Brad (Flet); Elka Pk., Aug (Drk); Oliverea, J1 (Shoe) ; Bear Mt., Je (Schott) ; Karner, Je (NYS); West Pt. (Robn); LI: Central Pk., Je (Shoe); Orient Pt., Je, Lath (Yg).

15536b B. lituratus Fab. var. lativittis Germ. West Pt. (Robn); White Plains, May-J1 (Sq) ; Nyack, Je (Ds) ; LI: Bayshore (O1s) ; Wading R., May (Ds) ; Yaphank, Je (Ds).

$15536 b$ B. lituratus var. recurvus Say. "NY" (Dietz); Oliverea, J1 (Shoe) ; SI, Je (Ds) ; LI: Cold Sp. Harb. (Brb); Massapequa, Je (Ds, Shoe); Floral Pk., Je (Schott).

\section{TRIACHUS LeConte}

15537 T. atomus Suffr. L. Placid, Sep, Shoe (Sf) ; Mosholu (Sf) ; LI: Pinelawn, Je (Ds) ; Brooktyn, Je (Shoe).

15538 T. cerinus Lec. LI: Rockaway Beach, Je (Sf, Ds); Amagansett (Sf); Cold Sp. Harb. (Brb).

\section{SUBFAMILY EUMOLPINAE}

\section{Chrysodina Baly}

15544 C. globosa Oliv. Windsor, May, Je (Not); Greenwood L. (Sf) ; West Pt., J1 (Eng); West Pt. (Robn); Ramapo, Ft. Montgomery, May (Ds) ; SI, Je, Aug-Sep (L\&D) ; LI: Pinelawn, Je (Eng, Ds) ; Cold Sp. Harb., J1 (Brb); Massapequa, J1 (Shoe); Huntington, May, Nov (Schott); Amagansett, Sep, Gardiners I., Aug, Wading R., May (Ds).

\section{Nodonota Lefevre}

15545 N. tristis Oliv. Olcott, Dec (Dt); Danby, J1 (Dt); Ithaca, J1, Yuasa (Vdyk); Albany (NYS); West Pt. (Robn) ; SI, on south shore, J1 (Ds).

15546 N. clypealis Horn. Mosholu (Sf); LI: Brooklyn, Aug (Shoe).

15549 N. puncticollis Say. Batavia, Je (Ds) ; Newport, Pike, Schenectady, Nassau, Albany, Je (NYS); Schoharie, Je (Not): Lagrangeville, Je (NYS) ; Ramapo, May (Ds) ; SI, May-J1 (Ds) ; LI: Pinelawn, Je (Eng); Bellport, Je (Nic); Cold Sp. Harb., Jl (Brb); Central Pk., Je (Shoe); Wyandanch, Je (Schott); Yaphank, J1 (Ds) ; Orient, Lath (NYS). 


\section{Colaspis Fabricius}

15555 C. brunnea Fab. Wells, J1 (Yg); Olcott, Je-J1 (Dt); Clyde, J1, Bish $(\mathrm{Yg})$; Fredonia (Felt); Ithaca, J1-Aug (Dt); Peekskill (Sherm); West Pt. (Robn) ; White Plains, Je-Aug, Sq (Fall); SI, Aug (Eng); LI: Rockaway Beach, Je (Eng); Brooklyn, J1-Aug (Shoe) ; Gardiners I., Aug, Orient, Sep, Amagansett, Aug (Ds).

15555 b C. brunnea var. flavida Say. "NY" (Dietz); SI, J1, Aug (L\&D) ; LI : Flatbush, J1 (Schott).

15559 C. favosa Say. SI, Je (L\&D) ; LI: Brooklyn, J1 (Shoe) ; Orient, Central Pk., J1 (Ds). On Myrica carolinensis (Sf).

\section{RHABDOPTERUS Lefevre}

15563 R. picipes Oliv. Elizabethtown, J1, Newport, Je (Yg) ; E. Aurora, J1-Aug (Dnd) ; Ithaca, Je (Felt); Schoharie, Je (Not); Kenwood, J1 (Yg); Poughkeepsie, Aug (Lath); West Pt. (Robn) ; SI: Aug, Je (Eng, Ds) ; Watchogue, Aug (Ds) ; LI: Gardiners I., Aug (Ds); Aqueduct, Je, Brooklyn, May (Shoe); Jamaica (Schott); Orient, Lath (NYS).

\section{GRAPHOPS LeConte}

15567 G. pubescens Melsh. Newport, May, Speculator, Aug (Yg) ; E. Aurora, Je (Dnd) ; Ithaca, Sep (Dt) ; Phoenicia, Aug (EPV); Clinton Hgts., Mar (Yg) ; Nassau, J1 (Yg) ; West Pt. (Robn) ; Van Cort. Pk., Sep (Yg); SI (L\&D); LI: Long Beach, May (Eng); Brooklyn, Rockaway Beach, May (Shoe); Huntington, May (Schott); Flatbush (Ds); Yaphank, May (Ds).

15568 G. curtipennis Melsh. LI: Pinelawn, Yaphank, Je (Eng) ; Wyandanch, J1, Montauk, Sep (Ds).

15569 G. marcassitus Cr. Rock City, Je (Ds) ; Windsor, May, Je (Not) ; Schuyler Co, Je (H) ; West Pt. (Robn) ; SI, May (L\&D) ; LI: Amagansett, Aug (Eng) ; Rockaway Beach, May-Je (Shoe); Flatbush, Aug (Zab); south of Smithtown, Je (Ds); Pinelawn, Je (Ds).

\section{Xanthonia Baly}

15573 X. decemnotata Say. Newport, May, Elizabethtown, Aug (Yg); Olcott, May-Aug (Dt) ; Pike, Sep (Yg) ; Batavia, Je (Ds) ; E. Aurora, MayJ1 (Dnd) ; Rock City, May (Ds) ; Westfield, May (Not) ; Schuyler Co, Je (H) ; Ithaca, Sep (Dt) ; Buttermilk Cr., May, Palm (Yg) ; Schoharie, Je (Not); Windsor, May, Je (Not); Karner, May (NYS); Clinton Hgts., Ap (NYS); Poughkeepsie, J1 (NYS); Oliverea, Je (Shoe) ; Nyack, May (Schott) ; Ramapo, May (Ds) ; Pine I., Je (Ds) ; SI (L\&D); LI: Central Pk., Ap (Eng); Yaphank, May, Oct (Ds, Eng) ; Manorville, Sep, Pinelawn, Je (Eng); Wyandanch, Je (Schott); Massapequa, Wading R., May (Ds).

15574 X. villosula Melsh. Newport, Je, Pike, Sep (NYS); Olcott, J1 (Dt) ; Letchworth Pk., J1 (Yg); Batavia,. Je (Ds); E. Aurora, J1 (Dnd); Genesee Co, Je (Ds) ; Ithaca, J1-Aug, Y uasa (Vdyk, Dt); Schoharie, Je (Not); West Pt., J1 (Eng); SI: Clove Val., J1 (Ds) ; LI: Yaphank, Je-J1 (Eng, Ds) ; Promised Land, Sep (Eng); Cold Sp. Harb., J1 (Brb) ; Brooklyn, Je, Aqueduct, J1 (Shoe); Central Pk., Jl (Schott) ; Selden, Aug, Wyandanch, J1 (Ds). 


\section{FIDIA Baly}

15577 F. viticida Walsh. Buffalo, Aug, Westfield, Je (NYS) ; Fredonia, Ripley, J1 (Felt); Albany, J1 (Lint); Highland, J1 (NYS); SI, J1 (L\&D); LI: Je (Eng) ; Brooklyn, Je, Aqueduct, JI (Shoe).

15578 F. longipes Melsh. West Pt. (Robn).

\section{METACHROMA LeConte}

15593 M. laevicollis Cr. LI: Central Pk., J1 (Sf, Ds) ; Coney I. (Linell).

15598 M. pallidum Say. Karner, J1, Hoffman (NYS) ; LI: Central Pk., J1 (Sf).

15598a M. pallidum var. lateralis Cr. LI: Central Pk., J1 (Sf).

15601 M. quercatum Fab. LI: Wyandanch (Sf); Yaphank, Je (Ds).

\section{Adoxus Kirby}

15604b A. obscurus L. var. villosulus Schrank. Mt. Whiteface, J1 (Shoe) ; Old Forge, Wells, Augur Flats, J1 (NYS) ; L. Placid, J1, Sep (Shoe-NYS); Wanakena, Aug (Drk); Cranberry L., J1-Aug (Drk); Horicon, Brant L., Aug (Doll); West Pt. (Robn); SI (L\&D); LI: Rockaway Beach; Je (Shoe, Eng).

\section{TYMNES Chapuis}

15605 T. tricolor Fab. Ithaca, Aug (Dt); Karner, J1 (Yg); West Pt. (Robn); Nyack, Pine I., Je (Ds); SI: Richmond, Je (Ds) ; LI: Flatbush, Yaphank, Je, Amagansett, Aug, Orient, Je (Eng); Jamaica, Je (Yg); Cold Sp. Harb., J1 (Brb); Brooklyn, Je (Shoe); Central Pk., Je (Shoe) ; Wyandanch, Long Neck, Je (Schott).

15607 T. metasternalis Cr. "NY" (Dietz); SI, on Crataegus (L\&D).

\section{Glyptoscelis LeConte}

15613 G. pubescens Fab. Pike (NYS) ; Erie Co, May-Je (Dnd) ; Ithaca, May, Aug (Dt); Cooperstown, May (Yg); Hudson, Je (Eng); West Pt., J1 (Ds); SI, Je (L\&D) ; LI: Massapequa, May (Shoe); Bayshore (Ols) ; Wading R., May (Schott); Wyandanch, J1, Central Pk., Yaphank, May (Ds).

15614 G. barbata Say. Pine I., Je (Ds) ; Ramapo (Sf) ; LI: Massapequa, J1 (Shoe); Greenport, Aug (Ds, Eng).

\section{PARIA LeConte}

15626 P. canella Fab. "NY" (Dietz) ; Erie Co, Je-J1 (Dnd); West Pt. (Robn) ; SI, Mar-Aug ( $\mathrm{I}_{\text {\& }}$ D) .

15626a P. canella var. aterrima Oliv. Gloversville, Ap, Alex (Yg); Buffalo, Aug (NYS) ; Chautauqua Co, J1 (Yg) ; Ithaca, May-Sep (Dt) ; Salem, Aug (NYS); Poughkeepsie, Je (Yg); SI, J1 (Ds); LI: Promised Land, Sep (Eng) ; Gardiners I., J1 (Ds) ; Orient, J1, Lath (Ds).

$15626 \mathrm{~b}$ P. Canella var. gilvipes Horn. Axton, Je (MacG); Onondaga Co, May-Je, Schuyler Co, May-Je (H) ; Rock City, Je (Ds); Ithaca, May-Aug (Dt) ; Oliverea, Je (Shoe); SI, J1 (Ds); LI: Aqueduct, Mar (Shoe); Brooklyn, May (Shoe). 
15626c P. canella var. pumila Lec. Mooers, Sep (Not); Pike, Mar (NYS) ; Ithaca, J1, Sep (Dt); Windsor, May; Je (Not); Poughkeepsie, Je (NYS); SI (L\&D); LI: Brooklyn, May (Shoe).

15626d P. canella var. quadriguttata Lec. Onondaga and Schuyler Counties, MayJe, flying $(\mathrm{H})$; Castile, Brad (Flet); Olcott, J1, Ithaca, Je (Dt); Mooers, Sep (Not); Ringwood, Jl, Yuasa (Vdyk); Windsor, Je (Not); LI: Orient, Lath (NYS).

15626e P. canella var. quadrinotata Say. Newport, May (Yg); Conesus L., Je (Ds) ; Pike, J1 (NYS); Rock City, Je (Ds); Ithaca, May-Aug (Dt); McLean, May (Ds); Albany, May (Yg); Slide Mt., Je (Shoe); Bangall, Sep (Eng); Pine I., Je (Ds); SI: Tottenville, Je (Ds); LI: Bellmore, Sep (Eng); Rockaway Beach, May (Shoe).

$15626 f$ P. canella var. sellata Horn. Cranberry Cr., Je, Pike, J1 (NYS) ; Windsor, May, Je (Not) ; SI, J1 (Ds); LI: Pinelawn, Je (Eng); Cold Sp. Harb., J1 (Brb); Wading R., May (Ds).

15626g P. canella var. sexnotata Say. Letchworth Pk., Sep (Dnd); Oliverea, Je (Shoe); Karner, May (NYS).

15626h P. canella var. thoracica Melsh. Speculator, Je (NYS); Northampton, Je $(\mathrm{Yg})$; Ithaca, Je (Dt); Schenectady, Je (NYS); Albany, Je (NYS); Ulster Co (Nic); SI (L\&D).

P. canella var. vittata Horn. Schuyler Co, Onondaga Co, May-Je (H) ; Ithaca, Je (Dt); Windsor, May, Je (Not); SI (L\&D); LI: Brooklyn, J1 (Shoe); Eastern Parkway, Brooklyn, J1 (Martin); Yaphank, Ap, Long Beach, May (Eng); Rockaway Beach, May (Shoe).

P. scutellaris Not. Windsor, Je (Not).

\section{CHRYSOCHUS Redtenbacher}

15627 C. auratus Fab. Wilmington, Aug, Long L. (Ds) ; Horicon, Aug (Doll); Mooers, Sep (Not); Onondaga Co, Oct (H); Olcott (Dt); Erie Co, Je-J1 (Dnd) ; Castile, Brad (Flet); Ithaca, Jl-Sep, Yuasa (Vdyk, Dt); Callicoon, J1 (Ds) ; West Pt., Sep (Ds); White Plains, J1 (Sq); SI, Je-J1 (Ds); LI: Yaphank, J1, Sep (Eng, Ds) ; Wading R., Aug (Sf, Ds); Brooklyn, J1-Aug . (Shoe); Orient, Lath (NYS).

\section{SUBFAMILY CHRYSOMELINAE}

\section{PRASOCURIS Latreille}

15636 P. vittata Oliv. Ogdensburg, J1 (Yg); Blue Mt., J1, Newport, Je, Cranberry L., J1 (Drk); Deer R., May, Pike, Mar (Yg); Castile, Brad (Flet); E. Aurora, Je, Oct (Dnd); Redford, J1 (Martin); Madison Co, May, herbage (H); Buffalo; Debruce, JI (Shoe); Avon, Geneva, May (Cls); Ithaca, Je-Aug, McLean, May (Dt); Debruce, J1 (Shoe); Pine I., May (Schott); West Pt., May (Robn); Ft. Montgomery, Je (Schott); White Plains, May-J1 (Sq); SI, Je (L\&D).

\section{LABIDOMERA Chevrolat}

15639 L. clivicollis Kby. Newport, Aug (Yg); Severance, J1 (Bks); New Russia, Je (Yg) ; Ogdensburg, J1 (Yg); Mooers, Sep (Not); Olcott, Je-J1 (Dt) ; Avon, May, Geneva, Jl-Aug (Cls) ; E. Aurora, May-Aug (Dnd); Castile, Brad (Flet); Rock City, Je (Ds); Ithaca, Ap, Je-Sep 
(Dt, Bish) ; McLean, May (Ds) ; Albany, Je (Yg) ; Waterford Reservoir, Aug (Leon) ; Thomson, May (Leon); Hudson Falls, Sep (Yg) ; Hudson, Aug (Eng) ; Oliverea, Je (Shoe) ; Debruce, J1 (Shoe) ; Sullivan Co (Ds) ; West Pt. (Robn) ; White Plains, May-Aug (Sq); SI, May-Aug (L\&D) ; LI: Yaphank, May, Jamaica, Jl, Greenport, Aug (Eng) ; Rockaway, May, Brooklyn, Aug (Shoe) ; Amagansett, Je (Sf) ; Orient, Jl, Lath, Maspeth, Aug (Ds).

\section{LEPTINOTARSA Stål}

15648 L. decemlineata Say. Colorado Potato Beetle. Big Moose, J1, Old Forge, Je (Yg); Cranberry L., Jl, Dec (Drk); Hamilton Co (Ds); Oswego, Je (Bks) ; Lockport, Sep (Yg); Avon (Cls) ; Castile, Brad (Flet); E. Aurora (Dnd); Rock City, May (Ds); Trenton Falls, Je (Bks); West Pt. (Robn); White Plains, all summer (Sq); SI (Ds); LI: Long Beach, Flatbush, May (Eng); Brooklyn, Rockaway Beach, May (Shoe); Orient, Je (Ds).

\section{ZYGOGRAMMA Chevrolat}

15655 Z. suturalis Fab. Onondaga Co, Oct $(\mathrm{H})$; Westfield, May (Not) ; Castile, Brad (Flet) ; Avon, Ap, Geneva, May, Aug (Cls); West Pt. (Robn); White Plains, May-Sep (Sq) ; SI, Aug (Ds) ; LI: Amagansett, Aug, Montauk Pt., May, Yaphank, May (Eng); Aqueduct, J1, Sep (Shoe, Ds).

15655 a Z. suturalis var. casta Rogers. White Plains, Je (Sq); SI (L\&D).

\section{Calligrapha Erichson}

15664 C. lunata Fab. Ilion, May ( $\mathrm{Yg})$; Schuylerville, Je ( $\mathrm{Yg}$ ); Ithaca, Ap (Bish); Scotia, Je (Yg); Karner, Aug (Yg); N. Chatham, Je (Yg); Poughkeepsie, J1 (Leon); Southfields, Aug (Schott); West Pt. (Robn); Mosholu, J1 (Yg); White Plains, J1-Aug (Sq); SI (L\&D); LI: Amagansett, Sep, Long Beach, May (Eng); Orient, Je, Lath (NYS); Rockaway Beach, May (Shoe); Montauk, Fire I., Promised Land, Sep, Wading R., Je (Ds).

15665 C. similis Rogers. Oswego, J1 (Bks); Olcott, Je-J1 (Dt); E. Aurora, Je-J1 (Dnd) ; Canandaigua, J1 (Felt) ; Westfield, May (Not) ; Ithaca, J1, Sep (Dt) ; Windsor, Je (Not) ; Albany, J1 (Bks); Windham, Oct (Dt) ; Pine I., May (Schott); Callicoon, J1 (Ds); West Pt. (Robn); White Plains, May-J1 (Sq); SI, May-Aug (L\&D); LI: Jamaica, J1 (Eng); Rockaway Beach, May, Aqueduct, J1 (Shoe); Orient, Lath (NYS); Flushing, Wading R., Aug (Ds); Brooklyn (Dt).

15669 C. elegans Oliv. Wells, J1, Newport, May (Yg); Clintonville, Ap (Yg); N. Pulaski, L. Ontario, Je (Ds); Williamsville, Je (Dnd) ; Ithaca, Ap-Sep (Dt, Vdyk) ; Windsor, Je (Not); Watervliet Reservoir, Aug (Leon) ; Hudson, J1 (Eng); Slide Mt., J1 (Shoe); West Pt. (Robn); Pine I., Je, Ramapo, Ap (Ds); White Plains (Sq, Bno); SI, Ap-Sep (Ds) ; LI: Long Beach, Rockaway Beach, May (Eng) ; North Beach, Dec, Queens, Je (Schott) ; Aqueduct, J1 (Shoe); Flushing, Wading R., Aug (Ds) ; Orient, Lath (NYS).

15671 C. scalaris Lec. Adirondack Lodge, J1 (Claas); Newport, May-Je, Keene Val., Je (Yg); Wilmington, J1 (Shoe); Ogdensburg (Yg); Clinton, May (Yg); Lowville, J1 (Ds); Westfield, May (Not); Oswego, Je 
(Bks); Avon, Je, Geneva, Oct (Cls); Ithaca, Je, Yuasa (Vdyk); Oliverea, Je (Ds); West Pt. (Robn); White Plains, J1-Aug -(Sq); SI, Oct (L\&D); LI: Brooklyn, May, Oct (Shoe); Oyster Bay, Aug (Ds).

15672 C. rhoda Knab. E. Aurora (Dnd).

15673 C. rowena Knab. Newport, Je (Yg); Wilmington, Je (Hoffman); Mt. Whiteface, J1 (Ds) ; E. Aurora, Je-J1 (Dnd) ; Avon, Geneva, Je (Cls) ; Ithaca, May, Aug (Dt) ; LI: Yaphank, May (Eng).

15674 C. philadelphica L. Newport, May-Je, Wells, Aug (Yg) ; Woodworth's L., J1 (Leon) ; Northampton, Je (Yg); Plattsburg, J1 (Shoe); Pike, Je (NYS); Genesee Co, Je (Ds); McLean, May (Dt); Schoharie, Je (Not); Nassau, Je (Yg) ; Voorheesville, Je (Bish); Thomson, May (Leon); N. Chatham, Je (Yg); White Plains, Je (Sq) ; LI: Bellport, Je-Aug (Nic); Bayshore, J1 (Ols).

15675 C. amelia Knab. Ellis, Je; SI (Knab).

15677 C. bigsbyana Kby. L. Placid, Sep (Eng) ; Keene Val., J1 (Yg) ; Long L., Aug (Yg) ; Severance, J1, Baker (Yg) ; Newport (Yg) ; Mooers, Sep (Not); Onondaga Co, May, herbage (H) ; Port Byron, Je (Dt) ; Olcott, Oct (Dt) ; western NY, Je-Aug (Dnd) ; Westfield, Aug (Yg) ; Castile, Brad (Flet) ; Ithaca, May, J1 (Dt) ; Groton, Je (Ds) ; Freeville, J1 (Chapman); Windsor, Je (Not); Schoharie, Je (Not); Callicoon, J1 (Ds) ; Oliverea, Je-J1 (Ds, Shoe) ; Albany, May, Karner, Sep (Yg) ; Catskills, J1 (Eng) ; LI: (Eng); Bayshore, J1 (Ols).

C. apicalis Not. Mooers, Clinton Co, Sep (Not).

\section{Chrysomela Linnaeus}

15692 C. subopaca Rogers. This is reported by Rogers as occurring in NY, but apparently this report is doubtful, as no specimens seem to have been taken by any one else.

\section{PLAGIODERA Redtenbacher}

15693 P. versicolora Laich. Windsor, Je (Not) ; NY Bot. Gardens, J1 (Hoffman) ; Van Cort. Pk., May (Nic); SI: Bulls Head, Sep-Oct, Dec (Ds); LI: Flushing, Jan (Bell); North Beach, Je (Schott). On willow (Sf).

\section{PHAEDON Latreille}

15699 P. viridis Melsh. Ilion (NYS); Newport, Je, Yg (Hoffman) ; Barneveld, Aug (Hoffman); Olcott, Oct (Dt); Ithaca, May-Aug (Dt) ; Catskills (Pears); Debruce, J1 (Shoe).

\section{Gastroidea Hope}

15701 G. polygoni L. Very common in the State (Sf). Batavia, Je (Ds) ; Ithaca, Ap, J1-Sep, Yuasa (Vayk, Dt) ; E Aurora, May, J1 (Dnd) ; Westfield, May (Not) ; Castile, Brad (Flet) ; Avon, Ap-May (Cls) ; Windsor, Je (Not); Schoharie, Je (Not); Kenwood, J1 (Yg); Albany, Fernow (Lint); Oliverea, Je (Ds); West Pt. (Robn); SI, Ap-Aug (Ds) ; LI: Rockaway Beach, Je, Long Beach (Eng); Wading R., May, Flatbush, Ap (Schott); Brooklyn, Ap, Je (Shoe).

15703 G. cyanea Melsh. SI, May-Je (Ds). On Rumex. 


\section{LINA Redtenbacher}

15708 L. interrupta Fab. Keene Val., J1 (Yg) ; Wilmington, J1 (Shoe) ; Gloversville, May, Alex (Yg) ; Castile, Brad (Flet); E. Aurora, May, Protection, Je (Dnd) ; Avon, May, Geneva, Je, Lodi, Jl (Cls); Ithaca, MarAug, Bish, Yuasa (div.); Corinth, Je, Karner, May (Yg); Pine I., Je (Sf) ; West Pt., Je (Ds) ; Ft. Montgomery, May (Schott) ; Southfields, May (Schott); SI: Je (Ds) ; Silver L. (Sf) ; LI: Wyandanch, Je (Sf); Rockaway Beach, May (Shoe, Schott); Flushing (Bell). Often in collections as lapponica L., which is European (Sf).

15709 L. tremulae Fab. Axton, Je (M\&H) ; Newport, Je, L. Clear, J1 (Yg); Mt. McIntyre, J1, Leon (Yg); Horicon, Aug (Doll); N. Pulaski, L. Ontario, Je (Ds); Mt. Whiteface, J1 (Shoe); Conesus L., Je (Ds) ; Elba, Je, Wild (Dnd); Ithaca, J1 (Dt) ; Ringwood, J1, Yuasa (Vdyk); Windsor, Je (Not) ; West Pt., J1 (Ds) ; SI, J1 (L\&D) ; LI: Rockaway Beach, Je (div.); Long Beach, Je (Eng, Schott); Flatbush, J1 (Schott).

15710 L. scripta Fab. Ausable R. (Lint) ; Liverpool, May (Lint) ; Northampton, Je (Yg) ; Cranberry L., Htly (H) ; Ithaca, May-Je (Dt) ; Albany, Je (Yg); West Pt. (Robn); SI, Je, Aug (Ds) ; LI: Wyandanch, Je (Sf) ; Long Beach, Je (Eng); Flatbush, May (Eng).

15711 L. obsoleta Rog. West Pt., Je (div.); Ramapo, Je (Ds).

\section{Phytodecta Kirby}

P. americana Sf. Axton, Je (M\&H) ; Keene Val., Je (Not); Mt. Whiteface, J1 (Shoe); Cranberry L., Htly $(\mathrm{H})$; Windsor, Je (Not); Oliverea, Je (Shoe); LI: Rockaway Beach, May (Schott).

P. notmani Sf. Keene Val., Je (Not).

\section{Phyllodecta Kirby}

15718 P. americana Sf. Wilmington, J1 (Shoe); Jay, J1 (Britton) ; Northampton, Je $(\mathrm{Yg})$; Schuyler Co, Je (H) ; Ithaca, Aug, Yuasa (Vdyk); Catskills (Pears) ; Oliverea, Je, Sep (Ds, Shoe) ; Callicoon, Sep (Ds); Schoharie, Je (Not); Windsor, Je (Not); West Pt., as vulgatissima (Robn). Wrongly identified as vitellinae or vulgatissima.

\section{SUBFAMILY GALERUCINAE}

\section{TRIRHABDA LeConte}

15724 T. tomentosa L. LI: Rockaway Beach, Jl (Shoe); Glen I. (Sf) ; Orient, J1, Montauk, Aug (Ds).

15725 ?T. brevicollis Lec. Avon, J1 (Cls).

15726 T. virgata Lec. L. Placid, Sep (Eng); Olcott, J1 (Dt); Wilmington, Aug (Ds) ; Avon, J1 (Cls) ; Ithaca, Aug-Sep (Dt) ; Callicoon, J1, E. Jewett, Aug, Oliverea, Sep (Ds) ; White Plains, J1 (Sq) ; SI, Sep (Ds); LI: Rockaway Beach, Aug-Sep (Shoe); Flushing, Aug (Ds).

15727 T. canadensis Kby. Mt. Marcy, J1, Clyde, J1 (Yg); Wilmington, Jl-Aug (Shoe, Ds) ; Olcott, J1 (Dt) ; Akron, Je (Dnd) ; L. George, Je (Shoe) ; Albany, Battle, Poughkeepsie, J1 (NYS) ; SI, Je (Ds) ; LI: Brooklyn; Aug (Shoe); Forest Pk., J1 (Martin); Rockaway Beach, J1 (Shoe); Jamaica, J1 (Yg); Wading R., Aug, Yaphank, J1, Montauk, Sep (Ds). 


\section{GaleRuCella Crotch}

15744 G. americana Fab. Gloversville, J1 (Yg); E. Aurora, J1 (Dnd) ; Windsor, Je (Not); Nassau, Poughkeepsie, J1 (Yg); Ulster Co (Nic); West Pt., J1 (Eng); White Plains, May, Aug (Sq); SI, Je-Oct (L\&D); LI: Brooklyn, Aug (Shoe).

15744 G. cribrata Lec. Long L. (Ds) ; Windsor, Je (Not) ; Nyack, Je (Ds) ; West Pt., J1 (Eng); White Plains, May-Aug (Sq); SI, J1 (Ds); LI: Central Pk., Ap (Eng); Cold Sp. Harb., J1 (Brb) ; Wyandanch, Aug, Little Neck, Dec, Huntington, Aug, Jamaica (Schott); Wading R., J1 (Ds).

15745 G. sexvittata Lec. Horicon (Doll); Ithaca, Je (Dt); Windsor, Je (Not).

15746 G. cavicollis Lec. Plattsburg, J1 (Ds) ; N. Elba, J1 (Shoe) ; Cranberry L., J1 (Drk); N. Pulaski, L. Ontario, Je (Ds) ; Buffalo; Portage, Je (Ds) ; Wyoming Co, Je (Ds) ; Rock City, Je (Ds) ; Ithaca, Je (Ds, Yuasa); Windsor, Je (Not); Corinth, Je (Yg) ; Schoharie, Je (Not) ; Slide Mt., Je-J1 (Shoe, Ds) ; E. Jewett, Aug, Oliverea, Je (Ds) ; West Pt. (Robn); White Plains (Sq); SI, Je (L\&D) ; LI: Yaphank, J1 (Sf).

15747 G. rufosanguinea Say. Keene Val., Aug, Newport, May (Yg) ; Batavia, Je (Ds); Peekskill, May 21 (Fall, Frost); West Pt. (Robn); Mosholu (Sf) ; SI, Ap, Je (Ds) ; LI: Wyandanch, Je (Sf) ; Rockaway Beach, May (Shoe).

G. kalmiae Fall. L. Clear, Je 6 (Yg) ; L. Placid, Sep (Eng) ; Saranac L., Sep (Shoe); Albany, May 6 (Yg); LI: Yaphank, May, J1 (Sf); Rockaway Beach, May (Shoe).

G. spiraeae Fall. Saranac L., Je, Speculator, Je, Wells, J1 23, Corinth, May 31, Nassau, Je 23 (Yg); Debruce, J1 (Shoe) ; Oliverea, Je, Sep (Ds) ; Slide Mt., Je (Ds) ; LI: J1 (Eng).

15749 G. notulata Fab. Cranberry L., Aug (Drk) ; Binghamton (Franck); West Pt., Je (Sf) ; SI (L\&D) ; LI: Rockaway Beach, May (Shoe).

15750 G. notata Fab. Letchworth Pk., Sep (Dnd); Westchester Co, Sep (Ds) ; Mosholu (Sf); Bronx Pk. (Sf) ; SI, J1 (Ds); LI: Aqueduct, Sep (Shoe); Wyandanch, Sep (Schott); Wading R., Aug (Sf, Ds); Cold Sp. Harb., Sep (Chitt).

15751 G. nymphaeae L. Saranac L., Sep (Shoe, Ndm) ; Bolton (Eng) ; Pike, J1 (Ndm) ; Ithaca, J1 (Dt) ; Kingston, J1 (Eng); West Pt. (Robn); White Plains, J1-Oct (Sq); Nyack, Nov (Schott); Van Cort. Pk., May (Sf) ; SI, May-Je, Aug (Ds) ; LI: Flatbush, J1 (Eng) ; Rockaway Beach, Je (Ds, Shoe); Wyandanch, L. Ronkonkoma, May (Schott); Wading R., Je, Flushing, Aug (Ds).

15752 G. tuberculata Say. Keene Val., Aug 3 (NYS) ; Newport, May 23 (Yg) ; E. Aurora, J1 (Dnd); Westfield, May (Not); Castile, Brad (Flet); Windsor, Je (Not); Debruce, J1 (Shoe).

15753 G. decora Say. Keene Val., Sep (Eng); Mt. Marcy, Je, Felt (Yg) ; Cranberry L., J1-Aug, Oct, Htly (H, Drk); Saranac L., Je (NYS); Cranberry Cr., Je (Yg) ; Mt. Whiteface, J1 (Shoe, Ds) ; Mooers, Sep (Not) ; Allegany Pk., J1 (Dnd) ; Ithaca, May-J1 (Dt) ; Windsor, Je (Not); Schoharie, Je (Not); Karner, May (NYS); Debruce, J1 (Shoe); Southfields, Aug (S'chott); Oliverea, Je (Shoe); White Plains, Sep (Sq); LI: Rockaway Beach, Je (Schott). 
15754 G. luteola Mull. (xanthomelaena Schrank). Elm LEAf-BeEtLe. This common species is undoubtedly generally distributed almost everywhere in the State, having been recorded from about seventy different localities (Sf). Elm. The name luteola has priority.

G. vaccinii Fall. Wells, J1 18 (Yg); Juanita I., L. George, Sep 13, Leon (NYS).

G. alni Fall. Northville, J1 (NYS).

\section{Monoxia LeConte}

15755 M. puncticollis Say. SI, Je-Aug, Nov (Ds) ; LI: Promised Land, Sep, Long Beach, Je (Eng); Rockaway Beach, Je, Aug (div.) ; Cold Sp. Harb., Je (Brb) ; Central Pk., Je, Aqueduct, J1 (Shoe); Jamaica (Schott); Wyandanch, J1 (Ds, Schott); Orient, Massapequa, J1 (Ds).

\section{DIABROTICA Chevrolat}

15769 D. duodecimpunctata Fab. Twelve-spotted Cucumber Beetle. Axton, Je $(\mathrm{M} \& \mathrm{H})$; Newport, Sep (Yg); Wells, J1 (Yg); Oswego, Sep, Bks (Yg) ; western NY, J1-Sep (Dnd) ; Olcott (Dt) ; Perry, Aug, Hawley (Vdyk); Castile, Brad (Flet); Geneva, Aug-Sep (Cls); Onondaga $\mathrm{Co}$, Oct, on herbage $(\mathrm{H})$; Westfield, $\mathrm{Jl}(\mathrm{Yg})$; Ithaca, JeNov, Yuasa (Vdyk, Dt) ; Albany Co, May (Yg) ; Catskills, J1 (Eng) ; Oliverea, Sep (Ds); West Pt. (Robn); White Plains, Aug-Sep (Sq) ; SI, Aug-Dec (Ds) ; LI: Rockaway Beach, Je (Eng) ; Brooklyn, Je, Aug (Shoe) ; Orient, Lath (NYS); Huntington (Schott).

15777 D. atripennis Say. Gloversville, Sep, Alex (Yg); Olcott, Je-J1 (Dt); Callicoon, J1 (Ds).

15782 D. vittata Fab. Striped Cucumber Beetle. Western NY, Je-Aug (Dnd) ; Lowville, (Ds) ; Schuyler Co, Onondaga Co, May-Je, on herbage (H); Ithaca, Ap-Sep (Dt, Ds) ; Windsor, Je (Not); Schoharie, Je (Not); West Pt. (Robn); White Plains, May-J1 (Sq); SI, Ap, Je, Aug (Ds) ; LI: Cold Sp. Harb., Sep (Eng); Rockaway Beach, Je (Eng); Creedmoor, May (Schott); Brooklyn, May (Shoe); Yaphank, J1, Central Pk., May, Sands Pt., Oct (Ds) ; Orient, Lath (NYS); Pinelawn, Sep (Ds).

\section{PhyLlobRotica Redtenbacher}

15787 P. vittata Horn. "NY" (Dietz); Ithaca, J1-Aug, Yuasa (Vdyk).

15788 P. decorata Say. Mt. Whiteface, J1 (Shoe); Speculator, Je (Yg); Cranberry L., J1 (Drk); W. Winfield, Je, Leon (Yg); E. Aurora, Je (Dnd) ; Ithaca, Je-Ji (Dt, Hoffman); LI (Eng).

15792 P. discoidea Fab. Cranberry L., J1 (Drk); Stony Pt., Je (Ds); SI, Je (Ds) ; LI: Brooklyn, Aqueduct, J1 (Shoe).

15794 P. limbata Fab. Newport, Je, Wells, J1 (Yg); Broadalbin, Je, Nassau, J1 (Yg); West Pt. (Robn); LI: Rockaway Beach, Je (Eng).

LUPERODES Motschulsky

15815 L. thoracicus Melsh. Broadalbin, Je (Yg); Gloversville, Sep (NYS); Conesus L., Je (Ds); Ithaca, Je-JI (Dt); Schoharie, Je (Not); E. Nassau, Aug; Callicoon, J1 (Ds). 
15823 L. meraca Say. Big Moose, Aug, Wells, J1, Newport, Je (Yg) ; L. Placid, Sep (Shoe); Cranberry L., Htly $(\mathrm{H})$; L. Pleasant, Je (Eng); Mt. Whiteface, J1 (Shoe); Batavia, Portage, Wyoming Co, Je (Ds); E. Aurora, Je (Flet, Dnd); Ithaca, Je (Ds, Chitt); W. Danby, May (Ds); Egleston's Glen, L. Keuka, Je (Ds) ; Cincinnatus, J1 (Flet, Dnd); Schoharie, Je (Not); Corinth, Je (Yg); Albany, Je (Yg); Catskills (Pears); Oliverea, Je (Ds); E. Jewett, Aug (Ds); Greenwood L., J1 (Sf); West Pt., J1 (Eng); Slide Mt., J1 (Shoe); Ft. Montgomery, Je (Schott); Pine I., Je (Ds) ; SI (L\&D).

15824 L. cyanellus Lec. Olcott, Je (Dt); Ithaca, J1-Aug, Yuasa (Vdyk); Windsor, Je (Not); Oliverea, Je (Ds); SI (L\&D).

\section{AgeLASTICA Redtenbacher}

15846 A. alni L. LI: Brooklyn, Oct (Eng).

Phyllecthrus LeConte

15850 P. gentilis Lec. Minnewaska, Aug 25, Nic (Frost).

\section{Cerotoma Chevrolat}

15854 C. trifurcata Forst. Bean Leaf-Beetle. Newport, May (Yg) ; E. Aurora, Je, Aug (Dnd); Ithaca, Aug-Sep (Dt); Schoharie, Je (Not) ; West Pt. (Robn); White Plains, J1-Aug (Sq); Mosholu, May (Yg); SI, May, J1-Sep (Ds) ; LI: Forest Pk., Jl (Martin); Wyandanch, May (Schott); Brooklyn, Aug (Shoe); Baldwin, Je (Yg).

\section{SUBFAMILY HALTICINAE}

\section{BLEPHARIDA Rogers}

15858 B. rhois Forst. Nassau, Je (NYS) ; Athens, J1 (Schott) ; Bangall, Sep (Eng); SI : south shore, Jl (Ds); Oct (Ds) ; LI: Pinelawn, Oct, Yaphank, Sep (Eng); Wyandanch, Massapequa, J1 (Schott); Orient Pt., Je, Lath (Yg).

HyPoLAMPSIS Clark

15861 H. pilosa Ill. Windsor, Je (Not); Poughkeepsie, Je (Yg) ; LI: Wyandanch, Dec (Schott).

\section{OEDIONYCHIS Latreille}

15865 O. gibbitarsa Say. Avon (Cls) ; LI: Rockaway Beach, Ap (Eng) ; Brooklyn, Ap (Shoe); Wading R., Je (Shoe, Ds); Gardiners I., West Hills, Je (Ds).

15867 O. thoracica Fab. Catskills, J1 (Eng); Slide Mt., Je (Shoe); West Pt. (Robn); Mosholu, May (Ds); White Plains, Je (Schott); SI, Ap, J1 (Ds) ; LI: Long Beach, May (Eng) ; Rockaway Beach, May, Wyandanch, Ap (Schott); L. Ronkonkoma, May (Schott); Selden, Aug, Farmingdale, Oct, Yaphank, J1 (Ds). 
15868 O. vians Ill. Newport, Je (Yg); Egypt's I., Nov (Ds) ; Olcott, J1 (Dt); Cayuga, May-Sep (Dt); Ithaca, May-Oct, Hoffman (Dt, Mat, Biss-CU) ; Voorheesville, J1, Leon (Yg); Nassau, Aug (Yg); White Plains, J1 4 (Sq) ; Pine I., West Pt., Je (Ds); SI, Je (Ds) ; LI: Rockaway Beach, May-Je (Eng, Shoe); Wading R., J1, Aqueduct, Sep (Shoe).

15868a O. vians var. scripticollis Say. West Pt. (Robn) ; LI: Jamaica, J1 (Eng) ; Rockaway Beach, May (Ds).

15873 O. fimbriata Forst. West Pt. (Robn) ; SI, Je (L\&D) ; LI: Promised Land, Je (Eng) ; Rockaway Beach, May-Je (Shoe); Shinnecock Hills (Ds); Half Way Hollow Hills, J1 (Ds).

15877 O. miniata Fab. West Pt. (Robn) ; SI: Great Kills, May (Ds) ; LI: Yaphank, Sep, Montauk Pt., May (Eng); Rockaway Beach, Je-J1 (div.); Wyandanch, J1, L. Ronkonkoma, May (Schott); Wading R., Sep (Ds, Nic) ; Fire I., J1, Amagansett, Sep (Ds).

15886 O. limbalis Fab. Newport, May-Je, Elm L., Aug, L. Clear, Je (Yg); Saranac L., Sep (Shoe); Cranberry L., J1-Aug (Drk); Big Woodhull, Je, Clms (Dt) ; Protection, Jl (Dnd); Ithaca, May-Je, McLean, May (Biss-CU); Ringwood, J1, Yuasa (Vdyk); Windsor, Je (Not); Corinth, Je, Karner, May (Yg); Oliverea, Je, Debruce, J1 (Shoe) ; West Pt. (Robn); ST, Je (Ds) ; LI: Amagansett, May, Yaphank, May, Sep (Eng) ; Bayshore, J1 (Ols) ; Central Pk., May (Shoe).

15886a O. limbalis var. subvittata Horn. Axton, Je (M\&H); Saranac L., Aug; Chipmunk Swamp, Vandalia, Je, Mix Cr. Val., Rock City, Je (BissCU) ; Westfield, May (Not); Ithaca, McLean, May (Biss-CU) ; Windsor, Je (Not); Greenwood L. (Sf); LI: Amagansett, May (Eng) ; Promised Land, Sep (Ds).

15887 O. sexmaculata Ill. Schuyler Co, Je (H) ; Ithaca, Je (Eng, Yg) ; Poughkeepsie, Je ( $\mathrm{Yg}$ ); Ft. Montgomery, Ji (Schott); West Pt., Je (Ds); Van Cort. Pk. (Pears).

15889 O. quercata Fab. Cranberry L., Htly (H); Ithaca, Ap (Biss-CU); Windsor, Je (Not); West Pt. (Robn); SI, Ap-Je (L\&D). These records probably refer to limbalis (Sf).

15890 O. scalaris Melsh. SI, J1 (L\&D).

\section{Disonycha Chevrolat}

15895 D. pennsylvanica Ill. Newport ( $Y g$ ); Saranac Inn, Aug (Yg); Mt. Whiteface, J1 (Shoe); Rochester, Je (Biss-CU); Ithaca, Ap-Sep (Dt) ; Nassau, May (Yg); Pine I., Je (Ds); Slide Mi., J1 (Shoe); West Pt. (Robn); SI, May-J1 (Ds).

15895 D. pennsylvanica var. pallipes Cr. Mt. Whiteface, J1 (Shoe) ; Mt. Seward, Je $(\mathrm{M} \& \mathrm{H})$; Sodus Pt., Sep (Yg); Rochester Jct., Je (Biss-CU); Castile, Brad (Flet) ; Ithaca, Mar-Sep, Y uasa (Vdyk, Biss-CU, Yg); Albany, Aug (Yg) ; SI (Ds) ; LI: Brooklyn, Rockaway Beach, May (Shoe); Cold Sp. Harb., J1 (Brb).

D. pennsylvanica var. parva Blatch. Madison Co, May, herbage $(\mathrm{H})$; Horicon, Aug (Doll) ; Montezuma, Aug, 1886 (Biss-CU); Callicoon, J1 (Ds); LI: Wading R., Je (Ds).

15898 D. caroliniana Fab. Ithaca, Je (Hoffman); West Pt. (Robn); SI, Je (L\&D) ; LI: Yaphank, Aug-Sep (Eng, Shoe); Long Beach, May, Amagansett, Aug, Cold Sp. Harb., Sep (Eng); Wyandanch, May 
(Sf); Wading R., May, Montauk (Ds); Rockaway Beach, May (Shoe) ; Huntington, Feb (Schott); Orient, May, Lath (Yg).

D. davisi Sf. LI: Yaphank, J1 (Ds); Wyandanch, J1 (Schott).

15906 D. triangularis Say. Three-Spotted Flea Beetle. Clyde, Severance, Aug, Blanke, Denmark, May ( $\mathrm{Yg}$ ) ; Rochester Jct., Je (Biss-CU) ; Honeoye Falls, Je (Ds) ; Olcott, May-Aug (Dt) ; Castile, Brad (Flet) ; Avon, May, Geneva, Oct (Cls) ; Westfield, Je (Yg); Ithaca, Aug (BissCU); Callicoon, J1 (Ds); West Pt. (Robn); SI (L\&D); LI: Brooklyn, J1-Aug (Shoe, Eng); Rockaway Beach, May, Dec (Shoe, Schott).

15907 D. xanthomelaena Dalm. SPInACH FleA BeEtle. Newport, May (Yg); Hubbardsville, Je (Biss-CU); Rochester Jct., Je (Biss-CU); Castile, Brad (Flet); Avon, Feb, and Geneva, Ap (Cls) ; E. Aurora, May-Je, Aug (Dnd); Westfield, May (Not); Ithaca, Ap-Nov 30 (Dt, BissCU) ; West Pt. (Robn) ; Bronx Pk. (Sf); SI, Mar-Dec (L\&D) ; LI: Rockaway Beach, Je, Aqueduct, J1 (Shoe); Huntington, Mar, Little Neck, Ap (Schott); Orient, Ap, Lath (Yg).

15911 D. collata Fab. SI, Je (L\&D) ; LI: Flatbush, Nov (Eng) ; Central Pk., Ap (Schott); Rockaway Beach, May (Shoe); Gardiners I., Aug, Pinelawn (Ds); Orient Pt., J1, Lath (Yg).

\section{HALTICA Geoffroy}

15916 H. bimarginata Say. Wilmington, J1 (Shoe); Plattsburg, J1 (Ds) ; N. Elba, J1 (Wat); L. Pleasant (Lint); Jay, J1 (Britton); Corinth, J1 $(\mathrm{Yg})$.

15917 H. chalybea I1l. Grapevinf, Flea Beetle. Newport, May, Canandaigua, Aug (Yg); Cranberry L., J1 (Drk); L. Ontario, near Pulaski, Je (Ds) ; Castile, Brad (Flet) ; Onondaga Co, May-Je (H) ; western NY, May-Sep (Dnd); Schuyler Co (?), May-Je (H) ; Conesus L., Je (Ds) ; Horicon, Aug (Doll); Slaterville, Je, Ithaca, May-Aug (Dt); West Pt. (Robn) ; SI, May (Ds) ; LI: Aqueduct, Mar, Rockaway Beach, May (Shoe); Gardiners I., Selden, Aug (Ds); Yaphank (Eng).

H. betulae Sf. Ithaca, Je, $D t$ (Sf); Chenango Co, Jl, Shoe (Sf); Oliverea, Je (Shoe). On birch (Shoe).

H. gloriosa Blatch. LI: Yaphank, Je (Sf). On wild rose (Sf).

15920 H. kalmiae Melsh. Mooers, Sep (Not); Olcott, May-Je (Dt) ; Ithaca, May-Je (Dt) ; Windsor, Je, Schoharie, Je (Not); Bear Mt., Aug (Schott); Stony Pt., Je (Ds); Peekskill, May (Sherm); SI, May, Aug-Sep (Shoe, Ds); LI: Yaphank, May-J1 (Eng). On laurel.

15920 H. ignita I11.56 West Pt., Je, Pine I. (Sf) ; Bronx Pk. (Sf). Recorded on Prunus.

15921 H. rosae Woods. Ithaca, May, Bish (Hoffman); Pomona, Ap (Hoffman); $\mathrm{SI}$, Je (Ds) ; LI: Amagansett, May (Sf). On wild rose.

H. woodsi Isely. Olcott, May, $D t$ (Sf); Niagara Falls; Ithaca, May, $D t$ (Sf) ; Ramapo, Je (Ds); LI: Mastic, Je (Murphy); Half Way Hollow Hills, J1 (Ds).

\footnotetext{
56 Records of the strawberry flea beetle in economic literature as $H$. ignita apparently refer to a complex of $H$. kalmiae, ignita, rosae, and possibly one or two other allied species (Sf).
} 
15922 H. corni Woods. Olcott, Ap-Je, Dt (Sf).

15923 H. carinata Germ. Mt. Whiteface, I1 (Shoe) ; Onondaga Co, Ap, hibernating about stump $(\mathrm{H})$; Grand I., Aug (Hoffman); Tompkins Co, Je (BM) ; Slaterville, Je; LI: Amagansett, May (Eng); Flushing, Dec (Bel1). On Ulmus.

15925 H. aenescens Blatch. Plattsburg (Shoe).

15926 H. litigata Fall. LI, J1 (Shoe); Maspeth, Long Beach, Je (Schott).

H. blanchardi Fall. LI: Promised Land (Eng); Wading R., May (Schott); Amagansett, Sep (Ds).

15937 H. evicta Lec. var. shoemakeri Sf. Plattsburg, Catskills, Je (Shoe).

15942 H. marevagans Horn. Karner, J1 (Yg); West Pt. (Robn); Rockland, Aug (Martin) ; SI, Je (Ds) ; LI: Manorville, Sep (Eng) ; Rockaway Beach, Je-J1 (Eng, Shoe); Brooklyn, Sep (Shoe); Cold Sp. Harb. (Chitt); Long Beach, Aug (Schott).

15949 H. fuscoaenea Melsh. Hope, Aug (Hoffman); Gloversville, Ap (Fall); McLean Bogs, Aug, Dt (Vdyk); Windsor, Je (Not); SI, Je (L\&D).

15950 H. knabi Blatch. SI, Je (Ds).

15955 H. rufa Ill. West Pt. (Robn); Greenwood L. (Sf); Van Etten, May (Hoffman); L. Katharina, Oct (Yg); SI (L\&D).

\section{Chalcoides Foudras}

15968 C. fulvicornis Fab. Widely distributed, occurring almost everywhere in the State (Sf). Peru, Je (Biss-CU); Mt. Whiteface, J1 (Shoe); Mooers, Sep (Not); Plattsburg, J1 (Ds); Oneida L., Aug (Drk); Westfield, May (Not); Onondaga and Schuyler Counties, May-Je, flying, beating trees $(\mathrm{H})$; Hemlock L., Aug, Honeoye Falls, May-Aug, Rochester Jct., Je, Canton, Je, Freeville, J1, Lyons, Je (Biss-CU); Howland I., Nov (Biss-CU); Olcott, Mar-Je (Dt); Yates Co (Ds); Ithaca, ApSep (Dt, Biss-CU); Durlandville, Je, FrostSW (Dt); Windsor, Schoharie, Je (Not) ; McLean, May-Je, Ringwood, Je (Biss-CU); E. Jewett, Aug (Ds); Debruce, J1 (Shoe); West Pt., Pine I., Je (Ds) ; Southfields, May (Schott); SI, Je (Ds) ; LI: Je (Eng); Brooklyn, May (Shoe); Huntington, Sep (Schott); Orient (Ds). As helxines in collections $(\mathrm{Sf})$

15968 C. fulvicornis var. violacea Melsh. Peru, Je, Rochester Jct., Je, Ithaca, Ap-J1, Ringwood, Je, McLean, May-Je, Ellis to Slaterville, Je, Caroline, Je, Rhinebeck, J1 (Biss-CU); Castile, Brad (Flet); Wyoming, Je (Ds); Rock City, Je (Ds); LI: Yaphank, Jl (Sf).

\section{CREPIDODERA Chevrolat}

15969 C. erythropus Melsh. Conesus L., Je (Ds); Ithaca, Ap-Je, Bish (Dt, Yg, Biss-CU) ; Poughkeepsie, May (Yg); Windsor, Schoharie, Je (Not); West Pt., Je (Sf); Westchester Co (Ds); SI, Je (L\&D) ; LI: Central Pk., Ap-May (Shoe, Eng) ; Yaphank, May (Eng, Ds); Huntington, Je, Wyandanch, May, Oyster Bay, Ji (Schott); Half Way Hollow Hills, J1, Wading R., May (Ds).

15974 C. atriventris Melsh. Newport, May (Yg); Johnstown, Ap (Alex-NYS); Olcott, Feb, Je (Dt); Ithaca, Aug-Sep (Dt, Hoffman); McLean, May (Biss-CU); Catskills (Pears); West Pt. (Robn); SI (Ds); LI: Brooklyn, Je, Rockaway Beach, May (Shoe). 
HIPPURIPHILA Foudras

15977 H. modeeri L. Albany (Hoffman); Ithaca, Frost, on Rumex.

\section{EPITRIX Foudras}

15982 E. cucumeris Harr. Potato Flea Beetle. Widely distributed, and occurring almost everywhere in the State (Sf) on a variety of field and garden products. Peru, Je, Wilmington, Aug, Pulaski, Je, Honeoye Falls, May-Aug, Rochester, May, Newark, J1, Indian Falls, Je, Waterville, May-Je, E. Bethany, J1, Dunkirk, Aug, Rock City, Je, Ithaca, May-Aug, McLean, May-J1, Ringwood, Je, Ellis to Slaterville, Je, Caroline, Je, Freeville, Aug, W. Danby, Aug, Spencer L., Je, Willseyville, May (Biss-CU); Schuyler, Onondaga, and Madison Counties, May-Je, herbage $(\mathrm{H})$ : Schoharie, Windsor, Je (Not); Ramapo, May (Ds); West Pt. (Robn); SI, Je-Sep (Ds) ; LI: Greenport, Aug, Flatbush, Ap, Yaphank, May (Eng); Rockaway Beach, May (Eng, Shoe); Wading R., Aug, Maspeth, May, Fire I., Aug, Central Pk., May (Ds); Massapequa, May (Shoe); Wyandanch, Jamaica, May (Schott); Farmingdale, J1, Nassau Co, May (Biss-CU).

15986 E. parvula Fab. Schuyler, Onondaga, and Madison Counties, May-Je, herbage $(\mathrm{H})$; Van Cort. Pk., Aug (Sf); LI: Wyandanch, Nov (Schott).

\section{ORThALTICA Crotch}

15988 O. copalina Fab. Newport, Je (Yg); Pike (Yg); Westfield, Je (Yg); Schuyler Co, Je (H) ; Ithaca, Je-Aug (Vdyk, Dt, Biss-CU); Schoharie, Je-J1 (Not, Lint) ; Poughkeepsie, Je (Yg); Middletown, J1 (Biss-CU) ; Debruce, J1 (Shoe); Oliverea, Je (Ds); West Pt., Je (Ds, Robn); Stony Pt., Je (Ds) ; SI, Je (Ds) ; LI: Brooklyn, J1 (Shoe); Cold Sp. Harb., Ji (Brb).

\section{LUPERALTICA Crotch}

15991 L. fuscula Lec. Ulster Co, Sep (Nic); Ft. Montgomery, Sep (Schott) ; Howland I., Je, Dt (Vdyk).

\section{MANTURA Stephens}

15993 M. floridana Cr. Onondaga Co, May, herbage (H); Pike, May (Yg); Ithaca, May-Nov 13 (Biss-CU); Windsor, Je' (Not); West Pt. (Robn); Mosholu (Sf); SI, Mar-Dec (L\&D).

\section{Chaetocnema Stephens}

15995 C. cribrata Lec. McLean, Je (Biss-CU).

15998 C. subcylindrica Lec. Westfield, May (Not); Ithaca, May, McLean, MayJe (Biss-CU); Debruce, J1 (Shoe); Howland I., Je (Dt).

16001 C. denticulata Ill. Olcott, May-J1 (Dt); E. Aurora, Je-Oct (Dnd); West Pt., Sep (Robn); Bronx Pk. (Sf); SI, J1 (Shoe); LI: Flatbush, Dec (Eng); Brooklyn, Rockaway Beach, May (Shoe).

16002 C. cribrifrons Lec. SI: Tottenville, J1 (Woodr). Determination queried by Sf. 
16006 C. minuta Melsh. Newport, May ( $\mathrm{gg}$ ); Windham, Oct (Dt) ; Onondaga Co, May (H); Westfield, May (Not); Ithaca, J1 (Dt, Vdyk); McLean, May (Biss-CU); Middletown, J1 (Biss-CU); Albany, May (Yg) ; Mosholu (Sf).

16013 C. parcepunctata Cr. Ithaca, May (Vdyk); SI, JI (L\&D).

16014 C. pulicaria Melsh. West Pt. (Robn) ; Suffern, May (Love) ; SI, J1, Nov (L\&D) ; LI: Flatbush, Mar (Eng); Rockaway Beach, May, Brooklyn, Aug-Sep (Shoe).

16016 C. confinis Cr. Sweet Potato Flea Beetle. Onondaga Co, May (H) ; Ithaca, Je-Aug (div.) ; Freeville (Biss-CU); Albany, Rensselaer, Poughkeepsie, May (Yg); SI, J1 (L\&D).

16020 C. quadricollis Schwz. LI: Glen I. (Sf).

\section{SySTENA Clark}

16022 S. hudsonias Forst. Smartweed Flea Beetle. Newport, Northampton, Je $(\mathrm{Yg})$; Canton, Je (Biss-CU); Rochester Jct., Je, Honeoye Falls, Sonyea, Je (Biss-CU) ; Portageville, Je (Ds) ; Buffalo (Forst) ; Westfield, Je (Yg) ; Fredonia (Felt) ; Castile, Brad (Flet) ; Crosby Landing, Je (Biss-CU) ; Ithaca, Je-J1 (div.) ; Ringwood, McLean, Je (Biss$\mathrm{CU})$; Windsor, Schoharie, Je (Not); Slingerland, J1, Albany, Aug, Schodack Landing, Je (Yg); Nyack, Je (Ds); West Pt. (Robn); Middletown, J1 (Biss-CU) ; Ramapo, May (Ds, Love) ; Van Cort. Pk., J1 (Biss-CU); SI, Je (Ds); LI: Yaphank, Rockaway Beach, Je (Eng); Cold Sp. Harb., J1 (Brb); Gardiners I., Aug, Wading R., Je (Ds).

$16023 \frac{1}{2}$ S. frontalis Fab. Red-Headed Flea Beetle. Mooers, Sep (Not) ; Saranac L., Sep (Shoe) ; Syracuse, Aug (Felt); Perry, Aug, Hawley '(Vdyk); Pike, Aug ( $\mathrm{Yg}$ ); Geneva, Aug, Pollier (Lint); Sheridan, Fredonia, Potter Swamp, Aug (Biss-Cu); Ithaca, Je-Sep (Dt, Biss-CU) ; Coy Glen, Michigan Swamp, Je (Biss-Cu); Schenectady, J1, Scotia, Aug (Yg); central NY, Aug-Sep (Chitt); Callicoon, J1 (Ds) ; Catskills (Pears); West Pt. (Robn); SI (L\&D); LI: Wyandanch, Je (Schott); Brooklyn, Sep (Shoe); Orient, Lath (NYS).

16027 S. elongata Fab. Oliverea, Sep (Ds) ; LI: Bay Shore, J1 (O1s).

16028 S. taeniata Say. Pale-striped Flea Beetle. Rochester Jct., Je-J1 (Biss-CU); Olcott, J1 (Dt); E. Aurora, J1-Aug (Dnd) ; Newark, Stuart; S. Byron (Felt) ; Ithaca, J1-Aug, Oct (Dt, Biss-CU) ; Middletown, J1 (Biss-CU); Albany, Oct (Yg); Schoharie, Je (Not); Alps, Aug (Martin); LI: Huntington, J1 (Schott); Rockaway Beach, Je (Ds) ; Central Pk., Je (Shoe).

16029 S. marginalis I1l. Hamburg, Aug (Yg) ; Ithaca, Aug-Sep (Dt) ; Coy Glen, Aug, Dt (Vdyk); L. George, Aug (Zab) ; Albany, Sep (Yg) ; Ulster Co, Sep (Sf, Shoe) ; West Pt. (Robn) ; Ft. Montgomery, Sep (Śchott); Westchester Co, Sep (Ds); SI, Je, Aug-Sep (Ds, Pears); LI: Yaphank, Sep (Eng); Bay Shore, J1 (Ols).

\section{LONGITARSUS Latreille}

16034 L. turbatus Horn. Ithaca, Ap-May (div.-CU); SI (L\&D).

16046 L. pygmaeus Horn. Schoharie, Je (Not).

16047 L. testaceus Melsh. Madison Co, May, on herbage (H) ; Honeoye Falls, Aug (Sf-CU); Olcott, Feb (Dt); Ellis, Tompkins Co, Je (CU) ; SI, Je (L\&D). 
16049 L. melanurus Melsh. Ithaca, Ap-Je (Biss-CU); Slaterville, May (Biss-CU) Willseyville, May (Biss-CU); New Baltimore, May (Yg); SI (L\&D); LI: Bellport, Je, Nic (Yg).

16051 L. insolens Horn. Newport, May-Je.(Yg); Suffern, May (Love); SI, J1 (L\&D).

\section{Glyptina LeConte}

16060 G. abbreviata Gent. Ithaca, Ap ( $\mathrm{Sf}-\mathrm{CU}$ ); Onondaga Co, May (H) ; Durlandville, May, FrostSW (Dt).

16061 G. spuria Lec. Ithaca, Ap-Je (Dt, Biss-CU) ; Albany, Je (Hoffman); Ft. Edward, Je (Biss-CU); Bronx Pk. (Sf); LI: Massapequa, May (Shoe).

\section{Phyllotreta Foudras}

16065 P. sinuata Steph. Sinuate-Striped Flea Beetle. Potsdam, Je (Yg); Hubbardsville, Je, Rochester Jct., May-Je, Ithaca, May-J1, Nov 13 (Biss-CU) ; Pike, Je (Yg); Ft. Montgomery, Je (Schott); SI, Je-J1 (L\&D) ; LI: Yaphank, May (Eng); Cold Sp. Harb. (Brb) ; Orient, Lath (NYS) ; Bay Shore (O1s).

16066 P. vittata Fab. Striped Cabbage Flea Beetle. Hubbardsville, Je (Biss-CU) ; Alexander, J1 (Biss-CU) ; Gloversville, Je (Yg) ; Clinton, May (Yg) ; Honeoye Falls, J1 (Biss-CU) ; Castile, Brad (Flet) ; Onondaga Co, May (H) ; Ithaca, May-Aug, Nov (Dt, Biss-CU); McLean, May-Je (Biss-CU) ; Ulster Co, Sep (Shoe); West Pt. (Robn); SI, Je, Aug (L\&D) ; LI: Nassau Co, Newtown, Ap (Biss-CU).

16068 P. robusta Lec. Cranberry. L., Htly $(\mathrm{H})$.

16074 P. bipustulata Fab. Newport (Yg); E. Aurora, May (Dnd); Westfield, May (Not) ; Ithaca, Ap, McLean, May (Biss-CU); West Pt. (Robn); Pine I., May (Schott); SI, Je (L\&D) ; LI: Rockaway Beach, May (Eng, Shoe); Brooklyn, Aug (Shoe).

16075 P. armoraciae Koch. Horse-Radish Flea BeEtle. Rochester Jct., Je (Biss-CU) ; Olcott, Ap-May (Dt) ; E. Aurora, May (Dnd) ; Schuyler Co, Je $(\mathrm{H})$; Ithaca, May, Bish (Yg, Biss-CU) ; McLean, Je (BissCU); Ringwood, J1, Y uasa (Vdyk); Schoharie, Je (Not); West .Pt. (Robn) ; LI: Rockaway Beach, May (Shoe); Flushing (Leon); Newtown, Ap (Biss-CU).

16076 P. chalybeipennis Cr. SI, Aug (Ds) ; LI: (Sf) ; Rockaway Beach, Aug, Oct (Schott, Shoe) ; Orient, Ap, Lath (Yg); Gardiners I., Je (Yg).

16081 P. picta Say. Ft. Montgomery, J1, Nyack, Je (Ds); SI (L\&D).

\section{Dibolia Latreille}

16086 D. borealis Chev. Peru, J1 (Biss-CU); Newport, May (Yg); Oneida L., Aug (Drk); N. Pulaski, Je (Ds) ; Rochester Jct., Je-J1 (Biss-CU); Olcott, Ap (Dt); Castile, Brad (Flet); Pike, Mar (NYS) ; Honeoye Falls, Ap, Je (Ds, Biss-CU) ; Westfield, May (Not); Mix Cr. Val. (Biss-CU) ; Rock City, Je (Ds, Biss-CU) ; Keuka Pt., Oct, Schurgers Gorge, Nov 24 (Biss-CU); Schuyler, Onondaga Co, Ap-Je, on herbage, flying $(\mathrm{H})$; Ithaca, 'Mar-Nov, Frost (Dt, Bks, Biss-CU); McLean, May-Je (Biss-CU); Schoharie, Je (Not); Slingerland, J1 (NYS); Albany, J1 (Yg) ; Cortland, J1 (Bks) ; Middletown, J1, Rhinebeck, J1 
(Biss-CU) ; West Pt. (Robn) ; Westchester Co (Ds) ; SI, Jan (Ds) ; LI: Rockaway Beach, Jl, Long Beach, May (Eng); Brooklyn, May, Massapequa, J1 (Shoe).

16088 D. libonoti Horn var. (?). Ringwood, Je ( $\mathrm{Sf}-\mathrm{CU}$ ); Slaterville, Je $(\mathrm{Sf}-\mathrm{BM})$.

\section{PSyLLIOdES Latreille}

16089 P. punctulata Melsh. Hop Flea Beetle. Newport, Glens Falls, Je (Yg); Hubbardsville, Je, Waterville, May (Biss-CU) ; L. Placid, Sep (Shoe) ; Howland I., Aug (Biss-CU) ; Honeoye Falls, Aug (Biss-CU) ; Rochester Jct., May-J1 (Biss-CU) ; Olcott, J1 (Dt) ; Westfield, May (Not) ; Potter Swamp, Aug (Biss-CU) ; Rock City, Je (Ds) ; Ithaca, Ap-Aug (Biss-CU) ; McLean, May-Je (Biss-CU) ; Madison Co, May, on herbage $(\mathrm{H})$; Ulster Co, Sep (Nic); Dormansville (Biss-CU); SI, Mar (L\&D) ; LI (Eng).

16090 P. convexior Lec. Ithaca, Ap, Nov (Biss-CU).

\section{SUBFAMILY HISPINAE}

\section{STENISPA Baly}

16094 S. metallica Fab. Poughkeepsie, May (Yg); Pine I., May (Schott) ; West Pt. (Robn); SI, May-Je (L\&D) ; LI: Brooklyn, Ji, Massapequa, Je (Shoe).

\section{ANOPLitis Chapuis}

16105 A. inaequalis Web. Newport, Je (Yg, Felt); Olcott, Je (Dt) ; Avon, Je, Aug (Cls); E. Aurora, Je (Dnd); Westfield, May (Not); Castile, Brad (Flet); Schuyler Co, Je (H); Ithaca, May-Sep, Yuasa (Vdyk, $\mathrm{Dt}$ ); Windsor, Je (Not); Trenton Falls, Je (Cy); Phoenicia, Aus (EPV); Albany, Je (Yg); Poughkeepsie, May (Yg); Nyack, Je (Ds) ; Ramapo, May, Ft. Montgomery, May (Ds); West Pt. (Robn); White Plains, all summer $(\mathrm{Sq})$, as nervosa Panz.; SI, Je (Ds) ; LI: Yaphank, May-Je (Eng); Flushing, Je, Aug (Eng, Ds) ; Fire I., Aug, Massapequa, J1 (Ds); Orient, Lath (NYS).

\section{Chalepus 'Thunberg}

16109 C. bicolor Oliv. West Pt. (Robn); Ft. Montgomery, Je (Schott); White Plains, May (Sq) ; SI, J1 (Ols) ; Watchogue, Sep (Ds) ; LI: Rockaway Beach, May (Eng).

16111 C. scapularis Oliv. White Plains, all summer ( Sq) ; Holersville, Je (Leon) ; West Pt. (Robn) ; SI, May-Je, Aug (Ds) ; LI: Yaphank, May, Flushing, Je, Rockaway Beach (Eng); Greenport, Aug (Eng, Ds); Wyandanch, J1 (Schott).

16113 C. dorsalis Thunb. E. Aurora, Sep (Dnd); McLean Bogs, Aug, Yuasa (Vdyk) ; Ithaca ; Schoharie, Je (Not) ; Nassau, Je (Hartman); Poughkeepsie, May. (Yg); West Pt. (Robn); SI, May-Je, Aug (Ds) ; LI: Hempstead Plains, May (Eng); south of Smithtown, Je, Wading R., May (Ds) ; Syosset, Aug (NYS) ; Central Pk., May, Yaphank, Aug (Ds) ; Rockaway Beach (Shoe); Oakdale, Aug (Schott) ; Wyandanch, J1, Oyster Bay, May (Schott); Jamaica, Aug (EPV); Orient, Lath (NYS).

16114 C. horni Sm. SI, on Tephrosia (Rbts). 


\section{Baliosus Weise}

16116 B. ruber Web. N. Pulaski, Je (Ds); Olcott, Je-J1 (Dt); E. Aurora, May-Je (Dnd); Conesus L., Je (Ds) ; Ithaca, May, Aug (Dt) ; Windsor, Je (Not); Bangall, Sep (Eng); West Pt. (Robn) ; SI, Ap-Oct (L\&D) ; LI: Yaphank, May, Promised Land, Sep (Eng) ; Rockaway Beach, May (Eng, Shoe); Wyandanch, J1, Little Neck, Ap (Schott); Orient, Sep (Ds, Schott); Wading R., Selden, Aug (Ds).

\section{UROPLATA Baly}

16126 U. porcata Melsh. "NY" (Dietz); Ithaca, Je (Hoffman); Karner, May (Yg); West Pt. (Robn); SI, Je-Ji (L\&D).

\section{MiCRORHOPALA Baly}

16129 M. vittata Fab. Honeoye Falls, Je (Ds); Onondaga Co, under stone (H) ; E. Aurora, Je, Wales, J1 (Dnd) ; Windsor, Je (Not); Thomson, May (Leon); Poughkeepsie, Jl, Kinderhook, Oct, Watervliet Reservoir, Nassau L., Sep (Yg); E. Jewett, Aug (Ds) ; West Pt. (Robn); White Plains, May-Je (Sq); SI, May, JI (Ds); LI: Jamaica (Vdyk); Forest Pk., Je ( $\mathrm{Lg})$; Rockaway Beach, Ap-May, Central Pk., Ap, Flushing, Je (Eng); Wading R., Je (Ds); Brooklyn, May (Shoe); Little Neck, Aug, Cedarhurst, Dec (Schott).

16130 M. xerene Newn. Greenwood L. (Sf) ; Oliverea, Je, Debruce, J1 (Shoe); West Pt. (Robn); Ramapo, May (Ds); White Plains, J1-Aug (Sq).

16133 M. excavata Oliv. Speculator, Je (Yg); L. Placid, Aug (EPV); Gloversville, J1 (Alex); Windsor, Je (Not); Oliverea, Je (Ds, Shoe); Debruce, J1 (Shoe); White Plains, May-J1 (Sq); West Pt., Je (Ds); Van Cort.

Pk. (Sf); SI, May (L\&D); LI: Cold Sp. Harb., J1 (Brb).

16135 M. cyanea Say. Cranberry L., Aug (Drk); Ithaca, Je (Dt).

\section{SUBFAMILY CASSIDINAE}

\section{CHELYMORPHA Boheman}

16139 C. cassidea Fab. Newport ( $\mathrm{Yg}$ ) ; Alder Cr., J1 ( $\mathrm{Yg}$ ); Ilion, May ( $\mathrm{Yg}$ ); Castile, Brad (Flet); Geneva, Utica, Aug (Cls); Ithaca, May-Ji (div.); Ringwood, J1, Y uasa (Vdyk); Schenectady, May, Poughkeepsie, May (Yg); Rensselaer. May (House); West Pt. (Robn); Ft. Montgomery, Aug (Schott); White Plains, May (Sq); SI, Ap, Je, Aug (Ds) ; LI: Flatbush, May-Je (Sf, Ds); Flushing, Dec, Wading R., Je (Ds); Rockaway Beach, May-Je, Sep (Shoe).

\section{PHYSONOTA Boheman}

16142 P. unipunctata Say. Blossom, Aug (Dnd) ; Ithaca, J1-Sep (Dt) ; Windsor, Je (Not).

16142a P. unipunctata var. quinquepunctata Walsh. LI (Eng).

Cassida Linnaeus

15147 C. flaveola Thunb. Suffern, May (Sf). 


\section{JONTHONOTA Spaeth}

16149 J. nigripes Oliv. Oneida L., Aug (Drk) ; West Pt. (Robn) ; LI: Rockaway Beach, May (Ds, Shoe).

\section{CHIRIDA Chapuis}

16152 C. guttata Oliv. Ilion, May ( Yg) ; Onondaga Co, May (H) ; E. Aurora, May-Sep (Dnd) ; Pike, Aug (Yg) ; Portageville, Je (Ds) ; Castile, Brad (Flet); Westfield, May (Not); Ithaca, Je-J1 (Dt, Vdyk) ; Windsor, Je (Not) ; Albany, May ( $\mathrm{Yg}$ ); West Pt. (Robn); White Plains, Je (Sq) ; Ramapo, Jl (Ds); SI, Je-Aug (Ds) ; LI: Long Beach, Je (Eng); Far Rockaway (Johnson); Flatbush, Mar, Orient, J1 (Ds) ; Rockaway Beach, May (Shoe); Wading R., May-Je (Ds, Schott); Glendale, Ap (Schott).

16152a C. guttata var. pennsylvanica Sp. Ithaca, Je (Dt); White Plains, Je (Sq); LI, Mar, Aug (Eng).

16152b C. guttata var. lucidula Boh. LI: Flatbush, Ap, Aug (Eng).

\section{DELOYALA Chevrolat}

16155 D. clavata Fab. Geneva, Mar-Je (Cls); Ithaca, Je-J1, Sep (Dt, Vdyk) ; Albany, Jl, Poughkeepsie, Je, Warwick, Jl (NYS); Amenia, Je (Yg) ; West Pt., Je-Jl (Eng, Ds); White Plains, Je (Sq); SI, Je (Ds); LI: Yaphank, Sep (Eng); Wading R., Je-J1 (Sf, Ds); Huntington, J1, Wyandanch, Je (Schott); Central Pk., May (Ds).

\section{Metriona Weise}

16157 M. bicolor Fab. Newport, Ogdensburg, Je, Ilion, May (Yg); Oneida L., Aug (Drk); western NY (Dnd); Schuyler, Onondaga Co, May-Je (H) ; Lowville (Ds) ; N. Pulaski, Je (Ds); Pike, J1 (Yg); Ithaca, May-Aug (Dt, Vdyk); Windsor, Je (Not); L. George, J1 (Yg); West Pt. (Rebn); White Plains, May (Sq); SI, May (Ds); LI: Flatbush, Mar (Eng); Whitestone, Sep (Hoffman); Rockaway Beach, May (Eng, Ds) ; Amagansett, Aug (Sf) ; Central Pk., May-Je (Shoe, Ds) ; Jamaica, Yaphank, J1 (Schott) ; Central Pk. (Ds) ; New Utrecht, Flushing, Gardiners I., Aug (Ds); Orient Pt., Je (Lath).

16159 M. purpurata Boh. Severance, Je (Bks); Syracuse, Sep (Drk); Rensselaer, May (Yg) ; Ilion, May (Yg) ; LI (Eng).

\section{FAMILY MYLABRIDAE}

\section{(Bruchidae)}

\section{MyLABRIS Mueller}

\section{(Bruchus Linnaeus)}

16165 M. pisorum L. PEA WEviL. Generally distributed throughout the State, infesting dried peas.

16166 M. rufimanus Boh. Broad BeAn Weevil. Buffalo (Z\&R); Poughkeepsie (WBDwight). 
16172 M. quadrimaculatus Fab. Four-spotted Bean WeEvil. NYC, in Russian peas. (Kaeber, Ent. News, vol. 24, p. 43, 1913.)

16175 M. discoideus Say. Cayuga L., Jl, swept from goldenrod and other flowers (Zab); SI, Je 19 (L\&D).

16188 M. cruentatus Horn. LI: Amagansett, Aug, Eng (Sf).

16190 M. nigrinus Horn. SI (L\&D); LI: Central Pk., Ap (Eng).

16211 M. longistilus Horn. LI, Eng (Sf).

16218 M. alboscutellatus Horn. SI, Je, Aug, Sep (L\&D) ; LI: Jamaica, J1, Eng (Sf).

16221 M. obtectus Say. Bean WeEvil. Generally distributed throughout the State, infesting dried beans.

16222 M. hibisci Oliv. SI, often common in seeds of the marsh mallow in Sep (L\&D) ; LI: Rockaway Beach, Dietz (Sf).

16231 M. calvus Horn. Ithaca, J1"23 (NYS) ; LI: Yaphank, J1, Oct, Hempstead Plains, May, Eng (Sf).

16236 M. musculus Say. Mosholu, Aug (NYS); SI, Je (L\&D).

$16244 \frac{1}{2}$ M. lentis Froel. Buffalo, in lentils in a store (Lint); Ithaca (CU).

\section{SPERMOPHAGUS Schönherr}

16246 S. hoffmannseggi Gyll. Ithaca, J1 (CU); SI, Nov, from seeds of honey locust (L\&D); Van Cort. Pk. (Sf).

\section{ZABROTES Horn}

16251 Z. subnitens Horn. LI: Yaphank, Je (Sf).

16254 Z. pectoralis Sharp. Perry, bred from S. Am. beans by I. M. Hawley (Vdyk).

\section{RHYNCHOPHORA 57}

\section{FAMILY BRENTIDAE}

\section{EUPSALIS Lacordaire}

16256 E. minuta Dru. Rochester (M\&W); Buffalo (Z\&R); Chautauqua, Je (Ds) ; Ithaca, Je, on oak and hemlock; West Pt., Je (Ds) ; Cairo, J1 (Lg) ; Bronx (Lg); SI, May-Sep, under oak, poplar, beech, maple, and chestnut (L\&D); LI, Je (Ds).

\section{FAMILY PLATYSTOMIDAE}

\section{ORMISCUS Waterhouse}

16263 O. saltator Lec. Rochester (M\&W) ; Buffalo (Z\&R); E. Schodack, J1 (NYS) ; Mosholu, Bronx, Je (Joutel); SI, Je, in dead wood of deciduous trees (L\&D); LI: Rosedale, Je (Nic).

57 The list in this group has, with the exception of the family Scolytidae, been compiled by Chas. W. Leng. He has checked all of the records as far as practicable, but is not to be held responsible for those not credited directly to him. About fifty species more have been recorded from neighboring States but are here omitted because of the lack of definite New York localities. Mr. Fall has looked over Apion; Mr. Satterthwait has contributed valuable records in Calendra; Mr. Nicolay and Mr. Buchanan have supplied many records throughout the group and have gone over the manuscript. 


\section{EUSPHYRUS LeConte}

16275 E. walshii Lec. Rochester (M\&W); Buffalo (Z\&R); Genesee Co, Je $(\mathrm{Lg})$; SI, Je, on hickory (L\&D).

\section{GoNot RopIs LeConte}

16278 G. gibbosus Lec. Big Moose, J1 (NYS).

\section{EURYMYCTER LeConte}

16279 E. fasciatus Oliv. Newport (NYS); Mt. Whiteface, J1 (Lg) ; Brant L., J1, Doll (Sf) ; Cranberry L. (H) ; Onondaga, Sep (NYS); Buffalo $(Z \& R)$; Pike (NYS); Castile, Je, Brad (Flet); Ithaca, Aug (Dt); Windsor, Je (Not) ; Harrison, J1 (Sf) ; SI, Mar, on fungus on dead beech.

\section{TROPIDERES Schönherr}

16282 T. bimaculatus Oliv. Rochester (M\&W) ; Bronx, Je (Joutel). On dead twigs (B\&L).

16283 T. rectus Lec. "NY".

AllandRus LeConte

16284 A. bifasciatus Lec. Rochester (M\&W) ; Buffalo (Z\&R); Ludlow (JCT); Washington $\mathrm{Co}, \mathrm{Jl}(\mathrm{Lg})$. On linden (B\&L).

\section{PIezocoRynus Schönherr}

16288 P. dispar Gyll. Buffalo, in fungus on dead beech (B\&L).

16290 P. mixtus Lec. Buffalo (Z\&R); Mosholu, J1 (Sf). From base of maple trees $(B \& L)$.

16291 P. moestus Lec. Mosholu, J1 (Sf).

\section{ANTHRIBUS Geoffroy}

16293 A. cornutus Say. Taughannock Falls, Aug (Hamilton); L. George, J1 (Zab); LI: Flatbush, Aug (Zab). On locust (Felt); on woody fungi (B\&L).

\section{EUPARIUS Schönherr \\ (Cratoparis Schönherr)}

16299 E. marmoreus Oliv. Streetroad, Je, Bks (NYS); Newport, May (NYS); Wells, Ap, Cy (NYS); Westport, Oct (NYS) ; Cranberry L., Je-Aug (H) ; Axton, Je (Houghton); Syracuse, on hickory (B1km) ; Rochester $(\mathrm{M} \& W)$; Olcott, May-Aug (Dt) ; Buffalo (Z\&R); Pike (NYS); Westfield, May (Not); Ithaca, May-Aug (Dt); West Pt. (Robn); Bronx, J1 (Joutel) ; SI, Ap-Jl, on woody fungus, Polyporus versicolor, on oak (L\&D) ; LI: Wyandanch (Sf). On fungi on maple and beech (Flet). 


\section{BRACHYTARSUS Schönherr 58}

16303 B. alternatus Say. Rochester (M\&W); SI, Sep (L\&D). Bred from fungus Cystopus.

16304 B. sticticus Boh. Potsdam, May (NYS); Mooers, Sep (Not) ; Cranberry L., Je $24(\mathrm{H})$; Rochester (M\&W) ; Buffalo (Z\&R) ; Westfield, May (Not); Ithaca, Ringwood, Howland I., May-Je (Dt); Shortsville (Lint) ; Ludlow, Sep (JCT) ; SI, Je, in smut of corn and wheat (L\&D).

16305 B. tomentosus Say. Cranberry L., Je (H) ; Rochester (M\&W); Ithaca, Aug; Windsor, Je (Not) ; Peekskill, Ludlow (JCT) ; West Pt. (Robn); Van Cort. Pk. (Sf) ; SI, Je, sometimes abundant on ragweed (L\&D) ; LI: Bellport, Aug (Nic).

16307 B. limbatus Say. "NY" (Sf).

16308 B. plumbeus Lec. "NY", Aug.

\section{ANTHRIBULus LeConte}

16315 A. rotundatus Lec. Bronxville, from gall of Drastrophus bassetti (AM); Van Cort. Pk., Aug (Sf); SI (L\&D); LI: Bellport, Aug (Nic).

\section{ARAEOCERUS Schönherr}

16316 A. fasciculatus DeG. NYC, introduced from Cayenne (B\&L).

\section{Choragus Kirby}

16317 C. sayi Lec. "NY", on beech (Jour. N. Y. Ent. Soc., vol. 1, p. 88).

16318 C. zimmermanni Lec. LI: Wading R., J1 (Sf). In fungus-infested twigs (B\&L).

[16320 C. nitens Lec. Mass. to D.C., on dead twigs (B\&L).]

\section{EUXENUS LeConte}

16322 E. punctatus Lec. Canada to D.C., on twigs (B\&L).

\section{FAMILY BELIDAE}

\section{ITHYCERUS Schönherr}

16324 I. noveboracensis Forst. The New York WeEvil. Newport, Je (NYS); Syracuse (Blkm); Buffalo (Z\&R); Ithaca, J1 (NYS); West Pt. (Ds, Robn) ; Rockland Co, Je (Joutel); West Farms (Zab); Van Cort. Pk., May-Je (Nic) ; SI, May-J1 (L\&D) ; LI: Jamaica (Nic); Bellport, May-Je (Nic); Yaphank, Wading R., Half Way Hollow Hills, May-J1 (Ds) ; Flatbush, J1 (Zab). On oak, hickory, beech $(B \& L)$; sometimes injurious to apple, pear, cherry, and plum orchards

${ }^{58}$ The larvae of Brachytarsus prey on Coccidae (Camb. Nat. Hist. Ins., p. 290). 


\section{FAMILY CURCULIONIDAE SUBFAMILY RHINOMACERINAE}

RHINOMACER Fabricius

16325 R. pilosus Lec. Karner, Ap (Felt); LI: Central Pk., Ap (Eng). On pine $(B \& L)$.

16326 R. elongatus Lec. Ringwood, Ithaca, May (Dt); Albany, Karner, Ap, Delmar, May (NYS); Broadalbin, Ap (NYS); LI: Pinelawn, Je (Eng).

16327 R. pallipennis Blatch. N.H. to Pa. (B\&L).

\section{SUBFAMILY RHYNCHITINAE}

\section{Auletes Schönherr}

16331 A. ater Lec. Buffalo ( $Z \& R)$; SI, on sweet fern and bayberry (L\&D); LI: Yaphank, Pinelawn, Je (Ds).

16338 A. albovestita Blatch. Cranberry L., Je (H); Oswego, J1-Aug (Pierce); Nassau, Salem, Aug (NYS); Peekskill, Sandy Hill (JCT); West Pt. (Robn); Dundee, Je (Hubbard\&Schwarz); LI: Bellport, Aug (Nic).

\section{EUGNAMPTUS Schönherr}

16343 E. collaris Fab. Newport, May-Je (NYS); Buffalo (Rein); Albany, Karner, Je (NYS); West Pt. (Robn); Mosholu, J1 (NYS); Van Cort. Pk. (AM) ; SI, Je-J1, on hickory (L\&D) ; LI: Cold Sp. Harb., $\mathrm{Jl}(\mathrm{H})$.

16343a E. collaris var. nigripes Melsh. SI, Je-J1, on hickory (L\&D); LI: Smithtown, Aug (Ds); Riverhead, Aug (Ds) ; Cold Sp. Harb., J1 (H).

16347 E. angustatus Hbst. Buffalo (Z\&R); Mosholu (Joutel); West Farms (Joutel); Bronxville (AM); SI, Je-Aug, on hickory leaves (L\&D); LI: Hempstead (AM).

\section{RHYNCHITES Herbst}

16348 R. bicolor Fab. The Rose Curculio of the Transition zone (B\&L). Rochester, J1 (AM); S. Byron, Je (NYS); Buffalo (Z\&R); Ithaca, Je; Karner, Je, Troy, J1 (NYS); Peekskill, J1 (Joutel) ; West Pt. (Robn); Yonkers, Ji (NYS); Mosholu, Je (Joutel); Bronx, Je (Joutel); SI, Je, on roses, the larvae in the hips (L\&D).

16351 R. aeneus Boh. Ft. Montgomery, J1 (B\&L); Rockland Co, Aug, Brorenell (CU).

16354 R. hirtus Fab. SI, Je-J1, on' blackjack oak (L\&D) ; LI: Yaphank, J1 (Ds).

16359 R. cyanellus Lec. Chazy L., L. Clear, Je (NYS); Fulton Co, May, on willow leaves; N. Chatham, Je, on willow (Felt); Callicoon (AM).

16361 R. aeratus Say. SI, J1, on oak (L\&D). 


\section{SUBFAMILy ATTELABINAE}

Attelabus Linnaeus 59

16365 A. analis Ill. Buffalo (Z\&R); Shokan (JCT) ; Poughkeepsie (Yg); West Pt. (Robn); SI, J1, on oak (L\&D); LI: Deep Pond, Wading R., May (Ds).

16365a A. analis var. similis Kby. Rochester (M\&W); White L., Sep (AM); Rock City, Je (Ds).

16366 A. nigripes Lec. White L., Aug (Zab); West Pt. (Robn) ; SI, May-Aug, on Rhus copallina (L\&D); LI: Yaphank, J1, Pinelawn, Aug (Ds); Half Way Hollow Hills, J1 (Ds).

16367 A. bipustulatus Fab. Buffalo (Z\&R); Albany (Felt); Sullivan Co (AM); West Pt. (Robn) ; LI: Yaphank, Pinelawn, Smithtown, Wading R., May-Aug (Ds). On oak (B\&L).

16369 A. rhois Boh. Windsor, Je (Not); Mt. Whiteface, J1 (Ds); West Pt. (Robn); Van Cort. Pk. (AM).

\section{SUBFAMILY PTEROCOLINAE}

\section{PTEROCOLUS Schönherr}

16370 P. ovatus Fab. Buffalo (Z\&R) ; Hastings, J1 (Joutel); Karner, Je (NNYS) ; West Pt. (Robn) ; SI, Aug, on oak (L\&D); LI: Yaphank, May, on oak (B\&L); Wyandanch, Deep Pond, Wading R., May-J1 (Ds).

\section{SUBFAMILY APIONINAE}

\section{APION Herbst}

16373 A. impeditum Fall. Winḋsor, Je (Not); Peekskill, type locality (Fall).

16376 A. impunctistriatum Sm. Breeds in stems of Heterotheca and Ambrosia (B\&L).

A. bischoffi Fall. SI (Fall).

16379 A. atripes Sm. Schoharie (Not).

16380 A. finitimum Fall. Mass. to D.C. (Fall).

16382 A. melanarium Gerst. Westfield, May (Not); SI, Je-J1 (L\&D); LI (Fall).

16389 A molestum Fall. Orangeburg, May (Nic).

16390 A. minutum Sm. "NY" (JBSm).

16392 A. pennsylvanicum Boh. Windsor, Je (Not); Shokan, Ludlow, Je-J1 (JCT).

16396 A. perminutum Sm. Mass. to Fla. (Fall).

16397 A. reclusum Fall. N. J. to D. C. (Fall).

16422 A. patruele Sm. Lake Bluff, Sep (NYS); LI: Rosedale, in sifting in winter (Nic).

16423 A. walshii Sm. (vicinum Sm.). Newport, May-Je (NYS); Cranberry L., Htly (Buch); Axton, Je (Houghton); Mt. Seward, Je (Houghton); Clinton Hgts., Je (NYS); NYC (AM); LI: Rosedale, Je (Nic).

59 The larvae in this genus roll leaves into compact thimble-shaped masses, on Corylus, Je-J1 (Fitch); also on oak, sumac, etc. (Blatch\&Lg). 
16429 A. turbulentum Sm. Ithaca (Fall); Schoharie (Not); Peekskill (Fall). Lives in seeds of Meibomia (B\&L).

16431 A. griseum Sm. Schoharie (Not); Peekskill (Fall); SI, Nov, in pods of Phaseolus polystachyus (L\&D). On Lespedeza under name of fraternum (Chitt).

16439 A. porcatum Boh. Schoharie (Not).

16441 A. rostrum Say. Buffalo (Z\&R); Ithaca (Chitt); Peekskill (B\&L) ; SI, Je-Oct, on Baptisia (L\&D).

16443 A. nigrum Hbst. Albany, Je (NYS); NYC (AM); SI, J1, in seeds of Robinia pseudacacia (L\&D); LI: Bellport, Je (Nic) ; Syosset, Aug (NYS).

16457 A. segnipes Say. SI, Sep, in pods of Tephrosia (L\&D); LI: Bellport, Je (Nic).

16468 A. decoloratum Sm. Canandaigua, Oct (NYS). Breeds in seeds of Meibomia (B\&L).

16469 A. emaciipes Fall. N. H. to Ill. (Fall).

16472 A. carinatum Sm. "NY" (Fall).

16473 A. attenuatum Sm. Canada to southern Cal. (Fall).

16474 A. hibisci Fall. "NY" ( $\mathrm{Lg}$ ). Lives in seeds of Hibiscus (Weiss).

16480 A. parallelum Sm. Mass. to D. C. (Fall).

16486 A. puritanum Fall. Buffalo (Fall); SI, Je, on Viburnum (L\&D).

16488 A. herculanum Sm. Genesee Co, Je ( $\mathrm{Lg}$ ); Letchworth Pk., J1 (NYS); West Pt. (Robn); Catskills (Nic); Peekskill (B\&L); SI, on witchhazel (Bischoff).

16489 A. molestus Fall. Orangeburg, May (Nic).

\section{PODAPION Riley}

16490 P. gallicola Rly. Mass. to Cal. ( $\mathrm{Lg})$. On pine (B\&L).

\section{SUBFAMILY TACHYGONINAE}

\section{TACHYGONUS Schönherr}

16492 T. lecontei Gyll. N. J. to Tex. ( Lg).

16493 T. spinipes Csy. LI: Bellport, Je, beating scrub oak (Nic); Yaphank, on oak (B\&L). T. tardipes of the Buffalo list is a western species.

\section{SUBFAMILY OTIORHYNCHINAE}

\section{PHYXELIS Schönherr}

16499 P. rigidus Say (glomerosus Boh.). Potsdam, Newport, Deer R., May (NYS) ; Gloversville, Aug (NYS) ; Albion, Sep (NYS) ; Lakeside, Orleans Co, Je, Cy (NYS); Buffalo (Z\&R); Pike (NYS); Genesee Co, Je (Lg); Ithaca, Ap, Bish (Dt-NYS); Binghamton, Ap (JCT); Windsor, Je (Not); Albany, Sep (NYS); Poughkeepsie, May (NYS) ; West Pt. (Robn); Ft. Montgomery, Jl (Ds) ; Nyack, Je (Ds); Mosholu, Je (Joutel); Ramapo, May (Ds); Carmel, Aug (AM) ; SI, Jan-Aug (L\&D); LI: Flatbush, Je (AM); Forest Pk., Je (NYS). 
ANAMETIS Horn

16547 A. granulata Say. Buffalo (Z\&R); Portage, May (B\&L) ; Kenwood, J1 (NYS).

\section{PANSCOPUS Schönherr}

16576 P. erinaceus Say. Buffalo (Pierce); Washington Co, Aug (Sherm); SI, J1 (L\&D) ; LI: Bellport, Aug, Wading R., May (Nic).

16578 P. maculosus Blatch. Washington Co (B\&L); Ft. Montgomery, May (Ds).

\section{TANYMECUS Germar}

16596 T. confertus Gyll. (canescens Gyll.). Buffalo (Z\&R); SI, Je-Jl, in stems of Ambrosia (L\&D).

\section{PANDELETEIUS Schönherr}

16605 P. hilaris Hbst. Buffalo (Z\&R) ; Letchworth Pk., J1 (NYS) ; Genesee Co (Lg) ; Ithaca, May, Aug (Dt); Albany, Karner, Je (NYS) ; Nassau L., May (NYS) ; Lansingburg, Canaan, Je (NYS) ; West Pt. (Robn) ; Ludlow, Sep (Lg) ; Ramapo, May (Lg) ; Mosholu (AM) ; SI, Je-Aug, abundant on oak (L\&D) ; LI: Wading R., Amagansett (Ds).

\section{POLYDRUSUS Germar}

\section{(Cyphomimus Horn)}

16621 P. americanus Gyll. (dorsalis Horn). Newport, May (NYS); Genesee Co, Je (Lg) ; Buffalo (Z\&R) ; Portage, May, on beech (B\&L) ; Westfield, May (Not); Westview, Jl, Cy (NYS); Ithaca, May (Dt, H) ; Otsego (Pierce); Schoharie (Not); Delmar, May (NYS) ; Orangeburg, Ap, very abundant, flying on certain days in spring (Nic).

16622 P. impressifrons Gyll. THE LEAF WeEvil. Introduced from Europe, and first found at Geneva and Lyons in 1906. Beetles feed on foliage of willow, poplar, birch, rose, apple, and pear, and are sometimes injurious in nursery plantings. Known only from N. Fair Haven $(\mathrm{H})$; Lyons, Sodus; Castile, Brad (Flet); Waterloo, Geneva, Seneca Castle, Rochester, Rochester Jct., Honeoye Falls, Indian Falls, Oak Orchard, Albion, LeRoy, Batavia, and Portage.

\section{SCYTHROPUS Schönherr}

16626 S. elegans Couper. Wilmington, Je (NYS) ; Rochester, Je (AM) ; Forest Lawn, J1 (AM) ; Buffalo (Lg); Ithaca, Ap-Jl, on pine (Chitt) ; Font Grove, Albany Co, Je (NYS); Nassau, Je (NYS); Callicoon, Je (AM).

\section{HORMORUS Horn}

16635 H. undulatus Uhl. Newport, Je (NYS); Axton (Houghton) ; Cranberry L., Je (H) ; Genesee Co, Je (Ds) ; Ithaca, Je (B\&L) ; Windsor, Je (Not); Albany, Je, Poughkeepsie, May (NYS) ; West Pt. (Robn); Van Cort. Pk. (Nic) ; SI, Je, Aug (L\&D); LI: Jamaica (Nic) ; Flatbush, Je (B\&L). 
SCIAPHILUS Schönherr

16640 S. muricatus Fab. Syracuse, under decayed hickory twigs (Blkm).

STROPHOSOMA Billberg

16641 S. coryli Fab. SI, Mar (L\&D); LI (B\&L).

EPICAERUS Schönherr

16646 E. imbricatus Say. LI: Flatbush (B\&L).

PANTOMORUS Schönherr

(Aramigus Horn)

16659 P. fulleri Horn. Fuller's Rose Beetle. Severance, Je, Bks (NYS); Herkimer Co (NYS); Little Falls, Oct, on greenhouse carnations (B\&L); Albany, Poughkeepsie (Lint); SI (L\&D); LI (AM).

\section{BARYNOTUS Germar}

16671 B. schönherri Zett. "NY" (Fitch).

BARYPEITHES Jacquelin-Duval

16672 B. pellucidus Boh. Syracuse, May (H, Blkm); Rochester, Je (NYS); Kendall, May (NYS); Genesee Co, Je ( $\mathrm{Lg})$; Batavia, Je (B\&L); Lakeside, Je, Cy (NYS); Letchworth Pk., Jl (NYS); Portage, Je (Ds) ; Geneva, Je (NYS) ; Ithaca, May, Bks (Dt) ; DeRuyter L., J1 (NYS); Albany, Voorheesville, Je, Leon (NYS); Van Cort. Pk. (AM) ; SI (div.) ; LI: Amityville, Astoria, May (Bt) ; Cold Sp. Harb., Je (Ds); Bellport, Je (Nic).

\section{AgRAPHUS Say}

16675 A. bellicus Say. SI: Tottenville, Je (NYS) ; LI: Rockaway, Babylon, JeSep (B\&L).

\section{BRACHYRHINUS Latreille}

\section{(Otiorhynchus Germar)}

16677 B. sulcatus Fab. Black Vine Weevil. Buffalo, Feb (NYS) ; Westfield, Aug (NYS) ; Ithaca, Mar, Sep (Dt) ; Albany, Mar (NYS); Hudson, Aug (Lg); Oliverea, Oct (NYS); Middle Hope, J1 (CU Econ); Peekskill, Ap (Hubbard\&Schwarz); Nyack, Sep (Lg); Van Cort. Pk. (AM) ; SI, J1, Nov (L\&D) ; LI: Greenport, Aug, Flushing, Jan-Dec (Ds); Westbury, larvae injurious to yew; Central Pk. (Southwick); Orient Pt., May (NYS). Larvae sometimes injurious to yew hedges.

16678 B. ovatus L. (ligneus Horn). Strawberry Crown-Girdier. Potsdam (NYS) ; Keene Val., J1 (NYS) ; Severance, J1, Bks, Wells, Jl (NYS); New Russia, Ap (NYS); Mooers, Sep (Not) ; Newport, May (NYS); Lyons Falls, JI (NYS) ; Long L. (Ds) ; Hastings (NYS) ; Clyde, Sep, Bish (NYS) ; Kendall, May (NYS) ; Niagara (Lg); Wyoming, May, 
infesting house (Lint); Chautauqua (Lg); Castile, Brad (Flet); Ithaca, McLean, Ringwood, Je-Nov (Dt); Ilion (NYS); L. George (AM) ; Appalachin, May (NYS) ; Nassau, Mar (NYS); Karner, Aug (NYS) ; Millerton, May (NYS) ; Carmel, Aug (AM) ; Huguenot, J1 (Zab); West Pt. (Robn) ; Peekskill (NYS) ; Mosholu, Je (Lg); Van Cort. Pk. (AM) ; SI, J1-Aug (L\&D) ; LI: Moriches (NYS); Orient Pt., Sep (NYS). Often injurious to strawberries.

16679 B. rugifrons Gyll. Genesce Co, Je ( $\mathrm{Lg})$; Letchworth Pk., J1 (NYS) ; Portage, Ap-Aug (B\&L); McLean, Mar, Aug-Sep (Dt); Westfield, May (Not); Ithaca, Ap-Aug (B\&L) ; Enfield Glen, Aug (NYS); Hubbardsville, Sep, Leon, injuring strawberries (CU Econ, NYS); Woodland, Aug (NYS); Ulster Co, general (CU Econ). Larvae sometimes injurious to strawberries.

CERCopeus Schönherr

16692 C. chrysorhoeus Say. SI (AM).

\section{Chaetechus Horn}

16694 C. setiger Horn. Barnerville, Aug (NYS).

TRACHYPHLOEUS Germar

16697 T. davisi Blatch. SI, type locality, on seashore, Ap (Ds).

\section{GEODERCES Horn}

16708 G. melanothrix Kby. Wilmington Notch, Aug, Cy (NYS).

\section{APHRASTUS Say}

16721 A. taeniatus Gyll. Hastings, J1 (Lg) ; Albany, Je, Slingerlands, J1 (NYS) ; Karner, J1 (Felt); Stony Pt., Je (AM); Mosholu, Je (NYS); Van Cort. Pk. (AM) ; SI (L\&D) ; LI: Flatbush (AM) ; Fishers I. (AM) ; Orient Pt., Je (NYS).

\section{BRACHYSTYLUS Schönherr}

16724 B. acutus Say. "NY" (Lg), on persimmon.

\section{PHYLLOBIUS Germar}

16726 P. glaucus Scop. "NY", Aug.

P. oblongus L. The European Snout Beetle. Found in 1923 in considerable numbers injuring elms at Rochester, this being the first record of its occurrence in this country (Leon).

\section{SUBFAMILY CURCULIONINAE}

\section{SitonA Germar}

16727 S. flavescens Marsh. Newport, May-Je (NYS); Saranac Inn, Aug, Gloversville, Mar (NYS) ; Sodus Pt., Aug (NYS) ; Buffalo (Z\&R); Westfield, J1 (NYS); Castile, May, Brad (Flet); Penn Yan, May, Cy 
(NYS) ; Ithaca, J1 (NYS) ; Juanita I., L. George, Sep, Leon (NYS) ; Voorheesville, Aug, Leon (NYS) ; Poughkeepsie, May (NYS); West Pt. (Robn) ; NYC, Je (Ds) ; SI, Je, Sep, on clover roots (L\&D) ; LI: Riverhead, Sep, Jamaica, Je (NYS).

16728 S. hispidulus Fab. Clover-Root Curculio. Cranberry L., J1 (H) ; Westfield, May (Not) ; Syracuse, Ap-May (H) ; Conesus L., Aug (Ds); Ithaca, Spencer, Ringwood, McLean, Je-Nov (Dt) ; L. George, J1 (AM) ; Schoharie (Not); New Baltimore (AM); West Pt. (Robn); Nyack, Tomkins Cove (AM) ; SI, Ap-Dec, on clover roots (L\&D) ; LI: Coney I., on beach (Linel1); Flatbush (AM).

16738 S. tibialis Hbst. Newport (NYS); Buffalo (Z\&R); Ithaca, J1 (NYS).

\section{LEPIDOPHORUS Kirby}

16752 L. setiger Ham. Letchworth Pk., J1 (NYS).

\section{HYPERA Germar}

16754 H. punctata Fab. Clover-Leaf Weevil. Mooers, Sep (Not) ; Cranberry L., J1 (H) ; Westfield, May (Not); White L. (AM) ; Forest Lawn (AM) ; Castile, Ap, Brad (Flet) ; Pike, May (NYS) ; Chautauqua (Lg); Canandaigua, J1 (NYS) ; Ithaca (Lg) ; McLean, Mar-Sep (Dt); Freeville, Aug, in beating spruce and heather; Albany, J1, Karner, Aug, Delmar, May, Phoenicia, Aug (NYS) ; West Pt. (Robn); Big I. (AM) ; Nyack, Sep (Brownell); SI, Je-Sep, feeds on clover; LI: Long Beach (AM); Rockaway, J1, Flatbush, Babylon, Fishers I. (AM). Thirty-eight more references (CU), four more (Ds).

\section{PHYTONOMUS Schönherr}

16755 P. eximius Lec. LI: Aqueduct Station, Brooklyn, May 16, 1925, on leaves of mullein, Nic, Mason, Quirs (Nic).

16757 P. comptus Say. Pulaski, Je (Ds) ; Buffalo (Z\&R) ; West Pt., Ap (Robn) ; Ft. Montgomery, May-JI' (Ds) ; Ramapo, May-Jl (Ds); SI, Ap-May, feeds on Polyg.onum in wet places (L\&D) ; LI: Flatbush, J1 (AM).

16764 P. meles Fab. Clover-Head Weevil. Franklinton, Je, Leon (NYS); Ithaca, Je (NYS); Windsor, Je (Not); Albany (CU); Schoharie (Not); New Baltimore (Felt); West Pt., Ap (Robn) ; Orangeburg, Je (Nic) ; Port Chester, Jl (NYS); White Plains (CU) ; Van Cort. Pk. (AM) ; SI, Je (L\&D) ; LI: Brooklyn (Lg); Rockaway Beach, May (Ds) ; Rosedale, Bellport, Je (Nic) ; Yaphank, Half Way Hollow Hills, May, J1 (Ds).

16765 P. nigrirostris Fab. Lesser Clover-Leaf Weevil. Axton (Houghton); Newport, May-Je (NYS) ; Bolton, Aug (JCT) ; Syracuse, Ap, flying (H) ; Westfield, May (Not); Ithaca (Detwiler); Ringwood, Ap-J1 (Dt); Windsor, Je (Not) ; Schoharie (Not) ; Albany, Je, Karner, Je, Clinton Hgts., Mar (NYS); West Pt. (Robn) ; Huguenot (AM); Van Cort. Pk. (AM) ; SI, Ap-Je, feeds on clover (L\&D) ; LI: Babylon, Flatbush (AM); Rockaway (Nic). Twenty-two more records (CU). five more (Ds). 


\section{LEPYRUS Germar}

16767 L. palustris Scop. (geminatus Say). Cranberry L., Je-J1, beaten from birch and willow $(\mathrm{H})$; Genesee $\mathrm{Co}, \mathrm{Je}(\mathrm{Lg})$; Batavia, J1 (B\&L); Buffalo (Z\&R).

\section{LISTRONOTUS Jekel}

16772 L. obliquus Lec. Barren I., J1 (AM).

16773 L. sordidus Gyll. Buffalo (Z\&R); Van Cort. Pk. (AM) ; SI, Aug, on subaquatic plants (L\&D).

16774 L. tuberosus Lec. Ithaca, Ap (B\&L); Orangeburg, May (Nic).

16775 L. squamiger Say. Buffalo (Say).

16776 L. callosus Lec. "NY" (Lec).

16778 L. inaequalipennis Boh. Buffalo (Z\&R); West Pt. (Robn); SI, May (L\&D) ; LI: Rockaway Beach, Je (Shoe).

16779 L. caudatus Say. Buffalo $(Z \& R)$; Ithaca, Je ( Lg) ; Albany, Sep (NYS) ; LI: Rosedale, Ap, Rockaway, Aug (Nic).

16783 L. appendiculatus Boh. N. Fair Haven, Aug (NYS) ; Buffalo (Z\&R) ; Peekskill (Lg); Van Cort. Pk. (Nic); SI, May-Aug (Woodr).

16786 L. frontalis Lec. "NY" (Lec).

16790 L. latiusculus Boh. Parsley Stalk-Weevil. Ithaca, Je (B\&L) ; Charlotte (AM) ; Nyack, Je (Ds); SI, J1, on Sagittaria (L\&D) ; LI: Valley Stream, Astoria, Suffolk Co, Ji, infesting carrots and arrowhead (CU); Babylon (AM); Rockaway, Je (Nic).

\section{HYPERODES Jekel}

\section{(Macrops Kirby)}

16801 H. solutus Boh. Buffalo (Z\&R); Ithaca, J1 ( $\mathrm{Lg})$; SI, Aug, on arrow $\operatorname{arum}(\mathrm{L} \& \mathrm{D})$.

16803 H. indistinctus Dietz. SI, Aug, on Sagittaria (Woodr) ; LI: Jamaica (Sirrine).

16819 H. delumbis Gyll. Buffalo ( $Z \& R)$.

16821 H. sparsus Say. Axton (Houghton); Buffalo (Z\&R); Monroe Co, May (H) ; Westfield, May (Not) ; Ithaca, Je (NYS); Windsor, Je (Not); New Baltimore (AM) ; Catskills (Nic); West Pt. (Robn); LI: Flatbush, May (AM); Jamaica (Nic).

16823 H. rotundicollis Dietz. Westfield, May (Not).

16824 H. obscurellus Dietz. Westfield, May (Not); Ithaca, Jan (Dt) ; LI: Brooklyn (B\&L).

16826 H. imbellis Dietz. Ithaca, Aug (Chitt).

16835 H. humilis Gyll. Axton (Houghton); Batavia, May (B\&L) ; Westfield, May (Not); West Pt. (Lg).

\section{PISSODES Germar}

16840 P. similis Hopk. Wallface Mt., J1 (Nic).

16845 P. strobi Peck. White-Pine Weevil. Undoubtedly present wherever white pine grows in the State. Representative localities are: Wells, Je, Harrisville, Jan (NYS) ; Cranberry L., J1 (H) ; Genesee Co, Je; Buf- 
falo (Z\&R) ; Ithaca ( $\mathrm{Lg})$; Albany, May, Salem, Ap, N. Chatham, May, Broadalbin, Ap (NYS) ; Poughkeepsie, Je (NYS); Slide Mt., J1 (Lg) ; New Baltimore (Hopk); West Pt. (Robn); Kiamesha (Hopk) ; Rhinebeck, Oct (NYS); SI, JI (L\&D); LI: Central Pk. (Eng).

16846 P. approximatus Hopk. Wallface Mt., J1 (Nic); near NYC (Hopk). On pine (B\&L).

16858 P. nigrae Hopk. Wallface Mt., J1 (Nic). On black spruce (B\&L).

16866 P. dubius Rand. Big Moose, on spruce (Felt); Saranac Inn, Jan (NYS); Cranberry L., Je-J1, common in dying balsam $(\mathrm{H})$; Old Forge, Je (NYS).

16868 P. affinis Rand. Wells, J1 (NYS) ; Buffalo (Z\&R) ; Genesee Co, Je (Lg) ; Ithaca, May, on white pine (B\&L, Chitt); Bath-on-Hudson, J1 (Felt); Callicoon (AM); West Pt. (Robn).

\section{PACHyLobius LeConte}

16872 P. picivorus Germ. (stupidus Boh.). Buffalo (Z\&R); southern NY (Fitch); LI: Yaphank, May-Sep, on pine (B\&L).

\section{Hylobrus Germar}

16873 H. pales Boh. The Pales Weevil. Severance, J1, Wells, Je-J1, Sep, Saranac Inn, Je (NYS); Old Forge, Je (NYS); Buffalo (Z\&R); Ithaca (Lg); Ringwood, Je-J1 (Dt); Karner, Oct (NYS); New Baltimore (AM) ; West Pt. (Robn); SI, Ap, Sep, on pine (L\&D); LI: Central Pk. (Eng) ; May-Sep (Ds).

16874 H. confusus Kby. Axton (Houghton) ; Cranberry L., Je-Jl (H) ; Saranac Inn, J1 (NYS) ; Buffalo (Kby); Albany, Je (NYS).

\section{HyPomoLyX LeConte}

16879 H. piceus DeG. (pinicola Couper). Windsor, Je (Not); Mt. Whiteface, Sep, Je ( $\mathrm{Lg})$; Wallface Mt., J1, crawling in deep dark woods and under bark at high elevations only (Nic); Mt. Marcy, Oct (B\&L); NYC, Je-J1 (B\&L).

\section{EUdocIminus Leng}

16880 E. mannerheimi Boh. "NY" (Chitt).

\section{EuCLYPTUS Dietz}

16883 E. testaceus Dietz. Buffalo, type locality (Dietz).

\section{DoRyTOMUs Germar}

16893 D. mucidus Say. Buffalo (Z\&R).

16894 D. laticollis Lec. Keene Val., Je (NYS) ; Cranberry L., Je (H) ; Buffal (Z\&R) ; Callicoon (AM) ; West Pt. (Robn) ; near NYC, on poplar LI: Rockaway Beach, wash-up, Je (B\&L). 
16897 D. parvicollis Csy. Ithaca, J1, Oct, Cy (NYS); Karner, under loose bark of poplar, May (Felt) ; Pomona, Ap (NYS) ; SI, on willow and aspen (L\&D).

16909 D. indifferens Csy. "NY" (B\&L).

16910 D. vagenotatus Csy. Karner (Felt); Albany, Ap (NYS); LI: Long Beach, Je 7 (Nic).

16913 D. fusciceps Csy. Buffalo (Z\&R); Callicoon (AM); West Pt. (Robn); Mosholu (AM) ; NYC, Mar-Nov, on pussy willow (B\&L); LI: Central Pk., Ap (Eng).

16914 D. brevicollis Lec. Cranberry L., Htly (Buch); Ithaca, May (Dt).

\section{GRYPIDIUS Schönherr}

16919 G. equiseti Fab. Batavia, Je (B\&L) ; Sport I., Je (B\&L) ; Westfield, May (Not).

\section{NOTARIS Germar \\ (Erycus Tournier)}

16922 N. puncticollis Lec. Newport, May-Je (NYS); Batavia, May-Je, on arrow arum (B\&L); Buffalo (Z\&R); Portage, Mar-Je, on arrow arum (B\&L) ; Montour Falls, Je (H); Westfield, May (Not); Ithaca, Aug, Nov (NYS); West Pt. (Robn); Albany, Ap (NYS); NYC, Mar-Ap (B\&L); LI: Brooklyn, May (Ds); Long Beach, Aug (Lg); Rosedale, May-Je (Nic).

\section{PACHYPhanes Dietz}

(Barytychius Jekel, Pachytychius LeConte not Jekel)

16925 P. discoideus Lec. "NY", May-J1 (Chitt, Dietz).

16929 P. lineolatus Csy. "NY" (?).

16931 P. amoenus Say. Van Cort. Pk. (AM); SI, Aug-Sep (L\&D).

\section{SMICRONYX Schönherr}

16972 S. connivens Csy. Mass. to Mo. (NY?).

16973 S. tychioides Lec. "NY" (Dietz).

16976 S. sculpticollis Csy. LI: Rosedale, May, sifting sphagnum in swamp (Nic), on dodder galls (B\&L).

16991 S. corniculatus Fahr. LI: Jamaica (Nic).

\section{BRACHYBAMUS Germar}

17012 B. electus Germ. NYC, May-Aug, on palustral plants (B\&L) ; LI: Wyandanch, J1 (Ds); Bellport, Je, in salt meadow (Nic).

ENDALUS Laporte

17014 E. limatulus Gyll. Buffalo (Z\&R); Aurora, J1 (Zab) ; Stony Pt., Je (Ds) ; NYC, May-Sep (B\&L); SI, May (L\&D); LI: Bellport, Je-J1, in sweeping salt meadow (Nic).

17019 E. ovalis Lec. Near NYC, J1-Aug, on palustral plants (B\&L); SI, Je (L\&D). 


\section{TANYSPHYRUS Germar}

17021 T. lemnae Fab. Old Forge, Je, Speculator, J1 (NYS) ; Ringwood, Je-J1 (Dt); Schoharie (Not); Albany, Je (NYS); Orangeburg, May (Nic); West Pt. (Robn); near NYC, on duckweed, May-Je (B\&L); SI, May, on duckweed (L\&D).

\section{ONYCHYLIS LeConte}

17022 O. nigrirostris Boh. Buffalo (Z\&R); Ramapo (AM); NYC, May-Dec (B\&L) ; SI, May, on arrowhead and pickerel weed (L\&D).

\section{ANCHODEMUS LeConte}

17025 A. angustus Lec. Buffalo (Z\&R).

\section{LISSORHOPTRUS LeConte}

17029 L. simplex Say (apiculatus Gyll.). The Rice Water Weevil. Near NYC, May-J1 (B\&L); SI, May, on salt meadow, feeds on roots of palustral plants $(L \& D)$.

\section{BAgous Germar}

17030 B. magister Lec. West Pt. (Robn).

17036 B. restrictus Lec. Ithaca, May 5 (B\&L).

17037 B. americanus Lec. Spencer L., Aug (Dt); L. George (Buchanan-US. Biol. Surv.).

17039 B. mammillatus Say. Windsor, Je (Not).

17042 B. planatus Lec. "NY", one of LeConte's type localities.

17043 B. obliquus Lec. Newport (NYS); Fulton Co (B\&L).

\section{PROCTORUS LeConte}

17057 P. decipiens Lec. Cranberry L., Je, beaten from willow $(H)$; Catskills (Nic-AM).

THYSANOCNEMis LeConte

17058 T. fraxini Lec. Buffalo (Z\&R); Ithaca, Je, in seeds of ash (B\&L) ; West Pt., Je (B\&L) ; LI: Rockaway, Aug (Nic); Long Beach, Aug (Lg).

17060 T. bischoff Blatch. Batavia, Je (Kngt); commonly confused with fraxini (B\&L).

17062 T. ocularis Csy. Menands, J1 (NYS).

\section{Plocetes LeConte}

17068 P. ulmi Lec. Batavia, Je (B\&L) ; West Pt., Je (B\&L) ; LI: Rosedale, Je (Nic).

\section{TychiUs Germar}

17074 T. picirostris Fab. (griseus Sf.). The Clover-Seed Weevil. Plattsburg, J1 (Ds); Newport, May-Je (NYS); Wells, J1 (NYS); Speculator (Felt); Mooers, Sep (Not); L. Champlain (Felt); Gouverneur 
(Felt); Waterville (B\&L); Oswego (Felt); Rochester, May (CU Econ); Batavia (B\&L) ; West Pt., May (Not); Ithaca, Ap-J1, in red clover (B\&L, Detwiler); McLean (B\&L) ; Windsor, Je (Not); Schoharie (Not); Corinth, May (NYS); Albany, May, J1 (NYS) ; New Baltimore, May (Felt); Orangeburg, May (Nic) ; Mosholu, Je (NYS).

\section{OTIDOCEPHALUS Chevrolat ${ }^{59 a}$}

17118 O. myrmex Hbst. (americanus Chev.). Newport, May-Je (NYS) ; Rochester (M\&W); Buffalo (Z\&R); Nassau, Je (NYS) ; Poughkeepsie, Je (NYS); West Pt. (Robn) ; SI, Je-J1, on oak (L\&D) ; LI: Jamaica (Nic).

17121 O. chevrolati Horn. Cranberry L., Htly (Buch); Rochester (M\&W) ; Buffalo (Z\&R); Schoharie (Not); Nyack, Je-J1 (Ds); SI (L\&D); LI: Yaphank, Je-J1 (Ds) ; Bellport, J1 (Nic).

17123 O. scrobicollis Boh. Buffalo (Z\&R) ; West Pt. (Robn) ; LI: Yaphank, J1 (Ds, B\&L).

17125 O. laevicollis Horn. Genesee Co, Je ( Lg) ; Buffalo (Horn) ; Bear Mt., I1 (Nic) ; Ft. Montgomery, J1 (Ds); Nyack, Je; Rockland Co, Je (Joutel) ; SI (L\&D).

\section{MAGDALIS Germar}

17129 M. barbicornis Latr. Newport, J1 (NYS) ; Lockport, J1 (NYS) ; Genesee Co, Je (Lg) ; Rensselaer, May (NYS); SI, Je, on elm (L\&D); LI (Fall).

17133 M. perforata Horn. Karner, Je-J1,, on pine (Felt); Lansingburg, Dec (NYS) ; SI, on pine (L\&D) ; LI: Bellport, Je-Aug (Nic); Yaphank, J1 (Ds).

17136 M. austera Fall. Wallface Mt., J1 (Nic); SI, J1, on pine (L\&D). ( $M$. lecontei records probably belong here.)

17136 a M. austera var. substriga Fall. Wallface Mt., J1 (Nic) ; Peekskill (B\&L) ; West Pt., on pine (Robn); Bear Mt., J1 (Nic); LI: Bellport, Je (Nic).

17141 M. alutacea Lec. Cranberry L., Htly (Buch); Ithaca, J1 (NYS) ; Karner, Je-J1 (Felt).

17146 M. olyra Hbst. Syracuse, on hickory (Blkm); Buffalo (Z\&R); Ithaca, May (Lg) ; Ilion, Je, on hickory (Felt) ; SI, May, on hickory (Chitt); LI: Wading R., May (Nic).

17147 M. pandura Say. Newport, May-Je (NYS) ; Syracuse, on hickory (B1km) ; Catskills (Nic) ; Poughkeepsie, J1 (NYS); Nyack, Je (Ds); SI, Je, on elm, oak, walnut, etc. (L\&D).

17148 M. inconspicua Horn. Buffalo (Rein); West Pt., Je, on butternut (Robn) ; Westchester Co, Je ( Lg); Congers, J1 (Lg).

17149 M. salicis Horn. Wells, May (NYS); Buffalo (Z\&R); Ithaca, J1 (Ham).

17150 M. barbita Say. Newport, May-Je (NYS) ; Buffalo (Z\&R) ; Ithaca, May (NYS) ; Albany, May-Je; Lansingburg, May (NYS); W. Nyack, Je.

17151 M. armicollis Say. Newport (NYS) ; Oswego, Je, Leon (NYS) ; Buffalo (Z\&R); Castile, Brad (Flet); Ithaca, Je (NYS); West Pt. (Robn); Catskills (Nic); Westchester Co, Je ( Lg).

17151a M. armicollis var. pallida Say. Westchester Co, Je (Lg).

59a Myrmex Sturm replaces Otidocephalus, fide H. S. Barber. 


\section{BALANINUS Germar}

17155 B. proboscideus Fab. (caryatrypes Boh.). The Large Chestnut Weevil. Glenmont, Sep (NYS); Albany, Oct, larva in chestnut (Fernow); Van Cort. Pk. (Nic); SI, nearly extinct, owing to death of trees (L\&D) ; LI: Rockaway; Kings Pk., Sep (Ds).

17156 B. caryae Horn. The Hickory-Nut Weevil. Batavia, Sep (B\&L) ; West Pt. (Robn) ; SI (L\&D) ; LI: Brooklyn (Horn).

17157 B. rectus Say (quercus Horn). THE ACORN WeEvIL. Rochester (M\&W) ; Buffalo (Z\&R) ; Ithaca, Sep (NYS) ; Karner, Sep (NYS); West Pt. (Robn) ; SI, Aug-Sep (L\&D) ; LI: Brooklyn (Okhurst); Rockaway Beach, Sep (Ds).

17158 B. algonquinus Csy. The Smaller Chestnut Weevil. Perry, J1 (H); West Pt. (Robn) ; possibly extinct on SI (L\&D).

17159 B. nasicus Say. Rochester (M\&W); Buffalo (Z\&R); Ithaca, Ap (Dt); Karner, Je (Felt); West Pt. (Robn); Huguenot (AM) ; SI, Je-Aug, on oak (L\&D); LI: Long Beach (AM); Rockaway, Je-Aug (Nic).

17160 B. pardalis Chitt. West Pt. (Robn).

17161 B. confusor Ham. Batavia, J1 (B\&L) ; West Pt., Je (Ds) ; Vań Cort. Pk., Je-Sep (AM) ; LI: Riverhead, Aug (Ds) ; Central Pk., Je, on Quercus minor (Ds) ; Babylon, Rockaway (AM).

17162 B. baculi Chitt. (uniformis auct. not Lec.). Buffalo (Lec) ; Ithaca, on Quercus alba (Chitt); Albany, J1 (NYS) ; Poughkeepsie, J1 (NYS) ; SI, Sep, on oak (L\&D) ; LI: Rockaway Beach, Sep, Central Pk. (Ds).

17164 B. obtusus Blanch. Taughannock Gorge, Aug (Ham); LI: Rockaway, Aug (Nic).

17170 B. strictus Csy. Bear Mt., J1, Nic (Chitt, with query).

B. auctus Csy. Buffalo, a single male (Csy).

\section{TACHYPTERELLUS Fall and Cockerell}

17186 T. quadrigibbus Say. The Apfle Curculio. Genesee Co, Je (Lg); Buffalo (Z\&R) ; Pike, J1 (NYS); Ithaca, May, Aug (Dt) ; McLean, May, Bish (NYS); West Pt. (Robn); SI, May-Je, on shadbush, thorn, and fruit trees (L\&D); LI: Bellport, Je (Nic).

\section{AnTHONOMUS Germar}

17189 A. scutellaris Lec. The Plum Gouger. Rochester (M\&W) ; Karner, May (NYS) ; SI, May, on beach plum (L\&D) ; LI: Yaphank, Je (Nic).

17194 A. pusillus Lec. "NY" (B\&L) ; Peekskill (Sherm), as A. hamiltoni Dietz.

17202 A. profundus Lec. Cranberry L., Je (H) ; Ringwood, May (Dt) ; SI, Je, on Crataegus Crus-galli (L\&D); LI: Massapequa, May, Shoe (Lg).

17203 A. rubidus Lec. Rochester (M\&W) ; Buffalo (Z\&R).

17204 A. nebulosus Lec. Batavia, May-J1, found on flowers of Crataegus (B\&L); Portage (B\&L); Ithaca, J1 (H); Ringwood, May (Dt).

17210 A. rufipennis Lec. Windsor, Je.(Not); LI: Rosedale, Je 3 (Nic).

17212 A. haematopus Boh. (sycophanta Walsh). Cranberry L., Aug, on willow (H) ; Newport, May-Je (NYS) ; Buffalo (Z\&R) ; Letchworth Pk., Je (NYS) ; Rock City, Je; on laurel (Ds) ; Danby, Freeville, Ithaca, Mar, Je-Jl (Dt); Windsor, Je (Not); Scotia, Je (NYS) ; Remsen, Je (NYS); Valley Falls, May (NYS); Albany, Je (NYS); W. Albany (Lint); LI: Jamaica (Nic). 
17213 A. suturalis Lec. Rochester (M\&W) ; Buffalo ( $Z \& R)$; Ithaca, May (Dt); West Pt. (Robn); Ramapo, Je (Ds) ; Orangeburg, May (Nic) ; West Farms (Lg); near NYC (B\&L); LI: Yaphank, Je (Ds).

17214 A. flavicornis Boh. Near NYC (B\&L).

17217 A. corvulus Lec. Axton (Houghton); Newport (NYS); Ithaca, May (Chitt); McLean, May (Dt); N. Chatham, Nassau, Je (NYS); near NYC (B\&L); SI, on dogwood, Prunus, Viburnum (L\&D).

17218 A. subguttatus Dietz. "NY" (Julich).

17219 A. signatus Say. The Strawberry Weevil. Axton (Houghton); Newport, May (NYS) ; Cranberry L., Je-J1, beaten from Rubus (H) ; Mt. Whiteface, Sep (Lg); Montour Falls, Je (H); Ithaca, Ringwood, May-Je (Dt) ; Ilion, May (NYS) ; Trenton Falls, Je (NYS); S. Bethlehem, May (NYS) ; Poughkeepsie, J1 (NYS); Red Hook, Je, Leon (NYS) ; Mosholu, Oct (NYS) ; SI, injurious in 1884 and 1885 (Chitt) ; LI: Bellport. Je (Nic); Forest Pk., Je. (NYS).

17221 A. consimilis Dietz. Windsor, Je (Not).

17224 A. musculus Say. Cranberry L., Je (H) ; Newport, May-Je (NYS) ; Buffalo (Z\&R); Windsor, Je (Not); Albany, Je (NYS); Orangeburg, May, the most common species in sifting (Nic); SI, on strawberry (Rly) ; LI: Wyandanch, Ap (Nic).

17231 A. interstitialis Dietz. Wallface Mt., J1 (Nic).

17236 A. nigrinus Boh. SI, on horse nettle (L\&D).

17245 A. scutellatus Gyll. Genesee $\mathrm{Co}, \mathrm{Je}(\mathrm{Lg})$; Batavia, Je (B\&L); Ithaca (Chitt); West Pt. (Robn) ; SI, Je-Aug (L\&D).

17247 A. orchestoides Dietz. Newport, May-Je (NYS); L. Clear, Je (NYS) ; N. Chatham, Je (NYS); Poughkeepsie, Je (NYS).

17250 A. ater Lec. Hamburg, Aug (NYS).

17258 A. rufipes Lec. Batavia, J1-Aug (B\&L).

17259 A. disjunctus Lec. Charlotte, Je (AM) ; Peekskill (Sherm) ; LI: Montauk, Sep (Ds).

17267. A. subfasciatus Lec. "NY" (Lec).

17268 A. robustulus Lec. (moleculus Csy.). Portage, May-J1 (B\&L); Windsor, Je (Not) ; Juanita I., L. George, J1 (NYS); Sandy Hill (B\&L).

17269 A. robinsoni Blatch. Batavia, Je (B\&L); West Pt., Ap (B\&L).

17270 A. ungularis Lec. "NY" ( $\mathrm{Lg}$ Cat.).

17273 A. nubilus Lec. Cranberry L., Je-J1, beaten from Rubus (H) ; Wallface Mt., J1 (Nic) ; Woodworth's L., J1 (NYS) ; New Lebanon, J1 (Frost).

17290 A. elongatus Lec. Canastota (B\&L); Peekskill (Lg); Orangeburg, May (Nic); Nyack, Je, Westchester Co (Lg).

\section{PSEUdANTHONOMUS Dietz}

17299 P. crataegi Walsh. Cranberry L., Htly (Buch); Wallface Mt., Jl (Nic); Buffalo (Z\&R); Westfield, May (Not); L. George, Aug (Zab); Albany, J1 (NYS).

17300 P. validus Dietz. Ithaca (Chitt) ; LI: Bellport, Je (Nic).

17301 P. hamamelidis Pierce. Genesee Co, Je ( $\mathrm{Lg})$; Conesus L., Je, on witchhazel $(\mathrm{B} \& \mathrm{~L})$; L. George, Aug (B\&L).

17303 P. incipiens Dietz. Speculator, J1, Newport, May-Je (NYS); Buffalo (Horn); Ithaca, May-Aug (Dt); Nassau, May (NYS); SI, on blueberry and laurel (Bischoff). 


\section{ELLESCHUS Stephens}

17333 E. bipunctatus L. Cranberry L., Htly (Buch); Fourth L., Je (NYS).

17334 E. scanicus Payk. West Pt. (Robn); LI: Bellport, Je, in sweeping salt marsh (Nic).

17335 E. ephippiatus Say. Buffalo (Z\&R); Nassau, May, on willow (Felt); SI, May-J1, bred by Walsh from willow galls (L\&D).

\section{ORCHESTES Illiger}

17337 O. ephippiatus Say. Cranberry L., Je-Aug, beaten from Salix (H) ; Newport, May (NYS) ; Herkimer Co, May-Je, on willow (Felt) ; Rochester (M\&W) ; Batavia, May-Sep (B\&L) ; Junius, Je (NYS) ; Buffalo $(Z \& R)$; Ithaca, May (NYS) ; Freeville, Je (Dt) ; Schoharie (Not); Rensselaer, May (NYS) ; Mosholu, Je (NYS); Van Cort. Pk. (Nic); SI, on Salix fragilis (L\&D); LI: Jamaica (Nic).

17338 O. salicis L. Cranberry L., Htly (Buch); North Creek, Aug (NYS).

17339 O. niger Horn. Newport, Labrador Pond, Je (NYS); Wells, J1 (NYS) ; Cranberry L., Je, on blossoms of Viburnum (H) ; Herkimer Co, MayJe, on willow (Felt) ; Rochester (M\&W) ; Woodworth's L., J1 (NYS) ; Batavia, May-Je (B\&L); Buffalo (Z\&R); Portage, May-Je (B\&L); Ithaca, Freeville, McLean, May-J1 (Dt); Windsor, Je (Not) ; Rensselaer, May (NYS) ; Ramapo, Je (Ds); West Pt. (Robn) ; SI, Je (L\&D) ; LI: Jamaica (Nic).

17343 O. canus Horn. Genesee Co, Je ( $\mathrm{Lg})$; Katonah (Sherm).

17345 O. pallicornis Say. Apple Flea-Weevil. Newport, Speculator, May-Je (NYS) ; Cranberry L., Je (H) ; Onondaga, May-Je, injuring apples, $C y$ (Flet) ; Wyoming, May, injuring apples, $C y$ (Flet); Ithaca, McLean, Ringwood, May (Dt); Windsor, Je (Not); Albany, May (NYS); Valley Falls, May (NYS); LI: Bellport, Je (Nic).

17347 O. rufipes Lec. Buffalo (Z\&R); Batavia, J1-Sep (B\&L) ; Chautauqua (JCT) ; Ithaca, J1, Bks (NYS).

\section{ACALYPTUS Schönherr}

17351 A. carpini Hbst. LI: Jamaica (Nic).

\section{PRIONOMERUS Schönherr}

17352 P. calceatus Say. Buffalo (Z\&R); West Farms (Lg); SI, Je-J1, larvae mining leaves of sassafras and tulip (L\&D) ; LI: Jamaica, Je, Mineola, J1 (NYS).

\section{PIAZORHINUS Schönherr}

17353 P. scutellaris Say. Newport, May-Je (NYS) ; Cranberry L., Je, Htly (Buch, H); Axton (Houghton); Rochester (M\&W); Genesee Co (Lg) ; Buffalo (Z\&R) ; Bear Mt., J1 (Nic) ; Peekskill (Lg) ; Nassau, Je (NYS) ; Ft. Montgomery (Lg); SI, May-Je (L\&D).

17354 P. pictus Lec. Buffalo (Z\&R); SI, Nov, on oak (L\&D); LI: Bellport, Aug (Nic). 


\section{GYMNETRON Schönherr}

17359 G. tetrum Fab. Cranberry L., Je (H); Chazy L., Je (NYS); Newport, Aug (NYS) ; Rochester (M\&W) ; Lakeside, Je, Cy (NYS) ; Buffalo (Z\&R); Pike, Je (NYS); Montour Falls, Je (H); Westfield, Je (NYS) ; Castile, Brad (Flet); Penn Yan, Je (NYS) ; Schuylerville, Je (NYS) ; Ithaca, J1 (Ham); W. Albany, Karner, Nassau, Je (NYS); Wappingers Falls, Je (NYS) ; Ludlow (JCT); West Pt. (Robn) ; SI, on mullein, larvae in seed pods (L\&D).

G. tetrum var. subrotundatum Reitter. Dunkirk (Pierce).

\section{MIARUS Stephens}

17360 M. hispidulus Lec. Carmel, Aug (AM).

\section{CLeonus Schönherr}

17376 C. plumbeus Lec. Gloversville, Ap, Aug (NYS); Mosholu (Sf).

17389 C. calandroides Rand. LI: Wading R., breeds on stems of the sea rocket (B\&L).

\section{LIXUS Fabricius}

17405 L. rubellus Rand. N. Fair Haven, Danby, McLean, May-J1 (Dt) ; Watervliet Reservoir, Aug, Leon (NYS).

17407 L. lupinus Blatch. Karner, May (NYS).

17414 L. marginatus Say. "NY".

17415 L. musculus Say. Ithaca (Lg); SI, May-Je, bred from Polygonum amphibium by Kellicott (L\&D).

17418 L. concavus Say. The Rhubarb Curculio. Syracuse, May, Schuyler Co, Je (H) ; Ithaca, Je (Lg); Albany, Kenwood, Je (NYS); Slingerlands, J1 (NYS) ; Coeymans ( $\operatorname{SmEB}$ ) ; Nassau, Je, Marlboro, May, Kingston, Mar (NYS) ; West Pt. (Robn) ; Florida, May (Ham) ; SI, May-Je, on stalks of dock and rye, Sep (L\&D); LI, J1 (Lg); Orient, J1 (NYS).

L. crassulus Not. Schoharie (Not), type.

17439 L. terminalis Lec. West Farms (B\&L); Bronxville (B\&L); LI (Csy).

\section{LAEMOSACCUS Schönherr}

17443 L. plagiatus Fab. Rochester (M\&W); Genesee Co, Je (L\&D); Buffalo (Z\&R) ; Ft. Montgomery, J1 (Ds) ; Bronx, Nic (Joutel) ; SI, Je-J1, also hatched from oak gall, Callirhytis cornigera, in Mar (L\&D); LI: Flatbush, Je-J1 (Zab); Half Way Hollow Hills, J1 (Ds).

\section{BARIS Germar}

B. fultoni Csy. West Pt. (Csy).

17447 B. umbilicata Lec. Huguenot, L. George (AM) ; West Pt. (Robn) ; SI, J1-Aug (L\&D); LI: (Csy); Brooklyn (Nic).

17454 B. callida Csy. (stremua Lec. of Buffalo list). Rochester (M\&W); SI, Sep, in stems of Xanthium (L\&D); LI: Rockaway, May (Ds). 
17458 B. tumescens Lec. "NY" (Lec, B\&L).

17462 B. subaenea Lec. Van Cort. Pk. (Nic); LI: Jamaica, Bellport (Nic).

17468 B. interstitialis Say. Schuylerville, Schenectady, J1 (NYS); W. Albany, J1, Karner, Je (NYS) ; Kenwood, J1 (NYS) ; West Pt., May (B\&L); Mosholu, Je (NYS).

17475 B. dolosa Csy. LI (Csy).

17489 B. confinis Lec. Ithaca, May-Je (Dt); LI: Long Beach (AM).

17497 ?B. aerea Boh. West Pt. (Robn).

17505 B. scolopacea Germ. Ithaca, J1, Ndm (Flet-CU); SI, J1 (L\&D); LI: Rockaway Beach, Je (Eng); Huntington (AM); Brooklyn, J1 (Nic). An introduced species.

\section{ONYCHOBARIS LeConte}

17539 O. pectorosa Lec. (subtonsa. Lec.). West Pt. (Robn).

\section{Madarellus Casey}

17542 M. undulatus Say. Rochester (M\&W) ; Forest Lawn, Sep (Zab) ; Buffalo (Z\&R) ; Ithaca (B\&L) ; Nassau, May (NYS); Catskills (Nic); West Pt. (Ds) ; Ludlow (JCT) ; Van Cort. Pk., Sep (NYS) ; SI, Aug-Sep, breeds in woodbine (L\&D); LI: E. Greenbush, May (NYS); Yaphank, Oct, Greenport (Ds) ; Amagansett, Sep (Ds) ; Shelter I. (Ds).

M. inconstans Csy. "NY" (Csy).

AUlobaris LeConte

17546 A. pusilla Lec: Mooers, Sep (Not); West Pt. (Robn).

\section{AMPELOGLyPTER LeConte}

17550 A. ater Lec. West Pt. (Robn).

\section{PSEUdobaris LeConte}

17561 P. angusta Lec. Batavia (B\&L); West Pt. (Robn); near NYC (AM).

17562 P. nigrina Say. Newport, May-Je (NYS); Johnstown, Sep (NYS); Buffalo (Z\&R) ; Voorheesville, Aug (NYS); Poughkeepsie, May (NYS); West Pt. (Robn); Mosholu, Oct (NYS); SI, Sep (L\&D).

\section{TRICHOBARIS LeConte}

17566 T. trinotata Say. The Potato Stalk-Borer. SI, Aug, in stem of Solanum (L\&D):

\section{Geraeus Pascoe \\ (Centrinus auct.)}

17580 G. picumnus Hbst. Nassau, Aug (NYS); West Pt. (Robn); Ludlow (JCT); Van Cort. Pk. (AM); SI, Je, Sep, in stems of grasses (L\&D); LI: Forest Pk., Je (NYS).

17591 G. penicellus Hbst. Mosholu, Sep (NYS). 


\section{ODONTOCORYNUS Schönherr}

\section{O. fultoni Csy. "NY" (Csy).}

O. semiruber Csy. "NY" (Csy).

17597 O. scutellum-album Say. Newport, May-Je (NYS); Rochester (M\&W); Buffalo (Z\&R); Point Breeze, Je (NYS); Schenectady, J1, Karner, Aug, Albany, J1, Poughkeepsie, Je (NYS); Catskills, J1 (Blkm); Peekskill (Sherm); Bolton (JCT); West Pt. (Robn) ; Van Cort. Pk., Aug (NYS); SI, Je, Aug (L\&D).

O. subabruptus Csy. LI (Csy).

O. amputatus Csy. "NY" (Csy).

O. parvus Csy. "NY" (Csy).

17599 O. salebrosus Csy. Buffalo (Csy) ; LI: Cold Sp. Harb., Je (H).

\section{Centrinopus Casey}

17602 C. alternatus Csy. Castile, Brad (Flet).

\section{NicentRus Casey}

17608 N. lecontei Champ. (lineicollis Csy.). Ithaca (Chitt).

17609 N. lineicollis Boh. Ithaca, J1 (Dt); Karner, Je (NYS).

\section{* Centrinites Casey}

17616 C. strigicollis Csy. Ithaca, Je $(\mathrm{H})$.

\section{LIMNOBARIS Bedel}

L. fultonica Csy. "NY" (Buch, Csy).

17634 L. confusa Boh. "NY" (Csy).

17637 L. confinis Lec. "NY" (B\&L).

17646 L. rectirostris Lec. Schoharie (Not); SI, Je, on sedges (L\&D).

17648 L. calva Lec. LI (B\&L).

\section{IDIOSTETHUS Casey}

17651 I. tubulatus Say. LI: Jamaica (Nic).

I. strigosicollis Csy. "NY" (Csy.)

\section{STETHOBARIS LeConte}

17659 S. ovata Lec. Ithaca, J1 (NYS).

ZAGLYPTUS LeConte

17662 Z. sulcatus Lec. SI, J1 (L\&D).

\section{DIORYMERELLUS Champion}

17694 D. laevimargo Champ. Has been introduced into greenhouses on orchids in one or two localities in the State. 


\section{Gelus Casey}

17699 G. oculatus Say. Speculator, Je (NYS); Genesee Co, Je (AM); Buffalo $(Z \& R)$; McLean, May (Dt); Nassau, May (NYS); L. George, Aug (AM) ; Big Ind. Val., Je, Pears (NYS); Catskills (Nic); LI: Jamaica (Nic).

17701 G. subfasciatus Lec. "NY" (Guex).

\section{CyLindRocoptuRus Heller}

17712 C. binotatus Lec. Ithaca, Aug (Dt); near NYC (B\&L).

17719 C. quercus Say. Rochester (M\&W) ; Ludlow (JCT); Bronx (Joutel); near NYC (B\&L); SI, Je-Aug, on oak (L\&D).

17721 C. longulus Lec. SI, J1, on oak (L\&D).

\section{EULECHRIOPS Faust}

17723 E. minutus Lec. Karner, Je; Mosholu, Aug (NYS); LI: Forest Pk., Je (NYS).

\section{Acoptus LeConte}

17726 A. suturalis Lec. Newport, May-Je, Wells, J1 (NYS) ; Cranberry L. (H) ; Rochester (M\&W); Wilmington, $\mathrm{Jl}(\mathrm{Lg})$; Genesee Co, Je ( $\mathrm{Lg})$; Buffalo. (Z\&R); Westfield, May (Not); Ithaca, Ringwood, May-Je (Dt) ; Big Ind. Val., J1, Pears (NYS) ; West Pt. (Robn) ; Orangeburg, Ap (Nic); Van Cort. Pk. (Nic); near NYC (B\&L); SI, May-Jl, on beech, hop hornbeam, and hickory (L\&D).

\section{Psomus Casey}

17727 P. armatus Dietz (politus Csy.). Schoharie (Not).

\section{MONONYCHUS Germar}

17728 M. vulpeculus Fab. Rochester (M\&W) ; Point Breeze, Je (NYS); Buffalo (Z\&R); Castile, May, Brad (Flet); Pike (NYS); Poughkeepsie, May (NYS) ; Peekskill (Lg); West Pt. (Robn); Bronx Pk (AM); SI, May-Je, breeds in seed pods of Iris (L\&D); LI, Je (Lg).

\section{CRAPONIUS LeConte}

17729 C. inaequalis Say. GraPe-Seed Curculio. West Pt. (Robn); near NYC $(B \& L) ; S I, J e(B \& L)$.

\section{Cnemogonus LeConte}

17730 C. lecontei Dietz. Mt. Whiteface, J1 (B\&L).

\section{ACANThoscelis Dietz}

17735 A. curtus Say. L. Placid, Aug (NYS) ; Newport, May-Je (NYS) ; Lakeside, Aug, Cy (NYS) ; Buffalo (Z\&R); Pike, Je (NYS); near NYC, on Polygonum in swamps, May-J1 (B\&L). 
17736 A. acephalus Say. Newport, Je (NYS); Rochester (M\&W); Buffalo $(Z \& R)$; West Pt., Je (Ds); S. Dover, J1 (Lg); near NYC, MaySep (B\&L) ; SI, on evening primrose (L\&D); LI: Rockaway, Coney I. $(\mathrm{Lg})$.

\section{Auleutes Dietz}

17743 A. asper Lec. Windsor, Je (Not).

17744 A. tenuipes Lec. West Pt., May (Robn); LI: Bellport; Je (Nic).

17745 A. ater Dietz. Bronx (Joutel); SI, J1 (L\&D).

17751 A. cruralis Lec. (related to European epilobii Payk.). Wallface Mt., J1 (Nic); Cranberry L. (H).

17753 A. nebulosus Lec. Axton (Houghton); Newport, Speculator, Aug (NYS); Remsen, Je (NYS); Genesee Co, Je (Lg); West Pt. (Robn) ; Mosholu, Sep (AM); SI, J1 (L\&D).

\section{HYPOCOELIODES Faust}

\section{(Orchestomerus Dietz)}

17755 H. ulkei Dietz. NY is type locality.

\section{Acallodes LeConte}

17757 A. ventricosus Lec. Orangeburg, May (Nic); near NYC, May-J1 (B\&L).

17758 A. lysimachiae Fall. LI: Rosedale, Ap, Je (Nic).

\section{COELIODES Schönherr}

17760 C. flavicaudis Boh. (apicalis Dietz). Newport, May-Je, Johnstown, Sep (NYS); Ithaca (Lg); Schoharie (Not); Nassau, May (CU).

\section{Ceutorhynchus Germar}

17762 C. rapae Gyll. (affluentus Dietz). Cabbage Curculio. Newport (NYS); Rochester (M\&W) ; Buffalo (Z\&R); Ithaca (Lg); Albany, Je (NYS); Albany, Je (NYS); Ludlow. (Lg); West Farms (Lg); SI, on cabbage seed-stalks, hedge mustard, peppergrass, etc. (L\&D).

17766 C. quadridens Panz. (seriesetosus Dietz). Cabbage Sefi-Stalk Weevil. Introduced. Peekskill (AM); LI: Cutchogue, bred from seed-stalks of kale (Sirrine); fairly common and often rather injurious in the cabbageseed-growing sections about Riverhead and Mattituck.

17767 C. marginatus Payk. Cranberry L. (H) ; Onondaga Co, May-Je, on herbage $(\mathrm{H})$; Wallface, J1 (Nic); Genesee Co, Je (Lg); Schuyler Co, May-Je (H) ; Ithaca, Mar-J1 (B\&L); Ringwood, McLean, Jan, May-Je (Dt) ; Catskills, Ap (Flet); Bear Mt., J1 (Nic).

17781 C. sulcipennis Lec. SI (L\&D).

17783 C. cyanipennis Germ. Johnstown (B\&L); Portage, May-J1, attacking seeds of cruciferous plants (B\&L); McLean, May, Je (Flet); Ithaca (div.); Ringwood, May (Dt); Windsor, Je (Not); Sport I. (B\&L).

17784 C. bolteri Dietz (erysimi auct. nec Fab.). Buffalo (Z\&R); Ithaca, May (B\&L). 
17791 C. omissus Fall. Cranberry L., Htly (Buch).

17797 C. neglectus Blatch. Gloversville, Je (NYS) ; Batavia, Je-J1 (B\&L) ; SI, Je-J1, on Polygonum (L\&D).

$17812^{\circ}$ C. semirufus Lec. Newport, May (NYS); Buffalo (Dietz); Batavia (B\&L) ; Windsor, Je (Not); Portage, May (B\&L); Poughkeepsie, May (NYS); Suffern (AM).

17817 C. septentrionalis Gyll. Newport, May-Je (NYS) ; Rochester (M\&W) ; Nassau, Poughkeepsie, May (NYS); West Pt. (Robn); Orangeburg, May (Nic).

17818 C. puberulus Lec. Newport, May (NYS) ; Clayville, Je, Leon (NYS); Schoharie (Not); Nassau, Poughkeepsie, May (NYS); West Pt. (Robn) ; Orangeburg, May (Nic).

17820 C. zimmermanni Gyll. West Pt. (Robn); LI: Riverhead, Ap (NYS).

C. punctiger Sahl. Westfield, May (Not); Windsor, Je (Not); Schoharie (Not).

\section{Dietzella Champion \\ (Coelogaster Schönherr)}

17822 D. zimmermanni Gyll. W. Hebron ( Lg); Peekskill, Je (Sherm); Bronx, Je (Joutel); near NYC (AM); LI: Flatbush, Je-J1 (Dietz).

\section{Perigaster Dietz}

17824 P. cretura Hbst. SI, Je-J1, on Ludwigia (L\&D) ; LI: Rockaway Beach, Je-J1, Flatbush (B\&L).

17825 P. obscurus Lec. Ithaca, Je-Sep (Dt).

\section{PELENOMUS Thomson}

17828 P. sulcicollis Fahr. Monroe Co, May (H) ; Ithaca, May, McLean, J1, E. Schenectady, J1 (NYS); Windsor, Je (Not); Poughkeepsie, Aug (NYS) ; Shandaken, Je, Cy (NYS); Orangeburg (Nic); Westchester Co, May (Lg); Van Cort. Pk. (AM); SI, Ap, Jl, on Polygonum (L\&D) ; LI: Jamaica (Nic) ; Rosedale, Je (Nic).

17831 P. griseus Blatch. Westfield, May (Not).

17832 P. squamosus Lec. Rochester (M\&W); Ithaca ( $\mathrm{Lg})$; LI: Rockaway, May (Ds).

\section{RHINONCUS Schönherr}

17841 R. pericarpius Fab. Ithaca, Je (NYS); Van Cort. Pk. (Nic); SI, Je, on Saponaria (L\&D).

17842 R. pyrrhopus Boh. Axton (Houghton); Speculator, Je, Newport, May, Wells, J1, Old Forge, J1 (NYS); Mt. Whiteface (Lg); Wallface Mt., J1 (Nic) ; Syracuse (H) ; Cranberry L. (H) ; Buffalo (Z\&R) ; Pike, Je (NYS) ; Ithaca, J1 (Lg); McLean, May-Aug (Dt); Windsor, Je (Not) ; Schoharie (Not); Albany, May, Karner, J1, Phoenicia, Aug, Corinth, Je, Cooperstown, May (NYS) ; Callicoon (AM) ; W. Hebron (Lg); Rockland Co (Lg); Ramapo (Ds) ; Ludlow (Lg); SI, Je-Jl, common on Rumex (L\&D); LI: Rockaway, J1, Sep (Ds); Yaphank (Ds) ; Aqueduct, Oct (Ds).

17843 R. longulus Lec. Ithaca, Je (B\&L); Van Cort. Pk. (Nic). 


\section{AMALUS Schönherr}

A. haemorrhous Hbst. Syracuse, $H^{\prime}$ (L.L.Buchanan). European; this is the first U. S. record.

\section{PhyRdenus LeConte}

17848 P. divergens Germ. (undatus Lec.). "NY" (Csy).

\section{CONOTRACHELUS Schönherr}

17851 C. nenuphar Hbst. Plum Curculio. Widely distributed and abundant in the State. Newport, Je (NYS); Onondaga Co, flying (H); E. Palmyra (Lint); Rochester (M\&W) ; Monroe Co (Lint) ; Genesee Co, Je (Lg); Buffalo (Z\&R); Pike (NYS); Westfield, Je (NYS); Castile, Brad (Flet); Penn Yan, Je (NYS); Schuyler Co, flying (H); Seneca Co (Felt) ; Ithaca, Ringwood, May-Aug (Dt); Enfield Falls, on wild cherry (CU) ; Petersburg (Lint) ; New Baltimore, Je (AM); Orange Co (Lint); SI, May-Aug; LI: Setauket (Felt). Breeds in plum, peach, cherry, etc., often causing great loss (L\&D).

17852 C. juglandis Lec. Walnut Curculio. Newport, Je (Felt); Rochester $(\mathrm{M} \& W)$; Genesee $\mathrm{Co}$, Je ( $\mathrm{Lg})$; Buffalo $(\mathrm{Z} \& \mathrm{R})$; Cattaraugus Co, J1 (Ds); Ithaca, Aug (Dt); Phoenicia, Aug (NYS); Poughkeepsie (Felt); West Pt., on walnut and butternut (Robn); Ft. Montgomery, May (Lg); Van Cort. Pk. (Nic); LI: Jamaica (Nic).

17856 C. affinis Boh. Albany, J1 (CU).

17858 C. seniculus Lec. Poughkeepsie, May-Je, on hickory (Felt); Mosholu, Oct (NYS).

17859 C. elegans Say. Rochester (M\&W) ; Buffalo (Z\&R); Ithaca, Ringwood, May-Je (Dt); W. Hebron (Lg); West Pt. (Robn) ; Orangeburg, Ap (Nic); West Farms (Lg); Bronx, Je ( $\mathrm{Lg})$; SI, Je, on pignut hickory (L\&D) ; LI: Wading R., May (Nic); Bellport, Aug (Nic).

17866 C. crataegi Walsh. Quince. Curculio. A common pest on quinces and pears wherever they are grown in the State.

17872 C. naso Lec. SI, Je-Jl, on Crataegus (Ham); LI: Long Beach, Je (B\&L).

17877 C. posticatus Boh. Rochester (M\&W) ; Buffalo (Z\&R); West Pt. (Robn); SI, Je-J1, on Crataegus (Ham); LI: Rockaway (Ds, Lg); Long Beach (Ds); Yaphank (Ds); Bellport (Nic); Orient Pt., May-J1 (Ds).

17882 C. pusillus Lec. LI: Bellport, J1-Aug, on oak (Nic).

17884 C. geminatus Dej. Rochester (M\&W); Genesee Co, Je ( Lg); SI, J1 (L\&D); LI: Brooklyn, J1 (Shoe).

17886 C. infector Boh. "NY" (Lec).

17887 C. cribricollis Say. "NY" (Lec).

17890 C. anaglypticus Say. Adirondacks ( $Y g$ ); Rochester (M\&W); Buffalo (Z\&R); Cattaraugus Co, J1 (Ds) ; Ithaca, Aug (Dt); Freeville, Aug (NYS); Enfield, May (NYS); Nassau, J1 (NYS); Big Ind. Val., Jl (NYS); Poughkeepsie, Je, on slippery elm (Felt); West Pt. (Robn) ; Ramapo, May (Ds); SI, Je, Aug, breeds in hickory (Say).

17893 C. fissunguis Lec. Pine I. (Ds); Ramapo, Je (Ds); SI, in seed pods of swamp rose mallow (L\&D); LI, Mar (Eng). 


\section{RySSEMATUS (Chevrolat) Schönherr}

17899 R. lineaticollis Say. Rochester (M\&W); Buffalo (Z\&R); Ilion, May (NYS) ; Schuyler Co, Je (H) ; Lansingburg, Ap-May (CU) ; Kinderhook, Aug (NYS); West Pt. (Robn); Huguenot, J1 (AM); Ft. Montgomery, J1 (Ds); SI, larva breeds in milkweed (L\&D); LI: Jamaica, Wolf (NYS).

17900 R. annectens Csy. ( $R$. palmacollis of Buffalo and NY lists may be this species.)

\section{Chalcodermus Schönherr}

17911 C. collaris Horn. Rochester (M\&W).

Tyloderma Say

(Analcis Horn)

17919 T. foveolata Say. Rochester (M\&W) ; Williamsville, Je (NYS); Buffalo $(Z \& R)$; Ithaca, J1 (Dt); Windsor, Je (Not); Karner, May, Sep (NYS) ; Clinton Hgts., Je, Nassau, May (NYS); West Pt. (Robn); Rockland Co, Je ( $\mathrm{Lg})$; White Plains, J1 (Lg); SI, Je, breeds in stems of evening primrose (L\&D) ; LI: Syosset, J1 (NYS) ; Rosedale (Nic); Bellport, Je (Nic).

17924 T. fragariae Rly. Strawberry Crown Borer. SI (L\&D).

17928 T. aerea Say. Axton, Je (CU) ; Newport (NYS) ; Buffalo (Z\&R) ; Pike (CU) ; Spring Lake, Je (H); West Pt. (Robn); White Plains, J1 $(\mathrm{Lg})$.

17928a T. aerea var. nigra Csy. Westfield, May (Not) ; Ithaca, J1 (Dt) ; Windsor, Je (Not); Gloversville, Nov, Pike, Nassau (NYS).

17929 T. punctata Csy. Peekskill, Nyack, Yonkers (B\&L).

\section{APTEROMECHUS Faust}

17968 A. ferratus Say. Ithaca, J1 (Dt); Poughkeepsie, Je (NYS); Bronx, Je (Joutel); SI, Je (L\&D) ; LI: Flatbush (AM).

\section{CRYPTORHYNCHUS Illiger}

17970 C. parochus Hbst. Newport, May (NYS) ; Buffalo (Z\&R) ; Ithaca, May, Aug-Sep (Dt) ; Albany, Sep, Nassau, Ap, Poughkeepsie, May (NYS); Ft. Montgomery, May (Lg); Ramapo, May, Mosholu (Lg).

17971 C. bisignatus Say. Cranberry L., Aug (H) ; Buffalo (Z\&R) ; Ramapo, Je (Ds); Bronx, Je (Joutel); SI, Je-J1 (L\&D); LI: Babylon, Je (AM) ; Jamaica (Nic).

17972 C. pumilus Boh. West Pt. (Robn); Ft. Montgomery, May (Lg) ; SI, J1 $(\mathrm{Lg})$; LI: Glendale, Aug ( $\mathrm{Lg})$.

17973 C. fuscatus Lec. Genesee Co, Je ( $\mathrm{Lg})$; Buffalo (Z\&R).

17974 C. lapathi L. Poplar and Willow Borer. Wilmington, Je (NYS) ; Cranberry L. (H) ; Newark, J1 (NYS); Honeoye Falls; Rochester $(\mathrm{M} \& W)$; Batavia (B\&L) ; Buffalo, Aug (Rein) ; Pike, Sep, N. Tonawanda, J1 (NYS) ; Ithaca (B\&L); McLean, May (Lg); Windsor, Je (Not); Schoharie (Not); Albany, J1, Phoenicia, Aug, Milton, May, 
Mosholu, Aug (NYS); Catskills, J1 (Nic); Van Cort. Pk. (Bt); Williams Bridge, 1882 (Julich); SI, Jl, Sep (L\&D); LI: Glendale, Aug ( $\mathrm{Lg})$.

17975 C. obliquus Say. Buffalo (Z\&R); LI: Yaphank, Sep (Ds).

17980 C. obtentus Hbst. Near NYC (Joutel, Julich); LI: Babylon (AM).

17981 C. fallax Lec. Syracuse (Blkm); Hastings, Je (Joutel); Buffalo (Z\&R); Ithaca, J1 (NYS) ; West Pt. (Robn); SI, bred from hickory (L\&D); LI: Long Beach (AM).

17986 C. tristis Lec. Mosholu, Je (NYS); SI, Je-Jl, on leaves of scarlet oak (L\&D) ; LI: Jamaica (Nic).

\section{SUBFAMILY COSSONINAE}

Acamptus LeConte

18006 A. rigidus Lec. Buffalo ( $Z \& R$ ).

\section{DRYOPHTHORUS Schönherr}

18013 D. americanus Bedel. Newport (NYS) ; Gloversville, Ap (NYS) ; Lakeside, Je, Cy (NYS); Rochester (M\&W); Buffalo (Z\&R); Cheektowaga (Zimm); Genesee Co, Je ( $\mathrm{Lg}$ ) ; Ithaca, Danby, Ap-May, J1 (Dt); Schoharie (Not); Albany, May, Delmar, Mar, Poughkeepsie, J1 (NYS) ; Catskills, Ap, Sep (Lg) ; West Pt. (Robn); Peekskill (Lg); White Plains, Mar (Lg); Bronxville (B\&L); Ramapo Mts. (B\&L); Van Cort. Pk. (B\&L) ; SI (L\&D); LI: Bellport, common under pine bark, Mar-Sep (Nic); Flatbush (B\&L); Mineola, J1 (NYS).

\section{Himatinum Cockerell}

18016 H. errans Lec. Rochester (M\&W).

\section{Carphonotus Casey}

18018 C. testaceus Csy. Cranberry L., J1, on Picea rubens (H).

\section{Cossonus Clairville}

18020 C. platalea Say. Newport, Sep-Oct, on butternut (Felt); Buffalo (Z\&R); Westfield, May (Not); Castile, Brad (Flet); Ithaca, Je (Dt); West Pt., May (Robn); Ludlow, Ji (JCT) ; Ramapo (AM).

18031 C. impressifrons Boh. Johnstown, Aug (NYS); Buffalo (Z\&R), as concinnus Boh.; Bronxville, J1 (B\&L).

18035 C. corticola Say. Rochester (M\&W) ; Buffalo (Z\&R) ; Ithaca (Lg); West Pt. (B\&L) ; Mosholu, Jl (NYS); Van Cort. Pk. (B\&L); SI, Ap, Aug (L\&D) ; LI: Bellport, Jl-Aug (Nic) ; Cold Sp. Harb., Je (H).

\section{Allomimus LeConte}

18039 A. dubius Horn. Westfield, May (Not); Crusoe L., May (NYS). 


\section{Mesites Schönherr}

18044 M. subcylindricus Horn. LI: Flatbush, from a Dutch dictionary (Zab) ; Bellport, J1, sometimes in great numbers in logs washed up by the ocean (Nic).

\section{PhLOEOPhagus Schönherr}

18047 P. apionides Horn. Newport (NYS); Catskills (Nic).

18049 P. minor Horn. Buffalo (B\&L).

18050 P. spadix Hbst. Introduced. LI: Rockaway Beach, Je, in water-soaked pine driftwood (Julich).

TOMOLIPS Wollaston

18051 T. quercicola Boh. "NY" (AM).

\section{PEnTARTHRInUs Casey}

18056 P. parvicollis Csy. NYC (AM). LI: Flatbush, May, from apple wood (Zab).

\section{HEXARTHRUM Wollaston}

18060 H. ulkei Horn. Rochester (M\&W) ; NYC, Dec, on white pine (Joutel).

\section{RHYNCOLUS Germar}

18070 R. brunneus Mann. Cranberry L., Je $(\mathrm{H})$; L. Clear, Je-Jl, on pine (Yg); Ithaca, Ap (B\&L) ; Catskills (Nic); Normanskill, Ap (NYS).

\section{STENOSCELIS Wollaston}

18073 S. brevis Woll. Newport, May, Severance, Aug, Bks (NYS) ; Olcott, J1Oct (Dt); Genesee Co, Je (Lg); Buffalo (Z\&R); Ithaca, Jl-Oct (Dt) ; Schoharie (Not); E. Schodack, Ap (NYS); Catskills (Nic); West Pt. (Robn); Warwick, J1 (NYS) ; Poughkeepsie, Je (NYS); SI, Je (L\&D) ; LI: Flatbush, Oct-J1, apple (Zab).

\section{SUBFAMILY CALENDRINAE}

\section{Rhodobaenus LeConte}

18087 R. tredecimpunctatus I11. Mosholu, Je, Bronxville, Aug (Ds); SI, MayOct (L\&D).

\section{SPHENOPHORUS Schönherr 60}

18101 S. inaequalis Say. Ithaca, Je (Dt); Van Cort. Pk. (B\&L) ; SI, Aug, etc. (L\&D) ; LI: Brooklyn, Ap (Shoe) ; Bellport (Nic) ; Rockaway, Je-J1 (Nic) ; Orient Pt., May (NYS); Aqueduct, Sep (Liebeck).

${ }^{60} \mathrm{~A}$. F. Satterthwait calls attention to the fact that Pierce has shown the priority of Calendra over Sphenophorus, and of Sitophilus Schön. over Calendra. (Proc. Ent. Soc. Wash. 21:21-26, 1919, and $27: 113-114,1925$.

Sphenophorus breeds in roots of grass-like plants. 
18102 S. aequalis Gy11. (ochreus Lec.). Rochester (M\&W); Buffalo (Z\&R); Ithaca (Satt); SI (L\&D); LI: Rockaway Beach (Shoe); Bellport, Je (Nic).

18107 S. striatipennis Chitt. N. Fair Haven, Je (H); McLean, May, J1 (Dt).

18108 S. pertinax Oliv. Rochester (M\&W) ; Buffalo (Z\&R); Cayuga L. (Satt); Cazenovia, Dunkirk, Ithaca (Satt); West Pt. (Robn); SI, Ap-May, Aug, in calamus, cattail, etc. (L\&D); LI: Flushing, Mar, pupae and adults in chambers gnawed in base of cattail stems. (Bel1); Long Beach, Je (Ds); Brooklyn, Bellport (Nic); Wading R., May-Je (Nic); Orient Pt., May (Ds). Breeds in Typha latifolia and Sparganium eurycarpum; adults feed also on corn (Satt).

18110 S. setiger Chitt. Ithaca (Chitt); Dunwoodie (Satt); NYC (Satt); SI, May (L\&D) ; LI: Cold Sp., Ap, Rockaway (Ds) ; Flatbush, Je (AM) ; Bellport, May-Je (Nic); Coney I. (Rbts).

S. setiger var. intervallatus Chitt. NY among type localities.

18112 S. peninsularis Chitt. var. nasutus Chitt. NYC, May (Satt).

18114 S. costipennis Horn. Buffalo (Horn); Pike, J1 (NYS); Renwick Swamp, Mar, Bish (NYS); Nassau, Je (NYS); Catskills (Wickham); West Pt. (Robn) ; SI, Mar, Je (L\&D) ; LI: Brooklyn, Rockaway (B\&L).

18114a S. costipennis var. callosipennis Chitt. Buffalo (Satt). Breeds in Carex lacustris (Satt).

18115 S. villosiventris Chitt. Buffalo (Chitt); Ithaca (Chitt) ; Orangeburg, May (Nic) ; LI: Rockaway, Je (Nic); Long Beach (Satt).

18120 S. cariosus Oliv. Buffalo (Rein); West Pt. (Robn); SI, Ap-Je (L\&D); LI: Jamaica, May-Sep (B\&L) ; Fire I. (B\&L); Babylon, Je (AM) ; Brooklyn, May-Je (Satt); Wading R., May (Nic); Rockaway, Je (Nic) ; Long Beach, Je (Nic). Breeds in Rynchospora corniculata, Scirpus Eriophorum, and Cyperus rotundus; adults feed also on corn and on Cyperus erythrorhizos (Satt).

18122 S. parvulus Gyll. Peekskill, Mar (Satt); West Pt. (Robn) ; NYC (Satt); SI, Ap-Je (L\&D) ; LI: Brooklyn, J1 (Nic) ; Babylon, Je (AM); Long Beach, Aug (Browne11); Southampton, Sep 24 (Satt). Breeds in wheat, timothy, blue grass, prop roots of corn, root bulbs of Panicum repens, and stalks of Agrostis alba; adults feed also on corn (Satt).

18123 S. minimus Hart. LI: Long Beach, Je 7 (Nic).

18124 S. marinus Chitt. West Pt., Sep (AM).

18126 S. retusus Gyll. Rochester (M\&W) ; Nyack (AM) ; LI: Rockaway Beach (B\&L) ; Fire I., J1-Sep (B\&L); Bellport, Aug (Nic); Long Beach, J1, Coney I. (Satt). Breeds in Panicum repens (Satt).

18132 S. zeae Walsh (sculptilis records probably belong here). Syracuse, May $(\mathrm{H})$; Rochester (M\&W) ; Batavia, Je, Buffalo, Aug (Satt); Silver Cr., J1, Hunt's Pt., Je (Satt); Ithaca, McLean, May-Sep (Dt); Schoharie (Not); Peekskill (B\&L); West Pt. (Robn); Dunwoodie, Sep (Satt) ; Bronx, Fel-Sep (B\&L) ; SI, J1 (L\&D); LI: Rockaway (AM); Rosedale, Je (Nic); Long Beach, Aug (Wick); Jamaica, Je (Wick). Breeds in timothy; adults feed also on corn (Satt).

18135 S. callosus Oliv. The Cuklew Bug of the southern States. Ithaca, Ringwood, May-Je, Nov (Dt). Breeds in sedges, Rynchospora corniculata, and, in the Carolinas, in corn; adults feed also on wheat and throughout their range on corn (Satt). 
18136 S. melanocephalus Fab. Buffalo (Z\&R); Pike (Liebeck); Ithaca, Je (Dt) ; Windsor, Je (Not) ; West Pt. (Robn) ; Van Cort. Pk. (Satt) ; SI, May-Je (L\&D) ; LI: Brooklyn, May (Nic, B\&L) ; Long Beach, Je (Nic) ; Woodside, Ap (Satt). Breeds in Lecrsia oryzoides; adults feed also on Carex sp., and occur, perhaps for feeding, in bunches of Elymus sp., Andropogon virginicus, and Sphenopholis obtusata (Satt).

18137 S. sayi Gyll. LI: Long Beach, Je (Nic).

18141 S. venatus Say (placidus Say). NYC (Satt); SI, May-Je, Aug (L\&D) ; LI: Rockaway Beach (Ds); Yaphank, Flatbush (AM); Long Beach, Aug (Brownell); Orient Pt., Je (NYS); Clason Pt., J1, Jamaica (Satt). Breeds in Cyperus esculentus, Bermuda grass, wheat, and timothy; adults feed also on corn (Satt).

\section{Calendra Clairville $60 \mathrm{a}$}

\section{(Calandra auct.)}

18157 C. granaria L. GRANARY WeEvil. Introduced. Generally distributed, infesting stored grain.

18159 C. oryzae L. RICE WeEvil. Introduced. Generally distributed, infesting stored grain.

\section{FAMILY PLATYPODIDAE}

Platypus Herbst

18166 P. compositus Say. SI, J1 25 (L\&D).

18167 P. punctulatus Chap. Long Island City, Aug (NYS) ; Astoria, Aug, on mahogany logs from Panama, Felt (Hopk).

\section{FAMILY SCOLYTIDAE 61}

\section{(Ipidae auct.)}

\section{SUBFAMILY SCOLYTINAE}

\section{Scolytus Geoffroy}

\section{(Eccoptogaster Herbst)}

18169 S. quadrispinosus Say. Hickory BARk BeEtle. Very common. Syracuse, Cazenovia (Blkm) ; Middletown (Dt, Cy); Tivoli (Felt) ; Rye (Bird); Bronxville, J1 (Felt); SI, Je-Aug, causes death of many hickory trees (L\&D) ; LI: Prospect Pk., Brooklyn (Felt).

18171 S. rugulosus Ratz. Shot-Hole Borer, or Fruit-Tree Bark Beetle. Probably generally distributed throughout the State, but somewhat less common in the northern part; often injurious to apple, cherry, plum, peach, and wild cherry. Representative localities are: Chazy; Plattsburg; Crittenden, Syracuse, Fulton, common, and Cranberry L., rare $($ Blkm) ; counties bordering L. Ontario shore; Ripley; Dansville;

60a A. F. Satterthwait calls attention to the fact that Pierce has shown the priority of Calendra over Sphenophorus, and of Sitophilus Schön. over Calendra. (Proc. Ent. Soc. Wash. 21:21-26, 1919, and 27:113-114, 1925.)

${ }_{61}$ The list for this family has been gone over by Dr. J. M. Swaine and Dr. M. W. Blackman, both of whom have supplied many records and made changes in synonymy. 
Elmira; Tompkins Co; Albany Co; Pittstown; Columbia Co; Mt. Vernon; Katonah; SI (L\&D).

18174 S. piceae Sw. Cranberry L., Je, not common (Blkm) ; Crittenden, Feb, J1, common $(B 1 \mathrm{~km})$. Larch, and red and white spruce $(B 1 \mathrm{~km})$.

18179 S. sulcatus Lec. "NY” (Sw); Rochester (M\&W).

\section{Cnesinus LeConte}

18186 C. strigicollis Lec. SI, Je (L\&D).

\section{SUBFAMILY HYLESININAE}

CRYPTURGUS Erichson

18188 C. atomus Lec. Cranberry L., Syracuse, Cicero Swamp, Elka Pk., fairly common (Blkm); Ithaca (Sw); Normanskill, Albany, Yg (NYS). Pine, spruces, fir, and larch (Blkm).

18191 C. corrugatus Sw. Cranberry L., Elka Pk., fairly common (Blkm).

\section{POLYGRAPHUS Erichson}

18193 P. rufipennis Kby. Mt. Seward, Je, $4500 \mathrm{ft} .(\mathrm{M} \& \mathrm{H})$; L. Pleasant (Lec) ; Big Moose (Felt); Cranberry L., Syracuse, Cicero Swamp, Elka Pk., very common except at Syracuse $(\mathrm{Bl} \mathrm{km})$; Ithaca (Sw). Pines, spruces, and larch $(B 1 \mathrm{~km})$.

\section{CARPHOBORUS Eichhoff}

18196 C. bicristatus Chap. Buffalo (Z\&R).

18197 C. bifurcus Eich. "NY" (Sw-Lec Coll.).

\section{XYLECHINUS Chapuis}

X. americanus Blkm. Cranberry L., not common (Blkm). Red and white spruce $(\mathrm{Blkm})$.

\section{Chramesus LeConte}

18202 C. hicoriae Lec. Syracuse, common (Blkm); Ithaca (Sw); West Pt. (Robn) ; SI (L\&D). Hickory.

\section{Phthorophloeus Rey}

18206 P. liminaris Harr. Peach Bark Beetle. Wanakena, Aug, Cranberry L., Je, fairly common (Blkm) ; Syracuse, not common (Blkm); LaSalle, Somerset (Lint); Buffalo (Kell); Penn Yan (Lint); Ithaca (Sw). Peach, wild cherry, and black cherry (Blkm).

18207 P. frontalis Oliv. "NY" (Sw) ; SI, on mulberry (L\&D).

18209 P. piceae Sw. Cranberry L., fairly common on spruce (Blkm). 


\section{DENDROCTONUS Erichson}

18221 D. simplex Lec. Eastern Larch Beetle. Crittenden, May 4-22, Stage $(B 1 \mathrm{~km})$. Larch.

18223 D. piceaperda Hopk. Eastern Spruce Beetle. "NY" (Hopk); L. Pleasant (Lec), as rufipennis. Spruce.

18229 D. punctatus Lec. Allegheny Spruce Beetle. Rochester (M\&W) ; Buffalo (Z\&R); Albany (Felt); LI: Manor (Felt); Islip (Hopk); Central Pk. (Eng). Swaine writes, "The Rochester, Buffalo, Albany, and Manor records I should think doubtful, unless checked by Hopkins."

18231 D. valens Lec. Red Turpentine Beetre. Cranberry. L., Syracuse, Elka Pk., common (Blkm); Ithaca (Hopk); West Pt. (Robn), as terebrans.

\section{Phloesinus Chapuis}

18237 P. canadensis Sw. Cranberry L., Syracuse, Jamesville, common to very common $(\mathrm{B} 1 \mathrm{~km})$. Arbor vitae.

18238 P. dentatus Say. Adirondacks (Felt); West Pt. (Robn); LI: Central Pk., Ap (Eng). Arbor vitae.

18239

18244

P. serratus Lec. "NY" ( $\mathrm{Sw}, \mathrm{Lec})$.

P. rugosus Sw. LI: Flatbush (Ds).

\section{LEPERISINUS Reitter}

18247 L. fasciatus Lec. "NY" (Sw). Ash.

18248 L. imperialis Eich. "NY" (Sw-Can. Nat. Coll.).

18252 L. aculeatus Say. Newport (NYS) ; Syracuse, Sep-Oct, not common $(B l \mathrm{~km})$; Olcott, Aug (Dt); Rochester (M\&W); Buffalo (Z\&R); Ithaca (Sw); Cornwall (NYS); SI, May 5 (L\&D). Ash.

\section{HyLASTinus Bedel}

18255 H. obscurus Marsh. Clover Root-Borer. Syracuse, May (Blkm); Buffalo, Je (Kell); Westfield, May (Not); Bristol Sps. (Lint); Ithaca, Je, Dt $(\mathrm{Sw})$; SI, Je (L\&D). Clover.

\section{HYLURGOPINUS Swaine}

18257 H. rufipes Eich. Rochester (M\&W) ; Buffalo (Z\&R); Ithaca, Je, Dt (Sw); Hoosick Falls, May (Blkm); West Pt. (Robn).

\section{HYLURGOPS LeConte}

18267 H. pinifex Fitch. Cranberry L., Syracuse, Elka Pk., very common (Blkm); Rochester (M\&W) ; Buffalo (Z\&R); Ithaca, Jl, Sep, Dt (Sw); West Pt. (Robn) ; LI: Central Pk., Ap. (Eng). Pines and spruce.

\section{HYLOCURUS Eichhoff}

H. biorbis Blkm. Syracuse, May 12, Stage, type. 


\section{HyLASTES Erichson}

(Tomicus Latreille)

18273 H. salebrosus Eich. Ithaca, Je, $D t$ (Sw). Pine.

18274 H. scaber Sw. "NY" (Sw).

18276 H. porculus Er. Ithaca (Sw). Pine.

\section{SUBFAMILY MICRACINAE}

\section{MicRacis LeConte}

18288 M. suturalis Lec. Buffalo (Z\&R); West Pt. (Robn).

18290 . M. rudis Lec. "NY" (Sw).

18291 M. opacicollis Lec. Ithaca (Sw); LI: Westbury (US). Oak and Castanea.

18292 M. asperulus Lec. "NY" (Sw) ; Windsor, Je (Not).

M. populi Sw. Ithaca ( $\mathrm{Sw})$, type. Poplar.

\section{ThySANOES LeConte}

18294 T. fimbricornis Lec. Rochester (M\&W); West Pt. (Robn).

\section{Pseudothysanoes Blackman}

P. drakei Blkm. Syracuse, type locality (Blkm). Basswood.

\section{PTEROCYCLON Eichhoff \\ (Monarthrum Kirsch)}

18298 P. fasciatum Say. Buffaio (Z\&R); Ithaca, J1, Dt (Sw) ; SI: Port Richmond (L\&D). Maple, beech, etc. (Sw).

18299 P. mali Fitch. Apple Wood Stainer. Axton, Floodwood (Felt); Cranberry L., Syracuse, Elka Pk., common (Blkm); Olcott, Dt (Sw) ; Buffalo (Z\&R) ; Ithaca, Dt $(S w)$; SI: Clove Val., May (L\&D). Oak, birch, and various hardwoods; apple.

\section{CORTHYLUS Erichson}

18301 C. punctatissimus Zimm. "Northeastern NY" ( $\mathrm{Sz})$. Rhododendron.

\section{XYLOTERINUS Swaine}

18304 X. politus Say. Axton, Floodwood (Felt); Cranberry L., Syracuse, Elka Pk., common (Blkm); Buffalo (Z\&R); Westfield, May (Not); Ithaca (Sw) ; Windsor, Je (Not); West Pt. (Robn). Various hardwoods and hemlock (B1km).

\section{TRYPODENDRON Stephens}

18305 T. scabricollis Lec. "NY" (Lg Cat., Sw). Pine (Sw).

18306 T. retusum Lec. Cranberry L., Syracuse, Elka Pk., J1-Aug, uncommon to fairly common $(\mathrm{Blkm})$; Ithaca $(\mathrm{Sw})$. Poplars. 
18307 T. betulae Sw. Cranberry L., Elka Pk., very common (B1km); Ithaca $(\mathrm{Sw})$. Birches.

18309 T. bivittatum Kby. Big Moose, J1, $Y g$ (Felt), as Xyloterus lineatus Oliv., probably belongs here (Sw). Cranberry L., Syracuse, not very common, Elka Pk., common (Blkm); Ithaca ( $\mathrm{Sw})$. Pines, spruces, firs, hemlock, larch, and juniper (Blkm, Sw).

\section{HYPOTHENEMUS Westwood}

18321 H. hispidulus Lec. SI, Mar (L\&D).

\section{STEPHANODERES Eichhoff}

18367 S. dissimilis Zimm. Ithaca, Sep (Frost, Sw), Nov (Chitt); SI, Je (L\&D).

\section{CRYPHALUS Erichson}

18377 C. balsameus Hopk. Cranberry L., Elka Pk., common (Blkm). Fir.

C. mainensis Blkm. Cranberry L., common (Blkm). Spruce.

\section{LETZNERELLA Reitter}

18379 L. jalappae Letzn. LI: Brooklyn, 1874, Hubbard and Sz, from Radix jalappae $(\mathrm{Sz})$.

\section{GNATHOTRICHUS Eichhoff}

18400 G. materiarius Fitch. Mt. Seward, 4500 ft., Je (M\&H) ; Big Moose (Felt); Cranberry L., Syracuse, Elka Pk., common (Blkm); Buffalo (Z\&R) ; Ithaca, Ap, J1, Dt (Sw) ; West Pt. (Robn) ; LI: Manorville, Oct (Felt). Pine, spruce, larch, and fir.

\section{CONOPHTHORUS Hopkins}

18405 C. resinosae Hopk. LI: Southold, Mar 1, in red-pine cones (Blkm).

18410 C. coniperda Sz. Axton, Upper Jay, Cranberry L., in white-pine cones (Blkm).

\section{PSEUdOPITYOPHTHORUS Swaine}

18417 P. minutissimus Zimm. Syracuse, May, fairly common (Blkm); West Pt. (Robn). Oaks.

18419 P. pruinosus Eich. Peekskill (Ds); LI: Rockaway Beach, in wash-up (Nic). Oak.

\section{PITYOPHTHORUS Eichhoff}

18421 P. ramiperda Sw. Niagara, Je 21, 1909 (Blkm).

18423 P. pulicarius Zimm. Ithaca (Sw). Pines.

18425 P. rhois Sw. Syracuse, rather common (Blkm); Ithaca (Frost, Sw). Sumach and poison ivy (B1km). 
18428 P. puberulus Lec. Cranberry L., Syracuse, common (Bikm); Ithaca ( Sw); Albany (Lint). Pine and fir.

18429 P. opaculus Lec. Cranberry L., very common (Blkm); Ithaca (Sw). Spruce and pine.

18437 P. cariniceps Lec. Syracuse, common in white pine (Blkm); Ithaca (Frost,Sw), Aug (Chitt).

18438 P. canadensis Sw. Axton, Sep, Cranberry L., common in white pine (Blkm).

18440 P. pulchellus Eich. LI: Westbury, Mar 23 (Blkm-US).

18442 P. pullus Zimm. "NY" (Sw). Pines.

18445 P. granulatus Sw. Cranberry L., Syracuse, infesting white pine similarly to $n u d u s$ (B1km).

18446 P. nudus Sw. Cranberry L., Syracuse, common in shaded-out limbs and small trees of white pine $(B 1 \mathrm{~km})$.

18447 P. consimilis Lec. Ithaca ( $\mathrm{Sw}$ ); Windsor, Je (Not).

P. dentifrons Blkm. Cranberry L., very common in red spruce (Blkm).

P. shepardi Blkm. Cranberry L., very common in red spruce $(B l \mathrm{~km})$.

\section{Pityogenes Bedel}

18455 P. hopkinsi Sw. Axton, Upper Jay, Cranberry L., Elka Pk., Syracuse, Cicero Swamp, Schaghticoke, mostly in Sep, very common in white pine (Blkm); Ithaca (Frost).

18459 P. plagiatus Lec. "NY", in Pinus banksiana (Sw).

\section{IPS DeGeer}

\section{(Tomicus Latreille)}

18462 I. calligraphus Germ. Adirondacks (Felt); Buffalo (Z\&R); Syracuse, Albany, not very common (Blkm); Ithaca (Sw); Lansingburg (Felt); West Pt. (Robn); LI: Central Pk., Ap (Eng); Manorville (Felt). Pines.

18463 I. grandicollis Eich. Cheektowaga (Lec); Ithaca ( $\mathrm{Sw}$ ); Windsor, Je (Not) ; Slingerlands, Karner, Aug-Sep (Felt) ; SI, Sep, on pine and spruce ( (L\&D) ; LI: Manorville, Oct (Felt).

18464 I. chagnoni Sw. "NY" (Sw). Pine.

18469 I. longidens Sw. Cranberry L., not common, Syracuse, fairly common in white pine $(\mathrm{Blkm})$; Ithaca, from hemlock, type locality (Sw).

18475 I. pini Say. Saranac, Round L., L. Clear Jct. (Felt); Axton, Cranberry L., Elka Pk., Syracuse, very common in pine (Blkm); Buffalo $(Z \& R)$; Ithaca, Ap-J1, Dt (Sw); Lansingburg, Rensselaer (Felt). Pine and hemlock ( $\mathrm{Sw})$.

18480 I. borealis Sw. Cranberry L., not common in white pine $(\mathrm{Bl} \mathrm{km})$.

Pityokteines Fuchs

18488 P. sparsus Lec. Mt. Seward, Je (M\&H); Cranberry L., Elka Pk., very common in fir (Blkm); Buffalo (Lec); West Pt. (Robn). 


\section{ORTHOTOMICUS Ferrari}

18492 O. caelatus Eich. Rochester (M\&W); Buffalo (Eich) ; Cranberry L., Elka Pk., Syracuse, Cicero Swamp, very common in stumps, in lower parts of trees, and in fallen trees of pines, spruces, and larch $(B 1 \mathrm{~km})$; Ithaca, Mar-J1, Dt (Sw); West Pt. (Robn).

18493 O. punctipennis Lec. "NY" ( $\mathrm{Nw})$.

\section{AMBROSIODMUS Hopkins}

18499 A. tachygraphus Zimm. "NY" (Sw).

\section{ANISANDRUS Ferrari}

18500 A. obesus Lec. Cranberry L., Elka Pk., not uncommon in beech (B1km); West Pt. (Robn).

18502 A. pyri Peck. Syracuse, May, not common, $H(\mathrm{~B} 1 \mathrm{~km})$; as Xyleborus dispar, a similar European species, Buffalo (Z\&R), Lockport, Geneva, Penn Yan (Lint), probably referring to this species. Hardwood and fruit trees.

18503 A. minor Sw. Syracuse, not common, in sugar maple (Blkm); Ithaca (Sw). A. swainei Drk. Cranberry L., J1-Aug, not common (Drk).

\section{XYLEBORUS Eichhoff}

18506 X. saxeseni Ratz. Syracuse, Dec, in oak, not common (Blkm); West Pt. (Robn). Fruit trees (Sw).

18507 X. xylographus Say. "NY" (Sw).

18511 X. pubescens Zimm. SI: Watchogue, Aug (L\&D).

$18512 \mathrm{X}$. celsus Eich. Syracuse, very common in dying hickory (Blkm) ; SI, Ap, in tulip $\log$ (L\&D).

18513 X. fuscatus Eich. Buffalo (Eich).

18524 X. fitchi Hopk. Described from LI (Hyslop), in Pinus.

\section{XYLOCLEPTES Ferrari}

18532 X. cucurbitae Lec. "NY" (Sw).

\section{DRYOCOETES Eichhoff}

18536 D. granicollis Lec. Cranberry L., Je 25, 1915, in spruce (Blkm); SI, Je (L\&D).

18538 D. americanus Hopk. Cranberry L., Elka Pk., Cicero Swamp, fairly common in spruce and firs $(\mathrm{B} 1 \mathrm{~km})$; Ithaca (Sw); Windsor, Je (Not). The D. septentrionis Mann. (18539), "NY" (Lec in Trans. Am. Ent. Soc., vol 2, p. 161, 1868, as Xyleborus), perhaps belongs here ( $\mathrm{Lg}$ ).

18540 D. betulae Hopk. Mt. Whiteface, $4000 \mathrm{ft}$., Aug, Dt (Sw) ; Big Moose, Yg (Felt) ; Cranberry L., Elka Pk., very common in yellow birch, and occasionally in white birch and beech, a few in pine and spruce (B1km).

18544 D. affaber Mann. (piceae Hopk., according to Sw). Cranberry L., Elka Pk., very common in spruce $(\mathrm{Blkm})$.

\section{LYMANTOR Lovell}

18547 L. decipiens Lec. Ithaca, in maple (Sw). 


\section{ORDER STREPSIPTERA \\ FAMILY STYLOPIDAE}

\section{XENOS Rossi}

X. wheeleri P. "NY," parasite of Polistes metricus (Pierce, 1918, p. 461).

X. peckii Kby. SI, based on a specimen in Ds Coll. of a stylopized Polistes $(\mathrm{Lg})$.

\section{ORDER MECOPTERA FAMILY PANORPIDAE}

\section{PANORPA Linnaeus}

\section{P. affinis Bks. LI: Sea Cliff (Bks).}

P. banksii Hine. LI: Sea Cliff (Hine). Type.

P. canadensis Bks. Adirondacks, Aug (Ds); Whiteface Mt., summit, J1 (Ds) ; Old Forge, Aug; Saranac L., Aug; Cranberry L., J1, div.; Axton, Je, MacG\&Houghton; trail to Mt. McIntyre.

P. claripennis Hine. Onteora Mt., Je, How (Bks).

P. confusa Westw. Ithaca, Je 26, 1915 (SmRC) ; Ft. Montgomery, J1 (Eng).

P. latipennis Hine. Mt. Whiteface, summit, J1, common (Ds); Axton, Je (Eng); Rock City, Je (Ds) ; Old Forge, J1 (NYS-CU).

P. maculosa Hagen.61a St. Lawrence Co, Aug ; Old Forge, Je (NYS-CU) ; Granberry L., J1; Morissey, Axton, Je, $M \mathcal{E} H$; Keene Val., Aug, Diven; Rock City, Je; Canandaigua L. (Felt) ; Ithaca, Je ; Freeville, J1; McLean, Je-J1; Caroline to Harford, Je; Trenton Falls, Je, Leon \& Fibs; Little Falls, J1; L. George, J1 (Ndm) ; Tackawasick Pond, Rensselaer Co., Je 25, Cy (SmRC) ; Nyack, Je, West Pt., Je, Ft. Montgomery, May (Ds) ; Mosholu, Je, Aug (Bno); SI, Je (Ds).

P. nebulosa Westw. ${ }^{61 a}$ Saranac Inn $(\mathrm{Ndm})$; Axton, Je, $M \mathcal{E} H$; Old Forge, Aug; Castile, Je, Brad; Rochester Jct., Je, Leon; Portageville, Je; Ithaca, May-J1 (div.) ; Ringwood, Je; Mud Cr., Tompkins Co, Je; McLean, Ap; Trenton Falls (Wlk); Onteora Mt., How (Bks); Ramapo, Je (Ds); West Pt, Je; SI: Watchogue, Je (Ds) ; LI: Yaphank, Half Way Hollow Hills, J1 (Ds).

P. rufa Gray (fasciata Klug). "NY" (Fitch). Probably a misidentification; occurs in Ga.

P. rufescens Ramb. N. Twin Br., Mt. Marcy, J1 (Fbs) ; Syracuse, Aug; Batavia, $\mathrm{J1}$, Kngt; Castile, Brad; Ithaca and vic., common everywhere (div.); Trenton Falls (Wlk); Sharon Sps. (Hagen); Onteora Mt., J1, How (Bks); Nyack, Je, Croton-on-Hudson, Je, Ramapo, May, Mosholu, May (Ds) ; SI: Tottenville, Richmond, Je-Aug (Ds) ; LI: Cold Sp. Harb., J1-Aug, Yaphank, J1, Pinelawn, Aug (Ds) ; Forest Pk., Je.

P. signifer Bks. Mt. Whiteface, summit, J1 (Ds) ; Saranac Inn (Ndm) ; E. Jewett, Aug (Eng); Greene Co, J1; Keene Val., Aug, Diven; Cranberry L., Drake; Axton, Je, MacG\&Houghton; Pine I., J1, Frost.

P. subfurcata Westw. Axton, Je, MacG\&Houghton; Cranberry L., J1, C.H.Foster (div.); McLean, May (Ds); Sullivan Co, Sep (Ds); Onteora Mt., J1, How (Bks); Ramapo, Je (Ds); Pine I., Je (Ds).

61a $P$. maculosa and $P$. nebulosa are much confused both in literature and in collections, and some records for each may be misplaced. The two species evidently fly together throughout the State. (Fbs). 
P. venosa Westw. Axton, Je (M\&H) ; Castile, May, Brad (Fbs); Ramapo, May (Ds) ; SI, Sep (Ds) ; LI: Cold Sp. Harb., J1-Aug, and Yaphank, J1 (Ds); Pinelawn, Aug.

\section{BOREUS Latreille}

B. brumalis Fitch. Washington Co (Hagen); SI: Clove Valley, Dec, on snow (Ds); LI: Sea Cliff (Eng-AM).

B. nivoriundus Fitch. Ithaca, Dec 18 (Eng-AM); Washington Co (Hagen); LI: East New York, early spring, on snow (Fitch).

\section{BitTacus Latreille}

B. apicalis Hagen. Canandaigua L., J1 21, Pettit (CU); Six Mile Cr., Ithaca, Aug, H. Yuasa, rare; Batavia, J1, Kngt.

B. pilicornis Westw. Trenton Falls, OS (Hagen); Mosholu, Je (Bno); Ithaca, J1.

B. stigmaterus Say. "NY" (Hine).

B. strigosus Hagen. Batavia, J1, Kngt; N. Hector, Aug; Ithaca, J1 (CU) ; SI: Richmond Hill, common in woods; LI: Cold Sp. Harb., Floral Pk., Jl (Ds); Queens, J1.

\section{MEROPE Newman}

M. tuber Newn. Cranberry L., J1 22 (CU) ; Ithaca, Aug 25, pair (Fbs); McLean, Aug 27 (Sib); Slaterville, J1 (Bdy); Trenton Falls, Dbl (Newn); Salem, J1 (Fitch); Sullivan Co, Aug (Jn).

\section{ORDER TRICHOPTERA ${ }^{62}$ FAMILY RHYACOPHILIDAE SUBFAMILY RHYACOPHILINAE}

\section{RHYACOPHILA Pictet}

R. carolina Bks. Old Forge, Beaver Meadow Br., J1 (Bet).

$\boldsymbol{R}$. fuscula Wlk. Old Forge, Ithaca, May-Aug (Bet).

R. nigrita Bks. (torva Bks. not Hagen). Colden, Je, Bet (EPV).

R. torva Hagen (terminata Bks., luctuosa Bks.). Woodworth's L., Gloversville, Je (Alex); Ithaca, Je (Bet); Trenton Falls (OS).

R. sp. Old Forge, J1 (Bet).

R. sp. Fulton Chain, Aug (Bet).

R. sp. Ithaca, Aug (Bet).

\section{SUBFAMILY GLOSSOSOMATINAE}

\section{MYSTROPHORA Klapálek}

M. americana Bks. Niagara Gorge (Bet) ; Newfield, Je (MacG); Ithaca (Lloyd); McLean, Je, Aug (Sib).

M. sp. Ogdensburg (Bet).

62 The list of Trichoptera has been compiled principally from Dr. Cornelius Betten's extensive manuscript dealing with a revision of the American Trichoptera. Many of the species in the present list recorded simply as "sp.," bear manuscript names in Dr. Betten's monograph, and for a number of species Dr. Betten has erected new genera. Since immediate publication of Dr. Betten's paper unfortunately is not assured, it has seemed impossible to indicate here the proper generic position of all species or to use his manuscript names for new species. 


\section{Agape'Tus Curtis}
A. minutus Sib. McLean, Sep (Sib).

\section{FAMILY HYDROPTILIDAE}

AgRAYLEA Curtis

A. multipunctata Curtis. Ogdensburg, Ithaca, J1-Sep (Bet) ; McLean, Je, Sep, very common (Sib).

\section{Protoptila Banks}

P. maculata Hagen. Ogdensburg, Buffalo, Ithaca, J1-Aug (Bet) ; Sacandaga Pk., Je, Alex (Bks).

\section{ORTHOTRICHIA Eaton}

O. americana Bks. Ithaca, J1 (Bet).

O. brachiata Morton. Ithaca, J1 (Bet).

O. pictipes Bks. Hales Cr., Johnstown, Je, Alex, type.

\section{ITHYTRICHIA Eaton}

I. clavata Morton. Ithaca, J1 (Bet).

I. confusa Morton. Ithaca, Aug (Bet) ; Cascadilla Cr., Ithaca (Lloyd) ; McLean, J1 (Sib). Should be a new genus.

\section{HYDROPTILA Dalman}

H. albicornis Hagen. Old Forge, Ithaca, Aug (Bet); McLean, Je-Sep, very common ( $\mathrm{Sib})$.

H. consimilis Morton. Ithaca, J1 (Bet); McLean, J1-Sep (Sib).

H. delineata Morton. Ithaca, J1 (Bet).

H. hamata Morton. Ithaca, J1 (Bet).

H. perdita Morton. Ithaca, J1 (Bet).

H. spatulata Morton. Ithaca, J1 (Bet).

\section{OXYETHIRA Eaton}

O. coercens Morton. Ithaca, J1 (Bet).

O. viminalis Morton. Ithaca, J1 (Bet).

O. dualis Morton. McLean, Aug-Sep (Sib).

O. sp. Old Forge, J1-Aug, Ithaca, Aug (Bet).

\section{NEOTRICHIA Morton}

N. collata Morton. Old Forge, Ithaca, J1-Aug (Bet). 


\section{FAMILY PHILOPOTAMIDAE}

\section{Philopotamus Leach}

P. americanus Bks. Gloversville, Mar, Alex (Bet); LI: Sea Cliff, Mar-Ap, Dec (Bks).

P. distinctus W1k. Woodworth's L., Je, Alex (Bks) ; West Falls, J1 (Bet) ; Ithaca, J1 (Bet); Trenton Falls (W1k).

\section{DOLOPHILUS Banks}

D. breviatus Bks. Old Forge, Buffalo, J1 (Bet); Ithaca, J1 (Bks).

\section{ChIMARRHA Leach}

C. aterrima Hagen. Ogdensburg, J1-Aug, very common (Bet); Johnstown, Sacandaga Pk., Aug, Alex (Bks); Ithaca, J1-Aug (Bet); McLean, May-Aug (Sib); SI, Je (Ds).

C. socia Hagen. Johnstown, Sacandaga Pk., Je, Alex (Bks); Tupper L., Niagara Falls, Lancaster, Ithaca, Je-Aug (Bet).

C. sp. Ogdensburg, Ithaca, Je-Aug (Bet).

\section{FAMILY HYDROPSYCHIDAE SUBFAMILY HYDROPSYCHINAE}

ARCTOPSYCHE McLachlan

A. apicalis Bks. Fourth L., Je (Dickerson); Old Forge, J1 (Bet); Sacandaga Pk., Je, Alex (Bks); Castile, Je, Brad (Sib); McLean, Je (Sib). Should be new genus.

\section{DiPLECTRONA Westwood}

D. modesta Bks. Woodworth's L., Je, Alex (Bks); Newfield, Je, Bet (MacG); McLean, Je-J1 (Lloyd, Sib).

\section{HYDROPSYCHE Pictet}

H. alternans Hagen. Old Forge, Fourth L., Ithaca, J1-Aug (Bet) ; McLean, J1Sep (Sib); Onteora Mt., How (Caudell).

H. chlorotica Hagen. Buffalo, Je, very common (Bet); Trenton Falls (OS).

H. incommoda Hagen. Saranac Inn, Old Forge, Ithaca, May-Sep (Bet).

H. phalerata Hagen. Saranac Inn, Ithaca, J1-Aug (Bet); McLean, Je-Sep (Sib).

H. recurvata Bks. McLean, Je-Aug (Sib).

H. scalaris Hagen. Ogdensburg, Buffalo, J1-Sep (Bet) ; Sacandaga Pk., Je, Alex (Bks).

H. venularis Bks. Old Forge, Jl (Bet).

H. sp. Ogdensburg, Aug (Bet).

H. sp. Niagara Falls, Ithaca, Aug (Bet).

H. sp. Ithaca, Je (Bet). 


\section{HYDROPSYCHODES Ulmer}

H. analis Bks. Ogdensburg, Old Forge, Saranac Inn, Buffalo, Ithaca, Je-Sep (Bet); McLean, Je-Sep (Sib).

H. sordida Hagen. Ogdensburg, Jl-Aug (Bet); Sacandaga Pk., Je, Alex (Bks); McLean, J1-Aug (Sib).

\section{SUBFAMILY MACRONEMATINAE}

MACRONEMA Pictet

M. zebratum Hagen. Saranac L., Je (NYS); Sacandaga Pk., Je, Alex (Bks); Niagara R., Buffalo, J1, very common (Bet).

\section{FAMILY POLYCENTROPIDAE SUBFAMILY POLYCENTROPINAE}

\section{NEURECLIPSIS McLachlan}

N. crepuscularis Wlk. Buffalo, Je-J1, common (Bet).

N. parvula Bks. Ogdensburg, Buffalo, Ithaca, J1-Aug (Bet).

\section{PHYLOCENTROPUS Banks}

P. lucidus' Hagen. Saranac Inn, Old Forge, West Falls, Je (Bet) ; Sacandaga Pk., Gloversville, Je, Alex (Bks); McLean, Je-Aug (Sib); Trenton Falls (OS).

P. placidus Bks. Saranac Inn, Buffalo, J1-Aug (Bet); Sacandaga Pk., Je, Alex (Bks); McLean, Je, Aug (Sib).

\section{Plectrocnemia Stephens}

P. adironica Bks. Axton, Je (MacG).

P. canadensis Bks. Ogdensburg, Old Forge, Ithaca, Je-J1 (Bet) ; Sacandaga Pk., Woodworth's L., Je, Alex (Bks); McLean, J1-Aug (Sib).

P. confusa Hagen. McLean, Aug-Sep (Sib); Trenton Falls (OS); SI: Silver . L., Je (Bks,Ds).

P. sp. Buffalo, J1 (Bet).

Polycentropus Curtis

?P. maculatus Bks. McLean, J1 (Sib).

P. sp. Ogdensburg, Aug (Bet).

Holocentropus McLachlan

H. flavus Bks. Long Pt., Cayuga L., Je (Bet); McLean, Je-J1 (Sib).

NyctiophyLAX Brauer

N. affinis Bks. Buffalo (EPV).

N. vestitus Hagen. Westport, Aug (NYS) ; Old Forge, Buffalo, Je, Aug (Bet); Woodworth's L., Sacandaga Pk., Je, Alex (Bks); McLean, Je-Aug (Sib); Nassau (Felt). 


\section{FAMILY PSYCHOMYIDAE}

LYPE McLachlan

L. sp. Old Forge, Je-J1 (Bet); McLean, Je-Aug (Sib).

\section{Psychomyia Latreille}

P. flavida Hagen. Gloversville, Johnstown, May-Je, Alex (Bks); Niagara Falls, Buffalo, J1 (Bet); McLean, Je-Aug, very common (Sib).

\section{FAMILY CALAMOCERATIDAE \\ GANONEMA McLachlan}

G. nigrum Lloyd. McLean, Je-J1 (Lloyd, Sib) ; New Rochelle (Mrs. N.F.Davis).

\section{HETEROPLECTRON McLachlan}

H. boreale Prov. Sacandaga Pk., Je, Alex (Bks); Ft. Montgomery (Ds) ; SI, May (Ds). Probably the same as Psilotreta.

\section{FAMILY ODONTOCERIDAE}

\section{PSILOTRETA Banks}

P. dissimile Bks. LI: Sea Cliff, Je-Jl (Bks).

P. frontalis Bks. Ogdensburg, Old Forge, J1 (Bet); Castile, Je, Brad (Sib) ; Freeville, May-Je (Lloyd); McLean, Je (Sib); Nassau (Felt); New Rochelle (Mrs. N.F.Davis); LI: Sea Cliff, Je (Bks). Probably this is boreale Prov.

\section{FAMILY MOLANNIDAE SUBFAMILY MOLANNINAE}

Molanna Curtis

M. blenda Sib. Old Forge, J1 (Bet); McLean, Aug-Sep (Sib).

M. cinerea Hagen. Ogdensburg, Old Forge, Buffalo, May-Aug (Bet); Woodworth's L., Sacandaga Pk., Je, Alex (Bks).

M. flavicornis Bks. Ogdensburg, Buffalo, J1 (Bet).

M. rufa Hagen. Trenton Falls (OS).

M. sp. Old Forge, Canoga, Cayuga L., J1-Aug (Bet); Oxford, J1 (Sib).

M. sp. Saranac Inn, Old Forge, Je-Aug (Bet); Nassau (Felt).

SUBFAMILY BERAEINAE

BERAEA Stephens

B. nigritta Bks. Ithaca (Bet); LI: Sea Cliff, Je (Bks). 


\section{FAMILY LEPTOCERIDAE}

\section{LEPTOCERUS Leach}

L. ancylus Vorhies. Buffalo, J1 (Bet); Ithaca (Lloyd).

L. dilutus Hagen. Buffalo, Ithaca, Je (Bet).

L. maculatus Bks. McLean, J1-Aug (Sib).

L. punctatus Bks. Ogdensburg, Ithaca, J1-Aug (Bet).

L. recurvatus Bks. Westfield, Je-J1 (Woglum).

L. submacula Wlk. Buffalo, Je, common (Bet).

L. tarsi-punctatus Vorhies. Saranac Inn, Ogdensburg, Old Forge, J1-Aug (Bet).

L. variegatus Hagen. Ogdensburg, Old Forge, Saranac Inn, Ithaca, Je-Aug (Bet).

L. sp. Buffalo, Je (Bet).

L. sp. Ogdensburg, Saranac Inn, Old Forge, Buffalo, Jl-Aug (Bet).

L. sp. Buffalo, J1 (Bet).

\section{LEPTOCELLA Banks}

L. albida Wlk. Ogdensburg, Buffalo, Je-Aug (Bet); Clayton, J1 (Felt) ; Niagara Falls, J1 (Ds).

L. exquisita Wlk. Ogdensburg, Buffalo, Ithaca, Je-Aug (Bet); Sacandaga Pk., Johnstown, Je, Alex (Bks); Albany (Felt).

L. pavida Hagen. Old Forge, J1 (Bet).

\section{Oecetis McLachlan}

O. avara Bks. Ogdensburg, Buffalo, Ithaca, J1-Aug (Bet); McLean, J1-Aug (Sib).

O. immobilis Hagen. Ogdensburg, Aug, common (Bet).

O. incerta Wlk. Westport (NYS); Ogdensburg, Old Forge, Buffalo, J1-Sep, very common (Bet); Woodworth's L., Sacandaga Pk., Je-Aug, Alex (Bks); McLean, Je-Aug (Sib); LI (Bks).

O. persimilis Bks. Saranac Inn, Old Forge, J1 (Bet).

O. resurgens Wlk. (fumosa Bks.). Ogdensburg, Old Forge, Buffalo, J1-Aug (Bet) ; SI (Ds) ; Silver L., Je (Bks, Ds).

O. sp. Old Forge, Clayton, J1 (Bet).

\section{SETODES Rambur}

S. americana Bks. McLean, J1-Aug, very common (Sib).

S. grandis Bks. Ogdensburg, Old Forge, Ithaca, Je-J1 (Bet).

S. vernalis Bks: Old Forge, Ithaca, Jl-Aug (Bet); Tonawanda (EPV).

S. sp. Ogdensburg, Buffalo, J1 (Bet).

\section{Mystacides Latreille}

M. longicornis L. Ogdensburg, Aug (Bet).

M. nigra L. Ogdensburg, J1 (Felt); Ramapo, May (Ds).

M. sepulchralis Wlk. Sacandaga Pk., Je-Aug, Johnstown, Je, Alex (Bks); Ithaca, McLean (Sib) ; L. George, L. Heart (Sib) ; Clayton, Nassau (Felt). Common at many points. Specimens reported as nigra doubtless belong here (Bet). 


\section{TRIAENODES McLachlan}

T. dentata Bks. Ogdensburg, J1 (Bet) ; Johnstown, Je (Alex).

T. ignita Wlk. Westport, Aug (NYS); Ogdensburg, Buffalo, Aug (Bet) ; Niagara Falls, J1 (Ds) ; Castile, Brad (Sib) ; Pine I., Je (Ds); SI: Clove Val., Aug (Ds).

T. injusta Hagen (flavescens Bks.). Ogdensburg, Saranac Inn (Bet); McLean, Aug ( $\mathrm{Sib})$.

T. sp. Ogdensburg, Buffalo, Aug (Bet).

T. sp. Ithaca (CU); McLean, J1-Aug (Sib).

\section{FAMILY PHRYGANEIDAE}

\section{PHRYGANEA Linnaeus}

P. cinerea Wlk. Ogdensburg, Old Forge, J1 (Bet).

P. improba Hagen. Catskills.

P. interrupta Say. Ogdensburg, J1-Aug (Bet); Clayton (Felt); Ithaca, May-Je (Lloyd), J1-Aug (Bet); McLean, J1-Aug (Sib); Sullivan Co (Ds); SI: Clove Val., J1 (Ds); LI: Deep Pond, Wading R., Je-J1 (Ds).

P. vestita Wlk. Saranac Inn (Bet); Ithaca (Bet), Je (Lloyd); McLean, Aug (Sib) ; SI, May (Ds).

\section{NEURONIA Leach}

N. concatenata Wlk. Ogdensburg, Keene Val., Old Forge, Jl (Bet) ; Johnstown, J1 (Alex).

N. dossuaria Say. Keene Val., Old Forge, J1 (Bet).

N. ocellifera Wlk. Batavia, J1 (Lloyd); SI, May-Aug (Ds).

N. pardalis Wlk. Gloversville, Je (Alex); McLean, Michigan Hollow (Lloyd); Ramapo, Je (Ds).

N. postica W1k. Woodworth's L., Je (Alex) ; Castile, Brad (Sib) ; Ithaca, McLean, May-Je (Lloyd); Onteora Mt., Je (Caudell).

N. semifasciata Say. Keene Val., Old Forge, Je-J1 (Bet); Mt. Marcy (NYS) ; Sacandaga Pk., Je (Alex); NYC, J1 (Ds).

N. stygipes Hagen. Castile, Brad (Sib); McLean (Lloyd); SI, Ap (Ds).

\section{AgRYPNiA Curtis}

A. glacialis Hagen. McLean, Sep (Sib).

A. straminea Hagen. Saranac Inn, Aug (Bet).

\section{FAMILY LIMNEPHILIDAE}

\section{ARCTOecia McLachlan}

A. consocia Wlk. Sacandaga Pk., Je (Alex); Ithaca, Je (Lloyd); McLean, J1Sep (Sib).

\section{Astenophylax McLachlan}

A. argus Harr. Keene Val., N. Elba, Je; McLean, Michigan Hollow, Je (Lloyd); Oxford (Sib); SI, Je (Ds). 


\section{CARBorius Navas}

C. punctatissimus Wlk. Newport, Sep (Bet); Woodworth's L., Aug, Alex (Bks); McLean, Sep (Sib); Onteora Mt., Je, How (Caudell).

\section{LIMNEPHILUS Leach}

L. indivisus W1k. Pinnacle Mt., Gloversville, J1-Aug (Alex); Castile, Brad (Sib) ; Hamburg, Aug (EPV); Lancaster, J1 (Bet); McLean, Aug (Sib) ; Ithaca, May (Lloyd).

L. ornatus Bks. Saranac Inn, Je (Bet); Castile, Brad (Sib); Onteora Mt., Je, How (Caudell).

L. pulchellus Bks. McLean, May-Je (Sib).

L. rhombicus L. McLean, Michigan Hollow, Je-J1 (Lloyd).

L. sublunatus Prov. Axton (Bet).

L. submonilifer Wlk. Mt. McIntyre, Je (Sib) ; Chazy L. (NYS) ; Ogdensburg, Newport, Pike, Albany, May-Je, Aug-Sep (Bet); Pinnacle Mt., Gloversville, Canada L., Je-Sep (Alex); Castile, Brad (Sib); McLean, Je (Sib). Should be a new genus in group of Colpotaulius.

L. sp. Saranac Inn, Jl-Oct (Bet).

L. sp. Old Forge, Ithaca, Aug (Bet).

L. sp. Long L., Aug (Bet).

L. sp. Ithaca (Bet).

STENOPHyLAX Kolenati

?S. divergens Wlk. W. Hebron, Sep (Ds); Delaware Co?, Sep (Bks, Ds); Debruce, Sep (Ds).

S. gentilis McL. McLean, Sep (Sib).

S. infernalis Bks. Pinnacle Mt., Sep (Alex).

S. limbatus McL. Mt. Marcy, J1 (Felt); Old Forge (Bet); Pinnacle Mt., Sep (Alex); McLean, Sep (Sib).

S. scabripennis Ramb. Keene Val., Saranac Inn, Old Forge, J1-Aug (Bet) ; Johnstown, Woodworth's L., May-Aug (Alex); Ithaca, McLean, Aug-Sep (Sib).

Halesus Stephens

H. guttifer Wlk. Saranac Inn, Keene Val., Wilmington, Aug (Bet); Michigan Hollow, Aug, Nov (Lloyd); McLean, Aug-Sep (Sib).

H. similis Bks. Sacandaga Pk., Gloversville, Aug-Sep, Alex (Bks).

\section{Platyphylax McLachlan}

P. lepidus Hagen. Livingston, Sep (Ds); McLean, Aug-Sep (Sib); W. Hebron, Sep, CWLevy (Ds); Palenville (Ds).

P. subfasciatus Say. Gloversville, Aug (Alex); Ithaca (Bet).

\section{Glyphotaelius Stephens}

G. hostilis Hagen. Ithaca (Lloyd); McLean (Sib).

Anabolia Stephens

A. bimaculata Wlk. Old Forge, Keene Val., Tupper L., Jl (Bet). 


\section{PlatycentRopus Ulmer}

P. indistinctus Wlk. Saranac Inn, Je, Aug (Bet).

P. maculipennis Kol. Saranac Inn, Keene Val., L. Pleasant, Old Forge, J1 (Bet) ; Castile, Je, Brad (Sib); McLean, J1-Aug (Lloyd, Sib); SI, Je, Aug (Ds).

\section{DRUSUS Stephens}

D. sparsus Bks. (calypso Bks.). Catskills, Je (Bks); Onteora Mt., Je, How (Caudell); LI, May (Bks).

D. sp. Hamburg, Ithaca, J1 (Bet). This and the preceding should form a new genus near Drusus.

HESPEROPHYLAX Banks

H. designatus Wlk. McLean, Ap-Aug (Lloyd).

\section{LEPTOPHYLAX Banks}

L. gracilis Bks. McLean, Je-J1 (Sib).

\section{GLYPHOPSYCHE Banks}

G. irrorata Fab. Gloversville, Mar, Bet (Alex).

\section{Chilostigma McLachlan}

C. difficile Wlk. N. Elba, Oct (Ds) ; Otto, Oct-Nov, Bet (Comst) ; McLean, OctDec (Lloyd); SI, Nov-Dec (Bks, Ds).

C. pallidum Bks. Ithaca (Bks); McLean, Nov (Sib).

\section{NEOPHYLAX McLachlan}

N. autumnus Vorh. Gloversville, Pinnacle Mt., Johnstown (Alex) ; McLean, Sep, Nov (Lloyd, Sib). This may be the same as concinnus McL.

N. concinnus McL. McLean, Sep, Nov (Lloyd, Sib).

N. fuscus Bks. Ogdensburg, Sep (How); McLean, Sep (Sib).

N. sp. Old Forge, Elizabethtown, J1-Aug (Bet).

\section{Apatania Kolenati}

A. incerta Bks. LI: Sea Cliff, Ap (Bks).

\section{FAMILY SERICOSTOMATIDAE} SUBFAMILY BRACHYCENTRINAE

\section{BRACHYCENTROPUS Curtis}

B. fuliginosus Wlk. St. Lawrence R., May, Bet (Comst).

B. lutescens Prov. Ithaca (Bks).

B. nigrosoma Bks. Ithaca (Bks); McLean, May (Lloyd). 


\section{SUbFAMILy SERICOSTOMATINAE}

AGARODES Banks

A. grisea Bks. Ogdensburg, Clayton, Je (Bet); Woodworth's L., as Schizopelex lobata Bks., Je, Alex, type.

\section{SUBFAMILY GOERINAE}

\section{GOERA Leach}

G. calcarata Bks. Old Forge, J1 (Bet); Gloversville, Johnstown, Je, Alex (Bks) ; Ithaca, Ap-May (Lloyd); McLean, Je-J1 (Sib) ; LI: Sea Cliff, Jl (Bks).

\section{SILO Curtis}

S. griseus Hagen. Trenton Falls (OS).

\section{SUBFAMILY LEPIDOSTOMATINAE}

MORMOMYIA Banks

M. vernalis Bks. Castile, Je, Brad (Sib); McLean, May (Sib); LI: Sea Cliff, May (Bks).

\section{AlePomyia Banks}

A. wisconsinensis Vorhies. Old Forge, Je, Aug (Bet); McLean, May (Sib).

A. sp. Pike (Bet). Perhaps these two should be in a new genus, as the spurs are 2-4-4.

THELIOPSYCHE Banks

T. parva Bks. Woodworth's L., Alex, type.

\section{Olemira Banks}

O. americana Bks. Woodworth's L., Aug, Alex (Bks); LI: Sea Cliff, J1 (Bks).

O. costalis Bks. Ogdensburg, Old Forge, J1-Aug (Bet); Woodworth's L., Aug (Alex); McLean, Sep (Sib).

PHANOPSYCHE Banks

P. grisea Bks. Ogdensburg, Aug (Bet); Woodworth's L., Alex, type; McLean, Aug-Sep (Sib).

\section{LEPIDOSTOMA Rambur}

L. pallidum Bks. Ithaca (Bks).

L. togatum Hagen. Ogdensburg, Aug (Bet); Woodworth's L., Sacandaga Pk., Johnstown, Je-Aug, Alex (Bks).

\section{SUBFAMILY HELICOPSYCHINAE}

\section{HELICOPSYCHE Hagen}

H. borealis Hagen. Ogdensburg, Old Forge, Je-Jl (Bet); Johnstown, Sacandaga Pk., Mountain L., Je, Alex (Bks); Buffalo, Lancaster, Ithaca, Je-J1 (Bet); L. George (Sib); Nassau (Felt). 


\section{ORDER LEPIDOPTERA}

Compiled by W. T. M. Forbes

The following list of Lepidoptera of New York is based on a tentative list drawn up by the compiler in 1916. That preliminary list was prepared without memoranda of collector or determiner unless there was something highly unusual in the records; and it proved impracticable later to insert these in the limited time available, owing to the amount of labor which would be involved in going through the various records again. The names of the collectors to be credited with all or the great majority of records at certain of these places are presented here:

Albany, Lintner et al.; Batavia, Knight; Bath, Lintner; Big Indian Valley, Pearsall; Brockport, Bruce; Buffalo, Kellicott; Center, Lintner et al.; Clayton, Stebbins; Debruce, Shoemaker; Evans Center, Grote; Fentons, Hill; Florida, S. W. Frost; Honeoye Falls, Leonard; Ilion, McElhose; Karner, Lintner et al.; Katonah, Beutenmüller; Lancaster, E. P. Van Duzee; Lewis County, Hill ; Liberty and New Windsor, Emily Norton; Oliverea, Pearsall ; Onteora Mt., L. O. Howard; Otto, Comstock and Slingerland; Plattsburg, Hudson; Rensselaer and Schoharie, Lintner; Sharon, Meske; Staten Island, Davis; Stony Clove, Watson; Trenton Falls, Doubleday; Waddington, Woodruff; West Farms, Angus.

Most of the specimens have been seen and determinations verified by the compiler. There are incorporated, however, a considerable number of local lists of very variable quality, and the compiler has had to use his judgment in including or omitting records of species which have perhaps been misdetermined. He hopes that no species which are really members of the New York fauna have been omitted in this way, though doubtless a few really correct records of stragglers from other faunas have been overlooked. Additions to the preliminary list have been made largely by correspondence or by collections of the Cornell University entomologists, and the collector or the determiner has been cited in all records of any particular interest.

The dates and food plants given are gathered from a wide variety of sources and not wholly from New York material. The dates given at the end of the series of records for each species are intended to apply especially to central New York; dates for southern New York are usually earlier in the spring and later in the fall, and in many cases, where definite records are available, have been cited in detail. When New York dates were fragmentary or lacking, dates were taken from outside the State.

The first entomologist to make a considerable collection of the Lepidoptera of New York was Edward Doubleday, who visited for a time at Trenton Falls and published an account of his visit. Augustus Radcliffe 
Grote was the first entomologist living in the State (at Evans Center) who worked especially on the Lepidoptera for a period of years. The collections of both these men are now in the British Museum. Doubleday's records were mainly published by Walker, while Grote published many records himself, besides those which were incorporated in Van Duzee's list of the Lepidoptera of the vicinity of Buffalo. In those families for which the British Museum material has been recently worked over in Hampson's Catalogue of the Lepidoptera Phalaenae, the records have been taken from this rather than from the earlier publications.

Manuscript of the list has been submitted to most of the lepidopterists of the State. There have thus been obtained many additional records from Mr. Davis in the entire order, from Messrs. Watson, Angle, and Bell in the butterflies, and from Mr. Wild in all groups but especially in the Microlepidoptera. Mr. Engelhardt has gone over the whole list, and has made numerous additions and corrections, but especially in the Ageriidae, and Mr. Bird has similarly concentrated on the P'apaipema group. We are particularly indebted to $\mathrm{Mr}$. Angle for allowing us to incorporate a manuscript list of the Rhopalocera of the State, with many records.

The arrangement of families follows the synopsis in Memoir 68 of the Cornell University Agricultural Experiment Station, pages 34 to 44 . For the species also that memoir is followed, with some corrections, as far as it goes-that is, through the Drepanidae. Beyond this point the order within each family is approximately that of Barnes and McDunnough's check list, sometimes reversed in order to start each family with the recognized primitive forms. The numbers attached are those of Barnes and McDunnough's list, more recently recognized segregates being designated by decimal numbers, and other recently described species by a dash in the number column.

County records are cited only when no more specific records from the county are at hand, save in the case of a few records from economic sources, where the citation is "___ Co, generally." In such cases the species is believed to occur wherever the food plant is commercially grown in the county. Local lists are treated similarly where detailed localities are not given in them, except in the case of Staten Island, which is generally taken as a unit.

In all, 2439 named forms are recognized as more or less authentically reported from the State, besides a few very doubtful ones passed over in footnotes. Of these, 169 are treated as varieties and 2270 as apparently valid species. There are also 11 undescribed Microlepidoptera of more than usual interest, mentioned in the list. 


\section{SUBORDER JUGATAE \\ FAMILY MICROPTERYGIDAE}

MICROPTERYX Hübner

8481 M. auricrinella W1sm. Mt. Marcy, $4000 \mathrm{ft} .(F b s)$ and Wehrle; Keene, Wild (Fbs); Caroline-Harford. May-J1.

\section{FAMILY ERIOCRANIIDAE}

ERIOCRANIA Zeller

8477 E. auricyanea W1sm. Karner (empty mine), Mar-Ap. Oak, chestnut, and chinquapin.

\section{FAMily HEPIALIdAE: The Swifts \\ SThenopis Packard}

8482 S. argenteomaculatus Harr. Castile, Brad; Catskills ; LI: Glendale; former breeding places in red maple and alder swamps near Brooklyn have been destroyed; not taken for thirty years (Eng). Midsummer. Alder.

8483 S. purpurascens Pck. form quadriguttatus Grt. Adirondack Lodge, Bish (Fbs) ; Lancaster, Buffalo (EPV); Albany.

8485 S. auratus Grt. Fentons; Lancaster; Ithaca; McLean; Granville, J1 4 (Eng); summit of Catskills, J1.

8484 S. thule Strk. Waddington, J1, Wood.

\section{Hepialus Fabricius}

8490 H. gracilis Grt. Allegany Pk., Aug 23, Wild (Fbs).

\section{SUBORDER FRENATAE \\ FAMLY INCURVARIIDAE}

\section{Tegeticula Zeller}

8471 T. yuccasella Rly. Ithaca (Fbs); LI, May-J1. Yucca filamentosa.

\section{INCURVARIA Haworth}

8430 I. russatella Clem. Ithaca. May; J1.

8429 I. taylorella Kf. Newcomb. May; J1. Ribes.

\section{PARACLEMENSIA Dyar}

8443 P. acerifoliella Fitch. St. Lawrence Co, generally; Black Br.; L. George (NYS); West Falls, Wild (Fbs); Ithaca; McLean; Deposit; Bolton (Felt); Albany. Maple.

\section{AdELA Latreille}

8449 A. ridingsella Clem. Rock City; Ithaca. Je-J1.

8454 A. purpura Wlk. Cranberry Cr., Ap.

8457 A. bella Cham. Gowanda (Wild) ; Crugers. Je. 


\section{FAMily NEPTICULIDAE \\ ECTOEDEMIA Busck}

8422 E. heinrichi Bsk. E. Aurora, Je (Wild).

\section{Nepticula von Heyden}

8384 N. slingerlandella Kf. Plum Leaf-Miner. Rochester. Plums and prunes, wild plum (Prunus americana), and occasionally sweet cherry.

8383 N. pomivorella Pck. Apple Serpentine Leaf-Miner. Castile, Je, Brad (Fbs); Albion; Geneva. Apple.

8373 N. latifasciella Cham. E. Aurora, Je, Wild (Braun).

\section{FAMILY MEGALOPYGIDAE}

\section{MEGALOPYGE Hübner}

\section{(Lagoa Harris)}

4858 M. opercularis S. \& A. A stray has been found at Ithaca.

4861 M. crispata Pck. Flannel Мотн. Le Roy (CU Econ); Onteora Mt.; New Baltimore; Bethlehem; Karner; Albany; Rhinebeck; Poughkeepsie; New Windsor; Rye; Croton-on-Hudson; Katonah; SI; LI: Brooklyn, Woodhaven, etc. Je-Jl. Various shrubs and trees.

\section{FAMILY EUCLEIDAE}

\section{Phobetron Hübner}

4832 P. pithecium S. \& A. HAG-Mотн. Bolton (larvae); New Haven, Albion (NYS) ; McConnellsville (CU Econ); Canandaigua (NYS) ; Lockport (NYS) ; Niagara Falls; E. Aurora, Buffalo; Sodus (NYS); Oswego; Lancaster; Randolph (CU Econ); Castile, Brad; Otsego (CU Econ); St. Johnsville (NYS); Hudson Val., from Rensselaer south; LI. Aug-Sep. A general feeder on shrubs.

\section{ISOCHAETES Dyar}

4833 I. beutenmulleri H. Edw. SI, pin oak (Joutel).

\section{SIBINE Herrich-Schaeffer}

4812 S. stimulea Clem. SAdDlE-BACK. Poughkeepsie; Highland Falls, Ossining, Athens, Catskill, Cementon, Kingston, Spring Val., Scarsdale (NYS); Rye; NYC; SI; LI: Brooklyn; Woodhaven; Millneck (NYS). On various trees; Indian corn, etc. (Ds).

\section{EuClea Hübner}

4816 E. delphinii Bdv. Plattsburg; Peru. Je-Jl. The typical form, with varieties interjecta Dyar and viridiclava Wlk., is rather common southward. Variety elliotii Pears. is recorded from Big Ind. Val.; also LI: Coney I.; Glendale; Maspeth (Olsen); Wyandanch, Wading R. (Ds). Variety paenulata Clem. is reported from NY by Pck. Various trees and shrubs. 


\section{PARASA Moore}

4813 P. indetermina Bdv. New Rochelle (NYS); SI; LI: Woodhaven, etc. Feeds on apple and many other trees and shrubs.

4814 P. chloris H-S. New Windsor; Rye, J1 (Ds); SI; LI: Forest Pk.; Brooklyn.

\section{ADONETA Clemens}

4824 A. spinuloides H-S. (leucosigma Pck.). Albany; New Windsor; SI: LI: Pinelawn; Yaphank. Jl-Aug. Various shrubs.

\section{SISYROSEA Grote}

4828 S. textula H-S. General from Plattsburg southward. J1-early Aug. Wild cherry and many other shrubs.

\section{NATADA Walker}

4830 N. nasoni Grt. LI: Ronkonkoma; Yaphank. Oak and other trees.

\section{PROLIMACODES Schaus}

4827 P. badia Wlk. (scapha Harr.). BeEchnut Box. Grand I., E. Aurora (Wild) ; Geneva ; Ithaca; Karner; Florida; Rhinebeck; West Haverstraw (NYS) ; SI; LI: Brooklyn, E. New York, Lynbrook, Newtown, etc. Judging from the larvae seen, the species will prove much more widespread. J1-Aug. Various trees and shrubs.

\section{Cochlidion Hübner}

4839 C. biguttata Pck. Plattsburg; Ithaca; SI (Ds) ; LI: Ronkonkoma; Yaphank. Je-Jl. Oak only.

4840 C. rectilinea G. \& R. Vic. NYC (Bt, G\&R). Northern records are perhaps based on C. latomia.

4840.1 C. latomia Harv. "NY."

4841 C. y-inversa Pck. Batavia; Lewiston; Buffalo; Ithaca; Rogers Rock, L. George, Jones; Big Ind. Val.; Poughkeepsie; New Windsor; NYC; SI; LI. J1. Hickory and blue beech.

\section{TORTRICIDIA Packard}

4852 T. flexuosa Grt. E. Aurora and ${ }^{\circ}$ W. Falls (Wild) ; Ithaca; McLean; Debruce; Big Ind. Val.; Onteora Mt.; Ilion; Sharon Sps.; Rhinebeck; Poughkeepsie; Windsor; Bronx Pk.; LI. J1. The dark form, caesonia Grt., is known from Big Ind. Val., ,Coeymans, and Poughkeepsie.

4853 T. pallida H-S. Plattsburg; Keene Valley; Ithaca; McLean; Rye; SI; LI: Brooklyn; Bellport; Coram, Kch (Fbs). J1. Willow oak and other shrubs.

4854 T. testacea Pck. Plattsburg; Saranac L.; Red L.; E. Aurora (Wild); Ithaca; McLean; Big Ind. Val.; Nyack, Aug, Ft. Montgomery, May (Ds) ; Rye; SI; LI: Merrick, Je (Eng). Oak, birch, wild cherry, etc. 
HETEROGENEA Knoch

4847 H. shurtleffi Pck. Vic. NYC (Eliot, Angus).

\section{LITHACODES Packard}

4842 L. fasciola H-S. Newport (NYS) ; Rochester (Angle); Buffalo; S. Wales, E. Aurora (Wild); Castile, Brad; Geneva; Ithaca; McLean; Rogers Rock, L. George, Jones; Schenectady; the southern Catskills; Rye; SI (Ds) ; LI: Greenport (Ds) ; Orient, Lath. Jl-Aug. Basswood.

PACKARDIA Grote and Robinson

4843 P. elegans Pck. Goat I.; E. Aurora; Ithaca; McLean; Newburgh; New Windsor; Katonah; NYC; LI. Je-J1. . On thin, shaded leaves of trees in dense woods.

4845 P. geminata Pck. Castile, Je, Brad (Fbs); Gowanda, Orchard Pk. (Wild); Rye (Bird) ; LI: Forest Pk.; Brooklyn. Je. On very small shrubs only a few inches high, especially on wild cherry.

4846 P. albipunctata Pck. Karner; Dutchess Co; Ft. Montgomery, May (Ds).

\section{FAMILY PYROMORPHIDAE}

\section{Acoloithus Clemens}

4876 A. falsarius Clem. Syracuse; Ithaca; Rhinebeck; Crugers; LI: Centerport; L. Ronkonkoma. May-Je. Grape and ampelopsis.

\section{PYROMORPHA Herrich-Schaeffer}

4867 P. dimidiata H-S. Coy Glen, Ithaca; West Pt. (Ds); SI; LI: Brooklyn; Pennequid (Eng). End of May-J1.

HARRISINA Packard

4881 H. americana Guér. Troy; Poughkeepsie; SI. May-Aug. Grape.

4881.1 H. texana Stretch. Poughkeepsie; Ft. Montgomery (Ds); SI (Ds) ; LI: Woodhaven; Yaphank; Wading R. (Ds); Orient, Lath. Je-Aug. Grape.

\section{FAMILY TINEIDAE SUBFAMILY ACROLOPHINAE}

ACROLOPHus Poey

8192 A. arcanellus Clem. Rhinebeck, Fordham (Dy); SI, Bt (Dy). Je-Jl. Corn.

8170 A. plumifrontellus Clem. Hastings (Grt) ; Parksville (Bt) ; LI: Forest Pk., Kch. J1.

8195 A. mora Grt. Niagara Falls and E. Aurora (Wild); LI: Woodhaven, Forest Pk., late Sep-Oct, Kch (Fbs). 


\section{SUBFAMILY TINEINAE}

\section{SCARDIA Treitschke}

8231 S. approximatella Dietz. Lime L. (Wild); Ithaca; McLean. J1-Aug. The larva has been found in a rotting sycamore log.

8223 S. anatomella Grt. Buffalo (Wild), Je-J1.

\section{TINEA Linnaeus}

8247 T. misella Z. Alexandria Bay, Thousand Isls., Je, Kch (Fbs).

8248 T. obscurostrigella Cham. McLean. May; Jl-Aug; Oct.

8254 T. trimaculella Cham. Otto, J1.

8249 T. fuscipunctella Haw. Ithaca, J1, a scavenger in birds' nests, Dobroscky (Fbs).

8258 T. pellionella L. Otd-fashioned Clothes Moth. Louisville; Canandaigua; Alfred Center. The larva is one of the clothes moths.

8257 T. grumella Z. Ithaca, Aug-Sep.

8271 T. ophrionella Dietz. Uphill Br., Mt. Marcy, Je; E. Aurora, J1, Wild (Braun).

8281 T. tylodes Meyr. (arcella auct.). Riceville, Cattaraugus Co (Wild) ; Ithaca (Dietz,Fbs). J1. Larva on fungi under bark of dead alder twigs.

8282 T. auropulvella Cham. Buffalo ( $W^{\text {ild }}$ ) ; Ithaca; McLean. Je-Aug.

8283 T. acapnopennella Clem. E. Aurora, Gowanda, Buffalo, Rock City (Wild); Ithaca; Albany. Je-Aug. Larva on Polyporus tulipiferus.

8289 T. granella L. European Grain-Moth. Batavia; Castile, Je, Brad (Fbs); Ithaca; Albany; LI: Orient, Lath. Aug. Grain, dried fruits, bracket fungi, and similar substances.

\section{TRICHOPHAGA Ragonot}

8284 T. tapetzella L. Case-bearing Clothes Moth. SI: New Dorp (Ds). A clothes moth and general scavenger, often breeding in owl pellets.

\section{MONOPIS Hübner}

8243 M. irrorella Dietz. Otto; lthaca. Je; Aug.

8241 M. dorsistrigella Clem. E. Aurora, Chautauqua L. (Wild) ; Ithaca; LI: Sea Cliff. Je-J1.

8242 M. crocicapitella Clem. E. Aurora, Buffalo (Wild); Ithaca; Morrisania, NYC; SI: Clove Valley; LI: Glendale, Kch (Fbs). Jl-Oct. The larva has been bred from seeds of absinth and from refuse.

8238 M. biflavimaculella Clem. Peru; Wilmington; E. Aurora (Wild); Castile, Brad; Ithaca; McLean; Big Ind. Val. Je-Sep.

8239 M. marginistrigella Cham. E. Aurora, Je, Wild (Braun).

8237 M. rusticella Hbn. Ithaca, probably introduced. May-midsummer; again in autumn. 
Tineola Herrich-Schaeffer

8298 T. bisselliella Hummel. Common Clothes Moth. Abundant everywhere. The commonest of the clothes moths.

\section{ISOCORYPHA Dietz}

8444 I. mediostriatella Clem. E. Aurora, Aug 29, Wild (Fbs).

\section{DIACHORISIA Clemens}

8307 D. argentistrigella Cham. Ithaca, Jl, Fbs (Busck).

8312 D. costisignella Clem. E. Aurora (Wild); Ithaca (Dietz). Je-J1.

8313 D. fasciella Cham. Chautauqua L., Je, EPV (Fbs); Ithaca, J1, Fbs (Busck).

\section{Choropleca Walsingham and Durrant}

8246 C. vesaliella Cham. "NY" (Dietz). Je.

\section{HYвROMA Clemens}

8301 H. servulella Clem. E. Aurora, Wild (Fbs); Ithaca. Je-Aug.

\section{XYLESTHIA Clemens}

8234 X. pruniramiella Clem. E. Aurora (Wild) ; Ithaca; McLean; West Farms; LI: Glendale, Kich (Fbs). May-Aug. The larva mines in woody excrescences on plum.

\section{AMYDRIA Clemens}

8197 A. effrenatella Clem. Ithaca; LI: Orient, Lath. Je, Aug.

\section{FAMILY PSYCHIDAE}

\section{SOLENOBIA Zeller}

4809 S. walshella Clem. Lockport, L. M. Dozener (Fbs, Wild); Niagara Falls, Wild (Braun); E. Aurora, Wild (Fbs); Ithaca; Bronxville (Wood). Ap-May. On lichens on trunks of trees.

\section{EURYCYTTARUS Hampson}

4803 E. confederata G. \& R. Ithaca; New Baltimore; SI; LI: Newtown. On low plants.

\section{THYRIDOPTERYX Stephens}

4798 T. ephemeraeformis Haw. BAG Worm. Geneva (cases); Ft. Edward; New Baltimore; Rye (Bird); Mt. Vernon; Yonkers (CU Econ); NYC; SI; LI, general. Introduction attempted, but species not able to survive at Ithaca though a pest on SI and LI. On various trees and shrubs, even arbor vitae and other evergreens, sometimes injurious. 


\section{FAMILY TISCHERIIDAE}

TISCHERIA Zeller

7873 T. malifoliella Clem. Apple Trumpet Leaf-Mrner. E. Aurora, Wild (Fbs); Schenectady; Menands; E. Greenbush; NYC (CU Econ). The larval work is seen throughout the State, but I have seen no other records. May. Apple.

7863 T. solidaginifoliella Clem. McLean; Albany. Aug. Solidago.

7861 T. tinctoriella Cham. Albany (NYS). Oak.

7857 T. citrinipennella Clem. Crosby; Ithaca. Aug. Oak.

7859 T. albotraminea W1sm. "NY" (type), Aug. White oak.

7876 T. zelleriella Clem. OAK Trumpet Leaf-Miner. "NY" (HEdw). MarAp. Oak.

\section{FAMILY LYONETIIDAE}

\section{BEDELLIA Staintor}

8120 B. somnulentella Z. NYC (Lint). Aug; mid-Sep to spring. Ipomoea.

\section{PhyLlocnistis Zeller}

8110 P. populiella Cham. Hemlock L. (bred). Aspen and other poplars.

8111 P. vitifoliella Cham. "NY" (Bt). Grape.

8112 P. vitigenella Clem. E. Aurora (Wild). May; Oct.

\section{Bucculatrix Zeller: The Ribbed-Cocoon Makers}

8133 B. niveella Cham. Rock City.

B. errans Braun. var. McLean, J1 30.

8141 B. ainsliella Murt. Ithaca, Aug (Fbs). Oak.

8135 B. pomifoliella Clem. Ribbed-Cocoon Maker of the Apple. Common everywhere in NY. Je. Apple.

8125 B. canadensisella Cham. Birch Leaf-Skeletonizer. Ogdensburg; L. Placid; Ausable Forks; Keene; Scottsville; Protection (Wild); Ithaca; Albany; Karner; Kinderhook; SI. Je-J1. White birch.

8153 B. crescentella Braun. Otto; Florida. J1. Aster, Solidago, and Erigeron.

8139 B. trifasciella Clem. E. Aurora, May, Wild (Braun).

B. cuneigera Meyr. E. Aurora, J1, Wild (Braun).

B. fusicola Braun. E. Aurcra, Jl, Wild (Braun).

Philonome Chambers

8101 P. clemensella Cham. "NY" (Bt). J1. Hickory and linden.

\section{FAMILY OPOSTEGIDAE}

\section{Opostega Zeller}

8419 O. albogaleriella Clem. Otto; Geneva; McLean. J1-Aug. Bast miner on gooseberry and on red and black currant.

O. albogaleriella var. quadristrigella Cham. McLean, J1 (Fbs). 


\section{FAMILY GRACILARIIDAE}

\section{PARORNIX Spuler}

\section{(Ornix)}

8023 P. inusitatumella Cham. E. Aurora, May-Je (Wild).

8013 P. guttea Haw. E. Aurora, Je (Wild).

8018 P. crataegifoliella Clem. E. Aurora, J1 (Wild). Cherry.

\section{GRACILARIA Haworth}

8041 G. sassafrasella Cham. Ithaca, J1-Aug. Sassafras.

8042 G. rhoifoliella Cham. E. Aurora; Ithaca; McLean. J1-Aug. Rhus toxicodendron and $R$. copallina.

G. elongella L. Cranberry L., Htly (Fbs); Wilmington; Mt. Whiteface; McLean. Alder; wild cherry (Htly).

G. pulchella Cham. Lancaster, Sep (Wild); Cranberry L., Htly (Bsk). Yellow birch.

8057 G. burgessiella Z. Ithaca; McLean. J1-Aug. Cornus.

8066 G. cornusella Ely. Buffalo (Wild); Ithaca. Ap; Aug. Cornus.

8065 G. bimaculatella Ely. Lancaster, Rock City (Wild); Ithaca. Red and soft maple.

8059 G. packardella Cham. E. Aurora (Wild) ; Ithaca. J1-Aug. Sugar maple.

8061 G. purpuriella Cham. Buffalo, E. Aurora, Wild; Ithaca; LI: Forest Pk., Aug, $K c h$ (Fbs). Aug; Oct-Ap. Willow and poplar.

8040 G. negundella Cham. E. Aurora, Wild (Braun); Ithaca. J1-Aug; Oct early spring. Acer negundo.

8056 G. azaleella Brants. (azaleae Bsk.). AzAleA LEAF-Roller. Rochester; Lockport (NYS); Yonkers. Evergreen azaleas.

8054 G. superbifrontella Clem. West Falls (Wild); Crosby; Ithaca. May-Sep. Hamamelis.

8071 G. fraxinella Ely (Coriscium). Ithaca, J1-Sep, May (the latter were doubtless specimens which had emerged in the preceding fall). Ash and apple.

8010 G. serotinella Ely (Parectopa). Ithaca, J1-early Sep. Prunus serotina.

\section{ACROCERCops Wallengren}

7997 A. strigifinitella Clem. Hamburg; Gowanda, E. Aurora, Wild (Fbs). Je. Breeds continually all summer. Chestnut, chinquapin, oak, and beech.

8001 A. astericola Frey \& Boll. Castile, Je, Brad (Fbs); Ithaca, May-Aug. Aster corymbosus, etc.

7993 A. albinotella Cham. "NY" (Bt). Ap-Je; Aug. Oak.

7991 A. venustella Clem. Ithaca, Aug 30. Late J1-Sep. Eupatorium.

\section{Marmara Clemens}

8033 M. elotella Bsk. Larval work has been seen in this State under bark of apple twigs and under skin of fruit. The species is apparently rather widely distributed in the apple belt of western NY, and possibly to a somewhat lesser extent in that of the Hudson R. 


\section{PARECTOPA Clemens}

8011 P. salicifoliella Cham. Lancaster, Sep, Wild (Braun).

8003 P. robiniella Clem. Ithaca (mines not rare). In a digitate mine on locust. 8006 P. pennsylvaniella Engel. Castile, Je, Brad (Fbs); Ithaca; McLean. MayOct. Aster cordifolius.

8000 P. plantaginisella Cham. E. Aurora, Wild (Braun). J1. Fleabane (Wild).

\section{CRemastobomycia Braun}

7974 C. ignota Frey \& Boll. Salamanca, J1 (Wild). Compositae.

\section{LiTHOCOLLETIS Hübner}

7877 L. fitchella Clem. Albany. Oak.

7885 L. argentifimbriella Clem. Ithaca, Aug. Oak, especially white oak.

7886 L. lucidicostella Clem. Buffalo, May (Wild). Sugar maple.

7879 L. hageni Frey \& Boll. Buffalo, May (Wild). Oak.

7891 L. ostryaefoliella Clem. Ithaca, Aug; E. Aurora, Buffalo, May (Wild). Hop hornbeam.

7898 L. propinquinella Braun. E. Aurora, May (Wild). Prunus serotina.

7899 L. crataegella Clem. "NY" (Bt). Rosaceae.

7932 L. salicifoliella Clem. Buffalo (Wild); Ithaca. Aug-Sep. Poplar and willow.

7917 L. morrisella Fitch. Delmar (NYS). Amphicarpa.

7915 L. robiniella Clem. Ithaca (CU). Locust.

7902 L. argentinotella Clem. Buffalo, Wild (Braun). Elm.

7894 L. basistrigella Clem. E. Aurora, Buffalo, Ap (Wild). Oak.

7920 L. lucetiella Clem. E. Aurora, J1; Ithaca, Aug. Basswood.

7969 L. desmodiella Clem. Big Ind. Val. Desmodium, etc.

7923 L. ostensackenella Fitch. E. Aurora, J1 (Wild). Locust (Robinia PseudoAcacia).

7924 L. tritaenianella Cham. Buffalo, May. Hop hornbeam.

7928 L. fragilella Frey \& Boll. Honeysuckle Leaf-Miner. Honeoye Falls, Leon (Braun). Honeysuckle.

7921 L. tiliacella Cham. Ithaca, Aug. Basswood.

7942 L. hamadryadella Clem. White-Blotch OAK LeAf-Miner. Syracuse (NYS) ; Buffalo, Grand I., Niagara Falls, May-J1 (Wild) ; Ithaca, Aug; Albany; New Hamburg, Crugers I., Coeymans, Goshen, Garrison, Sloatsburg, Mt. Vernon (NYS) ; LI: Forest Pk., May, Kch (Fbs). Oak, especially Quercus alba, and rarely, perhaps accidentally, on Magnolia and Ostrya.

7941 L. cincinnatiella Cham. New Baltimore (NYS).

7938 L. lentella Braun. E. Aurora, May (Wild); Ithaca, J1-Aug. Birch and hop hornbeam.

7951 L. cervina W1sm. "NY" (type).

7961 L. guttifinitella Clem. Buffalo, J1 (Wild). Poison ivy.

7962 L. obstrictella Clem. E. Aurora, Jl (Wild). Poison ivy. 
7964 L. aesculisella Cham. S. Wales; Gowanda, Je-J1 (Wild). Buckeye.

7966 L. aceriella Clem. S. Wales (Wild) ; Ithaca, J1. Maple, most commonly red maple.

7965 L. ostryarella Cham. E. Aurora, J1 (Wild). Ironwoods.

\section{FAMILY COLEOPHORIDAE}

Coleophora Hübner: The Case-Bearers

7757 C. cerasivorella Pck. E. Aurora, Chautauqua L., Tonawanda (Wild). Je-J1. Wild cherry.

7768 C. fletcherella Fern. Cigar Case-Bearer. Buffalo, E. Aurora, Je-J1 (Wild); Chautauqua Co (CU Econ); Oswego Co; S. Byron; Monroe Co; Albion (Felt); Geneva; Ithaca; Albany. Je-J1 (Wild). Apple, pear, and hawthorn (Wild).

7775 C. laricella Hbn. Larch Case-Bearer. E. Aurora, May-Je (Wild); Ithaca; Karner; Albany. Larch.

7802 C. unicolorella Cham. Buffalo and E. Aurora, May-Je (Wild). Juncus.

7785 C. pruniella Clem. Orchard Pk., Oct (Wild).

7759 C. cinerella Cham. Cranberry L., Htly (Bsk).

7762 C. coruscipennella Clem. E. Aurora, Gowanda (Wild); Ithaca; McLean; LI: Forest Pk., Glendale, Kch (Fbs). May-Aug.

C. spissicornis Haw. "NY". Reported by Zeller, no doubt in error for coruscipennella. Clover.

7792 C. rosacella Clem. E. Aurora, Je (Wild). Rose.

7754 C. albiantennaella Wild. Buffalo, Je-J1 (Wild). Dogwood.

7751 C. caryaefoliella Clem. E. Aurora; Ithaca. Je-J1. Hickory and pecan.

7788 C. ostryae Clem. E. Aurora, Je (Wild). Hornbeam.

7776 C. limosipennella Dup. (laticornella Clem.). Cranberry L., Htly (Bsk); E. Aurora, J1 (Wild) ; Ithaca; Karner; NYC; LI: Oyster Bay, Oakdale (Felt); Brooklyn (Bsk). Elm; hickory (Wild).

7764 C. cratipennella Clem. E. Aurora, Gowanda, May-J1 (Wild).

7758 C. caespititiella Z. LI: Coney I.; Flatbush, New Lots (Zab). Seeds of Juncus.

7803 C. vernoniaëlla Cham. McLean, Je-J1. Vernonia and Helianthus.

7769 C. fagicosticella Cham. (Iineapulvella Cham.). E. Aurora, Gowanda 7778 (Wild). Je-J1. Chestnut (Wild).

7811 C. vagans Wlsm. "NY" (type). Aug. Grass.

7777 C. leucochrysella Clem. Gowanda, Wild. Je. Chestnut.

7800 C. tiliaefoliella Clem. E. Aurora, Gowanda, Buffalo, Wild; Ithaca, J1. JeJ1. Basswood (Wild); linden.

7781 C. malivorella Rly. Pistol Case-Bearer. Walworth; Orleans Co; S. Byron; Geneva; Lansing. Early J1. Apple.

7791 C. querciella Clem. Albany; LI (Hein). Je. Oak.

C. atlantica Hein. E. Aurora, Je-J1 (Wild). Wild cherry. 


\section{FAMILY CYCNODIIDAE}

\section{(Elachistidae)}

\section{Aphelosetia Stephens}

\section{(Elachista)}

7827 A. orestella Bsk. Peru; Perry; E. Aurora (Wild); Ithaca; McLean. May-Sep. Hystrix.

A. cucullata Braun. Ithaca, May-early Je. Carex jamesii.

7844 A. albicapitella Engel. E. Aurora, Je (Wild).

A. n. sp. near trapeziella. Buffalo, J1, Wild.

\section{FAMILy DOUGLASIIDAE}

TinAGMa Zeller

7848 T. obscurofasciella Cham. Black Br.; Rock City. May.

\section{FAMILY HELIOZELIDAE}

ANTISPILA Hübner

7581 A. hydrangiaeella Cham. West Falls, J1, Wild (Fbs).

7578 A. ampelopsiella Cham. Ft. Edward, Aug. Ampelopsis.

7583 A. nyssaefoliella Clem. Buffalo, E. Aurora, Gowanda (Wild); Mohonk L. (larvae); Glens Falls; NYC (Lint); LI: Orient, Lath (NYS). May and Je.

7582 A. isabella Clem. Buffalo, J1, E. Aurora, Je, Wild (Fbs).

\section{COPTODISCA Walsingham}

7595 C. splendoriferella Clem. Resplendent Shield-Bearer. Ithaca; E. Greenbush; Albany; Fishkill. Crataegus, wild cherry, and apple.

\section{FAMILY GECOPHORIDAE}

\section{MARTYRINGA Busck}

6412 M. latipennis Wlsm. Ithaca, J1-Aug.

\section{EUMEYRICKIA Busck}

6414 E. trimaculella Fitch. Upper Ausable L.; Keene (Wild); E. Aurora, Salamanca (Wild). Je-J1.

\section{GERDANA Busck}

6416 G. carietella Bsk. E. Aurora, Wild (Braun); Ithaca; McLean. Jl-Aug.

\section{Cryptolechia Zeller}

6424 C. tentoriferella Clem. Potsdam; Rochester (Angle); E. Aurora (Wild); Ithaca; McLean; Big Ind. Val.; Schenectady; Karner; Rhinebeck; New Windsor; NYC; SI; LI: Glendale, Woodhaven, Kch (Fbs); Orient (Lath). Late Aug-Oct. Oak, cherry, maple (?), chestnut, etc. 


\section{PSILOCORSIS Clemens}

6425 P. quercicella Clem. Buffalo, Salamanca (Wild); New Windsor, Morton. Mar-Ap; J1. Oak and chestnut.

6426 P. obsoletella Z. Ithaca. Oak and chesmut.

6427 P. reflexella Clem. Ithaca; McLean. Je-J1. Oak.

6430 P. ferruginosa Z. Otto.

\section{AGONOPTERYX Hübner}

(Depressaria, in part)

6433 A. atrodorsella Clem. Fentons; E. Aurora, Buffalo (Wild) ; Crosby; Castile, Brad; Ithaca; McLean; Gloversville (Alex); Delmar; Schenectady; New Windsor; SI. Sep-Je. Bidens.

6437 A. canella Bsk. Wilmington; E. Aurora, Wild (Fbs); Catskills. J1-Sep. The related European species, alstroemeriana, feeds on Conium.

6449 A. pulvipennella Clem. Fentons; E. Aurora, Lancaster, Riceville (Wild) ; Ilion; Crosby; Ithaca; McLean; Schenectady; LI: Orient, Lath (NYS). Sep-May; J1. Eupatorium and Solidago.

6459 A. argillacea W1sm. E. Aurora, Wild (Fbs); Ithaca. Mar; Sep. This species is the American representative of yeatiana, which eats Umbelliferae.

6447 A. applana clemensella Cham. E. Aurora (Wild); Ithaca; New Windsor. Aug-Ap. Parsnip and wild parsnip (Heracleum).

6443. A. walsinghamella Bsk. Albany. Myrica.

6456 A. plummerella Bsk. Rock City.

A. n. sp. Ithaca, Ap.

6445 A. curvilineella Bt. E. Aurora (Wild) ; NYC. Oct-Ap.

6468 A. lythrella W1sm. McLean, J1-Aug. Lythrum alatum.

6463 A. flavicomella Engel. Ithaca, Je-J1.

6471 A. robiniella Pck. E. Aurora (Wild); Schenectady; Bridgetown; Rhinebeck; Crugers; Katonah. J1. Robinia.

6472 A. lecontella Clem. Ithaca; Schenectady; New Windsor; LI: Sea Cliff; Glendale, Sep, Kch (Fbs); Orient, Lath.

6489 A. allenella W1sm. (Semioscopis). Keene, E. Aurora (Wild); McLean; Rhinebeck; LI : Cold Sp., Kch (Fbs). Je-J1. Oak.

\section{DEPRESSARIA Haworth}

6479 D. groteella Robn. "NY" (Robn). Corylus.

6482 D. cinereocostella Clem. "NY" (Bsk). J1. Water parsnip (Sium) and caraway.

6480 D. betulella Bsk. Ithaca, Aug (Fbs).

6478 D. heracliana DeG. Parsnip Webworm. E. Aurora, Irving (Wild); Bath (CU Econ); Crosby; Ithaca; Albany; Greenville (NYS); Rhinebeck; New Windsor; West Farms; LI: Orient, Lath (NYS). Je-J1. Wild parsnip (Heracleum) and other Umbelliferae.

6473 D. emeritella alienella Bsk. Ilion. Artemisia.

18 
INGA Busck

6483 I. sparsiciliella Clem. West Farms, May-Je.

\section{SEMroscopis Hübner}

6488 S. inornata Wlsm. Ithaca; Karner.

6487 S. megamicrella Dyar. Ithaca; LI: E. New York. Ap-May.

6485 S. merriccella Dyar. Albany; Big Ind. Val. May.

6486 S. aurorella Dyar. Ithaca; Big Ind. Val.

6484 S. packardella Clem. E. Aurora (Wild) ; Ithaca; Big Ind. Val.; Albany. Ap-Je.

\section{ETHMIA Hübner}

6642 E. longimaculella Cham. Plattsburg; Ithaca. May; J1. Lithospermum.

\section{SCHIFFERMUELLERIA Hübner}

5490 S. argenticinctella Clem. Grand I., Rock City (Wild) ; Ithaca; McLean; Rhinebeck; NYC. End Je-Aug. Under bark of elder.

S. formosella Schiff. LI: Forest Pk., Aug 31, 1921, J1 17, 1923, Kch (Fbs). Heretofore known only from Europe, where the larva lives under bark of poplar.

FABIOLA Busck

6498 F. shaleriella Cham. Salamanca, J1 (Wild).

\section{DECANTHA Busck}

6497 D. borkhausenii Z. (boreasella Cham.). Ithaca, Je-J1. Under bark of pine.

\section{ECOPHORA Latreille}

6499 E. newmanella Clem. N. Elba; Batavia; E. Aurora, Gowanda (Wild) ; Rock City; Portage; Ithaca; Trenton Falls; Albany; New Windsor. Je.

\section{BORKHAUSENIA Hübner}

6505 B. ascriptella Bsk. Ithaca; McLean. Je-Aug.

\section{FAMILY XYLORICTIDAE}

STENOMA Zeller

6606 S. schlaegeri Z. Mt. Marcy; Thousand Isls., Kch; E. Aurora (Wild) ; Rock City; Castile, Je (Fbs) ; Ithaca; McLean; Big Ind. Val.; Bethlehem; New Windsor. May-Je. Oak.

6609 S. algidella W1k. (leucillana auct.). Buffalo (Wild) ; Rock City; Ithaca; McLean; Trenton Falls; Big Ind. Val.; Ft. Montgomery. Je-Aug. The caterpillar is common on maple and on various trees and shrubs, and has been found boring in an apple.

6614 S. querciella Bsk. Ithaca, J1 24 (Fbs); LI: Coram, J1 17, Kch (Fbs). 
Menesta Clemens

6397 M. albaciliaeella Cham. Peru (Fbs) ; Protection, Wild (Fbs). Early spring Je ; J1. Blackberry.

\section{FAMILY GELECHIIDAE}

\section{GELECHIA Hübner}

6208 G. trialbamaculella Cham. "NY" (Bt); late J1-early Aug. The caterpillar is found on many shrubs, such as locust, oak, Comptonia, Epigaea, and Vaccinium.

- G. psiloptera Barnes \& Bsk. var. Peru.

6211 G. continuella Z. L. Tear; Ithaca. J1-Sep.

6245 G. discoocellella Cham. E. Aurora, Je (Wild) ; Ithaca, J1 (Fbs) ; LI: Forest Pk., Aug 20, Kch (Fbs).

6236 G. fluvialella Bsk. Peru; Allegany Pk. (Wild); Rock City; Ithaca, Fbs (Bsk). Je-Jl.

6247 G. anarsiella Cham. var. E. Aurora, J1, Wild (Fbs). Wild cherry.

6253 G. albisparsella Cham. Ithaca.

6249 G. inquilinella Bsk. Karner. Spring. Larva inquiline in Rhabdophaga brassicoides gall on Salix.

6263 G. versutella Z. E. Aurora, J1, Wild (Braun).

6265 G. bicostomaculella Cham. Keene, Buffalo, E. Aurora, May-Je (Wild). Black oak.

6268 G. maculimarginella Cham. Cranberry L., Htly (Bsk); Buffalo (Wild); Salamanca, E. Aurora (Wild) ; Ithaca. Je-J1. Ash.

6269 G. biminimaculella Cham. E. Aurora, Buffalo, J1 (Wild).

6275 G. vernella Murt. Ithaca, J1. Laurel oak.

6266 G. nigrimaculella Bsk. Lancaster, EPV (Fbs); Ithaca. Late J1-early Oct.

6289 G. gilvomaculella Clem. E. Aurora; Ithaca. J1. Oak.

6279 G. fondella Bsk. E. Aurora, Je (Wild).

6296 G. pseudofondella Bsk. Ithaca. Je-JI.

6278 G. mediofuscella Clem. Peru; Amherst, E. Aurora, Gowanda (Wild); Rock City; Crosby; Potter Swamp; McLean. Early spring; J1. In seeds of Ambrosia trifida.

6280 G. walsinghami Dietz. Ithaca. May-J1.

- G. arenella Fbs. LI: Rockaway Beach. May-Aug.

6295 G. branella Bsk. Ithaca, J1, 1924 (Fbs).

\section{GNORIMOSCHEMA Busck}

6122 G. gallaesolidaginis Rly. Goldenrod Gall. Canandaigua (NYS); vic. Buffalo; Ithaca; McLean; Big Ind. Val.; Albany; LI: Richmond Hill. End Aug-Sep. In stems of goldenrod, forming a fusiform gall.

6126 G. gallaeasteriella Kell. E. Aurora, Protection, Aug-Sep (Wild).

6109 G. banksiella Bsk. Essex Co; Rochester (NYS); Batavia; E. Aurora; Otto; Ithaca; McLean; LI: Sea Cliff. J1-Aug. Solidago.

6117 G. batanella Bsk. E. Aurora, Buffalo, Je (Wild).

6119.1 G. chenopodiella Bsk. Ithaca, Je, Chap (Fbs). Chenopodium. 


\section{ANACAMPSIS Curtis}

6188 A. innocuella Z. Orchard Pk., Gowanda (Wild) ; Ithaca; Ilion; Pcarl R.; LI: Cold Sp. Harb. J1. Poplar.

6189 A. rhoifructella Clem. E. Aurora, Buffalo (Wild); West Farms; LI: Forest Pk., Kch (Fbs). May-Aug. Sumac; viburnum; spicebush, blackberry, and poplar (Wild).

6199 A. agrimoniella Clem. Otto; E. Aurora, Jc-J1 (Wild) ; Taughannock Falls; McLean; LI: Sea Cliff. Agrimony.

6195 A. nonstrigella Bsk. E. Aurora, Je-J1. Hop hornbeam (Wild).

6200 A. tristrigella W1sm. E. Aurora, J1, Wild (Fibs). Corylus; ironwood (Wild).

\section{ANARSIA Zeller}

6353 A. lineatella Z. PEAch Bud-Mотн. Rochester; Lockport; Clifton; Ithaca ; Jordan Station; Schoharie; Coxsackie, Blauvelt (NYS). Peach and plum.

\section{TRICHOTAPHE Clemens}

6355 T. flavocostella Clem. Rochester (Angle); Buffalo, E. Aurora (Wild) ; Ithaca; Ilion; Big Ind. Val.; Poughkeepsie; New Windsor; NYC. Je-J1. Solidago and Helianthus.

$6356 \mathrm{~T}$. inserrata Wism. Buffalo, Je (Wild).

6361 T. alacella Clem. Buffalo, E. Aurora (Wild) ; Ithaca; LI: Sea Cliff. Je-J1. Solidago (Wild).

6363 T. nonstrigella Cham. Keene, Je (Wild).

6364 T. juncidella Clem. E. Aurora, Wild (Fbs); Ithaca; McLean; Rhinebeck. Je-Sep. Solidago, Aster, Artemisia, and other Compositae.

6365 T. trinotella Bsk. Ithaca (Fbs); Colden, EPV (Fbs).

6366 T. leuconotella Bsk. E. Aurora (Bsk).

6368 T. levisella Fyles (ruashingtoniella Bsk.). Ithaca. Je; Aug. Aster.

6376 T. fernaldella Bsk. Peru, Je.

6367 T. setosella Clem. Ithaca; LI: Sea Cliff. J1-Aug.

\section{DICHOMERIS Hübner}

(Ypsolophus)

6380 D. ligulella Hbn. (pometella Harr.). PAlmer Worm. Buffalo, E. Aurora, Randolph (Wild) ; Geneva; Rock City; Ithaca; Byron; Albany. MayJ1. Apple, oak, azalea, and nettle; often injurious on apple.

6378 D. punctidiscella Clem. E. Aurora, Irving (Wild) ; Ithaca; Taughannock Falls; McLean. End of May-J1.

6389 D. ventrella Fitch (?gcorgiella W1k.). "NY" (Fitch); Gowanda (Wild).

6379 D. eupatoriella Cham. E. Aurora, Je (Wild); Crosby. May-J1; late Sep. Eupatorium ageratoides and Vernonia.

D. marginella Fab. JUniper Webworm. Ithaca; Tarrytown; NYC (NYS); LI: Westbury (NYS); Plandome. Aug. Juniperus communis. 


\section{GLYPHIDOCERA Walsingham}

6408 G. meyrickella Bsk. Ithaca.

G. (Sceptea) n.sp. Ithaca (Fbs).

\section{ANORThosia Clemens}

6351 A. punctipennella Clem. Taughannock Falls; Ithaca; McLean. Je-Aug.

\section{STROBISIA Clemens}

6398 S. emblemella Clem. Rock City; Taughannock Falls. Je-early J1. Caterpillar possibly on buckeye.

6399 S. irridipennella Clem. In Robinson Coll. (presumably from NY). Je-Aug.

\section{Duvita Busck}

6184 D. vittella Bsk. LI. Larva in "stunted cones" of Scotch and Austrian pine, and in "cecidomyid gall" on Pinus taeda.

6185 D. nigratomella Clem. Rock City; Ithaca. May-J1.

6186 D. concinusella Cham. E. Aurora, J1 (Wild); Rock City.

D. tahavusella Fbs. Uphill Br., Mt. Marcy; Peru. Je-early J1.

D. n.sp. near tahavusella. Ithaca (Fbs).

6283 D. conclusella W1k. E. Aurora, Protection (Wild) ; Peru; Castile, Je, Brad (Fbs); Rock City; Ithaca; McLean. Je-Aug.

\section{PARALECHIA Busck}

6166 P. pinifoliella Cham. Pine Leaf-Miner. Round L.; Ithaca; Karner. Je-J1. Leaf-miner in pine needles.

\section{AROGALEA Walsingham}

6167 A. cristifasciella Cham. Crosby; Ithaca, Babiy (Fbs) ; LI: Forest Pk., Kch (Fbs). Ap; J1. Oak.

\section{Telphusa Chambers}

6071 T. longifasciella Cham. E. Aurora; Crosby; Ithaca. Ap-May.

6074 T. latifasciella Cham. LI: Forest Pk., Jl 4, 1920, Kch (Fbs).

6080 T. basifasciella Z. Grand I., Buffalo (Wild); Ithaca (Fbs). Late May-J1.

6075 T. quercinigracella Cham. Ithaca, J1; LI: Forest Pk., Je, Kch (Fbs). Blackjack and other oaks.

6078 T. querciella Cham. E. Aurora, Wild (Fbs).

6077 T. palliderosacella Cham. Orchard Pk., West Falls (Wild); Rock City. Oak.

6083 T. belangerella Cham. Keene, Je, Wild (Fbs); E. Aurora, May, Wild (Fbs).

Glauce Chambers

6040 G. pectenalaeella Cham. Rock City; McLean. J1-Aug. 


\section{ARIstotelia Hübner}

6041 A. roseosuffusella Clem. E. Aurora, Gowanda, Protection (Wild) ; Ithaca; McLean; LI: Glendale, Kch (Fbs). May-Oct; commonest in J1 and Aug.

6042 A. pudibundella Z. Rock City, J1.

6052 A. absconditella W1k. E. Aurora, J1 (Wild); Lockport, Scotia, Albany (Felt). Sep.

6058 A. quinquepunctella Bsk. E. Aurora, Je, Wild (Braun); Ithaca, J1 (Fbs).

ENCHRYSA Zeller

6067 E. dissectella Z. Wells (NYS); Rock City (Wild).

\section{CHRYSOPORA Clemens}

6069 C. hermannella Fab. Ithaca. Chenopodium (Sling).

\section{RECURVARIA Haworth}

6135 R. apicitripunctella Clem. Wells; Ithaca (abundant). Je-J1. Hemlock.

6136 R. variella Cham. Yonkers, Je-J1. Bald cypress, and evidently other trees.

6141 R. thujaëlla Kf. LI: Westbury; Hicksville (NYS). Je. Thuja.

6138 R. piceaëlla Kf. E. Aurora, Hamburg, Je-J1 (Wild); Scarsdale (NYS). Hemlock.

6156 R. gibsonella Kf. Ithaca, Je-early J1. Juniper.

6143 R. crataegella Bsk. E. Aurora; Batavia; Albany. May-J1. Crataegus.

\section{EucoRdyleA Dietz}

6065 E. atrupictella Dietz. Ithaca, Aug-Sep 5.

\section{EPIthectis Meyrick}

6160 E. attributella Wlk. E. Aurora, Salamanca (Wild); Ithaca; McLean; Bronx Pk. (Wild). J1-Aug.

6162 E. sylvicolella Bsk. E. Aurora, May, Wild (Fbs).

\section{EvipPe Chambers}

6062 E. prunifoliella Cham. McLean, May-JI. Plum and peach; rarely on soft maple.

6063 E. leuconota Z. Has been reported from Albany.

\section{Sitotroga Heinemann}

6038 S. cerealella Oliv. Angoumors Grain Moth. Undoubtedly distributed throughout the warmer parts of the State, definite localities being: Geneva; Ithaca; Albany; Poughkeepsie; LI: Smithtown. Aug. In stored grain. 
PTycerata ,Ely

6035 P. busckiella Ely. Ithaca, Je 30, Fbs (Bsk).

\section{METZNERIA Zeller}

6021 M. lappella L. Burdock Moth; Triple-spotted Yellow. Peru; Newcomb; Alexandria Bay, Thousand Isls., Kch (Fbs); Rochester (NYS); E. Aurora, Irving (Wild) ; Lewistown; Castile, Brad; Ithaca; McLean; Katonah. May-Aug. In burs of burdock.

HELICE Chambers

5972 H. constrictella Z. Buffalo, Je (Wild); also Sep. Elm.

\section{FAMILY BLASTOBASIDAE}

EURESIA Dietz

6528 E. pulchella Dietz. Salamanca (Wild); Ithaca, J1-Aug 5 (Fbs).

\section{VALENTINIA Walsingham}

6520 V. glandulella Rly. Acorn Mотн. Ithaca, J1. In acorns.

6522 V. quaintancella Dietz. Ithaca, J1. Larva in mummy apples.

\section{HOLCOCERA Clemens (restricted) 63}

6531 H. dives Dietz. Ithaca, J1.

6.532 H. aphidiella W1sm. Rock City; Ithaca. J1. In Phylloxera gall on hickory.

6541 H. confluentella Dietz. Ithaca, Je-J1. On pitch pine.

65.36 H. purpurocomella Clem. Rock City; Vandalia. Je.

$6540 \mathrm{H}$. chalcofrontella Clem. Ithaca, J1. Larva in sumac heads.

-_._ H. n. sp. near villella Bsk. Ithaca ( $\mathrm{Fbs}$ ).

6549 H. funebra Dietz. Rock City, May-J1.

6555 H. elyella Dietz. Ithaca, J1-Aug.

6539 H. modestella Clem. E. Aurora, May-Je (Wild). Red oak (Wild).

\section{BLASTOBASIS Zeller}

6513 ?B. plummerella Dietz. Rock City, J1.

6514 B. sagittella Dietz. Rock City, Aug.

\section{PLOIOPHORA Dietz}

6578 P. fidella Dietz. Rock City, Je; Ithaca (Fbs).

\section{Pigritia Clemens}

6580 P. purpurella Dietz. Rock City, Je.

${ }^{63}$ All determinations in this genus are tentative ( $\mathrm{Fbs}$ ). 


\section{FAMILY LAVERNIDAE}

LAVERNA Curtis

6006 L. brevivittella Clem. Ithaca; Albany. Oct. In seed capsules of Oenothera.

6007 L. stellella Bsk. E. Aurora, Je-Sep, Wild (Braun).

— L. n. sp. Ithaca, Oct-Ap (Fbs).

6018 L. decorella Steph. (unifasciella Cham.). Ithaca (Fbs). Aug-Sep. Stem gall on Epilobium.

LYMNAECIA Stainton

5989 L. phragmitella Stt. Catrair Mотн. Lewiston; E. Aurora, Irving (Wild); Ithaca; McLean (larval work). May-Jl. Cattail.

WALSHIA Clemens

5985 W. amorphella Clem. Ithaca. May; J1. Amorpha fruticosa.

\section{Stilbosis Clemens}

5984 S. tesquella Clem. Irving, Wild (Fbs); Ithaca; LI: Sea Cliff. May-J1; Aug. Lespedeza.

\section{LOPHOPTILUS Sircom}

5990 L. tricristatella Cham. Uphill Br., Mt. Marcy. May-Je.

6008 L. eloisella Clem. E. Aurora, Wild (Braun); Ithaca, Je; LI: Forest Pk., Jl, Kch (Fbs). Stems of Oenothera.

5994 L. passerella Bsk. NYC (Wat).

\section{PSACAPHORA Herrich-Schaeffer}

5998 P. luciferella Clem. "NY" (Clem).

5996 P. terminella engelella Bsk. E. Aurora, Jamestown (Wild); Rock City; Castile, Je, Brad (Fbs); Hemlock L.; McLean. May; Je. Circaea lutetiana.

P. n. sp. E. Aurora, Wild.

\section{PERIMEDE Chambers}

5987 P. erransella Cham. Ithaca; McLean. J1-Aug.

P. falcata Braun. Ithaca, Je-J1.

\section{BLASTODACNA Wocke}

5986 B. curvilineella Cham. Ithaca (Wellhouse). The moth emerges in spring. In berries of Crataegus.

\section{BATRACHEDRA Stainton}

7821 B. salicipomonella Clem. Larva at Ithaca. Inquiline in willow galls, especially the sawfly gall on the leaves. 


\section{COSMOPTERYX Hübner}

5966 C. fernaldella W1sm. McLean, J1.

5957 C. qlemensella Stt. "NY" (US). Ipomoea.

5958 C. gemmiferella Clem. E. Aurora, Wild (Braun); LI: Sea Cliff (Bsk). Je-J1. Ipomoea.

C. magophila Meyr. Ithaca, Aug.

\section{FAMILY YPONOMEUTIDAE \\ YPONOMEUTA Latreille}

7723 Y. multipunctella Clem. E. Aurora, Je, basswood (Wild); Ithaca, late $\mathrm{J} 1$ - early Aug (Fbs). Evonymus.

7728 Y. padella L. Apple Ermine Mотн. Introduced on nursery stock in a number of localities in the vicinity of Geneva and Rochester and at Schoharie, but now exterminated. Apple and other trees of Rosaceae.

Atteva Walker

7731 A. punctella Cram. (aurea Fitch). LI: Newtown (Doll). Ailanthus.

URODUS Herrich-Schaeffer

U. n. sp. Cranberry L., pupa in suspended lace cocoon, Htly (Fbs).

\section{Plutella Schranck}

7683 P. maculipennis Curt. Diamond-Back Мотн. E. Aurora; Ithaca; McLean; Albany; NYC (Watson); LI: common and general. Aug-Sep. On various Cruciferae; often injurious to cabbage.

7675 P. porrectella L. Buffalo, May, on sweet rocket (Wild).

\section{Cerostoma Latreille}

7650 C. falciferella W1sm. Ithaca, Aug. Larva to be expected on Rosaceae.

7654 C. dentiferella Wlsm. Buffalo, J1, Wild (Kf).

C. dentiferella form canariella W1sm. McLean, Aug (Fbs).

C. xylostella L. European Honeysuckle Leaf-Roller. Ithaca, Je-J1 Lonicera.

\section{EPERMENIA Hübner}

8089 E. ramapoëlla Kf. Ramapo (type).

\section{ARGYRESTHIA Hübner}

7692 A. calliphanes Meyr. Cranberry L., Htly (Bsk); Ithaca, J1-Aug (Fbs); Albany. Alder.

7695 A. oreasella Clem. Wells; Rock City; Ithaca; McLean; Poughkeepsie; West Farms. J1-Aug. Larva possibly on oak.

7689 A. freyella Wlsm. Ithaca, Seeley (US). Je. Red cedar and arbor vitae.

7700 A. subreticulata Wlsm. West Falls, Aug (Wild); Ithaca, J1 (Fbs). Bass. wood (Wild). 
7687 A. alternatella Kf. Ithaca. May; J1. Juniper berries (Markowitz).

7703 A. conjugella Z. Mt. Marcy, 3000 ft.; Trenton Falls. J1. On fruits of apple and mountain ash.

7711 A. undulatella Cham. E. Aurora, Gowanda, Je (Wild).

7712 A. austerella Z. Otto; E. Aurora, Buffalo, Gowanda (Wild) ; Rock City; Ithaca. Je-J1.

7713 A. thuiella Pck. Arbor Vitae Leaf-Miner. Ithaca; LI: Hicksville: Westerly. Records in economic literature likely to refer also to Recurvaria thujaëlla.

\section{OCNEROSTOMA Zeller}

O. pinariella Z. European Pine Leaf-Miner. Ithaca, Jl, Comstock, Fbs (Fbs).

\section{ZELLERIA Stainton}

7721 Z. (Xyrosaris) celastrusella Kf. Buffalo (Wild); E. Aurora, Wiid (Braun). End of May-Je. Celastrus scandens.

\section{SCYTHRIS Hübner}

8079 S. impositella Z. Protection, E. Aurora, Niagara Falls (Wild) ; Rock City; West Farms. Je.

8080 S. eboracensis Z. E. Aurora, Gowanda (Wild); Rock City; Portage; Crugers; LI: Sea Cliff. Je-J1; end of Aug-Sep. Thistle.

8078 S. basilaris Z. Keene Val.; LI: Cold Sp. Harb. Je-J1.

\section{FAMILY GLYPHIPTERYGIDAE \\ SIMAËTHIS Leach \\ (Hemerophila Hübner)}

S. pariana Cl. Apple and Thorn Skeletonizer. First discovered by D. B. Van Buren, of NYS Dept. Farms and Markets, in Westchester Co in 1917. Throughout Hudson R. Val. and LI, southward from Whitehall. Je; Aug; Oct-May. Apple, rarely thorn and pear.

7607 S. pavonacella Clem. Ramapo. May; J1-Aug. Desmodium and Amphicarpa.

\section{ChOREutis Hübner}

- C. gnaphaliella Kearfott. Castile, Je, Brad (Fbs).

7608 C. inflatella Clem. Wilmington; Batavia; E. Aurora, Irving, Niagara Falls (Wild) ; Ithaca; West Farms. Je-J1; Sep. Scutellaria lateriflora. Most or all of these records are var. virginiella Clem.

7618 C. onustana Wlk. Mt. Whiteface; Uphill Br., Mt. Marcy; E. Aurora; Keene, Gowanda, Allegany Pk. (Wild) ; Ithaca. Je-J1.

\section{GLYPHIPTERYX Hübner}

G. impigritella Clem. McLean (Fbs).

7633 G. circumscriptella Cham. In Robinson Coll., presumably from NY. J1.

ABREnTHia Busck

7606 A. cuprea Bsk. Protection, Je 16, 1918, Wild (Fbs). 


\section{FAMILy HELIODINIDAE}

\section{EuClEMENSIA Grote}

7574 E. bassettella Clem. Ithaca (How, Fbs); Nyack (Zab); LI: Flatbush. J1-Aug. Larva in Kermes.

\section{CyClOPLASIS Clemens}

7558 C. panicifoliella Clem. NY(?). Late J1-Aug. Panicum clandestinum.

\section{SCHRECKENSTEINEA Hübner}

7573 S. erythriella Clem. Ithaca; Albany. Aug. Sumac.

7572 S. festaliella Hbn. Gowanda, Wild (Braun); McLean; Ithaca; Rock City. Ap-May; J1-Aug. Rubus.

\section{FAMILY $\mathbb{E}$ EGERIIDAE}

\section{MEMYThRUS Newman}

6755 M. asilipennis Bdv. Rochester Jct.; Buffalo; West Pt., Je (Ds); SI; LI: Brooklyn, Amagansett (Eng); Jamaica, Kch. Oak, in roots and in base of trunks (Eng).

6753 M. simulans Grt. var. luggeri H. Edw. Buffalo (Wild) ; SI; LI: Jamaica. Late May-J1; the moths flying in vic. NYC in odd-numbered years. Black and red oaks, saplings and branches (Eng); very rarely in white oak or chestnut.

6754 M. palmii H. Edw. LI: Flatbush; Yaphank. White oak (Eng).

6747 M. tricinctus Harr. Buffalo; Lancaster; Big Ind. Val.; Karner; LI: general. Je-Jl. Willow and poplar.

6757 M. dolli Neum. LI: Brooklyn. Poplar and willow (Eng).

6752 M. scepsiformis H. Edw. Suffern, Aug (Eng).

6744 M. pyramidalis Wlk. Axton; Niagara Falls, EPV; Big Ind. Val.; LI: Brooklyn; Bay Shore. Je-Aug.

M. pyramidalis form coloradensis H. Edw. LI: Brooklyn, Aug (Eng).

6745 M. fraxini H. Edw. (morrisoni H. Edw.). Suffern, Aug, a root borer in ampelopsis (Eng).

\section{Melittia Hübner}

6739 M. satyrinifromis Hbn. SQUASH VINE-BORER. Probably generally distributed throughout the State south of the Adirondacks. Definitely recorded from: Wells; Monroe, Wayne, Onondaga, Seneca, and Tompkins Counties; Rome; Johnstown; Schenectady; Albany; NYC; SI; LI. Injurious to squash, pumpkin, and other Cucurbitaceae.

\section{Podosesia Möschler}

6726 P. syringae Harr. LIlac Borer. Albany; Rye (Eng); NYC (Eng); LI: Brooklyn (Eng). Ash and lilac. 


\section{SANNINA Walker}

6725 S. uroceriformis Wlk. Albany. Persimmon.

\section{PARHARMonIA Beutenmüller}

6723 P. pini Kell. Oswego Co; Buffalo; Portage; Ithaca; Karner; Round L. (NYS) ; Hastings Center, East Schodack, Kinderhook (NYS); Hudson; Ulster Co. (NYS). J1. Pitch pine, white pine, and spruce.

\section{CONOPIA Hübner}

\section{(Sesia)}

6655 C. exitiosa Say (Samninoidea). Peach Tree Borer. Generally distributed and injurious in the Great Lakes strip including Genesee and Wyoming Counties; Trumansburg, Ithaca, Gloversville; Hudson Val. from Albany south; SI; LII, in western part. J1-Aug. Peach, plum, and cherry (Eng).

C. exitiosa var. edwardsi Bt. West Falls, Allegany Pk. (Wild); Phelps; Trumansburg; McLean. Jl-Aug. Largely replaces typical form in western and northern parts of State (Eng).

6674 C. rubrofascia H. Edw. Reported from NYC. This record is doubtful (Eng).

6675 C. bolteri H. Edw. Ithaca; Big Ind. Val.; Mosholu. Willow.

6715 C. pyralidiformis Wlk. Fairport; Batavia; E. Aurora, J1 28, Wild (Fbs); SI; LI: Amagansett; Woodhaven.

6686 C. corni H. Edw. LI: Woodhaven, May-Je, in rootstock of Doellingeria umbellata (Eng).

C. acerrubri Eng. Sharon Sps.; Karner; SI, Je (Ds) ; LI: Brooklyn and Newtown (Eng); Hempstead (CU). Maple, especially red maple (Eng).

6685 C. acerni Clem. Maple Callus-Borer. Speculator; Rochester (Angle); Buffalo; Irving ( Wild) ; Kenmore; Rock City; Ithaca ; Ilion; Schenectady; Albany; SI; LI: Brooklyn; Newtown. Je-JI. Maple.

6677 C. fulvipes Harr. Big Ind. Val.

6679 C. pictipes G. \& R. Lesser Peach-Tree Borer. L. Ontario strip from Hastings Center to Youngstown and Buffalo; Lyndonville; Hector; Ithaca; Catskills; Schenectady; Albany; Poughkeepsie; West Pt. (Ds) ; NYC; SI; LI: Brooklyn; Sands Pt. (Bs). Part of these records may belong to $C$. castaneae. Je. June berry and other Rosaceae.

6680 C. castaneae Bsk. Ithaca, Je 5, 1897 (CU); NYC (Eng); LI (Eng). Je-Jl. Chestnut.

C. viburni Eng. LI: Flatbush, Woodhaven, and vic. (Eng). Ap-May. Viburnum.

6694 C. rubristigma Kell. Mosholu; LI: Brooklyn. Oak galls.

6689 C. rhododendri Bt. RHODODENDRon BoRER. Rochester; Ithaca; NYC; LI: Brooklyn. Rhododendron.

6668 C. bassiformis Wlk. Tuckahoe; SI; LI: Sea Cliff. Aug-Sep. Rootstock of Eupatorium. 
6690 C. scitula Harr. Buffalo, Irving (Wild); Ithaca; Hudson; Pine I. (Ds); LI. Je-J1. Cornus; oak; hazel (Eng); hickory; chestnut; Crataegus (Wellhouse); and in oak galls, perhaps mixed with other species, certainly from Andricus galls on oak.

6669 C. tipuliformis L. Imported Currant Borer. Plattsburg; Orleans Co; Buffalo; Chautauqua Co; Ithaca; Kendall; Albany; U1ster Co; SI; LI: Pinelawn; Easthampton; Floral Pk., May (Ds); Brooklyn (Éng). Je. Gooseberry and currant stems.

$66 \varepsilon 8$ C. pyri. Harr. PeAR BorLR. Honeoye Fálls; Albion; Buffalo (Kell); Colden, Aug 3, EPV; Ithaca; SI; LI: Brooklyn. May; late J1. Apple, pear, etc.

6681 C. albicornis H. Edw. E. Aurora, Buffalo (Wild); Catskills; Karner; NYC; SI (Ds); LI: Brooklyn. Je-J1. Willow and poplar.

6691 C. ithacae Bt. Plattsburg (Ds); Ithaca (type). End of Je-early Aug. Roots of Heliopsis.

6660 C. rileyana H. Edw. SI: Tottenville, Aug (Eng, Ds) ; LI: Rockaway (Eng). Roots of Solanum (Eng).

6699 C. sigmoidea H. Edw. NYC (Eng); LI: Amagansett; Woodhaven. Aug. Black willow.

\section{ZEGERIA Fabricius}

6652 ÆE. apiformis C1. HoRnet Moth. Injurious in Brooklyn and NYC (Eng); LI: Glendale, Je, Kch; reported also from Syracuse. Poplar and willow.

6654 Æ. tibialis Harr. Plattsburg; Keene Val. (Eng); Goat I. (Kell). Je-J1. In poplar and willow.

\section{ALCATHO}

6650 A. caudata Harr. New Russia, Jones; NYC; LI: Brooklyn; Newtown. JeAug. In crown of Clematis.

A. caudata var. walkeri Neum. LI: Jamaica (Eng).

\section{BEMBECIA Hübner}

6649 B. marginata Harr. BlACKBERRY CROWN-Borer. Wilmington; Lancaster; E. Aurora, Buffalo (Wild) ; Allegany Pk., Wild (Fbs); Ithaca; West Pt., Sep, Robn (Ds); SI; LI. Blackberry and raspberry roots, often injurious.

B. marginata var. albicoma Hulst. Big Ind. Val.; LI: Brooklyn.

\section{FAMILY TORTRICIDAE}

\section{SUBFAMILY EUCOSMINAE}

HEMIMENE Hübner

7233 H. dana Kf. Black Br.; Batavia; Rock City; Crosby; Ithaca; McLean. May-Je.

7256 H. bittana Bsk. West Falls, Buffalo (Wild) ; Ithaca. Je-J1.

7255 H. simulana Clem. Buffalo (Wild); Ithaca; West Farms. End of May; end of Aug.

7254 H. incanana Clem. E. Aurora, Gowanda, Portage (Wild). 


\section{LASPEYRESIA Hübner}

\section{(Enarmonia)}

7231 \, lautana Clem. McLean; Ithaca; Ramapo. Ap-May.

7207 ᄂ. caryana Fitch. Pecan Shuck-Worm. Easton (type). May-Aug. Hickory and walnut.

7208 L. prunivora W1sm. Lesser Apple Worm. Buffalo, Je (Wild); Crosby; Ithaca, Aug (Hein). Young plums, Crataegus fruit, and crab apples.

7219 L. interstinctana Clem. Clover-Seed Caterpillar. Newport; E. Aurora, Gowanda (Wild) ; Castile, Brad; Ithaca; McLean; Big Ind. Val.; NYC. May-Je; late J1-Sep. Clover.

$7 \angle 15$ L. saundersana Kf. Karner (Fbs).

7213 L. fana Kf. E. Aurora, May 30, Dnd (Fbs).

7216 L. eclipsana Z. Castile, Brad; Ithaca. Ap-May. Larva on grape.

7235 L. nigricana Steph. (novimundi Hein.). PeA Mотн. Niagara Falls (Wild). Je-Aug. Larvae in pods of peas and other legumes.

7234 L. dandana Kf. E Aurora, Gowanda, Buffalo, Je-Aug (Wild).

L. molesta Bsk. Oriental Fruit Moth. Lockport, Aug, Strickland (larvae); White Plains (NYS); Larchmont (CU); LI: Flushing (CU). Injurious to peach, plum, apple, and other Rosaceae.

7250 L. multilineana Kf. Jamestown, Je.

7224 L. albimaculana Fern. Buffalo, Je-J1 (Wild).

7236 L. gallaesaliciana Rly. Peru; Buffalo, J1 (Wild); Big Ind. Val. Je-J1. Gall on willow.

7236 L. populana Bsk. Ithaca, J1, Fbs (Hein).

\section{CARPOCAPSA Treitschke}

7270 . C. pomonella L. Conling Moтн. Generally distributed. Spring; J1-Aug. Injurious chiefly to apple and pear.

\section{MeLissopus Riley}

7269 M. latiferreanus orichalceanus W1sm. Gowanda, E. Aurora (Wild); Ithaca; McLean; Karner. Jl-Aug; late Sep. On young acorns.

\section{GYMNANDROSOMA Dyar}

7252 G. punctidiscanum Dyar. Ithaca; NYC; LI: Maspeth. May-J1.

\section{ECDYTOLOPHA Zeller}

7253 E. insiticiana Z. Locust Gall. Peru; Lewiston; E. Aurora (Wild); Canandaigua (NYS) ; Baldwinsville; Rochester Jct.; Ithaca; Karner ; Rhinebeck; New Windsor; LI: Patchogue (NYS). May-Aug. Makes fusiform gall on twigs of locust. 


\section{EPINotia Hübner}

7163 E. nanana Tr. (piceafoliana Kf.). Portage Falls; Gowanda, Wild (Fbs); Scarsdale (NYS). Je. Spruce.

7140 E. lindana Fern. Ithaca; McLean. Sep. Cornus.

7084 E. signatana Clem. Newport (NYS); Buffalo (Wild); Castile, Je, Brad (Fbs) ; Rock City; Ithaca; McLean; New Windsor. Je. Maple.

7280 E. timidella Clem. Crosby; Ithaca (CU, bred); LI: Bellport (Dyar). May. Oak.

7028 E. similana Hbn. Pearl R. Hazel and birch.

6987 E. medioviridana Kf. McLean, Aug-Sep.

6984 E. vertumnana Z. McLean, Mar-May.

6979 E. walkerana Kf. Ithaca, late J1-Sep. Catkins of hazel.

6974 E. transmissana Wlk. Saranac Inn; Cranberry L., Htly (Bsk); summit of Mt. Marcy; E. Aurora, Buffalo (Wild) ; Rock City ; Ithaca ; McLean ; Albany; LI: Forest Pk., Kch (Fbs). J1-early Aug. Hazel (Wild).

6972 E. solicitana Wlk. Castile, Je, Brad (Fbs); E. Aurora, Niagara Falls $($ Wild $)$. May-Je. White birch.

6964 E. rectiplicana Wlsm. Peru (Fbs); Keene, Wild (Fbs); Ithaca; McLean. Je-J1. Willow.

E. signiferana Hein. Hemlock L., Sep (Fbs).

\section{ANCYLIS Hübner}

7203 A. tineana Hbn. McLean, Ap-J1. Poplar and various Rosaceae.

(7196) A. diminutana Haw. (uncana auct. not Hbn.). Peru; Black Br.; Crosby. Ap-J1. Willow.

7206 A. albacostana Kf. Black Br.; LI: Orient, May 5, Lath.

7173 A. mediofasciana Clem. Karner; Normanskill. May-Je.

7194 A. cornifoliana Rly. E. Aurora, Wild (Fbs); Ithaca; McLean. May-Je. Blackberry, cornel, and birch.

7193 A. apicana Wlk. Keene, Wild (Fbs) ; Buffalo, Je (Wild) ; Ithaca; McLean; Trenton Falls; Wellsville. May-Je. Raspberry.

7192 A. divisana W1k. Crosby; Ithaca; Trenton Falls. May. Oak and sycamore.

7189 A. floridana Z. Castile, May 16, Brad (Fbs); LI: Forest Pk., Aug 31, Kch (Fbs).

7185 A. comptana Froel. Strawberry Leaf-Roller. Reported from Buffalo (Wild), Glens Falls, Nassau, and West Farms. May. Injurious to strawberries in Europe. The strawberry leaf-roller in this State may be either this species or A. floridana Z.

\section{ANCHYLOPERA Stephens}

$$
\text { (Ancylis, in part) }
$$

7174 A. nubeculana Clem. N. Elba; Peru; Cranberry L., Htly; Palmyra; E. Bloomfield; Buffalo; E. Aurora; Fredonia; Buffalo ; Rock City ; Castile, Leon; Crosby; McLean; Ithaca; Albany (NYS); Niverville; LI: Glendale, Kch (Fbs). May-Jl. Apple and other trees. 
7175 A. subaequana Z. Black Br.; Ithaca. Je-J1; end of Aug.

7178 A. semiovana Z. Ithaca, May-early J1.

7184 A. maritima Dyar. LI: Speonk. Larva in summer on beach pea; moth in following spring and again in Aug.

7181 A. pulchellana Clem. Ithaca, May-J1.

7180 A. angulifasciana Z. Clover Leaf-Tier. E.Aurora (Wild) ; Castile, Brad; Crosby; Ithaca; McLean; LI: Forest Pk., Kch (Fbs). May-J1; late Aug. Clover.

7191 A. platanana Clem. Rock City; Big Ind. Val.; New Windsor. Je.

7186 A. burgessiana Z. Keene, E. Aurora (Wild) ; Rock City ; Ithaca ; McLean ; Karner. Je-J1. Oak; also plum (var. pruni).

7190 A. laciniana Z. E. Aurora, May-J1 (Wild).

7187 A. dubiana Clem. E. Aurora, Je (Wild).

- A. fuscociliana Clem. Rock City; Crosby; Ithaca; McLean; Trenton Falls. Je. Chestnut and elm.

7176 ?A. discigerana W1k. (spiracifoliana Hein. not Clem.). Newcomb; Rock City; Castile, Brad; Ithaca; McLean. Je-early J1.

NORMA Heinrich

7147 N. dietziana Kf. Rock City, May-Je. Crataegus.

\section{EPIblema Hübner}

6970 E. abbreviatana Wlsm. E. Aurora, Wild (Fbs); Buffalo, Niagara Falls (Wild); McLean. May-Je.

6981 E. strenuana W1k. Stanley; Poughkeepsie (NYS) ; NYC (Watson) ; LI: Glendale, Forest Pk., Kch (Fbs). May-Sep. Ambrosia.

E. strenuana var. minutana Kf. Buffalo, E. Aurora (Wild); Ithaca.

7026 E. otiosana Clem. Peru; Ithaca; Big Ind. Val.; LI: Forest Pk., Kch (Fbs) ; Orient, Lath. Je-Oct. Bidens.

6988 E. brightonana Kf. Ithaca, Aug.

7025 E. tripartitana Z. Southern NY, Aug. Larva in gall of Rudbeckia laciniata and Solidago.

7245 E. walsinghami Kf. Rock City, May.

7020 E. carolinana. W1sm. Buffalo (Wild); Ithaca. J1-Aug.

7014 E. scudderiana Clem. Lewiston; Irving (Wild); Buffalo; Castile, Brad; Ithaca; McLean; Albany; New Windsor. Je. Solidago.

7016 E. obfuscana Dyar. Rock City; Ithaca; McLean; SI: Clove Val. May-Sep. Solidago.

7013 E. illotana W1sm. E. Aurora (Wild); Kinderhook; LI: Orient, Lath. May-Je.

7015 E. desertana Z. Peru; E. Aurora, Buffalo (Wild) ; Ithaca; New Windsor ; Ramapo. May. Solidago.

\section{Eucosma Hübner}

6901 E. circulana Hbn. SI(?). This species presumably occurs northward, but northern records, so far as I have seen the specimens recently, are based on other species of the group. The type is said to have come from Penn. 
6901.1 E. scintillana Clem. Ithaca; McLean; LI: Woodhaven, Kch (Fbs). Je; Aug-Sep.

6902 E. dodecana Z. "NY", Je.

6884 E. adamantana Gn. "NY", Sep (coastal marshes).

6879 E. quinquemaculana Rob. Wells (NYS); LI: Bellmore. Je; Aug-Sep.

6880 E. robinsonana Grt. Buffalo, Je (Wild).

7036 E. sombreana Kf. Ithaca; LI: Brooklyn. J1-Aug. Helianthus.

6934 E. cataclystiana Wlk. Peru; E. Aurora, Wild (Fbs); Buffalo (Wild); Ithaca; McLean; Poughkeepsie; SI: Clove Val.; LI: Orient, Lath. Je-Aug. Ambrosia.

6917 E. pergandeana Fern. Peru; LI: Lynbrook; Gay I. (in LI Sound). Je-J1.

6926 E. comatulana Z. var. E. Aurora, Wild (Fbs); Ithaca. J1 21.

6967 E. juncticiliana W1sm. Otto; Ithaca; McLean. J1-Aug. Larva boring in Solidago.

7029 E. dorsisignatana Clem. Essex Co; McLean (Fbs); Ithaca; Dansville; New Windsor; Pine I.; NYC; SI; LI: Cypress Hills, May 25, Woodhaven, Sep, and Forest Pk., Aug, Kch (Fbs).

E. dorsisignatana Clem. var. diffusana Kf. Buffalo, Aug (Wild) ; LI: Coram.

7029.1 E. similana Clem. (confluana Kf). Buffalo (Wild) ; Ithaca; McLean; Big Ind. Val.; LI: Glendale, Kch (Fbs). Aug-Sep.

6950 E. eumaea Meyr. Ithaca, J1.

6941 E. womonana Kf. E. Aurora, Wild (Fbs); Ithaca; McLean, Aug 26.

E. palabundana Hein. Ithaca, J1-Aug. An atypical specimen, probably representing a good race.

E. tocullionana Hein. Peru, Je (Fbs). Larva on Picea, boring in cones.

\section{THIODIA Hübner}

7059 T. refusana W1k. LI: E. New York. Ap-May; Sep.

7239 T. parmatana Clem. (crispana Kf., etc.). Ithaca; LI: Forest Pk., Kch (Fbs). Je-Sep.

T. alterana Hein. Ithaca, Aug.

T. marmontana Hein. Ithaca, J1-Aug.

7054 T. formosana Clem. E. Aurora, Dnd (Fbs); Rock City; Ithaca; McLean; Karner; Ramapo; SI: Watchogue. End of May-Je.

7053 T. ferruginana Fern. Black Br.; E. Aurora; Rock City; Ithaca; McLean; SI; LI: Forest Pk., Kch (Fbs). May-Je.

7051 T. roseoterminana Kf. E. Aurora (Wild).

7052 T. awemeana Kf. Crosby; McLean; Ithaca. May-Je.

7048 T. radiatana Steph. N. Eiba; Castile, Je, Brad (Fbs); E. Auröra, Buffalo (Wild); McLean; Big Ind. Val.; Ramapo; Bronxville; LI: Oyster Bay. End of May-Je.

7065 T. striatana Clem. Peru; Oswego; E. Aurora, Niagara Falls; Crosby; Karner; Nassau; Ramapo; New Windsor; Katonah; Ithaca. End of May - Aug.

7067 T. clavana Fern. "NY" (Hein).

7063 T. ochroterminana Kf. Potter Swamp; Ithaca. Aug-Sep. 
7075 T. raracana Kf. E. Aurora, Aug, Wild (Fbs) ; LI: Forest Pk., Central Pk., Aug, Kch (Fbs).

- T. perfuscana Hein. Ithaca, Aug.

6976 T. tomonana Kf. E. Aurora, Wild (Fbs); Ithaca. Aug-Sep.

7062 T. imbridana Fern. LI: Forest Pk., Aug 31, Kch (Fbs).

7061 T. olivaceana Rly. McLean; Rhinebeck. Je-Aug.

\section{SpILonota Stephens \\ (Tmetocera Lederer)}

7170 S. ocellana Schiff. Bud-Mотн. Common and general wherever apples are grown. Je-early Aug. Apple and other Rosaceae.

\section{STREPSICRATES Meyrick}

7171 S. indentanus Dyar. "NY", J1 (AM). Myrica.

\section{SonIA Heinrich}

6971 S. constrictana Z. LI: Glendale, J1 1, 1923, Kch (Fbs).

\section{RHYACIONIA Hübner}

(Evetria auct. not Hübner; Retinia Guenée)

R. buoliana Schiff. European Pine-Shoot Moth. Buffalo (NYS); Tarrytown (NYS); NYC (NYS); LI: Great Neck; Westbrook; Lynbrook. In tips of pine shoots.

6760 R. frustrana Coms. Ithaca (Comstock); Karner (Felt). Shoots of scrub pine.

6761 R. rigidana Fern. Ithaca (Fern). Ap. Pinus rigida.

_ R. busckana Hein. LI: Bellmore, Central Pk.; Wyandanch, Kch (Fbs). Ap.

6762 R. comstockiana Fern. Ithaca (Comstock); Karner (Felt). In young twigs of Pinus rigida.

6765 R. gemistrigulana Kf. Ithaca, J1 10, 1924 (Fbs).

\section{PROTEOTERAS Riley}

7134 P. naracana Kf: Ithaca, end of May-J1.

7130 P. aesculana Rly. Ithaca, Je. Maple and buckeye.

7135 P. moffatiana Fern. Lancaster; E. Aurora; Ilion; Ithaca; Big Ind. Val. Je. Maple.

\section{GRETCHINA Heinrich}

- G. amatana Hein. Gowanda and E. Aurora (Wild); Castile, Je, Brad (Fbs); Ithaca; Crosby; McLean. May - early Je.

7116 G. deludana Clem. Buffalo, E. Aurora (Wild) ; Crosby; Ithaca. May-early Je.

7126 G. bolliana Sling. Gowanda, Buffalo, E. Aurora (Wild). Sep. Pecan.

- G. semialba McD. McLean, May (paratypes). 
EXEnTERA Grote

7120 E. maracana Kf. Ithaca; McLean. End of Mar-Ap.

6992 E. improbana W1k. Ithaca. Exact distribution uncertain because of confusion with E. spoliana.

7121 E. spoliana Clem. Ithaca, Mar-Ap. Chestnut.

7117 E. haracana Kf. Buffalo, Je (Wild).

7139 E. costomaculana Clem. Gowanda; Rock City; Portage; Crosby ; Ithaca. Ap-May.

7138 E. virginiana Clem. Ithaca; Pearl R.; Albany. Ap-May.

\section{Gypsonoma Meyrick}

7146 G. fasciolana Clem. Peru; McLean; Karner; New Windsor; LI: Wyandanch, May 30, Kch (Fbs). J1.

G. substitutionis Hein. Ithaca, J1.

7148 G. haimbachiana Kf. E. Aurora, Je, willow (Wild) ; LI: Forest Pk., Glendale, May and J1-Aug, Kch (Fbs).

7159 G. salicocolana Clem. Ithaca, Je-J1. Willow.

\section{Pseudogalleria Ragonot}

6878 P. inimicella Z. Ithaca, Je. Rootstocks of Smilax.

\section{EPISIMUS Walsingham}

7110 E. argutanus Clem. Speculator; E. Aurora (Wild); Crosby; Ithaca; NYC. May-Sep. Various shrubs, especially Rhus and Hamamelis; also Euphorbia.

E. tyrius Hein. LI: Westbury Sta., Queens Co (type). Maple.

\section{OLETHREUTES Hübner}

6869 O. bipartitana Clem. N. Elba; Fentons; Oswego; Jamestown; Rock City; Millerville; Otto; Castile; McLean; Ithaca; Big Ind. Val.; Albany; Ramapo; LI: Forest Pk., Kch (Fbs). May-Je; Aug.

6865 O. fuscalbana Z. Peru; N. Twin Br., Mt. Marcy; Newcomb; Rock City; Ithaca; McLean; Trenton Falls; Ft. Edward. Je-Aug.

O. castorana McD. Ithaca, Je 30 - Aug.

O. polluxana McD. N. Twin Br., Mt. Marcy; Peru. Je-J1.

6864 O. campestrana Z. Cranberry L., Htly (Bsk); Peru; Rock City; Ithaca; McLean; Trenton Falls; Albany (NYS). Je. Cherry.

6853 O. abietana Fern. Buffalo (CU Econ); Ithaca; Whitehall (CU Econ); LI: Woodmere, Leon (Fbs). Spruce.

6819 O. deceptana Kf. Peru; McLean. Je-Aug.

6862 O. instrutana Clem. Peru; Saranac Inn; Newport; Fentons; Batavia; E. Aurora and Buffalo (Wild); Otto; Ithaca; Schenectady; Rhinebeck; New Windsor; LI: Central Pk., Kch (Fbs). J1-Sep. Clover or buckeye; arrow-wood (Wild). 
6834 O. coruscana Clem. Fentons; Newport; Gowanda, E. Aurora (Wild) ; Rock City; Portage; Big Ind. Val.; Albany; Poughkeepsie; New Windsor; NYC; LI: Lynbrook, Je-J1.

6835 O. constellatana Z. Otto; Rock City; Portage; Ithaca; McLean; Trenton Falls; Big Ind. Val.; Schenectady. Je.

6843 O. astrologana Z. Mt. Marcy; Newcomb; E. Aurora, Buffalo (Wild); Ithaca. J1.

6838 O. coronana Kf. Trenton Falls, Je.

6839 O. major Wlsm. N. Twin Br., Mt. Marcy, J1.

- O. youngana McD. Wilmington, J1-early Aug.

6821 O. nimbatana Clem. Rose Bud-Worm. E. Aurora, Buffalo (Wild) ; Ithaca; McLean; Albany; Rhinebeck; Scarsdale. Je-Aug. Rose.

6825 O. separatana Kf. Ithaca; Big Ind. Val. May-Aug. Rose and blackberry.

6821 O. montanana Kf. Ithaca.

6859 O. duplex Wlsm. Peru; Cranberry L., Htly (Bsk); E. Aurora, Buffalo (Wild) ; Portage; Ithaca; Ilion. Je-J1. Poplar.

6816 O. albeolana Z. Big Ind. Val.; Karner; New Windsor; LI: Orient, Sep 9, Lath. Je-J1. White birch.

6847 O. chionosema Z. Buffalo, E. Aurora (Wild); Ithaca; Schenectady; Albany. Thorn and apple.

6871 O. impudens W1sm. Newcomb; Rock City; Batavia; McLean. Je-J1.

6827 O. hebesana Wlk. Castile, Brad (Fbs); E. Aurora (Wild) ; Crosby ; Ithaca; McLean; Nassau; New Windsor; LI: Forest Pk., Kch (Fbs). Je-end of Sep. General feeder.

6831 O. agilana Clem. Buffalo, Colden (Wild) ; Portage; Potter Swamp; Ithaca. May-J1. Impatiens.

6830 O. auricapitana W1sm. Ithaca. Je; Aug 1.

O. infuscata Hein. Ithaca.

O. approximana Hein. Rensselaer, May 26, 1916, Yg (NYS).

\section{PhaEcasiophora Grote}

6877 P. confixana Wlk. LI: Creedmoor, Kch (Fbs). May-Aug.

6873 P. niveiguttana Grt. "NY" (Fern). Late May. Sassafras and Hamamelis.

\section{CyMOLOMIA Lederer}

\section{(Exartema Clemens)}

6804 C. fasciatana Clem. E. Aurora, Gowanda, Lime L. (Wild) ; Otto ; Rock City; Salamanca (Wild) ; Ithaca; McLean; New Windsor; LI: Forest $\mathrm{Pk} ., K c h$. Je-Jl. Rumex.

C. rusticana McD. Peru; Ithaca; LI: Coram, Kch (Fbs). J1.

- C. electrofusca Hein. Ithaca, May-J1. Sweet fern.

C. micantana Kf. E. Aurora, Buffalo (Wild); Ithaca. Je. Dogwood.

6799 C. olivaceana Fern. Buffalo (Wila') ; Ithaca; New Windsor, Morton. Je-J1.

6803 C. appendicea Z. E. Aurora, Wild (Fbs); Rock City (aberration with pale antemedial fascia); Ithaca; Trenton Falls; LI: Forest Pk., Kch (Fbs). Je-J1. Oak; alder (Wild). 
6800 C. concinnana Clem. E. Aurora; Ithaca; McLean; West Farms, Angus. Je-J1. Blackberry.

C. concinnana var. terminana McD. E. Aurora, J1 (Wild).

6797 C. zelleriana Fern. Portage, J1-Aug. White birch.

6805 C. corylana Fern. Buffalo (Wild) ; E. Aurora; Ilion. Je-J1. Hazel.

6798 C. permundana Clem. E. Aurora, Buffalo (Wild); Ithaca; LI: Coram, Kch (Fbs). J1. Raspberry and Opulaster (Rosaceae), and huckleberry.

6796 C. sericorana W1sm. Elba and E. Aurora (Wild) ; Ithaca, J1; LI: Coram, Kch (Fbs).

C. melanomesa Hein. Ithaca. J1.

7545 C. merrickana Kf. Crosby; Ithaca; Portage (red variety). Je-J1. Witchhazel.

6801 C. punctana Wlsm. E. Aurora; Buffalo (Wild); Ithaca. Je-J1. Cornus.

- C. cornana Hein. E. Aurora, Wild (Fbs); Buffalo (Wild); Ithaca. J1. Cornus.

6806 C. exoleta Z. E. Aurora, Wild (Fbs); Ithaca. Je-Aug. Hazel or gooseberry.

C. tiliana Hein. Ithaca, Je-J1. Basswood.

6793 C. nitidana Clem. E. Aurora, Buffalo, J1 (Wild).

C. nigrana Hein. S. Wales, Wild (Fbs); Geneva; Ithaca. J1-Aug.

6807.1 C. clavana Wlk. S. Wales (Wild); Ithaca. J1-early Aug.

6807.2 C. quadrifida Z. Peru; Ithaca. Je-J1. Cornus.

6807 C. inornatana Clem. E. Aurora, Gowanda (Wild) ; Ithaca; LI: Forest Pk., Kch (Fbs). Je-Aug. Wild cherry.

6809 C. footiana Fern. Hemlock L.; Ithaca. Je-Aug. Witch-hazel.

C. tenebrica Hein. McLean, J1 (types). No other specimens are known.

\section{POLYCHROSIS Ragonot}

6784 P. yaracana Kf. Gowanda, Wild. May-Je.

6786 P. rhoifructana Kf. Near Rochester, May. Rhus, Cornus, and Kalmia.

6779 P. viteana Clem. Grape-Berry Moth. E. Aurora (Wild) ; Orleans Co, usually light infestation; Chautauqua $\mathrm{Co}$, usually scarce (Hartzell); Ithaca; Karner; SI: Stapleton, New Brighton (CU Econ) ; LI: Orient Pt., Latham. Grape.

\section{SUBFAMILY TORTRICINAE}

\section{SPARGANOTHIS Hübner}

7306 S. violaceana Rob. Peru; Rock City; Ithaca; McLean; Schoharie; Karner (Fbs). May-J1.

7303 S. irrorea Rob. Gowanda, E. Aurora (Wild) ; Otto; Ithaca. Je-early Aug.

7304 S. xanthoides Wlk. Newcomb; Portage; Gowanda, E. Aurora, Colden

(Wild); Potter Swamp; Ithaca; McLean; Liberty; Big Ind. Val.;

Putnam Co; New Windsor. Je-J1. Mint.

7302 S. cana Rob. LI: Glendale, J1 10, 1918, Kch (Fbs).

7315 S. caryae Rob. Lewis Co, Je-Sep. General feeder.

7307 S. unifasciana Clem. (puritana Rob.). Newport; North Creek; Wells; Ithaca; Trenton Falls; Karner. End of Je; Aug. 
7316 S. sulfureana Clem. Newport; Honeoye Falls; Buffalo; E. Aurora; Castile; Portage; Ithaca; McLean; Big Ind. Val.; Liberty; Pearl R.; New Windsor; LI: E. New York. Je-Sep. General feeder.

7322 S. tristriata Kf. "NY", Sep.

7296 S. diluticostana W1sm. Newark; Batavia; Otto; E. Aurora, Buffalo $(W i l d)$; Ithaca. Je-Aug. Oak, cherry, and syringa.

7294 S. reticulatana Clem. North Creek; McLean (Fbs).

7295 S. ferreana Bsk. Ilion, J1.

7293 S. karacana Kf. Batavia. Bayberry and scrub oak.

7288 S. pettitana Rob. Rochester; Batavia; Elba, E. Aurora (Wild) ; Rock City; Ithaca; Little Falls; Schenectady; Pine I.; LI: Great R. On various plants, especially maple.

7332 S. idaeusalis Wlk (sentana Clem.). North Creek; Buffalo, E. Aurora, Gowanda (Wild); Otto; Ithaca; McLean; Big Ind. Val.; New Windsor; SI: Clove Val.; LI: Forest Pk., Kch (Fbs). Je-Aug. Solanum, Solidago, clover, and other herbs.

7331 S. exasperatana Z. Ithaca, J1-Aug.

7323 S. flavedana Clem. Putnam Co; New Windsor; NYC; SI: Clove Val.; LI: Jamaica; Glendale, Kch (Fbs). General feeder.

\section{Amorbia Clemens}

7286 A. humerosana Clem. E. Aurora, Gowanda (Wild) ; Castile, Je, Brad (Fbs) ; Ilion; Crosby; Ithaca; McLean; Big Ind. Val.; New Windsor; LI: Forest Pk., Kch. May-Je.

\section{Coelostathma Clemens}

7281 C. discopunctanum Clem. Newcomb; E. Aurora; Otto; Ithaca; McLean ; Ilion; Schenectady; Rhinebeck; LI: Forest Pk., Kch (Fbs). Je-Aug. Clover.

ADoxophyes Meyrick

7282 A. furcatana W1k. Ithaca, Je-Sep.

\section{ARgYRotoxa Stephens}

7410 A. bergmanniana L. Buffalo (Wild).

7409 A. albicomana Clem. N. Elba; Batavia; Buffalo (Wild) ; Rock City ; Ithaca; Albany; Rhinebeck; Crugers; NYC; SI; LI: Glendale, $K c h$ (Fbs). Je-J1. Rose.

7409.1 A. curvalana Kf. LI: Cold Sp., Je 12, 1921, Kch (Fbs).

7409.2 A. semipurpurana Kf. Buffalo, Salamanca (Wild) ; Ithaca, abundant; LI: Forest Pk., Kch (Fbs). Je-J1. Rose and oak.

A. semipurpurana var. dorsipurpurana Kf. Ithaca, Je-J1.

\section{PERonea Curtis}

7419 P. minuta Rob. var. cinderella Rly. Yellow-Headed Cranberry Worm

McLean (cinderella only); Johnstown, Alex (Bsk). Sep.

7427 P. angusana Fern. West Falls, EPV (Fbs); Ithaca. End J1-Sep. 
7443 P. subnivana Wlk. Wilmington; E. Aurora; Ithaca; Albany. Late fall to spring. Vernonia.

7416 P. cervinana Fern. Cranberry L., Htly (Bsk).

7429 P. heindeliana Fern. Ithaca (?). In "pine-cone" gall on willow.

7422 P. trisignana Rob. Big Ind. Val.; Bronx, NYC; SI; LI. White birch.

7435 P. nigrolinea Rob, Boston, Sep (Wild).

P. placidana Rob. E. Aurora, Allegany Pk., Wild (Kf, as parisiana).

7440 P. chalybeana Fern. Ithaca; McLean. Aug; Oct-May.

7425 P. nivisellana Wlsm. Buffalo, E. Aurora, Aug (Wild); Ithaca. Crataegus.

P. variegana Schiff. Clinton, J1. Larvae on various trees and shrubs.

7423 P. logiana Schiff. Ithaca; Karner; New Windsor. Viburnum.

7439 P. hastiana L. Albany; New Windsor. Determinations doubtful. Vaccinium and Andromeda.

P. n. sp. near hastiana. Hemlock L.; Ithaca; McLean.

7431

P. maculidorsana Clem. McLean, Sep.

7426 P. variana Fern. McLean; Mt. Whiteface, just below tree line.

7413 P. ferrugana Schiff. Wilmington; Ithaca (US); Taughannock. White birch.

\section{TORTRICODES Guenée}

T. fragariana Bsk. Ithaca, common, Aug-Sep. Strawberry.

\section{CNephasia Curtis}

7402 C. argentana C1. Trenton Falls, J1.

7401 C. osseana Scop. var. niveosana Pck. Lewis Co, Je, Hill (Fern).

7374 C. peritana Clem. Newcomb; Otto; E. Aurora, Wild (Fbs); Ithaca; McLean; Ramapo; West Farms. May-J1; Sep.

7377 C. virescana Clem. Rock City; Portage; Crosby; Ithaca. Je.

7503 C. listerana Kf. E. Aurora, J1, Wild (Fbs).

\section{HARMOLOGA Meyrick}

7400 H. fumiferana Clem: Spruce Bud-Worm. Adirondack and Allegheny Highlands; Syracuse, Utica (NYS); E. Aurora, Buffalo (Wild) ; LI: Glendale, J1 23, 1913, Kch (Fbs). Spruce.

\section{EUlia Hübner}

7389 E. ministrana L. Summit and N. Twin Br., Mt. Marcy; N. Elba Twp.; Keene, Wild (Fbs); Castile, Brad; Ithaca; Big Ind.'Val. Je. Many trees and shrubs.

7390 E. velutinana Wlk. Rfn-bandes Leaf-Rolder. Newark; Honeoye Falls; Lewiston; E. Aurora; Salamanca; Ithaca; Delmar; Albany; New Windsor; Florida; LI: Jamaica, Forest Pk., Glendale, Kch (Fbs); Orient, Lath. Ap-Oct. Two or three broods, with maxima in May, J1. and late Aug. Almost any plants except conifers; even reported from balsam fir. 
7394 E. pinatubana Kf. Peru; Portage; Ithaca. Pine.

7393 E. gloverana W1sm. Lewis Co, May.

7396 E. mariana Fern. E. Aurora, Wild (Fbs); Jamestown; Castile, Brad; Ithaca; Ilion; Ramapo. May-J1. Vaccinium.

7399 E. juglandana Fern. Batavia, E. Aurora, Buffalo (Wild) ; Ithaca; NYC; LI: Brooklyn. Je-Aug. Hickory and Viburnum.

7398 E. quadrifasciana Fern. Lewis Co; Batavia; E. Aurora, Buffalo, Wild (Fbs); Albion; Ithaca. May-Jl.

7364 E. quercifoliana Fitch. Buffalo (Wild); Ithaca; Big Ind. Val.; Albany ; LI: Forest Pk., Coram, Kch (Fbs). Je-J1. Oak and buckthorn.

7397 E. alisellana Rob. Ithaca; Bethlehem; LI: Forest Pk., Kch (Fbs). Jeearly J1.

\section{TORTRIX Linnaeus}

7388 T. afflictana W1k. Ithaca; Albany; New Windsor. May. Fir.

7367 T. clemensiana Fern. Peru; Newport; Lewiston; Honeoye Falls; Buffalo; Rock City; Castile, Brad; Potter Swamp; Ithaca; McLean; Big Ind. Val.; Utica; Albany; New Windsor; SI; LI: Brooklyn. Var. nervosana Kf. occurs at Peru and Ithaca. May-Je; Aug-Sep. Golden$\operatorname{rod}$ (Wild).

7366 T. pallorana Rob. Newport; Honeoye Falls; Batavia; Buffalo (Wild) ; Castile, Brad; Potter Swamp; Ithaca; McLean; Albany; Hunt's Pt., Kch (Fbs) ; LI: Forest Pk., Kch (Fbs). Je-J1; Aug-Sep. Cherry. Silphium; Verbena; clover (Wild).

7369 T. alleniana Fern. Peru; Newcomb. J1. General feeder.

\section{ARCHIPS Hübner \\ (Cacoecia Hübner)}

7363 A. conflictana W1k. Peru; Batavia; Buffalo, E. Aurora (Wild); Ithaca. LI: Woodhaven; Forest Pk., Kch (Fbs). Je-J1.

7345 A. obsoletana Wlk. Strawberry Leaf-Roller. Peru; Essex Co; Ithaca; McLean; Albany; Putnam Co; Katonah; SI: Clove Val. J1-Aug (largely Sep in 1924). General feeder.

7360 A. parallela Rob. W. Albany; SI: Clove Val.

7358 A. purpurana Clem. Fentons; Rochester (Angle); E. Aurora, Salamanca $(W i l d)$; Castile, Brad; Ithaca; McLean; Schoharie; Albany. Geranium, probably a general feeder.

7356 A. fractivittana Clem. Buffalo (Wild); Castile, Brad; Ithaca; Trenton Falls. Je.

7361 A. rosaceana Harr. Oblique-banded Leaf-Roller. Common. Je (J1 in 1924). General on trees and shrubs; rarely on clover.

7340 A. rosana L. Thousand Isls.; Buffalo, E. Aurora, J1, Wild (Fbs) ; Johnstown (Alex); Albany; West Farms. General feeder.

7343 A. infumatana Z. Irving; Lime L., Allegany Pk., Je-J1 (Wild).

7348 A. fervidana Clem. OAK Webworm. Ithaca; East Branch (NYS) ; LI: Coram, Kch (Fbs); Westbury (NYS). Jl-Aug. Oak. 
7349 A. cerasivorana Fitch. Cherry Ugly-Nest Tortricid. Peru; Essex Co; Fentons; Forestport (NYS) ; Honeoye Falls; Erie Co, general; Little Val. (CU Econ); Ithaca; McLean; Alder Cr. (NYS); Big Ind. Val.; Schenectady; Albany; Chatham, Ellenville (NYS); Blauvelt (NYS); Albany; New Windsor; E. Greenbush (NYS). Jl-Aug. Wild cherry.

7342 A. persicana Fitch. Peru; N. Elba; Fentons; Newport; E. Aurora, Wild (Fbs); Rock City; Castile, Brad; Ithaca; Liberty; Debruce; Big Ind. Val.; Ballston; Schenectady. Je. Various plants.

7341 A. dissitana Grt. Uphill Br., Mt. Marcy; Buffalo; Jamestown; Ithaca; Ilion. J1. Probably on conifers.

7353 A. argyrospila Wlk. Fruit-Tree Leaf-Roller. Common and general. Je, rarely J1. General on trees and shrubs, often injurious to apple and pear.

7353b A. mortuana Kf. Castile, Brad; Ithaca; McLean. Je-J1.

7354 A. semiferana Wlk. Newport; Ithaca; Albany; LI: Forest Pk., Kch (Fbs).

7387 A. grisea Rob. Ithaca, Je. Oak and Rudbeckia.

7359 A. magnoliana Fern. E. Aurora, Wild (Fbs); Rock City; Ithaca. Je. Magnolia acuminata.

7357 A. melaleucana Wlk. Peru; Castile, Je, Brad (Fbs); N. Elba; Batavia; E. Aurora; Vandalia; Rock City; Portage; Ithaca; McLean; Big Ind. Val.; Schuyler; New Windsor; SI. May-Je. Trillium and Polygonatum.

\section{PANDEMis Hübner}

7336 P. limitata Rob. Buffalo (Wild) ; Ithaca; McLean; Schenectady. Je-Sep. General feeder, especially on trees.

7337 P. lamprosana Rob. E. Aurora, Buffalo (Wild); Ithaca; McLean; Schenectady; Albany; SI. Je-Aug.

7333 P. canadana Kf. E. Aurora; Buffalo, Je-J1 (Wild) ; Castile, Aug 3, 1924, Brad (Fbs).

\section{FAMILY PHALONIIDAE}

\section{Hysterosia Stephens}

7547 H. birdana Bsk. E. Aurora (Wild); Ithaca (Fbs); SI (Bird). Aug. Crown of Rudbeckia laciniata (Bird).

H. terminana Bsk. Buffalo (Wild); Ithaca; Slaterville; Catskills (Pears). Je-Aug.

H. modestana Bsk. Alexandria Bay, Thousand Isis., late Je, Kch (Fbs); Ithaca.

7550

H. baracana Bsk. Alexandria Bay, Thousand Isls., Kch (Fbs) ; Ithaca; McLean. Late Je-Aug.

7544 H. cartwrightiana Kf. E. Aurora (Wild); Slaterville; Ithaca; McLean. $\mathrm{J} 1$.

\section{PHARMACIS Hübner}

P. vitellinana Z. Peru, Black Br., Je (Fbs); Keene, Wild (Fbs).

\section{Phalonia Hübner}

7461 P. rutilana Hbn. Juniper Webworm. LI (introduced about 1878). Je. Juniper.

7468 P. labeculana Rob. Ramapo. May. 
7467 P. argentilimitana Rob. Ithaca. J1 28-Aug 1, and early Sep.

7513 P. biscana Kf. Peru; Ithaca; McLean. J1-Aug.

7462 P. dorsimaculana Rob. E. Aurora (Wild) ; Ithaca; Trenton Falls. Je-Sep.

7463 P. promptana Rob. Ithaca; LI: Forest Pk., Kch (Fbs). Je-Aug.

7466 P. angulatana Rob. Peru; Buffalo, Allegany Pk., Wild (Fbs) ; Slaterville; Ithaca; McLean. J1-Oct.

7510 P. rana Bsk. Rye, Aug-Sep. Ironweed.

7454 P. smeathmanniana Fab. Newcomb; E. Aurora, Wild (Fbs); Ithaca. $\mathrm{J} 1$ - early Aug.

7446 P. floccosana Wlk. Ithaca. Je.

7447 P. atomosana Bsk. Ithaca; McLean. Aug.

7456 P. louisiana Bsk. Ramapo (Kf). May-Jl.

7449 P. straminoides Grt. (zaracana Kf.). Buffalo; E. Aurora (Wild); Ithaca; Trenton Falls. Aug.

7259 P. plummeriana Bsk. Peru. Je.

7478 P. bunteana Rob. Wells; Ithaca; Poughkeepsie. Je-Sep. Lactuca scariola.

7509 P. albidana Wlk. (winniana Kf.). Rock City; Ithaca; Trenton Falls. May-J1.

7487 P. oenotherana Rly. Vic. NYC (Wat); LI: Glendale, Kch (Fbs). Ap; J1; Sep. Evening primrose.

7531 P. ednana Kf. McLean. Je-J1.

7481 . P. marloffiana Bsk. Ithaca. Ap; Aug.

7482 P. lavana Bsk. Ithaca. Je and Aug. Penstemon.

ComMOPHILA Hübner

7496 C. bana Kf. LI: Forest Pk., late Aug, Kch (Fbs).

\section{FAMILY CARPOSINIDAE}

CARPosina Herrich-Schaeffer

7556 C. fernaldana Bsk. E. Aurora, Buffalo, Colden (Wild) ; Ithaca. Je-Aug. Nearly ripe currants (Sling).

\section{BONDIA Newman}

7554 B. crescentella Wlsm. Rock City, not rare (Fbs); Castile; McLean (Fbs); Big Ind. Val. May.

\section{FAMLY COSSIDAE}

\section{Acossus Dyar}

8351 A. centerensis Lint. Peru; Batavia; Lestershire (Binghamton); Karner (type); LI: Brooklyn. Poplar.

\section{PRIONOXYSTUS Grote}

8354 P. robiniae Peck. Carpenter Worm. Plattsburg; Peru; Ogdensburg; Brockport; Buffalo; E. Aurora (Wild) ; Ithaca; Oneonta; Albany; SI; LI: Brooklyn, common. One Ithaca specimen is practically var. reticulatus Lint. Je. In many trees, preferring soft woods. 
8355 P. macmurtrei Guér. OAK CARPENTER Worm. Buffalo; Ithaca; Schoharie; Crugers; West Pt. (Ds); Newburgh; NYC; SI; LI: Brooklyn; Woodhaven; Newtown. Je. Oaks.

\section{ZEUZERA Latreille}

8339 Z. pyrina L. LEOPARD MOTH. NYC and north to Ossining in Westchester $\mathrm{Co}$; also reported injurious to apple in Orange Co (Felt). Je-J1. General feeder on trees.

\section{FAMILY THYRIDIDAE}

\section{THYRIS Laspeyres}

4888 T. maculata Harr. New Russia, Jones; Lancaster; E. Aurora, Gowanda (Wild) ; Castile, Brad; Newport; Debruce; Kingston; Poughkeepsie; Esopus; Ft. Montgomery, Ramapo (Ds) ; LI. End of May-J1. Larva probably on Clematis.

4889 T. lugubris Bdv. Ithaca (Eyer); Karner; Bronxville; Van Cort. Pk. (Wat) ; SI; LI: Brooklyn; Wading R. Late May-J1. Grape.

\section{FAMILY PYRALIDIDAE}

\section{SUBFAMILY SCHOENOBIINAE}

RUPELA Walker

5304 R. albinella Cr. SI; LI: Forest Pk., Kch (Fbs).

\section{Schoenobius Duponchel}

5309 S. unipunctellus Rob. LI: Forest Pk., J1 26, 1923, large var., Kch (Fbs).

5311 S. melinellus Clem. Buffalo, Wild; Otto; Ithaca; McLean; LI: Forest Pk., Kch (Fbs). Je-early Aug.

5314 S. longirostrellus Clem. Newport; Newcomb; North Creek; Niagara Falls; E. Aurora, Wild; Otto; Ithaca; McLean; Little Falls; Albany; New Windsor; LI: Orient, Lath. Je-J1.

\section{SUBFAMILY CHRYSAUGINAE}

\section{TOSALE Walker}

5277 T. oviplagalis W1k. Allegany Pk., giant var. (Wild) ; Ithaca; LI: Cypress Hills, Kch. May-Jl.

\section{GaLASA Walker}

5273 G. nigrinodis Z. Buffalo, EPV (Fbs); E. Aurora, Wild (Fbs); Ithaca; McLean; Big Ind. Val.; Schenectady; Nassau; Highland; New Windsor; Florida; Katonah; NYC. Je-early Aug; Sep.

\section{ARTA Grote}

5285 A. statalis Grt. LI: Coram, J1 17, 1921, Kch (Fbs). 


\section{Condylolomia Grote}

5297 C. participialis Grt. Allegany Pk., Wild (Fbs); Rock City; Ithaca; Katonah. Je-Aug.

Polloccia Dyar

5296 P. alticolalis Dyar. Jamestown, McElhose (Dyar); Ithaca (Fbs); McLean (Fbs). J1-Aug.

\section{SUBFAMILY GALLERIINAE}

GalleRIA Fabricius

5448 G. mellonella L. Beе Мотн. Commonly distributed throughout the State. Larvae in ill-kept beehives, eating and webbing up the comb; also a scavenger in waste wax, etc.

\section{PARALISPA Butler}

5450 P. terrenella Z.' Ithaca; McLean; vic. Albany. End Je-Aug.

5452 P. decorella Hulst. Buffalo (Grt).

\section{SUBFAMILY GLAPHYRIINAE}

GLAPHYRIA Hübner

4899 G. glaphyralis Guen. Rochester, Angle; West Falls (Wild); Otto; Ithaca. J1-Aug.

4900 G. sesquistrialis Hbn. Ithaca; U1ster Co. Je-Aug; Oct.

4902 G. psychicalis Hulst. LI: Coram, J1 17, 1921, Kch (Fbs).

4901 G. lentiflualis Z. LI: Coram, J1 17, 1921, Kch (Fbs).

4904 G. fulminalis Led. "NY" (Grt). Late Je-J1.

\section{LiPocosma Lederer}

4913 L. sicalis Wlk. "NY" (AM). May-J1.

4914 L. fuliginosalis Fern. Grand I. (Wild) ; Otto; Allegany Pk. (Wild) ; McLean; LI: Forest Pk., Kch (Fbs). Je; Sep.

Dicy Molomia Zeller

5444 D. julianalis W1k. Portage; Ithaca; New Windsor; Riverdale; Bronxville. Late Je-Aug. Cattail heads.

\section{SUBFAMILY PYRAUSTINAE}

\section{HYMENIA Hübner}

4919 H. perspectalis Hbn. Spotted Beet-Webworm. Keene, Buffalo, E. Aurora (Wild) ; Ithaca, Aug, Babiy (Fbs). Late autumn. Larva on beet and chard; in greenhouses also on Alternanthera. 
4920 H. fascialis Cr. Hawaiman Beet-Webworm. LI: Glendale, Sep, Kch. A stray from the South. Beet, chard, mangels, Amaranthus, and various weeds.

\section{DESMIA Westwood}

4921 D. funeralis Hbn. Grape LeAF-Roller. Lewiston; Honeoye Falls (Angle) ; Buffalo; Niagara Falls; E. Aurora (Wild) ; Ithaca; McLean; Big Ind. Val.; Onteora Mt.; Schenectady; Rhinebeck; New Windsor; Pearl R.; Katonah; LI: Forest Pk., Kch. May-Oct. Grape; more rarely on Oenothera and Cercis.

\section{DiAsTICHTIS Hübner}

4933 D. argyralis Hbn. Albany; New Windsor; Poughkeepsie; Crugers ; Pearl R.; SI: Clove Val.; LI: East N. Y.; Orient, Lath. JI.

\section{BLEPHAROMASTIX Lederer}

4955 B. ranalis Guen. Buffalo; E. Aurora, Gowanda (Wild); Ithaca; Poughkeepsie; New Windsor; NYC; LI: Forest Pk. and Glendale, Kch (Fbs). J1. Chenopodium.

4956 B. stenialis Guen. Buffalo, Gowanda (Wild) ; New Windsor. Je-J1.

\section{PANTOGRAPTA Lederer}

4960 P. limata G. \& R. Vic. Buffalo; Grand I., Allegany Pk. (Wild) ; Ithaca ; Big Ind. Val.; Ilion; Poughkeepsie; New Windsor; LI. Je-Aug. Linden.

\section{PILOCROCIS Lederer}

4937 P. ramentalis Led. Ithaca, Oct-Nov. A stray from the South.

\section{EUdioptis Hübner}

\section{(Diaphania)}

4974 E. nitidalis Cr. Pickle Worm. Albany, Aug. Cucurbitaceae.

4977 E. hyalinata L. Melon Worm. Buffalo; LI: Brooklyn. On melon.

4978 E. quadristigmalis Guen. Ithaca; New Windsor; LI: Cypress Hills, Kch.

\section{CONDYLORRHIZA Lederer}

5061 C. vestigialis Guen. Ithaca. Willow (Murt).

$$
\text { MetReA Grote }
$$

4980 M. ostreonalis Grt. Peru, J1, Everett.

\section{EVERGESTIS Hübner}

5004 E. straminalis Hbn. Purple-backed Cabbage Worm. Common everywhere. May-Sep. Cruciferae.

5000 E. rimosalis Guen. Cross-Striped Cabbage Worm. LI. 


\section{CROCIDOPHORA Lederer}

5005 C. serratissimalis Z. Newport; vic. Buffalo; Otto; Potter Swamp; Ithaca; Schenectady; Poughkeepsie; New Windsor; NYC. Je-Sep.

5008 C. tuberculalis Led. Buffalo; Lancaster; Ithaca; New Windsor; LI. Je-J1.

\section{SAMEODES Snellen}

4985 S. adipaloides G. \& R. Buffalo; Ithaca; McLean; Big Ind. Val.; Schenectady; Rhinebeck. J1-Sep.

\section{Nomophila Hübner}

5012 N. noctuella Schiff. Very common throughout the State. Je-Oct. General feeder on low plants, especially Polygonum.

\section{LoxosTEGE Hübner}

5101 L. helvalis Wlk. LI: Forest Pk., Glendale, Je, Kch (Fbs). Sep.

5025 L. similalis Guen. Garden Webworm. Saranac Inn; Ithaca; Albany; Nassau; LI: Woodhaven; Glendale and Coram, $K c h$ '(Fbs). General feeder.

5031 L. sticticalis L. Sugar-Beet Webworm. Ithaca; Big Ind. Val.; LI: E. New York. General feeder.

5032 L. commixtalis Wlk. "NY" (Grt).

5018 L. chortalis Grt. Black Br.; Uphill Br., Mt. Marcy; Alexandria Bay, Thousand Isls., Kch (Fbs); Clayton; Buffalo; Niagara Falls (Wild) ; Ithaca; Big Ind. Val.; Onteora Mt.; Karner; Albany; Rhinebeck; New Windsor. May-Aug.

5016 L. dasconalis W1k. New Windsor; LI: Central Pk., Kch (Fbs). Je-Aug. Baptisia.

5020 L. marculenta G. \& R. General. May-Aug. Solidago.

THOLERIA Hübner

5064 T. reversalis Guen. Ithaca; Oneonta; New Windsor; SI; LI: Glen Cove (in a conservatory). Je-J1. Baptisia and Lonicera.

\section{PERISPASTA Zeller}

5087 P. caeculalis $Z$. Ithaca, J1 3, '25 (Fbs). Je-Aug.

\section{CindaPHIA Lederer}

5103 C. bicoloralis Guen. Elba (Wild) ; E. Aurora, Buffalo (Wild) ; Lancaster ; Ithaca; McLean (Fbs); Big Ind. Val.; Albany; Rhinebeck; Poughkeepsie; New Windsor; NYC; LI: Orient, Lath. May-Sep.

\section{PhLyctaenia Hübner}

5088 P. rubigalis Gn. (ferrugalis auct.). CELERY LEAF-TIER. Rochester; Brockport; Buffalo; E. Aurora, Allegany Pk. (Wild); Crosby; Ithaca; Auburn; Big Ind. Val.; Albany; Hudson Falls (NYS); Pearl R.: 
New Windsor; NYC; LI: Forest Pk., Kch (Fbs); Lynbrook (NYS). Common all the year. General feeder on herbs; often injurious to greenhouse plants.

5098 P. acutella Wlk. Vic. Buffalo; Potter Swamp; Ithaca; McLean; Karner; Nassau; New Windsor. Je-Aug.

5099 P. terrealis Tr. Peru; North Creek; Adirondack Lodge, Bish; Alexandria Bay, Thousand Isls., Kch (Fbs); Buffalo; Lancaster; Jamestown; Crosby; Ithaca; McLean; Big Ind. Val.; Onteora Mt.; Ft. Edward; Schenectady; Rhinebeck; New Windsor; LI: Jamaica, Forest Pk., Kch (Fbs). May; Aug. Solidago.

5100 P. extricalis Guen. Peru; Newcomb; McLean; Big Ind. Val.; Schenectady; LI: Belmont Pk., Wyandanch, Glendale, Kch (Fbs). May-Je; J1; Sep. Alder.

5022 P. helvialis Wlk. Black Br.; Allegany Pk., Wild (Fbs); Ithaca; McLean; Neversink. J1-early Aug. Poplar.

5116

P. theseusalis W1k. Mt. Marcy; Lewis Co; Lewiston; Ithaca; Karner; Hudson Falls (NYS) ; Rhinebeck; Katonah; New Windsor. J1. Ferns.

P. tertialis Guen. Common everywhere. May-J1; Aug. Elder.

P. fumalis Guen. Wilmington; Saranac L.; Old Forge; Newcomb; North Creek; Fentons; Adirondack Lodge, Bish; Honeoye Falls; E. Aurora; Oak Orchard Swamp; Ithaca; Big Ind. Val.; Karner; New Windsor. Je-Sep.

\section{PyRAUSTA Schranck}

5111 P. pertextalis Led. Fentons; Rochester, Angle; Honeoye Falls; Buffalo; E. Aurora; Ithaca; Big Ind. Val.; Albany; New Windsor; NYC; LI: Lynbrook; Forest Pk., Kch (Fbs). Two broods, flying mainly in Je and Aug. Juglans, Asarum, Cornus, and nettle (Wild).

5114 P. thestealis W1k. Mt. Marcy; Lewis Co; Lewiston; Buffalo; E. Aurora; Ithaca; Karner; Rhinebeck; Katonah; New Windsor; LI: Orient, Oct, Lath. Je-J1. Corylus, linden, and Evonymus.

5112 P. fissalis Grt. Rochester, Angle; E. Aurora, Niagara Falls, Gowanda (Wild) ; Castile, Brad; Otto; Ithaca; McLean; Utica; Little Falls; Catskills; LI: Jamaica, Kch (Fbs). Je-Aug. Sassafras and goldenrod; wild bergamot (Wild).

5113 P. aeglealis W1k. Rochester, Angle; Buffalo (EPV) ; Liberty (Morton) ; Ithaca; McLean; SI: Buck's Hollow. Je-Aug. Phytolacca.

5119 P. flavidalis Guen. Florida; New Windsor; LI. Late J1-Aug. Vernonia.

P. penitalis Grt. Stuyvesant, Hopewell Jct. (NYS) ; LI: Lynbrook; Orient, Lath. Water lily and Nelumbo; rarely in other aquatic plants.

P. ainslei Hein. Dr. Felt reports the following distribution: Lockport; N. Collins; Ithaca; Schenectady; Ballston L.; Scotia; Albany; Nassau; Stuyvesant Falls; Hopewell Jct. The species is doubtless generally distributed south of the Adirondacks. May; Aug-Sep. Polygonum.

P. nubilalis Hbn. EUROPEAN CORN-Borer. This introduced pest was first discovered at Scotia, Schenectady Co., Jan 1919, and at N. Collins, Erie Co, Sep 1919. It now occurs in the western part of the State from L. Erie to Manroe, Livingston, Wyoming, and Allegany Counties. In the eastern part of the State it occurs in Washington, Rensselaer, Saratoga, Albany, Schoharie, Montgomery, Fulton, and southern Hamilton Counties. It has been recorded also from southern Herkimer, Mad- 
ison, Otsego, and U1ster Counties. Larva a serious pest in corn and other coarse herbaceous plants.

5125 P. illibalis arsaltealis Wlk. Buffalo; E. Aurora (Wild); Ithaca; Big Ind. Val.; New Windsor. May-J1.

5134 P. futilalis Led. Peru; Mt. Marcy; Lewis Co; Keene, Wild (Fbs) ; Lewiston; Alexandria Bay, Thousand Isls., Kch (Fbs); Crosby (NYS); Ithaca; Karner (yellow form); Albany; Poughkeepsie; New Windsor. Je-Jl.

5135 P. fumoferalis Hulst. Mt. Marcy; Lewis Co; Ithaca; Schenectady. Je-J1. Presumably on hickory.

5140 P. unifascialis subolivalis Pck. Peru; Axton; Fentons; Lancaster; West Falls; E. Aurora; Liberty; Portage; Potter Swamp; Ithaca; McLean; Big Ind. Val.; Karner; Nassau; Rhinebeck; New Windsor. Je-J1.

P. fodinalis Led. Lewiston; E. Aurora (Wild) ; Lancaster; Buffalo; Ithaca ; Big Ind. Val.; Onteora Mt.

5145 P. phoenicealis Hbn. "NY" (Wlk). Apple.

5148 P. acrionalis W1k. Old Forge; Newport; Honeoye Falls, Angle; Buffalo, E. Aurora (Wild) ; Speculator; Lancaster; Otto; McLean (Fbs); Ithaca; Big Ind. Val.; Utica; Rhinebeck; Ft. Montgomery; NYC. JeSep.

5155 P. ochosalis Hol. N. Elba; Oak Orchard Swamp; E. Aurora, Niagara Falls (Wild) ; Ithaca. Je-Aug.

5154 P. generosa G. \& R. Fentons; Newport; Lancaster; Buffalo; Karner; Sloatsburg. I have verified none of these records, which may belong wholly, or in part, to P. ochosalis.

5157 P. laticlavia G. \& R. "NY". (Grt). Aug.

5158 P. tyralis Gn. E. Aurora, Lancaster, Allegany Pk: (Wild).

5162 P. signatalis W1k. Lewiston; Niagara Falls, Buffalo (Wild) ; New Windsor, Morton. May; Aug-Sep. Horse mint.

5171 P. unimacula G. \& R. Ramapo; Brewster (Grt). End May-Aug.

5152 P. subsequalis Guen. Black Br. (male). Je-late J1. Thistle.

5152a P. subsequalis Guen. var. madestisalis Wlk. Black Br.; Saranac L.; Ithaca: Big Ind. Val.; Ilion; Schuyler; Nassau; Rhinebeck; New Windsor; Suffern; NYC; SI: Tottenville; LI: Cypress Hills, Kch. Ap-J1.

5176 P. funebris Ström. Very common everywhere. Je-J1. Solidago.

5175 P. niveicilialis Grt. Orchard Pk., West Falls, Gowanda (Wild) ; Crosby; Ithaca; Albany; Poughkeepsie; New Windsor; LI: Forest Pk., Kch. May-Je; Aug.

\section{BOEOTARCHA Meyrick}

B. demantrialis Druce. Ithaca; LI: Glendale, Kch (Fbs). J1-Aug.

\section{Loxostegopsis Dyar}

L. merrickalis B. \& McD. Ithaca, J1.

\section{THELCTERIA Lederer}

5178 T. pupula Hbn. Kinderhook, Sef, Bish; Poughkeepsie; New Windsor; IJI: Brooklyn; E. New York; Forest Pk., Je 24, Kch; Orient, Aug 1, Lath. 


\section{SUBFAMILY NYMPHULINAE}

\section{NYMPHULA Schranck}

5209 N. ekthlipsis Grt. Ithaca; Michigan Swamp, Danby; Albany. Sedge.

5208 N. icciusalis Wlk. Saranac L.; Saranac Inn; Lancaster; Buffalo, E. Aurora (Wild) ; Otto; Potter Swamp; Ithaca; McLean; Big Ind. Val.; Nassau; Rhinebeck; New Windsor; LI: Cold Sp. $(K c h)$. Je-Aug. Potamogeton, sedge, Lemna, Menyanthes, and other aquatic plants.

5204 N. obliteralis W1k. Water-LiLy Leaf-Cutter. Hemlock L.; Rhinebeck; LI: Forest Pk., Kch (Fbs). Usually a greenhouse pest northward, but the Hemlock L. specimens, at least, were taken outdoors. Water lilies, sometimes injurious.

5206 N. gyralis Hulst. Big Tupper L.; Saranac L.; Lewiston; Castile, Brad. Water lily.

5198 N. maculalis Clem. Saranac L.; Big Tupper L.; Lewis Co; Spencer L. Water lilies.

5200 N. obscuralis Grt. Alexandria Bay, Thousand Isls., Kch (Fbs) ; Waddington; Potter Swamp; Ithaca. Potamogeton and Vallisneria.

5202 N. badiusalis W1k. Saranac L.; Alexandria Bay, Thousand Isls., Kich (Fbs) ; Waddington; Lake Bluff; N. Fair Haven; Buffalo; Grand I. (Wild) ; Hemlock L. ; Ithaca; McLean; Rhinebeck; Ulster Co (Dyar). J1-Aug.

\section{Elophila Hübner}

5216 E. magnificalis Hbn. "NY" (Grt). J1.

5212 E. bifascialis Rob. Waddington; Honeoye Falls; Buffalo (Wild); Ithaca. Aug-Sep.

5217 E. fulicalis Clem. Peru; Waddington; Lancaster; Niagara Falls; Buffalo, E. Aurora (Wild) ; Jamestown; Honeoye Falls; Ithaca. Je-early Sep. Larva on rocks under water.

\section{DIATHRAUSTA Lederer}

5226 D. reconditalis W1k. Niagara Falls; Ithaca; Albany; Pearl R. Je-J1; Sep.

\section{Geshna Dyar}

5225 G. primordialis Dyar. Vandalia; Crosby; Ithaca; McLean; Big Ind. Val. Je-Jl.

\section{SUBFAMILY SCOPARIINAE}

\section{SCOPARIA Haworth}

5235 S. centuriella Schiff. Alexandria Bay, Thousand Isls., Kch (Fbs) ; Plattsburg; Peru; Newcomb; Essex Co; Saranac Inn; Fentons; Rochester; Buffalo; Ithaca; New Windsor. End Je-Jl.

5238 S. penumbralis Dyar. North Creek; Rock City; Vandalia; McLean. Je.

5244 S. basalis W1k. Adirondacks and Albany (unverified); Alexandria Bay, Thousand Isls., Kch (Fbs); Niagara Falls; Lewiston; Hemlock L.; Rock City; Otto; Crosby; Ithaca; McLean; Big Ind. Val.; Rhinebeck; New Windsor; NYC; LI: Forest Pk., Kch (Fbs). Je-Sep. Common on tree trunks. 
5239 S. cinereomedia Dyar. E. Aurora, West Falls (Wild) ; Otto; Ithaca. J!

5241 S. strigalis Dyar. Ithaca, J1-Aug.

5242 S. lugubralis Wlk. McLean(?), female, Je-early Aug.

\section{SUBFAMILY PYRALIDINAE}

\section{PYRALIS Linnaeus}

5253 P. farinalis L. Meal Snout-Мотн. Generally distributed, J1, etc. Larva injurious to meal, etc.

5254 P. costiferalis Wlk. Saranac Inn; Ithaca; Big Ind. Val.; Albany. J1-Aug. 5255 P. disciferalis Dyar. Ithaca, J1.

\section{AGLOSSA Latreille}

5250 A. cuprina Z. Alexandria Bay, Thousand Isls., Kch (Fbs); Buffalo (Wild); Ithaca; Rhinebeck. Je-Sep.

5249 A. suprealis Hbn. Mt. Marcy (Hill Coll.); Buffalo, EPV (Fbs); Ithaca; Poughkeepsie (NYS) ; New Windsor, Morton; LI: Brooklyn; Glendale (Kch); Orient, Lath. Je-Jl.

\section{HyPSOPYGIA Hübner}

5259 H. costalis Fab. Clover Hay Worm. Ogdensburg; Lewiston; E. Aurora; Buffalo; Castile, Brad; Sherwood, Cayuga Co; Ithaca; Gloversville; Big Ind. Val.; Onteora Mt.; vic. Albany; Amenia (NYS); Rhinebeck;

N. Hillsdale; New Windsor. Stored hay.

\section{HERCULIA Walker}

5261 H. intermedialis Wlk. Mt. Marcy; Fentons; Buffalo; Ithaca; Hastings; New Windsor; Katonah; LI: Glendale, Kch (Fbs). Je-early Aug.

5268 H. olinalis Guen. Lancaster; Peru; Ithaca; Big Ind. Val.; Schenectady; New Windsor; NYC; SI: Clove Val.; LI: Forest Pk., Kch (Fbs). Je-J1.

5264 H. thymetusalis Wlk. Saranac Inn; Mt. Marcy; Fentons; Albany; New Windsor.

\section{SUBFAMILY ANCYLOLOMIINAE}

PRIONAPTERYX Stephens

5322 P. nebulifera Steph. Karner; LI: Yaphank; Coram, Kch (Fbs). May-Aug, Oct. Sand-barren Ericaceae.

\section{SUBFAMILY CRAMBINAE}

\section{DiATRAEA Guilding}

5431 D. comptulalis Hulst. Ithaca.

5432 D. forbesellus Fern. Buffalo. Scirpus.

5440 D. alleni Fern. Newport; Big Ind. Val. 


\section{CHILO Zincken}

5430 C. plejadellus Zinck. Ithaca, J1. Rice and presumably other swamp grasses.

\section{ARGXRIA Hübner}

5419 A. nivalis Dru. Newcomb; North Creek; Newport; Locust L.; Lewiston; Lancaster; E. Aurora, Salamanca (Wild) ; Ithaca; McLean ; Big Ind. Val.; Utica; Schenectady; Little Falls; Hebron (W1s); Albany; Rhinebeck; New Windsor; Katonah; NYC; LI: Cypress Hills, Kch. Je-Aug.

5421 A. auratella Clem. Salamanca (Wild) ; Ithaca; McLean; Albany; Rhinebeck; Katonah. Jl.

A. critica Fbs. McLean; Ithaca; Utica; LI: Coram, Kch (Fbs); Orient, Sep, Lath. J1.

ThaUMATOPSIS Morrison

5403 T. pexella Z. New Windsor, Morton.

\section{CRAMBUS Fabricius}

5358 C. laqueatellus Clem. Common everywhere. Je-early J1. Larva on moss, refusing grass.

5356 C. myellus Hbn. Karner, J1.

5355 C. agitatellus Clem. N. Elba; summit of Mt. Marcy; Newcomb; North Creek; Rock City; Ithaca; McLean; Trenton Falls; Schenectady; New Windsor; Crugers; LI: Lynbrook. J1.

5353 C. alboclavellus Z. Adirondack Lodge; Keene; Newport; Lewiston; Buffalo; West Falls; Potter Swamp; Ithaca; McLean; Big Ind. Val.; Utica; Schenectady; New Windsor; Ft. Montgomery; Katonah; LI: Cypress Hills. Jl-Aug. Grass.

5348 C. labradoriensis Christoph. "NY" (type of dissectus Grt.).

5347 C. bidens Z. Niagara Falls; McLean. Aug.

5344 C. praefectellus Zinck. Common everywhere. Je; Aug.

5342 C. leachellus Zinck. Wilmington; Saranac L.; Newport; Ithaca; NYC; LI: Yaphank. Je-J1.

5343 C. unistriatellus Pck. Saranac Inn; North Creek; Newport; Trenton Falls; Schenectady.

5341 C. girardellus Clem. Saranac Inn; Adirondack Lodge; Newcomb; North Creek; Fentons; Rochester, Angle; Batavia; West Falls; Orchard Pk.; Allegany Pk.; Rock City; Otto; Castile; Ithaca; McLean; Trenton Falls; Liberty; Big Ind. Val.; Schenectady; Nassau; Ft. Montgomery; New Windsor; Katonah; NYC; LI: Cypress Hills. Je-J1.

5338 C. floridus Z. Ithaca; McLean. Je-J1. Grass.

5336 C. hamellus Thunb. Saranac Inn; Albany. Aug.

5362 C. albellus Clem. Buffalo; Orchard Pk.; Salamanca; Rock City; Ithaca; McLean; Trenton Falls; Big Ind. Val.; Schenectady; Poughkeepsie; New Windsor. Je-J1. Grass.

- C. biguttellus Fbs. Ithaca, Aug.

5332 C. satrapellus Zinck. Buffalo, Salamanca, J1 (Wild). 
5366 C. elegans Clem. Wilmington; Speculator; Buffalo, E. Aurora (Wild) ; Otto; Ithaca; McLean; Katonah; New Windsor; NYC; SI; LI: Forest Pk., Kch (Fbs). J1-Sep. Grass.

5367 C. polingi Kf. E. Aurora, Wild (Fbs); Ithaca; McLean. J1.

5365 C. turbatellus Wlk. E. Aurora, West Falls (Wild); Otto; Rock City; Allegany Pk. (Wild); Ithaca; McLean; Big Ind. Val. J1.

5364 C. perlellus innotatellus Wlk. Wilmington; N. Elba; Saranac Inn; Newcomb; Newport; Otto; Big Ind. Val.; Onteora Mt. J1-Aug. Grass.

5372 C. teterrellus Zinck. "NY" (AM). Je-J1. Grass.

5373 C. decorellus Zinck. Ithaca; New Windsor. J1-Aug.

5368 C. vulgivagellus Clem. Common and general. Aug-Sep. Various grasses and grains.

5371 C. ruricolellus Z. Ausable L.; Wilmington; Saranac Inn; Honeoye Falls; Batavia; Buffalo; E. Aurora; Allegany Pk.; Hemlock L.; Ithaca; McLean; Liberty; Big Ind. Val.; Onteora Mt. Aug-Sep. Grass and sorrel.

5384 C. mutabilis Clem. Peru; Newport; Lancaster; E. Aurora; Buffalo; Otto; Rock City; Ithaca; McLean; Big Ind. Val.; New Windsor; NYC; LI: Forest Pk., Kch (Fbs). End of Je-Sep. Grass.

5361 C. hortuellus topiarius Grt. Common and general. Je-Aug. Various low plants; sometimes injurious to cranberry.

5362 C. trisectus Wlk. Newport; Fentons; Newcomb; North Creek; Honeoye Falls; Batavia; Buffalo; Lewiston; Otto; Ithaca; McLean; Big Ind. Val.; Little Falls; Schenectady; Nassau; Rhinebeck; New Windsor; LI: Lynbrook. Je, Aug (J1, Sep, in 1924). Grass.

5397 C. caliginosellus Clem. E. Aurora; Buffalo; Irving; Lancaster; Otto ; Ithaca ; McLean; Saratoga Sps.; Nassau; Troy; Greenwich; Millerton, Pine Plains, Salt Pt. (NYS); New Windsor. Je-end of Aug. Sometimes injurious to young corn.

5398 C. zeellus Fern. Adirondack Lodge; Alexandria Bay, Thousand Isls., Kch (Fbs); Batavia; Ithaca; McLean; Big Ind. Val.; Little Falls; Albany; Rhinebeck; Katonah; SI. Je-Aug. Injurious to corn. This and $C$. caliginosellus are really only varieties of C. luteolellus.

5399 C. luteolellus Clem. Peru; Newcomb; Alexandria Bay, Thousand Isls., Kch (Fbs) ; Adirondack Lodge; E. Aurora ; Buffalo ; Gowanda ; Jamestown ; Auburn; Aurora; Ithaca; McLean; Utica; Hyndsville; Little Falls; Katonah. Je-Aug.

RAFHIPTERA Hampson

5331 R. argillaceella Pck. McLean Bogs.

\section{SUBFAMILY EPIPASCHIINAE}

Epipaschia Clemens

5467 E. superatalis Clem. New Windsor; Chappaqua; LI: Glendale, Kch (Fbs).

\section{ONEIDA Hulst}

5476 O. lunulalis Hulst. Ithaca, Je-Aug. 


\section{TETRALOPHA Zeller}

5490 T. asperatella Clem. E. Aurora; Allegany Pk.; Rock City; Ithaca; New Windsor, Morton. Je-Aug. Oak, maple, elm, and beech.

5483 T. melanogrammos Z. Ithaca; New Windsor, Morton. J1-early Aug. Shrubby Leguminosae (Gleditsia, Prosopis).

5489 T. militella Z. Ithaca; Ilion; Rhinebeck (Dyar); Onteora Mt. Platanus.

5496 T. baptisiella Fern. "NY" (Fern). Baptisia.

\section{SUBFAMILY PHYCITINAE}

Myelois Hübner

5509 M. bistriatella Hulst (bilineatella Rag.). "NY", Je (Ragonot, Hulst).

\section{RHodophaEA Guenée}

5518 R. exsulella Z. Ithaca, J1 10 (Fbs).

\section{ACROBASIS Zeller}

5532 A. angusella Grt. Ithaca(?) (female only); West Farms (type); LI: Brooklyn. Je-J1. Hickory.

5533 A. demotella Grt. Ithaca; West Farms. Je-J1. Black walnut.

5532 A. eliella Dyar. Ithaca. Je-J1; Aug.

5531 A. aurorella Ely. Ithaca; Ilion; LI: Glendale, Kch (Fbs). J1-Aug.

5530 A. stigmella Dyar. Buffalo, J1, Wild (Fbs); Ithaca, Aug (Fbs). Hickory.

5528 A. caryivorella Rag. Ithaca, Aug 23 (Fbs). Hickory.

5541 A. caryae Grt. "NY" (Grt). Je. Carya porcina.

5520 A. kearfottella Dyar. E. Aurora, Wild (Fbs); Ithaca. J1-Aug. Hickory.

5521 A. feltella Dyar. Warner (NYS).

5523 A. palliolella Rag. Ithaca, J1 (Fbs). Walnut and pecan.

5537 A. latifasciella Dyar. McLean (a little doubtful without breeding record). Je-early J1; Aug. Hickory.

5543 A. rubrifasciella Pck. Ithaca. Aug. Determination uncertain, lacking bred material.

5544 A. comptoniella Hulst. Rhinebeck. Sweet fern and bayberry.

5542 A. betulella Hulst. Buffalo, E. Aurora (Wild); Ithaca. End of J1. Birch.

5548 A. tricolorella Grt. ${ }^{64}$ Ithaca; Catskills. J1.

5553 A. indigenella Z. (Mineola). Apple Leaf-Crumpler. Ithaca; Big Ind. Val.; S. Byron (NYS); Schenectady; Albany. J1. Sometimes injurious to apple, and eats most other Rosales.

\section{DIORYCTRIA Zeller}

\section{(Pinipestis Hulst)}

5569 D. zimmermani Grt. Zimmerman's Pine Moth. Oswego Co; Gowanda; Cheektowaga; Hamburg; Clarence Center; Buffalo; Ithaca; Karner; Schenectady; Hastings; Tarrytown. Aug. Pine.

${ }^{64}$ This is the species so determined by Ragonot and others. It stands in the U. S. National Museum as scitulella Hulst. 
D. n. sp. Ithaca, Aug (div.) Predacious on white-pine weevil (TCBarnes).

5565 D. abietella Fab. Buffalo, J1, EPV; LI: Great Neck and Garden City, on pine (Hein).

\section{Glyptocera Ragonot}

5585 G. consobrinella Z. McLean. End of May-Aug.

\section{NEPHOPTERYX Hübner}

5596 N. ovalis Pck. Cincinnatus, Dnd (Fbs); Ithaca; McLean; Rhinebeck; Delmar. Late J1-Aug.

\section{TLASCALA Hulst}

5610 T. reductella Wlk. E. Aurora, Wild (Fbs); Ithaca; New Windsor. May. Gleditsia.

\section{MEROPTERA Grote}

5614 M. pravella Grt. E. Aurora; Ithaca; McLean; Lancaster; New Windsor. Mar-J1(?). Willow or Rhus Cotinus.

5615 M. unicolorella Hulst. Ithaca. Late May-Aug.

\section{SALEBRIA Zeller}

5631 S. contatella Grt. Peru; Keene, E. Aurora, Wild (Fbs); Ithaca; Albany; New Windsor; LI: Forest Pk., Kch (Fbs). May-J1; Aug. Locust.

S. leucochrysella Hulst ms. Ithaca, J1-Aug (Fbs).

5638 S. semiobscurella Hulst. "NY". J1. Sumac.

5630 S. carneella Hulst. Ithaca, J1 11, 1924 (Fbs). Willow.

\section{IMMYRLA Dyar}

5617 I. nigrovittella Dyar. Ithaca. May-J1.

\section{MYRLAEA Ragonot}

5650 M. vetustella Dyar. Ithaca, May.

\section{LAODAMIA Ragonot}

5653 L. fusca Haw. Waterville; Rochester; Ithaca. J1-Aug. Vaccinium and willow.

ElASMOPALPUS Blanchard

5657 E. lignosellus Z. Lesser Corn-Stalk Borer. Ithaca, Je 11, 1924 (a stray?).

Melitara Walker

5693 M. prodenialis W1k. Southern NY, end of J1. Opuntia. 
ZOPHODIA Hübner

5705 Z. grossulariae Rly. Gooseberry Fruit-Worm. Lancaster (EPV). Currants and gooseberries.

\section{EUZOPHERA Zeller}

5722 E. ostricolorella Hulst. "NY" (Hulst). May-J1. Bark of the trunk of tulip tree.

5721 E. ochrifrontella Z. Ithaca; McLean; LI: Forest Pk., Kch (Fbs). May; J1-Aug.

5720

E. semifuneralis Wlk. A'Merican Plum Borer. Lancaster (EPV); Ithaca; McLean; Kinderhook. Aug-Sep. Peach and plum.

\section{Vitula Ragonot}

5726 V. edmandsii Pck. Buffalo; Ithaca; McLean. Je-Oct. Larvae in bumblebee nests.

\section{LAETILIA Ragonot}

5730 L. coccidivora Comst. Albany. Larva feeding on soft scales (Pulvinaria).

\section{CANARSIA Hulst}

5734 C. ulmiarrosorella Clem. Peru; North Creek; Otto; Ithaca; McLean; New Windsor. End of Je-Aug. Elm, rarely hickory.

\section{Hulstia Ragonot}

5743 H. undulatella Clem. Niagara Falls; Kinderhook; LI: Forest Pk., Kch (Fbs). J1-Aug.

\section{Homoeosoma Curtis}

5772 H. stypticellum Grt. Fentons; Ithaca. Je-J1. Larvae apparently in the panicles of Rhus glabra.

5765 H. uncanale Hulst. Ithaca, Aug (Fbs).

5771 H. mucidellum Rag. Ithaca, J1-Sep. Gnaphalium and apparently in chicory seed.

\section{Ephestia Guenée}

5799 E. kuehniella Z. Mediterranean Flour-Moth. Castile; Ithaca; undoubtedly generally distributed. Sometimes in injurious numbers. Usually in meal, showing a decided preference for rolled wheat.

5801 E. cautella Wlk. Albany. Larva commonest in dried figs.

5804 E. elutella Hbn. Albany. Rotten wood.

\section{EPHESTIODES Ragonot}

E. n. sp. Ithaca; McLean. Aug.

5781 E. infimella Rag. Ithaca(?), Je. Seeds of Ambrosia. 


\section{MOODNA Hulst}

5795 M. ostrinella Clem. Rochester; E. Aurora (Wild) ; Otto; Ithaca. Late J1Aug. Sumac heads (Cy), and acorns (Murt).

5795 M. pelviculella Hulst. Irving, Wild (Fbs); Ithaca. J1-Aug.

\section{Plodia Guenée}

5807 P. interpunctella Hbn. Indian Meal Мотн. Common generally. Stored grain, occasionally on other dried foods.

\section{SUBFAMILY ANERASTIINAE \\ PEORIA Ragonot}

5834 P. bipartitella Rag. LI: Coram, J1 17, 1921, pink var., Kch (Fbs).

5835 P. approximella Wlk. Peru; Lancaster; E. Aurora; Buffalo; Allegany Pk.; Castile; Ithaca; McLean; Big Ind. Val.; Rhinebeck; New Windsor; Katonah. Je-Aug.

\section{Family PTERophoridae: The Feather-Wings}

\section{Platyptilia Hübner}

5881 P. pallidactyla Haw. Peru; Newcomb; North Creek; Black Br.; Adirondack Lodge; Rochester, Angle; Buffalo; Rock City; Castile, Je, Brad (Fbs); Potter Swamp; Portage; Ithaca; McLean; Karner; Albany; LI: Orient, Lath. Je-early Aug. Yarrow and Senecio.

- P. auriga B. \& L. Ithaca, May-Sep.

5870 P. tesseradactyla L. Castile, Je, Brad (Fbs); Crosby; Ithaca; W. Danby; Karner. May. Gnaphalium.

5869. P. carduidactyla Rly. LI: Glendale, Aug 24, 1919, Kch (Fbs). Thistle.

5864 P. acanthodactyla Hbn. E. Aurora; Buffalo; Otto, West Farms (doubtful). May. Ononis, Pelargonium, Euphrasia, and other mints:

\section{OxyPTILUS Zeller}

5857 O. periscelidactylus Fitch. Grape Plume-Moth. Buffalo; E. Aurora; Union Village; Batavia; Ithaca; Schenectady; Ballston; Albany; Menands; Poughkeepsie; New Windsor; Scarsdale; SI (NYS) ; LI: Forest Pk., Kch (Fbs); Mineola (NYS). End of May-Jl. Grape.

5860 O. tenuidactylus Fitch. Keene Val.; Wells; Geneva; Buffalo; E. Aurora; Rock City; Castile, Brad; Ithaca; Schenectady; Albany; Poughkeepsie. May; J1-Aug. Blackberry.

\section{TRICHOPTILUS Walsingham}

5856 T. lobidactylus Fitch. E. Aurora, Wild (Fbs); Ithaca; Ilion; NYC; LI: Glendale, $K c h$ (Fbs). Je; end of J1-early Aug. Solidago. 
Pterophoridae-OrNédidae-Epiplemidae-Lacosomidae 585

STENOPTILIA Hübner

5949 S. pterodactyla L. Reported from West Farms. Veronica.

5946 S. exclamationis W1sm. E. Aurora, Wild (Fbs); Ithaca (Fbs). J1-Aug; Oct.

OIDAEMATOPHORUS Wallengren

(Pterophorus auct.)

5924 O. cretidactylus Fitch. E. Aurora, Gowanda, Keene (Wild). Late Je-J1.

5925 O. eupatorii Fern. Elba, Wild (Fbs); Ithaca; McLean; Van Cort. Pk. J1early Sep. Eupatorium.

5928 O. inquinatus Z. E. Aurora, Buffalo (Wild); Ithaca. Je-Sep. Ambrosia.

5907 O. homodactylus Wlk. E. Aurora; Buffalo; Allegany Pk.; Ithaca. Solidago.

5908 O. elliottii Fern. Ithaca; McLean; Nassau(?). Je-J1. Eupatorium.

5896 O. stramineus W1sm. Ithaca, J1-Sep. Possibly on Anaphalis.

5914 O. lacteodactylus Cham. Portage; Potter Swamp; Ithaca; LI: Lynbrook. May-J1.

5923 O. kellicottii Fish. Ithaca; McLean. Je.

- O. balanotes Meyr. Ithaca, Je, Babiy (Fbs); LI: Orient, May, Lath (Fbs).

5942 O. monodactylus L. Wilmington; Buffalo; E. Aurora; Ithaca; Schenectady; Albany; LI: Orient, Lath (NYS). J1-Sep. Convolvulus and several other herbs.

AdAina Tutt

5890 A. montana Wlsm. Buffalo, J1. Solidago.

\section{FAMILY ORNEODIDAE}

ORNEODES Latreille

5954 O. montana Ckl. Ithaca (possibly introduced), Ap (overwintered adults). Probably on Centaurea or Knautia.

\section{FAMILY EPIPLEMIDAE}

Calledapteryx Grote

4790 C. dryopterata Grt. West Farms, Angus; LI: Corona. May-early Je; Aug. Viburnum prunifolium.

Callizzia Packard

4788 C. amorata Pck. Keene Val.; Mt. Marcy; N. Elba; Indian Pass; Newcomb; Ithaca; Big Ind. Val.; Albany. End of Je-J1. Lonicera dioica.

\section{FAMILY LACOSOMIDAE}

LAcosoma Grote

4793 L. chiridota Grt. Binghamton; Poughkeepsie; Ft. Montgomery, May (Ds): Rye (Bird); SI; LI: Yaphank. Je. Oak. 
Cicinnus Blanchard

4792 C. melsheimeri Harr. LI: Amagansett (Ds); Hempstead Plains; Yaphank. Je. ' Scrub oak.

\section{FAMILY CITHERONIIDAE}

\section{CitHERONIA Hübner}

810 C. regalis Fab. Brockport; Utica; Lansingburg; Albany; Oak Hill; Clermont; Poughkeepsie; New Windsor; Peekskill; Rye (Bird); S. Nyack; formerly not uncommon in Van Cort. Pk. (Wat); SI; LI: Mineola; Rockville Center (NYS). Je. Many shrubs and trees.

811 C. sepulcralis G. \& R. "NY", Je. Pine.

\section{EACLES Hübner \\ (Basilona Boisduval)}

813 E. imperialis Dru. Imperial Mотн. General from Peru southward. Je, in warmer part of State; J1-Aug at Ithaca. Formerly abundant in Van Cort. Pk., on sassafras (Wat). Many trees, especially pine and maple.

\section{Adelocephala Herrich-Schaeffer}

803 A. bicolor Harr. "NY" (Grt, presumably from Buffalo). May; J1; Aug. Locust and Kentucky coffee tree.

\section{Anisota Hübner}

802 A. rubicunda Fab. Spiny Maple Worm. General. One brood in the north, Je-early Aug; two broods southward, May-Je and Aug. Maple.

797 A. stigma Fab. Rochester, Angle; Buffalo (Wild) ; Karner; Lansingburg; Poughkeepsie; Rye (Bird); SI; LI: Brooklyn; Orient, Lath (NYS). May-Je. Oak.

800 A. virginiensis Dru. Plattsburg; Rochester, Angle; Buffalo; E. Aurora; Lancaster; Ithaca: Salem; Albany; Rye (Bird); SI; LI: Brooklyn. Je. Oak.

798 A. senatoria S. \& A. Karner; Albany (NYS); Rye (Bird) ; Bronxville; SI; LI: Lynbrook (NYS); Centerport (NYS); Yaphank. Je. Oak, rarely on beech.

\section{FAMILY SATURNIIDAE}

\section{HEMILEUCA Walker}

784 H. maia Dru. Crape Moth or Buck Moth. Karner; Albany; LI, general. Sep-Oct. Oak.

\section{Automeris Hübner}

779 A. io Fab. Iо Мотн. Common and generally distributed. Wilmington; North Creek; Mayfield; Buffalo; Allegany Pk.; Castile; N. Hector; Breesport; Ithaca; McLean; Gloversville; Oneonta; Ballston and southward. Two Ithaca specimens are transitional to variety lilith Str. Je-Aug. General feeder. 


\section{TROPAEA Hübner}

773 T. luna L. Luna Moth. Degrasse; New Russia; Hamilton; Rochester, Honeoye Falls (Angle); Buffalo; E. Aurora; Allegany Pk.; Rock City; Castile; Franklinville; Watkins; Ithaca; McLean; Trenton Falls; Gloversville; Oneonta; Schoharie; Albany; New Scotland; Saratoga Sps.; E. Chatham; New Windsor; Rye (Bird) ; NYC (Watson); SI; LI: Brooklyn. May-Je; Aug. Walnut, birch, beech, and many other trees.

T. luna form rubromarginata Ds. The first form to appear in the spring at Ithaca (CU), SI, and LI (Ds).

\section{TELEA Hübner}

774 T. polyphemus Cr. Common everywhere in the State. Je-Jl. Maple, birch, and many other trees.

Callosamia Packard

771 C. promethea Dru. New Russia; Red Cr.; Syracuse; Rochester, Angle; Buffalo; E. Aurora; Ithaca; Berkshire; Elmira; Frankfort; Hoosick Falls; Cropseyville; Albany; common southward. Je-Aug. Caterpillar preferring sassafras and spicebush, wild cherry, and tulip tree.

772 C. angulifera Wlk. Buffalo; Ithaca (rare); Dutchess Co; Rye (Bird); Bronx Pk. (Wat); SI; LI: Brooklyn. Je; Aug. Various trees, especially tulip tree.

\section{SAMIA Hübner}

766 S. cecropia L. General, not rare. Je. Many trees and shrubs.

\section{Philosamia Grote}

763 P. walkeri Fld. (cynthia auct. not Dru.). Fourth L., Fulton Chain, "about half a mile above lake at a clearing . . . bred two imagoes" (Wild); Rochester (Angle); Lansingburg (NYS); Livingston; NYC; SI. Ailanthus.

\section{FAMILY EUPTEROTIDAE}

\section{Apatelodes Packard}

3726 A. torrefacta S. \& A. Rochester, Angle; Kingston; Poughkeepsie; New Windsor; Rye (Bird); Greenwood L.; larvae formerly abundant on Prunis serotina in Van Cort. Pk. (Wat); SI; LI: Brooklyn; Lynbrook. Je-J1. Many trees and shrubs.

3728 A. angelica Grt. Plattsburg; Ithaca; Bath; Sharon Sps.; Albany ; Poughkeepsie; West Pt.; Coldenham; LI: Jamaica. May-J1. Ash and lilac.

\section{FAMILY LASIOCAMPIDAE}

TOLYPE Walker

3738 T. velleda Stoll. New Russia, Jones; Newport (NYS) ; Spier Falls; Rochester; Lancaster; Buffalo; E. Aurora; Ithaca; Oneonta; Albany; Lagrangeville (NYS) ; Rye (Bird) ; Bronxville; SI; LI: Southold; Bellport; Jamaica, Yaphank (Ds) ; Aqueduct (Wat). Sep-Oct. Apple, poplar, and other trees. 
3743 T. laricis Fitch. Plattsburg; Rochester; Cold Water; Buffalo; Ithaca: Albany; Rensselaer; Poughkeepsie; LI: Central Pk., Kch; Yaphank (Ds). Aug-Sep. Larch; pine (Kch).

\section{ARTACE Walker}

3737 A. punctistriga Wlk. LI: Brooklyn (Ottolengui).

\section{MALAcosoma Hübner}

3745 M. americana Fab. Apple-Tree Tent-Caterpillar. Common and general. Late Je-Aug. Apple, wild cherry, and other Rosaceae.

3752 M. disstria Hbn. Forest Tent-Caterpillar. McLean (Fbs). Common throughout the State, even on the summit of Mt. Marcy. J1, later than americana. Forest trees, especially maple.

I have seen var. sylvatica from Ithaca, Geneva, and Peru; var, thoracicoides from Ithaca and Peru (Fbs).

\section{EPICNAPTERA Rambur}

3754 E. americana Harr. American LAPPET. Old Forge; New Russia, Jones; Rochester; Lancaster; Buffalo; E. Aurora; Ithaca; Oneonta; Rhinebeck; Poughkeepsie; Rye (Bird) ; LI. May-early Je; J1-Aug. Apple, oak, maple, and other trees.

I have seen var. ferruginea Pck. from Ithaca and Karner (Fbs).

\section{FAMILY THYATIRIDAE}

\section{HABROSYNE Hübner}

3687 H. scripta Gosse. Plattsburg; Peru; Essex Co; L. Pleasant; Evans Center; Allegany Pk. (Wild); Rock City; Ithaca; Oliverea (Ds); Onteora Mt.; Schenectady; Poughkeepsie; LI: Brooklyn. Je; Aug. Blackberry, thimbleberry, and other Rosaceae.

3689 H. rectangula Ottol. LI: Brooklyn (type).

\section{Pseudothyatira Grote}

3690 P. cymatophoroides Gn. Wilmington (larva); Adirondack Lodge; Newcomb; Plattsburg; Mt. Marcy; Fentons; Clayton; Lancaster; Otto; Allegany Pk.; Ithaca; Debruce; Sharon; Schenectady; Poughkeepsie;

SI; LI: Newtown. Je-Aug. Oak and thimbleberry.

P. cymatophoroides var. expultrix Grt. Plattsburg; Fentons; Mt. Marcy; Adirondack Lodge; Newcomb; Clayton; Buffalo; E. Aurora (Wild); Lancaster; Allegany Pk. (Wild); Otto; Rock City; Ithaca; Sharon; Schenectady; Oliverea (Ds); Rye (Bird); SI (Ds); LI.

\section{EuthyatiRA Smith}

3693 E. pudens Gn. Brockport; Lancaster; Ithaca; Dutchess Co; LI: Queens (Bell and Eng). May. Cornus. 


\section{FAMILY DREPANIDAE}

EudeILINEA Packard

3756 E. herminiata Gn. N. Elba; Fourth L., Fulton Chain; Newcomb; Batavia; Oak Orchard Swamp; Otto; Potter Swamp; Crosby; Taughannock Falls; Ithaca; McLean; Big Ind. Val.; Nassau; Albany; Rhinebeck; Ft. Montgomery (Ds); New Windsor; SI. May-Je; J1-Aug. Cornus.

\section{DREPANA Schranck}

3760 D. arcuata W1k. Plattsburg; Peru; Old Forge; Mt. Marcy; Lewis Co; Lancaster; E. Aurora; Castile; Ithaca; McLean; Trenton Falls; Rhinebeck; Ulster Co (Shoe); Oliverea (Ds); Poughkeepsie; West Pt. and Ft. Montgomery (Ds) ; Rye (Bird) ; NYC; SI; general on LI. May-Je; Aug. Birch and alder.

\section{FALCARIA Haworth}

3761 F. bilineata Pck. Plattsburg; Wilmington; Albany; SI; LI: Brooklyn; Queens (Bell and Eng). May-Je. Birch.

F. bilineata var. levis Hudson. Known definitely from Cranberry L., Htly; Plattsburg; Wilmington; Alpine; Saratoga; Ulster Co (Shoe); Westchester Co; SI (Ds); LI: Brooklyn. Aug.

\section{ORETA Walker}

3757 O. rosea Wlk. Peru; Fentons; Mt. Marcy; Protection (Wild); Ithaca; McLean; Big Ind. Val.; Albany; Rhinebeck; Poughkeepsie; SI; LI: Brooklyn. May-Sep. Viburnum.

O. rosea var. marginata Wlk. Ithaca; Ulster Co; NYC and vic., Eliot; SI (Ds): May-Sep.

3758 O. irrorata Pck. Mt. Marcy; Ithaca. Je-Aug.

\section{FAMILY GEOMETRIDAE 65 SUBFAMILY BREPHINAE \\ BREPHOS Ochsenheimer}

3762 B. infans Mösch. Karner; Sloatsburg; Van Cort. Pk. (Wat) ; SI; LI: Wyandanch; Massapequa (Ds). A northern species that will certainly be found much more general. Mar-early Ap. White birch.

\section{LEUCOBREPHOS Grote}

3766 L. brephoides W1k. St. Lawrence Co, Ap (US, NYS). Aspen.

${ }^{65}$ Phalaena (Geometra) extenaria L., credited to NY, has never been recognized. 


\section{SUBFAMILY OENOCHROMINAE}

\section{Alsophila Hübner}

3767 A. pometaria Harr. Fall CANKer Worm. Records for the imago are: Rochester, Angle; E. Aurora (Wild) ; Castile; Ithaca; Mt. Vernon; SI; LI: Bellport. Larvae seem common anywhere in the State. A former pest in Brooklyn, it has almost disappeared since the introduction of the English sparrow about 1880 (Eng). Nov-Dec; Ap. General feeder on trees, often injurious to apple an delm.

\section{SUBFAMILY HEMITHEINAE}

Racheospila Guenée

3775 R. rubrolinearia Pck. Ithaca; LI (Hulst). May; J1. Birch.

3773 R. rubromarginaria Pck. Lancaster; LI: Central Pk., May 17, Kch (Doll).

\section{NEMORIA Hübner \\ (Aplodes Guenée)}

3781 N. bistriaria Hbn. New Windsor; Bronx Pk.; LI: Yaphank (Eng). If bifilata W1k. is a distinct species, the NY records should probably be credited to it. Black walnut.

3783 N. mimosaria Gn. Peru; Saranac L.; Cranberry L., Htly; Buffalo; Vandalia; Crosby; Ithaca; Danby; McLean; Big Ind. Val.; Trenton Falls; Schoharie; Albany; SI; LI: Brooklyn; Wyandanch, Central Pk., Kch (Doll) ; Pinelawn, Yaphank (Ds). End of May-J1.

3784 N. rubrifrontaria Pck. Buffalo, May (Wild); West Farms; SI; LI: Brooklyn; Maspeth; Central Pk., Kch; Yaphank (Eng). May.

\section{SYNCHLORA Guenée}

3800 S. aerata Fab. General and common. Je-Sep. General on low plants, especially the flowers and fruits.

3801 S. denticularia Wlk. Bronxville. Rudbeckia.

3802 S. rubrifrontaria Pck. Buffalo (Wild).

\section{DICHORDA Prout}

\section{(Anaplodes)}

3810 D. iridaria remotaria Hulst. Saranac Inn; Ithaca; Catskills; Katonah; SI; LI: Brooklyn; Orient, Lath. Jl-Aug. Sumac (Dimmock).

\section{ChLorissa Stephens}

\section{(Nemoria Hübner)}

3813 C. pistaciaria Gn. Ithaca; Karner; Poughkeepsie; Hastings; West Farms; SI (Ds); LI: Brooklyn; Amagansett; Yaphank. Late Je.

3815 C. subcroceata Wlk. Clayton; Ithaca; SI; LI: Brooklyn; Pinelawn; Yaphank. Late Je-Jl. Oak.

3815.1 C. dyari Hulst (auranticolorata Strk.). LI: Bellport, May-Je. 
Mesothea Warren

(Eucrostis Hübner)

3816 M. incertata W1k. Clayton; McLean; Big Ind. Val.; Karner; Albany.

\section{Chlorochlamys Hulst}

3818 C. chloroleucaria Gn. Peru; Newport; St. Ile; Rochester; Honeoye Falls; Buffalo; Otto; Castile; Potter Swamp; Ithaca; McLean; Catskills; Hebron; Schenectady; Albany; Rhinebeck; Katonah; West Farms; SI (Ds); LI: Brooklyn; Forest Pk., Coram, Kch (Fbs); Wading R. Late May-Sep. General on low plants.

\section{SUBFAMILY STERRHINAE}

\section{(Acidaliinae)}

\section{EUACIDALiA Packard}

(Eois Hübner)

3835 E. ossularia Hbn. Buffalo, E. Aurora, Aug (Wild) ; LI: Amagansett, Yaphank, Forest Pk., J1; Central Pk., Southold, Sep (Ds); Gardiners I., Je (Ds).

\section{SyNELYS Hulst}

\section{(Acidalia Treitschke not Hübner)}

3857 S. ennucleata Gn. (restrictaria W1k.). Common everywhere in the State; also the vars. relevata Swett and adornata Prout. Je-Aug. Wild cherry.

\section{Cinglis Guenée}

\section{(Acidalia)}

3856 C. quadrilineata Pck. (similaria auct., in part). Peru; N. Twin Br., Mt. Marcy; Newcomb; Otto(?) ; McLean; Trenton Falls; LI: Amagansett (Eng). Je-Jl.

3856 C. junctaria W1k. (persimilis Hulst, similaria auct.). E. Aurora, Wild (Fbs); Potter Swamp; McLean. These species have been universally confused; at least one of them occurs at Fentons, Newport, Big Ind. Val., and Liberty. Je-J1.

3901 C. inductata Gn. (Acidalia, Leptomeris). General and common. Je-Sep.

3903 C. australis Hulst. SI (Ds) ; LI: Yaphank; Wading R., May (Ds).

3853 C. purata Gn. Potter Swamp; West Farms; LI: Wading R., Je (Ds). Je-Aug.

3876 C. roseotincta Hulst. SI (AM). A stray from the Tropics.

\section{PTYCHOPODA Curtis}

(Eois Hübner)

3885 P. bonifata Hulst (ptelearia Rly., delicata Hulst). Albany. A herbarium pest, apparently not established. 
3888 P. demissaria Hbn. (ferrugata Pck.). Ithaca; Chappaqua; LI: Coram, Kch (Fbs); Pinelawn, Aug (Ds). Je-Aug. Var. russata Hulst was described from NY.

3902 P. productata Pck. LI: Yaphank (Pears).

\section{HAEMATOPIS Hübner}

3912 H. grataria Fab. General. Je-Sep. Chickweed, etc.

TIMANDRA Duponchel

(Erastria Hübner, Calothysanis Hübner)

3913 T. amaturaria W1k. Ithaca; Albany; SI; LI: Brooklyn; Jamaica; Lynbrook; Astoria; Maspeth (Ds). Je-Aug.

Pleuroprucha Möschler

(Deptalia Hulst)

3914 P. insulsaria Gn. Buffalo; Potter Swamp; Ithaca; Big Ind. Val.; Schenectady; Albany; West Farms; SI; general on LI. J1-Oct. Celastrus, Solidago flowers:

Cosymbia Hübner

3917 C. myrtaria Gn. Plattsburg; Ithaca, May, Babiy (Fbs) ; Poughkeepsie; West Farms; LI: Bellmore; Yaphank; Central Pk. (Kch). May-Sep. Oak.

3918 C. lumenaria Hbn. Fentons; Buffalo; Crosby; Ithaca; Big Ind. Val.; Karner; Albany; West Farms; SI: general on LI. May - early Aug. Sweet fern, Vaccinium, Psoralea.

\section{SUBFAMILY LARENTIINAE}

STAMNODES Guenée

3920 S. gibbocostata W1k. Buffalo and E. Aurora (Wild) ; Cascade L.; Onteora Mt. Rare. Aug-Sep.

\section{TRICHODEZIA Warren}

3942 T. albovittata Gn. Adirondacks to Buffalo and Gardiners I. (LI). May-Je; Aug.

3942a T. albovittata var. propriaria W1k. N. Twin Br., Mt. Marcy, J1 6, 1918 (Fbs).

ACASIS Duponchel

(Cysteopteryx Hulst)

3950 A. viridata Pck. Fourth L., Fulton Chain; Big Ind. Val. Ap-J1.

Nyctobia Hulst

3951 N. limitata Wlk. Plattsburg; E. Aurora, Lancaster (Wild) ; Ithaca; Karner; Albany ; E. Jewett (Ds) ; Big Ind. Val.; Ramapo. Also reported from Saranac L.; W. Seneca; Buffalo; Bronx Pk. Ap-May.

3952 N. anguilineata Grt. Saranac L.; Karner; Big Ind. Val.; Suffern; SI; LI: Hempstead Plains. Ap-May. 
Cladara Hulst

3955 C. atroliturata Wlk. Plattsburg; Brockport; Ithaca; Ilion; Karner; E. Jewett (Ds); Big Ind. Val.; Rio (NYS); LI: Jamaica. Ap-May, female to Je.

\section{LOBOPHORA Curtis}

(Philopsia Hulst)

3956 L. nivigerata W1k. Black Br.; Essex Co; Adirondack Lodge; E. Aurora (Wild); Ithaca; McLean; Big Ind. Val.; Karner; LI. May-J1.

\section{HETEROPHLEPS Herrich-Schaeffer}

(Nannia Hulst)

3959 H. refusata Wlk. Black Br.; Lancaster; Potter Swamp; Ithaca (Pck); Big Ind. Val.; Little Falls. Je-J1.

3961 H. triguttaria H-S. Common everywhere. Je-Aug. Maple.

\section{DySPTERIS Hübner}

3962 D. abortivaria H-S. BAD-Wing. Peru; New Russia; Lancaster; Gowanda and Irving (Wild); Ithaca; Trenton Falls; Rhinebeck; Poughkeepsie; Ft. Montgomery, Nyack (Ds); Florida; LI: Syosset; Wading R. (Ds); Orient, Lath. May-Aug. Grape.

\section{RACHELA Hulst}

3964 R. bruceata Hulst. Bruce's Canker-Worm. Mt. Marcy; E. Aurora; Geneva; Brockport; Kendall; Niagara Falls; Buffalo; Allegany Co; Castile, Brad; Ithaca; Big Ind. Val.; Ballston (NYS); Andes; SI (Ds); LI: Brooklyn; Woodhaven. Oct-Nov. Apple.

\section{TRIPHOSA Stephens}

3968 T. indubitata Grt. (affirmaria W1k. not Gn., dubitata auct.). Fentons; Brockport; Lancaster; Buffalo. May; Sep.

\section{Calocalpe Hübner}

\section{(Hydria Hübner)}

3971 C. undulata L. Peru; N. Elba; Newcomb; Adirondack Lodge; Fentons; Rochester, Angle; Brockport; E. Aurora; Buffalo; Ithaca; McLean; Utica; Schenectady; Poughkeepsie; West Farms; SI; LI: Sands Pt. (Bs); Orient (Ds). Je-Aug. Wild cherry, social in a web.

\section{LYGRIS Hübner}

\section{(Eustroma)}

3977 L. diversilineata Hbn. General. Late Je-Sep. Grape and ampelopsis.

3977.1 L. gracilineata Gn. Usually confused with L. diversilineata. General. Available records are: Peru; Otto; Ithaca; SI. J1-Aug. Ampelopsis. 
3979 L. testata L. Essex Co; Fentons; Protection, Dnd; Ithaca; McLean; Gloversville (Alex); Big Ind. Val.; Sharon Sps.; Poughkeepsie. Late Aug-Sep. Birch, willow, poplar, and bean.

3978 L. propulsata W1k. (populata auct.). Essex Co; Saranac L.; Fentons; Wells; Ithaca; Stony Clove, Greene Co.; Big Ind. Val.; Sharon Sps. Je.

3981 L. destinata lugubrata Mösch. Mt. Whiteface (Fbs); Mt. Marcy (Pck). Aug.

3983 L. explanata W1k. Lewis Co; L. Placid; Minnewaska.

L. explanata var. cunigerata Wlk. Mt. Whiteface; Old Forge; L. Placid; Fentons; Ithaca; Sharon; Albany. Aug.

3985 L. (Neolexia) xylina Hulst. "NY" (type). Presumably from the Adirondacks.

\section{Diactinia Warren}

3986 D. atrocolorata Grt. LI: Brooklyn. May; J1-Aug.

3987 D. silaceata albolineata Pck. Peru; Fentons; Ithaca; Onteora Mt.; Sharon. May-Je. Epilobium, Impatiens.

\section{THERA Stephens}

3989 T. contracta Pck. Old Forge.

\section{DYSSTROMA Hübner}

3992 D. truncata Hfn. The records listed here doubtless include some which belong to citrata (immanata), which has only recently been recognized in the East and can be certainly separated only by structure. Mt. Marcy; Fentons; Lancaster; Buffalo; McLean; Debruce; Big Ind. Val.; Trenton Falls; Sharon. Late Aug-Sep.

3993 D. citrata L. (immanata Haw.). Wilmington; Mt. Whiteface; also reported from Keene Val., and known from N.H. to the Pacific. Aug.

3992b D. brunneata Pck. Big Ind. Val., J1. A distinct species.

3995 D. walkerata Pears. Catskills, J1. Presumably a light form of truncata.

3998 D. hersiliata Gn. McLean (Fbs) ; Plattsburg; Essex Co; Fentons; E. Aurora (Wild) ; McLean; Big Ind. Val.; Onteora Mt.; Trenton Falls ; Sharon; Schenectady; Albany. Je-Jl.

\section{HYDRIOMENA Hübner}

This genus has been revised so recently that I have been unable to verify the NY records. Only the following can be treated as certain.

4015 H. frigidata W1k. "NY", May (B\&McD).

4016 H. transfigurata Swett. McLean Bogs, May (male).

4014a H. perfracta Swett. Catskills, May (type).

4014 H. pluviata Gn. Peru; Ithaca; McLean; Catskills. Je-J1.

4017 H. renunciata Wlk. Peru; E. Aurora (Wild) ; McLean (Fbs) ; LI: Central $\mathrm{Pk}$., Kch (Fbs). Late Je-J1. This is the American representative of autumnalis (coerulata). A member of this group occurs at Saranac Inn.

4024 H. ruberata Freyer. McLean (Fbs); Ithaca; Big Ind. Val. (not verified, but undoubtedly correct). May. 
PeRIzoma Hübner

4094 P. taeniata basaliata W1k. Mt. Marcy; Fentons; Big Ind. Val. Locally common.

\section{XANTHORHÖ̈ Hübner}

4037 X. lacustrata Gn. (intermediata Pck.). Black Br.; Peru; Lewis Co; Buffalo; Castile, Je, Brad (Fbs); Potter Swamp; Ithaca; McLean; Debruce; Big Ind. Val.; Sharon; Albany; Pearl R.; Bronx Pk.; NYC; SI: LI: Woodhaven; Orient, Lath. May-Aug. Impatiens.

$4038 \mathrm{X}$. designata Hbn. Common generally. Late May; late J1; late Aug. Herbs.

_ X. designata form gynandrata Pears. Ithaca; McLean; Catskills (types). Probably general with the type.

4039 X. ferrugata Cl. Adirondacks; E. Aurora; Castile, Je, Brad (Fbs) ; Rock City; LI. End of May - early Sep. Glecoma.

4041 X. munitata labradorensis Pck. N. Elba; Uphill Br., Mt. Marcy, 3000$4000 \mathrm{ft}$.

4043 X. incursata Hbn. L. Tear, Mt. Marcy, J1 12-13, 1918 (Fbs).

4046 X. montanata D. \& S. Buffalo (EPV).

4050 X. iduata Gn. (fluctuata). Mt. Marcy, Fentons (Hill); Ithaca; Catskills; Huguenot. End of Je-Sep.

4053 X. abrasaria congregata Wlk. Old Forge; Speculator; Mt. Marcy; Mt. Whiteface trail; Ithaca, Wehrle. J1.

ORThonama Hübner

(Percnoptilota Hulst)

4054 O. obstipata Fab. (fluviata Hbn.). Rather common all over the State. May-early Nov. Polygonum, etc.

\section{ENTEPHRIA Hübner}

4060 E. aurata Pck. (caesiata auct.). Mt. Marcy; Fentons; Big Ind. Val.' J1.

\section{PHILEREME Hübner}

4061

P. multivagata Hulst. Onteora Mt., Hore.

\section{SPARGANIA Guenée}

\section{(Coenocalpe)}

4068 S. magnoliata Gn. Mt. Marcy; Fentons; E. Aurora (Wild); Rock City; Allegany; Castile, Brad; Ithaca; McLean; Big. Ind. Val.; Stony Clove, Greene Co. Late May-J1 4; Aug 23.

\section{OPORINIA Hübner \\ (Epirrita Hübner)}

4069 O. autumnata henshawi Swett (dilutata auct.). Monroe Co; Big Ind. Val. Oct. Tsuga, etc. 
Euphyia Hübner

4072 E. intermediata Gn. (lacustrata Pck.). Peru; Mt. Marcy; Adirondack Lodge; Newcomb; Fentons; Moravia ; Castile; Crosby ; Geneva ; Rochester; Buffalo; Ithaca; Big Ind. Val.; Schenectady; Albany ; Brewsters; West Farms; SI; LI: Brooklyn. Je-Sep.

4075 E. immediata Grt. "NY". A lost species, perhaps the same as Xanthorhoë munitata Hbn.

4074 E. centrostrigaria Woll. (latirupta W1k.). Mt. Marcy; Newport; N. Fair Haven; Buffalo; E. Aurora; Otto; Potter Swamp; Ithaca; Catskills; Rhinebeck; New Windsor; SI; LI: Yaphank. May-Aug; Oct.

4076 E. multiferata W1k. Oneida; Trenton Falls; Buffalo; E. Aurora; Castile, Brad; Ithaca; McLean; Big Ind. Val.; Onteora Mt.; New Windsor; Brewster; SI; LI. Late Ap-Je.

4077 E. luctuata D. \& S. var. obductata Mösch. (lugubrata; transversata of European works). Plattsburg, late May.

Camptogramma Stephens

4085 C. stellata Gn. (sitellata). Ithaca, Aug-Sep (CU); LI: Glendale, Oct 7, Kch (Doll).

\section{EULyPE Hübner \\ (Rheumaptera Hübner)}

4088 E. hastata gothicata Gn. General, including LI: Rockaway, Yaphank (Eng). Commonest in Adirondacks. Je-J1; also May on SI. Birch, willow, and blueberry.

4092 E. alternata Mull. (sociata Borkh., unangulata Pck., Epirrhoë). Peru; Saranac Inn; Old Forge; N. Elba; Newcomb; Waddington; Vandalia; Oak Orchard Swamp; Castile, Brad; Potter Swamp; Ithaca; McLean; Catskills. Je-Aug.

\section{MESOLEUCA Hübner}

4086 M. ruficilliata Gn. Common, wherever it has been looked for, northward to Adirondack Lodge, Bish. Je; Aug.

\section{EAROPHILA Gumppenberg 66}

4109 E. vasiliata Gn. Plattsburg; Essex Co; Fentons; Brockport; Lancaster; Ithaca; Catskills; Ilion; Schoharie; Rhinebeck; Mt. Vernon; NYC; SI. Ap 30 - May.

E. vasiliata var. niveifasciata Hulst. Ithaca, Ap 19, 1915 (Fbs).

\section{Venusia Curtis}

4114 V. cambrica Curt. Mt. Marcy; Adirondack Lodge, Bish; Indian Pass; Fentons; Big Ind. Val.; Stony Clove, Greene Co; Catskill; Utica. Je-Jl. Mountain ash.

4116 V. comptaria W1k. (perlineata Pck., salienta Pears.). Lancaster; E. Aurora, Salamanca (Wild) ; Ithaca; Big Ind. Val.; Schoharie; Albany; Pear1 R. Ap - early May.

\footnotetext{
${ }^{68}$ Packard reports Zenophleps lignicolorata Pck. from NY, doubtless an error.
} 


\section{HYDRELIA Hübner \\ (Euchoeca Hübner)}

4119 H. inornata Hulst (exhumata Pears.). I. Tear, Mt. Marcy; Saranac Inn; N. Elba; Indian Pass; Fourth L., Fulton Chain; Big Moose; Newcomb; Fentons; Otto; Rock City; Ithaca; McLean; Big Ind. Val.; Stony Clove, Greene Co; Onteora Mt.; Sharon; Utica; Trenton Falls; Little Falls; Schenectady; SI, May-Je (Ds) ; LI: Jamaica, Kch (Fbs) ; Coram (Eng). Je-J1.

4118 H. lucata Gn. Lancaster; Ithaca; McLean; SI. May-Je.

4120 H. albifera W1k. Peru; Newcomb; Buffalo; E. Aurora; Oak Orchard Swamp; Ithaca; McLean; Trenton Falls; Big Ind. Val.; Albany; West Farms; SI; LI: Brooklyn. Je-J1; Aug-Sep.

\section{EUdULE Hübner}

4122 E. mendica W1k. Common everywhere, to the summit of Mt. Marcy. Je-J1. Violet.

4123 E. meridiana Sloss. SI, Je (Ds).

TEPHROCLYSTIS Hübner ${ }^{67}$

(Eupithecia Curtis)

4144 T. affinata Pears. Big Ind. Val. (type).

4208 T. albicapitata Pck. Ithaca, J1; Big Ind. Val. (Pears).

4210 T. borealis Hulst. Big Ind. Val. (Pears); Onteora Mt., How.

4153 T. catskillata Pears. Ithaca, McLean (Fbs); Big Ind. Val. (type). Je-J1.

4168 T. coagulata Gn. (fumosa Hulst). Catskills (Pears, Tayl).

4176 T. cretaceata Pck. L. Tear, Mt. Marcy, J1; summit of Mt. Marcy, J1; Castile, Je, Brad (Fbs); Ithaca, Je.

4250 T. dolorosata Pears. Big Ind. Val. (Pears).

4159 T. erpata Pears. Big Ind. Val. (Pears).

4145 T. filmata Pears. Ithaca (CU) ; Big Ind. Val.; Bronx Pk., NYC; LI. Ap.

4141 T. frostiata Swett (conformata Pears.). New Windsor (Swett); Big Ind. Val.; Bronx Pk.; LI: Central Pk. and Woodhaven (Pears).

4169 T. geminata Pck. (packardata Tayl., meritata Pears.). Potter Swamp and Ithaca (Fbs) ; Big Ind. Val. (Pears); LI: Cypress Hills, Aug 28, Kch (Doll). J1-Aug.

4261 T. indistincta Tayl. Catskills (Tayl).

4192 T. interruptofasciata Pck. Niagara Falls, Buffalo, E. Aurora (Wild) ; Ithaca (Fbs); Slaterville; Onteora Mt., How. Je-Oct. Juniper.

4187 T. latipennis Hulst. Ithaca; Big Ind. Val. Je.

4140 T. miserulata Grt. Ilion; Catskills; NYC; LI. Var. nebulosa Hulst is from: Big Ind. Val.; NYC; LI: Brooklyn (CU); Central Pk.; Bellport, Yaphank (BM). J1.

4209 T. mutata Pears. Big Ind. Val. (Pears).

${ }^{67}$ In this genus I have made few determinations; most of the records below are from specimens determined by Pearsall, Taylor, Swett, and Grossbeck (Fbs). 
4156 T. palpata Pck. Peru (CU); Ithaca (CU); Big Ind. Val. (Pears). Early Je.

4265 T. promulgata Pears. Big Ind. Val. (Pears).

4228 T. ravocostaliata Pck. Ithaca, May 9, 1915 (Fbs).

4191 T. youngata Tayl. Keene Val.; Catskills.

4173 T. strattonata Pck. (anticaria auct.). McLean; Big Ind. Val., Je.

HORISME Hübner

(Eucymatoge Hübner)

4280 H. intestinata Gn. Newcomb; Fentons; New Russia; Lancaster; Blossom; Ithaca; McLean; Onteora Mt., How; Rhinebeck; New Windsor; West Farms; SI; LI: Wading R. (Ds) ; Orient, Lath. May - early Je; Aug.

\section{SUBFAMILY GEOMETRINAE}

(Ennominae)

IsTURGIA Hübner

(Epelis Hulst, Fidonia)

4287 I. truncataria Wlk. L. Tear, Mt. Marcy; E. Concord; McLean, common on the bogs; Karner. May-J1. Bearberry.

\section{EMATURGA Lederer}

4288 E. amitaria Gn. (faxonii Minot). Black Br.; Catskills; Ft. Edward; Karner; Albany. Je. Pine(?).

\section{Eufidonia Packard}

4289 E. notataria W1k. Black Br.; Peru; Saranac L.; Buffalo; E. Aurora; Rock City; Dayton; Vandalia; Portage; Ithaca; McLean; Gloversville (Alex) ; Big Ind. Val.; Onteora Mt., How; Trenton Falls; Bethlehem; Karner; Albany; Ramapo (Ds). May-Jl. White pine.

\section{ORTHOFIDONIA Packard}

4290 O. exornata W1k. (tinctaria Wlk., Cymatophora deceptata Hulst). Saranac L.; Fourth L., Fulton Chain; Aurora Twp. (Wild); Rock City; Ithaca; McLean; Big Ind. Val.; Trenton Falls; Karner. The female form flavivenata Hulst occurs at E. New York, LI. May-Je.

\section{BAPTA Stephens \\ (Corycia, Orthofidonia)}

4292 B. semiclarata Wlk. N. Elba; Peru; Black Br.; Saranac Inn; Fourth L., Fulton Chain; Lewis Co; Rock City; Coy Glen and Six Mile Cr., Ithaca; McLean; Catskills; Karner. Late May-Je.

4293 B. vestaliata Gn. Very common everywhere. May-Jl; Aug. Apple and wild cherry.

4295 B. glomeraria Grt. Ithaca; McLean; Big Ind. Val.; LI: Coram (Eng). Ap-early May. 


\section{Heliomatä Grote}

4296 H. infulata Grt. LI: Brooklyn (Pck); Wading R. Je.

4298 H. cycladata Grt. Ithaca; Poughkeepsie; Bronxville; SI; LI: Brooklyn; Cold Sp. Harb.; Yaphank; West Hills, Wading R. (Ds); Orient, Lath. May-Je. Locust.

\section{Mellilla Grote}

4300 M. xanthometata W1k. (chamaechrysaria Grt.). Buffalo (Grt). Gleditsia.

\section{Physostegania Warren}

4303 P. pustularia Gn. Common and general. J1-Sep. Maple.

\section{Gueneria Packard}

4304 G. basiaria Wlk. General, but somewhat local and often mistaken for one of the Deilineas. Definite records are: Peru; Vandalia; Ithaca; McLean; Trenton Falls; Big Ind. Val.; Ft. Edward; West Farms; Hunt's Pt., Kch (Fbs) ; SI; LI: Woodhaven; Forest Pk. and Cold Sp., Kch (Fbs); Orient, Lath. Je-J1.

\section{DeILINEA Hübner \\ (Cabera Treitschke)}

4307 D. variolaria Gn. Common and general, but easily confused. I have verified the following records: Peru; Black Br.; Mt. Marcy; Adirondack Lodge, Bish; Wilmington; Fentons; Rochester, Angle; Vandalia; Portage; Potter Swamp; Ithaca; McLean; Binghamton; Big Ind. Val.; Schoharie; Karner; Albany; SI. Je-Aug, stragglers to Sep. Willow.

4309 D. erythremaria Scop. General. With the habits of the preceding species, but not always on the same stations. Trustworthy records are: Black Br.; Peru; Wilmington; Batavia; Portage; Egleston's Glen; Ithaca; McLean; Trenton Falls; Big Ind. Val.; Newport; Karner. End of May-early Sep. Willow.

\section{DREPANULATRIX Gumppenberg}

4325 D. liberaria W1k. Fentons; Buffalo; Seneca L.; Ithaca; Binghamton; Cedar Hill; Karner; Albany; Hartsdale; Ramapo, Oct (Ds); SI. Var. helena Hulst was described from Brooklyn. Aug-Sep. Possibly Ceanothus.

\section{Philobia Duponchel}

4332 P. ulsterata Pears. (notata auct.). Peru; Uphill Br. and N. Twin Br., Mt. Marcy; North Cr.; Shelving Rock, L. George; Utica; Karner; Big Ind. Val. (types); LI. J1.

4331 P. aemulataria W1k. (enotata auct.). Rock City; Potter Swamp; Ithaca; McLean; Catskills. "P. enotata" is reported also from: Fentons; Oneida; Buffalo; Albany; Poughkeepsie; Brewster; West Farms; SI; LI: Brooklyn. Late Jl-Aug. Lettuce (?). 
MACARIA Curtis

(Sciagraphia Hulst, Semiothisa)

4345 M. granitata Gn. Forms of this species in the broad sense are general. The commonest is dispuncta W'k. I have seen the typical form from Ithaca and the Catskills, and what seems to be var. unimodaria Morr. from Vandalia. May-Aug. Spruce(?).

4344 M. multilineata Pck. LI: Amagansett (BM).

4367 M. ocellinata Gn. Peru; Rochester, Angle; Ithaca; Rhinebeck; Florida; general south of New Windsor. Late May-early Sep. Locust.

4385 M. continuata Wlk. Keene, Wild. Celtis.

4387 M. orillata Wlk. LI(?).

4373 M. mellistrigata Grt. Plattsburg; Wilmington; Fentons; Waddington; Lancaster; McLean; Catskills; Karner; Albany ; Poughkeepsie. Je-Aug.

4372a M. trifasciata Pck. Peru, Je 11, 1916 (Fbs).

4380 M. infimata Gn. E. Aurora, Irving (Wild) ; Ithaca; NYC; SI; LI: Brooklyn; Glendale, Kch (Fbs.). The type of the synonym caesiaria Hulst was from NY. May-early Je, J1, Aug.

4389 M. eremiata Gn. Karner, May, Aug.

4336 M. aequiferaria Wlk. SI.

4338 M. minorata Pck. Newcomb; Ithaca; Poughkeepsie; LI: Forest Pk.; Brooklyn. Je-early Sep.

4341 M. praeatomata Haw. Ithaca, J1 4, 1891 (CU) ; Karner; West Pt., Je (Ds); West Farms; SI, May (Ds). Jl-Aug.

4339 M. bisignata Wlk. Plattsburg; Peru; Keene Val.; Oneida; Lewiston; Buffalo; Lancaster; Crosby; Ithaca; Ringwood; Catskills; Albany; West Farms; LI: Woodhaven; Yaphank. Je-Aug. Pine, etc.

\section{Hesperumia Packard}

4398 H. sulphurararia Pck. Peru, Mix\&Everett; Cranberry L., Htly; Catskills and West Pt. (Pears). J1-Aug; late autumn.

\section{ITAME Hübner}

\section{(Cymatophora Hübner, Diastichtis Hübner)}

4430 I. virginalis Hulst. Peru; Wilmington; Catskills; Ithaca; Karner; Albany; SI. Je; Aug.

4399 I. ribearia Fitch. Gooseberry Span-Worm. Peru; Ausable Forks (NYS); Fentons; Ogdensburg (NYS); Buffalo; Otto; Ithaca; McLean; Newport; Trenton Falls; Paris Hill; Schenectady; Saratoga Sps.; Salem; New Paltz. May, J1. .Currant and gooseberry, sometimes injurious.

4407 I. brunneata Thun. Fentons; Newcomb. J1.

4406 I. inceptaria Wlk. Plattsburg; Peru; Rock City; Ithaca; LI: Patchogue, $W l s$ (Fbs). Specimens from Big Ind. Val. are practically the same as the Mississippi Val. I. evagaria. End of Je-Jl.

4408 I. subcessaria Wlk. Sharon Sps.; Onteora Mt., How; also a Pearsall specimen perhaps from Big. Ind. Val. J1.

4409 I. coortaria enigmata B. \& McD. E. Aurora (Wild); Lancaster; Ithaca; DeRuyter. Je-J1. 
4411 I. anataria Swett. Adirondack Lodge, Bish.

- I. wauaria L. Lancaster; SI, Je (Ds) ; LI: Brooklyn, E. New York (Wasmuth).

4413 I. exauspicata Wlk. (bicolorata auct.). Ithaca; McLean; Sharon; Big Ind. Val. ; LI. J1.

\section{EUMACARIA Packard}

4425 E. latiferrugata W1k. (pustularia auct., brunnearia Pck.). Fentons; Buffalo; Karner; End of May-Je. Apple, plum, and wild cherry.

\section{DYSMIGIA Warren \\ (Sympherta Hulst)}

4453 D. loricaria julia Hulst. Mt. Whiteface trail; Big Ind. Val. J1.

HOMOCHLODES Hulst

4454 H. fritillaria Gn. (disconventa Wlk.). Saranac Inn; Fentons; Adirondack Lodge; E. Aurora; Lancaster; Castile, Je, Brad (Fbs) ; Rock City ; Ithaca; McLean; Liberty; Big Ind. Val.; Stony Clove, Greene Co; Oneida; Trenton Falls; Karner; Albany; Katonah; SI. Je-Jl.

\section{ApaEcasia Hulst}

\section{(Lithina)}

4455 A. detersata Gn. Black Br.; Buffalo; Vandalia; Castile, Je, Brad (Fbs); McLean; Ithaca; Ft. Edward; Karner; Albany; West Farms ; SI; LI: Yaphank, May (Ds); Merrick. Je-Jl.

4456 A. atropunctata Pck. (deductaria auct.). Ithaca, May 31, 1918; Poughkeepsie.

4457 A. subaequaria W1k. (defluata W1k.). Common everywhere in damp places. Je-early Aug.

4458 A. extremaria W1k. SI; LI: Amagansett; Woodhaven. Ap-Je. A saltmarsh species.

\section{CATOPYRRHA Hübner}

4460 C. coloraria Fab. Ithaca; Cedar Hill; Karner; Albany; LI: Brooklyn and E. New York, Ap-May. The summer form dissimilaria Hbn. is of course general with the species; it is reported definitely from: Trenton Falls; Esopus; Karner; Albany; Pocantico Hills; Katonah (Ds) ; Bronxville; LI. Je-J1. Clover.

\section{CARIPETA Walker}

4465 C. divisata Wlk. Jay; Mt. Marcy; Indian Pass; Big Moose; Fentons; Lancaster; Aurora; Ithaca; McLean; Big Ind. Val.; Schoharie; Albany. Late Je-Aug.

C. divisata var. nigraria Fbs. Ithaca (types).

4467 C. angustiorata W1k. (criminosa Swett). Plattsburg; Peru; Ithaca; Poughkeepsie; LI: Central Pk., May 6, Kch (Doll); Yaphank (Bell and Eng). Late J1-Aug. Pine (Kch).

4469 C. angustiorata var. aretaria Wlk. (angustiorata Pck., in part). Peru; Ithaca; Poughkeepsie; LI: Central Park (transition), Kch. Je-J1, Sep. 
Nepytia Hulst

4486 N. canosaria Wlk. (semiclusaria auct.). Keene Val.; L. Placid; E. Aurora; Otto; Ithaca; Big Ind. Val.; Catskills. A distinct species. Sep-Oct.

4488 N. semiclusaria Wlk. Albany, Pears; Cedar Hill? (Meske); LI: Bellmore; Delhaven; Amagansett; Yaphank. Oct.

\section{PARAPHIA Guenée}

4490 P. unipuncta Haw. (subatomaria Wood, deplanaria Gn., guttata Hulst). The numerous varieties of this species are not very sharply distinguished and I have not tried to record them separately. The species is general. Je-J1. Birch and willow, also variously reported on oak, elm, hickory, fir, and pine.

SPODOLEPIS Hulst

4493 S. subtriataria Hulst (Jubarella danbyi Hulst). Big Ind. Val.

\section{LYTROSIS Hulst}

4496 L. unitaria H.-S. Rochester, Angle; Ithaca; Pelham Manor; L. Mahopac (Ds). May-J1.

TORNOS Morrison

4506 T. scolopacinarius Gn. (abjectarius Hulst). LI: Flatbush. Aster.

\section{Cleora Curtis}

4567 C. sublunaria Gn. (atrolinearia Hulst, areataria Broad.). Pelham Manor; SI; LI: E. New York; Woodhaven. Ap.

\section{PSEUDOBOARMIA McDunnough}

4560 P. umbrosarium Hbn. Mt. Marcy (Hill); Lancaster (EPV); Albany (Lint); West Farms (Pck); SI; LI: Yaphank; Wading R. May-Je (Ds). Confusion with porcelaria makes the records slightly doubtful.

\section{PRotoboarmia McDunnough}

4560 P. porcelaria Gn. (indicataria Wlk.). Newcomb; Orchard Pk. (Wild); Ithaca; Catskills; Trenton Falls; Schoharie; SI. Je-Aug. Pinus virginiana $(P$. inops).

\section{GLenA Hulst}

4602 G. cognataria Hbn. (muricolor Hulst). LI: Amagansett; Yaphank (Ds). End of May-Aug.

4566 G. cribrataria Gn. Cedar Hill. Spruce.

\section{STENOPORPIA McDunnough}

4572 S. polygrammaria Pck. Ithaca; Rhinebeck. The confusion of this species with porcelaria in Dyar's list was probably a pure accident. May-Je. 


\section{VitRINELlA McDunnough}

4570 V. pampinaria Gn. Common throughout the State. May-Sep. A general feeder.

\section{ANACAMPTODES McDunnough}

4575 A. vellivolata Hulst. Portage; LI: Wading R. Je. Pine.

4585 A. larvaria Gn. Mt. Marcy; Buffalo; Ithaca; Big Ind. Val.; Albany; West Farms; SI; LI: West Hills (Ds). Rare, but general. May-Sep.

4574 A. humaria Gn. Big Moose; Karner; Poughkeepsie; Rhinebeck; NYC; SI, Ap-May (Ds) ; LI: Jamaica, Kch (Doll) ; Hempstead Plain. Late JeJ1. A general feeder.

4584 A. ephyraria Wlk. (takenaria Pears.). Cranberry L., Htly (Fbs); Ithaca; McLean; Catskills; SI. J1-Aug. Wild cherry.

\section{MELANOLOPHIA Hulst}

4599 M. canadaria Gn. Common everywhere. Castile, Je, Brad (Fbs) ; May-early J1; Aug 29(1). Spruce(?).

\section{ETHALOPTERA Hulst}

4601 A. anticaria W1k. (intextata auct.). General. Definite records are: N. Elba; Rock City; McLean; Ithaca; Big Ind. Val.; Albany; NYC; SI; LI: Woodhaven,; Central Pk., Kch (Doll). Ap-J1.

\section{ECTROPIS Hübner}

4604 E. crepuscularia D. \& S. Common throughout the State. I believe that our records apply rather to bistortata than to the true crepuscularia of Europe. May-Oct, mostly J1. Poplar, alder, etc.

E. crepuscularia ab. abraxaria Wlk. Ithaca, Je 9.

\section{EPIMECIS Hübner}

4607 E. hortaria F. (virginaria auct., not virginiaria $\mathrm{Cr}$.). Ithaca, probably a stray; Bronxville; SI; LI: Aqueduct; Forest Pk. May, Ji. Tulip tree and sassafras.

E. hortaria var. carbonaria Haim. SI, May-Aug (Ds) ; LI: Forest Pk., J1 4, $1923(K c h)$.

\section{LyCIA Hübner}

4608 L. ursaria W1k. Plattsburg; Lancaster; Buffalo; E. Aurora; Ithaca ; Albany; NYC; LI: Woodlawn. Ap-May. Poplar, plum, etc.

4609 L. cognataria Gn. (Amphidasys). Pepper-and-Salt Currant Moth. Peru; Wilmington (larva); Fentons; Geneva; Rochester, Angle; Lancaster; E. Aurora; Buffalo; Ithaca; Binghamton (NYS) ; Catskills; Poughkeepsie; Albany; New Windsor; SI; LI: Brooklyn; Glendale, black var. mesle; Maspeth (Ols). May-Aug. General on trees and shrubs. 
NACOPHORA Hulst

4615 N. quernaria S. \& A. (male phigaliaria and cupidaria). Keene, E. Aurora, Allegany Pk. (Wild) ; Ithaca; Oliverea, West Pt. (Ds); SI; LI: Brooklyn; Forest Pk., Kch; Glendale (black aberration), Kch (Fbs); West Hills, Cold Sp. (Ds). Je (Sep). Oak.

\section{Phigalia Duponchel}

4620 P. olivacearia Morr. Ithaca; Binghamton; Albany; Bronxville; Bronx Pk., Kch (Doll); SI; LI: Brooklyn. Rarer and even earlier than the other species. End of Mar-Ap.

4622 P. titea Cr. Half-winged Geometer. Peru; Rochester; Lancaster; Buffalo; Pike; Ithaca; Binghamton; Big Ind. Val.; Onteora Mt.; Albany; Rio (NYS) ; Ramapo (Ds) ; NYC; SI; LI: Jamaica. Sometimes very common in early spring. Ap-early May. A general feeder.

\section{PALEACRITA Riley}

4624 P. vernata Peck. Spring Canker-Worm. Old Forge; E. Aurora; Oakland; Castile; Ithaca; Cooperstown; Albany; Westchester Co; SI; LI: Brooklyn; Orient, Lath. The imago is regularly confused with that of the fall canker-worm, pometaria, especially in the female, and the larva is much like those of various geometers; therefore I can find only these few trustworthy records. The species is probably general but local in the State, and is often rare for several years together. Ap (rarely late fall). A general feeder.

\section{ERANNIS Hübner}

4628 E. tiliaria Harr. Lime-Tree Winter Moth. General and locally common in late fall. Oct-Nov. Linden, elm, apple, etc.

\section{Cingilia Walker}

\section{(Zerene)}

4620 C. catenaria Dru. Chain-dotted Geometer. General and locally common. Adirondack records are: Fentons; Peru. Sep-Oct. A general feeder, especially on shrubs.

4631 C. rubiferaria Swett. Peru, Sep 15-16, 1917, Mix\&Everett (CU).

\section{ANAGOGA Hübner}

\section{(Numeria)}

4641 A. pulveraria L. Black Br.; Mt. Marcy; Crosby; Ithaca; Debruce; Big Ind. Val.; Onteora Mt., How; Trenton Falls; Karner; Albany; SI; LI: Brooklyn; Yaphank. May-early J1.

\section{Sicya Guenée}

4644 S. macularia Harr. Peru; Mt. Whiteface; Fentons; Rochester, Angle; Lancaster; Lewiston, N. Evans, E. Aurora, and Allegany Pk., Wild; Castile, Brad; McLean (Fbs); Ithaca; DeRuyter; Big Ind. Val.; Sharon; Albany; Poughkeepsie; Katonah; Florida. Je-J1. Spiraea. 


\section{THERINA Hübner ${ }^{6} 8$ \\ (Ellopia B. \& McD.)}

4653 T. endropiaria G. \& R. Cranberry L.; Peru; Saranac L.; Rochester; Portage; Ithaca; Big Ind. Val.; West Pt., Ft. Montgomery, Nyack (Ds); SI; LI: Brooklyn; Bellport; Brookhaven; Yaphank. Je-J1. Oak and chestnut.

4651 T. pellucidaria G. \& R. LI: Brookhaven (Dyar); Bellport; Yaphank; Central Pk., Doll; Wading R., May (Ds). Yellow pine.

4652 T. athasiaria Wlk. Saranac L.; Buffalo; LI: Brookhaven; Central Pk.; Cold Sp., Je 12, Kch (Doll); Yaphank (Ds); Orient Pt., Aug 30, Lath. Ap-Je. Oak.

4654 T. fiscellaria Gn. Cranberry L.; Keene Val.; Cascade L.; Fentons; E. Aurora, Buffalo (Wild) ; Ithaca; McLean; Big Ind. Val.; Trenton Falls; LI: Yaphank. Sep-Oct. Yellow birch (Htly).

T. fiscellaria var. turbataria B. \& McD. (fervidaria auct.). Buffalo; Ithaca; Trenton Falls; Cedar Hill; Albany; SI; LI: Amagansett, Yaphank, Sep-Oct (Ds).

4655 T. fervidaria Hbn. LI: Farmingdale, Je 16, 1918 (CU).

CAMPAEA Lamarck

(Metrocampa Latreille)

4659 C. praegrandaria Gn. General and not rare. Je-early J1, Aug-Sep; two broods, the second dwarfed. White birch and willow.

\section{EUGONOBAPTA Warren}

4661 E. nivosaria Gn. General, and sometimes locally the commonest geometer. End of May-Aug.

ENNOMOS Treitschke

\section{(Eugonia Hübner)}

4664 E. subsignarius Hbn. SNow-white Linden Moth. Rochester; Buffalo; Ithaca; McLean (Fbs) ; Seager; Big Ind. Val.; Utica; Albany; Rhinebeck; Poughkeepsie; Katonah; SI; LI: Pinelawn, Yaphank (Ds). J1Aug. Trees and shrubs. Sometimes occurs in injurious numbers.

4665 E. magnarius Gn. (alniaria). General and sometimes in injurious numbers on maple. Aug-Oct. Maple, etc.

\section{XANTHOTYPE Warren}

4666 X. sospeta Dru. (crocataria Fab.). Common and general. Recently verified records are: Adirondack Lodge, Bish; Peru, Mix and Everett; Newcomb; Ithaca; McLean. Numerous records for southern NY should be verified, as possibly belonging to $X$. urticaria.

4666.1 X. urticaria Sw. (crocataria, in part). Recently separated from the preceding species and commoner than that. Definite records are: Potter Swamp; Ithaca; McLean.

\footnotetext{
${ }^{68}$ The species of this genus, except endropiaria, are difficult and sometimes impossible to determine, and $I$ am not sure that all the records here given are correct.
} 


\section{PLAGODIS Hübner}

4667 P. serinaria H.-S. Fourth L., Fulton Chain; Buffalo; E. Aurora (Wild) ; Rock City; Allegany Co; Ithaca; McLean; Binghamton; Catskills; Ilion; SI; LI: Forest Pk., Kch (Doll). May-early J1.

4669 P. keutzingaria Pck. (altruaria Pears.). Plattsburg; Fourth L., Fulton Chain; Crosby; Ithaca; Albany; LI: Yaphank (aberrant). Also reported from Fentons, but not verified. May-Je.

4468 P. nigrescaria Hulst (kuetzingi Grt., keutzingaria Pck. in part). Ithaca ; Onteora Mt., How; Poughkeepsie. May 31-Je 3.

4671 P. purpuraria Pears. LI: Merrick. (Hardly distinct from kentzingaria).

4673 P. schuylkillensis Grossb. SI, Ds (Grossb).

4674 P. alcoolaria Gn. Saranac L.; Cranberry L., Htly; Fourth L., Fulton Chain; Buffalo; E. Aurora; Portage; Ithaca; Catskills; SI; LI: Farmingdale; Yaphank. Late May-J1. Chestnut.

4676 P. phlogosaria Gn. Fentons (Lint); Thousand Isls. (Kch) ; Rochester, Angle; Lancaster (EPV); Ithaca; McLean; Big Ind. Val.; Albany (Pck); Poughkeepsie; SI; LI: Jamaica; Richmond Hill (Kch). J1-Aug.

4672 P. emargataria Gn. SI; LI: Valley Stream, May, Kch (Doll).

\section{Hyperitis Guenée}

4678 H. amicaria H.-S. Everywhere in the State. I have not tried to record the forms, but nyssaria Gn. occurs at Minnewaska and Fourth L., Fulton Chain, and a dark form of nepiasaria W1k. at Rock City. May-J1. Reported on Hypericum and beech.

\section{Nematocampa Guenée}

\section{(Ania Hulst not Stephens)}

4680 N. filamentaria Gn. Northern records are: Mt. Marcy; Old Forge; Fentons. The range of the species extends southward to the Tropics. Je-Sep. A general feeder.

\section{MetaRRANTHIS Warren}

\section{(Gonodontis, Epirranthis)}

4685 M. hypochraria H.-S. Common and general. The normal form on the Appalachian Highlands is much paler than the type. Late May-early J1. Apple and persimmon.

4685a M. hypochraria var. lateritiaria Gn. Trenton Falls (W1k).

4685b M. homuraria Grt. Sullivan Co; LI: Bellport, Nic (Fbs). A very distinct form or species.

4686 M. apiciaria Pck. Karner; Albany. Je-J1.

4687 M. warneri Harv. LI: Central Pk., Je 5, 1921, Kch (Doll).

4689 M. duaria Gn. Black Br.; Peru; Slaterville; Ithaca.

4689.1 M. duaria var. hamaria Gn. Black Br.; Newcomb; Ithaca; McLean. 
4689.2 M. duaria var. angularia B. \& McD. Ithaca.

All these forms or elementary species $(4689,4689.1$, and 4689.2) are May-Je. Also a good many records from all over the State recorded merely as "duaria."

4688 M. pilosaria Pck. Considered a good species by B. \& McD. Occurs in NY (Grossb).

4692 M. obfirmaria Hbn. Plattsburg; Peru; Black Br.; McLean; Catskills; Karner; Albany; SI; LI: Central Pk., Kch; Promised Land; Yaphank; Orient, Lath. Ap-Je. Vaccinium, Cassandra, etc.

\section{Euchlaena Hübner}

\section{(Endropia)}

4699 E. serrata Dru. Peru to Ithaca and Auburn, and southward to LI: not rare northward; uncommon on SI and LI (Ds). Je-early Aug. Maple.

4700 E. obtusaria Hbn. Peru; E. Aurora and Allegany Pk. (Wild); Ithaca; McLean; Big Ind. Val.; Albany (Pck); Katonah; SI. Je-J1. Rose, etc.

4701 E. effecta W1k. (effectaria auct.). Peru; Essex Co; Saranac L.; Fentons; Oneida; Lancaster; Allegany Pk. (Wild) ; Big Ind. Val.; Stony Clove, Greene Co; Albany. Var. muzaria Wlk., the southern form of this species, is credited to NY by Walker, probably from the vicinity of NYC. J1; Sep.

4702 E. johnsonaria Fitch (bilinearia Pck.). Peru; Essex Co; Fentons; Adirondack Lodge, Bish; Allegany Pk. (Wild) ; Lancaster; Ithaca; Binghamton (NYS); Big Ind. Val. ; Sharon; Hebron (W1s) ; Croton-onHudson (Ds); SI; LI: Bellport, Nic (Ds). Var. minoraria Hulst was described from NY. Je-J1; Aug-Sep. Oak.

4704 E. amoenaria Gn. Binghamton; SI; LI: Cypress Hills, Aug 7, Kch (Doll); Wading R. (atypical).

4705 E. vinulentaria G. \& R. Pearl R.; Binghamton; LI: Central Pk. and Coram, Kch (Doll) ; Riverhead; Yaphank. Je, J1.

4706 E. astylusaria W1k. LI: Yaphank. Various trees.

4707 E. marginata Minot. Fentons; Lancaster; Rock City; Ithaca; Schenectady; Karner; New Windsor; LI: Smithtown; Yaphank. May-Aug.

4710 E. tigrinaria Gn. (pectinaria auct.). Newcomb; Ithaca; Big Ind. Val.; Ramapo Mts. J1. Oak.

\section{SELENIA Hübner 69 \\ (Eutrapela Hübner)}

4711 S. alciphearia W1k. Big Ind. Val. The type form in May; var. ornata B. \& $\mathrm{McD}$. in $\mathrm{J} 1$.

4712 S. kentaria Grt. Brockport; Buffalo; Ithaca; Big Ind. Val.; Brewsters; LI: Brooklyn; Queens (Bell and Eng). The large typical form of this generally passes as alciphearia; the small summer form usually called kentaria is glaucata B. \& McD. May; autumn. Maple, beech, basswood, and birch.

${ }^{69}$ In this genus there are two broods, the second one much dwarfed. 


\section{Metanema Guenée}

4721 M. inatomaria Gn. Peru; Saranac L.; Adirondack Lodge, Bish; Buffalo, E. Aurora (Wild); Newcomb; Ithaca; Big Ind. Val.; Onteora Mt., How; Sharon; Albany; Rhinebeck; SI; LI: Brooklyn. Je-Aug. Poplar (?).

4722 M. determinata Wlk. Peru; Essex Co; North Cr.; E. Aurora; Ithaca; Oneonta; Poughkeepsie. Late May-early J1; late Aug.

4726 M. quercivoraria Gn. (textrinaria G. \& R.). Fourth L., "Fulton Chain; Buffalo; Ithaca; Overlook Mt., Catskills (G\&R); SI (Ds); LI: Wading R., Yaphank, Riverhead (Ds). May-Sep, two broods. Oak, elm, and poplar.

\section{PRIOCYCLA Guenée}

4728 P. armataria H.-S. Mt. Marcy; Buffalo; E. Aurora; Ithaca; McLean; Big Ind. Val.; Onteora Mt., How; Ilion; Rhinebeck; New Windsor; Ramapo, Fulda (Ds); SI; LI: Cypress Hills, Kch; Orient, Lath. Je-J1. Currant, birch, and maple.

4729 P. decoloraria Hulst (jucundaria Strk.). Peru; Ithaca; SI. Late Je-early J1. Raspberry(?).

\section{PERO Herrich-Schaeffer \\ (Azclina Guenée, in part)}

4744 P. honestarius W1k. (ancetaria auct.). Peru; Rochester; E. Aurora; Ithaca; common from the Mohawk Val. southward and westward. Ap-Je; Aug-Sep. Wild cherry.

4750 P. morrisonarius H. Edw. "Ithaca" (one old specimen at CU); Big Ind. Val. (Grossb); Pearl R. (Pears). Je-J1.

4752 P. marmoratus Grossb. Rock City; Ithaca; Ilion; New Windsor (Grossb); SI; LI: E. New York (Ds); Orient, Lath (Fbs). Je-J1.

\section{SYSSAURA Hübner}

\section{(Drepanodes)}

4755 S. puber G. \& R. (infensata auct.). Lewis Co; Albany; Catskills; Rhinebeck; West Farms; SI; LI: Brooklyn; Amagansett; Yaphank; Orient, Lath. Je; Aug-Sep. Juniper.

APICIA Guenée

(Caberodes Guenée)

4758 A. confusaria Hbn. (majoraria Gn.). Common throughout the State; the dark form metrocamparia Gn. almost as common as the type. May, J1-early Aug. General on low plants.

4759 A. irraria B. \& McD. (majoraria auct.). Ithaca; Catskills; SI. Je-Aug.

\section{TETRACIS Guenée}

4762 T. crocallata Gn. Peru; Fentons; Rochester, Angle; Ithaca; McLean; Little Falls; Gloversville; Albany; Oswego; Lancaster; Buffalo and southward. Late May-J1. Chestnut and sumac. 


\section{SABUlodes Guenée}

4763 S. arcasaria W1k. (sulphurata Pck.). Poughkeepsie; Ft. Montgomery (Ds); New Windsor; West Farms; SI; LI: Brooklyn; Jamaica; Woodside; Woodhaven; Forest Pk., Kch; Wading R. (Ds). The summer form furciferata Pck. is reported from: Ithaca, Je 9, 1922; LI: Brooklyn; E. New York; Newtown. Ap-May, Je-Aug. Sumac and maple.

4769 S. lorata Grt. (Tetracis). Black Br.; Essex Co; Fentons; Castile, Je, Brad (Fbs); Clayton; Geneva; Rochester; Buffalo; E. Aurora; Portage; Ithaca; McLean; Big Ind. Val.; Albany; Rhinebeck; Ft. Montgomery (Ds) ; West Farms ; SI ; LI: Wading R., Yaphank (Ds). General and not rare. Late May-J1; early Sep. Sweet fern (and other shrubs?).

4777 S. transversata Dru. General and common. J1-Oct (two broods). Oak, maple, etc.

\section{Aввотtana Hulst}

4780 A. clemataria S. \& A. General and not rare. May-J1. A general feeder, especially on maple.

SPHACELODES Guenée

4786 S. vulneraria Hbn. Buffalo; Ithaca, Benjamin; LI: Brooklyn. A stray from the Tropics.

\section{FAMILY SPHINGIDAE}

\section{HERSE Oken}

662 H. cingulata Fab. Ithaca (a stray); Kenwood (Albany); LI: Brooklyn; Glendale; E. Quogue. Convolvulaceae.

\section{Phlegethontius Hübner}

665 P. quinquemaculata Haw. (celeus Hbn.). Northern Tomato Worm. Plattsburg, Newport (NYS) ; Orleans, Genesee, Erie, and Chautauqua Counties, generally; Watertown; Castile; Ithaca; Cayuga and Onondaga Counties; E. Worcester; Cherry Valley; Saratoga Sps.; Watervliet; Schenectady; Coeymans; Albany; Poughkeepsie; Ulster Co, general; Woodstock and southward. Je-Ji. Solanaceae.

664 P. sexta Joh. (carolina L.). Southern Tomato Worm or Tobacco Worm. Ransomville (CU Econ); Clinton Corners (CU Econ); Yonkers; Rye (Bird); SI; LI, general (Wat). Solanaceae.

666 P. rustica Fab. SI and LI (Brooklyn) (strays). Bignonia, etc.

667 P. brontes Dru. "NY" (Westwood) (a stray).

\section{Chlaenogramma Smith}

670 C. jasminearum Bdv. Rye (Bird); Bronx Pk., occasional (Wat); West Farms; SI; LI. Ash.

\section{DoLBA Walker}

671 D. hylaeus Dru. Albany; Rye (Bird); New Windsor; LI: Brooklyn; Bellport, J1 (Nic). Ilex and papaw. 


\section{Ceratomia Harris}

674 C. amyntor Hbn. (quadricomis Harr.). Four-Horned SpHINx. Peru; Rochester; Buffalo; E. Aurora; Ithaca; Oneonta; Stuyvesant Falls; Gloversville; Albany; Poughkeepsie; Rye (Bird); NYC, common (Wat); SI; LI. Je-J1. Elm; white birch (Wat).

675 C. undulosa Wlk. (Daremma). Essex Co; Fentons; Rochester (Angle); Lancaster; Buffalo; Allegany Pk., abundant (Wild); Portage; Crosby; Ithaca; Onteora Mt., How ; Oneonta; Gloversville (Alex); Hebron, Wls; Edgewood, Greene Co (Wat); Van Cort. Pk., common (Wat); SI; LI: Brooklyn. May-J1; a straggler Aug 20. Ash and privet.

676 C. catalpae Bdv. (Daremma). Catalpa Sphinx. LI: Brooklyn, Wasmuth. Catalpa.

\section{SPHINX Linnaeus}

\section{(Hyloicus)}

693 S. kalmiae S. \& A. L. Placid; Saranac L.; Buffalo; E. Aurora, Allegany Pk. (Wild); Lake Forest; Nile; Ithaca; Trenton Falls; Oneonta; Gloversville; Hebron, Wls; Albany; Rye; Florida; SI; LI: Brooklyn. J1-Aug. Lilac, ash, and Chionanthus.

696 S. drupiferarum S. \& A. Plattsburg; Saranac L.; Newport; Geneva; Rochester; Buffalo; Westfield (NYS); Allegany Pk. (Wild) ; Ithaca; Oneonta; Ft. Plain; Albany; Rye; Van Cort. Pk., larvae formerly common on Prunus serotina (Wat); SI; LI. May-J1. Apple, plum, and cherry.

694 S. gordius Stoll. Plattsburg; Peru; Big Moose; Saranac L.; Buffalo; Lancaster; Crosby; Oneonta; Albany; Rye; Tappan (Wat); SI; LI: Brooklyn; Bellport (Nic); Wading R. (Ds). Je. Apple; Myrica; sweet fern and huckleberry (Eng).

695 S. Iuscitiosa Clem. Saranac L.; Ithaca; Albany; Karner; LI: Brooklyn. Je. Willow and poplar.

687 S. chersis Hbn. Great Ash Sphinx. Clayton; Peru; Buffalo; Otto; Ithaca; Trenton Falls; W. Winfield; Stuyvesant Falls; Oneonta; Gloversville; Hebron (W1s) ; Nassau; Rye; Van Cort. Pk. and Wash. Hgts., NYC, common (Wat); SI; LI: Cold Sp.; Brooklyn. Je-J1. Ash and lilac.

691 S. canadensis Bdv. Plattsburg; Adirondacks; Buffalo. Blueberry(?).

681 S. eremitus Hbn. Buffalo; E. Aurora (Wild) ; Ithaca; Rogers Rock, L. George, Jones; Rye; SI; LI: Brooklyn. Mints.

\section{Atreides Holland}

679 A. plebeja Fab. Ithaca; SI; LI: Brooklyn. Lilac; Bignonia; passion flower; trumpet vine (Eng).

\section{LAPARA Walker}

700 L. coniferarum S. \& A. U1ster Co (Seifert).

701 L. bombycoides W1k. Pine Tree Sphinx. Plattsburg; Peru; L. Ontario shore; Ithaca; Sharon Sps.; Oneonta; Albany; LI, in the pine barrens (Eng). Var. harrisii Clem. is known from Ulster Co. and is probably the form to which many of the other records should be referred. Var. pineum Lint., described from Schoharie, has been taken once at Ithaca. Je-Jl. Pine. 


\section{SMERINTHUS Latreille}

705 S. jamaicensis geminatus Say. Twin-Spotted Sphinx. Plattsburg; Peru; Wilmington; Rochester (Angle); Avon; E. Aurora; Allegany Pk.; Ithaca; McLean; Gloversville; Rye; SI; LI: Brooklyn. Je-Aug. Willow and poplar (Eng).

The Eliot Coll. has a bred series of typical jamaicensis, presumably from NY stock.

706 S. cerisyi Kby. Plattsburg; Peru; Saranac Inn; Ithaca; Oneonta; Karner. Late May-Je. Willow.

- S. hybr. interfaunus Neum. NYC. Bred from a wild male Paonias astylus and a captive female $S$. ocellatus from Europe.

\section{PAONIAS Hübner}

707 P. excaecatus S. \& A. Blind-Eyed Sphinx. Plattsburg; Peru; Newcomb; Axton; Mt. Marcy; L. Placid; Ogdensburg; Buffalo; Allegany Pk.; Union Sps. and southward. Je-Aug. Common on oak and wild cherry (Prunus serotina) (Wat); birch; apple.

708 P. myops S. \& A. Peru; Fentons; Mt. Marcy; Rochester (Angle); Lewiston, common (Wat \& W. P. Comstock); Buffalo; Allegany Pk. (Wild); Ithaca; Oneonta; Gloversville (Alex) ; Albany; Rye; Van Cort. Pk., formerly common (Wat) ; SI; LI: Brooklyn. Je-J1. 'Wild cherry, etc.

709 P. astylus Dru. Poughkeepsie; Rye; LI: Brooklyn. J1. Vaccinium.

\section{CRESSONIA Grote and Robinson}

710 C. juglandis S. \& A. Walnut Sphinx. Essex Co; Mt. Marcy; Wilmurt; Castile, Je, Brad (Fbs); Buffalo; E. Aurora, Allegany Pk. (Wild); Lancaster; Rock City; Ithaca; Trenton Falls; Oneonta; Rye; New Windsor; Pine I. (Ds); Van Cort. Pk. (Wat); SI; LI: Brooklyn; Wading R. (Ds). May 30-J1. Walnut and hickory.

\section{PACHYSPHINX Rothschild and Jordan}

\section{(Marumba)}

711 P. modesta Harr. Plattsburg; Adirondacks; Rochester (Angle); Tonawanda; Buffalo; Lancaster; E. Aurora, Allegany Pk. (Wild) ; Crosby ; Ithaca; Catskills (Ulster Co) ; Oneonta; Albany; SI; LI: Brooklyn. Late May - early J1. Poplar and willow.

\section{ERINNYIS Hübner}

\section{(Dilophonota Burmeister)}

715 E. ello L. Buffalo; Catskills; Ithaca. A stray from the Tropics.

\section{AËLlOPOS Hübner}

730 A. titan Cr. LI: Rockaway (Kunze). A stray from the Tropics.

729 A. tantalus L. Albany (RM \& WGray). A stray from the Tropics.70

\footnotetext{
${ }^{70}$ Cramer's type of Enyo japix was reported as from this State, almost certainly in error.
} 


\section{HEMARIS Dalman}

735 H. diffinis Bdv. Bumble-Bee Moth. General. May-J1, two broods. Transitional to var. aethra Strk. in cooler parts of the State, and nearly typical aethra from Axton. Je.

733 H. gracilis G. \& R. Trenton Falls; Karner; SI; LI: Smithtown. Early May-Je. Indian currant (Eng).

732 H. thysbe Fab. (cimbiciformis Steph.). Clayton; Rochester; Batavia; Buffalo; Castile, Brad; Ithaca; McLean; Trenton Falls; Ilion; Stuyvesant Falls; Gloversville; Hebron, Wls; Poughkeepsie; Crugers ; Bronxville ; SI; LI: Smithtown; Brooklyn. Var. ruficaudis Kby. (uniformis G. \& R.) is reported from: Fentons; Newcomb; Buffalo; Ithaca; McLean; Trenton Falls; Utica; Schoharie; Albany; Karner; Poughkeepsie; LI: Sands Pt. May-Aug, two broods. Viburnum.

\section{PhOLUS Hübner}

740 P. vitis L. SI; LI: Brooklyn, bred by Tepper (Doll). Grape and ampelopsis.

741 P. fasciatus Sulz. LI: Brooklyn, Wasmuth (Eng). Accidental.

737 P. satellitia pandorus Hbn. Pandorus Sphinx. Clayton; Newport, Cranberry Cr. (NYS); Lancaster; Buffalo; Ithaca; Mayfield (NYS); Hebron, Wls; Glenville (NYS); Albany and vic.; Rye; SI; LI: Brooklyn; Orient, Lath (Angle). Aug. Grape and ampelopsis.

738 P. achemon Dru. Lancaster; Buffalo; Ithaca; Newport; Albany (NYS) ; Poughkeepsie; Rye; SI; LI: Brooklyn; Orient, Lath (NYS). Je-J1. Grape and ampelopsis.

\section{AmpelophagA Bremer and Gray}

735 A. choerilis Cr. Azalea Sphinx. Plattsburg; Mt. Marcy; Clayton; Lake Forest; Buffalo; Otto; Ithaca; Oneonta; Sharon; Albany; Poughkeepsie; Rye; NYC; SI; LI: Brooklyn; Pine Haven. Je-J1. Azalea; Viburnum (Eng).

744 A. myron Cr. (pholus auct.). Hog Caterpillar of the Vine. Alder Co.; Port Kent; Oswego; Syracuse; Buffalo; E. Aurora; Ithaca; Ft. Plain;

Knox; Patria; Athens; Rome; Crown Pt.; Stuyvesant Falls; W. Troy; and southward. Je-Aug, two broods. Grape and ampelopsis.

743 A. versicolor Harr. Rochester; Buffalo; Lake Forest; Ithaca; Lestershire; Kenwood, Albany; LI: Brooklyn. Je-J1; Aug. Cephalanthus and Decodon (Nesea).

\section{SPHECODINA Blanchard}

746 S. abbottii Swains. Aввот's Sphinx. General on LI and northward to Hebron; Gloversville; Mt. Morris; Howe's Cave, Schoharie; Saranac L. May. Grape and ampelopsis.

\section{DEIDAMIA Clemens}

747 D. incripta Harr. Batavia; Ithaca; Brockport; Ft. Montgomery (Ds) : Rye; SI; LI: Brooklyn. May. Grape; ampelopsis (Eng). 


\section{AMPHION Hübner}

749 A. nessus Cr. Fentons; S. Bryon; Pike; Buffalo; E. Aurora (Wild); Ithaca; Trenton Falls; Oneonta; Ft. Edward; Albany; Cambridge; Athens; Rye; Pine I. (Ds); Bronx Pk. (Wat \& W.P.Comstock); SI; LI: Brooklyn; Smithtown; Orient, Lath. Je-J1. Ampelopsis; grape and evening primrose (Eng).

\section{PROSERPINUS Hübner (Lepisesia Grote)}

753 P. flavofasciata W1k. Harrisville, Elbridge Sibley (Quaintance). Epilobium.

\section{XYLOPHANES Hübner}

(Theretra)

760 X. tersa L. Kenwood, Albany; Rye; SI; LI : Brooklyn; Jamaica. Spermacoce.

\section{Celerio Oken}

\section{(Deilephila Ochsenheimer)}

761 C. gallii intermedia Kby. Plattsburg; Essex Co; Buffalo; E. Aurora; Ithaca; Oneonta; Schenectady; Albany; Poughkeepsie; LI: Brooklyn. Epilobium, etc.

762 C. lineata Fab. White-Lined Sphinx. Common southward. Definite records are; Buffalo; Ithaca; Oneonta; Stuyvesant Falls; Cedar Hill; Albany; Coeymans; Poughkeepsie; SI; LI: Brooklyn. A general feeder, preferring purslane and evening primrose.

\section{FAMILY NOTODONTIDAE}

\section{Melalopha Hübner}

\section{(Ichthyura)}

3589 M. apicalis W1k. Peru; Albany; Rensselaer. Reported also from Plattsburg and Poughkeepsie, but the species is too near the next following for the records to be quite certain. Je-J1 (a stray in Mar). Poplar.

3592 M. strigosa Grt. "S.N.Y.” J1 (?). Poplar.

3591 M. inclusa Hbn. Peru; Saranac L.; Buffalo; Ithaca; W. Coxsackie; Schoharie; Rensselaer; Putnam Co; Adams Corners; Mt. Vernon; NYC; SI. Ap-Je. Poplar.

3593 M. albosigma Fitch. Plattsburg; Peru; Essex Co; Fentons; Rochester; Batavia; Lancaster; Buffalo; Ithaca; Onteora Mt., How; Gloversville (Alex) ; Sharon; Álbany; Poughkeepsie; SI; LI: Jamaica. The var. specifica Dyar is general with the type, and is commoner at Ithaca at least. Je-Aug. Poplar.

3594 M. brucei H. Edw. Plattsburg (Pck). Willow. 


\section{DATANA Walker 71}

3595 D. ministra Dru. Yellow-Necked Apple Worm. Waddington; Massena; Potsdam; Chepachet; Medina; Chautauqua Co, scarce (Hartzell); Rock City and vic.; Bath; Windsor; Ithaca; Summitville; Sherburne; Stittville; Shushan; Albany; Clinton Corners, etc. ; Rye ; Middletown; Florida; Croton L.; Katonah; SI; LI. Je-Aug. Apparently the commonest Datana in the State. General on trees, sometimes injurious to apples in late summer.

3596 D. angusii G. \& R. Plattsburg; Peru; Buffalo (Wild) ; Lancaster ; Ithaca ; Sharon; McLean; Karner; Selkirk; Poughkeepsie; Rye; Katonah; West Farms; SI; LI: Brooklyn. Je-J1; stragglers in Aug. Walnut and hickory (?).

3597 D. drexelii H. Edw. Peru; Plattsburg; Ithaca; Rhinebeck; Poughkeepsie; SI. J1. Hamamelis; huckleberry (Eng).

3598 D. major G. \& R. Newburgh; Poughkeepsie; Ft. Montgomery (Ds) ; Rye ; Florida; SI; LI: Brooklyn. Jl. Andromeda.

3599 D. palmii Beut. Ithaca; Ulster Co (larvae); Poughkeepsie. J1. Will eat Vaccinium.

3600 D. floridana Graef. "NY" (N\&D). NYC and vic. (Eliot). I am suspicious of these records. Andromeda.

3603 D. perspicua G. \& R. Ithaca; Gloversville (Alex) ; Rhinebeck; Poughkeepsie; Newburgh; Katonah; Rye; West Farms; SI; LI: Brooklyn. J1. Sumac.

3604 D. intergerrima G. \& R. Walnut Datana. Plattsburg; Waddington; Rochester; Holley; Eden Center; Sinclairville; Westfield; Ithaca (larvae) ; Canastota; Moravia; Romulus; Windsor; Utica; Rhinebeck; Poughkeepsie; West Pt. (Ds); Rye; SI; LI: Brooklyn; Mineola; Wading R. J1-Aug. Walnut, usually black walnut; hickory (Eng).

3607 D. contracta Wlk. Buffalo; Ithaca; Newburgh; SI; LI: Brooklyn. J1-Aug. Oak; also reported from chestnut, hickory, and witch-hazel.

\section{HyPERAESCHRA Butler}

3608 H. stragula Grt. Plattsburg; Peru; Essex Co; Saranac Inn; Buffalo; E. Aurora; Ithaca; Catskills; Sharon; Hebron, Wls; Albany; Rhinebeck; Poughkeepsie; Rye; SI; LI. All summer, two broods (?). Poplar and willow.

3609 H. georgica H-S. Plattsburg; Ithaca; Poughkeepsie. May-J1. Oak.

\section{Odontosia Hübner}

3611 O. elegans Strk. Saranac L.; Ithaca (larva).

\section{Notodonta Ochsenheimer}

3613 N. basitriens W1k. Peru, Mix\&Everett; N. Elba; Keene Val.; Brockport; Buffalo; Lewiston (Wild) ; Onteora Mt., How; Ithaca; McLean; Trenton Falls; Sharon. Late May; Aug.

3614 N. simplaria Graef. Plattsburg; Peru, Aug 2, Mix EEverett.

${ }^{71}$ In this genus determinations based on the imago are usually a little doubtful, and therefore I have omitted records based on atypical imagoes (Fbs). 


\section{Pheosia Hübner}

3615 P. dimidiata H-S. Plattsburg; Wilmington (larva); Saranac L.; Lancaster; Buffalo; Rock City; Ithaca; North Branch; Onteora Mt., How; Utica; Albany; Poughkeepsie; Rye; LI: Brooklyn. J1-Aug. Poplar; willow (Eng).

\section{LOPHODONTA Packard}

3617 L. ferruginea Pck. Plattsburg; Peru; Keene Val.; Mt. Marcy; Ithaca (rare) ; Poughkeepsie; Catskills; Rye; SI; LI: Brooklyn: May; J1Aug. Birch.

3618 L. angulosa S. \& A. Plattsburg; Brockport; Ithaca; Ulster Co; Dutchess Co; SI; LI: Yaphank. May-Aug. Oak.

\section{NADATA Walker}

3602 N. gibbosa S. \& A. Common throughout the State. Je-J1 (stragglers to Sep). Oak.

\section{NERICE Walker}

3621 N. bidentata Wlk. Plattsburg; Peru; North Cr.; Geneva; Lancaster; E. Aurora; Ithaca; McLean; Trenton Falls; Sharon; Rhinebeck; Poughkeepsie; Rye; LI: Newtown. May-Aug. Elm.

\section{SYMMERISTA Hübner}

3622 S. albifrons S. \& A. Red-humped Maple Worm. Plattsburg; Peru; Lancaster ; E. Aurora; Castile, Je, Brad (Fbs); Gowanda; Leon; Ithaca ; McLean; Onteora Mt., How; Trenton Falls; Poughkeepsie; Rye; SI; LI: Brooklyn. Je-Jl. Maple, oak, and rarely, beech.

\section{DASYLOPHIA Packard}

3625 D. anguina S. \& A. Plattsburg; Big Ind. Val.; Albany; Poughkeepsie; New Windsor; Katonah; LI: Brooklyn (Doli). Je-Ji. Locust and other legumes.

3626 D. thyatiroides W1k. Plattsburg; Ithaca; Sharon. Reported from hickory, but probably in error.

\section{Heterocampa Doubleday}

3634.1 H. obliqua Pck. (trouvelotii Pck.). L. Placid; Ithaca; Big Ind. Val.; West Farms; LI: Newtown. Jl. Oak.

3640 H. umbrata Wlk. (pulverea G. \& R.). Ithaca; Poughkeepsie; SI; LI: Brooklyn. Jl-Aug. Oak.

3641 H. manteo Dbl. Plattsburg; Peru; Saranac L.; Castile, Je, Brad (Fbs); Buffalo; Ithaca; Onteora Mt., How; Trenton Falls; Utica; SI; general on LI. Je-Aug. Oak and other trees.

3642 H. biundata Wlk. Cincinnatus, Dnd; Ithaca; Big Ind. Val.; Trenton Falls (type); LI: Woodhaven. Credibly reported also from Plattsburg and Poughkeepsie. Maple and other trees. 
3643 H. guttivitta Wlk. Maple Prominent. Plattsburg; Peru; Saranac L.; Jamestown; Buffalo; E. Aurora (Wild); Ithaca; McLean; Big Ind. Val.; Johnstown; Berlin; Stephentown; Ramapo Mts.; SI; LI: Greenport, Aug (Ds). Reported also from Poughkeepsie, where it undoubtedly occurs. Rubbed or faded specimens are quite indistinguishable from biundata. May-J1, Sep. Maple and other trees, sometimes injuriouss.

3644 H. bilineata Pck. (Seirodonta). Plattsburg; Oswego; Buffalo (Wild) ; Lancaster; Castile; Ithaca; Rhinebeck; Poughkeepsie; Katonah; Irvington; SI; LI: Brooklyn. Je-Aug. Elm.

\section{MiSOGA:DA Walker}

3645 M. unicolor Pck. Brockport; Buffalo; Ithaca; Catskills; Poughkeepsie ; Rye; SI; LI: Brooklyn; Glencove; Woodhaven. Je. Sycamore.

\section{IANASSA Walker}

3649 I. lignicolor Walker. Plattsburg; Peru; Rochester, Angle; Buffalo; Aurora ; Rock City (Wild) ; Ithaca; Big Ind. Val.; Onteora Mt., How; Sharon; Poughkeepsie; Rye; SI; LI: Brooklyn. Je-Aug. Oak, beech, and birch.

\section{SCHizURA Doubleday}

3654 S. ipomoeae Dbl. Plattsburg; Wilmington (larva) ; Saranac L.; E. Aurora ; Geneva; Ithaca; Big Ind. Val.; W. Haverstraw; Poughkeepsie; Tarrytown; SI; LI: Woodhaven. The preceding records belong, either certainly or probably, to the typical form. Var. telifer Grt. is known from Poughkeepsie, Nyack (Ds), and NYC. Var. cinereofrons Pck. has been seen from Buffalo, Ithaca, Rhinebeck, and Bronxville. Je-Aug. Oak, maple, etc., not Ipomoea.

3655 S. concinna S. \& A. Red-humped Apple Worm. A pest all over the State. The imago is relatively seldom seen but the larva is unmistakable. Aug. Apple and other trees.

3652 S. semirufescens W1k. (eximia Grt.). Plattsburg; Peru; Buffalo (Wild) ; E. Aurora; Ithaca; Sharon Sps.; Poughkeepsie; SI. Je-early Aug. Maple, apple, and other trees.

3657 S. unicornis S. \& A. Unicorn Prominent. Common throughout the State. May-Aug. Various trees.

3659 S. apicalis G. \& R. Peru; Ithaca; Brockport; SI: Clove Val. Bayberry (Eng).

3656 S. badia Pck. Peru; Sharon; Dutchess Co; Florida; SI; LI: Brooklyn. JeAug. Viburnum.

3660 S. leptinoides Grt. Plattsburg; Peru; Brockport; Buffalo; E. Aurora, Wild, Dnd; Ithaca; McLean; Poughkeepsie; New Windsor; SI; LI (Doll). Je; Aug. Oak, etc.

\section{HyPARPAX Hübner}

3662 H. aurora S. \& A. Plattsburg; Protection (Wild) ; Ithaca, Je 9, 1918; Karner; New Windsor; Rye; Bronx Pk. (Ds); SI; common and general on LI. Je. Oak. 


\section{CERURA Schrank}

3666 C. scitiscripta multiscripta Rly. Poughkeepsie; Rye; SI; common and general on LI. Willow and poplar.

3670 C. occidentalis Lint. Plattsburg; Peru; Saranac Inn; Rochester; Buffalo; Ithaca; Poughkeepsie; SI; LI: Brooklyn; Woodhaven. May-Je; Aug. Willow.

\section{HARPYIA Ochsenheimer}

3669 H. borealis Bdv. Plattsburg; Fentons; Buffalo; Ithaca; Oneonta; Poughkeepsie; Rye; SI; common and general on LI. Wild cherry.

3671 H. cinerea Wlk. Plattsburg; Buffalo; Ithàca, Je 12, 1915; Poughkeepsie; Rye; SI; LI: Brooklyn. Willow and poplar.

3673 H. scolopendrina Bdv. LI: Flushing. Var. albicoma has been taken at Waddington.

3673a H. modesta Hudson. Plattsburg; Peru, Je; Ithaca, Ap (Benjamin) ; Poughkeepsie. This appears to be a distinct species. Willow and poplar.

\section{Fentonia Butler}

3674 F. marthesia Cr. Plattsburg; Buffalo; Ithaca; Big Ind. Val.; Sharon; Albany; Poughkeepsie; SI; LI. J1-Aug. Oak and maple.

\section{Gluphisia Boisduval}

3680 G. septentrionalis Wlk. Rochester; Buffalo (Wild) ; Ithaca; Big Ind. Val.; Sharon; Albany; SI; LI: Newtown. Late Mar-Ap, Je-Aug. Poplar.

3681 G. lintneri Grt. Plattsburg; Ithaca; Karner. Ap-May; Sep-Oct.

G. lintneri Grt. form avimacula Hudson. Plattsburg (type); Ithaca; Big Ind. Val.

ELLIDA Grote

3682 E. caniplaga W1k. Plattsburg; Ithaca; Utica; Karner. May 30-J1.

\section{FAMILY LIPARIDAE}

(Lymantriidae)

\section{Notolophus Germar}

$$
\text { (Orgyia, in part) }
$$

3701 N. antiqua nova Fitch. AntiQue Tussock-Moth. Ogdensburg, Newport, Gouverneur (NYS); Plattsburg; Keene Val.; Wilmington (larva); Fentons; Cranberry L., Htly; Rochester (NYS) ; Holland; Ontario; Ithaca; McLean; Silver Bay; Albany; Coxsackie (NYS). Aug. A general feeder on trees and shrubs.

\section{HEMEROCAMPA Dyar}

(Orgyia, in part)

3705 H. leucostigma S. \& A. White-Marked Tussock-Moth. Common throughout the State. End of $\mathrm{J1}$-early Oct. General on trees and shrubs, often injurious. 
$3706 \mathrm{H}$. inornata Beut. LI: Bellport, in a swamp (presumably now exterminated).

3707 H. definita Pck. Yellow-Headed Tussock. Rochester (NYS); Allegany Pk. (Wild) ; Ithaca; McLean; New Windsor. The species is probably rather general, but is easily recognized only in the larva. Sep.

\section{OLENE Hübner}

\section{(Parorgyia)}

3709 O. achatina S. \& A. Plattsburg; Rhinebeck; Poughkeepsie. These records probably belong to $O$. basiflava.

3710 O. basiflava Pck. Brockport; Rhinebeck; Poughkeepsie; LI: Yaphank (Do11). Late J1-early Aug. Hickory.

3712 O. vagans B. \& McD. Big Ind. Val. (Pears); LI: Yaphank (Doll). Oak.

3715 O. atomaria Wlk. Newburgh (Pck); Greene Co (Seifert). Aug.

O. atomaria var. parallela G. \& R. Ithaca; Big Ind. Val. (Pears); Rhinebeck. Je-Aug.

3716 O. cinnamomea G. \& R. Poughkeepsie. J1-Aug.

3717a O. interposita Dyar. Sharon. Aug 1.

3718 O. plagiata Wlk. Plattsburg. J1-Aug. Pine.

3720 O. pini Dyar. Albany (Dyar); Catskills (Franck). Pine.

\section{PORTHETRIA Hübner}

3722 P. dispar L. Gypsy Мотн. Dr. Felt states that infestation has been found at one time or another in the following localities, but that the species is now presumably exterminated: Geneva; Mt. Kisco; Garrison; a strip along the eastern border of the State from near L. George south to Dutchess Co, including the townships of Hampton, Stanford, Washington, Berlin, N. Greenbush, N. Albany, Gallatin, Austerlitz, Canaan, and Pine Plains; Moriah Twp. in Essex Co; LI: Prospect Pk., Brooklyn; Patchogue and vic.; Greenport. Aug.

\section{LIPARIS Ochsenheimer}

L. monacha L. Black Arches, or Nun Moth. LI: Brooklyn (Franck). Apparently never established.

\section{EUPROCTIS Hübner}

3723 E. phaeorrhoea Don. (chrysorrhoea auct.). Brown-taIl Moth. LI: Richmond Hill, J1 15, 1910, at light $(K c h)$; Hardscrabble; Orient Pt.; Fisher's I., breeding in small numbers in 1914; Shelter I. Now exterminated.

\section{FAMILY NOCTUIDAE SUBFAMILY HYPENINAE}

HYPENA Schrank

3579 H. humuli Harr. Hop Snout-Moth. General and common. May; Aug; Oct. A general feeder, sometimes injurious to hop. 


\section{Plathypena Grote}

3578 P. scabra Fab. Green Clover-Worm. General and common, in some seasons injurious to beans. Je-Aug; Sep-Oct; adult hibernates (Bird).

\section{ANEPHISCHETOS Smith}

3574 A. citata Grt. (Bomolocha). Ithaca. Aug-Oct.

\section{LOMANALTES Grote}

3576 L. eductalis W1k. Plattsburg; Peru; Fentons; Ithaca; McLean; Schenectady ; Karner; Albany; Rhinebeck; Rye. May-Je; Aug-Sep.

\section{BOMOLOCHA Hübner}

3560 B. manalis W1k. Elba (Wild) ; Geneva; Potter Swamp; Ithaca; Ulster Co; Poughkeepsie; Rye; SI; LI: Yaphank (Eng). Je-Aug.

3561 B. baltimoralis Gn. Throughout the State. Ap-Aug.

3562 B. bijugalis W1k. Peru; Saranac L.; Niagara Falls; Lancaster; Ithaca ; Big Ind. Val.; Onteora Mt., How; Newport; Coeymans; Poughkeepsie; SI; LI (Eng). Je; Aug-Sep.

3531 B. scutellaris Grt. Potter Swamp; Big Ind. Val.; Cedar Hill. Je-Aug.

3564 B. abalinealis Wlk. Peru; Saranac L.; Buffalo; E. Aurora; Ithaca; McLean; Big Ind. Val.; Newport; Oneonta; Sharon; Schenectady; Rhinebeck; Poughkeepsie; New Windsor; SI; LI (Eng). End of MayAug; apparently two broods. Elm.

3566 B. madefactalis Gn. (caducalis Wlk.). Peru; E. Onondaga; Lancaster; Buffalo; Ithaca ; Schenectady; Onteora Mt., How; Big Ind. Val.; Rye; Croton-on-Hudson (Ds); Filorida; Katonah. May-J1.

3567 B. sordidula Grt. Ithaca; Poughkeepsie; Rye; SI. Je. Alder.

3569 B. atomaria Sm. Peru; Potter Swamp; Ithaca. Je; Aug.

3571 B. toreuta Grt. Ithaca; Rye. May; J1-Aug.

3565 B. deceptalis W1k. Lancaster; E. Aurora, Niagara Falls (Wild) ; Castile, Brad; Ithaca; McLean; Trenton Falls; Big Ind. Val.; Onteora Mt., How; Sharon; Schoharie; Rhinebeck; Rye; Ramapo (Ds). May-Aug.

3572 B. edictalis W1k. Fentons; Potter Swamp; Ithaca; Little Falls; Big Ind. Val.; Sharon; Schenectady; Albany. J1-Aug.

SALIA Hübner

3559 S. interpuncta Grt. Poughkeepsie; Katonah. J1.

\section{DERCETIS Grote}

3556 D. vitrea Grt. Buffalo; Ithaca; West Farms (AM). J1.

\section{Capis Grote}

3555 C. curvata Grt. Saranac Inn; Buffalo; Castile, Brad; McLean; Poughkeepsie; Katonah. J1-early Aug. 


\section{Metalectra Hübner 72 \\ (Homopyralis Grote)}

M. praecisalis Hbn. LI: Lynbrook, Je 16.

3478 M. praecisalis discalis Grt. Saranac L.; Evans Center; Lancaster; Ithaca; Catskills; Karner; Albany; Rye; SI; LI: Woodhaven, Je-J1; Sep.

3479 M. contracta Wlk. (tactus Grt.). Fentons; Newcomb; Clayton; Buffalo; Otto; Ithaca; Catskills; Newport; Schenectady; Florida; Rhinebeck;

Rye; NYC; SI; LI: Lynbrook; Great Neck; Yaphank. Late May-Aug.

\section{Melanomma Grote}

3474 M. auricinctaria Grt. Rhinebeck; LI: Brooklyn; Glendale. J1. Huckleberry and Cephalanthus.

\section{SPARgALOMA Grote \\ (Hyamia Walker)}

3472 S. sexpunctata Grt. Plattsburg; Peru; Crosby; Ithaca; Karner; Poughkeepsie; Rye; SI. Je-Aug.

3473 S. perditalis Wlk. Buffalo; Catskills; Rhinebeck; SI, Ap, J1 (Ds) ; LI: Brooklyn. Cephalanthus.

\section{PANGRAPTA Hübner}

3471 P. decoralis Hbn. Saranac L.; Fentons; Minnewaska; Crosby; Ithaca; McLean; Karner; Albany; Rye; SI; general on LI. May-Aug.

\section{OXYCILla Grote}

3467 O. malaca Grt. SI, Je (Ds).

Phytometra Haworth

(Prothymia Hübner)

3459 P. rhodarialis W1k. Buffalo. Aug.

3460 P. semipurpurea W1k. Rhinebeck; Katonah; Rye; Mosholu; Woodbury (CU) ; SI, J1, Fulda. (Ds) ; LI: Amagansett; Yaphank. J1-Sep.

\section{Pleonectyptera Grote}

3445 P. pyralis Hbn. Rye; LI. Form geometralis Grt. is reported by Smith from Lahaway, N. J.

3449 P. habitalis Wlk. "N. Y." (Sm); Ithaca (Benjamin).

\section{MYCTEROPHORA Hulst}

3441 M. slossoniae Hulst. Big Ind. Val. J1. The members of this genus are generally mistaken for geometers.

${ }^{72}$ Caterpillars of this genus have been reported as living in rotten wood. 


\title{
PHIPROSOPUS Grote
}

3436 P. callitrichoides Grt. Rye; Fordham; SI; LI: common at Jamaica. May. Smilax.

Rivula Guenée

3434 R. propinqualis Gn. Common and general, especially in damp places. JeAug. Food probably grass.

\author{
GABARA Walker \\ (Eucalyptra Morrison)
}

3420 G. bipuncta Morr. Seen from Mosholu and Katonah. Reported also from: Rye; Albany; LI: Coram, J1, Kch.

\section{Scolecocampa Guenée}

3411 S. liburna Gey. Evans Center; E. Aurora; Lancaster; Ithaca; Rhinebeck; Rye; SI; LI: Queens; Yaphank. J1; larva hibernates (Bird). Dead wood.

\section{SUBFAMILY HERMINIINAE}

\section{Palthis Hübner}

3553 P. angulalis Hbn. Mt. Marcy; Saranac Inn; Fentons; Geneva; Buffalo; Castile, Je, Brad (Fbs) ; E. Aurora; Rock City; Crosby; Ithaca; McLean; Big Ind. Val.; Onteora Mt., How; Ft. Edward; Schenectady; Albany; Rhinebeck; Rye; Ramapo (Ds) ; Ft. Montgomery; SI; LI: Yaphank. May-early Sep.

3554 P. asopialis Gn. Honeoye Falls; Buffalo; E. Aurora; Ithaca; Rye; Florida. End of Je-Sep. Hop (Bird).

\section{LASCORIA Walker \\ (Gaberasa Walker)}

3550 L. ambigualis Wlk. Saranac L.; Hastings; Yorktown; Buffalo; Vandalia; Potter Swamp; Crosby; Ithaca; McLean; Newport; Schenectady; Rhinebeck; Rye; Ramapo (Ds) ; SI; LI: Yaphank; Forest Pk., Kch (Fbs). May-Sep.

\section{Phalaenophana Grote ${ }^{73}$}

\section{(Heterogramma)}

3546 P. pyramusalis Wlk. Common wherever looked for, northward to Adirondack Lodge, Bish, Saranac Inn, and Peru. Je-early J1; late Aug-Sep.

\section{Bleptina Guenée}

3536 B. caradrinalis Gn. Peru; Saranac Inn; Newcomb; North Cr.; Fentons; Buffalo (Wild); Lancaster; Otto; Ithaca; McLean; New Windsor; Rye; Katonah; SI; LI: Wading R.; Yaphank. The black-spotted form also occurs. J1-early Aug.

${ }^{73}$ Hypenula cacuminalis Wlk. is labeled "West Farms" in the Angus Collection, almost certainly in error. 


\section{RENIA Guenée}

3522 R. salusalis W1k. SI (Ds); LI (Eng). J1.

3524 R. discoloralis Gn. Fentons; Buffalo; E. Aurora; Ulster Co; Utica; Rye; SI; LI: Orient, May 10, Lath. J1-Aug.

3532 R. sobrialis W1k. Ithaca, J1 1, 1882; Rye; SI; LI (Eng). J1.

R. sobrialis var. larvalis Grt. E. Aurora, Gowanda, Wild; Lancaster (EPV); Allegany Pk. (Wild); SI, Jl, Ds (Sm). May.

3523 R. clitosalis W1k. Ithaca; Utica. I am not sure that I have this species rightly determined (Fbs).

3523 R. factiosalis W1k. Gowanda (Wild) ; E. Aurora; Ithaca; McLean; Karner; Schenectady; SI; LI (types of tilosalis Sm.). J1-Sep.

3528 R. flavipunctalis Gey. General and rather common. J1-Aug.

3530 R. atrimacula Sm. Ithaca, Aug 11.

\section{Chytolita Grote}

3519 C. morbidalis Gn. General wherever looked for. Late May-J1.

3519a C. morbidalis petrealis Grt. Peru; Black Br.; Adirondack Lodge; Saranac L.; Batavia ; Castile, Brad; Portage; McLean; Karner; SI; LI: Forest Pk., Kch (Fbs). Je.

\section{Philometra Grote}

3516 P. metonalis W1k. Common throughout the State. J1.

3517 P. eumelusalis Wlk. Peru; Fentons; Buffalo; Olean; Ithaca; Schenectady; Albany. Late Je-Aug.

3518 P. hanhami Sm. Karner (Sm).

\section{Phalaenostola Grote}

3470 P. larentioides Grt. Hastings; Cedar Hill; Karner; Kinderhook, Bish; Poughkeepsie; West Farms; SI; LI: Brooklyn. Aug. A scavenger in birds' nests (?).

\section{HORMISA Walker}

3512 H. absorptalis Wlk. Peru; Saranac; Newcomb; Fentons; Buffalo; Otto; Olean; Ithaca; Varna; McLean; Michigan Swamp, Danby; Albany; Bethelehem; Rye; SI. J1-Aug.

3513 H. litophora Grt. Ithaca; Little Falls; Albany; "West Farms" (Angus). J1.

3514 H. bivittata Grt. Waddington; Buffalo. J1.

3515 H. orciferalis Wlk. "NY" (Edw). A southern species, also known from Ct. and Mass.

\section{ZANCLOGNATHA Lederer}

3503 Z. laevigata Grt. Fentons; Buffalo; Otto; Allegany Pk. (Wild); Wells; Ithaca; McLean; Slaterville; Big Ind. Val.; Onteora Mt., How; Schenectady; Karner; Rye; SI; LI (Eng). Ji-early Aug.

Z. laevigata ab. obsoleta Sm. Ithaca, McLean (Fbs). 
Z. laevigata ab. reversata Dy. Ithaca, McLean (Fbs).

Z. laevigata ab. modestalis Fitch. Ithaca (Fbs).

3506 Z. pedipilalis Gn. Peru; Saranac L.; Rock City; Potter Swamp; Ithaca; Trenton Falls; Ft. Edward; Schenectady; Newcomb; North Creek; Portageville; New Windsor; Rye; SI. Je-early J1; earlier than ochreipennis.

3508 Z. cruralis Gn. Ithaca; McLean; Poughkeepsie; Fordham; SI; LI: Lynbrook. Late Je.

3507 Z. protumnusalis Wlk. Mt. Marcy; Syracuse; SI. Late Je; Aug-Sep.

3509 Z. marcidilinea Guen. Peru; Ithaca; Onteora Mt., How; SI (Ds) ; LI: Yaphank (Ds). Je-Aug.

3510 Z. ochreipennis Grt. Peru; Essex Co; Ithaca to SI. Species of this group are common all through the State, but are difficult to determine with certainty; therefore I have recorded only fairly typical specimens. J1-Aug (stragglers in Sep).

3499 Z. lituralis Hbn. Peru; Saranac L. ; Lancaster; E. Aurora; Gowanda; Rock City; Ithaca; McLean; Schoharie; New Windsor; LI (Eng). Jeearly Aug.

3500 Z. theralis Wlk. Peru; Mt. Marcy, Hill Coll.; Fentons (Lint); Schoharie; LI: Woodhaven (Eng). Je.

3502 Z. inconspicualis Grt. Peru; Mt. Marcy, Hill (Grt); Ithaca (Fbs); McLean. J1-Aug.

3501 Z. minoralis Sm. Schenectady. Aug.

\section{PARAHYPENODES Barnes and McDunnough}

P. quadralis B. \& McD. Newcomb, J1 5; McLean, J1 30.

\section{EPIZEUxis Hübner}

(With Psendaglossa Grote)

3485 E. americalis Gn. General and common. Larvae in birds' nests, Dobroscky (Fbs). Je-Sep.

3486 E. aemula Hbn. General and very common. Je-Sep. Larvae in birds' nests, Dobroscky (Fbs).

3496 E. lubricalis Gey. Peru; Jay; Fentons; Mt. Marcy; Rochester (Angle) ; Buffalo; Otto; Allegany Pk. (Wild); Castile, Brad; Ithaca; McLean; Debruce; Schenectady; Rhinebeck; Croton-on-Hudson, Je (Ds); Crugers; Katonah; SI; LI: Newtown (Eng); Orient, Lath. JI-Sep. Grass or dead leaves.

3494 E. denticulalis Harvey. Feru (CU); Ithaca; LI: Newtown (Eng). J1.

3487 E. scobialis Grt. Ithaca; McLean; E. Aurora (Wild); LI, general (Eng). J1-Aug.

3490 E. rotundalis W1k. Buffalo; Otto; E. Aurora (Wild); Wells; Ithaca ; McLean; LI: Orient, Lath. J1-Aug.

- E. julia B. \& McD. (Sm. MS.). Ithaca, not rare in 1922; Onteora Mt., Hoze. J1-early Aug.

3493 E. merricki Sm. Otto; E. Aurora (Wild).

- E. diminuendis B. \& McD. Ithaca; LI: Coram, Kch (Fbs). J1.

E. forbesi French. Ithaca, J1. 
DYSPYRALIS Warren

3475 D. illocata Warr. (humerata Sm.). E. Aurora (Wild); Ithaca; McLean. J1-Sep.

3477 D. nigellus Strk. (Epizenxis). E. Aurora (Wild); Ithaca. J1.

\section{SUBFAMILY EREBINAE}

Anomis Hübner

3407 A. erosa Hbn. Ithaca (Sep) ; Rye; SI, Oct (Ds) ; LI: Greenport. Cotton and other Malvaceae.

\section{Alabama Grote}

3406 A. argillacea Hbn. CоттоN Mотн. A subtropical and Gulf-strip species, straying north in the fall to Mt. Marcy, some seasons in large numbers. Cotton; at Rye the moth has been known to puncture thin-skinned grapes (Bird).

\section{SYLECTRA Hübner}

3402 S. ericata Cr. NYC. A stray from the Tropics.

\section{Plusiodonta Guenée}

3400 P. compressipalpis Gn. Plattsburg; Buffalo; Castile, Brad; Crosby; Ithaca; Albany; Rhinebeck; Poughkeepsie; New Windsor; Rye; SI. Late May-early Aug. Moonseed vine.

\section{SCOLIOPTERYX Germar}

3399 S. libatrix L. Herald Motr. Peru; Keene Val.; Saranac L.; Mountain L.; Fulton Co; Clayton; Buffalo; Otto; E. Aurora; Allegany Pk.; Castile; Ithaca; McLean; Schenectady; Hebron, Wls; Rhinebeck; Rye; Florida; Kitchawan; SI; LI: Aqueduct. Late J1-Aug; late fall. Willow.

CALPE Treitschke

3398 C. canadensis Beth. Plattsburg; Essex Co; Buffalo; Marilla (Wild); Ithaca; McLean; Trenton Falls; Rye; SI; LI: E. New York. Late Je-early Sep. Thalictrum.

\section{THYSANIA Dalman}

3392 T. zenobia Cr. Buffalo; Albany; NYC. Aug. A tropical wanderer.

\section{EREBUS Latreille}

3391 E. odora L. Black Witch. New Russia (Jones); Buffalo; Kendall; Ithaca; Albany (NYS) ; SI, J1 and Sep (Ds) ; LI: Jamaica; Parkville. Aug. A frequent stray from the Tropics. Larva on papilionaceous trees. 


\section{ANTIBLEMma Hübner}

3361 A. inexacta W1k. E. New York (Shoe). The type and var. guttula occurred together.

\section{ANTICARsia Hübner}

3359 A. gemmatilis Hbn. Velvet Bean Caterpillar. Ithaca; Rye; SI, Sep (Ds); LI: Orient Pt., Lath; Yaphank. Oct-Nov. A frequent stray from the South in autumn.

\section{PANOPODA Guenée}

3352 P. rufimargo Hbn. New Russia; Buffalo; Lancaster; E. Aurora; Castile, Brad; Ithaca; McLean; Rogers Rock, L. George; Albany and southward incl. LI and SI. Je-Aug. Oak. Vars. rubricosta Gn. and carneicosta Gn. from Ithaca.

\section{PHOBERIA Hübner}

3349 P. atomaris Hbn. Lancaster; Ithaca; Fordham; SI; LI: Brooklyn; Central Pk.; West Hills, Massapequa (Ds). Late Ap-early May. Common, but easily overlooked.

\section{Hypocala Guenée}

3347 H. andremona Cr. Brockport; Karner; Rye, Sep; SI, Sep (Ds); LI (Eng). A stray from the Tropics.

\section{SynEDA Guenée}

3328 S. limbolaris Gey. Mt. Whiteface; Clayton; Evans Center; Lancaster; Gowanda; Buffalo; Portage; Ithaca; Big Ind. Val.; Poughkeepsie; Katonah; Ramapo; SI; LI (Doll). May-J1.

3333 S. alleni Grt. Karner; Peru, Mix\&Everett (Fbs). Je.

\section{DRASTERIA Hübner (Syneda auct.)}

3326 D. atlantica B. \& McD. (graphica auct.). Saranac L. (McKnight, probably in error for alleni); SI; LI: Rockaway; Babylon; Wading R.; Yaphank; Montauk Pt.; Orient, Lath (Fbs). Ap-May; Aug.

\section{MELIPOTIS Hübner}

3308 M. nigrescens G. \& R. SI: Ft. Wadsworth, Je 5, 1924 (Ds) ; LI: Rockaway Pt., $K c h$.

3312 M. jucunda Hbn. SI, May, J1 (Ds) ; LI: Yaphank, Je (Ds).

\section{Cissusa Walker}

[3294 C. spadix Cr. This species ranges northward to Mass., and therefore it undoubtedly occurs in NY. May.] 


\section{RAPHIA Hübner}

3289 R. frater Grt. Plattsburg; Peru; Lancaster; Ithaca; Big Ind. Val.; Onteora Mt.; New Windsor; Rye; SI: LI: Flatbush; Newtown; Woodhaven. Ap-J1. Poplar.

3288 R. abrupta Grt. Buffalo. End of Ap-Je.

\section{SUBFAMILY PLUSIINAE}

ABRostola Ochsenheimer

3287 A. urentis Gn. Plattsburg; Peru; Saranac L.; Spier Falls; Rochester (Angle); Lancaster; Buffalo; Ithaca; McLean; Trenton Falls; Schenectady; Albany; LI (Dol1). May; J1-early Sep.

3286 A. ovalis Gn. Kenwood; Albany. Aug.

3285 A. formosa Grt. Fentons; Ithaca; Albany; Bear Mt., Erb (Eng); LI: Brooklyn. Je-J1. Vaccinium.

\section{PALEOPLUSIA Hampson}

3284 P. venusta W1k. Plattsburg; Peru; Saranac L.; Waddington. Aug.

\section{PSEUdEVA Hampson \\ (Panchrysia)}

3281 P. purpurigera W1k. Keene Val.; Saranac Inn; Lancaster; Ithaca. Late JeJ1. Thalictrum.

\section{EOSPHOROPTERYX Dyar}

3280 E. thyatyroides Gn. Fentons; New Russia (Jones); Buffalo; Lancaster; Ithaca; Albany; Rye; SI. Je-Sep.

\section{PLusia Hübner}

3276 P. aerea Hbn. Plattsburg; Keene Val.; Batavia; Buffalo; E. Aurora; Castile; Potter Swamp; Ithaca; Trenton Falls; Schoharie; Schenectady; Rye; Pine I. (Ds); SI; LI: Brooklyn; Orient, Lath. End of Je-Sep (one straggler in Oct).

3275 P. aeroides Grt. Plattsburg; Keene Val.; Wilmington; Mt. Marcy; Fentons ; Waddington; Lancaster; E. Aurora (Wild) ; Ithaca; McLean; Moravia; Little Falls; Stony Clove, Greene Co; Schoharie; Rhinebeck. J1-Aug. Spiraea.

3277 P. balluca Gey. General, not very common. J1-Aug. Hop.

\section{Autographa Hübner}

3265 A. mappa G. \& R. Plattsburg; Mt. Marcy; Fentons.

3264 A. bimaculata Steph. Keene Val.; Franklin Co; Fentons; Rhinebeck; LI: Jamaica. Aug.

3259 A. biloba Steph. Buffalo; E. Aurora (Wild) ; Ithaca, Aug 28 and Oct 28; Rye, Nov 5; SI; LI: Brooklyn; Jamaica; Wading R. A widespread southern species, straying northward. On various herbs; a greenhouse pest on LI. 
3248 A. verruca Fab. SI; LI: Woodhaven; Flatbush.

3260 A. oo Cr. (rogationis Gn.). Ithaca; W. Troy, a conservatory pest. Aug-Oct. Cabbage.

3261 A. precationis Gn. Common everywhere. Je-Nov, three broods. Hollyhock and other herbaceous plants.

3266 A. gamma pseudogamma Grt. Syracuse, J1.

3241 A. ni Hbn. LI: Brooklyn (Ottol). Jl. A stray from the Tropics.

3249 A. brassicae Rly. CABBAGE LOOPER. Rochester; Elba, somewhat injurious (Cy) ; Buffalo; E. Aurora; Ithaca; Albany; and general southward. J1-Nov. Cabbage and other herbs.

3254 A. oxygramma Gey. Ithaca, Oct; LI: Brooklyn, bred from Solidago by Wright (Eng).

3237 A. alias Ottol. Keene Val. ; Lewis Co; New Russia (Jones) ; Onteora Mt., How. The u-aureum records from Mt. Marcy and Keene Val, are also likely to belong here. Aug.

3239 A. octoscripta Grt. Mt. Marcy; Buffalo (Wild); Ithaca; LI: Glencove, Tepper. Sep.

3244 A. altera Ottol. Adirondacks (Ottol).

3236 A. rectangula Kby. Fentons; New Russia (Jones); Waddington; E. Aurora (Wild) ; Cascade L.; Ithaca; Trenton Falls; Albany; Poughkeepsie; LI: Central Pk., Je (Ds). J1-Aug.

3234 A. selecta W1k. Adirondacks, Aug (Ds).

3233 A. epigaea Grt. Franklin Co; Fentons.

3274 A. ampla W1k. Plattsburg; Mt. Marcy; Fentons; E. Aurora (Wild); Ithaca; McLean; Schenectady. J1.

3251 A. basigera W1k. Rochester (Angle); Buffalo, Oct (Wild); Rye; SI. Aug-Sep.

3230 A. falcigera Kby. Celery Looper. The type and the brown form simplex Gn. are common everywhere. May-Nov. General on herbaceous plants.

3257 A. contexta Grt. Plattsburg; Peru; Adirondacks; Lancaster; Ithaca; Oneonta; Hebron (W1s); Albany; Rye; SI; LI: Maspeth, Schott (Eng). Je, Aug-Sep 1. Grasses.

3255 A. festucae putnami Grt. Plattsburg; Peru; Newcomb; Fentons; E. Aurora (Wild); Cincinnatus (Dnd); Ithaca; Liberty; Stony Clove, Greene Co; Albany. Je-Aug. Larva on grasses and other monocotyledons.

\section{SUbFamily PANTHEINAE}

CHARADRA Walker

3215 C. deridens Gn. Fentons; Lake Forest; Evans Center; Lancaster; E. Aurora; Ithaca; Debruce; Big Ind. Val.; Sharon; Schenectady. LI: Forest Pk., Kch. May-Aug. Oak, birch, and elm.

\section{Colocasia Ochsenheimer}

\section{(Demas Stephens)}

3206 C. propinquilinea Grt. Ithaca; Oneonta; Albany; Oliverea (Ds). Je-J1. Walnut, maple, oak, and beech.

3205 C. flavicornis Sm. Saranac L.; Buffalo; Ilion; Albany; New Windsor. May.

C. flavicornis var. infanta Sm. Ilion; Ithaca, Eyer, transitional. 


\section{PANTHEA Hübner}

3211 P. furcilla Pck. Plattsburg; Peru; Franklin Co; New Russia (Jones); Buffalo; E. Aurora (Wild) ; Ithaca; Rogers Rock, L. George (Jones); Lestershire; Ulster Co; Sharon Sps.; Schoharie; Albany; Bethlehem; Poughkeepsie; LI (Doll). A northern species generally. Ap-Je; late J1-Aug. Pine and larch.

3207 P. acronyctoides W1k. Plattsburg, Hudson; Ithaca; Oneonta; Saratoga; Mt. Kisco, Hill. Je-J1.

\section{SUBFAMILY CATOCALINAE}

\section{ZALE Hübner}

\section{(Phaeocyma, Homoptera Boisduval)}

3175 Z. lunata Dru. Saranac L.; Buffalo; Lancaster; E. Aurora; Oneonta; Schenectady; Karner; Rhinebeck; Rye; SI; LI: Orient, Lath. Apparently rare northward. J1-Nov, mostly Sep. Willow, alder, etc.

3176 Z. unilineata Grt. Saranac L. ; E. Aurora (Wild) ; Ithaca; Karner ; Albany ; Crugers; SI; LI: Wyandanch (Ds). Late May-Je.

3180 Z. aeruginosa Gn. Fentons; Rock City; Schenectady; Karner; SI, May (Ds). LI: Yaphank.

3181 Z. undularis Dru. General and not rare. Var. umbripennis Grt. occurs at: Ithaca, Babiy; Rye; Fordham; Bronxville; SI; LI: Newtown; Yaphank. May-Aug.

3182 Z. coracias Gn. Rye; LI: E. New York (Barnes Coll.). Oak(?).

3184 Z. minerea Gn. Plattsburg; Clayton; Buffalo; Rock City; Ithaca ; Big Ind. Val.; Ilion; Sharon; Karner; New Windsor; LI: Yaphank. Late Ap-Je ("Sep"? error for lunata).

3185 Z. Iunifera Hbn. Franklin Co; Oneonta; Rye; SI, Oct (Ds) ; LI: Parkville; Newtown; Lynbrook. Pine.

3188 Z. duplicata Beth. Peru; Lake Forest; Hudson; LI: Merrick. Je.

3189 Z. helata Sm. Peru; Ithaca; Jamestown; Karner; Ft. Montgomery (Ds) ; LI: Brooklyn. May-Je.

3192 Z. obliqua Gn. Ithaca; Shelving Rock; Karner; SI; LI. Late Ap-Je; Aug. Pine(?).

Z. obliqua var. metata Sm. Karner. May; Aug.

Z. obliqua var. curema Sm. Karner (Sm). May.

3197 Z. cingulifera W1k. Saranac L.; White L.; Brockport; Ithaca; Karner; New Windsor; Rye; LI: Brooklyn. Late Ap-May; J1. Larva perhaps on Calycanthus (calycanthata S. \& A., not auct.).

3199 Z. horrida Hbn. Fentons; Clayton; Lake Forest; Lancaster; Castile, Brad; Ithaca; McLean; Potter Swamp; Karner; Rhinebeck; Rye; Nyack (Ds) ; SI; LI, generally. May-Aug.

\section{ARgYROSTROTIS Hübner}

\section{(Poaphila Guenée, Agnomonia Hübner)}

3163 A. quadrifilaris Hbn. Fentons; Karner; SI; LI: Yaphank. May-Je. Larva reported from cotton.

3164 A. anilis Dru. Ithaca; Rogers Rock (Jones); Albany; Bethlehem; SI; LI: Amagansett; Orient, Lath. J1-Aug. 


\section{Celiptera Guenée}

3153 C. frustulum Gn. Dutchess Co; Rye; Van Cort. Pk. (Ds) ; Bronxville; SI; LI: Brooklyn. May-Je; Aug.

\section{DORYODES Guenée}

3151 D. bistriaris Gey. Ithaca; Rye; SI, on salt meadow (Ds) ; LI: Rockaway Beach; Aqueduct; Glen Cove. Je-Aug.

\section{REMigia Guenée}

3142 R. latipes Gn. (repanda auct.). Ithaca; Big Ind. Val.; Rye; SI. Most probably transient from the South; taken in NY in Sep-Oct.

3138 R. marcida Gn. SI, Aug (Ds) ; LI: Forest Pk. (Eng). A good species.

3139 R. hexastylus Harv. (texana Morr.). Ithaca; SI, Oct, Ds (Grt); LI: Forest Pk. ; Brooklyn.

\section{CAENURGIA Walker \\ (Drasteria auct.)}

3131 C. convalescens Gn. "N.Y."

3135 C. erechtea Cr. Clover Looper. Very common everywhere. Ap-Je; AugSep. Clover and other herbs.

3136 C. crassiuscula Haw. Very common all over the State. Ap-Je; late J1-Sep. First brood dwarfed. Grass and other herbs.

\section{EUCLIDIA Ochsenheimer}

3130 E. cuspidea Hbn. Plattsburg; Black Br.; Saranac L.; Rochester (Angle); Buffalo; Portage; Ithaca; Liberty; Onteora Mt., How; Gloversville; Schenectady; Albany; Rhinebeck; Poughkeepsie; Katonah; Cornwall; Rye; Ramapo and Nyack (Ds); SI; LI: Brooklyn. May-Aug. Clover.

\section{PaRAllelia Hübner}

3127 P. bistriaris Hbn. Common and general, although the only LI record I happen to have is Brooklyn. Late May-Aug. Maple.

\section{Allotria Hübner}

3123 A. elonympha Hbn. Buffalo; Catskills (Ulster Co), Nic (Shoe Coll.); Millbrook; Katonah; SI; LI: Merrick. Two broods, early spring and J1-Aug. Reported from Glycine and from hickory.

\section{Euparthenos Grote}

3122 E. nubilis Hbn. Rather common all over the State. Two broods, May and Aug. Robinia. 


\section{CATOCALA Schrand 74}

3023 C. epione Dru. Evans Center; Buffalo; Ithaca; Albany; Poughkeepsie; Millbrook; Crugers; Rye; West Farms; SI; LI: Greenport (Eng). J1-Aug. Hickory; reported also from oak.

3044 C. lachrymosa Gn. Buffalo, EPV; Newburgh, Morton. J1. Walnut or possibly oak.

3043 C. viduata Gn. "NY" (Lint). Walnut and pecan.

3042 C. vidua S. \& A. Rochester; Ithaca; Sidney; Millbrook; New Windsor; Rye; Rockland L., Oct (Ds) ; Pearl R. (Wat) ; Central Pk. in NYC; SI; LI: Brooklyn; Amagansett; Yaphank. Aug-Sep. Walnut, hickory, pecan, and possibly oak.

3040 C. dejecta Strk. Bangall; Rye; LI: Brooklyn. Aug.

3039 C. retecta Grt. Saranac L.; Rochester; Buffalo; E. Aurora; Ithaca ; Sharon ; Albany; Bethlehem; Rhinebeck; Millbrook; Rye; West Farms; SI; LI: Brooklyn. Late Aug-Sep. Hickory.

3033 C. flebilis Grt. Hastings Center; Albany; Millbrook; Rye; West Farms; LI: Brooklyn; Yaphank. Hickory.

3031 C. robinsonii Grt. Buffalo; Ithaca; Kenwood, Albany; Millbrook; New Windsor; Rye; West Farms; Bronx Pk., formerly not uncommon (Wat) ; SI; LI: Newtown. Sep. Hickory.

3035 C. obscura Strk. Pulaski; Rochester (Angle) ; Buffalo; Binghamton; Millbrook; West Farms; SI, J1-Aug. Hickory.

3036 C. residua Grt. Lancaster; Buffalo; Ithaca; Sharon; Akhurst; Schenectady ; Rye; West Farms (type); SI; LI: Brooklyn. Aug-Sep.

3041 C. insolabilis Gn. Buffalo; Rye; West Farms; LI: Brooklyn. J1. Hickory.

3034 C. angusi Grt. West Farms (types of species and of var. lucetta H. Edw.). Hickory.

3032 C. judith Strk. Millbrook; West Farms; SI; LI: Maspeth, Olsen (Ds). Late J1. Hickory.

3095 C. andromedae Gn. (Hypogramma; C. tristis Edw.). Millbrook; West Pt., Robn (Ds) ; SI; LI: Amagansett; Yaphank. J1-Aug. Originally published as feeding on Andromeda.

3053 C. relicta W1k. The typical (dark) form is known from: Fentons; Rochester; Batavia; Buffalo; E. Aurora; Oneonta; S. Gilboa (NYS) ; Sharon Sps.; Schenectady; Albany; Karner; Millbrook; NYC; SI; LI: Brooklyn. Var. clara Beut. (relicta auct.) is from: N. Elba; Karner; Rhinebeck; West Farms; Battery Pk., NYC (Wat); LI: Floral Pk. Var. phrynia H. Edw. occurs at: Clayton; Karner; Ithaca. Aug. Poplar (will feed on willow). This and the next eight species all feed on willow or poplar.

3081 C. cara Gn. General from the Adirondacks to Buffalo and Yaphank. Late Aug-Sep. Willow.

3083 C. amatrix Hbn. Adirondacks; Rochester (Angle); Buffalo; Ithaca; Schenectady; Albany; Pittstown; Millbrook; Rye; Bronxville; SI; LI: Brooklyn; Maspeth (Olsen). Sep. Willow and poplar. Var. murus W1k. is from: Rochester; Elmira; Cedar Hill; SI.

\footnotetext{
74. The first thirteen species here listed in this genus belong to a group all of whose members seem to feed on hickory, or more rarely on walnut. The following species belong to a group limited to willow and poplar: relicta, cara, amatrix, marmorata, concumbens, unijuga, meskei, briseis, parta
} 
3054 C. marmorata Edw. Millbrook; West Farms; LI: Brooklyn, Schott (Ds).

3082 C. concumbens W1k. Plattsburg to Buffalo and SI, rare on LI. Sep. Willow and poplar.

3068 C. unijuga Wlk. General, including LI. Aug-Sep. Poplar and willow.

3067 C. meskei Grt. Schenectady ; Albany.

3065 C. briseis Edw. Plattsburg; Fentons; Clayton; Buffalo; E. Aurora; Evans Center; Castile, Brad; Ithaca (including var. albida Beut. in Benjamin Coll.); Catskills; Oneonta; Sharon; Saratoga; Schenectady; Millbrook; SI; LI: Glendale; Yaphank. J1-Aug.

3055 C. parta Gn. Throughout the State, common on LI. Aug-Sep. Willow and poplar.

3097 C. coccinata Grt. Fentons; Buffalo; Albany; Millbrook; Poughkeepsie; LI: Woodside, Metzger (Eng). Scrub oak (Quercus nana).

3096 C. herodias Strk. LI: Wading R. (Eng). Scrub oak.

3103 C. ultronia Hbn. General. Late J1-Aug. Wild cherry and plum; reported also from Cornus and oak.

3051 C. ilia Cr. General. The varieties have not been separately recorded, but seem to be generally distributed with the type. J1-Oct, mostly Aug. Oak.

3020 C. innubens Gn. Ithaca; Albany; Tarrytown; Bronx Pk. (Joutel); LI: Woodhaven, Erb (Eng). Gleditschia; (reported also from walnut).

3046 C. nebulosa Edw. Buffalo; Bronx Pk. (Joutel); LI: Brooklyn.

3021 C. piatrix Grt. Saranac L.; Buffalo; Ithaca; Schenectady; Bethlehem; Rhinebeck; Millbrook; Poughkeepsie; New Windsor; Rye; Bronx Pk. (Joutel) ; common on LI. Aug-Sep. Walnut, hickory, and persimmon.

3048 C. neogama S. \& A. Fentons; Rochester, common (Angle); Buffalo; Oneonta; Schenectady; Millbrook; Rye; SI; LI: Brooklyn. Walnut.

3047 C. subnata Grt. Clayton; Ithaca; Sharon; Bronxville (Ds) ; Bronx Pk. (Joutel) ; LI: Newtown; Montauk (Eng). Aug-early Oct. Walnut.

3052 C. cerogama Guen. Clayton; Fentons; Plattsburg; Sharon; Buffalo; E. Aurora; Ithaca; Trenton Falls; Millbrook; Rye; SI; LI, generally. Aug. Linden.

3045 C. palaeogama Gn. General and not rare. Var. phalanga Grt. occurs: SI; LI: Wading R.; Forest Pk. Var. annida Fager was found at Hemlock L., Sep 1, 1916, with the type. Aug-early Sep. Hickory and walnut.

3022 C. consors S. \& A. LI: Brooklyn; Woodhaven. Hickory.

3024 C. muliercula Gn. Rye; Van Cort. Pk., common (Wat); SI; common on LI. Myrica.

3030 C. serena Edw. Schenectady; West Farms. Aug. Hickory.

3025 C. antinympha Hbn. Saranac L.; Buffalo; Ithaca; Oneonta; Schenectady; Karner; Poughkeepsie; Millbrook; Rye; SI; LI: common and general. J1-Sep. Comptonia.

3027 C. badia G. \& R. Rye; Van Cort. Pk., common (Wat); SI; LI, common and general. J1-Sep. Myrica.

3026 C. coelebs Grt. Fentons; Schenectady. Aug.

3028 C. habilis Grt. Waddington; Rochester; Buffalo; Orchard Pk. and Gowanda (Wild) ; Ithaca; Binghamton; Hebron; Schenectady; Albany; Millbrook; New Windsor; Rye; SI; LI: Woodhaven (Eng). Aug-Sep. Hickory. 
3114 C. clintonii Grt. Evans Center; Buffalo; Ithaca; Sharon Sps.; Schenectady. J1. Rosaceae. This and the next six species represent a group whose members, so far as known, feed on Rosaceae.

3104 C. polygama crataegi Saund. Jamestown; Evans Center; Lancaster; Otto; Ithaca; Stony Clove, Greene Co; Ilion; Sharon Sps.; Schenectady; Albany; Croton-on-Hudson (Ds); LI: Brooklyn (Doll). Late Jl-Aug. Rosaceae, especially thorn.

3104.1 C. pretiosa Lint. Schenectady (type); Rhinebeck; Rye. Rosaceae.

3107 C. praeclara G. \& R. General. Aug. Rosaceae.

3113 C. dulciola Grt. "NY". J1. Rosaceae.

3106 C. grynea Cr. Sharon; Schenectady; Albany; Rhinebeck; Millbrook; Poughkeepsie; Rye; NYC; SI; LI. Apple and plum.

3094 C. gracilis Edw. Everywhere in the State; not rare. J1-Aug. Rosaceae.

3116 C. minuta Edw. Poughkeepsie; West Farms; NYC; SI; LI: Brooklyn; Woodhaven; Aqueduct; Yaphank. J1-Aug. Gleditsia.

3119 C. connubialis Gn. (amasia auct.). Rochester; Schenectady; NYC. Presumably on oak, originally figured on Cephalanthus.

3118 C. cordelia H. Edw. West Farms (Angus). Presumably on oak.

3115 C. similis Edw. Ithaca; West Farms; LI: Yaphank and Riverhead (Ds). J1. Oak.

3117 C. fratercula G. \& R. E. Aurora (Wild) ; Albany; Sullivan Co; LI: Yaphank and Fire Place (Ds). J1-Aug. Oak.

3120 C. amica Hbn. Ithaca, Aug 1, 1901; U1ster Co; Millbrook; Rye; SI; LI, generally. Oak.

\section{SUBFAMILY NYCTEOLINAE}

\section{(Sarrothripinae)}

BAILEYA Grote

3016 B. ophthalmica Gn. Peru; Saranac L.; Evans Center; Lancaster; E. Aurora (Wild); Ithaca; Big Ind. Val.; SI. Late May-early Jl.

3015 B. doubledayi Gn. Plattsburg; Saranac L.; E. Aurora (Wild); Castile, Brad; Karner; Newton; SI (Ds); LI: E. New York, Shoe (Ds). Je-Jl.

3018 B. dormitans Gn. Castile, Je, Brad (Fbs); Evans Center; Trenton Falls (type) ; Ilion; LI: Newtown (Doll); Queens (Bell and Eng). Je-early $\mathrm{J1}$.

\section{Nycteola Hübner}

3012 N. revayana lintnerana Spey. Keene Val.; Fentons; Chautauqua, Cy; Ithaca; Lancaster; Onteora Mt., How; Schenectady; NYC; LI: Brooklyn. Je 27; Aug. Willow.

\section{ChaRAcoma Walker}

3011 C. nilotica Rog. (proteella Dyar). Lewiston, EPV; Chautauqua; Ithaca: Utica. J1-Aug. Willow and tamarisk. 


\section{SUBFAMILY EUTELIINAE \\ PaEctes Hübner \\ (Ingura Guenée)}

3008 P. delineata Gn. LI: Glendale; Woodhaven. Jasmine.

3007 P. abrostoloides Gn. SI, J1-Sep (Ds).

3002 P. oculatrix Gn. Evans Center; Lancaster; Ithaca; Poughkeepsie; New Windsor; Rye; Katonah; NYC (Joutel); SI; LI: Brooklyn; Wading R. (Ds); Orient, Lath. Je-J1.

\section{Eutelia Hübner}

2997 E. pulcherrima Grt. Rye; Bronx Pk.; LI: Brooklyn. The moth has been beaten from sumac; larva reported to feed on hickory (Bird).

\section{MARASMALUS Grote}

3000 M. inficita Wlk. Peru; Rochester (Angle); Lancaster; E. Aurora, Wild; Ithaca; Big Ind. Val.; Debruce; Schenectady; Poughkeepsie; Katonah; Rye; Nyack (Ds) ; Bronxville; SI; LI: Jamaica. Je-Aug. Sumac (Reidy).

2999 M. basalis Grt. (ventilator Grt.). Rye; Bronxville; LI: Jamaica. Je-J1.

\section{SUBFAMILY ERASTRIINAE}

\section{TARACHE Hübner}

2989 T. terminimaculata Grt. Geneva; Lancaster; Ithaca; Sharon; Albany (type); Poughkeepsie; New Windsor. May-Jl.

2992 T. delecta Wlk. Rye; SI, salt meadow, Je (Ds); LI: Aqueduct; Woodhaven. Hibiscus.

\section{TARACHIDIA Hampson}

2952 T. ereastrioides Gn. General and common. Je-Sep. Ambrosia.

2956 T. candefacta Hbn. General, and as common as the preceding species. MayAug. Ambrosia.

2965 T. semiflava Gn. Rhinebeck; LI: Yaphank; Merrick. Je.

\section{SPRAgueIA Grote}

2930 S. leo Gn. SI, Sep.

\section{XANThoptera Guenée}

2922 X. nigrofimbria Gn. LI: Yaphank (Eng).

\section{EXYRA Grote}

2917 E. rolandiana Grt. Should be well distributed in the State on pitcher plant (Sarracenia purpurea). I have seen its work at McLean on pitcher plant (Fbs). May-Je. 
CRYPhia Hübner

C. pervertens B. \& McD. (secta auct., aetheria auct.). E. Aurora (Wild); Big Ind. Val.; Ilion; SI, Je (Ds) ; LI: Forest Pk., Glendale, Coram, Kch (Fbs). Late Je-Jl.

\section{LiTHACODIA Hübner}

\section{(Eustrotia Hübner)}

2898 L. bellicula Hbn. Fentons; McLean; Trenton Falls; S. Trenton; Nassau; Poughkeepsie; SI; LI: Brooklyn; Yaphank. Late Je-Aug.

2895 L. albidula Gn. Common everywhere. Je-early Aug.

2897 L. concinnimacula Gn. Plattsburg; Peru; Fentons; Keene (Wild) ; Geneva; Lancaster; Ithaca; Big Ind. Val.; Onteora Mt., How; Schenectady; Karner; LI: Jamaica; Glendale. May-J1.

2894 L. synochitis G. \& R. Plattsburg; Peru; North Cr.; E. Aurora, West Falls (Wild); Rochester (Angle); Castile, Brad; Ithaca; Big Ind.

Val.; Schenectady; Poughkeepsie; SI; LI: Lynbrook. May-Aug.

2899 L. musta G. \& R. Ithaca, Je 30, J1 2; Schenectady ; LI: Newtown .(Eng).

2902 L. muscosula Gn. Common and general. Je-Sep.

2901 L. apicosa Haw. Apparently general, including LI: Forest Pk. (Doll). Late May-Sep.

2900 L. carneola Gn. With the preceding species, and far more common. End of Je-early Oct. Yellow dock.

\section{CHAMYRIS Guenée}

2891 C. cerintha Tr. Plattsburg; Peru; Clayton; Geneva; Evans Center; Buffalo; E. Aurora; Ithaca; Binghamton; Sharon Sps.; Schenectady; Albany; Nassau; Rhinebeck; SI; LI, general. Je-J1. Wild cherry.

\section{ARGILLOPHORA Grote}

2890 A. furcilla Grt. Saranac L. (McKnight).

\section{SUBFAMILY ACRONYCTINAE}

\section{PSYCHOMORPHA Harris}

2832 P. epimenis Dru. Sloatsburg; SI; LI; Jamaica; Woodhaven; Massapequa. Ap-May. Grape.

\section{EUTHISANOTIA Hübner}

\section{(Eudryas)}

2829 E. unio Hbn. Waddington; Rochester (Angle); Buffalo; Lancaster; Moravia; Castile, Brad; Ithaca; McLean; Trenton Falls; Oneonta; Rhinebeck; Rye; SI; LI, general. Late Je-Aug. Epilobium and swamp loosestrife.

2827 E. grata Fab. General. Je-J1. Grape and ampelopsis. 


\section{Ommatostola Grote}

2812 O. lintneri Grt. LI: Rockaway Beach; Glen Cove; Long Beach; Fire I. Originally described from NY. In salt marshes.

\section{SEnTA Stephens}

2801 S. defecta Grt. SI, J1 8, 1912 (Ds).

\section{Amolita Grote}

2803 A. fessa Grt. Peru; Ithaca; McLean; Melrose; New Windsor; Rye; LI: Coram. Je-Aug.

Calamia Hübner

(Tapinostola Lederer)

2798 C. inquinata Gn. (orientalis Grt.). Buffalo. Aug.

2800 C. variana Morr. Saranac L.; Sharon; Nassau. Aug.

ARCHANARA Walker

(Nonagria)

2792 A. subflava Grt. (subcarnea Kell.). Buffalo; Rye. Aug-Sep. Scirpus occidentalis (Bird).

2791 A. oblonga Grt. Alexandria Bay; Buffalo; Brockport; Ithaca; Rye; LI: Newtown (Doll). J1-Sep 2. Cattail.

2794 A. laeta Morr. Plattsburg; W. Seneca; Brockport; Buffalo. J1-Aug. Sparganium (Bird).

\section{BELLURA Walker}

2788 B. gortynoides Wlk. Ithaca (larvae, Comstock); Albany; SI; LI: Newtown (Doll). Caterpillars in water lilies.

2789 B. melanopyga Grt. SI.

2790 B. diffusa Grt. Saranac L., Je; LI: Glendale, Aug, Kch.

\section{ARZAMA Walker \\ (Sphida Grote)}

2785 A. densa Wlk. LI: Sag Harbor; Orient, Lath. Cattail.

2784 A. obliqua Wlk. Buffalo; E. Aurora; Rock City (Ds); Ithaca; McLean (larvae); Schenectady; Albany; Rye; SI; LI. "Northern NY". End Ap-Je. Cattail.

Plagiomimicus Grote

2767 P. pitychromus Grt. Buffalo; Schenectady; Albany; Poughkeepsie; Rye; Florida; SI. Aug.

STIRIOdes Hampson

(Metoponia)

2763 S. obtusa H-S. Rye; SI, Je-J1 (Ds) ; LI: Forest Pk. 


\section{DERRIMA Walker}

2758 D. stellata W1k. The normal form, henrietta Grt., has been taken at: Rye;

LI: Amagansett; Yaphank. Je-Aug.

\section{ENARGIA Hübner}

(Cosmia Ochsenheimer)

2757 E. decolor W1k. (paleacea Esper). Cranberry L., Htly; New Russia; Spier Falls (NYS); Fentons; Lancaster; Chautauqua Co; Ithaca; McLean; Schenectady; Albany; Hudson; LI: Woodhaven. Aug-Sep. Poplar.

\section{CiRRHOPHANUS Grote}

2736 C. triangulifer Grt. Rye; SI, Aug, Fulda (Ds).

\section{Cosmia Ochsenheimer}

2735 C. orina Gn. Buffalo (Wild) ; Ithaca; LI: Wading R.; Yaphank. Jl-Aug. Oak and other trees, feeding by preference on other caterpillars.

\section{BAGISARA Walker \\ (Atethmia)}

2730 B. rectifascia Grt. Rye (Bird); LI: Central Pk. (Doll).

\section{IPIMORPHA Hübner}

2724 I. pleonectusa Grt. Wilmington; Fentons; Cranberry L., Htly; Seneca L.; Rochester; E. Aurora (Wild) ; Evans Center; Ithaca; Sharon; Schenectady. J1-early Sep. Poplar.

\section{OGDOCONTA Butler}

2704 O. cinereola Gn. Striped Green Bean Caterpillar. Evans Center; Lancaster; E. Aurora; Buffalo; Castile; Ithaca; Schenectady; Rhinebeck; New Windsor; Rye; SI; LI: Mattituck; Orient, Lath. Je-Sep.

\section{PAPAIPEMA Smith 75 \\ (Gortyna, Hydroecia)}

2658 P. cerina Grt. New Windsor; LI: Ft. Totten; Glendale. Sep 8-20 (Bird). Lilium superbum, Podophyllum peltatum (Bird).

2659 P. appasionata Harv. LI. Sep 2-19 (Bird). Sarracenia purpurea; bores in all species of Sarracenia in the South (Bird).

2661 P. inquaesita G. \& R. Lancaster; Ithaca; Sullivan Co. (Bird); New Windsor; Rye; SI (Ds); LI: Amagansett, Aug 15-Sep 19 (Bird). Oct. Onoclea sensibilis (Bird).

2662 P. speciosissima G. \& R. Buffalo (Bird). Sep 6-16 (Bird). Osmunda regalis and $O$. cinnamomea (Bird).

${ }^{75}$ The dates given are those of emergence; adults may be on the wing two weeks later (Bird). 
2663 P. marginidens Gn. (birdi Dyar). Buffalo and E. Aurora (Wild); Ithaca; Rye; SI; LI. Aug 20-Sep 12 (Bird). Cicuta maculata, occasionally in large umbellifers (Bird).

2664 P. baptisiae Bird. SI; LI: Central Pk. Aug 10-Sep 10 (Bird). Baptisia tinctoria, and B. alba farther south (Bird).

2666 P. nepheleptena Dyar (moeseri Bird). "NY" (type); Buffalo (Bird); West Seneca (Bird); SI (Bird). Sep 20 - Oct 15 (Bird). Chelone glabra (Bird).

2667 P. furcata Sm. "LI" (type) (Sm). Aug 30-Sep 17 (Bird). Terminal twigs of ash, Fraximus nigra, F. americana, $F$. pennsylvanica (Bird).

2668 P. circumlucens Sm. Rye; SI (Ds); LI. Sep 8-28 (Bird). Apocynum androsaemifolium, dogbane (Bird).

2669 P. humuli Bird. Poughkeepsie; Rye, type (Bird); SI (Bird); LI (Bird). Aug 10-28 (Bird). In gall in stem of hop (Bird).

2671 P. arctivorens Hamps. Buffalo (Bird). Aug 15-Sep 4 (Bird). Cirsium and Arctium.

2674 P. merriccata Bird. Ithaca, Sep. Podophyllum peltatum (Bird).

2676 P. harrisii Grt. Buffalo; Karner; SI (Ds); LI. Aug 20 -Sep 10 (Bird). Heracleum lanatum.

P. harrisii var. rubiginosa Bird. Buffalo (Bird) (type). Aug 17-Sep 7 (Bird). Angelica atropurpurea and Heracleum lanatum (Bird).

2677 P. astuta Bird. Rye (type); Buffalo (Bird); Sullivan Co (Bird); SI; LI. Sep 7-19 (Bird). Collinsonia canadensis.

2681 P. impecuniosa Grt. Buffalo and E. Aurora (Wild); Ithaca; Karner; Rye; SI; LI. Sep 13 -Oct 6 (Bird). Aster umbellatus, A. puniceus, and Helenium autumnale (Bird).

2683 P. purpurifascia G. \& R. Columbine Borer. Franklin Co; Canisteo; Ithaca; Cohoes (Weigel); Corning's Farm, Albany, Aug 15-Sep 10 (Bird); Rye. Aquilegia canadensis and cultivated varieties of columbine.

2684 P. lysimachiae Bird. Rye (type); Sullivan Co (Bird); SI; LI (Bird). Sep 8-28 (Bird). Lysimachia quadrifolia.

2685 P. pterisii Bird (triorthia Dyar). Ithaca; Ilion; Karner; Rhinebeck; Rye (type) (Bird); Sullivan Co (Bird); LI. Aug 23-Sep 8 (Bird). Pteris aquilina (Bird).

2686 P. cataphracta Grt. Burdock Borer. Gasport (Cy); Buffalo; E. Aurora (Wild) ; Caledonia (Cy) ; Ithaca; Reading Center. (Cy); Menands; Albany; New Windsor; Rye (Bird); SI (Ds). Sep 19-Oct 8 (Bird). Eupatorium, Rudbeckia, Lilium, Thalictrum, and generally in the larger perennials; Arctium and some cultivated plants (Bird).

P. cataphracta var. fluxa Bird. Buffalo (type) (Bird). Aug 28-Sep 14 (Bird). Burdock.

2687 P. duovata Bird. Rye (type); LI, Sep 25-Oct 10 (Bird). Solidago sempervirens (Bird).

2688 P. imperspicua Bird. Buffalo; W. Seneca. This reference is somewhat questionable, but the species may be expected in the Adirondacks region (Bird).

2689 P. rigida Grt. Buffalo; Lancaster; Ithaca; Cortland; Lestershire; Onondaga Val.; Rye. Sep 12-30 (Bird); early Oct. Heliopsis helianthoides and Zizia aurea (Bird). 
[2693 P. nelita Strk. Should be found in the State (Bird). Rudbeckia laciniata (Bird).]

2694 P. sciata Bird. Buffalo and Sullivan Co (Bird); Rye (type); Sep 15 - Oct 17 (Bird). Veronica virginica.

2695 P. frigida Sm. (thalictri Lyman). Buffalo (Bird); Rye; Westchester Co; Sullivan Co (Bird) ; LI. Sep 10-30 (Bird). Thalictrum.

2696 P. cerussata G. \& R. Buffalo; Rye; SI; LI: Woodhaven. Sep 12-30 (Bird). Vernonia noveboracensis.

2698 P. nebris Gn. and var. nitela Gn. Common Stalk-Borer. Buffalo; E. Aurora (Wild) ; Lancaster; Schenectady; Albany; Middletown; Rye; Monsey; SI; LI: Central Pk. and Wading R. (Ds). Sep 15-Oct 4 (Bird). Preferred food-plants of the subfamily Ambrosiaceae (Bird).

2699 P. duplicata Bird. Rye (type); SI; LI: Jamaica. Sep 28-Oct 18 (Bird). Collinsonia canadensis is the only known food-plant (Bird).

2700 P. necopina Grt. (impertubata Bird). Lancaster, Buffalo, E. Aurora, and Allegany $\mathrm{Pk}$. (Wild) ; Westchester Co and LI references almost surely belong to maritima (Bird). Aug 24-Sep 20. Helianthus divaricatus (Bird).

2701 P. maritima Bird. Lancaster; Ithaca; Rye (type) ; southern Westchester Co (Bird); SI (Ds). Sep 15-Oct 10 (Bird). Helianthus giganteus (Bird).

2703 P. eupatorii Lyman. Buffalo, Rye, and Sullivan Co (Bird). Sep 10-21 (Bird). Eupatorium purpureum (Bird).

\section{PyRRHIA Hübner}

P. umbra Hbn. Lancaster, E. Aurora, and Buffalo (Wild); Rock City; Ithaca; Karner; Poughkeepsie; SI, Aug (Ds) ; LI: Newtown; Aqueduct. Ononis, Geranium, Alnus, and cultivated Rhododendron (Wild).

P. exprimens W1k. Fentons; Clayton; Rochester; Buffalo; Ithaca; Schenectady; Albany; Rye (Bird); SI, J1 (Ds). The suffused var. angulata Grt. was described from Buffalo, and has been taken also at Utica (CU).

\section{RHodoecia Hampson}

R. aurantiago illiterata Grt. Poughkeepsie; Rye (Bird); SI; LI: Riverhead (Ds). J1-early Aug. Seeds of Afzelia macrophylla (Braun).

\section{GORTYNA Ochsenheimer}

G. immanis Gn. HOP BoRER. Plattsburg; Ithaca; Waterville; Sangerfield; Oneonta; New Windsor; Rye (Bird); LI: Yaphank (Ds). Aug 20Sep 12 (Bird). Works at the crown of Humulus Lupulus, but does not actually mine the stem; also at root of Silphium, in III. (Bird).

G. stramentosa Gn. Ithaca, Sep (Fbs); Rye (Bird) ; Sullivan Co (Bird) ; LI: Glendale, Kch. Aug 20-30 (Bird). Root of Scrophularia leporella (Bird).

Xanthoecia Hampson

(Ochria auct.)

X. buffaloënsis Grt. (latia Strk.). Buffalo, Sep 20 (Bird). Saururus cernuns. 


\section{Achatodes Guenée}

A. zeae Harr. Plattsburg; Fentons; Geneva (NYS); E. Aurora; Jamestown; Colden; Evans Center; Buffalo; Ithaca; McLean; Trenton Falls ; Rye (Bird); SI; LI: Brooklyn; Jamaica; Orient, Lath. Jl-Aug. Bores in elder.

Apamea Ochsenheimer

(Hydroecia)

A. u-album Gn. "NY" (Sm, Neumoegen).

A. velata W1k. Peru; Essex Co; Fentons; Keene (Wild) ; Clayton; Geneva; Evans Center; Buffalo; E. Aurora (Wild) ; Castile, Brad; Ithaca; McLean; Binghamton; Big Ind. Val.; Onteora Mt.; Schenectady; Albany; New Windsor; Sullivan Co (Ds) ; Florida; Durlandville; Katonah; Bronxville; Rye; SI; LI: Orient, Oct 30, Lath. Je-early Aug. Bores in Carex (Bird); recorded also from anemone, but this is undoubtedly an error (Bird).

A. nictitans Borkh. Common throughout the State. The var. erythrostigma Haw. is also common and general. End of J1-Sep. "Grass" (in Europe). The larvae probably work at roots of grass, or, more likely, of some sedges (Bird).

A. erepta ryensis Bird. Rye, Je 25-J1 6 (Bird). Tripsacum dactyloides (Bird).

\section{HELOTROPHA Lederer}

2636 H. reniformis Grt. Essex Co; Fentons; Buffalo (Wild) ; Lancaster; Ithaca; Oneonta; Sharon; Schenectady; Rye (Bird); SI. The var. atra Grt. is dominant southward: Saranac L.; Rochester (Angle); Lancaster;, Seneca L.; Ithaca; Sharon; Schenectady; Albany; SI; LI: Delhaven ; Yaphank. J1-Sep. Probably on Iris.

2634 H. caduca Grt. (Erastria). Rye. Nuphar.

2635 H. retis Grt. (Erastria). "NY" (Barnes Coll.), J1 2.

\section{XYLOMOEA Staudinger}

X. chagnoni B. \& McD. Ithaca (CU), Aug.

\section{MENopsimus Dyar}

2513 M. caducus Dyar. Peru; Mt. Marcy, trail; Adirondack Lodge; E. Aurora (Wild) ; Portage; Ithaca; McLean; Trenton Falls; Big Ind. Val. Our smallest noctuid. Probably general but overlooked. May-Sep.

\section{MONODES Guenée}

\section{(Oligia)}

2608 M. festivoides Gn. Peru; Adirondack Lodge; Evans Center; Rock City; Portage; Ithaca; Big Ind. Val.; Onteora Mt., How; SI; LI: Woodhaven; Amagansett; Yaphank. May-J1.

2607 M. chalcedonia Hbn. Reported from: Saranac L.; Lancaster; Buffalo. I have seen no specimens from the north, and suspect the records are based on brilliant individuals of the preceding. May-Je. 
2606 M. versicolor Grt. Buffalo; Lancaster. J1. Dead leaves of pine and balsam fir or oak(?).

2611 M. grata Hbn. Gowanda, Je (Wild).

BALSA Walker

2600 B. malana Fitch. Many-Spotted Apple Worm. General. May-Aug. Apple.

2601 B. tristrigella Wlk. General, and quite as common, as a rule, as the preceding species. Ap-Je, Aug.

2602 B. labecula Grt. Evans Center; Ithaca; McLean; Albany; Poughkeepsie; LI: Forest Pk.; Brooklyn. May-early Je; Aug.

\section{Platysenta Grote}

2598 P. videns Gn. Peru; Saranac L.; Waddington; Rochester; Ithaca; Poughkeepsie; Rye; SI; LI: Newtown; Woodside. Larvae exposed on panicles of several late-flowering perennials, where they eat the ovules (Bird). May-early Sep.

\section{Crambodes Guenée}

2595 C. talidiformis Gn. Saranac L.; Lancaster; Ithaca; McLean; Schenectady; Albany; Rhinebeck; Rye; SI; LI: Newtown. Je-Sep. Verbena.

\section{GALGula Guenée}

2592 G. hepara Gn. (o partita Gn.). Buffalo; E. Aurora; Gowanda; Ithaca; Schenectady; New Windsor; SI; LI: Brooklyn. Je-J1; Sep-Oct.

\section{PROXENUS Herrich-Schaeffer}

2590 P. miranda Grt. General and not rare. Je-early J1; Aug-early Oct. Apparently a cutworm.

CARADRINA Ochsenheimer 76

2579 C. meralis Morr. "NY" (Lint). Not seen.

2577 C. multifera Wlk. Plattsburg; Cascade L. (Adirondacks); Fentons; Ithaca; Big Ind. Val.; Onteora Mt., How; Sharon; Schoharie; Kaaterskill; LI: Brooklyn. Aug-Sep.

2578 C. extima Wlk. LI: Brooklyn (this may be the species reported as meralis).

2581 C. tarda Gn. (Anorthodes prima Sm.). E. Aurora (Wild); Ithaca; LI: E. New York. J1-early Sep.

\section{LAPHYGMA Guenée}

2574 L. frugiperda S. \& A. Fall Army Worm. General, northward to Schenectady, Utica, Ithaca, Buffalo; Fentons. The var. obscura Rly. is general with the species. J1-early Oct.

${ }^{76}$ The larvae of this genus are cutworms. 


\section{Prodenia Guenée}

2568 P. dolichos F. (commelinae S. \& A.). Seneca L.; Rye; SI; LI: Brooklyn (Ottol). Je; Aug-Oct. General on low plants.

2569 P. ornithogalli Gn. CotTon Cutworm. Lewis Co (a stray migrant) ; Lancaster; Buffalo; Rye; SI; LI: Brooklyn; Woodbury; Yaphank. AugOct. General on low plants.

\section{CATABENA Walker}

2564 C. lineolata Wlk. Plattsburg; Evans Center; Lancaster; E. Aurora; Ithaca; Sharon; Schenectady; Albany; LI: Orient, Lath. May-Je, late JlAug. Goldenrod, nettle (Wild).

\section{HyPPA Duponchel}

2499 H. xylinoides Gn. Common everywhere; on LI to Yaphank. May-Je, AugSep. A general feeder, probably a cutworm.

\section{ANDROPOLIA Grote}

(Polia)

2518 A. contacta Wlk. Fentons, Hill; Cranberry L., Htly (Dyar). Late Aug.

\section{Delta Hampson}

\section{(Actinotia)}

2515 D. ramosula Gn. Essex Co; Fentons; Buffalo; E. Aurora; Enfield; Ithaca; Lestershire; Pine Hill; Onteora Mt., How; Sharon; Schenectady; Albany; New Windsor; SI; LI: Central Pk.; Yaphank. May-Je; Aug-Sep.

\section{ARSILONCHE Lederer}

2514 A. albovenosa henrici Grt. Common everywhere. Definite records are: Plattsburg; Newport; Geneva; Buffalo; Ithaca; Binghamton; Lestershire; Albany; Kinderhook; Brewster; Rye; SI. Ap-Aug. Low plants, especially coarse grass-like plants.

\section{Merolonche Grote}

M. dolli B. \& McD. LI: Central Pk., Ap, Kch (types).

\section{Apatela Hübner \\ (Acronycta Ochsenheimer)}

2498 A. rubricoma Gn. "NY" (Guenée). LI: Woodhaven. Mar-May; J1-early Sep. Celtis.

2491 A. americana Harr. American Dagger Moth. Common throughout the State. May-J1. Maple.

2495 A. hastulifera S. \& A. Karner; SI, Je-J1 (Ds) ; LI: Newtown. The species is doubtless general, but in the imago it is very difficult to distinguish from the next. Alder. 
2492 A. dactylina Grt. Peru; Wilmington; Mt. Whiteface (larvae); Fentons; Rochester; Buffalo; E. Aurora; Castile, Brad; McLean (Fbs) ; Ithaca; Utica; Binghamton; Schenectady; Albany; New Windsor; SI; LI: Newtown (Doll). May-J1. Speckled alder (Wild).

2486 A. leporina vulpina Grt. Keene Val.; Catskills. Poplar, willow, and birch. The asymmetrical larva is distinctive and to be looked for all over the State.

2483 A. populi Rly. Waddington; Plattsburg; May-Je; Oct. Poplar.

2480 A. lepusculina Gn. Fentons; Ithaca; Buffalo. Je.77

2485 A. chionochroa Hamp. LI (Hamp).

2490 A. innotata Gn. Everywhere, Plattsburg to Evans and LI. Je-J1. Birch.

2492 A. betulae Rly. LI (Smith \& Dyar). May; Sep. Birch.

2473 A. morula Grt. Plattsburg; Fentons; Clayton; Rochester; Kendall; Lancaster; Evans Center; Otto; Ithaca; Schoharie; Schenectady; Rye ; SI; LI. May; J1. Elm, apple, and linden.

2474 A. interrupta Gn. (occidentalis G. \& R.). Peru; Fentons; Geneva; Rochester ; Kendall; Buffalo; E. Aurora; Ithaca; Big Ind. Val.; Trenton Falls; Oneonta; Schenectady; Albany; New Windsor; Mt. Vernon; SI; LI: Woodhaven; Greenport; Orient Pt.; Shelter I. May-Sep. Elm, apple, plum, and birch.

2466 A. elizabeta Sm. E. Aurora (Wild) ; Ithaca; LI: Prospect Pk., Brooklyn; Queens (Bell and Eng). Probably a dark variety of the preceding, which will occur rarely through the State. Aug.

2460 A. laetifica Sm. Evans Center, J1.

2464 A. lobeliae Gn. Fentons; Buffalo; Evans Center; Rock City (Ds) ; Ithaca ; Oliverea (Ds); Rhinebeck; Ft. Montgomery (Ds); Rye; SI; LI: Brooklyn; Aqueduct (Ds). Ap-Aug. Wild cherry (not Lobelia).

2463 A. furcifera Gn. E. Aurora (Wild) ; Crosby; New Windsor; LI: Newtown; Hempstead Plain. May; J1-Aug.

2461 A. hasta Gn. Lewis Co; Otto; Castile, Je, Brad (Fbs); E. Aurora ; Ithaca ; Oliverea (Ds) ; SI; LI: Hempstead Plain; Babylon. As no structural examination was made, the records for this group are slightly doubtful. Some Ithaca specimens, at least, are of var. telum Gn. May-Aug. (Oct, Mar). Wild cherry.

2470 A. radcliffei Harv. Fentons; Evans Center; Onteora Mt., How ; Schenectady; Karner; Albany; Oliverea (Ds); Rhinebeck; New Windsor; West Pt.; Rye; LI: Yaphank. Late Ap-Je. Wild cherry.

2475 A. spinigera Gn. (harveyana Grt.). E. Aurora, J1 13, 1918, Dnd; Oliverea and West Pt. (Ds); SI; LI: Wading R., Yaphank (Ds). May-J1.

2438 A. pruni Harr. (clarescens auct.). Ithaca; Schenectady; SI; LI: Brooklyn; Jamaica; Wading R. (Ds). May-J1 northward, May-Je and Aug-Sep on SI. Apple, cherry, etc.

2448 A. subochrea Grt. (hamamelis auct.). E. Aurora, Wild (Fbs); Ithaca; Oliverea. May-Aug. Hamamelis; beech (Wild).

2447 A. superans Gn. Mt. Whiteface (Ds); Keene Val.; Lewis Co; Clayton; Buffalo; E. Aurora; Evans Center; Rock City; De Bruce; Liberty; Oliverea (Ds); Onteora Mt., How; Schenectady; Albany; Rye; LI: Flatbush. Je-Áug. Apple, birch, etc.

77 The New York record for insita Smith was evidently due to a confusion of labels, as the New York specimens returned by him with the insita label are apparently lepusculina. 
2502 A. lithospila Grt. Evans Center; Ulster Co; Karner; Albany; SI; LI: Aqueduct; Bellport. May-Je; Aug. Chestnut and oak.

2465 A. tritona Hbn. Fentons; Evans Center; Karner; Albany; SI. May, Sep. Vaccinium and other Ericaceae.

2433 A. connecta Grt. Evans Center; Buffalo; Lancaster; Sharon Sps.; SI; LI: Brooklyn. Jl-Aug. Willow.

2472 A. funeralis Grt. Keene Val.; Ithaca; Schoharie; Sharon Sps.; Karner; Washington Hgts., NYC (Wat); SI; LI: Brooklyn. May-Je. Hickory, birch, elm, apple, etc.

2440 A. fragilis Gn. Plattsburg; Peru; Keene Val.; Indian Pass, Mt. Marcy; Fentons; Rochester; Lancaster; Salamanca (Wild); Ithaca; Onteora Mt., How; Big Ind. Val.; Schenectady; Rye. Je-Aug. Birch.

2428 A. vinnula Grt. Plattsburg; Evans Center; Buffalo; Rochester (Angle); Schenectady; Albany; Poughkecpsie; New Windsor; Rye; SI; LI. May-Aug. Elm.

2469 A. grisea Wlk. Plattsburg; Keene Val.; Fentons; Karner. Je-J1. Birch, etc.

2467 A. falcula Grt. "NY", very possibly an error. Hazel.

2450 A. afflicta Grt. (hamamelis auct.). Ithaca; Karner; Albany; Rye; NYC; LI: Brooklyn. Mar; late May-Je; Aug. Oak.

2432 A. albarufa Grt. Karner; Albany; LI: Central Pk. May-J1.

2452 A. increta Morr. Evans Center; E. Aurora, Irving, Rock City (Wild); Big Ind. Val.; West Pt. (Ds); Stony Clove; SI; LI: Brooklyn. Sep. Beech (Wild).

2451 A. hamamelis Gn. (tristis Sm.). Ithaca, May 31, 1918; LI: Aqueduct (Eng).

2435 A. modica Wlk. Schenectady; Albany; Rhinebeck; Rye; SI; LI: Forest Pk. (Doll). Je-J1. Oak.

2436 A. ovata Grt. Buffalo (Wild); Ithaca; Schenectady; Albany; New Windsor; West Pt. (Ds); SI; LI: Lynbrook; Bellport, Wading R. (Ds). May-J1. Oak.

2444 A. inclara Sm. (hamamelis Sm., brumosa auct.). Franklin Co; Onteora Mt., How; Ithaca (transitional to tristis Sm.) ; Florida; LI: Bellport. Je-J1.

2437 A. haesitata Grt. Ithaca; Little Falls; Albany; New Windsor; Florida; SI; LI: Cold Sp., Yaphank, Southold (Ds). May-Je; Jl.

2453 A. retardata Wlk. Everywhere in the State, Peru to Hempstead Plain, LI. Je-J1. Maple.

2454 A. caesarea Sm. E. Aurora, Je 9, 1918 (Dnd) ; LI: Brooklyn. Maple? (bred with retardata).

2434 A. exilis Grt. Schenectady; Albany; LI: Brooklyn.

2501 A. sperata Grt. Buffalo and E. Aurora (Wild); Ithaca; Schenectady; Karner; Albany; Liberty; Big Ind. Val.; Rhinebeck; New Windsor; Rye; LI: Bellport, Nic (Ds). May-Aug. Low bushes, such as poplar and alder.

2449 A. noctivaga Grt. General but not common. May-Aug. Low bushes.

2496 A. impressa Wlk. Buffalo and E. Aurora (Wild) ; Geneva; Ithaca; Garrison. The imago is hardly distinguishable from the next following species. May; J1; Aug-Sep. Willow. 
2497 A. distans Grt. Peru; Waddington; Onteora Mt., How; Karner; Bedford; LI: Queens; Yaphank. This is very generally determined as impressa, and may not be really distinct. May; J1 31-Aug 3. Willow.

2499 A. longa Gn. (xyliniformis Gn.). Lake Placid; Buffalo and West Falls $($ Wild $)$; Karner; New Windsor; SI; LI: Brooklyn; Bellport, Nic (Ds). May-J1. Low bushes.

2455 A. impleta Wlk. (luteicoma G. \& R.). In every part of the State, but not common. May-early J1; Aug-Sep. Prunus, poplar, etc.

2508 A. oblinita S. \& A. Smeared Dagger Moth. Evans Center; Lancaster; Buffalo; E. Aurora; Honeoye Falls; McLean; Binghamton; Albany; Poughkeepsie; and very common southward. End of Ap-Sep. Low shrubs and herbs.

[2509 A. lanceolaria Grt. Is to be expected in the State. Low bushes.]

\section{AGRIOPODES Hampson}

\section{(Diphthera)}

2419 A. fallax H-S. Fentons; Buffalo; Albany; Rye; SI; LI: Glendale, Southhaven. May-Aug. Viburnum.

2423 A. lepidula Grt. Adirondacks; Alexandria Bay, Kch (Fbs); Evans Center; Buffalo; Ithaca; Rhinebeck. Je-J1. Var. avirida Sm. is reported by Hampson from "NY".

2424 A. teratophora H-S. Lancaster; Ithaca; Catskill; Rhinebeck. Je-Aug.

LEUCONYCTA Hampson

(Microcoelia)

2418 L. diphteroides Gn. General. Var. obliterata Grt. is commoner than the type. May-J1. Solidago (Dyar).

PolygRammate Hübner

2417 P. hebraeicum Hbn. Rye; LI: Forest Pk., Kch; Flushing (Bell). J1. Nyssa.

\section{Cerma Hübner}

2415 C. galva Strk. Clyde. A lost species, most likely not from NY.

\section{HARRISIMEMNA Grote}

2404 H. trisignata W1k. New Russia, Jones; Buffalo; Ithaca; Catskills (Ulster Co) ; Coeymans; New Windsor; SI; LI: Brooklyn. Je-J1. Ilex, lilac.

\section{Chytonix Grote}

2400 C. palliatricula Gn. Mt. Marcy; Evans Center; Ithaca; Onteora Mt., How; Trenton Falls; De Bruce; Big Ind. Val.; Schenectady; Rhinebeck; Poughkeepsie; West Pt. (Ds) ; SI; LI: Central Pk. and Riverhead, Aug (Ds). The rather commoner var. iaspis Gn. does not figure in published records, but has been seen from Peru; N. Creek; Buffalo; Rock City; Ithaca; Big Ind. Val.; LI: Pine Haven. May-J1.

2401 C. sensilis Grt. LI: Riverhead. Early Aug.

2399 C. chlorostigma Harv. (viridimusca Sm.). Poughkeepsie. 
FAGitana Walker

2396 F. littera Gn. Adirondacks; Poughkeepsie; Rye.

Callopist Ria Hübner

(Eriopus)

2392 C. monetifera Gn. Plattsburg; Peru; Fentons; Newcomb; Evans; Buffalo; E. Aurora; Ithaca; Liberty; Onteora Mt., How; Lestershire; Utica; Karner; Poughkeepsie; Bronxville; SI; LI: Forest Pk., Kch; Wading R. (Bird). Je-J1. Larva on ferns.

2391 C. mollissima Gn. Peru; Fentons; Evans Center; Lancaster; E. Aurora; Salamanca; Ithaca; Liberty; De Bruce; Trenton Falls; Karner; Poughkeepsie; Rye; SI; LI (Ds). Larva on ferns. May-J1.

\section{Conservula Grote}

2386 C. anodonta Gn. Sharon Sps. J1-Aug.

\section{Chutapha Moore}

\section{(Trigonophora)}

2385 C. periculosa Gn. General, the var. v-brunneum Grt. from: Fentons; Buffalo; E. Aurora; Ithaca; McLean; Karner; LI: Woodhaven, Yaphank. Jl-early Sep. Food general, larva probably a cutworm.

\section{BROTOLOMIA Lederer}

2384 B. iris Gn. Plattsburg; Peru; Fentons; Clayton; Geneva; Evans Center; Lancaster; E. Aurora; Buffalo; Ithaca; McLean; Binghamton; Onteora Mt., How; Stony Clove; Big Ind. Val.; Utica; Schenectady; Albany; Rye; Van Cort. Pk. (Wat) ; SI; LI: Woodhaven. Je-Aug. Larva a cutworm (Wehrle).

\section{LUPERINA Boisduval}

\section{(Hadena)}

2380 L. passer Gn. General and common northward, including E. Aurora; Poughkeepsie; Big Ind. Val.; Katonah; SI. Var. conspicua Morr. is from: Peru; Ithaca. Je-J1, stragglers to Sep. Larva bores in root of Rumex obtusifolius (Bird).

2375 L. trigona Sm. Albany.

2369 L. burgessi Morr. LI: Brooklyn; E. New York; Jamaica. Sep-Oct.

\section{SIDEMIA Staudinger}

(Hadena)

2367 S. devastatrix Brace. Glassy Cutworm. A dominant species everywhere. The contrasty form speciosa Morr, is much rarer, but apparently general. J1-early Sep. A cutworm. 


\section{MACRONOCTUA Grote}

2365 M. onusta Grt. IRIS BORER. Lancaster; Ithaca; Saratoga; Ballston (Weigel); Schenectady (Cy); Albany (NYS); Catskills (NYS); Troy (Leon); Rye; Mt. Vernon (Weigel); LI: Brooklyn (Weigel). Sep. Bores in Iris, sometimes being injurious; also in Gemmingia chinensis.

\section{TAeniosea Grote \\ (Parastichtis)}

2364 T. discivaria W1k. Plattsburg; Fentons; Evans Center; Lancaster; Catskills; Newport; Sharon; Saratoga; Schenectady; Albany. Form perbellis Grt. has been taken at: Plattsburg; Fentons; Evans Center; Otto; Schenectady; Karner. Jl-Aug.

ERemobia Stephens

(Hadena)

2359 E. claudens W1k. (leucoscelis Grt., hillii Grt.). Essex Co; Fentons; McLean; Sharon. Late Jl-Aug.

\section{AGROPERINA Hampson}

(Hadena)

2346 A. lateritia Hbn. Peru; Fentons. A cutworm.

2344 A. dubitans W1k. General to Orient, LI. J1-Sep.

2357 A. helva Grt. Essex Co; Rochester; Honeoye Falls; E. Aurora; Ithaca; McLean; Oneonta; Schenectady; Rye; NYC; SI; LI: Brooklyn; Yaphank (Eng). Aug-early Sep.

2356 A. lutosa Andrews. Ithaca; Little Falls; Big Ind. Val.; Schenectady; Katonah; LI. J1. An obscure species which will probably prove commoner than here appears.

\section{Oligia Hübner}

\section{(Hadena)}

2333 O. bridghami G. \& R. Rye; LI: Aqueduct. Aug.

2335 O. vulgivaga Morr. (semicana Sm. not W1k.). Lined Corn Borer. Adirondacks; Hamburg (Wild) ; Ithaca; Rye; LI: Brooklyn; Newtown. Aug. The dark var. misera Grt. has been taken on LI: Wading River. Var. fractilinea Grt. is from: Saranac L.; Lancaster; Skaneateles; Ithaca; Binghamton (NYS); Stone Ridge (NYS); Sharon; Glen; Argyle; Schenectady; Karner; Albany; Eagle Bridge; Old Chatham; Poughkeepsie; Rockland; SI; LI: Orient, Lath (NYS). J1-Aug. Corn and coarse grasses; a minor pest on young corn.

2340 O. hausta Grt. "NY", Sep (Sm.). Should this be referred to exhausta?

2341 O. exhausta Sm. Schenectady (types); Rhinebeck; New Windsor; Katonah. Sep. 
2342a O. tonsa subjuncta Sm. Allegany Pk., Aug (Wild).

2339 O. diversicolor Morr. Ithaca (Fbs); Covert, Bdy; Rye (Bird). Sep-early Oct. Larva in Scirpus cyperinus (Bird).

2343 O. includens Wlk. Buffalo; New Windsor; Rye. Larva in Carex stricta (Bird).

\section{Perigea Guenée}

2323 P. xanthioides Gn. Rhinebeck; New Windsor; Rye; Katonah; Bronxville; SI. May-Sep.

2327 P. vecors Gn. Rochester; Evans Center; Lancaster; Ithaca; Onteora Mt., How; Schenectady; New Windsor; Rye; SI; LI: Newtown. May; Aug.

2324 P. epopea Cr. Rye; SI; LI: Woodhaven. J1-Sep.

2330 P. sutor Gn. SI; LI: Woodhaven; Newtown; Yaphank; Orient (Angle). Aug-Oct.

\section{Euplexia Stephens}

2317 E. lucipara L. Generally distributed. May-Sep. A climbing cutworm.

\section{TRACHEA Hübner}

\section{(Hadena)}

2271 T. delicata Grt. Evans Center; Buffalo; Ithaca; Schenectady; Poughkeepsie; Florida; Durlandville; Pine I. (Ds) ; LI: Brooklyn (Doll). Je-Aug.

2275 T. inordinata Morr. LI: Lynbrook; Central Pk.; Babylon. Je.

2286 T. mactata Gn. Keene Val.; Fentons; Lancaster; E. Aurora, Boston (Wild) ; Ithaca; Ulster Co; Trenton Falls; Schenectady; Albany ; Bangall; Storm King; De Bruce and Oliverea (Ds); LI: Jamaica (Doll). Sep-Oct.

2283 T. turbulenta Hbn. Albany; Rye; NYC; SI; LI: Brooklyn; Orient, Lath (NYS) ; Riverhead, J1 (Ds). Smilax.

2276 T. miselioides Gn. Evans Center; Buffalo; Lancaster; SI; LI: Newtown; Lynbrook; Orient (Angle). J1-Sep. Smilax.

2285 T. remissa indocilis W1k. A northern species. Mt. Marcy; Fentons; Evans Center; E. Aurora; Protection; Lancaster; Ithaca; Trenton Falls;

Schenectady; Liberty; Big Ind. Val. Je-Jl. Grass.

2312 T. enigra Sm. McLean, J1 (CU).

2288 T. separans Grt. ${ }^{78}$ Ithaca; Castile, Je, Brad (Fbs.).

2291 T. finitima Gn. Newcomb; Clayton; Lake Forest; Buffalo; E. Aurora; Rnck City; Castile, Brad; Portageville (Ds); Ithaca; McLean; Big Ind. Val.; Schenectady; Ảlbany; New Windsor; Rye; Florida; LI: Wading R. ; Montauk Pt. May-Ji.

2315 T. impulsa Gn. Saranac L.; Mt. Marcy; Newcomb; Franklin Co; Fentons ; Clayton; Evans Center; Buffalo; Lancaster; Rock City; Castile, Brad; Ithaca; McLean; Debruce; Liberty; Trenton Falls; Schenectady; SI; LI (Doll). Late Je-J1.

2308 T. modica Gn. Not rare; general. Je-Aug.

2270 T. illocata W1k. Fentons; Cascade L.; Karner; Ulster Co; LI: Yaphank.

${ }^{78}$ T. lona Strk., described from Clyde, is now considered the same as runata Sm., the western form of separans, with incorrect locality. 


\section{SEPTIS Hübner ${ }^{79}$}

(Hadena)

2268 S. arctica Lef. Yellow-Headed Cutworm. General; very common, especially northward. Late Je-Aug. A cutworm.

2246 S. verbascoides Gn. Newcomb; Fentons; Clayton; Evans Center; Buffalo; E. Aurora; Ithaca; De Bruce; Liberty; Big Ind. Val.; Onteora Mt., How; Trenton Falls; Ilion; Schenectady; Rye; SI; LI: Woodhaven (Doll). Je-Aug.

2247 S. nigrior Sm. Rock City (Ds); Ithaca; McLean; Evans Center; E. Aurora (Wild) ; Schenectady; E. New York. Late May-early Aug.

2248 S. cariosa Gn. Lancaster; E. Aurora (Wild); Ithaca; Trenton Falls; Schenectady; LI: Jamaica; Orient (Angle).

2251 S. vulgaris G. \& R. Evans Center; Buffalo (type) ; Lancaster; Rock City; Ithaca; De Bruce; Schenectady; New Windsor; Ramapo Mts.; LI: Woodhaven. Je-Ji.

2250 S. cristata Grt. Evans Center; Buffalo.

2253 S. lignicolor Gn. General and not rare. Je-Jl.

2269 S. suffusca Morr. (alia Gn.). Peru; North Creek; Batavia; Buffalo (Wild) ; E. Aurora, Wild; Lancaster; Ithaca; Liberty; Schenectady; Albany; Katonah. Je-Jl.

2269a S. rorulenta Sm. Peru; Franklin Co; Otto; Ithaca; Sharon; Albany. J1.

2260 S. vultuosa Grt. Mt. Marcy; Fentons; Evans Center; Buffalo; E. Aurora; Lancaster; Castile, Brad; Ithaca; McLean; Trenton Falls; Schenectady; Albany. Je-early J1.

2262 S. apamiformis Gn. Fentons; E. Aurora (Wild); Clayton; Schenectady. May-Aug. A cutworm.

\section{DipteRygia Stephens}

2243 D. scabriuscula L. Ithaca; Rogers Rock, L. George (Jones); Binghamton (NYS) ; New Russia (Jones); E. Aurora, Buffalo (Wild); Schenectady; Poughkeepsie; Rye; SI; LI: Lynbrook; Newtown; Greenport. Je-Sep. Rumex, Polygonum.

\section{MAGUSA Walker}

2242 M. orbifera W1k. (dissidens Felder). SI: Richmond Hill; LI. A stray from the Tropics. Condalia ferrea.

\section{PyROPHILA Hübner}

\section{(Amphipyra Ochsenheimer)}

2241 P. glabella Morr. Ithaca. Aug-early Sep.

2239 P. pyramidoides Gn. COPPER Underwing. Fentons; Rochester (Angle); Castile, Brad; Hebron (W1s). General in the Mohawk Val., southward and westward. Aug-early Oct. Grape, maple, and various other plants. Aug-early Oct.

${ }^{79}$ The larvae of this genus are cutworms. 
2240 P. tragopoginis L. Fentons; New Russia (Jones); Evans Center; Buffalo; E. Aurora; Lancaster; Seneca L.; Ithaca; Sharon; Schenectady; LI: Yaphank; Calverton (Ds). J1-early Sep. A general feeder.

\section{SUBFAMILY CUCULLIINAE}

HOMOGLAEA Morrison

2237 H. hircina Morr. Plattsburg; Saranac L.; Ithaca, May; Dutchess Co.

\section{XANTHIA Hübner}

2233 X. lutea Ström (flavago Fab.). Plattsburg; L. Placid; Fentons; Buffalo; Karner; Bangall. Willow, preferring the catkins.

BRACHYCOSMIA Hampson

(Anchocelis)

2232 B. digitalis Grt. Buffalo. Oct.

\section{IODIA Hübner}

2231 I. rufago Hbn. Rye; SI; LI: Central Pk.; West Hills (Doll) ; Bellmore; Massapequa; Yaphank. Ap-May. Willow.

\section{EUCIRRHOEDIA Grote (Atethmia)}

2230 E. pampina Gn. Wilmington; Fentons; Rochester; Buffalo; E. Aurora; Gowanda; Castile; Ithaca; Lestershire; Schenectady; Karner; Rye; SI; LI, general. Late Aug-Oct.

\section{PARASTICHTIS Hübner 80 \\ (Orthosia Ochsenheimer in part, Amathes)}

2224 P. ralla G. \& R. New Russia; Fentons; Trenton Falls; Seneca L.; Ithaca; McLean; Big Ind. Val.; Bangall. End of Aug-Sep.

2220 P. bicolorago Gn. E. Aurora (Wild). The var. ferruginoides Gn. is common and general; the type form occurs at: Lewis Co; Rochester (Angle); Ithaca; Karner; LI: Yaphank. Sep-early Nov.

[2226 P. inops Grt. (Caradrina insipida Strk.), described from Me. and Wis., should occur in the State. Je.]

2225 P. puta G. \& R. (euroa G. \& R.). Wilmington; L. Placid; Fentons; Buffalo; E. Aurora (Wild), Sep 20, 1882; Ithaca; McLean; Trenton Falls; Sharon; Karner. Late J1-Sep.

${ }^{80}$ P. americana Morr., described from New York, was a European species with incorrect locality. 
CONISTRA Hübner

(Scopelosoma Curtis, Glaea)

2216 C. indirecta W1k.81 (moffatiana Grt.). Franklin Co; Spier Falls; Lancaster; E. Aurora; Ithaca; Big Ind. Val.; Albany; Ramapo (Ds); NYC; SI; LI: Central Pk.; Yaphank. Oct-early May. Hamamelis.

2217 C. pettiti Grt. Saranac L.; Ithaca. Sep-late Ap.

2218 C. ceromatica Grt. Lancaster; Karner. Mar-Ap. Hamamelis.

2212 C. tristigmata Grt. Fentons; Cranberry L.; Geneva; Buffalo; Castile, Brad; Ithaca (Eyer, Fbs); McLean (seen); Newport; Oneonta; Karner; Albany; LI: West Hills, Jamaica (Ds). May. General on trees.

2213.1 C. walkeri Grt. Peru; Saranac L.; Geneva; Rochester; Lancaster; Buffalo; Ithaca; Albany; Rye; Fordham; SI; LI: West Hills (Ds). Ap-early May.

2213 C. sidus Gn. Karner; Rye; SI; LI: West Hills (Ds). Var. vinulenta Grt. is reported from Karner and Lancaster. Oct-spring.

2214 C. morrisoni Grt. Peru; Saranac L.; Geneva; Rochester; Kendall; Buffalo; Lancaster; Ithaca; Saratoga; Karner; Albany; Rye; SI; LI: West Hills (Ds). Oct-May.

2215 C. devia Grt. Saranac L.; Geneva; Lancaster; Buffalo, Hamburg (Wild) ; Ithaca; Big Ind. Val.; Albany; Dutchess Co; Rye; SI; LI: Brooklyn; Jamaica. Mar-May.

2210 C. viatica Grt. Karner; SI; LI: Yaphank. Sep. Oak, etc.

2209 C. inulta Grt. Fentons; Ithaca; Karner; Albany; SI; LI: Yaphank. SepOct. Viburnum.

\section{Psectraglaea Hampson}

2208 P. carnosa Grt. LI: Central Pk.; Yaphank. Late Sep-Oct.

\section{Epiglaea Grote}

2207 E. apiata Grt. Saranac L.; McLean; Karner; LI: Yaphank. Sep-Oct.

2206 E. decliva Grt. Karner; Lancaster; Rye.

\section{HARPAGLAEA Hampson}

\section{(Glaea)}

2203 H. sericea Morr. E. Aurora (Wild); Karner; SI; LI: Yaphank. Oct. The similarity between forms of this species and the next makes published records untrustworthy.

2205 H. pastillicans Morr. Karner (normal); LI: Yaphank (var. tremula Harv.). Oct.

${ }^{81}$ This name is applied to pale or faded spring specimens of the same species as moffatiana, and also to an apparently undescribed paler species (possibly graefiana Grt.). I cannot sort out the records. (Fbs.) 


\section{EUMICHTIS Hübner}

(Hadena)

2187 E. ducta Grt. Franklin Co; Saranac L. ; Farnham and E. Aurora (Wild) ; Buffalo. (The last record should be verified.) Aug-Sep.

2188 E. versuta Sm. "NY" (Wolley-Dod); E. Aurora, May (Wild); (Je in Alberta). Probably a cutworm. Elm and beech.

\section{EUROTYPE Hampson}

2178 E. medialis Grt. Schenectady (type).

XYLOTYPE Hampson

2177 X: capax G. \& R. Fentons; Big Ind. Val.; LI: pine barrens (Eng). Oct.

XYLENA Ochsenheimer

(Calocampa Stephens)

2169 X. nupera Lint. Fentons; Buffalo; E. Aurora (Wild); Ithaca; Schoharie; Karner; Albany; LI: Yaphank. Sep-May.

2174 X. thoracica Putn-Cr. Saranac L. (McKnight). The type was from "NY".

2173 X. cineritia Grt. Saranac L.; Lancaster; Karner; LI: Massapequa; Yaphank; West Hills (Ds). Early spring. Willow.

2171 X. curvimacula Morr. Plattsburg; Saranac L.; Buffalo; E. Aurora (Wild) ; Ithaca; McLean; Gloversville; Karner; Rye; Ramapo; SI; LI: West Hills (Ds). Sep-May. Cutworm.

\section{Graptolitha Hübner 82}

(Xylina Ochsenheimer, in part; Lithophane Hübner)

2136 G. disposita Morr. Fentons; Jamestown; Buffalo; Schenectady; Karner; Albany; Rye. Sep.

2138 G. petulca Grt. Plattsburg; L. Placid; Fentons; Lancaster; Schenectady; Karner; Albany; LI: Yaphank.

2134 G. hemina Grt. Lewis Co (Grt). Sep-Je.

2132 G. patefacta Wlk. Lewis Co; Monroe Co; Ithaca; Karner. Ap.

2153 G. antennata W1k. GREen FrUIT-Worm. Holley; Karner; Schenectady; Albany; NYC; SI; LI: Brooklyn to Yaphank. Oct-May. A green fruit-worm of apple, rarer than the following or than $G$. insciens.

2154 G. cinerosa Grt. (laticinerea Grt., grotei Rly.). GreEn FrUIT-Worm. Elma; Buffalo; E. Aurora; Geneva ; Ithaca ; Kingston; Schenectady; Karner; New Windsor; Rye; SI; LI: Brooklyn; Bellport; West Hills; Yaphank. Oct-early May. Apple, pear, and maple.

2137 G. ferrealis Grt. Fentons; Rochester; Lancaster; Ithaca; Catskills; Schenectady; Karner; Inwood; LI: Yaphank. Sep-May.

${ }^{82}$ All these species of Graptolitha emerge in late fall, overwinter, and reappear in the spring. 
2139 G. signosa W1k. (petulca Grt.). Ithaca; Karner; Saratoga; LI: Yaphank (Eng). Ap.

2133 G. innominata Sm. Lewis Co; E. Aurora (Wild); McLean; Catskills; Karner. Sep-May.

2141 G. amanda Sm. Karner.

2131 G. bethunei G. \& R. Common and general. Sep-May.

2130 G. semiusta Grt. Ithaca, Benjamin (Fbs); Sharon; Karner. Ap.

2156 G. fagina Morr. Saranac L.; Karner; Albany; LI: Yaphank.

2155 G. georgii Grt. Cascade L.; Fentons; Essex Co; Lancaster; Albany; Ulster Co (Nic).

2146 G. viridipallens Grt. "LI", Aug, Doll (Sm.).

2150 G. unimoda Lint. Loon L.; Fentons; Lancaster; E. Aurora, Sep (Wild); Karner; LI: Yaphank.

2160 G. tepida Grt. Fentons; Big Ind. Val.; Karner; Albany.

2144 G. baileyi Grt. Cascade L.; Fentons; Cranberry L., Htly (Dy) ; Buffalo; Lancaster; Karner; Albany; Oliverea (Ds). Sep-Ap.

2158 G. nigrescens Engel. Near Albany.

2149 G. querquera Grt. Karner.

2167 G. lepida Lint. Karner.

2168 G. lamda thaxteri Grt. Saranac L.; Karner; Albany; LI: Yaphank (Eng). Oct. Myrica (typical lamda).

2163 G. pexata Grt. Fentons; Lancaster; Buffalo (Wild) ; Schenectady; Karner; Albany; Rye; LI: Yaphank. Oct. Probably alder.

\section{Lithomoia Hübner}

2129 L. solidaginis germana Morr. Cascade L.; Fentons; Lancaster; McLean; Karner; Albany. Sep. Vaccinium.

\section{HILLIA Grote}

2127 H. crasis H-S. Fentons.

2126 H. algens Grt. Fentons; Catskills (Pears).

2125 H. discinigra Wlk. Karner.

\section{Litholomia Grote}

2124 L. napaea Morr. L. Placid; Fentons; LI: Yaphank. Ap-May.

\section{Copipanolis Grote}

2112 C. cubilis Grt. Ithaca; LI: L. Ronkonkoma; Central Pk.; Bellmore; Massapequa (Ds). Mar-Ap.

\section{Eutolype Grote}

2110 E. rolandi Grt. Ithaca; SI; LI: Queens (Eng). Late Mar-Ap.

2109 E. depilis Grt. (bombyciformis Sm.). Ithaca. Ap. Both the type commonly called bombyciformis, and the gray variety which passes for depilis.

2108 E. grandis Sm. Ithaca, Benjamin. Mar-Ap.

2107 E. electilis Morr. (Psaphidia). Ithaca, Ap. 30, 1916, Panoff (CU). Ap. 
PSAPHIDIA Walker

2105 P. grotei Morr. Ithaca; Bronx; SI. The commonest of the group, at Ithaca. Ap-May.

2104 P. resumens Wlk. Ithaca; Bronx; SI; LI: Brooklyn; Central Pk. Ap-May. 2106 P. thaxteriana Grt. Ithaca; Rye; LI: Brookhaven. Mar-May. Oak.

\section{Momophana Grote}

2098 M. comstocki Grt. Plattsburg; Saranac L.; Buffalo; E. Aurora (Wild); Portage; Ithaca (type); Big Ind. Val. May.

\section{Feralia Grote}

2096 F. jocosa Gn. Plattsburg; Fentons; West Seneca; Ithaca; Corinth; New Windsor; Ramapo; Bronx. Common locally. Ap. Hemlock.

2097 F. major Sm. Plattsburg; Saratoga; LI: Central Pk.; Massapequa. Ap-May.

\section{HomohaDENA Grote}

2080 H. badistriga Grt. Lewis Co; Lancaster; Ithaca; Schenectady; Albany. Lintner's record of infixa is undoubtedly based on the Lewis Co specimen. Je-Jl. Honeysuckle. Not a cutworm.

\section{ONCOCNEMIS Lederer}

2034 O. saundersiana Grt. Bangall (Eng). Sep-Mar.

2046 O. riparia Morr. Evans Center; Buffalo; Locust Val.; LI: Northport; Glencove. Harvey's record of chandleri is undoubtedly based on this species (Erie Co).

2061 O. piffardi W1k. (atrifasciata Morr.). Fentons; Long L.; LI. The last record should be verified.

\section{Cucullia Schrank 83}

2005 C. convexipennis G. \& R. Rather common throughout the State, the caterpillar being seen fully as often as the moth. Je-Aug.

2002 C. postera Gn. Mt. Marcy; Fentons; Ithaca, J1, Stedman; Liberty; Sharon; Rhinebeck; Poughkeepsie; New Windsor; LI: Glendale, Kch.

2003 C. asteroides Gn. Common everywhere, the caterpillar often seen. Je-J1 5; late Aug; late Sep.

1999 C. florea Gn. Ithaca; Newburgh; Trenton Falls; Sharon; Ulster Co; Poughkeepsie. May-early Jl.

1993 C. laetifica Lint. "NY" (Graef). I have seen the specimen; is it mislabeled? (Fbs).

1992 C. speyeri Lint. Rochester, Angle; Buffalo (Wild) : Lestershire; Albany; New Windsor; LI: Woodhaven; Pinehaven. J1.

1997 C. intermedia Speyer. General southward to Rochester, Angle; E. Aurora, the Catskills, and Albany. End of May-early Je; late J1-early Sep.

\footnotetext{
${ }^{83}$ The larvae in this genus are general feeders, usually on flowers.
} 
2139 G. signosa Wlk. (petulca Grt.). Ithaca; Karner; Saratoga; LI: Yaphank (Eng). Ap.

2133 G. innominata Sm. Lewis Co; E. Aurora (Wild); McLean; Catskills; Karner. Sep-May.

2141 G. amanda Sm. Karner.

2131 G. bethunei G. \& R. Common and general. Sep-May.

2130 G. semiusta Grt. Ithaca, Benjamin (Fbs); Sharon; Karner. Ap.

2156 G. fagina Morr. Saranac L.; Karner; Albany; LI: Yaphank.

2155 G. georgii Grt. Cascade L.; Fentons; Essex Co; Lancaster; Albany ; Ulster Co (Nic).

2146 G. viridipallens Grt. "LI", Aug, Doll (Sm.).

2150 G. unimoda Lint. Loon L. ; Fentons; Lancaster; E. Aurora, Sep (Wild); Karner; LI: Yaphank.

2160 G. tepida Grt. Fentons; Big Ind. Val.; Karner; Albany.

2144 G. baileyi Grt. Cascade L.; Fentons; Cranberry L., Htly (Dy); Buffalo; Lancaster; Karner; Albany; Oliverea (Ds). Sep-Ap.

2158 G. nigrescens Engel. Near Albany.

2149 G. querquera Grt. Karner.

2167 G. lepida Lint. Karner.

2168 G. lamda thaxteri Grt. Saranac L.; Karner; Albany ; LI: Yaphank (Eng). Oct. Myrica (typical lamda).

2163 G. pexata Grt. Fentons; Lancaster; Buffalo (Wild) ; Schenectady; Karner; Albany; Rye; LI: Yaphank. Oct. Probably alder.

\section{LithomoIA Hübner}

2129 L. solidaginis germana Morr. Cascade L.; Fentons; Lancaster; McLean; Karner; Albany. Sep. Vaccinium.

\section{HILLIA Grote}

2127 H. crasis H-S. Fentons.

2126 H. algens Grt. Fentons; Catskills (Pears).

2125 H. discinigra Wlk. Karner.

\section{Litholomia Grote}

2124 L. napaea Morr. L. Placid; Fentons; LI: Yaphank. Ap-May.

\section{Copipanolis Grote}

2112 C. cubilis Grt. Ithaca; LI: L. Ronkonkoma; Central Pk. ; Bellmore; Massapequa (Ds). Mar-Ap.

\section{Eutolype Grote}

2110 E. rolandi Grt. Ithaca; SI; LI: Queens (Eng). Late Mar-Ap.

2109 E. depilis Grt. (bombyciformis Sm.). Ithaca. Ap. Both the type commonly called bombyciformis, and the gray variety which passes for depilis.

2108 E. grandis Sm. Ithaca, Benjamin. Mar-Ap.

2107 E. electilis Morr. (Psaphidia). Ithaca, Ap. 30, 1916, Panoff (CU). Ap. 
PSAPHIDIA Walker

2105 P. grotei Morr. Ithaca; Bronx; SI. The commonest of the group, at Ithaca. Ap-May.

2104 P. resumens Wlk. Ithaca; Bronx; SI; LI: Brooklyn; Central Pk. Ap-May. 2106 P. thaxteriana Grt. Ithaca; Rye; LI: Brookhaven. Mar-May. Oak.

\section{Momophana Grote}

2098 M. comstocki Grt. Plattsburg; Saranac L.; Buffalo; E. Aurora (Wild); Portage; Ithaca (type); Big Ind. Val. May.

\section{FERAlia Grote}

2096 F. jocosa Gn. Plattsburg; Fentons; West Seneca; Ithaca; Corinth; New Windsor; Ramapo; Bronx. Common locally. Ap. Hemlock.

2097 F. major Sm. Plattsburg; Saratoga; LI: Central Pk.; Massapequa. Ap-May.

\section{HoMOHADENA Grote}

2080 H. badistriga Grt. Lewis Co; Lancaster; Ithaca; Schenectady; Albany. Lintner's record of infixa is undoubtedly based on the Lewis Co specimen. Je-J1. Honeysuckle. Not a cutworm.

\section{ONCOCNEMIS Lederer}

2034 O. saundersiana Grt. Bangall (Eng). Sep-Mar.

2046 O. riparia Morr. Evans Center; Buffalo; Locust Val.; LI: Northport; Glencove. Harvey's record of chandleri is undoubtedly based on this species (Erie Co).

2061 O. piffardi Wlk. (atrifasciata Morr.). Fentons; Long L.; LI. The last record should be verified.

\section{Cucullia Schrank 83}

2005 C. convexipennis G. \& R. Rather common throughout the State, the caterpillar being seen fully as often as the moth. Je-Aug.

2002 C. postera Gn. Mt. Marcy; Fentons; Ithaca, J1, Stedman; Liberty; Sharon; Rhinebeck; Poughkeepsie; New Windsor; LI: Glendale, Kch.

2003 C. asteroides Gn. Common everywhere, the caterpillar often seen. Je-J1 5; late Aug; late Sep.

1999 C. florea Gn. Ithaca; Newburgh; Trenton Falls; Sharon; Ulster Co; Poughkeepsie. May-early J1.

1993 C. laetifica Lint. "NY" (Graef). I have seen the specimen; is it mislabeled? (Fbs).

1992 C. speyeri Lint. Rochester, Angle; Buffalo (Wild): Lestershire; Albany; New Windsor; LI: Woodhaven; Pinehaven. Ji.

1997 C. intermedia Speyer. General southward to Rochester, Angle; E. Aurora, the Catskills, and Albany. End of May-early Je; late J1-early Sep.

\footnotetext{
${ }^{83}$ The larvae in this genus are general feeders, usually on flowers.
} 


\section{SUBFAMILY HADENINAE}

\section{(Mamestrinae)}

\section{LEUCANIA Ochsenheimer}

\section{(Heliophila Hübner)}

1972 L. pallens luteopallens Sm. Common throughout the State. Late May-Sep. Grass.

\section{NeLEuCANIA Smith \\ (Leucania, Heliophila)}

1959 N. albilinea auct. I have seen but one dark specimen from the State such as those which Smith considered typical of albilinea, from Yorktown. Specimens with dark hind wings have been seen from: Peru; Clayton; Albany; New Windsor; SI; LI: Brooklyn. They are probably merely a variety of the next species. May-early Je; J1-Aug. Grass.

1959a N. diffusa Wlk. WheAt-heAd Army Worm. Has been seen from: Waddington; Perry; E. Aurora; Gowanda; Ithaca; Sharon; Albany; New Windsor; Katonah; SI; LI: Amagansett; Orient, Lath. Will probably prove common wherever looked for. May-early Je; J1-Aug. Grass.

\section{Borolia Moore}

\section{(Lencania, Heliophila)}

1955 B. flabilis Grt. LI: Glen Cove. J1-Sep.

1957 B. linita Gn. (extincta Sm., not Gn.). "NY”. May, J1-Aug. A coastal species.

\section{CIRPHIS Walker 84 \\ (Leucania, Heliophila)}

1950 C. unipuncta Haw. Army Worm. Very common everywhere. Throughout the entire season.

1933 C. pseudargyria Gn. Common from the Adirondacks to Buffalo and LI. Je-Sep, with stragglers till Nov; two broods, the second dwarfed.

1941 C. insueta Gn. General, but less common than other species. May; J1-Aug.

1934 C. multilinea W1k. Peru; Fentons; Honeoye Falls; E. Aurora; Buffalo; Rock City; Ithaca; Schenectady; Karner; Albany; Cropseyville; Rhinebeck; Poughkeepsie; Katonah; SI; LI: Amagansett. Je-Sep.

1935 C. commoides Gn. Plattsburg; Newcomb; N. Creek; Essex Co; Peru; Fentons; Clayton; Rochester; Lewiston; Buffalo; E. Aurora; Otto; Castile; Ithaca; Onteora Mt.; Liberty; Debruce; Utica; Little Falls; Sharon; Schenectady; Karner; Albany; Rhinebeck; Poughkeepsie; Katonah. Je-Sep.

1936 C. phragmatidicola Gn. General throughout the State; easily confused with C. multilinea. May-early Oct.

${ }^{84}$ The larvae in this genus are cutworms feeding normally on grasses, and become army worms only when abundant. 


\section{XANTHOPASTIS Hübner}

\section{(Euthisanotia)}

1931 X. timais regnatrix Grt. West Farms (Angus); SI. A stray from the Tropics. Lily.

\section{Ceramica Guenée}

1930 C. picta Harr. (Mamestra). ZEBra Caterpillar. Common everywhere, Plattsburg to Buffalo and LI. The larva is much more often seen than the moth. May-Je; Aug-Sep. A general feeder on herbs.

\section{SIDERIDIS Hübner \\ (Crocigrapha Grote)}

1927 S. normani Grt. Plattsburg; Lancaster; Buffalo; Evans Center ; Rock City ; Ithaca; McLean; Karner; Dutchess Co; New Windsor; Fordham; SI; LI: Jamaica. End of Ap-early Je. Caulophyllum thalictroides.

1926 S. rosea Harv. Peru; Buffalo; E. Aurora; McLean (Fbs) ; Lancaster; Big Ind. Val.; Oneonta; Karner; SI; LI: Pinelawn. May-Je; Aug.

1928 S. congermana Morr. Karner; SI: Richmond; "LI". May-Aug.

1929 S. rubefacta Morr. Karner; Albany. Je-Oct. A general feeder. A cutworm?

ORTHOSIA Ochsenheimer

\section{(Taeniocampa auct., Monima Hübner)}

1917 O. revicta Morr. (subterminata Sm.). Plattsburg; Saranac L.; Ithaca; Karner; LI: Central Pk. Ap.

1919 O. hibisci Gn. (insciens Wlk., Xylina laticinerea Slingerland, in error for larvae and pupae). General; the form hibisci from Buffalo (Wild) and LI. Ap-May. A common "green fruit-worm" on apple.

1918 O. alurina Sm. Ithaca (two specimens). Ap.

1910 O. rubrescens Wlk. E. Aurora; Ithaca; New Windsor; LI: Central Pk. Ap-May.

1915 O. garmani Grt. LI: Brooklyn; Hempstead Plain. Ap-Je.

\section{XyLomiges Guenée}

1887 X. dolosa Grt. Plattsburg; Ithaca; Ilion. Ap-May.

1893 X. alternans Wlk. (tabulata Grt.). Fentons; Karner.

\section{MORRISONIA Grote}

1877 M. evicta Grt. (sectilis auct.). General. Var. vomerina Grt. is general with the type, and seen or reported from L. Placid; Saranac L.; Lancaster; E. Aurora; Ithaca; Oneonta; Karner; Albany; Nassau; LI. Ap-May.

1873 M. mucens Hbn. LI: Yaphank, Eng (Fbs). Mar-early Ap.

1878 M. confusa Hbn. Lancaster; Ithaca; McLean; Albany and vic.; New Windsor; Bronxville; SI; LI: Oyster Bay; Yaphank. Ap-early Je. Willow, etc., in a web. 
1695 P. latex Gn. Newcomb; Keene (Wild); Geneva; Evans Center; Buffalo; West Falls (Wild) ; Castile; Rock City; Randolph; Ithaca; Liberty; Big Ind. Val.; Oneonta; Schenectady; Rensselaer; New Windsor; Ramapo (Ds) ; SI; LI: Wyandanch (Eng). May-J1. A cutworm.

1700 P. adjuncta Bdv. Mt. Marcy; Fentons; Geneva; Buffalo; E. Aurora (Wild); Otto; Ithaca; Sharon; Schenectady; Onteora Mt.; Albany; Rye; SI; LI: Brooklyn; Jamaica; Ft. Hamilton; Flushing (Ds). Ap-Aug; two broods. Not a cutworm, usually resting above the ground. Roman wormwood (Wild).

[1709 P. tacoma Strk. Should occur in the State, as it is determined by Dod from Maine. It is probably confused with lilacina.]

1715 P. legitima Grt. Striped Garden Caterpillar. General in the State. Je-Aug. Not a cutworm.

1717 P. lilacina Harv. Peru; Newcomb; Fentons; Evans Center; Lancaster; Otto; Castile; Ithaca; Liberty; Oneonta; Poughkeepsie; Brewster. J1-Sep.

1718 P. goodellii Grt. Essex Co; Newcomb; Fentons; North Creek; Lancaster; Allegany Pk.; Evans Center; McLean; Sharon; Schenectady ; Katonah; LI. Jl-Aug. Apparently rare in the Appalachian highlands.

1720 P. ectypa Morr. Onteora Mt., How, Aug 7.

1750 P. renigera Steph. Bristly Cutworm. General, often our commonest Polia. Late May-early Sep. A cutworm.

1755 P. olivacea Morr. Keene Val.; Cascade L.; Fentons; Oak Orchard Swamp; Lancaster; Onteora Mt.; Sharon; Schenectady; Albany; NYC; LI. Je; Aug-Śep. A cutworm.

1756 P. laudabilis Gn. New Russia, Jones; E. Aurora, Portage (Wild); Ithaca; LI: Jamaica. J1-Oct. A cutworm.

1754 P. lorea Gn. Plattsburg; Peru; Mt. Marcy; Newcomb; Fentons; Clayton; Evans Center; Buffalo; Otto; Chautauqua (Ds); Ithaca; McLean; Catskills generally; Schenectady; Albany; Nassau; Rhinebeck; Rye; SI; LI. Late Je-J1. A cutworm.

1732 P. anguina Grt. Rye; SI; LI: Central Pk.; Hempstead Plain; Massapequa. May-Jl.

1734 P. vicina Grt. Peru; Fentons; Evans Center; Buffalo; Ulster Co; Sharon. J1.

ANARTA Ochsenheimer

1633a A. myrtilli acadiensis Beth. "NY" (Gerhard). Probably on heath.

Scotogramma Smith

(Mamestra)

1615 S. trifolii Rott. Clover Cutworm. Common all over the State. May-Je; Aug-Sep. The contrasty var. albifusa Wlk. is also general. A general feeder on herbs.

BARATHRA Hübner

1607 B. curialis Sm. Saranac L.; Albany. A cutworm. 


\section{SUBFAMILY AGROTINAE 86}

\section{EUERETAGROTIS Smith}

1597 E. sigmoides Gn. Plattsburg; Franklin Co; Evans Center; E. Aurora; Otto; Ithaca; McLean; Schenectady; Rye; LI: Great Neck. May; J1-Aug. A cutworm.

1598 E. perattenta Grt. Peru; Mt. Marcy; Saranac Inn; Fentons; Newcomb; Evans Center; Buffalo; E. Aurora; Debruce; McLean; Little Falls; Sharon; Schenectady; SI. Je-J1.

1599 E. attenta Grt. Peru; Franklin Co; Saranac L.; Ithaca; Karner. J1.

\section{CRYPTOCAla Benjamin}

1596 C. gilvipennis Grt. (Rhynchagrotis). Plattsburg; Peru; Fentons; Ithaca $(F b s)$. J1-Aug. Grass.

\section{LAMPRA Hübner 87}

\section{(Rhynchagrotis Smith)}

1565 L. rufipectus Morr. Essex Co; Fentons; Franklin Co; Buffalo (Wild); Lancaster; Ithaca; LI: Richmond Hill (Bj); Yaphank. J1-Aug. Grass.

1566 L. brunneicollis Grt. Peru; Brant L. (Bj); Evans Center; Buffalo; Ithaca; Albany; Dutchess Co (Bj) ; Poughkeepsie; SI; LI: Floral Pk.; Flushing, Richmond Hill, Yaphank $(\mathrm{Bj})$. Je-Ji.

1590 L. cupida Grt. LI: Yaphank (Bj).

L. cupida var. brunneipennis Grt. Carmel; NYC $(B j) ; S I, D s(B j) ; L I$ : Flatbush $(\mathrm{Bj})$. J1.

1593 L. minimalis Grt. Albany; Poughkeepsie.

1585 L. placida Grt. Fentons (type); Franklin Co (Bj); Rye (Bird); LI: Jamaica; Yaphank. The specimens seen lack the median shade.

1588 L. alternata Grt. Saranac L.; Buffalo; Salamanca; Ithaca (Bj) ; Onteora Mt.; Schenectady; Karner, Hill (Bj); Hudson $(\mathrm{Bj})$; SI; LI: Forest $\mathrm{Pk}$. Je-Oct. Oak, hickory, etc.

\section{Matuta Grote 8.8}

\section{(Semiophora)}

1552 M. elimata Gn. Buffalo; Karner; Rye; LI: Yaphank. Sep.

1552c M. janualis Grt. Franklin Co; Ulster Co; Schenectady; Karner; Albany; SI, Ds (Fbs) ; LI: Yaphank (Eng). Now considered a good species. Late Aug-Sep.

1420 M. opacifrons Grt. Karner. Aug-Sep.

1553 M. tenebrifera Grt. Saranac L., McKnight; Ithaca; LI: Brooklyn. Ap-May. 1551 M. youngii Sm. Ulster Co, Aug (red phase). A cutworm, injurious in Canada.

${ }^{86}$ The members of this subfamily to Porosagrotis inclusive, are cutworms; the remainder are feeders on flowers and seeds.

${ }^{s i}$ In this very difficult genus, records verified by Mr. Benjamin are marked with (Bj). All others should be viewed with suspicion.

${ }^{8}$ Mythimna oxalina Hbn. of Europe has been reported from Sharon Sps., undoubtedly in error. 
1560 M. prasina Fab. Keene Val.; Mt. Marcy and vic.; Fentons; Rochester; E. Aurora, Buffalo (Wild); Lancaster; Ithaca; Onteora Mt.; Oliverea; Utica; Little Falls; Trenton Falls; Schenectady; Karner; Poughkeepsie; Rye; SI; LI: Woodhaven; Forest Pk., Kch. Je-Sep. A cutworm.

\section{Anytus Grote}

1533 A. privatus W1k. Fentons; Buffalo; E. Aurora; Lancaster; Otto; Ithaca; Big Ind. Val.; Ilion; Schenectady; Karner; SI; LI. Many specimens are dark, approaching Smith's obscurus and teltowa. J1-Sep.

\section{UfEus Grote}

1522 U. plicatus Grt. Plattsburg (Dyar).

1523 U. satyricus Grt. Big Ind. Val. (Pears).

\section{ApHARETRA Grote}

1516 A. dentata Grt. Fentons.

\section{Aplectoides Butler}

(Platagrotis Smith)

1509 A. pressa Grt. Peru; Essex Co; Fentons; Ithaca; Karner; Buffalo; Utica. J1-Aug.

1505 A. speciosa Hbn. L. Tear, J1 7, 1918. Vaccinium and grasses.

1513 A. condita Gn. N. Elba Twp., near Indian Pass; Albany; Liberty.

\section{Adita Grote}

1504 A. chionanthi S. \& A. Fentons; Seneca; Ithaca. May; Aug. Chionanthus, Triosteum perfoliatum.

LYCOPHOTIA Hübner

(Peridroma Hübner)

1489 L. occulta L. Typically a northern species. Fentons; Buffalo; Schenectady; Big Ind. Val.; Rye; SI; LI: Amagansett. Je-Aug; Oct. A cutworm.

1487 L. astricta Morr. A northern species. Mt. Whiteface; Saranac Inn; Fentons; Big Ind. Val. Aug.

1490 L. margaritosa Haw. Variegated Cutworm. The type form and the var. saucia Hbn. are very common generally, northward to Albany and Ithaca. Aug-Nov. A cutworm.

1495 L. infecta Ochs. (incivis Gn.). Rye; SI; LI: Brooklyn; Newtown; Yaphank. Grass.

1502 L. lubricans Gn. Essex Co; Saranac L.; Adirondack Lodge; E. Aurora; Ithaca; New Windsor; SI; LI: Brooklyn. Late Je-Sep. 
EPISILIA Hübner

(Pachnobia Guenée; Choëphora Grote and Robinson)

1474 E. salicarum W1k. Saratoga; Dutchess Co. Ap-May.

1473 E. manifesta Morr. Rye; SI; LI: E. New York; Woodhaven; Yaphank; Babylon. May.

1478 E. fishii Grt. SI, Shoe; LI: Yaphank. Ap.

1471 E. fungorum G. \& R. Buffalo. Sep-Ap.

\section{ONYCHAGROTIS Hampson}

1467 O. rileyana Morr. Lancaster; Buffalo. A cutworm.

\section{EUCOPTOCNEMIS Grote}

1464 E. fimbriaris Gn. (Porosagrotis tripars Wlk.). SI; LI: Amagansett. Sep.

\section{AGROTIS Ochsenheimer}

1415 A. badinodis Grt. Saranac L.; Ithaca; Rye; SI; LI: Brooklyn; Yaphank. Sep-Oct.

1419 A. violaris G. \& R. Rye; SI; LI: Aqueduct; Woodhaven; Yaphank; Calverton. Sep-Oct.

1416 A. geniculata G. \& R. Wilmington; Allegany Co; Ithaca; New Windsor; Fordham; LI: Bellmore; Amagansett; Glencove; Yaphank. Aug-Sep.

1422 A. ypsilon Rott. Greasy Cutworm. Common everywhere. Je-early J1; Aug-Nov. A cutworm.

\section{NoctuA Linnaeus}

1430 N. baja smithii Snell. Generally distributed. Aug-Sep. A cutworm.

1428 N. normaniana Grt. Plattsburg; Keene Val.; Fentons; N. Fair Haven; Otto; Ithaca; McLean; Onteora Mt.; Trenton Falls; Schenectady; Stamford (Ds); Rye (Ds) ; SI; LI: Brooklyn; Newtown. Aug-Sep. A cutworm.

1425 N. bicarnea Gn. General, commoner northward. Late Jl-Sep. A cutworm.

1426 N. treatii Grt. (tennicola Morr. according to B. \& B.). Adirondacks (Sm); LI: Forest Pk. (Doll). Late J1-Aug.

1424 N. c-nigrum L. Spotted Cutworm. Very common everywhere; an important species. Je-Oct.

1443 N. jucunda W1k. (hospitalis Grt.). Peru; Franklin Co; Fentons; Clayton; Evans Center; Ithaca; McLean; Catskills; Karner; Poughkeepsie. J1. Rare.

1444 N. phyllophora Grt. Fentons; Ithaca; U1ster Co; Karner; Albany; Poughkeepsie; Rye; LI: Lynbrook. J1-Aug.

1438 N. rubifera Grt. Mt. Marcy; Franklin Co; Karner. Aug. 
1447 N. oblata Morr. Fentons. Aug. A climbing cutworm on willow.

1446 N. fennica Tausch. Black-LINEd Cutworm. Plattsburg; Fentons; Trenton Falls; Newport. Sep. A cutworm injurious in Canada.

1450 N. juncta Grt. Cranberry L., Htly.

1434 N. plecta L. General to Brooklyn, common northward. May-Sep. A cutworm.

1423 N. collaris G. \& R. Jay; Fentons; Clayton; E. Aurora (Wild) ; Seneca L.; Ithaca; North Branch; Newport; Rhinebeck; LI: Woodhaven. Je; Aug-Sep.

1458 N. haruspica Grt. Peru; Essex Co; Fentons; Clayton; Kendall; Lancaster; Buffalo; Castile, Brad; McLean (Fbs); Trenton Falls; Schenectady; Karner; Albany; Rhinebeck. J1-Aug.

1461 N. clandestina Harr. (unicolor Wlk.). Well-marked Cutworm. Common everywhere, Peru to Buffalo and Gardiners I, LI. Je-J1; Sep-early Oct. A cutworm.

1437 N. cynica Sm. Albany (Sm).

\section{FELTiA Walker 89}

1402 F. ducens W1k. (subgothica Sling. not Haw.). Dingy Cutworm. Very common everywhere; one of the common cutworms. Aug-Sep.

1403 F. jaculifera Gn. Dingy Cutworm. With the preceding species, and even more common. The var. herilis Grt. also is general, but is commoner northward. The form with pale hind wings is probably subgothica Haw., seen from: Ithaca; LI: Yaphank. J1-Sep.

1395 F. gladiaria Morr. Clay-Backed Cutworm. Rochester, Angle; Buffalo; Lancaster; Ithaca; Bangall; New Windsor; Rye; SI; LI: general (Eng). Sep-early Oct.

1397 F. venerabilis W1k. Fentons; Buffalo; E. Aurora; Ithaca; McLean; Newport; Oneonta; Schenectady; Bangall; Rye; SI; LI: Woodhaven (Eng). Common southward.' Sep-early Oct.

1409 F. volubilis Harv. Peru; Clayton; Buffalo; Castile, Je, Brad (Fbs); E. Aurora; Rock City; Ithaca; McLean; Oneonta; Schenectady; Karner; Albany; Rye; LI: Woodhaven. May-Je.

1413 F. annexa Tr. Granulated Cutworm. Franklin Co (McKnight); Ilion; Schenectady; SI; LI: Brooklyn. Common in the Austral zone. Oct.

1414 F. malefida Gn. Shagreened Cutworm. Essex Co; Brooklyn. Probably strays from the South.

\section{CHORIzAgRotis Smith}

1379 C. thanatologia Dyar. Peru, J1 1-7, Mix and Everett.

EUXOA Hübner 90

\section{(Paragrotis Pratt; Carneades Grote)}

1287 E. perpolita Morr. N. Fair Haven; Karner; Debruce (Ds); Big Ind. Val.; Rye; LI: Aqueduct. Aug-Sep.

${ }^{89}$ The species in this genus are all cutworms, and most of them are abundant. The western Rhizagrotis acclivis Morr. was originally described from New York in error.

90 So far as is known, all the species in this genus are cutworms. 
1312 E. fumalis Grt. Essex Co; Rochester, Angle; Ithaca; Sharon; Schenectady; Albany. Originally described from NY. Late J1-Aug.

1286 E. punctigera Wlk. "NY" (Hampson). In error for another black form?

1291 E. velleripennis Grt. Saranac L.; E. Aurora (Wild) ; Ithaca; Schenectady ; New Windsor; LI: Coram (Eng). Late Aug-Sep.

1486 E. scandens Rly. White Cutworm. Plattsburg; Peru; Essex Co; North Creek; Franklin Co; Little Falls; Evans Center; Schenectady; Albany. J1. A cutworm, sometimes climbing.

1272 E. detersa W1k. (pitychrous Grt.). Plattsburg; Peru; Fentons; Buffalo; Schenectady; Karner; Albany; SI; general on LI. This species favors sandy country. Late Aug-Oct.

1272b E. azif Strk. "NY." Another Strecker name, considered by B. \& McD. a variety of detersa.

1318 E. bostoniensis Grt. Rochester, Angle; Buffalo; Ithaca; Karner; SI; LI: Wyandach, Scheveyer (Eng). Sep-Oct.

1304 E. messoria Harr. DARK-Sided CUtworm. This and the following four species are not certainly determinable without dissection, and the records are therefore tentative. Typical messoria is generally common, and an important cutworm. J1-Sep.

1336 E. pleuritica Grt. "NY", type.

1304 E. friabilis Grt. Fentons, Hill (Lint).

1338 E. decolor Morr. (insulsa auct., verticalis Grt.). Karner; Schenectady; LI: Brooklyn.

1341 E. albipennis Grt. Fentons; Karner; LI: Newtown.

1329 E. tessellata Harr. STRIPed Cutworm. Common everywhere; usually recognizable by the yellow shoulders. Je-early Sep.

1363 E. ochrogaster Gn. Red-Backed Cutworm. Peru; Saranac L.; Fentons; Saratoga; LI: Mineola (NYS). This species is extremely variable and I have seen most of its forms from the State. Aug-Sep.

1356 E. obeliscoides Gn. New Russia; Peru; E. Aurora (Wild); Ithaca; Sharon; Schenectady; Albany; Coeymans; Sullivan Co; Rhinebeck; Bronxville; LI. J1-Sep.

1353 E. divergens Wlk. Peru; Ithaca; Liberty. J1-Aug.

1357 E. redimicula Morr. Generally distributed, apparently commoner northward. J1-Aug.

POROSAGROTIS Smith

1234 P. vetusta W1k. Spotted-LegGed Cutworm. Plattsburg; Peru; Franklin Co; Rochester; Schenectady; Albany; general on LI. Typically associated with sandy country. A cutworm. Aug-Sep.

1303 P. mimallonis Grt. Peru; Fentons; Karner. Aug.

\section{EUPANYCHIS Grote}

1195 E. spinosae Gn. Rye; SI; LI: Amagansett. Late Aug-Sep.

SCHINIA Hübner

1152 S. trifascia Hbn. Ithaca; Rhinebeck; Poughkeepsie; Rye; SI; LI: Amagansett. J1-Aug.

1163 S. nundina Dru. Gloversville; Rhinebeck; New Windsor; Rye (Bird); Hastings (Joutel); SI; LI: Brooklyn; Pinelawn (Ds); Yaphank. Late J1-Aug. On flowers of Solidago canadensis (Bird). 
1180 S. lynx Gn. Ithaca; Karner; Poughkeepsie; Rye (Bird); Ramapo, Nyack (Ds) ; SI; LI: E. Quogue (Ds) ; Orient, Lath; Amagansett, May-Aug.

1192 S. arcigera Gn. Lancaster; Ithaca; Rhinebeck; NYC; SI; general on LI. Aug-Sep. On flowers of Aster puniceus (Bird).

\section{LYGRANTHOECIA Grote and Robinson}

1127 L. thoreaui G. \& R. Rye; SI; LI: Brooklyn. Originally described from NY. Aug-Sep.

1130 L. marginata Haw. Rochester, Angle; Batavia; E. Aurora; Castile; Ithaca; Sharon; Hebron, Wls; Schenectady and southward. Var. constricta H. Edw. was taken at New Windsor; SI (Ds). J1-Sep. In flower head of Ambrosia elatior (Bird).

1122 L. brevis Grt. Karner; Mosholu; Rye (Bird); NYC; SI; general on LI. Aug 28-Sep 4. In flower head of Aster (Bird).

1120 L. obscurata Strk. Rye, J1 10 (Bird).

\section{RHODOPHORA Guenée}

1098 R. florida Gn. Evening Primrose Moth. Common and general in flowers of Oenothera, the larvae eating the seed pods. Je-Aug.

\section{ChLoridea Westwood}

1091 C. virescens Fab. Tовассо Budworm. Rye; SI; LI: Amagansett. Larvae on flowers of tobacco and geranium (CU).

\section{HELIOTHIS Ochsenheimer 91}

1090 H. obsoleta Fab. (armiger Hbn.). ConN-EAR Worm. Vic. of Buffalo and of NYC, and general and common on LI; in favorable years migrating northward as far as Watertown, Lowville, Gloversville, and Glens Falls. Je-J1. An important fruit feeder, frequently injurious to corn and tomatoes, and, in the South, to cotton.

\section{MELANOPORPHYRIA Grote}

1076 M. immortua Grt. Karner; Albany. Je.

\section{Heliophana Grote}

1054 H. bina Gn. "NY” (Lint).

\section{HeLiolonche Grote}

1052 H. modicella Grt. There is a specimen of this, labeled "West Farms," in the Angus Collection, probably in error.

${ }^{91} \mathrm{H}$. phlogophagus var. luteitinctus Grt. is credited to NY by Gerhard, doubtless in error. Larva on Grindelia. 


\section{FAMILY AGARISTIDAE}

\section{ALYPIA Hübner}

1042 A. octomaculata Fab. Eight-Spotted Forester. Saranac L. (possibly an error for males of the next species). Alder Creek; Fabius; Utica; Stanley; Geneva; Rochester, Angle; Buffalo; E. Aurora; Castile, Brad; Portage; Crosby; Ithaca; McLean; Oneonta; Gloversville; Schenectady; W. Troy; Albany; N. Nassau; West Pt. (Ds) ; Rye; Mosholu; NYC (Wat); SI; LI: Brooklyn, very common. Je-Aug (Ap-Aug on LI). Grape.

1046 A. langtoni Couper. Saranac L. (McKnight); N. Elba (Wat.) ; Mt. Whiteface (Shoe); Buffalo (EPV, probably in error for a variety of the preceding). Jl-Aug. Epilobium.

\section{FAMILy ARCTIIDAE 92}

\section{HAPLOA Hübner}

1031 H. clymene Brown (interruptomarginata DeB.). Buffalo; S. Wales (Wild); Lancaster; Ithaca; Bath; SI; LI: Richmond Hill. J1-early Aug. Low plants.

1032 H. colona Hbn. (clymene auct.). Buffalo (Grt). A general feeder.

H. colona var. reversa Stretch. Strawberry Tigfr Moth. Poughkeepsie (Dyar).

H. colona var. fulvicosta Clem. "NY" (Sm). A general feeder, rarely injurious to peach.

$\mathrm{H}$. colona var. triangularis Sm. Plattsburg.

1033 H. lecontei Bdv. Plattsburg; Keene Val.; Ilion; Buffalo. Cynoglossum.

H. lecontei var. militaris Harr. Fentons; Oneonta; Buffalo.

H. lecontei var. smithii Dyar. "NY", types.

$\mathrm{H}$. lecontei var. vestalis Pack. "NY" $(\mathrm{Sm})$.

1034 H. confusa Lyman. Plattsburg; Keene Val. ; Geneva; Lancaster; Salamanca, E. Aurora, S. Wales (Wild); Ithaca; McLean; Binghamton; Onteora Mt.; Trenton; Schoharie; Poughkeepsie. J1-Aug.

H. confusa var. lymani Dyar. Plattsburg; Ithaca; Poughkeepsie. J1.

1035 H. contigua Wlk. Buffalo; Schoharie; Rogers Rock, L. George, Jones; Ft. Montgomery, Jl (Ds).

\section{UTETHEISA Hübner}

1029 U. ornatrix bella L. Rattlebox Moth. Ausable Chasm; Buffalo; Castile, Brad; Ithaca (perhaps a stray); Gloversville (Alex); Albany; Poughkeepsie; Crugers; general southward. Var. nova Gross. is known from Rockaway Beach, LI. Je-Nov, two broods. Crotalaria; reported also from Lespedeza and Myrica.

92 The species in the family Arctiidae, except in the genera Haploa, Utetheisa, Hyphantria, Ammalo, Euchaetiás, and Halysidota, are general feeders. 


\section{ARCTIA Schrank}

1025 A. caja americana Harr. American Tiger Moth. Peru; Black Br. (larva); Essex Co; Allegany Pk. (Wild); Lockport (NYS); Canaseraga; Ithaca; McLean; Elmira; Catskills; Trenton Falls; Canajoharie; Gloversville (Alex); Sharon; Albany. J1-Aug.

\section{EUCHAETIAS Lyman}

1015 E. egle Dru. Milkweed Tussock Motн. Castile, Je, Brad (Fbs); Clayton; Kissena L.; Rochester, Angle; Buffalo; Otto; Castile, Brad; Ithaca; Newport; Oneonta; Little Falls; Coeymans; Albany; Poughkeepsie; Rye; Katonah; Kinderhook; Van Cort. Pk. (Eng); SI (Ds); LI: Lynbrook. Late Je-Aug. Milkweed.

1018 E. oregonensis Stretch. Peru; Severance; Ithaca (one specimen, slightly doubtful); Saratoga; Karner; Albany. May-Jl.

\section{APANTESIS Walker}

971 A. virgo L. Everywhere. Representative records are: Peru; Essex Co; Fentons; Clayton; Buffalo; E. Aurora; Allegany Pk.; McLean (Fbs); Ithaca; Sullivan Co; Ulster Co; Sharon; Oneonta; Gloversville; Johnstown; Albany; Rhinebeck; Poughkeepsie; Rye; SI. J1-Aug.

982 A. virguncula Kby. Plattsburg; Peru; Saranac L.; Clayton; Old Forge; Aurora; Geneva; Brockport; Lancaster; Buffalo; De Bruce; Utica; Big Ind. Val.; Sharon Sps.; Pittstown (NYS) ; Poughkeepsie; Rye;

LI: Newtown (Doll). Var. otiosa N. \& D. occurs at Plattsburg. J1.

973.1 A. circa Stk. (intermedia auct.). Allegany Co; Ithaca; Poughkeepsie. Sep.

973 A. parthenice Kby. Plattsburg; Peru; New Russia; Wilmington; Mt. Marcy; Fentons; Waddington; Buffalo; E. Aurora; Ithaca; McLean; Binghamton; Big Ind. Val.; Onteora Mt.; Schenectady. Aug-Sep. Larva partly carnivorous, eating other Lepidoptera.

974 A. oithona Strk. var. rectilinea Fr. LI: E. New York; Woodhaven.

979 A. anna Grt. Northern NY; Ithaca (male); Albany; New Windsor; LI. Typical specimens of var. persephone Grt. have been seen from: Ithaca; Utica; Ulster Co; Newburgh. Late Je-J1; Sep.

977 A. arge Dru. Plattsburg; Essex Co; Rochester; Brockport; Buffalo; Ithaca; Binghamton; Oneonta; Albany; New Windsor; Poughkeepsie; Rye; general southward. Var. nervosa N. \& D. occurs in the spring brood at Ithaca and on LI. Ap-Je; Aug-Sep.

989 A. phyllira Dru. Mt. Kisco; Rye; LI: Wyandanch, Sf. Var. excelsa Neum. was taken at LI: Rockaway Beach. May-Je.

991 A. celia Saund. Plattsburg; West Pt., Je (Ds).

990 A. figurata Dru. Lancaster; LI: Brentwood.

A. figurata form snowi Grt. LI: Bellport, Nic.

995 A. nais Dru. Ithaca; McLean; Albany; Rhinebeck; Poughkeepsie; Huguenot; Rye; Katonah; SI. Reported from Buffalo and Newburgh, but there is a little danger of confusion with forms of vittata. The species is not well understood. May-Aug.

996 A. vittata Fab. (phalerata Harr.). Harnessed Moth. Seen from N. Fair Haven; Rochester; Buffalo; E. Aurora; Potter Swamp; Poughkeepsie; Newburgh; Katonah; SI. Je-Sep. 


\section{ECPANTHERIA Hübner}

964 E. deflorata Fab. (scribonia Stoll). Great Leopard Moth. Buffalo; Lancaster; Oneonta; Rhinebeck; Poughkeepsie. Common about NYC.

\section{ESTIGMENE Hübner}

960 E. acraea Dru. Salt-Marsh Caterpillar. Common everywhere. Je-J1; Aug. Two broods.

E. acraea var. dubia Wlk. Ithaca, J1 1, 1915.

962 E. prima Sloss. Plattsburg; Chateaugay L.; LI: Pine Haven. May.

963 E. congrua Wlk. (antigone Strk.). Keene Val.; Brockport; Rock City; Ithaca; McLean; Catskills; Schenectady; Albany; Rhinebeck; Poughkeepsie; New Windsor; Sİ; LI: Bellport. Je-early J1.

\section{Hyphantria Harris: The Fall Webworms}

959 H. cunea Dru. Verified records are: Peru (one white specimen); Buffalo; E. Aurora, Wild; Ithaca; Utica; Albany; Florida; Yorktown; Rhinebeck; SI; LI: Brooklyn; Oyster Bay. More southern in dominance than textor, which may be a race of it. Var. pallida Pck. was described from "NY". Ap-May; Jl. Two broods. General on trees, sometimes injurious to apple.

958 H. textor Harr. Seen from Bronxville; SI; LI: Greenport. Reported also from: Fentons; Buffalo; Angola; Albany; LI: Brooklyn; Orient; but part of these latter records may be based on white specimens of $H$. cunea. Larva like the preceding. Je-J1. Normally one brood.

\section{ISIA Walker}

957 I. isabella S. \& A. Black-tipped BeAR. Common wherever collections have been made. Je-Aug.

\section{DIACRISIA Hübner}

954 D. virginica Fab. Yellow BeAR. Very common everywhere. May-Sep.

953 D. latipennis Stretch. Brockport; Buffalo; Potter Swamp; Ithaca; McLean; Newport; Karner; Albany; Big Ind. Val.; New Windsor; Poughkeepsie; Ft. Montgomery, Nyack (Ds); SI; LI: Flushing; Gardiners I. May-early J1.

\section{Phragmatobia Stephens}

948 P. fuliginosa L. Common everywhere. May-Aug.

949 P. assimilans W1k. Plattsburg; Hudson; Ithaca (one specimen of var. franconica) ; Big Ind. Val. May. The type (Doubleday Coll.) was presumably from Trenton Falls.

\section{HyPHORAIA Hübner}

943 H. hyperborea Curt. Catskills (Pears).

942 H. parthenos Harr. Saranac; Mt. Marcy; Mt. Whiteface; Schoharie; Catskills. Je. 


\section{EUBAPHE Hübner}

931 E. laeta treatii Grt. Ithaca; LI: Yaphank. J1.

937 E. opella Grt. E. Aurora; Ithaca; Poughkeepsie; LI: Bellport; Brookhaven; Yaphank. Je-Aug.

E. opella var. nigricans Reak. West Pt., not uncommon (Ds) ; LI: Wading R. (Ds).

934 E. aurantiaca Hbn. I am quite unable to sort out the various so-called forms of aurantiaca and immaculata Reak., which together cover the State. Specimens from Ithaca and the Adirondacks generally approach immaculata, while those from near NYC are more typical of aurantiaca. I have seen var. quinaria Grt. from Newcomb, Adirondack Lodge, and Big Ind. Val., and it is general, but relatively rare. Form trimaculosa Reak. is reported from Ft. Montgomery (Wat). Je-Aug.

\section{HaLysidota Hübner}

921 H. tessellaris S. \& A. Banded Tussock Mотн. Many of the following records are based on the imago, and may belong to the next species: Peru; Essex Co; Adirondack Lodge; Fentons; Saranac L. ; North Cr.; Clayton; Rochester; Honeoye Falls; Buffalo; E. Aurora; Allegany Pk.; Castile; Ithaca ; McLean; Newport; Oneonta ; Oliverea; Utica ; Little Falls; Schenectady; Albany; Rhinebeck; Poughkeepsie; Rye; Florida. Late Je-Aug. Maple and many other trees.

$922 \mathrm{H}$. harrisii Walsh. The following records have been verified by the larvae: Ithaca (CU); Ulster Co and Poughkeepsie (Dyar). Sycamore only.

918 H. maculata Harr. Sроттеd Tussock Mотн. Plattsburg; Peru; Wilmington (larvae); Fentons; Elizabethtown; Rochester; Castile; E. Aurora; Batavia; Buffalo; Lancaster; Ithaca; Big Ind. Val.; Oneonta; Gloversville; Little Falls; Albany; Rye; commoner southward. Je-J1. Apple and other trees.

915 H. caryae Harr. Hickory Tussock Motн. Common throughout the State. Je-J1. General on trees and shrubs.

\section{AMMALO Walker \\ (Tanada Walker)}

905 A. tenera Hbn. (collaris Fitch). Clayton; Buffalo; Rochester; Irving (Wild) ; Ithaca; Oneonta; Karner; Poughkeepsie; Katonah; Rye ; Bronxville; Astoria; Van Cort. Pk. (Wat); SI: LI: Brooklyn. Jeearly Aug. Apocynum.

907 A. inopinatus H. Edw. (eglenensis auct.). Big Ind. Val.; SI; LI, to Yaphank. The type occurs near NYC; the other records are based on the gray form that passes for eglenensis. J1-Aug. Milkweed.

\section{FAMILY LITHOSIIDAE}

\section{HYPOPREPIA Hübner}

899 H. miniata Kby. General. J1-Aug.

$900 \mathrm{H}$. fucosa Hbn. General with the preceding species, and commoner as a whole. J1-Aug. Lichens. 
Clemensia Packard

892 C. albata Pack. N. Elba; Saranac L.; S. Wales (Wild); Buffalo; Otto; E. Aurora $(W i l d)$; Ithaca; Big Ind. Val.; Onteora Mt.; Rhinebeck. Je-J1.

Ozodania Dyar ${ }^{93}$

881 O. tenuifascia Harv.? (unifascia Hamp.). "NY" (Hampson).

884 O. injecta Dyar. Ithaca, J1 14, 1892 (CU).

\section{CRAmbidia Packard}

856 C. pallida Pck. Plattsburg; Ithaca; McLean; Poughkeepsie. Late J1-early Sep.

860 C. casta Sanb. Ausable Chasm; Wilmington; Saranac L.; Fentons; LI: Yaphank. Aug-Sep.

\section{LEXIS Wallengren}

855 L. bicolor Grt. Plattsburg; Ithaca. Aug.

\section{FAMILY NOLIDAE}

ROESELIA Hübner

851 R. minuscula Zell. Keene (Wild); Buffalo (Wild) ; Ithaca; SI; LI: Long Island City; Lynbrook; Bellport; Cold Sp., Kch (Fbs). Our records seem to belong to var. phylla Dyar. May-J1.

\section{Nigetia Walker}

850 N. formosalis Wlk. Lancaster; Buffalo; Rhinebeck. Flies in Virginia in Je.

\section{NolA Leach}

847 N. clethrae Dyar. Castile, Je, Brad (Fbs); LI: Bellport (type), Brookhaven, South Haven, Quogue (Dyar). Clethra.

846 N. ovilla Grt. Plattsburg; SI; LI: Aqueduct (Ds). I am not sure what this species is (Fbs). Je. Oak.

\section{Celama Walker}

844 C. triquetrana Fitch. E. Aurora; West Falls; Portage; Ithaca; Dutchess Co; Pearl R.; Bronx; SI; LI: Wading R.; Yaphank. Late Ap-Je. Witch-hazel.

C. pustulata Wlk. Rhinebeck; Poughkeepsie.

842

C. cilicoides Grt. Ithaca, J1; McLean (Fbs).

${ }^{93}$ The larvae of this genus feed on lichens. 


\section{FAMILY EUCHROMIIDAE \\ (Syntomidae, Zygaenidae) \\ CTENucha Kirby}

838 C. virginica Charp. General, southward to Hunter, Big Ind. Val., Greene Co, and Ft. Montgomery. It should occur in the rest of the State, but I have not seen it. Late May-early Jl. Grass.

\section{LYCOMORPHA Harris}

831 L. pholus Dr. Wilmington; Fentons; Clayton; Clifton Sps.; Niagara Falls; Buffalo; Allegany Pk.; Hemlock L.; Ithaca; Hope; Big Ind. Val.; L. George; Schoharie; Gloversville (Alex); Pine I.; SI; LI: Woodhaven. J1-Aug. Lichens.

\section{SCEPSIS Walker}

824 S. fulvicollis Hbn. Peru; Fentons; Newport; Rochester; Honeoye Falls; Batavia; Lancaster; Protection; Ithaca; McLean; Schoharie; Albany; and southward. Je-early Sep. Grass.

\section{SUBORDER RHOPALOCERA \\ FAMILY HESPERIIDAE SUBFAMILY HESPERIINAE}

\section{EUdAMUS Swainson}

458 E. proteus L. Bean Leaf Roller. Central Pk. in NYC (Eng); LI: Brooklyn. Rarely breeding. Aug; late Sep-early spring. Legumes, rarely Cruciferae; in the South injurious to beans.

\section{EPARGYREUS Hübner}

468 E. tityrus Fab. Silver-Spotted Skipper. Everywhere, northward to Clayton and Keene Val. at least; LI: generally distributed and very common; Oakdale, Je 12; Hempstead, J1 3; Flushing, May 8, Aug 2, Sep 16; Coram, Sep 4; (all Bell). Je-Ji. Locust and other Leguminosae.

\section{RHABDOIDES Scudder}

473 R. cellus Bdv.-Lec. LI: Brooklyn, presumably a stray. Breweria aquatica.

\section{ACHALARUS Scudder}

472 A. lycidas S. \& A. Syracuse; Rochester (Angle) ; Portage; Karner; Greenwood L.; Riverdale (NYS) ; SI, uncommon forty years ago, but has gradually increased in numbers (Ds) ; LI: Flushing, Je 26 (G.L.Standfast); Jamaica, Je 18, '25 (Bell). May-J1. Meibomia paniculata (preferred) and Lespedeza (Wat). 


\section{THORYBES Scudder}

485 T. daunus Cr. (bathyllus S. \& A.). Katonah; Bronxville; SI; LI, well distributed and common: Coram, May 22, Jamaica, Je 4, Oakdale, J1 4 (Bell). Reported also from Keene Val., Not, Deposit and Binghamton (Blackburn), Oneonta, and Schoharie, but I have not seen the specimens, which may be heavily marked specimens of pylades. Je-early J1. Lespedeza capitata (preferred) and other legumes (Wat).

\section{Cocceius Godman and Salvin}

483 C. pylades Scud. Common everywhere. LI: Wyandanch, May 28, Jamaica, Je 4, Oakdale, Je 18 (Bell). Je; a partial brood in Aug. Clover and other legumes.

\section{PHOLISORA Scudder}

512 P. catullus Fab. Rochester, fairly common (Angle); Honeoye Falls; Buffalo; Crosby; Ithaca; Binghamton (Blackburn); Ulster Co; Ft. Edward; Karner; Albany; Bethlehem; Tappan; NYC; SI; LI, usually common: Flushing, May 26, Je 16, Aug 3, Jamaica, J1 16, Hempstead, Sep 5 (Bell); Lynbrook. Mid May-mid Je; Aug. Chenopodium album and Amaranthus (Wat).

\section{ThanAos Boisduval}

518 T. brizo Bdv.-Lec. Jamestown (EPV) ; Karner; Sloatsburg, Not; Bronxville; SI; LI, common locally: Central Pk., Ap 29, May 28, Coram, May 15 (Bell); Yaphank. May-Je. Oak.

517 T. icelus Lint. Black Br.; Keene Val., Not; Clayton; Gowanda; Rock City; Egleston's Glen; Crosby; Taughannock Falls; Ithaca; Karner ; Schoharie; Gloversville (Alex); Windsor, Not; Rhinebeck; Bronxville; SI; LI, generally distributed and locally common: Massaquogue; Smithtown; Flushing, May 8; Central Pk.; Coram; Oakdale (all Bell). Je. Willow and poplar (Wat).

520 T. lucilius Lint. Axton; Keene Val (NYS) ; Rochester, Conesus L. (Angle) ; Buffalo (EPV) ; Crosby; Ithaca; Hunter; Schoharie; Karner ; Bethlehem; Ramapo Mts.; Croton-on-Hudson (Ds); LI: Jamaica, May 28; Central Pk., May 29 (Bell) ; Coram, Je 15 (Eng); Flatbush. May; mid J1-mid Aug; occasional in Sep. Columbine.

521 T. persius Scud. Gloversville (Alex) ; Portage (EPV) ; E. Aurora, Wild; Rensselaer (Lint); Karner; SI: Watchogue; LI, locally common: Coram, May 13, Sep 2, Jamaica, Je 4, Flushing, J1 27 (Bell) ; Hempstead Plain, Aug 8 (Eng); Amagansett. I am not sure that any of these records belong to the true $T$. persius Scud. (1892), but they would be included in Scudder's later conception of the species (Fbs). May-Sep. Baptisia tinctoria (Wat); reported also from poplar and willow.

524 T. martialis Scud. Clayton; Rochester; E. Aurora, Dnd, Wild; Buffalo, Niagara Falls (Wild); Egleston's Glen; Ithaca; Raven Pass; Kaaterskill; Karner; Albany; Glen (Meske); Bethlehem, West Pt., Crotonon-Hudson, Ramapo, and SI (Ds); LI: Flatbush. Late May-Je; late J1-Aug. Red-root. Reported also from wild indigo.

T. martialis ab. ausonius Lint. Karner (type). 
525 T. juvenalis Fab. Clayton; Rochester, Conesus L. (Angle); Buffalo; Portage ; Rock City ; Crosby ; Ithaca ; McLean; Schoharie ; Karner ; Rensselaer; Sloatsburg, Not; Mosholu; Bronxville; SI; LI, generally distributed and very common in the pine barrens: Central Pk., May 3, Oakdale, Je 17, Flushing, May 8 (Bell) ; Oyster Bay ; Smithtown; Yaphank. May-early Je in central NY, and May-Oct, with two broods, on SI. Oak, less commonly legumes (Wat).

527 T. horatius Scud. \& Burg. Ramapo Mts.; Bronxville; SI; LI, generally distributed: Central Pk., Ap 19, May 3, Flushing, J1 12, Aug 15, Sep 29, Kings Pk., J1 26 (Bell); Amagansett; Wading R. May-Oct, two broods. Pin and other oaks, less commonly legumes.

\section{HESPERIA Fabricius}

495 H. tessellata Scud. (Pyrgus montivaga auct.). Pittsford, Boughton; Rochester (Angle); E. Aurora, Dnd, Wild; Hamburg, first record 1910 (Wild) ; Castile, Brad; Ithaca (abundant) ; SI; LI, a southern visitant, at times locally common, J1-Aug: Maspeth, Aug, 1898; Flatbush. Not known to hibernate on LI (Eng). J1-Sep, rarely Ap. Sida spinosa (Wat).

488 H. centaureae Ramb. E. Aurora, Dnd (Angle); Ramapo Mts ; LI (Calverly teste Edwards).

\section{SUBFAMILY PAMPHILINAE}

Carterocephalus Lederer

\section{(Pamphila)}

536 C. palaemon mandan Edw. Black Br.; Camp No. 3, Paul Smiths, Babiy; Lewis Co, Hill; not uncommon in the N. Elba region (div.); McLean. May-Aug. Females observed ovipositing on grass (Wat \& Coleman).

\section{ANCYLOXIPHA Felder}

544 A. numitor Fab. Common mostly in wet places. Definite records are: Honeoye; E. Aurora; Geneva; Lancaster; Castile; Ithaca; Karner ; Onteora Mt.; Bethlehem; Castleton; Coeymans; Rhinebeck; Poughkeepsie; Pine I.; SI; LI, generally distributed and very common in grassy fields: Flushing, May 28, Aug 2, Sep 10, Oct 1, Central Pk., Je 5, Farmingdale, J1 3, Oakdale, J1 4 (Bel1) ; Lynbrook; Gardiners I. (Ds). The absence of northern records may be accidental. Je-Sep, three broods. Grasses.

\section{PAMPHILA Fabricius}

(Erynnis etc.)

556 P. metea Scud. Karner, Hill (Fbs); Croton-on-Hudsòn (Ds); SI (Ds, Wainwright); LI, not common; Coram, May 13, Wyandanch, May 14 (Bell) ; Massapequa, May 23, Wading R., May 29 (Ds); Yaphank. May-Je. Grass.

[565a P. attalus seminole Scud. is not rare from Mass. to Fla., and therefore it must certainly occur in the State. J1.] 
566 P. leonardus Harr. Plattsburg; Keene Val., Not; Fentons; Alexandria Bay; Clayton; Catskills; Gloversville (Alex); Windsor, Not; Karner;

SI; LI, in the pine barrens, locally common: Coram, Sep 1 (Bell),

Sep 18 (Eng); Yaphank, Amagansett, Sep (Ds). Late Aug-Sep. Grass.

570 P. sassacus Harr. Mt. Whiteface (Ds); Peru; Axton (NYS) ; Rochester (Angle); Niagara Falls (Wild); Buffalo (EPV); Chipmunk Swamp, Vandalia; Rock City; Castile; Portage; McLean; Karner; Bethlehem (Lint) ; Oliverea (Ds); W. Hebron (Lg); Ft. Montgomery (Ds) ; Rhinebeck; Ramapo (Ds); SI; LI, local, common in the pine barrens: Coram, May 22, Jamaica, Je 5, Central Pk., May 29, Je 5 (Bell) ; Yaphank. Je-J1 in northern NY; May-Je in southern NY. Grass.

\section{HYLEPHILA Billberg}

571 H. phylaeus Dru. Katonah; NYC, var. locs. (div.) ; SI; LI: Brooklyn, J1-Aug (div.); Canarsie, in the fall (Doll); Flushing, J1 26, Aug 29, Sep 2 (Bell). Grass.

\section{Polites Scudder}

576 P. cernes Bdv.-Lec. (Limochores taumas). Common wherever looked for. LI, well distributed and very common: Sands Pt., May 21, Central Pk., Je 5, Flushing, J1 23, Aug 16, Oakdale, Je 12 (Bell); Gardiners I. (Ds). Je-Sep, two broods. Grass (Panicum, etc.).

577 P. manataaqua Scud. Rochester (Angle); Gowanda (Wild) ; Oliverea, E. Jewett (Ds) ; Albany, Karner, Bethlehem (Lint) ; Oneonta (Cleveland) ; Katonah; Van Cort. Pk.; SI; LI, locally distributed and usually not very common: Oakdale, J1 1, Hempstead, J1 3, Kings Pk., J1 27, Coram, Sep 5 (Bell) ; Wading R. J1-Aug. Grass.

581 P. mystic Scud. Mt. Whiteface (Ds); Axton; Fentons; Potsdam; Rochester (Angle) ; E. Aurora (Wild) ; Lancaster; Rock City; Castile, Brad; Portage; Ithaca; Rensselaer; Liberty; Oliverea, E. Jewett (Ds); Karner; Bethlehem; Ramapo Mts.; SI; LI, sometimes common locally: Oakdale, Je 12, J1 1 (Bell) ; Mt. Sinai Harb., Sep 3 (Eng) ; Gardiners I., Je, 1911 (Ds). Je and mid J1-Aug. Grass.

583 P. peckius Kby. Common wherever looked for. LI, usually very common: Sands Pt., May 21, Central Pk., Je 5, Flushing, J1 23, Aug 2, Sep 16 (Bell) ; Gardiners I. (Ds). Je-Aug. Grass.

587 P. brettus Bdv. LI, a stray (BM).

\section{AtAlopedes Scudder}

589 A. campestris Bdv. Ithaca; Van Cort. Pk., Blackburn. Strays. J1-Aug. Cynodon.

\section{CATIA Godman and Salvin}

590 C. otho egeremet Scud. Common southward. Keene Val., Wild; Clayton; Rochester, Angle; E. Aurora, Dnd, Wild; Ithaca, rare; Sharon Sps.; Karner; Bethlehem; SI; LI, usually common: Jamaica, Je 18, Flushing, J1 9, Oakdale, J1 10 (Bell); Wading R.; Gardiners I. (Ds). Aug. Panicum. 


\section{POANES Scudder}

591 P. massasoit Scud. Karner; Bethlehem (Lint) ; Orange Co; Tappan, J1-Aug, common (Wat and Friedle); Van Cort. and Bronx Pks. (Wat and W.P.Comstock) ; SI, J1-Aug (Ds); LI, local and not common, usually found in swampy locations: Flushing, J1 9, 1923 (Bell); Wyandanch, Aug 3 (Ds); Riverhead, Aug (Ds). Grass.

592 P. hobomok Harr. Everywhere. LI, generally distributed and very common: Sands Pt., May 21, Jamaica, Je 4, Coram, May 22, Oakdale, Je 19 (Bel1) ; Lynbrook, Je. Grass.

P. hobomok ab. pallida Wat. Dunwoodie, May 21 (type).

P. hobomok form pocahontas Scud. Plattsburg; Clayton; Buffalo (Wild); Castile, Brad; Windsor, Not; Ithaca; Freeeville; Greene Co; SI; LI: Central Pk., May 29, Je 5, Oakdale, Je 12, Jamaica, Je 18 (all Bel1).

P. hobomok form pocahontas ab. friedlei Wat. Van Cort. Pk., Sep 10 (type).

593 P. zabulon Bdv.-Lec. Washington Hgts., NYC (Wat and Friedle); SI; LI: Flushing, Aug 19, 26, 1916 (Bell). Practically all the published records for zabulon belong to hobomok. May-Oct. Grass.

\section{Atrytone Scudder}

597 A. logan Edw. (delaware Edw.). Bethlehem, Karner (Lint); northern Viestchester Co (W.C.Wood) ; Croton-on-Hudson, Ramapo (Ds). J1early Aug. Erianthus alopecuroides.

\section{PARATRYTONE Godman and Salvin}

602 P. aaroni Skin. Reported from Brooklyn, probably in error. Je; Aug. Grass in salt marshes.

\section{PHyCANASSA Scudder}

600 P. viator Edw. Mt. Marcy; Batavia; western NY, fairly common (Angle); SI; LI, locally distributed; often very common where it occurs: Flushing, J1 16 and 30, Aug 7 (Bell); Jamaica, Aug 11 (Eng); Aqueduct. J1. Grass, most often in salt marshes.

\section{EUPHYES Scudder}

604 E. vestris metacomet Harr. Newcomb; North Cr.; Fentons; Rochester (Angle) ; E. Aurora, Allegany Pk. (Wild) ; Newport; Clayton; Ithaca; McLean (Fbs) ; Schoharie; E. Jewett (Ds); Shelving Rock, L. George; Bethlehem; Beaver Kill; Ft. Montgomery, Croton-on-Hudson (Ds) ; SI; LI, generally distributed and common: Jamaica, Je 25, Oakdale, J1 4, Flushing, J1 23 (Bell); Coram, Aug 4 (Eng); Wading R. J1-Aug. Grass.

605 E. bimacula G. \& R. Michigan Swamp, Ithaca, Je 22 (Fbs) ; Karner; Cedar Hill. Reported also from: Bethlehem, Sharon Sps., Lint; Waterville, Not; Lancaster; Colden. Je-J1. Grass.

609 E. dion Edw. Batavia. J1; Sep. Grass. 
610 E. conspicua Edw. (pontiac Edw.). Buffalo; western NY, fairly common (Angle) ; Gloversville (Alex); Buffalo; Bronxville; SI; LI, common in swampy locations: Flushing, J1 2 and 23; Jamaica, J1 16 (Bell); Flatbush; Aqueduct. J1. Grass.

\section{ATRYTONOPSis Godman and Salvin}

613 A. verna Edw. Ithaca ? (female only) ; Sharon Sps.; Karner; Bethlehem; Katonah; Bronxville; Ramapo; Croton-on-Hudson; SI; LI, well distributed and fairly common: Jamaica, Je 18, Oakdale, Je 19, Kings Pk., J1 30 (Bell). Je-Aug. Grass.

615 A. hianna Scud. Karner (Fbs); Bethlehem (Lint); LI, apparently very scarce: Central Pk., May 29, 1921, Oakdale, Je 22 (Bell). End of May-Je. Grass.

\section{AMBLySCIRTES Scudder}

626 A. vialis Edw. Axton; Keene (Wild) and Keene Val., Not; Saranac L., Not; Bethlehem (Lint); Karner; Rhinebeck; Yonkers; LI, local in distribution and not very common: Coram, May 13, Je 10 (Bell); Wading R., May 31 (Ds). Je-Aug. Grasses (Avena, Poa).

635 A. hegon Scud. (samoset Scud.). Axton, Keene Val. (NYS); Windsor, Not; McLean; Poughkeepsie; Sloatsburg (div.). Late May-J1. Sorghum (Abbot).

Megistias Godman and Salvin

641 M. fusca G. \& R. (Stomyles, Oligoria). Van Cort. Pk. (Wat); SI, Je, rare (Ds); LI, apparently scarce: Rockaway Beach, Je 27 (Shoe). Je; Aug-Sep. Grass.

LEREMA Scudder

642 L. accius S. \& A. LI: New Lots, 1863 (Grote).

LERODEA Scudder

(Oligoria Scudder)

646 L. maculata Edw. Karner. J1.

\section{CALPodes Hübner}

647 C. ethlius Cr. Canna Skipper. Albany, 1893 (Bt) ; West Farms (according to Holland's Butterfly Book) ; LI, not indigenous, its occurrence in NY probably accidental; Bell states: "The first specimen was taken by Engelhardt at Yaphank, May 30, 1911, and the species was afterwards taken by a number of other collectors at various places, but did not survive the winter. Brooklyn, Sep 30, 1911; Middle Village, Oct 1911"; Maspeth; Floral Pk.; Glendale; Yaphank. Canna.

\section{PRENES Scudder}

653 P. ocola Edw. SI: Annadale, Sep, 1896, Capt. R. D. Wainwright (Ds). 


\section{FAMILY PAPILIONIDAE}

\section{LAËRTIAS Hübner}

1 L. philenor L. GReEN Swallow-TAIL. E. Aurora, Allegany Pk., strays (Wild) ; Ithaca, a stray; Tappan; Tarrytown (NYS); SI; LI, local, but often common: Brooklyn; Flushing, Sep 16 (Bell), J1 and Sep, two broods (Eng). A short-tailed var. similar to acauda, which is Mexican, occurs at Brooklyn; ab. wasmuthi Wks. was described from Flatbush, LI. Aristolochia.

\section{PAPILIO Linnaeus}

4b P. polyxenes asterius Cr. Black Swallow-TaIl. Common and general except perhaps in the higher Adirondacks: Newport (NYS); Keene Val. and Franklin Co, Not; Rochester; Buffalo; Crosby; Ithaca; Schoharie; Lestershire; Albany; SI; LI, generally distributed and usually common: Central Pk., May 5, Jamaica, Je 12, Flushing, J1 9, Aug 2, Hempstead, Sep 5 (Bell). The aberration calverleyi Grt. was described from Queens Co. Je-Sep, two broods. Umbelliferae (once found on cosmos by Fbs).

11 P. cresphontes Cr. Giant Swallow-Tail. Rochester (Angle); Albion; Batavia; Lancaster (EPV); La Salle (Wild) ; Buffalo; Ithaca ; Cortland, Wickwire; Schoharie (NYS); Altamont; Selkirk; New Baltimore; Albany; Athens; Rhinebeck; New Paltz (NYS) ; Poughkeepsie; Bear Mt., Arden, F. M. Brown (Wat) ; NYC-Bronx Pk., Van Cort. Pk., and Washington Hgts. (Wat and Comstock); SI; LI: Flushing, Aug 17 (W.J.Hamilton,Jr.) ; Brooklyn; Glencove; Yaphank, Aug, 1882 (Wks). Je; Aug-Sep. Prickly ash, Ptelea, and other woody Rutaceae.

13 P. glaucus L. The typical form is reported from: Buffalo; Nyack; SI, earliest date, Ap 22, 1919 (Ds) ; LI: Dyker Hgts., Brooklyn, May 9, 1919 (Pasch) ; Flushing, Aug 13 (Bel1) ; Yaphank, May 29, 1911 (Ds)'.

P. glaucus var. turnus L. Tiger Swallow-Tail. Everywhere in the State. LI, general and common: Flushing, Ap 28, Aug 2, Coram, May 13, Jamaica, Je 4, Hempstead, J1 3, Smithtown, Sep 10 (Bell). May-Je; mid J1-Aug. Wild cherry, lilac, and many other trees and shrubs.

18 P. troilus L. Blue Swallow-Tail. General except in the Adirondacks. Keene Val., Not; Gloversville (Alex); Utica (NYS); Rochester (Angle) ; E. Aurora (Wild); Rock City; Potter Swamp; Ithaca; McLean; Dutchess Co; Greenwood L.; Ramapo Mts.; SI; LI, generally distributed and usually common: Flushing, Oakdale, Coram (Bèll); Wading R. (NYS). May-Sep, two broods. Sassafras and spicebush.

19 P. palamedes Dru. SI: Ft. Wadsworth, Je 18, 1914, Bs (Ds); LI: Brooklyn, taken many years ago by Benjamin Meyer (Doll).

\section{IPHICLIDES Hübner}

20 I. marcellus Cr. (ajax auct.). ZeBra Swallow-TaIl. Mt. Marcy, one specimen on summit and another at $\mathrm{N}$. Elba, J1, both var. lecontei R. \& J. (Wat and Coleman); Rochester (Angle); Brockport, all forms (Angle) ; Lockport (Wild); Buffalo, all forms (EPV); E. Aurora, very rare (Wild); Cortland, Wickwire; Sloatsburg, Cook; SI (Ds and Fulda); LI: Flushing, J1 2, 1920 (W.J.Hamilton,Jr.); Eastern Parkway, Brooklyn, 1867 and 1911 (Doll) ; Brighton Beach, J1, 1912 (Eng); Rockaway. Papaw. 


\section{FAMILY PIERIDAE}

\section{APPIAS Hübner}

28 A. ilaire Gdt. (Tachyris margarita Hbn.). Lancaster, 1880 (EPV); two specimens seen and one captured Je 10, 1906, at Canarsie, LI, by Otto Mayer, in a field containing an occasional patch of scrub and partly hemmed in by truck gardens. The one captured was on blackberry blossoms (Bul. Brooklyn Ent. Soc., vol. 8, p. 45, 1913). Cruciferae.

\section{PIERIS Schranck}

\section{(Pontia)}

34 P. protodice Bdv.-Lec. Checkered White. Pittsford, Boughton; Brockport; Buffalo and vic. (EPV); E. Aurora (Wild); Ithaca; Schoharie; Karner (Cook and Wat); Bronx and Van Cort. Pks. (Wat); SI, but not in recent years (Ds); LI: City Line, Brooklyn, and common along the Aqueduct, Sep 12, 1910 (Ds); S. Jamaica, J1 28, 1912 (Ds) ; Long Pond, Wading R., Aug 8, 1912 (Ds) ; East NY, J1-Oct (Shoe); Glen Cove. Two broods. Cruciferae, especially Lepidium virginicum (Wat). The spring form vernalis Edw. has not been separately recorded.

$35 \mathrm{f}$ P. oleracea Harr. MUSTaRd White. Double-brooded. Formerly common and widespread, now replaced by rapae except in out-of-the-way places and open woods. Recent records are: Axton, 1901; Speculator, 1901; Keene Val., 1907, Not; Wilmington, Mt. Whiteface, 1916; N. Elba, 1918; Wilmurt; Malone (NYS). Last seen at Lancaster, 1878 (EPV). May; Aug. Cruciferae.

$35 i$ P. virginiensis Edw. Single-brooded. Authentic records are: Potsdam; vic. Buffalo (EPV); Slaterville (Fbs); Trenton Falls (Fbs); McLean, 1916-25, still very common locally. I do not know whether this or the preceding species was taken at Brooklyn, 1894, Sullivan Co, 1896, and Jay, 1911. May-Je. Slender Cruciferae.

37 P. rapae L. Cabbage Butterfly. Common everywhere in the open, introduced from Europe. Ap-Oct. Cruciferae, especially injurious to cabbage.

P. rapae var. immaculata Ck11. Occurs commonly in the spring brood.

ANTHOCHARIS Boisduval

(Synchloë Hübner in part)

43 A. genutia Fab. Orange Tip. Highlands of the Hudson, Eng; Greenwood, Tuxedo (Ds) ; Newburgh; Ramapo Mts.; LI: Canarsie (Blackburn). Ap-May. Arabis.

Callidryas Boisduval and LeConte

47 C. eubule L. Cloudless Sulphur. Rochester, rare (Angle); Wayne Co, Mable R. Wiggins; Washington Hgts., Crotona Pk., NYC (Wat); SI; LI, an annual visitor in late summer, Aug and Sep, but no breeding records on hand (Bel1); large migrating swarms were observed along the seashore at Massapequa and Patchogue, Aug, 1920 (Eng); Yaphank, Sep, 1911 (Wks) ; Bayshore, Sep 25, 1913 (Dow); Bellport, Sep, 1911 (Nic); Jamaica, Sep 14, 1903, Aqueduct and Rockaway Beach, Sep 14 and 22 (Shoe); Long Beach, Sep 21, 1916 (Ds). Cassia. 


\section{ZERENE Hübner}

55 Z. caesonia Stoll. Dog-FAced Butterfly. SI, J1, 1896, in numbers, but has not been seen since (Ds); LI: Prospect Pk.; Brooklyn (Doll).

\section{EURYMUS Swainson}

59 E. eurytheme Bdv. Alfalfa Butterfly. Rochester, fairly common in Oct (Angle) ; E. Aurora, Wild; Allegany Pk., W. Alex (Wild) ; Cortland, reported fairly common, Wickwire; Van Cort. Pk., ABKlots ; Washington Hgts. (Wat); SI; LI: "a single pair of this species was taken by the late SLEliot at Astoria" (Bt); Hicksville, Westbury (Doll); Brooklyn. All summer. The stray specimens in the northeast are mostly taken in Aug. Alfalfa, etc.

60 E. philodice Godt. Clouded Sulphur. Common everywhere. LI, common everywhere: Flushing, May 8, Aug 2, Sep 10, Oct 7, Oakdale, Je 12, Hempstead, J1 3, Syosset, Nov 19. (Bell). May-Oct. Clover, etc.

E. philodice ab. pallidice Scud. Common everywhere with the type. LI, common: Flushing, Je 30, J1 14, Aug 10, Coram, Sep 2 (Bell).

E. philodice ab. nigridice Scud. Binghamton; Palmyra (Scud).

63 E. interior Scud. Mt. Marcy (Wat and Coleman); Keene Val. (NYS); Osgood Pond; Camp Lou; Newcomb; L. Tear; Paul Smiths (W.P.Comstock). J1. Vaccinium.

\section{EUREMA Hübner}

76 E. nicippe Cr. Little Orange Sulphur. Ithaca; Pine I. (Lutz and Wat) ; SI, May-Oct, in 1880 appeared in great numbers (Ds); LI: Jamaica, common one season (Doll and Eng) ; Brooklyn; Glencove. All summer Cassia.

78 E. euterpe Mén. Little Sulphur. Wayne Co, rare, Wiggins; Pittsford, Boughton; Buffalo (EPV) ; Yonkers; Bronxville; SI: Fox Hills; LI, locally common: Flushing, J1 23, Aug 5, Sep 22, Coram, Sep 4, Jamaica, J1 16 (Bell); Brooklyn. Cassia.

\section{FAMILY LYCAENIDAE 94}

\section{Atrides Hübner}

334 A. halesus Cr. LI: Brooklyn, a stray from the Tropics. Oak.

\section{STRYMON Hübner}

\section{(Uranotes Scudder, Thecla)}

345 S. columella Fab. (eurytulus Hbn., ocellifera Grt.). E. Aurora (Scud) ; Buffalo. Both strays from the South. J1.

352 S. melinus Hbn. (humuli Harr.). Keene Val., Not; Wilmington; Rochester (Angle); Bethlehem; SI, May-Sep (Ds); LI: Wyandanch, Ap 29, May 14, Flushing, Je 30, Sep 16, Coram, Sep 4, Hempstead, Sep 5 (Bell) ; Amagansett; Greenport. Lespedeza hirta and other legumes (Wat); reported also from hops, probably in error.

\footnotetext{
${ }^{94}$ Most of the larvae in this family are feeders on flowers and fruits.
} 
355 S. autolycus ontario Edw. Ithaca, Je 24 (CU).

358 S. titus Fab. Fentons; New Russia, Jones; E. Aurora, Allegany Pk. (Wild); Lancaster; Buffalo; Ithaca; McLean; Karner; Albany, Cook; Bethlehem; Riverdale; NYC; SI; LI, locally common: Flushing, Je 29, Oakdale, J1 4 (Bel1); Aqueduct; Yaphank. J1-Aug. Wild cherry.

359 S. acadica souhegan Whitney. Keene Val., Not; Chilson L. (Wat and Cook); Rochester; Buffalo and vic. (EPV); Allegany Pk. (Wild); Stony Clove (Wat and Coleman); Boiceville (Blackburn); Karner; Bethlehem; Kingston; Gloversville (Alex); Sacandaga Pk. (Alex); Albany, Cook; Rhinebeck; Callicoon (Ds); Greenwood L., Joutel; Van Cort. Pk., one specimen (W.P.Comstock). Je-J1. Salix discolor.

S. acadica ab. swetti Wat. \& Comstock. Stony Clove, J1 10 (Wat).

363 S. edwardsi Saund. Keene Val., abundant $(N o t)$; Ithaca; Minnewaska; Oneonta; Karner; Albany; Bethlehem; Greenwood L.; Ramapo, J1 (Ds) ; LI: Brooklyn; Yaphank, J1 13 (Ds). Jl-Aug. Oak.

365 S. calanus Hbn. Rochester (Angle); Buffalo; E. Aurora (Wild) ; Castile, Brad; Ithaca; Kaaterskill; Sharon Sps.; Karner; Albany, Cook; Bethlehem (Lint); Esopus; Ft. Montgomery, Callicoon (Ds); Greenwood L.; SI; LI, locally common: Flushing, Je 28, Farmingdale, J1 3, Kings Pk, J1 27 (Bell); Half Way Hollow Hills, Wading R., Aug (Ds); Yaphank. Je-Aug. Hickory, butternut, and oak.

367 S. liparops strigosa Harr. This species is easily confused with forms of S. calanus. I have seen specimens from Ithaca, NYC, and Massapequa, LI. It is reported also from: Fentons; New Russia, Jones; Keene Val., abundant, Not ; Rochester (Angle) ; Lockport (Lint) ; E. Aurora, Dnd, Wild; Karner; Bethlehem, E. Jewett (Ds) ; all very probably correct; LI: Kings Pk., J1 (Bell); Riverhead (Ds). J1. Vaccinium corymbosum (Wat) and chokecherry (Wild).

\section{MITOURA Scudder}

377 M. damon Cr. Green Hatr-Streak. Clayton; Taughannock; Ithaca; Ramapo (Ds) ; NYC; SI, May 8, 1881, Lg (Ds) ; LI, local in its distribution: Brooklyn; Coram, May 15, Je 10, Wyandanch, May 28 (Bel1); Orient, Lath; Riverhead (Ds). May-Je; end J1-Aug. Will probably be found general and not rare wherever its food, the red cedar, grows. The only definite record for form patersonia Brehme is Van Cort. Pk., J1 (Wat and Comstock).

INCISALIA Minot

379 I. augustus Kby. Plattsburg; McLean; Karner (Lint); Albany (Cook) ; SI; LI, very common in the pine-barren district: Central Pk., Ap 19, Wyandanch, May 28, Coram, Je 10 (Bell). May-early Je. Vaccinium and Kalmia.

381 I. irus Godt. Karner (Lint); Albany (Cook); SI; LI: Flushing (W.J.Hamilton,Jr.) ; Coram, May 6; Central Pk., Je 4 (Bell); Wyandanch, Yaphank (Ds). This species is doubtless widespread and not rare, but records are so confused with $I$. henrici and $I$. polios that I have not dared to use them. Ap-May. Wild indigo and lupine (Wat).

383 I. henrici G. \& R. Karner; Albany (Cook); Schenectady, Harry Cook; Greenwood L. (Ds). Doubtless widespread, but confused with $T$. irus. May. Vaccinium. 
[385 I. polios Cook \& Wat. I have seen no NY records for this species, which certainly occurs, passing for henrici. End of Ap and early May. Arctostaphylos.]

388 I. niphon Hbn. Plattsburg; Keene Val., Not; Portage; Windsor, Not; Ithaca; McLean; Karner; Albany; Bethlehem; SI, 1881 (Ds); LI, locally distributed in the pine barrens: Central Pk., Ap 19, Wyandanch, May 28, Oakdale, Je 4 (Bell); Brooklyn; Massapequa, Yaphank, Wading R. (Ds). May-early Je, also Ap on LI. Pitch pine.

\section{ERORA Scudder}

393 E. laeta Edw. EArly Hair-Streak. Keene Val., Not; Cortland, Wickwire (Angle); Ithaca (Eyer-CU); Catskills (Edw). May; J1.

\section{FenisecA Grote}

396 F. tarquinius Fab. The WANDERER. Wilmington; Elizabethtown; Beedes, Essex Co; Keene (Wild) ; Elk L.; Sand L.; Rochester, fairly common and local (Angle); Buffalo (Wild); Castile, Brad; Binghamton; Ithaca; McLean; Raven Pass; Gloversville; Bethlehem, Karner (Lint) ; Albany (Cook); Rensselaer; Croton-on-Hudson (Ds); Katonah; SI; LI: Oakdale, J1 1 (Bell); Brooklyn Bot. Garden, Aug-Sep (Eng); Yaphank, Sep 3, 1910 (Ds) ; Orient Pt., Aug 2, 1913 (Ds) ; Grasmere. All summer, at least three broods. Mealy bugs or alder blight (Schizoneura) on alder.

\section{HEODES Dalman}

\section{(Chrysophanus Hübner)}

404 H. thoë Bdv.-Lec. Large Copper. Keene Val. (Not); Mooers (Not); Fentons; Clayton; Rochester, Angle; E. Aurora, Buffalo (Wild) ; Oak Orchard Swamp; Ithaca; McLean; Oneonta; Gloversville (Alex) ; Saratoga; Karner; Bethlehem; Callicoon (Ds) ; SI; LI, not overly common, found in or close by damp meadows, sometimes in fair numbers: Flushing, May 28, Je 30, J1 16, Aug 7, Sep 16, Jamaica, J1 16 (Bell); Brooklyn; Aqueduct. Je-Sep. Local in wet places. Rumex crispus; reported also from Polygonum.

409 H. epixanthe Bdv.-Lec. Small Copper. Keene Val. (NYS); Buffalo (EPV) ; McLean (on the bogs only) ; L. Ontario shore; LI: Yaphank, Manorville, Amagansett, Montauk, Je-J1 (Eng). J1-Aug. Cranberry.

410 H. hypophlaeas Edw. COMMON COPPER. Very common everywhere. LI; generally distributed and usually very common: Flushing, May 8, Aug 2, Sep 16, Jamaica, Je 18, Farmingdale, J1 3, Oakdale, J1 4, Coram, Sep 10 (Bell). May-Aug. Sorrel.

H. hypophlaeas ab. fasciatus Strk. Rochester, Franck (Angle); SI, MayOct (Ds) ; LI: Flushing, May 21, J1 2 (Bell) ; Amagansett.

H. hypophlaeas ab. obsoletus Scud. LI: Glendale, Aug 26, 1923, J.B.Mesle.

H. hypophlaeas ab. fulliolus Hulst. Orchard Pk., May 3 (Wild).

\section{EVERES Hübner}

424 E. comyntas Gdt. TAILED BluE. Common everywhere. LI, generally distributed and very common: Flushing, May 8, Aug 2, Sep 16, Oakdale, Je 12, Hempstead, J1 3, Central Pk., May 3 (Bell). All summer, two broods. Larvae on flowers of Lespedeza and other legumes. 


\section{Plebeius Linnaeus \\ (Rusticus Hübner)}

426 P. scudderi Edw. Clayton; Adirondacks; Karner (div.); Albany (Cook). May-Je; J1-Aug. Lupinus perennis (Wat).

\section{Glaucopsyche Scudder}

(Nomiades)

449 G. lygdamus Db1. Ithaca, May 17, 1914, female (Eyer); Binghamton. May. Probably a leguminous feeder.

\section{LyCAENOPSIS Felder}

\section{(Cyaniris)}

452 L. argiolus pseudargiolus Bdv.-Lec. (ladon auct.). Common everywhere. LI: Flushing, Ap 21, J1 30, Aug 13, Sep 13, Kings Pk., May 9, Jamaica, $\mathrm{Je} 18$, Coram, May 15 (Bell). Ap-Aug. General on flowers, but especially on Viburnum acerifolium and Cornus florida (Wat).

L. argiolus pseudargioius form lucia Kby. Reported from: Potsdam; Buffalo; Karner; Albany; West Pt.; Newburgh; Yonkers to SI; LI: Wyandanch, May 6, 1911 (Ds) ; Central Pk.; Glendale. Ap.

L. argiolus pseudargiolus form marginata Edw. N. Fair Haven; Rochester; Ithaca; Yonkers; SI; LI: Kings Pk., May 9, Flushing, Je 29 (Bel1). Ap-May.

The other forms are too confused to make records trustworthy, May-Aug.

\section{FAMILY ERYCINIDAE \\ (Riodinidae)}

CALEPHELIS Grote and Robinson

329 C. borealis G. \& R. Northern Metal-Mark. Upper Coldenham, Orange $\mathrm{Co}$, is the northern record for the species and family. May; J1.

\section{FAMILY NYMPHALIDAE SUBFAMILY LIBYTHEINAE}

Hypatus Hübner

320 H. bachmani Kirt. Snout Butterfly. Van Cort. Pk. (Wat); Central Pk., NYC (Coleman); SI; LI: Center I., Oyster Bay, Aug 15, 1896 (Ds); Dyker Meadows, Brooklyn, Aug 9, 1908 (Shoe); Coram, Je 10: Kings Pk., J1 20 (Bell) ; Bay Ridge; Woodhaven. Celtis.

\section{SUBFAMILY DANÁINAE}

\section{DANAUS Linnaeus}

84 D. archippus auct. (Anosia plexippus L.). Milkweed Butterfly. Common everywhere, but probably only as a migrant, not wintering in the State. SI, Ap 25, earliest date (Ds); LI, generally distributed and sometimes 
very common: Flushing, May 21, Je 4, J1 2, Aug 13, Sep 10, Oct 12, Hempstead, J1 3, Oakdale, J1 4 (Bell). J1-Sep, two broods northward. Milkweed.

\section{SUBFAMILY SATYRINAE}

\section{ENODIA Hübner}

90 E. portlandia Fab. (Debis). New Russia; Keene Val., Not; Clayton; Newcomb; Mendon (Deveson); Allegany Pk. (Wild); Ithaca; McLean; Cortland, Wickwire; Ulster Co; West Pt.; Sloatsburg (Cook); Pocantico Hills. Je-J1. Grass.

\section{SATYRODES Scudder}

100 S. canthus L. Essex Co; L. Waccabuc; Fentons; Clayton; Fulton; Rochester (Deveson) ; Buffalo (Wild) ; E. Aurora; Squaw I.; Potter Swamp; Ithaca; Freeville; McLean; Oneonta; Gloversville, Sacandaga Pk. (Alex); Hebron, $W l s$; Karner; Ushers; Durlandville; Crugers ; Yonkers; Bronxville; SI; LI, locally common: Flushing, J1 2 and 27, Oakdale, J1 10 (Bell). J1. Coarse swamp grass.

\section{Cissia Doubleday}

\section{(Neonympha)}

97 C. eurytus Fab. Common everywhere. Black Br.; Fentons; Clayton; Rochester; Wayne Co; Niagara Falls; Buffalo; Rock City; Egleston's Glen; Portage ; Ithaca ; Oneonta ; Kaaterskill ; Greenwood L. ; Karner ; Albany; Bethlehem; Stuyvesant Falls; Katonah; SI; LI, locally common: Coram, May 22, Huntington, Je 5, Farmingdale, J1 3, Kings Pk., Aug 13 (Bell); Wading R.; Gardiners I. End of Ap-Sep, two broods. Grass.

\section{CERCyonis Speyer}

111 C. alope Fab. (Satyrus). Blue-Eyed Satyr. The typical form is mainly southern, reported from: Essex Co; Fentons; Chain Lakes; Rochester, rarer than var. nephele; Lancaster; Buffalo; Liberty; Lexington; Ulster Co; Sharon Sps.; Oneonta; Schoharie; Gloversville, Sacandaga Pk. (Alex) ; Hebron, Wls; Karner; Stuyvesant Falls; Katonah; SI; LI, locally common: Farmingdale, J1 3, Flushing, J1 30, Kings Pk., Aug 13, Coram, Sep 4 (Bell); Gardiners I. (Ds). The records from the Adirondacks should be verified. The form has often been sought at Ithaca without success. J1-Aug. Grass.

111c C. alope nephele Kby. Blind-Eyed SAtyr. This northern form is known from: Waddington; Wilmington; Long L.; Fentons; Clayton; Geneva ; Rochester ; Scriba; Buffalo; Otto; Allegany Pk. ; Ithaca ; McLean; Sullivan Co, above $1000 \mathrm{ft}$.; Lexington; Hunter; Onteora Mt.; S. Jefferson; Summit; Sharon Sps.; Schoharie; Bethlehem. Skinner's record from NYC must have been a stray.

111a C. alope maritima Edw. occurs on SI and at Yaphank, LI, but the latter specimens are hardly typical. 


\section{SUBFAMILY HELICONIINAE}

\section{DIONE Hübner}

150 D. vanillae L. Gulf Fritmlary. Upper Twin L.; Central Val., Carleen Maley (Felt). A stray from the South.

\section{SUBFAMILY NYMPHALINAE}

Euptoieta Doubleday

151 E. claudia Dru. Variegated Fritillary. Lancaster (EPV); Pittsford, Boughton; Buffalo; Ithaca, Sep (Philip Miller); Oneonta; NYC (div.) ; SI; LI, not common: Aqueduct (Wat) ; Flushing, J1 29, Sep (Bell); Woodhaven (Pears); Rockaway Beach (Ds); Wading R., May 31, 1914 (Ds) ; Brooklyn, larvae on pansy plants, J1-Aug (Eng). A stray from the South. Aug. Passion flower; rarely Sedum (Wat) and violet.

\section{ARGYNNIS Fabricius}

\section{(Speyeria Scudder)}

153 A. idalia Dru. Binghamton (Blackburn); Hebron (W1s); Karner; Hudson Falls (Alex); Albany (Cook); Bethlehem (Lint); Boiceville (Blackburn) ; Nyack; Van Cort. Pk. (Wat and Coleman) ; SI; LI, locally common: Hempstead, J1 3, Flushing, J1 23, Aug 13, Coram, Sep 10 (Bell) ; Brooklyn; Aqueduct; Montauk, Riverhead, Gardiners I. (Ds). Local in wet places, and will probably prove more widespread. J1-Sep. Violets.

158 A. cybele Fab. Mt. Whiteface; Waddington; Fentons; Long L: ; Clayton; Rochester; Buffalo; E. Aurora; Allegany Pk.; Oak Orchard Swamp; Ithaca; McLean; Binghamton; Pittsfield; Oneonta; Hunter ; Onteora Mt.; Shelving Rock; Glen, Warren Co; Schoharie; Gloversville ; Bethlehem; Cropseyville; Newburgh; Greenwood L.; Katonah; SI; LI, common: Flushing, Je 16, J1 9, Aug 5, Oakdale, J1 4, Hempstead, J1 3 (Bell) ; Brooklyn. J1-Sep. Violets.

159 A. aphrodite Fab. Everywhere in the State from the Adirondacks and Buffalo to SI. Commoner northward than A. cybele. LI: Flushing, J1 30 (Bell); Richmond Hill (Doll). Franck reports the Mississippi Val. form, alcestis Edw., from Sullivan Co, probably in error. J1-Aug. Violets.

162 A. atlantis Edw. Keene; Ausable Chasm; Wilmington; Mt. Marcy and vic.; Newcomb; Saranac Inn; Long L.; Chain Lakes ; Fentons; Glen, Meske; Buffalo (EPV) ; E. Aurora (Dnd) ; Allegany Pk.; Ithaca; McLean (Fbs) ; Deposit; Warners L. (Cook) ; Stuyvesant Falls; Slide Mt. ; Stony Clove; Onteora Mt.; Mountain L., Johnstown (Alex). J1-Aug. Violets.

\section{BRENTHIS Hübner}

187 B. myrina Cr. Little Silver-Spot. St. Lawrence R. and Buffalo to LI; common in wet places. LI, common in damp meadows: Flushing, May 26, Je 4, J1 2, Aug 2, Sep 16, Oakdale, Je 19 (Bell); Yaphank. Je-Sep. Violets. 
199 B. bellona Fab. Marsh Fritillary. Everywhere with the preceding species. Specimens from the Appalachian highlands (Rock City) are lighter than usual. May-Sep. Violets.

\section{EUPHYDRYAS Scudder}

203 E. phaëton Dru. The Baltimore. Fentons; Keene; Rochester; Conesus L.; E. Aurora; Elma; Colden; Ithaca; McLean; Vestal; Oneonta; Sand L.; Raven Pass; Karner; Ulster Co; Gloversville; Dutchess Co; SI; LI, common in damp meadows: Flushing, May 28, Je 4, J1 15, Jamaica, J1 15 (Bell). Common, though decidedly local in wet places. Je-early J1. Turtlehead.

E. phaëton ab. superba Strk. LI: Brooklyn (type).

E. phaëton ab. phaëthusa Hulst. LI: Brooklyn (type).

E. phaëton ab. streckeri Ellsworth. Vestal (type).

\section{Cinclidia Hübner}

228 C. harrisii Scud. (ismeria of Harr.). Harris' Checker-Spot. Black Br.; Chilson. L. (Wat and Cook); Whiteface; N. Brookfield, Not; Rock City; Potter Swamp; McLean; Debruce; Raven Pass; Karner, Lint; Albany, Cook; Sloatsburg (Wat); N. Westchester Co (W.C.Wood); Bedford; LI: Brooklyn. The last two records should be verified. 'Late Je-early J1. Aster umbellatus.

\section{CHARIDRYAS Scudder}

243 C. nycteis Dbi. \& Hew. Silver Crescent. Whiteface; Fentons; Keene; Clayton; Rochester; Buffalo; Gowanda; E. Aurora; Ithaca; Oneonta; Ulster Co; Gloversville (Alex); Karner; Albany; Greenwood L.; Sloatsburg; Van Cort. Pk.; LI: Yaphank, May 30, 1911 (Grossbeck); Woodhaven, Je 7, 1919 (J. Kaiser). Usually common where it occurs. J1. Helianthus spp., especially $H$. divaricatus, and occasionally on aster (Wat).

\section{Phyciodes Hübner}

247 P. tharos Dru. Peart Crescent. Essex Co; Fentons; Honeoye Falls; Buffalo; Otto; Rock City; Potter Swamp; Ithaca; McLean; Oneonta; Hunter; Karner; Bethlehem; Stuyvesant Falls; Poughkeepsie; SI; LI, very common: Flushing, May 7 , Aug 2, Oct 1, Oakdale, Je 12, Jamaica, J1 16, Coram, Sep 4 (Bel1); Yaphank. The preceding are representative records; the species occurs everywhere. May-Oct. Aster.

P. tharos ab. reaghi Reiff. LI: Flushing, Aug 10, 1918 (Bell).

248 P. batesii Reak. Bates' Checker-Spot. Black Br.; Fentons; Keene Val. (NYS) ; between Chilson L. and Paradox L. (Cook); Gowanda; Rock City; Crosby; W. Danby (a suffused aberration); Ithaca; Schoharie; Sharon Sps.; Gloversville (Alex); Bethlehem; Karner, Lint; Albany, not now found (Cook); Hunter; LI (Bt), but probably in error. Je$\mathrm{J} 1$, one brood, at any given place flying between the two broods of $P$. tharos. Aster undulatus. 


\section{POLYGONIA Hübner}

268 P. interrogationis Fab. The Semicolon. Newport (NYS); N. Fair Haven; Centerport; Buffalo; E. Aurora; Rock City; Oneonta; Ithaca; Catskills; Ballston Spa (NYS) ; Coeymans; Stuyvesant Falls; NYC; SI; LI: Flushing, May 11, Je 20, J1 30, Aug 15, Sep 16, Oct 5, Farmingdale, J1 3 (Bell). The form umbrosa Lint. is definitely known from: Rochester; E. Aurora; Buffalo; Ithaca; McLean; Oneonta; Gloversville, Sacandaga Pk. (Alex); Albany; Bethlehem; LI: Woodhaven, Central Pk. Two broods, one in midsummer, the other emerging in the fall and overwintering. Elm, hop, and Celtis.

269 P. comma Harr. Hop Merchant. Fentons; Clayton; Geneva; Buffalo; E. Aurora; Irving; Portage; Ithaca; McLean; Oneonta; Catskills; Schoharie; Gloversville; Bethlehem; Hebron, Wls; Albany ; Rensselaer ; NYC; SI; LI: Brooklyn; Flushing, J1 14, Aug 12, Sep 16, Oct 1 (Bell); Gardiners I. Var. dryas Edw. reported from: Rochester; Buffalo; E. Aurora; Allegany Pk.; Ithaca; Oneonta; Catskills, Ulster Co; Schoharie; Albany; Katonah. J1; winter. Urticastrum divaricatum, and less commonly on elm and hop (Wat).

271 P. faunus Edw. Green Comma. Wilmington; Mt. Whiteface; Raquette L.; Elk L.; Fentons; Jay; L. Pleasant; Hamburg; Buffalo (EPV); Allegany Pk. (Wild); Cortland, Wickwire; Sidney; Debruce; Raven Pass, Catskills; Gloversville (Alex) ; Schoharie. Birch, willow, currant, etc. ${ }^{95}$

275 P. gracilis G. \& R. Keene Val. (NYS).

277 P. progne Cr. Gray Comma. Jay; Mt. Whiteface; Fentons; Geneva; Rochester ; Buffalo; Otto; E. Aurora; Allegany Pk.; Rock City; Ithaca; Oneonta; Onteora Mt.; Debruce; Cambridge; Schoharie; Bethlehem; Esopus; Ft. Montgomery; Sloatsburg; Big I., Pine I., Woodbury Falls (Wat); Van Cort. Pk.; SI; LI: Flushing, Je 23, Aug 11 (Bell); Brooklyn. Currant and gooseberry.

P. progne form 1-argenteum Scud. Ft. Montgomery, Je 24-J1 4 (Wat and Hall); Sloatsburg, J1 3 (Wat).

\section{Aglais Dalman}

\section{(Vanessa Fabricius in part)}

278 A. j-album Bdv.-Lec. Large Tortorse-Shels.. Plattsburg; Wilmington; Jay; Elk L.; Fentons; Newport (NYS) ; N. Elba (Wat and Coleman); Rochester (Angle); E. Aurora (Wild); Clayton; Lancaster, Buffalo (EPV); Allegany Pk. (Angle); Ithaca ; Cortland, Wickwire; Shelving Rock, L. George; Edgewood (Wat and Coleman); Debruce (Ds); Onteora Mt.; Gloversville and vic. (Alex); Corinth; Karner; Albany; Sloatsburg; Croton-on-Hudson (Ds) ; NYC (div.) ; SI ; LI: Flushing, Ap 7 (Bel1); Glendale (Kch); Yaphank, Oct 1 and 2, 1910 (Ds); Maspeth (Ols); Brooklyn. Sep-J1, one brood. Birch.

280 A. milberti Gdt. Small Tortoise-Shell. Ausable Chasm; Canton; Long L.; Fentons; Rochester (Angle) ; Buffalo; E. Aurora (Wild) ; Portage; Potter Swamp; Ithaca; Binghamton; Marathon; Catskills; Callicoon (Ds) ; Oneonta; Schoharie; Gloversville; Canajoharie; Lebanon Sps.; Hebron; Hudson; NYC; SI; LI: Flushing, J1 28 (Bell) ; Brooklyn; Astoria; Seacliff; Wyandanch. All the season, three broods. Nettle.

${ }_{95} P$. satyrus Edw. was reported from. Fentons, collected by Hill, doubtless in error for $P$. faunus. 
281 A. antiopa L. Mourning CloAk or Yellow Edge. Very common everywhere. LI, generally distributed and common, may be found hibernating throughout the winter: Flushing, Mar 26, Ap 7, May 8, Aug 2, Oct 22, Nov 19, Port Washington, Je 26, Farmingdale, J1 3, Kings Pk., Sep 4 (Bell). The ab. hygiaea Heyd. has been taken at Rochester, Albany (Scud), and Morrisania, NYC (Wat). All the year, two broods. Elm, willow, etc.

\section{VANESSA Fabricius}

282 V. atalanta L. Red Admiral. Common everywhere in the State. LI, generally distributed, some years very common, other years scarce: Flushing, Je 30, J1 23, Aug 18, Oct 5 (Bel1). J1-Aug; Sep-Je. Nettles, rarely elm, etc.

283 V. virginiensis Dru. (huntera Fab.). Painted Beauty. Saranac Inn; Mt. Marcy; Clayton; N. Fair Haven; Rochester; Buffalo; Allegany Pk. ; Ithaca; Oneonta; Ulster Co; Schoharie; Castleton; Gloversville; Hebron; Bethlehem; Katonah; SI; LI, generally distributed and usually common: Flushing, May 26, Aug 13, Jamaica, Je 18, Oakdale, J1 4, Coram, Sep 5 (Bell). Common everywhere, but rarely as common as the other two Vanessas. Everlasting.

284 V. cardui L. Painted Lady. Very common all over the State. LI: Flushing, J1 13, Sep 13 (Bell). Thistle, burdock, etc.

\section{JUNONIA Hübner}

\section{(Precis in part)}

286 J. coenia Hbn. Buck-Eye. Wayne Co, Wiggins; Lancaster (EPV); Buffalo; Squaw I.; Newburgh; Bronxville; SI; LI, common: Flushing, May 26, Je 30, J1 30, Aug 13, Sep 29, Oct 5 (Bel1) ; Brooklyn; Jamaica; Newtown; Corona; Bellmore; Rockaway; Aqueduct; Orient, Lath. All summer, several broods. Gerardia and other Scrophulariaceae.

\section{BASILARCHIA Scudder}

304 B. astyanax Fab. Red-Spotted Purple. General in the southern part of the State, replaced by $B$. arthemis northward: E. Aurora; Cattaraugus; Shandaken; West Kill; Catskills; Schoharie; Gloversville; Karner; Albany; Bethlehem; Stuyvesant Falls; Poughkeepsie; Pine I.; SI; LI, generally distributed and common: Jericho, May 29, Flushing, Je 9, Aug 2, Sep 16, Farmingdale, J1 3 (Bell) ; Brooklyn; Gardiners I. Wild cherry, etc.

B. astyanax form albofasciata Newc. This form is very close to arthemis in appearance, and not usually separated from it, but in distribution is associated rather with astyanax. Definite records are: Rochester (Angle); SI (Fulda); LI, not common: Flushing, Aug 5, 1922 (Bell); Glendale $(K c h)$. Part of the arthemis records probably should be credited to this form..

305 B. arthemis Dru. White Admiral. Elizabethtown; Big Moose; Mt. Marcy; N. Elba (Wat and Coleman); Newcomb; Keene (Wild) ; Fentons; E. Aurora; Buffalo; Allegany Pk. (Wild) ; Rock City ; Ithaca; Raven Pass; Hunter; Onteora Mt.; Big Ind. Val.; Lexington; Oneonta; 
Edgewood (Wat and Coleman); Schoharie; Mountain L., Johnstown (Alex); Karner; Bethlehem; Catskill. Je-J1. Black and yellow birch, basswood (Wat), and willow.

B. arthemis form proserpina Edw. N. Elba, scarce (Wat and Coleman); Rochester, Honeoye Falls, Buffalo (Angle); Jamestown (Wild) ; Rock City; Riceville (Wild); Ithaca; McLean; Catskills; Andes; Hebron (W1s) ; Edgewood, relatively common (Wat and Coleman). A report from LI is certainly in error. Je-Jl.

308 B. archippus Cr. (not archippus Fab.). The VICERoy. Common throughout the State. LI, common: Flushing, Je 4, J1 9, Aug 2, Sep 16, Oakdale, Je 19, Hempstead, Sep 5 (Bell). The var. pseudodorippus Strk., which is probably archippus $\times$ astyanax, was taken in the Catskills. Je-Sep. Willow, rarely poplar.

B. archippus form lanthanis Cook \& Wat. Rochester (Angle); Albany (Cook); Pearl R. (Wat). Transitional specimens occur generally.

Apatura Boisduval-LeConte

(Chlorippe Boisduval, Doxocopa)

311 A. celtis Bdv.-Lec. LI, formerly (Doll) reported by Angle. Celtis.

313 A. clyton Bdv.-Lec. The Emperor. Esopus; Newburgh; NYC, J1 15, form proserpina Scud., L. Johnson; SI; LI: Hempstead, J1 3, 1921 (Bell). Celtis.

\section{ORDER DIPTERA}

Edited by O. A. JOHANNSEN

The original draft of the list of Diptera was completed more than twelve years ago. It was based in large part on the specimens in the Cornell University collection and in the State Museum at Albany, and on published records. When Dr. M. D. Leonard assumed the editorship of the list of the insects of New York, and the prospect for publication became brighter, the parts relating to some of the families were sent to specialists and later the entire list of Diptera was submitted to C. W. Johnson and Dr. Joseph Bequaert. Many additional records were thus obtained. Acknowledgment to the various entomologists who have helped to make the list more complete, is made in the appropriate places.

The classification followed, in the main, is that given in Comstock's Introduction to Entomology. The arrangement of families and genera, with slight modifications, is that of Johnson's List of the Diptera of New England. Bibliographical references are not made, owing to the limitations of space. The reader is referred to Aldrich's catalog of the Diptera for those prior to 1905, and to Johnson's list for the more important recent ones. Synonymy is given only in a few cases, where otherwise a misunderstanding might arise. 


\section{FAMILY TANYDERIDAE 96}

Compiled by C. P. Alexander

PROTOPLASA Osten Sacken

P. fitchii O.S. Fulton Co, Je, 1909 and 1924 only, a total of ten specimens (Alex).

\section{FAMILY PTYCHOPTERIDAE SUBFAMILY PTYCHOPTERINAE \\ PTYCHOPTERA Meigen}

P. rufocincta O.S. Dunkirk, J1; Fulton Co, Je-Aug; Manlius, Aug; Batavia; Erie Co, Je-Aug; Ithaca, May-J1; Portage Falls; Hudson R. Val., May; LI: Wyandanch, May-Je, Ds (Jn).

\section{SUBFAMILY BITTACOMORPHINAE}

BITTACOMORPHA Westwood

B. clavipes Fab. Adirondacks, L. George; Essex Co; Fulton Co; Brookview; Erie Co; Manlius; Ithaca; Poughkeepsie; LI, etc. May-Sep.

\section{BitTACOMORPHELLA Alexander}

B. jonesi Jn. Fulton Co; S. Wales; Ithaca; Taylor; Karner; Brookview; Je-J1.

\section{FAMILY ANISOPODIDAE \\ (Rhyphidae) \\ SUBFAMILY ANISOPODINAE}

(Rhyphinae)

\section{ANISOPUs Meigen \\ (Rhyphus Latreille)}

A. alternatus Say. Foot of Cliff Mt., Essex Co (Beq) ; Speculator, Wells, Je-J1, Yg (Leon); Keene Val., Aug (Leon); Axton; E. Aurora; Ithaca; Albany, Ap-Je (NYS).

A. fenestralis Scop. Hamburg; Ithaca; New Hartford, Ap-May, Yg (Leon); Albany, Sep (Leon); Nassau, Je (Leon).

A. punctatus Fab. Speculator, Je-Aug, $Y g$ (NYS) ; Newport, Je, $Y g$ (NYS); Ilion, May, $Y g$; Johnstown, Aug (Leon) ; Erie Co; Ithaca, May-Oct; Nassau, May (Leon) ; Corinth, Je, $Y g$; Clinton Hgts., Ap (NYS) ; Albany, May-J1 (NYS); Normanskill, May (NYS).

\section{SUBFAMILY AXYMYINAE}

\section{AXYMYIA McAtee}

\section{A. furcata McAtee. Ithaca (Snn).}

\footnotetext{
${ }^{96}$ The list in this and in the families Ptychopteridae. Anisopodidae, Trichoceridae, and Tipulidae, commonly called the crane-flies, has been prepared by Dr. C. P. Alexander. All determinations are to be credited to him unless otherwise indicated.
} 


\section{SUBFAMILY MYCETOBIINAE}

MyсетовIA Meigen

M. divergens Wlk. Ithaca, May (Joh); Albany.

\section{FAMILY TRICHOCERIDAE}

\section{TRICHOCERA Meigen}

T. bimaculata Wlk. Fulton Co, Sep; Erie Co, Oct; Ithaca and vic., Oct, May.

T. brumalis Fitch. Fulton Co; Ithaca, Sep-Oct.

DiAzosma Bergroth

D. subsinuata Alex. Fulton Co, Je; Albany Co, near Altamont, Je (Crampton).

\section{FAMILY TIPULIDAE \\ SUBFAMILY LIMONIINAE \\ Tribe LIMONIINI \\ DICRANOMYIA Stephens}

D. adirondacensis Alex. Keene Val., Sep.

D. badia Wlk. Hamilton, Fulton, Schoharie, Erie, Herkimer, Niagara, Tompkins, and Cortland Counties; LI. Ap-Oct.

D. brevivena O.S. Herkimer, Fultom Erie, Niagara, and Cayuga Counties. Je-Oct.

D. gladiator O.S. Fulton Co, Aug

D. globithorax O.S. Fulton Co, Aug; Erie and Tompkins Counties. May-Sep.

D. haeretica O.S. Buffalo, Oct; vic. NYC; LI, J1-Oct.

D. halterata O.S. Fulton Co, Sep.

D. immodesta O.S. Adirondacks; Essex Co; Fulton Co; N. Fair Haven; Buffalo; Taylor; Ithaca; Rensselaer Co, Brookview, Je. Je-Oct.

D. liberta O.S. Fulton, Essex, Herkimer, Erie, Tompkins, Cortland, Onondaga, Albany, Rensselaer, and Westchester Counties; LI. Je-Sep.

D. longipennis Schum. Erie and Fulton Counties; Trenton Falls; Ithaca; W. Nyack; LI, etc. Je-Sep.

D. macateei Alex. Fulton and Essex Counties. Je-J1.

D. moniliformis Doane. LI.

D. monticola Alex. Hamilton, Fulton, Chenango, and Cortland Counties. Je-J1.

D. morioides O.S. Herkimer, Fulton, Tompkins, and Erie Counties. Je-J1.

D. pubipennis O.S. Hamilton, Fulton, Erie, Tompkins, Cortland, Chenango, Rensselaer, and Westchester Counties. May-Sep.

D. pudica O.S. Fulton, Erie, Tompkins, Cortland, Chenango, and Schenectady Counties. May-J1.

D. rara O.S. Genesee Co, Sep; Westchester Co, Je.

D. rostrifera O.S. Fulton and Cayuga Counties. Je-Sep.

D. simulans Wlk. Fulton, Herkimer, Erie, Tompkins, Cortland, Albany, Rensselaer, and Westchester Counties. May-Nov. LI, J1.

D. stulta O.S. Herkimer, Fulton, and Tompkins Counties. Je. 


\section{Geranomyia Haliday}

G. diversa O.S. Herkimer, Erie, and Tompkins Counties. Aug.

G. canadensis Westw. Herkimer and Fulton Counties, Je; Erie Co, Je-Sep; Onondaga Co, Aug; Tompkins Co, May-Oct.

G. rostrata Say. Fulton, Tompkins, and Cayuga Counties. May-Sep.

\section{RHIPIDIA Meigen \\ (Subgenus MonoRHipidia Alexander)}

R. fidelis O.S. Fulton, Essex, Erie, Tompkins, Cortland, Albany, and Schoharie Counties. May-J1.

\section{(Subgenus RHIPIdia Meigen)}

R. bryanti Jn. Erie Co, Je.

R. maculata Meig. Adirondacks; Essex, Fulton, Chenango, Cortland, and Tompkins Counties. Je-Aug.

\section{Limonia Meigen \\ (Limnobia Meigen)}

L. cinctipes Say. Adirondacks; Essex, Fulton, Onondaga, Erie, Cattaraugus, Chenango, and Tompkins Counties. May-Aug.

L. fallax Jn. Tompkins and Genesee Counties. May-J1.

L. hudsonica O.S. Erie Co, Je (MCV).

L. immatura O.S. Essex, Fulton, Herkimer, Erie, Tompkins, and Albany Counties. Je-J1.

L. indigena O.S. Adirondacks; Essex, Fulton, Onondaga, Erie, Niagara, Cattaraugus, Tompkins, and Saratoga Counties. May-Oct.

L. parietina O.S. Adirondacks; Fulton, Erie, and Tompkins Counties. Aug-Sep.

L. solitaria O.S. Adirondacks, J1; Essex and Fulton Counties, Je-Aug ; Tompkins Co, May-Je; Brookview, Rensselaer Co, Je; Catskills, J1.

L. triocellata O.S. Adirondacks; Essex, Fulton, Onondaga, Erie, Tompkins, Cortland, Putnam, and Rockland Counties. Je-Oct.

L. tristigma O.S. Adirondacks; Fulton, Niagara, Cortland, and Chenango Counties. J1-Aug.

\section{Discoвola Osten Sacken}

D. argus Say. Adirondacks; Essex Co; Gloversville; Rochester; Batavia; Erie Co; Little Valley; Keuka Pk.; Cincinnatus; Ithaca, Je-Oct.

\section{Helius St. Fargeau et Serville}

\section{(Rhamphidia Meigen)}

H. flavipes Macq. Fulton Co; Buffalo; Ithaca; Albany; Helderbergs; LI. May-Aug.

H. mainensis Alex. Ithaca, May. 


\section{DicRanoptycha Osten Sacken}

D. germana O.S. Adirondacks; Essex Co; Griffin; Sacandaga Pk.; S. Wales; Ithaca; Manlius ; Lower Cincinnatus; Taylor. Late Je-Jl.

D. septentrionalis Alex. Mayfield Mt.; N. Fair Haven; Ithaca. Aug-Sep.

\section{ANтосна Osten Sacken}

A. obtusa Alex. Genesee Co, Kngt.

A. opalizans O.S. Keene Val., Aug; Helderbergs, Albany Co, Je.

A. saxicola O.S. Adirondacks; Sacandaga Pk.; Manlius; Honeoye Falls; Buffalo; Ithaca; Taylor; Mohawk R. valley; Tarrytown. May-Sep.

A. septentrionalis Alex. Niagara Falls, Sep.

\section{Tribe ERIOPTERINI}

\section{ORMOSIA Rondani}

O. adirondacensis Alex. Fulton Co; Mountain L.; Woodworth's L.; Gloversville. Je.

O. apicalis Alex. Fulton Co, Je; Ithaca, Aug.

O. arcuata Doane. Hamburg; Ithaca. May.

O. bilineata Dietz. Holland, May.

O. dentifera Alex. Essex Co; Keene Val. May.

O. deviata Dietz. Fulton and Herkimer Counties, Je; N. Fair Haven, Sep; Hamburg, May; Onondaga, Tompkins, Rensselaer, and Westchester Counties, Je.

O. holotricha O.S. Johnstown; Ithaca. Ap-May.

O. innocens O.S. Hamburg; Ithaca; McLean; Albany; LI. May.

O. megacera Alex. Gloversville, Je.

O. meigenii O.S. Fulton Co; Erie Co, May; Ithaca, Ap-Je; Albany, May, Sep, $Y g$; Westchester Co, Je.

O. mesocera Alex. Essex Co, J1; Gloversville, Je.

O. monticola O.S. Adirondacks; Speculator; Fulton Co; Colden; Ithaca. Aug.

O. nigripila O.S. Essex Co; Fulton Co, Je-Aug; Onondaga; Ithaca, May-Je; Tarrytown; LI.

O. nimbipennis Alex. Hamilton Co, J1-Aug; Fulton Co, Aug; Wells, J1, Yg; Newport, Je, $Y g$; Elm L., J1-Aug, $Y g$.

O. notmani Alex. Keene Val., May; Albany, May, Yg.

O. nubila O.S. Johnstown; Erie Co, Sep-Oct; Ithaca, Ap-May; Trenton Falls; Albany; LI.

O. parallela Doane. Ithaca.

O. perplexa Dietz. Erie Co, May.

O. pygmaea Alex. Fulton Co, Aug; Hamburg, May; Ithaca.

O. rubella O.S. Fulton Co; Green L.; Indian Castle; N. Fair Haven; Erie Co; McLean; Albany, Sep, $Y g$; Tarrytown; West Pt. Je-Sep. 


\section{ERIOPTERA Meigen}

\section{(Subgenus ERIOPTERA Meigen)}

E. chlorophylla O.S. Adirondacks; Sacandaga Pk. Erie Co; Ithaca; Albany; LI. Je-Aug.

E. chrysocoma O.S. Fulton Co; Tarrytown. Je-J1.

E. ebenina Alex. Ontario, Gloversville, Je.

E. megophthalma Alex. Sacandaga Pk.; Indian Castle; Ithaca; Brookview; Tarrytown. Je.

E. septemtrionis O.S. Adirondacks; Essex, Fulton, and Erie Counties; Ithaca; Canastota; Sharon Sps.; Brookview. Ap-Aug.

E. straminea O.S. Sacandaga Pk.; Erie Co. Je.

E. vespertina O.S. Sacandaga Pk.; Hamburg; Vandalia; Ithaca. May-J1.

E. villosa O.S. Erie Co. May-J1.

\section{(Subgenus ILISIA Rondani)}

E. armillaris O.S. Fulton and Erie Counties; Ithaca; Trenton Falls; Lower Cincinnatus. Je-Aug.

E. graphica O.S. Ithaca. J1-Aug.

E. venusta O.S. Adirondacks; Gloversville; Rochester Jct.; Batavia; Erie Co; Gowanda; Ithaca; Hudson R. valley; LI. May-Sep.

(Subgenus Hoplolabis Osten Sacken)

E. armata O.S. Sacandaga Pk.; Indian Castle; Rochester; Erie Co; Ithaca; Tarrytown. May-Sep.

\section{(Subgenus Mesocyphona Osten Sacken)}

E. caloptera O.S. Sacandaga Pk. ; Indian Castle; N. Fair Haven; Erie Co; Ithaca; Lower Cincinnatus; Taylor; Albany; Brookview; LI. May-Sep.

E. needhami Alex. Sacandaga Pk.; Green L., Onondaga Co; Indian Castle; Ithaca; Cincinnatus. Je-J1.

E. parva O.S. Johnstown; N. Fair Haven; Colden; Ithaca. Aug-Sep.

\section{(Subgenus GonemPEDA Alexander)}

E. nyctops Alex. Fulton Co, Je.

\section{(Subgenus EMPEDA Osten Sacken)}

E. stigmatica O.S. Essex Co; Sacandaga Pk.; Erie Co; Ithaca; Lower Cincinnatus; Taylor; Trenton Falls; Helderbergs; Brookview. May-Sep.

\section{Molophilus Curtis}

\section{(Subgenus Molophilus Curtis)}

M. cramptoni Alex. Brookview, Rensselaer Co, Je.

M. forcipula O.S. Gloversville, J1; Niagara Falls, Oct; E. Aurora, Aug.

M. fultonensis Alex. Fulton Co; Lower Cincinnatus; Taylor. J1. 
M. hirtipennis O.S. Adirondacks; Essex Co; Fulton Co; Green L.; Erie Co; Ithaca; Hudson R. valley. Je-Aug.

M. pubipennis O.S. Adirondacks; Sacandaga Pk.; Green L.; Erie Co; Ithaca; Lower Cincinnatus; Taylor; Brookview; Tarrytown. May-Aug.

M. quadrispinosus Alex. Ithaca, Je.

\section{(Subgenus DASYMOLOPHILUS Goetghebuer)}

M. ursinus O.S. Adirondacks; Fulton Co; Lower Cincinnatus; Brookview. Je-J1.

\section{TRIMICRA Osten Sacken}

T. anomala O.S. Erie Co, May-J1; New Rochelle.

\section{Helobia St. Fargeau et Serville}

H. hybrida Meig. Adirondacks; Fulton and Erie Counties; Ithaca; Canastota; Karner; Clinton Hgts.; LI. Mar-Aug.

\section{GNophomyia Osten Sacken}

G. tristissima O.S. Fulton and Erie Counties; Niagara Falls; Ithaca; Hudson R. valley; LI. May-Sep.

Gonomyia Meigen

(Subgenus LiPophleps Bergroth)

G. alexanderi Jn. Sacandaga Pk.; Indian Castle; Johnson. Je-Aug.

G. manca O.S. Sacandaga Pk., Aug; Brookview, Je.

G. sacandaga Alex. Sacandaga Pk., Je-Aug.

(Stibgenus Ptilostena Bergroth)

G. blanda O.S. Ithaca; Taylor; Trenton Falls; Brookview; Albany. Je-J1.

G. mathesoni Alex. Essex Co; Indian Castle; Sacandaga Pk.; Ithaca; Taylor; Brookview. Je-Aug.

\section{(Subgenus Gonomyia Meigen)}

G. cognatella O.S. Sacandaga Pk.; Indian Castle; Gowanda. Je-Aug.

G. florens Alex. Sacandaga Pk. ; Indian Castle; McLean; Brookview. Je.

G. noveboracensis Alex. Sacandaga Pk. Je.

G. subcinerea O.S. Essex, Fulton, and Erie Counties; Green L.; Ithaca; Trenton Falls; Hudson R. valley. May-Aug.

G. sulphurella O.S. Peru; Fulton and Erie Counties; Ithaca; Trenton Falls; Tarrytown; LI. Je-Aug.

RHABDOMASTIX Skuse

(Subgenus SACANDAGA Alexander)

R. flava Alex. Adirondacks; Sacandaga Pk. Je--J1. 


\section{Teucholabis Osten Sacken}

T. complexa O.S. Trenton Falls, Je.

\section{CRYPTOLABIS Osten Sacken}

C. paradoxa O.S. Sacandaga Pk. ; Ithaca; Taylor; Brookview. Je-Jl.

\section{Neolimnophila Alexander}

N. ultima O.S. Hamilton and Fulton Counties; Rochester Jct.; Colden; Tompkins Co, Aug-Oct; Albany, May-Oct.

\section{Cladura Osten Sacken}

(Subgenus NeOcladuRA Alexander)

C. delicatula Alex. Hamilton Co, Sep; Fulton Co; Mayfield Mt., Oct.

\section{(Subgenus Cladura Osten Sacken)}

C. flavoferruginea O.S. Essex Co; Adirondacks; Fulton Co; Oswego; Manlius; Batavia; Niagara Falls; Ithaca; Trenton Falls; Highlands; LI; etc. Aug-Nov.

\section{Chionea Dalman}

C. gracilis Alex. Ithaca, Dec.

C. noveboracensis Alex. Ithaca, Feb.

C. primitiva Alex. Cascade; Owasco L. Nov.

C. valga Harr. Otto, Mar; Ithaca; L. Keuka, Manlius. Oct-Dec.

\section{TOXORHINA Loew}

T. muliebris O.S. Sacandaga Pk.; Hamburg; Ithaca; McLean; LI. Je-Jl.

\section{Tribe HEXATOMINI}

ULA Haliday

U. elegans O.S. Essex Co; Old Forge, Aug; Pinnacle Mt., Sep; Ithaca, May-Je.

U. paupera O.S. Johnstown; Erie Co; Ithaca. May-Je.

\section{EPIPHRAGMA Osten Sacken}

E. fascipennis Say. Adirondacks; Essex Co; Sacandaga Pk.; Green L.; Erie and Cattaraugus Counties; Ithaca; McLean; Hudson R. valley; LI. May-Je.

E. solatrix O.S. Sea Cliff, LI, Je.

\section{Dactylolabis Osten Sacken}

D. cubitalis O.S. Rock City, Je; Ithaca, May.

D. montana O.S. Mt. Buell; Essex Co; Spring Br.; Little Valley; Ithaca; Little Falls; Helderbergs. May-Je. 


\section{Pseudolimnophila Alexander}

P. contempta O.S. Sacandaga Pk., J1.

P. inornata O.S. Adirondacks; Sacandaga Pk.; Green L.; Oswego; Ithaca. May-Aug.

P. luteipennis O.S. Indian Castle; Sacandaga Pk.; Erie Co; Ithaca; Lower Cincinnatus; Taylor; Karner; New Baltimore; LI. May-Sep.

P. nigripleura A. \& L. Fulton Co; Indian Castle; Ithaca; Taylor; Lower Cincinnatus; LI. May-Aug.

P. noveboracensis Alex. Sacandaga Pk., Je; Tompkins, Cortland, Albany, and Rockland Counties. Je-J1.

P. toxoneura O.S. Adirondacks; Fulton Co; Ithaca; Karner; Helderbergs; Brookview. Je-Aug.

\section{LIMNOPHILA Macquart}

\section{(Subgenus Lasiomastix Osten Sacken)}

L. macrocera Say. Adirondacks; Sacandaga Pk.; Erie Co; Little Valley; Ithaca; Lower Cincinnatus; Taylor; Hudson R. valley; LI. Je-Aug.

L. subtenuicornis Alex. Tompkins Co, May-early Je; Helderbergs, Je; Brookview, Je.

L. tenuicornis O.S. Indian Castle; Gloversville; Ithaca; McLean; Brookview. May-Je.

(Subgenus IDIOPTERA Macquart)

L. fasciolata O.S. McLean; Albany. Je.

\section{(Subgenus EPhelia Schiner)}

L. aprilina O.S. Essex and Fulton Counties; Wells; Erie Co; Ithaca; Lower Cincinnatus; Taylor; Karner. May-J1.

L. johnsoni Alex. Fulton Co, Je; Ithaca, May.

L. solstitialis Alex. Fulton and Chenango Counties.

\section{(Subgenus DicRanophragma Osten Sacken)}

L. fuscovaria O.S. Adirondacks; Fulton and Erie Counties; Portage; Ithaca; Lower Cincinnatus; Taylor; Hudson R. valley; LI. May-Aug.

(Subgenus Eutonia Van der Wulp)

L. alleni Jn. Gloversville; Ithaca; Karner; Brookview. Je.

\section{(Subgenus PHYLIDOREA Bigot)}

L. adusta O.S. Adirondacks; Fulton Co; N. Fair Haven; Batavia; Erie Co;

Ithaca; Manlius; Schenectady. May-Sep.

L. auripennis Alex. Adirondack Mts., Aug; Ithaca, May; Brookview, Je.

L. caudifera Alex. Hamilton Co, Je.

L. fumidicosta Alex. Ithaca, Je.

L. lutea Doane. McLean, May. 
L. luteola Alex. Hamilton Co, Je.

L. neadusta Alex. Keene Val., J1, Not.

L. similis Alex. Wells, J1; Fulton Co, Je.

(Subgenus Limnophila Macquart)

L. albipes Leon. Fulton Co, J1; Tarrytown, Je.

L. areolata O.S. Essex Co; Adirondacks; Fulton Co; Green L.; Erie Co; Ithaca; Albany; Brookview. May-Je.

L. brevifurca O.S. Adirondacks; Essex Co; Gloversville; Erie Co; Ithaca; Brookview. May-Je.

L. emmelina Alex. Mt. Buell, Je.

L. fraterna O.S. E. Aurora, May.

L. fratria O.S. Essex Co.

L. laricicola Alex. Fulton Co, Je.

L. niveitarsis O.S. Old Forge, J1; Helderbergs, Je.

L. subcostata Alex. Indian Castle; Fulton Co; Ithaca; Brookview. May-early Je.

L. sylvia Alex. Fulton Co, Je; Brookview, Je.

\section{(Subgenus PRIONOLABIS Osten Sacken)}

L. munda O.S. Essex Co, J1; Fulton Co; Cattaraugus Co, Je.

L. rufibasis O.S. Adirondacks; Peru; Essex, Fulton, and Erie Counties; Ithaca; Hudson R. valley. May-Je.

L. simplex Alex. Fulton Co, Je.

PILARIA Sintenis

P. edwardi Alex. Gloversville, Je.

P. imbecilla O.S. Sacandaga Pk.; Batavia; Buffalo; Gowanda; Trenton Falls; Tarrytown. Aug.

P. lenta O.S. Wells; Johnstown; Green L.; N. Fair Haven; Erie Co; Ithaca; Taylor; Lower Cincinnatus; Corinth; Brookview. May-Sep.

P. quadrata O.S. Sacandaga Pk.; Green L.; Batavia; Ithaca; Lower Cincinnatus; Albany; Tarrytown. May-J1.

P. recondita O.S. Indian Castle; Sacandaga Pk.; Buffalo; Ithaca; McLean; Albany; Brookview; W. Nyack. Je.

P. stanwoodae Alex. Sacandaga Pk., Je; Brookview, Je.

P. tenuipes Say. Essex Co; Sacandaga Pk.; Albany; Brookview; W. Nyack.

\section{ULOMORPHA Osten Sacken}

U. pilosella O.S. Herkimer, Fulton, and Erie Counties; Ithaca; McLean; Taylor; Cincinnatus; Sharon Sps.; Brookview. Je-Jl.

\section{Elephantomyia Osten Sacken}

E. westwoodi O.S. Adirondacks; Fulton and Erie Counties; Lower Cincinnatus: Catskills; Brookview, Je. J1-Aug. 


\section{ATARBA Osten Sacken}

A. picticornis O.S. Sacandaga Pk.; S. Wales; Taylor; Trenton Falls; LI. Je-J1.

\section{PENTHOPTERA Schiner}

P. albitarsis O.S. Fulton and Erie Counties; Ithaca; Lower Cincinnatus; Brookview. Je-Sep.

\section{ERIOCERA Macquart}

E. brachycera O.S. Old Forge; Pinnacle Mt.; Erie Co; Lower Cincinnatus. J1-Aug.

E. cinerea Alex. Fulton Co; Ithaca and vic. May-Je.

E. fuliginosa O.S. Erie Co, May (MCV).

E. fultonensis Alex. Fulton Co; Ithaca. Je.

E. longicornis Wlk. Fulton Co; Dolgeville; N. Evans; Ithaca; Trenton Falls; Albany. May.

E. spinosa O.S. Sacandaga; Ithaca; Trenton Falls; Lower Cincinnatus; Brookview. May-Aug.

E. tristis Alex. Ithaca, Aug.

HeXATOMA Latreille

H. megacera O.S. Fulton Co; Ithaca and vic. May-Je.

Tribe PEDICIINI

ADELPHOMYIA Bergroth

A. americana Alex. Wells; Fulton Co; Ithaca; Taylor. J1-Sep.

A. cayuga Alex. Ithaca, Aug.

A. minuta Alex. Indian Castle; Sacandaga Pk.; Ithaca; McLean; Brookview. May-Je.

\section{Pedicia Latreille}

P. albivitta Wlk. Adirondacks; Fulton, Erie, and Broome Counties; Ithaca; Lower Cincinnatus; Taylor; Manlius; Hudson R. valley. May-Sep.

P. contermina Wlk. Essex Co; Ithaca and vic. May-early Je.

\section{TRICYPHONA Zetterstedt}

T. auripennis O.S. Indian Castle, Je.

T. autumnalis Alex. Adirondacks; Essex and Fulton Counties; N. Fair Haven; Grand I. Late J1-Sep.

T. calcar O.S. Essex and Fulton Counties; Newport; Mt. Marcy, J1; Erie and Cattaraugus Counties; McLean. May-Je.

T. inconstans O.S. Adirondacks; Essex and Fulton Counties; Rochester; Batavia; Erie Co; Portageville; Ithaca; Lower Cincinnatus; Taylor; Manlius; Hudson R. valley; Claverack; LI. May-Oct.

T. macateei Alex. LI: Wyandanch, May.

T. paludicola Alex. McLean, May.

T. vernalis O.S. Essex and Fulton Counties; Indian Castle; Ithaca; LI. Ap-Je. 


\section{RHAPHIDOLABINA Alexander}

R. flaveola O.S. Adirondacks; Fulton Co; Hamburg; Ithaca; Lower Cincinnatus; Brookview. May-Aug.

\section{RHAPHIDOLABIS Osten Sacken}

R. cayuga Alex. Fulton Co, Aug-Sep; Ithaca, Ap-May.

R. forceps Alex. Helderbergs, Je; Brookview, Je.

R. rubescens Alex. Gloversville, Je.

R. tenuipes O.S. Adirondacks; Gloversville; Erie Co; Little Valley; Ithaca; Saratoga; Hudson R. valley. May-Aug.

\section{(Subgenus Plectromyia Osten Sacken)}

R. confusa Alex. (previously recorded as modesta O.S.). Fulton, Erie, and Albany Counties; Holland, May; Helderbergs, Je.

\section{SUBFAMILY CYLINDROTOMINAE}

CylindRotoma Macquart

C. americana O.S. Essex Co, Mt. Marcy, J1.

C. tarsalis Jn. Fulton Co, Je-Aug; Herkimer Co, Je.

\section{LIOGMA Osten Sacken}

L. nodicornis O.S. Essex Co; Sacandaga Pk.; Green L.; Indian Castle; Erie Co; Ithaca; Brookview; Tarrytown. May-Je.

\section{Phalacrocera Schiner}

P. neoxena Alex. N. Fairhaven, May.

P. tipulina O.S. Essex Co, J1; Fulton, Je-Aug; Old Forge; Tompkins Co, J1.

\section{SUBFAMILY TIPULINAE}

\section{DOLICHOPEZA Curtis}

D. americana Ndm. Essex and Fulton Counties, Je; Old Forge, Aug; Little Valley, Je; LI: Flushing, Je.

\section{OROPEZA Needham}

O. albipes Jn. Old Forge; Fulton and Erie Counties; Four Mile; Taylor; Tarrytown; LI. Je-Aug.

O. obscura Jn. Fulton, Erie, and Cattaraugus Counties; Lower Cincinnatus; Taylor; Hudson R. valley. Je-Aug.

O. sayi Jn. Old Forge; Erie Co; Niagara Falls; Ithaca. Je-Aug.

O. subalbipes Jn. Erie Co, J1; Tarrytown, Je.

O. venosa Jn. Essex Co; Fulton Co; Indian Castle; S. Wales; Little Valley; Lower Cincinnatus; Brookview. Je-J1. 
TANYPTERA Latreille

T. frontalis O.S. Essex and Fulton Counties; Rock City; Ithaca. May-Je.

T. fumipennis O.S. Colden; Ithaca, May.

T. topazina O.S. Ithaca; Lancaster; Brookview, Je. May.

Ctenophora Meigen

C. apicata O. S. Mt. Buell, Je; LI, J1.

\section{LONGURIO Loew}

L. testaceus Lw. Gloversville; Lower Cincinnatus; LI. Je-J1.

\section{PRIONOCERA LOW}

P. fuscipennis Lw. Fulton Co; E. Aurora; Ithaca; Albany; Brookview. Je-Aug.

\section{NEPHROTOMA Meigen}

N. brevioricornis Doane. Sacandaga Pk., Je.

N. calinota Dietz. Fulton Co, Je.

N. eucera Lw. Essex Co; Sacandaga Pk.; Ithaca; Manlius; LI. Je-Jl.

N. euceroides Alex. Fulton Co; Sport I., Sacandaga R., Je; Brookview, Je.

N. ferruginea Fab. Adirondacks; Essex and Fulton Counties; Buffalo and vic.; Ithaca; Syracuse, Blkm (Hine); Hudson R. valley; LI. May-Sep.

N. gracilicornis Lw. Manlius, Aug.

N. incurva Lw. Adirondacks; Essex Co; Sacandaga Pk.; Batavia; Erie Co; Niagara Falls; Taylor; Ithaca; Manlius; Hudson R. valley. May-Aug.

N. lugens Lw. Adirondacks; Sacandaga Pk.; Batavia; Rock City; L. Keuka; Ithaca; Karner; Brookview. May-J1.

N. macrocera Say. Sacandaga Pk.; Tarrytown. Je.

N. pedunculata Lw. Adirondacks; Sacandaga Pk.; Taylor; Batavia; Four Mile; LI. Je-J1.

N. polymera Lw. Essex Co; Sacandaga Pk.; Niagara Falls; Ithaca; Brookview. Je.

N. sodalis Lw. Baldwinsville, Je.

N. tenuis Lw. Essex Co; Sacandaga Pk.; Batavia; Erie Co; Rock City; Ithaca; Cincinnatus; Hudson R. valley. Je-Aug.

N. virescens Lw. Fulton Co, Aug; Tompkins Co, J1.

N. xanthostigma Lw. Erie Co, Je-Sep; Sullivan; LI. Je-Aug.

Tipula Linnaeus

(Subgenus Cinctotipula Alexander)

T. algonquin Alex. Essex Co, Je-Aug.

T. unimaculata Lw. New Russia; Hamilton and Fulton Counties; Sodus; Ithaca; Manlius. Aug-Sep. 


\section{(Subgenus Odontotipula Alexander)}

T. unifasciata Lw. Norton's Landing; Manlius. Aug.

\section{(Subgenus TRICHOTIPULA Alexander)}

T. oropezoides Jn. Indian Castle; Fulton Co; Hamburg; Ithaca and vic. May-Je.

\section{(Subgenus TipUla Linnaeus)}

T. abdominalis Say. Adirondacks; Essex Co; Gloversville; Rochester Jct.; Gowanda, etc., May-Sep; Portage; Ithaca; Arkville; Sharon Sps.; Hudson R. valley; LI.

T. afflicta Dietz. S. Wales, J1.

T. angustipennis Lw. Essex Co; Sacandaga Pk.; Indian Castle; Holland; Ithaca; Albany and vic. Ap-Je.

T. annulicornis Say. LI: Riverhead, J1.

T. apicalis Lw. Essex Co; Sacandaga; Ithaca and vic.; Hudson R. valley. May-J1.

T. bella Lw. Sacandaga Pk.; Green L.; N. Fair Haven; Honeoye Falls; Batavia; Erie Co; Ithaca; Hudson R. valley; New Baltimore; LI. May-Sep.

T. bicornis Fbs. Potsdam; Sacandaga Pk.; Erie Co, May-Je; Ithaca and vic.; West Pt.

T. caloptera Lw. Gloversville; Sacandaga Pk.; Indian Castle; Erie Co; Niagara Falls; Wyoming; Ithaca and vic. May-Je. LI, Je-Sep.

T. cayuga Alex. Essex Co; Indian Castle; Gloversville; Ithaca; Brookview. May-Je.

T. centralis Lw. Mt. McIntyre, J1; Indian Ladder, Helderberg Mts., Albany Co, Je 19, ơ.

T. collaris Say. Gloversville; Indian Castle; Erie Co; Little Valley; Albany; Brookview; LI. May-J1.

T. cunctans Say. Alexandria Bay; N. Fair Haven; Clifton Sps.; Batavia; Niagara Falls; Buffalo and vic.; Owego; Ithaca and vic.; Brooklyn. Sep-Oct.

T. dejecta Wlk. Essex Co; Gloversville; Erie Co; Ithaca; Albany; Karner; LI. Ap-May.

T. eluta Lw. Essex Co; Sacandaga Pk. ; Indian Castle; Erie Co; Ithaca; Rhinebeck. May-Aug.

T. fragilis Lw. Adirondacks; Gloversville; Erie Co; Ithaca; New Baltimore. Sep-Oct.

T. fuliginosa Say. Sacandaga Pk. ; Indian Castle; Mt. Buell; Erie Co; Rock City; Portage; Conesus L.; Ithaca and vic.; Helderbergs; Brookview. Je-Jl.

T. fultonensis Alex. Essex and Fulton Counties. Je.

T. georgiana Alex. New Rochelle.

T. grata Lw. Old Forge, Je; Buffalo, Aug.

T. hebes Lw. Old Forge; Warren and Fulton Counties; Batavia; Erie Co; Ithaca Taylor; Cincinnatus; LI. Je-Aing.

T. helderbergensis Alex. Wells; Indian Ladder, Helderberg Mts. J1.

T. hermannia Alex. Essex and Fulton Counties; Adirondacks; Niagara Falls; Ithaca; Taylor; Cincinnatus; Sharon Sps.; New Baltimore; Hudson R. valley. Je-Aug. 
T. hirsuta Doane. Mayfield Mt., Fulton Co, Je.

T. ignobilis Lw. Ithaca; Helderbergs; Catskills. May-J1.

T. iroquois Alex. Essex and Fulton Counties; Indian Castle; Portage; Ithaca and vic. May-Je.

T. latipennis Lw. Sacandaga Pk.; Batavia; Grand I., Erie Co. Je-early Aug.

T. longiventris Lw. Fulton Co; E. Aurora; LI. May-Aug.

T. macrolabis Lw. Essex Co; Mt. Buell; Indian Castle; Helderbergs. Je-Jl.

T. manahatta Alex. LI: Yaphank, Sep.

T. margarita Alex. Ithaca, Je; Helderbergs, Je.

T. mingwe Alex. Hamilton Co; Sacandaga Pk.; Sodus; Batavia; Ithaca; Manlius; Sharon Sps. J1-Sep.

T. monticola Alex. Fulton Co; Indian Castle; Rock City; Ithaca and vic.; Wells, J1. Je.

T. nobilis Lw. Essex and Fulton Counties; Wyoming Co; McLean; Albany Co. Je.

T. noveboracensis Alex. Essex Co, May; Fulton Co, Je; Tompkins Co, May; Albany Co, May.

T. parshleyi Alex. Axton, Je; Essex Co.

T. penobscot Alex. Mt. Buell, Je.

T. perlongipes Jn. Fulton Co; LI: Flushing, Je.

T. sackeniana Alex. Ithaca, Aug.

T. sayi Alex. Adirondacks; Essex Co; Gloversville; N. Fair Haven; Batavia; Erie Co; Hudson R. val. J1-Sep.

T. senega Alex. Essex, Fulton, and Erie Counties; Ithaca and vic.; Helderbergs. May-Je.

T. serta Lw. Gloversville; Erie Co; Ithaca; Brookview. May-Je.

T. strepens Lw. Sacandaga Pk.; Indian Castle; Niagara Falls; Ithaca; Cincinnatus; Taylor; Corinth; Palisades. May-J1.

T. submaculata Lw. Essex and Fulton Counties; Batavia; N. Evans; Four Mile; Ithaca; Cincinnatus; Corinth; Albany Co. Je-J1.

T. sulphurea Doane. Fulton Co, Je; Green L., Je.

T. taughannock Alex. Fulton Co; Taughannock Falls; Helderbergs. May-Je.

T. tephrocephala Lw. Adirondacks; Sacandaga Pk.; Batavia; Ithaca and vic.; Hudson R. valley. May-Je.

T. tricolor Fab. Gloversville; Ithaca; Batavia; Cincinnatus; Niverville; LI. Late May-Sep.

T. trivittata Say. Sacandaga Pk. ; Batavia; Erie Co; Ithaca; Cortland Co; Hudson R. valley. May-Je.

T. ultima Alex. Essex Co; Adirondacks; Gloversville; N. Fair Haven; Batavia; Erie Co; Ithaca; Delhi; Peekskill; LI. Sep.

T. umbrosa Lw. Sacandaga Pk. ; Adirondacks; Ithaca. Jl-Aug.

T. valida Lw. Fulton Co; Green L.; Indian Castle; Erie Co; Rock City; Ithaca; Corinth; Helderbergs. Late May-early J1.

T. vicina Dietz. Erie Co, late May-early Je. 


\section{FAMILY DIXIDAE}

\section{DIXA Meigen}

D. centralis Lw. "NY" (Lw), type; Elizabethtown, J1 (Garr-NYS); Karner, Nov (Garr-NYS).

D. clavata Lw. Sulphur Sp. Jct., Sep (Joh); Elizabethtown, Je, Aug (GarrNYS) ; Speculator, J1 (Garr-NYS); Sodus Pt., Aug (Garr); Springville, Protection, Je (MCV) ; Karner, Nov, Albany, Oct (Garr-NYS).

D. cornuta Joh. Ithaca, Aug-Oct (Joh); McLean, Oct (Joh), holotype.

D. fusca Lw. "NY" (Lw), type; Colden, May, Aug (MCV); Ithaca, Je (Joh).

D. modesta Joh. Wells (Garr-NYS); Elizabethtown, Je (Garr-NYS); Ithaca, Ap-Oct (Joh), holotype; McLean, May (Joh).

D. similis Joh. Ithaca, Aug (Joh), holotype; Albany, May (Garr-NYS).

D. terna Lw. "NY" (Lw), type; Wells, J1 (Garr-NYS) ; Hamburg, Colden, May (MCV); Ithaca (Joh).

FAmily CULicidaE

ROBERT MATHESON

\section{SUBFAMILY CHAOBORINAE}

(Corethrinae auct.)

\section{EUCORETHRA Underwood}

(Pelorempis Johannsen)

E. underwoodi Underwood. Saranac L., Je, Ndm; Plattsburg, Aug (Dyar); Elizabethtown, Je (F\&Y); Ithaca, Ap-Oct (Mat); Utica, Je, Sib.

\section{CORETHRA Meigen}

\section{(Mochlonyx Loew)}

C. culiciformis DeG. (karnerensis Felt, linteri Felt). Elizabethtown, May-Aug (F\&Y); Plattsburg, Aug (Dyar); Old Forge, J1 (F\&Y); Karner, Je (F\&Y) ; Ithaca, Ringwood, May (Mat).

C. cinctipes Coq. Elizabethtown, Je (F\&Y); Ringwood, May-Je (Mat); Ithaca, May (Mat) ; Delmar, Karner, May (F\&Y).

C. fuliginosus Felt. Big Moose, Big Chief, Je (F\&Y); Nassau, Je (F\&Y).

\section{СнаовоRUS Lichtenstein}

\section{(Sayomyia Coq. ; Corethra auct.)}

C. crystallina DeG. (rotundifolia Felt). Ringwood, Je (Mat); Ithaca, Sep (Mat); Karner, J1-Aug ( F\&Y).

C. americanus Joh. (hudsoni Felt). Saranac Inn, Je (Joh); Elizabethtown, I1 $(\mathrm{F} \& \mathrm{Y})$; Old Forge, J1 (F\&Y); Karner, Je (F\&Y); Poughkeepsie, Je $(\mathrm{F} \& \mathrm{Y})$; SI, Ap (Mat).

C. albipes Joh. Clifton Sps., Aug-Sep (F\&Y); Ithaca, Sep (Joh); Bath-onHudson, Je ( F\&Y).

C. trivittatus Lw. Elizabethtown, Je-Aug (F\&Y). 
C. punctipennis Say. Old Forge, Je-Aug (F\&Y) ; Long L., Aug (F\&Y); Ithaca, Sep (Mat); McLean, Aug (Mat); Poughkeepsie, Je (F\&Y).

\section{SUBFAMILY CULICINAE}

\section{ANopheles Meigen}

A. barberi Coq. Ithaca, Sep, 1923 (Mat).

A. crucians Wied. LI: Bellport, Oct (Dyar).

A. maculipennis Meig. Raybrook, Buttermilk Falls on Raquette R., Franklin Co, and Old Forge, Aug (Mat\&Snn).

A. punctipennis Say. Elizabethtown, Je (F\&Y); Newport, Aug (F\&Y) ; N. Fair Haven, J1 (Mat) ; Sodus Pt., Aug (F\&Y) ; Clifton Sps., Aug (F\&Y) ; Erie Co, Sep-Oct (MCV); Ithaca, Jan-Dec (Joh, Mat) ; McLean, J1 (Mat) ; Freeville, J1 (Mat); Onteora Club, Ap (How) ; Albany, Aug-Sep (F\&Y); Nassau, Sep (F\&Y); Rensselaer, J1 (F\&Y); Poughkeepsie, May (F\&Y); White Plains (Beq) ; Pine I., Sep (F\&Y) ; Ramsey, Je (Mat); SI, J1 (Mat); LI: Oyster Bay, Glen Cove, J1 (F\&Y).

A. quadrimaculatus Say. Old Forge, Aug (F\&Y); Wells, J1 (F\&Y); N. Fair Haven, J1 (Mat); Sodus Pt., Je-Aug (F\&Y); Clifton Sps., Aug (F\&Y); Ithaca, Je-Nov (Joh, Mat) ; McLean, Aug (Mat) ; Albany, Aug-Sep (F\&Y) ; S. Salem, Je (F\&Y); SI, Sep (Mat) ; LI: Bellport, Aug (Dyar).

A. walkeri Theo. N. Fair Haven, Je-Aug (Mat \& Snn); Sodus Pt., Aug-Sep (Mat); Duck L., J1 (Mat); Dryden L., J1 (Mat).

\section{WYEOMYIA Theobald}

W. smithii Coq. Saranac Inn, J1 (F\&Y); Tupper L., Aug (Dyar); Old Forge, Je-J1 (F\&Y); E. Crooked Pond, JI (F\&Y) ; Big Moose, Je (F\&Y) ; Duck L., J1 (Mat); McLean, Je-Aug (Joh, Mat).

\section{Culex Linnaeus}

C. pipiens L. Sodus Pt., Sep (F\&Y) ; Erie Co, J1-Oct (MCV) ; Schuyler, Aug $(\mathrm{F} \& \mathrm{Y})$; Ithaca, all year (Joh, Mat) ; Albany, Je-Sep (F\&Y); Karner, Oct $(F \& Y)$; Voorheesville, Aug, Leon (Mat); Clinton Hgts., Sep (F\&Y); Nassau, Aug (F\&Y); SI, J1 (Ds) ; LI: Baldwin, May (Ds); Sheepshead Bay, Je-Sep ( F\&Y).

C. salinarius Coq. E. Sodus, May, Westfield, Aug, Karner, May, Nassau, Sep, E. Schodack, May, F\&Y (Mat); LI: Oyster Bay, J1, F\&Y (Mat).

C. territans W1k. (restuans Theo.). Elizabethtown, Je-J1 (F\&Y); Saranac Inn, J1 (F\&Y); Old Forge, Aug (F\&Y); Tupper L., Aug (Dyar); Newport, Aug $(F \& Y)$; Sodus, Aug $(F \& Y)$; Sodus Pt., Je-Sep $(F \& Y)$; Syracuse, J1 $(\mathrm{F} \& \mathrm{Y})$; Niagara Falls, Aug (F\&Y); Westfield, Aug (F\&Y); Ithaca, Je-Aug (Joh, Mat); Onteora Club (How); Mohawk, J1 (F\&Y); Albany, May-Sep (F\&Y); Karner, May-J1 (F\&Y); Clinton Hgts., Sep $(F \& Y)$; Nassau (F\&Y); Binnewater, May-Aug (F\&Y); Poughkeepsie, Je $(F \& Y)$; LI: Sheepshead Bay, Aug (F\&Y).

C. apicalis Adams (territans auct. not W1k., saxatilis Grossbeck, testaceus Dyar not Wulp). Big Moose, J1 (F\&Y); Newport, Aug (F\&Y); Old Forge, J1 (F\&Y) ; Tupper L., Aug (Dyar) ; Elizabethtown, J1 (F\&Y) ; Sodus Pt., Aug $(\mathrm{F} \& \mathrm{Y})$; N. Fair Haven, J1-Aug (Joh, Mat); Schuyler, Aug (F\&Y); Ithaca, 
Je-Oct (Joh, Mat) ; McLean, J1-Aug (Mat) ; Ringwood, Je-J1 (Mat); Albany, May-Sep (F\&Y) ; Karner, May-Sep (F\&Y) ; Bath, Je-J1 (F\&Y); Clifton Sps., Aug (F\&Y); Nassau, Sep (F\&Y); Poughkeepsie, May-Je $(\mathrm{F} \& \mathrm{Y})$; LI: Bellport, Aug (Dyar).

\section{URANOTAENIA Lynch Arribálzaga}

U. sapphirinus O.S. Sodus Pt., Aug (F\&Y); Ithaca, Aug-Oct (Joh); Albany, Aug $(F \& Y)$; Greenbush, Aug $(F \& Y)$; LI: Brooklyn (F\&Y); Bellport, Sep (Dyar).

Theobaldia Neveu-Lemaire

\section{(Culiseta Felt)}

T. dyari Coq. (brittoni Felt). Elizabethtown, May-Je (F\&Y); Big Moose, Je (F\&Y); Tupper L., Aug (Dyar) ; Ithaca, May-Je (Mat); Ringwood, Je (Mat); McLean, Je-J1 (Mat).

T. impatiens W1k. (absorbrinus Felt). Elizabethtown, Jl-Aug (F\&Y); foot of Cliff Mt., Essex Co (Beq); Saranac Inn, J1 (F\&Y); Mt. Marcy, Jl, Cy (Mat); Tupper L. (Dyar).

T. inornatus Will. (magnipennis Felt). Saranac Inn, Aug (F\&Y); Old Forge, Je $(\mathrm{F} \& \mathrm{Y})$; N. Fair Haven, J1 (Mat) ; Sodus Pt., Aug (F\&Y); Ithaca, Ap-Nov (Mat).

T. melanurus Coq. Tupper L., Aug (Dyar); Duck L., Aug (Mat).

\section{TAenioRhynchus Lynch Arribálzaga}

\section{(Mansonia Blanchard)}

T. perturbans W1k. Old Forge, J1 (F\&Y); N. Fair Haven, Je-Aug (Mat); Sodus Pt., Je-Aug (F\&Y) ; Sodus, Jl (F\&Y) ; LeRoy, Je (F\&Y) ; Dryden L., JeAug (Mat); Ithaca, J1-Aug (Mat), Je (Joh).

\section{PSOROPHORA Robineau-Desvoidy}

P. ciliata Fab. Valcour I. (Mat-NYS); Sodus Pt., Aug (F\&Y), J1-Sep (Mat); Duck L., Aug, West (Mat); N. Evans, Aug (MCV); Ithaca, Aug-Sep (Joh, Mat) ; Albany, Aug-Sep (F\&Y) ; Karner, Je-J1 (F\&Y) ; Clifton Sps., Aug (F\&Y); Arlington, J1 (F\&Y) ; Warwick, J1 (F\&Y); SI, J1-Aug (Ds);

LI: E. Quogue, Aug, Ds (Mat); Sheepshead Bay, J1-Aug, Cy (Mat).

P. columbiae D. \& K. LI: Cold Sp. Harb., J1 (Lutz).

P. sayi D. \& K. Poughkeepsie, Aug (F\&Y); LI: Sheepshead Bay, Jl, Cy (Mat).

\section{AEDES Meigen}

A. abserratus (F\&Y). Plattsburg, Ap (D\&H) ; Elizabethtown, Je (F\&Y); McLean, Ringwood, May (Mat); Onteora Club, Je (How); Nassau, May $(\mathrm{F} \& \mathrm{Y})$; Chapel Pond, Je, Leon (Mat).

A. atlanticus D. \& K. SI (F\&Y).

A. atropalpus Coq. Plattsburg, J1-Aug (Mat-NYS); Old Forge, Aug (Mat); McLean, J1 (Mat).

A. aurifer Coq. Elizabethtown, Je (F\&Y); Baldwinsville, May (F\&Y); N. Fair Haven, Je-J1 (Mat); Sodus Pt., Je (F\&Y); Ringwood, May (Mat). 
A. canadensis Theo. Keene Val., Je (F\&Y); Elizabethtown, May-J1 (F\&Y); Saranac Inn, J1 (F\&Y) ; E. Crooked Pond, J1 (F\&Y) ; Old Forge, Je-Aug $(\mathrm{F} \& \mathrm{Y})$; Big Moose, Big Chief, Je (F\&Y); Fulton Chain, Aug (F\&Y); Newport, Je (F\&Y); Herkimer, Aug (Mat); W. Winfield, Je (Mat); N. Fair Haven, Jl (Mat); Sodus Pt., Aug (Mat); Duck L., Je (Mat); Ithaca, May-Aug (Joh, Mat); McLean, Ringwood, May-Aug (Mat); Onteora Club, May-Je (How); Schodack, May (F\&Y); Delmar, May (F\&Y); Clarksville, Aug (F\&Y); Karner, May-Sep (F\&Y); Rensselaer, May (F\&Y); Nassau, May-Je (F\&Y); Poughkeepsie, J1 (F\&Y); SI, Ap (Mat), Je-Sep, Ds (Mat).

A. cantator Coq. SI, Aug (F\&Y), Sep (Mat); LI: Brooklyn, Cold Sp. Harb., May $(F \& Y)$; Sheepshead Bay, Je-Aug (F\&Y); Riverhead, Sep (Mat); Woodmere, Sep $(F \& Y)$.

A. cinereoborealis F. \& Y. Elizabethtown, May-Je (F\&Y); Old Forge, Je (F\&Y), Aug (Mat); Herkimer, Aug (Mat); Ithaca, May (Joh, Mat); Ringwood, May (Mat); Albany, May (F\&Y); Karner, May (F\&Y); Nassau, May $(F \& Y)$; Delmar, May (F\&Y); Normanskill, May (F\&Y).

A. cinereus Meig. (fuscus O.S.). Elizabethtown, Je-J1 (F\&Y); Old Forge, J1 (F\&Y); Big Moose, Je (F\&Y); Duck L., Je (Mat); N. Fair Haven, Je (Mat); Sodus Pt., Je, Aug (F\&Y); Buffalo, Sep-Nov (EPV); Ithaca, May-Je (Joh, Mat); Ringwood, May (Mat); McLean, May-Aug (Mat); Karner, May-Je (F\&Y); Bath, May (F\&Y); Nassau, May (F\&Y); Poughkeepsie, May (F\&Y).

A. diantaeus D. \& K. Ringwood, Je (Mat).

A. dorsalis Meig. (curriei Coq., onondagensis Felt, lativitattus Coq., quaylei D. \& K.). Syracuse, May-Sep (F\&Y); Baldwinsville, May (F\&Y); Ithaca, May, J1Aug (Joh, Mat); Albany, Aug-Sep (F\&Y).

A. excrucians W1k. (abfitchii Felt, siphonalis Grossbeck, sansoni D. \& K., enedes H. D. \& K.). Elizabethtown, May (F\&Y); Saranac Inn, J1 (F\&Y) ; Plattsburg, Ap (Dyar) ; L. George, Aug (Mat) ; Old Forge, Je-J1 (F\&Y); Herkimer, Aug (Mat); N. Fair Haven, J1 (Mat) ; Olcott, Je (Mat) ; Salamanca, Je (Mat); Watkins, May (Dyar); Ithaca, May-Aug (Joh, Mat); McLean, May-J1 (Mat); Ringwood, May-Aug (Mat); Onteora Club, Jl (How); Trenton Falls, Je (Mat); Albany, May-Je (F\&Y); Rensselaer, May (F\&Y); Karner, May (F\&Y) ; Delmar, May (F\&Y); Voorheesville, J1, Leon (Mat); Victory Mills, May, Leon (Mat); LI: Forest Pk., Je (F\&Y); Sheepshead Bay, Je-J1, Cy (Mat).

A. fitchii F. \& Y. Elizabethtown, May (F\&Y); Plattsburg, Ap (Dyar) ; Keene Val., Aug (F\&Y) ; Newport, Je-Aug (F\&Y); Wells, J1 (Mat); Herkimer, Aug (Mat); Syracuse, J1 (Mat); Sodus Pt., Je (F\&Y); Salamanca, Je (Mat) : Ithaca, Ap-Aug (Mat); McLean, Ringwood, May-Aug (Mat); Watkins, May (Dyar); Atwater, J1 (Mat); Trenton Falls, Je (Mat); Victory Mills, May, Leon (Mat); Meadowdale, May, Leon (Mat); Karner, May (F\&Y) ; Delmar, May (F\&Y) ; Nassau, May-Je (F\&Y) ; Niverville, Je (F\&Y) ; Onteora Club, Je-Aug (How); N. Salem, J1, Leon (Mat).

A. hirsuteron Theo. (pretans Grossbeck, aestivalis Dyar). Ithaca, May-Sep (Mat); Karner, Je $(F \& Y)$; Easton, May, Leon (Mat); LI: Sheepshead Bay, Ji $(\mathrm{F} \& \mathrm{Y})$.

A. impiger Wlk. (decticus H. D. \& K.). Elizabethtown, Ap (Dyar); Plattsburg, Ap (Dyar).

A. intrudens Dyar (impiger H. D. \& K. nec Wlk.). Elizabethtown, May-J1 (F\&Y) ; Big Moose, Je (F\&Y); Old Forge, Je, Aug (F\&Y); Saranac Inn, J1 (F\&Y); Duck L., Je (Mat); Sodus Pt., Jl (Mat); Ithaca, J1, Sep (Mat); Ringwood, 23 
May-Sep (Mat) ; McLean, Jl (Mat); Albany, May (F\&Y); Normanskill, May (F\&Y) ; Karner, May-Je (F\&Y) ; Delmar, May (F\&Y) ; Nassau, MayJe (F\&Y); Chapel Pond, Je, Leon (Mat); Onteora Club, Je (How).

A. lazarensis F. \& Y. Elizabethtown, May-J1 (F\&Y); Old Forge, Je (F\&Y); Big Moose, Je (F\&Y); Big Chief, Je (Mat); Plattsburg, Ap (Dyar); Saranac Inn, J1 (F\&Y); McLean, Ringwood, May (Mat); Delmar, Aug (F\&Y); Nassau, May (F\&Y).

A. punctor Kby. (auroides Felt, centrotus H. D. \& K.). Peru, Je (Mat) ; Elizabethtown, May-J1 (F\&Y); Big Chief, Big Moose, Je (F\&Y); Saranac Inn, J1 (F\&Y) ; Old Forge, Je-J1 (F\&Y) ; Duck L., Je (Mat); N. Fair Haven, J1 (Mat); Salamanca, Je (Mat); Ithaca, May-Aug (Mat); Ringwood, May-J1 (Mat) ; McLean, May (Mat); Thomson, May, Leon (Mat) ; Karner, May-Je (F\&Y); Voorheesville, May, Leon (Mat); Meadowdale, May, Leon (Mat); Nassau, May (F\&Y); Chapel Pond, Je, Leon (Mat); Thacher Pk., May, Leon (Mat).

A. sollicitans Wlk. Syracuse, Jl-Aug (F\&Y) ; SI: J1-Aug (F\&Y, Ds) ; Richmond, Je (Mat) ; LI: Woodmere, Sep (F\&Y) ; Sheepshead Bay, Forest Pk., Je-Sep (F\&Y) ; Wyandanch, J1 (Ds); Riverhead, Je, Sep (Mat); Farmingdale, J1 (Mat); Bellport (Dyar); Yaphank, Oct (Ds); Hog Pt., Aug (F\&Y).

A. stimulans Wlk. (subcantans Felt). Plattsburg, Ap (Dyar); Newport, Je (F\&Y) ; Piseco L., Aug (Mat) ; Duck L., Je (Mat) ; Sodus Pt., Je (F\&Y) ; Westfield, Je (Mat) ; Atwater, J1 (Mat) ; Ithaca, McLean, Ringwood, Ap-Aug (Mat) ; Karner, May-Aug (F\&Y) ; Delmar, May (F\&Y); Nassau, May-Je (F\&Y); Niverville, Je (F\&Y); Poughkeepsie, May (F\&Y); SI, May-Je (Ds).

A. taeniorhynchus Wied. NYC, J1 (Ds); SI, Aug (Ds); LI: Half Way Hollow Hills, J1 (Ds) ; Sheepshead Bay, J1-Aug (F\&Y); Hog Pt., Aug (F\&Y); Forest Pk., Je (F\&Y) ; Bellport, Aug (Dyar); Babylon, Sag Harb., J1 (Dyar).

A. triseriatus Say. Elizabethtown, J1-Aug (F\&Y); Old Forge, Aug (Mat); L. Mahopac, J1 (F\&Y) ; N. Fair Haven, Je-Jl (Mat) ; Erie Co, Je-Aug (MCV) ; Ithaca, May-Sep (Joh, Mat); McLean, J1 (Mat); Michigan Hollow, J1 (Mat); Albany, Aug (F\&Y); Karner, May (F\&Y); Ramapo, Oct (Ds); LI: Wyandanch, J1 (Mat); Yaphank, Half Way Hollow Hills, J1 (Ds); Cold Sp. Harb., Je (F\&Y).

A. trivittatus Coq. (inconspicuus Grossbeck, angustivittatus D. \& K.). Ithaca, Taughannock Falls, Sep (Mat); Albany, Je (F\&Y); Karner, Je (F\&Y); Bath, J1 (F\&Y).

A. vexans Meig. (sylvestris Theo.). Elizabethtown, J1 (F\&Y); Old Forge, J1 (F\&Y) ; Big Moose, J1 (F\&Y) ; Tupper L., Aug (Dyar); Newport, Je, Aug (F\&Y); Wells, J1 (Mat) ; Syracuse, J1 (F\&Y) ; Sodus Pt., Je-Aug (F\&Y) ; Erie Co, May-Aug (MCV); Ithaca, May-Sep (Joh,Mat); McLean, Sep (Mat); Albany, May-Je (F\&Y) ; Karner, Je-Sep (F\&Y); Nassau, Je, AugSep (F\&Y); Clifton Hgts., May, Sep (F\&Y); Rensselaer, J1 (F\&Y); Voorheesville, J1-Aug, Leon (Mat); Onteora Club (How); Poughkeepsie, Je $(F \& Y)$; Phoenicia, Aug (F\&Y); Pine I., Sep (F\&Y); SI: Je (Ds); Clove Valley, J1 (F\&Y); LI: Hog Pt., Aug (F\&Y); Riverhead, Sep (Ds) ; Sheepshead Bay, Je-Aug (F\&Y).

\section{FAMILY PSYCHODIDAE}

PERICOMA Walker

P. albitarsis Bks. S. Wales, Je-J1 (MCV); Ithaca, Ap-Jl.

P. basalis Bks. Rensselaer, May; Nassau, Je (NYS). 
PSyCHODA Latreille

P. alternata Say. Buffalo, Sep-Oct (MCV); Ithaca, Aug, Dec (Joh); Nassau, Sep; LI: Sea Cliff, J1 (Bks).

P. aterrima Bks. Ithaca (Bks).

P. bicolor Bks. LI: Sea Cliff (Bks).

P. marginalis Bks. Erie Co, J1 (MCV); LI: Sea Cliff, Je (Bks).

P. nitida Bks. Erie Co, May-Aug (MCV).

P. opposita Bks. Gowanda, Je (MCV).

P. phalaeroides L. (cinerea Bks., longifringa Has., mimuta Bks.). Hamburg, Oct, N. Evans, May, Erie, Je-Aug (MCV) ; Ithaca, Je, Aug (Joh); Albany, May; Nassau, Sep (NYS); Poughkeepsie, Je (NYS); LI: Sea Cliff (Bks).

P. slossoni Will. Watkins Glen (Will); Ithaca, Je, Aug (Bks).

P. superba Bks. Colden, May, J1, Buffalo, Oct (MCV); LI: Sea Cliff, Je (Bks).

MARUINA Müller

M. nigra Bks. Gowanda, Je, Little Valley, May (MCV); LI: Sea Cliff (Bks).

\section{FAMILY CHIRONOMIDAE} SUBFAMILY CERATOPOGONINAE

Culicoides Latreille

C. biguttatus Coq. Ithaca, J1 29 (Joh); McLean, J1, Sib (Joh).

C. guttipennis Coq. Ithaca, JI (Mall); McLean, Je, Sib (Joh).

C. sanguisuga Coq. Ithaca, Je-J1, Nov (Mall); McLean, Claas (Joh).

C. stellifer Coq. Speculator, Je (Hoff).

C. variipennis Coq. Ithaca, Je ( Joh).

C. venustus Hoff. Nassau, Je (Hoff).

\section{Ceratopogon Meigen}

C. culicoidithorax Hoff. Karner, May (Hoff).

C. fuscinervis Mall. Ithaca, Freeville, etc., Tompkins Co, J1 (Mall); McLean, Je, Sib. (Joh).

C. fusculus Coq. Ithaca, Je-J1, Joh (Mall).

C. levis Coq. Ithaca, Je-Jl (Mail).

C. peregrinus Joh. Old Forge; Ithaca, Jl-Aug (Joh); McLean, Claas (Joh).

\section{EUforcipomyia Malloch}

E. eques Joh. Old Forge, Je-J1; Ithaca, Je (Joh).

\section{PSEudoculicoides Malloch}

P. mutabilis Coq. Ithaca, J1 (Mal1); McLean (Joh).

P. major Mall. Ithaca (Mall). 


\section{FoRCIPOMYIA Meigen}

F. cilipes Coq. Ithaca, J1-Nov (Mall).

F. coquilletti Kieff. Adirondacks.

F. specularis Coq. Erie Co, Ap-Aug (MCV); Ithaca, McLean, Ap-Aug (Mall).

F. squamipes Coq. Fulton Co; Ithaca and vic., J1-Aug (Mall).

\section{PALPOMYIA Meigen}

P. illinoensis Mall. Ithaca.

P. longipennis Lw. Ithaca, J1 (Joh).

P. nebulosa Mall. McLean, J1 (Mall).

P. subasper Coq. Ithaca, J1 (Mall).

P. tibialis Meig. Hamburg, Je (MCV); Tompkins Co, Je-J1 (Joh).

\section{JOHANNSENOMYIA Malloch}

J. aequalis Mall. Hamburg, Je (MCV); Ithaca, Aug (Mall).

J. albaria Coq. var. magnipennis Joh. Old Forge, Je; Ithaca, J1 (Joh).

J. argentata Lw. Lancaster, Je, Aug (MCV); Ithaca, Je-J1 (Joh).

J. bimaculata Lw. "NY" (Lw).

J. caudelli Coq. N. Evans, Hamburg, May (MCV); Tonawanda, Aug (MCV). J. polita Coq. Ithaca, May (Joh).

\section{STILOBEZZIA Kieffer}

(Hartomyia Malloch)

S. antennalis Coq. Lewiston, Je (MCV).

S. mallochi Hoffm. McLean, Je, Sib (Joh).

\section{Heteromyia Say}

H. fasciata Say. "NY".

H. flavipes Meig. (flaviceps Joh., basalis Walk.). Ithaca, McLean (Joh).

H. plebeja Lw. Ithaca (Mall); McLean, Aug, Sib (Joh).

H. rufa Lw. Erie Co, Je, J1 (MCV); Ithaca (Joh).

H. trivialis Lw. Erie Co, Je-Aug (MCV); Ithaca, McLean, J1 (Mall).

\section{SERROMYia Meigen}

S. crassifemorata Mall. Lewiston, J1 (MCV).

S. femorata Meig. Ellis, Je (Joh).

\section{BEZZIA Kieffer}

B. flavitarsis Mall. Ithaca, Freeville, J1 (Mall).

B. setulosa Lw. Ithaca, J1 (Joh,Mall).

B. varicolor Coq. LI: Bellport (Coq). 


\section{Probezzia Kieffer}

P. albiventris Lw. Near Buffalo (MCV).

P. glaber Coq. Saranac Inn, J1; Ithaca, J1-Aug (Ma11).

P. obscura Mall. Ithaca, J1 (Mall).

P. pallida Mall. Lancaster, Je (MCV).

P. xanthogaster Kieff. (elegans Coq.). Fulton Co, Je; Portage, J1 (MCV); Ithaca, J1 (Joh).

\section{SUBFAMILY TANYPINAE \\ TANYPUS Meigen \\ (Ablabesmyia Johannsen)}

T. arietinus Coq. Plattsburg, Aug-Sep (Coq).

T. bifasciatus Coq. Lancaster, Ap, Hamburg, May (MCV).

T. carneus Fab. Fulton Co, J1; Ithaca, J1 (Joh); McLean, J1; Nassau, J1.

T. decoloratus Mall. McLean, Je, Sib (Joh).

T. dyari Coq. Fulton Co; Ithaca, Je (Joh); Albany; LI: Sheepshead Bay, Je; Bellport, Aug (Coq).

T. fastuosus Joh. Old Forge, J1; Ithaca, Ap (Joh); McLean, J1; Clinton Hgts.

T. florens Joh. Ithaca, McLean, J1 (Joh).

T. flavifrons Joh. Ithaca, Ap (Joh).

T. hirtipennis Lw. Old Forge, Jl-Aug, $N d m$ (Joh); Hamburg, May (MCV).

T. illinoiensis Mall. Erie Co, Je-Sep (MCV).

T. indecisus Will. McLean, Aug, Sib (Joh).

T. johnsoni Coq. Old Forge, J1 (Joh).

T. marginellus Mall. Ithaca, J1-Aug (Joh); McLean, J1, Sib (Joh).

T. melanops Wied. Erie Co, May-Sep (MCV); Ithaca, J1; McLean, J1 (Joh).

T. monilis L. Fulton Co, J1; Saranac Inn, Je; Buffalo, Sep (MCV); Ithaca, May-J1 (Joh); McLean, Aug, Sib (Joh); Nassau, Je.

T. ornatus Meig. Ithaca, J1 (Joh).

T. pallens Coq. Fulton Co; Niagara Falls, Je (Jn); Colden, May (MCV); Ithaca, Aug (Joh).

T. sinuosus Coq. Fulton Co (Joh).

\section{PROTENTHES Johannsen}

P. culiciformis L. Fulton Co; Old Forge, Je; Lewiston, May (MCV); Erie Co, Je-Sep (MCV) ; Ithaca, J1 (Joh); McLean, J1, Sib (Joh).

P. pulcher Joh. Old Forge, Je (Joh).

P. stellatus Coq. Ithaca, J1 (Joh); McLean, J1, Sib (Joh); Poughkeepsie.

P. vestitipennis Kieff. Long L. (Kieff).

\section{Coelotanypus Kieffer}

C. tricolor Lw. "NY" (Lw). 
TRICHOTANYPUS Kieffer

T. persimilis Joh. (posticalis Joh. nec Lundb.). Ithaca, McLean, Ap (Joh).

\section{Procladius Skuse}

P. adumbratus Joh. Ithaca, J1 (Joh).

P. bellus Lw. Old Forge, J1-Aug (Joh).

P. pusillus Lw. Fulton Co; Ithaca, J1 (Joh); McLean, J1, Sib (Joh).

\section{Clinotanypus Kieffer}

C. caliginosus Joh. Buffalo, Je (MCV); Ithaca, J1, Oct (Joh), J1, Sib (Joh).

C. pinguis Lw. Ithaca, Aug (Joh).

\section{SUBFAMILY CHIRONOIMINAE}

Diamesa Meigen

D. waltlii Meig. Fulton Co; Lancaster (MCV); Ithaca, Nov-Ap (Joh); Albany, Jan.

PSilodiamesa Kieffer

P. fulva Joh. Ithaca (Joh).

Prodiamesa Kieffer

P. notata St. McLean, Sep, Sib (Joh).

Cardiocladius Kieffer

(Thalassomyia Am. auct.)

C. fulvus Joh. Old Forge, Ji (Joh).

C. obscurus Joh. Old Forge, J1, Aug; Niagara Falls, Je, J1 (MCV); Ithaca, J1-Sep (Joh); McLean, Claas (Joh).

C. platypus Coq. Niagara Falls.

\section{ORTHOCladius Van der Wulp}

O. absurdus Joh. Ithaca (Joh).

O. atomarius Zett. Ithaca (Joh).

O. nivoriundus Fitch. Erie Co, May-Oct (MCV); Ithaca, Ap (Joh); McLean.

O. obumbratus Joh. Erie Co, Ap-Aug (MCV); Ithaca, Ap; McLean, Ap (Joh).

O. sordidellus Joh. (nec Eur. auct.). Old Forge, J1, Aug; Buffalo, May (MCV); Ithaca (Joh).

\section{TRICHOCLADIUS Kieffer}

T. fugax Joh. Buffalo, May (MCV); Ithaca (Joh).

T. nitidus Mall. McLean, Jl, Sib (Joh).

Psectrocladius Kieffer

P. flavus Joh. Ithaca, J1 (Joh). 
DACTYLOCLADIUS Kieffer

D. sordens Joh. Old Forge, J1; Ithaca (Joh); McLean, Sib (Joh).

CRicotopus Van der Wulp

C. bicinctus Meig. Fulton Co; Old Forge, J1; Erie Co, May-Oct (MCV) ; Ithaca, J1, Aug (Joh); McLean, Sep, Sib (Joh); Delmar, May.

C. exilis Joh. Ithaca, J1 ( Joh).

C. silvestris Fab. Buffalo, Oct (MCV).

C. tremulus Linn. Hamburg, Ap (MCV); McLean, Ap (Joh); Niagara Falls, Je ( Jn).

C. trifasciatus Panz. Fulton Co; Old Forge, J1; Erie Co, May-Je (MCV); Ithaca, J1, Aug (Jn); Albany, Sep.

C. varipes Coq. Fulton Co; Saranac Inn; Erie Co, Ap-Aug (MCV); Ithaca, May, Oct (Joh).

Camptocladius Van der Wulp

C. aterrimus Meig. Buffalo, Hamburg, Ap (MCV).

C. byssinus Schrank. Erie Co, Ap, Sep (MCV); Ithaca, Jl (Joh).

C. fumosus Joh. Old Forge; Erie Co, May (MCV); Ithaca, Ap (Joh); McLean (Joh).

C. graminicola Lundb. Buffalo, Oct (MCV).

C. minimus Meig. Lancaster, Buffalo, Ap (MCV); Ithaca, May (Joh).

Chasmatonotus Loew

C. bimaculatus O.S. Fulton Co, Je (Joh); Erie Co, May-Je (MCV); Little Valley, May (MCV); Poughkeepsie.

C. unimaculatus Lw. L. Placid (Joh).

CORYNONEURA Winnertz

C. celeripes Winn. Old Forge, J1; Ithaca, Aug; McLean, J1 (Joh).

METRIocnemus Van der Wulp

M. atratulus Zett. Old Forge, J1; Ithaca, McLean, J1 (Joh).

M. debilipennis Lundb. Old Forge, J1 (Joh); Buffalo, Aug, Oct (MCV).

M. exagitans Joh. Ithaca; McLean, J1 (Joh).

M. flavifrons Joh. Fulton Co; Old Forge, J1; Ithaca; McLean, J1 (Joh).

M. knabi Coq. Hamburg, Ap (MCV); McLean, J1 (Joh).

M. lundbeckii Joh. Old Forge, J1; Ithaca; McLean, J1 (Joh).

\section{BRILLIA Kieffer}

B. par Joh. Axton, J1; Old Forge, J1; Fulton Co (Joh).

\section{TANYTARSUS Van der Wulp}

T. deflectus Joh. Ithaca, Je (Joh).

T. dissimilis Joh. Ithaca, Je ( Joh).

T. dives Joh. Buffalo, May-Oct (MCV); N. Evans, May (MCV); Ithaca, Ap; McLean, J1 (Joh). 
T. pellucidus Walk. (exiguns Joh.). Old Forge, J1; Ithaca (Joh).

T. fatigans Joh. Ithaca, Ap; McLean, J1 (Joh).

T. flavellus Joh. Fulton Co; Erie Co, Je-Sep (MCV); Ithaca (Joh).

T. flavicauda Mall. McLean, Je, Sib. (Joh).

T. fulvescens Joh. Old Forge, J1; Ithaca, J1 (Joh).

T. gmundensis Egger. Buffalo, Ap-May (MCV); Ithaca, Ap, May, Oct (Joh).

T. johannsenii Bause. Saranac Inn.

T. muticus Joh. Ithaca, Ap-May (Joh).

T. neoflavellus Mall. Buffalo, Aug (MCV).

T. nigripilus Joh. Ithaca, Ap, J1 (Joh).

T. obediens Joh. Fulton Co; Old Forge, J1; Niagara Falls, Oct, Buffalo, Sep (MCV); Ithaca, J1 (Joh).

T. pusio Meig. Old Forge, J1; Hamburg, May, Lancaster, Sep (MCV); Ithaca, Ap (Joh).

T. tenuis Meig. Buffalo, May, Grand I., Je (MCV); McLean, J1, Sep (Joh); Ithaca, J1 (Joh).

PSEUdOCHIRONOMUS Malloch

P. richardsoni Mall. Ithaca, May (Joh).

\section{Calopsectra Kieffer}

C. americana Kieff. Long L.

\section{Chironomus Meigen}

C. aberrans Joh. Fulton Co; Lancaster, Je, Hamburg, Sep (MCV); Ithaca, May, Je (Joh); McLean, J1, Aug, Sib (Joh).

C. albimanus Meig. Fulton Co; Old Forge, J1; Springville, Je (MCV); Pike, Je; Ithaca (Joh); McLean, Je, Aug, Sib (Joh).

C. albipennis Meig. E. Aurora, May (MCV).

C. anonymus Will. LI: Bellport (Coq).

C. brachialis Coq. Fulton Co; Old Forge, J1; Lancaster, Je, E. Aurora, Je-Aug (MCV) ; Ithaca (Joh); Albany.

C. braseniae Leathers. Ithaca, Je.

C. brevitibialis Zett. Erie Co, May-Oct (MCV); Nassau, Je; LI.

C. brunneipennis Joh. Ithaca, J1 (Joh).

C. calopterus Mitch. N. Evans, May (MCV).

C. cayugae Joh. Ithaca, Je (Joh).

C. cristatus Fab. Niagara Falls, Je (Jn) ; Ithaca, Je, J1 (Joh) ; McLean, J1-Sep, Sib (Joh).

C. decorus Joh. Fulton Co; Erie Co, May-Sep (MCV); Ithaca, J1, Aug (Jn); McLean, J1, Sep, Sib (Joh); Clinton Hgts., Sep; Albany, Je.

C. devinctus Say. Old Forge, J1; Buffalo, Grand I., Je (MCV); Ithaca, J1 (Joh).

C. dux Joh. Buffalo, Lancaster, Williamsville, Je-Oct (MCV); Ithaca, Aug ; McLean, J1 (Joh).

C. excavatus Kieff. Adirondacks. 
C. fallax Joh. Ithaca, Je (Joh); McLean, Aug, Sep, Sib (Joh).

C. fasciventris Mall. Buffalo, J1 (MCV).

C. ferrugineovittatus Zett. Var. of plumosus? Ithaca (Joh).

C. festivus Say. E. Aurora, J1 (MCV).

C. flavicingula Wlk. Fulton Co; Erie Co, May-Sep (MCV); Ithaca, Sep (Joh); McLean, May, Aug, Sep, Sib (Joh).

C. flavus Joh. Niagara Falls, Oct (MCV); Erie Co, May-Oct (MCV); Ithaca, (Joh); McLean, Je-Jl, Sib (Joh).

C. frequens Joh. Old Forge, J1; Ithaca, J1 (Joh).

C. fulviventris Joh. Saranac Inn, Je; Ithaca, J1 (Joh).

C. fulvus Joh. Buffalo, Je, Hamburg, Sep (MCV); Ithaca, J1-Aug (Joh).

C. fumidus Joh. Ithaca, J1, Aug (Joh).

C. griseopunctatus Mall. Niagara Falls, Sep (MCV); Gowanda, May, Buffalo, May-Sep (MCV).

C. halteralis Coq. Fulton Co; Niagara Falls, Sep (MCV); Ithaca, J1, Aug (Joh).

C. hilaris Walk. (nephoterus Mitch.). Fulton Co; Old Forge, J1 (Joh).

C. hirtipes Mitch. Ft. Porter (EGM); Old Forge, J1 (Joh).

C. hyperboreus Staeg. Fulton Co; Old Forge, Jl (Joh).

C. indistinctus Mall. Ithaca, NY (Joh).

C. ithacanensis Joh. Ithaca (Joh).

C. lineatus Say. Old Forge, J1 (Joh); E. Aurora, Je-J1 (MCV).

C. lobiferus Say (Glyptotendipes). Albany (Joh); McLean, J1, Sib (Joh).

C. maturus Joh. Ithaca, Mar; McLean (Joh).

C. modestus Say. Fulton Co; Old Forge, J1; Ithaca, J1 (Joh); LI: Bellport (Coq).

C. naevus Mitch. Buffalo, Sep-Oct (MCV); Hamburg, May (MCV).

C. needhami Joh. Fulton Co; Niagara Falls, Je (Joh); Ithaca; Nassau.

C. nigricans Joh. Old Forge, J1; Niagara Falls, J1-Oct (Joh); Erie Co, Je-Sep (MCV); Ithaca, Je, J1; McLean, J1, Sib (Joh).

C. nubeculosus Meig. Buffalo, Je (MCV).

C. ornatipes Kieff. (devinctus Say?). Long L. (Kieff).

C. pallidus Joh. Niagara Falls, Oct (MCV) ; Erie Co, May-Sep (MCV) ; Ithaca, J1, Aug · (Joh).

C. pedellus DeG. Fulton Co; Erie Co, May-Sep (MCV); Ithaca, May, J1 (Joh); McLean, J1, Aug, Sib (Joh); Albany, Je.

C. plumosus L. Fulton Co; Niagara Falls, Je (MCV); Buffalo, May-J1 (MCV); Chautauqua L. (CVRiley); Ithaca (Joh); McLean, Je, Aug, Sep, Sib (Joh).

C. perpulcher Mitch. Fulton Co (Mitch).

C. poecilopterus Mitch. Old Forge, J1 (Joh); Lancaster, Je (MCV).

C. prasinus Meig. Ithaca, J1 (Joh).

C. pseudoviridis Mall. Ithaca (Joh).

C. pulchripennis Coq. Long L. (Coq); Grand I., Je (MCV).

C. quadripunctatus Mall. Old Forge, J1-Aug (Joh).

C. riparius Meig. Erie Co, Ap-Oct (MCV); Little Valley, Je (MCV); Ithaca (Joh).

C. similis Joh. Adirondacks (Joh). 
C. stigmaterus Say, N. Evans, Sep (MCV); LI (BDW).

C. stylifera Joh. Ithaca, J1 (Joh).

C. taeniapennis Coq. Rochester, May (MCV) ; Lewiston, J1, Grand I., Je (MCV); Nassau, Je; Ithaca (Joh); McLean, Je, Sib (Joh).

C. tenellus Zett. Old Forge, J1; Ithaca, May (Joh).

C. tentans Fab. Ithaca, J1 (Joh).

C. viridicollis Wulp. Fulton Co; Old Forge, J1; Ithaca, J1 (Joh).

\section{FAMILY THAUMALEIDAE}

(Orphnephilidae)

Thaumalea Ruthe

(Orphnephila Haliday)

T. americana Bezzi. Ithaca, Aug, Joh (Bezzi).

\section{FAMILY CECIDOMYIIDAE (Itonididae)}

Compiled by E. P. FELT

\section{SUBFAMILY LESTREMINAE \\ Tribe LESTREMINARIAE \\ LESTREMIA Macquart}

L. acerifolia Felt. Albany, May 21, on soft maple.

L. pini Felt. Albany, J1 16, on pine.

L. sambuci Felt. Albany, Aug 6, on elder (Sambucus canadensis); probably an inhabitant of decaying vegetable matter.

L. setosa Felt. Albany, J1 24, on maple.

L. spiraeina Felt. Albany, Je 15, on Spiraea.

\section{MicRocerata Felt}

M. borealis Felt. Speculator, J1 27, Yg.

M. corni Felt. Albany, J1 6, on Cornus.

M. diervillae Felt. Karner, Je 5, on bush honeysuckle (Diervilla trifida).

M. perplexa Felt. Albany, J1 19, on poison ivy.

\section{TRITOZYGa Loew}

T. borealis Felt. Keene Val., Sep 10-15, Not.

T. fenestra Felt. Albany, Je 3, on an office window.

Neptunimyia Felt

N. flavida Felt. Keene Val., Aug 26, Not.

N. tridens Felt. Albany, reared Ap 17. 
NEOCATOCHA Felt

N. sylvana Felt. Keene Val., Sep 6, Not.

KONISOMYIA Felt

K. borealis Felt. Keene Val., Aug 30, Not.

$\mathrm{K}$. fusca Felt. Albany, Je 2, on an office window.

Tribe CAMPYLOMYZARIAE

JOANNISIA Kieffer

J. boreaiis Felt. Keene Val., Aug 30, Not.

J. flavopedalis Felt. Newport, J1 25.

J. flavoscuta Felt. Nassau, J1 24, on a window.

J. photophila Felt. Albany, J1-Aug, on an office window; Poughkeepsie, in a trap lantern.

CAMPYLOMYZA Meigen

C. carpini Felt. Albany, Je 1, on ironwood.

C. cerasi Felt. Newport, J1 25.

C. flavoscuta Felt. Albany, Je 4.

C. gibbosa Felt. L. Clear, Je 7, on spruce.

C. modesta Felt. L. Clear, Je 7, on balsam.

C. monticola Felt. Keene Val., Aug 30, Not.

C. pomiflorae Felt. Karner, May 14, about flowers of shadbush and wild cherry.

C. pomifolia Felt. Nassau, May 6, on apple leaves.

C. producta Felt. Nassau, J1 31, on a window.

C. vitinea Felt. Albany, Aug 14, about grape or ash.

\section{PRIONELlus Kieffer}

(Prionota Kieffer)

P. defectiva Felt. Newport, J1 27, on hemlock.

P. graminea Felt. Karner, Ap 27, on grass.

P. hesperia Felt. Newport, J1 27, on hemlock.

P. latipennis Felt. Karner, Ap 27, on pine.

P. leguminicola Felt. Albany, Je 4, on red clover.

P. longipennis Felt. Albany, Aug 2, on an office window.

P. tsugae Felt. L. Clear, Je 7, on hemlock.

\section{MONARDIA Kieffer}

M. alexanderi Felt. Sport I., Sacandaga R., J1 25, Alex.

M. balsamicola Felt. L. Clear, Je 7, on balsam.

M. gilletti Felt. Albany, Sep 11, on a window.

M. karnerensis Felt. Karner, May 16.

M. populi Felt. Albany, Je 4, on large-toothed poplar.

M. toxicodendron Felt. Albany, Je 4, on poison ivy. 


\section{CoRdylomyia Felt}

C. brevicornis Felt. Nassau, J1-Aug, on a window.

C. luna Felt. Westfield, J1 11.

C. tumida Felt. Albany, Je 25, on a window.

C. versicolor Felt. Albany, J1 17.

\section{CoRINThomyia Felt}

C. gracilis Felt. Albany, J1 5, on an office window.

C. hirsuta Felt. Albany, Aug 1, on an office window.

\section{SUBFAMILY HETEROPEZINAE}

\section{MIASTOR Meinert}

M. americana Felt. Highland, Tivoli, Nassau, L. Placid. The larvae, frequently paedogenetic, are common in the decaying bark of various trees, especially maple, chestnut, oak, birch, beech, and hickory, and are more abundant in the early spring; probably widely distributed in the State.

\section{JOHNSONOMYIA Felt}

J. fusca Felt. Albany, Aug 9.

\section{LEPTOSYNA Kieffer}

L. americana Felt. Nassau, May 27, in trap lantern.

L. quercivora Felt. Reared Ap 20 from partly rotten red-oak bark collected at Nassau.

\section{KRONOMYIA Felt}

K. populi Felt. Reared May 5 from a whitish larva found in punky poplar wood at Nassau, Ap 12.

BRACHYNEURA Rondani

B. americana Felt. Albany, Aug 2, on a window.

B. eupatorii Felt. Reared Sep 14, possibly from a stem gall on thoroughwort (Eupatorium perfoliatum) taken at Poughkeepsie.

B. vitis Felt. Reared J1 13 from a jar containing Lasioptera vitis galls, collected probably at W. Nyack.

\section{Oligarces Meinert}

O. novaeboracensis Felt. Albany, J1 15, on a window.

O. ulmi Felt. Reared Mar 18, from larvae occurring in the thick, partly decaying bark of an old elm at Nassau.

\section{SUBFAMILY ITONIDIDINAE \\ Tribe PORRICONDYLARIAE \\ WINNERTZIA Rondani}

W. aceris Felt. Reared from white larvae occurring under the thin, decaying bark of sugar maple at Schodack. 
W. ampelophila Felt. Albany, J1 3, on Virginia creeper.

W. calciequina Felt. Albany, J1 24.

W. carpini Felt. Albany, Je 1, on ironwood.

W. hudsonica Felt. Reared Je 25 at Albany from a jar containing leaves of Crataegus bearing greenish, stout, cup-shaped, fimbriate galls.

W. karnerensis Felt. Karner, Je 26.

W. palustris Felt. Canada L., Caroga, J1 10, Alex.

W. pectinata Felt. Reared the following spring from larvae found under decaying chestnut bark at Nassau, Nov; Keene Val., Aug 27, Not.

W. solidaginis Felt. Albany, J1 6.

W. rubida Felt. Albany, Je 15.

\section{PARWINNERTZIA Felt}

P. notmani Felt. Keene Val., J1 17, Not.

\section{Didactylomyia Felt}

D. flava Felt. Albany, J1 6.

D. longimana Felt. Both sexes collected by Alex, Aug, at Woodworth's L.

D. maculata Felt. Poughkeepsie, Je 28, in a trap lantern; apparently the same form was captured in a similar manner at Newport, J1 11.

D. robusta Felt. Keene Val., Sep 14, Not.

\section{COLPODIA Winnertz}

C. alta Felt. Karner, J1 5.

C. americana Felt. Woodworth's L., Aug 14, Alex.

C. capitata Felt. Woodworth's L., Aug, Alex.

C. diervillae Felt. Karner, J1 5.

C. graminis Felt. Albany, J1 14, on quack grass.

C. maculata Felt. Albany, J1 16.

C. ovata Felt. Woodworth's L., Aug, Alex.

C. pectinata Felt. Reared in Aug from a jar containing irregular, subglobose leaf swellings on basswood collected at Albany.

C. porrecta Felt. Woodworth's L., Aug, Alex.

C. sanguinea Felt. Nassau, J1 15; later reared by Ald from an almost pure bluegrass sod.

C. sylvestris Felt. Woodworth's L., Aug, Alex.

C. temeritatis Felt. Reared Sep 9 from a jar containing ash leaves bearing a purplish green, subglobular gall collected near Albany.

C. terrena Felt. Albany, J1 7, on quack grass.

C. trifolii Felt. Albany, Jl 3, on white clover.

\section{ASYNAPTA Loew}

A. apicalis Felt. Woodworth's L., Aug 24, Alex.

A. borealis Felt. Keene Val., Aug 28, Not,

A. caudata Felt. Albany, Je, 
A. cerasi Felt. Albany, Je 21, on cherry.

A. dolens Felt. Keene Val., Sep 7, Not.

A. flavida Felt. Nassau, J1 6.

A. furcata Felt. Nassau, Je 21.

A. mediana Felt. Woodworth's L., Aug 28, Alex.

A. salciperda Felt. Keene Val., Aug 27, Not.

\section{PORRICONDYLA Rondani}

P. antennata Felt. Johnstown, J1 11, Alex.

P. bidentata Felt. Keene Val., Aug 26, Not.

P. borealis Felt. L. Clear, Je 7, on spruce.

P. caudata Felt. Nassau, J1 9, in a trap lantern.

P. dilatata Felt. Reared Sep 7, from a jar containing galls of Cecidonyia verrucicola O.S. collected at Highland.

P. dorsata Felt. Dug Mt., Aug 8, Yg.

P. flava Felt. L. Clear, Je 7, on red maple.

P. fultonensis Felt. Woodworth's L., Je, Alex.

P. juvenalis Felt. Elm L., Aug 7, $Y g$.

P. karnerensis Felt. Karner, May 16.

P. pini Felt. Albany, Je 11, on white pine.

P. setosa Felt. Woodworth's L., Aug 21, Alex.

P. spinigera Felt. Keene Val., Sep 4, Not.

P. tumidosa Felt. Keene Val., Aug 27, Not.

P. wellsi Felt. Wells, J1 5, Yg.

\section{CAMPTOMYIA Kieffer}

C. aestiva Felt. Keene Val., Sep 4, Not.

C. antennata Felt. Keene Val., Sep 15, Not.

C. dentata Felt. Keene Val., Sep 3, Not.

C. montana Felt. Wells, J1 5, Yg.

C. pectinata Felt. Keene Val., Aug 26, Not.

C. tsugae Felt. Reared from pinkish maggots inhabiting the larval galleries of Melanophila fulvoguttata working in dying hemlock in NYC; Scarsdale, Dec 2, larvae on hemlock.

\section{DIRHIZA Loew}

D. canadensis Felt. Keene Val., Aug 14, Not.

D. hamata Felt. Albany, Je 8; appears to be somewhat common; Keene Val., Aug 7, Not.

D. multiarticulata Felt. Keene Val., Aug 29, Not.

D. photophila Felt. Nassau, May 7, in a trap lantern.

D. sylvestris Felt. L, Clear, Je 7, in a balsam woods. 


\section{HOLONEURUS Kieffer}

H. altifilus Felt. Karner, Je 26, flying about skunk cabbage.

H. humulis Felt. Albany, J1 24, on low blackberry.

H. inflatus Felt. Keene Val., Sep 12, Not.

H. multinodus Felt. Nassau, J1 7, in a trap lantern.

H. photophilus Felt. Nassau, Je 3, in a trap lantern.

H. tarsalis Felt. Reared Aug 15 from pine needles and débris collected at North $\mathrm{Cr}$.

\section{Tribe DASYNEURIARIAE}

\section{RHABDOPHAGA Westwood}

R. absobrina Felt. Albany, May 17.

R. acerifolia Felt. Albany, May 17. Food plant unknown.

R. aceris Shim. Produces an irregular curling of white or silver maple leaves, and occurs at Albany ( $\mathrm{J} 1$, larvae and cocoons on soft maple) and probably other points in the State; Gloversville, Aug; Rensselaer, J1, larvae on soft maple.

R. batatas Walsh. Produces a gall which is common on willow in and about Albany, and is presumably widely distributed through the State; Canandaigua, Oct, gall on willow.

R. brassicoides Walsh. Inhabits the willow cabbage-gall, a deformity very common in certain parts of the State.

R. cephalanthi Felt. Nyack, Jan 7, 1884, reared from twigs on buttonbush (Cephalanthus occidentalis), Zab.

R. consobrina Felt. Albany, May 17.

R. cornuta Walsh. Galls provisionally identified as those of this species were observed at W. Nyack.

R. gemmae Felt. Albany, Je, reared from a peculiar stunted, elongate tip of a willow twig.

R. globosa Felt. Albany, May 7, reared from willow twigs bearing small, rounded galls, a part of the surface showing a discolored, dead area.

R. hirticornis Felt. Albany, Ap, Sep 20, reared from jars containing various galls, especially those of Caryomyia persicoides and Schizomyia pomum.

R. latebrosa Felt. Albany, May 7, reared from apparently normal willow buds.

R. marginata Felt. Albany, May 23, captured on choke cherry.

R. nodula Walsh. Produces a small, nodular swelling usually encircling the bases of smaller willow branches, and is somewhat common in the vic. of NYC.

R. persimilis Felt. Albany, Ap 11, reared from a rather typical though slender $R$. strobiloides Walsh gall.

R. podagrae Felt. Produces inconspicuous swellings $5-7 \mathrm{~cm}$. long in willow twigs. Appears to be common at E. Schodack.

R. populi Felt. Karner, May, reared from whitish cocoons on poplar. Appears to be a rather common species.

R. porrecta Felt. Gloversville, Ap 5, Alex.

R. pratensis Felt. Albany, Je 4, on red clover.

R. ramuscula Felt. Ap 22, from Salix twigs bearing galls closely resembling those of $R$. batatas Walsh. 
R. salicifolis Felt. Produces a characteristic thickened brownish midrib fold on meadowsweet, which is moderately common about Albany and at Nassau; Hudson Falls, Sep, on hardhack.

R. salicis Schrk. A European species which has become established in the vicinity of Rochester and is probably to be found in the important centers for the growing of European willow.

R. sodalitatis Felt. Albany, Ap 13, reared from what appeared to be a typical Phytophaga rigidae gall on Salix.

R. strobiloides Walsh. Produces the common pine-cone-like gall on willow, which appears to be widely distributed throughout the State; Albany; Trudeau, Aug 7.

R. triticoides Walsh. Appears to be common in vic. of Albany, and is probably widely distributed in the State, especially in localities favorable for the growth of its food plant, Salix. The gall is an irregular enlargement from 1 to nearly $3 \mathrm{~cm}$. thick and about $6 \mathrm{~mm}$. in diameter, and is evidently caused by the dwarfing of a number of adjacent buds.

\section{DASYNEURA Rondani}

D. aberrata Felt. Albany, May 19.

D. acerifolia Felt. Albany, May 21, on maple.

D. albohirta Felt. Albany, May 17.

D. albovittata Walsh. W. Nyack, reared from the common pine-cone galls of Rhabdophaga strobiloides Walsh.

D. ampelophila Felt. Albany, J1 13, on Virginia creeper.

D. anemone Felt. Kinderhook and Nassau, J1 12, reared from a loose bud gall on Anemone canadensis.

D. antennata Felt. Albany, Je 11, on sugar maple.

D. apicata Felt. Highland, Sep 7, reared from a deformed terminal growth on ash.

D. attenuata Felt. Albany, Je 9.

D. augusta Felt. Albany, Aug 6, on oak.

D. aurihirta Felt. Albany, J1 6, on New Jersey tea.

D. bidentata Felt. Albany, Je 21, on white oak.

D. borealis Felt. L. Clear, Je 7, found in collecting from spruce.

D. carbonaria Felt. Albany, J1 27, reared probably from a loose apical gall on the narrow-leaved Solidago.

D. caricis Felt. Albany, Je 4, on sedge.

D. cirsioni Felt. Albany, J1 17, on Canada thistle.

D. clematidis Felt. Produces an irregular, subglobular, axillary mass $1 \mathrm{~cm}$. in diameter, composed of deformed, enlarged, rudimentary leaves or young buds. Recorded from Highland, Albany, and Newport.

D. communis Felt. Produces thickened vein swellings on soft maple which are very common in different parts of the State. Canandaigua, Je, Oct; Goshen, Oct, 9, on red maple; Patterson, Je; Hudson Falls.

D. consobrina Felt. Albany, Je 11, on white pine.

D. denticulata Felt. L. Clear, Je 7, on white spruce.

D. filicis Felt. Albany, May 17.

D. flavescens Felt. Albany, J1 17, on ash. 
D. flavicornis Felt. Albany, latter part of J1, reared from a greenish or purplish pouch-like gall on the narrow-leaved Solidago.

D. flavoabdominalis Felt. Albany, Aug 6, on Solidago.

D. flavoscuta Felt. Albany, Je 11, on Solidago.

D. fraxinifolia Felt. Rensselaer, Aug 1, reared from tightly rolled leaves of ash.

D. fulva Felt. Nassau, Je 14, on huckleberry.

D. gleditschiae O.S. Produces characteristic pod-like swellings on the leaves of honey locust, and has been recorded as not common in Central Pk., NYC.

D. graminis Felt. Has been observed ovipositing in redtop or June grass; recorded from Albany, Kinderhook, and Poughkeepsie.

D. karnerensis Felt. Karner, Je 4, on Aster or Solidago.

D. leguminicola Lint. The common clover-seed midge. Appears to be widely distributed in the State, and materially restricts the production of clover seed.

D. lysimachiae Bt. Albany and Elm L., reared from a terminal conical leaf gall on the whorled loosestrife.

D. maculosa Felt. Nassau, Je 14, on a window.

D. meliloti Felt. Albany, Aug 6, on sweet clover.

D. modesta Felt. Albany, May 19, near a red oak.

D. multiannulata Felt. Nassau, Je 14, on hickory.

D. parthenocissi Steb. Produces a characteristic, sometimes winged, midrib gall on woodbine. Rather common at Nassau and in vic. of Syracuse.

D. pedalis Felt. Newport, Je 27, in a trap lantern.

D. photophila Felt. Nassau, Je 10, on a window.

D. piperitae Felt. Nassau, early Aug, reared from the enlarged terminal buds of peppermint.

D. pseudacaciae Fitch. Deforms the young leaves of black locust, and is common in various parts of NY from LI northward to Glens Falls, Je.

D. pudorosa Felt. Nassau, Je 14.

D. purpurea Felt. Karner and SI, Sep, reared from oval or fusiform bud-like flower galls of joe-pye weed.

D. quercina Felt. Albany, May 18, on oak.

D. rhodophaga Coq. Occurs in violet houses; recorded as causing considerable injury at Rhinebeck and presumably in other violet-growing centers; Beacon, Oct, on rose.

D. rhois Coq. Lebanon Sps., Mar, reared by Coquillett from galls on the roots of the common poison ivy; Whitehall.

D. rosarum Hardy. Albany, J1 11, reared from folded terminal leaves of rose.

D. rufipedalis Felt. Karner, Je 4, on red clover.

D. salicifolia Felt. Albany, Aug, reared from young terminal adherent willow leaves.

D. scutata Felt. Albany, J1 16.

D. semenivora Bt. Produces a plum-like gall on violet; recorded from Albany.

D. serrulatae O.S. Produces a bud gall on alder. Vic. Albany, rather common; Hudson Falls, Ap. Probably widely distributed throughout the State.

D. setosa Felt. Nassau, Aug 10, on a window.

D. simillima Kieff. (similis Felt). Albany, I1 7, on thistle.

D. simulator Felt. Nassau, J1 3 and 21.

D. smilacinae Bish. Ithaca, reared from root galls on false Solomon's seal. 
D. trifolii Lw. Newfane, Je 2; W. Haverstraw, W. Nyack, Je 14; Wappinger Falls, Je 28; Ghent, J1 29. Is probably moderately common where white clover (Trifolium repens) has been growing for a series of years. The larvae prevent the leaves from unfolding, and produce a yellowish or brownish discoloration of the affected parts.

D. tumidosae Felt. Reared in small numbers from jars containing numerous galls collected at Highland and generally identified as those of Cecidomyia pellex O.S.

D. unguicula Felt. Nassau, J1-Aug.

D. vernalis Felt. Nassau, Je 14, on New Jersey tea.

D. vitis Felt. W. Nyack, J1 16, reared from the common tomato gall of the grape, and presumed to be that of Lasioptera vitis O.S.

\section{LASIOPTERYX Felt}

L. carpini Felt. Albany, Je 21, on ironwood or blue beech.

L. coryli Felt. W. Nyack, J1 11, reared from a fuzzy, wrinkled, fold gall at the base of hazel leaves.

L. flavotibialis Felt. Nassau, May, reared from larvae in a partly decayed oak stump.

\section{NEUROMYiA Felt}

N. minor Felt. Nassau, Jl, on a window.

\section{CYSTIPHORA Kieffer}

C. canadensis Felt. Inhabits a blister leaf gall on white lettuce or rattlesnake root. LI: Orient, Aug, gall on Prenanthes.

C. viburnifolia Felt. Produces a very common, inconspicuous blister leaf gall on hobblebush. Albany.

\section{RHizomyia Kieffer}

R. absobrina Felt. Albany, J1 11 and 26, reared from a jar containing Crataegus leaves bearing cylindric, fimbriate, unicellular galls.

R. cerasi Felt. Albany, Je 21.

R. cincta Felt. Albany, J1 30, on Cornus.

R. hirta Felt. Rensselaer, Aug 17, reared from reddish, blister-like leaf mines on Crataegus.

R. hispida Felt. Albany, J1 6.

R. ungulata Felt. Albany, J1 6.

R. vitis Felt. Albany, J1 13, reared from the tomato grape gall, Lasioptera vitis.

\section{Tribe LASIOPTERARIAE}

\section{LASIOPTERA Meigen}

L. abhamata Felt. Karner, Je 4.

L. argentisquamae Felt. W. Nyack, J1, reared from Solidago bearing a characteristic, adhesive leaf gall.

L. basiflava Felt. Albany, J1 30, on grape. 
L. caulicola Felt. Vic. Albany, Je 6, reared from an apparently normal stem of bush honeysuckle. The larvae are found just under the bark in a small, oval cell.

L. cinerea Felt. Albany, May 21, on black alder.

L. clavula Bt. Appears to have been reared from the characteristic clavate twig galls on Cormus florida. The deformity is rather common in the vic. of Albany southward to NYC, and presumably occurs in other parts of the State. LI: Sea Cliff, Feb 4, galls.

L. consobrina Felt. Albany, Je 10, on maple-leaved arrowwood.

L. convolvuli Felt. Produces a monothalamous, fusiform stem gall $1.5 \mathrm{~cm}$. long, and $0.5 \mathrm{~cm}$. in diameter, on the hedge bindweed. Rensselaer Co, Hudson, the adults appearing the middle of May.

L. corni Felt. Apparently reared from a greenish or red-ringed blister leaf gall on Cormus paniculata. This gall has been received from: W. Nyack; Ellenville; Catskill; Hudson Falls, Sep; Canandäigua, Sep-Oct; and probably occurs in other parts of the State.

L. cylindrigallae Felt. Reared in early May from a uniform Solidago stem enlargement, with a length of about $10 \mathrm{~cm}$. and a diameter of $7 \mathrm{~cm}$., the central cavity being slightly enlarged and containing a number of larvae. Somewhat common at W. Nyack and Nassau, and on SI.

L. danthoniae Felt. White Church, reared by $\mathrm{Cy}$ in the spring of 1908 from a bunch of Danthonia. The gall is undescribed.

L. excavata Felt. Albany, Aug 17, reared presumably from a blister-like reddish brown leaf mine occurring rather commonly on Crataegus.

L. farinosa Bt. Produces a characteristic warty, usually pruinose, woody swelling at the base of the leaflets or midrib of the common blackberry. Probably widely distributed, not uncommon. Canandaigua, Oct; Albany; NYC; SI, Aug-Sep (Ds) ; LI: Orient, Sep.

L. flavipes Felt. Albany, J1 17, on white oak.

L. fraxinifolia Felt. Apparently reared from a purplish green, subglobular gall, $5 \mathrm{~mm}$. in diameter, on ash leaves and described by O.S. as Cecidomyia pellex. This deformity appears to be somewhat common at L. Onondaga, Westfield, Highland, Poughkeepsie, and NYC.

L. hamata Felt. Nassau, Je 14, on Solidago.

L. hecate Felt. Albany, Je 19.

L. humulicaulis Felt. Occurs in a uniform swelling of the hop stem about $1 \mathrm{~cm}$. in diameter and ranging in length from 4 to $50 \mathrm{~cm}$. Rye, Bird; probably occurs in other parts of the State.

L. impatientifolia Felt. Albany, reared from a tumid midrib fold on the leaves of touch-me-not or snapdragon. Probably rather widely distributed throughout the State.

L. juvenalis Felt. Newport, J1 25, on bouncing bet.

L. lactucae Felt. Karner, reared from a stem gall on the stalks of rabbit weed or wild lettuce, closely resembling the much more common gall produced by the cynipid, Aylax tumidus Bass.; probably occurs in other parts of the State.

L. linderae Bt. Produces an irregular, subcortical, eccentric stem gall 2 to $5 \mathrm{~cm}$. in length, on spicebush. Vic. NYC, rather common, adults, May-Je:

L. lycopi Felt. Inhabits a globose stem gall 8 to $10 \mathrm{~mm}$. long and $2.5 \mathrm{~mm}$. in diameter, on bugle weed (Lycopus communis). Albany; Shushan; White Plains; LI: Orient, Mar, Sep, Lath; probably widely distributed in the State. 
L. nassauensis Felt. Nassau, J1.

L. neofusca Felt. Albany, May 25.

L. nodulosa Bt. Produces an irregular, subfusiform or elongate swelling on the smaller branches of blackberry. The gall is reddish, usually of the same color as the adjacent bark, about $2 \mathrm{~cm}$. long, $1 \mathrm{~cm}$. in diameter, and polythalamous. Vic. Albany; appears to be much more common in and about NYC; SI (Ds).

L. palustris Felt. W. Nyack, Ap 18, reared from a subglobular to elongate, irregular, fusiform stem gall on an unknown plant.

L. panici Felt. Albany, Je 27, taken as the female was evidently about to oviposit on Panicum macrocarpon.

L. riparia Felt. Westfield, Ap 27, reared from an oval or fusiform petiole or tendril gall on $V$ itis bicolor.

L. serotina Felt. Albany, May 23, on choke cherry.

L. solidaginis O.S. Produces a rough, eccentric stem gall, length and diameter about $2.5 \mathrm{~cm}$., on Solidago rugosa. Albany, scarce; vic. NYC, common.

L. vernoniae Bt. NYC; SI. Reared from flower and leaf galls on ironwood. The leaf gall is oval, about $5 \mathrm{~mm}$. long, green tinged with red, and shows on both surfaces of the leaf.

L. viburni Felt. Albany, Je 10, on maple-leaved arrowwood.

L. vitis O.S. Produces a reddish, irregular, frequently lobulated, succulent enlargement of the leaf stalk, parts of the leaf, or even the tendrils, of grape, the mass being 2 or 3 inches long and polythalamous. Several species of midges have likewise been obtained from this gall. The insect is probably widely distributed in NY, excepting possibly the Adirondack region. Kingston, Je; Manorville, J1; Suffern, J1; Rensselaer, Je; Nassau, J1; Port Jervis, Aug ; NYC, Je 13; SI: New Brighton, Je (larvae).

\section{NeOLASIOPTERA Felt}

N. albitarsis Felt. Nassau, reared May 18 and 21 from fusiform, irregular stem galls $1 \mathrm{~cm}$. long and $6 \mathrm{~cm}$. in diameter on Diplopappus cornifolius.

N. albolineata Felt. Albany, Aug 5.

N. ambrosiae Felt. Ithaca, Jan, reared by Cy from apparently normal stems of the giant ragweed (Ambrosia trifida).

N. asclepiae Felt. Albany, Je 20, reared from an elongate, fusiform stem gall on the swamp milkweed (Asclepias incarnata).

N. basalis Felt. Albany, Aug 6, on hazel.

N. celastri Felt. Albany, J1 17, on bittersweet.

N. cinerea Felt. Albany, Je 21.

N. clematidis Felt. Newport, J1 17, captured as the female was attempting to oviposit in the stem of Clematis; Hudson Falls, Sep, gall.

N. cornicola Bt. Produces an irregular, nodular, polythalamous gall on the small twigs, the larger branches, and the old stems of Cornus stolonifera. The gall varies in length from 1 to $2 \mathrm{~cm}$., and is usually on one side of the twig. Hudson Falls, Mar, Nassau, abundant; W. Nyack; vic. NYC. Presumably widely distributed in the State. The midges appear in early May.

N. erigerontis Felt. Produces a fusiform stem gall on horse-weed (Erigeron canadensis). 
N. eupatorii Felt. Canandaigua, Sep; SI, reared from an oval or subglobular stem gall with a length of $1.5 \mathrm{~cm}$. and a diameter of $1 \mathrm{~cm}$., on white snakeroot (Eupatorium urticaefolium).

N. flavomaculata Felt. Albany, J1 11.

N. flavoventris Felt. Karner, Jl, flying about hard pine.

N. hamamelidis Felt. Albany, Je 10, on witch-hazel.

N. hamata Felt. Canandaigua, Sep; Albany, Ap-May, reared from an oval, polythalamous stem gall $2 \mathrm{~cm}$. long and $5 \mathrm{~cm}$. in diameter on an unknown weed.

N. helianthi Felt. Highland, Sep 3, from a gall on Helianthus strumosus supposed to be that of Asphondylia globulus O.S.

N. hibisci Felt. SI, Ap 25, reared from slightly enlarged stems of the swamp rose mallow, Hibiscus moscheutos.

N. liriodendri Felt. Albany, Je 8, on a tulip tree.

N. perfoliata Felt. Buffalo; Nassau. Midges in May. Produces an oval stem gall with a length of 1 to $1.5 \mathrm{~cm}$. and a diameter of $0.5 \mathrm{~cm}$., on boneset.

N. ramuscula Bt. Produces a fusiform branch gall with a length of $1 \mathrm{~cm}$. and a diameter of $4 \mathrm{~cm}$., on several species of Aster. Hudson Val.; probably widely distributed in State.

N. sambuci Felt. Produces an irregular, subcortical, polythalamous stem gall 2 to $6 \mathrm{~cm}$. in length on elder. Nassau; NYC; probably widely distributed in State.

N. sexmaculata Felt. Nassau, Je 14.

N. tenuitas Felt. Albany, J1 30.

N. tiliaginea Felt. Nassau, Je 14, on basswood.

N. tripunctata Felt. Nassau, Je 30.

N. viburnicola Bt. Produces an irregular, subcortical swelling from 2 to 5 or $6 \mathrm{~cm}$. long on the larger branches of arrowwood (Viburnum dentatum). Vic. NYC, common.

N. vitinea Felt. Highland Falls; Albany; W. Nyack; vic. NYC, common; SI, Je 15 , reared from a conical petiole gall on grape; probably widely distributed in the State. The gall is about $1.5 \mathrm{~cm}$. long and $0.5 \mathrm{~cm}$. in diameter, and contains from two to four larvae in a long central chamber.

\section{Asteromyia Felt}

A. abnormis Felt. Albany, J1 24.

A. albomaculata Felt. Jamesburg, reared from a diffuse, yellowish blister leaf gall 3 to $4 \mathrm{~mm}$. in diameter on Aster leaves; Albany, adult captured on Solidago.

A. agrostis O.S. (Lasioptera muhlenbergiae Martin, and probably Lasioptera carbonitens Ck11.). Produces a somewhat cone-shaped, aborted apical gall on Muhlenbergia mexicana. Several localities in State.

A. asterifoliae Bt. Highland, reared Je-J1 from a circular, white-ringed blister leaf gall.

A. canadensis Felt. Albany, May 21.

A. carbonifera Felt. Produces an oval, characteristic, jet-black, blister leaf gall on the narrow-leaved Solidago, Solidago graminifolia. Appears to be abundant in most localities where the food plant occurs.

A. convoluta Felt. Albany, Aug 11, reared from a loose, convolute tip gall on Solidago. 
A. divaricata Felt. Albany, Ap, reared from large, oval, blister leaf galls with a diameter of $1.2 \mathrm{~cm}$. on Aster divaricatus.

A. flavoannulata Felt. W. Nyack, J1 25, reared from a marginal, circular, golden gray, blister leaf gall with a diameter of $3.5 \mathrm{~mm}$. on Solidago canadensis.

A. flavolunata Felt. Albany, Mar and Ap, reared from a characteristic marginal, somewhat oval, yellowish blister gall on Solidago.

A. flavomaculata Felt. Albany, Mar 16, reared from a clouded blister gall, yellow beneath and dirty white above, with a length of $2 \mathrm{~cm}$. and a diameter of 1.2 $\mathrm{cm}$., presumably on aster.

A. flavoscuta Felt. Albany, J1 16.

A. laeviana Felt. Albany, Sep, reared from a greenish yellow blister leaf ga!l with a diameter of $1.75 \mathrm{~cm}$. on Aster laevis.

A. marginata Felt. Albany, Aug 7, reared from a yellowish white or light brown blister leaf gall on Aster paniculatus.

A. modesta Felt. Albany, reared from small, oval swellings appearing much like arrested buds on the stems of horseweed (Erigeron canadensis). The species is probably common.

A. nitida Felt. Meadowdale, Ap 17, reared from a large, smooth, glossy, yellowish, oval, blister leaf gall 6-8 $\mathrm{mm}$. in diameter, on the basal leaves of an aster.

A. paniculata Felt. Albany, Aug 10, reared from an oval, yellowish or brownish, blister gall on the leaves of Aster paniculatus.

A. pustulata Felt. Albany, reared from a small, pustulate swelling $4 \mathrm{~mm}$. long and $2 \mathrm{~mm}$. in diameter on the side of aster stems.

A. rosae Felt. Albany, May 16, reared from an oval, blister-like leaf gall with a diameter of $1 \mathrm{~cm}$., occurring on Solidago rugosa. The species is probably widely distributed.

A. rubra Felt. Albany, J1 21, reared from a more or less elongated blister leaf gall on Solidago rugosa. This species is probably widely distributed in the State and has been recorded from a number of localities in the US.

A. socialis Felt. Reared J1 26 from the adherent type of leaf gall on Solidago canadensis inhabited by Asphondylia monacha O.S. and Camptoneuromyia adhesa Felt. Albany.

A. squarrosae Felt. Rhinecliff, J1 20, reared from a grayish brown, black-margined, irregular blister leaf gall on Solidago squarrosa.

A. waldorfi Felt. Karner, reared in early May from a circular, brownish leaf gall with a diameter of $3 \mathrm{~mm}$., on a somewhat hairy unknown species of Aster.

\section{Clinorhyncha Loew}

C. eupatoriflorae Felt. Albany, reared in late Aug from apparently normal florets of thoroughwort.

C. filicis Felt. Nassau, Je 24, on a fern.

C. karnerensis Felt. Albany, J1 5, on willow.

C. millifolii Wachtl. Vic. Albany, Aug, reared from florets of yarrow.

\section{CAMptoneuromyia Felt}

C. adhesa Felt. Adirondacks; Nassau; W. Nyack, common, reared in J1 from an oval cell formed by the adhesion of two Solidago leaves.

C. brevicauda Felt. Nassau, Albany, J1-Aug. 
C. hamamelidis Felt. Albany, Je 12, on witch-hazel.

C. flavescens Felt. W. Nyack, J1 29, 30, reared from a closely adherent, pyriform, apical leaf gall on Solidago graminifolia.

C. fulva Felt. Albany, J1 3, on Cornus.

C. virginica Felt. Albany, Je 12, on witch-hazel.

\section{TROTTERIA Kieffer}

T. argenti Felt. Albany, J1 3, on New Jersey tea.

T. caryae Felt. Albany, Je 20, on hickory.

T. caudata Felt. Karner, J1 5, on bush honeysuckle.

T. karnerensis Felt. Karner, J1 5, on elder.

T. metallica Felt. Albany, Je 19, on hickory.

T. solidaginis Felt. W. Nyack, J1 2, reared from Solidago material containing galls of Camptoneuromyia adhesa Felt and Asphondylia monacha O. S. This species has also been reared in association with Asphondylia thalictri Felt, and is probably zoophagous.

T. squamosa Felt. Albany, J1 26, on New Jersey tea.

T. subfuscata Felt. Albany, J1 17, on Solidago.

T. tarsata Felt. Albany, J1 24, on scrub oak.

\section{Tribe OLIGOTROPHIARIAE}

\section{PHYTOPHAGA Rondani}

P. aceris Felt. Albany, May 21, on red maple.

P. azaleae Felt. Albany, May 18, on Azalea.

P. balsamifera Felt. L. Clear, Je 7, on balsam.

P. caudata Felt. Albany, May 9, on wild geranium.

P. destructor Say. THE Hessian FLy. Well known because of its occasionally very severe injury to wheat and barley, in particular. Is known to breed also in quack grass and Canadian rye grass. Generally distributed. Periodically destructive wherever wheat is grown. In Jefferson and St. Lawrence Counties, found in barley. One specimen found in Franklin Co.

P. electra Felt. Albany, May 18, on hazel.

P. fraxini Felt. Albany, Poughkeepsie, reared from leaf petioles of ash.

P. latipes Felt. Albany, J1 6, on New Jersey tea.

P. rigidae O.S. Produces a characteristic beaked gall on willow. Appears to be widely distributed in the State, the adults appearing in early spring.

P. socialis Felt. Albany, Je 1, on early meadow rue.

P. thalictri Felt. Albany, Je 1, on early meadow rue.

P. tsugae Felt. L. Clear, Je 7, on hemlock.

P. ulmi Felt. Canandaigua; deforms the small terminal leaves or leaf buds of the American elm about NYC and probably occurs at Albany.

P. violicola Coq. The Violet Midge. Has caused considerable injury in violet houses at Rhinebeck and presumably in other parts of the State.

P. virginiana Felt. Albany, May 23, on choke cherry. 
Janetiella Kieffer

J. acerifolia Felt. Albany, May 17.

J. americana Felt. Albany, J1 17.

J. breviaria Felt. Albany, May 21.

J. brevicornis Felt. Nassau, Je 14, on Solidago.

J. ligni Felt. Nassau, May 8, reared from larvae in decaying chestnut bark.

J. nodosa Felt. Albany, Ap 28.

J. parma Felt. Woodworth's L., Je 21, Alex.

J. pini Felt. Albany, May 26, on pine.

J. sanguinea Felt. Nassau, May 15.

J. tiliacea Felt. Westfield, May 23, on basswood.

\section{OLIGOTROPHUS Latreille}

O. betulae Winn. An introduced European species reared in 1886 from deformed white-birch catkins collected at Albany, the normal alate seed being transformed into a globose gall with rudimentary alae.

O. inquilinus Felt. Albany, Sep 11, reared from a large terminal rosette gall on Solidago.

O. salicifolius Felt. Karner, Mar-Ap, reared from irregular, yellowish, red-spotted leaf galls on Salix humilis collected in the preceding Sep; LI: Orient, Sep 22, from a gall, Lath.

O. vernalis Felt. Karner, May 19, on basswood.

\section{RHOPALOMYIA Rübsaamen}

R. abnormis Felt. Huguenot Pk., J1 14, in a trap lantern.

R. albipennis Felt. Albany, Aug 9-14, reared from a large terminal rosette gall on Solidago canadensis.

R. anthophila O.S. Produces a more or less cylindric, densely haired, flower-bud gall which appears to be widely distributed and occasionally locally abundant. Canandaigua, Sep, and Kings Bridge, on Solidago; LI: Orient, Sep.

R. apicata Felt. Nassau, J1 7, in a trap lantern.

R. arcuata Felt. Albany, Je 4.

R. asteriflorae Felt. Albany, Sep 25, from stunted, abnormally bunched heads of Aster paniculatus.

R. capitata Felt. Produces a conspicuous terminal rosette gall. Moderately common in vic. of Albany.

R. clarkei Felt. Undoubtedly occurs in the State, though no definite locality can be given.

R. fusiformis Felt. Produces a rather common, ribbed, fusiform gall about $6 \mathrm{~mm}$. long on the young leaves and flower stems of the narrow-leaved Solidago. Moderately common about Albany.

R. hirtipes O.S. Appears to be very common and widely distributed. Definite records are: Elizabethtown; Canandaigua; Nassau. Sep. Produces a characteristic apical bud gall on aerial stems and a variable deformation of rootstock buds.

R. inquisitor Felt. Produces a gall very similar to that of $R$. capitata Felt. 
R. lateriflori Felt. Produces a globose, axillary bud gall on Aster lateriflorus. L. George.

R. lobata Felt. W. Nyack, J1 17, reared from a subglobular stem gall on the narrowleaved Solidago.

R. 'major Felt. Huguenot Pk., May 31, in a trap lantern.

R. palustris Felt. Nassau, Je 7.

R. pedicellata Felt. Produces a stemmed, fusiform gall some $2 \mathrm{~cm}$. long on the narrow-leaved Solidago. Rather common in vic. of Albany.

R. pini Felt. Albany, Je 4.

R. racemicola O.S. Produces a firm, greenish or reddish, subglobular flower-bud gall with a diameter of about $2 \mathrm{~mm}$. Common about Albany and Poughkeepsie, and probably in other parts of NY. Canandaigua L., Sep, on Solidago caesia; LI: Orient, Sep, Lath, on Solidago.

R. solidaginis Lw. Probably occurs in the State, and $R$. capitata Felt may prove to be identical with it. Hudson Falls, Sep, on Solidago.

\section{SACKENOMYia Felt}

S. acerifolia Felt. Albany, May 17.

S. viburnifolia Felt. Produces purplish, fusiform vein swellings $5 \mathrm{~mm}$. long and $3 \mathrm{~mm}$. in diameter on arrowwood. Albany; Nassau; LI: Orient, Sep, from gall, Lath.

\section{Tribe ASPHONDYLIARIAE}

SCHIZOMYIA Kieffer

S. altifila Felt. L: Clear, Je 7, found in sweeping blueberry.

S. caryaecola Felt. Hamburg, reared from a jar containing a long, conic leaf gall on bitternut, or swamp hickory.

S. coryloides Walsh \& Riley. Produces a leafy apical gall on grape somewhat resembling a bunch of filberts or hazelnuts. Springwater, Aug, on grape; Nassau.

S. petiolicola Felt. Canadaigua, Aug; Watervliet, Feb; Westfield, Ap 24, reared from an oval or fusiform petiole or tendril gall on summer grape (Vitis bicolor).

S. pomum Walsh \& Rly. Produces a subglobose, polythalamous, chambered bud gall on grape. Presumably generally distributed; Westfield; Hamburg; vic. Albany; vic. NYC.

S. rubi Felt. Karner, J1 24, swept from high blackberry.

S. viburni Felt. Old Forge, Je 20.

\section{ASPHONDYLIA Loew}

A. azaleae Felt. Albany, Je 15-21, reared from unopened, brownish, fusiform buds of Azalea.

A. conspicua O.S. Produces an irregular, subglobular swelling of the flower heads some $5 \mathrm{~mm}$. in diameter, and has been taken at Rensselaer; apparently the same species was captured in a trap lantern at Newport.

A. diervillae Felt. Albany, infesting the unopened buds of bush honeysuckle (Diervilla trifida). 
A. eupatorii Felt. Canandaigua L.; Medina, Sep, reared by L. H. Weld from a green, fleshy stem gall on white snakeroot.

A. fulvopedalis Felt. Westfield, J1 11, swept from Solidago.

A. globulus O.S. Produces a globose stem gall on sunflower. Vic. NYC; LI: Orient, Aug, Lath, on Helianthus divaricatus.

A. helianthiflorae Felt. Highland, Aug-Sep, reared from apparently unmodified flower heads of Helianthus strumosus.

A. ilicioides Felt. Old Forge, inhabiting oval, green, bud galls on Ilicioides mucronata.

A. monacha O.S. A common, widely distributed species producing a small apical rosette gall on Solidago or developing in dwarfed flower-heads of several species of Solidago and at least one species of Aster. Has been reared also from an oval gall developing between two adherent leaves of Solidago.

A. thalictri Felt. Distorts the seed capsules of Thalictrum. Albany; Nassau.

\section{Cincticornia Felt 97}

C. americana Felt. Albany, Ap, reared from slight, blister-like swellings on the lateral veins of red oak.

C. cornifolia Felt. Albany, May 18, on flowering dogwood.

C. multifila Felt. Albany, Je 1, swept from white oak. Presumably a rather common species.

C. pilulae Walsh. Canandaigua, Oct, from gall on oak; Mt. Kisco, Sep 4; Albany, Je 1, on Rhus; LI: Orient, Sep 22, on oak.

C. rhoina Felt. Produces a reddish brown, coarsely reticulated, subglobose leaf gall. Occurs rather commonly in vic. of Albany, is presumably widely distributed in the State, and certainly has a considerable range in the eastern US.

C. simpla Felt. Vic. Albany, reared from irregular, oval, pustulate leaf swellings 5 to $6 \mathrm{~mm}$. in diameter on red oak.

C. transversa Felt. Albany, May 18. Probably occurs on oak.

\section{Tribe ITONIDIDINARIAE}

TOXOMYIA Felt

T. americana Felt. Woodworth's L., Aug 21, in a bog swamp, Alex.

\section{LOBOPTEROMYIA Felt}

L. abdominalis Felt. Albany, May 15, swept from pine.

L. apicalis Felt. Albany, May 18, on basswood.

L. caricis Felt. Karner, May 16, on sedge.

L. consobrina Felt. Karner, May 16, on fern.

L. filicis Felt. Karner, May 16, on ferns.

L. foetedi Felt. Karner, May 16, on skunk cabbage.

L. symplocarpi Felt. Karner, May 16, on skunk cabbage.

L. tiliae Felt. Karner, May 16, on basswood.

\footnotetext{
${ }^{97}$ Besides those here listed, there are undoubtedly a number of other species of this genus which occur in the State, some presumably undescribed, in addition to an excellent series of species which have been brought to light through the untiring labors of the late Miss Cora H. Clarke, of Boston.
} 


\section{CONTARINIA Rondani}

C. agrimoniae Felt. Bath, Sep 3, reared from florets of Agrimonia striata.

C. ampelophila Felt. Albany, Ap 26, on grape.

C. balsamifera Felt. L. Clear, Je 7, very common about balsam trees.

C. canadensis Felt. Southern Hudson R. Val., abundant; a possible maker of the familiar turgid midrib gall on ash generally ascribed to Cecidomyia pellex.

C. clematidis Felt. Newport, Aug 12, reared from an irregular, subglobular gall on virgin's bower.

C. divaricata Felt. Albany, Je 21, on hard pine.

C. flavolinea Felt. Albany, Je 11.

C. johnsoni Sling. Appears to be generally distributed in the Chautauqua grape region, and occasionally causes rather serious injury by blasting the blossom buds of grapes.

C. maculosa Felt. Albany, J1 17, on bittersweet.

C. obsesa Felt. Albany, May 9, in pine woods.

C. perfoliata Felt. Albany, Aug, reared from florets of thoroughwort.

C. pyrivora Riley. A pest of pears, well established in the Hudson R. Val.; New Baltimore, May.

C. rumicis Lw. Newport, J1 18, reared from reddish or brown seeds of curled dock, $Y g$.

C. sambucifolia Felt. L. Clear, Je 7, on elder.

C. trifolii Felt. Albany, Je 1, on white clover.

C. truncata Felt. Albany, May 25.

C. viatica Felt. Albany, Je 9, on clover.

C. virginianiae Felt. Infests the fruit of choke cherry (Prunus virginiana), producing an enlarged bladdery condition. Appears to be widely distributed. Has been recorded from Bethlehem and Keene Valley, and is known from a number of New England localities.

\section{TheCODIPLOSIS Kieffer}

T. hudsonici Felt. Poughkeepsie, Ap 19, on red cedar.

T. liriodendri O.S. Tulip Sрот Gall. Produces a dark, circular blister leaf gall on tulip. Canandaigua L., Sep; vic. Albany, sometimes common; Liverpool, Aug, H.D.House; Yonkers, J1 (Leon); Cornwall, J1; Tivoli, Aug.

T. mosellana Gehin. Batavia, reared from wheat chaff; Troy, Je, on rye heads.

\section{Dentifibula Felt}

D. caryae Felt. Albany, Je 19, on hickory.

D. viburni Felt. Albany, Je 11, on maple-leaved arrowwood.

\section{Group TRIFILI}

YOUNGOMYIA Felt

Y. podophyllae Felt. Albany, Je 11, on May apple.

Y. producta Felt. Karner, J1 5, on bush honeysuckle.

Y. rubida Felt. Nassau, Je 29, on sedge.

Y. umbellicola O.S. Produces a conspicuous, globose enlargement of the blossoms of elder, and occurs generally in the State. 


\section{DICRODIPLOSIS Kieffer}
D. androgynes Felt. Albany, Ap 28, on white pine.
D. annulata Felt. Albany, J1 6, swept from goldenrod and aster.
D. borealis Felt. L. Clear, Je 7, on spruce.
D. insolens Felt. Keene Val., Aug 12, Not.
D. longicornis Felt. Nassau, J1 9, in a trap lantern.
D. rubida Felt. Albany, J1 16, on elder.

\section{BREMIA Rondani}

B. caricis Felt. Nassau, Je 15, on sedge.

B. filicis Felt. Karner, Je 26, swept from low ferns.

B. podophyllae Felt. Albany, Je 21, on May apple.

B. sylvestris Felt. Keene Vàl., Sep 15, Not.

\section{Aphidoletes Kieffer}

A. basalis Felt. Albany, Oct 4, reared from tansy.

A. borealis Felt. Albany, Aug 7, reared from tulip leaves.

A. flavida Felt. Albany, J1 24, on maple.

A. fulva Felt. Albany, J1 17, on blackberry.

A. hamamelidis Felt. Albany, Je 27, on witch-hazel.

A. marginata Felt. Nassau, J1 15.

A. marina Felt. SI: Huguenot Pk., J1 14.

?A. meridionalis Felt. Rye, Aug, on sea lavender, Bird.

A. recurvata Felt. Keene Val., Sep 15, Not.

\section{LOBODIPLOSIS Felt}

L. acerina Felt. Appears to be widely distributed. Keene Val., Aug; Newport, J1 25; Johnstown, J1 20, Alex; Westfield, J1 11, on red cedar; Nassau, Je 11; Albany, Je 15, on soft maple and choke cherry. Is very probably predacious, possibly aphidivorous.

L. borealis Felt. Keene Val., Sep 4, Not.

L. cincta Felt. Newport, J1 23.

\section{Coquillettomyia Felt}

C. dentata Felt. Newport, J1 18, observed in numbers hanging from cobwebs.

C. lobata Felt. L. Clear, Je 7, on blueberry.

\section{Feltiella Ruebsaamen}

F. acerifolia Felt. Albany, May (Felt). On elm and maple.

F. americana Felt. Geneva, J1 (Felt). On plum.

F. emarginata Felt. Nassau, Je (Felt); Newport; Keene Val., Sep, Not.

F. ithacae Felt. Ithaca, Griswold (Felt). On rose.

F. minuta Felt. Nassau, Je (Felt).

F. pini Felt. Albany, Je (Felt). On Pinus rigida. 
KARSCHOMYIA Felt

K. viburni Felt. Keene Val., Aug 26, Not; Woodworth's L., Alex; Albany, Je 1, on sheepberry.

\section{Clinodiplosis Kieffer}

C. examinis Felt. Nassau, Je 19, occurring by the hundreds on a screen door or hanging from cobwebs attached thereto.

c. florida Felt. Tarrytown, Je 13, on pear.

\section{Mycodiplosis Rübsaamen}

M. acerifolia Felt. Albany, May 17.

M. aestiva Felt. Nassau, Je 25, in a trap lantern.

M. alternata Felt. Albany, Je 11, on May apple. Has been swept from sugar maple and white pine.

M. angulata Felt. Albany, Je 19, on hickory; Nassau; Poughkeepsie.

M. aurata Felt. Albany, Je 11, on maple-leaved arrowwood.

M. captiva Felt. Nassau, Je 10.

M. carolinae Felt. Keene Val., Aug 29, Not.

M. cerasifolia Felt. Ithaca, Aug, on Crataegus fruit.

M. cincta n. sp. Johnstown, J1 11, Alex.

M. contracta Felt. Albany, J1 24, on white pine.

M. coryli Felt. Albany, Je 12, on hazel.

M. corylifolia Felt. W. Nyack, J1 23, reared from a fuzzy, wrinkled leaf fold gall on hazel produced by Lasiopteryx coryli Felt; Brooklyn, Sep 7, from black birch, JJLevison.

M. cyanococci Felt. Karner, Je 4, on blueberry.

M. emarginata Felt. Newport, on burdock; Nassau, Je 10.

M. fibulata Felt. Newport, J1 23, in a trap lantern.

M. holotricha Felt. Albany, May, reared from hickory leaf galls supposed to be those produced by Caryomyia holotricha O.S.

M. hudsoni Felt. Albany, Je 10, on red maple.

M. impatientis Felt. W. Nyack, Je 19, reared from exposed larvae living on a fungus, Aecidium impatientis, occurring on the stems of snapdragon.

M. intermedia Felt. Keene Val., Aug 31, Not.

M. lenis Felt. Keene Val., Aug 31, Not.

M. minuta Felt. Nassau, Je 24.

M. modesta Felt. Nassau, Je 14.

M. obscura Felt. Albany, Je 11, on sugar maple.

M. packardi Felt. Round L., Je 24, reared from Pinus strobus.

M. perplexa Felt. Albany, J1 16, on low blackberry.

M. pini Felt. Albany, Je 21, on hard pine. 
M. populifolia Felt. Albany, Sep 3, reared from bullet-like swellings at the base of poplar leaves.

M. reducta Felt. Karner, J1 5, on hard pine.

M. robusta Felt. Albany, Je 11.

M. rotundata Felt. Newport, J1 20.

M. silvana Felt. Nassau, Je 14, on hemlock.

M. tenuitas Felt. Poughkeepsie, Je 14, in a trap lantern.

M. tsugae Felt. L. Clear, Je 7, on hemlock.

M. variabilis Felt. Keene Val., Sep 4, Not; Nassau, J1 24.

\section{Prodiplosis Felt}

P. floricola Felt. Albany, Aug 15, reared from somewhat enlarged, reddened flowers of the meadowsweet (Spiraea salicifolia); Karner, Aug 15, reared from enlarged blossoms of virgin's-bower (Clematis virginiana).

\section{CARYomyia Felt}

C. antennaria Wheeler. Little Falls, Sep, producing gall on Antennaria plantaginifolia.

C. antennata Felt. Produces one of the common hickory leaf galls. Albany and vic. Probably widely distributed.

C. caryae O.S. Produces a gall on hickory. Scarsdale, J1; Hudson Falls, Sep; vic. Albany; Mt. Kisco, J1 2; vic. NYC. Probably widely distributed.

C. caryaecola O.S. Produces a very slender, subglobose gall usually thickly clustered along the midrib of hickory leaves. Hamburg, Oct 17, on bitternut hickory. Appears to be relatively scarce.

C. consobrina Felt. Produces a small, thin-walled hickory leaf gall. Nassau.

C. glutinosa O.S. This is the name given to a larva living on the under surface of a hickory leaf, exposed except for the protection afforded by a viscid secretion. Vic. Albany and NYC, scarce.

C. holotricha O.S. Occurs commonly about Albany and NYC, and appears to be widely distributed.

C. inanis Felt. Nassau, Ap 29, reared from a hickory leaf gall having a more or less well-developed, empty chamber above the inhabited cavity.

C. nucicola O.S. Produces swellings in the green husks of shagbark hickory. Rhinebeck, Aug 9, 1860, O.S.

C. persicoides Bt. Produces a common, rather large, irregular, monothalamous, hairy gall on hickory, frequently 6 or $7 \mathrm{~mm}$. in diameter and usually clustered along the midrib. Albany; Barrytown, on gall, Sep; vic. NYC; probably widely distributed.

C. sanguinolenta O.S. Vic. Albany and NYC, the galls being occasionally numerous.

C. similis Felt. Nassau, reared the latter part of Ap and in early May from supposedly typical galls of Caryomyia caryae O.S.

C. thompsoni Felt. Produces a yellow, green, or brown, melon-shaped gall with a depressed center, apparently taken by LHWeld at Ithaca.

C. tubicola O.S. Produces a characteristic, tubular, common hickory leaf gall which appears to be widely distributed throughout the State; Canandaigua L., Sep. 


\section{ARTHROCNODAX Rübsaamen}

A. acerina Felt. L. Clear, Je 7, on maple.

A. cincta Felt. Albany, Je 13.

A. fenestra Felt. Nassau, J1 22.

A. filicis Felt. Karner, Je 4, on fern; Nassau, J1 9, in a trap lantern.

A. fraxini Felt. Albany, Je 10, on ash.

A. incisa Felt. Albany, May 21.

A. obscura Felt. Karner, Je 26, on blueberry.

A. rhoina Felt. Albany, Aug 27, reared from curled leafy heads of sumac.

A. rufa Felt. Albany, J1 24, on hickory.

A. sambucifolia Felt. Albany, Sep 13, reared from rolled elder leaves.

\section{HORMOMYIA Loew}

H. alexanderi Felt. Johnstown, J1 7, swept from palustral grasses, Alex.

H. americana Felt. Nassau, May 28 and 31, in a trap lantern. Appears to be fairly common.

H. canadensis Felt. Reared in early Ap from a subglobose, greenish or deep reddish, lipped leaf gall on shadbush. Fairly common in vic. of Albany.

H. clarkei Felt. L. Placid, inhabiting a terminal bud gall on Spiraea salicifolia.

H. consobrina Felt. Albany, Je 6, on Azalea.

H. crataegifolia Felt. Reared in early Ap from a cockscomb leaf gall on Crataegus. A very local species occurring in the vicinity of Nassau, though probably rather widely distributed; L. Placid Club, J1; Newport, J1, GSGraves; LI: Orient, Aug 12.

H. dilatata Felt. Newport, Je 27, in a trap lantern.

H. incisa Felt. Nassau, $\mathrm{J} 18$, in a trap lantern.

H. maxima Felt. Johnstown, Je 8, swept from palustral grasses, Alex.

H. palustris Felt. Ithaca, May 20, captured in considerable numbers on a lake marsh.

\section{MONARTHROPALPUS Rübsaamen}

M. buxi Lab. A European species which has become well established in several LI localities and is best known because of its injury to the foliage of ornamental box. Westchester Co, Jan 9, Atrood; New Rochelle, May; LI: Mineola, Ap 2, larvae; Woodlawn Cemetery, Ap 16, larvae, Bt; Roslyn, May, Aug; Sea Cliff, Ap 8, Levison.

\section{HYPERDIPLOSIS Felt}

H. insolens Felt. Keene Val., Aug 26, Not.

H. lobata Felt. Karner, Je 4, on white clover.

\section{GIARDOMYIA Felt}

G. emarginata Felt. Newport, J1 2, in a trap lantern.

G. hudsonica Felt. Poughkeepsie, Je 9, in a trap lantern.

G. menthae Felt. Karner, J1 27, reared from mint (Mentha canadensis) bearing pustule-like galls on the stem. 
G. montana Felt. Newport, Je 16, in a trap lantern; SI: Huguenot Pk., J1 14.

G. noveboracensis Felt. Nassau, Je 10.

G. photophila Felt. Newport, Je 16, in a trap lantern.

\section{Metadiplosis Felt}

M. spinosa Felt. Albany, J1 14, on quack grass.

\section{EPIDIPLosis Felt}

E. sayi Felt. Nassau, J1 1.

\section{LEstodipLosis Kieffer}

L. apocyniflorae Felt. Nassau, Aug 9, reared from apparently normal flower buds of dogbane.

L. asclepiae Felt. Highland and Albany, Aug, reared from rolled leaves of milkweed (Asclepias Cormuti).

L. asteris Felt. Albany, $\mathrm{J} 1 \mathrm{17}$, on aster.

L. basalis Felt. Albany, J1 6, on New Jersey tea.

L. caliptera Fitch. Undoubtedly belongs to this genus though insufficiently characterized.

L. cerasi Felt. W. Nyack, J1 26, reared from variable, oval or fusiform, twig swellings on wild cherry. The species is probably predacious.

L. cincta Felt. Albany, J1 3, on New Jersey tea.

L. clematiflorae Felt. Karner, Aug 28, reared from unopened flowers of virgin'sbower (Clematis virginiana).

L. crataegifolia Felt. Bath, J1 17, reared from an irregular, rather spiny gall on the upper surface of a Crataegus leaf. The species is probably predacious.

L. eupatorii Felt. Reared Sep 12 from globose galls in flower heads of Eupatorium ageratoides. South Bay; Glen L.; L. Champlain.

L. flavomarginata Felt. Albany, Je 1, on white clover.

L. hicoriae Felt. Nassau, Je 14, on hickory.

L. juniperina Felt. Nassau, Aug 7.

L. platanifolia Felt. NYC, Aug 1, reared from plane-tree leaves.

L. populifolia Felt. Albany, J1 22, reared from a marginal leaf roll on poplar. The species is probably predacious.

L. rugosa Felt. Albany, J1 15, reared from Solidago galls.

L. rumicis Felt. Newport, J1 29, reared from curled dock seeds infested by Contarinia rumicis.

L. satiata Felt. Keene Val., Aug 28, Not.

L. scrophulariae Felt. W. Nyack, Aug 8, reared from distorted flower buds of Scrophularia marilandica.

L. solidaginis Felt. Albany, Aug 20, reared from a gall in association with Rhopalomyia albipennis Felt.

L. spiraeafolia Felt. Albany, Aug 23, reared from a thickened leaf fold on meadowsweet, Spiraea tomentosa.

L. triangularis Felt. W. Lebanon, Aug 26, reared from Solidago leaves.

L. tsugae Felt. L. Clear, Je 7, on hemlock.

L. verbenifolia Felt. Karner, J1 20, from rolled leaves of Verbena urticaefolia. 
PARAdiplosis Felt

P. obesa Felt. L. Clear, Je 7, on hemlock.

PARALlelodiplosis Rübsaamen

P. acernea Felt. Nassau, Je 14, on red maple.

P. carpini Felt. Albany, Je 21, on ironwood.

P. caryae Felt. Albany, Je 19, on hickory.

P. coryli Felt. Albany, Je 11, on hazel.

P. extensa Felt. Albany, Je 11, on locust (Robinia Pseudo-Acacia).

P. montana Felt. Newport, J1 20, Yg.

P. pratensis Felt. Albany, Aug 6, on pine.

P. rubisolita Felt. Albany, J1 24, on low blackberry.

P. rubrascuta Felt. Albany, Je, on ash.

P. spirae Felt. Produces a variable yellowish or reddish marginal leaf roll on Spiraea salicifolia. Albany.

P. subtruncata Felt. Albany, J1 6, on Solidago.

P. triangularis Felt. Nassau, J1 2 and 6.

\section{OвоLODIPLOSIS Felt}

O. robiniae Hald. (orbiculata Felt). Albany, Je 10, on black locust; Kingston; LI: Orient, Aug 25. Presumably widely distributed in the State.

\section{ITONIDA Meigen}

I. abdominalis Felt. Albany, Je 1, on hickory.

I. agraris Felt. Newport, Nassau, Je-Jl.

I. albotarsa Felt. Albany, Je 19, on hickory.

I. americana Felt. Poughkeepsie, Je 28, in a trap lantern.

I. antennata Felt. Albany, May 21.

I. aphidivora Felt. Nassau, reared in numbers from apple leaves infested by the rosy aphis.

I. apicalis Felt. Newport, Je 27, in a trap lantern, $Y g$.

I. apocyni Felt. Nassau, Aug 21, reared from unopened, apparently normal blossoms of spreading dogbane.

I. aprilis Felt. Among the early-appearing forms collected at Albany.

I. catalpae Comst. Albany, J1.

I. claytoniae Felt. Albany, May 18.

I. emarginata Felt. Albany, May 17; Nassau, Je 30.

I. excavationis Felt. Albany, May 21, on soft maple.

I. explicata Felt. Albany, Ji 6.

I. flavoscuta Felt. Albany, May 21.

I. foliora Rssl. \& Hkr. Produces a somewhat characteristic marginal leaf fold on certain oak leaves. Common and widely distributed in NY. Canandaigua, J1 20; Hudson Falls, Sep.

I. fragariae Felt. Albany, Je 18, on strawberry. 
I. hartmania Felt. Albany, Ap 15, near a decaying hickory stump.

I. hudsoni Felt. Poughkeepsie, Ap 19, on red cedar.

I. infirma Felt. Albany, Je 15, on Syringa.

I. nixoni Felt. Albany, J1 6, on New Jersey tea.

I. opuntiae Felt. Reared from discolored, more or less decaying areas on various species of Opuntia collected in the NY Botanical Gardens at Bronx Pk.

I. paucifila Felt. Albany, Je 15, on Deutzia.

I. piperitae Felt. Nassau, Sep 4, reared from small, enlarged terminal buds on peppermint (Mentha piperita).

I. pugionis Felt. Nassau, May 4, reared from chestnut and maple bark infested with numerous Miastor americana Felt larvae.

I. putrida Felt. Reared from decaying birch wood inhabited by numerous larvae of Bolitophila cinerea Meign.

I. quercina Felt. Albany, Je 21, on swamp white oak.

I. ramuli Felt. Albany, May 25, reared from a small, monothalamous, almost imperceptible, twig enlargement on Cornus paniculata.

I. recurvata Felt. Poughkeepsie, Je 21, in a trap lantern.

I. ruricola Felt. Nassau, Je 15, on sedge.

I. sanguinia Felt. Nassau, Je 24, on fern.

I. setariae Felt. Albany, Aug 25, reared from seeds of the common fox-tail grass (Alopecurus pratensis).

I. spiraeaflorae Felt. Albany, Aug 27, reared from slightly cnlarged, reddened flowers of Spircea salicifolia.

I. tecomiae Felt. Albany, reared from larvae distorting and rolling leaves of the trumpet vine.

I. terrestris Felt. Poughkeepsie, Je 21, in a trap lantern.

I. tolhurstae Felt. Albany, Je 30, on sumac.

I. tritici Felt. Presumably the wheat midge, a well-known pest of wheat in earlier years and one widely distributed in this State and probably throughout the wheat-growing areas of the US. Belle Isle (NYS Rept 15).

I. verbenae Bt. Karner, J1 20, from rolled terminal leaves of Verbena urticaefolia.

\section{RETINODIPLOSIS Kieffer}

R. albitarsis Felt. Round L., Je 24, reared from Pinus strobus.

R. inopis O.S. Produces obscure, subcortical swellings in the smaller branches of scrub pine (Pinus rigida and $P$. virginiana). Common in vic. of Albany. Presumably widely distributed.

R. resinicola O.S. The Pitch MIDge. The common, widely distributed species inhabiting exuding masses of pitch. The larvae are remarkable because of their living within this resinous medium. Karner; Mechanicville, Sep.

\section{ODONTODIPLOSIS Felt}

O. americana Felt. Albany, J1 3 on alder, J1 24 on low blackberry.

O. karnerensis Felt. Karner, May 16.

O. montana Felt. Newport, J1 27, on hemlock, Yg. 


\section{Adiplosis Felt}

A. toxicodendri Felt. Nassau, Je 14, on poison ivy.

\section{Cecidomyia 98}

C. balsamicola Lint. Deforms balsam leaflets. Is widely distributed. Adult has not been definitely connected with its food plant. ?North Creek, Aug.

C. bedeguar Walsh. Shushan, Je 14, gall on Crataegus.

C. boehmeriae Bt. Produces a fusiform stem swelling on nettle (Boehmeria), and is probably a species of Lasioptera. Shushan.

C. celastri Stebb. Probably identical with the species producing a subcortical stem swelling on Celastrus at W. Nyack.

C. citrina O.S. Produces variable petiole and twig swellings on linden. Rhinebeck. Apparently widely distributed in the State.

C. collinsoniae Bt. Produces a green, onion-shaped, pubescent swelling on the under side of the leaves of Collinsonia. Garrison. Probably widely distributed.

C. erubescens O.S. Produces a common marginal leaf fold. Itonida foliora Rssl. \& Hkr. may prove to be a synonym.

C. eupatoriflorae Bt. Produces globose enlargements of the flower head of Eupatorium ageratoides. Vic. NYC.

C. fulva Bt. Produces a globular, succulent swelling of stem, petiole, or leaf. Recorded from Shushan. Probably widely distributed in the State. Has been provisionally referred to the genus Lasioptera.

[C. grossulariae Fitch. Inhabits gooseberries, and is presumably a NY species.]

C. impatientis O.S. Canantaigua, Aug 31; Newport, Aug, Yg. Appears to be common in the State. Inhabits a nearly globose, succulent swelling at the base of the flower of Impatiens fulva.

C. inimica Fitch. Observed by Dr. Fitch in wheat fields infested by the wheat midge.

C. majalis O.S. Produces a thin-walled, more or less wrinkled gall along the midrib of oak which may be identical with that from which Clinodiplosis florida Felt was reared. Moderately common in the State.

C. meibomiae Bt. Produces an elongate, fusiform, clustered bud growth on various species of tick trefoil. Vic. NYC. Probably widely distributed.

C. niveipila O.S. Produces a common swollen vein fold margined with a white pubescence, on various oaks. Hudson Falls, Je 6, on black oak and Quercus prinus.

C. nyssaecola Bt. Produces a narrow, marginal leaf fold on sour gum (Nyssa sylvatica). Vic. NYC, very common.

C. ocellaris O.S. Produces a common and widely distributed, circular, yellowish and red-margined discoloration on soft maple leaves.

C. pellex O.S. Produces a subglobular ash leaf gall, quite different from the gall usually considered as being produced by this species. Canandaigua, $\mathrm{Je} 4$; southern Hudson R. Val., moderately common.

C. pinirigidae $\mathrm{Pk}$. Produces deformed pine needles with a swollen base. The true gall-maker has not been reared. Moderately common on LI and in vic. of Albany. 
C. poculum O.S. Produces a moderately common, circular, flat stem gall on the leaves of post oak. Millbrook, Aug 24; NYC, Oct.

C. pudibunda O.S. Inhabits a red-tinged leaf fold on hornbeam. The gall is common and widely distributed.

C. serotinae O.S. Transforms the terminal buds on young shoots of wild cherry into globose, red-tinted swellings about the size of a cherry. Moderately common and widely distributed; LI: Fishers I., Jan 19, on gall.

C. tuba Stebb. Vic. Albany, scarce. Inhabits a slender, curved tube 10 to $16 \mathrm{~mm}$. long and about $2 \mathrm{~mm}$. in diameter, occurring on the leaves of Cornus amomum.

C. urnicola O.S. Produces an urn-shaped, subsessile, pale green, semitransparent, succulent leaf gall on Urtica gracilis. Ithaca; Trenton Falls. Probably widely distributed.

C. venae Stebb. Probably identical with Lobopteromyia venae Felt, a species recorded from Nassau.

C. verrucicola O.S. Produces a subglobular, green or brown, leaf gall on linden. Highland Falls, Nov 14; ?Newport, J1 23. Widely distributed, and occasionally very abundant.

C. sp. Little Falls, Sep 10, galls on Eupatorium ageratoides.

C. viticola O.S. Produces an elongate, conical, reddish or green leaf gall on wild grape. Canandaigua, Oct 5; Highland, rather common. Probably widely distributed in the State.

C. sp. Hudson Falls, J1 6, producing gall on Laportea canadensis.

\section{FAMILY MYCETOPHILIDAE SUBFAMILY BOLITOPHILINAE}

Bolitophila Meigen

B. cinerea Meig. Buffalo, May (MCV); Ithaca, May, Sep (Joh); Albany, ApMay ( $\mathrm{Yg})$.

B. disjuncta Lw. Albany, May ( $Y g$ ).

B. hybrida Meig. Wells, J1 (Yg); Little Valley, J1 (MCV); Albany, May; McLean, Je, Sib (Joh).

B. montana Coq. Ithaca, Sep (Joh).

PALAEOPLATYURA Meunier

P. johnsoni Joh. Ithaca, Je (Joh).

SYMMERUS Walker

S. lautus Lw. N. Evans, J1 (MCV); Little Valley, Je (MCV); Ithaca, Aug (Joh).

\section{SUBFAMILY DIADOCIDIINAE}

DIADOCIDIA Ruthe

D. ferruginosa Meig. Wells, J1 (Yg); Erie Co, May-Aug (MCV); Ithaca, Aug (Jn). 


\title{
SUBFAMILY CEROPLATINAE
}

\section{ASINDULUM Winnertz}

\begin{abstract}
A. montanum Roeder. Adirondacks, Aug (Joh); Speculator; Long L., Aug; Little Valley, J1 (MCV); Beaver Kill (CnJr); McLean, Sep, Sib (Joh); Oliverea (Beq).
\end{abstract}

\section{Ceroplatus Bosc}

C. clausus Coq. S. Wales, J1 (MCV); Ithaca (Joh).

C. militaris Joh. Ithaca (Joh).

\section{Platyura Meigen}

P. diluta Lw. Ithaca; McLean, J1 (Joh).

P. elegans Coq. Ithaca, J1 (Joh); Yonkers (AM).

P. fascipennis Say. Little Valley, Je (MCV).

P. genualis Joh. Lancaster, Hamburg, Je (MCV); McLean, J1, Sib (Joh).

P. inops Coq. Ithaca, Jl (Joh).

P. mendica Lw. Little Valley, Je (MCV); Ithaca (Joh).

P. mendosa Lw. Wells, J1 (Yg); S. Wales, J1 (MCV); Little Valley, Je (MCV); Ithaca (Joh).

P. moerens Joh. S. Wales (MCV).

P. subterminalis Say. Ithaca, J1 (Joh).

\section{SUBFAMILY MACROCERINAE}

MACROCERA Meigen

M. clara Lw. L. Placid, Je (Yg); Wells, J1 (Yg); S. Wales, J1 (MCV); Little Valley, J1; Ithaca (Joh); Albany, May.

M. formosa Lw. Little Valley, Jl (MCV); Ithaca, J1 (Joh); Beaver Kill, Aug $(\mathrm{CnJr})$.

M. geminata Joh. Buffalo, Je (MCV); Ithaca, Je (Joh).

M. hirsuta Lw. Mountain Lake, Je (Yg); Wells, J1 ; S. Wales; J1; Colden, Aug-Sep $(\mathrm{MCV})$.

M. immaculata John. Niagara Falls, Je ( Jn).

M. inconcinna Lw. Niagara Falls, Je (Jn).

M. nebulosa Coq. S. Wales, J1 (MCV).

\section{SUBFAMILY SCIOPHILINAE}

MONOCLONA Mik

M. elegantula Joh. Erie Co, Je-Sep (MCV); Ithaca, Aug (Joh).

EUDICRANA Loew

E. obumbrata Lw. Wells, J1 (Yg). 


\section{TETRAGONEURA Winnertz}

T. bicolor Coq, Ithaca, Je (Joh).

T. pimpla Coq. Speculator (Yg); Gowanda, Je (MCV).

\section{ScIOPHILA Meigen \\ (Lasiosoma Winnertz)}

S. galbana Joh. Ithaca (Joh); Albany, $\mathrm{Je}(\mathrm{Yg})$.

S. galbana var. socia Joh. Wells, Je (Yg); Grand I., Je (MCV).

S. habilis Joh. Ithaca (Joh).

S. incallida Joh. Gowanda, Je (MCV); Ithaca (Joh).

S. novata Joh. Hamburg, Oct (MCV); Ithaca (Joh).

S. nugax Joh. Ithaca, Aug (Joh).

S. pallipes Say. Hamburg, Mar; Colden, Aug (MCV); Ithaca (Joh).

S. severa Joh. Ithaca, J1 (Joh).

PARATINIA Mik

P. recurva Joh. Old Forge, Aug (Joh).

Polylepta Winnertz

P. obediens Joh. Wells, J1 (Yg); Colden, Aug (MCV).

\section{EMPALIA Winnertz}

E. tibialis Coq. McLean, J1, Sib (Joh).

\section{Diomonus Walker}

D. magnificus Joh. Hamburg, Aug, S. Wales, J1 (MCV); Ithaca, J1 (Joh).

D. pulcher Joh. Axton, Je (Joh); McLean, Sib (Joh).

D. subcaeruleus Coq. Protection (MCV); foot of Cliff Mt., $3000 \mathrm{ft}$. (Beq).

\section{Neoempheria Osten Sacken}

N. balioptera Lw. Erie Co, Je (MCV) ; Ithaca, J1 (Joh).

N. illustris Joh. Ithaca (Joh); Poughkeepsie, Aug (Yg).

N. impatiens Joh. Boston, Sep (MCV).

N. indulgens Joh. Erie Co, Je, J1 (MCV); Ithaca, J1 (Joh).

N. macularis Joh. Cranberry Cr., Je (Yg); Hamburg, J1 (MCV); Ithaca (Joh).

\section{Mycomya Rondani}

M. appendiculata Lw. "NY" (Lw).

M. brevivittata Coq. Wells, $\mathrm{Jl}(\mathrm{Yg})$; Ithaca (Joh).

M. imitans Joh. Wells, J1 (Yg); E. Aurora, Sep (MCV); Ithaca (Joh).

M. incompta Joh. Ithaca (Joh). 
M. littoralis Say. Old Forge; Wells, J1; Erie Co, May-Sep (MCV); Ithaca, J1 (Joh); Albany, May (Yg).

M. maxima Joh. E. Aurora, Sep (MCV).

M. mendax Joh. Wells, Jl (Yg).

M. nigricauda Adams. S. Wales, J1 (MCV).

M. obliqua Say. Erie Co, Aug, Sep (MCV); Ithaca, J1 (Joh).

M. sequax Joh. Wells, J1 (Yg); Ithaca (Joh).

\section{SUBFAMILY MYCETOPHILINAE}

GNORISTE Meigen

G. megarrhina O.S. Ithaca, May, Je (Joh).

\section{ACNemia Winnertz}

A. flaveola Coq. E. Aurora, Aug (MCV); Ithaca (Joh).

A. psylla Lw. Niagara Falls, Aug (MCV); Ithaca, Aug, Sep (Joh).

\section{RONDANIELLA Johannsen}

R. abbreviata Lw. Colden, May, S. Wales, J1 (MCV); Ithaca (Joh); Albany, Ap (Yg).

R. sororcula Lw. "NY" (Lw).

\section{LEPTOMORPHUS Curtis}

L. ypsilon Joh. Ithaca (Joh).

Boletina Staeger

B. cincta Joh. Old Forge, J1 (Joh); S. Wales, J1 (MCV).

B. imitator Joh. Erie Co, May, Sep (MCV).

B. notescens Joh. Adirondacks, Aug; Ithaca, Je (Joh).

B. obscura Joh. Ithaca (Joh).

B. sciarina Staeg. Lancaster, Ap; Hamburg, Ap-Oct (MCV).

B. tricincta Lw. Niagara Falls, Je (Jn); Erie Co, Je, Aug (MCV); Ithaca, Aug (Joh).

B. unusa Garr. Ithaca (Garr), monotype.

\section{LEIA Meigen \\ (Neoglaphyroptera Osten Sacken)}

L. bivittata Say. Colden, Aug (MCV) ; Ithaca, Aug, Sep (Joh); Albany, Oct $(\mathrm{Yg})$.

L. decora Lw. Ithaca, Je-Sep (Joh).

L. melaena Lw. Wells, $\mathrm{Jl}(\mathrm{Yg})$.

L. oblectabilis Lw. Ithaca, J1-Sep (Joh).

L. opima Lw. Niagara Falls, Je (MCV) ; Erie Co, Je-Sep (MCV); Ithaca, J1Sep (Joh). 
L. striata Will. Buffalo, Sep (MCV).

L. sublunata Lw. Erie Co, Je-Sep (MCV); Ithaca (Joh).

L. ventralis Say. Karner, Oct (Yg).

L. winthemi Lehmann. Saranac Inn; L. Tear, $4300 \mathrm{ft}$. (Beq); Erie Co, Je-J1 (MCV); Little Valley, Je (MCV); Ithaca, Je (Joh); Meadowdale, Aug $(\mathrm{MCV})$.

Phthinia Winnertz

P. curta Joh. Ithaca (Joh).

P. tanypus Lw. Old Forge (Joh).

Docosia Winnertz

D. dichroa Lw. Niagara Falls, Je (MCV); Hamburg, Colden, May (MCV); Ithaca, Je (Joh); New Baltimore, May (Yg).

SYNTEMMA Winnertz

S. polyzona Lw. McLean, J1, Sib (Joh).

ANATELla Winnertz

A. silvestris Joh. Wells, J1 (Yg); Ithaca, Mar, Aug (Joh).

TRICHONTA Winnertz

T. diffissa Joh. Ithaca, Aug (Joh).

T. patens Joh. Wells, J1 ( Yg); Ithaca, May, Aug (Joh).

T. triangularis Joh. Wells, J1 (Yg); Ithaca (Joh).

CORDYLA Meigen

C. manca Joh. Ithaca (Joh).

C. recens Joh. Colden, Aug (MCV); Ithaca, Caroline, Je-J1 (Joh).

C. volucris Joh. Ithaca, Oct (Joh); Meadowdale, Oct.

BRACHYPEZA Winnertz

B. bisignata Winn. var. divergens Joh. Little Valley, Je (MCV).

RHyMosia Winnertz

R. captiosa Joh. McLean, Sep, Sib (Joh).

R. inflata Joh. Erie Co, Je, Sep (MCV); Ithaca, Oct-Nov (Joh).

R. serripes Joh. Ithaca, Aug (Joh).

Allodia Winnertz

A. actuaria Joh. Colden (MCV); Ithaca (Joh).

A. beata Joh. Wells, J1 (Yg); Ellis, Ithaca, Aug (Joh).

A. bulbosa Joh. Wells, J1 (Yg); Ithaca, Je (Joh). 
A. crassicornis Stann. Wells, J1 (Yg); Niagara Falls, Sep (Joh); Ithaca, May, J1.

A. falcata Joh. Wells, J1 (Yg); Rochester, Sep (MCV); Ithaca, Sep (Joh).

\section{PHRONIA Winnertz}

P. difficilis Joh. Wells, J1; Ithaca, Aug (Joh); Albany, May ( $\mathrm{Yg}$ ).

P. insulsa Joh. Wells, Jl (Yg); Ithaca, Aug (Joh); McLean, Claas (Joh).

P. rustica Winn. Ithaca, Aug (Joh).

P. similis Joh. Wells, J1 (Yg); Ithaca, Sep (Joh).

P. venusta Joh. Albany, May (Yg).

\section{TELMAPHILUS Becker}

T. nebulosus Joh. Colden, Aug (MCV); Ithaca, Ap (Joh).

\section{EXECHIA Winnertz}

E. abrupta Joh. Ithaca (Joh).

E. absoluta Joh. Buffalo, Oct (MCV); Ithaca (Joh).

E. absurda Joh. Wells, J1 (Yg); Ithaca (Joh).

E. attrita Joh. Ithaca, Aug (Joh).

E. auxiliaria Joh. Ithaca (Joh).

E. bella Joh. Ithaca (Joh).

E. capillata Joh. Ithaca, May (Joh).

E. casta Joh. E. Aurora, Aug (MCV).

E. cincinnata Joh. Buffalo, Nov (MCV); Ithaca (Joh); Albany, May.

E. nexa Joh. Ithaca (Joh).

E. nativa Joh. Niagara Falls, Sep (MCV); Ithaca, Nov (Joh).

E. nugatoria Joh. Niagara Falls, Oct (MCV); Erie Co, May-Nov; Ithaca (Joh) ; Albany, May ( $\mathrm{Yg})$.

E. nugax Joh. Ithaca, Aug (Joh).

E. quadrata Joh. Ithaca, Aug (Joh).

E. repanda Joh. Ithaca, Aug (Joh).

E. satiata Joh. Ithaca (Joh).

E. umbratica Ald. Wells, J1 (Yg); Ithaca (Joh).

\section{DYNATOSOMA Winnertz}

D. errans Garr. Ithaca, J1 (Garr).

D. fulvida Coq. Ithaca, Je (Joh).

D. bifasciata Walk. (nigrina Joh.). S. Wales, J1 (MCV).

D. placida Joh. Niagara Falls, Aug (MCV).

Opistholoba Mik

O. ocellata Joh. Wells (Yg); Ithaca, Aug (Joh). 


\section{EPICYPTA Winnertz}

E. trinotata Staeg. Ithaca, Je-Aug (Joh).

\section{MYCOTHERA Winnertz}

M. fenestrata Coq. Buffalo, Mar, May (MCV).

M. fenestrata var. praenubila Joh. Ithaca, Ap (Joh).

M. impellans Joh. Ithaca, Aug (Joh).

M. paradoxa Joh. Wells, J1 (Yg); Ithaca (Joh).

M. paula Lw. Wells, J1; Ithaca, Oct (Joh); Albany, May (Yg).

M. recta Joh. Wells, J1 (Yg); Ithaca, Aug (Joh).

\section{MYCETOPHILA Meigen}

M. bipunctata Lw. Erie Co, May-J1; Niagara Falls, Sep (MCV); Ithaca, Je-Aug $(\mathrm{Yg})$.

M. edentula Joh. Wells, J1; Albany, May (Yg).

M. edura Joh. Ithaca, Aug (Joh).

M. extenta Joh. Ithaca, Ap (Joh); Albany, May (Yg).

M. exusta Joh. Colden, May (MCV).

M. falcata Joh. Ithaca, May (Joh).

M. fastosa Joh. Wells, J1; Albany, May (Yg); Ithaca (Joh); S. Wales, J1 (MCV).

M. foecunda Joh. Ithaca, Nov (Joh).

M. ichneumon Say. Niagara Falls, Je (Jn).

M. imitator Joh. Hamburg, Ap (MCV); Ithaca (Joh).

M. inculta Lw. Ithaca, May-Aug (Joh).

M. jucunda Joh. Ithaca, Aug (Joh).

M. lenta Joh. Wells, J1; Albany, May ( $Y g$ ).

M. monochaeta Lw. Colden, Aug (MCV).

M. mutica Lw. Wells, J1 (Yg) ; Erie Co, May, Je (MCV).

M. perita Joh. Ithaca, J1 (Joh).

M. perlonga Joh. Wells, J1; Ithaca, Aug (Joh) ; Albany, May.

M. polita Lw. Colden, Aug (MCV); Ithaca, J1 (Joh).

M. procera Lw. "NY" (Lw).

M. propinqua Wlk. "NY" (Wlk).

M. punctata Meig. Axton, Je; Erie Co, May-Nov (MCV); Ithaca, J1 (Joh).

M. quatuornotata Lw. Erie Co, Je-Oct (MCV).

M. scalaris Lw. Niagara Falls, Je; Erie Co, May, Aug; Rochester, Sep (MCV); Ithaca, Je-Oct (Joh).

M. socia Joh. Ithaca, Aug (Joh); Albany, May (Yg).

M. trichonota Lw. Ithaca, J1, Aug (Joh); Albany, May (Yg). 
DELOPSIS Skuse

D. anomala Joh. Hamburg, May (MCV); McLean, May; Ithaca, Aug (Joh).

PNYXIA Johannsen

P. scabiei Hopk. Rhinebeck (Joh).

SCEPTONIA Winnertz

S. nigra Meig. Wells, J1 (Yg); Colden, May (MCV); Ithaca, Aug (Joh).

ZYGOMYIA Winnertz

Z. ignobilis Lw. Wells, $\mathrm{Jl}(\mathrm{Yg})$; Ithaca (Joh).

Z. ornata Lw. Colden, May (MCV); Ithaca, Aug (Joh).

Z. varia Staeg. Wells, J1 (Yg).

\section{FAMILY SCIARIDAE}

EUGNoRISTE Coquillett

E. occidentalis Coq. Manlius; Ithaca (Joh).

\section{TRICHOSIA Winnertz}

T. hebes Lw. Ithaca, J1 (Joh).

\section{ZYGONEURA Meigen}

Z. flavicoxa Joh. Ithaca, J1 (Joh).

SCIARA Meigen

(Including Neosciara Pettey)

S. actuosa Joh. Niagara Falls, Oct; Erie Co, May, Je (MCV); Freeville, Ithaca, May, J1 (Joh).

S. agraria Felt. Albany ( $\mathrm{CnJr})$.

S. caldaria Lint. Ithaca (Joh).

S. coprophila Lint. Erie Co, Ap-Sep (MCV) ; Ithaca, Aug (Joh); Albany, Mar; LI: Glen Cove, Je.

S. diluta Joh. Freeville; Ithaca, J1 (Joh).

S. dolens Joh. Tompkins $\mathrm{Co}$, Je (Joh).

S. dux Joh. Ithaca, Je (Joh).

S. fatigans Joh. Ithaca (Joh).

S. femorata Say. Niagara Falls, Je (Jn) ; N. Evans, May (MCV).

S. fuliginosa Fitch. "NY" (Fitch); Erie Co, May, Je (MCV).

S. fumida Joh. Ithaca, Aug (Joh).

S. habilis Joh. Hamburg, May, Boston, Sep (MCV); Ithaca, Je (Joh).

S. hastata Joh. Tompkins Co, Je, J1 (Joh). 
S. impatiens Joh. Ithaca (Joh).

S. inconstans Fitch. Albany, Je (Fitch).

S. johannseni End. (nigricans Joh.). Hamburg, Oct (MCV); Ithaca, May, Aug (Joh).

S. jucunda Joh. Niagara Falls, May (Joh); Ithaca, Je-Aug.

S. lurida Wlk. Trenton Falls (Wik).

S. mali Fitch. "NY" (Fitch).

S. mellea Joh. Ithaca, Je (Joh).

S. mutua Joh. N. Evans, May (Joh); Ithaca, Je.

S. nacta Joh. Ithaca (Joh).

S. ocellaris Cmstk. Lancaster, Buffalo (Joh).

S. ochrolabis Lw. Ithaca, Je, Aug (Joh).

S. pauciseta Felt. Ithaca, J1-Oct (Joh); McLean, Ap, Claas (Joh).

S. parilis Joh. Ithaca (Joh).

S. prolifica Felt. Erie Co, May-Sep (MCV); Ithaca, Ap, Aug (Joh).

S. sciophila Lw. Ithaca, J1-Oct (Joh).

S. silvestrii Kieff. "NY" (Kieff).

S. similans Joh. LI: Cold Sp. Harb., Metz (Joh).

S. trivialis Joh. Ithaca, Aug (Joh); Bronx Pk.

S. varians Joh. Ithaca (Joh).

S. vicina Joh. Ithaca, J1 (Joh).

S. vulgaris Fitch. Karner, Oct.

\section{FAMILY BIBIONIDAE}

\section{Plecia Weidemann}

P. heteroptera Say. Saranac L., Sep, Ds (Leon); Hamburg, Oct, Buffalo, Oct. $M C V$ (Jn); Ithaca, Oct (Joh); McLean, Sep, Sib (Joh); Hebron, Sep, Ds (Leon); Debruce, Sep, Ds; Oliverea, Sep (Leon); West Pt., Nic; Ft. Montgomery, Schott.

\section{Bibio Geoffroy}

B. abbreviatus Lw. W. Nyack, Ols (Beq).

B. albipennis Say. Adirondacks, Je (Joh) ; Erie Co, $M C V$; Ithaca, common, May, Aug; New Baltimore; Suffern (AM); Dunwoodie (AM); SI (Beq); LI: Huntington, Rockaway Beach, Wading R., Yaphank, Ds (Jn), May.

B. basalis Lw. Mt. Marcy, $5344 \mathrm{ft}$. (Beq).

B. femoratus Wied. Mt. Whiteface, summit, Jl, Ds (Leon) ; Axton, Je; Newport, May; Hamburg. May, MCV (Jn) ; Tompkins Co, May. (Jn); White Plains, Bno (Beq); LI: Rockaway Beach, Ds (Leon); Orient, May, Lath; Wyandanch, May.

B. fraternus Lw... Tompkins Co (HHS),

B. fumipennis Wlk. Newport, May, Yg (NYS).

B. longipes Lw. Niagara Falls, Oct (MCV); Greene Co, How (Ald) ; McLean, Aug, Sib (Joh).

B. xanthopus Wied. Axton, Je (M\&H) ; Poug May, Oct (Joh); SI, May, Ds (Leon); LI: Rockaway Beach, May, Ds $(\mathrm{CnJr})$. 
DILOPHUS Meigen

D. breviceps Lw. L. Tear, $4600 \mathrm{ft}$. (Beq); Erie Co, Sep, Oct, MCV (Jn).

D. spinipes Say (dimidiatus Lw.). "NY" (Lw).

D. serraticollis Wlk. Axton, Je (Joh); McLean, Sep, Sib (Joh).

\section{FAMILY SCATOPSIDAE}

\section{SCATOPSE Geoffroy}

S. notata L. Ithaca, May (Joh).

S. pygmaea Lw. Erie Co, Mar, J1, MCV (Jn); Ithaca, Mar (Joh).

\section{RHEGMOCLEMA Enderlein}

R. atrata Say. Buffalo, Mar, Oct, $M C V(\mathrm{Jn})$; Saratoga Sps., Ap; Albany, Sep; Ithaca, J1 (Joh).

\section{SWAMMERDAMELLA Enderlein}

S. brevicornis Meig. Ithaca, May (Joh).

\section{Family SIMULIIDAE}

PRosimulium Roubaud

P. hirtipes Fries. L. Tear (Beq); Axton, Je; Erie Co, May (MCV); Ithaca, May, Je (Joh); McLean, J1, Sib (Joh); Albany, J1 (NYS); Yillburn, Schott (Beq); W. Nyack (Beq); SI (Beq).

\section{Eusimulium Roubaud}

E. bracteatum Coq. S. Wales (MCV); Ithaca, Oct (Joh).

E. johannseni Hart. Axton, Je (Joh).

\section{SIMULIUM Latreille}

S. parnassum Mall. Plattsburg, Aug, Dy (Dy\&Snn).

S. pictipes Hagen. Ausable R., Aug; Erie Co, May-Aug (MCV) ; Ithaca, Je-Aug (Joh).

S. piscicidium Rly. Mumford (Rly).

S. venustum Say. L. Tear, 4600 ft. (Beq) ; Adirondacks, Je-Aug; Colden, May (MCV) ; Ithaca, Je. (Joh); McLean, J1 (Joh); Wilmuth.

S. vittatum Zett. Axton, Je (Joh); Niagara Falls (Mall); Hamburg, May (MCV); Ithaca, Oct; E. Schodack, May.

\section{FAMILY BLEPHAROCERIDAE}

BLEPHAROCERA Macquart

B. tenuipes Wlk. Adirondacks, Je; Watertown; Colden, J1 (MCV); Ithaca, Je-J1 (Joh). 


\title{
FAMILY XYLOPHAGIDAE
}

\author{
Compiled by M. D. Leonard
}

SUBFAMILY XYLOPHAGINAE

\section{XYLOPHAGUS Meigen}

X. fasciatus Wlk. (abdominalis Lw.). Albany, May 15, Yg.

X. lugens Lw. Niagara Falls, May (MCV); Syracuse, Blkm (Hine); Ithaca, Ap; McLean, May; Slide Mt., Je 3, Leon; Albany, May 2, reared from under dead bark (Felt); Nassau, Ap 19, reared from under pine log (Felt); Mosholu, May 4, Ds; SI, Ap, Ds; LI, Ap 28; Queens, May, Ols; Flatbush; Bellport; Cypress Hills.

X. reflectens W1k. (rufipes Lw., persequus Walk.). Mt. Seward, Je 22, $4500 \mathrm{ft.}$, $M \mathcal{E} H$; Axton, $M \mathcal{E} H$ (Joh); L. Tear, J1 29, 4300-4600 ft., Beq; Ithaca, W. Danby, May; W. Winfield, Je 7, Leon; Slide Mt., Catskills, Je 3, Leon; Big Ind. Val., May-Je, Pears.

\section{SUBFAMILY ARTHROCERATINAE}

Solva Walker

(Xylomyia Rondani)

S. aterrima John. Rochester Jct., Jl 9 (Leon); N. Evans, J1 4 (MCV) ; Irving, J1 22 (MCV); Ithaca, J1 12, JLFrank.

S. pallipes Lw. Ithaca, May-Aug; Karner, Je; Albany, Je, $\mathrm{Yg}$; Clinton Hgts., Je 13, Yg; Kingston, Je 9; Poughkeepsie, Aug; Big Ind. Val., Je, Pears; Shandakin, Je 3, Leon; White Plains, Je 4; SI, Je, Ds; LI: Flatbush, Lutt Wood, Je-J1, Zab; Cold Sp. Harb., J1 25; Flushing, May 25, Beq; Sea Cliff, Bks.

S. tenthredinoides Wulp. Niagara Falls, Je 9 (MCV); S. Wales, Je 22 (MCV); Ithaca, Joh; Poughkeepsie, Je 3, Yg; NYC, Je 12; SI: Richmond Hill, J1 3.

ARTHROCERAS Williston

A. leptis O.S. Mt. Marcy, J1 31, 1913, Felt; L. Tear, J1 20, Beq.

\section{GLuTOPS Burgess}

G. singularis Burgess. Ithaca, Ap 14, 1922, male, McLean, 1924, female (Joh).

\section{FAMILY COENOMYIIDAE}

Compiled by M. D. Leonard

CoEnomyia Latreille

C. ferruginea Scop. Olcott, Je 5, Dt; Southfields (Beq); Protection, Je 8 (MCV); Ithaca, Je 22, SHEmerson; Pike; Labrador L., Je 9, Leon: Albany, May; West Pt., Je, Ds; Ft. Montgomery, May, Ds; Ramapo, May-Je, Ds; Suffern ; Pearl R., May 31, EEWatson; LI: Wading R., Je, Ds.

ARTHROPEAS Loew

A. americana Lw. L. Placid, Aug 7; Brant L. (Coq). 


\section{FAMILY STRATIOMYIIDAE}

\section{Allognosta Latreille}

A. brevicornis Jn. L. Tear, $4600 \mathrm{ft} ., \mathrm{Beq}(\mathrm{Jn})$.

A. fuscitarsis Say. Fulton Co; Trenton Falls; Erie Co, May-J1 (MCV); Little Valley (MCV); Ithaca and vic., May-Aug (Hine); Albany; Kiamesha (Mel); White Plains (Beq).

A. obscuriventris Lw. Ithaca and vic., Je-J1 (Hine) ; Catskills, Je (AM) ; Poughkeepsie, Je.

A. similis Lw. "NY" (Lw); Catskills, Je (AM).

\section{Actina Meigen}

(Hemiberis Enderlein, Allactina Curran)

A. viridis Say. Axton (Joh); Elba, Je (MCV); Oswego; Niagara Falls, May-Je (Hine); Erie Co (MCV); Little Valley, May-Je (MCV); Ithaca and vic.; Fulton Co; Catskills, Je (AM); Karner (NYS); Pine I. (Beq).

\section{SCOLIOPELTA Williston}

S. luteipes Will. Upper Ausable L., J1 (Beq).

\section{HERMetia Latreille}

H. illucens L. SI, Bs (Beq).

\section{Ptecticus Loew}

P. sackeni Will. Essex Co, J1 (NYS); W. Nyack, J1, Bs.

P. trivittatus Say. Ithaca (Hine); SI: Richmond, Aug (Bs); New Brighton, Je (Bs); LI: Sheepshead Bay, Aug (NYS); Flatbush, Schott (Beq); Sands Pt., Sep (Bs).

\section{CHRYSOCHROMA Williston}

C. nigricornis Lw. Norton's Landing, Cayuga L. (OS).

\section{Pedicelta Bigot \\ (Macrosargus Bigot)}

P. clavis Will. Ithaca, Ap-Je (Joh); Brooklyn, Je (Beq).

SARGUS Fabricius (not Klein)

(Geosargus Bezzi)

S. cuprarius L. Old Forge, Je; Niagara Falls, Je (Joh); Erie Co, Ap-Sep (MCV); Ithaca (Joh); Catskills, Je (AM) ; SI: Arlington, Aug, Bs; Richmond, Bs; Arrochar, J1, Bs; LI: Sands Pt., Jl, Bs ; Flushing (Beq) ; Central Pk. (Beq); Aqueduct, Eng (Beq); Queens, Schott (Beq); Glendale, Je, Bs. 
S. decorus Say. L. Tear, J1 (Beq) ; Fulton Co; Manlius; Niagara Falls, Je (Jn) ; Erie Co, May-Aug (MCV); Ithaca (Hine); Trenton Falls; Catskills, Je (AM) ; Albany, May (NYS) ; Kiamesha (Mel) ; SI: Scrub Oak, Aug (Bs);

LI: Greenport, May; Sands Pt., Sep (Bs); Cold Sp. Harb., Aug (Bs).

S. elegans Lw. Erie Co, Je-Aug (MCV); Ithaca (Hine); LI: Sands Pt., J1, Bs (Beq).

S. viridis Say. Fulton Co; Oswego, May; Niagara Falls, Je (Jn); Erie Co, Ap-Je (MCV); Ithaca (Hine); Albany (NYS); LI: Rockaway Beach (Jn); Huntington, Schott (Beq); Flatbush, May, Zab (AM).

\section{MICROCHRYSA Loew}

M. polita L. Oswego, Je (Jn); Erie Co (MCV); Ithaca, Je (Hine); Albany (NYS) ; Poughkeepsie; LI: Brooklyn (Beq); Sands Pt., Jl (Bs); Flatbush, J1 (AM).

\section{STRATIOMYIA Geoffroy}

S. apicula Lw. Lewiston, Little Valley, May-Je (MCV).

S. badia Wlk. Old Forge; Woodhull L., Aug ( Jn); Olcott, Aug (MCV); Erie Co, May-Je (MCV).

S. discalis Lw. Fulton Co; Erie Co, Je (MCV); Ithaca, J1 (Hine) ; Karner, May; Poughkeepsie (NYS); SI: (NYS); Moravian Cemetery, May (Bs); LI: Astoria (AM) ; Brooklyn, Eng (Beq); Maspeth (Beq); E. New York, Schott (Beq); Rockaway Beach (Jn).

S. lativentris Lw. Buffalo (Jn); E. Aurora, Je (MCV); Ithaca, Je (Hine) ; Woodhull L., Aug.

S. meigenii Wied. Erie Co, May-Je, Lewiston, Je (MCV); Ithaca (Hine); Albany, Aug (NYS); Woodworth's, J1; Forest Lawn, J1, Zab (AM).

S. norma Wied. Erie Co, Jl, Aug (MCV); Ithaca (Hine); LI: Astoria (AM), as normula.

S. normula Lw. Long L., Aug (NYS); Niagara Falls; Buffalo, Je (Jn); E. Aurora, Je (MCV); Ithaca (Joh).

S. quaternaria Lw. Ithaca, May (Hine).

\section{OdONTOMYiA Meigen}

O. cincta Oliv. Johnstown (NYS); Manitou Beach, Je, Zab; Buffalo, Je (Jn) ; Ithaca (Joh); SI: Watchogue, Je (Bs); Stony Pt., Je (AM) ; LI: Flatbush, Je, Zab; Long Beach (Beq); Maspeth, Rosedale, Flushing, Schott (Beq).

O. flavicornis Oliv. "NY" (Lw).

O. hydroleonoides Jn. McLean, J1 (Joh).

O. interrupta Oliv. Fulton Co (NYS); Springville, Je (MCV); Ithaca, Je (Hine); Trenton Falls; Albany; Poughkeepsie, May-J1; White Plains, Bno (Beq) ; SI: Moravian Cemetery, and Watchogue, May (Bs) ; LI: Wading R., May, Ds (Jn); Long Beach, J1 (Bs).

O. microstoma Lw. LI: Jamaica, Long Beach, Eng (Beq); Kings Pk., J1, Bell (Ds or Bs).

O. pallipes W1k. "NY". Not recognized.

O. pilimanus Lw. : E. Aurora, J1, Colden, Aug (MCV). 
O. pubescens Day. Oswego, May (Jn) ; Lewiston, May, Je (MCV); Erie Co; McLean; Albany (NYS); Fulton Co; SI (Beq); LI: Long Beach (Beq); Wading R., May, Ds (Jn).

O. vertebrata Say. Buffalo, Je (MCV); Norton's Landing, Cayuga L. (OS); Albany, Je (NYS); LI; Maspeth (Beq).

O. virgo Wied. Erie Co, Je-Jl (MCV); Pike, Je (NYS); Canandaigua L., J1; Ithaca, Je (Hine); Trenton Falls; Catskills, Je (AM); Poughkeepsie, J1; Ft. Montgomery, Schott (Beq); White Plains, Je (Beq); Stony Pt., Je (AM) ; LI: Maspeth (Beq).

\section{EUPARYPHUS Gerstaecker}

E. bellus Lw. Johnstown, Alex; Hamburg, May (Jn); Albany, May (NYS).

E. brevicornis Lw. Niagara Falls, Je (Jn) ; N. Evans, J1 (MCV); Rock Glen, JI (MCV); Ithaca (Joh).

E. greylockensis Jn. Ithaca (Joh).

E. stigmaticalis Lw. Ithaca, J1 (NYS).

E. tetraspilus Lw. Erie Co, Je-Jl (MCV); Ithaca, Je (Joh).

\section{Nemotelus Geoffroy}

N. canadensis Lw. LI: Sands Pt., Je (Bs).

N. carbonarius Lw. Erie Co, Je-J1 (MCV); Ithaca, Je; Johnstown, May (NYS).

N. melanderi Bks. "NY."

N. unicolor Lw. Ithaca, May, Je (Hine).

\section{OXYCERA Meigen}

O. approximata Mall. Ithaca, Je (Beq-Bs).

O. maculata Oliv. Niagara Falls, Je (Jn); Erie Co, Je-Jl (MCV); Ithaca (Hine); Johnstown.

\section{BERKSHIRIA Johnson}

(Johnsonomyia Malloch 1915, not Felt 1908)

B. aldrichi Mall. Ithaca, Je (Joh).

\section{PACHYGASTER Meigen}

P. pulcher Lw. Albany, Je (NYS); West Pt. (Mall).

NEOPAChyGaster Austen

$$
\text { (Veppo Latreille?) }
$$

N. maculicornis Hine. Ithaca, J1, MacG (Mall).

\section{ZABRACHIA Coquillett}

Z. polita Coq. Saranac Inn, Aug (Coq). 


\section{FAMILY TABANIDAE}

BuPLEX Austen

(Pangonia auct. not Latreille)

B. pigra O.S. "NY" (OS).

B. rasa Lw. Speculator, Aug (NYS) ; Old Forge, J1; Ithaca, J1-Aug; McLean, J1.

B. tranquilla O.S. N. Elba, J1; Old Forge, J1 (Hine-NYS); Long L., Ds; Woodhull L., Aug, EJLove (Johns); McLean, J1; Catskills (AM); Ramapo, Je $12, D s$.

\section{GoNIOPS Aldrich}

G. chrysocoma O. S. Trenton Falls; Batavia, J1; Little Valley (MCV); Ithaca, Aug (Joh); Covert (CU).

\section{CHRYSOPS Meigen}

C. brimleyi Hine. SI.

C. brunneus Hine. N. Fair Haven, J1; Lakeside Pk., Aug; Rochester, J1.

C. callidus O. S. Clyde, J1 21, Bish (Leon) ; Naples, J1 31, Bish (Leon) ; Erie Co ; Gowanda, May-J1, MCV ; Ithaca (Joh); Kenwood, Albany, J1 5 (Leon); Voorheesville, Je 19 (Leon); Poughkeepsie, J1 1 (Leon); Franklin, Je 20 (Leon) ; West Pt., Ds; Mosholu; Van Cort. Pk. (Osb) ; Pine I., Ds; Stony Pt., Ds; SI: Richmond, Je 16 (Leon) ; LI: Cold Sp. Harb., Aug (Hine).

C. carbonarius Wlk. (fugax O. S.). Axton (Joh); Chapel Pond, Essex Co, Je 28, Bish (Leon); Keene Val., Je-J1 (Leon) ; Old Forge, Je 2 (Leon); Big Moose, J1 24 (Leon); Saranac Inn, Je (Leon); Chazy L., Je 25 (Leon); Mt. McIntyre, May 30 (Leon) ; Hamilton Co, Ds; Thompson, May 25 (Leon) ; Erie Co, May-Je, $M C V$; Ithaca and vic. (Hine); Corinth, Je 2, Yg (Leon); Easton, May 25 (Leon) ; Rensselaer, May 30 (Leon); Albany, Je 7 (Leon) ; Karner, May 29 (Leon); Coeymans, Je 15 (Leon); Thacher Pk., Albany Co, May 2 (Leon); Cropseyville, May 23 (Leon); Big Ind. Val., May-Je, Pears; Oliverea, Ds ; Slide Mt., Catskills, Je 3 (Leon) ; West Pt., Ds ; Greenwood L. (Hine); Mosholu, Je (Hine).

C. celer O. S. Old Forge, J1; L. Placid, Je 27; Honeoye Falls, Je (Leon) ; Elba, Je (MCV) ; Niagara Falls, May-Je (Jn) ; Erie Co, May-Je (MCV); Ithaca, Je; Cooperstown, Je; Valley Falls, Je 19; Nassau, Je (NYS) ; Voorheesville, Je 19, Leon; West Pt., Je, Ds; Pine I., Je 19, Ds; Katonah, Je, Bt; Ramapo, Je, Ds; Mosholu, Je 11, Ds; NYC (Osb); LI, Je (Jn).

C. cuclux Whitn. Peru, Je ; Axton, Je ; Laborador L., Je; Wells, Je 30, Yg ; Johnstown, May 31 (Leon); Genesee Co, Je 25, Ds; Hamburg, May-Je; Ithaca, Je (Hine); McLean, May; Albany, Je 4, Bish; Voorheesville, Je 19, Leon; Thacher Pk., Albany Co, May 27, Leon; Karner, May 9 (Leon) ; Rensselaer, May 30, Leon; Hudson R. Val.; Poughkeepsie, Je 3 (Leon) ; West Pt., Je 18, Ds; Mosholu, Je (Hine).

C. cursus Ald. (cursim Whitn.). "NY".

C. delicatulus O. S. Big Moose, J1 3 (NYS) ; N. Fair Haven, J1; LI: Wading R., $\mathrm{Je}(\mathrm{Jn})$.

C. dimmocki Hine. Grand I., Erie Co, Je ( Jn); SI, Je 20, J1 12, Ds; LI: Southampton, J1 (NYS).

C. excitans Wlk. Axton, Je-J1 (Joh) ; Old Forge, Je, Yg; Keene Val., J1 31; Saranac Inn, J1 21; Speculator, J1 17, $Y g$; Long L., Ds; Big Moose, J1; Woodhull L., EJLove; Elba, MCV; Erie Co; Albany, J1 (NYS). 
C. geminatus Wied. Speculator, J1 17, $Y g$; Wells, J1 9 and 10, $Y g$; N. Fair Haven, $\mathrm{J1}$; Erie Co, J1-Aug (MCV); Otto, J1; Little Valley, J1 (MCV); Ithaca, Je-J1 (Joh); McLean, J1; Ringwood, J1; New Salem Swamp, Albany Co, J1 7, Bish; Watervliet Reservoir, Albany, Aug 2, Leon; Voorheesville, J1 7 and 15, Leon; Karner, J1 14, Leon; Catskills, J1 ; Mt. Merino, J1 (Jn) ; Huguenot, J1, $Z a b$; White Plains; Ramapo, Ds; Van Cort. Pk., J1, Zab; LI: Cold Sp. Harb., J1 19.

C. flavidus Wied. SI, Sep 2, Ds (Leon); LI: Riverhead, Je-Aug, Ds (Jn); Rockaway; Orient Pt., J1 12, Lath (Leon).

C. fuliginosus Wied. (plangens Wied.). SI: Watchogue, J1 4 (Ds) ; LI: Sands Pt., Je 15 (Ds) ; Flatbush, Je, Zab (AM); Sea Cliff (Hine).

C. frigidus O. S. McLean, J1-Aug (Hine); Cooperstown, J1.

C. lateralis Wied. Adirondacks, J1 (Hine); Speculator, Je, Aug 6, Yg; Old Forge, $\mathrm{Jl}$; Big Moose, J1, Yg (Leon); Saranac Inn, J1 17 (Leon); Upper Ausable L., J1 28 (Leon) ; Keene Val., J1 (Lint) ; Dug Mt., Aug, Yg; Wells, J1 10 and 27 , abundant, $Y g$ (Leon) ; Cooperstown, J1; Haines Falls, Aug; Elm L., Aug, Yg (Leon); Stony Clove, Catskills, J1, Ds; Big Ind. Val., Je-Aug, Pears; Huguenot, J1, $Z a b ;$ LI, Je.

C. indus O. S. Essex Co (NYS); Keene Val., J1 (Leon); Theresa, J1; Honeoye Falls, Je (Leon); Genesee Co, Je 26, Ds; Erie Co, Je-Aug, $M C V$; Ithaca and vic. (Hine); Trenton Falls, Je; McLean, J1; Hoversville, Schoharie Co, Je 20 (Leon) ; Franklinton; Je 20, Leon; Rensselaer, May 30, Leon; Albany, Je (Leon); New Salem Swamp, Albany Co, Je 1, Bish (Leon); Voorheesville, Je 19, J1 15, Leon; Hudson R. valley; Poughkeepsie, Je (Leon) ; Pine I., Je.

C. lugens Wied. "NY."

C. lugens var. morosus O. S. "NY."

C. mitis O. S. Axton, Je (Joh); Trenton Falls, Je 4, Leon; Cooperstown, Je; Ft. Montgomery, May $31, D s$.

C. moechus O. S. Lancaster, Je, $M C V$; Broadalbin, Je 29, Yg; Albany, Je 25 (Leon); White Plains, J1.

C. moerens W1k. N. Fair Haven, J1; Lakeside Pk., Aug; Olcott, J1; Freeville, J1 (Hine); Jamestown, J1 5 (Leon); Canandaigua L., Aug 21, Bish (Leon).

C. montanus O. S. Dug Mt., Aug, Yg; Old Forge, J1 17; Saranac Inn, J1 22; N. Fair Haven, J1; Catskills (O. S.) ; SI: Clove Val., Je 27; LI: Riverhead, Je-Aug, $D s$; Long Beach.

C. niger Macq. Throughout the State, May-Aug. Adirondacks; Old Forge, J1 11; Keene Val., Je 29; Big Moose, J1 2, Yg; Elm L., Aug 7, Yg; Speculator, Je, $Y g$; Saranac Inn, J1; Buffalo; Erie Co; Portage, Je-Jl, MCV (Jn); Castile, Brad (Joh); Ithaca and vic. (Joh); Woodhull L., EJLove; White L., J1, Zab; Corinth, May 26, Yg; Hoversville, Je 20 (Leon); Franklinton, Je 20 (Leon); Cropseyville, Coeymans, Aug 18, Sep 15 (NYS); Mudhole Pond, Albany Co (?), Je, Leon (NYS); Voorheesville, Je 19 (Leon); Karner, Je 18; Rensselaer, Je; N. Chatham, Je 6; Hudson R. Val.; Big Ind. Val., Je-J1, Pears; Yonkers; Poughkeepsie, Je 8; Pine I., Je 19, Ds; West Pt., Je 18, Ds; Huguenot, J1, Zab; SI: Richmond, Je (Leon); LI, Je.

C. nigribimbo Whit. LI, Je.

C. obsoletus Wied. (morosus O.S.). White Plains, J1 (Kröber).

C. wiedemanni Kr. Paradox L., J1 13, Ds; Big Moose, Aug 13, Yg; Speculator, Aug 9, Yg; Potsdam; Wells, J1 23, Yg; Clyde, J1 21, Bish; N. Fair Haven, $\mathrm{Jl}$; Erie Co, Je-Aug, $M C V$; Niagara Falls (Jn); Otto; Ithaca, Je (Hine); 
McLean, J1; Cooperstown, J1; Karner, J1 14, Leon; Voorheesville, J1 15, Leon; Watervliet Reservoir, Albany, Aug 2, Leon; Poughkeepsie, Je 30; Ramapo, Ds; White L., Zab; West Pt., Sep 2, Ds; Mosholu, J1; NYC (Osb) ; SI: Clove Val., J1; LI: Cold Sp. Harb., J1 (Hine).

C. parvulus Daecke. Callicoon, J1, Ds ( Lg).

C. pudicus O. S. LI, Je.

C. sackeni Hine. Pine I., Je 21, Ds; Mosholu, J1.

C. sordidus O. S. Adirondacks, Je (Joh); Saranac Jct., Je, Yg (NYS); Castile, Brad (Joh).

C. striatus O. S. Saranac Inn, J1 (NYS); Old Forge, J1 11; Fulton Co; L. Pleasant, J1 (Leon) ; Piseco L., Aug 31 (Leon) ; Sodus, J1 20, Lint (Leon); Clyde, J1 21, Bish; N. Fair Haven, J1; E. Aurora, Little Valley, Je-Aug, $M C V$; Ithaca (Joh); Canandaigua L., J1 31, Bish; Voorheesville, J1 15, (Leon); LI (Jn).

C. univittatus Macq. Naples, Canandaigua L., J1 31, Bish; Colden, J1 (MCV) ; Ithaca, Je-J1 (Hine); Albany, Je (Leon); Voorheesville, Je 19, J1 15 (Leon); Huguenot, J1, Zab; Ft. Montgomery, Je (MCV); Mosholu; SI: Richmond, Je, Leon; LI: Je-Aug (Jn); Yaphank, Je.

C. vittatus Wied. Throughout the State, Je-Aug. Big Moose, J1 7, Yg; Keene Val., J1; L. Pleasant, Aug; Piseco L., Aug 31; Elm L., Aug 7, Yg; Hague, Aug, Leon; Speculator, Aug 5, $Y g$; Wells, J1, $Y g$; Fulton Co; Sodus, Je, Lint; Clyde, J1 21, Bish; Erie Co; Otto; Little Valley, Je-Aug, MCV ; Ithaca and vic. (Hine) ; Watervliet Reservoir, Aug 2, Leon; Albany, Je-Aug; Karner, J1, Leon; Voorheesville, Je 19, J1 15, Leon; Ballston L., Aug 26, Leon; Big Ind. Val., J1, Pears; Poughkeepsie, Je; Van Cort. Pk., J1, Zab; LI: Lawrence, J1 27; Orient, Aug (NYS).

TABANUS Linnaeus

T. acteon O. S. Pine I., Sep 8, 1910 (Hine).

T. affinis Kby. Saranac Inn, Je (NYS); Potsdam, Je, Yg (Hine-NYS).

T. americanus Forster. "NY".

T. astutus O. S. Big Moose, Keene Val., Jl (Hine-NYS) ; Old Forge; McLean, Je, Sib (Joh).

T. atratus Fab. Abundant throughout the State. Adirondacks (Felt); Erie Co, Je-Aug, MCV ; Sardinia, Aug (Bish); Castile, Brad (Joh); Ithaca (Hine); Utica, J1, RWWells (Bish); Albany, May (NYS, Sep) ; Nyack, J1, Zab; Hudson R. valley (NYS) ; West Pt., Je 8, Ds; NYC, Bronx Pk., Ds; SI, common, Ds; Richmond; Ft. Wadsworth; Watchogue, Burns; LI: Flatlands, Aug, Zab; Rosedale, Schott; Wading R., Ds; Long Pond, Ds; Massepequa; Long Beach, Burns; Brooklyn, Eng; Flatbush.

T. atratus var. nantukensis Hine. LI: Long Beach (Beq).

T. bicolor Wied. E. Aurora, J1, $M C V$; Ithaca, Aug (Hine) ; McLean, J1; White Plains, J1; SI: Watchogue, Je 29, 1919, Burns; LI: Wading R.; Montauk, Aug 24, 1917, Ds.

T. carolinensis Macq. Rochester Jct., J1, Lcon; Ramapo, Je 12, Ds (Drury); West Pt., Je 4, Ds.

T. cinctus Fab. Karner, Je (Yg); SI, one male, Je 25, Ds; LI: Yaphank, Eng; Kings Pk., Bell; Babylon (BM).

T. coffeatus Macq. Adirondacks, J1 (Felt); Rhinebeck, J1; Poughkeepsie (NYS) ; SI: Clove Val., Ds; Watchogue, Bell; LI: Wading R., Eng. 
T. costalis Wied. Very abundant throughout the State. Erie Co., Je-Aug, $M C V$; Dunkirk (Hine); Gowanda (MCV); Ithaca, J1-Aug (Hine); Utica, J1, RWWells (Bish); W. Hebron, Aug, Lg; Hudson R. valley (NYS) ; Nyack, $Z a b$; Ft. Montgomery, J1 16, Ds; Ramapo, J1, Ds (Drury); Carmel, Aug; SI: Richmond; Watchogue; Ft. Wadsworth, Burns; LI: Jamaica, Osb; Long Beach; Flatbush; Coney I.; Flatlands, Zab; Aug, HMWells (Zab).

T. cymatophorus O. S. Canandaigua L., J1 (NYS).

T. daeckei Hine. SI.

T. epistates O. S. Adirondacks, J1 (NYS, Jn) ; Mt. Whiteface, Ds (Jn) ; Long L., $D s$; Rochester Jct., J1, Leon; SI: Huguenot Pk., Ds; LI: vic. of Brooklyn (Eng).

T. exul O. S. (sulcifrons Macq.?). New Windsor, J1, Edrw.

T. giganteus DeG. Ithaca, Aug; Huguenot, Burns; SI: Annadale, etc., Ds; LI: vic. Brooklyn, Eng, Sf.

T. hinei Jn. SI: Ds; Richmond; Watchogue, Burns; LI: Long Pond, Wading R., $D s$; Southampton, J1 (Hine-NYS).

T. illotus O. S. E. Aurora, Je; Rochester $(M C V)$; Franklinton, Je, Leon (HineNYS).

T. lasiophthalmus Macq. Abundant throughout the State; Adirondacks, Je (Joh); Saranac Jct. (NYS); Erie Co, May-Je, MCV; W. Winfield (Leon) ; Castile, Brad (Joh); Ithaca, May-Je (Hine); Burlington Flats, EEWehr (Bishopp); Rensselaerville; Big Ind. Val., Je, Pears; Port Chester; Katonah, Je, Bt; White Plains, Jl $(M B)$; Bedford Hills, Je (Bish); Orange Co, abundant, Wells; Pine I., Ds; NYC, Bronx Pk.; SI, common: Tottenville; Richmond, etc., Ds; Watchogue, Burns; LI: Wading R.; Gardiners I., Ds; Astoria; Montauk; Wyandanch, Schott; Jamaica, Nic.

T. lineola Fab. Abundant throughout the State; Adirondacks (Felt) ; Potsdam, J1, $Y g$ (Hine-NYS); Lewiston, J1; Erie Co, Je-J1 $M C V$; Otto (Hine); Ithaca; Nyack, J1, Zab(?) ; Hudson R. valley (NYS) ; Big Ind. Val., Aug, Pears; Bedford Hills, Je, WHSandies (Bish); Huguenot, Jl, Zab; SI, common, Ds, Burns; LI: Prospect Pk., Brooklyn; Flatbush, Eng; Jamaica; Yaphank; Aqueduct, Ds; Orient; Cold Sp. Harb., J1; Long Beach, Beq; Sands Pt., Burns; Wading R., Schott.

T. longus O. S. Saranac Inn, J1 (NYS).

T. microcephalus O. S. Adirondacks (Felt); Saranac Inn, J1 (Hine-NYS); Old Forge, J1; Wells, J1 (Hine-NYS); Colden, J1-Aug (MCV); McLean (Hine) ; South L., Catskills, Aug 11, Ds; Big Ind. Val., J1, Pears (Hine); Callicoon, J1 27, Ds?.

T. minusculus Hine. Oswego (Hine).

T. nigrescens Palisot de Beauvais. Rochester, $M C V$; Nyack, J1, Zab (Beq); ?Huguenot, J1, Zab (AM); SI: a female from Watchogue Aug 4, a male from there J1 21, 1920, Burns; a female found dead on Richmond Turnpike near Silver L., Aug 10, Ds.

T. nigrovittatus Macq. Gloversville, Aug (Hine-NYS) ; Ramapo, J1 2, Ds; Larchmont, Aug; SI : common on the beaches, Ds; Watchogue, Burns; LI: Rosedale, Schott; Sheepshead, Aug (Hine-NYS); Orient; Maspeth, Ds; Rockaway Beach, Osb; Long Beach; Gardiners I., Beq; Sands Pt., Burns; Cold Sp. Harb., J1 (Hine-AM).

T. nigrovittatus var. conterminus W1k. SI: Watchogue, Bs; LI: Rosedale, Schott (Beq) ; Rockaway Beach, Ols (Beq); Orient (Beq).

T. nivosus O.S. Hamilton Co (Ds); Saranac Inn, J1 (NYS); Big Moose, J1, Yg (Hine-NYS) ; Rochester Jct., Je, Leon; West Pt., Je 13, Ds. 
T. nudus McD. Saranac Inn, Je (Hine-NYS).

T. ohioensis Hine. Mt. Marcy, Je; Rochester Jct., Je, Leon; E. Aurora, J1, MCV; Ithaca, J1; McLean ; LI: Hempstead, J1.

T. orion O.S. NY (O.S.) ; Buffalo, $M C V$; Ithaca, J1; SI: Clove Val., one female, two males on railroad train, Richmond, Aug 13, 1920, Ds; Oakwood, J1 24, 1920, Burns; LI: vic. Brooklyn (Eng); Hempstead, J1.

T. pumilus Macq. Abundant throughout the State; Hamburg, J1 (MCV); Ithaca, J1 (Hine); near Albany, Je (NYS); Huguenot, J1, Zab; SI, common, Ds, Burns, Beq; LI: Rosedale, Schott; Pennequid Barrens, Beq; Cold Sp. Harb., Sep.

T. recedens W1k. NY; NYC, Ds; SI, Ds; Watchogue, Burns.

T. reinwardtii Wied. Adirondacks (Felt); Fulton Co, Ald; Ithaca, Je-Aug (Hine); Hudson R. Val. (NYS); Utica, J1, RWWells (Hine); SI: one male, J1 24, Ds; Huguenot, J1, Zab (Hine).

T. rhombicus O.S. Wells, Jl (NYS).

T. septentrionalis Lw. Old Forge, Aug (NYS); Keene Val. Je (Hine-NYS).

T. sparus Whitn. SI; LI.

T. stygius Say. Buffalo, Je, $M C V$; Olcott, Je; Ithaca and vic. (Joh) ; LI: Yaphank, Aug 16, 1921, Eng.

T. sulcifrons Macq. Coeymans (Lint); Palenville, Ds; SI: Watchogue, Ds; Richmond, Miriam Campbell; Arlington, Bs.

T. superjumentarius Whitn. S. Wales, J1; Colden, Aug, $M C V$; SI: Richmond, J1 13, 1919, Bs.

T. trimaculata Palisot de Beauvais. SI; LI.

T. thoracicus Hine. Oswego, Aug (Hine).

T. trepidus McD. Keene Val., Speculator, J1 (Hine-NYS).

T. trispilus Wied. Adirondacks, J1 (NYS); Long L., Ds; Irving, Je (MCV); Boston, Aug (MCV); Ithaca, Aug (Hine); Utica, J1, RWW ells (Hine); Croton, J1, Ds; SI: Huguenot, J1, Zab; LI: Queens, Schott; Sea Cliff (Hine).

T. trispilus var. sodalis Will. Ithaca, Je $(\mathrm{Jn})$; L. Pleasant, Elm L., Jl-Aug, Yg (Hine-NYS).

T. vivax O.S. Wells, Je-(Hine-NYS); Ithaca, Je-Aug (Joh).

T. zonalis Kby. Adirondacks, Je-J1 (Joh, Yg).

MERYCOMYIA Hine

M. whitneyi Jn. "NY" (Jn); SI: Clove Val., CLPollard.

FAMILY RHAGIONIDAE

(Leptidae)

Compiled by M. D. LeONARD

DiALYSIS Walker

(Triptotricha Loew)

D. elongata Say. E. Aurora, J1 (MCV); Olean, Aug 5 (MCV); White Plains, Aug 7, Bno; SI: Ft. Wadsworth, Richmond, Arrochar, J1, Bs; LI: Maspeth, Aug, Ols; Flatbush, J1-Aug, Zab. 


\section{RhaGio Fabricius}

\section{(Leptis Fabricius)}

R. gracilis Jn. Dug Mt., Aug, $Y g$; Upper Ausable L., J1, Beq; Wells, Newport, Speculator, J1-Aug, $Y g$; Colden, Aug (MCV) ; Olean, Aug (MCV); Dayton, Je (MCV); Rock City, J1; Trenton Falls (OS).

R. mystaceus Macq. Keeseville, Je; Peru, Je, Cy\&Fbs; Axton, $M \mathcal{E} H$ (Joh) ; N. Elba, J1, Wat; Keene Val., Je; Saranac Inn, Je; Speculator, Je, Yg; L. Clear, Je, Yg; Newport, May; W. Winfield, Je, Leon; Chapel Pond, Je, Bish; Wells, May-Je; Mt. McIntyre, 4000 ft., Je, Leon; Johnstown, May; Loon L., Je, Cy; Woodworth's L., Alex; Hamburg, May-Je; Lancaster, Je, Holland, May (MCV) ; Rock City, Mix Cr. Val., Je; Crosby, May, Cy; Ithaca, McLean, Danby, W. Danby, May-J1 (div.); Spencer, Je, And; Trenton Falls, J1, Yg. Leon; Corinth, Je, $Y g$; Albany, Je; Thacher Pk., Helderberg Mts., May, Leon; Cropseyville Je; Big Ind. Val., May-Je; Oliverea, Je, Ds; Greene Co, Je, $2500 \mathrm{ft} .$, Hore; Poughkeepsie, May, Yg; West Pt., Je, Ds; Wappingers Falls, J1, Leon; Nepperhan, Je, Bks; Ramapo, May; Ft. Edward, Je; LI: Oyster Bay, Je; Jamaica, May, Ols; Yaphank, May, Pollard; Flatbush, May-Je; Flushing, May, Beq; Central Pk.; Sands Pt; Cold Sp. Harb., Je, Ds; Orient Pt., Je, Lath.

R. plumbeus Say. Newport, Ilion, May, $Y_{g}$; Woodville, L. Ontario, Je, House ; Honeoye Falls, May, Leon; Hamburg, May (MCV); E. Aurora, May, Wild; Ithaca, May, Leon; Taughannock Falls, May ; Corinth, May, $\mathrm{gg}$; Albany, May, $\mathrm{Yg}$; Thacher Pk., Helderberg Mts., May, Rensselaer, May, Leon; Florida, Je, FrostSW ; Durlandville, May, FrostSW; LI: Richmond Hill; Sea Cliff, May, Bks.

R. punctipennis Say. Mt. Kisco, Je; Speculator, Je, $Y g$; Gloversville, Sep, Alex; Kiamesha; Tompkins Co; Sterling Forest, May; Poughkeepsie, May, $\mathrm{Yg}$; Nyack, Zab; White Plains, Je, Beq; Van Cort. Pk., Je, Bno; SI: Richmond, May-Je, Bs, Leon; New Dorp; LI: Richmond Hill, J1; Sea Cliff, Je, Bks; Flatbush, May-Je, Zab; Sands Pt., Je, Bs; Woodhaven, J1.

R. terminalis Lw. "NY", type; Taughannock Falls, J1, Leon; Valcour, J1.

R. vertebratus Say (scapularis Lw., hirta Lw.). Upper Ausable L., J1, Beq; pond at foot of Mt. Redfield, J1, Beq; Onteora Mt., J1, How; Newport, Wells, J1, Yg; Adirondack Lodge, J1, Leon; Augur Flats, J1, Yg; Hamburg, E. Aurora, Colden, Protection, Gowanda, Niagara Falls, Je-Jl (MCV); Little Valley, Je (MCV); McLean, J1, Leon; Ithaca, Je-J1, SmRC; Catskills, JeAug; Stony Clove, J1; SI, Je, Ds.

R. incisus Lw. Binghamton, Dietz.

\section{Chrysopilus Macquart}

C. basilaris Say. Hague, Aug, Leon; Batavia, J1, Kngt; Spring Lake, Cayuga Co, $\mathrm{J1}$; Ithaca, J1; Poughkeepsie, J1, $\mathrm{Yg}$; Mosholu.

C. fasciatus Say. Karner, J1; Katonah, Je, Bt; W. Nyack, J1, Beq; Van Cort, Pk., J1, Zab; West Farms, Angus; SI: Watchogue, J1, Bs; LI: Maspeth, J1, Ds.

C. modestus Lw. Niagara Falls, Aug (OS); Buffalo, J1-Aug (MCV); Goat I.

C. ornatus Say. Sacandaga Pk., Fulton Co, Je, Alex; Honeoye Falls, Je, Leon; Genesee Co, Ds; Niagara Falls, Je-Jl (MCV); Boston, Ji (MCV); Conesus L., Je, Ds; Ithaca and vic., Je-J1; Albany, J1; New Salem Swamp, Albanv 
Co, J1, Bish; Bethlehem, Je, Lint; Helderberg Mts., J1; Stony Clove, J1; Poughkeepsie, Je; West Pt.; Katonah, Je, Bt; Tarrytown, Mosholu, Je-Jl, Bno\&W $W$ at ; Ramapo, Je; Nyack, Je, Ds; SI: Richmond, Je, Ds; LI: Richmond Hill, J1; Flatbush, Je, Eng; Jamaica; Sea Cliff, Je, Bks.

C. proximus W1k. (propinquns W1k., simillimus W1k.). Speculator, Je, $\mathrm{Yg}$; Keene, Je, Leon; Adirondack Lodge, Je, Leon; Lawson's L., Je, Bish; Kirkland, Je, Leon; Clayville, Je, Leon; Ithaca, Je, SmRC; Corinth, Je, $Y g$; Salem, Je ; Karner, Je, $Y g$; Voorheesville, May, Bish (reared from sphagnum) ; Stony Clove, J1; Poughkeepsie, Je, Yg; Port Chester, Je, Haimbach; Ramapo, Je ; Suffern (AM); Mosholu, Van Cort. Pk., May, Bno; SI: Richmond, Watchogue, New Dorp, Je, Bs; LI: Flatbush, Je, Zab; Sea Cliff, Bks.

C. quadratus Say. Axton, Je, $M \mathcal{E} H$; Wilmington, Aug; New Russia, Aug, $B d y$; L. Placid, Aug; Saranac Inn, Je; Severance, J1, Bks; Keene, Je; Leon; Chazy L., J1; Wells, Speculator, J1, Yg; Hague, Aug, Leon; Upper Ausable L., J1, Beq ; Brant L. ; Sport I., Sacandaga R., Je, Alex; Lake Bluff, Sodus, J1 ; Honeoye Falls, Aug, Leon; Niagara Falls, Je (Bezzi); Batavia, Je-Aug (Kngt); Grand I., Sep (MCV) ; Canandaigua L., J1; Rock City, J1, Kngt ; Westfield, Je; Spring Lake, J1; Spencer L., Je; Ithaca and vic., Je-Sep; Clifton Sps., Aug; Karner, J1, Leon; Marlborough, 2500 ft., How; Catskills, Je-Aug ; Pine I., J1, SWFrost; SI: New Dorp, Bs; Watchogue, J1, Bs,Ds; LI: Lott Wood; Flatbush, Je, Pennequid Barrens, Coram, J1, Beq; Sands Pt., J1, Bs.

C. rotundipennis Lw. S. Wales, J1 (MCV).

C. thoracicus Fab. Honeoye Falls, Je, Leon; Rochester, Je (Ald); Batavia, Je, Kngt ; Portage, Je, Ds; Pike ; Colden, Je (MCV) ; Westfield, Je ; Ithaca and vic., May-Je, Leon; Interlaken, Je; Albany, Je, $Y g$; Voorheesville ; Castleton, Je, Lint; Nassau, Je; Bethlehem, May, Je, Ald; Clinton Hgts; N. Chatham, Je; Cropseyville, Je; Hudson, Je ; Poughkeepsie, May, $Y g$; Ramapo, May, Je, $D s$; Katonah, Je, Bt; Suffern; Nyack; Mosholu (AM) ; NYC?, Je, Angus; West Farms, Angus; SI: Richmond, New Dorp, Tottenville, May-Je, Bs; LI: Jamaica, Je, Ds.

\section{ATHERIX Meigen}

A. variegata W1k. Rochester Jct., Je, Leon; Pike, J1; Ithaca, May-Je, Leon,SmRC; Riders Mills, May, Bish (all males, resting on needles of a pine tree; common).

\section{SYMPHOROMYIA Frauenfeld}

S. cinerea Jn. Ithaca, Je, Leon.

S. hirta Johns. Niagara Falls, Je (MCV); Ithaca; Cooperstown, SmRC; LI: Richmond, Je, Ds.

Bolbomyia Loew

B. nana Lw. Colden, N. Evans, May (MCV); Danby, May, Snn.

\section{FAMILY CYRTIDAE}

Pterodontia Gray

P. flavipes Gray. LI: Montauk Beach, Schott (Beq). 


\section{OGCODES Latreille}

\section{(Oncodes auct.)}
O. costatus Lw. Niagara Falls, Aug (MCV); Honeoye Falls, J1 (Leon); Ithaca, Je (Joh).
O. dispar Macq. McLean, May, Sep (Joh).
O. incultus O.S. Potsdam; Grand I., Je (MCV); Keene, Je (MCV).

\section{Opsebius Costa}

O. pterodontinus O.S. Ithaca, Buys (Joh).

O. sulphuripes Lw. Sharon Sps., $O S(\mathrm{Lw})$.

\section{ACROCERA Meigen}
A. bimaculata Lw. McLean, Aug, Sib (Joh); West Pt., Je, Ds (Beq).
A. bulla Westw. Ithaca, Je, Hd (Joh); Big Ind. Val., Eng (Beq).
A. fasciata Wied. Poughkeepsie, May; McLean, Je, Sib (Joh).
A. liturata Will. (n. var.?). Juanita I., L. George, J1 26, Bish (Leon).
A. nigrina Westw. Newcomb, J1 6 (Joh); McLean, Aug, Sib (Joh).
A. subfasciata Westw. "NY".

\section{FAMILY BOMBYLIIDAE}

\section{Anthrax Scopoli}

\section{(Spogostylum Macquart)}

A. albofasciata Macq. Rochester (MCV); LI: Pennequid Barrens (Beq).

A. anale Say. Ithaca, Aug (Hine); Karner, J1-Aug; Albany, Oct; Croton-onHudson, J1, Ds (Jn); Unionport, Sep (AM) ; W. Nyack (Bs); Mosholu (AM); Van Cort. Pk. (Bs) ; SI, Aug-Sep (Bs); LI: Aqueduct, Sep, Ds (Jn); Sands Pt., Cold Sp. Harb., Jl-Aug (Bs); Yaphank, Aug-Sep, Ds (Jn); Riverhead, Aug (NYS).

A. argyropyga Weid. Inwood, Schott (Beq):

A. limatula Say. Essex Co, J1.

A. oedipus Fab. Essex Co; Little Valley, J1 (MCV); Niagara Falls, Je (Jn); Ithaca (Joh) ; Karner (NYS); Ft. Montgomery, Schott (Beq) ; Nyack, J1 (Beq) ; SI, J1 (Bs) ; LI: Cold Sp. Harb., Aug (Bs) ; Sands Pt., J1 (Bs); Half Way Hollow Hills, J1, Ds (Beq); Yaphank, May (CnJr-ANSP).

A. pauper Lw. Ithaca, Je (Hine); SI, Je-J1 (Beq,Bs); LI: Sands Pt., J1, Sep (Bs) ; Orient (Beq); Gardiners I. (Beq).

A. simson Fab. Coeymans (Lint); Mt. Ivy, Rockland Co, Aug (Ds); New Baltimore, J1 (AM) ; Nyack, J1 (AM); SI, Aug (Ds, Bs) ; LI: Setauket, Eng (Beq); Yaphank, Aug, Ds (Beq); Gardiners I. (Beq).

A. varium Fab. Reared from nest of Bomboides podilarius at Ithaca, Ap (Snn).

\section{EXOPROSOPA Macquart}

E. capucina Fab. Minerva, Aug.

E. decora Lw. Ithaca, J1 (Hine); Van Cort. Pk. (Bs) ; LI: Cold Sp. Harb., Aug (Bs). 
E. fasciata Macq. Ithaca, Aug (Hine, Jn) ; Niverville; W. Nyack (Beq) ; SI, Aug (Bs) ; LI: Aqueduct, Sep, Ds (Jn) ; Jamaica, Flushing, Canarsie, Long Beach, Rockaway Beach (Beq).

E. fascipennis Say. Newport, Aug (NYS); Irving, J1 (MCV); Ithaca, Aug (Hine); McLean, J1; Albany; Normanskill (CnJr-NYS); Clinton Hgts. (NYS) ; Nyack, J1 (AM) ; Mosholu (AM) ; Van Cort. Pk. (Bs); Allerton Ave., NYC (Ang); SI, Je-Aug (Bs); LI: Corona (AM); Manhattan Beach, Yaphank, Greenport, Gardiners I. (Beq); Wading R., Aug, Ds (Jn).

\section{VILla Lioy}

\section{(Anthrax auct. not Scopoli)}

V. alternata Say. Keene Val. (CnJr-NYS); L. Placid, Aug; Saranac, J1; Long L., Aug; Severance, Aug, CSBanks; L. Pleasant, J1; Erie Co, Aug-Sep (MCV); Mclean, Aug, Sib (Joh); Karner, Aug-Sep (NYS) ; SI (Beq);

LI: Cold Sp. Harb., Wyandanch, Orient (Beq); Wading R., Aug, Ds (Jn).

V. ceyx Lw. Karner, Aug (NYS) ; LI: Yaphank, Jl, Ds (Jn) ; Pennequid Barrens, Wading R., Wyandanch (Beq).

V. fulviana Say. Saranac Inn, J1-Aug (CnJr-NYS) ; Keene Val., Aug ; L. Pleasant, J1; Wilmington, Aug; Grand I., Sep (Jn); McLean, Sep, Sib (Joh); Oliverea (Beq).

V. fulvohirta Wied. Mosholu (AM) ; SI, Aug-Sep (Bs) ; LI: Cold Sp. Harb., Aug (Bs).

V. hypomelas Macq. Woodhull L., Aug (Jn); Keene Val., Long L., Wilmington, Aug (CnJr) ; Wells, J1, Yg (CnJr) ; Silver Bay, Aug (CnJr) ; SI, Sep (Bs).

V. lateralis Say. Keene Val., J1-Aug; L. Pleasant, J1-Aug; Wells, J1; Saranac Inn, Aug; Erie Co, Je-Aug (MCV); Tompkins $\mathrm{Co}$, including Ithaca, J1 (Hine) ; Haines Falls, J1; Beaverkill, Aug $(C n J r)$; E. Jewett, Aug, $D s$ (Jn) ; Albany, Dec(?) (NYS) ; Voorheesville, Aug; Kinderhook, Sep (CnJr); Poughkeepsie, J1; Ft. Montgomery, Schott (Beq); SI, J1-Sep (Bs) ; LI: Smithtown, Aug, Ds (Jn); Calverton, Yaphank, Amagansett, J1, Sep, Is $(\mathrm{CnJr})$; Cold Sp. Harb., Huntington, Orient, Gardiners I. (Beq) ; Half Way Hollow Hills, Yaphank, J1 (CnJr-ANSP).

V. lateralis var. gracilis Macq. L. Pleasant ( Jn).

V. lateralis var. nigra Cress. Adirondack L., Saranac Inn, J1-Aug, Wells, J1 (CnJr-NYS) ; Beaverkill, Aug ( $C n J r)$; Karner, Jl.

V. molitor Lw. LI: Huntington, Sep (Coq).

V. morio L. Adirondack L., J1, Saranac Inn, Je ( $\mathrm{CnJr}$ ) ; Rochester, Je (MCV); Niagara Falls (Jn) ; Lewiston, May (MCV) ; Corinth, May (CnJr) ; Karner, May-Je; Albany, May; Ft. Montgomery, Schott (Beq); Southfields, Schott (Beq).

V. nigricauda Lw. Beaverkill, Aug (CnJr-ANSP).

V. shawii Johns. LI: Amagansett, Sep, Ds (CnJr).

V. sinuosa Wied. Gowanda, Je (MCV); Karner, Je; Albany, Je-J1; Poughkeepsie, J1; Ft. Montgomery, Schott (Beq); Stony Pt., Je, Ds (Jn) ; Pine I., Je, Ds (Jn); SI, Je-Aug (Bs); LI: Kings Pk. (Beq); Huntington (Beq) ; Coney I., Rockaway (AM) ; Sands Pt., Je-Jl (Bs); Pennequid Barrens (Beq); Yaphank, May (CnJr-ANSP).

V. tegminipennis Say. Paradox L., J1, Ds ( Jn); Pine I., Sep, Ds (Jn) ; LI: Sands Pt., Wading R. (Bs) ; Huntington, Fire I., Sep (Jn); Promised Land, Sep, Ds (Jn); Gardiners I. (Beq).

V. webberi Johns. Ft. Montgomery, Southfields, Schott (Beq). 
BomвyLIUS Linnaeus

B. fraudulentus Johns. White Plains (Beq); SI: Ft. Wadsworth, May (Bs); LI: Cold Sp. Harb., Pennequid Barrens (Beq); Wading R., Je, Ds (Jn).

B. fulvibasis Macq. (atriceps Lw). Little Valley, Je (MCV) ; Tompkins Co (OS) ; Bethlehem Center, Je; Poughkeepsie, Jl; Mosholu (AM); SI: Watchogue, Bs (Beq) ; LI: Central Pk. (Beq); Farmingdale, Bs (Beq); Wading R., May, Ds (Jn).

B. incanus Johns. LI: Sands Pt., Je, Bs, Ds (Jn); Cold Sp. Harb., Je (Ds); Yaphank, Je (CnJr-ANSP).

B. major L. Pike; Erie Co, Ap-May (MCV); Ithaca, May (Hine); Karner, Ap-Aug, Albany (NYS) ; Ramapo, Mar 24, Ds (Jn) ; Mosholu (AM); SI, Mar 5-May (Bs) ; LI: Cold Sp. Harb., May (AM) ; Wyandanch, Yaphank, May, Ds (Jn).

B. mexicanus Wied. SI: Watchogue, Je (Bs); LI: Wading R., Schott (Beq); Yaphank, May (CnJr-ANSP).

B. pulchellus Lw. Nyack, Hillburn, Schott (Beq); Mosholu (Bks); SI, May (Ds) ; LI: Huntington, Amityville, Schott (Beq).

B. pygmaeus Fab. Hamburg, Colden, May (MCV); Ithaca, May, Sib (Joh); Ft. Montgomery, Schott (Beq); Ramapo Mts. (AM) ; Hillburn, May (Bs); SI: Watchogue, May (Bs); LI: Wyandanch, May, Ds (Jn); Yaphank, Melville (Beq).

B. subvarius Johns. Ft Montgomery, Schott (Beq); Mosholu, May (Ds).

B. validus $\mathrm{Lw}$. "NY" (OS).

B. varius Fab. Nyack, J1, Zab (AM); SI, Je, Ds (Jn); LI: Central Pk. (Beq); Farmingdale (Bs).

Anastoechus Osten Sacken

A. barbatus O.S. (nitidulus auct. not Fab.). LI: Amagansett, Sep (CnJr-ANSP); Wading R., Sep, Bs.

\section{Systoechus Loew}

S. candidulus Lw. LI: Yaphank, Ols, Coram, Wading R., Pennequid Barrens (Beq).

S. vulgaris Lw. Ramapo Mts. (AM).

PhthiRIA Meigen

P. sulphurea Lw. LI: Long Beach, Aug (Beq).

\section{LEPIDOPHORA Westwood}

L. aegeriformis Westw. Minnewaska, Ulster Co, Aug (Beq); SI: Watchogue, Sep 21, Ds (Jn).

\section{SPARNOPOLIUS Loew}

S. fulvus Wied. Erie Co, Aug-Sep (MCV) ; Tompkins Co, including Ithaca, J1Aug; Callicoon (AM); Phoenicia, Aug (MCV); White Plains (Beq); Pine I., Sep (AM) ; Mosholu (AM) ; SI, Aug-Sep (Bs) ; LI: Sands Pt. (Bs); Central Pk., Cold Sp. Harb., Flushing, Huntington, Little Neck, Southold, Sep (Beq). 
SystRopus Wiedemann

S. macer Lw. Erie Co, Aug-Sep (MCV); Ithaca, Aug (Joh); White Plains (Beq) ; Nyack, Aug (AM) ; Ramapo Mts. (AM) ; Stony Pt., Sep (Bs) ; SI, Aug (Bs) ; LI: Astoria (AM) ; Brookhaven, Sep, Schott (Beq) ; Riverhead, Aug, Ds (Jn).

\section{GERON Meigen}

G. calvus Lw. Erie Co, J1-Aug (MCV); Geneseo; Albany, J1.

\section{FAMILY THEREVIDAE 99}

TABUDA Walker

T. fulvipes Wlk. LI: Baiting Hollow, May (NYS).

\section{Psilocephala Zetterstedt}

P. amplifrons Cole (latifrons Cole). W. Danby, May, type.

P. flavipennis Cole. LI: Sands Pt. (Bs); Wyandanch, Schott (Beq) ; Riverhead, Jl-Aug (NYS).

P. frontalis Cole. Keene Val., Aug; Gloversville, Sep; Oswego, J1; Rochester Jct., J1, type, Leon (Cole) ; Lake Bluff, J1; Corinth, Je, Yg (NYS) ; Karner, Je, Yg (NYS) ; Hague, Aug (Leon-NYS); Ft. Montgomery, Schott (Beq); Poughkeepsie, Je, $Y g$ (NYS) ; SI; LI: Cold Sp. Harb.; Fishers I., Aug; Montauk, Aug, Ds (Felt).

P. grandis Jn. Ramapo Mts., Ds (Beq).

P. haemorrhoidalis Macq. Essex Co, Gloversville, Honeoye Falls, Rochester Jct., Je (Leon) ; Oswego; Lake Bluff; Erie Co, Je-Aug (MCV); Batavia, J1; Castile (Brad) ; Ithaca, May-Sep; Poughkeepsie; New Rochelle, Aug (AM); Ft. Montgomery (Schott) ; SI (Ds) ; LI: Flatbush, Sep; Sea Cliff, Bergen I., J1; Wyandanch (Schott); Sands Pt. (Bs); Jamaica, Bs (Beq).

P. melampodia Lw. Keene Val., Je-J1; Ithaca, May; Albany, May.

P. morata Coq. LI: Gardiners I., Jes Ds (Felt-Ds).

P. munda Lw. Axton, Je, Aug; Big Moose, L. Placid, Aug; N. Elba, J1; Middleport, J1 (MCV); Rock City, Je, Ds (Felt).

P. variegata Lw. Oswego, Je-J1; Pulaski, Je, Ds (Felt).

\section{Epomyia Cole}

E. pictipennis Wied. Oswego, J1; Sanford, $M C V$ (Cole); Ft. Montgomery, Schott (Beq) ; LI: Long Beach (Beq); Orient, J1, Zab (Cole).

E. rufiventris Lw. Essex Co, J1; LI: Sands Pt. (Bs) ; Long Beach; Riverhead, J1 (NYS).

E. scutellaris Lw. Mosholu, Je; Van Cort. Pk., Je-J1.

\section{THEREVA Latreille}

T. candidata Lw. Essex Co, Aug (JonesFM) ; Speculator, Je (NYS) ; Saranac Inn, J1; N. Elba, J1; Rochester Jct. (Leon) ; Lancaster, May-Je (MCV) ; Scotia, Je; Karner, J1 (Cole-NYS); Corinth, Je; Albany, May-J1.

\footnotetext{
${ }^{99}$ The manuscript for this family has been checked by Dr. F. R. Cole.
} 
T. duplicis Coq. Axton, Je (Cole).

T. flavicincta Lw. Adirondacks, Je; Speculator, Ang (NYS); Protection, Je, Little Valley, Je (MCV); Wanakena, Aug (Cole).

T. frontalis Say. Chazy, Je; Upper Ausable L. (Beq) ; foot of Mt. Redfield, Essex Co (Beq); N. Elba, J1; Wells, J1; Speculator, Aug; Genesee Co (Ds); Lancaster, Je; Gowanda, Je; Little Valley, Je (MCV); Yates Co, Ds (Beq); Ithaca, J1; Slaterville, Je; Albany, May.

T. senex Wlk. Ithaca, May.

T. strigipes Lw. Axton, Je.

\section{FAMILY SCENOPINIDAE}

\section{SCENOPINUS Latreille}

S. fenestralis L. Essex Co, J1; Ausable Forks, Jan (NYS) ; Middleport, J1 (MCV); Buffalo, Je-J1 (Lint); Salamanca, Little Valley, J1 (MCV); Ithaca (Joh) ; Amsterdam; Albany, Oct; Mosholu (AM) ; SI (Beq) ; LI: Flatbush, Je, $Z a b$; Gardiners I., J1 (Beq).

S. glabrifrons Meig. "NY", Ap (Jn) ; LI: Flatbush, Schott (Beq).

\section{FAMILY MYDAIDAE}

\section{MYDAS Fabricius}

M. carbonifer O.S. Ithaca, Je 30, Philip Miller (Joh) ; Norton's Landing, Cayuga L., J1, Comstock (OS).

M. clavatus Drury. Near Buffalo (MCV); Bronx Pk., Schott (Beq) ; SI: Wards Pt., J1 10 (Ds) ; Arlington, J1 24 (Ds); Watchogue (Ds) ; LI: Flatbush, Aug, Zab.

\section{FAMILY ASILIDAE \\ SUBFAMILY LEPTOGASTERINAE}

\section{LEPTOGASTER Meigen}

L. annulatus Say. "NY" (Back).

L. badius Lw. Essex Co, J1; Sacandaga Pk., Je; Niagara Falls, Je (Jn) ; Hamburg, J1.

L. flavillaceus Lw. LI: Wyandanch, Schott (Beq).

L. flavipes Lw. Common and widely distributed. Ft. Montgomery (Beq); SI, JI, Ds (Beq) ; LI: Aqueduct, J1, Riverhead, Aug, Ds (Beq); Flatbush, Jl, Zab; Cold Sp. Harb., J1 (AM) ; Central Pk., Wading R. (Beq).

L. incisuralis Lw. Mosholu (AM) ; LI: Wyandanch, Je (Jn).

L. loewi Bks. Ithaca (Bks).

L. pictipes Lw. LI: Aug (Back); Wading R., Je, Ds (Jn); Yaphank, J1, Ols, Ds (Beq); Pennequid Barrens (Beq).

L. testaceus Lw. Erie Co, $M C V$; Tompkins Co, Jl (Hine); Albany, Je; Pine I., Je, Ds (Beq) ; LI: Gardiners I. (Beq).

\section{SUBFAMILY DASYPOGONINAE}

LAPHYSTIA Loew

L. sexfasciata Say. "NY" (Back). 


\section{CeratuRgus Wiedemann}

C. aurulentus Fab. Van Cort. Pk. (Beq).

C. cruciatus Say. Tompkins Co, J1, Dt (Joh); Bethlehem; Albany; Poughkeepsie, Je; White Plains, Westchester (Beq) ; Mosholu, Sep, Ds (Beq); LI: North Beach, Schott (Beq).

C. similis Jn. Portage; Je, Ds (Beq).

\section{EсHTHODOPA Loew}

E. formosa Lw. LI: Gardiners I. (Beq).

\section{DIOCTRIA Meigen}

D. albius W1k. Upper Ausable L. (Beq); Adirondacks, J1; Erie Co, Je-Aug $(M C V)$; Otto, J1; Little Valley, Je; Rock City, Je (Ds); Tompkins Co, Je-J1 (Hine); Ft. Montgomery, Schott (Beq); Oliverea, J1, Ds (Beq) ; LI: Half Way Hollow Hills, J1 (Ds).

D. baumhaueri Meig. LI: Sands Pt., Bs (Beq).

D. brevis Bks. LI: Sea Cliff (Bks); Pennequid Barrens (Beq).

D. sackeni Will. Indian Pass, Essex Co, Not (Beq); Oliverea, Je, Ds (Beq).

\section{CyRtopogon Loew}

C. bimacula Wlk. Mt. Marcy (Beq).

C. falto Wlk. (chrysopogon Lw.). Adirondacks, Je (Back, Joh); Karner, May; LI: Central Pk., Kings Pk. (Beq).

C. lutatius Wlk. Hamburg, Je (MCV); Pike (Back); Cayuga L.; LI: Pennequid Barrens (Beq).

C. lyratus O.S. Adirondacks, Aug; Catskill, J1 (Back).

C. marginalis Lw. Karner, May; Catskills (Back); LI: Yaphank, Schott (Beq); Wyandanch, Schott (Beq); Pennequid Barrens (Beq).

\section{LASIOPOGON Loew}

L. terricola Jn. SI (Beq).

\section{HoLCOCEPHALA Jaennicke}

H. abdominalis Say. Potsdam, J1 (Hine); Erie Co, J1-Aug (MCV); Westfield, J1; Ithaca, J1-Sep (Joh); Trenton Falls, J1; SI, Aug, Ds (Beq).

H. calva Lw. Ithaca (Hine); Poughkeepsie, J1; SI, J1, Ds (Beq) ; LI: Flatbush, $\mathrm{J} 1, Z a b$.

\section{HOLOPOGON Loew}

H. guttula Wied. Oswego, J1 (Jn); Erie Co, Je-J1 (MCV); Ft. Montgomery, Schott (Beq) ; Nyack, Je, Ds (Jn) ; LI: Yaphank, May, Ds (Jn) ; Central $\mathrm{Pk}$. (Beq). 


\section{NEOPOGON Bezzi}

(Stichopogon auct. not Loew)

N. argenteus Say. SI, south shore, Ds (Beq); LI: Amagansett, Sep, Ds (Beq) ; Gardiners I. (Beq) ; Fire I., Sep, Ds (Beq) ; Rockaway (AM); Sands Pt., Back (Beq); Long Beach (Beq).

N. trifasciatus Say. Rochester (MCV)) ; Callicoon, J1, Lg; SI: Tottenville, Leon, Ds; Kreischerville, J1; LI: Sheepshead Bay, J1; Wading R., Aug-Sep, Ds; Fire I., Sep, Ds (Jn); Rockaway, Aug, Ols; Cold Sp. Harb. (Beq); Pennequid Barrens (Beq); Yaphank, J1, Orient, J1, Selden, Riverhead, Aug, Ds ; Manhattan Beach, J1, Southwick.

\section{HETEROPOGON Loew}

H. macerinus Wlk. (gibbus Lw.). Trenton Falls (Lw); Ft. Montgomery, Schott (Beq) ; LI: Wading R., Ds, Huntington, Schott (Beq).

\section{DEROMYiA Phillippi}

D. discolor Lw. Pittsford (Lint) ; Oliverea, Jl, Ds (Beq); Mosholu (AM) ; Van Cort. Pk., Aug, Ds (Beq) ; SI, Aug-Sep, Ds (Beq); LI: Brooklyn (Beq) ; Yaphank, Aug, Ds (Beq); Calverton, J1, Ds (Beq); Manhattan Beach (Beq) ; Fishers I., Aug, Zab; Baldwin, Sep; Flatbush, Aug (AM).

D. umbrina Lw. Keene Val., J1; Wells; Erie Co, Aug-Sep (MCV); Clifton Sps. (Hine) ; Pike; Ithaca, J1-Aug (Joh); McLean, J1, Sib (Joh); Albany, Je ; E. Jewett, Aug, Ds (Beq); Debruce, Sep, Ds (Beq); Dutchess Co (Beq); Carmel, Aug (Beq-AM) ; LI: Gardiners I. (Beq); Montauk, Aug, Ds (Beq).

D. winthemi Wied. (misellus Lw.). Larchmont, Aug, Beq (AM); W. Nyack (Beq) ; Woodbury Falls, Sep (AM) ; Carmel, Aug, Beq (AM) ; LI: Southold, Sep, Ds (Jn) ; Cold Sp. Harb., Oakdale, Wyandanch, Gardiners I. (Beq); Montauk, Aug, Ds (Jn).

TARACTICUS Loew

T. octopunctatus Say. Oswego, J1 (Jn); Mosholu (AM); SI, Aug, Ds (Beq); LI: Parkville (AM); Flatbush, Je-Sep, Zab.

NiCoCLES Jaennicke

N. pictus Lw. Gowanda, Je (MCV) ; LI: Selden, Aug, Ds.

\section{SUBFAMILY LAPHRIINAE}

Cerotainia Schiner

C. macrocera Say. E. Aurora, J1 (MCV).

\section{Atomosia Macquart}

A. puella Wied. Erie Co, Je-J1 (MCV); Otto, J1 (Hine); Ithaca, J1 (Hine) ; Albany, Aug; White Plains (Beq) ; SI: Clove Val., J1, Ds (Beq) ; LI: Flatbush, Jl, Zab (AM). 
Pogonosoma Rondani

P. dorsatum Say var. melanopterum Wied. LI: Montauk Beach, Schott (Beq).

DASYLECHIA Williston

D. atrox Will. Ithaca, Aug (Hine).

NusA Walker

N. fulvicauda Say. Elma, Aug (MCV).

LAMPRIA Macquart

L. bicolor Wied. Niagara Falls, Je (Jn); Tompkins Co.

\section{DASYLLIS Loew}

D. affinis Macq. Otto, Comst (Hine); LI:-Wyandanch, Schott (Beq).

D. champlainii Walton. LI: Baiting Hollow, May; Riverhead, Aug, Ds (Beq).

D. cinerea Back. Karner, Je (Back).

D. flavicollis Say. Mt. Whiteface, on trail, Jl, Ds (Jn); Adirondacks (Joh) ; Sodus Bay, Je; Niagara Falls; Erie Co, May-J1 (MCV); Conesus L., Portage, Je (Ds) ; Pike ; Rock City, Je (Ds) ; Ithaca (Hine) ; McLean, May, J1; Syracuse, Blkm (Hine); Albany, Nassau, Je; Slide Mt., Oliverea, Je (Ds); E. Jewett, Aug (Ds) ; Poughkeepsie, Je, $Y g$; Ramapo, Je, Ft. Montgomery, May (Ds) ; Highbridge, May (Ds) ; SI, Je (Ds) ; LI: Gardiners I., Je (Ds) ; Kings Pk., Jl, Bs (MCV); Flatbush, May, Zab; Port Jefferson (Beq); Wading R., May (Ds); Half Way Hollow Hills, J1 (Ds).

D. grossa Fab. (tergissa Say). Ithaca, J1; Poughkeepsie, J1; Yonkers, J1, Ang (Bs) ; SI: Clove Val., Je, Ds (Beq) ; LI: Flatbush, J1, Bs; Wyandanch, Aug, Ds (Beq); Kings Pk., J1 (Bs); Brooklyn, Rockaway Beach, Aqueduct, Ols (Beq).

D. posticata Say. Mt. Whiteface, J1, Ds (Jn); Adirondacks, Je-J1 (Joh); Paradox L., Jl, Ds (Jn); Gloversville, May; Erie Co, Je-J1 (MCV) ; Pike; Portageville, Ds (Beq) ; Rock City, Je, Ds (Jn, Beq) ; Tompkins Co, Je-J1 (Hine) ; Albany, May-Je; Karner, Je; Nassau, Sep; Rensselaer, May; LI: Pennequid Barrens (Beq).

D. sacrator Wlk. Adirondacks; N. Elba (Jn); Upper Ausable L. (Beq); Tompkins Co, Je-J1 (Hine); McLean, Jl (Joh).

D. thoracica Fab. Lowville, Aug (Ds); Oswego; Niagara Falls, Je-J1 (Jn) ; Erie $\mathrm{Co}$, Je-J1 (MCV) ; Ithaca (Hine); Syracuse, Blkm; Kenwood, J1; Coeymans, $\mathrm{J1}$; Albany, Je; West Pt., Je (Robn) ; Pine I., Je (Ds) ; Poughkeepsie, J1; Ft. Montgomery (Beq); W. Nyack (Beq) ; New Baltimore, Zab; SI: Tompkinsville, Je, Ds; Richmond, J1 (Ds) ; Clove Val., May-Je, Ds; Egypt's I., Je (Ds); Tottenville, Je (Ds) ; Ft. Wadsworth, Jl (Bs) ; LI: Brooklyn, Je, Shoe, Ds (AM); Flatbush, J1, Zab, Schott (Beq); Wading R. (Beq); Cold Sp. Harb., Je (Ds); Jamaica, Je (Ds).

\section{LAPHRIA Meigen}

L. aeatus W1k. (index McAtee). Erie Co, J1 (MCV); Genesee Co, Je, Ds (Beq); West Pt., Je, Ds (Beq).

L. aktis McAtee. Rock City, Je, Ds (Beq); Ft. Montgomery, Schott (Beq). 
L. altitudinum Bromley. L. Tear, $4300 \mathrm{ft.}$ (Beq).

L. canis Will. Keene Val., Upper Ausable L. (Beq); Erie Co, J1-Aug (MCV); Little Valley, Je, Aug; Tompkins Co, Je-Jl, Sep (Hine); Haines Falls; Oliverea (Beq) ; Ft. Montgomery, Aug, Ds (Beq); W. Nyack (Beq); SI: Ft. Wadsworth, J1, Watchogue, J1, Arrochar, J1 (Bs); Richmond, Aug, Ds (Beq) ; LI: Wyandanch (Beq).

L. dispar Bks. (canis Will.?). Ithaca (Bks).

L. gliva L. (bilineata Wlk.). Big Moose, J1, $Y g$; Pike.

L. sadalis Wlk. (pubescens Will.). "NY" (Wlk); Axton, Je (Joh).

L. scorpio McAtee. "NY".

L. sericea Say. Essex Co, J1; N. Elba, J1, 2000 ft., Wat (Beq) ; Speculator, J1, Yg ; Boston, J1-Aug (MCV); Pike; Portage, Je, Ds (Beq); Little Valley, Je; Tompkins Co, Je-J1 (Joh) ; Albany (Leon) ; Pine I., West Pt., Je (Ds) ; Ft. Montgomery, Schott (Beq); SI: W. New Brighton, Je, Ds (Beq).

\section{SUBFAMILY ASILINAE}

Ommatius Wiedemann

O. marginellus Fab. (tibialis Say). Poughkeepsie, J1; White Plains, W. Nyack (Beq) ; Mosholu (AM) ; SI: Watchogue (Bs) ; Richmond, J1 (Bs) ; LI: Wading R., Je (Ds) ; Sands Pt., Aug (Bs) ; Kings Pk., J1, Bell; Flatbush (AM) ; Riverhead, J1, Ds (Jn).

\section{Proctacanthus Macquart}

P. brevipennis Wied. Mosholu (AM) ; SI: Watchogue, Je, Bs (Beq) ; LI: Coram, Aug 18, Yaphank, J1 5, Riverhead, Aug 4, Ds (Beq).

P. nigriventris Macq. SI: Kreischerville, Bs; LI: Fire I., Sep 7, Gardiners I., Aug 20, Yaphank, Je, Riverhead, Je, Calverton, Sep, Coram, Aug, Ds (Beq); Kings Pk., Aug, Bell.

P. philadelphicus Macq. Severance, Aug; New Russia, Aug (Hine); Albany, Aug; Karner, Sep; Mosholu (AM) ; NYC, Van Cort. Pk. (Beq) ; SI: Arrochar, Sep 10, Ds; New Dorp, Ft. Wadsworth, Sep, Bs; LI: Cold Sp. Harb., Sep (Beq); Yaphank, Aug 26-Sep 3, Coram, Montauk, Aug 26, Ds (Beq); Wyandanch, Schott (Beq); Wading R., Bs; Gardiners I., Aug 20 (Beq).

P. rufus Will. SI: Watchogue, Aug, Bs (Beq); LI: Manhattan Beach (Beq); Coney I., Sep, Riverhead, J1 5, Ds (Beq); Gardiners I. (Beq).

\section{ERAX Scopoli}

E. aestuans L. (bastardii Macq.). Elizabeth, Je; Essex Co, J1; Erie Co, J1-Aug, S. Wales, J1 (MCV); Ithaca, Je-Aug (Hine); Clinton Hgts., Jl; Poughkeepsie, J1; Yonkers, Bs; White Plains, W. Nyack (Beq); NYC, Van Cort. Pk., Bs ; SI: Richmond, Aug 4, Ds (Jn) ; Reed's Val., J1 28, Ds (Beq); New Brighton, Ft. Wadsworth, Bs; LI: Sheepshead Bay, Aug; Yaphank, J1 10, Greenport, Orient, Je-Aug, Selden, Sep, Ds (Jn) ; Maspeth, Aug, Ols (Beq) ; Central Pk., Gardiners I. (Beq); Flatbush, Eng (Beq).

E. barbatus Fab. (cinerescens Bell; albibarbis Macq.). Bronx Pk., Mosholu (AM) ; SI: Ft. Wadsworth, Watchogue, Bs; LI: Wading R., Aug, Ds (Beq) ; Sands Pt., J1, Cold Sp. Harb., Bs (Beq); Sheepshead Bay, J1; Gardiners I., Orient, Rockaway Beach (Beq). 
E. rufibarbis Macq. W. Nyack, Bs; White Plains (Beq); SI: Ft. Wadsworth, Sep, Bs; LI: Patchogue, Sep, Montauk, Aug 24, Ds (Jn) ; Wyandanch, Ds (Beq) ; Cold Sp. Harb., Sep, Sands Pt., Bs; Gardiners I., Aug 21, Ds (Beq) ; Springs (Beq).

\section{MALLOPHORA Macquart}

M. clausicella Macq. LI: Oakdale, Huntington, Schott (Beq); Wading R., Aug, Ds (Jn) ; Yaphank, J1, Ds (Beq).

\section{PROMACHUS Loew}

P. bastardii Macq. Keene Val., Aug; Severance, Aug (Bks); New Russia, J1; Tonawanda, Aug (MCV) ; W. Hebron, Aug, Lg (Beq) ; Albany, J1 (Hine); Nassau; Clinton Hgts., J1; Nyack (AM) ; SI: Ft. Wadsworth, J1-Aug, Richmond, J1, Bs; LI: Maspeth, Ols (Beq); Sands Pt., Bs.

P. fitchii O.S. (apivorus Fitch). Nassau, May (Jn); White Plains (Beq) ; Pelham, J1; SI: south shore, Je-Aug, Clove Val., Ds (Beq) ; Richmond, J1, Arrochar, Jl, Bs; LI: Oakdale, Flatbush, Schott (Beq) ; Astoria (AM).

P. rufipes Fab. Nyack, Aug, Zab (AM).

\section{Asilus Linnaeus}

A. alethes W1k. "NY" (W1k) ; unrecognized.

A. antimachus W1k. Trenton Falls (W1k); W. Nyack, J1, Ds (Beq) ; SI: Ft. Wadsworth, New Dorp, Richmond, Watchogue, Arrochar, Je-J1 (Bs).

A. auricomus Hine. Ithaca, Aug (Hine); LI: Southold, Sep (Jn).

A. erythrocnemius Hine. Stony Pt., Schott, White Plains (Beq); SI: Richmond, Aug, Bs; LI: Wyandanch, Schott, Orient (Beq); Gardiners I., Je, Ds (Beq).

A. flavofemoratus Hine (flavipes Will.). Mt. Whiteface, summit, J1, Ds (Beq); Hamilton Co, Ds (Jn) ; Erie Co, J1 (MCV) ; Ithaca, Je-J1 (Hine) ; McLean, J1, Sib (Joh) ; Karner, Je; Pine I., Je, Ds (Jn); West Pt., Je, Ds (Beq); Nyack, Je, Ds (Jn); SI: Ft. Wadsworth, Je, Watchogue, J1 (Bs) ; LI: Farmingdale, Je (Bs); Central Pk., Cold Sp. Harb., Kings Pk. (Beq) ; Wading R., May, Ds (Jn); Yaphank, Je, Ds (Beq); Nissequogue, Je, Ds $(\mathrm{Jn})$; Sands Pt., Je (Bs).

A. fuscatus Hine (obscurus Hine). "NY" (Hine).

A. latipennis Hine. Ithaca, Aug (Hine).

A. maneei Hine. LI: Cold Sp. Harb., Aug (Bs); Huntington, Schott (Beq).

A. notatus Wied. Adirondacks, Je; Keene Val. (Beq) ; Oswego, J1 (Jn); Little Valley, Je (MCV) ; Ithaca, Je, Aug (Hine) ; McLean, J1, Sib (Joh) ; Karner, Poughkeepsie, Je-J1; Ft. Montgomery (Beq); Nyack, Je, Ds (Beq); Tomkins Cove, Sep, Carmel (AM); SI: Arrochar, Jl, Ds (Beq) ; Richmond, J1, Ft. Wadsworth, Je, Watchogue, Bs (Beq) ; LI: Cold Sp. Harb., Port Jefferson, Yaphank, Pennequid Barrens, Gardiners I. (Beq).

A. novae-scotiae Macq. Adirondacks, J1-Aug; Newport, J1; SI: Clove Val., J1, $D s$ (Beq) ; Tottenville, Je, Ds (Beq); Watchogue, Je, Ds (Beq); Ft. Wadsworth, J1, Richmond, Oakwood, Bs; LI: Yaphank, J1, Ds (Beq); Maspeth, J1, $D s$, Gardiners I. (Beq).

A. orphne Wlk. (neortamus, distinctus Will.). Mt. Whiteface, summit, J1, Ds (Beq) ; Adirondacks, J1; Niagara Falls, Je (Jn); Erie Co, Je-J1 (MCV) ; Tompkins Co, May, J1; W. Hebron, Aug, Lg (Beq) ; Albany, Je; West Pt., 
Je, Ds, Ft. Montgomery (Beq); White Plains, Bno (Beq) ; New Rochelle, Aug (AM) ; LI: Aqueduct, Sep, Ds, Huntington (Beq).

A. paropus Wlk. Ithaca, J1 (Beq); McLean; Clifton Sps., Aug (Hine).

A. sadyates Wlk. Alexandria Bay, Sep; Erie Co, Je (MCV); Little Valley; Tompkins Co, J1-Sep (Hine); Oliverea (Beq); West Pt., Je, Ds, Stony Pt. (Beq) ; Ramapo, Je, Ds (Beq); SI: Richmond, Je, Watchogue, J1, Ds (Beq) ; LI: Rockaway Beach, Je, Amagansett, Sep, Yaphank, Je, Fire I., Sep, Cold Sp. Harb., Ds (Beq); Wading R., Sep (Bs); Gardiners I., Aug, Ds (Beq).

A. sericeus Say. Saranac Inn; Hamburg, Je (MCV); Slingerlands, J1; Poughkeepsie; Ft. Montgomery (Beq) ; SI: Wadsworth, J1, Watchogue (Bs); LI: Kings Pk., J1, Bell, Queens, Central Pk., Farmingdale, Je (Beq); Flatbush, Je, Zab; Astoria (AM).

A. snowii Hine (annulatus Will.). Potsdam; Essex Co, Aug; Speculator, J1; Oswego (Jn) ; Erie Co, J1-Aug (MCV) ; Ithaca, J1-Aug (Hine); McLean, J1, Sib (Joh) ; Albany, Je; SI: Richmond, Ft. Wadsworth, Watchogue, J1 (Bs);

LI: Montauk, Aug, Ds; Flatbush, Aug, Zab.

\section{FAMILY DOLICHOPODIDAE 99a SUBFAMILY SCIAPINAE}

SCIAPUS Zeller

(Psilopodinus Aldrich not Bigot; Agonosoma Aldrich not Guérin; Psilopus Meigen not Poli)

S. banksii VanD. McLean, J1, Sib; LI: Sea Cliff, Bks (MCV).

S. calcaratus Lw. SI: Watchogue, J1, Bs (MCV); LI: Sands Pt., Je, Bs (MCV).

S. caudatus Wied. Lancaster, Je; Ithaca, J1; Albany, Je (MCV); SI: Richmond, Aug, Bs (MCV); LI: Sands Pt., Je, Bs (MCV); Wading R., Sep, Bs (MCV).

S. delicatus W1k. "NY" (W1k).

S. flavipes Ald. Erie Co, Je-Aug (MCV); Ithaca, J1; Karner, Je (Yg) ; Poughkeepsie, Je (MCV).

S. inermis Lw. LI: Pennequid Barrens, Jl (Beq).

S. novaboracensis VanD. Gowanda, Je (MCV); Rock Glen, J1 (MCV).

S. pallens Wied. Erie Co, Jl-Aug; Albany, May-J1 (NYS); Karner, Je, Comst (NYS) ; Bowling Green, Je-J1 (MCV); LI: Sag Harb.; Pennequid Barrens, J1 (Beq).

S. patibulatus Say. Manlius; Orleans Co, Je, Ds (MCV); Cattaraugus Co (MCV); Otto; Ithaca, J1; Trenton Falls; Poughkeepsie, Je-J1 (MCV); Pine I., Je 19-22, Ds (MCV); L. Waccabuc, Aug (Beq-AM) ; SI: Watchogue, $\mathrm{J} 1$ 2, Ds (MCV); LI: Sands Pt., Je, Bs (MCV); Farmingdale, Je, Bs $(\mathrm{MCV})$; Port Jefferson, J1 (Beq).

S. scaber Lw. Ithaca, J1 (MCV); Lowville, Aug (MCV-Ds); SI: Arrochar, Ft. Wadsworth, Je-Aug, Bs (MCV).

S. scintillans Lw. Manlius, Aug; Gowanda, Aug; Lancaster (MCV); McLean, I!, Sib; Ft. Montgomery, Schott (Beq).

99a The list for this family has been checked by M. C. Van Duzee. 
S. scobinator Lw. Axton; Niagara Falls; Erie Co, J1-Aug (MCV); Ithaca; Albany, Je (NYS) ; W. Nyack, Bs (MCV) ; SI, Jl, Bs (MCV) ; LI: Sands Pt., J1, Cold Sp. Harb., Aug, Bs (MCV); Wyandanch, Ols (Beq) ; Orient, $\mathrm{J1}$ (Beq).

S. sipho Say. Common and widely distributed throughout the State, Je-Sep (MCV).

S. unifasciatus Say. Olcott (MCV).

S. virgo Wied: "NY" (Wied).

\section{SUBFAMILY DIAPHORINAE}

\section{DIAPHORUS Meigen}

D. gibbosus VanD. Colden; Little Valley, May-Je (MCV) ; Dryden L., Je (MCVCU) ; SI: New Dorp, Bs (MCV); LI: Rosedale, May, Schott (MCV).

D. lamellatus Lw. Erie Co, May-J1 (MCV); SI: Richmond, Je, Bs (MCV).

D. leucostomus Lw. Manlius; Lewiston; Buffalo, Aug (MCV).

D. leucostomus var. infuscatus VanD. Erie Co, May-Je (MCV).

D. opacus Lw. Sacandaga R.; Erie Co, Je-Jl (MCV); Tompkins Co; Albany; SI: Richmond, Bs (Beq).

D. palpiger Wheel. (Chrysotus spinifer Mall.). Lewiston; Colden; Erie Co, JeAug (MCV); SI, Aug, Bs (MCV).

D. sodalis Lw. Springville, Je (MCV); Colden, J1 (MCV).

D. spectabilis Lw. Niagara Falls, Je (Ald); SI: Richmond, Bs (Beq).

D. variabilis VanD. N. Evans, Aug (MCV).

\section{Asyndetus Loew}

A. syntormoides Wheel. W. Nyack, J1, Bs (MCV) ; LI: Cold Sp. Harb., Aug, Sands Pt., Jl, Bs (MCV).

\section{Chrysotus Meigen}

C. affinis Lw. Elba; Tonawanda; Erie Co, Je-Sep; Ithaca, Je (MCV); Freeville, J1; W. Nyack, J1, Bs (MCV); Albany, Je (NYS); SI: Arrochar, JI, Richmond, Sep, Bs (MCV); LI: Farmingdale, Je, Bs (MCV).

C. anomalus Mall. McLean, J1.

C. auratus Lw. "NY" (Lw); Albany, Je, Sep; Voorheesville, J1 (Curr).

C. barbatus Lw. Lewiston, Aug; Buffalo, Je; E. Aurora, Aug; Salamanca, J1 $(\mathrm{MCV})$; SI, Sep (Beq).

C. bellus VanD. SI: New Dorp, Watchogue, Tottenville, Clove Val., May, Bs (MCV).

C. caudatus VanD. N. Evans, Aug (MCV).

C. choricus Wheel. (ciliatus Mall.). Niagara Falls, Je (Jn); Colden, J1; Elba; Erie Co, May-J1 (MCV); Trenton Falls, Je; Albany, J1 (Curr).

C. convergens VanD. Colden; S. Wales, May-J1 (MCV).

C. cornutus Lw. Colden; Salamanca, J1 (MCV). 
C. costalis Lw. Erie Co, Je-J1 (MCV); Tompkins Co; Wells, Je, Yg (Curr).

C. cressoni VanD. Beaver Kill, Aug (MCV).

C. currani VanD. Ithaca, J1 (MCV-CU).

C. discolor Lw. Keene Val., Aug (NYS) ; Speculator, J1-Aug, Yg (Ald) ; Ithaca, Je, Ringwood, J1 (MCV-CU); McLean, J1- (MCV); W. Nyack, J1, Bs (MCV) ; SI: Clove Val., Je 27, Ds (MCV); Richmond, Bs (MCV); LI: Sands Pt., J1, Bs (MCV); Long Beach, Je (Beq).

C. dividuus VanD. Elba, Hamburg, Lancaster, Je (MCV).

C. excisus Ald. Ringwood, J1 (MCV-CU).

C. exiguus VanD. Colden, J1 (MCV).

C. halteralis VanD. E. Aurora, J1 (MCV).

C. hastatus VanD. Ithaca, Aug.

C. hirtipes VanD. Olcott, J1 (MCV); McLean, J1; Ithaca, J1-Aug, Albany, Je, Yg.

C. hirtipes var. dubius VanD. Ringwood, J1 (MCV-CU).

C. magnicornis VanD. Ithaca.

C. major VanD. Erie Co, Je (MCV).

C. obliquus Lw. Olcott; Elba; Erie Co, May-Aug (MCV) ; Tompkins Co, MayAug, McLean, May, Ringwood, J1 (CU) ; Voorheesville, J1 (Curr).

C. pallipes Lw. Ithaca, Aug (MCV-CU) ; McLean, Sep, Sib; Van Cort. Pk., J1 (MCV-CU).

C. parvus VanD. Erie Co, J1.

C. parvicornis VanD. E. Aurora; Colden, May-Je (MCV).

C. perparvus VanD. Protection, J1 (MCV).

C. picticornis Lw. Lewiston, Aug: Erie Co, Je-Oct (MCV); Rock Glen, J1 (MCV) ; Tompkins Co, J1; W. Nyack, J1 (Beq).

C. sagittarius VanD. Buffalo, Ap (MCV).

C. subcostatus Lw. Albany, Je; Wells, J1 (Curr).

C. tibialis VanD. Clayville, Je, Leon (MCV); Silver Creek, Je; Wells, J1.

C. varipes VanD. Protection, J1 (MCV).

C. vividus Lw. Poughkeepsie, Je, Jl, Yg (Curr).

C. vulgaris VanD. Erie Co, Elba, Freeville, Harford, May-J1 (MCV); McLean, J1, Sib; Ithaca, Ellis, Je (MCV-CU); Newport, Je, Yg (Curr).

C. wisconsinensis Wheel. Peru, Je (MCV-CU) ; Lancaster; S. Wales, J1 (MCV); Cayuga L., Speculator, Aug; Wells, Jl, Yg (Curr).

\section{CAMPSICNEMUS Haliday}

C. americanus VanD. NY (Curr).

C. hirtipes Lw. Niagara Falls, Aug; Erie Co, May-Aug (MCV); Rock Glen, J1 (MCV) ; Ithaca, J1-Aug; Albany, Oct; SI: Watchogue, $B s$ (MCV).

\section{Kophosoma Van Duzee}

K. acuticornis VanD. Ithaca (MCV).

K. brevis VanD. Colden, Lancaster, Olcott, Irving, E. Aurora, J1; Portage; Middleport; Jamestown; Ithaca (MCV). 


\section{SUBFAMILY RHAPIINAE}

\section{ARGYRA Macquart}

A. albicans Lw. Erie Co, Je-Aug (MCV); Portage, J1 (MCV); Ithaca, Caroline, Taughannock, May; Thacher Pk., May (Curr); SI: Watchogue, Aug, Bs (MCV).

A. angustata VanD. L. Tear, $4500 \mathrm{ft}$. (Beq); Olean, Aug, $M C V$.

A. calceata Lw. Erie Co, J1-Sep (MCV); Little Valley, J1 (MCV); Ithaca, J1, McLean Bogs, Aug (MCV-CU); Manlius, J1; Wells, J1 (Curr).

A. calcitrans Lw. Erie Co, Je-J1 (MCV); Colden, Aug (MCV); Portage, J1 (MCV) ; Ithaca, Je (MCV-CU).

A. flavipes VanD. Hoversville, Je (Curr).

A. robusta Jn. Portage, J1 (MCV); Ithaca, May (MCV-CU); Ilion, May (Jn).

A. setipes VanD. Colden, Aug (MCV); Hamburg, J1 (MCV); Little Valley, J1 (MCV).

A. thoracica VanD. Newport, Je (MCV); Lewiston, Je (MCV); Gowanda, Je $(\mathrm{MCV})$; Speculator, Je (MCV); Protection, Je (MCV); Kiamesha (MCV).

\section{LEUCOSTOLA Loew}

L. cingulata Lw. Erie Co, Je-J1 (MCV); Hoversville, Je; Wells, J1 (Curr).

L. venustus Mall. NY.

\section{RHAPHIUM Meigen}

(Porphyrops Meigen)

R. armatum Curr. Colden (Curr).

R. canadense Curr. Poughkeepsie, May; Speculator, Je; Wells, J1, Yg (Curr).

R. effilatum Wheel. Niagara Co, J1 (MCV); Erie Co, Je-Sep (MCV); Rock Glen, J1 (MCV); Watervliet Reservoir, Aug (Curr).

R. fascipes Meig. Lewiston, Je (MCV); Erie Co, May-Aug (MCV); Ithaca, May (MCV).

R. longipes Lw. Olcott, J1 (MCV); Colden, May.

R. lugubre Lw. Albany, May, $Y g$.

R. melampus Lw. Erie Co, May-J1 (MCV) ; Rock Glen, J1 (MCV) ; Little Valley, Aug; Ithaca, May-Aug; Ellis, Je; Manlius, Sep; SI: Clove Val., Bs (MCV).

R. nigricoxa Lw. Lancaster, Je (MCV) ; Corinth, Je, $Y g$ (Curr).

R. ornatum VanD. Colden, J1 9-23, types (MCV) ; Lancaster, Je 2, types (MCV); Thomson, May (Curr).

R. signifer O.S. Manlius; Erie Co, Je-Aug (MCV); Ithaca; Tarrytown.

R. slossonae Jn. Nassau, May (Curr).

\section{NEMATOPROCTUS Loew}

N. venustus Mel. W. Nyack, J1 (MCV); Ringwood, J1 (MCV-CU).

\section{XIPHANDRIUM Loew}

X. femineum VanD. Rochester, May (MCV).

$\mathrm{X}$. femineum var. dubium VanD. Gasport, Aug (MCV). 


\section{SYNTORMON LOEW}

S. cinereiventris Lw. Niagara Falls, Aug-Oct (MCV); Gasport (MCV); Erie Co, Aug-Sep (MCV).

\section{SUBFAMILY SYMPYCNINAE}

\section{SYMPYCNUS Loew}

S. canadensis VanD. Protection, Aug, N. Evans, Je (MCV).

S. lineatus Lw. Speculator, Aug, $Y g$ (NYS) ; Albany, Oct, $Y g$; Rochester, Oct (MCV) ; Niagara Co; Erie Co; Tompkins Co, May-Aug; W. Nyack, J1 (Beq).

\section{NOTHOSYMPYCNUS Wheeler}

N. fortunatus Wheel. Newport, Je, $Y g$ (Curr-NYS); Wells, J1, Yg (Curr-NYS); Colden, Aug (MCV); Ithaca, Aug; Albany, Sep (Curr-NYS).

N. frontalis Lw. Erie Co, May-Aug (MCV); Ithaca, J1-Aug.

N. luteipes VanD. Wells, J1, Yg (MCV).

N. nodatus Lw. Niagara Falls, Je (Jn); Colden, Aug (MCV).

\section{SUBFAMILY NEURIGONINAE}

\section{NEURIgONA Rondani}

N. aestiva VanD. Lancaster, Je (MCV); Salem, Je (Curr).

N. aldrichi VanD. Lancaster, Je, E. Aurora, J1 (MCV).

N. arcuata VanD. E. Aurora, N. Evans, Je-Jl, Gowanda, Je (MCV).

N. carbonifer Lw. Erie Co, May-J1 (MCV).

N. deformis VanD. Buffalo, Gowanda, Je (MCV).

N. disjuncta VanD. E. Aurora, Little Valley, Je (MCV); Ithaca, J1.

N. dorsalis VanD. Elma, Irving, Aug (MCV); Ithaca, J1.

N. floridula Wheel. "NY" (MCV); LI: Pennequid Barrens, J1, Beq (MCV).

N. floridula var. infuscata VanD. Erie Co (MCV); Little Valley, Je.

N. lateralis Say. "NY" (Lw); Middleport, Jl (MCV); Albany, Je (Curr).

N. maculata VanD. Erie Co, Je-J1 (MCV); Little Valley, Je; Ithaca; LI: Sea Cliff.

N. minima VanD. Ithaca, Aug (MCV-CU), holotype.

N. nigricornis VanD. Colden, Aug (MCV) ; Wells, J1 (Curr); SI: Richmond, $\mathrm{Jl}, B s$ (MCV).

N. rubella Lw. Buffalo (MCV); LI: Sea Cliff, Bks; Sands Pt., Bs (Beq).

N. tarsalis VanD. E. Aurora, Je, Gowanda, Je (MCV) ; Ithaca; LI: Sea Cliff.

N. tenuis Lw. Niagara Falls, J1; Erie Co (MCV); E. Aurora, J1-Sep.

N. tibialis VanD. Lancaster, Aug (MCV); Ithaca, Aug (MCV-CU).

N. viridis VanD. S. Wales, J1; West Farms (MCV). 


\section{SUBFAMILY XANTHOCHLORINAE}

Chrysotimus Loew

C. delicatus Lw. "NY" (Lw).

C. flavicornis VanD. LI: Richmond Hill (MCV).

C. pusio Lw. Mt. Whiteface, Aug (MCV) ; Colden, Aug (MCV); Wells, J1 (Curr).

\section{XANTHOCHLORUS LOEW}

X. helvinus Lw. Wells, Speculator, J1-Aug, Yg (Curr); Niagara Falls, Aug (MCV) ; S. Wales, J1; Ithaca, Aug ; Albany, Aug (Curr-NYS).

\section{SUBFAMILY THINOPHILINAE}

THINOPHILUS Wahlberg

T. ochrifacies VanD. LI: Cold Sp. Harb., Sep, Long Beach, J1, Bs (MCV).

\section{Diostracus Loew}

D. prasinus Lw. "NY" (Ald); Wells, J1, Yg (Curr).

\section{SUBFAMILY MEDETERINAE}

MEDETERUS Fischer von Waldheim

M. aberrans Wheel. Gowanda, Je (MCV).

M. distinctus VanD. E. Aurora, Je, holotype (MCV).

M. emarginatus VanD. Peru, Oct (MCV-CU).

M. fimbriatus Lw. Sacandaga Pk. (MCV-CU).

M. frontalis VanD. Hamburg, May, Aug (MCV), holotype; Chautauqua Co, Je $(\mathrm{MCV})$.

M. nigripes Lw. E. Aurora, J1 (MCV) ; Little Valley, Je; Ithaca, Aug; L. Clear, Je (Curr).

M. obesus VanD. E. Aurora, J1 (MCV), holotype.

M. veles VanD. S. Wales, Little Valley, J1 (MCV).

M. vittatus VanD. Peru, Aug (MCV-CU); Erie Co, J1, Sep (MCV), paratype; Albany, Je; Little Valley, Je (MCV); LI: Long Beach, Je (Beq).

\section{PELOROPEODES Wheeler}

P. salax Wheel. Wells, J1, Yg (MCV).

\section{THRYPTICUS Gerstaecker}

T. comosus VanD. Ellis, J1 (MCV).

T. fraterculus Wheel. Lewiston, Aug (MCV); Voorheesville, J1.

T. muhlenbergiae Joh. \& Cy. Ithaca, May (Joh). Larva in stems of Muhlenbergia (type). 
T. tectus VanD. E. Aurora, J1 (MCV); Ringwood, J1 (MCV-CU); Albany, Je (Curr).

T. willistoni Wheel. Sacandaga Pk., Je (MCV-CU); Olcott, J1 (MCV); Manlius; Erie Co, May-Je (MCV); Ellis, Je; Ithaca, May, J1 (MCV-CU).

\section{SUBFAMILY HYDROPHORINAE}

\section{HYDROPHORUS Fallén}

H. aestuum Lw. Erie Co, Aug-Sep (MCV); SI: Watchogue, May, Bs (MCV).

H. agalma Wheel. Buffalo, Oct (MCV).

H. amplectens Ald. L. Tear, J1 (MCV).

H. chrysologus W1k. L. Tear, 4300-4600 ft., J1, Beq (MCV-Bs).

H. flavipennis VanD. Buffalo, Oct (MCV).

H. intentus Ald. SI: Watchogue, May, Sep, Bs (MCV); LI: Sands Pt., Jl, Cold Sp. Harb., Sep, Bs (MCV).

H. philombris Wheel. Rochester, Sep; Buffalo, Aug-Oct (MCV).

H. pirata Lw. Elma, Colden, Springville, J1-Oct (MCV); Karner, Oct.

\section{SCELlus Loew}

S. exustus Wlk. Buffalo, Je; Grand I., Aug (MCV).

\section{ORTHOCERATIUM Schrank}

\section{(Liancalus Loew)}

O. genuale Lw. Essex Co, J1-Aug; Elizabethtown, Aug (NYS) ; Keene Val., Aug (NYS) ; Niagara Falls, May-Oct (MCV); Erie Co, Je-Oct (MCV); Ithaca, Jl-Aug (Ald); Meadowdale, Aug; Poughkeepsie, Je (NYS).

\section{SUBFAMILY DOLICHOPODINAE}

\section{DoLICHOPUS Latreille}

D. abrasus V. C. \& A. Grand I., Aug.

D. abruptus Ald. L. Tear (Ald).

D. absonus V. C. \& A. Grand I., Aug; Buffalo, Sep.

D. acuminatus Lw. Lewiston; Lancaster; Erie Co, May-Oct (MCV); Rock Glen, J1 (MCV) ; Ithaca, J1 (VC\&A).

D. adultus V. C. \& A. Speculator, Je, Yg (Leon) ; Ilion, May, Yg (Curr) ; Lewiston, May; Colden; E. Aurora; Hamburg; Erie Co; McLean, May; Thomson, May 25 (Leon).

D. aequalis V. C. \& A. Wilmington Notch, J1, Leon (Curr); Wells, J1, Yg (Curr) ; Hoversville, Je, Leon (Curr); Erie Co, J1-Aug; Rock Glen, J1 (MCV); McLean, May; Franklinton, Je, Leon (Curr).

D. ainsleei V. C. \& A. Irving, Aug.

D. albiciliatus Lw. Manlius (OS) ; Olcott, J1 (MCV); western NY.

D. albicoxa Ald. Adirondack Lodge, Jl, Leon (Curr); Elm L., Aug, Yg (Curr); Elizabethtown, Je (Leon); Erie Co, Je; Portage, Jl. 
D. barbicauda V. C. \& A. Hoversville, Je, Leon (Curr); Franklinton, Leon (Curr).

D. batillifer Lw. Hoversville, Je, Leon (Curr); Portage, J1 (MCV); Trenton Falls, Je 20, $Y g$; Albany, Je 13 (Leon); West Pt.; SI: Richmond, Je, Bs (MCV).

D. bifractus Lw. Mt. McIntyre, summit, J1, Leon (Curr); Niagara Co, Aug; Erie $\mathrm{Co}$; Ithaca, Je-Sep (MCV); Long Beach, Je-J1, Bs (MCV).

D. brevimanus Lw. Ithaca and vic., May-Je (MCV).

D. burnesi V. C. \& A. Ballston L., Aug 26 (Leon); SI: Arrochar, Bs (MCV), holotype; LI: Wading R., Sep, Bs (MCV), allotype.

D. calcaratus Ald. Upper Ausable L. (Beq); Moss Pond, Essex Co, 4200 ft. (Beq); Erie Co, Je-Sep (Ald) ; Rock Glen, J1 (MCV) ; E. Aurora; Little Valley, J1 (MCV) ; Ithaca, J1, Bs (MCV) ; Voorheesville, Je 19 (Leon); Poughkeepsie, Je $5, \mathrm{Yg}$ (Leon).

D. coercens Wlk. "NY" (Wlk).

D. comatus Lw. Wells, Jl, Newport, Je, Yg (Curr); W. Winfield, Je, Leon (Curr) ; Niagara Co; Erie Co, May-Sep (MCV); Tompkins Co; Albany, May 22; Karner, Je 27, Yg; Poughkeepsie, Je; SI: Watchogue, Aug, New Dorp, Je, Clove Val., Bs (MCV); LI: Huntington, Oct, Schott (MCV); Cold Sp. Harb., Aug-Sep, Bs (MCV).

D. conterminus W1k. "NY" (W1k).

D. continguus Wlk. "NY" (Wlk).

D. cuniculus VanD. Grand I., Aug.

D. cuprinus Wied. Wells, J1, Yg (Curr); Blue Mt., J1 24, Yg; W. Winfield, Je, Leon (Curr) ; Manlius; Erie Co, Je-Sep; Little Valley, Aug (MCV) ; Tompkins Co, Je-J1 (Joh); Watervliet Reservoir, Aug, Leon (Curr); Albany, Je; Nassau L., Sep 3 (Leon); Nassau, J1 8; Voorheesville, J1, Leon (Curr); Poughkeepsie, Je; W. Nyack, J1, Bs (MCV); SI: Richmond, Je, New Brighton, $B s$ (MCV).

D. dakotensis Ald. Rochester, May (MCV); Trenton Falls, Je (Curr); Albany, Je (Curr).

D. dasypodus Coq. Moss Pond, $4200 \mathrm{ft} ., \mathrm{J1}$ (Beq).

D. decorus VanD. Buffalo, Je.

D. defectus V. C. \& A. Wells, Jl, Yg (Curr) ; Niagara Falls; Colden; E. Aurora, Il.

D. detersus Lw. Speculator, J1, Yg; Moss Pond, Essex Co, 4200 ft., J1 (Beq); Manlius; Olcott, J1; Erie Co, May-Aug (MCV); McLean, Je; Saratoga; Ballston L., Aug 26 (Leon); Voorheesville, Jl, Leon (Curr).

D. discifer Stan. (nigricornis Mg.?). Axton, Je; Dug Mt., Aug, Yg; Wells, Jl, Yg (Curr); Lewiston, Aug (MCV); Colden, Aug; McLean (MCV); Poughkeepsie, Je, $Y g$ (Curr).

D. discolor V. C. \& A. Lewiston, May; Tonawanda, Aug; Lancaster, Je; Nassau, Je (Curr-NYS).

D. distractus Wlk. "NY" (Wlk).

D. domesticus VanD. E. Aurora.

D. dorycerus Lw. Wells, J1, Yg; Moss Pond, Essex Co, J1, Bs (MCV); pond at foot of Mt. Redfield, $2500 \mathrm{ft} ., \mathrm{Jl}$ (Beq) ; L. Tear, $4300 \mathrm{ft} ., \mathrm{J1}$ (Beq) ; Lancaster, $\mathrm{Je}(\mathrm{Jn})$.

D. eudactylus Lw. Speculator, Wells, J1, Yg (Curr); Rochester, Je (MCV); Niagara Falls, Je; Erie Co, Je-J1 (MCV); Rock Glen, J1 (MCV); Ithaca (Joh); Trenton Falls, Je, $Y g$; Albany, Je, $Y g$; W. Nyack, J1, Bs (MCV) ; LI: Pennequid Barrens, Orient, J1 (Beq). 
D. finitus W1k. "NY" (W1k).

D. flagellitenens'Wheel. E. Aurora, Je.

D. flavicoxa V. C. \& A. McLean, J1 (Curr).

D. flavilacertus V. C. \& A. L. Tear (Beq) ; pond at foot of Mt. Redfield, J1 (Beq); LI: Farmingdale, Je, $B s$ (MCV).

D. fulvipes Lw. Cranberry Cr., Je, $Y g$; Erie Co, Je-J1 (MCV); Colden, May (MCV); Little Valley, J1 (MCV); Nassau, May.

D. funditor Lw. Niagara Falls, Sep (MCV).

D. gracilis Ald. Erie Co, Je-Sep (Ald\&MCV); W. Nyack, J1, Bs (MCV) ; LI: Wading R., Bs (Beq).

D. gratus Lw. Erie Co, Je-Aug (MCV); Rock Glen, J1 (MCV); Ithaca, Je; Trenton Falls; Voorheesville, J1, Leon (Curr) ; Poughkeepsie, Je, Yg; West Pt.; W. Nyack, J1, Bs (MCV); LI: Cold Sp. Harb., Aug, Bs (MCV).

D. harbecki Ald. Adirondacks (Ald).

D. idoneus VanD. E. Aurora, Je.

D. incisuralis Lw. Niagara Falls; Erie Co; Colden, J1 (MCV); Ithaca (MCV); Trenton Falls, J1; Karner, Je (Curr).

D. incongruus Wheel. Elizabethtown, J1 2 (Leon) ; Erie Co; Colden, May (MCV); Ellis, J1.

D. indigena V. C. \& A. Buffalo, Je; S. Wales, Je-J1; Wells, J1 (MCV).

D. laticornis Lw. Little Valley, J1 (MCV); Ithaca, May (MCV); Thacher Pk., Albany Co, May 27 (Leon).

D. latipes Lw. Manlius; Grand I., Aug-Sep (MCV); Ithaca, Aug (MCV); Pine I., Je 23 (Leon).

D. lobatus Lw. Manlius; W. Winfield, Je, Leon (Curr) ; Olcott, J1; Elba, Je-J1 (MCV) ; Buffalo, Je; Albany, Je (Leon).

D. longimanus Lw. Chazy L., Je; Keene Val., Aug (Lint); Niagara Falls, Je-Aug; Olean; West Pt. (OS).

D. longipennis Lw. Manlius; Irving, J1 (MCV) ; Ithaca, J1; W. Nyack, J1, Bs (MCV) ; SI: Richmond, J1, Bs (MCV).

D. luteipennis Lw. Manlius, Aug (OS) ; Little Valley, Jl (MCV).

D. marginatus Ald. Colden, Aug (MCV); McLean, May; Germantown, Je, Leon (Curr) ; SI: Watchogue, Bs (MCV); LI: Sands Pt., J1, Cold Sp. Harb., Aug, Bs; Orient, Sep (Beq).

D. melanocerus Lw. Niagara Falls, Je (Jn); SI: Watchogue, Je, Bs (MCV).

D. nigricornis Meig. (See $D$. discifer.)

D. nodipennis V. C. \& A. E. Aurora and several other localities in Erie Co, May-Je (MCV); McLean.

D. omnivagus V. C. \& A. Elba, Je; E. Aurora, Je.

D. ornatipennis V. C. \& A. McLean, J1.

D. ovatus Lw. Manlius, Aug (OS); Lewiston, May-Aug; Ithaca (MCV).

D. pachycnemus Lw. Speculator, J1 (Yg); E. Aurora, Je (MCV).

D. palaestricus Lw. Axton, Je (Joh); Keene Val., J1, Lint (Leon) ; Chapel Pond, Je, Leon (Curr) ; Wells, Jl, Yg (Curr) ; L. Placid, Aug (Curr-NYS) ; Cascade L., Je, Leon (Curr) ; Northampton, Je, Yg (Curr) ; Chazy L., Je 23, Yg (Leon); Lewiston, May; Niagara Falls, Je (Jn); Olean (MCV); Albany, Je (Curr-NYS). 
D. pantomimus M. \& B. Niagara Falls, Je.

D. penicillatus V. C. \& A. (Hygroceleuthus ciliatus Ald.). Albany, Je (Curr).

D. porphyrops V. C. \& A. Axton, Je (MCV) ; Upper Ausable L., J1 (Beq) ; Adirondack Lodge, J1 (Leon); Wells, J1, Yg (Curr) ; Lewiston, Aug; Corinth Je, Yg (Leon); W. Nyack, J1 (MCV); LI: Pennequid Barrens, Aug, Bs (MCV).

D. pugil Lw. Lancaster, J1; Buffalo (MCV); Karner, Je; SI: Watchogue, Bs (MCV) ; LI: Sands Pt., J1, Cold Sp. Harb., Sep, Bs (MCV).

D. quadrilamellatus Lw. Erie Co, J1; Buffalo (MCV); Ithaca, J1 (MCV) ; SI: Richmond, Watchogue, J1, Bs (MCV).

D. ramifer Lw. Beede's, Keene Val., Aug (Curr-NYS); Wells, J1, Yg (Curr) ; W. Winfield, Je, Leon (Curr); Clayville, Je, Leon (Curr); Alexandria Bay, Sep; Niagara Falls, J1; Erie Co, Je-Aug (MCV); Tompkins Co; Voorheesville, Aug, Leon (NYS); New Rochelle; LI: Sands Pt., Long Beach, Bs $(\mathrm{MCV})$.

D. reflectus Lw. Buffalo, Je (MCV); Ithaca, JI; SI: Richmond, Je, Bs (MCV); LI: Sands Pt., Je, Sep, Bs (MCV).

D. remus V. C. \& A. E. Aurora, Protection, Je-J1; Portage, J1; Gowanda ; Ithaca ; McLean, Je; Voorheesville, Je (Leon).

D. retinens V. C. \& A. Rock Glen, J1 (MCV); Tompkins Co, Je; SI: Richmond, Bs (MCV), paratype.

D. ruficornis Lw. Niagara Falls, J1; Grand I., Aug (MCV); E. Aurora, J1.

D. scapularis Lw. Lewiston, May; Erie Co, J1-Aug (MCV); Ballston L., Aug, Leon (Curr); Voorheesville, J1, Leon (Curr).

D. scoparius Lw. Adirondacks (Joh) ; Saranac Inn, J1; Manlius; Erie Co, Je-J1 $(\mathrm{MCV})$; Jamestown, Je (MCV); Tompkins Co, Je-Aug; Albany (Yg); Poughkeepsie.

D. serratus VanD. LI: Cold Sp. Harb., Je, Aug, Ds \&, Bs (MCV).

D. setifer Lw. N. Elba, J1, Leon (Curr) ; Niagara Falls, Sep; Erie Co, May-Aug (MCV) ; Little Valley, Aug; Manlius, Aug; Ithaca; Trenton Falls; Hoversville, Je, Ballston L., Aug, Leon (Curr); Albany, Je, Bish (Curr); SI : Arrochar, J1, Bs (MCV); LI: Wading R., Sep, Bs (MCV).

D. setosus Lw. Erie Co, Je (MCV); SI: Richmond, Bs (Beq).

D. slossonae V. C. \& A. Upper Ausable L., J1 (Beq) ; pond at foot of Mt. Redfield, 2500 ft., J1 (Beq) ; Colden, J1; Salamanca, J1 (MCV) ; Little Valley, Aug.

D. sincerus Mel. E. Aurora, Je (MCV); Johnstown, Aug (Curr-NYS).

D. sincerus var. subdirectus V. C. \& A. Elm L., Aug, Yg (Curr).

D. socius Lw. Winfield, Je, Leon (Curr); Salamanca, J1 (MCV).

D. socius var. gladius V. C. \& A. Tonawanda, Aug; Niagara Falls, J1; Erie Co, Je-Aug (MCV); Watervliet Reservoir, Aug, Leon (Curr).

D. splendidus Lw. Adirondacks, Je; L. Clear, L. Placid, Je (NYS) ; Buffalo, Je $(\mathrm{MCV})$; Albany, Je (NYS).

D. stenhammeri Zett. L. Tear, J1 (Beq).

D. tener Lw. Grand I., Aug (MCV) ; LI: Greenport, J1 (Leon).

D. terminalis Lw. Manlius, Aug; Grand I., Aug (MCV); Tonawanda, Aug; Genesee; Voorheesville, J1, Leon (Curr).

D. tonsus Lw. Irving, J1 (MCV); LI: Sands Pt., May-Je, Bs (MCV) ; Orient, $\mathrm{J1}(\mathrm{Beq})$. 
D. trisetosus VanD. Saranac Inn, J1 (NYS); Colden, May (MCV); McLean, Je-Jl.

D. variabilis Lw. Mt. Marcy, $4800 \mathrm{ft}$., J1 (Beq) ; L. Tear, $4300 \mathrm{ft}$. (Beq) ; Wells, J1, Yg (Curr) ; Essex Co, Aug; Keene Val., Aug (NYS); Elizabethtown, Aug, $Y g$ (NYS) ; Manlius, J1-Sep; Niagara Falls, Sep; Erie Co, J1-Sep (MCV) ; Little Valley, J1; Ithaca, J1, Sep; LI: Wading R., Cold Sp. Harb., Sep, Bs (MCV).

D. versutus V. C. \& A. Niagara Falls, Je (MCV).

D. vigilans Ald. Tonawanda, Aug; Erie Co, Je-J1 (MCV).

D. virga Coq. W. Winfield, Je, Leon (Curr-NYS); Wells, Jl, Yg (Curr-NYS); Albany, Je (Curr-NYS).

D. virginiensis V. C. \& A. Wells, J1, Yg (Curr).

D. vittatus Lw. Wells, J1, Yg (Curr); Manlius, Aug; Genesee Co; Clyde, J1 27, Bish (Leon) ; Erie Co, Je-Sep; Little Valley, Aug (MCV); Ithaca, Je-Aug; Syracuse, Blkm (Hine); Voorheesville, Jl, Leon (Curr); Nassau, J1 8 (Leon); Albany, Je (Curr) ; W. Nyack, J1, Bs (MCV, Beq) ; SI: Watchogue, Richmond, Bs (MCV).

\section{GyMNOPTERNUS Loew}

G. albiceps Lw. Erie Co, Je (MCV); Ithaca.

G. barbatulus Lw. Base of Mt. Redfield, 2500 ft., J1, Bs (MCV); Hague, J1, Leon (Curr) ; Tompkins Co, Je-J1; Clayville, Je, Leon (Curr); Voorheesville, J1, Leon (Curr); SI: Watchogue, May, Bs (MCV); Oakwood, Bs (Beq).

G. chalcochrus Lw. Erie Co, Je (MCV) ; Ellis; Tompkins Co.

G. coxalis Lw. Manlius (MCV).

G. crassicauda Lw. Wells, J1, Yg (Curr); Erie Co, Je-Aug (MCV); Tompkins Co, Je; Voorheesville, J1, Leon (Curr); Albany, Je, Yg (Curr); W. Nyack, $\mathrm{Jl}, B s(\mathrm{MCV})$.

G. debilis Lw. Tompkins Co, Je (MCV).

G. despicatus Lw. Erie Co, Je-Aug (MCV); W. Nyack, J1, Bs (MCV); LI: Farmingdale, Je, Bs (MCV).

G. difficilis Lw. Erie Co, Je-Aug (MCV); McLean, May; Albany, Je; Wells, J1; Chapel Pond, Je; SI: New Dorp, Je, Richmond, Je, Watchogue, Bs (MCV); LI: Orient, J1 (Beq).

G. exilis Lw. W. Nyack, J1 (Beq) ; Speculator, J1, Yg; Wells, J1; Adirondack. Lodge, J1.

G. flavitarsis VanD. Gloversville, Alex (MCV-CU), holotype.

G. flavus Lw. Speculator, Aug; Niagara Falls, Aug (MCV); Colden, Aug ; Ithaca, $\mathrm{J} 1$; Albany.

G. frequens Lw. Upper Ausable L., J1, Bs (MCV); Wells, Speculator, J1, Yg (Curr) ; Gloversville (MCV-CU) ; Hague, Aug, Leon (Curr) ; Peru (MCVCU); Northampton, Je, Yg (Curr); W. Winfield, Je, Leon (Curr); Erie $\mathrm{Co}$, Je-Aug (MCV); Tompkins Co, including Ringwood, Je-J1; Albany, Je (Curr-NYS).

G. humilis Lw. Erie Co, May-Jl (MCV); McLean, May; Albany, Je.

G. laevigatus Lw. Hamburg, Je (MCV).

G. lunifer Lw. W. Winfield, Je, Leon (Curr); Niagara Falls, J1; Erie Co, Je (MCV); Wells, J1, Yq. 
G. maculiventris VanD. LI: Cold Sp. Harb. (MCV).

G. meniscus Lw. Wells, J1, Yg (Curr).

G. nigricera VanD. SI: New Dorp, Watchogue, $B s$ (MCV).

G. nigrocoxa VanD. Poughkeepsie, J1, $Y g$; SI: Watchogue, J1, Bs (MCV).

G. obtusicauda VanD. Old Forge, Long L., Aug (MCV-CU), paratype; McLean Bogs, May 30 (MCV-CU), paratype.

G. opacus Lw. Erie Co, Je-Aug (MCV); McLean, J1; W. Nyack, J1 (Beq) ; Shrub Oak, Aug, Bs (MCV); LI: Sands Pt., J1, Bs (MCV).

G. parvicornis Lw. LI: Pennequid Barrens, J1 (Beq).

G. phyllophorus Lw. L. George; Wells, J1, $Y g$; Erie Co, Je (MCV); SI: Watchogue, Bs (MCV).

G. politus Lw. "NY" (Lw); Speculator, Aug; Northampton, Je, Yg; LI: Greenport, J1.

G. robustus VanD. Manlius, $\operatorname{SmHH}$ (MCV-CU), holotype.

G. scotias Lw. Mt. Whiteface, summit, J1, Fbs (MCV-CU); Grand I., Je (MCV); W. Nyack, Jl, Bs (MCV).

G. spectabilis Lw. Colden; Lancaster; Gowanda, Je (MCV); McLean, Je-J1; Voorheesville, Je, Leon (Curr).

G. subdilatatus Lw. W. Nyack, J1, Bs (MCV).

G. subulatus Lw. Upper Ausable L., Jl (Beq); Speculator, Je-Aug, Yg (Curr); Erie Co, Je-J1 (MCV); Ithaca, J1; Trenton Falls.

G. ventralis Lw. Erie Co, Je-Aug (MCV); Little Valley, Je; Albany, Je (CurrNYS) ; SI: Richmond, Je, Bs (MCV); LI: Orient, J1 (Beq).

G. vernaculus VanD. Erie Co, Je (MCV).

\section{HeRcostomus Loew}

H. ornatus VanD. Williamsville, Aug (MCV), types (Psyche, vol. 28, p. 129); Sands Pt., J1, Bs (MCV).

H. (Proarchus) violaceus VanD. Dayton, J1 (MCV), types; McLean, J1 (MCV-CU).

H. vestitus Mel, LI: Rosedale, May (MCV).

\section{PARACLIUS Loew}

P. albonotatus Lw. Colden, Aug (MCV) ; Dayton, Je (MCV); Ithaca, J1, Taughannock Falls, Je (MCV-CU).

P. alternans Lw. New Rochelle ( Lw).

P. angulatus VanD. Colden, Aug (MCV).

P. claviculatus Lw. New Rochelle (Lw); LI: Cold Sp. Harb., Aug, Bs (MCV).

P. hybridus Mel. LI: Sands Pt., Jl, Bs (MCV).

P. vicinus Ald. LI: Cold Sp. Harb., Sep, Bs (MCV); Sands Pt., J1, Bs (MCV).

\section{TACHYTRECHUS Loew}

T. binodatus Lw. Northville, Je, $Y g$ (Curr) ; E. Aurora, J1-Aug (MCV); Dayton, J1 (MCV); McLean, J1; Saratoga. 
T. moechus Lw. Wells, J1, Yg (Curr) ; Portage, J1; Erie Co, J1 (MCV); Rock Glen, J1 (MCV); Little Valley, Aug; Trenton Falls; Hoversville, Je, Leon (Curr); W. Nyack, J1 (Beq).

T. vorax Lw. Erie Co, Aug (MCV); Rock Glen, J1 (MCV); McLean, J1, Sib; Stony Pt., Je 27, Ds (MCV) ; W. Nyack, J1, Bs (MCV); SI: Richmond, Bs (MCV); LI: Wading R., Sep, Bs (MCV).

\section{Pelastoneurus Loew}

P. abbreviatus Lw. New Rochelle, Je (Lw); LI: Cold Sp. Harb., Sep, Sands Pt., $\mathrm{Jl}$, Bs (MCV).

P. laetus Lw. Buffalo, May-Aug (MCV).

P. lamellatus Lw. "NY" (Lw); LI: Gardiners I., Aug, Cold Sp. Harb., Oct (Beq).

P. longicauda Lw. "NY" (Lw); LI: Cold Sp. Harb., Sep (MCV).

P. lugubris Lw. Manlius; Buffalo, Aug (MCV); Trenton Falls; SI: Watchogue, Bs (Beq); LI: Sands Pt., Bs (MCV).

P. neglectus Wheel. Lewiston, Aug; Lancaster, Je. (MCV); Grand I., Aug; Albany.

P. ramosus VanD. Rock Glen, J1 (MCV).

P. vagans Lw. Wells, J1, Yg (Curr); W. Winfield, Je, Leon (Curr); Manlius, Aug; Erie Co, May-Aug (MCV); Jamestown, Je (MCV); Rock Glen, J1 (MCV) ; Ithaca, Je-Aug; Albany, Je (Curr-NYS); Watervliet Reservoir, Aug, Leon (Curr); Voorheesville, J1, Leon (Curr) ; Ballston L., Aug, Leon (Curr); W. Nyack, J1, Bs. (MCV); SI: Richmond, Bs (MCV); LI: Gardiners I., Aug (Beq).

\section{FAMILY EMPIDIDAE ${ }^{99 b}$}

\section{DRAPETIS Meigen}

D. aliternigra Mel. Cold Sp. Harb. (Mel).

D. pubescens Lw. "NY" (Lw).

D. septentrionalis Mel. Cold Sp. Harb. (Mel).

D. spectabilis Mel. Cold Sp. Harb. (Mel).

\section{Platypalpus Macquart}

P. aequalis Lw. Niagara Falls, Je; E. Aurora, Je, MCV; Ithaca, J1 (Mel); Manlius; Albany, Je.

P. apicalis Lw. Woodhull L., Aug; Cold Sp. Harb.

P. callithrix Mel. Niagara Falls (Mel).

P. coquilletti Mel. LI: Bayville, Bks (Mel).

P. crassifemoris Fitch. Ithaca; LI: Cold Sp. Harb.; Sea Cliff (Mel).

P. discifer Lw. Buffalo, Erie Co, Ap-Sep, MCV; McLean, Je-J1, Sib.

P. flammifer Mel. Ithaca; E. Aurora, $M C V$ (Mel).

P. flavirostris Lw. Erie Co, Je-J1, $M C V$.

P. hastatus Mel. Geneva ( $\mathrm{Mel})$.

99b The list for this family has been checked by Dr. A. L. Melander. 
P. hians Mel. var. fuscohalteratus Mel. Ithaca (Mel).

P. lateralis Lw. "NY" (Mel); McLean, Je, Sib.

P. mesogramma Lw. "NY" (Lw) ; E. Aurora, Aug, $M C V$.

P. pachycnema Lw. Tarrytown $(\mathrm{Lw})$.

P. pectinator Mel. Niagara Falls (Mel).

P. sutor Mel. "NY" (Mel).

P. tersus Coq. Woodworth's L., Aug.

P. trivialis Lw. Axton, Je; Erie Co, J1, $M C V$.

P. versutus Mel. Ithaca (Mel).

TACHYPEZA Meigen

T. fenestrata Say. Hamburg, $M C V$; Gowanda (Mel).

T. rapax Lw. Erie Co, May, Aug, $M C V$.

T. rostrata Lw. "NY" (Lw).

TACHYDROMIA Meigen

T. maculipennis Wlk. (pusilla Lw.). Erie Co, May, Je, $M C V$; Geneva (Mel); Albany, Je (NYS); LI: Sea Cliff (Mel).

Chersodromia Walker

C. houghii Mel. LI: Cold Sp. Harb., Oak I., Goose I. (Me1).

Coloboneura Melander

C. inusitata Mel. LI: Cold Sp. Harb., Oak I. (Mel).

Chelipoda Macquart

C. albiseta Zett. Ithaca (Mel).

Hemerodromia Meigen

H. capta Coq. Wells, J1, Yg.

H. empiformis Say. "NY" (Mel).

H. notata Lw. McLean, Sep, Sib.

H. palloris Coq. Woodworth's L., Aug; Hamburg, May, $M C V$.

H. rogatoris Coq. Ithaca, $\mathrm{Je}(\mathrm{Mel})$.

H. scapularis Lw. Old Forge (Ndm); Beaver Kill, Aug (CnJr).

H. valida $L w$. "NY".

Chiromantis Rondani

C. vocatoria Fall. Speculator, Aug (NYS); Ithaca.

ROEDERIODES Coquillett

R. juncta Coq. Saranac Inn, J1 29 (Coq). 


\section{Clinocera Meigen}

C. binotata Lw. "NY" (Lw).

c. conjuncta Lw. "NY" (OS-AM).

C. hamifera Mel. (MS.). Ithaca, Ap (Mel).

C. lineata Lw. Ithaca, Ap (Mel).

C. maculata Lw. "NY"; McLean, Sep, Sib.

C. taos Mel. "NY".

\section{SYNECHES Walker}

S. pusillus Lw. Ithaca, Je-J1 (Mel).

S. rufus Lw. Erie Co, May-Aug, $M C V$ (Mel).

S. simplex Wlk. Erie Co, Je; Tompkins Co, Je-Aug (Mel); Albany, Je; Freeville (Mel); LI: Cold Sp. Harb., J1 (Mel).

S. thoracicus Say. Tompkins Co, Je-Aug (Mel); Erie Co, Je-Aug; Ithaca, Je; Gowanda, MCV (Mel).

\section{SYNDYAS Loew}

S. dorsalis Lw. "NY" (Lw); Hamburg, $M C V$ (Mel).

S. polita Lw. Speculator, J1 (NYS); LI: Cold Sp. Harb. (Mel).

\section{Euнувоs Coquillett}

E. duplex Wlk. Ithaca, McLean (MCV).

E. nigripes Mel. Ithaca and vic., Je (Mel).

E. subjectus W1k. Slaterville, Je (Mel); McLean, J1 (Mel).

E. triplex Wlk. Erie Co, May, Je, $M C V$; Niagara Falls, Je (Mel); Manlius; Trenton Falls; Albany, J1; McLean, Je-J1, Sib.

\section{Нуво Meigen}

H. reversus W1k. Trenton Falls (W1k); S. Wales; Erie Co, J1-Aug, $M C V$; McLean, Aug, Sep, Sib; LI: Cold Sp. Harb.

H. slossonae Coq. Speculator, J1; Erie Co, J1-Aug, MCV; McLean, Aug-Sep, Sib; LI: Cold Sp. Harb.

\section{Oedalea Meigen}
O. ohioensis Mel. Ithaca (Mel).
O. pruinosa Coq. Gowanda, $M C V$, Ithaca (Mel).

\section{TRICHINA Meigen}

T. nura Mel. Gowanda, $M C V(\mathrm{Mel})$.

\section{ANTHALIA Zetterstedt}

A. bulbosa Mel. Kiamesha (Mel). 


\section{LEPTOPEZA Macquart}

L. compta Coq. Speculator, Aug; Niagara Falls, Je (CnJr); Erie Co, Je-Jl, $M C V$; Ithaca, Je-J1 (Mel); Albany, Je; McLean, Je, Sib.

L. disparilis Mel. Little Valley, Je, $M C V$.

L. rufipes Meig. Niagara Falls, Je ( $\mathrm{CnJr})$; Ithaca (Mel).

\section{OCYDROMIA Meigen}

O. glabricula Fall. Erie Co, May-Sep, MCV; Trenton Falls; Tompkins Co, Je, Aug-Sep, Sib (Mel); Hamburg.

\section{BLEPHAROPROCTA LOEW}

B. binummus Lw. Niagara Falls, Je (Mel); Ithaca.

B. serratula Lw. Erie Co, May-Je, $M C V$; E. Aurora, May-J1; Ithaca, Je; Niagara Falls, Je (Mel).

\section{PACHYMERIA Stephens}

P. pudica Lw. Nyack, Schott (Beq); Ithaca.

\section{EMPIS Linnaeus}

E. amystis Wlk. "NY" (Wlk).

E. armipes Lw. "NY" (Lw).

E. cucuminifer Mel. Hillburn, Schott (Beq).

E. humile Coq. McLean (Mel).

E. laevigata Lw. L. Tear, Mt. Skylight (Beq).

E. longipes Lw. L. George, OS (Lw); Niagara Falls, Je (Mel) ; Erie Co, May, $M C V$.

E. nuda Lw. Ithaca (Mel).

E. otiosa Coq. Hamburg, May, $M C V$.

E. pallida Lw. "NY" (Lw).

E. poeciloptera Lw. "NY" ( $\mathrm{Lw}$ ) ; Erie Co, Je.

E. rufescens Lw. Axton, L. Placid, Je (NYS).

E. sordida Lw. Rensselaer; E. Aurora, Je.

E. stenoptera Lw. Holland (Mel).

\section{Hilara Meigen}

H. femorata Lw. Niagara Falls, Je; Lancaster, Je, $M C V$; Ithaca (Mel).

H. gracilis Lw. Elizabeth, Aug (NYS); McLean, Aug, Sib; LI: Cold Sp. Harb. (Mel).

H. leucoptera Lw. LI: Sheepshead Bay, Je.

H. macroptera Lw. McLean, Je, Sib.

H. mutabilis Lw. Saranac Inn, Je (Coq); Albany.

H. testacea Lw. New Rochelle (OS). 
H. tristis Lw. Axton, Je (Joh); Erie Co, May-Je, MCV; McLean, Je, Sib; Upper Ausable L. (Beq).

H. umbrosa Lw. Elm L., J1 (NYS); Hamburg, Je, $M C V$.

H. unicolor Lw. Axton, Je.

OREOGETON Schiner

O. obscura Lw. Holland, May, $M C V$.

\section{CyRTOMa Meigen}

C. femorata Lw. Erie Co, May, MCV; McLean, Je, Sib.

C. halteralis Lw. Speculator, J1 (NYS) ; Erie Co, May-Je, MCV.

C. longipes Lw. Speculator, J1; Erie Co, Je-Aug, MCV; Albany, Sep (NYS); McLean, J1, Sib.

\section{RHAMPHOMYiA Meigen}

R. amplipedis Coq. LI: Yaphank (Mel).

R. angustipennis Lw. "NY" ( $\mathrm{Lw})$.

R. aperta Lw. Nineveh, Ap (NYS).

R. basalis Lw. Essex Co (NYS); Gowanda, Je, $M C V$.

R. brevis Lw. Nassau, May (NYS); N. Evans, May, MCV.

R. clavigera Lw. Nassau (NYS).

R. cophas W1k. "NY" (W1k).

R. corvina Lw. "NY" (Lw).

R. daria Wlk. "NY" (Wlk).

R. dimidiata Lw. Hamburg, E. Aurora, May, Aug, $M C V$.

R. frontalis Lw. Hamburg, May; N. Evans, May, MCV; Albany, May (NYS).

R. fumosa Lw. "NY" (Lw); Ithaca, Leon (Joh).

R. gilvipes Lw. Adirondacks, J1 (NYS); Buffalo, Je; Grand I., Je; L. Tear (Beq).

R. gracilis Lw. Elizabeth, Aug (NYS) ; McLean, Aug, Sep, Sib; Oliverea, Catskills (Beq).

R. irregularis Lw. Colden, May.

R. laevigata Lw. Erie Co, Je, $M C V$.

R. liturata Lw. Holland, May, $M C V$.

R. longicauda Lw. Albany, J1 (NYS); Poughkeepsie, Je.

R. luctifera Lw. "NY" (Lw); Ithaca (Mel).

R. luteiventris Lw. Holland, May, MCV; L. Tear (Beq).

R. macilenta Lw. Mt. Marcy, J1 (NYS).

R. manca Coq. LI: Cold Sp. Harb. (Mel).

R. minytus Wlk. Nassau, May (NYS).

R. mutabilis Lw. Nassau, May (NYS); McLean, Sep, Sib.

R. nigricans Lw. Axton, Je; Nassau, May (NYS).

R. polita Lw. Gowanda, Je, $M C V$.

R. pulchra Lw. "NY" (Lw).

R. pulla Lw. Albany, May (NYS); McLean, Je, Sib. 
R. rustica Lw. Axton, Je.

R. sordida Lw. "NY" (Lw).

R. testacea Lw. Karner, Ap (NYS).

R. umbrosa Lw. Mt. Marcy, L. Tear (Beq).

R. valga Coq. Poughkeepsie, May (NYS).

R. virgata Cog. Axton, Je.

\section{FAMILY LONCHOPTERIDAE}

LONCHOPTERA Meigen

L. furcata Fall. L. Tear, $4300 \mathrm{ft}$ (Beq); W. Nyack, Beq (Jn).

\section{FAMILy PHORIDAE 100 \\ TRUPHEONEURA Malloch}

T. microcephala Lw. Ithaca, Ap, Joh (Mall) ; Albany, Ap, Yg; Erie Co, Aug-Oct $(\mathrm{MCV})$.

\section{Chaetoneurophora Malloch}

C. luggeri Ald. Niagara Falls, Je; E. Aurora, Je, Aug (MCV); Ithaca, May, Joh (Mall).

C. olympiae Ald. Ithaca, Joh (Mall).

C. spinipes Coq. Ithaca, Joh (Mall).

\section{PARASPINIPHORA Malloch}

P. bergenstammi Mik. Ithaca, J1 (Brues).

P. multiseriata Ald. Wells, J1 (Yg); E. Aurora, Aug (MCV); Ithaca (Brues).

P. pachyneura Lw. Colden, May (MCV).

P. slossonae Mall. Ithaca, Je, Joh (Mall).

P. spinulosa Mall. Ithaca, Joh (Mall).

\section{DOHRNIPHORA Dahl}

D. concinna Meig. (nitidifrons Brues). Erie Co, J1-Sep (MCV); Rochester, Oct (MCV); Ithaca, J1-Sep (Brues).

D. venusta Coq. Ithaca, Feb (Brues).

\section{HYPOCERA Lioy}

H. ehrmanni Brues. Niagara Falls, May (MCV).

H. flavimana Meig. Gloversville, J1; Albany, Ap; McLean, Ap, Sep, Sib (Joh).

H. johnsoni Brues. LI: Wyandanch, Schott (Beq).

H. mordellaria Fall. Colden, May (MCV).

100 The manuscript of the list for this family has been checked by C. T. Brues, who has made several corrections and additions. 
Phalacrotophora Enderlein

P. speirae Brues. Grand I., Je (MCV).

P. longifrons Brues. Ithaca, Joh (Mall).

\section{MEgaselida Rondani}

\section{(Aphiochaeta Brues)}

M. agarici Lint. LI: Glen Cove, Oct (Mall).

M. albidohalteris Felt. Erie Co, May, Oct (MCV); Ithaca, Je.

M. aletiae Comst. Ithaca, Joh (Mall).

M. borealis Mall. N. Evans (MCV).

M. brunnipes Mall. Lancaster, Mar (MCV).

M. cayuga Mall. Ithaca, Aug, Joh (Mal1).

M. ciliata Zett. Ithaca, Aug, Joh (Mall).

M. difficilis Mall. Ithaca, Aug, Joh (Mall).

M. flava Fall. Buffalo, Grand I., J1; Niagara Falls, J1 (MCV); Ithaca, Aug (Mall).

M. fungicola Coq. Ithaca (Brues).

M. inornata Mall. Ithaca, Joh (Mall).

M. iroquoiana Mall. Ithaca, Joh (Mall).

M. johannseni Mall. Ithaca, Joh (Mall).

M. lutea Meig. Ithaca, Joh (Mal1).

M. minor Zett. Ithaca (Brues).

M. nigriceps Lw. Ithaca (Brues); NYC (Beq).

M. peregrina Mall. Niagara Falls, J1 (MCV).

M. picta Lehm. Colden; N. Evans, May (MCV); Ithaca (Mall).

M. pulicaria Fall. Buffalo (MCV); Ithaca (Mall).

M. ruficornis Meig. Ithaca, Joh (Mall).

M. rufipes Meig. Fulton Co; Buffalo, Je, Sep (MCV) ; Ithaca, J1 (Mall) ; McLean, Claas (Joh); Albany, Sep, Ap; NYC (Brues).

M. rusticata Mall. Ithaca, Ap, Joh (Mall).

M. scalaris Lw. Colden; Lancaster, May, Je (MCV).

M. straminea Mall. Ithaca, Aug, Joh (Mall).

M. straminipes Mall. Ithaca, Ap, Aug, Joh (Mall).

M. subatonella Mall. Ithaca, Joh (Mal1).

M. tertia Brues (inaequalis Mall.). Ithaca, Joh (Mall).

\section{Apocephalus Coquillett}

A. coquilletti Mall. Ithaca, Oct (Joh).

A. wheeleri Brues. Colden, Aug (MCV).

\section{GYMNOPHORA Macquart}

G. quartomollis Schmitz (arcuata auct. not Meig.). Niagara Falls, Je (Jn); Erie Co, Je, Oct (MCV); Ithaca, Je-J1. 
PHORA Latreille

(Trineura Meigen)

P. aterrima Fab. Fulton Co, Aug; Chapel Pond, Je, Leon (Yg); Ithaca, Mar, Sep, Joh (Mall); Albany, May; NYC (Brues).

P. velutina Meig. L. Placid, Aug (MCV).

\section{CONICERA Meigen}

C. dauci Meig. Westfield, J1; Ithaca, Aug; Sullivan Co (Brues).

\section{PULICIPHORA Dahl}

P. glacialis Mall. Ithaca.

\section{FAMILY PLATYPEZIDAE}

\section{Agathomyia Verrall}

A. canadensis Jn. McLean, Aug, Sib (Joh).

A. cushmani Jn. Boston, Sep (MCV).

A. notata Lw. Niagara Falls, J1; Erie Co, J1, Aug (MCV).

A. perplexa John. Elma, Aug 24 (MCV); McLean, Sep (Sib).

A. talpula Lw. Colden, May (MCV); Cayuga L. (Joh).

A. vanduzeei Jn. Springville, Sep (MCV); Colden, Aug (MCV); McLean, Sep, Sib (Joh).

\section{Callimyia Meigen}

C. venusta Snow. Elizabethtown, Aug ( $\mathrm{Yg})$; Lancaster, Je (MCV).

\section{Platypeza Meigen}

P. anthrax Lw. Niagara Falls, Sep (MCV); Little Valley, J1 (MCV); Ithaca, J1-Sep (Joh).

P. flavicornis Lw. Elma, Colden, E. Aurora, Niagara Falls, Aug-Sep (MCV).

P. infumata Haliday. E. Aurora, Gowanda, Colden, May-J1 (MCV).

P. minorata Bks. Colden, Aug (MCV).

P. pallipes Lw. (Calotarsa ornatipes Towns.). Ithaca (Bks); Ft. Montgomery, Schott (Beq).

P. taeniata Snow. Niagara Falls, Sep (MCV); E. Aurora, Aug; Boston, Sep, Little Valley, J1 (MCV); Elmira, Oct; Ithaca, Sep (Jn).

P. velutina Lw. Niagara Falls, Je-J1 (Jn) ; E. Aurora, Aug-Sep (MCV) ; Lancaster, Aug (MCV); Jamestown, Je (MCV); Ithaca, J1-Sep; SI (Beq).

Platypezoides Johnson

P. diversa Jn. Niagara Falls, Sep, $M C V$ (Jn), paratype.

Microsania Zetterstedt

M. stigmaticalis Zett (Platycnema imperfecta Lw.). Ithaca, May (Joh). 


\section{FAMILy PIPUNCULIDAE}

(Dorylaidae)

\section{ChalaRus Walker}

C. spurius Fall. Niagara Falls and vic., J1 ( $\left.\mathrm{MCV}^{\top}\right)$; Ithaca vic., May-J1 (Joh); Albany, May.

\section{VERRALLIA Mik}

V. pilosa Zett. LI: Wyandanch, J1 (Beq) ; foot of Cliff Mt., Essex Co, $3000 \mathrm{ft}$. (Beq).

\section{NEPHROCERUS Zetterstedt}

N. daeckei Joh. Rock City, Je (Snn); Poughkeepsie, Je; LI, J1 (Jn).

\section{Pipunculus Latreille}

P. aequus Cress. Ft. Montgomery, Schott (Beq).

P. affinis Cress. Rock City, Je; Vandalia, Je; Ithaca and vic., May-J1 (Joh).

P. appendiculatus Cress. Niagara Falls, Je (MCV); LI: Sea Cliff ( $\mathrm{CnJr}$ ).

P. atlanticus Hough. Axton, Je (Joh); Erie Co, May-Je; Niagara Falls, Je (MCV) ; Ithaca, Je, Sep (CnJr) ; Poughkeepsie, Je; LI.

P. caudatus Cress. LI: Sea Cliff, Sep $(\mathrm{CnJr})$.

P. cingulatus Lw. Johnstown, Je; Erie Co, Je, Sep (MCV); Vandalia; Ithaca, Aug, Sep (CnJr).

P. cingulatus var. velutinus Cress. Lancaster, Je, N. Evans, Aug (MCV).

P. confraternus Bks. LI: Sea Cliff (Bks).

P. coquilletti Kertesz. Long L., Aug (Kertesz).

P. discolor Bks. Ithaca, J1 (Bks).

P. fuscus Lw. Colden, Aug (MCV) ; Ithaca, Je (Joh); Poughkeepsie, Je.

P. houghi Kertesz (femoratus Cress.). Elma, Aug (MCV); Ithaca, Je-Sep $(\mathrm{CnJr})$; Colden, Aug (MCV).

P. horvathi Kertesz. Long L., Aug; Salamanca, J1 (MCV); Ithaca, May, J1, Sep $(\mathrm{CnJr})$.

P. minor Cress. Erie Co, May-Aug (MCV); Ithaca and vic., May-Aug (CnJr).

P. nigripes Lw. Ithaca ( $\mathrm{CnJr}$ ) ; SI (Beq).

P. pallipes John. Ithaca, Aug (CnJr); Upper Ausable L. (Beq).

P. republicae Wlk. (fuscus Lw.?). "NY" (Wlk).

P. scoparius Cress. Beaver Kill (CnJr).

P. similis Hough. Erie Co, Je-Sep (MCV); LI: Sea Cliff (CnJr).

P. sororius Cress. Ithaca ( $\mathrm{CnJr}$ ); LI: Sea Cliff.

P. subvirescens Lw. Axton, Je; E. Aurora, J1 (MCV); Ithaca, May-Aug.

P. tarsalis Cress. Ithaca, J1 $(\mathrm{CnJr})$.

\section{FAMILY SYRPHIDAE}

\section{MICRODON Meigen}

M. champlaini Curr. Ft. Montgomery, Schott.

M. cothurnatus Bigot. Ithaca, Je. 
M. globosus Fab. (fuscipennis auct. not Macq.). Keene Val., Je (Not); Hamburg, J1 (MCV); Ithaca; McLean; Katonah, Je ; Ft. Montgomery, Schott; SI, Bs.

M. megalogaster Snow (bombiformis Towns.). Little Valley, J1 (MCV); SI, Je, Ds; LI: Jamaica, Je, Ds; Jericho (Bell).

M. tristis Lw. Old Forge, Je; Axton, Je; Black Br., Je; Niagara Co, May-Je (MCV) ; Little Valley, Je (MCV) ; Ithaca, Je-J1; Binghamton, Ap; Hudson R. Val.; Ft. Montgomery (Schott); SI, Je, Bs; LI: Farmingdale, Je, Bs.

\section{Chrysotoxum Meigen}

C. derivatum Wlk. Saranac L., J1; Poughkeepsie, Je.

C. fasciolatum DeG. Keene Val. (Not).

C. laterale Lw. Keene Val.; McLean, Sep, Hd (Joh); Oliverea (Beq); LI: Huntington (Schott); Gardiners I. (Beq).

C. perplexum John. L. George, Sep, Leon (Snn).

C. pubescens Lw. (luteopilosum Curr.). Erie Co, Aug-Sep (MCV); Inwood, NYC (Schott); SI (Bs); LI (Bs).

\section{MIXOGASTER Macquart}

M. breviventris Kahl. W. Nyack, J1; Mosholu, J1-Aug (Bno); L. Oscawana, Interstate Pk., Sep (Bs) ; LI: Yaphank, Sep, Ds (CnJr); Cold Sp. Harb., Sep.

\section{Chrysogaster Meigen}

C. antitheus Wlk. "NY", type locality. Not recognized. Probably the same as C. nigripes $\mathrm{Lw}$.

C. ithaca Snn. Ithaca (Snn).

C. nigripes Lw. Abundant. Colden, Gowanda, Je (MCV); Ft. Potter, J1; Ithaca, J1; Poughkeepsie; W. Nyack, J1, Bs; Ft. Montgomery (Schott) ; SI, May (Bs) ; LI: Sea Cliff, Je; Cold Sp. Harb.

C. nitida Wied. Buffalo, Je (MCV); Ithaca, J1; SI, May-Sep (Bs); LI: Sea Cliff, Je-J1.

C. pictipennis Lw. Ithaca; Caroline, May-Je; Ellis, Je; Van Cort. Pk.

C. pulchella Will. Abundant. Old Forge, J1; Elba, Je (MCV); Ithaca and vic., May-Aug; Albany, May; Van Cort. Pk.

\section{PIPIZA Fallén}

P. femoralis Lw. Keene Val. (Not); Hamburg, Je (MCV); Ithaca, May-Je (Snn); Nyack, May, Bs; SI, May, Ds.

P. femoralis var. albipilosa Will. Essex Co, Aug; Rock City, Ds; Ithaca, J1; Poughkeepsie, Sep; Pine I.; Nyack (Schott).

P. nigropilosa Will. SI, J1, Bs.

P. puella Will. Nyack, Schott; SI (Beq).

P. quadrimaculata Panz. Keene Val., Je.

\section{Pipizella Rondani}

P. apisaon Wlk. (Pipiza modesta Lw., nigribarbis Lw., pulchella Will.). "NY", type locality; SI: $B s$; Richmond, Ds; LI: Sands Pt., Bs.

P. pubescens Lw. Hudson R. val.; Ithaca, Je-J1; Hillburn, Schott. 
HERINGIA Rondani

H. salax Lw. (Pipiza radicum Walsh \& Rly., P. pistica Will.). Ithaca; Ft. Montgomery, Schott; SI, Bs; LI: Farmingdale, Sands Pt., Wading R., Bs.

\section{CNEMOdON Egger}

C. calcarata Lw. Ithaca, May-J1.

C. coxalis Curr. Ithaca, Je, Snn.

C. pisticoides Will. Essex Co, J1; Axton, Je; L. Tear, 4300 ft., J1 (Beq) ; Ithaca, May.

\section{PARAGUS Latreille}

P. angustifrons Lw. Fulton Co; Ithaca, May; Ft. Montgomery, J1 (Ds) ; SI, JeSep (Bs,Ds) ; LI: Sea Cliff, Sep; Wyandanch (Schott).

P. bicolor Fab. Essex and Fulton Counties, Colden, Lewiston, May-J1 (MCV); Niagara Falls, Je; Ithaca, Je; Ft. Montgomery (Schott); SI, May (Bs).

P. tibialis Fall. Axton, Je; Ilion, May; Ithaca, Je; SI, Je (Bs); LI: Sea Cliff, J1.

\section{Chilosia Meigen}

C. orilliaensis Curr. Freeville; McLean; Caroline; Danby, Ap-May, on Caltha palustris; Ithaca, Snn.

\section{Cartosyrphus Bigot}

C. cyanescens Lw. Ithaca, Je-J1.

C. pallipes Lw. Keene Val., Je (Not); Long L., Ds; Cedarville, Je; Ithaca, J1; L. George, Sep.

C. similis Snn. L. Placid, Sep; Ithaca, Sep; SI.

C. slossonae Snn. Keene Val., May (Not).

C. tristis Lw. Keene Val., Je; Ithaca, May-J1.

Chalcomyia Williston

C. aerea Lw. McLean, May.

MyIOLEPTA Newman

M. nigra Lw. Keene Val., Je-J1 (Not); Hamburg, Je (MCV); Ithaca, May ; West Pt. (Ds).

M. varipes Lw. Keene Val., Je (Not); Ithaca.

\section{Eumyiolepta Shannon}

E. strigilata Lw. Ithaca, Je.

Callicera Panzer

C. johnsoni Hunter. Danby, Je; SI: Grasmere, Ap, Bs. 


\section{FERDINANDEA Rondani}

(Chrysochlamys Rondani)

F. buccata Lw. Ithaca, May-Je; Suffern, May, Schott.

F. dives O.S. Ithaca, Sep; Ft. Montgomery (Schott); SI, Aug (Bs).

F. nigripes O.S. Ithaca, May.

\section{ВассHA Fabricius}

B. cognata Lw. Ithaca, J1; SI, Aug (Bs).

B. fascipennis Wied. (aurinota Wlk.). Keene Val., J1-Aug; Niagara Falls, Sep, Erie Co, J1-Sep (MCV) ; Lake Ridge, Aug ; New Baltimore, Aug ; Mt. Vernon, Oct; Inwood, NYC (Schott); SI, Aug-Sep (Bs) ; LI: Sea Cliff, J1-Aug; Wading R., Sep, Sands Pt., J1-Sep (Bs).

B. tarchetius Wlk. Erie Co, Je, Aug, Lewiston, May (MCV); Van Cort. Pk.; SI, Sep (Bs).

\section{OCYPTAMUS Macquart}

O. fuscipennis Say. Niagara Falls, Sep (MCV); Ithaca; Ft. Montgomery, Schott; W. Nyack (Beq) ; Inwood, NYC, Schott; SI: Richmond, Jl-Aug, Ds (Beq) ; LI: Flushing, Aug, Ds (CnJr) ; Sands Pt., Bs; Cold Sp. Harb.; Little Neck, Schott.

\section{PyROPHAENA Schiner}

P. granditarsus Forst. (ocymi Fab.). Old Forge, Aug; Elba (MCV); Pine I., Je, Ds (Beq); SI, Je (Bs).

P. rosarum Fab. Caroline, May; LI: Queens (Schott).

\section{Platychirus St. Fargeau et Serville}

P. chaetopodus Will. Essex Co (Snn); Ithaca (Snn); Nassau, J1-Aug; Van Cort. Pk.; SI, Je (Bs); LI.

P. hyperboreus Staeger. Upper Ausable L.; Axton; Erie Co, May-Aug (MCV); Ithaca; Van Cort. Pk.; LI: Glendale (Schott); Queens (Schott); Sea Cliff.

P. peltatus Meig. L. Tear; Erie Co, May-Jl (MCV); Ithaca, May-Je, Aug (Snn) ; Karner; Nyack (Schott); LI: Cold Sp. Harb.; Flushing (Schott).

P. perpallidus Verrall. L. Tear (Beq).

P. quadratus Say. Saranac L.; Erie Co, May-Aug (MCV); Ithaca, Ap-Sep (Bs) ; Albany ; Van Cort. Pk.; SI, May-Aug (Bs) ; LI: Glendale (Schott) ; Queens (Schott).

\section{Melanostoma Schiner}

M. mellinum L. Adirondacks; Mt. Whiteface, J1, Ds (Jn); Upper Ausable; Erie Co, May-Sep (MCV) ; Ithaca, Ap-Sep; Hudson R. Val.; Pine I., Je, Ds (Jn) ; LI: Huntington (Schott).

M. montivagum Jn. Mt. Marcy, L. Tear, foot of Cliff Mt. (Beq).

M. obscurum Say. Essex Co; Mt. Skylight; L. Tear; Mt. Marcy; Wilmington, Aug, Ds; Erie Co, Ap-Oct (MCV) ; Rock City, Je, Ds; Ithaca, J1; Oliverea; SI, Ap-Nov; LI: Sea Cliff, May; Sands Pt., Ji. 


\section{LEUCOZONA Schiner}

L. lucorum L. var. americana Curr. Keene Val., Je (Snn); Mt. Skylight, 4800 ft. (Beq).

\section{DideA Macquart}

D. fasciata Macq. var. fuscipes Will. Erie Co, May-Je (MCV); Ithaca, Je; L. George, Sep; Karner, May; NYC; SI (Bs).

\section{SYRPHUS Fabricius}

S. amalopis O.S. Old Forge, J1; Axton, Je; Mt. Marcy, Je-J1 (Beq) ; Ithaca, May; Nassau, May; Oliverea.

S. arcuatus Fall. Adirondacks, Je; N. Evans, May (MCV); Axton, Je; Keene Val., May-J1; Mt. Marcy; Ithaca, Ap-Je; SI, May-Jl (Bs); LI: Kings Pk., $\mathrm{J1}(\mathrm{Bs}) ; \mathrm{J1}$-Sep.

S. disjectus Will. Keene Val., J1 (Not); N. Evans, May.

S. divisus Will. Niagara Falls, Je; Lancaster, Sep (MCV); Oliverea; SI, Sep (Bs).

S. emarginatus Say. Niagara Falls, Je, Gowanda, Aug (MCV); Ithaca, Je-Aug; Ft. Montgomery (Schott); Stony Pt. (Schott); Inwood, NYC (Schott); SI, Sep (Bs); LI: Cold Sp. Harb.

S. felix O.S. Piseco L., Aug; Grand I., Sep (MCV) ; Nassau, May; West Pt., Sep; Ft. Montgomery (Schott); SI, May-Sep (Bs); LI: Flushing.

S. knabi Snn. Ithaca, J1-Aug; Ft. Montgomery (Schott); SI, May-Sep (Bs); LI: Huntington (Schott).

S. latifasciatus Macq. (abbreviatus Will. not Zett.). SI, Je, Bs.

S. laxus O.S. Old Forge, J1; Essex Co, J1; Upper Ausable L.; Mt. Skylight; L. Tear; Woodhull L., Aug; L. George, Sep.

S. nitens Zett. Nyack (Schott); SI (Curr); LI: Farmingdale (Curr).

S. perplexus Osburn. Mt. Marcy; Keene Val., Ap-J1; Mt. Skylight; Herkimer; Niagara Falls, Sep (MCV); Buffalo, Colden, May (MCV); Rock City, Je, Ds (Osburn); Ithaca; Taughannock Falls; Nyack (Schott); Suffern (Schott); White Plains; SI, Ap-Sep (Bs); LI: Flushing; Queens (Schott).

S. rectus O.S. Herkimer, Aug; Erie Co, J1-Oct (MCV); Ithaca, Ap-Aug; Ft. Montgomery (Schotgt); SI, J1-Sep (Bs); LI: Sands Pt.; Cold Sp. Harb., Sep (Bs).

S. ribesii L. Adirondacks; Niagara Falls, Je; Erie Co, Sep (MCV); Manlius; Ithaca, J1; Catskills; West Pt.; Hillburn (Schott); Suffern, May (Bs); Bronx Pk., J1; SI: common (Bs); Clove Val., May, J1, Ds (Beq); Richmond, May, Ds (Beq); LI: Sea Cliff, J1; Cold Sp. Harb., Aug, Sands Pt., Je-Oct (Bs).

S. ribesii var. vittafrons Sh. Inwood, NYC (Schott).

S. sodalis Will. Mt. Marcy (Beq).

S. torvus O.S. Adirondacks, J1; L. Tear; Mt. Marcy; Niagara Falls, Je; Buffalo, Ap, Oct (MCV) ; Ithaca, May; Oliverea ; Ft. Montgomery (Schott); SI, ApMay (Bs); Clove Val., Ds; LI: Flatbush; Glendale (Schott).

S. venustus Meig. Keene Val., May (Not); Oliverea.

S. vitripennis Mg. Inwood, NYC (Schott); LI: Flatbush, Flushing (Schott). 
S. wiedemanii Jn. (americamus Wied.). Abundant. Essex Co, Aug; Niagara Falls, Je; Erie Co, Ap-Sep (MCV) ; Ithaca; LI: Cold Sp. Harb.; Flushing; Flatbush.

EPISTROPHE Walker

(Stenosyrphus Matsumura)

E. arcticus Zett. Mt. Marcy (Beq).

E. diversipes Macq. Old Forge, Je; Mt. Marcy, J1; L. Tear; Ithaca, May, Oct; L. George, Aug-Sep; Catskills, Aug; Oliverea; Inwood, NYC (Schott).

E. fisherii Walton. Ithaca, Je (Snn).

E. genualis Will. Keene Val., Je; L. Tear; Ithaca, May.

E. grossulariae Mg. L. Placid, Aug; Essex Co: Old Forge, J1; Long L., Ds, as Stenosyrphus lesueurii Macq.; L. Tear; Mt. Marcy; Erie Co, Ap; Ithaca, May-Je, Aug; L. George, Sep; Saratoga; South L., Catskills, Aug, Ds; Oliverea; Ft. Montgomery (Schott).

E. nudifrons Curr. Mt. Marcy, Je, Fbs (Curr).

E. xanthostomus Will. Hamburg, May (MCV) ; L. Keuka, May; Ithaca, MayAug; Trenton Falls, Je; Hillburn, Pine I. (Schott); SI: Richmond, May (Ds); LI: Sands Pt., Je.

\section{XANTHOGRAMMA Schiner}

X. flávipes Lw. Niagara Falls; Lewiston, May, Erie Co, May-J1 (MCV); Ithaca, Je-JI; McLean, Je ; Poughkeepsie ; Ft. Montgomery (Schott) ; Mosholu, May, Ds; SI, Je-Aug (Bs) ; LI: Sands Pt., Je, Cold Sp. Harb., Aug (Bs); Gardiners I.

\section{DoRos Meigen}

D. aequalis Lw. Keene Val., May-Je; Big Ind. Val. (Pears); Ramapo, May (Ds); LI: Wading R. (Ds).

\section{Allograpta Osten Sacken}

A. obliqua Say. Keene Val., Oct; Mt. Marcy; Erie Co, Je-Oct; Ithaca, J1-Nov; Albany; Nyack (Schott); Ft. Montgomery (Schott); SI, May-Sep (Bs) ; LI: Fire I., Glendale (Schott); Sea Cliff, J1.

\section{TOXOMERUS Macquart}

\section{(Mesogramma Loew)}

T. geminatus Say. Abundant. Fulton Co; Manlius; Otto; Erie Co, May-Aug (MCV) ; Ithaca, May-Aug; Albany; E. Jewett, Aug, Ds; West Pt., Je, Ds; SI, Je-Oct (Bs) ; LI: Sands Pt., J1 (Bs) ; Central Pk.; Glendale; Flatbush.

T. marginatus Say. Abundant. Adirondacks; Upper Ausable L.; Genesee Co, Je, Ds; Erie Co, Je-Oct (MCV) ; Ithaca, J1-Oct; SI, May-Je, Sep (Bs) ; LI: Sea Cliff; Aqueduct, Sep, Ds; Fire I. (Schott); Gardiners I.; Wyandanch.

T. politus Say. Erie Co, Aug-Oct (MCV); Ithaca, May-Oct; Albany, Sep; Poughkeepsie, Oct; Ramapo, May, Ds; Inwood, NYC (Schott); SI, AugSep (Bs) ; south shore, Jl, Ds; Watchogue, May (Ds); LI: Barker's Pt., Oct, Wading R., Aug (Bs); Glendale (Schott). 
SphaERophoria St. Fargeau et Serville

S. cylindrica Say. Abundant. Adirondacks; N. Elba, Oct, Wat; Manlius; Honeoye Falls, Je, Ds; Genesee Co, Je, Ds; Erie Co, May-Oct (MCV); Ithaca and vic., May, Oct; Albany; Oliverea ; Stony Pt., Je, Ds; Southfields (Schott); White Plains; SI, common, May-Oct (Bs); Tottenville, Je-Jl, Ds; LI: Wading R., Je, Ds; Flushing; Huntington (Schott); Sea Cliff.

S. menthastri L. Upper Ausable L.; Ithaca, Je-J1.

\section{SPHEgINA Meigen}

S. campanulata Robt. Keene Val., Je (Not); Ithaca, Je.

S. flavimanus Mall. Ithaca (Mall).

S. keeniana Will. Keene Val., Je; Upper Ausable L.; Erie Co, May-Je (MCV); Little Valley, Je (MCV); Ithaca, May.

S. lobata Lw. Keene Val., Je; Erie Co, May-Je; Ithaca, May-J1; Karner, J1; SI, May (Bs) ; LI: Sea Cliff, May.

S. rufiventris Lw. Keene Val., Je-J1; Saranac L.; Old Forge; Ithaca, May-J1; Ft. Edward; Ft. Montgomery (Schott).

\section{NEOASCIA Williston}

N. distincta Will. Colden, May (MCV); Ithaca, May.

N. globosa Wlk. W. Winfield, Je (Yg-NYS); N. Fair Haven, Ap-J1; Erie Co, May (MCV) ; Ithaca, May-J1; Freeville; Danby; Hudson R. Val., May; Yonkers; Ft. Montgomery (Schott); SI, Je-J1 (Bs) ; LI: Queens (Schott) ; Sea Cliff, May.

\section{RHINGIA Scopoli}

R. nasica Say. Abundant, May-Sep. Manlius; Conesus L., Je, Ds (Beq); Ithaca and vic., May-Aug; Hudson R. Val.; Helderbergs, J1, Ds; Oliverea, Je, Ds; Ft. Montgomery (Schott); SI, May-Sep (Bs); LI: Sea Cliff; Long Beach, etc. Common everywhere.

HAMMERSCHMIDTIA Schummel

H. ferruginea Scop. Keene Val., Je (Not).

\section{BRACHYOPA Meigen}

B. notata O.S. Keene Val., Je (Not); Ithaca, May.

B. perplexa Curr. "NY" (Curr).

B. vacua O.S. Old Forge, Je; Fourth L., Fulton Chain (Dickerson); Oswego (Snn).

\section{VoluCELla Geoffroy}

V. bombylans L. subsp. evecta Wlk. Adirondacks; N. Fair Haven; Gowanda, Je (MCV) ; Ithaca, Je-J1; Taughannock, Je-J1; Hudson R. Val.; Oliverea; Ft. Montgomery, Southfields (Schott); W. Nyack, J1 (Bs); LI: Sea Cliff.

V. bombylans subsp. evecta var. americana Jn. Oliverea; Ft. Montgomery; LI: Flushing, Wyandanch. 
V. bombylans subsp. facialis Will. var. lateralis Jn., Keene Val., J1 (Not).

V. fasciata Macq. LI: Gardiners I. (Beq).

V. obesa Fab. SI (Bs\&Beq).

V. vesiculosa Fab. Ithaca, Je-J1; Poughkeepsie; LI: Cold Sp. Harb.

\section{CONDIDEA Coquillett}

C. lata Coq. Ft. Montgomery, Je, Schott.

\section{SERICOMYIA Meigen}

S. bifasciata Will. Axton, Je.

S. chrysotoxoides Macq. Axton; Keene Val., Je-Oct; Adirondacks; Fulton Co; Upper Ausable; L. Tear; Old Forge; Ray Brook; Portage, Je, Ds; Erie Co, Jl-Sep (MCV) ; Ithaca, Je-Aug; McLean; Caroline; Trenton Falls; Albany; Manlius; Oliverea; Inwood, NYC (Schott); SI, Oct, Ds; LI: Wyandanch; Flatbush; Cold Sp. Harb., Sep (Bs).

S. militaris W1k. Mt. Whiteface, J1, Ds; Adirondacks, Je-Aug; Keene Val. (Not); Upper Ausable L.; Old Forge; Mt. Marcy; Hamilton Co, Ds; Lewiston, May $(\mathrm{MCV})$; Oswego; Erie Co, Je-Sep (MCV) ; Ithaca; L. George, Je-Sep.

\section{ERISTALIS Latreille}

E. aeneus Scop. Erie Co, Sep-Oct (MCV); Karner; NYC, Sep, Ds (Beq); SI: Ap-Sep (Bs) ; Watchogue, May, Ds (Beq) ; visits willow catkins, Ds (Beq); LI: Sea Cliff; Aqueduct, J1, Ds (CnJr); Long Beach; Flatbush; Fire I. (Schott); Sands Pt., J1 (Bs). Enters houses in fall like E. tenax (Bs).

E. anthorinus Fall. (montanus Will.). White Plains (Snn).

E. arbustorum L. Abundant. Genesee Co, J1, Ds; Ithaca, May-Oct; E. Jewett, Aug, Ds; Pine I., Ds; South L., Catskills, Aug, Ds; SI, taken in Mar on coltsfoot blossoms (Bs) ; Watchogue, Clove Val., J1-Aug, Ds; LI: Rockaway Beach, Je, Ds (CnJr); Half Way Hollow Hills, J1, Ds (CnJr). Common everywhere.

E. bastardi Macq. Keene Val.; Saranac L.; Mt. Skylight; Old Forge; Alexandria Bay; Manlius; N. Fair Haven; Erie Co, Aug-Sep (MCV) ; Ithaca, May-Sep; McLean; Albany, May-Oct; South L., Catskills, Aug, Ds (Beq) ; Southfields (Schott); NYC, Aug, Ds (Beq); Inwood; SI, May-Sep (Bs); LI: Canarsie; Oakdale (Bell).

E. brausi Will. (meigenii auct., not Wied.). Old Forge; Manlius; Wolcott; Erie Co., Je-Aug; Albany; LI: Brooklyn, May, $D s$.

E. compactus W1k. McLean; Freeville; Caroline, Ap-J1; Ithaca (Snn). Abundant on Caltha palustris.

E. dimidiatus Wied. Adirondacks; Mt. Marcy; Manlius, Ap-Sep; Erie Co, JeSep (MCV); Ithaca; McLean; Albany ; Pine I., Je, Ds; Southfields (Schott) ; Oliverea; Hillburn (Schott); Ft. Montgomery (Schott); SI, Je-Sep (Bs); visiting willow catkins, May, Ds; Arrochar, Sep, Ds; Richmond, May; LI: Maspeth, Sep; Sea Cliff ; Farmingdale; Brooklyn.

E. flavipes W1k. Fairly abundant. Piseco and Saranac Lakes, Ap-May; McLean, Aug; Erie Co, Sep-Oct (MCV); Oliverea; Poughkeepsie; SI, Jl-Sep (Bs), Aug, Ds (Jn); LI: Sea Cliff, Aug; Wyandanch (Schott); Orient. 
E. saxorum Wied. N. Fair Haven, May-Aug ; Erie Co, May-Oct (MCV) ; Ithaca, Je-J1; McLean; Poughkeepsie; Ft. Montgomery (Schott) ; SI, Je (Bs); LI: Sands Pt. (Bs); Gardiners I.

E. tenax L. Very abundant. Adirondacks; Mt. Whiteface, summit and trail, Ds; Mt. Marcy, Oct, Ds; Erie Co, J1-Sep (MCV) ; Ithaca, etc., Ap-Oct; Hudson R. Val.; Debruce, Sep, Ds; SI, Jan-Oct, Ds; LI: Central Pk., Yaphank, Montauk, Calverton, Amagansett, Ap-Sep, Ds (CnJr). Everywhere.

E. transversus Wied. Very abundant. Adirondacks; Batavia, Je, Ds; Erie Co, May-Sep (MCV) ; Ithaca, May-Sep; Manlius; Pine I., Je, Ds; Albany; Mosholu, May, Ds; White Plains; SI: Richmond, Clove Val., Arrochar, Tottenville, Je-Oct, Ds; May-Oct (Bs) ; LI: Wyandanch, May, Yaphank, Sep, Ds (CnJr) ; Gardiners I.; Orient; Sands Pt., Oct (Bs).

\section{Tropidia Meigen}

T. quadrata Say. Abundant. Erie Co, May-Sep (MCV) ; Ithaca, Je-Sep ; Albany, J1; Pine I., Je, Ds; Ft. Montgomery (O1s); White Plains; SI, May-Sep (Bs,Ds) ; LI: Brooklyn; Orient; Queens (Schott); Sea Cliff, May.

\section{Helophilus Meigen}

H. bilinearis Will. Ithaca, McLean, Freeville, Ap-May; Van Cort. Pk.; SI, Ap, on willow (Bs); LI: Flushing.

H. chrysostomus Wied. N. Fair Haven, J1-Aug; Manlius; Conesus L., Je, Ds (Beq) ; Ithaca and vic., J1 (Bs); Albany; SI.

H. conostoma Will. Saranac L.; Potsdam; N. Fair Haven; Erie Co, May-Aug (MCV) ; Ithaca; White Plains; Yonkers; SI, Je, Ds (Beq), May-Oct (Bs); LI: Brooklyn; Maspeth (Ols); Jamaica; Queens (Schott); Sea Cliff, Je-J1.

H. distinctus Will. Erie Co, Je-J1 (MCV).

H. divisus Lw. Ithaca, May; LI: North Beach (Schott).

H. hamatus Lw. Johnstown, May; Ithaca, J1 (Bs); SI, May-Aug (Bs) ; LI: Wading R., May, Ds (Jn).

H. integer Lw. "NY"; Ithaca, Je.

H. laetus Lw. Abundant. Johnstown; Buffalo, Je-Aug (MCV); McLean, J1; Ithaca, Je; Ft. Montgomery (Schott); White Plains; SI: May-JI (Bs); Watchogue, Je, Ds (Beq) ; LI: Brooklyn.

H. latifrons Lw. Abundant, May-Oct. Adirondacks; Buffalo, Je (MCV); Ithaca, May-Sep; Hudson R. Val.; LI: Flatbush (Schott).

H. lunulatus Mg. Ithaca, Caroline, May; Van Cort. Pk.; SI; LI: Forest Hills (O1s).

H. similis Macq. Abundant, May-Nov. Adirondacks; Piseco L., Aug; Manlius; Erie Co, May-Sep (MCV); Ithaca, May-Sep; Trenton Falls; Albany; SI: Sep (Bs) ; Richmond, J1, Arrochar, Tottenville, Sep, Ds (Beq) ; LI: Wyandanch (Schott); Jamaica, Huntington (Schott); Glendale (Schott); Wading R., Sep (Bs).

H. stipatus W1k. Trenton Falls, type locality; probably the same as H. conostoma Will. 


\section{MaLlota Meigen}

M. cimbiciformis Fall. Axton; Old Forge; Lewiston, May (MCV) ; Niagara; Ithaca; Albany, May-Aug; Oliverea; Poughkeepsie, Je-J1; Pine I. (Ds); SI, May-Aug (Bs); LI: Sea Cliff.

M. posticata Fab. Adirondacks; Axton; Old Forge; N. Fair Haven, May-Aug ; Niagara Falls, Je-J1; Erie Co, Je-Jl (MCV); Portage, Je, Ds (Beq) ; Otto; Ithaca; Albany; Oliverea ; Catskills; West Pt., Je, Ds (Beq) ; Ft. Montgomery (Schott); SI, May-Je (Bs) ; Clove Val., Jl, Ds (Beq) ; LI: Central Pk. ; Sea Cliff.

\section{MERODON Meigen}

M. equestris Fab. Keene Val., Je (Not); Ithaca; LI: Brooklyn (Eng).

\section{POLYDONTOMYIA Williston}

(Triodonta Williston not Bory)

P. curvipes Wied. SI, Je-Sep (Bs) ; LI: Sea Cliff, Lynbrook, Je; Long Beach ; Manhattan Beach; Kings Pk.; Flatbush (Eng); Rosedale (Schott) ; Jamaica; Queens.

\section{TEUCHOCNEMIS Osten Sacken}

T. bacuntius Wlk. SI, May (Bs); Richmond, Je, Ds; Willow Br., May, Ds.

T. lituratus Lw. Niagara Falls, Je (MCV); Gowanda, Je (MCV) ; Ithaca ; Windsor, May-Je; Poughkeepsie; Ft. Montgomery (Schott); SI, May (Bs).

\section{Pterallastes Loew}

P. thoracicus Lw. Hamburg, J1 (MCV); SI, May, Ds (Beq), Je-Sep (Bs) ; LI: Sea Cliff, May.

\section{SyRITTA St. Fargeau et Serville}

S. pipiens L. Very abundant. Upper Ausable L.; Adirondacks; Fulton Co; Manlius; Sodus Pt., Oct (Bs); Erie Co, May, Sep (MCV); Ithaca, Ap-Oct ; Hudson R. Val.; Pine I.; Ft. Montgomery (Schott); SI, J1-Oct (Bs); LI: Huntington; Brooklyn; Sea Cliff.

\section{EUMERUS Meigen}

E. strigatus Fall. Keene Val.; Buffalo, May-Je, Oct; Geneva, J1 (Felt) ; Ithaca (Joh); Saratoga Sps., Aug; Schenectady, J1 (Felt); Amsterdam, J1 (Felt) ; White Plains (Beq); SI, Je (Bs).

\section{XyLota Meigen}

\section{(Including Xylotomima Shannon)}

$\mathrm{X}$. analis Will. Ithaca, Je.

X. angustiventris Lw. Fulton Co; Niagara Falls, Je; Gowanda, Je (MCV) ; Ithaca; McLean, Aug; Albany, Je; Hudson R. Val.; W. Nyack; SI, Je-Jl, Ds.

$\mathrm{X}$. anthreas W1k. Ithaca, J1; Trenton Falls. 
X. bicolor Lw. Gowanda, Je (MCV); Ft. Montgomery, Southfields (Schott); SI, May-Je (Ds,Bs).

X. chalybea Wied. Niagara; Honeoye Falls, Ds; Niagara; Ithaca; Caroline; McLean; Poughkeepsie, May-J1; Pine I. (Ds); SI: Richmond, May, Ds (Beq) ; LI: Pennequid Barrens.

X. curvipes Lw. var. satanica Big. Old Forge; Axton; Keene Val., Je-Aug (Not); Mt. Whiteface, summit, J1, Ds (Beq).

X. ejuncida Say. Fairly abundant, May-Aug. Adirondacks; Lewiston, May (MCV) ; foot of Cliff Mt.; L. Tear ; Erie Co, Je-Jl (MCV) ; Ithaca, May-J1; Poughkeepsie; Oliverea; Ft. Montgomery (Schott); SI: Ap-Aug, Ds (Bs, Beq) ; Richmond, May, Ds (Beq) ; LI: Sands Pt., Je (Bs); Flushing.

X. ejuncida elongata Will. E. Aurora, J1 (MCV).

$\mathrm{X}$. fascialis Coq. (anthreas W1k., nemorum auct., not Fab.). Ithaca, Je-J1.

X. flavifrons Wlk. Adirondacks, Jl, Fbs (Snn).

X. fraudulosa Lw. (baton Wlk.). Ithaca; McLean; Freeville; Lakeside Park, Ap-Sep; Hudson R. Val., Ap-Je; W. Nyack (Beq) ; Nyack (Schott) ; LI: Flushing.

X. libo Wlk. (marginalis Will.). Axton, Je.

X. neobscura Snn. L. Tear, Fbs.

X. pigra Fab. Ithaca, May; Pelham Pk., Sep (Bs) ; LI, Ap.

X. plesia Curr. Mt. Whiteface, J1, Ald (Snn).

$\mathrm{X}$. subfasciata Lw. Essex Co, J1-Aug.

X. vecora O.S. Old Forge; Mt. Marcy, Je-J1; Ithaca; Caroline; Ft. Montgomery, Je $(\mathrm{MCV})$.

\section{BRACHYPALPUS Macquart}

B. frontosus Lw. Keene Val., Je; Ithaca, Ap-May; Trenton Falls; SI (Ds) ; LI: Massapequa; Yaphank (Not); Farmingdale.

B. rileyi Will. Caroline, May; SI, Mar-May (Bs).

\section{Criorhina Meigen}

C. verbosa Wlk. Keene Val., May ; Caroline, May ; Ithaca; Hartsdale, Ap; Ramapo, Mar, on willow catkins, Ds (Beq) ; SI: Ap, on willow catkins (Bs) ; Richmond, Mar, Ds (Beq); LI: Wyandanch (Schott).

\section{CyNORRHINA Williston}

\section{(Blera Billberg)}

C. analis Macq. Keene Val.; Ithaca; McLean; Poughkeepsie, May-Je; Ft. Montgomery, Southfields (Schott); SI, Je (Bs); LI: Yaphank (Ols); Sea Cliff.

C. badia W1k. (intersistens Wlk.). Axton; Adirondacks; foot of Cliff Mt.; Ithaca, Je-J1; Caroline ; Ringwood, Je; Danby (Emerson) ; Trenton Falls ; Oliverea; Pine I.; SI, May (Bs); LI: Yaphank.

C. confusa Jn. Axton; Mt. Marcy; Old Forge, Je-J1; foot of Cliff Mt.

C. nigra Will. Keene Val., May-J1; L. Tear.

C. pictipes Bigot. SI; LI: Yaphank (Eng).

C. umbratilis Will. LI: Yaphank. 


\section{SOMULA Macquart}

S. decora Macq. Fairly abundant. Lewiston, Je (MCV) ; Niagara Falls, May-Je; Erie Co, Je (MCV) ; Portage, Je, Ds; Ithaca; Hudson R. Val. ; Ft. Montgomery, May, Ds (Jn, Schott); Pine I. (Schott); Ramapo, Je, Ds; SI: May-Je, common on Spiraea blossoms (Bs) ; Clove Val., Je, Ds; LI: Wading R., May, Ds (Jn, Schott); Huntington (Schott).

\section{MiLesia Latreille}

M. virginiensis Dru. Ithaca, J1, Sep; Ft. Montgomery (Schott) ; SI: J1-Sep (Bs) ; Eltingville, Sep, Ds; Richmond, Oct, Clove Val., Sep, Ds; LI: Wading R., Sep (Bs) ; Pinelawn, Aug, Ds; Wyandanch, Flushing (Schott); Yaphank, Jl, Ds.

\section{SPILOMYia Meigen}

S. fusca Lw. Adirondacks, Aug-Sep; L. Pleasant, Aug, Love; Erie Co, Je-Aug (MCV) ; Ithaca; McLean; Oliverea; E. Jewett, South L., Catskills, Aug, Ds; Ft. Montgomery (Schott).

S. hamifera Lw. Manlius; Niagara Falls, Je-J1; Ithaca; Poughkeepsie; Ft. Montgomery (Schott) ; SI: J1 (Bs) ; St. George, J1, Ds; LI: Flatbush; Kings Pk., J1 (Bel1); Pennequid Barrens.

S. longicornis Lw. Erie Co, Aug-Sep (MCV); Ithaca, J1-Sep; Ft. Montgomery (Schott) ; SI, Aug (Bs) ; LI: Sands Pt., Sep (Bs) ; Gardiners I.; Rockaway Beach (Schott).

S. quadrifasciata Say. Elizabethtown; Essex Co, J1-Sep; Manlius; Erie Co, AugSep (MCV); Otto, J1-Sep ; Ithaca; McLean, Aug; Oliverea; SI, Sep (Bs); LI: Flushing.

SPHEComyia Latreille

S. vittata Wied. Axton, Je; Ithaca, Je, Aug; Karner; Ft. Montgomery (Schott) ; SI, Ap (Bs).

Temnostoma St. Fargeau et Serville

T. aequale Lw. Mt. Whiteface, summit, J1, Ds (Beq) ; Essex Co, Je-J1; Old Forge, Je-J1; Upper Ausable L.; Mt. Skylight; L. Tear; Oliverea.

T. alternans Lw. Axton; Keene Val., May-J1; Adirondacks, May-Je; Mt. Skylight; Cranberry L. (Drk); Boston, Aug (MCV); Ithaca; Albany ; Pine I. (Ds); LI: Flushing.

T. bombylans Fab. Adirondacks, May-Je; Ithaca and vic.; McLean; Trenton Falls; Poughkeepsie.

T. excentricum Harr. Keene Val. (Not) ; Old Forge, Je-J1; Boston, Aug (MCV); Ithaca, Je; McLean.

T. obscurum Lw. L. Tear, Mt. Skylight (Beq).

T. pictulum Will. Rochester (MCV).

T. venustum Will. Essex Co, J1.

TENTHREDOMYIA Shannon

(Ceriodes auct. not Rondani)

T. abbreviata Lw. (proxima Curr.). Lancaster, Je (MCV); Ithaca, Je; Taughannock Falls, J1; Karner. 


\section{Ceriodes Rondani}

C. signifera Lw. (willistoni Kah1). Rochester, Je-J1; Ithaca, Je; Albany.

\section{FAMILY CONOPIDAE}

CONOPS Linnaeus

C. brachyrhynchus Macq. ${ }^{10 j a}$ Tompkins Co (Will); Karner, J1; Poughkeepsie, Je (Yg); LI: Orient (Beq).

C. fulvipennis Macq. LI: Manhattan Beach (Beq).

C. gracilis Will. Beaver Kill, Aug (CnJr).

C. sylvosus Will. SI, $B s$ (Beq).

C. xanthopareus Will. ${ }^{10 j a}$ LI: Hempstead, J1 (Snn); Manhattan Beach (Beq).

\section{Physocephala Schiner}

P. affinis Will. Newport, J1 ( $\mathrm{Yg})$.

P. furcillata Will. New Russia, Aug (Snn); Wilmington, J1, Ds (Jn) ; Adirondacks, Aug, Ndm; Ithaca, J1 (Joh); Oliverea (Beq); Beaver Kill, Aug (CnJr-ANSP).

P. marginata Say. "NY".

P. sagittaria Say. Norton's Landing, Cayuga L. (Will); L. George, Aug, Zab (AM) ; New Baltimore, Aug, Nyack, Aug (AM); Rye, with Papaipema impecuniosa, perhaps as a parasite, Sep 21 (Bird).

P. tibialis Say. Ithaca, J1 (Joh); Albany, Sep (Yg); Mosholu (AM) ; SI: (Beq); Ft. Wadsworth, J1, Bs; LI: Wading R., J1, Sep, Bs, J1 27, Ds (Jn); Cold Sp. Harb., J1 (Snn), Sept, Bs; Sands Pt., Jl, Bs; Farmingdale, Je, Bs; Kings Pk., J1, Bs; Manhattan Beach, Flushing, Wyandanch, Orient, Jamaica, Coram (Beq); Yaphank, J1, Ds (CnJr-ANSP).

\section{ZODION Latreille}

Z. abdominale Say. LI: Cold Sp. Harb., J1, on Rhus glabra (Snn).

Z. fulvifrons Say. Adirondacks, Je, Aug; Erie Co, May-Aug (MCV) ; Tompkins $\mathrm{Co}$, Je-Aug (Joh); White Plains (Beq); SI: Moravian Cemetery, May, Bs; New Brighton, Je, $B s$; Richmond, Aug, Bs; LI: Sands Pt., Bs; Wading R., Sep, Bs; Newtown Hgts., Je (AM) ; Huntington, Schott (Beq); Orient (Beq).

Z. intermedium Bks. Oliverea (Beq); LI: Central Pk., Sep, Ds (CnJr-ANSP); Flushing, Cold Sp. Harb. (Beq).

Z. nanellum Lw. Erie Co, May-Aug, $M C V$; Little Valley, Je, $M C V$; LI: Little Neck (Snn).

Z. perlongum Coq. LI: Gardiners I. (Beq).

\section{STYLOGASTER Macquart}

S. biannulata Say. Wilmington, Aug (Snn); Grand I., Aug, $M C V$; Ithaca, Aug (Snn).

S. neglecta Will. Niagara Falls, J1; Springville, J1, MCV ; Canandaigua L. (Felt); Rhinebeck, J1 (Joh) ; LI: Cold Sp. Harb., J1 (Snn).

100a See Curran (Can. Ent., vol. 59, p. 32) on synonymy of brachyrhynchus and xanthopareus. 
DaLmannia Robineau-Desvoidy

D. nigriceps Lw. Norton's Landing, Cayuga L., Comstock (Will); Spencer, Je (Snn); Karner, Je (Yg).

THECOPHORA Rondani

(Occemyia Robineau-Desvoidy; Oncomyia Loew)

T. abbreviata Lw. Keene Val. (Beq); Bolton (Jn); Rochester, Sep; Olcott, Jl, $M C V$; Erie Co, J1-Sep (MCV); Perry, SHEmerson (Beq) ; Ithaca, Jl-Aug (Joh); Salem, Aug (Yg); Albany, Aug.

T. baroni Will. Hamburg, Sep (MCV); Lewiston, Je (MCV).

T. loraria Lw. Johnstown, Aug ; Erie Co, Je-Sep, $M C V$; Erie Co, Je-Sep, $M C V$; Ithaca, May-Aug (Joh); Oliverea (Beq) ; SI: Kreischerville, J1, Bs; LI: Wading R., Sep, Bs; Cold Sp. Harb., Aug, Bs (Beq).

T. modesta Will. Perry, Aug (Snn); Ithaca, Je-Aug (Snn); LI: Cold Sp. Harb. (Beq).

T. modesta var. melanopoda Will. Rochester, J1 (Joh) ; Freeville, Aug (MacG).

\section{MYopA Fabricius}

M. clausa Lw. Rock City, Je; Ithaca, Je (Joh); LI: Wading R., May, Schott (Beq); Wyandanch, Schott (Beq).

M. pilosa Will. Colden, May, $M C V$.

M. vesiculosa Say. Niagara Falls, May-Je; Erie Co, Je-J1, MCV ; Ithaca, May (Snn); Tompkins Co (Will, Joh); Karner, May (Yg); Poughkeepsie, Je $(\mathrm{Yg})$.

M. vicaria Wlk. Ithaca, Ap-May (Joh, Snn); Karner, Ap (Yg); Albany, May.

M. virginica Bks. Ithaca, Je-J1; Tompkins Co, Je (Snn) ; Hartford, Je; W. Nyack (Beq) ; Ft. Montgomery, Schott (Beq); LI: Cold Sp. Harb., J1, on Rhus glabra (Snn).

\section{FAMILY PYRGOTIDAE}

\section{PyRgotA Wiedemann}

P. chagnoni Jn. Elizabeth, Je; Colden, May (Jn) ; Ithaca (Joh); White Plains, May; LI: Huntington, May.

P. undata Wied. Fulton Co; Conesus L., Je (Ds) ; Ithaca, May-Je (Joh) ; Richfield, Je (AM); White Plains, Bno (Beq); Nyack, Aug, Zab (AM); W. Nyack, Ols (Beq) ; Mosholu, May (Ds) ; Bronx, NY Bot. Gardens, May (Ds) ; SI, Je (Ds); LI: Parkville (AM); Long Beach, Rockaway Beach, Schott (Beq).

P. valida Harr. Newport, May ; E. Aurora, May (MCV); Ithaca, May (Comstock); Pearl River, May 11, Wat; SI, Je (Ds); LI: Rockaway Beach, Je (Ds); Wading River, Je (Ds); Maspeth, Je (Ols).

\section{FAMILy CESTRIDAE}

Compiled by Jos. BEQUAERT

EETRUS Linnaeus

E. ovis L. The Sheep Botfly. Byron; Ballston Spa, J1 19, Yg (NYS): 


\title{
Cephenomyia Latreille
}

C. phobifer Clark (abdominalis Ald.). Mt. McIntyre, summit, J1, Leon (Beq); Mt. Whiteface, Ds (Ald) ; Mt. Skylight, J1, 4800-4900 ft. (Beq) ; Mt. Seward, Je (Joh); Wilmington, Sep 14 (Felt), Sep 9, Asa Lawrence (NYS).

\section{HYPODERMA Clark}

H. bovis L. Recorded from the following counties: St. Lawrence; Warren; Hamilton; Herkimer ; Lewis ; Jefferson ; Oneida; Onondaga ; Genesee; Erie ; Livingston; Cattaraugus; Ontario; Yates; Schuyler; Tompkins; Tioga ; Cortland; Broome; Chenango; Otsego; Greene; Columbia; Orange; Westchester. This and $H$. lineata are widely distributed in the State almost wherever cattle occur, and are often very troublesome. Many localities in each of the counties listed have been recorded, chiefly by $\mathrm{Mr}$. Wells and Mr. Brundrett through the courtesy of F.C.Bishopp of the U. S. Bur. Ent., as the result of extended survey in the State.

H. lineata De Vill. Recorded from the following counties: Clinton; Warren; Herkimer; Lewis; Jefferson; Onondaga; Monroe; Genesee; Erie; Allegany; Schuyler; Tioga; Cortland; Broome; Otsego; Delaware; Albany; Washington; Greene; Columbia; Sullivan; Orange.

\section{FAMILY CUTEREBRIDAE}

\section{Compiled by Jos. BequaERT}

\section{Cuterebra Clark}

C. cuniculi Clark (horripilum Clark). Albany, Nov, larva from rabbit (Bish); Ithaca, Je 28, J1 4, Philip Miller (Joh); SI: Clove Val., Je, Ang (Ds); LI: Sands Pt., J1 (Bs).

\section{BOGERIA Austen}

B. buccata Fab. SI: Tottenville, Je (Ds).

B. emasculator Fitch (Cuterebra scutellaris Brauer). "NY", type locality; Ithaca (Joh).

B. fontinella Clark. Chilson L., J1, Wat (Swenk) ; Lakeview, Aug; Schoharie, Sep (Lint); Oliverea, J1 (Swenk), neotype; Pine I., CWPollard (Jn).

\section{FAMILY PHASIIDAE 101}

\author{
Compiled by L. S. WeST
}

\section{Cistogaster Macquart}

\section{(Gymnoclytia Brauer and Bergenstamm)}

C. immaculata Macq. Axton; Keene Val.; Wells; Speculator; Johnstown; N. Fair Haven; Cattaraugus; Canandaigua; Manlius; Hemlock L.; Ithaca; McLean; Spencer L.; Ringwood; Owego; Freeville; Albany; Albany Co; Nor-

101 The lists for the families Phasiidae, Tachinidae, Megaprosopidae, and Dexiidae, as well as for the subfamilies Amobiinae and Miltogramminae of the Sarcophagidae, have been prepared by Dr. L. S. West. He has seen the great majority of the material on which these lists are based, and, in general, assumes authority for all records unless otherwise credited. In order to facilitate comparison, the grouping has been made to follow that of Johnson's "List of the Diptera of Ner England," but this does not necessarily indicate Dr. West's views on the relationships of these groups. 
manskill, Albany; L. Tackawasick; Debruce; Unionville; Rhinebeck; Oliverea (Ald) ; SI: Ft. Wadsworth; Moravian Cemetery; Richmond; New Dorp (Ald); Watchogue (Ald); LI: Riverhead; Long Beach, Bs. May-Aug.

C. occidua Wlk. Niagara Falls, Je; Lewiston, Je (MCV); Erie Co, Je-Sep $(\mathrm{MCV})$; White Plains (Beq); LI: Hempstead, Huntington, Jamaica (Beq).

\section{Gymnosoma Meigen}

G. dubia West. Ithaca; Lake Ridge; Spring Lake, Cayuga Co; Spencer L. ; Trenton Falls. Je-Sep.

G. fuliginosa Desv. Keene Val.; Axton; Old Forge; Saranac L.; Chapel Pond; Fulton Co; Lowville; Rochester Jct.; Erie Co (MCV) ; Hemlock L. ; Spencer L.; Ithaca; Freeville; McLean; Oliverea (Beq). Je-Oct.

\section{Phasia Latreille}

(Including Alophora Desv., Hyalomyia Desv., Phorantha Rond., Paraphasia Towns., etc.)

P. aeneoventris Will. Peru, Je; Keene Val., Aug; Chazy L., Je-J1; Rochester, Sep-Oct (MCV) ; Erie Co, Je-Aug (MCV); Ithaca, May, Snn; Sep, Mat; Ringwood, Oct, Leon; Owego, J1, West; Onteora Mt., J1, How (Ald \& Greene).

P. brevineura West. Ithaca, J1, Sep; McLean, Aug-Sep, Sib.

P. cara West. (?) Ithaca, J1; Karner, Oct-Nov.

P. fenestrata Big. Ithaca, May, Snn (Beq); Caroline, May, And; Owego, May, West; Albany, May; SI: Oakwood, Bs (Ald).

P. fumosa Coq. Elma, Aug (MCV); Ithaca; McLean, Sep, West; Albany.

P. furva West. McLean, Aug-Sep, Sib.

P. magnipennis Jn. Caroline, And (Beq) ; Karner, May.

?P. nigrens Wulp. Ithaca (probably a misidentification; the specimen is lost).

P. nitida Coq. McLean, J1, Sib; Ithaca, May-Je; Owego, J1, West; Poughkeepsie, J1.

P. opaca Coq. Ithaca, Je-Sep.

P. phasioides Coq. Keene Val., Aug.

P. pulverea Coq. Coy Glen, Ithaca, Aug; Ithaca, J1-Aug, JMStedman; SI, J1, Bs (Ald).

P. splendida Coq. Keene Val., Aug; Oliverea (MCV).

P. subopaca Coq. Erie Co, Je, Oct (MCV) ; Ithaca, J1.

\section{Phoranthella Townsend}

P. occidentis W1k. Honeoye Falls, J1 (Leon), from Miris dolabratus.

\section{EUPHORANTHA Townsend}

E. diversa Coq. Oswego; McLean, Sep, Sib; Karner, Oct. 
TRICHOPODA Latreille

T. cilipes Wied. Yonkers, Je, Ang.

T. pennipes Fab. Irondequoit; Ithaca, J1; Athens, J1, Bs; Yonkers, J1; SI: Clove Val., Je-J1, Ds (Jn); LI: Riverhead, J1, Huck; Baiting Hollow, J1, Oct, Huck; Cold Sp. Harb. (Beq).

T. radiata Lw. "NY".

\section{FAMILY TACHINIDAE}

Compiled by L. S. West

MYIOPHASIA Brauer and Bergenstamm

M. metallica Towns. (aenea auct.). Keene Val., J1; Old Forge, J1; Hemlock L., Aug; Ithaca, J1; SI (Beq) ; LI: Baiting Hollow, Aug-Sep, Huck; Riverhead, Sep.

MAUROMYia Coquillett

M. pulla Coq. Keene Val., J1.

\section{EMPHANOPTERYX Townsend}

E. eumyothyroides Town. Ithaca (Curr).

E. menapis Wlk. Mt. McIntyre, Adirondacks, Je; Ithaca, May; Victory Mills, May; Rensselaer, May; Thomson, May; Thacher Pk., Albany Co, May; Corinth, Je; Albany, May; Poughkeepsie, May; LI: Gardiners, E. Hampton, May; Montauk, May; Baiting Hollow, Je, Huck.

E. prisca Wlk. (Cryptomeigenia theutis auct., in part.). N. Evans, May (MCV); Lancaster, Je (MCV); Ithaca and vic., May; McLean, Je; Ringwood, Je, West ; Albany, May; Thacher Pk., May; Rensselaer, May; E. Jewett, Ap, Ds; SI, Ap-May ; LI: Baiting Hollow, Ap-May, Huck.

\section{EULASIONA Townsend}

E. catskillensis West. E. Jewett, Ds.

E. comstockii Towns. Mt. McIntyre; near Saranac L.; Woodville, L. Ontario; Ithaca; Ringwood; LI: Southampton, Huck. Je-J1.

E. neglecta West. Ithaca, Je.

OCYPTEROSOMA Townsend

O. polita Coq. Oswego, J1; Spring Lake, Cayuga Co.

\section{EUHYPERECTEINA Townsend}

E. nasoni Coq. LI: Baiting Hollow, Je, Aug, Huck.

\section{SCHIZOTACHINA Walker}

S. convecta Wlk. Karner, Sep.

\section{Clausicella Rondani}

?C. usitata Coq. Ithaca, Dobroscky. According to Johnson, this species is synonymous with Schizotachina convecta W1k. Those specimens to which I have referred here are certainly distinct; hence the query. (West.) 


\section{Phylacteropoda Townsend}

P. tarsalis Coq. Ithaca, J1 (Walton, as Clausicella).

\section{Plectops Coquillett}

P. melissopodis Coq. Ithaca, Je.

\section{NeAERA Robineau-Desvoidy}

N. longicornis Coq. Oswego, Aug, Sheldon.

\section{LISPIDEA Coquillett}

L. palpiger Coq. Keene Val., Aug.

\section{THRYPTOCERA Macquart}

T. americana Towns. Ithaca, Je (Walton, as Polychaetoneura elyii).

T. flavipes Coq. Rock City, Je; Ithaca, May-Je.

\section{Actia Robineau-Desvoidy}

A. palloris Coq. Long L., Aug; Ithaca, Je; LI, Aug.

A. pilipennis Fall. (i.e., pilipennis auct.; more than one form is most certainly included here). Ithaca, May-Je, Aug; McLean, Sep, Sib; Owego, J1, West ; Albany, Je; Amenia, Aug; SI: Watchogue, Aug (Ald).

\section{ACTIOPSIS Townsend}

A. autumnalis Towns. McLean, Aug, Sib.

\section{DichaETONEURA Johnson}

D. leucoptera Jn. Keene Val., J1; Ithaca, Je-J1, West; Owego, Aug, BeatriceRWest; Bainbridge, J1; Albany, J1; Rensselaer, J1; Nassau, Sep; SI, $\mathrm{J} 1, D s$ (Ald).

Chaetophleps Coquillett

C. setosa Coq. LI: Baiting Hollow, Je-J1, Huck; Selden, J1, Huck.

Celatoria Coquillett

C. spinosa Coq. Thompson L., Aug; SI (Beq).

\section{ADMONTIOPSIS Townsend}

A. tarsalis Coq. Ithaca, Je.

EUADMONTIA Townsend

E. pergandei Coq. Hamburg, Sep; Clinton Corners, Sep; Coy Glen, Ithaca, Sep ; McLean, May, Sep. 


\section{XENADMONTIA Townsend}

X. degeerioides Coq. Batavia, Je; Ithaca, J1, Sep; McLean, J1; Ringwood, J1; Spring Lake, Cayuga Co, J1; SI, Aug, Ds; LI: Baiting Hollow, Je, Huck.

\section{HYPERECTEINA Schiner}

H. aestivalis West. McLean, J1.

H. bishopi West. Clyde, J1, Bish; Ringwood, Je, Leon; LI: Baiting Hollow, Oct, Huck.

H. consternata West. LI: Baiting Hollow, J1, Huck.

H. duospinosa West. LI: Baiting Hollow, Aug, Huck.

H. extricata West. Albany, Aug.

?H. retiniae Coq. LI: Riverhead, Sep.

H. setigera Coq. Van Cort. Pk., Je, Ds.

\section{LIXOPHAGA Townsend}

L. variabilis Coq. (Hypostena variabilis Coq.). Elizabethtown, Aug; Ithaca, MaySep; L. Ridge, J1; McLean, Sep, Sib; LI: Baiting Hollow, Aug, Huck.

\section{Hypostena Meigen}

?H. gilvipes Coq. Ithaca.

\section{Methypostena Townsend}

M. barbata Coq. Axton, Je; Lowville, J1, West; Thompson L., Aug; Duck L., Conquest, Aug, Snn; Ithaca, J1; Coy Glen, May; Owego, May, West; McLean, Je-J1, West ; Ringwood, J1, West; LI: Cold Sp. Harb. (Beq).

\section{OEDEMATOCERA Townsend}

O. flaveola Coq. Ithaca, J1; Shelving Rock, L. George, Sep, Leon; LI: Jamaica, Je, $Y g$; Selden, Jl, Baiting Hollow, Sep, Huck.

\section{TACHINOPHYTO Townsend}

?T. maculosa Coq. Hague, Aug; McLean, Sep, Sib.

T. tortricis Coq. LI: Baiting Hollow, May, Huck.

\section{Didyma Van der Wulp}

D. setifacies West. Ithaca, Ap, West; Albany, Ap-May, Yg.

\section{Pseudenantha Townsend}

P. coquilletti Ald. Ithaca; LI: Baiting Hollow, J1, Huck; Southampton, J1, Huck; Selden, J1, Long Pond, Wading R., Je, Huck.

P. pristis Wlk. Mt. Marcy, $5300 \mathrm{ft}$. (Beq) ; Wells, Aug, $Y g$; Colden, J1; Ithaca and vic., May-Je; SI, Jl, Bs (Ald) ; LI: Baiting Hollow, J1-Sep, Huck: Selden, J1-Aug, Wading R., Aug, Southampton, J1, Riverhead, Je, Aug, Huck. 


\section{Polidea Macquart}

P. areos Wlk. Alexandria Bay, Sep; Erie Co, May-Sep (MCV) ; Cattaraugus, Sep; Ithaca and vic., May-Sep; Ringwood, Je; McLean, Aug-Sep, Sib; Taughannock Falls, May; Albany, May; Kenwood, May, Yg.

\section{Chaetophlepsis Townsend}

C. tarsalis Towns. (Hypochaeta endryae Sm.). Oswego, Je (HESmith).

ApINops Coquillett

A. atra Coq. Wilmington, 1000-2200 ft., Aug; Ithaca, Je, West.

\section{GeneA Rondani}

G. analis Say. Keene Val., Aug; Long L., Aug; Woodhull L.; Niagara Falls, J1 (MCV) ; Little Valley, J1 (MCV) ; Ithaca, J1-Aug; Rensselaer, Ap.

G. texensis Towns. Ithaca, NYC (Ald).

\section{MyobIOPSIS Townsend}

M. similis Towns. SI, Bs (Ald).

\section{Leskiomima Brauer and Bergenstamm}

L. tenera Wied. Keene Val., Aug; Saranac Inn, Aug; L. Placid, Aug; Ithaca, Je; LI: Wading R., Sep (Bs Coll.); ?Sands Pt., Aug (Bs Coll.).

\section{LESKIOPALPUS Townsend}

L. depilis Coq. Ithaca, Aug.

\section{PARADIONAEA Townsend}

P. atra Towns. Rochester Jct., J1, Leon; Hamburg, Je, Buffalo, Oct (MCV) ; Ithaca, Jl-Aug; Mud Cr., Tompkins Co, Je; LI: Baiting Hollow, Jl-Aug, Huck.

P. senilis Towns. "NY", Comstock (Towns); LI: Baiting Hollow, J1, Huck.

HYALOMYODES Townsend

H. triangulifera Lw. Speculator, Aug; Keene Val., Aug; Rock City, Je; Ithaca and vic., May, J1, Sep-Oct; Michigan Swamp, J1; Ringwood, Oct, Leon (Ald); Spencer L., Je; McLean, May, J1, Sep, Leon, Sib; Caroline to Harford, Je; Corinth, May; Owego, Jl, West; LI: Wading R., Sep, Bs (Ald); Pennequid Barrens (Beq).

\section{ORMIA Robineau-Desvoidy}

O. calva Coq. Karner, Aug; NY, Aug.

O. clausa B. \& B. Karner, May; LI: Selden, May, Huck.

O. signifera Wulp. LI: Central Pk. (Beq). 
Clistomorpha Townsend

C. hyalomoides Towns. "NY", Comstock (Towns).

\section{EUTRIXA Coquillett}

E. exile Coq. (masuria Coq. not Wlk.). Ithaca, Je-J1; Saratoga Sps., May, FTHartman.

\section{XaNTHOMELANODES Townsend}

X. arcuata Say. II: Riverhead, J1, Huck.

X. flavipes Coq. LI: Baiting Hollow, Aug, Huck.

\section{HEMYDA Robineau-Desvoidy}

H. aurata Desv. Saranac L., J1; Sodus Pt., Not (Beq) ; E. Aurora, Je (MCV); Ithaca, May, Sep; Poughkeepsie, Aug; LI: Flatbush, Eng (Beq); Riverhead. Aug, Huck; Baiting Hollow, Aug, Huck.

\section{SIPHOPHYTO Townsend}

S. floridensis Towns. Ringwood, Je; McLean, Aug; SI (Bs Coll.).

\section{EPIGRIMYIA Townsend}

E. polita Towns. Rochester Jct., J1, Leon.

E. geniculata Towns. Essex Co, Aug.

E. setigera Coq. Keene Val., Aug; Saranac Inn, Aug; Long L., Aug; Ithaca, J1.

\section{SiPHONA Meigen}

S. geniculata DeG. Keene Val., Aug, Diven; Wells, Aug, $Y g$; L. Placid, Aug; Elm L., Aug; Dug Mt., Aug, Yg ; Colden, May (MCV) ; Mix Cr. Val., Cattaraugus Co, Je; Little Valley, May (MCV); Ithaca, May-Je; McLean, May; W. Nyack, Jl, Bs (Ald); SI, B.s (Ald) ; LI: Sands Pt., Bs (Ald); Baiting Hollow, Je, Huck.

\section{GiNGLYMYIA Townsend}

G. acrirostris Towns. E. Aurora, Aug (MCV); Ithaca and vic., Aug-Sep, JTLloyd (Joh).

\section{Metaplagia Coquillett}

M. orientalis Towns. L. Charlotte, Je (Ald).

\section{Plagiprospherysa Townsend}

?P. parvipalpis Wulp. Speculator, Aug, $Y g$.

BLEPHARIGENA Rondani

B. spinulosa Big. Ithaca; Hamburg, Je (MCV). 


\section{Cyrtophloeba Rondani}

C. coquilletti Ald. (horrida Giglio-Tos not Coq.). Ilion, May, $\mathrm{Yg}$; Ithaca and vic., Ap-May, Aug; Ringwood, Je, West; McLean, May; L. George, Sep ; LI: Yaphank, May, Huck; Baiting Hollow, May, Huck.

\section{VORIA Robineau-Desvoidy}

V. ruralis Meig. (americana Wulp). Near Mt. McIntyre, J1, West; Deansboro, Je, West ; Chapel Pond, Je ; Ontario Beach, Aug, West; Ithaca, Aug; McLean, J1, West ; Slaterville, J1, West ; Owego, J1, West ; Albany, Aug; Poughkeepsie, Je, Yg; Wappingers Falls, Je, Leon; LI: Baiting Hollow, J1-Aug, Huck.

\section{STENOPLEURA Aldrich}

\section{(Siphoplagia Townsend)}

S. anomala Towns. LI: Baiting Hollow, Sep, Huck; Cold Sp. Harb., Sep (Bs Coll.), also (Beq).

S. latifrons Ald. (rigidirostris Wulp). Saranac L., Sep, Ds (Ald); Ithaca, J1, West ; Owego, J1, West (Ald) ; LI: Baiting Hollow, Sep, Huck.

S. similis Towns. LI: Baiting Hollow, Sep-Oct, Huck.

\section{PSEUdotractocera Townsend}

P. lachnosternae Towns. SI: Ft. Wadsworth, Sep, Bs (Ald).

\section{VIVIANIA Rondani}

?V. georgiae B. \& B. Near Saranac L., J1, West.

Belvosia Robineau-Desvoidy

B. bifasciata Fab. Buffalo, J1 (MCV); Ithaca, Je; near Albany, Je; Croton, J1, $D s$ (Ald) ; Pine I., Ds (Ald); SI, Je (Bs Coll.), also Ds (West); LI: Yaphank, Eng (Beq); Baiting Hollow, Sep, Huck.

\section{TRIACHORA Townsend}

T. unifasciata Desv. Ithaca, Je-J1; McLean, May, Ds; Taughannock Falls, J1; Michigan Swamp, J1; Owego, J1, West; Albany, J1, Nov; Ghent, Nov; W. Nyack, J1 (Beq) ; Pine I., Je, Ds; SI, Je-Oct (Bs) ; LI: Baiting Hollow, Sep, Huck; Wading R., Je, Ds; Flushing (Beq).

\section{MELANOPHRYS Williston}

M. insolita Wlk. Axton, Je (M\&H) ; Rock City, May-Je; Spencer, Je.

\section{APHRIA Robineau-Desvoidy}

A. ocypterata Towns. Ithaca, Aug, Oct, West; McLean, May, Jl, Leon; LI: Central Pk. (Beq); Farmingdale, Je, Bs (Ald), Morrow (West); Baiting Hollow; Je, Huck. 


\section{CylindRomyia Meigen}

C. carolinae Desv. A doubtful species, to which there are many references.

C. decora Ald. Ithaca (Ald).

C. dosiades W1k. Keene Val., Aug, Diven; Saranac L., J1; Erie Co, Je-J1 (MCV) ; North Cr., Aug; White Plains (Beq).

C. euchenor Wlk. Ithaca; Union Sps. (Aid).

\section{LINNAEMYIA Robineau-Desvoidy}

L. haemorrhoidalis Fall.' Keene Val., J1-Aug; Freeville, J1.

\section{Bonnetia Robineau-Desvoidy}

B. comta Fall. Keene Val., Ang; Wells, J1; Rochester Jct., J1, Leon; Niagara Falls, J1 (MCV); Buffalo, Oct (MCV); Walton, Sep; Ithaca, J1; McLean, J1-Aug; Owego, J1-Aug; LI: Baiting Hollow, J1-Oct, Huck.

\section{PyRaustomyia Townsend}

P. penitalis Coq. SI (Beq).

\section{ERNESTIA Robineau-Desvoidy}

E. ampelus W1k. Foot of Mt. Redfield, Essex Co (Beq); Ithaca, J1; Owego, J1, West (Curran); Big Ind. Val. (Beq) ; Oliverea, Sep, Ds (Ald); LI: Baiting Hollow, May, Huck. There are numerous records, from all parts of the State, for Panzeria radicum auct. A majority of these, upon verification, will probably be found to be referable to E. ampelus W1k. (West.)

E. arcuata Tothill. Ithaca, May, Ringwood, May, West (Curran).

E. flavicornis Brauer. Wells, J1, Yg (Curran).

E. platycarina Tothill. Mt. Marcy trail, J1, Fbs (Curran); Big Moose, Aug (Curran) ; Old Forge, J1 (Curran); Wells, J1, Yg (Curran); Karner, May (Curran).

\section{Metaphyto Coquillett}

M. genalis Coq. Potsdam, Sep.

MACromeigenia Brauer and Bergenstamm

M. chrysoprocta Wied. Lancaster (MCV); S. Wales, Gowanda, J1-Aug (MCV).

Chrysotachina Brauer and Bergenstamm

C. alcedo Lw. Niagara Falls, Je; Ithaca, Je; Ringwood, Je, West; SI (Bs) ; LI: Jamaica, Je, Yg; Baiting Hollow, J1, Huck; Selden, J1-Aug, Huck; Wading R., Aug, Huck.

C. ruficornis Will. Sport I., Sacandaga R., Alex.

LYPHA Robineau-Desvoidy

L. dubia Fall. Ithaca, Snn (Beq). 


\section{NEMORILLA Rondani}

N. insolens Ald. \& Webb. Albany, Je.

N. maculosa Meig. (Exorista pyste W1k. of Coq.). Rochester, Sep; Lockport, Je; Bainbridge, J1; Ithaca, May, J1-Aug; McLean, Sep, Sib, West ; Ringwood, J1; Owego, J1-Aug, West; Albany, Je-J1; LI: Baiting Hollow, Sep, Huck.

\section{ZENILLIA Robineau-Desvoidy}

\section{(Subgenus ZENILLIA Robineau-Desvoidy}

Z. amplexa Coq. Ithaca, J1 (Mues \& Leon); Poughkeepsie, Je-J1; SI: Ft. Wadsworth, J1-Aug, Bs (Ald); LI: Baiting Hollow, J1, Sep, Huck.

$Z$. eudryae Towns. Axton, Je, $M \& H$; Ithaca, Aug.

Z. formosa Ald. \& Webb. Poughkeepsie, Aug; SI, J1, Ds; LI: Baiting Hollow, J1, Sep, Huck; Wading R., Aug, Huck; Selden, Aug, Huck.

Z. helvina Coq. Big Moose, Aug, FTHartman; Ithaca, J1, Sep; Albany, Je-J1; Oliverea (Beq); SI: Ft. Wadsworth, Je, Bs (Ald); LI: Jamaica, Je.

Z. inflatipalpis Ald. \& Webb. Ringwood, J1.

Z. reclinata Ald. \& Webb. McLean, Sep, Sib; Ringwood, Je, West.

\section{(Subgenus PHRYXe Robineau-Desvoidy)}

Z. caesar Ald. Mt. Whiteface, 2000-4000 ft., Aug; Rochester, J1; Niagara Falls, Je; Ithaca and vic., May, Aug-Sep; McLean, Sep, Sib; Owego, J1, West ; Hoversville, Je, Leon; Albany, Je; LI: Riverhead, J1, Huck.

Z. crassiseta Ald. \& Webb. Ithaca, Aug; McLean, Sep, Sib; Owego, Aug, West.

Z. vulgaris Fall. Saranac Inn, Aug; Wells, J1-Aug, $Y g$; Honeoye Falls, J1; Ithaca, Je-J1; Taughannock Falls, May; McLean, Sep, Sib; Owego, J1, West (Ald).

\section{(Subgenus PAREXORISTA Brauer and Bergenstamm)}

Z. affinis Fall. McLean, J1, Sep, Sib; LI: Long Pond, Wading R., Je, Huck (identification doubtful).

Z. confinis Fall. N. Fair Haven, J1, West; Niagara Falls, Oct (MCV); Ithaca, J1-Aug; McLean, Sep, Sib; Ringwood, J1; Slaterville, Aug, West; Owego, May, J1, West; Lake Ridge, J1; Poughkeepsie, May; Middletown, J1, Spooner; SI: Clove Val., Aug, Bs (Ald); LI: Baiting Hollow, Aug, Huck.

\section{(Subgenus EUSISYROPA Townsend)}

Z. blanda O.S. E. Aurora, J1 (MCV); Boston, J1 (MCV); Ithaca, Je-Aug; McLean, J1-Sep, West; SI, Sep, Ds (West), Bs (Ald).

Z. blandita Coq. McLean, J1, West (Ald).

Z. futilis O.S. Niagara Falls, Je; Thompson, May; Ithaca and vic., Je-Aug, reared by C. L. Liu, from Malacosoma americana; Olean, J1, West ; Malloryville, Je; Caroline to Harford, Je; McLean, J1, West, Sep, Sib; Ringwood, May, Leon; L. Tackawasick, Je; Spencer L., Je; Michigan Hollow, J1; LI: Brooklyn, Eng (Beq); Flatbush (Beq). 
PHOROcERA Robineau-Desvoidy

(Subgenus PHOROCERA Robineau-Desvoidy)

P. assimilis Fall. LI: West Hills, Ap, Ds (Ald). Probably an old determination of slossonae.

P. slossonae Towns. Ithaca, Ap, Diven, May; LI: West Hills, Ap, Ds.

(Subgenus Parasetigena Brauer and Bergenstamm)

P. claripennis Macq. Niagara Falls, Je; Canandaigua, J1; Ithaca, Sep; Oakwood, Je, Ds, Bs (Ald) ; Owego, J1, West; Karner, Aug; White Plains, Bno (Beq); SI (Beq).

P. einaris Sm. SI, Aug (Bs); LI: Wading R. (Bs).

P. imitator Ald. \& Webb. LI: Baiting Hollow, Aug, Huck.

(Subgenus Patelloa Townsend)

P. leucaniae Coq. McLean, J1, West (Ald); Oliverea (Beq).

?P. silvatica Ald. \& Webb. Ithaca, May; Albany, May.

\section{EUPELECOTHECA Townsend}

E. celer Towns. Niagara Falls, Je; Ithaca (Walt, as rufilabris Wulp); SI (Bs); LI: Pennequid Barrens (Beq).

\section{EXORISTOIDES Coquillett}

E. slossonae Coq. (Exorista spinipennis Coq.). Slaterville, Aug, West; SI, Bs (Ald) ; LI: Baiting Hollow, Aug, Huck.

\section{Homalactia Townsend}

H. harringtoni Coq. Ithaca, May (Joh).

\section{DORYPHOROPHAGA Townsend}

D. aberrans Towns. LI: Baiting Hollow, Je-Sep, Huck; Wading R., Sep, Bs (Ald).

D. dorsalis Coq. SI, Bs (Ald).

D. doryphorae Rly. Buffalo, Sep, Hamburg, Oct (MCV); LI: Wading R., Sep, Bs (Ald); Baiting Hollow, Sep, Huck.

Frontina Meigen

F. aletiae Riley. Canandaigua L., Aug; NYC (Beq); SI, Sep (Bs); LI: Southampton, J1, Huck; Baiting Hollow, Aug, Wading R., Aug, Huck.

F. ancilla Wlk. LI: Sands Pt., Bs (Ald).

F. archippivora Will. Ithaca, Je-J1, reared by C.L.Liu from Malacosoma americana (Ald).

F. dubia Wlk. LI: Baiting Hollow, J1, Huck. 
F. frenchii Will. Wells, Jl, $Y g$; Ithaca, Je-Aug; Slingerlands, Je; Albany, Feb, Ap; Kingston, Mar; SI, Sep (Bs Coll.) ; LI: Fishers I., Feb; Westbury, Je;

Baiting Hollow, Jl-Sep, Huck; Wading R., Aug, Huck.

?F. spectabilis Ald. LI: Wading R., Aug, Huck.

F. violenta Wlk. LI: Baiting Hollow, Jl-Sep, Huck; Wading R., Aug, Huck.

MYRSINA Robineau-Desvoidy

M. tenthredinidarum Towns. (Masicera exilis auct.). Ithaca, J1; Owego, J1, West (Ald).

\section{EUMASICERA Townsend}

E. sternalis Coq. Boston, Aug (MCV); Ithaca (Will); SI: Richmond, J1, Bs (Ald).

STURMIA Robineau-Desvoidy

S. fraudulenta Wulp. SI (Beq).

S. nigrita Towns. Ithaca, Jl.

S. phyciodis Coq. Ithaca, J1, West (Ald).

PHRYNOLYDELLA Townsend

P. eufitchiae Towns. Hamburg, Je (MCV); Ringwood, J1 (Ald).

PARAPHOROCERA Brauer and Bergenstamm

P. myoidaea Desv. (Andrina radicis Towns.). Rye, Aug, Bird.

MASICERA Meigen

(Ceromasia Rondani)

M. albifacies Towns. Niagara Falls, Sep (MCV, as albifrons Towns., sic!) ; Ithaca, Aug.

M. arator Ald. Ithaca, J1, reared by C.L.Liu from Malacosoma americana.

M. celer Coq. LI: Wading R., Aug.

M. senilis Meig. Rye, Bird (Beq); LI: Sands Pt., Je (Bs); Long Beach (Beq).

\section{LYDELLA Robineau-Desvoidy}

L. lathami Curran. LI: Greenport.

PARAchaeta Coquillett

P. fusca Towns. (bicolor of Coq. not Macq.; inermis of Coq. not Big.). Ithaca, J1, West (Ald); NYC (Coq).

\section{BLEPHARIPEZA Macquart}

B. adusta Lw. Erie Co, Je-Aug (MCV) ; Ithaca; Freeville, J1; Onteora Mt., Hoze: (Ald\&Greene); Ramapo, Ds (Ald); Mooers, Sep, Not (Lutz). 
B. leucophrys Wied. Keene Val. (Beq); Wells, May, J1, Yg; Paradox L., J1, Ds (Ald) ; North Cr., J1, Fbs; Ithaca, Ap-Aug; McLean, May-Sep ; L. George, Sep, Leon; Karner, Ap; Schodack, Ap; Oliverea (Beq); Ramapo, Mar, Ds (Ald) ; SI, Mar-Sep, Ds, Bs; LI: Sands Pt., Sep (Bs Coll.); Wading R. (Bs Coll.); Baiting Hollow, May, Aug-Sep, Huck.

B. rufescens Towns. "NY". Probably not distinct from adusta Lw. (West).

\section{WinTHEMIA Robineau-Desvoidy}

W. fumiferanae Tothill. Rock City, Je; Ithaca, May-J1; McLean, J1; Ringwood, Je; Voorheesville, Aug, Leon; LI: Southampton, J1, Huck; Wading R., Aug, Huck. Placed by characters in Tothill's key (1912). It is not certain that these are truly diagnostic. (West.)

W. quadripustulata Fab. (auct.; more than one species is probably involved here). In this sense $W$. quadripustulata is the most abundant of all our species of Tachinidae. I have before me several hundred specimens from all parts of the State. Dates of collection range from May to October, falling mostly in J1-Sep. (West.)

\section{PARADIDYMA Brauer and Bergenstamm}

P. singularis Towns. McLean, J1; Shelving Rock Mt., L. George, Sep; SI, J1, Oct, Bs (Ald); LI: Sea Cliff (Bks).

\section{Metachaeta Coquillett}

M. atra Coq. (helymus W1k. and auct.). L. Placid, Aug; Ithaca and vic., Ap-J1; McLean, Aug, Sib; Owego, May, West ; Slingerlands, J1; Van. Cort. Pk., J1; LI: Wading R., Sep (Bs Coll.).

\section{ACEMYIA Robineau-Desvoidy}

A. dentata Coq. Ithaca, May; McLean, May; Owego, May, West; Middleton, J1; LI: Riverhead, Sep.

\section{PSEUdochaeta Coquillett}

P. argentifrons Coq. St. Huberts, Aug.

\section{EUTHERA Loew}

E. tentatrix Lw. "NY" (Lw); Gowanda, Je (MCV).

\section{ExORISTA Meigen \\ (Tachina auct.)}

E. larvarum L. (Tachina mella W1k., T. utilis Towns.). Keene Val., J1; Glens Falls, Je; Niagara Falls, Je-J1; Alder Cr., Sep; Ithaca, Je-Aug, reared by C. L. Liu from Malacosoma americana; McLean. Aug, Sib; Albany, J1; Hoversville, Je, Leon; Hudson R. Val.; White Plains (Beq); Southfields (Beq) ; SI (Bs Coll.) ; LI: Baiting Hollow, May-Sep, Huck; Wading R., Aug, Huck; Selden, J1, Huck. 
Chaetotachina Brauer and Bergenstamm

C. simulans Meig. (Tachina rustica auct.). Axton, Je, $M \& H$; Saranac L., Aug-Sep, Ds (Ald); Mt. Whiteface, 2000-4000 ft., Aug; Mt. McIntyre, J1; Northampton, Je; Wells, J1; Denmark, May ; Rainbow L., Je; Deansboro, Je; Ithaca and vic., May-Aug; McLean, J1, Sep; Owego, J1-Aug, West; L. Tackawasick, Je; W. Nyack, J1 (Bs) ; Oliverea, Je, Ds; White Plains (Beq) ; LI: Flushing (Beq).

\section{TACHINOMYIA Townsend}

T. robusta Towns. Potsdam; Rock City, Je, Ds; Ithaca, Ap-May; Ringwood, Je, West; McLean, Ap, Kngt; Thacher Pk., Albany Co.; Karner, Ap-May; Clinton Hgts., Ap; E. Schodack, Ap; Poughkeepsie, May; Bronxville, Mar, Ds (Ald); West Pt., Je; SI, Bs (Ald); LI: Pennequid Barrens (Beq); Wyandanch (Beq) ; Baiting Hollow, May, Huck; Selden, J1, Huck; Gardiners I. ; East Hampton, May; Montauk, May; Riverhead, Je, Huck.

NeOfischeria Townsend

N. flava Towns. SI: Watchogue, Sep (Bs).

\section{PhoRIChaEta Rondani}

P. sequax Will. L. Placid, Aug, $M C V$; near Mt. McIntyre, J1, West; Ithaca, May; McLean, May; Trenton Falls, Je.

P. vernata West (Wagneria vernata West). Ithaca, May-Je; LI: Baiting Hollow, May, Huck.

\section{EUTRICHOGENA Townsend}

E. setipennis Coq. Upper Ausable L. (Beq).

\section{GonIA Meigen}

G. aldrichi Toth. Karner, May (Curr); Oswego (Toth).

G. breviforceps Toth. Potsdam (Curr).

G. confusa Curr. Ithaca, Albany, Ap (Curr).

G. exul Will. "NY" (Will).

G. frontosa Say (capitata DeG. auct., in part). Wells, Ap; Black Br., Je, $C$, Fbs; Fulton Co; Colden (MCV); Hamburg, May (MCV); Ithaca, Ap-May; Ringwood, Je, West; Caroline to Harford, Je ; Albany, Ap-May; Albany Co, May, Leon; Karner, May; Clinton Hgts., Ap; Thompson, May, Leon; Wappingers Falls, Je, Leon; W. Nyack (Bs Coll.) ; SI, Mar-May, Bs, Ds; LI: Baiting Hollow, Je, Huck; Flushing (Beq); Long Beach, Central Pk. (Beq). I have credited to frontosa the majority of manuscript records pertaining to capitata DeG. (West).

G. longipulvilli Toth. Ithaca, Ap (Curr).

G. sagax Towns. Honeoye Falls, May; Ithaca and vic., May; Albany, May; Thacher Pk., May.

G. sequax Will. Wells, Aug, $Y g$ (Curr).

\section{(Subgenus Cnephalogonia Townsend)}

G. distincta Sm. Ithaca, Ap; Owego, J1, West; Karner, Ap; E. Schodack, Ap ; LI: Baiting Hollow, Ap-May, Huck. 


\section{SPALLANZANIA Robineau-Desvoidy}

S. hesperidarum Will. Wells, J1-Aug, $Y g$; Keene Val., Aug; Ithaca, J1; Karner, J1; SI, J1 (Bs Coll.) ; LI: Riverhead, Je; Baiting Hollow, Sep, Huck.

S. obsoleta Towns. Niagara Falls, Je (Towns).

\section{EUGAEDIOPSIS Townsend}

E. ocellaris Coq. L. Placid, Aug; Ithaca, Je-Aug; McLean, Aug-Sep, Sib; Owego, $\mathrm{J} 1$, West.

Chaetogaedia Brauer and Bergenstamm

C. analis Wulp. Newcomb, J1; Ithaca, J1-Sep; McLean, Aug-Sep, Sib; Owego, J1, West; Albany, Sep; Watervliet Reservoir, Aug, Leon; Nassau, Sep ; LI: Baiting Hollow, Sep, Huck.

\section{DICHOCERA Williston}

D. lyrata Will. Ithaca, Sep.

D. orientalis Coq. Juanita I., L. George, Sep, Leon (Ald).

\section{TRICHOPHORA Macquart}

T. ruficauda Wulp. Ithaca, J1; Owego, J1; Albany, Je-Aug; Poughkeepsie, J1; Ft. Montgomery, Schott (Beq) ; SI, Je-Sep, BS (Ald); LI: Cold Sp. Harb. (Beq).

\section{Cuphocera Macquart}

C. (Trichophora) stricklandi Curr. (possibly C. furcata Wulp and auct.). Poughkeepsie, Je (Curr); LI: Yaphank, J1, Ds (Curr).

\section{DEOPALPUS Townsend}

D. hirsutus Towns. LI: Yaphank (Beq).

\section{Peleteria Robineau-Desvoidy 102}

P. anaxias Wlk. Axton, Je; near Saranac L., J1, West; L. Placid, Aug; Newport, May, Yg; Old Forge, J1; Erie Co, May, Sep, as robusta (MCV); Ithaca and vic., J1-Oct; McLean, Je, Aug-Sep, Sib; Owego, Sep, West; Ballston L., Aug, Leon; Pearl Pt., L. George, Aug; Karner, May-Je; Rensselaer, May, Leon; Albany, May; Poughkeepsie, May; E. Jewett, Aug, Ds, as robusta (Ald); Oliverea, as robusta (Beq); SI, May, as robusta (Bs Coll.) ; LI: Baiting Hollow, Je-Sep, Huck; Sands Pt., Sep, as robusta (Bs Coll.) ; Cold Sp. Harb., Pennequid Barrens, as robusta (Beq). I have credited to anaxias all the manuscript records pertaining to $P$. robusta auct. Probably some should be referred to confusa Curr., but I am unable to decide which, not having seen Van Duzee's or Bequaert's material. (West.)

P. confusa Curr. Keene Val., Aug ; Elizabethtown, Aug ; Wells, Je, $\mathrm{Yg}$; Adirondack Lodge, J1, Bish; Saranac L., Sep, Ds; N. Fair Haven, Aug; Walton, Sep,

102 All Cornell material determined by C. H. Curran. 
West ; Ithaca, J1-Sep ; Ringwood, J1, Oct; McLean, J1-Sep, Sib; Owego, Aug, West; Pearl Pt., L. George, Aug, Shelving Rock, Sep, Leon; Karner, Oct; SI, Sep, Ds.

P. iterans Wlk. Axton, Je, $M \& H$; Keene Val., J1; Saranac Inn; Wells, J1, Lowville, J1, West; Long L., Aug; North Cr., J1, Fbs; Mt. Skylight (Beq) ; Gloversville, J1; Ground I., Sep (MCV) ; Hamburg, Oct (MCV); Cattaraugus, Sep; Ithaca, Aug; McLean, J1-Sep; Mud Cr., Je; LI: Gardiners I. (Beq).

\section{ARCHYTAS Jaennicke}

A. analis Fab. (amethystina Macq. of Curran's determination). Wells, J1-Aug, Yg; Long L., Ds; L. Pleasant, J1; Niagara Falls, Je; Erie Co, Je-Sep (MCV); Cattaraugus, Sep; North Cr., J1, Fbs; N. Fair Haven, J1, West; Ithaca, JeSep ; Lake Ridge, Aug; McLean, Jl-Sep; Owego, Jl, West; Michigan Hollow, Aug; Taughannock Falls, J1; Watervliet Reservoir, Aug, Leon; Kinderhook, Sep; Ballston L., Aug; E. Jewett, Aug, Ds; Big Ind. Val.,. Oliverea (Beq); Onteora Mt., Sep, How (Ald\&Greene); Poughkeepsie, Je; SI, J1-Aug (Bs Coll.) ; LI: Wading R., Sep (Bs Coll.); Canarsie (Beq).

A. aterrima Desv. Piseco L., Aug; Erie Co, J1-Sep (MCV) ; ?Ithaca, May ; Pine I., Je, Ds (Ald); White Plains (Beq); NYC (Beq); SI, Je-Sep (Bs Coll.); LI: Farmingdale, Aug, $D s$ (Ald); Wading R., Sep, $D s$ (Ald).

A. hystrix Fab. Ithaca, Aug, And (Snn Coll.) ; SI, Je, Aug (Bs Coll.) ; LI: Flushing, Manhattan Beach, Orient (Beq).

A. lateralis Macq. Manlius; Ithaca, Aug.

FABRICIELla Bezzi 103

F. algens Wied. L. Tear (Beq) ; Long L., Ds (Ald) ; Mt. Marcy (Beq) ; Oswego; Old Forge, J1; Speculator, Aug; Severance, J1, Bks; Mt. Whiteface,, 2000-4000 ft., Aug; Olcott, J1 (Dt) ; Ithaca, Je-Aug ; Big Ind. Val. (Beq) ; E. Jewett, Aug, Ds (West) ; Onteora Mt., Oct, How (Ald \& Greene).

F. dakotensis Towns. Keene Val., Aug; McLean, Aug, Sib (West) ; Albany, J1; LI: Farmingdale, Je, Bs (Ald); Baiting Hollow, Sep, Huck; Riverhead, Aug-Sep.

F. dakotensis var. orientalis Towns. (probably Echinomyia florum auct.). Axton, Je; L. Placid, Aug (Beq); Hamburg, Aug (MCV); Juanita I., Aug ; Shelving Rock, L. George, Sep, Leon; Debruce, Sep, Ds (Dru); LI: Baiting Hollow, Je, Sep, Huck.

F. hispida Toth. Summit of Mt. McIntyre, Jl, Bronson, Chrisp.

F. montana Towns. L. Placid, Aug; Mt. Marcy, 5000 ft., J1; Old Forge, J1.

F. piceifrons Towns. Keene Val., Not (Beq).

\section{Epalpus Rondani}

E. signiferus Wlk. Axton, Je, $M \& H$; Old Forge, Je-Jl; Adirondack Lodge, Je; Labrador L., Cortland Co, Je ; Bath, May ; Gowanda, Je (MCV); Ithaca, ApJe; L. George, J1; Karner, Ap; Albany, Je; Southfields, Je, Bs (Ald); E. Jewett, Ap, Ds (Ald) ; LI: West Hills, Ap, Ds (Ald) ; Huntington, Wyandanch (Beq); Baiting Hollow, Ap, Huck; Yaphank, May, Ds; Half Way Hollow Hills, May, Ds (Cress).

\footnotetext{
${ }^{103}$ Cornell material determined by C. H. Curran.
} 
BOMBYLIOPSIS Townsend

B. abrupta Wied. New Russia, Aug, Bdy; Big Moose, Aug, $Y g$; Gloversville, Je; N. Fair Haven, Aug; Niagara Falls, Sep (MCV) ; Erie Co, Je-Aug (MCV); Little Falls, J1; Canandaigua L.; Ithaca, Je-Aug; McLean, Aug-Sep, Sib; Albany; Wappingers Falls; Oliverea, Sep, Ds (Ald); Onteora Mt., Aug, How (Ald \& Greene) ; West Pt., Sep, Ds; SI, Je-Jl, Sep, Ds.

JURINIA Robineau-Desvoidy

J. decisa Wlk. (Echinomyia decisa auct.). Keene Val., Long L., Aug; Erie Co, J1-Sep (MCV); Pike; Ft. Plain, Oct; McLean, Aug-Sep, Sib.

\section{FAMLLY MEGAPROSOPIDAE}

Compiled by L. S. West

MicRophthalma Macquart

M. disjuncta Wied. E. Aurora, Sep (MCV) ; Little Valley, Aug (MCV) ; Oliverea, Je, Ds; SI, Je-Ji, Ds; LI: Baiting Hollow, Je-Aug, Huck; Wading R., Aug, Selden, J1, Huck; Wyandanch, Aug, Central Pk., Aug, Ds.

M. michiganensis Towns. (M. phyllophagae Curr. is probably a synonym.)

M. phyllophagae Curr. Keene Val., J1-Aug; Saranac Inn, Aug; McLean, Aug, Sib.

\section{FAMILY DEXIIDAE}

Compiled by L. S. WEST

RHAMphina Macquart

R. leonardi West. Shelving Rock, L. George, Sep, Leon.

\section{Mochlosoma Brauer and Bergenstamm}

M. valida B. \& B. Keene Val., J1.

\section{RHYNCHODEXIA Bigot}

R. confusa West. L. Placid; Keene Val.; Wells; Duck L., near Conquest; Ithaca; Freeville; McLean; L. George; Albany; Nassau; LI: Selden; Baiting Hollow; Riverhead; Fire I. J1-Sep.

R. elevata West. LI: Riverhead, Aug, Huck.

R. translucipennis West. Ithaca, J1, West; McLean, J1, West.

PARAPRosena Brauer and Bergenstamm

P. apicalis Desv. North Cr., Aug; Ithaca and vic., J1-Aug. Reared from tabanid larva (Joh).

DexiA Meigen

(Zelia Robineau-Desvoidy)

D. vertebrata Say. S. Wales, J1 (MCV) ; McLean, J1.VTPhillips; SI, J1, Ds; LI: Yaphank, Sep, Ds. 


\section{Ateloglossa Coquillett}

A. calyptrata West. LI: Selden, Aug.

A. glabra West. Wells,' J1, Yg.

PtilodexiA Brauer and Bergenstamm

P. abdominalis Desv. LI: Wading R., Aug, Ds (Jn).

P. harpasa W1k. Saranac Inn, J1; Old Forge, J1; Keene Val., J1; Mt. Jo, Je, West; L. Tackawasick, Je, Leon; Van Cort. Pk. (Beq) ; SI, Je, Ds; LI: Cold Sp. Harb., Sep; Orient (Beq).

P. hucketti West. Albany, Aug, Leon; LI: Yaphank, J1, Ds; Fire I., Sep, Ds; Riverhead, J1-Aug, Huck.

P. incerta West. West Pt., Sep, Ds; SI, Je, Ds; LI: Riverhead, Je, Aug, Huck; Southampton, J1, Wading R., Aug, Huck.

P. leucoptera West. LI: Riverhead, Sep, Huck.

P. minor West. North Cr., Aug; Duck L., near Conquest, Aug, West.

P. neotibialis West. Albany, J1; West Pt., Sep, Robn; LI: Fire I., Sep, Ds; Riverhead, J1, Huck, Sep; Selden, Aug-Sep, Ds.

P. obscura West. LI: Yaphank, J1, Wading R., Je, Ds.

P. proxima West. LI: Riverhead, Aug, Ds.

\section{MYIOCERA Robineau-Desvoidy}

M. amicabilis West. Ithaca, J1, FCWixom.

M. compacta West. Ringwood, J1, West.

M. protrudens West. Ringwood, J1, Leon.

\section{Phasiops Coquillett}

P. flava Coq. Caldwell (Coq); SI, Ds.

\section{Cholomyia Bigot}

C. inaequipes Big. Ithaca, J1 (Joh) ; LI: Richmond Hill, J1 (Jn) ; Cold Sp. Harb., May.

\section{SARDIOCERA Brauer and Bergenstamm}

S. valida Wied. (Theresia tandrec Desv.). Ithaca, J1; Kinderhook, Sep, Bish; Van Cort. Pk. (Bs) ; SI, Aug, Bs, Ds; LI: Yaphank, J1, Ds; Montauk, Aug, Aqueduct, J1, Wading R., Aug, Ds; Southampton, J1, Huck.

\section{Thelaira Robineau-Desvoidy}

T. nigripes Fab. (Iencozona Panz.). Deansboro, Je, West; Naples, J1; Erie Co, Je-Sep (MCV) ; Honeoye Falls, Je; Ithaca, Je-Aug; Coy Glen, Je; Taughannock Falls, J1; McLean, J1, Sep, Sib; Poughkeepsie, May-Je; SI, Aug, Ds; LI: Selden, J1; Montauk, Aug, Ds. 
EUTHERESIA Townsend

E. satisfacta West. SI, Aug, Ds.

E. sibleyi West. Mt. McIntyre, Je, Jl, Leon, Bish, Sib.

\section{OPSODEXIA Townsend}

O. bicolor Coq. Corinth, Je, Yg; Pearl Pt., L. George, Aug; Kaaterskill, Aug (Jn) ; LI: Cold Sp. Harb., Sep; Wading R., Riverhead, Aug, Huck.

\section{Chaetona Van der Wulp}

C. addenda West. Renwick (Stewart) Pk., Ithaca, Aug, West.

C. americana Big. Ithaca (Will).

C. flavipennis Coq. Niagara Falls, Je (Jn).

C. ordinaria West. Near Mt. McIntyre, J1, West.

\section{ARCTOPHYTO Townsend}

A. gillettei Towns. Gowanda, Aug, as Paraphyto (MCV).

\section{SPATHIDEXIA Townsend}

S. clemonsi Towns. Ithaca, J1 (Ald).

S. dunningi Coq. Potsdam, J1; Ithaca, May; McLean, Je.

Estheria Robineau-Desvoidy

E. tibialis Desv. Erie Co, Aug-Sep (MCV) ; LI: Fire I., Sep, Ds (Jn). I regard these records as rather uncertain, since Ptilodexia harpasa W1k. and Rhynchodexia confusa West have both been confused with E. tibialis in the past. I do not doubt that the species occurs in the State, but I have not myself seen any NY specimens. (West.)

\section{DINERA Robineau-Desvoidy}

D. futilis West. Rochester, Je-J1, Leon; Ithaca, Je, Aug, Sargent; Voorheesville, Je, Leon; LI: Baiting Hollow, Je-Aug, Wading R., Aug, Huck.

\section{MELANOPHORA Meigen}

M. roralis L. Rochester, Oct, Buffalo, Je-J1 (MCV); Niagara Falls, Je (Jn); Ithaca, Je-Jl, Sep-Oct; Albany, Je, Aug-Sep, Yg; LI: Southold, Sep, Ds; Baiting Hollow, Aug, Selden, J1, Huck; Sea Cliff; Flatbush, Hempstead (Beq).

\section{FAMILY SARCOPHAGIDAE SUBFAMILY AMOBIINAE}

Compiled by L. S. West

АмовIA Robineau-Desvoidy

A. aurata Coq. Ithaca, Owego, J1, West.

A. confundens Towns. Lancaster, May (MCV); Ithaca, J1, West (Ald). 
BRACHYCOMA Rondani

B. apicalis Coq. Black Mt., L. George, Sep (Ald).

OpPIOPSIS Townsend

O. sheldoni Coq. SI, Je, Ds (Ald).

\section{SUbFAMILy MILTOGRAMMINAE}

Compiled by L. S. WeST

\section{Metopia Meigen}

M. campestris Fall. Axton; Lowville; Broadalbin; N. Fair Haven; Upper Ausable L. (Beq) ; Duck L., near Conquest ; Ithaca; Slaterville; McLean; Ringwood; Trenton Falls; LI: Sands Pt. (Bs Coll.) ; Cold Sp. Harb. (Bs Coll.) ; Baiting Hollow. May-Sep.

M. lateralis Macq. Ithaca, Owego, Slaterville, Aug. West; LI: Wading R., Baiting Hollow, Aug, Huck.

M. leucocephala Rossi. Wells; Ausable R.; Broadalbin; Saranac L.; Axton; Upper Ausable L. (Beq); N. Fair Haven; Niagara Falls; Buffalo, Lancaster (MCV) ; Ithaca; Slaterville; McLean; Ringwood; Owego; Lake Ridge ; Albany; Middletown; SI; LI: Pennequid Barrens (Beq) ; Wading R.; Baiting Hollow; Riverhead; Jamaica. May-Sep, mostly J1-Aug.

M. tessellata Allen. Ithaca, J1 (Allen).

\section{Euaraba Townsend}

E. grisea Desv. N. Fair Haven, J1, West; Ithaca, Je-J1; Karner, Je; Rensselaer, May; LI: Baiting Hollow, May, Sep, Huck.

\section{OPSIDIA Coquillett}

O. gonioides Coq. SI, Je, Aug, Ds; LI: Long Beach (Beq); Selden, Aug, Riverhead, J1, Huck.

\section{Prosinella Robineau-Desvoidy}

P. fulvicornis Coq. Near Saranac L., J1, West; Batavia, Mar; Ft. Erie, J1 (MCV); Ithaca, Je-Aug; L. Tackawasick, Je; Owego, Aug; SI, Sep, Bs (Ald); LI: Riverhead, Je; Long Beach, Pennequid Barrens (Beq).

\section{GYMNOPROSOPA Townsend}

G. polita Towns. Ithaca, Aug; Middletown, J1, Spooner; SI, Je, Ds (West), Bs (Ald).

HILARELLA Rondani

H. hilarella Zett. (siphonina Zett.). LI: Wading R., Aug, Baiting Hollow, Sep, Huck.

Oestrohilarella Townsend

O. aristalis Coq. Oswego (Allen). 
PACHYophthalmus Brauer and Bergenstamm

P. distortus Allen. Ithaca, May, Sun (Allen).

P. floridensis Towns. SI, Ds; LI: Baiting Hollow, Je, Sep, Huck; Wading R., Sep, Ds (Beq).

P. signatus Meig. Speculator, Je, $Y g$; Niagara Falls, Je; Buffalo, Je (MCV); Ithaca, J1-Aug, Owego, J1, Ringwood, Je, West; Karner, Je; SI: New Dorp, Je, Bs (Ald) ; LI: Baiting Hollow, May-Sep, Wading R., Aug, Huck; Montauk, May; Gardiners I. (Beq).

\section{Senotainia Macquart}

S. rufiventris Macq. L. Charlotte, Je; L. Tackawasick, Je; Rhinebeck, J1, Cy; SI: Tottenville, Je, Bs (Ald); LI: Baiting Hollow, Je, Huck.

S. trilineata Wulp. Keene Val.; Mineola; Saranac L.; Ausable R., West Branch; N. Fair Haven; Duck L., near Conquest; Ithaca; Slaterville; Owego; Nassau; Poughkeepsie; W. Nyack (Bs Coll.) ; SI: Ft. Wadsworth, Watchogue, Arlington (Bs Coll.) ; LI: Gardiners I. (Beq) ; Sands Pt., Wading R. (Bs Coll.) ; Baiting Hollow, Huck. May-Aug; most abundant in Jl.

TAXigramma Perris

T. heteroneura Meig. (nasoni Coq.). Peru, Je.

\section{SUBFAMILY SARCOPHAGINAE}

WOHLFAHRTIA Brauer and Bergenstamm

W. vigil Wlk. Syracuse, Blkm (Hine); Rochester Jct., Leon (Ald) ; Ludlowville; Ithaca, J1 (Joh).

METOPOSARCOPHAGA Townsend

M. pachyprocta Park. (larga Ald.). LI: Oak I. (Ald) ; Sands Pt., J1 (Bs) ; Long Beach, Je, Bs (Ald).

\section{Chaetoravinia Townsend}

C. latesetosa Park. (Sarcophaga). Ithaca, Je (Park); LI: Cold Sp. Harb., Il.

C. quadrisetosa Coq. Yonkers (Beq); SI: Clove Val., Bs (Ald).

\section{HeLICobia Coquillett}

H. helicis Towns. Keene Val.; Buffalo, J1; Syracuse, Blkm (Hine) ; Ithaca, MayAug (Park); LI: Sea Cliff.

LACCHOPROSOPA Curran

L. avium Curr. Ithaca, Mar (Curr).

\section{SARCOPHAGA Meigen}

S. aldrichi Park. Axton, Je (Park); Mt. Whiteface, J1 13, Ds (Jn).

S. assidua Wlk. L. Erie (Park); Lancaster, May; Hamburg, Oct (MCV); SI, Sep 15, Ds (Ald); Ft. Wadsworth, Je, Bs (Ald). 
S. bisetosa Park. Ithaca, May-Sep (Park); Albany; White L., Sep, Zab; Greene Co.

S. bullata Park. Ithaca, J1 (Park) ; Nassau; Nyack; SI: Arrochar, J1, Bs (Ald); LI: Cold Sp. Harb., J1 (Ald).

S. cimbicis Towns. Erie Co, May, Aug (MCV); Ithaca and vic. (Park); Albany; Carmel; W. Nyack (Beq); NYC; SI: Watchogue, Sep, Bs (Ald); Ft. Wadsworth, Oct, Bs (Ald).

S. cingarus Ald. Ithaca, J1, Aug (Park); Greene Co (Ald).

S. cooleyi Ald. Ithaca, May, Rly (Bishopp).

S. communis Park. L. Tear (Beq); Potsdam; Unionport; Buffalo, May-Sep (Park); Ithaca and vic.; Mosholu; SI: New Brighton, May, Bs (Ald); Ft. Wadsworth, Je, Sep, Bs (Ald); LI: Flatbush; Sea Cliff; Cold Sp. Harb.; Gardiners I. (Beq) ; Wyandanch, Eng (Beq); Brooklyn (Beq).

S. dalmatina Schin. Buffalo, Oct (MCV); Albany (Park); NYC, Je; LI: Cold Sp. Harb.

S. dux exuberans Pand. Ithaca, Je (Park).

S. dux sarracenioides Ald. Axton, Je; Ithaca, Je, J1 (Park); NYC; SI, Ds (Beq); LI: Cold Sp. Harb.

S. falculata Pand. Ithaca, Aug, Sep (Park); Nyack.

S. fulvipes dissidia Park. Niagara Falls, $J_{n}$ (Park).

S. haemorrhoidalis Fall. Potsdam; Buffalo, Lancaster, Aug (MCV); Ithaca, Je, J1, Aug (Park) ; Albany (Jn) ; SI: Watchogue, J1, Bs (Ald) ; Ft. Wadsworth, Sep, Bs (Ald); LI: Flatbush; Cold Sp. Harb., Je, J1 (Park); Sands Pt., Aug, Bs (Ald).

S. houghi Ald. Buffalo, Je (MCV); NYC (Ald).

S. hunteri Hough. SI: Ft. Wadsworth, J1, Bs (Ald); New Dorp, Bs (Ald).

S. johnsoni Ald. W. Nyack (Beq); LI: Long Beach, Je, Bs (Ald).

S. latisterna Park. Grand I., Aug (MCV); Ithaca, May-J1 (Park) ; Karner; SI: Arrochar; Ft. Wadsworth, New Dorp, New Brighton, Watchogue, Richmond, May-J1 (Ald).

S. niagarana Park. Niagara Falls, Je 25 (Park).

S. parallela Ald. Inwood, Oct, pupa in dead Helix thyroides, Ds (Ald).

S. peniculata Park. L. Tear, Upper Ausable L. (Beq) ; Olcott, J1; Erie Co, MaySep (MCV); Ithaca and vic., Je, J1 (Park) ; LI: Cold Sp. Harb., J1.

S. reversa Ald. Greene Co (Ald) ; SI: New Dorp, Bs (Ald) ; Ft. Wadsworth, Sep, Bs\&Ds (Ald).

S. scoparia nearctica Park. Keene Val.; Hamburg, May (MCV); Cattaraugus, Je-Sep (Park); Ithaca; NYC, Sep, Bs (Ald); SI: Ft. Wadsworth, Sep, Bs (Ald); LI: Cold Sp. Harb.; Sea Cliff.

S. securifera Vill. Buffalo, Oct; Albany; NYC, Sturt (Ald, Beq) ; SI: New Brighton, J1, Bs (Ald) ; LI: Cold Sp. Harb., Je; Flatbush, Eng (Beq).

S. sinuata Meig. Buffalo; Aug (MCV); Cattaraugus; Ithaca and vic., May-Sep (Park); White Plains (Beq) ; Kiamesha; Mosholu; SI: (Beq) ; Richmond, Je, Bs (Ald); Ft. Wadsworth, Oct, Bs (Aid).

S. spuria Curr. Ithaca, Mar (Curr).

S. utilis Ald. Cattaraugus; Ithaca, Je-Sep (Park); LI: Cold Sp. Harb.

S. yorkii Park. Niagara Falls. 


\section{FAMILY CALLIPHORIDAE}

\section{SUBFAMILY PHORMIINAE}

COCHLIOMYiA Townsend

C. (Compsomyia) macellaria Fab. Buffalo, Oct, $M C V$; Cattaraugus, Sep; Ithaca, J1 (Joh); Albany, Sep; NYC (Beq); SI (Beq); LI: Riverhead, Flushing (Beq).

\section{PROTOCALLIPHORA Hough}

P. avium Snn. \& Dobr. Ithaca, Coutant (Snn\&Dobr).

P. splendida form splendida Macq. SI: Watchogue, Bs (Snn\&Dobr).

P. splendida form sialis Snn. \& Dobr. McLean, Joh; Caroline, And; Fall Cr., Ithaca (West) ; LI: Farmingdale, Beq (Snn\&Dobr).

\section{Phormia Robineau-Desvoidy}

(Euphormia Townsend)

P. regina Meig. Essex Co, May-Je; Syracuse, Oct; Buffalo, MCV; Ithaca, ApNov (Joh); Ramapo, May 31, Ds (Ald); White Plains (Beq) ; Albany; Nassau, Aug; SI: on dead goat, Oct 15, Ds (Ald); Ft. Wadsworth, Mar, Bs (Ald); Watchogue, Mar, Bs (Ald); LI: Long Beach (AM); Rockaway Beach; Promised Land, Sep, Ds (Ald).

\section{PROTOPHORMIA Townsend}

P. terrae-novae Desv. Potsdam; Ithaca, J1 (Joh) ; Clinton Hgts., Ap; N. Elba, Oct, Wat (Ald) ; Upper Ausable L. (Beq) ; Slide Mt., Je, Ds (Ald) ; Ft. Montgomery, Schott (Beq); LI: Aqueduct, Schott; Fire I., Schott (Beq).

\section{SUBFAMILY CALLIPHORINAE}

\section{Cynomyia Robineau-Desvoidy}

C. cadaverina Desv. Mt. Whiteface, J1, Ds (Ald); Upper Ausable L. (Beq); Saratoga, Je ; Syracuse, Oct (Bishopp) ; Buffalo, Oct, (MCV) ; Ithaca, ApMay (Joh); Oliverea, Je, Ds (Ald) ; Albany, Je; SI: Ft. Wadsworth, Clove Val., etc., Mar-Oct, Bs\& $\mathcal{E}$ (Ald); LI: Brooklyn, Ds (Ald); Flatbush, Flushing, (Beq).

C. mortuorum L. L. Tear (Beq).

\section{Calliphora Robineau-Desvoidy}

C. erythrocephala Meig. Essex Co, Aug; Niagara Falls, Je; Ithaca, May-Sep (Joh); Albany, Oct; Clinton Hgts., Ap-Je; NYC, Sep, Bs (Ald) ; SI, J1Aug, on dead goat, Oct 15, Ds (Ald) ; LI: Yaphank, Je, Ds (Ald).

C. viridescens Desv. Axton, Je; Ithaca, J1 (Joh); Schenectady, Aug; SI: Ft. Wadsworth, Mar, Bs (Ald).

C. vomitoria L. Mt. Marcy (Beq); Mt. Whiteface (Beq); Honeoye Falls, Je, Leon; Ithaca, May (Joh); Oliverea, Je 19, Ds (Ald); Slide Mt., Je 18, Ds (Ald); Albany, May; SI: on dead horse, Ds (Ald); Ft. Wadsworth, May, Oct, Bs (Ald).

C. vomitoria nigribarbis Snn. NY. 
LuCilia Robineau-Desvoidy

L. caesar L. Adirondacks, Je-J1; Erie Co, May-Sep, MCV; Ithaca, May-Sep (Joh), Zab (AM); SI: on dead goat, Oct 15, Ds (Ald); Ft. Wadsworth, May, Sep, Bs (Ald) ; Richmond, Jl, Bs (Ald); LI: Riverhead, Wyandanch, Sep.

L. pilatei Hough. SI: Ft. Wadsworth, Aug, Bs (Ald).

L. sericata Meig. Niagara Falls, Je (Jn) ; Ithaca, May-Aug (Joh) ; Albany, Aug;

SI, Oct 15, on dead goat, Ds (Ald); LI: Brooklyn (Beq); Rockaway Beach, Farmingdale, Je.

L. sylvarum Meig. Niagara Falls, Je (Jn); Ithaca, May-J1 (Jn).

\section{SUBFAMILY POLLENIINAE}

Pollenia Robineau-Desvoidy

P. rudis Fab. Common and widely distributed, Jan-Dec.

\section{FAMILY MUSCIDAE SUBFAMILY STOMOXYIDINAE}

\section{Stomoxys Geoffroy}

S. calcitrans L. Biting House-Fly. Common and widely distributed throughout the State, Ap-Nov.

LYPEROSIA Rondani

(Haematobia auct.)

L. irritans L. (serrata R.-Desv.). HORN Fly. Generally distributed throughout the State; frequently a serious pest of cattle in the dairy sections.

\section{SUBFAMILY MUSCINAE}

Musca Linnaeus

M. domestica L. House Fly. Common and widely distributed, Ap-Dec.

\section{Cryptolucilia Brauer and Bergenstamm}

(Pseudopyrellia Girschner)

C. caesarion Meig. (cornicina auct. not Fab.). Debruce, Sep 14, Ds (Beq); Unionport (AM); Nyack, Zab; SI, Mar 20, Ds (Beq); LI: Flatbush, Zab (AM).

\section{PyRELLIA Zetterstedt}

P. cyanicolor Zett. Oliverea (Beq) ; Ft. Montgomery, Schott (Beq) ; Woodhull L., Aug; Ithaca, Feb-Sep (Joh); Fair Haven, Sep; Beaver Kill, Aug (CnJr); E. Jewett, Ap 18, Ds (Ald).

\section{Mesembrina Meigen}

M. latreille Desv. (resplendens auct. not Wahlb.). Oliverea, Je, Ds (Ald). 
MORELlia Desvoidy

M. micans Macq. L. Tear, Upper Ausable, Mt. Skylight (Beq) ; Lancaster, MayJe (MCV); Rochester Jct., J1 (Leon) ; Ithaca, Ap-J1 (Joh) ; Albany, May; Rensselaer, Mar; SI: Ft. Wadsworth, Sep, Bs (Ald).

\section{GRAPHOMYIA Desvoidy}

G. maculata Scop. Adirondacks, Jl-Aug; Tompkins Co (Joh); Mosholu (AM) ; NYC, Aug, Bs (Ald) ; SI: Arrochar, Sep, Ds (Ald); Kreischerville, Ft. Wadsworth, Sep, Bs (Ald); LI: Riverhead.

\section{Muscina Desvoidy}

M. assimilis Fall. Axton, Je (Joh) ; Rochester Jct., Je, Leon; Ithaca, Ap-Je (Joh) ; NYC, Aug, Bs (Ald).

M. stabulans Fall. Stable Fly. Potsdam, Mar; Niagara Falls, Je (Jn); Erie $\mathrm{Co}, \mathrm{Je}(\mathrm{MCV})$; Syracuse, Blkm (Hine); Ithaca, Ap-Nov (Joh) ; Albany, $\mathrm{Jl}$; Rye, associate of Papaipema purpurfascia in decaying matter in burrows (Bird); Nyack; Ft. Montgomery, May, Ds (Ald); SI: Ft. Wadsworth, Je, Bs (Ald) ; LI: Cold Sp. Harb., Sep, Bs (Ald).

M. pascuorum L. Ithaca, Sep-Nov, 1922 (Joh); introduced from Europe.

\section{MYIOSPILA Rondani}

M. meditabunda Fab. Niagara Falls, Je (Jn); Erie Co, Aug-Oct (MCV); Tompkins Co, Ap-Sep (Joh) ; Albany, Je; Kenwood, Albany Co, J1; Bryn Mawr Pk., Sep (AM) ; Pine I., Je, Ds (Ald) ; SI: Ft. Wadsworth, Je-Sep, $B s$ (Ald).

\section{SUBFAMILY GASTEROPHILINAE}

\section{Gasterophilus Leach}

G. intestinalis DeG. (equi Clark). Horse Botfly. Coopersville, Je; Otto, Comstock; Baldwinsville, CPFitch (Bishopp); Ithaca, CPFitch (Bishopp); Utica, J1, Wells (Bishopp); Syracuse, Oct 6, eggs on horses (Bishopp); Cropseyville, Je (NYS Sep 15).

G. veterinus (nasalis L.). Utica, J1, Wells (Bishopp); Ithaca, CPFitch (Bishopp).

\section{FAMILY ANTHOMYIIDAE 104} SUBFAMILY PHAONIINAE

PSEUdophaONIA Malloch

P. orichalcea Stein. Ithaca, May (Huck).

\section{Bigotomyia Malloch}

B. houghi Stein. McLean Bogs, J1 (Huck); LI, Sep (Huck).

\footnotetext{
${ }^{104}$ This list has been checked by Dr. H. C. Huckett. The arrangement of subfamilies and genera, and the synonymy, express his latest opinion of relationships, which differs somewhat from that of Johnson's "List of the Diptera of New England."
} 


\section{DENDROPHAONIA Malloch}

D. hilariformis Stein. Ithaca, Joh (Stein).

D. querceti Bouché. Ithaca, Je, reared from a screech owl's nest, Snn \& Dobr (Huck).

\section{PhaOnIa Robineau-Desvoidy}

P. apicata Joh. Mt. Marcy (Beq) ; Ithaca, May-Sep, type; McLean, J1-Sep, Sib (Joh).

P. apta Stein (winnemanae Mall.). Herkimer, Aug (Huck); Ithaca, J1, Joh (Stein); McLean Bogs, Sep (Huck).

P. atlanis Mall. Peru, Je (Huck); LI, Aug-Sep (Huck).

P. azygos Mall. "NY" (Mall).

P. bysia Wlk. Adirondacks, Je-Sep, Ithaca, Je, LI, May-Sep (Huck).

P. curvipes Stein. McLean Bogs, Aug (Huck).

P. deleta Stein. Ithaca, Je (Ald); McLean, Aug, Sib (Joh).

P. diruta Stein. Ithaca, May-Aug (Joh).

P. errans Stein (errans Stein nec Meig.; tinctipennis Rond.?). Rock City, Je 5 (Joh); Ithaca, May 17 (Joh).

P. flavibasis Mall. Ithaca, May (Huck).

P. fusca Stein. Ithaca, May, LI, Aug (Huck).

P. harti Mall. Bryn Mawr Pk., Sep (Huck).

P. laticornis Mall. Adirondacks, Aug, LI, Ap-May (Huck).

P. morrisoni Mall. Adirondacks, Aug (Huck).

P. nigricans Joh. (nervosa Stein, cayngae Joh.). Ithaca, May-Aug (Joh); McLean, May, Aug, Sib (Joh).

P. pallicornis Stein. Greene Co, Aug, How (Huck).

P. pallidula Coq. LI, May (Huck).

P. prisca Stein. Ithaca, J1, Joh (Stein).

P. protuberans Mall. Mt. Marcy, 5300 ft. (Beq), paratype.

P. pulvillata Stein. LI, Aug (Huck).

P. rufibasis Mall. LI, Ap (Huck).

P. serva Meig. Woodhull L., Aug (Jn); L. Tear (Beq); Honeoye Falls, May, Leon; Ithaca, Je (Joh); McLean, May, Sib (Joh).

P. soccata W1k. Ithaca, May (Ald).

P. solitaria Stein. Ithaca, May (Stein).

P. subfusca Mall. Carmel, Aug (Huck).

P. tipulivora Mall. Adirondacks, Aug (Huck).

\section{Alloeostylus Schnabl and Dziedzicki}

A. diaphanus Wied. (Trichopticus signia Wlk.). Axton, Je 21 (Joh); Cranberry Cr., Broadalbin, Je (NYS); Upper Ausable L. (Beq) ; N. Elba, 2000 ft., J1 (Ald) ; Erie Co, May-Sep (MCV) ; Little Valley, J1 (MCV); Ithaca, May 19; Catskills; Albany, Je (NYS); Ft. Montgomery, Schott (Beq) ; LI: St. Albans, Je. 
TRICHOPTICUS Rondani

T. conformis Mall. Old Forge, J1 (NYS).

T. infestus Stein (latipennis Mall.). "NY" (Mall); Ithaca, Joh (Stein), as Alloeostylus.

T. innocuus Zett. Oliverea, Je 21, Ds (Ald); Upper Ausable L. (Beq).

T. johnsoni Mall. Adirondacks, J1 (Ald).

T. spiniger Stein. Mt. Marcy, 5300 ft. (Beq); L. Tear (Beq); Speculator (NYS).

\section{Dialyta Meigen}

D. flavitibia Joh. Adirondacks, Aug 17, type; Upper Ausable L. (Beq) ; McLean, J1, Sep, Sib (Joh).

\section{POGONOMYIA Rondani}

P. flavinervis Mall. (nitens Stein). Niagara Falls, Je (Jn); Lancaster, Je-Aug (MCV) ; Greene Co, How (Ald); Ithaca, Je 7 (Joh).

\section{SUBFAMILY MYDAEINAE}

\section{MydAEA Robineau-Desvoidy}

M. brevipilosa Mall. Adirondacks, J1; Ithaca, Je, LI, Ap-May (Huck).

M. discimana Mall. Adirondacks, J1; Ithaca, May-Aug, LI, Je-Sep (Huck).

M. flavicornis Coq. "NY" (Coq).

M. flavidipalpis Mall. Ithaca, Je, LI, J1-Sep (Huck).

M. neglecta Mall. Adirondacks, J1, Ithaca, Je, LI, Ap-Sep (Huck).

M. obscura Stein not Wulp (nubila Stein). Ithaca, Je (Ald).

M. pagana Fab. Tompkins Co; Albany, Sep (NYS).

M. persimilis Mall. Albany, May (Huck).

M. tincta Zett. N. Fair Haven, J1, Ithaca, Je (Huck).

M. urbana Meig. Erie Co, Je-Aug (MCV); Ithaca, May-Sep (Joh); McLean, May-Sep, Sib (Joh); Poughkeepsie (NYS); LI: Central Pk. (Beq).

M. winnemanna Mall. LI, Aug-Sep (Huck).

\section{HeLINA Robineau-Desvoidy}

(Spilogaster Macquart, in part)

H. consimilata Mall. Ithaca, J1 (Huck).

H. duplicata Meig. Ithaca, Je (Joh); LI: Long Beach (Beq).

H. johnsoni Mall. LI, Je (Huck).

H. lysinoe. Wlk. (varia Stein). Boston, Aug (MCV); Ithaca, May-Sep (Joh); McLean, May, Aug, Sib (Joh); Round I., Sep.

?H. nigripennis Zett. Lancaster, Aug (MCV).

H. obscurata Meig. (nasoni Mall.). Ithaca, Je-J1 (Huck).

H. obscurinervis Stein. Ithaca, May-J1; LI, J1-Sep (Huck).

H. obscuripes Zett. (cothurnata Rond.). Ithaca, Je (Joh). 
H. pectinata Joh. (biseriata Stein, mimetica Mall.). Upper Ausable L. (Beq); Adirondacks, J1 (Huck) ; Ithaca, Je 11, type; McLean, Je-J1, Sib (Joh).

H. procedens Wlk. (uniseta Stein). Elma, Aug 21 (MCV); Ithaca, Je-Aug (Joh).

H. rufitibia Stein. Ithaca (Joh); SI, Ap, Ds (Ald).

H. tinctipennis Stein. Ithaca (Stein).

H. uliginosa Fall. Ithaca, Mar (Joh); Albany, Sep, Yg (NYS).

\section{SPILARIA Schnabl}

S. lucorum Fall. Ithaca, Je (Joh); J1 (Huck); McLean, Je-J1, Sep, Sib (Joh) ; Niverville, Aug (Jn); LI, Ap-Sep (Huck).

S. marmorata Zett. N. Fair Haven, J1 (Huck).

S. multisetosa Schnabl. Adirondacks, J1-Sep.

\section{HEBECNEMA Schnabl}

H. affinis Mall. LI, Aug (Huck).

H. fumosa Meig. Essex Co, Je (Huck); LI, J1-Aug (Huck).

H. umbratica Meig. Saranac Inn, Sep, Ds (Ald); Erie Co, Je-Aug (MCV); Tompkins Co, May-Aug (Joh); SI, Aug 17, Ds (Ald); Ft. Wadsworth, Mar, on Forsythia, Bs (Ald).

H. vespertina Fall. Ithaca, Aug (Huck); LI, May-Je (Huck).

\section{SUBFAMILY HYDROTAEINAE}

\section{HydRotaEA Robineau-Desvoidy}

H. acuta Stein. Ithaca, Je, J1 (Huck).

H. armipes Fall. Ithaca, May (Huck) ; SI: Watchogue, Je, Bs (Ald) ; Richmond, $B s$ (Ald) ; LI: Farmingdale, Je, Bs (Ald).

H. comata Ald. Ithaca, May (Huck).

H. cristata Mall. Adirondacks, J1 (Ald).

H. houghi Mall. McLean, Aug, Sib (Joh); Oswego, Je (Coq).

H. metatarsata Stein. SI: Richmond, $B s$ (Ald).

H. meteorica Linn. LI, May (Huck).

H. militaris Meig. (impexa Lw.). Speculator (NYS); Rock City, Je; Tompkins Co, May-Aug (Joh).

H. nidicola Mall. Ithaca, Ap, Dobr (Mall).

H. occulta Meig. Ithaca, May, Sep (Joh).

H. orbitalis Ald. Ithaca, Je (Huck).

H. tuberculata Rond. Ithaca (Stein).

H. unispinosa Stein. Ithaca, May 29 (Joh); McLean, May-J1, Sep, Sib (Joh).

\section{OPHYRA Robineau-Desvoidy}

O. aenescens Wied. LI, J1 (Huck).

O. leucostoma Wied. Saranac L., Sep, Ds (Ald) ; Severance (NYS) ; Manlius, HHS ; Ithaca, May-Aug (Joh) ; McLean, Aug-Sep, Sib (Joh) ; Albany, Je ; LI: Massapequa, J1, Bs (Ald) ; Southold, Sep 6, Ds (Jn) ; Central Pk., Huntington, Orient (Beq). 


\section{SUBFAMILY FANNIINAE}

FANNIA Robineau-Desvoidy

F. aerea Meig. Ithaca, J1 (Huck).

F. canicularis L. Axton, Je; Northville, Je, $Y g$ (NYS); Buffalo (MCV); Tompkins Co, May-Aug (Joh); Albany, Mar; SI, Ds (Ald).

F. carbonaria Meig. (corvina Verr.). Tompkins Co, Je (Joh).

F. fuscula Fall. (tetracantha Lw.). Ithaca (Joh); SI: Watchogue, Je-J1, Ft. Wadsworth, Aug, Bs (Ald); LI: Sands Pt., Je, Bs (Ald).

F. genualis Stein. "NY" (Stein).

F. glaucescens Zett. Ithaca, May-J1 (Huck); LI, May, Sep (Huck).

F. incisurata Zett. "NY" (Meade); N. Evans, Nov 14 (MCV).

F. kowarzi Verr. Albany, May (Huck).

F. laevis Stein. LI, Je.

F. latipalpis Stein. Ithaca, Je (Huck); LI, May (Huck).

F. manicata Meig. Ithaca, Sep (Stein); McLean, Aug, Sib (Joh).

F. minutipalpis Stein. Caroline to Harford, Je (Huck); Ithaca, Je; LI, May.

F. ochrogaster Thoms. Adirondacks, J1 (Ald).

F. pallidiventris Ma11. McLean, J1 (Huck); LI, May (Huck).

F. parallela Joh. (sociella Zett.?). Wells, Je (NYS); Ithaca, Aug 30.

F. pellucida Stein. LI: Cold Sp. Harb., Sep (Huck).

F. postica Stein. "NY" (Stein); Adirondacks, J1 (Ald); Ithaca, Je (Hủck).

F. pusio Wied. Ithaca, J1 (Huck).

F. serena Fall. Upper Ausable L., Speculator, Keene Val., Saranac Inn, Je-Aug (Huck-NYS); Tompkins Co, May-Je (Joh).

F. scalaris Fab. Niagara Falls, Je; Ithaca, Ap-Nov (Joh).

F. spathiophora Mall. Adirondacks, J1 (Ald).

\section{Coelomyia Haliday}

C. subpellucens Hal. Mt. Marcy (Beq); L. Tear (Beq); Mt. Redfield (Beq).

\section{Platycoenosia Strobl}

P. mikii Strobl. LI: Pennequid Barrens (Beq).

\section{Azelia Robineau-Desvoidy}

A. cilipes Hal. Adirondacks, J1 (Ald); Gloversville, Alex; Caroline, Je (Joh); Ithaca, Aug (Huck).

A. gibbera Meig. Ithaca, May (Huck).

A. triquetra Wied. Ithaca, Je (Huck). 


\section{SUBFAMILY LISPINAE}

LISPA Latreille

L. albitarsis Stein. L. Tear (Beq); Erie Co, May-Jl (MCV); Tompkins Co, Aug (Joh); SI, Aug 18, Ds (Ald); LI: Sea Cliff (Joh); Brooklyn, Je 4, Ds (Ald).

L. nasoni Stein. Manlius, HHS (Joh); LI: Long Beach (Beq).

L. palposa Wlk. Erie Co, Je-Oct (MCV); Manlius, HHS (Joh) ; NYC, Je 15, Ds (Ald); LI: Long Beach (Beq).

L. sociabilis Lw. Ithaca, J1-Sep (Joh).

L. tentaculata DeG. Erie Co, May-Aug (MCV); L. George, Sep, Ndm (Joh); Ithaca, J1-Sep (Joh).

L. uliginosa Fall. L. Tear (Beq) ; Manlius, HHS; Buffalo, MCV (Ald) ; Ithaca, Aug (Joh); McLean, Aug, Sib (Joh).

\section{SUBFAMILY LIMNOPHORINAE}

LIMNOPHORA Robineau-Desvoidy

L. discreta Stein (incrassata Mall.). Ithaca, Aug-Sep (Joh); McLear, J1-Aug, Sib (Joh).

L. narona Wlk. Erie Co, Je-Aug (MCV); Ithaca, Aug 9 (Joh) ; LI: Sands Pt., Je-J1, Bs (Ald); Long Beach (Beq).

\section{LisPoides Malloch}

L. aequifrons Stein. Ithaca, Je-Sep (Joh); McLean, Sep, Sib (Joh).

\section{SpILogona Schnabl and Dziedzicki}

(Melanochelia Rondani)

S. acuticornis Mall. Ithaca, J1 (Huck).

S. (Gymnodia) arcuata Stein. LI, Aug (Huck).

S. alticola Mall. Adirondacks, J1 (Huck).

S. caroli Mall. Adirondacks, J1 (Huck).

S. magnipunctata Mall. Adirondacks, J1 (Huck).

S. monticola Mall. McLean, Sep, Sib (Joh).

S. parvimaculata Stein. Ithaca, Joh (Stein).

S. rufitibia Stein. LI (Huck).

S. surda Zett. Ithaca (Stein). (Cf. acuticornis Mall.)

S. tetrachaeta Mall. Ithaca (Huck).

S. torreyae Joh. Ithaca, May-J1 (Joh).

S. velutina Mall. (nigrifrons Stein). Caroline to Harford, Je (Huck); Tompkins Co, Ap (Stein), as nigrifrons.

\section{TETRAMERINX Berg}

T. unica Stein. LI: Sands Pt., Bs (Ald). 


\section{Lispocephala Pokorny}

L. alma Meig. Ithaca, May, Joh (Stein).

L. erythrocera Desv. N. Evans (MCV); McLean, J1-Sep, Sib (Joh).

\section{PENTACRICIA Stein}

P. aldrichi Stein. McLean, May (Joh).

L. albifrons Zett. "NY".

\section{LIMNOSPILA Schnabl}

\section{Phyllogaster Stein}

P. cordyluroides Stein. Chatham, Aug; LI: Cold Sp. Harb., Aug-Sep, Bs (Ald).

P. littoralis Mall. N. Fair Haven, J1 (West); LI: Long Beach, Je, Bs (Ald).

P. nudiseta Stein. LI: Sands Pt., Je, Bs (Ald).

P. robusta John. LI: Je (Huck); Cold Sp. Harb., Sands Pt., Sep, Bs (Ald).

\section{SUBFAMILY COENOSIINAE}

\section{MACRORCHIS Rondani}

M. ausoba W1k. (Coenosia aurifrons Stein). Alexandria Bay, Sep; Keene Val., Northampton, Broadalbin, Northville (NYS) ; Erie Co, May-Je, Aug, MCV (NYS); Tompkins Co, May-Sep (Joh) ; Albany, Corinth, Poughkeepsie, Je (NYS).

\section{Connosia Meigen}

C. antennalis Stein. LI, J1 (Beq).

C. basalis Stein. "NY."

C. canescens Stein. Tompkins Co, May-Aug (Stein), (See lata Wlk.)

C. compressa Stein. Wells, J1, $Y g$ (NYS).

C. errans Mall. LI, May (Huck).

C. flavicoxa Stein. Ithaca, Jl-Aug (Joh).

C. flavifrons Stein. McLean, J1, Sep, Sib (Joh).

C. fuscifrons Mall. Sport I., Sacandaga R. (Alex).

C. geniculata Fallén. Axton, Je (MacG); Alexandria Bay; Tompkins Co, MayAug (Joh).

C. humilis Meig. (nana Zett.). Ithaca, Mar-Sep (Joh); Lancaster, Mar, MCV (Ald) ; LI: Huntington, Schott (Beq).

C. hypopygialis Stein. E. Aurora, Aug (MCV); Peru, Je, Adirondacks, Aug (Huck).

C. lata Wlk. (?canescens Stein). Ithaca, Aug (Huck) ; Erie Co, Je-Oct (MCV); Greene Co, How (Ald); SI (Beq); LI: Long Beach (Beq).

C. modesta Lw. Ithaca, Aug (Joh); McLean, Aug, Sib (Joh).

C. nigrescens Stein. Ithaca, J1, McLean, Aug (Huck).

C. nivea Lw. Tompkins Co, Je (Joh); Niagara Falls, Je (Jn).

C. nudiseta Stein. Little Valley, Aug; Erie Co, Je-Aug (MCV); LI: Long Beach (Beq).

C. ovata Stein. Ithaca, J1; Otto (Joh). 
C. rufitibia Stein (tibialis Stein). Ithaca, May (Joh); Vandalia, Je (Joh).

C. solita W1k. Buffalo, Oct (MCV).

C. tigrina Fab. Speculator, Yg (NYS); LI: Valley Stream, Aug (Huck).

Macrocoenosia Malloch

M. triseta Stein. Upper Ausable L., Essex Co (Beq) ; McLean, J1, Speculator, J1 (Huck).

Xenocoenosia Malloch

X. calopyga Lw. Buffalo, Je (MCV) ; Ithaca, Joh (Stein) ; SI (Beq) ; LI: Long Beach (Beq).

X. floridensis Mall. Ithaca, J1, Poughkeepsie, J1 (Huck).

X. major Mall. LI: Cold Sp. Harb., Sep (Huck).

HOPLOGASTER Rondani

H. nigritarsis Stein. Upper Ausable L. (Beq); Speculator, Wells (NYS); W. Danby, MacG (Joh); Augur Flats, J1, Yg (NYS); Dug Mt., Aug, Yg (NYS).

\section{SCHOENOMYZA Haliday}

S. chrysostoma Lw. Niagara Falls, Je, MCV (Jn); Tompkins Co, Je-Aug (Joh).

S. dorsalis Lw. (litorella Fall.?). Buffalo, Je (MCV); Colden, May (MCV): Greene Co, How (Ald) ; LI: Riverhead, Sep (Joh).

S. litorella Fall. LI: Gardiners I., Aug (Beq).

\section{SUBFAMILY ANTHOMYIINAE}

ANTHOMYIA Linnaeus

A. pluvialis L. (amoena Meig., oculifera Bigot). Upper Ausable L. (Beq); Hamburg, May (MCV); Ithaca, May-Sep (Joh); McLean, Aug, Sib (Joh); L. George, Ap, Leon (Huck); LI: Hempstead, Ap (Huck).

Calythea Schnabl and Dziedzicki

C. separata Mall. Ithaca, May, Aug (Joh); McLean, Sep (Huck); Canandaigua L., Aug (Joh) ; LI: Hempstead, Ap, J1 (Huck).

ANTHOMYIELLA Malloch

A. pratincola Panz. Rochester Jct., Je, Leon; Ithaca, J1 (Joh) ; LI: Little Neck, Syosset, Riverhead, Je-J1 (Huck).

KINGIA Malloch

K. quintilis Mall. NYC (Beq).

EMMESOMYIA Malloch

E. apicalis Mall. LI: Cold Sp. Harb., Sep, Lakeville, Aug (Huck).

Macateeia Malloch

M. protuberans Mall. LI: Hempstead, Sep (Huck). 
NEOHYLEMYIA Malloch

N. mallochii Huck. Ithaca, Aug, types; LI: Hempstead, Glen Head, Cold Sp. Harb., Aug-Sep (Huck).

HYDROPHORIA Robineau-Desvoidy

H. ambigua Fall. Ithaca, Je-Sep (Huck); McLean, J1, Sib (Joh).

H. divisa Meig. Herkimer, Aug, Lcon (Huck); Ithaca, May (Huck); McLean, Aug, Sib (Joh).

H. flavohalterata Mall. SI: Watchogue, J1, Leon (Huck).

H. orientalis Huck. LI: Baiting Hollow, Aug, type (Huck).

H. ruralis Meig. Ithaca, Joh (Huck).

H. uniformis Mall. Ithaca, May (Huck); McLean, Sep, Sib (Joh); SI: Watchogue, Bs (Ald) ; LI: Cold Sp. Harb., Sep (Huck).

\section{Eustalomyia Kowarz}

E. festiva Zett. Mt. Whiteface, 2000-4000 ft., Aug; N. Fair Haven, Aug (Joh); Ithaca, Je (Joh); McLean, Sep, Sib (Joh); Syracuse, Blkm (Hine).

E. vittipes Zett. Old Forge, First L., Adirondacks, Aug (Huck); Buffalo, Je, $M C V$; Lake Ridge, Aurora, Coy Glen, Ringwood, Ithaca, May-Je (Huck); LI: Syosset, J1 (Huck).

HAMMOMYIA Rondani

H. johnsoni Stein. Ithaca, May-J1 (Huck); Meadowdale, May, Leon (Huck).

H. maculata Stein. Ithaca (Huck).

H. marylandica Mall. LI: Baiting Hollow, May.

H. obtusa Zett. (paludis Joh.). Ithaca, Ap-JI, type and paratype (Joh); LI: Glen Head, Ap (Huck).

H. unilineata Zett. Ithaca, Ap-May; Albany, May, Ig (Huck); Mosholu (HuckAM) ; SI (Beq) ; LI: Glen Head, Valley Stream, Ap (Huck).

H. uniseriata Zett. Ithaca, Ap (Huck); LI, Je (Huck).

\section{PRosalpia Pokorny}

P. angustitarsis Mall. L. George, Sep, Leon (Huck); Beaver Kill, Aug ( $C n J r)$.

P. silvestris Fall. Colden, Je (MCV); Oliverea, Ds (Beq); L. Tackawasick, Je, Leon.

\section{EREMOMYIA Stein}

E. humeralis Stein. Ithaca, Mar, Bish, May, Snn (Huck).

E. vernalis Huck. Ithaca, Mar-J1, type (Huck).

\section{EREMOMYOIDES Malloch}

E. cylindrica Stein. Ithaca, Mar-J1 (Joh) ; Albany, Delmar, Thacher Pk., Karner, Mar-May (NYS); Valley Falls, Yg (NYS); Nyack, W. Nyack (Beq); SI: New Dorp, Bs (Ald).

E. fuscipes Mall. Ithaca, Ap (Huck); SI: Cooper remetery, Mar, Bs (Huck).

E. similis Mall. Ithaca, Mar-Ap, Snn\&West (Huck). 
HyLEMYiA Robineau-Desvoidy

\section{(Including Phorbia Desvoidy and Chortophila Macquart)}

H. albula Fall. N. Fair Haven, J1, West (Huck); LI: Baiting Hollow, Sep (Huck); Cold Sp. Harb., Aug-Sep, Beq\&Bs (Huck).

H. alcathoe W1k. Adirondack Lodge, Keene, L. Placid, Speculator, Wells, Je-J1 (NYS) ; Upper Ausable L. (Beq) ; Buffalo, Je-Aug (MCV) ; Ithaca, AugSep (Joh) ; McLean, Aug-Sep (Beq); LI: Lakeville, May (Huck).

H. antiqua Meig. (Phorbia ceparum Meig.). Onion Maggot. Speculator; Erie Co (Leon) ; Ogdensburg (NYS) ; Williamson, Aug, Buys; Rochester ; Ithaca, McLean, May-Sep (Joh); Olean (Leon); Schenectady (NYS); Albany, Delmar, Kenwood (NYS); Durlandville, J1, FrostSW (Huck). Generally distributed, and in some seasons a serious pest on onions.

H. arnolitra Huck. Ithaca, May (Huck); LI: Hempstead, Ap (Huck).

H. betarum Lint. (substriata Stein). Ithaca, Ap-J1 (Huck); Albany (NYS) ; LI: Hicksville, Valley Stream, Ap (Huck), reared from beet leaves (Huck).

H. bicaudata Mall. Ithaca, Ap; LI, Ap (Huck).

H. brassicae Bouché. Cabbage Maggot. Clinton Co (Felt); Onondaga Co; Wayne Co; Orleans Co; Monroe Co; Ontario Co; Chautauqua; Ithaca; McLean; Albany Co; LI: general. Generally distributed. A serious pest of cabbage, cauliflower, brussels sprouts, and radishes.

H. cilicrura Rond. (Phorbia fusciceps Am. auct. not Zett.). SEED CoRN MAGgot. Chazy; Mooers; Rodman; Macedon; Rochester; Shortsville; Middleport; Lockport; Erie Co (MCV) ; Dansville; Watkins; Tompkins Co; Auburn; Cortland; Syracuse, Blkm (Hine); Stephentown; Oliverea (Beq); Yonkers; SI: Watchogue (Bs); LI: Pennequid Barrens, Great R. Widely, probably generally, distributed. In some seasons very injurious to sprouting beans.

H. coarctata Fall. "NY?" (Fallén).

H. coenosiaeformis Stein. Mt. Whiteface, 2000-4000 ft., Aug (Huck) ; Old Forge, First L., Aug; Saranac, Wells (NYS); Adirondack Lodge, Leon (Huck); Cranberry Cr. (NYS); L. Tear (Beq); Ithaca, Je-Aug (Huck).

H. curvipes Mall. Ithaca, May-J1 (Huck); Montezuma Marshes, Cayuga, J1 (Huck).

H. depressa Stein. Wells, Northampton, Je (NYS) ; Oswego, J1, West (Huck) ; Ithaca, J1-Aug (Huck); Albany, Scotia (NYS); Kinderhook, Je (NYS); Durlandville, Florida, Je, Frost $W$ (Huck).

H. florilega Zett. (planipalpis Stein, pp.). Ithaca, J1 (Huck).

H. fugax Meig. Ithaca, May, Sep-Oct (Huck); McLean, Sep (Huck). The larvae mine leaves of Chenopodium, Amaranthus, spinach, and beet (FrostSW).

H. grandivillosa Huck. LI: Hempstead, Ap (Huck).

H. inconspicua Huck. LI: Hempstead, Ap (Huck).

H. innocua Mall. Gloversville, J1, Alex (Huck); Wells, Cranberry Cr. (NYS) ; Ithaca, J1; McLean, J1-Aug, Sib (Joh).

H. inornata Stein. Ithaca, May (Huck); McLean, May-Sep, Sib (Joh) ; Shandaken, Je, Leon (NYS).

H. ithacensis Huck. Ithaca, Ap-May (Huck).

H. laevis Stein. Ithaca, Je, Sep (Huck); McLean, J1, Sib (Joh). 
H. lasciva Zett. Old Forge, Leon (Huck); Ithaca, Ap-J1 (Huck).

H. latifrontalis Huck. LI: Wading R., Cold Sp. Harb. (Huck).

H. latipennis Zett. Mt. McIntyre, Aug, SnnESib, Bish (Huck) ; Hamburg, Aug (MCV) ; Ithaca, May-Aug; McLean, May, Leon (Huck).

H. megacephala Mall. Ithaca, May; Montezuma Marshes, Cayuga, Je (Huck).

H. piloseta Mall. Mt. Marcy, J1, Fbs (Huck); Ithaca, Je (Huck).

H. pluvialis Mall. Wells (NYS); Buffalo, Je (MCV); Ithaca, J1-Aug (Huck); McLean, J1-Sep, Sib (Joh).

H. pullula Zett. Ithaca, May, West (Huck).

H. sepia Meig. LI: Hempstead, Ap (Huck).

H. setigera Joh. Rock City, Je; Ithaca, Je-J1 (Joh); Oliverea, Je, Ds (Ald) ; LI: Lakeville, Glen Head, May (Huck).

H. setitarsata Huck. Speculator, Je (NYS) ; Ithaca, Ap-May; McLean, May, Sib (Joh); LI: Hempstead, Ap (Huck).

H. simpla Coq. Adirondacks, J1, Ald (Huck).

H. spizellae Huck. Ithaca, reared from nests of chipping sparrow and robin by Dobroscky (Huck).

H. testacea Stein. Adirondacks (Joh); Oswego, J1 (Huck) ; Ithaca, Je; Kinderhook, Je; Durlandville, Je.

H. trichodactyla Rond. Speculator, Je (NYS) ; Peru, J1; Ithaca, Sep; McLean, May, J1, Sib (Joh) ; Clayville, Je, Leon (NYS) ; LI: Hempstead, Je (Huck).

H. trivittata Stein. Wells, Big Moose, Blue Mt., Yg (NYS); LI: Lakeville, Hempstead, J1, Sep (Huck).

H. unidorsalis Huck. Ithaca, Ap (Huck).

H. uniseriata Stein. Adirondacks, J1, Ald (Huck).

H. variata Fallén. Wells, J1 (NYS); Ithaca, Ap; McLean; Albany, Coeymans, Je (NYS) ; LI: Searingtown, Sep (Huck); Long Beach (Beq).

\section{Pycnoglossa Coquillett}

\section{(Pogonomyza Schnabl and Dziedzicki)}

P. cinerosa Zett. Ringwood, Je (Huck).

P. flavipennis Fall. Malloryville, Je, Freeville, J1 (Huck).

P. gleniensis Huck. Coy Glen, Ithaca, May (Huck); Karner, Je (NYS).

P. proboscidalis Mall. (campestris Huck.). Cayuga, Je (Huck); LI: Plandome, Hempstead, May-Je (Huck).

P. spinosissima Mall. Wells, J1 (NYS); Ringwood, J1 (Huck).

\section{EGLE Robineau-Desvoidy}

\section{(Xenophorbia Malloch)}

E. muscaria Fab. Ithaca, Ap (Joh); Karner, Ap (NYS); Nyack, W. Nyack, J1

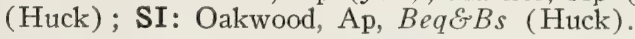

E. longipalpis Mall. Ithaca, Ap-May (Huck); Albany, Je, Karner, Ap (HuckNYS); SI: Oakwood, Bs (Huck); LI: Baiting Hollow, Ap (Huck). 
PAREGLE Schnabl and Dziedzicki

P. cinerella Fall. Speculator, Wells, Je-J1 (NYS); Hamburg, May (MCV); Otto, J1; Ithaca, May-Sep (Joh) ; McLean, Sep, Sib (Joh); Albany, Nassau, May (NYS) ; SI, Aug, Ds (Ald) ; LI: Franklin Sq., Ap; Searingtown, Long Beach, Sep (Huck).

P. radicum L. Keene Val., Aug (Huck-NYS) ; L. Tear (Beq) ; Speculator, Je, Wells, Jl (Huck-NYS) ; Ithaca, Sep (Huck) ; McLean, Aug-Sep, Sib (Joh) ; Ilion, May (NYS) ; Albany, Karner, Je (Huck-NYS) ; Poughkeepsie, Je (NYS) ; SI, Bs (Ald); LI: Glen Head, Ap, Aug; Central Pk. (Beq); Mineola, J1 (NYS).

\section{PEGomyia Robineau-Desvoidy}

P. acutipennis Mall. L. George, Sep, Leon (Huck).

P. affinis Stein. Speculator and Wells, Je-Jl (NYS); Colden, Je (MCV); Rock City (Joh) ; Ithaca, May-Sep (Joh) ; McLean, May, Aug-Sep, Sib (Joh) ; Karner, Easton, May-Je (NYS); Germantown, Je (NYS); White Plains (Beq).

P. bicolor Wied. Northville, Je, Yg (NYS) ; McLean, Aug-Sep, Sib (Joh) ; Syracuse, Blkm. (Hine); Albany, May (NYS); Niverville, May (NYS); Shandaken, Je, Leon (NYS) ; LI: Aug-Sep (Huck) ; Cold Sp. Harb. (Beq); Orient, J1 (NYS).

P. calyptrata Zett. L. George, Sep; Ithaca, May-Aug (Joh) ; Clinton Hgts., May, (NYS) ; LI: Hempstead, Ap (Huck).

P. connexa Stein (emmesia Mall.). Adirondack Lodge, Je, Leon (NYS); Ithaca, Ap; Riders Mills, Bish (NYS) ; Thacher Pk., Thompson L., Karner, May, Leon (NYS) ; LI: Cold Sp. Harb., Sep (Huck).

P. dissecta Meig. Mt. McIntyre, summit (NYS); McLean, May, Ringwood, J1 (Huck); Slide Mt., Catskills, Je, Leon (NYS).

P. fringilla Mall. Ithaca, May-Je (Huck); Albany, May (NYS).

P. fuscofasciata Mall. L. George, Aug, Leon; Karner, Je, Leon (NYS) ; Ithaca, Ringwood, Je; LI: Smithtown, Hempstead (Huck).

P. geniculata Bouché. Speculator, J1 (NYS); Herkimer, Aug, Lcon; Greene Co (Ald) ; Ithaca, Je; McLean Bogs, Sep; Albany, Je (NYS) ; LI: Cold Sp. Harb., Sep (Huck).

P. hyoscyami Panz. (vicina Lint.). Spinach Leaf Miner. Gouverneur; Morrisville; Ithaca ; Cobleskill ; Middleburg ; Middletown ; SI ; LI: Jamaica ; Elmont ; Hempstead. The larvae mine leaves of beets, spinach, chard, and Chenopodium; often injurious.

P. juvenilis Stein. Cranberry Cr., Wells, J1 (NYS); Ithaca, May; L. George, Sep, Leon; SI: Richmond, Watchogue, J1, Bs (Ald) ; LI: Lakeville, May (Huck).

P. lipsia W1k. Keene Val., Trenton Falls, Wells, Speculator, Je-J1 (NYS) ; Aurora, May (Huck); Ithaca, McLean, May-Sep (Joh); Thacher Pk., Easton, May, Leon (NYS); Poughkeepsie, Shandaken, Je (NYS) ; Yorktown Hgts., Ols (Beq).

P. littoralis Mall. Old Forge, Aug (Huck).

P. luteola Mall. L. George, Aug, Leon; Ithaca, Je; LI: Cold Sp. Harb., Lakeville (Huck).

P. nigritarsis Zett. Montezuma Marshes, Cayuga, Je; LI: Valley Stream, Ap (Huck). 
P. rubivora Coq. RASpberry CAne Maggot. Ithaca, Ringwood, May (Huck); L. George, Je; Rome, May.

P. ruficeps Stein. Ithaca, J1; Albany, Je (NYS); LI: Riverhead, J1 (Huck).

P. slossonae Mall. LI: Wading R., J1 (Huck).

P. unicolor Stein. Wells, J1 (NYS) ; Ithaca, Je-J1; McLean, J1, Sib (Joh) ; SI, J1 (Huck,Beq); LI: Lakeville, Little Neck, J1-Aug (Huck).

P. vanduzeei Mall. Ithaca, Taughannock Falls, Ap-May (Huck); Florida (Frost). The larvae mine leaves of Rumex.

P. vittigera Zett. Foot of Mt. Redfield, Essex Co (Beq).

P. winthemi Meig. Ithaca, Je-Sep; McLean, Aug-Sep, Sib (Joh) ; LI: Lakeville, May (Huck).

\section{PRoBoscimyia Bigot}

P. siphonina Bigot. Westfield, Sep (Stein).

\section{SUBFAMILy FUCELLIINAE \\ FUCELLIA Robineau-Desvoidy}

F. maritima Hal. Juanita I., L. George, Sep 1, Leon (Joh) ; LI: Rockaway, Long Beach (Beq).

\section{FAMILY SCATOPHAGIDAE}

(Cordyluridae)

CORDILURA Fallén

C. carbonaria Wlk. (gagatina Lw.). "NY" (Wlk); McLean, May, J1 (Cn.Jr).

C. cornuta Lw. Ithaca and vic., May (Joh).

C. gracilipes Lw. (Parallelomma gracilipes Lw.). Saranac L., J1; Erie Co, Je, Aug (MCV); Ithaca, May (CnJr); LI: Wyandanch, Ols (Beq).

C. latifrons Lw. (confusa Lw.). Van Cort. Pk., May (Bks); Erie Co, Je-Aug (MCV) ; Ithaca, McLean, Je (Joh); Catskills, Sep.

C. praeusta Lw. Erie Co, Je, Oct (MCV); Ithaca, Mày, Je, Sep (Joh) ; Karner (NYS).

C. setosa Lw. Mix Cr. Val.; Colden, May (MCV); Ithaca, Je (Joh); Mohawk R. Val. (Beq); W. Nyack (Beq).

C. terminalis Lw. McLean, J1 (CnJr); Albany (NYS).

C. variabilis Lw. Foot of Mt. Redfield, $2500 \mathrm{ft}$. (Beq).

PaRAllelomima Becker

P. banksi Mall. "NY" (Lw), type.

P. dimidiata Cress. Ithaca, May ( $\mathrm{CnJr})$.

P. munda Lw. L. Tear, Upper Ausable L. (Beq); Lancaster (MCV); Little Valley, Aug (MCV); Inwood; NYC, Schott (Beq).

P. pleuritica Lw. (slossonae Coq.). Erie Co, Je, Oct (MCV); Ithaca, J1 (Joh); McLean, May (CnJr) ; Nassau, Aug.

P. scapularis Lw. NYC, May (Sturt).

HeXamitocera Becker

H. flavida Coq. Caroline, Je ( $\mathrm{CnJr})$. 
Achaetella Malloch

A. varipes W1k. (bimaculata Wlk.). L. Tear (Beq); Fulton Co; Erie Co, MayAug (MCV); Ithaca, May-J1 (Joh); McLean, J1 (CnJr); Manlius; Albany (NYS); White Plains (Bks); Pine I.; Mosholu.

ORTHACHAETA Becker

O. amoena Cress. Ithaca, Ap-May ( $\mathrm{CnJr}$ ).

ACANTHOCNEMA Becker

A. capillata Lw. "NY" (Lw); Old Forge, J1.

Megophthalma Becker

M. lutea Lw. McLean, Sep, Sib (Joh).

SPATHIOPHORA Rondani

S. cincta Lw. Ithaca, Je ( $\mathrm{CnJr})$.

\section{MICROPROSOPA Becker}

M. haemorrhoidalis Meig. L. Tear (Beq); Buffalo, Oct (MCV); Karner.

\section{SCATOPHAGA Meigen}

S. furcata Say. Gloversville, Mar; Erie Co, May-Oct (MCV); Niagara Falls, Je (Jn); Syracuse, Blkm (Hine); Ithaca, Ap-J1; McLean, May (CnJr); W. Nyack (Beq); NYC, Mar-Nov (Sturt); SI (Beq); LI: Farmingdale, Flushing (Beq).

S. merdaria Fab. Niagara Falls, Je (Jn).

S. nigrolimbata Cress. Upper Ausable L. (Beq) ; Ithaca, Je; McLean, May (CnJr) ; Beaver Kill, Aug (CnJr).

S. stercoraria L. Common and widely distributed, representative localities being: Mt. McIntyre, J1; L. Tear (Beq) ; Axton ; Erie Co, Ap-Oct (MCV) ; Otto, May, Je (Joh) ; Clifton Sps., Aug; Ithaca; McLean, May; Manlius; Johnstown; Albany (NYS); NYC, Oct (Sturt); LI: Brooklyn (Beq); Flushing (Beq).

S. suilla Fab. L. Tear and foot of Cliff Mt. (Beq); Wells, J1 $(Y g)$; Niagara Falls, Je $(\mathrm{Jn})$.

HYDROMYZA Fallén

H. confluens Lw. L. George, J1 (CnJr).

\section{FAMILY HELOMYZIDAE 105}

\section{TEPHROCHLAMYS Loew}

T. canescens Meig. (rufiventris Meig.). Upper Ausable L. (Beq); Speculator, Je (Garr-NYS); Ithaca, Ap-May; Erie Co, Ap, Je, MCV (CnJr).

${ }^{105}$ The list for this family has been checked by C. B. D. Garrett, who has added records and made some changes in synonymy. 


\section{SUILlia Robineau-Desvoidy \\ (Helomyza Fallén 1820, not 1810)}

S. apicalis Lw. Taughannock Falls, Ap.

S. longipennis Lw. Upper Ausable L. (Beq); Erie Co, including Little Valley, Je-J1 (MCV) ; Ithaca, Je-Aug (CnJr).

S. plumata Lw. Newport, Je (Garr-NYS); N. Fair Haven; Manlius (OS); Ithaca, Je-Aug; Erie Co, May-Aug (MCV); Oswego.

S. quinquepunctata Say. Ithaca, Je (CnJr).

\section{Allophyla Loew}

A. laevis Lw. L. Tear, Upper Ausable L. (Beq); Niagara Falls, Je (Jn); Erie $\mathrm{Co}$, including Little Valley, J1-Sep (MCV); Ithaca, Aug.

\section{Oecothea Haliday}

O. fenestralis Fall. (possibly O. canadensis Garr.). N. Evans, Nov (MCV); Ithaca (CnJr) ; Albany, Nov (NYS) ; NYC, May (Sturt); LI: Cold Sp. Harb., May (Sturt).

AMoEBALERIA Garrett

A. defessa O.S. (possibly sackeni Garr.). Niagara Falls, Je (Ald); Ithaca, Feb $(\mathrm{CnJr})$.

A. flavotestaceus Zett. (biseta Lw.) (Leria Desv.). Boston, Sep, MCV.

A. sackeni Garr. Clarksville (Garr), paratype.

\section{Anorostoma Loew}

A. marginata Lw. Oswego, J1 (Ald); Buffalo, Je (MCV); LI: Yaphank (O1s) . Garrett writes that there are six species in the genus and that this is possibly A. jerseyi Garr.

\section{SCOLIOCENTRA Loew}

S. helvola Lw. Manlius; Erie Co, Je-J1 (MCV) ; Silver L., J1 (MCV) ; McLean; Ithaca, Je-Aug (Ald); Nassau, Oct; Poughkeepsie, J1 (Garr-NYS).

S. helvola var. angustifrons Bks. Ithaca.

S. tincta Wlk. var. pubescens Lw. Ithaca, Mar (Snn).

Postleria Garrett

(Neoleria Malloch)

P. leucostoma Lw. Ithaca $(\mathrm{CnJr})$.

HELEOMYZA Fallén 1810

(Leria auct., in part)

H. serrata L. Ithaca (Joh). 


\section{PSEUdOLERIA Garrett}

P. pectinata Lw. NYC (Joh); LI: Cold Sp. Harb., And (Sturt). Garrett writes that this is a composite containing ten forms or species.

LUTOMYiA Aldrich

L. spurca Ald. Ithaca, Oct, Joh (Ald), type.

\section{FAMILY CLUSIIDAE}

(Heteroneuridae)

Clusia Haliday

C. czernyi Jn. Sacandaga R., Je, Alex; Erie Co, May, Je; Holland, May (Jn) ; Little Valley, Je (MCV); Chautauqua L., Je (MCV); Ithaca (Mel) ; Albany, Je.

C. lateralis W1k. Erie Co, Je, Aug (MCV); Ithaca (Joh); Albany, Je; SI (Beq).

\section{Clusiodes Coquillett}

(Heteroneura Fallén 1823, not 1810)

C. albimana Meig. E. Aurora, Je.

C. americana Mall. Ithaca, May (Mel); Upper Ausable L. (Beq).

C. atra Mel. \& Argo. Ithaca (Mel).

C. johnsoni Mall. Cortland Co (Mel).

C. melanostoma Lw. Holland, May; Ithaca, Ap (Joh) ; E. Aurora, Je (MCV); L. Tear (Beq).

\section{FAMILY BORBORIDAE}

SPHAEROCERA Latreille

S. pusilla Fall. Buffalo (MCV); NYC, Oct (Sturt).

S. subsultans Fab. Ithaca (Joh); NYC, Mar-May (Sturt).

\section{LEPTOCERA Olivier}

\section{(Limosina Macquart)}

L. atra Adams. LI: Cold Sp. Harb. (Spuler).

L. brachystoma Stenh. LI: Cold Sp. Harb. (Spuler).

L. crassimana Hal. Bear Mt., Je (Sturt).

L. evanescens Tucker. NY.

L. ferruginata Stenh. Albany, Mar-Ap (Sturt); Bear Mt., Je (Sturt).

L. fontinalis Fall. McLean, J1; W. Nyack (Beq); SI, Oct (Sturt).

L. fuscipennis Hal. NY (Spuler).

L. levigena Spuler. LI: Cold Sp. Harb. (Spuler).

L. limosa Fall. Newport, Je, Yg; Albany, Ap.

L. longicosta Spuler. NY.

L. lutosa Stenh.' McLean, J1 (Sib); W. Nyack (Beq).

L. mitchelli Mall. Ithaca, Aug, Joh (Mall). 
BORBORUS Meigen

B. equinus Fall. Ithaca, common (Joh); McLean, J1-Aug; NYC, Feb, in greenhouse (Sturt); LI: Cold Sp. Harb., Mel (Spuler).

B. marmoratus Beck. (brevisetus Mall.). Bear Mt., Je (Sturt); Garrison, May (Sturt); LI: Cold Sp. Harb., Mel (Spuler).

SCATOPHORA Robineau-Desvoidy

(Olina Macquart)

S. carolinensis Desv. (geniculata Macq.). Niagara Falls, Je ( $\mathrm{Jn})$; Geneva; McLean, May-J1, Sib (Joh); Ramapo Mts. (Sturt); Garrison, May (Sturt); Pine I., Sep (AM) ; LI: Cold Sp. Harb., Mel (Spuler).

\section{FAMILY SCIOMYZIDAE \\ NEUROCTENA Rondani}

N. anilis Fall. Ogdensburg, Je (NYS) ; Johnstown; Niagara Falls; Wells, J1 (Yg) ; Ithaca, Aug (Joh) ; McLean, Ap; Karner, Je (Jn); Rensselaer, Je (NYS); Oliverea (Beq).

N. simplex Lw. Ithaca, Je, Aug (CnJr); Karner, May (NYS).

DRYOMYZA Fallén

D. dayi Cress. Ithaca, J1 ( $\mathrm{CnJr})$.

\section{Oidematops Cressun}

O. ferruginea Cress. Niagara Falls, Je (CnJr).

Sciomyza Fallén

S. aristalis Coq. Niagara Falls, J1 ( $\mathrm{CnJr})$.

\section{PTEROMICRA Lioy}

P. nigromana Mg. McLean, Aug, Sib (Joh).

\section{Atrichomelina Cresson}

A. pubera Lw. Ithaca, J1 ( $\mathrm{CnJr})$; Beaver Kill, Aug ( $\mathrm{CnJr})$.

Pherbellia Robineau-Desvoidy

(Melina Robineau-Desvoidy 1830, not Retzius 1788)

P. albovaria Coq. Saranac Inn, Je (NYS); L. Placid, Aug (NYS); Ithaca, Je $(\mathrm{CnJr})$; Niagara Falls, J1 (MCV).

P. annulipes Zett. "NY" (Mel).

P. grisescens Meig. LI: Cold Sp. Harb. (Beq).

P. nana Fall. Ithaca, May-Aug (Joh); W. Nyack (Beq); W. Winfield, Je, Leon (NYS) ; NYC, Oct (Sturt) ; SI, Oct (Sturt).

P. schoenherri Fall (maculata Cr.). Ithaca, J1; Mosholu, Sep 9, Lutz (AM). 


\section{ANTICHAETA Haliday}

A. melanostoma Mel. Ithaca, Je, J1; Beaver Kill, Aug ( $\mathrm{CnJr})$.

\section{RENOCERA Hendel}

R. longipes Lw. Ithaca, May ( $\mathrm{CnJr})$.

\section{Poecilographa Melander}

P. decora Lw. Gloversville; Rochester, Je, Il (Ald) ; Tompkins Co, Je-Aug (Mel) ; L. George, OS (Lw); Elba, Je (MCV); Rock Glen, J1 (MCV); Erie Co, Je-Aug (MCV); Voorheesville, Je (Leon).

Dictyomyia Cresson

D. ambigua Lw. McLean, Sep, Sib (Joh).

\section{EUTHYCERA Latreille}

E. arcuata Lw. (favescens Lw.). Speculator, Aug (Yg); Wells, J1 (Yg); Newport, J1 (Yg) ; Axton; Upper Ausable L. (Beq); Gowanda, Je (MCV); Ithaca; Poughkeepsie, Je-Aug.

E. arcuata var. uniformis Cress. Ithaca, Je (CnJr) ; LI: Forest Pk., Schott (Beq).

\section{TRYPETOPTERA Hendel}

T. canadensis Macq. ( $T$. pallida Lw.). Manlius; Erie Co; Ithaca, J1 ( $\mathrm{CnJr}$ ).

\section{HOPLODICTYA Cresson}

H. setosa Coq. Larchmont, Aug (NYS); LI: Cold Sp. Harb. (Beq).

\section{Dictya Meigen}

D. umbrarum L. (Tetanocera). Elm L., Aug, Yg (NYS); Hamburg (MCV); Little Valley, Aug (MCV); Ithaca; Albany and vic., Je-J1 (NYS); Carmel, Aug (AM); Poughkeepsie, May (NYS); Goshen, Sep (AM); White Plains, Beq; W. Nyack (Beq) ; NYC, Oct (Sturt); SI, Oct (Sturt) ; LI: Cold Sp. Harb. (Beq); Little Neck, Schott (Beq).

\section{LIMNIA Robineau-Desvoidy}

L. boscii Desv. (combinata Lw.). Saranac Inn, Je (Coq) ; Piseco L., Aug (NYS); Manlius (OS) ; Erie Co, Je (MCV) ; Gowanda, Je (MCV) ; Ithaca, Je-Aug; McLean, Je; Sacandaga Pk., Aug, Alex (NYS) ; Albany, Je (NYS); LI: Cold Sp. Harb. (Beq).

L. boscii Desv. var. sparsa Lw. Ithaca, J1 ( CnJr).

L. costalis Lw. Ithaca, Aug (CnJr) ; Albany, Aug (NYS).

L. saratogensis Fitch. Adirondacks, Saranac, Keene Val., North Cr., Aug (NYS); Erie Co (MCV) ; Ithaca; McLean, J1; Broadalbin, Je; Saratoga ; Albany, Je, Clinton Hgts., Je, Poughkeepsie, May (NYS)! Mosholu (Bks) ; Fishkill, J1; LI: Wyandanch, Ols (Beq). 


\section{Tetanocera Dumeril}

T. brevis Cress. Oswego, Aug ( $\mathrm{CnJr})$.

T. clara Lw. Saranac Inn (Coq); L. Placid, EPV (NYS) ; Upper Ausable L., J1 (NYS); Speculator, Wells, Jl-Aug, Yg (NYS); Johnstown, Aug (Yg); Wells, J1 (Yg); Trenton Falls, Je, Yg; Poughkeepsie, Je (NYS); W. Nyack (Beq).

T. elata Fab. Ithaca, Je-Aug ( $\mathrm{CnJr}$ ).

T. ferruginea Fall. Ithaca, May-Aug $(\mathrm{CnJr})$.

T. plebeja Lw. Northville, Speculator, Je-Aug, Yg (NYS); Elizabethtown, Je (NYS) ; Mt. Skylight, $4800 \mathrm{ft}$., Upper Ausable L. (Beq) ; Axton; Elm L., Aug, Chazy L., Je, Mt. Marcy, J1, 5000 ft. (NYS) ; Cranberry Cr., Je, Yg; Niagara Falls, Je (Jn); Erie Co (MCV); McLean; Ithaca (Joh); Trenton Falls, Je, Corinth, May, Yg; Albany, May, Je, Karner, Je, Rensselaer, Nassau, May, Poughkeepsie, Je (NYS) ; Pine I., Sep (AM) ; White Plains, Mosholu, May-Aug (Bezzi); W. Nyack (Beq).

T. rotundicornis Lw. Colden, May (Jn); Hamburg, May (MCV); Little Valley, Je (MCV).

T. triangularis Lw. Ithaca, May-Aug $(\mathrm{CnJr})$.

T. valida Lw. Erie Co, Je (MCV) ; Ithaca, Je, J1 (Jn) ; Manlius (OS) ; Dug Mt., Adirondacks, Aug, $Y g$; W. Nyack, Beq.

T. vicina Macq. (T. plumosus Lw.). Upper Ausable L., L. Tear (Beq) ; Essex Co, Keene Val., Aug (NYS); Blue Mt., J1, Yg; Saranac Inn, J1, Mt. Marcy, $1400 \mathrm{ft}$. (NYS); Manlius; Olcott, J1, Erie Co., J1-Sep (MCV); Otto, J1-Aug (Joh); Ithaca, May-Sep (CnJr); McLean (CnJr) ; Schodack Landing, Je (NYS); Albany, Je; Nassau, J1 (NYS); W. Nyack (Beq); Mosholu (Bezzi); SI, Sep (Sturt) ; LI: Huntington, Schott.

\section{HEDRONEURA Hendel}

H. rufa Panz. (T. lineata Day). Olcott, J1, Buffalo, Oct (MCV); Ithaca, Mar-Je (Joh); McLean, Je.

\section{SEPEDON Latreille}

S. armipes Lw. Dayton, J1, Lewiston, Aug (MCV) ; Manlius (Joh) ; McLean, J1; Ithaca, May; W. Nyack (Beq); Yaphank, Sep.

S. fuscipennis Lw. Saranac Inn (Coq) ; Erie Co, May-Sep (MCV); Ithaca, MayJe (CnJr); Fulton Co; Karner, Ap (Yg); Mosholu, May (Bks); L. Placid, Aug, EPV (NYS) ; Carmel, Aug (AM); W. Nyack (Beq); Pine I., J1 (NYS); LI: Bellport (Coq).

S. pusillus Lw. Saranac Inn, Aug (NYS); Rochester, Sep (NYS); Erie Co, Je-Oct (MCV); Ithaca, Je (CnJr); McLean, J1; Karner, Ap (NYS); Johnstown; LI.

\section{FAMILY SAPROMYZIDAE}

\section{LAUXANIA Latreille}

L. cylindricornis Fab. Upper Ausable L. (Beq); Chapel Pond, Je (NYS); L. Placid, J1; Speculator, Aug; Axton, Je; Wells, Jl (NYS) ; Niagara Falls, Je $(\mathrm{Jn})$; Ithaca, May (Joh); McLean, Ji (Joh).

\section{LAUXANIELLA Malloch}

L. manuleata Lw. Ithaca, J1 ( $\mathrm{CnJr})$. 


\section{CAMPTOPROSOPELLA Hendel}

C. verticalis $\mathrm{Lw}$. Lancaster, Je (MCV).

C. vulgaris Fitch. Essex Co, Aug (NYS); Erie Co, Je-Sep (MCV); Ithaca, Je; Albany, Je; Mosholu, May; NYC, Oct (Sturt); LI: Orient (Beq).

\section{DECEIA Malloch}

D. gracilipes Lw. Boston, Sep (MCV); Manlius (OS); Ithaca, J1, Aug (CnJr).

Caliope Haliday

C. flaviceps Lw. Ithaca, Ap (Joh); Ft. Edward (Lint); Mt. Vernon (Jn).

\section{Minettia Robineau-Desvoidy}

M. americana Mall. (longipennis auct. not Fab.). Axton; Ithaca and vic., Je; A1bany (NYS); Poughkeepsie (NYS); Bear Mt., Je (Stuart); LI: Central Pk. (Beq).

M. lupulina Fab. Upper Ausable L. (Beq); Chapel Pond, Je (NYS); W. Winfield, Je (NYS) ; Speculator; Trenton Falls, Je, J1 (Jn) ; Erie Co (MCV); Ithaca, Manlius (Joh); Albany (NYS); SI, Je (Sturt).

M. obscura Lw. Niagara Falls, Je (Jn); Erie Co, Je, J1 (MCV).

M. valida Wlk. NY.

\section{SAPROMYZA Fallén}

S. annulata Mel. Upper Ausable L. (Beq); Chazy L.; Karner; Axton; Niagara Falls, Je (Jn) ; Erie Co, Je (MCV); Caroline; Ithaca (CnJr).

S. quadrilineata Lw. S. Wales, J1 (MCV); Ithaca, Je, J1, Sep (Joh).

S. rotundicornis Lw. Poughkeepsie, Albany, Je ( $\mathrm{Yg}$ ).

\section{SAPROMYZOSOMA Malloch}

S. bispina Lw. Erie Co, Je (MCV); LI: Pennequid Barrens (Beq).

S. compedita Lw. Adirondacks, J1, Aug (NYS); Erie Co, Je, J1 (MCV); Ithaca, Je; Albany, Je.

S. conjuncta John. Ithaca, Je (Jn); McLean, J1, Aug (Joh).

S. disjuncta John. (similata Mall.). Ithaca (Jn); NYC (Beq).

S. fraterna Lw. Adirondacks, Je, J1 (NYS); Speculator, Aug; Honeoye Falls, J1. (Leon) ; Grand I., Je (MCV); McLean, Aug, Sep, Sib (Joh) ; Albany, Aug.

S. houghi Coq. Albany, Poughkeepsie, J1 (NYS).

S. incerta Mall. McLean, Aug, Sib (Joh).

S. philadelphica Macq. Erie Co, Je-Aug (MCV); Otto, J1; Ithaca; Manlius (OS); Oliverea (Beq); Bear Mt., Je (Sturt).

S. sheldoni Coq. Oswego, Aug (Coq); McLean, J1.

\section{FAMILY LONCHAEIDAE}

\section{LONCHAEA Fallén}

L. polita Say (rufitarsis Macq.). L. Placid (NYS); Axton (Joh); Syracuse, Blkm (Hine); Rochester (MCV); Niagara Falls, May-Jl (Jn); Erie Co, May-Sep; Ithaca; Johnstown.

L. vaginalis Fall. Durlandville, Je, Frost. 


\section{FAMILY PALLOPTERIDAE}

PALLOPTERA Fallén

P. superba Lw. Niagara Falls, Je (Jn); Erie Co, J1-Aug (MCV); Ithaca (Joh).

\section{FAMILY ORTALIDAE}

\section{AMPHICNEPHES Loew}

A. pertusus Lw. Karner, Je ( $\mathrm{Yg}$ ) ; Gowanda, Je (MCV) ; Mosholu, Je (Hendel) ; LI: Yaphank, Ols (Beq); Pennequid Barrens (Beq).

\section{RIVELLIA Robineau-Desvoidy}

R. cognata Cress. Ithaca, J1 (CnJr); Bear Mt., Je (Sturt).

R. flavimana Lw. Fulton Co; Niagara Falls, Je; Erie Co, May-J1 (MCV); Ithaca, Je-Aug (Joh); Poughkeepsie, May; Mosholu, May (Hendel); SI, Jé (Sturt).

R. metallica Wulp. Ithaca, J1 ( $\mathrm{CnJr}$ ).

R. pallida Lw. Erie Co, Je-Aug (MCV) ; Little Valley, J1 (MCV) ; L. Waccabuc, J1 (AM) ; Ft. Montgomery, Schott (Beq); W. Nyack (Beq) ; Mosholu, May (Hendel) ; LI: Flatbush, Je (AM).

R. quadrifasciata Macq. Ithaca, J1 (CnJr) ; LI: Long Beach (Beq).

R. variabilis Lw. Little Valley, J1 (MCV); Ithaca, J1 (CnJr); Poughkeepsie, May (Yg).

R. viridulans Desv. L. Pleasant, J1 (NYS) ; Newport (NYS) ; Olcott, J1 (MCV); Erie Co, J1 (MCV); Ithaca, J1; Oliverea (Beq); Albany; Red Hook, Je (Leon) ; Richmond Hill, J1 (Jn); West Pt., Je (AM); Rye Pt. Pond, Je (AM) ; Nyack (AM); Mosholu (AM); NYC, Je, EBSouthwick (AM); SI, Je (Sturt); LI: Central Pk. (Beq); Massapequa, J1 11, Yaphank, May 30, Ds ( CnJr); Orient (Beq).

\section{MYRMECOTHEA Hendel}

(Myrmecomyia, not of Robineau-Desvoidy)

M. myrmecoides Lw. LI: Sea Cliff; Wading R., Aug 6, Ds (Jn); Yaphank, Je 11, Ds (CnJr).

\section{TRITOXA Loew}

T. incurva Lw. Poughkeepsie, Jl (Yg); Mosholu, J1 (Hendel); SI (Sturt).

\section{Camptoneura Macquart}

C. picta Fab. Kingston, Aug (Yg); SI, Je (Beq); LI: Flatbush, Eng (Beq); Jamaica (Beq).

\section{IDANA Loew}

I. marginata Say. SI, Je (Sturt) ; LI: Rockaway, Je 4, Ds (Dru) ; Flushing, Wading R., Huntington, Pennequid Barrens, Flatbush, Schott \& Eng (Beq); Bergen Beach, Brooklyn, Eng (Beq); Yaphank, Je (CnJr). 


\section{TEPHRONOTA Loew}

T. canadensis John. Essex Co, J1 (NYS); Caroline, Je (CnJr).

T. narytia Wlk. (ruficeps Wulp). Gowanda, Je (MCV); Manlius, Ithaca, je (Joh) ; Poughkeepsie, Je; Mud Cr., Tompkins Co, Je (CnJr) ; LI: Yaphank, Ji ( $\mathrm{CnJr})$.

\section{Melieria Robineau-Desvoidy}

M. ochricornis Lw. Adirondacks, Aug (Joh).

M. similis Lw. Saranac Inn, J1 (Coq); Elba, Je (MCV); Ithaca, J1 (CnJr) ; Gloversville, Je, Alex (CU).

\section{TETANOPS Fallén}

T. luridipennis Lw. SI (Sturt).

\section{Callopistromyia Hendel}

C. annulipes Macq. Buffalo, May (MCV); Manlius (Joh); Tompkins Co, including Ithaca; Nassau, Ap; LI: Flushing, Mar; Half Way Hollow Hills, J1 2, Ds (CnJr); Little Neck, Schott (Beq); Yaphank, Je (CnJr).

\section{PSEUDOTEPHRITIS Johnson}

P. corticalis Lw. Ithaca (Joh).

P. cribrum Lw. Ithaca, $\mathrm{J} 1(\mathrm{CnJr})$.

P. vau Say. E. Aurora, Je (MCV) ; Colden, J1 (MCV) ; Ithaca, Ap, Aug (CnJr) ; Nassau, Ap; LI: Half Way Hollow Hills, J1 4, Amagansett, Sep 20, Ds $(\mathrm{CnJr})$; Pennequid Barrens (Beq); Cold Sp. Harb. (Sturt); Half Way Hollow Hills, J1 (CnJr).

P. vau var. approximata Bks. Ithaca, Aug ( $\mathrm{CnJr})$.

\section{Chrysomyza Fallén}

C. demandata Fab. Ft. Montgomery, Schott (Beq); NYC, Feb-Oct (Sturt); SI (Sturt); LI: Flatbush, Schott (Beq).

\section{Euxesta Loew}

E. notata Wied. Erie Co; Ithaca and vic. (CnJr); NY Bot. Gardens, J1; NYC, Oct (Sturt); LI: Brooklyn, Schott (Beq); Cold Sp. Harb. (Sturt).

\section{Chaetopsis Loew}

C. aenea Wied. Mosholu, Oct (Hendel); SI, Je (Sturt); LI: Kings Pk. (Beq); Maspeth, Je, Ols (Beq); Gardiners I., Cold Sp. Harb., Long Beach (Beq).

C. apicalis John. West Farms, NYC, Sep (AM); SI; LI: Cold Sp. Harb., May (Beq).

C. fulvifrons Macq. L. Tear (Beq); Ithaca, May, Aug (Jn, CnJr); NYC, Sep (Sturt) ; SI, Je, Oct (Sturt); LI: Sea Cliff (CnJr) ; Long Beach (Beq).

C. massyla W1k. Adirondacks, Aug (CnJr) ; Potsdam, J1 (CnJr) ; Oswego, J1 (Jn); Ithaca; McLean, J1 (CnJr); Dryden L., Je (CnJr); Carmel, Aug (AM) ; LI: Wyandanch, Ols (Beq). 


\section{SEIOPTERA Kirby}

S. albipes Cress. Ithaca, May, Je ( $\mathrm{CnJr}$ ).

S. vibrans L. Niagara Falls, Je; Buffalo, Je-J1 (MCV) ; Elba (MCV) ; Olcott, J1 (MCV); Syracuse, Blkm (Hine); Ithaca, Je (Joh); Albany, Je; White Plains, J1 (Bks); LI: Richmond Hill, J1 (Jn); Flatbush, Schott (Beq).

Eumetopiella Hendel

E. rufipes Macq. Erie Co, Je-Sep (MCV) ; Ithaca, May-Aug (Jn, CnJr) ; Mosholu, Je (Hendel); SI (Sturt).

\section{FAMILY TRYPETIDAE}

\section{STRAUSSIA Robineau-Desvoidy}

S. longipennis Wied. Potsdam; Newport; Niagara Falls, Je, J1; Rochester, May; Erie Co, Portage (MCV); Ithaca; Manlius; Albany; LI: Maspeth, Eng; Flatbush, Queens (Beq).

S. longipennis var. cornigera Wlk. (longitudinalis Lw.). Wells, Jl, Yg (Leon); Erie Co, Je, J1, Portage, J1 (MCV); Ithaca, Je-J1 (CnJr); Sacandaga Pk. (VTPhillips); Sharon Sps.; Albany, Je (Leon).

S. longipennis var. trimaculata Macq. (typica Lw.). Potsdam (VTPhillips); Tompkins $\mathrm{Co}$, including Ithaca, J1 (CnJr).

S. longipennis var. perfecta Lw. Ithaca, J1 (VTPhillips).

S. longipennis var. intermedia Lw. Ithaca, Je (VTPhillips).

ZoNosema Loew

(Spilographa Loew, in part)

Z. electa Say. "NY".

Z. flavonotata Macq. (basiolum O. S.). Ithaca, J1 ( $\mathrm{CnJr}$ ).

AcIdia Robineau-Desvoidy

A. fratria Lw. Tompkins Co.

EPochra Loew

E. canadensis Lw. Ausable Forks, May; Camden.

ACIURA Robineau-Desvoidy

A. nigricornis Doane. Axton, Je (M\&H); McLean, May (VTPhillips).

STENOPA Loew

S. vulnerata Lw. Protection, Sep (MCV).

\section{Rhagoletis Loew}

R. cingulata Lw. White-BAnded Cherry Fly. Common and frequently injurious wherever cherries are grown in the State.

R. fausta O.S. DARK-Bodied Cherry Fly. Common and frequently injurious wherever cherries are grown in the State. 
R. juniperina Marcovitch. Ithaca, Aug, reared from juniper berries.

R. pomonella Walsh. Apple MagGot. Common and frequently injurious wherever apples are grown in the State. J1-Oct.

R. suavis Lw. Ithaca, Aug, JMStedman (CnJr); Schuylerville, Sep (NYS); Middleburg; White Plains, Aug-Sep.

R. tabellaria Fitch. "NY" (Fitch); Hamburg, Je (MCV).

\section{Procecidochares Hendel}

(Oedaspis Loew, in part)

P. atra Lw. Erie Co, Je, Rock Glen, J1; Ithaca, Je-J1, Joh (VTP) ; Poughkeepsie, Je; Matteawan, Sep; White Plains (Beq); SI (Beq); LI: Maspeth (Beq).

P. penelope O.S. Manlius.

P. polita Lw. Keene Val., Aug (NYS); Ithaca, Aug; vic. NYC.

TERELLIA Robineau-Desvoidy

(Trypeta Loew not Meigen)

T. florescentiae L. (ruficauda Fab.). Essex Co; Bolton; Honeoye Falls, J1 (Leon) ; Buffalo, Je; Tonawanda, J1 (MCV) ; Otto, J1, Aug; Lancaster, Je ; Ithaca, Je, J1, Nov (CnJr); Albany.

Neaspilota Osten Sacken

(Aspilota Loew)

N. alba Lw. Ithaca $(\mathrm{CnJr})$.

Xanthomyia Phillips

(Tephritis auct., in part)

X. platyptera Lw. Johnstown, Je (NYS); Erie Co, May-Je; Albany, May (NYS) ; Nassau, May (NYS) ; Red Hook, Je (Leon); Poughkeepsie, May (NYS); Ithaca, Aug; McLean, Aug (CnJr).

\section{EUTRETA Loew}

E. sparsa Wied. Saranac L., Aug; Fulton Co; Niagara Falls, J1, Aug ; E. Aurora, J1 (MCV) ; Ithaca; McLean Bogs, Aug (Phillips); Manlius; Albany; Hudson R. val.; Pine I., Ramapo, Sep (AM); Tomkins Cove, Sep (AM) ; SI (Sturt); LI: Hempstead; Flushing.

\section{Eurosta Loew}

E. comma Wied. Keene Val. (Beq) ; Wilmington, Aug (Phillips); Karner, AugSep (NYS); Albany; White Plains, Sep; Ithaca (CnJr); Nyack (AM); Inwood (Schott); NYC (Sturt); SI, Sep (Sturt); LI: Brooklyn (Beq).

E. conspurcata Doane. Johnstown, Je; Ithaca; Albany, Aug; Wappingers Falls, Je (Leon) ; LI: Babylon, May (Sturt).

E. elsa Daecke. Fulton Co, Aug; Ithaca, May, Sep (CnJr); White Plains (Beq) ; NYC (Sturt); LI: Brooklyn (Beq); Richmond Hill, Oct; Forest Hills (Phillips); Wyandanch (Schott). 
E. reticulata Snow. Ft. Montgomery (Schott); Yonkers (Beq).

E. solidaginis Fitch. Speculator, Pike (NYS) ; Fulton Co; Geneva (Felt); Ithaca, Ap-May; Manlius; Hudson R. val.; Karner, including E. Schodack (NYS); Erie Co, May, Je (MCV); LI: Flushing.

\section{ICTERICA Loew}

I. circinata Lw. "NY".

I. seriata Lw. Irving, Aug (MCV); Ithaca; White Plains (Beq).

\section{EUARESTA Loew}

E. aequalis Lw. Erie Co. J1-Aug (MCV); Ithaca, J1-Aug (CnJr); Van Cort. Pk. (Phillips); SI, Aug; LI: Flatbush, Canarsie (Beq).

E. angustipennis Lw. "NY" (Lw), type; Sacandaga Pk. (Phillips).

E. bella Lw. Peru, Je, Cy (Phillips); Wilmington, Aug (Phillips); Mt. White. face, Aug (Phillips); Elizabethtown, Aug (NYS); L. Placid, Aug, EPV (NYS); Manlius; Honeoye Falls, Je (Leon); Clyde, Sep, Bish; Erie Co, Je-Aug (MCV) ; Westfield, J1 (NYS) ; Spring Lake, Cy (Phillips) ; Ithaca, Varna, McLean (Phillips); Albany; Karner, J1 (NYS);- Clifton Sps., Aug (Phillips); Middletown, ChasSpooner (Phillips); Salem, Aug (NYS); Germantown, J1 (NYS) ; Carmel, Aug (AM) ; Phoenicia, Aug, EPV (NYS); Rhinebeck, Cy (Phillips); Port Chester, J1 (NYS) ; Mosholu, J1-Aug; LI: Flatbush, Je, $Z a b$ (AM).

E. festiva Lw. L. Pleasant, J1 (NYS) ; Buffalo, Aug; Irving, Aug (MCV); Ithaca, Sep ; Middleburg, J1 (NYS) ; LI: Flushing.

E. pura Lw. Woodward L., Sep (Phillips); Ithaca, J1 (CnJr).

TRYPanea Schrank

T. abstersa Lw. Honeoye Falls, Je, Leon (VTP).

T. dacetoptera Phillips. Rock City, Karner, Hillside Pt., Sep (VTP).

T. daphne Wied. (solaris Lw.). Oswego, Je.

\section{TEPHRITIS Latreille}

T. albiceps Lw. Axton, Je, $M \& H$; Adirondacks, May; Mt. Whiteface, Aug (Phillips) ; Speculator, L. Pleasant, L. Placid, Chazy L., Newport, May-J1 (NYS) ; Spring Lake, J1 (Phillips) ; Caroline to Harford, Je (CnJr); McLean, Je-J1, Sib (Joh, CnJr); McLean Bogs, Aug (Phillips); Freeville, J1 $(\mathrm{CnJr})$; Albany, Aug.

T. euryptera Lw. West Pt.

\section{FAMILY MICROPEZIDAE}

TAENIAPTERA Macquart

T. antennaepes Say. Niagara Falls, Je ( $\mathrm{Jn})$; Erie Co, Je-Aug (MCV); Chautauqua L., Je (MCV) ; Potsdam, Jl, Ithaca, Jl (CnJr) ; Albany, Je; Ft. Montgomery, Schott (Beq); White Plains, J1 (Bks); LI: Flatbush, Eng (Beq); Pennequid Barrens (Beq).

T. bruneipes Cr. Ithaca, McLean, Jl, Oswego ( $\mathrm{CnJr}$ ).

T. divaricata Cr. LI: Brooklyn, Jl, Eng (Beq). 


\section{Calobata Meigen}

C. nasoni Cress. Ithaca, May (CnJr).

C. pallipes Say (alesia Wlk.). McLean, May, Dryden L., Tompkins Co, May, Je (CnJr); White Plains, May, Bno (Bks); Johnstown, Je; W. Winfield, Je (Leon-NYS); Erie Co, Je (MCV); Cayuga L. (OS).

C. univitta Wlk. Erie Co, May-J1 (MCV); Ithaca, May, Je, J1 (Sturt) ; Amity, Je; NYC, May (Sturt).

\section{TANYPEZA Fallén}

T. luteipennis Knab \& Snn. (longimana auct. not Hal.). Oswego, J1 (type); Dayton, J1; Jamestown, Je; Niagara Falls, Je (Jn).

T. picticornis Knab. West Nyack (Beq).

\section{FAMILY SEPSIDAE}

\section{SEPSIS Fallén}

S. cynipsea L. Buffalo, Lancaster, Mar-Ap, in greenhouse (MCV).

S. neocynipsea Mel. Buffalo, Mar; Manlius, Aug (Joh); McLean, J1, Sib; Ithaca, Ap (CnJr).

S. pectoralis Macq. Ithaca, J1, Aug; Manlius, Aug (Joh); LI: Cold Sp. Harb. (Mel).

S. signifera Mel. Alexandria Bay, Sep; Manlius, Aug (Joh); Ithaca, May-J1; LI: Cold Sp. Harb. (Mel).

S. signifera var. curvitibia Mel. McLean, J1, Sib (Joh); Ithaca; LI: Cold Sp. Harb. (Mel).

S. vicaria Wlk. Manlius, Aug (Joh); Ithaca, Je.

S. violacea Meig. Speculator; Erie Co, May-Oct (MCV); Geneva, J1, Aug; Ithaca, Je-Sep (Joh) ; Johnstown (NYS); Kingston; Southfields, Schott (Beq); W. Nyack (Beq); Bear Mt., Je (Sturt); LI: Cold Sp. Harb. (Mel).

S. violacea Meig. var. similis Macq. Ithaca, May-Aug ( $\mathrm{CnJr}$ ).

S. violacea Meig. var. hecata M. \& S. Ithaca, Ap, Je (CnJr).

\section{MEROPLIUS Rondani}

M. stercorarius Desv. Ithaca, J1 (CnJr); SI, Je (Sturt); LI: Cold Sp. Harb. (Mel).

\section{NEMOPODA Robineau-Desvoidy}

N. cylindrica Fab. Ausable; Manlius, May-J1 (Joh); Geneva; Niagara Falls (Mel) ; Erie Co, May-J1 (MCV); Fulton Co; Ithaca; W. Nyack (Beq); NYC, Sep (Sturt); LI (Mel).

\section{THEMIRA Robineau-Desvoidy}

T. flavicoxa Mel. LI: Cold Sp. Harb. (Mel).

T. incisurata M. \& S. var. latitarsata M. \& S. Beaver Kill, Aug (CnJr).

T. minor Hal. McLean, J1, Sib (Joh); LI (Mel).

T. putris L. Newport; Manlius; N. Evans, J1 (MCV); Buffalo, J1 (MCV); Ithaca and vic., May-Aug (Joh); Albany. 
SALTELla Robineau-Desvoidy

S. scutellaris Fall. E. Aurora, etc.; Erie Co, May, Je (MCV); Geneva (Mel) ; Hamburg, Je (Jn); Ithaca; NYC, May (Sturt); LI: Cold Sp. Harb.

S. scutellaris var. ruficoxa Macq. Hamburg, Je 20 (MCV); Ithaca, May, Je $(\mathrm{CnJr})$.

\section{FAMILY PIOPHILIDAE}

\section{PROChYLIZA Walker}

P. xanthostoma Wlk. Hamburg, Ap (MCV); Ithaca, Ap (Joh); NYC, Oct (Sturt).

\section{Piophila Fallén}

P. casei L. Cheese Skipper. General, infesting cheese and other stored food products.

P. nigriceps Meig. Ithaca, May (CnJr) ; LI: Cold Sp. Harb. (Mel).

P. oriens Mel. \& Spuler. Ithaca; Geneva (Mel).

P. privigna Mel. (pusilla auct. not Meig.). Ithaca, May; LI: Cold Sp. Harb. (Mel).

\section{FAMILY PSILIDAE}

\section{LOXOCERA Meigen}

L. collaris Lw. Erie Co, Je-Aug (MCV) ; Ithaca, J1 (Jn) ; Poughkeepsie, Je.

L. cylindrica Say. Blue Mt., Dug Mt. (Yg); Wells (Yg); Oswego, J1 (Coq); Erie Co, Je-Aug (MCV) ; Hamburg, Jl (Jn); Ithaca (CnJr) ; Poughkeepsie, $\mathrm{Je}(\mathrm{Yg})$.

L. cylindrica var. obsoleta Jn. Erie Co, Aug-Sep (MCV); LI: Cold Sp. Harb. (Beq).

L. cylindrica var. pectoralis Lw. Speculator, Aug (Yg); Ft. Montgomery, Schott (Beq).

L. cylindrica var. pleuritica Lw. Erie Co, J1-Sep (MCV); Olean, Aug (MCV); Ithaca and vic., Je-Aug (CnJr); Beaver Kill, Aug (CnJr-ANSP).

\section{PSEUdOPSILA Johnson}

P. angustata Cress. Lancaster, Sep 2 (MCV); Ithaca, Aug (CnJr).

P. bivittata Lw. Fulton Co, Je, Aug (Jn); Freeville, J1 (CnJr).

P. collaris Lw. Speculator, Je (Yg); Sport I., Sacandaga R., Ap (Alex); Erie Co, May-Je (MCV) ; Albany, Je, Poughkeepsie, Je, Yg; NYC, May (Sturt); SI (Sturt) ; LI: Cold Sp. Harb., May (Sturt).

P. fallax Lw. Axton, Je (Joh); Wilmington; Round I., Sep (CnJr) ; Speculator, Aug, $Y g$; Kaaterskill, Aug (Jn).

P. perpolita Johns. Colden, Je (MCV).

\section{Psila Meigen}

P. frontalis Coq. Fulton Co, Je; McLean, Aug-Sep, Sib (Joh).

P. lateralis Lw. Niagara Falls, Je (Jn); Ithaca, Aug; NYC, May (Sturt). 
P. rosae Fab. CARRot Rust-Fly. Broadalbin, Dec; Williamson, Je; Irondequoit; Lima; S. Lima; Niagara Falls; Wellsville; Erie Co, May-Je (MCV); Ithaca, Je (Joh).

P. sternalis Lw. Erie Co, Je-Aug (MCV); Caroline, Je ( $\mathrm{CnJr})$; Ithaca, Je.

Chyliza Fallén

C. apicalis Lw. Albany, Aug.

C. notata Lw. Speculator, J1, Yg; Rock City, Je; Ithaca, May-Je (Joh) ; Coy Glen, Ithaca, May.

STRONGYLOPHTHALMYIA Heller

S. angustipennis Mel. Wilmington, Aug (Snn).

\section{FAMILY DIOPSIDAE}

SPHYRACEPHALA Say

S. brevicornis Say. Wells, J1 (Yg); S. Wales, etc., J1-Aug (MCV) ; Erie Co; Ithaca, Je-Aug, Nov (Joh); Manlius; Johnstown, Sep (Yg) ; Saratoga Co; Hague, Aug (Leon); Nassau L., Sep (Leon); SI, Richmond, May (Bs).

\section{FAMILY EPHYDRIDAE 106}

\section{SUBFAMILY GYMNOPINAE}

GyMNOPA Fallén

G. tibialis Cress. LI: Cold Sp. Harb., Oak I., Sea Cliff, Bks.

\section{Athyroglossa Loew}

A. glabra Meig. Ithaca, May; Beaver Kill, Aug.

A. ordinata Beck. Beaver Kill, Aug, CnJr.

\section{OCHTHEROIDEA Williston}

O. glaphryopus Lw. LI: Cold Sp. Harb., Aug.

O. granulosa Cress. Norton's Landing, Cayuga L., Je, HHSmith.

\section{Clanoneurum Becker}

C. cimiciformis Hal. LI: Gardiners I. (Beq).

\section{SUBFAMILY PSILOPINAE}

\section{Psilopa Fallén}

P. atrimanus Lw. NYC, Sep (Sturt); Ithaca, Aug (CnJr).

P. flavida Coq. NYC, Sep (Sturt).

P. fulvipennis Hine. NYC, Sep (Sturt).

P. leucostoma Meig. Ithaca, Ap.

\footnotetext{
106 The list for this family has been checked by E. T. Cresson, Jr., and most determinations, unless otherwise indicated, should be credited to him.
} 
TRIMERINA Macquart

T. madizans Fall. Albany, May; Beaver Kill; Ithaca, Je (CnJr $)$.

RHYSOPHORA Cresson

R. robusta Cress. W. Nyack, Je (Beq).

\section{DITRICHOPHORA Cresson}

D. exigua Cress. Ithaca, May; Sport I., Sacandaga R.; Rensselaer, May.

\section{Discocerina Macquart}

D. obscura Will. LI: Cold Sp. Harb.

D. obscurella Fall. Niagara Falls, Je; Ithaca, May-Aug; Mud Cr., Tompkins Co, Je; Beaver Kill, Aug; Bear Mt., Je (Sturt); Van Cort. Pk., Je; LI: Cold Sp. Harb., Je-Aug; Babylon, May (Sturt).

D. parva Lw. Mud Cr., Je; Ithaca, Aug; Van Cort. Pk., J1.

\section{Diclasiopa Hendel}

D. xanthocera Lw. SI, Oct (Sturt).

\section{POLYTHICHOPHORA Cresson}

P. conciliata Cress. LI: Cold Sp. Harb., J1, Sheepshead Bay, Je, Yg.

P. orbitalis Lw. Beaver Kill, Aug, CnJr; LI: Cold Sp. Harb., Je (CnJr).

\section{SUBFAMILY NOTIPHILINAE}

\section{PHILYGRIA Stenhammar}

P. debilis Lw. Wells, Jl, Yg; Geneva, May; Ithaca, May; Albany, May, Yg; Mt. Whiteface, Aug; LI: Cold Sp. Harb., Aug (Claas).

P. fuscicornis Lw. SI, Je, Oct (Sturt).

P. opposita Lw. Wilmington, Aug; Buffalo, Je; Chipmunk Swamp, Vandalia, Je; Caroline, Je; Ithaca, J1; Freeville, J1; Mud Cr., Tompkins Co, Je; Albany, Je; NYC, Mar (Sturt); LI: Cold Sp. Harb., Aug (Sturt).

\section{PhILygRIola Hendel}

P. picta Fall. Wells, Je, $Y g$.

\section{HyDRELLIA Robineau-Desvoidy}

H. cruralis Coq. Grand I., Oct, $M C V$.

H. formosa Lw. Ithaca; NYC, Oct (Sturt) ; LI: Cold Sp. Harb., Je-Aug.

H. hypoleuca Lw. E. Aurora, Aug; Colden, Aug; Grand I., Oct; Ithaca, J1; LI: Ridgewood, Je.

H. ischiaca Lw. Ithaca, Aug. 
H. obscuripes Lw. Hamburg, Aug, $M C V$; Ithaca; Beaver Kill, Aug, $C n J_{r}$; LI: Cold Sp. Harb., Aug.

H. scapularis Lw. Saranac Inn, J1; Ithaca, May; LI: Cold Sp. Harb., Sheepshead Bay, Je-Aug.

H. tibialis Cress. SI, Oct (Sturt).

H. valida Lw. LI: Cold Sp. Harb., Je.

ILYTHEA Haliday

I. spilota Cut. Beaver Kill, Aug, CnJr; Ithaca, J1.

Paralimna Loew

P. appendiculata $\mathrm{Lw}$. Ithaca $(\mathrm{CnJr})$.

\section{Notiphila Fallén}

N. bispinosa Cress. LI: Cold Sp. Harb. (Beq).

N. loewi Cress. Franklinton, Je, Leon.

N. olivacea Cress. Ithaca.

N. riparia Meig. Ithaca.

N. scalaris Lw. L. Tear (Beq); Niagara Falls, Je (MCV); Colden, Je (MCV); Ithaca, and several other Tompkins Co localities, Je-J1; Beaver Kill, Aug; Gowanda, Je (MCV).

\section{Dichaeta Meigen}

D. caudata Fall. Ithaca, Ap-May; Albany, May, $Y g$; Karner, Ap, $Y g$.

\section{TyPOPSILOPA Cresson}

T. atra Lw. Ithaca, Je, J1 ( $\mathrm{CnJr})$.

\section{SUBFAMILY EPHYDRINAE}

\section{PELINA Haliday}

P. brevis Wlk. Niagara Falls, Je, $M C V$.

P. truncatula Lw. Buffalo, Oct, $M C V(\mathrm{Jn})$.

\section{LYTOGASTER Becker}

L. gravida Lw. Ithaca, Aug ( $\mathrm{CnJr})$.

\section{Hyadina Haliday}

H. albovenosa Coq. NYC, Mar (Sturt).

NAPAEA Robineau-Desvoidy

N. vanduzeei Cress. E. Aurora, J1 27, MCV. 


\section{PARYDRA Stenhammar}

P. bituberculata Lw. Manlius; Ithaca, Je-J1, Sep; Voorheesville, J1, Leon.

P. breviceps Lw. Erie Co, J1-Aug (MCV); Beaver Kill, Aug.

P. imitans Lw. LI: Cold Sp. Harb., J1.

P. paullula Lw. LI: Cold Sp. Harb., Aug.

P. pinguis Wlk. Niagara Falls, Je, $M C V$.

P. quadrituberculata Lw. Buffalo, E. Aurora, Hamburg, Lancaster, Je-Aug, $M C V$.

P. unituberculata Lw. LI: Cold Sp. Harb., Aug.

OCHTHERA Latreille

O. mantis DeG. Wilmington Notch, Je, Leon; L. Tear (Beq); Saranac Inn, J1; Manlius; Erie Co, May-Aug, MCV; Johnstown, Sep; Poughkeepsie, Je; White Plains, Aug; LI: Cold .Sp. Harb. (Beq).

BRACHYDEUTERA LOeW

B. argentata W1k. (dimidiata Lw.). Ithaca, J1 (Joh).

EPHYDRA Fallén

E. subopaca Lw. Syracuse, May; Ithaca, May-Oct (Joh) ; LI: Sheepshead Bay, Je.

E. atrovirens Lw. Ithaca ( $\mathrm{CnJr})$.

CirRula Cresson

C. gigantea Cress. LI: Sheepshead Bay, Aug, $Y g$.

Caenia Robineau-Desvoidy

C. bisetosa Coq. Niagara Falls, Aug, Oct ( $\mathrm{CnJr})$.

\section{Dimecaenia Cresson}

D. spinosa Lw. (Caenia). SI, Oct (Sturt); LI: Cold Sp. Harb., Gardiners I. (Beq).

\section{ScAtella Robineau-Desvoidy}

S. lugens Lw. Adams Center, May, Claas (Joh) ; Ithaca, Aug (CnJr) ; McLean, Je, Sib (Joh); Albany, Je; Rensselaer, May; Pawling, May; W. Nyack (Beq) ; LI: Sheepshead Bay, Je, $Y g$.

S. stagnalis Fall. Speculator, Aug, $Y g$; Broadalbin, Je, $Y g$; Ithaca, Aug (CnJr) ; Erie Co, May-Oct, $M C V$; Albany, Ap-May; Rensselaer, May-Je; Syracuse, Blkm; LI: Sheepshead Bay, Je, Yg.

S. quadrinotata Cress. Erie Co, Aug, Oct, $M C V$.

Scatophila Becker

S. despecta Hal. (variabilis Cress.). NYC, Mar (Sturt). 


\section{FAMILY CHLOROPIDAE}

MeRomyza Meigen

M. americana Fitch. Speculator; Axton; Ithaca; Scipioville; Albany; Karner, May-J1.

\section{EPICHLOROPUS Becker}

E. sp. Cayuga L., J1 (Ald).

\section{ChLoropisca Loew}

C. glabra Meig. (assimilis Macq.). Johnstown, Je; Ithaca, May, J1, Sep; Albany, Je; LI: Babylon, May (Sturt).

C. grata Lw. Beaver Kill, Aug, CnJr; LI: Rosedale, Schott.

C. rufescens Coq. Rhinebeck, J1, Cy (Ald); SI (Beq).

C. variceps Lw. (prolifica O.S.). Ausable Forks, Ap-Aug; Elizabethtown, Aug; Fulton Co; Alfred Center; Karner, Mar; Ithaca, Aug-Sep (Ald).

\section{Diplotoxa Loew}

D. microcera Lw. McLean, Aug, Sib (Joh).

D. recurva Adams. Saranac L., Aug (Ald).

D. versicolor Lw. Ithaca, J1 (Joh); Beaver Kill, Aug, CnJr; L. Tear (Beq).

\section{LASIOSINA Becker}

L. canadensis Ald. Saranac L. (Ald).

\section{Anthracophaga Loew}

A. sanguinolenta Lw. McLean, May (Ald).

\section{CHLOROPS Meigen}

C. antennalis Fitch. "NY".

C. certima Adams. Adirondacks (Ald); Sport I., Sacandaga R. (Alex).

C. hortensis Fitch. "NY". Not recognized (Beq).

C. liturata Adams. Albany, May.

C. melanocera Lw. Johnstown, Je; Niagara Falls, Je.

C. obscuricornis Lw. McLean, Je, Sib; L. Tear (Beq); LI: Huntington, Schott.

C. producta Lw. Eddyville, J1, FrostSW (Ald) ; Ithaca, Je-J1, Sib.

C. proxima Say. Ithaca, Je, And (All).

C. scabra Coq. Oswego.

C. tarsalis Becker. Ithaca, Je (Ald).

PARECTECEPHALA Becker

P. eucera Lw. Speculator, Aug; L. Placid, Je; Greene Co, How (Ald); Bear Mt. (Sturt); LI: Oyster Bay. 
Pseudogaurax Malloch

P. anchora Lw. Ithaca, May; NYC (Sturt).

\section{Eugaurax Malloch}

E. floridensis Mall. "NY".

\section{GauRaX Loew}

G. dorsalis Lw. "NY".

\section{Crassiseta von Roser}

C. aliena Becker. Adirondacks, Alex (Ald).

C. costata Lw. Speculator, J1 (NYS); Ithaca, May (Ald); Albany, May; NYC, Oct (Sturt); SI, Oct (Sturt).

C. nigriceps Lw. McLean, Je; Albany, May.

\section{Melanochaeta Bezzi}

M. (Doliomyia) longiventris Joh. Ithaca, Ap (Joh).

M. longula Lw. Saranac L., Aug (Ald); Schodack, Mar, NYC, Oct (Sturt); SI, Oct (Sturt).

\section{Hippelates Loew}

H. flavipes Lw. "NY".

H. nitidifrons Mall. Ithaca, Oct (Sturt) ; NYC, Sep (Sturt) ; LI: Cold Sp. Harb., May (Sturt).

H. nobilis Lw. Ft. Montgomery, Schott.

H. plebejus Lw. Ithaca, May (Ald).

H. subvittatus Mall. LI: Babylon, May (Sturt).

\section{MADiza Fallén}

(Siphonella Macquart)

M. cinerea Lw. Saranac L., Aug (Ald).

M. finalis Becker. Ithaca, Nov (Ald). Reared from decaying iris roots.

Notonaulax Becker

N. cincta Meig. Ithaca, Sep (Ald).

BotanobIa Lioy

(Oscinis auct.; Oscinosoma Lioy; Oscinella Becker)

B. anthracina Mg. McLean, J1 (Sib).

B. coxendix Fitch. Adirondacks, Aug; Greene Co, How (Ald); Ithaca, Ap, Oct (Ald) ; SI, Oct (Sturt).

B. coxendix var. obscura Coq. McLean, Aug.

B. dorsata Lw. (dorsalis Lw. 1869, not 1863). Ithaca, FrostSW (Ald). 
B. frit L. Ithaca, May-Aug (Ald).

B. incerta Becker. McLean, J1.

B. nitidissima Meig. (variabilis Lw.). McLean, May.

B. soror Macq. "NY".

B. tibialis Fitch. "NY".

B. umbrosa Lw. "NY".

\section{FAMILY DROSOPHILIDAE ${ }^{107}$}

\section{Amiota Loew}

(Phortica Schiner)

A. humeralis Lw. Wells, J1, Yg (Sturt); Axton, J1; Niagara Falls (Jn) ; Ithaca, Je, J1 (Joh) ; SI, J1 (Sturt); LI: Wyandanch, Schott (Beq).

A. leucostoma Lw. Wells, J1, Yg (Sturt); Ithaca, Je-Aug (CnJr); SI (Sturt). A. minor Mall. SI, Je (Sturt).

\section{STEGANA Meigen}

S. coleoptrata Scop. Wells, J1, Yg (Sturt); S. Wales, J1 (MCV) ; Cranberry Cr., Je (Sturt) ; Albany, May, Yg (Sturt) ; Ithaca (Joh), as vittata Coq; NYC, May (Sturt).

\section{Clastopteromyia Malloch}

C. inversa W1k. Ithaca (Sturt); NYC, Sep-Oct (Sturt).

\section{MyCODROSOPHILA Oldenberg}

M. dimidiata Lw. Ithaca ( $\mathrm{CnJr}$ ) ; SI (Sturt).

\section{Снymomyza Czerny}

C. amoena Lw. Speculator, Wells, J1-Aug, Yg (Sturt) ; Erie Co, Ap-Sep (MCV) ; Tompkins Co, May-Oct (Sturt) ; Karner, Aug (Sturt), and vic., May-Nov; Albany; Montgomery, J1; NYC, Je-Oct (Sturt); SI, Oct (Sturt); LI: Cold Sp. Harb. (Sturt).

C. procnemis Will. Albany, J1 (Sturt); NYC (Sturt).

\section{LEUCOPHENGA Mik}

L. maculosa Coq. NYC, Oct-Nov (Sturt); LI: Flatbush (Sturt).

L. varia Wlk. NYC, Sep (Sturt).

\section{DRosophila Fallén}

D. affinis Sturt. Wells, J1, Yg (Sturt); Niagara Falls (Jn); Ithaca, FrostSW; Albany, Je (Sturt); NYC, Je, Oct (Sturt); SI (Sturt).

D. busckii Coq. Ithaca, Nov 3 (Joh); Kingston, Nov; NYC, Je, Nov (Sturt).

D. colorata W1k. (sulcata Sturt.). Wells, J1, Yg (Sturt); Ithaca, May, Snn (Sturt). 
D. flaveola Meig. Buffalo, N. Evans, Je-Oct (MCV).

D. funebris Fab. Niagara Falls, Je (Jn); Buffalo, Ap, Nov (MCV); Ithaca, Aug, Sep (Sturt); Nassau, J1; Tarrytown; Nyack, Zab (Sturt); NYC, Je (Sturt); West Sand L., Oct, Nov (Sturt).

D. hydei Sturt. Albany, Oct (Sturt).

D. immigrans Sturt. (tripunctata Sturt.). Ithaca; White Plains (Sturt); NYC, Oct (Sturt); SI; LI: Cold Sp. Harb. (Sturt).

D. melanica Sturt. NYC (Sturt).

D. melanogaster Meig. (ampelophila Lw.). Common throughout the State all the year (Sturt).

D. ordinaria Coq. Erie Co, Je-Nov (MCV).

D. putrida Sturt. Speculator, Wells, Je, J1, Yg (Sturt); Cranberry Cr., Je, Yg (Sturt); Niagara Falls (Jn); Ithaca, Ap, May; Ramapo, Mar (Sturt); Albany, May, Yg (Sturt); NYC (Sturt); SI, Je (Sturt); LI: Cold Sp. Harb. (Sturt).

D. quinaria Lw. Speculator, Wells, Je, Aug, Yg (Sturt); Erie Co, May, Oct (MCV) ; Ithaca, May, Aug (Sturt) ; McLean, May (Joh) ; Albany, May, Oct, Yg (Sturt); Karner, May (Sturt); W. Nyack, Je (Sturt); NYC, Aug (Sturt); Rye, larvae in burrows of Papaipema spp. (Bird).

D. repleta Woll. Buffalo, Oct (MCV); Ithaca, Nov 3 (Joh); Albany, Sep-Nov (Mall); Meadowdale (Sturt); NYC, throughout the year (Sturt); LI: Maspeth (Sturt).

D. robusta Sturt. Ithaca, Ap 3, May, Aug, FrostSW (Sturt); SI (Sturt); LI: Cold Sp. Harb. (Sturt).

D. sigmoides Lw. NYC, Oct, Nov (Sturt); LI: Cold Sp. Harb. (Sturt).

D. simulans Sturt. SI (Sturt); LI: Cold Sp. Harb. (Sturt).

D. sulcata Sturt. Ithaca, Ap, J1 (Cress).

D. transversa Fall. Cranberry Cr., Je, $Y g$ (Sturt); Speculator, Wells, J1, Aug, Yg (Sturt); Niagara Falls, Je (Jn) ; Ithaca, May (Sturt); Albany, Je (Sturt) ; SI, Je (Sturt); LI: Cold Sp. Harb. (Sturt).

D. virilis Sturt. NYC, Nov (Sturt).

\section{SCAPTOMYZA Hardy}

S. adusta Lw. Ithaca, Tarrytown, Florida, reared from leaves of various mustards, Jl, Aug, FrostSW (Sturt) ; Valley Falls, Aug, Yg (Sturt); NYC, Sep (Sturt); SI, Oct (Sturt); LI: Cold Sp. Harb. (Sturt).

S. graminum Fall. Wells, J1, Yg (Sturt) ; Niagara Falls, Je (Jn) ; Buffalo, Sep, Oct (MCV); Ithaca, May-Sep (Sturt); Bear Mt., Je (Sturt); Albany, MarNov (Sturt); SI, Oct (Sturt); LI: Cold Sp. Harb. (Sturt); New Baltimore, May (Sturt).

S. terminalis Lw. Albany, Sep, $Y g$ (Sturt); Middletown, Cy (Sturt).

\section{CuRTonotum Macquart}

C. helvum Lw. Ithaca, May (Joh); Mosholu, May (Bks); LI: Orient, Zab (Sturt); Long Beach, Pennequid Barrens (Beq).

Aulacigaster Macquart

A. leucopeza Meig. Ithaca (Sturt). 


\section{FAMILy ASTEIIDAE}

Asteia Meigen

A. beata Ald. Wells (Yg-NYS).

\section{FAMILY GEOMYZIDAE}

Diastata Meigen

D. nebulosa Fall. Speculator, J1, $Y g$; Albany, Ap.

D. repleta Wlk. (pulchra Lw.). McLean, May, J1 (Joh).

ISCHNOMYIA Loew

I. albicosta Wlk. Erie Co, Je, Aug (MCV).

I. spinosa Hendel. Coy Glen, Ithaca, Je, Leon (Joh); McLean, Sep (Joh).

Anthomyza Fallén

A. gracilis Fall. Wells, J1 ( Joh); Slaterville (FrostSW).

A. tenuis Lw. "NY" ( $\mathrm{Lw})$.

A. variegata Lw. Buffalo, Je (MCV); McLean, Jl, Sib (Joh); Bear Mt., Je (Sturt).

\section{MuMETOPIA Melander}

M. occipitalis Mel. NYC, Oct (Sturt).

M. terminalis Lw. (nitens Mel.). Niagara Falls, Je 23 (Jn) ; Buffalo, Je (MCV); McLean, Je, Sib (Joh).

\section{TETHINA Haliday}

T. albula Lw. LI: Cold Sp. Harb., May (Sturt).

T. parvula Lw. (Rhicnoessa whitmani Mel.). LI: Rockaway Beach (Beq).

Pelomyia Williston

P. mallochi Sturt. (Tethina parvula auct. not Lw.). LI: Cold Sp: Harb., May (Sturt).

Chiromyia Robineau-Desvoidy

(Scyphella Robineau-Desvoidy)

C. flava L. Erie Co, Je-Aug (MCV).

\section{FAMILY MILICHIIDAE}

Pholeomyia Bilimek

P. indecora Lw. (Rhynchomilichia Hendel). "NY", Je, Bs (Mall); Ithaca (Joh).

Milichiella Giglio-Tos

M. arcuata Lw. LI (Me1).

M. lacteipennis Lw. SI, May (Sturt); Syracuse, Blkm (Hine). 
Eusiphona Coquillett

E. mira Coq. Rochester Jct., Leon; SI: Richmond (Beq).

PhyLlomyza Fallén

P. securicornis Fall. Ithaca, J1 (Joh).

\section{DESMOMETOPA Loew}

D. latipes Meig. New Baltimore, May 12 (Yg); Ithaca, Feb (Joh) ; NYC, Mar, Oct (Sturt).

D. m-nigrum Zett. NYC, Mar (Sturt).

MALLOCHIELLA Melander

M. glabra Fall. Geneva, Parr (Joh); Ithaca, May-Oct (Joh, Mall).

M. halteralis Coq. Erie Co, May-Oct. (MCV); NYC, Mar, Oct (Sturt); SI (Sturt).

MEONEURA Rondani

M. vagans Fall. (Agromyza tritici Fitch). NYC, May (Sturt).

\section{FAMILY OCHTHIPHILIDAE}

OснтнIPHILA Fallén

O. polystigma Meig. Erie Co, May-Aug (MCV); Niagara Falls, Je 22 ( Jn) ; Ithaca, May; McLean, May, J1, Sep (Joh); Honeoye Falls, Leon; Beaver Kill, Aug (CnJr); NYC, Sep (Sturt); SI (Sturt); LI: Cold Sp. Harb., May- (Sturt).

O. juncorum Fall. McLean, Ithaca (FrostSW).

\section{Chamaemyia Panzer}

C. elegans Panz. Dryden L., Tompkins Co, Je (Joh).

\section{LEUCOPIS Meigen}

L. bella Lw. "NY" (Lw).

L. nigricornis Egger. McLean.

L. simplex Lw. Nassau, Aug, $Y g$ (Ald); Erie Co, May-Je (MCV).

LEUCOPOMYia Malloch

L. pulvinaria Ma11. Shushan, J1 (Ma11).

\section{FAMILY AGROMYZIDAE 108 \\ ODINIA Robineau-Desvoidy}

O. maculata Meig. (ornata Zett.). LI: Westbury (Champlain).

108 The list for this family has been checked by Dr. S. W. Frost, who has made certain changes in nomenclature and added a number of records. 
TRAGINOPS Coquillett

T. irrorata Coq. Clinton Hgts., Je, $Y g$; NYC, May (Sturt).

\section{AgRomyza Fallén}

A. aeneiventris Fall. (plumipes Mall.). Lancaster, May, Aug (MCV); Albany, May.

A. aeneiventris var. cunctans Meig. Ithaca, Je 15.

A. allecta Mel. Erie Co, Je, Aug, Sep (MCV); Sheldrake, Trumansburg, Tarrytown, reared from Arctium, Bidens, and Eupatorium (FrostSW) ; Peru.

A. angulata Lw. (atra Meig.?). Ithaca (FrostSW); McLean, Sep (Joh).

A. borealis Mall. Ithaca, Florida, reared from Impatiens spp. (FrostSW).

A. burgessi Mall. Lancaster, Je (MCV).

A. curvipalpis Zett. (Ophiomyia Brochikow) var. texana Mall. McLean; Ithaca, FrostSW (Mel) ; Trumansburg, Florida, reared from aster, goldenrod, and Eupatorium (FrostSW) ; Peru; Gloversville.

A. eupatoriae Mall. Ithaca (FrostSW).

A. felti Mall. Hudson Falls, Ithaca, May (Ald); Albany, May.

A. flavonigra Coq. NY (Mall).

A. fragariae Mall. Ithaca, Tarrytown, Florida, reared from Rubus (FrostSW).

A. laterella Zett. (magnicornis Lw., grossicornis Zett.). Ithaca, Claas (Joh); McLean; Beaver Kill, Aug $(C n J r)$. In Johnson's List of the Diptera of New England, this species is listed as A. morosa Meig., with luctuosa Meig. and laterella Zett. as synonyms.

A. longicornis Lw. Niagara Falls, J1 (MCV).

A. longipennis $\mathrm{Lw}$. "NY" ( $\mathrm{Lw})$.

A. n. sp. near luctuosa Meig. Ithaca, reared from Cyperus acuminatus (FrostSW).

A. maculosa Mall. LI: Jamaica, Oct (Mall).

A. marginata Lw. McLean, J1, Sib (Joh).

A. maura Meig. Lewiston, Ithaca, LI, May (MCV).

A. maura var. setifrons Mel. Lancaster, May, Je (MCV); Ithaca (FrostSW).

A. maura var. simplex Lw. Asparagus Miner. Ithaca (Joh); LI.

A. melampyga Lw. (sorosis Will., flaviventris Jn.). Niagara Falls, Ithaca, on Plantago and Philadelphus; Hudson Falls (Felt).

A. nasuta Mel. Ithaca, Slaterville, McLean, mining in stems of dandelion, Je (FrostSW) ; Peru.

A. parvicella Coq. Ithaca (FrostSW).

A. parvicornis Lw. (dubitata Mall.). Buffalo, Je; Lancaster, Aug MCV (Mall); Ithaca, on grasses (FrostSW).

A. platyptera Thomson var. coronata Lw. Niagara Falls, Je (Mall); Erie Co, May, Sep, Oct (MCV); Ithaca, reared from goldenrod, aster, and violet (FrostSW); Wilmington.

A. platyptera var. jucunda Wulp. Ithaca, reared chiefly from aster, goldenrod, and sunflower (FrostSW); McLean, J1, Sep (Joh).

A. pusilla Meig. (trifolii Burgess, brassicac). Serpentine Leaf-miner. Middletown, Aug (Cy); Warwick, Aug (CU) ; Beaver Kill, Aug (CnJr) ; Ithaca (FrostSW); Freeville. One of the commonest leaf-miners, reared from fifteen host plants in NYS (FrostSW). 
A. posticata Meig. (?virgaureae Kalt., terminalis Coq., taeniola Coq.). Oswego, J1, Niagara Falls, Je (Mall); Ithaca, common on goldenrod (FrostSW).

A. pruni Gross. "NY" (Mall).

A. n. sp. near pruinosa Coq. Waterville, reared by I. M. Hawley from hop (FrostSW).

A. reptans Fall. var. subnigripes Mall. (kincaidi Mall., possibly also nitida Mall.). Ithaca, chiefly on Ambrosia, Urtica, and Helianthus (FrostSW).

A. setosa Lw. Ithaca, Ndm (Mel). Of doubtful occurrence in NY, most specimens probably being referable to either maculosa Mall. or fragariae Mall. (FrostSW).

A. salicis Mall. "NY" (Mall).

A. schineri Giraud. Rensselaer, May.

A. scutellata Fall. Lewiston, May; Erie Co, May (MCV).

A. subvirens Mall. Van Cort. Pk.; Ithaca (FrostSW).

A. superciliosa Zett. Buffalo, May (MCV).

A. ulmi Frost. Ithaca, Florida, mining leaves of American elm (FrostSW).

A. virens Lw. McLean, Sep, Sib (Joh); Ithaca (FrostSW).

A. waltoni Mall. Long L. (Mall).

A. youngi Mall. Albany, Ap, $\mathrm{Yg}$ (Mall).

Cerodontha Rondani

(Ceratomyza Schiner; Ceradonta Rondani)

C. femoralis Meig. (dorsalis Lw.). Ithaca, Sep, Oct, miner on various grasses (FrostSW); SI, Oct (Sturt) ; LI: Cold Sp. Harb. (Sturt); North Fair Haven.

Phyтomyza Fallén

P. affinis Fall. Ithaca (FrostSW).

P. albiceps Meig. (bipunctata Lw.). Ithaca, Florida, rather common on aster and Symphoricarpos (FrostSW); McLean, Sep (FrostSW).

P. angelicella Frost. From mines on Angelica atropurpurea, Ithaca (FrostSW).

P. aquilegiae Hardy. The Columbine LeAf-Miner. Ithaca, Tarrytown (FrostSW) ; Schenectady, Je 14 (Yg).

P. auricornis Frost. Ithaca, Jn, $D t$ (FrostSW).

P. bicolor Coq. Niagara Falls (Coq) ; Hamburg, May 28 (MCV); Ithaca, May 30 (Mel).

P. chrysanthemi (Napomyza) Kow. The Chrysanthemum Leaf-Miner. Ithaca (FrostSW) ; Albany, Feb, Yg.

P. ilicis Curt. (ilicicola Lw.). LI: Westbury, Ap 7 (Felt).

P. lactuca Frost. Ithaca, on wild lettuce (FrostSW).

P. loewii Hendel (clematidis Lw.). Lancaster, May (MCV); Beaver Kill, Aug ( CnJr).

P. marginalis Frost. McLean, Freeville, Ithaca (FrostSW).

P. nitida Mel. Florida, Ithaca, White Plains, Tarrytown (FrostSW). A fairly common leaf-miner on Nepeta, Ambrosia, Tanacetum, and Thalictrum.

P. obscurella Fall. var. nigritella Zett. Ithaca, leaf-miner on peach (FrostSW).

P. plantaginis Desv. Ithaca, on Plantago spp. (FrostSW).

P. plumiseta Frost. Ithaca, Florida, on Thalictrum (FrostSW). 


\section{FAMILY HIPPOBOSCIDAE}

ORNITHOICA Rondani

O. confluenta Say. Albany, Oct, Bish, on Strix varia varia.

\section{ORNITHOMYIA Latreille}

O. anchineura Speiser (pallida Say). Yankee L., Wurtsboro, Sullivan Co, Sep \&, on Maryland yellowthroat, JPChapin (Beq); SI, Sep 8, on Carolina wren, JPChapin (Beq) ; LI: Port Jefferson, J1, RCMurphy (Beq), on Toxostoma rufum.

\section{ORNITHOPONUS Aldrich}

O. americanus Leach (Olfersia). Buffalo, Oct-Nov (MCV); Hammondsport, Nov; Baldwinsville, on Bubo virginianus virginiamus, AGWhitney (Beq); Ithaca; Austerlitz, Oct, on Bonasa umbellus (Beq) ; Yankee L., Wurtsboro, Sullivan Co, Sep 6, on broad-winged hawk, JPChapin; SI, Nov 24 (Beq); LI: Freeport, Sep 13, on marsh hawk, HThurston (Beq) ; Orient Pt., May, Dec, Schott (Beq), on Buteo borealis borealis.

\section{OLFERSIA Wiedemann \\ (Psendolfersia Coquillett)}

O. fumipennis Sahib. (maculata Coq.). Clove Val., near Poughkeepsie, May 2, on fish hawk, JBBird (Bird). SI, May 20, Sep 1, on fish hawk, JPChapin (Beq)

MELophagus Latreille

M. ovinus L. NY (Fitch); Utica; Ballston Spa, Sep (NYS Rept., 15). On sheep.

\section{ORDER SIPHONAPTERA}

Compiled by M. A. Stewart

\section{FAMily PULICIDAE (sens. lat.)}

ECHIDNOPHAGA Olliff

E. gallinaceus Westw. NYC (Fox), on rats.

\section{Pulex Linnaeus}

P. irritans L. Springfield; Schenectady (Felt); LI: Brooklyn, on man.

\section{XENOPSYLLA Glink}

$\mathrm{X}$. cheopis Rothsch. NYC (Baker), on rats.

\section{Ctenocephalus Kolenati}

C. canis Curtis. Schenectady (Felt); NYC (Fox), on rats.

C. felis Bouché. Ithaca, on a cat; LI.

\section{SPILOPSYllus Baker}

S. simplex Baker. Ithaca, on cottontail rabbit (Sylvilagus floridanus mearnsi Allen). 


\section{Ctenophthalmus Kolenati}

C. gigas Kby. Ithaca. On red squirrel (Sciurus hudsonicus loquax Bangs).

C. pseudagyrtes Baker. Selkirk; Alpine; Ithaca. On Parascalops breweri, Fiber zibethicus zibethicus, Peromyscus leucopus, and Arvicola pennsylvanicus.

C. wenmanni Rothsch. Ithaca. On Peromyscus leucopus.

\section{TRICHOPSYLLA Kolenati}

T. lotoris Stew. Olcott. On raccoon (Procyon lotor lotor).

\section{Ceratophyllus Curtis}

C. acutus Baker. Ithaca.

C. arctomys Baker. McLean; Keeseville; Ithaca; Stamford. On woodchuck (Arctomys monax L.) and red squirrel (Sciurus hudsonicus loquax).

C. fasciatus Bosc. Ithaca. On rat and weasel (Putorius noveboracensis).

C. gallinae Schr. Perry.

C. leucopus Baker. Peterboro (Baker), on Peromyscus lencopus.

C. niger Fox. Ithaca. On bluebird (Sialia sialis L.).

C. pseudarctomys Baker. Ithaca. On flying squirrel (Sciuropterus volans).

C. riparius J. \& R. Ithaca, Olcott. From nests of bank swallow (Clivicola riparia) and kingfisher (Ceryle alcyon).

C. vison Baker. Ithaca. On Putorius noveboracensis.

C. wickhami Baker. Ithaca, Halsey Valley. On red squirrel, gray squirrel (Sciurus carolinensis lencotis), Northern flying squirrel (Sciuropterus volans), and Peromyscus leucopus.

\section{LEPTOPSYLLA Jordan and Rothschild}

L. herperomys Baker. Ithaca, Alpine. From vacated kingfisher's nest, mouse nest, and Peromyscus leucopus.

L. musculi Dugès. NYC (Fox), on rats.

\section{NEOPSYLLA Wagner}

N. similis Chapin. Ithaca. From grass siftings.

N. striata Stew. Ithaca. From nest of chipmunk (Tamias striatus).

N. testor Rothsch. Lansingburg (Rothsch), from nest (of mouse?).

Myodopsylla Jordan and Rothschild

M. insignis Rothsch. Homer; Ithaca. On Myotis lucifugus and M. sublatus. 


\section{ORDER HYMENOPTERA}

\section{Edited by J. Chester Bradley}

The classification adopted for the order Hymenoptera is that devised by Messrs. Rohwer, Bequaert, and Bradley, and employed by Professor Comstock in his Introduction to Entomology. Departures from this classification are minor, and consist chiefly in the division of the families Argidae and Chalcididae into more than a single family each. Where the strict application of the rules of nomenclature have compelled us, we have used other names for certain groups than those employed in Professor Comstock's book, although it is to be hoped that a way may yet be found for the retention of those old and well-known names. In all such instances we have introduced both names, so that the student will not be misled or puzzled. One serious source of uncertainty exists concerning these old group names. Twelve years ag: Messrs. Morice and Durrant published a study of an old and forgotten paper which has since come to be known as the "Erlangen List." It is expected that the International Commission on Nomenclature will rule as to whether this paper is to be allowed to affect our nomenclature. If so, numerous radical changes will occur, and all of these, or at least the most important, have been indicated in footnotes in the present list.

The present list may be looked upon as a fair representation of our knowledge of the hymenopterous fauna of the State, but as falling very far short of the actual fauna, particularly in certain groups. The sawflies, the gall-inhabiting cynipids, the Vespoidea, and the sphecoid wasps, probably list a large proportion of the species which actually occur here, but that can hardly be true of any of the parasitic groups.

The user of this list who wishes to discriminate between genera and species will encounter only one general manual. ${ }^{109}$ In dealing with the sawflies, the vespoid and sphecoid wasps, the ants, and to a lesser extent the bees, this manual will be helpful, but it is not of much assistance in the parasitic groups. Where there exist recent revisionary papers, these have usually been referred to by footnote in connection with the appropriate group.

The list in each family that has not been credited to another compiler, has been compiled by the editor for the order, who, with an editor's prerogative, has modified the form of individual lists to conform to the general

109 Guide to the insects of Connecticut. Part III. The Hymenoptera, or wasp-like insects, of Connecticut. By Henry Lorenz Viereck, with the collaboration of Alexander Dyer MacGillivray, Charles Thomas Brues, William Morton Wheeler, and Sievert Allen Rohwer. $1916.824 \mathrm{p} ., 10 \mathrm{pl}$. (State of Conn., Public document No. 47. State Geological and Natural History Survey, Bulletin No. 22.) 
plan, or has here and there added records or data of one sort or another, and who, therefore must be held responsible if in so doing he has unintentionally introduced any error, or anything that would not meet the approval of each individual contributor.

\section{SUBORDER CHALASTOGASTRA 110}

Compiled by S. A. ROHWER

FAMILY XYELIDAE

SUBFAMILY XYELINAE

XYELA Dalman

X. minor Nort. Ithaca, May (CU); Karner, Je (NYS).

\section{SUBFAMILY MACROXYELINAE}

\section{MACROXYELA Kirby}

M. aenea (Nort.). SI (Ds).

M. angulata (MacG). Axton, type.

M. distincta MacG. Ithaca, Ap 13-23, JOMartin, type (MacG-CU).

M. ferruginea (Say). Ithaca, Ap (MacG-CU).

M. infuscata (Nort.). Ithaca, Ap 28, 29, May 12, 1899 (CU).

M. obsoleta MacG. Ithaca, JOMartin, type.

\section{Pleuroneura Konow}

P. bruneicornis Roh. Gloversville, Ap 30, 1907, Alex, type.

P. borealis Felt. L. Clear, types.

\section{ODONTOPHYTES Konow}

O. avingrata (Dyar). Ithaca, Ap 29, Kngt (Bdy-CU) ; SI, Je 1; LI: Bedford Pk., May 25 (Dyar); Bellport, types. Larva feeds on immature leaves of hickory and butternut, appearing only early in spring.

110 This list has been prepared from records obtained from specimens in the collection of the National Museum (US); from records sent by Dr. Leonard from the collections in Cornell University (CU) and New York State Museum (NYS); from records sent by Dr. Frison, taken from specimens in the MacGillivray Collection (MacG-Ill); from records forwarded by $\mathbf{F}$. M. Schott (MacG-Schott); and from records taken from literature. I have not had an opportunity to study all the specimens from which the records were made. Those obtained from the collections of the National Museum are based, in the main, on identifications made by the writer. Nearly all of the other records are accepted as having been based on correct identifications, and are included with only the necessary nomenclatorial changes. The word "type" after a record does not necessarily mean "holotype," but is used in the sense of "one of the types." The systematic arrangement of the groups follows closely the classification proposed by the writer in 1911 (Proc. Ent. Soc. Wash., vol. 13, p. 215-226, 1911). It seems undesirable to make any radical changes from this arrangement in a list of this character. The list records 91 genera and 446 species and varieties from the State. 


\section{FAMILY PAMPHILIIDAE}

\section{ACANTHOLYDA A. Costa}

\section{(Subgenus Acantholyda A. Costa)}

A. atripes (Cress.). Greenwood L., May 16 (MacG-Schott).

A. bicolorata (Nort.). "NY", type.

A. ferruginea Felt. Mt. Marcy, type.

A. marginiventris (Cress.). "NY", type; Ithaca, Ap-May (CU, MacG-III).

A. modesta MacG. LI: Wyandanch, type.

\section{(Subgenus ITYCORSIA Konow)}

A. albomarginata (Cress.). Ithaca, Ap 12-Aug 15 (CU).

A. angulata MacG. Axton, Je, type; Ithaca, Je, Aug (MacG-I11).

A. brunniceps (Cress.). Ithaca, May 21, PESmith (CU).

A. Iuteomaculata (Cress.). Axton, Je (MacG-I11); Ithaca, Je-Aug (MacG-I11, CU).

A. maculiventris (Nort.). Ithaca, Ap 4 (MacG-Ill, CU); Karner, Je, 1901 (NYS).

A. ochrocera (Nort.). Plattsburg, Je (MacG-I11); Ithaca, Je 21 (CU); Karner, J1, 1901 (NYS).

A. zappei Roh. LI: Hicksville, Aug 24, 1916, ABChamplain (US).

Cephaleia Jurine

(Subgenus Cephaleia Jurine)

C. frontalis (Westw.). Ft. Montgomery, Aug 8 (MacG-Schott).

C. semidea (Cress.). Ithaca, May (US, CU), J1 (MacG-I11). MacGillivray usually places this species in the subgenus Caenolyda, but I prefer to assign it to the subgenus Cephaleia (Roh).

\section{NEUROTOMA Konow}

\section{(Subgenus NEUROTOMA Konow)}

N. fasciata (Nort.). "NY" (Nort); Ithaca, May (MacG-I11), J1 (CU). Larva feeds in webs on wild cherry.

N. inconspicua (Nort.). "NY" (Nort). Larva feeds in webs on plums.

\section{PAMPHILIUS Latreille}

(Subgenus PAMPHILIUS Latreille)

P. ocreatus (Say). Keene Val., Je 25, 1894, Dyar (US); Castile, EABradley (Bdy-CU) ; Ithaca, May 15-22 (CU); Poughkeepsie, May, 1903 (NYS).

Larva feeds on hazel and has been recorded from Cornus by Yuasa.

P. perseae MacG. Ithaca, Je 15 (CU).

P. semicinctus Nort. Stuyvesant Falls, Ap 29 (CU).

P. unalatus MacG. Ithaca, type. 


\section{(Subgenus AnOPlolyda A. Costa)}

P. excavatus (Nort.). "NY", Dbl (Kby 1882).

P. luteicornis (Nort.). "NY", Dbl (Kby 1882).

P. pallimaculus (Nort.). Plattsburg, Je 12-14, 1894, Dyar (US); Ithaca, May (MacG-I11), Je 9-J1 4 (MacG-CU).

P. perplexus (Cress.). Ithaca, Je 27 (CU) ; Caroline to Harford, Je 15, MacG $\mathcal{E} B d y$ (CU) ; LI: Maspeth, Je 6, Ols (MacG-Schott).

P. quebecensis (Prov.). Oswego, Je (MacG-Ill); Ithaca, Je (MacG-I11, CU).

P. rufocinctus (Cress.). Oswego (MacG-Ill); Taughannock Falls, May, Crampton (CU). MacGillivray assigns this species to the subgenus Pamphilius, but an examination of the type caused me to place it in the subgenus Anoplolyda (Roh).

P. scriptus (Say). Oswego, Je 9, 1896 (MacG-I11) ; Ithaca, Je (MacG-I11) ; Car.oline to Harford (MacG-Ill, CU); Taughannock, Ap 15 (CU).

\section{FAMILY CEPHIDAE 111}

\section{ADIRUS Konow}

A. trimaculatus (Say). "NY" (Nort); Slaterville (Bdy); Ithaca, Taughannock, J1 (Bdy-CU); SI, J1 (Bdy-Ds); LI: Brooklyn, J1 (Sf-BM); Yaphank, Eng.

\section{JANUS Stephens}

J. integer (Nort.). Currant-Stem Girdler. Childwold, Aug (Felt) ; Sodus, May (CU Econ) ; S. Byron, May (Lint-CU) ; Corning (Lint); Ithaca, Ap 12-Je 20 (Bdy-CU, MacG-Ill); Waverly, Oct (CU Econ); Ghent, Je (Lint); Troy (Felt).

J. abbreviatus (Say). Ithaca, J1-Aug (Bdy-CU); Ellis to Slaterville, Je (Bdy-CU). Larva feeds in stems of willow and has been recorded also from poplar.

\section{CEPHA Billberg}

(Trachelus Jurine)

C. tabida (Fab.). Black Grain-Stem Sawfly. Milo (DTRies) ; LI: Yaphank, May, Eng (Sf-BM). Larva feeds in stalks of wheat, rye, and barley, and perhaps also in certain grasses. Introduced from Europe.

\section{Cephus Latreille 112}

C. pygmaeus (L.). WheAT-Stem SAwfly. Recorded from the following counties: Oswego, Onondaga, Wayne, Monroe, Orleans, Niagara, Genesee, Erie, Wyoming, Livingston, Ontario, Yates, Schuyler, Steuben, Chemung, Tompkins, Seneca, Cayuga, Westchester (DTRies\&Cy). In addition it has been looked for but not found in Chautauqua, Tioga, Broome, Cortland, and Ulster Counties (DTRies\&Cy). Larvae feed in stems of wheat, and infestation up to ninety per cent occurs (Ries, Journ. Agr. Research, 1926, 32:284). Introduced.

111 If the Erlangen List is accepted, this family name will have to be changed to Astatidae, and the genus Cephus Latreille will fall as a synonym of the genus Astatus Jurine (Panzer).

${ }^{112}$ This name will be supplanted by Astatus, if the Erlangen List is accepted. 


\section{FAMILY XIPHYDRIIDAE}

XIPHYDRIA Latreille

X. attenuata Nort. Rochester Jct., Je, Leon (Roh-CU); Niagara Falls, Je (RohCU); Ithaca, Je-Aug (MacG-I11) ; LI: North Beach, Schott (Sf-BM). Larva feeds in Tilia americana.

X. erythrogastra Ashm. Niagara Falls (Roh-CU) ; Lancaster (MCV-US) ; Ithaca, Je-Jl (Roh-CU) ; Larva feeds in Carpimis caroliniana.

X. maculata Say. Newport, Je, 1899; Castile, EABradley (Bdy-CU) ; Portageville, Je (Roh-CU); Utica, Pergande (US) ; Albany, J1, 1909; Nassau, J1, 1902 (NYS) ; Ft. Montgomery, Je (Schott); LI: Yaphank (Roh-CU). Larva feeds in Acer.

X. hicoriae Roh. Syracuse, Blkm (US) ; LI: Westbury, Champlain (US). Larva feeds in Hicoria.

X. mellipes Harr. (provancheri Cress.). Cranberry L., Je 9, 13, 16, 1909, Drk (US) ; Saranac Inn, Feb 11, 1901, Drk (US, NYS Col. For.); Rock City, J1 (Roh-CU) ; Ithaca, May-Je (US, CU) ; LI: Rockaway Beach, Eng (Sf-BM).

X. tibialis Say. Ithaca, Je 15 (Roh-CU).

X. walshii Westw. "NY", type locality. Perhaps a synonym of X. mellipes Harr.

\section{FAMILY SIRICIDAE 113 SUBFAMILy SIRICINAE \\ SiREX Linnaeus \\ (Paururus Konow)}

S. edwardsii Brullé (zonatus Nort.). "NY" is one of the localities from which the types of zonatus Nort. were taken. Ithaca (MacG-I11) ; LI: Brooklyn, Sep, Eng (BM) ; Rockaway Beach, Oct, Eng-(BM).

S. juvencus L. race cyaneus Fab. "NY" (Nort). Mt. Whiteface, 2000-4000 ft., Aug 24 (Bdy-CU); Saranac L. (Ds).

S. nigricornis race nigricornis Fab. Ithaca (MacG-I11), Oct 26, 1917 (Bdy-CU) ; Binghamton, Franck (Sf-BM).

\section{UROCERUS Geoffroy}

U. flavicornis Fab. Ithaca (Bdy-CU).

U. albicornis (Fab.). Cranberry L., Aug, 1915 (Bdy-CU); Brant L., Aug, Doll (Sf-BM); Carthage (Felt); Ithaca, Ap 15-Sep 3 (Bdy-CU); Watervliet, Sep 25 (Felt); West Pt., Je, Sf (BM); SI (Ds).

U. cressoni var. cressoni Nort. Adirondacks, Aug (MacG-I11) ; Ithaca, Aug 22, Joh (Bdy-CU); Albany (Nort); White Plains, Aug, Schott (BM); LI: Prospect Pk., Jl (Sf-BM); Yaphank, Aug, Eng (BM).

U. cressoni var. tricolor Prov. Adirondacks, Houghton (Bdy-CU); Ithaca, Aug 7, 1907 (Bdy-CU); Troy, J1 18 (Felt).

U. taxodii Ashm. LI: Rockaway Beach, J1 3, 1909, Ds (Bdy). Known only from Ga. and Fla., where it breeds in cypress; this record may be only of accidental occurrence.

113 The Siricidae of North America. By J. C. Bradley. Journ. Ent. and Zool,, vol. 5, p. 1-30. 


\section{SUBFAMILY TREMICINAE}

\section{TREMEX Jurine}

T. columba race columba (L.). Common and widely distributed; representative localities are: Wilmington, 1000-2200 ft., Aug; New Russia, Aug 18 (Bdy); Newport, Ap, Aug 30 (Felt) ; Ogdensburg, Aug (Felt); Alder Cr., Aug (Felt) ; Syracuse, Sep; Perry; Rochester Jct., J1, Leon (Bdy) ; Cattaraugus, Sep; Fredonia, Sep 15 (Felt); Castile, EABradley (Bdy-CU); Ithaca, J1Sep; Trumansburg (Bdy-CU); Taughannock; Kinderhook (Bdy-CU); Albany, May-Aug (Felt); Mansville, Aug (Felt); Addison, Sep; Athens, Oct 15 (Felt) ; Ellenville (Felt) ; NYC (Felt); SI (Ds); LI: Brooklyn, Eng (BM).

T. columba race sericeus Say. Rochester Jct., J1 26, male, Leon (Bdy).

\section{FAMILY CRABRONIDAE 114}

(Cimbicidae auct.)

\section{SUBFAMILY CRABRONINAE}

\section{(Cimbicinae auct.)}

\section{CRABRO Geoffroy 115}

(Cimbex Olivier and auct.)

C. americanus (Leach) var. albus (Nort.). Ithaca (CU).

C. americanus var. americanus (Leach). ELM SAwfly. Keene Val., J1 (NYS) ; Axton, Je (MacG-I11); New Russia, Sep (Felt); Blue Ridge, J1 (Felt); Newport, Je (NYS); Elma (NYS); Jamestown, Collins, Windsor (CU Econ); Ithaca, May (CU); Albany, Je (NYS); White Sulphur Sps. and Montgomery (CU Econ).

C. americanus var. dahlbomi (Guér.). "NY" (Nort); Ithaca, Je (MacG-I11).

C. americanus var. decim-maculatus (Nort.). Ithaca (CU).

C. americanus var. laportei (Lep.). "NY" (Nort); Ithaca, May, Aug (CU).

C. americanus var. luciferus (Klug). "NY" (Nort); Ithaca, Je (CU).

C. americanus var. nortoni (MacG.). Ithaca, Je-J1 (MacG-I11, CU); Van Cort. Pk., Je (NYS), Jl (CU).

\section{TRICHIOSOMA Leach 116}

T. bicolor Nort. Saranac Inn, Je (MacG-Ill, as confusum MacG); Axton, Je (M\&H-CU).

T. crassum Kby. Ithaca, Je (MacG-I11).

T. cerasi (Fitch). Bred from cocoon on cherry by Fitch and described in the genus Abia; also recorded from Canton, Ap (Felt). Fitch's type has not been

\footnotetext{
${ }^{114}$ In Comstock's Introduction to Entomology this is termed Cimbicidae. In MacGillivray's classification it forms the subfamily Cimbicinae of the Tenthredinidae. (Bdy.)

${ }_{115}$ Bradley (Trans. Ent. Soc. Lond., p. 66, 1919) has pointed out that the generic name Crabro was first used by Geoffroy for the group of insects which has long been known under the name Cimbex Oliv. Cimbex is synonymized with Crabro Geoffroy, and the wasps which have for a long time been known under the generic name Crabro Fabricius are assigned to other genera. (Roh.) ${ }_{116}$ The species recorded by MacGillivray (New York State Mus. Bul. 47, 1901, p. 584) under the name angulatum Kirby from Saranac Inn, seems not to have been described.
} 
studied, but the description indicates that this species belongs to the genus Trichiosoma and it seems possible that it may apply to some other species more recently described.

T. spicatum MacG.' Ithaca, May, McLean, Je 13 (CU).

T. triangularum Kby. Keene Val., Dyar (US); Saranac Inn, Je (NYS). Larvae recorded as feeding on willow and wild cherry.

\section{SUBFAMILY ZARAEINAE \\ ZARAEA Leach \\ (Subgenus ZARAEA Leach)}

Z. americana Cress. L. Tear, J1 28, 1920 (US) ; LI: Jamaica, Ap 23, 1910 (US); Flatbush, Ap 20 (MacG-Schott).

Z. inflata Nort. Geneseo, J1 (Felt); Warner, Je (Felt); LI: Garden City, Je 8, 1911, Felt (NYS). Larva recorded as destroying the foliage of Lonicera.

\section{(Subgenus ABIA Leach)}

Z. lonicerae (L.). LI: Bayside (US). The record of this European species is based on two specimens reared from cocoons on honeysuckle.

FAMILY ARGIDAE 117

(Hylotomidae Ashmead)

\section{SUBFAMILY ARGINAE 117}

(Hylotominae Ashmead and MacGillivray)

ATOMACERA Say

A. debilis Say. Mt. Marcy, J1, 1913 (NYS).

A. decepta Roh. "NY" (type-US).

A. ruficollis Nort. LI: Wyandanch, Je 18 (MacG-Schott); Sea Cliff (MacG-CU).

\section{A RGE Schrank 118 \\ (Hylotoma Latreille)}

A. abdominalis (Leach). LI: Massapequa, May 4 (MacG-Schott) ; Farmingdale, Je 14, Morrow (Bdy-CU).

A. coerulea (Nort.). Ithaca, J1 (MacG-I11); LI: Centerport (CU).

A. clavicornis (Fab.). Plattsburg (Dyar); Rock City, Je 8 (Bdy-CU); Ithaca, May, J1 (MacG-CU); McLean, J1 29 (Bdy-CU); Albany (Nort).

A. dulciaria (Say). "NY" (Nort).

\footnotetext{
${ }^{117}$ If the Erlangen List is accepted, these names will be replaced by Cryptidae and Cryptinae, respectively, and the genus name Arge by Cryptus (Bdy).

118 The species of this genus are not satisfactorily defined, and, until a revision has been prepared, they cannot be satisfactorily determined. Larvae widely different in appearance produce adults which, according to the published keys, have been assigned to one species, and larvae which are superficially very similar produce adults which are very different in many characters. In this list no effort has been made to correct synonymy or verify identifications. (Roh.)
} 
A. erythrosoma (Leach), "NY" (Nort).

A. humeralis (Beauvois). "NY" (Nort); Ft. Montgomery, Aug 16 (MacG-Schott).

A. macleayi (Leach). Plattsburg, Keene Val., larvae reared by Dyar; Old Forge, (NYS); Ithaca, J1 (MacG-I11) ; Caroline to Harford, Je 15, MacG\&Bdy (Bdy-CU); McLean, May 30 (Bdy-CU) ; Karner, J1 (NYS) ; Ft. Montgomery, J1 19 (MacG-Schott). The larva of this species is recorded as feeding on Azalea, willow, black birch, white birch, hazel, mountain ash, wild cherry, and honeysuckle.

A. pectoralis (Leach). Keene Val., larva on Betula papyrifera (Dyar 1895); Loon L., J1 (Felt) ; Birch I., Upper Saranac (Felt) ; Plattsburg, larva on Betula lenta (Dyar 1895); Brille, Je 13, 1891 (CU); Essex Co, Je 1895, larva on birch (NYS); SI (Ds).

A. rubiginosa (Pal. de Beauv.). "NY" (Nort); LI: Roosevelt, J1 22 (MacGSchott).

A. rubra (Klug). Elizabethtown, Aug, 1904, Yg (NYS) ; Essex Co, J1, 1895, larva on oak, Yg (NYS) ; Ithaca, Je 10-J1 19. (MacG-CU) ; LI: Wyandanch, J1 14 (MacG-Schott). Larva recorded as feeding on Crataegus.

A. virescens (Klug). Ithaca, Je 19-Aug 8 (MacG-CU) ; Spencer L., Je 30 (Bdy$\mathrm{CU})$.

\section{SUBFAMILY STERICTIPHORINAE 119}

(Schizocerinae Ashmead and MacGillivray)

STERICTIPHORA Billberg

(Schizocera Lepeletier)

S. sericea (Nort.). Ithaca (MacG-I11, US).

S. prunivora (Dyar). LI: Bellport, Jefferson Highlands (Dyar). Larva recorded from Prunus serotina and $P$. pennsylvanica.

S. lineata (Roh.). LI: Richmond Hill, Aug 8, 1911 (US).

S. zabriskiei (Webster \& Malley). LI: Flatbush, J1 4, 1895, Aug 17, 1896, Zab (US); Huntington, Aug (MacG-Schott); Bellport (Dyar). Larva feeds on Portulaca oleracca.

S. cellularis (Say). "NY", type locality of a synonym.

S. plumigera (Klug). "NY", type locality.

\section{FAMILY DIPRIONIDAE 120}

(Lophyridae Ashmead, Lophyrinae MacGillivray)

DIPRION Schrank

(Lophyrus Latreille)

D. simile (Hartig). Introduced Pine Sawfly. Seven Sps. Farm, Mt. Kisco, Feb, 1925 (US) ; Pleasantville, Sep, larvae injurious (Roh); LI: Flushing (Middleton). An important enemy to pines, which has been introduced from Europe.

119 The species of this subfamily have been recently rearranged and a manuscript revision prepared. The present list gives only the specific names which will be accepted in this revision. (Roh.)

${ }^{120}$ In Comstock's Introduction to Entomology this family is included as part of the family Argidae. If the Erlangen List names are adopted, Diprionidae and Diprion will be replaced by Pteronidae and Pteronus, respectively. (Bdy.) 


\section{NEODIPRION Rohwer}

N. abietis (Harr.). Spruce Sawfly. Salem, Oct (NYS); Johnsburg, Ap (NYS). Larva a common defoliator of spruce and fir.

N. fabricii (Leach). Karner, Aug, 1902 (NYS) ; Nassau, May, 1902 (NYS) ; LI: Bellport, larva on pitch pine (Dyar). Larva on Pinus rigida. This species was originally described from Ga., and it is very probable that the above records are based on erroneous determinations.

N. lecontei (Fitch). Red-Headed Pine Sawfly. "NY", type locality; Axton, Aug (Felt) ; St. Huberts, J1 (Felt) ; Cazenovia, Aug 21 (NYS) ; Ithaca (MacGI11) ; Hyde Pk. (Middleton) ; Karner, May, 1902 (NYS) ; Poughkeepsie, Oct, 1895 (NYS) ; LI: Westbury, J1, Setauket, Oct (Felt). A common enemy of young pines, attacking many species.

N. pinetum (Nort.) (abbottii auct. not Leach). Saranac Inn, Sep (NYS) ; Hemlock L_., Aug, Big Moose, Sep (Felt) ; Johnsburg (Felt); Olmstedville (Felt 1910) ; Ballston Spa, Oct (Felt) ; Mt. Kisco, Sep (Felt) ; Karner, Aug, 1902 (NYS) ; Albany, Jan, 1902 (NYS). A rather common defoliator of hard pines.

\section{Monoctenus Dahlbom}

M. juniperinus MacG. Ithaca, reared from larvae on Juniperus virginiana by R. L. Junghanns (type).

M. fulvus (Nort.). "NY" (Kby 1882, Brit.Mus.).

\section{FAMILY TENTHREDINIDAE SUBFAMII.Y ALLANTINAE}

\section{(Emphytinae MacGillivray)}

\section{STRONGYLOGASTROIDEA Ashmead}

S. apicalis (Say). Axton, J1 (CU) ; Speculator, Aug, Yg (NYS) ; Old Forge, J1, $Y g$ (NYS) ; Cranberry L., J1 2, Htly (Roh-NYS Col. For.) ; Ithaca, May, Dyar (US, CU) ; Caroline to Harford, May (CU); McLean, Aug 3 (BdyCU) ; Albany, Nassau, Je, Yg (NYS) ; "LI" (US).

S. epicera (Say). Old Forge, J1, Yg (NYS) ; Hamburg, May, $M C V$ (US) ; Ithaca, May-Je (US, CU, MacG-I11); McLean, Je 9 (Bdy-CU) ; Poughkeepsie, Je, $Y g$ (NYS) ; LI: Sea Cliff, Bks (US).

S. pallidicornis (Nort.). Caroline to Harford, J1-Aug (CU); Ithaca (US, CU) ; SI (Ds); LI: Ashm (US) ; Brooklyn, Akhurst (Nort); Flatbush, J1, Zab (US).

S. potulenta MacG. Poughkeepsie, type.

S. rufocincta (Nort.). Ithaca (MacG-I11); McLean, J1 18 (Bdy-CU); LI: Sea Cliff, Bks (US).

S. rufocincta var. virginica Roh. Ithaca, Bks (US).

S. rufocinctana MacG. LI: Richmond Hill, type.

S. rufocula MacG. Ithaca, Je, McLean, May, type.

S. rufula MacG. Ithaca, type.

S. spiculata MacG. Axton, Je (MacG-Ill); Ellenville, type; Caroline to Harford, Je (MacG-I11). 
S. terminalis (Say). Axton, Je (MacG-I11) ; Newport, May (NYS) ; Upper Ausable L., J1 (US); Ithaca, J1 (US); Caroline to Harford, Je (MacG-Ill) ; Trenton Falls, Dbl (Kby); Nassau, May (NYS) ; LI (US); Flatbush, Je, $Z a b$ (US).

S. unicincta (Nort.). Tompkins Co, Je (MacG-Ill); Albany, Je (NYS) ; LI (US).

S. unicinctella MacG. Ithaca, Aug, type.

\section{DIMORPHOPTERYX Ashmead}

D. errans Roh. Ithaca, Je (US) ; LI: Bellport, Je, Dyar (US).

D. ithacus MacG. Ithaca, Je, type.

D. pinguis (Nort.). Speculator, J1, Yg (NYS).

\section{MACREMPHYTUS MacGillivray}

M. semicornis (Say). Ithaca, J1-Aug (US, MacG-Ill, CU) ; Ellis, Je (MacG-I11) .

M. tarsatus (Say). Syracuse, J1 (NYS); Rochester, Sep, larvae abundant on Cormus mascula, JHDodge (NYS, Ent. Rept. 1910, p. 59) ; Ithaca, Je-Aug (US, CU, MacG-I11) ; Caroline to Harford, J1 (MacG-I11) ; Old Chatham, Sep (NYS) ; Poughkeepsie, May-Je (NYS).

M. varians (Nort.). Plattsburg, Je (Dyar-US) ; Ellenville, Je (US, CU) ; Ithaca, Je-J1 (US, CU, MacG-I11) ; Ft. Edward, larvae feeding on Cormus stolonifera, MWBandenberg (NYS, Ent. Rept. 1889, p. 188).

M. versicolor (Nort.). Boonville, Sep (NYS); Tompkins Co, J1 (MacG-I11) ; Slaterville to Caroline, Je (MacG-I11); Dryden, J1 (MacG-I11); McLean, Sep (Bdy-CU) ; Poughkeepsie, May, Yg (NYS).

\section{ERIOCAMPA Hartig}

E. juglandis Fitch. "NY", larva on butternut (NYS). It is very probable that this is the same as rotunda (Nort.).

E. rotunda (Nort.). Ithaca, May (MacG-I11, CU).

\section{Pseudosiobla Ashmead}

P. cephalanthi Roh. Van Cort. Pk., Je 2, 1895, larvae on Cephalanthus occidentalis (Dyar-US), types.

P. excavata (Nort.). LI (US).

\section{Ametastegia A. Costa}

A. glabrata (Fall.). Ogdensburg, J1 (NYS); Old Forge, Aug (CU) ; Ithaca, May-Aug (US, CU, MacG-Il1) ; Freeville, J1 (CU) ; McLean, (CU, US) ; Albany, Karner, May (NYS) ; Poughkeepsie, J1 (NYS) ; LI: Flatbush, May (US). Larva feeds on dock, and pupates in brashy wood, fallen fruit, or similar substances.

A. equiseti L. var. bizonata (Zett.). Syracuse, Je, Aug, B\&S (US).

Polytaxonus MacGillivray

P. robustus (Prov.). Buffalo, Je, $M C V$ (US); LI: Yaphank (US). 


\section{UNITAXoNUS MacGillivray}

U. repentinus MacG. Ithaca, type.

U. rumicus MacG. Ithaca, type.

\section{PaRATAXonus MacGillivray}

P. multicolor (Nort.). Axton (US, MacG-I11) ; McLean, Ji (MacG-I11, Bdy-CU) ; Ellis, Je (MacG-I11) ; Slaterville, May, J1 (CU) ; LI: (US) ; Huntington, Jé (MacG-Schott).

P. multicolor var, erythrogaster (Roh.). LI (US).

\section{Monosoma MacGillivray}

M. inferentia (Nort.). Axton, J1 (US); Keene Val., Dyar; Castile, EABradlcy (Bdy-CU) ; Ithaca, May (US, CU, MacG-I11); McLean, May (US, CU, MacG-I11). Larva feeds on elder.

\section{Allantus Panzer \\ (Emphytus auctt.) \\ (Subgenus Emphytus Klug)}

A. cinctus cinctus (L.). This subspecies has often been intercepted at quarantine in NYC, but, so far as is known, has not yet become established. It is European and feeds on leaves of roses, pupating in the pith of the stalk.

A. cinctus cinctipes (Nort.). Ogdensburg, J1, $Y g$ (NYS); Ilion, May, $Y g$ (NYS) ; Poughkeepsie, J1, Yg (NYS); SI (Ds). This subspecies has sometimes been considered synonymous with the European cinctus. The feeding habits of the larva are similar to those of $A$ cinctus cinctus.

A. haustus MacG. Grand I., type.

A. heroicus MacG. Hamburg, type.

A. hiatus MacG. Ithaca, type.

A. mellipes Nort. Ithaca, May (CU) ; Clinton Mts., Ap, Yg (NYS); Albany, Je, Poughkeepsie, May, Yg (NYS).

A. yuasi MacG. Ithaca, type.

\section{(Subgenus Emphytina Rohwer)}

A. apertus (Nort.). Plattsburg, Je (US) ; Gloversville, J1 (NYS); Ithaca.(US, NYS, CU); Caroline to Harford (CU); W. Danby (CU) ; Albany, May (NYS).

A. pallipes (Spin.) (canadensis Kby.). Plattsburg (Dyar-US); Nassau, Felt (NYS) ; Rhinebeck, Pratt (Proc. Ent. Soc. Wash., vol. 4, p. 302, 1896). The larva feeds on the foliage of pansies and violets.

A. inornatus (Say). Speculator, J1, Yg (NYS); Karner, Albany, May, Yg (NYS) ; Van Cort. Pk., May (CU) ; LI (US).

A. tener (Fall.) (vanduzeei Roh.). Lancaster, $M C V$ (US). This European species is now known to occur in a number of localities in the United States. 


\section{SUBFAMILY DOLERINAE}

\section{Dolerus Panzer}

D. abdominalis Nort. Fulton Co, May (MacG-I11); Ithaca, Ap (US).

D. acritus MacG. McLean, Ithaca, types.

D. apricus Nort. Plattsburg, Je (Dyar-US) ; Speculator, Je, Battenville, May, Yg (NYS) ; Ithaca, May-Je (CU, US).

D. aprilis Nort. Ithaca, May (MacG-I11) ; Albany, Karner, May, Yg (NYS) ; Chatham, Aug (MacG-I11); Poughkeepsie, May, Yg (NYS); White Plains (US) ; SI (US, Ds).

D. apriloides MacG. Ithaca, Je, type.

D. bicolor (Pal. de Beauv.). Saranac Inn (MacG-Ill, NYS); Ithaca, Je (MacGI11) ; Albany, Ap-May (NYS); LI (US).

D. cohaesus MacG. Otto, Comstock, type.

D. collaris Say. Ithaca, May (US, MacG-I11) ; Karner, Ap (NYS).

D. conjugatus MacG. Fulton Co, Cy, types; Otto, Comstock, type.

D. dysporus MacG. Ithaca, type.

D. icterus MacG. Saranac Inn, $N d m$, type.

D. inspectus MacG. Ithaca, type.

D. luctatus MacG. Fulton Co, Ap (US); Ithaca, type.

D. maculicollis Nort. LI: Brooklyn (Nort).

D. minusculus MacG. Ithaca, type.

D. neoagcistus MacG. Southfields, Schoit, type.

D. neocollaris MacG. Fulton Co, $C y$, type; Ithaca, type.

D. neosericeus MacG. Ithaca, type; Southfields (MacG-I11); SI (US).

D. parasericeus MacG. Ithaca, type.

D. polysericeus MacG. Ithaca, type.

D. refugus MacG. Ithaca, Comst, type.

D. sericeus Say. Auburn, May (NYS); Ithaca, Ap-May (US, MacG-I11) ; Albany, Ap, Karner, May (NYS); Nassau, May (NYS).

D. similis Nort. Newport, May, Yg (NYS) ; Mallory, Je (US) ; Ithaca, J1 (US, MacG-I11) ; Albany, Je, Karner, J1, Yg (NYS) ; Chatham, Aug (MacG-I11); Ardsley, Je (MacG-Schott); SI, Ap (Ds).

D. stugnus MacG. Ithaca, type.

D. thoracicus (Pal. de Beauv.). "NY" (Kby).

D. unicolor (Pal. de Beauv.). Ithaca, Mar-May (US, MacG-Ill) ; Schenectady, May (NYS); Albany, Nassau, Poughkeepsie, Ap (NYS); Southfields, Ap (MacG-Schott); SI, Ap (Ds).

\section{LODERUS KonOW}

L. albifrons (Nort.). Newport, May, Yg (NYS) ; Axton, Je (MacG-I11) ; Ithaca, May-Je (CU, MacG-I11); McLean, May (MacG-Il1); Albany, May, Yg (NYS) ; SI, Je (Ds) ; LI: Yaphank, May, Ols (MacG-Schott). 
SUBFAMILY TENTHREDININAE

LEUCOPELMONUS MacGillivray

L. confusus (Nort.). Ithaca, May 28 (CU).

\section{Bivena MacGillivray}

B. delta (Prov.) (maria MacG.). Ithaca, J1 (US, CU); W. Danby, May (CU); McLean, May (CU) ; Van Cort. Pk., J1 (CU) ; LI: Astoria (MacG-Schott). B. quattrodecimpunctata (Nort.). L. Tear, J1 (US); Ithaca, May-Je (CU). Larva feeds on Podophyllum peltatum.

B. semilutea (Nort.). Ithaca, May (CU).

\section{LAGIUM Konow}

L. atroviolaceum (Nort.). Castile, EABradley (Bdy); Ithaca, J1 (CU) ; Southfields, Ap (MacG-Schott).

L. planifrons Roh. LI: Gardiners I., Je 11 (MacG-Schott).

PACHYPROTASIS Hartig

P. omega Nort. Saranac Inn, $N d m$ (MacG); Ithaca (CU).

\section{RHOGOGASTER Konow}

R. evansii (Harr.). Mt. Skylight, Je (US); Cranberry L., Htly (US).

\section{ZALAGIUM Rohwer}

Z. cinctulum (Nort.). LI (US).

Z. clypeatum Roh. LI (US).

\section{MACROPHYA Dahlbom}

M. alba MacG. Ithaca, May (CU, US); Van Cort. Pk., Je, Dyar (US) ; LI: Forest Hills, May, Ols (US); Maspeth, May, Ols (MacG-Schott).

M. albomaculata (Nort.). Ithaca, McLean, May (CU).

M. bilineata MacG. Ithaca, Je 28 (CU).

M. cassandra Kby. "NY", $D b l$ (Kby); Ithaca, J1 (CU).

M. confusa MacG. Ithaca, Je 10 (CU).

M. contaminata Prov. LI (US).

M. crassicornis Prov. Ithaca, J1 (CU); LI (US).

M. dejecta (Nort.). LI: Brooklyn, JAkhurst (Nort).

M. dyari Roh. Ithaca (MacG-I11); Van Cort. Pk., Dyar (US), type.

M. epinota (Say). Old Forge, J1, Yg (NYS) ; Poughkeepsie, J1, Yg (NYS) ; LI: Flatbush, May (MacG-Schott). Larva feeds on Sambucus canadensis and $S$. racemosa. 
M. externa (Say). Ithaca, May-Je (CU) ; Albany (NYS) ; Bronx Pk., J1, Dyar; LI: Bellport, Dyar (Ashm, Can. Ent., vol. 30, p. 174, 1898). Larva feeds on hickory.

M. fascialis Nort. Big Moose, Jl, Yg (NYS); Mt. Marcy, J1 (US).

M. fistula MacG. Ithaca, type.

M. flaccida MacG. Ithaca, type.

M. flavicoxae (Nort.). Newport, Je, Yg (NYS); Axton, J1 (CU); Castile, EABradley (Bdy); Ithaca, May-Je, McLean, May-J1 20 (Bdy-CU); Albany, Nassau, May, Yg (NYS); Morrisville, May (CU) ; Poughkeepsie, May, Yg (NYS).

M. flicta MacG. Ithaca, type.

M. formosa (Klug). Old Forge, Je, $Y g$ (NYS); Poughkeepsie, J1, Yg (NYS); LI (US).

M. goniphora (Say). Poughkeepsie, J1, Yg (NYS) ; Ft. Montgomery, Je (MacGSchott); Van Cort. Pk., Je, Dyar (US); LI (US).

M. intermedia (Nort.). Ithaca, J1 (CU) ; Poughkeepsie, May, J1, Yg (NYS); LI: Huntington, Je (MacG-Schott).

M. lineata Nort. Johnstown, May (NYS); Ithaca, May, J1 (US, CU) ; Albany, May (NYS). Larva feeds on Aster prenanthoides.

M. minuta MacG. Plattsburg, Dyar, type; Ithaca, May 28 (CU).

M. mixta MacG. "NY" (US).

M. nigra (Nort.). Johnstown, Aug (NYS); Newport, Je (NYS); Ithaca, Je (US) ; Poughkeepsie, J1, Yg (NYS) ; LI: Flatbush, Je, Zab (US).

M. pannosa (Say). "NY" (Nort).

M. propinqua Harr. Ithaca, Je (CU):

M. proximata Nort. Albany, Je, 1909 (NYS).

M. pulchella (Klug). Ithaca, J1 (CU) ; Albany, Je, $Y g$ (NYS) ; Big Ind. Va1., Je, Pears (US) ; Kingston, Aug, Poughkeepsie, May, Yg (NYS). Larva feeds on Solidago juncea and Rudbeckia laciniata.

M. punctata MacG. Plattsburg, type.

M. simillima Roh. Ithaca, May 7, 1897 (US).

M. succincta Cress. Ithaca (US, CU).

M. tibiator Nort. Newport, May, Yg (NYS) ; Ithaca, May-Je (CU) ; Albany, Je, Yg (NYS); Van Cort. Pk., Dyar; LI: Rockaway Pk., May (MacG-Schott). Larva feeds on Sambucus.

M. trisyllaba (Say). Axton, J1 (CU) ; Ithaca, Aug (CU) ; McLean, Je 12-Sep 24 (Bdy-CU); LI: Flatbush, Je-J1, Zab (US). Larva feeds on Sambucus canadensis.

M. trosula Nort. Poughkeepsie, J1, Yg (NYS); LI (US).

M. varia (Nort.). Adirondacks, Je (US) ; Axton, J1 (CU) ; McLean, Je 12-J1 19 (Bdy-CU) ; Nassau, May (NYS).

M. zabriskiei Roh. LI (type-US).

M. zonalis Nort. Axton, Je-J1 (US, CU) ; Cranberry L., Je-J1, Htly (Roh); Old Forge, J1 (CU) ; McLean, Ithaca, J1 (CU) ; Slaterville, J1 (CU) ; Malloryville $(\mathrm{CU})$. 


\section{TENTHREDO Linnaeus}

(Allantus auctt.)

\section{(Subgenus TENTHREDO Linnaeus)}

(Allantus auctt.)

T. basilaris Say. L. Pleasant, J1 (NYS) ; Castile, EABradley (Bdy); Ithaca, J1Aug (CU, US); McLean, Aug 19-Sep 3 (Bdy-CU); L. George, Aug, Zab (US) ; Albany (NYS) ; Karner, Aug (NYS); Salem, Aug (NYS); Southfields, Aug (MacG-Schott); SI (Ds).

T. dubius (Nort.). McLean, J1 18 (Bdy-CU).

\section{(Subgenus LABIDIA Provancher)}

T. originalis (Nort.). L. Tear, J1 (US), Fbs (Bdy-CU); Mt. Skylight, J1 (US).

\section{TENTHREDELLA Rohwer 123}

(Tenthredo auctt.)

T. angulifera (Nort.). Ausable L., J1, Bks (NYS); Severance, Aug (NYS); Ithaca, J1 (CU); Albany (Nort).

T. antennata (Kby.). Castile, EABradley (Bdy).

T. bilineata (MacG.). Ithaca, J1, types; larva feeds on Geranium maculatum.

T. causata (MacG.). Ithaca, Junghanns, type.

T. cressoni (Kby.). Keene Val., Dyar (MacG). Larva feeds on white birch.

T. eximia (Nort.). Cranberry L., J1 28, 1924, Htly (Roh-NYS).

T. flavomarginis (Nort.). Canandaigua L., May, Poughkeepsie, J1, Yg (NYS).

T. grandis (Nort.). Speculator (Smulyan); Canandaigua L., J1, Yg (NYS); Ithaca (Smulyan); McLean, May; Poughkeepsie, J1 (Smulyan-NYS).

T. hyalina (MacG.). Plattsburg, Dyar, type.

T. junghannsii (MacG.). Ithaca, Junghanns, type.

T. leucostoma (Kby.) (rufipes Say). Keene Val., J1, Yg (NYS); Old Forge, Jl, $Y g$ (NYS) ; Saranac Inn, Ndm; McLean, May (Bdy-CU); Poughkeepsie, Je (Smulyan); Ft. Montgomery, Je (MacG-Schott).

T. lobata lobata (Nort.). Cranberry L., Htly (US); Ithaca, Je 14-J1 4 (BdyCU) ; LI: Wyandanch, J1 (MacG-Schott).

T. macgillivrayi Smulyan. Gloversville, Aug (Smulyan).

T. mellina (Nort.). Keene Val., J1, Yg (NYS); Karner, Je, Yg (NYS).

T. nigricollis (Kby.). "NY" (Smulyan).

T. piceocincta (Nort.). LI: Brooklyn, JAkhurst, type.

T. rubripes (MacG.). Ithaca, Junghanns, type.

T. rufipes (Say). McLean, May 31, 1913 (Bdy-CU).

T. rufopecta (Nort.). Newport, May, Yg (NYS); Corning, Lint (Marlatt); Ithaca, McLean (Smulyan); N. Chatham, Je, Yg (NYS); Rensselaer, J1, Yg (NYS).

T. rufopediba (Nort.). Karner, J1 (NYS).

${ }^{128}$ The New England sawflies of the genus Tenthredella Rohwer. By M. T. Smulyan. Proc. Bost. Soc. Nat. Hist., vol. 36, p. $383-465.1923$. 
T. signata (Nort.). Speculator, May, Yg (NYS); Newport, J1, Yg (NYS); Ithaca (Smulyan); McLean, May (Bdy-CU); Poughkeepsie, May, Yg (NYS).

T. smectica (MacG.). Ithaca, type.

T. verticalis (Say). Saranac Inn, Ndm; L. Pleasant, Je, Yg (NYS); Sacandaga Pk. (Bdy-CU) ; Albany, Jl, Karner, Je, $Y g$ (NYS); N. Chatham, Je, $Y g$ (NYS); Rock City, Je 9 (Bdy-CU); Rochester Jct., J1 7, Leon (Bdy-CU); LI (Ds) ; Huntington, Je (MacG-Schott).

T. yuasi (MacG.). Ithaca, type.

\section{SUBFAMILY MESSINAE 124}

(Phyllotominae, Fenusinae, and Scolioneurinae, MacGillivray)

Caliroa O. Costa

C. aethiops (Fab.). Rose SAwfly. Ithaca, May (CU); Geneseo, Je (NYS). This is a well-known European enemy of roses which has become well established in the US; it is probably widely distributed throughout NY; in literature it is often referred to as Endelomyia rosae or Monostegia rosae.

\section{ERIOCAMPOIDES Konow}

E. fasciata (Nort.). Plattsburg, Dyar. Larva feeds on black oak.

E. lata (MacG.). Ithaca, type.

E. limacina (Retz.). Pear Slug. Albion, Felt; Syracuse (CU Econ); Angola (CU Econ) ; Corning, Je (NYS) ; Binghamton (CU Econ); Schenectady, Rensselaer (Felt); Kinderhook, Felt; Athens, Aug (Felt); West Farms. This species is undoubtedly widely distributed throughout the State. The larva is injurious to plums, cherries, pears, and allied plants, skeletonizing the leaves.

E. lobata (MacG.). Oswego, CSSheldon, type.

E. lunata (MacG.). Ithaca, type.

E. nortonia (MacG.). McLean, May 30, 1890, type. I do not know this species and find no reference to it since the original description. It was described in the genus Caliroa (Can. Ent., vol. 26, p. 324, 1894).

E. obsoleta (Nort.). Albany, May, Karner, Je (NYS); Nassau, May (NYS) ; Poughkeepsie, May (NYS). Larva feeds on leaves of wild cherry.

\section{Profenusa MacGillivray}

P. collaris MacG. Cherry and Hawthorn Sawfly Leaf-Miner. Rochester (Parr\&Fult) ; Geneva, May, Parr \& Fult (US) ; Ithaca, May, types; Germantown (Parr\&Fult); Botanical Gardens, NYC (Parr\&Fult). Larva mines the leaves of cherry and hawthorn.

\section{MEssa Leach}

M. alsia MacG. Ithaca, May, type.

M. amica MacG. N. Evans, Aug, type.

M. appota MacG. Buffalo, Je, type.

\footnotetext{
${ }^{124}$ Messinae is used for this subfamily by Rohwer because Messa is the oldest contained generie name. Those who believe, with the editor, that the subfamily name should be based on the oldest usage of a contained generic name for the stem of the name of a group higher than genus, will substitute Phyllotominae for Messinae. (Bdy.)
} 


\section{FENUSA Leach}

F. dohrnii (Tischb.). Ithaca, Je, Aug (US, CU) ; LI: Prospect Pk., Brooklyn, Ap (US). Larva mines the leaves of European elm, also of European alder.

F. pumila Klug. Birch LeAf-Miner. Generally present on gray birch from Chatham northward to Hudson Falls and westward to Binghamton (Felt, litt. Feb. 7, 1925) ; "very abundant on trees less than six feet high at Stephentown," (Felt, May 28, 1925). A European species recently found in the US.

\section{KALIOFEnUSA MacGillivray}

K. ulmi (Sundewall). Elm Lenf-Miner. Syracuse, Je (Felt); Johnstown, Je (Felt); Clinton, Je (Felt); Rochester (Felt); Ticonderoga, Je, Wilton, Je (Felt); Baldwinsville, Pompey, Kenwood (Felt); L. George, Je (Felt); Ithaca, Je (US, CU) ; Albany, Troy (Felt) ; Tivoli, May (Felt) ; Cambridge, Greenwich, Je (Felt). Larva mines the leaves of American and European elms.

Polybates MacGillivray

P. slossonae MacG. Niagara Falls, Sep 4, 1911, MCV (US).

\section{METALLUS Forbes}

M. capitalis (Nort.). LI: Brooklyn, JAkhurst, type.

M. rubi Forbes. BlackberRy LeAF-Miner. "NY" (CU). Larva mines the leares of Rubus.

\section{SUBFAMILY EMPRIINAE 125}

(Blennocampinae, Lycaotinae, part of Emphytinae, MacGillivray)

EMPRIA Lepeletier

E. caeca MacG. Ithaca, type.

E. callida MacG. Ithaca, type.

E. callosa MacG. Ithaca, type.

E. candidula MacG. Ithaca, type.

E. caprina MacG. Ithaca, type.

E. cariosa MacG. Slaterville to Caroline, type.

E. casta MacG. Phoenix, type $\delta^{\prime}$.

E. cauduca MacG. Ithaca, type.

E. cauta MacG. Ithaca, type.

E. cava MacG. Lancaster, $M C V$, type.

E. cavata MacG. Oswego, CSSheldon, type.

E. celebrata MacG. Buffalo, $M C V$, type.

E. celsa MacG. Ithaca, type.

E. cerina MacG. Ithaca, type.

E. cetaria MacG. Ithaca, type.

E. columna MacG. LI: Wyandanch, Sichott, type.

125 As with Messinae (see footnote on page 885), Emprimae is based on the oldest contained genus. But the oldest group name is Blennocampinae. (Bdy.) 
E. coryli (Dyar). Plattsburg (Dyar); Van Cort. Pk., Dyar. Larva feeds on hazel.

E. ignota (Nort.). Glens Falls, Je 2 (NYS).

E. maculata (Nort.). Black-Marked Strawberry Slug. Ithaca (CU); Saratoga Sps., May 30 (Felt) ; LI (US). Larva feeds on leaves of cultivated strawberries and often does some damage.

\section{Aphanisus MacGillivray}

A. muricatus MacG. Ithaca, type.

A. odoratus MacG. Ithaca, type.

\section{BLENNOCAMPA Hartig}

B. abnorma MacG. Ithaca, type.

B. abjecta MacG. Ithaca, type.

B. spiraeae Dyar. Keene Val. (Dyar-US), type. Larva feeds on Spiraea salicifolia.

\section{ERYTHRASPIDES Ashmead}

E. pygmaea (Say). Albany, Nassau, May (NYS); West Park, J1, Felt, Poughkeepsie, May (NYS). Larva feeds on leaves of grape.

E. caryae (Nort.). Malden-on-Hudson, Aug 20 (Felt).

\section{HYPARGYRICUS MacGillivray}

H. fumipennis (Nort.). Newport, May (NYS); Ithaca, May-Je (CU, MacG-I11); Kingston, Aug (NYS); Poughkeepsie, J1, MCV (NYS); Ft. Montgomery, Je (MacG-Schott); SI, J1 (Ds). Larva feeds on leaves and flowers of Smilacina racemosa.

H. infuscatus MacG. Ithaca, type.

\section{MONOPHADNOIDES Ashmead}

M. collaris MacG. Ithaca, type.

M. concessus MacG. Ithaca, type.

M. conspersus MacG. Ithaca, type.

M. conspiculata MacG. Ithaca, type.

M. conspicuus MacG. McLean, type.

M. rubi (Harr.). RaspberRy SAwfly. Newark, CHStuart (NYS); Oneida (Felt-NYS); Naples, Je (CU Econ). Larva is sometimes destructive to foliage of Rubus and has been recorded as feeding on ragweed.

\section{MONOPHADNUS Hartig}

M. aequalis MacG. Ithaca, type.

M. bipunctatus MacG. Ithaca, type.

M. tiliae Nort. Ithaca, McLean, May (CU, MacG-I11) ; Rensselaer, Je (NYS); Poughkeepsie, Je (NYS). Larva feeds on leaves of Tilia. 
PARACHARACTUS MacGillivray

P. obscuratus MacG. Ithaca, type.

P. rudis (Nort.). "NY" (Nort).

\section{PERICLISTA Konow}

(Isodyctium Ashmead)

P. albicollis (Nort.). LI: Bellport (Dyar-US); Yaphank, Dyar (US). Larvae feed on leaves of Quercus tinctoria.

P. caryicola (Dyar). Bronx Pk. (Dyar-US); LI: Bedford, Dyar. Larva feeds on hickory.

P. confusa MacG. Ithaca, type.

P. emarginata (MacG.). Pelham Manor, May, Van Cort. Pk., May, Dyar. Larvae feed on leaves of Quercus coccinea.

P. infrequens (Nort.). LI: Brookhaven, Je, Bellport, Je, Dyar. Larva feeds on foliage of Quercus alba.

P. marginicollis (Nort.). "NY" (Nort).

P. media (Nort.). Pelham Manor, Van Cort. Pk., Dyar (US) ; LI: Brookhaven, Bellport, Yaphank, Dyar (US). Larva feeds on foliage of Quercus alba.

P. purpuridorsum Dyar. Ithaca, May-Je (CU, MacG-I11); Pelham Manor, May, Dyar (US) ; LI: Bellport, Brookhaven, Je, Dyar (US).

P. similaris Roh. LI: Brookhaven, Dyar. Larva feeds on Quercus alba.

P. subgregaria (Dyar). Ithaca, May (CU); Pelham Manor, Dyar (US). Larva feeds on Quercus prinus.

P. subtruncata Dyar. Van Cort. Pk., May, Dyar (US). Larva feeds on Quercus coccinea.

P. xanthognatha Roh. Ithaca, type.

RHADINOCERAEA Konow

R. similata MacG. Ithaca, type.

\section{SUBFAMILY PHYMATOCERINAE ${ }^{126}$}

Neotomostethus MacGillivray

N. hyalinus MacG. McLean, type.

\section{TOMOSTETHUS Konow}

T. bardus (Say). Keene Val., Je (Dyar-US); Rouses Pt., Je, Dyar; Ithaca, May-Je (CU) ; Greenwood L., May (MacG-Schott). Larva feeds on foliage of ash.

T. inhabilis (Nort.). McLean, Je 13-J1 15 (Bdy-CU).

\section{SUBFAMILY SELANDRIINAE}

\section{CORYNA Lepeletier}

C. vanduzeei (Roh.). Buffalo, Je 4, 1890, MCV (US), type.

${ }^{126}$ Included in Blennocampinae by MacGillivray. 


\section{PolyselandRIA MacGillivray}

P. flavipes (Nort.). Saranac Inn, J1 (NYS); Axton, J1 (CU); Oswego, J1 (CU); Ithaca, J1 (CU); Karner, J1, N. Chatham, Je (NYS); LI (US). Larva feeds on Pteris aquilina.

\section{Hemitaxonus Ashmead}

H. albidopictus (Nort.) (innominatus MacG.). Saranac Inn, Ndm, type of innominatus; Syracuse, Aug (MacG-I11) ; Boston, Aug, $M C V$ (US) ; Ithaca, Sep (MacG-CU) ; LI: Wyandanch, May (MacG-Schott).

H. amicus (Nort.). Rouses Pt., Je 13, 1895 (Dyar-US). Larva feeds on Onoclea sensibilis.

H. dubitatus (Nort.). Ellenville, J1 (CU); Ithaca, May (US,CU); McLean, May (CU) ; W. Nyack, J1 (US); Van Cort. Pk., J1 (US); LI: (US); Maspeth, Je, Ols (MacG-Schott). Larva feeds on Onoclea.

\section{STRONGYLOGASTER Dahlbom}

S. annulosus Nort. Saranac Inn, Ndm (MacG); Axton, Je (US, CU); Woodworth, Je (NYS) ; Ilion, May (NYS); Albany, Je (NYS); LI: Wyandanch, May (MacG-Schott). Larva feeds on Pteris aquilina.

S. longulus Nort. McLean, Je 5 (Bdy-CU); Albany, May (NYS) ; LI: Wyandanch, May (MacG-Schott).

S. luctuosus Prov. Keene Val., Dyar (MacG).

S. politus Cress. Otto (CU); McLean, Je 5 (Bdy-CU). Larva feeds on Pteris aquilina.

S. soriculatipes Cress. Cranberry L., Je 19, 1924, Htly (Roh).

S. tacitus (Say). Castile, EABradley (Bdy); McLean, Ap 30 (Bdy-CU) ; Karner, May, Albany, Je (NYS); LI: (US); Yaphank, J1, Ols (MacG-Schott).

S. unicus Nort. LI: Brooklyn, JAkhurst (Nort).

\section{THRINAX Konow}

T. pullatus MacG. Ithaca, type. Larva feeds on fern.

\section{SUBFAMILY CLADIINAE}

\section{TRICHIOCAMPUS Hartig}

T. gregarius (Dyar). Keene, J1 1, 1894, Dyar (US). Larva feeds gregariously on Populus tremuloides and $P$. deltoides.

T. pacatus MacG. Ithaca, type.

T. viminalis (Fall.). Poplar Sawfly. Greenfield Center, Chester, Aug, New Milford, Aug (Felt); Binghamton, Sep (Felt); Albany and vic. (NYS,CU, US, MacG-I11); Cambridge, Felt (NYS); NYC, May, Dyar (US); LI: Brooklyn (US). This European species was first recorded from US by Lintner, who reared it from larvae feeding on leaves of Populus monilifera collected at Albany, and described it as Aulacomerus lutescens. It has since been observed in a number of localities in the northeastern part of the US and is sometimes recorded as injurious to Carolina poplar. Lintner observed that adults of the first brood emerge in July and the second brood appears in August. 


\section{PRIOPHORUS Dahibom}
P. aequalis (Nort.). Castile, EABradley (Bdy); Albany, Ap, Yg (NYS).
P. infuscatus (MacG.). Ithaca, type.
P. palliolatus (MacG.). Ithaca, type. Larva feeds on leaves of Salix.
P. rubi Roh. Hamburg, May 14, 1911, MCV (US).
P. solitarius (Dyar). Ithaca, Aug (CU).

\section{Cladius Rossi}

C. isomerus Nort. (pectinicornis auctt. not Fourc.). Bristly Rose Slug. Ellenville, Je (MacG-I11) ; Ithaca, Aug (Griswold); Van Cort. Pk., Je, Dyar (US). This is a very common leaf skeletonizer of roses, and undoubtedly is widely distributed throughout the State.

\section{SUBFAMILY NEMATINAE}

\section{Platycampus Schi申dte}

P. americanus (Marl.). Plattsburg, Dyar. Larva feeds on foliage of poplar.

\section{Ceraterocercus Rohwer}

C. albidovariatus (Nort.). LI: Bellport, May, Dyar (US). Larva feeds on foliage of Quercus coccinea.

C. phytophagicus (Dyar). Van Cort. Pk., Ap (Dyar-US); Bronx Pk., May, det. from larva (Dyar). Larva feeds on foliage of Quercus alba.

C. quercivorus Roh. Bronx Pk., May (Dyar-US); Pelham Bay Pk., May, Dyar (US); Franklin Pk., Je (Dyar). Larva feeds on foliage of Quercus alba.

\section{HEMICHROA Stephens}

H. americana (Prov.). Ithaca, Aug (CU); Fulton, J1 (CU); Ellis, J1 (CU) ; McLean, May (CU).

H. dyari Roh. Rhinebeck, determined from larva (Dyar). Larva feeds on Alnus.

\section{Hoplocampa Hartig}

H. halcyon Nort. Niagara Falls, May, $M C V$ (US); Ithaca (US); McLean, May, CFBaker (US). Larva feeds within the fruit of Amelanchier.

\section{DINEURA Dahlbom}

D. litura (Klug). Karner, Ap-May, "found on willow" (NYS).

\section{Pontania A. Costa}

P. borealis Marl. Plattsburg, Dyar. Larva causes galls on leaves of Salix sericea, and according to Dyar is single-brooded.

P. consors Marl. Plattsburg, Dyar. Larva causes folds on leaves of Salix sericea, and pupates in decayed wood.

P. daedala MacG. Ithaca, type. 
P. decrepita MacG. Ithaca, type.

P. dedocora MacG. Ithaca, type.

P. demissa MacG. Ithaca, type.

P. derosa MacG. Ithaca, type.

P. desmodioides (Walsh). Buffalo, J1 (CU Econ); Richfield Sps. (Marlatt).

P. dotata MacG. Ithaca, type.

P. gracilis Marl. Gouverneur (Lint); Van Cort. Pk., Dyar. Larva causes galls on leaves of Salix petiolata.

P. hyalina (Nort.). Saranac Inn, Ndm (MacG-I11); Johnstown, May (NYS); Canandaigua, Oct (Felt) ; Ithaca, J1 (CU) ; Richfield Sps., Pergande (Marlatt); Hudson Falls, J1 (Felt) ; Nassau, May (NYS); Dunkirk, J1 (NYS); LI: Bellport, Dyar. Larva causes galls on leaves of smooth-leaved willow, according to Dyar; according to records from Cornell collections, the gall occurs on Salix fragilis.

P. pallicornis (Nort.). Plattsburg, Aug (Dyar-US); Van Cort. Pk. (Dyar).

P. pisum (Walsh). Ithaca, May (CU, MacG-I11) ; Richfield Sps., Pergande (Marlatt); E. Steamburg, ELHorton (Marlatt). Larva causes galls on the leaves of Salix discolor.

P. pomum (Walsh). Canandaigua, Oct (Felt); Ithaca (CU); Albany, Yg (NYS); W. Albany, Lint (NYS) ; Hudson Falls, Je (Felt) ; Easton Hills, Sep (NYS); vic. of NYC ( $\mathrm{Bt})$. Larva causes galls on leaves of Salix cordata and S. discolor.

P. populi Marl. Vic. NYC, Dyar (type-US). Larva folds the edge of the leaf of Populus grandidentata.

P. robusta Marl. Vic. NYC, Dyar. Larva folds the edge of the leaf of Populus tremuloides.

P. terminalis Marl. Van Cort. Pk., Dyar; NYC, Dyar (US), type.

\section{PTERONIDEA Rohwer}

(Pteronus auctt.)

P. cornelli Marl. Ithaca, J1 (CU) ; SI, Je (Ds); LI: Montauk, J1 (MacG-Schott). Larva feeds on foliage of Salix.

P. coryli (Cress.). Plattsburg, J1, Dyar; SI, May (Ds). Larva feeds on foliage of Corylus rostrata.

P. dyari Marl. "NY", Dyar (type-US).

P. edita MacG. Ithaca, type.

P. edura MacG. Ithaca, type.

P. erythrogastra (Nort.). Ithaca, Bks (US) ; Karner, Aug (NYS). Yuasa records the larva as feeding on foliage of Carpinus caroliniana, Morus alba, and Alnus rugosa.

P. fulvicrus (Prov.). Ithaca (MacG-I11).

P. hudsonii-magna (Dyar). Plattsburg, Aug 31, 1893, Dyar (US). Larva feeds on foliage of poplar.

P. hyalina (Marl.). Plattsburg, Je 6, Dyar (US). Larva feeds on foliage of white birch.

P. integra (Say). LI: Brookhaven, J1 7, Dyar (US). 
P. lateralis (Nort.). Plattsburg, Keene Val., Dyar; Albany, Peck (Nort); Central Pk., NYC, Dyar. Larva feeds on foliage of Betula populifolia.

P. latisfasciata (Cress.). Keene Val. (Dyar). Larva feeds on foliage of Betula papyrifolia.

P. lombardae (Mar1.). Plattsburg, Sep 6, 1893, Dyar (US). Larva feeds on foliage of Populus grandidentata.

P. longicornis (Marl.). "NY" (types-US); Ithaca, Ap-May (CU,US) ; LI: Flatbush, May, Dyar (US).

P. longulicornis (Nort.). "NY" (Nort).

P. marlatti (Dyar). "NY", Dyar, type.

P. mendica (Walsh). Plattsburg, Dyar (US); Ithaca, Ap, Aug (CU) ; Jefferson Highlands (US) ; Central Pk., NYC, Dyar (US). Larva feeds on foliage of Salix.

P. militaris (Cress.). Ithaca, May, Aug (CU, MacG-I11).

P. odorata (Dyar). Bluff Pt., near Plattsburg, Aug (Dyar-US); Ithaca, J1 (MacG-I11).

P. ostiaria MacG. Ithaca, Yuasa, type.

P. pinguidorsum (Dyar). Keene Val., Dyar (type-US). Larva feeds on foliage of Betula papyrifera.

P. plesia Roh. LI: Westbury (type-US). Larva feeds on foliage of Populus grandidentata.

P. quercus (Marl.). Ithaca, Trelease, type; Albany, May (NYS); LI: Brookhaven (Dyar-US). Larva feeds on foliage of Quercus alba.

P. ribesii (Scop.). Imported CURrant Worm. Indian L., Watertown (NYS); Oswego, May (MacG-I1l) ; Fulton (NYS) ; Rochester (Fitch) ; Lyons, western NY (NYS); Ithaca (CU); Utica (NYS); Binghamton, May, Ballston Spa, Je (NYS); Rhinebeck, Albany, Johnson City (NYS); LI: Flatbush, Dyar (US). Larva feeds on foliage of Ribes. This is the common, introduced, currant and gooseberry sawfly. It is undoubtedly very widely distributed throughout the State, and may at times become injurious to cultivated species of Ribes. It has a number of broods each year.

P. trilineata (Nort.). Newburgh, Edwards (Nort); Ithaca, J1 (CU) ; LI: Maspeth, Je (MacG-Schott). Larva feeds on foliage of Salix.

P. ventralis (Say). Central Pk. in NYC (Dyar-Us); LI: Flatbush, J1, Dyar (US). Larva is common on foliage of willow, and feeds gregariously.

P. vertebrata (Say). Plattsburg (Dyar-US); Jefferson Highlands, Je (Dyar-US); LI: Flatbush, J1, Zab (US).

AMAURONEMATUS Konow

A. azaleae Marl. Rochester, Je (Felt); Ithaca (MacG-I11). Larva feeds on foliage of Azalea.

A. brunneus (Nort.). Ithaca, May 5, 1899 (CU).

A. comstocki Marl. Ithaca, May 2, 1891 (CU).

A. concolor (Nort.). Albany, Peck (Nort).

A. fulvipes (Nort.). Ithaca, May (MacG-I11).

A. gracilis Marl. Ithaca, May (type-CU); LI: Wyandanch, Ap (MacG-Schott).

A. lineatus (Harr.). Ithaca, Ap 26, 1896 (CU). 
A. luteotergus (Nort.). Keene Val., Dyar (US); Ithaca, May (MacG-I11). Larva feeds on foliage of Alnus.

A. nigrofemoratus (Cress.). Ithaca, May (CU). This is an unusual record and may be based on an erroneous determination.

A. oregonensis Marl. Keene Val. (Dyar). Larva feeds on foliage of "woolly willow."

A. $\operatorname{rapax}$ (Cress.). Ithaca, Ap (MacG-I11). This species was previously reported only from Nevada.

A. similis Marl. Plattsburg and Keene Val., Dyar. Larva feeds on foliage of "woolly willow."

CRoesus Leach ${ }^{127}$

C. latitarsis (Nort.). Plattsburg (Dyar); Ithaca, Aug (CU); Glen, J1 (CU). Larvae feed gregariously on foliage of birch.

NEMATINUS Rohwer

(Nematus Konow and auctt.)

N. chloreus (Nort.). LI: Bellport, Je 5, 1897, Dyar (US). Larva feeds on foliage of black oak.

N. unicolor (Dyar). Keene Val., type. Larva feeds on white birch.

\section{Nematus Panzer 128}

\section{(Holconeme Konow)}

N. erichsonii Hartig. Larch Sawfly. New Russia, Je (NYS); Alder Cr., Je (NYS) ; Horicon, Pottersville, Schroon L., DeKalb Jct., Cherry Val., Newton's Corners to Sageville, St. Lawrence Co, Wilmington Notch to Mountain View House in Essex Co (Felt) ; Crosby, Je (NYS); Ithaca, Ap-J1 (CU, MacGI11) ; Ballston Spa (Felt); Albany, May-Aug (Felt). Larvae feed on foliage of larch, and are often an important pest.

N. suratus Fitch. "NY", type. Larva is recorded as feeding on cherry. This species has not been examined since it was originally described, and very probably does not belong to the genus Nematus as at present restricted.

\section{PaChynematus Konow}

P. aurantiacus Marl. McLean (CU).

P. corniger (Nort.). McLean, Je-Aug (Bdy-CU); Johnstown, Ji, 1908 (NYS).

P. corticosus MacG. Saranac Inn, type.

P. extensicornis (Nort.). "NY" (Nort); Ithaca (CU); W. Danby, May 31 (CU). Larva feeds on foliage of wheat.

P. gregarius Marl. Ithaca (MacG-I11).

P. inconspicuus (Kby.). "NY", Dbl (type-Brit. Mus.).

${ }^{127}$ If the Erlangen List is accepted, this generic name will have to be changed to Nematus Jurine (Panzer).

${ }_{12}$ If the Erlangen List is accepted, the generic name Nematus will have to be assigned to the group here treated under the name Croesus, and the group treated as Nematus will have to be called Holconeme Konow. 
P. palliventris (Cress.). Oswego, May 15 (CU); Ithaca, AMShaw (CU).

P. pleuricus (Nort.). Ithaca, J1 3 (CU).

P. remissus MacG. Ithaca, type.

P. repertus MacG. Ithaca, type. Larva feeds on Carex.

P. robustus Marl. Ithaca, May 26, Houghton (CU).

P. roscidus MacG. Adirondacks, type.

P. subalbatus (Nort.). Ithaca, May 9, 1895 (CU). Larva feeds on Carex.

\section{PRISTIPHORA Latreille}

P. banski Marl. Ithaca (US), type; LI: Sea Cliff (US), type; Huntington, Je (MacG-Schott).

P. dyari Marl. Keene Val., Je, Dyar, type; Ithaca, J1 (MacG-I11).

P. idiota Nort. Speculator, May (NYS); Karner, J1, Yg (NYS).

P. murtfeldtiae Marl. Ithaca, Aug 19 (CU). Larva feeds on foliage of Salix.

P. ostiaria MacG. Ithaca, type.

P. allicoxa Roh. Lancaster, Je 4, 1911, MCV (US).

P. sycophanta (Walsh). Keene Val., Dyar; Jefferson Highlands, Dyar (Marl). Larva is recorded as feeding on foliage of Betula papyrifera and Salix.

\section{DIPHADNUS Hartig}

D. appendiculatus (Hartig). "NY" (Nort); Ithaca (US). Introduced; sometimes abundant enough to be considered a pest; larva feeds on foliage of cultivated currants and gooseberries.

\section{EUURA Newman}

E. nigria Prov. Ithaca (US, CU, MacG-I11).

E. orbitalis (Nort.). L. Placid, Ap, $M C V$ (NYS); Karner, Je, $M C V$ (NYS) ; Phoenicia, Aug, $M C V$; LI: Brooklyn, Akhurst (Nort).

E. salicis-nodus Walsh. "NY" (Nort).

E. salicis-ovum Walsh. "NY" (Nort).

\section{FAMILY PTERYGOPHORIDAE 129}

\section{ACORDUleCERA Say}

A. dorsalis Say. Albany, May, 1910 (NYS); Poughkeepsie, May, 1903 (NYS). Larva feeds on oak.

A. maculata MacG. Ithaca, type locality; Caroline to Harford, Je (US,CU); McLean, J1, Slaterville to Caroline, Je (MacG-I11).

A. maura MacG. Ithaca (CU), type; Caroline to Harford, Je (US).

A. maxima MacG. Ithaca, type. Larva feeds on Quercus alba.

A. meleca MacG. Ithaca, type.

A. minima MacG. Ithaca, type.

129 This family is composed of the subfamilies Pterygophorinae and Acordulecerinae of the family Tenthredinidae, in MacGillivray's classification. In Comstock's Introduction to Entomology, it is included in the Argidae. (Bdy.) 
A. mixta MacG. Ithaca, paratype (US) ; Mud Cr., Je 17, 1920 (US).

A. munda MacG. Ithaca, type (CU); Greenwood L., Je (MacG-Schott).

A. musta MacG. Ithaca, type (CU). Larva feeds on hickory.

A. nigritarsis Roh. "LI", paratype. Larva feeds on Quercus marilandica.

A. parva Roh. Van Cort. Pk., type.

A. quercus Roh. "NY", type. Larva feeds on oak.

A. saginata Prov. Ithaca, Je (MacG-I11).

\section{SUBORDER IDIOGASTRA}

Compiled by S. A. ROHWER

\section{FAMILY ORYSSIDAE}

\section{ORYSSUS Latreille}

O. affinis Harr. Karner, Je (Yg-NYS); Poughkeepsie, Je (Yg-NYS).

O. sayii Westw. Peru, Sep 19, 1916 (Bdy-CU); Ithaca, Je 3, 1925 (Fbs-CU); SI, May-Je (Beq-Ds).

O. terminalis Newman. Trenton Falls (Db1), type; Michigan Hollow Swamp, Lloyd (Bdy-CU); West Pt. (Beq-Ds).

\section{SUBORDER CLISTOGASTRA Superfamily ICHNEUMONOIDEA FAMILY BRACONIDAE 130 \\ Compiled by C. F. W. Muesebeck}

New York specimens of all the species of Braconidae in the following list have not yet been obtained, or at least have not yet been identified; but on the basis of their known general distribution and hosts, they must certainly occur in the State, and for that reason are included. ${ }^{131}$ Many of the subfamilies are still in an extremely unsatisfactory condition from a taxonomic standpoint. This is true particularly of Alysiinae, Blacinae, Sigalphinae, Braconinae, Macrocentrinae, Hormiinae, and Aleiodinae. Revision of these groups, and the more thorough working of material already in museum collections, will certainly reveal a much larger braconid fauna in the State of New York. Practically all the species of this family, with the exception of certain Alysiinae, are primary parasites, and many of them are very important agents in the natural control of injurious insects.

\section{SUBFAMILY COENOCOELIINAE}

This group has sometimes been held as a distinct family because of the unusual attachment of the abdomen far above the level of the posterior coxae, in which respect it somewhat resembles the Evaniidae. But the wing venation and general structure, as well as the habits and manner of development of the various species, are typically braconid. It is nearest the Helconinae.

\footnotetext{
130 At the suggestion of the editors, Mr. Muesebeck has retained the sequence of subfamilies developed by Szepligeti in Genera Insectorum, vol. 22, Hymenoptera, Fam. Braconidae, 1904.

131 Although in other groups species not actually recorded from the State have not been listed, in view of the great pains taken by Mr. Muesebeck in preparing the list of this family, and his feeling, shared by ourselves, that the omission of such species would greatly curtail the value of the list, they have been included but placed in brackets and set in italics. As so much of the literature dealing with our species of Braconidae has been published subsequent to any catalog, Mr. Muesebeck has kindly included the reference to the original description of each species.-Editors.
} 


\section{Coenocoelius Provancher}

\section{(Aulacodes Cresson)}

[C. ashmeadi D.T. Cat. Hymen., 1898, 4:72. A parasite of Leiopus (Cerambycidae) in sumac.]

C. erythrogaster Roh. Can. Ent., 1914, 46:317. Syracuse. Reared from Leiopus alpha (Say) and ?Saperda discoidea Fab. (both Cerambycidae), in hickory.

[C. populator (Say). Keating's Narrative of an expedition to the source of St. Peter's River, etc., 1824, 2: 323.]

C. provancheri Roh. Can. Ent., 1914, 46:321. Śyracuse, reared by Blackman and Stage from ?Neoclytus acuminatus (Fab.) (Cerambycidae) in hickory. Also parasitic on Leiopus larva in sumac.

C. rugosus Prov. Addit. fauna Can. Hymen., 1886, p. 122. Ithaca, Je 29, from the elm borer, Saperda tridentata Oliv. (Cerambycidae) (Ashm-CU).

C. saperdae (Ashm.). Proc. U.S. Nat. Mus., 1889(1888), 11:653. Ithaca, Sep (Mues-CU); Slaterville to Caroline, Je, MacG\&Bdy (Mues-CU). A parasite of the round-headed apple-tree borer, Saperda candida Fab. (Cerambycidae).

\section{SUBFAMILY VIPIINAE}

\section{(Braconinae auctt.)}

(Many species of this group are parasitic on Coleoptera of various types, while others are important enemies of injurious Lepidoptera.)

\section{ATANYCOLUS Foerster \\ (Melanobracon Ashmead)}

A. epicus (Cress.). Trans. Amer. Ent. Soc., 1872, 4: 183. Norton's Landing, Cayuga L., SmHH (Ashm-CU).

A. charus (Rly.). Seventh Ann. Rep. Ins. Mo., 1875, p. 75. Ithaca, Aug (Ashm$\mathrm{CU}$ ). A parasite of the larva of Chrysobothris femorata (Oliv.) (Buprestidae).

A. rugosiventris (Ashm.). Proc. U.S. Nat. Mus., 1889(1888), 2:613. Syracuse (B\&S). Reared from Chrysobothris femorata (Oliv.) (Buprestidae).

A. simplex (Cress.). Trans. Am. Ent. Soc., 1872, 4: 184. Syracuse (B\&S); Ithaca, May (Ashm-CU); SI, J1 31, Ds (Ashm); LI: Huntington, Schott (Roh). Probably throughout the State. Recorded as a parasite of Saperda discoidea Fab. and the elm borer, S. tridentata Oliv. (both Cerambycidae).

\section{Coeloides Wesmael}

[C. pissodis (Ashm.). Proc. U.S. Nat. Mus., 1889(1888), 11:617. A parasite of the white pine weevil, Pissodes strobi (Peck).]

C. scolytivorus (Cress.). Riley, C.V., Fifth Rep. Ins. Mo., 1873, p. 106. Geneseo Reared from the hickory bark-borer, Scolytus quadrispinosus Say.

\section{CAMPYloneurus Szepligeti}

[C. rugator (Say). Bost. Journ. Nat. Hist., 1836, 1 : 251.] 


\section{IPHIAULAX Foerster}

I. agrili (Ashm.). Proc. U.S. Nat. Mus., 1889(1888), 11: 612. Recorded from NY. A parasite of Agrilus arcuatus fulgens Lec. (Buprestidae), and Neoclytus acuminatus (Fab.) (Cerambycidae).

I. erythrogaster Br. Hist. Nat. Ins. Hymen., 1846, 4: 400. Ithaca, May-Sep (CU). A parasite of the larvae of various longicorn beetles.

\section{MiCROBRACON Ashmead ${ }^{132}$}

\section{(Bracon auctt. not Jurine)}

As here considered this genus includes the species formerly placed in both Habrobracon and Microbracon. It is a large and very difficult group. Most of the species are parasites of lepidopterous larvae, but some attack Coleoptera, while a few are found parasitizing both lepidopterous and coleopterous larvae which occur in similar situations.

M. apicatus (Prov.). Nat. Can., 1880, 12:142. Ithaca, Aug (Ashm-CU); LI; probably occurs throughout the State.

[M. atricollis (Ashm.). Proc. U.S. Nat. Mus., 1889(1888), 11:622.]

[M. auripes (Prov.). Addit. fauna Can. Hymen., 1888, p. 372. A parasite of lepidopterous larvae boring in such weeds as Amaranthus and Ambrosia.]

[M. brachyurus (Ashm.). Can. Ent., 1891, 23:1.]

M. caulicola Gahan. Proc. U.S. Nat. Mus., 1922, 61:2. Schenectady, reared by E.M.Searls from Pyrausta ainslei (Hein.) (Pyralidae). Probably throughout the State. A parasite of various species of Pyrausta including mubilalis Huebner, the imported European corn borer.

[M. cushmani Mues. (variabilis Cush.). Proc. U. S. Nat. Mus., 1925, 67:29. A widely distributed species. Parasitic on Psorosina hammondi Rly., Acrobasis indiginella nebulella (Rly.), A. indiginella Zell. (all Pyralidae), and Laspeyresia prunivora Wals. (Tortricidae).]

[M. furtivus (Fyles). Can. Ent., 1892, 24:34. A very common parasite of the goldenrod gall-maker, Gnorimoschema gallaesolidaginis Rly. (Gelechiidae).]

[M. gastroideae (Ashm.). Proc. U.S. Nat. Mus., 1889(1888), 11:617. Parasitic on larva of Gastroidea cyanea Melsh.]

M. gelechiae (Ashm.). Proc. U.S. Nat. Mus., 1889(1888), 11:623. Ithaca, May (Ashm-CU) ; McLean, Aug 1 (Mues-CU); Oswego. Undoubtedly throughout the State; very widely distributed, occurring from Me. to Calif. Its recorded hosts include Psorosina hammondi Rly. (Pyralidae), the oriental peach moth (Laspeyresia molesta Busck), the grape leaf-roller (Desmia funeralis Hbn.) (Pyralidae), the grape berry moth (Polychrosis viteana Clemens), the appleleaf-roller (Archips argyrospila W1k.) (Tortricidae), and Gelechia spp. (Gnorimoschema?) (Gelechiidae).

M. hebetor (Say). Bost. Journ. Nat. Hist., 1836, 1:252. Ithaca, J1 (Ashm-CU). A very abundant cosmopolitan species, occurring throughout the range of its hosts. Its principal hosts are the flour and meal moths, Ephestia kuhniella 'Zell. and Plodia interpunctella Hbn. It also parasitizes other species of Ephestia, the angoumois grain moth (Sitotroga cerealella Oliv.) (Gelechiidae), Vitula edmandsii Pack., and the bee-moth (Galleria mellonella L.) (both Pyralidae).

${ }^{132}$ A revision of the North American species of this genus has just been published by Mr. Muesebeck (Proc. U. S. Nat. Mus., 1925, vol. 67, art. 8, 85 pp. (Bdy.) 
[M. juncicola (Ashm.). Proc. U.S. Nat. Mus., 1889 (1888), 11:620. Apparently parasitic on the larvae of various species of Coleophora (Coleophoridae).]

M. lixi (Ashm.). Insect Life, 1892, 4:257. Rye, J1 15-25 (Bird), a primary parasite on Papaipema (Noctuidae) in early larval stages.

M. lutus (Prov.). Nat. Can., 1880, 12:142. McLean, Aug 12, Sib (Mues-CU); Rye, reared by HCBird from Papaipema nebris Guenée (Noctuidae). Also recorded as a parasite of Lixus scrobicollis Boh. (Curculionidae), in Ambrosia.

[M. melanaspis (Ashm.). Can. Ent., 1891, $23: 1$.

M. mellitor (Say). Bost. Journ. Nat. Hist., 1836, 1:256. Manlius, SmHH (Ashm-CU). A very widely distributed species. In the Cotton States it is an exceedingly important parasite of the cotton-boll weevil, Anthonomus grandis Boh., and of the pink boll-worm, Pectinophora gossypiella Saunders. In the northern States it has been abundantly reared from the grape berry moth, Polychrosis viteana Clemens.

[M. meromyzae (Gahan). Proc. U.S. Nat. Mus., 1913, 46:432. A parasite of Meromyza americana Fitch.]

M. nigridorsum (Ashm.). Can. Ent., 1891, 23:2. Ithaca, May (CU).

M. nuperus (Cress.). Trans. Amer. Ent. Soc., 1872, 4:187. Thousand Isls., J1 (Ashm-CU).

[M. oenotherae Mues. Proc. U.S. Nat. Mus., 1925, 67:62. A parasite of lepidopterous larvae feeding in the seed capsule of the evening primrose.]

M. papaipemae Gahan. Proc. U.S. Nat. Mus., 1922, 61:4. Rye, type locality, reared by HCBird from the larva of Papaipema frigida Smith (Noctuidae).

[M. pini Mues. Proc. U.S. Nat. Mus., 1925, 67:52. A rather common parasite of the white-pine weevil, Pissodes strobi Peck, in the northeastern States.]

[M. politiventris (Cush.). Proc. U.S. Nat. Mus., 1919, 55:517. A common gregarious parasite of the grape berry moth, Polychrosis viteana Clem., and of various leaf-rollers.]

M. punctatus Mues. Proc. U.S. Nat. Mus., 1925, 67:24. Nassau Co, type locality, reared from the parsley stalk-weevil, Listronotus latiusculus (Boh.), by Chittenden.

M. pygmaeus (Prov.). Nat. Can., 1880, 12:144. McLean, Sep 1 (Mues-CU). A very common and widely distributed species, occurring from Me. to Calif. Parasitic on various species of Coleophora (Coleophoridae).

[M. quinnipiacorum Vier. Conn. Geol. and Nat. Hist. Surv., 1916, Bull. 22, p. 207.]

[M. sanninoideae Gahan. Proc. U.S. Nat. Mus., 1917, 53:196. A widely distributed parasite of the peach-tree borer, Conopia exitiosa Say.]

[M. scanticorum Vier. Conn. Geol. and Nat. Hist. Surv., 1916, Bull. 22, p. 207.]

[M. sesiae Mues. Proc. U.S. Nat. Mus., 1925, 67:53. A parasite of the currant borer, Conopia tipuliformis (L.) (Aegeriidae).]

M. tachypteri Mues. Proc. U.S. Nat. Mus., 1925, 67:68. Stony I. (Mues-US). Parasitic on the apple curculio, Tachypterellus quadrigibbus (Say).

[M. tenuiceps Mues. Proc. U.S. Nat. Mus., 1925, 67 : 46.]

M. variabilis (Prov.). Addit. fauna Can. Hymen., 1888, p. 382. McLean, Aug 12, Sib (Mues-CU). A very common, widely distributed species. Recorded as a parasite of the grape berry moth, Polychrosis viteana Clem., and the plum curculio, Conotrachelus nemuphar (Herbst).

[M. xanthonotus (Ashm.). Proc. U.S. Nat. Mus., 1888, 11:618. Recorded from N. H. to Calif.] 


\section{SUBFAMILY SPATHIINAE}

larvae.)

(The species belonging to this group are parasites of wood-boring coleopterous

\section{Spathius Nees}

[S. brachyurus Ashm. Can. Ent., 1893, 25:71-73. Recorded as a parasite of the white-pine weevil, Pissodes strobi Peck.]

[S. canadensis Ashm. Can. Ent., 1891, 23:2. A parasite of Phloeosinus dentatus (Say) and Ips grandicollis (Eich.) (both Scolytidae).]

[S. claripennis Ashm. Can. Ent., 1893, 25:70-72. On record as a parasite of Polygraphus rufipennis (Kby.) (Scolytidae).]

S. laflammei Prov. Nat. Can., 1880, 12:164. Ithaca, Je (Ashm-CU).

[S. pallidus Ashm. Can. Ent., 1893, 25:70-72. Recorded as parasite of Ips grandicollis (Eich.) (Scolytidae).]

S. simillimus Ashm. Can. Ent., 1893, 25:70-73. Recorded from NY, as a parasite of Agrilus bilineatus (Weber) (Buprestidae).

S. tomici Ashm. Can. Ent., 1893, 25:71. Recorded from Erie Co. A parasite of Orthotomicus punctipennis (Lec.), Scolytus piceae (Swaine), and Polygraphus rufipennis (Kby.) (all Scolytidae).

S. trifasciatus Rly. Fifth Ann. Rep. Ins. Mo., 1873, p. 106. Syracuse; Geneseo; Ithaca, Je (Vier-CU). A parasite of the hickory bark-borer, Scolytus quadrispinosus Say; probably attacks various species of Scolytus.

S. unifasciatus Ashm. Can. Ent., 1893, 25:70-72. Geneseo. A parasite of the hickory bark-borer, Scolytus quadrispinosus Say.

Caenophanes Foerster

C. aciculatus Ashm. Can. Ent., 1893, $25: 76$. Ithaca, May 31 (Ashm-CU).

\section{SUBFAMILY HECABOLINAE}

HECABOLUS Curtis

[H. lycti (Cress.). Amer. Ent., 1880, 3:24. A parasite of Lyctus linearis (Goeze) (Lyctidae).]

[H. minimus (Cress.). Amer. Ent., 1880, 3:24.]

H. utilis (Cress.). Amer. Ent., 1880, 3:24. NY, type locality.

\section{SUBFAMILY DORYCTINAE}

\section{GLYPTODORYCTES Ashmead}

G. caryae (Ashm.). Trans. Am. Ent. Soc., 1896, 23:214. Syracuse. From a coleopterous larva in Hicoria glabra $(\mathrm{B} \& \mathrm{~S})$.

DORYCTES Haliday

D. disjunctus Cress. Trans. Am. Ent. Soc., 1872, 4:186. Syracuse. From a coleopterous larva in hickory (B\&S).

[D. exhalans (Say). Contrib. Maclur. Lyc. Phila., 1828, 2:78.]

[D. pallipes Prov. Addit. fauna Can. Hymen., 1888, p. 375.]

D. radiatus Cress. Trans. Amer. Ent. Soc., 1872, 4: 185. Ithaca, Je 25, J1 12, Yuasa (Bdy-CU). 


\section{ODONTOBRACON Cameron}

[O. elaphidiovorus Roh. Proc. U.S. Nat. Mus., 1917, 53:168. A parasite of Elaphidion sp. (Cerambycidae).]

[O. pectinator (Say). Bost. Journ: Nat. Hist., 1836, 1:251. A parasite of Melanophila fulvoguttata (Harr.) (Buprestidae).]

\section{SUBFAMILY HORMIINAE 133}

EcPHyLus Foerster

E. hicoriae Roh. Can. Ent., 1919, 51:161. Syracuse, type locality. Reared from a coleopterous larva in Hicoria glabra.

[E. hypothenemi Ashm. Trans. Am. Ent. Soc., 1896, 23:215. A parasite of Hypothenemus sp. (Scolytidae).]

[E. johnsoni Roh. Proc. U.S. Nat. Mus., 1913, 45: 539.]

[E. pallidus Ashm. Trans. Am. Ent. Soc., 1896, $23: 216$.

\section{ChREMylus Haliday}

[C. terminalis Ashm. Trans. Am. Ent. Soc., 1893, 20:39. Widely distributed.]

\section{HORMIUS Nees}

H. americanus Ashm. Bull. Colorado Biol. Assoc., 1890, 1:16. "Ithaca, Aug (Ashm-CU).

[H. completus (Prov.). Addit. fauna Can. Hymen., 1886, p. 156.]

\section{AtoReutes Foerster}

A. astigmus (Ashm.). Trans. Am. Ent. Soc., 1896, 23:215. Erie Co. Reared from a coleopterous larva in American larch.

\section{Heterospilus Haliday}

[H. anthaxiae (Ashm.). Can. Ent., 1893, 25:77. A parasite of Anthaxia viridifrons (Gorey) (Buprestidae).]

H. blackmani Roh. Can. Ent., 1919, 51: 161. Syracuse, type locality. From a coleopterous larva in Hicoria glabra.

H. cephi Roh. Ithaca, DRies (types US, CU), bred from the wheat-stem sawfly, Cephus pygmaeus (L.) ; recorded from the following counties: Wayne, Monroe, Onondaga, Orleans, Genesee, Niagara, Erie, Wyoming, Ontario, Yates, Tompkins, Schuyler, and Cayuga (DTRies).

[H. hylotrupidis (Ashm.). Can. Ent., 1893, 25:78. A parasite of Anacomis lignea (Fab.) (Cerambycidae).]

[H. languriae (Ashm.). Can. Ent., 1893, 25:77. A parasite of Anacomis lignea (Fab.) (Cerambycidae).]

\footnotetext{
${ }^{133}$ This and the following subfamily are greatly in need of revision. A thorough study of them will probably result in a decidedly different grouping of the genera.
} 
[H. mordellistenae Vier. Proc. U.S. Nat. Mus., 1911, 39:403. A parasite of Mordellistena pustulata (Melsh.) (Mordellidae).]

[H. pityophthori (Ashm.). Can. Ent., 1893, 25:76. Parasitic on Polygraphus rufipennis (Kby.) (Scolytidae).]

\section{SUbFAMILy ALEIODINAE \\ (Rhogadinae auctt.) \\ EPIRHYSSALUS Ashmead \\ (Rhyssalus Haliday)}

E. atriceps (Ashm.). Proc. U.S. Nat. Mus., 1888, 11:628. Ithaca, from Cacoecia cerasivorana (Tortricidae) (CU Exp. Sta.). A parasite of various leafrollers, including Archips rosaceana Harr., and of Gelechia confusella Chamb. (Gelechiidae).

E. loxoteniae Ashm. Proc. U.S. Nat. Mus., 1888, 11:628. Perry, IMHawley, reared from the clover Hypena, Plathypena scabra Fab. (Noctuidae) (Mues$\mathrm{CU})$; Ithaca, May (Ashm-CU).

\section{NOSERUS Foerster}

[N. pomifoliellae (Ashm.). Proc. U.S. Nat. Mus., 1888, 11:620. Parasitic on Bucculatrix pomifoliella Clem. (Lyonetiidae).]

\section{Clinocentrus Haliday}

[C. americanus Weed. I11. State Lab. Nat. Hist., 1890, Bull. 3, p. 43.]

\section{BUCCULATRIPLEX Viereck}

[B. bucculatricis (Ashm.). Proc. U.S. Nat. Mus., 1888, 11:618. A parasite of Bucculatrix sp. (Lyonetiidae).]

[B. secundus Vier. Conn. Geol. and Nat. Hist. Surv., 1916, Bull. 22, p. 765. A parasite of Bucculatrix canadensisella Chamb. (Lyonetiidae).]

\section{Aleiodes Wesmael (Rogas Nees)}

A. abdominalis (Cress.). Trans. Am. Ent. Soc., 1869, 2:379. Axton, Je (M\&HCU) ; Norton's Landing, Cayuga L., SmHH (Cress-CU); McLean, Aug 11 (Mues-CU).

A. aciculatus (Cress.). Trans. Am. Ent. Soc., 1869, 2: 381. Ithaca, Aug (AshmCU) ; Norton's Landing, Cayuga L., SmHH (Cress-CU); McLean, Je 24 (Mues-CU).

A. canadensis (Cress.). Trans. Am. Ent. Soc., 1869, 2:380. McLean, Aug 31, Sep 1 (Mues-CU).

[A. hyphantriae Gahan. Proc. U.S. Nat. Mus., 1922, 61:1. A parasite of the fall web-worm, Hyphantria cunea Drury.]

A. lectus (Cress.). Trans. Am. Ent. Soc., 1869, 2: 379. Ithaca, J1 (Mues-CU). 
[A. melleus (Cress.). Trans. Am. Ent. Soc., 1869, $2: 382$.

A. parasiticus Norton. Trans. Am. Ent. Soc., 1869, 2:327. Ithaca, Aug (Ashm$\mathrm{CU})$; Norton's Landing, Cayuga L., SmHH (Cress-CU); W. Danby, May (Ashm-CU). Recorded as a parasite of a sawfly, Neodiprion abietis (Harr.).

[A. platypterygus Ashm. Proc. U.S. Nat. Mus., 1888, 11:633. Recorded as a parasite of the oriental peach moth, Laspeyresia molesta Busck.]

A. rileyi (Cress.). Trans. Am. Ent. Soc., 1869, 2:382. Recorded from Acronycta oblinita S. \& A. (Noctuidae), and the bronzed cutworm, Nephelodes minians form violans Guenée (Noctuidae). Ithaca, at light (Ashm-CU).

[A. scrutator (Say). Bost. Journ. Nat. Hist., 1836, $1: 254$.

A. stigmator (Say). Keating's Narrative of an expedition to the source of St. Peter's River, etc., 1824, $2: 324$. Otto (CU) ; Ithaca, Ap-Aug, at light (CU) ; Norton's Landing, Cayuga L., SmHH (Cress-CU) ; LI: Orient, Zab (AM). Our most common species, occurring throughout NY. A gregarious parasite of various species of Acronycta (Noctuidae).

A. terminalis (Cress.). Trans. Am. Ent. Soc., 1869, 2:379. Axton, Je (M\&HCU) ; Ithaca, Je-J1, at light (Ashm-CU); LI: Farmingdale, J1, Morrow (CU). A very common and widely distributed species, occurring throughout NY. A parasite of the army worm, Cirphis unipuncta (Haworth).

\section{Heterogamus Wesmael}

[H. fumipennis (Cress.). Trans. Am. Ent. Soc., 1869, 2:378. Recorded as a parasite of various sphingid larvae.]

\section{SUBFAMILY SIGALPHINAE 134}

(Some of the species in this subfamily are valuable parasites of injurious Lepidoptera; others are important parasites of Coleoptera.)

\section{TRIASPIS Haliday}

T. curculionis (Fitch). Country Gentleman, 1859, p. 221. Ithaca, J1 (Mues-CU) ; LI: Cold Sp. Harb., Ds (Beq). A widely distributed parasite of the plum curculio, Conotrachelus nenuphar (Herbst).

T. texanus (Cress.). Trans. Amer. Ent. Soc., 1872, 4:179. Ithaca, Comstock (Ashm-CU).

\section{UROSIGALPHUS Ashmead}

[U. barberi Crawf. Insecutor Inscitiae Menstruus, 1914, $2: 23$.]

[U. femoratus Crawf. Insecutor Inscitiae Menstruus, 1914, 2:26.]

[U. otidocephali Cush. Proc. U.S. Nat. Mus., 1922, 60:25. A parasite of Otidocephalus (Curculionidae) in sycamore.]

[U. robustus Ashm. Proc. U.S. Nat. Mus., 1888, 11:638.]

\section{SIgALPHUS Latreille}

S. bicolor (Cress.). Trans. Am. Ent. Soc., 1880, 8: 17. Ithaca, J1 (Mues-CU). Occurs throughout the eastern States. A parasite of certain noctuid larvae. Has been reared from Apatela clarescens Guen. (Noctuidae), and probably attacks related forms.

${ }^{134}$ This group includes the Cheloninae and Sigalphinae of Szepligeti and other authors. 


\section{Chelonus Panzer}

C. aughei (La Munyon). Proc. Nebr. Assoc. Adv. Science, 1877. Thousand Is1s., Sep 4 (Ashm-CU).

C. basicinctus Prov. Nat. Can., 1881, 12:198. White L., Zab (AM) ; LiI: Flatbush, Zab (AM).

[C. basilaris (Say). Keating's Narrative of an expedition to the source of St. Peter's River, etc., 1824, $2: 322$.]

[C. brevipennis Melander \& Brues. Biol. Bull., 1903, 5 :20.]

C. concaputus Vier. Carmel (AM).

C. fissus Prov. Nat. Can., 1881, 12:199. Ithaca, Je (Ashm-CU) ; Norton's Landing, Cayuga L., SmHH (Ashm-CU); White L., Zab (AM); LI: Coney I., $Z a b$ (AM).

C. iridescens Cress. Proc. Ent. Soc. Phila., 1865, 4: 294. Manlius, SmHH (Cress$\mathrm{CU})$; Ithaca, Aug (Ashm-CU); Norton's Landing, Cayuga L., SmHH (Cress-CU).

C. lunatus Hald. Proc. Acad. Nat. Sci. Phila., 1849, 4:203. Rochester, Aug 25 (Ashm-CU).

[C. parvus Say. Bost. Journ. Nat. Hist., 1836, 1:265.]

C. sassacus Vier. Conn. Geol. \& Nat. Hist. Surv., Bull. 22, 1916, p. 233. LI: Flatlands, Fishers I., Zab (AM).

C. sericeus (Say). Keating's Narrative of an expedition to the source of St. Peter's River, etc., 1924, 2:321. Manlius, SmHH (Cress-CU).

[C. sobrinus Hald. Proc. Acad. Nat. Sci. Phila., 1849, 4 : 203.]

\section{Ascogaster Wesmael}

[A. carpocapsae (Vier.). Proc. Ent. Soc. Wash., 1909, 11:43. A widely distributed parasite of the larva of the codling moth, Carpocapsa pomonella L. It is also recorded as having been reared from Gelechia confusella Cham. (Gelechiidae), and on a single occasion it was reared from the oriental moth, Cnidocampa flavescens Wlk. (Eucleidae).]

A. flaviceps Ashm. Proc. U. S. Nat. Mus., 1888, p. 636. Norton's Landing, SmHH (Ashm-CU).

A. provancheri D.T. Cat. Hymen., 1898, 4:196. Ithaca, May-J1, Comstock (Ashm-CU).

\section{PhaneRotoma Wesmael}

P. tibialis (Hald.). Proc. Acad. Nat. Sci. Phila., 1849, 4:203. Ithaca, reared from clover-seed caterpillar by L.P.Wehrle; Freeville, J1-Aug (Ashm-CU).

[P. recurvariae Cush. Proc. Ent. Soc. Wash., 1914, 16:78. Parasitic on the larva of Recurvaria crataegella Busck (Gelechiidae).]

\section{SUBFAMILY NEONEURINAE 1.35}

The subfamily Neoneurinae constitutes a very rare group, represented in the United States by four known species of the genus Elasmosoma. Not more than a very few specimens of any of these occur in any museum collection. They have alalways been found associated with ants, and observations on European species indicate that they are probably parasitic on them.

135 This subfamily is not recognized by Szepligeti. For a recent revision, see footnote under Microgasterinae, page 904. 
Elasmosoma Ruthe

[E. bakeri Ashm. Proc. Ent. Soc. Wash., 1895, 3:382. Occurs from Me. to N. Mex.]

[E. pergandei Ashm. Proc. Ent. Soc. Wash., 1895, 3:283. Known to occur from Kans. to NJ, and doubtless is present in NY.]

[E. schwarzi Ashm. Proc. Ent. Soc. Wash., 1895, 3:283. Probably occurs throughout the eastern States.]

\section{SUBFAMILY MICROGASTERINAE 136}

The subfamily Microgasterinae is the richest in species of all the subfamilies in the Braconidae. The genus Apanteles is a particularly large group, containing nearly two hundred known North American forms. All the species, so far as known, are endoparasites of lepidopterous larvae, some being gregarious parasites, others solitary; and many of them rank among our most valuable natural enemies of injurious insect species.

\section{MiRAx Haliday}

[M. aspidiscae Ashm. Psyche, 1893, 6:378. Recorded as a parasite of Coptodisca splendoriferella Clem. (Heliozetidae), and Laspeyresia prunivora Walsh (Tortricidae).]

M. lithocolletidis Ashm. Psyche, 1893, 6:378. Ithaca (CU). Bred from Lithocolletes aceriella Clem. (Gracilariidae).

\section{Adelius Haliday}

[A. nigripectus Mues. Proc. U.S. Nat. Mus., 1922, 61:13. A parasite of the poplar leaf-miner.]

\section{MiCRogaster Latreille}

M. auripes Prov. Addit. fauna Can. Hymen., 1886, p. 141. Vandalia, Je (MuesCU); Ithaca, J1, Caroline to Harford, J1, MacG $\mathcal{E} B d y$ (Mues-CU). A parasite of Nelencania albilinea Hbn. (Noctuidae).

M. brittoni Vier. Conn. Geol. and Nat. Hist. Surv., 1916, Bull. 22, p. 202. Rochester Jct., Otto, Ithaca, Taughannock, Spencer L. (all Mues-CU).

M. carinata Pck. McLean, Aug 31 (Mues-CU).

M. canadensis Mues. Proc. U.S. Nat. Mus., 1922, 61:38. Nyack, Zab.

M. congregatiformis Vier. Conn. Geol. and Nat. Hist. Surv., 1916, Bull. 22, p. 202. LI, $Z a b$.

M. ecdytolophae Mues. Proc. U.S. Nat. Mus., 1922, 61:24. Mt. Whiteface, 2000-4000 ft.; Freeville; Ithaca (all Mues-CU). A parasite of Ecdytolopha insiticiana Zell. (Tortricidae), and Psorosina hammondi Rly. (Pyralidae).

M. epagoges Gahan. Proc. U.S. Nat. Mus., 1917, 53:197. Ithaca, Aug (MuesCU). Described as a parasite of Sparganothis sulfureana Clem. (Tortricidae).

M. facetosa Weed. Trans. Am. Ent. Soc., 1888, 15:296. Cranberry L., Htly (Mues) ; Rock City, Je ; Caroline to Harford, Je, MacG\&Bdy ; Ellis to Slaterville; Slaterville to Caroline, Je, $M a c G \mathcal{E} B d y$; Freeville, J1; Rock City, Je; W. Danby, May; Waterville, May (all Mues-CU). A very common species. Recorded as a parasite of Plathypena scabra Fab. (Noctuidae).

136 For a recent revision of this subfamily, see $A$ Revision of the North American Ichneumon-Flies Belonging to the Subfamilies Neoneurinae and Microgasterinae, by C. F. W. Muesebeck. Proc. U. S. Nat. Mus., 1922, 61:1-76. 
M. garmani (Ashm.). Proc. U.S. Nat. Mus., 1900, 23:132. LI: Flatbush.

M. gelechiae Rly. First Ann. Rep. Ins. Mo., 1869, p. 178. Otto (Mues-CU). A parasite of the goldenrod gall-maker, Gnorimoschema gallaesolidaginis Rly. (Gelechiidae).

M. lateralis Prov. Addit. fauna Canad. Hymen., 1886, p. 141. Cranberry L., Htly (Mues).

M. pantographae Mues. Proc. U.S. Nat. Mus., 1922, 61:34. Ithaca, May-Aug (Mues-CU). Described as a parasite of Pantographa limata G. \& R. (Pyralidae).

M. rubricoxa Prov. Addit. fauna Can. Hymen., 1886, p. 143. Cayuga Co, J1.22 (Mues-CU); Spring Lake; LI.

[M. schizurae Mues. Proc. U.S. Nat. Mus., 1922, 61:30. A parasite of Schizura unicornis S. \& A. and S. concinna S. \& A. (Notodontidae).]

M. zonaria Say. Bost. Journ. Nat. Hist., 1836, 1:263. N. Fair Haven, Sep (MuesCU) ; Ithaca, Sep (Mues-CU) ; LI: Flatbush. Recorded as a parasite of species of Recurvaria (Gelechiidae).

\section{MiCROPLitis Foerster}

M. alaskensis Ashm. Proc. Wash. Acad. Sci., 1902, 4:249. Manlius, Aug, SmHH (Mues-CU); Ithaca, May-J1 (Mues-CU). A very widely distributed species. Recorded as a parasite of Hadena and Plusia (Noctuidae).

M. ceratomiae Rly. Trans. St. Louis Acad. Sci., 1881, 4:303. Wilmington, Aug 20 (Mues-CU). Doubtless occurs throughout the State. A common parasite of sphingid larvae, particularly Ceratomia amyntor Hbn., Paonias excaecatus S. \& A., Sphinx drupiferarum S. \& A., and S. gordius Stoll.

M. confusus Mues. Proc. U.S. Nat. Mus., 1922, 61:69. Rock City, Je 9, McLean, May (Mues-CU). A parasite of cutworms.

[M. croceipes (Cress.). Trans. Am. Ent. Soc., 1872, 4:183. Occurs from Colo. to the Atlantic coast. Recorded as a parasite of the corn ear-worm, Heliothis obsoleta Fab., and the tobacco bud-worm, Chloridea virescens Fab. (Noctuidae).]

M. gortynae Rly. Trans. St. Louis Acad. Sci., 1881, 4: 304. Waterville, Je 11, reared from Gortyna immanis Gn. (Noctuidae), by IMHawley (Mues-CU); Rye, reared from Papaipema nebris Guenée (Noctuidae), by Bird.

M. hyphantriae Ashm. Proc. Ent. Soc. Wash., 1897, $4: 164$. Ithaca, Je-J1 (MuesCU). A parasite of fall web-worm, Hyphantria cunea Drury (Arctiidae).

M. kewleyi Mues. Proc. U. S. Nat. Mus., 1922, 61: 51. Geneva, Melander; Ithaca, May, PettyFW (Mues-CU). A parasite of cutworm larvae.

M. laticinctus Mues. Proc. U. S. Nat. Mus., 1922, 61:67. Oswego, type locality ; Rock City; Forest Lawn; McLean; Hornerstown; Nyack.

M. mamestrae Weed. Ill. State Lab. Nat. Hist., 1887, Bull. 3, p. 2. Dryden, Caroline to Harford, Je, $M a c G \mathcal{E} B d y$ (Mues-CU) ; Albany, reared from Ceramia picta Harr. (Noctuidae), by Lintner.

M. maturus Weed. Trans. Am. Ent. Soc., 1888, 15: 294. Waterville, May, Ithaca, May-J1 (Mues-CU). A common and widely distributed species.

M. melianae Vier. Proc. U.S. Nat. Mus., 1911, 40: 185. Axton, Je (M\&H-CU) ; Caroline to Harford, Je, MacG\&Bdy (Mues-CU). Recorded as a parasite of Neleucania albilinea Hbn., and the armyworm, Cirphis unipuncta Haworth (Noctuidae). 
[M. perplexus Mues. Proc. U.S. Nat. Mus., 1922, 61:49. A widely distributed species.]

M. quadridentatus (Prov.). Addit. fauna Can. Hymen., 1886, p. 140. Ithaca (Mues-CU). Recorded as a parasite of Simyra henrici Grt. (Noctuidae).

M. varicolor Vier. Conn. Geol. and Nat. Hist. Surv., 1916, Bull. 22, p. 204. Peru, Rock City, Caroline, Slaterville (all Mues-CU). A parasite of Cirphis unipuncta Haworth, the army worm, also of Cirphis sp. and Plathypena scabra Fab. (Noctuidae).

\section{Apanteles Foerster ${ }^{137}$}

[A. acronyctae Rly. Second Rep. Ins. Mo., 1870, p. 120. Recorded as occurring from Calif. to Me. A gregarious parasite of various species of Acronycta (Noctuidae).]

[A. alypiae Mues. Proc. U.S. Nat. Mus., 1922, 61:18. A parasite of the larva of a common grape pest, the eight-spotted forester, Alypia octomaculata Fab. (Agaristidae).]

A. anisotae Mues. Proc. U.S. Nat. Mus., 1920, 58:555. Elmira, CLGriswold. A gregarious parasite of Anisota senatoria S. \& A. (Citheroniidae).

A. argynnidis Rly. Scudder, S. H., Butterflies eastern U. S., 1889, p. 1904. Westport; Ithaca, May (Mues-CU) ; Ashokan; Rhinebeck; Glens Falls. Reared from Epargyreus tityrus Fab. (Hesperiidae), Sep, 1890, at CU Agr. Exp. Sta.

A. aristoteliae Vier. Proc. U.S. Nat. Mus., 1912, 42:613. Cranberry L., Htly (Mues-CU) ; Ithaca, May (Mues-CU) ; Ellis to Slaterville, and Caroline to Harford, Je, MacG\& $B d y$ (Mues-CU). Occurs throughout the northeastern States, at least from Ill. to Me. A parasite of Aristotelia fungivorella Clem., Gelechia trialbamaculella Chamb., and G. confusella Chamb. (Gelechiidae).

A. atalantae (Pck.). Proc. Bost. Soc. Nat. Hist., 1881, 21:27. Waterford, Hebron, $J V S c h a f f n e r, J r$. A very abundant parasite of the red admiral, Vanessa atalanta L., and the small tortoise, Aglais milberti Godart. The cocoons are grouped in small masses, completely inclosed in a ball of white silk, much resembling a spider-egg cocoon.

A. bedelliae Vier. Proc. U.S. Nat. Mus., 1911, 40:174. LI: Westbury. Recorded as a parasite of Bedellia minor Busck, B. somnulentella Zell. (Lyonetiidae), Recurvaria thujaeella Kf. (Gelechiidae), and Anomis erosa Hbn. (Noctuidae).

A. cacoeciae Rly. Trans. St. Louis Acad. Sci., 1881, 4:306. Hilton, Je (MuesCU); Ithaca (Mues-CU) ; Cohoes, reared from Tortrix sp. by THSoden. A common parasite of leaf-rollers from Mo. to Me. Recorded hosts include Cacoecia semiferana Wlk. (Tortricidae), Acrobasis caryae Grt. (Pyralidae), and Tortrix spp.

A. canarsiae Ashm. Proc. Ent. Soc. Wash., 1897, 4:127. Ithaca, J1 (CU). Occurs from Ia. to Me. A parasite of the grape leaf-folder, Desmia funeralis Hbn., and the apple leaf-skeletonizer, Canarsia hammondi Rly.

[A. carduicola (Pck.). Proc. Bost. Soc. Nat. Hist., 1881, 21:27. Recorded as a parasite of the painted lady, Vanessa cardui L.]

[A. carpatus (Say). Bost. Journ. Nat. Hist., 1836, 1:263. A widely distributed parasite of various clothes moths, Tinea pellionella L., Trichophaga tapetzella L., and Tineola biselliella L.]

${ }^{137}$ A revision of the North American ichneumon-flies belonging to the genus Apanteles. By C. F. W. Muesebeck. Proc. U. S. Nat. Mus., 1920, 58: 483-576. 
[A. charadrae Mues. Proc. U.S. Nat. Mus., 1920, 58:567. A gregarious parasite of Charadra deridens Guen. (Noctuidae).]

[A. chorenti Vier. Proc. U. S. Nat. Mus., 1912, 42:627. Recorded as a parasite of Choreutis carduiella Kf. (Glyphipterygidae), and the grape leaf-roller, Desmia funeralis Hbn.]

A. cinctiformis Vier. Proc. U. S. Nat. Mus., 1911, 40:176. Ithaca; McLean, Je-J1; Malloryville, Je, Caroline to Harford, Ellis to Slaterville, Slaterville to Caroline, Je, MacG\&Bdy ; Mud Cr., Tompkins Co, Je.

A. clisiocampae Ashm. N. H. Agr. Exp. Sta., 1903, Bull. 6, p. 229. Auburn, BAPorter; Ithaca, CLLiu (Mues-CU); LI: Brooklyn, Aug, Schott (Gahan). A rather uncommon solitary parasite of the small larvae of the apple-tree tent-caterpillar.

[A. compressus Mues. Can. Ent., 1919, 51:114. Occurs throughout the New England States and undoubtedly is to be found in NY. Recorded as a parasite of Hypoprepia sp.]

[A. conanchetorum Vier. Conn. Geol. and Nat. Hist. Surv., 1916, Bull. 22, pp. 191, 199.I

A. congregatus (Say). Bost. Journ. Nat. Hist., 1836, 1:262. Cranberry L., Htly (Mues) ; Ithaca, Aug (Mues-CU). An exceedingly abundant and widely distributed gregarious parasite of the larvae of a great variety of Sphingidae. Among its hosts are the following species: the tomato worm, Phlegethontius quinquemaculata Haworth; the tobacco worm, P.. sexta Joh.; Dolba hylaeus Drury; the hog caterpillar, Ampeloeca myron Cramer; Abbott's sphinx, Sphecodina abbotti Swainson; and the catalpa sphinx, Sphinx catalpae Boisd.

A. consimilis Vier. Proc. U.S. Nat. Mus., 1911, 40:177. McLean, J1 (Mues$\mathrm{CU})$; LI, type locality.

[A. crambi Weed. Ill. State Lab. Nat. Hist., 1887, Bull. 3, 3:8. Rather widely distributed east of the Mississippi. A parasite of various species of Crambus.]

A. crassicornis (Prov.). Addit. fauna Can. Hymen., 1886, pp. 139, 142. McLean, Aug 1 (Mues-CU).

A. cyaniridis Rly. Scudder, S. H., Butterflies eastern U. S., 1889, p. 1903. Ellis to Slaterville, Je 13, MacGEBdy (Mues-CU). A parasite of Lycaenopsis pseudargiolus B. \& L.

A. delicatus How. U.S. Bur. Ent., Tech. Ser., 1897, Bull. 5, p. 54 . Ithaca, Je 25 (Mues-CU); LI: Brooklyn. A parasite of the white-marked tussock moth, Hemerocampa leucostigma S. \& A.

[A. diacrisiae Gahan. Proc. U.S. Nat. Mus., 1917, 53:198. Throughout the eastern States. A gregarious parasite of certain Arctiidae, including the common yellow bear, Diacrisia virginica Fab.]

[A. dolicocephalus Mues. Proc. U.S. Nat. Mus., 1920, $58: 503$.

[A. edwardsii Rly. Scudder, S. H., Butterflies eastern U.S., 1889, p. 1901. A parasite of the red admiral, Vanessa atalanta L.]

[A. empretiae Vier. Proc. U.S. Nat. Mus., 1913, 44:562. A gregarious parasite of the saddle-back caterpillar, Sibine stimulea Clem.]

A. ensiger (Say). Bost. Journ. Nat. Hist., 1836, 1:260. Axton, Je, $M \& H$ (MuesCU) ; Oswego; Spencer L., Je (Mues-CU) ; LI.

A. euchaetis Ash. Proc. Ent. Soc. Wash., 1897, 4:159. Cambridge, Meadowbrook, JVSchaffner. A very abundant parasite of the milkweed tussock caterpillar, Euchaetias egle Drury. 
A. euphydryidis Mues. Proc. U. S. Nat. Mus., 1920, 58:564. McLean, May 27, 1909 (Mues-CU). A parasite of the Baltimore, Euphydryas phaeton Drury.

A. femur-nigrum (Prov.). Addit. fauna Can. Hymen., 1886, pp. 139, 142. Axton, Je (M\&H-CU); Peru, Je (Mues-CU) ; Spencer L., Je (Mues-CU) ; Ithaca, Je-Aug, Mud Cr., Je, Dryden L., Je (Mues-CU) ; McLean, J1 (Mues-CU) ; Slaterville to Caroline, Je, Ellis to Slaterville, Je, MacG\&Bdy (Mues-CU); Middletown, J1, CSSpooner (Mues-CU). Common in the northeastern States and in Canada.

[A. fiskei Vier. Proc. U.S. Nat. Mus., 1910, 38:379. A parasite of various species of Olene (Lymantriidae).]

[A. flaviconchae Rly. Trans. St. Louis Acad. Sci., 1881, 4:308. Parasitic on the army worm, Cirphis unipuncta Haworth, the sulphur butterfly, Eurymus philodice Godart, and the clover hypena, Plathypena scabra Fab.]

A. forbesi Vier. Proc. Ent. Soc. Wash., 1910, 11:208. Ithaca, J1 (Mues-CU). Doubtless to be found throughout the State. A common species. Recorded as a parasite of the cutworms, Cirphis unipuncta Haworth, Polia renigera Stephens, and Feltia sp.

A. fumiferanae Vier. Proc. U.S. Nat. Mus., 1912, 42:139. Cranberry L., Htly (Mues). A parasite of Harmologa fumiferana Clem. (Tortricidae).

A. glomeratus (L.). Syst. Nat., Ed. 10, 1758, 1: 568. Ithaca, J1-Aug (Mues-CU). A cosmopolitan and very important parasite of the larvae of the cabbage butterfly, Pieris rapae L.

A. griffini Vier. Proc. U.S. Nat. Mus., 1911, 40:177. Waterville (Mues-CU). A very common and widely distributed parasite of various cutworm larvae.

[A. harti Vier. Proc. Ent. Soc. Wash., 1910, 11:209. A parasite of Pyrausta penitalis Grt. and $P$. ainsliei Hein. (Pyralidae).]

A. hemileucae Rly. Trans. St. Louis Acad. Sci., 1881, 4:309. Karner, Felt, reared from Hemileuca maia Drury (Saturniidae).

A. hydriae Mues. Proc. U.S. Nat. Mus., 1920, 58: 561. Ithaca, Sep 9 (Mues-CU). A common parasite of Calocalpe undulata L. (Geometridae).

A. laeviceps Ashm. Bull. Colo. Biol. Assoc., 1890, 1:17. Ithaca, bred from a noctuid (Ashm-CU). Widely distributed, but apparently restricted to the higher elevations. A gregarious parasite of cutworms.

A. limenitidis (Rly.). Third Ann. Rep. Ins. Mo., 1871, p. 158. Ithaca to Ellis, Je, $M a c G \mathcal{E} B d y$ (Mues-CU). Occurs at least from Mo. to Me. A not uncommon parasite of the small larvae of the viceroy, Basilarchia disippe Godd.

A. lunatus (Pck.). Proc. Bost. Soc. Nat. Hist., 1881, 21:28. Recorded from NY. A rather widely distributed solitary parasite of the small larvae of various species of Papilio. The cocoon is large and deep yellow in color.

A. militaris Walsh. Trans. I11. State Agric. Soc., 1861, 4:27. Occurs throughout NY. A common and widely distributed gregarious parasite of the army worm, Cirphis unipuncta Haworth.

A. murtfeldtae Ashm. Proc. Ent. Soc. Wash., 1897, 4: 159. Cranberry L., Htly (Mues); Ithaca (Mues-CU). Found from Mass to Mo. A gregarious parasite of geometrid larvae.

[A. nemoriae Ashm. Proc. Ent. Soc. Wash., 1897, 4:160. A parasite of various Geometridae, including Haematopis grataria Fab., Eupithecia miserulata Grt., and Pleuroprucha insulsaria Guen.] 
[A. nephoptericis (Pck.). Proc. Essex Inst., 1864, 4:122. A parasite of the larva of Vitula edmansii Pck. (Pyralidae), which feeds on the comb in nests of bumblebees.]

[A. nigrovariatus Mues. Proc. U.S. Nat. Mus., 1920, $58: 523$.

A. parastichtidis Mues. Homer, J1 2, 1908 (Mues-CU). On Parastichtis bicolorago Guen. (Noctuidae).

A. ornigis Weed. I11. State Lab. Nat. Hist., 1897, Bull. 3, p. 6. Throughout the State. Reared by GWHerrick from the maple case-bearer, Paraclemensia acerifoliclla Fitch; also recorded from Ornix geminatella Pck. (Gracilariidae), and various species of Lithocolletes. A common parasite of many different leaf-mining Lepidoptera.

[A. orobenae Fbs. Twelfth Rep. Nox. Ins. I11., 1882, p. 104. Parasitic on the larvae of the cross-striped cabbage-worm, Evergestis rimosalis Guen.]

A. paleacritae Rly. Trans. St. Louis Acad. Sci., 1881, 4:313. Dryden L., Je (Mues-CU). Throughout at least the northeastern States. A solitary parasite of the cankerworms, Alsophila pometaria Harr. and Paleacrita vernata Peck.

A. papaipemae Mues. Proc. U.S. Nat. Mus., 1920, 58:526. Rye, type locality, reared by HCBird from Papaipema nebris Guen. and $P$. maritima Bird (Noctuidae).

[A. phigaliae Mues. Can. Ent., 1919, 51:113. A parasite of Phigalia titea Cram. (Geometridae). The cocoons are grouped and are rather strongly ribbed, resembling cocoons of Microplitis.]

A. phobetri Roh. Proc. U.S. Nat. Mus., 1915, $49: 228$. Cranberry L., Htly (Mues). A common gregarious parasite of the banded tussock caterpillar, Halisidota tesselaris S. \& A.

[A. pholisorae Rly. Scudder, S. H., Butterflies eastern U.S., 1889, p. 1904. A parasite of Pholisora catullus Fab. (Hesperiidae).]

A. polychrosidis Vier. Proc. U.S. Nat. Mus., 1912, 42:615. Ithaca; McLean; Ellis to Slaterville, Je, MacGE $B d y$ (Mues-CU) ; Rhinebeck, J1, Cy (Mues$\mathrm{CU})$. Probably throughout the State. A parasite of the grape berry moth, Polychrosis viteana Clem., $P$. liriodendrana $\mathrm{Kf}$., the oblique-banded leaf-roller, Cacoecia rosaceana Harr., and other species with similar habits.

[A. pyralidis Mues. Proc. U.S. Nat. Mus., 1920, 58:552. Occurs at least from I1l. to Mass. A parasite of Nomophila noctuella Denis \& Schiff. (Pyralidae), and the garden web-worm, Loxostege similalis Guen. The cocoons are very small, pure white, and in groups.]

[A. pyranstae Vier. Proc. U.S. Nat. Mus., 1912, 42:626. A common parasite of Pyrausta futilalis Lederer (Pyralidae). The cocoons are white, in groups, and usually formed within the thin cocoon made by the mature host larva.

A. radiatus Ashm. Proc. Ent. Soc. Wash., 1897, 4: 162. Ithaca, Je 20 (Ashm-CU).

A. rohweri Mues. (nigripes Rohw.). Proc. U.S. Nat. Mus., 1920, 58 : 550. Ithaca, $\mathrm{J1}$ (Mues-CU); Ellis to Slaterville, Je, MacGE $\mathcal{E} B d y$ (Mues-CU); McLean, Aug 31 (Mues-CU).

A. robiniae (Fitch). Fifth Rep. Ins. N. Y., Trans. N. Y. State Agric. Soc., 1858, p. 836. Described from NY. A parasite of Recurvaria robiniella Fitch (Gelechiidae).

A. rufocoxalis Rly. Trans. St. Louis Acad. Sci., 1881, 4:310. Batavia. A parasite of the army worm, Cirphis unipuncta Haworth. 
[A. sarrothripae Weed. Ill. State Lab. Nat. Hist., 1897, Bull. 3, p. 3. A gregarious parasite of Nycteola revayana Scop. (Noctuidae), Melalopha inclusa Hbn. (Notodontidae), and Peronea permutana Duponchel (Tortricidae).]

A. schizurae Ashm. Proc. Ent. Soc. Wash., 1897, 4:162. Rochester. A parasite of Schizura unicornis S. \& A. (Notodontidae).

[A. scitulus Rly. Trans. St. Louis Acad. Sci., 1881, 4:310. Widely distributed, at least east of the Mississippi. A gregarious parasite of the yellow bear, Diacrisia virginica Fab., and Acronycta spp. (Noctuidae).]

[A. smerinthi Rly. Trans. St. Louis Acad. Sci., 1881, 4:311. A widely distributed and common gregarious parasite of the larvae of various Sphingidae, including the twin-spotted sphinx (Smerinthus jamaicensis geminatus Say), the blindeyed sphinx (Paonias excaecatus S. \& A.), and Ceratomia undulosa Wlk.]

A. terminalis Gahan. Proc. Ent. Soc. Wash., 1912, 14:2. LI, type.

[A. tischeriae Vier. Proc. U.S. Nat. Mus., 1912, 42:140. A parasite of the trumpet miner, Tischeria malifoliella Clem.]

[A. xylinus (Say). Bost. Journ. Nat. Hist., 1836, 1:262. A very widely distributed and very abundant parasite of cutworms. The cocoons are in groups, and are encased in a ball of white silk.]

\section{SUBFAMILY BRACONINAE}

\section{(Agathidinae auctt.)}

(The host relations and habits of only a comparatively small number of species of this subfamily are known; but apparently all are parasitic on lepidopterous larvae.)

\section{Agathis Latreille}

A. femorator Prov. Nat. Can., 1880, 12:177. Ithaca, Aug 20-Sep 1 (Morr-CU).

A. perforator Prov. Nat. Can., 1880, 12:177. Oswego; Ithaca, Aug-Sep (Morr-CU).

A. rubripes Cress. Trans. Am. Ent. Soc., 1872, 4:183. Slaterville to Caroline. Je, Ellis to Slaterville, Je, Dryden L., Mud Cr., Malloryville, Je, MacG\&Bdy (Morr-CU).

[A. tibiator Prov. Nat. Can., 1880, 12:177.]

BRACON Jurine, Fab., not auctt. ${ }^{138}$

(Cremnops auctt.)

B. comstocki Morr. Proc. U.S. Nat. Mus., 1917, 52:323. Ithaca, Je-J1 (MorrCU) ; Ithaca to Ellis; Freeville.

B. montrealensis Morr. Proc. U.S. Nat. Mus., 1917, 52:326. Ithaca, J1-Sep (Morr-CU).

[B. nigrosternum Morr. Proc. U.S. Nat. Mus., 1917, 52 : 332.]

B. virginiensis Morr. Proc. U.S. Nat. Mus., 1917, 52:341. Ithaca, Aug 16 (Morr-CU).

\footnotetext{
${ }^{138}$ Monograph of the nearctic Hymenoptera of the genus Bracon Fabricius. By Harold Morrison.
} Proc. U. S. Nat. Mus., 1917, 52:305-343. 


\section{Crassomicrodus Ashmead}

C. divisus (Cress.). Can. Ent., 1873, 5:52. Ithaca, Aug (Ashm-CU).

\section{Bassus Fabricius}

(Microdus auctt.)

B. agilis (Cress.). Can. Ent., 1873, 5:52. Ithaca, Je-J1 (Ashm-CU). Apparently the most common species in this genus. A widely distributed parasite of leafrollers, particularly of species of Archips.

[B. albocinctus (Ashm.). Proc. U.S. Nat. Mus., 1888, 11:639. Occurs throughout the eastern States.]

B. annulipes (Cress.). Can. Ent., 1873, 5:53. Ithaca, Aug (Mues-CU) ; Mud Cr., Je, MacGEBdy (Mues-CU).

B. bicolor (Prov.). Nat. Can., 1880, 12:179. McLean, Sep 19 (Mues-CU).

B. calcaratus (Cress.). Can. Ent., 1872, 5:51. McLean, Sep 15 (Mues-CU). Common in the northeastern States at least. A parasite of Acrobasis caryivorella Rag., A. betulella Hulst (Pyralidae), and Gelechia trialbamaculella Chamb. (Gelechiidae).

[B. carpocapsae Cush. Proc. U.S. Nat. Mus., 1915, 48:508. A parasite of the codling moth, Carpocapsa pomonella L.]

B. cinctus (Cress.). Can. Ent., 1873, 5:53. McLean, Aug 1 (Mues-CU) ; NYC.

[B. coleophorae Roh. Proc. U.S. Nat. Mus., 1915, 49:230. Parasitic on the larva of Coleophora leucochrysella Clem., and doubtless also on other species of that genus.]

[B. discolor (Cress.). Can. Ent., 1873, 5: 52.]

B. earinoides (Cress.). Can. Ent., 1873, 5:52. New Russia, Aug 11, Bdy (Mues$\mathrm{CU}$ ); Baldwinsville, J1 (Ashm-CU) ; Ithaca, Je-J1 (Ashm-CU) ; Norton's Landing, Cayuga L., SmHH (Ashm-CU) ; LI: Farmingdale, J1, Morr (MuesCU). A parasite of Spilonota ocellana Schiff., the common bud moth on apple.

[B. grapholithae (Ashm.). Proc. U.S. Nat. Mus., 1888, 11:639. Widely distributed over at least the eastern half of the US.]

B. imitatus (Cress.). Can. Ent., 1873, 5:51. Ithaca, J1-Aug (Ashm-CU).

B. nigricoxus (Prov.). Addit. fauna Can. Hymen., 1886, pp. 137-138. Oswego.

B. perforator (Prov.). McLean, Aug 22 (Mues-CU).

B. sanctus Say. Bost. Journ. Nat. Hist., 1836, 1:249. Ithaca, J1 (Ashm-CU). Commonly collected in the eastern States. Nothing is known of its host relations.

[B. simillimus (Cress.). Can. Ent., 1873, 5: 51.]

B. laticinctus Cress. Can. Ent., 1873, 5:53. Ithaca, reared from the clover-seed caterpillar, Laspeyresia interstinctana $\mathrm{Cl}$., by LPWehrle.

\section{EARINUS Wesmael}

E. limitaris (Say). Bost. Journ. Nat. Hist., 1836, 1:250. Waterville (Mues-CU) ; Ithaca, abundant in early spring (Bdy-CU); McLean, Apr 17, Babiy (Bdy$\mathrm{CU})$; SI, Ap 16 (Ds). 


\section{SUBFAMII, BLACINAE 139}

The Blacinae are only imperfectly known from the standpoint of classification, and also as regards habits and biology. The species of some genera appear to be exclusively parasites of lepidopterous larvae, while those of other genera parasitize Coleoptera, and, in a few instances, Diptera.

\section{Blacus Nees}

B. lactucaphis (Fitch). First Rep. Ins. N.Y., 1854, p. 136. Described from NY, as an Aphidius.

[B. orchesiae (Ashm.). Proc. U.S. Nat. Mus., 1888, 11:645. Recorded as a parasite of Orchesia castanea Melsh. (Melandryidae).]

\section{ORGILUs Halidiay}

O. gelechiaevora Cush. Proc. U.S. Nat. Mus., 1920, 58:291. A parasite of Gelechia trialbamaculella Chamb.

[O. kearfotti Ashm. Journ. N. Y. Ent. Soc., 1903, 11:144. A parasite of Recurvaria juniperella $\mathrm{Kf}$.

\section{EuBAdizon Nees}

[E. americanus Cress. Can. Ent., 1872, 4:230. Occurs throughout the northeastern States and in Canada.]

[E. gracilis Prov. Nat. Can., 1880, 12:171. Range extends at least from Canada to $\mathrm{Va}$. It has been recorded as a parasite of the oriental peach moth, Laspeyresia molesta Busck.]

[E. phymatodis Ashm. Proc. U.S. Nat. Mus., 1888, 11: 644.]

E. pleuralis Cress. McLean, Je 24, Sib (Mues-CU).

\section{BRACHISTES Wesmael}

B. magdali (Cress.). Psyche, 1878, 2: 189. Syracuse. A parasite of the larvae of Magdalis barbita Say and M. olyra (Hbst.) (Curculionidae).

B. major (Cress.). Can. Ent., 1872, 4:229. Occurs in NY.

[B. tibiator (Cress.). Can. Ent., 1872, 4:229. Said to be a parasite of the strawberry weevil, Anthonomus signatus Say.]

\section{SYRRHIZUS Foerster}

[S. diabroticae Gahan. Ohio Journ. Sci., 1922, 22:141. A parasite of the adult striped cucumber-beetle, Diabrotica vittata (Fab.).]

\section{LEIOPIron (Nees) Haliday}

[L. americanus (Ashm.). Journ. Cincinnati Soc. Nat. Hist., 1895, 17:47. A parasite of the Hessian fly.]

\section{SUBFAMILY ICHNEUTINAE}

The subfamily Ichneutinae constitutes a small group, containing but few described species. Those forms the habits of which are known, attack sawfly larvae.

\footnotetext{
${ }^{139}$ Includes Calyptinae, Leiophroninae, and Blacinae of Szepligeti's classification.
} 


\section{ICHNEUTES Nees}

[I. bicarinatus (Ashm.). Can. Ent., 1891, 23:4.]

[I. bicolor Cress. Can. Ent., 1872, $4: 230$.

I. fulvipes Cress. Can. Ent., 1872, 4:231. Ithaca, May 26 (Mues-CU) ; McLean, Aug 12, SRobinson (Mues-CU).

\section{ICHNEUTIDEA Ashmead}

I. secunda Roh. Ent. News, 1908, 19:149. Saranac L., Aug (Mues-CU); Rochester, J1, Leon (Mues-CU); Ithaca, Sep (Mues-CU).

\section{SUBFAMILY CARDIOCHILINAE}

\section{CARDIOCHILES Nees}

The genus Cardiochiles comprises the subfamily Cardiochilinae. But little is known regarding the habits and host relations of the various species.

C. tibiator (Say). Keating's Narrative of an expedition to the source of St. Peter's River, etc., 1824, $2: 323$. Ithaca, J1 (Mues-CU). Occurs from Canada to Fla.

[C. viator (Say). Bost. Journ. Nat. Hist., 1836, 1:258. Distributed over most of the US.]

\section{SUBFAMILY MACROCENTRINAE}

\section{MACROCENTRUS Curtis}

M. aciculatus Prov. Addit. fauna Can. Hymen., 1888, p. 380. Thousand Isls., Sep (Ashm-CU).

M. amicroploides Vier. Proc. U.S. Nat. Mus., 1913, 43:579. Bethany Center, type locality. A parasite of the apple leaf-roller, Archips argyrospila Wlk.

M. cerasivoranae Vier. Proc. U.S. Nat. Mus., 1912, 42:623. Ithaca, type locality. The type was reared from the ugly-nest tortricid, Archips cerasivorana Fitch, by GWHerrick. This is a very large species, and a very common parasite of the above-named host.

[M. delicatus Cress. Trans. Am. Ent. Soc., 1872, 4:178. Recorded as a parasite of Mineola indigenella Zell. (Pyralidae), the codling moth, Carpocapsa pomonella L., and Archips fervidana Clem. (Tortricidae).]

M. iridescens French. Can. Ent., 1880, 12:43. Ithaca, J1-Sep, at light (Ashm$\mathrm{CU})$. A parasite of Ennomos subsignarius Hbn. (Geometridae).

M. nuperus Cress. Trans. Amer. Ent. Soc., 1872, 4:178. Ithaca, J1, at light (Ashm-CU).

M. plesius Vier. McLean, Je 24-Aug 23 (Mues-CU).

M. pyraustae Vier. McLean, Aug 20-24, Sib (Mues-CU).

M. terminalis (Ashm.) (Zele terminalis Ashm.). Proc. U.S. Nat. Mus., 1888, p. 300. Ithaca, Je, from Archips (CU).

M. uniformis Prov. Nat. Can., 1880, 12:173. Norton's Landing, Cayuga L., SmHH (Ashm-CU); LI: Flatbush, Zab (AM). 


\section{SUBFAMILY ZELIINAE 140}

\section{ZELE Curtis}

Z. truncator (Say). Contrib. Maclur. Lyc. Phil., 1828, 2: 78. Ithaca, J1, at light (Ashm-CU).

\section{SUBFAMILY HELCONINAE}

(The species of this rather poorly known group appear on the whole to be parasitic on the larvae of wood-boring Coleoptera.)

\section{EuMACROCENTRUS Ashmead}

[E. americanus (Cress.). Can. Ent., 1873, 5:84. Occurs at least from Canada to Va.]

HELCONIDEA Viereck

H. albitarsis (Cress.). Can. Ent., 1873, 5:83. Huguenot Pk. (Mues-CU).

H. borealis (Cress.). Can. Ent., 1873, 5:83. Indian Falls, Je (Mues-CU). Northeastern States. Parasitic on cerambycid larvae.

H. ligator (Say). Keating's Narrative of an expedition to the source of St. Peter's River, etc., 1824, 2:323. Syracuse, reared by B\&S from coleopterous larvae in hickory; New Baltimore, from Neoclytus acuminatus (Fab.) (Cerambycidae) (AM); Nyack, Zab (AM).

\section{HELCON Nees \\ (Gymnoscelus Foerster)}

[H. pedalis Cress. Can. Ent., 1873, 5:85. Throughout the northeastern States. Recorded as a parasite of a larva of Xylotrechus (Cerambycidae) in dead hemlock.]

\section{SUBFAMILY OPIINAE 141}

(The members of this subfamily are mostly parasitic on phytophagous Diptera belonging to the families Agromyzidae, Trypetidae, and Anthomyiidae.)

\section{OpIUs Wesmael}

O. anthomyiae Ashm. Proc. U.S. Nat. Mus., 1888, 11:654. Honeoye Falls, J1, Leon (Mues-CU) ; Ithaca, Aug (Mues-CU). Parasitic on an anthomyiid mining the leaves of dock.

O. apicalis Gahan. Proc. U.S. Nat. Mus., 1915, 49: 85 . Ithaca, Je-J1 (Mues$\mathrm{CU}$ ) ; Ellis to Slaterville, Caroline to Harford, Je, MacG\&Bdy (Mues-CU).

O. bruneipes Gahan. Can. Ent., 1913, 45: 148. Chipmunk Swamp, Mix Cr. Val., Cattaraugus Co, Je (Mues-CU); Caroline to Harford, Je, MacG\& $B d y$ (Mues-CU).

O. cincticornis Gahan. Proc. U.S. Nat. Mus., 1915, 49:78. Oswego, type locality.

${ }^{140}$ Included in Macrocentrinae by Szepligeti.

${ }^{141} \mathrm{~A}$ revision of the North American ichneumon-flies of the subfamily Opiinae. By A. B. Gahan. Proc. U. S. Nat. Mus., 1915, 49:63-95. 
O. cinctus Prov. Addit. fauna Can. Hymen., 1886, p. 124. Potsdam, J1 (Mues$\mathrm{CU})$; Norton's Landing, Cayuga L., SmHH (Mues-CU) ; Ellis to Slaterville, Caroline to Harford, Je, MacG\&Bdy (Mues-CU).

O. cupidus Gahan. Proc. Ent. Soc. Wash., 1919, 21:162. NYC, type locality, HBShaw; LI: Brooklyn, reared from Pegomyia hyoscyami (Panzer) by Montague Free.

O. dimidiatus (Ashm.). Proc. U.S. Nat. Mus., 1888, 11:647. Ithaca, J1 (MuesCU). A common parasite of Agromyza pusilla Meig.

[O. ferrugineus Gahan. Proc. U.S. Nat. Mus., 1915, 49:75.]

O. foveolatus Ashm. Proc. U.S. Nat. Mus., 1888, 11:654. Norton's Landing, Cayuga L., SmHH, Ithaca, J1 (Mues-CU); Ellis to Slaterville, Je, $M a c G \& B d y$ (Mues-CU).

O. fuscipennis Gahan. Proc. U.S. Nat. Mus., 1915, 49:79. McLean, Aug 19, SRobinson (Mues-CU); Ithaca, J1 (Mues-CU).

O. luteiceps Vier. Trans. Kans. Acad. Sci., 1905, 19:271. Mix Cr. Val., Cattaraugus Co, Je (Mues-CU).

O. mandibularis Gahan. Proc. U.S. Nat. Mus., 1915, 49:81. Pine I. (FrostSW), from Pegomyia pusilla Meig.

O. politus Prov. Nat. Can., 1883, 14:16. Ithaca, May-Je (Mues-CU).

O. provancheri D.T. Cat. Hymen., 1898, 4:64. Axton, Je, $M \mathcal{E} H$ (Mues-CU); Caroline to Harford, Je, MacG\&Bdy (Mues-CU) ; Freeville, J1 (Mues-CU); McLean, Aug 31 (Mues-CU).

O. quebecensis (Prov.). Nat. Can., 1881, 12:201, fig. 28. Ithaca, reared from Pegomyia calyptrata Zett. by FrostSW.

O. sanguineus Ashm. Proc. U.S. Nat. Mus., 1888, 11:655. Recorded from NY. A parasite of Trypetidae.

O. striativentris Gahan. Proc. U.S. Nat. Mus., 1915, 49:89. LI: Westbury, Ap 7, from Phytomyza ilicis Curtis (Felt).

O. succineus Gahan. Can. Ent., 1913, 45:149. Slaterville to Caroline, Je, $M a c G \mathcal{E} B d y$ (Mues-CU) ; Florida (FrostSW), from Agromyza parvicornis Lw.; Pine I. (FrostSW), from A. pusilla Meig.

O. tibialis Ashm. Can. Ent., 1893, 25:79. Durlandville (FrostSW), from Agromyza melanopyga Lw.

O. utahensis Gahan. Can. Ent., 1913, 45:145. Florida (FrostSW), from Agromyza parvicornis Lw.

\section{SUBFAMILY EUPHORINAE}

But little is known regarding the habits and biology of members of this group, but most of the species are apparently parasitic on various Coleoptera.

\section{Perilitus Nees}

[P. epitricis Vier. Proc. U.S. Nat. Mus., 1912, 42:625. Reported from N. J., and probably occurs in NY. A parasite of the tobacco flea-beetle, Epitrix parvula (Fab.).]

[P. gastrophysae Ashm. Proc. U.S. Nat. Mus., 1888, 11:641. Recorded as a parasite of Gastroidea cyanea Melsh. (Chrysomelidae).] 


\section{COSMOPHORUS Ratzeburg}

C. hopkinsi Ashm. Trans. Am. Ent. Soc., 1896, 23:216. Reported from NY as a parasite of Pityokteines sparsus (Lec.). The type, from N. J., was reared from Polygraphis rufipennis (Kby.). (Both Scolytidae.)

\section{WESMAELia Foerster}

W. americana Myers. Proc. U.S. Nat. Mus., 1917, 59:293. Ithaca, Aug 30 (Mues-CU), allotype. This is the only species of this genus known to occur in the US.

\section{EUPHORUS Nees}

E. mellipes Cress. Can. Ent., 1872, 4:227. Ithaca, May (Ashm-CU) ; Norton's Landing, Cayuga L., SmHH. (Ashm-CU).

E. scitulus Cress. Can. Ent., 1872, 4:227. Norton's Landing, Cayuga L., SmHH (Ashm-CU).

\section{EUPHORIANA Gahan}

[E. uniformis Gahan. Proc. U.S. Nat. Mus., 1913, 46:433, fig. 1. A parasite of Meromyza americana Fitch.]

\section{DinOCAMPUS Foerster}

D. coccinellae (Schr.). Fauna Boica., 1802, 2:310. Axton, Je, M\&H (Mues$\mathrm{CU})$; Victor, Je (Mues-CU). A cosmopolitan species, which is an internal parasite of adult Coccinellidae. It has been referred to in literature under a variety of names, including Dinocampus terminatus Nees, Perilitus americamus Rly., and Euphorus sculptus Cress.

\section{SUBFAMILY METEORINAE 142}

This subfamily is comprised of the single genus Meteorus, most of the species of which are internal parasites of lepidopterous larvae, although a few are apparently parasitic on Coleoptera. Some of them are very abundant, and exert a decided check on the increase of injurious species.

\section{Meteorus Haliday}

M. angustipennis Mues. Proc. U.S. Nat. Mus., 1923, 63:20. This species has been taken at Ithaca.

[M. autographae Mues. Proc. U.S. Nat. Mus., 1923, 63:30. Occurs throughout the eastern part of the US, having been recorded from Canada to Fla. It has been reared from larvae of the cabbage looper (Autographa brassicae Rly.), the army worm (Cirphis unipuncta Haworth), the alfalfa borer (Eurymus eurythemae Boisd.), and the clover hypena (Plathypena scabra Fab.).]

M. bakeri Cook \& Ds. Mich. Agr. Exp. Sta., 1891, Bull. 73, p. 9. Oswego. A parasite of the fall web-worm, Hyphantria cunea Drury, but not nearly so abundant as $M$. hyphantriae, which also attacks this host.

112 A revision of the North American species of ichneumon-flies belonging to the genus Meteorus Haliday. By C. F. W. Muesebeck. Proc. U.S. Nat. Mus., 1923, 63:1-144. 
M. communis (Cress.). Can. Ent., 1872, 4:82. Occurs throughout the State. A widely distributed and abundant parasite of a variety of hosts, but most frequently reared from Noctuidae, especially the army worm (Cirphis unipuncta Haworth) and cutworms. Also recorded from the tent caterpillar (Malacosoma americana Fab.) and the green-fruit worm (Grapholitha laticinerea Grt.).

[M. datanae Mues. Proc. U.S. Nat. Mus., 1923, 63:38. A parasite of Datana integerrima G. \& R., D. ministra Drury, and D. angusii G. \& R. (Notodontidae).]

M. dimidiatus (Cress.). Can. Ent., 1872, 4:83. Throughout the State. Occurs from Calif. to Me. Recorded as a parasite of the grape-leaf folder, Desmia funeralis Hbn.

[M. hicoriae Mues. Proc. U.S. Nat. Mus., 1923, 63:25. Type locality is Harrisburg, $\mathrm{Pa}$. The species is undoubtedly in NY.]

M. humilis (Cress.). Can. Ent., 1872, 4:84. Oswego, Spencer L. The species has also been referred to in literature under the names $M$. robustus (Prov.), $M$. orchesiae Ashm., and $M$. agilis. The recorded hosts include Orchesia castanea Melsh. (Melandryidae), Platydema ellipticum (Fab.) (Tenebrionidae), and Tinea oregonella Busck.

M. hyphantriae Rly. U.S. Dept. Agric. Div. Ent., 1887, Bull. 10, p. 49. Occurs throughout NY. A parasite of the fall web-worm, Hyphantria cunea Drury, especially; but has been reared from various hosts, including the tent caterpillar (Malacosoma americana Fab.), the forest tent caterpillar ( $M$. disstria Hbn.), the white-banded tussock caterpillar (Hemerocampa leucostigma S. \& A.), Neleucania albilinea Hbn. (Noctuidae), the fall cankerworm (Alsophila pometaria Harr.), and the spring cankerworm (Paleacrita vernata Peck).

[M. indagator (Rly.). Fourth Ann. Rep. Ins. Mo., 1872, p. 43. Widely distributed from Calif. to Mass. A parasite of leaf-rollers, especially of various species of Acrobasis and Mineola.]

[M. loxostegei Vier. Proc. U.S. Nat. Mus., 1911, 39:401. Occurs from Colo. to Mass. Must certainly occur in NY. Parasitic on the larvae of the sugar-beet web-worm, Loxostege sticticalis L., Pyrausta futilalis Lederer, and the European corn-borer, $P$. nubilalis $\mathrm{Hbn}$.]

M. maximus Mues. Proc. U.S. Nat. Mus., 1923, 63:13. New Salt, J1, 1903 (ESSouthworth).

[M. niveitarsis (Cress.). Can. Ent., 1872, 4:81. Throughout the northeastern States. Host unknown.]

[M. pallitarsis (Cress.). Can. Ent., 1872, 4:80. Distribution same as for niveitarsis.]

M. politus (Prov.). Addit. fauna Can. Hymen., 1886, p. 126. McLean, Aug 24-31, Sib, SRobinson (Mues-CU). Occurs in the Canadian provinces Quebec and Ontario, and in the northeastern US.

M. proximus (Cress.). Can. Ent., 1872, 4:83. Oswego; Stony I.; Thousand Is1s.

M. tauricornis (Prov.). Nat. Can., 1880, 12:168. Oswego; Saranac L.; Spring Lake; Ithaca; Slaterville; McLean, Aug 26, SRobinson (Mues-CU) ; LI: Flatbush.

M. tibialis Mues. Proc. U.S. Nat. Mus., 1923, 63:23. Syracuse, type locality, Drk.

M. trachynotus Vier. (archipsidis Vier.). Proc. U.S. Nat. Mus., 1912, 42: 142. Spring Lake, Bdy (Mues-CU); Youngstown; Bethany Center, Genesee Co. (type of archipsidis), reared from Archips argyrospila W1k. ; McLean, July 20- 
Aug 24 (Mues-CU) ; Little Falls. Additional hosts are Harmologa fumiferana Clem., and the strawberry leaf-roller, Ancylis comptana Froelich (both Tortricidae).

M. vulgaris (Cress.). Can. Ent., 1872, 4:83. McLean, J1 10-Aug 24 (Mues-CU). Probably the most common and most widely distributed of the North American species. Occurs throughout the US and much of Canada. An abundant parasite of Noctuidae of the cutworm type. Recorded from various species of Feltia, Euxoa, and Scotogramma.

\section{SUBFAMILY APHIDIINAE 143}

All the species of the Aphidiinae are internal parasites of aphids. The entire developmental period is spent within the body of the host, the adult parasite finally emerging by cutting a circular hole in the aphid shell. The host is killed at the time when the parasitic larva completes its growth, and accordingly aphids which contain pupae or adults of the parasite are readily distinguished in the field. As a rule development is very rapid, most species passing through many generations in a season. Some of them are very important parasites of particularly injurious aphids. But they are themselves subject to extensive parasitism by certain secondary parasites, especially by cynipoids belonging to the genus Charips and by various species of chalcidoids.

\section{EPHEDRUS Haliday}

E. incompletus Prov. Addit. fauna Can. Hymen., 1886, p. 156. Lockport, Je 14, from Myzus cerasi (Fab.) (Bdy-CU). Widely distributed over the eastern half of the US and in Canada. Recorded as a parasite of Myzus cerasi (Fab.), Macrosiphum rosae (L.), and Tritogenaphis rudbeckiae (Fitch).

[E. nitidus Gahan. Proc. U.S. Nat. Mus., 1917, 53:195. A parasite of the cabbage aphis, Brevicoryne brassicae (L.).]

\section{PRAON Haliday}

[P. americanus (Ashm.). Proc. U.S. Nat. Mus., 1888, 11:669. Recorded as attacking the apple grain aphid, Rhopalosiphum prunifoliae (Fitch).]

P. cerasaphis (Fitch). First Rep. Ins. N.Y., 1855, p. 138. Described from NY material reared from the cherry aphid.

P. humalaphidis Ashm. Proc. U.S. Nat. Mus., 1888, 11:657. Richfield Sps., type locality.

P. simulans (Prov.). Addit. fauna Can. Hymen., 1856, p. 153. Ithaca, J1, Griswold (Gahan-CU). A parasite of Tritogenaphis rudbeckiae (Fitch); also reared at Ithaca by Miss Griswold from Illinoia pisi (Kalt.) and Macrosiphum cornelli Patch.

P. viburnaphis Fitch. First Rep. Ins. N.Y., 1856, p. 137. Described from NY. The identity of the species is somewhat uncertain. Gahan (Md. Agr. Exp. Sta., 1911, Bull. 152, p. 197) states that a specimen in the U.S. Nat. Mus., which is labeled as type but which does not fully agree with the description, is a species of Lysiphlebus. The species is here provisionally retained in the genus Praon.

\section{Aphidius Nees}

[A. avenaphis (Fitch). Sixth Rep. Ins. N. Y., 1865, p. 98. A parasite of the apple grain aphid, Rhopalosiphum prunifoliae (Fitch), and Macrosiphum cerealis (Kalt.).]

${ }^{143}$ Aphidinae of North America. By A. B. Gahan. Md. Agr. Exp. Sta., 1911, Bull. 152, p. $147-200$. 
[A. crassicornis Ashm. Can. Ent., 1891, $23: 5$.

A. nigripes Ashm. N.Y. Mus., 1901, Bull. 47, p. 588. Saranac Inn, type locality, Ndm. Recorded as an efficient parasite of Macrosiphum granaria (Kby.).

A. phorodontis Ashm. Proc. U.S. Nat. Mus., 1888, 11:662. Ithaca (CU). A parasite of Phorodon mahaleb Koch and Rhopalosiphum dianthi (Schr.).

[A. polygonaphis (Fitch). First Rep. Ins. N.Y., 1856, p. 136. This species has been referred to in literature under the names Aphidius canadensis Prov., $A$. obscurus Prov., A. pallidus Ashm., A. nigriceps Ashm., and Lysiphlebus succineus Vier. It is a parasite of Tritogenaphis rudbeckiae (Fitch) and Illinoia liriodendri (Monell).]

[A. procephalis Ashm. Proc. U.S. Nat. Mus., 1888, 11:659. Recorded as a parasite of Procephalus sp.]

[A. ribis Haliday. Ent. Mag., 1835, 2:101. A widespread parasite of Cryptomyzus ribis (L.).]

A. rosae Haliday. Ent. Mag., 1833, 1:261, and 1835, 2:197. Ithaca, Griswold (Gahan-CU), from Illinoia pisi (Kalt.). A common parasite of Macrosiphum rosae (L.).

\section{LySIPHLEBUS Foerster}

L. testaceipes (Cress.). Rep. U.S. Com. Agr. for 1879, p. 208. Buffalo (Mues$\mathrm{CU})$. A very common species, occurring throughout the US as a parasite of a great variety of hosts, including Aphis gossypii Glov., A. maidis Fitch, Hysteroneora setariae (Thos.), Brevicoryne brassicae (L.), Anuraphis maidiradicis (Fbs.), Cryptomyzus ribis (L.), Macrosiphum granaria Kby., M. cerasi (Fab.), M. rosae (L.), M. cucurbitae (Thomas), and Rhopalosiphum prunifoliae (Fitch). The species has been referred to under a large number of names. For a complete record of this synonymy see Gahan, Md. Agr. Exp. Sta., 1911, Bull. 152, p. 186.

\section{SUBFAMILY ALYSIINAE 144}

The Alysiinae, in so far as their habits are known, are parasitic on the larvae and pupae of Diptera. Some exert an important degree of control on injurious species, while others live as hyperparasites, attacking primary parasites of the family Tachinidae. But the group as a whole is very poorly understood, from the standpoint of biology as well as that of classification. Unquestionably there are many species belonging to this subfamily in New York, but the identification of most of these must await revision of the group.

\section{Aphaereta Foerster}

[A. muscae Ashm. Proc. U.S. Nat. Mus., 1888, 11:646. Common throughout the eastern States as a parasite of various Diptera.]

[A. oscinidis Ashm. Proc. U.S. Nat. Mus., 1888, 11:647.]

[A. pallipes (Say). Contrib. Maclur. Lyc. Phila., 1828, 2 : 77.]

\section{Cratospila Foerster}

[C. ridibunda (Say). Contrib. Maclur. Lyc. Phila., 1828, 2 : 77.]

${ }^{144}$ Includes Dacnusinae and Alysiinae of Szepligeti's system. 


\title{
GRAMMOSPILA Foerster
}

G. triticaphis (Fitch). Sixth Rep. Ins. N.Y., Trans. N.Y. Agr. Soc., 1860, p. 840. NY, type locality. According to Gahan (Md. Agr. Exp. Sta., 1911, Bull. 152, p. $152)$, this species, which was described as an aphidiine in the genus Toxares, is probably a Grammospila.

\section{BRACHYSTROPHA Foerster}

B. quadriceps Ashm. N. Y. State Mus., 1901, Bull. 47, p. 587. Saranac Inn, type locality, $N d m$.

\section{COELINIDEA Viereck}

[C. ferruginea Gahan. Proc. U.S. Nat. Mus., 1913, 46:434. A parasite of Meromyza americana Fitch.]

[C. meromyzae (Fbs.). Thirteenth Rep. Ins. I11., 1884, 13:26. An abundant and widely distributed parasite of Meromyza americana Fitch. It attacks the egg of its host, but the parasitic larva does not mature until after the Meromyza has pupated.]

\section{RHIZARCHA Foerster}

R. astigma Ashm. N.Y. State Mus., 1901, Bull. 47, p. 587. Saranac Inn, type locality, $N d m$.

\section{DACNUSA Haliday}

D. agromyzae Gahan. Can. Ent., 1913, 45:153. Tarrytown, reared from Phytomyza obscurella Fall. by FrostSW.

D. scaptomyzae Gahan. Can. Ent. 1913, 45:150. Reared at Ithaca, as a parasite of Pegomyia calyptrata Zett. (FrostSW); recorded also as a parasite of Scap-. tomyza flaveola Meig.

\section{FAMILY ICHNEUMONIDAE 145}

\author{
Compiled by R. A. Cushman
}

\section{SUBFAMILY LYSIOGNATHINAE ${ }^{146}$}

\section{LYSIOGNATHA}

L. comstocki Ashm. Ithaca, SmHH (types-US, CU).

\section{SUBFAMILY JOPPINAE}

\section{(Ichneumoninae auctt.)}

(So far as is known, the members of this subfamily are parasitic in the pupae of Lepidoptera.)

\footnotetext{
145 The nomenclature and arrangement of this family, with the exception of the inclusion of Lysiognathinae as a subfamily, have been entirely in the hands of Mr. Cushman, to whom the editors furnished such records, published and otherwise, as were available to them.

${ }_{146}$ The editors are responsible for ranking the genus Lysiognatha as a subfamily of Ichneumonidae, rather than as a separate family as placed in Mr. Cushman's manuscript. This was done to bring it into conformity with the classification adopted for the order as a whole.
} 


\section{Tribe JOPPINI}

\section{Psilomastax Tischbein}

P. vulpinus (Grav.) (Trogus exesorius Brullé). Keeseville, Je, Fisher (Cush-US) ; Albion (Hasey-CU); Ithaca, Je-J1 (Hasey-CU); Karner, Je, Nassau, May (NYS); New Baltimore, Zab (Hasey-AM); Nyack, Zab (AM); West Farms, Angus (AM); LI: Flatbush, Zab (AM); Long Beach (Hasey-AM). Parasite on Papilio.

\section{TROGUS Panzer}

T. brullei Cress. SI, J1 (Ashm-Ds). A parasite of hawk-moths.

T. canadensis Prov. Ithaca, Je, Aug (Gahan-NYS, US); Poughkeepsie, Aug (NYS) ; SI, Aug-Sep (Ashm-Ds).

T. copei Cress. New Russia, Aug 12, Bdy (Hasey-CU).

T. elegans Cress. Oswego, J1 (Vier-US); Ithaca, J1-Aug (Hasey-CU).

T. nubilipennis Hald. LI: Long Beach, J1 (Cush-Schott, US).

T. obsidianator Brullé. New Russia, Aug, Bdy (Hasey-CU); L. Pleasant, J1 (NYS); Forest Lawn, Zab (AM) ; Ithaca, J1-Aug (Hasey-CU) ; Poughkeepsie, Aug (NYS) ; Rensselaer, Je (NYS) ; LI (Vier-US). Parasite on Papilio polyxenes Fab.

TROGOMORPHA Ashmead

T. ferrugator (Fab.) (trogiformis Cress.). Ilion, May, Yg (NYS).

\section{Tribe AMBLYTELINI}

\section{(Ichneumonini auctt.)}

\section{TRACHICHNEUMON Viereck}

T. confirmatus (Cress.). Waterville (Martin-CU); Oswego, Ap (US); Ithaca, Mar (Martin-CU, US) ; McLean, Sep 16, 1924, Sib (Bdy-CU) ; Normanskill, Ap, $Y g$ (NYS); Port Chester, Jl, Yg (NYS).

\section{ECTOPIMORPHA Viereck}

E. anceps (Cress.). Ithaca, J1 31, 1918, Yuasa (Bdy-CU) ; Norton's Landing, Cayuga L., May 30, 1872, SmHH (Cress-CU) ; LI: Rockaway (Martin-AM).

\section{Amblyteles Wesmael}

\section{(Ichneumon auctt. and Amblyteles auctt.)}

A. acerbus (Cress.). Saranac Inn. J1, Keene Val., Aug, Yg (NYS); Axton, Je, $M \& H$ (Martin-CU); Adirondacks, Aug (Martin-CU); McLean, May 30Sep 24, Sib, SRobinson (Bdy-CU).

A. agnitus (Cress.). Ithaca, Je (Martin-CU); White L., Zab (Martin-AM).

A. annulipes (Cress.). Mt. Whiteface, 3800 ft., J1, Ald (Cush-US) ; Cranberry L., Je 11-J1 26, Htly (Cush-US, NYS Col. For.) ; Manlius, Aug, Norton's Landing, Je, SmHH (Cress-CU); McLean, Sep 3, Sib (Cush-CU); Poughkeepsie, May (NYS); Nyack (AM). 
A. apertus (Cress.). New Russia, Aug 18, Bdy (Martin-CU); Albany, Je-J1 (NYS).

A. ater (Cress.). West Farms, Angus (AM). Locality may be doubtful (Leon).

A. atrifrons (Cress.). LI: Flatbush, Zab (Martin-AM).

A. atrox (Cress.). Ithaca, Mar (Martin-CU).

A. azotus (Cress.). Karner, Aug (NYS); Nyack (Martin-AM).

A. bimembris (Prov.). LI (Ashm-CU).

A. blandii (Cress.). LI (Ashm-US).

A. brevicinctor (Say) (extrematis [Cress.]). Newport, Je (NYS); Ilion, May (NYS); Manlius, Sep, SmHH (Cress-CU) ; Oswego, Je (US); Norton's Landing, Cayuga L., Je, SmHH (Cress-CU); Ithaca, May-Aug (Martin, Cress-CU) ; McLean, May (Martin-CU), Aug 28, Sib (Bdy-CU); Caroline to Harford, Je 15, MacG\&Bdy (Martin-CU) ; Dryden L., Je, MacG\&Bdy (Martin-CU) ; Albany, Je (NYS); Pine I. (AM); Nyack (Martin-AM); West Farms (Martin-AM); SI (Vier) ; LI: Flatbush (Martin-AM). Parasite on Pyrausta mubilalis Hueb., the European corn-borer.

A. brevipennis (Cress.). Axton, Je, $M \& H$ (Ashm-CU) ; Johnstown, Ap, Kenwood, J1 (NYS).

A. bronteus (Cress.). Old Forge, J1, Yg (NYS).

A. caeruleus (Cress.). Elizabethtown, Johnstown, Aug (NYS); Mt. Whiteface, 3800 ft., J1, Ald, Cranberry L., Je, Htly (Cush-US); Oswego, May, Aug (Ashm-US); Forest Lawn (Martin-AM); Norton's Landing, Cayuga L., Je, SmHH (Cress-CU) ; Ithaca, May (Martin-CU); Nyack, Zab (Ashm-US, Martin-AM).

A. caliginosus (Cress.). Ithaca, Je, Freeville, J1 (Martin-CU).

A. centrator (Say) (flavicornis [Cress.]). Newport, J1, Yg (NYS) ; Ithaca, Mar-

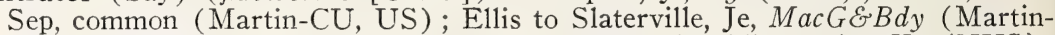
CU) ; McLean, Ap 17, Babiy, Je 20, Sib (Bdy-CU); Albany, Ap, Yg (NYS); Rensselaer, May (NYS); Poughkeepsie, May, J1 (NYS) ; Hillburn, Dec, Schott (US) ; Nyack (Martin-AM) ; West Farms (Martin-AM); SI, Nov (Martin-AM, Ashm-Ds); LI: Flatbush (Martin-AM); "NY" (type of flavicornis-ANSP). Host: Isia isabella S. \& A. (Arctiidae).

A. cincticornis (Cress.) (galemus [Cress.]). Albion (Martin-CU); Manlius, Sep, SmHH (Martin-CU); Rochester Jct., Je, Leon (Martin-CU) ; Ithaca, Ap-Aug (Martin-CU) ; McLean, J1 (Martin-CU) ; Newfield, Je, MacG (Martin-CU); Albany, Sep, Rensselaer, Mar (NYS); Normanskill, Ap (NYS); Big I., Mosholu (AM) ; West Farms (Martin-AM) ; SI, Ap (Martin-AM, AshmUS); LI: Flatbush, Zab (Martin-AM); Prospect Pk. (Martin-AM); Cold Sp. Harb. (Martin-AM) ; Riverhead, Oct, Russell (Cush-US); Farmingdale, J1, Morrow (Martin-CU). Host, Autographa brassicae Riley (Noctuidae).

A. cinctitarsis (Prov.). New Russia, Aug, Bdy (Martin-CU).

A. comes (Cress.). New Russia, Aug, Bdy (Martin-CU); Keene Val., J1, Yg; Saranac Inn, J1, $Y g$; Manlius, Aug, SmHH (Martin, Cress-CU) ; Cattaraugus, Sep (Martin-CU) ; Norton's Landing, Cayuga L., Je, SmHH (Cress-CU); McLean, J1 11-25, Sib (Bdy-CU); Albany, Je, Yg; Poughkeepsie, Je, Yg.

A. comptus (Say). Speculator, Aug (NYS); Ithaca, Aug (Martin-CU); Poughkeepsie, Je, Yg (NYS); West Farms (Martin-AM); SI (Vier).

A. corvinus (Cress.). Albany, Je (NYS).

A. creperus (Cress.). Keene Val., J1 (NYS); Albion (Martin-CU); Ithaca. Sep (NYS). 
A. detritus (Brullé). Coy Glen, Ithaca, Sep (Martin-CU); Barneveld, Wolcott.

A. devinctor (Say). Ithaca, Jan-Je (Martin-CU) ; McLean, Apr 17, 1925, Babiy (Bdy-CU) ; SI (Vier, Ashm-Ds); LI: Parkville (Martin-AM).

A. duplicatus (Say) (signatipes [Cress.]). New Russia, Aug, Bdy (Martin-CU); Rock City, Je (Martin-CU); Mud Cr., Je, MacG $\mathcal{}{ }^{\prime} B d y$ (Martin-CU) ; Ithaca, May-Aug (Martin-CU); Norton's Landing, Cayuga L., May-Je, SmHH (Martin\&Cress-CU) ; McLean, J1 25-Sep 17, Sib, SRobinson (Bdy-CU) ; Albany, Je, Poughkeepsie, May, $Y g$ (NYS); Nyack; LI: Flatbush, Zab, Rockaway (Martin-AM).

A. electus (Cress.). Ithaca, May (Martin-CU).

A. feralis (Cress.). Keene Val., Aug (NYS).

A. finitimus (Cress.). McLean, Aug 22, 1924, Sib (Cush-CU).

A. funestus (Cress.). Ithaca, Taughannock, Norton's Landing, McLean (Bdy-CU) ; New Russia, Axton (Bdy-CU); Oswego, Sep (Gahan-US); Nyack, Zab (US) ; LI (Vier).

A. fuscifrons (Cress.). McLean, Sep 16, 1924, Sib (Cush-CU).

A. germanus (Cress.). McLean, Aug 12, 1924, Sib (Bdy-CU); Johnstown, Aug, Poughkeepsie, May, $Y g$ (NYS).

A. grandis (Brullé). New Russia, Aug, Bdy (Martin-CU) ; Johnstown, Sep ; Canandaigua L., J1 (NYS) ; Otto, Sep, Comst (Martin-CU) ; Olean, Sep (Martin$\mathrm{CU})$; Norton's Landing, Cayuga L., Je, SmHH (Martin\&Cress-CU); McLean, Sep 3, Sib (Bdy-CU); Nyack, West Farms (Martin-AM).

A. helvipes (Cress.). Oswego, J1 (Ashm-US); LI: Flatbush, Je, Zab (MartinAM, Ashm-US).

A. humillimus (D.T.) (humilis [Prov.]). Gloversville, Ap (NYS).

A. improvisus (Cress.). McLean, Aug 26-Sep 4, Sib, SRobinson (Bdy-CU) ; LI (Vier).

A. inconstans (Cress.). Rochester Jct., J1, Leon (Martin-CU) ; Norton's Landing, Cayuga L., Je, SmHH (Martin-CU) ; Ithaca, Taughannock, Greenville, J1 (Martin-CU).

A. instabilis (Cress.). Axton, Je, $M \mathcal{E} H$ (Martin-CU); Ithaca, May-J1 (Martin$\mathrm{CU})$; Huguenot, $Z a b \cdot(\mathrm{AM})$.

A. jejunus (Cress.). Albany, Je, Aug (NYS); Nyack, Zab (Martin-AM); West Farms, Angus (Martin-AM); LI (Vier, Cush-US).

A. jucundus (Brullé) (flavizonatus [Cress.]). Keene Val., J1-Aug (NYS) ; Albion (Martin-CU) ; Gloversville, Mar, Bks (NYS) ; Oswego, Aug (US); Rochester Jct., J1, Leon (Martin-CU) ; Pike, Je (NYS) ; Otto, Je, Comst (Martin$\mathrm{CU})$; Ithaca, Ap-Aug (Ashm,Martin-NYS,CU) ; Norton's Landing, Cayuga L., Je, SmHH (Martin\&Cress-CU) ; West Farms, Angus (Martin-AM) ; LI: Flatbush, Zab (Martin-AM). Host: Papaipema (Noctuidae).

A. lachrymans (Prov.). Utica, Pergande (Cush-US); McLean, J1 25-Aug 19, SRobinson (Cush-CU).

A. laetus (Brullé) (canadensis [Prov.]). New Russia, Aug, Bdy (Martin-CU); Newport, Aug, $Y g$ (NYS); Axton, Je, $M \mathcal{E} H$ (Martin-CU) ; Oswego, Aug (US) ; Rochester, Aug (Martin-CU) ; Manlius, Jl-Sep, SmHH (Martin-CU) ; Buffalo, Nov, $M C V$, Stony I., J1 (US) ; Pike, Je, $Y g$ (NYS) ; Clifton Sps., Aug, Comstock (Martin-CU); Otto, Sep, Comstock (Martin-CU); Ithaca, Je-Aug (Martin-CU, NYS) ; Taughannock, J1 (Martin-CU) ; Norton's Landing, Cayuga L., Je, SmHH (Martin-CU) ; McLean, J1-Sep 20 (Martin-CU) ; Binghamton, Sep, Ellsworth (Martin-CU); Albany, Sep, Yg (NYS) ; Stock- 
port, May, Currie (US) ; Poughkeepsie, Je, Yg (NYS) ; Nyack (MartinAM) ; Rye, Sep, Bird; West Farms, Angus (Martin-AM); SI, J1 (Vier, Ashm-Ds) ; LI: Bird (Crawford) ; Flatbush, Zab, Fishers I., J1 (MartinAM) ; Farmingdale, Je, Morrow (Martin-CU). Parasite on pupa of most Papaipema which pupate in burrows (Bird); also on the army worm (Kngt).

A. leucaniae (Fitch) (seminiger [Cress.]). Rochester Jct., J1, Leon (Martin-CU); Clifton Sps., Aug (Martin-CU) ; Ithaca, Ap-J1 (Martin-CU) ; Forest Home, Ap (NYS); Nyack (Martin-AM) ; West Farms (Martin-AM) ; LI: Huntington, Ap, Schott (Cush) ; Rockaway, Long Beach, Flatbush, Zab (Martin-AM). Parasite on the army worm.

A. leviculus (Cress.). Ithaca, Je (Martin-CU); Poughkeepsie, May (NYS).

A. lewisii (Cress.). Ithaca, May, J1 (Martin-CU) ; Nassau, May (NYS); West Farms (Martin-AM); SI, Zab (Martin-AM); LI: (Vier) ; Flatbush, Zab (Martin-AM).

A. lewisii var. sandix (Cress.). "NY" (type-ANSP) ; LI (Cush-US).

A. libens (Cress.). "NY" (Cress.).

A. luctus (Cress.). Axton, Je, $M \mathcal{E} H$ (Martin-CU); Canandaigua L., J1 (NYS), from a noctuid larva.

A. malacus (Say). Peru, May (Martin-CU); Manlius, Sep, SmHH (Martin\&Cress-CU) ; Clifton Sps., Sep, Comstock (Martin-CU) ; Ithaca, Aug (Martin-CU); McLean, Aug 30-Sep 20, Sib (Bdy-CU); West Farms (Martin-AM); SI (Martin-AM) ; LI: (Vier-US) ; Flatbush, Zab (MartinAM) ; Rockaway (Martin-AM).

A. maurus (Cress.). Saranac Inn, Wells, J1 (NYS); SI (Vier); LI (Ashm-Ds).

A. mellicoxus (Prov.). Oswego, Aug (Cush-US).

A. mellipes (Prov.). "NY", Baker (Cush-US).

A. mendax (Cress.). Gloversville, Pike, May, $Y g$ (NYS); Ithaca, Feb-May (Martin-CU); Rensselaer, May, $Y g$ (NYS).

A. merus (Cress.). Poughkeepsie, Je, $Y g$ (NYS).

A. milvus (Cress.). New Russia, Aug, Bdy (Martin-CU) ; Canandaigua L., J1 (NYS) ; McLean, Aug 30-Sep 1, SRobinson (Bdy-CU).

A. mimicus (Cress.). Axton, Je, $M \mathcal{E} H$ (Martin-CU) ; Ithaca, May-Aug (MartinCU).

A. montanus (Cress.). Manlius, Sep, SmHH (Martin\&Cress-CU).

A. munificus (Cress.). Manlius, Sep, SmHH (Martin-CU); Ithaca, Aug-Sep (Martin-CU) ; McLean, Sep 3, Sib (Bdy-CU); Nyack, Zab (Cush-US).

A. nanodes Cush. (nanus [Cress.]). L. George (AM); LI: Flatbush, Zab (Martin-AM).

A. navus (Say). Old Forge, J1 (NYS) ; Ithaca, Mar, J1 (Martin-CU, NYS) ; Nyack, Zab (Cush-US); SI, Ap-May (Vier, Ashm-Ds); LI: Flatbush (Martin-AM).

A. nortoni (Cress.). Nyack (Martin-AM).

A. nubivagus Cress. Nyack, Zab (AM); LI: Flatbush, Zab (Ashm).

A. nuncius (Cress.). SI, Ap (Ashm-Ds).

A. ormenus (Cress.). Ithaca, Aug (Martin-CU).

A. ornatus (Cress.). "NY" (Cress-Am.Ent.Soc.). This is obviously not an Amblyteles, but its proper placing without study of specimens is impossible.

A. orpheus (Cress.). McLean, Aug 31, 1925, SRobinson (Bdy-CU) ; Delmar, Ap, Poughkeepsie, Je (NYS); West Farms (Martin-AM). 
A. otiosus (Say). Gloversville, Ap (NYS); Ithaca, May (Martin-CU).

A. paratus (Say). New Russia, Aug, Bdy (Martin-CU) ; Manlius, Je, Norton's Landing, Cayuga L., J1, SmHH (Martin\&Cress-CU); Ithaca, May-Aug (Martin-CU) ; McLean, Aug 22, Sib (Bdy-CU) ; Ft. Montgomery, Aug, Schott (Cush); Poughkeepsie, JI (NYS); Bear Mt., Schott (Cush-US); Nyack (Martin-AM); LI: Long Beach (Martin-AM); Flatbush, Zab (Martin-AM).

A. parvus (Cress.). Elizabethtown, Aug (NYS); Lancaster, Sep, MCV (CushUS) ; Ithaca, Sep (Martin-CU,NYS) ; McLean, Je 13-Sep 3, Sib, SRobinson (Bdy-CU); West Farms, Angus (AM).

A. pepticus (Cress.). Nyack (Cush-US).

?A. pequoitorum Vier. LI (Cush-US).

A. pervagus (Cress.). Ithaca, J1 (Cush-US); LI: Flatbush, Zab (Martin-AM).

A. pilosulus (Prov.). Ithaca, J1 (Martin-CU).

A. provancheri Cush. (bifasciatus [Prov.]). New Baltimore (Martin-AM).

A. proximus (Cress.). "NY" (Martin-AM).

A. pulcher (Brullé). LI: Rockaway. (Martin-AM).

A. pullatus (Cress.) (subcyaneus [Cress.]). New Russia, Je, Bdy (Martin-CU); Manlius, Sep, SmHH (Martin-CU); Oswego, Je (Cush-US); Syracuse, Blkm (Cush-US) ; Poughkeepsie, J1 (NYS) ; Nyack (Martin-AM) ; West Farms (Martin-AM) ; LI: Flatbush, Zab (Cush-US). Host: Diacrisia virginica Fab.

A. putus (Cress.). "NY", Baker (Cush-US).

A. quadrizonatus Vier. Ithaca, Sep (Martin-CU).

A. quebecensis (Prov.). Newport, Jl, Gloversville, Aug, Yg (NYS); McLean, Je 20-J1 11, SRobinson (Bdy-CU).

A. restrictus (Cress.). "NY" (Cress).

A. rubicundus (Cress.). Ithaca, May-Aug (Martin-CU); Norton's Landing, Cayuga L., Je, SmHH (Cress\&Martin-CU).

A. rufiventris (Brullé). Axton, Je, $M \& H$ (Martin, Ashm-CU); Mt. Marcy, J1, Sacandaga R., Aug (NYS); Ithaca, J1 (Martin-CU); Norton's Landing, Cayuga L., Je, SmHH (Martin\&Cress-CU) ; Ft. Montgomery, J1 (CushSchott) ; LI: Brooklyn (Martin-US).

A. rufizonatus (Cress.). Oswego, Sep (Ashm-US); Ithaca, J1 (Martin-CU).

A. sagus (Cress.). LI: Rockaway (Martin-AM).

A. saucius (Cress.). Albany, Aug (NYS); LI (Vier).

A. scelestus (Cress.). Oswego, Je (Ashm-US); Ithaca, Ap-Je (Martin-CU); Rensselaer, Mar, $Y g$ (NYS) ; Normanskill, Ap, Yg (NYS) ; Rye, Aug, Bird, on Papaipema impecuniosa; LI: (Ashm-US); Flatbush (Martin-AM).

A. scriptifrons (Cress.). LI (Vier).

A. solitus (Cress.). LI (Vier).

A. soror (Cress.). Ithaca, Chitt (Cush-US); LI: Wyandanch, Dec (CushSchott).

A. stadaconensis (Prov.). Old Forge, Je (NYS).

A. stygicus (Prov.). Oswego, J1 (Gahan-US); McLean, J1 18 (Cush-CU).

A. subdolus (Cress.). Potsdam (Martin-CU); Johnstown, J1, Alex (Cush-US); Oswego, Je (Cush-US) ; Caroline to Harford, Je, MacG\&B Bdy (Martin-CU);

West Farms, Ang (Martin-AM); SI (Vier); LI (Vier). 
A. subfuscus (Cress.). New Baltimore, Zab (AM); SI, Oct (Vier, Ashm-Ds).

A. sublatus (Cress.) (pravus [Cress.] + ). Oswego, Je (Cush-US) ; Manlius, J1Sep, SmHH (Martin\&Cress-CU) ; Canandaigua L., J1, Yg (NYS); Rock City, Je (Martin-CU) ; Gowanda, Aug, EPV (Ashm-US) ; Norton's Landing, Cayuga L., Je, SmHH (Martin\&Cress-CU); Ithaca, Aug (Martin-CU, NYS) ; McLean, ㅇ, Sep 20-24, 1924, Sib (Bdy-CU), ó, J1 28-Aug 29 (BdyCU) ; Poughkeepsie, Je, $Y g$ (NYS); Nyack (Ashm, Martin-US, AM) ; SI (Vier, Ashm-Ds); LI (Cush-US); Long Beach (Martin-AM); Flatbush, Zab (Martin-CU). Reared from Heterocampa guttivitta Wlk. (Notodontidae), in Me. and Mass.

A. subrufus (Cress.). Potsdam (Martin-CU); Ithaca, Ap (Martin-CU); West Farms, Angus (AM).

A. succinctus (Brullé). Rochester Jct., Je, Leon (Martin-CU) ; Ithaca, J1-Sep (Martin-CU, US) ; Taughannock, J1, McLean (Martin-CU) ; Albany, Sep (NYS); West Farms, Angus (Martin-AM) ; LI (Vier).

A. suturalis (Say). Keene Val., J1, Gloversville, Mar (NYS) ; LI: Huntington, Nov, Schott (Cush-US).

A. tetricus Prov. "NY" (Ashm).

A. truculentus (Crẻss.). "NY" (Martin-AM); Ithaca, Je (NYS).

A. ultimus (Cress.). Ithaca, Je (Martin-CU). Determination doubtful.

A. ultus (Cress.). Axton, Je, $M \mathcal{E} H$ (Martin-CU) ; Norton's Landing, Cayuga L., Je, SmHH (Martin-CU); Ithaca, Je (Martin-CU); Nyack (Martin-AM).

A. ultus var. regalis (Cress.). "NY" (Cress).

A. uncinatus (Cress.). Albany, Je (NYS).

A. unifasciatorius (Say). Oswego, Aug-Sep (Ashm-US) ; Manlius, Sep, SmHH (Martin\&Cress) ; Niagara Falls, Aug, $M C V$ (Cush-US) ; Ithaca, Je-Sep (Martin-CU, NYS) ; McLean, Aug 11-Sep 24, Sib, SRobinson (Bdy-CU); Nyack (Martin-AM); West Farms (Martin-AM); LI: (Ashm-US); Sea Cliff (CU); Sands Pt., Ap (Martin-AM); Flatbush, Zab (Martin-AM). Parasite on Apatela oblinita S. \& A. (Noctuidae), and the hickory tussock caterpillar, Halisidota caryae Harr.

A. utilis (Cress.). "NY" (Cress).

A. velox (Cress.). Johnstown, Sep (NYS) ; Norton's Landing, Cayuga L., Je, SmHH (Martin\&Cress-CU) ; Ithaca, J1 (Martin-CU) ; Poughkeepsie, May (NYS) ; West Farms, Angus (Martin-AM); LI: Brooklyn (Martin-AM).

A. versabilis (Cress.). "NY" (paratype-US) ; Ithaca, Je (Martin-CU) ; Mud Cr., Je, $M a c G \mathcal{E} B d y$ (Martin-CU).

A. vescus (Prov.). New Russia, Aug, $B d y$ (Martin-CU); Axton, Je $M \mathcal{E} H$ (Martin-CU) ; Cranberry L., Aug, Htly (Cush-NYS Col. For.) ; Norton's Landing, Cayuga L., May-Je, SmHH (Martin-CU); Ithaca, Je (Martin-CU).

A. vinnulus (Cress.). Ithaca, May-Oct (Martin-CU); Katonah (Martin-AM) ; New Baltimore, Nyack, West Farms (Martin-AM); LI: Flatbush (Martin-AM).

A. viola (Cress.). Saranac Inn, J1 (NYS); LI (Vier-US).

A. vitalis (Cress.). Ithaca, Sep (NYS); LI (Vier).

A. vittifrons (Cress.). LI (Cush-US).

A. vivax (Cress.). Ithaca, J1 (Martin-CU).

A. volens (Cress.). "NY" (Cress).

A. volesus (Cress.). Axton, Je, $M \mathcal{E} H$ (Martin-CU). 
A. w-album (Cress.). Mt. Whiteface, 3800 ft., Ald (Cush-US) ; Norton's Landing, Cayuga L., May, SmHH (Martin\&Cress-CU) ; Ithaca, Je (Martin-CU) ; Mc-

Lean, Aug 24-Sep 3, Sib (Bdy-CU) ; Albany, Je, Karner, J1, Yg (NYS) ; Bear Mt., J1 (Cush-Schott) ; Poughkeepsie, Je, Yg (NYS) ; Nyack (AM) ; SI, Je (Vier, Ashm-Ds). LI (Ashm-Us).

A. wilsoni (Cress.). Poughkeepsie, J1, Yg (NYS); Rhinebeck, J1, Cy (Martin$\mathrm{CU}$ ) ; West Farms, Angus (Martin-USS).

\section{EuRylabus Wesmael}

E. agilis Cress. "NY" (Cress).

Platylabus Wesmael

P. thoracicus (Cress.). "NY" (Cress).

\section{HOPLISMENUS Gravenhorst}

H. morulus (Say). Manlius, Aug, SmHH (Cress-CU); Ithaca, J1-Aug (CU); SI, Ds (Ashm-Ds). Hosts: Aglais antiopa L. and Polygonia interrogationis Fab.

\section{Tribe PHAEOGENINI}

HeRpestomus Wesmael

H. pyriformis (Prov.). "NY" (Cress).

\section{Colpognathus Wesmael}

C. helvus (Cress.). Manlius, Aug, SmHH (Cress-CU); Barneveld, Ap, Wolcott (Cush-US); LI (Ashm-US).

\section{Centeterus Wesmael}

C. tuberculifrons (Prov.). LI (Ashm).

\section{EPARCES Foerster}

E. quadriceps (Cress.). Ithaca, J1, Yuasa, Spring Lake, J1 (Bdy-CU) ; McLean, Sep 19, 1924, Sib (Bdy-CU).

\section{PhaEogenes Wesmael}

P. fungor (Cress.). Ithaca, Chitt (Cush-US); LI (Ashm-US).

P. gelechiae Ashm. Ithaca, Leiby (Cush-US). Host: the goldenrod gall-maker, Gnorimoschema gallaesolidaginis Rly.

P. hebe (Cress.). NY (Cress); LI (Ashm).

P. hebrus (Cress.). Ithaca, Je-J1 (Bdy-CU); McLean, Ap 17, 1925 (Cush-CU).

P. mellinus (Prov.). Ithaca (Bdy).

P. pimploides Bdy. Ithaca, J1 12 (type-CU).

P. vincibilis (Cress.). Norton's Landing, Cayuga L., Je 13, 1872 (Cress-CU). 


\section{SUBFAMILY CRYPTINAE 147 \\ Tribe STILPNINI \\ Mesoleptus Grav. \\ (Exolytus Holmberg and auctt.)}

M. aciculatus (Davis). "NY" (GCDavis).

M. glaucus (Davis). "NY" (GCDavis).

M. ithacae (Ashm.). Ithaca (type-US).

ATRACTODES Gravenhorst

A. compressus (Cress.). "NY" (Ashm).

A. sepedontis Ashm. Saranac Inn, Aug, 1900 (type-US).

STILPNUS Gravenhorst

S. americanus Cress. SI (Vier).

\section{Tribe PHYGADEUONINI}

\section{THYSIOTORUS Foerster}

T. smithi Cush. McLean, Aug 18-Sep 16, SRobinson (Cush-US) ; Hamden, Je 30, 1924 (Cush-US). A secondary parasite of the ophionine wasp, Hyposoter geometrae (Ashm).

\section{GLyPHICNEMIS Foerster}

G. crassipes (Prov.). Newport, J1, Yg (NYS); Ithaca, Je (Cush-US).

\section{STYLOCRYPTUS Thomson}

S. inflatus (Prov.). McLean, Aug 18, 1925, SRobinson (Cush-CU).

S. maculatus (Prov.). Old Forge, Jl, $Y g$ (NYS).

S. mucronatus (Prov.). "NY" (Ashm).

S. pubescens (Prov.). McLean, Aug 1, 1925, SRobinson (Cush-CU).

S. subclavatus (Say) (Phygadeuon rotundiceps Prov.). Nassau, Je (NYS).

S. vulgaris (Cress.). LI (Ashm-US).

\section{PEZOPORUS Foerster}

P. tenthredinarum Roh. Geneva (type-US). On Profenusa collaris (MacG.) (Tenthredinidae).

\section{Aptesis Foerster}

A. pterygia Brad. Ithaca, Je, Yuasa (Bdy-CU) ; Taughannock, J1, 1925, Bdy\& Guerlac (Cush-CU).

${ }^{147}$ If the Erlangen List is accepted, this subfamily should be called Gelinae, from Gelis Thunberg, the next oldest name in the subfamily (Cush), or Itamopleginae, from Itamoplex, replacing the homonym Cryptus Fab. (Bdy). 


\section{PlectocRyptus Thomson}

P. contiguus (Cress.). Oswego, Je, Aug (Cush-US).

P. major (Cress.). Poughkeepsie, Je, Yg (NYS).

\section{PHYGADEUON Gravenhorst}

(Subgenus PhygadeuON Gravenhorst)

P. brevicornis (Prov.). Beaver Cr., McLean, Sep 14, 1924, Sib (Cush-CU).

P. orbitalis (Prov.). McLean, Sep 17, 1924, Sib (Cush-CU).

P. subfuscus Cress. LI (Ashm).

(Subgenus Stibeutes Foerster)

P. yuasai Bdy. Ithaca, J1, Yuasa (type-CU).

\section{PolytRIBAX Foerster}

P. pallescens Vier. Ithaca, Chitt (Cush-US); McLean, Sep 16, 1924, Sib (Cush-CU).

\section{Tribe HEMITELINI}

\section{ACROLYTA Foerster}

A. smerinthi Ashm. Ithaca, Sep 13 (Ashm-CU), from sphinx larvae.

\section{ISDROMAS Foerster}

I. aletiae (Ashm.). Ithaca (Ashm-CU). On Apanteles.

I. bucculatricis (Ashm.). Ithaca (Ashm-US).

\section{ASTOMASPIS Foerster}

A. nanus (Grav.) (fulvipes [Grav.]). Rye, May-Sep, Bird. Bird says there seem to be two broods. A parasite on many species of Apanteles.

\section{HEMITELES Gravenhorst}

H. humeralis Prov. LI: Flatbush, Zab (AM).

H. ruficoxis Prov. McLean, Aug 26, 1925 SRobinson (Cush-CU).

H. sessilis Prov. Albany, May, 1895 (NYS).

H. tenellus (Say) (melitiae Ashm., utilis Nort., periliti Ashm.). Genoa, Nov (Ashm-CU) ; Ithaca, J1 (Bdy, Ashm-CU); Corinth (Felt); Rye, May, Bird (CU) ; LI: Flatbush, Zab (AM). A secondary parasite attacking many species of primary parasites.

\section{AENOPLEX Foerster}

A. rubricornis (Ashm.). LI (Ashm). 


\title{
Chrysopoctonus Cushman
}

(All the species of this genus are parasitic within the cocoons of Chrysopa.)

C. atriceps (Ashm.). Ithaca, Chitt (Cush-US).

C. rileyi (Ashm.). Cranberry L., Aug, Htly (Cush-NYS Col. For.); Ithaca (Cush-US).

\author{
Tribe GELINI \\ (Pezomachini auctt.) \\ GeLIS Thunberg \\ (Pezomachus Gravenhorst)
}

(The species of this genus are either secondary parasites or parasitic within the egg cocoons of spiders.)

G. bucculatricis (Ashm.). Chapel Pond, Je, Cy (Cush-US) ; Artist's Br., Jan, Leon (Cush-US); McLean, Sep 16, 1924, Sib (Mues-CU).

G. dimidiatus (Cress.). Ithaca, Mar, May (Ashm-CU), from a spider's egg-cocoon.

G. dispar (Strick1.). Ithaca, Sep 11, 1925, Babiy (Cush-CU).

G. flavocinctus (Ashm.). Ithaca (Strickland).

G. meabilis (Cress.). SI (Ashm).

G. microplitidis Gahan. Rye, May-Sep, Bird (type-US), from Microplitis gortynae Rly.

G. ottawaensis (Harr.). Penn Yan, Aug 2, 1925, Cy (Cush-CU).

G. thripites (Taylor). "NY" (Taylor).

G. uniformis (D.T.). Ithaca, Nov 19, 1925, Cy (Cush-CU), found ovipositing on spider cocoon.

G. urbanus (Brues). Taughannock, J1, 1925, Bdy, Guerlac (Cush-CU); Ithaca, Mar 7 (Cush-CU); McLean, Ap, 1917, Babiy (Cush-CU).

\section{Tribe CRYPTINI 148}

(Including Mesostenini)

\section{ACRORICNUS Ratzeburg}

A. junceus (Cress.). Norton's Landing, Cayuga L., Je, SmHH (Cress-CU); McLean, Aug 30, 1924, Sib (Bdy-CU); SI (Ds); LI: Fishers I. (AM). Parasitic in nests of Odynerus.

\section{SPILOCRYPTUS Thomson}

S. atricollaris (Walsh). Norton's Landing, Cayuga L., J1, SmHH (Cress-CU); McLean, J1 25-Sep 12, SRobinson, Sib (Cush-CU).

S. canadensis (Prov.). McLean, Aug 31, 1925, SRobinson (Cush-CU).

S. extrematis (Cress.). Albany, Je (NYS); LI: Flatbush, Zab (AM), Je (US) ; Jamaica, Je (US). Host: Samia cecropia.

S. nuncius (Say). McLean, Aug 23-30, SRobinson, Sib (Cush-CU); Albany, J1 (NYS); Nyack, $Z a b$ (AM).

S. polychrosidis Cush. Ithaca, Sling (Cush-US). Host: grape berry moth.

\footnotetext{
${ }^{148}$ If the Erlangen List is accepted, this tribe should be known as the Mesostenini, from the next oldest name, Mesostenus Grav. (Cush), or Itamoplegini, from Itamoplex Foerster, replacing the homonym Cryptus Fab. (Bdy).
} 
COMPSOCRYPTUS Ashmead

C. retentor (Brullé). SI, Sep (Ashm-Ds).

\section{MEgaplectes Förster}

M. blakei (Cress.). McLean, Je 30, 1921 (Bdy-CU). This is undoubtedly identical with the European monticola Grav. I find no distinction between the McLean and European specimens. (Bdy.)

\section{CRyptus Fabricius \\ (Itamoplex Foerster)}

C. altoni D.T. Cranberry L., Je, Htly (Cush-US); Coeymans (NYS).

C. persimilis Cress. Oswego, May-J1, Stony I., J1, LI (Cush-US).

C. vinctus (Say) (americanus Cress.). Old Forge, Je (NYS); Norton's Landing, Cayuga L., May-Je, SmHH (Cress-CU) ; Stony I., J1 (Cush-US) ; LI: Flatbush, Zab (AM, US).

\section{Chromocryptus Ashmead}

C. planosae (Fitch). "NY" (type-US), (Cush-US). The type was bred from a cocoon of Tolype laricis Fitch, the other specimen from T. vellida Stoll. (Lasiocampidae).

\section{IDIOLISPA Foerster}

I. limata (Cress.). Oswego, Je (US); Manlius, J1, Norton's Landing, Cayuga L., May-Je, SmHH (Cress-CU); LI: (US) ; Flatbush, Zab (AM, US).

\section{TRYCHOSIS Foerster}

T. rufoannulata (Prov.). Oswego, May (Cush-US).

T. similis (Cress.). Ft. Montgomery, Je (Cush-Schott); LI Vier-US).

\section{HOPLOCRYPTUS Thomson}

H. apicatus (Prov.). Oswego, Jl-Aug (Cush-US).

H. incertulus Cush. LI (Vier-US).

\section{CRyptoideus Ashmead}

C. agrili (Vier.). LI: Huntington, Ap (Cush-Schott). Host: Agrilus vittaticollis Rand.

ECHTHRUS Gravenhorst

E. abdominalis Cress. Cranberry L., A. Gottlicb (Cush-US).

Chaeretymma Foerster

C. velox (Cress.). "NY" (type-ANSP). 
Crypturopsis Ashmead

C. candidus (Cress.). LI: Orient, $S_{e p}$ (Cush-Schott).

C. fortis (Cress.). "NY", Comstock (type-ANSP).

ListRognATHUS Tschek

L. albomaculatus (Cress.). Manlius, Aug 2, 1872, SmHH (Cress-CU); "NY" (Vier-US).

\section{Polyaenus Cresson}

P. spinarius (Brullé). LI (Cush-US).

\section{Mesostenus Gravenhorst}

M. erythrogaster Ashm. Oswego, Je (Cush-US); Ithaca, Chitt (Cush-US).

M. gracilis Cress. Ithaca, Chitt (Cush-US).

M. thoracicus Cress. Manlius, Sep, Norton's Landing, Cayuga L., May-Je, SmHIII (Cress-CU) ; Ithaca, Je (Ashm-CU); Poughkeepsie, Je, $Y g$ (NYS).

\section{Polistiphaga Cushman}

P. arvalis (Cress.). Nyack, $Z a b$ (AM) ; LI (US). Host: Polistes.

\section{SUBFAMILY ICHNEUMONINAE 149}

\section{(Pimplinae auctt.)}

\section{Tribe LISSONOTINI}

(The members of this tribe are very largely parasitic on lepidopterous larvae, although a few are recorded as reared from sawfly larvae.)

\section{Exetastes Gravenhorst}

E. albitarsis Prov. Cranberry L., J1, Htly (Cush-US).

E. fascipennis Cress. Manlius, Sep, SmHH (Cress-CU); Nyack, Zab (CushUS); SI, Oct (Ashm-Ds).

E. obscurus Cress. Nyack, Zab (AM).

E. suaveolens Walsh. Saranac Inn, Yg (NYS); ?Manlius, SmHH (Cress-CU); Oswego, Aug (Ashm-US); Ithaca, J1, EHSargent (Ashm-CU); McLean, J1 20-Aug 24, Sib (Bdy-CU) ; L. George, Zab (AM) ; Ft. Montgomery, Aug, Schott (AM); LI, Aug (Ashm-US).

\section{AREnetra Holmgren}

A. nigrita Cress. Caroline, Ap (Cush-CU) ; Nassau, May (NYS) ; SI (Ashm-DsUS).

A. ventralis Cress. "NY" (Cress).

\footnotetext{
${ }^{149}$ Holarctic tribes of the ichneumon-flies of the subfamily Ichneumoninae (Pimplinae). IRv Robert A. Cushman and S. A. Rohwer. Proc. U. S. Nat. Mus., 1920, 57:379. 396.
} 
Meniscus Schiødte

M. agilis (Cress.). Manlius, Aug, SmHH (Cress-CU); Waterville (Cush-US).

M. scutellaris (Cress.). Adirondacks, J1, Aug (Cush-CU); Essex Co, J1 (NYS); Ithaca, JI (Cush-CU). Recorded as a parasite of Gelechia psendacaciella Chamb.

M. superbus (Prov.). Oswego, May (Cush-US); Rock City, Je (Cush-CU); Caroline to Harford, Je, MacGEBdy (Cush-CU); McLean Bogs, Ithaca, May-Je (Cush-CU).

\section{LISSONOTA Gravenhorst}

\section{(Lampronota Cresson not Curtis)}

\section{(Subgenus Lissonota Gravenhorst)}

L. americana (Cress.). Essex Co, Aug (NYS); Potsdam, Albion (Bdy-CU); Cranberry L., Aug, Spooner (Cush-NYS); Middletown, J1, Spooner (CushCU) ; Manlius, Sep, SmHH (Cress); L. Placid, Aug (Cush-US); Thousand Isls., Sep (Cush-CU) ; Rochester, Aug, Summers (Cush-CU) ; Oswego, Sep (Vier) ; Otto, Sep, Comstock (Cush-CU) ; Delhaven, Aug (Cush-CU) ; Spencer Summit, Aug, Comstock (Maidl-CU); Ithaca, May-Sep (Cush-CU); Spring Lake, J1, Bdy (Cush-CU); Freeville, Aug (Cush-CU); McLean, J1 18-Aug 1, SRobinson (Bdy-CU); LI: Riverhead, Sep (Cush-CU). Very common, probably everywhere in the State.

L. angusta (Davis). Ithaca, Ap (Cush-US, CU).

L. brunnea Cress. Rye, Bird (Cush-US).

L. cressonii Vier. (occidentalis Cress.). Manlius, Aug, SmHH (Cress-CU); Norton's Landing, Cayuga L., Je, SmHH (Cress-CU); Spring Lake, J1 (Cush-CU) ; Ithaca, J1 (Cush-CU) ; LI: Jamaica (Roh-US), on Podosesia syringae Harr. (Aegeriidae).

L. exilis (Cress.). New Russia, Aug, Bdy (Cush-CU); North Cr., J1, Fbs (Cush-US) ; Axton, Je, $M \& H$ (Ashm-CU) ; Rochester Jct., J1, Leon (CushCU) ; Stony I., J1 (US); Ithaca, Sep (Maidl-CU).

L. frigida (Cress.). Old Forge (NYS) ; Watertown, Lint (Cress-NYS); Ithaca ; Nassau, Jl-Sep (NYS) ; Manlius, J1-Sep, SmHH (Cress-CU); Middletown, Jl, Spooner (Cush-CU). Recorded as a parasite of Crambus vulgivagellus Clem.; specimens from this host are also in the U. S. Nat. Mus.

L. humeralis (Prov.). Oswego, J1 (Roh-US).

L. jocosa (Cress.). Portageville, Je (Cush-CU) ; Norton's Landing, Cayuga L.,

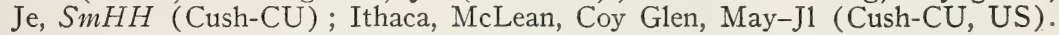

L. laevigata (Cress.). McLean, Sep 12-17, Sib (Cush, Bdy-CU).

L. parva (Cress.). Oswego, Ap (Cush-US); Ithaca, from leaf-miner, Apr-May (Ashm, Cush-CU, US); McLean, Aug 20-Sep 3, Sib, SRobinson (Bdy-CU); LI: Orient Pt., Ap (Cush-NYS).

L. philipi Vier. Ithaca, Aug (Cush-CU, US).

L. pleuralis (Cress.). Potsdam, J1 (Cush-CU) ; Oswego, Sep (Cush-US) ; Manlius, Sep, $\mathrm{SmHH}$ (Cress-CU); Ithaca, Je-Aug (Cush-CU, US). Recorded as a parasite of Eulia velutinana W1k. (Tortricidae).

L. punctata (Cress.) (albifacies [Prov.] ${ }^{\star}$ ). Potsdam (Cush-CU, US); Utica, Pergande (Cush-US) ; N. Fair Haven, Sep (Cush-CU) ; Albion (Cush-US); Manlius, Sep, SmHH (Cress-CU); Oswego, Sep (Vier-US); Cattaraugus, Sep (Cush-CU); Ithaca, Ap-Sep (Cush-CU); Freeville, Sep (Maidl-CU). 
L. rubrica (Cress.). Norton's Landing, Cayuga L., Je, SmHH (Cress-CU); Spring Lake, J1 (Cush-US); Spring Cr., J1, Fbs (Cush-US); Ithaca, Aug, Foster (Cush-CU); Middletown, Jl, Spooner (Cush-CU); LI' (Vier-US).

L. rufipes (Cress.) (tegularis [Cress.], male). Manlius, Aug, Sep, SmHH (CressCU); Utica, Pergande (Cush-US); N. Fair Haven, Sep, Bdy (Cush-CU); Otto, Sep, Comstock (Cush-CU); Pearl Pt., Aug (Cush-CU).

L. varia (Cress.). Potsdam (Cush-CU) ; Adirondacks, Aug (Cush-CU) ; L. Placid, Aug (Roh-US); Thousand Isls., Sep (Cush-CU); Manlius, Sep, SmHH (Cress-CU); Otto, Comst (Cush-CU) ; Varna, Sep (Cush-CU); Ithaca, J1Sep (Cush-CU, US); McLean, Sep 1, SRobinson (Bdy-CU), Sep 24, Sib (Cush-CU).

\section{(Subgenus Asphragis Foerster)}

L. mirabilis (Cress.). Cranberry L., J1, Htly (Cush-SyracuseU) ; McLean, Aug 7, 1925, SRobinson (Bdy-CU). Specimens in the U.S. Nat. Mus. were reared from Recurvaria robiniella Fitch and Gelechia trialbamaculella Chamb. (both Gelechiidae).

\section{SYZEUCTUS Foerster}

S. elegans (Cress.). Ithaca, J1, Yuasa (Bdy, Cush-CU). Specimens in the U.S. Nat. Mus. are said to have been reared from Archips fervidana Clem. (Tortricidae) and Psilocorsis sp. (Oecophoridae).

\section{CYLLOCERIA Schiødte}

C. occidentalis Cress. Axton, J1, $M \mathcal{\&} H$ (Cush-CU, US) ; Keene Val., Aug (CushUS) ; Rock City, Je (Cush-CU, US) ; Ithaca, May, J1 (Cush-CÜ); Coy Glen, Taughannock, J1, Sep (Cush-US); McLean, May-Sep 24 (Cush, Bdy-CU, US).

\section{Tribe GLYPTINI}

(The members of this tribe are parasitic on the larvae of Lepidoptera.)

\section{GLYPTA Gravenhorst}

G. animosa Cress. "NY" (Cress). Recorded as a parasite of Eucosma scudderiana Clem. (Tortricidae).

G. borealis Cress. Johnstown, Aug (NYS).

G. canadensis Cress. Oswego, Je (Cush-US) ; McLean, Aug 31, Sib (Cush-US).

G. erratica Cress. Cranberry L., Jl, Htly (Cush-N.Y.S. Col. For.); Axton, Je, $M \mathcal{E} H$ (Cush-CU); Peru, Je (Cush-CU); Ithaca, Je-Sep (Cush-CU); Mud Cr., Je (Cush-CU) ; Caroline to Harford, Je, MacG EBdy (Cush-CU) ; Karner, Nassau, Albany, May, J1, Yg (NYS). Parasite on Grapta comma Harris.

G. inversa Cress. Ithaca, Aug (Cush-CU).

G. phoxopteridis Weed. McLean, Aug 31, 1925, SRobinson (Bdy-CU). Parasite of the strawberry leaf-roller, Ancylis comptana Froelich.

G. pulchripes Cress. Ithaca, Je-Sep (Cush-CU).

G. rufiscutellaris Cress. New Russia, Aug, Bdy (Cush-CU); Rochester Jct., J1, Leon (Cush-CU); Ithaca, Ap-J1 (Cush-CU); McLean, Je (Cush-CU); Cohoes, Je (Cush-US) ; LI: Farmingdale, Je, Morrow (Cush-CU). A native parasite of the introduced oriental peach moth, Laspeyresia molesta Busck. 
G. rufofasciata Cress. Spring Lake, J1 (Cush-US) ; LI: Farmingdale, J1, Morrow (Cush-CU).

G. simplicipes Cress. Rochester Jct., Je, Leon (Cush-CU) ; Ithaca, J1-Aug (CushCU) ; Nassau, Ap (NYS); Albany, Feb (NYS). Recorded as a parasite of Peronea mimuta Rob. and its variety cinderella Rly., Archips rosaceana Harr. (all Tortricidae), and Heterocampa bilineata Pack. (Notodontidae). Specimens in the U.S. Nat. Mus. are from the first of the above-named hosts, Cymolomia footiana Fern. (Tortricidae), and Zotheca tranquilla Grt. (Noctuidae).

G. varipes Cress. Ithaca, May (Cush-CU). Specimens in the U.S. Nat. Mus. are from the lesser codling worm, Laspeyresia prunivora Walsh.

G. vulgaris Cress. Norton's Landing, Cayuga L., Je, SmHH (Cress-CU) ; Ithaca, May-Aug (Cush-CU). Specimens in the U.S. Nat. Mus. are recorded as reared from Trichotaphe juncidella Clem. (Gelechiidae), Eulia velutinana Wlk., Oletherentes interruptolineana Fern. (Tortricidae), the oriental peach moth (Laspeyresia molesta Busck), and Cymolomia punctana Walsh.

\section{Tribe PHYTODIETINI 150}

Phytodietus Gravenhorst

P. annulatus (Prov.). Oswego (Roh-US) ; Ithaca, Mar-May (Roh, Cush-CU, US) ; Watkins (Roh); Mix Cr. Val., Je (Cush-CU). Specimens in the U.S. Nat. Mus. were reared from Archips argyrospila Wlk. and Ancylis fragariae Walsh \& Rly. (both Tortricidae).

P. burgessi (Cress.). Rock City, Je (Cush-CU); Ithaca, May, Aug (Maidl-CU, Roh-US); McLean Bogs, May, J1 20, Sib (Cush-CU). Specimens in the U.S. Nat. Mus. were reared from Exartema myricanum, Peronea logiana Schiff., Ancylis platana Clem. (Tortricidae), and Acrobasis betulella Hulst (Pyralidae).

P. vulgaris Cress. Manlius, Sep, $\mathrm{SmHH}$ (Cress-CU) ; Portageville, Je (Cush-CU) ; Ithaca, Je-J1 (Cush-CU), from Archips argyrospila Wlk. (Tortricidae); McLean, Aug 22, Sib (Bdy-CU) ; Albany, Oct (NYS) ; LI: Riverhead, Sep (Cush$\mathrm{CU})$. A specimen in the U.S. Nat. Mus. was reared from Peronea minuta Rob. (Tortricidae).

\section{Tribe THERONIINI 151}

(The members of this tribe are parasitic in the pupae of Lepidoptera, sometimes, at least, as secondary parasites.)

\section{THERONIA Holmgren}

T. fulvescens (Cress.). Ithaca, Aug (Mues-CU); Manlius, Sep, SmHH (CressCU) ; Pike, Jl (NYS); Glens Falls (Cush-US); Albany, Aug (NYS); Slingerlands (Felt-NYS); Dutchess Co., Sep, Dyar (Cush-US). Specimens in the U.S. Nat. Mus. were reared from the pierid butterfly, Neophasia menapia Felder, the spruce budworm, Hormologe fumiferana Clem., the white-marked tussock, Hemerocampa leucostigma S. \& A., Oreta rosea Wlk. (Drepanidae), and Malacosoma sp. Also recorded from the tent caterpillar Malacosoma americana Harris, and the gipsy moth, Porthetria dispar L. Specimens in NYS were bred from the forest tent caterpillar, Malacosoma disstria Hbn.

150 See work cited under Rhyssini, page 938.

151 The North American ichneumon-flies of the tribes Lycorini, Polysphinctini, and Theroniini. By Robert A. Cushman. Proc. U. S. Nat. Mus., 1920, 58:7-480. 
T. melanocephala (Brullé). New Russia, Aug, Bdy (Mues-CU) ; Cranberry L., Aug, Htly (Cush-NYS Col. For.); Manlius, J1-Sep, SmHH (Cress-CU); Rock City, Je (Mues-CU) ; Norton's Landing, Cayuga L., May-Je, SmHH (Cress-CU) ; Ithaca, May-J1 (Mues, Cush-CU); McLean, Aug 20, Sep 4, Sib (Bdy-CU) ; Nyack (?Brues-AM); Ft. Montgomery, Sep (Cush-Schott) ; LI: Flatbush (?Brues-AM). Recorded as reared from the gipsy moth, Porthetria dispar L., the silver-spotted skipper, Epargyreus tityrus Fab., the spotted tussock caterpillar, Halisidota maculata Harr., and the hickory tussock caterpillar, H. caryae Harris.

\section{Tribe EPHIALTINI 152}

\section{(Pimplini auctt.)}

(The species of this tribe are primary parasites within the pupae of Lepidoptera.)

EPHIALTES Schrank

( $P$ impla auctt., in part)

\section{(Subgenus Ephialtes Schrank)}

E. aequalis (Prov.). Newport, Je, Yg (NYS) ; Cranberry L., Je, Aug, Htly (Cush-NYS Col. For.); Labrador L., May (Cush-CU) ; Manlius, J1-Sep, SmHH (Cress-CU); N. Fair Haven, Sep (Baird-CU); Oswego, Aug (Cush-US) ; Rochester Jct., Je, Leon (Baird-CU) ; Rock City, Je (Cush-US) ; Norton's Landing, Cayuga L., May, Je, SmHH (Cress-CU); Ithaca, Sep (Cush, Bdy, Baird-US, CU) ; Taughannock, May (Baird \& Bdy-CU) ; Ringwood, J1 (Cush-US); Caroline, Je (Baird-CU); McLean Bogs, May, Sep 3 (Cush, Bdy-US, CU); Spring Lake, J1, Bdy (Cush-US) ; Middletown, J1, Spooner (Cush-US); Trenton Falls, Je (Cush-NYS); Albany, Je, Yg (NYS) ; Poughkeepsie, Je (Cush-NYS); SI, Bird (Ashm-Ds). A common parasite of the codling moth (Carpocapsa pomonella L.), and recorded from a great variety of other hosts among both macrolepidoptera and microlepidoptera.

E. aquilonia (Cress.). Ithaca, J1 (Cush-CU); Albany, J1 (Cush-US).

E. pedalis (Cress.). New Russia, Aug, Bdy (Baird-CU) ; Potsdam, J1 (BairdCU) ; Manlius, Sep, SmHH (Cress-CU); Oswego, Sep (Cush-US) ; Utica, Pergande (Cush-US); Honeoye Falls (Cush-CU); Otto, J1, Comstock (Baird-CU); Ithaca, Ap-Sep (Cush, Bdy-US, CU); McLean Bogs, Spring Lake, Taughannock, Je-Aug (Baird, Bdy-CU); Glens Falls, Albany (NYS); New Baltimore, Zab (?Brues-AM); SI (Ashm-Ds); LI: Flatbush, Zab (?Brues-AM) ; Fishers I. (?Brues-AM); Melville, May (Cush-Schott) ; Riverhead, Sep (Baird-CU). A common parasite of the tent caterpillars (Malacosoma spp.), the gipsy moth (Porthetria dispar L.), the brown-tailed moth (Euproctis chrysorrhoea L.), and many other Lepidoptera.

E. tenuicornis (Cress.). Newport, $1200 \mathrm{ft}$., May-Je (Cush-NYS) ; Cranberry L., Je, Aug, Htly (Cush-NYS Col. For.); Potsdam, J1 (Baird-CU) ; Axton, Je, $M \mathcal{E} H$ (Ashm-CU) ; Utica, Pergande (Cush-US); Oswego, Jl (Cush-US); N. Fair Haven, Sep (Baird-CU); Stony I., J1 (Cush-US); Olean, Sep (Baird-CU); Norton's Landing, Cayuga L., J1, SmHH (Cress-CU); Mud Cr., Je, MacG\&Bdy (Bdy-CU); McLean Bogs, May-Sep 16 (Baird, Bdy, Maidl-CU); Seneca Jct., Je (Baird-CU); Spring Lake, J1 (Baird-CU);

${ }^{152}$ The North American ichneumon-fies of the tribe Ephialtini. By Robert A. Cushman. Proc. U. S. Nat. Mus., 1920, 58:327-362. 
Ithaca, J1 (Cush-NYS) ; Albany, Je, Sep (Cush-NYS). Recorded as a parasite of the gipsy moth (Porthetria dispar L.) and Alcathoe caudata Harr. (Aegeriidae).

\section{(Subgenus ITOPLECTIS Foerster)}

E. conquisitor (Say). Peru, Je, $M \mathcal{E} E$ (Baird, Gahan-CU) ; Rochester Jct., Je, Leon (Baird-CU); Waterville (Baird-CU); Manlius, Sep-Oct, SmHH (Cress-CU) ; N. Fair Haven, Sep (Baird-CU) ; Portageville, Je (Baird-CU) ; Rock City, Je (Baird-CU) ; Chipmunk Swamp, Je (Baird-CU); L. Keuka, Je, $C y$ (Baird-CU) ; Ithaca, May-Sep (Bdy-CU), from Malacosoma americana $A L L i u$; Malloryville, Je, $M a c G$ (Bdy-CU) ; Slaterville to Caroline, and Caroline to Harford, Je, MacG (Bdy-CU); Freeville, McLean, Spring L., J1 (Baird-CU) ; Bainbridge, J1 (Cush-NYS) ; Albany, Je, $Y g$ (NYS) ; Slingerlands (Felt); SI: W. Brighton (Lint); LI (US). Throughout the State. Parasitic on pupae of many Lepidoptera. One of the most common of ichneumonids.

E. leavitti Cush. Richfield Sps., Oct 14, 1888, Perg (Cush-US).

\section{Aреснтнis Foerster}

A. picticornis (Cress.). Cranberry L., Aug, Htly (Cush-NYS Col. For.) ; Ithaca, Yuasa (Bdy-CU); McLean, Aug 20-31, Sib, SRobinson (Bdy-CU).

\section{Tribe POLYSPHINCTINI 153}

(The species of this tribe are externally parasitic on spiders.)

Clistopyga Gravenhorst

C. canadensis Prov. Oswego, Aug (Cush-US).

\section{POLYSPHINCTA Gravenhorst}

\section{(Subgenus PoLysphinctiA Gravenhorst)}

P. burgessi Cress. Cranberry L., Aug, Htly (Cush-NYS Col. For.); Oswego, Je (Cush-US) ; Ithaca, Aug (CU).

P. koebelei How. Ithaca, J1 (Maidl-CU); Ellis to Slaterville, Je, MacGE Bdy (Cush-CU). The type of this species was reared from a spider of the genus Epeira (strix or sclopetaria).

P. slossonae Davis. Cranberry L., Aug, Htly (Cush-NYS Col. For.); Chapel Pond, Je (Cush-NYS Col. For).

P. strigis How. Oswego (Cush-US); Ithaca, Je (Cush-CU); McLean, Aug 26, SRobinson (Cush-CU); LI: Sea Cliff (How-US), type, on Epeira strix.

P. texana Cress. Ithaca, J1 (Cush-US) ; McLean, Aug 24, 1925, SRobinson (Bdy$\mathrm{CU})$. Recorded as a parasite of Steatoda borealis.

\section{(Subgenus ZAтуротA Foerster)}

P. crosbyi Cush. Oswego, J1 (holotype-US); McLean, Nov, Cy (allotype, CushUS).

?P. granulosa Davis. Ithaca, J1 (Cush-CU).

P. nigrita Walsh. SI (Ashm).

P. parva (Cress.). Bolton (Cush-US).

\footnotetext{
${ }^{153}$ See work cited under Theroniini, page 935.
} 


\section{Tribe LABENINI 155}

\section{LABENA Cresson}

(The species of this genus are parasitic on the larvae of beetles of the families Buprestidae and Cerambycidae.)

L. apicalis Cress. Ithaca, Je (NYS), bred from Chrysobothris femorata (Oliv.). Specimens in the U.S. Nat. Mus. are from Saperda puncticollis Say, Stenosphemus notatus (Oliv.), Psendibidion unicolor (Rand), and Agrilus sp. (all Cerambycidae).

L. confusa Roh. Ithaca, J1 31 (Cush-CU). Recorded by Rohwer as a parasite of Thrincopyge alacris Lec. (Buprestidae), and Chion cinctus (Drury) (Cerambycidae).

L. grallator (Say). Ithaca, Sep (NYS); LI (Vier). Recorded as parasite of Chrysobothris femorata (Oliv.) (Buprestidae) and Chion cinctus (Drury) (Cerambycidae).

\section{GroteA Cresson}

G. anguina Cress. Indian Ladder, J1 (Cush-CU) ; Ithaca, Je (Cush-CU) ; Nyack, $\mathrm{Zab}$ (?Brues-AM); West Farms, New Baltimore (?Brues-AM).

\section{Tribe RHYSSINI 155}

\section{RHysSA Gravenhorst}

(The species of this genus are parasitic on horntails of the genera Xeris and Sirex.)

R. lineolata (Kby.) (albomaculata Cress.). New Russia, Aug, Bdy (Cush-CU); Mt. Whiteface, 2000-4000 ft., Aug (Cush-CU); Erie Co, Blackburn (Roh); Ithaca, May-Sep (Cush-CU). Recorded by Rohwer as parasitic on Xeris sp.

R. persuasoria (L.). Saranac Inn, J1 (NYS) ; Gloversville, Sep (NYS) ; Oswego, Je (Roh-US); Port Byron, Je (Cush-CU); Castile, EABradley (Bdy); Ithaca, Yuasa (Bdy-CU). Recorded by Rohwer as parasitic on Xeris sp. and Sirex sp. Records associating this species with Coleoptera as hosts are probably erroneous.

\section{RHYSSELLA Rohwer}

(The species of this genus are parasitic on wood wasps of the genus Xiphydria.)

R. humida (Say). Ithaca, May, Aug (Cush-CU) ; Ft. Montgomery, Sep (CushSchott). Recorded by Rohwer as reared from Xiphydria attenuata Norton, $X$. abdominalis Say, and $X$. erythrogaster Ashm.

R. nitida (Cress.). New Russia, Aug, Bdy (Cush-CU) ; Mt. Whiteface, 2000-4000 ft., Aug (Cush-CU); Castile, EABradley (Bdy); Ithaca, May-Aug (CushCU); Taughannock, J1 (Cush-CU) ; McLean, May-Aug (Cush-CU); Caroline to Harford, Je, MacG\&Bdy (Cush-CU). A common parasite of the larva of Xiphydria maculata Say (Rohwer).

$15 \overline{5}$ The North American ichneumon-flies of the tribes Labenini, Rhyssini, Xoridini, Odontomerini, and Phytodietini. By S. A. Rohwer. Proc. U. S. Nat. Mus., 1920, 57:405-474. 


\section{MEgarhyssa Ashmead}

(The species of this genus are parasitic on horntails of the genus Tremex.)

M. atrata (Fab.). Axton, Je, $M \& H$ (Ashm-CU) ; Raquette R. (Lint) ; Ithaca, Je-J1 (CU) ; Spencer, Je (Cush-CU) ; Taughannock, May, Bdy (Cush-CU) ; Trenton Falls, Leon,Fbs (Cush-CU); Livingston (Clarkson); Albany, Je (LintNYS) ; Menands, J1, Mattimore (Lint-NYS) ; SI (Ashm); LI (Roh-US). Common probably everywhere in the State.

M. greenei Vier. Cranberry L., J1, Htly (Cush-NYS Col. For.) ; Ithaca, Je, Aug (Cush-CU) ; Ft. Montgomery, Je (Cush-Schott). Parasitic on larva of Tremex columba (L.).

M. lunator (Fab.). Wilmington, Aug, 1000-2000 ft. (Cush-CU) ; Rochester Jct., Je, Leon (Cush-CU); Castile, EABradley (Bdy); Portageville, Je (Cush$\mathrm{CU})$; Schuyler Co, Aug, Burner (Roh-US) ; Meridian, Williams (LintNYS) ; Taughannock, May (Cush-CU) ; Ithaca, May-Aug (Cush-CU) ; Elmira, Sep, Diven (Cush-CU); Albany, Sep (Lint); Loudonville (Felt); Livingston (Clarkson); Port Chester, J1, Sample (Roh-US); SI (Ashm). Common probably everywhere in the State. Parasitic on larva of Tremex columba (L.).

\section{Tribe ACOENITINI 156}

\section{COLEOCEnTRUS Gravenhorst}

(Very little is known of the host relations of this genus, but the species are probably parasitic on the larvae of wood-boring Coleoptera.)

C. harringtoni Cush. Ithaca, J1 (Cush-CU) ; LI: Rockaway (AM).

C. pettitii Cress. Cranberry L., Je, Htly (Cush-NYS Col. For.).

C. rufus Prov. Johnstown, Ap, Poughkeepsie, Je, Yg (NYS).

C. similis Cush. Cranberry L., Je, Gray (Cush-CU); Buck I., Je, Htly (Cush-US) ; Ithaca, May-Je (Cush-CU); Ft. Montgomery, Je, Schott (Cush-CU).

\section{AROTES Gravenhorst}

A. amoenus Cress. Mt. Whiteface, 4872 ft., J1, Ald (Cush-US) ; Cranberry L., Je, Htly (Cush-NYS Col. For.) ; Labrador L., Je (Cush-CU) ; Batavia, Je (Cush-CU) ; Ithaca, Je (Mues-CU) ; Caroline to Harford, Je, MacGEBdy (Mues-CU); Trenton Falls, Je, Leon \&Fbs (Cush-CU) ; Ft. Montgomery, Je, Schott (Cush-US) ; LI: E. Hampton, Je (Mues-CU) ; Gardiners I., Je (Schott).

A. decorus (Say). Albany, Aug (NYS); Poughkeepsie, Je (NYS); Bronx Pk. (Ashm). Recorded as a parasite of Xylotrechus colonus (Fab.) (Cerambycidae), and Tomoxia bidentata (Say) (Mordellidae).

A. occiputalis Cress. (apicatus Ds.). LI: Cold Sp. Harb., J1 (Cush-CU). Recorded as a parasite of Ophistomis bicolor (Swed.).

A. vicinus Cress. New Russia, Aug, $B d y$ (Mues-CU) ; Long L., Aug (NYS) ; Ithaca, J1 (NYS). Recorded as a parasite of Strangalepta vittata (Oliv.), Brachyleptura rubrica (Say), and B. vagans (Oliv.) (all Cerambycidae).

${ }^{158}$ The North American ichneumon-flies of the tribe Acoenitini.

S. A. Rohwer. Proc. U.S. Nat. Mus., 1920, 57: 503-523.

By Robert A. Cushman and 


\section{Tribe XORIDINI 157}

(The members of this tribe are parasitic on the larvae of wood-boring Coleoptera.)

XORIDES Latreille

X. albopictus (Cress.). Cranberry L., Je, Aug, Htly (Cush-NYS Col. For.); Poughkeepsie, May-Je, $Y g$ (NYS). Recorded as parasitic on the cerambycid Saperda discoidea Fab.

X. frigidus (Cress.). Big Moose, J1, Yg (NYS); Keene Val., Aug (CU).

$\mathbf{X}$. humeralis (Say). Keene Val., Aug (Cush-CU); Ithaca, Je, Aug (Cush-CU); Utica, Pergande (Cush-US). Recorded as parasitic on the longicorn Phymatodes varius (Fab.).

X. stigmapterus (Say). New Russia, Aug, Bdy (Mues-CU); Cranberry L., Je, Drk (Cush) ; Wilmurt, Je, Comstock (Cush-CU) ; Spencer L., Je, Rly \&MacG (Cush-CU) ; Norton's Landing, Cayuga L., Je, SmHH (Cress-CU); Ithaca, $\mathrm{Je}$ (Cush-CU). Recorded as a parasite of the longicorn Leptura proxima Say.

\section{DEUTEROXORIDES Viereck}

D. borealis (Cress.). Malone, Je (Cush-CU). Recorded as a parasite of the longicorn beetles Atimia dorsalis Lec., Anacomis lignea (Fab.), and Tetropium velutinum Lec.

D. caryae (Harr.). Syracuse, Blkm (Cush-US). Recorded as a parasite of the longicorn borer Saperda discoidea Fab., and, according to Rohwer, probably confined to this host.

D. vittifrons (Cress.). Cranberry L., J1, Htly (Cush-US); Johnstown, Aug (NYS) ; Norton's Landing, Cayuga L., J1, SmHH (Cress-CU). Recorded as a parasite of the longicorn beetle Urographis fasciata (DeG.).

\section{PoEMENIA Holmgren}

P. albipes (Cress.). Cranberry L., Aug, Htly (Cush-US); Oswego, Je (Roh-US); Honeoye Falls, Aug, Leon (Cush-CU); Ithaca, Je-Aug (Cush-CU, NYS).

P. americana (Cress.). Ithaca, J1 (Cush-CU). Recorded as a parasite of Paratimia conicola Fisher and Laspeyresia toreuta Grt.; according to Rohwer the latter record may be an error.

\section{Tribe ODONTOMERINI 157}

(The members of this tribe are probably always parasitic on the larvae of woodboring Coleoptera.)

\section{ODONTOMERUS Gravenhorst}

O. abdominalis Cress. Norton's Landing, Cayuga L., SmHH (Cress-CU); Ithaca, Yuasa (Bdy-CU).

O. albotibialis Bdy. Ithaca, J1, Yuasa (Bdy-CU).

O. atripes Roh. Ithaca to Ellis, Je (Cush-CU); L. George, Zab (?Brues-AM); Utica, Pergande (Cush-US).

O. bicolor Cress. Elizabethtown, J1, Yg (NYS); Big Moose, Aug, Yg (NYS).

${ }^{157}$ See work cited under Rhyssini, page 938. 
O. canadensis Prov. Rock City, Je (Cush-CU); Ithaca, May, Je (Cush-CU); Freeville, J1 (Cush-CU); McLean, Aug .31, 1925, SRobinson (Bdy-CU); L. George, Jl, Fisher (Cush-US) ; Black Mt., L. George, Sep 4, Leon (BdyCU). Recorded by Rohwer as parasitic on Dicerca divaricata (Say), D. sp. (Buprestidae), Leptura sp. (Cerambycidae), and Serropalpus sp. (Melandryidae).

O. dichrous Roh. McLean Bogs, May, Sep 14, Sib (Cush, Bdy-CU).

O. mellipes (Say). Rock City, Ringwood (Bdy-CU) ; Ithaca, J1-Sep (Cush-CU); McLean, Aug 8, Sib (Bdy-CU); Tioga Co (Bdy-CU); SI (Viereck); LI: Flatbush, J1, Zab (Roh-US). Recorded as a parasite of Parandra brunnea (Fab.) (Cerambycidae).

O. strangaliae Roh. Oswego, J1 (Roh-US). The type was reared from Ophistomis luteicornis (Fab.) (Cerambycidae).

O. vicinus Cress. Ithaca, Je (Bdy-CU); McLean, May 31-Sep 21 (Cush, Bdy-CU).

\section{Tribe ICHNEUMONINI}

(Pimplini auctt.)

\section{ICHNEUMON Linnaeus}

(Ephialtes auctt.)

\section{(Subgenus ICHNEUMON Linnaeus)}

(The species of this subgenus are usually, if not always, parasitic on wood-boring or twig-boring Coleoptera.)

I. irritator Fab. Rock City, Je (Cush-CU); Ithaca, May-Aug (Bdy, Cush-CU); Coy Glen, May (Cush-CU) ; McLean, May, Je 23, Sib (Cush, Bdy-CU); Albany, May (NYS) ; LI: W. Bergen, Juelich (Cress) ; Wyandanch, May, (Cush-Schott). Specimens in the U.S. Nat. Mus. were reared from the longicorn Obrium rufulum Gahan. Also recorded as a parasite of Leiopus variegatus (Hald.) (Cerambycidae), and Cryptorhynchus lapathi (Lec.) (Curculionidae).

I. macer (Cress.). New Russia, Aug, Bdy (Cush-CU).

I. occidentalis (Cress.). Cranberry L., J1, Htly (Cush-NYS Col. For.) ; Ithaca, J1 (Cush-US) ; Johnstown, May (NYS) ; Albany, J1 (NYS).

I. pusio (Walsh). New Russia, Aug, Bdy (Cush-CU). A specimen in the U.S. Nat. Mus. is said to have been reared as a parasite of Xiphydria in maple, which, however, was infested also by a coleopterous larva.

\section{(Subgenus Calliephialtes Ashmead)}

(The species of this subgenus are parasitic on lepidopterous and coleopterous larvae living in twigs, weed stems, etc.)

I. comstockii (Cress.). Ithaca, Comst (holotype-US) from Retinia comstockiana, Aug (Cush-CU) from Pinipestis zimmermani; Port Chester, May (Cush-US). Specimens in the U.S. Nat. Mus. were reared as parasites of Rhyacionia taxifoliella Busck, $R$. buoliana, and $R$. frustrana Comst. (Tortricidae).

I. grapholithae (Cress.). Ithaca, Je (Cush-CU). Recorded as a parasite of Laspeyresia caryana Fitch (Tortricidae), Acrobasis hebescella Hulst (Pyralidae), and the sawfly Eunra salicis-nodus Walsh. 
I. notandus (Cress.). Ithaca, Ap-May (Baird-CU); West Farms, Angus (Cush$\mathrm{CU})$; LI (Cush-US). A common parasite of the goldenrod gall-maker Gnorimoschema gallaesolidaginis Rly. and other similar gall-making lepidopterous larvae. The U.S. Nat. Mus. has specimens from the above-named host and from G. gallaeastericlla Kell., G. baccharisella Busck, and Walshia amorphella Clem. (Lavernidae).

\section{ScAmbus Hartig}

\section{(Pimpla auctt., in part)}

(The species of this genus are parasitic on lepidopterous, and, rarely, coleopterous, larvae.)

\section{(Subgenus ISEROPUS Foerster)}

S. coelebs (Walsh). New Russia, Aug, Bdy (Baird-CU) ; Potsdam, Je (Baird$\mathrm{CU})$; Speculator, Aug (Cush-NYS) ; Batavia, Mar (Cush-CU) ; Manlius, Aug-Sep, SmHH (Cress-CU) ; Fredonia, J1 (Baird-CU); Spring Lake, J1 (Baird-CU) ; Ithaca, Mar-J1 (Baird, Maidl-CU) ; Enfield, J1 (Maidl-CU); Ringwood, Aug (Maidl-CU); Motts Corners, Aug (Baird-CU); Labrador L., May (Maidl-CU) ; Freeville, Jl (Baird-CU) ; Norton's Landing, Cayuga L., J1, SmHH (Cress-CU); McLean, Aug 3-31, SRobinson (Bdy-CU); Slingerlands, Albany, Karner, Gloversville, Je-Aug (NYS) ; NYC, Sep, Cy (Baird-CU) ; LI: Flatbush, Sep, Zab (Cush-US); Melville, May (CushSchott). An abundant parasite of Orgyia, Malacosoma, Thyridopteryx, etc., its larvae feeding gregariously on prepupae of the host.

\section{(Subgenus EPIURUS Foerster)}

S. alborictus (Cress.) (investigatrix Walsh). Adirondacks, Aug (Baird-CU); Cranberry L., J1, Htly (Cush-NYS Col. For.) ; Ithaca, Je-Nov (Cush, BairdCU) ; Spring Lake, J1 (Baird-CU) ; Albany, Je (Cush-NYS). Parasitic on leaf-rolling lepidopterous larvae such as the apple leaf-roller, Archips argyrospila Wlk. Specimens in the U.S. Nat. Mus. are from Eulia pinitubana, Episimus argutanus $\mathrm{Cl}$. (both Tortricidae), and Zotheca tranquilla Grt. (Noctuidae).

S. indagator (Cress.). Ithaca, Ap-Aug (Cush-CU, US) ; Spring Lake, J1 (BairdCU) ; Van Cort. Pk., Jl (Baird-CU). Parasitic on larvae of many internallyfeeding microlepidoptera. The U.S. Nat. Mus. has specimens reared from the grape berry moth (Polychrosis viteana Clem.), Simaethis pariana (Glyphipterygidae), a leaf-roller on box-elder, Acrobasis indiginella Zell., Acrobasis caryella (Pyralidae), Dichomeris ligulella Hbn. (Gelechiidae), the codling moth (Carpocapsa pomonella L.), Coleophora sp. on wild cherry, Eulia pinitubana (Tortricidae), and Phycis juglandis S. \& A. (Pyralidae).

S. nigrifrons Vier. Albany, J1, Aug (Cush-NYS). On the white-marked tussock caterpillar, Hemerocampa leucostigma S. \& A.

S. pterophori (Ashm.) (pterelas auctt.). L. Pleasant, Je (NYS) ; Gloversville, Aug (NYS); Oswego, May (Cush-US, NYS); Buffalo, EPV (GCDavis); Rochester Jct., J1, Leon (Baird-CU); Honeoye Falls, N. Fair Haven, Sep (Baird-CU); Clifton Sps., Aug, Cy (Baird-CU); Perry, Aug, Emerson (Cush-CU) ; Spring Lake, J1 (Baird-CU) ; Norton's Landing, Cayuga L., May-Je, SmHH (Cress-CU); Ithaca, Mar-Aug (Baird, Cush-CU, NYS); Mud Cr., J1, MacG\&Bdy (Baird-CU); McLean Bogs, May (Cush-CU); 
Poughkeepsie, May (Cush-NYS); LI (Cush-US). Parasitic on larvac of stem-boring and stem-gall-making Lepidoptera and Coleoptera such as Gnorimoschema gallaesolidaginis (Rly.), G. gallaeasteriella Kell., the European corn-borer (Pyrausta nubilalis Hueb.), Stagmatophora ceanothiella Cosens, Lophoptilus eloisella Clem. (Lavernidae), Eucosma scudderiana Clem. (Tortricidae), and the weevil Mononychus vulpeculus (Fab.).

S. tecumseh Vier. McLean, Aug 26, 1925, SRobinson (Cush-CU).

\section{TROMATOBIA Foerster}

(The species of this genus parasitize the egg cocoons of spiders.)

T. rufopectus (Cress.) (scriptifrons Cress.). Cranberry L., Je-Aug, Htly (CushNYS Col. For.); Ithaca, May-Aug (Bdy, Baird-CU); McLean Bogs, May (Baird-CU); Poughkeepsie, Je, $Y g$ (NYS). A common parasite in the egg cocoons of the spiders of the genus Argiope. It was probably this species that Clarkson (Can. Ent., 1890, p. 122) recorded from NY under the name Ichneumon aranearum Ratz.

\section{ZAGLYPTUS Foerster}

(The species of Zaglyptus are parasitic within the egg cocoons of spiders.)

Z. incompletus (Cress.). Saranac L., Olean, Ithaca, Spring Lake, J1-Sep (CushCU) ; McLean, Aug 30, 1925, SRobinson (Cush-CU). In the U.S. Nat. Mus. there is a series of specimens from Dallas, Texas, reared from the egg cocoon of a spider.

\section{DELOMERISTA Foerster}

(The species of this genus are parasitic within the cocoons of sawflies.)

D. novita (Cress.). Cranberry L., Je, Htly (Cush-US). There is no authentic published record of the hosts of this species. Such records as are published should be credited to one or another of the species of Epiurus.

\section{PERIThous Holmgren}

(The species of Perithous are parasitic in the nests of solitary wasps.)

P. pleuralis Cress. Ithaca, Je-Aug (Cush-CU, NYS); Freeville, J1 (Cush-CU); Slaterville to Caroline, Je, MacG\&Bdy (Cush-CU); Taughannock, May (Cush-CU) ; Albany, Sep (NYS); Nyack, Aug, Zab (Cush-US). Specimens in the U.S. Nat. Mus. were reared from the nests of the wasp Pemphredon concolor Say, while others were reared from a sassafras stem containing the nest of a bee or a wasp.

\section{SUBFAMILY TRYPHONINAE 159}

With a few exceptions the members of the tribes Catoglyptini, Tryphonini, and Cteniscini are parasitic on the larvae of sawflies. The members of the other tribes show a wide diversity in host relations.

159 The classification adopted for this subfamily is that of Thomson as elaborated by Schmiedeknecht in his Opuscula Ichneumonologica, with some slight modifications and the addition of the tribe Paniscini. 


\section{Tribe CATOGLYPTINI}

\section{(Mesoleptini)}

\section{(Subtribe EUCEROSINA)}

(Eumesiina Thompson)

EUCERos Gravenhorst

E. canadensis Cress. Cranberry L., Je, Htly (Cush-NYS Col. For.) ; Ithaca, Je (Cush-CU) ; McLean Bogs, May (Cush-CU).

E. couperi Cress. Rock City, Je (Cush-CU); McLean Bogs, May (Cush-CU).

E. flavescens Cress. Ithaca, Aug (NYS); Bear Mt., J1 (Cush-Schott).

E. frigidus Cress. Cranberry L., Je, Htly (Cush-US).

E. medialis Cress. Norton's Landing, Cayuga L., Je, SmHH (Cress-CU); Ithaca, Je-J1 (Cush-CU) ; Caroline to Harford, Je, MacG $\mathcal{B} B d y$ (Cush-CU); McLean, Je 30 (Bdy-CU).

E. thoracicus Cress. McLean Bogs, May (Cush-CU); Ithaca, Je (Cush-CU).

\section{(Subtribe THYMARIDINA)}

\section{NeLiopisthus Thomson}

N. densatus (Say). Rock City, Je (Cush-CU, US); McLean, Aug 1-18, SRobinson (Cush-CU).

N. luggeri (Ashm.). LI: Melville, May, Schott (Cush-US). Recorded as a parasite of a tineid larva on Comptonia.

HyвOPHANES Foerster

H. nasutus (Cress.). L. Placid (Cush).

ZAGRYPHUS Cushman

Z. nasutus (Cress.). "NY" (Ashm).

\section{(Subtribe OXYTORINA)}

Callidiotes Foerster

C. albopleuralis (Prov.). Ithaca, J1 (Cush-US).

\section{(Subtribe PERILISSINA)}

(The members of this subtribe are parasitic on sawfly larvae. There are apparently no recorded hosts of American species.)

ECLYTUS Holmgren

E. perennis Davis. "NY" (GCDavis).

E. pleuralis (Prov.). Cranberry L., Je, Htly (Cush-US), from larva on yellow birch; Waterville, May, Hawol (Cush-CU) ; Ithaca, Ap-J1 (Cush-CU) ; Van Cort. Pk., J1 (Cush-CU). 


\section{SPANOTECNUS Foerster}

S. bicolor (Cress.). Ithaca (Ashm-US), type of Prionopoda ruficornis; Poughkeepsie, Je (NYS).

S. concolor (Cress.). Waterville (Cush-CU); Johnstown, May, Yg (NYS); Oswego, May; Lancaster, Je, EPV (Vier-US); Rochester Jct., Je, Leon (Cush-CU) ; Rock City, Je (Cush-CU); Pike, Je, Yg (NYS); Norton's Landing, Cayuga L., May-Je, SmHH (Ćress-CU); Ithaca, May-Je (CushCU); McLean, May-Je (Cush-CU); Spencer L., Je (Cush-CU).

S. decoloratus (Cress.) (Perilissus southrvicki Ashm.). Ithaca, J1 (Cush-CU); Central Pk., NYC, Southwick (Ashm), type of Perilissus southwicki.

S. discolor (Cress.). McLean, Je 18, 1924, Sib (Bdy-CU).

S. iladus Davis. "NY" (Ds).

TREMATOPYGUS Holmgren

T. semirufus (Cress.). Norton's Landing, Je, SmHH (Cress-CU); Ithaca, May (Maidl-CU).

\section{(Subtribe CATOGLYPTINA)}

STIPHROSOMUS Foerster

S. fucatus (Cress.). Saranac Inn, J1 (NYS).

Provancherella Dalla Torre

P. rhopalocera (Prov.). "NY" (Ds).

\section{(Subtribe NOTOPYGINA)}

CTENopelma Holmgren

C. sanguineum (Prov.). Oswego, Je (Cush-US).

Polycinetis Foerster

P. limata (Cress.). "NY" (GCDavis).

Notopygus Holmgren

N. cymbaformus (Davis). Geneva, Je (Cush-US).

HomaSPIS Foerster

H. albipes Davis. "NY" (type-Amer. Ent. Soc.).

\section{(Subtribe HADRODACTYLINA)}

(Mesoleptina Thomson)

(The members of this subtribe are parasites on the larvae of sawflies.)

Mesoleptidea Viereck

M. decens (Cress.). Rock City, J1 (Cush-CU); Norton's Landing, Je, SmHH (Cress-CU); Caroline, Ithaca, Je (Cush-CU); Delmar, Albany, May, Je (NYS).

M. zebrata (Davis). "NY" (type-Am. Ent. Soc.). 
HADRODACTYLUS Foerster

H. elongatus (Cress.). Albany, Nassau, May-Je, EPV (NYS).

H. elongatus var. coxatus Davis. "NY" (type-Am. Ent. Soc.).

H. elongatus var. femoratus Davis. McLean, J1 1, 1924, Sib (Cush-CU).

\section{(Subtribe EURYPROCTINA)}

\section{EURYPROCTUS Holmgren}

E. moyeni (Prov.). Saranac Inn, J1 (NYS); Poughkeepsie, May (NYS).

E. petiolatus Davis. Ithaca, Chitt (Cush-US).

\section{DIALGES Foerster}

D. frontalis Davis. Oswego, Je (Cush-US); Rock City, Je (Cush-CU); Ithaca, May-Je (Cush-US).

\section{POLYTERUS Foerster}

P. olympiae (Ashm.). Ithaca, Aug, Taughannock, J1 (Cush-CU). A series of specimens in the U.S. Nat. Mus. were reared as parasites of the sawfly Pteronidea ventralis (Say).

\section{(Subtribe MESOLEIINA)}

\section{Opheltes Holmgren}

O. glaucopterus var. flavipennis (Prov.). Gloversville, Aug (NYS); Ithaca, J1Aug (CU) ; McLean, Aug 20, SRobinson (Bdy-CU) ; Richfield Sps., Oct, Pergande (Cush-US) ; Karner, Aug; West Farms, Angus (AM); LI (CushUS). Parasite of the elm sawfly, Cimbex.

\section{ABSYRTUS Holmgren}

A. paniscoides (Ashm.). Central Pk., NYC (type-US).

\section{Alexeter Foerster}

A. albotarsatus (Prov.). Ithaca, Je-Sep (Ashm-CU).

A. canaliculatus (Prov.). Spring Lake, I1, Bdy (Cush, Maidl-CU); Norton's Landing, Je, SmHH (Cush, Maidl-CU); Ithaca, J1 (Bdy-CU); Ellis to Slaterville, Je, $M a c G, B d y$ (Cush-CU).

A. honestus (Cress.). "NY" (GCDavis); Rensselaer, J1 (NYS).

A. riparius (Davis) (?Mesoleptus). McLean, Je 30, 1921 (Cush-CU).

\section{Mesoleius Holmgren}

M. conformus Davis. "NY" (GCDavis).

M. convergens Davis. Rock City, Je (Cush-CU); McLean, May (Cush-CU).

M. mellipes (Prov.). Oswego, J1 (Cush-US); McLean, Aug 1, 1925, SRobinson (Cush-CU).

M. pulchronotus Davis. Portageville, Je (Cush-CU). 
M. submarginatus (Cress.). Adirondacks, Aug (Cush-CU) ; Rock City, Je (CushCU) ; Norton's Landing, Cayuga L., Je, SmHH (Cress-CU); Ithaca, May-Je (Cush-CU); McLean, Aug 27, 1925, SRobinson (Cush-CU).

SPHECOPHAGA Westwood

S. burra (Cress.). Cranberry L., J1, Htly (Cush-US) ; Bolton (US) ; New Baltimore, Zab (GCDavis); Central Pk., NYC, Ap, Zab (AM). Parasitic in nests of Vespa.

\section{Tribe TRYPHONINI \\ (Subtribe TRYPHONINA) \\ (Including Polyblastina Schmiedeknecht) \\ SyNOECETES Foerster}

S. sedulus (Cress.). "NY" (Davis).

Cosmoconus Foerster

C. canadensis (Prov.). Saranac Inn, Je, $Y g$ (NYS).

\section{OTLOPHORUS Foerster}

O. innumerabilis Davis var. feria Davis. Waterville, Mix Cr. Val., Je, Rock City, Je, Ithaca, May (all Cush-CU).

\section{EPAchthes Foerster}

E. basilicus Davis. Rock City, Je, Ithaca, May-Je (Cush-US).

\section{QUADRIGANA Davis}

Q. americana (Cress.). Ithaca, May, Van Cort. Pk., J1 (Cush-CU).

\section{TRYPHON Fallén}

T. communis Cress. Elizabethtown, J1 (NYS); Rock City, Mix Cr. Val., Je (Cush-CU) ; Norton's Landing, Cayuga L., Je, SmHH (Cress-CU); Ithaca, J1, Spencer L., Je (Cush-CU, US).

T. communis var. clypeatum Prov. Oswego, Je (Cush-US); Rock City, Mix Cr. Val., Je (Cush-CU); Ithaca to Ellis, Je, $M a c G, B d y$ (Cush-CU); Ithaca, May-Je (Cush-CU); Center Lisle, Je (Cush-CU).

T. seminiger Cress. Rock City, Je, Ithaca, Je-Jl (Cush-CU); Dryden L., Je, $M a c G \mathcal{E} B d y$ (Cush-CU) ; Slaterville to Caroline, Je, MacGE्EBdy (Cush-CU); Spencer L., Je (Cush-CU) ; Nyack, Zab (Cush-US); LI (Cush-US).

\section{Polyblastus Hartig}

P. pedalis (Cress.). Black Br., Je (Cush, Maidl-CU); Shelving Rock Br., L. George, Sep 2, Leon (Bdy-CU).

P. tibialis (Cress.). "NY" (Ashm). 
SCOPIORUS Foerster

S. analis (Cress.). McLean, Aug 31-Sep 20, Sib, SRobinson (Bdy-CU).

S. subcrassus (Cress.). Peru, Ithaca, Je (Cush-CU).

ERROMENUS Holmgren

E. dimidiatus (Cress.). L. Placid, Aug (NYS) ; Norton's Landing, Cayuga L., Je, SmHH (Cress-CU); Homer, Ithaca, McLean, Slaterville to Caroline, May-J1 (Cush-CU).

\section{Monoblastus Hartig}

M. varifrons (Cress.). McLean, May (Cush-CU).

(Subtribe SCOLOBATINA)

Scolobates Gravenhorst

S. auriculatus (Fab.). "NY" (Ashm.).

\section{Tribe CTENISCINI}

\section{MICROPLECTRON Foerster}

M. albilineatum (Walsh). Cranberry L., Je, Htly (Cush-US).

\section{Cteniscus Haliday}

C. clypeatus Cress. Peru, Je (Cush-CU). Determination queried.

C. consors Cress. Ithaca, Aug (Cush-CU), Determination queried.

C. flavicoxae Cress. W. Danby, Je, MacG (Cush-CU).

ANECPHYSIS Foerster

A. curvineura Davis. Ithaca, Je, Chitt (Cush-US, CU).

\section{EXYSTON Schiødte}

E. abdominalis Cress. var. rufinum Davis. "NY", cotype.

E. variatum Prov. "NY" (Davis).

\section{Tribe PANISCINI}

(The members of this tribe are externally parasitic on lepidopterous larvae. The egg is relatively large and is attached by a stalk to the skin of the host. The feeding larva uses the egg shell as a means of keeping its hold on the active host.)

\section{PANISCUS Schrank}

P. brevicornis Cush. "NY" (Cush-US).

P. geminatus (Say). Newport, May (NYS); Manlius, Sep, Norton's Landing, Cayuga L., Je, SmHH (Cress); Ithaca, common (Ashm-CU); Croton-onHudson (Felt); Poughkeepsie, J1 (NYS); Nyack, Zab; SI, May (AshmDs) ; II: Fishers I., Zab (Ds). 
P. geminatus var. sayi Cush. Oswego, Je, Ithaca, J1 (Cush-US).

P. leo Cush. Oswego, Ludlow (type-US); McLean, Aug 31, 1924, Sib (Cush-CU).

P. smithi Cush. Beaver Cr., J1 10, 1924, Sib (Cush-CU).

P. unguicularis Cush. Ithaca, J1 (Cush).

PARABATES Foerster

P. crassus Cush. Oswego, May (type-US).

P. latungula Thomson subsp. deceptor (Morl.) Bdy. Ithaca, Yuasa (Bdy-CU).

P. monticola Cush. Oswego, Oct (Cush-US).

\section{Tribe DIPLAZONINI}

\section{(Bassini Ashmead)}

(The members of this tribe are parasitic on syrphid flies. The egg, at least in some cases, is deposited in the egg of the host, the adult parasite emerging from the host puparium.)

\section{DIPLAZON Nees}

\section{(Bassus auctt.)}

D. concinnus (Cress.). Oswego, Je (Cush-US); Ithaca, May-Sep (Cush-CU).

D. laetatorius (Fab.). Cranberry L., Aug, Htly (Cush-NYS Col. For.); Oswego (US) ; Rochester Jct., J1, Leon (Cush-CU); Honeoye Falls, May (Cush-CU); Spring Lake, J1 (Cush-CU); Norton's Landing, Cayuga L., Je, SmHH (Cress-CU), det. as sycophanta Cr.; Ithaca, May-Sep, McLean Bogs, May 29 (Cush, Maidl-CU) ; Mud Cr., Slaterville to Caroline, Je, MacG \& Bdy (Cush$\mathrm{CU}$ ); Binghamton, Aug, Jackson (Cush-US); Albany, May, Yg (NYS). This is a cosmopolitan and very variable species. It has a long list of synonyms and named varieties. The latter serve no useful purpose, as they are not well defined but are merely steps in a gradual variation.

D. orbitalis (Cress.). "NY" (GCDavis).

D. pulchripes (Prov.). Cranberry L., J1, Htly (Cush-NYS Col. For.); Ithaca, May-Sep (Cush-CU).

D. scutellaris (Cress.). Manlius, SmHH (Cress-CU); Rock City, Ithaca, Je (Cush-CU).

\section{ENIZEMUM Foerster}

E. tibiale (Cress.). Poughkeepsie, May, Yg (NYS).

\section{HOMOTROPUS Foerster}

H. bicapillaris (Walsh). Olean, May, $Y g$ (NYS).

H. bicapillaris var. albopictus Davis. Cranberry L., Je, Htly (Cush-NYS Col. For.) ; Rock City, J1 (Cush-CU) ; Ithaca, J1-Aug (Cush-CU) ; McLean, Je 18, $\mathrm{Sib}$ (Bdy-CU).

H. decoratus (Cress.). Sport I., Sacandaga R., Je, Alex (Cush-CU).

H. humeralis (Prov.). Caroline to Harford, Je, MacG\&Bdy (Cush, Maidl-CU). 


\section{PROMEThES Foerster}

P. costalis (Prov.). Oswego, J1 (Cush-US); Mix Cr. Val., Je (Cush-CU); Spring Lake, J1, Bdy (Cush-CU) ; Ithaca, McLean, Taughannock, May-J1 (Cush-CU); Mud Cr., Ellis to Slaterville, Je, MacG\& $B d y$ (Cush-CU); Rhinebeck, J1, Cy (Cush-CU).

P. elongatus (Prov.). Newport, May (NYS); Ithaca, May, Dryden L., Je (Cush-CU).

P. ruficrus (Walsh). Sodus Pt., Aug (NYS); Dryden L., Je, Ellis to Slaterville, Je, MacG\& $\mathcal{E} B y$ (Cush-CU) ; Freeville, J1 (Cush-CU); Ithaca, May-Aug (Cush-CU, US).

\section{SYRPHOCTONUS Foerster}

S. agilis (Cress.). Oswego, Je (Cush-US); Ithaca, May-Jl, McLean, May, Van Cort. Pk., J1 (Cush-CU).

S. minimus (Cress.) (pacificus Cress.). Ithaca, May-J1 (Cush-CU, US); Mud Cr., Slaterville to Caroline, Je, MacG\& $\mathcal{B} B d y$ (Cush-CU); McLean, May (Cush-CU) ; W. Danby, Je, MacG (Cush-CU) ; LI (Cush-US).

S. pleuralis (Cress.). Ithaca, May (Cush-CU).

\section{ZOOTREPHES Foerster}

Z. antennatus Davis. Ellis to Slaterville, Dryden L., Je, MacG\&Bdy (Cush-CU) ; Ithaca, Mar-Sep (Cush-CU); Taughannock, May (Cush-CU).

Z. compressiventris (Cress.). Axton, Je, MacG\&H (Cush-CU) ; Cranberry L., Je, Htly (Cush, Maidl-NYS Col. For.); Waterville, Honeoye Falls, May, Ithaca, Ap-Sep (Cush-CU); Mud Cr., Je, MacGE $\mathcal{B} B d y$ (Cush-CU) ; McLean Bogs, May (Cush-CU) ; W. Danby, Je, MacG (Cush-CU); Stuyvesant Falls, May (Cush-CU).

\section{Tribe ORTHOCENTRINI}

Atmetus Foerster

A. tetrazonatus Ashm. Ithaca, Ap-Aug (Maidl-CU).

\section{DeLETER Foerster}

D. suffuscus Davis. Wilmington, Aug (Cush-CU).

D. undulatus Davis. Mt. Whiteface, 2000-4000 ft., Aug (Cush-CU); Ellis to Slaterville, Je (Cush-CU).

\section{TAPINOPS Foerster}

T. pusillus (Walsh). Mt. Whiteface, 2000-4000 ft., Aug (Cush-CU) ; L. Placid, Aug (Ashm-US); Gloversville, Mar (NYS); Oswego, J1 (Ashm-US); Ithaca, Mar (Maidl-CU); McLean, Aug 17-Sep 30, Sib, SRobinson (Bdy-CU).

\section{Tribe EXOCHINI}

(The members of this tribe are, so far as is known, parasitic in the pupae of Lepidoptera.) 


\section{Chorinaeus Holmgren}

C. carinatus Cress. Oswego, J1 (Roh-US).

C. cariniger (Walsh). Piseco L., Aug (NYS).

C. costatus Davis. Rock City, May (Cush-US); W. Danby, Je (Cush-CU).

C. onitis Davis. Rock City, Je (Cush-US); W. Danby, May (Cush-CU).

\section{Colpotrochia Holmgren}

C. trifasciata (Cress.). "NY" (GCDavis).

\section{Exochus Gravenhorst}

E. atriceps Walsh. Ellis to Slaterville, Je, MacG\&Bdy (Cush-CU).

E. dorsalis Cress. McLean, Sep 12, Sib (Bdy-CU) ; Pelham Bay Pk., Aug (Cush$\mathrm{CU})$.

E. dorsalis var. annulicrus Walsh. Cranberry L., Aug, Htly (Cush-NYS Col. For.) ; LI: Flatbush, May, Zab (Bdy-CU) ; Rockaway (Bdy-AM). Recorded as a parasite of Archips rileyana Gr. (Tortricidae).

E. pallipes Cress. Essex Co, J1 (NYS) ; Norton's Landing, Cayuga L., Je, SmHH (Cress-CU); McLean, Aug 12, SRobinson (Bdy-CU); Ithaca, May-J1, Van Cort. Pk., J1 (Cush-CU) ; LI: Flatbush, Je, Zab (Bdy-CU). A parasite of Archips rileyana $\mathrm{Gr}$. and $A$. cerasivorana Fitch (Tortricidae).

E. pleuralis Cress. Oswego, J1 (Ashm-US).

E. semirufus Cress. Norton's Landing, Cayuga L., Je, $\mathrm{SmHH}$ (Cress-CU) ; Ithaca, Sep (Cush-CU); Albany, May (NYS).

\section{Metacoelus Foerster}

M. laevis (Cress.). Manlius, Aug, SmHH (Cress-CU) ; Ithaca, Je, Aug (Cush, Ashm-CU); Albany (NYS); LI: Flatbush, Je, Zab (Cush, Bdy-CU).

M. mansuetor (Grav.). Ithaca, May-J1 (Ashm,Cush-CU,US). A cosmopolitan parasite of the clothes moth. Also recorded from Pyralis farinalis L.

\section{TRICLISTUS Foerster}

T. curvator (Fab.). Wilmington, Aug (Cush-CU) ; Ilion, Je, Yg (NYS) ; Oswego, Je (Cush-US) ; Spring Lake, J1 (Cush-CU) ; Ithaca, Je-J1, Taughannock, J1, McLean, May (Cush-CU) ; Karner, May, Yg (NYS); Van Cort. Pk., J1 (Cush-CU). Recorded in the U.S. INat. Mus. as a parasite of Eulia velutina Wlk., Archips rileyana Gr. (Tortricidae), and the European Nothris verbascella. In the U.S. Nat. Mus. are also specimens reared from Simaethis pariana Clerck (Glyphipterygidae), and the grape berry moth, Polychrosis viteana Clem.

T. pygmaeus (Cress.). Ithaca, Je (Vier-CU). Recorded by Herrick as a parasite of the ugly-nest tortricid, Archips cerasivorana Fitch.

T. propinquus (Cress.). Saranac L., Aug (Cress-CU); Manlius, Sep, SmHH (Cress-CU); Waterville, Rock City, Je, Ithaca, May-J1 (Maidl-CU) ; Ellis to Slaterville, Je, MacG\&Bdy (Maidl-CU); McLean Bogs, May-Aug, Sib (Mues-CU). 


\section{Tribe METOPIINI}

(The members of this tribe are parasitic in lepidopterous pupae.)

\section{Metopius Panzer}

M. pollinctorius (Say). LI: Wading R., Aug (Ds).

\section{SUBFAMILY OPHIONINAE 160 Tribe OPHIONINI}

(The members of this tribe are internal parasites of lepidopterous larvae.)

\section{ENICOSPILUS Stephens}

E. flavus (Fab.) (appendiculatus Felt). "NY" (Felt-NYS).

E. purgatus Say. Ithaca, Je-Sep (Felt); McLean, Aug 19-Sep 4, SRobinson, Sib (Bdy-CU); Albany, Je-Aug (NYS); West Farms (Hooker-AM); LI: Flatbush, Sep, Zab (Hooker-US); Parkville (Hooker-AM). Recorded as a parasite of Scotogramma trifolii Rott., Ceramica picta Harr. (both Noctuidae), Schizura unicornis S. \& A. (Notodontidae), and the army worm, Cirphis unipuncta Haw.

E. purgatus var. arcuatus Felt. Ithaca, J1, Stedman (Felt); Albany, May, Hill (Felt-NYS); Poughkeepsie, Yg (Felt); West Farms; NYC (Felt,Joutel) ; LI (Hooker-US). A specimen in the U.S. Nat. Mus. was reared from Scoliopteryx libatrix L. (Noctuidae).

\section{EREMOTYLUS Foerster}

E. glabratus (Say) (arctiae Ashm.). Ithaca, Aug (Felt-NYS,CU). Parasite of the fall web-worm, Hyphantria cunea Dru., Isia isabella S. \& A., Diacrisia virginica Fab., and Halisodota agassizii Pck. (all Arctiidae).

E. macrurus (L.). Gloversville (NYS), from Samia cynthia Dr.; Ithaca, J1, Aug, Stedman (Felt); McLean, Sep 13, Sib (Bdy-CU); Albany, Mar (NYS); West Farms; NYC, Joutel (Felt); LI (Hooker-US). Recorded as parasite of Apatelodes torrefacta S. \& A. (Eupterotidae), Telea polyphemus Cr., Samia cecropia L., and Callosamia promethia Dru.

\section{OPHION Fabricius}

O. bifoveolatum Brullé. Norton's Landing, Cayuga L., Je, SmHH (Cress-CU); Ithaca, May-Je (Cress,Felt-NYS,CU); McLean, Fbs (CU); West Farms (?Brues-AM); LI (Hooker-US).

O. bilineatum Say. Newport, Johnstown, Ilion, May-J1 (NYS); Oswego, Je (Hooker-US) ; Norton's Landing, Cayuga L., SmHH (Cress-CU) ; Ithaca, May-Oct (Felt-NYS,CU); McLean, Je 29-Sep 22 (Bdy-CU); Albany, Karner, May (Felt-NYS); Nyack, Zab (Hooker-AM); West Farms (HookerAM); SI (Vier); LI: (Hooker-US); Flatbush, Je, Zab (Hooker-US). Parasite on the silver-spotted skipper, Eudamus tytyrus Fab.

\footnotetext{
${ }^{160}$ The ichneumon-flies of America belonging to the tribe Ophionini. By Charles W. Hooker. Trans. Am. Ent. Soc., 38: 1-176.
} 
O. slossonae Davis (ferruginipennis Felt). Ithaca, rare, Aug 19, 1924, one male, Babiy, Sep 1, 1925, one female, $B d y$, Sep 3, 18, 1924, Fbs, a male and a female at light (CU); NYC, Joutel (Felt-NYS).

\section{THYREODON Brullé}

T. atricolor (Oliv.) (brullei Vier., morio auctt.). Peru, Je, Mix (CU); Ithaca. Je-J1 (CU) ; McLean, Aug 11-Sep 16, Sib, SRobinson (Bdy-CU); Nyack (Hooker-US); West Farms (Hooker-AM); LI: Flatbush, J1, Zab (AM). Parasitic on larvae of sphinx moths such as Lapara coniferarum S. \& A. anis Paonias excaecatus S. \& A.

\section{Tribe ANOMALINI}

(Nototrachini Ashmead)

ANOMALON Jurine not auctt.

(Nototrachys Marshall)

A. ejuncidum Say. "NY" (Ashm).

NEOGREENEIA Viereck

N. picticornis Vier. Ithaca, Chitt (Cush-US).

\section{Tribe THERIONINI}

\section{(Anomalini Ashmead)}

(The species of this tribe are internai parasites of lepidopterous pupae.)

\section{HABRONYX Foerster}

H. flavistigma Davis. "NY", type.

\section{HETEROPELMA Wesmael}

H. datanae Rly. LI: Long Beach, J1 (Gahan-Schott). Recorded as a parasite of the black-walnut caterpillar, Datana integerrima G. \& R.

H. flavicorne (Erullé). Saranac Inn, Canandaigua L., J1 (NYS); Ithaca, Aug (US); McLean, Aug 28, Sib (Bdy-CU); Nyack, Zab (AM); SI (Ashm); LI (US). Specimens in the U.S. Nat. Mus. are from the yellow-marked apple-worm, Datana ministra Dr., and other species of Datana.

\section{AGRYPON Foerster}

A. chlamidatum (Prov.). McLean (Cush-US).

\section{LABRORYCHUS Foerster}

L. analis (Say). Ithaca, J1 (Mues-CU,NYS); McLean, J1 18-Sep 7, Sib (CushCU) ; Poughkeepsie, May-Jl, Yg (NYS). This is a common parasite of the tent caterpillars, specimens in the U.S. Nat. Mus. having been reared from the American tent caterpillar, Malacosoma americana F., M. fragilis Stretch, and the forest tent caterpillar, $M$. disstria Hbn. 


\section{PARANOMALON Viereck}

(Anomalon auctt.)

P. ambiguum (Nort.). Woodworth's L., May (NYS).

P. clavipes (Davis). Oswego, Je (Gahan-US).

P. curtum (Davis). Manlius, $\mathrm{SmHH}$ (Cress-CU).

P. edwardsii (Cress.). LI (Gahan-US).

P. exile (Prov.). Lyons Falls (Felt); Glens Falls, J1 (NYS), both from cocoons of the forest tent caterpillar, Malacosoma disstria $\mathrm{Hbn}$.

P. ferrugineum (Nort.). Manlius, $\mathrm{SmHH}$ (Cress-CU).

P. filiforme (Prov.). Big Moose, J1 (NYS).

P. hyalinum (Nort.). Mt. Whiteface, J1, Ald (Gahan-US).

P. laterale (Brullé). "NY" (Nort).

P. nigrorufum (Nort.). "NY" (Nort). A specimen in the U.S. Nat. Mus. is said to have been reared from Datana contracta Wlk. (Notodontidae).

P. relictum (Fab.). "NY" (Nort). Specimens in the U.S. Nat. Mus. were reared from Datana sp.

P. semirufum (Nort.). Albany (Nort); Newport, May, $Y g$ (NYS).

\section{THERION Curtis}

T. fuscipenne (Nort.). McLean, J1 20-Aug 20, SRobinson (Bdy-CU); LI: Fire I., Aug (Gahan-Schott). Recorded as a parasite of Merolonche lupini Gr. and Apatela hastulifera S. \& A. Specimens in the U.S. Nat. Mus. were reared from Acronycta americana Harr., A. hesperida Sm., and the "salt-marsh caterpillar".

T. hyaline (Nort.). McLean, Je 30, 1921 (Cush-CU).

T. morio (Fab.) (Exochilum mundum Say). Manlius, SmHH (Cress-CU); Ithaca, Je-Aug (CU,US) ; Freeville, Jl, Comstock (CU) ; McLean, J1 20Sep 17, Sib (Bdy-CU); Binghamton, Jackson (Cush-US) ; SI (Ashm-Ds); LI: Flatbush, $Z a b$ (US) ; Fishers I., $Z a b$ (AM). Recorded as a parasite of Vanessa cardui L., Papilio marcellus Cr., Zerene catenaria Dr. (Geometridae). A specimen in the U.S. Nat. Mus. is said to have been reared from Halisidota maculata Harr. (Arctiidae).

T. occidentale (Cress.). SI, Aug (Gahan-Schott).

T. tenuipes (Nort.). "NY" (Cress-CU). Recorded as a parasite of Diacrisia virginica Fab. (Arctiidae).

\section{Tribe CAMPOPLEGINI}

(With a few exceptions, the members of this tribe are internal parasites of lepidopterous larvae.)

Campoplegidea Viereck

C. alia (Nort.). Ithaca, J1 (NYS).

C. argentea (Nort.). Ithaca, Je (NYS); McLean, Aug 31, 1925, SRobinson (Cush-CU). 
C. diversa (Nort.). Cranberry L., Je, Htly (Cush-US); Manlius, Aug, SmHH (Ashm-CU); Ithaca, Chitt (Cush-US) ; Ft. Montgomery, Je (Cush-Schott); SI (Ashm-Ds).

C. laticincta (Cress.). LI: Flatbush, Je, J1, Zab (Cush-US, AM).

C. minor (Prov.). Ithaca, Karner, Sep-Oct (NYS).

VIERECKIANA Strand

V. bellulus (D.T.). McLean, Aug 26, 1924, Sib (Cush-CU).

V. brachiator (Say). McLean, Aug 18-24, SRobinson (Cush-CU).

V. villosa (Nort.). Albany, Aug, Poughkeepsie, May (NYS).

V. vitticollis (Nort.). Oswego, Aug (Gahan-US); McLean, Aug 28, 1924, Sib (Cush-CU); Karner, Poughkeepsie, Je, $Y g$ (NYS).

\section{ZASTENOMORPHA Viereck}

Z. lamina Vier. Ithaca, J1 (Cush-US).

\section{Casinaria Holmgren}

C. orgyiae (How.). Corinth (Felt). A parasite of the white-marked tussock-moth, Hemerocampa leucostigma S. \& A.

\section{HYPOSOTER Foerster}

H. annulipes (Cress.). Oswego, J1 (Ashm-US); Ithaca, Chitt (Cush-US); LI: Huntington, Ap (Cush-Schott). Recorded as a parasite of Mamestra picta Harr. (Noctuidae), Gelechia pseudoacaciella Chamb. (Gelechiidae), and Acrobasis indigenella Zell. (Pyralidae). A specimen in the U.S. Nat. Mus. was reared from Autographa precationis Guen., and another from Heliothis obsoleta Fab. (Noctuidae).

H. clisiocampae (Weed). Ithaca, Liu (Mues-CU); Crataegus Hill, Je, Htly (Cush-NYS Col. For.). The types and the Ithaca specimens were reared from the tent caterpillar Malacosoma americana Fab., and there is a specimen in the U.S. Nat. Mus. reared from the brown-tail moth, Euproctis chrysorrhoea L.

H. fugitivus (Say). Lockport, Aug (NYS); Ithaca, Lin (Mues-CU), from Malacosoma americana; Albany, J1 (NYS) ; Haines Falls, Rusk (Lint-NYS), from Oedemasia concinna. Recorded as a parasite of the tent caterpillars Malacosoma americana $\mathrm{Fab}$. and $M$. disstria Hbn. (Lasiocampidae), Anisota senatoria S. \& A., A. stigma Fab., A. virginiensis Dru., $A$. rubicunda Fab. (Citheroniidae), Hemileuca maia Dru., Euchaetes egle Dr. (Arctiidae), Apatura clyton B. \& LeC., A. celtis B. \& LeC. (Nymphalidae), the skipper Pholisora catullus Fab., and Acrobasis indigenella Zell. (Pyralidae).

H. geometrae (Ashm.). Cranberry L., Aug, Htly (NYS Col. For., US) ; Hamden, Je (Cush-US); McLean, Aug 1 (Cush-CU).

H. pallipes (Prov.). Oswego, May (Cush-US); Buffalo, Sep, MrsNEDavis (Cush-US). Two specimens in the U.S. Nat. Mus. were reared at Washington, DC, from a Hemerocampa, probably leucostigma.

H. pilosulus (Prov.). McLean, Aug 1, 1925, SRobinson (Cush-CU). 
Cymodusa Holmgren

C. distincta (Cress.). Oswego, Je, Aug (Cush-US); Ithaca, Chitt (Cush-US); Slingerlands, J1 (NYS); LI (Cush-US).

\section{SAGARITIS Holmgren}

S. consimilis (Ashm.). Oswego, Je (Cush-US); Ithaca, Chitt (Cush-US); LI (Cush-US). Parasitic on cutworms, specimens in the U.S. Nat. Mus. having been reared from Feltia sp. and Agrotis ypsilon Rott.

S. oxylus (Cress.). Mt. Whiteface, summit, J1, Ald (Cush-US); Ithaca, Chitt (Cush-US); McLean, Aug 1 (Cush-CU). Recorded as a parasite of the army worm, Cirphis unipuncta Haworth. Specimens in the U.S. Nat. Mus. also from Epiglaea apiata and Laphygma frugiperda S. \& A. (Noctuidae).

S. provancheri (D.T.). Rotterdam, Aug, Searls (Gahan-US). Recorded as a parasite of Laphygma frugiperda S. \& A. (Noctuidae). In the U.S. Nat. Mus. also specimens reared from the European corn-borer, Pyrausta mubilalis Hbn., Heliothis obsoleta F., Neleucania sp. probably diffusa, Prodenia ornithogall Guen., Polia stricta Wlk., Hadena procincta Gr., Triphaena confusa (all Noctuidae).

OLESICAMPE Foerster

(The members of this genus are parasitic on sawfly larvae.)

O. argentifrons (Cress.). Ithaca, Je, Albany, Sep (NYS).

\section{PYRACMON Holmgren}

(The species of Pyracmon are parasitic on wood-boring coleopterous larvae.)

P. macrocephalum Prov. Syracuse, Blkm (Cush-CU).

NEMERITIS Holmgren

N. canescens (Grav.). Ithaca, Nov (Mues,Gahan-US,CU); LI, Aug (US). A cosmopolitan parasite of lepidopterous pests in stored products.

N. ruficoxa (Prov.). Manlius, Sep, SmHH (Ashm-CU); Oswego (Cush-US); LI: Barren I., Flatlands, Aug, Zab (Cush-US).

\section{IDECHTHIS Foerster}

I. nigrocoxalis Cush. Youngstown (type-US). Bred from Euzophera semifuneralis Wlk. (Pyralidae).

CAMPOPLEX Gravenhorst

C. ferrugineipes (Ashm.). McLean, Sep 19, 1924, Sib (Cush-CU).

C. nigricincta (Ashm.). L. Placid, Aug (Ashm-US); Freeville, Aug (Ashm-CU).

C. polychrosidis Vier. LI: Richmond Hill, Jl, Kf (paratype-US).

ECHTHRONOMAS Foersicr

E. ochreofrons Cush. McLean (Cush-US). 
ANGITIA Holmgren

A. plutellae Vier. Ithaca, J1, Mues (Cush-US). Host: Plutella maculipennis Curt. (Yponomeutidae).

A. tibiator (Cress.). Albany, Je (NYS).

\section{SESIOPLEX Viereck}

S. depressus Vier. Ithaca, Chitt (Cush-US). Parasites of larva of the goldenrod gall-maker, Gnorimoschema gallaesolidaginis (Rly.).

EULIMNERIA Schmiedeknecht

E. valida (Cress.). Cranberry L., Je, Htly (Cush-NYS Col. For.) ; Ithaca, J1 (Vier-US, NYS) ; Poughkeepsie, Je (NYS). A common parasite of the fall web-worm, Hyphantria cunea Dru.

\section{Droctes Foerster}

D. obliteratus (Cress.) (Thymaris slingerlandana Ashm.). Ithaca, Aug, Sling (Ashm-CU, US) ; Westchester Co, Oct (Gahan-US). A common parasite of the grape berry moth. Specimens in the U.S. Nat. Mus. are from the oriental peach moth (Laspeyresia molesta Busck), an introduced apple leaf-roller (Simaethis pariana Clerck), and Eulia velutinana Wlk.

D. salicicola (Ashm.). Albion, Felt (Ashm), from Ypsolophus ligulellus Hbn. The type was reared from a gall on willow.

\section{BATHYPLECTES Foerster}

(Several species of this genus are known to be parasitic on curculionid larvae, and it is probable that all are.)

B. exigua (Grav.). Ithaca, Mues (Cush-US). Specimens in the U.S. Nat. Mus. from Phytonomus nigrirostris (Fab.) and Hypera punctata (Fab.), the cloverleaf weevils.

\section{Tribe CREMASTINI 161}

(With the exception of a few species of the subgenus Zaleptopygus, all the species of this tribe the habits of which are known are parasites of lepidopterous larvae.)

\section{EipHosoma Cresson}

(Subgenus BRACHIXIPHOSOMA Viereck)

E. pyralidis Ashm. Ithaca, J1 (Ck11-US); Nyack, Zab (Ckll-US) ; LI: Flatbush, $Z a b$ (AM). The type was reared from a "pyralid on Solidago."

\section{PRISTOMERUS Curtis}

P. fuscipennis Cush. Oswego, Aug (paratype-US).

${ }^{161}$ A revision of the hymenopterous insects of the tribe Cremastini of America, north of Mexico. By Robert A. Cushman. Proc. U.S. Nat. Mus., 1917, 53: 503-551. 
PSEUDERIPTERNUS Viereck

P. radiolatus (Prov.). Larchmont (AM); LI: (Vier-US); Flatbush, Zab (AM).

\section{CREMASTUS Gravenhorst}

\section{(Subgenus CREMASTUS Gravenhorst)}

C. decoratus Grav. LI (Cush-US). Host: Rhyacionia buoliana Schiff. (Tortricidae).

C. facilis (Cress.). Oswego, Aug (Cush-US).

C. forbesii Weed. Mt. Whiteface, summit, Jl, Ald (Cush-US); Oswego, Aug (Cush-US). Specimens in the U.S. Nat. Mus. reared from Gelechia trialbamaculella Chamb., G. confusella Chamb. (Gelechiidae), and Eulia velutinana Wlk. (Tortricidae).

C. graciliventris Cush. McLean, Aug 28, 1924, Sib (Cush-CU).

\section{(Subgenus ZALEPTOPYGUS Viereck)}

C. hartii (Ashm.). Ithaca, Je-Sep (Ashm).

C. dorcaschemae Cush. Syracuse, Blkm, Drk (type-US). Types reared from pupal cells of the longicorn beetle Dorcaschema nigrum (Say).

C. incompletus (Prov.). Oswego, J1 (Cush-US).

C. retiniae (Cress.). Ithaca (type-US), from Rhyacionia rigidana Fern.; SI (Vier, Ashm-Ds).

C. rostratus Cush. "NY", Aug (type-US).

\section{Tribe PORIZONINI}

\section{ORTHOPELMA Taschenberg}

(These live as parasites in the galls of Cynipidae.)

O. luteolator (Grav.). Ithaca, May (Ashm-CU). A common parasite of Rhodites rosae (L.).

O. minutum (Ashm.). LI: Jamaica Plains, May (type-US), from Rhodites gall.

\section{LEPTOPYGUS Foerster}

L. politus (Ashm.). Stony I., J1 (Cush-US).

\section{THERSILOCHUS Holmgren}

T. conotracheli Rly. Oswego, May, Je (Cush-US); LI (Ashm-US). A parasite of the plum curculio.

T. provancheri (Ashm.). LI (Ashm).

Heterocola Foerster

H. americana Brues. Oswego, Aug (Cush-US). 


\section{Tribe PLECTISCINI}

\section{Megastylus Schiødte}

M. albocollaris (Cress.). Oswego, Aug (Ashm-US).

\section{APERILEPTUS Foerster}

A. clypeatus Cush. McLean, Aug 25, 1925, SRobinson (Cush-CU).

A. delicatus Cush. Bear Mt., Sep (Cush-Schott).

\section{DIACRITUS Foerster}

D. muliebris (Cress.). Cranberry L., Aug, Htly (Cush-NYS Col. For.) ; N. Fair Haven, Sep (Cush-CU); Ithaca, Chitt (Cush-US); McLean, Aug 7-Sep 16, Sib, SRobinson (Cush-CU).

\section{Tribe BANCHINI}

\section{BANCHUS Fabricius}

(The members of this genus are parasitic in lepidopterous pupae.)

B. borealis Cress. Speculator, Remsen, Karner, Je-Aug, $Y g$ (NYS) ; LI: Flatbush, $Z a b$ (?Brues-AM).

B. canadensis Cress. Old Forge, J1, Yg (NYS).

B. ferrugineus Prov. Bear Mt., Je (Cush-Schott).

B. flavovariegatus Prov. Saranac Inn, Je (NYS); LI: Huntington, Ap (CushSchott, US).

B. inermis Prov. Ithaca, Sep (Bdy-CU).

B. pallescens Prov. Poughkeepsie, May (NYS).

\section{Ceratogastra Ashmead}

C. ornata (Say). Nyack, Zab (AM); SI, Aug (Vier, Ashm-Ds, Schott); LI: (Cush, Gahan-US) ; Southampton, Aug, Gabrielson (Cush, Gahan-US).

\section{Tribe MESOCHORINI}

\section{Cridaphus Foerster}

C. paniscoides (Ashm.). "NY", Pergande (Cush-US).

\section{AstiphROMMA Foerster}

A. uniformis (Cress.). Ithaca, Sep (Ashm-CU).

\section{MESOCHORUS Gravenhorst}

(Most, if not all, of the species of this genus are secondary parasites attacking other Ichneumonidae and Braconidae.)

M. agilis Cress. Newport, May, $Y g$ (NYS). 
M. aprilinus Ashm. LI: Flatbush, Aug, Zab (AM). Ashmead records it as bred from Apanteles.

M. luteipes Cress. Manlius, Oct, SmHH (Ashm-CU); Nyack, Sep, Oct, Zab (Ashm-AM, US).

M. obliquus Cress. Albany, May (NYS).

M. provancheri D.T. (politus Prov.). Ithaca (Sling\&Flet).

\section{FAMILY TRIGONALIDAE}

\section{LYCOGASTER Shuckard}

L. pullata hollensis (M. \& B.). Ithaca, J1 19, 1919, And (Bdy-CU). One of the rarest insects recorded from the State. This species has been recorded from a cocoon of Telea polyphemus, and is believed to be a parasite on Eremotylus macrurus contained therein (Bdy).

\section{ORTHOGONALOS Schultz}

O. pulchellus (Cr.). Ithaca, J1 3, 1904, RSWoglum, J1 21-23, numerous individuals, Yuasa (Bdy-CU); Taughannock, J1 21, 1905, GCCrampton (Bdy-CU); Karner, J1, 1902 (Bdy-NYS).

\section{TAPINOGONALOS}

[T. costalis (Cress.). Hewitt, N. J., near the NY border, J1 (DS).]

\section{FAmily AULACIDAE}

(With the exception of species of Pammegischia, these are parasites of woodboring coleopterous larvae.)

\section{OdONTAULACUS Kiefier}

O. abdominalis (Cress.). Keene Val., J1 (Bdy-NYS); Big Moose, J1 (BdyNYS); Black Br., Je 21 (Bdy-CU) ; Norton's Landing, Cayuga L., SmHH (Bdy-CU) ; Karner, Je (Bdy-NYS).

O. bilobatus (Prov.). L. Clear, J1 9, 1903 (Bdy-NYS); Ithaca, J1-Aug (BdyCU); Scarsdale, Mar 14-26, 1913 (Bdy-NYS); Albany, Aug 25, 1913 (Bdy-NYS) ; Broadalbin, Je 29, 1915 (Bdy-CU).

\section{PRISTAULACUS Kieffer}

(Subgenus PRIStaulacus Kieffer)

P. flavicrurus (Bdy.). Keene Val., J1 24 (Bdy-NYS).

P. fuscalatus (Bdy.). Rensselaer, J1 26, 1901 (Bdy-NYS).

P. niger (Shuckard). L. Pleasant, J1 20 (Bdy-NYS); Albany, Je 21, Sep (Bdy-NYS).

\section{(Subgenus OLEISOPRISTER Bradley)}

P. abbottii (Westw.). Ft. Montgomery; Je 9, Schott (Cush).

P. glabrescens Bdy. Albany, Je 5, 1909 (holotype-NYS).

P. stigmaterus (Cress.). Geneva (Bdy-CU) ; Norton's Landing, Cayuga L., Je 21, SmHH (Cress, Bdy-CU); West Pt., Je 17, Ds (Beq).

P. subfirmus (Vier.). LI: Bellport, Nic (Sf-BM).

P. taughanic Bdy. Taughannock Falls, Aug 3, 1925, Guerlac (holotype-CU). 


\section{Aulacidae-Stephanidae-Gasteruptionidae-Ropronitdae 961}

\section{PAMMEGISCHIA : Provancher}

(The species of this genus are parasites of the wood-boring larvae of the horntails of the family Xiphydriidae.)

P. burquei Prov. Essex Co, Jl (Bdy-NYS); McLean, Je 30, 1921 (Bdy-CU); Nassau, J1 (Bdy-NYS); LI: Yaphank, Je (Bdy-BM).

P. pallipes Cress. Saranac Inn (US), type of xiphydriae Ashm.; Caroline to Harford, Je 15, MacG (Bdy-CU).

\section{FAMILY STEPHANIDAE}

(Regarded as extremely rare insects.)

SCHLETTERERIUS Ashmead

S. cinctipes Cress. "NY" (Schletterer).

S. rufipes Say. LI, Eng (Sf-BM).

\section{FAMILY GASTERUPTIONIDAE}

\section{(Foenidae)}

The eastern species of this family have usually been identified as Gasteruption incertum or G. tarsatorium, according to whether they have short or long ovipositors. Kieffer has shown that, in fact, other species of Gasteruption, as well as of his genus Trichofoenus, are involved, and pending definite determination of the species to which each record should apply we are obliged to omit most records, despite the fact that specimens are numerous, attracted by the flowers of wild parsnip and other plants, in late summer. Numerous specimens from New York State were in the hands of L'Abbé Kieffer at the time of his death, and had he lived to determine these the records below would doubtless be much more extensive. The insects are parasites of bees.

\section{Gasteruption Latreille}

\section{(Foemus Fabricius)}

G. assectator (L.). According to Kieffer a holarctic species. He records it from NY.

G. tarsatorium (Say). Ithaca, Je 25 (Bdy-CU).

G. intricatum Kf. Ithaca, J1 24-Aug 24 (Bdy-CU).

\section{TRICHOFOENUS Kieffer}

T. canadensis Kf. Ithaca, Je 13-17 (Bdy-CU) ; Spencer L., Je 30 (Bdy-CU).

T. micrurus Kf. New Russia, Aug 18 (Bdy-CU); Niagara Falls, Je 27, Vier (Bdy-CU) ; Crosby Landing on L. Keuka, Je 26 (Bdy-CU); Ithaca, Je 14 J1 25 (Bdy-CU); Taughannock, J1 4 (Bdy-CU). This seems to be the common form usually identified as Gasteruption incertum.

\section{Superfamily PROCTOTRUPOIDEA or SERPHOIDEA \\ FAMILY ROPRONIIDAE}

ROPRONIA Provancher

R. garmani Ashm. Ithaca, JI 22, 1918, Yuasa (Bdy-CU).

R. ashmeadi Bdy. Ithaca, J1 9, 1904 (MacG) (type-CU). 


\section{FAMILY HELORIDAE \\ HELORUS Latreille}

H. paradoxus (Prov.). Ithaca, Smith (Bdy-CU), bred from Chrysopa; L. George. Aug 3, 1903 (Bdy-AM).

\section{FAMILY VANHORNIIDAE}

VANHORNIA Crawford

V. eucnemidarum Crawford. Ithaca $(B d y$-CU). Two specimens found at Ithaca in different years are the only records of the occurrence of this anomalous family, except the type series, taken from cells of eucnemid larvae in Maryland and Virginia.

\section{FAMILY DIAPRIIDAE 162}

(Belytidae)

Compiled by Robert M. Fouts

\section{SUBFAMILY BELYTINAE}

Belyta Nees

B. robustior Fouts. Proc. Ent. Soc. Wash., 1925, $27: 152$. Ithaca, J1, Bdy (type-CU).

B. rugifrons Fouts. McLean, Aug 19 (type-CU).

\section{ACLISTA Foerster}

A. excavata Fouts. McLean, Jl-Aug (type-CU).

A. obliterata Fouts. McLean, Aug 31 (type-CU).

A. palustra Fouts. McLean, Aug 31 (type-CU).

A. simulans Fouts. McLean, J1 26 (type-CU).

\section{Xenotoma Foerster}

X. antennalis Fouts. McLean, Aug 20 (type-CU).

X. curvicaudis Fouts. McLean, Aug 19 (type-CU).

X. palustra Fouts. McLean, Aug 17 (type-CU).

X. pilosa Fouts. McLean, Aug 31 (type-CU).

\section{Cinetus Jurine}

C. pleuralis Fouts. Proc. Ent. Soc. Wash., 1925, $27: 151$. McLean Bogs (type).

\footnotetext{
162 Comparatively little material from New York State of this and the five succeeding families has been determined in collections. In the collections of Cornell University, $\mathrm{Mr}$. Fouts has recognized unidentified or undescribed species in the following genera: Acerota (1 n. sp.), Aclista, Anectata (2 n. spp.), Aneurhynchus (1 n. sp.), Aparamesius, Ashmeadopria, Belyta ( 3 n. spp.), Cinetus (7 n. spp.), Cryptoserphus, Exallonyx, Galesus (2 n. spp.), Leptorhaptus ( 3 n. spp.), Oxylabis ( 1 n. sp.), Pantoclis (6 n. spp.), Paramesius, Phaenopria (1 n. sp.), Platygaster (1 n. sp.), Proctotrupes (1 n. sp.), Spilomicrus, Trichopria (1 n. sp.), and Xenotoma.
} 
Miota Foerster

M. rufopleuralis Ashm. Can. Ent., 1897, 29:589. Ithaca, J1 (Fouts-CU).

\section{SUBFAMILY DIAPRIINAE}

PhaEnopria Ashmead

P. aptera Ashm. Ithaca, Nov 19, Cy; McLean, May 16, Leon (Fouts).

\section{DIAPRIA Latreille}

D. conica (Fab.). Syst. Ent., 1775, p. 343. Malloryville, Je 18-20, Mud Cr., $M a c G \mathcal{E} B d y$ (Fouts-CU) ; McLean, J1 3 (Fouts-CU); Albany; LI (Ashm). Host: Eristalis tenax (L.).

\section{Galesus Haliday}

G. viereckii Brues. Bul. Wisc. Nat. Hist. Soc., 1905, (2) 3:187. Ithaca, J1 12, Yuasa (Bdy-CU).

\section{IDIOTYPA Foerster}

I. pallipes Fouts. Proc. Ent. Soc. Wash., 1925, $27: 102$. McLean, Je, $C y$ (holotype).

\section{LOXOTROPA Foerster}

L. flavipes Ashm. Bul. U.S. Nat. Mus., 1893, 45:415. LI, Bird (Crawf), hyperparasite bred from cocoons of Papaipema maritima Edw.

\section{PaRAmesius Westwood}

P. pallidipes Ashm. Bul. U.S. Nat. Mus., 1893, 45:395. Ithaca, Aug 16, Yuasa (Bdy-CU).

APARAMESIUS Kieffer

A. nigriclavis Fouts. McLean, May 16, Leon (type-CU).

\section{ANEURHYNCHUS}

A. mellipes Ashm. Can. Ent., 1888, 20:52. McLean, Aug 31, 1925, SRobinson (Fouts-CU).

TRICHOPRIA Ashmead

T. agromyzae (Fitch). Second Rep. Ins. N. Y., 1856, p. 303. LI, type locality, from Meoneura vagans (Fall.).

\section{SPILOMICRUS Westwood}

S. kiefferi Fouts. Proc. Ent. Soc. Wash., 1925, $27: 150$. Saranac L., Aug 26, 1916 (types-CU). 


\section{FAMILY PROCTOTRUPIDAE or SERPHIDAE}

Compiled by Robert M. Fouts

PROCTOTRUPES Latreille

\section{(Serphus Schrank)}

P. clypeatus (Ashm.). Bul. U.S. Nat. Mus., 1893, 45:339. Ithaca, type locality.

P. linellii (Ashm.). Bul. U.S. Nat. Mus., 1893, 45:337. LI, type locality.

P. rufigaster (Prov.). Nat. Can., 12:263. Onteora Mt., 2500 ft., How (Crawf).

P. terminalis (Ashm.). Bul. U.S. Nat. Mus., 1893, 45:337. Axton, Je, $M \mathcal{E} H$ (Ashm, Fouts); Ithaca, Je-Aug (Fouts-CU); Rhinebeck, J1 27, Cy (Fouts-CU).

\section{Family CALLICERATIDAE}

\section{CALliceras Nees}

C. melanocerus (Ashm.). Bul. U.S. Nat. Mus., 1893, $45: 129$. Ithaca, bred from pine-cone willow-galls (Ashm).

\section{FAMILY SCELIONIDAE}

Compiled by Robert M. Fouts

\section{Acolus Foerster}

A. zabriskiei Ashm. Bul. U.S. Nat. Mus., 1893, 45:175. LI, type locality. A parasite of spiders' eggs.

\section{ANTERIS Foerster}

A. nigriceps Ashm. Bul. U.S. Nat. Mus., 1893, 45:226. Manlius, Aug 19, SmHH (Ashm-CU).

\section{BAEUS Haliday}

B. americanus How. Ins. Life, 1890, 2:270. LI, type locality. A parasite of spiders' eggs.

\section{GRYON Haliday}

G. flavipes Ashm. Bul. U.S. Nat. Mus., 1893, 45:208. McLean Bogs, May, Cy\&LLeon (Fouts).

\section{HOPLOGRYON Ashmead \\ (Prosacantha Nees)}

H. brachypterus (Ashm.). Can. Ent., 1898, 30:50. McLean, May, J1, Cy\&Leon (Fouts); Cinnamon L., Je 5, Cy (Fouts).

H. coxalis Fouts. Proc. Ent. Soc. Wash., 1925, 27:103. Suffern, May, Cy (holotype).

H. flavipes (Ashm.). Bul. U.S. Nat. Mus., 1893, 45 :208. McLean, Jl, Cy (Fouts).

H. grandis Brues. Bul. Wisc. Nat. Hist. Soc., 1907, 5: 102. LI, type locality.

H. linellii (Ashm.). Bul. U.S. Nat. Mus., 1893, 45: 195. LI, type locality.

H. obscuripes Ashm. Bul. U.S. Nat. Mus., 1893, 45:204. Cinnamon L., Je 5, Cy (Fouts).

H. rufipes Ashm. Bul. U.S. Nat. Mus., 1893, 45:205. McLean, J1, Cy (Fouts). 
Hoploteleia Ashmead

H. noveboracensis Brues. Bul. Wisc. Nat. Hist. Soc., 1908, 6:49. NYC, type locality.

MACROTELEIA Westwood

M. virginiensis Ashm. Bul. U.S. Nat. Mus., 1893, 45:218. Manlius, Aug, SmHH (Ashm-CU).

Opisthacantha Ashmead

O. mellipes Ashm. Bul. U.S. Nat. Mus., 1893, $45: 221$. LI: Riverhead, Je 28, Cy (Fouts).

\section{TELEAS Latreille}

T. lineaticeps Ashm. Bul. U.S. Nat. Mus., 1893, 45 : 199. Manlius, Aug 19, SmHH (Ashm-CU).

\section{TELEnomus Haliday}

T. hemerocampae Wilcox. Psyche, 1920, 27 : 78. LI: Brooklyn, from the whitemarked tussock caterpillar, Hemerocampa leucostigma S. \& A.

T. fiskei Brues. Psyche, 1910, 17: 106. "NY", type locality. Host: Hemerocampa leucostigma S. \& A.

T. longicornis Ashm. Bul. N.Y. State Mus., 1901, 47:586. Adirondacks.

T. clisiocampae Rly. Bul. U.S. Nat. Mus., 1843, 45:160. Ithaca, Spring Val., White Plains, Suffern, Tallman, CLLiu. (Mues), reared from eggs of the tent caterpillar Malacosoma americana $\mathrm{F}$.

T. orgyiae Fitch. Eighth Rep. N.Y. State Ent., p. 197. "NY" (Fitch), from eggs of Notolophus antiqua L. subsp. nova Fitch.

T. podisi Ashm. Bul. U.S. Nat. Mus., 1893, 45:158. Enfield Glen, Ap 22, Cy\&Seeley (Fouts).

\section{Tiphodytes Bradley}

T. gerriphagus Marchal. Ann. Soc. Ent. France, 69:171. Ithaca, Je 11 (Cy\&Mat-CU), reared from the eggs of Gerris remigis Say.

\section{TRISSOLCUS Ashmead}

T. podisi Ashm. Bul. U.S. Nat. Mus., 1893, 45: 162. Ithaca, J1 22 (Brues-CU), bred from pentatomid eggs.

T. thyantae Ashm. Bul. U.S. Nat. Mus., 1893, 45:163. Colonie (Gahan). Host: Thyanta custator (Fab.) (Pentatomidae).

\section{FAMILY PLATYGASTERIDAE 163}

Compiled by Robert. M. Fouts

ACEROTA Foerster

A. leonardi Fouts. Proc. Ent. Soc. Wash., 1925, $27: 147$. McLean Bogs, May 16, Leon (type).

${ }^{163}$ Revision of the North American wasps of the subfamily Platygasterinae. By Robert M. Fouts. Proc. U.S. Nat. Mus., 1924, $63: 1-145$. 


\section{ERITRISSOMERUS Ashmead}

E. noveboracensis Brues. Bul. Wisc. Nat. Hist. Soc., 1910, 8: 48. Albany, type locality. Bred from a cecidomyiid gall.

\section{Fidiobia Ashmead}

F. flavipes Ashm. (Rosneta tritici Brues). Journ. Cincinnati Soc. Nat. Hist., 1894, 17:171. Middleport, types of Rosneta tritici Brues; Westfield, parasite of grape root-worm, Sling.

\section{INOSTEMMA Haliday}

I. leguminicolae Fouts. Proc. Ent. Soc. Wash., 1923, $25: 64$. Ithaca, type locality; reared by Wehrle from the clover midge, Dasyneura leguminicola (Lint).

\section{LEPTACIS Foerster}

L. hopkinsi (Crawf. \& Bdy.) (Dolichotrypes hopkinsi). Proc. Ent. Soc. Wash., 1911, 13:124. Ithaca, Comstock (types-CU, US), many females inserting slender part of abdomen into intercellular spaces of wood near the bark of a freshly cut oak stump along Fall Creek.

L. bradleyi Fouts. Proc. U.S. Nat. Mus., 1824, 63:132. Ithaca, Comstock (typeCU, US); collected under the same circumstances as, and in company with, $L$. hopkinsi.

\section{Platygaster Latreille}

P. anormis Brues. Bull. Wisc. Nat. Hist. Soc., 1910, 8:49. Albany, type locality.

P. aphidis Ashm. Bull. U.S. Nat. Mus., 1893, 45:325. Richfield Sps. (type); reared from an aphis on Chenopodium album.

P. error Fitch. Sixth Rep. N.Y. State Ent., p. 76. Branchport, from the clover midge, Dasyneura leguminicola (Lint.), also a parasite of the wheat midge, Thecodiplosis mosellana Gahan.

P. pallida Fouts. Proc. Ent. Soc. Wash., 1925, 27:148. McLean Bogs, May 16, Leon (holotype).

P. herrickii Pck. Third Rep. U.S. Ent. Comm., p. 220. Hilton, Fancher, Knowlesville, Waterport, Sanborn, $C y$ (Myers\&Hill).

P. hiemalis Fbs. Psyche, 1888, 5:39. Evans Mills, Theresa, McConnell (Hill); Albion, McConnell, Newfane, Lima, $C y$ (Hill); Stafford, Berne, $C y$ (Hill).

P. salicicola (Ashm.). Bull. U.S. Nat. Mus., 1893, 45: 305. Brighton (Felt), bred from the European willow gall-midge, Rhabdophaga salicis Schrank (Cecidomyiidae).

P. leguminicolae Fouts. Proc. Ent. Soc. Wash., 1920, 22:69. Ithaca, Wehrle (Fouts), from the clover midge, Dasyneura leguminicola Lintner.

P. vernalis (Myers). Proc. U.S. Nat. Mus., 1917, 53:255. Generally distributed in central and western NY south of the Mohawk Val. and in the Hudson R. Val. north of Fishkill. The following definite records have been supplied by W. R. McConnell, P. R. Myers, C. C. Hill, and H. D. Smith, of the Hessian Fly Laboratory of the U.S. Bur. Ent., based largely on material supplied them by C. R. Crosby: Marion, Elbridge, Camillus, Borodino, Williamson, Sodus, Sodus Pt., Wolcott, Rose, Palmyra, Fruitland, Clyde, W. Walworth, Walworth, W. Henrietta, Spencerport, Scottsville, Rush, N. Greece, N. Cambric Center, 
Mumford, Mendon, Macedon Center, Hilton, Hamlin, Fairport, Charlotte, Chili, Churchville, Brockport, Fancher, Albion, Kendall, Orleans, Holley, Knowlesville, Waterport, Pavilion, Medina, LeRoy, Indian Falls, Honeoye Falls, Griswold, Geneseo, Newfane, Alden, Lockport, Lima, Alexander, Batavia, Bergen, Corfu, Indian Falls, E. Aurora, Ashantee, Canawaugus, E. Avon, York, Silver Sps., Geneva, Manchester, Victor, Canandaigua, Flint, Cayuga, Auburn, Skaneateles, Skaneateles Falls, Waterloo, Ithaca, Lodi, Niobe, Covert, Blockville, Romulus, Waterloo, Berne, Brunswick, Wallkill, Highland, Rhinebeck, and Fishkill. Host: the Hessian fly, Phytophaga destructor Say.

P. rubi (Ashm.). Bull. U.S. Nat. Mus., 1893, 45:315. Ithaca, bred from a cecidomyiid gall on blackberry (Brues-CU).

P. semiglaber Gir. Proc. U.S. Nat. Mus., 1920, 58:177. Albany, type locality; from Rhopalomyia hirtipes O.S.

\section{FAMILy PELECINIDAE \\ Pelecinus Latreille}

P. polyturator Drury. New Russia, Aug 8 ( $B d y$-CU) ; Otto, Potsdam, Manlius (Bdy-CU) ; Oswego, Sep 7, JGLindsey (Lint-NYS) ; Hamburg ; Ithaca, Sep, Guerlac; McLean, Aug 19-30 (Bdy-CU) ; Albany, Aug (NYS) ; Coeymans, EBSm (Lint-NYS); New Riverside (CU) ; L. George, Zab (AM) ; Hague, West Farms (AM); Huguenot, Tomkins Cove (AM) ; LI: Flatbush, Fishers I. (AM). Common, probably in all parts of the State (Bdy).

\section{FAMILY CYNIPIDAE}

Compiled by L. H. WELD

SUBFAMILY IBALIINAE

(Members of this subfamily are parasites on horntails.)

IBALIA Latreille

I. ensiger Nort. Oswego, J1 22.

I. maculipennis Hald. Batavia; Norton's Landing, Cayuga L.; Ithaca; Albany, Je 4, SCBishop (Bdy-NYS); Poughkeepsie, Je 1 (Bdy-NYS); Port Chester, Sep 24 (Bdy-NYS); LI, Je.

\section{SUBFAMILY ANACHARITINAE}

(Species of this and the three following subfamilies-Aspicerinae, Figitinae, and Eucoilinae-are parasites of dipterous puparia.)

ANACHARIS Dalman

A. marginata Prov. Ellis; Slaterville.

- Xyalaspis Hartig

X. flavipes Ashm. Manlius, Aug 19 (Weld-CU). 


\section{SUBFAMILY ASPICERINAE}

Callaspidia Dahlbom

C. provancheri Ashm. Oswego, Je; Rochester, J1 7; Ithaca, Aug 7, Sep 13 (Weld) ; Caroline to Harford, Je 15; L. George, Aug 22.

\section{SUbFAMILy FIGITINAE}

Figites Latreille

F. floridanus Ashm. Norton's Landing, Cayuga L., SmHH (Weld); Ithaca, Je 26.

\section{SUBFAMILY EUCOILINAE \\ COTHONASPIS Hartig \\ (Subgenus CотHonaspis Hartig)}

C. rapae (West.). Geneva; Medina; Carlton; LI. A parasite of the cabbage root-maggot.

\section{PSILODORA Foerster}

P. impatiens (Say). Oswego, Aug 17.

\section{KLEIDOTOMA Westwood}

K. avenae (Fitch). "NY" (type). From Aphis avenae Fab.

K. marginata (Gill.). Spencer L., Je 30.

\section{Eucoilidea Ashmead}

E. canadensis Ashm. Oswego, J1 1; LI.

\section{SUBFAMILY CHARIPINAE}

(Species of this subfamily are secondary parasites of plant lice.)

\section{CHARIPS Haliday \\ (Subgenus ChaRIPS Haliday)}

C. brassicae (Ashm.). Medina; Carlton; Ithaca; Groton. From cabbage aphis.

Alloxysta Foerster

(Subgenus Alloxysta Foerster)

A. tritici (Fitch). "NY" (type). Probably from grain aphis.

\section{SUBFAMILY CYNIPINAE}

(Members of this subfamily are producers of galls on plants or are inquilines in these galls.) 


\section{GALL-MAKERS}

\section{NEUROTERUS Hartig}

N. batatus (Fitch). OAK Potato Gall. Hemlock L.; Rochester (Felt); Ithaca; Albany; Easton Hills (Felt); SI; LI: Flatbush; Southold. Galls on white oak.

N. distortus Bass. "NY". (Bt). Galls on swamp white oak.

N. exiguus Bass. "NY" (Bt). Galls on post oak.

N. floccosus (Bass.). OAK Flake Gall. Nyack; NYC; LI: Manorville. Galls on white oak.

N. irregularis (O.S.). "NY" (Bt.). Galls on post oak.

N. majalis (Bass.). Ithaca; LI: Southold; E. Hampton. Galls on white oak.

N. minutus (Bass.). "NY" (Bt.). Galls on white oak.

N. niger Gill. Ithaca; NYC; LI: Glen Cove. Galls on white oak.

N. noxiosus (Bass.). Noxious OAK Gall. Medina; Geneva; Albany; Spring Val.; Mt. Vernon; SI; LI: Greenport. Galls on swamp white oak.

N. pallidus Bass. "NY" (Bt). Galls on staminate flowers of swamp white oak.

N. pallipes Bass. "NY" (Bt.). Galls on white oak.

N. papillosus Bt. OAK Blister Gall. NYC (Bt). Galls on swamp white oak.

N. saltarius Weld. Eastern Jumping Neuroterus Gall. Medina. Galls on bur oak.

N. tectus Bass: "NY" (Bt). Galls on scrub chestnut oak.

N. umbilicatus Bass. Ithaca; Hudson Falls (Felt); Albany; Mt. Kisco (Felt); NYC; LI: Sea Cliff (Felt). Galls on swamp white oak.

N. verrucarum (O.S.). "NY" (Bt). Galls on post oak.

N. vesicula (Bass.). Soft OAK-Bud Gall. Nyack. Galls on white, bur, and swamp white oak.

\section{Diplolepis Geoffroy}

(Dryophanta Foerster)

D. centricola (O.S.). West Farms; LI: Farmingdale. Galls on post oak.

D. cinereae (Ashm.) (saccularius Bass.). Medina; LI: E. Hampton. Galls on red and scarlet oak.

D. clarkei (Bass.). "NY" (Bt). Galls on white oak.

D. erinacei (Walsh). OAK HEDGehog Gall. Buffalo; Waterport; Penn Yan; Ithaca (CU) ; Syracuse; Albany; NYC; SI; LI: Southold. The early spring bud-scale galls of the sexual generation have been taken at Ithaca. Galls on white oak.

D. gemula (Bass.). Brittle Bud-Gall. NYC. Galls on white oak.

D. ignota (Bass.). "NY" (Bt). Galls on swamp white oak.

D. notha (O.S.). NYC; LI: E. Hampton. Galls on scarlet, red, and pin oak.

D. pallipes (Bass.). "NY" (Bt). Galls on white oak.

D. palustris (O.S.). Succulent OAK Gall. Hudson Falls; Ithaca; Nyack; West Farms; NYC; LI: Cypress Hills; Southold; E. Hampton. Galls on red, scarlet, black, scrub black, pin, willow, and blackjack oak.

D. pedunculata (Bass.). LI: E. Hampton. Galls on scarlet oak.

D. polita (Bass.). Polished OAK Gall. LI: Southold. Galls on post oak. 
Disholcaspis Dalla Torre and Kieffer

D. globulus (Fitch). Round Bullet Gall. Syracuse; Indian Fails; Eden; Hemlock L.; Canandaigua; Ithaca; Nyack; West Farms; SI; LI: Flatbush; Manorville; Southold; E. Hampton; Orient. Galls on white, chestnut, and scrub chestnut oak.

D. globosa Weld. Ithaca; Bartow. Galls underground on white oak.

D mamma (Walsh). Pointed Bullet Gall. Ithaca; Karner; West Farms; SI. Galls on bur and swamp white oak.

\section{DRYOCOSMUS Giraud}

D. decidua (Bt.). Deciduous Oak Gall. LI: Riverhead. Galls on red, black, and scrub black oak.

D. floridensis (Bt.) (peltatus Wells and Metcalf). Rosette Gall. LI: Flatbush. Galls on blackjack and pin oak.

D. imbricariae (Ashm.) (fasciatus Bass.). Banded Bullet Gall. Bronxville; SI; LI: Riverhead, Flanders. Galls on scrub black and blackjack oak.

D. piperoides (Bass.). Medina; Canandaigua; Ithaca. Galls on red oak.

D. rileyi (Ashm.). LI: Riverhead. Galls on red oak.

\section{TRIGONASPIS Hartig}

T. radicola (Ashm.). Fleshy OAK Fig Root-Gall. NYC; LI: Flatbush. Galls on white oak.

BIORHIZA Westwood

B. rubina Gill. Syracuse; Medina. Galls on white oak.

\section{XANTHOTERAS Ashmead}

X. forticorne (Walsh). OAK FIg Gall. Ithaca; Karner; Albany; West Farms; Nyack; SI; LI: Flatbush; Riverhead; Southold. Galls on white oak.

\section{XYSTOTERAS Ashmead}

X. nigrum (Fitch). Albany. Galls on white oak.

X. poculum Weld. Oak Spangle Gall. Hemlock L.; Ithaca. Galls on white oak.

\section{ZOPHEROTERAS Ashmead}

Z. compressum (Gill.). Katonah; LI. Galls on scarlet oak.

Z. hubbardi (Ashm.) (Parateras). LI: Bellmore. Gall unknown.

\section{ACRASPIS Mayr}

A. hirta (Bass.). Ithaca; Storm King; West Pt.; High Bridge; NYC. Galls on chestnut oak.

A. macrocarpae Bass. Jewel OAk Gall. St. Lawrence Co; Medina; LI: Manorville; Farmingdale. Galls on bur oak. 
A. pezomachoides (O.S.). OAK PEA GALL. Syracuse; Albany; Nyack; West Farms; LI: Flatbush; Manorville; Riverhead; Southold. Galls on white oak.

A. prinoides (Bt.). Spiny OAK Gall. Oriskany Falls; SI. Galls on scrub chestnut oak.

A. villosa Gill. HaIry OAK Gall. Medina; Seneca L.; Ithaca. Galls on bur oak.

\section{Philonix Fitch}

P. fulvicollis Fitch. "NY" (Fitch). Gall unknown.

P. nigra (Gill.). Ithaca; NYC; LI: Riverhead. Galls on white and bur oak.

P. nigricollis Fitch. "NY" (Fitch). Gall unknown.

\section{CYNIPS Linnaeus}

C. dimorphus Bt. Ithaca; LI. Galls on white and bur oak.

C. strobilana O.S. Pine-Cone OAK Gall. Ithaca; Albany; Mt. Vernon; Bronxville; NYC; SI. Galls on swamp white oak.

C. weldi Bt. Syracuse; Ithaca; LI: Riverhead. Galls on white oak.

\section{ANDRICUS Hartig}

A. chinquapin (Fitch) (fusiformis O.S., capsula Bass.). OAK CAPSUle Gall. Ithaca; NYC; SI; LI: Farmingdale; E. Hampton. Galls on scrub chestnut, swamp white, and white oak.

A. coronus Bt. NYC, Bronx Pk. Galls on pin oak.

A. excavatus Ashm. Hudson Falls. Galls on black oak.

A. flocci (Walsh). OAK Wool Gall. Genoa; Canandaigua (Felt); Ithaca; Troy (Felt) ; NYC (Bt) ; LI: Cold Sp. Harb. (Felt) ; Riverhead. Galls on white oak.

A. osten sackenii (Bass.). Ithaca; LI: Farmingdale; E. Hampton. Galls on scarlet oak.

A. pattoni (Bass.). SI; LI: Pinelawn. Galls on post oak.

A. petiolicola (Bass.). OAK Petrole Gall. Medina; Watkins Glen; Ithaca; Albany; Hudson Falls; Storm King; West Farms; Scarsdale (Felt) ; SI; LI: Cold Sp. Harb. (Felt) ; Ravenswood; Farmingdale; Manorville; Southold ; E. Hampton. Galls on swamp white, white, bur, chestnut, scrub chestnut, and post oak.

A. rugulosus Bt. NYC. Galls on red and black oak.

A. singularis (Bass.). Small OAK Apple. Medina; Ithaca; Albany; Shushan; Katonah; SI; LI: Cold Sp. Harb. (Felt). Galls on red oak.

A. utricula (Bass.). Ithaca; LI: Orient. Galls on white and post oak.

\section{CALliRhytis Foerster}

C. balanoides Weld. Ithaca. Gall on immature acorn of black oak.

C. bignelli (D.T.) (punctata Ashm., montana Bt.). Nyack. Gall unknown.

C. clavula (O.S.). OAK Club Gall. Syracuse; Indian Falls; Hemlock L. ; A1bany; White Plains; Hudson Falls; NYC; SI; LI: Farmingdale. Galls on white oak. 
C. cornigera (O.S.). Horned Knot Gall. Ithaca; Nyack; West Farms; NYC; SI; LI: S.outhold; Gardiners I. Galls on red, pin, shingle, and scrub black oak.

C. elliptica Weld. Nyack. Root gall on white oak.

C. flavohirta (Bt.). Medina; Ithaca. Galls on swamp white and bur oak.

C. fructuosa Weld. Ithaca. Galls inside black-oak acorns.

C. futilis (O.S.). OAK WART Gall. Medina; Indian Falls; Ithaca; Hudson Falls; NYC; LI: Manorville; Farmingdale; Southold. The alternating agamic generation in the thick bark underground has been taken at Ithaca and Indian Falls. Galls on white oak.

C. gallaestriatae Weld. NYC. Galls on red oak.

C. gemmaria (Ashm.). Ithaca; LI: Flatbush; Pinelawn. Galls on red oak.

C. gemmiformis (Bt.). NYC: Woodlawn. Galls on white oak.

C. lanata (Gill.) (Dryophanta). Medina; Hemlock L.; LI: Riverhead. Galls on red, scarlet, black, and scrub black oak.

C. lustrans (Bt.) (impositus Bt.). N.YC. Galls on post oak.

C. modesta (O.S.) (papula Bass.). Medina; NYC; LI: Riverhead. Galls on red, black, and scrub black oak.

C. operator (O.S.). Woolly Flower Gall. Ithaca; Schenectady; Albany; SI; LI: Massapequa; Riverhead; E. Hampton. Galls on red, scarlet, black, and scrub black oak. The acorn pip gall of the alternating agamic generation has been taken at Medina, Ithaca, and Riverhead.

C. phellos (O.S.) (similis Bass.). Scrub-oak Club Gall. SI; LI: Farmingdale; Riverhead; Flanders; E. Hampton. Galls on scrub black and willow oak.

C. pigra (Bass.). OAK MID-RIB Gall. Ithaca; NYC; Galls on black and scarlet oak.

C. pulchra Bass. Smooth Flower Gall. Poughkeepsie; LI: Flatbush. Galls on red and black oak.

C. punctata (Bass.). OAк Knot Gall. Watkins; Loudonville; W. Albany; Mt. Vernon; SI; LI: Cold Sp. Harb.; Southold. Galls on red, scarlet, black, scrub black, and blackjack oak.

C. rugosa (Ashm.) (Andricus). LI: E. Hampton. Galls on red oak.

C. scitula (Bass.) (Andricus). Ithaca; New Rochelle; Nyack; LI: Flatbush. Galls on red and black oak.

C. seminator (Harr.). Wool Sower. Rochester; Indian Falls; Ithaca; Glens Falls; Hudson Falls; Cheviot; W. Nyack; NYC; LI: Farmingdale; Southold; E. Hampton. Galls on white oak.

C. seminosa Bass. Peekskill; Nyack; NYC; SI. Galls on red oak.

C. tumifica (O.S.). Mid-Rib Tumor Gall. Ithaca; Dobbs Ferry; NYC; LI: Farmingdale. Galls on red and black oak.

\section{АMPHIBOLIPS Reinhard}

A. coelebs (O.S.). Smaller Spindle Gall. SI; LI: Yaphank; Riverhead; E. Hampton. Galls on red and scarlet oak.

A. cookii Gill. Medina; Atwater; Ithaca; West Point; Katonah. Galls on red and black oak. 
A. confluentus (Harr.) (spongifica O.S.). SPONGY OAK Apple. Waterport; Ithaca; New Baltimore; Hudson Falls; West Farms; White Plains; NYC; SI; LI: New Lots; Flatbush; Farmingdale; Cold Sp. Harb. (Felt) ; Manorville; Riverhead; Southold; E. Hampton. Galls on red and black oak.

A. ilicifoliae (Bass.). Larger Oak Spindle Gall. SI; LI: Cold Sp. Harb.; Riverhead; Flanders; Southold; E. Hampton. Galls on scrub black oak.

A. inanis (O.S.). Larger Empty OAK Apple. Medina; Ithaca; Hudson Falls; SI. Galls on red oak.

A. nubilipennis (Harr.). Translucent Oak Gall. Indian Falls; Ithaca; LI: Farmingdale; E. Hampton. Galls on red, black, and scarlet oak.

A. prunus (Walsh). AcoRn Plum Gall. Lowville; Ithaca; SI; LI: Moriches. Galls on red oak.

A. tinctoriae Ashm. KeEled OAK Apple. Katonah; LI: Port Jefferson. Galls on scarlet and black oak.

\section{DIASTROPHUS Hartig}

D. bassetti Bt. Bronxville; West Farms; SI. Galls on roots of dewberry.

D. cuscutaeformis O.S: Blackberry Seed Gall. Newport; Salamanca; Ithaca; West Farms; SI; LI: Flatbush; Southold. Galls on stem of blackberry.

D. fragariae Bt. Cylindrical Strawberry Gall. Ithaca; NYC. Galls on petiole of wild strawberry.

D. nebulosus (O.S.). Gouty Blackberry Gall. Waterford; Albany; Poughkeepsie; White Plains; Mt. Vernon; Nyack; West Farms; SI : Ft. Wadsworth, on a cultivated climbing blackberry (Ds) ; LI: Farmingdale; Southold. Galls on wild blackberry.

D. niger Bass. Ithaca; SI; LI: Southold. Galls on Potentilla.

D. radicum Bass. Blackberry Root Gall. NYC (Bt); LI: Amagansett (Ds); Pinelawn. Galls on wild blackberry.

D. turgidus Bass. Ithaca. Galls on stems of wild raspberry.

\section{Aylax Hartig}

A. glechomae (L.). Ground Ivy Gall. Medina; Ithaca; Albany (Felt); LI. Galls on Nepeta hederacea. Introduced from Europe.

A. taraxaci (Ashm.). Medina; Hudson Falls. Galls on dandelion. Introduced from Europe.

\section{Aulacidea Ashmead}

A. harringtoni (Ashm.) (bicolor Gill., mulgediicola Ashm., cavicola Ashm.). Katonah. Galls on Lactuca.

A. nabali (Brodie). Nyack. Galls on Prenanthes.

A. podagrae (Bass.). Ithaca; SI. Galls on Lactuca.

A. tumida (Bass.) (sonchicola Ashm.). Lettuce Tumor Gall. Ithaca; Albany; E. Schodack; Nassau; Waldorf; Nyack; West Farms; NYC; LI: Southold; Orient (Felt). Galls on Lactuca.

\section{GoNASPIS Ashmead}

G. potentillae (Bass.). Cinguefoil Axil Gall. Norton's Landing, Cayuga L. (Weld-CU); Ithaca; E. Jewett; West Farms; NYC; SI; LI: Astoria; Southold; Gardiners I. Galls on Potentilla. 


\section{RHODites Hartig}

(Members of this genus produce galls on roses.)

R. bicolor (Harr.). Spiny Rose Gall. Medina; Canandaigua; Ithaca; Albany; Shushan; Nyack; Hudson Falls; West Farms; NYC, Central Pk., Inwood; SI; LI: Hempstead Plains; Bellport; Southold; E. Hampton.

R. dichlocerus (Harr.). Long Rose Gall. Medina; Canandaigua; Hudson Falls; Nassau; Niverville; Nyack; West Farms; SI; LI: Flatbush; Wyandanch; Orient.

R. gracilis Ashm. Regal Rose Gall. Canandaigua: Ithaca.

R. ignotus O.S. Mealy Rose Gall. Rochester; Albany; West Farms; NYC, Inwood (Ds) ; SI; LI: Richmond Hill; Southold; Shelter I.; Wading R. (Ds) ; Orient.

R. nebulosus Bass. NYC; SI.

R. radicum O.S. Rose Root Gall. Albany; Nyack; NYC; SI. On Rosa carolina.

R. rosae (L.). Mossy Rose Gall, or Bedeguar. Ogdensburg; Medina; Albion; Rochester; Honeoye Falls; Batavia; Ithaca; Taughannock; L. George (Felt) ; E. Jewett (Ds) ; Athens; West Farms; Nyack; NYC; SI. On Sweetbrier. Introduced from Europe.

R. rosaefolii Ckll. Rose Lentil Gall. Medina; Canandaigua; NYC.

R. semipiceus (Harr.) (fulgens Gill.). Ithaca; Bronxville; NYC; SI; LI: Flatbush.

R. vernus (O.S.). Knotty Rose Gall. Hudson Falls; SI; LI: Southold.

\section{GUESTS, OR INQUILINES \\ PERICLISTUS Foerster}

(Members of this genus have been reared from galls on roses.)

P. piratus (O.S.). Nyack; SI.

P. silvestris (O.S.). NYC, Bronx Pk.

\section{SYNERGUS Hartig}

(Members of this genus have been reared from galls on oak.)

S. campanula O.S. Buffalo; Nyack.

S. dimorphus O.S. SI.

S. erinacei Gill. Buffalo; Ithaca, Feb, Ap 3, J1 1-Aug 8 (Weld).

S. lana (Fitch). "NY" (Fitch).

S. lignicola (O.S.). Nyack.

S. oneratus (Harr.). Ithaca.

Ceroptres Hartig

(Members of this genus have been reared from galls on oak.)

C. arbos (Fitch). "NY" (Fitch). Bred from gall of Callirhytis clavula (O.S.).

C. ficus (Fitch). Axton, Je; SI. Bred from gall of Xanthoteras forticorne (Walsh).

C. petiolicola (O.S.). Nyack; SI. Bred from gall of Andricus petiolicola (Bass.).

C. pisum (Fitch)。 "NY" (Fitch). Bred from gall of Acraspis pezomachoides (O.S.).

C. tuber (Fiten). Nyack. Bred from gall of Callirhytis clavula (O.S.). 


\section{Superfamily CHALCIDOIDEA}

Compiled by A. B. Gahan, C. R. Crosby, and M. D. Leonard

\section{FAMILY CALLIMOMIDAE \\ (Torymidae)}

Callimome Spinola

(Torymus Dalman)

C. aea (W1k.). "NY" (Wlk).

Ditropinotus Crawford

D. aureoviridis Crawf. Waterloo (PhillipsWJ); Victor, Clifton (CU). Carlton (Crawf-CU); Penn Yan (PhillipsWJ); Middletown (Cy). Bred from larvae in wheat.

\section{ERIDONTOMERUS Crawford}

E. isosomatis (Rly.) (primus Crawf.). Geneva (PhillipsWJ); Victor, Ithaca, $(\mathrm{Cy}-\mathrm{CU})$; Elmira (Crawf-CU); Albany (Cy-CU). Bred from larvae in timothy.

\section{Megastigmus Dalman}

M. aculeatus (Swederus). Ithaca ( $\mathrm{Cy}-\mathrm{CU})$, reared from rose seeds.

M. brevicaudis Ratzeburg. Oswego, Weedsport (Cy) ; Jamesville, Cy-CU) ; Wayland $(\mathrm{Cy})$; Ithaca $(\mathrm{Cy}-\mathrm{CU})$. All reared from seeds of mountain ash.

M. laricis Marcovitch. Ithaca (types), reared from seeds of larch.

M. nigrovariegatus Ashm. Wellsville, Ithaca, White Church, Stuyvesant Falls $(\mathrm{Cy}-\mathrm{CU})$, reared from rose seeds.

\section{MONODONTOMERUS Westwood}

M, dentipes (Boheman). LI: Farmingdale, J1, reared from cocoons of Lophyrus sp. on pine (Gahan).

\section{ORMYRUS Westwood}

O. ventricosus Ashm. Watkins, Ithaca (CU). Bred from galls of Diastrophus nebulosus O.S. and Diplolepis erinacei (Walsh).

\section{SYNTOMASPIS Foerster}

S. druparum (Boheman). Apple Seed Chalcis. Clinton Co (Cy) ; Cranberry Cr., Hornby, Geneva, Monterey (Cy) ; Rochester, Penn Yan, Ithaca (Cy-CU); Kingston (Cy); Red Hook and Poughkeepsie (CU Econ). Lives only in apple seeds.

S. racemariae Ashm. SI, Schott (Gahan).

S. thalassinus Cy. Cranberry Cr., Lowville, Remsen, Victor, Byron, Bluff Pt., Penn Yan, Elmira (Cy); Taughannock Falls (Cy-CU); Ithaca (Cy); Wilseyville (Cy-CU) ; McLean, Cortland, Amsterdam, Kingston (Cy). From timothy and orchard grass. 
WeBSTERELlus Ashmead

W. harrisi (Fitch). "NY" (Fitch).

\section{FAMily PERILAMPIDAE}

PERILAMPUS Latreille

P. subcarinatus Crawf. Norton's Landing, Cayuga L., SmHH (CU).

\section{FAMILY CHALCIDIDAE}

BRACHYMERIA Westwood

B. compsilurae (Cwfd.). LI: Pinelawn, Aug 24, Schott (Gahan).

B. ovata (Say). Axton, $M \& H$ (Ashm-CU); Ithaca (Ashm-CU), from $V$ anessa antiopa.

\section{Diplodontia Ashmead}

D. secunda Gir. LI (Gir).

\section{LEUCOSPIS Fabricius}

L. affinis Say. Crosby Landing, L. Keuka, Je, $C y$, Taughannock (C. Weld-CU) ; common at Ithaca (Bdy); McLean, Sep 7, 1924, Sib (Bdy-CU); Potsdam, Je (C. Weld-CU) ; LI: Central Pk., Je (Schott).

\section{PHASGONOPHORA Westwood}

P. sulcata Westw. Syracuse (B\&S), from dead hickory; Crittenden (B\&S), from larch; Ithaca (CU), from Agrilus axius Gory. A well-known parasite of flat-head borers.

\section{SpILochalcis Ashmead}

S. mariae (Rly.). SI (Ds); LI: Brooklyn. Parasite of Samia cecropia Dr. and the bag-worm, Thyridopteryx ephemeraeformis Steph.

S. albifrons (Walsh). Oswego; Medina.

\section{TRIGONURA Sichel}

T. hicoriae Roh. Syracuse, $B \mathcal{E} L$ (type-US). Bred from hickory, and presumably from Chrysobothris femorata (Oliv.) and C. sexsignata (Say).

\section{FAMILY EURYTOMIDAE \\ BRUCHOPHAGUS Ashmead}

B. funebris (How.). Clover Seed Chalcid. Probably rather generally distributed throughout the State. Ithaca, Harford (Cy-CU).

\section{Decatoma Spinola}

D. amsterdamensis Gir. Geneva, Penn Yan (PhillipsWJ), reared from Harmolita material. 
D. flava Ashm. Ithaca (Cy-CU), from galls of Diplolepis crinacei (Walsh).

D. nubilistigma Walsh. Ithaca, J1 ( $\mathrm{Cy}-\mathrm{CU})$.

D. querci-lanae-dorsalis (Fitch). Ithaca, J1-Aug (Cy-CU). Specimens in CU bred from galls of Diplolepis erinacei (Walsh) and Andricus petiolicola (Bass.).

D. varians Walsh. Ithaca (Cy-CU), from galls of Diplolepis erinacei (Walsh).

\section{EURY'TOMA Illiger}

E. auriceps Walsh. Ithaca (Cy-CU). Bred from galls of Diplolepis erinacei (Walsh).

E. bolteri Rly. Ithaca, Mar, WAStocking,Jr (Ashm-CU), bred from gall on goldenrod.

E. bolteri var. parva W. J. Phillips. Waterloo (Gir).

E. gigantea Walsh. Ithaca, Mar 11, 1898, WAStocking,Jr (Ashm-CU), bred from gall on goldenrod.

E. juniperinus Marcovitch. Ithaca (types), reared from juniper berries.

E. lanulae Fitch. "NY" (Fitch).

E. orchidearum (Westw.) (Isosoma). The ORchid Fly. Occasionally infesting greenhouse orchids. Rochester; West Nyack (CU); LI: College Pt. (Leon).

E. pater Gir. Penn Yan, Auburn, Waterloo (Gir).

E. querci-globuli (Fitch). "NY" (Fitch).

E. querci-pisi (Fitch). "NY" (Fitch).

E. rhois $\mathrm{Cy}$. Ithaca (types-CU), reared from seeds of sumac.

E. studiosa Say. Ithaca (Cy-CU). Bred from galls of Diplolepis erinacei (Walsh).

\section{EURYTOMOCHARIS Ashmead}

E. triodiae How. Kingston (Cy).

Evoxysoma Ashmead

E. vitis (Saunders). Ithaca, $C y$ (How-CU), reared from grape seeds.

HARMOLITA Motschulsky 164

(The species of this genus are all phytophagous, either occupying the center of the stem, or causing warty or gall-like swellings on the stem, of definite species of grasses.)

H. agropyrophila P. \& E. Lowville, Geneva, Bluff Pt., Albany, Amsterdam (PhillipsWJ-CU). Lives only in species of quack grass, Agropyron. Lives in the center of the stem.

H. albomaculata (Ashm). Richland (Cy); Carthage (PhillipsWJ-CU); Lowville (Cy); Cranberry Cr. (PhillipsWJ-CU); Remsen; Victor; Bluff Pt.; Penn Yan (Cy); Elmira (PhillipsWJ-CU); Ithaca (Cy); Cortland (PhillipsWJ-CU) ; Oneonta (PhillipsWJ-CU); Albany, Kingston (Cy). Lives only in timothy, and inhabits the center of the stem. Probably occurs wherever timothy is grown.

${ }^{161}$ A revision of the chalcid-flies of the genus Harmolita of America, north of Mexico. By W. J. Phillips and W. T. Emery. Proc. U.S. Nat. Mus., 1919, 55:433-471. 
H. atlantica P. \& E. Auburn (Cy-CU); Weedsport; Waterloo (PhillipsWJ); Ge neva (Phillips WJ-CU); Canastota; Sea Breeze; Byron (Cy); Bluff Pt. (Phillips WJ-CU) ; Penn Yan (type-OS) ; Taughannock Falls; Ithaca (Cy); Amsterdam (PhillipsWJ-CU). Breeds only in quack grass, Agropyron, making inconspicuous galls, or occasionally galls in the sheath that surrounds the head. Males are rare.

H. dactylicola P. \& E. Penn Yan, Geneseo, Waterloo, Geneva (P\&E) ; Ithaca and Hancock $(\mathrm{Cy})$. Breeds only in orchard grass, and inhabits the center of the stem. Males normally occur.

H. elymi (French). Sea Breeze, Middleport, Wellsville, Ithaca, Avoca, Port Jervis, Tuxedo $\mathrm{Pk}$. $(\mathrm{Cy})$. Lives only in spurs of Elymus, probably wherever it grows. Occupies the center of the stem.

H. elymicola P. \& E. Cranberry Cr., Ithaca, Weedsport, Taughannock Falls, Tuxedo Pk. (Cy). Breeds only in species of Elymus, making very conspicuous galls at the second or third internode from the base of the plant. Males regularly occur.

H. festucae P. \& E. Watertown, Geneseo, Syracuse, Geneva (PhillipsWJ). Breeds only in Festuca, making galls in the second to fourth internode from the base of the plants. Males rarely occur.

H. grandis (Rly.) form minuta (Rly.). Wheat Straw Worm. Victor (Cy). Lives only in wheat and is usually found wherever wheat is grown. Inhabits the center of the stem.

H. hordei (Harr.). Barley JoInt Worm. Watertown; Little Falls (PhillipsWJ); Syracuse (PhillipsWJ); Auburn (P\&E). Breeds only in barley, making galls above the second to fourth nodes. Males rarely occur.

H. maculata (How.). Watertown, Little Falls, Syracuse, Geneva, Penn Yan, Ithaca (PhillipsWJ). Breeds only in cheat and other species of Bromus, inhabiting the center of the stem. Males regularly occur.

H. phalaridis P. \& E. Ithaca, Hornby $(\mathrm{Cy})$.

H. tritici (Fitch). Wheat JoInt Worm. Axton, Je $M \& H$ (Ashm-CU) ; Evans Mills; Theresa; Vernon; Clifton; Carlton; Albion; Barre Center; Kent; Pt. Breeze; Wilson; Victor; Middleport; Byron; Elba; E. Bethany; Pavilion; Geneseo; Avon; Perry; Perry Center; Cuylerville; Canandaigua; Second Milo; Alpine; Sheridan; Bradford; Lodi; Ovid; Romulus; Ithaca; Newfield; Cayuta; Niverville; LaGrange (Cy-CU) ; LI: Miller's Place (Cy). Lives only in wheat; makes conspicuous galls, usually about the second or third internode from the base of the stem, though they may occur at every internode.

H. vaginicola (Doane). Wheat-Sheath Gall Joint Worm. Evans Mills; Theresa; Vernon; Clifton; Carlton; Albion; Barre Center; Kent; Pt. Breeze; Wilson; Johnson Cr.; Victor; Middleport; Byron; Elba; E. Bethany; Pavilion; Geneseo; Avon; Perry; Perry Center; Cuylerville; Canandaigua (Cy) ; Crosby (PhillipsWJ-CU) ; Second Milo; Alpine; Sheridan; Bradford ; Lodi; Ovid; Romulus; Newfield; Cayuta (Cy-CU); Ithaca (PhillipsWJCU) ; La Grange; Niverville (Cy); LI: Miller's Place (Cy). Breeds only in wheat, forming a conspicuous gall in the sheath surrounding the head of wheat. The head rarely grows out of the sheath or develops grain. Males never have been found.

\section{PRODECATOMA Ashmead}

P. phytophaga Cy. Taughannock Falls (Cy), reared from seeds of Virginia creeper. 


\section{FAMILy ENCYRTIDAE}

ANABROLEPIS Timberlake

A. zetterstedtii (Westw.). Ithaca, Griswold (Gahan-CU), on Lepidosaphes ulmi (L.).

COECIDENCyRtus Ashmead

C. ochraceipes Gahan. Ithaca, in greenhouse, Griswold (Gahan-US), from Diaspis boisduvalii Sign. on Cattleya sp.

\section{ApHyCus Mayr}

A. rileyi Timberlake. Ithaca (Sling), on Lecanium corni Bouché.

\section{Atropates Howard}

A. collinsi How. LI: Brooklyn, Roslyn (Collins), on Pulvinaria innumerabilis Rath.

BOTHRIOTHORAX Ratzeburg

B. novaeboracensis How. Richfield Sps., Pergande (How); McLean Bogs, Je, Leon (Gahan).

\section{Cheiloneurinus Mercet}

C. microphagus (Mayr.). Ithaca, Griswold (Mues-CU), from Lepidosaphes ulmi (L.).

\section{ENCYRTUS Latreille}

E. marylandicus var. alpinus Gir. McLean, Aug 1 (Gahan-CU).

\section{APHIDENCYRTUS Ashmead}

A. inquisitor (How.). Ithaca, in greenhouse, Griswold (Gahan-CU, US), from Macrosiphum cornelli Patch on geranium, and Trialeurodes vaporariorum (Westw.) on squash. A hyperparasite.

\section{Copidosoma Ratzeburg}

C. gelechiae How. Ithaca (Leiby). Host: Gelechia gallaesolidaginis Rly.

C. truncatellum (Dalm.). Rye, Aug, oviposit in egg of Autographa sp., emerge from larva. 1732 adults have been reared from one host larva and presumably from one parasite egg (Bird).

\section{OOENCYRTUS Ashmead}

O. clisiocampae (Ashm.). Ithaca; Spring Val.; Suffern; Tallman; White Plains, CLLiu (Mues-CU); reared from egg of apple-tree tent-caterpillar.

PSEUdency RTUS Ashmead

P. cecidomyiae (How.). Ithaca (Ashm). 


\section{Psylledontus Crawford}

P. insidiosus Crawf. Geneva (Parrott), from pear psylla.

ZaRHOPALUS Ashmead

Z. sheldoni Ashm. L. Placid, Sheldon (Ashm).

\section{FAMILY SIGNIPHORIDAE}

SIGNIPHORA Ashmead

S. coquilletti Ashm. Ithaca, in greenhouse, Griswold (Gahan-CU, US), from Diaspis boisduvalii Sign. on orchid.

\section{FAMILY EUPELMIDAE}

\section{Anastatus Motschulsky}

A. pearsalli Ashm. LI: Brooklyn, bred by Pearsall as an egg parasite of Smerinthus astylus Dru.

\section{EUPELMUS Dalman}

E. allynii (French). Camillus; Ira (D. T. Ries); Waterloo (PhillipsWJ); Clifton (PhillipsWJ-CU); Victor; Brockport; Bergen (DTRies); Byron (PhillipsWJ-CU); Albion; Middleport; Wyoming; Mt. Morris; Penn Yan (PhillipsWJ); Newfield (DTRies); Ithaca (PhillipsWJ-CU); Owasco (DTRies). Reared from Cephus pygmaeus by D. T. Ries; and from larvae in orchard grass and timothy by $\mathrm{Cy}$.

\section{Metapelma Westwood}

M. spectabilis Westw. Syracuse $(\mathrm{B} \& \mathrm{~S})$; Batavia, Kngt (Cy-CU); Ithaca (Cy$\mathrm{CU})$; Ilion; Albany (Cy).

\section{FAMILY PTEROMALIDAE}

\section{ASAPHES Walker}

A. americana Gir. Ithaca, in greenhouse, Griswold (Gahan-CU, US), from Macrosiphum cornelli Patch on geranium, and from Myzus persicae Sulz. on Chrysanthemum frutescens.

A. rufipes Brues. Ithaca (Herrick and Hungate), on cabbage aphis.

\section{CyRTOGASTER Walker}

C. liqueata Ashm. Durlandville (FrostSW), from Agromyza parvicornis Loew.

\section{DIBRACHYS Foerster}

D. boucheanus (Ratz.). Ithaca, CLLiu (Mues-CU), from cocoons of Apanteles clisiocampae Ashm.; Glens Falls, J1 (Felt).

EUPTEROMALUS Kourdjumoff

E. viridescens (Walsh). Rye (Bird), from Microplitis cocoons. 
EUTELUS Walker

E. onerati (Fitch). "NY" (Fitch).

HABROCYTUS Thomson

H. dux Gir. Ithaca, CLLin (Mues-CU), from Hyposoter fugitivus (Say).

H. piercei Crawf. Ithaca (Mues), from Anthonomus nebulosus Lec. (Curculionidae).

HOMOPORUS Thomson

H. chalcidiphagus (W. \& R.). Penn Yan (PhillipsWJ); Weedsport, Cy (PhillipsWJ-CU), Victor (Cy-CU), from larvae in wheat.

Hypopteromalus Ashmead

H. tabacum (Fitch). LI: Brooklyn, Schott (Gahan).

LARIOPHAGUS Crawford

L. distinguendus (Foerst.). LI: Brooklyn, on bags of grain, Schott (Gahan).

MERISUS Walker

M. destructor (Say). Ithaca (CU).

M. febriculosus Gir. Cranberry Cr.; Remsen; Geneva; Victor; Taughannock Falls; Ithaca (Cy-CU); Oneonta; Amsterdam; Albany, from timothy and Muhlenbergia.

MoRMONIELla Ashmead

M. brevicornis Ashm. Ithaca (Cy), from Protocalliphora avium Shannon.

P. altiscuta How. Ithaca.

PACHYNEURON Walker

P. siphonophorae (Ashm.) (micans How.). Ithaca (Herrick \& Hungate), on cabbage aphis.

\section{PSYCHOPHAGUS Mayr}

P. omnivorus (Wlk.). Ithaca, CLLin (Mues-CU), reared from pupae of appletree tent-caterpillar.

Pteromalus Swederus

P. puparum (L.). Esperance; Schenectady.

P. vanessae How. Waterville (Gahan-CU), from pupae of Polygonia interrogationis Fab.; ?Ithaca (CU), from pupae of Aglais antiopa L.

RHAPHitelus Walker

R. maculatus W1k. Ithaca (Cy).

\section{FAMILy APHELINIDAE}

ABLERUS Howard

A. clisiocampae (Ashm.). Tallman; Spring Val.; Suffern, CLLiu (Mues-CU), reared from eggs of apple-tree tent-caterpillar. 


\section{Aphelinus Dalman}

A. diaspidis How. Several counties (Hodgkiss \& Parrott). Host: San José scale.

A. fuscipennis How. Ithaca, Griswold (Gahan-CU), on Lepidosaphes ulmi (L.). Several counties (Hodgkiss \& Parrott), from San José scale.

A. jucundus Gahan. Ithaca, in greenhouse, Griswold (Gahan-CU, US), from Macrosiphum cornelli Patch on geranium.

A. mali (Hald.). Ithaca (Lundie), on Eriosoma lanigera.

A. mytilaspidis LeB. Rochester (CU), from Diaspis coerulea; Ithaca, Griswold (Mues-CU), on Lepidosaphes ulmi (L.).

A. semiflavus (How.). Ithaca, in greenhouse, Griswold (Gahan-CU, US), from Francoa rosarum (Kalt.), an aphid on rose.

\section{ENCARSIA Foerster}

E. formosa Gahan. Ithaca, greenhouse, Griswold (Gahan-CU, US), from Trialeurodes vaporariorum (Westw.) on squash and Solanum.

E. pergandiella How. Ithaca, greenhouse, Griswold (Gahan-CU, US), from Trialeurodes vaporariorum (Westw.) on squash.

\section{ASPIDIOTIPHAGUS Howard}

A. citrinus (Crawf.). Ithaca, Griswold (Gahan-CU), on Lepidosaphes ulmi (L.).

\section{CENTRODORA Foerster}

C. speciosissimus (Gir.). Brockport: Lyons. Reared from Hessian fly, $C y$ (Myers).

Coccophagus Westwood

C. fletcheri How. Ithaca, CLLiu (Gahan-CU).

C. gossypariae Gahan. Ithaca, May, July, Griswold (Gahan-CU, US), from Gossyparia spuria (Modeer) on American elm.

C. lecanii (Fitch). Ithaca (How); LI: Flatbush, Roslyn (How); parasite on scale insects.

\section{PERISSOPTERUS Howard}

P. pulchellus (How.). Several counties (Hodgkiss \& Parrott). Host: San José scale.

\section{Prospaltella Howard}

P. aurantii (How.). Ithaca (How), parasitic on scale insects.

\section{FAMILY EULOPHIDAE}

\section{APTEROLOPHUS Gahan}

A. pulchricornis Gahan. Leeds (WMMann).

\section{CHRYSOCHARIS Foerster}

C. parksi Crawf. Durlandville, from Agromyza taxana Mel., Pine I., from Agromyza pusilla Meig., and Tarrytown, from Phytomyza obscurella Fall. (FrostSW). 
CHRYSOCHAROMYIA Girault

C. elegantissima Gir. Tarrytown (FrostSW), from Phytomyza obscurella Fall.

Cirrospilus Westwood

C. flavicinctus Rly. Western NY (Lint), on Bucculatrix pomifoliella Clem.

C. ovisugosus Cy. \& Mat. Ithaca (types-CU), as an egg parasite of the four-lined leaf-bug, Poecilocapsus lineatus (Fab.).

\section{Closterocerus Westwood}

C. tricinctus (Ashm.). Tarrytown (FrostSW), from Phytomyza obscurella Fall.

COMEDO Schrank

C. orgyiae (Fitch.). The Grave-Stone Chalcid. Ringwood, Ndm (Cy\&LeonCU) ; McLean, Aug 19 (Gahan-CU).

\section{DEROSTENUS Westwood}

D. salutaris Cy. Rochester (Cy), from Nepticula slingerlandella Kf.

\section{DiAulinus Schulz}

D. begini (Ashm.). Pine I. (FrostSW), from Agromyza pusilla Meig.

D. pulchripes Crawf. Pine I. (FrostSW), from Agromyza posticata Meig.

D. websteri Crawf. Durlandville (FrostSW), from Agromyza parvicornis Loew; Tarrytown (FrostSW), from Phytomyza obscurella Fall.

\section{DimmockiA Ashmead}

D. incongrua (Ashm.). Ithaca, CLLin (Mues-CU), from cocoons of Apanteles clisiocampae Ashm., also from Psychophagus omnivorus (W1k) and Tachina mella Wik.(?).

\section{ELACHeRtus Spinola}

E. benefactor Crawf. Karner (Crawf), from Rhyacionia comstockiana Fern.

\section{PLEUROTROPIS Foerster}

P. benefica Gahan. Honeoye Falls; Stafford; LeRoy; Bethany; Caledonia; Batavia; Canandaigua; Holcomb; Moravia; Newfield (DTRies); reared from Cephus pygmaeus.

\section{Secodella Girault}

S. argyresthiae Crawf. Ithaca, from Argyresthia alternatella Zell. (Yponomeutidae).

\section{SyMPIESIS Foerster}

S. felti Crawf. Hudson Falls (Felt), reared from Agromyza melampyga Loew; LI: Westbury, Ap (Felt), from Phytomyza ilicis Curt.

S. maculipes Crawf. Geneva (BBFulton), from Lithocolletes sp. 


\section{TETRASTichus Haliday}

T. agrili Crawf. Geneva, from Agrilus sinuatus (Oliv.).

T. asparagi Crawf. LI: Riverhead (Russell \& Johnson).

T. juniperi (Crawf.). Ithaca (Marcovitch), reared from juniper berries.

T. malacosomae Gir. Ithaca, Spring Val., Suffern, Tallman, White Plains, CLLiu (Mues-CU), reared from eggs of apple-tree tent-caterpillar.

T. marcovitchi (Crawf.). Ithaca, Marcovitch (types), reared from juniper berries.

T. rapae Wlk. Ithaca, Mues (Gahan-CU), from Apanteles glomeratus (L.).

\section{FAMILY TRICHOGRAMMIDAE}

HYDROPHYLAX Matheson and Crosby

H. aquivolans Mat. \& Cy. Ithaca (types-CU).

\section{TRICHOGRAMMA Westwood}

T. minutum Rly. Utica; Ithaca; Schoharie; Albany; Youngstown.

\section{FAMILY MYMARIDAE}

ANAGRUS Haliday

A. ovijentatus Cy. \& Leon. Ithaca, egg parasite on Lygus pratensis L.

CARAPHRACTUS (Haliday) Walker

C. cinctus W1k. Ithaca (Mat\&Cy), reared from eggs of Notonecta.

Gonatocerus Nees

G. ovicenatus Cy. \& Leon. Ithaca (types-CU), egg parasite of. Idiocerus scurra Germ.

P. piceipes Gir. Oswego.

$$
\text { Polynema Haliday }
$$

\section{FAMILY EVANIIDAE}

EVANIA Fabricius

(Subgenus Evania Fabricius)

E. appendigaster L. NYC; LI: Brooklyn (Sf). This and the following species are parasites of domestic cockroaches, and are found in cities.

E. punctata Brullé (urbana Bdy.). Albany, Je 3-Aug 6 (Bdy-NYS); Helderbergs, SCBishop (Bdy-NYS) ; NYC; SI, Ds (Bdy); LI: Brooklyn, Je, Eng (Sf); Flatbush, Zab (Bdy-AM); Bellport, Nic (Sf).

\section{(Subgenus Evaniella Bradley)}

E. semaeoda Bdy. LI: Wading R. (Sf). 
HYртіA Illiger

H. harpyoides Bdy. Ithaca, J1 9-17 (types-CU) ; LI: Flatbush, J1 2, Zab (BdyCU) ; Central Pk., J1 (Sf) ; Cold Sp. Harb., J1 31 (Bdy-AM); Wading R. (Sf).

H. mylacridomanesa Bdy. Ithaca, Comstock (type-CU).

H. reticulata Say. Huguenot, Zab (Brues-AM); LI: Bellport, Nic (Sf-AM).

H. prosetethetra Bdy. LI: Flatbush, Fishers I., Zab (Brues-AM).

H. thoracica Blanch. LI: Wading R. (Sf).

Superfamily VESPOIDEA

Family PSAMmochaRIdAE: The Spider-Hunting Wasps 165

(Pompilidae)

SUBFAMILY PSAMMOCHARINAE

(Pompilinae)

(The members of this subfamily construct subterranean nests which they provision with spiders.)

Tribe PSAMMOCHARINI

(Pompilini)

Agentordeus Ashmead

A. humilis (Cress.). Keene Val., Not (Beq); Ithaca, J1 1-7 (Bks-MCZ); LI (Bks-MCZ).

Allocyphonyx Așmead

A. maurus (Cress.). Saranac, J1, 1905, Yg (NYS).

APORINELlus Banks

A. fasciatus (F. Sm.). LI: Pennequid Barrens (Beq). Austral species.

A. ferrugineipes (Vier.). LI: Bayville (Bks-Beq, 1919). Austral species.

\section{ARACHNOPHROCTONUS Ashmead}

A. ferrugineus (Say). Ithaca, Aug 7 (Bks-CU) ; Ft. Montgomery, Schott (Beq) ; SI (Beq). Widely distributed; a common southern species. Ithaca must be nearly its northern limit.

\section{Batazonus Ashmead}

B. interruptus (Say). Gloversville, Sep, 1908 (NYS) ; Rochester Jct., J1 7, Leon (Bks-CU) ; White Plains (Beq) ; LI: Jamaica, Springs, Mastic, Wyandanch, Orient (Beq). Austral form.

165 The manuscript for this family has been submitted to Messrs. Banks, Bequaert, and Rohwer, and we are indebted to these gentlemen for their criticisms, suggestions, and additions. Mr. Banks has kindly indicated the proper generic position of certain sfecies. 


\section{EPISYRON Schiødte}

E. biguttatus (Fab.). Keene Val., Aug 3, Not (AM) ; New Russia, Aug 18, Bdy (Bks-CU) ; Potsdam, Je (Bks-CU) ; Buffalo (Beq); Cattaraugus, Sep 4 (Bks-CU) ; Ithaca, J1 25 (Bks-CU); Norton's Landing, May 25-J1, SmHH (Cress-CU) ; McLean, J1 9-Aug 17, Sib (Bdy-CU); Oliverea (Beq) ; L. George, Aug, Zab (AM); Rhinebeck, J1 27, Cy (Bks-CU) ; Katonah, Aug, Bt (AM) ; West Farms, Angus (AM) ; LI: Wyandanch, Orient, Gardiners I. (Beq) ; Barren I., Aug, Zab (AM). Chiefly Canadian. Provisions its nest with Epeira labyrinthea.

E. quinquenotatus (Say). Wilmington, Aug 20-25 (Bks-CU); Keene Val., Not (Beq) ; Ithaca, J1 25-Aug 8 (Bks-CU) ; L. George, Aug 22, Zab (AM) ; Ft. Montgomery, Schott (Beq) ; LI: Rosedale, Schott (Beq); Forest Hills, Sep 11, Frost (Bks-CU); Flatlands, Zab (AM); Wading R., J1 (Ds). Canadian. Provisions its nest with Epeira strix.

E. snowi (Vier.). Rochester Jct., Je 7, Leon (Bks-CU). A southern form ranging into Canada.

\section{PEDINASPIS Kohl}

P. niger (Cress.). Boston, Niagara Falls, Lancaster, J1-Aug, MCV (Bks-MCZ); LI: Orient, Aug, Zab (Bridwell-AM).

PSAMMOCHARES Latreille

(Pompilus Fabricius)

(Subgenus PSAMmochaRes Latreille)

P. atlanticus Bks. LI: Long Beach, Aug (types-AM).

P. astur Bks. New Russia, Aug 18, $B d y$ (Bks-CU). Canada to Va.

P. lasiope Bks. Essex Co, Aug 18, Bdy (paratype-CU) ; Saranac L., Aug 25 (typeCU) ; Ithaca, May, 1907 (paratype-CU).

P. relativus (Fox). New Russia, Aug 18, Bdy (Bks-CU); White L. (AM); Manlius, Sep 1-21, SmHH (Cress-CU) ; LI: Fire I., Aug 7 (AM); Wading R., Ds; Orient, Long Beach, Gardiners I. (Beq).

P. pretiosa Bks. LI: Sea Cliff, on the beach (types-MCZ).

P. scelestus (Cress.). Speculator, Aug, 1912, Yg (NYS) ; Harford to Caroline, Je 15, MacG, Bdy (Bks-CU).

P. sublaevis Bks. LI: Sea Cliff (one of the types-MCZ).

P. tropicus (Fab.). SI: Watchogue (Beq); LI: Orient (Beq).

P. ventralis Bks. Ithaca, J1 1 (Bks-CU). Known also from Ga.

\section{(Subgenus LOPHOPOMPILUS Radoszkovszky)}

P. aethiops (Cress.). Round I. (Bks-CU) ; Gloversville, Sep, $Y g$ (NYS) ; Albion (Bks-CU) ; Cattaraugus, Sep 4 (Bks-CU) ; Otto, Sep 1-15, Comstock (BksCU) ; Ithaca, J1 24-Aug 28 (Bks-CU); McLean, J1 18-Sep 14, Sib (BdyCU) ; Oliverea (Beq) ; Nyack, West Farms, Mosholu (AM); Atwater, Sep 17; SI (Beq) ; LI: Sea Cliff (Bks-CU); Flatbush (AM); Long Beach, Gardiners I. (Beq).

P. atrox (Dahlbom). Ithaca, J1 25 (Bks-CU) ; LI: Flushing, Jamaica, Rockaway Beach, Wyandanch (Beq).

P. bengtssoni Regan. LI: Riverhead, Sep 14 (Bdy-CU); Fishers I., Long Beach (paratype); Wyandanch, Gardiners I. (Beq). 
P. cleora Bks. Regan records this species from Ithaca and from Rockaway and Wading R., LI; but Banks thinks these eastern records of cleora are really his ilione.

P. ilione Bks. LI: Sea Cliff (one of the type series).

\section{(Subgenus Anoplius Lepeletier)}

P. depressipes (Bks.). Ithaca, J1 21 (type-CU).

P. illinoiensis (Robertson). New Russia, Aug 18, Bdy (Bks-CU) ; Cattaraugus, Sep 4 (Bks-CU) ; Otto, Comstock (Bks-CU) ; Ithaca, J1 4-Aug 24 (Bks-CU); McLean, J1 18-Sep 12 (Bks-CU); Oliverea (Beq) ; Middletown, J1 2-20, Spooner (Bks-CU); SI (Beq).

P. ithaca Bks. New Russia, Aug 18, Bdy (Bks-CU) ; L. Keuka, Je 28, Cy (Bks$\mathrm{CU})$; Ithaca, J1 (type), common, May 23-Aug 26 (Bks-CU) ; Thousand Isls. (Bks-CU).

P. luctuosus (Cress.). SI (Beq). This is a western and northern species.

P. similaris Bks. Ithaca, J1 19, 25 (types-CU).

P. tenebrosus (Cress.). Keene Val., Not (Beq); Saranac, J1, Yg (NYS); Old Forge, J1, Yg (NYS); Ithaca, Aug 15, McLean, J1 29, Malloryville, Je 18-20, $M a c G, B d y$ (Bks-CU) ; LI: Coney I., Oct 6, Zab (AM). Chiefly a Canadian and Rocky Mt. species.

P. virginiensis (Cress.). New Russia, $B d y$ (Bks-CU); Ithaca, Je 10-Aug 7, McLean, J1 27-Aug 12, W. Danby, Aug 15, Harford to Caroline, Je 15, MacG, $B d y$, Spencer, Je 30 (Bks-CU) ; Norton's Landing, Cayuga L., $\mathrm{SmHH}$ (Cress-CU) ; Ft. Montgomery, Schott (Beq); SI (Beq); LI: Gardiners I. (Beq). Transitional to austral.

\section{(Subgenus Pompiloides Radoszkovszky)}

P. americanus (Pal. de Beauv.). New Russia, Aug 18, Bdy (Bks-CU); Keene Val., Not (Beq); SI (Beq); LI: Brooklyn (Beq); Farmingdale, J1 18, Morrow (Bks-CU). A southern species.

P. cylindricus (Cress.). New Russia, Aug 18, Bdy (Bks-CU); Keene Val., Not (Beq) ; Wilmington, Aug 20-25, 1000-2000 ft. (Bks-CU); Speculator, Aug 12, Yg (NYS) ; Ithaca, May-Aug 31, Spencer, Je 30, McLean, J1 3-Sep 24, $S i b$ (Bks-CU) ; Norton's Landing, Cayuga L., Je 22, SmHH (Cress-CU); LI: Wading R., Orient (Beq). Essentially a Canadian species.

P. marginatus (Say). Keene Val., Not (AM) ; New Russia, Aug 18, Bdy (BksCU) ; Potsdam, Aug 14 (Bks-CU); White L., Zab (AM); Rochester Jct., J1 14, Leon (Bks-CU) ; Manlius, Sep 6, SmHH (Cress-CU); Ithaca, Taughannock, J1 15-Sep 1 (Bks-CU); Homer, J1 2 (Bks-CU); Oliverea (Beq); White Plains (Beq); Mosholu (AM); West Farms, Angus (AM); SI (Beq) ; LI: Forest Hills, Sep 11, Frost (Bks-CU) ; Riverhead, Sep 17 (BksCU) ; Coram, Fishers I. (AM); Mastic, Yaphank, Gardiners I. (Beq).

P. rectus (Bks.). Black Br., Je 21 (Bks-CU) ; E. Aurora, $M C V$ ( $\mathrm{MCZ}$, one of type series); Ithaca, J1 9-25 (Bks-CU); LI: Orient (Beq).

P. reducta (Bks.). Wilmington, Aug 20-25, 1000-2200 ft. (Bks-CU); McLean, J1 18, 1921 (Bdy-CU). Known chiefly from Canada.

P. semirufus (Cress.). LI: Long Beach (Beq).

(Subgenus SeRICopompilus Ashmead)

P. humilis (Cress.). "NY", type. 


\section{(Subgenus NANNopompILUS Ashmead)}

P. argenteus (Cress.). White L., Aug 25, Zab (AM); McLean, J1 18, 1921 (Bdy-CU); Catskills (AM); Rhinebeck, J1 27, Cy (Bks-CU). An austral species.

P. rufibasis (Bks.). Ithaca, early J1 (holotype).

\section{(Subgenus SoPHROPOMPILUS Ashmead)}

P. brevicornis (Cress.). Niagara Falls, J1, $M C V$ (Bks).

P. hyacinthinus (Cress.). E. Aurora, Elma, J1-Aug, $M C V$ (Bks) ; Ithaca, J1 25, Otto, Comstock (Bks-CU); Oliverea (Beq); LI: Orient (Beq).

P. ingenuus (Cress.). New Russia, Aug 18, Bdy (Bks-CU); SI (Beq); LI: Sea Cliff, Aug (Bks-Mus. Comp. Zool., Harvard Univ.); Long Beach (Beq); Rockaway Beach (Beq).

P. quadrispinosus Bks. LI: Long Beach, Aug (Bks); Orient, Gardiners I. (Beq).

P. subviolaceus (Cress.). Albany, J1, 1906, Yg (NYS); LI: Cold Sp. Harb. (AM) ; Gardiners I. (Beq). Mr. Banks considers that this is probably identical with $P$. hyacinthinus.

\section{Tribe PEPSINI}

\section{AgENIELla Banks}

A. accepta (Cress.). LI: Riverhead (Beq).

A. bombycina (Cress.). Rochester Jct., J1 11, Leon (Bks-CU); Ithaca, bred from mud nest found under stone, adults emerged Je 14, Comstock (Rly-CU); West Farms, Angus (Cress-AM); LI: Brooklyn (Beq); Rockaway (AM).

A. calcarata (Cress.). Ithaca, J1 16 (Bks-CU).

A. eximia Bks. Ithaca, J1 2-Aug 10 (paratype-CU).

A. iridipennis (Cress.). Ithaca, J1 22 (Bks-CU).

A. norata Bks. Niagara Falls, $M C V$ (one of type series-Mus. Comp. Zool., Harvard Univ.).

A. perfecta (Prov.). E. Aurora, J1, MCV (Bks); Ithaca, J1 10 (Bks-CU).

A. petiolata (Cress.). LI: Gardiners I. (Beq).

A. tenella Bks. Niagara Falls, J1 31, $M C V$, Boston, Aug 1, MCV (types).

\section{Agriogenia Banks}

A. brevis (Cress.). LI: Springs (Beq).

A. pulchripennis (Cress.). Albany, Je, $Y g$ (NYS); Poughkeepsie, Je, 1908, Yg (NYS); West Farms, Angus (AM).

\section{CRyptocheilus Panzer}

C. fulvicornis (Cress.). LI: Sheepshead Bay, Aug, 1903, Yg (NYS).

C. magnus (Cress.). LI: Riverhead (Beq).

C. minorata (Bks.). Ithaca, May 13-Sep 7 (Bks-CU).

C. unifasciatus (Say). New Baltimore (AM); Albany, Aug, 1907 (NYS); Ft. Montgomery, Aug 19, Schott (Beq) ; Stony Pt. (Beq); Bear Mt., Ols (Beq); Thiells, Aug 22, Wat (Ds); SI, Aug 25, Ds (Beq); LI: Cold Sp. Harb. (AM); Mastic (Beq). 


\section{PRIOCNEMIS Schiødte}

P. alienatus (Sm.). Manlius, Aug 29, SmHH (Cress-CU); Taughannock, Aug 22, HJones (Bdy); Ithaca, J1 9, Mud Cr., Je 17-20, MacG, Bdy (Bks-CU); Ft. Montgomery, W. Nyack, Schott (Beq); West Farms, Angus (Bks-AM) ; SI: Watchogue, Sep (Ds) ; LI: Sea Cliff, Sep (Bks) ; Port Jefferson, Cold Sp. Harb., Orient, Long Beach (Bks-AM); Pennequid Barrens, Gardiners I. (Beq).

P. conicus (Say). New Russia, Aug 18, Bdy (Bks-CU) ; Rock City, Je 9 (BksCU) ; Ithaca, May 2-Je 8, common, McLean Bogs, May 30, Taughannock Falls, May 15, Slaterville to Caroline, Je 14 (Bks-CU); Norton's Landing, Cayuga L., May 25-Je 5, SmHH (Cress-CU); Oliverea (Beq). Essentially a transitional species.

P. fortis (Cress.). "NY" (type-AES).

P. germanus (Cress.). Keene Val., Not (Beq); Keene (Bks-CU); New Russia, Aug 18, Bdy (Bks-CU) ; Old Forge, J1, Yg (NYS) ; White L., Aug 16, Zab (Bks-AM); Ithaca, J1 19 (Bks-CU); Oliverea (Beq); NYC (Beq); LI: Cold Sp. Harb., Gardiners I. (Beq).

P. nebulosus (Dahlb.). "NY" (Cress).

P. nothus (Cress.). E. Aurora, $M C V$ (Bks); Otto, Comstock (Bks-CU); Ithaca. J1 28.

P. pompilius (Cress.). Ithaca, Aug 10-Sep 1 (Bks-CU); Ft. Montgomery, Schott (Beq). Mostly austral; also Pacific Coast.

P. relictus (Bks.). LI: Sea Cliff, Sep (type).

\section{Pseudagenia Kohl}

P. albopilosa (Cress.). Ithaca, J1 22-Sep, and Otto, Comstock (Bks-CU).

P. architecta (Say). Ithaca, Mar 20-J1 22 (Bks-CU); Poughkeepsie, Je, Yg (NYS) ; LI: Huntington, Schott (Beq). Builds mud cells under stones.

P. caliptera (Say). New Russia, Aug 18, Bdy (Bks-CU) ; Niagara Falls, J1, MCV (Bks); Ithaca, Je 9-J1 26 (Bks-CU); Ft. Montgomery, Schott (Beq); Mosholu, Je 18 (AM); LI: Port Jefferson, Gardiners I. (Beq).

P. mellipes (Say). Pike (NYS).

P. nanella Bks. Ithaca (paratype); LI: Sea Cliff (type).

P. nigrella Bks. Ithaca, J1 1 (Bks-CU).

P. variitarsata D.T. New Russia, Aug 18, Bdy (Bks-CU); Keene Val., Not (Beq) ; Otto, Aug, Comstock (Bks-CU) ; Ithaca, Je 25-Aug 19 (Bks-CU); McLean, Aug 7-18, 1925, Robinson, Aug 23-31, 1924, Sib (Bdy-CU).

\section{SUBFAMILY CEROPALINAE}

(The members of this subfamily live as social parasites in the nests of other Psammocharidae.)

Ceropales Latreille

C. bipunctata Say. Newport, Aug, 1909 (NYS) ; Clifton Sps., Aug 18 (Bdy-CU); Otto, Sep, Comstock (Bdy-CU); Cattaraugus, Sep 14 (Bdy-CU); Johnstown, Aug (NYS); Ithaca, not rare (Bks-CU); Freeville, Aug 17 (Bdy-CU); Stony Pt., NÝC (AM); SI (Beq); LI: Long Beach, Gardiners I. (Beq). 
C. fraterna Sm. Peru, Je 10 (Bks-CU); New Russia, Aug 18 (Bdy-CU); Wilmington, Aug 20-25, 1000-2200 ft. (Bks-CU); Honeoye Falls, Aug 15, Leon (Bks-CU); Otto, Sep, 1886, Comstock (Bdy-CU); Ithaca, Je 7-Ji 25, frequent (Bks-CU); Malloryville, Je 18-20, MacG (Bdy-CU); McLean, J1 18, 1921 (Bdy-CU). A common Canadian and transitional form.

C. robinsonii Cress. McLean, J1 19, visiting Cicuta maculata, And (Bdy-CU). A rare species, mostly southern and central.

\section{Family CHRYSIDIDAE: Cuckoo Wasps, or Gold Wasps}

Compiled by Leland H. TAYloR

(These wasps live as social parasites in the nests of solitary bees and wasps.)

\section{OMalus Jurine \\ (Elampus Spinola)}

O. corruscans (Nort.). Aurora (E. Aurora?) (AM); Manlius, Aug 4, Norton's Landing, Cayuga L., May 25-Je 22, SmHH (MacG).

O. laeviventris Cress. Albany, Je, Karner, May (NYS).

O. sinuosus (Say). Norton's Landing, Cayuga L., Je 2, SmHH (MacG-CU).

\section{HOLOPHRIS Mocsáry}

H. iridescens (Nort.). Norton's Landing, Cayuga L., May 25-Je 15, Ithaca, MayJ1 16, SmHH (MacG-CU): McLean, Sep 12, 1924, Sib (Bdy-CU).

\section{Notozus Foerster}

N. marginatus Patton. N. Fair Haven, J1 4 (Taylor-CU) ; Penn Yan, J1 12, 1925, Babiy (Taylor-CU) ; Ithaca, J1 16-19 (MacG, Bdy-CU).

N. viridicyaneus (Nort.). Herkimer, Aug 8, 1921, Leon (Taylor-CU).

\section{HOLOPYGA Dahlbom}

H. ventralis (Say). Norton's Landing, Cayuga L., J1, SmHH (MacG-CU); Ithaca, Je-J1 21 (MacG-CU).

\section{HEDYCHRIDIUM Perrin}

H. dimidiatum (Say). Manlius, Aug 4, SmHH (MacG-CU); Ithaca, J1 25 (Bdy-CU).

\section{HEDYCHRUM Latreille}

H. violaceum Brullé. Long L., Aug (NYS) ; N. Fair Haven, J1 4, 1922, Spring Lake, J1 22, 1918, Bdy (Taylor-CU) ; Ithaca, J1 5-Aug 26 (MacG, Bdy-CU); Karner, Je (NYS).

PARNOPES Fabricius

P. aglaspidula Melander and Brues. "NY" (Taylor). 


\section{Chrysidea Bischoff}

(Chysogona auctt. not Foerster)

C. verticalis (Patton). Wilmington, White L. (Taylor-CU); Canandaigua L., Je (NYS) ; Otto, Comstock (MacG-CU) ; Penn Yan, J1 12, 1925, Babiy (TaylorCU) ; Norton's Landing, Cayuga L., May 31-Je 21, SmHH (MacG-CU); Ithaca, Je 8-Aug 31 (Taylor-CU) ; Caroline to Harford (Taylor-CU); New Baltimore (AM) ; Albany, Je, Nassau, May (NYS) ; West Farms (TaylorCU) ; LI: Cold Sp. Harb., Sea Cliff (Taylor-CU).

\section{CHRYSIS Linnaeus}

(Subgenus OLOCHRYsis Lichtenstein)

C. hilaris Dahlb. Norton's Landing, Cayuga L., SmHH (MacG-CU); Ithaca, May 11 (MacG-CU).

C. pacifica Say. Ithaca (Taylor-CU).

\section{(Subgenus GoNochrysis Lichtenstein)}

C. perpulchra Cress. Keene (Taylor).

\section{(Subgenus MONOCHRYSIS Lichtenstein)}

C. discreta Aaron. Ithaca, Sonyea, SI (Taylor-CU).

(Subgenus TRICHRYSIS Lichstenstein)

C. tridens (Lep.) (parvula Fab.). Wells, J1, Yg (NYS); Ithaca, New Windsor (Taylor-CU).

\section{(Subgenus TETRACHRYSIS Lichstenstein)}

C. cessata duBuysson. N. Fair Haven, J1 4, 1922, Snn (Taylor-CU) ; Portageville, Ithaca, J1 5-Aug 2, Babiy, Norton's Landing, Cayuga L., May 30-Je 27, SmHH (Taylor-CU); McLean. Je 20, 1924, Sib (Taylor-CU); Manlius, Sep 15 (Taylor-CU); West Farms (Taylor-CU).

C. coerulans Fab. Summit of Mt. McIntyre, Sep 2, 1922, Snn\&Sib (Taylor-CU); Ithaca, Je 26-Sep 18 (Taylor-CU). It is not certain that these really represent the Fabrician species.

C. conserta duBuysson. "NY" (duBuysson).

C. nitidula Fab. Ithaca, Je 26-J1 29 (Taylor-CU); McLean, Aug 18, 1925, Sib (Taylor-CU). "There is considerable doubt that this is the Eur. nitidula, but it is provisionally so determined." (L. H. T.)

C. pellucidula Aaron. Manlius, Aug 2, SmHH (MacG-CU); Ellis to Slaterville, Je 13, 1904, MacGEBdy (Taylor-CU) ; LI: Cold Sp. Harb. (Taylor).

\section{(Subgenus HEXACHRYSIS Lichstenstein)}

C. inaequidens Dahl. "NY" (Brullé).

C. smaragdula Fab. Ithaca, Je 18 (Bdy-CU); LI: Sea Cliff (Taylor). 


\section{FAMILy ANTHOBOSCIDAE}

\section{SiEROLOMORPHA Ashmead}

S. ambigua Ashm. Ithaca, J1 20, 1904, one specimen (Bdy-CU). An extremely rare insect, of unknown habits.

\section{FAMILY SAPYGIDAE}

(These are extremely rare social parasites of aculeate Hymenoptera.)

SAPYGA Latreille

S. americana Cress. "NY" (type-AES); Ithaca ( $B d y$-CU); Taughannock Falls, Je 6, 1925 (Bdy-CU); Center Lisle, Je 10, PBFletcher (Bdy-CU); Nyack (Bdy-AM); West Farms, Angus (Bdy-AM).

S. centrata Say. Chappaqua, Je 15, 1919, Wat (Bdy-AM).

S. martini Sm. Ithaca, Je 6 (Bdy-CU).

\section{FAMILY TIPHIIDAE SUBFAMILY TIPHIINAE \\ Tiphia Fabricius ${ }^{166}$}

(The members of this genus live in the larval stage on white grubs, Phyllophaga.)

T. clypeata Robertson. Ithaca, J1 19 (Bdy-CU) ; LI: Sea Cliff, Bks (Mall-Mus. Compar. Zool., Harvard Univ.).

T. clypeolata Mall. Ithaca, J1 20, 1904 (Mall-CU).

T. punctata Robertson. Ithaca, Aug 7, 1897 (Mall-CU).

T. similis Mall. Ithaca, J1 20, 1904 (Mall-CU).

T. transversa Say. Ithaca, J1 19-24 (Bdy-CU). This seems to be the common local species at Ithaca.

T. robertsoni Mall. Ithaca, Aug 4, 1885 (Mall-CU).

T. inornata Say. Ithaca, J1 17-Aug 18 (Bdy-CU).

$\mathrm{T}$. illinoiensis Robertson. Ithaca, J1 (Bdy-CU).

\section{ELIS Fabricius \\ (Myzine auctt.)}

(The members of this genus are southern, and are confined in New York to the sandy coastal region. The adults are found on flowers of Compositae and Umbelliferae in late summer. The larvae live on white grubs.)

E. interrupta (Say). SI (Beq); LI: Springs, Rosedale, Huntington, Pennequid Barrens, Gardiners I. (Beq).

E. quinquecincta (Fab.) (sexcincta auctt.). SI (Beq); LI: Springs, Wyandanch, Huntington, Long Beach, Gardiners I. (Beq).

${ }^{166}$ The North American species of the genus Tiphia in the collection of the Illinois State Natural History Survey. By J. R. Malloch. Ill. Nat. Hist. Survey. Bul. 13. 


\section{SUBFAMILY METHOCINAE}

Methoca Latreille

M. stygia (Say). Rochester Jct., Je 30, Leon (Bdy-CU); Taughannock, Aug 1, a male, Guerlac (Bdy-CU); Ithaca, Aug 12, 1925, Babiy (Bdy-CU). The larvae live on the larvae of tiger beetles.

\section{SUBFAMILY MYRMOSINAE \\ Myrmosa Latreille \\ (Subgenus Myrmosa Latreille)}

M. unicolor Say. Chilson L., J1 15 (Ds) ; Rochester Jct., Je 19-30, Leon (Bdy-CU) ; Ithaca, Je 24-Aug 6 (Bdy-CU); Taughannock, Aug 15, a female, Guerlac (Bdy); McLean, Aug, 1916 (Bdy-CU) ; L. George, Zab (Bdy-AM) ; Ft. Montgomery, J1 7, Schott (Roh); Rhinebeck, J1 27, Cy (Bdy-CU); West Farms (AM); LI: Cold Sp. Harb., Flatbush, Parkville (Bdy-AM). The females are found in gravel pits among burrows of Halictus, in which they doubtless lay their eggs.

M. blakei Bdy. "NY", a female (type-AES).

\section{FAMILY MUTILLIDAE 167}

PSEUDOMETHOCA Ashmead

P. canadensis (Blake). Rocky Glen, Aug 23 (Mickel) ; Cinnamon L., Je 5, Babiy (Bdy) ; Ithaca, J1 19-Aug 10 (Mickel-CU) ; McLean, Aug 2 (MickelCU); Nyack, West Farms (Mickel); LI: Yaphank (Mickel). Occurs throughout eastern US.

P. geryon (Fox). LI: Sea Cliff, Aug, Bks (Bdy-MCZ). An austral species.

P. simillima (Sm.). SI, Aug 8, Ds (Bdy) ; LI: Flatbush, May 16-Sep 6 (Mickel); Selden, Aug 30, Ds (Mickel) ; Wyandanch, May (Mickel) ; Kissena L., Aug 20 (Mickel). An austral species.

P. sanbornii (Blake). New Russia, Sep 12, Bdy (Mickel-CU); LI: Cold Sp. Harb., J1 27 (Mickel). Occasional in the Carolinian zone, extending slightly into the Transitional.

\section{DAsymutilla Ashmead 168}

(Subgenus BRUESIA Ashmead)

D. bexar (Blake) (harmonia Fox, 9 ). Karner, Je 28-J1 24, $\delta$ ㅇ (Bdy-NYS) ; Albany, J1 9, ㅇ (Bdy-NYS) ; LI: Selden, Sep 1, O⿱㇒ Ds (Mickel-CU) ; Cold Sp. Harb., Je 17-Sep 14, And, SHEmerson (Mickel-CU).

\section{(Subgenus DASymutilla Ashmead)}

D. occidentalis (L.). Mt. Vernon, Sep 1 (Bdy-NYS) ; LI: Selden, Amagansett, Aug 10, thirty-one ơ, Ds (Mickel-CU); Montauk, Aug, Ang (Bdy-NYS). A very common austral species.

${ }^{167}$ Contributions toward a monograph of the Mutillidae and their allies of America north of Mexico, II. The Mutillidae of the eastern United States. By J. C. Bradley. Trans. Amer. Ent. Soc., 1916, 42:309-336.

${ }_{168}$ Dr. C. E. Mickel, who is revising the genus Dasymutilla, has kindly furnished the records known to him for this genus. 
D. gibbosa (Say). Ithaca, J1 27, male (Mickel, Bdy-CU); Rhinebeck, J1 27, male, Cy (Mickel-CU); Middletown, J1 2-10, male, Spooner (Mickel-CU); LI: Sea Cliff, J1 23, HTBasset (Bdy-AES) ; Cold Sp. Harb., J1 16, male (Mickel$\mathrm{CU})$. A scarce species of the Transition zone, extending into the Carolinian.

D. cariniceps (Fox). Ithaca, Je 23-Aug 7, female (Bdy, Mickel-CU) ; LI: Sea Cliff, Bks (Bdy-Mus. Compar. Zool., Harvard Univ.) ; Cold Sp Harb., J1 16Sep 15, females, SHEmerson, And (Mickel-CU). Probably the female of gibbosa; range the same.

D. agenor (Fox). Mosholu, Aug 9, male (Mickel-Penn. State Col.) ; LI: Cold Sp. Harb., Je 27, male, And (Mickel-CU).

D. rugulosa (Fox). LI: Sea Cliff, J1, female, Bks (Bdy, Mickel-CU) ; Cold Sp. Harb., Je 19-Sep 13, females, And, SHEmerson (Mickel-CU).

D. cypris (Blake). LI: Yaphank, Bellport, Wading R., Aug 27, females, Ds (MickelCU) ; Cold Sp. Harb., Je 17-27, female, And (Mickel-CU).

D. ferrugata (Fab.). LI: Wading R., Selden, Yaphank, Aug 27-Sep 1, females, Ds (Mickel-CU) ; Cold Sp. Harb., Je 22-Sep 12, females, And, SHEmerson (Mickel-CU) ; Amityville, J1 14, female, SHEmerson (Mickel-CU).

D. lepeletieri (Fox). LI: Cold Sp. Harb., Je 22-J1 18, males, SHEmerson, And (Mickel-CU) ; Yaphank, J1 4 (Mickel-CU).

D. champlaini Roh. New Russia, Aug 12, female, Bdy (Mickel-CU) ; LI: Yaphank, Aug 29, female, Ds (Mickel-CU); Selden, Amagansett, female, Aug 10-30 (Mickel-CU) ; Cold Sp. Harb., J1 8-Aug 4, females, And, SHEmerson (Mickel-CU).

D. zella Roh. LI: Cold Sp. Harb., Je 17-Aug 25, females, And, SHEmerson (Mickel-CU).

D. alesia Bks. LI: Yaphank, Aug 16, female, Ds (Mickel-CU).

D. hirticula Mickel. Pelham, Aug 7 (paratype); LI: Amagansett, Aug 10 (BdyBM) ; Sea Cliff, J1 1, male, Bks (Bdy-MCZ, Mickel-CU) ; Cold Sp. Harb., J1 9-18, male, And, SHAnderson (Mickel-CU). A species of the Carolinian zone.

D. permista Mickel. LI: Flatbush, J1 21 (paratype); Central Pk., Selden, Yaphank, Aug 27-30, males, Ds (Mickel-CU) ; Cold Sp. Harb., J1 23-27 (paratype), J1 9, male, And (Mickel-CU). This seems to belong chiefly to the Carolinian zone.

D. obscura (Blake). LI: Greenport, Aug 3, female, Eng (Bdy); Wading R., female (Bdy-BM). A scarce species of the Carolinian zone.

\section{Mutilla Linnaeus}

(Subgenus Timulla Ashmead)

M. briaxus Blake. New Russia, Aug 18, female (Bdy-CU) ; Crown Pt., Aug, female (Bdy) ; Selkirk, female, Sep 15, SCBishop (Bdy); Rochester Jct., male, J1 11, Leon (Bdy-CU); Ithaca, male and female, J1 (Bdy-CU); LI: Queens, J1 23, male (Bdy-BM) ; Roslyn, female, Yaphank, J1 10, female, Eng (Bdy); Amagansett, Aug 11, Eng (Bdy) ; Sea Cliff, female, Bks (Bdy); E. Hampton, Sep 26, female, Eng (Bdy); Cold Sp. Harb., Sep 9, female, Eng (Bdy). Ranges from the Rocky Mts. and Canada southward throughout the eastern States to Florida.

M. rufa Lepeletier. LI: Sep 5, male, Eng (Bdy); Sea Cliff, female, Bks (Bdy). An austral species not known from north of $\mathbf{L I}$. 
M. hexagona Say. LI: Central Pk., Ap 17, female, Eng (Bdy); Yaphank, Sep 22, Oct 11, male and female, Eng (Bdy-CU). Known chiefly from the northern Carolinian zone and not from north of LI.

\section{EPHUtA Say}

E. puteola (Blake). LI: Yaphank, Sep 24, female, Eng (Bdy). An austral species unknown from north of LI.

\section{FAMILY SCOLIIDAE}

(The members of this family construct loose cells around the subterranean larvae of Scarabaeidae, paralyzing them and laying their eggs upon them. The species are confined to the warmer parts of the State, and almost exclusively to Long Island and Staten Island.)

\section{SCOLIA Fabricius}

S. dubia Say. LI: Flushing (Beq). An austral species.

S. nobilitata Fab. SI, Ds (Bdy); LI (Bartlett). An austral species, certainly not to be found in New York State outside of the SI and LI regions.

S. bicincta Fab. L. George, Aug 22, Zab (Bdy-AM); Nyack (AM); LI: Flatbush, Aug 11, Zab (AM).

\section{CAMPSOMERIS Lepeletier}

(Elis auctt.)

C. plumipes (Drury). SI, Ds (Bdy); LI: Manhattan Beach, Flushing, Rockaway Beach, Wading R. (Beq); Yaphank (Bdy-AM).

C. quadrimaculata (Fab.). West Farms, Angus (AM); SI, Ds (Bdy); LI: Riverhead, Ds (Bdy); Pennequid Barrens (Beq).

\section{FAMILY FORMICIDAE}

\section{Compiled by Joseph BeQuaert}

\section{SUBFAMILY PONERINAE}

\section{STIGMATOMMA Roger}

S. pallipes (Hald.). Mill Cr., Lewis Co, Not (Beq); Ithaca, occasional (Bdy); Walhalla, Schott (Beq); White Plains, Dt (Beq); Ramapo (Beq) ; Bronxville (Wheel) ; Inwood, NYC, Ds (Beq); SI: Arlington, Je (D\&B) ; LI: West Hills, Wading R., North Beach, Yaphank, Cold Sp. Harb. (D\&B). This and the following species form very small colonies under stones in woodlands.

\section{PONERA Latreille}

P. coarctata (Latr.) subsp. pennsylvanica Buck. Thousand Isls.; Manlius, SmHH (CU) ; Ithaca, common everywhere (Bdy) ; Bronxville (Wheel) ; SI: Arrochar, Watchogue, Clove Val., St. George, and many other places (D\&B) ; LI: Wyandanch, E. New York, West Hills, Newtown Hgts., Cold Sp. Harb. (D\&B).

\section{SySPHINCTA Roger}

S. pergandei Emery. SI: Arrochar near Old Town Road, Je (D\&B). 


\section{PROCERATIUM Roger}

P. crassicorne Emery. SI: Old Town Road, May-Nov (D\&B); LI: Cold Sp. Harb., Aug (D\&B).

\section{SUBFAMILY MYRMICINAE}

MYRMECINA Curtis

M. graminicola (Fab.) subsp. americana Emery. SI: Arrochar, Je (D\&B); LI: Amagansett, Cold Sp. Harb. (D\&B). A rare and local species nesting in rotten wood, or under stones, in damp, shady woods.

M. graminicola (Fab.) subsp. americana Emery var. brevispinosa Emery. "NY" (teste Emery). Nests in small colonies under stones in shady woods, feigning death when disturbed.

\section{MONOMORIUM Mayr}

M. minimum (Buck.). SI: Clove Val., and elsewhere (L\&B); LI: Rockaway, Wading R., Baldwin, Pinelawn, Wyandanch, Gardiners I., Cold Sp. Harb. $(D \& B)$. Constructs crater nests in sandy or gravelly places.

M. pharaonis (L.). The Little Yellow House Ant. Ithaca; NYC. Introduced. Occurs in houses, warehouses, etc.

\section{SOLENOPSIS Westwood}

S. molesta (Say). The Thief Ant. SI: Tottenville, Long Neck (D\&B); LI: Baldwin, Wyandanch, Yaphank, Brooklyn, E. New York, Cold Sp. Harb., Forest $\mathrm{Pk}$. (D\&B). In open grassy places, nesting under stones, or living in the walls of the galleries of nests of large ants.

\section{CREMATOGASTER Lund}

C. lineolata (Say). The Shed-Builder Ant. Wyoming Co (Ds); Ithaca, abundant (Bdy); McLean (Wheel) ; Debruce, Ds (Beq) ; SI: Long Neck, Arlington, Watchogue, and many other places (D\&B) ; LI: Oakdale, Calverton, Yaphank, Half Way Hollow Hills, Wading R., Wyandanch, Pinelawn, Amagansett, Flushing, Shelter I., Southold, West Hills (D\&B). A very abundant species, nesting under stones in open places, stumps, boards, the bark of old logs, etc.

C. lineolata (Say) var. cerasi Fitch. "NY" (Fitch); Ithaca (Comstock).

\section{Pheidole Westwood}

(These are harvesting ants, and are confined to the sandy coastal regions.)

P. davisi Wheel. LI: Wading R., Je (D\&B). Makes small craters in pure white sand of the pine barrens.

P. morrisi Forel. LI: Wading R., Yaphank, Selden (D\&B). Forms low mounds in pure sand, sometimes covering one or two square feet, and often about the roots of a plant (Whieel).

P. pilifera (Roger). Southfields, Schott (Beq); Hillburn, Schott (Beq); SI: Tottenville, Arrochar, and elsewhere (D\&B) ; LI: Aqueduct, Pinelawn, Baldwin, Wyandanch, Cold Sp. Harb. (D\&B). Constructs obscure craters with several openings in sandy or gravelly soil, more rarely lives under stoncs (Wheel).

P. tysoni Forel. Bronxville (Wheel). 
P. vinelandica Forel. SI: Watchogue, Richmond Val., Rossville, Tottenville $(D \& B)$. Constructs diminutive craters in pine barrens where the soil is mixed with clay (Wheel).

\section{STENAmma Mayr}

S. brevicorne (Mayr). Norton's Landing, Cayuga L. ( $\mathrm{SmHH})$; Ithaca, Caroline to Harford (MacG\&Bdy) ; SI, in a salt meadow, May, Nov; LI: Cold Sp. Harb., May (D\&B). This is a rare ant, nesting under stones or in vegetable mold in rich woods (Wheel).

S. brevicorne (Mayr) subsp. diecki Emery. W. Nyack (Beq).

S. brevicorne (Mayr) subsp. diecki (Emery) var. impressum Emery. Richfield Sps. (Emery), type.

\section{Aphaenogaster Mayr}

(The species of this genus nest under bark or stones, or the rotten wood in woodlands.)

A. tennesseensis (Mayr). McLean (Beq); Ithaca.

A. treatae Forel. SI: Long Neck, and elsewhere (D\&B) ; LI: Pinelawn, Sep, Long Pond, Deep Pond, Wading R., Riverhead, Central Pk., Calverton, Yaphank, Gardiners I. (D\&B).

A. fulva Roger. Ithaca; Bronxville (Wheel); Ramapo (Wheel).

A. fulva Roger subsp. aquia Buck. Portage, Rock City, Ds (Beq); McLean (Wheel); Oliverea, Ds (Beq); Southfields, Hillburn, Schott (Beq); LI: Little Neck, Huntington, Cold Sp. Harb. (D\&B).

A. fulva Roger subsp. aquia var. undescribed. Van Cort. Pk. (Beq); SI: New Dorp, Clove Val. (D\&B); LI: Valley Stream, Wyandanch, Half Way Hollow Hills, Amagansett, Baldwin, Yaphank, Gardiners I., Selden (D\&B).

A. fulva Roger subsp. aquia var. picea Emery. SI: Arrochar, Je (D\&B); LI: Smithtown, Cold Sp. Harb. (D\&B).

\section{MYRMICA Latreille}

M. punctiventris Roger. SI: Long Neck, and other places (D\&B); LI: Wyandanch, Yaphank, Amagansett, Gardiners I., Cold Sp. Harb. (D\&B). A rare species, nesting in small colonies under stones or moss in shady woods (Wheel).

M. punctiventris Roger subsp. pinetorum Wheel. LI: Yaphank, Wading R. (D\&B). Nests in pure sand.

M. scabrinodis Nyl. var. fracticornis Emery. Buffalo (teste Emery).

M. scabrinodis Nyl. var. sabuleti Mein. Debruce, West Pt., Ds (Beq) ; Bronxville (Wheel); SI, found several times (D\&B) ; LI: Maspeth, Orient Pt., Fire I., Amagansett, Wyandanch, Yaphank (D\&B). Nests in sandy or gravelly, sunny places, such as open pastures, roadsides, etc. (Wheel).

M. scabrinodis Nyl. subsp. schencki Emery var. emeryana Forel. Wilmington, Ds (Beq); Genesee Co, Ds (Beq); Ithaca; White Plains (Beq); Bronxville (Wheel) ; Inwood, NYC, Ds (Beq) ; SI: Tottenville, Arrochar, Long Neck, and many other places (D\&B); LI: Pinelawn, Wading R., Gardiners I., Rockaway Beach, Montauk, Yaphank, Central Pk., Massapequa, Wyandanch, Half Way Hollow Hills, Amagansett, Orient Pt., Smithtown, Brooklyn, Cold Sp. Harb. (D\&B).

M. brevinodis Emery var. canadensis Wheel. McLean (Wheel-CU) ; E. Jewett, Oliverea, $D s$ (Beq) ; LI: Pinelawn (D\&B). 


\section{LEPTOTHORAX Mayr}

L. curvispinosus Mayr. McLean (Wheel-CU) ; SI: Richmond, Watchogue, Arrochar, and many other places (D\&B); LI: Half Way Hollow Hills, Wyandanch, Yaphank, Amagansett, Pinelawn, Cold Sp. Harb. (D\&B). Nests in hollow twigs and galls (Wheel).

L. curvispinosus Mayr subsp. ambiguus Emery. "NY" (teste Emery).

L. longispinosus Roger. McLean (Wheel-CU); West Pt., Ds (Beq) ; SI: Willow Br., May, Tottenville, Arrochar (D\&B) ; LI: Amagansett, Half Way Hollow Hills, Gardiners I. (D\&B). Nests under small stones lying on large boulders, in the clefts of rocks, in hollow nuts lying on the ground, and more rarely under bark (Wheel).

L. fortinodis Mayr. Newport, Genesee (Beq); SI: Richmond, May-Je (D\&B); LI: Calverton (D\&B). Rare on SI and LI. Has been found nesting in the bark of a living pitch pine (Wheel).

L. acervorum (Fab.) subsp. canadensis Prov. Mt. Marcy, and foot of Cliff Mt. (Beq) ; Mt. Whiteface, $D s$ (Beq). A rather rare boreal form nesting in bark in small colonies (Wheel).

L. schaumii Roger. SI: Richmond. Nests in bark of large elms and willows (Wheel).

\section{HARPAGOXENUS Forel}

H. americanus (Emery). Bronxville (Wheel).

\section{TETRAMORIUM Mayr}

T. caespitum (L.). The Lawn Ant. NYC (Beq); Bronx Pk., Ds (Beq); Bronxville (Wheel); SI: Arrochar, New Brighton, and elsewhere (D\&B); LI: Baldwin, Forest Pk., Rockaway Beach, and Cold Sp. Harb. (D\&B). Introduced.

T. guineense (Fab.). LI: Brooklyn, Jan, in greenhouse; introduced (D\&B).

\section{STRUMigenys F. Smith}

S. pergandei Emery. LI: Forest Pk., Brooklyn, Sep (D\&B). Very rare ants, making subterranean nests, often mixed with larger species (Wheel).

\section{TRACHYMYRMEX Forel}

(These are the fungus-growing ants.)

T. septentrionalis (McC.). Milford (Ds) ; LI: Wading R., May-Je, Hauppauge, Smithtown, Selden (D\&B). The colonies of two to seven spherical chambers are built only in sand, and after a few weeks in early spring are closed until after the heat of summer has passed.

\section{SUBFAMILY DOLICHODERINAE}

\section{TAPINOMA Foerster}

T. sessile (Say). Ithaca, extremely abundant; McLean; SI: Old Place, Tottenville, Todt Hill, and Watchogue (D\&B) ; LI: Rockaway Beach, Riverhead, Yaphank, Wading R., Orient Pt., Fire I., Wyandanch, Pinelawn (D\&B). One of the most abundant of ants. Nests under stones, logs, etc., mostly in open places. 
T. pruinosum Roger. LI: Yaphank, Wyandanch, Montauk (D\&B). A southern species reaching here its extreme northern limit. Forms single or clustered craters in pure white sand (Wheel).

\section{DORYMYRMEX Mayr}

D. pyramicus (Roger). LI: Wading R. (D\&B). Forms a small crater in white sand, three or four inches in diameter (Wheel). This is probably the northern extremity of the range of this species also.

\section{DOLICHODERUS Lund}

D. mariae Forel. LI: Pinelawn, Riverhead, Long Beach, Wyandanch, Calverton, Yaphank, Selden, Massapequa (D\&B). Forms large colonies, nesting in sandy places about the roots of grasses and bushes (Wheel). The workers are commonly met with on plants, attending aphids.

D. taschenbergii (Mayr) var. aterrimus Wheel. (taschenbergii var. gagates Wheel.). Upper Ausable L. (Beq) ; Mt. Whiteface, Ds (Beq); SI: near seashore (D\&B) ; LI: Wyandanch, Calverton, Central Pk., Amagansett, Yaphank, Pinelawn, Massapequa, Coram (D\&B).

D. plagiatus (Mayr). Oliverea, Ds (Beq); SI: Long Neck, Tottenville, Watchogue (D\&B) ; LI: Wyandanch, Yaphank, Amagansett, Smithtown, Massapequa, West Hills (D\&B). Nests in the ground in small colonies (Wheel). These workers also attend aphids.

D. plagiatus (Mayr) subsp. plagiatus Mayr var. inornatus Wheel. SI (D\&B); LI: Pinelawn, Yaphank, Smithtown (D\&B).

D. plagiatus (Mayr) subsp. pustulatus Mayr. SI: Tottenville (D\&B) ; LI: Yaphank, Smithtown, Massapequa, Wyandanch (D\&B).

D. plagiatus (Mayr) subsp. pustulatus Mayr var. beutenmuelleri Wheel. McLean (Wheel-CU) ; LI: Coram, Wyandanch, Massapequa, Farmingdale, south of Smithtown, Selden (D\&B).

\section{SUBFAMILY FORMICINAE}

\section{BRACHYMYRMEX Mayr}

B. heeri Forel subsp. depilis Emery. Ithaca; Bronxville (Wheel); SI: New Springville, Ap (D\&B) ; LI: Cold Sp. Harb. (D\&B). Nests under stones and attends root Coccidae (Wheel).

\section{PRENOLEPIS Mayr \\ (Subgenus PRENolepis Mayr)}

P. imparis (Say). Conesus L., Ds (Beq); Clinton Hgts. (Beq); Callicoon, Ds (Beq); White Plains, Van Cort. Pk. (Beq); SI: Tottenville, New Brighton, Watchogue, Long Neck, Annadale (D\&B); LI: Amagansett, Yaphank, Calverton, Montauk, Pinelawn, Wading R., Gardiners I., Cold Sp. Harb. (D\&B). Makes small crater nests in shady oak woods in soil usually containing more or less clay (Wheel).

P. imparis (Say) var. testacea Emery. SI: Tottenville (D\&B) ; LI: Wading R., Amagansett (D\&B). Nests only in sandy regions, where it replaces the preceding (Wheel). 


\section{(Subgenus NyLANDERIA Emery)}

P. parvula Mayr. SI: Long Neck, Arrochar, Tottenville, Watchogue (D\&B) ; LI: Pinelawn, Wyandanch, Central Pk., Cold Sp. Harb. (D\&B). Under stones in gravelly and sunny places (Whee1).

P. longicornis (Latr.). NYC (D\&B). Introduced.

LASIUS Fabricius 169

(Subgenus LAsIUs Fabricius) 170

(The members of this subgenus are not exclusively subterranean in habit.)

L. niger (L.) var. americanus Emery. Common everywhere. McLean (WheelCU) ; Ithaca; Slide Mt. (Beq) ; SI: Arrochar, Arlington, Watchogue, Long Neck, etc. (D\&B) ; LI: Flatbush, Rockaway Beach, Maspeth, Brooklyn, Yaphank, Fire I., Gardiners I., Pinelawn, Wyandanch, Jamaica, Cold Sp. Harb. $(D \& B)$. The most abundant of ants and hence of all insects. Nests indifferently in rotten wood, under bark, logs, or stones, or in soil. Cultivates root aphids, but also visits the foliage of trees and bushes in search of aphids (Wheel).

L. niger (L.) var. neoniger Emery. Otto; Ithaca (CU).

\section{(Subgenus Chтhonolasius Ruszky)}

(These ants are exclusively subterranean, except for the mating flight. They cultivate root aphids and coccids [Wheel]).

L. brevicornis Emery. Ithaca; Bronxville (Wheel); LI: Long Beach, Yaphank (D\&B).

L. flavus (L.) subsp. nearcticus Wheel. (myops Forel). Ithaca; Garrison (Ck11); LI: Cold Sp. Harb. (D\&B). The American representative of the European flavus. The colonies, which are rather small, are formed under stones on hill slopes and in pastures, where the soil is rather dry and sandy (Wheel).

L. umbratus (Nyl.) subsp. mixtus Nyl. var. aphidicola Walsh. Ithaca, Bdy (Beq); McLean (Wheel); Cedar Hill, Albany (Beq); Richford Sps.; Bronxville (Wheel) ; SI: south shore, and Mariner's Harb. (D\&B); LI: Gardiners I. (D\&B) ; Bergen Beach, VKrochow (Beq). Populous colonies under stones, or in logs and stumps in damp shady woods (Wheel).

L. umbratus (Nyl.) subsp. minutus Emery. McLean, SRobinson (Wheel-CU); SI: New Springville (D\&B).

\section{(Subgenus Acanthomyops Mayr)}

(These are also subterranean, "cultivating root aphids [Wheel]).

L. interjectus Mayr. Canandaigua L. (Beq); SI, found many times (D\&B); LI: Coram (D\&B). Nests in old logs and stumps in open woods, and occasionally makes rough mounds, or excavates its galleries under large stones (Wheel).

L. claviger (Roger). Ithaca; Tarrytown (Beq); Bronxville (Wheel); Van Cort. Pk. (Beq) ; SI: Clove Val., Princess Bay, etc. (D\&B) ; LI: Selden, Amagansett, Riverhead, Wading R., Gardiners I., Huntington, Flatbush, North Beach, Maspeth, Jamaica (D\&B). Nests under stones along the margin of woods where there is plenty of warmth and moisture (Wheel).

109 If the Erlangen List is accepted, the name Lasius will be replaced by Acanthomyops Mayr.

170 If the Erlangen List is accepted, the name of the subgenus Lasius will be replaced by Donisthorpea Morice and Durrant. 
L. latipes (Walsh). SI: Tottenville, and elsewhere (D\&B) ; LI: Selden, Yaphank, Riverhead, Rockaway, West Hills, Wyandanch, Gardiners I., Cold Sp. Harb. (D\&B).

L. murphyi Forel. Bronxville (Wheel); West Farms (Angus); SI: St. George, New Brighton, Clove Val., Watchogue (D\&B); LI: Rockaway, Baldwin, Cold Sp. Harb. (D\&B).

\section{FORMICA Linnaeus ${ }^{171}$}

\section{(Subgenus FoRMICA Linnaeus)}

F. sanguinea Latr. subsp. aserva Forel. Wilmington, Mt. Whiteface, Ds (Beq); Mt. Skylight, Cliff Mt. (Beq); Rock City, Ds (Beq). One of the slavemakers.

F. sanguinea Latr. subsp. rubicunda Emery. Ithaca (Beq); Oliverea, Ds (Beq); Slide Mt., Ds (Beq) ; Bronxville (Wheel) ; LI: Coram (D\&B). A slavemaker, capturing $F$. subsericea. Nests under stones in grassy places along the edges of woods (Wheel).

F. sanguinea Latr. subsp. subintegra Emery. White Plains (Beq); Mosholu, Bronxville (Wheel); SI: New Brighton, Clove Val., Watchogue, etc. (D\&B); LI: Yaphank, Half Way Hollow Hills, Riverhead, Pinelawn (D\&B). Also enslaves $F$. subsericea.

F. sanguinea Latr. subsp. subintegra var. gilvescens Wheel. Tuckahoe (types). Elsewhere known only from Wis.

F. truncicola Nyl. subsp. integra Nyl. Keene Val., Not (Beq); Mt. Whiteface (Beq) ; Ithaca, Debruce, E. Jewett, Oliverea, Ds (Beq) ; Ashokan, VKrochore; High Bridge, West Farms (Angus); Mosholu (Wheel); Bronxville; SI: Richmond, Bradley's Road, Reed's Val., Great Kills (D\&B) ; LI: Rockaway Beach, Riverhead, Yaphank, Wyandanch, Wading R., Half Way Hollow Hills, Long Beach, West Hills (D\&B); Bergen Beach, VKrochow (Beq). Forms great colonies in piles of large stones, or in old logs or stumps, stuffing the crannies with dead grass and leaves. Prefers sunny glades or clearings in the forests of hilly regions (Wheel).

F. truncicola Nyl. subsp. obscuriventris Mayr. Ithaca (Beq); Saugerties, VKrochow (Beq) ; LI: Riverhead, Coram, Pinelawn, Wading R., Amagansett, Gardiners I., Cold Sp. Harb. (D\&B). Under large stones in open woods, often banking the edges of the stones in the vegetable débris. The colonies are much smaller than those of the preceding (Wheel).

F. truncicola Nyl. subsp. obscuriventris var. gymnomma Wheel. LI: Cold Sp. Harb. (types).

F. difficilis Emery. Ithaca (Beq) ; Bronxville (Wheel); SI: Long Neck, Watchogue, Clove Val., etc. (D\&B) ; LI: Coram, Gardiners I., Calverton, Yaphank, Selden, Orient Pt., Pinelawn, Half Way Hollow Hills, Wyandanch, Riverhead $(D \& B)$. Nests under stones, which it banks with vegetable detritus. Occurs sporadically in open woods from NY to N. Car. (Wheel).

F. exsectoides Forel. The Mound-Building Ant of the Alleghenies. Rock City, Ds (Beq) ; Ithaca; Slaterville (Bdy) ; Garrison-on-Hudson, Ckll (Beq); Oliverea, Ds, Palenville (Beq) ; Ramapo Mts., Bronxville (Wheel) ; Southfields, Schott (Beq); West Farms (Angus); SI: Richmond, Kreischerville, Annadale, Mariner's Harb., Bradley's Road, Tottenville (D\&B); LI: Wyandanch, Amagansett, Yaphank, Wading R., Selden, Montauk, Farmingdale

${ }^{171}$ A revision of the ants of the genus Formica. By William Morton Wheeler. Bul. Mus. Comp. Kool., Harvard Univ., 1913, 53:379-565. 
(D\&B). Constructs domes three to four feet in diameter and one to two feet high, in open glades of wooded hilltops. These domes are often partly covered with growing grass.

F. fusca L. var. fusca L. Upper Ausable L., Cliff Mt. (Beq) ; Niagara Falls (Beq) ; McLean (Beq) ; Clinton Hgts, (Beq) ; Ramapo Mts. (Wheel). Nests under stones or logs, or in rude craters or small earthen mounds (Wheel).

F. fusca L. var. subsericea Say. Wilmington, $D s$ (Beq) ; Portageville, Ds (Beq) ; Ithaca, Dryden L., Danby Hills (Beq); McLean (Bdy-CU); Greenville (Wheel); Garrison-on-Hudson (Ck11); Saugerties, VKrochow (Beq); Kiamesha, Brues (Beq) ; Debruce, Ds (Beq) ; Mosholu, Bronxville (Wheel); White Plains (Beq) ; Central Pk., NYC, VKrochow (Beq) ; SI, very common (D\&B) ; LI: Bergen Beach, VKrochow (Beq) ; Flatbush, Central Pk., Wading R., Gardiners I., Wyandanch, West Hills, Amagansett, Yaphank, Flushing, Cold Sp. Harb. (D\&B). A very abundant ant. Prefers open sunny places, and may construct mounds or build under stones, etc. The host of many slave-making species.

F. fusca L. var. gelida Wheel. Cliff Mt., Redfield Mt. (Beq). "The most stenothermal and alpine of all our forms of fusca. Nests in rather small colonies under stones or in logs in woods at high altitudes, just below timberline" (Wheel).

F. fusca L. var. argentea Wheel. Essex Co; Gloversville; Ithaca. Belongs to the colder part of the Transition zone (Wheel).

F. fusca L. var. subaenescens Emery. Ithaca, Bdy (Beq); Bedford (Wheel). A rare species, nesting under logs and stones in shady woods.

F. neogagates Emery. Niagara Falls, Ithaca (Beq) ; Kiamesha, Brues (Beq) ; LI: Gardiners I., Cold Sp. Harb. (D\&B). In small colonies under stones, on hilis at an altitude of $1000 \mathrm{ft}$. or more (Wheel).

\section{(Subgenus NEOFORMICA Wheeler)}

F. pallidefulva Latr. subsp. pallidefulva. Ithaca; White Plains (Beq) ; Bronxville (Wheel). A southern form, very rare as far north as this State.

F. pallidefulva Latr. subsp. schaufussi Mayr. Ithaca, Karner (Beq) ; W. Nyack (Beq) ; Bronxville, Mosholu (Wheel) ; SI, common (D\&B) ; LI: Pinelawn, Amagansett, Yaphank, Wading R., Wyandanch, Gardiners I. (D\&B). A common species, nesting in small colonies under stones or in small obscure mound-nests in sunny and grassy fields.

F. pallidefulva Latr. subsp. schaufussi Mayr var. incerta Emery. Niagara Falls (Wheel); Albany, White Plains (Beq); Tuckahoe (Wheel); Bronxville (Wheel); West Farms (Angus) ; SI: Todt Hill (D\&B) ; LI: Wyandanch, Hempstead, Smithtown (D\&B). Common in the same situations as the preceding (Wheel).

F. pallidefulva Latr. subsp. nitidiventris Emery. Clifton Sps.; Ithaca; W. Danby ; Mosholu, Bronxville. (Wheel); SI (D\&B); LI: Amagansett; Calverton, Southold, Gardiners I., Montauk, Cold Sp. Harb. (D\&B). Habits similar to those of the preceding species.

F. pallidefulva Latr. subsp. nitidiventris var. fuscata Emery. Bronxville (Wheel) ; SI: Todt Hill (D\&B); LI: Wyandanch, Amagansett, Gardiners I., Central $\mathrm{Pk}$., Montauk (D\&B). Rarer than any of the other forms, nests only in woods, usually in hilly country (Wheel). 


\section{Polyergus Latreille}

P. lucidus Mayr. The Shining Slave-Maker. Ithaca, very rare (Bdy); White Plains, W. Nyack (Beq) ; Bronxville (Wheel); LI: Long Pond, Wading R. (D\&B) ; Pinelawn; Selden. Aug-Sep. Enslaves Formica schaufussi.

\section{Camponotus Mayr 172}

C. castaneus (Latr.). West Farms (Angus); SI, Je (D\&B); LI: Central Pk. (D\&B). Nests under stones and logs in small colonies.

C. castaneus (Latr.) subsp. americanus Mayr. Conesus L., Ds (Beq); Ithaca; Norton's Landing, Cayuga L. (SmHH) ; Ft. Montgomery, Schott (Beq) ; SI: New Brighton, and many other places (D\&B) ; LI: Wyandanch, Yaphank, Half Way Hollow Hills, Wading R., Melville, Rockaway Beach, Pinelawn, Gardiners I., Cold Sp. Harb. (D\&B).

C. herculeanus (L.) var. whymperi Forel. Cliff Mt., $3000 \mathrm{ft}$. (Beq).

C. herculeanus (L.) subsp. pennsylvanicus DeG. The Carpenter Ant. Saranac L., Big Moose, Keene Val., Oswego (Beq); Turin, Lucy Armstrong (Beq); Batavia, Portage, Ds (Beq) ; Rock City, Ds (Beq); Ithaca (Beq); McLean (Bdy); Nassau, Cedar Hill (Beq) ; Oliverea, Slide Mt., Ds (Beq); Garrisonon-Hudson (Ckll); White Plains (Beq); W. Nyack, Ols (Beq); Bronxville (Wheel) ; West Farms (Angus) ; SI, common (D\&B) ; LI: Central Pk., Yaphank, Fire I., Smithtown, Gardiners I. Southold, Huntington Sps., Cold Sp. Harb. (D\&B). Abundant in all woodlands, nesting in solid dead wood.

C. herculeanus (L.) subsp. pennsylvanicus DeG. var. ferrugineus Fab. Mosholu, Bronxville (Wheel) ; Ft. Montgomery, Schott (Beq); West Farms (Angus); Manhattan, NYC, Ds (Beq) ; SI: Watchogue, Clove Val. (D\&B) ; LI: Richmond Hill, Jamaica, Wyandanch, Yaphank, Amagansett, Coram, Melville, Wading R., Southold, Cold Sp. Harb., Half Way Hollow Hills, Smithtown (D\&B) ; Forest Pk., VKrochow (Beq). Of similar habits to the preceding, but less common (Wheel).

C. herculeanus (L.) subsp. pennsylvanicus DeG. var. noveboracensis Fitch. L. Tear, Cliff Mt. (Beq) ; Keene Val., Elizabethtown, Saranac L., Ds, Boonville, Titus (Beq); Wilmington, Ds (Beq); Plattsurg (Beq) ; Orleans Co, Pulaski, Ds (Beq) ; Batavia, Genesee Co (Beq) ; Rock City, Ds (Beq); Potter Swamp (Beq) ; Ithaca (Beq) ; Tarbel, W. Danby, McLean, Ds (Beq); Karner, Cedar Hill (Beq) ; Oliverea, Callicoon, Slide Mt., E. Jewett, Ds (Beq) ; West Pt., Debruce, $D s$ (Beq). Of similar habits to the two preceding species.

C. caryae (Fitch) (fallax Nyl. var. nearcticus Emery). Wilmington, Ds (Beq); Honeoye Falls, Ds (Beq); Niagara Falls, $D s$ (Beq) ; Batavia, Ds, Wyoming, $D s$ (Beq) ; Taughannock, Ithaca, Ds (Beq) ; Ithaca (Beq) ; Albany (Beq); White Plains, Bno (Beq); Valhalla, Schott (Beq); West Farms (Angus); Bronx Pk. (Beq); SI: Long Neck, Tottenville, etc. (D\&B); LI: Brooklyn, Queens, Wading R., Yaphank, Gardiners I., Riverhead, Pinelawn, Amagansett, Orient Pt., Central Pk., Half Way Hollow Hills, Cold Sp. Harb. (D\&B). Wheeler says that this ant seems to be associated with pine trees.

C. caryae (Fitch) var. minutus Emery. SI: Long Neck, Clove Val., etc. (D\&B); LI: Melville, Jamaica, Central Pk., Massapequa, Yaphank, Amagansett, Wading R., Coram (D\&B). Nests in dead twigs of oak.

C. caryae (Fitch) var. pardus Wheel. White Plains, Mosholu (Wheel) ; Bronxville (Wheel); West Farms (Angus) ; LI: Queens, Jamaica (D\&B).

${ }^{172}$ The North American ants of the genus Camponotus Mayr. By William Morton Wheeler. Ann. N. Y. Acad. Sci., 1910, 20:295-354. 


\section{FAMILY BETHYLIDAE}

PARASIERola Cameron

P. cellularis (Say). Spring Lake, J1 23 (Fouts-CU); Ithaca (Fouts-CU).

\section{PLASTANOXUS Kieffer}

P. chittendeni (Ashm.). Ithaca, Chitt (type-US). From Cis fuscipes Melsh. in fungus.

\section{PRISTOCERA Klug}

P. armifera (Say). White L., Zab (AM); LI: Flatbush, Zab (AM); Yaphank, May $8, D s$ (Beq).

\section{FAMILY VESPIDAE 173}

\section{Compiled by Joseph Bequaert}

\section{SUBFAMILY ZETHINAE}

\section{ZETHUS Fabricius}

Z. spinipes Say. LI: Gardiners I., Ds; Cold Sp. Harb., Brues. The Gardiners I. specimen appears to belong to var. substrictus Hald.

\section{SUBFAMILY EUMENINAE}

EUMENES Latreille

E. fraterna Say. New Russia, Bdy (CU) ; Trenton Falls, Leon, Fbs (CU) ; Spring Lake, $B d y(\mathrm{CU})$; Ithaca, McLean (CU) ; Manlius, $\mathrm{SmHH}$ (CU) ; Oliverea ; White Plains; SI; LI: Long Beach; Central Pk.; Gardiners I.; Orient Pt.; Springs; Hempstead Plains, Wyandanch, Schott; Wading R., Eng; West Hills, Riverhead, Rockaway Beach, Yaphank, Ds.

E. globulosa Sauss. New Russia, Bdy (CU) ; Keene Val., Not ; Potsdam; Manlius, SmHH (CU); Ithaca (CU); McLean; Oliverea (Beq).

E. verticalis Say. LI: Rockaway Beach, Schott.

\section{PACHyMENES Saussure}

P. symmorphus (Sauss.). Ithaca, $B d y$; White Plains; W. Nyack.

\section{MoNOBIA Saussure}

M. quadridens (L.). White Plains; SI, Ds; LI: Wading R., Eng; Orient Pt.; Aqueduct, Eng; Wyandanch, Schott; Sands Pt., Bs; Flushing, Schott; Gardiners I.; Yaphank; Farmingdale, Morrow (CU).

\section{ANCISTROCERUS Wesmael}

\section{(Subgenus ANCISTROCERUS Wesmael)}

A. albophaleratus (Sauss.). Keene Val., Not; Potsdam; Cranberry L., Drk; Ithaca, Bks; Oliverea.

A. birenimaculatus (Sauss.). LI: Massapequa, Ds; Flushing, Bell.

A. campestris (Sauss.). Cattaraugus (CU); Ithaca; SI, Ds.

A. capra (Sauss.). New Russia, Bdy; Wilmington, Ds; Keene Val., Not; Indian L., Brb; Cranberry L., Drk; Potsdam; Honeoye Falls (CU); Cattaraugus

\footnotetext{
${ }^{173}$ All records in this family not otherwise indicated have been verified by Dr. Bequaert.
} 
(CU) ; Elmira, Diven; Ithaca, Bdy; McLean (CU); Manlius, $S m H H$ (CU) ; Rhinebeck, $C y$ (CU) ; Big Ind. Val.; E. Jewett; Oliverea; Debruce, Ds; Ft. Montgomery, Schott; Southfields, Schott; West Pt., Ds; SI; LI: Cold Sp. Harb.; Long Beach; Gardiners I.; Sea Cliff, Bks; Rockaway Beach, Long Pond, Wading R., Aqueduct, Ds.

A. catskillensis (Sauss.). Keene Val., Not; Potsdam; Wilmington; Manlius, SmHH; Portageville; L. Keuka; Ithaca; McLean; West Pt., Ds ; Rocky

Glen; Oliverea; W. Nyack; LI: Riverhead; Cold Sp. Harb.; Jamaica; Sea Cliff, Bks; Forest Hills.

A. parietum (L.). Ithaca; LI: Flatbush, Schott.

A. pertinax (Sauss.). Described from New York State. Unknown to me. H. de Saussure has doubtfully synonymized it with $A$. tigris. (Beq.)

A. spinolae (Sauss.). LI: Long Beach; Yaphank, Ds.

A. tigris (Sauss.). Keene Val., Not; New Russia, Bdy; Manlius, SmHH; Albion; Portage, Ds; Rock City; Otto; Ithaca, Bdy, Morse; Taughannock; Homer ; McLean; Center Lisle; Oliverea; Kaaterskill High Peak, Morse; West Pt., Ds; Ft. Montgomery, Ds; Rhinebeck, Cy; W. Nyack; SI, Ds; LI: Springs; Gardiners I.; Cold Sp. Harb.; Central Pk.; Riverhead; Long Pond, Wading R., Pennequid Barrens, Ds.

A. unifasciatus (Sauss.). Keene Val., Not; Mt. Marcy; Otto (CU) ; Ithaca, $B d y$; Spring Lake, $B d y$ (CU) ; Kinderhook; Nyack, $D s$; SI: Eltingville, $D s$; LI: Cold Sp. Harb.; Orient; Roslyn, Bks; Sea Cliff, Bks.

A. waldenii (Vier.). Ithaca, $B d y$; Manlius, $S m H H$ (CU); W. Danby; Ft. Montgomery, Schott; Ramapo, $\dot{D} s$.

\section{(Subgenus PARANCISTROCERUS Bequaert)}

A. clypeatus (Robt.). Otto; Rhinebeck, $C y$; LI: Long Pond, Wading R., Ds.

A. fulvipes (Sauss.). SI: Emerson Hill, J1, Ds (Beq).

A. proximus (Sauss.). Rock City; Ithaca; W. Danby; Caroline to Harford; Thousand Isls.; Rocky Glen; Rhinebeck, $C y$; W. Nyack; Ft. Montgomery, $D s$; SI: Tottenville, Ds; LI: Deep Pond, Long Pond, Wading R., Ds; Yaphank, Ds; Gardiners I.; Springs; Orient; Central Pk.

\section{SYMMORPHUS Wesmael}

S. albomarginatus (Sauss.). Ausable L.; Potsdam, $B d y$; Slaterville, $B d y$; Ithaca ; Caroline to Harford; Mud Cr.; Oliverea, Ds; Ft. Montgomery, Schott.

S. canadensis (Sauss.). Otto; Ithaca.

S. debilis (Sauss.). Keene Val., Not; Mt. Whiteface; Ithaca, Bdy; Mud Cr.; Caroline to Harford; McLean (Bdy-CU) ; Spencer; W. Nyack; LI: Gardiners I.

S. philadelphiae (Sauss.). Rock City; Ithaca; Slaterville to Caroline; SI: Ft. Wadsworth, $D$ s.

S. walshianus (Sauss.). Ithaca, $B d y$.

\section{ODYNERUS Latreille}

\section{(Subgenus Rygchium Spinola)}

O. arvensis Sauss. Cattaraugus; SI.

O. boscii Lep. NY State, according to H. de Saussure. I have seen it from N.J. (Beq). 
O. dorsalis (Fab.). White Plains: Inwood, NYC, J1, Schott; LI: Brooklyn, Eng; Canarsie, Wiegmann; Sands Pt., Ds; Flatbush, Eng; Jamaica, Schott.

O. foraminatus Sauss. Potsdam; Peru; New Russia; Manlius, SmHH; Cattaraugus; Spring Lake; Ithaca, McLean, Bdy; Spencer; Norton's Landing, Cayuga L., SmHH; Ft. Montgomery, Schott; White Plains; W. Nyack; Inwood, NYC, Schott; SI: Great Kill, Ds; Tottenville; LI: Flushing; Gardiners I.; Mastic; Aqueduct, Ds; Yaphank, Wading R., Ds; Orient; Cold Sp. Harb.

O. hidalgi Sauss. LI: Orient Pt.; Cold Sp. Harb.

O. leucomelas Sauss. Caroline to Harford; Coy Glen, Ithaca, Je, Ds; White Plains.

O. megaera Lep. SI: Clove Val., Ds.

\section{(Subgenus STENodynerus Saussure)}

O. anormis (Say). Manlius, SmHH; Cattaraugus; Ithaca; LI: Wyandanch, Schott.

O. conformis Sauss. NY, according to H. de Saussure. Unknown to me (Beq).

O. illinoensis Robertson. Ithaca; West Pt., Ds; White Plains.

O. pedestris Sauss. Peru; Manlius, SmHH ; Ithaca; Nyack, Ft. Montgomery, Ds; W. Hebron, Ds; White Plains; SI, Ds; LI: Gardiners I.; Cold Sp. Harb.; Central Pk.; Orient; Flushing, Eng.

O. pennsylvanicus Sauss. NY, according to H. de Saussure. Unknown to me (Beq).

O. perennis Sauss. NY, according to $H$. de Saussure. Unknown to me (Beq).

O. vagus Sauss. NY, according to H. de Saussure. I have seen it from N.J. and Vt. (Beq).

O. zendaloides Robertson. LI: Mastic, Nichols; Cold Sp. Harb., Ds.

(Subgenus uncertain)

O. mohicanus Sauss. Described from NY by de Saussure. Unknown to me (Beq).

\section{SUBFAMILY POLISTINAE}

Polistes Latreille

P. canadensis (L.) var. annularis (L.). Ft. Montgomery, Schott; Hillburn, Schott. A specimen was taken years ago by Ds on SI, but I have never seen this species near NYC (Beq).

P. pallipes Lep. (metricus Say, variatus Say). Keene Val., Castile, EABradley; Taughannock, Guerlac, Bdy; Ithaca, abundant, Bdy; Oliverea; Yorktown Hgts., Ols; Southfields, Schott; W. Nyack; NYC; SI; LI: Orient Pt.; Gardiners I.; Wyandanch, Little Neck, Schott; Mastic, Nichols; Bay Shore, Kissena Pk., Ols.

\section{SUBFAMILY VESPINAE \\ VESPA Linnaeus \\ (Subgenus VESPA Linnaeus)}

V. crabro L. European Hornet. Rye, Felt; Poughkeepsie; Nyack; W. Nyack; White Plains, Bno; Mahopac; Brewster; Yonkers; West Farms, Edw; Van Cort. Pk.; SI, Ds; LI: Brooklyn; Jamaica; Flatbush; Flushing; Bay Shore; Mineola; Far Rockaway. Introduced from Europe; gnaws twigs of various 
trees, especially birch, and is sometimes injurious to lilac. Generally selects a hollow tree or an attic in which to nest.

\section{(Subgenus VESPULA Thomson)}

V. carolina L. Pelham Parkway; SI, Je 3, Ds; LI: Rockaway Beach, Sep 20, Schott. An austral species, practically reaching its northern limit in N.J.

V. maculifrons Buy. (communis Sauss.). Upper Ausable L.; New Russia, Bdy; Cranberry L., Drk; Keene Val, Not; Ithaca, Clifton Sps. (CU) ; McLean, $B d y$ (CU) ; Spencer (CU) ; Taughannock, Guerlac (Bdy); Shelving Rock Mt., L. George, Leon ; Oliverea; W. Nyack; Yorktown, Ols; Hillburn, Schott; Van Cort. Pk.; LI: Rockaway Beach, Bay Shore, Ols; Jamaica; Flushing; Gardiners I.; Port Jefferson; Coram; Queens, Schott; Wading R.; Riverhead. An extremely common wasp, known as yellow-jacket.

$V$. vulgaris L., $V$. germanica Fab., and $V$. occidentalis Cress. do not occur in the eastern US. All specimens under those names in collections are $V$. maculifrons. Bequaert writes: "I have found underground nests of both $V$. communis and $V$. diabolica; they are generally not at a great depth, and when the cavity chosen is small, part of the nest eventually is more or less exposed; often it is merely covered above by dry leaves or dense grass. Of both species I have seen nests in a hole of a dead tree stump."

V. consobrina Sauss. New Russia, Bdy; Saranac L., Axton (M\&H) ; Moose L.; Upper Ausable L.; Keene Val., Not; South L.; Lowville, Ds; Mt. Skylight; McLean; Shelving Rock, L. George, Leon; Oliverea; Debruce, Ds; Ft. Montgomery, Schott; Stony Pt. Nests under the ground (Beq).

V. vidua Sauss. Cliff Mt., Essex Co; Ithaca (CU) ; New Baltimore; Ft. Montgomery, Schott; W. Nyack, Ds; SI; LI: Huntington, Wyandanch, Schott ; Jamaica; Bay Shore, Ols; Central Pk.; Merrick, Eng; Coram, Ds; Wading R., Yaphank, Ds. Not a common species.

\section{(Subgenus Dolichovespula Rohwer)}

V. arctica (Roh.) (borealis Lewis). Keene Val., Not; Castile, EABradley; Ithaca, Newfield; Oliverea; SI, $D s$. Lives as an inquiline in the nest of $V$. diabolica.

V. diabolica Sauss. Upper Ausable L.; Keene Val., Not; Manlius, SmHH ; N. Fair Haven (CU) ; Geneva; Castile, EABradley; Conesus L., Ds; Ithaca; McLean; Newfield; Norton's Landing, Cayuga L., SmHH; W. Danby (CU) ; Oliverea; Ft. Montgomery, Schott; Nyack: Inwood, NYC, Schott; SI, Ds; LI: Cold Sp. Harb. A very common species, known as yellow-jacket. For nesting habits, see under $V$. maculifrons. But diabolica also builds aerial nests similar to those of $V$. maculata, often about the eaves of buildings.

V. maculata L. Axton ( $\mathrm{M} \& \mathrm{H})$; New Russia, Bdy; Upper Ausable L.; Keene Val., Not ; Ithaca; McLean; Otto; L. George ; Oliverea ; Yorktown Hgts., Ols ; SI, Ds; LI: Little Neck, Wyandanch, Schott; Hempstead, Ols; Flushing; Cold Sp. Harb.; Gardiners I.; Jamaica; Queens, Schott. Probably occurs at all points in the State and is everywhere abundant. Builds strictly aerial nests in trees, under porches, in barns, etc. (Beq).

V. norwegica Fab. Mt. Marcy, 3000 ft.; Upper Ausable L.; Keene Val., Diven; Oliverea. Generally builds a nest similar to that of diabolica, but placed in low, dense bushes, four to five inches above the ground (Beq).

\section{(Subgenus Pseudovespa Schmiedeknecht)}

V. austriaca Panz. SI, Hine. 


\section{Superfamily SPHECOIDEA FAMILY AMPULICIDAE}

So far as their habits are known, the species of this family utilize crevices or crannies of some sort for their nests, stocking them with imperfectly paralyzed cockroaches.

\section{RHINOPSis Westwood}

R. canaliculata (Say). LI: Central Pk., Aug 13, 1913, Yaphank, Sep 4, 1910, Ds (Beq). An extremely rare insect.

\section{FAMILY DRYINIDAE}

\section{Bocchus Ashmead}

B. atriceps Brues. Mosholu, J1 25 (Bno) (type); LI: Flatbush, J1 27, Zab (AM).

\section{PSILODRYINUS Kieffer}

P. nigrellus (Brues). LI: Parkville, $B t$ (type-AM).

\section{Gonatopus Ljungh}

G. flavifrons Ashmead. Albany, EPV (type-US).

G. contortulus Patton. Black Br., Je 21 (Mues-CU).

\section{Chelogynus Haliday}

C. henshawi Ashmead. Ithaca, Je 28, Yuasa (Bdy-CU).

C. vivariensis Bdy. McLean, J1 26, 1925, SRobinson (type-CU).

C. xanthothorax Bdy. Taughannock Falls, Guerlac\&Bdy (type-CU) ; Penn Yan, J1 12, 1925, Babiy (paratype-CU).

\section{FAMILY SPHECIDAE 174 \\ SUBFAMILY LARRINAE \\ Tribe ASTATINI}

\section{Astata Latreille}

(The members of this genus burrow in the ground and provision their nests with Pentatomidae.)

A. unicolor Say. Manlius, Sep 20, SmHH (Cress-CU) ; Honeoye Falls, J1 23 (Bdy-CU) ; Albion (Bdy-CU) ; Canandaigua L., Je (NYS) ; Ithaca, J1 14 (Bdy-CU) ; Slaterville (Bdy-CU) ; Karner, Sep (NYS); Nyack, Mosholu (AM) ; Pelham Bay Pk., Aug 20 (Bdy-CU) ; LI: Queens, J1 21 (Schott); Cold Sp. Harb., Fishers I. (AM).

A. bicolor Say. Rochester Jct., J1 15 (Bdy-CU). A southern species.

${ }^{174}$ The manuscript for this family was submitted to Messrs. Banks, Bequaert, and Rohwer, who added many records. H. T. Fernald furnished records in the Sphecinae, and is responsible for the nomenclature and most of the records in the genus Sphex. 


\section{Tribe LARRINI}

(The members of this tribe burrow in sandy soil, and provision their nests with Orthoptera. A few small species build nests in brambles.)

\section{LARRA Fabricius}

L. analis Fab. "NY" (Fox), A southern species.

\section{NotogonidEA Rohwer}

N. argentata (Pal. de Beauv.). Ithaca, J1 16 (Bdy-CU). Occurs from the Atlantic to the Pacific, but chiefly southern. Provisions its nest with crickets.

N. nigripennis (Fox) (aequalis Fox). "NY", type.

\section{LARROPSIS Patton}

L. distincta (Sm.). Ithaca, Aug 9-Sep 12 (Bdy-CU); Oliverea, W. Nyack (Beq) ; SI (Beq) ; LI: Springs, Cold Sp. Harb., Huntington, Gardiners I. (Beq); Forest Hills, Sep 11 (Bdy-CU); Wyandanch, Sep 17 (Schott).

\section{TAChytes Panzer}

T. mandibularis Patton. Ithaca, J1 14 (Bdy-CU) ; White Plains (Beq) ; SI (Beq) ; LI: Bay Shore, Wyandanch, Rosedale, Schott (Beq). Chiefly austral.

T. breviventris Cress. Forest Hills, Sep 11 (Bdy-CU). An austral species.

T. columbiae Fox. Ithaca, Aug 1 (Bdy-CU). An austral species.

T. propinquus Rohw. Van Cort. Pk. (paratype-Milwaukee Mus.).

T. calcaratiformis Rohw. Van Cort. Pk. (types-Milwaukee Mus.).

\section{TACHYSPHEX Kohl}

T. tarsatus (Say). Ithaca, J1 9-Sep 21 (Bdy-CU); LI: Orient (Beq).

T. terminatus (Sm.). Ithaca, Aug-Sep 8 (Bdy-CU) ; Norton's Landing, on Cayuga L., J1 1-14, SmHH (Cress-CU) ; Union Sps., Sep 20, And (Bdy-CU) ; Trenton Falls, SaundersWW (F.Sm-Brit. Mus.); Rochester Jct., Je 16, Leon (Bdy-CU) ; LI: Pennequid Barrens, Orient (Beq). Provisions its nest with small and medium-sized nymphs of grasshoppers, especially of Syrbula admirabilis Uhl., according to the Raus.

\section{Tribe DINETINI}

(The habits of these species are similar to those of the preceding tribe.)

\section{SILAON Piccioli}

S. niger Roh. LI: Yaphank, Ds (Beq). Nests in elder stems according to the Raus.

SOLIERELLA Spinola

S. plenoculoides (Fox) (Niteliopsis). "NY", type. 
Plenoculus Fox

P. allanticus Vier. LI: Cold Sp. Harb. (Beq).

\section{LYRODA Say}

L. subita (Say). Keene Val. (Beq); Piseco L., Aug (NYS); Ithaca, Je 27-Sep 20, common (Bdy-CU); White Plains (Beq) ; LI: Jamaica (Beq). Provisions its nests with crickets of the genus Nemobius.

\section{SUBFAMILY TRYPOXYLONINAE}

\section{TRYPOXYLON Latreille}

(The species of this genus provision their nests with spiders. Most species build in twigs and stems, but some burrow in posts; one species builds clay nests; another utilizes deserted nests.

T. albopilosum Fox. Ithaca, J1 19-Aug 14 (Bdy-CU) ; Albany, Aug (NYS); SI, Sep 26, Ds (Ashm) ; LI: Huntington, J1 9 (Schott); Sea Cliff, J1 29-Aug 25 (Bks) ; Greenport, Flatbush, Eng (Beq) ; Rosedale, Schott (Beq) ; Yaphank, Ds (Beq); Gardiners I. (Beq).

T. bidentatum Fox. Essex Co., Wilmington, Aug 20-25 (Bdy-CU); Shelving Rock Mt., Sep 2 (Bdy-CU) ; Ithaca, May 30 (Bdy-CU) ; McLean, Aug, SRobinson $(\mathrm{Bdy}-\mathrm{CU})$; LI: Sea Cliff (Bks); Orient. (Beq). An essentially Canadian species.

T. carinatum Say. LI: Sea Cliff (Bks).

T. clavatum Say. Ithaca, Aug 18 (Bdy-CU) ; Albany, J1 (NYS); West Farms (Pck) ; SI, Ds (Beq) ; LI: Cold Sp. Harb., Wading R. (Beq) ; Huntington, Brookhaven, Schott (Beq). Utilizes the abandoned nests of mud-daubers, remodeling, partitioning, and resealing them. Can also burrow into wood and make its own nests, according to the Raus.

T. excavatum Sm. "NY" (Fox).

T. frigidum Sm. Keene Val., Not (Beq) ; Old Forge, J1, Newport, J1, Ilion, J1, Karner, May, $Y g$ (NYS) ; Ithaca, common, Je 10-Aug 4, Slaterville (BdyCU) ; Norton's Landing, on Cayuga L., SmHH (Cress-CU) ; McLean, Aug 20-31, SRobinson (Bdy-CU) ; W. Nyack (Beq) ; West Farms (Angus), reared from stems of Syringa; LI: Sea Cliff (Bks); Cold Sp. Harb., Central Pk., Gardiners I. (Beq). Very common inhabitant of elder stems.

T. politum Say (albitarse auctt.). Karner, Ds (Beq) ; Clinton Hgts., Ap (NYS); SI, Ds (Ashm). A common austral species. This species builds large "pipeorgan" nests, consisting of parallel tubes of mud attached to walls, etc., and provisions them with spiders.

T. rubrocinctum Pck. Honeoye Falls, Je 27, Leon (Bdy-CU); Ithaca, J1 14-Aug 30 ; W. Nyack (Beq); SI, Ds (Ashm); LI: Sea Cliff (Bks); Gardiners I. (Beq).

\section{SUBFAMILY SPHECINAE}

\section{CHLORION Latreille 175}

(The species of this genus burrow in the ground and store their nests with grasshoppers. They are large and handsome wasps and are common visitors of flowers during the late summer.)

${ }^{175}$ The digger-wasps of North America and the West Indies belonging to the subfamily Chlorioninae. By Henry T. Fernald. Proc. U. S. Nat. Mus., 1906, 31:291-423. 


\section{(Subgenus CHLORION Latreille)}

C. cyaneum Dahlbom subsp. aerarium Patt. SI, J1, Ds (Beq) ; LI: Flatbush, Aug 2, Zab (Bdy-AM); Wading R., Ds (Beq); Cold Sp. Harb., Jamaica, Ds (Beq).

\section{(Subgenus Palmodes Kohl)}

C. dimidiata (DeG.) (abdominalis Cress.). Ludlowville (Fern). An austral species.

\section{(Subgenus PRIONONYX Dahlbom)}

C. atratum (Lep.). Dansville, Sep 10 (Fern-CU) ; Cattaraugus, Sep 11 (Bdy-CU) ; New Baltimore, Nyack (AM); West Farms, Angus (Fern); SI, J1 29, Ds (Beq) ; LI: Gardiners I. (Beq) ; Cold Sp. Harb., Fishers I. (AM) ; Southold, Sep 6, Ds (Beq). Common and generally distributed over the US from Me. and Canada southward and westward.

C. bifoveolatum (Tasch.). Ithaca, Je-J1 (Fern-CU); SI (Beq) ; LI: Flushing (AM) ; Rockaway, Ds (Beq); Sea Cliff (Fern-CU) ; Orient (Beq). A widely distributed common southern species, extending northward well into NY and Mass.

\section{(Subgenus Isodontia Patton)}

C. macrocephala (Fox) (aztecum [Sauss.]). Manhattan I., Sep 2, Ds (Beq); LI: Flatbush, J1 30, Zab, Yaphank, J1 12 (Bridwell-AM). A scarce austral species, not known from north of LI.

C. auripes Fernald. New Baltimore, NYC (Bridwell-AM); White Plains (Beq); Nyack, West Farms, Angus (Fern) ; SI (Beq); LI: Flatbush, Cold Sp. Harb. (Bridwell-AM). A rather common austral species, not known from north of the Catskills.

C. harrisii Fern. Ogdensburg, White L. (Bridwell-AM); Rochester Jct., J1 4, Leon (Bdy-CU) ; Long L., Aug, Felt (NYS) ; Canandaigua L., J1, Karner, Je, Felt (NYS) ; L. George (Fern) ; Ithaca, J1 22 (CU), J1 19, 1887 (Fern); White Plains (Beq); New Baltimore, Nyack, West Farms (Bridwell-AM); LI: Flatbush, Cold Sp. Harb. (Bridwell-AM) ; Flatbush, Cold Sp. Harb., Orient, Gardiners I., Wyandanch (Beq).

\section{(Subgenus АммовіA Billberg)}

C. icheumoneum (L.). Keene Val. (Beq); New Russia, Aug 18 (Bdy-CU) ; Albany, J1, Felt (NYS); New Baltimore, Crugers, L. George, Nyack, Carmel, West Farms, Mosholu (AM); White Plains (Beq); SI, J1-Aug, Ds (Beq); LI: Cold Sp. Harb., Flushing (AM) ; Fishers I. (NYS) ; Springs, Jamaica, Rosedale, Orient, Gardiners I. (Beq). Distributed over nearly the entire US, from Canada southward and also over North America. More abundant in the Austral zone and scarce in the Transition zone.

C. pennsylvanicum (L.). Karner, Sep, Felt (NYS) ; Ramapo Mts., West Farms, Mosholu (AM) ; SI: Rossville, Ds (Beq) ; LI: Fishers I. (AM) ; Gardiners I., Aqueduct, Flushing (Beq).

\section{SPHEX Linnaeus}

(The members of this genus are common flying along roads, paths, and bare ground, in which they nest. They store their burrows with caterpillars. The habit of some of using a pebble as a mallet for pounding down the soil on their burrows, has drawn widespread attention to them.) 


\section{(Subgenus Sphex Linnaeus)}

(Ammophila Kirby)

S. arvensis (Dahlb.). Keene Val. (Beq); Wilmington; Mt. Marcy (Beq) ; Piseco L. (Fern) ; Elk L. (Fern) ; Long L., L. Pleasant, Stony I., White L., Oswego, Otto, Clifton Sps., Ithaca, L. George (all Fern); Karner; Oliverea (Beq); Van Cort. Pk. (Fern,Beq) ; SI (Beq) ; LI: Forest Pk. (Fern) ; Orient (Beq) ; Long Beach (Beq). This species usually goes as urnarius Klug. Present indications are that urnarius and inepta Cress. may be synonyms of arvensis, and that among the above records may be included a second valid species very similar to arvensis, to which none of these names can be properly applied (Fern). May-Sep.

S. pictipennis (Walsh). Ithaca (Fern); SI (Beq); LI: Farmington (Fern); Rockaway (Beq). S. extremitata has been included among these records as a probable synonym, but there is a possibility of there really being two species here (Fern). J1-Oct.

S. procerus (Dahlb.). Keene Val. (Beq) ; Oliverea (Beq); LI: (Fern) ; Wyandanch (Beq).

S. aureonotatus (Cam.). Keene Val., Not (Beq); Ithaca (Fern) ; Oliverea (Beq) ; LI: Roslyn (Fern) ; Gardiners I., Springs, Cold Sp. Harb. (Beq). J1-Aug. This species has usually, but incorrectly, been determined as abbreviatus Fab.; sometimes as gracilis St. Farg. (Fern).

S. nigricans (Dahlb.). Evidently generally distributed (Fern). Ithaca, Binghamton, Mosholu (Fern); White Plains (Beq); SI (Beq); LI: Cold Sp. (Fern); Jamaica (Fern,Beq) ; Sea Cliff (Fern); Orient (Beq). Aug-Oct.

S. argentatus (Hart). Albany, Je (Fern-NYS); Ithaca, Sep (Fern-CU); LI: Far Rockaway Beach, Aug, Snn (Fern).

\section{(Subgenus Podalonia Spinola) \\ (Psammophila Dahlbom)}

S. violaceipennis (Lep.). Mt. Whiteface, Aug 22-24, New Russia, Aug 18 (Fern); Keene Val. (Beq) ; Taughannock, J1 14 (Fern); LI: Mastic, Orient (Beq).

S. luctuosa (Sm.). Keene Val., Not (Beq) ; Norton's Landing, Cayuga L., SmHH (Fern-CU).

\section{SCELIPHRON Klug}

(These are the well-known mud-daubers, which plaster their clay nests over walls and ceilings, provisioning them with spiders.)

\section{(Subgenus ScELIPHRON Klug) \\ (Pelopaeus Latreille)}

S. coementarium (Dru.). The Black-ANd-Yellow Mud-Dauber. Common and generally distributed, except possibly in the colder regions of the State. Color forms A, B, C, D, E (nigriventre Costa), and F (coementarium), are all represented by Ithaca specimens (Bdy-CU).

\section{(Subgenus Chalybion Dahlbom)}

S. cyaneum Dahlb. The Blue Mud-Dauber. With the foregoing, possibly more restricted to the warmer districts: Otto, Castile, Ithaca (Bdy-CU); Albany, etc.; common on SI and LI (Beq). 


\section{SUBFAMILY PSENINAE \\ Tribe PSENINI}

Diodontus Curtis not auctt.

(Neofoxia Viereck)

(Subgenus PSENULUS Kohl)

D. trisulcus (Fox). Otto, bred from nests in sumac stems, adults emerging May.18, Comstock (Bdy-CU).

PSEN Latreille

(Subgenus PSEN Latreille)

P. monticola (Pck.). Ithaca, J1 18, 1922 (Bdy-CU), J1 7 (Bks).

\section{(Subgenus Mimesa Shuckard)}

P. argentifrons (Cress.). Ithaca, Aug 18 (Bdy-CU); Gloversville, Sep (NYS). Nests in sandy places, and Williams has observed a cone of agglutinized sand grains surmounting the entrance; the nests are provisioned with leaf hoppers, Williams states of the species Athysanus exitiosa Uhler.

P. borealis (Sm). Enfield Falls, Sep 10 (Bdy-CU).

P. cressoni (Pck.). Niverville, Aug 24 (Bks).

P. denticulata (Pck.). "NY", Nort (Pck).

P. johnsoni (Vier.). Dryden L., Je 16, MacG (Bdy-CU).

P. kohlii Fox. LI: Gardiners I. (Beq).

P. leucopus Say. Ithaca, J1 16-Aug 9 (Bdy-CU).

P. longicornis Fox. Ithaca, J1 16 (Bdy-CU).

P. mellipes Say. "NY" (Fox).

P. niger Pck. Axton, Je 12-22, $M \& H$ (Ashmead-CU) ; Norton's Landing, Cayuga L., Je 13, SmHH (Cress-CU); LI: Long Beach (Beq).

P. pauper (Pck.). Albion (Bdy-CU) ; Hamburg, J1 19, $M C V$ (Bks) ; Ithaca, Aug 23 (Bdy-CU); LI: Fishers I., Aug, Zab (AM).

P. trisulcus Fox. LI: Sea Cliff, Sep (Bks).

P. unicincta (Cress.). Ithaca, J1 5 (Bdy-CU).

\section{Tribe PEMPHREDONINI}

\section{STIGMus Panzer}

(The members of this genus build in wood, two or three wasps building, in some cases at least, chambers near together, which have a common entrance gallery. They provision their nests with aphids.)

S. americanus Pck. Ithaca (Bks); McLean, Sep 16, 1924, Sib (Bdy-CU).

S. fraternus Say. L. Placid, Je, $Y g$ (NYS) ; Silver Bay, Je, $Y g$ (NYS) ; Elm L., Aug, $Y g$ (NYS) ; Norton's Landing, Cayuga L., Je 4, SmHH (Cress-CU); Ithaca, J1, nest in pithy stem, adults emerged J1 22 (CU) ; McLean, Sep 16, 1924 (Bdy-CU); Albany, Aug, $Y g$ (NYS) ; Rensselaer, Je, $Y g$ (NYS); W. Nyack (Beq); Dobbs Ferry, J1, Yg (NYS); West Farms, Angus (Pck); II: Gardiners I. (Beq); Sea Cliff, Je, Roslyn (Bks). 


\section{PEMPHREDON Latreille}

(The members of this genus build chiefly in decaying wood, provisioning their nests with aphids.)

\section{(Subgenus Diphlebus Westwood)}

P. bipartior Fox. LI: Sea Cliff, Aug-Sep (Bks).

P. inornatus (Say). L. Placid, Je, Elm L., Aug (NYS) ; Ithaca, J1 2-Aug 4 (Bdy$\mathrm{CU})$; Mud Cr., Je 17-20, MacG ( $B d y$-CU) ; Norton's Landing, Cayuga L., May 29-Je 3, SmHH (Cress-CU) ; Albany, Je, Dobbs Ferry, Je, $Y g$ (NYS); Rensselaer, J1, Yg (NYS) ; LI: Flatbush, Zab (AM); Sea Cliff (Bks).

P. tenax Fox. Ithaca, May 30-J1 25 (Bdy-CU) ; Mud Cr., Je 17-20, Slaterville to Caroline, Je 14, Dryden L., Je 16, MacG (Bdy-CU); McLean, Sep 20, Sib (Bdy-CU) ; Bear Mt., Je 2, Schott (Roh); LI: Sea Cliff (Bks); Flatbush (AM). The nests of this species have been observed by the Raus in elder stems, provisioned with an aphid, Macrosiphum rudbeckiae (Fitch).

\section{(Subgenus PEMPHREDON Latreille)}

P. angularis Fox. LI: Sea Cliff, Je (Bks).

P. concolor Prov. Big Moose, Aug, Speculator, May, Yg (NYS) ; Newport, J1, $Y g$ (NYS) ; Ithaca, Aug, Yg (NYS); Albany, Sep, Yg, Lint (NYS); Karner, May, Yg (NYS); Inwood, Schott (Beq); SI, May 30, Ds (Ashm).

\section{Passaloecus Shuckard}

(These wasps prefer rotten wood for their nesting site, or utilize the galleries of wood-boring insects, or hollow stems of plants, building clay cells within the cavity. They provision their nests with aphids.)

P. annulatus (Say). Ithaca, J1 10. (Bdy-CU); Mud Cr., Je 17-20, MacG (BdyCU) ; LI: Sea Cliff, J1 (Bks).

P. distinctus Fox. Ithaca, Je 28 (Bdy-CU).

P. mandibularis Cress. L. Placid, Je, $Y g$ (NYS); Ithaca, Je 5-27 (Bdy-CU); Albany, Je, Yg (NYS); West Farms, Angus (AM); LI: Sea Cliff (Bks).

\section{SUBFAMILY BEMBECINAE Tribe NYSSONINI}

\section{Alyson Jurine 176}

A. conicus Prov. Wilmington, 1000-2200 ft., Aug 20-25 (Bdy-CU); Lancaster, Aug 15, MCV (Bks); Middletown, J1 2-20, SpoonerCS (Bdy-CU) ; Rhinebeck, J1 27, Cy (Bdy); Van Cort. Pk., J1 20 (Bdy-CU).

A. guignardi Prov. Mud Cr., Je 17-20, MacG (Bdy-CU) ; McLean, J1 2-3 (BdyCU); Ithaca, J1 31 (Bdy-CU); SI (Beq).

A. melleus Say. Ithaca, Aug 10-31 (Bdy-CU); LI: Wading R., Je 27, Schott (Rohwer). An essentially southern species. Builds, according to the Raus, always in a cool damp bank of mud or sandy clay near a body of water, but at least the surface of the sand may be hot and dry. Provisions its nest with leaf hoppers.

${ }^{176}$ If the Erlangen List is accepted, this name will have to be spelled "Alysson." 
A. oppositus Say. Wilmington, Aug 20-25, 1000-2200 ft. (Bdy-CU); Old Forge, J1. $Y g$ (NYS); Ithaca, J1 19 (Bdy-CU); LI: Sea Cliff (Bks).

A. striatus Fox. Ithaca, J1 7 (Bks).

A. triangulifer Prov. Ithaca, Je 23-Aug 14, Dryden L., Je 16, Mud Cr., Je 17-20, $M a c G(B d y$-CU).

DIDINEIS Wesmael

D. texana (Cress.). Ithaca, J1 14 (Bdy-CU).

Mellinus Fabricius

M. bimaculatus Pck. "NY" (AM). This is a rare insect.

GoRYTES Latreille not auctt.

(Hoplisus Lepeletier)

(These wasps nest in sand or soil and provision their nests with leaf-hoppers.)

(Subgenus PSEUdoplisus Ashmead)

G. phaleratus Say. New Russia, Aug 18 (Bdy-CU); Manlius, Aug 1-15, SmHH (Cress-CU) ; Potsdam, J1-Aug 14 (Beq-CU) ; Rochester Jct., J1 11, Leon (Bdy-CU) ; Ithaca, J1 14-Aug 6 (Bdy-CU) ; McLean, J1 18, 1921 (Bdy-CU) ; L. George, Aug 28, Zab (AM); New Baltimore, Zab (AM); Oliverea (Beq); Nyack (AM); Stony Pt. (Beq); SI, Ds (Ashm, Beq); LI: Huntington (Beq) ; Flushing (AM).

\section{(Subgenus GoRytes Latreille)}

(Hoplisus Lepeletier)

G. atricornis Pck. Keene Val. (Beq); Ithaca, J1 4-21 (Bdy-CU).

G. canaliculatus Pck. Newport, Je, Yg (NYS) ; Ithaca, J1 17 (Bdy-CU).

G. costalis Cress. LI: Cold Sp. Harb., J1 23 (Bridwell-AM).

G. nebulosus Pck. Ithaca, J1 15-Aug 10 (Bdy-CU).

G. simillimus (Sm.). Keene Val., Not (Bridwell, Beq); Ithaca, Je 26-Aug 10 (Bdy-CU) ; McLean, Aug 1, 1925, SRobinson (Bdy-CU); Oliverea (Beq); W.

Nyack (Beq) : Yonkers (Bridwell-AM); LI: Sea Cliff (Bks).

ARpactus Jurine

(Gorytes auctt.)

A. nigrifrons Sm. Huguenot, J1 6, Zab (Bridwell-AM).

Ammatomus Spinola

(Subgenus PARAMEllinus Rohwer)

A. bipunctatus (Say). Albany, Ap 12, Yg (NYS); Nyack, Aug (AM). 


\section{NySSON Latreille}

(Subgenus Nysson Latreille)

N. aequalis Patt. W. Nyack (Beq); SI (Beq).

N. daeckei Vier. LI: Pennequid Barrens (Beq).

N. lateralis Pck. Poughkeepsie, J1 (NYS).

N. plagiatus Cress. SI (Beq); LI: Flatbush, Eng, Springs (Beq); Gardiners I. (Beq).

\section{(Subgenus BRACHYSTEGUS Costa)}

N. opulentus Gerst. "NY" (Cress, Fox).

\section{SPHECIUS Dahlbom}

S. speciosus (Dru.). The Cicada Killer. Albany (FelteNYS); Karner, Aug (NYS) ; Kinderhook, Aug 26 (Bdy-CU) ; Tarrytown, PineJA (Lint-NYS); SI, numerous (Ds); LI: Roslyn, Bks (Bdy-CU); Flatbush, Gardiners I. (Beq); Sea Cliff (Bks). An austral form, not likely to be found elsewhere in the State than LI and the lower Hudson Val. and such an austral station as the sand hills around Karner. Nests in sandy soil and provisions its nest with cicadas.

\section{Tribe PHILANTHINI}

\section{Philanthus Fabricius}

(These wasps burrow in the ground, provisioning their nests with bees.)

P. albifrons Cress. SI, Aug 9, Ds (Beq).

P. bilunatus Cress. Saranac Inn, Aug (NYS); Keene Val. (Beq) ; Rochester Jct., J1 7, Leon (Bdy-CU) ; Norton's Landing, Cayuga L., J1 1-15, SmHH (CressCU); New Baltimore, Zab (Bks-AM); W. Nyack (Beq); SI (Beq).

P. consimilis Bks. Boston, $M C V$, types.

P. gibbosus (Fab.) (punctatiis Say). Niagara Falls, Aug 17, Ds (Beq); Ithaca, J1 5-Oct 27 (Bdy, Bks-CU) ; Norton's Landing, Cayuga L., J1 10, SmHH (Cress); New Baltimore (Bks-AM) ; Ft. Montgomery, J1, Ds, Schott (Beq); W. Nyack (Beq); White Plains (Beq); Van Cort. Pk., J1 20 (Bdy-CU); SI, Jl 4, Ds (Beq); LI: Sea Cliff, Bks (Beq); Orient (Beq); Flatbush, Fishers I. (Bks-AM); Gardiners I. (Beq) ; Farmingdale, J1 15, Morrow (Bdy-CU). Provisions its nests with species of Halictus.

P. lepidus Cress. Aurora (E. Aurora?), J1 21, Zab (Bks-AM).

P. politus Say. Karner, J1 (NYS).

P. sanborni Cress. Ft. Montgomery, Schott (Beq); LI: Forest Lawn, J1 27, Zab (Bks-AM).

P. solivagus Say. New Russia, Aug 18 (Bdy-CU); Keene Val. (Beq); Ithaca, Aug 8-31 (Bdy-CU); Cattaraugus, Sep 11 (Bdy-CU); Enfield Falls (BdyCU) ; Binghamton, Sep 5, Ellsworth (Bdy-CU) ; L. George, Zab (Bks-AM) ; White Plains (Beq); SI (Beq); LI: Fishers I., Zab (Bks-AM); Gardiners I. (Beq) ; Orient (Beq).

P. vertilabris Fab. Nyack, $Z a b$ (Bks-AM). Provisions its nest with several species of bees. 


\section{ApHilanthops Patton}

A. frigidus (Sm.). New Russia, Aug 18 (Bdy-CU); Ithaca, Aug (Bdy-CU); Norton's Landing, Cayuga L., J1 1-15, SmHH (Cress); McLean, Aug 20-26, 1925, SRobinson (Bdy-CU) ; Montgomery, J1 20, Frost (Bdy-And) ; Inwood, Schott (Beq) ; SI, Aug 15, Ds (Beq) ; LI: Elden, Aug 30, Ds (Beq) ; Bay Shore, Ols, Wyandanch, Schott (Beq). This species provisions its nest with queen ants.

\section{Tribe CERCERINI}

\section{EUCERCERIS Cresson}

E. laticeps Cress. Gloversville, Aug (NYS); White Plains, Bno (Beq).

\section{CERCERIS Latreille}

(Wasps of this genus usually burrow in the ground and provision their nests with weevils or buprestids. But at least one species, sometimes if not always, builds in weed stalks.)

C. alaope Bks. LI: Sea Cliff (Bks).

C. arelate Bks. New Russia, Aug 18, 1912, Bdy (Bks-CU); Ithaca, J1 27-Aug 19 (Bks-CU).

C. bicornuta Guer. SI, J1 1-Aug 19, Ds (Beq); LI: Rosedale, Schott (Beq) ; Jamaica, Eng (Beq); Flushing (Beq); Huntington, Schott (Beq).

C. chrysippe Bks. Ithaca, J1 13 (Bdy-CU).

C. clypeata Dahlb. Johnstown, Aug (NYS); Ithaca, J1 9-Aug 18 (Bdy-CU); W. Danby, Aug 15, Summers (Bdy-CU); McLean, Aug 31, 1925, SRobinson (Bdy-CU); W. Nyack, White Plains (Beq) ; SI, Ds (Cress, Beq) ; LI : Cold Sp. Harb., Jamaica, Huntington, Port Jefferson (Beq) ; Farmingdale, J1, Morrow (Bks-CU).

C. compacta Cress. Elizabethtown, Je, Yg (NYS) ; LI: Gardiners I. (Beq).

C. compar Cress. Ithaca (Bks) ; Rhinebeck, J1 27, 1907, Cy (Bks-CU).

C. dentifrons Cress. Essex Co, Aug; LI: Sea Cliff (Bks).

C. deserta Say. New Russia, Aug 18, Bdy (Bks-CU) ; Saranac L., Aug 26 (BksCU) ; Ithaca, Je 28-Sep 4 (Bdy, Bks-CU); White Plains (Beq) ; Chatham, Morse (Bks) ; SI, J1, Ds (Beq) ; LI: Sea Cliff (Bks); Springs (Beq); Gardiners I. (Beq).

C. fumipennis Say. Albany (NYS); Karner; W. Nyack (Beq) ; LI: Yaphank, Aug 5, Comstock (Grossbeck); Wading R., Eng, Wyandanch, Schott, Yaphank, Aug, Ds (Beq).

C. nigrescens Sm. Keene Val. (Beq); Manlius, Aug 1-15, SmHH (Bdy-CU); Otto, Sep 15-30, Comstock (Bdy-CU) ; Rochester Jct., Je 29, Leon (Bdy-CU); Ithaca, J1 22-Sep (Bdy, Bks-CU); Norton's Landing, Cayuga L., Je 1-15, $\mathrm{SmHH}$ (Bdy-CU).

C. nigritulus Bks. Colden, $M C V$, type.

C. occipitomaculata Pck. (fulvipediculata Schletterer, fulvipes Cress.). Long L., Aug (NYS) ; Keene Val., Not (Beq); Wiimington, Aug 20-25, 1000-2200 ft. (Bks-CU) ; Cattaraugus, Sep 11 (Bdy-CU).

C. robertsoni Fox. Ithaca, J1 16 (Bdy-CU).

C. salome Bks. Nyack, NYC, types.

C. venator Cress. "NY", Angus (Cress, Pck). 


\section{Tribe BEMBICINI 177}

(Sand wasps. All build in sand.)

STICTIA Illiger

S. carolina (Fab.). SI: Watchogue, Aug 10, Ds (JBParker, Ds, Sf). An austral species reaching in SI the extreme northern limit of its known range. Davis states that it occurred in some abundance one season but has not been observed since. The species nests in colonies and provisions its nests with horseflies, but lays its eggs in the empty cell, not bringing in food until the larva has hatched.

\section{Stictiella Parker}

S. emarginata Cress. Center, Ji, 1870, 10 (Roh-NYS).

\section{BICyRTES Lepeletier}

(These species store their nests with nymphs of Hemiptera, mostly stinkbugs, filling and sealing the cells, instead of providing daily provender.)

B. quadrifasciata (Say). New Windsor, Nyack (AM); White Plains (Beq); SI, Aug (JBParker, Ds) ; LI: Cold Sp. Harb. (Beq). An austral species reaching the northern limit of its range in the coastal region of Conn.

B. ventralis (Say). Wilmington, Aug 20-25 (Bdy-CU) ; Keene Val., Not (Beq); White L. (AM) ; L. Waccabuc (AM) ; Manlius; Aug 18-23, SmHH (CressCU) ; Castile, EABradley; Ithaca, J1 15-Aug 18 (Bdy-CU); Norton's Landing, Cayuga L., J1 1-14, SmHH (Cress-CU); L. George (AM); Rhinebeck, J1 27, Cy (Bdy-CU) ; Ft. Montgomery, J1, Ds (IBParker, Ds); W. Nyack (Beq) ; White Plains (Beq) ; NYC (AM) ; SI, Jl-Sep (JBParker, Ds) ; LI: Yaphank, J1, Aqueduct, Sep (JBParker, Ds); Farmingdale, Je 17, Morrow (Bdy-CU) ; Sea Cliff (Bks); Jamaica, Flushing (Beq). Extends throughout the Carolinian and Transition zones, northward into Canada.

\section{BEMBEX Fabricius}

(The species of Bembex nest in colonies in sand. They feed their larvae from day to day with flies. The egg is laid on a paralyzed fly.)

B. pruinosa Fox. Karner, Sep (Bdy-NYS); "NY" (Parker). An austral species which probably reaches its northern limit in the coastal district of New York, and such Carolinian islands as the sand hills around Karner.

B. spinolae Lep. Mt. Whiteface, 2000-4000 ft., Aug 23 (Bdy-CU); Norton's Landing, Cayuga L., J1 1-14, SmHH (Cress-CU); Ithaca, J1 10-Sep 9 (Bdy-CU) ; New Riverside (Bdy-CU) ; Karner, J1 (Parker, Ds) ; Debruce, Sep (Parker, $D s$ ) ; SI, Aug (Parker, Ds) ; LI: Aqueduct, Wading R., Rockaway, Je-Sep (Parker, Ds). The commonest species of the genus throughout the greater part of the Austral and especially of the Transition zone, reaching northward into Canada.

\section{MICROBEMBEX Patton}

M. monodonta (Say). Karner, J1 (NYS) ; SI, Je-Sep (Parker,Ds) ; LI: Sea Cliff, Bks (Parker-CU). An abundant species of the sea beaches, both Atlantic and Pacific, nesting in white sand, but also found more sparingly

${ }^{177}$ A revision of the bembecine wasps of America, north of Mexico. By John Bernard Parker. Proc. U.S. Nat. Mus., 1917, 52:1-155. 
inland on sandy areas. Its northern limit, so far as known, is the coast of Conn. It provisions its nest with a variety of dead insects, which it gathers up on the sand.

\section{SUBFAMILY OXYBELINAE}

\section{(Crabroninae)}

\section{Tribe SOLENIINI}

\section{(Crabronini)}

Some species of this tribe nest in the ground, some excavate the pith of plants, some build in solid wood, and some use deserted burrows or any suitable hole. Mostly the nests are provisioned with flies. The genera and subgenera recognized in this tribe correspond to those recognized by Kohl in his recent monograph of the Palearctic forms. The "Artengruppen" of Crabro are here recognized as subgenera, but his "Untergruppen" are not so recognized.

\section{SOLENIUS Lepeletier}

(Crabro auctt.)

\section{(Subgenus SolEnIUs Lepeletier)}

S. brunneipes Pck. Ithaca, Je 25-J1 (Bdy-CU).

S. corrugatus Pck. Ithaca (Bks).

S. montanus Cress. Norton's Landing, Cayuga L., Je 17, SmHH (Cress-CU); Dryden L., Je 16, Ithaca to E1lis, Je 9-12, MacG (Bdy-CU). Nests in logs.

S. parvulus Pck. Rock City, Je 7 (Bdy-CU); Ithaca, J1 25, McLean, May 31-J1 18 (Bdy-CU).

S. obscurus Sm. Ithaca (Bks); Manlius, Aug, SmHH (Cress-CU); McLean, May 30-Je 30, Ithaca to Ellis, Ap 22-Aug 1, MacG (Bdy-CU); Oliverea (Beq) ; LI: Orient (Beq). Has been observed nesting in logs.

S. nigrifrons Cress. Ithaca, J1 (Bks).

S. septentrionalis Pck. Ithaca, Je 13-Aug 30, Freeville, J1 4 (Bdy-CU).

S. sayi Ckll. (sexmaculatus Say). Wilmington, 1000-2000 ft., Aug 20-25, New Russia, Aug 8, Potsdam, Aug 14, Peru, Je 21, Taughannock, Aug 13, Ithaca, Ap-Aag 28 (Bdy-CU); Alexandria Bay, Sep 4 (Bdy-CU); Malloryville, Je 18-20, MacG (Bdy-CU); Rock City, Je 5 (Bdy-CU); Ithaca (Bks); Caroline to Harford, Je 15, McLean, May 31-Aug 30, Sib, Mud Cr., Je 17-20, Ellis to Slaterville, Je 13, MacG (Bdy-CU) ; Norton's Landing, Cayuga L., J1 1-14, $\mathrm{SmHH}$ (Cress-CU) ; Spencer, Je 30 (Bdy-CU) ; Center Lisle, Je 10 (BdyCU) ; Oliverea (Beq) ; Ft. Montgomery, Schott (Beq) ; Pine I. (Beq) ; Bear Mt., Sep 7, Schott (Roh) ; SI, J1, Ds (Beq); LI: Maspeth (Beq). Known to nest in logs.

S. trifasciatus (Say). Newport, J1, Saranac Inn., J1 (NYS); Manlius, Aug, SmHH (Cress-CU); Otto, Sep 1-15, Comst (Bdy-CU); Geneva, Je 26, Comstock (Bdy-CU); Ithaca, May-J1 9, Spencer, Je 30, Comstock (Bdy$\mathrm{CU}$ ); Ellis to Slaterville, Je 13, MacG; McLean, J1 18 (Bdy-CU) ; Taughannock, J1 13, Homer, J1 2, MacG (Bdy-CU) ; Norton's Landing, Je, SmHH (Cress-CU) ; Albany, Sep, Poughkeepsie, Jl (NYS); SI, Aug, Ds (Ashm); LI: Gardiners I. (Beq). 
S. stirpicola Pck. Spencer L., Je 30, Ithaca, J1 7-Aug 1, McLean, J1 18 (Bdy-CU). Nests in brambles and elder twigs, provisioning them with a variety of flies. They have been observed to utilize tunnels in a railroad tie for a nesting site.

S. decemmaculatus Say. West Farms, Angus, Nort (Pck); LI: Springs (Beq). Preys on horseflies.

S. chrysargyrus Lep. (chrysarginus Pck.). Keene Val., Not (Beq); Manlius, Sep 6, SmHH (Cress-CU); New Russia, Aug 18 (Bdy-CU); Caroline to Harford, Je 10, Ithaca, Je-J1 9, MacG (Bdy-CU); Ft. Montgomery, Schott (Beq) ; SI, Ds (Ashm). Very widely distributed from Ga northward to Canada and westward to the Pacific Coast. Nests in logs.

S. rufifemur Pck. Ithaca, J1-Aug 4 (Bdy-CU); SI (Beq).

S. singularis Sm. (maculatus Fab.?). Keene Val. (Beq); Old Forge, Wells, Saranac Inn, J1 (NYS); Malloryville, Je 18-20, MacG (Bdy-CU); Otto; Ithaca, J1 14-30, McLean, J1 9-Aug 30, SRobinson (Bdy-CU); Trenton Falls, $\mathrm{Dbl}$ (FSm); Oliverea (Beq); LI: Orient (Beq).

\section{(Subgenus Ceratocolus Lepeletier)}

S. producticollis Pck. Keene Val. (Beq); Wells, J1, Long L., Aug (NYS); Portageville, Je 13, Ithaca, Je 13-Aug 6 (Bdy-CU); Mud Cr., Je 17-20, MacG $(B d y-\mathrm{CU})$; Norton's Landing, Cayuga L., J1, SmHH (Cress-CU) ; LI: Sea Cliff (Bks); Pennequid Barrens (Beq); Huntington, Aug 19, Schott (Roh). A Canadian species.

S. interruptus Lep. Canandaigua L., J1 (NYS); Manlius, Sep, SmHH (CressCU) ; Geneva, Je 26, $C y$ (Bdy-CU) ; Otto, Sep 1-15, Comstock (Cress-CU); Ithaca, May-Sep (Bdy-CU) ; Taughannock, J1 2, Spencer, Je 30, Comstock (Bdy-CU) ; Norton's Landing, Cayuga L., J1, SmHH (Cress-CU); Ft. Montgomery, Schott (Beq); Middletown, J1 2-20, Spooner (Bdy-CU); SI, J1 20, Ds, Ashm (Beq); LI: Springs, Gardiners I. (Beq).

\section{(Subgenus THYREOPUS Lepeletier)}

S. argus Pck. LI, Ashm (Fox); Gardiners I. (Beq).

S. cribellifer Pck. LI, Ashm (Fox); Gardiners I. (Beq).

S. latipes Sm. Axton, Je 12-22, MacG\&H (Bdy-CU); Long L., Aug (NYS). A Canadian species.

S. monticolus Pck. Old Forge, J1, Piseco L., Aug (NYS).

S. provancheri Fox. Ithaca, Aug 12 (Bdy-CU).

S. advenus Sm. Batavia, Je 18, Ithaca, Je 20-Oct 2 (Bdy-CU); LI, Ashm (Fox).

S. tumidus Pck. LI: Jamaica (Beq).

\section{(Subgenus Crossocerus Lepeletier)}

S. nitidiventris Fox. Ithaca, Je 5-J1 (Bdy-CU).

S. maculipennis Sm. Speculator, Aug (NYS) ; Rochester Jct., Je 28, Leon (BdyCU) ; Black Br., Je 21 (Bdy-CU); Ithaca, Je 5-J1 22 (Bdy-CU); L. Keuka, Je 28, $C y$ (Bdy-CU); Albany, May (NYS).

S. minimus Pck. Wilmington, 1000-2000 ft., Aug 20-25 (Bdy-CU); Ithaca, Je 27, Guerlac (Bdy); LI: Sea Cliff (Bks).

S. tarsalis Fox. "NY" (Fox). 
S. harringtonii Fox. New Russia, Aug 18 (Bdy-CU).

S. impressifrons Sm. New Russia, Aug 18, Ithaca, J1 9-Aug 27 (Bdy-CU); LI: E. New York, Schott. (Beq).

S. nigricornis (Prov.). Ithaca, Je 22, McLean Bogs, May 29 (Bdy-CU); LI: Gardiners I. (Beq).

S. ater (Cress.). McLean, Aug 19-31, Sib, SRobinson (Bdy-CU).

S. pedicellatus Pck. Otto (Bdy-CU); Ithaca, Je 27-Jl 22 (Bdy-CU); Albany, May, Nort, West Farms, Angus (Pck); W. Nyack (Beq); SI (Beq). Ithaca specimens bred from nest in a hollow stem. The Peckhams say they build in old stumps, provisioning with Chironomus.

\section{ANACRABRO Packard}

A. ocellatus Pck. Ithaca, J1 21 (Bdy-CU); Taughannock, J1 21 (Bdy-CU); Karner, Kenwood, Albany, J1 (NYS) ; Bear Mt., Je 2, Schott (Roh) ; SI, Je 30, Ds (Ashm, Beq); LI: Cold Sp. Harb., Pennequid Barrens (Beq). Builds in sand banks, and provisions its nest with the plant-bug Lygus pratensis (L.).

\section{Tribe OXYBELINI}

OXYBELUS Latreille

O. quadrinotatus Say. Old Forge, J1 (NYS); Ithaca, Aug 10, Comstock, found nesting in sandy place, the cells provisioned with flies, from two to four inches below surface (Bdy-CU) ; McLean, J1 18 (Bdy-CU); Manlius, Aug 15, SmHH (Cress-CU); Dublin (Pck); Karner, J1, Rensselaer, J1 (NYS). Common. Nests in sandy places, using flies for provender, which it transports by impaling them on its sting.

FAMILY HYLAEIDAE 178

(Prosopidae)

Compiled by M. D. LenNard

SUbFamily COLLETINAE

Colletes Latreille

(The members of this genus build characteristic nests in the ground, often in colonies.)

C. americanus Cress. Ithaca, J1-Aug (Rich-CU) ; New Baltimore (Schw); Nyack (Schw) ; Tappan, Oct (Schw); Mosholu, Sep (Schw); Bryn Mawr Pk., Sep (Schw) ; LI: Pine I., Sep (Schw).

C. armatus Patt. "NY" (Patt-AES), type; Hague, Sep (Schw); L. George, Aug (Schw) ; Forest Lawn, Sep (Schw); White L., Sep (Schw); Mosholu, Sep (Schw); Nyack (Schw); LI.

C. compactus Cress. Tomkins Cove, Sep (Schw); Nyack, Mosholu, Suffern, Sep, Ramapo, Van Cort. Pk., Sep (all Schw); LI: Flatbush, Sep, Pine I., Sep (Schw).

178 The list of bees, with the exception of Bombidae, has been compiled from literature and from material which has been determined in the Cornell collection by E. A. Richmond and others. H. E. Schwarz has contributed records of Colletes and Hylaeus. The editors are under obligations to Miss Grace Sandhouse, and especially to T. D. A. Cockerell, for assistance and suggestions as to synonymy, etc., and for additional records. $H$. L. Viereck has compiled the list of the genus Andrena. 
C. inequalis Say. Ithaca, Ap-May (Rich-CU); Albany, Karner, Poughkeepsie, Ap-May (NYS); New Baltimore, Nyack (Schw); LI, May (Schw).

C. latitarsis Robt. Huguenot, Aug 27, one $\delta$ (Schw).

C. productus Rob. Chazy L., Je; Trenton Falls, J1; Newport, J1, Yg; Ithaca, May; Karner, Ap-Sep; Clinton Hgts., Ap (all NYS).

C. validus Cress. LI: Sea Cliff, Bayville (Swenk).

\section{SUBFAMILY HYLAEINAE}

\section{HYLAEUS Fabricius}

\section{(Prosopis Fabricius)}

(The members of this genus usually nest in pithy stems, but some in the ground, or in crevices in walls.)

H. basalis (Sm.). Cranberry L., J1 (Schw); Ithaca, J1 (Rich-CU).

H. cressoni (Ckll.) (pygmaeus Cress.). Ithaca, Je-J1 (Rich-CU).

H. modestus Say. Keene Val, J1-Aug (Schw); L. George, Aug (Schw); Ithaca, Caroline to Harford, Taughannock Falls, Je-Aug (Rich-CU) ; Bushnellsville, Sep (Schw) ; Oliverea, Je (Schw) ; Stony Pt., Je (Schw); LI: Flatbush, Je, Pine I., Sep, Cold Sp. Harb., J1 (Schw). Visits milkweed, goldenrod, parsnip, meadowsweet, and day lily.

H. pygmaeus (Cress.). Keene Val., Aug (Schw); L. George, Aug (Schw); White L., Aug (Schw); LI: Cold Sp. Harb., J1 (Schw); Fishers I., Aug (Schw). On flowers of goldenrod and red raspberry.

H. varifrons (Cress.). Ithaca, Caroline, Je (Rich-CU); LI: Pine I. (Schw).

H. verticalis (Cress.). Mud Cr., Je (Rich-CU) ; McLean, Aug 20-31 (Roh-CU) ; Catskills, Aug (Schw).

H. ziziae (Robts.). Ithaca, Taughannock, Ringwood, Spencer L., Slaterville, MayJe (Rich-CU); McLean, Aug 1-7 (Roh-CU).

\section{FAMILY ANDRENIDAE 179}

\section{Compiled by M. D. LEONARD}

\section{SUBFAMILY ANDRENINAE}

\section{ANDRENA Fabricius 180}

(The very numerous species of Andrena build their nests in the ground where the vegetation is sparse. They often group their nests into colonies.)

A. accepta Vier. White Plains, Aug, Sep, on Helianthus giganteus, Beq.

A. angusi Vier. West Farms, NYC.

A. arabis Rob. McLean, Ap, Morr.

A. asteris Rob. White Plains, Sep 10, Beq; LI: Montauk, Sep, Ds. Visits aster.

A. barbarica Vier. LI: Brooklyn, Beq.

A. bisalicis Vier. Ithaca, Sep; SI, Ap, Beq; LI: Flushing; Farmingdale, Ap, on Salix.

A. braccata Vier. White Plains, Sep, on Aster ericoides, Beq. Found also on goldenrod.

179 See footnote under family Hylaeidae, page $102 i$.

180 The list for this genus has been prepared by. H. L. Viereck. 
A. bradleyi Vier. SI, May, on Salix, Beq. Also on gooseberry and huckleberry.

A. canadensis D.T. Keene Val., Aug, Lint.

A. carlini Ckll. Newport, May, Yg; Ripley, Ap; Ithaca, Ap-May, MacG, Morr ; McLean, Ap 17-Je 18; Easton, May; Albany, Ap-May; Delmar, May ; Clinton Hgts., Ap-May; Garrison, Ckll; Poughkeepsie, May; SI: Watchogue, May ; LI: Flatbush, May, Zab; Flushing; Yaphank, May, on Antennaria plantaginifolia and Salix; Half Way Hollow Hills, May; Wyandanch, May, Ds; Southold, Ap.

A. ceanothi Vier. LI: Central Pk., Je 27, on Cornus candidissima, Beq.

A. crataegi Rob. Speculator, Je, Yg; Keene Val., J1, Lint; Saranac Inn, Je; Ilion, May, $Y g$; Oswego, Je; Northville, Je, $Y g$; Niagara Falls, Je, Jn; Pike, Je; Ithaca, Fawaugh; Corinth, May, Yg; Salem, Je; Albany, May-Je; Karner, Mar, May, Yg; Bath, Je; Red Hook, Je, Leon; White Plains, Je, Beq; Garrison, Cattell; LI: Cold Sp. Harb., Je, Beq. On fruit-tree flowers.

A. cressoni Rob. Ithaca, Ap; McLean, Ap, Morr; Nassau, May; Albany, Je; Poughkeepsie, May; LI: Flushing, Beq. Visits chokeberry.

A. erythrogastra var. subaustralis Ck11. Nyack, Feb, Zab.

A. erythronii Rob. Ithaca, Ap, Morr.

A. fenningeri Vier. Ithaca, Bks.

A. fimbriata Ill. SI, Aug, Ds (Ashm).

A. flavoclypeata Sm. Speculator, Je, $Y g$; Ithaca, May, Bish, MacG; McLean, Ap, wild cherry ; Coy Glen, May, Morr; Nassau, May ; Clinton Hgts., Ap ; Karner, Ap-May; Bath, May; Delmar, May; Poughkeepsie, May; Red Hook, Je, Leon; Nyack, Oct, Zab; Garrison, Cattell (Ck11) ; SI ; LI: Orient Pt., May; Central Pk., on Cornus candidissima, Beq; Farmingdale, Ap, on Antennaria plantaginifolia, Beq. Common on flowers of fruit-trees.

A. forbesi Rob. LI: Flushing, Ap; Farmingdale, Ap, on Salix, Beq. On pear, currant, willow.

A. fragariana Graen. Garrison, Cattell (Ck11).

A. fragilis Sm. Newport, Je, $Y g$; Northampton, Je, $Y g$; Ithaca, Aug; Poughkeepsie, Je; LI: Sea Cliff, Bks.

A. geranii maculati Rob. LI: Sea Cliff, Bks.

A. helianthi Rob. var. White Plains, Sep; LI: Flushing, on Helianthus giganteus, Beq.

A. hippotes Rob. Ithaca, May; Coy Glen, Ithaca, May; McLean, Ap, Morr. Visits apple, Japanese plum, and sweet cherry.

A. hirticincta Prov. Ithaca, Aug; McLean, Aug 23 (Roh-CU) ; Garrison, Cattell; SI, Aug-Sep, Ds. On goldenrod and other late summer flowers.

A. imitatrix Cress. Pike, Je.

A. imitatrix var. claytoniae Rob. Ithaca, Ap-May, Morr; McLean, Ap, wild cherry.

A. imitatrix var. texana Cress. Ithaca, Ap, Morr; McLean, Ap, wild cherry.

A. impunctata Kby. Cumberland, type; SI, Ap 30, Ds (Ashm).

A. integra Sm. "NY", Nort. On golden alexanders, Zisia aurea (L).

A. lata Vier. Adirondacks; Axton, Je $(\mathrm{M} \& \mathrm{H})$.

A. mandibularis Rob. Niagara Falls, Je, $J n$.

A. mariae Rob. var. concolor Rob. Coy Glen, Ithaca, Ap-May; McLean, Ap. Visits blackberry, currant, and gooseberry flowers. 
A. milwaukeensis Graen. Mt. Skylight, 4800-4920 ft., J1, Beq; Oswego, Je; Albany, May; Nyack, Zab; Garrison, Cattell; SI, Ds.

A. miranda Sm. Ithaca, May; Salem, Je; Rensselaer, May.

A. moesta Sm. LI: Farmingdale, Ap-May, on Salix; Flushing, Beq; Half Way Hollow Hills, $D s$.

A. nasoni Rob. Oswego (NYS); Niagara, Je, $J n$; Ithaca, Ap-May, Morr ; McLean, Ap, on wild cherry; Albany, Je (NYS) ; Poughkeepsie, May '(NYS); Garrison, Cattell (Ck11) ; LI: Cold Sp. Harb., Je, Beq. On fruit-tree blossoms, also currant and gooseberry.

A. nubecula Sm. Van Cort. Pk., Beq.

A. nuda Rob. LI: Cold Sp. Harb., Je, Beq.

A. perplexa Sm. "NY" (Pck).

A. placida Sm. (macgillivrayi Ckll.). Albany, Ap (NYS); SI, May 15, on Rubus canadensis, Beq; LI: Farmingdale, Ap, on Salix; Flushing, Ap, Beq; Orient, Ap (NYS). Gooseberry and Japanese plum.

A. regularis Mall. Speculator, Je, $Y g$; Ithaca, Ap-Je, $B d y$; Rensselaer, May.

A. robertsoni D.T. Niagara Falls, Je, Jn; White Plains, Je; Garrison, Cattell (Ck11); LI: Cold Sp. Harb., Aug, on Clethra alnifolia, Beq.

A. rugosa Rob. Garrison, Cattell.

A. salictaria Rob. Newport, Je, $Y g$; Albany, Ap ; Karner, Ap-May, $Y g$; W. Nyack, Mar, Beq.

A. sigmundi Ck1l. Ithaca, Bks; McLean, Ap, Morr; Karner, Ap-May; Nassau, Ap; W. Nyack, Mar; LI: Farmingdale, on Salix; Flushing, Ap, Beq; Central Pk., Ds.

A. spireana Rob. White Plains, Je, on Ceanothus americamus, Beq; West Farms, NYC, Angus.

A. tridens Rob. Nyack, Oct, Zab.

A. vicina Sm. Ilion, May, $Y g$; Oswego, May (US); Ithaca, Ap-May, Morr; McLean, Ap, on wild cherry; Albany, May; Bath, Je; SI, May, Beq; LI: Half Way Hollow Hills, May, Ds; Central Pk., Ap, Ds; Farmingdale, Ap, on Salix; Flushing, Beq. On fruit-tree and gooseberry flowers.

A. wilkella Kby. Mt. Skylight, 4800-4920 ft., J1, Beq; Mt. Marcy, 4800-5344 ft.., J1, on Potentilla tridentata, Beq; Rochester Jct.; Honeoye Falls, Je, Ds; Rock City, Je, Ds; Ithaca, May-Je, on wild cherry, Bks, Morr; McLean Bogs; Albany, May-Je; Karner, May, $Y g$; N. Chatham, Je; Niverville, Je, Yg; Poughkeepsie, May; Loomis; SI, May, on Rubus canadensis; LI: Farmingdale, Ap, on Salix; Wyandanch, May, $D s$.

\section{Calliopsis Smith}

C. andreniformis Sm. Keene Val., Aug (Rich-CU); Newport, Je, Yg; Ithaca, Je-Jl (Rich-CU).

\section{EPEolus Latreille}

(Social parasites in the nests of other bees.)

E. mercatus Fab. SI, Sep 2, Ds (Ashm).

E. scutellaris Say. LI: Huntington, Sep 16 (Schott).

E. vernoniae Ckll. Mooers, Sep 9-13, Not (Cwfd). 


\section{TRIEPEOLUS Robertson}

T. donatus (Sm). LI: Huntington, Sep 16 (Schott).

\section{Halictus Latreille}

(These are gregarious mining bees which excavate their nests in bare level ground or in vertical banks, such as old gravel pits. Several bees of some species build their cells opening from a common gallery, the entrance to which is guarded by a bee whose head is used as a portal.)

\section{(Subgenus Halictus Latreille)}

H. inconspicuus Sm. SI, J1-Aug, Ds (Ashm).

H. lerouxii Lep. Ithaca, May-Aug (Rich-CU); Scotia, Je (NYS). On gooseberry.

H. similis Sm. Ithaca, May (Rich-CU).

H. zonulus Sm. Ithaca, Je (Rich-CU).

\section{(Subgenus ODONTALICTUS Robertson)}

H. ligatus Say. Ithaca, Aug (Rich-CU); McLean, Sib (Bdy-CU); SI, Aug, Ds (Ashm). On goldenrod.

\section{(Subgenus Agapostemon Guérin)}

H. radiatus Say. Ithaca, Je (Cress-CU) ; McLean, Aug 27 (Roh-CU); Garrisori Cattell (Ck11). On goldenrod, New Jersey tea, etc.

H. virescens (Fab.). Mooers, Sep 11, Not (Lutz); Ithaca, Je (Rich-CU); McLean. Sep 24 (Roh-CU).

\section{(Subgenus Oxystoglossa Smith)}

H. confusus Smith. Ithaca, Je-J1 (Rich-CU); McLean, Aug 19-31 (Roh-CU; Garrison, Cattell (Ck11).

H. purus Say. Ithaca, J1 (Rich-CU); McLean, Sep 16 (Roh-CU).

\section{(Subgenus Lasioglossum Curtis)}

H. fuscipennis Sm. SI, Je 15, Ds (Ashm).

\section{(Subgenus ChloRalictus Robertson)}

H. cattellae E1lis. Garrison, Cattell (type, Ck11).

H. hortensis Lovell. McLean, Aug 1-Sep 24, SRobinson, Sib (Roh-CU) ; Garrisr, Cattell (Ellis).

H. laevissimus Sm. SI: Prince's Bay Sta., Ds (Ashm).

H. subconnexus Ellis. Niagara, Je (Ck11); Garrison, Cattell (Ellis).

H. zephyrus Sm. Garrison (Ellis-CU). On fruit flowers.

\section{(Subgenus CURTISAPIS Robertson)}

H. coriaceus Sm. Ithaca, Je-J1 (Rich-CU); SI, Ap 30, Ds (Ashm). 
(Subgenus SELAdonia Robertson)

H. flavipes Fab. SI, J1, Ds (Ashm).

H. provancheri D.T. Ithaca, Ap (Rich-CU); McLean, Aug 24-Sep 16 (Roh-CU).

\section{MACROPIS Panzer}

M. ciliata Patt. Newport, Old Forge, Je, $Y g$ (NYS); Elm L., Aug (NYS); Karner, J1 (NYS). On laurel, loosestrife, and ginseng.

M. morsei Patt. Elizabethtown (NYS); McLean, J1 (Rich-CU) ; Poughkeepsie. J1 (NYS).

M. patellata Patt. Ithaca, J1 (Rich-CU) ; McLean, J1 19 (Roh-CU). Visits sumac, water hemlock, and fringed loosestrife.

NOMADA Fabricius

(Social parasites in the nests of other bees.)

(Subgenus Gnathias Robertson)

N. albofasciata Sm. "NY" (Ckll-US).

N. bella Cress. Ithaca, Ap-May (Rich-CU); McLean, May (Rich-CU) ; Nyack, Zab (Ckil).

N. cornelliana Ckll. Ithaca, Bks (Ck11).

N. cuneata (Robt.). "NY" (Ckl1).

N. cuneata (Robt.) var. quadrisignata Robt. McLean, Ap 17-Je 18 (Roh-CU).

N. lepida Cress. Ithaca, Ap-May (Rich-CU); W. Danby, May (Rich-CU).

N. perplexa Cress. Ithaca, Je-J1 (Rich-CU).

(Subgenus HOLONOMADA Robertson)

N. affabilis Cress. Ithaca (Rich-CU); W. Danby, May (Rich-CU).

N. vincta Say. Ithaca (Rich-CU).

(Subgenus Heminomada Cockerell)

N. obliterata Cress. Ithaca, May (Rich-CU).

(Subgenus Xanthidium Robertson)

N. luteola Lep. Ithaca, May (Rich-CU).

N. luteoloides Robt. Nyack, Zab (Ck11).

\section{(Subgenus Nomada Fabricius)}

N. bisignata Say. Ithaca, Ap-May (Rich-CU). Cannot be identified, according to Robertson, 1898.

N. cressoni Robt. Ithaca, Je (Rich-CU).

N. denticulata Robt. Ithaca, Ap 26 (Roh-US); W. Danby, May (Rich-CU).

N. gracilis Cress. Ithaca, Ap (Rich-CU).

N. pygmaea Cress. Ithaca, Ap-May (Rich-CU); McLean, May (Rich-CU).

N. sayi Robt. Ithaca, Ap-Je (Rich-CU).

N. xanthura Ck11. LI: Sea Cliff, May, Bks. 


\section{PANURGINUS Nylander}

P. pauper (Cress.). "NY" (Cress).

\section{PERDita Smith}

P. octomaculata (Say) Prov. Keene Val., Aug (Rich-CU); Ithaca, Aug (Rich$\mathrm{CU})$. Visits goldenrod and aster.

\section{SPHECODES Latreille}

(These are social parasites on the species of Halictus.)

S. arvensis Patt. McLean, J1 18 (Roh-CU). On goldenrod, sunflower, parsnip, and meadowsweet.

S. dichropa Sm. Ithaca, Je (Rich-CU).

S. mandibularis Cress. Ithaca, $S m$ (CU). Visits goldenrod, New Jersey tea, and day lily.

S. persimilis Lov. \& Ck11. Trenton Falls (Ck11).

S. trentonensis Ck11. Trenton Falls, type (Ck11).

\section{SUBFAMILY ANTHOPHORINAE}

ANTHOPHORA Latreille ${ }^{181}$

(The species of Anthophora build in vertical or steeply inclined banks, their entrance galleries protected by cylindrical chimneys. They build in colonies.)

\section{(Subgenus ANThemoessa Robertson)}

A. abrupta Say. Ithaca, Je-J1, Smith (CU). On rose, milkweed, bindweed, beardtongue, Virginia water-leaf, Virginia cowslip, ipecac, and New Jersey tea.

\section{(Subgenus ANTHOPHORA Latreille)}

A. bomboides Kby. Ithaca, J1-Aug (Rich-CU). On dogbane.

A. pyralitarsis Dours. "NY", type.

A. ursina Cress. Garrison, Cattell (Lutz\&Ck11). On Virginia cowslip, blue-eyed Mary, milk vetch, and wild honeysuckle.

\section{(Subgenus Clisodon Patton)}

A. terminalis Cress. Ithaca, J1-Aug (Rich-CU) ; McLean, J1 18-Aug 31 (Roh-CU) ; Canandaigua, Je, Felt (NYS). Nests in wood. Visits aster, hedge nettle, pickerel-weed, etc. Considered identical with the European A. furcata (Panz.).

\section{MELISSOdES Latreille}

\section{(Subgenus MELissodes Latreille)}

M. agilis Cress. (aurigenia Cress.). Hope, Aug, 1900 (NYS) ; Gloversville, Aug, 1906; Ithaca, Je-Aug (Rich-CU); Albany, Ap, 1905. On sunflower and other composites.

${ }^{181}$ If the Erlangen List is accepted, the name Anthophora will be replaced by Lasius. 
M. bimaculata (Lep.). SI, Aug, Ds (Ashm); LI: Orient, Sep, Ds (Beq). On milk vetch, milkweed, wild potato, bindweed, figwort, speedwell, ironweed, Joe-pye weed, other composites, etc.

M. desponsa Sm. Ithacd, Jl-Aug (Rich-CU); SI, Ds (Ashm); LI: Yaphank, Sep, Ds (Beq). On goldenrod, thistle, sunflower, and other composites, and on pickerel-weed.

M. perplexa Cress. Ithaca, Aug (Rich-CU). On Jimson weed, goldenrod, aster, sunflower, and other composites.

M. rustica (Say). Ithaca, Aug (Rich-CU). On goldenrod.

Tetralonia Spinola 182

(Synhalonia Patton)

T. robertsoni Ckll. Garrisọn, Cattell (Lutz\&Ckl1).

XENOGLOSSA Smith

(Subgenus PEPONAPIS Robertson)

X. pruinosa (Say). Ithaca, J1-Aug (Rich-CU). On pumpkin and other cucurbits, also milkweed.

\section{SUBFAMILY XYLOCOPINAE}

\section{Xylocopa Latreille: Carpenter Bees}

$\mathrm{X}$. virginica (Dru.). Ithaca, not common, Bdy (Cress-CU). Builds in beams and posts.

\section{SUBFAMILY CERATININAE}

Ceratina Latreille: The Little Carpenter Bees

C. dupla Say. Ithaca, Ap-J1 (Rich-CU); McLean, May, Sep 16-20 (Roh-CU); Taughannock Falls, May (Rich-CU); SI, Ds (Ashm). The commonest dweller in sumac branches. Visits red raspberry and goldenrod flowers.

\section{FAMILY MEGACHILIDAE 183}

Compiled by M. D. LEONARD

\section{ANDRONICUS Cresson}

A. cylindricus Cress. Ithaca, Taughannock, Je-J1 (Rich-CU).

\section{Alcidamea Cresson}

A. producta Cress. Ithaca, May, Comstock (Rich-CU). Nests in sumac and other pithy plants.

\section{Hoplitis Klug}

H. truncata (Cress.). Ithaca, Je-J1 (Rich-CU).

\footnotetext{
${ }^{182} \mathrm{Lutz}$ and Cockerell point out that Synhalonia is probably the correct name for this genus and that Tetralonia Spin. is probably a synonym of Eucera.

$1{ }_{1}$ See footnote under family. Hylaeidae, page 1021.
} 
Coelioxys Latreille

(These bees are social parasites in the nests of Megachile.)

C. dubitata Sm. Ithaca, J1-Aug, Comstock (Rich-CU); Taughannock, J1 (RichCU).

C. modesta Sm. Ithaca, J1 (Rich-CU).

C. octodentata Say. Ithaca, Aug (Rich-CU); SI, J1 30, Ds (Ashm). On mountain mint and goldenrod.

\section{DiAnThidium Cockerell}

(These bees cement fragments together with resin to form their nests.)

D. jugatorium (Say). Ithaca, J1-Aug (Rich-CU).

D. notatum (Latr.). LI: Huntington, Aug 19, Schott (Roh).

HERIADES Spinola

H. carinatus Cress. Ithaca, Je-J1 (Rich-CU); Spring Lake. J1 (Rich-CU).

\section{Megachile Latreille: Leaf-Cutter Bees}

(Subgenus Megachile Latreille)

M. brevis Say. Ithaca, Je-Aug (Rich-CU); SI, Ds (Ashm). On sunflower, goldenrod, and milkweed.

M. exilis Cress. Ithaca, Sep (Rich-CU).

M. generosa Cress. Ithaca, J1-Aug (Rich-CU).

M. mendica Cress. Ithaca, J1-Sep (Rich-CU).

\section{(Subgenus Anthemois Robertson)}

M. infragilis Cress. Ithaca, Je-Aug (Rich-CU): On milkweed and burdock.

M. decipiens Lov. \& Ck11. McLean, Aug 31, 1925, SRobinson (Roh-CU).

(Subgenus XANTHOSARUS Robertson)

M. latimana Say. Ithaca, J1-Aug (Rich-CU); McLean, J1 19 (Roh-CU); SI, J1, Ds (Ashm).

M. melanophaea Sm. Ithaca, Je-J1 (Rich-CU).

\section{(Subgenus CyPHOPyga Robertson)}

M. montivaga Cress. Ithaca, McLean, Je-Aug (Rich-CU).

(Subgenus SAYAPIS)

M. pugnata Say. Ithaca, J1-Aug (Rich-CU).

M. vidua Sm. "NY"?

(Subgenus Delomegachile Viereck)

Monumetha Cresson

M. albifrons (Kby.). Ithaca (Rich-CU). 


\section{Osmia Panzer}

(Some Osmiae, known as mason bees, construct their nests of a mixture of sand and clay and some fluid which makes a cement-like mass. Some use vegetable fragments, some nest in snail shells; others line their nests with flower petals.)

O. albiventris Cress. Ithaca, Je-J1 (Rich-CU); W. Danby, May (Rich-CU).

O. atriventris Cress. (proxima Cress.). Ithaca, Je-J1 (Rich-CU).

O. bucephala Cress. (latitarsus Cress., megacephala Cress.). Ithaca, May-Je (Rich-CU).

O. canadensis Cress. Ithaca, Je (Rich-CU).

O. chalybea Sm. SI, Ap, Ds (Ashm).

O. collinsiae Robt. McLean, May 30 (Sand-CU).

O. conjuncta Cress. Ithaca, Ap-J1 (Rich-CU).

O. faceta Cress. Ithaca, Ap-Aug, Norton's Landing, Cayuga L., SmHH, Danby (Rich-CU).

O. lignaria Say. Ithaca, Ap-May (Rich-CU). Quince and apple.

O. major Robts. "NY"? On honeysuckle flowers.

O. pumila Cress. (vicina Cress.). Ithaca, Ap-May (Rich-CU); McLean, Ap-May (Rich-CU), J1 15 (Sand-CU). On red-raspberry flowers.

O. rustica Cress. Ithaca, Slaterville, Je (Rich-CU). Lilac.

O. simillima Sm. Ithaca, May-J1 (Rich-CU).

\section{PRochelostoma Robertson}

P. philadelphi Robt. Ithaca, Je (Rich-CU).

\section{STELIS Panzer}

\section{(Subgenus MicRostelis)}

S. foederalis Sm. Ithaca, Je (Rich-CU).

\section{(Subgenus Stelis Panzer)}

S. lateralis Cress. Norton's Landing, Cayuga L., SmHH (CU) ; Ithaca, Comstock (CU) ; Albany, Je (NYS); Karner, Je (NYS). A parasite in the nests of Alcidamia producta Cress.

\section{FAMILY BOMBIDAE 184}

Bombus Latreille: Bumblebees

(Subgenus ВомвUs Latreille)

B. terricola Kby. New Russia (Bdy-CU); Keene Val. (Bdy, Beq) ; Long L. (Bdy-CU); L. Tear (Beq); Essex Falls, Nic (Beq); Mt. McIntyre (BdyNYS); Mt. Skylight (Beq); Labrador Hollow (Bdy); Crater Club, Aug (Fris) ; Cranberry L., J1 (Fris) ; St. Lawrence Co, Aug (Fris) ; Hermon, Aug (Fris) ; L. Pleasant (Bdy-NYS) ; Oneida (Franklin) ; Syracuse, May, J1, Sep (Fris) ; Geneva, May (Fris) ; Ithaca (Bdy, Beq) ; Castile, EABradley;

\footnotetext{
${ }^{184}$ The editors are under obligations to Dr. Joseph Bequaert for extensive records in this family. T. H. Frison has also kindly sent in the records known to him.

If the Erlangen List is accepted, the family name Bombidae will become Bremidae, and the genus and subgenus name Bombus will become Bremus.
} 
Gannett Hill, Ontario Co, 2000 ft., Guerlac\&Bdy; Taughannock (Bdy-CU) ; Slaterville (Adams); L. George, Zab (Beq) - Albany (Bdy-NYS); Blackhead Mt., Greene Co (Beq); Big Ind. Val. (Beq); Oliverea (Beq); Ft. Montgomery, Schott (Beq); W. Nyack (Beq); LI (Franklin). In the Adirondacks I have taken it as high as 4800 to $5000 \mathrm{ft}$., where it visits Ledum groenlandicum, Oxycoccus oxycoccus, and Potentilla tridentata (Beq). Essentially a boreal species, and moderately common in the colder part of the State; only one record from the LI district (Bdy).

B. affinis Cress. Keene Val., Not (Beq) ; Crater Club, Aug (Fris) ; Newport (BdyNYS) ; Castile, EABradley; Gannett Hill, Ontario Co, $2000 \mathrm{ft}$. (Guèrlac) ; Covert (Bdy-NYS); Ithaca, J1-Aug (Bdy,Fris); Taughannock Bdy-CU); Albany (Bdy-NYS); Big Ind. Val. (Beq); New Kingston (Bdy-CU); Woodbury (Beq) ; Ft. Montgomery, Schott (Beq) ; Ramapo Mts. (Beq) ; SI, Ds (Beq) ; LI: Huntington, Schott (Beq) ; Pennequid Barrens (Beq) ; Wyandanch, Schott (Beq) ; Massapequa, Schott (Beq) ; Brooklyn (Beq). A transition and upper austral species, moderately abundant in the eastern to southern parts of the State, less common northward (Bdy).

B. affinis var. novae-angliae Beq. Covert, Labrador Hollow (Bdy-CU); LI: Brooklyn, Schott (Beq).

B. borealis Kby. Keene Val. (Bdy, Beq); Mt. Skylight, $4800 \mathrm{ft}$., on Potentilla tridentata (Beq); Brant L. (Beq); Canton, Aug (Fris); Hermon, Aug (Fris) ; Syracuse, Aug (Fris) ; Covert, one female (Bdy-CU) ; Taughannock, one female, CarlWillsey (Bdy); Ithaca (Bdy-CU); Labrador Hollow, four females $(\mathrm{Bdy}-\mathrm{CU})$. A boreal and uncommon species, known only from the colder regions of the State $(B d y)$.

B. bimaculatus Cress. Keene Val. (Bdy-NYS) ; Onondaga Co, May (Fris) ; Syracuse, May, J1 (Fris); Glenora (Guerlac\&Bdy); Ithaca (Rich-CU); Covert (Bdy-CU); Albany (Bdy-NYS); SI, Ds (Beq); LI: Orient, Farmingdale, Central Pk., Cold Sp. Harb., Oakdale, Yaphank (Beq). A transition and austral species, scarce in NY and largely confined to the warmer districts (Bdy).

B. impatiens Cress. New Russia (Bdy-CU) ; Crater Club, Aug (Fris) ; Hermon, Aug, Sep, Canton, Aug (Fris); Newport (Bdy-NYS); Gloversville (BdyNYS) ; Syracuse, May, Aug-Sep (Fris) ; Oak Orchard Swamp, Ds (Beq); Otto; Castile, EABradley, Gannett Hill, Ontario Co, 2000 ft., Guerlac (Bdy); Covert (Bdy-CU); Ithaca; Taughannock (Bdy-CU); Labrador Hollow (Bdy-CU) ; Ft. Plain (Bdy-NYS); Delmar, Albany, Karner, Cropseyville, Easton (Bdy-NYS) ; White Plains (Beq) ; Ramapo Mts. (Beq) ; Ft. Montgomery (Beq); W. Nyack (Beq) ; Yonkers (Beq); Van Cort. Pk. (Beq) ; SI (Beq) ; LI: Coram, Schott (Beq) ; Orient, Springs, Gardiners I., Brooklyn, Eng, Rockaway, Schott, Wyandanch, Amagansett, Yaphank (all Beq); Forest Hills (Bdy-CU). A very abundant species everywhere except in the mountains, where it is less common and probably absent from the higher altitudes (Bdy).

B. ternarius Say. Keene Val. (Bdy, Beq); New Russia (Bdy-CU) ; Mineville, DReis (Bdy); Crater Club, Aug (Fris); Chapel Pond (Bdy); Plattsburg (Beq) ; Saranac L. (Beq); north face of Mt. Marcy (Bdy-CU); Cranberry L., J1 (Fris) ; St. Lawrence Co, Aug (Fris) ; Canton, Aug (Fris) ; Hermon, Aug (Fris); Syracuse, May (Fris); Castile, EABradley; Gannett Hill, Ontario Co, 2000 ft., Guerlac; Cinnamon L., Lake Bluff (Bdy); Ithaca (BdyCU) ; Labrador Hollow (Bdy-CU) ; E. Jewett (Beq) ; Oliverea, Ds (Beq) ; Cooks Falls (Beq); Debruce (Beq). Essentially a Canadian and northern transition species, abundant in the Adirondacks, scarce about Ithaca, absent 
from the LI districts (Bdy). Smith's record for SI was based upon misidentification; the species is not found near NYC (Beq).

B. ternarius expallidus Ckll. Wilmington, Keene Val. (Fris-NYS).

B. perplexus Cress. Keene Val., Not (Beq); New Russia (Bdy-CU); Cliff Mt. (Beq); Upper Ausable L. (Beq); Crater Club, Aug (Fris); Mt. Marcy (Beq) ; Cranberry L., J1 (Fris) ; St. Lawrence Co, Aug (Fris); near Pulaski, Ds (Beq) ; Hermon, Aug (Fris) ; Syracuse, May, J1 (Fris); Ithaca, Sep (Fris, Bdy) ; Slaterville (AAdams); Labrador Hollow (Bdy-CU); New Salem, Delmar, Bish (Bdy-NYS); Big Ind. Val. (Beq) ; Oliverea (Beq); New Kingston (Bdy-CU); Debruce, Ds (Beq); Ft. Montgomery, Schott (Beq) ; W. Nyack (Beq) ; LI: Cold Sp. Harb., Wyandanch, Schott (Beq). In the Adirondacks, I have taken it at 4800-5000 ft., where it visits $V$ accinium uliginosum (Beq). A Canadian species running well south in the Allegheny Mts. Moderately common in the northern and central part of the State, but rare in the LI district (Bdy).

B. vagans F. Sm. Keene Val., Diven, Not (Bdy, Beq) ; New Russia (Bdy) ; Long L. (Bdy) ; Mt. Marcy, Upper Ausable L., Mt. Skylight (Beq) ; Mt. Whiteface, Ds (Beq); Mt. McIntyre (Bdy-CU); Crater Club, Aug (Fris); Cranberry L., Jl (Fris); Saranac (Bdy-NYS); Wells, Yg (Bdy-NYS); Newport (Bdy-NYS) ; Old Forge (Bdy-NYS); Hermon, Canton, Aug (Fris) ; St. Lawrence, Aug (Fris); Syracuse, J1-Aug (Fris); Clyde (Bdy-NYS); Gannett Hill, Ontario Co, 2000 ft., Guerlac; Castile, EABradley; Cattaraugus (Bdy-CU) ; Covert (Bdy-CU) ; Ithaca, Sep (Fris); Taughannock (Bdy$\mathrm{CU}$ ) ; McLean (Bdy-CU) ; Labrador Hollow (Bdy-CU) ; Karner, New Salem (Bdy-CU) ; Oliverea, Big Ind. Val. (Beq) ; Poughkeepsie (Bdy-CU) ; New Kingston (Bdy-CU) ; Callicoon, Ds (Beq) ; Pine I., Ds (Beq) ; Ft. Montgomery, Schott (Beq) ; SI, Ds (Beq) ; LI: Cold Sp. Harb., Oakdale, Schott, Gardiners I., Fiatbush. (Beq); Wyandanch, Schott (Beq) ; Half Way Hollow Hills, Montauk, Amagansett, Orient, all Ds (Beq); Jamaica, Schott (Beq) ; Riverhead (Bdy-CU). The most abundant species of Bombus throughout the greater part of the State. Common, although perhaps less so than others in even the high Adirondacks (Bdy). I have taken this species in the Adirondacks at an altitude of 5000-5200 ft., where it visits Solidago alpestris, Vaccinium uliginosum, Oxycoccus oxycoccus, and Potentilla tridentata (Beq).

B. vagans var. coctus Beq \& Plath. LI: Flushing, Eng (Beq\&Plath).

B. fervidus (Fab.). Canton, May, Hermon, Aug (Fris); Otter L. (Bdy-CU); DeRuyter (Bdy-CU) ; Pike (Bdy-NYS) ; Geneva, May (Fris) ; Covert (Bdy$\mathrm{CU}$ ); Ithaca, Sep (Bdy, Fris); Labrador Hollow (Bdy-CU); Monticello, Schott (Bdy-CU); Debruce, Ds (Beq); Mosholu, Ds (Beq) ; White Plains (Beq); Van Cort. Pk. (Beq); SI (Beq); LI: E. New York, Aqueduct, Greenport, Ds (Beq) ; Flatbush, Jamaica, Little Neck, Oakdale, Schott (Beq); Sands Pt., Bs (Beq). Essentially a southern species, and one of our most abundant in southern and central NY. Not known from the Adirondacks and Catskills (Bdy).

B. fervidus var. dorsalis Cress. Covert, Labrador Hollow (Bdy-CU).

B. americanorum (Fab.) (pennsylvanicus Geer). Mt. Marcy (Bdy-NYS); Otter L. (Bdy-CU) ; Syracuse, Oct (Fris); Canandaigua L. (Bdy-NYS); Covert (Bdy-CU); Ithaca (Bdy-CU); Taughannock (Bdy-CU); Slaterville (AAdams); Albany (Bdy-CU); White Plains (Beq); SI (Beq); LI: Oakdale, Coram, Schott (Beq) ; Long Beach, Orient, Gardiners I. (Beq) ; Wyandanch, Schott (Beq); Aqueduct, Montauk, Amagansett, Ds (Beq). An abundant southern species, common as far north as central NY, but rare in the mountains (Bdy). 


\section{(Subgenus Bombias Robertson)}

B. auricomus Robts. Manlius, $\mathrm{SmHH}$ (Richmond-CU); Covert (Bdy-CU).

B. separatus Cress. New Russia (Bdy-CU); Otter L. (Bdy-CU); Canandaigua L. (Bdy-NYS) ; Covert (Bdy-CU) ; Itháca (Rich, Bdy-CU) ; Norton's Landing, Cayuga L.; west shore, Cayuga L. (AAdams); Labrador Hollow (BdyCU) ; Karner (Bdy-NYS) ; SI, Ds (Beq) ; LI: Bellmore, Eng (Beq) ; Long Beach, Orient, Pennequid Barrens, Gardiners I. (Beq); Wyandanch, Schott (Beq); Montauk, Yaphank, Ds (Beq). A southern species, rare in central NY and the Adirondacks, more common in the LI district (Bdy).

B. rufocinctus Cress. Crater Club (Fris-SyracuseU); Lake Bluff, Sep 3, 1912 (color var. 6, Bdy); Castile, one male, May, ECBradley (Bdy-CU). Essentially a Rocky Mountain species, although known from eastern Canada and Vt. To be counted as our rarest species of Bombus.

\section{Psithyrus Lepeletier: Usurper BeEs}

(Social parasites of bumblebees.)

P. laboriosus (Fah).). Crater Club, Aug (Fris); St. Lawrence Co, Aug (Fris) ; Hermon, Aug (Fris) ; Cranberry L., J1 (Fris); Otter L. (Bdy-CU) ; Syracuse, Sep (Fris) ; Geneva, May (Fris) ; Covert (Bdy-CU) ; Norton's Landing, Cayuga L., SmHH (Cress-CU) ; west shore, Cayuga L. (AAdams); Ithaca, AAdams, RLounsberry (Bdy-CU); Spring Lake (Bdy-CU); Taughannock (Bdy-CU); Hudson Falls (Bdy-NYS); SI (Beq); LI: Kings Pk., Orient (Beq) ; Wyandanch, Huntington, Schott (Beq); Wading R. (Schott). A transition and upper austral species extendirg well into Canada. Our most common species. A form of this species with all-black abdomen in the female is not uncommon around Ithaca. It has been taken by Armand Adams, Victor Skiff, Royden Lounsberry, J. C. Bradley, Henry Guerlac, and others, at several localities (Bdy).

P. insularis (Sm.). Keene Val., Not (Beq, Fris); Ithaca (Franklin-CU).

P. ashtoni (Cress.). Keene Val., Not (Beq); Mt. Skylight, $4800 \mathrm{ft}$., on Potentilla tridentata (Beq) ; Hermon (Fris) ; Gannett Hill, Ontario Co, 2000 ft., Guerlac : Covert (Bdy-CU); west shore, Cayuga L. (AAdams); Ithaca (Cress, Franklin-CU); Taughannock (Bdy-CU); Labrador Hollow (Bdy-CU); Big Ind. Val. (Beq) ; Oliverea, Ds (Beq); Ft. Montgomery (Schott). This species seems to be confined to the central and northern part of the State; elsewhere its chief distribution seems to be in the Canadian and Northern Transition zones (Bdy).

P. fernaldae Franklin. St. Lawrence Co, Aug (Fris) ; Brant L. (Franklin) ; Cranberry L., J1 (Fris); Otto (Franklin); Gannett Hill, Ontario Co, 2000 ft., one male, Guerlac $\mathcal{G} B d y$; Ithaca (Franklin); McLean (Beq). A boreal species. known here only from the central and Adirondack regions and very rare (Bdy).

\section{FAMILY APIDAE}

\section{Aprs Linnaeus}

A. mellifica L. Honey BeE. Generally distributed throughout the State. 


\section{CLASS ARACHNIDA}

ORDER ARANEAE

Compiled by C. R. Crosby and S. C. Bishop

\section{SUBORDER ARANEAE THERAPHOSAE \\ FAMILY ATYPIDAE}

ATYPUS Latreille

A. niger Htz. Cornwall, May (Em).

\section{SUBORDER ARANEAE VERAE FAMILy ULOBORIDAE}

Hyрtiotes Walckenaer

H. cavatus Htz. Plattsburg, Sep; Wilmington Notch, Aug; Chapel Pond, Je; Ar' st's Brook, Je; Manlius, Aug ; Lake Bluff, Sep ; Lewiston, Sep, Plm; Silver Ct Sep; Montour Falls, Sep-Oct; Perry City, Sep; Taughannock Falls, Oct; Ith ca, Sep; Waldorf, Sep, Yg; Burden L., Aug; Valcour I., Aug; Elizabeth I., Aug; Juanita I., Pearl Pt., J1; Catskill, Aug, Chap; Poughkeepsie, vanIngen; Highland, Aug, Wlf; LI: Riverhead, Sep; Sea Cliff, Sep, $W l f$, Taub.

\section{ULOBORUS Latreille}

U. americanus Wlck. Onondaga Co (Britcher) ; Penn Yan, May; Montour Falls, Sep; Ithaca, Aug, Nov; Enfield Glen, Oct; Taughannock Falls, Oct; Valcour I., Aug; Oakland Val., May; LI: Cold Sp. Harb., Je, Anderson.

\section{FAMILY DICTYNIDAE}

\section{AmAuRobius C. Koch}

A. americanus Em. Onondaga Co (Britcher); Ithaca, May; Enfield Glen (Bks); Paradise, May; LI: Bayville, Aug (Bks).

A. bennetti Blackw. Wilmington Notch, Aug; Wilmington, Aug; Mt. Whiteface, Aug; L. Placid, Sep, Plm; Saranac L., Sep, Plm; Uphill Br., Opalescent R., J1; Paul Smiths, Je, Babiy; Raquette L., Sep, Plm; Chateaugay L., Sep, Plm; Big Burnt L., J1; Gloversville, Sep, Alex; Onondaga Co (Britcher); Lake Bluff, Sep; Lewiston, Sep, Plm; Clarksburg, Sep; Stow, Sep; Olcott, Sep ; Rock City, Sep; Savona, Sep; Oak Ridge, Canandaigua L., Aug; L. Keuka, Sep; Taughannock Falls, Oct; Connecticut Hill, Nov; Spencer, Aug; Ithaca, Mar-Sep; McLean, May; Voorheesville, Je ; Kenwood, May, Plm; Crystal L., J1; S. Westerlo, May; Feura Bush, Dec; Queechy L. May, Schoon; Vrooman's Nose, May; Valcour I., Aug; Pearl Pt., Juanita I., J1; Elizabeth I., Aug ; Riders Mills, May, Chrisp; L. Charlotte, Je ; Oakland Val., May ; Cragsmoor, May; Poughkeepsie (Em) ; Hunter, Je, Aug, Schoon; Stamford, May, Chrisp; Sterlington, May; Larchmont, Sep, Wlf; LI, Nov-Dec (Bks).

A. borealis Em. Mt. Whiteface, Aug; Wilmington Notch, J1-Aug; Wilmington, Aug; Newcomb, J1; Charley L., Ap; Old Forge, Oct, Sib; Italy Hill, Oct; Connecticut Hill, Aug; McLean, May, J1; Meredith, May; Stamford, Oct; Maratanza L., May; Pinekill, May.

A. ferox Walck. Onondaga Co (Britcher) ; Clyde, Ap, May; Ithaca, Mar, May; Albany, Je; Vrooman's Nose, May; LI, Sep (Bks).

A. tibialis Em. Mt. Whiteface, Aug (Em). 


\section{Dictyna Sundevall}

D. bicornis Em. Lake Bluff, Sep; LI: Sea Cliff, Je; Bayville (Bks); Watermill, Je; Wading R., Je; Riverhead, Sep.

D. bostoniensis Em. Sodus Pt., J1; SI: Tottenville, Je; LI: Bay Shore, J1 (Bks); Quogue, Je.

D. brevitarsus Em. Ithaca (Em); Little Pond, Orange Co, May.

D. cruciata Em. Ithaca (Bks) ; McLean, May-J1; Labrador Pond, Je; LI: Flushing, Je; Long Pond, Suffolk Co, Je.

D. foliacea Htz. Wilmington Notch, Aug; Wilmington, Aug; Sacandaga Pk., Je, Alex; Wells, May, $Y g$; Trenton Falls, Je; Onondaga Co (Britcher) ; Southwest Oswego, J1; Williamson, Je; Clyde, May; Crusoe L., May; Lake Bluff, Ap; Marilla, J1; Pt. Breeze, Je-J1; Letchworth Pk., J1; Penn Yan, Aug; Taughannock Falls, Oct; Enfield Glen, Je ; Cinnamon L., J1; Ithaca, May, Je, Oct; Ringwood, J1; McLean, May, J1; Caroline Center, May; E. Greenbush, Je; Albany, May-Je; Kenwood, May, Yg; Tackawasick Pond, Je ; Waldorf, Sep, $Y g$; Juanita I., Pearl Pt., J1; Cossayuna L., May, Schoon; Valley Falls, May, Yg; Schodack Landing, Je; L. Charlotte, Je; Riders Mills, May, Chrisp; Wappingers Falls, May; Port Jervis, May; Oakland Val., May; Sterlington, May; LI: Cold Sp. Harb., Je, Anderson; Sea Cliff, Je.

D. frondea Em. Newcomb, J1; Cranberry L. (Barrows); Taughannock Falls, J1; Ithaca (Bks); Clayville, Je; L. Erie beach, Je; Shaker Road, Albany Co, Je; LI: Sea Cliff, Aug (Bks); Long Pond, Suffolk Co, Je.

D. Iongispina Em. Sacandaga Pk., Je, Alex; Trenton Ealls, Je; Ithaca (Bks).

D. maxima Bks. Old Forge, Oct (immature); Paul Smiths, Je, Babiy; Ithaca (Bks) ; Caroline Center, May; McLean, May; Labrador Pond, Je; Stamford, May, Chrisp.

D. minuta Em. Cinnamon L., Je; Ithaca, Ap-May; McLean, Je; Riders Mills, May, Chrisp; Sterlington, May; LI, Sep (Bks).

D. quadrispinosa Em. Black Br., Je; Vrooman's Nose, May.

D. rubra Em. Onondaga Co (Britcher).

D. sublata Htz. Cranberry L. (Barrows); Raquette Falls, Aug; Gloversville, Mar, Alex; Johnstown, J1; Saratoga, Je; Labrador Pond, May, Tarris; Savannah, May; Lake Bluff, Ap; Pt. Breeze, Je; Olcott, Feb, Dt; Buffalo, Je; Keuka Pk., Nov; Stanley, May; Crosby, Ap; Ithaca, May; West Winfield, Je; Red Hook, J1, Plunk; Poughkeepsie; Germantown, Je; Stamford, May, Chrisp; LI (Bks).

D. volucripes Key. Wilmington Notch, Aug; Newcomb, J1; Speculator, J1, Yg; Cranberry L. (Barrows); Peru, Je; Gloversville, Je-J1, Alex; Onondaga Co (Britcher) ; Chautauqua Co, Palmer; Sodus, J1; Oak Ridge, Canandaigua L., Aug; Junius, Je; Penn Yan, Sep; Ithaca, J1-Aug; Ringwood, J1; Deruyter L., J1; Trenton Falls, Je; Albany, May; Thacher Pk, Je; Valcour I., Aug; Germantown, Je; Little Pond, Orange Co, May; Oakland Val., May; LI (Bks).

\section{LATHYS Simon}

L. foxii Marx. Barrington, Oct; Ithaca, Comst; Enfield Glen, Ap; Altamont, Ap ; Vrooman's Nose, May; Silver Bay, Sep, Leon; Juanita I., Aug; L. Charlotte, Je ; Cragsmoor, May; Sam's Pt., Ulster Co, May. 


\section{SCOTOLATHyS Simon}

S. maculata Bks. LI: Riverhead, Ap-May.

S. pallida Marx. Newfane, Oct; West Barre, Sep; Rock City, Sep; Rye Pt., Sep; Savona, Sep; Ithaca, Aug-Oct; Freeville, Aug ; Labrador Pond, Je; Meadowdale, May; Feura Bush, Dec; Voorheesville, May-Je; Karners, Mar; Vrooman's Nose, May; Valcour I., Aug; Juanita I., J1-Aug; Storm King Mt., J1; Sam's Pt., and Maratanza L., May; Oakland Val., May; LI: Baiting Hollow, Ap-May; Southold, Ap; Riverhead, May.

\section{FAMILY SICARIIDAE}

\section{SCYTODES Latreille}

S. thoracica Lat. Ithaca, J1; Onondaga Co (Britcher); Rensselaer, Aug, Schoon; Troy (Htz); LI: Sea Cliff (Bks).

\section{FAMILY OONOPIDAE}

\section{ORCHESTINA Simon}

O. saltitans Bks. LI: Sea Cliff, J1 (Bks).

\section{FAMILY DYSDERIDAE}

ARIADNA Audouin

A. bicolor Htz. Wappingers Falls, May; LI (Bks).

DYSDERA Latreille

D. crocata C. Koch. Syracuse (Britcher); Ithaca, J1 (Const); Rensselaer, Ap, Kilf; Poughkeepsie, vanIngen.

\section{FAMILY GNAPHOSIDAE}

\section{CALlilepis Westring}

C. imbecilla Key. Ithaca (Bks); LI: Cold Sp. Harb., Bryant; Sea Cliff (Bks).

\section{CESONIA Simon}

C. bilineata Htz. Spafford (Britcher); L. Keuka; Ithaca, Aug (Comst); Oak Ridge, Canandaigua L., Aug; Valcour I., Aug; Elizabeth I., Aug; Poughkeepsie, vanIngen; Waterford, Ap; Larchmont, Sep, Wlf; LI, Sep (Bks).

\section{DRASSODES Westring}

D. neglectus Key. Adirondack Lodge, Jr; Onondaga Co (Britcher); Lake Bluff, Sep; Rochester, Nov, Chad; Crocketts, Sep, Chap; Connecticut Hill, Oct; Ithaca, Aug-Oct, Comstock (Bks); McLean, Je; Lawson L., May; Crystal L., J1; Mud Hollow Pond, Je; S. Westerlo, May ; Dormansville, Je ; Vrooman's Nose, May; Hunter, Je, Plm; Elizabeth I., Aug ; Juanita I., Peari Pt., Aug; Black Mt., Aug; Stamford, Je; LI: Gardiners I., May. 


\section{DRASSYLLUS Chamberlin}

D. aprilinus Bks. LI: Sea Cliff, Bks.

D. blandus Bks. Ithaca (Bks).

D. depressus Em. Onondaga Co (Britcher) ; Dunkirk; Ithaca, Oct (Bks) ; Danby, May; Albany, Je; Voorheesville, Je; Rensselaer, J1; Dormansville, Je; LI: Sea Cliff, Bks.

D. fallens Chamb. LI: Sea Cliff (Chamb).

D. frigidus Bks. Gloversville, Mar; Ithaca, Feb (Bks); LI: Sea Cliff, Sep (Bks).

D. rufulus Bks. Onondaga Co (Petrunkevitch); Ithaca, Sep-Oct (Bks) ; Lake Bluff, Sep; LI: Cold Sp. Harb. (Em); Sea Cliff (Em) ; Riverhead, Sep.

D. virginianus Chamb. LI: Sea Cliff (Chamb).

\section{Geodrassus Chamberlin}

G. auriculoides Barrows. Greenville, Ap; Wappingers Falls, May.

G. phanus Chamb. LI: Sea Cliff (Chamb).

\section{GNAPHosA Latreille}

G. brumalis Thor. Mt. Marcy, J1, 5000 ft.; Mt. Whiteface, Aug.

G. fontinalis Key. Ithaca (Bks).

G. gigantea Key. Mt. Whiteface, Aug; Adirondack Lodge, J1; N. Elba, J1; Upper Jay, Sep, Plm; Chapel Pond, Je; Paul Smiths, Je, Babiy; Gloversville, Ap, Aug, Alex; Onondaga Co (Britcher) ; Ithaca, Jan, J1 (Comstock) ; Freeville, Bks; McLean, Je, Bissell; Danby, May; Valcour I., Aug; Dormansville, Je ; Voorheesville, Je, Aug; Normansville, Je; Mud Hollow Pond, Je; L. Charlotte, Je; Vrooman's Nose, May; Juanita I., J1; Black Mt., Washington Co, Aug; Elizabeth I., J1-Aug; Hunter, Je, Plm; Sullivan Co (Key); Poughkeepsie (Em); NYC (Bks).

G. parvula Bks. L. Placid, Sep, Plm; Gloversville, Mar, Alex; Crystal L., J1; Mt. Lebanon, May, Chrisp; LI: Cold Sp. Harb., J1, Bryant; Riverhead, Je; Montauk Pt., May.

G. sericata L. Koch. SI: Tottenville, Je; LI: Sea Cliff, Bks.

\section{HAPLODRASSUS Chamberlin}

H. hiemalis Em. Ithaca, May; Freeville; McLean, Je.

H. signifer C. Koch. Onondaga Co (Britcher); Ithaca; Pike Pond, Rensselaer Co, Je; LI: Sea Cliff, Bks; Gardiners I., May.

\section{HERPYLLUS Hentz}

H. vasifer Walck. Wells, Je-J1, Yg; Sacandaga Pk., Je, Alex; Gloversville, May, Alex; Onondaga Co (Britcher); Lake Bluff, J1, Sep; Clyde, Aug; Otto, Jan, Comstock; Wilmurt, Aug, Plm; Rochester, Nov; Hemlock, Aug; L. Keuka, Ap, Sep-Oct; Oak Ridge, Canandaigua L., Aug; Taughannock Falls, Oct; Ithaca, Sep, Nov; Freeville, Sep; Juanita I., J1; Albany, J1, Hartman; Poughkeepsie, vanIngen; II: Lynbrook, Oct; Sea Cliff, Bks. 
LITOPYLLUS Chamberlin

L. rupicolens Chamb. LI: Sea Cliff (Chamb).

POECILOCHROA Westring

P. montana Em. Ithaca, Mar (Bks).

\section{SERGIOLUS Simon}

S. variegatus Htz. Silver Bay, Sep, Leon; Ithaca, May, Bks, J1, Bryant; Feura Bush, Dec; LI: Long Pond, Suffolk Co, Je.

Sosticus Chamberlin

S. insularis Bks. LI: Sea Cliff (Bks).

\section{ZELOTES Gistel}

Z. duplex Chamb. LI: Sea Cliff.

Z. subterraneus C. Koch. Mt. Whiteface, Aug; Mt. Marcy, J1; Crater Club, Essex Co, Aug ; Cranberry Cr., Aug; Onondaga Co (Britcher); Lake Bluff, Sep; Ithaca (Bks) ; Karner, Ap; Mud Hollow Pond, Je; Voorheesville, Je; Pearl Pt., Aug; Juanita I., J1; Riders Mills, May; Stamford, May, Chrisp; Hunter, Aug; LI: Sea Cliff, Bks; Montauk Pt., May; Gardiners I., May.

\section{FAMILY PHOLCIDAE}

Pholcus Walckenaer

P. phalangioides Fuess. Ithaca, all months in buildings; Syracuse (Britcher); Albany, May, Sep, $Y g$; Cobleskill; Catskill, Aug-Sep, Chad; Poughkeepsie, vanIngen; LI: Brooklyn (Bks).

\section{SPERMOPHORA Hentz}

S. meridionalis Htz. Ithaca, J1; Poughkeepsie, vanIngen; LI (Bks).

\section{FAMILY THERIDIIDAE}

\section{ANCYLORRHANIS Simon}

A. hirsutum Em. Wolcott, May; Olcott, Sep-Oct, Dt; Clarksburg, Sep; L. Keuka, Sep-Oct, Dec; Alpine, Nov; Rye Pt., Sep; Connecticut Hill, Aug, Oct-Nov; Enfield Glen, May, Oct; Ithaca, May, Aug, Nov; Freeville, Oct; McLean, J1; Jenksville, Oct; Delaware L., May ; Accord, Mar; SI: Clove Val., Nov; LI: Sea Cliff (Em).

\section{ARGYRODES Simon}

A. cancellatus Htz. Onondaga Indian Reservation (Britcher); LI: Sea Cliff (Bks); Cold Sp. Harb. (Em).

A. trigonum Htz. Penn Yan, May, Aug; Onondaga Co (Britcher); Letchworth Pk., J1; Enfield Glen, Je, Oct; Ithaca, J1, Oct; Albany (Em); Riders Mills, May; Oakland Val., May; LI, J1 (Bks). 


\section{ASAGENA Sundevall}

A. americana Em. Gloversville, Mar-Ap, Alex; Barneveld, May; Onondaga Co (Britcher); Lake Bluff, Sep; Ithaca, May-Je, Oct, Nov; Schoharie, May (Em) ; Albany, Ap; Poughkeepsie, vanIngen; LI: Sea Cliff, Je (Bks) ; Baiting Hollow, May; Long Pond, Suffolk Co, Je; Riverhead, Je.

\section{Crustulina Menge}

C. guttata Wider. Cranberry Cr., Aug; Onondaga Co (Britcher); Ithaca, Nov-Dec; Black Mountain Pt., Washington Co, Aug; Kenwood, Ap, Chad; LI: Sheepshead Bay, May; Southold, Ap; Baiting Hollow, Ap; Riverhead, Ap-May; Montauk Pt., May.

C. sticta Cambr. Weedsport, Ap; Ithaca, Mar-May; Saratoga Sps., Ap; Rensselaer, Ap, Schoon; Guilderland Center, May; Thacher Pk., May; Trenton Falls, Je; Little Pond, Orange Co, May.

\section{DiPOENA Thorell}

D. buccalis Key. Enfield Glen, Aug, Wlf, Taub; NY (Marx); LI: Long Pond, Suffolk Co, Sep, Chap.

D. nigra Em. Adirondack Lodge, J1; Newcomb, J1; Johnstown, J1; Onondaga Co (Britcher) ; Westview, J1 ; Letchworth Pk., J1; Howard, J1; Connecticut Hill, Je, Oct; Ithaca, Je, Aug; McLean, May, J1; Labrador Pond, Je; Ringwood, Tompkins Co, Je, Oct; Trenton Falls, Je; Valcour I., Aug; Juanita I., J1; Oakland Val., May; Larchmont, Sep, Wlf; LI: Long Pond, Suffolk Co, Je.

D. pallida Em. LI: Riverhead, Ap.

\section{EnOPLOGNATHA Pavesi}

E. marmorata Htz. Johnstown, May, Alex; Onondaga Co (Britcher) ; Sodus Pt., J1; Taughannock Falls, Oct; Ithaca, May, J1; Rensselaer, Je, Schoon; Albany, Ap, Aug, Yg, House; Normansville, J1; Claverack, Mar; Wappingers Falls, May; Highland, Ap; Oakland Val., May; SI: Tottenville, Je; LI (Bks).

E. pallida Em. Ithaca, May.

\section{EURYOPIS Menge}

E. argentea Em. SI: Clove Val., Nov.

E. funebris Htz. Newcomb, J1; Onondaga Co (Britcher); Letchworth Pk., J1; Ithaca, Aug, Oct; Thacher Pk., Je; Valcour I., Aug; LI: Wading R., Je; Long Pond, Suffolk Co, Je.

E. quinque-maculata Bks. LI: Wading R., Sep, Chap.

E. spinigera Cambr. LI: Riverhead, Sep.

\section{LATRODECTUS Walckenaer}

L. mactans Fab. Lewiston, Sep, Plm; LI: Woodhaven, Ditmars.

\section{LITHYPHANTES Thorell}

L. corollatus L. LI: Glen Cove, Jan, Oct (Bks); Bayville, Jan, Oct (Bks) ; Baiting Hollow, May. 
Mysmena Simon

M. guttata Bks. LI, Je, Oct (Bks).

PAIDISCA Bishop and Crosby

P. marxi Cros. LI: Riverhead, Ap-May.

\section{Pedanostethus Simon (See Robertus) \\ RHOMPHAEA L. Koch}

R. fictilium Htz. Onondaga Co (Britcher); LI: Sea Cliff, Sep-Oct (Bks).

\section{ROBERTUS Cambridge}

R. fuscus Em. Mt. Whiteface, Aug; Uphill Br. and Opalescent R., J1; High Falls, Aug; Wilmington Notch, Aug.

R. palustris Bks. Old Forge, Oct, Sib; Howland I., Nov; Ithaca, Feb, Bks; McLean, May; Rensselaer, May, Schoon; Maratanza L., May; Suffern, May; LI: Flushing, Nov.

R. pumilus Em. Auger Pond, Nov; Cranberry Cr., Aug; Clarksburg, Sep; West Barre, Sep; Savona, Sep ; Italy Hill, Oct; Egleston's Glen, Sep; Ithaca, May ; McLean, Ap-May; Ringwood, May; Larchmont, Sep, Wlf.

R. riparius Key. Upper Jay, Sep, Plm; Wilmington Notch, J1-Aug; High Falls, Aug; Wilmington, Aug; Elizabethtown, Aug; Old Forge, Oct; Howland I., Nov; Cascade, Owasco L., Nov; Connecticut Hill, Oct; Guyanoga, Je; Enfield Glen, Aug ; Ithaca, Mar, Dec; Freeville, Sep; Ringwood, Je; McLean, Ap-Je; Labrador Pond, Je; W. Winfield, Je; Rensselaer, May; Silver Bay, Sep, Leon; Pearl Pt., Je; Nassau, Aug; Highland, Mar; Maratanza L., May; Pinekill, May; Oakland Val., May; LI: Southold, Ap ; Riverhead, Ap, Sep ; Long Pond, Suffolk Co, Je.

R. spiniferus Em. Karner, Mar; Goshen, May.

R. terrestris Em. Ithaca, Em; Cornwall, Em.

\section{SPINTHARUS Hentz}

S. flavidus Htz. Taughannock Falls, Oct; Ithaca, Aug-Sep; LI, Aug (Bks).

\section{Steatoda Sundevall}

S. borealis Htz. Wilmington, Aug; L. Placid, Sep, Plm; Watertown, Sep, Plm; Green L., Fulton Co, Jan, Dt; Gloversville, Mar-Ap, Alex; Wells, J1, Yg; Onondaga Co (Britcher); Clyde, Aug, Dec; Cayuga, Aug; Rochester, Nov; Silver Cr., Sep; L. Keuka, May, Je, Oct; Bluff Pt.; Cayuta L., Nov; Ithaca, all months; Dormansville, Je; Albany, May, Aug, Chrisp; Thacher Pk., May-Je; Kenwood, Ap, Chad; Greenville, Ap; Rensselaer, Jan, Oct, Schoon; Nassau, Je, Schoon; Valcour I., Aug ; Juanita I., J1; Cossayuna L., May, Schoon; Waterford, Jan, Chrisp; Stockport Sta., Sep; Catskill, Je, Sep, Dec, Chad; Poughkeepsie, vanIngen; Suffern, May; Goshen, May; Hunter, Je; SI: Jan, Ds; Clove Val., Nov; LI: Sheepshead Bay, Je-J1; Jamaica, Nov; Riverhead, Aug. 


\section{Teutana Simon}

T. triangulosa Walck. Onondaga Co (Britcher); Ithaca, J1-Sep; Poughkeepsie, vanIngen; LI: Sea Cliff (Bks).

\section{TheONOE Simon}

T. stridula Cros. Paul Smiths, Je, Babiy; West Barre, Sep; Penn Yan, May; Alpine, Nov ; Enfield Glen, Oct ; Ithaca, Oct; McLean, Ap-J1; Labrador Pond Je; Altamont, Ap; Voorheesville, May-Je; Little Pond, Orange Co, May; LI: Southold, Ap; Riverhead, Je, Aug.

\section{THERIDION Walckenaer}

T. albidum Bks. LI: Sea Cliff (Bks).

T. aurantium Em. Axton (Em); Wilmington Notch, Je, Aug (Em); Newcomb, J1; Labrador Pond, Je.

T. blandum Htz. LI, J1 (Bks).

T. cinereum Em. Peru, Je; Danby, May; LI: Sea Cliff (Em).

T. differens Em. Wilmington, Aug; Wilmington Notch, J1; L. Tear, J1; Axton (Em) ; Black Br., Je; Peru, Je; Wells, May, Yg; Saratoga, Je; Gloversville, Je, Alex; Thacher Pk., May; Sacandaga Pk., Je, Alex; Onondaga Co (Britcher) ; Webster, J1; Pt. Breeze, Je ; Howard, J1; N. Lansing, Je ; Penn Yan, May, Aug; Taughannock Falls, May; Enfield Glen, May ; Ithaca, May-J1; Freeville, Aug; McLean, May, Sep; Labrador Pond, May, Je, Tarris; Trenton Falls, Je; Clayville, Je; Dormansville, Je; S. Westerlo, May; Vrooman's Nose, May; L. Charlotte, Je; Burden L., Aug, Schoon; Tackawasick Pond, Je; Valcour I., Aug; Pearl Pt., Juanita I., J1-Aug; Bumps Pond, J1; Little Pond, Orange Co, May; Oakland Val., May; LI: Baiting Hollow, May ; Shinnecock Hills, Je; Long Pond, Suffolk Co, Je.

T. frondeum Htz. Mt. Marcy, Sep; Wilmington Notch, J1; Black Br., Je; Cranberry L. (Barrows); Wells, J1, Yg; Sacandaga Pk., Je, Alex; Mountain L., Je, Alex; Woodworth's L., May, Alex; Johnstown, Aug, Alex; E. Caroga L., Aug, Hoffman; Baldwinsville, Bks; Trenton Falls, Je; Oneida, J1; Southwest Oswego, J1; Lake Bluff, Sep; Letchworth Pk., J1; Lakeside Pk., Orleans Co, J1; Westview, Livingston Co, J1; Bean Station, Steuben Co, J1; Cinnamon L., J1; Taughannock Falls, Je ; Ithaca, Je, Aug; McLean, J1; Deruyter L., J1 ; Labrador Pond, Je; Elmira, J1; Schenectady, Je; Albany, Je, Sep; Thacher Pk., Je; Rensselaer, Je-J1; Central Nassau, J1; Tackawasick Pond, Je; Valcour I., Aug; Hague, Aug ; Poughkeepsie, vanIngen; Yonkers, J1; SI: Richmond, J1, Burns; LI: Cold Sp. Harb., Je; Sea Cliff, Je; Smithtown, Je; Jamaica, Je.

T. globosum Htz. Onondaga Co (Britcher); Central Nassau, J1; LI: Cold Sp Harb. (Em).

T. kentuckyense Key. Letchworth Pk., J1; Enfield Glen, Aug; Thacher Pk., Je; Stamford, May, Chrisp; LI: Long Pond, Suffolk Co, Je.

T. maxillare Em. Valcour I., Aug.

T. montanum Em. Mt. Whiteface, Aug; Mt. Marcy, Sep; L. Tear, J1; Mt. McIntyre, J1; Wilmington Notch, Aug ; A'rtist's Brook, Je; Bumps Pond, Ji; Slide Mt., Je.

T. murarium Em. Wilmington, Aug; Cranberry Cr., Aug; Cranberry L. (Barrows) ; Trenton Falls, Je; Onondaga Co (Britcher); Southwest Oswego, J1; Webster, J1; Lakeside Pk., J1; Marilla, J1; Letchworth Pk., J1; Penn Yan, 
May, Aug; Cinnamon L., J1; Cayuta L., J1; Ithaca, Je ; McLean, J1; Spencer, Aug; Ringwood, Je; Albany, J1; Dormansville, Je; Shelving Rock Mt., J1; Elizabeth I., J1; Red Hook, J1; Wappingers Falls, May; LI: Sheepshead Bay, J1; Yaphank, Aug, Shoe; Riverhead, Aug; Shinnecock Hills, Je; Water Mill, Je.

T. pennsylvanicum Em. LI: Cold Sp. Harb., Aug (Em).

T. rupicola Em. Onondaga Co (Britcher); Ithaca, Jan, J1, Sep, Dec; Freeville, Aug ; S. Westerlo, May; Poughkeepsie, vanIngen; Larchmont, Sep, Wlf.

T. sexpunctatum Em. Mt. Whiteface, Aug; Wilmington, Aug; Mt. Marcy, Sep; Mt. McIntyre, J1; Avalanche Lake, J1; Chapel Pond, Je; Uphill Br., Opalescent R., J1; Onondaga Co (Britcher); Guyanoga, Je ; Egleston's Glen, Sep ; Connecticut Hill, Nov; Enfield, Aug, Wlf, Taub; Ithaca, May; Ringwood, May, Dt; Tackawasick Pond, Je; Pearl Pt., J1.

T. spirale Em. Axton, MacG\&Houghton; Keeseville, Je; Onondaga Co (Britcher) ; Howard, J1; Loucks Pond, J1; Stanley, May; Penn Yan, Aug; Levanna, J1; Cinnamon L., J1, Sep; Enfield Glen, Aug, Oct; McLean, J1; Freeville, Aug; Trenton Falls, Je; Albany, Je, J1; Vrooman's Nose, May; Riders Mills, May; Central Nassau, J1; Tackawasick Pond, Je; Valcour I., Aug; Juanita I., J1; Shelving Rock Mt., J1; LI: Cold Sp. Harb., Je.

T. tepidariorum C. Koch. Wells, J1, Yg; Gloversville, Aug, Oct, Alex; Baldwinsville (Bks); Cazenovia, Sep; Lake Bluff, Aug; Clyde; Rochester; Hemlock L., Aug; Irving, Sep, Chad; Cayuga, Oct; Naples, Aug; Ithaca ; Albany, Jan, Aug-Oct; Slingerlands, Mar, Wlf; Rensselaer, Je, Oct, Schoon; Valcour I., Aug; Cossayuna L., May, Schoon; Poughkeepsie, vanIngen; Highland, Aug, Wlf; Kingston, Aug, Schoon; Catskill, Aug-Sep; Saugerties, Aug; LI: Sea Cliff, Je (Bks) ; Forest Hills, Sep; Jamaica, Oct-Nov, Wlf.

T. unimaculatum Em. Ithaca, Ap, Nov; Albany, Je; Voorheesville, Je; Rensselaer, J1, Schoon; Tackawasick Pond, Je; Oakland Val., May; LI: Cold Sp. Harb., Je, Anderson; Shinnecock Hills, Je ; Long Pond, Suffolk Co, Je.

T. zelotypum Em. Adirondacks (McCook): Bumps Pond, J1.

\section{THERIDULA Emerton}

T. opulenta Walck. Cranberry L. (Barrows); Johnstown, Aug, Alex; Ithaca, Je; McLean, Je, J1; Labrador Pond, Je; Apulia, Onondaga Co (Britcher) ; Albany, Je, J1; Central Nassau, J1; Oakland Val., May; LI: Cold Sp. Harb., Je, Anderson.

\section{ULESANIS L. Koch}

U. americana Em. Jamesville (Britcher); Marilla, J1; Ithaca, Aug, Wlf, Taub; Mt. Marion, Oct, Chad; Oakland Val., May; LI: Riverhead, May.

\section{FAMILY LINYPHIIDAE}

\section{ARAEONCUS Simon}

A. pedalis Em. Mountain L., Ap; Altamont, Ap.

\section{Bathyphantes Menge}

B. alboventris Bks. Wilmington Notch, Aug; Mt. Whiteface, Aug; Malone, Oct; Little Falls, J1; Castile, May; Enfield Glen, Oct; Sheldrake Sps., Nov; Ithaca, Oct, Nov; McLean, Sep; Rensselaer, Nov, Schoon; Riders Mills, May. 
B. alpinus Em. Wilmington, Aug; Mt. Whiteface, Aug; Newcomb, J1; Uphill Br., Opalescent R., Jl.

B. angulatus Em. Ithaca (Bks); Rye Pt., Sep; Crosby, Nov; Little Pond, Orange Co, May; LI, J1 (Bks).

B. bihamatus Em. Maratanza L., May.

B. brevis Em. Wilmington Notch, Aug (Em).

B. canadensis Em. Mt. Marcy, Jl.

B. complicatus Em. Uphill Br., Opalescent R., J1.

B. concolor Reuss. Lake Bluff, Sep; Wayne Center, Mar; Ithaca, Mar-Sep, Nov-Dec; Albany, J1; Rensselaer, May, Schoon; Coxsackie, Mar; Kingston, Aug, Schoon; Poughkeepsie, vanIngen; LI: Aug-Oct (Bks); Bergen Beach, Ap; Brooklyn, Oct; Sea Cliff (Em).

B. conicus Em. Ithaca, Ap, Nov-Dec; Little Pond, Orange Co, May.

B. crosbyi Em. Uphill Br., Opalescent R., J1.

B. decoratus Bks. Ithaca, Sep (Bks); LI (Bks).

B. formicus Em. Honeoye Falls, J1; Ithaca, Aug-Sep ; Freeville, Aug (Bks) ; LI: J1-Aug (Bks); Sea Cliff, Em.

B. furcatus Em. Mt. Whiteface, Aug (Em) ; Avalanche L., J1.

B. inornata Em. (not B. inornata Bks.). Mt. Whiteface, Aug (Em).

B. intricatus Em. Crosby, Dec; Egleston's Glen, Oct; Shurger's Gorge, Nov; McLean, Ap.

B. luteolus Bks. Ithaca, Nov (Bks).

B. micaria Em. Guilderland Center, May; LI, J1 (Bks).

B. nigrinus Westr. Wilmington, Aug; Uphill Br., Opalescent R., J1; Lake Bluff, Sep; Newfane, Oct; Richburg, Sep; Enfield Glen, Ap, Sep, Nov; Ithaca, Ap, Aug-Sep, Nov; McLean, J1; Rensselaer, May, Aug, Nov, Schoon; Tackawasick Pond, Je; Riders Mills, May; Stamford, May; LI, Oct (Bks).

B. pallidus Bks. Ithaca, Ap, Oct, Dec (Bks).

B. subalpinus Em. Wilmington, Aug; Mt. Whiteface, Aug; Ithaca (Bks); Thacher Pk., Je.

B. tristis Bks. Ithaca, Nov (Bks).

B. unimaculatus Bks. Weedsport, Ap; Ithaca, J1, Feb; LI: Riverhead, Ap; Montauk Pt., May.

B. zebra Em. Old Forge, Oct; Wilmington Notch, J1-Aug; Wilmington, Aug; Mt. Whiteface, Aug; Auger Pond, Nov; Howland I., Nov; Richburg, Sep; Crosby, Dec; Enfield Glen, Oct; Ithaca, Sep-Oct; Freeville, May; McLean, Ap-May; Egleston's Glen, Oct; Silver Bay, Sep, Leon; Juanita I., J1; Poughkeepsie, vanIngen; Slide Mt., Je; Maratanza L., May; Cragsmoor, May; Oakland Val., May; Stamford, May, Chrisp; Tuxedo, Oct, Wlf.

B. zygios Key. Ithaca, J1; McLean, Ap; Poughkeepsie, vanIngen; LI, Oct (Bks).

CASEOLA Emerton

C. alticeps Em. Thacher Pk., May.

\section{Ceraticelus Simon}

C. alticeps Fox. LI: Baiting Hollow, Ap-May; Long Pond, Suffolk Co, Je; Cold Sp. Harb., J1-Aug, Bryant; Riverhead, Ap, Sep. 
C. atriceps Cambr. Wilmington Notch, Aug; Mt. Whiteface, Aug; Adirondack Lodge, J1; Mt. McIntyre, J1; L. Tear, Jl, Sep; Mt. Marcy, Sep ; Chapel Pond, Je; Uphill Br., Opalescent R., J1; Wells, Ap-May, Yg; Bumps Pond, J1; Mountain L., Ap, Alex; L. Keuka, Dec; Enfield Glen, Oct; Ithaca, Feb-Ap, Oct; McLean, Ap-May, Oct; Jenksville, Oct; Onondaga Val., Je (Britcher); Hunter, Aug; Little Pond, Orange Co, May; Oakland Val., May.

C. bulbosus Em. McLean Bogs, May-Je; Tully, Oct (Britcher).

C. carinatus Em. Oakland Val., May.

C. emertoni Cambr. L. Tear, J1; Black Br., Je; Sacandaga Pk., Je, Alex; Barneveld, J1-Sep; Canastota, Nov; Pt. Breeze, Je; Letchworth Pk., J1; Honeoye, J1; Geneva, Nov; Bluff Pt., Sep; L. Keuka, Oct, Dec; Horseheads, Ap ; Ithaca, Mar-Nov; McLean Bogs, Sep; Ringwood, May; Nassau L., Ap; Coxsackie, Mar; LI: Cold Sp. Harb., J1-Aug; Oyster Bay, Chamb.

C. fissiceps Cambr. Wilmington Notch, Aug; Mt. Whiteface, Aug; Mt. Marcy, Aug; Chapel Pond, Je; Northumberland Township, Saratoga Co, Je; Cranberry Cr., Aug; Bumps Pond, J1; Mountain Lake, Ap; Trenton Falls, Je; Syracuse (Britcher); N. Fair Haven, J1; Lake Bluff, Sep; East Bay, Sep; Stow, Sep; Rock City, J1-Aug ; Letchworth Pk., J1; Guyanoga, Je; Penn Yan, Aug; Crosby, Ap, Oct, Dec; L. Keuka, Je, Dec; Cinnamon L., J1; Montour Falls, Oct; Taughannock Falls, Aug, Oct; Enfield Glen, May, Aug; Ithaca, Ap-Dec; Ringwood, J1, Oct ; Freeville, J1-Aug, Oct; McLean Bogs, Ap-May, J1; Spencer, Aug; Labrador Pond, Je; Owego, Dec; Tully, Oct; Jenksville, Oct; Apalachin, May; Voorheesville, Je; Riders Mills, May; Schodack Landing, Je; Tackawasick Pond, Je; Valcour I., Aug; Poughkeepsie, vanIngen; Slide Mt., Je; Highland, Mar; Oakland Val., May; Mountainville, May; Mt. Utsayantha, Oct; Larchmont, Sep, Wlf; LI: Farmingdale, Je ; Riverhead, ApMay, Aug; Long Pond, Suffolk Co, Je; Cold Sp. Harb., J1-Aug; Oyster Bay, Chamb; Montauk Pt., May.

C. formosus Bks. Ithaca, Sep (Bks); LI: (Bks); Sea Cliff, Fox.

C. laetabilis Cambr. Wilmington Notch, Aug; High Falls, Aug; Auger Pond, Nov; L. Pleasant, Ap; Pinnacle Mt., Aug; Clarksburg, Sep; Stow, Sep; Rock City, Sep; Letchworth Pk., J1; Penn Yan, May, Aug ; Egleston's Glen, Yates Co, Sep; L. Keuka, Ap, Dec; Rye Pt., Sep; Savona, Sep; Interlaken, J1; Taughannock Falls, J1; Montour Falls, Oct; Alpine, Nov; Connecticut Hill, Oct-Nov; Enfield Glen, Aug, Oct; Ithaca, all months; Danby, Oct; Freeville, May, Oct; McLean Bogs, Sep; Deruyter L., J1; Cascade, Nov; W. Winfield, Je; Apalachin, May; Belden Hill, Broome Co, May; Delaware L., May; Meredith, May; Valcour I., Aug; Silver Bay, Jabe Pond, Sep, Leon; Juanita I., Pearl Pt., J1; S. Westerlo, May; Mt. Utsayantha, Oct; Cragsmoor, May ; Sam's Pt., May; Slide Mt., Je; Oakland Val., May; Pinekill, May; Tuxedo, Oct, $W l f ;$ LI (Bks).

C. laetus Cambr. McLean Bogs, May-Je.

C. laticeps Em. Ithaca, Aug; LI: Long Pond, Suffolk Co, Je.

C. limnologicus Cros. and Bish. Crusoe L., May.

C. micropalpis Em. Barneveld; Ithaca, Nov (Bks); Ancram, Je; LI (Bks).

C. minutus Em. Wilmington Notch, Aug; Elizabethtown, Aug, Oct; High Falls, Aug ; L. Pleasant, Ap; Mountain L., Ap; Raquette Falls, Aug ; Gilman L., Ap. ; Sylvan Beach, J1; Wolcott, May; Lake Bluff, Sep; Mendon, Oct; Newfane, Oct; Rock City, Sep; Richburg, Sep; Savona, Sep; Rye Pt., Sep ; L. Keuka, Sep, Dec; Crosby, Oct; Guyanoga, Je; Egleston's Glen, Sep; Penn Yan, May; Cinnamon L., Jl; Interlaken, Jl, Nov; Connecticut Hill, Oct; Ithaca, all 
months; Enfield Glen, Je, Oct; McLean Bogs, Ap ; Danby, Oct; Freeville, May, Oct; Deruyter L., J1; Cascade, Nov; Meredith, May; Belden Hill Broome Co, May; Altamont, Ap; Vrooman's Nose, May; Silver Bay, Sep, Leon; Pearl Pt., J1; Juanita I., J1, Aug ; L. Charlotte, Je ; Accord, Mar ; Sam's Pt., May; Oakland Val., May; Pine Island, May; Mt. Utsayantha, Oct; English Church, Rockland Co, May; Tuxedo, Oct, Wlf; LI: Flushing, Je; Cold Sp. Harb., Aug; Patchogue, Ap ; Southold, Ap; Baiting Hollow, May; Great Pond, Riverhead, May.

C. ornatulus Cros. and Bish. Mt. Whiteface, Aug.

C. paschalis Cros. and Bish. Oakland Val., May.

C. pygmaeus Em. LI: Sea Cliff, Bks; Cold Sp. Harb., Ap, Bryant.

C. similis Bks. Clayville, Je; Olcott, Feb; Ithaca, Ap, Sep-Nov; Ringwood, J1; Putnam, Sep; Poughkeepsie, vanIngen; LI: Sea Cliff, Em; Cold Sp. Harb., J1-Aug, Bryant; Oyster Bay, Chamb; Riverhead (Sound Beach), Sep ; Wading R. (Sound Beach), Je; Bridgehampton (Ocean Beach), Aug.

\section{Ceratinella Emerton}

C. brunnea Em. Wilmington Notch, Aug; Mt. Whiteface, Aug; Mt. McIntyre, J1; Wawbeek, J1; Uphill Br., Opalescent R., J1 ; Raquette Falls, Aug; Gilman I.., Ap; L. Pleasant, Ap ; Cranberry Cr., Aug; Barneveld; Howland I., Nov ; Olcott, Mar; Penn Yan, May, Aug; Interlaken, J1; Ithaca, Feb, Ap-May, J1Aug, Oct; Enfield Glen, Oct; Danby, Oct; Freeville, Oct; Valcour I., Aug; Juanita I., J1-Aug; Silver Bay, Sep, Leon; Maratanza L., May; Slide Mt., Aug; Oakland Val., May; Pine I., Orange Co, May.

C. placida Bks. Ithaca, Feb, Sep (Bks).

C. sphaerica Em. LI: E. Hampton, May.

\section{Ceratinopsis Emerton}

C. formosa Bks. (alternata Em.). Wilmington, Aug; Wilmington Notch, Aug; Mt. Whiteface, Aug; Elizabethtown, Aug ; Peru, Je ; Cranberry Cr., Aug-Sep; Woodworth's L., Aug-Sep, Alex; Lake Bluff, Sep ; Lancaster ; Rock City, Aug; Penn Yan, Aug; Montour Falls, Sep; Enfield Glen, Aug; Ithaca, J1-Aug; Juanita I., Shelving Rock Mt., J1; Pearl Pt., Aug; Queechy L., Aug, Schoon; Hunter, Aug; LI: Riverhead, Sep.

C. auriculata Em. Ithaca $(\mathrm{Em})$; Freeville, May; Ringwood, May, Dt.

C. interpres Cambr. Wilmington Notch, Aug (Em); Peru, Je; Rock City, Je; Ithaca, Ap, Oct (Bks); Little Pond, Orange Co, May; Oakland Val., May; LI, Je (Bks).

C. laticeps Em. Olcott, Feb, Dt; Ithaca, May, Aug; LI: Montauk Pt., May.

C. nigriceps Em. Saratoga, Je; Clyde, Ap; McLean, J1; Shelving Rock Mt., J1; Tackawasick Pond, Je; Oakland Val., May; LI: Riverhead (Sound Beach), Sep; Cold Sp. Harb., J1.

C. nigripalpis Em. Ithaca, Sep (Bks) ; LI: Riverhead, Aug; Baiting Hollow, May

C. tarsalis Em. LI: Cold Sp. Harb., Ap, Bryant; Oyster Bay, Chamb; Riverhead (Sound Beach), Sep.

C. unicolor Cros. Egleston's Glen, Je; LI: Yaphank, Aug, Shoe; Baiting Hollow, May. 


\section{Cornicularia Menge}

C. auranticeps Em. Albany, Je; Little Pond, Orange Co, May.

C. clavicornis Em. Mt. Whiteface, Aug (Em).

C. placida Bks. Ithaca, Oct (Bks).

\section{DELORRHIPIS Simon}

D. unicornis Bks. Ithaca, Mar, Nov; Crosby, Ap; Barrington, Oct.

\section{Dicymbium Menge}

D. pectinatum Em. McLean Bogs, May.

\section{Diplocephalus Bertkau}

D. abruptus Em. McLean, J1; Slaterville, Aug; LI: Riverhead, Je.

D. arenarius Em. Mt. McIntyre, J1; Clyde, Ap ; Pine I., Orange Co, May.

D. castaneus Em. Mt. Whiteface, Aug; L. Tear, Sep.

D. crenatus Em. Woodworth's L., Aug, Alex; Ithaca, Aug-Sep; LI: Sea Cliff (Bks) ; Cold Sp. Harb., J1, Bryant.

D. cuneatus Em. Sylvan Beach, J1; Crusoe L., May.

D. erigonoides Em. Mt. McIntyre, J1; Olcott, Feb, Dt; Keuka Pk., Ap; Crosby, Nov; Hammondsport, J1; Ithaca, Mar, May, Oct-Dec; Etna, Nov; McLean, May, Sep; Sacandaga Pk., Je, Alex; Middletown, Ap; SI: Tottenville, Nov; LI: Sheepshead Bay, Sep; Sea Cliff, Je; Gardiners I., May; Montauk Pt., May.

D. exiguus Bks. Ithaca, Jan (Bks).

D. latus Em. Cranberry Cr., Aug; Ithaca (Bks); McLean, May; Poughkeepsie (Bks); LI, Mar (Bks).

D. littoralis Em. LI: Cold Sp. Harb., Aug (Em); Riverhead, Sep.

D. minutus Em. L. Pleasant, Ap.

D. pallidus Em. Auger Pond, Aug; Jabe Pond, Sep, Leon; Karner, Mar.

D. simplex Em. Wilmington Notch, Aug; Mt. Whiteface, Aug; Auger Pond, Nov; Silver Bay, Sep, Leon; Egleston's Glen, Sep; Connecticut Hill, Aug, Oct-Nov ; McLean; Ringwood, May, Dt; Labrador Pond, Je.

\section{DRAPETISCA Menge}

D. alteranda Chamb. Crown Pt., Sep, Plm; Wilmington Notch, Aug; L. Placid, Sep, Plm; Upper Jay, Sep, Plm; Chateaugay L., Sep, Plm; Raquette L., Sep; Big Moose, Aug, Plm; Onondaga Co (Britcher); Lake Bluff, Sep; Glen Haven, Sep, Plm; Clarksburg, Sep; Richburg, Sep ; Rock City, Sep ; Wellsville, Sep; Hornell, Sep; Painted Post, Sep; Cinnamon L., Sep; Geneva, Sep ; Ithaca, Sep; Bronx Pk., Sep, Plm; LI, Oct (Bks).

\section{ERIGONE Audouin}

E. atra Blackw. Mt. McIntyre, J1; Wilmington, Aug; Geneva, J1; Cayuta L., J1; Taughannock Falls, J1-Aug, Dt; Ithaca, Mar, Je-Aug, Oct; Waterville, Aug; Tackawasick Pond, Je. 
E. autumnalis Em. Barneveld, Aug; Ithaca, J1-Aug; McLean, May; LI: Oyster Bay, Chamb; Cold Sp. Harb., J1, Bryant; Sea Cliff (Em).

E. brevidentata Em. Ithaca, J1.

E. dentigera Cambr. Chapel Pond, Je; Ithaca, Aug; McLean, May; Tackawasick Pond, Je; Kingston, Mar; LI: Sheepshead Bay, Je-Jl.

E. tridentata Em. L. Kauka, Sep; Ithaca, Feb, Nov; Rensselaer, Nov, Schoon; SI: Clove Val., Nov; LI, Oct-Nov (Bks).

E. tristis Bks. Ithaca, Ap, Oct (Bks).

\section{EXECHOPHYSIS Simon}

E. nigriceps Bks. Ithaca, May (Bks); LI: Sea Cliff, May (Bks).

E. plumalis Cros. Sylvan Beach, J1; Ithaca, May; Juanita I., Aug; Little Pond, Orange Co, May; LI: Riverhead, May.

\section{Gonatium Menge}

G. rubens Blackw. Pinnacle Mt., Sep; Painted Post, Sep; Taughannock Falls, Oct; Ithaca, Aug, Nov; Freeville, Aug, Nov (Bks) ; Juanita I., Aug-Sep ; Larchmont, Sep, Wlf.

\section{GONGYLIDIELLUM Simon}

G. minutum Bks. Ithaca, Mar (Bks); Varna (Bks).

G. pallidum Em. Wells, Ap; Mountain L., Ap; Sylvan Beach, J1; Wolcott, May; L. Keuka, Dec; Ithaca, Feb-Ap, Dec; Freeville, Oct; Belden Hill, Broome Co, May; Juanita I., Aug; Accord, Mar; Pomona, Ap; English Church, Rockland Co; Larchmont, Sep, Wlf; LI: Oct (Bks) ; Riverhead, Aug; Jamaica, Ap ; Southold, Ap.

\section{Grammonota Emerton}

G. gigas Bks. L. Tear, J1; Ithaca, May (Bks) ; Freeville, Ap-May; Cinnamon L., J1.

G. inornata Em. Barneveld, Aug; Ithaca, May, Aug; LI: May-Je (Bks) ; Sea Cliff, Je; Mattituck, Aug; Patchogue, Aug; Orient Pt., Aug.

G. ornata Cambr. Ithaca, Mar (Bks); SI: Clove Valley, Nov; LI, Sep-Oct (Bks).

G. pallipes Bks. LI, Oct (Bks).

G. pictilis Cambr. Wilmington, Aug; Ithaca, Mar, Sep (Bks); Valcour I., Aug; Bumps Pond, J1; Stamford, May, Chrisp; Little Pond, Orange Co, May.

G. trivittata Bks. LI: Sea Cliff, Nov-Dec (Bks).

\section{HYPoмMA Dahl}

H. bilobata Bks. McLean Bogs, May.

H. trilobata Bks. McLean Bogs, May; Ringwood, May; Ramapo Mts., May.

\section{Hypselistes Simon}

H, florens Cambr. Axton, MacG, Houghton; Wells, J1, Yg; Black Br., Je ; Cranberry L. (Barrows); Johnstown, J1-Aug, Alex; Onondaga Co (Britcher); Clyde, Ap; Walworth, Je; Rock City, Aug; Bluff Pt., Sep; Ithaca, May, Sep, 
Oct; McLean Bogs, May-J1; Albany, May; Karner, J1; Guilderland Center, May; Rensselaer, May; Central Nassau, J1; Tackawasick Pond, Je; Valley Falls, May, $Y g$; Queechy L., May, Schoon; Maratanza L., May; Little Pond, Orange Co, May; Pine I., Orange Co, May; LI: Millneck, Ap (Bks).

\section{Lephthyphantes Menge}

L. minutus Blackw. Keuka Pk., Oct; Ithaca, Nov (Bks); Poughkeepsie, vanIngen; LI, Aug (Bks).

L. nebulosus Sund. Gloversville, Oct; Onondaga Co (Britcher); Clyde, Aug; Lake Bluff, Sep; Geneva, Nov; Bluff Pt., Sep; Ithaca, Mar, Nov (Bks); Albany, Aug, Hartman; Rensselaer, Nov; Oakland Val., May; LI, Sep, Nov (Bks).

\section{LINYPHIA Latreille}

L. cayuga Em. Ithaca, Mar, Dec (Em).

L. clathrata Sund. Black Br., Je; Ithaca, Je, Nov (Bks); McLean, May, J1; Mt. Lebanon, May; Rensselaer, May-Je, Schoon; SI, Jan, young; LI: Oct-Dec (Bks); Sea Cliff (Em).

L. communis Htz. Wilmington, Aug; Axton; Keene Val., Sep; Paul Smiths, J1; Speculator, J1; Newcomb, J1; Peru, Je; Pompey (Britcher) ; Saratoga, Je; Ithaca, J1; Freeville, May; McLean, May-Je; Pearl Pt., J1; Shelving Rock Mt., J1; Bumps Pond, J1; Central Nassau, J1; Little Pond, Orange Co, May.

L. conferta Htz. LI, Oct (Bks).

L. insignis Blackw. Wilmington Notch, Aug; Wilmington, Aug; Marble Mt., Aug; Cranberry L. (Barrows); Gloversville, Ap, Alex; Johnstown, Aug-Sep, Alex; Sacandaga Pk., Je, Alex; Woodworth's L., Aug-Sep; Burke, Oct; Lake Bluff, Sep ; Rochester, Nov, Chad; Stow, Sep ; Olcott, Sep ; Ceres, Sep ; Richburg, Sep; Wellsville, Sep; Painted Post, Sep; Ithaca, Sep-Nov; Owego, Dec; Indian Head Mt., L. George, Sep, Leon.

L. lineata L. Gloversville, Mar, Alex; Fishers Landing, Sep, Plm; Onondaga Co (Britcher); Lake Bluff, Sep; Glen Haven, Sep, Plm; Rochester, Nov, Chad; Lewiston, Sep, Plm; Bluff Pt., Sep, Heck; Ithaca; McLean, Ap; Copeland Hill, Albany Co, May; Rensselaer, Nov; Mill Cr., May; Coxsackie, Mar; Poughkeepsie, vanIngen; LI, Oct-Nov (Bks).

L. maculata Em. Ithaca, Mar-Ap, Oct; McLean, J1; Oakland Val., May.

L. mandibulata Em. Wilmington, Aug; L. Tear, J1; Black Br., Je; Barneveld; Johnstown, May, Alex; Onondaga Co (Britcher) ; L. Keuka, May-Je; Ithaca, May; McLean, May; Albany (Em) ; Voorheesville, May; Rensselaer, May, Aug, Schoon; Grafton, Aug; Vrooman's Nose, May; Black Mt., Washington Co, Aug; Bumps Pond, J1; Cragsmoor, May; Stamford, May, Chrisp; LI (Bks).

L. marginata C. Koch. Wilmington, Aug; Axton, MacG, Houghton; Paul Smiths, J1; Chapel Pond, Je; Speculator, J1, Yg; Old Forge, Jl, Plm ; Cranberry L. (Barrows); Raquette L., Je; Wells, J1, Yg; Johnstown, J1, Alex; Gloversville, Aug, Alex; Woodworth's L., May, Alex; Onondaga Co (Britcher); Ithaca; Oak Ridge, Canandaigua L., Aug; Albany; Thacher Pk., Je-J1; Thompson L., J1; Dormansville, Je; Grafton, Aug; Rensselaer, Ap, Schoon; Tackawasick Pond, Rensselaer Co, Je; Riders Mills, May, Chrisp; Queechy L., May, Schoon; Valcour I., Aug; Pearl Pt., Juanita I., J1; Sleeping Beauty Mt., J1; Bumps Pond, J1; Tongue Mt., Warren Co, Sep, Leon; Poughkeepsie, 
vanıngen; Mt. Marion, Ulster Co, Oct, Chad; Vrooman's Nose, May; Stamford, May, J1; Little Poind, Orange Co, May; Highland, Wlf; Paradise, May; Oakland Val., May.

L. nearctica Bks. Mt. Whiteface, Aug; Wilmington, Aug; Marble Mt., Aug ; Uphill Br., Opalescent R., J1; Mt. Marcy, Sep ; L. Tear, Sep; Avalanche L., J1.

L. phrygiana C. Koch. Wilmington, Aug; North Cr., Je, Plm; Paul Smiths, J1, Plm; Gloversville; Mar-Ap, Aug, Alex; Onondaga Co (Britcher) ; Clyde, Ap ; Cayuga, Oct, Chad; Ithaca (Bks) ; McLean, May; Schenectady, Dec, Dt ; Albany, Je; Kenwood, May, Plm; Thacher Pk., Je; E. Schodack, Je, Chad; Cossayuna L., May, Schoon; Valley Falls, May, $\mathrm{Yg}$; Riders Mills, May, Chrisp; Waterford, Jan, Chad; Valcour I., Aug; Bumps Pond, J1; Catskill, Sep; Stamford, May, Chrisp; Cragsmoor, May; Bronx Pk., Sep, Plm; LI, Ap, Oct (Bks).

L. variabilis Bks. Wilmington, Aug; Newcomb, J1; Ithaca, Feb, Oct (Bks) ; Rensselaer, Nov, Schoon; Stamford, May, Chrisp.

\section{LOPHOMMA Menge}

L. elongatum Em. Raquette Falls, Aug; Ringwood, May, Dt; McLean Bogs, May; Pinekill, May.

L. longitarsum Em. Wilmington Notch, Aug; LI: Montauk Pt., May.

\section{MASO Simon}

M. frontata Bks. Wilmington, Aug; Wilmington Notch, Aug (Em); Ceres, Sep; Rock City, Sep; Ithaca, Mar (Bks) ; McLean, J1; Labrador Pond, Je; L. Charlotte, Je; LI: Long Pond, Suffolk Co, Je ; Baiting Hollow, May.

\section{Microneta Menge}

M. clavata Em. Wilmington Notch, Aug.

M. complicata Bks. Michigan Hollow Swamp, Ap (Bks).

M. cornupalpis Cambr. Wilmington Notch, Aug; Crosby, Dec; Tthaca, Mar-Ap, Nov (Bks) ; Shurger's Gorge, Nov; McLean, May; Juanita I., J1; Oakland Val., May; Pomona, Ap; Larchmont, Sep, Wlf; SI: Clove Val., Nov; LI: Flushing, Nov; Jamaica, Ap.

M. denticulata Em. LI: Baiting Hollow, Ap.

M. fabra Key. (bowditchiae Bish., wana Chamb.). Ithaca, J1 (Chamb) ; LI: Sheepshead Bay, Ap; Oyster Bay (Chamb).

M. latidens Em. Italy Hill, Oct; Accord, Mar.

M. longibulbus Em. McLean Bogs, May; Little Pond, Orange Co, May; LI (Bks).

M. minutissima Bks. Fall Cr., Ithaca, Oct.

M. multesima Cambr. Auger Pond, Nov; Lake Bluff, Sep; Stow, Sep; Olcott, Sep; Honeoye Falls, Nov; Lockport, Nov; Connecticut Hill, Nov; Savona, Sep; Rye Pt., Sep; Italy Hill, Yates Co, Oct; Ithaca, Feb (Bks); Owego, Dec; Altamont, Ap.

M. persoluta Cambr. Wilmington Notch, Aug; Auger Pond, Nov; Old Forge, Oct; Burke, Oct; Crosby, Dec; L. Keuka, Oct; Egleston's Glen, Oct; Enfield Glen, Oct; McLean, May-J1; Meredith, May; Slide Mt., Je ; Cragsmoor, May; Maratanza L., May; Sam's Pt., May; Silver Bay, Sep, Leon; Indian Head Mt., L. George, Sep, Leon.

M. quinquedentata Em. Malone, Oct; Burke, Oct; Howland I., Nov; Egleston's Glen, Sep; Enfield Glen, Oct; Ithaca, Nov; McLean Bogis, Sep; Saratoga Sps., Ap ; Rensselaer, Sep, Nov; LI: Baiting Hollow, Ap. 
M. serrata Em. Crosby, Oct; Keuka Pk., Nov.

M. viaria Blackw. Wilmington Notch, J1-Aug; Howland I., Nov; Rock City, Sep; Italy Hill, Oct; Connecticut Hill, Oct; Ringwood, Oct; Enfield Glen, Oct; Ithaca, Oct-Nov; Voorheesville, Mày; Juanita I., Pearl Pt., J1; Meredith, May; Slide Mt., Je; Delhi, May; Pomona, Ap; Cragsmoor, May; Tuxedo, Oct, Wlf; Larchmont, Sep, Wlf; SI: Clove Val., Nov; LI: Sea Cliff (Em).

\section{MINYRIOLUS Simon}

M. scopuliferus Em. Ithaca, Nov-Dec; Wappingers Falls, May.

\section{OEDOTHORAX Bertkau}

O. bidentatus Em. Mt. Whiteface, Aug; Mt. McIntyre, J1; Uphill Br., Opalescent R., J1; Little Pond, Orange Co, May.

O. bostoniensis Em. Woodwardia Swamp, Sep-Oct.

O. brunneus Em. Ithaca, Aug (Bks).

O. concavus Em. LI: Black Swamp, Oct (Bks).

O. corticarius Em. Woodworth's L., J1, Alex.

O. debilis Bks. Ithaca, Mar, Dec (Bks).

O. decemoculatus Em. Newcomb, May; Ithaca, May.

O. flaveolus Bks. Otto, Oct; Geneva, Nov; Ithaca, Feb, Oct (Bks); LI, Oct.

O. limatus Cros. Ithaca, Nov; McLean, May, J1.

O. microtarsus Em. Wilmington, Aug; Mt. Whiteface, Aug.

O. montanus Em. High Falls, Aug; Mt. Whiteface, Aug; Mt. McIntyre, J1; L. Tear, Sep ; Uphill Br., Opalescent R., J1.

O. montiferus Em. Shurger's Gorge, Nov; Ithaca, Mar-Ap, Dec.

O. ornatus Em. Ithaca (Em); Crusoe L., May; Montauk Pt., May.

O. oxypaederotipus Cros. Old Forge, Oct; Clarksburg, Sep; Ceres, Sep; Letchworth Pk., J1; Taughannock Falls, J1; Guyanoga, Je; Penn Yan, May; L. Keuka, Ap; Connecticut Hill, Aug; Ithaca, Feb, May-J1; Danby, Oct; Ringwood, May, Dt; Freeville, May, J1, Oct; McLean, May; Labrador Pond, Je; Deruyter L., J1; Trenton Falls, Je; Altamont, Ap ; Apalachin, May; Belden Hill, Broome Co, May; Meredith, May; Delaware L., May; Slide Mt., Je; Paradise, May; Mt. Utsayantha, Oct; Oakland Val., May.

O. plumosus Em. Mt. McIntyre, J1; Ithaca, Mar, J1, Oct-Nov; Kingston, Mar.

O. rusticus Bks. Ithaca, Dec (Bks), Ap, Seeley, Flet.

O. spiniferus Cambr. Ithaca, Oct (Bks).

O. tarsalis Em. LI: Great Pond, Riverhead, May.

O. terrestris Em. LI: Black Swamp, Oct (Bks).

O. truncatus Em. Mt. Whiteface, Aug; Auger Pond, Nov.

O. undulatus Em. Ithaca, May.

O. unidentatus Em. Mt. Whiteface, Aug.

\section{PARERIGONE Crosby and Bishop}

P. contorta Em. Ithaca, May; McLean Bogs, May-Je; LI: Jamaica, Ap; Montauk Pt., May.

P. entomologica Em. Mt. Whiteface, Aug (Em); McLean Bogs, May; Mt. Whiteface, Aug. 
P. index Em. Cinnamon L., J1; Freeville, May; McLean Bogs, Ap-May; Ringwood, May, Dt; Suffern, Ramapo Mts., May ; LI: Flushing, Je.

P. probata Cambr. Wilmington, Aug; Sylvan Beach, J1; Wolcott, May; Lake Bluff, Sep; Olcott, Sep; Richburg, Sep; Rock City, Sep; Italy Hill, Oct; Guyanoga, Je; L. Keuka, Je; Rye Pt., Sep; Barrington, Oct; Interlaken, J1; Connecticut Hill, Oct; Taughannock Falls, J1; Enfield Glen, Je, Aug ; Ithaca, Feb, May, J1-Aug, Oct; Ringwood, May, Dt; Freeville, J1; McLean Bogs, May, J1; Deruyter L., J1; Labrador Pond, Je; Jenksville, Oct; Delaware L., May; Silver Bay, Sep, Leon; Pearl Pt., J1; Altamont, Ap; Mountainville, May; Pine Hill, May; Tuxedo, Oct, Wlf; Larchmont, Sep, Wlf; Pomona, Ap; SI: Clove Val., Nov; LI: Southold, Ap; Great Pond, Riverhead, May; Baiting Hollow, May; Riverhead, Ap-Aug; Long Pond, Suffolk Co, Je; Montauk Pt., May.

P. rectangulata Em. Mt. Whiteface, Aug; McLean Bogs, May; Ringwood, Oct; Maratanza.L., May; Little Pond, Orange Co., May; LI: Great Pond, Riverhead, May.

P. simplex Em. Little Pond, Orange Co, May.

P. trilobata Em. Trenton Falls, Je; Olcott, Sep; Lockport, Nov; Cinnamon L., $\mathrm{J} 1$; Ithaca, Mar-May, Oct-Nov; Freeville, Oct; Ringwood, May, Dt; Albany, J1; Voorheesville, Je; Little Pond, Orange Co, May; SI: Clove Val., Nov; LI: Jamaica, Ap, Wif.

\section{PELECOPSis Simon}

\section{(Lophocarenum Menge)}

P. excavatum Em. Summit of Mt. Whiteface, Aug.

P. moestum Bks. Ithaca, Nov (Bks); Poughkeepsie (Bks); Pine I., Orange Co, May.

P. rugosum Em. Juanita I., Aug; LI: Baiting Hollow, May.

\section{POCADICNEMIS Simon}

P. pumila Blackw. (equals longituba Em.). Sylvan Beach, J1; Howard, J1; Penn Yan, May; Cinnamon L., Je-J1; Ithaca, May, J1-Aug; Freeville, Aug; McLean, May-J1; Vrooman's Nose, May; L. Charlotte, Je; Schodack Landing, Je; Riders Mills, May; Ancram, Je; Little Pond, Orange Co, May; LI: Wading R. (Sound Beach), Je; Baiting Hollow, May; Long Pond, Suffolk Co, Je.

\section{ProsopotheCA Simon}

P. brevicornis Em. LI: Riverhead, Ap.

P. communis Em. Mt. Whiteface, Aug; West Barre, Sep; Ithaca, Feb, Ap-May; McLean Bogs, May; LI (Bks).

P. digitata Em. (Tmeticus). LI: Cold Sp. Harb., Je (Em).

P. directa Cambr. Stow, Sep; Ithaca, Sep-Oct; Freeville, May; McLean, ApMay; Italy Hill, Oct; Egleston's Glen, Sep; Karner, Mar; Poughkeepsie, vanIngen; Meredith, May.

P. indirecta Cambr. Ithaca, Ap (Bks); LI (Bks).

P. minuta Em. Lake Bluff, Sep; LI: Riverhead, Ap ; Baiting Hollow, Ap.

P. pallida Em. Ithaca, Oct (Bks); Auger Pond, Nov. 
P. spiralis Em. Ithaca, Aug, Nov; McLean, May-Je; Freeville, Aug (Bks);

LI: Jamaica, Sep.

P. tibialis Em. Little Pond, Orange Co, May.

TAPINOCYBA Simon

T. distincta Bks. Varna, Mar (Bks); Freeville, May (Bks); McLean Bogs, J1.

TAPINOPA Westring

T. bilineata Bks. Ithaca (Comst); Freeville, Aug; LI: Sea Cliff (Bks); Long Pond, Suffolk Co, Je.

\section{TMeticus Menge}

T. cultigerus L. Koch (armatus Bks.). Mt. Whiteface, Aug (Em); Hague, Sep, Leon.

TORTEMBOLUS Crosby

T. alpinus Bks. Voorheesville, Nov, Leon.

TRACHELOCAMPTUS Simon

T. rostratus Em. Olcott, Sep; Ithaca, Mar; LI: Sea Cliff, Dec (Bks); Southold, Ap; Riverhead, Ap-May; Cold Sp. Harb., Ap, Bryant; Baiting Hollow, May.

\section{UTOPIELLUM Strand}

U. curvitarsis Em. Mt. Whiteface, Aug (Em).

WABANA Chamberlin

W. drassoides Em. Mt. Whiteface, Aug.

\section{FAMILY ARGIOPIDAE}

\section{ACACESIA Simon}

A. foliata Htz. Sacandaga Pk., J1, Alex; LI: Sea Cliff, Sep (Bks).

\section{ARANEUS Clerck}

A. angulatus Cl. Mt. Whiteface, Aug; Wilmington, Aug; Wilmington Notch, Aug; Mt. Marcy, Sep; Uphill Br., Opalescent R., J1; Saranac L. (McCook); Keene Val., Sep; Gloversville, Sep; W. Caroga L., Aug, Alex; Johnstown, Aug, Alex; Spring Brook, Sep; Slingerlands, Oct, Goldring.

A. arenatus Walck. LI: Sea Cliff, Sep (Bks).

A. cavaticus Key. Keene Val., Aug; Ausable Chasm (Em); Wells, J1, Yg; Ithaca, Sep, Nov (Bks); Thacher Pk., May-Je; Kattskill Bay, Aug; Black Mt., Washington Co, Sep, Leon; Shelving Rock Mt., J1; Shelving Rock Br., Sep, Leon.

A. cornutus Cl. Axton, MacG, Houghton; Saranac L., Aug; Wilmington, Aug; Deer I., Thousand Isls., Aug; Fishers Landing, Sep, Plm; Johnstown, May, Alex; Onondaga Co (Britcher); Lake Bluff, Sep; Rochester, Nov, Chad; Irving, Sep, Chad; Dunkirk; Barcelona, Sep; Crosby, Ap; Taughannock Falls, Aug; Enfield Glen, Oct; Ithaca, Aug, Nov (Bks); Glenwood, Aug, 
Leon; Vrooman's Nose, May; Plattsburg, Sep; Valcour I., Aug; Elizabeth I., Je; Juanita I., J1; Albany, Jan, Je-J1, Sep; Rensselaer, May, Sep; Thompson L., Sep; Voorheesville, Je; Cossayuna L., May, Schoon; Stockport, Sep; Poughkeepsie; L. Katrine, Oct; LI: Sea Cliff, May-Je (Bks).

A. corticarius Em. Wilmington Notch, Aug; Saranac L., Aug; Chateaugay I.., Sep, Plm; Raquette L., Sep, Plm; Johnstown, Aug, Alex; Woodworth's L, Aug, Alex; Onondaga Co (Britcher); Cinnamon L., Sep; Ithaca (McCook); Dryden, Sep (Bks).

A. cucurbitinus C1. Mt. McIntyre, J1; Axton, MacG, Houghton; Wilmington, Aug ; L. Tear, J1; Wells, May, Yg; Speculator, Aug, $Y g$; Old Forge, Je-J1; Gloversville, May-Je, Aug, Oct, Alex; E. Caroga L., Aug ; Johnstown, Ji, Sep, Alex; Sacandaga Pk., Je-J1, Alex; Baldwinsville (Bks); Rochester; Ithaca, Aug-Oct (Bks); Connecticut Hill; McLean, May; Waterford, Nov; Kenwood, J1, Chad; Albany, Sep-Nov; Alps, Chrisp; Queechy L., May, Schoon; Vrooman's Nose, May; Catskills, J1, Chad; Stamford, May-Je, Chrisp; SI: New Brighton, Nov; LI: Sea Cliff, Oct (Bks).

A. ectypus Walck. LI: Sea Cliff (Bks).

A. emertoni Bks. LI: Sea Cliff (Bks).

A. juniperi Em. Ithaca (Bks); LI: Sea Cliff (Bks).

A. marmoreus C1. Wilmington, Aug; Saranac, Aug; Saranac L., Sep, Plm; Plattsburg, Sep; Chateaugay L., Sep; Cranberry L. (Barrows) ; Fishers Landing, Sep, Plm; Mountain L., Je, Alex; Gloversville, Aug-Sep, Alex; E. Caroga L., Aug; Deer I., Thousand Isls., Aug; Watertown, Sep; Onondaga Co (Britcher) ; Clyde, Aug; Lake Bluff, Sep; Hemlock L., Aug; Rochester; Glen Haven, Sep-Oct; Barcelona, Sep; Richburg, Sep; West Barre, Sep; Dunkirk; Irving, Sep; Oak Ridge, Canandaigua L., Aug; Painted Post, Sep ; Watkins Glen; Rock Stream, Oct; Cinnamon L., Sep; Enfield Glen, Oct; Ithaca (Bks); Ringwood, Oct; McLean, Aug; Crown Pt., Sep, Plm; Sleeping Beauty Mt., J1; Waterford, Sep; Albany, Je, Aug-Oct; Kenwood, Oct, Plm; Altamont, Oct; Rensselaer, J1, Sep, Oct; Waldorf, Sep; Queechy L., Aug, Schoon; Catskill, Aug; Poughkeepsie, vanIngen; West Park, Aug, Wlf; Fishkill, Sep; Van Cort. Pk., Sep, Plm; Bronx Pk., Sep, Plm; LI: Sea Cliff, J1 (Bks).

A. miniatus Walck. LI: Sea Cliff, J1, Dec (Bks).

A. nordmanni Thorell. Wilmington Notch, Aug; L. Placid, Sep, Plm; Woodworth's L., Aug, Alex; Caroga L., Aug, Alex; Onondaga Co (Britcher); Glenwood, Aug, Leon; Ithaca, Sep, Nov; Thacher Pk., Je; Rensselaer, Oct, Schoon; Tackawasick Pond, Je; Riders Mills, May; Catskill Mts., Aug, Schoon; Sam's Pt., May; Oakland Val., May.

A. patagiatus Cl. Plattsburg, Sep; Adirondack Mts. (Em\&McCook) ; Gloversville, Aug, Alex; Wells, J1, Yg; Lake Bluff, Aug-Sep; Rochester; Hemlock L., Aug; Glen Haven, Sep, Plm; Irving, Sep; Charlotte, Sep; Naples, J1; Ithaca, Nov (Bks) ; Cascade, Nov; Cazenovia, Sep; Valcour I., Aug; Juanita I., J1-Aug; Elizabeth I., J1; Waterford, Ap; Albany, Aug ; Kenwood, Ap, Chad; Nassau, Je, Schoon; Cossayuna L., May, Schoon; Kinderhook L., Aug, Schoon; Fishkill, Sep.

A. pegnia Walck. Ithaca (Bks); Catskill, Sep, Chad; Poughkeepsie, vanIngen; LI, Sep (Bks).

A. sericatus C1. Plattsburg, Sep; Alexandria Bay (McCook) ; Sacandaga Pk., J1, Alex; Gloversville, Aug, Alex; Johnstown, May, Alex; Baldwinsville (Bks) ; Clyde, Ap, Aug; Lake Bluff, Aug-Sep; Cayuga, Oct, Chad; Rochester, Je, 
Chad; Dunkirk; Naples, J1, Aug; Ithaca, Aug, Nov; Elizabeth I., May, Aug ; Albany, J1, Nov; Kinderhook L., Aug, Schoon; Cornwall, May; LI: Bay Shore, Sep, Plm.

A. solitarius Em. Chateaugay L. (Em) ; Saranac L. (McCook) ; Mt. Marcy, Sep; Munsonville, Oct, Alex; Onondaga Co (Britcher); Rochester, Sep-Oct; Ithaca, Oct (Bks).

A. stellatus Walck. Axton, MacG, Houghton; Wells, J1, Yg; Woodworth's L., J1, Alex; Gloversville, Ap-May, Alex; Johnstown, May ; Barneveld, Aug; Onondaga Co (Britcher) ; Connecticut Hill, Oct; McLean, J1; Dryden, Aug (Bks) ; Ringwood, Oct; Hudson Falls, Je; Poughkeepsie, vanIngen; LI: Sea Cliff (Bks).

A. thaddeus Htz. Wilmington, Aug; Cranberry L. (Barrows) ; Gloversville, Aug, Alex; E. Caroga L., Aug, Alex; Onondaga Co (Britcher); Rochester, Franck; Ithaca, Aug-Oct; Kenwood, Sep-Oct; Waldorf, Sep ; Alps, Oct; LI: Sea Cliff, J1 (Bks).

A. trifolium Htz. Plattsburg, Sep; Wilmington, Aug; Chateaugay L., Sep, Plm; Saranac L., Sep, Plm ; Cranberry L. (Barrows) ; Cranberry Cr., Aug ; Gloversville, Ap, Aug-Sep, Alex; Johnstown, Sep, Ale.r; E. Onondaga, Sep, Plm; Lake Bluff, Sep; Rochester, Sep-Oct; Clarksburg, Sep; Richburg, Sep; Hornell, Sep; West Barre, Sep; Hemlock L., Aug; Irving, Sep, Chad; Dunkirk; Ithaca, Sep-Oct; Valcour I., Aug; Albany, Sep-Oct; Waldorf, Sep, $Y g$; Schodack, Sep; Castleton, Sep; Clinton Hgts., Aug-Sep; Queechy L., Aug, Schoon; Kinderhook L., Sep; Poughkeepsie, vanIngen; NYC (Bks); LI: Sea Cliff, Sep-Oct (Bks).

A. trifolium var. candicans McCook. Wilmington Notch, Aug; Ithaca (Comstock) ; Taughannock Falls, Aug; Juanita I., Sep, Leon.

\section{ARGIOPE Audouin}

A. aurantia Lucas. Saranac L., Aug; Gloversville, Aug, Alex; Onondaga Co (Britcher) ; Rochester, Sep-Oct; Irving, Sep; Naples, Aug; Trumansburg, Aug; Taughannock Falls, Aug, Dt; Valcour I., Aug; Ithaca, Aug-Sep; Albany, Aug-Sep; Menands, Aug ; Rensselaer, Sep; Kinderhook, Sep; Kinderhook L., Aug, Schoon; Poughkeepsie, vanIngen; Beacon, Aug; Brooklyn, Pike; NYC, Sep-Oct; LI: Sea Cliff (Bks).

A. trifasciata Forskal. Wilmington, Aug; Saranac L., Aug; Crater Club, Aug; Watertown, Sep, Plm; Johnstown, Aug, Alex; Onondaga Co (Britcher); Clyde, Aug; Lake Bluff, J1, Sep; Rochester, Sep-Oct; Irving, Sep, Chad; Ithaca, Sep-Oct; Albany, Sep-Oct; Leeds, Aug, Chad; Rensselaer, Sep-Oct; Schodack, Sep; Kinderhook, Sep; Kinderhook L., Aug, Schoon; Valcour I., Aug; Poughkeepsie, vanIngen; Highland, Aug; LI, Aug-Sep (Bks).

\section{Cyclosa Menge}

C. conica Pallas. L. Placid, Sep; Onondaga Co (Britcher); Rochester; Watkins Glen, Oct; Enfield Glen, Oct; Ithaca; Vrooman's Nose, May; Stamford, May; Gloversville, Oct; Albany, Sep; Meadowdale, May; Thacher Pk., Je; Queechy L., May, Schoon; E. Schodack, Sep, Yg; Waldorf, Sep, Yg; Riders Mills, May, Chrisp; Copake Falls, Ap; Valcour I., Aug; Oakland Val., May; LI: Baiting Hollow, May.

C. turbinata Walck. Mountain L., Je, Alex; Gloversville, Oct, Alex; Tully (Britcher) ; Ithaca (Bks); Albany (Htz); LI: Sea Cliff (Bks). 


\section{Eucta Simon}

Compiled by R. M. SeELey

E. lacerta Walck. Wilmington, Aug; Saranac L., Aug (Em); Tully (Britcher); Long Branch, Sep, Britcher; Sodus Bay, Sep; Lake Bluff, Sep; Rochester, Franck; Pt. Breeze, Je; Cinnamon L., Sep; Ithaca, Aug.

E. vermiformis Em. Clyde, Aug; Lake Bluff, Aug; Cinnamon L., J1; Ithaca, Bks; Labrador Pond, Je; Voorheesville, Je; LI, J1 (Bks).

\section{Eugnatha Audouin}

Compiled by R. M. Seeley

E. pallescens Cambr. Cinnamon L., Sep; Ithaca, May; Labrador Pond, Je; Watervliet, Leon; Voorheesville, Je; Rensselaer, Sep, Schoon; Burden L., J1, Schoon.

E. straminea Em. Trenton Falls, Je; Onondaga Co (Britcher); Painted Post, Sep; Interlaken, J1; Enfield Glen, May; Ithaca, J1, Oct; McLean, May; Labrador Pond, May-Je, Tarris; Apulia, Oct, Britcher; Tully, Oct, Britcher; Pompey, Sep, Britcher; Jamesville, Oct, Britcher; Onondaga Val., Britcher; Baldwinsville, Sep, Britcher; Thompson L., May; Normansville, J1; Voorheesville, Je; Rensselaer, Sep, Schoon; Tackawasick Pond, Je.

\section{Eustala Simon}

E. anastera Walck. Axton, MacG, Houghton; Saranac L., Aug; Gloversville, May, Alex; Onondaga Co (Britcher); Rochester, Franck; Irving, Sep, Chad; Ithaca, Sep-Oct; Poughkeepsie, vanIngen; LI: Sea Cliff, Je (Bks).

\section{Glenognatha Simon}

G. foxi McCook. Ithaca, J1.

\section{GLYPTOCRANIUM Simon}

G. bisaccatum Em. Eastwood (Britcher); Larchmont, Feb; LI, Sep (Bks).

G. cornigerum Htz. Ithaca, Nov, Ilg.

\section{LARINIA Simon}

L. borealis Bks. LI: Sea Cliff, Je (Bks).

\section{LEUCAUGE White}

L. venusta Walck. Gloversville, Ap, Aug, Alex; Johnstown, Sep, Alex; Onondaga Co (Britcher) ; Otto, Sep; Rochester, Franck; Taughannock Falls, Je; Ithaca, Je, Aug-Sep; Labrador Pond, Je; Deruyter L., J1; Crystal L., J1; Poughkeepsie, vanIngen; LI: Cold Sp. Harb., And.

\section{MANGORA Cambridge}

M. gibberosa Htz. Wilmington Notch, Aug; Wilmington, Aug; Crater Club, Aug ; Wells, J1, Yg; Gloversville, Aug-Sep, Alex; Onondaga Co (Britcher); Barcelona, Sep ; Ithaca, Aug-Sep; Albany, J1; Central Nassau, J1; Valcour I., Aug; Poughkeepsie, vanIngen; NYC (Bks); LI (Bks). 
M. maculata Key. Albany, J1; Poughkeepsie, vanIngen; LI (Bks).

M. placida Htz. Cranberry L. (Barrows); Onondaga Co (Britcher); Ithaca, Je, Sep; McLean, May-Je; Enfield Glen, Oct; Queechy L., May, Schoon; Riders Mills, May, Chrisp; Little Pond, Orange Co, May; Oakland Val., May; LI (Bks).

\section{META C. Koch}

M. menardi Latreille. Avalanche L., J1; Sacandaga Pk., Je, Alex; Ausable Chasm, Sep, Leon; Onondaga Co (Britcher); Ithaca, Aug-Oct ; Elmira, J1; Binghamton; Thacher Pk., May-Je; E. Berne, Je; Valcour I., Aug; Sam's Pt., May.

\section{Metepeira F. Cambridge}

M. labyrinthea Htz. Wilmington, Aug; Saranac L., Aug; Baldwinsville (Britcher) : Glen Haven, Sep, Plm; Niagara Falls, Sep, Kngt; Ithaca, Sep-Oct; Elmira, J1; Willow Pt., Canandaigua L., Aug; Poughkeepsie, vanIngen; LI: Aug-Sep (Bks); Sea Cliff, Je (Bks).

\section{MicrathenA Sundevall}

M. gracilis Walck. Ithaca (Bks); Poughkeepsie, vanIngen; Mt. Vernon, NYC (Marx) ; LI: Wading R., Sep, Burns; Riverhead (Sound Beach), Sep.

M. reduviana Walck. LI: Riverhead (Sound Beach), Sep.

M. sagittata Walck. Middletown, J1, Meyer; Mt. Vernon, Sep, Felt; LI: Riverhead (Sound Beach), Sep.

\section{NEOSCONA Simon}

N. arabesca Walck. Axton, $\operatorname{Mac}$, Houghton; Wilmington, Aug; Mt. Marcy, Sep; Cranberry L. (Barrows) ; Wells, J1, Yg; Gloversville, Ap, Aug, Alex; Woodworth's L., J1, Alex; Onondaga Co (Britcher) ; Rochester; Hemlock L., Aug ; Cinnamon L., Sep; Ithaca, Sep-Oct; Ringwood, Oct; McLean, J1-Aug; Taughannock Falls, Aug, Dt; Oak Ridge, Canandaigua L., Aug ; Albany, AugSep; Grafton, Aug, Chad; Valcour I., Aug; Juanita I., J1; Pearl Pt., J1; Elizabeth I., J1; Bumps Pond, J1; Tongue Mt., Warren Co, Sep, Leon; Oakland Val., May; Stamford, Je; Poughkeepsie (Bks); Highland, Aug, Wlf; NYC, Plm; LI: Sea Cliff, Je (Bks).

N. benjamina Walck. Wilton, J1; Gloversville, Aug, Alex; Onondaga Co (Britcher); Ithaca, Sep-Oct (Bks); Albany, Sep; Rensselaer, Sep; Grafton, Aug; Valcour I., Aug; Catskill, Aug, Chad; Highland, Aug, Wlf; LI: Sea Cliff, Oct (Bks).

N. pratensis Htz. Ithaca (Bks); Albany, Sep.

\section{Nesticus Thorell}

N. pallidus Em. Dormansville, Je.

\section{PACHYGNATHA Sundevall}

P. autumnalis Key. Syracuse (Britcher); Ithaca, May, Oct, Dec; LI (Bks).

P. brevis Key. Green L., Fulton Co, Jan, Dt; Syracuse (Britcher); Tully (Britcher) ; Ithaca, Mar, Nov-Dec; McLean, May; Queechy L., Aug, Schoon; Poughkeepsie, vanInqen: Little Pond. Orange Co, May; LI, Oct (Bks). 
P. mccooki Bks. Ithaca (Bks).

P. tristriata C. Koch. Cranberry Cr., Aug; Gloversville, Mar-May, Sep, Oct; LI, Key.

SingA C. Koch

S. pratensis Em. Pompey Hill, Onondaga Co (Britcher) ; Riders Mills, May.

S. maculata Em. Tully (Britcher); Jamesville (Britcher); Ithaca (Bks) ; Poughkeepsie, vanIngen; LI (Bks).

S. variabilis Em. Axton, MacG, Houghton; Wilmington, Aug; Saranac L., Aug; Newcomb, J1; Tully (Britcher); Ithaca (Bks); Ringwood, Oct; LI, Oct (Bks).

\section{TETRAGNATHA Latreille}

\section{Compiled by R. M. Seeley}

T. elongata Walck. Cranberry L. (Barrows) ; Beaver R., J1, Yg; Old Forg.e, J1; Wells, J1, $Y g$; Trenton Falls Je; Pompey (Britcher) ; Lake Bluff, Aug-Sep ; Clyde, J1-Aug; Rochester, Franck; Warsaw, Aug; Oak Ridge, Canandaigua L., Aug; Cinnamon L., J1; Ithaca, J1-Oct; McLean, Je, Palmer; Valcour I., Aug; Elizabeth I., J1; Juanita I., J1; Kattskill Bay, Aug; Pearl Pt., J1, AugSep; L. George, J1-Aug; Voorheesville, J1-Aug,. Leon; Rensselaer, May, Schoon; Burden L., J1, Schoon; Tackawasick Pond, Je; Kinderhook L., Je, Sep, Schoon; Cossayuna L., May, Schoon; Poughkeepsie, vanIngen; Saugerties, J1-Aug; W. Shokan, J1; Ashokan, Aug, Treadwell; NYC (Bks); LI: Cold Sp. Harb., Je, Dt; Rockaway Beach, Sep, Pike; Hoversville, Je.

T. extensa L. Paul Smiths, J1, Babiy; Mt. McIntyre, Je; Axton, MacG, Houghton; Adirondack Lodge, J1; Keene Val., Je, Not; Chapel Pond, Je; Upper Ausable L., J1, Not ; Artist's Br., Je ; Speculator, Je-J1, Yg; Wells, J1; Old Forge, J1-Aug, Ndm; Sacandaga Pk., Je, Alex; Johnstown, J1-Aug, Alex; Mountain L., Je, Alex; Trenton Falls, Je; Clayville, Je; Oneida L., Aug; Crocketts, Sep, Chap; Sodus Bay, Sep; Rochester, Franck; Charlotte, Plm ; Hemlock L., Aug; Letchworth Pk., J1; Chautauqua Co, Palmer; Howard, J1; Penn Yan, J1, Babiy; Cinnamon L., Je-J1; Montour Falls, Sep; Alpine, Nov; Enfield Glen, May; Glenwood, Aug; Ithaca, Je-Oct; Caroline Center, May; McLean, May-J1; Freeville, Sep; Ringwood, J1; Owasco L., Nov; Baldwinsville, Sep, Britcher; Long Branch, Sep, Britcher; Jamesville, Oct; Apulia, Oct, Britcher; Tully, Oct, Britcher; Labrador Pond, May-Je, Tarris; Deruyter L., J1; Valcour I., Aug; Pearl Pt., J1; Juanita I., J1; Tongue Mt., Warren Co, Sep, Leon; Shelving Rock, L. George, J1, Sep; Cossayuna L., May, Schoon; Bumps Pond, J1; Thompson L., Sep, Chad; Mud Hollow Pond, Je; Voorheesville, Je-J1, Leon; E. Berne, Je; Waldorf, Oct; Poestenkill, Je, Yg; Tackawasick Pond, Je; Riders Mills, May; Kinderhook, Je, Aug, Schoon; L. Charlotte, Je; Hunter, Aug; Wappingers Falls, May; L. Katrine, Sep; Ashokan, Aug, Treadwell; Oakland Val., May; Pinekill, May; LI: Cold Sp. Harb., Je, And; Smithtown, Je, Leon; Long Pond, Suffolk Co, Je.

T. laboriosa Htz. Black Br., Je; Peru, Je; Keene Val., J1, Not; Ausable R., Aug; Mt. McIntyre, Aug; Paul Smiths, J1, Babiy; Saranac L., Aug . Em) ; Cranberry L. (Barrows); Wells, J1, Yg, Babiy; Sacandaga Pk., Je, Alex; Bleecker, Sep; Woodworth's L., Je, Alex; Trenton Falls, Je; Waterville, Aug ; Clayville, J1; Smithville, Aug; Baldwinsville (Bks); Lake Bluff, Sep; Honeoye Falls, J1; Pt. Breeze, Je; Lakeside, J1; Barre Center, Je; Irving, Sep ; Penn Yan, Aug; Cinnamon L., Je-J1, Sep; Taughannock Falls, J1; Ithaca, J1-Aug ; 
Ringwood, J1, Dt; Otisco, Aug, Britcher; Onondaga Val., Je, Britcher; Pompey, Sep, Britcher; Apulia, Oct, Britcher; Tully, Oct, Britcher; Valcour I., J1-Sep; Juanita I., J1-Sep; Watervliet Reservoir, Aug, Leon; Mud Hollow Pond, Albany Co, Je; Tackawasick Pond, Je; Pike Pond, Je; Stamford, Je; Columbia Co; Kingston; Sam's Pt., May; Rhinebeck, Oct; Ashokan, Aug, Treadwell; Lagrangeville, May; Wappingers Falls, May; Oakland Val., May; NYC (Bks); LI: Bergen Beach, Je; Flatbush, J1, Pike; Cold Sp. Harb., Je, And; Rockaway Beach, Sep, Pike; Shinnecock Hills, Je.

\section{Theridiosoma Cambridge}

T. gemmosum L. Koch. Wilmington Notch, Aug; Mt. Whiteface, Aug; Chapel Pond, Je ; L. Colden, J1; Cranberry L. (Barrows) ; Woodworth's L., J1., Alex ; Johnstown, J1, Alex; Jamesville; Ithaca, Aug ; Enfield Glen, Oct ; Rensselaer, J1, Schoon; Larchmont, Sep, Wlf; Central Nassau, J1; LI, Je (Bks).

\section{ZILLA C. Koch}

Z. montana C. Koch. Mt. McIntyre, J1; Caroline Center, May; Thacher Pk., Je ; Dormansville, Je.

Z. x-notata Cl. NY (McCook).

\section{FAMILY MIMETIDAE}

ERo C. Koch

E. furcata Vill. Wilmington Notch, Aug; Rye Pt., Sep; Penn Yan, Aug; Savona, Sep; Cinnamon L., May; Ithaca, Mar; Canastota, Mar, Faure; Vrooman's Nose, May; Dormansville, Je; Juanita I., Aug; LI, Sep (Bks).

\section{Mimetus Hentz}

M. interfector Htz. Pompey (Britcher) ; Crosby, Ap; Freeville, Aug-Sep ; Enfield Glen, Oct; Ithaca, J1-Sep; Karner, Ap, $Y g$; LI: Sheepshead Bay, J1.

\section{FAMILY THOMISIDAE}

\section{Eво Keyserling}

E. latithorax Key. Onondaga Co (Britcher); LI, Sep-Oct (Bks).

Misumena Latreille

M. vatia C1. Wilmington, Aug; Crater Club, Aug; Axton, MacG, Houghton; Saranac L., Aug; Cranberry L. (Barrows) ; Little Sandy Pond, Jefferson Co, Je, House; Wilton, J1 (Hoffman); Gloversville, Ap-May, J1, Alex; Woodworth's L., May, J1, Alex; Johnstown, J1-Aug, Alex; Mountain L., May, Alex; Onondaga Co (Britcher); White L., Oneida Co, J1; Lake Bluff, Aug; Rochester, Franck; Howard, J1; Ithaca, May, J1, Sep; Delaware L., May; McLean, May; Slaterville, May; Kirkland, J1; Schenectady, J1, $Y g$; Valley Falls, May, $Y g$; Central Nassau, J1; Valcour I., Aug; Poughkeepsie, vanIngen; LI, May-Je, Oct. 


\section{Misumenoides Cambridge}

M. aleatorius Htz. Old Forge, Je; Woodworth's L., May, Alex; Onondaga Co (Britcher); Rochester; Irving, Sep, Chad; Taughannock Falls, Aug, Dt; Ithaca, Aug (Bks); McLean, May; Albany, Aug; Catskill, Sep, Chad; Castleton, Sep, Plm; Poughkeepsie, vanIngen ; Patterson, Aug; SI: Tottenville, Aug, Ds; LI, Aug (Bks).

\section{MISUMENOPS Cambridge}

M. asperatus Htz. Wilmington, Aug; Gloversville, Aug, Alex; Sacandaga Pk., Je, Alex; Ithaca, Oct; Oakland Val., May; LI (Bks).

M. celer Htz. Ithaca (Bks).

M. oblongus Key. Onondaga Co (Britcher); LI, J1 (Bks).

\section{OXYPTILA Simon}

O. americana Bks. Ithaca, Oct (Bks).

O. conspurcata Thor. Wilmington Notch, Aug; Ithaca, Je; McLean, May; Riders Mills, May; Poughkeepsie (Bks); Paradise, May; Oakland Val., May; LI: Sea Cliff, Oct (Bks).

O. monroensis Key. LI: Sea Cliff (Bks).

\section{PHILODROMUS Walckenaer}

P. aureolus Cl. Axton, MacG, Houghton; Sacandaga Pk., J1, Alex; Oakland Val., May; LI, J1 (Bks).

P. bidentatus Em. Valcour I., Aug.

P. canadensis Em. Rochester; Plattsburg, Sep, PIm; Juanita I., J1.

P. exilis Bks. Freeville, May (Bks); Albany, Je.

P. infuscatus Key. Interlaken, Nov; Ithaca, Sep (Bks); Poughkeepsie, vanIngen.

P. lineatus Em. Onondaga Co (Britcher).

P. maculatus Blackw. Ithaca (Bks).

P. minutus Bks. Enfield, Je; Ithaca (Bks).

P. ornatus Bks. Onondaga Co (Britcher); Howard, J1; Ithaca (Bks); Poughkeepsie, vanIngen.

P. pernix Blackw. Newcomb, J1; Sacandaga Pk., Je, Alex; Onondaga Co (Britcher) ; Rochester, Je; Ithaca, Mar, Je-J1, Nov-Dec; Albany, Ap; Thacher Pk., Je; Poughkeepsie, vanIngen; LI: Sea Cliff, Je (Bks).

P. placidus Bks. Ithaca (Bks); LI, J1 (Bks).

P. robustus Em. LI: Bayville (Bks).

P. rufus Walck. Axton, $\operatorname{Mac} G$, Houghton; Wilmington Notch, J1; Sacandaga Pk., Je, Alex; Onondaga Co (Britcher) ; Ithaca, May, Oct; Vrooman's Nose, May; Riders Mills, May; Valcour I., Aug; Mt. Utsayantha, May, Chrisp; Stamford, May; Oakland Val., May; LI (Bks).

\section{SyNAEMA Simon}

S. bicolor Key. Sacandaga Pk., Je, Alex. 


\section{Thanatus C. Koch}

T. coloradensis Key. Onondaga Co (Britcher); Rochester, Nov, Chad; McLean, J1; Voorheesville, Je; Clinton Hgts., Yg; Valcour I., Aug; Rensselaer, Dec, Schoon; Defreestville, Mar; L. Katrine, Oct.

T. rubicundus Key. LI, Oct (Bks).

\section{TibEllus Simon}

T. oblongus Walck. Axton, MacG, Houghton; Cranberry L. (Barrows); Saranac L., Aug; Sacandaga Pk., Je,. Alex; Johnstown, Je, Alex; Woodworth's. L., J1; Onondaga Co (Britcher) ; Pt. Breeze, Je; Junius Bog, Je; Cinnamon L., Je; Ithaca, May, J1; McLean, May; Valcour I., Aug; Albany, Je; Rensselaer, Jan, Schoon; LI, J1 (Bks).

\section{TMARUS Simon}

T. angulatus Walck. Onondaga Co (Britcher); Ithaca, Sep; McLean, May; Central Nassau, J1; LI, J1 (Bks).

\section{Xysticus C. Koch 185}

X. acquiescens Em. Scotia, Je, $Y g$.

X. elegans Key. Johnstown, J1, Alex; Ithaca, J1; McLean, May; Menands, J1; Rensselaer, Je, Schoon; L. George, Aug; LI: Brooklyn (Em).

X. ferox Htz. Johnstown, Sep, Alex; Sacandaga Pk., Je, Alex; Onondaga Co (Britcher); Geneva, Je; Ithaca, Ap, Je, Oct-Nov; Spencer, Je; Ringwood, J1; Albany, Je; Rensselaer, Je, Schoon; Poughkeepsie, vanIngen; SI: Tottenville, Je; LI: Sheepshead Bay, J1.

X. formosus Bks. Wilmington, Aug; Varna, Mar; McLean, May; Dormansville, Je; Tackawasick Pond, Je; Oakland Val., May.

X. fraternus Bks. LI, May (Bks).

X. graminis Em. Ithaca (Bks); McLean, May; LI (Bks).

X. gulosus Key. Gloversville, Ap, Aug, Oct, Alex; Barnevelt, Sep; Onondaga Co (Britcher) ; Lake Bluff, Sep; Ithaca, Oct-Nov; Manlius, Smith; Dormansville, Oct; Rensselaer, Oct, Schoon; Coxsackie, Mar; Poughkeepsie, vanIngen; LI: (Pike); Sea Cliff, Je (Bks).

X. limbatus Key. Wilmington Notch, Aug; Cranberry I.. (Barrows) ; Saratoga, Je; Onondaga Co (Britcher); Bath, Aug; Enfield, May; Ithaca (Bks); Stamford, May, Chrisp; Dormansville, Je ; Schenectady, Dec, Dt; L. Charlotte, Je; Poughkeepsie, vanIngen; LI: Brookiyn (Pike) ; Quogue, Ap ; Long Pond, Suffolk Co, Je.

X. luctans C. Koch. Johnstown, Aug, Alex; Gloversville, Oct, Alex; Onondaga Co (Britcher); Ithaca, May, Aug; Clayville, Je; Rensselaer, Oct, Schoon; Nassau L., Sep; Wappingers Falls, May.

X. nervosus Bks. Gloversville, Ap, Oct, Alex; Pulaski, Nov; Lake Bluff, Sep; Lewiston, Sep, Plm; Howard, J1; Hall, Sep, Glasgow; Ithaca, Aug-Nov; Crosby, Sep; Kenwood, Oct, Plm; Poughkeepsie, vanIngen; L. Katrine, Oct, Plm; LI: Aug-Sep (Pike, Bks); Sea Cliff, Je (Bks).

185 Miss Augusta Wolf is responsible for many of the determinations in this genus. 
X. triguttatus Key. Axton, MacG, Houghton; Johnstown, May, Alex; Sacandaga Pk., Je, Plm; Barnevelt, May; Hilton, Je; Ithaca, Je-J1, Sep; Ringwood, $\mathrm{J1}$; Trenton Falls, Je; Clayville, Je; Central Nassau, J1; Burden L., Je;

Tackawasick Pond, Je; Poughkeepsie, vanIngen; LI: Sea Cliff, Je.

X. versicolor Key. Cranberry L. (Barrows) ; Gloversville, Ap, Oct, Alex; Onondaga Co (Britcher); Geneva, Nov, Glasgow; L. Keuka, Sep; Ithaca, May, Nov; W. Winfield, Je; Valcour I., Aug; Scotia, Je, $Y g$; Karner, Nov; Albany, Je; Poughkeepsie, vanIngen; LI, Mar, Nov (Bks).

\section{FAMILY CLUBIONIDAE}

\section{AGROECA Westring}

A. minuta Bks. LI: Sea Cliff, May (Bks).

A. ornata Bks. Wilmington, Aug; Olcott, Sep; Clarksburg, Sep; Stow, Sep; Letchworth Pk., J1; Ithaca, Ap, Oct; Elizabeth I., Aug; Oakland Val., May;

Sterlington, May; LI, May (Bks).

A. pratensis Em. Wilmington Notch, Aug; Elizabethtown, Aug; Onondaga Co (Britcher) ; Enfield, May; Ithaca, Feb-Ap, Oct, Dec; McLean, May; Juanita I., J1; Albany (Em) ; Guilderland Center, Dec; Poughkeepsie, vanIngen ; LI: Forest Hills, Mar.

\section{AnyphaEna Sundevall}

A. celer Htz. Onondaga Co (Britcher); L. Keuka, Oct; Ithaca, Jan-Mar, Je, Oct-Nov; McLean, May; Normansville, Nov; Feura Bush, Dec; Rensselaer, Mar, Schoon; Poughkeepsie, vanIngen; Larchmont, Sep, Wlf; Dunwoodie, May, Pioneer Youth; SI: Clove Val., Nov; LI: Flushing, Nov.

A. fraterna Bks. LI: Roslyn, May (Bks); Sea Cliff, Je (Bks).

A. gracilis Htz. LI, Oct (Bks).

A. pectorosa L. Koch. Albany, Je; LI: Farmingdale, Je.

A. rubra Em. LI: Cold Sp. Harb., Je, And.

A. saltabunda Htz. Enfield Glen (Bks) ; Ithaca, Sep (Bks) ; LI: Cold Sp. Harb., Je, And.

\section{Apostenus Westring}

A. acutus Em. LI (Em).

CASTANEIRA Keyserling

C. cingulata C. Koch. Onondaga Co (Britcher); Cinnamon L., J1; Ithaca, Aug; Juanita I., J1, Sep; S. Westerlo, May; Poughkeepsie, vanIngen; LI: Sea Cliff, May (Bks).

C. descripta Htz. Wilmington, Aug; N. Elba, J1; Cranberry Cr., Aug; Onondaga Co (Britcher) ; Ithaca, May, Aug, Oct; Valcour I., Aug; Kenwood, Sep; S. Rensselaer, J1; Poughkeepsie, vanIngen; LI, Aug.

C. longipalpus Htz. Onondaga Co (Britcher); Ithaca (Bks); Burden L., Aug, Schoon; LI, Aug (Bks).

C. trilineata Htz. LI: Sea Cliff, J1-Aug (Bks).

\section{Chiracanthium C. Koch}

C. inclusum Htz. Cranberry L. (Barrows). 


\section{Clubiona Latreille}

C. abboti L. Koch. Wilmington Notch, J1; Gloversville, Ap, Alex; Lake Bluff, Sep ; Cinnamon L., J1; Spencer, Je; Ithaca, Feb, May, Oct; McLean, May; Juanita I., J1; Albany, Je (Hartman) ; Rensselaer, J1, Schoon; Wappingers Falls, May; Kingston, Aug, Schoon; Stamford, May, Chrisp; Slide Mt., Je; Cragsmoor, May; Maratanza L., May; Pine I., May; Hunter, Je, Plm ; LI: Gardiners I., May; Montauk Pt., May.

C. canadensis Em. Wilmington, Aug; Mt. Whiteface, Aug; Mt. McIntyre, J1; L. Keuka, Oct; Cinnamon L., J1; Ithaca, Oct; Riders Mills, May; Slide Mt., Je; Stamford, May, Chrisp.

C. emertoni Petrunk. Lake Bluff, Sep; Freeville, May; Juanita I., Aug; Little Pond, Orange Co, May; LI, Oct (Bks).

C. lenta Bks. Ithaca, Sep (Bks).

C. littoralis Bks. LI: Sea Cliff, Je (Bks).

C. minutissima Petrunk. Ithaca, Nov (Bks); Interlaken, Aug; LI: Sea Cliff, J1 (Bks).

C. obesa Htz. Sacandaga R., Je, Alex; Lake Bluff, Sep; Clyde, Sep; Rochester; Youngstown, Oct; Crosby, Ap ; Ithaca, May, Oct, Nov; Newfield, Je; McLean, May; Albany, Je; Thacher Pk., May; Cossayuna L., May, Schoon; Queechy L., May, Schoon; Juanita I., J1; Cragsmoor, May; Oakland Val., May; LI: Sea Cliff, Dec (Bks); Roslyn, Dec (Bks).

C. pallens Htz. Oak Ridge, Canandaigua L., Aug; Ithaca, Nov; Voorheesville, Je; Juanita I., J1; Elizabeth I., Aug; Shelving Rock, Sep, Leon; Wappingers Falls, May; LI: Oct, Dec (Bks); Baiting Hollow, May.

C. pygmaea Bks. Ithaca, Sep, Nov (Bks); LI, J1 (Bks).

C. riparia L. Koch. Sacandaga Pk., Je; Onondaga Co (Britcher); Ithaca (Bks); McLean, May; Scotia, Je, $Y g$; Albany, Je; Delmar, J1; Riders Mills, May; Poughkeepsie, vanIngen; LI, Dec (Bks).

C. tibialis Em. Ithaca, Aug (Bks); LI (Bks, Em).

\section{LIOCRANUM L. Koch}

L. calcaratum Em. Mt. Whiteface, Aug (Em); Wilmington, Aug; Auger Pond, Nov; Uphill Br., Opalescent R., J1; Connecticut Hill, Oct.

\section{MICARIA Westring}

M. agilis Bks. LI: Sea Cliff (Bks).

M. aurata Htz. Onondaga Co (Britcher); Ithaca, Aug-Sep (Bks); SI (Bks); LI $(\mathrm{Em})$.

M. gentilis Bks. Clyde, Ap.

M. montana Em. Onondaga Co (Britcher); LI (Bks).

\section{PhruRolithus C. Koch}

P. alarius Htz. Malone, Oct; Stow, Sep; Lakeside, J1; Letchworth Pk., J1; Cheshire, Oct; Guyanoga, Je; L. Keuka, Sep; Barrington, Oct ; Taughannock Falls, Mar, J1; Ithaca, May-Je, Aug, Dec; Deruyter L., J1; Labrador Pond, Je; Tackawasick Pond, Je; Dormansville, May; Albany, J1; Crystal L., J1; S. Westerlo, May; Sam's Pt., May; LI: Montauk Pt., May. 
P. britcheri Petrunk. Onondaga Co, Britcher.

P. formica Bks. Silver Bay, Sep, Leon; Spencerport, May; Sam's Pt., May; LI, Dec (Bks).

P. minutus Bks. Ithaca, Sep (Bks).

P. palustris Bks. Lake Bluff, Sep; Barcelona, Sep; Stow, Sep; Richburg, Sep; Little Valley, Sep; Alabama, Je; Rye Pt., Sep; Barrington, Oct; Savona, Sep ; Cinnamon L., J1; Taughannock, Aug ; Enfield, Je; Ithaca, Aug ; McLean, May-Je; L. George, Aug; Feura Bush, Dec; Crystal L., J1; Thacher Pk., May; Vrooman's Nose, May; Little Pond, Orange Co, May; Tuxedo, Oct, Wlf; Larchmont, Sep, Wlf; SI: Clove Val., Nov; LI: Flushing, Je; Sea Cliff, Je; Garden City, Je; Baiting Hollow, Ap.

P. pugnatus Em. Ithaca (Bks); Karner, Mar; Little Pond, Orange Co, May; Sam's Pt., May.

P. similis Bks. McLean, May; LI: Sep-Oct (Bks); Gardiners I., May.

\section{Trachelas L. Koch}

T. deceptus Bks. LI: Sea Cliff, Sep-Oct (Bks) ; Gardiners I., May; Montauk Pt., May.

T. tranquillus Htz. Onondaga Co (Britcher); Clyde, Aug-Sep; Glen Haven, Sep; Rochester; Hall, Sep, Glasgow; Geneva, Nov, Glasgow; L. Keuka, Oct; Taughannock Falls, Oict; Ithaca, Jan, Aug-Oct; Utica; Albany, Sep-Oct; Rensselaer, J1, Sep, Oct, Schoon; Poughkeepsie, vanIngen; NYC (Bks); LI: Sea Cliff, Sep, Wlf, Taub.

\section{ZORA C. Koch}

Z. spinimana Sund. LI: Baiting Hollow, May.

\section{FAmily AGELENIDAE}

\section{AgelenA Walckenaer}

A. naevia Walck. Mt. Marcy, Aug; Wilmington, Aug; Upper Jay, Sep, Plm; Chateaugay L., Sep, Plm; Cranberry L. (Barrows); Watertown, Sep, Plm; Big Moose, Aug, Plm; Raquette Falls, Aug; Gloversville, Aug-Sep, Alex; Johnstown, Aug, Alex; Cazenovia, Sep; Baldwinsville; E. Onondaga, Sep; Clyde, Aug; Lake Bluff, J1, Sep; East Bay, Sep; Cayuga, Oct, Chad ; Rochester, Nov, Chad; Geneva, Nov, Glasgore; Hornell, Sep; Glen Haven, Sep; Alpine, Sep; Cinnamon L., Sep; Interlaken, Nov; Ithaca, Jl-Nov; Ringwood, J1-Aug; Albany, J1, Sep ; Kenwood, Sep-Oct; Rensselaer, J1-Sep, Schoon; Lyon's L., Aug ; Plattsburg, Sep, Plm; Valcour I., Aug; Elizabeth I., J1-Aug; Juanita I., J1; Black Mt., Washington Co; Jabe Pond, Sep, Leon; Stockport, Sep, Plm; Poughkeepsie, vanIngen; Wappingers Falls, May; Catskill, Sep, Nov; Kingston, Aug-Sep, Schoon; L. Mahopac, Sep, Fox; Hunter, Aug, Mills; Tuxedo, Oct, Wlf; Van Cort. Pk., Sep, Plm; SI: Clove Val., Nov; Silver L., Aug; LI: Brooklyn (Em) ; Jamaica, Oct; Forest Hills, Nov; Sea Cliff ; Mattituck, Aug.

\section{CICURINA Menge}

C. arcuata Key. Old Forge, Oct, Sib; Gloversville, Oct, Alex; Mountain L., Ap; Lake Bluff, Sep; Olcott, Sep, $D t$; Newfane, Oct; Little Valley, Sep; Richburg, Sep; Clarksburg, Sep; W. Barre, Sep; Guyanoga, Je; Penn Yan, May; 
Egleston's Glen, Sep; Cayuga, Oct, Chad; Alpine, Nov; Connecticut Hill, Aug-Oct; Interlaken, Nov; Enfield Glen, Ap; Danby, Oct; Ithaca, Ap, Oct; McLean, Ap-May; Cascade, Nov; Pearl Pt., J1; Meadowdale, Ap-May; Crystal L., J1; Riders Mills, May, Chrisp; Schodack, Ap; Oakland Val., May; Sterlington, May; Larchmont, Sep, Wlf.

C. brevis Em. Wilmington, Aug; Wilmington Notch, Aug; High Falls, Aug; Auger Pond, Nov; Adirondack Lodge, J1; Northville, Ap; Gilman L., Ap ; Elizabethtown, Aug; Lake Bluff, Sep; Olcott, Sep, Dt; Stow, Sep; Richburg, Sep; Clarksburg, Sep; Little Valley, Sep; Rock City, Sep; Castile, May; Potter, May; Guyanoga, Je; Rye Pt., Sep; Egleston's Glen, Sep; Crosby, Dec; Penn Yan, May; Montour Falls, Sep; Connecticut Hill, Aug, Oct; Enfield, Aug, Oct; Danby, Oct; Ithaca, Ap-May, Oct; Freeville, May; McLean, ApMay; Labrador Pond, Je; W. Winfield, Je; Valcour I., Aug; Slide Mt., Je; Juanita I., Aug; Trenton Falls, Je; Waterford, Ap ; Altamont, Ap ; Karner, Mar; Rensselaer, Nov; Cragsmoor, May; Pinekill, May; Tuxedo, Oct, Wlf; LI: Roslyn, Oct (Bks).

C. pallida Key. Newcomb, J1; Charley L., Ap; Mt. Rouge, Hamilton Co, J1, Yg ; Rye Pt., Sep; Egleston's Glen, Sep; Guyanoga, Je; Penn Yan, Aug; Alpine, Nov; Ithaca, Mar-Ap; McLean, May; Cascade, Nov; Labrador Pond, Je; Altamont, Ap; S. Westerlo, May; Rensselaer, May, Schoon; Valcour I., Aug; Silver Bay, Sep, Leon; Poughkeepsie, vanIngen; Tuxedo, Oct, Wlf; Larchmont, Sep, Wlf.

C. placida Bks. Ithaca (Bks) ; Meadowdale, May; Crystal L., J1; Sam's Pt., May; Cragsmoor, May.

\section{Coelotes Blackwall}

C. calcaratus Key. Wilmington Notch, Aug; Charley L., Ap; Lake Bluff, Sep ; Olcott, Sep; Richburg, Sep; Cheshire, Oct; Penn Yan, May; Rye Pt., Sep; L. Keuka, Dec; Savona, Sep; Connecticut Hill, Oct; Ithaca, Ap-May, Aug ; McLean, Ap-May; Cascade, Nov; Valcour I., Aug; Pearl Pt., J1; Silver Bay, Sep, Leon; Poughkeepsie, vanIngen; Suffern, Ramapo Mts., May; Pinekill, May; Larchmont, Sep, Wlf.

C. fidelis Bks. Uphill Br., Opalescent R., J1; Chateaugay L., Sep, Plm; E. Onondaga, Sep, Plm; Olcott, Ap; W. Barre, Sep; Bath, Mar; Painted Post, Sep; Rochester, Nov, Chad; Cayuga, Oct, Chad; Taughannock Falls, Mar; Enfield Glen; Spencer, Aug; Ithaca, Feb-May, Aug, Nov; McLean, Ap-May, Oct; Delhi, May; Albany, Ap; W. Berne, Ap; Delmar, Ap; S. Westerlo, May; Thacher Pk., Je; Meadowdale, May; Greenville, Ap; Vrooman's Nose, May; Averill Pk., Je, Plm; Rensselaer, Nov, Schoón; Riders Mills, May; Crown Pt., Sep, Plm; Juanita I., J1; Shelving Rock, Sep, Leon; Delhi, May; Turner, Aug; Wappingers Falls, May; Hunter, Aug; Paradise, May ; Pine I., May; Pinekill, May; Sam's Pt., May; Mt. Utsayantha, Oct; Tuxedo, Oct, Wlf.

C. hybridus Em. Chateaugay L. (Em); Adirondack Lodge, J1; Gloversville, Je, Oct, Alex; E. Onondaga, Sep; Rochester, Sep-Oct; Glen Haven, Sep, Plm; Potter, May; Montour Falls, Oct; Enfield Glen, Ap; Ithaca, May, Aug (Bks) ; McLean Bogs, Ap; Altamont, Ap; New Salem, Ap; Thacher Pk., Je; Rensselaer, Ap; E. Schodack, Sep; Catskill, Oct, Chad; Elizabeth I., Aug; Poughkeepsie, vanIngen; Bronx Pk., Sep, Plm; LI: Sea Cliff (Bks).

C. montanus Em. Beaver R., J1; Chateaugay L. (Em); Gilman L., Ap; McLean, Oct; Lake Bluff, Sep; Voorheesville, Ap; Valcour I., Aug.

C. nigriceps Bks. LI: Roslyn, Oct (Bks). 


\section{CORAS Simon}

C. medicinalis Htz. Chateaugay L. (Em); Wilmington, Aug; Wilmington Notch, Aug; Gloversville, Oct, Alex; E. Onondaga, Sep; Hemlock L., Aug; Ithaca, Oct-Nov; Deruyter L., J1; Schoharie (Em); Valcour I., Aug ; LI, J1 (Bks).

\section{CRYPHOECA Thorell}

C. montana Em. Mt. Whiteface, Aug; Wilmington, Aug; Wilmington Notch, J1Aug; Uphill Br., Opalescent R., J1; High Falls, Aug ; Adirondack Lodge, J1; Guyanoga, Je; Montour Falls, Sep; McLean, May; Slide Mt., May-Je; Mt. Utsayantha, May, Chrisp.

Cybaeus L. Koch

C. giganteus Bks. Ithaca (Bks).

HAHNIA C. Koch

H. agilis Key. Wilmington Notch, Aug; Onondaga Co (Britcher); Lake Bluff, Sep; Otto, Aug; Olcott, Sep; Richburg, Sep; Rock City, Sep; Ithaca, MayJe, Aug; Slaterville, Aug; McLean, Ap; Deruyter L., J1; Dormansville, Je ; French Mills, Albany Co, Mar; Vrooman's Nose, May; Juanita I., J1; Pine I., May; LI: Baiting Hollow, Ap.

H. brunnea Em. Cinnamon L., Je-J1; Freeville, Ap, Aug; Ithaca (Em) ; McLean, May; Little Pond, Orange Co, May.

H. cinerea Em. Wilmington Notch, Aug; Wilmington, Aug; Newcomb, J1; Olcott, Sep; Ithaca, Ap; Freeville, Aug; Labrador Pond, Je; Karner, Mar; Juanita I., J1; Little Pond, Orange Co, May; Sam's Pt., May; LI: Riverhead, Ap.

H. radula Em. Ithaca (Bks).

\section{TEGENARIA Latreille}

T. domestica Cl. Tahawus, J1; Onondaga Co (Britcher) ; Ithaca, May; Rochester; Albany, Mar; Kingston, Sep, Schoon; Poughkeepsie (Em).

\section{FAMILy PISAURIDAE}

\section{DOLOMEDES Latreille}

D. scriptus Htz. Newcomb, Sep, House; Chilson L., Essex Co; Chapel Pond, J1; Cazenovia; Onondaga L., Sep, Plm; Clyde, Ap; Rochester, Franck; Locke; Ithaca, May, J1, Sep; Enfield Glen, Je, Aug, Oct, $N d m$; Glenwood, Aug, Leon; N. Lansing, Je; Elizabeth I., L. George, J1-Aug; Juanita I., Pearl Pt., $\mathrm{J1}$; Bolton Landing, L. George, J1; Kattskill Bay, Aug; Albany; Dormansville, Je; Slingerlands, J1; Rensselaer, Mar, Schoon; Pike Pond, Rensselaer Co, Je; Nassau L., Mar, Schoon; E. Greenbush, Je, Plm; Burden L., Jl, Schoon; Tackawasick L., Je; E. Nassau, Je, Wlf; Kinderhook L., Sep, Schoon; Merrill; Valcour I., L. Champlain, Aug; Hoversville, Je; Riders Mills, May; Ashokan; Stamford, Je.

D. striatus Giebel. Ithaca; Ringwood; McLean Bogs, Sep; Kinderhook, Sep; Pine I., Orange Co., Sep.

D. tenebrosus Htz. Axton, MacG, Houghton; Keene Val., J1; Deer I., Thousand Isls.; Saratoga (McCook) ; Johnstown, Aug, Alex; Gloversville, Aug, Alex; Mountain L., Alex; Manlius; Batavia (McCook); Hemlock L., Je; Ithaca, 
Ap (Comst) ; McLean, Je; Berkshire; Thacher Pk., May; Valcour I., Aug ; Elizabeth I., J1; Juanita I., Aug; LI: Sea Cliff (Bks) ; Cold Sp. Harb.

D. triton sexpunctatus Htz. Brittons; Long Branch; Clyde, Sep; Sodus Bay, Sep; Sodus Pt., Sep; Rochester, Franck; Cinnamon L., J1; Ithaca, Aug; McLean Bogs, May, J1; Albany; Kenwood, May, Plm; Rensselaer, Je, Schoon; SI: Grasmere Sta; LI: Baldwin.

D. urinator Htz. Ithaca, Ap (Bks); Rensselaer, Aug, Schoon; Debruce; LI: Jamaica.

D. vittatus Walck. Green L., Onondaga Co; Ithaca, Mar; Voorheesville, Ap; Rensselaer, Je, Schoon; Nassau L., Sep, Schoon; New Rochelle, Ap; L.I (Bks).

\section{PISAURINA Simon}

P. brevipes Em. SI: Watchogue, J1, Burns; LI: Sands Pt., Sep, Burns.

P. mira Walck. Saratoga, Je; Brittons; Jamesville; Baldwinsville; Long Branch; Rochester, Franck; Otto, May; Honeoye Falls, May; Keuka L., Je; Taughannock Falls, Oct; Enfield, Oct; Ithaca, J1, Sep, Nov; McLean Bogs, May; Albany, May-Je, Oct; Voorheesville, Dec; Riders Bridge, May; Selkirk, Sep; Rensselaer, Mar, Schoon; Valcour I., Je, Hudson; Bumps Pond, J1; Fishkill Landing, Sep, Plm; SI: Hyde Pk.; LI: Flatbush; Oyster Bay, Ap, Plm; Cold Sp. Harb., Je.

P. mira, var. subinflata Htz. Onondaga Val.; Baldwinsville; Indian Reservation, Onondaga Co; Enfield Glen, Aug; Riders Mills.

\section{FAMILY LYCOSIDAE}

Allocosa Banks

A. funerea Htz. LI: Montauk Pt., May.

A. rugosa Key. Little Pond, Orange Co, May; LI (Em).

\section{Alopecosa Simon}

A. beanii Em. Gloversville, May, Alex.

\section{Arctosa C. Koch}

A. littoralis Htz. N. Fair Haven, J1; Albany, May (Em) ; Rensselaer, Sep, Schoon; SI: Tottenville, J1, Ds; LI: Cold Sp. Harb.

A. rubicunda Key. Upper Jay, Sep, Plm; Gloversville, Ap-May, Alex; Taughannock Falls, Aug, Dt; Ithaca, Mar; Caroline Depot, Ap; Albany; Clarksville, May; Pike Pond, Rensselaer Co, Je; E. Nassau, Je, Wlf; Wappingers Falls, May; LI, Oct (Bks).

\section{LYcosA Latreille}

L. alpigena Dol. Mt. Marcy, Adams.

L. aspersa Htz. Hope, Aug; Wells, Oct, $Y g$; Chautauqua Co, Je ; Ithaca, Aug ; Naples, Aug; Burden L., Aug, Schoon; Elizabeth I., J1; Tackawasick Pond, Je; LI (Chamb).

L. avara Key. Onondaga Co (Britcher) ; Rochester, Franck; Ithaca, Jan, Mar (Bks); L. Katrine, Oct, Chad; LI: Bayville, Dec (Bks). 
L. avida Walck. Ballston L., J1; Lake Bluff, Sep; Taughannock Falls, Aug; Ithaca, May, Jl-Aug, Oct; Spencer, Je, Frost; Trenton Falls, Je; Albany, Je; Thacher Pk., Je; Delmar, J1; Voorheesville, Oct; Dormansville, Je; Thompson L., Je; Medusa, Ap; Mud Hollow Pond, Je; Rensselaer, May, Schoon; Hudson, May; Ancram, Je; Paradise, May; Goshen, May; SI: Tottenville, Je; Watchogue, J1; LI: Wading R., Je.

L. baltimoriana Key. Gloversville, May, Alex.

L. carolinensis Walck. Schroon L., Aug; Chilson L., Sep; Wells, J1, Yg ; Galway, May, Fuller; Gloversville, Oct, Alex; Onondaga Co (Britcher); LI: Riverhead, Aug, Ds.

L. fatifera Htz. Sea Breeze, Sep.

L. frondicola Em. Wilmington, Aug; Upper Jay, Sep, Plm; Newcomb, J1; Wells, Oct, $Y g$; Onondaga Co (Britcher) ; Otto, Sep; Penn Yan, Sep ; Ithaca, May, Aug; Caroline Depot, Ap; Freeville, Sep; Slaterville, May; McLean, May; Deruyter L., J1; Dormansville, Je; Thompson L., Je; Valcour I., Aug; Spruce Mountain, L. George, Sep, Leon; Elizabeth I., J1-Aug; Vrooman's Nose, May ; Catskill, Aug, Chad; Spooky Hollow, Greene Co, Chad; Stamford, Oct; Milton, Ap; L. Charlotte, Je; Wappingers Falls, May; L. Katrine, Oct; Paradise, May; Pinekill, May.

L. gulosa Walck. Wilmington, Aug; Upper Jay, Sep, Plm; Gloversville, Sep, Alex; Rock City, Sep; Ithaca; McLean, May; Rensselaer, Mar, Je, Oct, Schoon; Silver Bay, Sep, Leon; Elizabeth I., Aug; LI.

L. helluo Walck. Upper Jay, Sep, Plm; Gloversville, Aug, Alex; Onondaga Co (Britcher); Clyde, Aug; Rochester, Franck; Tyrone, Sep; Oak Ridge, Canandaigua L., Aug; Glenwood, Aug, Leon; Taughannock Falls, J1; Ithaca, Jan, Ap-Aug, Dec; McLean, Ap-May, J1; W. Winfield, Je; Cranberry Cr., Aug; Kenwood, May; W. Albany, Je; Voorheesville, Ap, Je; Slingerlands; J1; Dormansville, Je ; Featherstone L., Schenectady Co, J1; Meadowdale, Ap; Menands, Je, Peck; E. Berne, Je; Rensselaer, Jan, Ap-J1, Sep-Oct, Schoon; Pike Pond, Rensselaer Co, May-Je; Tackawasick Pond, Je; Schodack, Ap; Riders Mills, May; Juanita I., J1; Elizabeth I., J1; Wappingers Falls, May; Saugerties, Aug; Cornwall, May, Wlf; Goshen, May; Paradise, May; Hancock, May; SI: Watchogue, Sep ; LI: Montauk Pt., May.

L. mordax Walck. NY (Walckenaer, Marx).

L. nidifex Marx. Albany (Em) ; Sand Plains, near Albany, May; SI: Tottenville, Je; LI: Great Pond, Riverhead, May; Bridgehampton, Aug; Long Pond, Suffolk Co, Je.

L. pikei Marx. Rochester (McCook); Albany (Em); SI: Tottenville, Je-J1; LI: Bayville, Aug-Sep, Bks; Shinnecock Hills (Marx); Bridgehampton, Aug; Montauk Pt., May; Baiting Hollow, May.

L. pratensis Em. Wilmington, Aug; Mt. Whiteface, Aug; Axton, MacG, Houghton; Plattsburg, Sep, Plm; Sacandaga Pk., J1, Alex; Baldwinsville (Bks); Clyde, Ap; Rochester; Charlotte, Sep, Plm; Barcelona, Sep; Olcott, Ap; Ithaca, Mar-May; McLean, May; Caroline Depot, Ap; Valcour I., Aug; Juanita I., J1; Pearl Pt., J1 ; Bumps Pond, J1; Albany, Ap, Schoon ; Dormansville, Je; Medusa, Ap; Rensselaer, Ap, Oct, Schoon; E. Schodack, Sep, Yg; Wappingers Falls, May; Hudson, May ; Alsen, May; Copake Falls, Ap, Chap; Stamford, May, Chrisp; Tuxedo, Oct, Wlf; LI: Baiting Hollow, Ap.

L. punctulata Htz. LI, Oct (Bks).

L. rabida Walck. Ithaca (Bks); LI, Sep (Bks). 


\section{PARDOSA C. Koch}

P. atlantica Em. LI: Fire I. (Em).

P. banski Chamb. LI: Mill Neck, Je (Bks) ; Sheepshead Bay, Aug; Gardiners I.. May.

P. distincta Blackw. Mt. Whiteface, Aug; Wilmington, Aug; Mt. Marcy, J1-Aug; L. Tear, J1; N. Elba, J1.

P. emertoni Chamb. Keeseville, Je; Wells, J1, $\mathrm{g}$; Speculator, J1, $\mathrm{Yg}$; Woodworth's L., J1, Alex; Onondaga Co (Britcher); Ithaca, Aug-Sep; McLean, Ap; Deruyter L., J1; Juanita I., J1; Pear1 Pt., Aug; Black Mt. Pt., J1; Dormansville, Je.

P. lapidicina Em. Axton, MacG, Houghton; Onondaga Co (Britcher) ; Lake Bluff, Sep; Sodus Pt., Sep; East Bay, Sep; N. Fair Haven, J1; Crocketts, Sep; Rochester, Franck; Bluff Pt., Sep; Egleston's Glen, Je; Rye Pt., Sep; Elmira, J1; Oak Ridge, Canandaigua L., Aug; Montour Falls, Sep; Cayuta L., J1 ; Enfield Glen, Oct; Ithaca, May-J1, Aug, Oct; Caroline Center, May; Valcour I., Aug; Juanita I., J1 ; Pearl Pt., J1; Kenwood, May ; Wells, Je, Babiy ; Dormansville, Je ; Rensselaer, May; Schodack, Je, $W$ lf; Pike Pond, Rensselaer Co, Je; Tackawasick Pond, Je; LI: Gardiners I., May.

P. mackenziana Key. Mt. Whiteface, Aug; Uphill Br., Opalescent R., J1; Wilmington, Aug; Crystal L., J1; Burden L., Aug, Schoon; Catskills, Aug, Schoon.

P. milvina Htz. Uphill Br., Opalescent R., J1; Sacandaga Pk., Je, Alex; Gloversville, Aug, Alex; N. Rose, Je; Clyde, Aug; Lake Bluff, Sep; East Bay, Sep; Rochester, Franck; Irving, Sep, Chad; Naples, Aug; Tyrone, Sep; Ithaca, May-Je, Oct; Alsen, Sep; Vrooman's Nose, May; Schenectady, J1; Ravena; Albany, Je; Slingerlands, Mar, Wlf; Rensselaer, May, J1, Schoon; Mill Cr., May, Plm; Vail Gate, May; Ashokan, Je; Pine I., May; Dunwoodie, May; SI, J1; LI: Sheepshead Bay, J1.

P. modica Blackw. Baldwinsville (Em) ; Clyde, May; Ithaca (Bks) ; Little Utica, Je; Albany, Ap; New Salem, Ap. LI: Mill Neck (Bks).

P. modica var. brunnea Em. Waterford, Ap, Chad.

P. moesta Bks. Mt. Whiteface, Aug; L. Tear, J1; Keene Val., Sep; Newcomb, Oct; Saratoga, Je; Rochester, Franck; Cinnamon L. ; Ithaca, May; McLean, Je-Jl; Penn Yan; Wemple, May; Voorheesville, Je; Hoversville, Je ; Featherstone L., Schenectady Co, J1; Rensselaer, Ap-Je, Schoon; Tackawasick Pond, Je; Valley Falls, May, Yg; Wappingers Falls, Je; Stamford, Je, Aug, Chrisp; Mt. Utsayantha, May; Dunwoodie, May; LI.

P. muscicola Em. Mt. Whiteface, Aug (Em); Mt. Marcy, J1; Mt. McIntyre, J1.

P. saxatilis Htz. L. Tear, J1; Newcomb, J1; Wells, J1, Yg; Gloversville, Aug-Sep, Alex; Onondaga Co (Britcher) ; Loucks Pond, J1; L. Keuka; Cinnamon L., Je-J1; Ithaca (Bks) ; Vrooman's Nose, May; Voorheesville, Je; Scotia, MayJe, Yg; Dormansville, Je; Myosotis L., Albany Co., J1; Rensselaer, May-J1, Schoon; E. Schodack, Je, Chad; Riders Mills, May; Black Mountain Pt., J1; Wappingers Falls, May; Red Hook, Je; Goshen, May; Paradise, May; Dunwoodie, May; LI (Bks).

P. xerampelina Key. Wilmington, Aug; Avalanche L., J1; Keene Val., Sep; Chapel Pond, Je; Paul Smiths, J1, Plm; Raquette Falls, Aug; Cranberry Cr., AugSep; Gloversville, Aug, Alex; Howard, J1; Penn Yan, May ; Egleston's Glen, Je; Elmira, J1; Ithaca, May; McLean, May, J1; Freeville, Ap, Aug ; Deruyter L., Jl; Delhi, May; Albany, Je; Shakers, May; Voorheesville, MayJe; Thacher Pk., May; Dormansville, Je; Clarksville, May; Mud Hollow Pond, Je; Rensselaer, Mar-J1, Schoon; Tackawasick Pond, Je; Pike Pond, 
Rensselaer Co, Je; E. Schodack, Je, Chad; Queechy L., May, Schoon; Nassau, Je, Schoon; Riders Mills, May, Chrisp; Pearl Pt., Aug; Sleeping Beauty Mt., J1; Cossayuna L., May, Schoon; Wappingers Falls, May; Maratanza L., May; Hunter, Je; Little Pond, Orange Co, May; Stamford, Je.

\section{Pirata Sundevall}

P. arenicola Em. Cranberry Cr., Aug; Schenectady, Je.

P. aspirans Chamb. Ithaca (Bks); Voorheesville, Je.

P. humicola Mont. NY (Petrunk).

P. insularis Em. Long L. (Em); Axton, MacG, Houghton; Cranberry L. (Barrows); Paul Smiths, J1, Plm; Saratoga, Je; Onondaga Co (Britcher) ; Sodus Bay, Sep; Lake Bluff, Sep; Lakeside Pk., Orleans Co, J1; Hemlock L., Aug; Loucks Pond, J1; Cayuta L., J1; Cinnamon L., J1; W. Danby, J1; Spencer, Je, Aug; Ithaca, Je-Aug; Ringwood, Oct; Freeville, Ap, Aug; McLean, May; Venice Center, Je; Juanita I., J1; Kenwood, Je; Dormansville, Je; E. Nassau, Je, Wlf; Pike Pond, Rensselaer Co, J1; Tackawasick Pond, Je; LI: Riverhead, Aug.

P. marxi Stone. Saratoga, Je; Gloversville, Aug, Alex; Ithaca. (Bks) ; Cinnamon L., Je-J1; Central Nassau, J1; LI: Flushing, Nov.

P. minutus Em. Mt. Whiteface, Aug; L. Tear, J1; Cranberry Cr., Aug ; Gloversville, Sep, Alex; Barneveld, J1; Tyrone, Sep ; Cinnamon L., J1; Taughannock Falls, J1; Ithaca, Aug; McLean, Je; Slaterville, May; Irving, Sep, Chad; Crystal L., J1; Rensselaerville, J1; Dormansville, Je; Rensselaer, J1, Schoon; Central Nassau, J1; Red Hook, Je; Pike Pond, Rensselaer Co, Je; Mt. Lebanon, May, Chrisp; Mt. Utsayantha, May, Chrisp.

P. montanus Em. Long L. (Chamb); Mt. Marcy, Sep; Gloversville, Sep, Alex; Lake Bluff, Sep; Interlaken, J1; Connecticut Hill, Aug; Rye Pt., Sep; Watkins, May; Ithaca, May-Je; Labrador Pond, Je; Kenwood, May-Je; Thacher Pk., May-Je; Pearl Pt., J1; Oakland Val., May; Paradise, May; Little Pond, Orange Co, May.

P. wacondana Scheffer. Upper Jay, Sep, PIm; Beaver R., J1; Hemlock L., Aug; Cinnamon L., J1; Spencer, Aug; Ithaca, Je; Kenwood, Je; Rensselaerville, J1; Tackawasick Pond, Je; Riders Mills, May.

\section{Schizocosa Chamberlin}

S. bilineata Em. Stuyvesant Falls, Je; Rensselaer, May, Schoon; Schodack, Ap; Vrooman's Nose, May; LI: Sea Cliff (Bks); Harbor Hill, J1 (Bks) ; Long Pond, Suffolk Co, Je.

S. crassipalpis Em. North Cr., Je, Plm; Genoa, Je; Spencer, Je; McLean, Ap; Schenectady, Je.

S. crassipes Walck. North Cr., Je, Plm; Gloversville, Ap, Alex; Ithaca, Aug-Oct (Bks) ; McLean, Ap; Schenectady, Je; Albany, Je; Rensselaer, Je ; LI: Cold Sp. Harb. (Em).

S. saltatrix Htz. Gloversville, Je, Alex; Penn Yan, May; Ithaca (Bks) ; McLean, May; Egleston's Glen, Je; Vrooman's Nose, May; Kenwood, May; Thacher Pk., Je; Voorheesville, Je; Meadowdale, Ap-May; Rensselaer, Ap, Schoon; Schodack, Ap; Alsen, May, Chad; Oakland Val., May; LI: Sheepshead Bay, Sep. 
TRABAEA Simon

T. aurantiaca Em. Onondaga Co (Britcher) ; McLean, May-J1; Schodack, Je; LI: Baiting Hollow, May; Long Pond, Suffolk Co, Je.

\section{FAMILY OXYOPIDAE}

OXYOPES Latreille

O. salticus Htz. LI, Je (Bks, Em).

O. scalaris Htz. LI, Oct (Bks).

\section{FAMILy SALTICIDAE}

Admestina Peckham

A. tibialis C. Koch. Ithaca (Bks); Elizabeth I.; LI (Bks).

AGASSA Simon

A. cyanea Htz. Ithaca (Bks); LI, Oct (Bks).

\section{Ballus C. Koch}

B. youngi Peckham. LI, Aug (Bks).

\section{EVARCHA Simon}

E. hoyi Peckham. Wilmington, Aug; Axton, MacG, Houghton; Cranberry L., (Barrows); Saranac L., Aug; Speculator, Jl, $Y g$; Wells, Jl, $Y g$; Sacandaga Pk., Je, Alex; Johnstown, Aug, Alex; Gloversville, J1, Alex; Cranberry Cr., Sep; Barneveld, J1, Wolcott; Onondaga Co (Britcher); Lake Bluff, Sep; Ithaca, Aug; Valcour I., Aug; Juanita I., Aug; Thacher Pk., Je; LI.

\section{EVOPHRYS C. Koch}

E. diminuta Bks. LI: Bayville, Je (Bks).

\section{Fuentes Peckham}

F. lineata C. Koch. Onondaga Co (Britcher); L. Charlotte, Je; LI (Bks).

\section{HABRoCESTUM Simon}

H. parvulum Bks. L. Keuka, Sep; Ithaca (Bks, Peckham); Coy Glen, Ithaca (Bks).

H. pulex Htz. Gloversville, Aug, Alcx; Onondaga Co (Britcher); Clyde, Ap; Crocketts, Sep; Lake Bluff, Sep; Rochester, Franck; Olcott, Ap, Sep; Silver Cr., Sep; Spring Brook, Sep; Richburg, Sep; Clarksburg, Sep; L. Keuka, May-Je; Ithaca, Mar, May, Aug; Caroline Center, May; Valcour I., Aug; Juanita I., Jl-Aug; L. George, Aug; Dormansville, Je ; Voorheesville, Je ; Poughkeepsie, vanIngen; LI, Sep (Bks). 
Hyctia Simon

H. bina Htz. Long Branch (Britcher); Ithaca (Bks).

H. pikei Peckham. SI, Nov; LI: Sea Cliff, J1 (Bks); Bayville (Bks).

ICIUS Simon

I. formicarius Em. McLean, May; LI: Montauk Pt., May.

I. hartii Em. Johnstown, J1, Alex; Sport I., Sacandaga R., Je, Alex; Ithaca (Bks); Columbia Co; LI, Aug (Bks).

I. similis Bks. Paul Smiths, Je, Babiy; McLean, May-Je.

MAEVIA C. Koch

M. vittata Htz. Onondaga Co (Britcher); Ithaca, May, Sep, Nov; LI (Bks).

MARPISSA C. Koch

M. undata DeGeer. Onondaga Co (Britcher); Clyde, Aug; Rochester, Franck; Glenwood, Aug, Leon; L. Keuka, Sep; Ithaca, Aug-Nov; LI, J1 (Bks).

Metacy Rba Cambridge

M. taeniola Htz. NY (Peckham).

\section{Metaphidippus Cambridge}

M. capitatus Htz. Axton, MacG, Houghton; Wells, May, Yg; Cranberry L. (Barrows) ; Mountain L., Je, Alex; Woodworth's L., May, Alex; Sport I., Sacandaga R., Je, Alex; Gloversville, May, Alex; Johnstown, Alex; Onondaga Co (Britcher) ; Enfield Glen, Oct; Ithaca, May, Oct; Freeville, Ap; McLean, May; Schenectady, J1; Albany, May-Je; Rensselaer, Sep, Schoon; Tackawasick Pond, Je; Central Nassau, J1; Juanita I., J1; Sam's Pt., May; Paradise, May; Oakland Val., May; LI: Sea Cliff, J1 (Bks); Sheepshead Bay, J1.

M. flavipedes Peck. Wilmington, Aug; Uphill Br., Opalescent R., J1; Mt. Whiteface, Aug; Ithaca (Bks); Wells, Je, Babiy; Valcour I., Aug; Juanita I., J1; Tackawasick Pond, Je; Wappingers Falls, May; Paradise, May; Little Pond, Orange Co, May; Sam's Pt., May; Oakland Val., May.

M. flavus Peck. Ithaca (Bks).

MYRMARACHNE MacLeay

M. albocinctus C. Koch. NY (Peck, Marx, Bks); LI (Bks).

\section{NEON Simon}

N. nellii Peck. Wilmington, Aug; Wilmington Notch, Aug; Uphill Br., Opalescent R., J1; Onondaga Co (Britcher) ; Lake Bluff, Sep; Olcott, Sep; Richburg, Sep; Letchworth Pk., J1; Alabama, Je; Connecticut Hill, Oct; Ithaca, Mar, May-Aug; Freeville, Aug-Sep; Labrador Pond, Je; Trenton Falls, Je: Voorheesville, Aug; Karner, Mar; Schodack, Je; Rensselaer, J1; Valcour I.: 
Aug; Juanita I., J1; Little Pond, Orange Co, May; Sam’s Pt., May; Maratanza L., May; Slide Mt., Je; Stamford, May, Chrisp; Oakland Val., May; Sterlington, May; Dunwoodie, May; LI: Garden City, Je, Wlf; Riverhead, May; Baiting Hollow, Ap; Long Pond, Suffolk Co, Je; Montauk Pt., May.

\section{PARAPHIDIPPUS Cambridge}

P. marginatus Walck. Axton, MacG, Houghton; Wilmington Notch, Aug (Em); Wilmington, Aug; Saranac L., Aug; Cranberry L. (Barrows); Barcelona, Sep; Geneva, Nov, Glasgow; Ithaca, Oct-Nov, Bks; Valcour I., Aug; Juanita I., J1; Poughkeepsie, vanIngen; Lagrangeville, May; Oakland Val., May; LI: Sea Cliff, J1 (Bks).

\section{PECKHAMiA Simon}

P. picata Htz. Onondaga Co (Britcher); Ithaca, Ap; Juanita I., Aug; LI, Sep (Bks).

P. scorpionea Htz. LI: J1-Aug (Bks, Peck); Cold Sp. Harb. (Em).

\section{Pellenes Simon}

P. agilis Bks. Ithaca (Bks); LI (Em).

P. borealis Bks. Mountain L., Je, Alex; Lake Bluff, Sep; East Bay, Sep; Ithaca (Bks).

P. coronatus Htz. Axton, MacG, Houghton; Ithaca (Bks); LI (Bks, Em).

P. decorus Blackw. Saranac L., Aug; Sacandaga Pk., Je, Alex; Onondaga Co (Britcher) ; Ithaca, Aug-Sep (Bks); Albany (Peck); Clarksville (Em).

P. longimanus Em. LI: Sea Cliff (Bks).

P. peregrinus Peck. Onondaga Co (Britcher); Ithaca, May, Sep; LI (Bks).

P. roseus Htz. Taughannock Falls, May (Em).

P. viridipes Htz. Vrooman's Nose, May; Wappingers Falls, May.

\section{Phidippus C. Koch}

P. audax Htz. Sacandaga Pk., Je, Alex; Onondaga Co (Britcher) ; Clyde, Ap; Lake Bluff, Sep; Rochester, May, Nov; Crosby, May; Ithaca, Aug; Albany, Dec; Greenville, Ap; Poughkeepsie, vanIngen; Wappingers Falls, May; Middletown, May; NYC, Sep, Hoffman; LI: Sea Cliff, Je (Bks) ; Gardiners I., May.

P. brunneus Em. Scotia, Je, Yg; Sacandaga Pk., Je, Alex; Vrooman's Nose, May; Oakland Val., May.

P. cardinalis Htz. Ithaca (Bks); NY (Peck).

P. clarus Key. Wilmington, Aug (Em); Cranberry L. (Barrows) ; Rochester, Franck; Ithaca, Jl-Aug; Deruyter L., Je; Ravena; Scotia, Je, Yg; Schenectady, J1, $Y g$; Black Mountain Pt., Pearl Pt., Aug; SI, J1; LI: Bridgehampton, Aug.

P. insolens Htz. LI (Peck).

P. mccooki Peck. Wilmington, Aug; Rochester, Franck; Elizabeth I., Aug.

P. princeps Peck. Gloversville, Ap, Alex. 
P. purpuratus Key. Mt. Whiteface, Aug (Em); Speculator, J1, Yg; Gloversville, Mar, Alex; Woodworth's L., Je, Alex; Onondaga Co (Britcher) ; Bluff Pt., Sep; Ithaca, Ap, Sep, Nov; Guilderland, May; Greenville, Ap; Vrooman's Nose, May; Poughkeepsie, vanIngen; Wappingers Falls, May.

P. putnami Peck. Ithaca (Bks).

P. variegatus Lucas. LI: Valley Stream, May, Fitch.

P. whitmanni Peck. Onondaga Co (Britcher); Ithaca (Bks); LI: Sea Cliff, J1 (Bks).

\section{Poultonella Peckham}

P. alboimmaculata Peck. NY (Bks, Peck, Marx).

\section{Salticus Latreille}

S. albocinctus Peck. NY (Bks, Peck).

S. scenicus Cl. Cranberry Cr.; Sacandaga Pk., Je, Alex; Gloversville, May, Alex ; Baldwinsville (Em); Clyde, Dec; Lake Bluff, Sep; Rochester, Nov; Wilson; Ithaca, Je, Aug, Nov; Dormansville, Je; Albany, Je; Rensselaer, May-Je, Schoon; Nassau, Je, Schoon; LI: Sea Cliff, Je (Bks) ; Sheepshead Bay, J1.

\section{SIDUSA Peckham}

S. borealis Bks. Ithaca (Bks).

\section{SitTicus Simon}

S. palustris Peck. Wilmington, Aug (Em); Saranac L., Aug (Em) ; Cranberry L. (Barrows) ; L. Tear, J1; Sacandaga Pk., Je-J1, Alex; Onondaga Co (Britcher); Enfield Glen, May; Ithaca, Aug (Bks); Venice Center, May; Freeville, Aug (Bks); Poughkeepsie, vanIngen; LI: Mill Neck, Oct (Bks).

S. striatus Em. Cranberry L. (Barrows); Little Pond, Orange Co, May.

\section{SYNEMOSYNA Hentz}

S. formica Htz. Wilmington, Aug (Em); Indian Reservation, Onondaga Co (Britcher) ; Howland I., Nov ; Penn Yan, May, Aug ; Ithaca, Je, Nov ; Albany, Je, $Y g$; Rensselaer, May, Schoon; Elizabeth I., Aug; Sterlington, May; LI: Riverhead, Ap; Long Pond, Suffolk Co, Je.

\section{TALAVERA Peckham}

T. minuta Bks. LI: Sea Cliff, May (Bks).

\section{Tutelina Simon}

T. elegans Htz. Adirondacks (Em); Onondaga Co (Britcher); Ithaca (Bks); Scotia, Je, $Y g$; Albany, Je-J1; Lagrangeville, J1; NYC (Bks); LI, Aug (Bks).

WALA Keyserling

W. mitrata Htz. Onondaga Co (Britcher); Ithaca, Sep-Oct (Bks); LI, J1 (Bks). W. palmarum Htz. Onondaga Co (Britcher); W. Barre, Sep; Ithaca, Aug (Bks); LI, J1 (Bks). 


\section{ZygoBALLUS Peckham}

Z. bettini Peck. Onondaga Co (Britcher); Manlius; Ithaca, Je; Oakland Val., May; LI, Oct (Bks).

Z. nervosus Peck. Onondaga Co (Britcher); Ithaca (Bks); LI (Bks).

Z. sexpunctatus Htz. NY, Aug (Bks).

\section{ORDER OPILIONES}

Compiled by C. R. Crosby, Augusta Wolf, and S. C. Bishop

\section{SUBORDER PALPATORES}

FAMILY NEMASTOMATIDAE Simon

\section{Crosbycus Roewer}

C. dasycnemus Cros. Egleston's Glen, Sep; Freeville, Oct; LI: Montauk Pt., Je.

\section{FAMILY ISCHYROPSALIDAE Simon}

\section{SABACON Simon}

S. cavicolens Pack. Ithaca, Aug.

\section{Family PHALANGitdaE Simon \\ CADDO Banks}

C. agilis Bks. Olcott, Sep, Dt; Woodwardia Swamp, Aug; Covert, J1, Bdy; Delhi, May; LI: Jamaica, Je, $W l f$; Great Pond, Riverhead, May; Montauk Pt., May.

C. boopis Cros. W. Barre, Sep; Ithaca, Aug; Ballston L., Aug, Wlf; Voorheesville, Aug.

\section{Mitopus Thorell}

M. morio Fabr. Mt. Whiteface, Aug; Artist's Br., Je; Mt. Marcy, J1, Sep; Uphill Br., Opalescent R., J1; Mt. McIntyre, J1; Avalanche L., J1.

\section{OdIELlUS Roewer}

O. pictus Wood. Mt. Whiteface, Aug; Marble Mt., Aug; Mt. McIntyre, Je-J1; Mt. Marcy, Sep; Wilmington, Aug ; Wilmington Notch, Aug ; Upper Jay, Sep ; Saranac L., Sep, Plm; Plattsburg, Sep, Plm; Elizabethtown, Aug; Valcour I., Aug; Raquette L., Sep, Plm; L. Placid, Sep, Plm; Chapel Pond, Je-J1; Watertown, Sep, Plm; Brownsville, Sep, Plm; Pinnacle Mt., Aug; Woodworth's L., Aug, Alex; Big Moose, Aug; Little Falls, J1; Lake Bluff, Sep; Charlotte, Sep, Plm; Glen Haven, Sep, Plm; Lewiston, Sep, Plm; Silver Cr., Sep; Spring Brook, Sep; Barcelona, Sep; Olcott, Sep; Stow, Sep; Rock City, Aug-Sep; Richburg, Sep; Wellsville, Sep; Hornell, Sep; Painted Post, Sep; Hemlock L., Aug; Crosby, Sep-Oct; Watkins, Oct; Montour Falls, Sep ; Covert, Aug; Interlaken, Nov; Taughannock Falls, Aug, Oct; Connecticut Hill, Aug; Enfield, Aug; McLean, J1; Kenwood, Oct; Thompson L., Je; Voorheesville, Je-J1; Pearl Pt., J1; Rensselaer, Aug ; Nassau L., Sep, Wlf. 


\section{PhaLANGIU M Linnaeus}

P. opilio L. Malone, Oct; Plattsburg, Sep; Norwood, Oct; Watertown, Sep, Plm; Evans Mills, Oct; Syracuse, Sep; Lake Bluff, Aug-Sep; East Bay, Sep; Rochester, Sep-Oct; Charlotte, Sep, Plm; Carlton, J1; Newfane, Oct; Lockport, Aug; Lewiston, Sep, Plm; Hamburg, Sep; Silver Cr., Je, Sep, Leon; Spring Brook, Sep; W. Barre, Sep; Holley, Oct; Hornell, Sep; Honeoye Falls, J1, Sep, Nov; Geneva, Nov; Stanley, Sep; Penn Yan, Je, Nov; Crosby, Sep; Cinnamon L., J1; Ithaca, Sep-Nov; McLean, Sep; Canastota; Trenton Falls, Je; Watervliet, Aug; Albany, Aug; Normansville, J1; Myosotis L., Albany Co, J1; Rensselaer, J1, Nov, Schoon; Dormansville, Je; Elizabeth I., J1; Kinderhook, Sep; LI: Manhasset, Je; Jamaica, Sep.

\section{OPILIO Herbst}

O. parietinus DeG. Plattsburg, Sep, Plm; Wilmurt, Aug, Plm; Indian L., Aug, Schoon; Otto, Sep; Keuka L., Aug; Ithaca, Aug, Oct-Nov; LI (Bks).

\section{LEIOBUNUM C. Koch}

L. calcar Wood. Wilmington Notch, J1-Aug; Chapel Pond, J1; Big Moose, Aug, Plm; Woodworth's L., Je, Alex; Letchworth Pk., J1; Factory Hollow, Ontario Co, Aug; Taughannock Falls, J1-Aug; Cinnamon L., J1; Enfield, J1, $W l f$; Ithaca, Je-Aug; Spencer, Aug; Woodwardia Swamp, Aug; McLean; J1; Slaterville, Aug; Ringwood, J1; Deruyter L., J1; Trenton Falls, Je ; Little Falls, J1; Ballston L., Aug, Wlf; High Mills Gorge, Aug, Wlf; Lawson L., Je; Black Mt., Washington Co, Aug; Hague, Aug, Leon.

L. formosum Wood. LI: Sea Cliff (Bks).

L. longipes Weed. Plattsburg, Sep, Plm; Uphill Br., Opalescent R., Aug; Wilmington, Aug; L. Placid, Sep, Plm; Chapel Pond, J1; Upper Jay, Sep, Plm; Saranac L., Sep, Plm; Woodworth's L., Aug; E. Onondaga, Sep ; Cayuga, Aug; Barcelona, Sep; Silver Cr., Sep; Stow, Sep; Rock City, Sep; Richburg, Sep; Wellsville, Sep; Hornell, Sep; Montour Falls, Sep; Elmira, Aug; Enfield Center, May, Rea; Ithaca, Jan, Aug, Dec; Caroline, May; McLean, ApMay; Ballston L., Aug, Wlf; High Mills Gorge, Aug, Wlf; Albany, Aug, Leon; Thacher Pk., May; Voorheesville, Je-J1; Thompson L., Sep; Normansville, J1; Kenwood, J1-Aug ; Delmar, Aug; Watervliet, Aug ; Valcour I., Aug ; Hague, Sep, Leon; Pearl Pt., J1; Black Mt., Washington Co, Aug ; Catskill, J1, Plm; Rhinebeck, J1; Highland, Aug, $W l f$.

L. nigropalpi Wood. Wilmington, Aug; Hemlock L., Aug; Elmira, J1; Taughannock Falls, Aug; Enfield, J1, Wlf, Taub; Ithaca, J1-Aug; Naples, J1; L. Keuka, Sep; Ballston L., Aug, Wlf; Albany, J1; Kenwood, J1; Valcour I., Aug; LI: Jamaica, Je, Wlf.

L. politum Weed. Mt. Whiteface, Aug; Ausable Chasm, Sep, Leon; Wilmurt, Aug; Woodworth's L., Aug ; Clyde, J1; Penn Yan, Aug ; Naples, J1; Montour Falls, Sep; Taughannock Falls, J1-Aug; Spencer, Aug; Ithaca, J1-Sep ; Woodwardia Swamp, Aug; High Mills Gorge, Aug, Wlf; Ballston L., Aug, Wlf; Albany, J1; Voorheesville, J1; Kenwood, Aug, Plm; Rensselaer, J1; Nassau L., Sep, Wlf; Watervliet Reservoir, Aug, Leon; Valcour I., Aug; Hague, Aug, Leon; Pearl Pt., J1; Black Mt., Washington Co., Aug ; Rhinebeck, J1; Hunter, Aug; Van Cort. Pk., Sep, Plm; LI: Forest Hills, Sep, Plm; Amityville, Aug, Plm; Riverhead, Sep.

L. serratipalpe Roewer. Long L., Cold R. (Roewer). 
L. speciosum Bks. LI: Sound Avenue, Suffolk Co, Aug, Lath; Peconic, Sep, Lath; Orient, Sep, Lath.

L. ventricosum Wood. Adirondack Lodge, Je-J1; Chapel Pond, Je-J1; Indian L., Je ; Upper Jay, Sep ; Johnstown, Je; Old Forge, J1 ; Clyde, J1; Howland I., Nov; Otto, Jan, Aug, Comstock; Rock City, Je ; Portage, Je ; Letchworth Pk., J1; Oak Orchard Swamp, Je; L. Keuka, Je; Cinnamon L., Je; Taughannock Falls, J1-Aug, Comstock; Enfield, J1, Wlf; Ithaca, May, J1-Sep; McLean, J1; Slaterville, Aug; W. Winfield, Je; Thacher Pk., May; Voorheesville, Je-J1; Normansville, Nov; Kenwood, May, J1, Plm; Karner, Mar, Aug; Rensselaer, May, Plm; E. Greenbush, Je ; Central Nassau, J1; Schodack Landing, Je ; Red Hook, Je; Sleeping Beauty Mt., J1; Queechy L., May, Schoon; Riders Mills, May, Chrisp; Hunter, Je, Plm; Hunter Mt., Je, Plm; Goshen, May; Big Indian, Je; Tompkins Corners, J1, Wlf; Mosholu; LI: Cold Sp. Harb., J1, Plm; Riverhead, Sep; Sanford Club, Je; Gardiners I., May.

L. verrucosum Wood. Rensselaer, Aug, Schoon; Orangeburg, Je, Giles von Krockow; SI: Watchogue, J1, Barnes; LI: Ronkonkoma, Sep; Yaphank, J1, von Krockow; Riverhead, Aug.

L. vittatum Say. Ausable Chasm, Sep, Leon; Wilmington, Aug; Upper Jay, Sep; Johnstown, Aug, Alex; Gloversville, Ap, Alex; Sacandaga Pk., Aug ; Woodworth's L., Aug; Mountain L., Sep; Brownsville, Sep, Plm; E. Onondaga, Sep, Plm; Lake Bluff, Sep; Clyde, J1-Aug; Charlotte, Sep, Plm; Glen Haven, Sep, Plm; L. Erie Beach, Chautauqua Co, Je; Silver Cr., Sep; Richburg, Sep; Wellsville, Sep; Hornell, Sep; Hemlock L., Aug; Naples, J1-Aug; Montour Falls, Sep; Enfield, J1, Taub ; Ithaca, J1, Aug-Sep ; High Mills Gorge, Aug, Wlf; Ballston L., Aug, Wlf; Albany, J1-Aug; Voorheesville, J1, Leon; Normansville, J1; Plattsburg, Sep; Valcour I., Aug; Pearl Pt., J1; Shelving Rock Mt., J1; Spruce Mt., Sep, Leon; Elizabeth I., J1; Juanita I., J1; Hague, Aug-Sep, Leon; Watervliet Reservoir, Aug, Leon; Nassau L., Sep, Wlf; Rensselaer, Aug, Plm; Kinderhook L., Aug, Schoon; Saugerties, Aug ; Turner, Aug ; Kingston, Sep; Highland, Aug, Wlf; Fishkill, Sep, Plm; Craryville, Aug, Wlf; Middletown, Aug; Hunter, Aug; Van Cort. Pk., Sep, Plm; LI: North Beach, Queens Co, J1; Forest Hills, Sep, Plm; Amityville, Aug, Plm; Riverhead, Sep.

\section{HADROBUNUS Banks}

H. grandis Say. Otto, Aug; Taughannock Falls, J1; Cinnamon L., Je; Covert, Aug; Enfield, J1, Taub; Ithaca, May-Sep; Slaterville, Aug ; Cranberry Cr., Aug; Voorheesville, J1; Ancram, Je; L. Charlotte, Je; Saugerties, Aug; Poughkeepsie, vanIngen; LI: Long Pond, Suffolk Co., Je; Shinnecock Hills, Je.

\section{ORDER ACARINA FAMILY ERIOPHYIDAE 186}

\section{Compiled by H. E. Hodgkiss}

The Eriophyidae, or gall mites, are widespread as regards distribution within New York. Although this has been recognized for some years and attempts have been made to classify the species, there is very little

180 Of the eriophyid galls occurring within New York State, there are many the identity of whose causal agents has not been disclosed through the medium of published references to the species. Such galls have been found on the following genera of plants: Artemisia, Betula, Bromus, Celtis, Crataegus, Fagus, Nyssa, Sambucus, Sorbus, Verbena, Viburnum, and Vitis. It is not improbable that many of the mites, when identified, will prove to be of European origin, although in this mass of material there must be a wealth of undescribed forms. 
information as to the mites which cause the various gall formations. There are also a number of free-living forms, which apparently do not make galls but are associated with the gall-forming individuals.

Chadwick's list (1908) is a very important contribution to the knowledge of the mite galls, but it is probable that many of these structures were described originally from material collected at different periods of development and are the work of the same species of mite.

Some species, as the mites attacking apple, pear, maple, elm, linden, beech, willow, and the wild and cultivated stone fruits, are found wherever these plants are growing. In most instances serious damage is caused by the deforming of the leaves, or by the dropping of leaves and deforming of fruits.

The apple and pear mites are a menace in orchards of these fruits. Eriophyes phloeocoptes is of only local importance, but wherever it occurs it deforms the fruit spurs. Phyllocoptes cornutus is responsible for "silver leaf" on peach, plum, and apricot. The species was very abundant during one season at Geneva, but no serious harm to trees or fruit was noted.

\section{SUBFAMILY ERIOPHYINAE}

\section{ERIOPHYES Siebold}

E. abnormis Garm. Geneva. On Tilia americana.

E. avellanae Nal. Nassau; Albany. On Corylus americana.

E. brevitarsus Fock. "NY" (?), reference in Chadwick (N. Y. State Mus., Bul. 12, p. 124, 1907). On Alnus glutinosa.

E. caulus Cook. Irving. On Juglans nigra.

E. confusus Hodg. Geneva. On Acer saccharinum.

E. elongatus Hodg. Geneva. On Acer saccharum.

E. fraxiniflora Felt. Albany; LI: Brooklyn. On Fraxinus americana.

E. fraxini Garm. Geneva; Poughkeepsie; NYC. On Fraxinus americana.

E. malifoliae Parr. Geneva. On Pyrus malus.

E. maculatus Hodg. Geneva. On Acer saccharum.

E. marginatus Conn. Albany. On Salix alba.

E. major Hodg. Geneva. On Acer rubrum.

E. modestus Hodg. Bug L., Adirondacks. On Acer saccharum.

E. negundi Hodg. Geneva. On Acer negundo.

E. ornatus Hodg. Geneva. On Acer rubrum.

E. padi var. Nal. Blackhead Mt., Catskills; Poughkeepsie. On Prunus serotina.

E. parallelus Hodg. Seventh L., Adirondacks. On Acer spicatum.

E. phloeocoptes Nal. Hudson Val. On Prunus domestica.

E. populi Nal. Lebanon Sps. On Populus tremuloides.

E. pruni Schoene. Geneva. On Prumus americana.

E. pruni-crumena Walsh. Geneva. On Prunus americana. 
E. piri Pagnst. Pear Leaf Blister Mite. Generally distributed through the State, and often injurious to apple and pear.

E. piri var. variolata Nal. Geneva. On Pyrus malus.

E. quadrisetus juniperinus Nal. Ithaca. On juniper berries.

E. quadrisetus typicus F. Thom. Ithaca. On juniper berries.

E. querci Gar. NY. On Quercus macrocarpa.

E. regulus Hodg. Geneva; Phelps. On Acer saccharum.

E. rhois Stebbins. "NY". On Rhus toxicodendron.

E. ryderi Bks. "NY". On Acer sacicharum.

E. salicicola Gar. Irving. On Salix amygdaloides.

E. semen Walsh. "NY". On Salix nigra.

E. serotinae Beut. Blackhead Mt., Catskills; Poughkeepsie. On Prunus serotina.

E. thujae Gar. "NY" (Felt). On Thuja occidentalis.

E. ulmi Gar. Geneva; Catskill. On Ulmus americana.

\section{SUBFAMILY PHYLLOCOPTINAE}

\section{Anthocoptes Nalepa}

A. transitionalis Hodg. Geneva. On Acer rubrum.

\section{EPITRIMERUS Nalepa}

E. piri Nal. Geneva. On Pyrus communis.

\section{OXyPleurites Nalepa}

O. dentatus Hodg. Geneva. On Acer platanoides.

O. dentilobus Hodg. Geneva. On Acer saccharum.

O. serratus Nal. Geneva. On Acer platanoides.

\section{Phyllocoptes Nalepa}

P. aceris-crumena Rly. Phelps; Geneva; Seneca Castle. On Acer saccharum and A. saccharinum.

P. brevisetosus Hodg. Geneva. On Acer negundo.

P. constrictus Hodg. Geneva. On Acer saccharum.

P. cornutus Bks. Geneva. On Prunus persica.

P. gymnaspis Nal. Geneva. On Acer platanoides.

P. magnificus Hodg. Geneva. On Acer platanoides.

P. minutissimus Hodg. Geneva. On Acer rubrum.

P. quadripes Shim. "NY”. On Acer saccharinum.

P. quinquilobus Hodg. Geneva. - On Acer rubrum.

P. schlechtendali Nal. "NY". On Pyrus malus and P. communis.

P. splendidus Hodg. Geneva. On Acer negundo.

P. trilobis Hodg. Geneva. On Acer platanoides.

P. variabilis Hodg. Geneva. On Acer saccharum. 


\section{CLASS DIPLOPODA}

Compiled by John W. BaIley

\section{ORDER CHILOGNATHA}

\section{FAMILY ISOBATIDAE}

BLANIULUS Gervais

B. guttatus (Bosc). NYC, Je, 1917.

\section{Nopoiulus Menge}

N. minutus (Brandt). Freeville, Oct, Ithaca, Ap, Bail; West Farms, Sep; Bronx Pk., Sep (Plm).

\section{FAMILY JULIDAE}

\section{BRACHYIULUS Berlese}

B. pusillus (Leach) (virgatus [Wood]). Forbes Manor, Ap (Plm); Vischer Ferry, May.

\section{Diploiulus Berlese}

D. londinensis coeruleocinctus (Wood) (Julus hortensis Wood). Lyons Falls, J1; Linlithgo, Je, Oneida, Je, Brownville, Sep (Plm); Buffalo, Ap, Warren; Ellington, Oct; Jamestown, Ap, Rumple; Montour Falls, Oct, Cy; Stockton, May; Albany; Rensselaer; Ithaca, Mav, J1, Nov, Bail; Danby, Oct, Cy; McLean, May, Babiy; Caroline, Ap, Diven; Freeville, Oct, Schenectady, Oct (Plm) ; Scarsdale, Oct, Larth; Delmar, Ap, Yg; Fishkill, Sep (Plm); LI: Valley Stream, Mar, Weston.

D. luscus (Mein.). West Farms, Sep.

\section{OpHyiulus Berlese}

O. pilosus (Newport). Ithaca, Mar, Bail ; Rensselaer, Fishkill, Van Cort. Pk., Washington Pk., Bronx Pk.; SI: Castleton; LI: Cold Sp. Harb., Mar-Sep; (all Plm).

\section{FAMILY PARAJULIDAE}

\section{PaRAjulus Humbert and Saussure}

P. canadensis (Newport). Upper Jay, L. George, Old Forge, Saranac L., Crown Pt., North Cr., Paul Smiths, Lewiston, Wilmurt, Copeland, E. Onondaga, Chautauqua L., Big Moose, Raquette L., Brownville, L. Placid, Plattsburg, Chateaugay L., Hunter Mt., Watertown, Glen Haven, Thompson L., Kenwood, Rensselaer, Pine Hills, May-Sep (all Plm); McLean, Cy, Sib; Ithaca, Freeville, Crown Hill, Oct, Bail.

P. immaculatus (Wood). Mt. Marble, Mt. Whiteface, Wilmington, Aug, Bail; Kenwood, Oct (Plm).

P. impressus (Say). Upper Jay, Crown Pt., Brownville, Plattsburg, Lewiston, E. Onondaga, Charlotte, Glen Haven, Schenectady, Kenwood, Albany, Poughkeepsie, Fishkill, Linlithgo, May-Oct (all Plm).

P. pennsylvanicus (Brandt). Ithaca, Dt; McLean, Queechy L., E. Onondaga, Glen Haven, Kenwood, Bronx Pk., Van Cort. Pk.; SI: Arrochar, Castleton, MayOct; (all Plm). 


\section{FAMILY SPIROBOLIDAE}

\section{SPIROBOLUS Karsch}

S. marginatus (Say). Chilson L., Sep; Indian Ladder, Je, Crown Pt., Sep, Plattsburg, Sep, Port Douglas, Aug (Plm); Gloversville, Ap, Alex; E. Onondaga, Sep (Plm) ; Ithaca, May, J1, Bail, Dt, Bizzell; Cortland, J1, McLean Bogs, Taughannock, Freeville (Dt); L. George, Aug; Peekskill, Fishkill, Je, Sep (Plm).

\section{FAMILY XYSTODESMIDAE}

\section{FONTARIA Gray}

F. corrugatus (Wood). Upper Jay, Sep, E. Onondaga, Sep, and Howe Cave, Je (Plm) ; Ithaca, May, J1, Dt; McLean, May ; Enfield Gorge, Slaterville, May (Bizzell) ; Coy Glen, Mar; Aqueduct, Je, Peekskill, Je (Plm).

F. trimaculata (Wood). North Cr., Lewiston, Indian Ladder, May-Sep (Plm); Coy Glen, J1, Dt; Gloversville, May, Aug, Alex; Saratoga, Je, Whitlock.

\section{FAMILY STRONGYLOSOMIDAE}

\section{ORTHOMORPHA Bollman}

O. gracilis (Koch). Ithaca, May, J1, Schenih; McLean Bogs, Aug, Sib; Albany, Mar, Brown.

\section{FAMILY POLYDESMIDAE}

\section{Polydesmus Latreille}

P. canadensis (Newport). Wilmington, Aug, Bail; Wilmurt, May (Plm).

P. granulatus Say. Bronx Pk., Sep (Plm).

P. moniliaris (Koch). West Farms, Ap, Oct, Cy.

P. serratus (Say). Big Moose, North Cr., Paul Smiths, Jl-Aug (Plm); Mt. Marion, Ulster Co, Oct, Chadrvick; Wilmurt, E. Onondaga, Aug-Sep (Plm) ; Warner, Jl, Wlf; Gloversville, Sep, Alex; Chautauqua L., Sep (Plm) ; Connecticut Hill, Ithaca, Freeville, Oct, Bail ; L. George, Hunter Mt., Catskill, Kenwood, Van Cort. Pk. (Plm); LI: Cold Sp. Harb., Amityville, Je-Sep (Plm).

P. testi (Bollman). Rouses Pt., Crown Pt., Plattsburg, Old Forge, North Cr., Forbes Manor, Lewiston, Charlotte, Rensselaer, Hunter, Van Cort. Pk. (Plm) ; LI: Amityville, Mar-Nov (Plm).

Scytonotus Koch

S. nodulosus (Koch). E. Onondaga, Glen Haven, Cayuga (Plm); LI: Cold Sp. Harb., J1-Oct (Plm).

S. granulatus (Say). Plattsburg, Kenwood, Aug, Sep (Plm).

\section{FAMILY CRASPEDOSOMIDAE}

\section{TRICHOPETALUM Harger}

T. album (Cook). Glen Haven, Sep (Plm).

T. sp. Hunter, Hunter Mt., Je (Plm). 
Cleidogona Cook and Collins

C. caesioannulata (Wood). Danby, Oct, Cy\&Chap.

\section{FAMILY POLYZONIIDAE}

POLYZONIUM Brandt

P. rosalbum (Cope). Crown Pt., E. Onondaga, Sep (Plm); Danby, McLean, Ap, Oct, Cy\&Chap; Connecticut Hill, Oct, Bail.

\section{FAMILY LYSIOPETALIDAE}

CAllipus Risso

C. lactarius (Say). Ithaca, May, J1, Bail; Taughannock, Aug; Coy Glen, J1, Dt.

\section{FAMILY POLYXENIDAE}

POLYXENUS Latreille

P. fasciculatus (Say). Ithaca, Mar-Ap, Bail, Babiy; Valley Cr. Swamp and Voorheesville, Aug, Bish; Greenville, Ap.

\section{CLASS CHILOPODA}

Compiled by John W. BaILEy

\section{ORDER SCHIZOTARSIA}

FAMILY SCUTIGERIDAE

SCUTIGERA Lamarck

S. forceps Raf. The House Centipede. Ithaca, May, Bail; Albany, Feb, Oct (NYS).

\section{ORDER ANAMORPHA SUBORDER LITHOBIOMORPHA \\ FAMILY ETHOPOLIDAE}

BOTHROPOLYS Wood

B. multidentatus (Newport). Wilmington, Aug, Bail; Upper Jay; Old Forge; Crown Pt.; Indian Head; L. Placid; Raquette L.; Big Moose; Saranac L.; Forbes Manor; North Cr.; Hunter Mt.; Wilmurt; Thousand Isls.; E. Onondaga; Glen Haven; Chautauqua L.; Penn Yan; Ithaca; Caroline; Albany; Karner; Rensselaer; Kenwood; Linlithgo; Fishkill; SI: Castleton; LI: Cold Sp.

\section{FAMILY LITHOBIIDAE}

\section{LITHOBIUS Leach}

L. forficatus (L.). Rouses Pt.; Saranac L., Aug, Bail; Upper Jay; Charlotte; Plattsburg; Crown Pt.; Raquette L.; Lewiston; Forbes Manor; Fishkill; Marion; Old Forge; Gloversville; Syracuse; Niagara; Penn Yan; Hornell; 
Howe Cave; Ithaca, May; Glen Haven; Caroline; L. George; Albany; Kenwood; Altamont; Rensselaer; Cedar Hill; Hunter; Pine Hill; Waterford; Bronx Pk.; Van Cort. Pk.; SI: Arrochar; Silver L.; LI: Bay Shore; Amityville; Oyster Bay; Cold Sp. Harb.

\section{NADABIUS Chamberlin}

N. aristeus (Chamb.). Montour Falls; Ithaca; Freeville, Oct, Bail; LI: Baiting Hollow.

\section{NAMPABIUS Chamberlin}

N. lundii Mein. Upper Jay; Wilmurt, Aug, Plm; Forbes Manor; Chautauqua L. ; NYC (Lund).

N. fungiferopes (Chamb.). Very common throughout the State. Montour Falls, Oct, $C y$; Ithaca, Dec, Chamb; McLean Bogs; Hunter Mt., Je, Plm.

SoNIBIUS Chamberlin

S. parvus (Chamb.). Big Tupper L. (Thomas, FKBarbour).

S. numius (Chamb.). Forbes Manor, May, Plm.

Sozibius Chamberlin

S. providens (Bollman). SI: Castleton, Sep, Plm.

Paitobius Chamberlin

P. exiguus (Mein.). NYC, Lund.

\section{FAMILY HENICOPIDAE}

ZYGETHOBIUS Chamberlin

Z. sp. McLean Bogs, Ap, $C y$.

\section{ORDER EPIMORPHA \\ SUBORDER SCOLOPENDROMORPHA}

FAMILY CRYPTOPIDAE

CRYPTOPS Leach

C. hyalinus (Say). Rensselaer, Sep, Plm; Bronx Pk.; SI: Castleton; LI: Cold Sp. Harb.

\section{OTOCRYPTOPS Haase}

O. sexspinosus (Say). Syracuse; E. Onondaga; Ithaca, May, Bail; Thompson L.; Caroline; Freeville; Glen Haven; Rensselaer; Hunter Mt.; Fishkill ; Bronx Pk.; SI: Castleton; Arrochar; LI: Cold Sp. Harb. 
Geophilidae-Linotaenimae-Schendylidae-Eosentomidae $108 \hat{\jmath}$

\section{SUBORDER GEOPHILOMORPHA}

\section{FAMILY GEOPHILIDAE}

\section{Geophilus Leach}

G. deducens (Chamb.). LI: Sea Cliff, Bks.

G. rubens (Say). Forbes Manor; Wilmurt; Gloversville; Caroline; Ithaca, May, Bail; Kenwood; Rensselaer; Altamont; Fishkill; Bronx Pk.; LI: Bay Shore.

G. mordax (Mein.). LI, Chamb.

\section{ARENOPHILUS Chamberlin}

A. bipuncticeps (Wood). NYC, Oct; LI: Oyster Bay, Ap, Barlow, May, Plm.

\section{PACHYMERIUM Koch}

P. ferrugineum (Koch), E. Greenbush, Je, Plm.

\section{FAMILY LINOTAENIIDAE}

LINOTAENIA Koch

L. fulva (Sager). Forbes Manor; Syracuse; Montour Falls; Ithaca, J1, Bail; Freeville; McLean Bogs; Danby ; Hunter Mt. ; Kenwood ; Rensselaer ; Fishkill; Bronx Pk.; Van Cort. Pk.; SI: Silver L.

L. chionophila (Wood). Rouses Pt.; Saranac L.; Raquette L.; Grass L.; Connecticut Hill, Oct, Bail; McLean Bogs; Ithaca; Schenectady; Kenwood; Van Cort. Pk., Port Washington Pk., Bronx Pk.; SI: Arrochar.

\section{FAMILY SCHENDYLIDAE}

\section{ESCARYUS Cook and Collins}

E. liber (C.\&C.). Kirkville, Ap, $C \mathcal{E} C$.

E. urbicus (Mein.). Syracuse, Jan, $C \& C$; Ithaca, Dec-Mar.

Schendyla Bergsve and Meinert

S. nemorensis (Koch). Clyde, Dec; Hunter Mt.; Ithaca; Kenwood; SI; LI: Baiting Hollow.

\section{CLASS MYRIENTOMATA \\ ORDER PROTURA FAMILY EOSENTOMIDAE}

EOSENTOMON Berlese

E. wheeleri Silvestri. Collected by Silvestri in NY, probably in the vicinity of NYC. 
LIST OF ORDERS, AND NUMBER OF FAMILIES, GENERA, AND SPECIES RECORDED

\begin{tabular}{|c|c|c|c|c|}
\hline Class & Order & Families & Genera & $\begin{array}{l}\text { Species } \\
\text { and varieties }\end{array}$ \\
\hline $\begin{array}{l}\text { Arachnida } \\
\text { Diplopoda } \\
\text { Chilopoda } \\
\text { Myrientomata }\end{array}$ & $\begin{array}{l}\text { Thysanura } \\
\text { Collembola } \\
\text { Orthoptera } \\
\text { Isoptera } \\
\text { Neuroptera } \\
\text { Ephemerida } \\
\text { Odonata } \\
\text { Plecoptera } \\
\text { Corrodentia } \\
\text { Mallophaga } \\
\text { Thysanoptera } \\
\text { Anoplura } \\
\text { Hemiptera } \\
\text { Homoptera } \\
\text { Dermaptera } \\
\text { Coleoptera } \\
\text { Strepsiptera } \\
\text { Mecoptera } \\
\text { Trichoptera } \\
\text { Lepidoptera } \\
\text { Diptera } \\
\text { Siphonaptera } \\
\text { Hymenoptera } \\
\text { Araneae } \\
\text { Opiliones } \\
\text { Acarina (Eriophyidae) } \\
\text { Chilognatha } \\
\text { Schizotarsia } \\
\text { Anamorpha } \\
\text { Epimorpha } \\
\text { Protura }\end{array}$ & $\begin{array}{r}3 \\
3 \\
7 \\
1 \\
11 \\
1 \\
3 \\
4 \\
9 \\
4 \\
4 \\
2 \\
32 \\
10 \\
1 \\
88 \\
1 \\
1 \\
13 \\
59 \\
71 \\
1 \\
58 \\
19 \\
3 \\
1 \\
11 \\
1 \\
3 \\
4 \\
1\end{array}$ & $\begin{array}{r}5 \\
22 \\
62 \\
1 \\
25 \\
24 \\
49 \\
19 \\
18 \\
17 \\
38 \\
8 \\
264 \\
219 \\
4 \\
1,356 \\
1 \\
4 \\
68 \\
800 \\
909 \\
11 \\
649 \\
176 \\
9 \\
5 \\
16 \\
1 \\
8 \\
8 \\
1\end{array}$ & $\begin{array}{r}7 \\
71 \\
136 \\
1 \\
61 \\
61 \\
159 \\
59 \\
36 \\
53 \\
77 \\
11 \\
727 \\
864 \\
4 \\
4,546 \\
2 \\
20 \\
174 \\
2,439 \\
3,615 \\
26 \\
2,300 \\
553 \\
19 \\
52 \\
27 \\
1 \\
10 \\
12 \\
1\end{array}$ \\
\hline - & Grand total & 430 & 4,797 & 16,124 \\
\hline
\end{tabular}




\section{LIST OF THE MORE IMPORTANT COLLECTING STATIONS NOT IN THE UNITED STATES OFFICIAL POSTAL GUIDE}

Adirondack Lodge, Essex Co.

Allegany Park, Cattaraugus Co.

Alps, Rensselaer Co.

Annadale, Richmond Co.

Aqueduct, Queens Co.

Arlington, Richmond Co.

Arrochar, Richmond Co.

Artist's Brook, Essex Co.

Auger Pond, Essex Co.

Ausable Lakes, Essex Co.

Avalanche Lake, Essex Co.

Axtorı, Franklin Co.

Baiting Hollow, Suffolk Co.

Bay Ridge, Kings Co.

Bear Mountain, Rockland Co.

Belden Hill, Broome Co.

Bergen Beach, Kings Co.

Black Brook, Clinton Co.

Black Mountain, Washington Co.

Bleecker, Fulton Co.

Brant Lake, Warren Co.

Brighton Beach, Kings Co.

Bronx Park, Bronx Co.

Bryn Mawr Park, Westchester Co.

Bumps Pond, Washington Co.

Burden Lake, Rensselaer Co.

Canada Lake, Fulton Co.

Canarsie, Kings Co.

Cascade Lakes, Essex Co.

Cedar Hill, Albany Co.

Center, old name for Karner

Center Island, Nassau Co.

Central Nassau, Rensselaer Co.

Chapel Pond, Essex Co.

Charley Lake, Hamilton Co.

Cheektowaga, Erie Co.

Cicero Swamp, Onondaga Co.

Cinnamon Lake, Schuyler Co.

Clinton Heights, Rensselaer Co.

Clove Valley, Richmond Co.

Conesus Lake, Livingston Co.

Connecticut Hill, Tompkins Co.

Cossayuna Lake, Washington Co.

Coy Glen, Tompkins Co.

Crater Club, Essex Co.

Crocketts, Cayuga Co.

Crosby, Yates Co.

Crusoe Lake, Wayne Co.

Crystal Lake, Albany Co.

Cypress Hills, Kings Co.

Danby, Tompkins Co.
Deep Pond, Wading River, Suffolk Co.

Deruyter Lake, Madison Co.

Dryden Lake, Tompkins Co.

Dunwoodie, Westchester Co.

East Caroga Lake, Fulton Co.

Egleston's Glen, Yates Co.

Elizabeth Island, Lake George, Warren Co.

Elm Lake, eastern border of township of Lake Pleasant, Hamilton Co.

Enfield Glen, Tompkins Co.

Fall Creek, Tompkins Co.

Fentons, Lewis Co.

Fire Island, Suffolk Co.

Forest Park, Queens Co.

Fort Wadsworth, Richmond Co.

Fourth Lake, Fulton Chain, Herkimer and Hamilton Cos.

Gardiners Island, Suffolk Co.

Gilman Lake, Hamilton Co.

Guyanoga, Yates Co.

Half Way Hollow Hills, Suffolk Co.

Hebron, Washington Co.

Hemlock Lake, Livingston Co.

High Falls, Essex Co.

Hillside Park, Johnstown, Fulton Co.

Howland Island, Cayuga Co.

Hunter Mt., Greene Co.

Indian Castle, Herkimer Co.

Italy Hill, Yates Co.

Jabe Pond, Warren Co.

Juanita Island, Lake George, Warren Co.

Karner, formerly Center, Albany Co.

Kenwood, Albany Co.

Kissena Park, Queens Co.

Kreischerville, Richmond Co.

Labrador Pond, Onondaga and Cortland Cos.

Lake Charlotte, Columbia Co.

Lake Keuka, Yates Co.

Lake Mahopac, Putnam Co.

Lake Minnewaska, Ulster Co.

Lakeside Park, Orleans Co.

Lake Tear on Mt. Marcy, Essex Co.

Lawson Lake, Albany Co.

Letchworth Park, Wyoming Co.

Little Neck, Queens Co.

Long Branch, Onondaga Co.

Long Pond, Wading River, Suffolk Co.

Loucks Pond, Steuben Co.

Lowerre, Westchester Co.

Ludlow, U1ster Co. 
Madison Barracks, Jefferson Co.

Manhattan Beach, Junction, Kings Co.

Maratanza Lake, Ulster Co.

Melville, Suffolk Co.

Menands, Albany Co.

Michigan Hollow Swamp, Tompkins Co.

Mill Creek, Rensselaer Co.

Monterey, Schuyler Co.

Mooers Junction, Clinton Co.

Mosholu, Van Cortlandt Park, Bronx Co.

Moss Pond, Essex Co.

Mountain Lake, Fulton Co.

Mt. Buell, Fulton Co.

Mt. Colden, Essex Co.

Mt. Dix, Essex Co.

Mt. Haystack, Essex Co.

Mt. Hopkins, Essex Co.

Mt. McIntyre, Essex Co.

Mt. Marcy, Essex Co.

Mt. Redfield, Essex Co.

Mt. Seward, Franklin Co.

Mt. Skylight, Essex Co.

Mt. Utsayantha, Delaware Co.

Mt. Whiteface, Essex Co.

Mud Creek Valley, Cattaraugus Co.

Mud Hollow Pond, Albany Co.

Murray Bay, Essex Co.

Nepperhan, Westchester Co:

New Brighton, Richmond Co.

New Salem, Albany Co.

Newtown, Queens Co.

Nine-corner Lake, Fulton Co.

Nissequogue, Suffolk Co.

Normanskill, Albany Co.

Normansville, Albany Co.

North Beach, Queens Co.

North Lansing, Tompkins Co.

Norton, Tompkins Co.

Norton's Landing, Tompkins Co.; now known as Portland Point.

Oak Orchard, Swamp and Creek, Orleans Co.

Oak Orchard Swamps, near Batavia, Genesee and Orleans Cos.

Oak Ridge, Canandaigua Lake, Yates Co.

Oakwood, Richmond Co.

Old Place, Richmond Co.

Onteora Mt., Greene Co.

Orient Point, Suffolk Co.

Paradise, Orange Co.

Parkers, Chautauqua Co.

Parkers, Lewis Co.

Parkville, Kings Co.

Pearl Point, L. George, Washington Co. Pennequid Barrens, Suffolk Co.
Penny Bridge, Queens Co.

Pike Pond, Rensselaer Co.

Pinekill, Sullivan Co.

Pinnacle Mountain, Fulton Co.

Pittstown, Rensselaer Co.

Point Breeze, Orleans Co.

Portage, Livingston Co.

Potter Swamp, Yates Co.

Promised Land, Suffolk Co.

Queechy Lake, Rensselaer Co.

Ravenswood, Queens Co.

Ringwood, Tompkins Co.

Riverdale, NewYork City.

Rock City, Cattaraugus Co.

Rosedale, Queens Co.

Rye Beach, Westchester Co.

Rye Point, Keuka Lake, Steuben Co.

Sam's Point, Ulster Co.

Sancy Brook, Richmond Co.

Sands Point, Nassau Co.

Sandy Hill, old name for Hudson Falls, Washington Co.

Saranac Inn, Franklin Co.

Scotchtown, Orange Co.

Second Milo, Yates Co.

Shakers, Albany Co.

Shelving Rock, Washington Co.

Shelving Rock Mountain, Washington Co.

Shurgers Gorge, Tompkins Co.

Silver Lake, Richmond Co.

Six Mile Creek, Tompkins Co.

Slaterville, Tompkins Co.

Sleeping Beauty Mt., Washington Co.

Slide Mt., Ulster Co.

Somerset, Niagara Co.

Southwest Oswego, Oswego Co.

Spafford, Onondaga Co.

Spier Falls, Saratoga Co.

Spring Lake, Cayuga Co.

Springs, Suffolk Co., north of Amagansett.

Stanford, Schenectady Co.

Stony Clove, Greene Co.

Stony King, Dutchess Co.

Streetroad, Essex Co.

Sturgeon Point, Lake Erie.

Sunny Side, Chautauqua Co.

Tackawasick Pond, Rensselaer Co.

Tarbel, Tompkins Co.

Taughannock Falls, Tompkins Co.

Thacher Park, Albany Co.

The Vlei, Fulton Co.

Thompson Lake, Albany Co.

Turkey Hill, Tompkins Co.

Unionport, New York Co. 
Uphill Brook and Opalescent River, Essex Co.

Valcour Island, Clinton Co.

Van Cortlandt Park, Bronx Co.

Vandalia, Cattaraugus Co.

Vandenburgs Pond, Fulton Co.

Vermillion, Oswego Co.

Vienna, Oneida Co.

Vrooman's Nose, Schoharie Co.

Waldorf, Albany Co.

Watchogue, Richmond Co.

Waterloo, Orange Co.

Wawbeek, Franklin Co.
West Hills, Suffolk Co.

Westriew, Livingston Co.

White Church, Tompkins Co.

White House, Cattaraugus Co.

White Lake, Onondaga Co.

Willets Point, Queens Co.

Wilmington Notch, Essex Co.

Wilmurt, Herkimer Co.

Windom, Erie Co.

Winfield, Queens Co.

Woodwardia Swamp, near Freeville, Tompkins Co.

Woodworth's Lake, Fulton Co. 


\section{LIST OF COOPERATORS AND AUTHORITIES}

Academy of Natural Sciences, Philadelphia...... ANSP

Aldrich, J. M........... Ald

Alexander, C. P.......... Alex

American Entomological Society.

American Museum of Natural History ........... AM

Anderson, E. G............ And

Angell, J. W. . . . . . . . . Ang

Angle, J. L.............

Angus, James............

Ashmead, Wm. H........ Ashm

Atwood, G. G...........

Babiy, P. P

Back, E. A .

Bailey, John W.......... Bail

Baker, A. C. . . . . . . . . . .

Baker, A. W............ BakerAW

Ball, E. D..............

Banks, Nathan.......... Bks

Barber, H. G. . . . . . . . . . Brb

Barber, H. S............

Bell, E. L. . . . . . . . . .

Benjamin, F. H........ Bj

Bequaert, Joseph......... Beq

Betten, C............... Bet

Beutenmüller, Wm....... Bt

Bird, Henry . . . . . . . . . . .

Bishop, S. C............ Bish

Bissell, T............ Biss

Blackman, M. W......... Blk or B1km

Blackman, M.W., and Stage, H. H................ B\&S

Blaisdell, F. E. ......... Blais

Blatchley, W. S., and Leng, C. W

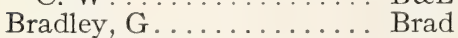

Bradley, J. C........... Bdy

Britcher, H. W..........

Britton, W. E............

Brooklyn Museum......... BM

Brues, C. T

Buchanan, L. L............ Buch

Bueno, J. R. de la Torre-... Bno

Burns, E. J............ Bs

Busck, August.......... Bsk

Buys, John L..........

Calvert, P. P........... Calv

Carnochan, F. G......... Carn

Casey, Thomas L. . . . . . . Csy
Caudell, A. M.......... Caud

Chamberlin, R. V........ Chamb

Champlain, A. B.

Chapman, P. J.......... Chap

Chapman, R. N.........

Chittenden, F. H........ Chitt

Chrisp, H. P...........

Claassen, P. W........... Claas

Clemens, Lucy Smith...... . .

Clemens, W. A........... Clms

Cockerell, T. D. A........ Ck11

Cole, F. R.............

Collins, Donald. . . . . . . . Cis

Comstock, J. H . . . . . . . Comstock or

Coquillett, D. W........ Coq

Cornell University........ CU or

CU Econ

Crawford, David L.

Cwfd or Crawf

Cresson, E. T............ Cress

Cresson, E. T., Jr......... CnJr

Crosby, C. R.......... Cy

Curran, C. Howard........ Curr

Cushman, R. A......... Cush

Davis, G. C

Davis, John June......... JJDs

Davis, W. T............ Ds

Davis, W. T., and Bequaert, Joseph............. D\&B

Dawson, R. W.......... Daw

DeLong, D. M........... DeL

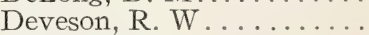

Diamond, A. G.......... Dnd

Dickerson, Edgar L........

Dietrich, Alice Stout (Mrs. Henry Dietrich)........ DtAS

Dietrich, Henry.......... Dt

Dietz, W. J.............

Diven, E. L. . . . . . . . . .

divers, used to indicate several collectors or determiners.............. div.

Dobroscky, Irene.......... . Dobr

Doll, Jacob............. .

Doubleday, Edward...... . Dbl

Dow, R. P. . . . . . . . . .

Drake, C. J ............. Drk or Dke

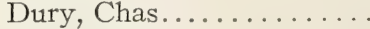

Dyar, H. G............. Dy 
Emerton, J. H. ......... Em

Engelhardt, G. P........ Eng

Everett, Dr. W. H........

Eyer, J. R

Fall, H. C.

Farley, J. M., Jr. . . . . . .

Faure, J. C.............

Felt, E. P............

Felt, E. P., and Young, D. B. F\&Y

Fenyes, A."

Fernald, H. T.......... Fern

Ferris, G. F. . . . . . . . .

Fisher, W. S............

Fitch, Asa.............

Fletcher, F. C.......... Flet

Foerster, J. R........... Foerst or

Folsom, H. J.......... Fols

Forbes, Wm. T. M . ...... Fbs

Fouts, R. M...........

Frison, T. H........... Fris

Frost, C. A............

Frost, S. W............ FrostSW

Funkhouser, W. D....... Funk

Gahan, A. B

Garrett, C. B. D ......... Garr

Giffard, W. M. . ........ WMG

Girault, A. A.......... Gir

Good, Henry . . . . . . . . .

Greene, Chas. T..........

Griswold, Grace $\mathrm{H}$.

Grossbeck, John A.......... Grossb

Grote, A. R.......... Grt

Grote, A. R., and Robinson, C. T.............. G\&R

Hagen, H. A..

Haldeman, S. S. . . . . . . . Hald

Hamilton, C. C. ........... Ham

Hampson, Sir George F . . . Hamp

Harris, E. D............ Harr or

Hartley, E. A ............ Htly

HarrED

Hartzell, F. Z . . . . . . . .

Hasey, W. H. . . . . . . . .

Hatch, M. H. . . . . . . . . H

Hawley, I. M............... Hawl

Hebard, Morgan..........

Heidemann, Otto............ Heid

Heinrich, Carl ........... Hein

Henshaw, Samuel . . ...... Hw

Herrick, Glenn W . ........ Herr

Hill, W. W . . . . . . . . . .

Hine, J. A
Hodgkiss, H. E. . . ...... Hodg

Hoffman, W. A . . . . . . . . . Hoff

Hood, J. D........... Hd

Hopkins, A. D . . . . . . . . . Hopk

Horn, Geo. H.

Houghton, C. O . ........... Hough

Howard, L. O............. How

Hubbell, Theo. H. . . . . . . .

Huckett, H. C. . . . . . . . Huck

Hudson, G. H

Hungerford, H. B . . ........ Hgfd

Hussey, R. F . . . . . . . . . Huss

Hyslop, J. A...........

Johannsen, O. A ........ Joh

Johnson, Chas. W........ Jn

Jones, Frank Morton......

Joutel, Louis A.

Jülich, Wm.............. Julich

Kearfott, W. D......... Kf

Kellicott, D. S. . . . . . . . . Kell

Kircher, Chas.............. Kch

Knight, H. H. . . . . . . . Kngt

Latham, Roy.......... Lath

Lawson, P. B . ............. Laws

Leng, Chas. W............. Lg

Leng, C. W., and Beutenmüller, $W m . . . . . . . . . . L \& B$

Leng, C. W., and Davis, W $T$. L\&D

Leonard, M. D............ Leon

Liebeck, Chas . . . . . . . . . .

Liljeblad, Emil.......... Lilj

Linell, M. L. . . . . . . . . .

Lintner, J. A . . . . . . . . Lint

Lloyd, J T...............

Lutz, F. E.............

McAtee, W. L...........

MacGillivray, A. D....... MacG

MacGillivray, A. D., and Houghton, C. O......... M\&H

Malloch, J. R............. Mall

Martin, J. O............

Mason, P. W ...........

Matausch, Ignatz......... Mtch

Matheson, R.......... Mat

Melander, A. L . . . . . . . . Mel

Mickel, C. E............

Mitchell, Evelyn G........ EGM

Mix, A. J. ...............

Mix, A. J., and Everett, W. H. ............... M\&E

Moore, R. M ............ 
Moore, R. M., and Wendt, Geo................. M\&W

Morgan, Anne H........ Morg

Morrison, Harold.......... Morr

Morrow, N. D..........

Morton, Emily........... .

Muesebeck, C. G. W...... Mues

Mundinger, F. G......... Mund

Museum of Comparative

Zoology, Harvard Univ... MCZ

Mutchler, A. J...........

Nadler, A. M........... Nad

Needham, J. G.......... Ndm

Newman, Edward......... Newn

New York State College of Forestry.............

New York State Museum... NYS

Nicolay, Alan S......... Nic

Notman, Howard........ Not

Olsen, C. E............ Ols

Osborn, Herbert.......... Osb

Osborn, H., and Ball, E. D. . O\&B

Osborn, H., and Drake, C. J. O\&D

Osborne, C. E............

Osten Sacken, Baron C. R. von............. OS or O.S.

Ottolengui, R.......... Ottol

Palm, Chas.

Panzer, G. W. F.......... Panz

Parker, J. B . . . . . . . . .

Parker, J. H........... Park

Parrott, P. J........... Parr

Parshley, H. M . . . . . . . . Parsh

Patch, Edith M.........

Paulmier, F. C........... Plm

Pearsall, R. F............ Pears

Pergande, Theo......... Perg

-Pettit, R. H . . . . . . . . .

Phillips, Venia Tarris...... VTP or

Phillips, W. J............ PWJ

Phillips, W. J., and Emery, W. T............ P\&E

Plunkett, Chas. R....... Plunk

Quaintance, A. L........ Q

Quirsfeld, E. D.......... Quirs

Rees, I..............

Rehn, J. A. G...........

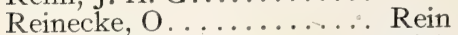

Richmond, E. A......... Rich
Riley, C. V............. Rly

Roberts, Chris. H. . . . . . Robts or Rbts

Robinson, Col. Wirt....... Robn

Rohwer, S. A.......... Roh

Sanders, J. G., and DeLong, D. M................ S\&DeL

Sandhouse, Grace. . . . . . . Sand

Satterthwait, A. F....... Satt

Say, Thos.............

Schaeffer, Chas......... Sf

Schaupp, F. G...........

Schoonmaker, J. W....... Schoon

Schott, F. M...........

Schwartz, E. A........... Sz

Schwartz, H. E. . . . . . . . Schw

Scudder, S. H........... Scud or

Shannon, R. C........... Snn

Sherman, Franklin....... Sherm

Sherman, J. D., Jr........ . ShermJr

Shoemaker, Ernest. ....... Shoe

Sibley, C. K. . . . . . . . . Sib

Sirrine, F. A............

Slingerland, M. V . . . . . . Sling

Smith, H. H. ......... SmHH or

Smith, John B......... Sm HHS

Smith, Roger C.........

Snyder, T. E..........

Southwick, E. B.........

Squire, Eben H. . . . . . . . Sq

Stage, H. H. . . . . . . . . .

Staten Island Museum..... SI Mus

Sturtevant, A. H. . . . . . . . Sturt

Swaine J. M. ......... Sw

Taub, Esther

Taylor, Leland $\mathrm{H}$.

Thompson, Joseph C..... J J T

Uhler, Philip........... . Uhl

United States Bureau of Entomology. ............. USBE

United States National Museum.

US

Van Duzee, E. P........ EPV

Van Duzee, M. C......... MCV

Van Dyke, E. C.......... Vdyk

Viereck, H. L........... Vier

Walker, E. M........... Wlk

Walsingham, Lord........ Wls 
Watson, F. E .......... Wat

Weeks, Archibald C...... Wks

Weiss, H. B . . . . . . . .

Weld, L. H. . . . . . . . . . .

Wells, R. W . . . . . . . . .

Wendt, Geo............

West, L. S............

Wheeler, Wm. Morton..... Wheel

Wickham, H. F ......... Wick

Williamson, E. B......... Willn

Williston, W. R. . . . . . . Will

Wilson, J. E. P. . . . . . . W1s

Winters, Fred E.........
Wolcott, A. B.......... Wolc

Wolf, Augusta ......... Wlf

Woodruff, Lewis B....... Wood or

Young, Chester..........

Young, D. B........... Yg

Yuasa, H.............

Zabriskie, Rev. J. L. ..... Zab

Zesch, F. H., and Reinecke,

O ................ Z\&R

Zimmerman, C. D........ Zimm

\footnotetext{
Memoir 100 A Study of Pogoniris Varieties, the next preceding number in this series of publications, was mailed on May 25, 1927.
} 

A

Abacidus, 223

Abbottana, 609

Abia, 876

Ablabesmyia, 709

Ablerus, 981

Abrenthia, $\mathbf{5 5 4}$

Abrostola, 626

Abstrulia, 408

Absyrtus, 946

Acacesia, 1052

Acallodes, 507

Acalypta, 100

Acalyptus, 502

Acamptus, 511

Acanalonia, 178

ACANalonitnaE, 178

Acanthocephala, 86

ACANTHOCERINAE, 422

Acanthocinus, 453

Acanthocnema, 842

Acantholoma, 75

Acantholyda, 872

Acanthomyops, 1000

$\Lambda$ canthoscelis, 506

ACAnTHosomatinaE, 84

Acanthothrips, 72

ACARINA, 1076

Acasis, 592

Acemyia, 817

Acerota, 965

Achaetella, 842

Achalarus, 670

Achatodes, 639

ACHILINAE, 176

Acholerops, 276

Acholla, 106

Achorutes, 12

ACHORUTINAE, 12

Acidalia, 591

Acidalinae, 591

Acidia, 851

Acidoproctinae, 65

Acidoproctus, 65

Acidota, 279

Acilius, 262

Aciura, 851

Aclista, 962

Acmaeodera, 355

Acmaeops, 438

Acnemia, 743

Acoenitini, 939

Acoloithus, 537

Acolus, 964

Aconura, 167

Acoptus, 506

Acordulecera, 894

Acossus, 570

Acraspis, 970

Acratrichis, 313

ACRIDIDAE, 29

Acritus, 318

Acrobasis, 581

Acrocera, 761

Acrocercops, 541
ACROLOPHINAE, 537

Acrolophus, 537

Acrolyta, 929

Acroneuria, 58

Acronycta, 641

ACRONYCTINAE, 634

Acropteroxys, 383

Acroricnus, 930

Acrosternum, 83

Acrotona, 304

Actenodes, 361

Actia, 808

Actidium, 312

Actina, 751

Actinotia, 641

Actiopsis, 808

Actium, 308

Actobius, 290

Acucephalus, 163

Acupalpus, 250

Icutalis, 149

Acylomus, 392

1cylophorus, 294

Idaina, 585

Adalia, 397

Adela, 534

Adelges, 192

DELGINAE, 192

Idelius, 904

Adelocephala, 586

1delocera, 342

Adelphocoris, 131

Adelphomyia, 697

Aderocharis, 286

Adiplosis, 739

Adirus, 873

Adistemia, 389

Adita, 660

Admestina, 1070

Admontiopsis, 808

Adoneta, 536

Adoxophyes, 566

Adoxus, 468

Adranes, 312

Aedes, 704

Ægeria, 557

EgERIIDAE, 555

Aegialia, 418

Aegomorphus, 451

Aellopos, 611

Aenoplex, 929

AEOLOTHRIPIDAE, 66

AEOLOTHRIPOIDEA, 66

Aeolothrips. 66

Aeolus. 343

Aesalinae, 432

Aeschna, 51

Aeschnidae, 49

Ethaloptera, 603

Aethus, 77

Agabetes, 259

Agabus, 258

Agallia, 156

Agapetus, 523

Agaporus, 258
Agapostemon, 1025

Agaristidae, 665

Agarodes, 531

Agašsa, 1070

Agathidinae, 910

Agathidium, 274

Agathis, 910

Agathomyia, 790

Agelastica, 475

Agelena, 1063

Agelenidae, 1063

Ageniella, 988

Agenioideus, 985

Aglais, 685

Aglossa, 578

Aglyptinus, 274

Agnomonia, 628

Agonoderus, 251

Agonopteryx, 545

Agonosoma, 771

Agraphus, 492

Agraylea, 523

Agrilaxia, 359

Agrilus, 361

Agriogenia, 988

AGRIONIDAE, 45

Agriopodes, 644

Agriotes, 348

Agroeca, 1061

A gromyza, 865, 866

AGROMYZIDAE, 865

Agroperina, 646

Agrotinae, 659

Agrotis, 661

Agrypnia, 528

Agrypon, 953

Airora, 374

Alabama, 624

Alaus, 342

Alcathoe, 557

Alcidamea, 1028

Alebra, 173

Aleiodes, 901

Aleiodinae, 901

Aleochara, 306

Aleocharinae, 298

Aleodorus, 306

Alepidia, 122

Alepomyia, 531

Aleurochiton, 193

Alexeter, 946

Aleyrodes, 193

ALEYRODIDAE, 193

Allactina, 751

Allandrus, 486

Allantinae, 878

Allantus, 880,884

Alleculidae, 398

Alleculinae. 398

Allochrysa, 40

Allocosa. 1066

Allocvphonyx, 985

Allodia, 744

-1lloeostylus, 8.30

Alognosta, 751 
Allograpta, 796

Allomimus, 511

Alloperla, 57

Allophyla, 843

Allopoda, 411

Allotria, 629

Alloxacis, 332

Alloxysta, 968

Alobates, 405

Alobus, 423

Aloconota, 303

Alopecosa, 1066

Alophora, 806

Alphitobius, 404

Alphitophagus, 403

Alsophila, 590

Alveotingis, 103

Alypia, 665

ALYSIINAE, 919

Alyson, 1014

Amalopota, 179

Amalus, 509

Amara, 227

Amartus, 375

Amathes, 649

Amaurobius, 1034

Amauronematus, 892

A mbl Y CERA, 63

Amblycorypha, 21

Amblyctis, 410

Amblyderus, 340

Amblyscirtes, 675

Amblyteles, 921

AmbLytelinI, 921

Ambrosiodmus, $\quad 520$

Ameletus, 42

Amerinus, 250

Amerizus, 218

Ametastegia, 879

Amiota, 862

Amischa, 303

Ammalo, 668

Ammatomus, 1015

Ammobia, 1011

Ammodonus, 401

Ammophila, 1012

Amnestus, 77

Amobia, 823

AMOBIINAE, 823

Amoebaleria, 843

Amolita, 635

Amorbia, 566

Ampeloglypter, 504

Ampelophaga, 612

Amphasia, 248

Amphiagrion, 48

Amphibitherion, 303

Amphibolips, 972

Amphicerus, 415

Amphicnephes, 849

Amphicoma, 422

Amphicrossus, 378

Amphidasys, 603

Amphigerontia, 60

Amphion, 613

Amphionycha, 457

Amphipyra, 648

Amphorophora, 188

Amphotis, 378

Ampulicidae, 1008

Amydria, 539

Anabolia, 529

Anabrolepis, 979

Anacaena, 267
Anacampsis, 548

Anacamptodes, 603

Anacharis, 967

ANACHARITINAE, 967

Anacrabro, 1021

Anadaptus, 248

Anaedus, 406

Anaferonia, 222

Anagoga, 604

Anagrus, 984

Analcis, 510

Anametis, 491

ANAMORPHA, 1081

Anamorphus, 391

Anaphothrips, 68

Anaplodes, 590

Anarsia, 548

Anarta, 658

Anasa, 87

Anaspis, 335

Anastatus, 980

Anastoechus, 763

Anatella, 744

Anatis, 397

Anatrichis, 243

Anaulascaspis, 306

Anax, 51

Anaxipha, 28

Anchicera, 386

Anchocelis, 649

Anchodemus, 498

Anchylopera, 559

Anchytarsus, 368

Ancistrocerus, 1004

Ancylis, 559

ANCYLOLOMIINAE, 578

Ancylorrhanis, 1038

Ancyloxipha, 672

Ancyronyx, 367

Ancyrophorus, 280

Andrena, 1022

ANDRENIDAE, 1022

ANDRENINAE, 1022

Andricus, 971

Andrimus, 400

Andrina, 816

Androchirus, 400

Andronicus, 1028

Andropolia, 641

An cphysis, 948

Aneflomorpha, 436

Anephischetos, 619

AnERASTIINAE, 584

Aneurhynchus, 963

Aneurus, 91

Angitia, 957

Ania, 606

Anisandrus, 520

Anisodactylus, 247

Anisolabis, 202

ANISOPODIDAE, 688

ANISOPODINAE, 688

AnISOPTERA, 49

Anisopus, 688

Anisosticta, 395

Anisostoma, 273

Anisota, 586

Anisotarsus, 248

AnisotominaE, 273

Anisoxya, 411

ANOBIIDAE, 412

ANOBIINAE, 412

Anocomis, 444

Anoecia, 184
Anomala, 426

Anomalagrion, 49

Anomalini, 953

Anomalon, 953, 954

Anomis, 624

Anomoglossus, 243

Anopheles, 703

Anoplitis, 482

Anoplium, 436

Anoplius, 987

Anoplolyda, 873

ANOPLURA, 73

Anorostoma, 843

Anorthodes, 640

Anorthosia, 549

Anosia, 681

Anotia, 179

Anteris, 964

Anthalia, 785

Anthaxia, 359

Anthemoessa, 1027

Anthemois, 1029

Antherophagus, 385

ANTHICIDAE, 340

Anthicus, 340

Anthobium, 278

AntновоSCIDAE, 992

Anthoboscus, 447

Anthocharis, 677

Anthocomus, 326

Anthocoptes, 1078

ANTHOCORIDAE, 109

ANTHOCORINAE, 109

Anthocoris, 109

Anthomyia, 836

Anthomyiella, 836

ANTHOMYIIDAE, 829

ANTHOMYIINAE, 836

Anthomyza, 864

Anthonomus, 500

Anthophilax, 439

Anthophora, 1027

ANTHOPHORINAE, 1027

Anthracophaga. 860

Anthrax, 761, 762

Anthrenus, 372

Anthribulus, 487

Anthribus, 486

Antiblemma, 6?5

Anticarsia, 625

Antichaeta, 846

Antillocoris, 97

Antipus, 462

Antispila, 544

Antocha, 691

Anuraphis, 186

Anurida, 13

Anurophorus, 13

Anyohaena, 1061

Anytus, 660

Aonidia, 201

Apaecasia, 601

Apamea, 639

Apanteles, 906

Apantesis, 666

Aparamesius, 963

Apatania, 530

Apatela, 641

Apatelodes, 587

A pateticus, 85

Apatura, 687

A pechthis, 937

Apenes, 240

Aperileptus, 959 
Apha,enogaster, 997

Aphaereta, 919

Aphalara, 182

Aphanisus, 887

Aphanus, 98

Apharetra, 660

ApHelinidae, 981

Aphelinus, 982

Aphelonema, 178

Aphelosetia, 544

Aphidencyrtus, 979

APHIDIINAE, 918

Aphidius, 918

Aphidoletes, 732

ApHiIdAe, 184

Aphinae, 184

APHIINI, 186

Aphilanthops, 1017

Aphiochaeta, 789

Aphis, 186

Aphodinae. 419

Aphodius, 419

Aphonus, 428

Aphorista, 391

Aphrastus, 493

Aphria, 812

Aphrophora, 145

APHROPHORINAE, 145

Aphycus, 979

Apicia, 608

APIDAE, 1033

Apinops, 810

APIOMERINAE, 105

Apiomerus, 105

Apion, 489

Apioninae, 489

Apis, 1033

Aplectoides, 660

Aplodes, 590

Apocellus, 280

Apocephalus, 789

Aporinellus, 985

Apostenus, 1061

Appias, 677

Apristus, 238

Apterolophus, 982

Apteromechus, 510

Aptesis, 928

Aptinothrips, 68

ArachNidA, 1034

Arachnophroctonus, 985

ARADIDAE, 89

Aradus, 89

Araeocerus, 487

Araeoncus, 1042

Aramigus, 492

Araneae, 1034

Araneae Theraphosae, 1034

Araneae Verae, 1034

Araneus, 1052

Archanara, 635

Archasia, 152

Archimerus, 87

Archips, 568

Archytas, 820

Arctia, 666

Arctididae, 665

Arctocorixa, 141

Arctoecia, 528

Arctophyto, 823

Arctopsyche, 524

Arctosa, 1066

Ardistomis, 213

Arenetra, 932
Arenophilus, 1083

Arge, 876

Argia, 47

ARgIDAE, 876

Argillophora, 634

ARgINAE, 876

Argiope, 1054

ARgIOPIDAE, 1052

Argynnis, 683

Argyra, 774

Argyresthia, 553

Argyria, 579

Argyrodes, 1038

Argyrostrotis, 628

Argyrotoxa, 566

Arhopalus, 446

Ariadna, 1036

Arilus, 106

Arisota, 304

Aristotelia, 550

Arogalea, 549

Arotes, 939

Arpactus, 1015

Arpedium, 279

Arphia, 31

Arsilonche, 641

Arta, 571

Artace, 588

Arthroceras, 750

Arthroceratinae, 750

Arthrocnodax, 735

Arthromacra, 407

Arthropeas, 750

Arzama, 635

Asagena, 1039

Asaphes, 980

AscALAPHidAe, 42

Asclera, 332

Ascogaster, 903

Asemum, 434

Asilidae, 765

Asilinae, 769

Asilus, 770

Asindulum, 741

AsopinaE, 84

Asphondylia, 729

ASPHONDYLIARIAE, 729

Asphragis, 934

ASPICERINAE, 968

Aspidiotiphagus, 982

Aspidiotus, 200

Aspilota, 852

Astata, 1008

Astatini, 1008

Asteia, 864

AsteIIDAE, 864

Astenophylax, 528

Astenus, 288

Asterolecanium, 194

Asteromyia, 725

Asthenidea, 109

Astiphromma, 959

Astomaspis, 929

Astylidius, 452

Astylopsis, 451

Asynapta, 717

Asyndetus, 772

Ataenius, 420

Atalopedes, 673

Atanycolus. 896

Atarba, 697

Ateloglossa, 822

Atethmia, 636, 649

Atherix, 760
Atheta, 301

Athous, 344

Athyroglossa, 856

Atimia, 448

Atlanticus, 24

Atlides, 678

Atmetus, 950

Atomacera, 876

Atomaria, 386

AtomariInAE, 386

Atomosia, 767

Atoperla, 58

Atoreutes, 900

Atractodes, 928

Atractotomus, 113

Atranus, 235

Atreides, 610

Atrichomelina, 845

Atropates, 979

Atrytone, 674

Atrytonopsis, 675

Attagenus, 371

Attalus, 326

Attelabinae, 489

Attelabus, 489

Atteva, 553

Atymna, 153

AtyPidAe, 1034

Atypus, 1034

Aulacaspis, 199

Aulacidae, 960

Aulacidea, 973

Aulacigaster, 863

Aulacizes, 160

Aulacodes, 896

Auletes, 488

Auleutes, 507

Aulobaris, 504

Aulonium, 388

Aulonothroscus, 354

Autographa, 626

Automeris, 586

Autoserica, 423

Axinopalpus, 239

Axion, 398

Axymyia, 688

AXYMYINAE, 688

Aylax, 973

Azelia, 833

Azelina, 608

\section{B}

Babia, 462

Bacanius, 318

Baccha, 794

Bactridium, 380

Badister, 229

Baeocera, 314

BaEtinae, 42

Baetis, 43

Baetisca, 42

Baeus, 964

Bagisara, 636

Bagous, 498

Baileva, 632

Balaninus, 500

Balclutha, 172

Baliosus, 483

Baliothrips. 69

Ballus, 1070

Balsa, 640

Banasa, 83

BANCHINI, 959

Banchus, 959 
Bapta, 598

Baptolinus, 289

Barathra, 658

Barce, 104

Baris, 503

Barynotus, 492

Baryodma, 306

Barypeithes, 492

Barytychius, 497

Basiaeschna, 51

Basilarchia, 686

Basilona, 586

Bassareus, 466

BAssinI, 949

Bassus, 911, 949

Batazonus, 985

Bathona, 276

Bathyphantes, 1042

Bathyplectes, 957

Batrachedra, 552

Batrisodes, 309

Batyleoma, 449

Bedellia, 540

Belidae, 487

Bellamira, 442

Bellura, 635

Belonochilus, 93

Belonuchus, 292

Belostoma, 140

Belostomatidae, 140

Belvosia, 812

Belyta, 962

Belytinae, 962

Bembecia, 557

BEMBECINAE, 1014

Bembex, 1018

Bembicini, 1018

Bembidion, 214

Benacus, 140

Beraea, 526

Beraeinae, 526

Berkshiria, 753

Berosus, 265

BEROTHIDAE, 40

Berytidae, 91

Betarmon, 349

BETHYLIDAE, 1004

Bezzia, 708

Bibio, 748

BibionidAE, 748

Bibloplectus, 308

Bibloporus, 308

Bicyrtes, 1018

Bidessus, 255

Bigotomyia, 829

Biorhiza, 970

Bitoma, 387

Bittacomorpha, 688

Bittacomorphella, 688

PitTACOMORPHINAE, 688

Bittacus, 522

Bivena, 882

Blacinae, 912

Blacus, 912

Blaniulus, 1079

Blaps, 401

Blapstinus, 401

Blaptinae, 401

Blastobasidae, 551

Blastobasis, 551

Blastodacna, 552

Blasturus, 44

Blatta, 19

Blattella, 18
Blattidae, 18

Blechrus, 238

Bledius, 281

Blennocampa, 887

BLENNOCAM PINAE， 886

Blepharida, 475

Blepharigena, 811

Blepharipeza, 816

Blepharocera, 749

BLEPHAROCERIDAE, 749

Blepharomastix, 573

Blepharoprocta, 786

Bleptina, 621

Blera, 801

Blethisa, 210

BLISSINAE, 94

Blissus, 94

Bocchus, 1008

Boeotarcha, 576

Bogeria, 805

Bolbocerosoma, 421

Bolbomyia, 760

Boletina, 743

Bolitobius, 297

Bolitochara, 300

BOLITOPHAGINAE, 402

Bolitophagus, 402

Bolitophila, 740

BolitopHilinaE, 740

Bolitotherus, 402

Bolothrips, 71

Bombias, 1033

BOMBIDAE, 1030

Bombus, 1030

BомвYLIIDAE, 761

Bombyliopsis, 821

Bombylius, 763

Bomolocha, 619

Bondia, 570

Bonnetia, 813

BORBORIDAE, 844

Borboropora, 305

Borborus, 845

Boreaphilus, 279

Boreus, 522

Boriomyia, 40

Borkhausenia, 546

Borolia, 654

Boros, 406

BOSTRICHIDAE, 415

BostrichidAe. 415

Botanobia, 861

Bothrideres, 388

Bothriopterus, 225

Bothriothorax, 979

Bothropolys, 1081

Bothrotes, 400

Bourletiella, 16

Boyeria, 51

Brachinus, 241

Brachistes, 912

Brachixiphosoma, 957

Brachyacantha, 393

Brachybamus, 497

BrACHYCENTRINAE, 530

Brachycentropus, 530

Brachycis, 417

Brachycoma, 824

Brachycosmia, 649

Brachydeutera, 859

Brachy gluta, 309

Brachyiulus, 1079

Brachyleptura, 439

Brachylobus, 243
Brachymeria, 976

Brachy myrmex, 999

Brachynemurus, 41

Brachyneura, 716

Brachyopa, 797

Brachypalpus, 801

Brachypeza, 744

Brachypterolus, 374

Brachypterus, 375

Brachyrhinus, 492

Brachys, 364

Brachysius, 13

Brachystegus, 1016

Brachystropha, 920

Brachystylus, 493

Brachytarsus, 487

Bracon, 897, 910

BRACONIDAE, 895

BRACONINAE, 896, 910

Bradytus, 226

Brathinidae, 270

Brathinus, 270

Bremia, 732

Brenthis, 683

Brentidae, 485

BREPHINAE, 589

Brephos, 589

Brevicoryne, 187

Brillia, 711

Brochymena, 78

Brontes, 382

Brotolomia, 645

BRUCHIDAE, 484

Bruchomorpha, 178

Bruchophagus, 976

Bruchus, 484

Bruesia, 993

BrYOCORINAE, 115

Bryoporus, 298

Bucculatriplex, 901

Bucculatrix, 540

Buenoa, 139

Buplex, 754

BuPRESTIDAE, 355

Buprestis, 357

BYRRHIDAE, 372

BYRRHINAE, 372

Byrrhus, 373

Bythinopsis, 311

BYTHOSCOPINAE, 156

Byturinae, 370

Byturus, 370

\section{C}

Cabera, 599

Caberodes, 608

Cacoecia, 568

Cacoplia, 450

Caddo, 1074

Caecilitidae, 61

Caecilinnae, 61

Caecilius, 61

Caenia. 859

Caeniella, 319

Caenis, 43

Caenocara, 414

Caenophanes, 899

Caenoscelis, 386

Caenurgia, 629

Caerosternus, 318

Cafius, 292

Cainosterum. 273

Calamia, 635

Calamoceratidide, 526 
Calandra, 514

Calaphis, 185

Calathus, 230

Calendra, 514

Calendrinae, 512

Calephelis, 681

Caliope, 848

Caliroa, 885

Calitys, 374

Callaspidia, 968

Calledapteryx, 585

Calleida, 239

Callibaetis, 43

Callicera, 793

Calliceras, 964

Calliceratidae, 964

Callicerus, 307

Callicorixa, 141

Callidiotes, 944

Callidium, 444

Callidrvas, 677

Calliephialtes, 941

Calligrapha, 470

Callilepis, 1036

Callimome, 975

Callimomidae, 975

Callimoxys, 443

Callimyia, 790

Calliopsis, 1024

Calliphora, 827

CALLIPHORIDAE, 827

CALlipHORINAE, 827

Callipterini, 184

Callipus, 1081

Callirhytis, 971

Callizzia, 585

Calloides, 446

Callopistria, 645

Callopistromyia, 850

Callosamia, 587

Calobata, 854

Calocaipe, 593

Calocampa, 651

Calochromus, 320

Calocoris, 131

Calophya, 183

Calopsectra, 712

Calopteron, 319

Calopteryx, 45

Calosoma, 209

Calotarsa, 790

Calothysanis, 592

Calpe, 624

Calpodes, 675

Calyptocephalus, 320

Calythea, 836

Camnula, 31

Campaea, 605

Campodea, 11

Campodeidae, 11

Camponotus, 1003

Campoplegidea, 954

CAMPOPLEGINI, 954

Campoplex, 965

Campsicnemus, 773

Campsomeris, 995

Camptobrochis, 116

Camptogramma, 596

Camptomyia, 718

Camptoneura, 849

Camptoneuromvia, 726

Camptoprosonella, 848

Campylenchia, 155

Campylomma, 110
Campylomyza, 715

CAMPYLOMYZARIAE, 715

Campyloneurus, 896

Canarsia, 583

Canifa, 411

CANTHARIDAE, 322

Cantharis, 324

Canthon, 417

Canthydrus, 254

Capis, 619

Capitophorus, 189

Capnella, 59

Capnia, 59

CAPNIIDAE, 59

Capnochroa, 399

Capsinae, 125

Capsus, 126

Carabidae, 207

CARABINAE, 207

Carabus, 208

Caradrina, 640, 649

Caraphractus, 984

Carborius, 529

Carcinops, 317

Cardiochiles, 913

CARDIOCHILINAE, 913

Cardiocladius, 710

Cardiola, 305

CARDIOPHORINAE, 352

Cardiophorus, 352

Caripeta, 601

Carneades, 662

Carphoborus, 515

Carphonotus, 511

Carpocapsa, 558

CarpophilinaE, 375

Carpophilus, 376

Carposina, 570

CARPOSINIDAE, 570

Carterocephalus, 672

Carthasis, 108

Cartodere, 389

Cartosyrphus, 793

Carynota, 149

Caryomyia, 734

Caseola, 1043

Casinaria, 955

Casnonia, 235

Cassida, 483

CASSIDINAE, 483

Castaneira, 1061

Catabena, 641

Cateretes, 375

CAteretinae, 374

Catharellus, 250

Cathartus, 381

Catia, 673

Catocala, 630

Catocalinae, 628

Catogenus, 381

Catoglyptina, 945

CATOGLYPTINI, 944

Catonia, 177

Catopyrrha, 601

Catorama, 414

Cavariella, 187

Cecidomyia, 739

Cecidomyitdae, 714

Cedius, 311

Cedusa, 179

Celama, 669

Celatoria, 808

Celerio, 613

Celetes, 319
Celia, 227

Celina, 255

Celiptera, 629

Celithemis, 56

Cenchrea, 179

Centeterus, 927

Centrinites, 505

Centrinopus, 505

Centrinus, 504

Centrodera, 437

Centrodora, 982

Centrotinae, 156

Cepha, 873

Cephaleia, 872

CEPHALOIdAe, 331

Cephaloon, 331

Cephennium, 276

Cephenomyia, 805

CEPHIDAE, 873

Cephus, 873

Ceracis, 417

Ceradonta, 867

Ceraleptus, 88

Ceramb y CIDAE, 432

Ceramica, 655

Cerastipsocus, 60

Cerataphis, 192

Ceraterocercus, 890

Ceraticelus, 1043

Ceratina, 1028

Ceratinella, 1045

CERATININAE, 1028

Ceratinopsis, 1045

Ceratocapsus, 121

Ceratocolus, 1020

Ceratocombus, 135

Ceratogastra, 959

Ceratographis, 453

Ceratomegilla, 395

Ceratomia, 610

Ceratomyza, 867

Ceratophyllus, 869

Ceratopogon, 707

CeratopogoninaE, 707

Ceraturgus, 766

Cercerin I, 1017

Cerceris, 1017

Cercometes, 375

Cercopeus, 493

Cercopidae, 145

Cercopinae, 145

Cercyon, 269

Cercyonis, 682

Ceresa, 147

Ceriodes, 802, 803

Cerma, 644

Cerococcus, 194

Cerodontha, 867

Ceromasia, 816

Ceropales, 989

CeropalinaE, 989

Ceroplastes, 196

Ceroplatinae, 741

Ceroplatus, 741

Ceroptres, 974

Cerostoma, 553 ,

Cerotainia, 767

Cerotoma, 475

Ceruchus, 432

Cerura, 617

Cerylon, 388

Cesonia, 1036

Cetoninnae, 428

Ceuthophilus, 25 
Ceutorhynchus, 507

Chabuata, 656

Chaeretymma, 931

Chaetarthria, 269

Chaetechus, 493

Chaetocnema, 479

Chaetogaedia, 819

Chaetona, 823

Chaetoneurophora, 788

Chaetophleps, 808

Chaetophlepsis, 810

Chaetopsis, 850

Chaetoravinia, 825

Chaetotachina, 818

Chaitophorus, 186

Chalarus, 791

Chalastogastra, 871

ChalcididaE, 976

ChalcidoideA, 975

Chalcodermus, 510

Chalcoides, 478

Chalcomyia, 793

Chalcophora, 355

Chalcophorella, 355

Chalepus, 482

Chalybion, 1012

Chamaemyia, 865

Chamyris, 634

CHAOBORINAE, 702

Chaoborus, 702

Characoma, 632

Charadra, 627

Charidryas, 684

Chariessa, 330

Chariesterus, 87

Charipinae, 968

Charips, 968

Charisalia, 440

Chartolampra, 138

Chartoscirta, 138

Chasmatonotus, 711

Chauliodes, 38

Chauliognathus, 322

Cheiloneurinus, 979

Chelipoda, 784

Chelogynus, 1008

Chelonus, 903

Chelymorpha, 483

Chermidae, 182

Chersodromia, 784

Chevrolatia, 276

Chilo, 579

Chilocorus, 398

Chilognatha. 1079

Chilopoda, 1081

Chilopora, 308

Chilosia, 793

Chilostigma, 530

Chimarrha, 524

Chion, 435

Chionaspis, 198

Chionea, 694

Chiracanthium, 1061

Chirida, 484

Chiromantis, 784

Chiromyia, 864

Chironomidae, 707

Chironominae, 710

Chironomus, 712

Chirotenetes, 42

Chirothrips, 68

Chlaenius, 242

Chlaenogramma, 609

Chlamydatus, 110
Chlamydinae, 463

Chlamys, 463

Chloealtis, 29

Chloralictus, 1025

Chloridea, 664

Chlorion, 1010, 1011

Chlorissa, 590

Chlorochlamys, 591

Chlorochroa, 79

Chloroperla, 58

Chloropidae, 860

Chloropisca, 860

Chlorops, 860

Chlorotettix, 171

Choëphora;, 661

Choeridium, 418

Choleva, 272

Cholomyia, 822

Choragus, 487

Choreutis, 554

Chorinaeus, 951

Chorizagrotis, 662

Choropleca, 539

Choroterpes, 42

Chorthippus, 30

Chortophaga, 31

Chramesus, 515

Chremylus, 900

Chromagrion, 48

Chromatia, 399

Chromocryptus, 931

ChrYSAUginae, 571

Chrysidea, 991

Chry Sididae, 990

Chrysis, 991

Chrysobothris, 359

Chrysocharis, 982

Chry socharomyia, 983

Chrysochlamys, 794

Chrysochroma, 751

Chrysochus, 469

Chrysodina, 466

Chrysogaster, 792

Chrysomela, 471

CHRYSOMELIDAE, 458

Chry SOMELiNAE, 469

Chrysomphalus, 201

Chrysomyza, 850

Chrysopa, 40

Chrysophanus, 680

ChrYSOPIDAE, 40

Chrysopilus, 759

Chrysopoctonus, 930

Chrysopora, 550

Chrysops, 754

Chrysotachina, 813

Chrysotimus, 776

Chrysotoxum, 792

Chrysotus, 772

Chthonolasius, 1000

Chutapha, 645

Chyliza, 856

Chymomyza, 862

Chysogona, 991

Chytolita, 622

Chytonix, 644

Cicada, 144

Cicadella, 160

Cicadellidae, 156

Cicadellinae. 160

Cicadidae, 142

Cicadula, 172

Cicindela, 204

Cicindelidae, 204
Cicinnus, 586

Cicurina, 1063

Cidaphus, 959

Cimbex, 875

Cimbicidae, 875

CimbiCINAE, 875

Cimex, 108

Cimicidae, 108

Cinclidia, 684

Cincticornia, 730

Cinctotipula, 699

Cindaphia, 574

Cinetus, 962

Cingilia, 604

Cinglis, 591

Cinyra, 357

Circotettix, 33

Cirphis, 654

Cirrhophanus, 636

Cirrospilus, 983

Cirrula, 859

Cis, 416

Cisidae, 416

Cisinae, 416

Cissia, 682

Cissusa, 625

Cistogaster, 805

Citheronia, 586

Citheronitidae, 586

CixiINAe, 177

Cixius, 177

Cladara, 593

Cladiinae, 889

Cladius, 890

Cladura, 694

Clambidae, 274

Clambus, 274

Clanoneurum, 856

Clastoptera, 146

Clastopteromyia, 862

Clausicella, 807

Clavigeridae, 312

Clavigerus, 186

Cleidogona, 1081

Cleis, 397

Clemensia, 669

Cleonus, 503

Cleora, 602

Cleotus, 422

Cleridae, 327

Clerinae, 327

Climacia, 39

Clinidium, 373

Clinocentrus, 901

Clinocephalus, 30

Clinocera, 785

Clinodiplosis, 733

Clinorhyncha, 726

Clinotany pus, 710

Clioperla, 58

Clisodon, 1027

Clistogastra, 895

Clistomorpha, 811

Clistopyga, 937

Clivina, 212

Clivineminae, 115

Cloeon, 44

Closterccerus, 983

Clubiona, 1062

Clubionidae, 1061

Clusia, 844

Clusiidae, 844

Clusiodes, 844

Clytoleptus, 447 
Clytrinae, 462

Clytus, 448

Cnemodon, 793

Cnemodus, 97

Cnemogonus, 506

Cnephalogonia, 818

Cnephasia, 567

Cnesinus, 515

Cocceius, 671

Coccidae, 193

Coccidula, 394

CoccinaE, 196

Coccinella, 396

COCCINELlidAe, 392

CoccinellinaE, 392

Coccobaphes, 126

Coccophagus, 982

Coccus, 196

Cochlidion, 536

Cochliomyia, 827

Coecidencyrtus, 979

Coelambus, 255

Coelinidea, 920

Coeliodes, 507

Coelioxys, 1029

Coelogaster, 508

Coeloides, 896

Ccelomyia, 833

Coelostathma, 566

Coelostethus, 413

Coelotanypus, 709

Coelotes, 1064

Coenagrion, 48

Coenocalpe, 595

COENOCOELIINAF, 895

Coenocoelius, 896

Coenomyia, 750

COENOMYIIDAE, 750

Coenosia, 835

Coenositnae, 835

Coenus, 81

Colaspis, 467

Colenis, 273

Coleocentrus, 939

Coleophora, 543

Coleophoridae, 543

Coleoptera, 203

Collaria, 123

Collembola, 12

Colletes, 1021

Colletinae, 1021

Collops, 326

Coloboneura, 784

Colobopterus, 42

Colocasia, 627

Colon, 273

Colopha, 189

Colopterus, 375

Colpocephalum, 63

Colpodia, 717

Colpodota, 305

Colpognathus, 927

Colpotrochia, 951

ColydiddaE, 387

Colydium, 388

Colymbetes, 260

Colymbetinae, 258

Comedo, 983

Commophila, 570

Compsocryptus, 931

Condidea, 798

Condylolomia, 572

Condylorrhiza, 573

Conicera, 790
Coninomus, 389

CONIOPTERYGIDAE， 42

Coniopteryx, 42

Conistra, 650

Connophron, 275

Conocephalus, 24

Conophthorus, 518

Conopia, 556

CONOPIDAE, 803

Conops, 803

Conosoma, 297

Conotelus, 376

Conotrachelus, 509

Conservula, 645

Contarinia, 731

Conwentzia, 42

Copelatus, 260

Copidita, 332

Copidosoma, 979

Copipanolis, 652

COPRINAE, 417

Copris, 418

Coprothassa, 305

Coptodera, 238

Coptodisca, 544

Coptotomus, 260

Coquillettomyia, 732

Coras, 1065

Cordilura, 841

Cordulegaster, 49

Cordulia, 53

Cordyla, 744

Cordylomyia, 716

Cordyluridae, 841

Coreidae, 86

COREINAE, 86

Corethra, 702

Corethrinae, 702

Corimelaena, 76

Corinthiscus, 330

Corinthomyia. 716

CORISCINAE, 87

Coriscus, 88

Corixa, 142

CORIXIDAE, 141

CORIZINAE, 89

Corizus, 89

Cornicularia, 1046

Corrodentia, 60

Corthylus, 517

Corticaria, 389

Corticoris, 135

Corticotomus, 373

Corycia, 598

CORYDALINAE, 38

Corydalis, 38

Corylophodes, 276

Coryna, 888

CORYNETINAE, 330

Corynoneura, 711

Corythaica, 102

Corythucha, 100

Coscinoptera, 462

Cosmia, 636

Cosmoconus, 947

Cosmopepla, 82

Cosmophorus, 916

Cosmopteryx, 553

Cossidae, 570

CossoninaE. 511

Cossonus, 511

Cosymbia. 592

Cotalpa, 427

Cothonaspis, 968
Cotinis, 428

Coxelus, 388

Crabro, 875, 1019

Crabronidae, 875

CRABRONINAE, 875, 1019

Crabronini, 1019

Crambidia, 669

CRAMBinae, 578

Crambodes, 640

Crambus, 579

Craponius, 506

Craspedosomidae, 1080

Crassiseta, 861.

Crassomicrodus, 911

Cratacanthus, 244

Crataraea, 307

Cratoparis, 486

Cratospila, 919

Cregya, 330

CREMASTINI, 957

Cremastobomycia, 542

Cremastocheilus, 429

Cremastus, 958

Crematogaster, 966

Cremnops, 910

Crenitis, 268

Creophilus, 294

Crepidodera, 478

Cressonia, 611

Cricotopus, 711

Crigmus, 348

Criocephalus, 434

CRIOCERINAE, 461

Crioceris, 461

Criocoris, 113

Criorhina, 801

Crocidophora, 574

Crocigrapha, 655

Crophius, 95

Crosbycus, 1074

Crosimus, 385

Crossocerus, 1020

Crustulina, 1039

Cryobius, 223

Cryphalus, 518

Cryphia, 634

Cryphoeca, 1065

Cryphula, 99

Cryptarcha, 379

CRYPTARCHINAE, 379

CRYPTINAE, 928

CRYPTINI, 930

Cryptobiella, 285

Cryptocala, 659

CRYPTOCEPHALINAE, 463

Cryptocephalus, 464

Cryptocercus, 20

Cryptocheilus, 988

Cryptohypnus, 347

Cryptoideus, 931

Cryptolabis, 694

Cryptolechia, 544

Cryptoleon, 41

Cryptolucilia, 828

Cryptomeigenia, 807

Cryptomyzus, 189

CRYPTOPHAGIDAE, 385

CRYPTOPHAGINAE, 385

Cryptophagus, 385

CRYPTOPIDAE, 1082

Cryptopleurum, 270

Cryptops, 1082

Cryptorhophalum, 371 .

Cryptorhynchus, 510 
Cryptostematidae, 135

Cryptothrips, 71

Crypturgus, 515

Crypturopsis, 932

Cryptus, 931

Crytophyllaspis, 201

CTENISCINI, 948

Cteniscus, 948

Ctenocephalus, 868

Ctenolepisma, 11

Ctenopelma, 945

Ctenophora, 699

Ctenophthalmus, 869

Ctenothrips, 69

Ctenucha, 670

CUCUJIDAE, 380

Cucujus, 381

Cucullia, 653

CuCUllitnaE, 649

Culex, 703

Culicidae, 702

Culicinae, 703

Culicoides, 707

Culiseta, 704

Cupes, 331

Cupesidae, 331

Cuphocera, 819

CurCulionidaE, 488

Curculioninae, 493

Curtisapis, 1025

Curtonotum, 863

Curtonotus, 226

Cuterebra, 80.5

Cuterebridae, 805

Cyaniris, 681

Cybaeus, 1065

Cybister, 262

CYBISTERINAE, 262

Cychramus, 378

Cycloneda, 396

Cycloplasis, 555

Cycloptilum, 28

Cyclosa, 1054

Cyclotrachelus, 222

Cycnoditdae, 544

CYDNIDAE, 76

CYDNINAE, 77

Cylapinae, 115

Cylapus, 115

Cylindrocharis, 221

Cylindrocopturus, 506

Cylindromyia, 813

Cylindrotoma, 698

CYLINDROTOMINAE, 698

Cylistix, 316

Cyllene, 445

Cylloceria, 934

Cyllodes, 378

Cymatodera, 327

Cymatophora, 598, 600

Cymbiodyta, 268

Cyminae, 93

Cymindis, 240

Cymodusa, 965

Cymolomia, 564

Cymus, 93

CYNIPIDAE, 967

CYNIPINAE, 968

Cynips, 971

Cynomyia, 827

Cynorrhina, 801

Cyphomimus, 491

Cyphon, 369

Cyphopyga, 1029
Cyrtidae, 760

Cyrtinus, 449

Cyrtogaster, 980

Cyrtolobus, 152

Cyrtoma, 787

Cyrtomenus, 77

Cyrtophloeba, 812

Cyrtophorus, 448

Cyrtopogon, 766

Cyrtorhinus, 119

Cyrtusa, 273

Cysteopteryx, 592

Cystiphora, 722

Cytilus, 372

\section{D}

Dacne, 384

Dacnusa, 920

Dactylocladius, 711

Dactylolabis, 694

Dactylopinnae, 194

Dactylopius, 195

Dalmannia, 804

Danainae, 681

Danaus, 681

Daremma, 610

DAscillidae, 368

DASCILLINAE, 368

Dasyglossa, 307

Dasylechia, 768

Dasyllis, 768

Dasylophia, 615

Dasymolophilus, 693

Dasymutilla, 993

Dasyneura, 720

DASYNEURIARIAE, 719

DASYPOGONINAE, 765

Datana, 614

Datomicra, 303

Debis, 682

Decantha, 546

Decarthron, 311

Decatoma, 976

Deceia, 848

Dectes, 454

Degeeriella, 65

Deidamia, 612

Deilephila, 613

Deilinea, 599

Deinopsis, 298

Deleter, 950

Delomegachile, 1029

Delomerista, 943

Delopsis, 747

Delorrhipis, 1046

Deloyala, 484

DELPHACINAE, 180

Delphacodes, 181

Delphastus, 394

Delta, 641

Deltocephalus, 165

Deltometopus, 353

Demas, 627

Dendrocoris, 83

Dendroctonus, 516

Dendroides, 339

Dendroleon, 41

Dendrophagus, 382

Dendrophaonia, 830

Dendrophilus. 317

Dendrotettix, 34

Dentifibula, 731

Deopalpus, 819

Depressaria, 545
Deptalia, 592

DERAEOCORINAE, 115

Deraeocoris, 116

DERBINAE, 179

Dercetis, 619

Dermaptera， 202

Dermestes, 370

DERMESTIDAE, 370

DERMESTINAE, 370

Derobrachus, 433

DERODONTIDAE, 384

Derodontus, 384

Deromyia, 767

Derostenus, 983

Derrima, 636

Desmia, 573

Desmocerus, 443

Desmometopa, 865

Desmopachria, 254

Deuteroxorides, 940

Dexia, 821

DexiIDAE, 821

Diabrotica, 474

Diachorisia, 534

Diachus, 465

Diacrisia, 667

Diacritus, 959

Diactinia, 594

Diadocidia, 740

Diadocidinae, 740

Dialges, 946

Dialysis, 758

Dialyta, 831

Dialytes, 420

Diamesa, 710

Dianous, 283

Dianthidium, 1029

DIAPERINAE, 402

Diaperis, 402

Diaphania, 573

Diapheromera, 20

Diaphnidia, 121

DIAPHORINAE, 772

Diaphorus, 772

Diapria, 963

DIAPRIIDAE, 962

DIAPRIINAE, 963

DIASPINAE, 198

Diaspis, 199

Diastata, 864

Diastichtis, 573, 600

Diastrophus, 973

Diathrausta, 577

Diatraea, 578

Diaulinus, 983

Diazosma, 689

Dibolia, 481

Dibolocelus, 266

Dibrachys, 980

Dicaelus, 229

Dicerca, 355

Dichaeta, 858

Dichaetoneura, 808

Dichelonyx, 425

Dichocera, 819

Dichomeris. 548

Dichorda, 590

Dichromorpha, 30

Dichrooscytus. 129

Diclasiopa, 857

Dicranomyia, 689

Dicranophragma, 695

Dicranopselaphus. 368

Dicranoptycha, 691 
Dicrodiplosis, 732

Dictya, 846

Dictyna, 1035

DICTYNIDAE, 1034

Dictyomyia, 846

Dictyophara, 176

DICTYOPHARINAE, 176

Dicymbium, 1046

Dicymolomia, 572

DICYPHINAE, 114

Dicyphus, 114

DicyrtominaE, 17

Didactylomyia, 717

Didea, 795

Didineis, 1015

Didyma, 809

Didymops, 52

Dietzella, 508

Dikraneura, 173

Dilachnus, 184

Dilophonota, 611

Dilophus, 749

Dimecaenia, 859

Dimetrota, 304

Dimmockia, 983

Dimorphopteryx, 879

Dinaraea, 303

Dinera, 823

Dinetini, 1009

Dineura, 890

Dineutes, 262

Dinocampus, 916

Diochus, 290

Dioctes, 957

Dioctria, 766

Diodontus, 1013

Dioedus, 403

Diomonus, 742

Dione, 683

DropsIDAE, 856

Dioryctria, 581

Diorymerellus, 505

Diostracus, 776

Diphadnus, 894

Diphlebus, 1014

Diphthera, 644

Diplazon, 949

DIPLAZONINI, 949

Diplectrona, 524

Diplocephalus, 1046

Diplocoelus, 385

Diplodontia, 976

Diploiulus, 1079

Diplolepis, 969

DIPLOPODA, 1079

Diplotaxis, 423

Diplotoxa, 860

Dipoena, 1039

Diprion, 877

DIPRIONIDAE, 877

DiPTERA, 687

Dipterygia, 648

Dircaea, 411

Dirhiza, 718

Discobola, 690

Discocerina, 857

Discoderus, 247

Disholcaspis, 970

Disonycha, 476

Dissosteira, 32

Distenia, 433

Ditrichophora, 857

Ditropinotus, 975

Ditylus, 332
Dixa, 702

DIXIDAE, 702

Docosia, 744

Dohrniphora, 788

Dolba, 609

DOLERINAE, 881

Dolerus, 881

DOLICHODERINAE, 998

Dolichoderus, 999

Dolichopeza, 698

DOLICHOPOIIDAE, 771

DOLICHOPODINAE, 777

Dolichopus, 777

Dolichosoma, 327

Dolichotrypes, 966

Dolichovespula, 1007

Dolomedes, 1065

Dolophilus, 524

Dolopius, 348

Donacia, 458

DONACIINAE, 458

Dorcatoma, 414

Dorchaschema, 450

DORCINAE, 431

Dorcus, 431

Dorocordulia, 53

Doros, 796

Doru, 203

Doryctes, 899

DORYCTINAE, 899

Dorylaidae, 791

Dorymyrmex, 999

Doryodes, 629

Doryphorophaga, 815

Dorypteryx, 62

Dorytomus, 496

DOUGLASIIDAE, 544

Draeculacephala, 161

Drapetes, 354

Drapetis, 783

Drapetisca, 1046

Drassodes, 1036

Drassyllus, 1037

Drasteria, 625, 629

Drepana, 589

Drepanaphis, 185

DREPANIDAE, 589

Drepanodes, 608

Drepanosiphum, 185

Drepanulatrix, 599

Driotura, 167

Dromaeolus, 353

Dromius, 238

Dromogomphus, 50

Drosophila, 862

DROSOPHILIDAE, 862

Drusus, 530

DRYINIDAE, 1008

Drymus, 98

Dryobius, 434

Dryocoetes, 520

Dryocosmus, 970

Dryomyza, 845

Dryophanta, 969

Dryophthorus, 511

DRYOPIDAE, 366

DUFOURIELLINAE, 110

Dufouriellus, 110

Duvita, 549

Dynastes, 428

DYNASTINAE, 427

Dynatosoma, 745

Dyschirius, 211

Dyscinetus, 427
Dysdera, 1036

DYSDERIDAE, 1036

Dysidius, 225

Dysmigia, 601

Dyspteris, 593

Dyspyralis, 624

Dysstroma， 594

DytiscidAe, 254

Dytiscinae, 261

Dytiscus, 261

\section{E}

Eacles, 586

Earinus, 911

Farophila, 596

Ebo, 1058

Eburia, 435

Eccoptogaster, 514

Ecdyonurus, 45

Ecdytolopha, 558

Echidnophaga, 868

Echinomyia, 820, 821

Echinothrips, 67

Echmepteryx, 62

Echthodopa, 766

Echthronomas, 965

Echthrus, 931

Eclytus, 944

Ecpantheria, 667

Ecphylus, 900

Ectamonogonus, 251

Ectoedemia, 535

Ectopimorpha, 921

Ectopria, 368

ECTRICHODIINAE, 105

Ectropis, 603

Ecyrus, 454

Egle, 839

Eiphosoma, 957

Elachertus, 983

Elachista, 544.

ElACHISTIDAE, 544

Elampus, 990

Elaphidion, 436

Elaphrothrips, 72

Elaphrus, 209

Elasmopalpus, 582

Elasmosoma, 904

Elasmostethus, 84

Elater, 349

ELATERIDAE, 342

Elaterinae, 348

Eleates, 402

Elephantomyia, 696

Eleusis, 277

Flipsocus, 62

Elis, 992, 995

Elleschus, 502

F1lida, 617

Ellopia, 605

Elonus, 341

Elophila, 577

Elytroleptus, 449

Ematurga, 598

Emblethis, 98

Emesa, 103

Emesaya, 103

Emmesa, 410

Emmesomyia, 836

Empalia, 742

Empeda, 692

Emphanopteryx, 807

Emphytina, 880

EMPHYTINAE。 878,886 
Emphytus, 880

Empicoris, 103

EMPIDIDAE, 783

Empis, 786

Emplenota, 307

Empoa, 174

Empoasca, 173

Empria, 886

EMPRIINAE, 886

Enallagma, 47

Enargia, 636

Enarmonia, 558

Encarśia, 982

Enchenopa, 156

Enchodes, 410

Enchrysa, 550

Encoptolophus, 31

Encyclops, 443

ENCYRTIDAE, 979

Encyrtus, 979

Endalus, 497

Endecatomus, 415

Enderleinellus, 74

ENDOMYCHIDAE, 390

ENDOMYCHINAE, 390

Endomychus, 391

Endropia, 607

Enicmus, 389

ENICOCEPHALIDAE, 103

Enicospilus, 952

Enizemum, 949

Ennearthron, 417

ENNOMINAE, 598

Ennomos, 605

Enochrus, 268

Enoclerus, 328

Enodia, 682

Enoplognatha, 1039

Entephria, 595

Entomobrya, 15

ENTOMOBRYIDAE, 13

ENTOMOBRYINAE, 15

Entomophthalmus, 353

Entylia, 155

Eois, 591

EOSENTOMIDAe, 1083

Eosentomon, 1083

Eosphoropteryx, 626

Epachthes, 947

Epalpus, 820

Evarces, 927

Epargyreus, 670

Epelis, 598

Epeolus, 1024

Epeorus, 45

Epermenia, 553

Ephalus, 401

Ephedrus, 918

Ephelia, 695

Ephemera, 44

Ephemerella, 44

EPHEMERIDA, 42

EPHEMERIDAE, 42

EPHEMERINAE, 44

Ephestia, 583

Ephestiodes, 583

Ephialtes, 936, 941

Ephialtini, 936

Ephistemus, 386

Ephuta, 995

Ephydra, 859

EPHYDRIDAE, 856

EPHYDRINAE, 858

Epia, 657
Epiaeschna, 52

Epiblema, 560

Epicaerus, 492

Epicauta, 337

Epichloropus, 860

Epicnaptera, 588

Epicordulia, 52

Epicypta, 746

Epidiaspis, 200

Evidiplosis, 736

Epierus, 317

Epiglaea, 650

Epigrimyia, 811

Epilachna, 398

EPILACHNINAE, 398

Epimecis, 603

EPIMORPHA, 1082

Epinotia, 559

Epipaschia, 580

EPIPASCHIINAE, 580

Epiphanis, 354

EPIPHRAGMA， 694

EPIPLEMIDAE, 585

Epiptera, 176

Epirhyssalus, 901

Epirranthis, 606

Epirrita, 595

Episcopellus, 249

Episilia, 661

Episimus, 563

Epistrophe, 796

Episyron, 986

Epithectis, 550

Epitrimerus, 1078

Epitrix, 479

Epiurus, 942

Epizeuxis, 623, 624

Epochra, 851

Epomyia, 764

Epurea, 377

Erannis, 604

Erastria, 639

ERASTRIINAE, 633

Erax, 769

Erchomus, 296

EREBINAE, 624

Erebus, 624

Eremobia, 646

Eremocoris, 99

Eremomyia, 837

Eremomyoides, 837

Eremotylus, 952

Eridontomerus, 975

Erigone, 1046

Erinnyis, 611

Eriocampa, 879

Eriocampoides, 885

Eriocera, 697

Eriococcus, 194

Eriocrania, 534

ERIOCRANIIDAE, 534

Eriopeltis, 196

Eriophyes, 1077

ERIOPHYIDAE, 1076

ERIOPHYINAE, 1077

Erioptera, 692

ERIOPTERINI. 691

Eriopus, 645

Eriopyga, 656

Eriosoma, 190

ERIOSOMATINAE, 189

ERIOSOMATIN I, 189

Eristalis, 798

Eritettix, 29
Eritrissomerus, 966

Ernestia, 813

Ernobius, 413

Ero, 1058

Erora, 680

Eros, 320

EROTYLidAe, 383

EROTYLINAE, 383

Erromenus, 948

ERYCINIDAE, 681

Erycus, 497

Erynnis, 672

Erythemis, 55

Erythraspides, 887

Erythrodiplax, 55

Erythroneura, 175

Escaryus, 1083

Estheria, 823

ESTHIOPTERINAE, 65

Esthiopterum, 65

Estigmene, 667

Ethmia, 546

ETHOPOLIDAE, 1081

Euacidalia, 591

Euadmontia, 808

E.uaesthetinae, 284

Euaesthetus, 284

Euaraba, 824

Euaresta, 853

Eubadizon, 912

Eubaphe, 668

EUBRIINAE, 368

Eucalymnatus, 196

Eucalyptra, 621

Eucanthus, 421

Euceraphis, 185

Eucerceris, 1017

Euceros, 944

EuCEROSINA, 944

Euchaetias, 666

Euchlaena, 607

Euchoeca, 597

EUCHROMIIDAE, 670

Eucicones, 387

Eucinetinae, 368

Eucinetus, 368

Eucirrhoedia, 649

Euclea, 535

EUCLEIDAE, 535

Euclemensia, 555

Euclidia, 629

Euclyptus, 496

EUCNEMINAE, 352

Eucoilidea, 968

EucoIlinAe, 968

Euconnus, 274

Eucoptocnemis, 661

Eucordylea, 550

Eucorethra, 702

Eucosma, 560

EU CosMINAE, 557

Eucrada, 412

Eucrostis, 591

Eucta, 1055

Eucymatoge, 598

Eudamus, 670

Eudeilinea, 589

Euderces, 448

Eudicrana, 741

Eudioptis, 573

Eudociminus, 496

Eudryas, 634

Eudule, 597

Eueretagrotis, 659 
Euferonia, 222

Eufidonia, 598

Euforcipomyia, 707

Eugaediopsis, 819

Eugaurax, 861

Euglenidae, 341

Eugnamptus, 488

Eugnatha, 1055

Eugnathodus, 173

Eugnoriste, 747

Eugonia, 605

Eugonobapta, 605

Euhaematopinus, 74

Euhybos, 785

Euhyperecteina, 807

Eulachnus, 184

Eulasiona, 807

Eulathrobium, 286

Eulechriops, 506

Eulia, 567

Eulimneria, 957

Eulimnichus, 373

EulopHidae, 982

Eulype, 596

Eumacaria, 601

Eumacrocentrus, 914

Eumasicera, 816

Eumenes, 1004

Eumeninae, 1004

Eumerus, 800

Eumesiina, 944

Eumetopiella, 851

Eumeyrickia, 544

Eumichtis, 651

Eumicrota, 300

Eumolops, 222

EUMOLPINAE。 466

Eumyiolepta, 793

Eupactus, 414

Eupanychis, 663

Euparius, 486

Euparthenos, 629

Euparyphus, 753

Eupelecotheca, 815

EUPELMIDAE, 980

Eupelmus, 980

Euphorantha, 806

Euphoria, 428

Euphoriana, 916

EUPHORINAE, 915

Euphormia, 827

Euphorus, 916

Euphydryas, 684

Euphyes, 674

Euphyia. 596

Eupithecia, 597

Euplectus, 308

Euplexia, 647

Eupogonius, 454

Eupristocerus, 361

Euproctis, 618

Eupsalis, 485

Eupteromalus, 980

EUPTEROTIDAE, 587

Eupteryx, 174

Euptoieta, 683

Eurema, 678

Euresia, 551

Europs, 380

Eurosta, 852

Eurctype, 651

Eurychilopterella, 116

Eurycyttarus, 539

Eurygaster, 76

Eurylabus, 927
Eurymus, 678

Eurymycter, 486

Euryophthalmus, 99

Euryopis, 1039

Eurypogon, 368

EURYPROCTINA, 946

Euryproctus, 946

Euryscelis, 447

Eurytoma, 977

EURYTOMIDAE, 976

Eurytomocharis, 977

Euscelis, 167

Euschistus, 80

Eusimulium, 749

Eusiphona, 865

Eusisyropa, 814

Eusphyrus, 486

Eustala, 1055

Eustalomyia, 837

Eustictus, 115

Eustroma, 593

Eustrophinus, 408

Eustrophus, 409

Eustrotia, 634

Eutelia, 633

Eutelinae, 633

Eutelus, 981

Eutettix, 168

Euthera, 817

Eutheresia; 823

Euthisanotia, 634, 655

Euthochtha, 87

Euthyatira, 588

Euthycera, 846

Eutochia, 404

Eutolype, 652

Eutonia, 695

Eutrapela, 607

Eutreta, 852

Eutrichogena, 818

Eutrixa, 811

Eutylistus, 414

Euura, 894

Euxenus, 487

Euxesta, 850

Euxoa, 662

Euzophera, 583

Evacanthus, 161

Evania, 984

Evaniella, 984

EvaniIDAE, 984

Evarcha, 1070

Evarthrus, 222

Everes, 680

Evergestis, 573

Evetria, 562

Evippe, 550

Evodinus, 438

Evophrys, 1070

Evoxysoma, 977

Exartema, 564

Exechia, 745

Exechophysis, 1047

Exema, 463

Exentera, 563

Exetastes, 932

ExосHINI, 950

Exochomus, 398

Exochus, 951

Fxoprosopa. 761

Exorista, 815, 817

Exoristoides, 815

Exyra, 633

Exyston, 948
F

Fabiola, 546

Fabriciella, 820

Fagitana, 645

Falagria, 306

Falcaria, 589

Fannia, 833

FANNIINAE, 833

Feltia, 662

Feltiella, 732

Feniseca, 680

Fentonia, 617

Fenusa, 886

Fenusinae, 885

Feralia, 653

Ferdinandea, 794

Fidia, 468

Fidiobia, 966

Fidonia, 598

Figites, 968

FigitinaE, 968

Fiorinia, 199

Fitchia, 105

Fitchiella, 178

Flatinae, 178

Foenidae, 961

Foenus, 961

Folsomia, 13

Fontaria, 1080

Forcipomyia, 708

Forda, 191

FordinI, 191

Forficula, 203

FORFICULIDAE， 202

FORFICULINAE, 203

Formica, 1001

FORMICIDAE, 995

FormicinaE, 999

Fornax, 353

Francoa, 188

Frankliniella, 68

Frenatae, 534

Frontina, 815

Fucellia, 841

Fucellitnae, 841

Fuentes, 1070

Fulgoridae, 176

Fulvius, 115

\section{G}

Gabara, 621

Gaberasa, 621

Galasa, 571

Galeatus, 101

Galerita, 236

Galerucella, 473

GALERUCINAE, 472

Galesus, 963

Galgula, 640

Galgupha, 76

Galleria, 572

GalleritnaE, 572

Ganonema, 526

Garganus, 132

Gargaphia, 102

Gasterophilinae, 892

Gasterophilus, 829

Gasteruption, 961

GASTERUPTIONIDAE，961

Gastrellarius, 222

Gastroidea, 471

Gastrolobium, 284

Gastrosticta, 223

Gaurax, 861

Gauropterus, 288 
Gaurotes, 438

GELASTOCORIDAE, 141

Gelastocoris, 141

Gelchossa, 102

Gelechia, 547

GELECHIIDAE， 547

GELIN I, 930

Gelis, 930

Gelus, 506

Genea, 810

Geocorinae, 94

Geocoris, 94

Geoderces, 493

Geodrassus, 1037

Geodromicus, 279

Geoica, 191

Geometridae, 589

GeOMETRINAE, 598

GEOMYZIDAE, 864

Geophilidie, 1083

GEOPHULOMORPIIA, 108,3

Geophilus, 1083

Geopinus, 244

Georyssidae, 368

Georyssus, 368

Geosargus, 751

Geotomus, 77

Geotrupes, 421

Geotrupinae, 421

Geraeus, 504

Geranomyia, 690

Gerdana, 544

Geron, 764

GerRiddae, 135

GERRINAE, 135

Gerris, 135

Geshna, 577

Giardomyia, 735

Gibbium, 412

Ginglymyia, 811

Glaea, 650

Glaphyria, 572

GLAPHYRIINAE, 572

GLAPHyRINAE, 422

Glauce, 549

Glaucopsyche, 681

Glena, 602

Glenognatha, 1055

Glischrochilus, 379

Glossonotus, 150

GLOSSOSOMATINAE, 522

Gluphisia, 617

Giutops, 750

Glycerius, 249

Glycobius, 446

Glyphicnemis, 928

Glyphidocera, 549

GLYPHIPTERYGIDAE， 554

Glyphipteryx, 554

Glyphonyx, 349

Gly phopsyche, 530

Glyphotaelius, 529

Glypta, 934

Glyptina, 481

GLYPTINI, 934

Glyptocera, 582

Glyptocranium, 1055

Glyptodoryctes, 899

Glyptoscelis, 468

Glyptothrips, 72

Gnaphosa, 1037

GNaphosidae, 1036

Gnathias, 1026

Gnathocerus, 403
Gnathoncus, 319

Gnathotrichus, 518

Gnophomyia, 693

Gnorimella, 429

Gnorimoschema, 547

Gnoriste, 743

Gnypeta， 305

Gobaishia. 190

Goera, 531

Goerinae, 531

Goes, 450

Gomphaeschna, 51

Gomphoides, 49

Gomphus; 50

Gonaspis, 973

Gonatium, 1047

Gonatccerus, 984

Gonatopus, 1008

Gonempeda, 692

Gongylidiellum, 1047

Gonia, 818

Goniocotes, 64

Goniodes, 64

GONIODINAE, 64

Goniolophus, 250

Goniops 754

Gonocallus, 444

Gonochrysis, 991

Gonodontis, 606

Gonomyia, 693

Gonotropis, 486

Gortyna, 636, 638

Gorytes, 1015

Gossyparia, 194

Gracilaria, 541

GRACILARIIDAE, 541

Gracilia, 436

Grammonota, 1047

Grammospila, 920

Graphisurus, 453

Graphocephala, 161

Graphoderes, 262

Graphomyia, 829

Graphops, 467

GRAPHOSOMATINAE, 78

Graptolitha, 651

Gretchina, 562

Gronevus, 276

Grotea, 938

Gryli.idae, 26

Gryllotalpa, 28

Gryllus, 26

Gryon, 964

Grypidius, 497

Gueneria, 599

Gymnandrosoma, 558

Gymnaspis, 201

Gymnetron, 503

Gymnoclytia, 805

Gymnococcus, 195

Gymnopa, 856

Gymnophora, 789

GYMNOPINAE, 856

Gymnoprosopa, 824

Gymnopternus, 781

Gymnoscelus, 914

Gymnosoma, 806

Gymnusa, 298

Gynandropus, 246

Gypona, 162

GYPONINAE, 162

Grpsonoma, 563

GYRINIDAE, 262

Gyrinus, 263
Gyrohypnus, 289

Gyronycha, 299

Gyrophaena, 300

H

HABROCERINAE, 298

Habrocerus, 298

Habrocestum, $107 \mathrm{C}$

Habrocytus, 981

Habronyx, 953

Habrophlebia, 44

Habrosyne, 588

Hadena, 645, 646, 647, 648, 651

HADENINAE, 654

Hadrobregmus, 413

Hadrobunus, 1076

HADRODACTYLINA, 945

Hadrodactylus, 946

Hadronema, 118

Haematobia, 828

HAEMATOPINIDAE. 73

Haematopinus, 73

Haematopis, 592

Haemodipsus, 74

Haemonia, 460

Hagenius, 49

Hahnia, 1065

Halesus, 529

Halictus, 1025

HALIPLidAe, 252

Haliplus, 252

Hallomenus, 409

Halobatinae, 136

Haltica, 477

Halticinae, 475

Halticus, 118

Halysidota, 668

Hamamelistes, 191

Hammerschmidtia, 797

Hammoderus, 450

Hammomyia, 837

Hapalosalia, 437

Hapithus, 28

Haplandrus, 405

Haploa, 665

Haplodrassus, 1037

Haplothrips, 69

Harmolita, 977

Harmologa, 567

Harmostes, 89

HarpactcrinaE, 105

Harpaglaea, 650

Harpagoxenus, 998

HARPALINAE. 213

Harpalus, 244

Harpyia, 617

Harrisimemna, 644

Harrisina, 537

Hartomyia, 708

Hebecnema, 832

Hebridae, 106

Hebrus, 106

Hecabolinae. 899

Hecabolus, 899

Hecalus, 163

Hedroneura, 847

Hedvchridium, 990

Hedvchrum, 990

Heidemannia, 135

Helcon, 914

Helconidea, 914

HELCONINAE. 914

Heleomyza, 843

Helice, 551 
Helichus, 366

Helicobia, 825

HeliconitinaE, 683

Helicopsyche, 531

HelicopsychinaE, 531

Helina, 831

HeLIOdinidae, 555

Heliolonche, 664

Heliomata, 599

Heliophana, 664

Heliophila, 654

Heliothis, 664

Heliothrips, 67

Heliozelidae, 544

Heliria, 150

Helius, 690

Helluomorpha, 240

Helmidae, 366

Helmis, 366

Helobia, 693

Helochara, 160

Helochares, 268

Helocombus, 269

Helocordulia, 52

Helodes, 368

Helodidae, 368

Helodinae, 368

Helomyza, 843

Helomyzidae, 842

Helophilus, 799

HeLOPHORINAE, 265

Helophorus, 265

Helopinae, 407

Helops, 407

HeLoridae, 962

Helorus, 962

Helotropha, 639

Hemaris, 612

HEMEROBIIDAE, 39

Hemerobius, 39

Hemerocampa, 617

Hemerodromia, 784

Hemiberis, 751

Hemichionasnis, 199

Hemichroa, 890

Hemicrepidius, 346

Hemileuca, 586

Hemimene, 557

Heminomada, 1026

HEMIPTERA, 74

Hemirhipus, 343

Hemitaxonus. 889

Hemiteles, 929

HeMitelini, 929

Hemitheinae, 590

Hemyda, 811

HENICOPIDAE. 1082

Henoticus, 385

Heodes, 680

Hepialidaf. 534

Hepialus, 534

Heptagenia, 45

HEPTAGENINAE, 45

Heraeus, 96

Hercostomus. 782

Herculia, 578

Heriades, 1029

Heringia, 793

Hermetia, 751

HER MINIINAE, 621

Herpestomus, 927

Herpyllus, 1037

Herse, 609

Hesperia, 672
Hesperitdae, 670

HesPeRIINAE， 670

Hesperobium, 284

Hesperophylax, 530

Hesperotettix, 34

Hesperotingis, 102

Hesperumia, 600

Hesperus, 292

Hetaerina, 46

Hetaerius, 318

Heterachthes, 436

Heterelmis, 367

Heterocampa, 615

Heteroceridae, 367

Heterocerus, 367

Heterocola, 958

Heterocordylus, 121

Heterogamus, 902

Heterogenea, 537

Heterogramma, 621

Heteromyia, 708

Heteroneura, 844

HeTERONEURIDAE, 844

Heteropelma, 953

HETEROPEZINAE, 716

Heterophleps, 593

Heteroplectron, 526

Heteropogon, 767

Heteropsylla, 182

Heterospilus, 900

HETEROTARSINAE, 406

Heterothops, 294

Heterothrips, 67

Heterotoma, 121

Hetoemis, 450

Hexachrysis, 991

Hexagenia, 44

Hexamitocera, 841

Hexarthrum, 512

Hexatoma, 697

Hexatomini, 694

Hilara, 786

Hilarella. 824

Hillia, 652

Himatinum, 511

Hindsiana, 70

Hippelates, 861

HIPPOBOSCIDAE, 868

Hippodamia. 395

Hippopsis, 455

Hippuriphila, 479

HISPINAE. 482

Hister, 314

HISTERIDAE, 314

Histrinae, 314

Holcocephala, 766

Holcocera, 551

Holocentropus, 525

Hololepta, 314

Hololeptinae, 314

Holoneurus, 719

Holonomada, 1026

Holoparamecus, 388

Holophris, 990

Holopogon, 766

Holopyga, 990

Holostrophus. 409

Homaemus, 76

Homalactia, 815

Homalota, 300

Homaspis, 945

Homochlodes, 601

Homoeosoma, 583

Homoeusa, 307
Homoglaea, 649

Homohadena, 653

Homoporus, 981

HOMOPTERA, 142

Homoptera, 628

Homopyralis, 620

Homotropus, 949

Hoplandria, 301

Hopland rothrips, 72

Hoplia, 426

Hoplismenus, 927

Hoplisus, 1015

Hoplitis, 1028

Hoplocampa, 890

Hoplocephala, 402

Hoplocryptus, 931

Hoplodictya, 846

Hoplogaster, 836

Hoplogryon, 964

Hoplolabis, 692

Hoplopleura, 74

Hoplosia, 454

Hoploteleia, 965

Hoplothrips, 71

Horcias, 130

Horisme, 598

Horistonotus, 352

HORMAPHIINAE, 191

Hormaphis, 191

HORMIINAE, 900

Hormisa, 622

Hormius, 900

Hormomyia, 735

Hormorus, 491

Howardia, 198

Hulstia, 583

Hyadina, 858

Hyaliodes, 114

Hyalomyia, 806

Hyalomyodes, 810

Hyalopterus, 187

Hyamia, 620

Hybophanes, 944

Hybos, 785

Hybroma, 539

Hyctia, 1071

HydATICINAE, 261

Hydaticus, 261

Hydnobius, 273

Hydnocera, 329

Hydraena, 264

HYDRAENINAE, 264

Hydrelia, 597

Hydrellia, 857

Hydria, 593

Hydriomena, 594

Hydrobius, 267

Hydrocanthus, 254

HYDROCHINAE, 265

Hydrochus, 265

Hydroecia, 636, 639

Hydrometra, 135

Hydrometridae. 135

Hydromyza, 842

HYDROPHILIDAE, 264

HYDROPHILINAE, 265

Hydrophilus, 266

Hydrophoria, 837

HYDROPHORINAE, 777

Hydrophorus, 777

Hydrophylax, 984

HYDROPORI NAE, 254

Hydroporus, 256

Hydropsyche, 524 
HYDROPSYCHIDAE, 524

HYDROPSYCHINAE, 524

Hydropsychodes, 525

Hydroptila, 523

HYDROPTILIDAE, 523

Hydrosmecta, 303

Hydrotaea, 832

Hydrotaeinae, 832

Hydrous, 266

Hydrovatus, 254

Hylaeidae, 1021

Hylaeinae, 1022

Hylaeus, 1022

Hylastes, 517

Hylastinus, 516

Hylecoetus, 331

Hylemyia, 838

Hylephila, 673

HyLESININAE, 515

Hylobius, 496

Hylocurus, 516

Hyloicus, 610

Hylota, 307

Hylotoma, 876

HYLOTOMIDAE, 876

HYLOTOMINAE, 876

Hylotrupes, 444

Hylurgopinus, 516

Hylurgops, 516

Hymenarcys, 81

Hymenia, 572

HYMENOPTERA, 870

Hymenorus, 399

Hymetta, 175

Hypargyricus, 887

Hyparpax, 616

Hypatus, 681

Hypena, 618

HYPENINAE, 618

Hypenula, 621

Hypera, 494

Hyperaeschra. 614

Hyperaspis, 392

Hyperdiplosis, 735

Hyperecteina, 809

Hyperitis, 606

Hypermallus, 435

Hyperodes, 495

Hyperplatys, 453

Hyphantria, 667

Hyphoraia, 667

Hypnoidus, 347

Hypocala, 625

Hypocera, 788

Hypochaeta, 810

Hypocoeliodes, 507

Hypocoelus, 354

Hypodacne, 384

Hypoderma, 805

Hypogeocoris, 95

Hypolampsis, 475

Hypomedon, 287

Hypomma, 1047

Hypomolyx, 496

Hypophloeus, 404

Hypoprepia, 668

Hypopteromalus, 981

Hyposoter, 955

Hypostena, 809

Hypothenemus, 518

Hyppa, 641

Hypselistes, 1047

Hypsopygia, 578

Hyptia, 985
Hyptiotes, 1034

Hypulus, 411

Hyssia, 656

Hysteroneura, 187

Hysterosia, 569

Ianassa, 616

Ibalia, 967

IBALIINAE, 967

Icerya, 193

Ichneumon, 921, 941

ICHNEU MONIDAE, 920

ICHNEUMONINAE, 920, 932

ICHNEUMONINI, 921,941

ICHNEUMONOIDEA, 895

Ichneutes, 913

Ichneutidea, 913

ICHNEUTINAE, 912

Ichthyura, 613

Icius, 1071

Icterica, 853

Idana, 849

Idechthis, 965

Idiobates, 405

Idiocerus, 157

IDIOGASTRA, 895

Idiolispa, 931

Idioptera, 695

Idiopterus, 189

Idiostethus, 505

Idiotypa, 963

Ilisia, 692

Illinoia, 188

Ilnacora, 118

Ilybiosoma, 259

Ilybius, 259

Ily thea, 858

Immyrla, 582

Incisalia, 679

Incurvaria, 534

INCURVARIIDAE， 534

Inga, 546

Ingura, 633

Inostemma, 966

INSECTA, 11

Iodia, 649

Iphiaulax, 897

Iphiclides, 676

Iphthimus, 405

IPIDAE, 514

Ipimorpha, 636

Ips, 379, 519

Iron, 45

Ischalia, 338

Ischnaspis, 202

ISCHNOCERA, 64

Ischnodemus, 94

Ischnoglossa, 307

Ischnomyia, 864

Ischnorhynchus, 93

Ischnura, 48

ISCHYROPSALIDAE, 1074

Ischyrus, 383

Isdromas, 929

Iseropus, 942

Isia, 667

ISOBATIDAE, 1079

Isochaetes, 535

Tsocorypha, 539

Isodontia, 1011

Isodyctium, 888

Isogenus, 57

Isohydnocera, 329
Isolomalus, 317

ISOMETOPIDAE. 135

Isomira, 399

Isoperla, 58

ISOPTERA, 38

Isorhipis, 353

Isosoma, 977

Isotoma, 14

ISOTOMINAE, 13

Isotomurus, 14

ISSINAE, 178

Isturgia, 598

Itame, 600

Itamoplex, 931

Ithycerus, 487

Ithytrichia, 523

Itonida, 737

ITONIDIDAE, 714

ITONIDIDINAE, 716

ITONIDIDINARIAE, 730

Itoplectis, 937

Itycorsia, 872

\section{J}

Jalysus, 92

Janetiella, 728

Janus, 873

JASSINAE, 163

Jassus, 172

Joannisia, 715

Johannsenomyia, 708

Johnsonomyia, 716, 753

Jonthonota, 484

TOPPINAE, 920

JOP PIN I, 921

Tudolia, 439

JugataE, 534

JULIDAE, 1079

Junonia, 686

Jurinia, 821

K

Kaliofenusa, 886

Karschomyia, 733

Kelisia, 180

Kermes, 194

Kingia, 836

Kleidotoma, 968

Kolla, 160

Konisomyia, 715

Kophosoma, 774

Kronomyia, 716

\section{L}

Labena, 938

LABENINI, 938

Labia, 203

Labidia, 884

Labidomera, 469

LABIDURINAE, 202

LABIINAE, 203

Labops, 118

Labrorychus, 953

Lacchoprosopa. 825

Laccobius, 269

Laccocera, 181

LACCOPHILINAE, 254

Laccophilus, 254

I. achesilla, 61

I.ACHNINI, 184

Tachnocrepis, 243

Lachnus, 184

Lacon, 342 
Lacosoma, 585

LACOSOMIDAE, 585

Ladona, 54

Laemophloeus, 381

Laemosaccus, 503

Laertias, 676

Laetilia, 583

Lagium, 882

LAGRIIDAE, 407

Lampra, 659

Lampracanthia, 138

Lampria, 768

Lampronota, 933

LAMPYRIDAE, 320

Languria, 383

LANGURIINAE, 383

Lanthus, 50

Laodamia, 582

Lapara, 610

Laphria, 768

LAPHRIINAE, 767

Laphygma, 640

Laphystia, 765

LARENTIINAE, 592

Largidea, 115

Laricobius, 384

Larinia, 1055

Lariophagus, 981

Larra, 1009

LARRINAE, 1008

LARRINI, 1009

Larropsis, 1009

Lasconotus, 388

Lascoria, 621

LASIOCAM PIDAE, 587

Lasiochilus, 109

Lasioderma, 414

Lasioglossum, 1025

Lasiomastix, 695

Lasiopogon, 766

Lasioptera, 722

LASIOPTERARIAE, 722

Lasiopteryx, 722

Lasiosina, 860

Lasiosoma, 742

Lasius, 1000

Laspeyresia, 558

LATHRIDIIDAE, 388

Lathridius, 389

Lathrobiella, 286

Lathrobioma, 285

Lathrobium, 285

Lathrolepta, 286

Lathrotaxis, 286

Lathys, 1035

Latrodectus, 1039

Lauxania, 847

Lauxaniella. 847

Laverna, 552

LAVERNIDAE, 552

Lebia, 236

Lecanium, 197

Leia, 743

Leiobunum, 1075

Leiocnemis, 226

Leiodes, 274

Leiophrcn, 912

Leiopus, 452

Leironotus, 226

Lema, 462

Leperisinus, 516

Lephthyphantes. 1048

Lepidocyrtus, 16

Lepidophora, 763
Lepidophorus, 494

Lepidopsallus, 113

LEPIDOPSOCIDAE, 62

LEPIDOPTERA, 532

Lepidosaphes, 202

Lepidostoma, 531

LEPIDOSTOMATINAE, 531

Lepisesia, 613

Lepisma, 11

LEPISMIDAE, 11

LEPISMINAE, 11

Leptacinodes, 289

Leptacinus, 289

Leptacis, 966

LEPTIDAE, 758

Leptinotarsa, 470

Leptis, 759

Leptobamona, 299

Leptobyrsa, 102

Leptocella, 527

Leptocera, 844

LEPTOCERIDAE, 527

Leptocerus, 527

Leptogaster, 765

LEPTOGASTERINAE, 765

Leptoglossus, 86

Leptolinus, 289

Leptomorphus, 743

Leptopeza, 786

Leptophlebia, 43

Leptophylax, 530

Leptopsylla, 869

Leptopygus, 958

Leptoschema, 344

Leptostylus, 451

Leptosyna, 716

Leptothorax, 998

Leptothrips, 70

Leptotrachelus, 235

Leptoypha, 102

Leptura, 441

Lepturges, 452

Lepturoides, 345

Leptusa, 299

Lepyronia, 146

Lepyrus, 495

Lerema, 675

Leria, 843

Lerodea, 675

Leskiomima, 810

Leskiopalpus, 810

Lestes, 46

Lestodiplosis, 736

Lestremia, 714

LESTREMINAE, 714

L.ESTREMINARIAE, 714

Lethocerus, 140

Letznerella, 518

Leucania, 654

Leucaspis, 199

Leucauge, 1055

Leucobrephos, 589

Leuconycta, 644

Leucoparyphus, 296

Leucopelmonus, 882

Leucophenga, 862

Leucopis, 865

Leucopomyia, 865

Leucorhinia, 56

Leucospis, 976

Leucostola, 774

Leucozona, 795

Leuctra, 59

Lexis, 669
Liancalus, 777

Libellula, 54

LibELLULidae, 52

Liburnia, 181

Liburniella, 181

LIBYTHEINAE, 681

Lichenomima, 61

Lichenophanes, 415

Ligyrocoris, 96

Ligy rodes, 428

Ligyrus, 428

LIMNEBIINAE, 264

Limnebius, 264

LIMNEPHILIDAE, 528

Limnephilus, 529

Limnia, 846

LIMNICHINAE, 373

Limnichites, 373

Limnius, 367

Limnobaris, 505

Limnobia, 690

Limnophila, 695, 696

Limnophora, 834

LIMNOPHORINAE, 834

Limnoporus, 136

Limnospila, 835

Limochores, 673

Limonia, 690

LIMONIINAE, 689

LIMONIINI, 689

Limonius, 343

Limosina, 844

Limothrips, 67

Limulodes, 313

Lina, 472

Linnaemyia, 813

Linognathus, 73

Linotaenia, 1083

LINOTAENIIDAE, 1083

Linyphia, 1048

LINYPHIIDAE, 1042

Liocranum, 1062

Liogma, 698

Liosomaphis, 187

Liothrips, 70

LIPARIDAE, 617

Liparis, 618

Lipeurus, 66

Lipocosma, 572

Lipophleps, 693

LIPOSCELIDAE, 63

Liposcelis, 63

Lispa, 834

Lispidea, 808

LISPINAE, 834

Lispinus, 277

Lispocephala, 835

Lispoides, 834

Lissonota, 933

Lissonotini, 932

Lissorhoptrus, 498

Lissothrips, 71

Listrognathus, 932

Listronotus, 495

Litargus, 387

Lithacodes, 537

Lithacodia, 634

Lithina, 601

LITHOBIIDAE, 1081

LITHOBIOMORPHA, 1081

Lithobius, 1081

Lithocharis, 287

Lithocolletis, 542

Litholomia, 652 
Lithomoia, 652

Lithophane, 651

LithosIIDAE, 668

Lithyphantes, 1039

Litolathra, 285

Litopyllus, 1038

Livia, 182

LIVIINAE, 182

Lixophaga, 809

Lixus, 503

Loberus, 385

Lobiopa, 378

Lobodiplosis, 732

Lobophora, 593

Lobopoda, 398

Lobopteromyia, 730

Loderus, 881

Lomamyia, 40

Lomanaltes, 619

Lonchaea, 848

LONCHAEIDAE, 848

Lonchoptera, 788

LONCHOPTERIDAF, 788

Longistigma, 184

Longitarsus, 480

Longurio, 699

Lopheros, 319

Lophocarenum. 1051

Lophodonta,' 615

Lophoglossus, 224

Lophomma, 1049

Lophopompilus, 986

Lophoptilus, 552

LOPHYRIDAE, 877

LOPHYRINAE, 877

Lophyrus, 877

Lopidea, 118

Lopus, 113

Loricera, 210

Lorinota, 306

Loxandrus, 225

Loxocera, 855

Loxostege, 574

Loxostegopsis. 576

Loxotropa, 963

LUCANIDAE, 430

LUCANINAE. 430

Lucidota, 320

Lucilia, 828

Ludius, 345

Luperaltica, 479

Luperina, 645

Luperodes, 474

Lutomyia, 844

LYCAENIDAE, 679

Lycaenopsis, 681

LYCAOTINAE, 886

Lycia, 603

Lycidae, 319

Lycogaster, 960

Lycomor ha, 670

Lycoperdina, 390

Lycophot:a, 660

Lycosa, 1066

LYCOSIDAE, 1066

LyctidAe, 416

LYCTOCORINAE, 109

Lyctocoris, 109

Lyctus, 416

Lydella, 816

LYGAEIDAE, 92

LYGAEINAE 92

Lygaeus, 92

Lygidea, 126
Lygranthoecia, 664

Lygris, 593

Lygus, 127

Lymantor, 520

LYMANTRIIDAE, 617

LYMEXYLIDAE, 331

Lymnaecia, 552

LYONETIIDAE, 540

Lype, 526

Lyperopherus, 223

Lyperosia, 828

Lypha, 813

Lypsimena, 455

Lyroda, 1010

Lysiognatha, 920

LYSIOGNATHINAE, 920

LySiOPETALIDAE, 1081

Lysiphlebus, 919

Lytogaster, 858

Lytrosis, 602

Lytta, 337

\section{M}

Macaria, 600

Macateeia, 836

Machilidae, 11

Machilis, 11

Macratria, 340

Macremphytus, 879

Macrobasis, 337

Macrocentrinae, 913

Macrocentrus, 913

Macrocera, 741

MACROCERINAE, 741

Macrocoenosia, 836

Macrodactylus, 426

Macrolophus, 114

Macromeigenia, 813

Macromia, 52

Macronema, 525

Macronematinae, 525

Macronoctua, 646

Macronychus, 367

Macrophya, 882

Macropis, 1026

Macrops, 495

Macropsis, 158

Macrorchis, 835

Macrosargus, 751

Macrosiagon, 336

Macrosiphoniella, 188

Macrosiphum, 188

Macroteleia, 965

Macrotracheliella, 109

Macrotylus, 113

Macroxyela, 871

Macroxyelinae, 871

Madarellus, 504

Madiza, 861

Maevia, 1071

Magdalis, 499

Magicicada, 144

Magusa, 648

MalachitnaE, 326

Malachius, 326

Malacomyza, 42

Malacosoma, 588

Malezonotus, 98

Mallochiella, 865

MALLOPHAGA, 63

Mallophora. 770

Mallota, 800

Malthinus, 325

Malthodes, 325
Mamestra, 655, 657, 658

MAMESTRINAE, 654

Mangora, 1055

Manomera, 20

Mansonia, 704

Mantidae, 20

Mantis, 20

Mantispa, 39

MantisPidae, 39

Mantura, 479

Marasmalus, 633

Margarodinae, 193

Marmara, 541

Marpissa, 1071

Martyringa, 544

Maruina, 707

Marumba, 611

Masicera, 816

Maso, 1049

Mastogenius, 365

Matsucoccus, 193

Matus, 259

Matuta, 659

Mauromyia, 807

Meadorus, 84

Mecomma, 119

Mecoptera, 521

Mecynotarsus, 340

MEDETERINAE, 776

Medeterus, 776

Megachile, 1029

MEGACHILIDAE, 1028

Megalodacne, 384

Megalopyge, 535

MEgalOPygidaE, 535

Megalothrips, 72

Megalotomus, 87

Megamelanus, 180

Megamelus, 180

Megapenthes, 251

Megaplectes, 931

Megaprosopidae, 821

Megarhyssa, 939

Megarthrus, 278

Megaselida, 789

Megastigmus, 975

Megastilicus, 288

Megastylus, 959

Megistias, 675

Megophthalma, 842

Melalopha, 613

Melanactes, 347

Melandrya, 409

MelandRYidaE, 408

Melanobracon, 896

Melanochaeta, 861

Melanochelia, 834

Melanolestes, 104

Melanolophia, 603

Melanomma, 620

Melanophila, 358

Melanophora, 823

Melanophrys, 812

Melanophthalma, 389

Melanoplus, 34

Melanoporphyria, 664

Melanorhopala, 102

Melanostoma, 794

Melanotus, 351

Melaphini, 191

Melaphis, 191

Melasidae, 352

Melasis, 352

Melba, 308 
Meleoma, 41

Melieria, 850

Meligethes, 375

Meligethinae, 375

Melina, 845

Melipotis, 625

Melissodes, 1027

Melissopus, 558

Melitara, 582

Melittia, 555

Melittomma, 331

Mellilla, 599

Mellinus, 1015

Meloe, 337

Meloidae, 336

Meloinae, 336

MeLOLONTHinAE, 422

Melophagus, 868

Melyridae, 326

Melyrinae, 327

Membracidae, 147

Membracinae, 155

Memythrus, 555

Menecles, 82

Menesta, 547

Meniscus, 933

Menopon, 63

MenOPONIDAE, 63

Menopsimus, 639

Meoneura, 865

Meracantha, 407

Meracanthinae, 407

Merinus, 404

Merisus, 981

Merium, 444

MerocorinaE, 86

Merocoris, 86

Merodon, 800

Merolonche, 641

Meromyza, 860

Meronera, 305

Merope, 522

Meroplius, 854

Meroptera, 582

Merothripidae, 69

Merothrips, 69

Merragata, 106

Merycomyia, 758

Mesamia, 164

Mesembrina, 828

Mesites, 512

Mesochorus, 959

Mesocyphona, 692

Mesogramma, 796

Mesoleinna, 946

Mesoleius, 946

Mesoleptidea, 945

Mesoleptina, 946

Mesoleptini, 944

Mesoleptus, 928

Mesoleuca, 596

Mesomiris, 124

Mesopsocidae, 62

Mesopsocinae, 62

Mesopsocus, 62

Mesostenini, 930

Mesostenus, 932

Mesothea, 591

Mesovelia. 107

Mesovelitidae, 107

Messa, 885

Messinae, 885

Meta, 1056

MetacanthinaE, 92
Metachaeta, 817

Metachroma, 468

Metacoelus, 951

Metacyrba, 1071

Metadiplosis, 736

Metalectra, 620

Metallus, 886

Metanema, 608

Metapelma, 980

Metaphidippus, 1071

Metaphyto, 813

Metaplagia, 811

Metapterus, 104

Metarranthis, 606

Metatropiphorus, 108

Metaxya, 303

Meteorinae, 916

Meteorus, 916

Metepeira, 1056

Methoca, 993

Methocinae, 993

Methypostena, 809

Metopia, 824

Metopinin, 952

Metopius, 952

Metoponia, 635

Metoposarcophaga, 825

Metrea, 573

Metriocnemus, 711

Metriona, 484

Metrobates, 136

Metrocampa, 605

Metzneria, 551

Mezium, 412

Miarus, 503

Miastor, 716

Micaria, 1062

Micracanthia, 138

MicracinaE, 517

Micracis, 517

Micrathena, 1056

Microbembex, 1018

Microbracon, 897

Microcara, 369

Microcentrum, 22

Microcentrus, 156

Microcerata, 714

Microchrysa, 752

Microcoelia. 644

Microdon, 791

Microdus, 911

Microedus, 279

Microgaster, 904

Microgasterinae, 904

Microgoes, 451

Microlathra, 286

Micromaseus, 225

Micromus, 40

Microneta, 1049

MiCROPE PLINAE, 277

Micropeplus, 277

MicROPEZIDAE, 853

Microphthalma, 821

Microphthirus, 74

Microphylellus, 110

Microplectron, 948

Microplitis, 905

Microprosopa, 842

MICROPTERYGIDAE, 534

Micropteryx, 534

Microrhagus, 353

Microrhopala, 483

Microsania, 790

Microscapha, 409
Microstelis, 1030

Microsynamma, 113

Microtonus, 411

Microvelia, 136

Microweisea, 393

Micrutalis, 149 .

Milesia, 802

Milichiella, 864

Milichitdde, 864

Milfogramminae, 824

Mimesa, 1013

Mimetidae, 1058

Mimetus, 1058

Mimoceps, 123

Mindarinae, 189

Mindarus, 189

Minettia, 848

Mineus, 84

Minthea, 416

Minyriolus, 1050

Miogryllus, 26

Miota, 963

Mirax, 904

MiridAE, 110

MirinaE, 123

Miris, 124

Miscodera, 241

Misogada, 616

Misumena, 1058

Misumenoides, 1059

Misumenops, 1059

Mitopus, 1074

Mitoura, 679

Mixogaster, 792

Mochlonyx, 702

Mochlosoma, 821

Molamba, 277

Molanna, 526

Molannidae, 526

Molanninae, 526

Molophilus, 692

Molorchus, 443

Momophana, 653

Monachulus, 464

Monalocoris, 115

Monardia, 715

Monarthropalpus, 735

Monecophora, 145

Monellia, 185

Monima, 655

Monobia, 1004

Monoblastus, 948

Monochamus, 449

Monochrysis, 991

Monoclona, 741

Monocrepidius, 343

Monoctenus, 878

Monodes, 639

Monodontomerus, 975

Monoferonia, 222

Monomorium, 996

Mononychus, 506

Monophadnus, 887

Monophlebinae, 193

Monophylla, 327

Monopis, 538

Monorhipidia, 690

Monosoma, 880

Monotoma, 380

Monotomidae, 380

MONOTOMINAE, 380

Monoxia, 474

Monumetha, 1029

Moodna, 584 
Mordella, 333

Mordellidae, 332

Mordellistena, 333

Mordwilkoja, 190

Morellia, 829

Mormidea, 80

Mormomyia, 531

Mormoniella, 981

Morrisonia, 655

Mumetopia, 864

Murgantia, 82

Musca, 828

Muscidae, 828

Muscina, 829

MuscinaE, 828

Mutilla, 994

Mutillidae, 993

Myas, 221

Mycetaea, 390

Mycetaeidae, 390

Mycetina, 391

Mycetobia, 689

Mycetobiinae, 689

Mycetochara, 400

MYCETOPHAGIDAE, 386

Mycetophagus, 386

Mycetophila, 746

MYcetopHILIDAE, 740

MYCETOPHILINAE, 743

Mycetoporus, 298

Mycodiplosis, 733

Mycodrosophila, 862

Mycomya, 742

Mycothera, 746

Mycterophora, 620

Mycterus, 412

Mydaea, 831

MYDAEINAE, 831

MYdAIDAE, 765

Mydas, 765

Myelois, 581

Myiocera, 822

Myiolepta, 793

Myiospila, 829

MylabRidae, 484

Mylabris, 484

Myllaena, 298

MYMARIDAE, 984

Myndus, 177

Myobiopsis, 810

Myodocha, 96

Myodopsylla, 869

Myopa, 804

MyOPSOCIDAE, 61

MYOPSOCINAE, 61

MYRIENTOMATA, 1083

Myrlaea, 582

Myrmarachne, 1071

Myrmecina, 996

Myrmecomyia, 849

Myrmecothea, 849

Myrmedonia, 301

Myrmeleon, 41

MYRMELEONidAe, 41

Myrmica, 997

MyrmicinaE, 996

Myrmosa, 993

Myrmosinae, 993

Myrsidea, 63

Myrsina, 816

Mysmena, 1040

Mystacides, 527

Mystaxus, 410

Mystrophora, 522
Myzine, 992

Myzocallis, 185

Myzus, 189

\section{$\mathrm{N}$}

NabidaE, 107

Nabis, 107

Nacerda, 332

Nacophora, 604

Nadabius, 1082

Nadata, 615

Naemia, 395

NAEOGEIDAE, 106

Nampabius, 1082

Nannia, 593

Nannopompilus, 988

Nannothemis, 55

Nanosella, 312

Napaea, 858

Napomyza, 867

Nasiaeschna, 52

Natada, 536

NAUCORIDAE, 139

Nausibius, 381

Neaera, 808

Neanura, 13

NEANURINAE, 13

Neaspilota, 852

Neatus, 406

Nebria, 211

Necrobia, 330

Necrophilus, 272

Necrophorus, 270

Necydalis, 443

Nehalennia, 48

Neichnea, 330

Neides, 91

NeIdidAe, 91

NeidinaE, 91

Neleucania, 654

Neliopisthus, 944

Nemastomatidae, $10 \%$

Nematinae, 890

Nematinus, 893

Nematocampa, 606

Nematodes, 354

Nematolinus, 289

Nematoproctus, 774

Nematus, 893

Nemeritis, 965

Nemobius, 26

Nemognatha, 338

Nemopoda, 854

Nemoria, 590

Nemorilla, 814

Nemotelus, 753

Nemoura, 59

NeMouridae, 59

Neoascia, 797

Neobisnius, 290

Neoborus, 125

Neocatocha, 715

Neocladura, 694

Neoclytus, 447

Neoconocephalus, 22

Neodiprion, 878

Neoempheria, 742

Neofischeria, 818

Neoformica, 1002

Neofoxia, 1013

Neoglaphyroptera, 743

Neogreeneia, 953

Neohaematopinus, 74

Neohylemyia, 837
Neolasioptera, 724

Neolecanium, 197

Neoleria, 843

Neolimnophila, 694

Neomysia, 398

Neon, 1071

NeOneurinae, 903

Neony mpha, 682

Neopachygaster, 753

Neoperla, 58

Neophylax, 530

Neopogon, 767

Neoprociphilus, 191

Neopsylla, 869

Neopyrochroa, 339

Neosciara, 747

Neoscona, 1056

Neotettix, 37

Neothomasia, 186

Neothrips, 70

Neotomostethus, 888

Neotrichia, 523

Neottiglossa, 82

Neoxabea, 28

Nena, 140

Nephanes, 313

Nephelodes, 656

Nephopteryx, 582

Nephrocerus, 791

Nephrotoma, 699

NePidde, 140

Nepticula, 535

NePticUlidae, 535

Neptunimyia, 714

Nepytia, 602

Nerice, 615

Nesticus, 1056

Neureclipsis, 525

Neurigona, 775

NEURIGONINAE, 775

Neurocolpus, 132

Neurocordulia, 52

Neuroctena, 845

Neuroctenus, 91

Neuromyia, 722

Neuronia, 528

Neuroptera, 38

Neuroterus, 969

Neurothrips, 72

Neurotoma, 872

Nicagus, 432

Nicentrus, 505

Nicocles, 767

Nigetia, 669

Nionia, 163

Nisaxis, 309

Niteliopsis, 1009

Nitidula, 376

Nitidulidae, 374

NitidulinaE, 376

Noctua, 661

NoctuidaE, 618

Noctuocoris, 121

Nodonota, 466

Nola, 669

Nolidae, $6 i 9$

Nomada, 1026

Nomaretus, 207

Nomiades, 681

Nomius, 214

Nomophila, 574

Nonagria, 635

Nopoiulus, 1079

Norma, 560 
Noserus, 901

NOSODENDRINAE, 372

Nosodendron, 372

Notaris, 497

NOTERINAE, 254

Nothodes, 344

Nothosympycnus, 775

Notiophilus, 210

Notiphila, 858

NotipHILINAE, 857

Notodonta, 614

NotodontidaE, 613

Notogonidea, 1009

Notolophus, 617

Notonaulax, 861

Notonecta, 138

Notonectidiae, 138

Notonectinae, 138

NotopyginA, 945

Notopygus, 945

NototrachinI, 953

Nototrachys, 953

Notoxus, 340

Notozus, 990

Nudobius, 288

Numeria, 604

Nusa, 768

Nycteola, 632

Nycteolinae, 632

Nyctiophylax, 525

Nyctobia, 592

Nylanderia, 1000

NyMPHALIDAE, 681

NYMPHALINAE, 683

Nymphopsocus. 62

Nymphula, 577

NyMPHULINAE, 577

Nysius, 93

Nysson, 1016

NYSSONINI, 1014

\section{$\mathrm{O}$}

Oberea, 456

Obolodiplosis, 737

Obrium, 436

Occemyia, 804

Ochria, 638

Ochrosidia, 427

Ochteridae, 141

Ochterus, 141

Ochthebius, 264

Ochthera, 859

Ochtheroidea, 856

Ochthiphila, 865

OCHTHIPHILIDAE, 865

Ocnerostoma, 554

Octotemnus, 417

Ocydromia, 786

Ocyptamus, 794

Ocypterosoma, 807

Ocypus, 293

Odiellus, 1074

Odinia, 865

ODONATA, 45

Odontaeus, 421

Odontalictus, 1025

Odontaulacus, 960

Odontobracon, 900

ODONTOCERIDAE, 526

Odontocorvnus, 505

Odontodiplosis, 738

ODONTOMERINI, 940

Odontomerus, 940

Odontomyia, 752
Odontophytes, 871

Odontosia, 614

ODONTOTARSINAE, 76

Odontotipula, 700

Odynerus, 1005

Oecanthus, 27

Oecetis, 527

Oeciacus, 108

Oecleus, 177

Ecophora, 546

ECOPHORIDAE, 544

Oecothea, 843

Oedalea, 785

Oedancala, 95

Oedaspis, 852

Oedematocera, 809

OEDEMERIDAE, 332

OEDEMERINAE, 332

Oedionychis, 475

Oedostethus, 347

Oedothorax, 1050

Oeme, 434

OENOCHROMINAE, 590

Oestodes, 348

CEsTRIDAE, 804

Oestrohilarella, 824

Estrus, 804

Ogcodes, 761

Ogdoconta, 636

Oidaematophorus, 585

Oidematops, 845

Okanagana, 145

Oleisoprister, 960

Olemira, 531

Olene, 618

Olesicampe, 965

Olethreutes, 563

Olfersia, 868

Oliarus, 177

Olibrus, 391

Oligarces, 716

Oligia, 639,646

Oligomerus. 413

Oligoria, 675

Oligota, 299

OLIGOTROPHIARIAE, 727

Oligotrophus, 728

Olina, 845

Olisthaerus, 278

Olisthopus, 235

Olla, 397

Olochrysis, 991

Olophrum, 279

OMALIINI, 278

Omalium, 279

Omalus, 990

Omaseus, 224

Omethes, 322

Ommatius, 769

Ommatostola, 635

OMOPHLINAE, 400

Omophron, 252

OMOPHRONIDAE, 252

Omosita, 376

Oncorotrachelus, 104

Oncideres, 455

Oncocnemis, 653

Oncodes, 761

Oncometopia, 160

Oncopeltus. 92

Oncopsis, 159

Oneida. 580

Onthophagus, 418

Onthophilus, 318
Onychagrotis, 661

ONYCHIURINAE, 13

Onychiurus, 13

Onychobaris, 504

Onychylis, 498

Oodes, 244

Ooencyrtus, 979

OONOPIDAE, 1036

Oosternum, 270

OPATRINAE, 401

Opatrinus, 401

Opheltes, 946

Ophiderma, 154

Ophiogomphus, 49

Ophion, 952

OPHIONINAE, 952

OPHIONINI, 952

Ophistomis, 442

Ophyiulus, 1079

Ophyra, 832

OpIINAE, 914

Opilio, 1075

OPILIONES, 1074

Opisthacantha, 965

Opistheuria, 125

Opistholoba, 745

Opius, 914

Oporinia, 595

Opostega, 540

OPOSTEGIDAE, 540

Oppiopsis, 824

Opresus, 276

Opsebius, 761

Opsidia, 824

Opsodexia, 823

Orchelimum, 23

Orchesella, 16

Orchesia, 409

Orchestes, 502

Orchestina, 1036

Orectoderus, 114

Oreogeton, 787

Oreta, 589

Orgilus, 912

Orgyia, 617

Orius, 109

Ormenis, 178

Ormia, 810

Ormiscus, 485

Ormosia, 691

Ormyrus, 975

Orneodes, 585

ORNEODIDAE, 585

Ornithobius, 65

Ornithoica, 868

Ornithomyia, 868

Ornithoponus, 868

Ornix, 541

Oropeza, 698

OROPHIINAE. 417

Orphilus, 372

Orphnephila, 714

ORPH NEPHILIDAE, 714

Orphulella, 29

Orsodacne, 460

ORSODACNINAE, 460

Ortalidae, 849

Orthachaeta, 842

Orthaea 97

Orthaltica, 479

Orthezia, 194

ORTHEZIINAE, 194

ORTHOCENTRINI, 950

Orthocephalus, 118 
Orthoceratium, 777

Orthocis, 416

Orthocladius, 710

Orthodes, 656

Orthofidonia, 598

Orthogonalos, 960

Ortholomus, 92

Orthomorpha, 1080

Orthonama, 595

Orthopelma, 958

ORTHOPERIDAE, 276

Orthoperus, 276

Orthopleura, 330

ORTHOPTERA, 17

Orthosia, 649, 655

Orthotomicus, 520

Orthotrichia, 523

ORTHOTYLINAE, 117

Orthotylus, 120

ORYSSIDAE, 895

Oryssus, 895

Oryzaephilus, 380

Oscinis, 861

Oscinosoma, 861

Osmia, 1030

Osmoderma, 429

Osphya, 412

Ostoma, 374

Ostomidae, 373

Otidocephalus, 499

Otiocerus, 179

OTIORHYNCHINAE, 490

Otiorhynchus, 492

Otlophorus, 947

Otocryptops, 1082

OXYBELINAE, 1019

OXYBELINI, 1021

Oxybelus, 1021

OXYCARENINAE, 95

Oxycera, 753

Oxycilla, 620

Oxycnemus, 378

Oxyethira, 523

Oxygonus, 348

Oxyomus, 420

Oxyopes, 1070

OXYOPIDAE, 1070

Oxypleurites, 1078

Oxypoda, 307

OXYPORINAE, 295

Oxyporus, 295

Oxyptila, 1059

Oxyptilus, 584

Oxystoglossa, 1025

OXYTELinae, 277

OXYTELINI, 280

Oxytelus, 280

OXYTORINA, 944

Ozodania, 669

Ozophora, 97

\section{$\mathrm{P}$}

Pachnobia, 661

Pachybrachys, 463

Pachycerota, 308

Pachydiplax, 56

Pachygaster, 753

Pachygnatha, 1056

Pachygronthinae, 95

Pachylobius, 496

Pachymenes, 1004

Pachymeria, 786

Pachymerium, 1083

Pachynematus, 893
Pachyneuron, 981

Pachyophthalmus, 825

Pachyphanes, 497

Pachyprotasis, 882

Pachypsylla, 183

Pachyschelus, 364

Pachysphinx, 611

Pachystethus, 426

Pachystilicus, 288

Pachyta, 438

Pachytychius, 497

Packardia, 537

Paectes, 633

Paederinae, 284

Paederus, 285

Pagaronia, 161

Pagasa, 107

Paidisca, 1040

Paitobius, 1082

Palaeoplatyura, 740

Palaminus, 284

Paleacrita, 604

Paleoplusia, 626

Pallodes, 379

Palloptera, 849

Pallopteridae, 849

Palmacorixa, 142

Palmodes, 1011

Palorus, 403

PalPatores, 1074

Palpomyia, 708

Palthis, 621

Pammegischia, 961

Pamphila, 672

PAMPHILIIDAE, 872

Pamphilinae, 672

Pamphilius, 872

Panagaeus, 213

Panchlora, 20

Panchrysia, 626

Pancota, 304

Pandeleteius, 491

Pandemis, 569

Pangaeus, 77

Pangonia, 754

Pangrapta, 620

Paniscini, 948

Paniscus, 948

Panopoda, 625

Panorpa, 521

PANORPIDAE, 521

Panscopus, 491

Pantala, 56

Panthea, 628

Pantheinae, 627

Pantograpta, 573

Pantomorus, 492

Panurginus, 1027

Paonias, 611

Papaipema, 636

Papilio, 676

PAPILIONIDAE, 676

Parabates, 949

Parabolocratus, 163

Paracalocoris, 131

Parachaeta, 816

Paracharactus, 888

Paraclemensia, 534

Paraclius, 783

Paracoelidea, 172

Paracymus, 267

Paradidyma, 817

Paradionaea, 810

Paradiplosis, 737
Paragrotis, 662

Paragus, 793

Parahypenodes, 623

PARAJ Ulidae, 1079

Parajulus, 1079

Paralechia, 549

Paralesteva, 279

Paralimna, 858

Paralispa, 572

Parallelia, 629

Parallelina, 440

Parallelodiplosis, 737

Parallelomma, 841

Parallelostethus, 348

Paramellinus, 1015

Paramesius, 963

Parancistrocerus, 1005

Parandra, 432

Paranomalon, 954

Paraphasia, 806

Paraphia, 602

Paraphidippus, 1072

Paraphorocera, 816

Paraprosena, 821

Parasa, 536

Parasetigena, 815

Parasierola, 1004

Paraspiniphora, 788

Parastichtis, 646, 649

Parataxonus, 880

Paratenetus, 406

Paratenodera, 20

Parateras, 970

Paratettix, 37

Paratinia, 742

Paratrytone, 674

Paraxenetus, 134

Parcoblatta, 18

Pardalophora, 31

Pardosa, 1068

Parectecephala, 860

Parectopa, 542

Paregle, 840

Parerigone, 1050

Parexorista, 814

Parharmonia, 556

Paria, 468

Parlatoria, 202

Parnopes, 990

Paromalus, 317

Parorgyia, 618

Parornix, 541

Paroxya, 34

Parthenicus, 117

Parthenothrips, 67

Parwinnertzia, 717

Parydra, 859

Pasimachus, 211

Passalidae, 432

Passaloecus, 1014

Passalus, 432

Patara, 179

Patelloa, 815

Patrobus, 220

Pauropsyllinae, 182

Paururus, 874

Peckhamia, 1072

Pedanostethus, 1040

Pedicella, 751

Pedicia, 697

Pedicilni, 697

Pediculidae, 73

Pediculus, 73

Pedilidae, 339 
Pedilus, 339

Pedinaspis, 986

Pedininae, 401

Pediopsis, 158

Pegomyia, 840

Pelastoneurus, 783

Pelecinidae, 967

Pelecinus, 967

Pelecopsis, 1051

Pelecotoma, 336

Pelenomus, 508

Peleteria, 819

Pelidnota, 427

Pelina, 858

Pellenes, 1072

Pelocoris, 139

Pelomyia, 864

Pelopaeus, 1012

Peloropeodes, 776

Peltodytes, 253

Peltoperla, 58

Pemphigini, 190

Pemphigus, 190

Pemphredon, 1014

Pemphredonini, 1013

Pentacora, 137

Pentacricia, 835

Pentagramma, 182

Pentaphyllus, 403

Pentarthrinus, 512

Pentatomidae, 78

Pentatominae, 78

Penthe, 408

Penthoptera, 697

Penthimia, 162

Peoria. 584

Peponapis, 1028

Pepsini, 988

Percnoptilota, 595

Percosia, 226

Perdita, 1027

Peribalus, 79

Periclista, 888

Periclistus, 974

Pericoma, 706

Pericompsus, 218

Peridroma, 660

Perigaster, 508

Perigea, 647

Perigenes, 96

Perillampidae, 976

Perilampus, 976

Perilissina, 944

Perilitus, 915

Perillus, 84

Perimede, 552

Periphyllus, 186

Periplaneta, 19

Peripsocinae, 61

Peripsocus, 61

Perispasta, 574

Perissopterus, 982

Perithemis, 55

Perithous, 943

Peritrechus. 98

Perizoma, 595

Perla, 57

Perlesta, 58

Perlidae, 57

Perlinella, 58

Perlodes, 57

Pero, 608

Peronea, 566

Perothopinae, 354
Perothops, 354

Petalium, 414

Pezomachin I, 930

Pezomachus, 930

Pezoporus, 928

Phaecasiophora, 564

Phaedon, 471

Phaenonotum, 269

Phaenopria, 963

Phaeocyma, 628

Phaeogenes, 927

Phaeogenini, 927

Phalacridae, 391

Phalacrocera, 698

Phalacrotophora, 789

Phalacrus, 391

Phalaenophana, 621

Phalaenostola, 622

Phalangiidae, 1074

Phalangium, 1075

Phaleria, 401

Phaleritnae, 401

Phalonia, 569

Phalonitdae, 569

Phanaeus, 418

Phanerotoma, 903

Phanopsyche, 531

Phaonia, 830

Phaonitnae, 829

Pharmacis, 569

Phasgonophora, 976

Phasia, 806

Phasitidae, 805

Phasiops, 822

Phasmidae, 20

Pheletes, 344

Phelister, 317

Phellopsis, 401

Phenacoccus, 195

Phengodes, 322

Phengodidae, 322

Phenolia, 378

Pheosia, 615

Pherbellia, 845

Phidippus, 1072

Phigalia, 604

Philaenus, 146

Philanthini, 1016

Philanthus, 1016

Philaronia, 146

Philereme, 595

Philobia, 599

Philodes, 250

Philodromus, 1059

Philometra, 622

Philonix, 971

Philonome, 540

Philonthus, 290

Philophuga, 239

Philopotamidae, 524

Philopotamus, 524

Philopteridae, 64

Philopterinae, 64

Philopterus, 64

Philosamia, 587

Philotarsus, 62

Philotermes, 301

Philothermus, 388

Philygria, 857

Philygriola, 857

Phiprosopus, 621

Phlaeothiripidae, 69

Phlaeothripoidea, 69

Phlaeothrips, 71
Phlegethontius, 609

Phlegon, 354

Phlegyas, 95

Phlepsius, 169

Phloeobiothrips, 72

Phloeonomus, 279

Phloeophagus, 512

Phloeopora, 308

Phloeotrya, 411

Phloesinus, 516

Phlyctaeria, 574

Phoberia, 625

Phobetron, 535

Pholcidae, 1038

Pholcus, 1038

Pholeomyia, 864

Pholisora, 671

Pholus, 612

Phora, 790

Phorantha, 806

Phoranthella, 806

Phorbia, 838

Phorichaeta, 818

Phoridae, 788

Phormia, 827

Phorminae, 827

Phorocera, 815

Phorodon, 189

Phortica, 862

Photinus, 321

Photuris, 322

Phragmatobia, 667

Phronia, 745

Phrurolithus, 1062

Phryganea, 528

Phryganeidae, 528

Phrynolydella, 816

Phryxe, 814

Phthinia, 744

Phthiria, 763

Phthirius, 73

Phthorophloeus, 515

Phycanassa, 674

Phyciodes, 684

Phycitinae, 581

Phygadeuon, 928, 929

Phygadeuonini, 928

Phylacteropoda, 808

Phylidorea, 695

Phylinae, 110

Phyllaphis, 184

Phyllecthrus, 475

Phyllobaenus, 330

Phyllobius, 493

Phyllobrotica, 474

Phyllocnistis, 540

Phyllocoptes, 1078

Phyllocoptinae, 1078

Phyllodecta, 472

Phyllodinus, 181

Phyllodrepa, 278

Phyllogaster, 835

Phyllomyza, 865

Phyllophaga, 424

Phylloscelis, 176

Phylloscyrtus, 28

Phyllotominae, 885

Phyllotreta, 481

Phylloxera, 192

PhylloXeridae, 192

Phylloxerinae, 192

Phylocentropus, 525

Phymaphora, 390

Phymata, 103 
Phymatidae, 103

Phymatocerinae, 888

Phymatodes, 445

Phyrdenus, 509

Physatocheila, 102

Phy socephala, 803

Physocnemum, 444

Physokermes, 198

Physonota, 483

Physostegania, 599

Phytodecta, 472

Phytodietini, 935

Phytodietus, 935

Phytometra, 620

Phytomyza, 867

Phytonomus, 494

Phytophaga, 727

Phyxelis, 490

Piazorhinus, 502

Pieridae, 677

Pieris, 677

Piesma, 99

Piesmidae, 99

Piestini, 277

Piezocorynus, 486

Pigritia, 551

Pilaria, 696

Pilocrocis, 573

Pilophorus, 122

Pilopius, 311

Pimpla, 936, 942

Pimplinae, 932

Pimplini, 936, 941

Pinacodera, 239

Pinipestis, 581

Pinnaspis, 199

Pinophilus, 284

Pinotus, 418

Piophila, 855

Piophilidae, 855

Pipiza, 792

Pipizella, 792

Pipunculidae, 791

Pipunculus, 791

Pirata, 1069

Piratinae, 104

Pisauridae, 1065

Pisaurina, 1066

Pisenus, 408

Pissodes, 495

Pissonotus, 180

Pithanus, 123

Pityobius, 343

Pityogenes, 519

Pityokteines, 519

Pityophthorus, 518

Placopterus, 328

Placusa, 299

Plagiodera, 471

Plagiomimicus, 635

Plagiprospherysa, 811

Plagodis, 606

Plastanoxus, 1004

Platagrotis, 660

Plateros, 320

Plathemis, 54

Plathypena, 619

Platidius, 221

Platycampus, 890

Platycentropus, 530

Platycerus, 431

Platychirus, 794

Platycnema, 790

Platycoenosia, 833
Platydema, 403

Platygaster, 966

Platygasteridae, 965

Platylabus, 927

Platylygus, 127

Platymetopius, 165

Platynus, 230

Platypalpus, 783

Platypeza, 790

Platypezidae, 790

Platypezoides, 790

Platyphylax, 529

Platy Podidae, 514

Platyptilia, 584

Platypus, 514

Platysenta, 640

Platysoma, 316

Platystethus, 281

Platystomidae, 485

Platytylellus, 125

Platyura, 741

Plea, 139

Plebeius, 681

Plecia, 748

Plecoptera, 57

PlectiscinI, 959

Plectocryptus, 929

Plectops, 808

Plectrocnemia, 525

Plectromyia, 698

Plegaderus, 318

Pleinae, 139

Plenoculus, 1010

Pleonectyptera, 620

Plesiocis, 417

Pleuroneura, 871

Pleurophorus, 421

Pleuroprucha, 592

Pleurotropis, 983

Plinthisus, 98

Plocetes, 498

Plochionus, 239

Plodia, 584

Ploiaritinae, 103

Ploiariola, 103

Ploiophora, 551

Plusia, 626

Plusiinae, 626

Plusiodonta, 624

Plutella, 553

Pnyxia, 747

Poanes, 674

Poaphila, 628

Pocadicnemis, 1051

Pocadius, 378

Podabrus, 323

Podalonia, 1012

Podapion, 490

Podisma, 34

Podisus, 85

Podops, 78

Podosesia, 555

Podura, 12

Poduridae, 12

Podurinae, 12

Poecilocapsus, 130

Poecilochroa, 1038

Poecilographa, 846

Poecilonota, 357

Poecilothrips, 72

Poecilus, 224

Poemenia, 940

Pogonocherus, 454

Pogonomyia. 831
Pogonomyza, 839

Pogonosoma, 768

Polemius, 325

Polia, 657

Polidea, 810

Polistes, 1006

Polistinae, 1006

Polistiphaga, 932

Polites, 673

Pollenia, 828

Pollenimae, 828

Polloccia, 572

Polyaenus, 932

Polybates; 886

Polyblastina, 947

Polyblastus, 947

POLYCENTROPIDAE, 525

Polycentropinae, 525

Polycentropus, 525

Polychrosis, 565

Polycinetis, 945

Polydesmidae, 1080

Polydesmus, 1080

Polydontomyia, 800

Polydrusus, 491

Polyergus, 1003

Polygonia, 685

Polygrammate, 644

Polygraphus, 515

Polylepta, 742

Polymerus, 130

Polymitarcys, 44

Polymoechus, 427

Polynema, 984

Polyphylla, 425

Polyplax, 74

Poly psocinae, 61

Polypsocus, 61

Polyselandria, 889

Polysphincta, 937

Polysphinctini, 937

Polystoechotes, 40

Polystoechotidae, 40

Polytaxonus, 879

Polyterus, 946

Polythichophora, 857

Polytribax, 929

Polyxenidae, 1081

Polyxenus, 1081

Polyzonitidae, 1081

Polyzonium, 1081

Pomphopoea, 336

Pompilidae, 985

PoMPILINAE, 985

PoMpilin I, 985

Pompiloides, 987

Pompilus, 986

Ponera, 995

Ponerinae, 995

Pontania, 890

Pontia, 677

Popillia, 427

Porcinolus, 373

Porizonini, 958

Porosagrotis, 661, 663

Porphyrops, 774

Porricondyla, 718

PORRICONDYLARIAE, 716

Porthetria, 618

Postleria, 843

Potamanthus, 45

Poultonella, 1073

Praon, 918

Prasocuris, 469 
Prateus, 406

Precis, 686

Prenes, 675

Prenolepis, 999

Priocera, 327

Priocnemis, 989

Priocycla, 608

Prionapteryx, 578

Prionellus, 715

Prioninae, 432

Prionocera, 699

Prionochaeta, 272

Prionocyphon, 369

Prionolabis, 696

Prionomerus, 502

Priononyx, 1011

Prionota, 715

Prionoxystus, 570

Prionus, 433

Priophorus, 890

Pristaulacus, 960

Pristiphora, 894

Pristocera, 1004

Pristodactyla, 230

Pristomerus, 957

Probezzia, 709

Proboscimyia, 841

Procecidochares, 852

Proceratium, 996

Prochelostoma, 1030

Prochyliza, 855

Prociphilin I, 191

Prociphilus, 191

Procladius, 710

Proctacanthus, 769

Proctorus, 498

Proctotrupes, 964

Proctotrupidae, 964

Proctotrupoidea 961

Prodecatoma, 978

Prodenia, 641

Prodiamesa, 710

Prodiplosis, 734

Profenusa, 885

Prokelisia, 180

Prolimacodes, 536

Promachus, 770

Promethes, 950

Prometopia, 378

Prosacantha, 964

Prosalpia, 837

Proserpinus, 613

Prosimulium, 749

Prosinella, 824

Prosminthurus, 16

Prosopidae, 1021

Prosopis, 1022

Prosopotheca, 1051

Prospaltella, 982

Prostemminae, 107

Proteinus, 278

Protenor, 87

Protenthes, 709

Proteoteras, 562

Prothalpia. 409

Protheca, 414

Protoboarmia, 602

Protocalliphora, 827

Protophormia, 827

Protoplasa, 688

Protoptila, 523

Protopulvinaria, 196

Protura, 1083

Provancherella, 945
Proxenus, 640

Psacaphora, 552

Psallus, 112

Psammobius, 420

Psammochares, 986

PSAM MOCHARIDAE, 985

PSAMMOCHARINAE, 985

PSAMMOCHARIN I, 985

Psammoecus, 382

Psammophila, 1012

Psapharochrus, 451

Psaphidia, 652, 653

Psectra, 39

Psectraglaea, 650

Psectrocladius, 710

Pselaphidae, 308

Pselaphus, 311

Pselliopus, 105

Psen, 1013

Pseninae, 1013

Psenini, 1013

Psenocerus, 449

Psenulus, 1013

Psephenidae, 366

Psephenus, 366

Pseudachorutes, 13

Pseudactium, 308

Pseudagenia, 989

Pseudaglossa, 623

Pseudamphasia, 248

Pseudanthonomus, 501

Pseudaonidia, 201

Pseudargutor, 225

Pseudenantha, 809

Pseudencyrtus, 979

Pseuderipternus, 958

Pseudeva, 626

Pseudibidion, 436

Pseudischnaspis, 201

Pseudobaeus, 326

Pseudobaris, 504

Pseudoboarmia, 602

Pseudochaeta, 817

Pseudochironomus, 712

Pseudocnemodus, 97

Pseudococcus, 195

Pseudoculicoides, 707

Pseudogalleria, 563

Pseudogaurax, 861

Pseudoleria, 844

Pseudolfersia, 868

Pseudclimnophila, 695

Pseudolucanus, 430

Pseudomedon, 287

Pseudomenopon, 63

Pseudomethoca, 993

Pseudophaonia, 829

Pseudophilippia, 196

Pseudopityc pthorus, 318

Pseudoplisus, 1015

Ps udonomala. 29

Pseudopsila, 855

Pseudopsis. 278

Pseudopyrellia, 828

Pseudosiobla, 879

Pseudota, 304

Pseudotephritis, 850

Pseudothyatira, 588

Pseudothysanoes, 517

Pseudotractocera. 812

Pseudovespa, 1007

Pseudoxenetus, 123

Psila, 855

Psilidae, 855
Psilocephala, 764

Psilocorsis, 545

Psilodiamesa, 710

Psilodora, 968

Psilodryinus, 1008

Psilomastax, 921

Psilopa, 856

Psilopinae, 856

Psilopcdinus, 771

Psilopus, 771

Psiloscelis, 317

Psilotreta, 526

Psinidia, 33

Psithyrus, 1033

Psocidae, 60

Psocinae, 60

Psocus, 60

Psomus, 506

Psoquillidae. 62

Psorophora, 704

Psychidae, 539

Psychoda, 707

Psychodidae, 706

Psychomorpha, 634

Psychomyia, 526

PSYCHOMYIDAE, 526

Psychophagus, 981

Psylledontus, 980

Psyllia, 183

Psyllidae, 182

Psyllinnae, 183

Psylliodes, 482

Psyllipsocidae, 62

Psyllipsocus, 62

Psyllobora, 394

Psyllopsis, 183

Ptecticus, 751

Ptenidium, 312

Ptenothrix, 17

Pterallastes, 800

Pterocolinae, 489

Pterocolus, 489

Pterocyclon, 517

Pterodontia, 760

Pteromalidae。 980

Pteromalus, 981

Pteromicra, 845

Pteronarcidae, 57

Pteronarcys, 57

Pteronidea, 891

Pteronus, 891

Pterophoridae, 584

Pterophylla, 22

Pterostichus, 221

PTERYGOPHORIDAE, 894

Pteryx, 313

Ptilitdae, 312

Ptilitanae, 312

Ptilinus, 415

Ptiliola, 312

Ptilium, 312

Ptilodactyla, 370

Ptilodactylinae, 370

Ptilodexia, 822

Ptilostena, 693

Ptinella, 313

Ptinidae, 412

Ptinus, 412

Ptochiomera, 97

Ptomaphagus, 272

Ptycerata. 551

Ptychopoda, 591

Ptychoptera, 688

PTYCHOPTERIDAE, 688 
Ptychopterinae, 688

Publilia, 155

Pulex, 868

Pulicidae, 868

Puliciphora, 790

Pulvinaria, 196

Purpuricenus, 449

Pycnocelus, 19

Pycnoderes, 115

Pycnoglossa, 839

Pycnoglypta, 278

Pycnophus, 275

Pycnotomina, 380

Pygolampis, 104

Pyracmon, 965

Pyractomena, 321

Pyralididae, 571

Pyralidinae, 578

Pyralis, 578

Pyrausta, 575

Pyraustinae, 572

Pyraustomyia, 813

Pyrellia, 828

Pyrgota, 804

Pyrgotidae, 804

Pyrgus, 672

Pyrochroidae, 338

Pyromorpha, 537

PyromorPhidae, 537

Pyrophaena; 794

Pyrophila, 648

Pyrophorinae, 342

Pyrrhia, 638

PyrRhocoridae, 99

Pythidae, 338

Pytho, 338

\section{Q}

Ouadrigana, 947

Quedius, 294

\section{$\mathrm{R}$}

Rachela, 593

Racheospila, 590

Rallicola, 65

Ramecia, 309

Ranatra, 140

Raphia, 626

Raphiptera, 580

Recurvaria, 550

Reduvindae, 103

ReduvitnaE, 104

Reduviolus, 107

Reduvius, 104

Refonia, 223

Reichenbachia, 310

Rembus, 228

Remigia, 629

Renardia, 279

Renia, 622

Renocera, 846

Reticulitermes, 38

Retinia, 562

Retinodiplesis, 738

Reuteria, 121

Reuteroscopus, 113

Rhabdoides, 670

Rhabdomastix, 693

Rhabdonhaga, 719

Rhabdopterus, 467

Rhadinoceraea, 888

Rhaeboscelis, 364

Rhagio, 759
RHAGIONIDAE, 758

Rhagium, 437

Rhagoletis, 851

Rhagovelia, 137

Rhamphidia, 690

Rhamphina, 821

Rhamphomyia, 787

Rhanis, 390

Rhantus, 260

Rhaphidolabina, 698

Rhaphidolabis, 698

Rhaphitelus, 981

Rhaphium, 774

RHAPIINAE, 774

Rhegmoclema, 749

Rheumaptera, 596

Rheumatobates, 136

Rhexidius, 309

Rhexius, 309

Rhicnoessa, 864

Rhiginia, 105

Rhingia, 797

Rhinocapsus, 113

Rhinomacer, 488

RHINOMACERINAE, 488

Rhinoncus, 508

Rhinopsis, 1008

Rhinosimus, 338

RHIPICERIDAE, 342

RHIPIDANDRINAE, 402

Rhipidandrus, 402

Rhipidia, 690

RHIPIPHORIDAE, 336

Rhipiphorus, 336

Rhizarcha, 920

Rhizomyia, 722

Rhizopertha, 416

RHIZOPHAGIDAE, 379

Rhizophagus, 379

Rhodites, 974

Rhodobaenus, 512

Rhodoecia, 638

Rhodophaea, 581

Rhodophora, 664

RHOGAdINAE, 901

Rhogogaster, 882

Rhomphaea, 1040

RHOPALOCERA, 670

Rhopalomyia, 728

Rhopalosiphum, 187

Rhyacionia, 562

Rhyacephila, 522

RHYACOPHIIIDAE, 522

RHYACOPHILINAE, 522

Rhymbus, 391

Rhymosia, 744

Rhynchagrotis, 659

Rhynchites, 488

Rhynchitinae, 488

Rhynchodexia, 821

Rhynchomilichia, 864

RHYNCHOPHORA, 485

Rhynchothrips, 70

Rhyncolus, 512

RHYPAROCHROMINAE, 96

RHYPHIDAE, 688

RHYPHINAE; 688

Rhyphus, 688

Rhysodes, 373

RHYSODIDAE, 373

Rhysophora. 857

Rhyssa, 938

Rhyssella, 938

Rhyssemus, 420
Rhyssini, 938

Rhytidolomia, 79

RicinidaE, 64

Ricinius, 64

RiOdinidae, 681

Ripersiella, 195

Rivellia, 849

Rivula, 621

Robertus, 1040

Rocconota, 105

Roederiodes, 784

Roeselia, 669

Rogas, 901

Romaleum, 435

Rondaniella, 743

Ropalopus, 444

Ropronia, 961

ROPRONIIDAE, 961

Rosneta, 966

Rupela, 571

Rushia, 410

Rusticus, 681

Rutelinae, 426

Rybaxis, 310

Rygchium, 1005

Rypobius, 276

Ryssematus, 510

\section{$\mathrm{S}$}

Sabacon, 1074

Sableta, 304

Sabulodes, 609

Sacandaga, 693

Sacium, 277

Sackenomyia, 729

Sagaritis, 965

SaicinaE, 104

Saissetia, 197

Salda, 137

SAldidae, 137

Saldula, 137

Salebria, 582

Salia, 619

Salpingus, 338

Saltella, 855

Salticidae, 1070

Salticus, 1073

Sameodes, 574

Samia, 587

Sandalus, 342

Sannina, 556

Sanninoidea, 556

Saperda, 455

Saprinus, 318

Sapromyza, 848

SAPROMYZIDAE, 847

Sapromyzosoma, 848

Sapyga, 992

SAPYgIDAE, 992

Sarcophaga, 825

SARCOPHAGIDAE, 823

SARCOPHAGINAE, 825

Sardiocera, 822

Sargus, 751

SARROTHRIPINAE. 632

SATURNIIDAE, 586

SATYRINAE, 682

Satyrodes, 682

Satyrus, 682

Saxinis, 462

Sayapis, 1029

Scambus, 942

Scaphidema, 402

SCAPHIDIIDAF, 313 
Scaphidium, 313

Scaphinotus, 207

Scaphisoma, 314

Scaphoideus, 164

Scaptomyza, 863

SCARABEIDAE, 417

Scardia, 538

Scarites, 211

Scatella, 859

Scatophaga, 842

Scatophagidae, 841

Scatophila, 859

Scatophora, 845

Scatopse, 749

SCATOPSIDAE, 749

SCELIONIDAE, 964

Sceliphron, 1012

Scellus, 777

SCENOPINIDAE, 765

Scenopinus, 765

Scepsis, 670

Sceptonia, 747

Schendyla, 1083

SCHENDYLIDAE, 1083

Schiffermuelleria, 546

Schinia, 663

Schistocerca, 33

Schizocera, 877

SCHIZOCERINAE, 877

Schizocosa, 1069

Schizogenius, 213

Schizomyia, 729

Schizophilus, 354

Schizotachina, 807

Schizotarsia, 1081

Schizotus, 339

Schizura, 616

Schlettererius, 961

Schoenicus, 400

Schoenobilnae, 571

Schoenobius, 571

Schoenomyza, 836

Schreckensteinea, 555

Sciagraphia, 600

Sciaphilus, 492

SCIAPINAE, 771

Sciapus, 771

Sciara, 747

SCIARIDAE, 747

Sciocoris, 78

Sciomyza, 845

Sciomyzidae, 845

Sciophila, 742

Sciophilinae, 741

Scirtes, 370

Scirtetica, 33

Scobicia, 415

Scolecocampa, 621

Scolia, 995

SCOLIIDAE, 995

Scoliocentra, 843

SCOLIONEURINAE, 885

Scoliopelta, 751

Scoliopteryx, 624

Scolobates, 948

Scolobatina, 948

SCOLOPENDROMORPHA, 1082

Scolopostethus, 99

Scolops, 176

Scolothrips, 68

SCOLYTIDAE, 514

Scolytinae. 514

Scolytus, 514

Scopaeoma, 287
Scopaeopsis, 287

Scopaeus, 287

Scoparia, 577

SCOPARIINAE, 577

Scopelosoma, 650

Scopiorus, 948

Scotobates, 404

Scotochroa, 410

Scotogramma, 658

Scotolathys, 1036

Scraptia, 411

Scudderia, 21

SCUTELleRIdAe, 75

Scutigera, 1081

ScutigeridAe, 1081

Scutopterus, 260

SCYDMAENIDAE, 274

SCYDMAENiNAE, 274

Scydmaenus, 276

Scymnus, 394

Scyphella, 864

Scythris, 554

Scythropus, 491

Scytodes, 1036

Scytonotus, 1080

Secodella, 983

Sehirus, 77

Seioptera, 851

Seladonia, 1026

SElandritnae, 888

Selenia, 607

Selenophorus, 246

Semiophora, 659

Semioscopis, 546

Semiothisa, 600

Senotainia, 825

Senta, 635

Sepedon, 847

SePsidae, 854

Sepsis, 854

Septis, 648

Sergiolus, 1038

Serica, 422

Sericoderus, 277

Sericomyia, 798

Sericophanes, 123

Sericopompilus, 987

Sericostomatidae, 530

Sericostomatinae, 531

Sericothrips, 67

Sericus, 348

SERPHIDAE, 964

SERPHOIDEA, 961

Serphus, 964

Serromyia, 708

Serropalpus, 410

Sesia, 556

Sesioplex, 957

Setodes, 527

Siagonium, 277

SiALIDAE, 38

SiALINAE, 38

Sialis, 38

Sibine, 535

SICARIIDAE, 1036

Sicya, 604

Sidemia, 645

Sideridis, 655

Sidusa, 1073

Sierolomorpha, 992

SigalPHinAE, 902

Sigalphus, 902

Signiphora, 980

SIGNIPHORIDAE, 980
Silaon, 1009

Silis, 325

Silo, 531

Silpha, 27i

SILPHIDAE, 270

Silusa, 299

Silvanus, 381

Simaëthis, 554

SimULIIDAE, 749

Simulium, 749

Sinea, 106

Siphlonisca, 43

Siphlonurus, 43

Siphloplecton, 43

Siphona, 811

SIPHONAPTERA, 868

Siphonella, 861

Siphophyto, 811

Siphoplagia, 812

Sira, 16

Sirex, 874

SiricidAE, 874

Siricinae, 874

Sisyra, 39

Sisyridae, 39

Sisyrosea, 536

Sitodrepa, 413

Sitona, 493

Sitotroga, 550

Sitticus, 1073

Sixeonotus, 115

Smerinthus, 611

Smicronyx, 497

Smicrus, 313

Smilia, 152

SMILIINAE, 147

SMINTHURIDAE, 16

SMinthuridinaE, 16

SMinthurinae, 16

Sminthurinus, 16

Sminthurus, 17

Smodicum, 433

SoleniInI, 1019

Solenius, 1019

Solenobia, 539

Solenopotes, 74

Solenopsis, 996

Solierella, 1009

Solubea, 80

Solva, 750

Somatium, 299

Somatochlora, 53

Somula, 802

Sonia, 562

Sonibius, 1082

Sophropompilus, 988

Soronia, 378

Sosticus, 1038

Sozibius, 1082

Spallanzania, 819

Spangbergiella, 163

Spanotecnus, 945

Spargaloma, 620

Spargania, 595

Sparganothis, 565

Sparnopolius, 763

Spathidexia, 823

Spathindae, 899

Spathiophora, 842

Spathius, 899

Spermophagus, 485

Spermophora, 1038

Speyeria, 683

Sphacelodes, 609 
Sphaeriditinae, 269

Sphaeridium, 269

Sphaerobius, 96

Sphaerocera, 844

Sphaeroderus, 207

Sphaerophoria, 797

Spharagemon, 32

SPHecidae, 1008

SPHECINAE, 1010

Sphecius, 1016

Sphecodes, 1027

Sphecodina, 612

Sphecoidea, 1008

Sphecomyia, 802

Sphecophara, 947

Sphegina, 797

Sphenophorus, 512

Sphex, 1011, 1012

Sphida, 635

SPHindidae, 416

Sphindus, 416

SPHINGIDAE, 609

Sphinx, 610

Sphragisticus, 98

Sphyracephala, 856

Spilaria, 832

Spilochalcis, 976

Spilocryptus, 930

Spilogaster, 831

Spilogona, 834

Spilographa, 851

Spilomicrus, 963

Spilomyia, 802

Spilonota, 562

Spilopsyllus, 868

Spilotus, 410

Spintharus, 1040

SPIRobolidae, 1080

Spirobolus, 1080

Spodolepis, 602

Spogostylum, 761

Spongopus, 249

Spragueia, 633

Stachyocnemus, 88

Stamnoderus, 288

Stamnodes, 592

STAPHYLINIDAE, 277

Staphylininae, 288

Staphylinus, 293

Statira, 407

Steatoda, 1040

Stegana, 862

Stelidota, 377

Stelis, 1030

Stenamma, 997

Stenelmis, 366

Steninae, 282

Stenispa, 482

Stenocellus, 250

Stenocorus, 437

Stenocranus, 180

Stenodema, 124

Stenodynerus, 1006

Stenolophus, 251

Stenoma, 546

Stenopa, 851

Stenophylax, 529

Stenopleura, 812

Stenopoda, 104

STENOPODINAE, 104

Stenoporpia, 602

Stenoptilia, 585

Stenoscelis, 512

Stenosphenus, 448
Stenosyrphus, 796

Stenotarsus, 391

Stenotus, 131

Stenus, 282

STEPHANIDAE, 961

Stephanitis, 101

Stephanoderes, 518

Stephanopachys, 415

Stephanucha, 429

Stereocerus, 226

Stereopalpus, 340

Sterictiphora, 877

STERICTIPHORINAE, 877

STERRHINAE, 591

Stethaulax, 75

Stethobaris, 505

Stethon, 353

Stethophyma, 30

Stethorus, 393

Sthenopis, 534

Stibeutes, 929

Stichoglossa, 307

Stichopogon, 767

Stictia, 1018

Stictiella, 1018

Stictocephala, 148

Stictocranius, 284

Stictolinus, 289

Stigmatomma, 995

Stigmus, 1013

Stilbosis, 552

Stilbus, 392

Stilicus, 287

Stilobezzia, 708

STILPNINI, 928

Stilpnus, 928

Stiphrosomus, 945

Stiretrus, 84

Stiriodes, 635

Stobaera, 181

STOMOXYIDINAE, 828

Stomoxys, 828

Stomyles, 675

Strangalepta, 440

Strangalia, 441

Strategus, 428

Stratiomyia, 752

STRATIOMYIIDAE, 751

Straussia, 851

Strepsicrates, 562

STREPSIPTERA, 521

Strigoderma, 427

Strigodermella, 427

Strigota, 305

Strobisia, 549

Stroggylocephalus, 163

Stromatium, 435

StrongYliINAE, 407

Strongylium, 407

Strongylocoris, 118

Strongylogaster, 889

Strongylogastroidea, 878

Strongylophthalmyia, 856

Strongylosomidae, 1080

Strophiona, 441

Strophosoma, 492

Strumigenys, 998

Strymon, 678

Sturmia, 816

Stygnocoris, 98

Stylocryptus, 928

Stylogaster, 803

STYLOPIDAE, 521

Suillia, 843
Sulcacis, 417

Swammerdamella, 749

Sylectra, 624

Symmerista, 615

Symmerus, 740

Symmorphus, 1005

Sympetrum, 55

SYMPHEROBIIDAE, 39

Sympherobius, 39

Sympherta, 601

Symphora, 411

Symphoromyia, 760

Sympiesis, 983

SYMPYCNINAE, 775

Sympycnus, 775

Synaema, 1059

Synaptina, 304

Syncalypta, 373

Synchita, 387

Synchloë, 677

Synchlora, 590

Synchroa, 408

Syndyas, 785

Syneches, 785

Syneda, 625

Synelys, 591

Synemosyna, 1073

Synergus, 974

Syneta, 461

Synhalonia, 1028

Synoecetes, 947

Synstrophus, 409

Syntemma, 744

Syntomaspis, 975

Syntomidae, 670

Syntomium, 280

Syntormon, 775

Syritta, 800

SYRPHIDAE, 791

Syrphoctonus, 950

Syrphus, 795

Syrrhizus, 912

Sysinas, 115

Sysphincta, 995

Syssaura, 608

Systelloderus, 103

Systena, 480

Systoechus, 763

Systropus, 764

Syzeuctus, 934

\section{$\mathrm{T}$}

TABANIDAE, 754

Tabanus, 756

Tabuda, 764

Tachina, 817,818

TACHINIDAE, 807

Tachinomyia, 818

Tachinophyto, 809

Tachinus, 295

Tachistodes, 251

Tachopteryx, 49

Tachycellus, 249

Tachydromia, 784

TACHYGONINAE, 490

Tachygonus, 490

Tachymenis, 220

Tachypeza, 784

TACHYPORINAE, 295

Tachyporus, 296

Tachyris, 677

Tachys, 219

Tachysphex, 1009 
Tachyta, 220

Tachytes, 1009

Tachytrechus, 783

Tachyura, 219

Tachyusa, 305

Taeniaptera, 853

Taeniocampa, 655, 656

Taeniopteryx, 59

Taeniorhynchus, 704

Taeniosea, 646

Taeniothrips, 68

Talavera, 1073

Tanada, 668

Tanilotes, 341

TANYDERIDAE, 688

Tanymecus, 491

Tanypeza, 854

TANYPINAE, 709

Tanyptera, 699

Tanypus, 709

Tanysphyrus, 498

Tanytarsus, 711

Taphrocerus, 265

Tapinocyba, 1052

Tapinogonalos, 960

Tapinoma, 998

Tapinopa, 1052

Tapinops, 950

Tapinostola, 635

Tarache, 633

Tarachidia, 633

Taracticus, 767

Tarpela, 407

Taxigramma, 825

Tegenaria, 1065

Tegeticula, 534

Telamona, 150

Telea, 587

Teleas, 965

Telenomus, 965

Teleorhinus, 114

Telephanus, 382

Telmaphilus, 745

Telmatophilus, 385

Telphusa, 549

Temnochila, 374

Temnostoma, 802

Tenebrio, 406

TENEBRIONIDAE, 400

TENEBRIONINAE, 404

Tenebroides, 374

Tenthina, 864

Tenthredella, 884

TENTHREDINIDAE, 878

TENTHREDININAE, 882

Tenthredo, 884

Tenthredomyia, 802

TENTYRIINAE, 400

Tephritis, 852,853

Tephrochlamys, 842

Tephrociystis, 597

Tephronota, 850

Teratocoris, 124

TEREBRANTIA, 66

Terellia, 852

Teretriosoma, 316

Teretrius, 316

TERMITIDAE, 38

Tessaropa, 434

Tetanocera, 846, 847

Tetanops, 850

'Tetartopeus, 286

Tethina, 864

Tetracha, 204
Tetrachrysis, 991

Tetracis, 608

Tetragoneura, 742

Tetragoneuria, 53

Tetragonoderus, 236

Tetraleurodes, 193

Tetralonia, 1028

Tetralopha, 581

Tetramedon, 287

Tetramerinx, 834

Tetramorium, 998

Tetraneura, 190

Tetraopes, 457

Tetraphleps, 109

Tetrastichus, 984

Tetratoma, 408

Tetropium, 434

Tettigidea, 37

TETTIGONIIDAE, 21

Tetyra, 75

TETYRINAE, 75

Teuchocnemis, 800

Teucholabis, 694

Teutana, 1041

Thalassomyia, 710

Thamnotettix, 170

Thanaos, 671

Thanasimus, 327

Thanatus, 1060

Thaumalea, 714

THAUMALEIDAE, 714

Thaumatopsis, 579

Thecodiplosis, 731

Thecophora, 804

Thectura, 299

Thecturota, 299

Thelaira, 822

Thelcteria, 576

Thelia, 150

Theliopsyche, 531

Themira, 854

Theobaldia, 704

Theonoe, 1041

Thera, 594

Theresia, 822

Theretra, 613

Thereva, 764

THEREVIDAE, 764

Therididdae, 1038

Theridion, 1041

Theridiosoma, 1058

Theridula, 1042

Therina, 605

Therioaphis, 185

Therion, 954

THERIONINI, 953

Thermobia, 11

Thermonectes, 262

Theronia, 935

THERONIINI, 935

Thersilochus, 958

Thiasophila, 307

Thinobius, 281

THINOPHILINAE, 776

Thinophilus, 776

Thiodia, 561

Thionia, 178

Tholeria, 574

THOMISIDAE, 1058

Thoracophorus, 278

Thorybes, 671

Thrinax, 889

THRIPIDAE, 67

THRIPOIDEA, 67
Thrips, 69

THROSCIDAE, 354

Throscus, 354

Thrypticus, 776

Thryptocera, 808

Thyanta, 82

THYATIRIDAE, 588

Thylodrias, 370

THYLODRIINAE, 370

Thymalus, 374

THYMARIDINA, 944

THYREOCORINAE, 76

Thyreodon, 953

Thyreopus, 1020

THYRIDIDAE, 571

Thyridopteryx, 539

Thyris, 571

Thysania, 624

Thysanocnemis, 498

Thysanoes, 517

THYSANOPTERA， 66

THYSANURA, 11

Thysiotorus, 928

Tibellus, 1066

Tibicen, 142

Tilloclytus, 448

Timandra, 592

Timulla, 994

Tinagma, 544

Tinea, 538

TINEIDAE, 537

TINEINAE, 538

Tineola, 539

TingItidda, 100

Tinotus, 301

Tiphia, 992

TIPHIIDAE, 992

TipHIINAE, 992

Tiphodytes, 965

Tipula, 699, 700

TiPUlidae, 689

Tipulinae, 698

Tischeria, 540

TISChERIIDAE, 540

Tlascala, 582

Tmarus, 1066

'Tmesiphorus, 312

Tmeticus, 1052

Tmetocera, 562

Tollius, 88

Tolype, 587

Tomarus, 385

Tomicus, 519

TOMOCERINAE, 15

Tomocerus, 15

Tomoderus, 340

Tomolips, 512

Tomostethus, 888

Tomoxia, 332

Tornos. 602

Tortembolus, 1052

TORTRICIDAE, 557

Tortricidia, 536

TORTRICINAE, 565

Tortricodes, 567

Tortrix, 568

TORYMIDAE, 975

Torymus, 975

Tosale, 571

Toumeyella, 197

Toxidium, 314

Toxomerus, 796

Toxomyia, 730

Toxorhina, 694 
Trabaea, 1070

Trachea, 647

'Trachelas, 1063

Trachelocamptus, 1052

Trachelus, 873

Trachichneumon, 921

Trachykele, 355

Trachymyrmex, 998

Trachyphloeus, 493

Trachysectus, 287

Tragidion, 449

Traginops, 866

Tragosoma, 433

Tramea, 56

Trapezonotus, 98

Traumoecia, 302

Trechus, 221

Trematopygus, 945

Tremex, 875

TrEMicinaE, 875

Trepobates, 136

Triachora, 812

Triachus, 466

Triaena, 228

Triaenodes, 528

Trialeurodes, 193

Triaspis, 902

Tribolium, 403

Trichadenotecnum, 60

Trichina, 785

Trichiocampus, 889

Trichiosoma, 875

Trichiotinus, 430

Trichiusa, 301

Trichobaris, 504

Trichocellus, 249

Trichocera, 689

TRICHOCERIDAE, 689

Trichocladius, 710

Trichoclea, 657

Trichodectes, 64

TrichodectidAe, 64

Trichodes, 328

Trichodesma, 413

Trichodezia, 592

Trichofoenus, 961

Trichogramma, 984

TRICHOGRAM MIDAE, 984

Tricholita, 656

Trichonta, 744

Trichopepla, 79

Trichcpetalum, 1080

Trichophaga, 538

Trichophora, 819

Trichophya, 298

TRICHOPHYINAE, 298

Trichopoda, 807

Trichopria, 963

Trichopsylla, 869

TRICHOPTERA, 522

Trichopticus, 831

Trichoptilus, 584

Trichosia, 747

Trichotany pus, 710

Trichotaphe, 548

Trichothrips, 71

Trichotipula, 700

Trichrysis, 991

Triclistus, 951

Tricorythus, 44

Tricrania, 338

Tricyphona, 697

Tridactylus, 29

Triepeolus, 1025
Trifili, 731

Triga, 277

TRIGONALIDAE, 960

Trigonaspis, 970

Trigonodemus, 278

Trigonogenius, 412

Trigonophora, 645

Trigonotylus, 124

Trigonura, 976

Triliarthrus, 249

Trimerina, 857

Trimerotropis, 33

Trimicra, 693

Trimiomelba, 308

Trimioplectus, 308

Trineura, 790

Trinoton, 63

Triodonta, 800

Trionymus, 195

Trioza, 183

TrIOZINAE, 183

Triphleps, 109

Triphosa, 593

Triplax, 384

Triplectrus, 247

Triptotricha, 758

Trirhabda, 472

Trissolcus, 965

Tritogenaphis, 188

Tritoma, 383

Tritoxa, 849

Tritozyga, 714

Trochiloecetes, 64

TrogIIDAE, 62

Trogitnae, 62

TROGINAE, 422

Trogium, 62

Trogoderma, 371

Trogomorpha, 921

'Trogophoeus, 280

Trogus, 921

Tromatobia, 943

Tropaea, 587

Tropideres, 486

Tropidia, 799

Tropidosteptes, 125

Tropisternus, 266

Trotteria, 727

Trox, 422

Trupheoneura, 788

Trychosis, 931

Trypanea, 853

Trypeta, 852

TRYPETIDAE, 851

Trypetoptera, 846

Trypherus, 325

Tryphon, 947

TRYPHONINA, 947

TRYPHONINAE, 943

TRYPHONINI, 947

Trypodendron, 517

Trypopitys, 413

Trypoxylon, 1010

TRYPOXYLONINAE, 1010

Tryxalis, 29

TubuliferA, 69

Tullbergia, 13

Tutelina, 1073

Tychius, 498

Tychus, 311

Tyloderma, 510

Tylonotus, 434

Tymnes, 468

Tympanophorus, 294
Typhaea, 387

Typhlocyba, 174

TYPHLOCYBINAE, 173

Typitium, 331

Typocerus, 442

Typopsilopa, 858

Tyrus, 311

Tytthonyx, 322

U

Ufeus, 660

Ula, 694

Ulesanis, 1042

ULOBORIDAE, 1034

Uloborus, 1034

Ulolonche, 656

Uloma, 404

UlOMINAE, 403

Ulomorpha, 696

Ululodes, 42

Unitaxonus, 880

Upis, 405

Uranotaenia, 704

Urocerus, 874

Urodus, 553

Uroplata, 483

Urosigalphus, 902

Utetheisa, 665

Utopiellum, 1052

\section{V}

Valentinia, 551

Valgus, 430

Vanduzea, 155

Vanessa, 685, 686

Vanhornia, 962

VAN HORNIIDAE, 962

Vanonus, 341

VELIIDAE, 136

Venusia, 596

Veppo, 753

Verrallia, 791

Vespa, 1006

VESPIDAE, 1004

VESPINAE, 1006

VESPOIDEA, 985

Vespula, 1007

Viereckiana, 955

Villa, 762

VipiInAE, 896

Vitrinella, 603

Vitula, 583

Viviania, 812

Volucella, 797

Voria, 812

W

Wabana, 1052

Wagneria, 818

Wala, 1073

Walshia, 552

Websterellus, 976

Wesmaelia, 916

Williamsonia, 53

Winnertzia, 716

Winthemia, 817

Wohlfahrtia, 825

Wyeomyia, 703

$\mathrm{X}$

Xanthia, 649

$X$ anthidium, 1026

XANTHOCHLORINAE, 776

Xanthochlorus, 776 
Xanthoecia, 638

Xanthogramma, 796

Xantholobus, 154

Xanthomelanodes, 811

Xanthomyia, 852

Xanthonia, 467

Xanthopastis, 655

Xanthoptera, 633

Xanthorhoë, 595

Xanthosarus, 1029

Xanthoteras, 970

Xanthotype, 605

Xenadmontia, 809

Xenoborus, 126

Xenocoenosia, 836

Xenodusa, 301

Xenoglossa, 1028

Xenophorbia, 839

Xenopsylla, 868

Xenorhipis, 358

Xenos, 521

Xenotoma, 962

Xenylla, 12

Xerophloea, 162

Xestipyge, 317

Xestobium, 412

Xestocephalus, 163

Xestocis, 417

Xestocoris, 99

Xestoleptura, 441

Xestonotus, 248

Xiphandrium, 774

Xiphydria, 874

XIPHYDRIIDAE, 874

Xorides, 940

XORIDINI, 940

Xuthia, 388

Xyalaspis, 967

Xyela, 871

XYELIDAE, 871

XYelinAE, 871

Xyleborus, 520

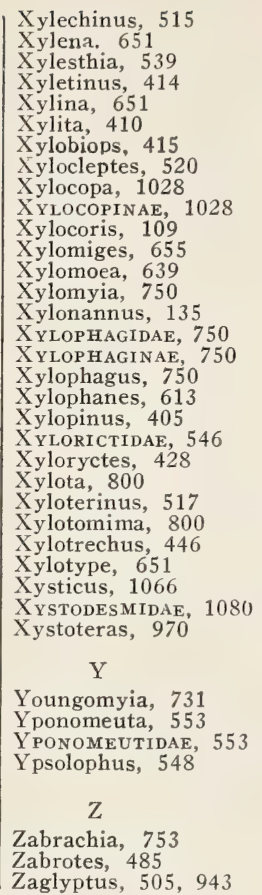

Zagryphus, 944

Zalagium, 882

Zale, 628

Zaleptopygus, 958

Zanclognatha, 622

Zaraea, 876

ZARAEINAE, 876

Zarhopalus, 980

Zastenomorpha, 955

Zatypota, 937

Zele, 914

Zelia, 821

Zelinae, 914

Zelleria, 554

Zelotes, 1038

Zelus, 105

Zenillia, 814

Zenodosus, 329

Zerene, 604, 678

Zeridoneus, 97

ZETHINA AE, 1004

Zethus, 1004

Zeugophora, 461

Zeuzera, 571

Zilla, 1058

Zilora, 410

Zodion, 803

Zonantes, 341

Zonitis, 338

Zonosema, 851

Zootrephes, 950

Zopheroteras, 970

Zophodia, 583

Zora, 1063

ZYGAENIDAE, 670

Zygethobius, 1082

Zygoballus, 1074

Zygogramma, 470

Zygomyia, 747

Zygoneura, 747

ZYGOPTERA, 45

Zygothrips, 70 





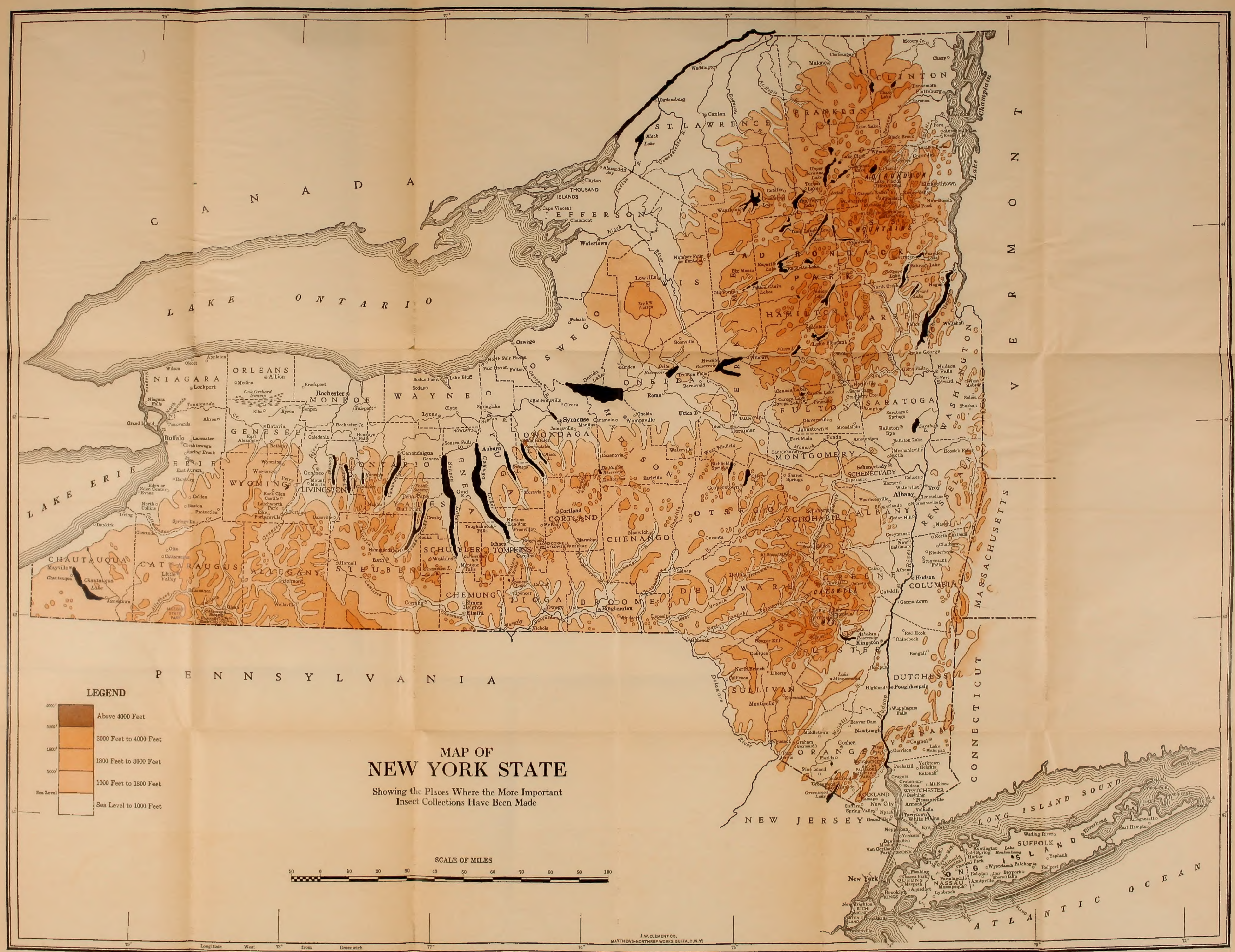




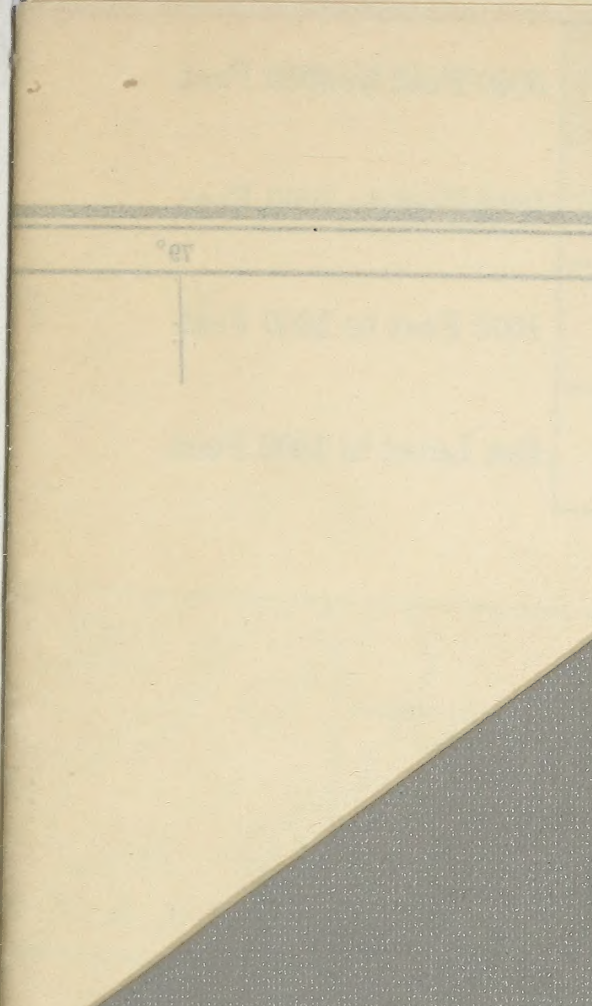
